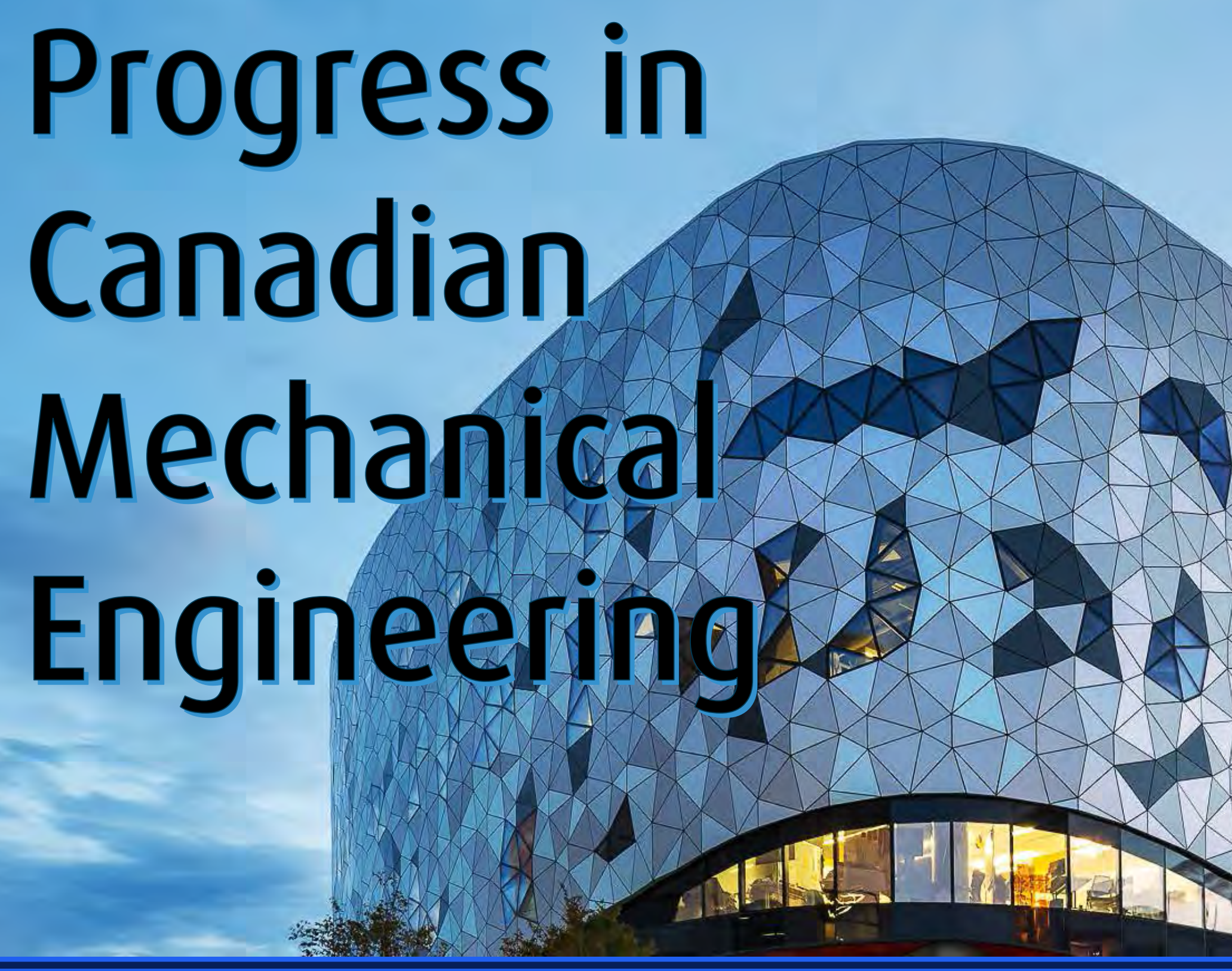

Proceedings of the 2018

Canadian Society for Mechanical Engineering (CSME)

International Congress

Sunday May $27^{\text {th }}-$ Wednesday May $30^{\text {th }}, 2018$

York University, Toronto, Ontario, Canada 


\section{CSME International Congress 2018 \\ "Progress in Canadian Mechanical Engineering" \\ Sunday May $27^{\text {th }}-$ Wednesday May $30^{\text {th }}, 2019$ \\ York University \\ Toronto, Ontario, Canada}

\section{SPONSORED BY}

The Canadian Society of Mechanical Engineering

\section{EDITED BY}

Aleksander Czekanski, MBA, PhD., P.Eng., FCSME

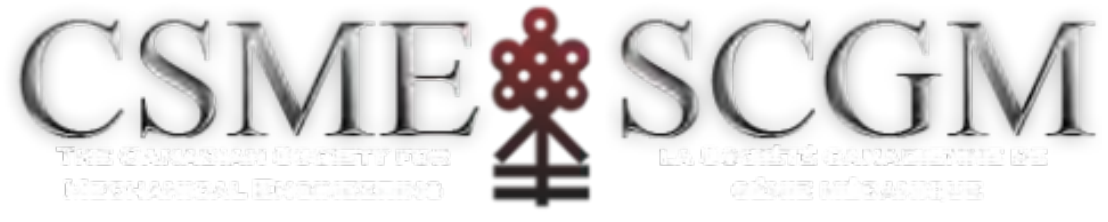

Published by

York University Libraries 
The CSME International Congress promotes a technical program that focus on topics and themes relevant to Mechanical Engineers. One of the primary roles of CSME is to train and update the mechanical engineering community on new advances and emerging fields. It is through the Congress that we provide a platform for community members to acquire and disseminate such information to each other as well as discuss recent research challenges and practical importance to the profession and its related field.

The papers in the proceedings are on the following topics:

- Fluid Mechanics

- Mechatronics, Robotics and Control

- Advance Energy Systems

- Manufacturing

- Computational Mechanics

- Student Paper Competition

- Heat Transfer

- Biomechanics and Biomedical Engineering

- Engineering Analysis \& Design

- Materials Technology

- Environmental Engineering

- Mechanical Engineering Education

\section{Acknowledgments}

Preparation for the 2018 CSME International Congress was made possible by the dedication, time and effort from the members of the CSME Organization Committee and the York University Libraries.

Sincerely,

Aleksander Czekanski MBA, PhD, P.Eng, FCSME York University

Chair, 2018 CSME International Congress

Any statements expressed in these materials are those of the individual authors and do not necessarily represent the views of CSME, which takes no responsibility for any statement made herein. No reference made in this publication to any specific method, product, process, or service constitutes or implies an endorsement, recommendation, or warranty thereof by CSME. The materials are for general information only and do not represent a standard of CSME, nor are they intended as a reference in purchase specifications, contracts, regulations, statutes, or any other legal document. CSME makes no representation or warranty of any kind, whether express or implied, concerning the accuracy, completeness, suitability, or utility of any information, apparatus, product, or process discussed in this publication, and assumes no liability therefor. The information contained in these materials should not be used without first securing competent advice with respect to its suitability for any general or specific application. Anyone utilizing such information assumes all liability arising from such use, including but not limited to infringement of any patent or patents.

Published by York University Libraries

Copyright (C) 2018 by the Canadian Society of Mechanical Engineering

All Rights Reserved

DOI: $10.25071 / 10315 / 35193$

ISBN (ebook) 978-1-77355-023-7

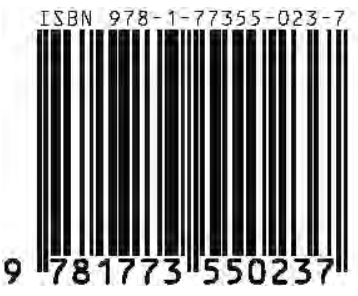


Note: Conference proceedings in italics are not included in printed proceedings.

Wind Loads on Cyclists Due to Passing Vehicles.

William Lubitz and Bryan Rubie

Numerical Investigations of Aerodynamic Noise Due to Flow Past a Bluff Body.

Sultan Alqash and Kamran Behdinan

Vibration Analysis of a Double Circular Pzt Actuator for a Valveless Micropump

Behzad Parsi, Lihong Zhang and Vlastimil Masek

Microfluidic Platform for Studying Biofilm Formation of Oil-Degrading Bacteria.

Saeed Rismani Yazdi, Corey Stevens, Peter Davies and Carlos Escobedo

Pneumatic Pressure Control Droplet Microfluidic System: an Open-Source Droplet Microfluidic

System.

Run Ze Gao, Marie Hebert, Carolyn L. Ren

Drag Reduction and Heating.

Jerzy M Flöryan, S Shadman and M.Z. Hossain

A Comparison Between Cyclone Separator Efficiency Enhancement Using Ferrous Powder and Additional Tangential Chamber.....

Walid Mazyan, Ali Ahmadi and Mina Hoorfar

Changes in The Leidenfrost Transition Point During the Evaporation of Water and N-Heptane Droplets on Hot,

Porous Stainless Steel Surfaces.

Nick Lipson and Sanjeev Chandra

Computational Fluid Dynamics Simulations of Flow in the Renal Arteries After Stent Graft Implantation

Tianyi Xia, Matthew Doyle, Thomas Forbes and Cristina Amon

Numerical Simulation of an Over-Expanded Supersonic Nozzle Flow.

Faisal AI Qurooni, Ali Vakil, Ehab Elsaadawy and Sheldon Green

Motion of Pigments in Thin Paint Films.

Marina Curak, Nazli Saranjam and Sanjeev Chandra

Particle Image Velocimetry Data Processing on a GPU Cluster.

Cameron Dallas and Pierre Sullivan

A Droplet Deformation and Fragmentation in a High Speed Air Jet.

Maryam Medghalchi and Nasser Ashgriz

Dynamics of a Free Pitching Flexible Cantilever Naca0012 Airfoil at Transitional Reynolds Numbers

Crystal Itwar Barrett, Dr. Dominique Poirel and Dr. Asad Asghar

The Evaluation of a Free-Flow Electrophoresis Device with a Velocity Gradient for Enhanced Protein Fractionation Matthew Courtney, Tomasz Glawdel and Carolyn Ren

A Prediction Tool for Spatially Decaying Freestream Turbulence.

Dwaipayan Sarkar and Eric Savory

Water Droplet Impact And Spreading on a Narrow Gap.

Dr. Jordan Bouchard and Sanjeev Chandra 
Flow Over a Wall-Mounted Finite-Length Square Cylinder

Junting Chen, Ram Balachandar and Ronald Barron

Piercing a Liquid Droplet by A Gas Jet.

Maryam Ebrahimiazar, Amirreza Amighi and Nasser Ashgriz

Shear Layer Instabilities in a Counter-Flowing Wall Jet

Sachin Sharma, Vimaldoss Jesudhas, Ram Balachandar and Ron Barron

An Immersed-Boundary (IB)-Based 3D Tornado Model and its Application.

Xixiong Guo and Jun Cao

A Model to Predict the Residual Layer Thickness in the Injection Molding Process.

Roozbeh Mollaabbasi, Ehsan Behzadfar and Seyed Mohammad Taghavi

An Experimental Study of Yield Stress Fluid Displacement Flows in a Moving Annulus

Amin Amiri, Roozbeh Mollaabbasi, Faïçal Larachi and Seyed Mohammad Taghavi

Modeling the Effect of Produced Water on Asphaltene Deposition in a Production Tubing

Using Population Balance.

Roozbeh Mollaabbasi, Dmitry Eskin and Seyed Mohammad Taghavi

Gust Response of a Two-Dimensional Nonlinear Wing.

Xiaoyang Zhang, Mojtaba Kheiri and Wen-Fang Xie

A Multi-Color Technique for Three-Dimensional Flow Characterization.

Kadeem Dennis and Kamran Siddiqui

Estimation of Shock Stand-Off Distance Using the Curved Shock Theory and its

Validation via Numerical Modelling....

Zelalem Arega Worku, Evgeny Timofeev and Sannu Molder

An Experimental and Theoretical Investigation into Airflow Through Air Box

of a Potato-Rock Separator.

Prabir Basu, Jacob Melvin Bobby Sagayaraj, Akash Kulshreshtha and Lyle Gauthier

MECHATRONICS, ROBOTICS AND CONTROL

Effect of Photoacoustic Radar Chirp Parameters on Profilometric Information.

Zuwen Sun and Natalie Baddour

Using Visual Feedback Assisted Active Control to Investigate the Hydrodynamic Resistance of Nanoliter-sized Droplets in a Microchannel

Marie Hébert, Jan Huissoon and Carolyn Ren

Bearing Fault Signature Extraction Under Time-Varying Speed Conditions via Oscillatory Behavior-Based Signal Decomposition (OBSD).

Huan Huang, Natalie Baddour and Miang Liang

Optimization of Interaural Intensity Difference Based Binaural Sonar Sensing System for Object Detection......144 Payman Rajai and Mohammed Jalal Ahamed

Design of Electronic Stability Control (ESC) Systems for Car-Trailer Combinations

Mutaz Keldani and Yuping He

Dry Surface Micromanipulation Using an Untethered and Magnetic Microrobot 153

Omid Youssefi and Eric Diller

Adaptive Yaw Control of Three-Axle Road Vehicles Based on Mass, Yaw Inertia and CG Position

Identification.

Yubiao Zhang, Ami Woo, Baris Fidan and Amir Khajepour 
Discrete 2D Fourier Transform in Polar Coordinates.

Xueyang Yao and Natalie Baddour

Sensor Filtering and Position Tracking of a Simulated Planar Bipedal Robot

Stefano Rossi and Stephen Andrew Gadsden

Modeling and Estimation of Reaction Constants in Hydrogen Peroxide Deterioration Reactions

Hung Yi Chiang, Andrei Buin, Faraz Ahmadpour and Stephen Andrew Gadsden

Online Tuning Rule Based Adaptive Speed Control Algorithm for DC Motors Using Recursive Least Squares with Forgetting.

Kwang-Seok Oh and Jaho Seo

Modeling of Structural and Environmental Effects on Microelectromechanical (MEMS)

Vibratory Gyroscopes.

Md. Kaes, D.S-K Ting and Mohammed Jalal Ahamed

Lagrangian, Hamiltonian, and Energy-Based Control for Space Tethered System.

Junjie Kang and Zheng Hong Zhu

Piezoresistive chopped carbon fiber/polydimethylsiloxane composite yars for deformation sensing in textile fabrics Hossein Montazerian, Abbas S. Milani and Mina Hoorfar

Direct Switching Position Control Algorithms For Pneumatic Actuators Using On/Off Solenoid Valves. 197

Yile Zhang and Gary M. Bone

Design and Simulation of the First Joint for a Prospective 5 Degrees of Freedom Manipulator With MR

Actuators

Sergey Pisetskiy and Mehrdad Kermani

Comparative Analysis of Optitrack Motion Capture Systems

Joshua Furtado, Hugh Liu, Gilbert Lai, Herve Lacheray and Jason Desouza-Coelho

Impact Force Reduction Strategies to Achieve Safer Human-Robot Collisions.

Behrad Rouzbeh and Gary M. Bone

Estimation and Control of a Double-Inverted Pendulum

Bowen Xu, You Lyu and Stephen Andrew Gadsden

Comparison of Sliding Mode Controller and State Feedback Controller Having

Linear Quadratic Regulator (LQR) on a Two-Wheel Inverted Pendulum Robot: Design and Experiments .225

Mikail S. Arani, Hamid Ebrahimi Orimi, Wen-Fang Xie and Henry Hong

Robust Cooperative Close Formation Flight Control Of Multiple Unmanned Aerial Vehicles

Qingrui Zhang and Hugh H. T. Liu

A Decentralized Model Reference Adaptive Controller for Modular Reconfigurable Robots

Gokhan Gungor, William Melek and Baris Fidan

Propeller Performance in Presence of Freestream. 235

Mojtaba Hedayatpour, Mehran Mehrandezh and Farrokh Janabi-Sharifi

\section{ADVANCED ENERGY SYSTEMS}

Temperature Measurements of N-Heptane Injection and Combustion

in the Ignition Quality Tester (IQT $\left.{ }^{\mathrm{TM}}\right)$....

Osama Hmood, Edgar Matida, Dave Gardiner, Luc Menard, Omar Ramadan, Aaron Wilcox and Gary Webster

A New Mechanical Band Pass Filter Design for Energy Harvesting 
Analysis of Thermal Losses in Air-Water Interfacial Solar Heating Systems

Shuzhe Wang and Paul O'Brien

Inlet Channel Effects on Archimedes Screw Generators.

Adil Khan, Scott Simmons, Murray Lyons and William Lubitz

Investigation of Short-Side-Chain lonomer and Membrane for Proton Exchange Membrane Fuel Cells....

Samaneh Shahgaldi, Adnan Ozden and Xianguo Li

Optimization of Advanced Space Propulsion Technique: Solar Sail

Ujjwal Grover and G. Vidya Sagar

Thermal Management for Efficient Fast Refill of Compressed Natural Gas

Aiqing Lan, Ri Li and Joshua Brinkerhoff

Energy Benefits of Integrating Transportation Energy with a Net Zero Energy Solar Building Using Captured Waste Hydrogen from Electrochemical Plants and Bio-Gas from Various Bio-Gasification Processes.

Shahryar Garmsiri and Marc A. Rosen

Predicting the Consumption Speed of a Premixed Flame Subjected to an Unsteady Stretch Rate.

Meysam Sahafzadeh, Larry Kostiuk and Seth Dworkin

Wind Turbine Wake Effect Visualization and Lidar Measurement Techniques.

Michael Mckinnon and David Johnson

Polymeric Triboelectric Nanogenerator: Effects of Polymer Type, Geometry, and Porosity on

Triboelectrification.

Hossein Abdoli and Siu N. Leung

Effects of Steam Injection on the Performance of Natural Gas Combined Cycle Power Generation System......292 Nitin Varia, Dipal Patel, Bale Reddy and Tangellapalli Srinivas

Numerical Simulation of Gas-Liquid Phenomena in the Cathode of a Stirred Tank Reactor Proton Exchange Membrane Fuel Cell.

Xichen Wang, Biao Zhou and Mengcheng Jiang

Gas-Liquid Phenomena with Dynamic Contact Angles in The Cathode of a Parallel Proton Exchange Membrane Fuel Cell

Xichen Wang, Biao Zhou and Mengcheng Jiang

Synthesis of Diamond-Like Carbon by PECVD on Different Substrates for Fuel Cell Applications.

Ibrahim Alaefour, Samaneh Shahgaldi, Jian Zhao and Xainguo Li

Effects of Oxyfuel Combustion on The Performance of Natural Gas Combined Cycle Power Generation

System.

Nitin Varia, Dipal Patel, Bale Reddy and Tangellapalli Srinivas

Advanced Exergy Analysis of $\mathrm{LiCl}-\mathrm{H}_{2} \mathrm{O}$ Absorption Air Conditioning System.

Julia Aman, Paul Henshaw and David S-K Ting

State of Charge and Parameter Estimation of Electric Vehicle Batteries.

Richard Bustos, Abu Raihan Mohammad Siddique,

Taranjit Cheema, Stephen Andrew Gadsden and Shohel Mahmud

Optimal Charging of Li-Ion Batteries Based on an Electrolyte Enhanced Single Particle Model. 323

Xianke Lin, Xiaoguang Hao, Zhenyu Liu and Weiqiang Jia

3D Numerical Study of Hill Mounted VAWT

Belkacem Belabes and Marius Paraschivoiu 
The Effect of Nanoparticle Concentration on MQL Performance When Machining Ti-6Al-4V Titanium

Alloy....

Abdelkrem Eltaggaz and Ibrahim Deiab

Multi-Objective Optimization During Machining Ti-6Al-4V Using Nano-Fluids.

Hussien Hegab, Waleed Abdelfattah, Shahryar Rahnamayan, Atef Mohany and Hossam Kishawy

Comparison of Wc-10Co-4Cr Coatings Properties Produced by HVOF and HVOLF Processes for Application in

Petrochemical Industry.

Anas Atieh, Abdulaziz Alhazaa and Rohit Upadhyaya

Compressive Instabilities in Metal-Coated Polymer Microtrusses.

Bharat Bhaga and Craig Steeves

Simulating Layup Defects During Tow Steering in Automated Fiber Placement

Nima Bakhshi and Mehdi Hojjati

Computational Modelling of Radiofrequency Cardiac Ablation to Study the Effect of Cooling on Lesion

Parameters.

Carolyn Berkmortel, Hamed Avari and Eric Savory

FEM Assisted Observations of Cutting Forces, Temperature and Chip Curling in Oblique Machining 365

Salman Pervaiz and Sathish Kannan

3D Printing of Metallic Structures from a Green Ink

Chao Xu, Arslane Bouchemit, Gilles L'Espérance, Louis Laberge Lebel and Daniel Therriault

A Study of the Thermal and Mechanical Properties of Recycled Crosslinked HDPE

Jardel Nobrega Dos Santos and Raghavan Jayaraman

Non-Contact Auxiliary Fixture for Better Machining of Thin Flexible Workpiece Using Eddy Currents.

Robinderpal Singh and Ibrahim M. Deiab

Shaker Vibration Test of Epoxy Composites Reinforced with Pristine and Functionalized Carbon Nanotubes...384 Mohammad Rafiee, Fred Nitzsche and Michel Labrosse

The Effect of High Strain Rate on the Piezo-Resistance of Polydimethylsiloxane with Carbon Nanotubes 388

Farouk Wahsh and Aleksander Czekanski

Design of Hard Compaction Rollers for Automated Fiber Placement on Complex Mandrel Geometries.

Tom Trung Zacchia, Farjad Shadmehri, Jeffrey Fortin-Simpson and Suong Van Hoa

The Effect of Minimum Quantity Lubrication on the FSW Process Performance 396

Wisam Al-Wajid, Ibrahim Deiab and Fantahun Defersha

Review Paper On Digital Manufacturing \& Design

Mukhtiar Singh

Fabrication of SnO2 Composite Nanofiber-Based Gas Sensor Using Elecrospinning Method

Pouria Mehrabi, Justin Hui, Mohammad Paknahad, Allen O'Brien and Mina Hoorfar

\section{COMPUTATIONAL MECHANICS}

A Novel Finite Strain Visco-Hyperelasticity Based Constitutive Model for Elastomers 408

Rohan Thakkar and Aleksander Czekanski

Modal Participation Factors and their Potential Applications in Aerospace: A Review

Michelle Nieto, Mostafa Elsayed and Denis Walch 
Meso-Scale Computational Modeling of Hypervelocity Impact Damage in Advanced Materials

Aleksandr Cherniaev and Igor Telichev

Design, Fabrication, and Calibration of a MEMS Based Sensing Rosette for Quantifying the Influence of Strain

Engineering on the Piezoresistive Coefficients. 425

Amr Balbola and Walied Moussa

Heat Conduction in a Layered Structure with an Interface Crack Using the Dual Phase Lag Model

Zengtao Chen and Keqiang Hu

Scaling and Machine Learning Analysis of Turbulent Fluxes of Momentum and Heat in the Microclimate of an Urban Canyon.

Mohsen Moradi, William D. Lubitz, E. Scott Krayenhoff and Amir A. Aliabadi

Mechanical Analysis of Multi-Directional Functionally Graded Cellular Plates.

Hamed Niknam, Hamid Akbarzadeh, Daniel Therriault and Denis Rodrigue

Gradient Elasticity Modelling for the Analysis of Fiber Composites with Fiber Resistant to Flexure.

Mahdi Zeidi and Chun II Kim

3D Dynamical Model for Liquid Sloshing Simulation in a Partially Filled Elliptical Tank.

Omar Noui, Mohamed Bouazara and Marc J. Richard

Improved Modal Contribution Factors as Response Tracking Mechanisms for Dynamic Systems During Design

Optimization.

Michelle Nieto, Mostafa Elsayed and Denis Walch

A Higher Order Theory of Beams and its Application to the MEMS/NEMS Analysis and Simulations.

Aleksander Czekanski and Volodymyr V Zozulya

Coupled Orbital-Attitude Dynamics Of Flexible Electric Solar Wind Sail.

Gangqiang Li and Zhenghong Zhu

Buckling and Post Buckling Behavior for Unsymmetrical Laminates Part I: Curing Cycle.

A. Y. Elruby, Tawsif Ahmed and Sam Nakhla

Buckling and Post Buckling Behavior for Unsymmetrical Laminates Part II: Stability Characteristics. 475

Tawsif Ahmed, A. Y. Elruby and Sam Nakhla

Inclusion of Aerodynamic Effects in Multibody Dynamics

Bruce Minaker, Mingyu Sun and Jeff Defoe

Numerical Study of Inlet Gas Velocity Effects on Droplet Behaviors in Microchannel.

Mengcheng Jiang and Biao Zhou

Using Visco-Plastic Lubrication for Suspension Transportation.

Saeed Garmeh, Ali Dolatabadi and Ida Karimfazli

Vortex-Induced Vibrations of a Low-Tension Cable-Sheave System Modeled Using Nonlinear Finite

Elements.

Cassidy Westin and Rishad A. Irani

Convergence Control for Topology Optimization.

Dylan Bender and Ahmad Barari

Incorporating Asymmetric Hardening Behavior in Modeling the Cold Expansion Process of AZ31B

Sheet.....

Sasan Faghih, Seyed Behzad Behravesh and Hamid Jahed

Effect of Additives on Cutting Temperature of Glass Fibers Reinforced Polymers

Honoré Kuate Togue, Jean-François Chatelain and Claudiane Ouellet-Plamondon 
Energy Transfer Mechanisms in the Ranque-Hilsch Vortex Tube

Nolan Dyck and Anthony Straatman

Thermal Conductivity of Advanced Architected Cellular Materials

Armin Mirabolghasemi, Hamid Akbarzadeh, Denis Rodrigue and Daniel Therriault

Displacement Flows of Viscoplastic Fluids in Near-Horizontal Channels: Numerical and Analytical

Simulations.

Ali Eslami, Roozbeh Mollaabbasi and Seyed Mohammad Taghavi

Development and Validation of a Numerical Model for Vibration of Power Lines.

Mohammad Hadi Jalali and Geoff Rideout

On the Finite Element Modeling of Turbo Machinery Rotors in Rotor Dynamic Analysis.

Mohammad Hadi Jalali, Nima Nouri and Saeed Ziaei-Rad

\section{STUDENT PAPER COMPETITION}

Assessment of Aliphatic Based Soot Inception in Laminar Diffusion Flames

Nemanja Ceranic and Seth Dworkin

Glass Micro-Cutting by Spark Assisted Chemical Engraving

Lucas A. Hof and Rolf Wuthrich

A Finite Element Model to Study the Effect of Porosity Location on the Elastic Modulus of a Cantilever

Beam.

Stephen Handrigan and Sam Nakhla

Microfluidic Method of Viscosity Measurement in Propane-Saturated Bitumen

Mira Kim, Nader Mosavat and David Sinton

Effect of Body Forces Due to Aircraft Acceleration on Ventilation Airflow and Contaminant Dispersion in Passenger Aircraft Cabins.

Hossam A. Elmaghraby, Yi Wai Chiang and Amir A. Aliabadi

Quantitative Natural Gas Discrimination for Pipeline Leak Detection Through Time-Series Analysis of an MOS

Sensor Response

Matthew Barriault, Mahyar Mohaghegh Montazeri, Allen O'brien, Homayoun Najjaran and Mina Hoorfar

The Influence of Powder Size and Packing Density on the Temperature Distribution in Selective Laser

Melting.

Diego Augusto de Moraes and Aleksander Czekanski

Experimental Results and Proposed Electromechanical Model for Vibrational Communication in Mining and Oil-

Gas Drill Strings.

Md Shahriar Islam, Nathan Peter and Travis Wiens

Investigation into The Influence of Fused Deposition Modeling (FDM) Process Parameters on the Thermal Properties of 3D-Printed Parts.

Ahmed Elkholy and Roger Kempers

Sonification of Flow Instabilities in CFD Aneurysm Models

Daniel E. MacDonald, Thangam Natarajan, Richard C. Windeyer, Peter W. Coppin and David A. Steinman

Droplet Impact, Spreading And Freezing on Metallic Surfaces of Varying Wettability 
Preparation and Properties of Nanoparticle-Enhanced Composite Phase Change Material with Ceramic Porous

Media.

Runfeng Li, Yang Zhou and Xili Duan

Effect of Dispersing Copper Oxide Nanoparticles to RT-35 Filled Circular Thermal Energy Storage System

Soroush Ebadi, Mohammadhossein Hajiyan, Syeda Tasnim, Amir Abbas Aliabadi and Shohel Mahmud

Experimental Investigation of Ice Accretion on Horizontal Axis Wind Turbines

Mustafa Elhajare, Kevin Pope and Xili Duan

Testing and Analysis of Freezing Phenomenon in Conventional Carbon Steel Pipes.

Milad Rezvani Rad, Rick Marsden and André McDonald

A New Three-Layer Model for Horizontal Slurry Flow.

Alireza Sarraf Shirazi and lan Frigaard

Adsorbent-Adsorbate Pairs for Solar Thermal Energy Storage in Residential Heating Applications:

A Comparative Study....

Kapil Narwal, Roger Kempers and Paul O'Brien

Thermal Management of Electrical and Electronic Systems Using PCM

Yazeed Alomair, Muath Alomair, Syeda Tasnim, Hussien Abdullah and Shohel Mahmud

Investigation of Latent Heat Thermal Energy Storage System for Airconditioning Applications

Muath Alomair, Yazeed Alomair, Syeda Tasnim, Hussein Abdullah and Shohel Mahmud

Development of Absorbents with High Contact Area for Applications in Desiccant Coated Heat Exchanger......652

Yunxi Li, Benwei Li and Zhigang Wang

Effectiveness-NTU Relationships of Parallel-Plate Moving Bed Heat Exchangers

Pedro Isaza and Markus Bussmann

Numerical Simulation of Fluid Flow and Heat Transfer of the Supercritical Water in Different Fuel Rod Channels.

Huirui Han and Chao Zhang

Feasibility Study of Synthetic Oil Based Nanofluids for Use in Thermal Oil Heaters

lan Mullett, Xili Duan, Zongming Wang and Yishan Li

Effects of High Pressure Coolant on Machining Temperature and Machinability of AISI 304 Stainless Steel

Yassmin Seid Ahmed and Stephen Clarence Veldhuis

Thermal and Hydraulic Performance of a Novel Evaporator Coil for Refrigeration Systems

Hossam Elkady, Mostafa Elsharqawy, Sameh Saad and Kazi Ahmed

One-Dimensional Fully Coupled Heat and Moisture Transfer Using the Finite Volume Method

Lam Dang, Wey Leong and Alan Fung

Thermal Management of PQ Transformer for a Passively Cooled Power Module

Anshuman Dey, Navid Shafiei, Rahul Khandekar, Kevin Lau, Wilson Eberle and Ri Li

Analytical Heat Conduction Model of Annular Composite Fins.

Chen Feng, Subramaniam Yugeswaran, Michael Gibbons and Sanjeev Chandra

Continuous Fiber Polymer Composites (CFPCS) for Thermal Applications.

Yehia Elsayed, Ahmed Elkholy, Garrett Melenka and Roger Kempers

Thermal Conductivity of Beta-Arsenene Under Biaxial Tensile Strain: A First Principle Study

Armin Taheri, Carlos Da Silva and Cristina Amon

The Design of Infrared Mirror Coatings for the Enhanced Performance of Incandescent Lighting .713

Mohsen Rostami, Nima Talebzadeh and Paul O'Brien 
Integration and Simulation of Microfluidic Platforms and Terahertz Time-Domain Spectroscopy Systems......722 Rasha Al-Hujazy and Christopher Collier

In Pursuit of an Optimum Optical Imaging Technology for Early Detection of Dental Caries 726

Elnaz Baradaran Shokouhi and Nima Tabatabaei

Helical Spring Design Optimization for Endoscopic Devices Using a Design-Of-Experiments and Response Surface Approach.

Hamid Ebrahimi, Albert Yee and Cari Whyne

Focused Ultrasound Surgery of Breast Cancer: A Computational Study on a Realistic Patient Model. .734

Moslem Sadeghi-Goughari, Soo Jeon, Toktam Beheshtian and Hyock-Ju Kwon

Controlled Deflection Of Diamagnetic Biocompatible Aqueous Droplets.

Stephanie Buryk-Iggers, Jennifer Kieda and Scott Tsai

Characterization of the Electrodes of DEP-Based Micro-Separator.

Arash Dalili, Erfan Taatizadeh and Mina Hoorfar

A Novel Growth Chamber for the Discovery of New Natural Products Using Bacteria Microencapsulation In Agarose Microbeads

Tartela Alkayyali, Tiffany Cameron, Ali Ahmadi, Russell Kerr, Bradley Haltli and James Tweel

Generation of Dendritic Microparticles by lonic Crosslinking in an Aqueous Two-Phase System

Niki Abbasi, Maryam Navi and Scott S. H. Tsai

Investigation of Bioprintability of Starch-Chitosan Composite Materials for Fabrication of Tissue Engineering Scaffolds Haley Butler and Ali Ahmadi

Wyatt MacNevin, Matthew Dunlop, Bishnu Acharya and Ali Ahmadi, Development Of A Multi-Stimuli-Responsive Nanocrystalline Cellulose-Based Material For 3D And 4D Bioprinting Applications

Automation in High Throughput/Content Screening for Cancer Stem Cell Drug Discovery

Eleftherios Sachlos

Investigation of Epithelial-to-Mesenchymal Transition Through Microcontact Printing.....

Huma Inayat, Scott Tsai and Andras Kapus

Kinetics Analysis of Multi-Segment Trunk After Experimental Errors Minimization.

Alireza Noamani, Albert H. Vette, Richard Preuss, Milos R. Popovic and Hossein Rouhani

Development of a Dynamic, Reconfigurable Finger-Hand Skeletal Structure.

Shreya Patki and Dr. R. Jill Urbanic

A Novel Concussion Active Prevention Testing Device for Neck Strength Evaluation During Sub-Concussive Impacts Milad Nazarahari, Jordan Arthur and Hossein Rouhani

Development of a Rehabilitation Mobility Assistive Device

Xueqiao Han, Reza Fotouhi and Doug Bitner

Design and Rapid Prototyping of Novel Ventricular Assist Device

Sayedali Mousavi and Afshin Enteshari 
Pouria Mehrabi, Justin Hui, Mahyar Mohaghegh Montazeri, Kim Tien Nguyen

Abigail Logel, Allen O'Brian and Mina Hoorfar

In-Vitro Investigation of the Effect of a Dysfunctional Bileaflet Mechanical Aortic Valve on Flow Characteristics in the Ascending Aorta.

Ahmed Darwish, Wael F. Saleh, Giuseppe Di Labbio and Lyes Kadem

Experimental Measurements of Far Field Cough Airflows Produced by Healthy and Influenza-Infected

Subjects.

Nicholas Dudalski, Ahmed Mohamed, Eric Savory and Samira Mubareka

On the Validity Extent of Linear Viscoelastic Models of Human Brain.

Hesam Hoursan, Mohammad Taghi Ahmadian, Reyhaneh Kazemiasfeh and Ahmad Barari

\section{ENGINEERING ANALYSIS \& DESIGN}

Vibration Isolation of a Carbon Nanotube Filled with a Mass Chain

Lu Lu and C.Q. Ru

Vibration Control of Downtown Toronto High-Rise Development.

Ali Noormohamed, Oya Mercan and Amirahmad Fathieh

Modelling Thermal Conductivity of Porous Thermal Barrier Coatings for High-Temperature Aero Engines Using Five Phase Model...

Ramandeep Singh Ghai, Dr. Kuiying Chen and Dr. Natalie Baddour

Inter-Laminar Strain Energy Continuity in Orthotropic Face Sandwich and Composite Plate Analysis Using Improved Higher-Order Theory.

Temesgen Takele Kasa

The Libration Stabilization of a Partial Space Elevator System Using Analytical Reel Rate Control.

Gefei Shi, Zhanxia Zhu and Zheng H Zhu

Design, Analysis And 3D Printing of Architected Sandwich Panels.

Hamidreza Yazdani Sarvestani, Hamid Akbarzadeh, Hamed Niknam and Kyle Hermenean

The Evaluation of Modelling Techniques for Lubricant Cavitaion in the Application of Squeeze Film

Dampers.

Tieshu Fan and Kamran Behdinan

Design and Optimization of a Novel Beam-Based Shimmy Damper.

Mohsen Rahmani and Kamran Behdinan

Gas Thruster Calibration for Satellite Simulators on an Air Bearing Table.

Joshua Cookson and Zheng Zhu

Predicting the Thermal Conductivity of Epoxy/MWCNT Composites Using Analytical Modelling.

Mohammed Ghuzi and Pierre Mertiny

Application of FBG Optical Sensors to In-Situ Monitoring the Thermo-Mechanical Behaviour of Cold Spray Coated Samples.

Bahareh Marzbanrad, Farid Ahmed, Hamid Jahed and Ehsan Toyserkani

Buckling Paradox and Anisotropic Plastic Plate Bifurcation

Surersh Shrivastava 
Effect of Loading Strain Rates on Unloading Behavior of Shot Peened Materials

The Design and Validations of the Ultrasonic Tactile Sensor

Yanjun Qian and Hyock Ju Kwon

Investigating the Effect of a Speckle Pattern on Measurement Uncertainty in a Three-Dimensional Digital Image Correlation (3D-DIC) System.

Calvin Jee, Gabriel Salata, David Nobes and Kajsa Duke

Natural Frequencies of Internally-Doubly-Tapered Laminated Composite Beams

Amin Fazili and Rajamohan Ganesan

Modeling of Magneto-Mechanical Response of Magnetorhological Elastomers Having Different Arrangement of Magnetic Particles.

Mostafa Asadi Khanouki and Ramin Sedaghati

On-Line Determination of a Go-Nogo State Using a Continuous Estimation of the System Response .883

Johanna McPhee and Rishad Irani

Application of Fiber Bragg Grating Sensor for Strain Measurement at the Notch Tip Under Cyclic Loading.....889 Amirhossein Pahlevanpour, Bahareh Marzbanrad, Seyed Behzad Behravesh and Hamid Jahed

Evaluation of Energy Efficient Propulsion Technologies for Unmanned Aerial Vehicles 894

Jay Matlock, Philipp Sharikov, Stephen Warwick, Jenner Richards and Afzal Suleman

Dynamic Testing System for Rocks Under in Situ Stresses

Wei Yao and Kaiwen Xia

A Two-Stage Conversion Process for AZ31B Corrosion.

Jie Wang, Hamid Jahed and Xin Pang

Development of a Ground Based Testbed for Studying the Libration Dynamics of Orbiting Tethered Satellite

Systems

Udai Bindra and Zheng Hong Zhu

Anisotropic Fatigue Modeling of Wrought Magnesium Alloys

Amirhossein Pahlevanpour, Seyed Behzad Behravesh and Hamid Jahed

Finite Element Analysis of Modified Conebolt Under Static and Dynamic Loadings

Benjamin Nguyen, Ming Cai and Krishna Challagulla

Effect of the Error Propagation Rates in Developing a Skin Model of Measured Surfaces in Coordinate

Metrology.

Cody Berry, Lillian Goodwin and Ahmad Barari

Constrained Topology Optimization for Additive Manufacturing of Structural Components in ANSYS ${ }^{\circledR}$

Davin Jankovics, Hossein Gohari and Ahmad Barari

Vision-Based Relative State and Inertia Ratio Estimation of Unknown Targets.

Qian Feng, Quan Pan, Zheng Hong Zhu, Xiaolei Hou and Yong Liu

Generating Reuse Possibilities for Retired Wind-Turbine Blades.

S R X Jiang, David Inkermann, Thomas Vietor and L H Shu

Collapse Surfaces of the Octet-Truss Lattice at Different Lattice Angles

Mohamed Abdelhamid and Aleksander Czekanski

New Experimental Setup Design for Applying Physical Blowing Agent in Rapid Rotational Foam Molding Technology.

Isha Raktim and Remon Pop-lliev 
A Procedure for Performance Experimental Analysis of a Globe Control Valve 963

Hesam Hoursan, Mohamad Moradi, Mohammad Omid Hadjiazim,

Mohammad Taghi Ahmadian and Ahmad Barari

Graphene Oxide Reinforced Bio-Epoxy Polymers.

Anthony Loeffen, Duncan Cree, Mina Sabzevari and Lee Wilson

High Density Polyethylene/Carbon Nanotube Nanocomposite Foams:

Electrical Conductivity and Percolation Threshold

Trong Linh Hoang, Mohammadmehdi Aghelinejad, Siu N. Leung and Zheng H. Zhu

\section{MATERIALS TECHNOLOGY}

A Finite Element Model to Predict the Effect of Porosity on Elastic Modulus in Low Porosity Materials

Liam Morrissey and Sam Nakhla

Evaluation of Anti-Icing Polymer Coatings

Nick Tepylo, Xiao Huang, Marc Budinger, Pierrick Rouset and Valérie Pommier-Budinger

Low-Velocity Impact Response and Experimental Optimization of Modified Fiber Metal Laminates with Integrated Mechanical Interlock Bonding System.

Tran-Vu Nong, Mostafa Elsayed and Thomas Letellier

Corrosion Performances of Micro-Arc Oxidation Coatings on AZ31B, AZ80 And ZK60 Cast Mg Alloys

Yuna Xue, Xin Pang, Bailing Jiang and Hamid Jahed

Influence of Porosity and Alloy Addition on the Wear Behaviour of a Sinter-Forged C45 Steel Using Taguchi

Method.

Easwaran Naveen and Sripada Raghavendra Kesava Narasimha

Effect of the Interlacing Pattern on the Compaction Behaviour of 3D Carbon Fibre Textile Reinforcements...1009 Rasool Salekrostam and Francois Robitaille

Investigation of Fraying in Textile Reinforcements for Composites

Yousef Badripour and François Robitaille

Modeling Viscoelastomers with Nonlinear Viscosity

Jianyou Zhou, Liying Jiang and Roger Khayat

Strategies for Improving Electrospinability of Polysulfone.

Iman Azarian Borojeni, Biao Zhou and S. Holger Eichhorn

A Hybrid Manufacturing Approach for Low Volume High Temperature Thermoplastic / Thermoset Material Molds.

Hamed Kalami and Jill Urbanic

Influence of Flow Velocity on Erosion-Corrosion Performance of $90^{\circ}$ Carbon Steel Elbows in Potash

Brine.

Raheem Elemuren, Richard Evitts, Ikechukwuka Oguocha,

Glyn Kennell, Akindele Odeshi and Regan Gerspacher

Preparation and Characterization of Graphene Oxide Cross-Linked Composites 1037 Mina Sabzevari, Duncan Cree and Lee Wilson 
On Microstructure and Corrosion Properties of Selective Laser Melted 316L Stainless Steel

Mostafa Kazemipour, Mohsen Mohammadi and Ali Nasiri

High Strain-Rate Behavior of Mechanoluminescent Material Dispersed in a Soft Polymer Matrix 1046

Chukwubuikem Omeziri and Aleksander Czekanski

Effects of Unit-Cell Boundary Type on the Electromechanical Properties of Randomly Distributed Multifunctional Composite Structures.

Joshua Martin and Krishna Challagulla

\section{ENVIRONMENTAL ENGINEERING}

Wave Interaction of Partially Immersed Semicircular Breakwater Suspended on Piles Using Flow-3D

Ahmed Abozaid, Wael Mahmod, Ayman Koraim, Elsadek Heikal and Hassan Fath

An Experimental Evaluation pn Air Conditioning Performance with Vairtex Air Director Applied on Residential Condenser Unit

Vien Nguyen, Edgar Matida and Gerard Godbout

The Spanwise Variation of Roof-Level Turbulence in a Street Canyon Flow

Tomek Jaroslawski, Eric Savory, Laurent Perret and Karin Blackman

Development of Raw and Treated Flax Straw for Oil Spill Cleanup

Pooya Ghasemi, Duncan Cree and Alvin Ulrich

\section{MECHANICAL ENGINEERING EDUCATION}

Preparing and Coordinating Undergraduate Engineering Design Projects

Roger Carrick and Aleksander Czekanski

\section{A Practical Approach for Teaching Finite Element Analysis for Senior Undergraduate Mechanical Engineering}

Students

Khaled Al-Athel

Towards a Conceptual Model of Design Team Learning in Mechanical Engineering Education 1087

Minha $\mathrm{Ha}$ and Aleksander Czekanski

Graduate Attributes and Threshold Concepts: Are We Measuring What's Important?..... .1096 Nancy Nelson and Bob Brennan 
FLUID MECHANICS 


\title{
Wind Loads on Cyclists due to Passing Vehicles
}

\author{
William David Lubitz*, Bryan Rubie \\ School of Engineering \\ University of Guelph \\ Guelph, Canada \\ *wlubitz@uoguelph.ca
}

\begin{abstract}
Cyclists on rural highways travel at much lower speeds than motor vehicles. There is concern that unsteady aerodynamic loads produced as a large vehicle passes a cyclist can cause instability and loss of control, potentially initiating a traumatic accident for the cyclist. Large lateral spacing can be provided between cyclists and motor vehicles using wider paved shoulders, however this adds cost to road construction, so there is a need to balance the needs of cyclist safety and paved shoulder width. Understanding the nature of the unsteady wind loads experienced by a cyclist when a motor vehicle passes is a necessary first step in determining optimum paved shoulder widths. An experiment was conducted that directly measured the lateral forces on a full scale model cyclist, static pressure and wind speed as motor vehicles passed a cyclist. As a motor vehicle passed, the cyclist first experienced a large transient lateral forcing, followed by lower magnitude forcing. The magnitude of the force was well correlated to the measured static pressure, while induced transient wind speeds were relatively low (on the order of $1 \mathrm{~m} / \mathrm{s}$ ). As would be expected, the magnitude of forcing increases with vehicle size and speed, and decreases as lateral spacing between cyclist and vehicle increases. The results were used to develop an expression to predict tipping moment as a function of passing vehicle characteristics and offset distance.
\end{abstract}

\section{Keywords-bicycle; wind load; aerodynamics; passing vehicles}

\section{INTRODUCTION}

The passing of a large vehicle like a truck can be an unsettling experience for a cyclist travelling on a rural highway. The wakes of large vehicles produce aerodynamic forces on cyclists and other road-side objects. There is concern that unsteady lateral forces on a cyclist produced by a large passing vehicle could cause a cyclist to become unstable and lose control, potentially initiating a traumatic accident.

If available, cyclists on rural highways generally utilize paved shoulders for travel, allowing them to travel outside the regular travel lane. Wider paved shoulders allow cyclists to travel with greater lateral separation from large vehicles, reducing the aerodynamic forces experienced by a cyclist induced by passing vehicles. However, shoulder paving cost increases with width, and the safety benefits of increased shoulder paving width must be balanced with costs [1]. Currently, information is still needed on minimum safe lateral spacing between the travel paths of bicycles and large vehicles on rural highways. Minimum safe spacing depends on the aerodynamic forces induced on a cyclist by large vehicles passing at highway speeds, and the maximum such forces that a cyclist can experience and remain stable [2].

There is little quantitative information available on the forces induced on cyclists by wakes generated by passing large vehicles. Several publications on bicycle infrastructure design [3-5] reproduce the same plot of lateral aerodynamic "force" exerted on a cyclist by a passing truck as a function of speed and separation distance (Fig. 1). A 1976 US government report [6] was the earliest incarnation of this plot that was found. No information on the source of the plot was provided except a note that it was from "prior research."

It is apparent Figure 1 is at best an idealization. While force is approximately proportional to the square of the vehicle velocity, the linear relationship between separation distance and force seems physically unlikely. The reporting of a force in units of mass $(\mathrm{kg})$ is worrisome (note that lbs was used by Smith [6]), and it is unclear if average or peak lateral forces are reported. No guidance is given on how the estimated tolerance limit of $1.73 \mathrm{~kg}$ $(17 \mathrm{~N})$ was derived. Despite these limitations, the $17 \mathrm{~N}$ lateral force limit and Fig. 1 appear to be the current accepted standard in the bikeway design literature for aerodynamic forces on cyclists due to passing vehicles. For example, Llorca et al. [2] recently applied this same linear relationship with zero effect at $3.0 \mathrm{~m}$ in their analysis of motor vehicles passing cyclists.

Only one prior study was located that attempted to directly measure the wind loads that would be induced on a cyclist (as opposed to other object) by a passing vehicle [7]. Two propeller anemometers were placed at the roadside, and the induced wind was measured as a heavy goods truck was driven past at a range of speeds and offset distances. However, the impact on a cyclist was estimated using assumptions of cyclist area and drag coefficient, rather than by a more direct form of measurement. They observed that as the truck passed, a wind speed "pulse" occurred in the lateral direction that was first away from, and then towards, the direction of the vehicle. As vehicle speed increased, the amplitude of this pulse increased while the time duration decreased, and it was noted that the rapid change in wind direction was likely to be particularly destabilizing. 


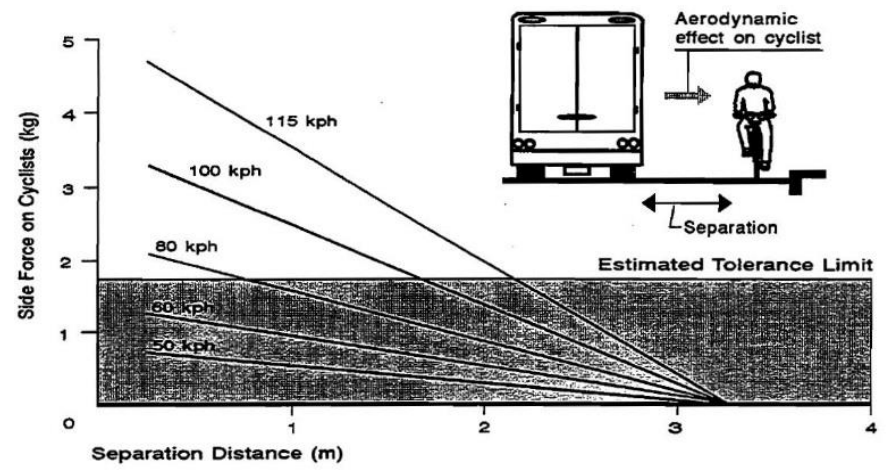

Figure 1. Aerodynamic force on a cyclist due to a passing large truck. From [4].

Research has been conducted in the similar areas of wind loads caused by large trucks on overhead sign bridges [8], temporary roadside signs and barriers [9] and pedestrians [10]. Recently, Lichtneger and Ruck [11] experimentally measured forces on a range of plates beside and above passing vehicles ranging from passenger cars to large buses and trucks (lorries). The winds induced on railway station platforms due to passing express trains have also been studied [12,13].

While these studies encompass a range of situations and investigative methods (field measurements, simulations, theory), they agree on the features of the aerodynamic forcing experienced by a stationary or relatively slow moving object (such as a bicycle) being passed closely by a high-speed vehicle. The most concerning effect of a passing large vehicle is an initial sharp pulse of increased pressure, which occurs over a time period of a few tenths of seconds, and is followed immediately by a period of decreased pressure. This is accompanied by wind speed gusts on the order of $1 \mathrm{~m} / \mathrm{s}$, also over a time period of order $0.1 \mathrm{~s}$. The magnitude of these pressures and gusts increases with frontal area of the passing vehicle and decreases with vehicle streamlining. The initial pressure pulse is followed by increased turbulence and a return to pressures nearer ambient. The passage of large gaps in vehicles (e.g. between truck cab and trailer) and the blunt tail of a vehicle also induce pulses of pressure and wind speed variation from ambient levels, although these are typically lower than those associated with the passing of the front of the vehicle. An example of the transient forcing, pressure and air speed from the experiments for this project is shown later in Fig. 4.

There are a few important differences between the case of lateral forcing on a moving cyclist that could cause loss of balance, and forcing of stationary objects such as signs and walls. First, while all prior literature has focused on forces on cyclists or other surfaces, arguably the most important phenomena from the passing of a large vehicle would be not a transient lateral force on the cyclist, but rather the resulting transient lateral moment around the contact line between the wheels and the road surface. A lateral force exerted only a centimeter above the ground would do much less to unbalance a traveling cyclist than the same force exerted at the level of the cyclists shoulders or head (provided the wheels do not slide sideways, which given the forces we will discuss below is a reasonable assumption). In the experiments and discussion that follow, it will be lateral moments that are measured and considered, rather than just lateral force, although at times "force" will be used because moment will be the force measured by a load cell multiplied by a known moment arm.

The time periods of transient forcing of the cyclist are also very important. Unlike signs or walls, cyclists actively respond to transient forcing, by adjusting steering and body position, in order to maintain a balanced riding position. A cyclist could easily respond to a large force (or moment) being applied smoothly and gradually over a long period of time. However, the same forcing applied in a few milliseconds could cause an immediate instability that would be very difficult to correct and could lead to a loss of balance. Unlike for fixed objects, forcing timing matters, as does the nature of the cyclist's response.

Bicycle-specific measurements of the aerodynamic forces induced by large passing vehicles are still needed. It is not feasible to calculate highly accurate wind and pressure forces on a bicycle and rider because they are a geometrically complex bluff-body, unlike the uniform flat plate or cylindrical tubes typical of highway signs and sign bridges, immersed in a highly transient flow. While there is a large body of research on the aerodynamics of both cyclists and motor vehicles, most has been conducted with the goal of reducing the steady state aerodynamic drag of the vehicle itself. These studies don't address crosswinds or unsteady flow, and are of little use for this research. Also, studies that do investigate crosswinds are generally not directly applicable. For example, Moulton et al. [14] investigated the crosswind loads on a streamlined bicycle, and Tew and Sayers [15] measured crosswind effects on bicycle wheels. However, different forces due to crosswind effects would be expected for a conventional upright non-streamlined bicycle (which is essentially a collection of bluff bodies), rather than a rotating wheel or streamlined human powered vehicle. The authours could find no direct measurements of lateral aerodynamic forces on a non-racing cyclist due to transient events such as passage of a large vehicle.

Given the temporal and spatial transience of the flow, this situation cannot be reliably modelled using CFD tools unless field data is available to validate the CFD simulations. The geometry of an unstreamlined bicycle and rider is quite complex, and it is not feasible to directly measure the induced pressures using pressure taps. Therefore, measuring the forces induced on a full size bicycle was selected as the most feasible approach. This research project sought to address this lack of fundamental data by directly measuring the lateral force caused by passing vehicles on upright, diamond frame, single rider, non-streamlined bicycles. It should be noted that the experiment documented below was a relatively modest, resource-constrained affair. While some conclusions are provided to some of the questions and issues raised above, significant scope remains for additional experimental study.

\section{Methodology}

A stationary instrumented bicycle with full scale model rider, three-dimensional sonic anemometer (RM Young 81000) and omni-directional static pressure sensor were placed in a line adjacent to the vehicle travel path in Fig. 2. The test vehicle would first pass the model cyclist, than the pressure probe, and last the sonic anemometer. Horizontal spacing was $1.20 \mathrm{~m}$ between the 
centers of the anemometer and pressure probe, and $1.00 \mathrm{~m}$ between the pressure probe center and the leading edge of the front bicycle tire. The anemometer and pressure probe were mounted on vertical poles at a sampling height of $1.15 \mathrm{~m}$. Lateral moment on the bicycle, static pressure and three-dimensional wind speed were measured simultaneously as vehicles passed.

Static pressure was measured using a parallel plate two-port static pressure probe like that of Robertson [16]. A Setra 265 differential pressure transducer was mounted immediately below the pressure probe. The reference side of the transducer was connected to a rigid tank housed within an insulated container (the white box in Fig. 2) that included a small rigid syringe to allow setting of the reference pressure, allowing recording of both positive and negative pressures relative to ambient pressure.

Characteristics of the truck and the model bicyclist are summarized in Table 1. Projected areas and centroids were determined by analysis of photographs taken specifically for measurement purposes using a long telephoto lens to minimize parallax errors. ImageJ (https://imagej.nih.gov/ij/) was used for the actual image analysis.

All testing was done on a closed course. Cones and pavement markings located prior to the bicycle and sensors allowed the vehicle driver to laterally position the vehicle as it passed the instruments. Two optical switches placed on the roadway $10 \mathrm{~m}$ apart adjacent to the instruments directly measured the speed of the passing vehicle. The distance between vehicle path and the line of instruments was measured directly by wetting the roadway before the vehicle passed, and measuring the distance to the tire tracks left by the vehicle immediately after it passed. The method was accurate to $\pm 1 \mathrm{~cm}$.

The bicycle wheels were set in a low-profile heavy aluminum channel, and the tires were inflated to a very high pressure to produce minimal resistance to rotation in the lateral direction. The bicycle brakes were permanently set to prevent forward or backward rolling of the bicycle. A dummy rider was constructed incorporating a solid wood, metal and plastic frame, plus additional packing material inside a full length $8 \mathrm{~mm}$ neoprene wetsuit. Boots, a foam head and cycling helmet and diving gloves were attached rigidly to the body frame at the locations of feet, head and hands. The dummy was rigidly affixed to the bicycle at the handlebars and pedals, positioned in a semi-upright riding position. The dimensions of the combination bicycle and rider are also given in Table 1.

Lateral force on the model cyclist was directly measured using a custom-built load cell. A temperature-compensating load cell was constructed by placing four surface-mount strain gauges wired in a full Wheatstone bridge on the surface of a thin wall aluminum tube. Spherical rod-end joints were placed at each end of the tube. The tube was oriented horizontally with one end connected to the downtube of the bicycle above the pedals at a height of $0.59 \mathrm{~m}$ above the ground. The other end was affixed to a heavy low-profile rigid frame. The load cell provided the only lateral support of the bicycle. Before each set of tests the load cell was calibrated in-situ using precise weights and a pulley system to apply tensile and compressive lateral forces up to $15 \mathrm{~N}$. The
Wheatstone bridge was connected via shielded cables to a custom-built amplifier.

All instrumentation was connected to a netbook computer using a National Instruments USB-6008 data acquisition device (for switches, barometer, load cell, lateral component of wind speed) and serial-to-USB converter (all anemometer channels). A custom-written LabView application simultaneously collected and stored measurements of optical switch output, pressure, force and lateral wind speed component at $1000 \mathrm{~Hz}$. Note that the update rate of the anemometer was $32 \mathrm{~Hz}$, meaning that the lateral wind speed is oversampled. Additionally, all channels of anemometer output (three wind speed components and temperature) were recorded at a rate of $32 \mathrm{~Hz}$ by recording the anemometer serial output.

Table 1. Vehicle and model cyclist parameters.

\begin{tabular}{|c|c|c|}
\hline Parameter & Model Bicyclist & Truck \\
\hline Description & $\begin{array}{c}\text { Semi-upright, with } \\
\text { helmet, on 26" } \\
\text { wheel mountain bike }\end{array}$ & $\begin{array}{c}\text { 2001 Dodge Ram, 4 } \\
\text { wheel drive, full size } \\
\text { pickup truck }\end{array}$ \\
\hline Drag Coefficient & $0.9-1.1[17]$ & $0.47[18]$ \\
\hline Frontal Area & $0.74 \mathrm{~m}^{2}$ & $3.17 \mathrm{~m}^{2}$ \\
\hline Overall Height & $1.72 \mathrm{~m}$ & $1.84 \mathrm{~m}$ \\
\hline Overall Length & $1.67 \mathrm{~m}$ & $5.49 \mathrm{~m}$ \\
\hline Side Area & $0.71 \mathrm{~m}^{2}$ & \\
\hline $\begin{array}{c}\text { Centroid Height Above } \\
\text { Ground }\end{array}$ & $0.87 \mathrm{~m}$ & \\
\hline $\begin{array}{c}\text { Centroid Horizontal } \\
\text { Distance from Front }\end{array}$ & $0.94 \mathrm{~m}$ & \\
\hline
\end{tabular}

\section{RESULTS}

Data was collected for passes of the truck at a range of offset distances and vehicle speeds, as shown in Fig. 3. Maximum speeds were limited by the need to bring the vehicle up to test speed, settle at that speed, pass through the test area, and decelerate safely. Pressure, wind and loading fluctuations were negligible when the truck passed at a speed of $U_{v}$ less than 30 $\mathrm{km} / \mathrm{h}$.

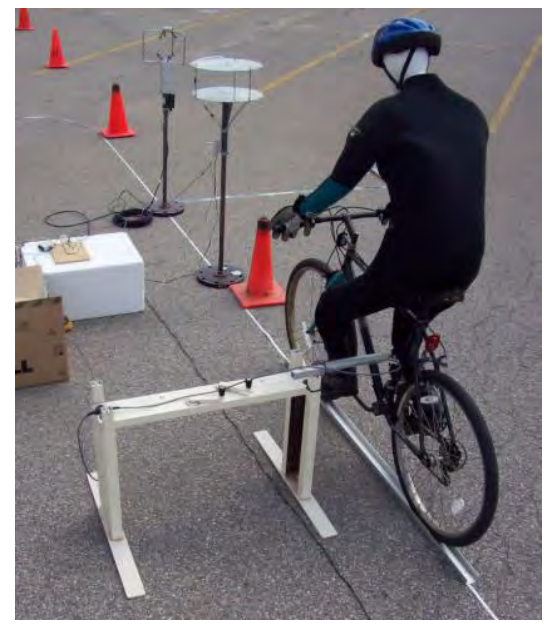

Figure 2. Overview of experimental setup. Vehicles passed on right side, in same direction as cyclist. 


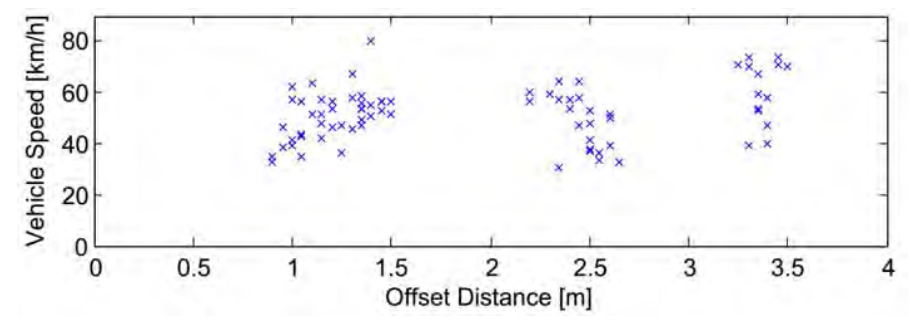

Figure 3. Test data speed and offset distances.

Measurements of lateral force, lateral wind speed component and static pressure from a typical run are shown in Figure 4. The data is presented time-offset based on the vehicle speed, so that the three traces represent measurements that would be observed at a single location for any given time.

Lorca et al. [2], and Lichtneger and Ruck [11] suggest that the lateral force $F$ on a roadside object might be modeled as

$$
C_{f}=2 F / \rho A_{v} U_{v}^{2}
$$

where $\rho$ is air density, $A_{v}$ is frontal area of the passing vehicle and $U_{v}$ is the relative velocity of the passing vehicle. The coefficient $C_{f}$ is a dimensionless value that accounts for the streamlining and shape of both the passing vehicle and the cyclist. A peak pressure difference coefficient $C_{p}$ can be similarly defined as

$$
C_{p}=2 P / \rho U_{v}^{2}
$$

where $P$ is $\rho$ is air density and $U_{v}$ is the relative velocity of the passing vehicle. The coefficient $C_{p}$ is a dimensionless force coefficient with a similar role to $C_{f}$.

The initial pulse of pressure and air velocity (and resulting transient moment on the cyclist) are larger than any of the fluctuations that followed, both in terms of overall magnitude, and in terms of time rate of change of the quantities. In this study, the magnitude of the difference between the first positive peak and negative "peaks" of pressure, air speed and moment were extracted from the time-series data for each run. (For example, in Fig. 4, the peak differences in pressure, force and wind speed are $20 \mathrm{~Pa}, 6.4 \mathrm{~N}$ and $2.0 \mathrm{~m} / \mathrm{s}$, all occurring within the first second.)

Fig. 5 shows the measured peak force difference versus measured peak pressure difference. (While the experiment was configured to measure a torque, all tests had the same moment arm and the load cell itself measures force.) The relationship between the two is approximately linear, within measurement uncertainty. This suggests measurements of pressure, which are simpler experimentally and can be found in the literature (e.g. Lichtneger and Ruck [11] or Sanz-Andrés and Santiago-Prowald [12]) can be used to give reasonable approximations of the side force (or moment) that could be expected on a cyclist.

Fig. 6 shows the peak differences of pressure, force and wind speed $U$ as a function of offset distance. It can be argued that offset distance could be normalized by the square root of the vehicle frontal area, however, verification of this theory was not possible since only a single vehicle was tested.

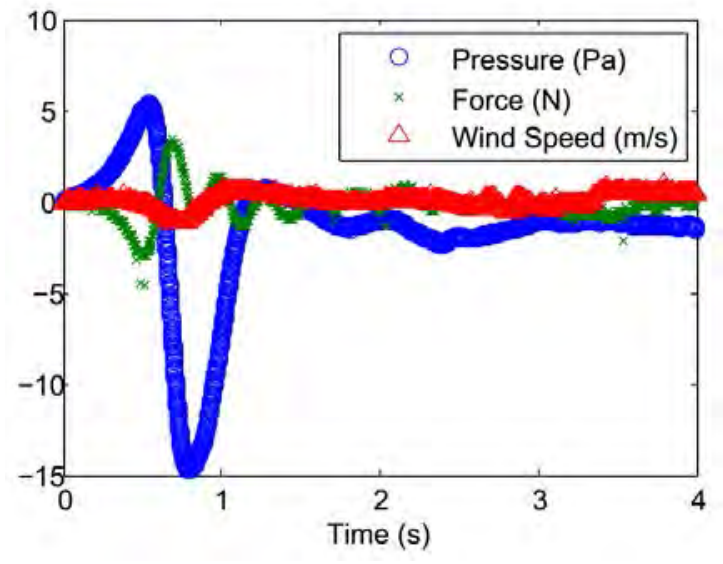

Figure 4. Example pressure, load cell force and wind speed magnitude. Vehicle speed $53.3 \mathrm{~km} / \mathrm{h}$, offset distance $1.35 \mathrm{~m}$.

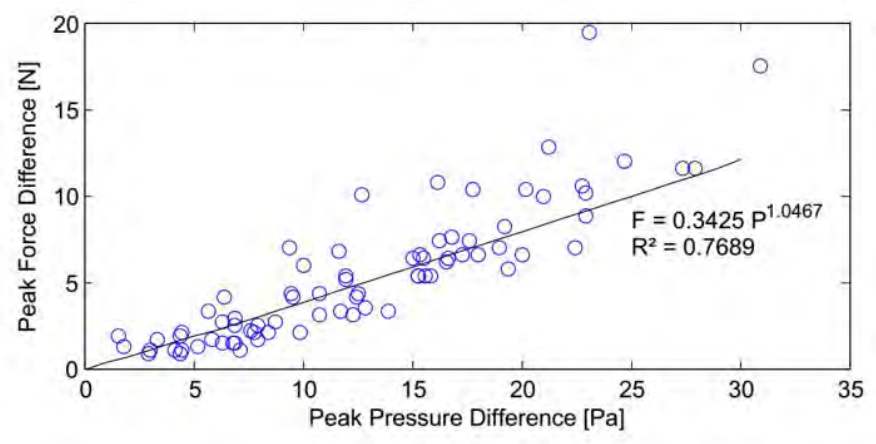

Figure 5. Peak differences in pressure and force observed for each test case.

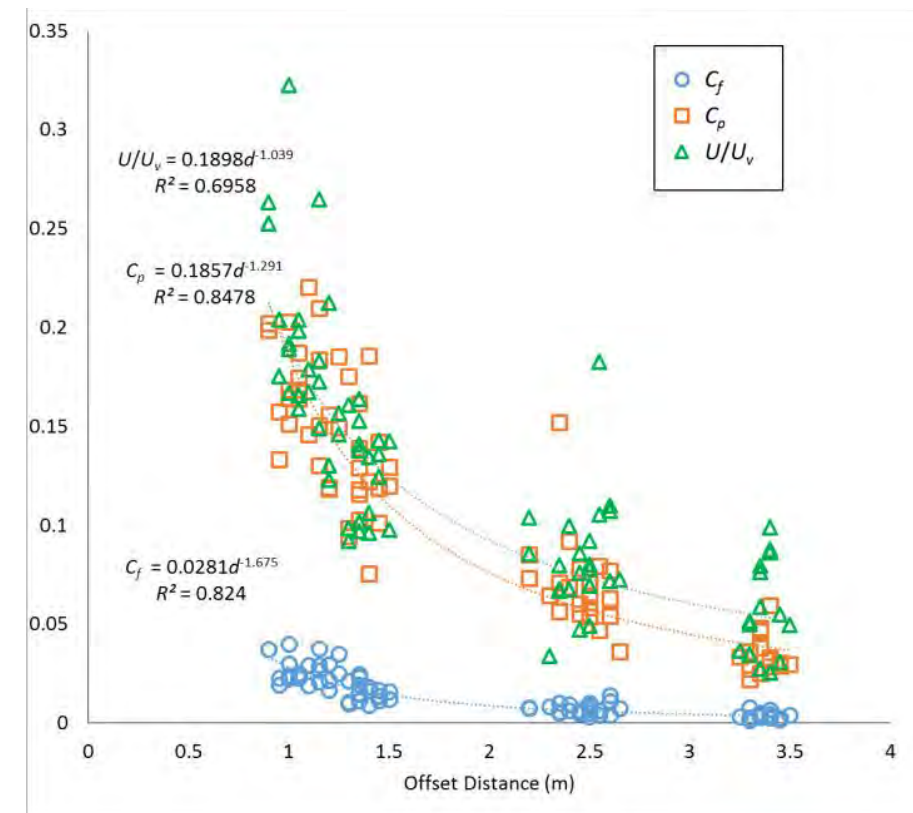

Figure 6. Measured peak differences versus offset distance. 
Fig. 7 shows an updated version of Fig. 1 based on the experimental measurements presented here. Peak force was assumed to vary with vehicle velocity squared, and the measured force was used with Eqn. 2 and the fit equation for $C_{f}$ in Fig. 6. The resulting forces were multiplied by $0.59 \mathrm{~m}$ (the experimental moment arm) to get tipping moments, in N.m. This gives a predictive equation for tipping moment $M$ of

$$
M=\frac{1}{2} \rho A_{v} U_{v}^{2}\left(0.0281 d^{-1.675}\right) L
$$

where $L$ is the experimental moment arm $(0.59 \mathrm{~m})$ and $d$ is the offset distance in units of meters.

Although no supporting evidence was found for the $17 \mathrm{~N}$ "tolerance limit" reported (and re-reported in the literature) [3-6], here it has been converted to a moment by multiplying by the 0.87 $\mathrm{m}$ centroid height of the bike and rider in this experiment (Table 1) and is included in Fig. 7 for comparison purposes. Due to the power law relation used to fit the data, the model shown in Fig. 7 is unrealistic at offset distances less than about $1 \mathrm{~m}$. Since offset distance is measured from vehicle side to the centerline of the cyclist, vehicles passing with offsets much less than $1 \mathrm{~m}$ would risk physically contacting the cyclist.

Both experimental results (Fig. 6) and the tipping moment model (Eqn. 3; Fig. 7) suggest that tipping moment decreases asymptotically with offset distance. No supporting evidence was found to support the assumption of no forcing at offset distances greater than $3.0 \mathrm{~m}$ shown in Fig. 1. The magnitudes of the experimental forces and moments, and those predicted by the resulting model, were of similar magnitude to those in Fig. 1 and those observed by Lichtneger and Ruck [11] for a small flat surface alongside a roadway at cyclist height.

Finally, readers are cautioned that the uncertainty of Fig. 7 is high, owing to the scatter in the experimental data from which it was derived and the limited nature of these preliminary experiments (e.g. only one test vehicle).

\section{CONCLUSIONS}

The results of this experimental study suggest that the peak forcing on a bicycle is reasonably well correlated to the peak static pressure pulse (within experimental scatter), and also to the peak wind speed magnitude. This means that (1) measurements of wind speed and/or pressure may be sufficient in future experiments, and (2) extrapolation from studies of similar objects, such as signs and walls near roadways, should likely give representative forcing for bicycles.

The relationship in Fig. 1 appears to be a reasonably practical approximation for general planning. The most notable deficiency is that vehicle size and aerodynamic considerations are not illustrated. Fig. 7 presents a possible improvement to this relationship, however, it is not yet validated. During this study, it became apparent that there is little understanding of what levels and types of transient forcing cyclists can safely compensate for in practice. This would be an interesting and relevant area for future investigation.

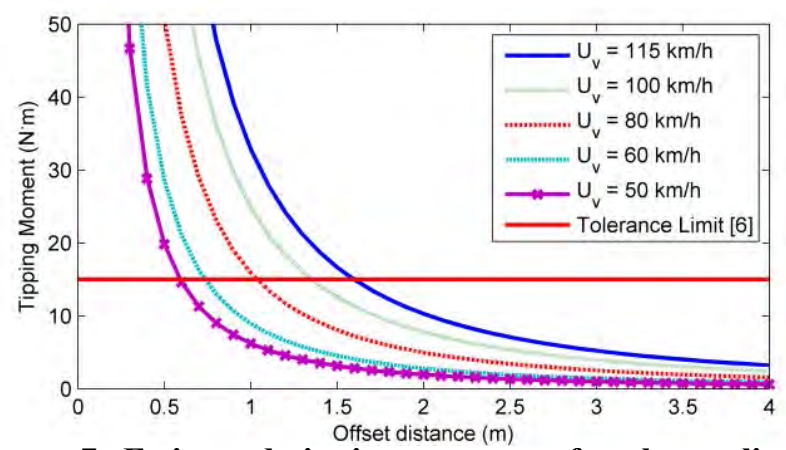

Figure 7. Estimated tipping moments for the cyclist and vehicle from the current study.

\section{ACKNOWLEDGMENT}

Mr. Rubie's work on this project was supported by a University of Guelph Undergraduate Research Assistant grant.

\section{REFERENCES}

[1] A. M. Khan, A. Bacchus, "Bicycle use of highway shoulder, " Transportation Research Record 1502. Nat. Academy Press, pp. 8-21, 1995.

[2] C. Llorca, A. Angel-Domenech, F. Agustin-Gomez, A. Garcia, "Motor vehicles overtaking cyclists on two-lane rural roads: analysis on speed and lateral clearance," Safety Science, vol. 92, pp. 302-310, 2017. doi: 10.1016/j.ssci.2015.11.005.

[3] M. Hudson, Bicycle Planning, London: Architectural Press, 1982.

[4] MTO (Ministry of Transportation, Ontario). Ontario Bikeways Planning and Design Guidelines, March 1996.

[5] C. Bernard, R. Boivin, L. Moreau, J.F. Pronovost, P. Ryffranck, Vélo Québec, Technical Handbook of Bikeway Design: Planning, Design, Implementation, Montréal : Vélo Québec, Ministère des transports du Québec and the Canadian International Development Agency, 1992.

[6] D. T. Smith, Safety \& Locational Criteria for Bicycle Facilities User Manual Vol. 1 Bicycle Facility Locational Criteria. Report FHWA-RD-75-113. U. S. Federal Highway Administration, February 1976.

[7] D. Walton, V.K. Dravitzki, B.S. Cleland, J.A. Thomas, R. Jackett, Balancing the Needs of Cyclists and Motorists, Land Transport New Zealand Research Report 273, 2005.

[8] P.M. Cali, E. E. Covert, "Experimental measurements of the loads induced on an overhead highway sign structure by vehicle-induced gusts, J. Wind Eng. \& Ind. Aero., vol. 84, pp. 87-100, 2000. doi:10.1016/S0167-6105(99)00045-8.

[9] A.D. Quinn, C.J. Baker, N.G. Wright, "Wind and vehicle induced forces on flat plates - part 2: vehicle induced force," J. Wind Eng. \& Ind. Aero., vol. 89, pp. 831-847, 2001. doi: 10.1016/S0167-6105(01)00071-X.

[10] A. Sanz-Andrés, A. Laverón, A. Cuerva, C. Baker, "Vehicle-induced force on pedestrians," J. Wind Eng. \& Ind. Aero., vol. 92, pp. 185-198, 2004. doi: 10.1016/j.jweia.2003.11.002.

[11] P. Lichtneger, B. Ruck, "Full scale experiments on vehicle induced transient loads on roadside plates," J. Wind Eng. \& Ind. Aero., vol. 136, pp. 73-81, 2015. doi: 10.1016/j.jweia.2014.10.010. 
[12] A. Sanz-Andrés, J. Santiago-Prowald, "Train-induced pressure on pedestrians," J. Wind Eng. \& Ind. Aero., vol. 90, pp.1007-1015, 2002.

doi: 10.1016/S0167-6105(02)00216-7.

[13] H. J. Gerhardt, O. Krüger, "Wind and train driven air movements in train stations," J. Wind Eng. \& Ind. Aero. , vols. 74-76, pp. 589-597, 1998.

doi: 10.1016/S0167-6105(98)00053-1.

[14] A. E. Moulton, A. Hadland, D.L. Milliken, "Aerodynamic research using the Moulton small-wheeled bicycle," Proc. I. Mech E Part A: J. Power and Energy, vol. 220, pp. 189-193, 2006. doi: 10.1243/09576509A01305.

[15] G.S. Tew, A.T. Sayers, "Aerodynamics of yawed racing cycle wheels," J. Wind. Eng. \& Ind. Aero., vol. 82, iss. 1-3, pp. 209-222, 1999.

doi: 10.1016/S0167-6105(99)00034-3.

[16] P. Robertson, "A direction-insensitive static head sensor," Journal of Physics E (Scientific Instruments), vol. 5, no. 11, pp. 1080-1082, 1972.

doi: 10.1088/0022-3735/5/11/016.

[17] C.R. Kyle, "Reduction of wind resistance and power output of racing cyclists and runners travelling in groups," Ergonomics, vol. 22, issue 4, pp. 387-397, 1979. doi: 10.1080/00140137908924623.

[18] V. Eowsakul, T. Ortolani, "Improving the aerodynamic characteristics of a Dodge Ram pickup truck," ASME IMECE Paper No. 97-WA/DE-18, 8p, 1997. 


\section{Numerical Investigations of Aerodynamic Noise due to Flow Past a Bluff Body}

\author{
Sultan Ibrahim Alqash \\ PhD Student, ARL-MLS Lab \\ MIE Department, University of Toronto \\ Toronto, Canada \\ sultan.alqash@mail.utoronto.ca
}

\author{
Kamran Behdinan \\ Professor, ARL-MLS Lab \\ MIE Department, University of Toronto \\ Toronto, Canada \\ behdinan@mie.utoronto.ca
}

\begin{abstract}
Landing gears (LG) are primarily designed to support all the loads of an aircraft during landing, taxiing, and taking off. To ease inspection and maintenance, the aerodynamic design is not refined, where many components are exposed to the air flow generating what so-called aeroacoustics noise. To understand the underline physics and investigate both the flow field and its associated acoustic field, a two-dimensional (2D) case of flow past a circular cylinder was simulated using ANSYS Fluent. Two different Reynolds numbers, $R e, 150$ and 90,000 were examined. For low $R e$, two distinct numerical conditions steady and unsteady flow were simulated and compared to examine the effect of the time dependency on the associated acoustic field. For high $R e$, the acoustic field was computed using the built-in Ffowcs William and Hawkings (FW-H) acoustic analogy solver in Fluent. The results show the importance of considering the time variable to extract the corresponding flow data. The far-field noise prediction highly depends on the location of the near-filed data and its associated integral source terms (surface/volume).
\end{abstract}

Keywords-Reynolds number; acoustic analogy; near-field; farfield; pressure fluctuations

\section{INTRODUCTION}

The aircraft noise was recognized as an environmental problem in the early 1950 s, when the turbo-jet powered aircraft was introduced to the markets. Since then, the aircraft noise issue has been dominated by jet noise, known as propulsive noise. In the early 1970 s, aircraft noise was successively reduced after the introduction of the first twin cycle bypass turbofan engines [1]. Due to the noise reduction of the propulsive noise that originates from jet-engines, the airframe itself becomes the major contributor to the overall noise. As a result of decades of research and development into noise reduction, by the year 2000 the aircraft noise level had become 20-30 dB quieter compared to the first generation of turbofan powered aircraft. The US and European communities have set a target to minimize the noise level by $50 \%$ near airport areas by 2020 [2]. By 2050, the overall noise level emission of flying aircraft should be further reduced by $65 \%$ [3]. When the aircraft approaches the airport, the engines are almost at low speed which reduces its noise contribution. That yields to put a high portion of the generated noise on the airframe itself. Among the airframe components, the landing gears have been identified as a main source of noise during the approach phase, when they are fully deployed prior to landing for both aircraft types: long range and short range. It has a significant noise level due to its complex structural design that consists of many components with different lengths and sizes.

For a commercial aircraft, the noise generated from the airframe has received much attention in recent years. This has led the Federal Aviation Administration (FAA) to impose strict regulations on the aircraft noise level [4]. All these demands created pressure on industries and academic communities to develop improved techniques for noise reduction and better methods for its prediction. To ease inspection and maintenance, the aerodynamic design is not refined. As a result, many components such as, hydraulic cables, electric wiring, torque links, front and rear braces are exposed to the air flow. Therefore, the flow separation over the LG components constitutes the main noise source mechanism through unsteady wake flow and large-scale vortex instability and deformation [5]. In the literature, there are three distinct approaches to study flow field and associated acoustic field around the LG within certain limitations. Those approaches are experimental, empirical, and numerical. The main difference between flow around landing gears and that around simple geometries, like single or tandem circular cylinders, is the three-dimensionality flow characteristic. For that reason, the experimental studies for the isolated or installed LG system are recommended but too expensive. Thus, the numerical approach becomes an alternative candidate to resolve this issue. Although current computational approaches are infeasible to handle all the turbulence scales that inevitably exist in cases of complex geometry such as a LG, there are some computational fluid dynamic (CFD) approaches that have been utilized to resolve this issue. The most popular methods are Direct Numerical Simulations (DNS), Large-Eddy Simulations (LES), and Reynolds Averaged Navier-Stokes (RANS). Recently, there is a common CFD method known as Detached-Eddy Simulations (DES). The DES approach is a combination of LES and RANS 
solvers, the LES takes care of the large-scale separated flow regions, while RANS handles the modeling of the turbulence boundary layer [6].

Any aeroacoustics problem consists of two fields as its name implies fluid dynamics and acoustics, respectively. It is a two-step process: 1) sound generation based on the study of the aerodynamic flow which generates sound/noise sources and then, 2) sound propagation based on the investigation of the acoustic field spreading in a medium at rest that generated from step 1. The Lighthill's acoustic analogy is considered as the first pioneering work in sound generated aerodynamically and is named after Jims Lighthill [7] in 1952. In 1954, he assumed that the turbulent fluctuations of the stream are equivalently represented by a distribution of quadrupole sources in the same volume of the flow. In 1955, Curle [8] modified the Lighthill's analogy to include the effect of solid boundaries. In 1969, Ffowcs William and Hawkings [9] considered both the influence of the solid surfaces and the turbulence in arbitrary motion and developed the FW-H analogy. The FW-H analogy is considered as the generalized Lighthill's acoustic analogy which makes it more common and applicable for a wide range of applications. Van Mierlo et al. [10] studied the influence of the main landing gear (MLG) inclination angle on the noise level using ANSYS Fluent. However, they did not include the contribution of the quadrupole sources corresponds to the volume integral due to its high computational cost. Thus, the selection of the surface always coincides with the wall of the bluff body. The same approach was used by Long et al. [11] to study the far-field noise of a simplified nose landing gear (NLG). They noticed that the directivity of the noise is a dipole like source. Another method based on CFD and the FW-H equation with penetrable surface has advanced considerably. For instance, Lockard et al. [12] compared the results and noticed that the noise predicted based on solid surface agreed well with the near-field CFD results, and that noise predicted based on the penetrable surface was highly suspected to be corrupted by the pseudo-sound created at the closing FW-H surface in the wake region. Also, Spalart et al. [13] and Sanders et al. [14] argued that by calculating the noise based only on a solid surface would lead to inaccurate results even at a Mach number as low as 0.115 . The results revealed a typical discrepancy of $3 \mathrm{~dB}$ compared to the permeable surface.

In this study, since the LG system is too complex and contains multiple bluff bodies, 2D flow past a circular cylinder with two different Re numbers were simulated. This simplified case will pave the road to understand the underlying physics of both flow structure (near-field) as well as its associated noise propagation (far-field). Different turbulent models were examined according with the flow anticipated behavior (laminar/turbulent). The FW-H solver in Fluent was utilized to study the far-field noise prediction.

\section{ACOUSTIC ANALOGY FORMULATIONS}

\section{A. Lighthill Acoustic Analogy}

Lighthill formulated a wave equation of acoustic analogy where the source derived by comparing the exact equations of motion of a fluid with the equations of sound propagation in a medium at rest as follows [7],

$$
\begin{gathered}
\frac{1}{c_{o}^{2}} \frac{\partial^{2} \rho^{\prime}}{\partial t^{2}}-\nabla^{2} \rho^{\prime}=\frac{\partial^{2} T_{i j}}{\partial x_{i} \partial x_{j}} \\
\mathrm{~T}_{\mathrm{ij}}=\rho v_{\mathrm{i}} \mathrm{v}_{\mathrm{j}}+\mathrm{P}_{\mathrm{ij}}-\mathrm{c}_{\mathrm{o}}^{2} \rho^{\prime} \delta_{\mathrm{ij}} .
\end{gathered}
$$

where $\left(\rho^{\prime}=\rho-\rho_{\mathrm{o}}\right)$ indicates density perturbation, $\mathrm{T}_{\mathrm{ij}}$ is the Lighthill's stress tensor, $\mathrm{P}_{\mathrm{ij}}=\mathrm{p}^{\prime} \delta_{\mathrm{ij}}-\tau_{\mathrm{ij}}$ refers to the compressive stress tensor with $\mathrm{p}^{\prime}$ being the fluctuating pressure and $\tau_{\mathrm{ij}}$ being the total stress tensor equal to $\sigma_{\mathrm{ij}}-\mathrm{p} \delta_{\mathrm{ij}}$ with $\sigma_{\mathrm{ij}}$ refers to the residual stress tensor. Note that $\mathrm{v}_{\mathrm{i}}$ and $\mathrm{v}_{\mathrm{j}}$ indicate the velocity components in $x_{\mathrm{i}}$ and $x_{\mathrm{j}}$ directions, respectively, and $\mathrm{c}_{\mathrm{o}}$ is the speed of sound.

\section{B. Ffowcs Williams and Hawkings Acoustic Analogy}

Curle [8] expanded the Lighthill's analogy considering a rigid surface; after that, Ffowcs Williams and Hawkings further generalized the theory considering a rigid object in arbitrary motion as follows [9],

$$
\begin{gathered}
\left(\frac{\partial^{2}}{\partial \mathrm{t}^{2}}-\mathrm{c}_{\mathrm{o}}^{2} \frac{\partial^{2}}{\partial \mathrm{x}_{\mathrm{i}}^{2}}\right)\left(\mathrm{H}(f) \rho^{\prime}\right)=\frac{\partial^{2}}{\partial \mathrm{x}_{\mathrm{i}} \partial \mathrm{x}_{\mathrm{j}}}\left(\mathrm{T}_{\mathrm{ij}} \mathrm{H}(f)\right)-\frac{\partial}{\partial \mathrm{x}_{\mathrm{i}}}\left(\mathrm{F}_{\mathrm{i}} \delta(f)\right)+\frac{\partial}{\partial \mathrm{t}}(\mathrm{Q} \delta(f)) \\
F_{i}=\left(P_{i j}+\rho v_{i}\left(v_{j}-u_{j}\right)\right) \frac{\partial f}{\partial x_{j}} \\
Q=\left(\rho_{o} u_{i}+\rho\left(v_{i}-u_{i}\right)\right) \frac{\partial f}{\partial x_{i}}
\end{gathered}
$$

Where $f$ represents the body surface as function of $f(x, t)=$ 0 ; in which $f<0$ and $f>0$ refer to inside and outside of rigid body, respectively. The right-hand side of (3) is the source terms; the first term shown explicitly in (2) physically means unsteadiness inside the fluid expressed in quadruple source as Reynolds stress which is called turbulent-induced term, the second term is dipole source due to local fluctuating stresses exerted by the surface on the fluid and third term is monopole source generated by mass-flux fluctuating around the surface. The last two terms are represented in (4) and (5) which are called loading noise and thickness noise, respectively.

The differential form of FW-H shown in (3) can be written in terms of fluctuating pressure based on the following relationship $\left(p^{\prime}=c_{o}^{2} \rho^{\prime}\right)$ which shows the isentropic relation.

There are three important functions used in the above equations namely Kronecker delta, $\delta_{i j}$, Heaviside, $H(f)$, and the derivative of the Heaviside function $H^{\prime}(f)=\delta(f)$ is the Dirac delta. The turbulent term associated with the quadrupole source is in volume integration. Hence, the decision of including the contribution of the volumes in fluid around rigid body to 
integrate and evaluate the integration kernel is difficult. Thus, it requires a better algorithm/numerical technique to handle the computational cost and memory management. This approach is still under investigation and deserves more developments.

\section{NUMERICAL SET UP}

A two-dimensional case of flow past a circular cylinder was simulated using ANSYS Fluent. First, the flow domain was designed using ANSYS Workbench. The computational domain was extended 5 times and 20 times the cylinder of unity diameter, $D$, upstream and downstream, respectively. The vertical dimension is $5 D$ equally in both Y-directions from the cylinder center. Then, the domain was meshed using an unstructured method of a triangular. However, close to the cylinder wall the structured mesh with rectangular shape elements were utilized to account for the boundary layer (BL) effect. The total number of cells is 22370. After that, the boundary conditions (B.C's) were assigned within corresponding input values. All the numerical modeling process is shown in Fig. 1(a), (b), and (c) starts from the domain, mesh, and B.C's, respectively, including the values of B.C's as shown in Table I.

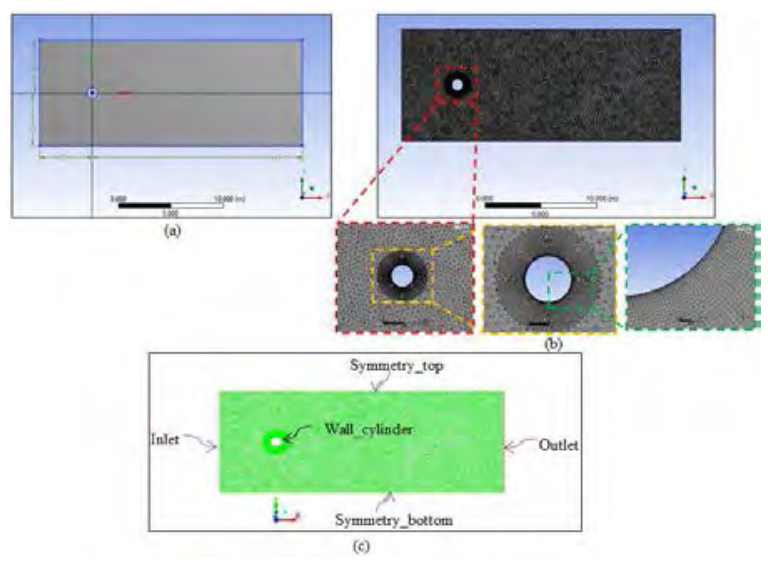

Figure 1. Numerical Modeling: (a) Computational domain, (b) Mesh generation, and (c) Boundary conditions

TABLE I. BOUNDARY CONDITIONS

\begin{tabular}{|l|l|l|}
\hline \multicolumn{1}{|c|}{ B.C. } & \multicolumn{1}{c|}{ Value } & \multicolumn{1}{c|}{ Remarks } \\
\cline { 1 - 2 } Inlet & $\mathrm{V}=1[\mathrm{~m} / \mathrm{s}]$ & $\begin{array}{l}\text { They are common for } \\
\text { incompressible flows }\end{array}$ \\
\cline { 1 - 2 } Outlet & Gauge Pressure $=0[\mathrm{~Pa}]$ & With no-slip condition \\
\hline Wall_cylinder & Stationary wall, $\mathrm{V}_{\text {wall }}=0[\mathrm{~m} / \mathrm{s}]$ & $\begin{array}{l}\text { To ensure that flow } \\
\text { does not form B.L. }\end{array}$ \\
\cline { 1 - 2 } Symmetry_top & No values needed to be entered &
\end{tabular}

Two different Reynolds numbers were examined $R e=150$ and 90,000. In case of low $R e$, two distinct numerical conditions steady and unsteady flow were simulated and compared to examine the effect of the time dependency. For both flow conditions the viscous laminar model was utilized. In case of high $R e$, the acoustic field was computed using the built-in FW-H solver available in Fluent. For this case, the same computational domain is utilized, but the DES with a Spalart-Allmaras (one-equation) model is used to capture the turbulent flow.

\section{RESULTS AND DISCUSSION}

The pressure distribution around the cylinder was computed for both cases and validated with analytical as well as experimental data [16] and showed good agreement as shown in Fig. 2. The analytical pressure coefficient, $C_{p}=1-4 \sin ^{2} \theta$, with respect to different azimuthal angles around the cylinder $\left(0^{\circ} \leq \theta \leq 180^{\circ}\right)$ depicts the symmetrical pattern of the inviscid flow. This symmetry indicates that the integrated surface pressure force in streamwise direction is zero (i.e., no drag force effect). This is an example of the d'Alembert paradox for inviscid flow past an immersed body. However, the experimental data show completely different flow behavior upstream and downstream the bluff body. It strongly depends on the Reynolds numbers as shown in Fig. 2 below, where both the numerical results of laminar and turbulent successfully reproduce the flow behavior upstream and downstream. For the laminar, the separation occurs at around $\theta=87^{\circ}$ which creates a broad wake and very low pressure within large drag coefficient. In contrast, the turbulent flow has a boundary layer that is more resistant which delays the separation point to occur at about $\theta=120^{\circ}$ and then a smaller wake and higher pressure within about $75 \%$ less drag coefficient is resulted.

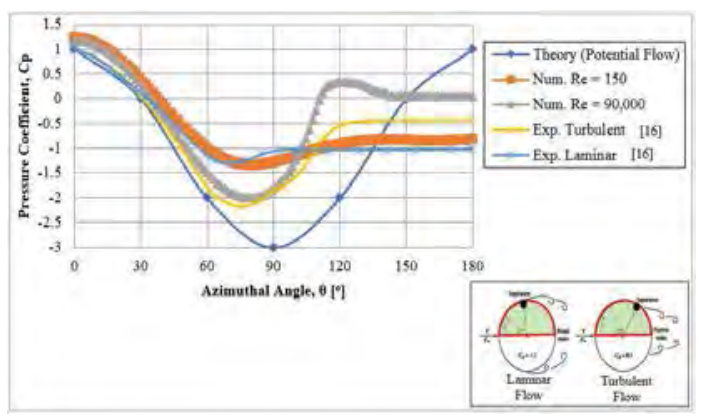

Figure 2. Pressure coefficient profile around the upper surface

The purpose of steady-state simulation is to investigate the mean flow patterns around the bluff-body. The results showed that only the mean velocity and pressure can be captured as shown in Fig. 3(a) and (b), respectively. This is evidence that the acoustic field cannot be extracted, but the results could be utilized as an initial value for simulating unsteady case. On the other hand, the unsteady flow simulation includes the transient behavior which then clearly depicts the flow motion as shown in Fig. 3(c), (d), (e) and (f). The flow instability downstream known as von Karman Street was successfully captured.

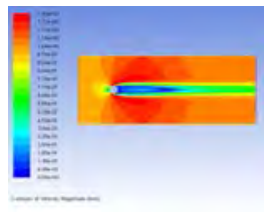

(a)

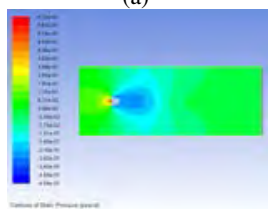

(b)

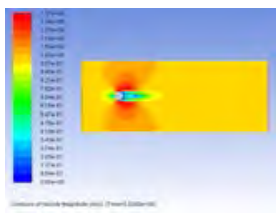

(c)

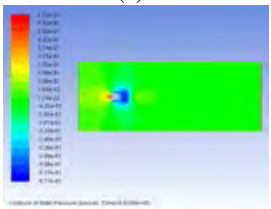

(d)

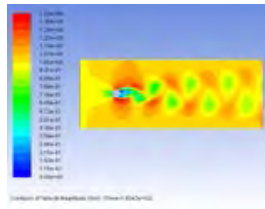

(e)

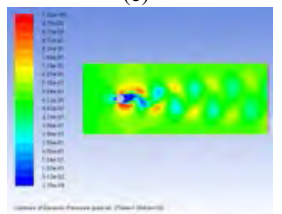

(f)
Figure 3. Near-field results of flow around circular cylinder with $R e=150$ 
Because the flow depends on time, it is important to calculate the time step. For example, in the region behind the circular cylinder a vortex shedding will be created. From the experiments [15], the Strouhal number, $\mathrm{St}=f D / \mathrm{v}$ is 0.172 , then the period, $\mathrm{T}=1 / f$ is found to equal 5.8139 seconds if the frequency, $f$ is calculated. By assuming the number of time steps is $\mathrm{n}=20$, then $\Delta \mathrm{T}=\mathrm{T} / \mathrm{n}=0.291$ seconds, so then, the first vortex shedding can be captured. Note that the lift coefficient, $\mathrm{C}_{\mathrm{L}}$, has a sinusoidal pattern which is an indication of the sustained vortex shedding that takes place in the wake region as shown in Fig. 4.

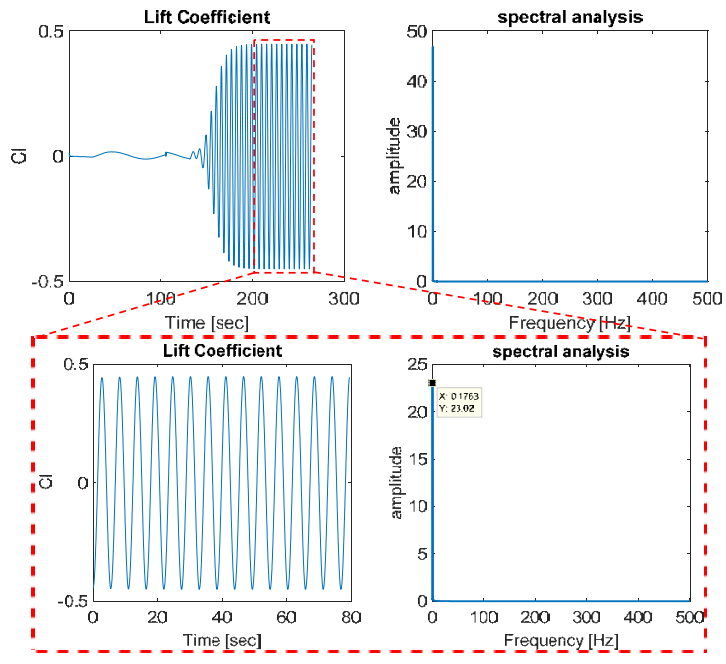

Figure 4. Lift Coefficient time history and frequency content for $R e=150$

The associated acoustic pressure fluctuations for $R e=150$ were collected at two receivers, receiver 1 and receiver 2 located at $90^{\circ}$ above the circular cylinder within $0.665 \mathrm{~m}$ and $2.432 \mathrm{~m}$, respectively. Fig. 5 shows that the far-field pressure fluctuations at both locations preserved the periodicity pattern. That because the near-field flow pressure oscillated with sinusoidal type of behavior when the flow instability occurs.

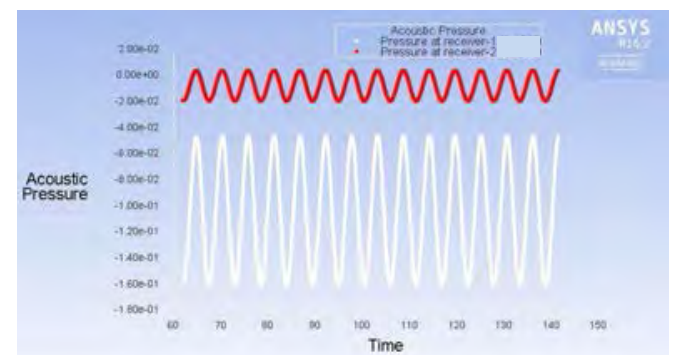

Figure 5. Acoustic pressure fluctuations at two different locations

From Fluent the drag force, $\mathrm{F}_{\mathrm{d}}$, is found to equal $0.696 \mathrm{~N}$, then the drag coefficient, $\mathrm{C}_{\mathrm{d}}$, was calculated using this relation: $\mathrm{C}_{\mathrm{d}}=2 \mathrm{~F}_{\mathrm{d}} / \rho \mathrm{V}^{2} A$, where $A$ is the projected area normal to the flow direction (i.e. for a $2 \mathrm{D}$ cylinder is equal to the diameter), and it turned out to be equal to 1.392 which differs by about $7.2 \%$ from the experimental value [15].

For $R e=90,000$, the near-field data that corresponds to the far-field acoustic prediction is captured as seen in Fig. 6. The wall of the circular cylinder is selected to extract all the relevant acoustic source date such as velocity and pressure. In case of $2 \mathrm{D}$, the source correlation length is assigned to be about $5 D$ since the Fluent assumes that the sound sources are perfectly correlated over the specified correlation length and zero outside. The acoustic pressure signals at the two receiver locations were detected and shown in Fig. 7(a). Also, the spectral of the receiver signals are plotted using the Fast Fourier Transform (FFT). Then, the sound pressure level (dB) versus the frequency $(\mathrm{Hz})$ is calculated as shown in Fig. 7(b) and (c). Due to the turbulence behavior, different size of eddies generated, but the small-scale eddies are not captured within this number of cells. The results prove that the noise generated is a broadband as detected by receivers 1 and 2 . In this case, the pressure fluctuations are no more preserving the periodicity and revealed asymmetric behavior. This indicates that a higher frequency creates a more rapid variation in air pressure and results in a higher pitch.

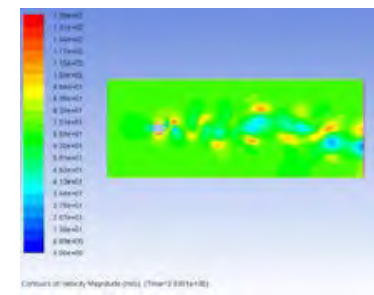

(a)

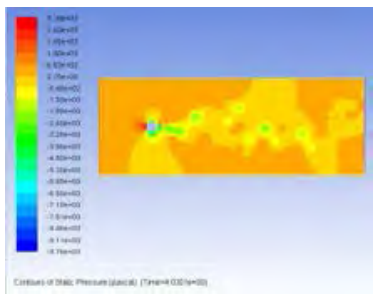

(b)
Figure 6. Numerical results of flow around circular cylinder with $R e=90,000$

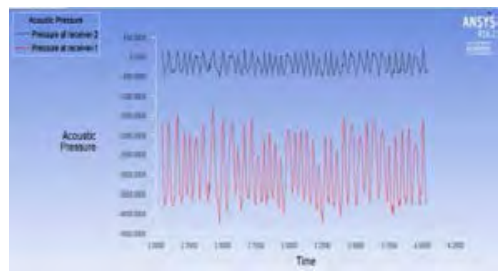

(a)

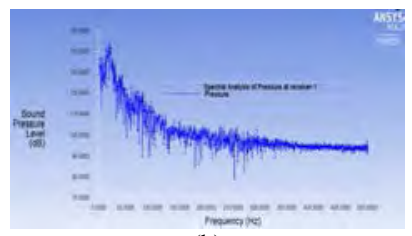

(b)

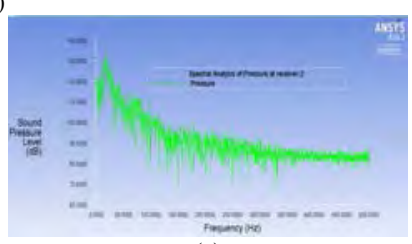

(c)
Figure 7. Acoustic results of flow around circular cylinder with $R e=90,000$

\section{CONCLUSION}

In order to understand the physics of both flow and acoustic fields of landing gear systems, 2D flow past a bluff body (circular cylinder) was simulated using ANSYS Fluent. Two distinct $R e$ numbers 150 and 90,000 were examined. The time dependency consideration affects the extraction of near-field flow data as well as the far-field noise prediction. The FW-H solver in Fluent does not include the contribution of the quadrupole sources (volume integration). Thus, type and location of the surface data are crucial in capturing the far-field noise level. Further investigations are needed to adjust for all source terms. An efficient algorithm/numerical technique in terms of computational cost and memory management are highly required to tackle such complex case like a LG. 


\section{ACKNOWLEDGMENT}

The author is grateful to Prof. Behdinan for supporting and supervising this research. The computing facility provided by his ARL-MLS lab at University of Toronto is gratefully acknowledged. I acknowledge Saudi Arabia government (Taibah University) for granting a scholarship.

\section{REFERENCES}

[1] H. G. Morgan and J. C. Hardin, "Airframe Noise-The Next Aircraft Noise Barrier,” J. Aircr., vol. 12, no. 7, pp. 622-624 . Jul. 1975. doi: 10.2514/3.59844.

[2] P. Argüelles et al., European Aeronautics: A vision for 2020, Advisory Council for Aeronautics Research in Europe, Report., Jan. 2001.

[3] Flightpath, A.C.A.R.E., 2050-Europe's Vision for Aviation, Advisory Council for Aviation Research and Innovation in Europe, 2011.

[4] FAA 2009 Airport noise and access restrictions, In FAA Airport Compliance Manual, 5190.6B.

[5] B. Lazos, "Surface Topology on the Wheels of a Generic Four-Wheel Landing Gear," AIAA Journal, vol. 40, no. 12, pp. 2402-2411, Dec. 2002. doi: $10.2514 / 2.1608$.

[6] L. S. Hedges, A. K. Travin, and P. R. Spalart, "Detached-Eddy Simulations Over a Simplified Landing Gear," J. Fluids Eng., vol. 124, no. 2, p. 413, 2002. doi: 10.1115/1.1471532.

[7] "On sound generated aerodynamically I. General theory," Proc. R. Soc. London, Ser. A, vol. 211, no. 1107, pp. 564-587, Mar. 1952. doi: 10.1098/rspa.1952.0060
[8] "The influence of solid boundaries upon aerodynamic sound," Proc. R. London, Ser. A, vol. 231, no. 1187, pp. 505-514, Sep. 1955. doi: $10.1098 /$ rspa.1955.0191

[9] J. E. F. Williams and D. L. Hawkings, "Sound Generation by Turbulence and Surfaces in Arbitrary Motion," Philos. Trans. R. Soc., A, vol. 264, no. 1151, pp. 321-342, May 1969. doi: 10.1098/rsta.1969.0031.

[10] K. van Mierlo, K. Takeda, and E. Peers, "Computational Analysis of the Effect of Bogie Inclination Angle on Landing Gear Noise," in 16th AIAA/CEAS Aeroacoustics Conference, 2010. doi: 10.2514/6.2010-3971.

[11] S. L. Long, H. Nie, and X. Xu, "Aeroacoustic Study on a Simplified Nose Landing Gear," Appl. Mech. Mater., vol. 184-185, pp. 18-23, Jun. 2012. doi: 10.4028/www.scientific.net/AMM.184-185.18.

[12] D. Lockard, M. Khorrami, and F. Li, "High Resolution Calculation of a Simplified Landing Gear," in 10th AIAA/CEAS Aeroacoustics Conf., 2004. doi: 10.2514/6.2004-2887.

[13] P. R. Spalart, M. L. Shur, M. K. Strelets, and A. K. Travin, "Initial noise predictions for rudimentary landing gear," J. Sound and Vibration, vol. 330, no. 17, pp. 4180-4195, Aug. 2011. doi: 10.1016/j.jsv.2011.03.012.

[14] L. Sanders, E. Manoha, S. Ben Khelil, and C. Francois, "LAGOON : CFD/CAA Coupling for Landing Gear Noise and Comparison with Experimental Database," in 17th AIAA/CEAS Aeroacoustics Conference (32nd AIAA Aeroacoustics Conference), 2011. doi: 10.2514/6.2011-2822.

[15] I. H. Shames, Mechanics of Fluid, 3rd ed., Ch.13: pp. 669-675, McGraw-Hill, New York, 1992.

[16] Y. Nakayama and R. Boucher, Introduction to Fluid Mechanics, Elsevier, 1998. doi: 10.1016/B978-0-340-67649-3.X5000-3. 


\title{
Vibration Analysis of a Double Circular PZT Actuator for a Valveless Micropump
}

\author{
Behzad Parsi, Lihong Zhang, Vlastimil Masek \\ Department of Electrical and Computer Engineering, Faculty of Engineering and Applied Science \\ Memorial University of Newfoundland \\ St. John's, Canada \\ bparsi@mun.ca, lzhang@mun.ca,masek@mun.ca
}

\begin{abstract}
In the conventional mechanical micropumps, the motion from one component (such as gear or diaphragm) produces pressure difference, which is needed to move fluid. In this paper, we propose a new method to use double PZT layers as an actuator for a valveless micropump, which is designed for pumping blood. Due to the high surface tension of blood, one PZT actuator is not strong enough to produce sufficient displacement. So instead of one PZT layer, a double-PZT layer is utilized to produce movement force. In this research, the analytic equations of a double circular PZT layer are derived to express the natural frequency of the system. The finite element method (FEM) simulation is utilized to verify the analytic equations. Then the required input voltage is applied to maximize the functionality of the system, whose simulation results are compared with the experimental measurement setup. Finally, a typical valveless micropump with the proposed double PZT layer is simulated by using COMSOL Multiphysics software. The flow rate of this configuration is compared with a single PZT actuator to demonstrate the effect of the proposed double-PZT actuator on the application to the valveless micropumps.
\end{abstract}

Keywords-component; circular PZT actuator; mechanical vibration; valveless micropump; FEM modeling

\section{INTRODUCTION}

The concept of microfluid was introduced 40 years ago, with the advent of inkjet printing nozzle by IBM (E. Bassous et al. 1977 and Petersen K E 1979) [1]. In 1993, Peter Gravesen et al. published a review paper about micro pumping technology and different actuating principles [2]. Afterwards many new fabrication technologies and in turn new micropump technologies have been developed, such as the positive displacement micropump by Cunneen et al in 1997 [3], the electro-osmotic micro-pump by Chen et al. in 2005 [4], the pneumatic PDMS micropump by Jeong and Konishi in 2007 [5], the piezoelectric micro-pump by Samira Kaviani et al in 2014 [6], and the high pressure peristaltic micropump by Loth and Förster in 2016 [7]. These are just some examples of the new technologies and fabrication methods in the microfluidic area, where the micropump design is highly dependent on specific applications.

In this paper, a valveless micropump with a double PZT layer actuator is developed to pump blood sample. In general, being applied a voltage, a piezoelectric (PZT) layer bends inwards into the micropump [8]. This action pushes the fluid out of the chamber through the outlet valve. In suction mode, when the voltage is removed, the PZT layer would back up to allow the fluid to enter the chamber. This reciprocating process causes the pumping action. A recent attempt of improving PZT micropump was done by $\mathrm{Hu}$ et al. in 2017 [9], who added a new layer to the previous design to decrease the resonant frequency as an advantage of the newly designed device. The authors verified the FEM model with experimental setup. An accurate analytic modeling for a single circular piezoelectric actuator was developed by Mo et al. [10]. They investigated the effect of the thickness ratio and the radius of the piezoelectric layer, which is bonded to a metallic layer with the maximum deflection by using their proposed analytic model. A recent analytic modeling based on the thin plate theory and KelvinVoigt laws for a single piezoelectric actuator was proposed by Esfahani and Bahrami [11]. They also studied the vibration analysis of an edge clamp for a rectangular PZT actuator in a fluid environment. They considered the effect of fluid on the system by adding a damping coefficient to their equations, and then solved the governing equation by a numerical method. In [12], they also investigated the effect of the input voltage, viscoelastic elements, damping coefficient, and excitation signal on the system response. They verified their proposed modeling with the FEM method by using COMSOL Multiphysics software.

This paper is organized as follows. In Section 2, the governing equations of double PZT layer with a bonding layer will be presented and then the natural frequency will be calculated. In Section 3, the FEM simulation will be presented to verify the analytic solution. Then the required voltage, which can maximize the functionality of the system, will be computed. By utilizing a high voltage amplifier and a signal generator, we construct the test bench of our prototype system. The measurement result will be compared with the FEM result. In Section 4, we use COMSOL Multiphysics software to help design a typical valveless micropump. With reference to the 
computated natural frequency of the system, a high AC voltage with the same frequency is applied to the system to investigate the effect of the double PZT layer on the output flow rate. Finally, we draw a conclusion in Section 5.

\section{DYNAMIC MODEL}

The primary purpose of a double PZT layer actuator is to provide large bending displacement in the transverse direction. As illustrated in Fig. 1, two piezoelectric layers are glued with each other by a bonding layer. Two PZT layers are assumed to connect each other by a massless and linear bonding layer. The radius of the bonding layer and PZT layers is $r_{O}$ and the thicknesses of PZT layers and bonding layer are $t_{p}$ and $t_{b}$. In this model, because of the thickness of electrodes is less than $0.5 \mu \mathrm{m}$, the effect of the electrode on deflection of the PZT layers will be ignored [13].

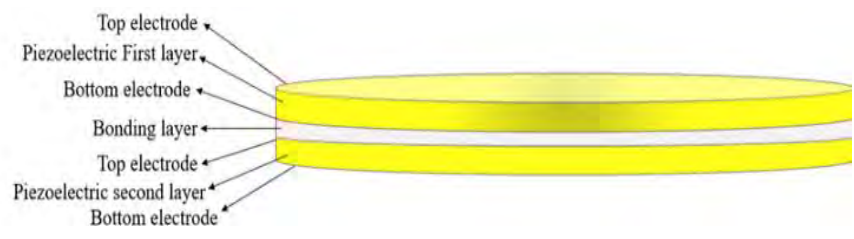

Figure 1. The schematic view of a double PZT layer with a bonding layer.

The axisymmetric governing equations can be obtained by utilizing LaGrange method as follows:

$$
\begin{gathered}
T_{1}\left(\frac{\partial^{2} w_{1}(r, t)}{\partial r^{2}}+\frac{1}{r} \frac{\partial w_{1}(r, t)}{\partial r}\right)=m_{1} \frac{\partial^{2} w_{1}(r, t)}{\partial t^{2}}+ \\
k\left(w_{1}(r, t)-w_{2}(r, t)\right)+f_{1}(t) \\
T_{2}\left(\frac{\partial^{2} w_{2}(r, t)}{\partial r^{2}}+\frac{1}{r} \frac{\partial w_{2}(r, t)}{\partial r}\right)=m_{2} \frac{\partial^{2} w_{2}(r, t)}{\partial t^{2}}- \\
k\left(w_{1}(r, t)-w_{2}(r, t)\right)+f_{2}(t)
\end{gathered}
$$

where $T_{1}$ and $T_{2}$ are the tension, $w_{1}$ and $w_{2}$ are displacement, $k$ is the stiffness coefficient of the bonding layer, $m_{1}$ and $m_{2}$ are the mass per unit area. To compute natural frequencies, $f_{1}$ and $f_{2}$ should be equal to zero. The PZT layers are clamped along the edge such that there is no displacement and as a result the boundary equations can be written as follows:

$$
w_{i}\left(r_{o}, t\right)=0, w_{i}(0, t) \neq \infty .(\mathrm{i}=1 \text { or } 2)
$$

Moreover, the PZT layers have no initial displacement:

$$
w_{i}(r, 0)=w_{0}, \frac{\partial w_{i}}{\partial t}(r, 0)=0
$$

By applying the separation variation method, $w_{1}$ and $w_{2}$ can be rewritten as follows:

$$
\begin{gathered}
w_{1}(r, t)=R_{1}(r) Z(t) \\
w_{2}(r, t)=R_{2}(r) Z(t) \\
\mathrm{Z}(\mathrm{t}) T_{1}\left(\frac{\partial^{2} R_{1}(r)}{\partial r^{2}}+\frac{1}{r} \frac{\partial R_{1}(r)}{\partial r}\right)=m_{1} R_{1}(r) \frac{\partial^{2} Z(t)}{\partial t^{2}}+
\end{gathered}
$$

$$
\begin{gathered}
k\left(R_{1}(r) Z(t)-R_{2}(r) Z(t)\right) \\
\mathrm{Z}(\mathrm{t}) T_{2}\left(\frac{\partial^{2} R_{2}(r)}{\partial r^{2}}+\frac{1}{r} \frac{\partial R_{2}(r)}{\partial r}\right)=m_{2} R_{2}(r) \frac{\partial^{2} Z(t)}{\partial t^{2}}- \\
k\left(R_{1}(r) Z(t)-R_{2}(r) Z(t)\right)
\end{gathered}
$$

Let's assume that $Z(t)=Z_{0} e^{-i \omega t}$ then:

$$
\begin{gathered}
Z_{0} e^{-i \omega t} T_{1}\left(\frac{\partial^{2} R_{1}(r)}{\partial r^{2}}+\frac{1}{r} \frac{\partial R_{1}(r)}{\partial r}\right)= \\
m_{1} R_{1}(r) Z_{0} e^{-i \omega t}\left(-\omega^{2}\right)+k\left(R_{1}(r) Z_{0} e^{-i \omega t}-\right. \\
\left.R_{2}(r) Z_{0} e^{-i \omega t}\right) \\
Z_{0} e^{-i \omega t} T_{2}\left(\frac{\partial^{2} R_{2}(r)}{\partial r^{2}}+\frac{1}{r} \frac{\partial R_{2}(r)}{\partial r}\right)= \\
m_{2} R_{2}(r) Z_{0} e^{-i \omega t}\left(-\omega^{2}\right)-k\left(R_{1}(r) Z_{0} e^{-i \omega t}-\right. \\
\left.R_{2}(r) Z_{0} e^{-i \omega t}\right)
\end{gathered}
$$

These equations can be rewritten as:

$$
\begin{gathered}
Z_{0} e^{-i \omega t} T_{1}\left(\frac{\partial^{2} R_{1}(r)}{\partial r^{2}}+\frac{1}{r} \frac{\partial R_{1}(r)}{\partial r}\right)= \\
m_{1} R_{1}(r) Z_{0} e^{-i \omega t}\left(-\omega^{2}\right)+k Z_{0} e^{-i \omega t}\left(R_{1}(r)-\right. \\
\left.R_{2}(r)\right) \\
Z_{0} e^{-i \omega t} T_{2}\left(\frac{\partial^{2} R_{2}(r)}{\partial r^{2}}+\frac{1}{r} \frac{\partial R_{2}(r)}{\partial r}\right)= \\
m_{2} R_{2}(r) Z_{0} e^{-i \omega t}\left(-\omega^{2}\right)-k Z_{0} e^{-i \omega t}\left(R_{1}(r)-\right. \\
\left.R_{2}(r)\right)
\end{gathered}
$$

With simplification:

$$
\begin{gathered}
T_{1}\left(\frac{\partial^{2} R_{1}(r)}{\partial r^{2}}+\frac{1}{r} \frac{\partial R_{1}(r)}{\partial r}\right)=-m_{1} \omega^{2} R_{1}(r)+ \\
k\left(R_{1}(r)-R_{2}(r)\right) \\
T_{2}\left(\frac{\partial^{2} R_{2}(r)}{\partial r^{2}}+\frac{1}{r} \frac{\partial R_{2}(r)}{\partial r}\right)=-m_{2} \omega^{2} R_{2}(r)- \\
k\left(R_{1}(r)-R_{2}(r)\right)
\end{gathered}
$$

Let's define $\nabla R_{i}=\frac{\partial^{2} R_{i}(r)}{\partial r^{2}}+\frac{1}{r} \frac{\partial R_{i}(r)}{\partial r}$. By substituting $\nabla R_{i}$ into the equations, the following equations will be obtained:

$$
\begin{aligned}
& T_{1} \nabla R_{1}+\left(m_{1} \omega^{2}-k\right) R_{1}(r)+k R_{2}(r)=0, \\
& T_{2} \nabla R_{2}+\left(m_{2} \omega^{2}-k\right) R_{2}(r)+k R_{1}(r)=0 .
\end{aligned}
$$

By eliminating $R_{2}$ from these equations, the following equation can be obtained:

$$
\left(\nabla+C_{1}^{2}\right)\left(\nabla+C_{2}^{2}\right) R_{1}=0
$$

where $C_{1}$ and $C_{2}$ are equal to:

$$
\begin{gathered}
C_{1,2}^{2}=\frac{1}{T_{1} T_{2}}\left(-\left(m_{2} T_{1}+m 1 T_{2}\right) \omega^{2}+\left(T_{1}+T_{2}\right) k\right) \pm \\
\left(2 T_{1} T_{2} k^{2}+T_{2}^{2} m_{1}^{2} \omega^{4}+T_{1}^{2} m_{2}^{2} \omega^{4}+T_{2}^{2} k^{2}+T_{1}^{2} k^{2}-\right. \\
2 m_{2} \omega^{2} T_{1}^{2} k+2 m_{1} T_{2}^{2} \omega^{2} k-2 m_{2} m_{1} T_{1} T_{2} \omega^{4}+
\end{gathered}
$$




$$
\left.\left.2 m_{1} T_{1} T_{2} k \omega^{2}\right)^{\frac{1}{2}}\right)
$$

The solution of this equation can be represented as Bessel function as follows:

$$
R_{1 i}=a_{i} J_{i}\left(C_{1} r\right)+b_{i} Y_{i}\left(C_{1} r\right)
$$

By applying the first boundry condition, since $R_{1 i}(0, t) \neq$ $\infty$. $b_{i}$ should be equal to zero and also by applying the second B.C, $R_{1_{i}}\left(r_{o}, t\right)=0$, it can be shown that:

$$
J_{i}\left(C_{1} r\right)=0 \text { or } J_{i}(\delta)=0
$$

where $\delta$ is the root of Bessel function. Now by solving this simple algebraic equation, natural frequencies $\omega$ of the system can be calculated. Material properties of the system are shown in Table I.

TABLE I. MATERIAL PROPERTIES OF PZT AND BONDING LAYER.

\begin{tabular}{|c|c|c|}
\hline Mechanical property & $\begin{array}{c}\text { Bonding layer } \\
\text { (Epoxy Resin) }\end{array}$ & $\begin{array}{c}\text { PZT (Lead } \\
\text { Zirconate Titanate) }\end{array}$ \\
\hline Mass density & $2000\left(\mathrm{Kg} / \mathrm{m}^{3}\right)$ & $7500\left(\mathrm{Kg} / \mathrm{m}^{3}\right)$ \\
\hline Elastic modulus & $5.17(\mathrm{GPa})$ & $9.5(\mathrm{GPa})$ \\
\hline Poisson's ratio & 0.31 & 0.30 \\
\hline
\end{tabular}

\section{FEM MODELING AND EXPERIMENTAL SETUP}

To validate the analytic solution, FEM method and experimental setup are utilized. Two piezoelectric actuators with the radius of $14 \mathrm{~mm}$, which are glued to each other by a bonding layer, are simulated in Comsol Multiphysics software. Fig. 2 shows the mode shapes of the system that are calculated by FEM simulation.

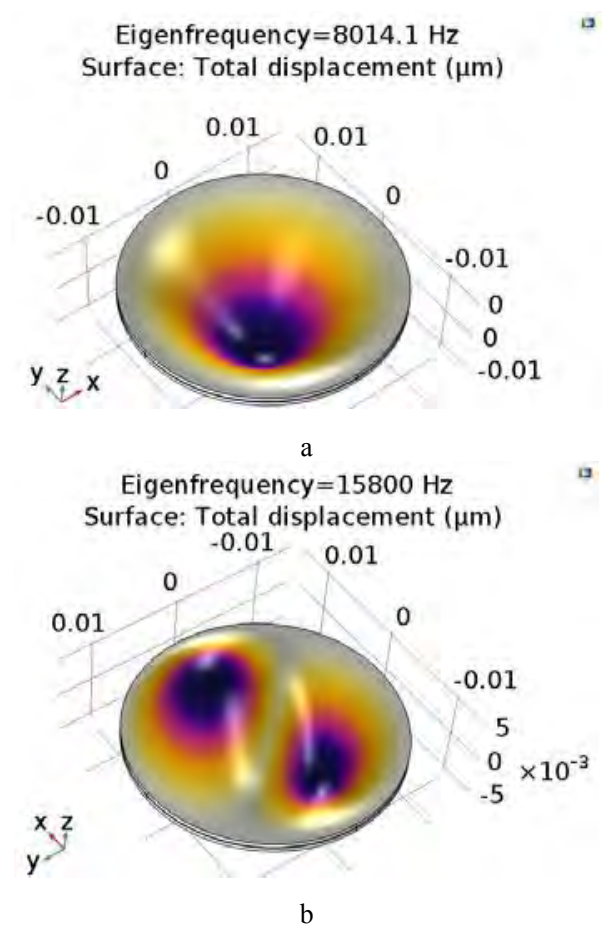

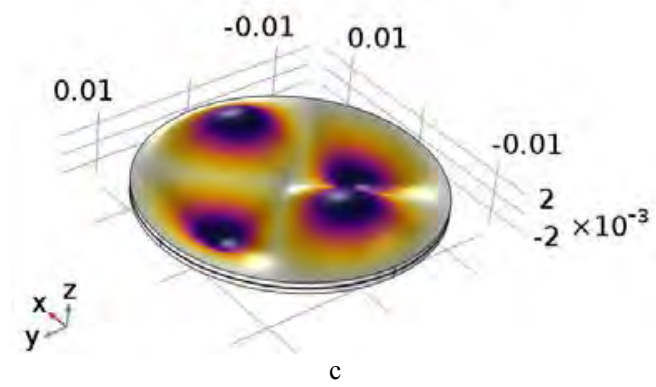

Figure 2. The natural frequency of double PZT layer with bonding layer in Comsol Multiphysics software a) The first mode shape, b) The second mode shape, c) The third mode shape.

The maximum stress occurs at the boundary condition. The Tresca stress at the boundary as a function of frequency is shown in Fig. 3. The result demonstrates that the maximum displacement and maximum stress will occur at the first natural frequency $\omega_{1}$. As a result, the maximum functionality of the system is at the first natural frequency. Table II shows the natural frequency, which is calculated by analytic solution and FEM modeling. The absolute percentage error (APD) is calculated by the following equation [14-15]:

$$
\epsilon_{i}=100\left|\frac{\left(\omega_{F_{i}}-\omega_{A_{i}}\right)}{\omega_{F_{i}}}\right|
$$

where $\omega_{F_{i}}$ is natural frequencies calculated by FEM simulation, and $\omega_{A_{i}}$ is natural frequencies calculated by analytic solution.

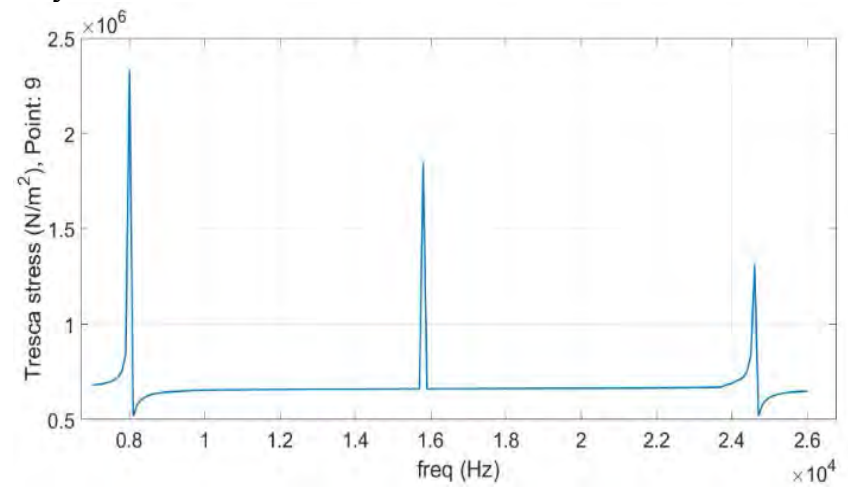

Figure 3. The Tresca stress at the boundary of the system for the first, second, and third natural frequencies.

TABLE II. THE FIRST, SECOND AND THIRD NATURAL FREQUENCY

\begin{tabular}{|c|c|c|c|}
\hline $\begin{array}{c}\text { Resonant } \\
\text { frequency }\end{array}$ & $\begin{array}{c}\text { Analytical } \\
\text { Solution (HZ) }\end{array}$ & $\begin{array}{c}\text { FEM } \\
\text { Modeling } \\
\text { (HZ) }\end{array}$ & $\begin{array}{c}\text { Absolute } \\
\text { percentage } \\
\text { Error (APD) }\end{array}$ \\
\hline 1 & 7947.35 & 8014.1 & 0.83 \\
\hline 2 & 15678.86 & 15800 & 0.76 \\
\hline 3 & 24507.32 & 24637 & 0.52 \\
\hline
\end{tabular}

The result shows the accuracy of the analytic model. We can see that this analytic model is very valuable, since the designer can use it to estimate the natural frequency of a new double PZT layer very quickly for different dimensions without 
any expensive FEM simulation. The transverse vibration of the double PZT layer will be turned into a time-dependent vibration when the input voltage signal changes as a function of time. The important point is to find the best input signal, which can maximize the efficiency of the system. In order to reach this goal, after calculating the natural frequencies, the first natural frequency will be chosen to apply to the system. So a high voltage signal with the same frequency $\omega_{1}$ will be applied to the system and the maximum deflection will be measured by FEM simulation and experimental setup. Fig. 4-a shows the excitation voltage, which is equal to $200 \sin \left(\omega_{1} t\right)$. The system response is shown in Fig. 4-b. The maximum stress, which occurs at edge, is shown as a function of time in this figure. This figure reveals that the maximum deflection of the system with respect to that excitation signal is equal to $121 \mu \mathrm{m}$.

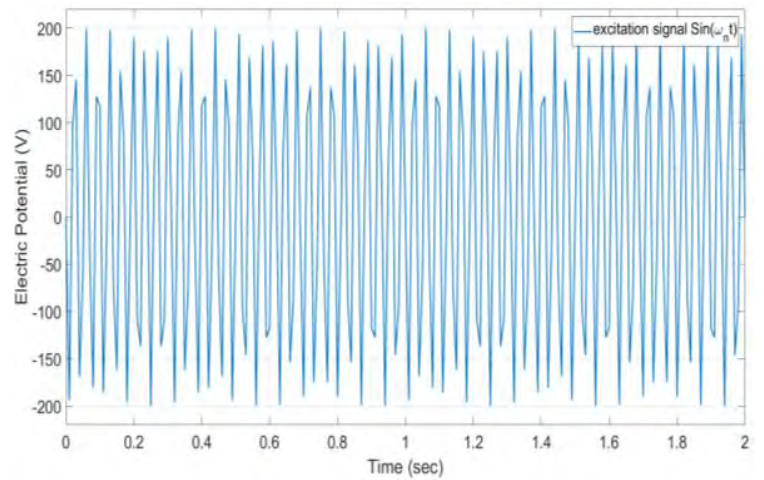

(a)

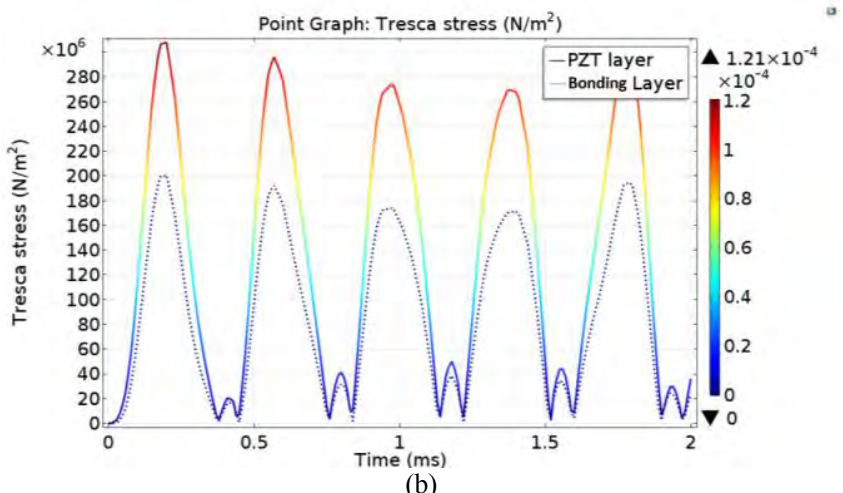

Figure 4. a) The excitation signal at the first natural frequency, b) The Tresca stress at the boundary and displacement profile.

For the experimental setup, the main instruments include a fixture, a high precision laser measurement sensor (LK-H022, resolution of $0.02 \mu \mathrm{m}$ ), a signal generator, a high-precision high voltage power amplifier (TEGAM-2350), and a data acquisition system.. The experimental setup is shown in Fig. 5. In order to verify the FEM simulation along with the experimental measurement results, an excitation signal the same as Fig. 4-a was applied to the system. Fig. 6 shows the center deflection of the double PZT layer. As can be seen in this figure, the maximum value for deflection is equal to 117 $\mu m$, which is close to the FEM result with the error of $3.3 \%$.

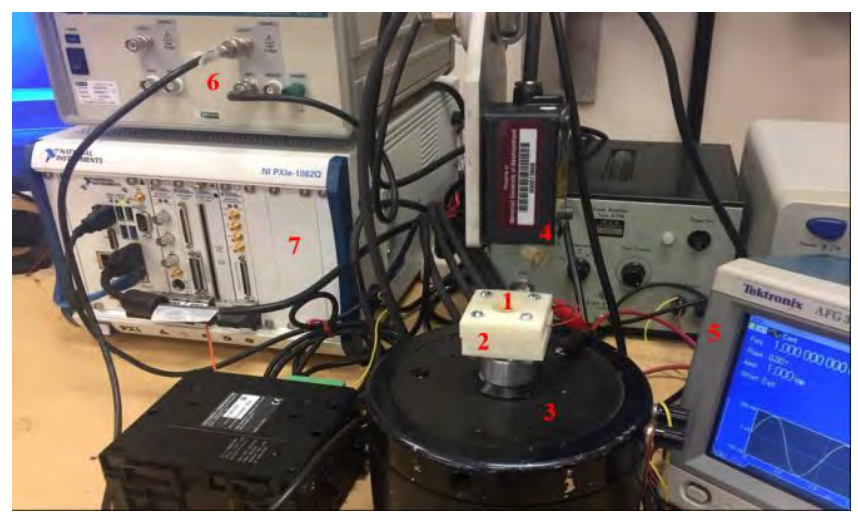

Figure 5. 1) Double PZT layer, 2) Fixture, 3) Vibration Shaker Systems, 4) a high precision laser measurement sensor (LK-H022, resolution of $0.02 \mu \mathrm{m}$ ), 5) Signal generator, 6) Precision power amplifier high voltage (TEGAM-2350), 7) Data acquisition system.

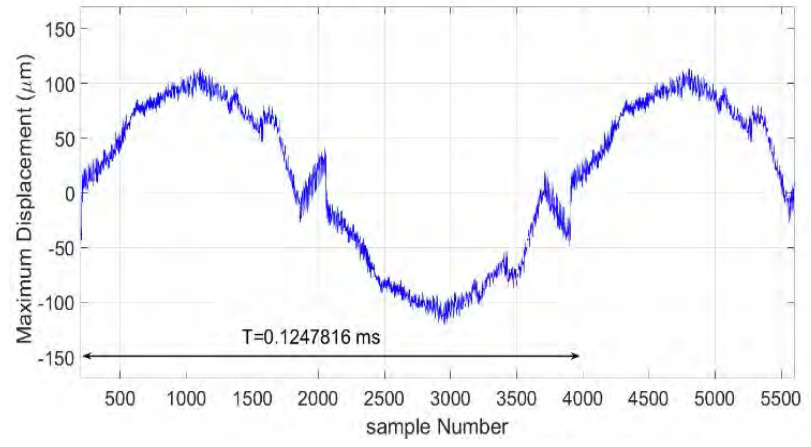

Figure 6. The center displacement response of the system under excitation signal of $200 \sin \left(\omega_{1} t\right)$

\section{APPLICATION OF DOUBLE PZT LAYER IN MICROPUMP}

In this section, the double PZT actuator will be installed on a valveless micropump to see the effect of this proposed actuator on the flow rate of the valveless micropump. In the conventional mechanical micropump, the motion of one component such as gear or diaphragm can produce pressure difference, which is needed to move fluid sample. Mechanical micropumps can be classified into the following categories: rotary micropumps, moving diaphragm micropumps, and peristaltic micropumps. The actuation methods of the moving diaphragm micropumps include piezoelectric, electrostatic, thermopneumatic, shape memory alloys, and other novelties. In this research, we choose the double piezoelectric actuator to move the diaphragm of a valveless micropump. A typical configuration of the valveless micropump with the double piezoelectric actuator in COMSOL Multiphysics software is shown in Fig. 7. COMSOL Multiphysics is powerful software that can simulate the interface between solid structure, fluid environment, and electrical field.

In a typical valveless micropump, there is no moving part. It works base on geometry property of nozzle and diffuser. The purpose of this section is not focused on the principle of this type of micropumps. More detail about the micropump design can be found in Stemme's and Cui's papers [16,17]. Fig. 7-a 
shows the micropump chamber in suction mode and Fig. 7-b shows the chamber of micropump in pump mode.

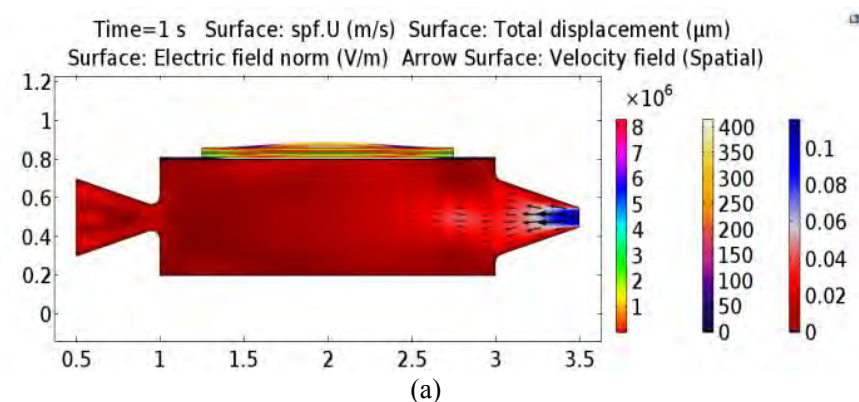

Time $=0.9 \mathrm{~s}$ Surface: $\mathrm{spf} . \mathrm{U}(\mathrm{m} / \mathrm{s})$ Surface: Total displacement $(\mu \mathrm{m})$ Surface: Electric field norm (V/m) Arrow Surface: Velocity field (Spatial)

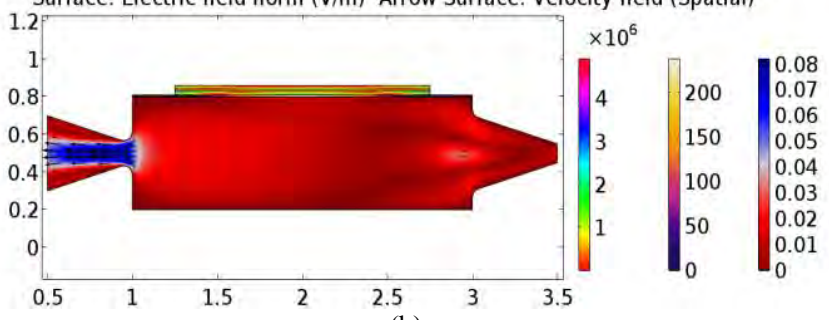

(b)

Figure 7. The FEM modeling of the valveless micropump with double PZT actuator a) The suction mode, b) The pump mode.

In order to investigate the effect of the double PZT actuator on the system, the flow rate of the system is calculated by FEM simulation. Fig. 8-a shows the flow rate of a valveless micropump with a regular single PZT actuator. As can be seen from this figure, the maximum flow rate reaches up to $1.47 *$ $10^{-5} \mathrm{~m}^{2} / \mathrm{s}$. On the other hand, Fig. 8-b shows the flow rate of the same microvalve in the same condition, but with a double PZT actuator and a bonding layer. As can be seen from this figure the maximum flow rate would reach $12.1 * 10^{-5} \mathrm{~m}^{2} / \mathrm{s}$, which is suitable for pumping biological samples such as blood. In other words, by utilizing our proposed double PZT actuator, the flow rate of a valveless micropump can increase up to 8.23 times.

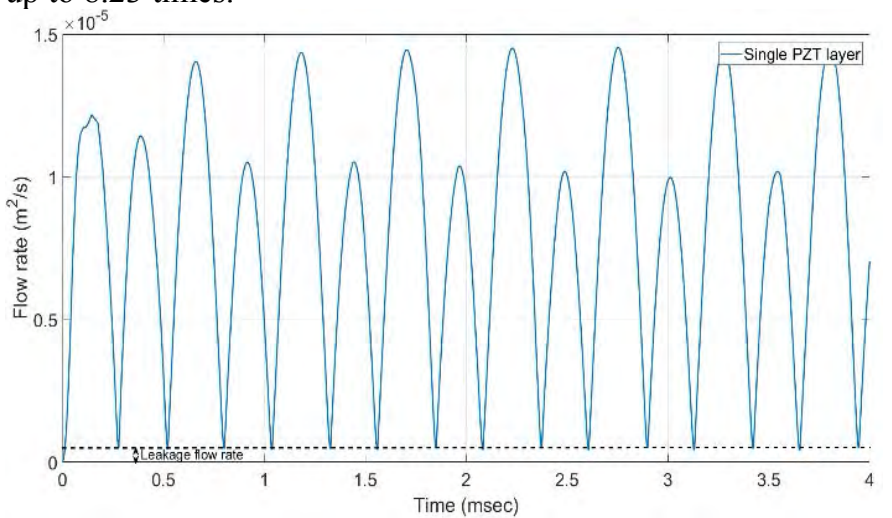

(a)

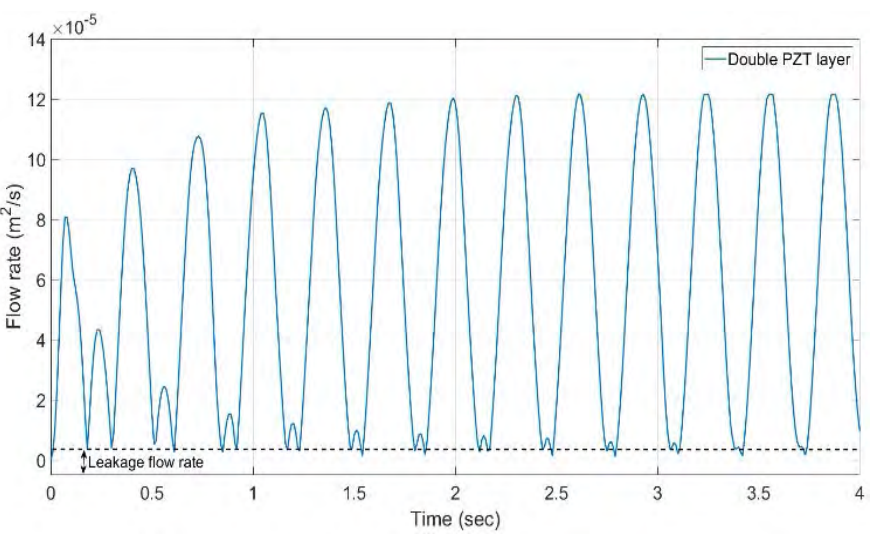

(b)

Figure 8. The output flow rate of the valveless micropump a) With a single PZT actuator, b) With a double PZT actuator.

\section{CONCLUSION}

In this research, we designed a double PZT layer as an actuator for a valveless micropump. The analytic analysis of this newly proposed actuator was first conducted and then verified with FEM simulation. The comparison between COMSOL Multiphysics simulation and analytic computation reveals the high accuracy of our deducted analytic solution, whose error is less than $1 \%$. This is valuable since the designer can estimate the natural frequency of a new double PZT layer quickly for different dimensions by using our analytic solution without depending on any expensive FEM simulation. Moreover, the experimental setup was established to measure the maximum displacement for a comparison with the FEM simulation. The result shows that the difference between FEM simulation and our experimental measurement is less than $4 \%$. Furthermore, the proposed actuator was applied to a typical valveless micropump to evaluate the functionality of the actuator. The comparison between a regular valveless micropump with a single PZT actuator and the same micropump with a double PZT actuator reveals that the flow rate will increase up to 8.23 times.

\section{ACKNOWLEDGEMENT}

This work was supported in part by the Natural Sciences and Engineering Research Council of Canada, in part by the Canada Foundation for Innovation, in part by the Research and Development Corporation of Newfoundland and Labrador through the Industrial Research and Innovation Fund and ArcticTECH R\&D Award, and in part by the Memorial University of Newfoundland.

\section{REFERENCES}

[1] E. Bassous, H. H. Taub, and L. Kuhn, "Ink jet printing nozzle arrays etched in silicon," Appl. Phys. Lett., vol. 31, no. 2, pp. 135-137, 1977. doi: $10.1063 / 1.89587$.

[2] P. Gravesen, J. Branebjerg, and O. S. Jensen, "Microfluidics-a review," J. Micromech. Microeng., vol. 3, no. 4, pp. 168-182, Jan. 1993.

doi: 10.1088/0960-1317/3/4/002. 
[3] J. Cunneen, Y.-C. Lin, S. Caraffini, J. G. Boyd, P. J. Hesketh, S. M. Lunte, and G. S. Wilson, "A positive displacement micropump for microdialysis," Mechatronics, vol. 8, no. 5, pp. 561-583, 1998. doi: 10.1016/S0957-4158(98)00008-7s .

[4] Z. Chen, P. Wang, and H.-C. Chang, "An electro-osmotic micro-pump based on monolithic silica for micro-flow analyses and electro-sprays," Anal. Bioanal. Chem., vol. 382, no. 3, pp. 817-824, Jan. 2005. doi: $10.1007 / \mathrm{s} 00216-005-3130-7$

[5] O. C. Jeong and S. Konishi, "Fabrication and drive test of pneumatic PDMS micropump," Sens. Actuators, A, vol. 135, no. 2, pp. 849-856, 2007. doi: 10.1016/j.sna.2006.09.012.

[6] S. Kaviani, M. Bahrami, A. M. Esfahani, and B. Parsi, "A modeling and vibration analysis of a piezoelectric micro-pump diaphragm," C. R. Mec., vol. 342, no. 12, pp. 692-699, 2014. doi: 10.1016/j.crme.2014.06.005.

[7] ]A. Loth and R. Forster, "Disposable high pressure peristaltic micro pump for standalone and on-chip applications," in 2016 IEEE 11th Annual International Conference on Nano/Micro Engineered and Molecular Systems (NEMS), 2016. doi: 10.1109/NEMS.2016.7758193.

[8] S. Nabavi and L. Zhang, "Design and Optimization of Piezoelectric MEMS Vibration Energy Harvesters Based on Genetic Algorithm," IEEE Sens. J., pp. 7372-7382, 2017. doi: 10.1109/JSEN.2017.2756921.

[9] Y. Hu, X. Liang, and W. Wang, "A theoretical solution of resonant circular diaphragm-type piezo actuators with added mass loads," Sens. Actuators, A, vol. 258, pp. 74-87, 2017. doi: 10.1016/j.sna.2017.02.029.

[10] C. Mo, R. Wright, W. S. Slaughter, and W. W. Clark, "Behaviour of a unimorph circular piezoelectric actuator," Smart Mater. Struct., vol. 15, no. 4, pp. 1094-1102, Jul. 2006. doi: 10.1088/0964-1726/15/4/023.

[11] A. M. Esfahani and M. Bahrami, "Vibration analysis of a circular thin polymeric piezoelectric diaphragm with fluid interaction," Int. J. Mech. Mater. Des., vol. 12, no. 3, pp. 401-411, Oct. 2015. doi: 10.1007/s10999-015-9308-z.

[12] A. M. Esfahani, M. Bahrami, and S. R. G. Anbarani, "Forced vibration analysis of a viscoelastic polymeric piezoelectric microplate with fluid interaction," Micro \& Nano Letters, vol. 11, no. 7, pp. 395-401, Jan. 2016. doi: 10.1049/mnl.2016.0043.

[13] M. Arik, S. M. Zurn, A. Bar-Cohen, and D. L. Polla, "Design, fabrication, and characterization of thin film PZT membranes for high flux electronics cooling applications," Smart Mater. Struct., vol. 14, no. 6, pp. 1239-1249, Nov. 2005. doi: 10.1088/0964-1726/14/6/017.

[14] B. Parsi, M. Bahrami, A. M. Esfahani, and B. S. Sany, "Calibration verification of a low-cost method for MEMS accelerometers," Trans. Inst. Meas. Control, vol. 36, no. 5, pp.579-587, 2014.

doi: $10.1177 / 0142331213513607$

[15] M. H. Jalali, B. Shahriari, O. Zargar, M. Baghani, and M. Baniassadi, "Free Vibration Analysis of Rotating Functionally Graded Annular Disc of Variable Thickness Using Generalized Differential Quadrature Method," Scientia Iranica, 2017.

[16] Q. Cui, C. Liu, and X. F. Zha, "Modeling and Numerical Analysis of a Circular Piezoelectric Actuator for Valveless Micropumps," J. Intell. Mater. Syst. Struct., vol. 19, no. 10, pp. 1195-1205, 2007. doi: $10.1177 / 1045389$ X07084204.

[17] E. Stemme and G. Stemme, "A valveless diffuser/nozzle-based fluid pump," Sens. Actuators, A, vol. 39, no. 2, pp. 159-167, 1993. doi: 10.1016/0924-4247(93)80213-Z. 


\section{Microfluidic Platform for Studying Biofilm Formation of Oil-Degrading Bacteria}

\author{
Saeed Rismani Yazdi, Carlos Escobedo \\ Department of Chemical Engineering \\ Queen's University \\ Kingston, Ontario, Canada \\ carlos.escobedo@queensu.ca
}

\author{
Corey A. Stevens, Peter L. Davies \\ Department of Biomedical and Molecular Sciences \\ Queen's University \\ Kingston, Ontario, Canada
}

\begin{abstract}
Crude oil spill is a significant threat to marine wildlife and human health. Understanding the interaction of oil-degrading bacteria at aqueous-oil interfaces at submillimeter scale is crucial to biodegradation and bioremediation. Here we present a microfluidic system that allows real-time and parallel observation of bacteria interaction with crude oil during biofilm formation and degradation of the oil. A simple and robust microfluidic platform is designed, fabricated and tested. Stable and controllable oil-bacterial solution interfaces are formed. The theory of the capillary valves used in the device and the microfabrication steps are presented. Experimental results show the accumulation of Marinobacter hydrocarbonoclasticus -an oil degrading bacteria- at the bacterial solution interface with paraffin oil. After $\sim 3 \mathrm{hr}$ of incubation, bacteria start to form microcolony at the oil interface. Results show that presented microfluidic device can be successfully used for research on bacteria-oil interactions and other applications requiring an interface formed by two immiscible liquids.
\end{abstract}

Keywords- microfluidics; oil-degrading bacteria; biofilm; interfaces; capillary valve

\section{INTRODUCTION}

Crude oil spill is a significant threat to marine wildlife and human health. Each year, 700 million tons of crude oil is introduced to aquatic ecosystem through human related activities and by natural seepage from reservoirs [1], [2]. This, therefore, has motivated more progress in marine oil spill remediation studies [3], [4]. Nature has evolved an effective way to rectify the problem using indigenous microorganisms to clean up the oil contaminants [5]. Marinobacter hydrocarbonoclasticus (MH) is an aquatic bacterium with the ability to form biofilms at the interface between water and oil [6]. MH uses this ability to increase the bioavailability of hydrocarbons, which serve as its main energy source, and further to degrade the oil. Biofilms have been previously studied using macroscopic approaches [7]. Analysis at microscales, however, can provide key information on the dynamics of biofilm formation and disruption [8], [9]. A central challenge for studying $\mathrm{MH}$ is to form a stable and observable oil-bacterial solution interface that mimics those found in natural settings.

Over the last decade, the lab-on-a-chip technology and in particular microfluidics has been maturing, leading to tremendous applications in life science and engineering [10]. In general, microfluidics can help to overcome challenges and limitations of convectional assays, for example in biology and chemistry, and also allow development of approaches that can lead to better understanding of natural or engineered phenomenon at submillimeter scale [11]. Microfluidics offers unique advantages such as low sample and reagent volumes ( $\mu \mathrm{L}$ to $\mathrm{pL}$ ), precise control of spatial and temporal factors with high sensitivity, low-cost, with simplified fabrication and operation steps [10], [12]. Moreover, the use of biocompatible and optically transparent materials such as Polydimethylsiloxane (PDMS) facilitates the culture of biological samples and image acquisition using conventional microscopy [13]. These advantages make microfluidics an ideal tool for studying the interaction of Marinobacter hydrocarbonoclasticus with oil.

Here we present a simple and versatile microfluidic device for studying bacteria with oil degrading ability as a model for marine bacteria interaction with crude oil during biofilm formation. Using capillary valves incorporated in the microfluidic device, the oil-bacterial solution interface is realized. The principle behind the capillary valves used in the microfluidic device is presented. Use of such microfluidic device with passive valves is advantageous because it does not require complex setup, thus increasing the simplicity of the fabrication and liquid handling in the device. The design of the device allows for experimental parallelization with different conditions at a time to investigate, for instance, the effects of two different oils or promoting/inhibitory reagents on the bacteria interaction with the oil.

\section{RESULTS AND DISCUSSION}

\section{A. Microfluidic device}

Fig. 1 shows the schematic representation of the microfluidic device for studying bacteria interaction with oil. 
The device consists of three parallel channels that allow the formation of immiscible liquid-liquid interfaces. The middle and the two adjacent channels are interconnected through 100 $\mu \mathrm{m}$ gaps between 20 trapezoidal posts located in series (Fig. 1). All channels have $40 \mu \mathrm{m}$ height. The abrupt angle of the posts geometry increases the physical contact angle between the solid-liquid interface, resulting in a capillary valve action [14], which consequently retains the meniscus between the microposts and prevents the liquid in the middle channel from flowing into the side channels. The middle channel can be filled with oil and side channels with bacteria solution to bring the two liquids into contact (Fig. 1). Fig. 2 shows the PDMS microfluidic device fabricated using photolithography and soft lithography techniques as described in the method section [15].

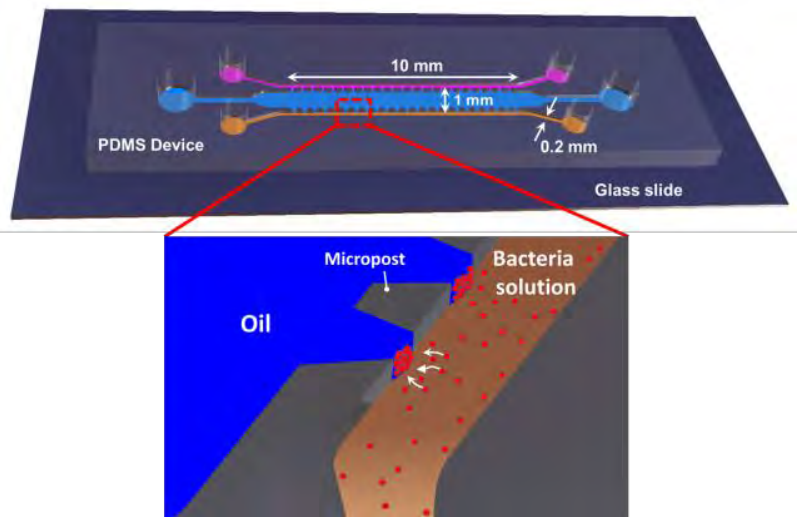

Figure 1. Architecture of the microfluidic device developed to study interaction of Marinobacter hydrocarbonoclasticus and oil. Liquid-liquid interfaces are achieved at the meniscuses formed as a result of the gaps between the microposts.

\section{B. Capillary valves}

The capillary valves stop the liquid from flowing to a certain region of the microfluidic device using a capillary pressure barrier [14]-[16]. For this particular type of valve, the capillary pressure barrier develops when abrupt enlargement of the channel happens. The valve consists of a region of the channel that is necked down followed by a sharp expansion of the cross-section that locally increases of the static contact angle. In the proposed microfluidic device, the implemented micorposts work as capillary valves (Fig. 2).

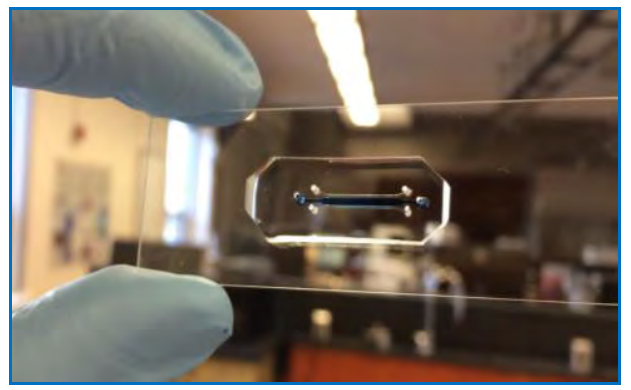

Figure 2. Image of fabricated PDMS microfluidic device. The loaded blue liquid is stopped from flowing to the side channels by the microposts acting as capillary valves.

The capillary valves stop the liquid from flowing to a certain region of the microfluidic device using a capillary pressure barrier [14]-[16]. For this particular type of valve, the capillary pressure barrier develops when abrupt enlargement of the channel happens. The valve consists of a region of the channel that is necked down followed by a sharp expansion of the cross-section that locally increases of the static contact angle. The loaded liquid in the microfluidic device drives by the combination of the external pressure and capillary action in the channel and between microposts (necked regions, Fig. 3a). The abrupt enlargement of the edge of the microposts $\left(145^{\circ}\right)$ prevented the liquid from flooding to the side channel using the pressure barrier (Fig. 3b). This can be explained in terms of changes in the energy for the liquid-solid-air interface system. The total interfacial energy $\left(U_{T}\right)$ of capillary system can be presented by:

$$
\mathrm{U}_{\mathrm{T}}=\mathrm{ASL}_{\mathrm{SL}} \gamma_{\mathrm{SL}}+\mathrm{ASA}_{\mathrm{SA}} \gamma_{\mathrm{SA}}+\mathrm{A}_{\mathrm{LA}} \gamma_{\mathrm{LA}}
$$

where $A_{S L}$ and $\gamma_{S L}, A_{S A}$ and $\gamma_{S A}, A_{L A}$ and $\gamma_{L A}$ are solid-liquid, solid-air, and liquid-air interface areas and surface energies per unit area, respectively [16], [17]. The relation between surface energies of triple interfaces and the contact angle of liquid $\theta_{\mathrm{C}}$ with the surface of microfluidic channel at the meniscus can be expressed by Young's equation:

$$
\gamma_{\mathrm{SA}}=\gamma_{\mathrm{SL}}+\gamma_{\mathrm{LA}} \cos \theta_{\mathrm{C}}
$$

The $U_{T}$ of the system is a function of loaded volume liquid $\left(V_{L}\right)$ into the device as wetted surfaces change by increase of the $V_{L}$.

$$
P=-\frac{d U_{\mathrm{T}}}{d V_{\mathrm{L}}}=\gamma_{\mathrm{LA}}\left(\cos \theta_{c} \frac{d A_{\mathrm{SL}}}{d V_{\mathrm{L}}}-\frac{d A_{\mathrm{LA}}}{d V_{\mathrm{L}}}\right)
$$

When liquid is loaded, it wets the surfaces of the channels which increases the liquid-solid interface and thus the positive pressure moves the liquid forward and fills the channel. In order to stop the liquid from going to the side channels, pressure as driving force should be controlled. This can be achieved by geometry change of the valve. The abrupt geometry change of valve results in an increase of liquid-air interface more than solid-liquid interface at the end of the tapered edge, which leads to opposite sign of the pressure at the meniscus. The negative pressure is a repulsive force and thus stops the filling front at the edge of the valve. When the liquid reaches the sharp edge of the valve, the filling front stops and meniscus remains at the edge unless higher pressure is applied to the liquid which breaks the pressure barrier and liquid flows to the side channels. The Young-Laplace equation expresses the inverse relation of pressure across the liquid-air interface and the radius of meniscus. According to the equation 4 , the pressure at the liquid-air interface increases when the meniscus radius (r) gets smaller or in other words when meniscus becomes more convex [16], [18].

$$
\Delta p=p-p_{n}=\frac{2 \gamma_{\mathrm{LA}}}{\mathrm{r}}
$$

Depending on the difference between internal pressure $(p)$ of the liquid and normal pressure in the side channel $\left(p_{n}\right)$, the liquid-air interface bulges more or less. For a given volume, if further illiquid is gradually loaded to the channel, the liquid continues to move and meniscus shape changes from concave to flat and then to convex (Fig. 3). If the volume of the liquid decreases, the meniscus shape changes back to the concave. 
This is a reversible action until the meniscus is pinned and valve is not open. Here we assume that all the surfaces in the microchannel for PDMS and glass are homogenous and have same surface properties (e.i. hydrophobic).
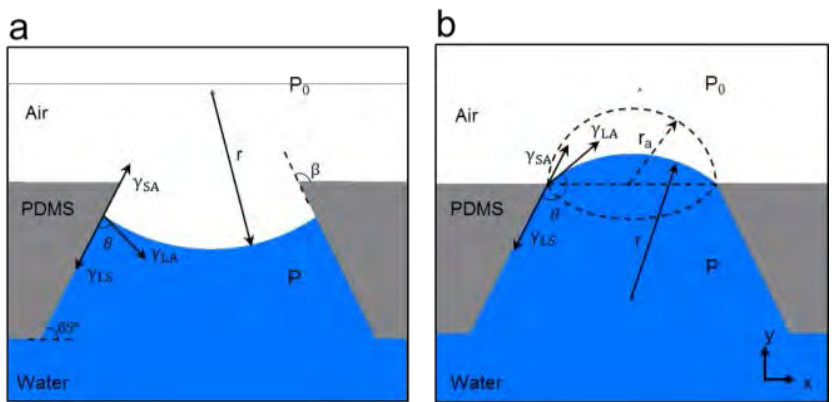

Figure 3. Principle of the capillary valves. (a) Filling front of the liquid while reaching the sharp edge of the valve and (b) the pinned meniscus due to the pressure barrier from the valve.

\section{Formation of liquid-liquid interface}

To test the functionality of the device, first the middle channel was filled with the oil (colored in green in Fig. 4) and after the oil filling front between the microposts was stopped by the valves, the side channels were filled with the water (colored in pink in Fig. 4). Fig. 4 shows the well-defined oilwater interfaces formed between microposts. Similar interfaces achived for all the 20 gaps between microposts. By modulating the volume of liquid loaded into the middle channel, meniscus shape can be altered and concave, flat, and convex meniscus can be achieved (Fig. 5).

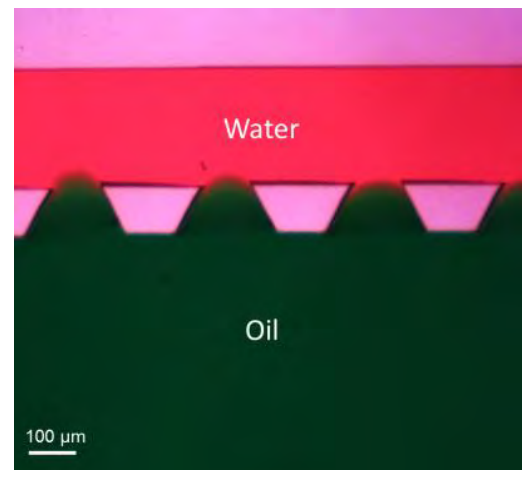

Figure 4. Stable and well-defined oil-water interfaces formed between microposts. Oil is colored in green and water in pink.

The effectiveness of the valves to pin and maintain the meniscus during the experiments were tested and results showed that oil-bacterial solution interfaces were stable over the course of the experiment for $>5 \mathrm{hr}$. Using this approach, it is possible to bring two immiscible liquids into contact and form a liquid-liquid interface, such as oil-water, between the microposts.
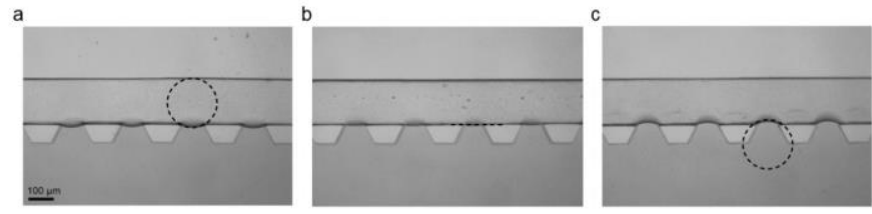

Figure 5. Zoomed view of the middle channel filled with water. By varying the volume of liquid loaded to the channel, the meniscus shape can be controlled to be a) convex, b) flat, and c) concave. Side channel was left empty. The dashed line and circles show the direction of the meniscus.

\section{Bacteria interaction with oil}

In order to study biofilms formation at the oil-water interface, the middle channel was loaded with metabolisable hydrocarbon and the side channels were filled with highly concentrated salt solution containing FITC-labeled $M$. hydrocarbonoclasticus. The experimental results demonstrate observable oil-bacterial solution interfaces between all microposts and substantial bacteria accumulation at the oil interfaces (Fig. 6). Due to the migration of the bacteria towards the oil and their accumulation there, higher number of bacteria were found close to the oil interface (Fig. 6).
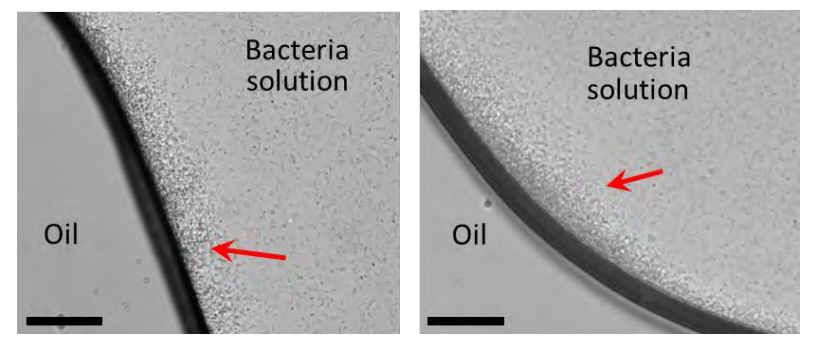

Figure 6. Accumulation of Marinobacter hydrocarbonoclasticus at the bacterial solution interface with paraffin oil. Scale bars, $50 \mu \mathrm{m}$. Red arrows show the area with bacteria accumulated at the oil interface.

Fig. 7 shows the images of the oil-bacteria interface at time zero (Fig. 7-left) and at time $\sim 3 \mathrm{hr}$ (Fig. 7-right). The accumolation of the bacteria and formation of the microcolony in the microfluidc device was imaged over time. After 3 hours of incubation, marinobacter bacteria started to form biofilm/microcolony at the interface with the parafin oil. Results show the successful formation of stable and observable oil-bacterial solution interfaces that can mimics those found in natural settings. The posibility to have 20 interfaces at each side of the middle channel improves the number of data points and statistical analysis to support the observed phenomenons.
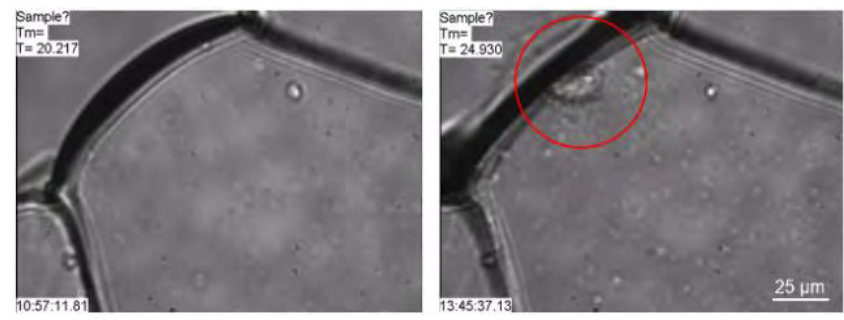

Figure 7. Formation of a biofilm/microcolony between marinobacter and parafin oil after $\sim 3$ hours of incubation in $20^{\circ} \mathrm{c}$ in the microfluidic device. The microcolony is shown by red circle.

\section{CONCLUSION}

In summary, a simple and robust microfluidic device allowing the formation of oil-water interfaces was presented and used to study interaction of Marinobacter hydrocarbonoclasticus - an oil degrading bacteria - with parafine oil. The device utilized capillary valves formed by the sharp edges of 20 microposts that connect the two channels 
together and allowed for controllable and observable interfaces between oil and bacterial solution. The proposed microfluidic device enabled experimental parallelization with different conditions at a time to investigate, for instance, the effects of two different oils or promoting/inhibitory reagents on the biofilm formation. In order to study biofilm formation at the oil-water interface, the middle channel can be loaded with a highly concentrated salt solution containing Marinobacter hydrocarbonoclasticus, while the side channels can be filled with a degradable and non-degradable oil such as Hexadecane and 1-Octanol, respectively. This versatile platform has great potential not only for studying oleolytic biofilms, but also for applications that require interfaces formed by two immiscible liquids.

\section{MATERIALS AND METHODS}

\section{A. Fabrication of microfluidic device}

The microfluidic device was fabricated using photolithography and soft lithography techniques [19]. Microfluidic networks were designed in CATIA (Dassault Systems, V19). For maximum process reliability, the silicon wafer was cleaned with acetone and isopropanol, and then rinsed with water for 5 minutes. The wafer was then dried on a $100{ }^{\circ} \mathrm{C}$ hotplate. A $40 \mu \mathrm{m}$ layer of SU-8 50 (MicroChem, USA) was spin-coated on a silicon wafer and selectively exposed to ultraviolet light using a maskless photolithography system (Intelligent Micro Patterning Co., USA). SU-8 crosslinking was obtained at near UV (350-400 nm) radiations for 3 $\mathrm{s}$ with $\sim 35 \mathrm{~mW} / \mathrm{cm}^{2}$ exposure energy. The unexposed photoresist was developed in the SU-8 developer (MicroChem, USA) for 20 minutes. The exposure and development time were optimized to achieve sharp edges for the microposts. The developed SU-8 mold was silanized with (tridecafluoro-1,1,2,2-tetrahydrooctyl)-1-trichlorosilane (Sigma-Aldrich, Switzerland) to reduce adhesion between the SU-8 mold and PDMS. PDMS precursor and crosslinking agent (Sylgard 184 Silicone Elastomer, Dow Corning Corp., USA) were mixed at a ratio of 10 to 1 , based on weight, and degassed in a vacuum chamber for $30 \mathrm{~min}$. Then, the PDMS was poured on the mold, degassed, and cured at $80{ }^{\circ} \mathrm{C}$ for at least 1 hour. The cured PDMS layer was peeled off from the SU-8 mold and a hole puncher (1 $\mathrm{mm}$ in diameter) was used to punch the inlet and outlet ports. A microscope slide (Microscopes America, Inc., USA) was bonded to the PDMS layer using a plasma cleaner (Harrick Plasma Inc., USA) to complete the microfluidic device.

\section{B. Experimental procedure and microscopy}

Microchannel inlets were connected to syringes containing the oil and bacteria solutions using silicon tubes (TYGON R3607, Ismatec) and 21-gauge 90-degree-bent needle (APM Technica). Marinobacter hydrocarbonoclasticus culture of 2 days old was used for the experiments. Prior to the experiments, bacteria were centrifuged for $10 \mathrm{~min}$ at $2000 \mathrm{rpm}$ and then resuspended in $200 \mu \mathrm{l}$ of the culture medium. After loading the channels, microfluidic device was placed into the incubator stage of an inverted microscope for temperature and humidity control. An inverted microscope (Olympus IX83,
Germany) equipped with a CMOS camera (Zyla-4.2-CL10, ANDOR, Ireland) was used to capture 10x (numerical aperture $(\mathrm{NA})=0.13$, UPLanFL $\mathrm{N}$ ) and 40x (NA 0.6, LUCPlanFL N) magnification bright-field image sequences of bacteria interacting with the oil in the microfluidic device. Freely available image processing software ImageJ (US National Institutes of Health) [20] was used to perform image analysis.

\section{ACKNOWLEDGMENT}

This work was supported by Natural Sciences and Engineering Research Council of Canada (NSERC) Discovery Grants to CE and PLD, and a Canada Foundation for Innovation Leaders Opportunity Fund program.

\section{REFERENCES}

[1] S. Kleindienst, J. H. Paul, and S. B. Joye, "Using dispersants after oil spills: impacts on the composition and activity of microbilal communities.," Nat. Rev. Microbiol., vol. 13, no. 6, pp. 388-396, 2015.

https://doi.org/10.1038/nrmicro3452

[2] I. R. MacDonald et al., "Transfer of hydrocarbons from natural seeps to the water column and atmosphere," Geofluids, vol. 2, no. 2, pp. 95-107, 2002. https://doi.org/10.1046/j.1468-8123.2002.00023.x

[3] H. P. Bacosa, Z. Liu, and D. L. Erdner, "Natural sunlight shapes crude oildegrading bacterial communities in northern Gulf of Mexico surface waters," Front. Microbiol., vol. 6, no. Dec. 2015.

https://doi.org/10.3389/fmicb.2015.01325

[4] T. J. McGenity et al., "Marine crude-oil biodegradation: a central role for interspecies interactions," Aquat. Biosyst., vol. 8, no. 1, p. 10, 2012. https://doi.org/10.1186/2046-9063-8-10

[5] J. G. Leahy and R. R. Colwell, "Microbial degradation of hydrocarbons in the environment," Microbiol. Rev., vol. 54, no. 3, pp. 305-315, 1990.

[6] B. Klein et al., "Cytoplasmic wax ester accumulation during biofilm-driven substrate assimilation at the alkane-water interface by Marinobacter hydrocarbonoclasticus SP17," Res. Microbiol., vol. 159, no. 2, pp. 137-144, 2008. https://doi.org/10.1016/j.resmic.2007.11.013

[7] M. Salta et al., "Anti-biofilm performance of three natural products against initial bacterial attachment," Int. J. Mol. Sci., vol. 14, no. 11, pp. 21757-21780, 2013. https://doi.org/10.3390/ijms141121757

[8] M. T. Meyer et. al., "Development and validation of a microfluidic reactor for biofilm monitoring via optical methods," J. Micromechanics

Microengineering, vol. 21, no. 5, p. 54023, 2011.

https://doi.org/10.1088/0960-1317/21/5/054023

[9] G. E. Kapellos, "Microscale study of biofilm dynamics at the oil-water interface," George E. Kapellos Department of Chemical Engineering, University of Patras, 26504 Patras, Greece," pp. 4-6, 2015.

[10] G. M. Whitesides, "The origins and the future of microfluidics," Nature, vol. 442, no. 7101, pp. 368-373, Jul. 2006. https://doi.org/10.1038/nature05058

[11] S. Guo et al., "Structure of a 1.5-MDa adhesin that binds its Antarctic bacterium to diatoms and ice.," Sci. Adv., vol. 3, no. 8, p. e1701440, 2017. https://doi.org/10.1126/sciadv.1701440

[12] A. Tay, A. Pavesi, S. R. Yazdi, C. T. Lim, and M. E. Warkiani, "Advances in microfluidics in combating infectious diseases," Biotechnology Advances. 2016. https://doi.org/10.1016/j.biotechadv.2016.02.002 
[13] S. Rismani Yazdi et al., "Magnetotaxis Enables Magnetotactic Bacteria to Navigate in Flow," Small, vol. 1702982, p. 1702982, Dec. 2017.

https://doi.org/10.1002/smll.201870019

[14] S. R. Yazdi, et al, "Microfluidic hanging-drop platform for parallel closed-loop multi-tissue experiments," in 2015 28th IEEE International Conference on Micro Electro Mechanical Systems (MEMS), pp. 535-538, Feb. 2015.

https://doi.org/10.1109/MEMSYS.2015.7051010

[15] M. Zimmermann, P. Hunziker, and E. Delamarche, "Valves for autonomous capillary systems," Microfluid. Nanofluidics, vol. 5, no. 3, pp. 395-402, 2008. https://doi.org/10.1007/s10404-007-0256-2

[16] J. Melin, et al., "A liquid-triggered liquid microvalve for onchip flow control," Sensors Actuators B Chem., vol. 100, no. 3, pp. 463-468, 2004. https://doi.org/10.1016/j.snb.2004.03.010

[17] M. I. Mohammed, E. Abraham, and M. P. Y. Desmulliez, "Rapid laser prototyping of valves for microfluidic autonomous systems," J. Micromechanics Microengineering, vol. 23, no. 3, p. 35034, 2013. https://doi.org/10.1088/0960-1317/23/3/035034

[18] T. Wu and Y. Suzuki, "Design, microfabrication and evaluation of robust high-performance superlyophobic surfaces," Sensors Actuators, B Chem., vol. 156, no. 1, pp. 401-409, 2011. https://doi.org/10.1016/j.snb.2011.04.065

[19] S. Rismani Yazdi, A. Shadmani, S. C. Bürgel, P. M. Misun, A. Hierlemann, and O. Frey, "Adding the 'heart' to hanging drop networks for microphysiological multi-tissue experiments," Lab Chip, vol. 15, no. 21, pp. 4138-4147, 2015.

https://doi.org/10.1039/C5LC01000D

[20] C. Schneider, W. S. Rasband, and K. W. Eliceiri, "NIH Image to ImageJ: 25 years of image analysis.," Nat. Methods, vol. 9, no. 7, pp. 671-5, Jul. 2012. https://doi.org/10.1038/nmeth.2089 


\title{
Pneumatic Pressure Control: An Open-Source Droplet Microfluidic System
}

\author{
Run Ze Gao, Marie Hébert, and Carolyn L. Ren \\ Mechanical and Mechatronics Engineering \\ University of Waterloo \\ Waterloo, Canada \\ c3ren@uwaterloo.ca
}

\begin{abstract}
Open-source droplet microfluidic systems are envisioned to decrease the barriers to entry for exploration in new microfluidic research areas and increase adoptions in existing areas such as micro total analysis systems. At the heart of droplet microfluidics systems is a pneumatic pressure control system, which produces pulseless pressure-driven flow to manipulate nanoliter-sized droplet movement in microfluidic environments. Currently, this device remains a barrier regarding cost and performance. Commercially available off-the-shelf and customized systems offer high performance at a premium price and are proprietary which cannot be upgraded or modified. In-house built systems often do not exist due to long development cycles from the ground up. Hence, other than dedicated droplet microfluidics groups, compromises regarding the cost to performance ratio and development flexibility are taken by most.
\end{abstract}

This project presents a pneumatic pressure control system based on open-source hardware and software at a fraction of the cost and similar performance compared to premium commercial systems. All components are widely available through online suppliers. The code, design, and bill of material are made available for everyone on GitHub. The open-source nature of this project provides a gateway for many on a tight budget to tryout droplet microfluidics with the pulseless flow. This project is a small step in creating an open ecosystem for droplet microfluidics. Nonetheless, it enables an alternative to the rigid and non-standardized methodology that exists in the overarching microfluidics and lab on chip community today.

Keywords - Microfluidics, pnuematic control, open-source

\section{INTRODUCTION}

Fluigent [1], Elveflow [2] and Dolomite [3] produces premium pneumatic controlled actuators commercially. These systems have high performance but lack flexibility due to proprietary and closed-source nature of commercial products. The cost is also much higher than the open-source pneumatic pressure control system introduced here. This prevents the adoption of precision microfluidic control which is one the key area that must be developed for broad adoption of microfluidic and lab-on-chip technology.

\section{Methodology}

The making of the open-source pneumatic control system for microfluidics involves two critical factors, component selection and procedure for assembly and testing as it must be reproducible by others. Fig. 1 shows the open-source system development steps.

Component selection is crucial due to the very nature of open-source projects. All parts are selected to be easily obtainable and widely available via reputable online sellers. The procedure for assembly and performance testing are streamlined.

\section{A. Materials}

The components are purchased from online sellers, which are DigiKey, McMaster Carr, and Amazon. The pressure actuator is purchased from ControlAir. All components can be acquired worldwide.

\section{B. Procedure}

The building procedure will be outlined in the standard operating procedure available on GitHub. The complete bill of material, software code, printed circuit board/soldered breadboard design, housing design and performance testing procedure will be available on GitHub as well. All resources available on GitHub for this pneumatic pressure control

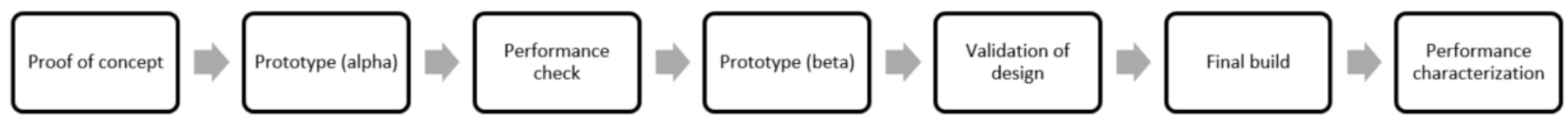

Figure 1. Open-source pneumatic pressure control system development process 


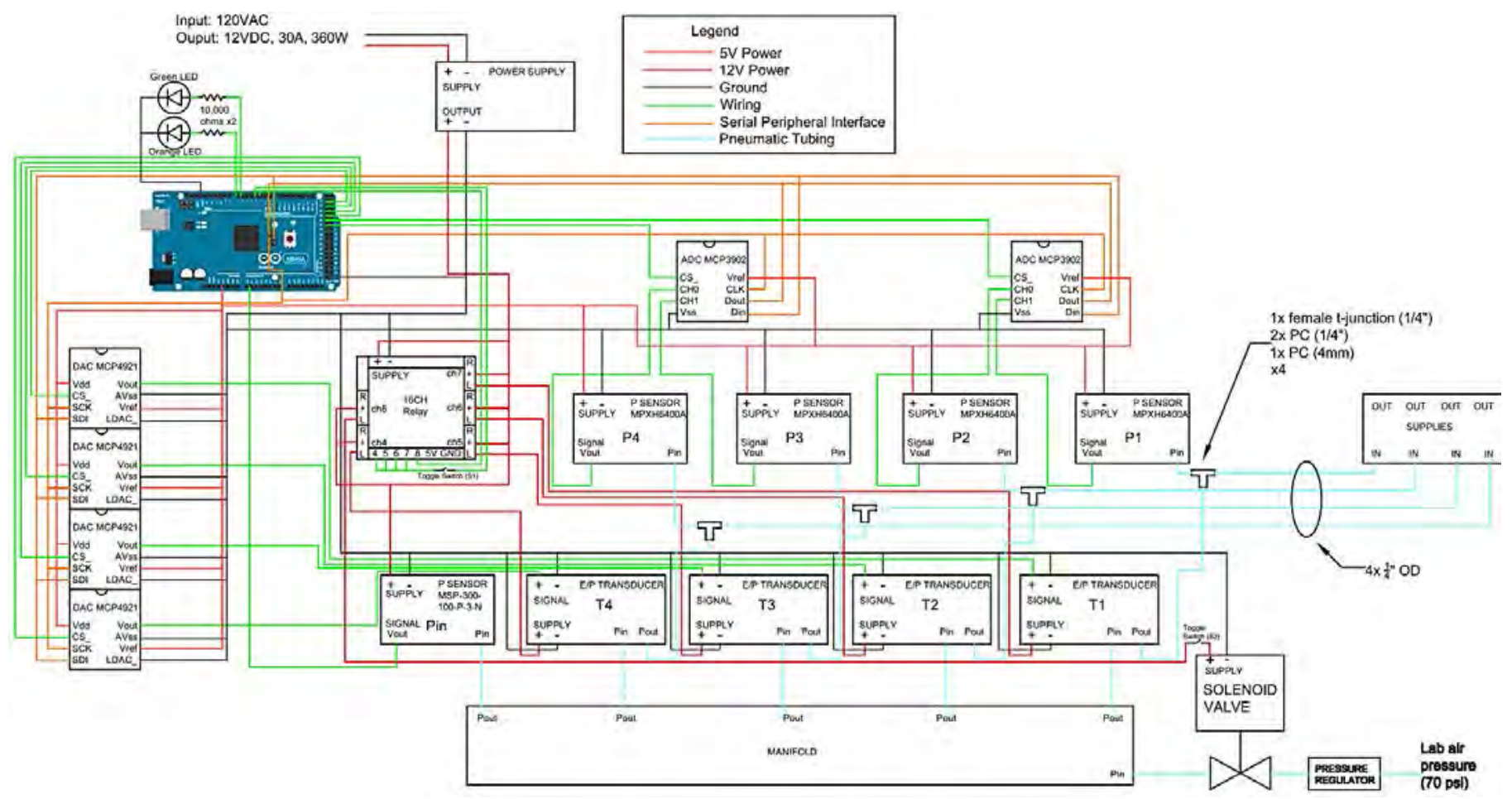

Figure 2. Open-source pneumatic pressure control system schematic

system will be open to everyone with General Public License.

The building procedure consists of populating the soldered breadboard or printed circuit board with electronic components, and system integration.

The performance and criteria validation procedure includes a signal to noise ratio testing, response testing, stability testing, and real-world performance testing.

\section{DESIGN}

Due to the open-source nature of the project, the performance to cost ratio is crucial. Ideally, the performance should be on par with the premium commercial systems while being much cheaper than commercial units. Table 1 and Table 2 shows the design constraints and criteria. Fig. 1 shows the design schematic.

Table 1. Pneumatic pressure control system design constraints

\begin{tabular}{|c|c|c|}
\hline $\begin{array}{c}\text { Design } \\
\text { Constraint }\end{array}$ & $\begin{array}{c}\text { Constraint } \\
\text { Value/Range }\end{array}$ & Description/Reasoning \\
\hline $\begin{array}{l}\text { Pressure } \\
\text { range }\end{array}$ & $\begin{array}{c}0 \text { to } \\
2000 \mathrm{mbar}\end{array}$ & $\begin{array}{l}\text { Most microfluidics operates within this } \\
\text { range. }\end{array}$ \\
\hline Resolution & $\leq 0.5 \mathrm{mbar}$ & $\begin{array}{l}\text { For most application's sensitivity } \\
\text { requirement, this resolution is sufficient. }\end{array}$ \\
\hline Accuracy & $\pm 5 \mathrm{mbar}$ & $\begin{array}{l}\text { Physical flow is not affected by accuracy } \\
\text { smaller than this (hypothesis). }\end{array}$ \\
\hline Precision & $\pm 5 \mathrm{mbar}$ & $\begin{array}{l}\text { Physical flow is not affected by precision } \\
\text { smaller than this (hypothesis). }\end{array}$ \\
\hline $\begin{array}{l}\text { Response } \\
\text { time }\end{array}$ & $\leq 1$ second & $\begin{array}{l}\text { Passive microfluidics does not require } \\
\text { stringent response time as it operates } \\
\text { mostly based on steady state pressure per }\end{array}$ \\
\hline
\end{tabular}

\begin{tabular}{|c|c|c|}
\hline $\begin{array}{c}\text { Settling } \\
\text { time }\end{array}$ & $\leq 1$ second & $\begin{array}{c}\text { channel. Active-control systems are the } \\
\text { same as it operates based on closed-loop } \\
\text { feedback control of droplet position. } \\
\text { Passive microfluidics does not require } \\
\text { stringent settling time for the same reason } \\
\text { as response time. Active-control systems } \\
\text { operate based on droplet location rather } \\
\text { than droplet velocity/acceleration, thus } \\
\text { settling time is determined to be sufficient } \\
\text { at this constraint. }\end{array}$ \\
\hline
\end{tabular}

Table 2. Pneumatic pressure control system design criteria

\begin{tabular}{|c|c|}
\hline $\begin{array}{c}\text { Design } \\
\text { Criteria }\end{array}$ & Description/Reasoning \\
\hline $\begin{array}{c}\text { Safe to } \\
\text { operate }\end{array}$ & $\begin{array}{c}\text { The system must have redundancy built-in to ensure } \\
\text { electrical safety and pneumatic pressure safety for users. }\end{array}$ \\
\hline Pulseless & $\begin{array}{c}\text { The control system must produce pulseless flow during } \\
\text { real-world operations in any microfluidics systems with } \\
\text { any fluid, }\end{array}$ \\
\hline $\begin{array}{c}\text { Modular } \\
\text { hardware } \\
\text { design }\end{array}$ & $\begin{array}{c}\text { Allow the user to build the system, change components, } \\
\text { and upgrade hardware with ease. }\end{array}$ \\
\hline $\begin{array}{c}\text { Open source } \\
\text { software }\end{array}$ & $\begin{array}{c}\text { The open source software allows the user to continuously } \\
\text { improve the software, and use any suitable control } \\
\text { algorithm, and integrate with their system seamlessly. }\end{array}$ \\
\hline $\begin{array}{c}\text { Ease of } \\
\text { maintenance }\end{array}$ & $\begin{array}{c}\text { The user has the flexibility to carry out maintenance of } \\
\text { any hardware by themselves with no patent or proprietary } \\
\text { restrictions. }\end{array}$ \\
\hline
\end{tabular}

\section{A. Control System and Data Acquisition}

The control system consists of an Arduino Mega, which is open-source electronic incorporating a microcontroller ATmega 2560, and four MCP 4921 which are single channel 12-bit digital to analog converters (DAC). The DAC converts 
digital control signals to analog signals for pneumatic pressure actuation through ControlAir T900.

The data acquisition system consists of two MCP 3202, which are two channel analog to digital converter (ADC) that converts analog signals from the external pressure sensor, MPX4250D, to digital signals feed into Arduino microcontroller used in feedback in the active-controlled microfluidic system, and user interface display in both passive and active-controlled microfluidic systems. Fig. 2 shows the full schematic of the four channel open-source pneumatic pressure control system.

\section{B. Pneumatic System}

The pneumatic system consists four pressure actuators, ControlAir T900, which has a range of 0 to $2000 \mathrm{mbar}$, with \pm 2 mbar accuracy. Four pressure sensors, MPX4250D, are used to measure the pressure provided by the actuators, one sensor for each actuator. MPX4250D has a range of 0 to 2500 mbar, with an overall minimum accuracy of \pm 34.5 mbar. Various tubing is used to deliver pneumatic pressure to fluid reservoirs, which will be included in the bill of material on GitHub.

\section{Software and User Interface}

The software is written in $\mathrm{C}++$. Arduino IDE is used for the control and operation algorithm of the open-source pneumatic pressure control system. This includes the system start-up sequence, system calibration sequence, a signal converting algorithm, serial peripheral interfacing, pressure command code for the pressure actuators, and pressure read code for the pressure sensors.

The graphical user interface (GUI) and computer-tomicrocontroller communication are coded using MS Visual Studios based on QT framework. The design of the GUI is based on end-user suggestion as well as emulating premium commercial unit's GUI. GUI is designed to be minimalistic to streamline the user experience. However, due to the opensource nature of the software, the GUI can be modified with additional or fewer functions, and the GUI can be integrated with other Windows-based operating system GUI. Fig.3 shows the graphical user interface.

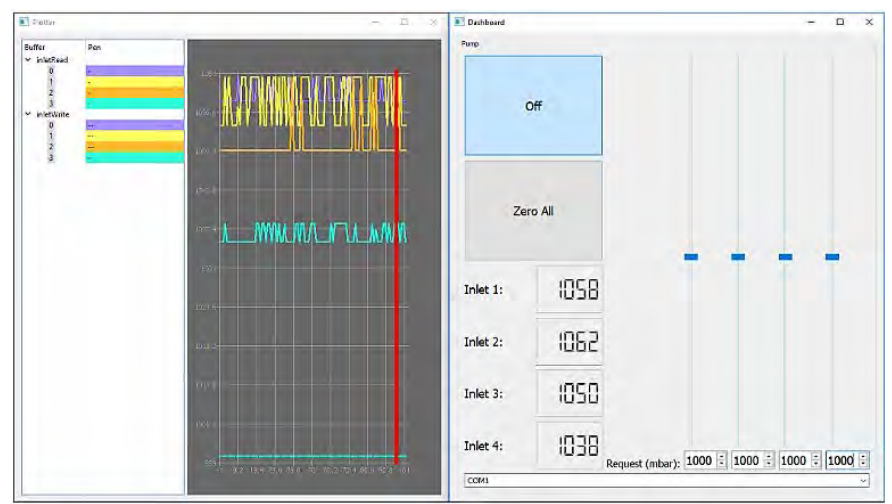

Figure 3. Open-source pneumatic pressure control system graphic user interface

\section{TESTING AND VALIDATION}

The signal testing is done using digital phosphor oscilloscope. The pressure performance testing and benchmarking is done using the prototype setup. Two different types of pressure sensors, MPX4250DP and MPXV5004 are used to conduct performance testing. A flow sensor, Sensirion SLG1430-480 is used to conduct real-world performance testing and benchmarking.

\section{A. Signal Noise Ratio}

Noise is minimized by using eigth-order Butterworth, low pass filters, MAX7480, which provides ADC anti-aliasing and post-DAC filtering. Also, noise is further reduced by using precision $5 \mathrm{~V}$ voltage reference, LM4040, which has an accuracy of $5 \mathrm{mV}$. The result of which is that the pressure sensors and pressure actuators are operating within their manufacture's specifications.

\section{B. Response Time}

Fig. 4 shows the step response time of all four transducers increasing from $1000 \mathrm{mbar}$ to $2000 \mathrm{mbar}$. The response time for each of the four transducers over this range is less than one second, which satisfies the response time and settling time constraints.

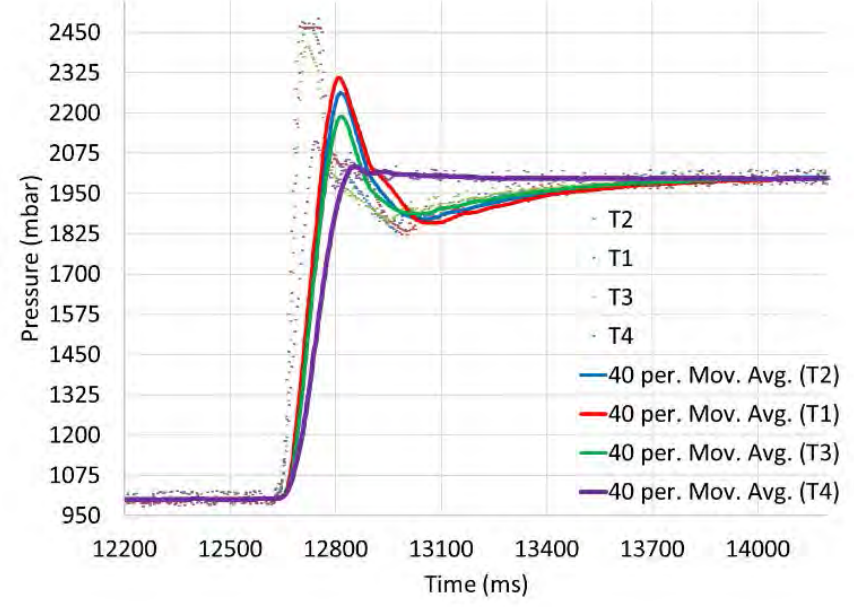

Figure 4. Response time and settling time of all four channels of the open-source pneumatic pressure control system

\section{Pressure Stability}

Fig. 5 shows the pressure stability comparison between one of pneumatic pressure control system's channels and one of Fluigent MFCS EZ's channels. The data is taken at $20 \mathrm{mbar}$. The two systems have similar performance regarding pressure stability. The accuracy and precision design constraints for pneumatic pressure control system are achieved. 


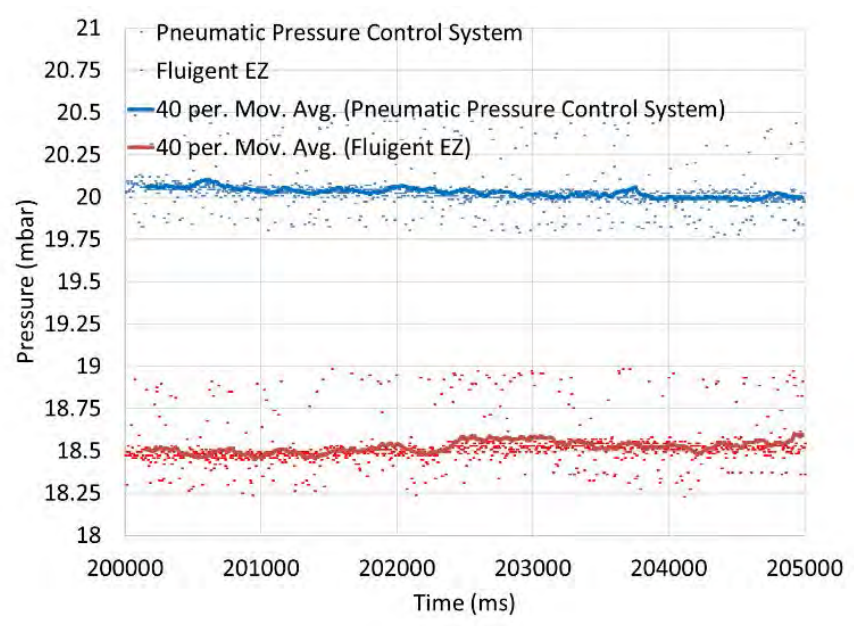

Figure 5. Pressure stability comparison between open-source pneumatic pressure control system and Fluigent MFCS EZ

\section{Flow Rate Stability}

Fig. 6 shows the flow rate stability comparison between one of pneumatic pressure control system's channels and one of Fluigent MFCS EZ's channels. The flow rate data are taken at $300 \mathrm{mbar}$. Both systems display similar flow rate stability.

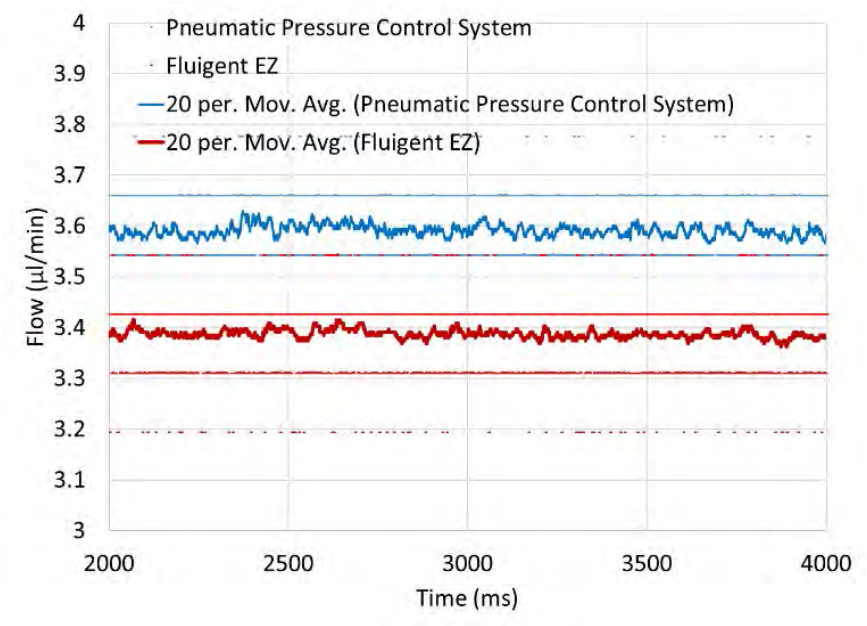

Figure 6. Flow Rate stability comparison between open-source pneumatic pressure control system and Fluigent MFCS EZ

\section{DISCUSSION}

Safety features are built into the design. Soft stops are incorporated into the code which stops the communication when the pressure sensor reads sudden unexpected pressure spikes or drops or higher than operating limit pressure levels. Hard stops include physical toggle switches that shut the supply power to the pneumatic solenoid and pressure actuators.

The testing and validations results show that the opensource pneumatic pressure control system can take full advantage of pressure actuator performance without sacrifice or compromise due to an analog signal to noise ratio. In other words, the precision and accuracy are limited by the pressure actuator. Also, precision is determined to be more important than accuracy as pulseless criteria is mostly concerned with precision. Overall, all of the design constraints and criteria are met.

The pressure stability test and flow rate stability test show that the open-source pneumatic pressure control system can achieve the same performance as Fluigent MFCS EZ. The difference between the two systems lies in the consistency in performance between channels/pressure actuators.

\section{CONCLUSION}

Safety features regarding pneumatic pressure and electricity have been incorporated into the design and build of the opensource pneumatic pressure control system. The testing and validation results show that the open-source pneumatic pressure control system can take full advantage of pressure actuator performance. Overall, all of the design constraints and criteria are met. The benchmarking shows that open-source pneumatic pressure control system has similar performance compared to Fluigent MFCS EZ while costing much less than a commercial system.

\section{ACKNOWLEDGMENT}

The authors would like to thank Natural Science and Engineering Research Council, Canada, and Ontario Center of Excellence for the grants awarded to Professor Carolyn Ren.

\section{REFERENCES}

[1] C.-A. Kieffer et al., "A High Precision Fluid Handling System Based on Pressure Actuation," in 3rd European Conference on Microfluidics, Heidelberg, 2012.

[2] Elveflow. (2018). OB1 MK3 - Microfluidic Flow Control System [Online]. Available: https://www.elveflow.com/microfluidic-flowcontrol-products/flow-control-system/pressure-controller/. Accessed: 13 Mar 2018.

[3] Dolomite. (2018). Mitos P-Pump Basic [Online]. Available: https:// www.dolomite-microfluidics.com/product/mitos-p-pump-basic/. Accessed: 13 Mar 2018. 


\title{
Drag Reduction and Heating
}

\author{
J.M. Floryan, S. Shadman, M.Z. Hossain \\ Department of Mechanical and Materials Engineering \\ Western University \\ London, Ontario, N6A5B9, Canada
}

\begin{abstract}
An external force is required to maintain the relative movement of horizontal plates. It is shown that this force is reduced when the plates are subject to a spatially distributed heating. The largest reduction occurs for heating wavelengths of the order of distance between the plates with its magnitude increasing proportionally to the second power of the relevant Rayleigh number. It is shown that a sufficiently strong heating eliminates the need for the driving force altogether. The effect is active only in small Reynolds number flows.
\end{abstract}

\section{Keywords - drag reduction; heating}

\section{INTRODUCTION}

Friction between moving shafts found in numerous machines contributes to the energy cost of operating these devices. This cost may be estimated by determining force required to maintain the relative motion between these parts. Similar processes can be found in other applications, e.g. towing of a free-floating body in a shallow basin. In gen flows which form in the space between two infinite plate: relative motion are well approximated by the Couette $f$ model, which is one of the family of simple flows freque used in analyses.

Couette flow is characterized by the absence 0 : streamwise pressure gradient, a linear velocity distribu1 across the fluid layer and the lack of the linear stability li [1]. The nonlinear stability analyses are well reviewed in and demonstrate various routes to secondary finite-amplit states as well as to turbulence. Surface modifications eithe the form of transverse grooves [3], longitudinal grooves [4 wall transpiration [5] can lead to centrifugal instabilit Replacing the plane Couette flow with the annular Cou flow leads to shear instabilities [6]. Transition to seconc states leads to an increase in the wall shear and the neec increase the externally-imposed driving force. Such sti. should be avoided if minimization of energy cost is of interest.

Analyses of non-isothermal Couette flows are rather limited. They typically involve fluids with temperaturedependent material properties in a flow system exposed to a spatially uniform heating [7]. The addition of gravity brings in buoyancy effects which may generate secondary flows through the Rayleigh-Bénard (RB) instability $[8,9]$ if the relevant critical conditions are met. Analyses of the resulting mixed convection are well reviewed in [10]. The use of spatially nonuniform heating leads to structured convection which occurs regardless of the heating intensity, but which is yet to be studied in the case of Couette flow. It is known that such heating leads to the reduction of pressure losses in pressuregradient driven flows [11-13].

The present work is part of a wider search for drag reducing methods which, in the present case, manifests itself in the form of reduction of the external force required to maintain the relative plate movement. One of the approaches is to assure stability of the flow so that transition to secondary states is avoided. Another approach, which is followed here, is to create spatial flow modulations which could lead to the reduction of shear and, thus, reduction of the driving force. The use of grooves for this purpose has been explored in [14-16]. This paper explores modulations created by spatially distributed heating.

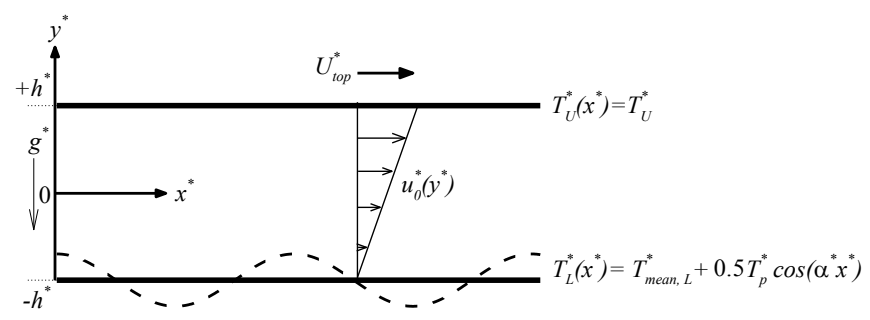

dynamic viscosity $\mu^{*}$, thermal expansion coefficient $\Gamma^{\circ}$ and with variations of density $\rho^{*}$ described by the Boussinesq approximation. The gravitational acceleration $g$ acts in the negative $y$-direction. The upper plate is pulled in the positive $\mathrm{x}$ direction with a constant velocity $U_{\text {top }}^{*}$ while the lower plate is stationary. When the system is isothermal, the drag force and the resulting flow field can be easily determined: 
$v_{0}(x, y)=\left[u_{0}(y), 0\right]=\left[\frac{(1+y)}{2}, 0\right], p_{0}(x, y)=C, F_{0}=0.5(1)$

where velocity has been scaled with $U_{\text {top }}^{*}$ as the velocity scale, $Q_{0}$ stands for the flow rate scaled with the same velocity scale, $\tau_{0}$ stands for the shear acting on the upper plate scaled with $U_{\text {top }}^{*} \mu^{*} / h^{*}, F_{0}$ denotes the force per unit length and width required to drag the upper plate scaled with $U_{\text {too }}^{*} \mu^{*} / h^{*}$ and the relevant Reynolds number is defined as $R e=U_{t o p}^{*} h / v$.

Introduce an external heating resulting in sinusoidal temperature variations along the lower plate,

$$
T_{L}^{*}\left(x^{*}\right)=T_{\text {mean }, L}^{*}+0.5 T_{p}^{*} \cos \left(\alpha^{*} x^{*}\right), T_{U}^{*}\left(x^{*}\right)=T_{U}^{*},
$$

where the subscripts "mean" and " $p$ " refer to the mean and periodic parts, respectively, $T_{p}^{*}$ is the peak-to-peak amplitude of the periodic component, and subscripts $L$ and $U$ refer to the lower and upper plates, respectively. Using the upper plate's temperature for reference and introducing the relative temperature $\theta * T^{*}-T_{U}^{*}$ lead to plates' temperatures of the form

$$
\theta_{L}^{*}(x)=\theta_{\text {uni }}^{*}+0.5 \theta_{p}^{*} \cos \left(\alpha^{*} x^{*}\right), \quad \theta_{U}^{*}(x)=0 \text {, }
$$

where $\theta_{\text {uni }}^{*}=T_{\text {mean } L}^{*}-T_{U}^{*}, \theta_{p}^{*}=T_{p}^{*}$. Using half of the gap height $h^{*}$ as the length scale and $\kappa^{*} v^{*} /\left(g^{*} \Gamma^{*} h^{*}\right)$ as the temperature scale results in the temperature boundary conditions of the form

$$
\theta_{L}(x)=R a_{\text {uni }}+0.5 R a_{p} \cos (\alpha x), \quad \theta_{U}(x)=0,
$$

where $R a_{\text {uni }}=g^{*} \Gamma^{*} h^{*} T_{\text {uni }}^{*} /\left(\kappa^{*} v^{*}\right)$ is the uniform Rayleigh number measuring the intensity of the uniform heating, $R a_{p}=g^{*} \Gamma^{*} h^{*} T_{p}^{*} /\left(\kappa^{*} v^{*}\right)$ is the periodic Rayleigh number measuring the intensity of the periodic heating, and all material properties are evaluated at the reference temperature $T_{U}^{*}$.

The field equations take the form

$\frac{\partial u}{\partial x}+\frac{\partial v}{\partial y}=0, \quad u \frac{\partial u}{\partial x}+v \frac{\partial u}{\partial y}=-\frac{\partial p}{\partial x}+\nabla^{2} u$,

$u \frac{\partial v}{\partial x}+v \frac{\partial V}{\partial y}=-\frac{\partial p}{\partial y}+\nabla^{2} v+P^{-1} \theta, u \frac{\partial \theta}{\partial x}+v \frac{\partial \theta}{\partial y}=P^{-1} \nabla^{2} \theta$,

where $(u, v)$ are the velocity components in the $(x, y)$ directions, respectively, scaled with $U_{v}^{*}=v^{*} / h^{*}$ as the velocity scale, $p$ stands for the pressure scaled with $\rho^{*} U_{v}^{* 2}$ as the pressure scale and $P r=v^{*} / \kappa^{*}$ is the Prandtl number. These equations are subject to boundary conditions (2.4) combined with

$u(-1)=0, u(1)=R e, v(-1)=0, v(1)=0$,

where $U_{\text {top }}^{*} / U_{W}^{*}=R e$. As the flow is driven by the movement of the upper plate, any effects associated with the mean pressure gradient are eliminated through imposition of constraint of the form

$$
\left.\frac{\partial p}{\partial x}\right|_{\operatorname{man}}=0
$$

Heating alters the flow resulting in the change of the driving force, $\Delta F^{*}=F_{0}^{*}-F^{*}$, which, when scaled with $\rho^{*} U_{v}^{* 2}$, can be evaluated using the following relation

$$
\Delta F=F_{0}-F=\operatorname{Re}\left(\frac{1}{2}-\left.\operatorname{Re}^{-1} \lambda^{-1} \int_{0}^{\lambda} \frac{d u}{d y}\right|_{y=1} d x\right) .
$$

\section{DISCUSSION OF RESULTS}

Identical mean temperatures of both plates correspond to $R a_{u n i}=0$. When the upper plate is stationary $(R e=0)$, a purely periodic heating results in the formation of convective counterrotating rolls with the fluid moving upwards above the hot spots and downwards above the cold spots, as illustrated in Fig.2a, and its temperature rising above the mean in most of the fluid volume. Slow movement of the upper plate $(R e=1)$ results in a competition between the plate-driven and the buoyancy-driven motions. The flow topology is simple in the zones with the clockwise-rotating rolls as the roll movement is kinematically consistent with the plate movement, resulting in the formation of a single stream of fluid moving in the positive $x$-direction located in the immediate vicinity of the moving plate. A complex flow topology forms in the zones with the counterclockwise-rotating rolls as the fluid stream splits into two branches, one flowing above the rolls and one flowing beneath them. The upper branch is dominated by the plate effect, and the lower branch is dominated by the roll effect (see Fig.2b). Most of the fluid remains trapped in the rolls, i.e. either in the clockwise rolls attached to the lower plate or in the counter-clockwise rolls bounded by the two branches of the stream moving to the right. A further increase of the plate velocity $(R e=5)$ results in the dominance of the plate-driven movement with most of the fluid moving to the right, the elimination of the counterclockwise rolls and the reduction of the size of the clockwise rolls (see Fig.2c) but with the buoyancy effects still providing a significant contribution to the overall flow dynamics. A further increase of $R e$ results in the eventual elimination of the rolls (see topology for $R e=50$ in Fig.2D). The sequence of plots displayed in Fig.2 illustrates the process of formation of both the flow and(4heank boundary layers near the lower plate as $R e$ increases.

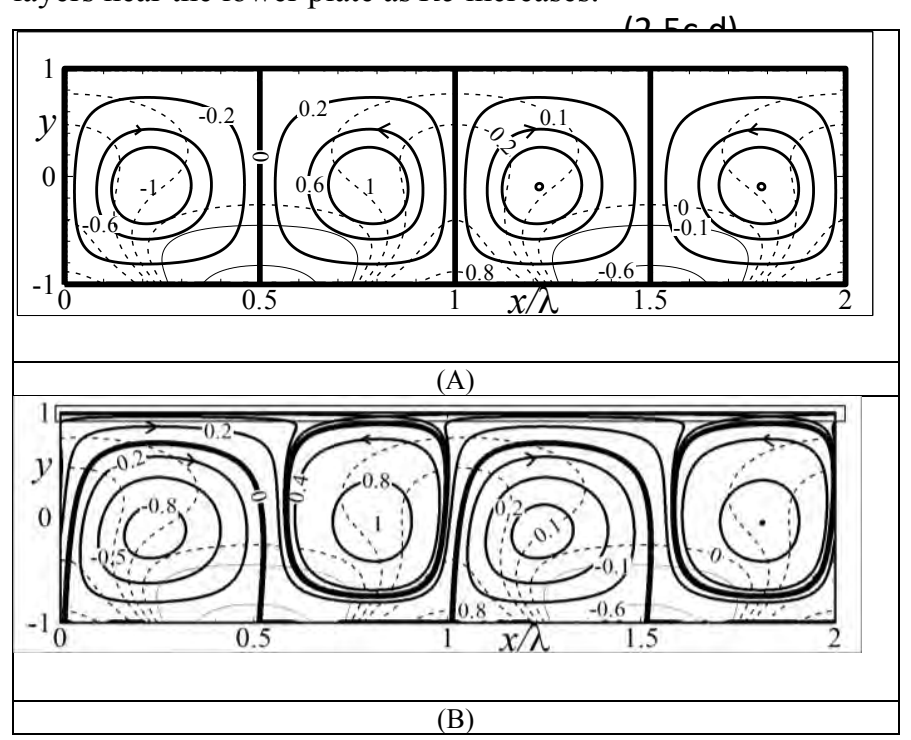




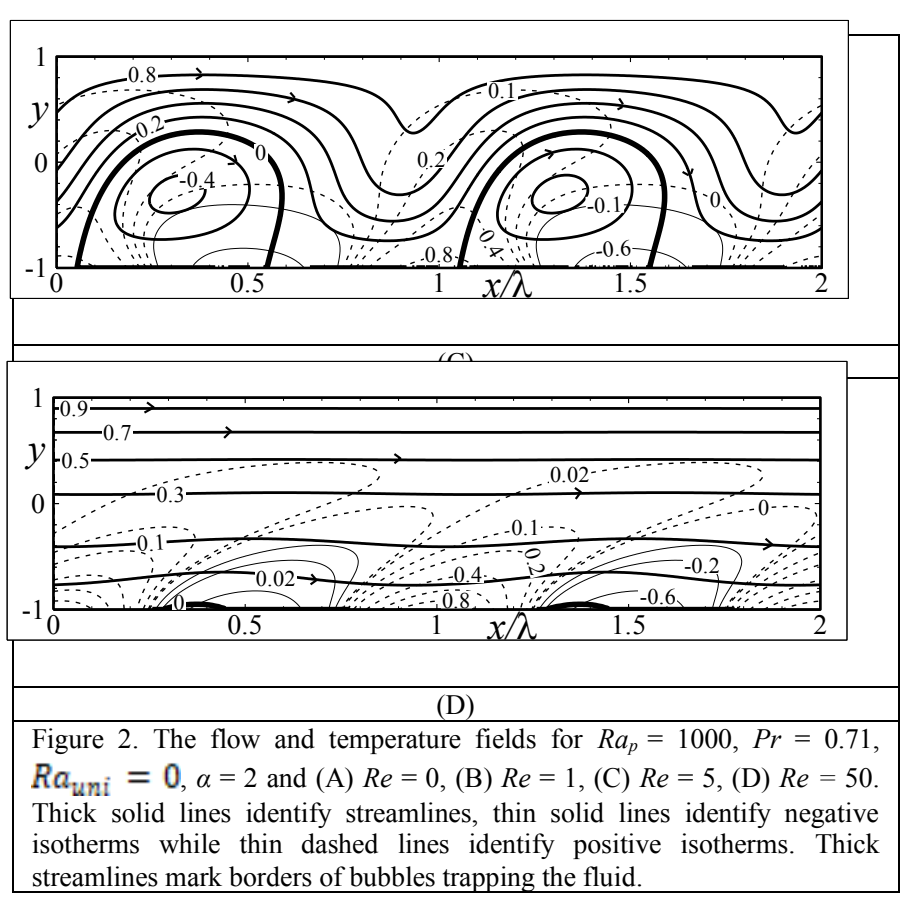

The results presented in Fig. 3 demonstrate that all heating wave numbers lead to a decrease of the driving force with the magnitude of this reduction being a strong function of $\alpha$. The largest reduction occurs for $\alpha \approx 1-2$ with its magnitude decreasing proportionally to $\alpha^{4}$ if an excessively small $\alpha$ is used. The flow and temperature fields (not shown) are qualitatively similar to those displayed in Fig. 2. The use of an excessively large $\alpha$ also results in a reduction of $\Delta F$ but at a much higher rate, i.e. proportionally to $\alpha^{-7}$.

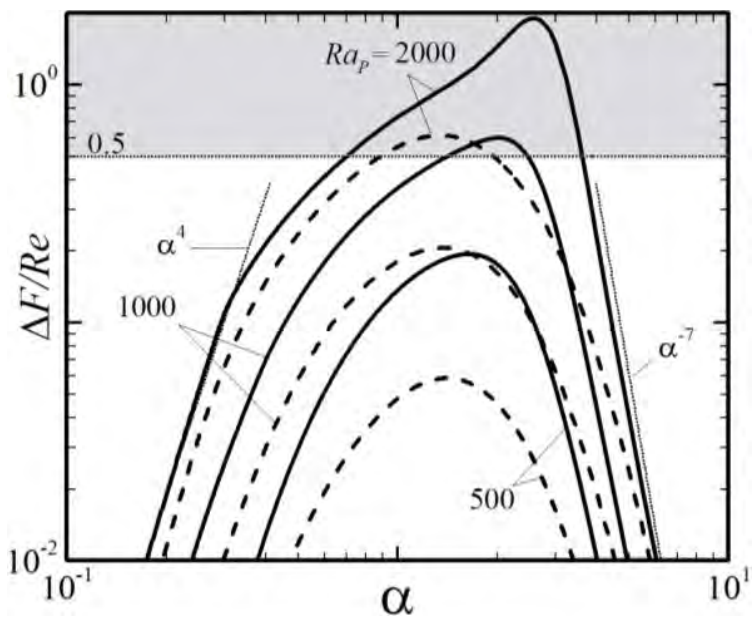

Figure 3. Variations of $\Delta F / R e$ as a function of $\alpha$ for $P r=0.71, R a_{\ln R}=0, R e$ $=1$ (solid lines) and $R e=10$ (dashed lines). Thin dotted lines identify asymptotes. The shaded area identifies conditions where the driving force must change direction and becomes a braking force.

The force-reducing effect is a strong function of $R e$ (see Fig.4). The magnitude of $\Delta F$ increases proportionally to $R e$ for small $R e$ 's, reaches a maximum at $R e \sim 5-6$ and then decreases at a rate proportional to $R e^{-2}$. The flow topologies displayed in Fig. 2 show that the elimination of $\Delta F$ is associated with the reduction of convection bubbles and confinement of convection effects to a thin boundary layer near the lower plate.

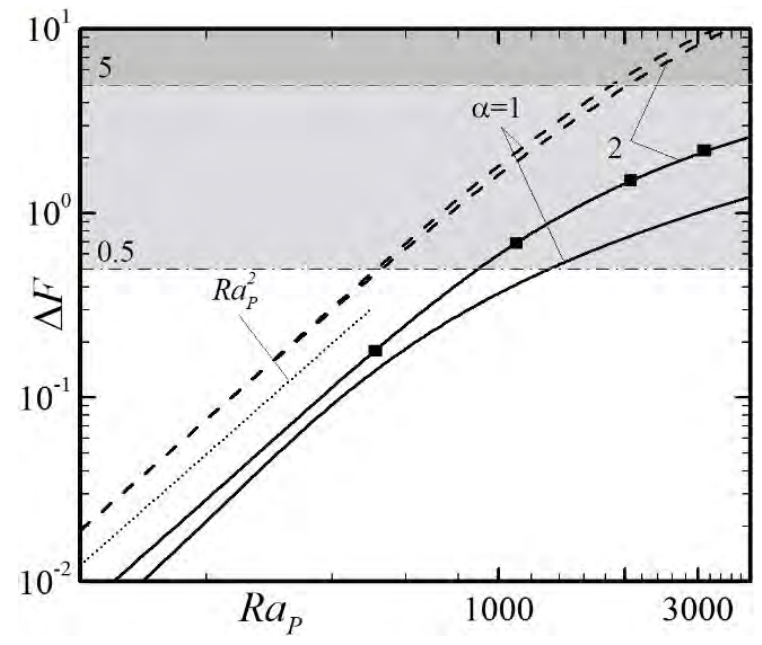

Figure 4. Variations of $\Delta F$ as a function of $R a_{p}$ for $P r=0.71, R a_{\text {bint }}=0, R e=$ 1 (solid lines) and $R e=10$ (dashed lines). The shaded area identifies conditions where the driving force must change direction and becomes a braking force when $R e=1$ and the double shaded area identifies such conditions for $R e=10$.

As it is unlikely that the mean temperatures of both plates can be kept equal, it is of interest to determine how their difference may affect the system response. This difference is expressed in the analysis as the uniform Rayleigh number $R a_{\text {wni }}$ whose positive (negative) values correspond to the lower plate being hotter (cooler). The results displayed in Fig.5 demonstrate that the uniform heating increases $\Delta F$ while cooling decreases it, and the change is approximately linear with $R a_{\text {uni }}$.

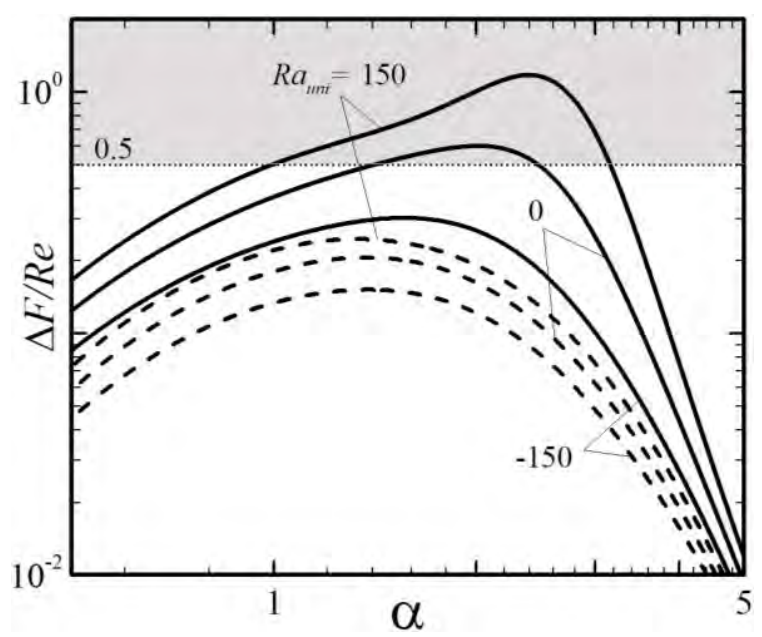

Figure 5. Variations of $\Delta F / R e$ as a function of $\alpha$ for $R e=1$ (solid lines) and $R e$ $=10$ (dashed lines), $R a_{P}=1000, P r=0.71$. The shaded area identifies conditions where the driving force changes direction and becomes a braking force. 


\section{SUMMARY}

An external force is required to generate a relative motion between horizontal plates with the rate of this motion expressed in terms of the Reynolds number Re. The effects of spatially distributed heating on the magnitude of this force have been analyzed. Heating resulting in the lower plate temperature varying sinusoidally in the horizontal direction, with its amplitude expressed in terms of a periodic Rayleigh number $R a_{p}$ and the spatial distribution described by the wave number $\alpha$, has been considered. The analysis has been limited to $R a_{p}<$ 3000 to avoid condition leading to a potential formation of secondary flows. The difference between the mean plates' temperatures has been expressed in terms of the uniform Rayleigh number $R a_{\text {uni }}$ with positive values corresponding to a warmer lower plate. The fluid motion results from a competition between the buoyancy-driven effects and the platedriven movement. The former has the form of counter-rotating rolls whose distribution is dictated by the heating pattern. The latter one adds a rectilinear motion which leads to the reduction and eventual elimination of the rolls if $R e$ is large enough.

It has been shown that periodic heating always reduces the driving force, regardless of whether the heating is applied to the lower or upper plate, but the magnitude of this reduction is a strong function of the heating wave number. The largest reduction is achieved for $\alpha=1-2$ with a rapid decrease of this effect when either too small or too large $\alpha$ 's are used. An increase in $R a_{\text {wni }}$ magnifies this effect. An increase in $R e$ eliminates the rolls and reduces this effect, leading to its practical elimination for $R e>30-50$ depending on the heating intensity. The use of proper heating intensity and distribution results in the complete elimination of the driving force as the plate movement can be supported by the buoyancy effects only. Conditions where an external braking force needs to be used to prevent the plate from accelerating have also been identified.

\section{ACKNOWLEDGMENT}

This work has been carried out with the support of NSERC of Canada.

\section{REFERENCES}

[1] V. A. Romanov, "Stability of plane-parallel Couette flow," Funct. Anal. Appl., vol. 7, no. 2, pp. 137-146, 1973. doi: 10.1007/BF01078886.

[2] K. DEGUCHI and M. NAGATA, "Bifurcations and instabilities in sliding Couette flow,” J. Fluid Mech., vol. 678, pp. 156-178, Apr. 2011. doi: $10.1017 / \mathrm{jfm} .2011 .103$.

[3] J. M. Floryan, "Centrifugal instability of Couette flow over a wavy wall," Phys. Fluids, vol. 14, no. 1, pp. 312-322, Jan. 2002. doi: 10.1063/1.1416185.

[4] H. V. Moradi and J. M. Floryan, "Sliding Couette flow in a ribbed annulus," Phys. Fluids, vol. 28, no. 7, p. 74103, Jul. 2016. doi: 10.1063/1.4955101.

[5] J. M. Floryan, "Wall-transpiration-induced instabilities in plane Couette flow,” J. Fluid Mech., vol. 488, pp. 151-188, Jul. 2003.

doi: 10.1017/S0022112003004804.

[6] P. Gittler, "Stability of axial Poiseuille-Couette flow between concentric cylinders," Acta Mech., vol. 101, no. 1-4, pp. 1-13, Mar. 1993. doi: 10.1007/BF01175593.

[7] A. W. O. B. A. Peter, "Analytical Solution of Non-Isothermal Couette Flow between two Plates.," IOSR J. Math., vol. 10, no. 2, pp. 44-48, 2014. doi: 10.9790/5728-10264448.

[8] H. Bénard, "Les tourbillons cellulaires dans une nappe liquide. Méthodes optiques d'observation et d'enregistrement," J. Physique Théorique et Appliquée, vol. 10, no. 1, pp. 254-266, 1901. doi: 10.1051/jphystap:0190100100025400.

[9] Lord Rayleigh, "LIX. On convection currents in a horizontal layer of fluid, when the higher temperature is on the under side," Philos. Mag. (1798-1977), vol. 32, no. 192, pp. 529-546, Dec. 1916. doi: $10.1080 / 14786441608635602$.

[10] R. E. Kelly, "The Onset and Development of Thermal Convection in Fully Developed Shear Flows," Adv. Appl. Mech., 1994, pp. 35-112. doi: 10.1016/S0065-2156(08)70255-2.

[11] M. Z. Hossain, D. Floryan, and J. M. Floryan, "Drag reduction due to spatial thermal modulations," J. Fluid Mech., vol. 713, pp. 398-419, Oct. 2012. doi: $10.1017 / \mathrm{jfm} .2012 .465$.

[12] D. Floryan and J. M. Floryan, "Drag reduction in heated channels," J. Fluid Mech., vol. 765, pp. 353-395, Jan. 2015.

doi: $10.1017 / \mathrm{jfm} .2014 .683$.

[13] M. Z. Hossain and J. M. Floryan, "Drag reduction in a thermally modulated channel," J. Fluid Mech., vol. 791, pp. 122-153, Feb. 2016. doi: $10.1017 / \mathrm{jfm} .2016 .42$.

[14] M.J. Walsh, "Drag Characteristics of V-Groove and Transverse Curvature Riblets," in AIAA Viscous Flow Drag Reduction, 1980, pp. 168-184.

[15] M. J. Walsh, "Riblets as a Viscous Drag Reduction Technique," AIAA Journal, vol. 21, no. 4, pp. 485-486, Apr. 1983.

doi: $10.2514 / 3.60126$.

[16] A. Mohammadi and J. M. Floryan, "Effects of longitudinal grooves on the Couette-Poiseuille flow," Theor. Comput. Fluid Dyn., vol. 28, no. 5, pp. 549-572, Oct. 2014. doi: 10.1007/s00162-014-0331-8. 


\section{A Comparison between Cyclone Separator Efficiency Enhancement using Ferrous Powder and Additional Tangential Chamber}

\author{
W. Mazyan; A. Ahmadi \\ School of Sustainable Design Engineering \\ University of Prince Edward Island \\ Charlottetown, Canada
}

\author{
M. Hoorfar \\ School of Engineering \\ University of British Columbia \\ Kelowna, Canada \\ mina.hoorfar@ubc.ca
}

\begin{abstract}
This paper provides a comparison between the separation efficiency in cyclones separators using two optimization techniques. Separation optimizations using ferrous powder, and additional tangential chamber were investigated. The proposed techniques show separation efficiency enhancements compared to the basic cyclone separator design. The separation ratio efficiency, $\varepsilon_{\mathrm{sp}}$, is evaluated by calculating the outlet to inlet count ratio. Similar to experimental studies in the literature, the inlet and outlet particle counts were evaluated using discretization techniques with the help of a microscope and a membrane collecting the dust at the outlet. It is observed from the two optimization techniques that the addition of ferrous powder while attracting it through magnetic forces provides an additional separation enhancement by $2 \%$ for $11 \mu \mathrm{m}$ particles sizes. This advantage comes with a total of $25 \%$ efficiency enhancement for $4 \mu \mathrm{m}$ particles compared to conventional designs. This study gives an insight on the different approaches proposed.
\end{abstract}

Keywords-component; cyclone separators; efficiency; ferrous powder; tangential chambers

\section{INTRODUCTION}

There has been an increasing demand for natural gas extraction in the last 50 years [1]. To meet this demand efficiently, there have been advances in all the natural gas processes. A special attention had been given to natural gas treatment process by which the natural gas is cleaned from residues and unwanted particles [2]. The efficiency enhancement in gas treatment leads to reduction in energy consumption, which in turns reduces the overall $\mathrm{CO}_{2}$ emissions into the environment.

The removal of solid and liquid particles is among the most important processes in natural gas treatment [2]. It is essential to remove the solid particles from the gas stream as they are considered to be the main reason in damaging the downstream equipment such as compressors, pressure vessels and heat exchangers. Many techniques have been innovated to remove these particles from the natural gas stream. Among the evolving technologies used to remove solid particles are the cyclone separators. Cyclone separators consist of a major barrel and a conical chamber by which the solid particles are separated from the main stream by centrifugal forces [3]. Compared to other solid-gas separators, the main drawback of cyclone separators would be its inability to separate small sized particles (i.e. particles smaller than $3 \mu \mathrm{m}$ at efficiencies higher than $75 \%$ ). On the other hand, cyclone separators have an advantage among other types of solid-gas separators (such as cartridge filters) and that is its low operating cost as well as its simple design [3].

Many researchers have focused on enhancing the cyclone separator efficiency. Among those, Kim et al. [4] proposed the introduction of guiding vanes to enhance the separation efficiency. Their results indicate an increase by $27 \%$ in separation efficiency for $4 \mu \mathrm{m}$ particles. Another study conducted by Lim at al. [5] investigated splitting the inlet of the cyclone separators into two parts. Their results showed a separation efficiency increase by $15 \%$ as compared to the conventional one inlet cyclone separator. In a similar study, Xiong et al. [6] attached vortex finders at the inlet of the cyclone separator. Their analysis showed that helical profile vortex finders could enhance the separation efficiency by $9 \%$ compared the straight profile vortex finders. Xiang et al. [7] also studied the effect of the conical chamber dimensions on the separation efficiency. Their study findings indicate that a decrease in the bottom radius of the cone from $19.4 \mathrm{~mm}$ to 11.6 $\mathrm{mm}$ would enhance the separation efficiency by $16 \%$. Yoshida et al. [8] suggested the study of the conical length effect on the separation efficiency. Their analysis showed that the $50 \%$ cut size was reduced. Park et al. [9] also investigated the effect of allocating cyclone separators in series with step-up cyclone chamber diameters. The first cyclone could segregate particle sizes ranging from $4.5-11 \mu \mathrm{m}$, the second cyclone could segregate particle sizes ranging from $3.4-7.9 \mu \mathrm{m}$, and the third cyclone could segregate particle sizes ranging from $1.8-$ $4.3 \mu \mathrm{m}$. The injection of mist was another proposed study analyzed by Yang and Yoshida [10]. In their analysis, they were able to achieve a $20 \%$ efficiency enhancement for $1 \mu \mathrm{m}$ particle size capturing at $20 \mathrm{~m} / \mathrm{s}$ inlet velocity.

Although many investigators have reached an enhancement in the separation efficiency, this study compares between two novel and simple techniques for separation efficiency enhancement. 


\section{Methodolgy}

In this study, a conventional cyclone separator based on Stairmand's design [11] was modified. Two modification techniques were applied. The first method studies the effect of adding ferrous powder along with magnets to enhance the separation efficiency. The magnetic force will act in the direction of the centrifugal force, and hence, separation efficiency will be enhanced. The second method studies the effect of adding a tangential collecting chamber at the conical section of the separator. As particle velocities increase in this section of the cyclone separator, the tangential collecting chamber will increase the tendency of capturing smaller solid particles at a higher rate, and hence, separation efficiency will be enhanced.

\section{A. Ferrous Powder Addition}

This novel technique involves mixing the gas-solid inlet mixture with adhesive ferrous powder. Particles entering with the gas flow get attached to ferrous powder through a helical mixing chamber. The solid particles will stick to the adhesive ferrous powder and then get attracted by permanent magnets attached to the walls of the cyclone separator in the major barrel [12]. The additional magnetic force is applied radially in the same direction as the centrifugal force; therefore, separation forces would increase and increase the separation efficiency. Figure 1 shows a schematic of the attraction process.

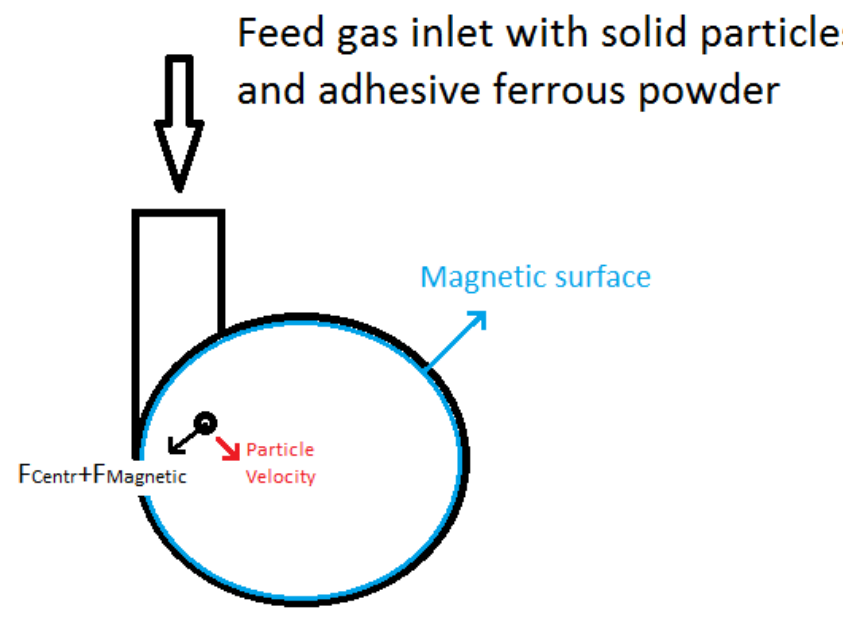

Figure 1: Schematic of ferrous powder addition

\section{B. Additional Tangential Chamber}

This novel technique suggests the use of an additional opening (collecting chamber) located at the conical section of the cyclone separator. The aim of the additional chamber is to collect particles at their highest angular velocities. Higher velocities conclude that the particles have higher tendency to reach outer walls of the conical section [13]. The additional collecting chamber at the conical section would increase the chances of collecting more particles reaching the walls of the conical chamber. This approach will reduce the possibility of escaping particles after bouncing off the conical chamber walls and escape with clean gas. Figure 2 shows an image of the additional tangential chamber located at the conical part of the cyclone separator.
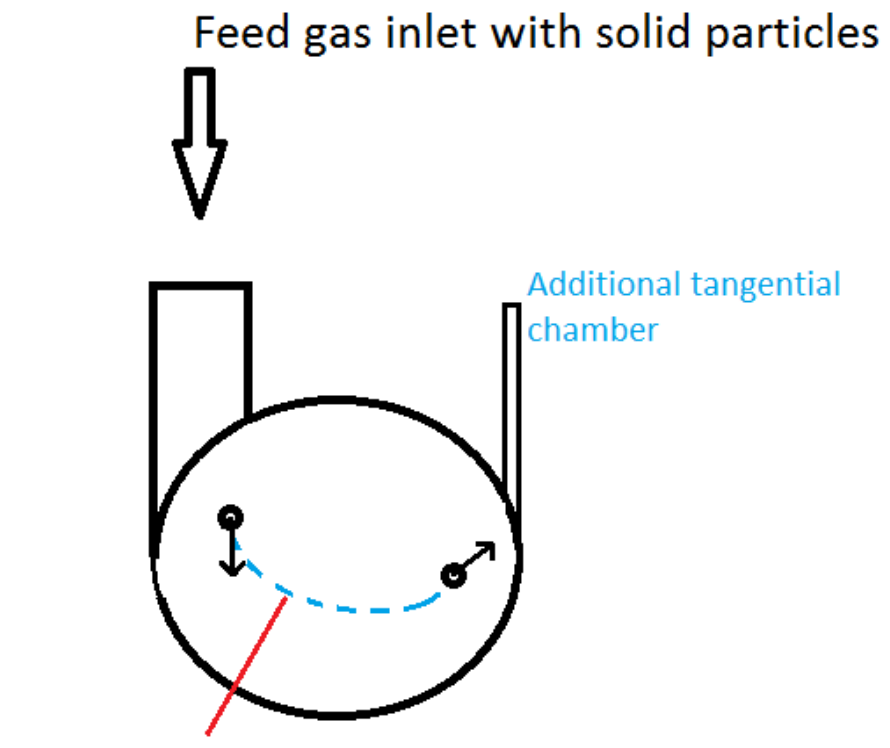

\section{Particle trajectory}

Figure 2: Additional conical tangential chamber

In both setups, the escaped solid particles are captured on a Whatman GE Healthcare membrane at the outlet stream. The exit outlet particles are also analyzed using the Qualitest Digital Microscope.

\section{ReSULTS AND Discussions}

The separation efficiency evaluated in this study $\left(\varepsilon_{\mathrm{sp}}\right)$ is an indication of the cyclone separator performance. Therefore, higher values of the separation efficiencies indicate an enhanced cyclone separator and vice versa.

\section{A. The Effect of Ferrous Powder Addition}

Adding ferrous powder to the solid particles stream and recapturing the mixed (ferrous-solid) combination adds to the centrifugal force behaving on the solid particles in traditional cyclone separators. The additional force increases the chances of solid particles to reach the outer walls and get separated. Thus, the separation efficiency enhancement shown in Figure 3 explains the effect of the additional magnetic force compared to tradition cyclone separator performance.

\section{B. The effect of adding a tangential chamber}

Adding a tangential collection chamber at the conical section of the cyclone separator plays the role of capturing particles which reach the walls of the cone. This method raises the opportunity of capturing these particles while reducing the percentage of escaping particles with the fluid flow after hitting the walls. Thus, the separation efficiency enhancement shown in Figure 3 explains the effect of the additional tangential chamber compared to tradition cyclone separator performance

The separation efficiency results of both proposed techniques are shown in Figure 3. It is very clear that both techniques add to the separation efficiency. The minimum enhancement that both techniques provide is $25 \%$ at $4 \mu \mathrm{m}$ 
particles and $3 \%$ at $11 \mu \mathrm{m}$ particle sizes. Moreover, the separation efficiency enhancement using ferrous powder show better performance as compared to tangential chamber performance (i.e. ferrous powder technique shows a $2 \%$ higher separation performance compared to the tangential chamber addition). This enhancement could be due to a consistent force applied to all particles via the magnetic field. However, in the additional chamber process, not all particles will reach the outer walls of the cone at the chamber's opening. Therefore, the reduction of the tangential chamber performance compared to the ferrous powder is explained.

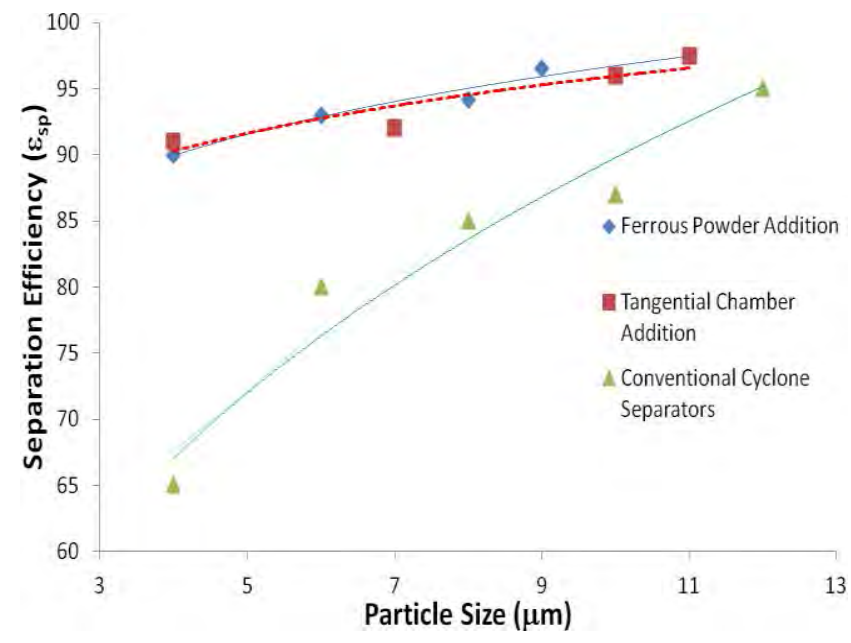

Figure 3: Comparison between proposed novel methods

\section{CONCLUSIONS}

The comparison between two novel techniques was investigated in this paper. Results show that these techniques, the ferrous powder addition and the tangential chamber addition enhance the separation efficiency of solid particles. On the other hand, the ferrous powder addition shows a better performance for larger particles as compared to the tangential chamber addition. Ferrous powder addition shows a $2 \%$ enhanced efficiency over the addition of the tangential chamber. This could represent the nature of the continuous attraction force exerted by the magnets on solid-ferrous particles combination. None the less, both approaches provide a minimum enhancement of at least $25 \%$ for $4 \mu \mathrm{m}$ particles. Thus, further research in the future could allow cyclone separators to have higher separation efficiencies and could allow cyclones separators to replace cartridge filters. Therefore, lower maintenance time and cost would be saved in the filtration industry.

\section{REFERENCES}

[1] M. Mei, "North American Natural Gas Market Dynamics. Canadian Energy Research Institute". Global LNG: A review study no. 123Section II, 2011.

[2] M. Scholes, G. Stevens, and S. Kentish, "Membrane gas separation applications in natural gas processing," Fuel, vol. 96, pp. 15-28, 2012. doi: 10.1016/j.fuel.2011.12.074.

[3] A. Bahadori, Natural Gas Processing-technology and Engineering Design, Waltham, MA: Elsevier inc, 2014.

[4] H. Kim, K. Lee, and M. Kuhlman, "Exploratory design modifications for enhancing cyclone performance," Aerosol Sci., vol. 32, pp. 11351146, 2001. doi: 10.1016/S0021-8502(01)00040-4.

[5] K. Lim, S. Kwon, and K. Lee, "Characteristics of the collection efficiency for a double inlet cyclone with clean air," Aerosol Sci., vol. 34, pp. 1085-1095, 2003. doi: 10.1016/S0021-8502(03)00079-X.

[6] Z. Xiong, Z. Ji, X. Wu, "Investigation on the Separation Performance of a Multicyclone Separator for Natural Gas Purification," Aerosol and Air Qual. Res., vol. 14, pp. 1055-1065, 2014. doi: 10.4209/aaqr.2013.09.0298.

[7] R. Xiang, R. Park, S. Lee, and W. Lee, "Effects of cone dimension on cyclone performance," J. Aerosol Sci., vol. 32, pp. 549-561, 2001. doi: 10.1016/S0021-8502(00)00094-X.

[8] H. Yoshida, Y. Hayase, K. Fukui, T. Yamamoto, "Effect of conical length on separation performance of sub-micron particles by electrical hydro-cyclone," Powder Technol., vol. 219, 29-36, 2012. doi: 10.1016/j.powtec.2011.12.002

[9] C. Park, D. Song, S. Yook, "Development of a single cyclone separator with three stages for size-selective sampling of particles," J. Aerosol Sci., vol. 89, pp. 18-25, 2015. doi: 10.1016/j.jerosci.2015.07.001.

[10] K. Yang, H. Yoshida, "Effect of mist injection position on particle separation performance of cyclone scrubber," Sep. Purif. Technol., vol. 37 pp. 221-230, 2004. doi: 10.1016/j.seppur.2003.09.009

[11] L. Brar, R. Sharma, K. Elsayed, "The effect of the cyclone length on the performance of Stairmand high-efficiency cyclone, Powder Technol. vol. 286, pp. 668-677, Dec. 2015. doi: 10.1016/j.powtec.2015.09.003.

[12] W. I. Mazyan, A. Ahmadi, R.D. Jesus, H. Ahmed and M. Hoorfar, "Use of ferrous powder for increasing the efficiency of solid particle filtration in natural gas cyclones," Sep. Sci. Technol., vol. 51, pp. 2098-2104. doi: 10.1080/01496395.2016.1200085.

[13] W. I. Mazyan, A. Ahmadi, H. Ahmed and M. Hoorfar, "Increasing efficiency of natural gas cyclones through addition of tangential chambers," J. Aerosol Sci., vol. 110, pp. 36-42, 2017. doi: 10.1016/j.jaerosci.2017.05.007. 


\section{Changes in the Leidenfrost Transition Point During the Evaporation of Water and N-Heptane Droplets on Hot, Porous Stainless Steel Surfaces}

\author{
N. Lipson \\ Dept. of Mechanical \& Industrial Engineering \\ University of Toronto \\ Toronto, Canada
}

\author{
S. Chandra \\ Dept. of Mechanical \& Industrial Engineering \\ University of Toronto \\ Toronto, Canada
}

\section{INTRODUCTION}

\begin{abstract}
The impact and evaporation of droplets impinging on a heated porous substrate is relevant to applications such as fire suppression by sprinkler systems, spray cooling of heated surfaces, and the deposition of fuel droplets on combustor walls. Design involving these sub systems requires an understanding of the heat and mass transfer between the droplet and porous surface. An experimental study was done in which pure water and n-heptane droplets were deposited onto porous, stainless steel surfaces made from sintered powders with varying pore size $(5 \mu \mathrm{m}$ and $100 \mu \mathrm{m})$. n-Heptane was chosen to compare the effects of surface tension on the evaporation process. Initial surface temperatures were varied from $60^{\circ} \mathrm{C}$ to $300^{\circ} \mathrm{C}$. Results were compared with those for droplet evaporation on a solid, impermeable stainless steel surface. On porous surfaces, it is difficult to determine when a droplet has completely evaporated from video images, since liquid penetrates into the surface pores. At low wall temperatures $\left(60^{\circ} \mathrm{C}\right.$ to $\left.120^{\circ} \mathrm{C}\right)$, droplet evaporation was measured by placing the heated surface on a digital scale and recording the weight decrease as a function of time. At high wall temperatures (above $120^{\circ} \mathrm{C}$ ), video techniques were employed to capture evaporation times. At high wall temperatures, the porous surfaces were the most effective at vaporizing both the pure water, and n-heptane droplets, resulting in the lowest evaporation times. At low wall temperatures the porous surfaces became less effective at transferring heat to both the water and n-heptane. For n-heptane the evaporation times increased on the porous surfaces as compared to the impermeable surface. Similar results could be seen with the water on the $100 \mu \mathrm{m}$ surface. The Leidenfrost transition point was shown to increase with porosity. Droplet levitation was not achieved with the water on the porous surfaces, however due to the lower heat of vaporization of n-heptane it was achieved on the $5 \mu \mathrm{m}$ and $100 \mu \mathrm{m}$ substrates at surface temperatures of $225^{\circ} \mathrm{C}$ and $285^{\circ} \mathrm{C}$ respectively.
\end{abstract}

Keywords; Porous media, Evaporation, Stainless steel, Water, nHeptane, Pore size, Leidenfrost point
Liquid droplet impact on hot porous surfaces is studied due to its significance in a wide variety of spray cooling applications. These include, cooling of reactors in nuclear power plants; fire suppression by sprinkler systems; and the deposition of fuel droplets on combustion chamber walls. In the fire extinguishment process, water can be used to both extinguish flames, and prevent the flame spread by cooling surfaces that have not yet been ignited. However, the use of large amounts of water can cause secondary fire damage encouraging research into this area to reduce the amount of water used. As fires can involve the burning of porous materials (e.g. wood, fabrics, papers), understanding the thermal effects of droplet impingement on hot porous surfaces will assist in predicting the quantity of water needed for sufficient cooling of the surface while minimizing secondary damage. In addition to fire suppression systems, the evaporation of fuel droplets on heated surfaces is of critical importance in the design of combustion devices. Optimizing droplet evaporation times is important in ensuring improved combustion efficiencies, and reducing gaseous emissions during the combustion process.

The total evaporation time of a droplet is dependent on several key parameters. Reference [1] stated that the heat transfer rate to the droplet is governed by the fluid and solid thermal properties, as well as the surface roughness and temperature. The surface temperature was described as the most significant parameter as it is used to define the heat transfer regime the droplet resides in (i.e. single phase evaporation, nucleate boiling, and film boiling). However, porous surfaces substantially alter the impact dynamics and heat transfer to the droplet. A droplet impinged on a porous substrate will not only spread over the surface, but penetrate into the surface pores. This pore penetration can enhance the heat transfer to the fluid and is known to depend on the properties of both the liquid and the porous substrate (i.e. pore size, porosity, wettability) [2]. The critical parameter influencing the impact behavior of the droplet is the Weber number. The Weber number, We, is defined as the ratio of the droplets inertial to surface tension forces. 


$$
W \mathrm{e}=\frac{\rho_{f} u_{o}^{2} d_{o}}{\sigma}
$$

The present work reports the results of an experimental study involving the evaporation of pure water and n-heptane $\left(\mathrm{C}_{7} \mathrm{H}_{16}\right)$ droplets deposited on hot porous substrates with different pore sizes, and varying surface temperatures. n-Heptane was chosen since it has lower surface tension and higher wettability compared to water and penetrated more easily into surface pores. A digital scale was the measurement technique used at low wall temperatures, where the evaporation times were captured from weight decrease as a function of time plots. There were two main objectives for this work: (1) measure droplet evaporation times using a weight-time approach at low wall temperatures to remove the uncertainty associated when using imaging techniques, and (2) to compare the effects of fluid surface tension on the impact and evaporation of a droplet when impinged on a porous substrate with different surface temperatures.

\section{EXPERIMENTAL METHOD AND CONDITIONS}

Fig. 1 shows a schematic of the experimental setup employed to observe the droplet evaporation of a pure water and n-heptane droplet on a heated sintered 316 stainless steel porous substrate (5 $\mu \mathrm{m}$ and $100 \mu \mathrm{m}$ average pore size, see Fig. 2). The results were compared with those for droplet evaporation on a solid, impermeable 316 stainless steel substrate. The arithmetic average surface roughness Ra for the impermeable, $5 \mu \mathrm{m}$ and $100 \mu \mathrm{m}$ surfaces are $0.100 \pm 0.004 \mu \mathrm{m}, 4.7 \pm 0.1 \mu \mathrm{m}$ and 12.2 $\pm 1.6 \mu \mathrm{m}$ respectively. All surfaces were square, $45 \mathrm{~mm}$ x $45 \mathrm{~mm}$ in size and $1.5 \mathrm{~mm}$ thick. Surface temperatures were varied from $60^{\circ} \mathrm{C}$ to $300^{\circ} \mathrm{C}$. A gauge $24 \mathrm{~K}$-type thermocouple was fixed using Omega CC high temperature cement to the surface of each of the substrates. The surface temperatures were monitored using a wireless thermocouple connector (MWTC-D-K-915, Omega Engineering, Laval, Quebec, Canada) and droplet evaporation was experimentally observed in the evaporative, nucleate boiling, and film boiling regimes.

Using a syringe pump (NE-1000, New Era Pump Systems, Farmingdale, New York, United States), $2.5 \mathrm{~mm}$ diameter droplets were formed with a gauge 26 blunt hypodermic needle for pure water, and a gauge 16 blunt needle for n-heptane. A larger needle was used for n-heptane due to its lower surface tension. Measurements of the droplet weight showed a drop-todrop variation of less than $\pm 2 \%$. The droplets formed on the needle tip, and once large enough would detach under their own weight. Due to n-heptane's solvent characteristics, a glass syringe, along with stainless steel fittings and chemical resistant PVC tubing were used to prevent contamination of the working fluid.

The impact velocity was controlled by adjusting the vertical distance from the tip of the needle to the top of the substrate, and was fixed at $50 \pm 1 \mathrm{~mm}$ for the experiments. The impact velocities for the pure water and $n$-heptane droplets at the time of impact were $0.9 \mathrm{~m} / \mathrm{s}$. The velocity measurements were determined from high speed imaging where they varied by $\pm 5 \%$

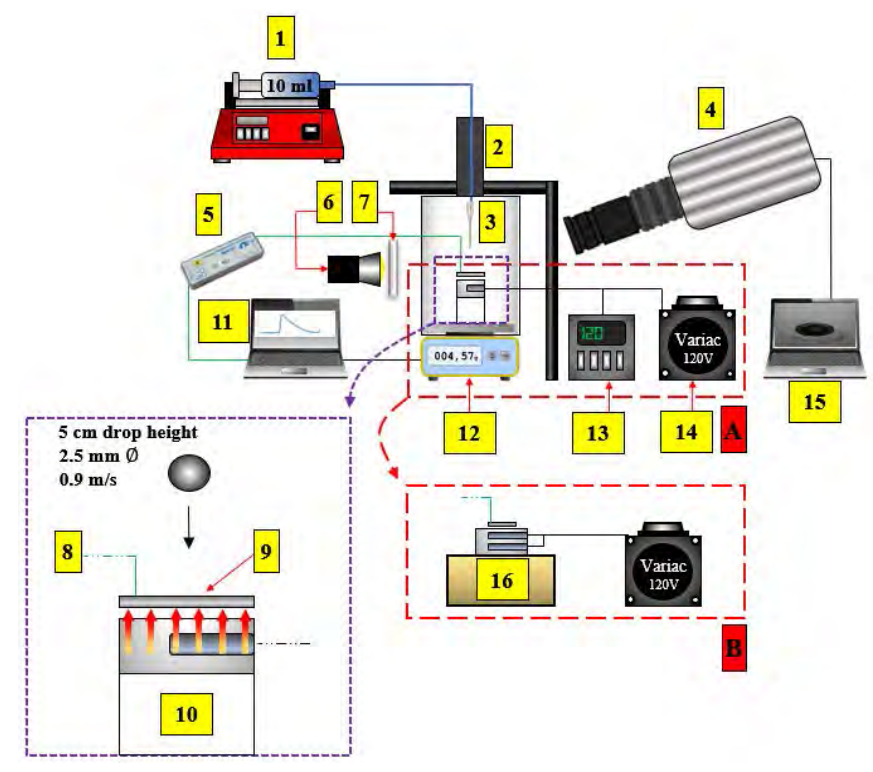

Figure 1. Schematic of experimental setup: (1) Syringe pump coupled with a $10 \mathrm{ml}$ syringe, (2) Vertical height adjustment, (3) Hypodermic needle, (4) Highspeed camera, (5) Wireless thermocouple connector, (6) Light source, (7) Light diffuser, (8) Thermocouple, (9) Substrate, (10) Thermal mass coupled with $100 \mathrm{~W}$ cartridge heater, (11) PC logging scale data, and monitoring substrate temperature, (12) Digital scale, (13) Temperature controller, (14) 120V Variac, (15) PC capturing high-speed camera images, (16) Thermal mass coupled with two, $200 \mathrm{~W}$ cartridge heaters. (a) Setup used at surface temperatures ranging from $60^{\circ} \mathrm{C}$ to $120^{\circ} \mathrm{C}$, (b) Setup used at surface temperatures above $120^{\circ} \mathrm{C}$.

for the water and $\pm 9 \%$ for the $\mathrm{n}$-heptane. The Weber numbers associated with these drop velocities are 29 and 65 respectively.

At surface temperatures ranging from $60^{\circ} \mathrm{C}$ to $120^{\circ} \mathrm{C}$, setup Fig. 1a was employed to capture droplet evaporation. A 3D printed plastic holder was created to hold an aluminum thermal mass ( $45 \mathrm{~mm}$ x $45 \mathrm{~mm}$ x $20 \mathrm{~mm}$ thick) in which the substrate would sit, and was heated using a single $100 \mathrm{~W}$ cartridge heater. The voltage applied to the heaters was adjusted with a $120 \mathrm{~V}$ variac and the substrate surface temperature was regulated using a temperature controller (CN9000A, Omega Engineering, Laval, Quebec, Canada) and could be controlled to $\pm 0.1^{\circ} \mathrm{C}$. Droplet evaporation was measured by placing the heated apparatus equipped with the surface on a digital scale (AG245, Mettler Toledo, Mississauga, Ontario, Canada) and recording the weight decrease as a function of time as the droplet evaporated.
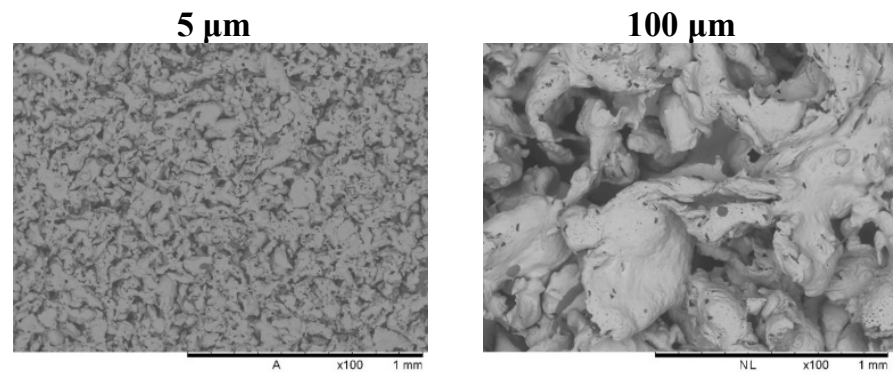

Figure 2. SEM images of the sintered porous stainless steel samples ( $5 \mu \mathrm{m}$ and $100 \mu \mathrm{m}$ average pore size) used during experimentation. Substrate dimensions: $45 \mathrm{~mm} \times 45 \mathrm{~mm}$ by $1.5 \mathrm{~mm}$ thick. 
With surface temperatures above $120^{\circ} \mathrm{C}$, due to limitations with the 3D printed polylactic acid (PLA) substrate holder having a maximum operating temperature of approximately $150^{\circ} \mathrm{C}[9]$, and the scale having a maximum sample rate of $3 \mathrm{~Hz}$, setup Fig. $1 \mathrm{~b}$ was employed. This setup used a larger aluminum thermal mass ( $70 \mathrm{~mm}$ wide x $75 \mathrm{~mm}$ long x $40 \mathrm{~mm}$ thick) equipped with two, $200 \mathrm{~W}$ cartridge heaters wired in parallel and powered by a $120 \mathrm{~V}$ variac used to regulate the heater output. The high-speed camera (Fastcam SA5, Photron, Tokyo, Japan) equipped with a bellows and $105 \mathrm{~mm}$ lens, was used to capture evaporation times at frame rates of 1000 to $5000 \mathrm{fps}$.

Surface temperatures of $150^{\circ} \mathrm{C}$ and above were in the nucleate boiling regime for both water and $n$-heptane, and evaporation was fast enough to prevent significant spreading of the absorbed liquid. As a result, the evaporation time of the deposited liquid coincided with the disappearance of the dry out front. All experiments were performed at an ambient room temperature of $23^{\circ} \mathrm{C} \pm 3^{\circ} \mathrm{C}$.

\section{RESULTS AND DISCUSSION}

The difference in evaporation times observed between the pure water and n-heptane are closely tied to the differences in fluid properties and how that affects the behavior of the droplet as it impacts on the porous surfaces. Figs. 3 and 4 show image sequences of the first 25 milliseconds immediately after impact of pure water (Fig. 3) and n-heptane (Fig. 4) droplets impinging on (a) an impervious surface, (b) a surface with $5 \mu \mathrm{m}$ pores and (c) a surface with $100 \mu \mathrm{m}$ pores. The initial surface temperature in all cases was $250^{\circ} \mathrm{C}$. Droplets were deposited from a $50 \mathrm{~mm}$ drop height. The water and n-heptane droplets evolution on all three surfaces starts off similar during the first $2.4 \mathrm{~ms}$ except for the spread factor which is larger with the n-heptane as compared to the water on the same surface due to n-heptane's lower surface tension. The spread factor $(\boldsymbol{\beta})$ is the dynamic spread diameter of the droplet after impact normalized by the initial droplet diameter $\left(\boldsymbol{\beta}_{\text {film }}=D_{\text {film }} / d_{0}\right)$. The lower surface tension allows inertial forces to play the dominant role during droplet impact where the formation of the liquid jet can span a larger distance before viscous dissipation, surface tension and surface roughness effects dissipate the kinetic energy and bring the droplet to a stop. The surface roughness can be seen impeding the droplet spread as porosity is increased with both water and $\mathrm{n}$-heptane. The water droplet begins its upwards levitation at 10 ms on the impermeable surface (see Fig. 3a) while this process is observed to be delayed with the n-heptane (see Fig. 4a). This appears to be due to n-heptane's larger contact area, where the droplet has a longer distance to recoil before levitation of the bulk fluid occurs. Similarly, instability at the rim of the lamella for n-heptane takes effect early in the spreading process at $1 \mathrm{~ms}$ for all three surfaces. The fingering is a source for satellite droplets which can be seen being ejected from the bulk of the fluid at 10 to $25 \mathrm{~ms}$. The Leidenfrost point (LP) was achieved with the water on the impervious surface only, and with the nheptane on all surfaces, porous and impervious. The delayed

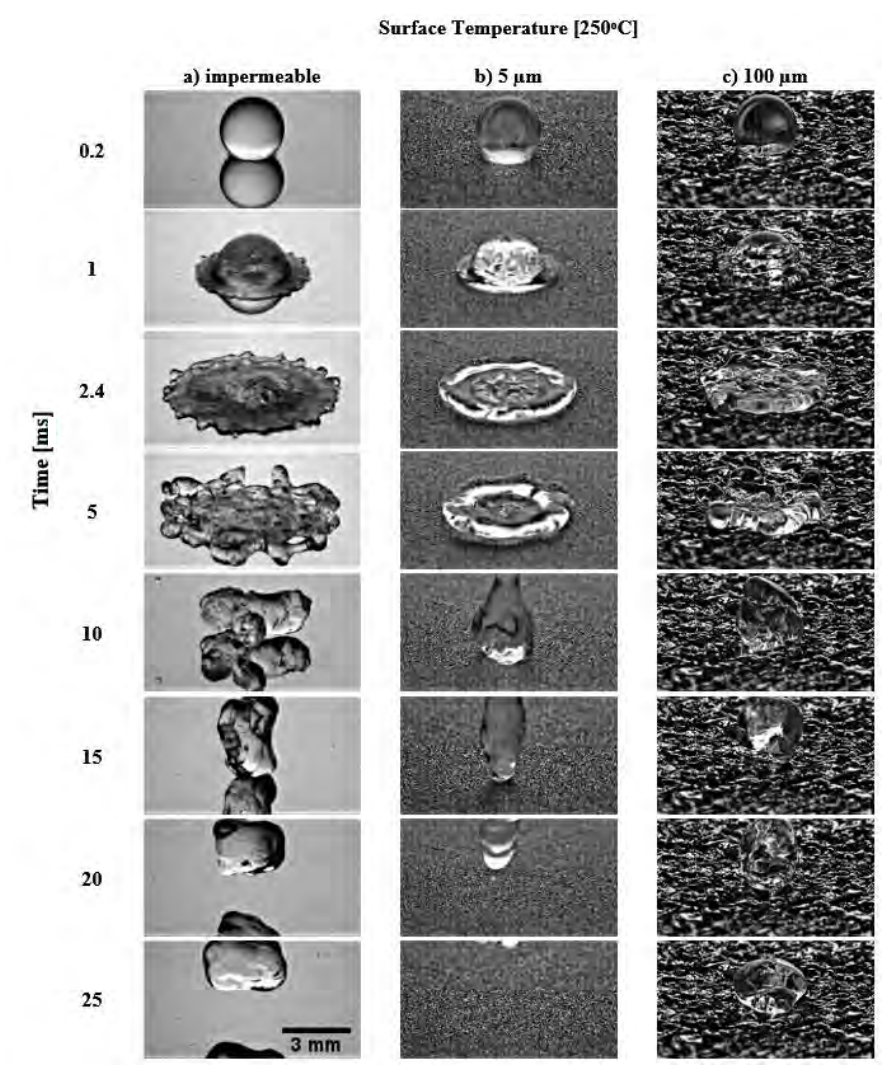

Figure 3. Water droplet impact on the (a) impermeable, (b) $5 \mu \mathrm{m}$ and (c) 100 $\mu \mathrm{m}$ surfaces. Surface temperature $\mathrm{Tw}=250^{\circ} \mathrm{C}$ (film boiling regime), $\mathrm{Vi}=0.9$ $\mathrm{m} / \mathrm{s} \pm 5 \%$, do $=2.5 \mathrm{~mm} \pm 2 \%$, We $=29$.

onset of the LP is due to the vapors produced during evaporation being able to permeate through the porous structure preventing vapor pressures from building between the solid-liquid interface, and allowing the fluid to remain in contact with the surface at increased temperatures. Direct contact heat transfer across a solid-liquid interface is higher than across a vapor gap improving the evaporation times in the film boiling regime. Droplet levitation occurred with the n-heptane on the porous surfaces due to its lower heat of vaporization. It takes less energy to cause a phase change, where the rate of vapor production as a result would increase. The pressures build underneath the droplet providing the necessary forces required for levitation.

Curves of the droplet lifetime as a function of surface temperature can be used to determine when changes occur during the evaporation and impact process [4]. For both pure water and n-heptane, the droplet lifetime curves as a function of surface temperature followed similar trends and are shown in Figs. 5 and 6 respectively. A graph insert is shown in each of the plots, utilizing a log time scale to clearly illustrate the difference in evaporation times between the three surfaces at temperatures ranging from $150^{\circ} \mathrm{C}$ to $300^{\circ} \mathrm{C}$. As the surface temperature was raised, evaporation times decreased until a minimum was reached, corresponding to the critical heat flux (CHF) point. For pure water on the impervious and the $100 \mu \mathrm{m}$ surfaces, the CHF occurred at approximately $200^{\circ} \mathrm{C}$. The CHF for the $5 \mu \mathrm{m}$ surface occurred at $150^{\circ} \mathrm{C}$. Similarly, for the n-heptane the CHF for all 


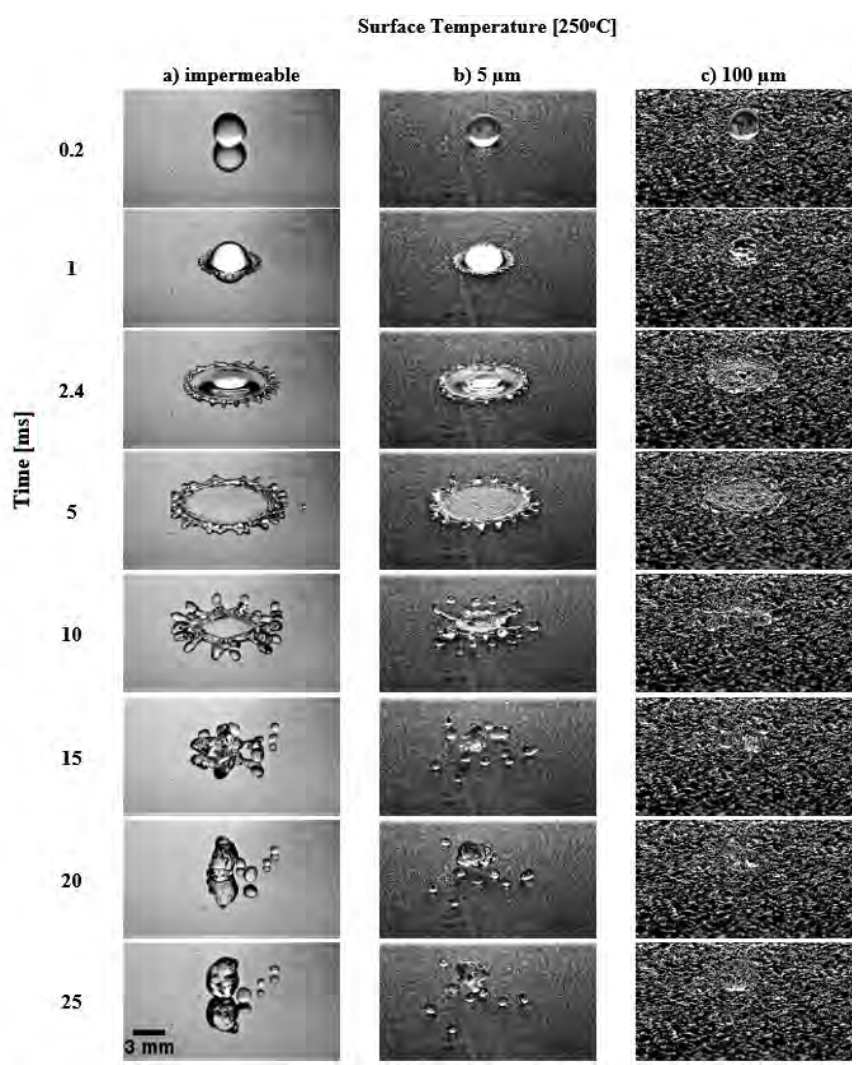

Figure 4. n-Heptane droplet impact on the (a) impermeable, (b) $5 \mu \mathrm{m}$ and (c) $100 \mu \mathrm{m}$ surfaces. Surface temperature $\mathrm{Tw}=250^{\circ} \mathrm{C}$ (film boiling regime), $\mathrm{Vi}=$ $0.9 \mathrm{~m} / \mathrm{s} \pm 9 \%$, do $=2.5 \mathrm{~mm} \pm 2 \%, \mathrm{We}=65$.

three surfaces happened at approximately $150^{\circ} \mathrm{C}$. At low wall temperatures ranging between $60^{\circ} \mathrm{C}$ to $120^{\circ} \mathrm{C}$ the ability of the porous substrates to effectively vaporize the water and $n$-heptane droplets displayed significantly different results. The water droplet evaporated the fastest on the $5 \mu \mathrm{m}$ surface while the nheptane droplet on average evaporated the slowest as compared with the impervious and $100 \mu \mathrm{m}$ surfaces. The reduced times

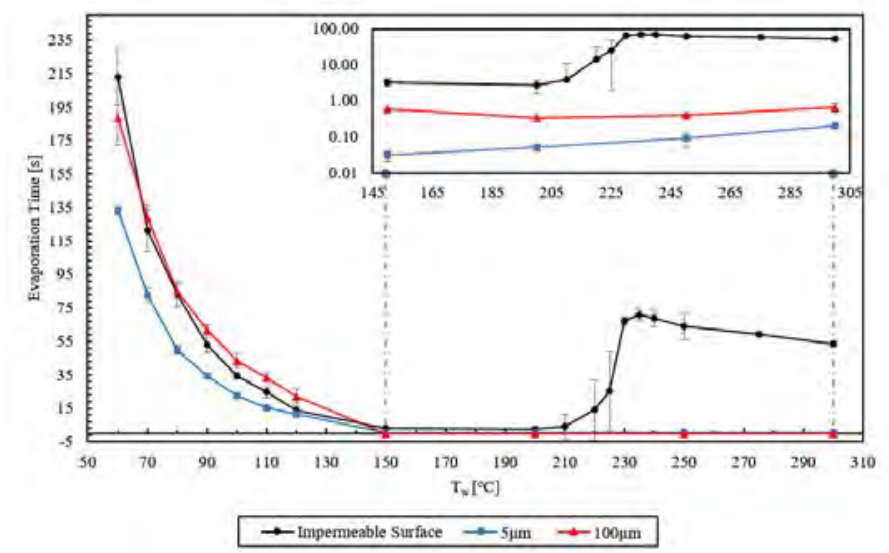

Figure 5. Evaporation time as a function of the surface temperature for the (a) impermeable, (b) $5 \mu \mathrm{m}$ and (c) $100 \mu \mathrm{m}$ surfaces using pure water as the working fluid. A graph insert is shown at surface temperatures ranging from $150^{\circ} \mathrm{C}$ to $300^{\circ} \mathrm{C}$ using a log time scale to show evaporation time differences between the surfaces.

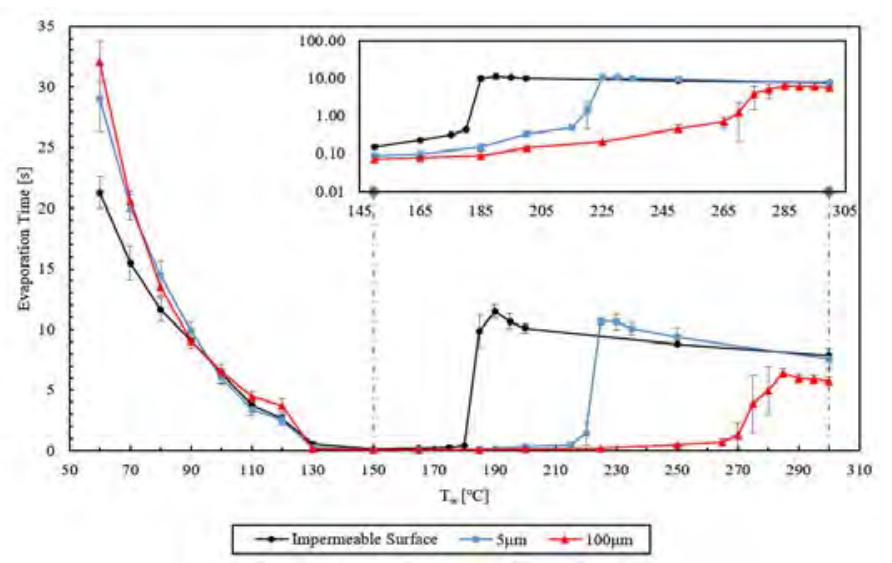

Figure 6. Evaporation time as a function of the surface temperature for the (a) impermeable, (b) $5 \mu \mathrm{m}$ and (c) $100 \mu \mathrm{m}$ surfaces using n-heptane as the working fluid. A graph insert is shown at surface temperatures ranging from $150^{\circ} \mathrm{C}$ to $300^{\circ} \mathrm{C}$ using a log time scale to show evaporation time differences between the surfaces.

with the water is attributed to an increase in the total contact area that can be achieved as the water permeates into the porous structure. As the primary mode of heat transfer within this temperature range is heat conduction, this would increase the rate of heat transfer to the fluid reducing overall evaporation times. Moreover, the increased evaporation times observed with the n-heptane can be attributed to the fluids lower surface tension. The reduction in surface tension substantially improves the rate at which n-heptane penetrates the surface pores causing the fluid to pool at the bottom of the substrate before fully evaporating. Due to n-heptane's low heat of vaporization and the lower permeability of the $5 \mu \mathrm{m}$ surface, vapor pressures build up more rapidly within the substrate, reducing heat transfer and increasing the time required for complete evaporation. Similar behavior can be observed with the water and n-heptane on the $100 \mu \mathrm{m}$ surface within this temperature range. As wall temperatures were raised, ranging from $150^{\circ} \mathrm{C}$ to $300^{\circ} \mathrm{C}$, the porous substrates were the most effective at vaporizing both the water and n-heptane droplets before the LP was reached. This behavior suggests that the substrate structure plays a critical role in droplet evaporation. Water droplets evaporated the quickest on the $5 \mu \mathrm{m}$ surface due again to an increased contact area that can be achieved as the vapors permeate away from the solidliquid interface allowing the droplet to remain in contact with the surface at the increased temperatures. Additionally, the nheptane evaporated the fastest on the $100 \mu \mathrm{m}$ surface. Due to the low heat of vaporization of $n$-heptane, and the higher porosity, and permeability of the $100 \mu \mathrm{m}$ surface compared to the $5 \mu \mathrm{m}$ surface meant a larger quantity of vapor could flow into the porous structure. As a result, less vapor will build up within the porous cavities hindering the rate of evaporation. The LP was reached with the water droplet on the impervious surface at $235^{\circ} \mathrm{C}$. Similarly, the LP was successfully reached with the nheptane at surface temperatures of $190^{\circ} \mathrm{C}$ (impervious surface), $225^{\circ} \mathrm{C}(5 \mu \mathrm{m}$ surface $)$, and $285^{\circ} \mathrm{C}$ (100 $\mu \mathrm{m}$ surface) respectively. 


\section{ACKNOWLEDGMENT}

The authors gratefully acknowledge funding for this project provided by the Natural Sciences and Engineering Research Council of Canada.

\section{REFERENCES}

[1] J. D. Bernardin, C. J. Stebbins, and I. Mudawar, "Mapping of Impact and Heat Transfer Regimes of Water Drops Impinging on a Polished Surface," Int. J. Heat Mass Transfer, vol. 40, no. 2, pp. 247267, Jan. 1997. doi: 10.1016/0017-9310(96)00119-6.

[2] K. P. Hapgood, J. D. Litster, S. R. Biggs, and T. Howes, "Drop Penetration into Porous Powder Beds," J. Colloid Interface Sci., vol. 253, no. 2, pp. 353-366, Sep. 2002. doi: 10.1006/jcis.2002.8527.

[3] C. T. Avedisian and J. Koplik, "Leidenfrost Boiling of Methanol Droplets on Hot Porous/Ceramic Surfaces," Int. J. Heat Mass Transfer, vol. 30, no. 2, pp. 379-393, Feb. 1987. doi: 10.1016/0017-9310(87)90126-8.

[4] S. Chandra and C. T. Avedisian, "Observations of Droplet Impingement on a Ceramic Porous Surface," Int. J. Heat Mass Transfer, vol. 35, no. 10 , pp. 2377-2388, Oct. 1992. doi: 10.1016/0017-9310(92)90080-C.

[5] M. Abu-Zaid, "An Experimental Study of the Evaporation of Gasoline and Diesel Droplets on Hot Surfaces," Int. Commun. Heat Mass Transfer, vol. 21, no. 2, pp. 315-322, Mar. 1994. doi: 10.1016/0735-1933(94)90029-9.

[6] W. S. Kim and S. Y. Lee, "Behavior of a Water Drop Impinging on Heated Porous Surfaces," Exp. Therm. Fluid Sci., vol. 55, pp. 6270, May 2014. doi: 10.1016/j.expthermflusci.2014.02.023.

[7] G. Liang and I. Mudawar, "Review of Drop Impact on Heated Walls," Int J. Heat Mass Transfer, vol. 106, pp. 103-126, Mar. 2017. doi: 10.1016/j.ijheatmasstransfer.2016.10.031

[8] H. Hu and R. G. Larson, "Evaporation of a Sessile Droplet on a Substrate," J. Phys. Chem. B, vol. 106, no. 6, pp. 1334-1344, Feb. 2002. doi: 10.1021/jp0118322.

[9] MakerBot. Safety Data Sheet: PLA 3D Printer Filament/MakerBot PLA [Online]. Available: https://images.makerbot.com/support/ production/SDS-000002ENA.pdf. [Accessed 8 Sept 2017]. 


\section{Computational Fluid Dynamics Simulations of flow in the Renal Arteries After Stent Graft Implantation}

\author{
Tianyi Xia, Matthew G. Doyle \\ Dept. of Mechanical and Industrial \\ Engineering, University of Toronto \\ Toronto, Canada
}

\author{
Thomas L. Forbes \\ Division of Vascular Surgery, \\ UHN, and University of Toronto \\ Toronto, Canada
}

\author{
Cristina H. Amon \\ Dept. of Mech. and Industrial Eng., \\ and IBBME, University of Toronto \\ Toronto, Canada
}

\begin{abstract}
The objective of this work is to report a computational fluid dynamics study assessing the hemodynamic effects of fenestration misalignment, towards understanding post-surgical complications of fenestrated endovascular aneurysm repair for abdominal aortic aneurysms. Idealized models were constructed based on geometries from a patient with an infrarenal aortic aneurysm. Fenestrated stent grafts were simulated in the models, with combinations of different fenestration misalignments and takeoff angles. Computational fluid dynamics simulations were performed by solving the governing equations for blood flow under physiologically realistic boundary conditions. Hemodynamic results of renal artery flow rate and time-averaged wall shear stresses were analyzed to build connections between the degree of fenestration misalignment, the takeoff angle, and changes in flow dynamics.
\end{abstract}

Keywords-abdominal aortic aneurysms; computational fluid dynamics; fenestrated endovascular aneurysm repair; hemodynamics; time-averaged wall shear stress

\section{INTRODUCTION}

An abdominal aortic aneurysm (AAA) is a weakening and progressive enlargement of the aorta, which is the body's main artery. If left untreated, AAAs will continue to grow and potentially rupture, leading to death in $80 \%$ to $90 \%$ of cases [1]. Fenestrated endovascular aneurysm repair (FEVAR) is a minimally invasive treatment option for AAAs that involve the branch vessels such as the renal arteries. FEVAR involves inserting a fenestrated stent graft into the aorta at the site of the aneurysm and can include additional stenting of the branch vessels. FEVAR has been continuously evolving over the past two decades [2]; however, post-surgical complications including acute kidney injury (AKI) and renal artery thrombosis [3] were reported, and in some cases, at greater rates than for open aneurysm repair [3], leading to secondary interventions and renal function deterioration [4]. Some cases of post-surgical renal dysfunction were associated with aortic stent graft misalignment [4], where the fenestration was not aligned with the renal artery ostium; however, associations between fenestration misalignment and post-surgical complications have not been investigated in vivo due to difficulties with data acquisition [5].
As a first step towards understanding post-surgical complications of FEVAR, including AKI and renal artery thrombosis, this study aims to use computational fluid dynamics (CFD) to determine how fenestration misalignment impacts renal artery hemodynamics. Two factors, renal artery flow rate and renal artery time-averaged wall shear stress (TAWSS) were adopted as hemodynamics indices [6-10] to indicate the risks of $\mathrm{AKI}$ and renal thrombosis.

\section{METHODS}

\section{A. Geometry}

An idealized 3-D computational model of a post-surgical AAA was built using ANSYS DesignModeler (ANSYS Inc., Canonsburg, PA, USA). The lengths and diameters of the vessels were taken from the geometry of a patient with an infrarenal aortic aneurysm [11]. The patient was treated with endovascular aneurysm repair instead of FEVAR. However, this prototype was chosen due to availability of both the AAA geometry and physiological boundary conditions. It is assumed that neither the post-surgical model boundary conditions nor the post-surgical geometry changes with aortic aneurysm type. Taking advantage of the assumed symmetry between the left and right sides of the model, a half-symmetric geometry was adopted, including half of the aorta, one renal artery, and one iliac artery (Fig. 1). A fenestrated stent graft was then simulated in the model, with a flared renal stent that opens in the renal artery.

Both a reduction of the effective area of the renal artery orifice and the takeoff angle (TOA) between the aorta and the renal artery were considered in our model. Reduction of effective area on the proximal end of renal artery is caused by fenestration misalignment [4]. TOA is defined as the angle between the centreline of the renal stent and centreline axis of aorta [4].

A total of 15 cases were simulated, consisting of all combinations of five different fenestration alignments and three different TOAs. A fully aligned case, along with four cases of misalignment were considered. For the misaligned cases, the fenestration was positioned either $2.8 \mathrm{~mm}$ above or $2.8 \mathrm{~mm}$ below the proximal end of the renal artery, referred to as 'vertical misalignment', or rotated relative to the proximal end of the renal artery by either $17.5^{\circ}$ clockwise or $17.5^{\circ}$ counterclockwise, referred to as 'horizontal misalignment' 

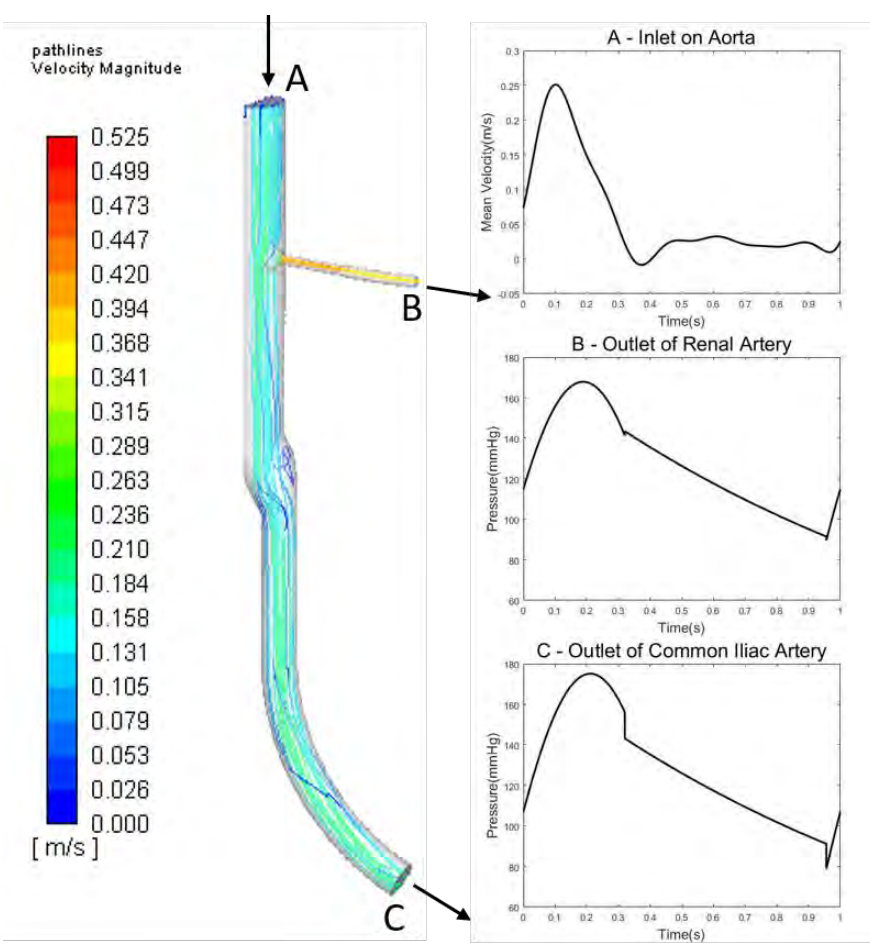

Figure 1. Post-surgical model with boundary conditions and velocity pathlines

(Fig. 2). These four misalignment cases were designed to have the equivalent of $45 \%$ effective area reduction in the proximal end of renal artery.

For the aligned case and each misaligned case, three different TOA were considered: $87.25^{\circ}$ to model the average $\mathrm{TOA}$, and $60.95^{\circ}$ and $113.55^{\circ}$ to model the maximum and minimum TOAs (Fig. 3), as reported by Jiale et al. [4].

\section{B. Boundary Conditions}

Patient-specific flow data were considered to define the inlet and outlet boundary conditions. Supraceliac-level flow rates, acquired from cine phase-contrast magnetic resonance imaging (PC-MRI) [11], were adapted to a Womersley velocity profile [12] applied at the inlet.

For the outlets of the renal and iliac arteries, 2-element Windkessel models [13] were used. These models account for arterial compliance and total peripheral resistance through analogies between fluid flow and electric circuits. The parameters for these models were fit to pressure data for the renal and iliac artery, approximated from brachial pressure cuff measurements immediately after the PC-MRI scan (Fig. 4) [11].

No-slip boundary conditions were specified for all vessel and stent graft walls, which were assumed to be rigid. A symmetry boundary condition was applied to the centre of the aorta. To delineate the effects of fenestration misalignment on renal artery flow, identical boundary conditions were defined for all simulations. Blood was assumed to be non-Newtonian fluid based on Quesmada viscosity model.
A

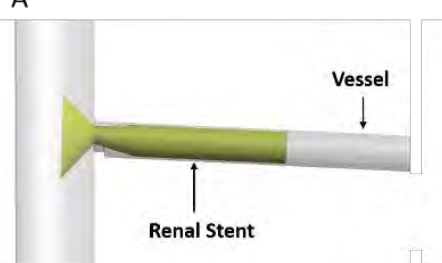

B

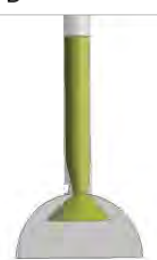

Figure 2. Post-surgical geometry with (A) vertical misalignment; and (B) horizontal misalignment.

A

C

C

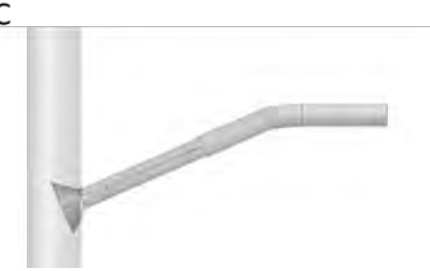

Figure 3. (A) Minimum (60.95 $)$, (B) mean $\left(87.25^{\circ}\right)$, and (C) maximum $\left(113.55^{\circ}\right)$ takeoff angles

\section{Numerical Analysis}

Simulation of pulsatile flow in this 3-D post-surgical geometry were performed by solving the continuity and momentum equations using a finite volume approach in ANSYS FLUENT v. 18.

The computational geometries were discretized by tetrahedral patch-conforming methods with advanced functions of proximity and curvature in ANSYS ICEM CFD. Mesh layers were refined on the walls of the renal arteries to accurately resolve the renal artery WSS.

Mesh independence studies were carried out for two steady state simulations, conducted at $t=0.1 \mathrm{~s}$, corresponding to the maximum inlet velocity, and $t=0.5 \mathrm{~s}$, corresponding to a low inlet velocity and high outlet pressure. With the number of elements increasing by $50 \%$ for each refinement, 6 different mesh sizes were applied on each geometry, and grid independence was deemed to be reached when both the change of renal artery flow rate and the change of renal artery WSS were within $\pm 5 \%$ between two successive meshes. Among the 15 studied geometries, element numbers required to achieve grid independence varied from 199,708 to 450,033 .

Relative residuals of continuity and velocities in the $x, y$, and $z$ coordinates were set to $1 \times 10^{-4}$. Based on monitoring the Courant number in pulsatile flow simulations, a uniform $0.02 \mathrm{~s}$ time-step was adopted. As suggested by Kandail et al. [7], simulations for all geometries were performed for four cardiac cycles to achieve periodic results. 


\section{RESULTS AND DISCUSSIONS}

Renal artery flow rate and renal artery TAWSS were used in our study as indices of risks of AKI and renal thrombosis, respectively. A decrease of renal artery flow rate leads to a decrease in kidney blood supply; hence it is an indicator of potential AKI. A decrease in renal TAWSS is suggestive of increased chance of renal thrombosis according to the Low Shear Theory [6].

\section{A. Comparison of Renal Artery Flow Rate between Misaligned and Aligned Cases}

Renal artery flow rates between cases with aligned and misaligned fenestrations were compared to determine the influence of fenestration misalignment on the amount of blood flow to the kidney. As is summarized in Table 1 and Fig. 4, a decrease of renal artery flow rate was observed by comparing flow rates in the misaligned models and the aligned model with the same TOA. The maximum decrease was found in the case where the fenestration was $2.8 \mathrm{~mm}$ below the proximal renal artery with a TOA of $60.95^{\circ}(11.0 \%)$, while the minimum was found where the fenestration was $2.8 \mathrm{~mm}$ above the renal artery with a TOA of $113.55^{\circ}(7.1 \%)$. Fenestration misalignment caused a decrease of renal artery flow rate in all studied renal TOAs, hence an increased opportunity for post-surgical renal AKI.

\section{B. Comparison of Renal Flow Rate between Different TOAs}

Results of renal flow rates for different TOAs were studied to determine the influence of renal artery position on renal flow rate. As shown in Fig. 4, for each group with the same alignment, the case with the $60.95^{\circ} \mathrm{TOA}$ had a higher renal flow rate than the case with the $87.25^{\circ} \mathrm{TOA}$; while the case with the $113.55^{\circ}$ TOA had the lowest renal artery flow rate. Within misaligned cases, by comparing renal flow rates of cases with $133.55^{\circ}$ TOAs to cases with $60.95^{\circ}$ TOAs, the maximum decrease of flow rate was found in the case where the fenestration was rotated $17.5^{\circ}$ clockwise $(18.0 \%)$, while the minimum was found where the fenestration was $2.8 \mathrm{~mm}$ below the renal artery (17.4\%). Comparing to the percentage changes due to misalignment effects on renal flow rate (maximum of $11.0 \%$ ), change of TOA had a larger effect on the decrease of renal flow rate.

\section{Comparison of Renal TAWSS between Misaligned and Aligned Cases}

Renal artery TAWSS between cases with aligned and misaligned fenestrations were compared to delineate the influence of fenestration misalignment on TAWSS. As is summarized in Table 2 and Fig. 5, decrease of renal artery TAWSS was observed by comparing cases of the misalignment models against the aligned models with constant TOAs. The maximum percentage decrease was found in the case where the fenestration was $2.8 \mathrm{~mm}$ below the renal artery with a TOA of $87.25^{\circ}(21.0 \%)$, while the minimum was found where the fenestration was rotated $17.5^{\circ}$ counterclockwise with a TOA of $60.95^{\circ}(17.7 \%)$.

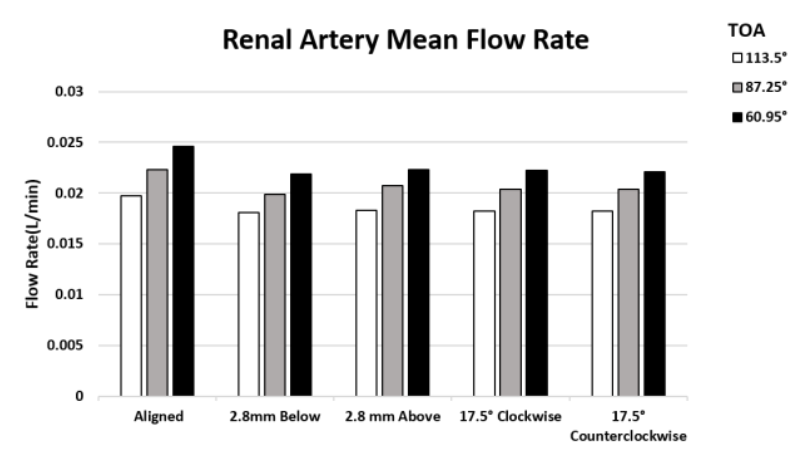

Figure 4. Renal artery flow rate $(\mathrm{L} / \mathrm{min})$ in different misalignment cases.

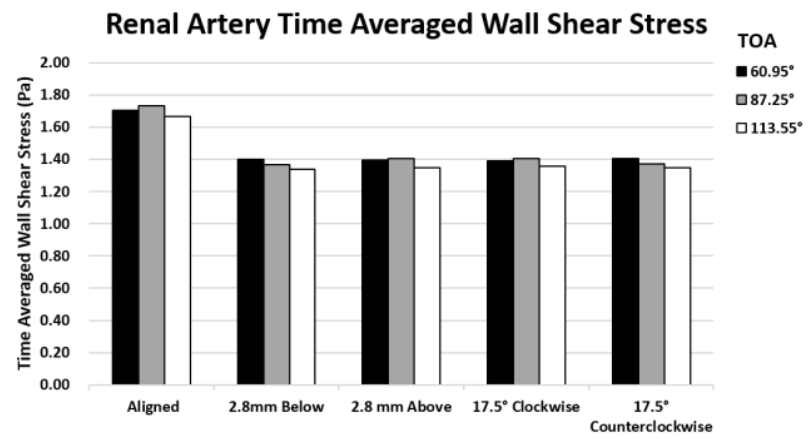

Figure 5 Renal artery time averaged wall shear stress in different misalignment cases.

In our study, we showed that fenestration misalignment and changes in TOA cause changes in renal hemodynamics, including the decrease of renal flow rate and renal TAWSS, which are suggestive of increased risk for post-surgical complications, including AKI and renal thrombosis.

Georgakarakos et al. [14] studied the impact of fenestration misalignment on renal WSS, but without considering the flaring of the renal stents or the reduction of the effective area of the proximal renal stent. In our institution, flaring of the renal stent is standard practice during FEVAR. Kandail et al. [7, 15] simulated blood flow with different TOAs and studied effects of flaring stent graft, but without considering either reduction of effective area or its combined effect with changes in TOA.

We were surprised to find that misalignment cases with constant reduction of effective renal artery area had different reductions in renal artery flow compared to the aligned case. Within the 2 cases of vertical misalignment (the fenestration above or below the renal artery) and the 2 cases of horizontal misalignment (the fenestration rotated either clockwise or counterclockwise), the case with the fenestration below the renal artery caused the largest decrease in renal artery flow, while the case with the fenestration above the renal artery caused the smallest decrease. A major limitation of our research is the lack of patient-specific geometries. Although we have previously validated our idealized model by comparing renal artery flow rates in our model with data reported in literature by Figueroa et al. [11], a more precise hemodynamic result would be obtained by studying models with patient-specific geometries applied with the same boundary conditions. 
TABLE I. Mean Flow Rate of Different Takeoff Angles and Alignement Cases

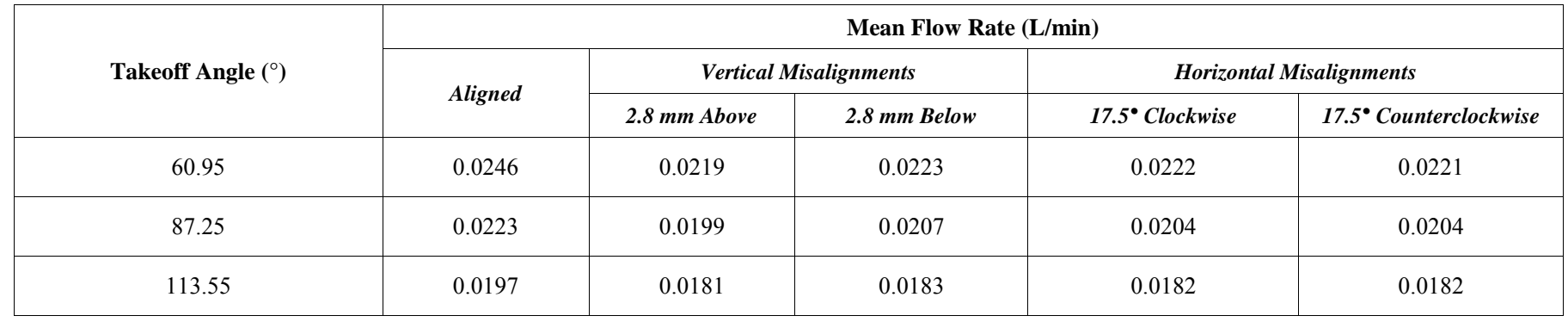

TABle II. Time Averaged Wall Shear Stress in Different TaKeoff ANGle and Alignment Cases

\begin{tabular}{|c|c|c|c|c|c|}
\hline \multirow{3}{*}{ Takeoff Angle $\left(^{\circ}\right)$} & \multicolumn{5}{|c|}{ Time Averaged Wall Shear Stress (Pa) } \\
\hline & \multirow{2}{*}{ Aligned } & \multicolumn{2}{|c|}{ Vertical Misalignments } & \multicolumn{2}{|c|}{ Horizontal Misalignments } \\
\hline & & $2.8 \mathrm{~mm}$ Above & $2.8 \mathrm{~mm}$ Below & $17.5^{\circ}$ Clockwise & $17.5^{\circ}$ Counterclockwise \\
\hline 60.95 & 1.7043 & 1.3993 & 1.3964 & 1.3931 & 1.4034 \\
\hline 87.25 & 1.7305 & 1.3669 & 1.4041 & 1.4051 & 1.3706 \\
\hline 113.55 & 1.6667 & 1.3396 & 1.3465 & 1.3556 & 1.3483 \\
\hline
\end{tabular}

The study has built connection between fenestration misalignments and changes in hemodynamics, indicating that FEVAR with misalignment occurring could cause further renal dysfunction and possible post-operative impairment [4]. At the clinical level, it is suggested that closer attention should be put on possible fenestration misalignments when determining therapeutic strategies, and at the same time, more attention should be placed on studies to reduce misalignments.

\section{CONCLUSIONS}

Our study clearly showed that fenestration misalignments caused decreases of hemodynamic indices including renal artery flow rate and renal TAWSS. In the perspective of renal artery flow rate, among misalignments with same reduction of effective area, the case with the fenestration $2.8 \mathrm{~mm}$ below the renal artery caused the highest decrease in renal flow rate, while the case with the fenestration $2.8 \mathrm{~mm}$ above caused the lowest decrease of the index. The TOA also affects indices of renal artery flow rate. Cases with TOAs of $113.55^{\circ}$ had the lowest renal flow rate while cases with TOAs of $60.95^{\circ}$ had the highest. TOA has larger effects than misalignments on renal artery flow rate.

\section{REFERENCES}

[1] A. N. Assar and C. K. Zarins, "Ruptured abdominal aortic aneurysm: a surgical emergency with many clinical presentations," Postgrad. Med. J., vol. 85, pp. 268-273, 2009. doi: 10.1136/pgmj.2008.074666.

[2] M. G. A. Norwood, G. M. Lloyd, M. J. Bown, G. Fishwick, N. J. London, and R. D. Sayers, "Endovascular abdominal aortic aneurysm repair," Postgrad. Med. J., vol. 83, pp. 21-27, 2007.

doi: $10.1136 /$ pgmj.2006.051177.

[3] A. N. Saratzis, M. F. Bath, S. C. Harrison, R. D. Sayers, and M. J. Bown, "Impact of fenestrated endovascular abdominal aortic aneurysm repair on renal function," Nephrol. Dial. Transplant., vol. 30, pp. 889-896, 2015. doi: 10.1177/1526602815605311.

[4] J. Ou, Y. C. Chan, C. Y. T. Chan, and S. W. Cheng, "Geometric alteration of renal arteries after fenestrated grafting and the impact on renal function," Ann. Vasc. Surg., vol. 41, pp. 89-95, 2017. doi: 10.1016/j.avsg.2016.09.036.

[5] L. R. de Souza and G. S. Oderich, "Renal function deterioration in complex aortic repair," in Endovascular Aortic Repair: Current Techniques with Fenestrated, Branched and Parallel Stent-grafts, G. S. Oderich, Ed. Springer, 2017, pp. 726-728.

[6] V. Peiffer, S. J. Sherwin, and P. D. Weinberg, "Does low and oscillatory wall shear stress correlate spatially with early atherosclerosis? A systematic review," Cardiovasc. Res., vol. 99, pp. 242-250, 2013. doi: $10.1093 / \mathrm{cvr} / \mathrm{cvt} 044$.

[7] H. Kandail, M. Hamady, and X. Y. Xu, "Comparison of blood flow in branched and fenestrated stent-grafts for endovascular repair of abdominal aortic aneurysms," J. Endovasc. Ther., vol. 22, pp. 578-590, 2015. doi: 10.1177/1526602815587261. 
[8] D. Bluestein, L. Niu, R. T. Schoephoerster, and M. K. Dewanjee, "Steady flow in an aneurysm model: correlation between fluid dynamics and blood platelet deposition," J. Biomech. Eng., vol. 118, p. 280, 1996.

doi: $10.1115 / 1.2796008$.

[9] V. L. Rayz, L. Boussel, M. T. Lawton, G. Acevedo-Bolton, L. Ge, W. L. Young, R. T. Higashida, and D. Saloner, "Numerical modeling of the flow in intracranial aneurysms: prediction of regions prone to thrombus formation," Ann. Biomed. Eng., vol. 36, pp. 1793-1804, 2008.

doi: 10.1007/s10439-008-9561-5.

[10] M. Jackson et al., "Low wall shear stress predicts subsequent development of wall hypertrophy in lower limb bypass grafts," Artery Res., vol. 3, pp. 32-38, 2009. doi: 10.1016/j.artres.2009.01.001.

[11] C. A. Figueroa, C. A. Taylor, V. Yeh, A. J. Chiou, and C. K. Zarins, "Effect of curvature on displacement forces acting on aortic endografts: a 3dimensional computational analysis," J. Endovasc. Ther., vol. 16, pp. 284-294, 2009. doi: 10.1583/08-2667.1.

[12] J. R. Womersley, "Method for the calculation of velocity, rate of flow and viscous drag in arteries when the pressure gradient is known," $J$. Physiol. (Lond.), vol. 127, pp. 553-563, 1955.

doi: 10.1113/jphysiol.1955.sp005276.

[13] D. Chemla, E. M. Lau, Y. Papelier, P. Attal, and P. Hervé, "Pulmonary vascular resistance and compliance relationship in pulmonary hypertension," Eur. Respir. J., vol. 46, pp. 1178-1189, 2015

doi: 10.1183/13993003.00741-2015.

[14] E. Georgakarakos, et al., "The hemodynamic impact of misalignment of fenestrated endografts: A computational study," Eur. J. Vasc. Endovasc.

Surg., vol. 47, pp. 151-159, 2014. doi: 10.1016/j.ejvs.2013.09.029.

[15] H. Kandail, M. Hamady and X. Y. Xu, "Effect of a flared renal stent on the performance of fenestrated stent-grafts at rest and exercise conditions," J. Endovasc. Ther., vol. 23, pp. 809-820, 2016.

doi: $10.1177 / 1526602816651425$. 


\title{
Numerical Simulation of an Over-Expanded Supersonic Nozzle Flow
}

\author{
Faisal Al Qurooni ${ }^{1}$, Ali Vakil ${ }^{1,2}$, Ehab Elsaadawy ${ }^{3}$, Sheldon I. Green ${ }^{1}$ \\ ${ }^{I}$ Department of Mechanical Engineering, The University of British Columbia - Vancouver \\ 6250 Applied Science Ln, Vancouver, BC, Canada V6T $1 Z 4$ \\ ${ }^{2}$ Coanda Research and Development Corporation \\ 6741 Cariboo Rd, Burnaby, BC, Canada, V3N $4 A 3$ \\ ${ }^{3}$ Saudi Aramco, Research \& Development Center, Dhahran 31311, KSA \\ Email:faisal90@alumni.ubc.ca(F.AlQurooni),alivakil@mech.ubc.ca(A.Vakil), \\ ehab.elsaadawy@aramco.com (E.Elsaadawy),Sheldon.Green@ubc.ca (S.I. Green)
}

\begin{abstract}
Flaring in oil and gas production is the controlled burning of unwanted exhaust gases to enhance safety. Obtaining complete combustion and thus eliminating or reducing smoke is critical to meet increasingly strict environmental regulations. To improve flare combustion, gas flares are equipped with air nozzles that introduce extra oxygen and improve mixing in the combustion zone. These nozzles are operated in the subsonic, sonic, or supersonic regimes. In this paper we are concerned with turbulence modeling of the jet flow exiting from a particular convergent-divergent nozzle used in flare systems. The Realizable $k-\varepsilon$ and SST $k-\omega$ models are used to study the compressible flow within that specific nozzle, which has an exit diameter of $3.38 \mathrm{~mm}$ and has convergent and divergent sections that are connected via a throat section with a finite length and constant diameter. The velocity profiles and turbulent kinetic energy predicted by both turbulence models, in the vicinity of the nozzle outlet and along the symmetry axis of the nozzle, are compared for nozzle pressure ratios in the range $1.18 \leq \mathrm{NPR} \leq 1.78$. It is shown that for $M a \leq 1$, both turbulence models predict nearly identical flow evolution along the nozzle. When the flow becomes supersonic, the shock surface, and consequently nozzle outlet velocity profiles, predicted by the SST $k-\omega$ model deviates slightly from the other model. The differences, however, become negligible a couple of diameters downstream of the nozzle outlet. Computed entrainment rate coefficients vary slightly when changing the turbulence model, and this difference remains insignificant with increasing downstream distance.
\end{abstract}

Keywords: CD nozzle flow, compressible flow, turbulence models, entrainment rate, gas flaring

\section{INTRODUCTION}

Gas flaring is a technique used in several industries such as gas and oil separation plants (GOSPs), oil refineries, gas plants and other petrochemical plants in which unwanted or excess gases are burnt safely. Gas flares are also used to protect the plants against the danger of over pressurizing in case of emergency or critical equipment trip. The gas flaring system is very important and plants operations should cease if it is not working properly. While gas flaring is required for safety reasons, it has many drawbacks, including negative impacts on the environment (primarily due to incomplete combustion of the gas) and the waste of potentially useful resources. To mitigate these negative impacts, companies seek to eliminate or reduce gas flaring. In situations when flaring is unavoidable, it is desirable to have smokeless gas flaring (Figure 1). Saudi Aramco, the largest oil company in the world, has made a thorough analysis and study [1] to investigate a cost effective and efficient solution to retrofit its hundreds of gas flares in Saudi Arabia. Several options were explored such as Steam-Assisted Flare tips, sonic flare tips and LowPressure Air-Assist. However, none of these solutions was deemed to be cost effective and, therefore Saudi Aramco developed a new flare design named the High Pressure Air Assist System (HPAAS). Briefly, the system consists of convergent-divergent nozzles that surround the circumference of the flare exit tip. As these nozzles inject compressed air to the combustion zone, the resulting air entrainment from the atmosphere due to the high exit velocity provides the required air mass to yield smokeless flaring (Figure 1). 
For a given nozzle shape, the flow controlling parameter is the nozzle pressure ratio (NPR). In converging nozzles $(\mathrm{CN})$, as the NPR increases, the maximum flow rate is achieved when the flow speed at the nozzle exit is sonic, corresponding to the choked flow condition. A converging-diverging nozzle geometry (CDN) allows the flow to achieve supersonic speeds. For a $\mathrm{CDN}$ that undergoes an isentropic expansion the sonic condition is reached at the throat connecting an upstream subsonic condition to a downstream supersonic condition. In contrast, an isentropic compression within the nozzle connects an upstream supersonic condition to a downstream subsonic condition via a sonic throat [2]. If the flow is choked at the throat but the NPR differs from that required to yield isentropic flow, a combination of normal and oblique shocks is formed downstream of the throat [3]. Numerical predictions of the compressible flow within $\mathrm{CDN}$, shocks behavior, and the compressible turbulent shear flow of supersonic jets has been subject of extensive studies in the last century.

Eggers [4] conducted an analytical and experimental investigation of a supersonic jet (Mach 2.22 nozzle) to understand turbulence quantities and jet entrainment. He concluded that an eddy viscosity formulation independent of the radial coordinate, employed by other researchers, for a compressible turbulent jet was not justified. Birch and Eggers [5] collated experimental data on developed free turbulent shear layers to facilitate the validation of the turbulence models and numerical predictions. They concluded that the available data were not sufficient to clearly establish the effect of density gradients on mixing rate. Lau et al. [6] conducted measurements of a $51 \mathrm{~mm}$ diameter turbulent jet at Mach 0.28, 0.90, and 1.37 using a LDV and a hot-wire anemometer. Their data indicate a decrease in the spreading rate of the mixing layer with increasing Mach number. Seiner et al. [7] investigated the effect of jet temperature on the mixing rate, and concluded that high temperatures resulted in enhanced density fluctuations to increase the mixing rate.

Reynolds-averaged Navier-Stokes (RANS) solvers have been used by many researchers to simulate supersonic exhausts. A comprehensive review of various turbulence modelling, advantages and limitations of each approach is presented in [8]. Koch conducted a study on subsonic axisymmetric jets with flow separation using a two-equation $k-\varepsilon$ turbulence model [9]. While the mean flow values showed a good agreement with experiment, the mixing rate predicted by the numerical modelling was lower than the experimental results, and so were the turbulent kinetic energy levels. To incorporate compressibility effects in turbulence models, and consequently to better predict the decrease in the growth rate with increasing Mach number, an additional compressibility term should be included in the turbulence transport equations $[10,11]$. Georgiadis et al. $[12,13]$ assessed the accuracy of modified two-equation turbulence models in flow field predictions of a subsonic (Mach number 0.5 ) heated and unheated jet. They showed that all the modified equations provided improved predictions compared to the standard models. However, all the models underestimated initial jet mixing rate and the turbulence kinetic energy fields.

Xiao et al. [14] examined experimentally and numerically the effect of both the nozzle expansion ratio and the NPR on the entrainment from CDN. The computations showed that the nozzle expansion ratio has a greater effect on the entrainment than the NPR. Among the RANS models, the SST $k-\omega$ model compared the best with experiment, which was attributed to its ability to predict the flow separation after the shock correctly. For a fixed nozzle expansion ratio of 1.5, their experimental study showed the best mixing was achieved when the NPR was in the range of 1.4-1.6. In a numerical study conducted on CDN with Mach number in the range $0.9<M a<1.2$ and NPR of 4 and 6, DalBello et al. [15] found that the SST $k-\omega$ model could match well with experimental measurements. The RANS models prediction accuracy decreases as the flow compressibility effects become more important. To account for these effects, modified RANS models were considered and their prediction performance were compared against experiment [16] [13].

Sarkar and Sarkar et al. [17, 18] studied compressible shear layers using a Reynolds Stress Model (RSM). When the compressibility effects were not considered explicitly, the model failed to predict the dependence of the shear layer growth rate on the Mach number. In comparison, when the compressibility effects were considered explicitly, the computed results compared better with experiment. Lijo et al. [19] used RSM to study transient flows in a rocket propulsion nozzle which involves free and restricted shock separation, and they found RSM model results were in good agreement with experiment. Balabel et al. [20] assessed RSM and different RANS models for gas flow in a two-dimensional CDN with steady, compressible and turbulent flow. In their investigation to predict the separation point and the shock wave location, they found that the SST $k-\omega$ model gave the best results when compared to experiment. The objective of this paper is to study the compressible turbulent flow picture within a specific converging- 
diverging nozzle with a finite length throat of constant area, and specifically to compare the nozzle flow field including the axial velocity, mass entrainment, Mach number and turbulent kinetic energy, predicted by different turbulence models.

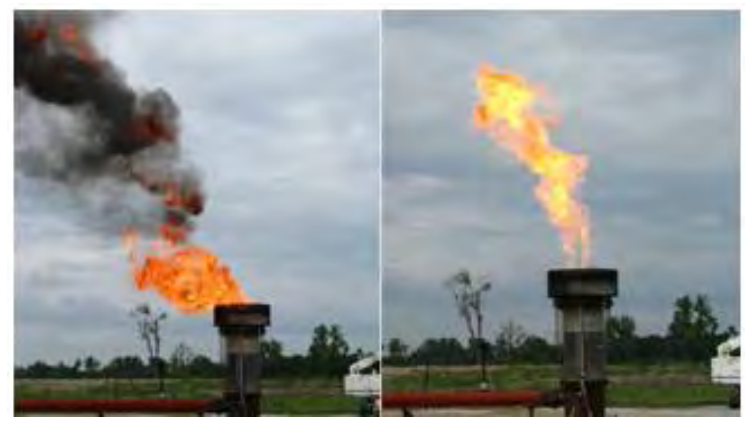

Figure 1: A flare with (right) and without (left) smokeless air, which shows the nozzles' advantage [1].

\section{NUMERICAL METHOD}

In this section, the numerical formulations employed to obtain the CFD results using ANSYS Fluent 18.0 are presented.

\subsection{Governing Equations}

The governing-equations require special treatment, i.e. closure, when the flow is turbulent. In the timeaveraging of the governing equations, the physical quantity $\phi$ is decomposed into its mean component $\bar{\phi}$ and fluctuating component $\phi^{\prime}$ via the Reynolds decomposition as $\phi=\bar{\phi}+\phi^{\prime}$. To account for the effects of the density fluctuations due to turbulence, Favre-averaging or density-weighed averaging is adopted in turbulent compressible flows. The densityweighted average of $\phi$ is obtained as $\phi=\tilde{\phi}+\phi^{\prime \prime}$, where $\tilde{\phi}=\overline{\rho \phi} / \bar{\rho}$. The details of the Favre averaging can be found in [21].

The mass-averaged conservation of mass in terms of mean velocity vector $\tilde{u}_{i}$ is given by Equation (1). The governing equations for the transport of momentum is given by Equation (2). $p$ is the mean pressure and related to mean temperature and density as $p=\bar{\rho} R \tilde{T}$. The viscous stress tensor $\tau_{i j}$ for an isentropic Newtonian fluid is defined as given by Equation (3) where $\delta_{i j}$ is the Kronecker delta function, and $\mu$ is the dynamic viscosity. The Reynolds stresses $\bar{R}_{i j}=$ $-\bar{\rho} \widetilde{u_{\imath}^{\prime \prime} u_{\jmath}^{\prime \prime}}$, arising from the Favre-averaging of the momentum equations, require modelling. One of the most common approaches to close the set of equations is to use the Boussinesq hypothesis to relate the Reynolds stresses to the mean deformation rates and the turbulent kinetic energy $k$ as given in Equation (4). The turbulent viscosity $\mu_{t}$ is given by $\mu_{t}=\rho C_{\mu} k^{2} / \varepsilon$, where $\varepsilon$ is the dissipation of turbulent kinetic energy and $C_{\mu}$ is a constant.

The Realizable $k-\varepsilon$ employs Equation (5) and Equation (6) as transport equations for $k$ and $\varepsilon$, respectively. $\bar{R}_{i j} \partial \tilde{u}_{i} / \partial x_{j}$ is the generation of turbulence kinetic energy due to the mean velocity gradients. $Y_{M}$ is the contribution of fluctuating dilation to the dissipation rate. $S=\sqrt{2 S_{i j} S_{i j}}$ is the modulus of the mean deformation rate tensor. $\sigma_{k}=1.0, \sigma_{\varepsilon}=$ 1.2 are, respectively, the turbulent Prandtl numbers for $k$ and $\varepsilon$. In contrast to the standard $k-\varepsilon$ model, $C_{\mu}$ is no longer a constant but rather depends on the mean deformation and rotation rates, the angular velocity of the rotation frame, and the turbulence quantities [22].

The SST $k-\omega$ employs Equations (8) and (9), respectively, as transport equations for $k$ and the specific dissipation rate $\omega . \sigma_{k}$ and $\sigma_{\omega}$ are the turbulent Prandtl numbers for $k$ and $\omega$, respectively. The turbulent viscosity is computed as $\mu_{t}=f \rho k / \omega$, where $f$ is a function of the strain rate magnitude, the specific dissipation rate, a limiter function, and a Reynolds number defined as $R e_{t}=\rho k / \mu \omega$. The details of the model constants can be found in [23]. This formulation accounts for the transport of the turbulence shear stress in the definition of the turbulent viscosity. The Favre-averaged energy equation is given by Equation (10). The turbulent flux $-\overline{\rho u_{\jmath}^{\prime \prime} h}$ is given based on the averaged enthalpy, turbulent Prandtl number, and the turbulent eddy viscosity as given in Equation (11). The Favreaveraged material derivative is defined in Equation (12).

\subsection{Meshing and Boundary Conditions}

Figure 2 shows the geometry of the specific nozzle studied in this paper. This geometry is used commonly in gas flares. The converging and diverging sections of this nozzle are connected via a throat with a constant diameter and a finite length. Figure 2 also shows the computational domain used for the simulations with the associated boundary conditions. The domain extends from $50 r_{\text {exit }}$ downstream of the nozzle exit, where $r_{\text {exit }}$ is the nozzle exit radius. The domain dimensions are given in Figure 2. The lateral walls, 
Table 1: List of Governing Equations

Conservation

of Mass

$$
\frac{\partial \bar{\rho}}{\partial t}+\frac{\partial}{\partial x_{i}}\left(\bar{\rho} \tilde{u}_{i}\right)=0
$$

$\frac{\partial}{\partial t}\left(\bar{\rho} \tilde{u}_{i}\right)+\frac{\partial}{\partial x_{j}}\left(\bar{\rho} \tilde{u}_{j} \tilde{u}_{i}\right)=-\frac{\partial p}{\partial x_{i}}+\frac{\partial}{\partial x_{j}}\left(\bar{\tau}_{i j}-\bar{R}_{i j}\right)$

Conversation

of

$$
\bar{\tau}_{i j}=\mu\left(\frac{\partial \tilde{u}_{i}}{\partial x_{j}}+\frac{\partial \tilde{u}_{j}}{\partial x_{i}}-\frac{2}{3} \frac{\partial \tilde{u}_{k}}{\partial x_{k}} \delta_{i j}\right)
$$

Momentum

Realizable

$k-\varepsilon$

$$
\begin{gathered}
\bar{R}_{i j}=-\bar{\rho} \widetilde{u_{\imath}^{\prime \prime} u_{\jmath}^{\prime \prime}}=\mu_{t}\left(\frac{\partial \tilde{u}_{i}}{\partial x_{j}}+\frac{\partial \tilde{u}_{j}}{\partial x_{i}}\right)-\left(\frac{2}{3} \rho k+\mu_{t} \frac{\partial \tilde{u}_{k}}{\partial x_{k}}\right) \delta_{i j} \\
\frac{\partial}{\partial t}(\bar{\rho} k)+\frac{\partial}{\partial x_{j}}\left(\bar{\rho} \tilde{u}_{j} k\right)=\frac{\partial}{\partial x_{j}}\left(\left[\mu+\frac{\mu_{t}}{\sigma_{k}}\right] \frac{\partial k}{\partial x_{j}}\right)+\bar{R}_{i j} \frac{\partial \widetilde{u}_{i}}{\partial x_{j}}-\rho \varepsilon-Y_{M} \\
\frac{\partial}{\partial t}(\bar{\rho} \varepsilon)+\frac{\partial}{\partial x_{j}}\left(\bar{\rho} \tilde{u}_{j} \varepsilon\right)=\frac{\partial}{\partial x_{j}}\left(\left[\mu+\frac{\mu_{t}}{\sigma_{\varepsilon}}\right] \frac{\partial \varepsilon}{\partial x_{j}}\right)+\rho C_{1} S \varepsilon-\rho C_{2} \frac{\varepsilon^{2}}{k+\sqrt{v \varepsilon}} \\
C_{1}=\max \left[0.43, \frac{\eta}{\eta+5}\right], \eta=S \frac{k}{\varepsilon}, C_{2}=1.9 \\
\mu_{t}=\rho C_{\mu} k^{2} / \varepsilon
\end{gathered}
$$

SST $k-\omega$

$$
\begin{gathered}
\frac{\partial}{\partial t}(\bar{\rho} k)+\frac{\partial}{\partial x_{j}}\left(\bar{\rho} \tilde{u}_{j} k\right)=\frac{\partial}{\partial x_{j}}\left(\left[\mu+\frac{\mu_{t}}{\sigma_{k}}\right] \frac{\partial k}{\partial x_{j}}\right)+\bar{R}_{i j} \frac{\partial \widetilde{u}_{i}}{\partial x_{j}}-\rho \beta^{*} k \omega \\
\frac{\partial}{\partial t}(\bar{\rho} \omega)+\frac{\partial}{\partial x_{j}}\left(\bar{\rho} \tilde{u}_{j} \omega\right)=\frac{\partial}{\partial x_{j}}\left(\left[\mu+\frac{\mu_{t}}{\sigma_{\omega}}\right] \frac{\partial \omega}{\partial x_{j}}\right)+\frac{\alpha \alpha^{*}}{v_{t}} \bar{R}_{i j} \frac{\partial \widetilde{u}_{i}}{\partial x_{j}}-\rho \beta \omega^{2}+\gamma \rho \frac{1}{\omega \sigma_{w, 2}} \frac{\partial k}{\partial x_{j}} \frac{\partial \omega}{\partial x_{j}} \\
\frac{\mu_{t}=f \rho k / \omega}{\partial t}(\bar{\rho} \tilde{h})+\frac{\partial}{\partial x_{j}}\left(\bar{\rho} \tilde{u}_{j} \tilde{h}\right)=\frac{\widetilde{D} \bar{p}}{\partial t}+\overline{u_{\jmath}^{\prime \prime} \frac{\partial p}{\partial x_{J}}}+\frac{\partial}{\partial x_{j}}\left(-\overline{\rho u_{\jmath}^{\prime \prime} h}\right)+\bar{\tau}_{i j} \frac{\partial \tilde{u}_{j}}{\partial x_{j}}+\overline{\tau_{l \jmath} \frac{\partial u_{J}^{\prime \prime}}{\partial x_{J}}}+\frac{\partial}{\partial x_{j}}\left(\frac{\mu}{P r} \frac{\partial \tilde{h}}{\partial x_{j}}\right)
\end{gathered}
$$

Conservation of Energy

$$
\begin{gathered}
-\overline{\rho u_{\jmath}^{\prime \prime h}}=\frac{\mu_{t}}{P r_{t}} \frac{\partial \tilde{h}}{\partial x_{j}} \\
\frac{\widetilde{D}}{D t}=\frac{\partial}{\partial t}+\tilde{u}_{j} \frac{\partial}{\partial x_{j}}
\end{gathered}
$$
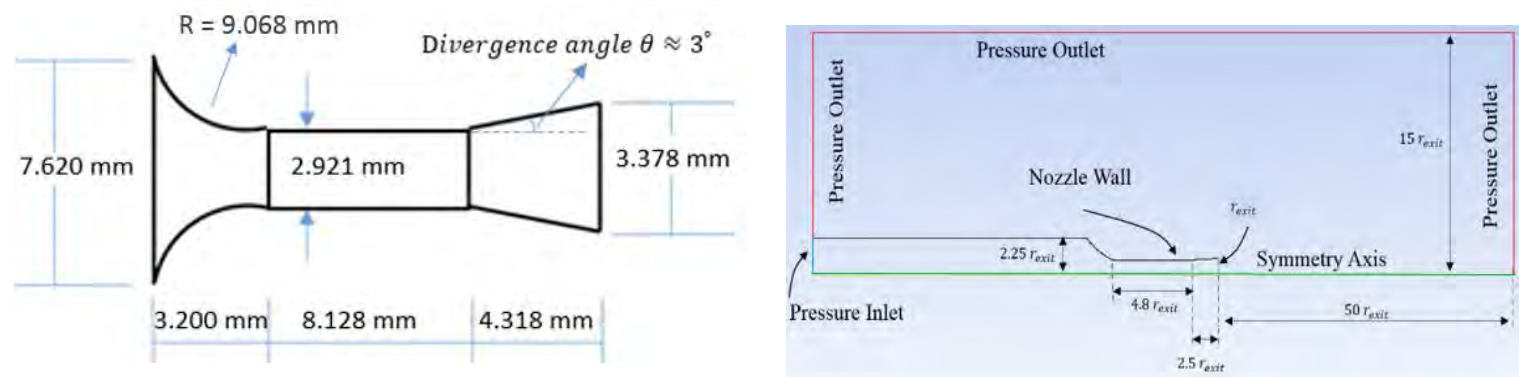

Figure 2: (Left) The geometry of the nozzle. (Right) Schematic of the computational domain used for the simulations with the associated boundary conditions; the dimensions of the computational domain are also shown in terms of nozzle exit radius $r_{\text {exit }}$ (not shown to scale). 
considered as the pressure outlet boundary condition, are extended to $15 r_{\text {exit }}$ to assure their impact on the jet flow is insignificant. The typical computational mesh used in the simulations, shown in Figure 3, highlights the small mesh size used in regions of high velocity gradients and around the shock location.

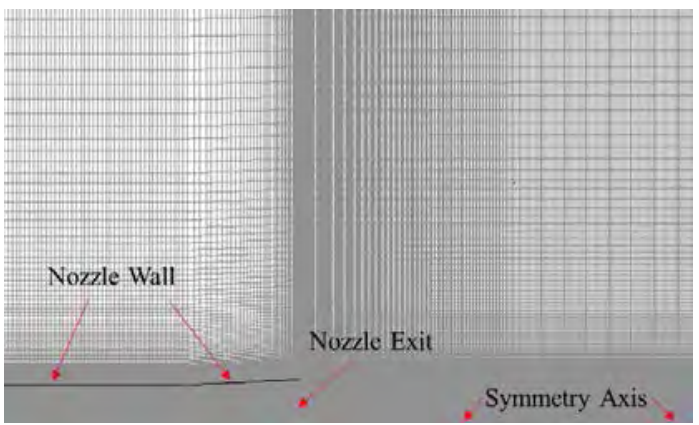

Figure 3: The typical computational mesh used in the simulation; the mesh around the nozzle exit is only shown for the sake of illustration.

\subsection{Mesh Independency Check}

To ensure that the simulations are mesh-independent, several flows were simulated with different mesh densities (number of cells was $1 \times 10^{5}, 1.5 \times 10^{5}$ and $3 \times 10^{5}$ for the coarse, medium and fine mesh, respectively). In the refinement procedure, we refined the boundary layer mesh close to the nozzle wall, the flow region around the jet shear layer, and the shock areas. Additionally, we slightly refined the mesh in the outer region of the flow. The velocity profiles at $\mathrm{x}=2.256 r_{\text {exit }}$ downstream of the nozzle exit for $\mathrm{P}_{\text {in }}=$ $160 \mathrm{kPaa}$ for different mesh sizes are shown in Figure 4. To estimate the discretization error, we followed the procedure recommended in [24] for the velocity profiles. The numerical uncertainty in the fine-grid solution was found to be $1.6 \%$ for the velocity profiles.

\subsection{D vs. 3D Comparison}

For a highly over-expanded flow within a converging -diverging nozzle, the flow is inherently not axisymmetric [25]. The complex pattern of the shock surfaces (oblique and normal shocks) is threedimensional, which means that the azimuthal derivatives of quantities should be accounted for in the governing equations. However, for the range of operating conditions of interest in this study, the flow remains attached to the nozzle walls and no flow separation was observed. These characteristics should permit one to use with reasonable accuracy a $2 \mathrm{D}$ axisymmetric model instead of full three-dimensional modelling. Using the 2D model avoids long computational times.

To verify that the $2 \mathrm{D}$ axisymmetric modelling is capable of capturing the shock surfaces and flow details, some $3 \mathrm{D}$ simulations were conducted to compare the simulations results with those of the corresponding $2 \mathrm{D}$ axisymmetric simulations. The SST $k-\omega$ turbulence model was chosen for both sets of simulations. The Mach number and the pressure coefficient profiles along the nozzle symmetry axis for the same upstream pressure are shown in Figure 5. The $2 \mathrm{D}$ profiles are almost identical to their 3D counterparts.

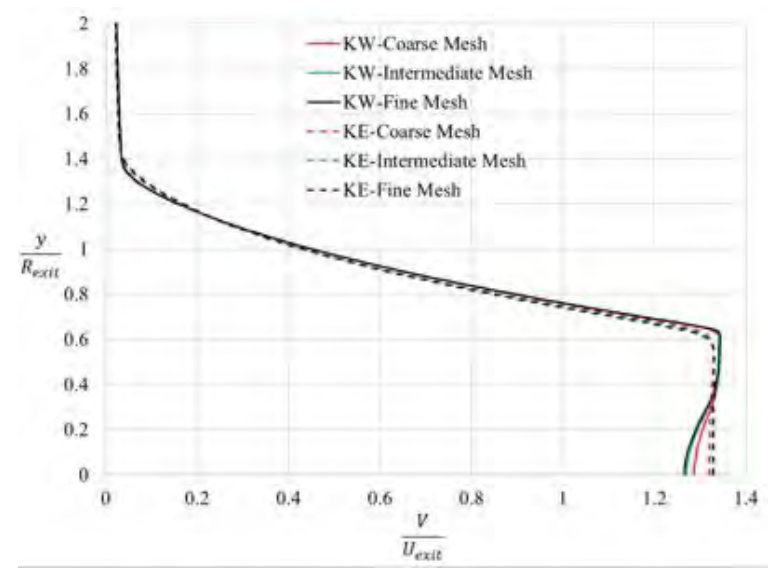

Figure 4: Velocity profile at $\mathrm{x}=2.256 \mathrm{r}_{\text {exit }}$ downstream of the nozzle exit for $P_{\text {in }}=160 \mathrm{kPaa}$.

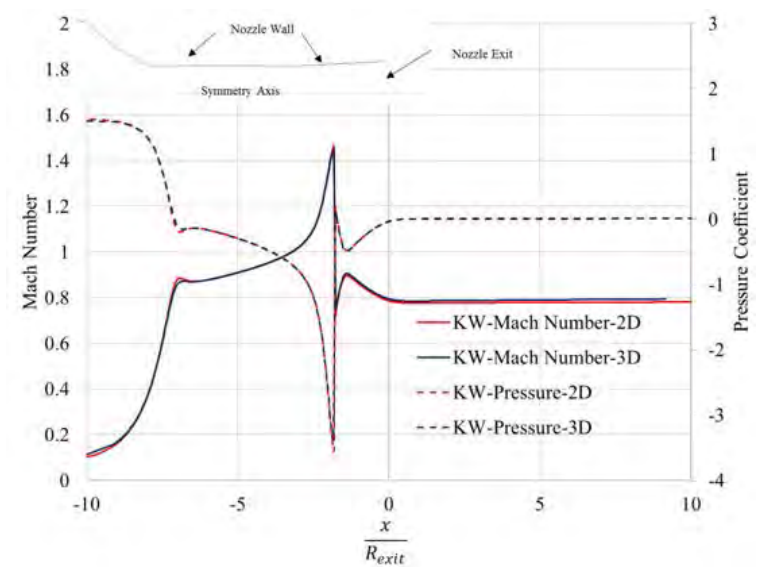

Figure 5: Mach number and pressure coefficient profiles along the nozzle symmetry axis for 2D axisymmetric and 3D simulation. The upstream pressure is $P_{\text {in }}=160 \mathrm{kPaa}$. 


\subsection{Model Validation against Experimental Data}

This section contains a comparison between the simulation results on the Acoustic Reference Nozzle (ARN) [26] from the ANSYS Fluent solver and those reported in [13] and [26]. The geometry details are reported in [26]. The jet is issued from a nozzle with an exit diameter of $50.8 \mathrm{~mm}$. The Setpoint 3, where the jet is unheated $\left(\mathrm{T}_{\mathrm{j}} / \mathrm{T}_{\infty}=0.950\right)$ and the compressibility effects $(\mathrm{Mach}=0.513)$ on the flow evolution could be insignificant, was chosen for the comparison reported here. The NPR of this point is 1.197 .

Figure 6 and Figure 7, respectively, show a comparison of the axial velocity and turbulent kinetic energy along the symmetry axis between the current simulations and those reported in [13] and [26]. In Figure 6, the present simulation shows a good agreement with both the experimental data and previous simulation [13] results.

Both the present simulation and the previous simulation [13] predict a longer potential core than that observed experimentally. The current simulation, however, predicts a potential core shorter than the previous simulation [13]. Furthermore, the current simulation predicts a decay rate faster than both the experimental results and the previous simulation [13]. On the other hand, the turbulent kinetic energy (TKE) predicted by the current simulation using the SST $k-$ $\omega$ turbulence model is in better agreement with experimental data than the previous simulations [13].

A comparison of the velocity profile at different positions downstream of the nozzle exit is shown in Figure 8. Very good agreement exists in the dimensions of the potential core and shear layer between the experimental data, the earlier simulation [13], and the current simulation. The above results show that the utilized modeling approach in this paper is adequate to capture the flow features of the flow field studies in this investigation, especially at Mach number of 0.5 .

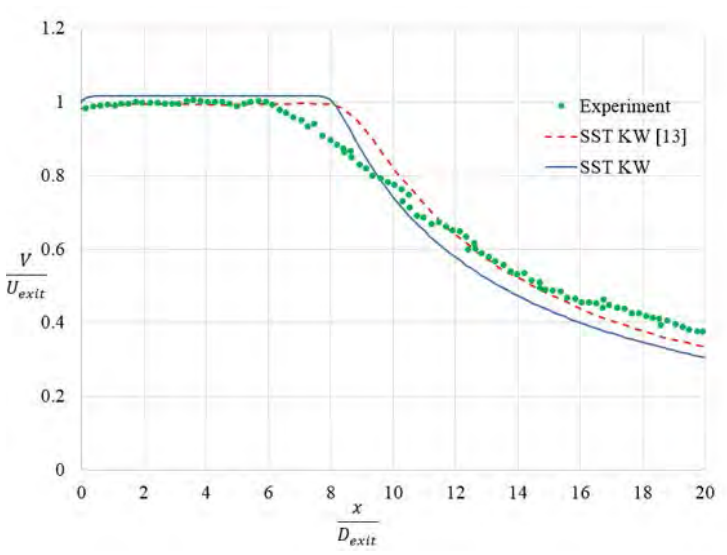

Figure 6: Comparison of axial velocity profile along the symmetry axis between current simulations, those in [13], and experiments [26].

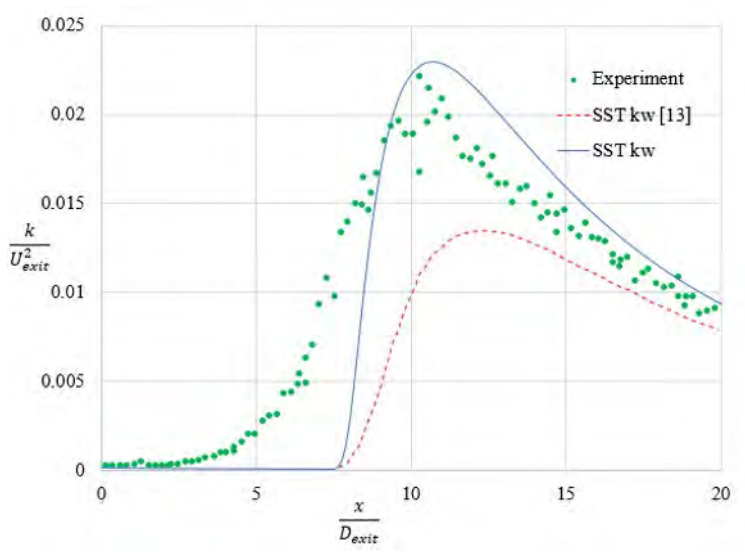

Figure 7: Comparison of turbulent kinetic energy profile along the axis of symmetry between current simulations, those in [13], and experiments [26].

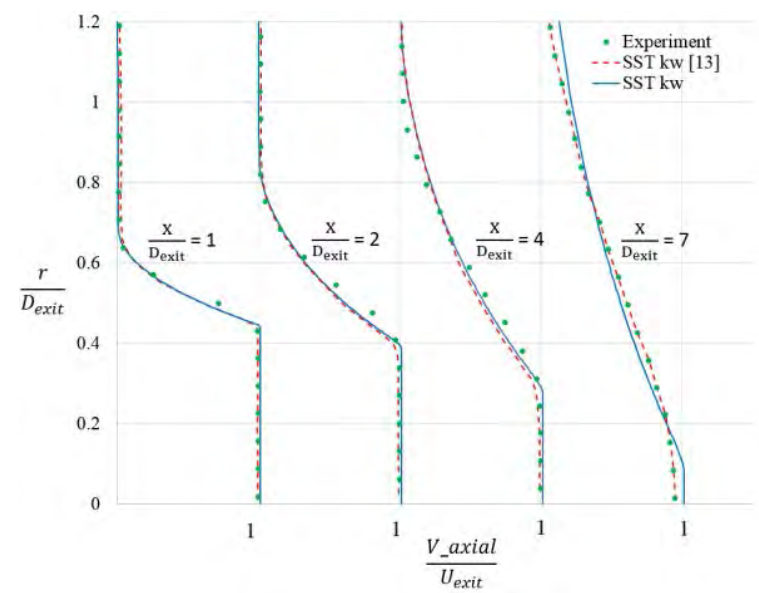

Figure 8: Comparison of velocity profile downstream of the nozzle exit between current simulations and results in [13] and [26]. 


\section{RESULTS AND DISCUSSIONS}

In this section, simulation results of the convergingconstant throat-diverging nozzle, as shown in Figure 2 , are presented. The nozzle has an exit to throat area ratio of 1.34. The flow fields are studied at different pressure ratios (NPR) in the range $1.18 \leq \mathrm{NPR} \leq$ 1.78 , corresponding to $120 \mathrm{kPa} \leq \mathrm{P}_{\text {in }} \leq 180 \mathrm{kPa}$. The downstream pressure is set to standard atmospheric pressure at sea level $(101 \mathrm{kPa})$. The working fluid is air. Velocity, TKE, and Mach number variations within and downstream of the nozzle are presented.

\subsection{Mean Axial Velocity Profiles}

Figure 9 shows a comparison of the normalized axial velocity profiles as computed by the different turbulence models for two upstream pressures: $\mathrm{P}_{\mathrm{in}}=$ $140 \mathrm{kPaa}$ and $\mathrm{P}_{\mathrm{in}}=160 \mathrm{kPaa}$. The profiles are extracted at two locations downstream of the nozzle exit, $\mathrm{x}_{1}=2.256 \mathrm{r}_{\text {exit }}$ and $\mathrm{x}_{2}=11.278 \mathrm{r}_{\text {exit }}$.

The normalized velocity profiles predicted by different turbulence models follow each other closely at $\mathrm{P}_{\text {in }}=140 \mathrm{kPaa}$; they start with a potential core close to the jet centerline and transition to the slow moving outer flow through a shear layer. The potential core at $\mathrm{x}=2.256 \mathrm{r}_{\text {exit }}$ exists in $0<\mathrm{y} / \mathrm{r}_{\text {exit }}<0.6$. The extent of the potential core in the cross-stream direction at $\mathrm{x}=11.278 \mathrm{r}_{\text {exit }}$ shrinks to $0<\mathrm{y} / \mathrm{r}_{\text {exit }}<$ 0.1 due to the mixing. The SST $\mathrm{k}-\omega$ model predicts a slightly faster decay in the potential core compared to the Realizable $\mathrm{k}-\varepsilon$ model. The shear layer is contained in $0.6<y / \mathrm{r}_{\text {exit }}<1.5$ at $\mathrm{x}=2.256 \mathrm{r}_{\text {exit }}$, while it has a far-reaching extent $0.1<\mathrm{y} / \mathrm{r}_{\text {exit }}<$ 3.5 at $\mathrm{x}=11.278 \mathrm{r}_{\text {exit }}$ (for comparison purposes the profiles at $\mathrm{x}=11.278 \mathrm{r}_{\text {exit }}$ are clipped to $\mathrm{y} / \mathrm{r}_{\text {exit }}=$ 2.0). The flow at this inlet pressure approaches sonic conditions at the throat.

At $\mathrm{P}_{\text {in }}=160 \mathrm{kPaa}$ the velocity profiles predicted by the Realizable $\mathrm{k}-\varepsilon$ model are similar to those at the lower pressure except that the potential core reaches a higher normalized peak velocity. The SST $\mathrm{k}-\omega$, however, predicts a velocity deficit in the near field in the potential core, which seems unphysical. This can be seen at the centerline $(\mathrm{y}=0)$ as velocity profile is dented backward between $0<y / r_{\text {exit }}<0.4$. Note that the flow within the nozzle at this pressure is supersonic (both turbulence models predict a peak Mach number of $\approx 1.45$ in the diverging section), where the compressibility effects play a critical role in the flow evolution. This velocity deficit, however, disappears in the far field (after $\mathrm{x} \geq 11.278 \mathrm{r}_{\text {exit }}$ ). In fact, both turbulence models predict almost the same normalized axial velocity profile in the far field at $x=11.278 r_{\text {exit }}$ regardless of the flow condition.
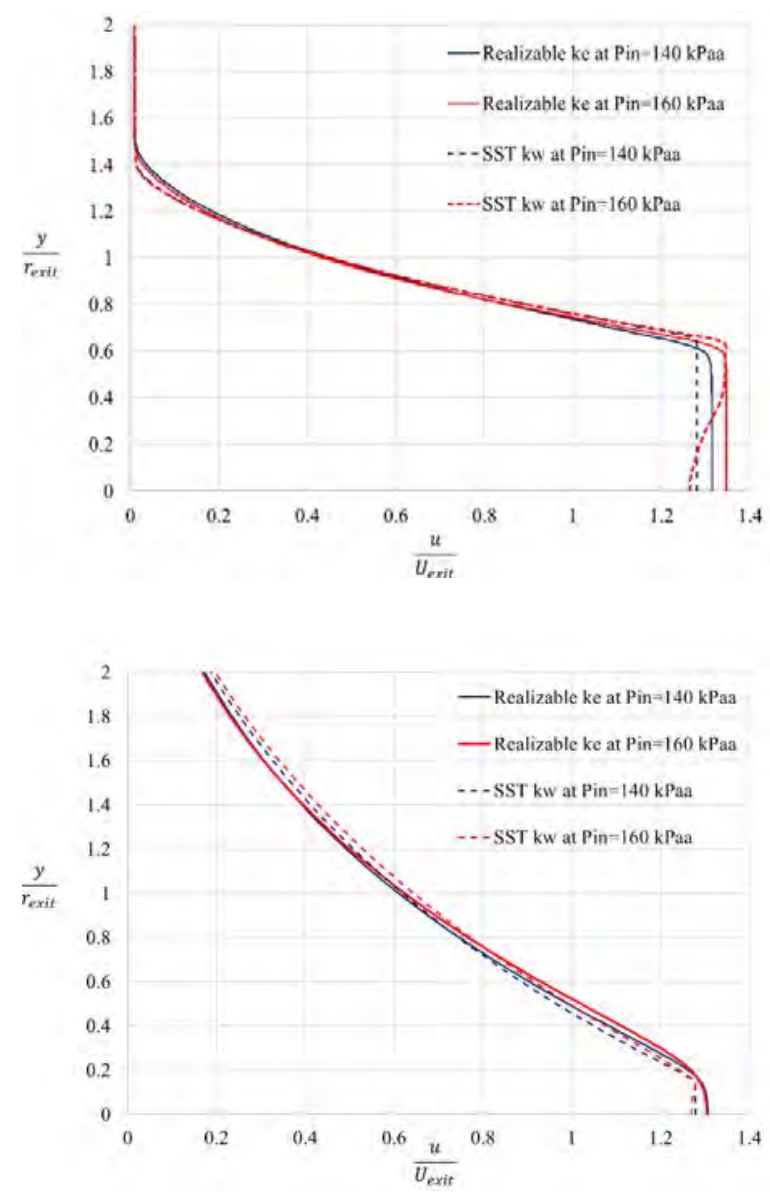

Figure 9: The normalized axial velocity profiles downstream of the nozzle exit for the different turbulence models; Extraction line is at (top) $\mathrm{x}=2.256 r_{\text {exit }} \quad$ (bottom) $\quad \mathrm{x}=11.278 r_{\text {exit }}$ downstream of the nozzle exit.

\subsection{Turbulent Kinetic Energy Profiles}

The normalized turbulent kinetic energy (TKE) along the axis of symmetry at $\mathrm{P}_{\text {in }}=140 \mathrm{kPaa}$ and $\mathrm{P}_{\text {in }}=$ $160 \mathrm{kPaa}$ is compared between different turbulence models in Figure 10. The transport of TKE to the jet centerline and the evolution of TKE predicted by the two turbulence model is almost the same. The Realizable $\mathrm{k}-\varepsilon$ model, however, predicts some turbulence upstream and downstream of the nozzle exit for $\mathrm{x} / \mathrm{r}_{\text {exit }} \lesssim 12$. This level of turbulence is absent in the predictions of the SST $\mathrm{k}-\omega$ model, which 
predicts almost zero turbulence along the symmetry axis up to $\mathrm{x} / \mathrm{r}_{\text {exit }} \approx 12$, where the potential core exists.

After the end of the potential core, in the shear layer region, the diffusion of turbulence reaches to its maximum value at a downstream distance of $\mathrm{x} / \mathrm{r}_{\text {exit }} \approx$ 18, where the normalized TKE graph attains a maximum value. This maximum value is slightly greater than 0.04 for the Realizable $\mathrm{k}-\varepsilon$ model and slightly less than 0.04 for the SST $\mathrm{k}-\omega$ model. The dissipation of the TKE after the peak point is slightly higher for the SST $\mathrm{k}-\omega$ model for $\mathrm{x} / \mathrm{r}_{\text {exit }}<22$, and thereafter the Realizable $\mathrm{k}-\varepsilon$ model predicts a slightly faster decay, and consequently more mixing, farther downstream.

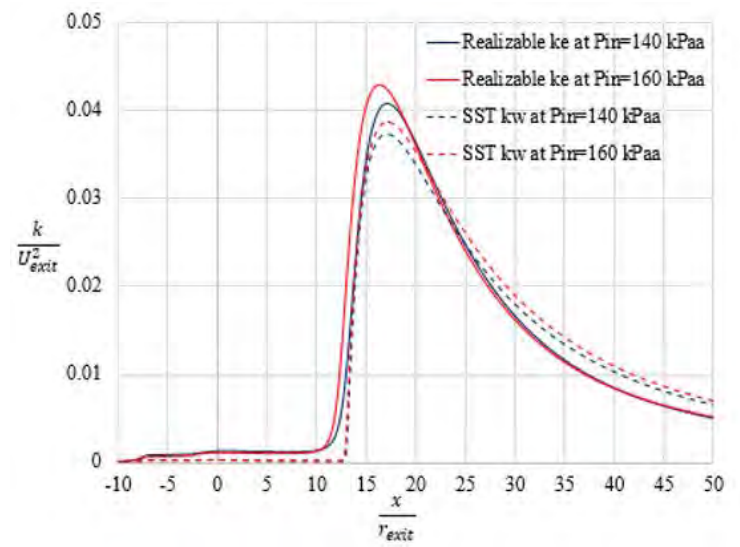

Figure 10: The normalized turbulent kinetic energy along the axis of symmetry predicted by the turbulence models at $P_{\text {in }}=140 \mathrm{kPaa}$ and $P_{\text {in }}=160 \mathrm{kPaa}$.

\subsection{Mach Number Profiles}

Figure 11 shows the Mach number profiles along the symmetry axis predicted by both turbulence models at $P_{\text {in }}=140 \mathrm{kPaa}$ and $P_{\text {in }}=160 \mathrm{kPaa}$. Generally, both turbulence models predict almost the same Mach number evolution inside and outside the nozzle. The location of the shocks in the nozzle (when $P_{\text {in }} \geq$ $160 \mathrm{kPaa}$ ) predicted by the two models are the same. In addition, both models show that the sonic flow condition along the centerline is always reached at the constant throat around $x / r_{\text {exit }}=-3.2$, just upstream of the divergent section (which starts at $\left.x / r_{\text {exit }}=-2.556\right)$.

However, when the flow is subsonic $\left(P_{\text {in }} \leq\right.$ $140 \mathrm{kPaa}$ ), the SST $k-\omega$ model predicts slightly higher flow velocities than the Realizable $k-\varepsilon$ model in the converging and constant-throat sections (between-9.3<x/ $r_{\text {exit }}<-2.6$ ). The difference disappears after that and the profiles perfectly match.

When the flow is supersonic ( $P_{\text {in }} \geq 160 \mathrm{kPaa}$ ), both turbulence models predict the same Mach number profiles inside the nozzle. Outside the nozzle, there are some slightly higher values predicted by the Realizable $\mathrm{k}-\varepsilon$ model within the potential core, i.e, $0 \leq x / r_{\text {exit }} \leq 10$, followed by a slightly earlier decay in velocity. The difference disappears after that and the profiles match. Another difference between the SST $k-\omega$ and Realizable $k-\varepsilon$ models is that the former model predicts a sharp velocity decay; whereas the latter model predicts a much smoother velocity decay at the end of the potential core.

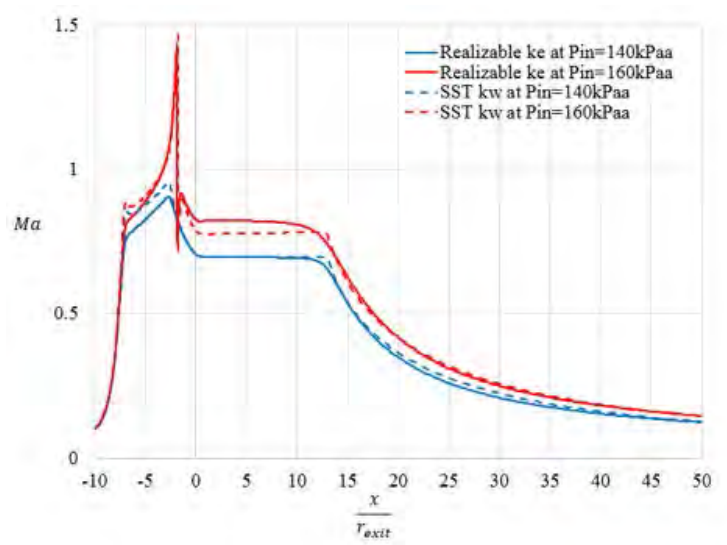

Figure 11: Mach number profile along the jet symmetry axis predicted by different turbulence model at $P_{\text {in }}=140 \mathrm{kPaa}$ and $\mathrm{P}_{\text {in }}=160 \mathrm{kPaa}$.

\subsection{Entrainment Rate Profiles}

This specific nozzle is used to provide gas flares with air to facilitate smokeless combustion. Therefore, it is important to quantify the variation of the mass entrainment along the jet centerline with the upstream pressure. The total mass flow rate through a given cross-section downstream of the nozzle is denoted by $M$ and the mass flow rate at the nozzle exit is denoted by $M_{\text {exit }}$. The entrainment rate coefficient, defined as $K_{e}=\frac{\mathrm{dM}}{\mathrm{dx}} \frac{r_{\text {exit }}}{M_{e}}\left(\frac{\rho_{e}}{\rho_{x}}\right)^{0.5}$ [27], where $\rho_{e}$ and $\rho_{x}$ are the initial and entrained fluid densities, respectively, increases linearly as shown in Figure 12. For sake of illustrations, $K_{e}$ graphs at lower pressure (where nozzle flow is subsonic with $0.5<M a<0.9$ ) and higher pressure $(1.5<M a<2.0)$ are not shown on the graph. However, the normalized entrainment graphs at operating pressures of $120 \mathrm{kPaa} \leq \mathrm{P}_{\text {in }} \leq$ $180 \mathrm{kPaa}$ almost collapse on the presented graphs with insignificant difference. 


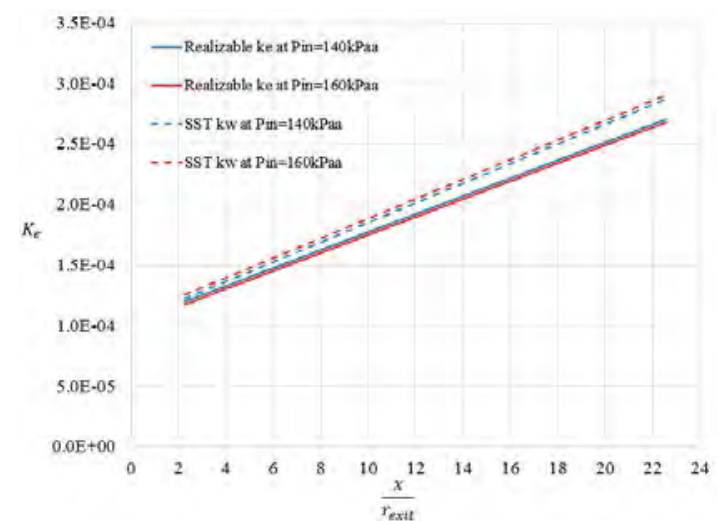

Figure 12: The entrainment rate coefficient $K_{e}$ as a function of distance along the jet for different turbulence models.

\section{CONCLUSIONS}

Numerical studies of compressible flow in a specific converging diverging nozzle were conducted. The nozzle consists of converging and diverging sections connected via a throat section with a finite length and constant diameter. The jet generated by the nozzle is used to control the performance of typical industrial flare systems by entraining the right amount of air into the flame to assure a smokeless flame. The numerical studies characterized the nozzle performance at various pressure ratios (NPR) in the range $1.18 \leq$ NPR $\leq 1.78$ for two different turbulence models. The mean axial velocity profiles at different streamwise locations, mass entrainment rates, the turbulent kinetic energy and Mach number along the jet centerline were presented.

In the case of subsonic nozzle flow, the mean axial velocity profiles were found to be insensitive to the turbulence models used. However, for supersonic flow this insensitivity was seen only downstream of the nozzle exit and outside the potential core region, while within this region the SST $k-\omega$ model showed an unexpected deficit in the mean axial velocity profiles. The turbulent kinetic energy profiles for different turbulence models follow each other closely, with the Realizable $\mathrm{k}-\varepsilon$ model predicting a slightly higher peak value.

For this nozzle design, the flow along the centerline becomes supersonic at the end of the finite-length throat right before entering the diverging part. The turbulence models capture the same normal shock location along the nozzle symmetry axis. The SST k$\omega$ predicts a slightly higher Mach number in the converging and constant-throat parts of the nozzle when the flow is subsonic. The Realizable $\mathrm{k}-\varepsilon$ model, on the other hand, predicts a slightly higher velocity in the potential core when the flow is supersonic. The entrainment rate coefficient increases linearly with the distance along the jet centerline, as reported in [27].

Finally, it is found that the SST k- $\omega$ model, which is a superior model when the flow contains a separation region, predicts an unrealistic velocity deficit in the potential core when the flow became supersonic inside the nozzle. This velocity deficit was not associated with any flow separation at the wall as the diverging section angle in this nozzle was relatively small and no flow separation was observed for the flow.

\section{ACKNOWLEDGEMENTS}

The authors would like to thank Saudi Aramco and NSERC (Natural Sciences and Engineering Research Council of Canada) for their support of this research.

\section{REFERENCES}

[1] M. Mashour, S. Smith, N. Palfreeman, G. Seefeldt, "New Technology: Saudi Aramco High Pressure Air Assist System (HPAAS) for upgrading existing flares to smokeless combustion". International Flame Research Foundation 16th Members Conference, June 2009.

[2] A.H. Shapiro, The Dynamics and Thermodynamics of Compressible Fluid Flow, Vol. 1, John Wiley \& Sons, 1953.

[3] D. Anderson, J.D Anderson, Fundamentals of Aerodynamics, third edition, McGraw-Hill International Editions, 2001.

[4] J. E. Eggers, "The velocity profiles and eddy viscosity distributions downstream of a Mech 2.22 nozzle exhausting into quiescent air", NASA Technical Note, NASA TN D-3601, September 1966.

[5] S.F. Birch, J.E. Eggers, "A critical review of the experimental data for developed free turbulent shear layers", Free Turbulent Shear Flows, NASA SP 321, January 1973.

[6] J.C. Lau, P.J. Morris, M.J. Fisher, "Measurements in subsonic and supersonic free jets using a laser velocimeter", J. Fluid Mech., vol. 93, no. 1, 1-27, 1979. doi:10.1017/S0022112079001750.

[7] J.M. Seiner, M.K. Ponton, B.J. Jansen, N.T. Lagen, "The effects of temperature on supersonic jet noise emission", DGLR/AIAA 14th

Aeroacoustic Conference, Aachen, Germany, paper 92-02-046, February 1992.

[8] N.J. Georgiadis, J.R. DeBonis. "Navier-Stokes analysis methods for turbulent jet flows with application to aircraft exhaust nozzles", Prog. Aeronaut. Sci., vol. 42, 377-418, 2006.

doi:10.1016/j.paerosci.2006.12.001.

[9] L.D. Koch, J.E. Bridges, A. Khavaran, "Flowfield comparisons from three Navier-Stokes solvers for an axisymmetric separate flow jet", AIAA Paper 2002-0672, 2002.

[10] S. Sarkar, G. Erlebacher, M.Y. Hossaini, H.O. Engblow, "The analysis and modelling of dilatational terms in compressible turbulence ", J. Fluid Mech, vol. 227, 473-493, 1991. doi:10.1017/S0022112091000204 
[11] S. Sarkar, "Modeling the pressure-dilatation correlation", NASA Technical Report, May 1991.

[12] N.J, Georgiadis, T.W Aerodynamic Design and Analysis of High Performance Nozzles for Mach 4 Accelerator Vehicles", 44th AIAA Aerospace Sciences Meeting and Exhibit, Reno, Nevada, AIAA paper 490, January 2006.

[13] N.J. Georgiadis, D. A. Yoder, W.A. Engblom, "Evaluation of modified two-equation turbulence models for jet flow predictions", AIAA J.,, vol. 44, no. 12, De. 2006. doi:10.2514/1.22650.

[14] Q. Xiao, H.M. Tsai, D. Papamoschou, A. Johnson, "Experimental and numerical study of jet mixing from a shockcontaining nozzle," J. Propul. Power, vol. 25, o.3, May-June 2009. doi: $10.2514 / 1.37022$.

[15] T. DalBello, N. Georgiadis, D. Yoder, T. Keith, "Computational study of axisymmetric off-design nozzle flows", 42nd Aerospace Sciences Meeting and Exhibit, January 2004. doi:10.2514/6.2004-530.

[16] D.S. Tandra, A. Kaliazine, D.E. Cormack and H.N Tran, "Numerical simulation of supersonic jet flow using a modified model", Inter. J. Computational Fluid Dyn., vol 20; 19-27, August 2006. doi:10.1080/10618560600587204

[17] S. Sarkar, B. Lakshmanan, "Application of a Reynolds Stress turbulence model to the compressible shear layer", AIAA Journal, May 1991. doi:10.2514/3.10649.

[18] S. Sarkar, B. Lakshmanan, "Modelling the pressure-strain correlation of turbulence: an invariant dynamical systems approach", J. Fluid Mech., vol. 227, 245-272, June1991. doi:10.1017/S0022112091000101.

[19] V. Lijo, H.D. Kim, T. Setoguchi, S. Matsuo, "Numerical simulation of transient flows in a rocket propulsion nozzle", Int. J. Heat Fluid Flow, 31(3):409-417, June 2010. doi: 10.1016/j.ijheatfluidflow.2009.12.005.

[20] A. Balabel, A. M. Hegab, M. Nasr, and S. M. ElBehery, "Assessment of turbulence modeling for gas flow in two-dimensional convergent-divergent rocket nozzle," Applied Mathematical Modelling, vol. 35, no. 7, pp. 34083422, Jul. 2011. doi:10.1016/j.apm.2011.01.013.
[21] D.C. Wilcox, Turbulence Modeling in CFD, DCW Industries, Inc. 1994.

[22] W.C. Reynolds, "Fundamental of turbulence and turbulence modelling and simulation", Lecture notes for Von Karman Institute Agard Report No. 775, 1987.

[23] F. R. Menter, "Two-equation eddy-viscosity turbulence models for engineering applications," AIAA Journal, vol. 32 no. 8, pp. 1598-1605, Aug. 1994. doi:10.2514/3.12149.

[24] P. J. Roache, "Quantification of uncertainty in computational fluid dynamics", Annual Review of Fluid Mechanics, vol. 29, no. 1, pp. 23-60, Jan. 1997. doi:10.1146/annurev.fluid.29.1.123.

[25] A. T. Nguyen, H. Deniau, S. Girard, and T. Alziary de Roquefort, "Unsteadiness of Flow Separation and EndEffects Regime in a Thrust-Optimized Contour Rocket Nozzle," Flow, Turbul. Combust. (formerly Appl. Sci. Res.), vol. 71, no. 1-4, pp. 161-181, 2003. doi:10.1023/B:APPL.0000014927.61427.ad.

[26] J. Bridges, M. Wernet. "Measurements of the aeroacoustic sound source in hot jets", Tech. Rep. NASA/ TM-2004-212508, NASA, Cleveland, OH, February 2004.

[27] F. Medrano, Y. Fukumoto, C.M. Velte, A. Hodzic, "Mass entrainment rate of an ideal momentum turbulent round jet", J. Phys. Soc. Jpn., vol. 86, no. r, pp 3440, Feb.2017. doi:10.7566/JPSJ.86.034401. 


\title{
Motion of Pigments in Thin Paint Films
}

\author{
Marina Curak, Dr. Nazli Saranjam, Dr. Sanjeev Chandra \\ Department of Mechanical and Industrial Engineering \\ University of Toronto \\ Toronto, Canada \\ mcurak@mie.utoronto.ca, nazlis@mie.utoronto.ca, \\ chandra@mie.utoronto.ca
}

\begin{abstract}
When thin paint layers are exposed to heat, evaporation of the solvent begins, and significant movement within the paint film occur, causing temperature and concentration gradients. A clear coat paint was used, which had a density of $988 \mathrm{~kg} / \mathrm{m}^{3}$, viscosity of $240 \mathrm{cP}$ and a surface tension of $26 \mathrm{mN} / \mathrm{m}$ and was mixed with spherical solid blue particles with a mean diameter $26 \mu \mathrm{m}$ were added to the paint at $2 \%$ concentration of the total mass. A knife edge was used to coat stainless steel substrates at different thicknesses and placed in a convection oven at $121^{\circ} \mathrm{C}$ for 30 minutes. Images of the samples during the curing process were taken, showing how the pigments moved from low to high surface tension areas. Colour measurements were taken to show that increasing the thickness of the same paint significantly decreases the lightness value.
\end{abstract}

Keywords-component; Mass transfer and drying, colour, boiling and evaporation, two-phase flow, surface-tension-driven, concentration gradient, self-organizing patterns, automotive paint

\section{INTRODUCTION}

The paint applied on a car serves two functions: to appear attractive and uniform in colour, as well as protecting the car from abrasion and corrosion. In recent years, the automotive industry has been investigating the incorporation of metallic pigments within solvent based paints, due to their visual appeal and increased ability to protect against the aforementioned factors [1]. However, the manufacturing process of spraying this paint onto car parts does not yield consistent colour reading results [1]. This problem was approached in two directions; varying the composition of the paint, and varying the coating process of the paint.

The location of the pigments within the paint film significantly impacts the colour of a sample [2]. Kirchner and Houweling [2] conducted a study on changing the paint composition to use different types of metallic pigments of different shape and surface roughness with various toners and metallic pigment orientation controlling agents. Their results for solvent based paints stated that flop controlling agents had limited success on improving the orientation of the metallic pigments, with just $1^{\circ}$ decrease in the flop angle [2]. The conclusions were that other alternative mechanisms are responsible for the disorientation of pigments, but it was still unclear as to what those mechanisms were [2]. Kirchner [3] then conducted another study, which was focused on the orientation of the pigments during the curing process from a cross sectional perspective. It was determined that the evaporation of the solvent does significantly influence the orientation of the pigments, however is again is not the only mechanism causing it [3].

This, film shrinkage, that Kirchner [3] investigated is caused by a well-researched effect. Once a sample is exposed to heat in a convection oven, convective cells (Bénard-Marangoni cells) begin forming as a result from concentration and temperature gradients $[4,5]$. These convective cells move from low to high surface tension areas [4]. In paint applications, once the BénardMarangoni cells occur and all the solvents has evaporated, the paint beings to harden in these hexagonal structures, creating an effect known as "orange peel", which is defined by a surface resembling the skin of an orange [6]. It is not desirable to this effect occur, as the increased roughness due to the waves from the hardened Bénard-Marangoni cells adversely impacts the colour readings of the paint samples [7].

This paper will focus on changing the thickness of the paint with solid spherical nonmetallic pigments, as these pigments used in this study are within the same order of magnitude of the metallic pigments used in the automotive industry.

\section{EXPERIMENTAL SYSTEM}

A clear coat model paint was used, which had a density of 988 $\mathrm{kg} / \mathrm{m}^{3}$, viscosity of $240 \mathrm{cP}$ and a surface tension of $26 \mathrm{mN} / \mathrm{m}$ [6]. Spherical solid blue particles of a mean diameter $26 \mu \mathrm{m}$ were added to the paint, at a concentration of $2 \%$ of the total mass of the paint. A knife edge, shown in Figure 1, was used to coat reflective stainless steel substrates with a diameter of $50.8 \mathrm{~mm}$ and a surface roughness of $0.1 \mu \mathrm{m}$.

Funding for this project was provided by NSERC and Magna Exteriors. 


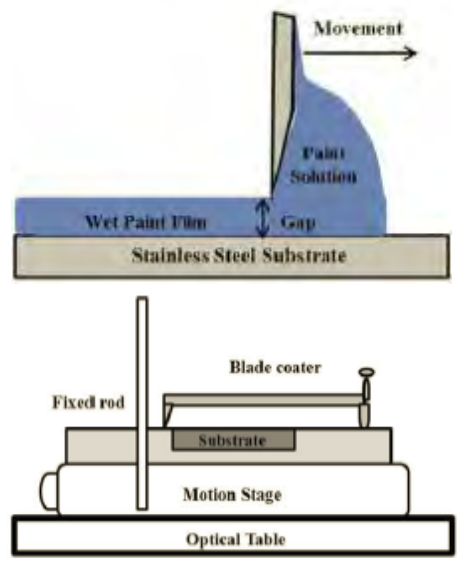

Figure 1. Knife edge coating apparatus [6]

After each sample was coated, it was given a flash off time of 20 seconds before it was placed in the oven, to avoid air entrapment within the sample. The sample was then placed in the convection oven to bake for 30 minutes at approximately $121.1^{\circ} \mathrm{C}^{+} / .1 .5^{\circ} \mathrm{C}$.

Figure 2 shows the schematic of the experimental system of the convection oven. Two flexible heating sheets at $1.55 \mathrm{~W} / \mathrm{cm}^{2}$ were placed on opposing sides of the oven and secured with an adhesive. Each heating sheet was connected to a variac, which was then connected to a temperature controller. This controller received readings from the thermocouple placed behind each heating sheet. An additional thermocouple was placed right beside the sample in the oven, to continuously measure the temperature. Excluding the oven's legs and the observation window, the rest of the oven was covered with thermal insulation to reduce the heat loss. The sample was then observed with a Pixelink PL-D7715CU-T camera with a resolution of $4608 \times 3288$ [8], which took images during the curing process. Mass measurements were collected every 60 seconds with a resolution of $0.1 \mathrm{mg}$.
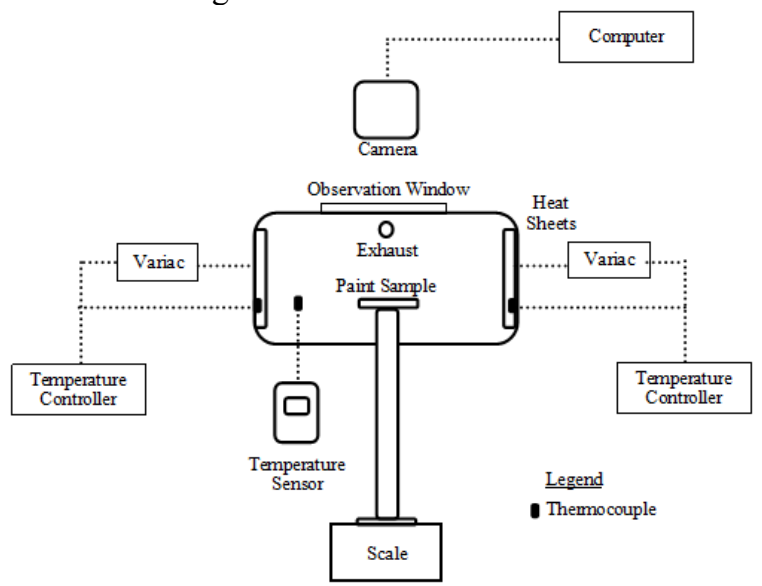

Figure 2. Convection oven curing system

\section{RESULTS AND DISCUSSION}

Once the sample is placed in the oven, the solvent begins to evaporate, causing the paint resin and pigments to move. This creates concentration and density gradients within the paint film, which results in surface tension gradients.

Figures 3 to 5 (with Figures 4 and 5 on the following page) show how the pigments move within the Bénard-Marangoni cells throughout this curing time. The initial thicknesses were extrapolated by determining the volume of the paint on the substrate and measuring the mass of the substrate. The field of view in each photo is $5.7 \mathrm{~mm}$ wide.
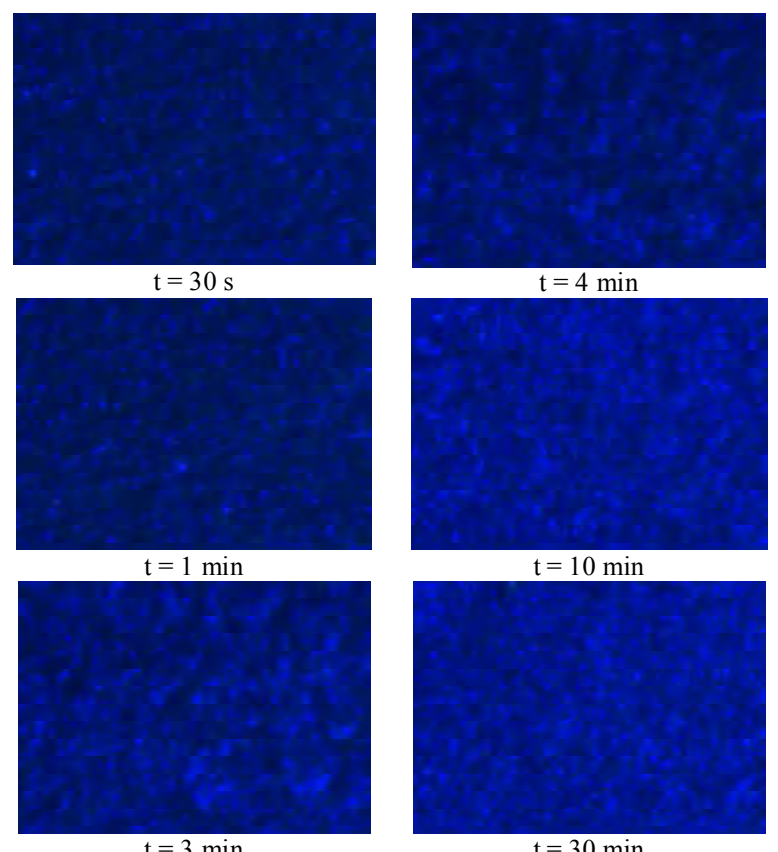

Figure 3. $300 \mu \mathrm{m}$ sample blue pigments moving within the Bénard-Marangoni cells

In Figure 3, at $\mathrm{t}=30$ seconds, the boundaries of the BénardMarangoni cells at the $300 \mu \mathrm{m}$ sample are present. After $\mathrm{t}=3$ minutes, the $300 \mu \mathrm{m}$ sample appears to have a significant decrease in magnitude, as the changes between the images in minutes 3 to 4 are negligible. At $540 \mu \mathrm{m}$, Figure 4 shows that there is continuous movement of the pigments for the first 4 minutes before the velocity magnitude decreases, with significantly larger Bénard-Marangoni cells than shown in Figure 3. Lastly, Figure 5 shows the pigments moving within the Bénard-Marangoni cells within the first 6 minutes of the curing process. Similarly seen in Saranjam [6], a secondary quasi-stationary period, with elongated cells are observed after the primary Bénard-Marangoni cells. 


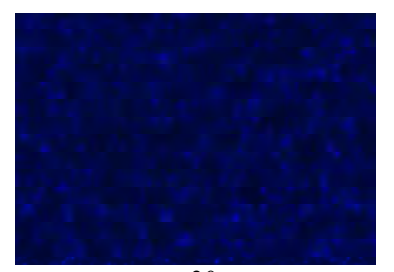

$\mathrm{t}=30 \mathrm{~s}$

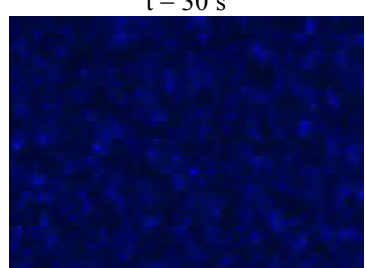

$\mathrm{t}=1 \mathrm{~min}$

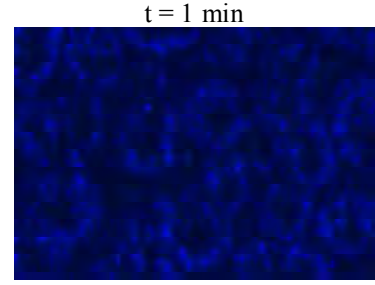

$\mathrm{t}=2 \min$

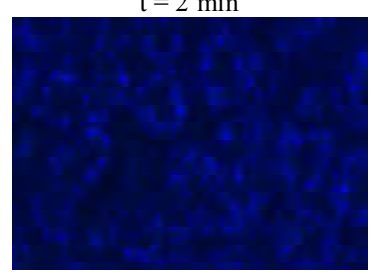

$\mathrm{t}=2.5 \mathrm{~min}$

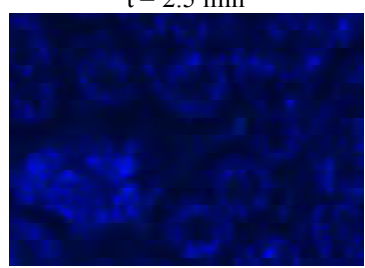

$\mathrm{t}=3 \min$

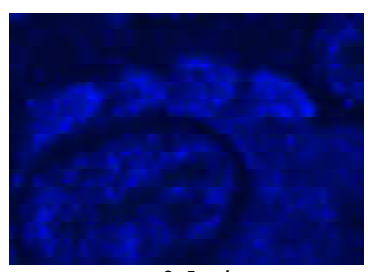

$\mathrm{t}=3.5 \mathrm{~min}$

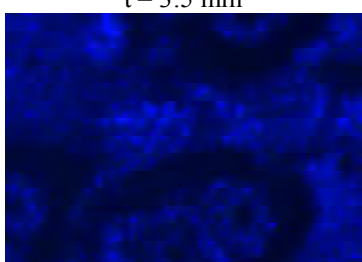

$t=4 \min$

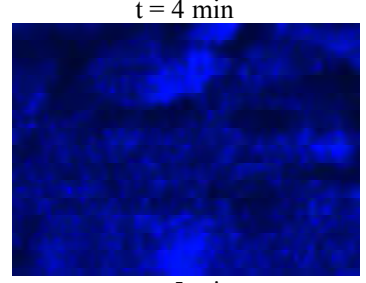

$\mathrm{t}=5 \mathrm{~min}$

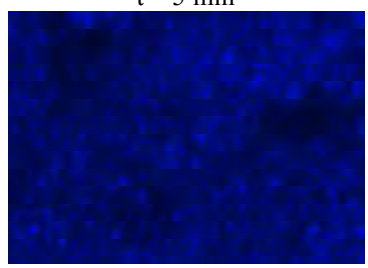

$\mathrm{t}=10 \mathrm{~min}$

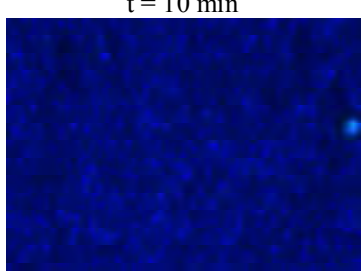

$\mathrm{t}=30 \mathrm{~min}$

Figure 4. $540 \mu \mathrm{m}$ sample blue pigments moving within the Bénard-Marangoni cells

All the images collected during the curing process were then analyzed using Particle Image Velocimetry (PIV) in Matlab [9, $10,11]$, which calculated the velocity magnitude between frames throughout the 30 -minute curing process, which is shown in Figure 6.

This PIV analysis concludes what Figures 3-5 already show- the larger the paint film thickness, longer the pigments move within their cells. Additionally, Figure 6 shows that the velocity of these pigments increases with increasing paint thicknesses. This is expected as there is more solvent and pigments that would cause greater concentration gradients and therefore higher surface tension gradient.

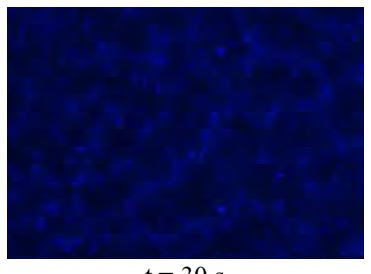

$\mathrm{t}=30 \mathrm{~s}$

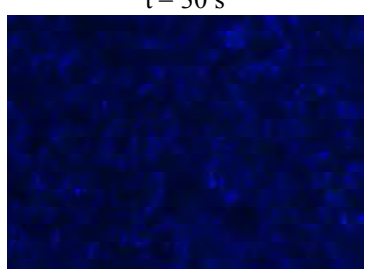

$\mathrm{t}=2 \min$

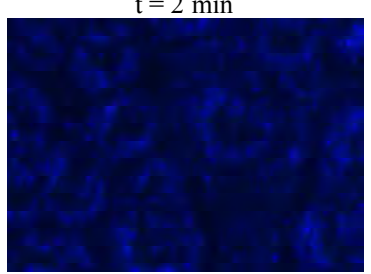

$\mathrm{t}=2.5 \mathrm{~min}$

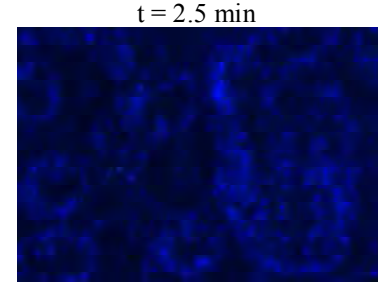

$\mathrm{t}=3 \mathrm{~min}$

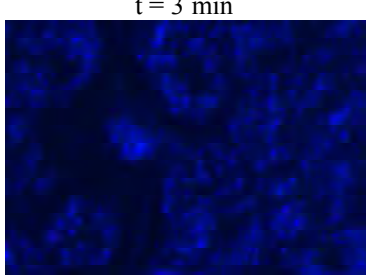

$\mathrm{t}=3.5 \mathrm{~min}$

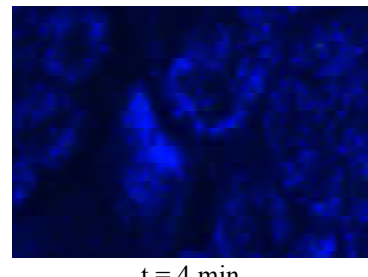

$\mathrm{t}=4 \min$

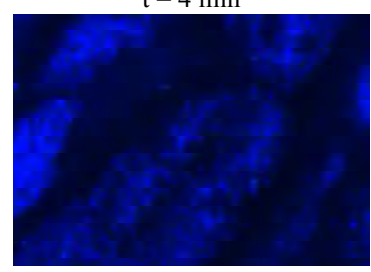

$\mathrm{t}=5 \mathrm{~min}$

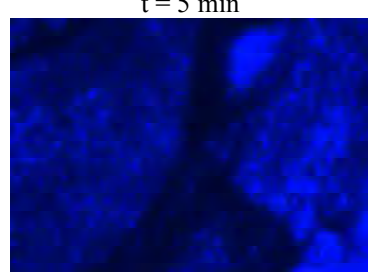

$\mathrm{t}=6 \mathrm{~min}$

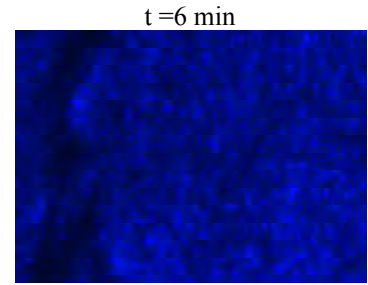

$\mathrm{t}=10 \mathrm{~min}$

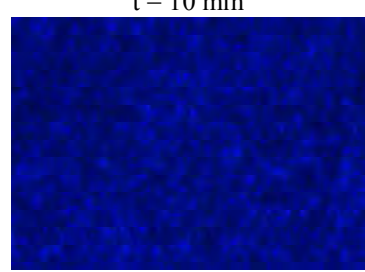

$\mathrm{t}=30 \mathrm{~min}$

Figure $5.750 \mu \mathrm{m}$ sample blue pigments moving within the Bénard-Marangoni cells

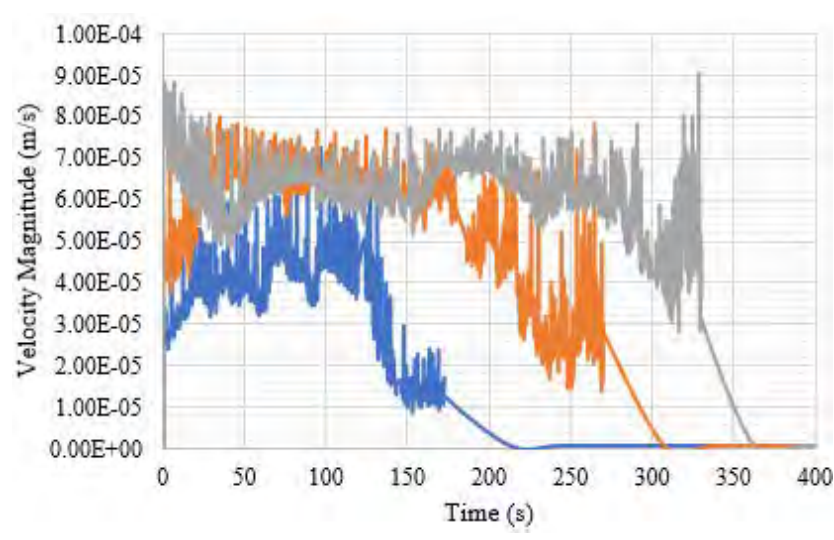

Figure 6. Velocity magnitude of the pigments within the paint. $300 \mu \mathrm{m}$ in blue, $540 \mu \mathrm{m}$ in orange and $750 \mu \mathrm{m}$ in grey. 
After the samples were cooled, their colour was measured using the Nix Color Pro Sensor [12]. This sensor can be used under different lighting and observation angle conditions. The lighting condition was set to illuminate at the $\mathrm{D} 65^{\circ}$ setting, which simulates daylight, and both observation angles of $2^{\circ}$ and $10^{\circ}$ were used [12]. This is to simulate the multiple different observation angles that is used in the automotive industry [13]. The sensor measured colour using the CIELAB colour space, as it is the standard used in industry [13], and the colour was measured in 5 different places on the sample. In the CIELAB colour space, parameters are linearly proportional to the change in colour. Figure 7 shows how many units the parameters, lightness (L), the red to green value (a), and yellow to blue value (b) change from the initial colour of the substrate, where an increase in any parameter corresponds to the name of the parameter, and a decrease corresponds to the converse of the parameter's name [13]. Since the human eye is most susceptible to changes in the lightness value, it is that change in parameter that has the most significance in colour standards [14]. Figure 7 shows the change in CIELAB colour readings from the sample's colour reading minus the reflective substrate's colour reading. Since the difference in colour readings between the observation angles are negligible, the readings taken at an observation angle of $10^{\circ}$ is shown in Figure 7.

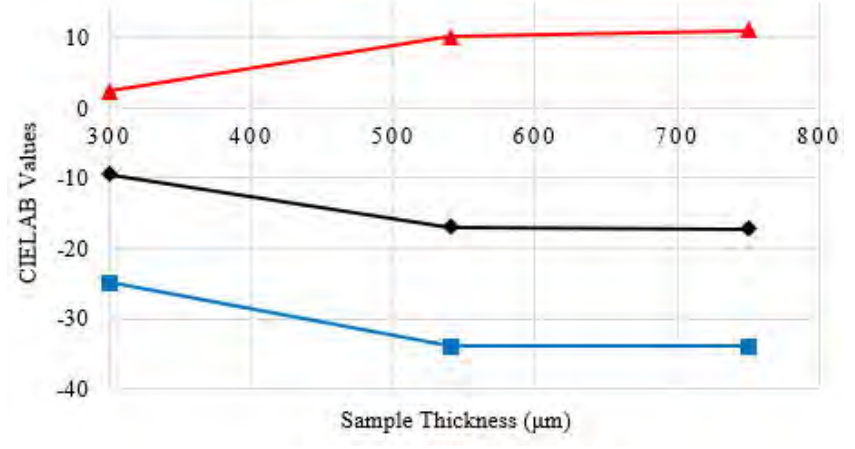

Figure 7. Change in colour readings reading at observation angle $10^{\circ} \mathrm{L}$ is grey, a is green, and b is yellow.

The most significant change colour change occurred by increasing the paint's thickness from 300 to $540 \mu \mathrm{m}$. This increase made the $540 \mu \mathrm{m}$ appear to be darker, redder and bluer. Meanwhile, the increase in paint thickness from 540 to $750 \mu \mathrm{m}$ did not have as large of an impact, with the $750 \mu \mathrm{m}$ sample appearing darker and redder.

\section{CONCLUSION AND FUTURE WORK}

During the paint curing process, evaporation of the solvent creates concentration and temperature gradient throughout the paint film. The thickness of the paint moves the pigments within the paint, outlining the Bénard-Marangoni cells which occurs due to the fluid moving from low to high surface tension areas. The velocity magnitude of the pigments moving between these low to high surface tension areas increases as the paint thickness increases. The understanding of this flow will be essential for the next sets of tests involving metallic pigments-with a significantly larger size and shape at different amounts.

The thicker paint films resulted in significantly darker colour readings. These set of tests will be repeated with a lowered percentage of pigments to total paint mass; to observe this effect on the velocity magnitude and colour.

With these variation of the paint composition, an analytical mass diffusion analysis will be compared to the experimental results and the concentration gradients will be calculated, to see the paint composition's effect on the mass diffusion.

\section{REFERENCES}

[1] H. Streitberger and K. Dossel, Eds., Automotive paints and coatings, 2nd ed., Weinheim, DE: Wiley-VCH, 2008

[2] E. Kirchner and J. Houweling, "Measuring flake orientation for metallic coatings," Prog. Org. Coat., vol. 64, no. 2-3, pp. 287-293, Feb. 2009. doi: 10.1016/j.porgcoat.2008.08.023.

[3] E. Kirchner, "Film shrinkage and flake orientation", Prog. Org. Coat vol. 65, no. 3, pp. 333-336, Jul. 2009. doi: 10.1016/j.porgcoat.2009.01.006.

[4] H. Bénard, "Les tourbillons cellulaires dans une nappe liquide. - Méthodes optiques d'observation et d'enregistremen," J. Phys. Theor. Appl., vol. 10, no. 1, pp. 254-266, 1901. doi: 10.1051/jphystap:0190100100025400.

[5] C. Hansen and P. Pierce, "Cellular convection in polymer coatings: An assessment", Ind. Eng. Chem. Prod. Res. Dev, vol. 12, no. 1, pp. 67-70, Mar. 1973. doi: 10.1021/i360045a010.

[6] N. Saranjam, "Transport Phenomena in Drying Paint Films", Ph.D, dissertation, Dept. Mech. Ind. Eng., Univ. of Toronto, Toronto, ON, 2016.

[7] F. Fensterseifer. "Visual evaluation and instrumental measurement of Orange Peel and DOI," in BYK-Gardner Catalog 2010/2011. Geretsried, DE: BYK-Gardner GmbH, 2010, pp. 33-36. Accessed on Oct., 25, 2013.[Online]. Available: https://www.byk.com/en/ support/instruments/technical-information/appearance.html

[8] "PL-D7715CU-T Aptina MT9F002 Machine Vision Camera," (n.d.). [Online]. Accessed on: Feb. 14, 2017. Available: http://pixelink.com/product/pl-d7715cu-t-aptina-mt9f002/

[9] W. Thielicke and E. J. Stamhuis, "PIVlab - Towards user-friendly, affordable and accurate digital particle image velocimetry in MATLAB," J. Open Res. Software, vol. 2, Oct. 2014. doi: 10.5334/jors.bl.

[10] W. Thielicke and E. J. Stamhuis, "PIVlab - Time-Resolved Digital Particle Image Velocimetry Tool for MATLAB". Figshare, Mar. 1, 2019. doi: 10.6084/m9.figshare.1092508.v12.

[11] W. Thielicke, "The flapping flight of birds: Analysis and application," Ph.D. thesis, Univ. of Groningen, Groningen, NL, 2014.

[12] "Nix Pro Tech Specs," (July 2017). [Online]. Accessed on: Jan. 10, 2017. Available: http://www.ghinstruments.com/wp-content/ uploads/2017/08/Nix-Pro-Tech-Specs-Digital_8-10-17.pdf.

[13] "BYK-mac i COLOR," (n.d.). [Online]. Accessed on: Aug. 25, 2016. Available: http://www.kohsieh.com.tw/PDF_Files/BYKGardner/03_Color/02_Metallic_Color/BYK-mac_i_COLOR.pdf.

[14] Standard Test Method for Multiangle Color Measurement of Interference Pigments, ASTM E2539-14, 2017. 


\section{Particle Image Velocimetry Data Processing on a GPU Cluster}

\author{
C. Dallas \\ Department of Mechanical and Industrial Engineering \\ University of Toronto \\ Toronto, Canada
}

\author{
P.E. Sullivan \\ Department of Mechanical and Industrial Engineering \\ University of Toronto \\ Toronto, Canada
}

\begin{abstract}
Particle image velocimetry (PIV) data processing is a computationally expensive process. The immense time taken to analyze data can limit the maximum dataset size. Using graphics processing units (GPUs) has been shown to drastically decrease the processing time for PIV image pairs. The open-source PIV data processing software OpenPIV has been ported to run on a GPU to boost speed and efficiency and has outperformed the CPU version of the software. A multipass method is being implemented in OpenPIV to improve both speed and accuracy. The completed algorithm will be tested on an embedder CPU-GPU device, a desktop computer, and the SOSCIP GPU-accelerated supercomputing cluster. Ultimately, OpenPIV will run on a wide variety of computer platforms an enable larger datasets to be collects, leading to better statistics on the resulting velocity fields.
\end{abstract}

\section{Keywords-PIV; GPU}

\section{INTRODUCTION}

Particle image velocimetry (PIV) has become a powerful tool for studying aerodynamic flow. However, the time required to process PIV data can be considerably large which limits the maximum size of a dataset, leading to concerns about statistical convergence. Normally, PIV data is processed on serial computers, utilizing multiple cores and multithreading technology to speed up calculations. Despite advances in serial computing hardware, the processing remains too slow for large datasets. Therefore, PIV algorithms are being developed to run on parallel architectures equipped with graphics processing units (GPUs).

Most PIV algorithms use cross-correlation-based methods which are extremely parallelizable and therefore can be considerably accelerated using GPUs. Executing a PIV window deformation algorithm with multiple GPUs on a $256 \times 256$ pixel image, researchers were able to increase processing speed by 120 times, resulting in a processing speed of 30 pairs per second [1]. In another study, by storing matrices as texture maps on the GPU, images were processed at a speed of 13 pairs per second for a $1024 \times 1024$ pixel image [2]. Taking a different approach, researchers using a gradient-based cross-correlation algorithm (FOLKI) on an NVIDIA Tesla C1060 GPU achieved a $50 \times$ speedup on $1376 \times 1040$ pixel images, resulting in a processing speed of 5 image pairs per second [3]. This is an immense speedup, considering that analyzing a single image pair on serial computers can take on the order of one minute.

It is clear that GPUs can be used in various PIV algorithms to accelerate data processing. Some commercial PIV software has added support for GPUs, but the code is proprietary and thus cannot be developed, optimized for specific architectures, or ported to different architectures such as ARM processors or supercomputing clusters. Therefore, the open-source PIV data processing code OpenPIV has been developed to utilize GPUs. Transferring the cross-correlation portion of the algorithm to the GPU resulted in a $10 \times$ speedup when compared to running on a CPU. The total processing time only decreased by a factor of 2. The majority of processing time was spent on the CPU performing sub-pixel peak approximations and velocity field calculations, both of which could be accelerated using a GPU. Another shortcoming of OpenPIV is it does not have full support for multi-pass. A PIV multi-pass algorithm first calculates a coarse velocity field (usually on a $64 \times 64$ pixel grid), then uses that information to calculate a finer velocity field. This process repeats until noise levels becomes too great and the highest resolution velocity field is achieved. Furthermore, for complex flows with turbulence and regions of high shear, the absence of a multi-pass PIV algorithm results in more spurious vectors in the final velocity field. Correcting for the spurious vectors is computationally expensive and can not be ported to a GPU, inevitably slowing down the computations. Developing the multi-pass portion of OpenPIV to execute on the GPU would greatly reduce the number of spurious vectors as well as the processing time.

Having a fast, open-source platform for PIV data processing using GPUs would be a valuable tool for multiple researchers. The code can run on any platform, and can be optimized for specific systems. For this to be fully realized, more of the code must be rewritten to run on the GPU, and full support for multipass must be added. To ensure universality of the code across 
platforms, the OpenPIV software will be tested on a desktop computer with a GPU, an embedded CPU-GPU development system, and the SOSCIP GPU-accelerated supercomputing cluster at SCINET.

\section{METHODS}

All software development is done using an NVIDIA Jetson TX1, referred to from now on as the TX1, which is an embedded CPU-GPU platform for developing GPU accelerated software. The TX1 includes a quad-core ARM Coretx-A57 processor (host) with 4GB of RAM, a 256-core NVIDIA Maxwell GPU, and a variety of $\mathrm{I} / \mathrm{O}$ ports for peripherals. The host runs the algorithm and transfers certain calculations to the GPU. The data transfer between the host and the GPU is time consuming and often comprises most of the GPU related processing time. Therefore, maximal speed is obtained by strategically and efficiently managing data transfer between the host and GPU. For further testing, the software will be run on a desktop CPU with 16GB of RAM and an NVIDIA GEFORE GT 710 GPU, as well as the SOSCIP cluster at SCINET which includes 14 IMB servers, each with $2 \times 10$ core CPUs with $512 \mathrm{~GB}$ of RAM and 4 NVIDIA Telsa P100 GPUs.

The version of OpenPIV being developed was originally written in Python. However, Python is unable to execute on the GPU. To run code on an NVIDIA GPU, the CUDA interface must be used. CUDA is a layer of software that allows certain languages, such as $\mathrm{C}, \mathrm{C}++$, and Fortran, to execute on the GPU. Therefore, the library PyCUDA [4], a Python wrapper for CUDA functions, was used to insert $\mathrm{C}$ code into OpenPIV and interface with the GPU. Additionally, some functions from the GPU accelerated Scikits-CUDA library were used. For increased speed, OpenPIV was compiled using Cython.

The PIV algorithm works by cropping an image pair into smaller overlapping regions called interrogation windows (IW) and then uses a FFT-based cross-correlation algorithm on each IW pair to calculate the velocity field. A depiction of the process is shown in Figure 1. This portion of the algorithm has already been written for the GPU, resulting in a $10 \times-35 \times$ speedup depending on IW properties. To further improve OpenPIV, multi-pass capabilities will be added to the software.

A PIV multi-pass algorithm iteratively refines the resolution of the velocity field until the maximum resolution is achieved. First, a coarse velocity field is calculated over the domain. Then, the grid is refined and corrections to the velocity on the finer grid are calculated. This process is then repeated until the noise becomes excessive, or the properties in each grid cell are not optimal for PIV calculations. The iterative method being developed in OpenPIV is based on the work done by Scarano and Riethmuller [5]. The multi-pass algorithm in OpenPIV must be parallelized to run on the GPU. The algorithm contains multiple loops for bounds checking, correction calculations, interpolations, and velocity calculations that can be massively accelerated using the GPU.

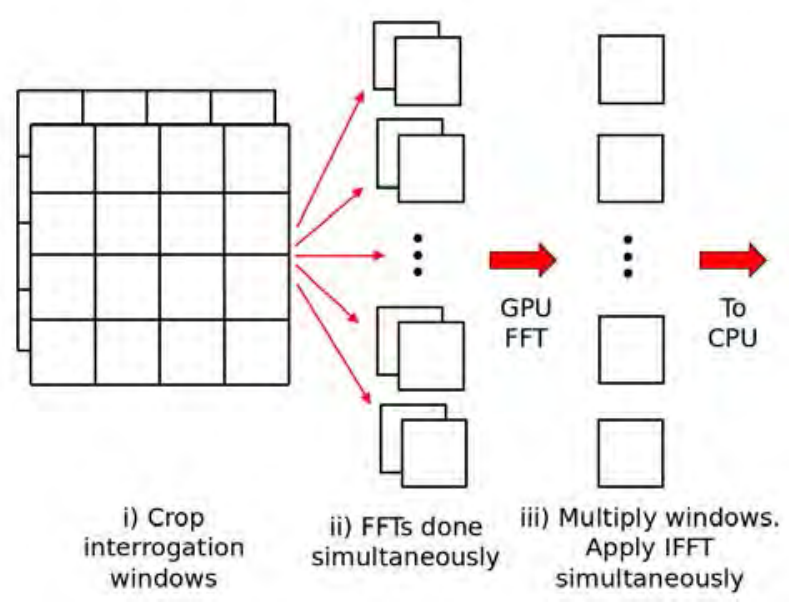

Figure 1. Scheme for calculating FFTs on the GPU

\section{EXPECTED RESULTS}

The OpenPIV software will be tested on two datasets. The first contains data on a flow control experiment of low Reynolds Number airflow over an airfoil. The experiment was done at a range of angles of attack using a variety of flow control parameters. Details of the experimental setup can be found in [6]. 1000 image pairs were taken at a resolution of $2560 \times 2160$ pixels. This data was originally processed using the commercial software package LaVision, which provides a set of results for comparison.

The second dataset is open-source and was originally intended to test PIV uncertainty quantification algorithms [7]. The experiment consists of a single turbulent jet with multiple sets of images taken at different regions of the jet, including the jet-core and shear layer. The dataset at each location contains an accurate velocity field calculated from ultra-high resolution images as well as a set of lower resolution images to use with PIV software.

For both datasets, three properties will be investigated. First, the difference in processing time between the GPU-accelerated and CPU version of OpenPIV will be calculated. It is expected that the GPU-accelerated version will be more than $2 \times$ faster than the CPU version as more portions from the previously accelerated algorithm are being moved to the GPU. Second, the number of spurious vectors produced in the final velocity field with and without multi-pass will be compared. It is expected that the multi-pass version will produce far less spurious vectors as it should reduce the relative noise level in the calculations. Third, velocity field results of the full GPU-accelerated, multi-pass algorithm will be compared to the LaVision and high resolution velocity field in each dataset. Comparisons for each image pair, the resulting velocity field, and other statistics will be made. When previously compared to LaVision, the CPU version of OpenPIV which did not use multi-pass performed well, except in regions of high shear, such as the boundary layer over the airfoil. The addition of multi-pass is expected to greatly improve 
the accuracy of the algorithm, especially in regions of turbulence and high-shear.

The processing time will be tested on three different computer platforms to investigate how the algorithm performs across architectures. The platforms, mentioned before, are the Jetson TX1, a desktop computer equipped with an NVIDIA GPU, and the SOSCIP GPU-accelerated supercomputing cluster at SCINET. The comparison between the TX1 and the desktop computer will be interesting, as the TX1 has a two times more powerful GPU, but the desktop has four times more RAM. It is still expected that the TX1 will outperform the desktop computer, as the bulk of the calculations will be done on the GPU.

Despite the fact that the SCOSCIP cluster is expected to massively outperform the other platforms, the results can be used to infer a maximum PIV dataset size when using supercomputing clusters. When using normal desktop computers, reasonable PIV datasets range from 500 - 1000 images. This puts severe limits on the statistical convergence of some calculations, and increasing the image size by an order of magnitude or more using a supercomputer cluster would be extremely advantageous.

\section{CONCLUSION}

Running PIV algorithms on GPUs can greatly decrease processing time. The open-source PIV data processing software OpenPIV is currently being developed to run on a GPU with added support for multi-pass. The software is written in a combination of Python and CUDA $\mathrm{C}$ which is then compiled using Cython, which can run on any architecture that has a
CUDA capable GPU. Once complete, OpenPIV will be tested in a variety of datasets for speed and accuracy. The software will also be run on an embedded computer, desktop computer, and supercomputing cluster to compare performance. Ultimately, this project will provide an open-source tool for PIV analysis that will operate on a wide variety of computing platforms.

\section{REFERENCES}

[1] S. Tarashima, S. Someya, and K. Okamoto, "Acceleration of Recursive Cross-Correlation PIV Using Multiple GPUs," Proceedings of the ASME/ JSME 2011 8th Thermal Engineering Joint Conference, 2011. doi: 10.1115/AJTEC2011-44442.

[2] T. Schiwietz and R. Westermann, "GPU-PIV," Vision, Modeling and Visualization, 2004, pp. 151-158.

[3] F. Champagnat, A. Plyer, G. Le Besnerais, B. Leclaire, S. Davoust, and Y. Le Sant, "Fast and accurate PIV computation using highly parallel iterative correlation maximization," Experiments in Fluids, vol. 50, no.4, pp. 1169 1182, 2011. doi: 10.1007/s00348-011-1054-x.

[4] A. Klöckner, N. Pinto, Y. Lee, B. Catanzaro, P. Ivanov, and A. Fasih, "PyCUDA and PyOpenCL: A scripting-based approach to GPU run-time code generation," Parallel Computing, vol. 38, no. 3, pp. 157-174, 2012. doi: 10.1016/j.parco.2011.09.001.

[5] F. Scarano and M. L. Riethmuller, "Iterative multigrid approach in PIV image processing with discrete window offset," Experiments in Fluids, vol. 26, no. 6, pp. 513-523, 1999. doi: 10.1007/s003480050318.

[6] M. Feero, S. D. Goodfellow, P. Lavoie, and P. E. Sullivan, "Flow Reattachment Using Synthetic Jet Actuation on a Low-Reynolds-Number Airfoil," AIAA Journal, vol. 53, no. 7, pp. 1-10, 2014.

[7] A. Sciacchitano, D. R. Neal, B. L. Smith, S. O. Warner, P. P. Vlachos, B. Wieneke, and F. Scarano, "Collaborative framework for PIV uncertainty quantification: the experimental database," Measurement Science and Technology, vol. 26, no. 7, p. 074004, 2015.

doi: $10.1088 / 0957-0233 / 26 / 7 / 074004$. 


\title{
A Droplet Deformation and Fragmentation in a High Speed Air Jet
}

\author{
Maryam Medghalchi, Nasser Ashgriz \\ Department of Mechanical and Industrial Engineering \\ University of Toronto \\ Toronto, Canada \\ medghalchi@mie.utoronto.ca, ashgriz@mie.utoronto.ca
}

\begin{abstract}
Impingement of a gas jet on a suspended water droplet is modeled numerically. The jet velocity and jet diameter effects on the penetration length and the shape of the gas tunnel inside the droplet are studied. The gas tunnel inside the droplet is smaller on the windward and larger on the leeward of the droplet. Different types of breakup processes are identified and categorized according to jet to droplet diameter ratio, as well as the droplet Weber number.
\end{abstract}

\section{Keywords; droplet breakup; fragmentation; jet ;}

\section{INTRODUCTION}

The droplet deformation and fragmentation are involved in many different systems, such as in fuel injection systems and powder production system. The process of droplet fragmentation is a complex one involving large interface deformations and interface breakups, and mostly in turbulent conditions. In order to characterize this complex process, usually the droplet breakup process is studied in a more controlled condition. For instance, a single droplet is injected into a high velocity gas flow and its deformation and breakup is determined at different gas flow velocities. Almost all prior studies on the droplet deformation and breakup have considered a droplet in a gaseous crossflow, where the gaseous crossflow is much larger than the droplet diameter. The present study has taken a different approach. Here, the deformation and a breakup of a droplet subject to a cross flow that its diameter is in the order of the droplet diameter is considered.

The droplet behavior is generally characterized using a Reynolds, a Weber and an Ohnesorge number defined as [1]:

$$
\begin{gathered}
R e=\frac{\rho_{l} U D_{\text {drop }}}{\mu_{l}} \\
W e=\frac{\rho_{l} U^{2} D_{\text {drop }}}{\sigma} \\
O h=\frac{\sqrt{W e}}{R e}=\frac{\mu_{l}}{\sqrt{\rho_{l} D_{\text {drop }} \sigma}}
\end{gathered}
$$

where $D_{\text {drop }}$ is the droplet diameter, $U$ is the relative velocity between the gas and the droplet, $\rho_{l}$ is the liquid density, $\mu_{l}$ is the liquid viscosity, and $\sigma$ is the coefficient of surface tension.
Gelfand [2] categorized the droplet breakup into several different modes as a function of $W e$ and $O h$. The following modes are identified: Vibrational, bag breakup, multimode, sheet thinning and catastrophic modes. In the vibrational mode, the drop oscillates until it divides into smaller droplets. In the bag breakup mode, a thin shell is generated, which later bursts forming many small fragments. The multimode breakup is similar to the bag breakup except that it includes a stamen, which forms in the leeside of the droplet (opposite to the gaseous flow). In the sheet thinning breakup, the surface of the droplet is sheered forming a thin liquid sheet, which later breaks forming small fragments. In the catastrophic breakup, the droplet instantly fragments into many small droplets. Guildenbacher et al. [3] reviewed the droplet secondary breakup and they found that viscous forces do not play a significant role at moderate Weber numbers. There are many other studies on the droplet breakup, and readers are referred to see the following references [4-10].

In the present study, we consider the impingement of a gas jet on a droplet, where the gas jet diameter is about the size of the droplet.

\section{MethodOLOGY}

Figure 1 shows the problem parameters. An initially stationary droplet with diameter $D_{\text {drop }}$ is exposed to a gas jet with diameter and velocity. The dynamics of the droplet deformation and breakup is simulated numerically.

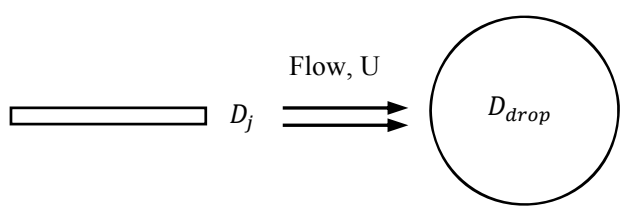

Figure 1: Schematic diagram of the nozzle and droplet

The numerical code used is ANSYS/FLUENT. The gasliquid interface is tracked by Volume Of Fluid (VOF) method. Continuity, momentum and energy conservation equations are solved with presence of the gravity. The turbulence model is RANS, standard $k-\varepsilon$ [11]. 
The governing equation are:

$$
\begin{gathered}
\frac{1}{\rho_{q}}\left[\frac{\partial}{\partial t}\left(\alpha_{q} \rho_{q}\right)+\nabla \cdot\left(\alpha_{q} \rho_{q} \vec{u}_{q}\right)=0\right] \\
\frac{\partial}{\partial t}(\rho \vec{u})+\nabla \cdot(\rho \vec{u} \vec{u})=-\nabla p+\nabla \cdot\left[\mu\left(\nabla \vec{u}+\nabla \vec{u}^{T}\right)\right]+\rho g+\vec{F} \\
\frac{\partial}{\partial t}(\rho E)+\nabla \cdot(\vec{u}(\rho E+p))=\nabla \cdot\left(k_{e f f} \nabla T\right)
\end{gathered}
$$

where in equation (4), $\alpha_{q}$ is the volume fraction of the secondary phase, $\rho_{q}$ is the density of the secondary phase and $u$ represents the velocity. In equation (6), $k_{e f f}$ is the effective thermal conductivity.

The continuous phase volume fraction is determined by, $\sum_{q=1}^{n} \alpha_{q}=1$ and $\rho=\sum \alpha_{q} \rho_{q}$, and $\mu=\sum \alpha_{q} \mu_{q}$. In equation (6), $E$ is the mass average of specific heats of phases.

$$
E=\frac{\sum_{q=1}^{n} \alpha_{q} \rho_{q} E_{q}}{\sum_{q=1}^{n} \alpha_{q} \rho_{q}}
$$

\section{A. Numerical Setup}

To reduce the computational time the numerical simulations are run on a $3 \mathrm{~cm} \times 1 \mathrm{cmrectangular} 2 \mathrm{D}$-axisymmetric geometry with the total of $1,140,000$ computational cells in fine mesh cases. In coarse mesh cases, the number of cells is reduced to 58,000 .

The air density is determined using the ideal gas equation of state and the droplet density is assumed to be constant.

Three different nozzle sizes with different jet velocities and droplet sizes are simulated. Results are presented in the following section.

\section{RESULTS AND DISCUSSION}

Two different droplet sizes of $2.4 \mathrm{~mm}$ and $4.8 \mathrm{~mm}$, and three different air jet diameters of $0.2 \mathrm{~mm}, 0.4 \mathrm{~mm}$ and $3.8 \mathrm{~mm}$ with two different gas velocities of $49.7 \mathrm{~m} / \mathrm{s}$ and $99.4 \mathrm{~m} / \mathrm{s}$ are considered.

Figure 2 shows the impingement of a $0.2 \mathrm{~mm}$ air jet with jet velocity of $49.7 \mathrm{~m} / \mathrm{s}$ on a $2.4 \mathrm{~mm}$ water droplet. The Weber number based on droplet diameter for this case is $W e_{\text {Drop }}=124$. The air jet impinges on the droplet and penetrates into the droplet forming an air tunnel. The liquid surface tension forces resist the jet flow. Therefore, the incoming gas goes through a circulation pattern forming a large cavity inside the droplet (see the evolution of the cavity in Figure $2 \mathrm{a}$ to $2 \mathrm{~d}$ ). In addition, the liquid at the windward of the droplet is pushed together to close the jet opening zone. Moreover, Figure 2 shows that droplet oscillates between circular and oval shape.

Figure 3 shows the droplet deformation for a gas jet velocity of $99.4 \mathrm{~m} / \mathrm{s}$ (Weber number 496) for the same jet and droplet diameters as in Figure 2. In this case, the air jet is strong enough to overcome droplet inertia and surface tension forces, and pierces through the droplet.
Figure 4 shows the velocity vectors on the droplet plane for a jet diameter of $0.4 \mathrm{~mm}$ and a droplet diameter of $4.8 \mathrm{~mm}$. Both the jet diameter and droplet diameter are increase such that their ratio is kept the same as those shown in Figure 2 and Figure 3. The jet velocity in this case is $49.7 \mathrm{~m} / \mathrm{s}$ (same as Figure 2, however, the Weber number is 248). In Figure 4 , the jet impinges, penetrates and pierces through the droplet. A similar type of cavity as in Figure 2 is observed in this case. However, in this case, because of higher inertia, the jet can force through the droplet. The bursting of a liquid layer that is formed at the time that the gas jet emerges out of the droplet results in the formation of several small droplets.
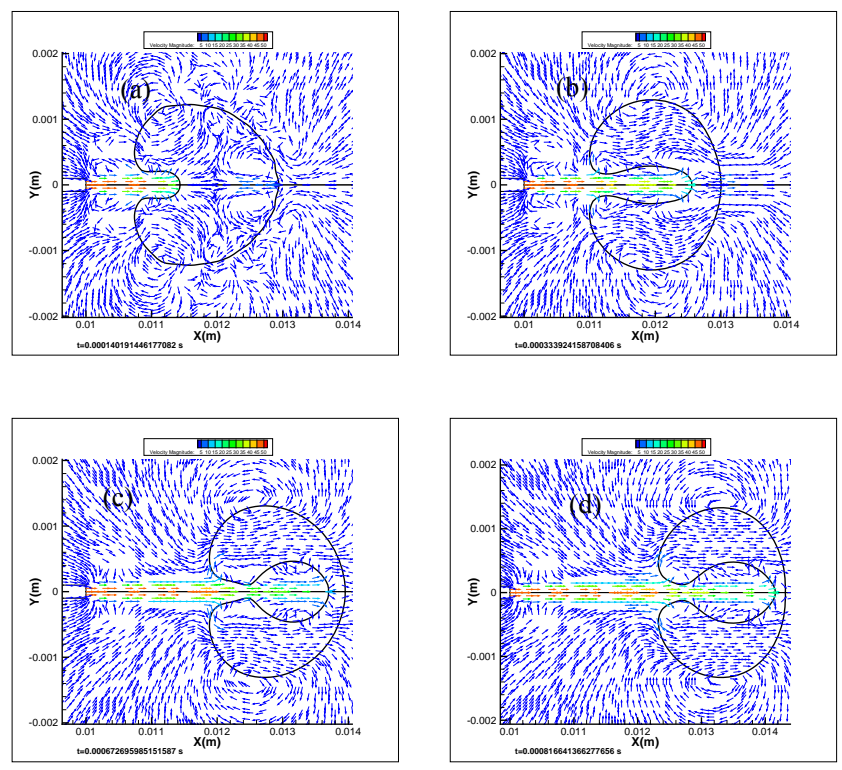

Figure 2: Velocity vectors and droplet for the jet diameter of $0.2 \mathrm{~mm}, U=49.7 \mathrm{~m} / \mathrm{s}$ and $W e_{\text {Drop }}=124$
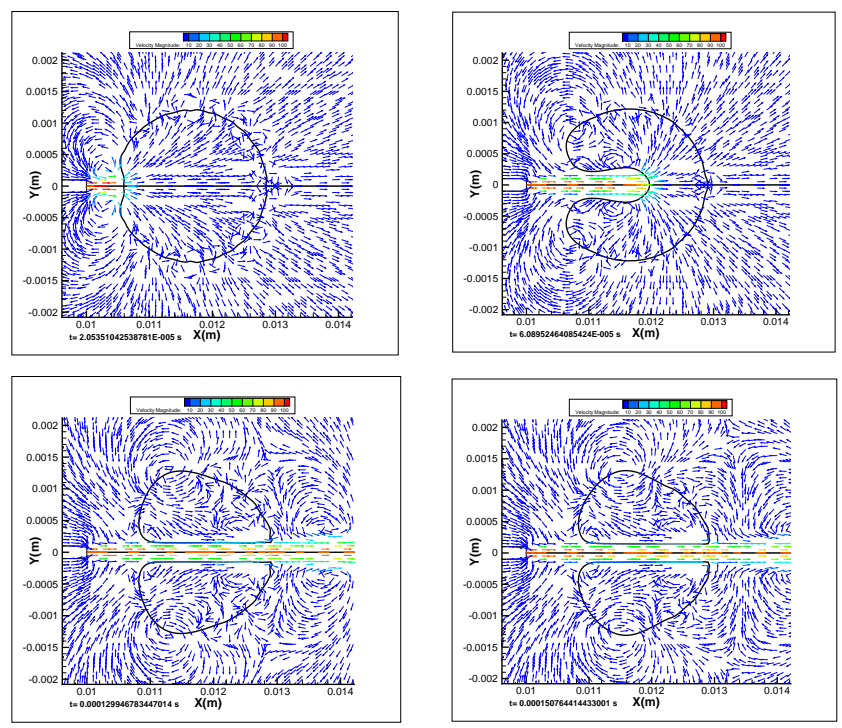

Figure 3: Velocity vectors and droplet for the jet diameter of $0.2 \mathrm{~mm}, U=99.4 \mathrm{~m} / \mathrm{s}$ and $W e_{\text {Drop }}=496$ 

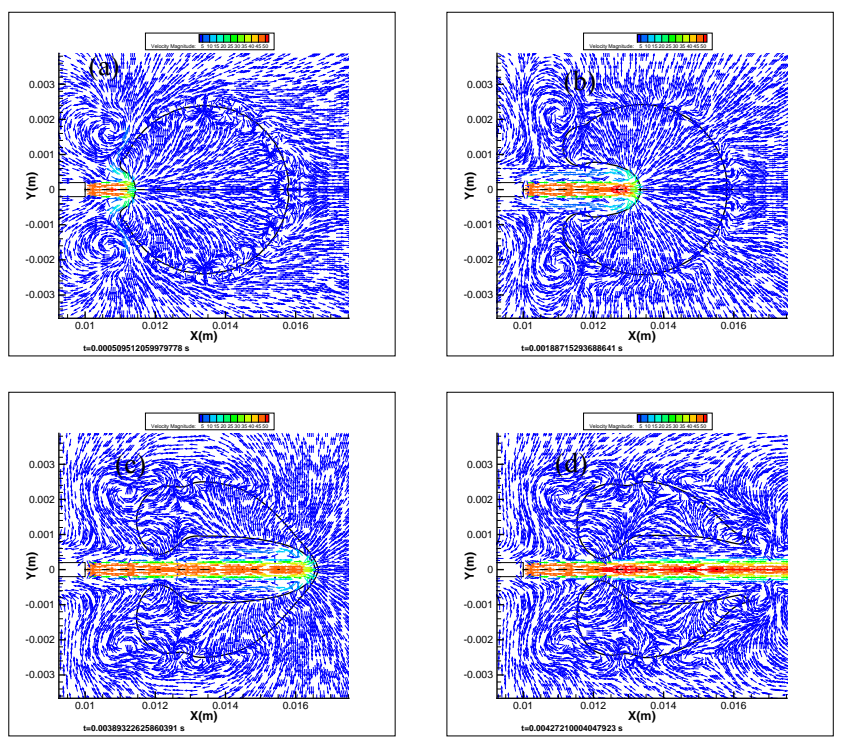

Figure 4: Velocity vectors and droplet for the jet diameter of $0.4 \mathrm{~mm}$, droplet diameter of $4.8 \mathrm{~mm}, U=49.7 \mathrm{~m} / \mathrm{s}$ and $W e_{\text {Drop }}=248$

Figure 5 shows droplet breakup for the same jet as in Figure 4 but with a smaller droplet size $(2.4 \mathrm{~mm})$. The jet diameter to droplet diameter ratio and the Weber number are reduced by $50 \%$. Comparing two figures shows that the larger droplet breaks earlier than the smaller droplet. Moreover the shape of the air tunnel inside the droplet is different in two cases. For the larger droplet (Figure 4) the air tunnel is long and thin, however, in the smaller droplet, the air tunnel is wide and short. Furthermore, the droplet in Figure 5, is significantly deformed, indicating that the inner flow behavior influences the droplet shape.

Comparing Figure 2 and Figure 5, which have the same droplet size and different gas jet sizes, shows that by doubling the jet size and keeping the Weber number constant the droplet stretched by the flow and its thickness is decreased until it bursts.

Figure 6 shows the droplet breakup for the jet diameter of $D_{j e t}=3.8 \mathrm{~mm}$ and the droplet size of $D_{\text {Drop }}=2.4 \mathrm{~mm}$. Air jet flattens the windward of the droplet and does not pierce the droplet. The droplet deforms from circular shape to a thin disk and the fragments are released from the top and bottom of the droplet.
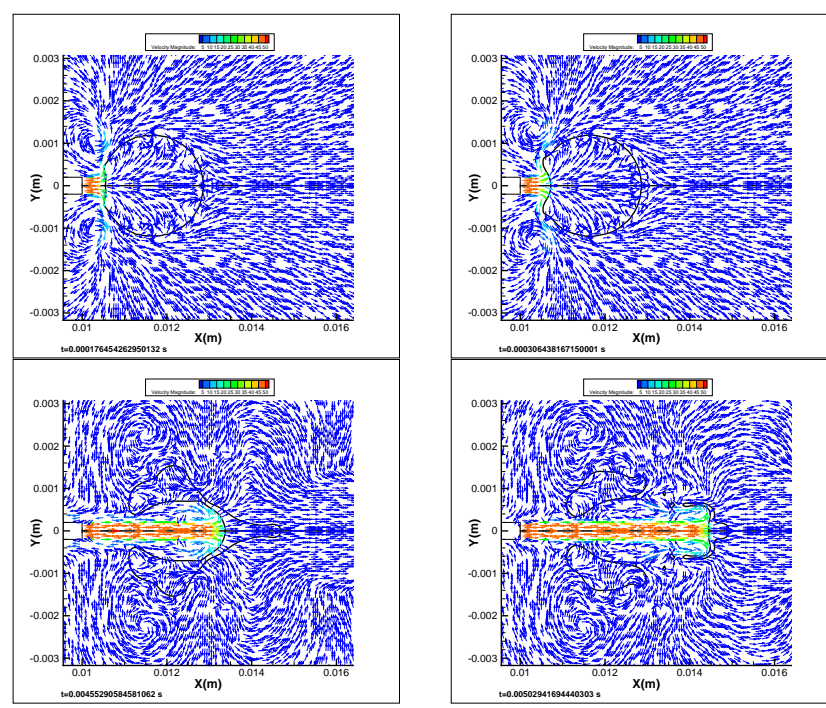

Figure 5: Velocity vectors and droplet for the jet diameter of $D_{\text {jet }}=0.4 \mathrm{~mm}$, droplet diameter of $D_{\text {drop }}=2.4 \mathrm{~mm}, U=$ $49.7 \mathrm{~m} / \mathrm{s}$ and $W e_{\text {Drop }}=124$
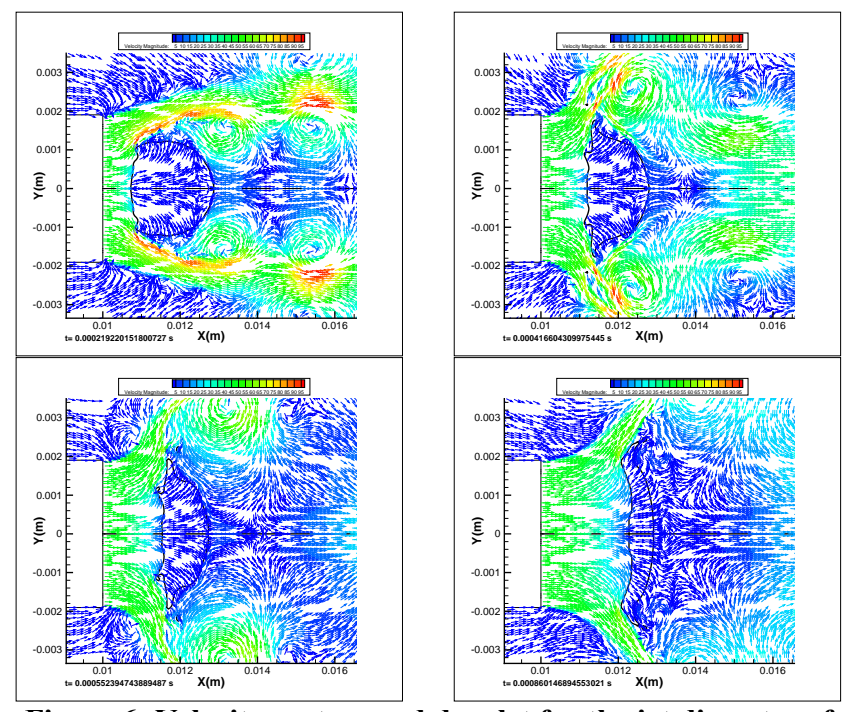

Figure 6: Velocity vectors and droplet for the jet diameter of $3.8 \mathrm{~mm}, U=49.7 \mathrm{~m} / \mathrm{s}$ and $W e_{\text {Drop }}=124$

\section{CONCLUSION}

Impingement of a gas jet of a liquid droplet for cases with gas jet diameter being smaller or in the order of droplet diameter are simulated numerically. The results indicate that the droplet deformation behavior is quite different than those reported in the literature for droplet deformation in a gaseous cross flow. The results show that for the jet to droplet diameter ratios less than one, a gas tunnel is formed inside the droplet that may have different configurations. At low gas jet velocities, a closed tunnel is formed which may result in an air entrapment inside the droplet. At larger gas velocities, the jet may penetrate through the droplet with less of an effect on the droplet deformation. 


\section{REFERENCES}

[1] G. Strotos et al., "Predicting droplet deformation and breakup for moderate Weber numbers," Int. J. Multiphase Flow, vol. 85, pp. 96-109, 2016. doi: 10.1016/j.jimultiphaseflow.2016.06.001.

[2] B. E. Gelfand, "Droplet breakup phenomena in flow with velocity lag," Prog. Energy Combust. Sci., vol. 22, pp. 201-265, 1996.

doi: 10.1016/S0360-1285(96)00005-6.

[3] D. R. Guildenbecher, C. Lo'pez-Rivera, and P. E. Sojka,

"Secondary atomization," Exp. Fluids, vol. 46, pp. 371-402, 2009.

doi: 10.1007/s00348-008-0593-2.

[4] G. M. Faeth, L. P. Hsiang, and P. K. Wu, "Structure and breakup properties of sparayes," Int. J. Multiphase Flow, vol. 21, pp. 99-127, 1995. doi: 10.1016/0301-9322(95)00059-7.

[5] A. Ashgriz, Handbook of atomization and sprays: Theory and applications. New York: Springer, 2011. doi: 10.1007/978-1-4419-7264-4.

[6] M. Pilch and C. A. Erdman, "Use of breakup time data and velocity history data to predict the maximum size of stable fragments for accelerationincluded breakup of a liquid cdrop," Int. J. Multiphase Flow, vol. 13, no. 6, pp. 741-757. 1987. doi: 10.1016/0301-9322(87)90063-2

[7] A. Mashayekh and N. Ashgriz, "Model for deformation of drops and liquid jets in gaseous crossflows, " AIAA journal, vol. 47, no. 2, pp. 303-313, 2009. doi: 10.2514/1.36148.

[8] T. Kékesi, G. Amberg, and L. Prahl Wittberg, "Drop deformation and breakup," Int. J. Multiphase Flow, vol. 66, pp. 1-10, 2014.

doi: 10.1016/j.ijmultiphaseflow.2014.06.006.

[9] S. A. Krzeczkowski, "Measurment of liquid droplet disintegration mechanisms, " Int. J. Maltiphase Flow, vol. 6, pp. 227-239, 1979. doi: 10.1016/0301-9322(80)90013-0.

[10] Z. Liu and R. D. Reitz, "An Analysis of the distortion and breakup mechanisms of high speed liquid drops," Int. J. Multiphase Flow, vol. 23, no. 4, pp. 631-650, 1997. doi: 10.1016/S0301-9322(96)00086-9.

[11] ANSYS Inc. Ansys/Fluent theory guide, 2017. 


\section{Dynamics of a Free Pitching Flexible Cantilever NACA0012 Airfoil at Transitional Reynolds Numbers}

\author{
Crystal Itwar Barrett \\ Department of Mechanical and \\ Aerospace Engineering \\ Royal Military College of Canada \\ Kingston, Ontario, Canada \\ Crystal.Itwar-Barrett@rmc.ca
}

\author{
Dr. Dominique Poirel \\ Department of Mechanical and \\ Aerospace Engineering \\ Royal Military College of Canada \\ Kingston, Ontario, Canada \\ poirel-d@rmc.ca
}

\author{
Dr. Asad Asghar \\ Department of Mechanical and \\ Aerospace Engineering \\ Royal Military College of Canada \\ Kingston, Ontario, Canada \\ Asad.Asghar@rmc.ca
}

\begin{abstract}
The dynamics of a free pitching flexible cantilever NACA 0012 airfoil were investigated at transitional Reynolds numbers. This work builds on previous investigations based on a quasi-2D rigid wing, moving elastically in pitch and heave. Wind tunnel tests were performed at various speeds, and three limit cycle oscillation (LCO) branches were observed. Further work is required to supplement this preliminary analysis, such as modeling, FEA simulation, and evaluation of the strain and acceleration information of the wing deformation
\end{abstract}

Keywords-transitional Reynolds numbers, flexible wing, LCO, free pitching, NACA0012, flexible cantilever

\section{INTRODUCTION}

The purpose of this series of experiments is to characterize the dynamics of a free pitching flexible cantilever NACA0012 wing at transitional Reynolds numbers. The wing experiences both rigid body and flexible body mode dynamics. It is well noted that the aerodynamics at transitional Reynolds numbers are complex, and include such phenomena as laminar boundary layer separation, which subsequently forms a laminar separation bubble (LSB) [1]. This paper is an extension of previous work performed on a rigid, elastically mounted in pitch and heave, quasi-2D NACA0012 wing.

\section{A. Wind Tunnel}

The tests were performed using the RMC wind tunnel, which is a closed circuit low speed tunnel powered by a $75 \mathrm{~kW}$ threephase motor. The flow velocity is controlled by varying the fan speed. The freestream velocity was measured with a pitot static tube (located approximately 9 chords upstream of airfoil), which was connected to an analog pressure transducer. The test section inner dimensions are $1.07 \mathrm{~m} \times 0.76 \mathrm{~m}$. The turbulence intensity of the tunnel is below $0.2 \%$ [1]. There is also a safety net located approximately 3 chords downstream from the wing.

\section{B. Flexible Wing Configuration}

The wing testing apparatus consists of the flexible NACA 0012 wing, mounted over a thin cantilever steel beam. Its kinematics can be seen in Fig. 1. The beam is fixed at its root to a free rotary base. The beam provides the structural stiffness, while the wing provides the aerodynamics loads. The wing and beam parameters can be seen in Tables I and II, respectively. The mass moments of inertia of the wing, beam and base can be seen in Table III.

TABLE I. WING PARAMETERS

\begin{tabular}{|c|c|}
\hline Material & Fiberglass epoxy, foam, and plastic \\
\hline Span & $0.445 \mathrm{~m}$ \\
\hline Chord & $0.15 \mathrm{~m}$ \\
\hline Aspect Ratio & 3 \\
\hline Mass & $0.145 \mathrm{~kg}$ \\
\hline
\end{tabular}

TABLE II. BEAM PARAMETERS

\begin{tabular}{|c|c|}
\hline Material & AISI O1 steel \\
\hline Length (exposed) & $0.45 \mathrm{~m}$ \\
\hline Width & $0.051 \mathrm{~m}$ \\
\hline Thickness & $0.0014 \mathrm{~m}$ \\
\hline Young's Modulus & $200 \mathrm{GPa}$ \\
\hline Mass & $0.222 \mathrm{~kg}$ \\
\hline
\end{tabular}

TABLE III. MASS Moments of Inertia (ABout Pitch AXIS)

\begin{tabular}{|c|c|}
\hline $\begin{array}{c}\text { Mass Moment of Inertia of } \\
\text { Beam }\end{array}$ & $5.5 \times 10^{-5} \mathrm{kgm}^{2}$ \\
\hline $\begin{array}{c}\text { Mass Moment of Inertia of } \\
\text { Rotary Base }\end{array}$ & $1.6 \times 10^{-4} \mathrm{kgm}^{2}$ \\
\hline $\begin{array}{c}\text { Mass Moment of Inertia of } \\
\text { Wing }\end{array}$ & $6.6 \times 10^{-4} \mathrm{kgm}^{2}$ \\
\hline
\end{tabular}

The free rotary base that holds the beam is connected to translational springs on a pulley. The pitch stiffness, $K_{\theta}$, was found to be $0.24 \mathrm{Nm} / \mathrm{rad}$. 
Pitch data was measured using contactless potentiometers. The potentiometer used for the large amplitude oscillation (LAO) tests was the RLS RMA05A3A00 contactless rotary magnetic actuator, and the corresponding RM22VA0010B10F1B00 encoder body, (resolution of $0.5^{\circ}$ ). The potentiometer used for the small amplitude oscillation (SAO) tests utilized the Contelec Co-Vert-X 22E2 $836 \quad 221 \quad 505 \quad 78107 \quad 1307 \quad 1$ (resolution of $0.18^{\circ}$ ). LAO and SAO will be further discussed in section 3 .

The beam was outfitted with two PCB Piezoelectronics $352 \mathrm{C} 22$ accelerometers and eight Vishay CEA-13-240UZ-120 strain gauges (four for bending and four for torsion). The data from the accelerometers fed into a PCB 483C signal conditioner. The strain gauge data fed into a model 2120 Strain Gauge signal conditioner. The conditioned strain gauge and accelerometer data, along with the potentiometer data, fed into the National Instruments cDAQ 9174, which connected to the computer and LabVIEW via USB; see Fig. 2.

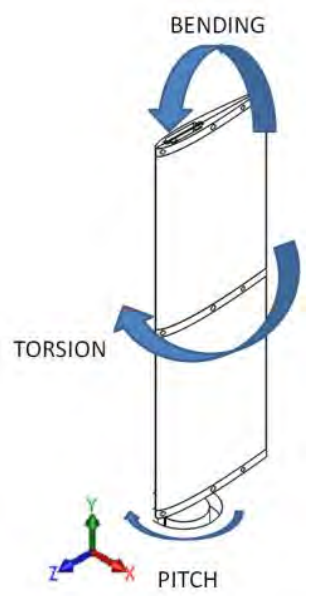

Figure 1. Illustration of Pitch, Bending and Torsion Motion

\section{Considerations for Design and Testing}

The wing was supported in the wind tunnel such that the wing support was flush with the wing tunnel floor. The ratio of wing span to tunnel height was calculated and found to be less than 0.6 , which ensured that the effects of the tunnel wall were negligible. [2].

The centre of gravity (CG) of the wing and beam combination was found to be $0.057 \mathrm{~m}$ aft of the leading edge (LE). Fig. 3 depicts the relative locations of the aerodynamic centre (AC), centre of gravity (CG), elastic axis (EA), and pitch axis (PA). In the current configuration, the $\mathrm{EA}, \mathrm{PA}$, and $\mathrm{AC}$ are all aligned.

The maximum blockage was calculated to be $8.3 \%$ (wing at $90^{\circ}$ pitch angle) while the minimum blockage was calculated to be $1.0 \%$ (wing at $0^{\circ}$ pitch angle).

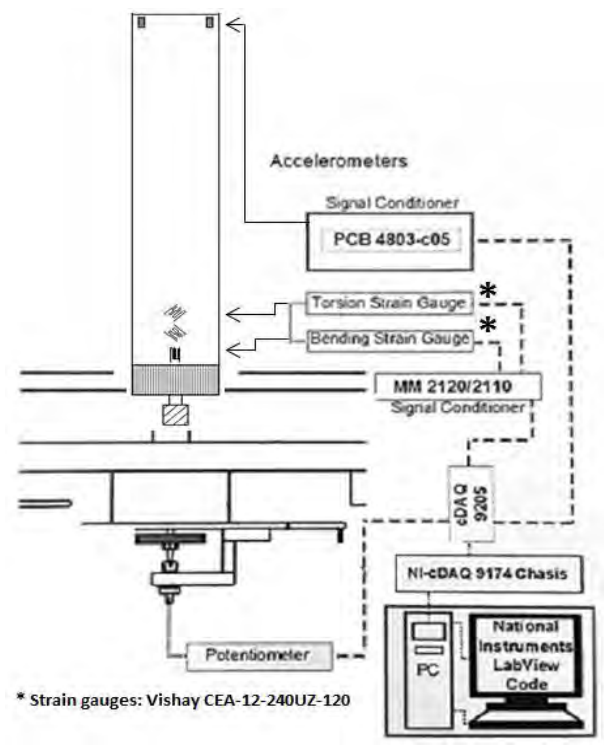

Figure 2. Experimental Test Set-Up

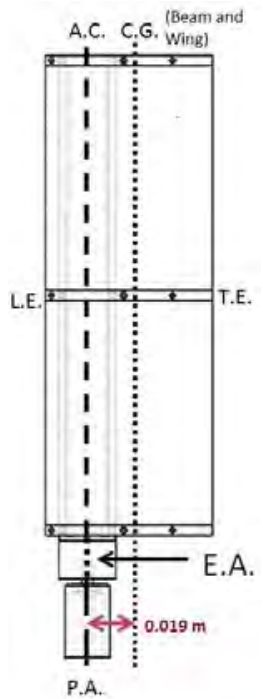

Figure 3. Relevant System Axes

\section{METHODOLOGY}

\section{A. Testing Procedure}

A total of six valid tests were performed. The test section was installed and lined up to ensure symmetry with the wind tunnel; the wing was set to zero angle of attack and set vertically straight.

The instrumentation was turned on to allow at least 20 minutes before testing, which allowed time for steady state to be reached. The wires were hooked up, isolated, and loosely taped to create support but still mitigate adding to the damping of the wing. In order to assess the ambient and equipment noise for each test, three recordings were done with the wind tunnel off, motor on, and motor and clutch on at zero RPM. 
The sampling frequency was $1000 \mathrm{~Hz}$. This data would be used two-fold: to find the baseline noise frequencies of the system and calculate the mean bias of the wing. Following this, the first type of ground vibrational testing (GVT1) was performed. GVT1 consisted of two free decay tests: one test with the pitch lock off (allowing for bending, torsion, and pitch, with a pitch initial condition) and one test with the pitch lock on (bending and pitch only, with a bending initial condition). With the pre-test GVT1 complete, tests were performed at various airspeeds, in combinations of increasing or decreasing airspeed sweeps, with the goal of obtaining the behavior of different amplitudes of LCO's. In an effort to locate the LCO branches, initial conditions were applied to the wing in the form of pitch perturbations and changes in airspeed. When changing sweep direction, hysteresis was noted in response due to the change in initial conditions via airspeed [3]. Following the airspeed tests, GVT1 (i.e. no-flow) was once again performed, wherein the purpose was to check if the system parameters changed. Following GVT1, the second type of ground vibrational was performed (GVT2), which was comprised of two free decay tests (with pitch initial conditions) for each of the three configurations, defined as such: S1 (rotary base, shaft, pulley, and encoder), S2 (rotary base, steel beam, eight strain gauges, two accelerometers, wires, shaft, pulley, encoder, and adhesives), and S4*(rotary base, steel beam, NACA 0012 wing, eight strain gauges, two accelerometers, wires, shaft, pulley, encoder, and adhesives) configurations as seen in Fig. 4-6, respectively. This data would allow us to find the decay rate and damping coefficient of the system in future work.

The $1^{\text {st }}$ and $2^{\text {nd }}$ analytical natural frequencies were calculated for bending and torsion of the S2 configuration (based on elastical beam theory), and the pitch damped natural frequency was experimentally determined for the S2 configuration as well, as seen in Table IV.

TABLE IV.

NATURAL FREQUENCIES (S2 CONFIGURATION)

\begin{tabular}{|l|l|l|}
\hline \multicolumn{3}{|l|}{ Natural Frequencies (Hz) based on [4], [5], [6], and [7] } \\
\hline Motion & Mode 1 & Mode 2 \\
\hline Pitch & 5.3 & n/a \\
\hline Bending & 6.0 & 38 \\
\hline Torsion & 102 & 308 \\
\hline
\end{tabular}

Note that the bending and torsion modes (as defined by the motion of the EA) are uncoupled due to the beam uniformity. The pitch motion is also considered to be uncoupled from the torsion motion due to the large difference in frequency scale. This assumption was confirmed from results of a finite difference (FD) solution of the S2 configuration. FD modeling of the S4* configuration resulted in the first three modes at $2.56 \mathrm{~Hz}, 5.0 \mathrm{~Hz}$ and $29.4 \mathrm{~Hz}$. These numerical results also match those obtained from the GVT. The first mode is pitch dominated, and the second mode is bending dominated.

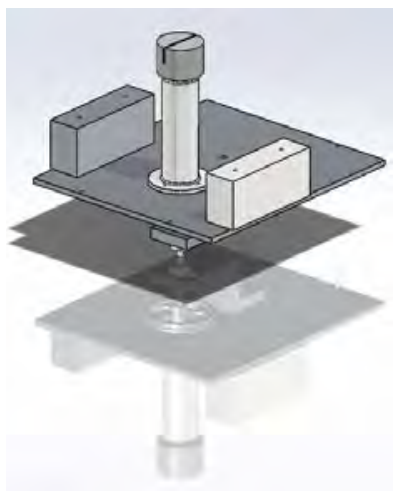

Figure 4. S1 Configuration (Rotary base only)

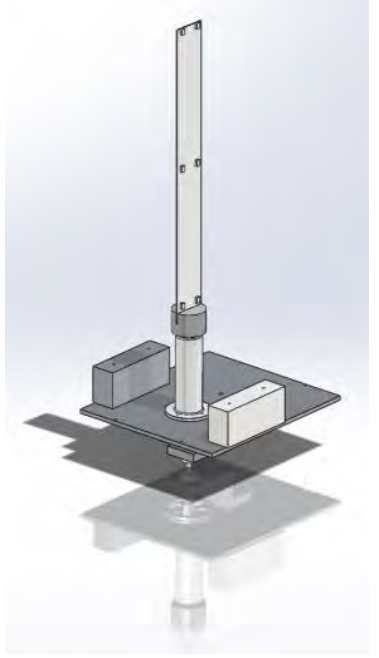

Figure 5. S2 Configuration (Rotary base and Beam)

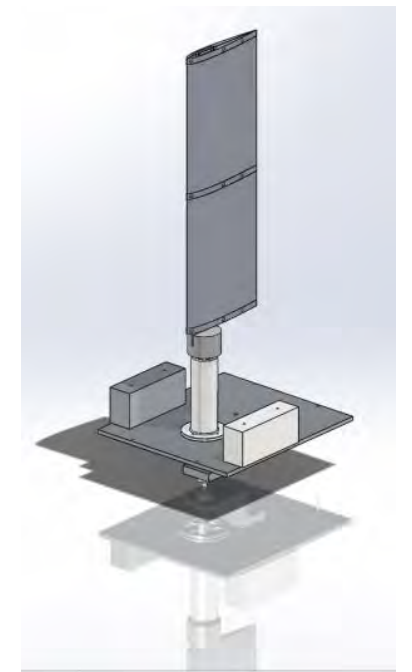

Figure 6. S4* Configuration (Rotary base, Beam and Wing) 


\section{B. Analysis Procedure}

The data obtained from testing was recorded into text-files using LabView, which were then manipulated into suitable excel files for post-processing with Matlab code. The aforementioned codes first took the potentiometer noise data, and converted it into root pitch angle (in degrees). The data was sorted in ascending order to find the fundamental frequency, which in turn was used to filter the data and find the mean bias of the filtered data portion which had a Gaussian distribution.

The ambient test data (temperature, pressure) were used to find the air density, airspeed, and Reynolds number. The varying airspeed data was then filtered and adjusted using a low pass filter, and the mean bias. The time histories were filtered to remove high frequency noise, since they have no impact on the dynamics. The time history served to visually indicate where the LCO behavior [3] occurred and select an appropriate portion of the signal, as well as to confirm if steady-state had been attained. If no global trends indicating increasing/decreasing root pitch angle was observed, the data was assumed to be gleaned during steady state. Histograms served to provide information on the data spread and whether the dynamics were symmetrical, along with the even harmonics of the PSD's which further confirmed symmetry. Once the data was deemed properly selected and filtered, the mean and mode positive pitch angle peak amplitudes were located using the filtered time history data, while the LCO frequency was found using the filtered root pitch angle PSD's. This was performed for all five tests and yielded final values of mean and mode positive root pitch angle peak amplitudes, pitch LCO frequency, airspeed, and Reynolds number.

\section{RESULTS AND DISCUSSION}

\section{A. Positive Mean Peak Amplitude of Root Pitch Angle Results}

Values of the mean positive peak amplitude of the root pitch angle were plotted versus airspeed for all five tests (Fig. 7). The mode positive peak amplitude of the root pitch angle values were also found, and determined to be sufficiently close to the mean values, thus further substantiating that the time response was symmetrical.

It is worthy to confirm that the range of airspeeds corresponded to a Reynolds number range of $9.11 \times 10^{4}<\mathrm{Re}_{\mathrm{c}}$ $<1.19 \times 10^{5}$, which is indeed in the range of transitional Reynolds number. Previous research has observed that the range of Reynolds numbers where LCO's occur is $5.5 \times 10^{4}<$ $\mathrm{Re}_{\mathrm{c}}<1.2 \times 10^{5}[8]$. Hitherto for the quasi-2D rigid wing, elastically mounted in pitch and heave, two LCO regions (also called branches) had been observed [8]. These regions can be described as SAO and LAO. In this paper, we tentatively define the ranges of $0^{\circ}-15^{\circ}$, and $30^{\circ}-70^{\circ}$ for SAO and LAO, respectively.
Referring to Fig. 7, it is substantial to notice that along with the evident SAO and LAO branches, a third branch is observed, which we have deemed very large oscillations (VLAO) and tentatively defined as the range of $70^{\circ}-120^{\circ}$.

It is important to note that the LAO region across all tests did not appear to be stationary and at steady state, i.e. there was significant amplitude modulation and a slight global increase in peak amplitude over time. No explanations are offered at this time.

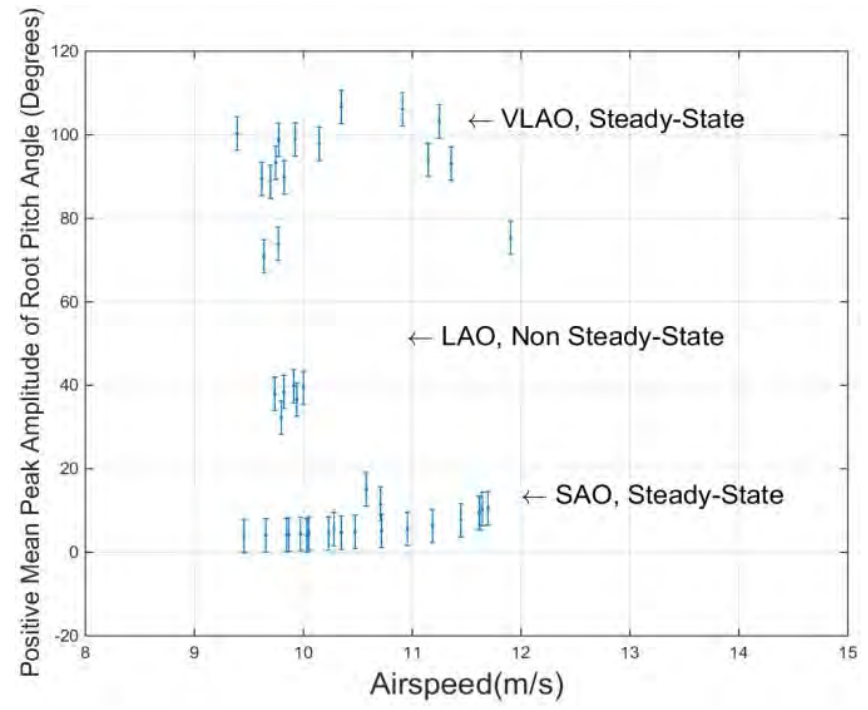

Figure 7. Positive Mean Peak Amplitude of Root Pitch Angle Versus Airspeed

It is meaningful to state that the wing could possibly be experiencing coalescence flutter or stall flutter for the LAO and VLAO branches. For the possibility of coalescence flutter, this could be a result of the coupling of the pitch and $1^{\text {st }}$ bending mode as these two frequencies are close to each other (Table IV); ergo, data reduction of the torsion and bending information is an important part of future work ponderations. The information can then be compared to the bending and torsion natural frequencies in Table IV.

This preliminary focus on pitch data is important because we use it as a guide; if we ascertain that it is well behaved, we thusly assume that the bending and torsion data will also be well behaved.

While we theorize that $\mathrm{SAO}$ is caused by laminar flow separation, along with the free pitching wing motion which causes negative aerodynamic damping, the physical mechanisms which cause LAO and VLAO are still to be confirmed. Hence, LAO and VLAO will be the primary focus, with the SAO serving to confirm that the Reynolds number effect is present, which provides information on the state of the flow. For our testing, the existence of the Reynolds number effect confirms that the flow is laminar and the wing is smooth. 


\section{B. LCO Frequency Results}

The LCO frequencies as measured from the root pitch angle were also found, and shown in Fig. 8. For the LCO frequency behaviour (Fig. 8), an average frequency resolution $f=1 / F_{\mathrm{s}} \Delta t$ $=0.0305 \mathrm{~Hz}$ was used, since $\Delta t$ varied for each test. From Fig. 8 , we see that once again, there are three distinct branches, corresponding to SAO, LAO and VLAO. All LCO frequencies are within the small frequency spectrum of $2.5-4.5 \mathrm{~Hz}$. Note that these are lower than the experimental natural pitch frequency of approximately $5 \mathrm{~Hz}$. Overall, as the mean peak amplitude of the root pitch angle increases, the LCO frequency decreases. Also for consideration is that the work from the quasi-2D wing [8] observed that the SAO had an approximate frequency range of $2.5-5 \mathrm{~Hz}$, whereas $\mathrm{LAO}$ had an approximate frequency range of $2-2.5 \mathrm{~Hz}$ (for a frequency ratio of 0.74 ). This is parallel to the results shown in this paper, where the frequency ranges are approximately $3.7-4.5$ $\mathrm{Hz}$ for SAO, 3.2 - 3.7 Hz for LAO and 2.8 - 3.1 Hz for VLAO.

While this work provides a good basis in the ongoing research to characterize the dynamics of a free pitching flexible cantilever NACA0012 wing, more detailed work is required. For instance, FEA modelling will be performed, where we will attempt to couple finite elements with a simple aerodynamic model, using Theodorsen's or Wagner's functions for instance for the linear case, as well as performing a modal analysis. Additionally, the strain gauge and accelerometer data will be fully analyzed. LAO tests will be redone in order to try and attain steady-state results. Post-work calculations will also include assessing the damping and stiffness values.

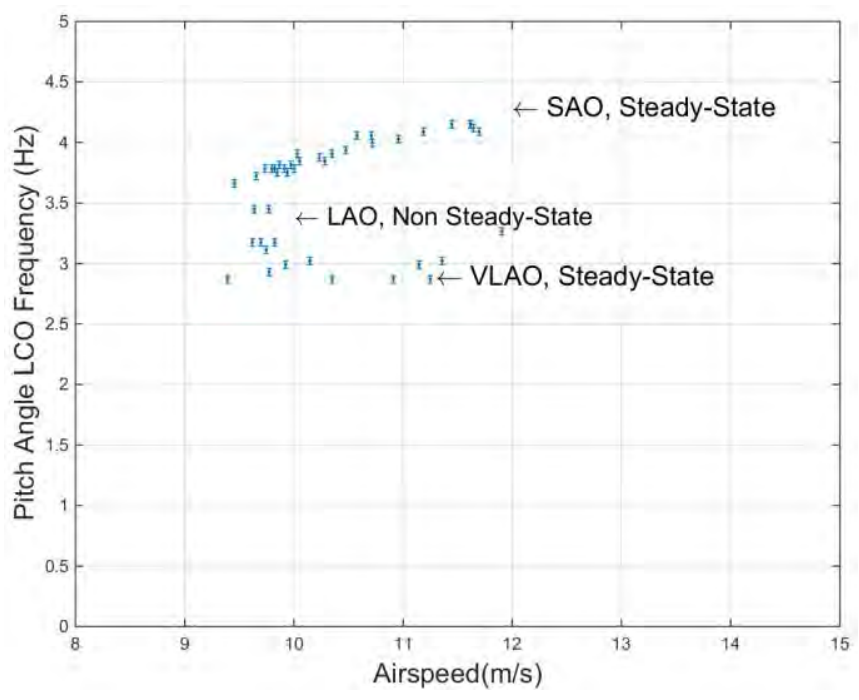

Figure 8. Pitch Angle LCO Frequency Versus Airspeed

\section{CONCLUSIONS}

A series of tests were performed on a free pitching flexible cantilever NACA0012 wing, in order to characterize the dynamics at transitional Reynolds numbers. Data was recorded using a potentiometer, strain gauges (in bending and torsion) and accelerometers. The potentiometer data was filtered and used to generate plots of the mean positive peak amplitude of the root pitch angle versus airspeed and LCO frequency versus airspeed. Three LCO branches were found corresponding to SAO, LAO and VLAO. It is theorized that the SAO is caused by flow separation at small angles of attack due to transitional Reynolds number and wing free pitching, which in turn causes negative aerodynamic damping. The mechanisms responsible for LAO and VLAO have yet to be confirmed through future testing and analysis. The range of Reynolds numbers where LCO's are observed, as well as the LCO frequencies are consistent with previously published work suggesting either coupled or stall flutter in these cases. Further tests will be performed to better capture LAO. Furthermore, the strain gauge and accelerometer data will be analyzed. FEA and numerical modelling will be done and used for corroboration of results, and post-work damping and stiffness values will be calculated.

\section{ACKNOWLEDGMENTS}

The authors gratefully acknowledge the help and support of Dr. Azemi Benaissa, M. Lavoie, Jeff Fleming, Brendan Freeman, Charles Sadiq, Pierre Seguin, Pierre Bissonnette, and David Babcock, and the other members of the Design Laboratory.

\section{REFERENCES}

[1] D. Poirel, Y. Harris, and A. Benaissa, "Self-sustained aeroelastic oscillations of a NACA 0012 airfoil at low-to-moderate Reynolds numbers," J. Fluids Struct., vol. 24, no.5, pp. 700-719, 2008. doi: 10.1016/j.jfluidstructs.2007.11.005.

[2] J. B Barlow, W. H. Rae, Jr., and A. Pope, Low-speed wind tunnel testing, 3rd ed. Toronto, ON: John Wiley and Sons Inc., 1999.

[3] E. H. Dowell, J. Edwards, and T. Strganac, "Nonlinear aeroelasticity," J,. Aircr., vol.40, no.5, pp.857-874, Sept.-Oct. 2003. doi: $10.2514 / 2.6876$

[4] A. Bedford and W. Fowler, Engineering mechanics statics and dynamics. Upper Saddle River, NJ: Prentice Hall, 2002.

[5] D. H. Hodges and G. Alvin Pierce, Introduction to structural dynamics and aeroelasticity. New York, NY: Cambridge University Press, 2002, pp. 35-36. doi: 10.1017/CBO9780511809170.

[6] P. P. Benham, R. J. Crawford, and C. G. Armstrong, Mechanics of engineering materials. Harlow, Essex, England: Longman Group, 1996, pp.115-116,

[7] W. H. Thomson, Theory of vibration with applications. Englewood Cliffs, NJ: Prentice Hall Inc., 1972, pp. 273.

[8] D. Poirel and F. Mendes, "Experimental small amplitude selfsustained pitch-heave oscillation at transitional Reynolds numbers," AIAA Journal, vol. 52, no. 8, pp.1581-1590, Aug. 2014. doi: $10.2514 / 1 . J 052541$. 


\title{
A Prediction Tool for Spatially Decaying Freestream Turbulence
}

\author{
Dwaipayan Sarkar, Eric Savory \\ Dept. of Mechanical \& Materials Engineering \\ The University of Western Ontario \\ London, Canada \\ dsarkar3@uwo.ca
}

\begin{abstract}
A set of new correlation equations characterizing the spatial decay of isotropic homogeneous turbulence has been formulated in the current study. The correlation equations are based on the results from the current numerical simulations combined with data from earlier relevant experimental studies [10], [15], [16]. The correlation equations can be effective for predicting the local values of turbulent properties (turbulence intensity (TI) and integral length scale $\left(\mathrm{L}_{\mathrm{u}}\right)$ ) from well specified initial conditions and, similarly, the magnitudes of inlet turbulence ( $\mathrm{TI}$ and $\mathrm{L}_{\mathrm{u}}$ ) can be estimated from the local magnitudes of length scale and turbulent kinetic energy (TKE) using the same prediction methodology. The set of prediction tools should be useful in the design of wind tunnel experiments to characterize bluff body aerodynamics in response to different incident turbulence conditions.
\end{abstract}

Keywords- isotropic, homogeneous, integral length scale, turbulence intensity

\section{INTRODUCTION}

The streamwise dissipation of isotropic, homogenous, free stream turbulence is one of the fundamental and widely explored topics in turbulence theory and its effects on various bluff body aerodynamics and thermal physics problems has been discussed over the past few decades [1-4]. In the absence of any external turbulent kinetic energy (referred to as TKE in the text hereafter) generation mechanism, the kinetic energy carried by the integral length scales of motion will decay, mostly due to inertial eddy interaction at high turbulent Reynolds number. Similarly, for low turbulent Reynolds number flows, molecular viscous forces dominate which causes the decay of TKE carried by the small scale dissipative eddies [1]. This decay of turbulent velocity fluctuations has an influential impact on the development of laminar and turbulent boundary layers over bluff bodies which in turn alters the heat and mass transfer rates from them [2-4]. Therefore, quantitative prediction of the free stream turbulent flow parameters incident on any bluff body, based on upstream inlet conditions, becomes an important factor for accurate determination of the dynamic properties of fluid and thermal responses in laminar, turbulent and transitional boundary layers.
There have been inconsistencies observed from the studies carried out for homogeneous and isotropic flows of freely decaying turbulence that have created disparities in the estimates of the spatial and temporal decay rates of TKE. Experimental grid-generated turbulence decays with a typical power law of the form, $\frac{\mathrm{k}}{\overline{\mathrm{U}}^{2}}=\mathrm{D} *\left(\frac{\mathrm{x}-\mathrm{x}_{0}}{\mathrm{M}}\right)^{-\mathrm{n}}[1],[5]$, where $\mathrm{k}$ is the TKE in the streamwise direction, $\bar{U}$ is the mean velocity, $D$ is the dimensionless dissipation constant, $\mathrm{x}$ is the streamwise distance, $\mathrm{x}_{0}$ is the virtual origin, $\mathrm{M}$ is the mesh width of the grid, and $\mathrm{n}$ gives the decay exponent. However, it was observed that different structures of geometrical grids generate turbulence with different decay rates and, hence, there is a need to achieve consistency, since the large-scale decay structures are known to affect the structure of turbulence at small scales [6], [7]. Numerous wind tunnel experiments have focused on generating homogeneous turbulence by different grids; passive [8], [9], active [10], [11], and multiscale (fractal) [12-15]. Numerical steady-state simulations [16] have also been carried out to reconcile the differences but no quantitative data have been presented at the inlet that would characterize the decay of turbulence downstream of a grid based on those specified initial conditions. A variation in the observed decay exponent $\mathrm{n}$ (from 1.0 to 1.4 ) implies that there might not be a universal state of decay mechanism.

It is generally agreed that the large scale turbulent properties, notably turbulence intensity (\%) (referred to as TI in the text, hereafter, given by $\left.\left(\mathrm{u}^{\prime} / \overline{\mathrm{U}}\right) \times 100\right)$ and integral length scale $\left(\mathrm{L}_{\mathrm{u}}\right)$ are the decisive factors governing the decay of quasi-static homogeneous isotropic turbulence, since both these parameters evolve according to power laws [1] and play an intrinsic role in dictating the dissipation rate of the TKE given by $\varepsilon \approx-\left(\mathrm{u}^{\prime 3} / \mathrm{L}_{\mathrm{u}}\right)$ , where $\mathrm{u}^{\prime}$ is the root-mean square of the characteristic turbulent velocity fluctuation and $L_{u}$ is the size of the average energy containing eddy. Therefore, the decay of free stream turbulence should be reflected in the values of $\mathrm{u}^{\prime}$ and $\mathrm{L}_{\mathrm{u}}$ at any point in the flow system and both parameters should be considered in the formulations that characterize the spatial decay of TKE. 
Hence, the aim of the current study is to employ Large Eddy Simulation (LES) models to develop simple yet powerful correlation equations that systematically quantify the spatial decay of turbulence, based on upstream inlet conditions, in a nearly isotropic and homogenous regime of turbulence. The final goal from the current study is to use those correlation functions as a prediction tool to estimate local and initial values of TKE and length scale which can then assist in numerous wind tunnel experiments of the flow over bluff bodies.

\section{MethodOLOGY}

\section{A. LES equations}

In LES the largest scales of turbulent motions are solved, whereas the smallest fluctuation scales are modelled. A spatial filtering is then applied to the continuity and the Navier-Stokes equations and the corresponding equations of the filtered velocity fields are obtained. The equations are then solved numerically using the concept of sub-grid viscosity $v_{\mathrm{t}}$ proposed by Smagorinsky [17], at each time-step, for each computational cell of the flow domain. More information about the numerical models associated with the LES formulation can be found in [18].

Among the sub-grid scale models that are available for LES, the dynamic sub-grid scale model by Smagorinsky [17], and Germano et al. [19] was used in the present work for simulating spatially decaying homogeneous isotropic turbulence.

\section{B. Solution parameters}

Different solution parameters used for handling the flow variables in LES are summarized in Table I. In order to maintain the convergence and the accuracy of the solution, the Courant Fredrichs-Lewy (CFL) number [20] is maintained at less than 1.0 by setting the time-step accordingly, based on the gridspacing. The LES simulations were carried out for 20,000 timesteps and statistically averaged results are presented. The simulations were performed using SHARCNET [21] a highcomputing cluster facility available at Western University.

TABLE I. Solution Parameters used in LES

\begin{tabular}{|c|c|}
\hline Time discretization & Second order implicit \\
\hline Momentum discretization & Bounded central difference \\
\hline Pressure discretization & Second order \\
\hline Pressure-velocity coupling & Segregated \\
\hline Under relaxation factors & $\begin{array}{c}0.5 \text { for the velocity and } 0.2 \\
\text { for the pressure }\end{array}$ \\
\hline
\end{tabular}

\section{CFD MODELLING DETAILS}

\section{A. Computational Domain and Boundary Conditions}

A three dimensional (3D) computational domain was created with sufficient length in the streamwise direction to allow for spatial decay of turbulence to develop fully. The geometry, along with the mesh, was generated using ICEM v.16.0 [22].
The domain was discretized using a perfect structured hexahedral mesh in all the three directions for accurate interpolation of the mean flow quantities and to be consistent with the cut-off scales for the spatial filtering. The physical dimensions of the grid were $\mathrm{L}_{\mathrm{x}}=4 \mathrm{~m}, \mathrm{~L}_{\mathrm{y}}=1 \mathrm{~m}, \mathrm{~L}_{\mathrm{z}}=1 \mathrm{~m}$.

Generating a proper inflow boundary condition for LES simulations is important [23-25] as the inflow should maintain proper spectra and coherency to replicate the spatial turbulent flow characteristics throughout the domain. Hence, to develop an isotropic homogeneous inflow boundary condition, the Consistent Random Flow Generation (CDRFG) technique was used. More information about this technique can be found in [26]. Pure slip wall conditions with zero shearing were imposed at the walls to negate the effect of the physical boundaries on the decay of TKE. A pressure outlet was specified as a suitable boundary condition for the outlet boundary. The parameters for generating the inlet velocity conditions are summarized in Table II.

TABLE II. Parameters used for generating the inlet velocity field

\begin{tabular}{|l|c|}
\hline Parameters & Values \\
\hline Mean velocity & $4 \mathrm{~m} / \mathrm{s}$ \\
\hline Integral length scale & $0.1 \mathrm{~m}$ \\
\hline Turbulence intensity & $10 \%$ \\
\hline Cut-off frequency & $100 \mathrm{~Hz}$ \\
\hline No. of samples generated & 20000 \\
\hline
\end{tabular}

\section{B. Grid-Independency}

Three different grid resolutions M1, M2 and M3 were created and used for the present grid-independence tests. The properties of these three grids are summarized in table III. The grid-independence study was carried out for a mean inlet velocity $(\bar{U})$ of $4 \mathrm{~m} / \mathrm{s}$, initial TI of $10 \%$ and integral length scale $\left(\mathrm{L}_{\mathrm{u}}\right)$ of $0.1 \mathrm{~m}$.

TABLE III. Properties of the employed grids in the current study

\begin{tabular}{|c|c|c|c|}
\hline Grid & Type & Element size ( $\mathbf{\Delta x})$ & No. of cells \\
\hline M1 & Coarse & $40 \mathrm{~mm}$ & 250,000 \\
\hline M2 & Medium & $20 \mathrm{~mm}$ & 500,000 \\
\hline M3 & Fine & $10 \mathrm{~mm}$ & $1,000,000$ \\
\hline
\end{tabular}

For LES, the variability in non-dimensional TKE going from M1 to M2 grid in proportion to its average value over the constant region where the TKE becomes fairly uniform (i.e. $\mathrm{x}=2 \mathrm{~m}$ to $\mathrm{x}=4 \mathrm{~m}$ ) is $13.1 \%$, whereas the variability of the same variable going from M2 to M3 is $4.8 \%$. On a similar note, the variation of the magnitude of $\mathrm{L}_{\mathrm{u}}$ in proportion to its average value over the constant region from M1 to M2 is 5.9\% and from $\mathrm{M} 2$ to M3 it is 2.3\%.

Hence, the grid (M3) with 1,000,000 cells was chosen for the LES computations, corresponding to the least relative discretization error with an acceptable value of convergence [27]. The employed grid also resolves the most energy 
containing eddies (ensuring that $80 \%$ of the TKE is resolved in the present case).

\section{Validation of the current CFD model}

The time-averaged, non-dimensional TKE profiles along the centreline of the domain in the streamwise direction using the Dynamic-Smagorinsky LES model is compared with the previous existing experimental and numerical data of [10], which are more recent than the classical Comte-Bellot and Corrsin experiments [28], [29] in high turbulent Reynolds number flows (fig. 1). It is clear from figure (1) that the quantitative agreement between the LES and different sets of data presented from that study [10] is remarkably good with a proper choice of virtual origin $\mathrm{x}_{0}$ value (which refers to the distance downstream of an experimental grid origin, where the wake vortices from the grid has decayed sufficiently and the turbulence has started to become well-developed and nearly isotropic and homogeneous in nature). It should be noted that the choice of the virtual origin is sensitive to the decay rate of TKE and, in this comparison, the virtual origin of the experiments was typically taken at 20 mesh widths i.e. $\mathrm{x}_{0}=20 \mathrm{M}$, for comparison [1].

The agreement between these two studies show that in the absence of mean shear and boundary layer wall effects, welldeveloped turbulent flow decays at a similar rate and there exists a universal decay mechanism of turbulence that would fit a single non-dimensional curve.

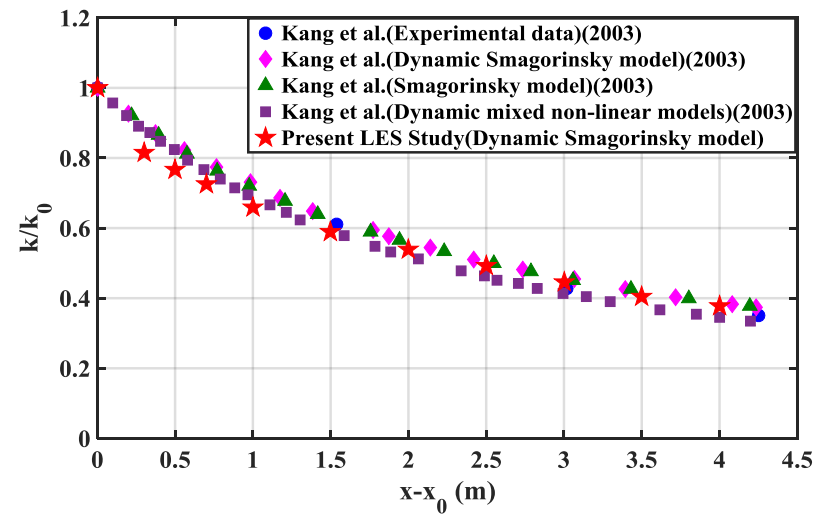

Figure 1. Comparison of decay of TKE in the streamwise direction between the studies of Kang et al. (2003) and present LES results.

Further comparison between the two studies reveals that the magnitude of $\frac{\mathrm{dk}^{\prime}}{\mathrm{dx}}$ at the virtual origin $\left(\mathrm{x}_{0}\right)$, computed from the values of $\mathrm{D}, \mathrm{u}^{\prime}, \overline{\mathrm{U}}$ and $\mathrm{L}_{\mathrm{u}}$ gives an approximate value of $0.014 \mathrm{~m}^{-}$ ${ }^{1}$ for the experiments [10] and $0.010 \mathrm{~m}^{-1}$ for the present LES study which are close to each other. Since the initial spatial TKE gradient rate controls the downstream decay of the TKE, a similar tendency of its decay in the nearly isotropic and homogeneous region of turbulence is observed.

\section{RESUlTS \& DISCUSSIONS}

\section{A. Predictive Methods for Spatial Decay of TKE}

A new predictive algorithm based on the current methodology has been formulated in the present study which provides an estimate of the local and initial turbulence levels in the uniform flow approaching the leading edge of a bluff body. The predictive algorithm is based on the simple inviscid estimation of the turbulence dissipation rate $\varepsilon$ at a given length scale, represented by $\varepsilon=\mathrm{dk} / \mathrm{dt}$. Starting from the inviscid estimation of $\varepsilon$ given by $\varepsilon=-\mathrm{D}\left(\mathrm{u}^{\prime 3} / \mathrm{L}_{\mathrm{u}}\right.$ ) (where $\mathrm{D}$ is the dimensionless dissipation constant, taken here as being unity, for convenience) and invoking Taylor's hypothesis of frozen turbulence, both these forms of $\varepsilon$ can be related and a new power law equation derived (which includes both TKE and $\mathrm{L}_{\mathrm{u}}$ ) that gives the spatial variation of TKE in the streamwise direction presented in the form

$$
\frac{\mathrm{k}}{0}=\frac{\mathrm{k}^{\prime}}{\mathrm{k}_{0}{ }^{\prime}}=\left(\frac{\mathrm{A}\left(\mathrm{k}_{0}{ }^{\prime}\right)^{0.5} \mathrm{x}}{\mathrm{L}}+1\right)^{-\mathrm{n}}
$$

where $\mathrm{k}$ and $\mathrm{k}_{0}$ are the local and initial TKE (at $\mathrm{x}=0$ ), $\mathrm{k}^{\prime}$ and $\mathrm{k}_{0}{ }^{\prime}$ are the dimensionless local and initial TKE (at $\mathrm{x}=0$; $\mathrm{k}$ is normalized using the mean velocity $\overline{\mathrm{U}}, \mathrm{A}$ is a dimensionless constant having a magnitude of 0.27 (where $A=0.27 \times D ; D=1)$, $\mathrm{n}$ is the decay exponent of magnitude $2, \mathrm{x}(\mathrm{m})$ is the streamwise distance starting from the origin of the physical grid and $\mathrm{L}_{\mathrm{u}}$ is the integral length scale (m) which is taken as a constant in this derivation for simplicity. This correlation equation (1) assumes turbulence is strictly isotropic and Taylor's hypothesis has been invoked while deriving spatial functions of the velocity field. However, in reality, the integral length scale $\left(\mathrm{L}_{\mathrm{u}}\right)$ grows in the streamwise direction as turbulence decays and cannot be treated as a constant which then alters the magnitude of the power law exponent, $n$, obtained from the correlation function presented in equation (1). Hence, to incorporate the effects of the spatial evolution of the length scale $\left(\mathrm{L}_{\mathrm{u}}\right)$, the correlation equation (1) is rewritten in a general form as

$$
\frac{\mathrm{k}}{0}=\frac{\mathrm{k}^{\prime}}{\mathrm{k}_{0}{ }^{\prime}}=\left(\frac{\mathrm{A}_{1}\left(\mathrm{k}_{0}^{\prime}\right)^{0.5}\left(\mathrm{x}-\mathrm{x}_{0}\right)}{\mathrm{L}}+1\right)^{-\mathrm{n}_{1}}
$$

where $\mathrm{x}_{0}$ is the virtual origin, and $\mathrm{A}_{1}, \mathrm{n}_{1}$ are the decay coefficient and decay exponent, respectively, for the above correlation (eq. 2).

Replacing $\mathrm{L}_{\mathrm{u}}$ with $\mathrm{L}_{\mathrm{u} 0}$ another form of decay power law can be achieved with the following form

$$
\frac{\mathrm{k}}{{ }_{0}}=\frac{\mathrm{k}^{\prime}}{\mathrm{k}_{0}{ }^{\prime}}=\left(\frac{\mathrm{A}_{2}\left(\mathrm{k}_{0}{ }^{\prime}\right)^{0.5}\left(\mathrm{x}-\mathrm{x}_{0}\right)}{\mathrm{L}_{\mathrm{u} 0}}+1\right)^{-\mathrm{n}_{2}}
$$

where $L_{u 0}$ is the initial value of the integral length scale (at $\mathrm{x}=$ $0), A_{2}$ and $n_{2}$ are the new decay coefficient and decay exponent, respectively, for the above correlation equation (3).

The correlations presented in equations $(2,3)$ clearly depict a power law form analogous to the grid-generated TKE decay law given in [1].

The correlation equation obtained in equation (2) is tailored specifically to include the effects of the TKE and the integral length scale into a single correlation equation, since the 
evolution of length scales influence the energy decay of the turbulent velocity fluctuations [9], [30].

The range of decay exponent (n) values obtained for different types of grids and numerical simulations is large because it depends on the initial conditions of the flow, the physical characteristics of the experimental grids (shape, size, width and porosity) and the choice of the virtual origin $\left(\mathrm{x}_{0}\right)$ [7], [31]. A faster or slower rate of decay of TKE is also possible depending upon the solidity of the grid which depends on the porosity $(\beta)$ which, in turn, is a function of the rod or wire dimeter used and the mesh width (M), as reported in [6].

Hence, the present work does not aim to address the question of determining definitive and precise values of the decay exponent (n) exhibited in eq. (2), but, rather, to come up with a global decay exponent (n) and dimensionless constant (A), that best fits the correlation function when using all the relevant previous experimental studies and the present numerical simulations, within an acceptable degree of accuracy, in the region of well-developed, homogeneous and isotropic turbulence.

The correlation (eq.2 and eq. 3) can be used to obtain an estimate of the local statistical turbulence parameters $\left(k\right.$ and $\left.L_{u}\right)$ at any location downstream of a grid based on the specified initial turbulence conditions $\left(\mathrm{k}_{0}\right.$ and $\left.\mathrm{L}_{\mathrm{u} 0}\right)$ at the inlet. Similarly, the same set of prediction equations (2 and 3) also helps to recover the initial free-stream turbulence levels relevant to any bluff-body aerodynamics problem knowing the local magnitudes of turbulence scales, thus making this prediction method a complete closure solution to the choice of experimental turbulence generating grid required to achieve a particular level of turbulence.

\section{B. Prediction equation based on the local value of the integral length scale $\left(L_{u}\right)$}

Figure (2) shows the direct comparisons between the earlier experimental studies of Kang et al. (2003) [10], Krogstad and Davidson (2011) [15], Torrano et al. (2015) [16] and the present LES with a best fit curve for the correlation function presented in equation (2). The streamwise evolution of non-dimensional TKE profiles $\left(\mathrm{k} / \mathrm{k}_{0}\right)$ are plotted against a non-dimensional parameter $\left(\mathrm{x}-\mathrm{x}_{0}\right) *\left(\mathrm{k}_{0}\right)^{0.5} / \mathrm{L}_{\mathrm{u}}$ where, $\left(\mathrm{x}-\mathrm{x}_{0}\right)$ is

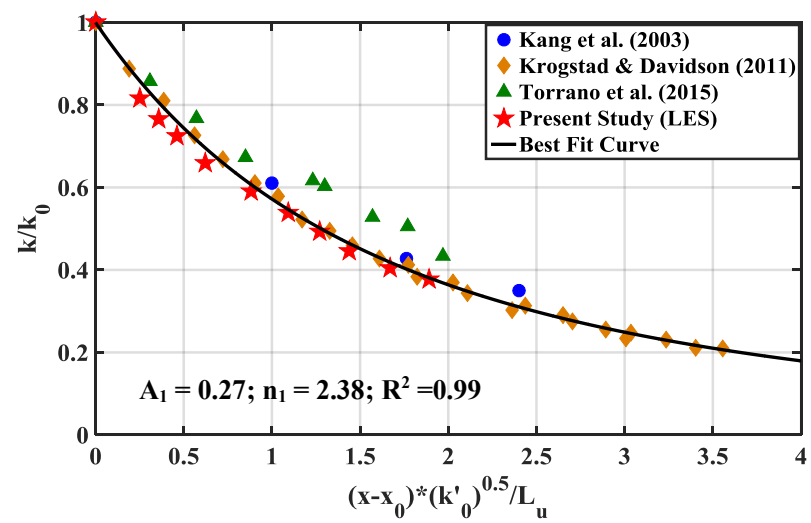

Figure 2. Spatial decay of TKE profiles of earlier experimental studies and present LES , plotted with a solid line that shows the best-fit power law the effective origin, $\left(\mathrm{k}_{0}{ }^{\prime}\right)$ is the non-dimensional initial TKE value and $\left(\mathrm{L}_{\mathrm{u}}\right)$ is the local value of the integral length scale. The magnitude of the initial normalized TKE and integral length scale values are digitized and extracted from the previous studies to achieve a direct comparison between the variables used in the plots by using the above scaling, even though the initial conditions of the different experiments and the LES are different. The fitted coefficient values of $A$ and $n$ estimated from the best-fit curve are $\left(A_{1}=0.27\right.$ and $\left.n_{1}=2.38\right)$. The goodness of the fit is excellent at $\mathrm{R}^{2}=0.99$. Figure (4) also reveals that the numerical results are fairly consistent (within 3\%) with the experimental data and both the studies reproduce a similar tendency of the predicted turbulence energy decay.

\section{Prediction equation based on the initial value of the integral length scale $\left(L_{u 0}\right)$}

Figure (3) shows the comparison of the spatial decay of the TKE profiles for different experiments stated earlier and the LES study, with the $\mathrm{x}$ axis variable $\left(\mathrm{x}-\mathrm{x}_{0}\right) *\left(\mathrm{k}_{0}\right)^{0.5} / \mathrm{L}_{\mathrm{u} 0}$ now normalized with the initial constant value of $\mathrm{L}_{\mathrm{u}}$, i.e. $\left(\mathrm{L}_{\mathrm{u} 0}\right)$, instead of local values of $\mathrm{L}_{\mathrm{u}}$. The scaling parameter has been changed to see its effect on the quantitative agreement between the LES and the experimental studies. Here $\mathrm{R}^{2}=0.97$, which is not as good as the previous scaling, whilst the values of $\mathrm{A}_{2}$ and $\mathrm{n}_{2}$ are 0.44 and 1.16 , respectively. It turns out that the $\mathrm{A}_{2}$ and $\mathrm{n}_{2}$ values obtained from the regression fit (eq. 3) have different

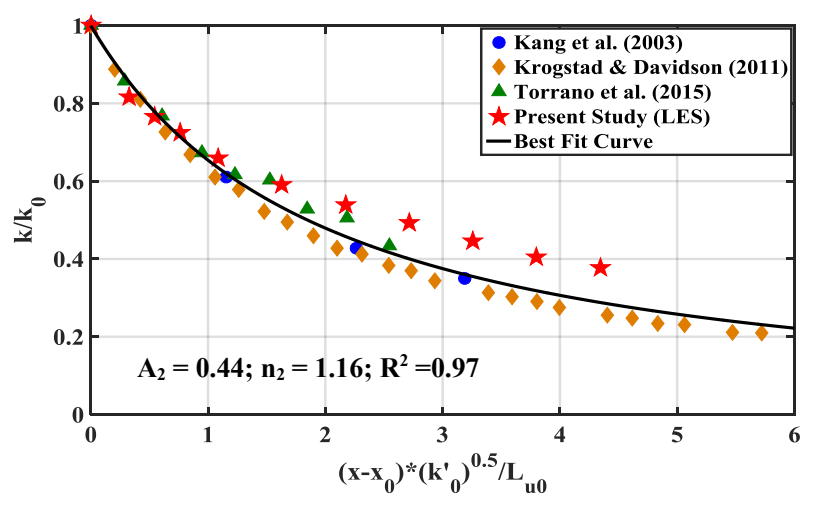

Figure 3. Spatial decay of TKE profiles for different experiments and the present LES study scaled with initial integral length scale $\left(\mathrm{Lu}_{0}\right)$ plotted with a solid line that shows the best-fit power law

magnitudes compared to the $\mathrm{A}$ and $\mathrm{n}$ obtained while deriving equation (1) even though both correlations assume a constant $\mathrm{L}_{\mathrm{u}}$. The values of the decay coefficient $\mathrm{A}$ and the decay exponent $\mathrm{n}$ obtained from equation (1) are 0.27 and 2 respectively.

There are reasons for this observed variation, between the magnitudes of decay coefficient $\mathrm{A}$ and decay exponent $\mathrm{n}$, obtained from the simple inviscid estimation of $\varepsilon$ (eq. 1) and the best-fit curve over all the experimental data and LES results (eq. $3)$.

Firstly, strictly isotropic turbulence has been assumed while deriving the correlation (eq. 1), which in reality is difficult to 
achieve in turbulence generated in computer simulations [32]. However, this is not surprising as such inhomogeneity of the flow has already been observed in the earlier experiments of [33], [34]. Secondly, while deriving the correlations presented in equations (1), (2) and (3), it was assumed that D is a nondimensional constant of magnitude unity, having no temporal or spatial dependency in the streamwise direction, but $\mathrm{D}$ is seen to have spatial dependency in the studies reported in [9]. Therefore, the estimate of $\mathrm{A}_{2}$ and $\mathrm{n}_{2}$ obtained from the best fit curve shown in figure (3) inherently incorporates the variability of $\mathrm{D}$ if any, present in the current simulations.

\section{Prediction equation based on the local magnitude of TKE \\ $(k)$ and local magnitude of integral length scale $\left(L_{u}\right)$}

Figure (4) shows another form of the spatial decay of TKE profiles from both the experiments and LES data, but with the $\mathrm{x}$ ordinate scaled with the local non-dimensional TKE, $\mathrm{k}$, and the local magnitude of the integral length scale $\left(\mathrm{L}_{\mathrm{u}}\right)$. Figure (6) reveals a linear relationship between $\left(\mathrm{k} / \mathrm{k}_{0}\right)$ and $\left(\mathrm{x}-\mathrm{x}_{0}\right) *\left(\mathrm{k}^{\prime}\right)^{0.5} / \mathrm{L}$ which implies

$$
\frac{\frac{\mathrm{d}}{\mathrm{dx}}\left(\mathrm{k} / \mathrm{k}_{0}\right)}{\frac{\mathrm{d}}{\mathrm{dx}}\left(\mathrm{x}-\mathrm{x}_{0}\right)^{*}\left(\mathrm{k}^{\prime}\right)^{0.5} / \mathrm{L}} \approx \mathrm{C}
$$

This holds true and is manifest through the power law dependency of $\mathrm{k}$ and $\mathrm{L}_{\mathrm{u}}$ along the streamwise direction which is given by the following equation

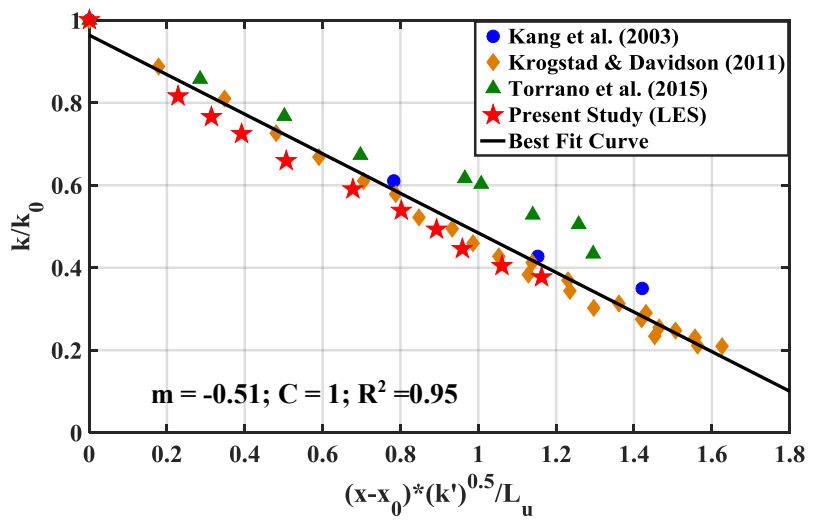

Figure 4. Spatial decay of TKE profiles for different experiments and the present LES data with the $\mathrm{x}$ ordinate scaled with local $\mathrm{k}$ ' and $\mathrm{L}_{\mathrm{u}}$

$\overline{\mathrm{k}_{0}}=\frac{\mathrm{k}^{\prime}}{\mathrm{k}_{0}{ }^{\prime}}=\left(\mathrm{a}_{1} \mathrm{x}+1\right)^{-\mathrm{p}}$

$\frac{\mathrm{L}_{\mathrm{u}}}{\mathrm{L}_{\mathrm{u} 0}}=\left(\begin{array}{ll}\mathrm{a}_{2} \mathrm{x} & 1\end{array}\right)^{\mathrm{q}}$

In order to arrive at linearity; $\left(\mathrm{a}_{1} \approx \mathrm{a}\right)$ and a slight deviation from the numbers can affect the linearity of the experimental data sets and the LES results seen in figure (4), which is simply a consequence of the curve fitting performed for the best fit for equations (11), (12). Similarly, $\mathrm{p}$ and $\mathrm{q}$ are closely related to each other in the form of $q / 2-p=1$ for the LES results, which is verified by the exponents derived in [9]. The left-hand side of equation (4) can be then solved to arrive at a constant value. The best linear fit line is drawn in the figure which gives a $\mathrm{R}^{2}=0.96$ with the slope $\mathrm{m}$ being -0.51 and the intercept $\mathrm{C}$ being 1 . Hence the final equation of the spatial decay of TKE stands in the form of

$\left(\frac{\mathrm{k}}{\mathrm{k}_{0}}\right)=\left(\frac{\mathrm{k}_{0}^{\prime}}{\mathrm{k}_{0}}\right)=\mathrm{m}\left(\frac{\left.\mathrm{x}-\mathrm{x}_{0}\right)^{*}\left(\mathrm{k}^{\prime}\right)^{0.5}}{}\right)+\mathrm{C}$

It is worthwhile mentioning that the correlation functions presented in equation (3) along with equation (7) also helps one to predict the initial TKE $\left(\mathrm{k}_{0}\right)$ and length scale values $\left(\mathrm{L}_{\mathrm{u}}\right)$, provided one knows the local values of TKE (k) and length scale $\left(\mathrm{L}_{\mathrm{u}}\right)$, thus giving another method of estimating the statistical turbulence parameters in the domain.

There are, altogether, 3 sets of data, which have been used here for the predictive methodology, in accordance with the previous experimental studies, to estimate the magnitude of the TKE and $\mathrm{L}_{\mathrm{u}}$ values. The values of the fitted coefficients for all sets of data are summarized in table IV and V.

TABLE IV Constants obtained from best regression curve fitting procedure using the method of non-linear least squares

\begin{tabular}{|c|c|c|c|c|}
\hline $\begin{array}{c}\text { Normalization } \\
\text { parameter of the } \\
\mathbf{x} \text { ordinate }\end{array}$ & $\mathbf{R}^{2}$ & $\begin{array}{c}\text { Decay } \\
\text { coefficient } \\
(\mathbf{A})\end{array}$ & $\begin{array}{c}\text { Decay } \\
\text { exponent } \\
\text { (n) }\end{array}$ & $\begin{array}{c}\text { Equation } \\
\text { number }\end{array}$ \\
\hline $\mathrm{k}_{0}{ }^{\prime}$ and $\mathrm{L}_{\mathrm{u}}$ & 0.99 & 0.27 & 2.38 & 2 \\
\hline $\mathrm{k}_{0}{ }^{\prime}$ and $\mathrm{L}_{\mathrm{u} 0}$ & 0.97 & 0.44 & 1.16 & 3 \\
\hline
\end{tabular}

TABLE V Constants obtained from best regression curve fitting procedure using the method of non-linear least squares

\begin{tabular}{|c|c|c|c|c|}
\hline $\begin{array}{c}\text { Normalization } \\
\text { parameter of the } \\
\mathbf{x} \text { ordinate }\end{array}$ & $\mathbf{R}^{\mathbf{2}}$ & $\mathbf{m}$ (slope) & $\begin{array}{c}\text { C (intercept } \\
\text { on the } \mathbf{y} \\
\text { ordinate) }\end{array}$ & $\begin{array}{c}\text { Equation } \\
\text { number }\end{array}$ \\
\hline $\mathrm{k}^{\prime}$ and $\mathrm{L}_{\mathrm{u}}$ & 0.95 & -0.51 & 1 & 4 \\
\hline
\end{tabular}

It is worth mentioning that these correlations $(2,3)$, while written in logarithmic form along with the correlation given by equation (7), each represent a simple linear form of equations involving one known and one unknown variable which can then be used to estimate either the local turbulence parameters at any downstream location or to predict the initial turbulent parameters for any turbulent flow. Thus, the equations (2), (3) and (7) complete the prediction tool for designing turbulence generating grids. More information about the applicability of these prediction equations can be found in [35].

\section{CONCLUSIONS}

The present work attempts to simulate freely decaying isotropic homogenous turbulence using Large Eddy Simulation (LES). The current results show good quantitative agreement with the previous experimental results from [10], [15], [16]. Three different correlations were formulated which can be useful in estimating the local and initial values of turbulent parameters (i.e. TKE and $\mathrm{L}_{\mathrm{u}}$ ) which can then aid the design of wind tunnel experiments. 


\section{ACKNOWLEDGMENT}

This work was made possible by the facilities of the Shared Hierarchical Academic Research Computing Network (SHARCNET: www.sharcnet.ca) and Compute/Calcul Canada.

\section{REFERENCES}

[1] S. B. Pope, Turbulent Flows. Cambridge, UK: Cambridge University Press, 2000. doi: 10.1017/CBO9780511840531.

[2] J. C. Simonich and P. Bradshaw, "Effect of free stream turbulence on heat transfer through a turbulent boundary layer," J. Heat Transfer, ASME, vol.100, no. 4, pp. 671-677, 1978. doi: 10.1115/1.3450875.

[3] M. F. Blair, "Influence of free stream turbulence on turbulent boundary layer heat transfer and mean profile development, Part-I-experimental data," J. Heat Transfer, vol. 105, pp. 33-40, 1983. doi: 10.1115/1.3245555.

[4] M. F. Blair, "Influence of free stream turbulence on turbulent boundary layer heat transfer and mean profile development, Part-II-experimental data," J. Heat Transfer, vol. 105, pp. 41-47, 1983. doi: 10.1115/1.3245557.

[5] G. K. Batchelor and A. A. Townsend, "Decay of isotropic turbulence in the initial period," Proc. R. Soc. London, Ser. A, vol. 193, no. 1035, pp. 539-558, 1948. doi: 10.1098/rspa.1948.0061.

[6] J. Tan-Atichat, H. M. Nagib, and R. I. Loehrke, "Interaction of freestream turbulence with screens and grids: a balance between turbulence scales." J. Fluid Mech., vol. 114, pp. 501-28, 1982. doi: $10.1017 / \mathrm{S} 0022112082000275$.

[7] P. Lavoie et al., "An effect of initial conditions on decaying grid turbulence at low R $\lambda$," Exp. Fluids, vol. 39, pp. 865-874, 2005. doi: $10.1007 / \mathrm{s} 00348-005-0022-8$.

[8] T. Ishida, P. A. Davidson, and Y. Kaneda, "On the decay of isotropic turbulence," J. Fluid Mech., vol. 564, pp. 455-475, 2006. doi: $10.1017 / \mathrm{S} 0022112006001625$.

[9] P. A. Krogstad and P. A. Davidson, "Is grid turbulence Saffman turbulence?," J. Fluid Mech., vol. 642, pp. 373-394, 2009.

[10] H. S. Kang, S. Chester, and C. Meneveau, "Decaying turbulence in an active-grid-generated flow and comparisons with large-eddy simulation," J. Fluid Mech., vol. 480, pp. 129-160, 2003. doi: $10.1017 / \mathrm{S} 0022112002003579$.

[11] N. Mordant, "Experimental high Reynolds number turbulence with an active grid," Am. J. Phys., vol. 76, no. 12, pp. 1092-1098, 2008. doi: $10.1119 / 1.2980582$.

[12] B. Mazzi, and J. C. Vassilicos, "Fractal-generated turbulenc," J. Fluid Mech., vol. 502, no. 1, pp. 65-87, 2004. doi: 10.1017/S0022112003007249.

[13] R. E. Seoud, and J. C. Vassilicos, "Dissipation and decay of fractalgenerated turbulence," Phys. Fluids, vol. 19, no. 10, pp. 105-108, 2007. doi: $10.1063 / 1.2795211$.

[14] D. Hurst and J. C. Vassilicos, "Scalings and decay of fractalgenerated turbulence," Phys. Fluids, vol. 19, no. 3, pp. 1-31, Mar. 2007. doi: $10.1063 / 1.2676448$.

[15] P. A. Krogstad and P. A. Davidson, "Freely decaying, homogeneous turbulence generated by multi-scale grids," J. Fluid Mech., vol. 680, pp. 417-434, 2011. doi: 10.1017/jfm.2011.169.

[16] I. Torrano et al., "Comparison of experimental and RANS-based numerical studies of the decay of grid-generated turbulence," J. Fluids Eng., vol. 137, no. 6, pp. 1-12, 2015. doi: 10.1115/1.4029726.

[17] J. Smagorinsky, "General circulation experiments with the primitive equations: I. The basic experiment," Mon. Weather Rev., vol. 91, pp. 99-164, 1963. doi: 10.1175/1520-0493(1963)091\%3C0099:GCEWTP\%3E2.3.CO;2
[18] Ansys, ANSYS FLUENT Theory Guide. Canonsburg, PA: Ansys Inc., 2005, pp. 1-814.

[19] M. Germano et al., "A dynamic subgrid-scale eddy viscosity model," Phys. Fluids, vol. 3, no. 7, pp.1760-1765, 1991. doi: 10.1063/1.857955.

[20] R. Courant, K. Friedrichs, and H. Lewy, "On the partial difference equations of mathematical physics," IBM J. Res. Dev., vol. 11, no. 2 , pp. 215-234, 1967. doi: 10.1147/rd.112.0215.

[21] SHARCNET (2015). A consortium of colleges, universities and research institutes operating a network of high-performance computer clusters across southwestern, central and northern Ontario [Online]. Available : www.sharcnet.ca

[22] ICEM v.16.0. Ansys. Product [Online], 2016. Available: http:// www.ansys.com

[23] J.-L. Aider and A. Danet, "Large-eddy simulation study of upstream boundary conditions influence upon a backward-facing step flow," C.R. Mecanique, vol. 53, no. 334, pp. 447-453, 2006. doi: 10.1016/j.crme.2006.05.004.

[24] P. Sagaut et al., "Turbulent in-flow conditions for LES of subsonic and supersonic wall-bounded flows," AIAA J., vol. 42, pp. 469-478, 2003. doi: $10.2514 / 1.3461$.

[25] G. R. Tabor and M. H. Baba-Ahmadi, "Inlet conditions for large eddy simulation: A review," Comput. Fluids, vol. 39, pp. 553-567, 2010. doi: 10.1016/j.compfluid.2009.10.007.

[26] H. Abasosha et al., "Consistent inflow turbulence generator for LES evaluation of wind-induced responses for tall buildings," J. Wind Eng. Ind. Aerodyn., vol. 142, pp. 198-216. 2015.

doi: 10.1016/j.jweia.2015.04.004.

[27] N. J. Georgiadis, D. P. Rizzetta, and C. Fureby, "Large-eddy simulation: Current capabilities, recommended practices, and future research," AIAA J., vol. 48, no. 8, pp.1772-1784, 2010. doi: $10.2514 / 1 . J 050232$

[28] G. Comte-Bellot and S. Corrsin, "The use of a contraction to improve the isotropy of grid-generated turbulence," J. Fluid Mech., vol. 25, no. 4, pp. 657-682, 1966. doi: 10.1017/S0022112066000338.

[29] G. Comte-Bellot and S. Corrsin, "Simple Eulerian time correlation of full-and narrow-band velocity signals in grid-generated, 'isotropic'turbulence," J. Fluid Mech., vol. 48, no. 2, pp. 273-337 (1971). doi: 10.1017/S0022112071001599.

[30] H. L. Dryden, "A review of the statistical theory of turbulence," Q. Appl. Math., vol 1, no. 1, pp.7-42, 1943. doi: 10.1090/qam/8209.

[31] M. S. Mohamed and J. C. LaRue, "The decay power law in gridgenerated turbulence," J. Fluid Mech., vol. 219, pp. 195-214, 1990. doi: $10.1017 / \mathrm{S} 0022112090002919$.

[32] T. Ishida, P. A. Davidson, and Y. Kaneda, "On the decay of isotropic turbulence," J. Fluid Mech., vol. 564, pp. 455-475, 2006. doi: $10.1017 / \mathrm{S} 0022112006001625$.

[33] J. C. Bennett and S. Corrsin, "Small Reynolds number nearly isotropic turbulence in a straight duct and a contraction," Phys. Fluids, vol. 21, no. 12, pp. 2129-2140, 1978. doi: 10.1063/1.862168.

[34] P. Lavoie, L. Djenidi, and R. A. Antonia, "Effects of initial conditions in decaying turbulence generated by passive grids," J. Fluid Mech., vol. 585, pp. 395-420, 2007. doi: 10.1017/S0022112007006763.

[35] D. Sarkar, "A numerical tool for predicting the spatial decay of freestream turbulence," M.Eng.Sci. thesis, Dept. Mech. Mater. Eng., Western Univ., London, ON, 2018. 


\title{
Water Droplet Impact and Spreading on a Narrow Gap
}

\author{
D. Jordan Bouchard and Sanjeev Chandra \\ Department of Mechanical Engineering \\ University of Toronto \\ Toronto, Canada
}

\begin{abstract}
The impact and spreading of a water droplet on a gap between two parallel plates has been studied experimentally. A deionized water droplet $(2.03 \mathrm{~mm}$ diameter) impacted the plates at velocities of $0.06,0.5,1.0$, and $1.5 \mathrm{~m} / \mathrm{s}$; the tested gap spacings of the parallel glass plates were 50 , 100 , and $150 \mu \mathrm{m}$. Using a high-speed camera, we simultaneously photographed the drop spreading both above and within the gap. We show that water begins to penetrate the gap immediately after impact. On the largest spacing tested, up to $10 \%$ of the initial drop volume can penetrate the gap before the maximum spreading diameter is reached.
\end{abstract}

\section{drop spreading; drop impact; parallel plates;}

\section{INTRODUCTION}

Many porous surfaces that droplets can interact with paper, soil, textiles and fabrics - are opaque. This opaqueness makes it difficult to observe how the porous surface interacts with the drop during droplet impact and spreading. In addition, an impacting drop will spread to its maximum diameter in a few milliseconds, well below the temporal resolution of nondestructive scanning techniques.

There remains some uncertainty as to the amount of water that absorbs into a porous media during droplet impact and spreading, and if this volume of water influences the maximum spread of the droplet. Chandra \& Avedisian [1] and Lee et al. [2] analyzed photographs of the drop impacting on a porous surface to estimate the volume of water that penetrated a porous material. They conclude that the volume of water is negligibly small for the materials they studied. Roisman et al. [3] find that a porous surface will increase the probability of drop deposition instead of splashing, which they suspect is due to some liquid penetration during impact. Lembach et al. [4] and Yamamoto et al. [5] show that water penetration can occur during droplet impact on their fabricated porous medias. Their medias were fabricated in such a way that allowed them to simultaneously photograph the drop above and below the surface, but the focus of their papers was not on the maximum spread of the droplet.

Thus, there is a need for more experiments that can simultaneously photograph a droplet above and below a surface during impact in order to understand the role the pore size has on droplet spreading. This is the first experiment in a series designed to investigate how porous media affects how a droplet spreads and penetrates a porous media. Here, we study the influence of a long narrow pore, created by two closely spaced glass plates.

\section{EXPERIMENTAL APPARATUS}

Two uncoated N-BK7 borosilicate glass plates $(50 \mathrm{~mm} \times 50$ mm x $4 \mathrm{~mm}$ ) (Edmund Optics, Inc; stock\# 47-944) were used to create a narrow gap between two parallel surfaces. The gap spacing between the two plates was set by inserting two steel feeler gauges between the plates and then clamping the plates together. Between individual trials, the surfaces of the glass plates were rinsed with deionized water and dried with compressed air. Periodically, the surfaces were cleaned with ethanol and thoroughly rinsed with tap water and then deionized water.

A $10 \mathrm{ml}$ BD syringe was used in a syringe pump to dispense deionized water through a 33-gauge syringe needle (\#91033, Hamilton) at a flow rate of $10 \mu \mathrm{l} / \mathrm{min}$. A separate test found the average diameter of a drop generated by this method to be $2.03 \mathrm{~mm}(\mathrm{~N}=50,95 \% \mathrm{CI})$. The three gap sizes tested were 50,100 , and $150 \mu \mathrm{m}$. The plates were also clamped together with no feeler gauges to get a fourth gap size of approximately $0 \mu \mathrm{m}$. Drop impact velocities of $0.06,0.5,1.0$, and $1.5 \mathrm{~m} / \mathrm{s}$ were tested by increasing the height at which the drop detached from the syringe needle. Each of the impact velocity-gap spacing pairs was repeated between three and five times. Very good repeatability of the data was achieved.

A high-speed camera (FASTCAM SA5, Photron, USA) equipped with a $105 \mathrm{~mm} \mathrm{f} / 2.8$ Nikon lens, an extension bellow, and a $55 \mathrm{~W}$ LED light (AOS Technologies AG) were used to photograph the droplet spread at a resolution $16.8 \mu \mathrm{m} /$ pixel. Photographs were taken at $4000 \mathrm{fps}$, shutter speed of 1/6000 s. A schematic of the experiment is shown in Figure 1.

Image analysis was performed in MATLAB using an automated script to convert the photographs from the highspeed camera to binary images. The measurement of interest was obtained by counting the number of pixels and multiplying it by the image resolution. 


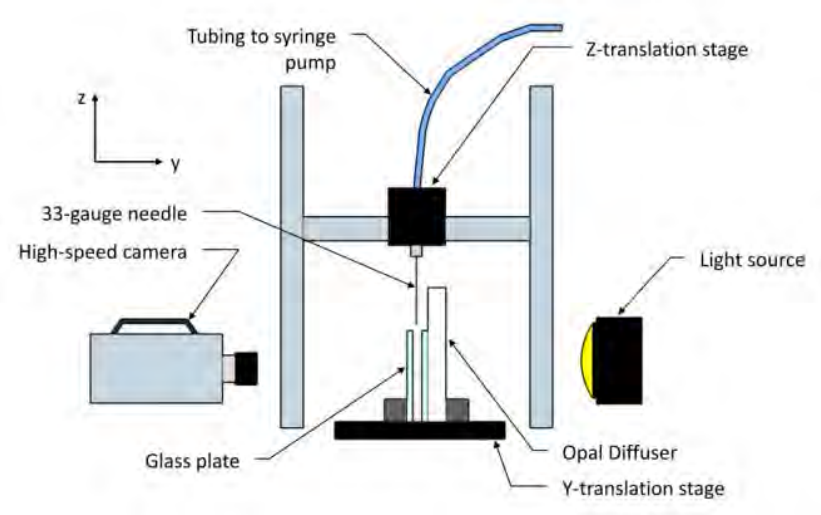

Figure 1. Schematic representation of experiment apparatus

\section{RESULTS AND DISCUSSION}

We compare the maximum spreading diameter by first making the spreading diameter and time dimensionless. The droplet spreading diameter is made dimensionless by dividing it by the initial drop diameter, $\beta=\mathrm{D} / \mathrm{D}_{0}$; time is made dimensionless by multiplying it by the impact velocity and dividing by the initial drop diameter, $\tau=\mathrm{Ut} / \mathrm{D}_{0}$, where $\mathrm{U}$ is the impact velocity. Figure 2 . shows the dimensionless spreading coefficient, $\beta$, plotted against dimensionless time, $\tau$. The top, middle, and bottom charts of Figure 2. show the effect of the gap spacing at the constant impact velocities of $0.5,1.0$, and $1.5 \mathrm{~m} / \mathrm{s}$, respectively.

At each impact velocity, the maximum spreading diameter increases as the gap spacing decreases. In the $1.5 \mathrm{~m} / \mathrm{s}$ impact velocity graph of Figure 2 ., where this observation is most pronounced, the maximum spreading diameter doubles as the gap spacing is reduced from $150 \mu \mathrm{m}$ to $0 \mu \mathrm{m}$.

Also, the time required to reach the droplet maximum spreading diameter increases as the gap size decreases. At all three impact velocities the drop takes the longest time to spread at zero gap spacing. For example, at an impact velocity of 1.5 $\mathrm{m} / \mathrm{s}$, the maximum spreading diameter is reached shortly after $\tau$ $=1$ for the $150 \mu \mathrm{m}$; the $100 \mu \mathrm{m}$ and $50 \mu \mathrm{m}$ gap sizes take progressively longer, and the longest time of $\tau=2.7$ is reached for the at $0 \mu \mathrm{m}$.

The maximum spreading diameter and time to maximum spread increase with a decreasing gap size because there is less kinetic energy and liquid available for the drop to spread when a gap is present. We observe that the drop starts to penetrate the gap almost immediately after impact. Photographs of the droplet spreading on the gap at $\tau=0.5$ are shown in Figure 3 . At every gap spacing and impact velocity a semi-circular profile can be seen developing below the drop before the maximum diameter is reached. With less water remaining in the drop it is unable to spread as far. Time to the maximum spreading diameter is reduced because there is less distance to travel.

It is also interesting to point out in Figure 3. that the shape of the drop is very similar for each impact velocity at each gap spacing. This is despite there being an increasingly larger volume of water within the gap at successively larger gap spacings. What this means is that immediately after impact the droplet behavior is dominated by inertia, but the influence of the gap spacing becomes apparent before maximum spread is reached. We can see this transition in Figure 2. : the spreading coefficient for each gap size lie on a single curve immediately after impact and then start to diverge before the maximum spreading diameter is reached.

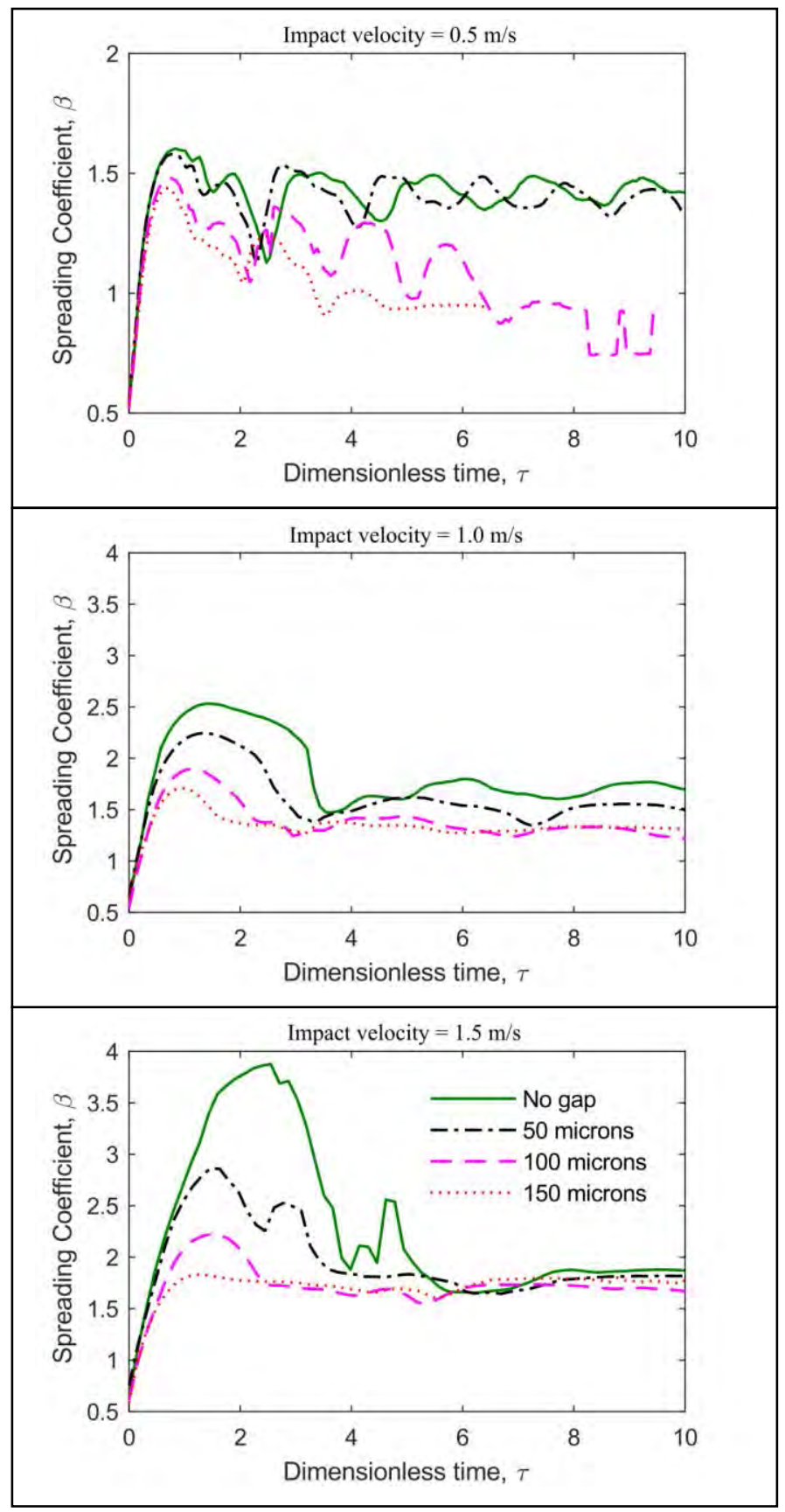

Figure 2. Comparison of dimensionless spreading diameter for impact velocities of $0.5,1.0$, and $1.5 \mathrm{~m} / \mathrm{s}$. 
Shortly after the drop contacts the glass plates and begins to spread, water wicks into the gap. The width of the profile grows at the same rate as the droplet spreads, but the depth of the profile grows at a different rate. For the $50 \mu \mathrm{m}$ spacing in Figure 3., the profile depth reached at $\tau=0.5$ decreases slightly as the impact velocity increases. This suggests that the growth rate of the profile width depends on the impact velocity, and that the growth rate of the profile depth depends on the gap spacing, which determines the developed capillary pressure.

Figure 4. shows the fraction of the initial drop volume that penetrates between each plate spacing. The top, middle, and bottom chart are plotted with the gap spacing held constant at 50,100 , and $150 \mu \mathrm{m}$, respectively. In the initial stages of the droplet impact and just prior to the maximum spreading diameter being reached, the volume of water in the gap is approximately the same for the $0.5,1.01$ and $1.5 \mathrm{~m} / \mathrm{s}$ impact velocities. 0also shows that the flow rate into the gap is constant shortly after impact, times greater than $\tau=0.25$. Water penetration into the gap during droplet spreading is mostly capillary driven.

The volume of water that can penetrate the gap during droplet spreading is significant for the larger gap spacings. For the 100 and $150 \mu \mathrm{m}$ gap spacing, between $5-10 \%$ of the drop's initial volume has already entered the gap at $\tau=0.5$. For the 50 $\mu \mathrm{m}$ gap spacing the volume of water that has left the drop by this time is less significant. Despite this large difference in the volume of water that has penetrated the gap, the drops in Figure 3. look very similar for a constant impact velocity. This highlights the difficulty of estimating the volume of water that has penetrated a porous medium during the droplet spreading phase.

\section{CONCLUSIONS}

When a drop impacts a narrow gap, the maximum spreading diameter will be less than the maximum spreading diameter that could be attained on a solid surface. This is partly due to water penetrating the gap during the spreading phase, so there is less water available within the drop to spread. The volume of water that penetrates the gap is mostly dependent on the width of the gap. A higher fraction of the initial drop volume will be lost to the gap at the time of maximum spread the larger the gap spacing is. The water penetrates mostly due to capillary forces - the impact forces only have a minor influence

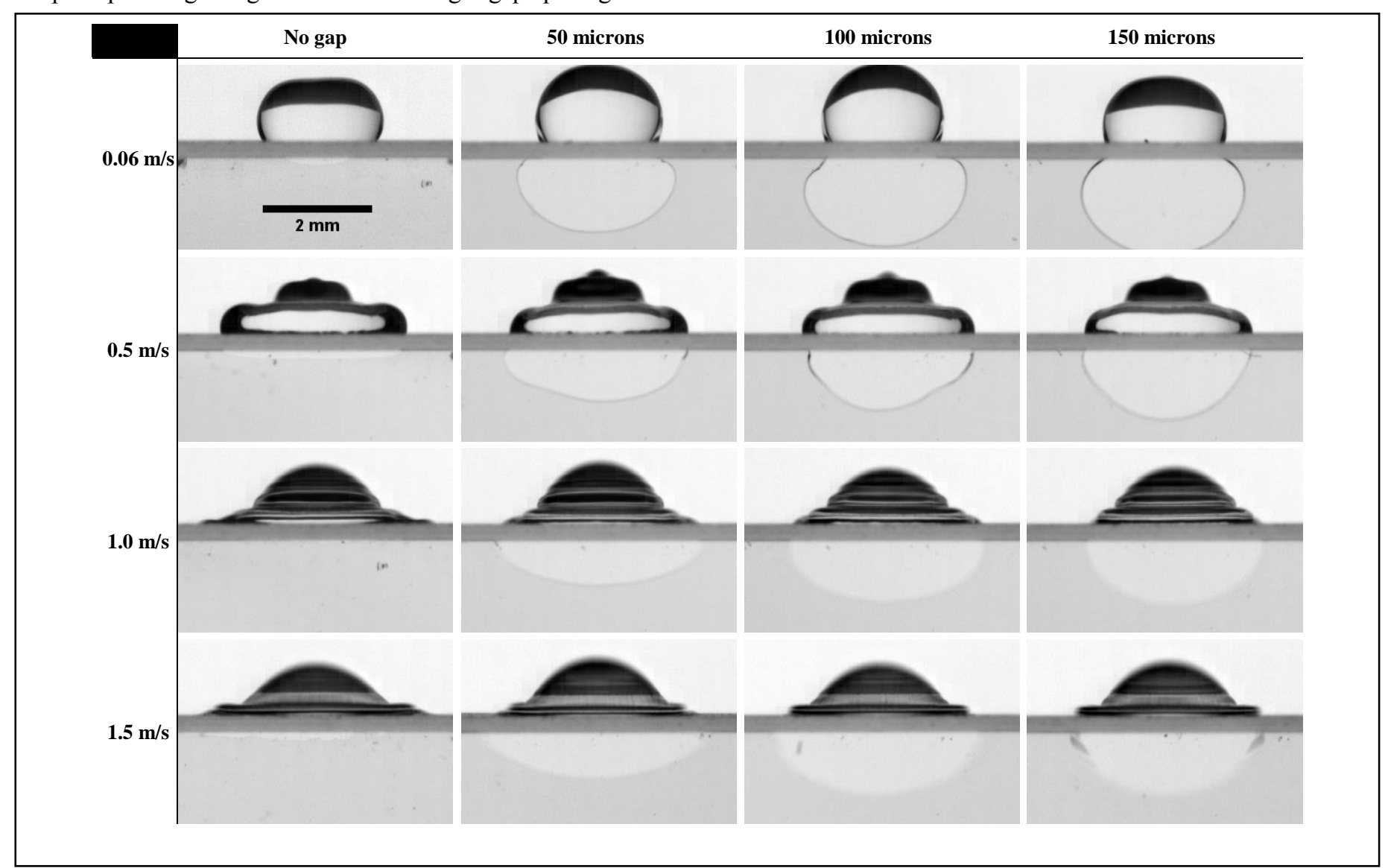

Figure 3. Photographs of a drop impacting on a narrow gap. Rows are a constant impact velocity; columns are a constant gap spacing. Each photograph was taken at dimensionless time, $\tau=0.5$. The images were cropped, scaled, and adjusted for contrast and brightness in ImageJ [6] 


\section{REFERENCES}

[1] S. Chandra and C. T. Avedisian, "Observations of droplet impingement on a ceramic porous surface," Int. J. Heat Mass Transf., vol. 35, no. 10, pp. 23772388, Oct. 1992. doi: 10.1016/0017-9310(92)90080-C.

[2] J. B. Lee, D. Derome, and J. Carmeliet, "Drop impact on natural porous stones," J. Colloid Interface Sci., vol. 469, pp. 147-156, May 2016. doi: 10.1016/j.jcis.2016.02.008.
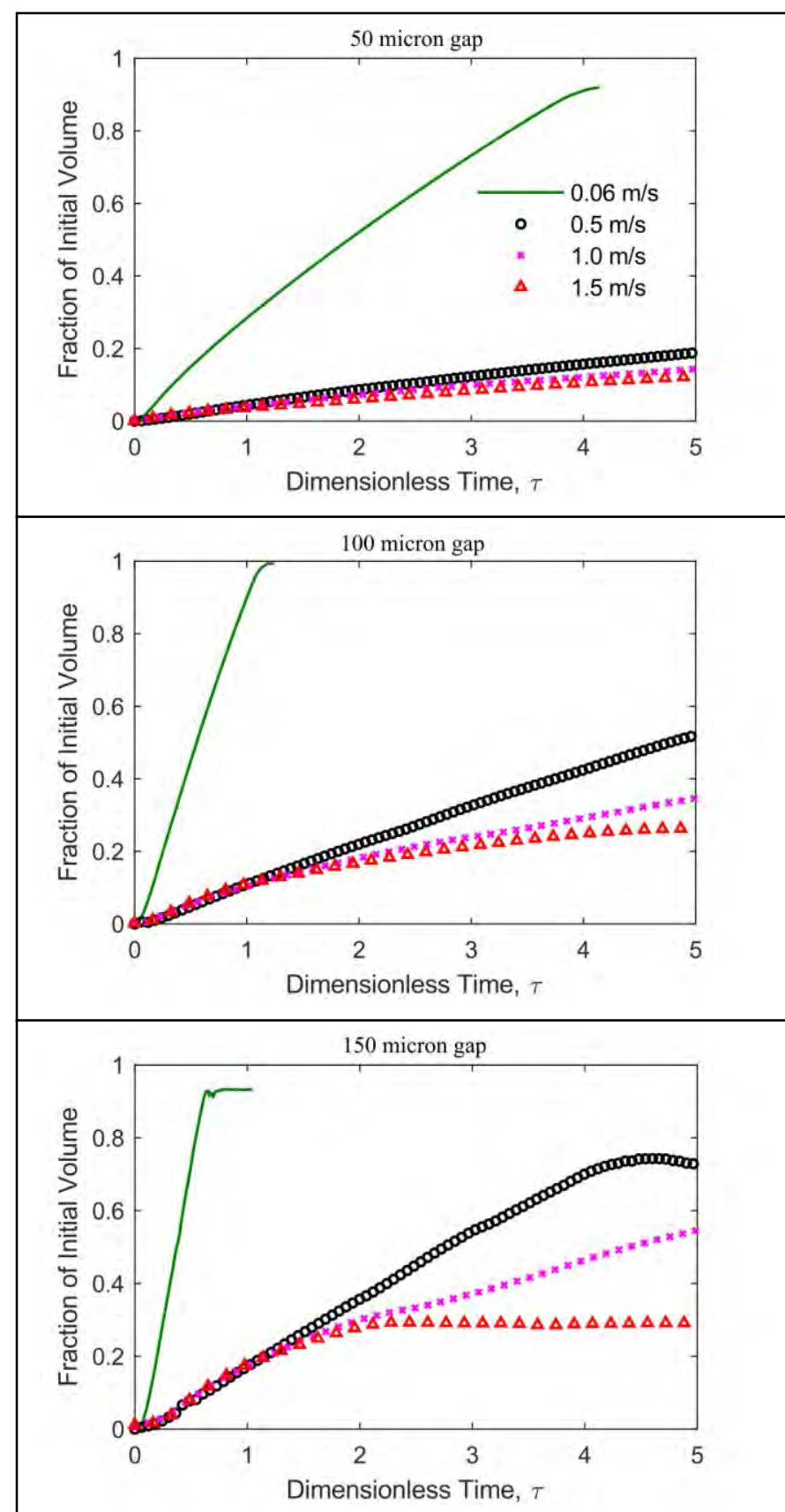

Figure 4. Fraction of initial drop volume that penetrates the plates for gap spacings of 50,100 , and $150 \mu \mathrm{m}$.
[3] I. V. Roisman, A. Lembach, and C. Tropea, "Drop splashing induced by target roughness and porosity: The size plays no role," Adv. Colloid Interface Sci., vol. 222, pp. 615-621, Aug. 2015. doi: 10.1016/j.cis.2015.02.004.

[4] A. N. Lembach, I. V Roisman, and C. Tropea, "Drop impact on porous media," in DIPSI Workshop, Bergamo, Italy, May 2011, pp. 1-5.

[5] K. Yamamoto, H. Takezawa, and S. Ogata, "Droplet impact on textured surfaces composed of commercial stainless razor blades," Colloids Surf., A, vol. 506, pp. 363-370, Oct. 2016. doi: 10.1016/j.colsurfa.2016.06.024.

[6] C. A. Schneider, W. S. Rasband, and K. W. Eliceiri, "NIH Image to ImageJ: 25 years of image analysis," Nat. Methods, vol. 9, no. 7, pp. 671-675, June 2012. doi: 10.1038/nmeth.2089. 


\title{
Flow Over a Wall-mounted Finite-length Square Cylinder
}

\author{
J. Chen \\ Mechanical, Automotive and Materials Engineering \\ University of Windsor \\ Windsor, Canada \\ chen112p@gmail.com
}

\author{
R.M. Barron \\ Mechanical, Automotive and Materials Engineering \\ University of Windsor \\ Windsor, Canada
}

\author{
R. Balachandar \\ Mechanical, Automotive and Materials Engineering \\ University of Windsor \\ Windsor, Canada \\ rambala@uwindsor.ca
}

\begin{abstract}
A conventional way of studying the flow characteristics of flow over a bluff body is by extracting the velocity distribution in horizontal planes crossing the bluff body near the bed, mid-height and close to the free-end location. The intention of selecting the planes at these levels is to examine the horseshoe vortex and the bed effect, flow separation at the mid-height, and the downwash flow from the free-end. In some cases, where a relatively short cylinder is studied along with a taller cylinder, the mid-height level of each may present very different flow structures. It is proposed in this study that a better way of selecting the planes of interest could be based on the pressure distribution imposed on the front surface of the bluff body by the approaching flow. This paper uses a sample numerical study of wall-mounted finitelength square cylinder with three aspect ratios of 1,2 and 4 , immersed in the air flow with a thin boundary layer. The horizontal planes based on the front face pressure distribution are selected and examined to show the advantage of the proposed method.
\end{abstract}

Keywords: Bluff body; flow separation; Detached-eddysimulation

\section{INTRODUCTION}

A wall-mounted finite-length square cylinder immersed in a flow field has many engineering applications in the real world, such as flow over low-rise buildings, skyscraper stacks, chimney stacks, etc. The aspect ratio $(A R=h / d$, where $h$ and $d$ are the height and width of the object, respectively) plays an important role in establishing the flow features around the body (Kawamura et al. [1]). As AR increases, the shear layer in the overhead region induced by the top edge becomes more energetic and then interacts with the base vortices. This phenomenon slowly diminishes when AR approaches a critical value.
When an oncoming flow with a boundary layer impinges on the front surface of a square cross-section cylinder, a stagnation point (or zone) is induced on the front side of the cylinder surface (Fig. 1). The location of the stagnation point $\left(\mathrm{h}_{\text {stg }}\right)$ is defined by the maximum value or the middle point of the near-constant region on the pressure distribution of the front surface. The flow below the stagnation streamline rolls up into the horseshoe vortex (HSV), while the flow above it separates from the top edge of the body. The separated flow forms the wake region as indicated in the schematic.

When discussing the wake structures behind the cylinder of different $A R$, researchers tend to present results in different horizontal planes. These planes usually include the one at middepth $(50 \% \mathrm{~h})$, one near the wall $(25 \% \mathrm{~h})$ and one near the free-end of the cylinder $(75 \% \mathrm{~h})$. However, the induced flow structures are not simply decided by the cylinder's height. When comparing flow past cylinders of different height, it is quite possible that the flow structures are different at a certain value of the cylinder height to width ratio. For instance, the mid-height level of a short cylinder may be heavily influenced

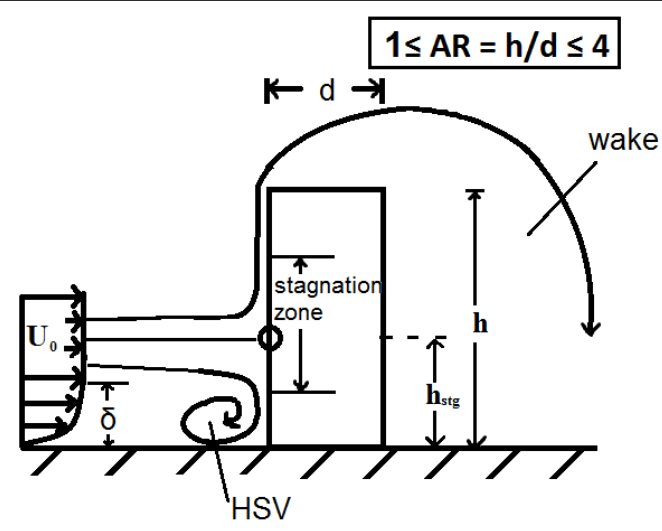

Fig. 1 Central plane schematic of flow over a wall-mounted finitelength square cylinder 
by the bed-effect, but the mid-height level of a taller object may not be influenced either by the distance to the wall or to the top edge of the body. A new scheme based on the pressure distribution on the object's front surface which could be potentially used in the analysis of the flow over a bluff body problem is introduced in this paper.

\section{COMPUTATIONAL DOMAIN}

The schematic of the flow field is shown in Fig. 2. A wallmounted square cylinder with the width (d) of $12.7 \times 10^{-3} \mathrm{~m}$ is immersed in an air flow. The air flow is at a Reynolds number of 12,000 (based on freestream velocity and cylinder width), with the free stream velocity of $15 \mathrm{~m} / \mathrm{s}$ and dynamic viscosity of $1.855 \times 10^{-5} \mathrm{~Pa}$-s. Three aspect ratios $(\mathrm{AR}=1,2$ and 4$)$ of the square cylinder are studied.

The origin of the coordinate system is located at the center of the square at the bed level. The $\mathrm{x}-, \mathrm{y}$ - and $\mathrm{z}$ - coordinate directions correspond to the streamwise, spanwise and normal directions, respectively. The two side-walls and the ceiling are slip-walls, and the bottom bed and the cylinder faces are noslip walls. The square cylinder is placed $6 \mathrm{~d}$ downstream of the inlet, $40 \mathrm{~d}$ upstream of the pressure outlet, $7 \mathrm{~d}$ under the ceiling (9d and $10 \mathrm{~d}$ for $A R=2$ and $A R=1$, respectively), and $15 \mathrm{~d}$ away to each side-wall. The side length of the computational domain is guided by the work of Nasif et al. [2], which showed the need for a distance of $15 \mathrm{~d}$ between each side-wall and the cylinder to maintain a small enough blockage ratio. This length is larger than that used by Saeedi and Wang [3] in their direct numerical simulation (DNS) of a similar flow field.

In the experimental study by El Hassan et al. [4], the cylinder was placed at the location along the bottom wall where the boundary layer thickness was $9.1 \times 10^{-3} \mathrm{~m}(72 \% \mathrm{~d})$ in the absence of the cylinder. To properly benchmark the numerical setup with the experiment, the inlet condition is set to reproduce the velocity profile at the same location in the absence of the cylinder. Based on a grid independency study, the proper grid was chosen to simulate the flow field using Detached Eddy Simulation. The results were validated using the experimental results of El Hassan et al. [4] in several horizontal and vertical planes through the wake.

\section{RESULTS AND DISCUSSION}

Fig. 3 shows the pressure distribution on the front surface of the cylinder along the central plane $(y / d=0)$. Two points of

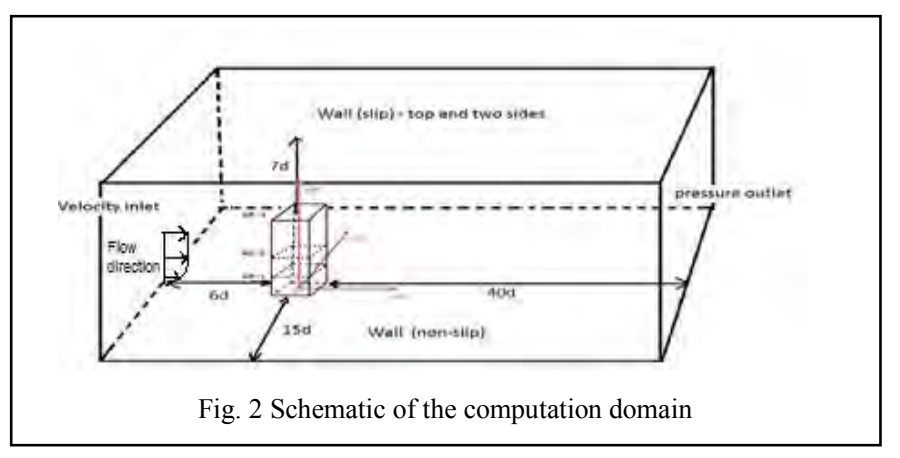

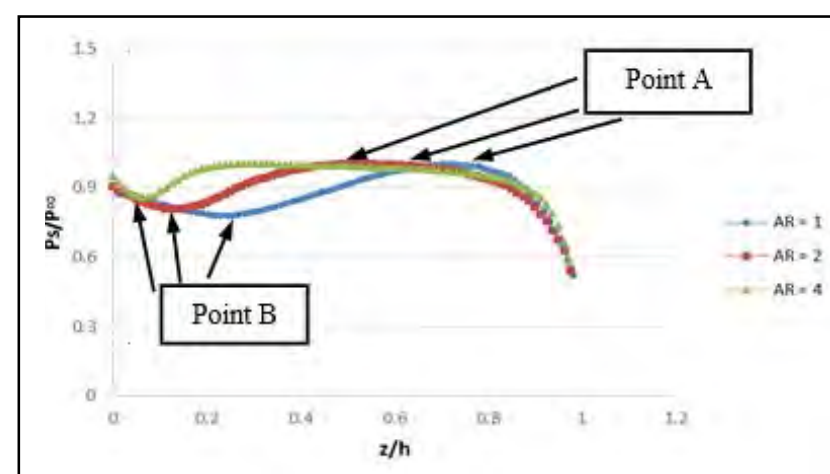

Fig. 3 Pressure coefficient distribution on the front surface of the cylinder at central plane $\mathrm{y} / \mathrm{d}=0$

interest are labeled as Point $\mathrm{A}$ and Point B, which are the maximum pressure (or maximum pressure zone) on the surface and local minimum pressure, respectively. Point A characterizes the stagnation point in each case. Only in the case of $\mathrm{AR}=1$, a distinctive maxima is found on the cylinder's front surface at $\mathrm{z} / \mathrm{d}=0.727$, while the cylinders $\mathrm{AR}$ $=2$ and 4 have a near constant pressure region. In the cases of $\mathrm{AR}=2$ and 4, Point $\mathrm{A}$ is marked at the center of the constant pressure region, which are $\mathrm{z} / \mathrm{d}=1.2$ and 2 , respectively.

As seen in Fig. 3, the constant pressure region increases in size as the cylinder AR increases. Since the free-end of the cylinder moves further away from the bed, there is more space in the middle section that is not influenced by either end. The stagnation point's location with respect to the cylinder width increases as the AR increases, and the stagnation point's location with respect to the cylinder's height decreases as the cylinder's AR increases.

Point B describes the local minimum pressure on the front surface and lies closer to the bed region. The location of Point $\mathrm{B}$ with respect to the cylinder's width are relatively close for all three ARs. Therefore, Point B can be understood as a critical point induced by the bed effect, namely the horseshoe vortex. The core of HSV (where the highest vorticity is found) is roughly half of the distance between Point B and the bed. If the HSV is seen as a symmetric structure about its core, the incoming flow at the level of Point B in each case is at the upper boundary of the HSV.

By extracting the velocity field on the horizontal planes passing through Point A and Point B, flow structures for each AR are expected to share similar features. Therefore, the comparison between different ARs would be more meaningful than only considering the comparison at a certain cylinder height to width ratio, especially when one cylinder is significantly shorter than the other.

Figs. 4 (a), (b), and (c) show the time-averaged streamwise velocity on a horizontal plane passing through Point A for AR $=1,2$, and 4 , respectively. The flow separates at the sides of the cylinder and accelerates in the downstream direction. The flow with high velocity slowly reduces as it moves downstream. Even though the size of the wake structure and 


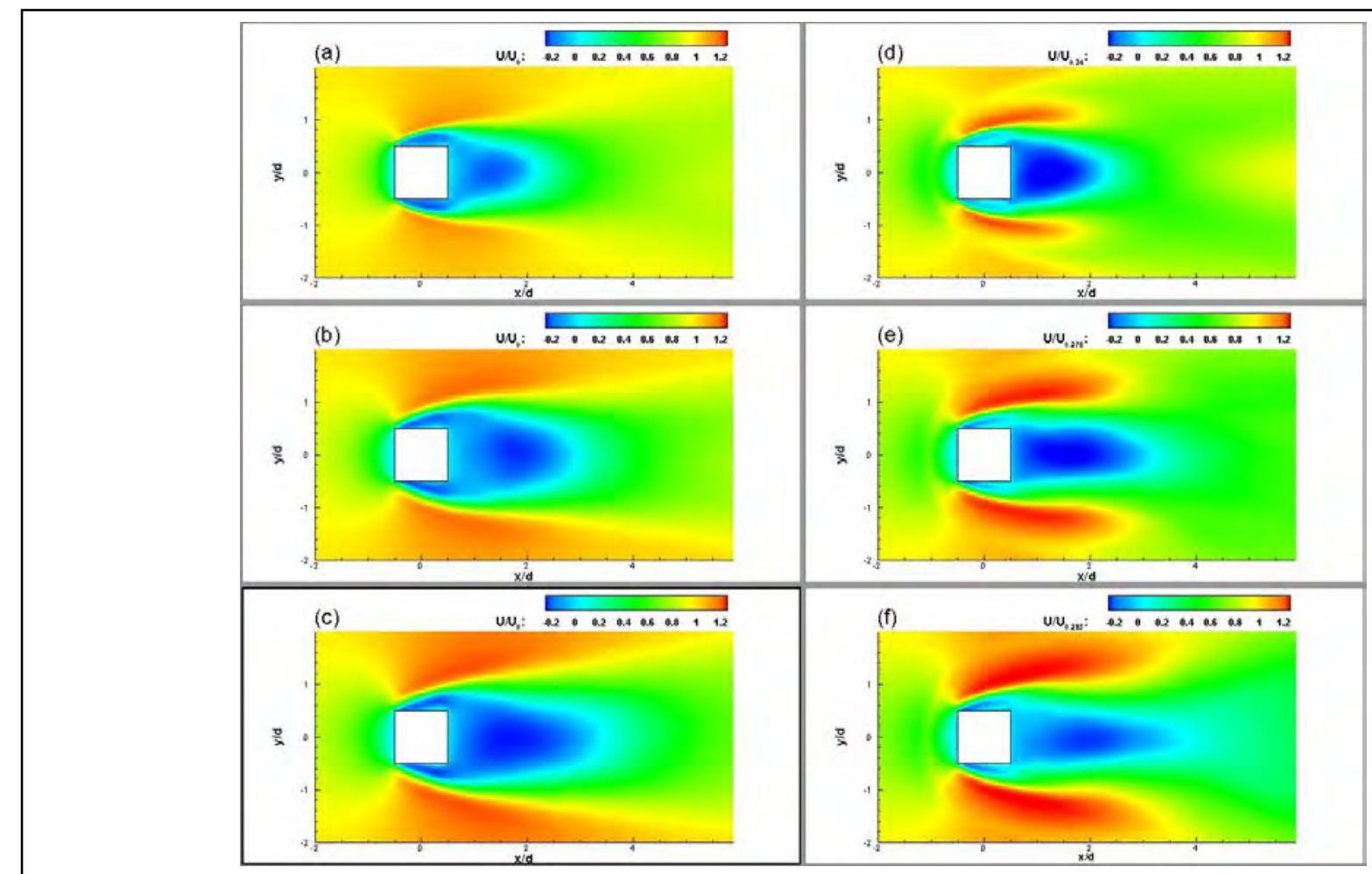

Fig. 4 Horizontal planes shaded by the time-averaged streamwise velocity at the location of Point A: (a), (b) and (e) for the cylinder AR $=1,2$, and 4 respectively; and Point B: (d), (e), and (f) for the cylinder AR $=1,2$, and 4 respectively.

the value of the streamwise velocity are different, the contour of the flow structures shows many similarities.

Figs. 4 (d), (e), and (f) show the time-averaged streamwise velocity on a horizontal plane passing through Point B for AR $=1,2$, and 4 , respectively. The planes at Point B show many different features than the planes at the stagnation point (Point A), while the planes at Point B share a lot of similarities among themselves. Two zones (red and blue regions), which represent the separating shear layer (outer) and the collar vortex (inner) (Nasif et al. [2]), lie on each side of the cylinder. Although the characteristics of the flow structures are different due to the increase of AR, the flow structures presented are similar.

\section{CONCLUSION}

The comparison made by selecting the planes based on the pressure distribution show similar structures in the flow field at all aspect ratios. The pressure distribution on the front face is clearly a function of the aspect ratio. The choice of the horizontal planes based in the maximum and minimum pressure values enables a direct comparison at different value of AR. Further analysis of the use of the front face pressure distribution is necessary to understand the characteristics of the flow past bluff objects.

\section{REFERENCES}

[1] T. Kawamura, et al., "Flow around a finite circular cylinder on a flat plate: Cylinder height greater than turbulent boundary layer thickness," Bull. JSME, vol. 27, no. 232, pp. 2142-2150, 1984. doi: 10.1299/jsme1958.27.2142.

[2] G. Nasif, R. Balachandar, and R. M. Barron, "Characteristics of flow structures in the wake of a bed-mounted bluff body in shallow open channel," J. Fluids Eng., vol. 137, no. 10, pp. 101207-1, Oct. 2015. doi: $10.1115 / 1.4030537$.

[3] M. Saeedi and B. C. Wang, "Large-eddy simulation of turbulent flow around a finite-height wall-mounted square cylinder within a thin boundary layer," Flow, Turbul. Combust., vol. 97, no. 2, pp. 513-538, Sept. 2016. doi: 10.1007/s10494-015-9700-7.

[4] M. El Hassan, J. Bourgeois, and R. Martinuzzi, "Boundary layer effect on the vortex shedding of wall-mounted rectangular cylinder," Exp. Fluids, vol. 56, no. 33, Feb. 2015. doi: 10.1007/s00348-014-1882-6. 


\title{
Piercing a Liquid Droplet by a Gas Jet
}

\author{
Maryam Ebrahimiazar, Amirreza Amighi, Nasser Ashgriz \\ Department of Mechanical and Industrial Engineering \\ University of Toronto \\ Toronto, Ontario, Canada
}

\begin{abstract}
Impingement of a gas jet on a suspended water droplet is studied experimentally. The jet penetration process depends on the jet velocity and jet to droplet diameter ratio. At small jet to diameter ratios and large enough jet velocities, the jet penetrated through the droplet. At larger jet to droplet diameter, the droplet fragmentation becomes more significant, with large droplet deformation. For this experiment, different types of breakup process are categorized, and theoretical explanations are provided.
\end{abstract}

Keywords-droplet breakup, atomization,Rayleigh-Taylor instability

\section{INTRODUCTION}

Fragmentation of a liquid into small droplets by a gaseous jet has numerous industrial applications. Although the fragmentation process is studied extensively, it still cannot be predicted accurately. All of the presently available models are based on empirical correlations and/or simplified physical models. For instance, the breakup of a liquid droplet is experimentally studied and the breakup process is categorized based on a Weber number:

$$
W e=\frac{\rho U^{2} D_{d r o p}}{\sigma}
$$

where $\rho$ can be the density of the gas or the liquid, $U$ is the relative velocity between the gas and droplet, $D_{\text {drop }}$ is the droplet diameter, and $\sigma$ is the surface tension. It is also found that the viscosity of the fluid that the droplet is composed of also plays a role in preventing the deformation and dissipating the energy from the aerodynamic forces imposed on the droplet. The ratio of droplet viscous forces to surface tension forces can be quantified using the Ohnesorge number [2]:

$$
O h=\frac{\mu_{l}}{\sqrt{\rho_{l} D_{\text {drop }} \sigma}}
$$

where $\mu_{l}$ and $\rho_{l}$ are the droplet viscosity and density,respectively. When the $O h$ umber is greater than 0.1 , drop viscous forces are significant and should be accounted for.Different types of droplet breakups are identified and categorized based on the Weber number: Vibrational $(0<$ We $<\sim 11)$, bag $(\sim 11<W e<\sim 35)$, multimode $(\sim 35$ $<W e<\sim 80)$, sheet thinning $(\sim 80<W e<\sim 350)$, and catastrophic $(W e>\sim 350)$.
There are a wide variety of methods to perform the fragmentation process. The most common method is by applying a large relative velocity between an external gas and the liquid, thus shearing off small drops from the liquid surface. The relative velocity can be induced by either accelerating the liquid and shooting it out of a nozzle, thus having a high velocity liquid jet with respect to the surrounding gas, or by applying a high velocity gas onto the surface of the liquid. Both these effects have been studied extensively. The atomization method that is introduced here is within the later category, and in particular, on the breakup of a liquid droplet by a high velocity gaseous flow.

\section{EXPERIMENTAL SETUP}

The experimental system comprises of a suspended droplet, a gaseous jet and an imaging system. A droplet is suspended at the tip of a capillary needle (see Fig.1). The droplet is suspended so that it is fixed in space and its dynamics can be studied. A capillary needle is used to continuously and easily replace the droplet. The capillary needle is connected to a syringe pump to provide the mass needed for the droplet. The size of the droplet is controlled by the syringe pump. The liquid is pumped to the needle until a desired droplet size is obtained.

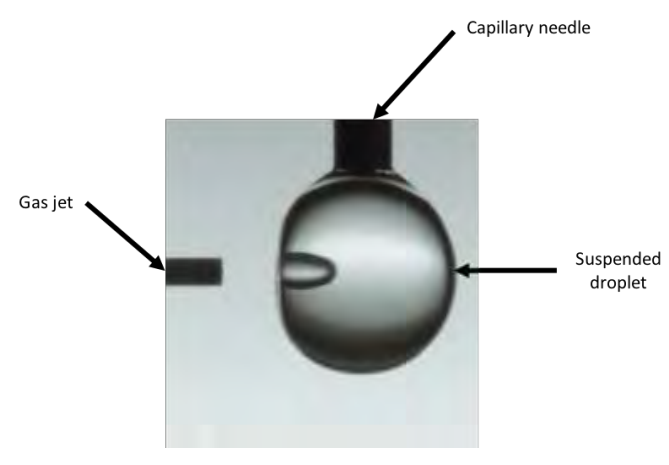

Fig. 1. Experimental Setup

A capillary needle is positioned horizontally next to the suspended droplet. The needle is connected to a highpressure air-line, through a solenoid valve. Pressure gauges are installed on the line to control the flow conditions. The 
flow is controlled by a processor. Direct visualization is used to study the droplet dynamics. Both time lapsed photography, using a still camera with a short duration flash, and a high speed video camera are used in this study. The photographs provided in this paper are from still camera. The schematic of experimental setup is shown in Fig.2.

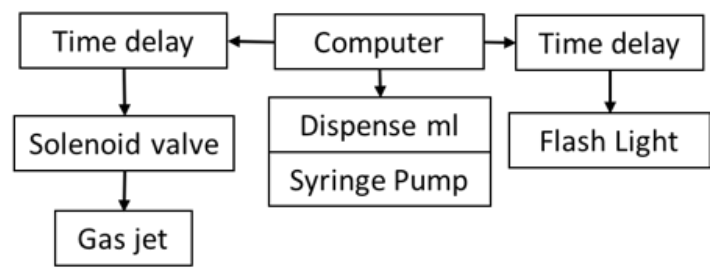

Fig. 2. Schematic of Experimental Setup

\section{Results And Discussion}

\section{A. Observations}

In our experimental study, a droplet with an average diameter of $2.6 \mathrm{~mm}$ is exposed to an air jet, and the dynamics of the droplet is studied. Droplet dynamics differ according to jet to droplet diameter ratio, as well as droplet Weber number based on air density. By progressively increasing the nozzle diameter from $0.2 \mathrm{~mm}$ to $0.8 \mathrm{~mm}$, the following dynamics were observed (Fig. 3).

1) An air tunnel is formed inside the droplet (Fig.3-1). Contrary to one's expectation, the gas jet does not just form a cylindrical tunnel inside the droplet (e.g., bullet through an apple). The gas jet slightly expands as it penetrates into the droplet. By the time the jet reaches to the end of the droplet, the front opening of the droplet is almost closed, forming a conical shape at the front side (Fig. $3-1, \mathrm{t}=0.8 \mathrm{~ms}$ ). At this time, the air jet pushes through the droplet forming another inner jet, which has the same diameter as the initial gas jet. Therefore, two different size cavities are formed inside the droplet.

2) The jet can reach to the end of the droplet and pierce through. Once the jet pierces through, another liquid front forms on the upstream side of the tunnel. In addition, capillary waves form on the drop surface. These capillary waves interact with the front opening and distort the incoming jet (Fig.3-2, $\mathrm{t}=2 \mathrm{~ms}$ ). Magnified images are shown in Fig.4.

3) In the third row, the jet reaches to the end of the droplet and then stretches the water forming a balloon (Fig.3$3, \mathrm{t}=1.2 \mathrm{~ms}$ ); the balloon burst (Fig.3-3, $\mathrm{t}=1.6 \mathrm{~ms}$ ); the balloon front retreats collapsing on itself (Fig.3-3, $t=2$ $\mathrm{ms}$ ), and the incoming jet bursts it again (Fig.3-3, $\mathrm{t}=2.4$ ms). Magnified images are shown in Fig.5.

4) In this case, the jet diameter is so large that it generates a large cavity inside the droplet. Therefore, the liquid thickness around the cavity is small and susceptible to disturbances. In this case, the drop becomes very wavy in the beginning. As the balloon is formed and stretches, the liquid thickness becomes thinner. The liquid thickness around the cavity is small and susceptible to disturbances. In this case, the drop becomes very wavy in the beginning. As the balloon is formed and stretched, the liquid thickness becomes thinner and thinner and also smoother. The balloon can stretch for a long distance before it bursts. This is shown in Fig.34.

\section{B. Flow Regime Map}

In this part, the dynamics of the droplet are characterized based on the jet to droplet diameter ratio and air pressure. In regime (a), two separated cavities (bubbles) are observed in the droplet and there is a clear boundary between the two cavities (bubbles). When $\frac{D_{\text {jet }}}{D_{\text {drop }}}=0.08$ and $p=70 \mathrm{psi}$ the dynamics of the droplet starts to change. In regime (b), still two bubbles are seen in the droplet; however, there is not a clear boundary between them. In regime (c), $\frac{D_{\text {jet }}}{D_{\text {drop }}}$ is large enough to stretch the droplet and form a bag shape; meanwhile, a bubble is seen at the back of the bag shape. In regime (d), the air jet goes through the droplet, forming a large cavity inside it. The four different regimes are shown in Fig. 6.

\section{Rayleigh-Taylor Waves}

In large jet to drop diameter ratios, the air jet forms a large cavity inside the droplet, the droplet stretches, forming a front rim (windward). This liquid rim goes through RayleighTaylor (RT) instability and breaks into small droplets. The size of the droplets can be estimated based on the RT instability theory. For a RT instability, the wavelength, $\lambda_{\max }$ , corresponding to the maximum growth rate is found to be

$$
\lambda_{\text {max }}=2 \pi\left(\frac{3 \sigma}{\rho a}\right)^{1 / 2}
$$

where $a$ is the acceleration, and $\rho$ is the density of the denser fluid. To consider the effect of viscosity and surface tension, Eq.(3) changes to the following:

$$
\lambda_{\text {max }}=2 \pi\left[\left(\frac{3 \sigma}{\rho a}\right)^{1 / 2}+A_{2}\left(\frac{\mu^{2}}{\rho^{2} a}\right)^{1 / 2}\right]
$$

where $\mu$ is the dynamic viscosity of the denser fluid, and $A_{2}$ is a coefficient. This simplified equation was obtained by Aliseda et al[1], which agrees favorably with the results of their experiments. For small $O h$, the viscosity effects can be neglected, and Eq. (3) can be used instead of Eq. (4). In the present study, for large enough jet to droplet diameter ratios and large enough jet velocities, Rayleigh-Taylor waves appear at the front rim of the droplet (Fig. 7). In our case $\left(\mathrm{Oh}=2.2 \times 10^{-3}\right)$, Ohnesorge number is small enough to ignore the viscosity effects. Thus, we will use Eq.(3) to find the critical wavelength of Rayleigh-Taylor Instability. In Eq. (3), all the parameters are known except for the acceleration. Drop acceleration is found using a force balance[3]. The drag force is found through:

$$
F_{\text {drag }}=\frac{1}{2} \rho_{g} u^{2} C_{D} A
$$




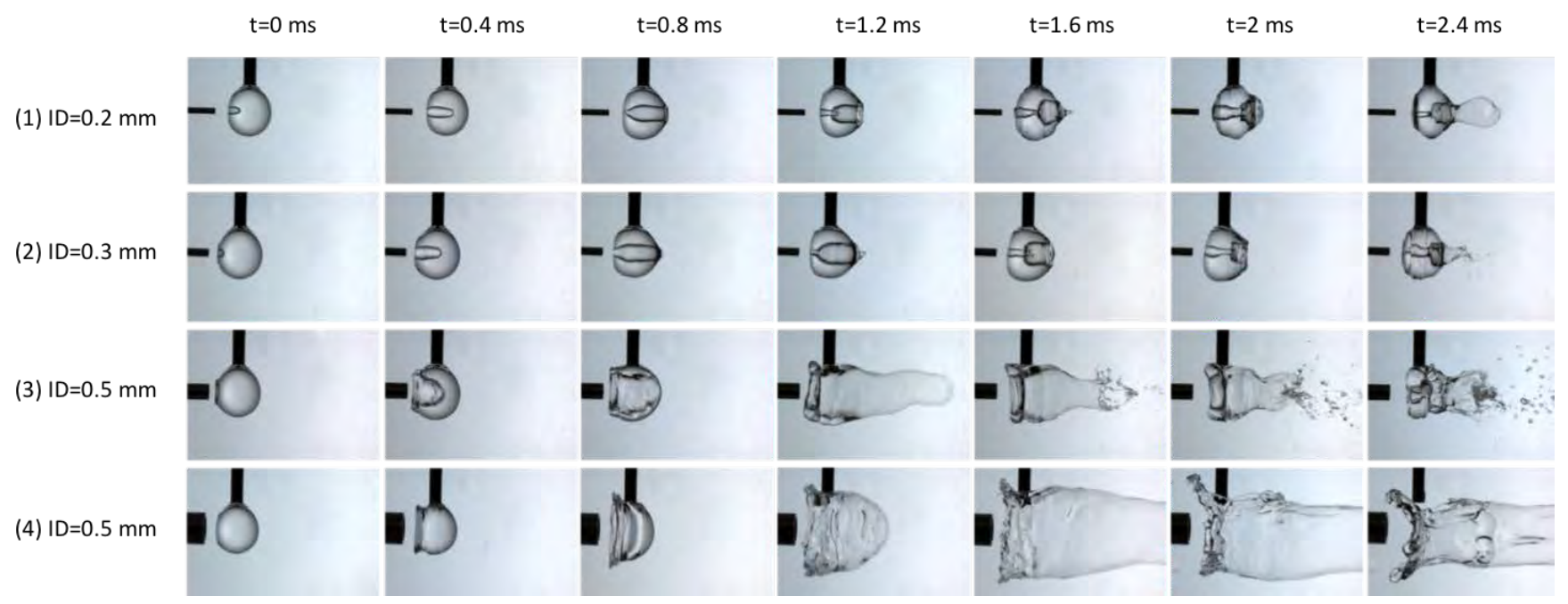

Fig. 3. Droplet dynamics for different nozzle diameters at $\mathrm{p}=10 \mathrm{psi}$

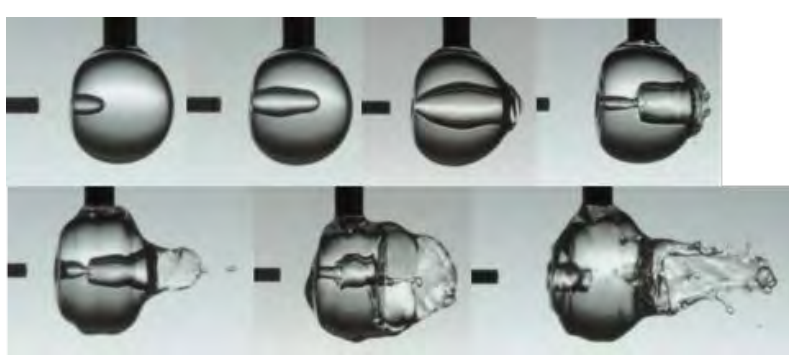

Fig. 4. Droplet dynamics for nozzle with diameter of $0.3 \mathrm{~mm}$

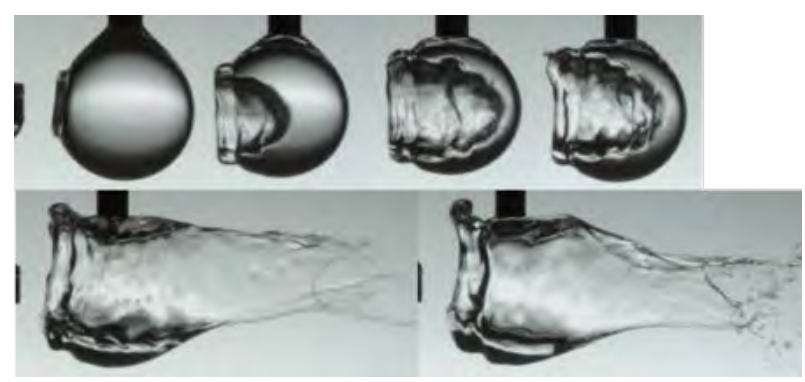

Fig. 5. Droplet dynamics for nozzle with diameter of $0.5 \mathrm{~m}$

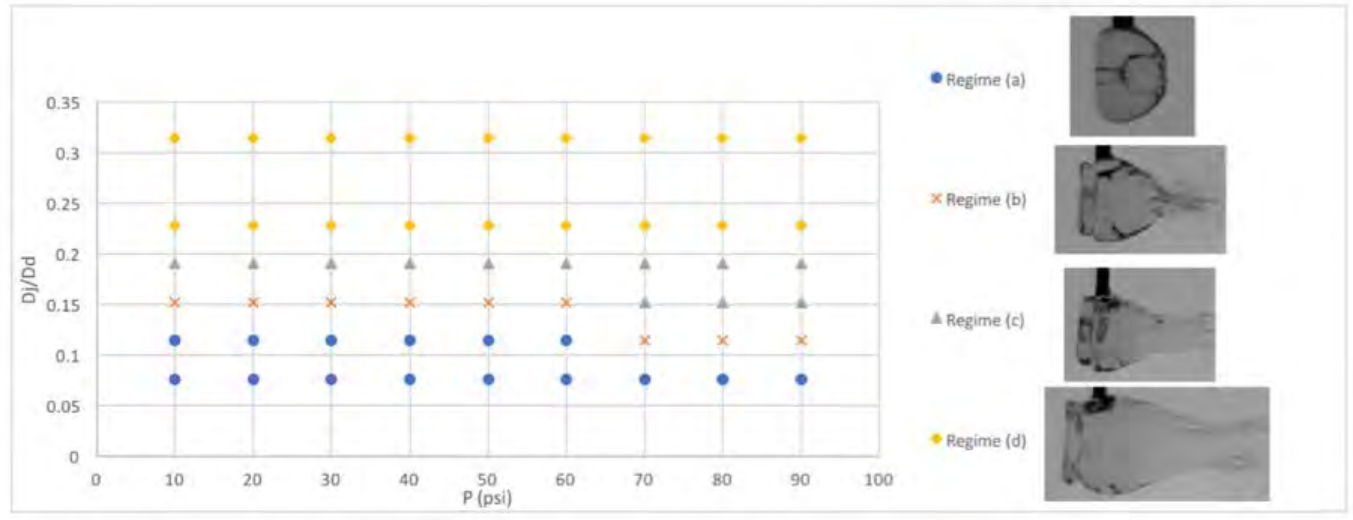

Fig. 6. Flow Regime Map

where $\rho_{g}$ is the gas density, $u$ is the air jet velocity, $C_{D}$ is the drag coefficient, and $A$ is the cross sectional area. This area is shown in Fig. 8. By this definition, the drag force equation will be

$$
F_{\text {drag }}=2 \rho_{g} u^{2} C_{D} \pi\left(R^{2}+r R\right)
$$

The other force acting on the droplet is surface tension, which can be found from Eq. (7)

$$
F=\frac{2 \sigma}{R} \times A
$$

where $\frac{1}{R}$ is the surface curvature, and $A$ is the surface on which the surface tension force is acting. Thus, the surface tension force can be written as:

$$
F=8 \pi \sigma(R+r)
$$




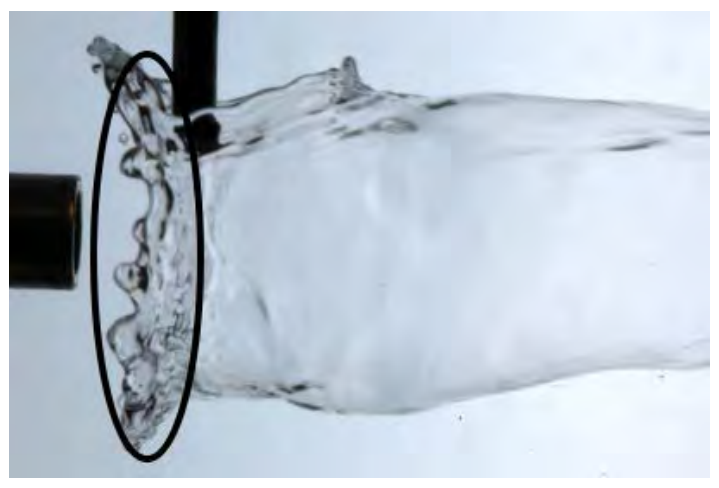

Fig. 7. Rayleigh-Taylor Waves for nozzle with diameter of $1.6 \mathrm{~mm}$ L

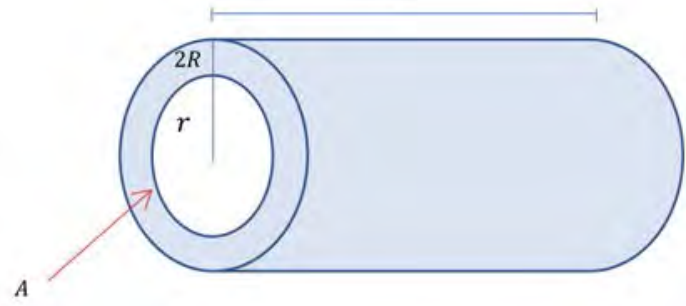

Fig. 8. Hollow cylinder forming after the jet injection

The front rim of the droplet is assumed to have a torus shape with inlet radius of $r$ and outlet radius of $R$. The mass of this torus can be found from Eq. (9).

$$
m=\frac{1}{4} \pi^{2}(2 r+2 R)(2 R)^{2}
$$

Then, the drop acceleration can be written as:

$$
a=\frac{\rho_{g} u^{2} C_{D} R+4 \sigma}{\pi R^{2} \rho_{l}}
$$

In Eq. (10), all the parameters are defined except $R$. In order to find the value of $R$, mass balance is written. The mass of the initial droplet is equal to the mass of the hollow cylinder which is formed after the air jet injection. This is shown in Eq. (11) .

$$
\pi \frac{D_{\text {drop }}^{3}}{6}=4 \pi\left(R^{2}+r R\right) L \rightarrow R=\frac{-r+\sqrt{r^{2}+\frac{D_{\text {drop }}^{3}}{6 L}}}{2}
$$

RT instabilities are observed for the $1.6 \mathrm{~mm}$ jet diameter case. For smaller nozzle diameters the front rim is smooth. The calculations are done for nozzle with diameter of 1.6 $\mathrm{mm}$, and the results are shown in Fig. 9. As the figure shows, the experimental data compare favorably with the theoretical study.

\section{Penetration Velocity}

Penetration velocity is defined as the velocity of the air jet when penetrating through the droplet from one side to the other side. By processing the images, and measuring the distance traveled by the air jet, the penetration velocity is

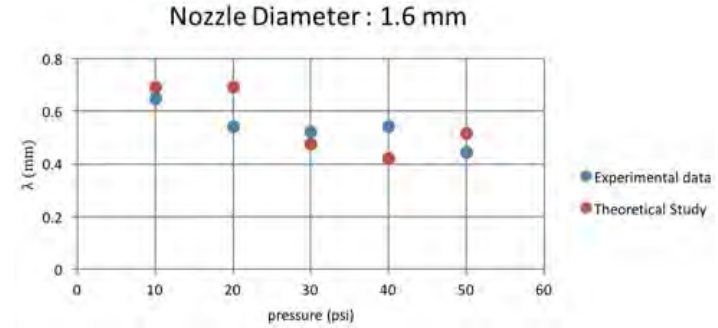

Fig. 9. Experimental and theoretical results for Rayleigh-Taylor waves

calculated. The results are shown in Fig. 10. As the figures show, the air jet is penetrating through the droplet with constant velocity. Moreover, as it is expected, by increasing the air pressure, the penetration velocity is increasing as well.

By writing a pressure balance, the penetration velocity can be found theoretically.

$$
\frac{1}{2} \rho_{a} V_{j}^{2}=\frac{1}{2} \rho_{V_{l}}^{2}+\frac{4 \sigma}{D_{d r o p}}+\frac{4 \sigma}{D_{j}}
$$

where $\rho_{a}$ and $\rho_{j}$ is air and water density, $V_{j}$ is jet velocity, $\sigma$ is the surface tension of water, $D_{d r o p}$ and $D_{j}$ are droplet and jet diameter, respectively. For nozzle with diameter of 0.2 $\mathrm{mm}$, the penetration velocity is calculated theoretically.Table 1 shows both theoretical and experimental results. As the table shows, for low pressure, the theoretical and experimental velocity are in good agreement. However, by increasing the pressure, the theoretical penetration velocity is no longer a good estimate.

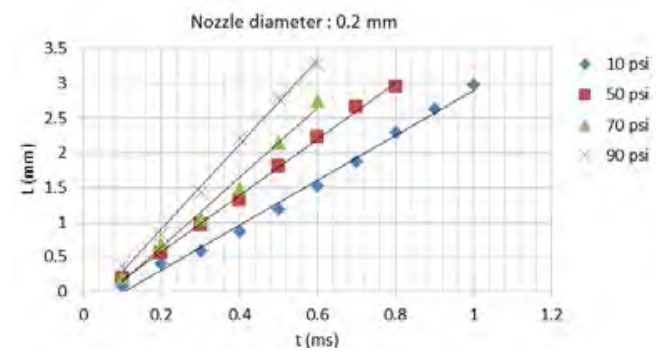

Fig. 10. Experimental penetration velocity

TABLE I

Experimental and theoretical values of penetration velocity for nozzle with diameter of $0.2 \mathrm{~mm}$

\begin{tabular}{ccc}
\hline $\begin{array}{c}\text { Nozzle Pressure } \\
(\mathrm{psi})\end{array}$ & $\begin{array}{c}\text { Experimental } \\
\text { Penetration } \\
\text { Velocity }(\mathrm{m} / \mathrm{s})\end{array}$ & $\begin{array}{c}\text { Theoretical Penetration } \\
\text { Velocity }(\mathrm{m} / \mathrm{s})\end{array}$ \\
\hline 10 & 3.24 & 3.32 \\
50 & 4.06 & 6.34 \\
70 & 5.04 & 7.52 \\
\hline
\end{tabular}




\section{REFERENCES}

[1] A. Aliseda et al., "Atomization of viscous and non-newtonian liquids by a coaxial, high-speed gas jet experiments and droplet size modeling," Int. J. Multiphase Flow, vol. 34, no. 2, pp. 161-175, 2008.

doi: 10.1016/j.ijmultiphaseflow.2007.09.003.

[2] G. I. Taylor, "The instability of liquid surfaces when accelerated in a direction perpendicular to their planes," Proc. R. Soc. A., vol. 201, no.

1065, pp. 192-196, Mar. 1950. doi: 10.1098/rspa.1950.0052.

[3] H. Zhao et al., "Breakup characteristics of liquid drops in bag regime by a continuous and uniform air jet flow," Int. J. Multiphase Flow, vol. 37, no. 5, pp. 530-534, June 2011. doi: 10.1016/j.ijmultiphaseflow.2010.12.006. 


\section{Shear Layer Instabilities in a Counter-flowing Wall Jet}

\author{
Sachin Sharma \\ Mechanical, Automotive \& Materials Engineering \\ University of Windsor \\ Windsor, Canada \\ sharm14d@uwindsor.ca
}

\author{
Ram Balachandar \\ Civil and Environmental Engineering \\ University of Windsor \\ Windsor, Canada \\ rambala@uwindsor.ca
}

\author{
Vimaldoss Jesudhas \\ Civil and Environmental Engineering \\ University of Windsor \\ Windsor, Canada \\ jesudha@uwindsor.ca
}

\author{
Ron Barron \\ Mathematics and Statistics \\ University of Windsor \\ Windsor, Canada \\ az3@uwindsor.ca
}

\begin{abstract}
Counter-flowing wall jets are often used in heat transfer and combustion applications. While several experimental and numerical studies have been conducted to evaluate the characteristics of counter-flowing wall jets, their internal turbulence structure is not well understood. This paper presents the results of a three-dimensional, unsteady, Improved Delayed Detached Eddy Simulation conducted on a counter-flowing wall jet. The mean flow features captured by the simulation are presented with relevant discussions. The shear layer instabilities caused by the vortical structures in the recirculation zone are analyzed by examining the instantaneous flow field. The flow mechanism that causes the fluctuations in the penetration length is also evaluated. The results depict the complexity of the turbulence structure in a counter-flowing wall jet.
\end{abstract}

Keywords- Counter-flowing jets; turbulent wall jet; IDDES; shear layer instability; penetration length fluctuations

\section{INTRODUCTION}

A turbulent wall jet issued opposite to the direction of a moving main / ambient stream is known as a counter-flowing wall jet (CFWJ). Counter-flowing wall jets are particularly effective as mixing devices, such as effluent mixing in rivers and streams, air-fuel mixture for combustion, etc. This is due to the fact that the turbulence in a counter-flowing wall jet is more enhanced than cross and co-flowing jets or even a counter-flowing free jet (CFFJ). Fig. 1a shows the schematic of a typical CFWJ flow field. The higher momentum at the inlet enables the CFWJ to penetrate the main flow. The momentum of the CFWJ reduces gradually as it approaches the stagnation point. The main flow pushes the jet backwards, thereby creating a recirculation zone. The recirculation zone enhances the turbulence in the CFWJ, thereby improving its mixing efficiency. The distance from the nozzle exit to the stagnation point is known as the penetration length of the jet $\left(x_{s}\right)$. The dark black line in Fig. 1a shows a streamsurface, which is the loci of $U=U_{o}$. This streamsurface divides the wall jet and main flow region. The maximum height of this streamsurface gives the width $(h)$ of the recirculation zone. The $U=0$ line divides the forward and backward flow. While several experimental studies [1-4] have been conducted on CFFJ, the studies on CFWJ are relatively limited. However, the studies of CFFJ can be used to interpret the salient characteristics of a CFWJ flow field. One of the prominent aspects of the CFFJ is the fluctuating nature of the penetration length $[2,4]$. The stagnation point oscillates about a mean position. The presence of the wall in a CFWJ further enhances the complexity of the flow. In experimental studies, it is difficult to capture the penetration length accurately. Typically, experimental researchers have employed dye or wool turfs to identify the instantaneous location of the stagnation point $[1,5]$, leading to uncertainties in the measurements. Also, in experiments the complete threedimensional (3D) flow field is not available for analysis, thereby posing challenges in identifying the flow physics that are responsible for the oscillations of the penetration length.

Numerical studies of CFWJ are sparse. "Reference [6]" studied the mean characteristics of CFWJ using the ReynoldsAveraged Navier-Stokes (RANS) $k-\varepsilon$ turbulence model. They analyzed the mean characteristics of the flow for different velocity ratios $\alpha=U_{j} / U_{o}$ where $\alpha$ is the ratio of jet velocity $\left(U_{j}\right)$ to main flow velocity $\left(U_{o}\right)$.However, due to the limitations of RANS turbulence models, a rigorous investigation of the turbulence characteristics was not carried out. To address these limitations, 3D, unsteady, Improved Delayed Detached Eddy Simulation (IDDES) of a planar CFWJ is conducted in this study. After validating the simulation results with available experimental data, the instantaneous flow field is analyzed. The shear layer instabilities in the CFWJ flow field are identified. The flow 
mechanism responsible for the penetration length fluctuations is also identified.

\section{MODEL SETUP}

In the present study, a hybrid RANS-LES approach was employed to model turbulence. IDDES uses RANS near the walls and LES away from it. The complete formulation is available in several publications [7,8] and hence not repeated here. The commercial CFD solver STAR-CCM+ was used to setup and run the simulations. A two-dimensional representation of the $3 \mathrm{D}$ computational domain is shown in Fig. 1b. The computational domain is modeled based on the experiments of [9]. A hexahedral mesh was used, with refinements in the regions of interest. Based on the grid dependency study, the mesh selected for the present simulation consists of about 10 million cells. .Fine prism layers were added near the walls. The boundary conditions used in the simulation are also shown in Fig. 1b. The side walls were treated as no-slip walls; the complete details of the simulated case are presented in Fig. 1b. Shear stress transport (SST) $k-\omega$ is used along with LES in the present simulation. IDDES uses a blending function to switch between the RANS and LES regions; this was monitored to ensure that LES was executed in the regions of interest. The solution was considered to be converged when the residuals for continuity and momentum fell below $10^{-6}$. The mean quantities presented here were obtained by averaging for a time of 50s following convergence
The simulation results were validated based on the experiments of [9]. The validation procedure was based on the American institute of Aeronautics and Astronautics (AIAA) guidelines for verification and validation of CFD simulations (2002). The penetration length, width of the recirculation zone and velocity profiles were compared with the experimental results. The simulation results agreed well with the experimental results. Only after establishing sufficient confidence in the simulation results, the analysis presented here was carried out.

\section{RESULTS AND DISCUSSION}

Fig. 2 shows the velocity vectors in the mean flow field of the CFWJ predicted by the simulation. The velocity vectors are colored based on the mean streamwise velocity. As the jet exists the nozzle at $\left(x / h_{j}=0\right)$, where $h_{j}$ is the size of jet, a shear layer is created between the jet and recirculation zone. The mean velocity of the jet decreasing as it approaches the stagnation point. At the stagnation point $\left(x / h_{j}=72\right)$ the jet turns in the opposite direction due to the influence of the main flow, forming the recirculation zone. The $U=0$ line divides the forward and backward momentum in the flow field. Due to the interaction between the two flows, the main flow readjusts by flowing around the recirculation zone. Figs. 3(a-d) depict Instantaneous velocity at four time instances. As the jet emanates from the nozzle, the vortical structures from recirculation region interact with the shear layer and create

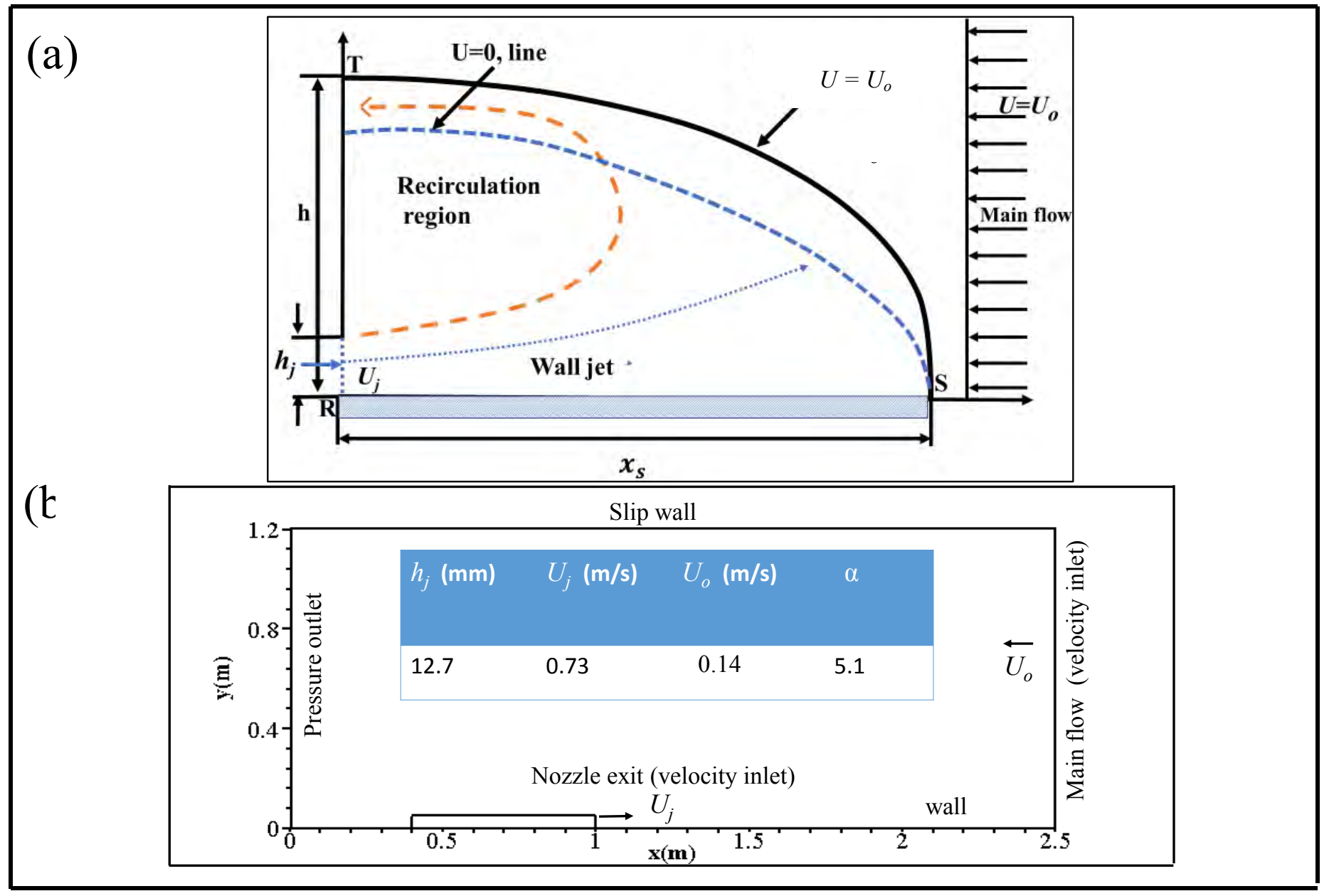

Fig. 1(a) Schematic of counter-flowing wall jet (b) Computational domain 
instabilities. The vectors are also superimposed to depict this interaction. The downward motion of the fluid in the recirculation zone (marked by red arrow in Fig. 3a) interacts with the jet between $x / h_{j}=20$ to 30 . This excites the jet causing instabilities making it wavy in nature. The influence of the recirculation zone and the adverse pressure gradient it encounters enables it to detach from the wall. This allows the main flow to penetrate further into the jet in the near bed region (marked by the dashed circle in Fig. 3a). As the fast moving jet negotiates the slow moving fluid particles (light blue region) arriving from the near-wall main flow, it bifurcates in to an upward moving flow towards the $U=0$ line and the wall (Fig. 3b). The fluctuating nature of the wall jet continues (Fig. 3c and Fig. 3d) and the corresponding main flow adjusts itself to the jet characteristics, reducing the penetration length of the jet. The penetration length is $x / h_{j}=$ 55 in Fig. 3d. The phenomenon of jet fluctuations and the corresponding change in the penetration length enhances the mixing. This analysis clearly shows the complex nature of the turbulence in the CFWJ flow field and the feedback

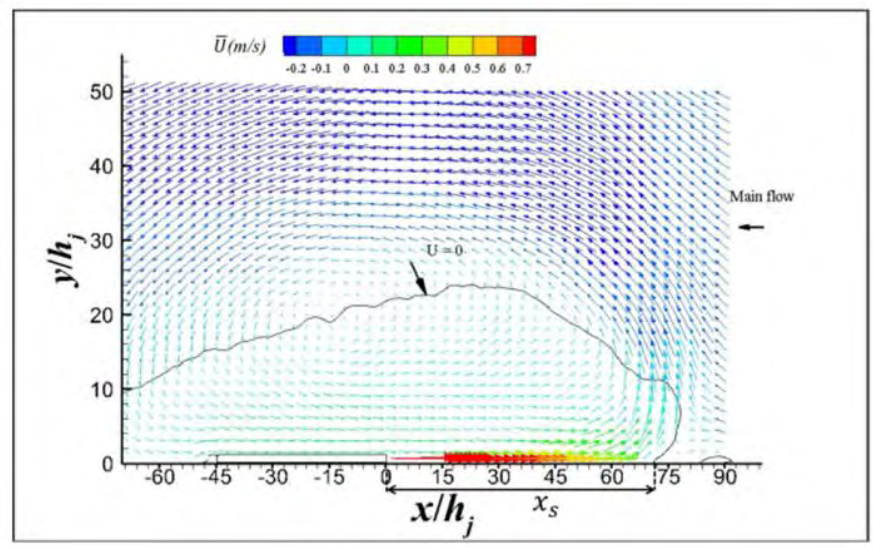

Fig. 2 Mean flow field of counter-flowing wall jet mechanism that exists between the recirculation zone and the wall jet. Further analysis is needed to explore this phenomena.

\section{CONCLUSION}

A computational study of a counter-flowing wall jet is carried out using the 3D, unsteady, Improved Delayed Detached Eddy Simulation. The simulation captures the relevant flow features of the CFWJ flow field. The mechanism causing the fluctuations in the penetration length has been identified. The analysis of the instantaneous velocity field shows a feedback mechanism that exists between the recirculation zone and the jet, causing the oscillation of the jet penetration length. This mechanism contributes to the enhanced mixing in the CFWJ flow field.

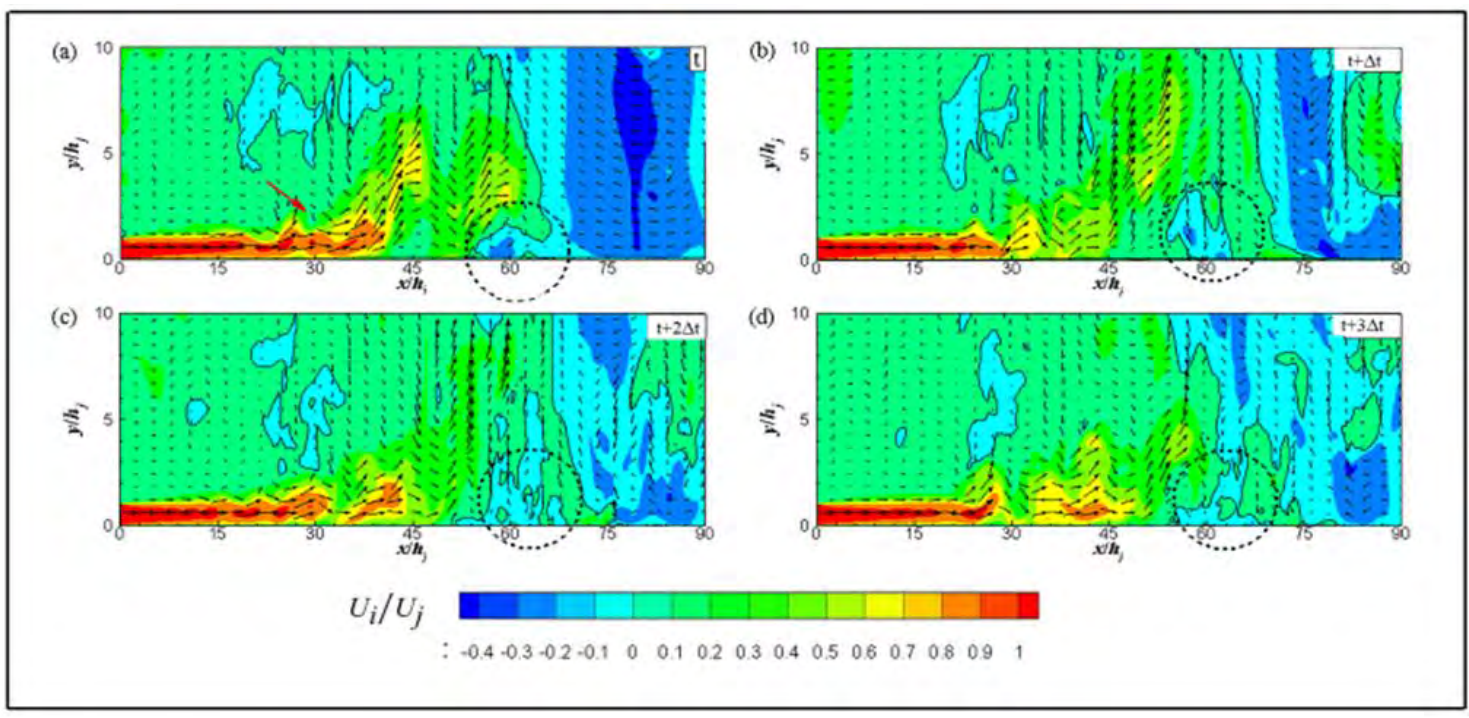

Fig. 3 Instantaneous streamwise velocity at (a) $120 \mathrm{~s}$, (b) $120.5 \mathrm{~s}$, (c) $121 \mathrm{~s}$, (d) $121.5 \mathrm{~s}$ 
[1] W. D. Morgan, B. J. Brinkworth, and G. V. Evans, "Upstream penetration of an enclosed counterflowing jet," Ind. Eng. Chem. Fundam., vol. 15, no. 2, pp. 125-127, 1976. doi: 10.1021/i160058a008.

[2] M. Yoda and H. E. Fiedler, "The round jet in a uniform counterflow: flow visualization and mean concentration measurements," Exp. Fluids, vol. 21, no. 6, pp. 427-436, 1996. doi: 10.1007/BF00189045.

[3] K. M. Lam and H. C. Chan, "Round jet in ambient counterflowing stream," J. Hydraul. Eng., vol. 123, no. 10, pp. 895-903, 1997. doi: 10.1061/(ASCE)0733-9429(1997)123:10(895).

[4] S. Bernero and H. E. Fiedler, "Application of particle image velocimetry and proper orthogonal decomposition to the study of a jet in a counterflow," Exp. Fluids, vol. 29, no. 1, pp. S274-S281, 2000.

doi: $10.1007 / \mathrm{s} 003480070029$.

[5] R. Balachandar, L. Robillard, and A. S. Ramamurthy, "Some characteristics of counter flowing wall jets," J. Fluids Eng., vol. 114, no. 4, pp. 554-558, 1992. doi: 10.1115/1.2910067.

[6] H. Kordi, A. Abedini, and E. Kordi, "Numerical modeling of the penetration area of reversed submerged jet by the methods of finite elements and finite volumes," Water Resour., vol. 43, no. 3, pp. 472-477, 2016. doi: 10.1134/S0097807816030064.

[7] M. L. Shur, P. R. Spalart, M. K. Strelets, and A. K. Travin, "A hybrid RANS-LES approach with delayed-DES and wall-modelled LES capabilities," Int. J. Heat Fluid Flow, vol. 29, no. 6, pp. 1638-1649, 2008. doi: 10.1016/j.ijheatfluidflow.2008.07.001.

[8] V. Jesudhas, Modelling of Free-surface Flows with Air Entrainment, Ph.D. Dissertation, Univ. of Windsor, Windsor, ON, Canada, (2016).

[9] Tudor, M., Turbulent Characterstics of a counter flowing wall jets, Master's Thesis, University of Concordia, (2003). 


\section{An Immersed-Boundary (IB)-Based 3D Tornado Model and Its Application}

\author{
Xixiong Guo \\ Department of Mechanical and Industrial Engineering \\ Ryerson University \\ Toronto, Ontario, Canada, M5B 2K3 \\ xixiong.guo@ryerson.ca
}

\author{
Jun Cao \\ Department of Mechanical and Industrial Engineering \\ Ryerson University \\ Toronto, Ontario, Canada, M5B 2K3 \\ jcao@ryerson.ca
}

\begin{abstract}
A tornado features kinematically combining three major co-existent components, namely, updraft, translation, and rotation, which involve all the three dimensions in space while transient in time. For numerical simulation of a tornadobuilding interaction scenario, it looks quite challenging to seek a set of physically-rational and meanwhile computationallypractical boundary conditions to accompany traditional CFD approaches; thus, little literature can be found, as of today, in three-dimensional (3D) computational tornado dynamics study. Inspired by the development of immersed boundary (IB) method, this study employed the re-tailored Rankinecombined vortex model (RCVM) that applies the "relative motion" principle to the translational component of tornado, such that the building is viewed as "virtually" translating towards a "pinned" rotational flow that remains time-invariant at the far field region. This revision renders a steady-state kinematic condition applicable to the outer boundary of a large tornado simulation domain, successfully circumventing the boundary condition updating process that the original RCVM would have to suffer, and tremendously accelerating the computation. Here, this re-tailored RCVM was extended to its $3 \mathrm{D}$ version with the aid of logarithm law that describes the vertical flow evolution. Eventually, this tornado model was embedded in Incompact3D, an academic high-order finite difference turbulent flow solver, resulting in a practical powerful 3D tornado-building interaction simulation tool. A case study examined the tornadic wind induced loadings on a prismatic building; over all three directions, the vertical force component was found dominant, which effectively suggests the uprooting effect as observed in many reported scenes after a devastating tornado swept over.
\end{abstract}

Keywords: Re-tailored Rankine-combined vortex model (RCVM); Immersed boundary (IB); Large eddy simulation (LES); Finite difference method; Wind loadings.

\section{INTRODUCTION}

Tornadoes have been worldwide observed, and more frequently reported in United States and Canada. Compared to a straight-line wind, a tornadic flow is much more complex, since it is a type of airflow that essentially combines translational, vertical, and rotational velocities, and all components have to be considered during the investigation. Since the study of tornado dynamics through field observations or laboratory experiments tends to be time-consuming, laborious, and expensive, more research efforts have been made in the direction of mathematical modeling and numerical simulations. Historically, Wilson [1] first applied a two dimensional (2D) tornado model to examine the effects of tornadoes on rectangular-shaped buildings. Lewellen et.al. ${ }^{[2-3]}$ employed a three-dimensional (3D) large eddy simulation (LES) turbulence model to investigate the dynamics of a tornado-like vortex near the surface with particular attention paid to the turbulent flow characteristics in the corner region. Natarajan and Hangan ${ }^{[4]}$ also used LES model to study the effects of surface roughness and translation on the mean tangential velocity of the tornadic wind for different swirl ratios. On the other hand, tornado-induced wind loadings exerted on constructions have been also a practical topic. Based upon the Rankine-combined vortex model (RCVM), Selvam et.al. ${ }^{[5-6]}$ used the finite difference discretization of the NavierStokes (N-S) equations to conduct a series of studies focusing on the tornado-induced loadings on 2D sections of a cylinder and multi-cubic buildings and, more recently, some $3 \mathrm{D}$ cases ${ }^{[6]}$ at elevated Reynolds numbers also with the aid of LES ${ }^{[7]}$.

However, most aforementioned computational tornado dynamics studies mainly relied on conventional numerical methods, which need to update the time-dependent boundary conditions due to the dynamic nature of tornado. To overcome that tedious updating process, Guo et.al. ${ }^{[8-9]}$ re-formulated the RCVM to model the two-dimensional (2D) tornado-building interaction with the aid of immersed boundary (IB) method and the "relative motion" principle, resulting in a "virtual" scenario in which the building "moves" at a velocity that is the negative of the translation velocity of the tornado, while the background airflow has only a rotational component about its "pinned" center. Thus, as long as the computational domain is large enough for its outer boundary to little affected by the inner flow evolution induced by the "virtual translation" of the building, the time-dependency and, hence, the kinematic condition updating process, can be effectively eliminated at the outer boundary of the computational domain. 
This study aims to extend the $2 \mathrm{D}$ tornado simulation framework ${ }^{[8]}$ to the $3 \mathrm{D}$ version by taking the vertical velocity component into account. A combination of direct forcing IB approach with a centered finite difference scheme of high accuracy is performed. Meanwhile, the LES turbulence model is coupled with the N-S solver in order to better simulate the tornadic flow at relatively high Reynolds numbers. The advantage of this framework was discussed in terms of computational efficiency, accuracy, and simplicity [10]. Considering the large computational domain along with fine grid resolution required by $3 \mathrm{D}$ tornado simulations, this framework has been adapted to be portable on massive parallel computing platforms via Message Passing Interface (MPI) techniques ${ }^{[10]}$.

The rest of this paper will unfold as follows: the numerical method, including the tornado model, governing equations, and some other numerical treatment details, is presented in Section II. Section III provides a tornado-building interaction case with tornadic wind loadings analyzed in all directions. Finally, remarks are made in Section IV, addressing the conclusion and future extension of this study.

\section{NUMERICAL METHOD}

\section{A. Tornado Model}

The simplest model that can satisfy the Navier-Stokes equations while describing a tornadic wind field is the Rankine-combined vortex model (RCVM). In RCVM, tornado velocity profile can be decomposed into a constant-translation component, $\vec{V}_{t}$, a component of rotation with respect to the wind centre, $\vec{V}_{\theta}$, which denotes the tangential velocity component, and a vertical velocity component that obeys the logarithmic law to account for the boundary layer growth. The RCVM velocity profile along the radial direction from the tornado center consists of two distinct regions. The forcedvortex region refers to a circular area in which the radius from the tornado centre, $r$, is less than the $r_{c}$, the maximum radius of the forced-vortex region, $V_{\theta}$ varies linearly with $r$ and reaches the maximum tangential velocity at $r_{c}$. At radii larger than $r_{c}$, i.e., in the free-vortex region, $V_{\theta}$ decreases with the radius. The detailed mathematical expression of $V_{\theta}$ is as follows:

$$
V_{\theta}= \begin{cases}r \omega, & r \leq r_{c} \\ r_{c}^{2} \omega / r, & r>r_{c}\end{cases}
$$

where $\omega$ is the constant angular velocity. If taking the translational velocity and counter-clockwise rotational velocity into consideration, the resultant velocity on a horizontal plane can be re-projected to the Cartesian coordinate system, resulting in ${ }^{[5]}$ :

$$
u= \begin{cases}\left(V_{t}-y \cdot \omega\right) Z_{f}, & r \leq r_{c} \\ \left(V_{t}-y \cdot r_{c}^{2} \omega / r\right) Z_{f}, & r>r_{c}\end{cases}
$$

This study is fully funded by the Discovery Grant of the Natural Sciences and Engineering Research Council (NSERC) of Canada.

$$
v= \begin{cases}\left(\left(x+V_{t} \cdot t\right) \omega\right) Z_{f}, & r \leq r_{c} \\ \left(\left(x+V_{t} \cdot t\right) r_{c}^{2} \omega / r\right) Z_{f}, & r>r_{c}\end{cases}
$$

Remark that in Eqs. (2) and (3) $Z_{f}$ denotes a vertical kinematics adjustment parameter, which is a logarithmic law based function of vertical coordinate, $z$, as follows:

$$
Z_{f}=\frac{u^{*}}{k} \operatorname{In}\left(\frac{z+z_{0}}{z_{0}}\right)
$$

where $k$ and $z_{0}$ are constants, with $k=0.4, z_{0}=0.00375$, respectively. When using the re-tailored RCVM in which the rotational flow component is virtually viewed as pinned, the translational velocity $\vec{V}_{t}$ of the air vanishes, and Eqs. (2) and (3) reduce to:

$$
\begin{aligned}
& u= \begin{cases}(-y \cdot \omega) Z_{f}, & r \leq r_{c} \\
\left(-y \cdot r_{c}^{2} \omega / r\right) Z_{f}, & r>r_{c}\end{cases} \\
& v= \begin{cases}(x \cdot \omega) Z_{f}, & r \leq r_{c} \\
x \cdot\left(r_{c}^{2} \omega / r\right) Z_{f}, & r \quad r_{c}\end{cases}
\end{aligned}
$$

\section{B. Governing Equations}

The simulations are performed with Incompact $3 \mathrm{~d}$, an opensource code developed mainly by Laizet \& Lamballais ${ }^{[11]}$. All governing equations are solved on a collocated velocity grid via the six-order central compact finite different scheme ${ }^{[12]}$, while the pressure is on a staggered grid. A second-order Adams-Bashforth (AB) scheme is used for time-advancement and continuity is verified at the end of each sub-time step by solving a pressure Poisson equation. This Poisson equation is solved through a spectral solver to avoid the expensive cost incurred by the high-order discretization along with iterative schemes. The massively parallel version of the code was employed through an MPI implementation based on pencil domain decomposition strategy ${ }^{[10]}$.

The mass and momentum conservation principles are represented by the Navier-Stokes equations, which have the following form for an incompressible fluid:

$$
\begin{gathered}
\rho\left(\frac{\partial \vec{u}}{\partial t}+(\vec{u} \cdot \vec{\nabla}) \vec{u} \mid=-\vec{\nabla} p+\mu \vec{\nabla}^{2} \vec{u}+\overrightarrow{\mathrm{f}}\right. \\
\vec{\nabla} \cdot \vec{u}=0
\end{gathered}
$$

where $\vec{u}(\vec{x}, t)=(u(\vec{x}, t), v(\vec{x}, t), w(\vec{x}, t))^{t}$ is the fluid velocity, $p(\vec{x}, t)$ is the pressure field, and $\rho$ is constant $(\rho=1)$ in the present framework. $\overrightarrow{\mathrm{f}}$ is the external forcing term caused by the interaction between the air and the immersed building.

\section{Time Advancement}

The time integration for solving Eq. (7) is performed using a second-order Adams-Bashforth scheme. A fractional step method ${ }^{[13]}$ is applied to decouple the velocity and pressure, and detailed steps are as follows: 


$$
\begin{aligned}
\frac{\vec{u}^{*}-\vec{u}^{n}}{\Delta t}=\frac{3}{\vec{N}^{n}-}-\frac{\vec{N}}{2} \vec{N}^{n-1}-\vec{\nabla} \tilde{p}^{n}+\tilde{\overrightarrow{\mathrm{f}}}^{n} \\
\frac{\vec{u}^{* *}-\vec{u}^{*}}{\Delta t}=\vec{\nabla} \tilde{p}^{n} \\
\vec{\nabla} \tilde{p}^{n+1}=\frac{\vec{\nabla} \cdot \vec{u}^{* *}}{\Delta t} \\
\frac{\vec{u}^{n+1}-\vec{u}^{* *}}{\Delta t}=-\vec{\nabla} \tilde{p}^{n+1}
\end{aligned}
$$

with

$$
\vec{N}^{n}=-\vec{u}^{n} \cdot \vec{\nabla} \vec{u}^{n} \quad \mu \vec{\nabla}^{2} \vec{u}^{n}
$$

and

$$
\tilde{p}^{+1}=\frac{1}{t} \int_{t_{n}}^{t_{n+1}} p d t, \quad \tilde{\overrightarrow{\mathrm{f}}}^{n+1}=\frac{-}{\Delta t} \int_{t_{n}}^{t}{ }^{1} \overrightarrow{\mathrm{f}} d t,
$$

where $\vec{u}^{* *}$ and $\vec{u}^{*}$ are the intermediate velocities obtained between time steps $n$ and $n+1$

\section{Evaluation of Forcing Term}

In order to solve the Eq.(9), the forcing term $\tilde{\vec{f}}^{n+1}$ needs to be determined in advance. This term is generated by the IB approach, and reflects the interaction between the immersed object and the fluid. In this study, a direct method is adopted to calculate, the forcing term as follows:

$$
\tilde{\overrightarrow{\mathrm{f}}}^{n+}=\varepsilon\left(-\frac{3}{2} \vec{N}^{n}+\frac{1}{2} \vec{N}^{n-1}+\vec{\nabla} \tilde{p}^{n}+\frac{\vec{u}_{0}^{n+1}-\vec{u}^{n}}{\Delta t}\right)
$$

with $\varepsilon=1$ in the solid body region, and $\varepsilon=0$ elsewhere else in the computational domain. The target velocity $\vec{u}_{0}$ is calibrated in order to satisfy the no-slip condition at the surface of solid body.

\section{E. Entire Procedure}

Details about the spatial discretization using sixth-order compact finite difference method can be found in ${ }^{[10]}$. Here, a typical solution procedure for each time step within the simulation framework is described as follows:

1. Set up a computational domain, and initialize the velocity field using the RCVM as aforementioned in Eqs. (5) and (6);

2. Based on the location of the boundary of the immersed body at time step $n+1$, identify the fluid, solid and solid boundary points on the Eulerian grid;

3. Calculate the target velocity $\vec{u}_{0}^{n+1}$ in Eq.(15);

4. Obtain the forcing term using Eq.(15);

5. Compute the velocity field $\vec{u}^{*}$ by solving Eq.(9);

6. Compute intermediate velocity $\vec{u}^{* *}$ by solving Eq.(10);

7. Compute the pressure $\vec{\nabla} \tilde{p}^{n+1}$ by solving pressure Poisson equation Eq.(11);

8. Correct divergence-free fluid velocity $\vec{u}^{n+1}$ at the $(n+1)^{\text {th }}$ time step by solving Eq. (12);

9. Go back to Step 2 if time-marching is still needed; otherwise, stop the computation.

\section{NUMERICAL RESULTS}

\section{A. Initial condition and Boundary condition}

As aforementioned in the beginning of the precedent section, this study re-tailored the original RCVM, such that the scenario of a tornado passing through a building (prism) is interpreted as a superposition of a "pinned" rotational airflow and a "virtual translation" of the building, which is opposite to the physical translation direction of the tornado. Then, the retailored RCVM serves to set up the initial velocity background, and note that the outer boundary condition has been rendered time-independent owing to this re-tailored model.

Figure 1 illustrates the computational domain with $\left(\mathrm{L}_{\mathrm{x}}, \mathrm{L}_{\mathrm{y}}\right.$, $\left.\mathrm{L}_{\mathrm{z}}\right)=(24,16,16)$. The length (in the $x$-direction) and the height (in the $z$-direction) of the prism are equal to 1 , which is equal to the radius of the forced vortex region, $r_{c}$, and the depth (in the $y$-direction) of prism is set at 12 . The translation velocity remains one unit. The Reynolds number based on the height of the prism is $R e=10000$. No slip boundary condition is applied on the bottom surface, as well as the prism walls, and the velocity on all other surfaces is governed by the re-tailored time-independent RCVM.

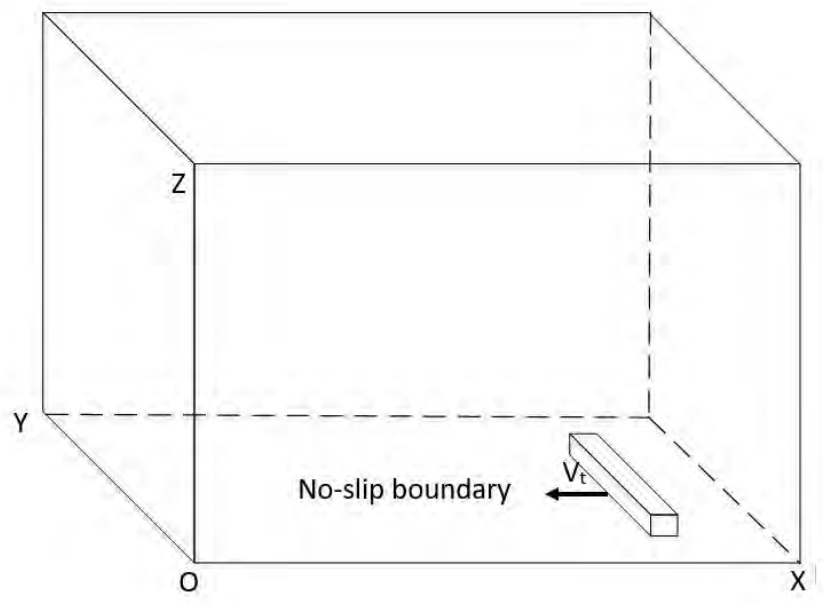

Fig.1 Schematic of the computational domain

\section{B. Force Coefficient Definition}

The dimensionless force coefficients in all three directions are defined as:

$$
\begin{aligned}
& C_{x}=\frac{F_{x}}{0.5 \rho V_{t}^{2} S} \\
& C_{y}=\frac{F_{y}}{0.5 \rho V_{t}^{2} S} \\
& C_{z}=\frac{F_{z}}{0.5 \rho V_{t}^{2} S}
\end{aligned}
$$

where $F_{x}, F_{y}, F_{z}$ and $S$ is the components of the resultant force in three directions and projection area, respectively. Using the 
$x$-direction as example, $F_{x}=-\int_{\Omega_{0}} f_{1} d \mathbf{x}$, and $f_{1}$ is the $x$ component of the force density, the calculation of which is based upon the following integral form of the $x$-component of the momentum equations on any fluid domain $\Omega_{0}$ :

$$
\begin{aligned}
& \frac{\partial}{\partial t} \int_{\Omega_{0}} u_{1} d \mathbf{x}+\int_{\partial \Omega_{0}} \rho u_{1} \mathbf{u} \cdot \mathbf{n} d s \\
& =-\int_{\Omega_{0}} p n_{1} d \mathbf{x}+\int_{\partial \Omega_{0}} \mu\left(\frac{\partial u_{1}}{x_{j}}+\frac{\partial u_{j}}{\partial x_{1}}\right) n_{j} d s+\int_{\Omega_{0}} f d \mathbf{x}
\end{aligned}
$$

The computations of $F_{y},{ }_{z}$ are similar to $F_{x}$.

\section{Case Study}

In this case, the initial position of the prism is placed at 3 units from right boundary of the computational domain at time step $\mathrm{t}=0$, as shown in Fig. 1 . When employing the re-tailored RCVM, the building is "virtually moving" towards left, while the tornado central vertical line remains located at the center of the computational domain. Fig. 2 illustrates the evolution of the force coefficients in all three directions $C_{x}, C_{y}$, and $C_{z}$. Note that the horizontal coordinate, $\mathrm{X}$, is arranged such that, while $\mathrm{X}<0, \mathrm{X}=0$ and $\mathrm{X}>0$, the tornado center physically approaches the prism, nominally coincides with the prism, and leaves the prism, respectively.

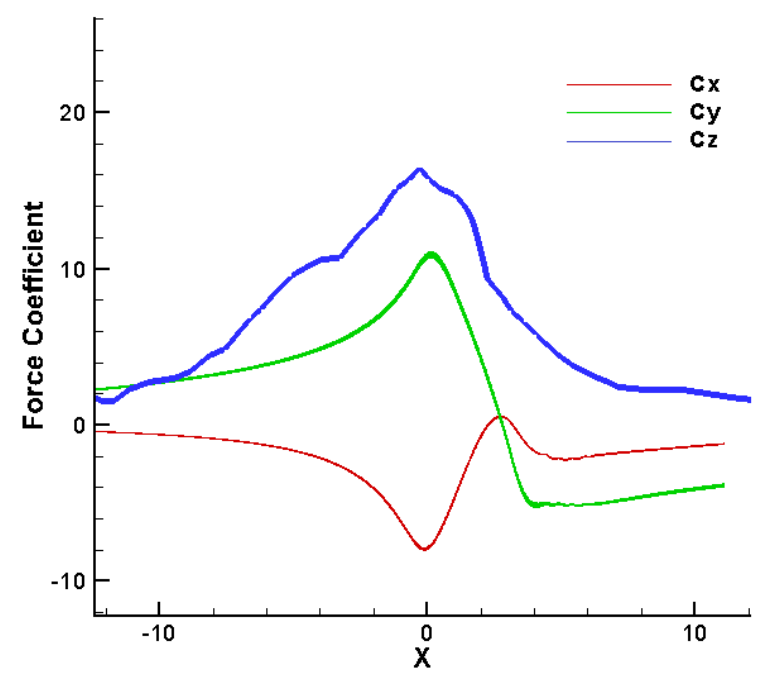

Fig. 2 Evolution of force coefficients in three directions

When the translating tornado center approaches the prism, $C_{x}$ keeps decreasing and stays negative, while both $C_{y}$ and $C_{z}$ keep positive and continuously go up. This indicates the following tornado characteristics in the approaching stage: (1) the building is attracted by the tornado in the $x$-direction, and the closer between the tornado center of the building, the larger attractive force; (2) the building is pushed by the tornado towards the positive $y$-direction due to the counter-clockwise rotation direction of the airflow, and the closer between the tornado center of the building, the larger pushing force; and (3) the building experiences an upward suction force in the $z$ direction, and the closer between the tornado center of the building, the larger suction force.
When the tornado is in the stage of nominally coinciding with the building, the three force coefficients consistently reach their respective extreme values, challenging the wind-resistant capability of the building to the utmost extent.

Afterwards, in the stage of tornado leaving the building, the three force components are consistent, again, in exhibiting their magnitude decreases. However, the decreasing trend for the magnitude of $C_{z}$ remains unchanged all the way in this stage, tending to vanish at the end; on the other hand, $C_{x}$ and $C_{y}$ experience up and down in terms of their magnitudes. In detail, the $C_{x}$ magnitude drops until it reaches its second extreme, which is numerically very close to zero and, locally, the tornado center is about 3 units away from the building, then rerises during the period in which the distance between the tornado center and the building goes approximately from 3 units to 5 units, and then re-drops and gradually diminishes at the end; for the $C_{y}$ magnitude in this stage, it steeply drops and goes to zero when $C_{x}$ reaches its second extreme (at about $\mathrm{X}=3$ ) and, then, the direction of $F_{y}$ changes to the negative $y$ direction, and the $C_{y}$ magnitude remains increasing until approximately $\mathrm{X}=4$, followed by a decreasing trend. The complex up-and-down trend for $C_{x}$ and $C_{y}$ is attributed to the strongly disturbed wake behind the building after the tornado sweeps it over. The three force coefficients calm down only after the core zone of the tornado has been completely away from the building.

Compared with each other, the magnitude of the $z$-direction force component is the highest, suggesting that the influence caused by the given logarithm law based vertical adjustment parameter $Z_{f}$ is a dominant factor for the tornado dynamics study.

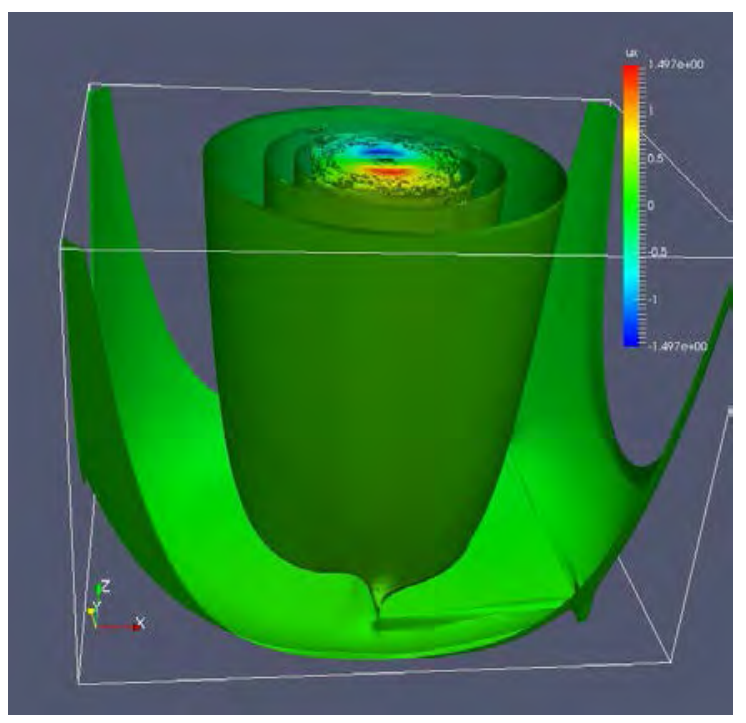

Fig.3 Velocity profile when tornado center coincides with prism

Figure 3 provides a $3 \mathrm{D}$ view of tornadic flow when the tornado center coincides with the building. A number of eddies are found in the tornado core area on a horizontal section near the top face of the computational domain, while beyond the core zone the streamlines overall remain circular about the center of tornado. Figure 4 zooms into the vicinity of the 
building, indicating the no-slip condition on the surfaces of the prism are satisfactorily guaranteed as no penetration is observed across the interface of the prism and air.
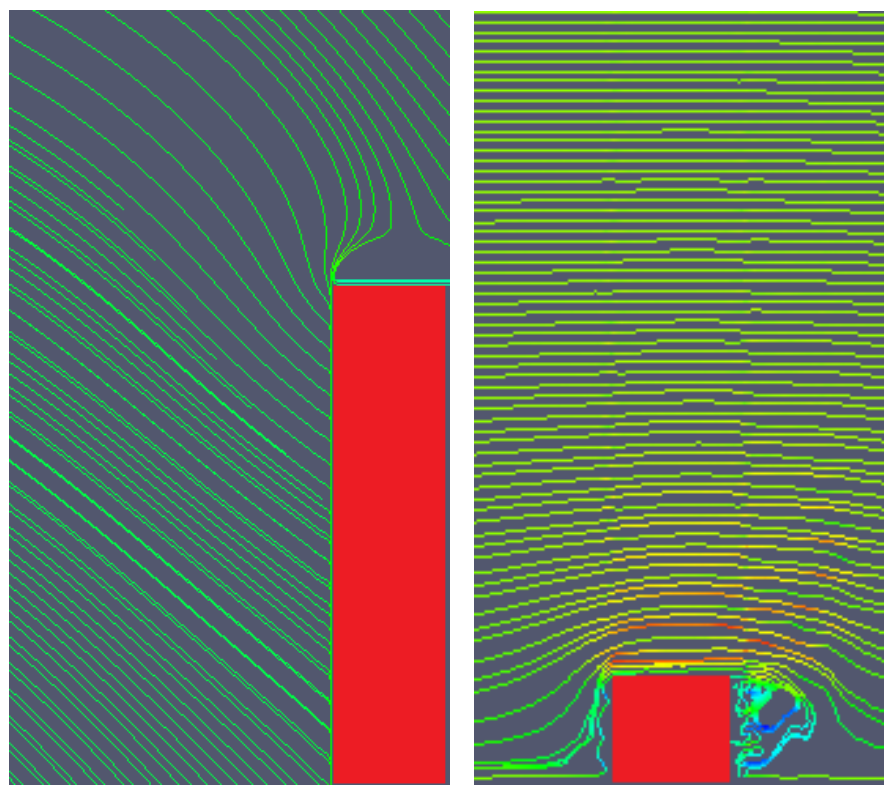

Fig.4 Streamlines near the prism on a horizontal $(x-y)$ and a vertical $(x-z)$ sections

\section{CONCLUSIONS}

In this paper, a novel computational framework was established, aiming at extending previously built 2D tornado simulation model to its $3 \mathrm{D}$ version by introducing a vertical kinematics adjustment parameter, $Z_{f}$, for describing the boundary layer growth in the $z$-direction. The re-tailored Rankine-Combined Vortex Model (RCVM) was employed with the aid of the immersed boundary (IB) approach, so that the numerical investigation of tornado-construction interaction can be made without requesting kinematic condition updating process at the outer boundary of the $3 \mathrm{D}$ computational domain. The results obtained in the present study has shown that the vertical component of a tornado plays a significant role in the tornado dynamics study since the largest force coefficient was found in the $z$-direction. Extensive simulations are underway to investigate the interaction between a variety of tornadic wind scenarios and differently deployed multi-constructions owing to the embedment of the IB strategy.

\section{ACKNOWLEDGMENT}

In addition to the Discovery Grant from the Natural Sciences and Engineering Research Council (NSERC) of Canada for the present study, the authors also wish to thank the Compute Canada for providing the computational facilities in support of the computations reported here.

\section{REFERENCES}

[1] N. B. Ward, "The Exploration of Certain Features of Tornado Dynamics Using a Laboratory Model," J. Atmos. Sci., vol. 29, no. 6, pp. 1194-1204, Sep. 1972. doi: 10.1175/1520-0469(1972)029<1194:TEOCFO >2.0.CO;2.

[2] W. S. Lewellen, D. C. Lewellen, and R. I. Sykes, "Large-Eddy Simulation of a Tornado's Interaction with the Surface,” J. Atmos. Sci., vol. 54, no. 5, pp. 581-605, Mar. 1997.. doi: 10.1175/1520-0469(1997)054<0581:LESOAT>2.0.CO;2.

[3] D. C. Lewellen and W. S. Lewellen, "Near-Surface Intensification of Tornado Vortices," J. Atmos. Sci., vol. 64, no. 7, pp. 2176-2194, Jul. 2007. doi: 10.1175/JAS3965.1.

[4] D. Natarajan and H. Hangan, "Large eddy simulations of translation and surface roughness effects on tornado-like vortices," J. Wind Engineering and Industrial Aerodynamics, vol. 104-106, pp. 577-584, May 2012. doi: 10.1016/j.jweia.2012.05.004.

[5] R. P. Selvam and P. C. Millett, "Computer modeling of tornado forces on buildings," Wind and Structures, vol. 6, no. 3, pp. 209-220, Jun. 2003. doi: 10.12989/was.2003.6.3.209.

[6] P. Gorecki and R. P. Selvam, "Rankine combined vortex interaction with a rectangular prism," Inter. J. Computational Fluid Dynamics, vol. 29, no. 1, pp. 120-132, Jan. 2015. doi: 10.1080/10618562.2015.1010524.

[7] J. Smagorinsky, "General circulation experiments with the primitive equations," Monthly Weather Review, vol. 91, no. 3, pp. 99-164, Mar. 1963. doi: 10.1175/1520-0493(1963)091<0099:GCEWTP>2.3.CO;2.

[8] X. Guo and J. Cao. An IB-LBM investigation into the aerodynamic coefficients in relation to the rotation intensity of a tornado-like wind. Computers and Mathematic with Applications, 2016. doi: 10.1016/j.camwa.2016.07.016 (In press).

[9] X. Guo, R. Palanisamy J. Cao, "A two-dimensional IB-LBM framework combined with re-tailored RCVM for assessing the rotation intensity of a tornadic wind over a building configuration. Engineering Structures, vol. 131, pp. 57-68, 2017.

[10] S. Laizet S and N. Li. "Incompact3d: a powerful tool to tackle turbulence problems with up to O (105) computational cores," Int. J. Numer. Methods Fluids., vol. 67, no. 11, pp. 1735-1757, Nov. 2010. doi: 10.1002/fld.2480.

[11] S. Laizet and E. Lamballais, "High-order compact schemes for incompressible flows: A simple and efficient method with quasi-spectral accuracy," J. Comput. Phys., vol. 228, no. 16, pp. 5989-6015, Sep. 2009. doi: 10.1016/j.jcp.2009.05.010.

[12] S. K. Lele, "Compact finite difference schemes with spectral-like resolution," J. Comput. Phys., vol. 103, no. 1, pp. 16-42, Nov. 1992. doi: 10.1016/0021-9991(92)90324-R.

[13] J. Kim and P. Moin, "Application of a fractional-step method to incompressible Navier-Stokes equations," J. Comput. Phys., vol. 59, no. 2, pp. 308-323, Jun. 1985. doi: 10.1016/0021-9991(85)90148-2. 


\section{A Model to Predict the Residual Layer Thickness in the Injection Molding Process}

\author{
R. Mollaabbasi \\ Chemical engineering department \\ Université Laval \\ Québec, Canada \\ Roozbeh.mollaabbasi.1@ulaval.ca
}

\author{
E. Behzadfar \\ Husky Injection Molding Systems \\ Bolton, Canada
}

\author{
S. M. Taghavi \\ Chemical engineering department \\ Université Laval \\ Québec, Canada
}

\begin{abstract}
The effects of the wall temperature on the residual layer thickness of the polymers are studied numerically in the filling process in the injection molding process. A lubrication approximation model is developed to furnish a semi-analytical solution. The shear viscous heating terms are neglected to simplify the governing equations. The Cross-WLF viscosity model is used to capture the residual layer. There is a good agreement between the results of the developed model and experiments.
\end{abstract}

Keywords-Heat transfer; Cross-WLF model; non-Newtonian fluid; residual layer thickness; blocking time

\section{INTRODUCTION}

The mold filling process is the main stage in the injection molding process, which is the most common process in manufacturing plastic goods. During the filling process, a melted polymer fills the cavity part [1]. The temperature reduction due to the heat transfer of the mold with the walls during the filling process directly affects the viscosity of the polymer and, consequently, the filling flow rate that strongly influences the quality of the molded part [2]. The probability of the residual layer formation negatively affects the quality of the final products.

Several researchers have modeled the momentum and heat transfer in the injection molding process to find the velocity and temperature profiles [3, 4]. Newtonian [5], power-law [6, 7] and the Cross-WLF models [4, 8] have been used mainly to simulate the rheological properties of the polymers in the filling stage. The Cross-WLF model offers the best fit to the most viscosity data of polymers in the filling process [9] because the effects of shear rate and temperature on the viscosity of the polymers are considered simultaneously. The combination of heat-transfer analysis coupled with nonNewtonian fluid mechanics approach, also, can predict the velocity and temperature profiles of the polymer in the filling process [10].

Formation of the residual layer depends on the balance between the main forces that involve in the movement of fluid. In a different context, Allouche et al. [11] have investigated the residual layer in the displacement of two visco-plastic fluids in a plane channel. Their study quantifies the sufficient conditions for removing the residual layer basde on the value of the
Bingham number (as the ratio yield to viscous stresses). In general, the minimum and maximum thickness of the residual layer depends on the competition of yield stress and viscous stress $[12,13]$.

In this paper, we study the residual layer thickness following two scenarios, A. constant injection mass flux B. constant imposed pressure to simulate the formation and removing the residual layer in the filling process.

\section{MODELING}

The governing equations in the Cartesian coordinates $(\mathrm{x}, \mathrm{y})$ assuming a symmetric 2D channel flow in the dimensionless form are:

$$
\begin{aligned}
& \operatorname{Re}\left(\frac{\partial u}{\partial t}+u \frac{\partial u}{\partial x}+v \frac{\partial u}{\partial y}\right)=-\frac{\partial p}{\partial x}+\left[\frac{\partial}{\partial x} \tau_{x x}+\frac{\partial}{\partial y} \tau_{y x}\right] \\
& \operatorname{Re}\left(\frac{\partial v}{\partial t}+u \frac{\partial v}{\partial x}+v \frac{\partial v}{\partial y}\right)=-\frac{\partial p}{\partial y}+\left[\frac{\partial}{\partial x} \tau_{x y}+\frac{\partial}{\partial y} \tau_{y y}\right]+\frac{1}{S t}, \\
& \frac{\partial u}{\partial} \quad \frac{\partial v}{\partial y}=0 \\
& \left(\frac{\partial \theta}{\partial t}+u \frac{\partial \theta}{\partial x}+v \frac{\partial \theta}{\partial y}\right)=-\frac{1}{\operatorname{Re} \operatorname{Pr}}\left(\frac{\partial \theta}{\partial x}+\frac{\partial^{2} \theta}{\partial y^{2}}\right) \\
& -\frac{E c}{\operatorname{Re}}\left(\tau_{x x} \frac{\partial u}{\partial x}+\tau_{x y} \frac{\partial u}{\partial y}+\tau_{y x} \frac{\partial v}{\partial x}+\tau_{y y} \frac{\partial v}{\partial y}\right)
\end{aligned}
$$

Here, $u, v$ denote the dimensionless velocity components, $p$ is the pressure, $\tau$ represents the stress in the fluid. Prandtl, Reynolds, Stokes and Eckert numbers are defined as:

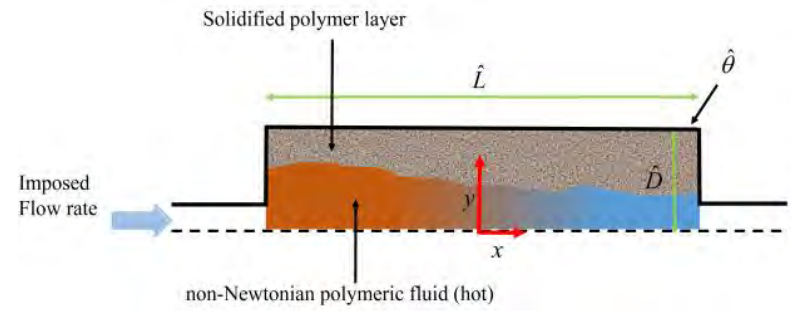

Figure 1. A schematic of problem. 


$$
\begin{aligned}
& \operatorname{Pr}=C \hat{p} \hat{\eta}_{00} / \hat{k}, \operatorname{Re} \hat{\rho} u_{0} \hat{D} / \hat{\eta}_{00}, \\
& S t=\hat{\eta}_{00} \hat{u}_{0} / \rho \hat{g} \hat{D}, E c=\hat{u}_{0}{ }^{2} / C \hat{p}\left(\hat{\theta}-\hat{\theta}_{w}\right),
\end{aligned}
$$

where, $\hat{u}_{0}, \hat{D}, \hat{g}$ are the mean imposed velocity, the half depth of the cavity and the gravitational acceleration respectively. $C \hat{p}, \hat{k},{ }_{w}, \hat{\theta}_{i}$ are the specific heat capacity, the thermal conductivity, the wall temperature and the initial fluid temperature. $\hat{\eta}_{00}$ is the constant parameter of the Cross-WLF model. The dimensionless temperature is defined as $\theta=\left(\hat{\theta}-\hat{\theta}_{w}\right) /\left(\hat{\theta}_{i}-\hat{\theta}_{w}\right)$. We assume that the geometry is full of fluid when the molten polymer is injected to the cavity (see Figure 1). Based on the small aspect ratio of the problem ( $\delta=\hat{D} / \hat{L}$ ) we re-scale as follows:

$$
\delta x=X, \delta t=T, \delta p=P, \delta V=v .
$$

Applying the lubrication approximation $(\delta \operatorname{Re} \rightarrow 0)$ and neglecting the viscous dissipation terms, we can rewrite the governing equations as:

$$
\begin{aligned}
& \frac{\partial}{\partial y} \tau_{y X}=\frac{\partial P}{\partial X}, 0=\frac{\partial}{\partial y}, \\
& \frac{\partial u}{\partial X}+\frac{\partial V}{\partial y}=0, \frac{\partial \theta}{\partial T}=\left(\frac{\partial^{2}}{\partial y^{2}}\right),
\end{aligned}
$$

where, $T^{*}=T / \delta \operatorname{Re} \operatorname{Pr}$. The Cross-WLF model interprets the rheological properties of the polymer as;

$$
\begin{aligned}
& \tau_{y X}=\eta \dot{\gamma}, \eta=\frac{\eta_{0}}{1+\left(B \eta_{0} \dot{\gamma}\right)^{1-n}}, \\
& \eta_{0}=\exp \left(\frac{\left.A_{1} \theta-\theta^{*}\right)}{A_{2}+\left(\theta-{ }^{*}\right.}\right),
\end{aligned}
$$

where, $\frac{\theta_{w}-\hat{\theta}^{*}}{\hat{\theta}_{i}-\hat{\theta}_{w}}=-\theta^{*}, \frac{\hat{A}_{2}}{\hat{\theta}_{i} \hat{\theta}_{w}}=A_{2}, \quad{ }_{0}=\frac{\hat{\eta}_{0}}{\hat{\eta}_{0}}$,

$$
\eta \frac{\hat{\eta}}{\hat{\eta}_{00}}, B=\frac{\hat{u}_{0}}{\hat{\tau}^{*}} \frac{\hat{\eta}_{00}}{\hat{D}} .
$$

$\hat{\eta}, \hat{\tau}^{*}, \hat{\eta}_{0}, \hat{\theta}^{*}, \mathrm{n}$ are the melt viscosity, the critical stress level at the transition to shear thinning, the zero shear viscosity, the glass transition temperature and the power law index respectively. $\hat{A}_{1}, \hat{A}_{2}$ and $\hat{\eta}_{00}$ are data-fitted coefficients.

\section{RESULTS AND DISCISSIONS}

Solving the momentum and energy equations considering the Cross-WLF model gives the velocity profile at different times. To calculate the temperature profile of the polymer along the filling process, we solve the energy equation $\left(\frac{\partial \theta}{T^{*}}=\frac{\partial^{2} \theta}{\partial y^{2}}\right)$ analytically. Velocity profile obtained based on scenario A (constant injection mass flux) and scenario B (constant imposed pressure). The latter is to simulate the formation and removing the residual layer in the filling process.

\section{A. Constant mass flux}

In the first scenario, we considered the mass flux is constant along the process and the pressure drop is variable. We can then calculate the velocity profile during the filling process at different time.

Figure $2 \mathrm{a}$ and Figure $2 \mathrm{~b}$ demonstrate the temperature and velocity profiles of a polymer during the filling process versus time. The maximum temperature gradient exists at the beginning where the dimensionless melt temperature is 1 . It takes a finite amount of time for a temperature "wave" to propagate through the polymer and the melt temperature decreases. At longer times, the temperature gradient goes to zero and the polymer temperature becomes equal to the wall temperature.

The polymer layers close to the wall have small overall heat resistance and become cool faster in comparison to the layers close to the middle of the cavity. This is because the conduction heat transfer is the main mechanism in the heat transfer between the wall and melt in the filling process. Consequently, the viscosity of the polymer layers increase adjacent the wall and a residual layer appears. The temperature gradient decreases with time and the thickness of residual layer increases. The maximum velocity at the middle of the cavity increases with time since the mass flux needs to remain constant.

Figure 3 shows three different zones in which the residual layer thickness changes with time. In short times, the residual layer increases with time. Then the thickness decreases gradually and it becomes zero at longer times. This phenomenon can be explained by looking back at Cross-WLF model. The viscosity of the polymer depends on the temperature and the shear rate. In a short filling process, the temperature difference between the wall and polymer is considerable. Therefore, the viscosity of the polymer layer close to the wall increases dramatically and the residual layer thickness increases. Along the time, the temperature difference inside the polymer decreases and the shear rate effect increases. It removes the residual layer with time. In a long time process, the temperature gradient inside the polymer tends to zero and the shear rate is able to move the polymer completely.

The viscosity of the polymer increases by cooling the polymer. The pressure drop increases with time dramatically due to the constant mass flux in the filling process. The pressure drop becomes constant when the residual layer is removed completely. Note that in this stage the polymer temperature is close to the wall temperature; therefore, the viscosity does not change. 

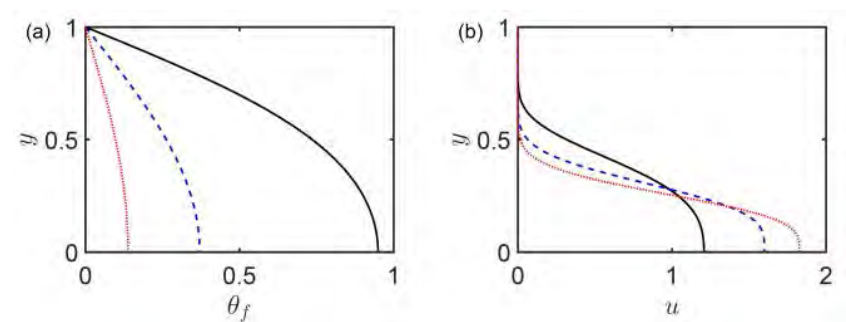

Figure 2. Temperature and velocity profiles at $T^{*}=0.1,0.5,0.9$ for the blacksolid, blue dashed and red dotted line. $A_{1}=31, \mathrm{~A}_{2}=0.2, \theta^{*}=0.12, \mathrm{n}=0.3, \mathrm{~B}=5 \times 10^{11}$.

\section{B. Constant imposed pressure}

The results of the previous section shows that the residual layer is removable at longer times if the pressure is increased dramatically. Looking back at Figure 3b, the imposed pressure must be increased almost ten orders of magnitude to remove the residual layer completely, which is not feasible for the industry. Therefore, we are considering the second scenario (constant imposed pressure) to understand the behavior of the residual layer during a more realistic filling process.

Figure 4 shows the velocity profile evolution of the polymer during the filling process in which the imposed pressure is constant. As we have discussed, the viscosity of the polymer increases due to the heat transfer between the wall and the melt. Therefore, the residual layer thickness increases with time in the case of constant imposed pressure (compare the residual layer in the inset of Figure 4 a for $T^{*}=0.01$ (blue), 0.2 (black) and 0.4 (red)). In addition, increasing the viscosity of fluid causes the maximum velocity to decrease with time. Increasing the imposed pressure augments the driving force that enhances the maximum velocity (for instance compare the blue lines in the Figures $4 a$ and $4 b$ ). In addition, higher pressure decreases the thickness of the residual layer. The dashed lines in the insets of Figures $4 \mathrm{a}$ and $4 \mathrm{~b}$ show the residual layer thickness reaching 0.52 and 0.48 at $P$ of $3 \times 10^{-12}$ and $4.2 \times 10^{-12}$ respectively when $T^{*}=0.4$.
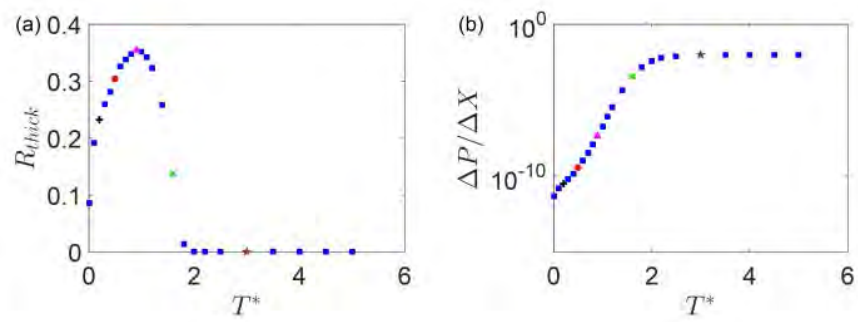

Figure 3. Dimenssionless residual layer thickness and pressure drop with time for $A_{1}=31, \mathrm{~A}_{2}=0.2, \theta^{*}=0.12, \mathrm{n}=0.3, \mathrm{~B}=5 \times 10^{11}$.
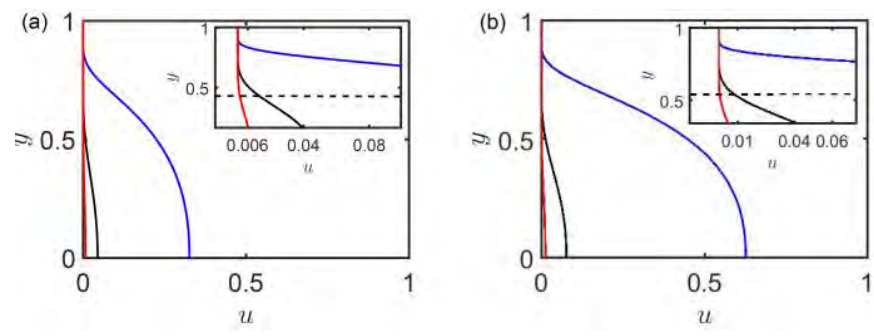

Figure 4. Velocity profile evolution along the time with the constant impose pressure. $A_{1}=31, \mathrm{~A}_{2}=0.2, \theta^{*}=0.12, \mathrm{n}=0.3, \mathrm{~B}=5 \times 10^{11}$. The imposed pressure of (b) is $40 \%$ greater than (a).

\section{Comparison with experiments}

In order to evaluate the developed model, a pilot tool was made to produce sheets with dimensions of $130 \mathrm{~mm} \times 30 \mathrm{~mm}$ $\times 3 \mathrm{~mm}$. A gate was located in the center of the cavity as shown in Figure 5. The cavity was equipped with two Kistler 61923 thermocouples flush with the surface and $44 \mathrm{~mm}$ apart from each other. The presence of the thermocouples enabled not only the measurement of the temperature of the cavity wall overtime, but also monitoring the advancement of the melt front inside the cavity. The response time of the thermocouples was $1 \mathrm{~m} / \mathrm{s}$. Using a thermolator, adjustments were made to the temperature of the cooling water and hence the cavity walls.

By controlling the injection pressure, we deliberately produced short shots to monitor the melt front advancement at different injection pressures. We used the traveling time of the melt front between two thermocouples to calculate the average velocity of the melt inside the cavity and calculate the blocking time (the time that the residual layer thickness becomes half of the cavity).

Table 1 shows the comparison between the blocking time coming from the present work, the model of Chen [14] and the experimental results. The model of Chen is an empirical method for the calculation of the blocking time. In this model, the blocking time is independent of the imposed pressure and depends on the thermal diffusivity, the melt temperature, the mold temperature and the no-flow temperature. Table 1 shows that there is a good agreement between the results of the present work and the experimental one.

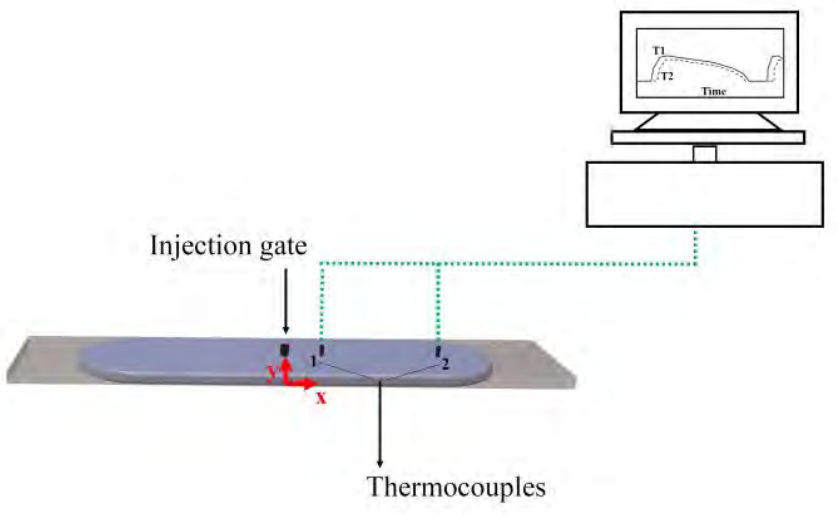

Figure 5. Schematic of the experimental setup. 
TABLE I. BLOCKING TIME COMPARISION

\begin{tabular}{|c|c|c|c|}
\hline \multirow{2}{*}{$\left.A_{2},-\theta^{*}\right]$} & \multicolumn{3}{|c|}{ Blocking Time } \\
\cline { 2 - 4 } & Experiments & Present work & Chen [14] \\
\hline$[0.19,0.08]$ & $0.725 \pm 0.10$ & 0.836 & 0.248 \\
\hline$[0.21,0.09]$ & $0.907 \pm 0.10$ & 0.915 & 0.215 \\
\hline$[0.28,0.11]$ & $0.915 \pm 0.10$ & 0.791 & 0.284 \\
\hline
\end{tabular}

\section{CONCLUSIONS}

Through developing a semi-analytical model, we have studied the residual layer that appear in the injection molding process. We have assumed that the geometry is full of the fluid when the molten polymer is injected to the cavity. We have relied on the Cross WLF to predict the rheological properties of the polymer. We have followed two scenarios when the momentum equation: Scenario A: constant mass flux; Scenario $\mathrm{B}$ : constant imposed pressure. The model is able to predict the blocking time of the cavity in the constant imposed pressure condition and there is a good agreement between the results of the model and experiments. Based on the developed model the residual layer disappears completely in the filling process if the imposed pressure increases several orders of magnitude in scenario A. However, this pressure is much higher than the pressure that is typically used in industrial cases.

\section{ACKNOWLEDGMENT}

This research has been carried out at Université Laval, supported financially by the NSERC Engage grant. The authors acknowledge Husky injection molding systems for the support of this work.

\section{REFERENCES}

[1] Z. Tadmor, C. Gogos, Principles of Polymer Processing, John Wiley and Sons. 2013.

[2] J. Primo Benitez-Rangel, A. Dominguez-G, G. Herrera-Ruiz, and M. DelgadoRosas, "Filling process in injection mold: A review,"Polym-Plast Technol., vol. 46, no. 7, pp. 721-727, 2007. doi: 10.1080/15583720701271641.

[3] M. Kamal and P. Laeur, "A structure oriented computer simulation of the injection molding of viscoelastic crystalline polymers, Model predictions and experimental results," Polym. Eng. Sci., vol. 26, pp. 103, Jan. 1986. doi: 10.1002/pen.760260115.

[4] B. Chen and W. Liu, "Numerical simulation of the post filling stage in injection molding with a two phase model," Polym. Eng. Sci., vol. 34, pp. 835, May 1994, 34, 835. doi: 10.1002/pen.760341009.

[5] R. Chang, W. Yang, "Experimental and theoretical studies of shrinkage, warpage, and sink marks of crystalline polymer injection molded parts," Int. J. Numer. Methods Fluids, vol. 37, pp. 125, Sept. 2001. doi: 10.1002/fld.166.

[6] M. Kamal and S. Kenig, "The injection molding of thermoplastics part I: theoretical mode," Polym. Eng. Sci., vol. 12, pp. 294, Jul. 1972. doi: 10.1002/pen.760120408.

[7] M. Barone and C. Tucker, Fundamentals of computer modeling for polymer processing, Hanser 1989.

[8] Y. Liu, "Heat transfer process between polymer and cavity wall during injection molding," Ph.D dissertation, Technischen Universität, Chemnitz, 2014.

[9] C. A. Hieber and H. H. Chiang, "Shear-rate-dependence modeling of polymer melt viscosity," Polym. Eng. Sci., vol.32, no. 14, pp. 931-938, Jul. 1992. doi: 10.1002/pen.760321404.

[10] T. Osswald, L.-S. Turng and P. Gramann, Injection Molding Handbook, Hanser, 2008.

[11] M. Allouche, I. Frigaard and G. Sona, "Static wall layers in the displacement of two visco-plastic fluids in a plane channel," J. Fluid Mech., vol. 424, pp. 243-277, 2000. doi: 10.1017/S0022112000001956.

[12] I. Frigaard, O. Scherzer and G. Sona, "Uniqueness and Non-uniqueness in the Steady Displacement of Two Visco-plastic Fluids," J. Appl. Math. Mech. vol. 81, no. 2, pp. 99, Feb. 2001.

doi: 10.1002/1521-4001(200102)81:2<99::AID-ZAMM99>3.0.CO;2-Q.

[13] I. Frigaard, S. Leimgruber, O. Scherzer, "Variational methods and maximal residual wall layers," J. Fluid Mech., 2003, vol. 483, pp. 37-65, May 2003. doi: 10.1017/S0022112003004105.

[14] Z. Chen, A. Giacomin and L. Turng, "Flash," Polym. Eng. Sci., vol. 46, pp. 241. 2006. doi: 10.1002/pen.20467. 


\title{
An Experimental Study of Yield Stress Fluid Displacement Flows in a Moving Annulus
}

\author{
Amin Amiri", Roozbeh Mollaabbasi, Faï̧al Larachi, Seyed Mohammad Taghavi \\ Chemical engineering department \\ Université Laval \\ Québec, Canada \\ *amin.amiri.1@ulaval.ca
}

\begin{abstract}
In this paper, the displacement flow of a viscoplastic fluid by a non-Newtonian fluid in a vertical annulus is experimentally studied. The two fluids have a very small density difference. The light displaced fluid is placed below the heavy displacing fluid. Buoyancy, the imposed flow velocity and the annulus motion are the important flow parameters. In regard to the annulus motion, two scenarios are considered: first, the annulus is stationary; Second, the annulus slowly reciprocates with an amplitude of $100 \mathrm{~mm}$ and the frequency of $0.1 \mathrm{~Hz}$. Our experimental results demonstrate that the displacement flow efficiency increases significantly when the annulus is in motion.
\end{abstract}

Keywords- Miscible displacement flows; Non-Newtonian; Moving geometry; Annulus

\section{INTRODUCTION}

Primary cementing is a key operation in the construction of oil\&gas wells. This operation is executed in oil\&gas wells for a number of reasons, such as providing a mechanical support for the well, preventing water penetration into the well, protecting the casing from corrosive materials, to name a few. Failure in primary cementing may results in a decrease in production [1] and/or environmental consequences.

Removal of drilling mud from the annulus and its replacement with cement slurry is the foundation of the primary cementing process. The efficiency of the process depends on how successfully cement is able to displace mud out the flow geometry (i.e., an annulus). Therefore, to improve the process, displacement flows need to be studied [2].

There is currently no robust method to fully and completely displace the drilling mud in the casing and more importantly in the annulus section of the well. Recently, in the industrial setting it is becoming more common to use certain movements of the casing to improve the displacement. The current casing movements are slow reciprocation and/or rotation, aiming to increase the displacement efficiency. There exist various types of casing heads and special adaptors which make the rotation and/or reciprocation of the casing possible. Typical casing reciprocations are between 6-12 $\mathrm{m}$ over a few minutes and casing (or liner) rotations vary between $10-40 \mathrm{rpm}$. Although the precise effects of these movements are not known, they are thought to be able to break the gel strength of the drilling mud to finally mobilize the slowly moving and static mud in the annulus $[3,4,5]$.

Displacement flows of primary cementing are hard to fully understand due to the presence of various parameters. For example, the parameters that describe the geometry (inclined pipe and annulus), the inclination angle, gravitational acceleration and the flow rate require at least eight dimensionless groups to describe the flow. Considering twofluid displacement flows, this increases to 10-12 dimensionless groups [2]. Rheological properties of the fluids involved also further contribute to the complexity of the system $[6,7]$. To date, several studies have been conducted on different aspects of displacement flows to gain insights crucial understanding. Miscible displacement flows of Newtonian fluids in uniform pipes have been studied in detail in an inclined pipe [8,9], a stationary vertical pipe [10] and a moving pipe [11]. Displacement flows of non-Newtonian fluids have also been studied experimentally and numerically $[12,13,14]$. Buoyant miscible displacement flows in nearly-horizontal, inclined and vertical ducts have also received attention $[15,16,17]$. Annular displacement flows have been investigated in only a few studies $[18,19]$. There even fewer studies in literature which can be directly relevant to the displacement flows in moving geometries [7, 20]. To fill an obvious lack in the literature, in this novel work, we experimentally investigate the displacement of a viscoplastic fluid in a moving annulus. In addition to providing essential scientific knowledge, this work is also technically important as it considers for the first displacement flows in a moving annulus with moving parameters similar to those used in industrial settings.

\section{EXPERIMENTAL SETUP, Procedures AND DETAILS}

Our experiments were performed in a $270 \mathrm{~cm}$ long annulus. The inner cylinder had an outer diameter of $40 \mathrm{~mm}$ and the outer cylinder had a diameter of $50 \mathrm{~mm}$, creating a gap of 5 $\mathrm{mm}$ between two cylinders. The cylinders were transparent 
(plexiglass clear acrylic cylinders) and they positioned vertically. They system had a manual plastic socket-weld gradual On/Off gate valve located at $40 \mathrm{~cm}$ from the top end (see figure 1). The gate valve initially separated the two parts of the annulus setup and completely segregated the displacing and displaced fluids. The gate valve system was designed in a way that it could be manually opened at the beginning of each experiment. All the experiments were performed at room temperature. The displacement flow setup was held on a fixed platform and it was divided into two parts: fixed and mobile sections, which enabled us to investigate the displacement flow in a stationary and moving geometry. The fixed part of the annulus setup included the outer cylinder, the clear cube vessel and all connections above the gate valve. The mobile section contained a stepper motor connected to the inner cylinder to produce the reciprocation motion. The maximum amplitude and the maximum frequency of reciprocation motion could be $15 \mathrm{~cm}$ and $0.3 \mathrm{~Hz}$, respectively. The LabVIEW was used to control all parameters. Initially, the lower part of the annulus was filled using a peristaltic pump with a Carbopol solution (light fluid or displaced fluid) colored with a small amount of ink (Fountain Pen India black ink) for visualization purposes. Light absorption calibrations were performed in usual fashion, for which the amount of $2 \mathrm{~g}$ of ink per one liter of liquid was found to be a reasonable trade-off. The upper part of the annulus, above the gate valve, was filled with displacing fluid (heavy fluid). The densities of our experimental fluids were measured by a high-precision density meter (Anton Paar DMA 35). The viscosity and the yield stress of the solutions were measured in usual fashion, using AR-G2 TA Instrument digital controlled shear stress-shear rate rheometer. The maximum error in measurement of viscosity was $5 \%$. To avoid pump disturbances and ensure a smooth steady inflow, the displacing upper fluid was fed by gravity from a large elevated tank filled with the displacing fluid. The flow rate was controlled by a needle valve and it was measured by a flow meter (Omega FTB 421, low flow plastic turbine), located downstream of the annulus setup. At the start of each experiment, the gate valve was opened and the displacement process started. Images of the displacement flow were recorded using a high-speed camera (Basler acA2040, with 4096 gray-scale levels) covering around $120 \mathrm{~cm}$ below the gate valve and they were subsequently postprocessed using in-house Matlab codes, to quantify different aspects of the flow. To enhance quality of the images, the annulus setup was back-lit using Light-Emitting Diode (LED) strips. In addition, to improve light homogeneity, a diffusive layer was placed between the annulus setup and the LED strips.

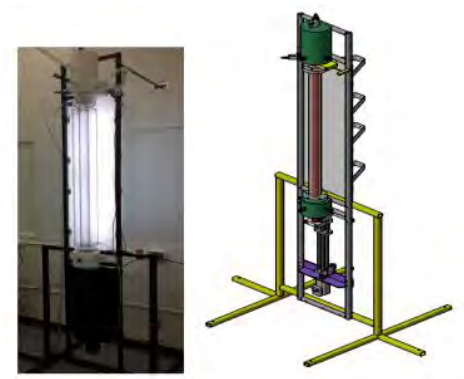

Figure1. Real and schematic views of experimental annulus setup.

\section{RESUlTS AND DiscUSIONS}

The experiments were performed at a fixed density difference and at a small Atwood number $\left(A t=\frac{\hat{\rho}_{H}-\hat{\rho}}{\hat{\rho}+\hat{\rho}_{L}}\right.$ 0.0005). $\hat{\rho}_{H}$ and $\hat{\rho}_{\mathrm{L}}$ represent the densities of the displacing and displaced fluids, respectively. The displaced fluid was a Carbopol solution as a viscoplastic fluid with a $1.4 \mathrm{~Pa}$ yield stress. The displacing fluid was a Xanthan solution. Carbopol and Xanthan compositions and determined parameters from rheological measurements, assuming the Herschel-Bulkley model (Eq. 1), and power low model (Eq. 2) are listed in Table.1, respectively.

$$
\begin{array}{r}
\hat{\tau}=\hat{\tau}_{\mathrm{Y}}+\hat{\kappa} \hat{\dot{\gamma}}^{\mathrm{n}} . \\
\hat{\tau}=\hat{\kappa} \hat{\gamma}^{\mathrm{n}} .
\end{array}
$$

TABLE I. RHEOlOGicAl MEASURMENTS For CARBopol $\&$ XANTHAN

\begin{tabular}{|c|c|c|c|}
\hline \multicolumn{4}{|c|}{ Rheological measurements for Carbopol composition } \\
\hline & Consistency & $\begin{array}{c}\text { Power-law } \\
\text { index }\end{array}$ & $\begin{array}{c}\text { Yield } \\
\text { stress }\end{array}$ \\
\cline { 2 - 4 } & $\hat{\kappa}\left(\mathrm{Pa} . \mathrm{s}^{\mathrm{n}}\right)$ & $\mathbf{n}$ & $\hat{\tau}_{\mathrm{Y}}(\mathrm{Pa})$ \\
\hline Carbopol $0.07 \%(\mathrm{wt} / \mathrm{wt})$ & 1.34 & 0.45 & 1.4 \\
\hline Xanthan $0.25 \%(\mathrm{wt} / \mathrm{wt})$ & 0.81 & 0.31 & ---- \\
\hline
\end{tabular}

The Herschel-Bulkley and power low models are appropriate rheological models that describes the shear behavior of the Carbopol gel and Xanthan solutions, respectively. Figure 2 shows an example of the rheometer flow curve for our Carbopol solution, in which the Herschel-Bulkley model is fitted to the datapoint.

To study the effect of the reciprocation motion on the displacement flow, the annulus setup was used to impose a reciprocation motion of $100 \mathrm{~mm}$ at a small frequency $(0.1 \mathrm{~Hz})$.

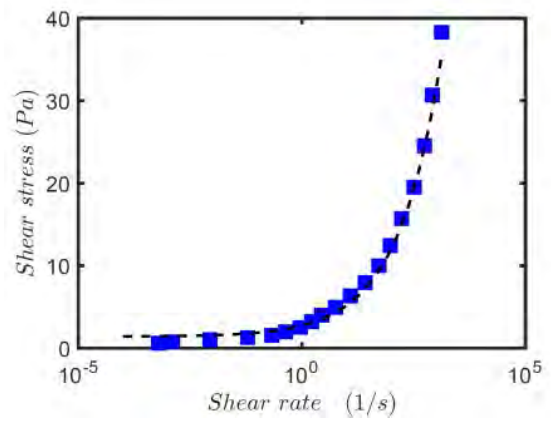

Figure 2. Rheological results for low concentration of the Carbopol solution. The dashed line indicates the Herschel-Bulkley model parameters fitted to the data. 


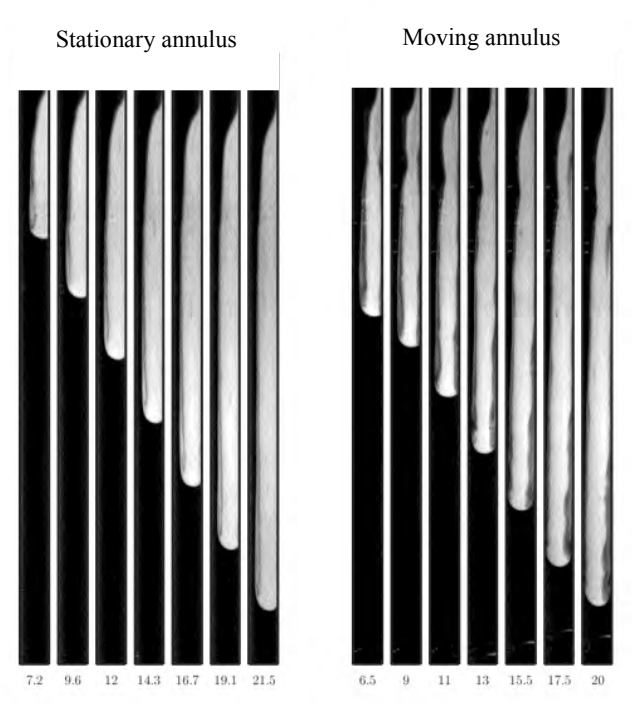

Figure 3. Sequence of images showing the displacement flow in a stationary and moving geometry (with a frequency of $0.1 \mathrm{~Hz}$ over $100 \mathrm{~mm}$ amplitude with respect to vertical). The field of view is $46 \times 1200 \mathrm{~mm}^{2}$ right below the gate valve. From left to right, the results of stationary and moving geometry are shown at $\hat{\mathrm{V}}_{0} \approx 14(\mathrm{~mm} / \mathrm{s})$. The experimental times in each image sequence panel are provided in second (s), below each snapshot.

Figure 3 shows examples of our annular displacement flow results. This figure (left side) shows a sequence of images of a flow in stationary annulus at a low imposed flow rate, i.e., $\hat{\mathrm{V}}_{0}=13.74(\mathrm{~mm} / \mathrm{s})$. As time grows, the heavy fluid displaces the light fluid in the downward direction and there is not much mixing between the two fluids. In addition, there is a static residual layer of the Carbopol solution on the wall. The same behavior is observed in figure 3 (right side) and it shows that the displacement process is not as efficient as the case without motion at short time. However, it should be mentioned that the story is different at extended times.

Figure 4 shows a sequence of experimental images with the same parameters of figure 3 but for an extended time. This figure indicates the effects of a moving geometry on the flow at longer times. It is obvious that the static residual layer is evidently stable and the amount of the Carbopol solution remains unchanged for the stationary geometry (figure 4). Figure 4 (right side) shows that by adding a reciprocation motion, the overall displacement improves: the amount of the Carbopol displaced by Xanthan solution is increased. In fact, the reciprocating motion of the inner cylinder is able to remove the static residual layers of the displaced phase, in the downward direction. Due to the shear exerted by the inner wall motion, the reciprocation enhances the removal of Carbopol from the walls and consequently the displacement flow efficiency increases.
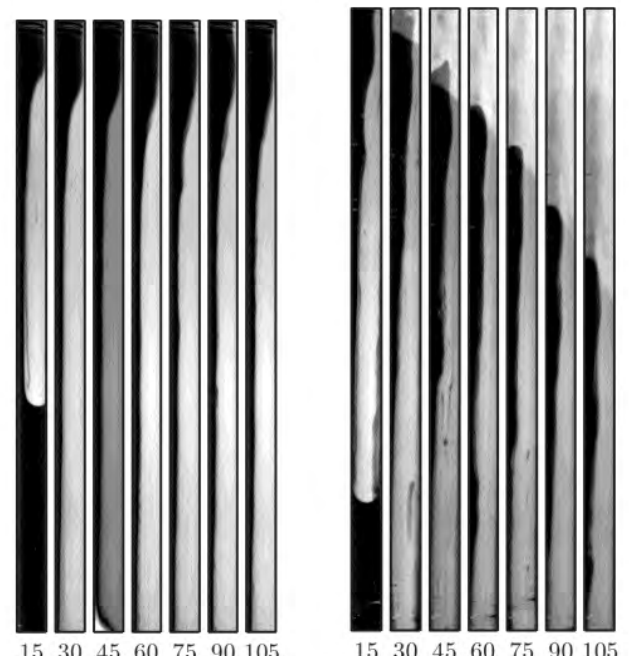

Figure 4. Sequence of images showing the displacement flow in a stationary and moving geometry (with a frequency of $0.1 \mathrm{~Hz}$ over $100 \mathrm{~mm}$ amplitude with respect to vertical) at long time. The field of view is $46 \times 1200 \mathrm{~mm}^{2}$ right below the gate valve. From left to right, the results of stationary and moving geometry are shown at $\hat{\mathrm{V}}_{0} \approx 14(\mathrm{~mm} / \mathrm{s})$. The experimental times in each image sequence panel are provided in second (s), below each snapshot.

To explain this feature more quantitatively, it is necessary to introduce the depth-averaged concentration profiles of our displacements (shown by $\mathrm{C}$ in the following figures). Figure 5 shows an example of the evolution of the average concentration profile with time and streamwise location. Note that the experimental snapshots are calibrated between 0 (dark displaced fluid) and 1 (transparent displacing fluid). Therefore, in these diagrams, when the concentration value is around 1 , the displacement is complete and there is no layer of the displaced fluid on the wall. On the other hand, when the concentration value is smaller than 1 a layer of the Carbopol solution remains on the wall.

There are interesting results delivered through analyzing depth-averaged concentration diagrams. First, the depthaveraged concentration profiles show the displacement flow is not complete and a layer of the displaced fluid remains on the wall at all times. Second at short times, the motion does not seem to affect the displacement efficiency. 

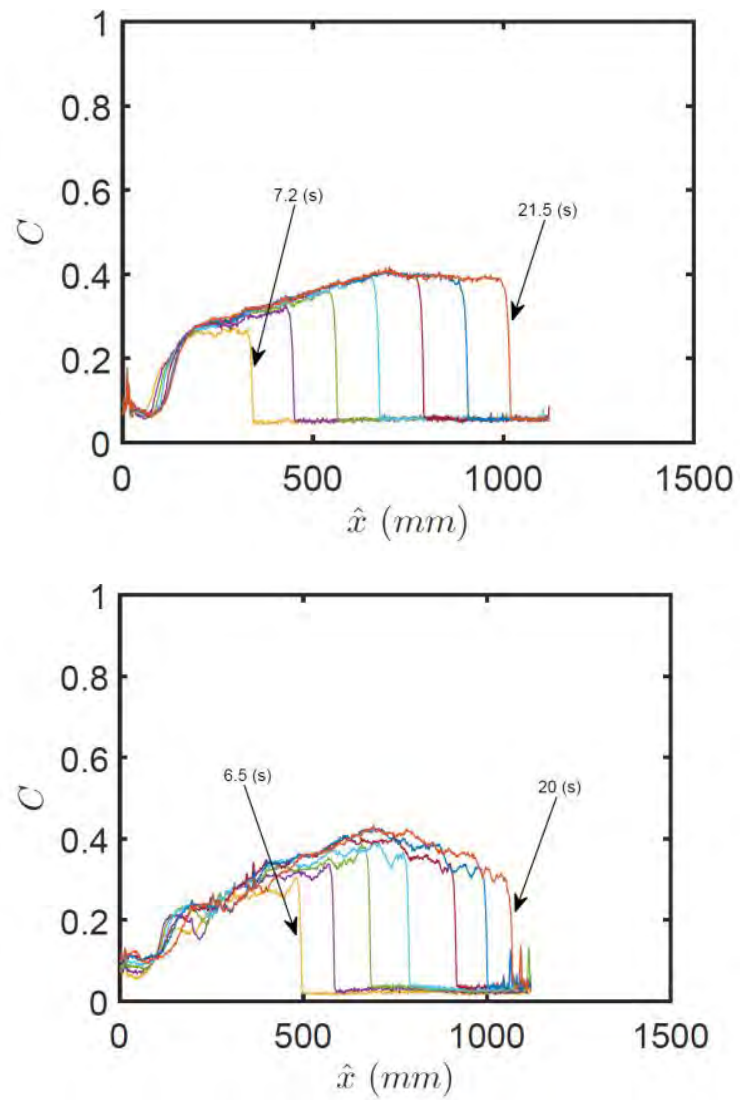

Figure 5. Evolution of the depth-averaged concentration field with time $\hat{t}$, and streamwise location, $\hat{x}$ measured from the gate valve. From top to bottom, the geometry is stationary $\quad(\hat{\mathrm{t}}=[7.2,9.6,12,14.3,16.7,19.1,21.5](\mathrm{s})) \quad$ and moving $(\hat{\mathrm{t}}=[6.5,9,11,13,15.5,17.5,20](\mathrm{s}))$, respectively.

To give a global vision of the displacement efficiency at longer times, the depth-averaged concentration diagrams with the same parameters are presented in figure 6. It can be seen that when the Carbopol solution is displaced by the Xanthan solution in the moving geometry, the amount of Carbopol layer on the wall decreases, significantly. In the stationary geometry of figure 6 (top), the concentration values are approximately below 0.5 and all profiles nearly collapse. Therefore, as time grows, the efficiency of the displacement flow does not change. However, in the moving geometry of figure 6 (bottom) the concentration value is under 0.8 . Therefore, the amount of the Carbopol solution on the wall decreases significantly and the displacement efficiency increases at an extended time.
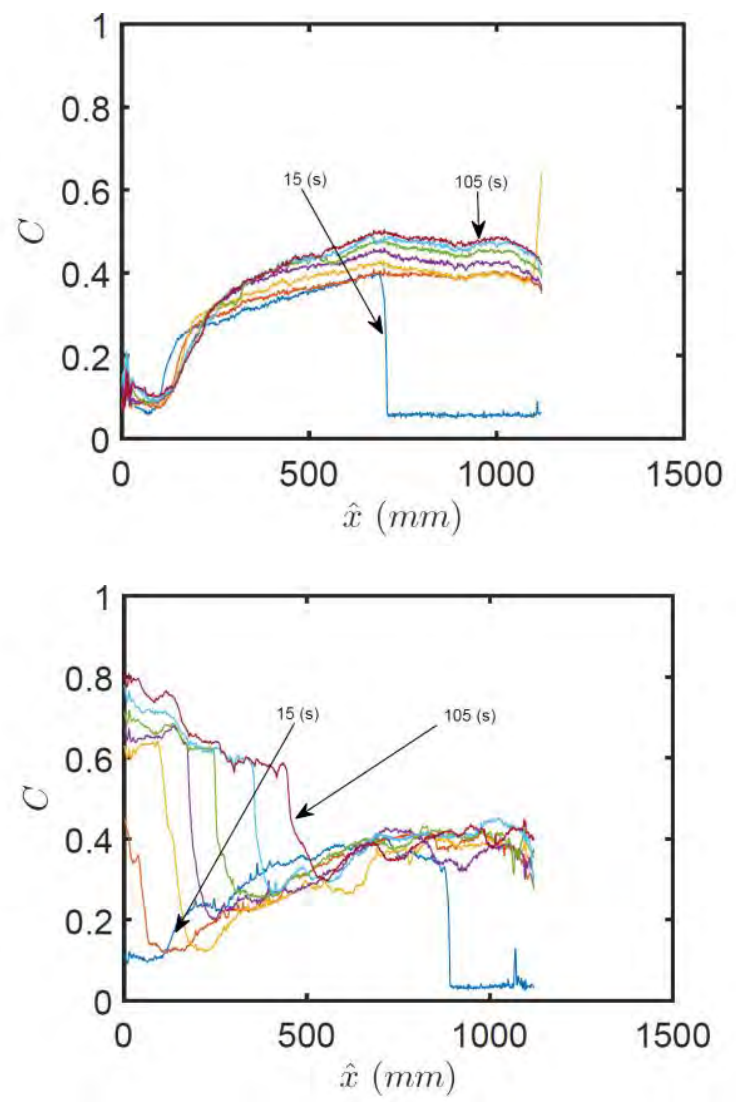

Figure 6. Evolution of the depth-averaged concentration field with time $\hat{\mathrm{t}}=[15,30,45,60,75,90,105](\mathrm{s})$, and streamwise location, $\hat{\mathrm{x}}$ measured from the gate valve. From top to bottom, the geometry is stationary and moving, respectively.

\section{CONCLUSION}

We experimentally studied miscible displacement flows in a long, vertical moving annulus. The experimental fluids were Xanthan solution (displacing fluid) and non-Newtonian viscoplastic (displaced fluid) and they had a very small density difference. The main finding was that the displacement flow efficiency was a function of the geometry motion. In the cases studied, the displacement flow in the moving annulus (reciprocation motion) was more efficient in comparison to the stationary annulus.

\section{REFERENCES}

[1] E. B. Nelson and D. Guillot, Well cementing, 2nd ed., Sugarland, TX: Schlumberger, 2006.

[2] I. A. Frigaard, K. G. Paso and P. de Souza Mendes, "Binghams model in the oil and gas industry," Rheol. Acta, vol. 56, no. 3, pp. 259-282, Mar. 2017. doi: 10.1007/s00397-017-0999-y.

[3] N. Moroni, K. Ravi, T. Hemphill, and P. Sairam, "Pipe rotation improves hole cleaning and cement-slurry placement: Mathematical modeling and field validation," In: Offshore Europe, Aberdeen, UK: Society of Petroleum

Engineers, 2009. doi: 10.2118/124726-MS. 
[4] M. Carrasco-Teja and I. Frigaard, "Displacement flows in horizontal, narrow, eccentric annuli with a moving inner cylinder," Phys. Fluids, vol. 21, no. 7, 073102, 2009. doi: 10.1063/1.3193712.

[5] M. Carrasco-Teja and I. Frigaard, "Non-newtonian fluid displacements in horizontal narrow eccentric annuli: effects of slow motion of the inner cylinder," J. Fl. Mech.,vol. 653, pp. 137-173, 2010. doi: $10.1017 / \mathrm{S} 0022112010000212$.

[6] R. C. Haut and R. J. Crook, "Primary cementing: The mud displacement process," in: SPE Annual Technical Conference and Exhibition, Society of Petroleum Engineers, 1979. doi: 10.2118/8253-MS.

[7] C. Holt, N. Lahoti, and V. Fortier, "New cementing method uses pipe movement to maximize displacement," in Drilling Contractor - Official Magazine of the International Association of Drilling Contractors, 2013. $\mathrm{http}: / /$ www.drilling contractor.org

[8] K. Alba, S. Taghavi, and I. Frigaard, "Miscible density-stable displacement flows in inclined tube," Phys. Fluids., vol. 24, 23102, 2012. doi: 10.1063/1.4766197.

[9] T. Séon, J. Znaien, B. Perrin, E. J. Hinch, D. Salin, and J. P. Hulin, "Front dynamics and macroscopic diffusion in buoyant mixing in a tilted tube," Phys. Fluids, vol. 19, no. 12, p. 125105, Dec. 2007.

doi: $10.1063 / 1.2821733$.

[10] A. Amiri, F. Larachi and S. Taghavi, "Buoyant miscible displacement flows in vertical pipe," Phys. Fluids, vol. 28, 102105, 2016. doi: $10.1063 / 1.4965250$.

[11] A. Amiri, F. Larachi, and S. Taghavi, "Displacement flows in periodically moving pipe: understanding multiphase flows hosted in oscillating geometry," Chem. Eng. Sci., vol. 170, Mar. 2017. doi: 10.1016/j.ces.2017.01.058.

[12] M. Ben Amar and E. Poire, "Pushing a non-newtonian fluid in a hele-shaw cell: From fingers to needles," Phys.Fluids, vol. 11, no. 7, pp. 1757-1767, 1999. doi: 10.1063/1.870041.

[13] I. A. Frigaard and D. P. Ryan, "Flow of a visco-plastic fluid in a channel of slowly varying width," Journal of Non-Newtonian Fluid Mechanics, vol. 123, no. 1, pp. 67-83, Oct. 2004.

doi: 10.1016/j.jnnfm.2004.06.011.

[14] S. M. Taghavi, K. Alba, M. Moyers-Gonzalez, and I. A. Frigaard, "Incomplete fluid-fluid displacement of yield stress fluids in nearhorizontal pipes: Experiments and theory," J. Non-Newtonian Fluid Mechanics, vol. 167-168, pp. 59-74, Jan. 2012. doi: 10.1016/j.jnnfm.2011.10.004.

[15] K. Alba, S. Taghavi and I. Frigaard, "Miscible density-unstable displacement flows in in inclined tube," Physics of Fluids, vol. 25, no. 067101, 2013. doi: 10.1063/1.4808113.

[16] S. Taghavi, et. al., "Influence of an imposed flow on the stability of a gravity current in a near horizontal duct," Phys. Fluids, vol. 22, 031702, 2010. doi: 10.1063/1.3326074.

[17] R. Mollaabbasi and S. Taghavi, "Buoyant displacement flows in slightly non-uniform channels," J. Fluid. Mech., vol 795, pp. 876-913, May, 2016. doi: $10.1017 / \mathrm{jfm} .2016 .232$.

[18] M. Tehrani, S. Bittleston and P. Long, "Flow instabilities during annular displacement of one non-newtonian fluid by another," Exp. Fluids, vol. 14, no. 4, pp. 246-256, 1993. doi: 10.1007/BF00194015.

[19] S. Pelipenko and I. Frigaard, "Two-dimensional computational simulation of eccentric annular cementing displacements," J. Engng. Math., vol. 64, no. 6, pp. 557-583, 2004. doi: 10.1093/imamat/69.6.557.

[20] M. Carrasco-Teja, I. A. Frigaard, B. R. Seymour, and S. Storey, "Viscoplastic fluid displacements in horizontal narrow eccentric annuli: stratification and travelling wave solutions," J. Fluid Mechanics, vol. 605, May 2008. doi: 10.1017/S0022112008001535. 


\section{Modeling the Effect of Produced Water on Asphaltene Deposition in a Production Tubing Using Population Balance}

\author{
R. Mollaabbasi \\ Chemical engineering department \\ Université Laval \\ Québec, Canada \\ Roozbeh.mollaabbasi.1@ulaval.ca
}

\author{
D. Eskin \\ Schlumberger-Doll Research \\ Cambridge, USA
}

\author{
S. M. Taghavi \\ Chemical engineering department \\ Université Laval \\ Québec, Canada
}

\begin{abstract}
An engineering model evaluates the effect of produced water in a production tubing on the reduction of asphaltene deposition on the tube (pipe) wall. We rely on an industrial hypothesis that droplets colliding with the pipe partially prevent the deposition of the asphaltene particles on the wall surface. First, we use a population balance model to calculate the droplet size evolution along a production tubing. Then, we study the fraction of the pipe wall surface dynamically coated by water droplets. To do so, we consider the wall bombardment by droplets that fluctuate in a turbulent flow. The results demonstrate that the dynamically coated area by droplets increases gradually (max by order of $10^{-4}$ ), the effects of which on asphaltene deposition is negligible.
\end{abstract}

\section{Keywords-Asphaltene precipitation; droplet breakup; population balance model}

\section{INTRODUCTION}

The interaction between asphaltene particles and droplets is an important problem of flow assurance in the oil industry. Asphaltenes are molecular substances, which compose crude oil along with resins, aromatic hydrocarbons and saturated hydrocarbons such as alkanes that are not soluble in straightchain solvents. Asphaltenes precipitate from a hydrocarbon fluid when the local pressure drops below the so-called asphaltene onset precipitation pressure. Further downstream pressure decrease is accompanied with further precipitation. Complex deposition phenomenon consists of different processes. Precipitated particles interact with each other, grow due to agglomeration, are transported to the wall and stick to its surface with some probability [1, 2]. Asphaltene deposition represents a significant concern only for a vertical production tubing. Here, a substantial pressure drop required for intense asphaltene precipitation is obtained due to hydrostatic pressure difference. Asphaltene deposition has received significant attention in the literature [3, 4]. Numerous articles have considered the problem of asphaltene deposition in reservoirs $[5,6]$, in pipelines $[1,2]$ and in the Couette flows [1]. Some of published asphaltene deposition models demonstrated a reasonable agreement with experimental data. It is important to emphasize that those models were developed for a single-phase (hydrocarbon) flow, whereas, in practice, water is usually produced along with oil. The effects of droplets on the asphaltene deposition in turbulent flow are an unanswered question due to the physical complexity of the problem.

\section{MODELING}

\section{A. Basic concept of accounting for an effect of dispersed water on asphaltene deposition}

Figure 1 illustrates asphaltene deposition on the tubing wall in the presence of dispersed water. The smaller particles and the larger spheres denote the asphaltene aggregates and the water droplets, respectively. According to the comprehensive approach to asphaltene deposition modeling, this phenomenon is a complex process, which consists of the following components: 1) precipitation of primary asphaltene particles; 2) particle agglomeration; 3) particle transport to the pipe wall; 4) interaction of particles with the wall. Eskin et al. [1,2] showed that the asphaltene deposition rate is a strong function of the particle size. Therefore, modeling of the particle size distribution evolution along a production tubing is a key deposition model element. Brownian motion as well as turbulent diffusion (dispersion) govern both agglomeration and deposition of asphaltene particles. However, Eskin et al. [2] showed that turbulent diffusion can be neglected because only very small submicron particles are able to deposit on the pipe wall.

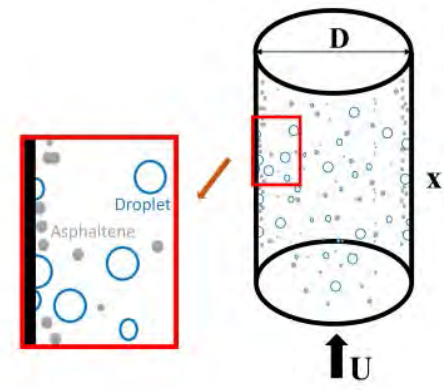

Figure 1. Diagram illustrating asphaltene deposition in a pipe in presence of asphaltene particles and water droplets. 
Here, we consider the effect of water on asphaltene deposition. Water droplets absorb asphaltene molecules. It leads to a modification of droplet surfaces causing coalescence suppression, i.e., droplet stabilization. Non-coalescing droplets are intensely fragmented by turbulence and can reach rather small sizes. At high water holdups ( $\sim 50 \%)$, the droplet specific surface may reach very high values. For example, this surface can be up to 3 orders of magnitude larger than the corresponding specific surface of the pipe wall. However, a simple analysis shows the amount of asphaltene absorbed by droplets is small. Indeed, absorbed asphaltene molecules form a monolayer on a droplet surface. The thickness of this monolayer is approximately equal to the diameter of an asphaltene molecule, which does not exceed a few nanometers [3]. Because the monolayer thickness is so small, the total volume concentration of asphaltenes absorbed on droplets is always much lower $\left(<\sim 10^{-4}\right)$ than the volume concentration of asphaltenes in a fluid, which can cause a significant deposition rate $\left(\sim 10^{-2}\right)$ [3]. Therefore, an effect of water on asphaltene deposition is limited to only droplets-pipe wall interactions. Droplets, driven by turbulence eddies, collide with the wall (deposit surface) and bounce from it. In the course of a dropletwall interaction, a deformed droplet coats some surface area of the wall. This area can vary during the bouncing process. Evidently, the area coated by a droplet is unavailable for depositing aspahltene particles. Thus, the problem of accounting for an effect of water on asphaltene deposition can be reduced to determining the mean fraction of the pipe wall surface that is dynamically coated with water droplets. Then, the deposition flux in the presence of dispersed water is calculated as: ${ }_{a v}=q_{a}(1-\mathrm{S})$, where, $q_{a}$ is the asphaltene deposition flux and $S$ is the mean fraction of the pipe wall surface dynamically coated by water droplets. To determine the parameter $S$ we need to evaluate the droplet size distribution in a pipe flow and consider a process of droplet interaction with the pipe wall (deposit surface).

\section{B. Droplet dispersion in a pipe flow}

We have relied on the population balance model (PBM) in order to predict the droplet size evolution along the time. A disbalance between the viscous stress of the fluid inside the droplets, the surface tension of the droplets and the external stress causes droplet breakup from the continuous phase. The competition of these stresses destroys the surface of the droplets and droplet breakup occurs. A transient diameter based on PBM holding the breakage and the coalescence terms can be written as follows in turbulent uniform conditions $[7,8]$ :

$$
\frac{d f_{i}}{d t}=-B_{D}(\mathrm{i}, \mathrm{t})+B_{B}(\mathrm{i}, \mathrm{t})-C_{D}(\mathrm{i}, \mathrm{t})+C_{B}(\mathrm{i}, \mathrm{t}),
$$

where $f_{i}$ is the mass based distribution of the $i_{t h}$ droplet. $B_{D}, B_{B}, C_{D}$ and $C_{B}$ are the breakage death, the breakage birth, the coalescence death and the coalescence birth of droplets respectively in the PBM model. The breakage death has been defined as:

$$
B_{D}(i, t)=b(i) f(i, t) \text {. }
$$

The breakage frequency function $b(i)$ and daughter size distribution function are investigated by different methods (e.g., Coulaloglou and Tavlarides [9]). The breakage birth is calculated as:

$$
B_{B}(i, t)=v(i) \int_{d_{i}}^{d_{i \max }} h(i, j) b(j) \frac{f(j, t)}{v(j)} d\left(d_{j}\right)
$$

where $v(i)$ is the volume of the $i_{t h}$ droplet and daughter size redistribution function $h(i, j)$ is calculated by Coulaloglou and Tavlarides [9]. The coalescence birth term is:

$$
\begin{aligned}
& C_{B}(i, t)=\frac{d^{2}(\mathrm{i}) v(i)}{2} \times \\
& \int_{d_{\min }}^{\left.d_{i}^{3}-d_{\min }{ }^{3}\right)^{1 / 3}} \frac{C\left(\left[d_{i}^{3}-d_{j}{ }^{3}\right]^{1 / 3}, d_{j}\right)}{\left.d_{i}{ }^{3}-d_{j}{ }^{3}\right]^{2 / 3}} \frac{f(i, t)}{\rho_{d} v(j)} \frac{f\left(\left[d_{i}^{3}-d_{j}{ }^{3}\right]^{1 / 3}, t\right)}{v(i)-v(j)} d\left(d_{j}\right) .
\end{aligned}
$$

The coalescence death term is [10]:

$$
C_{D}(i, t)=f(i, t) \int_{d_{\min }}^{\left(d_{\max } \int^{3} d_{i}^{3 / 3}\right.} C\left(d_{i}, d_{j}\right) \frac{f(j, t)}{\rho_{d} v(j)} d\left(d_{j}\right) .
$$

Figure 2 shows the evolution of pure droplet with time in oil, $\rho_{d}=965.3 \mathrm{~kg} / \mathrm{m}^{3}, \mu_{d}=0.3 \mathrm{mPa} / \mathrm{s}$ and liquid-liquid surface tension is $0.0056 \mathrm{~N} / \mathrm{m}$. We have considered the monodispersed initial condition to solve PBM. The initial mass fraction of droplets is $\phi=0.1$ (assuming a very diluted system to neglect the coalescence terms) and the initial droplet size is $d_{d}=150 \mu \mathrm{m}\left(V_{i+1} / V_{i}=0.5\right.$ and $\left.\mathrm{i}=1: 20\right)$. The results show that the droplet size is alleviated with time. Figure $2 \mathrm{~d}$ illustrates the evolution of the mean droplet size with time in a log-log scale, showing that there are three different regions in the droplet breakage with time in case of initially mono-dispersed droplets.
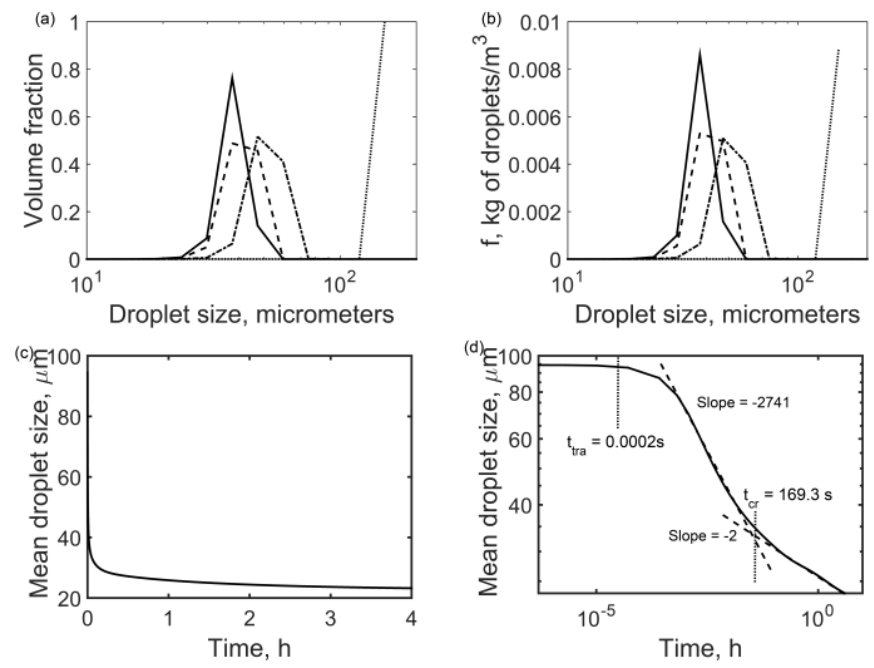

Figure 2. Size evolution of pure droplet with time in turbulent system. Subfigures in the top row show the volume fraction and mass distribution function with time from left to right (the breakage time is $0 \mathrm{~h}, 0.1 \mathrm{~h}, 1.5 \mathrm{~h}$ and $4.0 \mathrm{~h}$ in the dotted line, dash-dotted line, dashed line and solid line respectively). The bottom row shows the average droplet size with time in linear and logarithmic axis. The continuous and dispersed phases are considered as oil and water respectively. 
The mean droplet size does vary much with time in the first region before reaching the transition time $\left(t_{\text {tra }}\right)$, which is in the order of $10^{-4} \mathrm{~s}$. The transition time represents the time required for the imbalance between the viscous stress of the fluid inside the droplet and surface tension to break the droplets considering the mono dispersed droplet distribution as an initial condition. Another important time is the critical time $\left(t_{c r}\right)$ which is quantified by the inflection point of the mean droplet size and it demonstrates the time when the droplet breakup mechanism is changed. Rapid size reduction implies that eddies have more effects on the turbulent mechanism of droplet breakup due to the large size of droplets (first zone). Brownian motion has more effects on collision of droplets in the case of the small droplet size; therefore, the rate of size reduction decreases in tiny droplets. The coalescence terms do not have a significant effect on the PBM in diluted systems because the coalescence terms are 5 orders of magnitude smaller than the breakup terms. Solsvik et al. [8] have stated the coalescence terms are negligible compared to droplet breakup in a dilute dispersed system. The results of our simulation confirm this statement.

\section{Droplet deposition on the wall}

To evaluate the cumulative wall coating effect caused by multiple droplets-wall interactions, a collision of a single droplet with the wall should be considered. In practice, a pipe wall is often characterized by a rather significant roughness. This circumstance complicates the interaction analysis. However, a reasonable estimate of the interaction phenomenon could be obtained assuming that the wall is smooth. The larger the ratio between the droplet diameter and the wall roughness, the closer this is to reality. The only significant mechanism causing a droplet-wall collision is a droplet fluctuation caused by a turbulent eddy. Following Kuboi [11], we assume that droplet fluctuations are mainly controlled by eddies, whose scales are equal to droplet sizes. Usually, the droplet fluctuation is relatively low. Therefore, we assume that the droplet deformation during the interaction process is small. Obviously, after a collision with the wall the droplet should ricochet. Thus, the droplet-wall interaction process consists of the two stages: droplet compression (deformation) and relaxation (shape restoration). Due to the smallness of droplet deformation, we do not consider a hydrodynamic flow field inside the droplet at the compression stage. Then, we can formulate the momentum equation for the droplet-wall interaction, assuming that all fluid elements inside a droplet have the same velocity (see Figure 3). Also, we make additional assumptions to derive the momentum equation for a droplet interacting with the wall. We assume that the droplet interacting with the wall maintains a shape of a sphere with a cut segment. The volume of this segment is neglected due to the assumed deformation smallness. We also assume that during the compression stage, the wall reaction force acting on a droplet is comprised of the two components. First, a hydrodynamic reaction force appears because a droplet interacting with the wall loses momentum component normal to the wall. We evaluate this force by considering an interaction of an imaginary jet of radius a. We consider that this this force is absent during the relaxation stage. The second component of the reaction force is the capillary force applied also to the same droplet wall contact area. For the sake of simplicity, we neglect the drag force acting on the droplet during its interaction with the wall. Because a turbulent eddy transporting the droplet is also decelerated in the wall vicinity, this assumption should not significantly affect the droplet-wall interaction process. Thus, the momentum conservation equation for a droplet of the $\mathrm{i}$-th size fraction interacting with the wall during the compression stage is:

$$
\left(\rho_{d}+\frac{-}{2} \rho_{o}\right) v_{i} \frac{d V_{i y}}{d t}=-\rho_{d} V_{i y} \pi a_{i}^{2}-\frac{2 \sigma}{R_{i}} \pi a_{i} .
$$

$V_{i y}$ is the velocity component normal to the wall for the i-th size fraction droplet. In this equation, the left-hand side represents the inertial force that is equilibrated by the forces expressed by the terms on the right-hand side, the hydrodynamics reaction force and the capillary force, respectively. Let us formulate equation 5 in the dimensionless form as follows:

$$
\frac{d \tilde{y}_{i}}{d_{i}^{2}}=-\frac{3}{4} \frac{1}{1+s}\left(1-\tilde{y}_{i}^{2}\right)\left(\frac{d \tilde{y}_{i}^{2}}{d \tau_{i}}+1\right),
$$

where $\tilde{y}_{i}=y_{i} / R_{i}, \quad \tilde{V}_{i}=V_{i} / \Omega_{i}, \quad \Omega_{i}=\sqrt{2 \sigma / \rho_{d} R_{i}}$ and $s=\rho_{o} / 2 \rho$. This equation can be solved numerically considering $\tau_{i}=0, \tilde{y}_{i}=1$ and $\frac{d \tilde{y}_{i}}{d \tau}=\tilde{V}_{i y o}$. We assume that the fluid fluctuation velocity distribution is Maxwellian. In addition, we assume that a droplet exactly follows the fluid in a fluctuation motion. The root-mean-square fluid fluctuation velocity can be calculated by the following empirical correlation [1]:

$$
\sqrt{\bar{V}^{2+}}=\frac{0.005 y^{+2}}{1+0.002923 y^{+2.128}} .
$$

Here, $\bar{V}_{y}^{\prime 2}\left(\frac{d_{i}}{2}\right)$ is the mean-square fluid fluctuation velocity at the moment of touching the wall. $y^{+}=y / \delta^{+}$is the normalized distance to the wall.

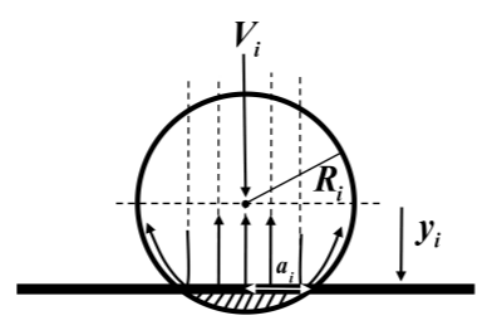

Figure 3. The schematic of the droplet interacting with the wall. 
$\delta^{+}$is the wall layer thickness. Then, it is considered that the initial value of normal to the wall droplet fluctuation velocity is equal to the most probable fluid fluctuation velocity at $y=0.5 d_{i}$. Equation 6 is easily integrated up to the moment when $\tilde{V}_{i y}=0$ indicating the end of the compression stage. The specific pipe wall area dynamically covered by droplets of the i-th size fraction due to their multiple collisions with the wall is: $S_{i}=\operatorname{fr}_{i} S_{i} A_{i}$, where $A_{i}$ is the mean droplet-wall contact area during a single collision; $\zeta_{i}$ is the mean droplet wall contact time; $f r$ is the frequency of droplet-wall collisions. The frequency of droplet-wall collisions per surface area unit is determined, assuming the Maxwellian droplet fluctuation velocity distribution. The total specific area (per unit pipe surface area) covered by droplets is calculated as:

$$
S=\sum_{i=1}^{m} f r_{i}[\underbrace{\int_{R_{i}}^{y_{i f}} \pi\left(R_{i}^{2}-y_{i}^{2}\right) \frac{d y_{i}}{V_{i y}}}_{\text {compression }}+\underbrace{\int_{y_{i f}}^{R_{i}} \pi\left(R_{i}^{2}-y_{i}^{2}\right) \frac{d y_{i}}{V_{i y}}}_{\text {relaxation }}\rfloor
$$

The problem of accounting for the effect of water on asphaltene deposition can be reduced to determining the mean fraction of the pipe wall surface that is dynamically coated with water droplets. Then, the deposit flux in the presence of dispersed water is calculated as: $q=q_{a}(1-\mathrm{S})$ which $q_{a}$ is the asphaltene deposition flux and $\mathrm{S}$ is the mean fraction of the pipe wall surface dynamically coated by water droplets.

Let us illustrate the results of the model developed by a few examples. We consider a dispersion of water in oil flowing in a production tubing of the diameter $D=0.0625 \mathrm{~m}$ with the mean velocity $U$. The water holdup is $\phi=0.1$. The oil viscosity and density are $\mu_{o}=1.3 \mathrm{mPa} / \mathrm{s}$ and $\rho_{o}=760 \mathrm{~kg} / \mathrm{m}^{3}$, respectively. The interfacial tension is $0.0025 \mathrm{~N} / \mathrm{m}$. The steady-state droplet size distribution, calculated by the dispersion model developed, is employed for this analysis. Figure 4 illustrates the dropletwall interaction process. Figure $4 \mathrm{a}$ shows that how the velocities of droplets of different sizes change during the droplet compression stage.
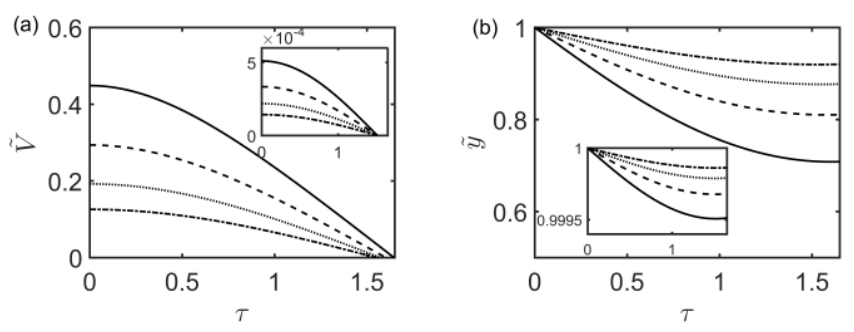

Figure 4. The dimensionless velocity (a) and the dimensionless distance from the droplet center to the wall surface (b) versus dimensionless time during the droplet compression stage. The droplet size decreases for the line, dashed line, dotted line and dash-dotted line, respectively. The results are shown for the four first larger droplets and the insets show the results of the four last small droplets.
Figure $4 \mathrm{~b}$ shows how the distance from the droplet center to the wall changes during the compression stage. The largest deformation is observed for the droplet of the largest size. However, even in this case, the maximum deformation value does not exceed $30 \%$ of the droplet radius. Thus, the assumption on the small droplet deformation accepted for modeling is satisfactory. Moreover, the fraction of larger droplets in the entire population is relatively small, whereas the deformation rapidly decreases with a reduction in droplet size.

For the same mixture, we also calculate the specific pipe surface, dynamically coated by droplets, for the different mean flow velocities $U=0.8,1,1.5$ and $2 \mathrm{~m} / \mathrm{s}$. Droplets are initially assumed to be monodispersed with the size $d_{o}=150 \mu \mathrm{m}$. Figure 5 illustrates the distribution of the sought parameters along a tubing of $4 \mathrm{~km}$ long. Only a negligible fraction of the pipe surface is dynamically coated with droplets. The droplet sizes decrease along a tubing due to their fragmentation. The droplet size reduction leads to an increase in the droplet number concentration causing an increase in the droplet-pipe wall collision frequency. As a result, the pipe surface area, coated with droplets, slowly increases along the tubing. In addition, the droplet coated surface expectedly increases with an increase in the mean flow velocity because of the two effects. First, droplet sizes decrease with an increase in the mean flow velocity due to a corresponding increase in the turbulence energy dissipation rate. Second, a higher mean flow velocity results in a larger droplet fluctuation velocity. An increase in the fluctuation velocity causes an enhancement in frequency of droplet-wall collisions as well as in stronger droplet deformations. These factors lead to an increase in a dropletwall contact area. A significant increase in the water holdup (see Figure 5b) leads to a relatively slight increase in the specific wall area dynamically coated by droplets. An increase in the holdup causes a reduction in the droplet breakup rate. Therefore, an effect of water holdup on the pipe area, dynamically coated by droplets, is relatively small.

The major outcome of our modeling is a demonstration that the effect of water dispersed in oil on the process of asphaltene deposition in a production tubing is negligible.
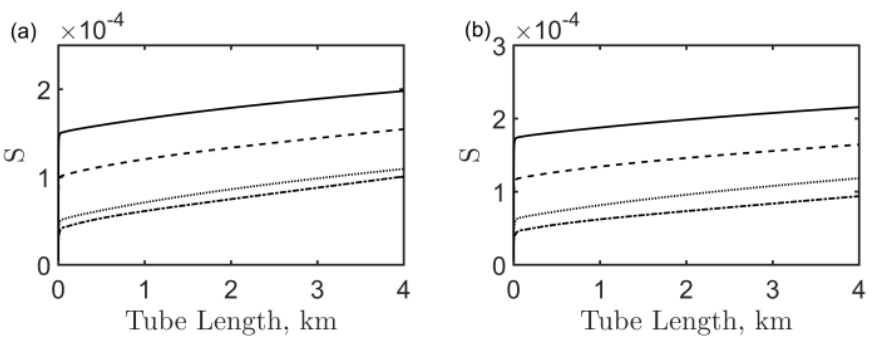

Figure 5. Distribution of the specific pipe wall area covered by droplets, along the pipe. Flow velocities: $U=0.8,1.0,1.5,2.0 \mathrm{~m} / \mathrm{s}$ corresponding to the dash-dotted, dotted line, dashed line and line, respectively. Water hold up: $=$ $\phi=0.1$ (a) and 0.4 (b). 


\section{CONCLUSIONS}

An engineering model was developed to estimate the pipe wall surface area dynamically coated by droplets, which collide with the wall due to their fluctuations in a turbulent flow. An evolution of the droplet size distribution along production a tubing was modeled by a population balance equation. A droplet-wall collision process was modeled assuming that a droplet deformation in a single collision was small. It was also assumed that droplets follow the fluid in fluctuation motion caused by turbulence. The frequencies of the droplet-wall collisions were calculated assuming that the droplet fluctuation velocity distribution was Maxwellian. Calculations of the specific pipe surface area dynamically coated with water droplets, at different mean flow velocities and water holdups, showed that the coated specific area remains small and, therefore, the effect of produced water on asphaltene deposition can be ignored in practical computations of asphaltene deposition.

\section{ACKNOWLEDGMENT}

This research has been carried out at Université Laval, supported financially by the NSERC Engage grant. The authors acknowledge Schlumberger Ltd. for the support of this work.

\section{REFERENCES}

[1] D. Eskin, J. Ratulowski, K. Akbarzadeh, and S. Pan, "Modelling asphaltene deposition in turbulent pipeline flows", Can. J. Chem. Eng., vol. 89, no. 2, Apr. 2011. doi: 10.1002/cjce.20507.

[2] D. Eskin, J. Ratulowski, K. Akbarzadeh, and S. Andersen, "Modeling of asphaltene deposition in a production tubing", AlChE J., vol. 58, pp. 2936, Oct. 2011. doi: 10.1002/aic. 12800 .

[3] J. Davies, "Drop sizes of emulsions related to turbulent energy dissipation rates", Chem. Eng. Sci., vol. 40, no. 5, pp. 839-842, 1985.

doi: 10.1016/0009-2509(85)85036-3.

[4] D. Eley and M. Hey and J. Symonds, "Emulsions of water in asphaltenecontaining oils 1 . Droplet size distribution and emulsification rates", $J$. Colloids Surf., vol. 32, pp. 87-101, 1988. doi: 10.1016/0166-6622(88)80006-4.

[5] A. S. Kurup et al., "Development and application of an asphaltene deposition tool (ADEPT) for well bores", Energy Fuels, vol. 25, no. 10, pp. 4506, 2011. doi: $10.1021 /$ ef200785v.

[6] G. Mansoori, "Modeling of asphaltene and other heavy organic depositions", J. Petrol. Sci. Eng., vo1. 17, nos. 1-2, pp. 101-111, Feb. 1997. doi: 10.1016/S0920-4105(96)00059-9.

[7] Solsvik, J and Jakobsen, H, "On the solution of the population balance equation for bubbly flows using the high-order least squares method: implementation issues", Rev. Chem. Eng. vol. 29, no. 2, pp. 263, Apr. 2013. doi: 10.1515/revce-2012-0018.

[8] Solsvik, J and Becker, J and Sheibat-Othman, $\mathrm{N}$ and Jakobsen, H, "Population balance model: Breakage kernel parameter estimation to emulsification data," Can. J. Chem. Eng., vol. 92, no. 6, pp. 1082, June 2014. doi: 10.1002/cjce.21928.

[9] C. Coulaloglou and L. Tavlarides, "Description of interaction processes in agitated liquid-liquid dispersions", Chem. Eng. Sci., vol. 32, no. 11, pp. 1289-1297, 1977. doi: 10.1016/0009-2509(77)85023-9.

[10] M. J. Prince and H. W. Blanch, "Bubble coalescence and break-up in air-sparged bubble columns," AlChE. J., vol. 36, no. 10, pp. 1485-1499, Oct. 1990. doi: 10.1002/aic.690361004.

[11] R. Kuboi, I. Komasawa and T. Otake, "Behavior of dispersed particles in turbulent liquid flow," J. Chem. Eng. Jpn., 1972, 5, 349.

doi: $10.1252 /$ jcej.5.349. 


\title{
Gust Response of a Two-Dimensional Nonlinear Wing
}

\author{
Xiaoyang Zhang, Mojtaba Kheiri, Wen-Fang Xie \\ Department of Mechanical, Industrial and Aerospace Engineering \\ Concordia University \\ Montreal, QC, Canada \\ xiaoyang.zhang@mail.concordia.ca,mojtaba.kheiri@concordia.ca,wfxie@encs.concordia.ca
}

\begin{abstract}
In this paper, the equations of motion for a twodimensional (2-D) wing encountering a gust are presented. The wing has two degrees of freedom, i.e. pitch and plunge, and its motion is restrained by nonlinear translational and rotational springs. Two different stiffness nonlinearities are examined: (i) cubic and (ii) free play. For given system parameters, the responses of the nonlinear system to the sharp-edged and 1cosine gust profiles are obtained at different flow velocities and compared to the time response of the system with no gust input.
\end{abstract}

Keywords: aeroelasticity; gust response; cubic nonlinearity; freeplay nonlinearity; flutter analysis

\section{INTRODUCTION}

Aeroelasticity is an interdisciplinary field of study dealing with interactions between inertia, aerodynamic and elastic forces. Classical theories in aeroelasticity assume linear aerodynamics and structural dynamics resulting in a set of linear equations. These equations can be fairly easily solved in time or frequency domain and used for examining the aircraft stability and response to external excitation. However, linear aerodynamic theories start to break down at high airspeed. Flow separation and shock oscillations may also introduce aerodynamic nonlinearities. Moreover, structural nonlinearities may arise from, for example, large deformations, material behavior, worn hinges of control surfaces, and loose control linkages $[1,2]$.

Woolston et al. may be the first researchers that investigated the effects of structural nonlinearities on the flutter of a wing [1]. Two different wing models were studied: a wing capable of bending and twisting (i.e. two-degree-of-freedom (2-DOF) system) and, also, a wing with a control surface (i.e. 3-DOF system). They considered structural nonlinearities as concentrated in the torsional stiffness. Three different types of nonlinear springs, namely flat spot (representing free play), hysteresis, and cubic were examined. They found that there is a strong connection between the stability of a nonlinear system and the initial conditions - in many cases the flutter speed was decreased as the initial disturbance was increased.

A comprehensive review of different types of structural and aerodynamic nonlinearities encountered in aeronautical engineering was conducted by Lee et al. [2]. They discussed several techniques, such as finite difference and describing function, for solving equations with structural nonlinearities. They found that even a 2-DOF system with a single nonlinearity in the pitch degree of freedom may show a complex dynamical behavior. For example, they showed that a system with a freeplay nonlinearity may undergo period-1, period-2, and period-4 limit-cycle oscillations (LCO), as well as, chaotic motion, depending on the airspeed. For a more recent and quite shorter review of nonlinear aeroelasticity, the reader is referred to [3].

Several researchers have studied the response of a lifting surface to a time-dependent external excitation. For example, Poirel and Price investigated the effect of longitudinal atmospheric turbulence on the dynamics of an airfoil with a hardening cubic structural nonlinearity in pitch [4]. They found that flutter occurred at a lower velocity for the excited case than the so-called non-excited one, whereas the onset of LCO occurred at a higher velocity. Marzocca et al. investigated the aeroelastic response to a time-dependent external excitation of a 2-D rigid/elastic-lifting surface in incompressible flow field, featuring plunging-pitching coupled motion [5]. Haddadpour et al. examined the effects of the sharp-edged gust on the dynamics of a flexible high-aspect ratio wing [6]. In their model, they used linear structural dynamics and linear quasi-steady aerodynamics.

In reality, almost all lifting surfaces involve some sort of structural nonlinearity, and they may also encounter with atmospheric turbulence during a normal flight. Nevertheless, very few studies on the nonlinear aeroelastic response to a timedependent excitation can be found in the body of the literature. This is, in fact, the principal objective of this paper, namely studying the effects of a time-dependent excitation, such as sharp-edge and 1-cosine gusts on the dynamics and stability of a 2-DOF typical airfoil section with structural nonlinearities.

\section{NONLINEAR AEROELASTIC MODEL WITH GUST EXCITATION}

\section{A. Governing Equations Including the Gust Input}

Fig. 1 shows the cross-section of a 2-D rigid wing with degrees of freedom in the plunge and pitch directions. The plunge displacement is measured from the elastic axis and is represented by $h$ (positive downward); $\alpha$ is the pitch angle about the elastic axis (positive nose up). The elastic axis is located at a distance $a b$ from the mid-chord, while the mass center is located at a distance $x_{\alpha} b$ from the elastic axis, $b$ being the semi-chord. The wing is supported by a translational and a rotational spring, attached to the elastic axis, which generally have a nonlinear stiffness. 


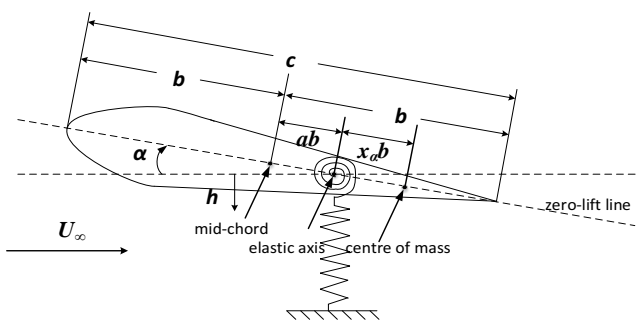

Figure 1. Schematic showing geometry of the wing section.

Here, we follow formulations presented in $[2,5]$. The dimensionless aeroelastic equations featuring coupled plungingpitching motion of a typical airfoil section exposed to a gust input are expressed as

$$
\begin{gathered}
\xi^{\prime \prime}+x_{\alpha} \alpha^{\prime \prime}+2 \zeta_{\xi} \frac{\bar{\omega}}{U^{*}} \xi^{\prime}+\left(\frac{\bar{\omega}}{U^{*}}\right)^{2} G(\xi)-l_{a}(\tau)=l_{g}(\tau), \\
\left(\frac{x_{\alpha}}{r_{\alpha}}\right) \xi^{\prime \prime}+\alpha^{\prime \prime}+2 \zeta_{\alpha} \frac{1}{U^{*}} \alpha^{\prime}+\frac{1}{U^{* 2}} M(\alpha)-m_{a}(\tau)=m_{g}(\tau),
\end{gathered}
$$

where $\xi=h / b$ is the dimensionless plunge displacement, and $\zeta_{\xi}$ and $\zeta_{\alpha}$ are, respectively, viscous damping ratios in pitch and plunge; $U^{*}=U_{\infty} / b \omega_{\alpha}$ is the dimensionless velocity, and $\bar{\omega}=$ $\omega_{\xi} / \omega_{\alpha}$ is the frequency ratio, $U_{\infty}$ being the freestream velocity, $\omega_{\xi}$ and $\omega_{\alpha}$ also being natural frequencies in pitch and plunge, respectively; also, $l_{a}(\tau)$ and $m_{a}(\tau)$ are the aerodynamic lift and pitching moment about the elastic axis, respectively, while $l_{g}(\tau)$ and $m_{g}(\tau)$ are the lift and moment about the elastic axis due to the gust; $G(\xi)$ and $M(\alpha)$ represent the nonlinear plunge and pitch structural stiffness terms, respectively; moreover, ()$^{\prime} \equiv$ $\partial() / \partial \tau$ denotes the time derivative with respect to dimensionless time $\tau$.

The aerodynamic lift $l_{a}(\tau)$ and pitching moment $m_{a}(\tau)$ may be expressed as

$$
\begin{gathered}
l_{a}(\tau)=-\frac{2}{\mu} \int_{0}^{\infty} \phi(\tau-\sigma)\left(\alpha^{\prime}+\xi^{\prime \prime}+\left(\frac{1}{2}-a\right) \alpha^{\prime \prime}\right) d \sigma- \\
\frac{1}{\mu}\left(\alpha^{\prime}+\xi^{\prime \prime}-a \alpha^{\prime \prime}\right), \\
m_{a}(\tau)=\frac{2}{\mu}\left(\frac{1}{2}+a\right) \int_{0}^{\infty} \phi(\tau-\sigma)\left(\alpha^{\prime}+\xi^{\prime \prime}+\left(\frac{1}{2}-a\right) \alpha^{\prime \prime}\right) d \sigma \\
+\frac{1}{r_{\alpha}^{2} \mu} a\left(\xi^{\prime \prime}-a \alpha^{\prime \prime}\right)-\frac{1}{r_{\alpha}^{2} \mu}\left(\frac{1}{2}-a\right) \alpha^{\prime}-\frac{1}{8} \frac{1}{r_{\alpha}^{2} \mu} \alpha^{\prime \prime},
\end{gathered}
$$

where $\mu=m / \pi \rho b^{2}$ is the mass ratio, and $r_{\alpha}=I_{\alpha} / m b^{2}$ is the dimensionless radius of gyration; $m, \rho$, and $I_{\alpha}$ being, respectively, mass of the wing per unit span, air density, and mass moment of inertia per unit span. Also, $\phi(\tau)$ is called the Wagner function and is given by

$$
\phi(\tau)=1-A_{1} e^{-b_{1} \tau}-A_{2} e^{-b_{2} \tau},
$$

where the constants are $A_{1}=0.165, A_{2}=0.335, b_{1}=$ $0.0455, b_{2}=0.3$.

By considering $w_{G}(\tau)$ as a gust variable velocity and using Duhamel's integral concept, the lift and pitching moment about the elastic axis due to the penetration into the gust, $l_{g}(\tau)$ and $m_{g}(\tau)$, respectively, may be written as

$$
\begin{gathered}
l_{g}(\tau)=\frac{2}{\mu} \int_{0}^{\tau} \psi^{\prime}(\tau-\sigma) \frac{w_{G}(\tau)}{U_{\infty}} d \sigma \\
m_{g}(\tau)=\left(\frac{1}{2}+a\right) \frac{2}{r_{\alpha}^{2} \mu} \int_{0}^{\tau} \psi^{\prime}(\tau-\sigma) \frac{w_{G}(\tau)}{U_{\infty}} d \sigma
\end{gathered}
$$

where $\psi(\tau)$ is called the Küssner function.

A widely-used approximation for Küssner's function is the following two-term exponential expression [2]:

$$
\psi(\tau)=1-A_{3} e^{-b_{3} \tau}-A_{4} e^{-b_{4} \tau},
$$

where the constants are $A_{3}=0.5, A_{4}=0.5, b_{3}=0.130$ and $b_{4}=1$.

In this paper, two different gust profiles, implying specific time variations of their velocity distribution, will be used. They are: (a) sharp-edged gust, and (b) 1-cosine gust. Their analytical expressions are:

$$
\begin{gathered}
\text { sharp - edged gust: } w_{G}(\tau)=H(\tau) w_{0}, \\
1-\text { cosine gust: } w_{G}(\tau)=H(\tau) \frac{1}{2} w_{0}\left(1-\cos \frac{\pi \tau}{\tau_{G}}\right) \\
-H\left(\tau-2 \tau_{G}\right) \frac{1}{2} w_{0}\left(1-\cos \frac{\pi \tau}{\tau_{G}}\right),
\end{gathered}
$$

where $w_{0}$ is the gust maximum amplitude, and $H(\tau)$ represents the Heaviside step function.

\section{B. State-space Equations}

In order to deal with the integral terms in (3-4) and (6-7), we introduce six new variables or states as

$$
\begin{array}{r}
w_{1}=\int_{0}^{\tau} e^{-b_{1}(\tau-\sigma)} \alpha(\sigma) d \sigma, \quad w_{2}=\int_{0}^{\tau} e^{-b_{2}(\tau-\sigma)} \alpha(\sigma) d \sigma, \\
w_{3}=\int_{0}^{\tau} e^{-b_{1}(\tau-\sigma)} \xi(\sigma) d \sigma, \quad w_{4}=\int_{0}^{\tau} e^{-b_{2}(\tau-\sigma)} \xi(\sigma) d \sigma, \\
w_{5}=\int_{0}^{\tau} e^{-b_{3}(\tau-\sigma)} \frac{w_{G}(\sigma)}{U_{\infty}} d \sigma, \quad w_{6}=\int_{0}^{\tau} e^{-b_{4}(\tau-\sigma)} \frac{w_{G}(\sigma)}{U_{\infty}} d \sigma .
\end{array}
$$

Using variables in (11), the aeroelastic equations (1-2) may be re-written as

$$
\begin{gathered}
c_{0} \xi^{\prime \prime}+c_{1} \alpha^{\prime \prime}+c_{2} \xi^{\prime}+c_{3} \alpha^{\prime}+c_{4} \xi+c_{5} \alpha+c_{6} w_{1}+c_{7} w_{2} \\
+c_{8} w_{3}+c_{9} w_{4}+c_{10} w_{5}+c_{11} w_{6}+\left(\frac{\bar{\omega}}{U^{*}}\right)^{2} G(\xi)=f(\tau) \\
d_{0} \xi^{\prime \prime}+d_{1} \alpha^{\prime \prime}+d_{2} \xi^{\prime}+d_{3} \alpha^{\prime}+d_{4} \xi+d_{5} \alpha+d_{6} w_{1}+d_{7} w_{2} \\
+d_{8} w_{3}+d_{9} w_{4}+d_{10} w_{5}+d_{11} w_{6}+\left(\frac{1}{U^{*}}\right)^{2} M(\alpha)=g(\tau),
\end{gathered}
$$

where $G(\xi)$ and $M(\alpha)$ are nonlinear functions of $\xi$ and $\alpha$, respectively. $f(\tau)$ and $g(\tau)$ are functions of initial conditions and terms in the Wagner function, which are given by:

$$
\begin{gathered}
f(\tau)=\frac{2}{\mu}\left(\left(\frac{1}{2}-a\right) \alpha(0)+\xi(0)\right)\left(A_{1} b_{1} e^{-b_{1} \tau}+A_{2} b_{2} e^{-b_{2} \tau}\right) \\
g(\tau)=-\frac{(1+2 a) f(\tau)}{2 r_{\alpha}^{2}}
\end{gathered}
$$


The coefficients $c_{i}(i=0, . .9)$ and $d_{i}(i=0, . .9)$ in (12) and (13) were given in [2] and will not be repeated here for the sake of brevity; however, $c_{10}, c_{11}, d_{10}$ and $d_{11}$ are given as

$$
\begin{gathered}
c_{10}=-\frac{2}{\mu} A_{3} b_{3}, c_{11}=-\frac{2}{\mu} A_{4} b_{4}, \\
d_{10}=-\left(\frac{1}{2}+a\right) \frac{2}{r_{\alpha}^{2} \mu} A_{3} b_{3}, d_{11}=-\left(\frac{1}{2}+a\right) \frac{2}{r_{\alpha}^{2} \mu} A_{4} b_{4} .
\end{gathered}
$$

After introducing a variable vector $X=\left(x_{1}, x_{2}, \ldots, x_{10}\right)^{T}$ defined as

$$
\begin{gathered}
x_{1}=\alpha, x_{2}=\alpha^{\prime}, x_{3}=\xi, x_{4}=\xi^{\prime}, x_{5}=w_{1}, x_{6}=w_{2}, \\
x_{7}=w_{3}, x_{8}=w_{4}, x_{9}=w_{5}, x_{10}=w_{6} .
\end{gathered}
$$

(12) and (13) can be written as a set of ten first-order ordinary differential equations.

\section{NUMERICAL EXAMPLES AND ANALYSIS}

The dimensionless parameters of the linear system used in this paper are: $a=-0.5, \mu=100, x_{\alpha}=0.25, r_{\alpha}=0.5, \zeta_{\alpha}=\zeta_{\xi}=$ 0.0 , and $\bar{\omega}=0.2$. We set $\alpha^{\prime}(0)=\xi^{\prime}(0)=\xi(0)=0, \alpha(0)=1 \mathrm{deg}$ as the initial conditions. Using the eigenvalue solution method with the given parameters, we obtain the linear dimensionless flutter speed $\left(U_{L}^{*}\right)$ as 6.28 , which agrees well with results in [2].

Since in this paper we consider nonlinearities only for the pitch DOF, plunging oscillations display very much expected features and are not worthy to be discussed here. Thus, throughout the paper, we only discuss the response in the pitching direction.

\section{A. Gust Response with Cubic Nonlinearity}

We consider a cubic nonlinearity in the structural stiffness for the pitch and a linear stiffness for the plunge degree of freedom. Thus, functions $G(\xi)$ and $M(\alpha)$ in (12) and (13), respectively, can be set to $G(\xi)=\xi$ and $M(\alpha)=\beta_{1} \alpha+\beta_{3} \alpha^{3}$. First, we examined a system with a weak cubic nonlinearity, where $\beta_{1}=1$ and $\beta_{3}=10$. Assuming a sharp-edged gust with $w_{0}=10 \mathrm{~m} / \mathrm{s}$, the response of the system was obtained at $U^{*}=$ 6.27 (not shown here because of space limitation). It was found that the oscillations gradually decay with time, and the system regains its static equilibrium. Thus, even a strong excitation at an airspeed very close to the linear flutter speed cannot change the stability of the system with a weak cubic nonlinearity.

Next, we consider a system with a strong cubic nonlinearity $\left(\beta_{1}=0.01, \beta_{3}=10\right)$ and keep other system parameters the same. The linear flutter speed for this system is $U_{L}^{*}=0.91$. The response of the system to a sharp-edged gust and also a 1-cosine gust with the maximum amplitude of $w_{0}=1 \mathrm{~m} / \mathrm{s}$ at $U^{*}=$ $0.84, U^{*}=U_{L}^{*}=0.91$ and $U^{*}=2 U_{L}^{*}=1.82$ are shown in Figs. 3-5, respectively. Note that for the 1-cosine gust, $\tau_{G}=20$ throughout the paper.

As seen from Fig. 2, both the sharp-edged and 1-cosine gusts result into LCO with comparable amplitudes at $U^{*}=0.84<U_{L}^{*}$, while for the system without the gust input, oscillations decay with time. ${ }^{1}$ This means that the nonlinear system may undergo a Hopf bifurcation leading to $\mathrm{LCO}$ at a speed below the linear flutter speed, provided that a sufficiently strong gust excites it. Fig. 3 shows the dynamic response of the system with and without the gust input at the linear flutter speed, $U_{L}^{*}$. From the figure, one may infer that except for the transient part of the response, the gust does not make any significant changes to the dynamics of the system at $U^{*}=U_{L}^{*}$.

Fig. 4 Shows time responses of the system with and without the gust input at $U^{*}=2 U_{L}^{*}=1.82$. As seen from the figure, the amplitude of oscillations when the system encounters a gust is obviously higher than that when there is no gust. In addition, the system undergoes period-2 motion in response to the gust input, while it performs period- 1 motion when there is no gust. It is also interesting to see that the sharp-edged gust tends to increase the pitch angle in the negative direction, whereas the 1-cosine gust does the opposite.
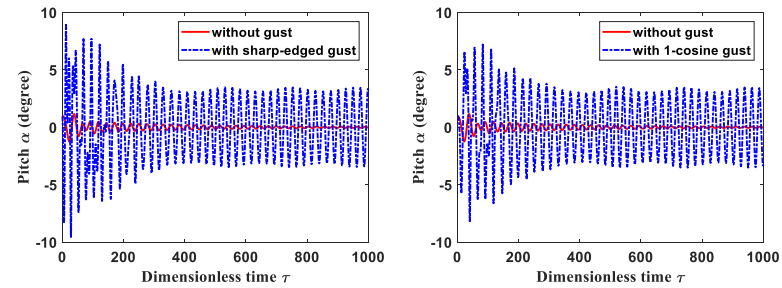

Figure 2. Sharp-edged (left) and 1-cosine (right) gust responses of a system with the strong cubic nonlinearity in pitch at $U^{*}=0.84 ; w_{0}=1 \mathrm{~m} / \mathrm{s}$.
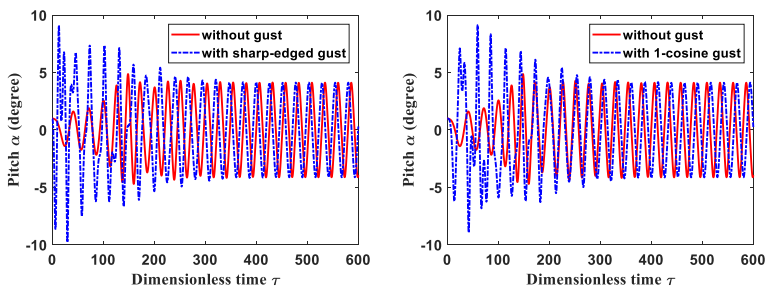

Figure 3. Sharp-edged (left) and 1-cosine (right) gust responses of a system with the strong cubic nonlinearity in pitch at $U^{*}=U_{L}^{*}=0.91 ; w_{0}=1 \mathrm{~m} / \mathrm{s}$.
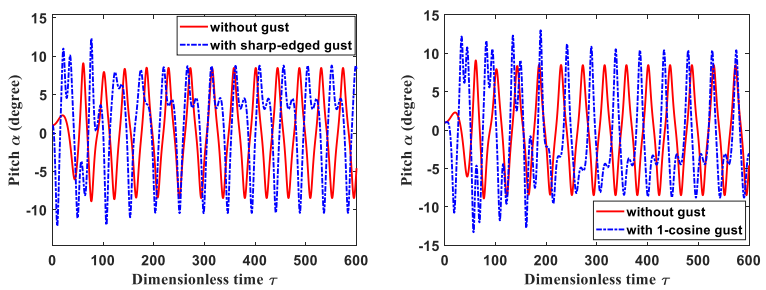

Figure 4. Sharp-edged (left) and 1-cosine (right) gust responses of a system with the strong cubic nonlinearity in pitch at $U^{*}=2 U_{L}^{*}=1.82 ; w_{0}=1 \mathrm{~m} / \mathrm{s}$.

\section{B. Gust Response with Free-play Nonlinearity}

The free-play nonlinearity, in its general form, may be represented by the trilinear stiffness model, as shown in Fig. 5. In the figure, $M_{0}$ represents the preload, $M_{f}$ the stiffness in the free-play zone, $\alpha_{f}$ the pitch angle offset, and $\delta$ the free-play range. We examine the dynamics of a system with free-play

\footnotetext{
${ }^{1}$ The time responses to the gust inputs were obtained for a longer time range (not shown in Fig. 2) where period-1 LCO were observed.
} 
parameters $\delta=0.5^{\circ}, M_{0}=M_{f}=0, \alpha_{f}=-0.25^{\circ}$ for the pitch stiffness. As also previously mentioned, the linear flutter speed for this system is $U_{L}^{*}=6.28$.

Fig. 6 shows the bifurcation diagram for the free vibration (i.e. no gust) of a typical airfoil section with free-play type nonlinearity in pitch, where the peak values of the pitch angle, $\alpha$, are plotted against the normalized dimensionless airspeed, $U^{*} / U_{L}^{*}$. In the bifurcation diagram, a single dot/point represents a static equilibrium position (region I); two dots represent a period-1 motion (regions II and IX), and four dots show a period2 motion (regions III and VIII); also, eight dots represent a period-4 motion (regions V and VII), and finally, multiple scattered dots show a chaotic motion (regions IV and VI). Table I summarizes the dynamical behavior of the system shown in the bifurcation diagram. It is noted that, as seen in Fig. 6, in region IX, the pitch amplitude increases sharply with $U^{*} / U_{L}^{*}$ and goes to very large values for $U^{*} / U_{L}^{*}>1$ (not shown in the figure). These indicate the occurrence of divergent or violent flutter in region IX (for more details, see [1, 2]).

The important message of Fig. 6 is that the free-play nonlinearity may cause the system to lose the static equilibrium at a much lower flow velocity than the linear flutter speed (e.g. at 0.95 instead of 6.28 for the given system parameters). The bifurcation diagram also suggests that the route to chaos is via period-doubling, as also observed by Lee et al. [2].

Fig. 7 shows the typical phase-plane diagrams of four types of motion, which are period-1, period-2, period-4 and chaotic, respectively, at $U^{*} / U_{L}^{*}=0.8,0.56,0.45$, and 0.48 .

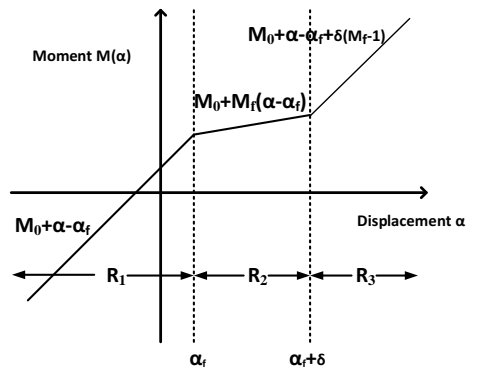

Figure 5. General free-play stiffness model; $M_{0}$ represents the preload; $M_{\mathrm{f}}$ is the stiffness in the free-play zone, $\alpha_{\mathrm{f}}$ is the pitch angle offset, and $\delta$ is the freeplay range.

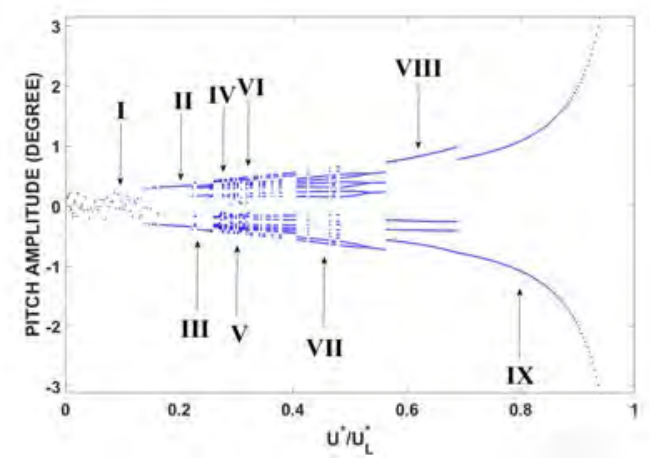

Figure 6. Bifurcation diagram of a system with a free-play nonlinearity in pitch $\left(\delta=0.5^{\circ}, M_{0}=M_{f}=0, \alpha_{f}=-0.25^{\circ}\right)$, where the peak values of the pitch angle is plotted versus the normalized dimensionless airspeed, $U^{*} / U_{L}^{*}$.
TABLE I. DYNAMICAL BEHAVIOUR IN DIFFERENT REGIONS OF THE BIFURCATION DIAGRAM SHOWN IN FIG. $6 ;\left(U^{*} / U_{L}^{*}\right)$ IS THE NORMALIZED DIMENSIONLESS AIRSPEED.

\begin{tabular}{|c|c|c|}
\hline Region & $\left(\boldsymbol{U}^{*} / \boldsymbol{U}_{\boldsymbol{L}}^{*}\right)$ & Type of motion \\
\hline Before I & $(00.151)$ & Static Equilibrium \\
\hline I and VIII & $(0.1510 .221)$ and $(0.6881)$ & Period-1 \\
\hline II and VII & $(0.2210 .255)$ and $(0.5290 .688)$ & Period-2 \\
\hline IV and VI & $(0.3310 .463)$ and $(0.4840 .529)$ & Period-4 \\
\hline III and V & $(0.2550 .331)$ and $(0.4630 .484)$ & Chaos \\
\hline
\end{tabular}
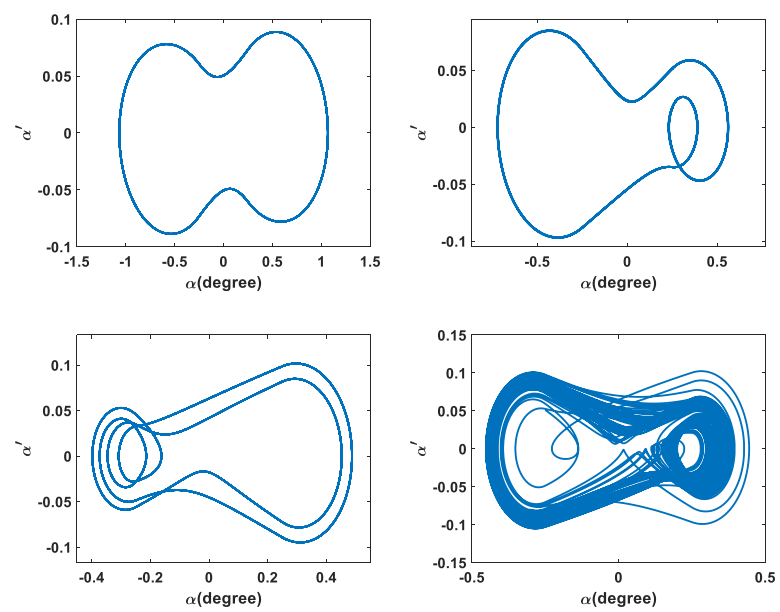

Figure 7. Phase-plane diagrams for different regions shown in Fig. 6; where $U^{*} / U_{L}^{*}=0.8,0.56,0.45$, and 0.48 for the top-left, top-right, bottom-left, and botom-right subfigures, respectively.

Fig. 8 shows time responses of the system in the pitch direction to a sharp-edged (left) and a 1-cosine (right) gust at $U^{*}=0.93$. For comparison purposes, the gust response is plotted over the time response in the absence of the gust. As seen, the system undergoes LCO under the gust input, while in the absence of the gust it regains static stability. In other words, the gust input causes the system to lose its static stability at a lower airspeed, i.e. at $U^{*}=0.87$ instead of 0.95 .

Fig. 9 shows the time responses at $U^{*}=1.30$ which lies in region II in the bifurcation diagram in Fig. 6. As discussed, within this region, the airfoil section undergoes period-1 LCO in the absence of a gust. However, this changes to period-2 LCO when the airfoil encounters a sharp-edged or 1-cosine gust. Thus, the gust may change the type of the oscillatory motion. In addition, it increases the amplitude of oscillation, especially in the transient part of the response.

Fig. 10 shows time responses at $U^{*}=3.06$. Here also, the period-4 motion, which occurs in the gust absence, changes to a chaotic type motion due to the gust input. This is more evident from Fig. 11 which shows the phase-plane plots at the same airspeed without (left) and with (right) a sharp-edged gust input. Fig. 12 shows time responses at $U^{*}=6.00$. Very large amplitudes due to gust effects are noticeable in the transient part of the responses. As seen from Fig. 13, when $U^{*}$ approaches $U_{L}^{*}$, the amplitude of LCO due to the gust input increases rapidly to very large values (of the order of $35 \mathrm{deg}$ ). When $U^{*}$ is greater than $U_{L}^{*}$, namely in the supercritical region, the time responses increase exponentially, indicating divergent flutter (see Fig. 14). 
As seen from the figure, the gust response amplitude is always greater than the one in the absence of the gust.
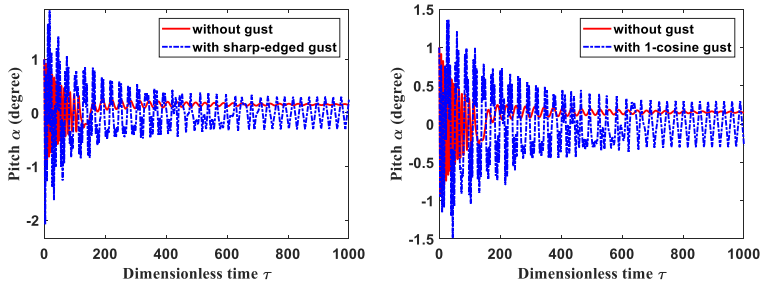

Figure 8. Sharp-edged (left) and 1-cosine (right) gust responses of a system with the free-play nonlinearity in pitch at $U^{*}=0.93 ; w_{0}=1 \mathrm{~m} / \mathrm{s}$.
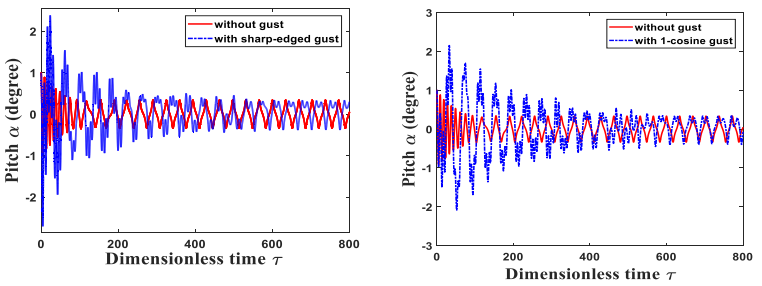

Figure 9. Sharp-edged (left) and 1-cosine (right) gust responses of a system with the free-play nonlinearity in pitch at $U^{*}=1.30 ; w_{0}=1 \mathrm{~m} / \mathrm{s}$.
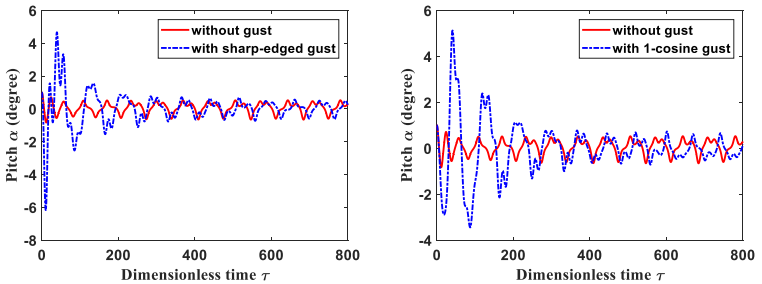

Figure 10. Sharp-edged (left) and 1-cosine (right) gust responses of a system with the free-play nonlinearity in pitch at $U^{*}=3.06 ; w_{0}=1 \mathrm{~m} / \mathrm{s}$.
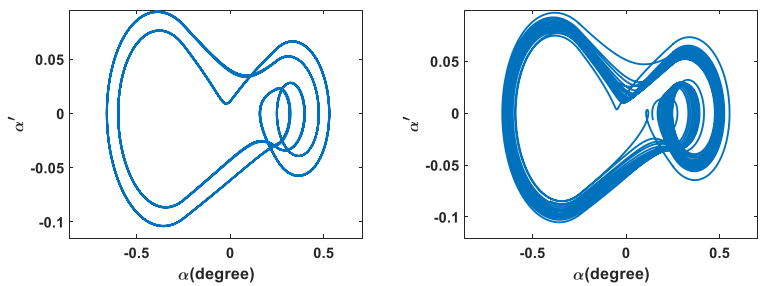

Figure 11. Phase-plane diagrams with gust and without gust (sharp-edged) at $U^{*}=3.06$; without (left) and with (right) a sharp-edged gust, featuring period-4 and chaotic motions, respectively; $w_{0}=1 \mathrm{~m} / \mathrm{s}$
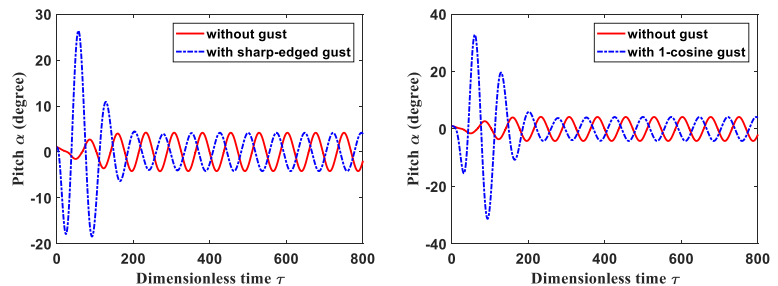

Figure 12. Sharp-edged (left) and 1-cosine (right) gust responses of a system with the free-play nonlinearity in pitch at $U^{*}=6.00 ; w_{0}=1 \mathrm{~m} / \mathrm{s}$.
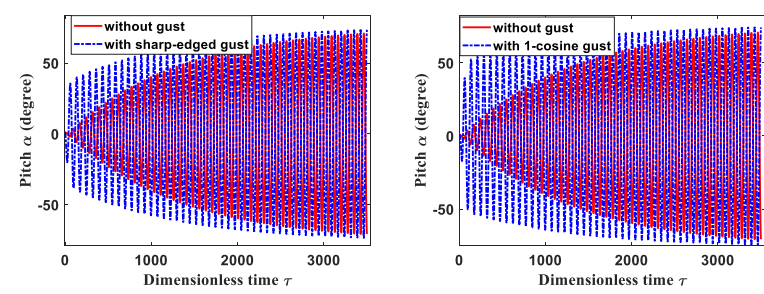

Figure 13. Sharp-edged (left) and 1-cosine (right) gust responses of a system with the free-play nonlinearity in pitch at $U^{*}=6.27 ; w_{0}=1 \mathrm{~m} / \mathrm{s}$.
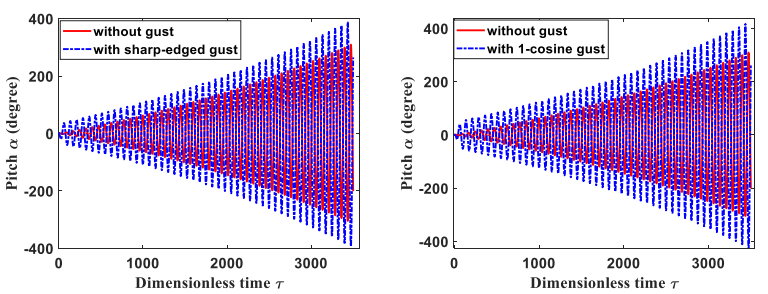

Figure 14. Sharp-edged (left) and 1-cosine (right) gust responses of a system with the free-play nonlinearity in pitch at $U^{*}=6.29 ; w_{0}=1 \mathrm{~m} / \mathrm{s}$.

\section{ACKNOWLEDGMENT}

The authors are grateful to the Faculty of Engineering and Computer Science of Concordia University for the FRS funding. The second author also greatly appreciates the Faculty for a Start-Up grant.

\section{CONCLUDING REMARKS}

The numerical results presented in this paper show that a sharp-edged or a 1-cosine gust input may influence the dynamics and stability of a two-dimensional wing with a strong cubic or free-play stiffness nonlinearity in three different ways: (1) it may alter (strictly speaking, lower) the onset of instability, (2) it may change the mode of oscillatory motion (e.g. from period-1 to period-2 motion), and (3) it may increase the amplitude of vibrations. Thus, studying the effects of time-dependent excitation on the dynamics and stability of lifting surfaces seems essential for their design. These studies are particularly crucial for the new generation of aircraft which are likely to be lighter, faster, more flexible, and more agile.

\section{REFERENCES}

[1] D. S. Woolston, H. L. Runyan, and R. E. Andrews, "An investigation of effects of certain types of structural nonlinearities on wing and controlsurface flutter," J. Aeronaut. Sci., vol. 24, no. 1, pp.57-63, 1957. doi: $10.2514 / 8.3764$.

[2] B. H. K. Lee, S. J. Price, and Y. S. Wong, "Nonlinear aeroelastic analysis of airfoils: bifurcation and chaos," Prog. Aeronaut. Sci., vol. 35, no. 3, pp. 205-334, 1999. doi: 10.1016/S0376-0421(98)00015-3.

[3] E. H. Dowell and D. Tang, "Nonlinear aeroelasticity and unsteady aerodynamics," AIAA J., vol. 40, no. 9, pp. 1697-1707, 2002. doi: $10.2514 / 2.1853$

[4] D. C. Poirel and S. J. Price, "Post-instability behavior of a structurally nonlinear airfoil in longitudinal turbulence," J. Aircr., vol. 34, no. 5, pp. 619-626, 1997. doi: 10.2514/2.2238.

[5] P. Marzocca, L. Librescu, and G. Chiocchia, "Aeroelastic response of 2-D lifting surfaces to gust and arbitrary explosive loading signatures," Int. J. Impact Eng., vol. 25, no. 1, pp. 41-65, 2001. doi: 10.1016/S0734-743X(00)00033-6

[6] F. Haddadpour, S. Shams, and M. Kheiri, "Sharp edge gust effects on aeroelastic behavior of a flexible wing with high aspect ratio," in 43rd AIAA Aerospace Sciences Meeting and Exhibit, 2005, pp. 838. 


\section{A Multi-Color Technique for Three-Dimensional Flow Characterization}

\author{
Kadeem Dennis \\ Department of Mechanical Engineering \\ University of Western Ontario \\ London, Ontario, Canada
}

\begin{abstract}
Turbulent flows are fundamentally threedimensional. Due to their inherent complexity, turbulent flow characterization is heavily relies on experimental research. A multi-color grid technique was recently proposed by the authors as a novel method for characterizing and measuring three-dimensional flows.

In this paper, further improvements of this technique are reported by implementing a new method based on color permutations. Several new advances in the experimental implementation and analysis algorithm are then presented and detailed. Results are presented using color streak images and three-dimensional particle trajectories.
\end{abstract}

Keywords: 3D; flow visualization; multi-color; flow measurement;

\section{INTRODUCTION}

Turbulent flows are one of the fundamental topics of classical fluid mechanics. A majority of practical engineering systems encounter turbulent flows, including the wake of buildings and flows in pumps, turbines, and heat exchangers. In these systems turbulence manifests as either a desirable trait, such as enhanced heat transfer, or non-desirable trait, such as dynamic loading on buildings. Complex threedimensional motion and non-linear dissipative processes, two defining characteristics of turbulent flows make them extremely difficult to study numerically. This makes experimentation the primary means of turbulence research. However, this presents the challenge of developing a technique to measure all three velocity components simultaneously as all components are needed to fully define key quantities in the turbulent kinetic energy budget.

Numerous techniques have been developed in the past to measure flow velocity while few have been used quite often for turbulent flow measurement. Hot-wire anemometry is one of the simplest techniques where velocity is determined from flow passing over a very thin heated wire attached to a probe. By deploying several probes at orthogonal angles, three-dimensional velocity can be determined. This technique has the benefit of excellent temporal resolution but lacks the ability to capture the flow field with good spatial resolution. Furthermore, the probe array intrudes upon the flow. Planar Particle Image Velocimetry (PIV) is a non-

\author{
Kamran Siddiqui \\ Department of Mechanical Engineering \\ University of Western Ontario \\ London, Ontario, Canada
}

intrusive technique that generates a two-dimensional flow field by taking images of a laser-illuminated seeded flow. This produces a flow field with high spatial resolution but is limited to planar measurements. Stereoscopic PIV builds upon this by utilizing two off-axis cameras viewing the light sheet [1]. From the captured images, a 3D flow field is constructed; however, the out-of-plane velocity has been shown to have questionable accuracy and no out-of-plane gradients can be determined [2,3]. Tomographic PIV is a further advancement of the PIV technique where four or more cameras observe an illuminated volume [4]. By applying a computational scheme to the images of the seeded flow, all three velocity components can be estimated. It has been shown that the calculated third velocity component is of questionable accuracy, especially in high shear flows, due to ambiguities in identifying seed particles, called ghost particles $[5,6]$.

Recently, wavelength-encoding techniques have been developed to measure flow velocity. Rainbow Volumic Velocimetry (RVV) is one such technique where one camera views a seeded flow passing through a volume illuminated with the polychromatic light spectrum [7]. In this method, two velocity components are found from tracking particles while the third is derived from color changes corresponding to the particles' motion in the direction normal to the cameras viewing plane. This technique produces a threedimensional flow field but is restricted to small volumes as the out-of-plane velocity components accuracy is strongly dependent upon of the width of each color in the volume [7]. A new method of volumetric color illumination was recently proposed by the present authors utilizing a grid pattern [8]. In this grid-based technique, color changes occur over multiple planes with respect to the camera viewing plane. With many colors and permutations of those colors, high spatial resolution in the out-of-plane direction is possible. The initial implementation of this grid-based technique was able to resolve three-dimensional motion while simultaneously identifying challenges.

In this study, the limitations of the grid-based technique are presented along with progress on a series of advances both in experimental implementation and in analysis algorithm design. Findings of the grid-based technique are first reviewed and weaknesses are identified. Then, recent progress on improvements are presented with a refined 
experimental method and analysis algorithm leading to a newly developed technique. The new technique is then tested and 3D particle trajectory results are presented.

\section{REVIEW OF THE MULTI-COLOR GRID TECHNIQUE}

The multi-color grid technique for flow characterization divides the measurement volume intro an $\mathrm{N} \times \mathrm{N}$ grid where $\mathrm{N}$ colors are used [8]. The color pattern is designed such that each color appears only once in each row and column. Hence when a particle is observed to change color without a corresponding pixel shift in the camera view plane, it can be concluded the particle moved perpendicular to the camera view plane. Similarly, the particle would change color in addition to a pixel shift if the motion is parallel to the camera view plane. One of the advantages of the grid technique is that the number of permutations of colors, exceeds the number of colors enabling the grid pattern to be extended. This allows for improved spatial resolution in the out-ofplane direction.

The multi-color grid technique was implemented using a simple experiment of tracking particle motion underwater using a three-color grid and a basic analysis procedure [8]. Captured streaks were discretized with a simple streak technique that was able to capture many points along each streak allowing for the general shape to be well-resolved. The three-dimensional position of the streak was found using linear interpolation of color described with hue from the Hue-Saturation-Value (HSV) color model. This demonstrated that a grid technique was able to capture basic three-dimensional motion [8].

The three-color grid pattern used only contained red, green, and blue however resulted showed other colors such as pink were present [8]. This is due to neighboring colors interfering with each other. When this technique was implemented with a six-color grid, this color cross-talk effect was stronger and began to interfere with three-dimensional position determination. Hence, increasing the number of colors, thus reducing grid size, is a major obstacle in improving the possible spatial resolution of the technique due to cross-talk between neighboring colors.

The LCD projector used in experiments produced a highly divergent beam, resulting in a rectangular frustum shaped measurement volume. The calibration of the measurement volume was very simple based on a hue threshold [8]. The basic streak discretization technique had issues in accurately capturing the ends of streaks and could not handle streaks with high curvature.

\section{Developing the Color Permutation Method}

Several advancements and improvements have been completed to address these limitations and to refine the technique. The first improvement is in the light beam delivery to overcome the beam divergence issue. This was achieved by placing a planoconvex lens in front of the LCD projector. This has the effect of producing an easily modelled measurement volume in the shape of a parallelepiped. This improvement to reduce beam divergence is illustrated in Fig. 1 where the new approach is compared with the lens-free case. From observing the thickness of each colored region in the image, it is clear that the lens significantly reduces the beam divergence improving the spatial uniformity of each single-colored region.

A new calibration technique has been developed for more robust analysis. The new method fits a normal distribution to hue values using the HSV model from a single-colored volume. The region within three standard deviations of the mean hue is defined as the "core" and the region beyond this is defined as the "boundary". An example of the core and boundary regions for a three-colored volume identified by the technique are shown in Fig. 2. Each core region is now separated by two boundary regions. .

The previous streak discretization method would have issues near ends of streaks where points were mis-aligned and could not handle streaks with significant curvature. These issues have been overcome with a new discretization technique that follows the shape of a streak. In this approach, a given streak is divided into many streak subdivisions. Each subdivision is identified by a white region where segmented streak and semi-circle intersect, as shown in Fig. 3. The centroid of the subdivision, as marked by the red asterisk, and the median hue are stored for each subdivision.

The issue of cross-talk between colors has been addressed by defining the mid-plane between neighboring single-colored volumes. This leads to the case where if a particle appears as an undefined hue in the whole measurement volume, it must exist between neighboring volumes. By identifying the volumes where the particle was last found before and after transition, it is possible to determine which mid-plane the particle passed through. This approach is dependent upon proper identification of volumes before and after transition.

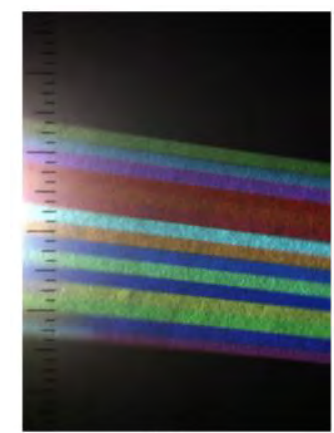

(a)

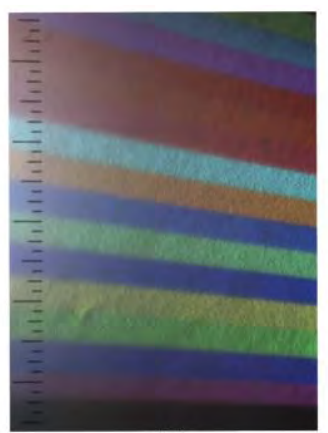

(b)
Figure 1. Comparison of illuminated volume (a) with planoconvex lens and (b) without lens showing the effect of the lens on beam divergence. 


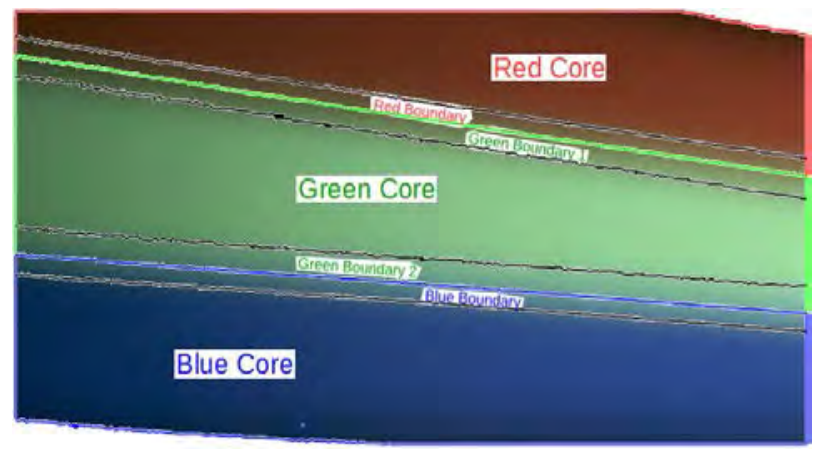

Figure 2. Cross-section of three-color grid pattern using the new calibration technique. The core and boundary regions for each color are outlined and labeled.

The robustness of volume identification and spatial resolution in the out-of-plane direction can be greatly improved via color permutations. For a selection of $\mathrm{N}$ colors, they can be arranged such that each color neighbors every other color only once. This allows for each color to appear several times in the volume where each appearance features unique neighbors. The result of this configuration is that once a particle changes color, e.g. a sufficiently long streak is captured by the camera, the three-dimensional position is known. As colors can repeat in the volume and mid-planes between colors are defined, this leads to a substantial increase in spatial resolution compared to the previous method for the same number of colors.

The culmination of these improvements have led to the color permutation method where color permutations drive the resolving of motion in the third dimension. For planar motion in the camera's field of view, the resolution provided by pixels far surpasses what is offered by the grid pattern. Hence, color changes are only needed in the out-of-plane direction. Moreover, this simplifies analysis as color crosstalk is limited to one direction.

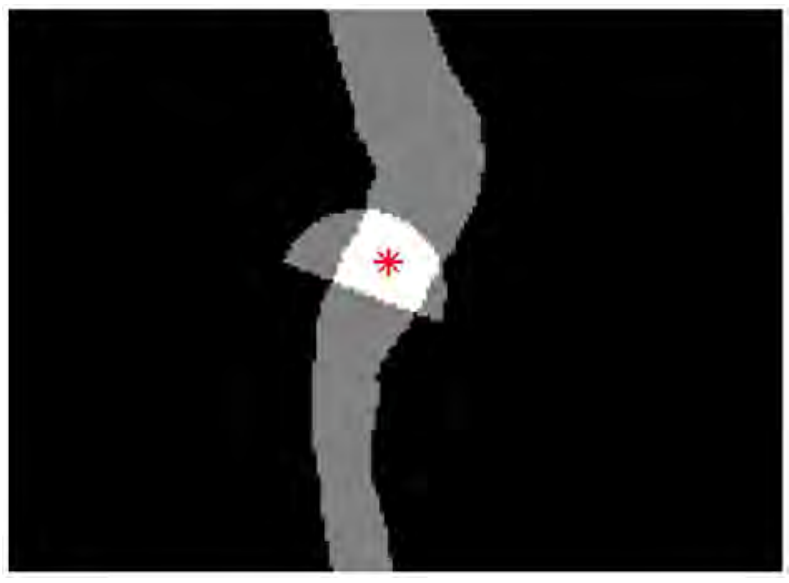

Figure 3. One step in the process of streak discretization. The subdivision is the white intersection area between semi-circle and segmented streak. The red asterisk denotes the subdivision centroid.

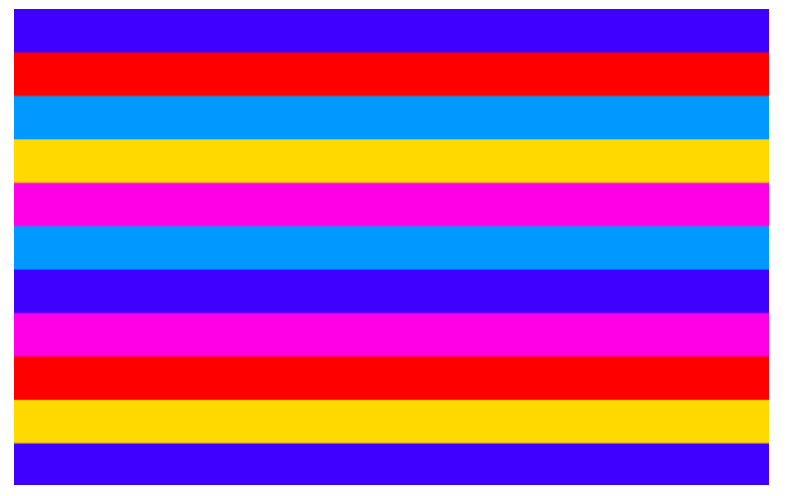

Figure 4. Five-color pattern with permutations to produce 11 layers used in experiments.

\section{Results}

The color permutation technique was tested using a fivecolor permutation pattern as shown in Fig. 4. This image contains one of many possible permutations of five colors. There are 11 single-colored volumes and by utilizing midplanes, this gives 21 possible out-of-camera-plane positions, over 4 times larger than the 5 positions possible with the previous technique. The experimental setup and conditions were the same as those previously reported [8].

Fig. 5a shows a two-frame composite image of a particle traveling from left to right. The corresponding twodimensional discretized streak is shown in Fig. 5b. Comparing the discretized streak to the original image shows the new path-following discretization method successfully captures local curvatures in the streak. This is evidenced by features such as the drop in $\mathrm{Z}$ position where $\mathrm{Y} \approx 1200$ pixels, matching the left-most orange part of the streak composite image. Similar features along the streak image are well captured by the new discretization technique. There were no mis-aligned points at the ends of each streak showing the new technique has improved over the previous method in this aspect.

Fig. 6 shows the discretized streaks in three dimensions describing the particle trajectory. The left-most part of the streak at $Y \approx 900$ pixels was found to be in layer 11, then continuing along the streak showed the streak moving threedimensionally up to layer 6 . At this point, the second frame in the composite image begins where the streak continues from $\mathrm{Y} \approx 1700$ pixels and layer 6 up to $\mathrm{X} \approx 2500$ pixels in layer 3 . The additional depth positions obtained by color permutations and mid-planes showed good resolution of the particle's motion in the out-of-camera-plane direction.

\section{ANALYSIS OF NEW Findings}

Numerous changes have been made to the multi-color grid flow visualization technique. A planoconvex lens placed in-front of the projector greatly reduces beam divergence. 


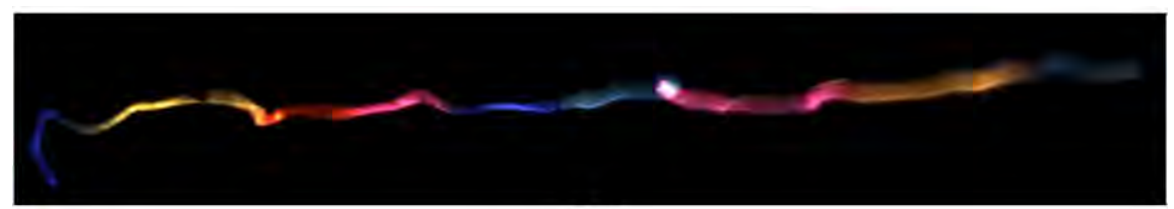

(a)

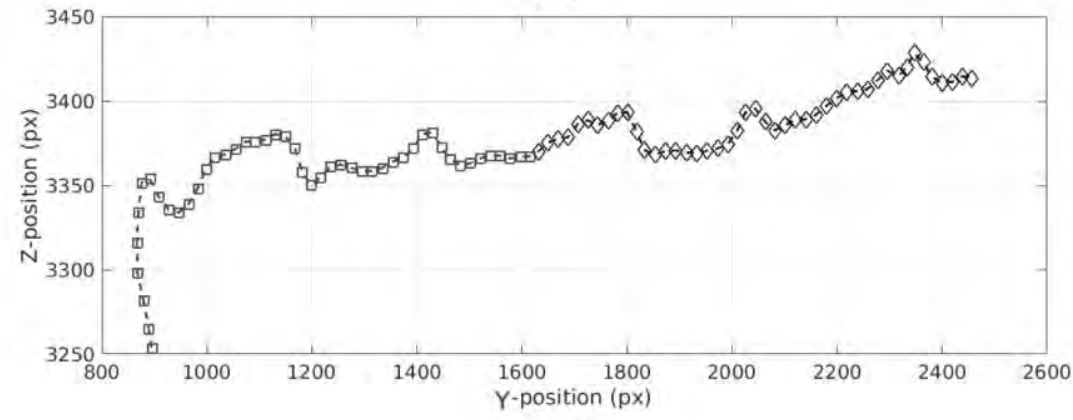

(b)

Figure 5. Two-frame composite image (a) of streak captured and two-dimensional view of discretized streak (b). The image has been rotated to match the coordinate system of the plot

This changed the shape of the illuminated volume from a rectangular frustum to a parallelepiped which is very simple to describe mathematically. Furthermore the size of each single-colored volume is smaller compared to the divergent beam setup, providing an increase in spatial resolution in one direction. With precise alignment of the planoconvex lens, a rectangular prism shaped volume is possible.

The two analysis algorithm changes, improved calibration and discretization, lay the ground work for high quality flow characterization. In the new calibration technique, using a normal distribution to define the hue threshold enables the technique to be implemented with many different projectors and clearly defines the calibration core and boundary regions. The new discretization technique demonstrated an ability to follow the shape of a streak with somewhat complex geometry, such as local curvatures that characterizes the three-dimensionality of the flow. These advancements provide an opportunity for the future growth of the analysis algorithm.

The addition of mid-planes and color permutations together provided a very significant increase in out-ofcamera-plane spatial resolution. The introduction of midplanes mitigated the effects of cross-talk between neighboring volumes as hues not defined by the grid pattern do not need to be explicitly determined. Mid-planes also increased the out-of-plane resolution by a factor of just under two.

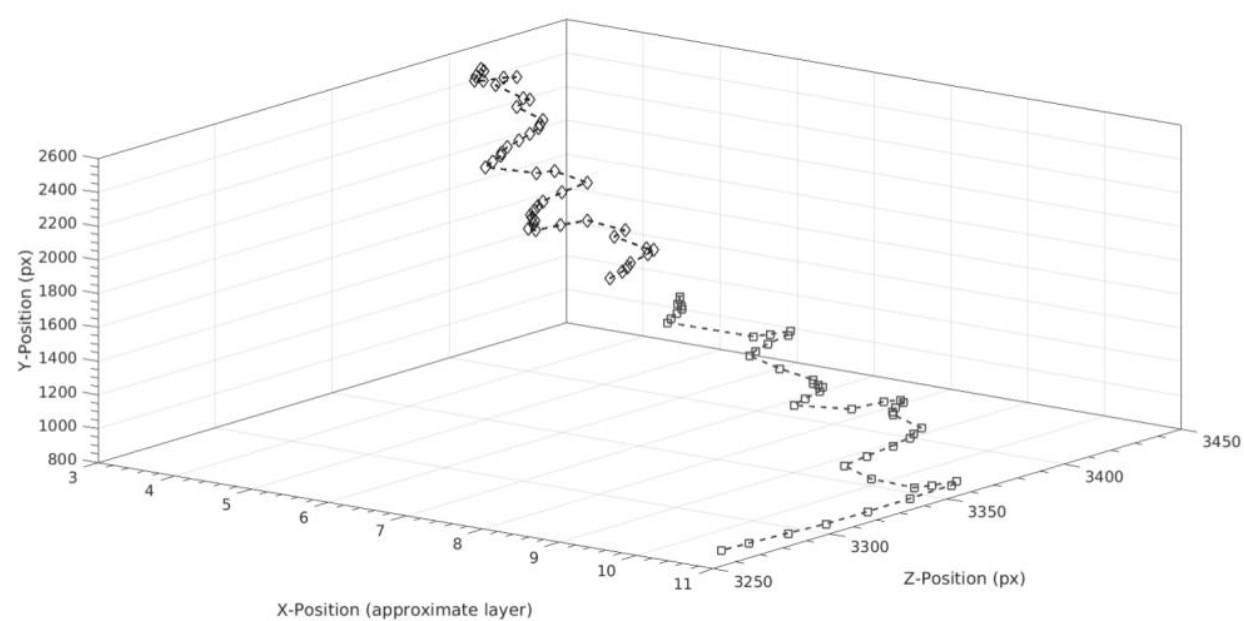

Figure 6. Three-dimensional reconstructed particle path corresponding to the two-dimensional discretized streak in Fig. 5. 
Color permutations demonstrate that a large increase in spatial resolution is possible with only a few colors via permutations. As tested in the present study, 5 colors were able to produce 11 different core depth positions and with mid-planes, generated 21 possible positions. The cost for this improvement is loss of color uniqueness in the volume as a red region could end up in two different positions. This is an ambiguity that cannot be resolved until the streak changes color.

This color permutation method has shown strong potential for future growth. With additional colors, more permutations are possible, producing further improvements in out-of-plane spatial resolution. Work is currently in progress in this area of identifying valid color permutations for six or more colors.

Thus far the color permutation method has presented results from the $3 \mathrm{D}$ path traversed by a single particle. While this is useful to build a framework and test analysis methods in isolation, it is not practical. Multiple particles must be present for meaningful flow characterization. In a test where many particles are present, the streaks left behind by their motion may overlap or otherwise interact. Current work is focused on implementing analysis methods for cases where streaks interact.

Finally, the three-dimensional reconstruction work has been performed manually to date. Currently an automatic reconstruction technique is in development to convert hue data along streaks into depth positions. There is also ongoing work in more robust preprocessing methods to better separate streaks from background noise and select streaks with color changes to ensure compatibility with the color permutation method.

\section{CONCLUSION}

In conclusion, the multi-color grid technique was successful at resolving $3 \mathrm{D}$ particle motion but did reveal many flaws in the method and early implementation. The present paper serves as a progress update that identifies and resolves these limitations while advancing the technique utilizing color permutations and improved algorithm design. There are new challenges found in the updated method that join unresolved issues. Presently, work continues on addressing these issues and further refining the technique. These findings using the color permutation method will drive future research and development on this technique.

\section{ACKNOWLEDGMENT}

The authors would like to thank Natural Sciences and Engineering Research Council of Canada (NSERC) and the University of Western Ontario for their support.

\section{REFERENCES}

[1] M. P.Arroyo, and C. A. Greated, "Stereoscopic particle image velocimetry," Meas. Sci. Technol., vol. 2, pp. 1181-1186, 1991. doi:10.1088/0957-0233/2/12/012.

[2] R. Fei and W. Merzkirch, "Investigations of the measurement accuracy of stereo particle image velocimetry," Exp. Fluids, vol. 37, pp. 559-565, Oct. 2004. doi:10.1007/s00348-004-0843-x.

[3] K. H. Peterson, "Single camera, three-dimensional particle tracking velocimetry," Ph.D. dissertation, Dept. Mech. Eng., Univ. of Michigan, 2012.

[4] G. E. Elsinga et al., "Tomographic particle image velocimetry," Exp. Fluids, vol. 41, no. 6, pp. 933-947, Dec. 2006. doi: 10.1007/s00348-006-0212-z.

[5] G. E. Elsinga, J. Westerweel, and F. Scarano, "On the velocity of ghost particles and the bias errors in Tomographic PIV," Exp. Fluids, vol. 50, pp. 825-838, Apr. 2011. doi: 10.1007/s00348-010-0930-0.

[6] D. Michaelis and B. Wieneke, "Comparison between tomographic PIV and stereo PIV," in Proc. 14th Int. Symp. of Laser Techniques to Fluid Mech., Libson, Portugal, July 7-10, 2008.

[7] J. P. Prenel and Y. Bailly, "Recent evolutions of imagery in fluid mechanics: From standard tomographic visualization to 3D volumic velocimetry," Opt. Lasers Eng., vol. 44, pp. 312-334, Mar.-Apr. 2006. doi: 10.1016/j.optlaseng.2005.04.007.

[8] K. Dennis and K. Siddiqui, "A multicolor grid technique for volumetric velocity measurements," in Proc. ASME FEDSM, Waikoloa, HI, USA, July 30 Aug. 2, 2017, pp. V01BT06A020. doi: 10.1115/FEDSM2017-69546. 


\section{Estimation of Shock Stand-off Distance Using the Curved Shock Theory and Its Validation via Numerical Modelling}

\author{
Z. Worku, E. Timofeev \\ Department of Mechanical Engineering \\ McGill University \\ Montreal, Canada \\ zelalem.worku@mail.mcgill.ca
}

\author{
S. Mölder \\ Professor Emeritus \\ Ryerson University \\ Toronto, Canada \\ smolder@ryerson.ca
}

\begin{abstract}
Curved shocks that are locally oriented normal to the direction of the pre-shock flow vector appear on bluff and blunt bodies in supersonic flow and at the center lines of axisymmetric air intakes. There have been numerous studies to analytically approximate the shock stand-off distance associated with these curved normal shock waves; however, in view of the absence of satisfactory results across the entire range of freestream Mach numbers, further efforts are warranted. In this study, the Curved Shock Theory (CST) is used to derive analytical expressions for the pressure gradient right behind convex normal shocks in uniform upstream flow. This pressure gradient can be converted to gradients of other variables using the conservations laws and the isentropic relations. Using these gradients at the curved normal shock and an additional assumption on their variation between the shock and the blunt body it is possible to develop relations for the ratio of the shock stand-off distance to the radius of curvature of the shock surface as a function of freestream Mach number and the specific heat ratio. CST predictions are compared with experimental data from the literature and the CFD results obtained in the present study for freestream Mach numbers ranging from 1.2 to 8 .
\end{abstract}

Keywords-shock stand-off distance; curved shock theory; blunt body; radius of shock curvature

\section{INTRODUCTION}

The shock stand-off distance from blunt bodies is an important parameter to estimate the effects of shock waves on objects moving at supersonic and hypersonic speeds. The change in the shock stand-off distance can be an effective indicator of the changes in other important properties such as the drag on an aircraft or the heat flux on a re-entry vehicle. There have been continuous efforts over many decades to come up with handy expressions to quickly calculate the shock stand-off distance from blunt bodies. However, rather limited success is achieved in formulating expressions that can be applied over a wide range of Mach numbers.

Several assumptions were implemented by different researchers to simplify the problem and formulate widely applicable expressions for the shock stand-off distance as a function of the freestream Mach number. In an earlier work by Moeckel [1], for instance, it is assumed that the detached shock wave can be represented by a hyperbola and that the sonic curve between the shock and the body is a straight line. In a recent study, Sinclair and Cui [2] made use of the variation of the size of the sonic zone bounded by the bow shock and the fore part of the body to relate the shock stand-off distance to the freestream Mach number.

In this paper, the Curved Shock Theory (CST) is used to derive an expression for the pressure gradient right behind convex bow shocks that appear in supersonic and hypersonic flows with uniform pre-shock flow. This pressure gradient relation is combined with the momentum conservation law and the isentropic relations for various flow variables, such as Mach number, density, temperature, etc. to obtain the gradients of those variables at the curved bow shock. By assuming the gradients to be constant within the shock layer between the bow shock and the blunt body or considering their average values, the ratio of the shock stand-off distance to the shock radius of curvature is obtained as a function of freestream Mach number. The aim of this study is to provide such alternative theoretical relations for the shock stand-off distance using CST and to investigate their validity. Billig's experimental correlations [3, 4], Liepmann and Roshko's experimental data [5], and our numerical simulation results are used as tools for validation.

\section{THEORETICAL FORMULATION}

\section{A. Curved Shock Theory as Applied to Curved Normal Shocks in Uniform Pre-shock Flow}

Theory that relates the pressure gradient, $P$, the streamline curvature, $D$, and vorticity, $\Gamma$, to the shock curvatures, $S_{a}$ and $S_{b}$, on the pre-shock and post-shock sides of a doubly curved shock wave is developed in [6]. Such theories are also found in $[7,8]$. When applied to a normal shock facing uniform upstream flow [8], the CST relations become:

$$
\begin{gathered}
0=A_{2}^{\prime} P_{2}+C^{\prime} S_{a}+G^{\prime} S_{b} \\
P=\frac{\partial p / \partial s}{\rho V^{2}}=\frac{\partial p / \partial s}{\gamma p M^{2}}
\end{gathered}
$$




$$
\begin{gathered}
A_{2}^{\prime}=-\frac{M_{1}^{2}-1}{M_{1}^{2}} \cdot \frac{(\gamma-1) M_{1}^{2}+2}{2 \gamma M_{1}^{2}-\gamma+1} \\
C^{\prime}=G^{\prime}=-\frac{2}{\gamma+1} \cdot \frac{M_{1}^{2}-1}{M_{1}^{2}}
\end{gathered}
$$

where subscripts 1 and 2 correspond to the upstream and downstream sides of the bow shock at the center line, respectively; $M_{1}$ is the freestream Mach number; the derivative in (2) is taken along the streamline; other notations are conventional.

For the blunt body case both shock surface curvatures, $S_{a}$ and $S_{b}$, are negative and the corresponding radii of curvature, $R_{a}=-1 / S_{a}$ and $R_{b}=-1 / S_{b}$, are positive. The total curvature is $S_{a}+S_{b}$, the 'average curvature' is $\bar{S}=\left(S_{a}+S_{b}\right) / 2$ and the 'harmonic average radius of curvature' is $\bar{R}$, where $1 / \bar{R}=\left(1 / R_{a}+1 / R_{b}\right) / 2$, so that $\bar{S}=$ $-1 / \bar{R}$.

Substituting (3) and (4) into (1), it is possible to show that,

$$
P_{2}=-\frac{4 \bar{S}}{(\gamma+1) M_{2}^{2}}=\frac{4}{(\gamma+1) M_{2}^{2} \bar{R}}
$$

Furthermore, applying (2) at the downstream face of the shock wave it can be written as,

$$
\left(\frac{\mathrm{d} p}{p}\right)_{2}=\frac{4 \gamma}{(\gamma+1) \bar{R}} \mathrm{~d} s
$$

\section{B. Shock Stand-off Distance from a Blunt Body in Uniform Pre-shock Flow}

Expressions for the shock stand-off distance, $\Delta$, from blunt and bluff bodies in a uniform pre-shock flow can be obtained using the post-shock pressure gradient (6) from CST, along with appropriate assumptions regarding flow variables in the downstream region. One such assumption is the constant pressure gradient assumption, in other words - the linear pressure profile in the shock layer. Equation (6) can be then written as,

$$
\begin{gathered}
\left(\frac{\mathrm{d} p}{\mathrm{~d} s}\right)_{2}=\frac{p_{0}-p_{2}}{\Delta}=\frac{4 \gamma p_{2}}{(\gamma+1) \bar{R}} \\
\frac{\Delta}{\bar{R}}=\frac{\gamma+1}{4 \gamma}\left(\frac{p_{0}}{p_{2}}-1\right) \\
\frac{p_{0}}{p_{2}}=\left(1+\frac{\gamma-1}{2} M_{2}^{2}\right)^{\frac{\gamma}{\gamma-1}} \\
\frac{\overline{\bar{R}}}{\bar{R}}=\frac{\gamma+1}{4 \gamma}\left[\left(\frac{M_{1}^{2}(\gamma+1)^{2}}{4 \gamma M_{1}^{2}-2(\gamma-1)}\right)^{\frac{\gamma}{\gamma-1}}-1\right]
\end{gathered}
$$

Here, $p_{0}$ is the stagnation pressure for the post shock flow at the centerline. Equation (10) gives a relation of the ratio of the shock stand-off distance to the radius of curvature as a function of the freestream Mach number and the specific heat ratio only.

A similar equation can be obtained by using an average pressure gradient instead of the constant gradient assumption. It can be shown that the pressure gradient at the stagnation point is zero, i.e., the pressure gradient varies across the shock layer from the value given by (6) at the shock to zero at the stagnation point. Therefore, the average pressure gradient within the shock layer can be taken as an arithmetic average, i.e., one half of the value given by (6),

$$
\left(\frac{\mathrm{d} p}{\mathrm{~d} s}\right)_{\mathrm{avg}}=\frac{1}{2}\left[\left(\frac{\mathrm{d} p}{\mathrm{~d} s}\right)_{2}+0\right]=\frac{1}{2}\left(\frac{\mathrm{d} p}{\mathrm{~d} s}\right)_{2}
$$

It is easy to show that such an assumption is mathematically equivalent to the parabolic pressure profile in the shock layer. This leads to,

$$
\frac{\Delta}{\bar{R}}=\frac{\gamma+1}{2 \gamma}\left[\left(\frac{M_{1}^{2}(\gamma+1)^{2}}{4 \gamma M_{1}^{2}-2(\gamma-1)}\right)^{\frac{\gamma}{\gamma-1}}-1\right]
$$

Another estimation of $\Delta / \overline{\mathrm{R}}$ can be obtained by assuming a constant velocity gradient in the downstream flow and using the conservation of momentum equation for steady, inviscid, onedimensional flow,

$$
\rho V\left(\frac{\mathrm{d} V}{\mathrm{~d} s}\right)=-\left(\frac{\mathrm{d} p}{\mathrm{~d} s}\right)
$$

Substituting the pressure gradient from CST into the right-hand side of (13) and applying the constant velocity gradient assumption on the left-hand side result in,

$$
\frac{\Delta}{\bar{R}}=\frac{\gamma+1}{4}\left(\frac{2+(\gamma-1) M_{1}^{2}}{2 \gamma M_{1}^{2}-\gamma+1}\right)
$$

The third flow variable that can be used to estimate the ratio of the shock stand-off distance to the shock radius of curvature is the Mach number. The equation that allow to achieve that follows from (9),

$$
\left(\frac{\mathrm{d} M}{M}\right)_{2}=-\frac{2+(\gamma-1) M_{2}^{2}}{2 \gamma M_{2}^{2}}\left(\frac{\mathrm{d} p}{p}\right)_{2}
$$

Equation (15) used with (6), the normal shock relation for Mach number, and the constant Mach number gradient assumption results in,

$$
\frac{\Delta}{\bar{R}}=\frac{(\gamma-1) M_{1}^{2}+2}{2(\gamma+1) M_{1}^{2}}
$$

It is also possible to relate the density gradient at the downstream face of the shock wave with the pressure gradient using the fact that the flow in this region is isentropic:

$$
\left(\frac{\mathrm{d} p}{p}\right)_{2}=\gamma\left(\frac{\mathrm{d} \rho}{\rho}\right)_{2}
$$

Equation (17) can be used either with the constant density gradient assumption or the average density gradient assumption to find relations for $\Delta / \overline{\mathrm{R}}$. The respective results are,

$$
\frac{\Delta}{\bar{R}}=\frac{\gamma+1}{4}\left[\left(\frac{M_{1}^{2}(\gamma+1)^{2}}{4 \gamma M_{1}^{2}-2(\gamma-1)}\right)^{\frac{1}{\gamma-1}}-1\right]
$$




$$
\frac{\Delta}{\bar{R}}=\frac{\gamma+1}{2}\left[\left(\frac{M_{1}^{2}(\gamma+1)^{2}}{4 \gamma M_{1}^{2}-2(\gamma-1)}\right)^{\frac{1}{\gamma-1}}-1\right]
$$

The isentropic nature of the flow in the downstream flowfield can also be used to obtain an equation that relates the temperature to the pressure gradient,

$$
\left(\frac{\mathrm{d} T}{T}\right)_{2}=\frac{\gamma-1}{\gamma}\left(\frac{\mathrm{d} p}{p}\right)_{2}
$$

Applying (20) at the downstream face of the shock wave and employing the constant temperature gradient assumption and the average temperature gradient assumption in the downstream flow field, it is possible to show that the respective equations that relate $\Delta / \bar{R}$ to the freestream Mach number are,

$$
\begin{aligned}
& \frac{\Delta}{\bar{R}}=\frac{\gamma+1}{8}\left(\frac{2+(\gamma-1) M_{1}^{2}}{2 \gamma M_{1}^{2}-\gamma+1}\right) \\
& \frac{\Delta}{\bar{R}}=\frac{\gamma+1}{4}\left(\frac{2+(\gamma-1) M_{1}^{2}}{2 \gamma M_{1}^{2}-\gamma+1}\right)
\end{aligned}
$$

\section{NUMERICAL INVESTIGATION OF RATIO OF SHOCK STAND-OFF DISTANCE TO RADIUS OF CURVATURE}

Flows over a sphere, representing an axisymmetric flow case, and over a cylinder, representing a planar flow case, are numerically simulated for Mach numbers ranging from 1.2 to 8 using an adaptive unstructured total variation diminishing (TVD) finite volume Euler flow solver [9]. The shock stand-off distance and the radius of curvature for each case are obtained, and their ratio is compared against the CST results and Billig's experimental correlations.

The relations for $\Delta / \bar{R}$ presented in Section II are adapted for different flow types by substituting the average radius of curvature, $\bar{R}$, with $R$ for axisymmetric flows and with $2 R$ for planar flows, where $R$ is the radius of shock curvature.

Experimental correlations of the shock stand-off distance and the radius of curvature with the freestream Mach number are presented in [3, 4]. These correlations, in addition to the numerical studies conducted, are used to validate the CST-based estimations of $\Delta / \overline{\mathrm{R}}$. Billig's experimental correlations for spherical and cylindrical bodies, respectively, are,

$$
\begin{aligned}
& \frac{\Delta}{R}=\frac{0.143 \exp \left[3.24 / M_{1}^{2}\right]}{1.143 \exp \left[0.54 /\left(M_{1}-1\right)^{1.2}\right]} \\
& \frac{\Delta}{R}=\frac{0.386 \exp \left[4.67 / M_{1}^{2}\right]}{1.386 \exp \left[1.8 /\left(M_{1}-1\right)^{0.75}\right]}
\end{aligned}
$$

\section{A. Comparison of Analytical and Numerical Results}

The ratios of the shock stand-off distance to the radius of shock curvature obtained for the axisymmetric and planar flow cases are presented in Fig. 1 and Fig. 2, respectively. It is seen that the theoretical curves form two distinct groups. One of the groups shows reasonable agreement with the present numerical results and Billig's experimental correlations for freestream Mach numbers higher than $\sim 2$ for axisymmetric flow and higher than $\sim 3$ for planar flow. The lines of this group are for the average gradient (parabolic profile) assumption applied to the flow variables having zero gradient at the stagnation point (pressure, density, temperature) or for the constant gradient (linear profile) assumption applied to the flow variables with non-zero gradient at the stagnation point (Mach number, velocity). The second group of theoretical lines gives two times lower values of $\Delta / R$. The lines of this group are for the constant gradient (linear profile) assumption applied to the flow variables having zero gradient at the stagnation point (pressure, density, temperature). It is clear that the constant gradient (linear profile) assumption is not suitable for flow variables having zero gradients at the stagnation point because for gradient to become zero at the stagnation point, it should significantly deviate from its value at the shock.

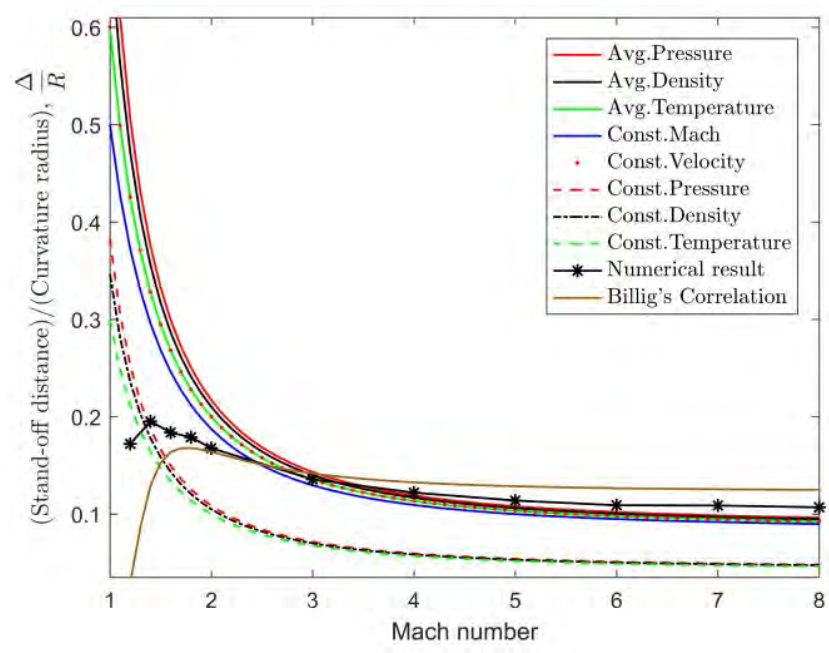

Figure 1. Ratio of the shock stand-off distance to the radius of shock curvature vs. the freestream Mach number for axisymmetric flow (over a sphere)

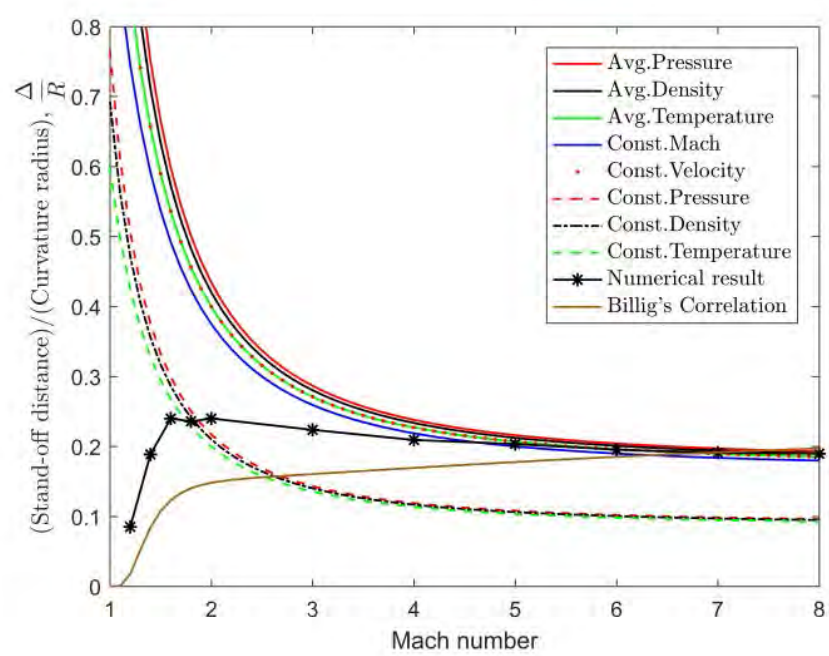

Figure 2. Ratio of the shock stand-off distance to the radius of shock curvature vs. the freestream Mach number for planar flow (over a cylinder) 


\section{B. Evaluation of Assumptions Using Numerical Solutions}

To shed some light on the accuracy of the constant pressure gradient (linear pressure profile) and average pressure gradient (parabolic pressure profile) assumptions for various Mach numbers, the average pressure gradients in the flow downstream from the bow shock at the center line are obtained from the numerical solutions. The deviation of the constant and average pressure gradient assumptions from these numerical pressure gradient values are shown in Figs. 3 and 4. It is evident from these figures that for higher Mach numbers ( $>2$ or 3 ) the average pressure gradient assumption results in the pressure gradients which are much closer to the numerically determined values. On the other hand, at lower Mach numbers the constant pressure gradient assumption is more accurate. This is consistent with the results for the shock stand-off distance in Figs. 1 and 2.

\section{Weighted Average Pressure Gradient Approximation}

The above discussion suggests that the CST predictions for the shock stand-off distance would be more accurate if one would come up with a better approximation of gradients in the downstream flow field. To demonstrate that, a weighting factor,

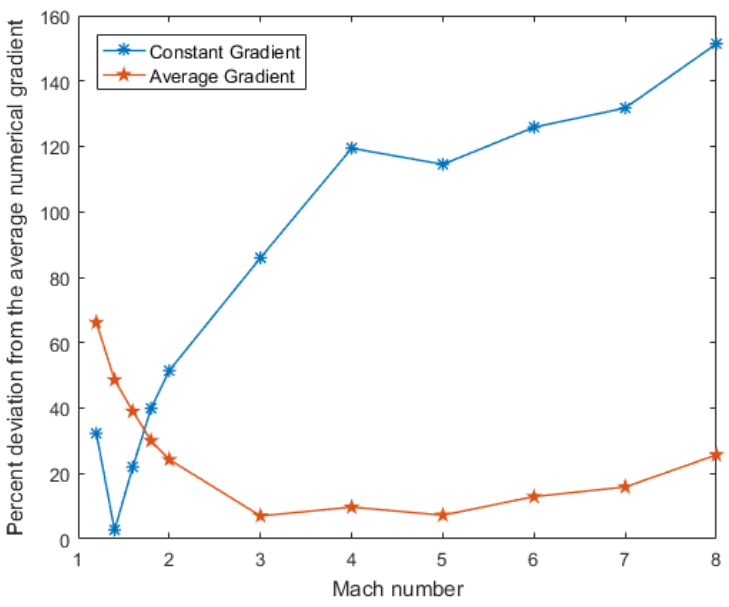

Figure 3. Percent deviation of the average and constant pressure gradient assumptions from the numerical average pressure gradient for axisymmetric flow

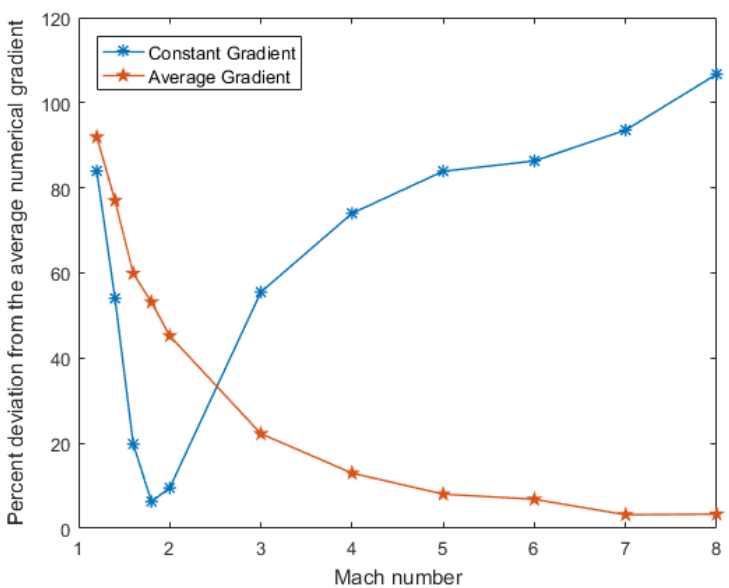

Figure 4. Percent deviation of the average and constant pressure gradient assumptions from the numerical average pressure gradient for planar flow
$W$, is introduced in the calculation of the average pressure gradient. The weighting factor is calculated as the ratio of the average numerical pressure gradient downstream from the shock to the pressure gradient at the downstream face of the shock wave. The results are presented in Fig. 5.

Using the weighting factor, the averaging equation (11) is modified as follows,

$$
\left(\frac{\mathrm{d} p}{\mathrm{~d} s}\right)_{\mathrm{avg}}=\frac{1}{2}\left[W\left(\frac{\mathrm{d} p}{\mathrm{~d} s}\right)_{2}+0\right]=\frac{W}{2}\left(\frac{\mathrm{d} p}{\mathrm{~d} s}\right)_{2}
$$

This allows to get (compare with (12)),

$$
\frac{\Delta}{\bar{R}}=\frac{1}{W} \cdot \frac{\gamma+1}{2 \gamma}\left[\left(\frac{M_{1}^{2}(\gamma+1)^{2}}{4 \gamma M_{1}^{2}-2(\gamma-1)}\right)^{\frac{\gamma}{\gamma-1}}-1\right]
$$

The values $\Delta / \overline{\mathrm{R}}$ based on the weighted average pressure gradient approximation for axisymmetric and planar flows are shown in Fig. 6 and 7, respectively. It is seen that this approximation leads

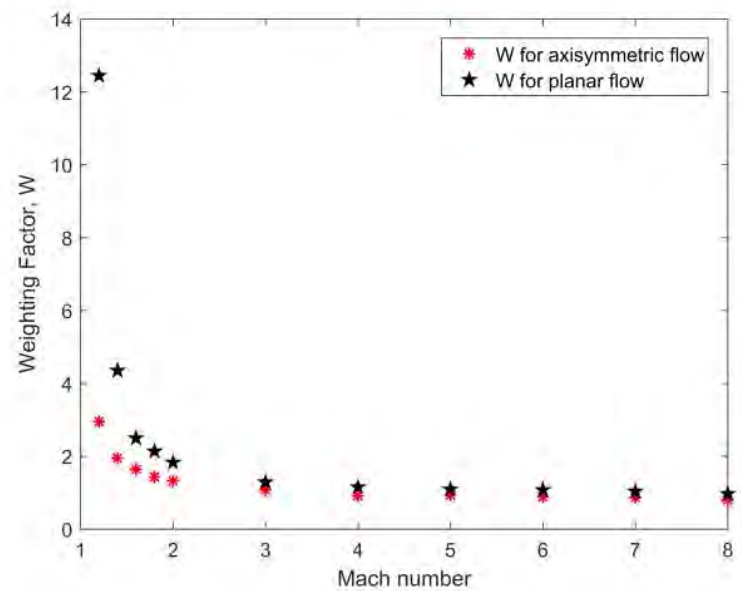

Figure 5. Weighting factor at different Mach numbers for axisymmetric and planar flows

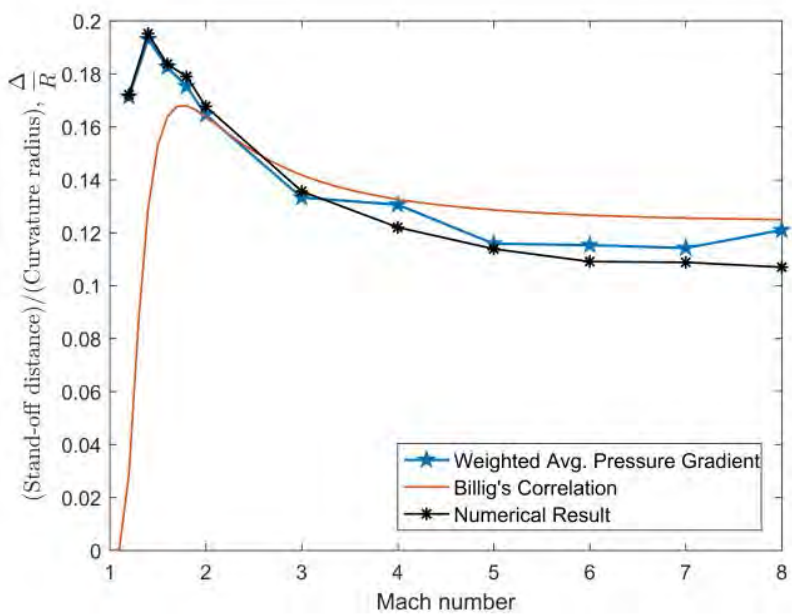

Figure 6. Ratio of the shock stand-off distance to the radius of shock curvature vs. freestream Mach number for axisymmetric flow using the weighted average pressure gradient 


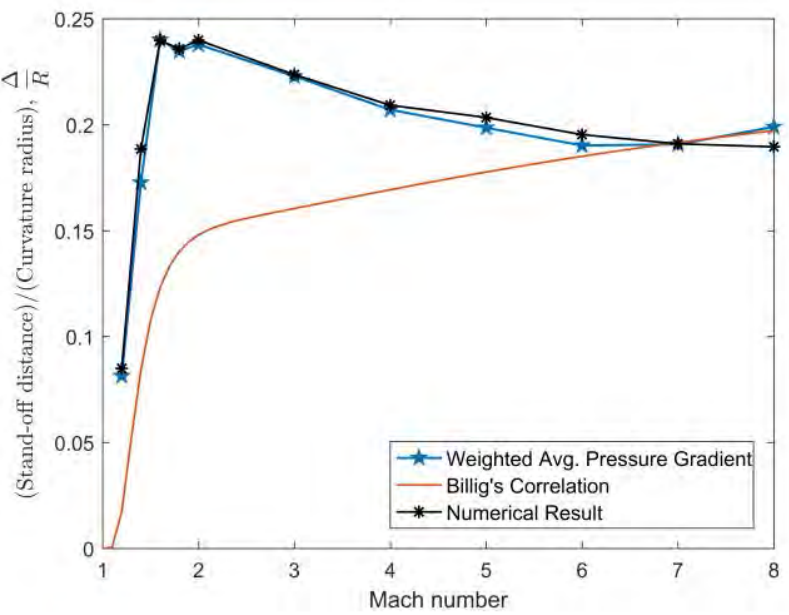

Figure 7. Ratio of the shock stand-off distance to the radius of hock curvature vs. freestream Mach number for planar flow using weighted average pressure gradient

to the results which are close to the values produced via numerical modelling (the difference is less than $10 \%$ for the whole Mach number range considered). The predictions based on the weighted average pressure gradient approximation cannot be considered as an independent theoretical treatment because numerical simulation data are used when calculating the weighting factor. However, Figs. 6 and 7 demonstrate clearly that better approximation of downstream flow gradients would certainly lead to more accurate results for $\Delta \bar{R}$.

\section{Comparison of Shock Stand-off Distance with Results in Literature Using Approximated Radius of Curvature}

Most published data on the shock stand-off distance is in terms of the body radius, $R_{\text {body }}$, rather than the shock radius of curvature, $R$. Hence, an approximation of the radius of curvature is needed to facilitate the comparison of the CST results with other data. In the present work, the radius of curvature is assumed to be the sum of the body radius and the shock standoff distance, i.e.,

$$
R=R_{\mathrm{body}}+\Delta
$$

or

$$
\frac{R}{R_{\text {body }}}=1+\frac{\Delta}{R_{\text {body }}}
$$

Then the CST predictions for $\Delta / R$ can be converted to $\Delta / R_{\text {body }}$ as follows,

$$
\frac{\Delta}{R_{\text {body }}}=\frac{\Delta}{R} \times \frac{R}{R_{\text {body }}}
$$

As seen from Figs. 8 and 9 it is a reasonable approximation at high Mach numbers when the bow shock lies close to the body surface. At low Mach numbers, equation (27) significantly underestimates the radius of shock curvature.

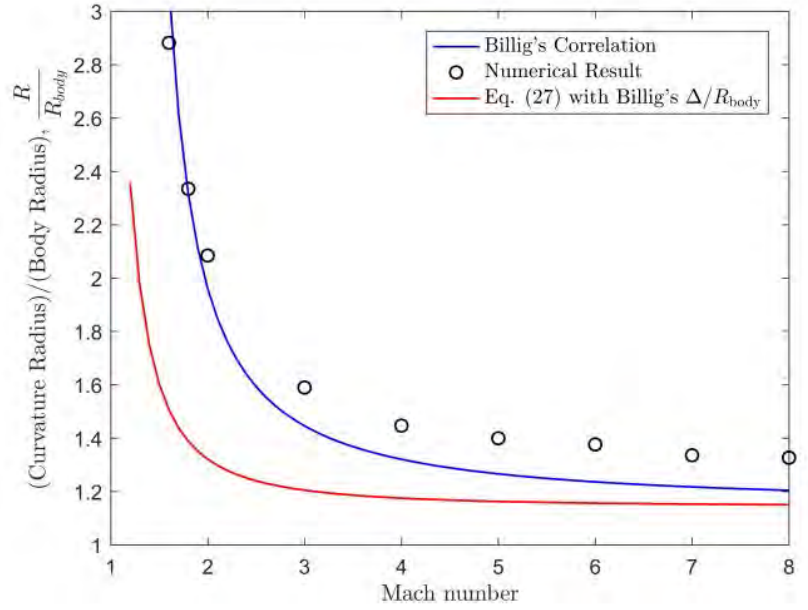

Figure 8. Comparison of the radius of shock curvature given by (27) with experimental data and numerical results for axisymmetric flow

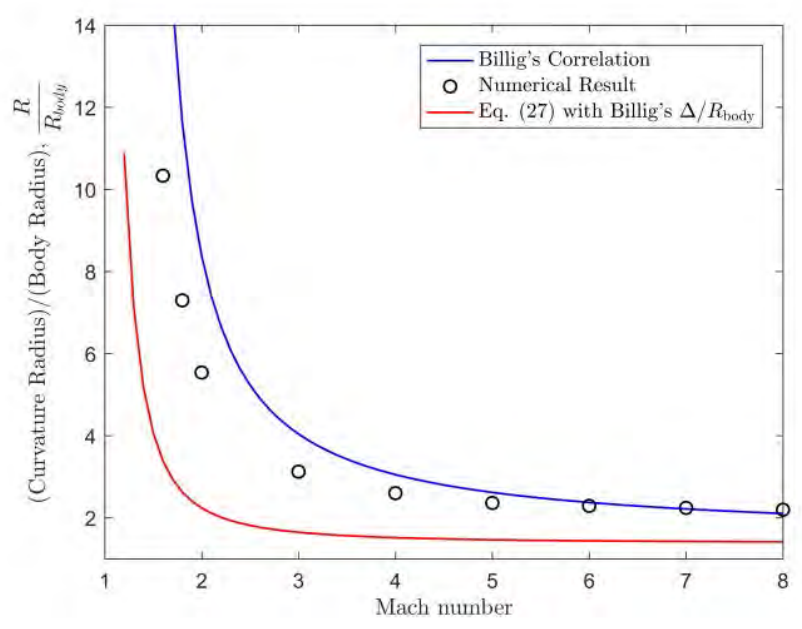

Figure 9. Comparison of the radius of shock curvature given by (27) with experimental data and numerical results for planar flow

The results for $\Delta / R_{\text {body }}$ are shown in Figs. 10 and 11 for axisymmetric and planar flows, respectively. The CST predictions are represented by the same two groups of curves as in Figs. 1 and 2. Experimental results include Billig's correlations and data from Liepmann \& Roshko [5]. Numerical results from the present work are also shown. The analytical prediction by Sinclair \& Cui [2] is provided as well (for the planar case only).

It is seen in Figs. 10 and 11 that the CST relations correctly predict the overall monotonic variation of the stand-off distance (in terms of the body radius) with increasing Mach number (as opposed to Figs. 1 and 2 where non-monotonic behavior of the ratio of the stand-off distance to the radius of shock curvature is not reproduced by the CST predictions). This is because for low Mach numbers CST overpredicts the ratio of $\Delta / R$ (see Figs. 1 and 2) while assumption (26-27) underestimates the radius of curvature $R / R_{\text {body }}$ (see Figs. 8 and 9 ) so that their product (28) serendipitously exhibits correct behavior (Figs. 10 and 11). 
As seen in Figs. 10 and 11, among various assumptions regarding the gradients in the post-shock flow along the center line the average pressure gradient assumption (in other words, the parabolic pressure distribution in the shock layer) leads to the best CST-based result in comparison with experimental and numerical simulation data. Nevertheless, CST somewhat underestimates the shock stand-off distance for the whole range of Mach number considered. This may be attributed to underprediction of the radius of shock curvature by (27). It is to be noted that the alternative theory by Sinclair \& Cui [2] appears to overestimate the shock stand-off distance.

\section{CONCLUDING REMARKS}

It may be concluded that the CST-based approach shows a good promise in predicting the shock stand-off distance. Further improvements might be achieved by finding better approximations for the post-shock flow gradients and the radius of shock curvature.

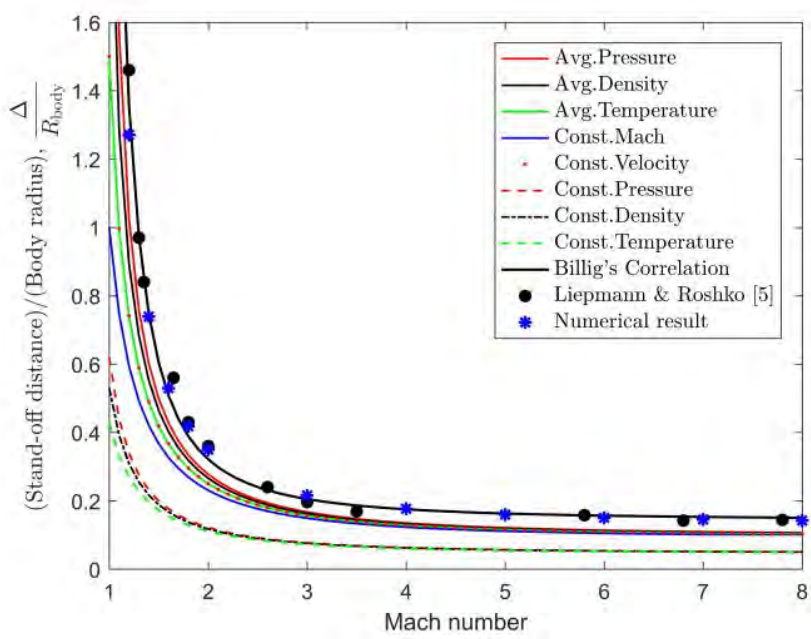

Figure 10. Comparison of CST estimates of the shock stand-off distance with experimental data and numerical results for axisymmetric flow

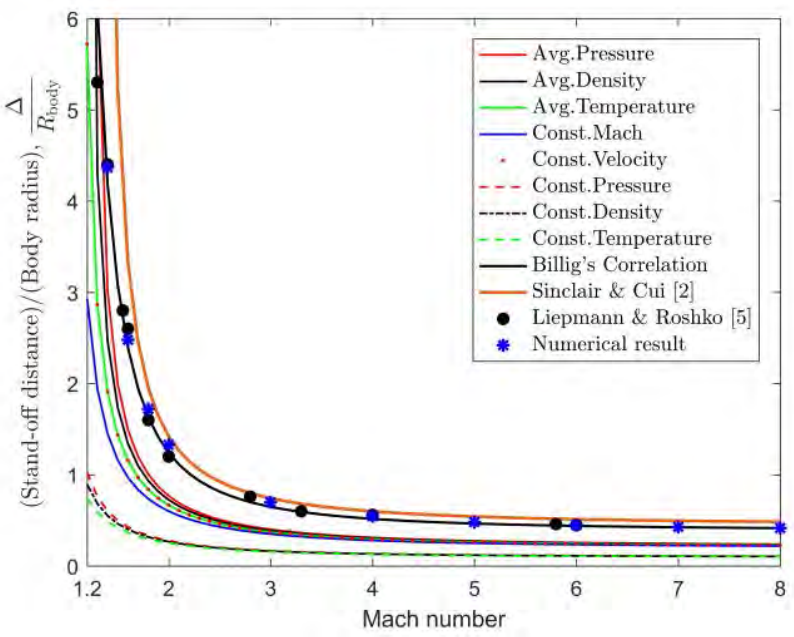

Figure 11. Comparison of CST estimates of the shock stand-off distance with experimental data and numerical results for planar flow

\section{REFERENCES}

[1] W. E., Moeckel, "Approximate method for predicting form and location of detached shock waves ahead of plane or axially symmetric bodies," NACA Tech. Note no. 1921, July 1949.

[2] J. Sinclair and X. Cui, "A theoretical approximation of the shock standoff distance for supersonic flows around a circular cylinder," Phys. Fluids, vol. 29, no. 026102, 2017. https://doi.org/10.1063/1.4975983

[3] F. S. Billig, "Shock-wave shape around spherical- and cylindrical-nosed bodies, J. Spacecr. Rockets, vol. 4, no. 6 , pp. 822-823, 1967.

https://doi.org/10.2514/3.28969

[4] J. D. Anderson Jr., Hypersonic and High-Temperature Gas Dynamics, 2nd ed., Reston, VA: AIAA, 2006. https://doi.org/10.2514/4.861956

[5] H. W. Liepmann, and A. Roshko, Elements of Gas Dynamics, London, U. K.: Wiley, 1956.

[6] S. Mölder, "Curved shock theory," Shock Waves J., vol. 26, pp. 337-353, 2016. doi: 10.1007/s00193-015-0589-9.

[7] G. Emanuel, Analytical Fluid Dynamics, 3rd ed., Boca Raton, FL: CRC Press, 2016.

[8] S. Mölder, "Curved Aerodynamic Shock Waves," Ph.D. Thesis, McGill University, Montreal, QC, 2012.

[9] Masterix (2003-2015), Version 3.40, RBT Consultants, Toronto, Ontario. 


\title{
An Experimental and Theoretical Investigation into the Flow Through Air Box of a Potato-rock Separator
}

\author{
Jacob Bobby Sagayaraj \\ Akash Kulshreshtha \\ Prabir Basu \\ Mechanical Engineering Department, Dalhousie University, Halifax \\ Lyle Gauthier \\ Allan Equipment, York, Prince Edward Island
}

\begin{abstract}
-
Velocity and pressure distribution in the air duct system and that over the grate of a potato-rock separator of Alan Equipment, PEI was analysed using both experimental measurements and computational fluid dynamics analysis with appropriate boundary conditions To calibrate the CFD simulations the velocity, pressure and flow rates were measured on the full-scale unit of the potato-rock separator. The effect of duct geometry on the velocity distribution through the grate was established through this exercise. Finally one design was implemented in the full scale unit. Velocity distribution obtained through this modification was checked through experimental measurements.
\end{abstract}

\section{INTRODUCTION}

Potato harvesting is a major agricultural activity of Prince Edward Island, where the need for faster yet efficient means of harvesting is of growing importance. Potatoes are harvested from the field by large machineries. Such mechanical means of digging potato from the ground picks up from the field lots of undesired stuff like rocks vines and dirt along with the potato. Separation of rocks from harvested potato is thus a critical step in this production cycle.

Allan Equipment is developing a new type of machine to separate rocks from potato-rock mixture. The potatoes, rocks, vines are picked up from the field and sent into a conveyor. This mixture potatoes and rocks along with the vines it falls onto a 'wave-bed' below which an air duct system is present (Figure 1). A centrifugal fan feeds air into the air plenum. The separation of potatoes from rock above the wave bed is dependent on various factors like shape and density of rock particles. The separation is achieved by a high flow of air, resulting in floatation of the potatoes. This high flow of air results in the levitation of potatoes and eventual removal through the exit because of the wave action of the wave of the wave bed. The inclined angle of the wave bed makes the rock exit the separator with the help of pinch rollers. Uniform distribution of air over the large area of its plenum of critical importance for proper operation of this potato-rock separator.

A radial fan, mounted at the inlet of the duct system provides the air to the air plenum. The fan, designed for 17,200 ACFM $\left(29222 \mathrm{~m}^{3} / \mathrm{h}\right)$ and a static pressure 9.8 inches of water $(2439 \mathrm{~Pa})$, is driven by a $38.9 \mathrm{HP}$ motor. This particular model of fan is chosen as it is being used in most of harvesting machines at Allan equipment. This high flow fan provides enough air to levitate lighter potatoes above the wave bed. As the potatoes are levitated, the heavier rocks stay at the bottom due to its higher density. The wave action of the belt facilitates the separation of the rock at the side with help of pinch rollers. The fan is capable of functioning at different speeds with the help of programmable logic controller and a variable frequency drive. The research was carried out at an $80 \%$ load, which is the maximum load used when the harvester is used in the field.

In addition to the main fan, there is also a secondary fan (back fan) which is connected to a rear duct that sends air through ducts and into the pinch rollers present at the rock removal section. This back fan ensures that the potatoes do not come out of the pinch rollers when the separation through levitation takes place.

The Wave-bed is a unique mechanism which is developed by Allan Equipment that generates a wave motion through a cam mechanism whose speed is also controlled. The belt is made up of steel rods coated with rubber which is thinly spaced. The portion below the belt consists of disks attached to two individual rods which move along an elliptical track and meet the wave bed along its width. This produces the wave like action on the bed

Difficulty in separation was observed due to the uneven air flow distribution above the wave bed, which resulted in the clogging of potatoes in certain regions. This led to inefficient separation of the potatoes from rocks. Thus uniform flow distribution in the air plenum below the wave bed is a primary requirement of this system.

The present research was made to achieve even air flow by using theoretical and experimental means

\section{EXPERIMENTAL}

A series of experiments were conducted in a full-scale commercial rock separator developed and used by Allan Equipment of Prince Edward Island. Primary objective of these experiments was to calibrate results of theoretical analysis and then check the results of some design improvements attempted to meet the requirements of this machine. 


\subsection{Air flow measurement above the wave bed}

The flow region above the wave bed was analysed both theoretically and experimentally. Results of the theoretical analysis is discussed in section 3.1. Present section discusses the experimental results.

The purpose of this test was to understand the behavioural pattern of air flow above the wave bed. The experiment was done by placing a Pitot tube above the wave bed and measuring the velocity and pressure by connecting the pitot tube to a digital manometer. The velocity corresponding to dynamic and static pressures at each measuring location was then calculated. The measurement was done by moving the pitot tube along the length of the air plenum exit above the wave bed and recording the readings every six inches $(152 \mathrm{~mm})$ apart. The wave bed is shown in Fig 1.

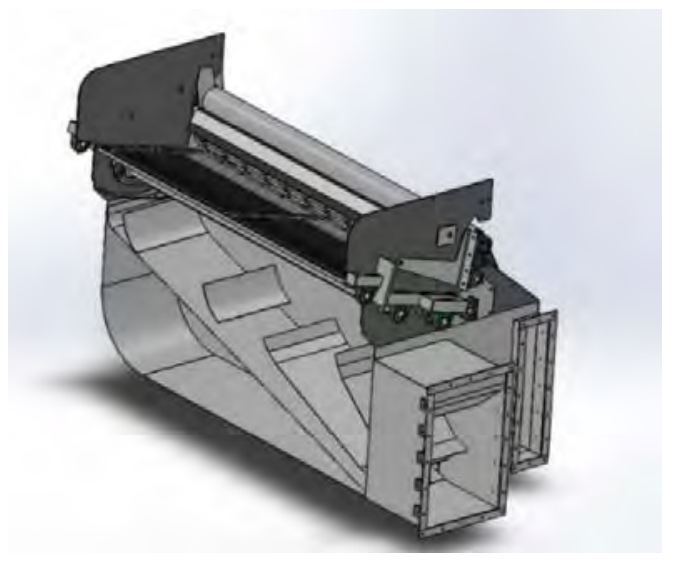

Fig 1 Design of the air plenum with baffles and wave bed

\subsection{Introduction of a Perforated plate}

Several attempts were made earlier to bring in flow uniformity by installing varied designs of flow straighteners in the air box, but they did not give the desired result. So, to achieve uniform air flow at the exit of the air plenum, a perforated sheet was introduced between the wave bed and the airbox exit. The velocity-pressure at the exit of the wave bed was measured with the help of a pitot tube and a manometer. The initially used perforated plate was made up of $1 / 4$ inch $(6.35 \mathrm{~mm})$ holes equally spaced 1 inch apart throughout the entire area of 66x12 inches (1676 mm x 305 $\mathrm{mm})$.

\subsection{Adjustment of hole size in perforated plate}

The velocity of the air escaping through the wave bed improved after the introduction of the perforated plate but it still appeared to uneven. So, an increase in the hole size in specific sections of low velocity was explored. The perforated plate was subdivided into 4 sections. These 4 sections were made into perforations of different sizes. (Fig 2)

\subsubsection{Layout 1}

This design option (layout 1) consists of 4 sections in which section 1 has $3 / 8$ inch $(9.52 \mathrm{~mm})$ holes, the centre region has a combination of alternate $1 / 4$ inch $(6.35 \mathrm{~mm})$ holes and $3 / 8$ inch $(9.52 \mathrm{~mm})$ holes and the section 3 has $1 / 2$ inch $(12.7 \mathrm{~mm})$ holes. There is an entire section $(254 \mathrm{~mm} \mathrm{x} 1676$ $\mathrm{mm})$ consisting of $1 / 4$ inch $(6.35 \mathrm{~mm})$ holes.

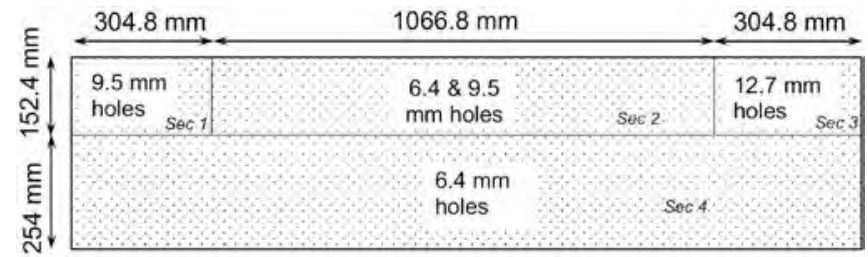

Fig 2 Layout 1 of the perforated plate

\subsection{Removal of the wave bed}

To allow access for measuring the pressure difference across the perforated plate the wave bed had to be removed from the air plenum. The testing was done along the plate in the near end (closest to air inlet), middle and far end. The far end is the inlet of the potatoes. The perforated plate had the layout 1 sized holes (Fig 3).

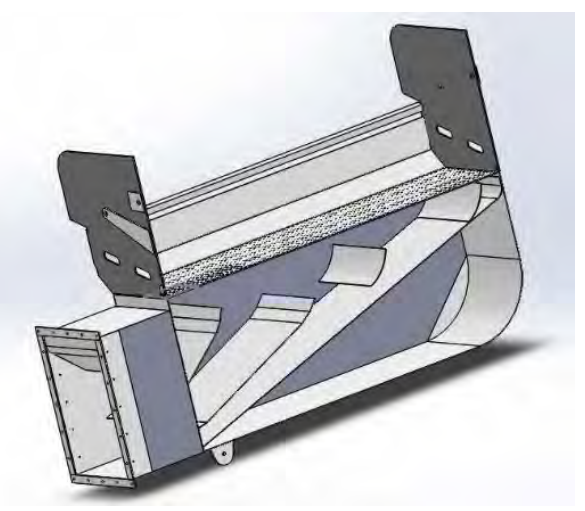

Fig 3 Perforated plate above the baffles with the wave bed removed

\subsection{Removal of the Baffles}

The desired uniformity of velocity distribution was achieved by the perforated plate. So, the role of air box baffles or flow straighteners was limited. So, they were removed to examine its impact. Experiment was carried out again after the baffles were removed (Fig 4). The layout of the perforation was the same as in Layout 1. (Fig 3)

The test was carried out in three regions; the near (To air entry) end, middle and far end. The Pitot tube was traversed across the perforated plate and the velocity pressure was measured.

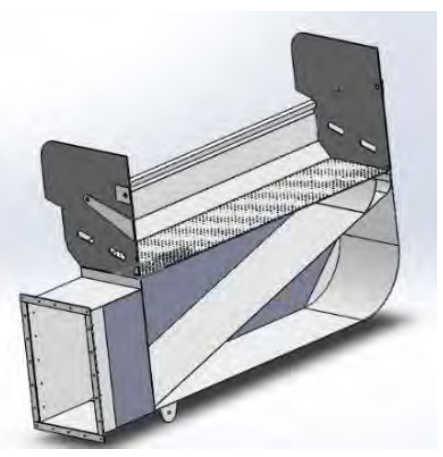

Fig 4 Perforated plate with both the baffles and wave bed removed

\subsubsection{Factional opening for layout 1}

The fractional opening is important to understand the overall porosity of the perforated plate. This is calculated by dividing the total hole area by the total area. 
Table 1 Layout 1

\begin{tabular}{|l|c|c|c|}
\hline \multicolumn{4}{|c|}{ Factional opening calculation } \\
\hline & $\begin{array}{c}\text { Nos of } \\
\text { holes }\end{array}$ & $\begin{array}{c}\text { Hole size } \\
(\mathrm{mm})\end{array}$ & Area $\left(\mathrm{mm}^{2}\right)$ \\
\hline Section 1 & 72 & 9.52 & 5127 \\
\hline Section 2 & 252 & 7.93 & 12467 \\
\hline Section 3 & 72 & 12.7 & 9124 \\
\hline Section 4 & 680 & 6.35 & 21543 \\
\hline Total void area & 48262 & $\mathrm{~mm}^{2}$ \\
\hline Plate Length & 1727 & $\mathrm{~mm}$ \\
\hline Plate Width & 406 & $\mathrm{~mm}^{2}$ \\
\hline Total Area & 701162 & $\mathrm{~mm}^{2}$ \\
\hline Total solid area & 652899 & $\mathrm{~mm}^{2}$ \\
\hline \multicolumn{4}{|l|}{} \\
\hline Fractional opening & $\mathbf{0 6 8}$ \\
\hline
\end{tabular}

\subsection{Increase in perforated plate hole size (Layout 2)}

Introduction of the perforated plate in air plenum brought about grater uniformity of velocity across the plenum but it significantly reduce the overall air flow rate into the plenum. This happened due to the additional flow resistance that the perforated plate imposed on the centrifugal fan use. To increase the flow rate of air above the perforated plate, the hole size was increased further without changing the hole pattern. The hole size was increased to $40 \%$ of the layout 1 . The test was carried out in the presence of a flowmeter to measure the flow rate of air through the fan suction inlet. The flow rate through the fan had reduced much from the previous test, carried out under similar fan load conditions. This reduction in flow rate could be attributed to the high resistance in new venturi type flow meter used at the entrance of the fan.

The layout of the perforation is shown in Fig 5

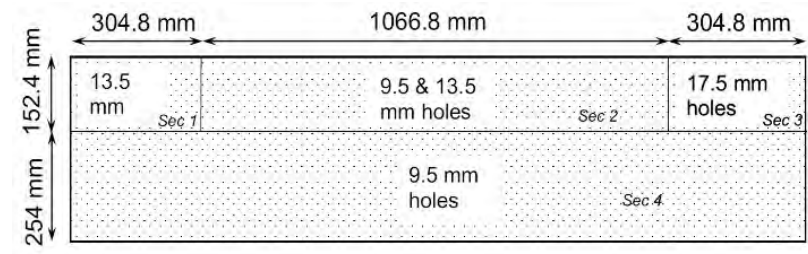

Fig 5 Layout 2

\subsubsection{Fractional opening for layout 2}

The fractional opening of the perforated plate was calculated. This calculation is important to make sure that the hole size is not increased too much.

\begin{tabular}{|l|c|c|c|}
\hline & $\begin{array}{l}\text { Nos of } \\
\text { holes }\end{array}$ & $\begin{array}{l}\text { Hole } \\
\text { size }(\mathrm{mm})\end{array}$ & Area $\left(\mathrm{mm}^{2}\right)$ \\
\hline Section 1 & 72 & 13.5 & 10294 \\
\hline Section 2 & 252 & 11.5 & 26219 \\
\hline Section 3 & 72 & 17.4 & 17249 \\
\hline Section 4 & 680 & 9.5 & 48473 \\
\hline Total void area & 102237 & $\mathrm{~mm}^{2}$ \\
\hline Length & 1727 & $\mathrm{~mm}$ \\
\hline Width & 406 & $\mathrm{~mm}$ \\
\hline Total Area & 701162 & $\mathrm{~mm}^{2}$ \\
\hline Total solid area & 598924 & $\mathrm{~mm}^{2}$ \\
\hline \multicolumn{4}{|c|}{$\mathbf{0 . 1 4 5}$} \\
\hline Fractional opening &
\end{tabular}

\subsection{Attaching the wave bed}

When potatoes are fed through the conveyor, they fall directly onto the wave bed shown in Fig 1. This wave bed is placed few inches above the perforated plate. So, it is desirable that we measure the velocities above the wave bed and make needed changes to the hole size in the perforated plate and thereby ensure that the flow is even in the region above the wave bed. The wave bed is mounted at an inclination and bands of different sized rubbers wound upon the bars in the wave bed. This design of different size rubbers bands made more air flow at the rear end, compared to that near end. The rear end of the wave bed is where the separation of the rocks takes place with the help of the pinch rollers. This region requires much more air compared to that near end. The movement of the potatoes to the outlet is mostly due the wave-like action of the bed, assisted by the slight bubbling provided by air.

\subsubsection{Layout 3}

The layout of the perforated plate was changed based on preliminary experiment. This change in design was to provide more air flow towards the rear end and provide an even flow of air, so that there is no clogging up of clods in the regions above the wave bed. in Fig 6

The layout of the modified perforated plate is shown

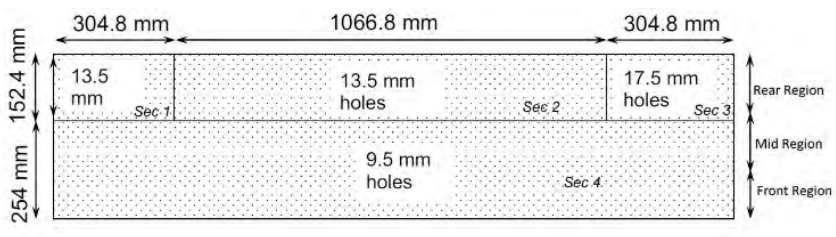

Fig 6 Layout 3

\subsubsection{Opening fraction for layout 3}

The opening fraction calculation was done for Layout 3 to make sure that the overall opening (or)Voidage does not exceed the nominal range of $20 \%$. 
Table 3. Increase in Voidage by $20 \%$

\begin{tabular}{|l|c|c|c|}
\hline & $\begin{array}{l}\text { Nos of } \\
\text { holes }\end{array}$ & $\begin{array}{l}\text { Hole size } \\
(\mathrm{mm})\end{array}$ & $\begin{array}{l}\text { Area } \\
\left(\mathrm{mm}^{2}\right)\end{array}$ \\
\hline Section 1 & 72 & 13.49 & 10295 \\
\hline section 2 & 252 & 13.49 & 36032 \\
\hline section 3 & 72 & 17.46 & 17250 \\
\hline section 4 & 680 & 9.52 & 48473 \\
\hline Total void area & 112050 & $\mathrm{~mm} 2$ \\
\hline Length & 1727 & $\mathrm{~mm}$ \\
\hline Width & 406 & $\mathrm{~mm}$ \\
\hline Total Area & 701162 & $\mathrm{~mm} 2$ \\
\hline Total solid area & 589111 & $\mathrm{~mm} 2$ \\
\hline \multicolumn{4}{|l|}{0.16} \\
\hline Opening fraction & \\
\hline
\end{tabular}

\subsection{Removing the flow meter}

The velocity measurements were carried on the Layout 5 design without the flow meter at fan inlet. The isolation of the flow meter from the suction section of the fan is to record the velocity at which the harvester will function under ideal load conditions in the field.

\section{RESULTS AND DISCUSSION}

The velocities at region above the wave bed was computed using the commercial CFD code of COMSOL whose results are shown in Fig 7. These results were compared with those measured on the full scale unit. Figure 5 shows a reasonably good correlation between the flow predicted by CFD simulation and that measured. This verified validity of the present simulation.

\subsection{Results from COMSOL CFD}

The simulation was also used to find out the best possible design configuration by using appropriate boundary condition. These boundary conditions were obtained by keeping the fan setting at initial load conditions and measuring the value of velocity with air exits from the centrifugal fan. Thus, the need for hardware changes in the rock separator was avoided, thereby saving usage of raw materials and man power.

For simulation, the initial boundary conditions and the turbulence condition must be specified for the k- $\varepsilon$ model. Parameters like the turbulent intensity and turbulent length scale can be determined with the help of the turbulent kinetic energy [1]. The modelling method employed was the RANS turbulence flow model. The parameters were chosen based on previous studies, showing the difference in computational cost when switching between different models for CFD analysis [2]. The figures shown here are for a few conditions in which the simulations results matched the physically conducted experiments.

Figure 7 shows the region above the bed when the baffles, the wave bed and the perforated plate are not present. This condition shows that without any resistance and the fan attached at the inlet, the velocity with which air exits the bed is concentrated at the back and not much at the fan inlet side. This design shows uneven air flow and hence there will be inefficient separation of potato. This simulation was, however, run at a lower velocity of $33 \mathrm{~m} / \mathrm{s}$ as opposed to the nominal velocity with which the fan operates.

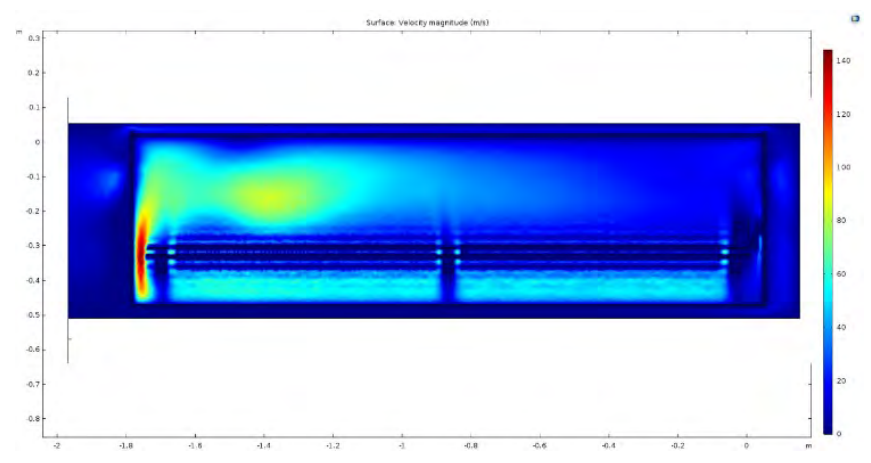

Fig 7 Velocity distribution above the air chamber with no perforated plate $\&$ baffles

The simulation was carried out with both baffles and the wave bed but without perforated plate, Fig 8 . Based on the fan setting at $80 \%$, the velocity of air coming through the inlet was kept at $43 \mathrm{~m} / \mathrm{s}$. Since there is no perforated plate, the velocity is high as indicated in the velocity scale. This pattern shows that in the centre region of the rear end, there is slight dip in the velocity.

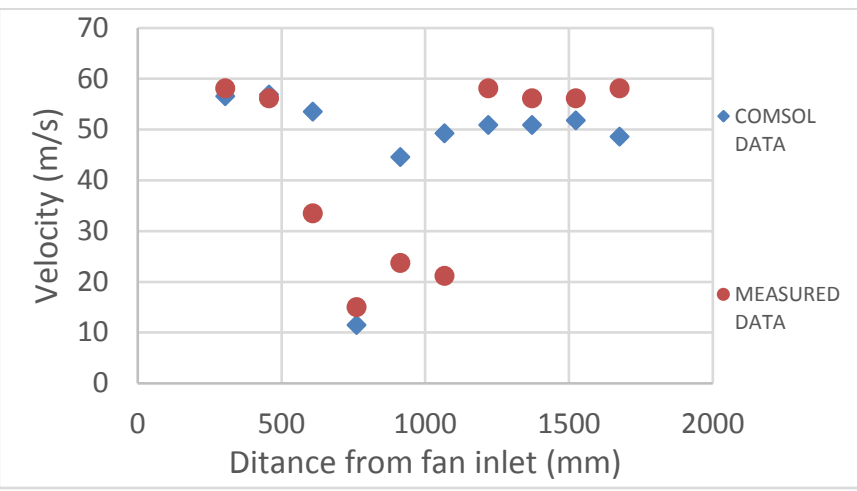

Fig 8 Simulated and experimental values of velocity above the wave bed with baffles and without the plate.

A simulation was run for the layout 3 and this is compared with the experimental results in Fig 9. which shows that the CFD result obtained, closely resembles and matches with the measured values at the rear end. The velocity pattern is shown in Fig 11. The analysis was carried out in the region above the perforated plate and hence the simulation's velocity profile is obtained at the region right above the perforated plate. 


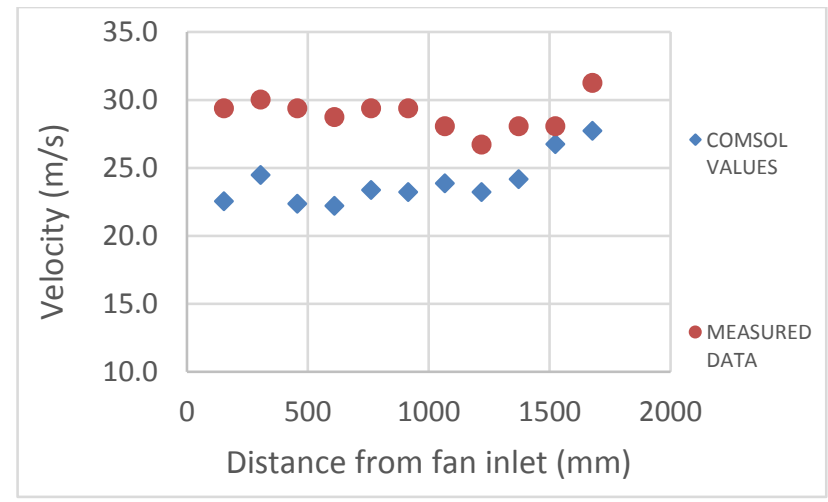

Fig 9 Simulated and experimental velocity above the perforated plate without the baffles

\subsection{Distance vs velocity for different conditions}

Case 1: Above wave bed-with baffles-without perforatedwithout flow meter-layout 1

The purpose of this test was to understand the behavioural pattern of air flow above the wave bed region in the air bed. The testing was done by placing a Pitot tube above the region and measuring the velocity pressure by connecting the Pitot tube to a digital manometer. The measurement was done by moving the instrument along the air plenum exit above the wave bed and recording the readings every six inches. The measurement was done only at the rear end. In this experiment the baffles were present inside the air chamber, as shown in Fig 1.

From the readings obtained it can be inferred that the air velocity above the wave bed was not uniform. The centre region of the air plenum appears to have a significantly low velocity, shown in Fig 10. COMSOL simulation was run with the required boundary conditions, and the obtained simulation result was close to the experimental result, as shown in Fig 8.

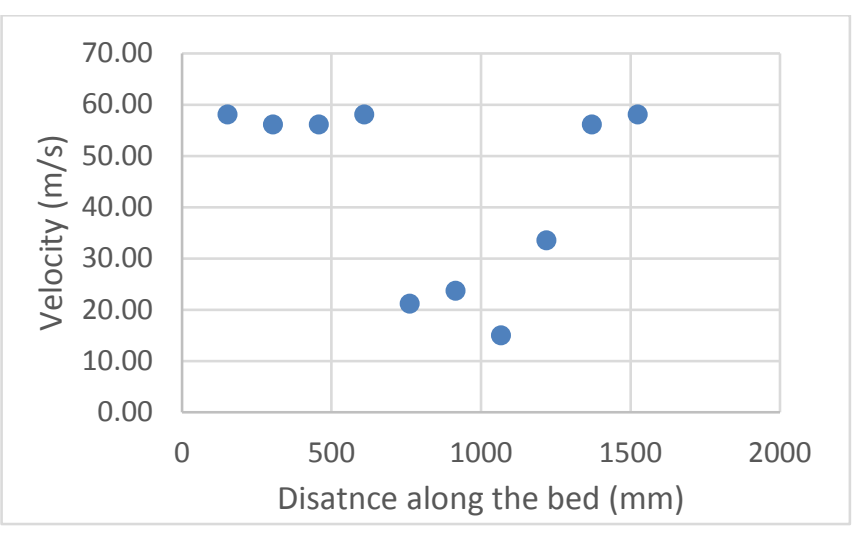

Fig 10 Experimental values of velocity plotted against distance from fan end of wave bed - case 1

Case 2: Flow above wave bed-with baffles-with perforatedwithout flow meter-layout 1

When the perforated plate of uniform holes was introduced in the air chamber, the flow was uniform in few regions but not entirely. This was influenced by the design of the wave bed. After conducting initial testing on the air chamber, a change in design of the perforated plate was required. The initial stage of testing was carried out on the updated design shown in Fig 11. The region above the wave bed tested to find out the velocity pressure using a pitot tube. The pitot tube was moved along the wave bed and the velocity pressure was measured.

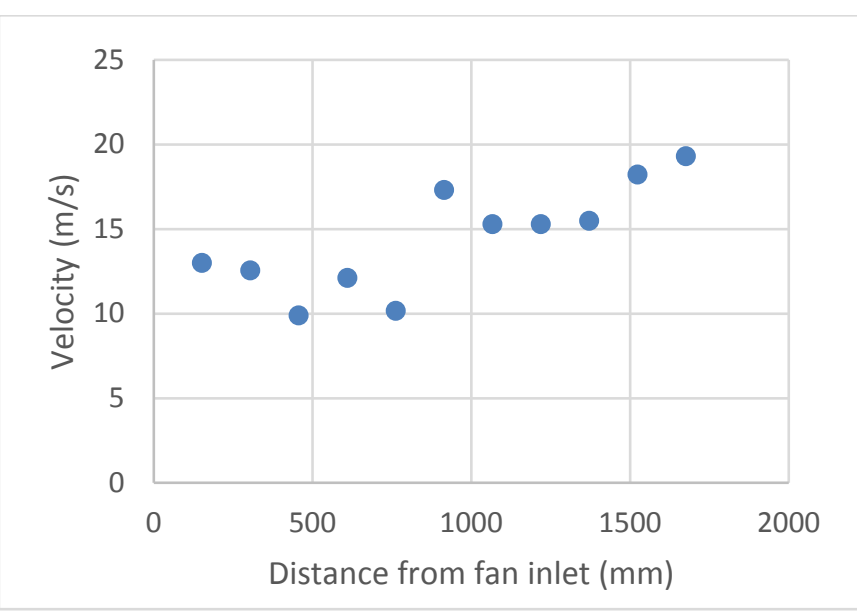

Fig 11 Measured values of velocity plotted against distance from fan end curve - case 2

Case 3: flow above perforated-without baffles and wave bed but with flow meter -Layout 1

A duct flowmeter of 12 inch $(25.4 \mathrm{~mm})$ diameter was attached at the suction section of the centrifugal fan and the flow rate was found to be $4834 \mathrm{CFM}\left(8213 \mathrm{~m}^{3} / \mathrm{h}\right)$ and $5.2 \mathrm{inch}$ of water $(1294 \mathrm{~Pa})$, which reduced considerably from the original value of 17,200 ACFM $\left(29223 \mathrm{~m}^{3} / \mathrm{h}\right)$.

The test was carried out again after removal of the baffles inside the airbox. The pitot tube measurements were taken at a height above the perforated plate and at distances along the place. A more uniform velocity distribution was noted after removal of the wave bed and baffles. The test was carried out in three regions: the near end, middle and far end. The pitot tube was traversed across the perforated plate and the velocity pressure was measured. The pressure difference across the perforated plate was also measured and found to be $1040 \mathrm{~Pa}$.

The velocity was more uniform above the perforated plate compared with that measured above the wave bed (Fig 12). 


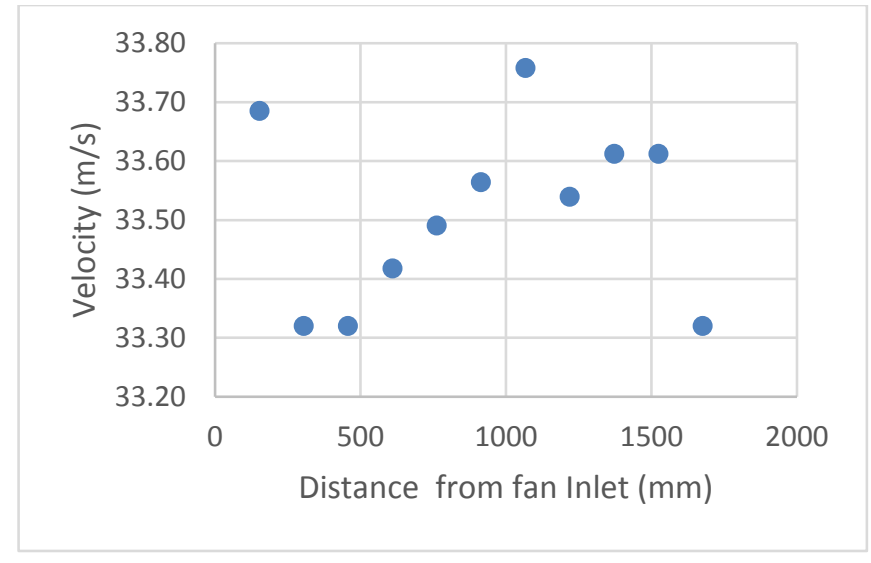

Fig 12 Distance vs Velocity above perforated plate without baffles - case 3

Case 4: Flow above perforated plate without baffles-with flow meter-layout 2

The analysis being focused on improving the flow distribution of air above the perforated plate, the hole size was increased further without changing the hole pattern. The hole size was increased from that of the layout 1 . The bed pressure drop across the perforated plate was $600 \mathrm{~Pa}$. The test was carried out in the presence of a flow meter installed at suction inlet of the fan. It measured the total flow rate of air through the fan. The flow rate through the fan had drastically reduced from the previous test, carried out under similar fan load conditions but without the flow meter. The fan was operated at the default load setting and the flow rate was measured to be 4834 CFM $\left(8213 \mathrm{~m}^{3} / \mathrm{h}\right)$. This reduction in flow rate could be attributed to the high pressure drop of 5.2 inch WG (1294 $\mathrm{Pa}$ ) across the flowmeter at fan inlet.

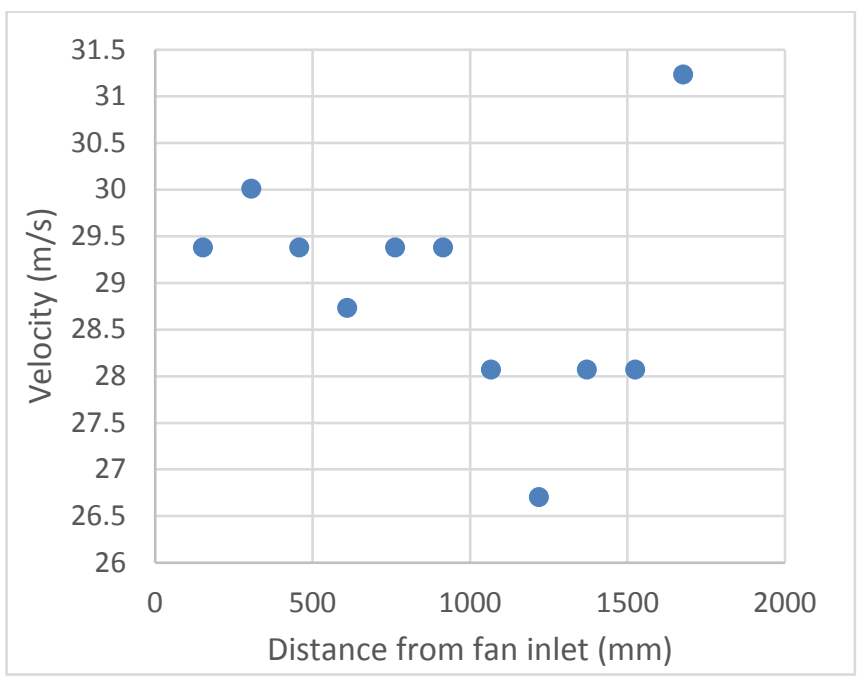

Fig 13 Distance vs Velocity above perforated plate without baffles - case 4
Case 5: Measurements above wave bed-without baffleswithout flowmeter- Layout 3

Velocity measurements were carried on the Layout 3 design without the presence of the duct flowmeter. The flowmeter was removed from the suction of the fan to measure the flowrate the harvester will have under actual operating conditions in the field. This showed no change in velocity distribution pattern but the magnitude of the velocity had increased. Some drop in air velocity after passage through the wave bed is noted.

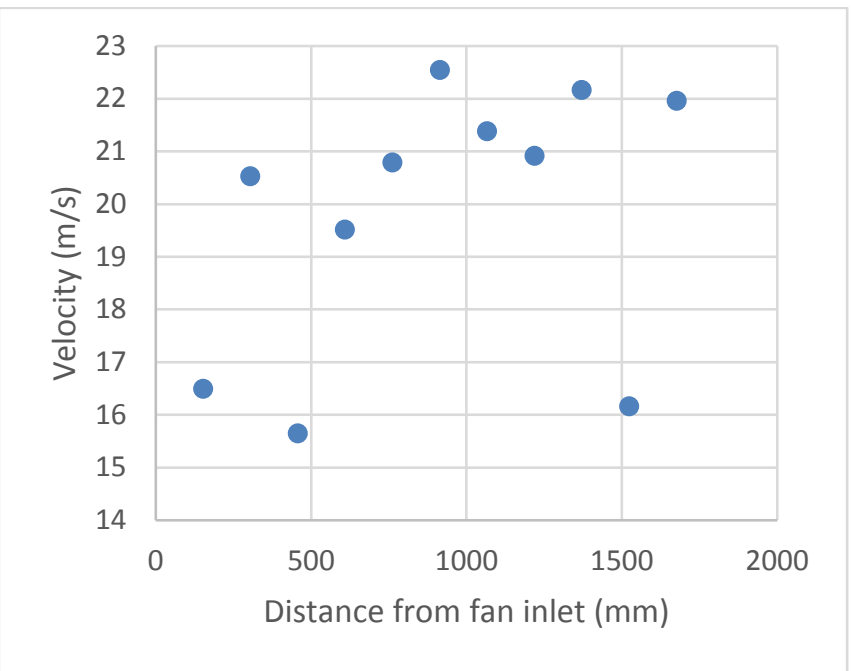

Fig 14 Distance vs Velocity above wave bed without flow meter- case 5

\section{CONCLUSION AND RECOMMENDATION}

CFD simulations were successfully utilized to analyse the air flow through plenum of a commercial potatorock separator. Predicted values of velocity distribution around the air distribution chamber was validated against values measured on a full scale model.

The Simulation model was used to predict flow distribution of several design configurations, but none gave the desired improvement.

To obtain the desired flow pattern a perforated plate was inserted above the plenum to get uniform flow distribution the size and percentage open areas of the perforated plate was varied and resulting air velocity distribution was checked through measurement.

\section{REFERENCES}

[1] A. E. Byl, "Characterization of airflow through an air handling unit using computational fluid dynamics," M.Sc., Dept. Mec. Eng., Mondatna State University, Bozeman, USA, 2015. [Online]. Available: https://scholarworks.montana.edu/xmlui/handle/1/9429.

[2] A. Kayne, "Computational Fluid Dynamics (CFD) Modeling of Mixed Convection Flows in Building Enclosures," M.A. dissertation, Dept. of Eng. And Mat. Sci., Washington University in St. Louis, USA, 2012. [Online] doi: $10.7936 /$ K7X928CQ. 
MECHATRONICS,

ROBOTICS \& CONTROL 


\section{Effect of Photoacoustic Radar Chirp Parameters on Signal to Noise Ratio}

\author{
Zuwen Sun \\ Department of Mechanical Engineering \\ University of Ottawa \\ Ottawa, Canada \\ zsun046@uottawa.ca
}

\author{
Natalie Baddour \\ Department of Mechanical Engineering \\ University of Ottawa \\ Ottawa, Canada \\ nbaddour@uottawa.ca
}

\begin{abstract}
Continuous wave photoacoustics has been under investigation for more than a decade. Matched-filtering and pulse compression techniques, which use a chirp waveform as input light source, have been implemented into this imaging modality to improve signal to noise ratio (SNR) and resolution. However, the chirp parameters' effects on SNR are still not clear. The theory behind chirp parameters' effects on SNR is discussed in this paper. It is found that in order to get a higher SNR, chirp duration $T$ should be maximized, chirp center frequency $f_{0}$ should be controlled to make the chirp spectrum overlap with absorber spectrum, and chirp bandwidth $\Delta$ should be smaller or equal to the absorber bandwidth.
\end{abstract}

Keywords-Continuous wave photoacoustics, SNR, chirp

\section{INTRODUCTION}

Photoacoustic imaging, also called optoacoustic imaging, for biomedical applications has attracted a lot of interest during the past several decades. The principle of this imaging method can be found in recent work [1], [2].

The most common excitation source for photoacoustics has been a pulsed electromagnetic wave [3]-[5]. The key advantage of using a short pulse to irradiate the tissue is that the distribution of heat sources can be directly ascertained from the shape of the photoacoustic response signal [6]. However, the pulsed laser modality is limited by incident energy levels that must meet safety standards for in-vivo tissue imaging [1], as well as the difficulty in generating and detecting very short pulses.

An alternative excitation modality, which uses a continuous incident laser wave, is often referred to as Frequency Domain photoacoustics (FD-PA) where the acoustic wave is generated by periodic modulation of a laser [1], [2], [7]-[11]. More recently, the idea of implementing a pulse compression approach via matched filtering was introduced and investigated [2], [7]-[10], [12]-[14]. This is sometimes referred to as Photoacoustic Radar (PAR). The matched filter approach enables detection of a pre-known signal immersed in Gaussian white noise, therefore a long duration coded waveform with moderate power could potentially replace short high-power pulses.
Signal to Noise Ratio (SNR) is one important aspect to assess the performance of an imaging system. Many theoretical and experimental investigations have been done on the SNR of FD-PA [2], [8], [10], [12]. However, the theory behind optimizing input optical waveform parameters is still not clear. In this paper, the one-dimensional theory of FD-PA will be developed, and the incident wave parameters' effects on SNR will be discussed.

\section{1-D THEORY OF CONTINUOUS WAVE PHOTOACOUSTICS SIGNAL TO NOISE RATIO}

Diebold [15] gives a concise explanation of the governing equation for the pressure that results from launching a photoacoustic wave. The governing equation is given by

$$
\nabla^{2}-\frac{1}{c_{s}^{2}} \frac{2}{t^{2}} \mid p(\vec{r}, t)=-\frac{p_{0}}{c^{2}} A(\vec{r}) \frac{\partial I(t)}{\partial t}
$$

where $p_{0}=\frac{\beta c_{s}^{2}}{C_{p}} \mu_{a} \quad$ and $\beta$ is the thermal expansion coefficient, $c_{s}$ is the speed of sound, $C_{p}$ is the specific heat, $\mu_{a}$ is the optical absorption coefficient of the chromophore absorber that has been heated by an optical pulse with fluence $F$. $p(\vec{r}, t)$ is the pressure of the acoustic wave, a function of space and time. $A(\vec{r})$ is a function of space that describes the geometry of the absorber and $I(t)$ is a function that describes the time dependence of the incident optical pulse.

In one-dimensional model as shown in Figure 1. a), the space variable $\vec{r}$ becomes $z$. The incident laser waveform $I(t)$ is transmitted from the negative side of $z$ axis, outside of the absorbing medium. The absorber is considered as an infinite sheet with a thickness $l$. The speed of sound $c_{s}$ is considered to be the same in all three layers [16]. The pressure response $p(z, t)$ is then detected by a transducer and be sent 
to a receiver-filter. The output of the receiver-filter $y(z, t)$ is the final photoacoustic signal.
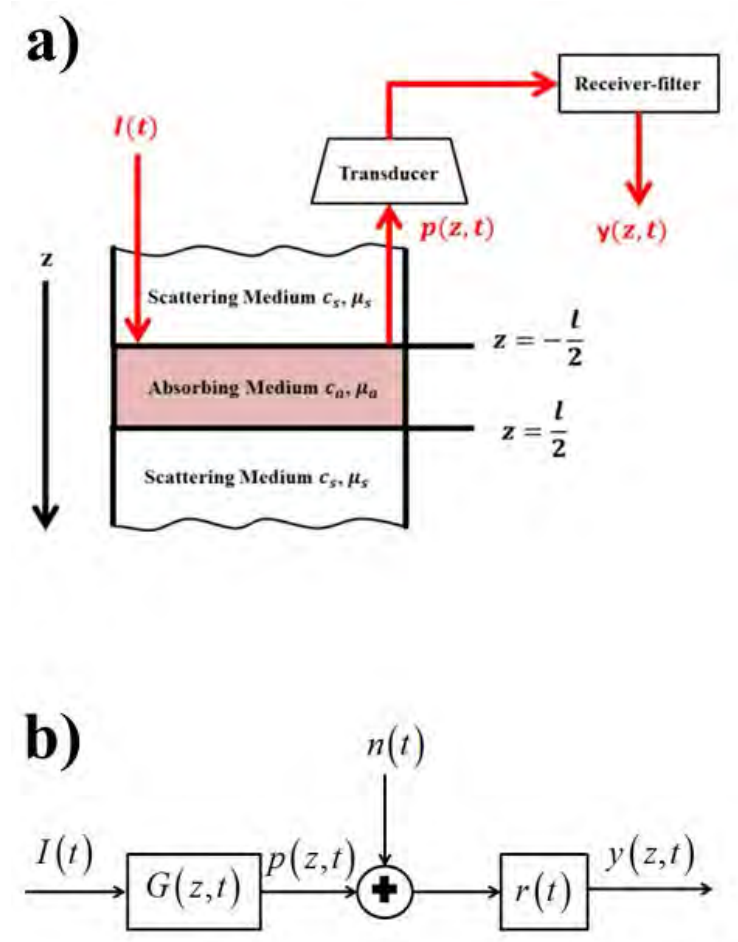

Figure 1. a) Model of absorber and surroundings; b) block diagram

When viewing the system shown in Figure 1. a) as a linear system, as shown in Figure 1. b), the transfer function concept is very useful. $G(z, t)$ in Figure 1. b) denotes the absorber impulse response, and is given by

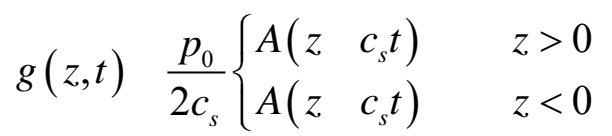

where $A(z)$ is the spatial shape of the absorber, $r(t)$ is the receiver-filter impulse response. The signal received at the receiver-filter also contains noise $n(t)$ which is assumed to be a zero-mean additive Gaussian white noise [17] which is assumed to have a double-sided power spectral density of $\tilde{S}_{n n}(\omega)=\frac{N_{0}}{2}$. The output of receiver-filter $y(z, t)$ contains the photoacoustic signal $y_{s}(z, t)$ and the noise signal $y_{n}(z, t)$ and is given by

$$
y(z, t)=y_{s}(z t)+y(t)
$$

where

$$
\begin{aligned}
y_{s}(z, t) & =r(t) * g(z, t) * I(t) \\
\text { or } \quad \tilde{y}_{s}(z, \omega) & =\tilde{R}(\omega) \tilde{G}(z, \omega) \tilde{I}(\omega)
\end{aligned}
$$

$$
\begin{aligned}
y_{n}(t) & =r(t) * n(t) \\
\text { or } \quad \tilde{y}_{n}(\omega) & =\tilde{R}(\omega) \tilde{S}_{n n}(\omega)
\end{aligned}
$$

Given a chosen waveform/receiver filter pair, the instantaneous signal-to-noise ratio (SNR) at time $t_{0}$ is defined as

$$
S N R=\left(\frac{S}{N}\right)_{t_{0}}=\frac{\left|y_{s}\left(z, t_{0}\right)\right|^{2}}{E\left|y_{n}\left(t_{0}\right)\right|^{2}}
$$

where $E\left|y_{n}\left(t_{0}\right)\right|$ refers to the noise expectation value.

\section{MATCHED-FILTER AND THE CHIRP}

Matched-filtering is a traditional way to improve SNR in radar technology, and the transfer function of the receiver-filter for the ideal matched-filter for any arbitrary input waveform $I(t)$ is given by [17]

$$
\tilde{R}(\omega)=k \tilde{G}^{*}(z, \omega) \tilde{I}(\omega) e^{-i \omega t_{0}}
$$

where $k$ is a scaling constant. However, (7) is not necessarily implementable for a typical photoacoustic system since $\tilde{G}(z, \omega)$ (determined by the absorber profile) is not known apriori. In this case, the receiver-filter is implemented as

$$
\tilde{R}(\quad)=k \tilde{I}^{*}(\omega) e^{-i \omega t_{0}}
$$

Under the condition of (8), (4) becomes

$$
\tilde{y}_{s}(z, \omega)=k \tilde{G}(z, \omega) \underbrace{\tilde{I}(\omega) \tilde{I}^{*}(\omega)}_{\tilde{I}^{S D}(\omega)} e^{-i \omega t_{0}}
$$

In (9), $k$ is simply a scaling factor and the $e^{-i \omega t_{0}}$ is just a time delay. Hence, (9) can be interpreted in input/output form as a pressure response to an input pulse $\tilde{I}^{S D}(\omega)$ where $\tilde{I}^{S D}(\omega)=|\sim()|^{2}$ is the spectral energy density of $I(t)$. The inverse Fourier transform of $|\tilde{I}(\omega)|^{2}$ in time domain is known to be

$$
R_{I I}(t) \int_{-\infty}^{\infty} I(t+\tau) \overline{I(\tau)} d \tau
$$

which is the autocorrelation of $I(t)$. The SNR in (6) can then be then written 


$$
S N R=\frac{\left.\left.\frac{1}{\pi}\left|\int_{-\infty}^{\infty} \tilde{G}(z, \omega)\right| \tilde{I}(\omega)\right|^{2} d \omega\right|^{2}}{N_{0} \int_{-\infty}^{\infty}|\tilde{I}(\omega)|^{2} d \omega}
$$

By implementing matched-filtering, the SNR is improved. However, $R_{I I}(t)$ generally has a longer duration than $I(t)$, which may leads to a worse resolution. However, there exist classes of specific waveforms which possess the property of pulse compression, which means that the waveform will have shorter duration after autocorrelation. The chirp is one such waveform that can be compressed and is given by

$$
I_{T}(t)= \begin{cases}\cos \left(2 \pi f_{0} t+\frac{\pi \Delta t^{2}}{T}\right) & |t|<\frac{T}{2} \\ 0 & |t|>\frac{T}{2}\end{cases}
$$

where $T$ is the duration of the chirp. During the $T$ second interval of the pulse, the instantaneous frequency changes linearly from $\left(f_{0}-K T / 2\right) \mathrm{Hz}$ to $\left(f_{0}+K T / 2\right) \mathrm{Hz}$. The sweep $\Delta$ is the difference in these two values. The $|\tilde{I}(\omega)|^{2}$ of the chirp shown in (11) can be approximated as a rectangular function and is given by [18]

$$
\left|\tilde{I}_{T}(f)\right|^{2} \approx\left\{\begin{array}{lr}
\frac{T}{4 \Delta} & f-\frac{-}{2} \leq f \leq f_{0}+\frac{\Delta}{2} \\
0 & \text { otherwise }
\end{array}\right.
$$

\section{CHIRP PARAMETER EFFECTS ON SNR}

From (11), it becomes clear that the SNR is determined by the absorber frequency spectrum that lies within the frequency interval bounded by the chirp sweep range $f_{0}+\frac{\Delta}{2}$ and $f_{0}-\frac{\Delta}{2}$. Now consider a simple bandlimited absorber.

The bandlimited absorber is considered as a square function in the frequency domain as

$$
\hat{A}(f)= \begin{cases}1 & |f| \leq \frac{\Delta_{a}}{2} \\ 0 & \text { otherwise }\end{cases}
$$

Taking the chirp rectangular approximation shown in (13), the equation of SNR for a bandlimited absorber can be written as

$$
S N R=\frac{T C}{\Delta^{2}} \quad(\text { Overlapping Area })^{2}
$$

where $C=\frac{p_{0}^{2}}{16 \pi N_{0} c_{s}^{2}}$ is a constant factor which is independent of the chirp, and the overlapping area is the shaded area shown in Figure 2. It is obvious from (15) that SNR is proportional to chirp duration $T$.

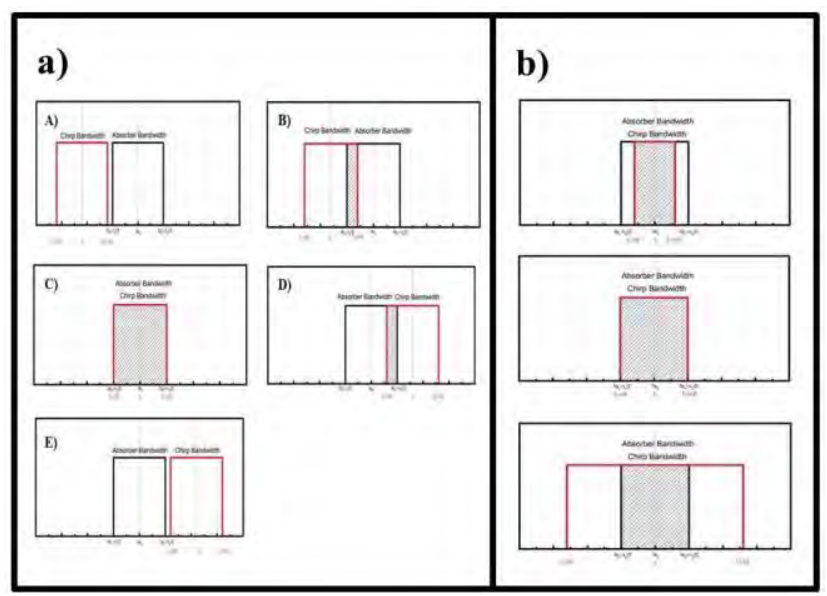

Figure 2. Bandlimited absorber and chirp frequency spectrum

In Figure 2., the black boxes denote the bandlimited absorber frequency spectrum, and the red boxes denote the rectangular approximation of the chirp spectrum. Figure 2. a) shows that the chirp bandwidth $\Delta$ remains constant and the chirp center frequency $f_{0}$ moves from left to right of the absorber. The relationship between SNR and $f_{0}$ is shown in Figure 3. a). The SNR first increases with $f_{0}$ until a maximum value is reached and then decreases, corresponding to the overlapping conditions. Figure 2. b) visualize the overlapping conditions when the chirp center frequency remains constant and bandwidth increases. The trend of the SNR is shown in Figure 3. b). The overall trend is that SNR decreases with increasing chirp bandwidth, and the maximum value occurs when the chirp bandwidth is smaller or equal to the absorber bandwidth, $\Delta \leq \Delta_{a}$. 
a)

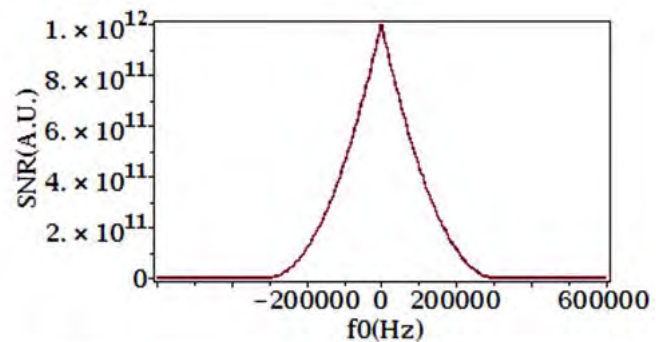

b)

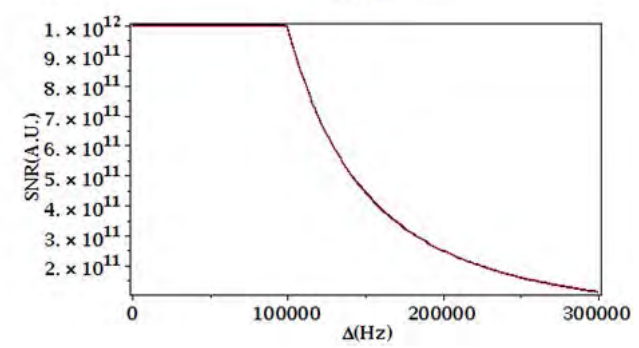

Figure 3. Bandlimited absorber and chirp frequency spectrum

In conclusion, by controlling the chirp parameters, the SNR for a photoacoustic radar signal can be optimized. In order to get a higher SNR, chirp duration $T$ should be maximized, chirp center frequency $f_{0}$ should be controlled to make the chirp spectrum overlap with that of the absorber spectrum, and chirp bandwidth $\Delta$ should be smaller or equal to the absorber bandwidth.

\section{ACKNOWLEDGMENT}

This work was financially supported by the Natural Sciences and Engineering Research Council of Canada.

\section{REFERENCES}

[1] Y. Fan, A. Mandelis, G. Spirou, I. A. Vitkin, and W.M. Whelan, "Laser photothermoacoustic heterodyned lock-in depth profilometry in turbid tissue phantoms," Phys. Rev. E. Stat Nonlin Soft Matter Phys., vol. 72, no. 5, p. 1, 051908, Nov. 2005. doi: 10.1103/PhysRevE.72.051908.

[2] S. Telenkov and A. Mandelis, "Signal-to-noise analysis of biomedical photoacoustic measurements in time and frequency domains," Rev. Sci. Instrum., vol. 81, no. 12, p. 124901, Dec. 2010. doi: 10.1063/1.3505113.

[3] G. Ku and L. V. Wang, "Scanning thermoacoustic tomography in biological tissue," Med. Phys., vol. 27, no. 5, pp. 1195-1202, May 2000. doi: $10.1118 / 1.598984$.

[4] M. Xu and L. V. Wang, "Time-domain reconstruction for thermoacoustic tomography in a spherical geometry," Med. Imaging IEEE Trans. On., vol. 21, no. 7, pp. 814-822, 2002.

doi: 10.1109/TMI.2002.801176.

[5] M. Xu, Y. Xu, and L. V. Wang, "Time-domain reconstruction algorithms and numerical simulations for thermoacoustic tomography in various geometries," IEEE Trans. Biomed. Eng., vol. 50, no. 9, pp. 10861099, Sep. 2003. doi: 10.1109/TBME.2003.816081.

[6] A. A. Karabutov, N. B. Podymova, and V. S. Letokhov, "Time-resolved optoacoustic measurement of absorption of light by inhomogeneous media," Appl.Opt., vol. 34, no. 9, pp. 1484-1487, Mar. 1995. doi: 10.1364/AO.34.001484.
[7] S. A. Telenkov and A. Mandelis, "Photothermoacoustic imaging of biological tissues: maximum depth characterization comparison of time and frequency-domain measurements," J. Biomed. Opt., vol. 14, no. 4, pp. 044025, 2009. doi: 10.1117/1.3200924.

[8] B. Lashkari and A. Mandelis, "Linear frequency modulation photoacoustic radar: Optimal bandwidth and signal-to-noise ratio for frequency-domain imaging of turbid media," J. Acoust. Soc. Am., vol. 130, no. 3, pp. 1313-1324, Sep. 2011. doi: 10.1121/1.3605290.

[9] B. Lashkari and A. Mandelis, "Comparison between pulsed laser and frequency-domain photoacoustic modalities: Signal-to-noise ratio, contrast, resolution, and maximum depth detectivity," Rev. Sci. Instrum., vol. 82, no. 9, p. 094903, Sep. 2011. doi: 10.1063/1.3632117.

[10] B. Lashkari and A. Mandelis, "Photoacoustic radar imaging signal-tonoise ratio, contrast, and resolution enhancement using nonlinear chirp modulation,” Opt. Lett., vol. 35, no. 10, pp. 1623-1625, May 2010. doi: 10.1364/OL.35.001623.

[11] Y. Fan, A. Mandelis, G. Spirou, I. A. Vitkin and W. Whelan, "Threedimensional photothermoacoustic depth profilometric imaging by use of linear frequency sweep heterodyne method", SPIE vol. 5320, Photons plus Ultrasound: Imaging and Sensing, A. A. Oraevsky and L. V. Wang, Eds., Bellingham WA, 2004, pp. $113-127$.

[12] S. A. Telenkov, R. Alwi, and A. Mandelis, "Photoacoustic correlation signal-to-noise ratio enhancement by coherent averaging and optical waveform optimization," Rev. Sci. Instrum., vol. 84, no. 10, p. 104907, Oct. 2013. doi: $10.1063 / 1.4825034$.

[13] B. Lashkari and A. Mandelis, "Features of the frequency- and timedomain photoacoustic modalities," Int. J. Thermophys., vol. 34, no. 8-9, pp. 1398-1404, 2013. doi: 10.1007/s10765-013-1462-7.

[14] N. Baddour and A. Mandelis, "The effect of acoustic impedance on subsurface absorber geometry reconstruction using $1 \mathrm{D}$ frequency-domain photoacoustics," Photoacoustics, vol. 3, no. 4, pp. 132-142, Dec. 2015. doi: 10.1016/j.pacs.2015.10.001.

[15] Gerald J. Diebold, "Photoacoustic Monopole Radiation," in Photoacoustic Imaging and Spectroscopy, Lihong V. Wang Ed. Boca Raton: CRC Press, 2009, pp. 3-17. doi: 10.1201/9781420059922.

[16] M. Xu and L. V. Wang, "Photoacoustic imaging in biomedicine," Rev. Sci. Instrum., vol. 77, no. 041101, 2006. doi: 10.1063/1.2195024.

[17] J. R. Klauder, A. C. Price, S. Darlington, and W. J. Albersheim, "The Theory and Design of Chirp Radars," Bell Syst. Tech. J., vol. 39, no. 4, pp. 745-808, 1960. doi: 10.1002/j.1538-7305.1960.tb03942.x.

[18] B. Lashkari, "Photoacoustic Imaging Using Chirp Technique: Comparison with Pulsed Laser Photoacoustics," Ph.D dissertation, Dept. Mech. Ind. Engineering, University of Toronto, 2011. 


\section{Bearing Fault Signature Extraction Under Time-varying Speed Conditions via Oscillatory Behavior-based Signal Decomposition}

\author{
Huan Huang \\ Department of Mechanical Engineering \\ University of Ottawa \\ Ottawa, ON, Canada K1N 6N5
}

\author{
Natalie Baddour \\ Department of Mechanical Engineering \\ University of Ottawa \\ Ottawa, ON, Canada K1N 6N5
}

\author{
Ming Liang \\ Department of Mechanical Engineering \\ University of Ottawa \\ Ottawa, ON, Canada K1N 6N5
}

\begin{abstract}
Oscillatory Behavior-based Signal Decomposition (OBSD) is a new technique which decomposes a signal according to oscillatory behavior, instead of frequency bands. It has been used for bearing fault signature extraction under constant speed conditions, where the bearing fault-induced vibration signal can be regarded as a low oscillatory component and the interference can be regarded as a high oscillatory component. However, its effectiveness for bearing fault signature extraction under time-varying speed conditions has not been evaluated. Theoretically, the OBSD is a frequency-independent method and should thus be effective under time-varying speed conditions. In this paper, the performance of the OBSD for bearing fault signature extraction under time-varying speed conditions is examined. The results show that the OBSD can be effectively utilized to extract the bearing fault signature under time-varying speed conditions.
\end{abstract}

Keywords- Bearing fault feature extraction; Time-varying speed; Oscillatory behavior-based signal decomposition

\section{INTRODUCTION}

Bearing fault diagnosis is a useful means to prevent early bearing faults from developing into severe faults, which may lead to machine breakdown. Bearing faults can be detected and diagnosed by observing the Fault Characteristic Frequency $(\mathrm{FCF})$ and its harmonics in the frequency domain of the vibration signal [1]. Each type of fault has a specific FCF, which is proportional to the rotational speed [2]. However, the collected bearing vibration signal is often contaminated by random noise and interferences transmitted from other sources, such as gears. Therefore, bearing fault signature extraction is an important step to insure the accuracy of bearing fault diagnosis.

Band-pass filtering is a commonly used method to extract the bearing fault signature [3]-[5]. The essence of this method is to remove the interference signal by using band-pass filters. However, the effectiveness of band-pass filters can be suppressed if the bearing fault-induced signal and the inference signal have similar frequency features. Additionally, in reality bearings are often operated under time-varying speed conditions which may also reduce the effectiveness of bandpass filters. Therefore, it is necessary to implement frequencyindependent methods to extract bearing fault signatures under time-varying speed conditions.

Oscillatory Behavior-based Signal Decomposition (OBSD) is a newly developed method which can be used to decompose a signal according to oscillatory behavior, instead of frequency bands [6]. It can be implemented to extract the bearing fault signature from the signal contaminated by interferences since the bearing fault-induced signal can be considered as a low oscillatory component and the inference signal can be considered as a high oscillatory component. Its effectiveness for bearing fault signature extraction under constant speed condition has been validated [7]. However, the performance of the OBSD for bearing fault signature extraction under timevarying speed conditions has not been examined. Theoretically, the OBSD should be still effective under time-varying speed conditions since it is frequency-independent.

In this paper, the performance of the OBSD for bearing fault signature extraction under time-varying speed conditions is investigated. Signals collected from experimental apparatus are used to examine the effectiveness of the OBSD.

\section{OSCILLATORY BEHAVIOR-BASED SIGNAL DECOMPOSITION}

The essence of the OBSD method is utilizing two sets of wavelets with two different oscillatory behaviors to estimate a given signal [6]. The signal is decomposed into a low oscillatory component and a high oscillatory component by the OBSD. Therefore, the OBSD method can be used to extract the bearing fault signature from a signal obscured by interferences.

Compared to frequency or scale based methods for interference removal, the OBSD is superior since the signal decomposition is based on oscillatory behavior instead of frequencies. A Q-factor, defined as the ratio of the center 
frequency to the bandwidth of the frequency response, is used to describe the oscillatory behavior of a wavelet [8]. A higher value of the Q-factor indicates a higher level of oscillation. Four wavelets and their frequency spectra are shown in Figure 1. The Q-factors of the waveforms are also given. It can be seen that waveform 1 and waveform 2 have different oscillatory behaviors, however, their frequency spectra share the same center frequency. The same can be observed for waveform 3 and waveform 4 . Under such circumstances, frequency-based band-pass filters would not be able to separate waveforms 1 and 2, nor waveforms 3 and 4. However, they can be separated according to their Q-factors. It is calculated that waveforms 1 and 3 have a Q-factor of 1 since they exhibit low oscillatory behavior, and waveforms 2 and 4 have a Q-factor of 5 since they have relatively high oscillatory behavior. Additionally, this demonstrates that waveforms that have the same oscillatory behavior have the same the Q-factor, even if the center frequencies are different. This makes the OBSD effective for capturing the true features that are useful for bearing fault signature extraction under time-varying speed conditions.
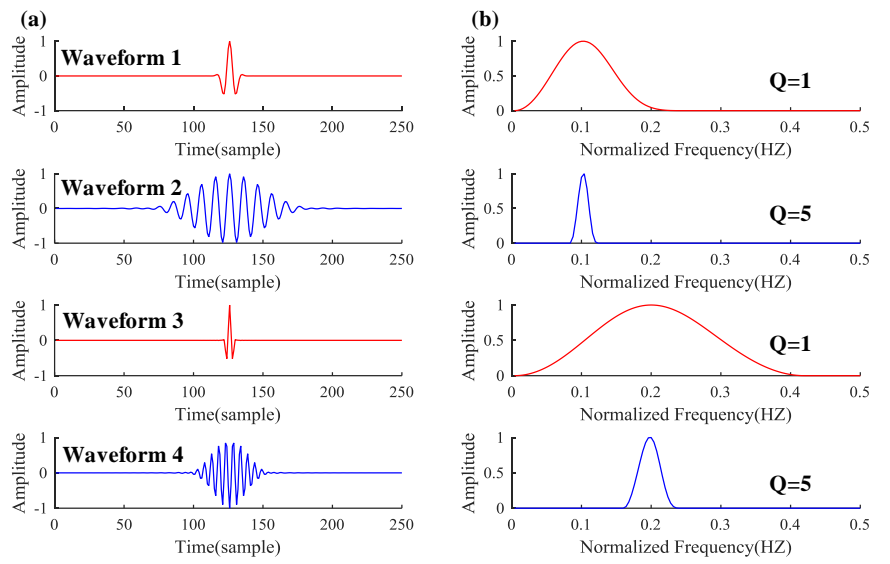

Figure 1 Wavelets and their frequency spectra

The OBSD method employs the Tunable Q-factor Wavelet Transform (TQWT) and Morphological Component Analysis (MCA) to realize the signal decomposition [6]. The TQWT is used to generate a set of wavelets that have the same Q-factor, i.e. the same oscillatory behavior. The wavelets can be obtained with the selection of three parameters, $Q$ (Q-factor), $r$ and $P$, where $Q$ is related to the oscillatory behavior, $r$ is related to the redundancy of the frequency responses, and $P$ is the number of wavelets. By setting up two sets of wavelets, one with low oscillatory behavior with parameters $Q_{l}, r_{l}$, and $P_{l}$, and the other set with high oscillatory behavior with parameters $Q_{h}, r_{h}$, and $P_{h}$, then the signal decomposition can be completed by MCA. The wavelet coefficients are obtained via optimization [6] as

$$
\left[\begin{array}{l}
\mathbf{w}_{h}^{\text {opt }} \\
\mathbf{w}_{h}^{\text {opt }}
\end{array}=\underset{\mathbf{w}_{l}, \mathbf{w}}{\arg \min }\left\|\mathbf{y}-\mathbf{S}_{l} \mathbf{w}_{l}-\mathbf{S}_{h} \mathbf{w}_{h}\right\|_{2}^{2}+\lambda_{l}\left\|\mathbf{w}_{l}\right\|_{1}+\lambda_{h}\left\|\mathbf{w}_{h}\right\|_{1}\right.
$$

where $\mathbf{w}_{l}$ refers to wavelet coefficients for the low oscillatory component, $\mathbf{w}_{h}$ stands for the wavelet coefficients for the high oscillatory component, $\mathbf{w}_{l}^{\text {opt }}$ and $\mathbf{w}_{h}^{\text {opt }}$ are results after optimization, $\mathbf{y}$ is the signal to be decomposed, $\mathbf{S}_{l}$ refers to wavelets obtained via the TWQT for the low oscillatory component, $\mathbf{S}_{h}$ represents wavelets for the high oscillatory component, $\lambda_{l}$ is the regularization parameter for the low oscillatory component, $\lambda_{h}$ is the regularization parameter for the high oscillatory component, and \|\|$_{1}$ and \|\|$_{2}$ are the norm1 operation and the norm-2 operation, respectively. This optimization problem can be solved using an iterative algorithm called the Split Augmented Lagrangian Shrinkage Algorithm (SALSA), obtained as [6]

$$
=f\left(\mathbf{w}_{i}^{k-1}, \mu\right), i=l, h, k=1,2, \ldots, K
$$

where $\mu$ is the penalty parameter and $K$ is the maximum number of iterations. Details of the solution can be found in the appendix in [7]. By selecting the maximum number of iterations, the optimal wavelet coefficients are obtained as

$$
\mathbf{w}_{i}^{\text {opt }}=\mathbf{w}_{i}^{K}, i \quad l, h
$$

With the calculated wavelet coefficients, the decomposed low oscillatory and high oscillatory components can then be obtained by inverse TQWT with the optimized wavelet coefficients.

\section{IMPLEMENTATION OF OBSD ON BEARING FAULT FEATURE EXTRACTION UNDER TIME-VARYING SPEED CONDITIONS}

According to the characteristics of the bearing faultinduced signal and the interference signal, the bearing faultinduced signal is more impulsive. which can be considered as low oscillatory behavior, and the interference is smoother which can be considered as high oscillatory behavior [9]. Therefore, the decomposed low oscillatory component and the high oscillatory component via the OBSD are taken to be the bearing fault signature and interference, respectively.

The bearing fault-induced signal can be simulated as impulse responses which occur at the FCF along the time span [10]. For a bearing operating under time-varying speed conditions, the equation is given as [11]

$$
x(t)=\sum_{m=1}^{M} L_{m} e^{-\beta\left(t-t_{m}\right)} \sin \left[\omega_{r}\left(t-t_{m}\right)+\phi_{m} \quad u\left(t-t_{m}\right)\right.
$$

where $M$ is the number of impulse responses which is determined by the signal length $T$ and Instantaneous Fault Characteristic Frequency (IFCF), $L_{m}$ is the amplitude of the $m$ th impulse response, $\beta$ is the coefficient related to damping, $\omega_{r}$ is the excited resonance frequency or damped frequency of the vibration system, $\phi_{m}$ is the phase of the $m$ th impulse response, and $u(t)$ is unit step function. In the previous equation, $t_{m}$ is the occurrence time of the $m$ th impulse response which is calculated as

$$
\left\{\begin{array}{l}
t_{1} \quad\left(1+\delta_{1}\right)\left[1 / f_{c}\left(t_{0}\right)\right\rfloor \\
\left.t_{m}=\left(1+\delta_{m}\right) 1 / f_{c}(0)+\ldots+1 / f_{c}\left(t_{m-1}\right)\right] m=2,3, \ldots, M
\end{array}\right.
$$

where $t_{0}=0, \delta_{m}$ is the random slippage ratio with the average varying between 0.01 and $0.02, f_{c}(t)$ denotes the IFCF, and the time interval between the $(m-1)$ th impulse response and the $m$ th impulse response is $(1+\delta) / f_{c}\left(t_{m-1}\right)$. 
The interference signal can be simulated as the sum of sinusoidal functions of the frequency of the interference and its harmonics, given as [11]

$$
h(t)=\sum_{n=1}^{N} B_{n} \sin \left(2 \pi n f_{h} t\right)
$$

where $N$ is the number of sinusoidal functions, $B_{n}$ is the amplitude, and $f_{h}$ is the time-varying frequency of the interference, called Instantaneous Interference Frequency (IIF) in this paper.

(a) Fault-induced signal $x(t)$

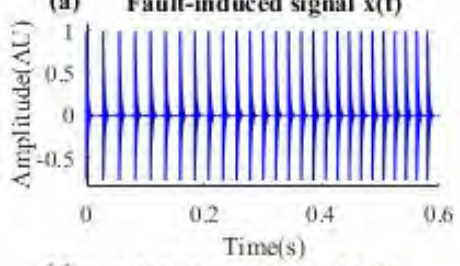

(c) Interfer ence signal h(t)

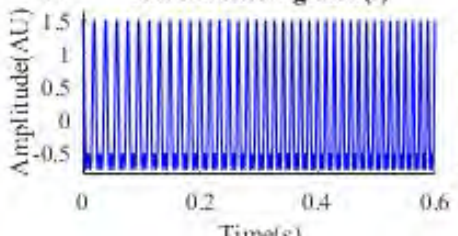

(e) Mixture of $x(t)$ and $h(t)$
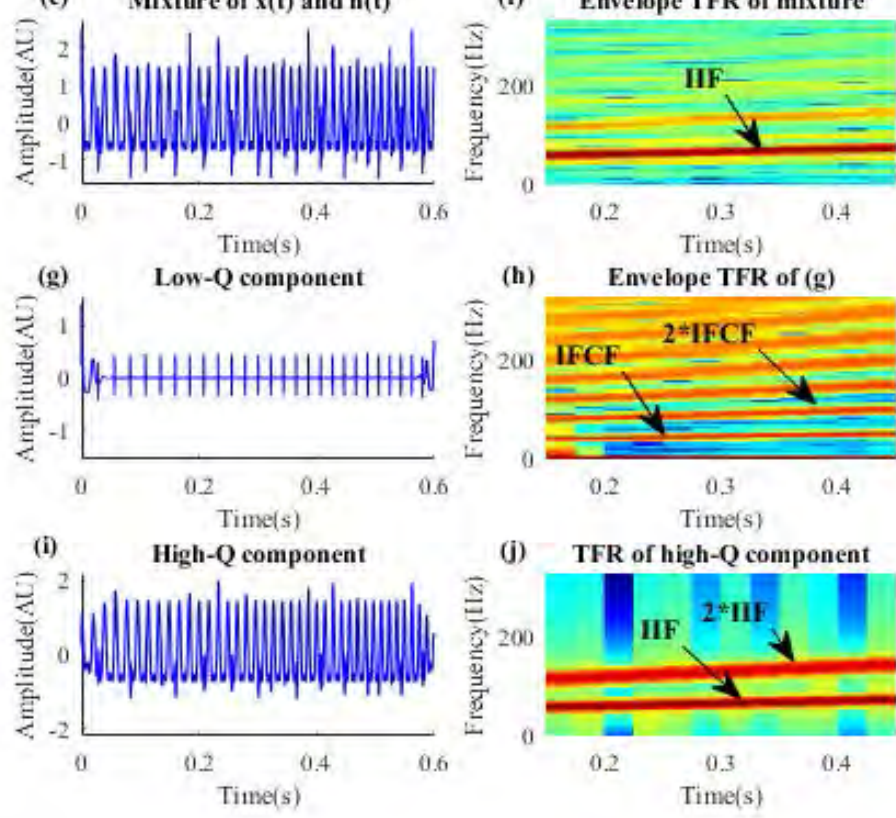

Figure 2 Implementation of the OBSD on bearing fault signature extraction under time-varying speed conditions

An example of the implementation of the OBSD on bearing fault signature extraction is illustrated in Figure 2. The original signal, shown in Figure 2(e), is a mixture of bearing fault impulses (Figure 2(a)) and interference (Figure 2(c)). The bearing fault signal is modeled by equation (4) with IFCF $f_{c}(t)=35 t+35 \mathrm{~Hz}, L_{m}=1, \beta=500, \omega_{r}=4000 * 2 \pi \mathrm{rad} / \mathrm{s}, \quad \phi_{m}=0$, $\delta_{m}=0.01$ and signal length $T=0.6 \mathrm{~s}$. In addition, the interference is modeled by equation (6) with $f_{h}=50 t+50 \mathrm{~Hz}, N=2$ and $B_{n}=[1$, $0.5]$. Under constant speed, the bearing fault is generally detected by the envelope spectrum which is the spectrum of the envelope preceded by the computation of a Hilbert transform [2]. Under time-varying speed, since the IFCF and its harmonics are time-varying, they can be observed in the Time-Frequency Representation (TFR) obtained via ShortTime Fourier Transform (STFT) [12]. As shown in Figure 2(b), the envelope TFR of the bearing fault-induced signal is composed of time-frequency curves at IFCF and its multiples, which can be used to detect the presence of a bearing fault. Similarly, the frequency of interference IIF and its $2^{\text {nd }}$ harmonic show curves in the TFR of the interference signal, shown in Figure 2(d). However, it can be seen from Figure 2(f) that the envelope TFR of the original signal is dominated by the IIF, which implies that the bearing fault cannot be detected without additional signal treatment. By applying the OBSD to the contaminated original signal with OBSD parameters $Q_{l}=1$, $r_{l}=6, P_{l}=40, Q_{h}=6, r_{h}=6, P_{h}=124, \lambda_{l}=\lambda_{h}=0.3, \mu=2$ and $K=150$, the signal is decomposed into a low oscillatory component (shown in Figure 2(g)) and a high oscillatory component (shown in Figure 2(i)). The bearing fault-induced impulses, which is the fault signature, are clearly seen in the low oscillatory component (Figure 2(g)). Furthermore, the envelope TFR of the low oscillatory component, shown in Figure 2(h), is dominated by the IFCF and its harmonics which is the same case as in Figure 2(b). The bearing fault can thus be easily detected. Additionally, the TFR of the high oscillatory component shown in Figure 2(j) is dominated by the IIF and its $2^{\text {nd }}$ harmonic, which is the same case as in Figure 2(d). It can be seen from this implementation that the OBSD can be effectively used to separate the bearing fault signature from a signal contaminated by interference under time-varying speed conditions.

\section{EXPERIMENTAL EVALUATION}

To test the performance of the OBSD method for bearing fault signature extraction under time-varying speed conditions, it is applied to signals collected from an experiment. The experiment is conducted on a SpectraQuest machinery fault simulator (MFS-PK5MT) to collect the bearing vibration signal which is contaminated by interference transmitted from a gearbox and noise.

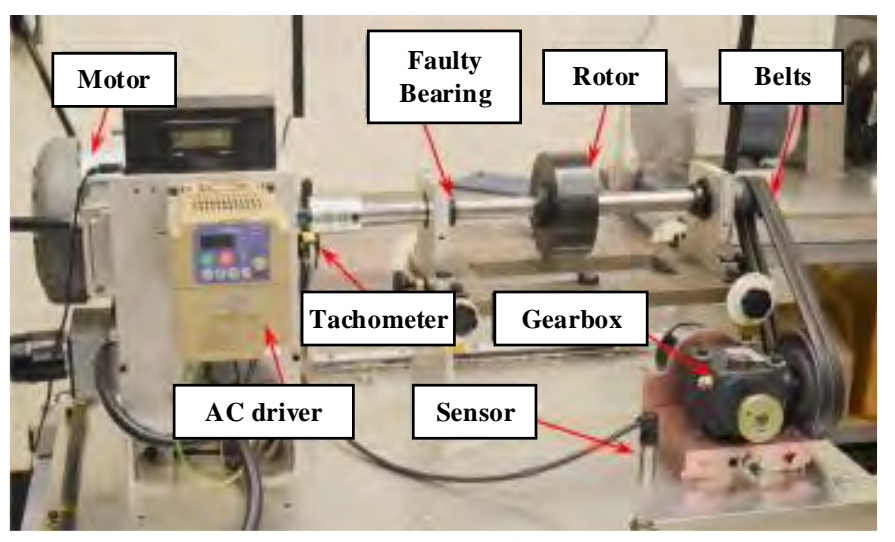

Figure 3 Experimental set-up

The set-up of this experiment is shown in Figure 3. The shaft is supported by two bearings and one of them is a faulty bearing with an outer race fault. The shaft is driven by a motor 


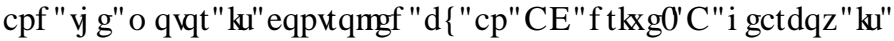

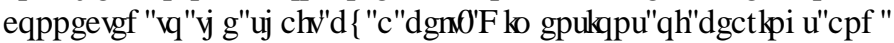

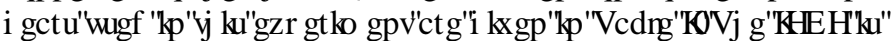

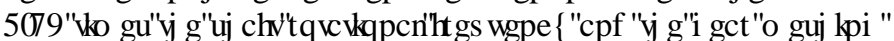

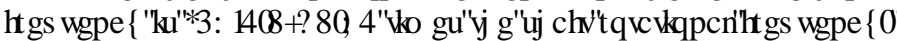

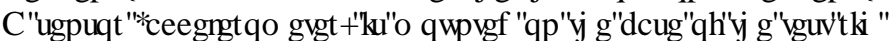

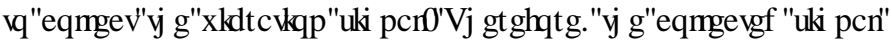

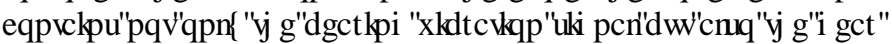

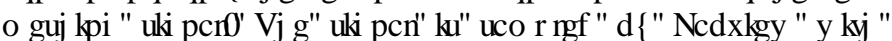

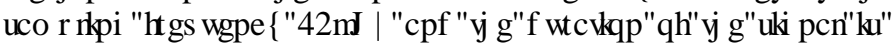

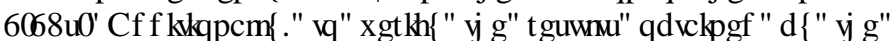

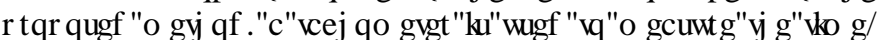

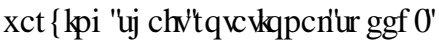

7 DEOH,【 IP HQMRQVIRIEHDWD VIOQGJ HDW

\begin{tabular}{|c|c|c|c|c|}
\hline $\begin{array}{c}\text { Bearing } \\
\text { type }\end{array}$ & $\begin{array}{c}\text { Pitch } \\
\text { diameter }\end{array}$ & $\begin{array}{c}\text { Ball } \\
\text { diameter }\end{array}$ & $\begin{array}{c}\text { Number of } \\
\text { balls }\end{array}$ & BPFO \\
\hline (500.0 & 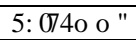 & पिएP P & पि & पष्व $f_{r} \square$ \\
\hline \multicolumn{2}{|c|}{ Diameter ratio of sheaves } & $\begin{array}{c}\text { Number of } \\
\text { teeth }\end{array}$ & \multicolumn{2}{|c|}{$\begin{array}{l}\text { Gear meshing } \\
\text { frequency }\end{array}$} \\
\hline \multicolumn{2}{|c|}{ ०समप } & प०० & \multicolumn{2}{|c|}{ पार $f_{r} \square$} \\
\hline
\end{tabular}
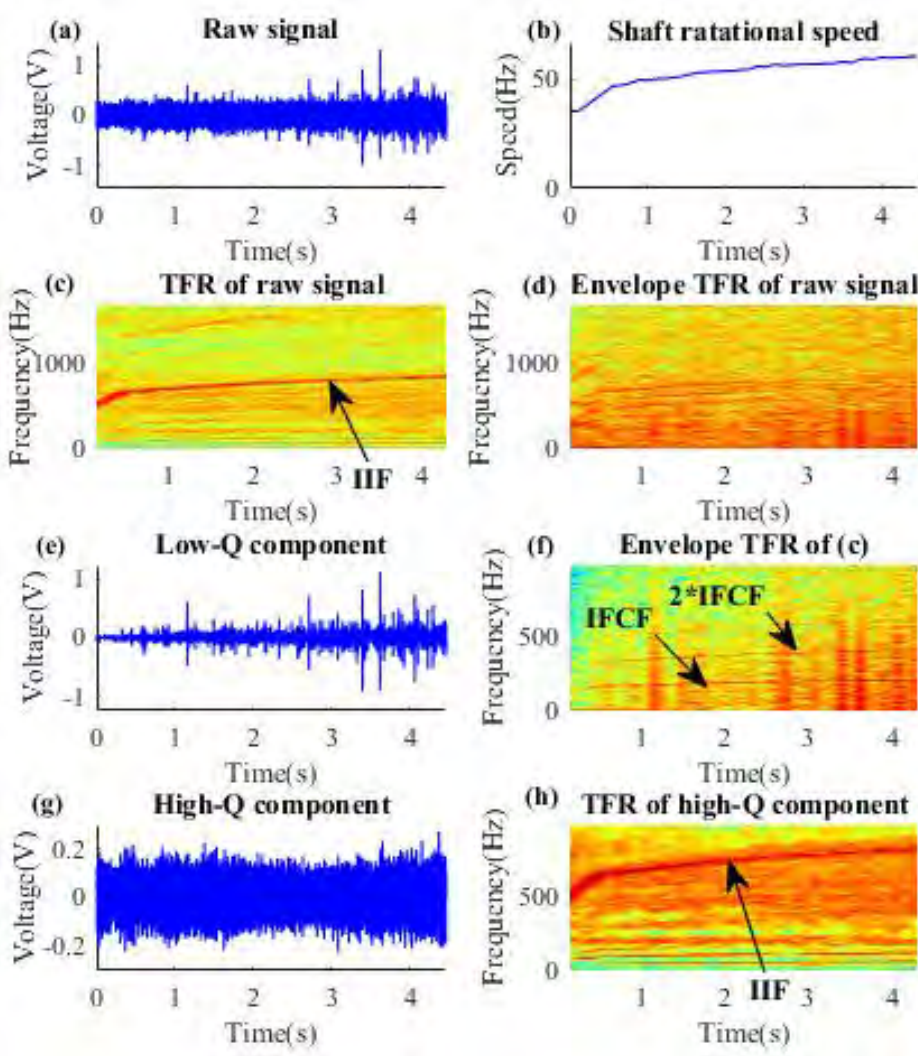

Time(s)

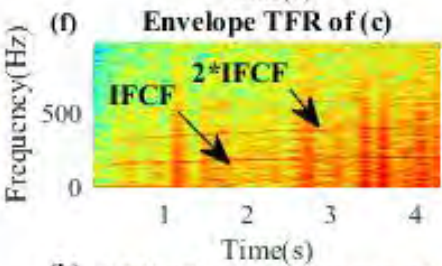

) UJ XUHD 15 HXOVRILMKH SHIP HQW

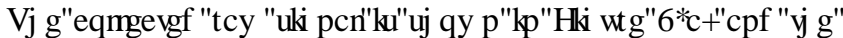

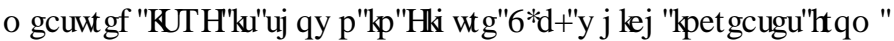

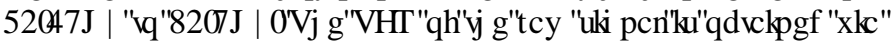

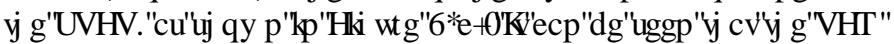

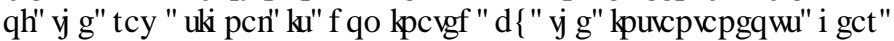

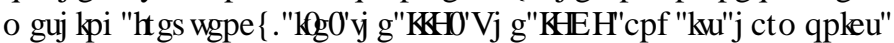

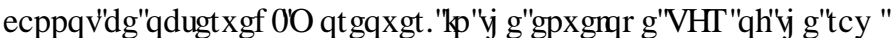

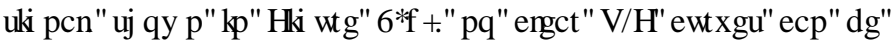

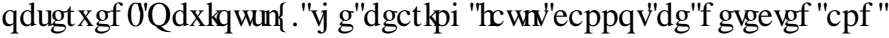

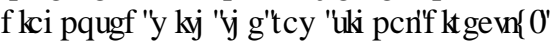

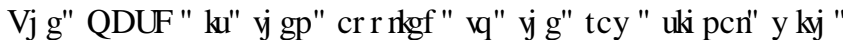

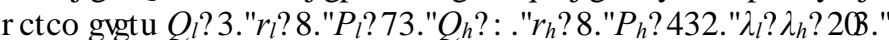

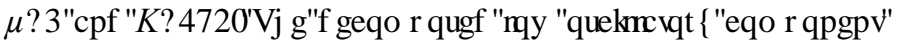

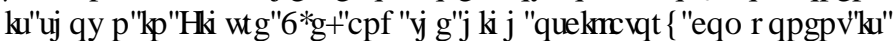

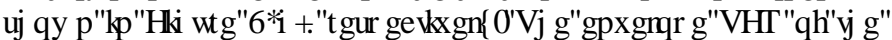

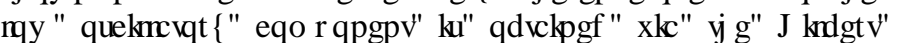

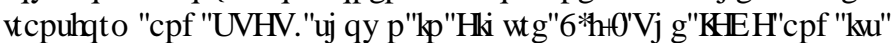

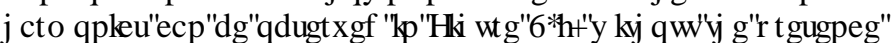

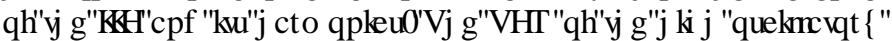

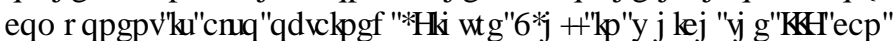

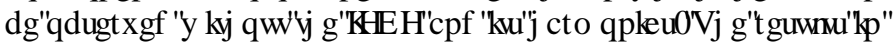

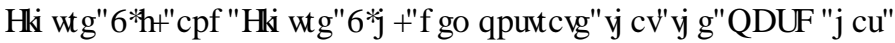

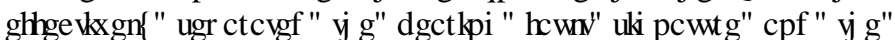

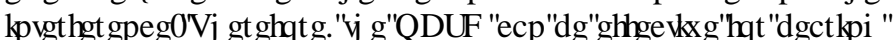

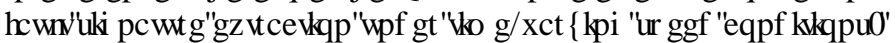

\section{0 \&2 $1 \& / 86,216$}

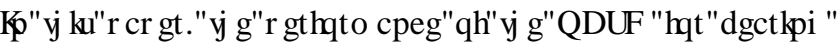

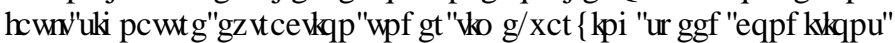

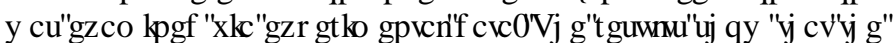

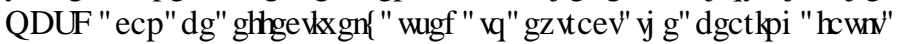

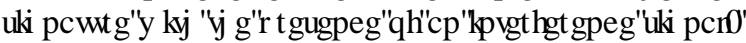

$$
\text { \$\&. } 12: /(' *(0) 17 \text { 口 }
$$

7 KVV ZRLV ZDV IIQDOFIDOI WXSRLWG EI I WKH 1 DXWO 6FIHFHV DOG ( Q IQHUDI 5 HMDFK \&RXQFID RI \&DODOD

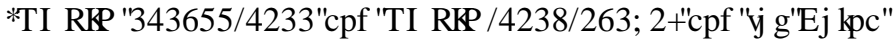
6FKR(DUWSLRRXQFID

\section{5( ) ( 5 ( $1 \&(6 \square$}

[1] N. Tandon and A. Choudhury, "A Review of Vibration and Acoustic Measurement Methods for the Detection of Defects in Rolling Element Bearings," Tribol. Int., vol. 32, no. 8, pp. 469-480, Aug. 1999. doi: 10.1016/S0301-679X(99)00077-8.

[2] R. B. Randall and J. Antoni, "Rolling Element Bearing Diagnostics-a Tutorial," Mech. Syst. Signal Process., vol. 25, no. 2, pp. 485520, Feb. 2011. doi: 10.1016/j.ymssp.2010.07.017.

[3] P. D. McFadden and J. D. Smith, "Vibration Monitoring of Rolling Element Bearings by the High-Frequency Resonance Technique a Review," Tribol. Int., vol. 17, no. 1, pp. 3-10, Feb. 1984. doi: 10.1016/0301-679X(84)90076-8.

[4] J. Antoni and R. B. Randall, "The Spectral Kurtosis: Application to the Vibratory Surveillance and Diagnostics of Rotating Machines," Mech. Syst. Signal Process., vol. 20, no. 2, pp. 308-331, Feb. 2006. doi: 10.1016/j.ymssp.2004.09.002.

[5] T. Barszcz and A. JabŁoński, "A Novel Method for the Optimal Band Selection for Vibration Signal Demodulation and Comparison with the Kurtogram," Mech. Syst. Signal Process, vol. 25, no. 1, pp. 431-451, Jan. 2011. doi: 10.1016/j.ymssp.2010.05.018. 
[6] I. W. Selesnick, "Resonance-Based Signal Decomposition: a New Sparsity-Enabled Signal Analysis Method," Signal Process, vol. 91, no. 12, pp. 2793-2809, Dec. 2011. doi: 10.1016/j.sigpro.2010.10.018.

[7] H. Huang, N. Baddour, and M. Liang, "Auto-OBSD: Automatic Parameter Selection for Reliable Oscillatory Behavior-Based Signal Decomposition with an Application to Bearing Fault Signature Extraction," Mech. Syst. Signal Process., vol. 86, pp. 237-259, Mar. 2017. doi: 10.1016/j.ymssp.2016.10.007.

[8] I. W. Selesnick, "Wavelet Transform with Tunable Q-Factor," IEEE Trans. Signal Process., vol. 59, no. 8, pp. 3560-3575, Aug. 2011. doi: 10.1109/TSP.2011.2143711.

[9] L. Cui, D. Mo, H. Wang, and P. Chen, "Resonance-Based Nonlinear Demodulation Analysis Method of Rolling Bearing Fault," Adv. Mech. Eng., vol. 5, pp. 1-13, Jan. 2013. doi: 10.1155/2013/420694.
[10] T. Wang, M. Liang, J. Li, and W. Cheng, "Rolling Element Bearing Fault Diagnosis Via Fault Characteristic Order (FCO) Analysis," Mech. Syst. Signal Process., vol. 45, no. 1, pp. 139-153, Mar. 2014. doi: 10.1016/j.ymssp.2013.11.011.

[11] J. Shi, M. Liang, and Y. Guan, "Bearing Fault Diagnosis Under Variable Rotational Speed Via the Joint Application of Windowed Fractal Dimension Transform and Generalized Demodulation: a Method Free From Prefiltering and Resampling," Mech. Syst. Signal Process., vol. 68-69, pp. 1533, Feb. 2016. doi: 10.1016/j.ymssp.2015.08.019.

[12] Z. Feng, M. Liang, and F. Chu, "Recent Advances in Time-Frequency Analysis Methods for Machinery Fault Diagnosis: a Review with Application Examples," Mech. Syst. Signal Process., vol. 38, no. 1, pp. 165-205, Jul. 2013. doi: 10.1016/j.ymssp.2013.01.017. 


\title{
Optimization of Interaural Intensity Difference based Binaural Sonar Sensing System for Object Detection
}

\author{
Payman Rajai, Mohammed Jalal Ahamed \\ MicroNano Mechatronics Laboratory, Mechanical, Automotive and Materials Engineering Department, \\ University of Windsor \\ Windsor, ON, Canada \\ email: jahamed@uwindsor.ca
}

\begin{abstract}
Interaural Intensity Difference (IID) in binaural sonar systems is used for echolocation and obstacle sensing. In this article, we show by simulation the optimized system's parameters in terms of frequency, sensor separation distance and the working range for an IID based binaural sonar sensing system. Our result shows that the best performances with a frequency range between 100 to $300 \mathrm{kHz}$ and a separation distance, depending on the size of the microphone, in our case between $2 \mathrm{~cm}$ to $5 \mathrm{~cm}$ within the working range of $1 \mathrm{~m}$. The approach developed in this paper could be useful for mobile localization and mapping, particularly in compact size mobile devices.
\end{abstract}

Keywords-component; acoustic sensor, binaural system, Interaural Intensity Difference.

\section{INTRODUCTION}

Ultrasonic sensors are robust and low-cost, well suited for identifying objects/obstacles within a few meters, typically configured with an array of emitters and receivers to achieve an accurate mapping of the surrounding environment. Binaural ultrasonic sensors are inspired by natural hearing systems where two ultrasonic receivers flank an ultrasonic emitter. In a binaural ultrasonic the object's distance and bearing angle can be estimated from the differences in interaural time [1]-[5], amplitude [6], intensity [7]-[9] and/or phase [10], [11]. It is known that interaural time and phase difference are appreciable with low-frequency sounds. With high-frequency sounds, the interaural difference in amplitude or intensity is more convenient. For instance, bat's echolocation system uses Interaural Intensity Difference (IID) at two different frequencies as the cue to the direction of a prey [12].

In a binaural system, the degree of accuracy depends on the separation distance between the two receivers for interaural time difference cue. The sonar separation must be much larger than the wavelength of the sonic wave in use. The separation distance is usually 10's of centimeters to 10's of meters [13]. When the separation between the receiver microphones is large compared to the object's distance, there is a risk that one of the receivers does not pick up the signal. If the microphones are too close to each other (similar to miniature animals with little separation between the two ears), both microphones record essentially the same acoustic signal. This results in very small interaural amplitude and time cues, posing substantial challenges to directional sensitivity of the auditory system [14]. To amplify the interaural difference, fly-ear inspired acoustic sensors consisting of two wings mechanically coupled at the middle have been recently developed [15].

In this article, we will show by simulation that the approach using IID makes it possible to place the receivers as close together as their size permits and thus making small size sonar system where interaural time difference measurement is not convenient. Our binaural system consists of a single emitter scanning across the range of incident angle flanked by two receivers (Figure 1). In this paper, we develop a mathematical model relating systems parameters in an IID based binaural sonar sensing system. We later utilized the model to demonstrate that the sensor performance for object identification using IID is enhanced under certain frequency and separation range. In the next sections, we will establish the concept from a theoretical perspective, and subsequently, simulate results to evaluate the model.

\section{MATHEMATICAL FORMULATION}

The geometry of our binaural sonar system is depicted in Error! Reference source not found., which consists of a transmitter flanked by two fixed receivers. In the model, the transmitter and receivers are assumed to be of piston shape, with the transmitter located at the origin, and the two receivers at distance $d$ to its left and right sides. As illustrated in Fig. 1, the object coordinate with respect to the emitter placed at the center of the polar coordinate system is $(r, \beta)$, where $r$ is the radial distance and $\beta$ is the azimuth angle. The angular deviation is measured with respect to the normal line through the emitter, and it takes positive values in the clockwise direction. Likewise, the object coordinate with respect to the receiver is $\left(r_{r}, \beta_{r}\right)$. Note that $\beta_{r}$ is measured with respect to the line that passes through the center of the object. It is parallel to the emitter's normal and is positive in the clockwise direction. 


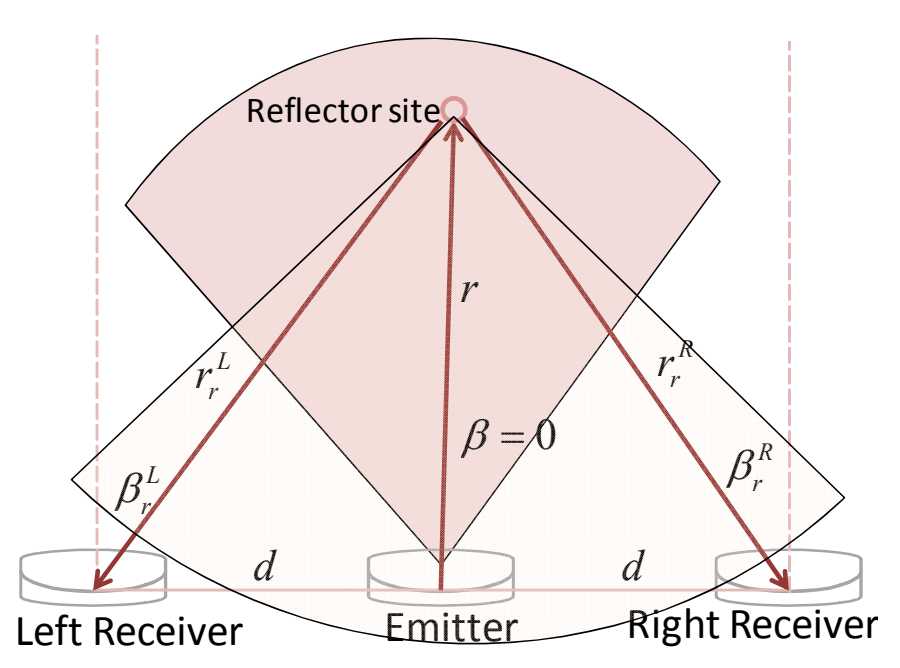

Figure 1. The geometry of the binaural sonar system with rotating emitter.

If a small object is ensonified by the emitter, it re-emits a spherical field that can be detected by a receiver. For pistonshaped emitter and receiver, the pressure field identified by the receiver can be described by [16], [17]

$$
\begin{aligned}
& P_{D}\left(k, r, \beta, r_{r}, \beta_{r}\right)=\frac{\rho c \sigma}{2} U_{0} \frac{k a_{e}^{2} a_{r}^{2}}{r_{e} r_{r}} \\
& \ldots \frac{2 J_{1}\left[k a_{e} \sin (\beta)\right]}{k a_{e} \sin (\beta)} \cdot \frac{2 J_{1}\left[k a_{r} \sin \left(\beta_{r}\right)\right]}{k a_{r} \sin \left(\beta_{r}\right)},
\end{aligned}
$$

where $a_{e}$ and $a_{r}$ are, respectively, the radius of the circular emitter and of the receiver surface, $k$ is the wavenumber relates to the frequency via $2 \pi \mathrm{f} / \mathrm{c}, c$ is the speed of sound, $\rho$ is the air density, $J_{1}$ is the Bessel function of the first kind, and $\sigma$ is the scattering coefficient, the ratio of the scattering and the incident fields. As depicted in Figure 1., the position of the object to the left or right of the receivers is obtained from

$$
r_{r}^{L, R}=\sqrt{d^{2}+r^{2}-2 d r \cos (\pi / 2 \pm \beta)}
$$

and

$$
\beta_{r}^{L, R}=\left|\sin ^{-1}\left(\frac{d \cos (\beta)}{r_{r}^{L, R}}\right) \pm \beta\right|,
$$

where the superscriptions $\mathrm{L}$ and $\mathrm{R}$ denote the left and the right receivers also are shown in Figure 1. Once the echo is detected by both receivers on the two sides of the emitter, the Interaural Intensity Difference (IID) is measured from absolute difference of the detected intensities that is the squared of amplitudes defined by $\mathrm{Eq}(1)$.

\section{SIMULATION}

The theoretical model developed in the previous section is simulated by sweeping the parameters of the system to evaluate the performance of the model in detecting an obstacle. A typical example of the detected intensities and the IID is presented in Figure 2. . In this example plot, we set $a_{e}=a_{r}=4.25 \mathrm{~mm}, \mathrm{U}_{0}=1000, r=30 \mathrm{~cm}, d=5 \mathrm{~cm}$ at $50 \mathrm{kHz}$ frequency.

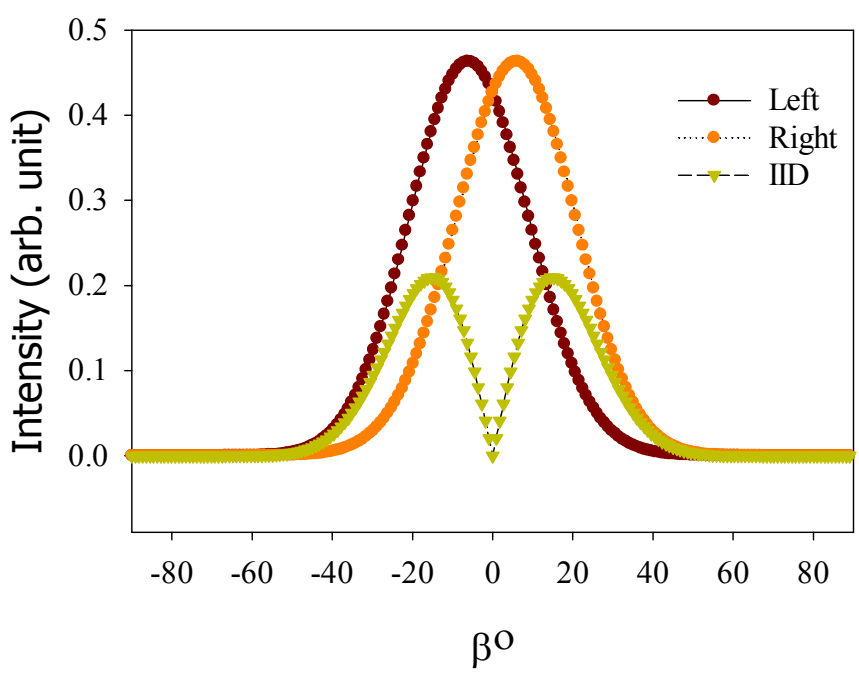

Figure 2. The detected intensities by the left and the right microphones and the IID.

In Figure 2. a point object is scanned for a range of azimuth angle from $-\pi / 2$ to $+\pi / 2$. It is observed that the intensity captured by each microphone is largest when the object is just above the microphone. The quantity of our interest is the IID that is zero when the object is aligned with the sonar axis as the sensor scans the environment. We use this property of the IID to scan the environment searching for obstacles for various system's parameters as frequency, the separation distance between the emitter and the receiver and working range.

The crucial condition here is that the zero point of the IID must be differentiable from other parts of the signal. In other words, the contrast between the zero and the maximum of the IID signal and the slope of the curve from the maximum towards the zero point defines the performance of such system to identify the obstacle effectively. In the next section, we will evaluate necessary conditions for best performance including the system's parameters of frequency $(f)$, separation distance $(d)$ and working range $(r)$.

\section{A. Tuning system's parameters}

In this section, we identify optimal parameters for the emitter frequency $(f)$, the separation between the microphones and the emitter $(d)$, and the working range $(r)$. To realize this, various combinations of $f, d$, and $r$ were simultaneously explored while looking for systematic variations in the IID contrast. 


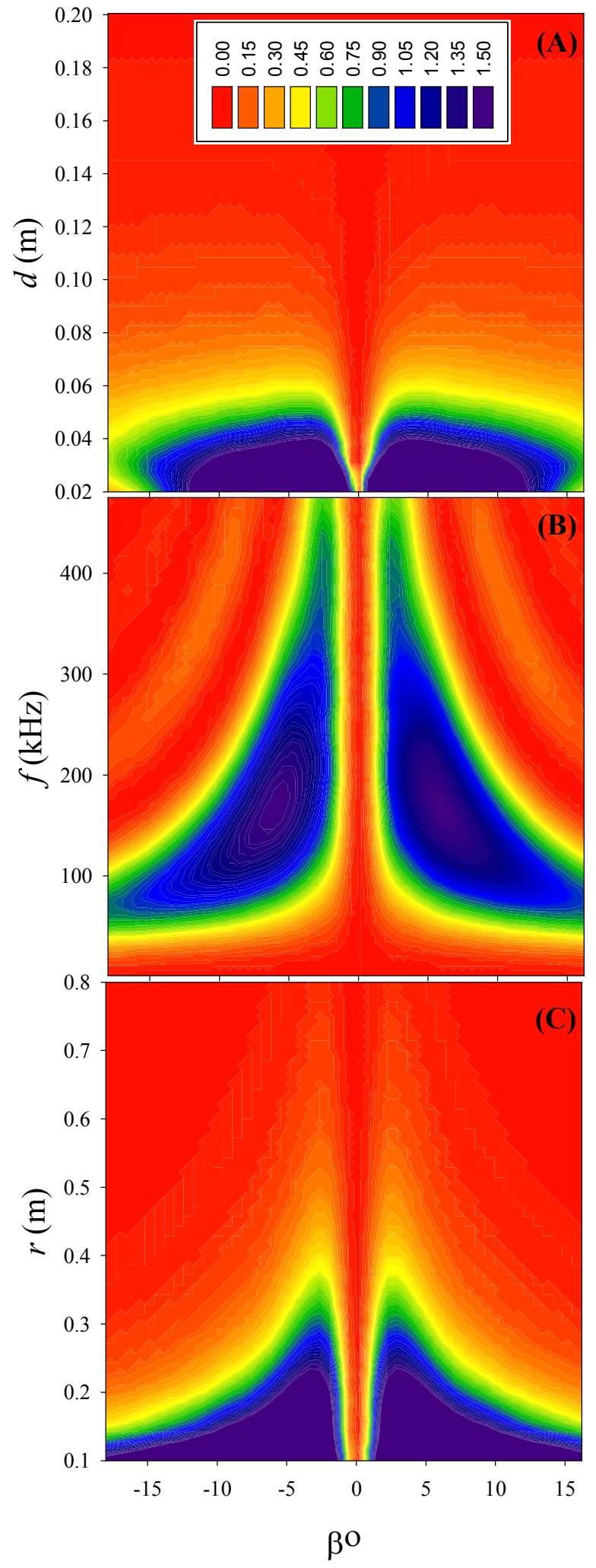

Figure 3. Color map of IID for a range of separation distance (A), frequency (B) and working range $(\mathrm{C})$ respectively.
The simulation test results for the IID variation with system's parameters are plotted in Figure 3 . where the horizontal axis corresponds to the scanning angle $\beta$. In Figure 3 . , red signifies low contrast, and blue denotes higher contrast desirable for better performance.

Figure 3. corresponds to the IID map with respect to varying separation distance between the emitter and the microphone $(d)$ that is swept from $2 \mathrm{~cm}$ to $20 \mathrm{~cm}$. From Figure 2. Figure 3. (A) it is observed that the best performance of the sonar system corresponds to the small separation between the emitter and microphones i.e. up to $6 \mathrm{~cm}$. The large separation will degrade the performance of the system especially with high-frequency ultrasonic waves where they are highly directive and therefore the IID contrast gradually fades away with increasing the separation distance. In other words, large separation is permissible with low-frequency source but will be impractical with high frequencies. This fact is shown in Figure 4. where the IID contour is plotted for a range of frequency versus separation distance. As the figure shows, with increasing the frequency, the separation distance that gives appreciable IID contrast would be limited to small distances.

In Figure 3. (B), the IID map is provided with respect to varying frequency from $1 \mathrm{kHz}$ up to $500 \mathrm{kHz}$. In this figure, the suitable frequency range is observed to be between 100 to $300 \mathrm{kHz}$ with the best performance around $200 \mathrm{kHz}$. The system is impractical for frequencies lower than $20 \mathrm{kHz}$ since the acoustic wave loses its directivity and the microphones record almost similar pressure field regardless of the separation distance. The system also works with frequencies higher than $300 \mathrm{kHz}$; however, side lobes appear in the recorded fields as well as the IID, which may make it difficult to distinguish the main IID zero from the side lobes. One possible approach with high-frequency emitter source is to reduce the separation distance as suggested in Figure 2. Figure 3. (A).

Regarding the working range of the system in Figure 2. Figure 3. (C), the radial distance from the emitter is swept from $10 \mathrm{~cm}$ up to $100 \mathrm{~cm}$. It is observed that the performance degrades with distance with the best performance correspond to distances lower than $50 \mathrm{~cm}$. This is also due to the ultrasonic natural degradation with distance. This limitation, however, can be easily overcome by increasing the emitter's power to generate stronger pressure fields. 


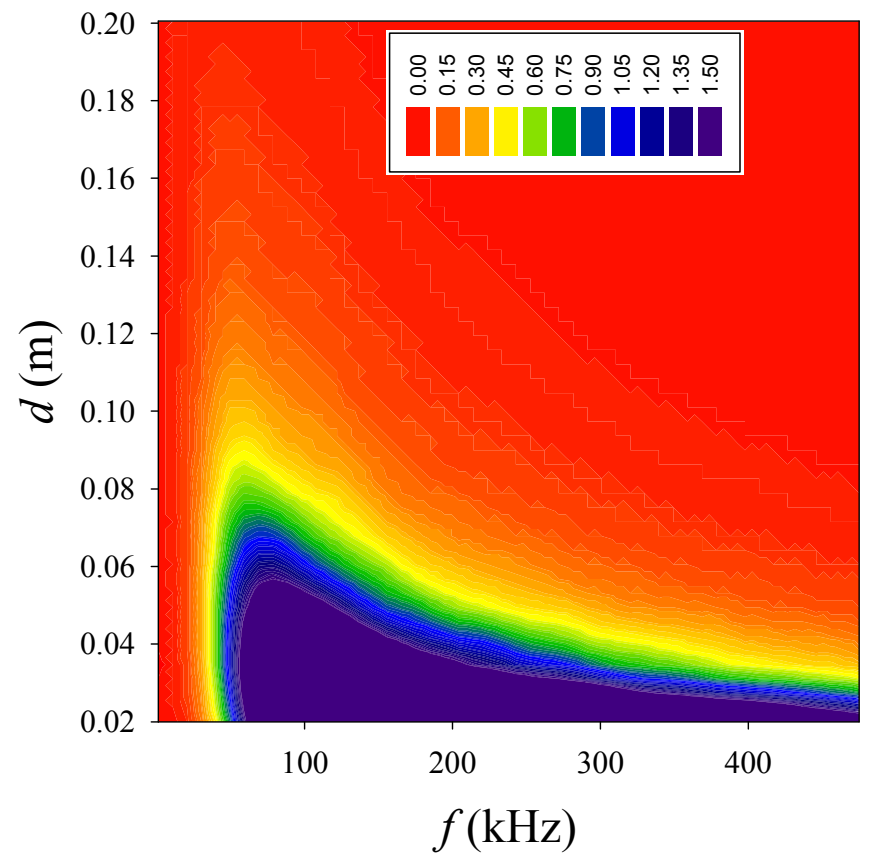

Figure 4. The IID contour for a range of frequency versus separation distance.

With respect to the above-findings, a suitable sonar system for working range within $1 \mathrm{~m}$ would consist of a transducer that emits ultrasonic pulses of $100 \mathrm{kHz} \leq f \leq 300 \mathrm{kHz}$, flanked by two microphones separated by $d<5 \mathrm{~cm}$.

\section{CONCLUSION}

In this paper, we presented the optimization of binaural sonar systems for object detection using IID measurement. This system as showed in this article is suitable for sonar systems where time of flight measurement is not practical. The simulation result showed that the performance of such system is enhanced with a frequency range between 100 to $300 \mathrm{kHz}$ with the best performance around $200 \mathrm{kHz}$ and the separation distance between the emitter and receiver up to maximum $6 \mathrm{~cm}$ within the working range of $1 \mathrm{~m}$.

\section{ACKNOWLEDGEMENTS}

The authors would like to thank Natural Sciences and Engineering Research Council of Canada (NSERC) and University of Windsor for financial supports.

\section{REFERENCES}

[1] B. Barshan and R. Kuc, "A bat-like sonar system for obstacle localization," IEEE Trans. Syst. Man Cybern., vol. 22, no. 4, pp. 636-646, July. 1992. doi: 10.1109/21.156577.

[2] R. Kuc, "Binaural sonar electronic travel aid provides vibrotactile cues for landmark, reflector motion and surface texture classification," IEEE Trans. Biomed. Eng., vol. 49, no. 10, pp. 1173-1180, Oct. 2002. doi: 10.1109/TBME.2002.803561.

[3] W.-Y. Mu, G.-P. Zhang, Y.-M. Huang, X.-G. Yang, H.-

Y. Liu, and W. Yan, "Omni-directional scanning localization method of a mobile robot based on ultrasonic sensors," Sensors, vol. 16, no. 12, Dec. 2016. doi: 10.3390/s16122189.
[4] R. Kuc, "Biomimetic sonar locates and recognizes objects," IEEE J. Ocean. Eng., vol. 22, no. 4, pp. 616-624, Oct. 1997. doi: $10.1109 / 48.650828$.

[5] C. Baumann, C. Rogers, and F. Massen, "Dynamic binaural sound localization based on variations of interaural time delays and system rotations," J. Acoust. Soc. Am., vol. 138, no. 2, pp. 635-650, Aug. 2015. doi: $10.1121 / 1.4923448$.

[6] H. Peremans, A. Walker, and J. C. T. Hallam, "3D object localisation with a binaural sonarhead, inspirations from biology," in Proceedings. 1998 IEEE International Conference on Robotics and Automation (Cat. No.98CH36146), vol. 4, pp. 2795-2800 1998. doi: 10.1109/ROBOT.1998.680472.

[7] R. Kuc, "Sensorimotor model of bat echolocation and prey capture," J. Acoust. Soc. Am., vol. 96, no. 4, pp. 1965-1978, Oct. 1994. doi: $10.1121 / 1.410140$.

[8] B. A. Edmonds and J. F. Culling, "Interaural correlation and the binaural summation of loudness," J. Acoust. Soc. Am., vol. 125, no. 6, pp. 3865-3870, Jun. 2009. doi: 10.1121/1.3120412.

[9] Y. Zhang and B. A. Wright, "An influence of amplitude modulation on interaural level difference processing suggested by learning patterns of human adults," J. Acoust. Soc. Am., vol. 126, no. 3, pp. 1349-1358, Sep. 2009. doi: 10.1121/1.3177267.

[10] M. I. Mandel and D. P. W. Ellis, "A probability model for interaural phase difference," ISCA Tutor. Res. Workshop Stat. Percept. Audit. SAPA2006 16 Sept. 2006 Pittsburgh PA, pp. 1-6, 2006.

[11] C. Faller and J. Merimaa, "Source localization in complex listening situations: selection of binaural cues based on interaural coherence," J. Acoust. Soc. Am., vol. 116, no. 5, pp. 3075-3089, Nov. 2004. doi: 10.1121/1.1791872.

[12] A. W. Mills, "Lateralization of high frequency tones," J. Acoust. Soc. Am , vol. 32 no. 1 , pp. 132-134, Jan. 1960.
doi: $10.121 / 1.1907864$.

[13] A. Saxena and A. Y. Ng, "Learning sound location from a single microphone," in Proceedings of the 2009 IEEE International Conference on Robotics and Automation, Piscataway, NJ, USA, 2009, pp. 4310-4315. doi: 10.1109/ROBOT.2009.5152861.

[14] D. Robert, R. N. Miles, and R. R. Hoy, "Directional hearing by mechanical coupling in the parasitoid fly Ormia ochracea," $J$. Comp. Physiol. A, vol. 179, no. 1, pp. 29-44, Jul. 1996. doi: $10.1007 /$ BF00193432.

[15] D. Wilmott, F. Alves, and G. Karunasiri, "Bio-inspired miniature direction finding acoustic sensor,” Sci. Rep., vol. 6, p. 29957, Jul. 2016. doi: 10.1038/srep29957.

[16] "Sensorimotor model of bat echolocation and prey capture," J. Acoust. Soc. Am., vol. 96, no. 4, pp. 1965-1978, Oct. 1994. doi: 10.1121/1.410140.

[17] R. Kuc, "Three-dimensional tracking using qualitative bionic sonar," Robot. Auton. Syst., vol. 11, no. 3, pp. 213-219, Dec. 1993. doi: 10.1016/0921-8890(93)90026-9. 


\title{
Design of Electronic Stability Control (ESC) Systems for Car-trailer Combinations
}

\author{
Mutaz Keldani, Yuping He \\ University of Ontario Institute of Technology \\ Department of Automotive Engineering \\ Oshawa, Canada \\ Mutaz.keldani@uoit.net, Yuping.he@uoit.ca
}

\begin{abstract}
This paper presents the design of an electronic stability control (ESC) system based on trailer differential braking for increasing safety and improving handling performance of car-trailer combinations. Numerical simulations were performed using CarSim software to assess the performance of the proposed control strategies of the ESC system. To validate the virtual design of the ESC system for car-trailer combinations, a small scaled physical prototype $(1: 10)$ of a car-trailer combination was fabricated and tested. The prototype consists of an aluminum frame, accelerometer, and two wheels equipped with an electromagnetic differential braking. Numerical simulation and prototype testing demonstrated the effectiveness of the proposed ESC system for car-trailer combinations.
\end{abstract}

Keywords: electronic stability control; lateral acceleration; differential braking; car-trailer combination

\section{INTRODUCTION}

Car-trailers are easy to own recently for several reasons, such as, their low cost, availability, and simplicity of the design. However, trailer safety systems and technology are outdated and not increasing at the same rate as passenger vehicles. For example, In Ontario, towing a trailer with a gross vehicle weight of up to 4,600 Kilograms does not require a higher driving license class. In addition, the Ministry of Transportation of Ontario does not have any regulations regarding the braking system required to ensure the safety of the trailer [1].

Car trailers usually face two of the most dangerous scenarios, known as sway and jackknifing [2]. In addition, rollovers have a huge impact on the trailer's stability. Rollovers occur due to different reasons such as; high speeds, crosswinds, road conditions and center of gravity of the trailer, which could be, affected by the way the trailer was loaded.

Understanding why each of sway, jackknifing and rollovers occur will result in an effective robust ESC controller. First, sway is defined as the unusual lateral motion that the trailers go through due to several reasons, such as; side passing wing on open roads, bow wind produced by commercial vehicles and lastly, the load distribution inside the trailer which has an effect on the tongue load. Moving sideways will result in pendulum motion [3], which cause the car-trailer combination to lose stability. Load distribution has an impact on the braking distribution force, as the trailer could lose traction and gripping the road, which allows the trailer to swing and skid [4].

The second major hazard is the rollover, which happens due to sharp turns, high speeds and load distribution. Rollover often occurs when the lateral acceleration of the trailer is larger than the threshold value.

Jackknifing is the third major unstable motion mode. Jackknifing may be caused by several factors, such as momentum of the combination, weather condition, and low friction of road surfaces [5].

To address the aforementioned problems and avoid severe accidents, conventional braking systems may be enhanced by introducing active trailer differential braking systems. An electronic stability control system (ESC) is developed as an important safety feature in passenger vehicles. ESC is an active safety technology that improves the vehicle's stability by assisting the driver to keep the car-trailer combination on the desired path [6].

To implement the functionality of ESC, several vehicle dynamic states can be controlled, such as yaw rate of vehicle units [7], lateral motions of vehicle units [8]. Each of the above mentioned methods requires a car-trailer combination equipped with several sensors to ensure a responsive and accurate system.

Lateral acceleration control has been used for the design of active trailer differential braking system. A small-scale prototype was fabricated and examined to validate the active trailer differential braking system designed in this project, and the lateral-acceleration rearward amplification (RWA) was used as an essential lateral stability measure of the car-trailer combination [9]. Controlling the lateral acceleration of the vehicle units may effectively prevent the occurrence of rearward amplification, in which the trailer has a greater lateral acceleration than the leading unit, which can lead to rollover of the trailer [10]. 


\section{DESIGN OF AN ACTIVE TRAILER DIFFERENIAL BRAKING SYSTEM}

The design of the electronic stability control (ESC) system involves the modeling of the car-trailer system for controller design, a small-scaled physical prototype fabrication, and performance evaluation. The design process may be divided into two different phases. The first is the design of a virtual prototype, while the second phase is the fabrication of the smallscaled physical prototype to validate the virtual design.

\section{A. Mathematical Modeling}

As shown in Fig, 1, 3 degrees of freedom (DOF) yaw-plane car-trailer model is generated for the ESC controller design.

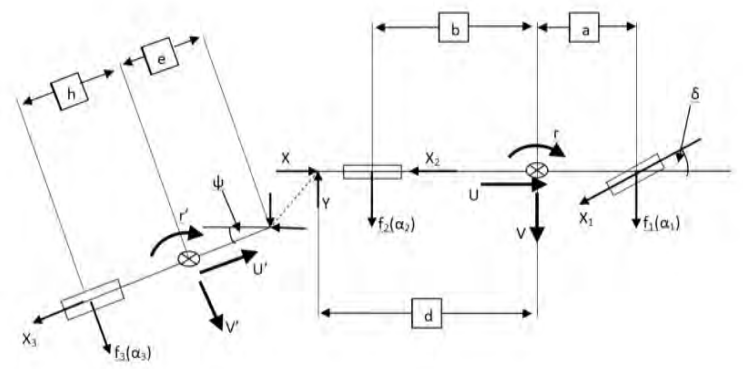

Figure 1. Schematic representation of the 3 DOF yaw-plane car-trailer model and the parameters used [11].

The equations of motion of the leading vehicle are expressed as follows [11]:

$$
\begin{gathered}
m_{1}(\dot{U}-V r)=-X_{1} \cos \delta-X_{2}+X \\
m_{1}(\dot{V}+U r)=f_{1}\left(\alpha_{1}\right)+f_{2}\left(\alpha_{2}\right)+X_{1} \sin \delta-Y \\
I_{1} \dot{r}=a f_{1}\left(\alpha_{1}\right)-b f_{2}\left(\alpha_{2}\right)+a X_{1} \sin \delta+d Y
\end{gathered}
$$

The trailer's equations of motion are expressed as [11]:

$$
\begin{gathered}
m_{2}\left(\dot{U}^{\prime}-V^{\prime} r^{\prime}\right)=-X_{3}-Y \sin \psi-X \cos \psi \\
m_{2}\left(\dot{V}^{\prime}+U^{\prime} r^{\prime}\right)=f_{3}\left(\alpha_{3}\right)+Y \cos \psi-X \sin \psi \\
I_{2} \dot{r}^{\prime}=-h f_{3}\left(\alpha_{3}\right)-e(-Y \cos \psi+X \sin \psi)
\end{gathered}
$$

Due to fact that the trailer is connected to the leading vehicle with an articulated joint, the forward velocity and acceleration of the leading and trailing units are assumed the same. This assumption simplifies the trailer equations of motion and helps linearize them.

In addition, the angle $\psi$ is approximated to be small, which leads to [11]:

$$
\begin{aligned}
& \cos (\psi)=1 \\
& \sin (\psi)=\psi
\end{aligned}
$$

Also, the following equation is determined at zero initial conditions [11].

$$
\dot{\psi}=r-r^{\prime}
$$

Using all the above assumptions and approximations, a linearized equation of motion can be expressed as [11]:

$$
\boldsymbol{M}\{\dot{x}\}+\boldsymbol{D}\{x\}+\boldsymbol{F} \delta=0
$$

\begin{tabular}{|c|c|c|}
\hline \multicolumn{3}{|c|}{ Parameters } \\
\hline Description & Symbol & Value \\
\hline Leading vehicle mass & $M_{L}$ & $1521 \mathrm{Kg}$ \\
\hline Trailer mass & $M_{T}$ & $602 \mathrm{Kg}$ \\
\hline $\begin{array}{l}\text { Distance between CG and front } \\
\text { axle }\end{array}$ & $a$ & $0.972 \mathrm{~m}$ \\
\hline $\begin{array}{l}\text { Distance between CG and rear } \\
\text { axle }\end{array}$ & $b$ & $1.807 \mathrm{~m}$ \\
\hline $\begin{array}{l}\text { Distance between vehicle } \mathrm{CG} \\
\text { and contact point with the trailer }\end{array}$ & $d$ & $4.835 \mathrm{~m}$ \\
\hline $\begin{array}{l}\text { Distance between the trailer CG } \\
\text { and contact point with the vehicle }\end{array}$ & $e$ & $2.00 \mathrm{~m}$ \\
\hline $\begin{array}{l}\text { Distance between the CG and the } \\
\text { rear axle }\end{array}$ & $h$ & $0.6 \mathrm{~m}$ \\
\hline Height of vehicle CG & $H 1_{C G}$ & $0.325 \mathrm{~m}$ \\
\hline Height of trailer CG & $H 2_{C G}$ & $0.676 \mathrm{~m}$ \\
\hline Slip angle & $\delta$ & \\
\hline $\begin{array}{l}\text { Longitudinal force on car's front } \\
\text { wheel, rear wheel and trailer } \\
\text { wheel respectively }\end{array}$ & $\mathrm{X}_{1,2,3}$ & \\
\hline Car,trailer forward velocity & $\mathrm{U}, \mathrm{U}$ & \\
\hline $\begin{array}{l}\text { Lateral velocity of car and trailer } \\
\text { respectively }\end{array}$ & $\mathrm{V}, \mathrm{V}^{\prime}$ & \\
\hline $\begin{array}{l}\text { Lateral force on car's front } \\
\text { wheel, rear wheel and trailer's } \\
\text { wheel respectively }\end{array}$ & $\mathrm{f}_{1,2,3}$ & \\
\hline $\begin{array}{l}\text { Car, trailer yaw angle } \\
\text { respectively }\end{array}$ & $\mathrm{r}, \mathrm{r}^{\prime}$ & \\
\hline Hitch angle & $\psi$ & \\
\hline
\end{tabular}

where the state variables are written as shown in "(11)" [11]:

$$
\{x\}=\{\operatorname{Vr} \dot{\psi} \psi\}
$$

Matrices M,D and F are listed in Appendix A.

The parameters listed in Table 1 are used in the mathematical modeling and CarSim model generation.

TABLE I. PARAMETERS USED TO DESIGN AND TEST A VIRTUAL PROTOTYPE.

Equations (1) to (11) show how to express a mathematical model of a car-trailer combination with some assumptions to linearize the system. However, the trailer differential braking system is designed to generate a counter moment to stabilize the car-trailer combination [10], which will result in the shwon equation [11]:

$$
I_{2} \dot{r}^{\prime}=-h f_{3}\left(\alpha_{3}\right)-e(-Y \cos \psi+X \sin \psi)+\Delta M_{z}
$$


Using the same approximations and assumptions, the governing equations of motion of the car-trailer can be linearized and simplified as the following form [11]:

$$
M\{\dot{x}\}+D\{x\}+C_{B} u+F \delta=0
$$

where,

$$
u=-K_{p} * a_{y}^{\prime}
$$

The lateral acceleration of the trailer $\left(a_{y}^{x}\right)$ can be formulated using mathematical manipulations and operations. However, an accelerometer sensor was used to measure the lateral acceleration of the physical prototype.

\section{B. ESC Controller Design}

There are several types of control techniques that can be used to design an ESC system, such as, PID and LQR [12]. The purpose of this research is not only to design an ESC controller but also to fabricate a small-scaled car-trailer prototype equipped with the ESC system. Thus, a feasible ESC controller is designed. A P-control scheme is used for simplicity of tuning, proving the concept design, and fabricating a small-scaled cartrailer prototype with the ESC.

The block diagram of the controller design is shown in Fig. 2, representing the P-control scheme.

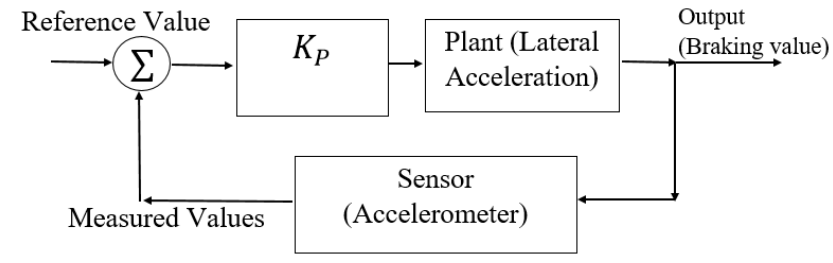

Figure 2. Block diagram representation of the designed system with a Pcontroller.

\section{Virtual Car-Trailer Prototype with the ESC system}

The virtual car-trailer prototype with the ESC system is designed and tested using CarSim software package. CarSim provides various vehicle and trailer models that could be used to simulate selected testing maneuvers.

Since the trailer is the focus of the research, the ESC controller designed in Matlab was integrated in the car-trailer model developed in CarSim. The designed ESC is built upon the existing Anti-Locking Braking System (ABS) of trailers. The ESC controls the right and left brakes of the trailer independently. This is known as differential braking [13]. Differential braking is used to control the yaw moment, which affects the lateral acceleration of the trailer.

To ensure that the ESC is functioning as desired, several testing maneuvers are simulated, such as a double lane change maneuver.
Fig. 3, shows the integration of the ESC controller designed in Matlab/Simulink software and the car-trailer model generated in CarSim software.

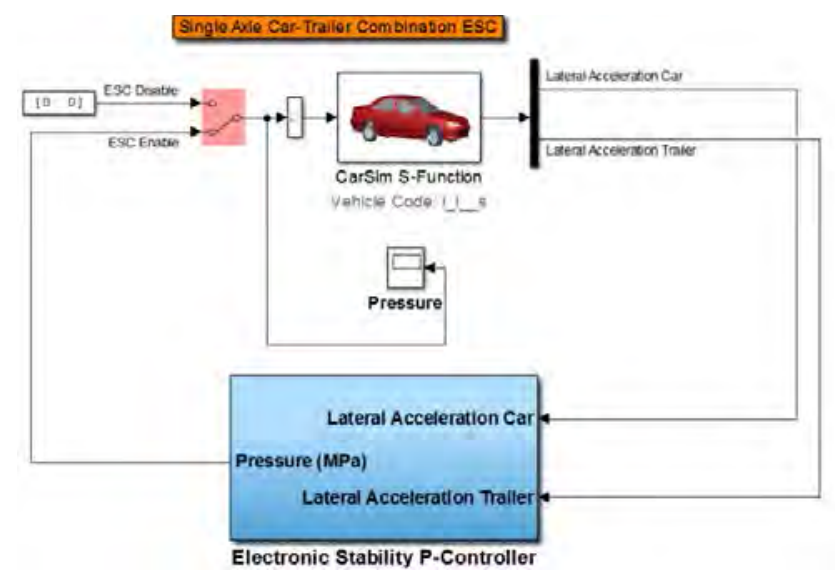

Figure 3. CarSim model of the Car-trailer combination.

The car-trailer block in CarSim exports the lateral acceleration of the leading vehicle and the trailer into the PController based ESC system. The controller multiplies the input by the gain $K_{p}$, and then a generated brake pressure value is sent back to the car-trailer block to apply brakes on a specific wheel. This process continues until the error between the desired and actual lateral accelerations is minimized. The ESC controller is designed to meet the requirements of a differential braking where brakes are applied on each wheel independently. In addition, the controller applies braking force in form of brake pulsing to prevent wheels from locking.

\section{Small-Scaled Physical Car-Trailer Prototype with the ESC System}

Once all the results are justified based on the numerical results of the virtual car-trailer prototype with the ESC system, a 1:10 small-scaled physical car-trailer prototype with the ESC system is designed and fabricated. The purpose of building the physical prototype is to conduct similar tests that have been done in CarSim and to compare the performance measures derived from both cases.

Since the physical prototype is equipped with a P-controller, a microcontroller is required on board. An Arduino Uno is used as the brain of the module to simulate all the data gathered by the accelerometer. The designed ESC is stored in the microcontroller to apply the required braking force based on the input of the lateral acceleration. In addition, the fabricated trailer is equipped with differential braking using two electromagnetic brakes. Fig. 4, shows the fabricated prototype with all the mentioned components needed to achieve a functional ESC. 


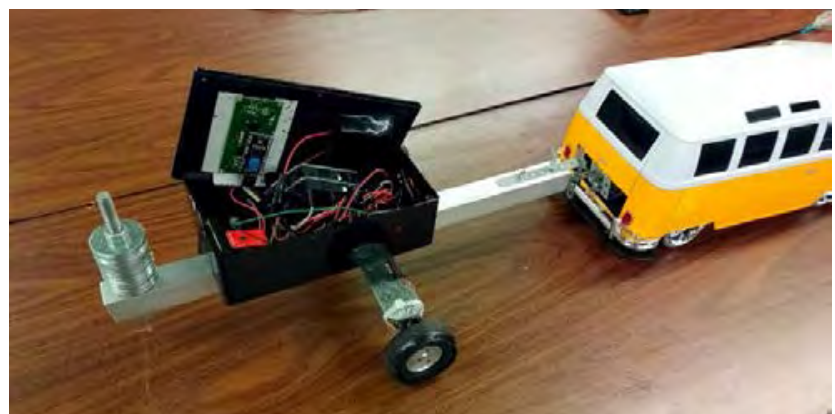

Figure 4. The fabricated 1:10 scale prototype for testing the functionality of the ESC controller.

\section{RESULTS AND DISCUSSION}

As mentioned earlier, the car-trailer model developed in CarSim is used to simulate the double lane change maneuver to test the effectiveness of the designed ESC. A representation of double lane change maneuver can be seen in Fig. 5.

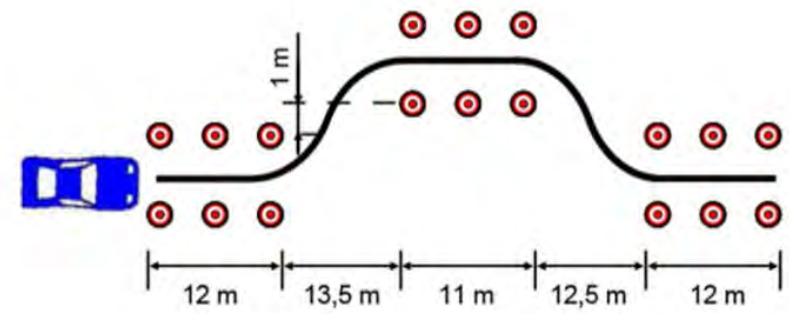

Figure 5. A representation of a double lane change maneuver [14].

The first test is done using the combination of a car towing a trailer, which is not equipped with an ESC. The Fig. 6, shows the graphs representation of the lateral acceleration over time.

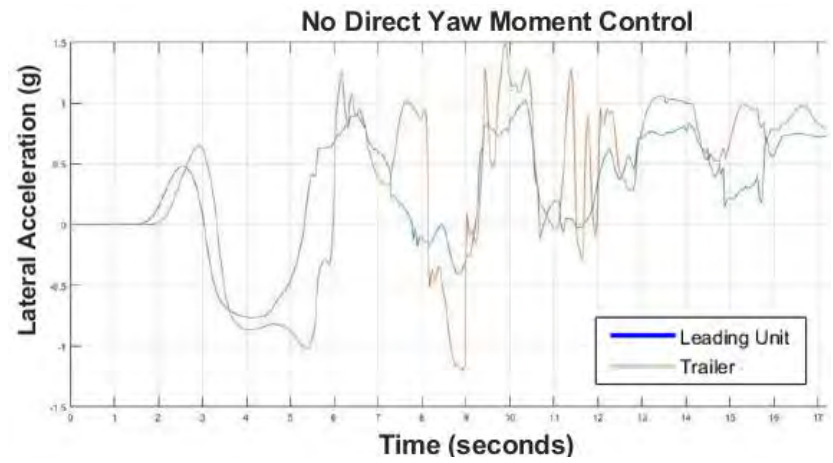

Figure 6. Graph generated from CarSim, representing the behavior of the car-trailer combination when ESC is OFF.

By looking at the graph in Fig. 6, the blue line represents the lateral acceleration of the leading vehicle which gets affected by the trailer in similar scenarios, such as double lane change maneuver, whereas the orange line represents the lateral acceleration of the trailer. It is concluded that the trailer's reaction has a time delay and then the combination lost stability. In addition, it is also determined that the trailer has a higher lateral acceleration at all times which means the trailer has a larger sway rate than the leading car which results in losing control over the combination.

To examine the system, the same test is done. However, the car-trailer combination is now equipped with the designed ESC. Fig. 7, represents the lateral acceleration over time of the leading vehicle and trailer with the ESC controller.

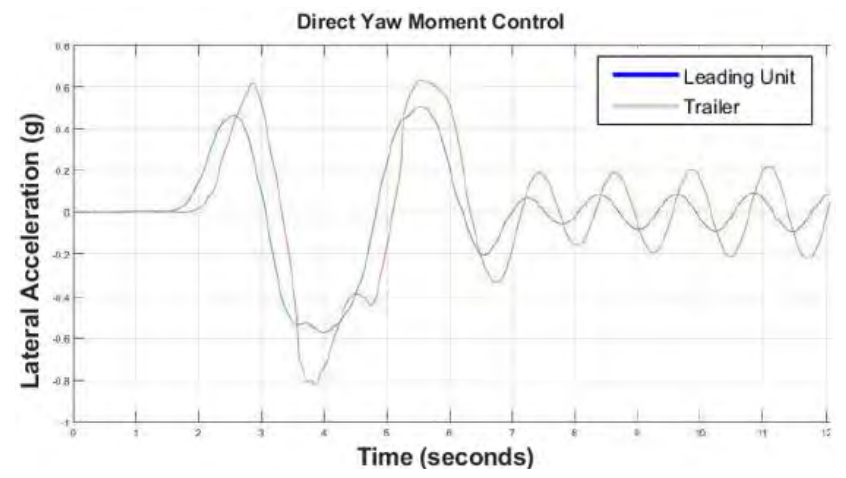

Figure 7. Graph generated from CarSim, representing the behavior of the car-trailer combination when ESC is ON.

By looking at the plots in Fig. 7, the blue curve represents the response of the leading vehicle, where the orange curve represents the trailer's behavior. It is observed that the combination has its lateral acceleration peaks at the turning points. As expected, the trailer has a larger lateral acceleration compared to the leading vehicle. However, the combination does not lose stability and sway is controlled. By looking at the graph between time intervals of 7-18 seconds, the combination has a minor oscillation and that was due to the use of Pcontroller.

By comparing the behavior of controlled and uncontrolled combination, we conclude that the trailer's lateral acceleration has an impact on the leading vehicle as it loses stability in the first case when the trailer loses stability. In addition, the lateral acceleration of leading vehicle and trailer have a larger amplitude when the combination is not equipped with a controller.

As stated previously, the testing phase for the physical prototype defers from the virtual prototype. To compare the results of the virtual and physical prototype, two test have been done using the physical prototype. Both tests have the same scenario where the vehicle would accelerate to a certain speed using a treadmill until it loses stability. To exert graphs from the physical prototype, a gyroscope is used to calculate the articulation angle between the leading vehicle and the trailer with reference to the leading car. Fig. 8, shows the representation of the articulation angles over time in both controlled and uncontrolled situations based on the testing results using the small-scaled physical car-trailer prototype. 


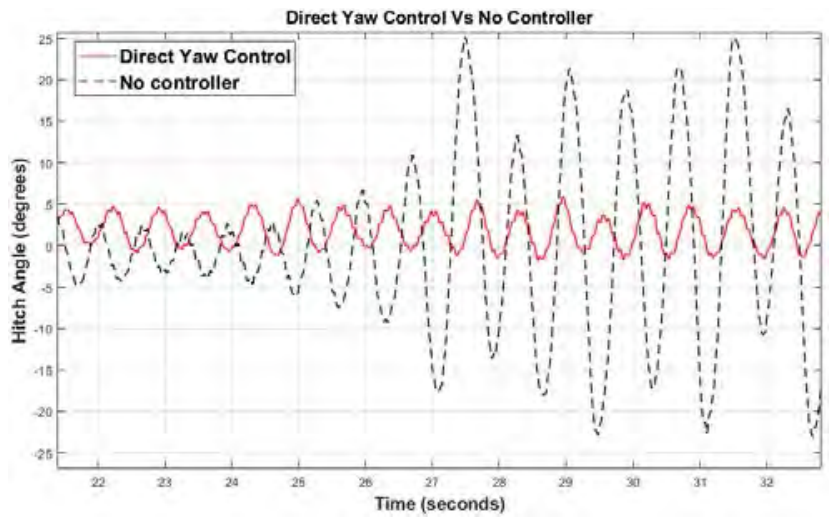

Figure 8. Comparison between the responses of the controlled and uncontrolled car-trailer combination based on the test with the small-scaled car-trailer prototype.

\section{CONCLUSION}

An active trailer differential braking controller is designed using the P-control technique based on a 3-DOF yaw-plane cartrailer model. The ESC controller designed in Matlab is then integrated with the car-trailer model generated for cosimulation. Built upon the virtual car-trailer with the ESC, a 1:10 small scaled physical car-trailer prototype with the ESC is fabricated. In both virtual and physical prototype tests, the ESC improves the stability by controlling the yaw moment of the combination. Although the results based on the P-control scheme are promising, an advanced control scheme for the ESC could be used to further improve the performance of the ESC. Since the project is done under limited resources and time, there is room to improve in a few aspects, such as building a fullscale car-trailer prototype and implementing an advanced control strategy for the ESC.

\section{ACKNOWLEDGEMENT}

The lead author would like to thank his team members for their time and efforts in this project.

\section{REFERENCES}

[1] Ministry of Transportation (MTO), Driver's Handbook. Ministry of Transportation (MTO). April 4.2017. [Online]. Available: https:// www.ontario.ca/document/official-mto-drivers-handbook/towing\#section-1. Accessed on: October 9, 2017.

[2] Automatic trailer sway sensing and brake applying system by H.A. Reise. (1981, Mar 24). USRE30550 [Online]. Available: https://www.google.com/ patents/USRE30550. Accessed on: October 9, 2017.

[3] sway control means for a trailer, by J. T. Kasselmann, H. Dorsett, H. J. Burkett, and T. B. Corporation. (1976, Apr. 06). US3948567 [Online]. Available: https://www.google.com/patents/US3948567. Accessed on: October 10, 2017.

[4] S. U. J. Smith, "Avoid a Jackknife: Mirage trailers: TNT trailer. [Online]. Available: http://www.miragetrailers.com/avoid-a-jack-knife.html. Accessed on: October 7, 2017.

[5] 2010. TheTruckersPlace, 2010. [Online]. Available: http:// www.thetruckersplace.com/jackknife.aspx. Accessed on: October 11, 2017.
[6] E.K. Liebemann, K. Meder, J. Schuh, G. Nenninger, "Safety and Performance Enhancement: The Bosch Electronic Stability Control (ESP)," 2005 [Online]. https://

pdfs.semanticscholar.org/976e/85445ed0ddfa95788c79b1474203d39e1f15.pd f. Accessed on: October 9, 2017.

[7] Method and apparatus for controlling a trailer and an automotive vehicle with a yaw stability control system, by E. E. Tseng (2005, Nov. 1). United States Patent US 6,959,970 B2.

[8] Trailer oscillation detection and compensation method for a vehicle and trailer combination by A. L. Getman, P.R. MacNeille, A. D. Shaffer, (2012, April 24). United States Patent (10) Patent No.: US 8,165,770 B2 Patent US $8,165,770$ B2.

[9] Md M. Isalm, Y. He, S. Zhu, and Q.Wang, . 2014. "A comparative study of multi-trailer articulated heavy-vehicle models," Proc. Inst. Mech. Eng., Part D, 2014. doi: $10.1177 / 0954407014557053$.

[10] M. F. J. Luijten, "Lateral Dynamic Behaviour of Articulated Commercial Vehicles," M.A. thesis, Dept. of Mech. Eng., Eidnhoven University of Technology, Netherlands, 2010. [Online].

Available: http://www.mate.tue.nl/mate/pdfs/12050.pdf.

[11] R. Shamim, M. Islam and Y. He, "A Comparative Study of Active Control Strategies for Improving Lateral Stability of Car-Trailer Systems," SAE Technical Paper 2011-01-0959, 2011, doi: 10.4271/2011-01-0959.

[12] E. Lee, T. Sun, and Y. He,"Lateral stability analysis for car-trailer combinations," ASME 2016 International Mechanical Engineering Congress and Exposition. Arizona, 2016. doi: 10.1115/IMECE2016-65648.

[13] R. Rajamani, 2006. "Electronic Stability Control," in Vehicle Dynamics and Control. Mechanical Engineering Series," Boston, MA: Springer, 2006. doi: 10.1007/0-387-28823-6_8.

[14] I. J. Neubeck, "Road test," 2017. [Online.] Available: http://www.fkfs.de/ en/automotive-engineering/leistungen/driving-dynamics/road-test/. Accessed on October 9, 2017.

\section{APPENDIX A}

$$
M=\left[\begin{array}{cccc}
m_{1}+m_{2} & -m_{2} d & -m 2_{e} & 0 \\
-m_{2} d & I_{1}+m_{2} d^{2} & m_{2} e d & 0 \\
-m_{2} e & m_{2} e d & I_{2}+m_{2} e^{2} & 0 \\
0 & 0 & 0 & 1
\end{array}\right]
$$

$$
F=\left[\begin{array}{c}
c_{1} \\
c_{1} a \\
0 \\
0
\end{array}\right]
$$

$$
\begin{aligned}
& D=\frac{1}{u} \times \\
& {\left[\begin{array}{cccc}
-c_{1}-c_{2}-c_{3} & -c_{1} a+c_{2} b+c_{3} d+\left(m_{1}+m_{2}\right) u^{2} & c_{3}(h+e) & -c_{3 u} \\
-c_{1} a+c_{2} b+c_{3} d & -c_{1} a^{2}-c_{2} b^{2}-c_{3} d^{2}-m_{2} d u^{2} & -c_{3} d(h+e) & c_{3} d u \\
c_{3}(h+e) & -c_{3} d(h+e)-m_{2} e u^{2} & -c_{3}(h+e)^{2} & c_{3}(h+e) u \\
0 & -u & u & 0
\end{array}\right]}
\end{aligned}
$$




\section{Dry Surface Micromanipulation Using an Untethered Magnetic Microrobot}

\author{
Omid Youssefi \\ Department of Mechanical and Industrial Engineering \\ University of Toronto \\ Toronto, ON \\ omidy@mie.utoronto.ca
}

\author{
Eric Diller \\ Department of Mechanical and Industrial Engineering \\ University of Toronto \\ Toronto, ON \\ ediller@mie.utoronto.ca
}

\begin{abstract}
Precise micromanipulation tasks are typically performed using micromanipulators that require an accessible workspace to reach components. However, many applications have inaccessible or require sealed workspaces. This paper presents a novel magnetically-guided, and untethered, actuation method for precise and accurate positioning of microcomponents on dry surface within a remote workspace using a magnetic microrobot. By use of an oscillatory and uniform magnetic field, the magnetic microrobot can traverse on a dry surface with fine step size and accurate open-loop vector following, $3 \%$ and $2 \%$ of its body-length, respectively (step size of $7 \mu \mathrm{m}$ ). While maintaining precise positioning capability, the microrobot can manipulate and carry other microcomponents on the dry surface using direct pushing or grasping using various attachments, respectively. We demonstrate and characterize the untethered micromanipulation capabilities of this method using a $3 \mathrm{~mm}$ cubic microrobot for use in applications such as microassembly and cargo transport.
\end{abstract}

Keywords: Microrobotics; Microfactory; Microassembly; Magnetic Micromanipulation; Untethered Actuation.

\section{INTRODUCTION}

Industrial robotic systems, such as robotic arms, part positioners, and sorters, have enabled rapid and precise manufacturing of large-scale assemblies. Extension of such systems into centimeter-scale manufacturing, such as in part pick-and-place for printed circuit board assembly, has also proven to be practical. However, significant challenges exist for manufacturing at the microscale level (characteristic dimensions of less than $10 \mathrm{~mm}$ ). Flexure-based mechanisms, such as the delta [1] and MEMS-based [2] positioners, can position microcomponents within a few micrometer and nanometer accuracies, respectively. High speeds and precise operation make tethered and traditional manufacturing methods suitable for widespread use. Tethered systems require an entry area for access to the workspace, which may not be possible in some applications requiring sealing. Tethered assembly of microcomponents, such as the $200 \mu \mathrm{m}$ optical prism used in [3], require a cleanroom environment. On the other hand, untethered microassembly can be performed within a smaller and sealed workspace, which is a cost-effective approach as the workspace can meet the requirements of a clean environment.

Due to the small scale of untethered microrobots, onboard power and actuation is impractical and thus, most designs in the literature rely on external actuation such as using magnetic fields [4], [5], and [6]. Most in-liquid micromanipulation methods use magnetic gradient pulling to perform micromanipulation tasks [7], [8], and [9]. In-liquid pick-and-place operations, using bubbles [10] and a magnetic microgripper [7], have proven effective in performing autonomous tasks and are well studied. However, micromanipulation on dry surface is required for many applications and it is challenging due to the strong friction and adhesion present on the small scale [5]. To avoid the stiction phenomenon altogether, levitated micromanipulation techniques, using diamagnetic [11] and acoustic [12] levitations, are used. However, to overcome the stiction forces on the surface, methods such as the stick-slip [13] and tumbling motions [14], use varying magnetic fields to induce torque instead of direct gradient pulling. Magnetic torque enables lifting and rolling of the magnetic microcomponents which indirectly result in step-wise motion on a surface.

Dry surface micromanipulation methods in the literature either have coarse stride step sizes (at least a body-length) [14], [15], and [16] that are imprecise or are uncontrolled during some portion of their stride cycle [13]. In addition, a pushing force cannot be applied using these for direct micromanipulation. Accuracy, precision, and speed are the core requirements for applications involving dry surface microassembly, where assembly force, as well as precise and efficient positioning and orienting are necessary. Most methods require complex actuation and control schemes and thus, become sensitive to external disturbances. Use of closed-loop controllers can alleviate the positioning inaccuracies, however; ability of applying pushing force is insufficient or lacking. Dry surface micromanipulation tasks, in inaccessible workspaces, require untethered, precise, and accurate locomotion with strong force application, which have not been achieved so far.

In this work, we introduce a novel method for precise positioning of a magnetic microrobot on dry surface. Unlike 
previous work, which aim to overcome the surface stiction forces, the new method of walking takes advantage of friction forces to apply strong pushing forces required for microassembly. The open-loop operation of this walking method provides accurate path following (within $2 \%$ of body-length) and has walking step sizes as small as 3\% of the microrobots largest dimension, which enable precision micromanipulation and handling of various microcomponents.

\section{CONCEPT}

In this section, we introduce the new principle behind the actuation, the stepwise, and precise locomotion of a cubic magnetic microrobot on a dry surface. By taking advantage of high surface friction force, the microrobot can manipulate other microcomponents either by carrying or direct pushing.

\section{A. Method of Walking}

Walking is achieved via remote magnetic actuation which is a uniform and oscillatory field. A torque, $\boldsymbol{\tau}_{\mathrm{B}}$, is induced on the magnetic microrobot resulting in change of its kinematic state. As shown in Fig. 1(a), the external magnetic field vector, $\mathbf{B}$, rotates at a frequency, $\mathrm{f}$, about an axis defined by angles $\theta$ and $\beta$ which are the heading and the tilt angles, respectively. This field profile cone, as shown in Fig. 1(a), has an opening angle of $\phi$ degrees. The microrobot, with its volume magnetization vector, $\mathbf{M}$, follows this field orientation because of the induced torque, which is defined by

$$
\mathbf{\tau}_{\mathrm{B}}=\mathbf{M} \times \mathbf{B} \text {. }
$$

This two-degree-of-freedom (2-DoF) actuation, along with the dynamic interaction of the microrobot with the dry surface, result in a stepwise motion with a full-cycle stepping shown in Fig. 1(a). The motion characteristics (i.e. step precision, speed, and path accuracy) are finely tunable by varying the field parameters. To achieve a consistent stepping motion, a suitable pose must be established such that the microrobot contacts the surface with exactly two defined points. This is the case for most flat-edged contacts where the line contact can be approximated with two points dictated by surface roughness profile. However, for accurate and repeatable motion, the two points can be physically fabricated to be more prominent to ensure repeatable contact over successive stepping cycles and during micromanipulation tasks such as pushing. In this work, an attachment, with two sharp corners, is glued underneath the microrobot for use in the experiments. Unlike tumbling and rolling methods for locomotion of flat-edged geometries, [14] and [15], the point contact reduces the contact friction, however; it enables pushing of microcomponents with precision using sharp-point anchorage, controllable steering, and precise positioning.

\section{B. Method of Force Application}

Two significant parameters of interaction between the microrobot and the surface, especially at the microscale level, are: the friction, $\mathbf{F}_{\mathbf{N}}$; and the surface adhesion, $\mathbf{F}_{\mathbf{a}}$, forces. These correlated forces enable the microrobot to use the
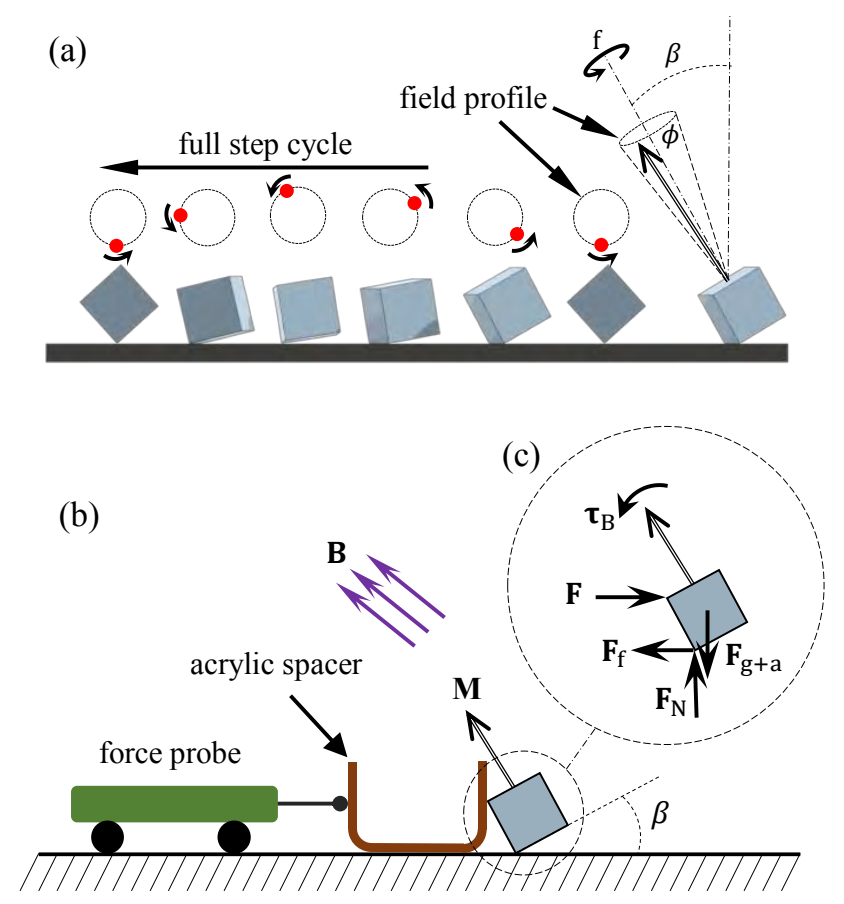

Figure 1. Conceptual schematic of walking and pushing tasks: (a) walking motion sequence during a full step cycle; (b) schematic of the force measurement setup; and (c) free-body diagram showing the forces and torque induced on the block at an instant of a pushing task.

friction force, $\mathbf{F}_{\mathbf{f}}$, to apply an equal and opposite force, $\mathbf{F}_{\mathbf{p}}$, to other microcomponents. A free-body diagram showing forces during a pushing task (Fig. 1(b)) is shown in Fig. 1(c). By adjusting the heading angle, the microrobot can push microcomponents around on a surface on a specific path, or towards a goal pose in a semi-holonomic fashion.

\section{MODELLING AND CHARACTERIZATION}

In this section, we present the magnetic field actuation scheme, and show the characteristics of a pushing task.

\section{A. Time Varying Magnetic Field}

A uniform magnetic field (field with no gradient) is necessary for the 2-DoF actuation of the microrobot. The smooth rotation of this field along the circular profile shown in Fig. 1(a), is necessary to prevent jerks, which would result in undesired movement or jumping of the microrobot. Thus, a central controller, with accurate time keeping capability, is essential to ensure the smooth actuation. The magnetic field is defined by

$$
\mathbf{B}=\mathrm{B}\left(\mathrm{R}_{\mathrm{z}}(\theta) \mathrm{R}_{\mathrm{y}^{\prime}}(\beta)\right)\left[\begin{array}{c}
\sin (\phi / 2) \cos (2 \pi \mathrm{ft}) \\
\sin (\phi / 2) \sin (2 \pi \mathrm{ft}) \\
\cos (\phi / 2)
\end{array}\right]
$$

where $\mathrm{t}$ is time, $\mathrm{B}$ is the magnitude of the magnetic flux density, $R_{z}$ is the rotation matrix about the $Z$ axis for the world frame, and $\mathrm{R}_{\mathrm{y}^{\prime}}$ is the rotation matrix about the $\mathrm{Y}$ axis for the new rotated frame. From (1), given an object with a fixed magnetization, a strong magnitude field is necessary for full rolling motion because a higher magnetic torque is 


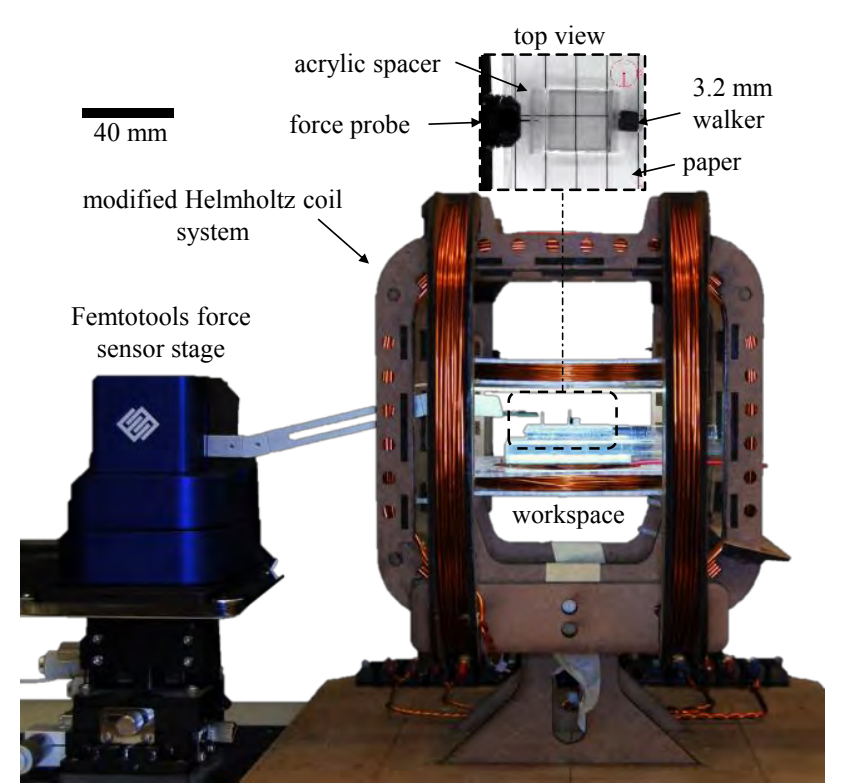

Figure 2. Experiment setup: the microrobot, the acrylic spacer, and the force sensor are placed on a paper surface within the workspace which is in the middle of custom-designed 3-axis Helmholtz coil system.

required to overcome the torque due to the weight and the surface adhesion forces from a lay-flat pose. However, with this method, since the microrobot needs to follow only the direction of the varying field with a short span, lowermagnitude field can be used. This becomes apparent for the case when a cubic microrobot is used and when $\beta=45^{\circ}$ where the magnetic torque is mostly overcoming the inertia (minimal at the microscale level and low frequency) as the weight is balanced by $\mathbf{F}_{\mathbf{N}}$. Therefore, unlike positioning achieved by rolling, a lower magnitude magnetic field can be used in certain configurations.

\section{B. Pushing Force Characterization}

Using the same magnetic field actuation in (2), the microrobot can exert a pushing force on microcomponents with a flat and vertical surface. The friction and adhesion forces anchor the contact point of the microrobot to the surface and thus, an equal and opposite force can be applied to the microcomponent. This force is applied during a portion of a full-step cycle corresponding to the configuration shown in Fig. 1(b), where the pushing force $\mathbf{F}_{\mathbf{p}}$ is achieved because of the magnetic torque $\tau_{\mathrm{B}}$. As the microrobot relies on the point of contact to apply a force, the magnitude of the force is limited by the friction force between the microrobot and the surface. A pushing experiment was performed, and the results are presented in section IV(B).

\section{RESULTS AND DISCUSSION}

\section{A. Experimental setup - Magnetic Field and Force Sensing}

A custom-designed setup was fabricated to actuate, sense, and measure the performance of the microrobot. The prototype coil system is comprised of three pairs of nested and mutually-orthogonal square Helmholtz coils which can generate a nearly uniform magnetic field of up to $3 \mathrm{mT}$ in a workspace with dimensions of $30 \times 40 \times 40 \mathrm{~mm}$. The

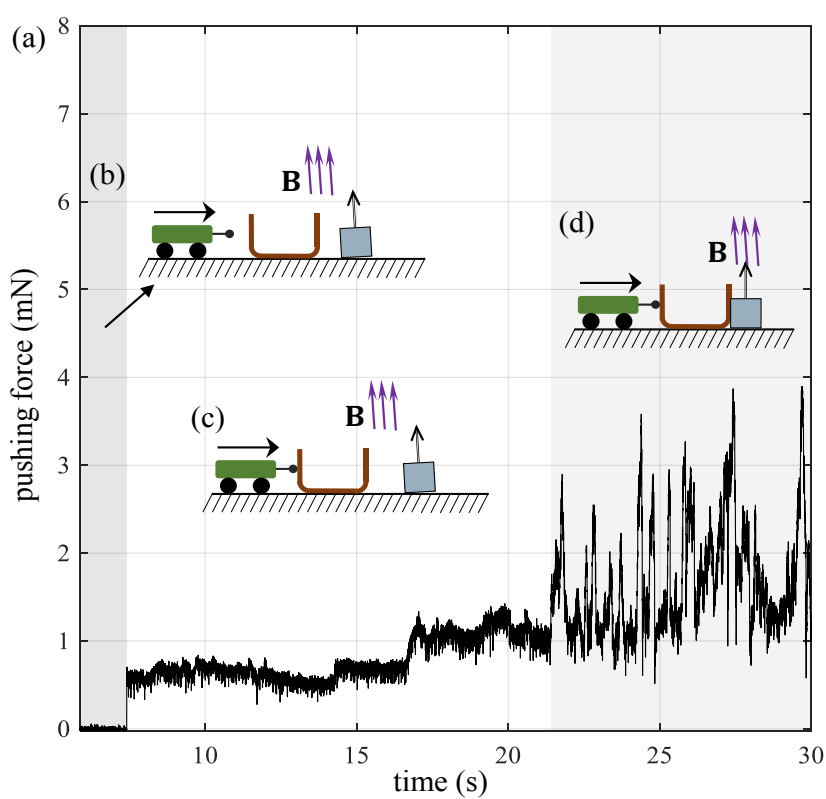

Figure 3. Friction force measurement between the microrobot and the paper surface, measured by a moving force sensor: (a) pushing force raw data; (b) interval of no contact, force sensor approaching the spacer; (c) interval of force sensor contact with the spacer; and (d) interval of microrobot contact with the spacer.

magnitude of the field is directly proportional to the current flowing in the coils which are controlled by six amplifiers (Advanced Motion Controls, 30A8) and commanded by a digital-to-analog converter card (Sensoray, Model s826). The commands are sent from a custom $\mathrm{C}$-code executed on a Linux-based PC. A top-view camera (FOculus, FO123TB) is used to record position information of the microrobot. The proof-of-concept microrobot is composed of a cubic N52 magnet with side length of $3.22 \mathrm{~mm}$. A two-point attachment is glued on the bottom surface of the magnet to ensure consistent contact with the surface. To measure the pushing and friction forces, a microforce sensing probe (Femtotools FT S10000) was mounted on a moving stage with a constant feed rate.

\section{B. Pushing Friction Test Results}

To test the pushing capability of the microrobot, it was necessary to first determine the friction force present between the microrobot and the surface. This friction force was measured indirectly by using the acrylic spacer placed in between the force probe and the microrobot. The acrylic spacer provided two vertical surfaces for accurate transfer of force. Using a $3 \mathrm{mT}$ constant magnetic field, the microrobot was tilted $5^{\circ}$ towards the acrylic spacer as shown in Fig. 3(b). The force probe is moving to the right with a constant federate. During the initial approach (Fig. 3(b)), a small amount of noise is observed. Upon reaching the acrylic spacer, the sensor measured an averaged friction force of about $0.5 \mathrm{mN}$ which is the friction of the acrylic spacer with the surface. At around 17 seconds, the acrylic spacer contacted the top edge of the microrobot which corresponds to the increase in force value at that time. After the microrobot was rotated to a flat pose by the acrylic spacer, the magnetic field was turned off and the microrobot was 
pushed on the surface by the force probe (Fig. 3(c)). An average force of $1.70 \mathrm{mN}$ was measured during this interval. By subtracting the acrylic friction force, the average friction force of the microrobot was calculated to be $1.2 \mathrm{mN}$.

The highly varying friction force profile, as shown in Fig. 3 (c), is due to the stick-slip characteristic of friction on dry surfaces. Thus, unlike sliding by gradient pull, a step-wise motion is preferred on dry surface. Further, the high impulse and oscillatory force application of the microrobot on other microcomponents enables the reliable micromanipulation.

\section{Open-loop Dead Reckoning Experiment and Locomotion Characterization}

To characterize the motion, the microrobot was placed on a piece of copy paper inside the coil system and the position of the microrobot was tracked over time using vision analysis post-experiment. The tilt angle, opening angle, and frequency parameters were varied to characterize the effects of each on the walking accuracy and speed. The result of the first experiment, with $\beta=20^{\circ}, \phi=8^{\circ}$, and $\mathrm{f}=1 \mathrm{~Hz}$, is shown in Fig. 4(a). A heading angle of $0^{\circ}$ (travelling to east) was set and the experiment was conducted for a duration of $34.8 \mathrm{~s}$, effectively corresponding to about 35 full stepping cycles. A distance of $24.81 \mathrm{~mm}$ was covered during this duration in which, the effective per-step resolution is $0.7 \mathrm{~mm}(22 \%$ of body-length). The on-course path deviation error was characterized by RMSE, as shown as an inset in Fig. 4, which is $0.059 \mathrm{~mm}$ ( $1.8 \%$ of body-length). The end position of the microrobot was too close to the expected position for characterizing the offset using vision. Despite an open-loop actuation, the vector following is precise and accurate. This is as a result of the oscillatory and fine stepping of the microrobot on the dry surface. A separate experiment, using $0.25 \mathrm{~mm}$ cubic magnet on glass, was performed with step size and path deviation of $2 \%$ and $3 \%$ of body-length, respectively.

To characterize the speed, three opening angles $\left(\phi=8^{\circ}\right.$, $10^{\circ}$, and $15^{\circ}$ ) were selected and a magnetic field of $3 \mathrm{mT}$ was applied. The speed depends on all field parameters, however; the most notable dependability is on the frequency and the opening angle. The distance of the CoM was measured using post-experiment vision analysis and the results are shown in Fig. 4(b). Linear fits were calculated, and the average speeds were found to be $0.714,1.004$, and $1.542 \mathrm{~mm} / \mathrm{s}$ for $\phi=8^{\circ}$, $10^{\circ}$, and $15^{\circ}$, respectively. These sub-body length speeds and walking steps are finely adjustable making them suitable for precision positioning and locomotion. Using this microrobot, a maximum linear speed of $4.23 \mathrm{~mm} / \mathrm{s}$ (131\% of body length) was achieved with $\phi=45^{\circ}, \beta=30^{\circ}$, and $\mathrm{f}=1 \mathrm{~Hz}$. The speed can also be increased by increasing the frequency. However, at high frequencies, a higher magnitude magnetic field is required because of the inertia of the microrobot. The distance plot, shown in Fig. 4(b), shows the reliability of open-loop actuation on a uniform surface. Presence of external disturbances will affect the performance, however; it can be compensated by using active feedback.
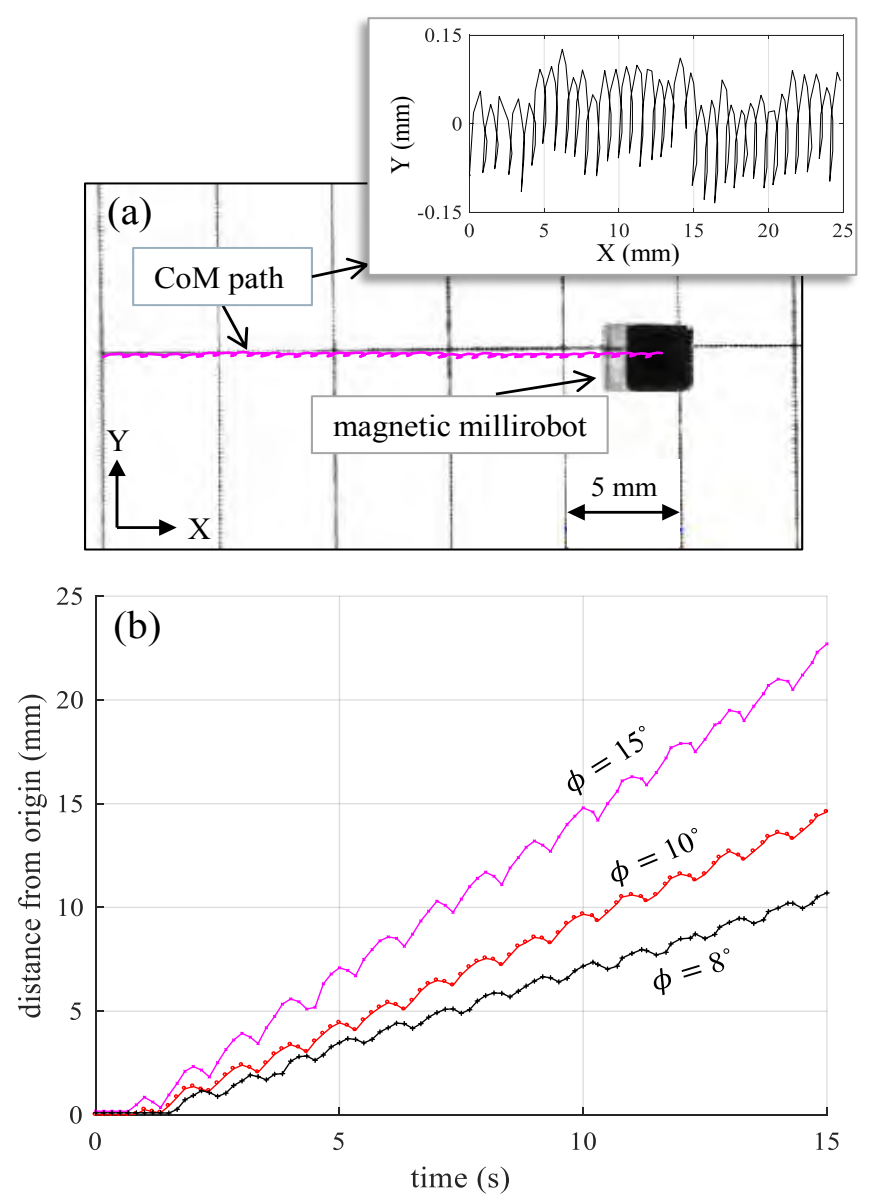

Figure 4. Precision open-loop walking characteristics of the magnetic walker: (a) top view of the microrobot at the end position with the walking path traced, $\beta=20^{\circ}, \phi=8^{\circ}, \mathrm{f}=1 \mathrm{~Hz}$; and (b) plot of distance of center of mass from the starting location for span angles, $\phi=8^{\circ}, 10^{\circ}$, and $15^{\circ}$ where $\mathrm{f}$ $=1 \mathrm{~Hz}$.

\section{CONCLUSION AND FUTURE WORK}

The new micromanipulation method presented in this paper allows for simple yet, precise open-loop and untethered positioning and micromanipulation of various microcomponents on a dry surface actuated by an external magnetic field. Unlike previous work, the presented microrobot can push other components while maintaining a reasonable positioning accuracy, and it can accurately traverse on a dry surface with different stepping size and speeds, which are finely tunable. In-place change of heading is possible by using some tilt angle giving this microrobot a holonomic characteristic to its otherwise nonholonomic motion. Due to the precise nature of the locomotion, highspeed travel is limited to 1-2 body lengths per second. For high speeds, rolling motion can be used by trading off stepping resolution and precision pushing capability. In addition, a reasonably flat surface is required for repeatable and open-loop path following, however; significant potential exists for integrating a closed-loop controller for making this method suitable for use in many industrial applications such as microassembly and cargo transport at the milli- and microscale levels. 


\section{REFERENCES}

[1] H. McClintock, F. Temel, N. Doshi, J. Koh and R. Wood, "The milliDelta: A high-bandwidth, high-precision, millimeter-scale Delta robot", Science

Robotics, vol. 3, no. 14, p. eaar3018, 2018.

[2] H. Chen, D. Jiang and I. Hagiwara, "Development of Novel XY Micropositioning Stage", Key Engineering Materials, vol. 407-409, pp. 103-106, 2009. doi:10.4028/www.scientific.net/KEM.407-408.103.

[3] B. Li et al., "Hybrid Intravascular Ultrasound and Optical Coherence Tomography Catheter for Imaging of Coronary Atherosclerosis," Catheterization and Cardiovascular Interventions, vol. 81, pp. 494-507, 2013. doi: $10.1002 /$ ccd.24295.

[4] Kummer, Michael P., et al. "OctoMag: An electromagnetic system for 5DOF wireless micromanipulation." IEEE Transactions on Robotics vol. 26, no. 6, pp. 1006-1017, 2010. doi: 10.1109/TRO.2010.2073030.

[5] E. Diller and M. Sitti, "Micro-Scale Mobile Robotics," Foundations and Trends in Robotics, vol. 2, no. 3, pp. 143-259, 2013. doi: 10.1561/2300000023.

[6] E. Diller, J. Giltinan, G. Lum, Z. Ye, and M. Sitti, "Six-degree-of-freedom magnetic actuation for wireless microrobotics," International Journal of Robotics Research, vol. 35, no. 1, pp. 114-128, 2016.

doi: $10.1177 / 0278364915583539$.

[7] J. Zhang, O. Onaizah, K. Middleton, L. You, and E. Diller, "Reliable Grasping of Three-Dimensional Untethered Mobile Magnetic Microgripper for Autonomous Pick-and-Place", IEEE Robotics and Automation Letters, vol. 2, no. 2, pp. 835-840, 2017. doi: 10.1109/LRA.2017.2657879.

[8] Pieters, Roel, et al. "RodBot: A rolling microrobot for micromanipulation," 2015 IEEE International Conference on Robotics and Automation. doi: 10.1109/ICRA.2015.7139764.

[9] Fluid-based micromanipulation: Z. Ye, E. Diller and M. Sitti, "Micromanipulation using rotational fluid flows induced by wireless magnetic micro-,anipulators," J. Appl. Phys. (Melville, NY, U. S.) vol. 112, no. 6, 064912 , 2012. doi: $10.1063 / 1.4754521$.

[10] J. Giltinan, E. Diller, and M. Sitti, "Programmable Assembly of Heterogeneous Microparts by an Untethered Mobile Capillary Microgripper," Lab Chip, vol. 16, pp. 4445-4457, 2016. doi: 10.1039/C6LC00981F.

[11] A. Hsu, et al. "Automated 2D micro-assembly using diamagnetically levitated milli-robots," Manipulation, Automation and Robotics at Small Scales (MARSS), 2017 International Conference on. IEEE, 2017.

doi: 10.1109/MARSS.2017.8001926.

[12] D. Foresti, M. Nabavi, M. Klingauf, A. Ferrari and D. Poulikakos, "Acoustophoretic contactless transport and handling of matter in air", Proc. Natl. Acad. Sci. U. S. A., vol. 110, no. 31, pp. 12549-12554, 2013.

doi: 10.1073/pnas.1301860110.

[13] C. Pawashe, S. Floyd, and M. Sitti. "Modeling and experimental characterization of an untethered magnetic micro-robot," The International Journal of Robotics Research vol. 28 no. 8. pp. 1077-1094, 2009. doi: $10.1177 / 0278664909341413$.

[14] Jing, Wuming, Nicholas Pagano, and David J. Cappelleri. "A novel microscale magnetic tumbling microrobot." Journal of Micro-Bio Robotics vol. 8, no. 1, pp. 1-12, 2013. doi: 10.1007/s12213-012-0053-1.

[15] C. Bi, M. Guix, B. Johnson, W. Jing and D. Cappelleri, "Design of microscale magnetic tumbling robots for locomotion in multiple environments and complex terrains", Micromachines, vol. 9, no. 2, p. 68, 2018. doi: $10.3390 / \mathrm{mi} 9020068$.

[16] S. Jeon, "A sphericon-shaped magnetic microrobot rolling on a surface actuated by an external wobbling magnetic field", $A I P A d v$., vol. 7, no. 5, p. 056708, 2017. doi: 10.1063/1.4974019. 


\title{
Adaptive Yaw Control of Three-axle Road Vehicles Based on Mass, Yaw Inertia and CG Position Identification
}

\author{
Yubiao Zhang*, Ami Woo, Baris Fidan, Amir Khajepour \\ Department of Mechanical and Mechatronics Engineering \\ University of Waterloo \\ Waterloo, Ontario, Canada
}

\begin{abstract}
This paper introduces an adaptive yaw control scheme based on the estimation of the vehicle mass, yaw inertia and center of gravity (CG) position. The control deigns for three-axle road vehicles, which can be trucks, buses, or even three-axle passenger cars. System parameters of these vehicles vary significantly due to varying conditions, such as unloading and fully-loading of payloads. As a result, control references and fixed-model-based controller lose efficacy. The proposed adaptive yaw control compensates these issues, utilizing the integration of a least-square based parameter identification algorithm and a Model Reference Adaptive Control (MRAC) law. Simulation test results verify the effectiveness of the proposed adaptive control scheme.
\end{abstract}

Keywords-three-axle vehicle, vehicle dynamics, parameters identification, adaptive yaw control

\section{INTRODUCTION}

Advances in low cost sensors and computation technology expedited the progress on the performance and control of modern vehicles. One of the active areas of automobile research so far has been vehicle stability and handling characteristics. A practical and proven solution to improve the vehicle dynamics during critical driving conditions has been to produce corrective yaw moment to the vehicle. Various control methods to direct yaw-moment control (DYC) has been proposed in literature including electronic stability program based on differential braking $[1,2]$ and differential traction or torque vectoring $[3,4]$. Indirect yaw moment control systems such as active steering control, which produces corrective steering angle to the wheels, have been alternative way to stabilize yaw moment $[5,6]$.

In vehicle control designs, the vehicle dynamics are often estimated using single track model. To simplify the controller design process, assumptions such as the negligible effect of the vehicle parameter variations on the dynamics and the performance region of the tire forces in the linear range are often imposed. Therefore, the performance of many existing control designs are promising specifically on two axle vehicles travelling within the linear range of the tires. However, when the effect of the vehicle parameter variations on the performance becomes substantial, the controller becomes less effective even under the same operating conditions.
One of the important parameters to take into consideration in control design is the vehicle mass. With varying mass, the center of gravity and inertia of vehicle vary, which has direct effect on the overall vehicle dynamics. For some vehicles such as passenger cars where the variation of the payload compared to the mass of the vehicle is insignificant, such changes may be tolerable. These variations are more critical in situations when the variation of the loading conditions and configurations impacts more on the overall dynamics [7-9]. The three axle heavy load trucks and commercial buses typically involve in multiple loadings and unloading of payloads more significantly compared to two axle passenger cars. These variations affect the stability and performance of the overall vehicle motion even with the appropriate control design. A controller which adapts to such changes and responds accordingly is desired. The apparent difficulty with variation of parameters in the process of control design is that these parameters are not directly measurable.

In this paper, an adaptive DYC is proposed on three-axle vehicles utilizing least-square (LS) estimation of unknown parameters of the vehicle, for improving the trajectory tracking of yaw dynamics. Section II explains the three-axle vehicle system dynamics. A single track model is developed to describe the DYC system. In section III, parameter identification and control design are developed. The mass, inertia, and center of gravity of vehicle are treated as unknown parameters and estimated indirectly in the section. The model reference adaptive control design is proposed with the reference model. The performance and effectiveness of the control strategy is proposed in Section IV. Finally, the paper is summarized and concluded in Section V.

\section{VEHICLE MODELING}

\section{A. Single Track Model}

The vehicle dynamics, in general, is non-linear and is difficult to formulate precisely. For vehicle state estimation and motion control design, linearized models are preferred. A three-axle vehicle single track model $[10,11]$, depicted in Fig. 1 is used in this paper The equations for lateral dynamics and yaw motion are expressed by

$$
\begin{gathered}
m a_{y}=F_{y f}+F_{y c}+F_{y r}, \\
I_{z} \dot{r}=F_{y f} l_{f}-F_{y c} l_{c}-F_{y r} l_{r}+M_{e}+M_{d},
\end{gathered}
$$

*email: gary.zhang@uwaterloo.ca 


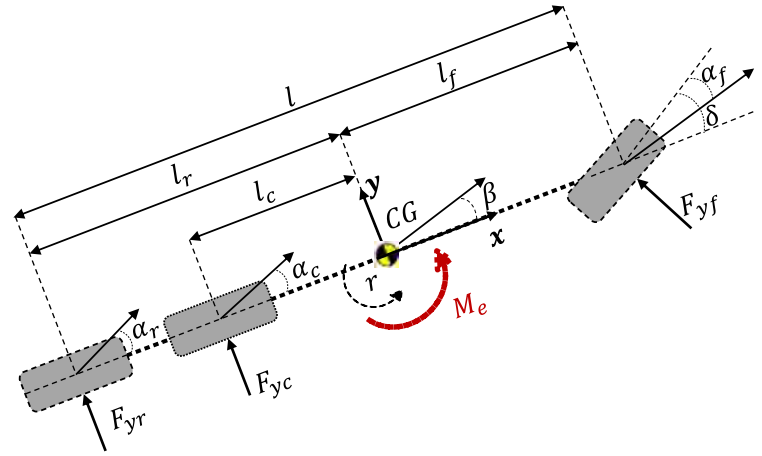

Fig. 1 The single track model

and the lateral acceleration is given by

$$
a_{y}=\dot{v}_{y}+v_{x} r=v_{x}(\dot{\beta}+r),
$$

where, assuming the slip angles are small, tire lateral forces have a linear relationship to the tire slip angle as

$$
\begin{gathered}
\left.F_{y f}=C_{f} \delta-\beta-\frac{l_{f} r}{v_{x}}\right) \\
\left.F_{y c}=C_{c}-\beta+\frac{l_{c} r}{v_{x}}\right) \\
F_{y r}=C_{r}\left(-\beta+\frac{l_{r} r}{v_{x}}\right)
\end{gathered}
$$

The terms in (1)-(6) are defined as follows:

$m, I_{z}$ - the vehicle mass and the moment of inertia about $\mathrm{z}$ axis;

$v_{x}, v_{y}, v$ - vehicle longitudinal speed, lateral speed and vehicle resultant speed;

$\alpha_{y}, \beta, r$ - lateral acceleration, body slip angle and yaw rate at vehicle's CG (center of gravity);

$F_{y f}, F_{y c}, F_{y r}$ - lateral force of front, central and rear tires;

$l_{f} / l_{c} / l_{r} / l$ - the distance from CG to front/central/rear axle, the distance between front axle to rear axle;

$C_{f} / C_{c} / C_{r}$ - the front/central/rear wheels cornering stiffness,

$\delta$ - the front wheels steering angle;

$M_{e}$ - the extra yaw moment from the controller;

$M_{d}$ - the disturbance moment, the side wind causes this disturbance moment and affect to the vehicle's stability in cornering maneuver, It is assumed to be zero in this research.

Rearranging (1)-(6), the yaw dynamics at a certain operating point can be represented by the following state space

$$
\begin{gathered}
\dot{x}=A x+B u \\
y=x
\end{gathered}
$$

where $x=\left[\begin{array}{ll}\beta & r\end{array}\right]^{T}, u=\left[\begin{array}{ll}\delta & M_{e}\end{array}\right]^{T}$, and the output $y$ is as the same as the system state;

$$
\left.A=\mid \begin{array}{cc}
-\frac{C_{f}+C_{c}+C_{r}}{m v_{x}} & -1-\frac{l_{f} C_{f}-l_{c} C_{c}-l_{r} C_{r}}{m v_{x}^{2}} \\
-\frac{l_{f} C_{f}-l_{c} C_{c}-l_{r} C_{r}}{I_{z}} & \frac{l_{f}^{2} C_{f}+l_{c}^{2} C_{c}+l_{r}^{2} C_{r}}{I_{z} v_{x}}
\end{array}\right], B=\left[\begin{array}{cc}
\frac{C_{f}}{m v_{x}} & 0 \\
\frac{l C_{f}}{I} & \frac{1}{I_{z}}
\end{array}\right] .
$$

\section{B. Model Validation}

The derived model (7) was simulated for verification with the high-fidelity model of TruckSim with step-steer input (constant speed of $80 \mathrm{~km} / \mathrm{h}$ ) with bus parameters presented in Table 1. The result in Fig. 2 shows that the vehicle dynamics has a fast response and small overshoot. But they are quite similar with the results of the TruckSim model, which verifies the reliability of the derived model for control design.
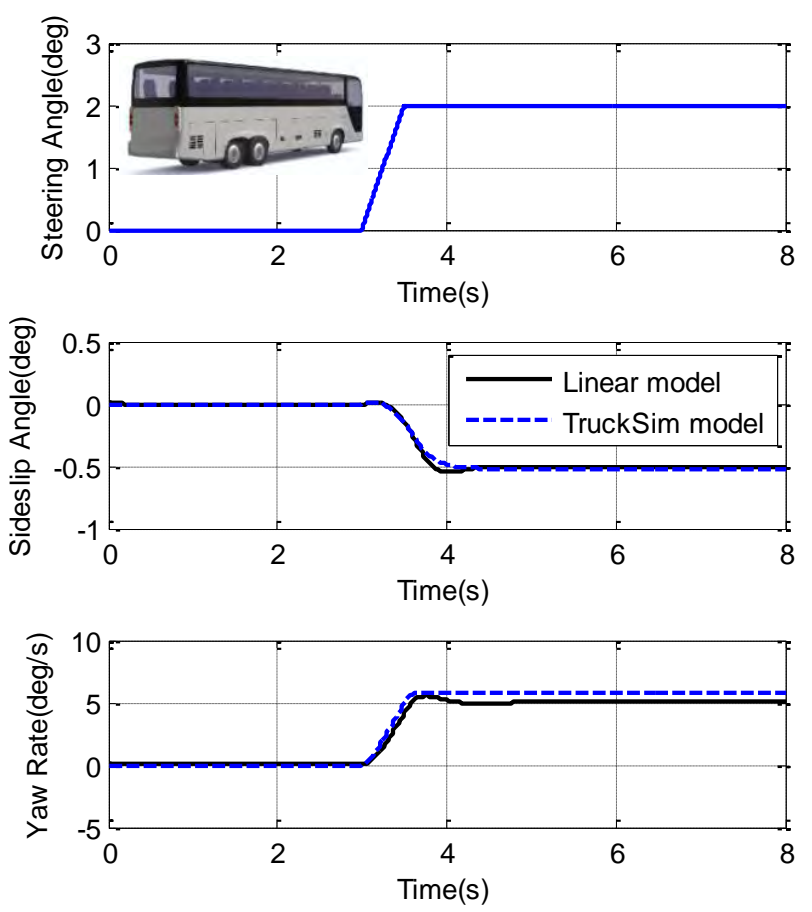

Fig. 2 Validation of the derived model by step steer

TABLE I PARAMETERS FOR A GENERIC THREE-AXLE VEHICLE

\begin{tabular}{lll} 
Parameter & Description(units) & Value \\
\hline$m$ & Vehicle mass(kg) & 9415 \\
\hline$l_{f}$ & Distance of front axle from CG(m) & 3.5 \\
\hline$l_{r}$ & Distance of rear axle from CG(m) & 3.47 \\
\hline$l_{c}$ & Distance of central axle from CG(m) & 2.29 \\
\hline$l$ & Distance of front axle from rear axle(m) & 6.97 \\
\hline$l_{r c}$ & Distance of central axle from rear axle $(\mathrm{m})$ & 1.22 \\
\hline$I_{z}$ & Yaw moment of inertia(kgm2) & 34685 \\
\hline$C_{f}$ & Total front wheels stiffness(N/rad) & $3.35 \mathrm{e} 5$ \\
\hline$C_{c}$ & Total central Wheels stiffness $(\mathrm{N} / \mathrm{rad})$ & $2.75 \mathrm{e} 5$ \\
\hline$C_{r}$ & Total rear wheels stiffness $(\mathrm{N} / \mathrm{rad})$ & $2.45 \mathrm{e} 5$ \\
\hline
\end{tabular}




\section{PARAMETER IDENTIFICATION AND CONTROL DESIGN}

\section{A. Parameter Identification}

The parameter identification (PI) of the unknown parameters is performed using an LS estimator. The estimator uses the measurements of steering angle $\delta$, extra yaw moment $M_{e}$, and yaw rate $r$, assuming available. In reality, these parameters are available for vehicles with advanced vehicle sensing technologies such as vehicles with GPS/IMU. Although these advanced sensing systems are not widely available for commercial cars yet, they could be equipped on other specific applications such as autonomous and military surveillance vehicles [12-13]. As for the vehicle side slip angle, it is usually estimated by other measurements such as the lateral acceleration and longitudinal speed of the vehicle from (1). In this research, we assume the side slip angle is available for measurement. Detailed explanations of the following basic procedures can be found in [14].

\section{1) Parametric Model}

The model (7) is in the form,

$$
\left[\begin{array}{l}
\dot{\beta} \\
\dot{r}
\end{array}\right]=\left[\begin{array}{ll}
a_{11} & a_{12} \\
a_{21} & a_{22}
\end{array}\right]\left[\begin{array}{l}
\beta \\
r
\end{array}\right]+\left[\begin{array}{cc}
b_{11} & 0 \\
b_{21} & b_{22}
\end{array}\right]\left[\begin{array}{c}
\delta \\
M_{e}
\end{array}\right]
$$

where $b_{11}=k_{11} a_{11}, k_{11}=-\frac{C_{f}}{C_{f}+C_{c}+C_{r}}, l_{c}=l-l_{f}-l_{r c}$,

$$
l_{r}=l-l_{f},
$$

$$
\begin{aligned}
& a_{21}=-\frac{l_{f} C_{f}-l_{c} C_{c}-l_{r} C_{r}}{I_{z}} \\
& =-\frac{l_{f}\left(C_{f}+C_{c}+C_{r}\right)}{z}+\frac{\left(C_{c}+C_{r}\right) l-C_{c} l_{r c}}{I} \\
& =k_{21} b_{21}+k_{22} b_{22}
\end{aligned}
$$

where $k_{21}=-\frac{C_{f}+C_{c} C_{r}}{C_{f}}, k_{22}=\left(C_{c}+C_{r}\right) l-C_{c} l_{r c}$.

Separating the known terms from the unknown ones and applying the Laplace transform to $\beta$ and $r$ yields (10) and (11), respectively,

$$
\begin{gathered}
s \beta=a_{11} \beta+a_{12} r+k_{11} a_{11} \delta=a_{11}\left(\beta+k_{11} \delta\right)+a_{12} r \\
s r=a_{22} r+b_{21}\left(\delta+k_{21} \beta\right)+b_{22}\left(M_{e}+k_{22} \beta\right)
\end{gathered}
$$

Filtering both sides of the equation with the filter $\frac{1}{\Lambda(s)}=\frac{1}{s+l}$ ,we can express the system in the form of the static parametric model (SPM) as

$$
\begin{aligned}
& z_{1}=\theta_{1}^{* T} \phi_{1} \\
& z_{2}=\theta_{2}^{* T} \phi_{2}
\end{aligned}
$$

where $z_{1}=\frac{s}{\Lambda(s)} \beta, \quad \theta_{1}^{*}=\left[\begin{array}{ll}a_{11} & a_{12}\end{array}\right]^{T}$,

$$
\begin{aligned}
& \phi_{1}=\left[\frac{1}{\Lambda(s)} \beta+\frac{k_{11}}{\Lambda(s)} \delta \quad \frac{{ }^{T}}{\Lambda(s)} r^{T} ;\right. \\
& z_{2}=\frac{s}{\Lambda(s)} r, \quad \theta_{2}^{*}=\left[\begin{array}{lll}
a_{22} & b_{21} & b_{22}
\end{array}\right]^{T} \text {, } \\
& \phi_{2}=\left[\frac{1}{\Lambda(s)} r \quad \frac{1}{\Lambda(s)} \delta+\frac{k_{21}}{\Lambda(s)} \beta \quad \frac{1}{\Lambda(s)} \quad e^{+}+\left.\frac{22}{\Lambda(s)} \beta\right|^{T} .\right.
\end{aligned}
$$

In this case, $z_{i}$ and $\phi_{i}(i=1,2)$ are available for measurement or estimation since then can be generated by filtering the measurements $u$ and $x$.

\section{2) Estimation Model and Adaptive Law}

The estimation model has the same form as the SPM with the exception that the unknown parameter $\theta^{*}$ is replaced with its estimate at time $\mathrm{t}$, denoted by $\theta(t)$, i.e.,

$$
\hat{z}_{1}(t)=\theta_{1}^{T}(t) \phi_{1}(t)
$$

$$
\hat{z}_{2}(t)=\theta_{2}^{T}(t) \phi_{2}(t)
$$

and the estimation errors,

$$
\varepsilon_{i}(t)=\frac{z_{i}(t)-\hat{z}_{i}(t)}{m_{s i}^{2}},(
$$

where $m_{s i}$ is the normalizing signal designed to bound $\phi$ from above. A straightforward choice for $m_{s i}$ is $m_{s i}{ }^{2}=1+\alpha_{i} \phi_{i}^{T} \phi_{i}$ for any $\alpha_{i}>0$.

We use the recursive least-squares algorithm with forgetting factor to minimize the cost function,

$$
\begin{gathered}
\dot{\theta}_{i}(t)=P_{i}(t) \varepsilon_{i}(t) \phi_{i}(t), \theta_{i}(0)=\theta_{i 0} \\
\dot{P}_{i}(t)=\beta P_{i}(t)-P_{i}(t) \frac{{ }_{i}^{T} \phi_{i}}{m_{s i}{ }^{2}} P_{i}(t), P_{i}(0)=P_{i 0}=Q_{i 0}{ }^{-1},(i=1,2)
\end{gathered}
$$

\section{3) Stability and Convergence}

To guarantee the convergence of $\theta_{i}(t)$ to their actual values $\theta_{i}^{*}$, the input (for this application the steering angle) should include at least two different frequencies to ensure it is sufficiently rich signal and $\frac{\phi_{i}}{m_{s i}}$ is persistently exciting [14]. Although the scenario of varying vehicle mass and yaw inertia which affects the CG position of the vehicle is considered for the paper, the varying range of the two parameters are relatively fixed small range. Table 2 shows the range of the unknown parameters to be estimated. This is used as a parameters projection for a better estimation of convergence.

\begin{tabular}{lccc}
\multicolumn{4}{c}{ TABLE II RANGES OF UNKNOWN PARAMETERS } \\
\hline Parameters & Mass(kg) & Yaw inertia(kgm2) & lf(m) \\
\cline { 2 - 3 } & & & 3 \\
minimum & 9415 & 34685 & 4
\end{tabular}




\section{B. Adaptive Reference Model}

The desired vehicle behavior is can be described by a reference model. The desired vehicle performance from the reference model is to have zero vehicle sideslip angle at the center of gravity in steady state and the desired yaw rate to be determined by the front steering angle and vehicle speed.

In steady state, variables can be solved in terms of the control input as

$$
\begin{gathered}
{\left[\begin{array}{c}
\beta_{s s} \\
r_{s s}
\end{array}\right]=-A^{-1}\left[\frac{C_{f}}{m v_{x}} \frac{l_{f} C_{f}}{I}\right\rfloor} \\
r_{\mathrm{ss}}=G_{r} \delta
\end{gathered}
$$

where, $G_{r}=-\frac{-a_{21} b_{11} a_{11} b_{21}}{a_{11} a_{22}-a_{21} a_{12}}$ is the steady-state gain of yaw rate, which leads to an under-steering behavior of the vehicle's stability. The reference model proposed is assumed to be a first-order system [15] as follows,

$$
r_{d} W(s) \delta=\frac{G_{r}}{1+\tau s} \delta
$$

where $\tau$ is the desired time constant of yaw rate. This low-pass filter is used to filter out the noise in the yaw rate signal [16]. Applying the adaptive reference model from the parameters estimation results in $\hat{G}_{r}=-\frac{-\hat{a}_{21} \hat{b}_{11} \hat{a}_{11} \hat{b}_{21}}{\hat{a}_{11} \hat{a}_{22}-\hat{a}_{21} \hat{a}_{12}}$.

\section{Controller Design}

The overall system including PI and control scheme is shown in Fig. 3. For yaw control, a model reference adaptive control (MRAC) is proposed. The desired vehicle behavior which is an LTI first order transfer function (15) is described by a reference model and is driven by a reference input. The control law is then developed so that the closed-loop plant has a transfer function equal to the reference model.

Consider the yaw rate of the plant,

$$
\dot{r}=a_{21} \beta+a_{22} \gamma+b_{21} \delta+b_{22} M_{e}
$$

where $a_{21}, a_{22}, b_{22}$ are constants but unknown. The control objective is to determine a bounded function $M_{e}=$ $f(t, \beta, r, \delta)$ such that the yaw rate is tracking the desired yaw rate from the reference model of (14), Rewriting the reference model yields

$$
(1+\tau s) r_{d}=G_{r} \delta \rightarrow \dot{r}_{d}=-\frac{1}{\tau} r_{d}+\frac{G_{r}}{\tau} \delta
$$

If the plant parameters are known, control law can be considered as

$$
M_{e}=k_{1} \beta+k_{2} \gamma+k_{3} \delta
$$

Substituting (17) into (15), results in the closed-loop system as

$$
\begin{aligned}
& \dot{r}_{c}=a_{21} \beta+a_{22} r_{c}+b_{21} \delta+b_{22}\left(k_{1} \beta+k_{2} r_{c}+k_{3} \delta\right) \\
& =\left(a_{21}+k_{1} b_{22}\right) \beta+\left(a_{22}+k_{2} b_{22}\right) r_{c}+\left(b_{21}+k_{3} b_{22}\right)
\end{aligned}
$$

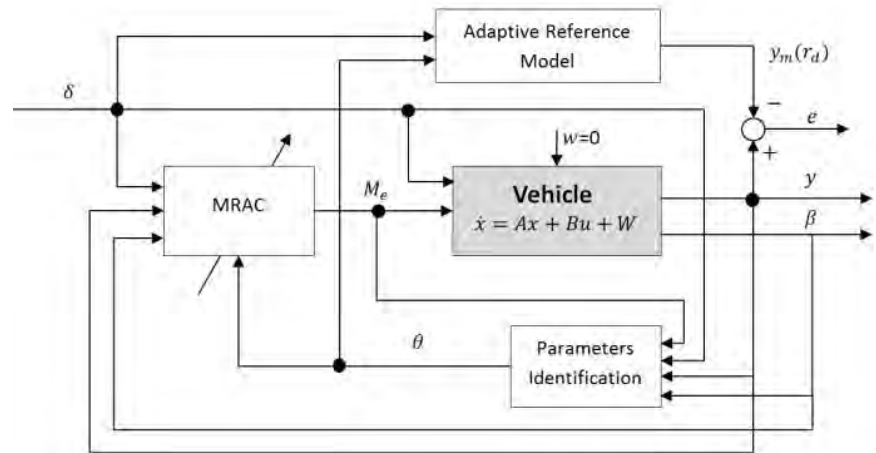

Fig.3 The PI and control scheme of whole system

The control objective is satisfied if controller parameters $k_{1}, k_{2}, k_{3}$ are carefully chosen, in a way that the closed-loop poles are stable and $\frac{\gamma_{c}}{\delta}=\frac{\gamma_{d}}{\delta}$, as follows:

$$
k_{1}=-\frac{a_{21}}{b_{22}}, k_{2}=-\frac{a_{21}}{b_{22}} k=-\frac{a_{21}}{b_{22}}
$$

Replacing the unknown plant parameters with their estimates, the adaptive control law is established as follows:

$$
\begin{aligned}
& \hat{G}_{r}=-\frac{-\hat{a}_{21} \hat{b}_{11} \hat{a}_{11} \hat{b}_{21}}{\hat{a}_{11} \hat{a}_{22}-\hat{a}_{21} \hat{a}_{12}}, \\
& \hat{k}_{1}=-\frac{\hat{a}_{21}}{\hat{b}_{22}} \hat{k}_{2}=-\frac{1}{\tau \hat{b}_{22}}-\frac{a_{22}}{\hat{b}_{22}}, \hat{k}_{3}=\frac{\hat{G}_{r}}{\hat{b}_{22}}-\frac{\hat{b}_{21}}{\hat{b}_{22}} .
\end{aligned}
$$

\section{Simulations}

To verify the proposed MRAC, simulations are performed in MATLAB and Simulink. The simulation results include vehicle responses with different parameters sets (Fig. 4), parameters identification (Fig. 5). The behavior of yaw rate, and side slip angle using adaptive yaw control is also presented (Fig. 6).

\section{A. Different Parameter Sets}

To demonstrate the influence of varying parameters, different sets of mass, yaw inertia and CG position (lf) are simulated and the vehicle dynamics response and reference

\begin{tabular}{|c|c|c|c|}
\hline Para. Set & Mass(kg) & Yaw inertia(kgm2) & lf(m) \\
\hline 1 & 9415 & 34685 & 3.5 \\
\hline 2 & 10945 & 36185 & 3 \\
\hline
\end{tabular}
model are observed. Table 3 presents two possible parameters sets and were applied to the same bus. Fig. 4 shows a significant offset for both vehicle sideslip angle and yaw rate responses due to parameters changes. For the reference of yaw rate, the similar error happened between Para. Set 1 (reference 1) and Para. Set 2. This implies that the reference model will continuously change the values for reference output resulting in the controller to track inaccurate reference. However, an adaptive reference model is an on-line estimation and will adapt to the change of vehicle parameters. 

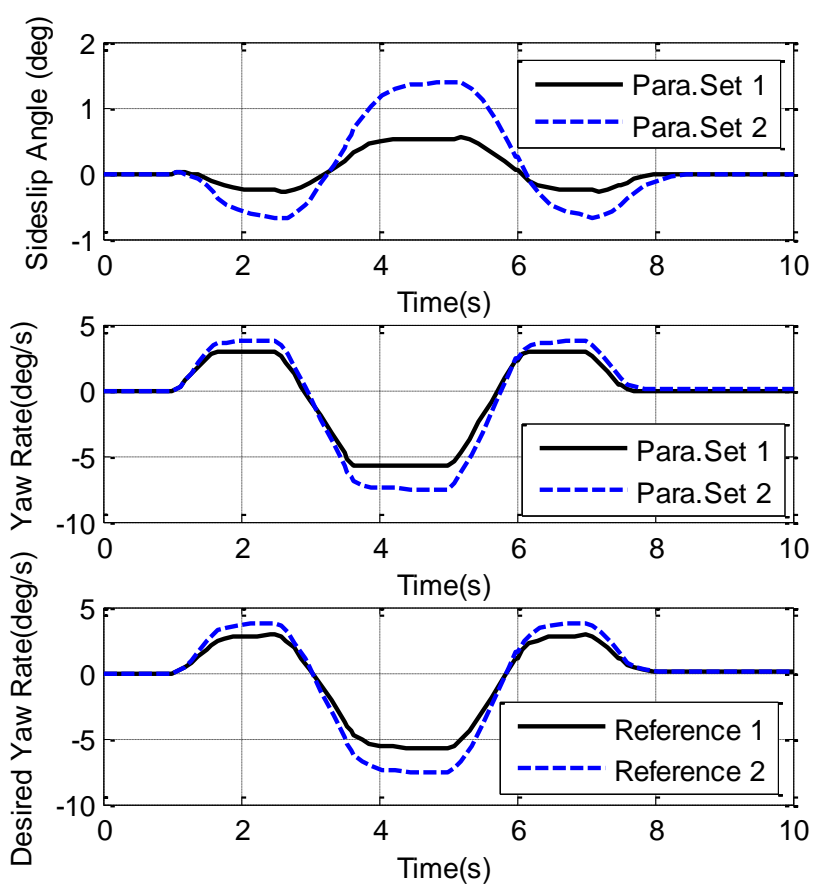

Fig.4 Vehicle responses with different parameters sets

\section{B. Results of PI and Adaptive Control}

Persistently exciting signal is desired to ensure that the unknown parameters converge to the actual values using the adaptive scheme. In practical driving condition, the driver's steering input is not a simple step input or sinusoidal input, but a combination of complex shaped inputs. For the sake of simulation, we apply the front wheel a slight steering angle incas follows,

$$
\delta=0.05+0.05 \sin 0.5 t+0.1 \sin t(\mathrm{deg})
$$

The unknown parameters were estimated using the steering input (20), which contains two different frequencies. Estimation results show that all parameters converge to their actual values within or around 35 seconds. Hence, the accuracy of the reference model is guaranteed. In Fig. 6, the first 10 seconds of the actual yaw rate behavior shows large fluctuations. This is due to the large fluctuations from parameters estimation module. They could be constrained within reasonable bounds in real application. However, after the 10th second, the actual yaw rate tracks the desired yaw rate precisely. From $36^{\text {th }}$ to $43^{\text {rd }}$ second, a steering input of double lane change is applied to the vehicle integrating with (20). Results show the actual yaw rate tracks the reference as well by generating an active yaw moment as the control action.

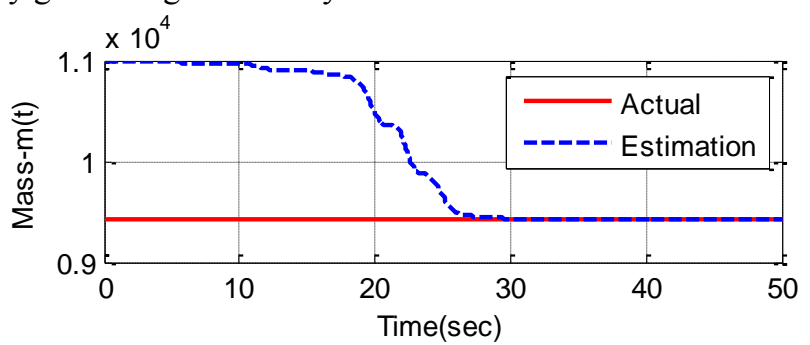

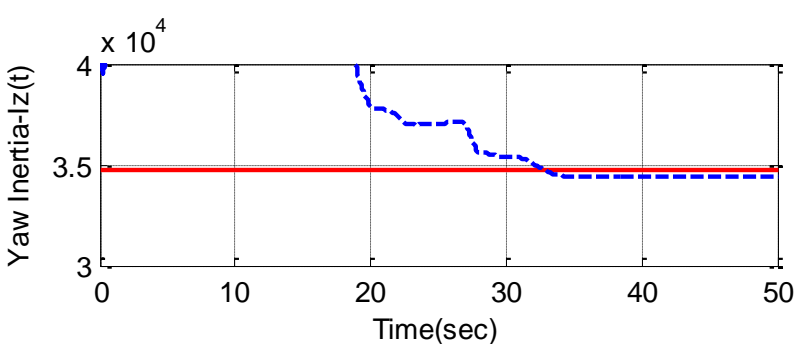

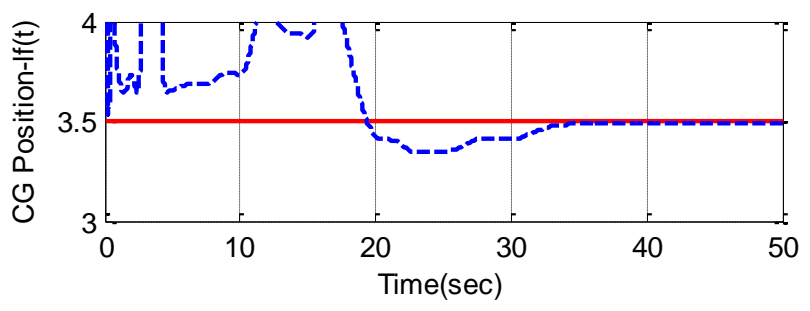

Fig.5 Parameters identification
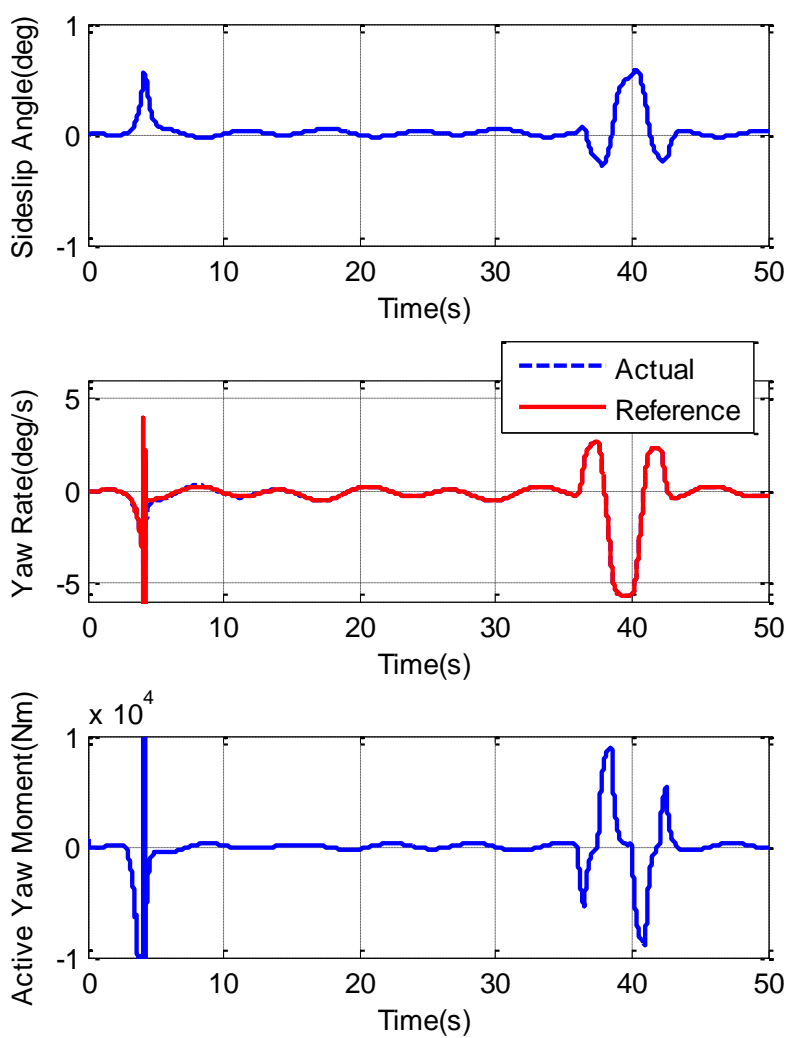

Fig.6 Adaptive yaw control

\section{CONCLUSIONS}

In this paper, the vehicle mass, yaw inertia and CG position identification and a MRAC with DYC were proposed, modeled and verified. By comparison, a reference model without adaptive parameters estimation is not accurate.

To estimate the unknown parameters and verify the MRAC, an interrelated system model was built and simulated with MATLAB and Simulink. The estimation results and time response of the yaw rate and vehicle sideslip show that the proposed method was effective in improving vehicle's yaw 
stability. For further study, a more comprehensive and realistic vehicle dynamic model is recommended and the plant should be tested in a real car or commercial software like TruckSim. Deeper merits of adaptive control strategy, along with robustness with parameter changes, i.e. tire nonlinear effects, will be also investigated and compared with the previous DYCs, i.e optimal control.

\section{REFERENCES}

[1] M. Shino and M. Nagai, "Yaw-moment control of electric vehicle for improving handling and stability," JSAE Review, vol. 22, no. 4, pp. 473480, 2001.doi:10.1016/S0389-4304(01)00130-8.

[2] A. Goodarzi, Avesta, N. Babak and E. Esmailzadeh, "Direct yaw moment controller design for vehicle dynamic control systems." Modelling, Simulation, and Optimization, pp. 120-124, 2003.

[3] M. Canale, L. Fagiano, M. Milanese, and P. Borodani, "Robust vehicle yaw control using an active differential and IMC techniques," Control Engineering Practice, vol. 15, no. 8, pp. 923-941, 2007. doi: 10.1016/j.conengprac.2006.11.012.

[4] B. Tabbache, A. Kheloui, and M. E. H. Benbouzid, "An adaptive electric differential for electric vehicles motion stabilization," IEEE Transactions on Vehicular Technology, vol. 60, no. 1, pp. 104-110, 2011. doi:10.1109/TVT.2010.2090949.

[5] J. Tjonnas and T. A. Johansen, "Stabilization of automotive vehicles using active steering and adaptive brake control allocation," IEEE Transactions on Control Systems Technology, vol. 18, no. 3, pp. 545558, 2010. doi: 10.1109/TCST.2009.2023981.

[6] Y. Zhang, A. Khajepour, and B. Zhang, "Vehicle yaw dynamics control using pulsed active rear steering," IEEE International Conference on Mechatronics and Automation, pp. 263-268, 3-6 Aug. 2014. doi: 10.1109/ICMA.2014.6885706.
[7] M. Russo, R. Russo, and A. Volpe, "Car parameters identification by handling manoeuvres," Vehicle System Dynamics, vol. 34, no. 6, pp. 423-436, 2000. doi: 10.1076/vesd.34.6.423.2053.

[8] D. Wesemeier and R. Isermann, "Identification of vehicle parameters using stationary driving maneuvers," Control Engineering Practice, vol. 17 , no. 12 , pp. 1426-1431, 2009. doi: 10.1016/j.conengprac.2008.10.008.

[9] S. Hong, C. Lee, F. Borrelli, and J. K. Hedrick, "A novel approach for vehicle inertial parameter identification using a dual Kalman filter," IEEE Transactions on Intelligent Transportation Systems, vol. 16, no. 1, pp. 151-161, 2015. doi: 10.1109/TITS.2014.2329305.

[10] A. Soltani, A. Goodarzi, M. H. Shojaeefard, and A. Khajepour, "Vehicle dynamics control using an active third-axle system," Vehicle System Dynamics, vol. 52, no. 11, pp. 1541-1562, Aug. 2014. doi: 10.1080/00423114.2014.946520.

[11] Y. Zhang, A. Khajepour, and Y. Huang, "Multi-axle/articulated bus dynamics modeling: a reconfigurable approach," Vehicle System Dynamics, pp. 1-29, Sep. 2018. doi: 10.1080/00423114.2017.1420205.

[12] J. Wang, and M.F. Hsieh. "Vehicle yaw-inertia-and mass-independent adaptive steering control." Proceedings of the Institution of Mechanical Engineers, Part D: Journal of Automobile Engineering vol. 223, no.9, pp.1101-1108, 2009. doi: 10.1243/09544070JAUTO1135.

[13] J. Wang, L. Alexander, and R. Rajamani, "Friction Estimation on Highway Vehicles Using Longitudinal Measurements," Journal of Dynamic Systems, Measurement, and Control, vol. 126, no. 2, p. 265, 2004.doi: 10.1115/1.1766028.

[14] P. Ioannou and B. Fidan, "Adaptive Control Tutorial." Society for Industrial and Applied Mathematics, 2006.

[15] O. Mokhiamar and M. Abe, "Effects of model response on model following type of combined lateral force and yaw moment control performance for active vehicle handling safety," JSAE Review, vol. 23, no. 4, pp. 473-480, 2002.

[16] $\mathrm{C} . \mathrm{Fu}$, "Direct yaw moment control for electric vehicles with independent motors," PhD thesis, RMIT University, 2014. 


\section{Discrete 2D Fourier Transform in Polar Coordinates}

\author{
Xueyang Yao \\ Department of Mechanical Engineering \\ University of Ottawa \\ Ottawa, Canada \\ xyao011@uottawa.ca
}

\author{
Natalie Baddour \\ Department of Mechanical Engineering \\ University of Ottawa \\ Ottawa, Canada \\ nbaddour@uottawa.ca
}

\begin{abstract}
The discrete Fourier transform in Cartesian coordinates has proved to be invaluable in many disciplines. However, less theory has been developed for functions that are best described in polar coordinates. In this paper, a discrete 2D-Fourier transform in polar coordinates is proposed and tested by numerical simulations with respect to accuracy and precision. Guidelines for choosing sample size are developed.
\end{abstract}

Keywords-2D Fourier Transform, discrete, polar coordinates

\section{INTRODUCTION}

The Fourier transform is a powerful analytical tool and has proved to be invaluable in many disciplines such as physics, mathematics and engineering. The development of the Fast Fourier Transform (FFT) algorithm [1], which computes the discrete Fourier transform with a fast algorithm, established the Fourier transform as a practical tool in diverse areas, most notably signal and image processing.

In two dimensions, the FFT can still be used to compute the discrete Fourier transform in Cartesian coordinates. However, in many applications such as photoacoustics [2] and tomography ([3], [4], [5]), it is often necessary to compute the Fourier transform in polar coordinates. Moreover, for functions that are naturally described in polar coordinates, a discrete version of the 2D Fourier transform in polar coordinates is needed.

There have been some attempts to calculate the Fourier transform in polar coordinates. Nonequally spaced FFTs (NUFFT, see [6], [7], [8], [9]) is one of the methods that can be implemented to compute the FFT in polar coordinates. However, due to local interpolations, the NUFFT can be prohibitively slow for large input sizes and is not easily inverted. Averbuch et al. [10] proposed a new discrete polar Fourier transform, where the original function was sampled on a near-polar grid called a pseudo-polar grid followed by 1D equispaced FFT and 1D interpolations. Based on Averbuch's work, this new method was more accurate than the NUFFT method.

It should be noted that prior work has focused on numerically approximating the continuous transform. This stands in contrast to approaches that have been taken with the continuous/discrete Fourier transform in two aspects: 1. both functions in the space and frequency domain of the DFT are in the same coordinates. 2. The DFT is defined as a transform in its own right, which means the existence of the DFT is valid even if without the existence of the continuous Fourier transform. In this paper, a new discrete 2D-Fourier transform in polar coordinates is proposed and tested by numerical simulations with respect to accuracy and precision.

\section{DEFNITION OF THE DISCRETE 2D FOURIER TRANSFORM IN POLAR COORDINATES}

\section{A. Kernel of the discrete 2D Fourier transform in polar coordinates}

To propose and work with a 2D polar DFT, the following kernels are proposed

$$
\begin{aligned}
& E^{-}(q l ; p k)=\frac{2}{N_{2}} \sum_{n=-M}^{M} \frac{J_{n}\left(\frac{j_{n k} j_{n l}}{j_{n N_{1}}}\right)}{j_{n N_{1}} J_{n+1}^{2}\left(j_{n k}\right)} i^{-n} e^{-i n \frac{2 \pi p}{N_{2}}} e^{+i n \frac{2 \pi q}{N_{2}}} \\
& E^{+}(q l ; p k)=\frac{2}{N} \sum_{n=-M}^{M} \frac{J_{n}\left(\frac{j_{n l} j_{n k}}{j_{n N_{1}}}\right.}{j_{n N_{1}} J_{n+1}^{2}\left(j_{n l}\right)} i^{n} e^{+i \frac{2 \pi n p}{N_{2}}} e^{i \frac{\pi n q}{}}
\end{aligned}
$$

where $p, k, q, l, n, N_{1}$ and $N_{2}$ are integers such that $-M \leq n \leq M$, where $2 M+1=N_{2}, 1 \leq l, k, \leq N_{1}-1$ and $-M \leq p, q \leq M . J_{n}()$ is the Bessel function of $n t h$ order and $j_{n k}$ is the kth zero of the Bessel function of order $n$. The integers $N_{1}$ and $N_{2}$ represent the size of the spaces in which we work, with $N_{2}$ representing the dimension in the angular direction and $N_{1}$ represents the dimension in the 
radial direction. Since $N_{2}=2 M+1, N_{2}$ must be an odd integer.

\section{B. Definition of the discrete transform}

The 2D-Discrete Fourier Transform in polar coordinates is defined as the discrete transform that transforms the matrix (or double-subscripted series $f_{p k}$ to the matrix (double-subscripted series) to the matrix $F_{q l}$ according to

$$
\begin{aligned}
& F_{q l}=\mathbb{F}\left(f_{p k}\right) \\
& F_{q l}=\sum_{k=1}^{N_{1}-1} \sum_{p=-M}^{M} f_{p k} E^{-}(q l ; p k) \\
& =\sum_{k=1}^{N_{1}-1} \sum_{p=-M}^{M} f_{p k} \frac{2}{N_{2}} \sum_{n=-M}^{M} \frac{J_{n}\left(\frac{j_{n k} j_{n l}}{j_{n N_{1}}}\right) i^{-n}}{j_{n N_{1}} J_{n+1}^{2}\left(j_{n k}\right)} e^{-i n \frac{2 \pi p}{N_{2}}} e^{+i n \frac{2 \pi q}{N_{2}}}
\end{aligned}
$$

The notation for $E^{-}(q l ; p k)$ and $E^{+}(q l ; p k)$ are the discrete kernels for the forward and inverse transform, respectively. The subscript ( + or -$)$ indicates the sign on the exponent containing the $p$ variable; the $q$ variable exponent then takes the opposite sign. From a matrix point of view, both $f_{p k}$ and $F_{q l}$ are $N_{2} \times\left(N_{1}-1\right)$ sized matrices. The inverse transform is then given by

$$
\begin{aligned}
f_{p k} & =\mathbb{F}^{-1}\left(F_{q l}\right) \\
f_{p k} & =\sum_{l=1}^{N_{1}-1} \sum_{q=-M}^{M} F_{q l} E^{+}(q l ; p k) \\
& =\sum_{l=1}^{N_{1}-1} \sum_{q=-M}^{M} F_{q l} \frac{2}{N_{2}} \sum_{n=-M}^{M} \frac{J_{n}\left(\frac{j_{n l} j_{n k}}{j_{n N_{1}}}\right) i^{n}}{j_{n N_{1}} J_{n+1}^{2}\left(j_{n l}\right)} e^{+i \frac{2 \pi n p}{N_{2}}} e^{-i \frac{\pi n q}{N_{2}}}
\end{aligned}
$$

\section{Approximation to the continuous Fourier transform}

To approximate the continuous Fourier transform, the function needs to be sampled at some specific points. For a space limited function defined over continuous $(r, \theta)$ space where $0 \leq r \leq R$ and $0 \leq \theta \leq 2 \pi$ and the function is effectively zero elsewhere (that is the function can be made as close to zero as necessary, elsewhere in the plane), the sampling points are defined in the spatial domain as

$$
r_{p k}=\frac{j_{p k} R}{j_{p N_{1}}} \quad \theta_{p}=\frac{p 2 \pi}{N_{2}}
$$

And in the spatial frequency domain as

$$
\rho_{q l}=\frac{j_{q l}}{R} \quad \psi_{q}=\frac{q 2 \pi}{N_{2}}
$$

Equations (4) and (5) give the sampling grid in regular $(r, \theta)$ and frequency $(\rho, \psi)$ space. Clearly, the density of the sampling points depends on the numbers of points chosen, that is on $N_{1}$ and $N_{2}$. Also clear is the fact that the grid is not equi-spaced in the radial variable. It can be shown that the approximate relationship between sampled values of the continuous function $f(r, \theta)$ and sampled values of its continuous forward 2D transform $F(\rho, \psi)$ is given by

$F\left(\frac{j_{q l}}{R}, \frac{2 \pi q}{N_{2}}\right) \approx 2 \pi R^{2} \sum_{k=1}^{N_{1}-1} \sum_{p=-M}^{M} f\left(\frac{j_{p k} R}{j_{p N_{1}}}, \frac{2 \pi p}{N_{2}}\right) E^{-}(q l ; p k)$

Similarly, the inverse transform relationship is given as

$$
f\left(\frac{j_{p k} R}{j_{p N_{1}}}, \frac{2 \pi p}{N_{2}}\right) \approx \frac{1}{2 \pi R^{2}} \sum_{l=1}^{N_{1}-1} \sum_{q=-M}^{M} F\left(\frac{j_{q l}}{R}, \frac{2 \pi q}{N_{2}}\right) E^{+}(q l ; p k)
$$

Consider the case of a bandlimited function, such that the function is defined over continuous $(\rho, \psi)$ space where $0 \leq \rho \leq W_{p}$ and $0 \leq \psi \leq 2 \pi$ and the function is effectively zero elsewhere (that is the function can be made as close to zero as necessary, elsewhere in the plane), the sampling points are defined as

$$
r_{p k}=\frac{j_{p k}}{W_{p}} \quad \theta_{p}=\frac{p 2 \pi}{N_{2}}
$$

and

$$
\rho_{q l}=\frac{j_{q l} W_{p}}{j_{q N_{1}}} \quad \psi_{q}=\frac{q 2 \pi}{N_{2}}
$$

The approximate relationship between sampled values of the continuous function and sampled values of its continuous forward 2D transform $F(\rho, \psi)$ can be shown to be given by

$$
F\left(\frac{j_{q l} W_{p}}{j_{q N_{1}}}, \frac{2 \pi q}{N_{2}}\right) \approx \frac{2 \pi}{W_{p}^{2}} \sum_{k=1}^{N_{1}-1} \sum_{p=-M}^{M} f\left(\frac{j_{p k}}{W_{p}}, \frac{2 \pi p}{N_{2}}\right) E^{-}(q l ; p k)
$$

Similary, the inverse transform is given as 


$$
f\left(\frac{j_{p k}}{W_{p}}, \frac{2 \pi p}{N_{2}}\right) \approx \frac{W_{p}^{2}}{2 \pi} \sum_{l=1}^{N_{1}-1} \sum_{q=-M}^{M} F\left(\frac{j_{q l} W_{p}}{j_{q N_{1}}}, \frac{2 \pi q}{N_{2}}\right) E^{+}(q l ; p k)
$$

\section{DisCRETE 2D Fourier TRANSFORM TEST AND RESUltS}

\section{A. Method for testing the Algorithm}

In this section, the ability of the 2D discrete Fourier transform is evaluated for its ability to estimate the continuous Fourier transform at the selected special sampling points in the spatial and frequency domains.

1) Accuracy

In order to test the accuracy of the 2D-DFT and 2D-IDFT to calculate the continuous counterpart, the dynamic error has been used. It is defined as [11]

$$
E(\mathrm{v})=20 \log _{10}\left[\frac{|C(\mathrm{v})-D(\mathrm{v})|}{\max |D(\mathrm{v})|}\right]
$$

where $C(\mathrm{v})$ is the continuous forward or inverse $2 \mathrm{D}$-Fourier transform and $D(\mathrm{v})$ is the values obtained from the discrete counterpart. The dynamic error is defined as the ratio of the absolute error to the maximum amplitude of the function, which is calculated on a log scale. Therefore, a large negative value represents an accurate discrete transform. The dynamic error is used instead of the percentage error in order to avoid division by zero.

\section{2) Precision}

The precision of the algorithm is another important evaluation criterion, which is tested by sequentially performing a pair of forward and inverse transforms and comparing the result to the original function. High precision indicates that the transform does not add much error by the calculations. An average of absolute error of each sample points between the original function and the calculated counterpart was used to measure the precision. It is given by

$$
\varepsilon=\frac{1}{\left(N_{1}-1\right) \cdot N_{2}} \sum_{n=1}^{\left(N_{1}-1\right) \cdot N_{2}}\left|f-f^{*}\right|
$$

where $f$ is the original function and $f^{*}$ is the calculated counterpart. An ideal precision would result in the absolute error being zero.

\section{B. Test Functions}

\section{1) Gaussian}

The first function chosen for evaluation is a circular symmetric function which is Gaussian in the radial direction. The function in the space domain is defined as

$$
f(r, \theta)=e^{-a^{2} r^{2}}
$$

where $a$ is some real constant. Since the function is circularly symmetric, the 2D-DFT is actually a zeroth-order Hankel Transform [12] and can be written as

$$
F(\rho, \psi)=\frac{\pi}{a^{2}} e^{\frac{-\rho^{2}}{4 a^{2}}}
$$

The graphs for the original function and its continuous 2DDFT (which is also a Gaussian) are shown with $a=1$ and shown in Figure 1.
Oripinal Function

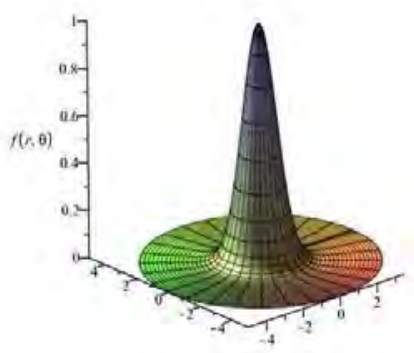

2D-Fourier Trantón

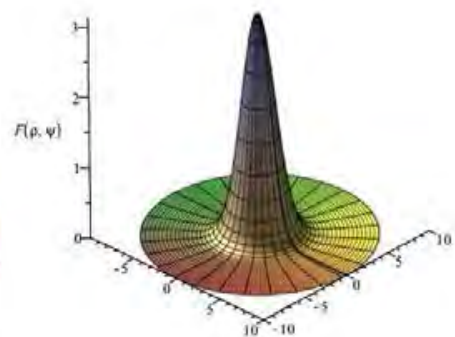

Figure 1. The original Gaussian function and its 2D-Fourier Transform
From Figure 1, the function is circular symmetric in the angular direction and fairly smooth in the radial direction. Moreover, the function can be considered as an effectively space limited function or an effectively band limited function. For the purposes of testing it, it shall be considered as a space limited function and (6) and (7) will be used to proceed with the forward and inverse transform in sequence.

To perform the transform, the following variables need to be chosen: $N_{2}, R$ and $N_{1}$. In the angular direction, since the function in the spatial domain is circularly symmetric, $N_{2}$ can be chosen to be small. Thus, $N_{2}=15$ is chosen.

In the radial direction, the effective space limit $R=40$ and effective band-limit $W_{p}=30$ are chosen, which gives $j_{0 N_{1}} \geq R \cdot W_{p}=1200$ [13]. Therefore, $N_{1}=383$ is chosen to satisfy this constraint.

\section{a) Forward Transform}

Test results for the forward transform are shown in Figure 2 and Figure 3. The error gets bigger at the center as expected. However, the maximum value of the error is $E_{\max }=-8.3842 \mathrm{~dB}$ and this occurs at the center. The average value of the error is $E_{\text {avg. }}=-63.8031 \mathrm{~dB}$. 


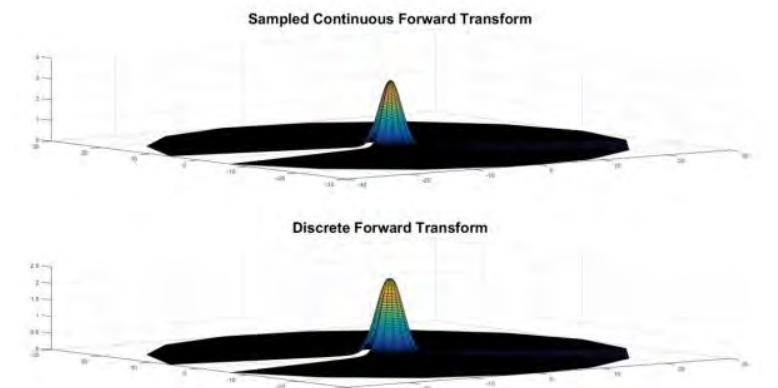

Figure 2. Sampled Continuous forward transform \&. Discrete forward transform of Gaussian Function with $\mathrm{R}=40, \mathrm{~N}_{2}=15, \mathrm{~N}_{1}=383$

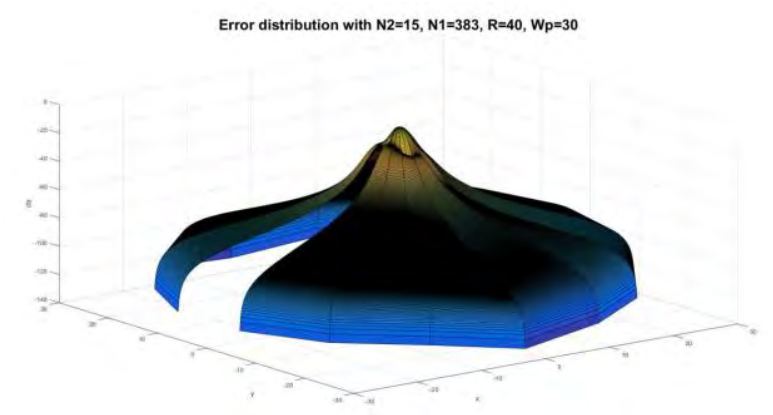

Figure 3. The error distribution of the forward transform of Gaussian Function with $\mathrm{R}=40, \mathrm{~N}_{2}=15, \mathrm{~N}_{1}=383$

\section{b) Inverse Transform}

Figure 4 and Figure 5 show the test results for the inverse transform. The maximum value of the error is $E_{\max }=-12.2602 d B$ and this occurs at the center. The average of the error is $E_{\text {avg. }}=-98.0316 \mathrm{~dB}$.

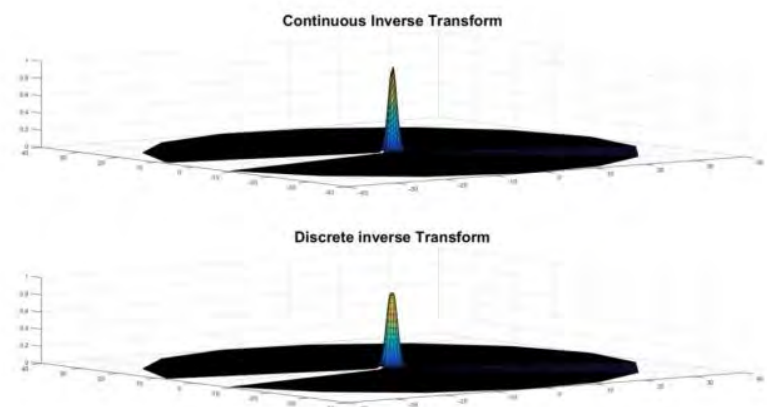

Figure 4. Sampled continuous inverse transform and discrete inverse transform of the Gaussian function with $\mathrm{R}=40, \mathrm{~N}_{2}=15, \mathrm{~N}_{1}=383$

Performing sequential 2D-DFT and 2D-IDFT results in $\varepsilon=4.1656 \times e^{-17} \quad$ where $\varepsilon$ is calculated with (13). Therefore, performing the forward and inverse transforms does not add much error.

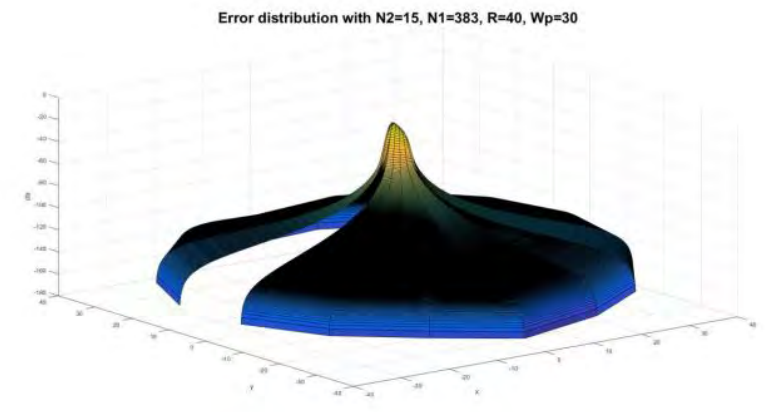

Figure 5. The error distribution of the inverse transform of Gaussian Function with $\mathrm{R}=40, \mathrm{~N}_{2}=15, \mathrm{~N}_{1}=383$

\section{2) Square Donut}

The second chosen function is a circularly symmetric function which is a square wave in the radial direction. The function is given by

$$
f(r, \theta)= \begin{cases}0, & r<5 \text { and } r>10 \\ 1, & 5 \leq r \leq 10\end{cases}
$$

The continuous 2D-FT can be written as:

$$
F(\rho, \psi)=\frac{2 \pi}{\rho}\left[10 J_{1}(10 \rho)-5 J_{1}(5 \rho)\right]
$$

where $J_{n}(x)$ is Bessel function of order $n$.

The graphs for the original function and its continuous 2DDFT (which is also a Gaussian) are plotted and shown in Figure 6.

Original Function 2D-Fourier Transform
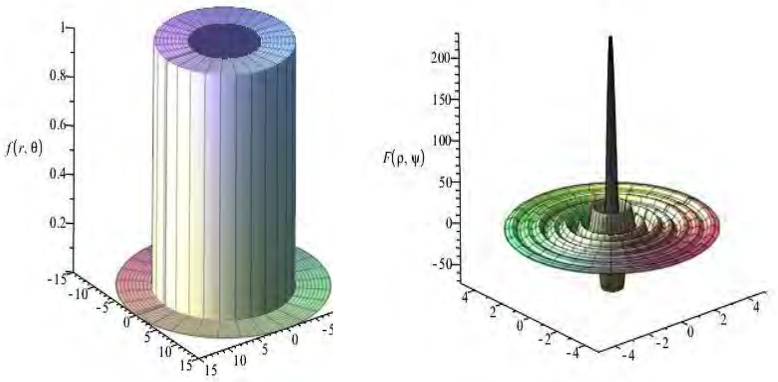

Figure 6. The original 'Square Donut' and its 2D-Fourier Transform

\section{a) Forward Transform}

Test results for the forward transform are shown in Figure 7 and Figure 8. Similar to the previous case, the error gets bigger at the center as expected. The maximum value of the error is $E_{\max }=-8.1664 d B$ and occurs at the center area. The average of the error is $E_{\text {avg. }}=-34.5471 \mathrm{~dB}$.

\section{b) Inverse Transform}

Figure 9 and Figure 10 show the test result for the inverse transform. The maximum value of the error is 


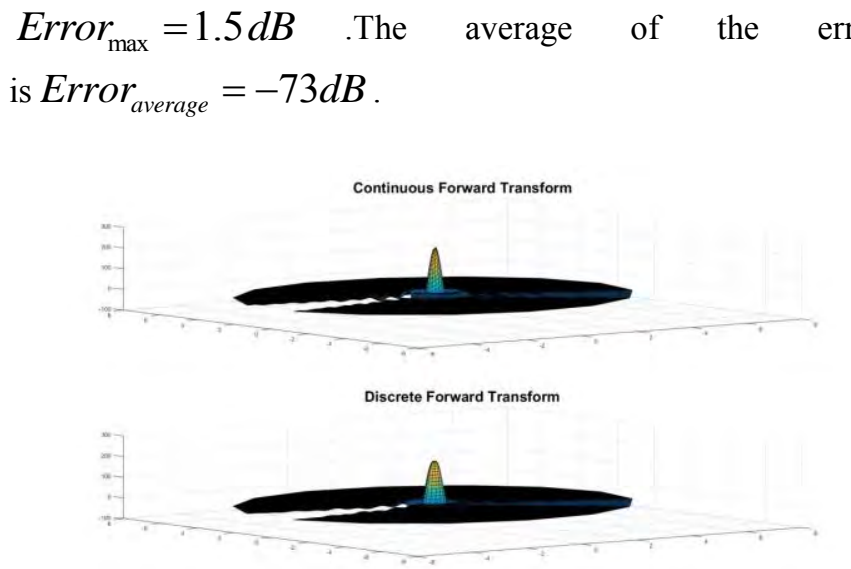

Figure 7. Sampled Continuous forward transform \&. Discrete forward transform of 'Square Donut' Function with $\mathrm{R}=40, \mathrm{~N}_{2}=15, \mathrm{~N}_{1}=383$

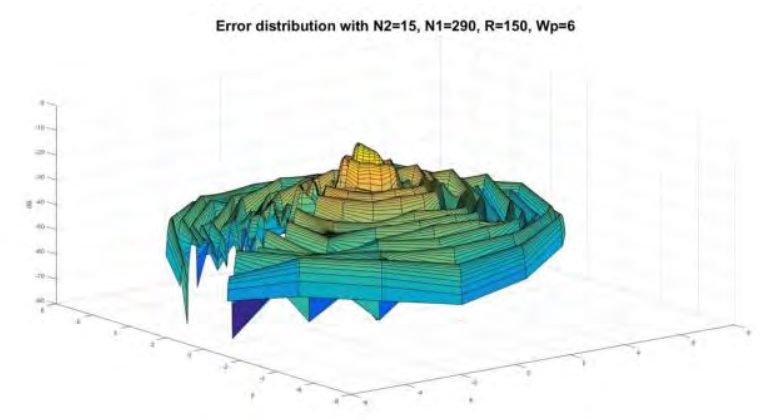

Figure 8. The error distribution of the forward transform of 'Square Donut' Function with $\mathrm{R}=40, \mathrm{~N}_{2}=15, \mathrm{~N}_{1}=383$

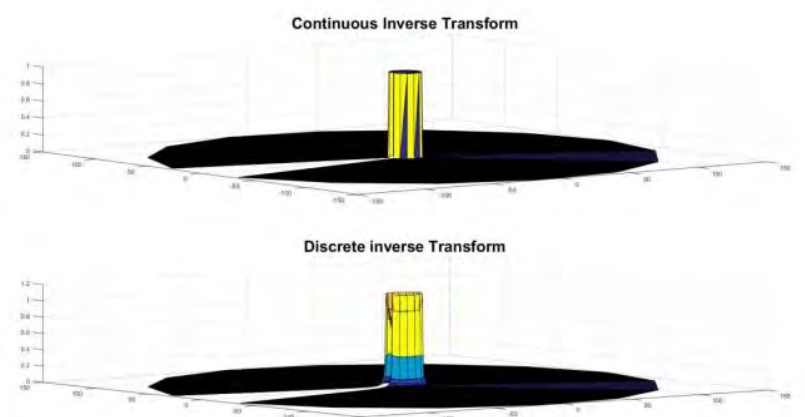

Figure 9. Sampled continuous inverse transform and discrete inverse transform of the 'Square Donut' function with $\mathrm{R}=40, \mathrm{~N}_{2}=15, \mathrm{~N}_{1}=383$

Performing sequential 2D-DFT and 2D-IDFT results in $\varepsilon=6.7253 \times e^{-14}$ where $\varepsilon$ is calculated with (13). Therefore, performing forward and inverse transform does not add much error.

\section{SUMMARY AND CONLUSION}

From the two test cases, both the forward and inverse transform showed good accuracy to approximate the continuous Fourier transform. Moreover, the good precision results indicated that the transform itself does not add much error.

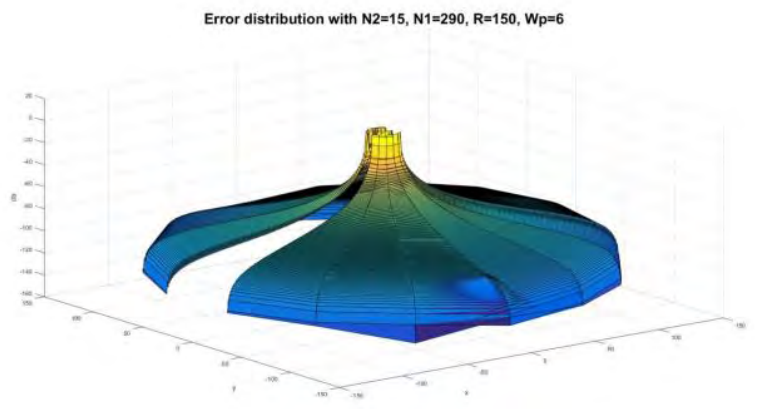

Figure 10. The error distribution of the inverse transform of 'Square Donut' Function with $\mathrm{R}=40, \mathrm{~N}_{2}=15, \mathrm{~N}_{1}=383$

\section{REFERENCES}

[1] J. W. Cooley and J. W. Tukey, "An Algorithm for the Machine Calculation of Complex Fourier Series," Math. Comput., vol. 19, no. 90, pp. 297-301, 1965. doi: 10.1090/S0025-5718-1965-0178586-1.

[2] Y. Xu, D. Feng, and L. V. Wang, "Exact frequency-domain reconstruction for thermoacoustic tomography. I. Planar geometry," Med. Imaging IEEE Trans. On, vol. 21, no. 7, pp. 823-828, 2002. doi: 10.1109/TMI.2002.801172.

[3] M. C. Scott et al., "Electron tomography at 2.4- ngström resolution," Nature, vol. 483, no. 7390, p. 444, Mar. 2012. doi: 10.1038/nature10934.

[4] B. P. Fahimian et al., "Radiation dose reduction in medical x-ray CT via Fourier-based iterative reconstruction," Med. Phys., vol. 40, no. 3, Mar. 2013.

[5] E. Lee et al., "Radiation dose reduction and image enhancement in biological imaging through equally-sloped tomography," J. Struct. Biol., vol. 164, no. 2, pp. 221-227, 2008. doi: $10.1016 /$ j.jsb.2008.07.011.

[6] E. Suli and A. Ware, "A Spectral Method of Characteristics for Hyperbolic Problems," SIAM J. Numer. Anal., vol. 28, no. 2, pp. 423445, 1991. doi: 10.1137/0728024.

[7] A. Dutt and V. Rokhlin, "Fast Fourier Transforms for Nonequispaced Data," SIAM J. Sci. Comput., vol. 14, no. 6, pp. $1368-$ 1393, Nov. 1993. doi: 10.1137/0914081.

[8] C. Anderson and M. Dahleh, "Rapid computation of the discrete Fourier transform," SIAM J. Sci. Comput., vol. 17, no. 4, pp. 913-919, Jul. 1996. doi: 10.1137/0917059.

[9] A. F. Ware, "Fast approximate Fourier transforms for irregularly spaced data," SIAM Rev., vol. 40, no. 4, p. 838, Dec. 1998. doi: $10.1137 / \mathrm{S} 003614459731533 \mathrm{X}$.

[10]A. Averbuch, R. R. Collman, D. L. Donpho, M. Elad, and M. Tsiaeni, "Fast and accurate Polar Fourier transform," Appl. Comput. Harmon. Anal., vol. 21, no. 2, pp. 145-167, 2006. doi: 10.1016/j.acha.2005.11.003.

[11] M. Guizar-Sicairos and J. C. Gutiérrez-Vega, "Computation of quasi-discrete Hankel transforms of integer order for propagating optical wave fields," JOSA A, vol. 21, no. 1, pp. 53-58, Jan. 2004. doi: 10.1364/JOSAA.21.000053.

[12] A. D. Poularikas, The Transforms and Applications Handbook. Boca Raton, Fla. : [S.1.]: CRC Press; IEEE Press, 2000.

[13] N. Baddour and U. Chouinard, "Theory and operational rules for the discrete Hankel transform," JOSA A, vol. 32, no. 4, pp. 611-622, Apr. 2015. doi: 10.1364/JOSAA.32.000611. 


\title{
Sensor Filtering and Position Tracking of a Simuated Planar Bipedal Robot
}

\author{
Stefano Rossi* and S. Andrew Gadsden \\ College of Engineering and Physical Sciences, \\ University of Guelph, \\ Guelph, Ontario, Canada \\ *srossi@uoguelph.ca
}

\begin{abstract}
The development of a humanoid robot is a very prevalent area of research today. Legged robots have many advantages over wheeled robots on rough or uneven terrains, and are more suitable for an everyday household setting, however they possess many design and control challenges. The spring loaded inverted pendulum (SLIP) is frequently used as a fundamental model to analyze bipedal locomotion. In general, it consists of a stance phase and a flight phase, employing different strategies during these phases to control speed and orientation. Due to the underactuation and hybrid dynamics of bipedal robots, estimating the state of the robot and its appendages can be challenging. In this paper, various Kalman estimation techniques are used to predict the state of a simulated planar SLIP model. The state estimations utilize only simulated sensor data, as if the simulated model was a physical one.
\end{abstract}

Keywords-Kalman filter; bipedal robot; legged robot; robotics; simulation; inverted pendulum; SLIP; hybrid dynamics; advanced estimation; sensor filtering.

\section{INTRODUCTION}

The simulated model consists of a planar biped robot designed and simulated in Virtual Robotic Experimentation Platform (VREP), using Open Dynamics Engine (ODE) for the physics. In flight phase, when there is no ground contact, the dynamics of the system is simply ballistic flow and has well known and analytically solved dynamics. However, due to gravity, the stance phase dynamics is much more complicated and includes coupled equations and non integratable terms. Therefore, an accurate estimation of the state based on sensor measurements becomes much more valuable than closed form analytical motion planning. The main controller design goals of the robot are:

1. To achieve dynamically stable running.

2. To be able to accelerate and decelerate to achieve a desired running speed.

3. To control its angular momentum to maintain a desired body attitude during running.

These design goals are achieved through the main control loop, however the controller relies on accurate measurements of the body attitude, leg positions and angular and linear velocity of the robot. The Kalman filter (KF) is applied to estimate positions and velocities of these components [1]. The KF is an optimal state estimation strategy, and is widely used in the field of control and estimation theory $[2,3]$.

\section{A. SLIP Model}

To study running in its simplest form, a planar running machine was built at MIT [4]. This robot only had one leg, but the principle is identical to a biped. The leg behaved as a passive spring and was telescopic. It used pneumatics to simulate a spring and could also apply thrust force. During one cycle there was a stance and flight phase. During the stance phase, the leg supports the body and remains in a fixed location on the ground. In this phase, the robot tips like an inverted pendulum and there is no chance to move the foot placement to control position. In order to change the foot position, the robot jumps to flight phase where the leg is unloaded and free to move. Marc Raibert developed a simple control strategy for simple legged robots which decomposes into the parts, the hopping height, the forward speed and the body attitude [5].

\section{B. Previous Research}

Many methods of state estimation for planar legged locomotion exist currently [6]. Stance mapping is a commonly used approach in state estimation of linkages. It has been proven that stance dynamics are nonlinear, coupled and contain nonintegratable terms [7]. Many assumptions and approximations need to be made to derive a stance map. Common assumptions include ignoring gravity, no inputs, constant speed, small spring compressions and small sweep angles [8]. Other methods use a mean value theorem derived from interactive performance testing [9]. Kalman filtering has also extensively been applied to the linkages of bipedal walking models [10], however a walking model lacks the complex hybrid dynamics of running, and again many assumptions are made to reduce the model, such as constant height CoG [6]. Applying these methods to real life models is challenging and impractical, with the introduction of system and measurement noise, uncertain impact dynamics and imperfect limb coordination and model design. An alternative to a closed form stance map is real-time adaptive state estimation from sensor data, which is implemented in this project. 


\section{Kalman Filter}

In order for the Raibert controller to function, the orientation, linear and angular velocity of the main body needs to be known, as well as the positions of the legs relative to the body. Two Kalman filters are applied to the entire model to determine the state relative to the robots' constituents. The first is used to determine the attitude of the body of the model. It utilizes a gyroscope and the control torque applied during stance. The second is a sensor fusion Kalman filter utilizing an accelerometer and gyroscope on the pivot points of the leg.

\section{SIMUlATED MODEL}

The model created in VREP originally based on Raibert planar biped [10]. The model consists a rectangular main body, with two actuated hip joints, which connect to telescopic legs (Fig. 1). The legs act as a passive spring/dampers during compression, and are capable of applying a thrust force. The robot is attached to a spherical joint at the hip by a $5 \mathrm{~m}$ massless boom (Fig. 1). This eliminates 3DOF from the model, its yaw, roll and lateral movement. The feet consist of spheres and have perfect friction with the ground $(\mu=1)$.

TABLE I. SYMBOLS AND DESCRIPTIONS

\begin{tabular}{|c|c|}
\hline Symbol & Description \\
\hline$\theta$ & Leg angle relative to body vertical \\
\hline$\varphi$ & Body angle with respect to horizontal \\
\hline$\varphi_{d}$ & Desired body angle \\
\hline$r$ & Leg length at equilibrium \\
\hline$z$ & Body position on track \\
\hline$x$ & Body velocity \\
\hline$\dot{x}$ & Desired body velocity \\
\hline$\dot{x}_{d}$ & Moot distance from body CoG \\
\hline$x_{r}$ & Mass of leg \\
\hline$M_{B}$ & Body mass moment of inertia \\
\hline$M_{L}$ & Gravity \\
\hline$I_{B}$ & Time of Stance \\
\hline$g$ & Spring constant of leg \\
\hline$T_{S t}$ & Control torque of active hip \\
\hline$K_{L}$ & \\
\hline$\tau$ & \\
\hline
\end{tabular}

The simulation used Open Dynamics Engine as its physics engine due to its accuracy modelling spring damper systems. The main control loop is implemented directly in VREP via a child script. The running motion of the robot can be described in 5 phases: flight, touchdown, compression, thrust and takeoff. The main working principle behind the speed control is foot placement. Because the leg acts as a spring-damper, the time of the stance phase (compression and thrust) can be approximated by:

$$
T_{s t}=\frac{\pi}{\omega}=\pi \sqrt{\frac{M_{B}+M_{L}}{K_{L}}}
$$

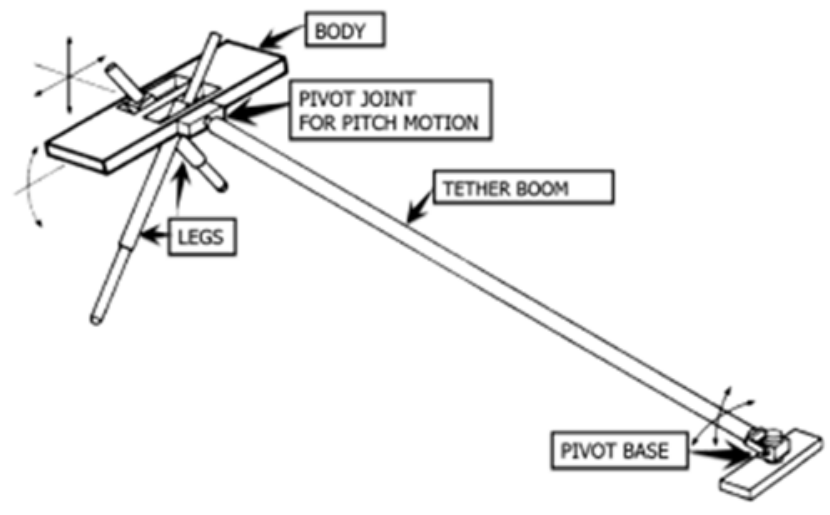

Figure 1. Biped model assembly.
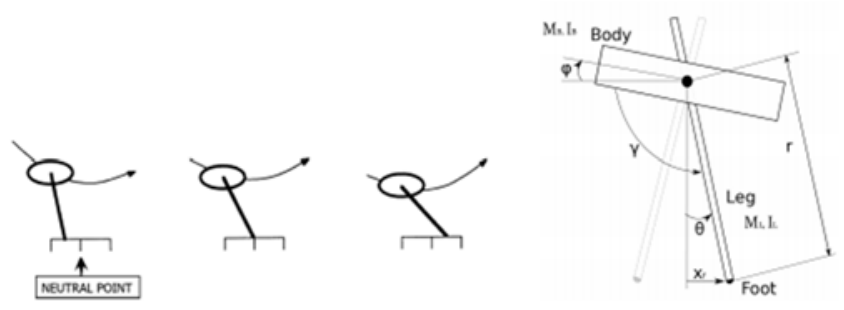

Figure 2. Foot placement effects and free body diagram.

Foot placement has a direct effect on the resultant velocity at takeoff. If the foot is placed directly at the halfway point throughout the stance (neutral point), the stance phase is symmetric and the takeoff velocity is the same as the touchdown.

$$
x_{f 0}=\frac{\dot{x} T_{s t}}{2}
$$

Any deviation from the neutral point results in a non-zero horizontal acceleration. Placing the foot before the neutral point results in acceleration, as more of the vertical velocity is converted to horizontal, and vice versa (Fig. 2). To accelerate to a desired speed, proportional control is used.

Therefore, the algorithm for foot placement and the corresponding hip angle is:

$$
\begin{gathered}
x_{f}=\frac{\dot{x} T_{s t}}{2}+k_{\dot{x}}\left(\dot{x}-\dot{x_{d}}\right) \\
\gamma_{d}=\emptyset-\sin ^{-1}\left(\frac{\dot{x} T_{s t}}{2}+\frac{k_{\dot{x}}\left(\dot{x}-\dot{x_{d}}\right)}{r}\right)
\end{gathered}
$$

Body attitude is maintained by applying a torque about the hip during the stance phase. Since angular momentum is conserved during flight, the friction between the foot and the ground provides an opportunity to correct the angular momentum of the entire system. To servo the body to a desired attitude, this control torque is applied:

$$
\tau=-k_{p}\left(\varphi-\varphi_{d}\right)-k_{v}(\dot{\varphi})
$$




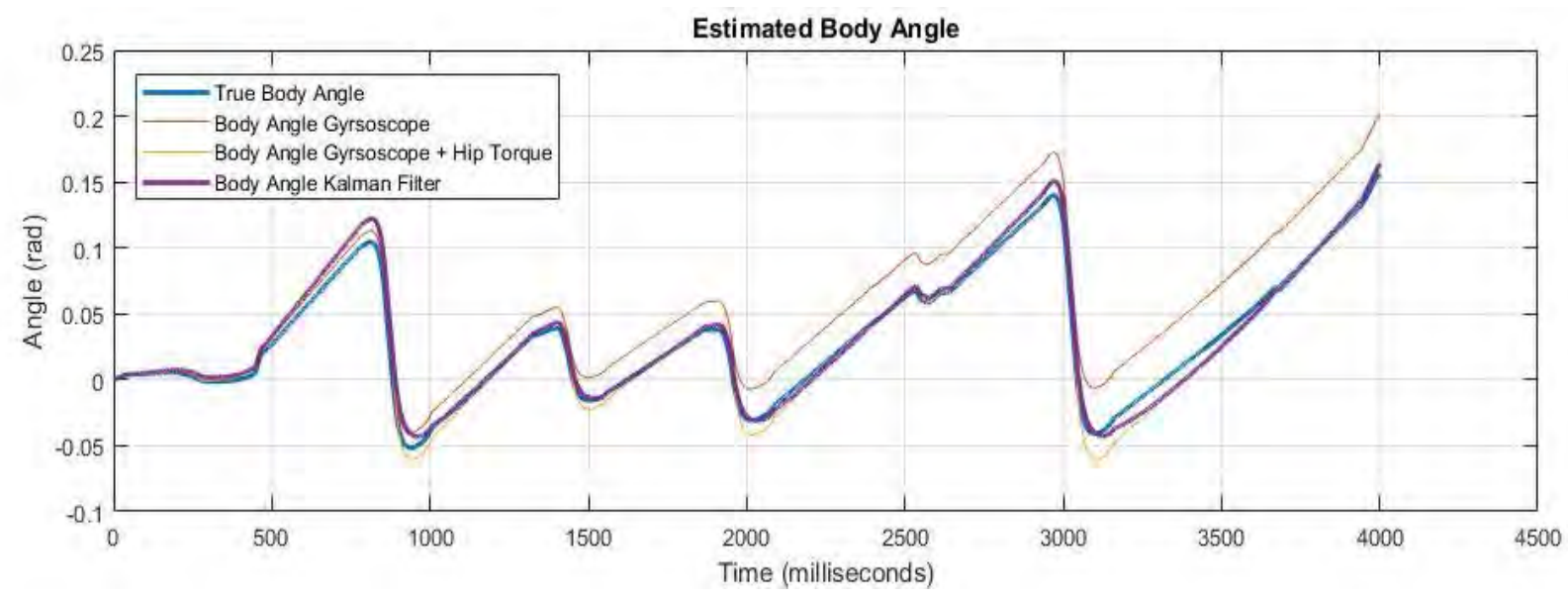

Figure 3. True and estimated body angle.

where $k_{p}$ and $k_{v}$ are constants, 80 and 20 respectively. During the stance and flight phases, the idle leg mirrors the active leg to cancel out any angular momentum.

\section{BODY STATE ESTIMATION}

The body angle of the robot is determined through a gyroscope and the applied control torque during stance phase. The gyroscope was simulated using a reference point where the Euler angles were read, and simulation noise was added. The control torque is an input based on the current body angle and angular velocity. These values were streamed from VREP in real time to MATLAB using a remote API. They were then filtered and streamed back into VREP to calculated the control inputs for the next time step. The true angle and angular velocity of the body were also streamed to MATLAB for comparison.

TABLE II. SIMULATION PARAMETERS

\begin{tabular}{|c|c|c|}
\hline Parameter & Value & Description \\
\hline$t$ & 0.001 & Simulation time step, in sec \\
\hline$I$ & $4.708 \mathrm{e}-02$ & Body mass moment of inertia \\
\hline$\phi_{d}$ & 0 & Desired body angle, in rad \\
\hline$r$ & 0.5575 & Leg length uncompressed \\
\hline$\dot{x}_{d}$ & 2 & Desired body velocity, in $\mathrm{m} / \mathrm{s}$ \\
\hline$M_{B}$ & 9.246 & Mass of body, in $\mathrm{kg}$ \\
\hline$M_{L}$ & 0.478 & Mass of leg, in $\mathrm{kg}$ \\
\hline$T_{s t}$ & 0.178 & Time of Stance, in sec \\
\hline$K_{L}$ & 2700 & Spring constant of leg, in N/m \\
\hline
\end{tabular}

\section{A. Estimation Methods}

Three methods were used to estimate the angle of the body. The angle was estimated just from the gyroscope measurement of the angular velocity.

$$
\varphi_{i}=\varphi_{i-1}+t \dot{\varphi}_{\iota}
$$

The body angle was also calculated using the input torque applied during the stance phase to correct the body angle.

$$
\varphi_{i}=\varphi_{i-1}+t \dot{\varphi}_{\imath}+\frac{\tau}{2 I} t^{2}
$$

The body angle was also estimated using a Kalman filter.

$$
\begin{gathered}
{\left[\begin{array}{c}
\varphi \\
\dot{\varphi}
\end{array}\right]=\left[\begin{array}{ll}
1 & t \\
0 & 1
\end{array}\right]\left[\begin{array}{l}
\varphi \\
\dot{\varphi}
\end{array}\right]+\left[\begin{array}{c}
\frac{t^{2}}{2 I} \\
t / I
\end{array}\right]\left[\begin{array}{l}
\tau \\
\tau
\end{array}\right]} \\
C=\left[\begin{array}{ll}
1 & 0 \\
0 & 1
\end{array}\right]
\end{gathered}
$$

Measurement and system noise were then added to the system to simulate real world conditions. These values were assumed to be Gaussian and white. These values were approximated, and it was assumed most of the noise would stem from the sensors, so the system noise was several orders of magnitude less than the measurement noise.

\section{B. Results}

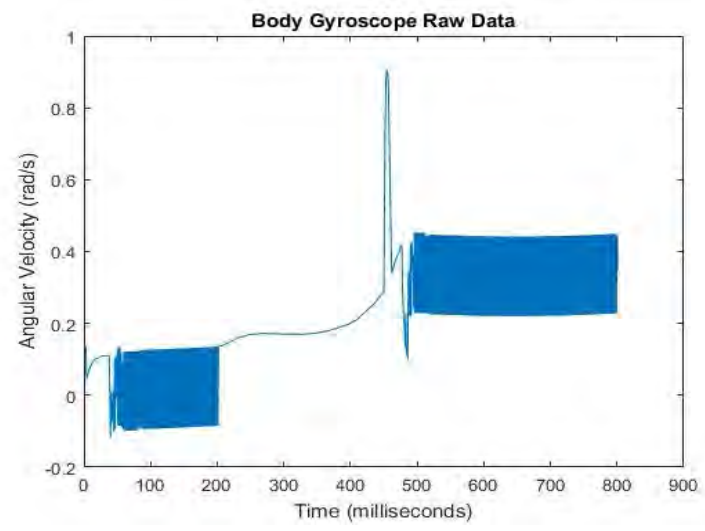

Figure 4. Body gyro data without Gaussian noise. 


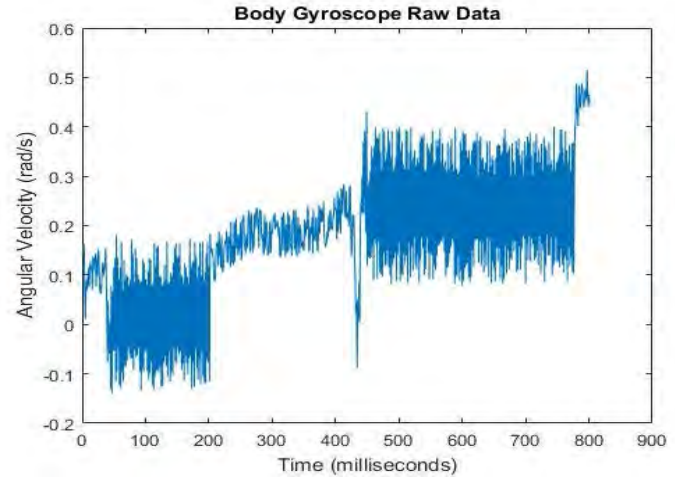

Figure 5. Body gyro data with Gaussian noise.

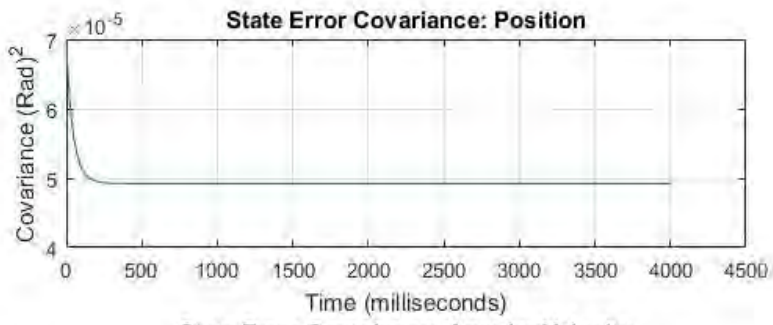

State Error Covariance: Angular Velocity

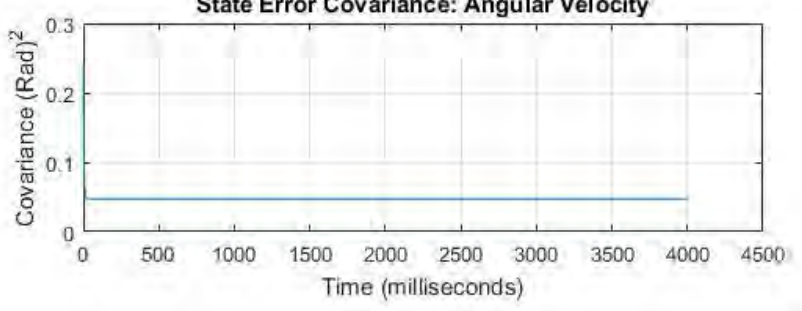

Figure 6. Position and angular velocity state error covariance.

TABLE III. ROOT MEAN SQUARE ERROR RESULTS

\begin{tabular}{|c|c|c|c|}
\hline & $\begin{array}{c}\text { Gyroscope } \\
\text { Angle } \\
\text { Estimation }\left(\mathrm{rad}^{2}\right)\end{array}$ & $\begin{array}{c}\text { Gyro + Torque } \\
\text { Angle Estimation } \\
\left(\mathrm{rad}^{2}\right)\end{array}$ & $\begin{array}{c}\text { Kalman Filter } \\
\text { Angle Estimation } \\
\left(\mathrm{rad}^{2}\right)\end{array}$ \\
\hline $\boldsymbol{R M S E}$ & 0.0393433 & 0.0184116 & 0.0071413 \\
\hline
\end{tabular}

The KF performed the best with an RMSE approximately five times lower than that of the gyroscope estimation alone, and 2.5 times lower than that of the gyroscope and the torque estimation. As per the position estimation results, the gyroscope estimation drifts over time, while the combined estimation overshoots the extreme tilt angles. The KF accurately accounts for these deviations and best represents the true angle of the body. Although the Kalman filter is able to filter out most of the noise in the angular velocity estimation, it does seem to have a bias. This may be due to the Kalman filter counteracting the bias of the gyroscope, as the state error covariance of both the position and the velocity converge quickly over time. The estimated state of the body was streamed back into the VREP simulation to predict the next control outputs, and the robot performed just as well as if reading the true states, running at its desired speed of $2 \mathrm{~m} / \mathrm{s}$ with a deviation of $0.2 \mathrm{~m} / \mathrm{s}$.

\section{LEG State Estimation}

The state estimation of the leg consists of a sensor fusion between an accelerometer and gyroscope located at the hip joint of the model. The virtual accelerometer consists of a reference mass attached to a force sensor. These values were streamed from VREP in real time to MATLAB using a remote API. They were then filtered and streamed back into VREP to calculated the control inputs for the next time step. The true angle and angular velocity of the leg were also streamed to MATLAB for comparison.

\section{A. Estimation Methods}

Due to the impact forces of touchdown and the rising and falling motion of the model, the calculated accelerometer angle of the leg is very noisy and very biased during the stance phase (Fig. 7). The signal is processed through two corrective algorithms before it is filtered to provide better estimations. The first one uses accelerometer data from the body rather than the leg. Because the body of the biped remains relatively level $( \pm 5$ deg) compared to the sweep range of the leg $( \pm 40 \mathrm{deg})$, the calculated accelerometer angle of the body is subtracted from that of the leg to correct for the rising and falling motion of the model. The data is then corrected for the large spikes occurring during touchdown (Fig. 8). The angle of the leg was estimated using two filters, a complimentary and a Kalman. The complementary filter combined the calculated angle from the gyroscope sensor and the accelerometer sensor.

$$
\theta_{\text {comp }}=\alpha\left(\theta_{\text {gyro }}\right)+(1-\alpha)\left(\theta_{\text {accel }}\right), \alpha=0.9
$$

The state of the leg was also estimated using a Kalman filter. Measurement and system noise were then added to the system to simulate real world conditions. These values were assumed to be Gaussian and white. These values were approximated, and it was assumed most of the noise would stem from the sensors, so the system noise was several orders of magnitude less than the measurement noise.

$$
\begin{aligned}
& {\left[\begin{array}{c}
\theta \\
\dot{\theta}
\end{array}\right]=\left[\begin{array}{ll}
1 & t \\
0 & 1
\end{array}\right]\left[\begin{array}{l}
\varphi \\
\dot{\varphi}
\end{array}\right], C=\left[\begin{array}{ll}
1 & 0 \\
0 & 1
\end{array}\right]} \\
& Q=\left[\begin{array}{cc}
\frac{t^{3}}{3} & \frac{t^{2}}{2} \\
\frac{t^{2}}{2} & t
\end{array}\right], R=\left[\begin{array}{cc}
10^{-4} & 0 \\
0 & 10^{-1}
\end{array}\right]
\end{aligned}
$$

\section{B. Results}

As seen in the results (Fig. $10 \& 11$ ), the KF outperformed the complementary filter, with an RMSE less than half that of the complementary (Table IV). The complementary performed fairly well, however after a certain time the gyroscopic drift had too large of an effect on the estimation. The Kalman filter was able to filter out much of the noise from the accelerometer reading (Fig. 10). While the position tracking estimation was smooth, very little of the velocity estimation noise was filtered out. This is primarily due to the low measurement noise covariance applied to the gyroscope data, and a large noise covariance applied to the accelerometer. The estimated state of the active leg was streamed back into the VREP simulation to predict the next control outputs in real time. The robot performed just as well as if reading the true states, running at its desired speed of $2 \mathrm{~m} / \mathrm{s}$ with a deviation of $0.2 \mathrm{~m} / \mathrm{s}$. 


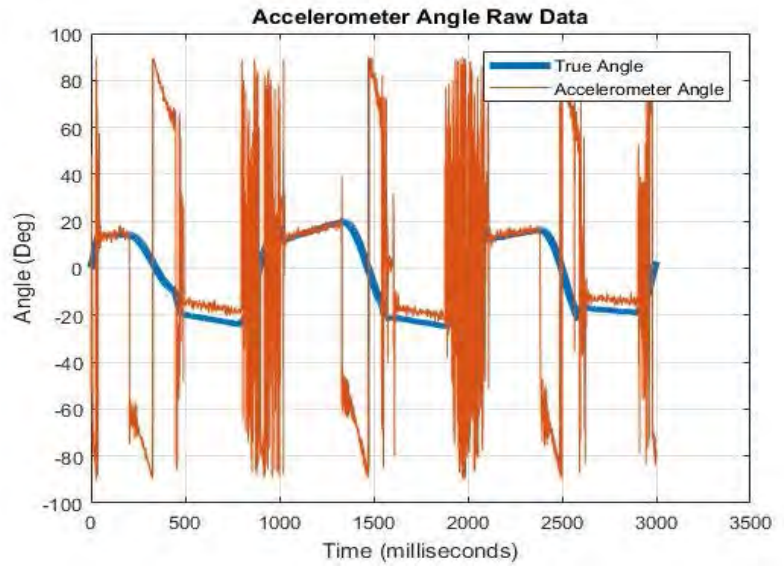

Figure 7. Raw accelerometer data from leg.

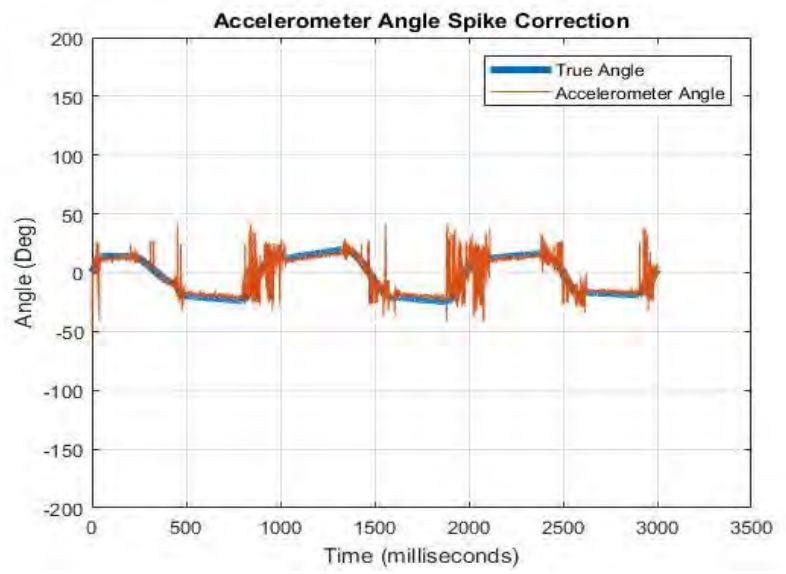

Figure 8. Filtered accelerometer data from leg.
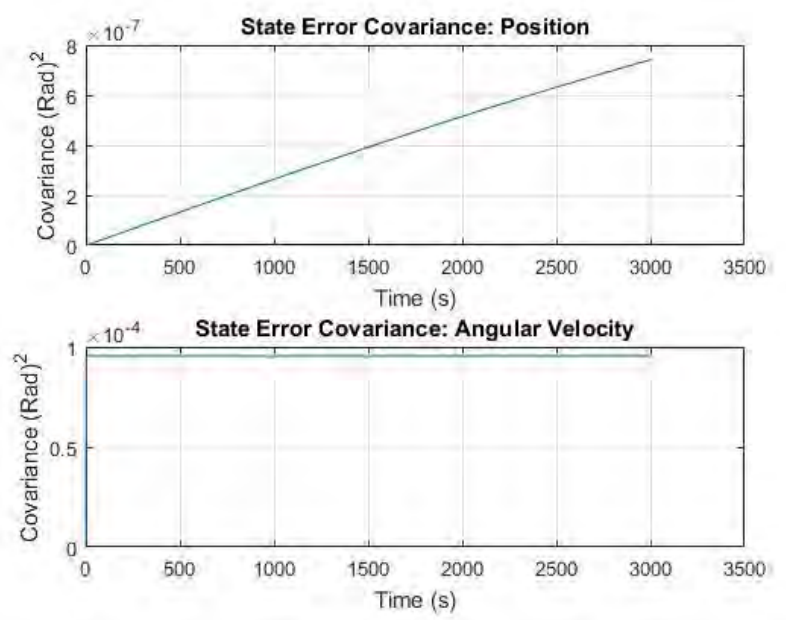

Figure 9. State error covariance of leg estimation.
TABLE IV. ROOT MEAN SQUARE ERROR RESULTS

\begin{tabular}{|c|c|c|}
\hline & $\begin{array}{c}\text { Complementary Filter } \\
\text { Angle Estimation }\left(\mathrm{rad}^{2}\right)\end{array}$ & $\begin{array}{c}\text { Kalman Filter Angle } \\
\text { Estimation }\left(\mathrm{rad}^{2}\right)\end{array}$ \\
\hline $\boldsymbol{R M S E}$ & 0.0562632 & 0.0276804 \\
\hline
\end{tabular}

\section{CONCLUSIONS}

Experimentation showed that the implementation of a Kalman filter is very effective at estimating of the states of the model. The Kalman filter worked best when the system was linear and known. The system matrix has the largest effect on the performance of the Kalman filter. Despite the challenges of predicting the overall state of the model, it has been demonstrated that it is possible to use Kalman filtering techniques to estimate the state of this model just using sensor data, allowing for a physical implementation.

\section{FUTURE WORK}

While the Kalman estimations for the states of the body and the legs were very successful, the state estimation of the entire model did not yield desirable results. Linearized state equations for the stance phase would be very useful in improving the filters, and allowing the implementation of an EKF and IMMEKF strategy. Although the simulated model is easier to work with, a physical model would be the next step in experimentally tuning these filters. Ideally, one large filter would predict the changing center of gravity and hybrid dynamics would be state of the entire system, however the equations integrating highly complex. Finally, this system lends itself to a neural net configuration. Applying a Kalman filter to adaptively train the neural net may yield a very successful control strategy. 


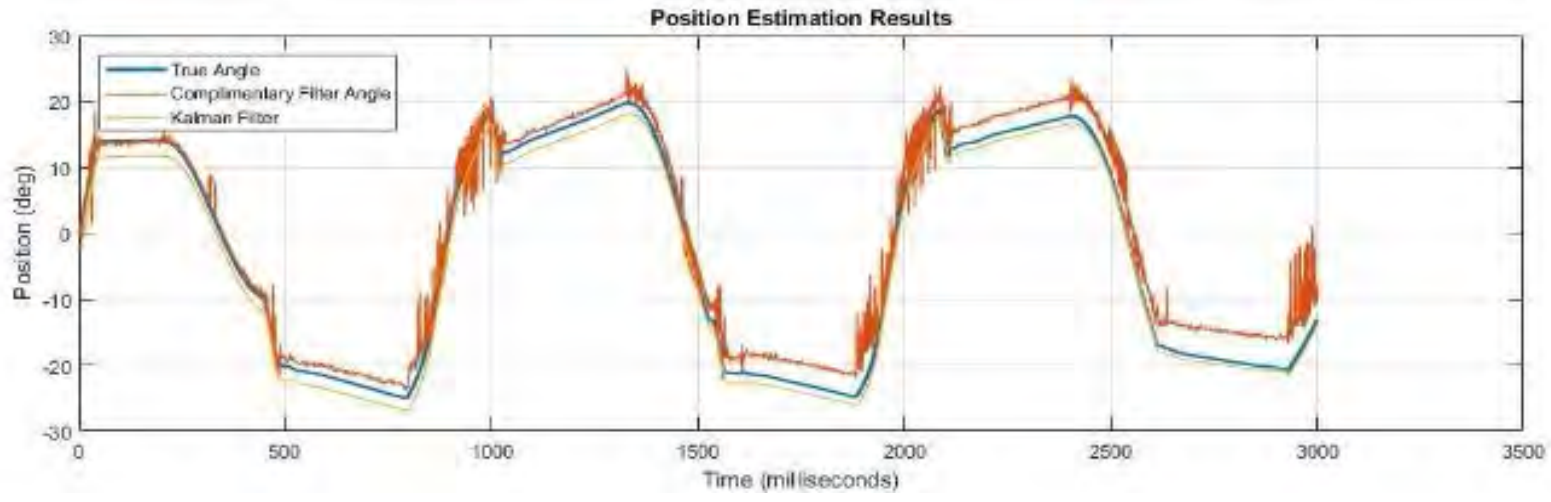

Figure 10. Position estimation results for leg angle.

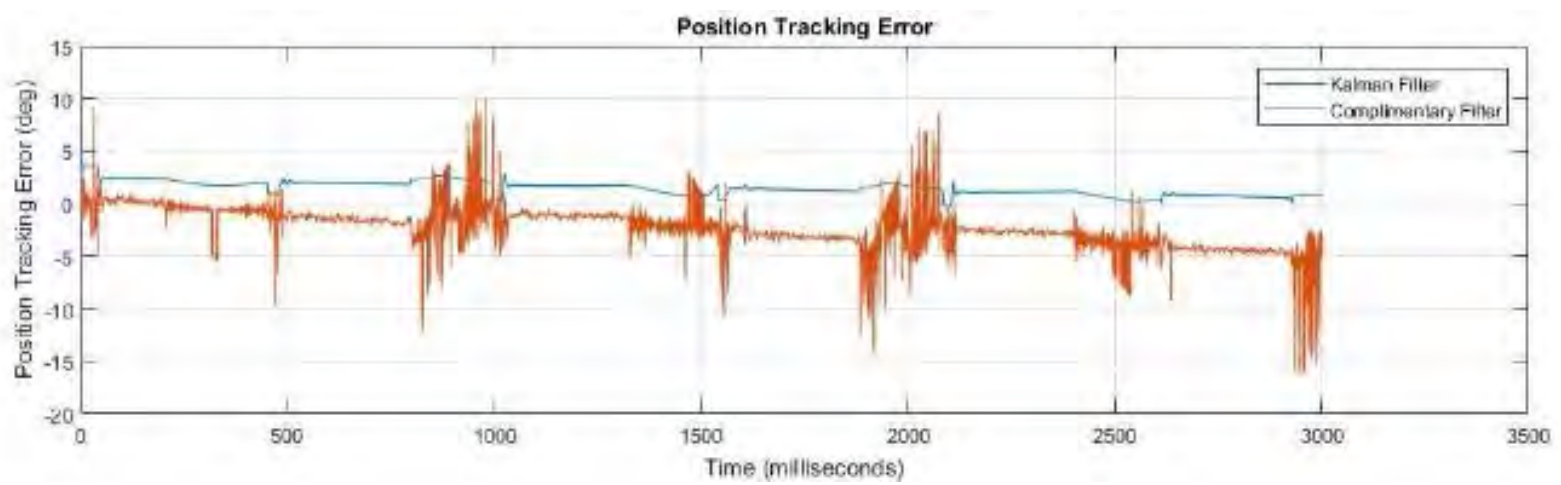

Figure 11. Position tracking error for leg angle estimation.

\section{REFERENCES}

[1] S. A. Gadsden and A. S. Lee, "Advances of the Smooth Variable Structure Filter: Square-Root and Two-Pass Formulations," $J$. Appl. Remote Sens., vol. 11, no. 1, pp. 1-19, Mar. 2017. doi: 10.1117/1.JRS.11.015018.

[2] H. H. Afshari, S. A. Gadsden, and S. Habibi, "Gaussian Filters for Parameter and State Estimation: A General Review of Theory and Recent Trends," Signal Process., vol. 135, pp. 218-238, Jun. 2017. doi: 10.1016/j.sigpro.2017.01.001.

[3] S. A. Gadsden, Y. Song, and S. R. Habibi, "Novel Model-Based Estimators for the Purposes of Fault Detection and Diagnosis," IEEE-ASME T. Mech., vol. 18, no. 4, pp. 1237-1249, Aug. 2013. doi: 10.1109/TMECH.2013.2253616.

[4] M. H. Raibert, "Hopping in Legged Systems — Modeling and Simulation for the Two-Dimensional One-Legged Case," IEEE Trans. Syst. Man Cybern., vol. 14, no. 3, pp. 3, May 1984. doi: 10.1109/TSMC.1984.6313238.

[5] M. H. Raibert, Legged Robots That Balance, Cambridge, MIT Press, 1986.

[6] X. Xinjilefu, "State Estimation for Humanoid Robots," Carnegie Mellon University Robotics Institute, Pittsburgh, Pennsylvania, 2015

[7] Ömür, "Spring Loaded Inverted Pendulum (SLIP) Approximate Stance Map for Nonsymmetric Motions and Variable Stiffness," Electrical \& Electronics Engineering Department, Bilkent University, 2008.
[8] H. Geyer, A. Seyfarth, and R. Blickhan, "Spring-Mass Running: Simple Approximate Solution and Application to Gait Stability," J. Theor. Biol., vol. 232, no. 3, pp. 315-328, Feb. 2005. doi: 10.1016/j.jtbi.2004.08.015.

[9] W. J. Schwind, "Spring loaded inverted pendulum running: A plant model," Ph.D. dissertation, University of Michigan, Ann Arbor, Michigan, 1998.

[10] J. Hodgins, "Planar Biped," MIT Leg Laboratory, Boston, Mass., 1985. 


\title{
Modeling and Estimation of Reaction Constants in Hydrogen Peroxide Deterioration Reactions
}

\author{
Hung Yi Chiang*, Andrei Buin, Faraz Ahmadpour \\ and S. Andrew Gadsden \\ College of Engineering and Physical Sciences, \\ University of Guelph, \\ Guelph, Ontario, Canada \\ *hchiang@uoguelph.ca
}

\begin{abstract}
In this paper, a new model in augmented state space allowing for faster prediction of hydrogen peroxide decay rates is proposed. This information is important for the development of new hydrogen peroxide solutions used to fight pathogens and harmful germs. The well-known Kalman filter and the unscented Kalman filter are applied on developed mathematical models and experimental measurements. The results are compared and discussed.
\end{abstract}

Keywords-Hydrogen peroxide; unscented Kalman filter;
interacting multiple models

\section{INTRODUCTION}

Virox Technologies Inc. (Oakville, Ontario) specializes in the development of innovative cleaning and disinfection technologies based on hydrogen peroxide as the active ingredient. Hydrogen peroxide is an optimal ingredient for use in disinfectant formulations as it has a wide spectrum of antimicrobial efficacy, has a highly preferred environmental profile, and does not leave toxic residues on surfaces upon application (i.e., it breaks down to water and oxygen). More specifically, Virox Technologies Inc. is an industry leader in innovating peroxide based antimicrobial formulations wherein very low concentrations of hydrogen peroxide (less than $1 \%$ ) is synergistically made to be highly effective against pathogenic organisms. This selective acceleration of hydrogen peroxide's activity against pathogens has been possible by blending hydrogen peroxide with specific types and amounts of readily available and safe inert ingredients; such as anionic surfactants, cyclic carboxylic acids, and more. The innovative discoveries on synergistic mixtures of hydrogen peroxide at low concentrations with the select inert additives has led to development of the multi-patented accelerated hydrogen peroxide (AHP) technology platform. Today, products based on the AHP technology platform are found in over 60 countries worldwide, and in about $75 \%$ of Canadian healthcare institutions.

Experiments performed at Virox have shown that collecting statistically significant data regarding hydrogen peroxide decay requires weeks of incubation time. The need of an accelerated degradation method is required to speed up the determination of hydrogen peroxide lifetime. This paper suggests a hypothetical setup using estimation strategies that predict two parameters: temperature variation, and hydrogen peroxide concentration.

State and parameter estimation theory is an important field in mechanical and electrical engineering. The strategies are used to predict, estimate, or smooth out important system state and parameters $[1,2]$. The Kalman filter (KF) is the most popular estimation strategy, and yields a statistically optimal solution to the linear estimation problem [3]. The goal of the KF is to minimize the state error covariance, which is a measure of the estimation accuracy and is defined as the expectation of the state error squared [4]. The state error is defined as the difference between the true state value and the estimation state value. Although the KF yields a solution for linear estimation problems, it is based on a few strict assumptions: the system and measurement models must be known, the noise distribution is Gaussian, and the behavior is linear $[5,6]$. If any of these assumptions are not held by the actual system, then the KF may yield inaccurate or unstable estimation results [7]. A popular nonlinear form of the Kalman filter is the unscented Kalman filter (UKF). It utilizes statistics to approximate the nonlinear probability density function (PDF) [8].

For the experiments performed in this paper, the following assumptions have been made: (1) reactions are first order only. In the event that this is not the case, one can still consider reactions as pseudo-first order but with a different reaction constant $\boldsymbol{k}_{\text {obs }}(\boldsymbol{t})$ [9]; and, (2) the loss of hydrogen peroxide is independent on the concentration when the concentration is low. This applies to the experiments in this paper due to the fact that hydrogen peroxide concentrations were relatively low, ranging between $0.5 \%$ to $2 \%$ of total solution weight. The experimental data is described in Section 2, the methods and analysis are described in Section 3, the results are found in Section 4, and the papers is then concluded.

\section{EXPERIMENTAL DATA}

Experimental data was obtained within the facilities at Virox and averaged in order to determine initial reaction constant and variances that needed to be fed into matrix $R$, the measurement noise covariance. A summary of data is available in Tables I through III. 
Based on this data, it is sufficient to determine the reaction constant $k$, activation energy $E_{\text {a }}$ (also known as the enthalpy of decomposition), and the initial parameter $\boldsymbol{A}_{0}$ which come into the Arhenius equation in (1). In (1), $R$ is the universal gas constant and $T$ is the temperature.

$$
k\left[\mathrm{H}_{2} \mathrm{O}_{2}\right]=A_{0} \exp (-\mathrm{Ea} / \mathrm{RT})
$$

In order to determine $\boldsymbol{k}$, one linearly fits the natural $\log$ of peroxide loss over time via following relationship:

$$
\ln \left[C(t) / C_{0}\right]=-k\left[H_{2} \mathrm{O}_{2}\right] t
$$

where $\mathrm{C}(\mathrm{t})$ and $\mathrm{C}_{0}$ are the concentrations of the current and initial time steps respectively. Analogously, one fits reciprocal temperature to natural $\log$ of $\mathrm{k}$ to determine $\boldsymbol{E}_{a}$ and $\boldsymbol{A}_{0}$ :

$$
(-E a / R T)+\ln \left(A_{0}\right)=\ln (k(T))
$$

Plots of the curve fitting described by (2) and (3) are shown in Fig. 1 and 2.

TABLE I. PERCENT LOSS OF $\mathrm{H}_{2} \mathrm{O}_{2}$ AT $50^{\circ} \mathrm{C}$

\begin{tabular}{|c|c|c|c|c|c|c|c|c|}
\hline \multirow{2}{*}{$\begin{array}{c}\text { Time } \\
(\mathbf{H r})\end{array}$} & \multicolumn{2}{|c|}{$\mathbf{p H}=\mathbf{2}$} & \multicolumn{2}{c|}{$\mathbf{p H}=\mathbf{3}$} & \multicolumn{2}{c|}{$\mathbf{p H}=\mathbf{4}$} & \multicolumn{2}{c|}{$\mathbf{p H}=\mathbf{5}$} \\
\cline { 2 - 9 } & $\begin{array}{c}\mathbf{L} \\
\text { Loss }\end{array}$ & $\begin{array}{c}\text { Err } \\
\text { or }\end{array}$ & $\begin{array}{c}\text { \%o } \\
\text { Loss }\end{array}$ & $\begin{array}{c}\text { Err } \\
\text { or }\end{array}$ & $\begin{array}{c}\text { \% } \\
\text { Loss }\end{array}$ & $\begin{array}{c}\text { Err } \\
\text { or }\end{array}$ & $\begin{array}{c}\text { \% } \\
\text { Loss }\end{array}$ & $\begin{array}{c}\text { Err } \\
\text { or }\end{array}$ \\
\hline 120 & 0.16 & 0.27 & 0.00 & 0.00 & 0.02 & 0.03 & 0.00 & 0.00 \\
\hline 240 & 1.43 & 0.55 & 0.00 & 0.00 & 0.49 & 0.47 & 0.06 & 0.10 \\
\hline 360 & 2.92 & 1.06 & 0.06 & 0.08 & 0.47 & 0.41 & 0.21 & 0.27 \\
\hline
\end{tabular}

TABLE II. PERCENT LOSS OF $\mathrm{H}_{2} \mathrm{O}_{2}$ AT $54^{\circ} \mathrm{C}$

\begin{tabular}{|l|c|c|c|c|c|c|c|c|}
\hline \multirow{2}{*}{$\begin{array}{c}\text { Time } \\
(\mathbf{H r})\end{array}$} & \multicolumn{2}{|c|}{$\mathbf{p H}=\mathbf{2}$} & \multicolumn{2}{c|}{$\mathbf{p H}=\mathbf{3}$} & \multicolumn{2}{c|}{$\mathbf{p H}=\mathbf{4}$} & \multicolumn{2}{c|}{$\mathbf{p H}=\mathbf{5}$} \\
\cline { 2 - 9 } & $\begin{array}{c}\text { Loss } \\
\text { Lrr }\end{array}$ & $\begin{array}{c}\text { or } \\
\text { Loss }\end{array}$ & $\begin{array}{c}\text { Err } \\
\text { or }\end{array}$ & $\begin{array}{c}\text { \%oss } \\
\text { Loss }\end{array}$ & $\begin{array}{c}\text { Err } \\
\text { or }\end{array}$ & $\begin{array}{c}\% \\
\text { Loss }\end{array}$ & $\begin{array}{c}\text { Err } \\
\text { or }\end{array}$ \\
\hline 120 & 1.37 & 0.70 & 0.07 & 0.07 & 0.03 & 0.04 & 0.00 & 0.00 \\
\hline 240 & 2.41 & 0.85 & 0.35 & 0.60 & 0.51 & 0.51 & 0.04 & 0.03 \\
\hline 360 & 2.97 & 0.95 & 0.21 & 0.36 & 0.24 & 0.41 & 0.00 & 0.00 \\
\hline
\end{tabular}

TABLE III. PERCENT LOSS OF $\mathrm{H}_{2} \mathrm{O}_{2} \mathrm{AT} 70^{\circ} \mathrm{C}$

\begin{tabular}{|l|c|c|c|c|c|c|c|c|}
\hline \multirow{2}{*}{$\begin{array}{c}\text { Time } \\
\text { (Hr) }\end{array}$} & \multicolumn{2}{|c|}{$\mathbf{p H}=\mathbf{2}$} & \multicolumn{2}{c|}{$\mathbf{p H}=\mathbf{3}$} & \multicolumn{2}{c|}{$\mathbf{p H}=\mathbf{4}$} & \multicolumn{2}{c|}{$\mathbf{p H}=\mathbf{5}$} \\
\cline { 2 - 9 } & $\begin{array}{c}\text { Loss } \\
\text { Lor }\end{array}$ & $\begin{array}{c}\text { Er } \\
\text { Loss }\end{array}$ & $\begin{array}{c}\text { Err } \\
\text { or }\end{array}$ & $\begin{array}{c}\text { \%o } \\
\text { Loss }\end{array}$ & $\begin{array}{c}\text { Err } \\
\text { or }\end{array}$ & $\begin{array}{c}\% \\
\text { Loss }\end{array}$ & $\begin{array}{c}\text { Err } \\
\text { or }\end{array}$ \\
\hline 120 & 1.680 & 1.94 & 0.03 & 0.06 & 0.03 & 0.05 & 0.00 & 0.00 \\
\hline 240 & 7.85 & 1.29 & 0.07 & 0.07 & 0.00 & 0.00 & 0.12 & 0.21 \\
\hline 360 & 10.98 & 1.05 & 0.30 & 0.52 & 0.71 & 0.72 & 0.04 & 0.07 \\
\hline
\end{tabular}

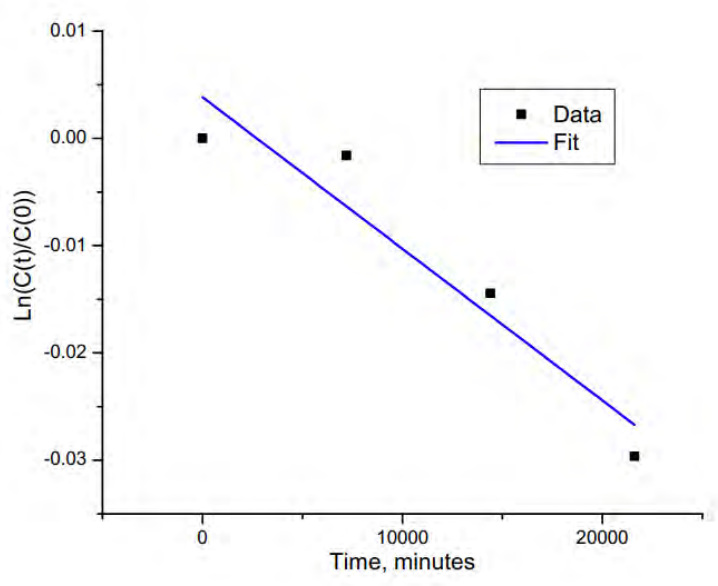

Figure 1. Linear fit of (2).

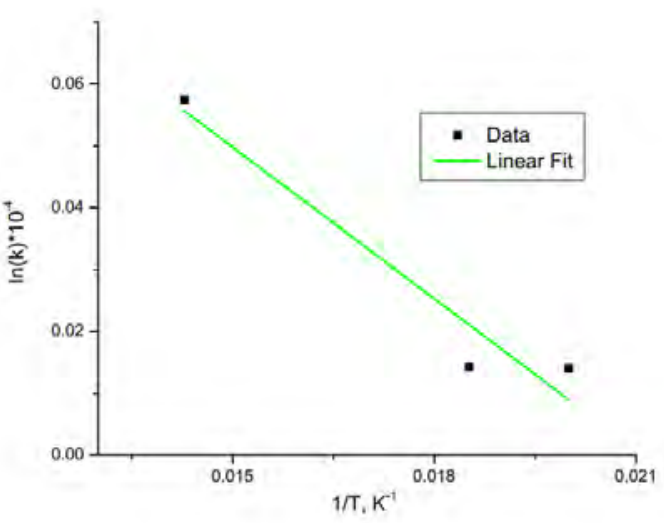

Figure 2. Linear fit of (3).

Both of these fits from Fig. 1 and 2 can provide all required ingredients for the determination of $k$. The constants have been determined as follows:

$$
\begin{gathered}
E_{a}=70 \mathrm{~kJ} \times \mathrm{mol}^{-1} \\
A_{0}=0.4 \times 10^{5.8} \mathrm{~min}^{-1} \\
k=1.45 \times 10^{-6} \mathrm{~min}^{-1} \text { at } 50^{\circ} \mathrm{C}
\end{gathered}
$$

The obtained values are within reasonable agreement with results published elsewhere for aqueous solutions, keeping in mind the $\mathrm{pH}$ levels in this study [10]. Additionally, analysis of $k$ constants was performed on various $\mathrm{pH}$ levels. Results are depicted in Fig. 3. As there are only 4 points, there is no clear relationship between $k$ and $\mathrm{pH}$. Therefore, it will be omitted in calculations. 


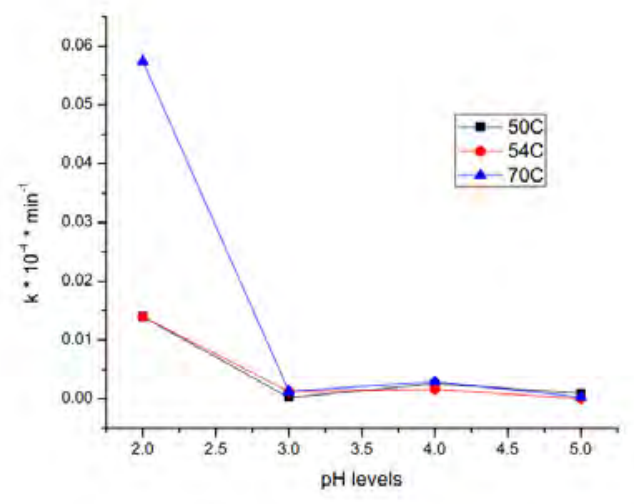

Figure 3. $\mathrm{K}$ at various $\mathrm{pH}$ Levels.

\section{METHODS AND ANALYSIS}

\section{A. Linear System of Equations}

The augmented state space system is based on three variables, $\boldsymbol{c}$ - concentration, $\boldsymbol{T}$ - temperature, and $\boldsymbol{k}$ - reaction rate constant. The discretized augmented state space system is written as:

$$
\left[\begin{array}{c}
c_{i+1} \\
T_{i+1} \\
k_{i+1}
\end{array}\right]=\left[\begin{array}{ccc}
-k_{i} \Delta t+1 & 0 & 0 \\
0 & 1 & 0 \\
0 & 0 & 1
\end{array}\right] \times\left[\begin{array}{c}
c_{i} \\
T_{i} \\
k_{i}
\end{array}\right],
$$

where $\Delta \mathrm{t}$ is sampling time (200 minutes). Initial conditions for all simulations are shown in (5).

$$
\left[\begin{array}{c}
c_{0} \\
T_{0} \\
k_{0}
\end{array}\right]=\left[\begin{array}{c}
0.1615 \mathrm{M} \\
323.50 \mathrm{~K} \\
1.45 \times 10^{-6} \mathrm{~min}^{-1}
\end{array}\right]
$$

\section{B. Determination of $Q$ and $R$ matrices}

In most cases, only $\boldsymbol{c}$ and $\boldsymbol{T}$ are measurable during experiments. Thus, $\mathrm{H}$, or measurement matrix, is written as:

$$
H=\left[\begin{array}{lll}
1 & 0 & 0 \\
0 & 1 & 0
\end{array}\right]
$$

Additionally, the noise covariance matrices $Q$ (system noise) and $R$ (measurement noise) are required to run any type of Kalman filter. The determination of $R$ is straightforward and it is obtained through experimental setup. For this study, it is determined to be:

$$
R=\left[\begin{array}{cc}
5 \times 10^{-8} & 0 \\
0 & 0.01
\end{array}\right]
$$

The determination of the $\mathrm{Q}$ matrix is usually the trickiest part of Kalman filtering. Matrix elements of Q are treated as tunable parameters which are determined based on the uncertainties that may arise in a system. With this experiment, these uncertainties might be temperature and humidity variations. Additionally, for the augmented state space system, matters become more complicated due to the fact that the augmented part of the system, shown below:

$$
\left[\begin{array}{l}
T_{i+1} \\
k_{i+1}
\end{array}\right]=\left[\begin{array}{l}
T_{i}+w_{T}(i) \\
k_{i}+w_{k}(i)
\end{array}\right]
$$

This becomes a Wiener process with unbounded variance shown in (9). One solution to this problem is to set variances $\mathrm{w}_{\mathrm{T}}, \mathrm{w}_{\mathrm{K}}$ to zero. However, doing so would change the controllability of the system.

$$
\lim _{i \rightarrow \infty} \operatorname{Var}(T, k)=\infty
$$

There is another way, however, to keep variances nonzero while having bounded covariances of augmented state variables. This is described in [2], and the procedure is as follows:

- Choose matrix elements of $\mathrm{Q}$ to be a few percent of estimated values

- Run simulations for true and Kalman estimated systems

- Calculate variances of simulated variables

- Adjust matrix elements of Q such that (10) is satisfied

$$
\operatorname{Var}(x)=\lim _{i \rightarrow \infty} \mathrm{P}_{i}(x)
$$

The procedure from [3] was adopted and the resulting Q matrix was determined to be:

$$
Q=\left[\begin{array}{ccc}
10^{-9} & 0 & 0 \\
0 & 2 \times 10^{-8} & 0 \\
0 & 0 & 10^{-16}
\end{array}\right]
$$

\section{System Setup and Kalman Estimators}

The estimators used in this study were the Kalman filter (KF), unscented Kalman filter (UKF), and the interacting multiple model (IMM) setup based on the KF and the UKF. For the IMM algorithm, (12) was used as the probability transition matrix:

$$
R=\left[\begin{array}{ll}
0.95 & 0.05 \\
0.05 & 0.95
\end{array}\right]
$$

Temperature profiles were treated as unknown parameters fed into the Kalman estimators. The main objective was to assess the accuracy of predicted reaction rate constants compared to their true counterparts. 


\section{RESUlts}

\section{A. Profiles with Rising Temperatures}

Rising temperature profiles were simulated through raising the temperature by a certain amount at $p H=2$ as demonstrated in Fig. 4, Fig. 5, and Fig. 6. The three state variables (Peroxide concentration, temperature, and k-constant) are shown in each of these figures, along with the KF, UKF, and IMM results. The probabilities $(\mathrm{Mu})$ of the IMM system being in the KF or UKF mode are also shown.
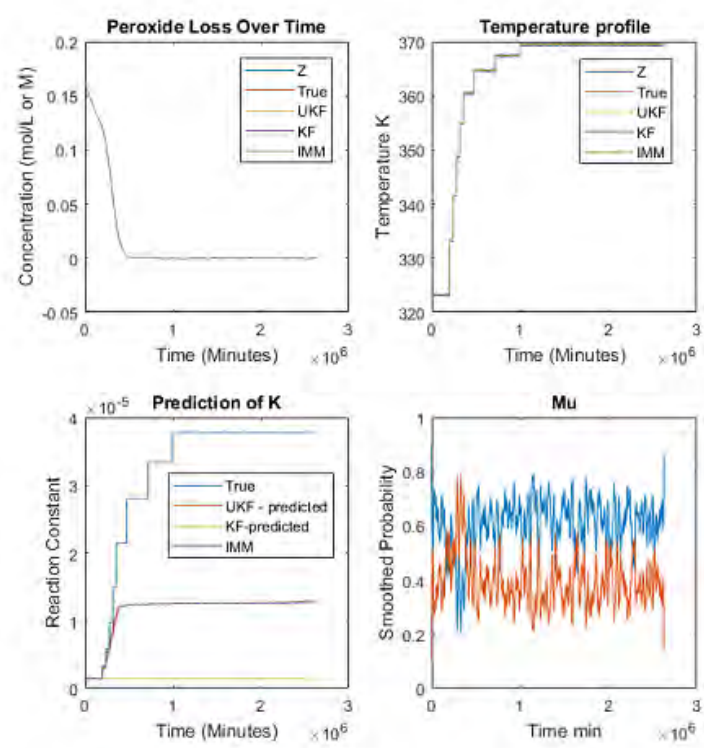

Figure 4. Temperature profile with a $50^{\circ} \mathrm{C}$ change.
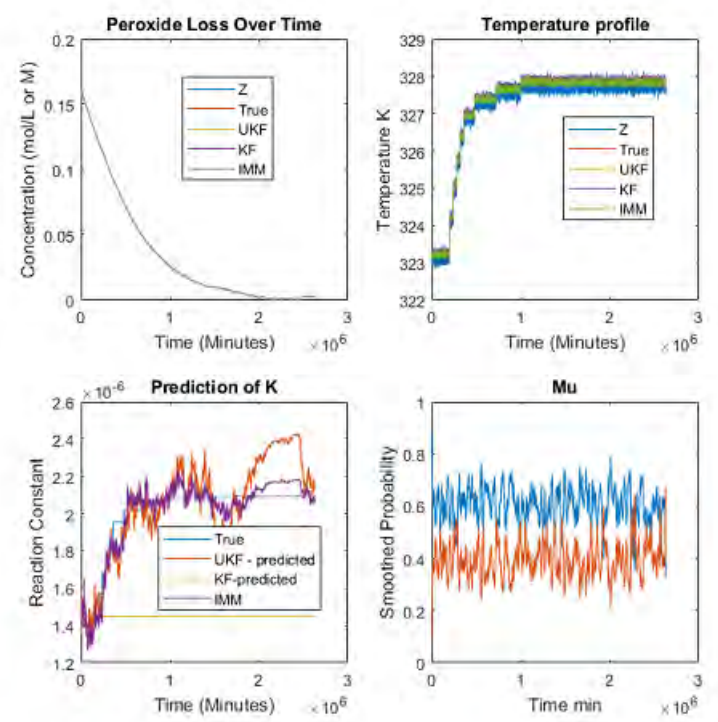

Figure 5. Temperature profile with a $5^{\circ} \mathrm{C}$ change, late onset.
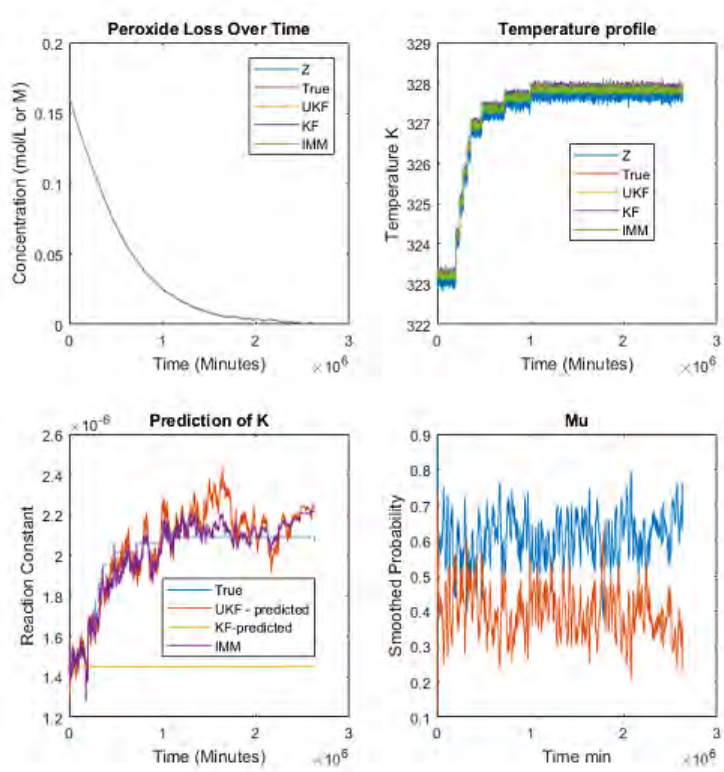

Figure 6 . Temperature profile with a $5^{\circ} \mathrm{C}$ change, early onset.

It is interesting to note that in the case of large temperature change, neither one of the Kalman estimators provided correct values of $\mathrm{k}$. At lower changes in temperature and based on the root mean square errors (RMSE) seen in Tables IV and V, one can see that the IMM and the UKF are similar in accuracy. The plain $\mathrm{KF}$ is unable to estimate the non-linear $\mathrm{k}$ constant of the system.

TABLE IV. RMSE'S FOR TEMPERATURE PROFILE WITH A $5^{\circ} \mathrm{C}$ CHANGE, LATE ONSET

\begin{tabular}{|c|c|c|c|}
\hline \multirow{2}{*}{ System States } & KF & UKF & IMM \\
\cline { 2 - 4 } & $8.64662 \times 10^{-5}$ & $8.60678 \times 10^{-5}$ & $8.41062 \times 10^{-5}$ \\
\hline $\begin{array}{c}\text { Concentration } \\
(\mathrm{M})\end{array}$ & $5.25555 \times 10^{-1}$ & $1.41209 \times 10^{-1}$ & $7.67782 \times 10^{-2}$ \\
\hline $\begin{array}{c}\text { Temperature } \\
(\mathrm{K})\end{array}$ & $5.85417 \times 10^{-7}$ & $1.60082 \times 10^{-7}$ & $6.5447 \times 10^{-8}$ \\
\hline $\begin{array}{c}\mathrm{K} \text { Constant } \\
\left(\mathrm{min}^{-1}\right)\end{array}$ & & \\
\hline
\end{tabular}

TABLE V. RMSE'S FOR TEMPERATURE PROFILE WITH A $5^{\circ} \mathrm{C}$ CHANGE, EARLY ONSET

\begin{tabular}{|c|c|c|c|}
\hline \multirow{2}{*}{ System States } & KF & UKF & IMM \\
\cline { 2 - 4 } & $8.14059 \times 10^{-5}$ & $8.15825 \times 10^{-5}$ & $7.98528 \times 10^{-5}$ \\
\hline $\begin{array}{c}\text { Concentration } \\
(\mathrm{M})\end{array}$ & $5.26052 \times 10^{-1}$ & $1.401 \times 10^{-1}$ & $7.6568 \times 10^{-2}$ \\
\hline $\begin{array}{c}\text { Temperature } \\
(\mathrm{K})\end{array}$ & $5.84784 \times 10^{-7}$ & $1.09314 \times 10^{-7}$ & $6.8593 \times 10^{-8}$ \\
\hline $\begin{array}{c}\mathrm{K} \text { Constant } \\
\left(\mathrm{min}^{-1}\right)\end{array}$ & \multicolumn{3}{|c|}{} \\
\hline
\end{tabular}

\section{B. Profiles with Random Temperature Changes}

Experimental setup remains the same as described in the preceding section, but here the temperature profiles have random temperature variations to simulate hydrogen peroxide products being used and stored under real world conditions. 
The simulation results are shown in Fig. 7 through Fig. 9 and Tables VI through VIII.
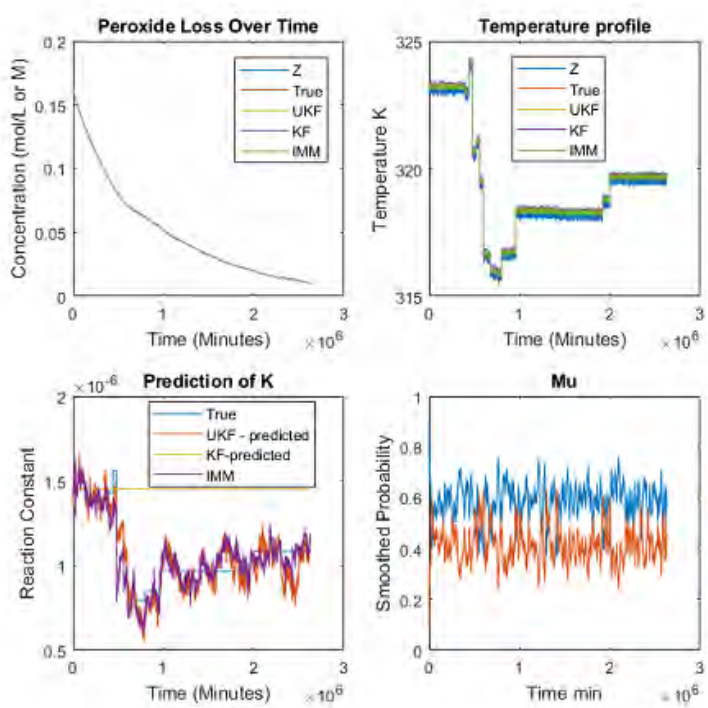

Figure 7. First profile with random temperature variation.
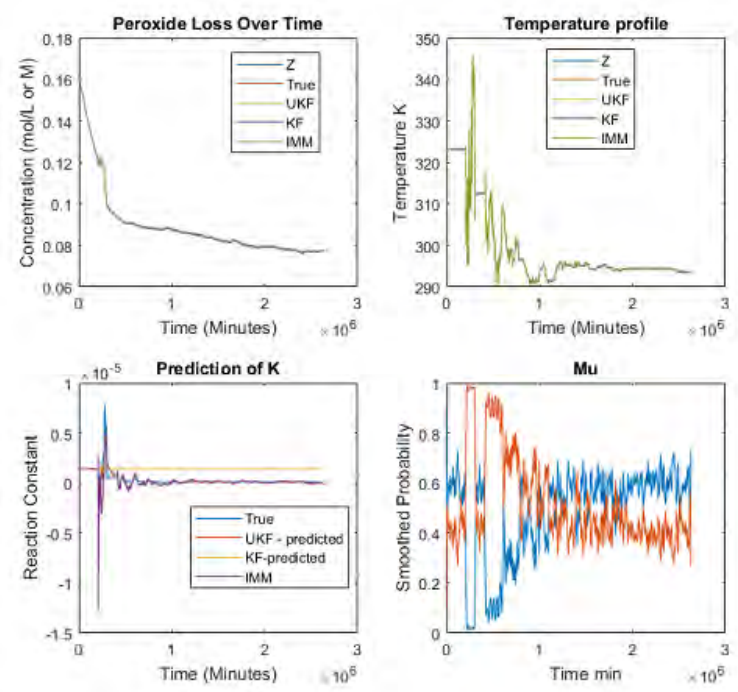

Figure 8. Second profile with random temperature variation.
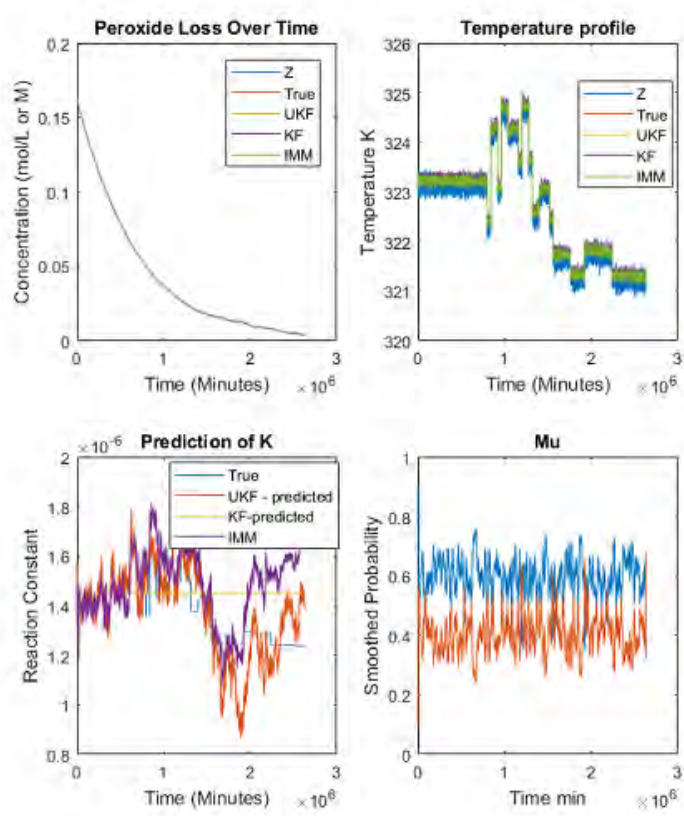

Figure 9. Third profile with random temperature variation.

TABLE VI. RMSE'S FOR FIRST PROFILE WITH RANDOM TEMPERATURE VARIATION

\begin{tabular}{|c|c|c|c|}
\hline \multirow{2}{*}{ System States } & \multicolumn{3}{|c|}{ Estimator RSME } \\
\cline { 2 - 4 } & KF & UKF & IMM \\
\hline $\begin{array}{c}\text { Concentration } \\
(\mathrm{M})\end{array}$ & $8.60051 \times 10^{-5}$ & $8.54607 \times 10^{-5}$ & $8.3524 \times 10^{-5}$ \\
\hline $\begin{array}{c}\text { Temperature } \\
(\mathrm{K})\end{array}$ & 1.02656 & $1.51515 \times 10^{-1}$ & $9.73733 \times 10^{-2}$ \\
\hline $\begin{array}{c}\mathrm{K}_{\text {Constant }} \\
\left(\mathrm{min}^{-1}\right)\end{array}$ & $1.34067 \times 10^{-7}$ & $1.29145 \times 10^{-7}$ & $1.71801 \times 10^{-7}$ \\
\hline
\end{tabular}

TABLE VII. RMSE'S FOR SECOND PROFILE WITH RANDOM TEMPERATURE VARIATION

\begin{tabular}{|c|c|c|c|}
\hline \multirow{2}{*}{ System States } & \multicolumn{3}{|c|}{ Estimator RSME } \\
\hline & KF & UKF & IMM \\
\hline $\begin{array}{l}\text { Concentration } \\
\text { (M) }\end{array}$ & $1.74623 \times 10^{-4}$ & $2.27619 \times 10^{-4}$ & $2.25101 \times 10^{-4}$ \\
\hline $\begin{array}{c}\text { Temperature } \\
(\mathrm{K})\end{array}$ & 4.55449 & $6.321 \times 10^{-1}$ & $6.21528 \times 10^{-1}$ \\
\hline $\begin{array}{c}\text { K Constant } \\
\left(\mathrm{min}^{-1}\right)\end{array}$ & $1.29936 \times 10^{-6}$ & $2.91613 \times 10^{-7}$ & $6.26771 \times 10^{-7}$ \\
\hline
\end{tabular}

TABLE VIII. RMSE'S FOR THIRD PROFILE WITH RANDOM TEMPERATURE VARIATION

\begin{tabular}{|c|c|c|c|}
\hline \multirow{2}{*}{ System States } & \multicolumn{3}{|c|}{ Estimator RSME } \\
\cline { 2 - 4 } & KF & UKF & IMM \\
\hline $\begin{array}{c}\text { Concentration } \\
(\mathrm{M})\end{array}$ & $8.13347 \times 10^{-5}$ & $8.34285 \times 10^{-5}$ & $8.16195 \times 10^{-5}$ \\
\hline $\begin{array}{c}\text { Temperature } \\
(\mathrm{K})\end{array}$ & $4.56238 \times 10^{-1}$ & $1.47175 \times 10^{-1}$ & $8.8286 \times 10^{-2}$ \\
\hline $\begin{array}{c}\mathrm{K}_{\text {Constant }} \\
\left(\mathrm{min}^{-1}\right)\end{array}$ & $1.34067 \times 10^{-7}$ & $1.29145 \times 10^{-7}$ & $1.71801 \times 10^{-7}$ \\
\hline
\end{tabular}


Based on Fig. 7 through Fig. 9 and Tables VI through VIII, one can see that the general trend is preserved. The UKF and IMM do the best job at predicting the reaction constant $k$, in terms of state estimation error. Additionally, the probability of switching between KF and UKF modes are more pronounced during large temperature variations. This indicates the robustness of IMM predictions with respect to random temperature spikes.

\section{CONCLUSION}

This paper proposes a new augmented state space model for faster prediction of hydrogen peroxide decay under any userdefined temperature profile. It was shown that the best state estimation accuracy was achieved by the UKF and IMM methods. Additionally, it was shown that for the IMM and UKF to have the best accuracy, low temperature changes and shorter durations of particular temperature variations are required. In terms of future work, more temperature profiles will be tested, and the $\mathrm{pH}$ level will be incorporated into the model. As the team was unable to find equations that relate the $\mathrm{pH}$ level to the rate of reaction, the effects of $\mathrm{pH}$ level would have to be incorporated through experimental data. The team will utilize a small set of experimental data with combined estimation and deep learning algorithms to create a more comprehensive model in the future.

\section{ACKNOWLEDGEMENTS}

The authors would like to express gratitude for the technical, financial, and in-kind support from Virox Technologies Inc. (Oakville, Ontario). The authors would also like to thank NSERC and the Ontario Centres of Excellence for their financial support.

\section{REFERENCES}

[1] B. D. O. Anderson and J. B. Moore, Optimal Filtering, Englewood Cliffs, NJ: Prentice-Hall, 1979.

[2] Y. Bar-Shalom, X. Rong Li and T. Kirubarajan, Estimation With Applications to Tracking and Navigation, New York: John Wiley and Sons, Inc., 2001.

[3] S. A. Gadsden, "Smooth variable structure filtering: Theory and applications," Ph.D. Thesis, McMaster Univ., Hamilton, ON, 2011.

[4] M. S. Grewal and A. P. Andrews, Kalman Filtering: Theory and Practice Using MATLAB, 3rd ed., New York: Wiley, 2008.

[5] S. A. Gadsden and S. R. Habibi, "A new form of the smooth variable structure filter with a covariance derivation," in IEEE Conference on Decision and Control, Atlanta, GA, 2010.

[6] R. E. Kalman, "A New Approach to Linear Filtering and Prediction Problems," Journal of Basic Engineering, Transactions of ASME, vol. 82, pp. 35-45, 1960.

[7] D. Simon, Optimal State Estimation: Kalman, H-Infinity, and Nonlinear Approaches, Hoboken, NJ: Wiley, 2006.

[8] S. A. Gadsden and A. S. Lee, "Advances of the smooth variable structure filter: Square-root and two-pass formulations," J. Appl. Remote Sens., vol. 11, no. 1, pp. 15018, Jan. 2017. doi: 10.1117/1.JRS.11.015018

[9] S. S. Lin and M. Gurol, "Catalytic decomposition of hydrogen peroxide on iron oxide: Kinetics, mechanism, and implications," Environ. Sci. Technol., vol. 32, no. 10, pp. 1417-1423, May 1998. doi: $10.1021 / \mathrm{es} 970648 \mathrm{k}$

[10] J. Tajagi and K. Ishigure, "Thermal decomposition of hydrogen peroxide and its effect on reactor water monitoring of boiling water reactors," Nucl. Sci. Eng., vol. 89, no. 2, pp. 177-186, Feb. 1985. doi: 10.13182/NSE85-A18191

[11] H. H. Afshari, S. A. Gadsden and S. R. Habibi, "Gaussian Filters for Parameter and State Estimation: A General Review and Recent Trends," Signal Process., vol. 135, pp. 218-238, June 2017. doi: 10.1016/j.sigpro.2017.01.001 


\section{Online Tuning Rule Based Adaptive Speed Control Algorithm or DC Motors Using Recursive Least Squares with Forgetting}

\author{
Kwang-seok Oh \\ Department of Mechanical Engineering \\ Hankyong National University \\ Anseong-si, 17579, Korea \\ oks@hknu.ac.kr
}

\author{
Jaho Seo \\ Department of Automotive, Mechanical and \\ Manufacturing Engineering \\ University of Ontario Institute of Technology \\ Oshawa, L1H 7K4, Canada \\ Jaho.Seo@uoit.ca
}

\begin{abstract}
This paper presents an online tuning rule-based adaptive control algorithm to control DC motor speed. For this, a method of recursive least squares with forgetting was proposed to approximate the relation between single input (voltage) and single output (angular speed) of the DC motor system as a first order differential equation. Using this approximated first order system and Lyapunov method-based disturbance observer with the online turning rule, a voltage input of the DC motor was generated to track a desired value of rotational speed of the motor. A performance evaluation of the proposed algorithm was conducted in the MATLAB/Simulink environment. The results show that the designed algorithm enables to track the reference speed successfully using only a single input and a single output of DC motor system.
\end{abstract}

Keywords- Lyapunov direct method; recursive least squares; single input single output; disturbance observer; DC motor system

\section{INTRODUCTION}

Modeling of dynamics in many systems including the DC motor is crucial for the purpose of dynamic analysis and control. However, the actual DC system has uncertainties, which cause a modeling error and thus have a negative effect on control performance. To deal with this issues, there are several existent studies to control the DC motor system that have been mainly based on model basis control approaches.

Ali et al. presented a model-based adaptive PID controller for the DC motor. The presented controller was able to secure robust and optimized control performance despite parameter variation and uncertainties [1]. Dursun and Durdu, proposed a speed control algorithm in the DC motor using a sliding mode observer that has been widely adopted in various industry control applications with uncertainties [2]. In addition, Meena and Bhushan suggested a DC motor speed control algorithm using fuzzy logic techniques [3]. In their study, a comparative analysis of the developed controller's performance with the PID control was conducted. The above studies require a mathematical model or knowledge of the systems to predict its dynamic behaviors and to derive an optimal input for successful control. However, it is hard to derive an accurate mathematical model (or obtain knowledge) for an optimal control in the actual system due to its nonlinearity and complexity. To address this issue, Xue et al. investigated a fractional order PID controller for a position servomechanism control system that dealt with actuator saturation and shaft torsional flexibility [4]. For an actual implementation, a modified approximation method was introduced in their study where some fixed parameters for the approximation were defined for the target system. However, the parameters need to be tuned whenever the target system is changed.

To overcome the above limitation, this study proposes a controller design method based on a model approximation that does not require parameters of the target system. In the method, an adaptive speed control algorithm with the online tuning was developed to control the DC motor speed using only single input (voltage) and single output (angular speed) of an approximated mathematical model (first order system). Also, this study introduces a disturbance observer using the Lyapunov direct method [5] that was designed to estimate disturbance.

The remainder of this paper is organized as follows. Section 2 provides a description of control concept. Section 3 describes the online tuning rule-based speed control algorithm. Section 4 provides a simulation-based performance evaluation results. Finally, Section 5 concludes the paper.

\section{CONCEPT OF CONTROL ALGORITHM}

A control algorithm proposed in this study was designed based on an approximated mathematical model that was assumed to have no restoring force due to frictional force existent in the actual system and thus can be finally considered as a damped first order system (Eq. (3)).

In the typical mechanical second order system, its dynamics can be expressed by the following equation.

$$
m \ddot{x}+c \dot{x}+k x+f=u
$$


where $m, c, k$ represent mass, damping coefficient, and stiffness, respectively. $x, u, f$ are the state variable, input and frictional term. If the stiffness $k$ can be assumed to have zero (no restoring force due to frictional force), the above equation can be arranged by Eq. (2) as follows.

$$
\ddot{x}+\frac{c}{m} \dot{x}+\frac{f}{m}=\frac{1}{m} u
$$

Since Eq. (2) does not include $k$ (restoring force) and thus it can be considered to be in a neutral equilibrium state. Then Eq. (2) can be rewritten as Eq. (3) by replacing $\dot{x}$ by $y$, which becomes a first order system.

$$
\dot{y}+\frac{c}{m} y+\frac{f}{m}=\frac{1}{m} u
$$

Since conditions of the first order damped system could be changed by various factors such as temperature, operating points, etc., the recursive least squares (RLS) was employed in this study to deal with an adaptive approximation of the damped system. Detailed explanation is provided in Section 3.

\section{ONLINE TUNING RULE BASED SPEED CONTROL ALGORITHM}

\section{A. RLS with forggeting based approximation}

For the adaptive approximation of damped system based on current input and output data, the RLS with forgetting method was applied. The first order differential equation used for this approximation is expressed in Eq. (4).

$$
\dot{y}+a y=u_{a}
$$

where $y$ is the system output that is controlled by the input $\left(u_{a}\right) . a$ is a time-varying parameter that represents the characteristic of the first order differential equation (4). To estimate the parameter $a$ adaptively using the system input and output, $u_{a}-\dot{y}, y$ and $a$ are defined as the output for the $\operatorname{RLS}\left(y_{r}\right)$, regressor $(\Phi)$, and estimate $(\hat{\theta})$, respectively. Fig. 2 shows a model schematic for the applied RLS with forgetting.

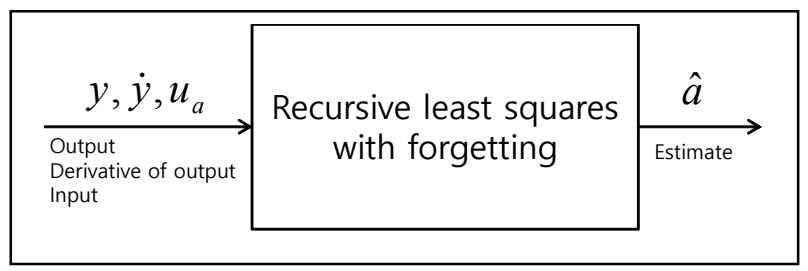

Figure 1. Model schematic for the RLS with forgetting
The following equations were used to compute the optimal gain $(L)$, and covariance $(P)$, and estimate $(\hat{\theta})$ that minimize the cost function $\left(J_{r}\right)$ with forgetting factor $(\lambda)$ in Eq. (8).

$$
\begin{gathered}
\hat{\theta}_{k}=\hat{\theta}_{k-1}+L_{k}\left(y_{r, k}-\Phi_{k} \hat{\theta}_{k-1}\right) \\
L_{k}=P_{k-1} \Phi_{k}\left(\lambda+\Phi_{k}^{T} P_{k-1} \Phi_{k}\right)^{-1} \\
P_{k}=\left(I-L_{k} \Phi_{k}^{T}\right) P_{k-1} / \lambda \\
J_{r}\left(\hat{\theta}_{k}, k\right)=\frac{1}{2} \sum_{i=1}^{k} \lambda^{k-i}\left(y_{r}(i)-\Phi(i) \hat{\theta}_{k}\right)^{2}
\end{gathered}
$$

\section{B. Disturbance observer}

The role of the Lyapunov method-based disturbance observer [5] adopted in the study is to estimate the disturbance that is a main source of modeling error between the approximately modeled damped system and actual one. The disturbance $(d)$ in the approximated first order equation is shown in the (9).

$$
\dot{y}=-\hat{a} y+u+d
$$

The used disturbance observer requires two stages of precorrection and estimation correction with the Lyapunov method. The following equation shows the disturbance observer's dynamics.

$$
\dot{e}_{y}=-\hat{a} e_{y}-e_{d}+K_{d}(y-\hat{y})
$$

where $e_{y}, e_{d}$, and $K_{d}$ represents the output error, disturbance error, and observer gain, respectively.

\section{Lyapunov direct method based control input}

The Lyapunov candidate function $(J)$ defined for control input is as follows.

$$
J=\frac{1}{2} e^{2}
$$

where $e$ represents the error $\left(e=y_{d e s}-y\right)$.

Using Eq. (12) below, a proper control input is derived so that the value of derivative of $J$ is always negative.

$$
\begin{aligned}
\frac{d J}{d t}=e \dot{e} & =\left(y_{d e s}-y\right)\left(\dot{y}_{d e s}-\dot{y}\right) \\
& =-W\left(y_{d e s}-y\right)^{2}
\end{aligned}
$$

where $W$ is the positive weighting factor that represents a change rate in the Lyapunov candidate function. Based on Eq. 
(9) and (12), The proper control input, $u_{p}$ that minimizes $J$ in Eq. (11) is derived as follows.

$$
u_{p}=\dot{y}_{d e s}+W y_{d e s}+(\hat{a}-W) y_{d e s}+\hat{d}
$$

The derived proper input can enable the output $(y)$ to track its desired output $\left(y_{d e s}\right)$. The derivative of the desired output $\left(\dot{y}_{d e s}\right)$ was estimated using a linear Kalman filter.

\section{Online tuning rule}

To cover the issue of degradation of control performance due to disturbance, the online tuning rule for $W$ with the error defined in Eq. (11) was utilized as shown in Eq. (14). While $W$ has a relatively large value in case of a large value of error, $\mathrm{W}$ has a relatively smaller value in the opposite case.

$$
W=m_{w} e^{2}+n_{w}
$$

where $m_{w}$ and $n_{w}$ are coefficients with negative values. Figure 2 shows the tuning rule in the $W$ and $e$ plane.

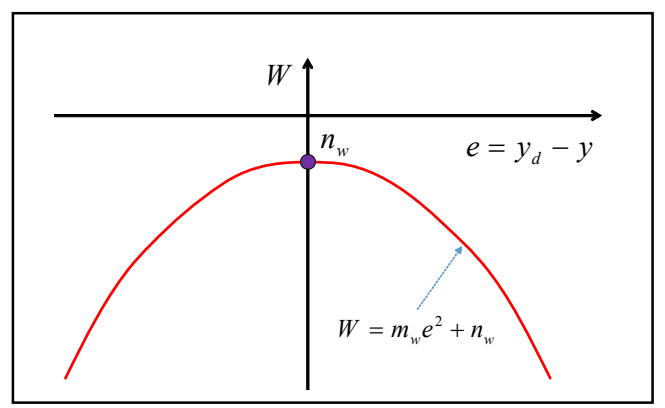

Figure 2. Model schematics for simulation model

\section{SIMULATION BASED PERFORMANCE EVALUATION}

Simulation was conducted to evaluate the designed controller's performance with a mathematical model of DC servo motor in the MATLAB/Simulink environment. Figure 3 shows a model schematic for the control performance evaluation.

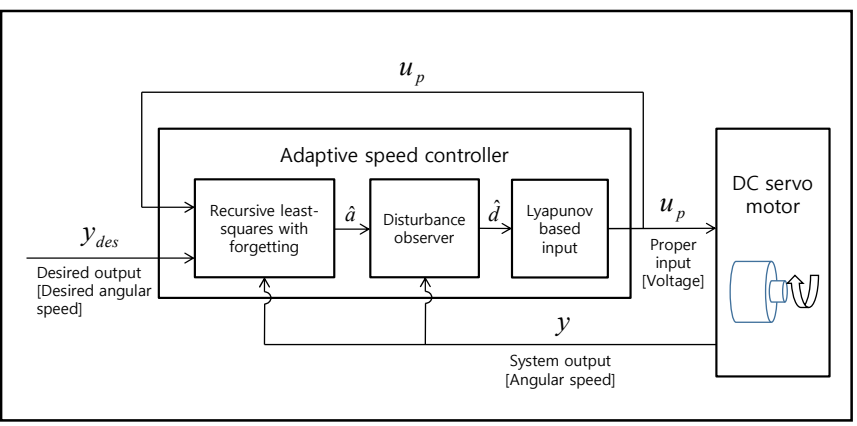

Figure 3. Model schematic for the control peformance evaluation
Figure 4 shows a typical circuit diagram of DC servo motor used in this study for the performance evaluation.

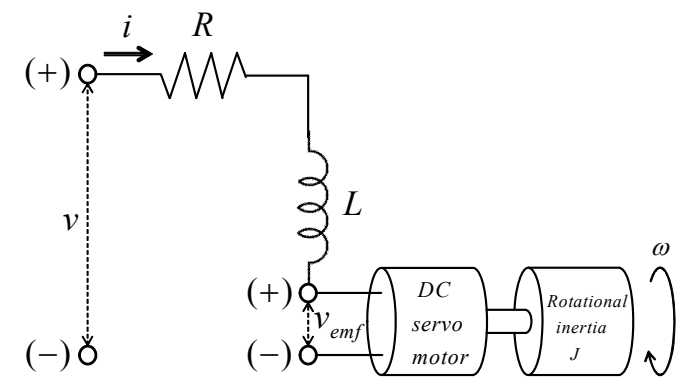

Figure 4. Circuit diagram of DC servo motor

In the figure, $R, L, \omega, i, v, v_{\text {emf }}, J$ represent the resistance, inductance, angular velocity, current, input voltage, counter electromotive force, and rotational inertia, respectively. Mathematical models of DC servo motor used for the performance evaluation are given in the following equations.

$$
\begin{gathered}
\frac{d i}{d t}=\frac{v}{L}-\frac{R}{L} i-\frac{K_{\Phi}}{L} \omega \\
\frac{d \omega}{d t}=\frac{K_{\Phi}}{J} i-\frac{b}{J} \omega
\end{gathered}
$$

where $K_{\Phi}$ represents the electromagnetic field constant. Based on the (15) and (16), the relationship between input (voltage, $v$ ) and output (angular speed, $\omega$ ) can be rewritten as follows.

$$
\dot{\omega}+\left(\frac{K_{\Phi}^{2}}{J R}+\frac{b}{J}\right) \omega+\left(\frac{K_{\Phi} L}{J R} \frac{d i}{d t}\right)=\left(\frac{K_{\Phi}}{J R}\right) v
$$

Using the RLS-based approximation method, the DC motor system model was approximated as shown in Fig. (1) and (3). Based on the approximated model and Lyapunov direct method in Eq. (13) and (14), a proper voltage input for DC motor speed control was derived. Table 1 shows parameter values used for the simulation-based evaluation.

TABLE I. THE PARAMETER VALUES FOR SIMULATION-BASED EVALUATION

\begin{tabular}{|c|c|c|}
\hline Division & Unit & Value \\
\hline Lambda $(\lambda)$ & - & 0.99 \\
\hline$R$ & $\Omega$ & 2 \\
\hline$L$ & $H$ & 0.1 \\
\hline$b$ & $N m s / r a d$ & 0.01 \\
\hline$J$ & $\mathrm{kgm}^{2}$ & 0.1 \\
\hline$K_{\Phi}$ & - & 0.3 \\
\hline$m_{w}$ & - & 0.7 \\
\hline
\end{tabular}




\begin{tabular}{|c|c|c|}
\hline Division & Unit & Value \\
\hline$n_{w}$ & - & -10 \\
\hline$W_{\text {constant }}$ & - & -10 \\
\hline
\end{tabular}

The performance of adaptive controller with the online tuning rule was compared with one with a constant weighting factor. Figs. 5-8 describes the evaluation results.

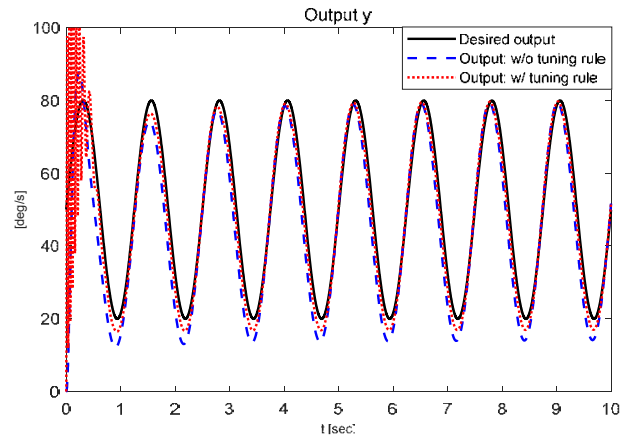

Figure 5. Evaluation results: output

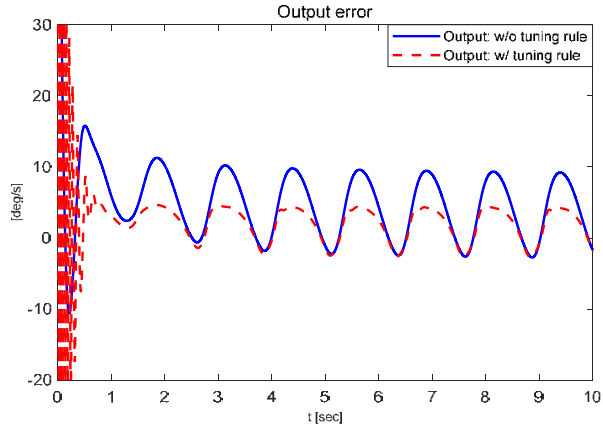

Figure 6. Evaluation results: output error

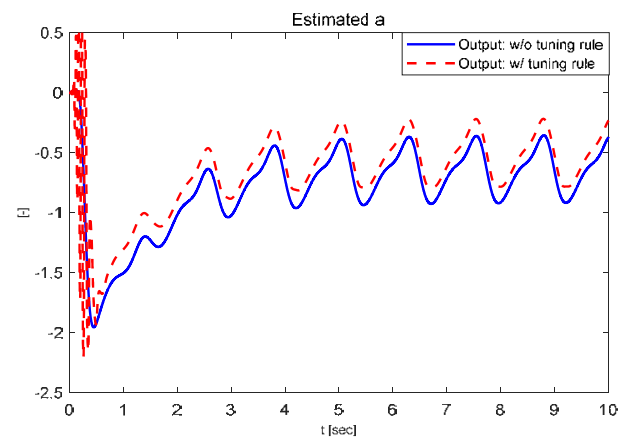

Figure 7. Evaluation results: estimated $a$

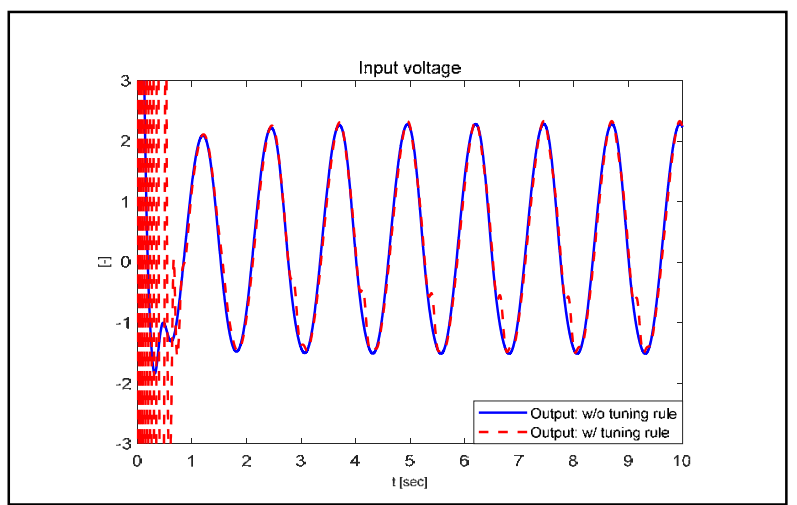

Figure 8. Evaluation results: voltage input

As seen in Figs. 5-6 and the Table 2 that shows the RMS values of output errors, the controller with the online tuning rule has smaller error than one with a constant weighting factor. However, the output $y$ in the online tuning rule-based control algorithm shows more oscillations compared to one with a constant weighting factor in the early stage of the simulation. Also, there are steady state output errors in both cases (Fig. 6). Oscillations in the estimated $a$ in both cases are found in Fig. 7, which are associated with oscillation of the desired output $y_{d}$. A shown in Fig. 8, there is no significant difference in the value of input voltage between both cases during the simulation time except for the beginning (i.e., transient state).

Table 2. RMS values of output errors

\begin{tabular}{|c|c|c|}
\hline Division & Unit & $\begin{array}{c}\text { RMS value } \\
\text { (Output error) }\end{array}$ \\
\hline $\begin{array}{c}\text { Without tuning rule } \\
\text { (With a constant weighting factor) }\end{array}$ & $\mathrm{deg} / \mathrm{s}$ & 7.69 \\
\hline With online tuning rule & $\mathrm{deg} / \mathrm{s}$ & 5.29 \\
\hline
\end{tabular}

\section{CONCLUSION}

In this study, the online tuning rule-based adaptive control algorithm was developed for controlling the DC motor system with single input (voltage) and single output (angular speed). For this, the approximated first order system and Lyapunov method-based disturbance observer with the online turning rule were utilized to design the control algorithms with the MATLAB/Simulink tool. The simulation results show that the system output with the designed controller accurately tracks the desired output. In addition, the RMS value of the output errors in the designed controller is smaller than the controller without tuning rule (i.e., constant weighting factor). However, both cases show oscillations at the beginning of simulation and steady state output errors. Therefore, a further study is required to improve the control performance to cope with this issue. It is expected that the proposed adaptive controller can be used as an alternative solution for the DC motor speed control in the related industrial applications. 


\section{REFERENCES}

[1] A. Ali, E. Tayeb, and B. Mohd, "Adaptive PID controller for DC motor speed control," Inter. J. Eng. Inventions, vol. 1, no. 5, pp. 26-30, 2012.

[2] E. Dursun and A. Durdu, "Speed control of a DC motors with variable load using sliding mode control," Inter. J. Compu. Elec. Eng., vol. 8, no. 3, pp. 219-226, 2016. doi: 10.17706/IJCEE.2016.8.3.219-226.

[3] P. Meena and B. Bhushan, "Simulation for position control of dc motor using fuzzy logic," Inter. J. Electron. Electri. Compu. System, vol. 6, no. 6, pp. 188-191, 2017.
[4] D. Xue, C. Zhao, and Y. Chen, "Fractional order PID control of a DCmotor with elastic shaft: A case study," Proc. Am. Control Conf., Minneapolis, Minnesota, USA, June 14-16, pp. 3182-3187, 2006. doi: 10.1109/ACC.2006.1657207.

[5] C. Liu and H. Peng, "Disturbance observer based tracking control," J. Dyn. Syst., Meas., Control, vol. 122, pp. 332-335, 2000. doi: 10.1115/1.482459. 


\title{
Modeling of Structural and Environmental Effects on Microelectromechanical (MEMS) Vibratory Gyroscopes
}

\author{
Md. Imrul Kaes, D.S-K Ting, Mohammed Jalal Ahamed \\ Micro/Nano-Mechatronics Laboratory, Mechanical, Automotive \& Material Engineering \\ University of Windsor, Windsor, ON, Canada \\ Email: jahamed@uwindsor.ca
}

\begin{abstract}
-
In this paper we investigate the effects of stiffness, damping and temperature on the performance of a MEMS vibratory gyroscope. The stiffness and damping parameters are chosen because they can be appropriately designed to synchronize the drive and sense mode resonance to enhance the sensitivity and stability of MEMS gyroscope. Our results show that increasing the drive axis stiffness by $50 \%$ reduces the sense mode amplitude by $\sim 27 \%$ and augments the resonance frequency by $\sim 21 \%$. The stiffness and damping are mildly sensitive to typical variations in operating temperature. The stiffness increases by $1.25 \%$, while the damping decreases by $3.81 \%$, when the temperature is raised from $0^{\circ} \mathrm{C}$ to $45^{\circ} \mathrm{C}$. Doubling the damping reduces the oscillation amplitude by $80 \%$, but $\sim 1 \%$ change in the frequency. The predicted effects of stiffness, damping and temperature can be utilized to design a gyroscope for the desired operating condition.
\end{abstract}

Keywords- MEMS gyroscope, Resonator, Spring Stiffness Coefficient, Damping Coefficient, Temperature, Coriolis

\section{INTRODUCTION}

Microelectromechanical systems (MEMS) based inertial sensors such as accelerometers and gyroscopes are the most commercially successful MEMS sensors to date. With the rapid advancement of semi-conductor based mass manufacturing, the cost, size and power consumption are continuously decreasing, enabling new applications in automotive, aerospace, biomedical and consumer electronics. MEMS vibratory gyroscopes are physical sensors that can detect and measure the angular motion of an object relative to an inertial frame of reference. MEMS vibratory sensors show promising vibration characteristics. Fabrication accuracies are comparable to macro-scale high performance sensors [1]-[4]. A vibratory gyroscope uses a vibrating structure (proof mass) to determine the rate of rotation [5]. The vibrating proof mass tends to vibrate along the same plane even if its support rotates. The Coriolis Effect causes the object to exert a force on its support, and the rate of rotation can be determined from the exerted force. The energy is transferred from the vibrating drive axis to the sense axis through Coriolis force. The sense mode response detects the angular velocity. The sensing performance of the MEMS gyroscope can deteriorate because of the influence of time-varying parameters such as damping, cross stiffness, and environmental variations. They generate a frequency of oscillation mismatch between the two vibrating axes [6]. Due to fabrication imperfections and environmental effects, true values of resonant frequencies deviate from their nominal values (which is known as frequency mismatching). These lead to considerable reduction in the sensitivity of the gyroscope. Therefore, it is necessary to design proper stiffness and damping for achieving a robust gyroscope.

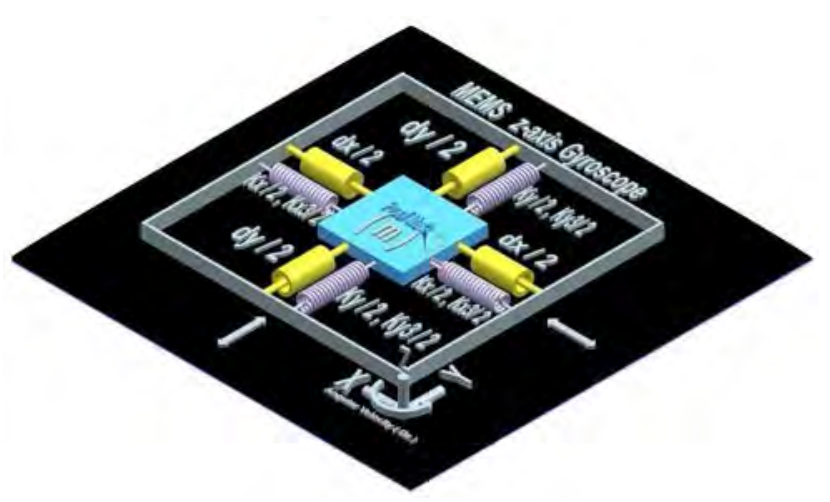

Figure 1. Schematic showing MEMS gyroscope model.

Optimization of time varying system parameters for better performance can be achieved by iterative modelling and simulation procedure. Various modeling techniques, including finite element analysis (FEA) modeling, analytical modeling and simplified lumped parameter modeling are employed [7]. FEA based modeling are robust, flexible and accurate in solving the complete multi-physics problem. Additionally, it can be coupled with controllers and electronics for selecting an appropriate controller [8].

Usually gyroscope displacement measurement varies under open loop scheme which can cause undesirable performance characteristics such as scale factor nonlinearity, limited dynamic range and narrow bandwidth [9]. In MEMS gyroscopes, 
manufacturing imperfection and noises often exist which negatively influence the resolution and performance. Errors due to noise are inevitable in actual MEMS gyroscopes, and thus, the controller has to be robust and effectively designed [10]. Postfabrication modeling of gyroscope using lumped-parameter techniques can shed light to the influence of system parameters, noise and environmental parameters on the gyro performance. This will allow iteration and design of a proper controller.

In this paper, a lumped-parameter model for predicting the gyroscope performance with the influence of various system parameters such as stiffness, damping and environmental condition (temperature) is analyzed. The results can be utilized to design a robust high performance gyroscope with efficient controller.

\section{MATHEMATICAL MODEL}

In a vibratory gyroscope the main sensing element is the proof mass, which is suspended above the substrate by several flexible beams. The overall dynamic system is usually modeled as a 2 degrees-of-freedom (2-DOF) spring-mass-damper system, as shown in Figure 1. The proof mass is suspended on the substrate using four springs (Figure 1), the other end of the springs are anchored to the substrate. An electrostatic actuation is used to give an oscillatory motion to the proof mass in the drive axis. When the proof mass is subject to an angular velocity, energy is transferred from drive axis to sense axis, causing it to oscillate. The mechanism for sensing position and velocity of the proof mass along the sense axis is present. Constant velocity of the proof mass and changing angular velocity of gyroscope about the $z$-axis are assumed. The MEMS vibratory gyroscope model includes proof mass $(m)$, drive axis displacement $(x)$, the angular velocity input $\left(\Omega_{z}\right)$, and sense axis displacement $(y)$.

According to Lagrange's [11] equation, the dynamics of the gyroscope can be described by the equations of motion below [12]:

$\mathrm{m}_{\ddot{x}}+\mathrm{d}_{\mathrm{xx}} \dot{x}+\mathrm{k}_{\mathrm{xx}} \mathrm{x}+\mathrm{k}_{\mathrm{xy}} \mathrm{y}+\mathrm{d}_{\mathrm{xy}} \dot{y}=\mathrm{u}_{\mathrm{x}}+2 \mathrm{~m} \Omega_{\mathrm{z}} \dot{y}$
$\mathrm{~m} \ddot{y}+\mathrm{d}_{\mathrm{yy}} \dot{y}+\mathrm{k}_{\mathrm{yy}} \mathrm{y}+\mathrm{k}_{\mathrm{xy}} \mathrm{x}+\mathrm{d}_{\mathrm{xy}} \dot{x}=\mathrm{u}_{\mathrm{y}}+2 \mathrm{~m} \Omega_{\mathrm{z}} \dot{x}$

Manufacturing imperfections are translated mainly to the asymmetric spring and damping terms, $\mathrm{k}_{\mathrm{xy}}$ and $\mathrm{d}_{\mathrm{xy}}$. The drive and sense axes spring and damping terms $\mathrm{k}_{\mathrm{xx}}, \mathrm{k}_{\mathrm{yy}}, \mathrm{d}_{\mathrm{xx}}$ and $\mathrm{d}_{\mathrm{yy}}$ are mostly known, but have small unknown variations from their nominal values. The proof mass can be determined accurately, and $\mathrm{u}_{\mathrm{x}}, \mathrm{u}_{\mathrm{y}}$ are the control forces in the $\mathrm{x}$ and $\mathrm{y}$ directions.

Dividing gyroscope dynamics by the reference mass, the following equation can be obtained [13]:

$q * *+\frac{D *}{m} q *+\frac{k 1 *}{m} \mathrm{q} *+\frac{k 3 *}{m}+\mathrm{q}^{* 3}$

$=\Omega^{*} \mathrm{q}^{*}+S^{*} * \mathrm{q}^{*}+2 \mathrm{~S}^{*} q^{*} *+\mathrm{u}^{*}$

where

$\mathrm{q}^{*}=\left[\begin{array}{l}x^{*} \\ y^{*}\end{array}\right], \mathrm{u}^{*}=\left[\begin{array}{l}u_{x^{*}} \\ u_{y^{*}}\end{array}\right], \mathrm{d}^{*}=\left[\begin{array}{cc}d_{x^{*}} & d_{x y^{*}} \\ d_{x y^{*}} & d_{y^{*}}\end{array}\right], \mathrm{k}_{1} *=\left[\begin{array}{cc}k_{x^{*}} & k_{x y^{*}} \\ k_{x y^{*}} & k_{y^{*}}\end{array}\right]$, $\mathrm{k}_{3} *=\left[\begin{array}{cc}k_{x^{3 *}} & 0 \\ 0 & k_{y^{3 *}}\end{array}\right], \mathrm{S} *=\left[\begin{array}{cc}0 & \Omega_{z^{*}} \\ -\Omega_{z^{*}} & 0\end{array}\right]$

Angular rate variation may be considered to be negligible. The equations of motion of the gyroscope are normalized to generalize the model. The final non-dimensional equation is derived in terms of normalized time, $t^{*}=\omega_{0} t$. With this, dividing both sides of the equation by the reference frequency and length gives [13]:

$\frac{\ddot{q}^{*}}{q_{0}}+\frac{d^{*}}{m \omega_{0}} \frac{\dot{q}^{*}}{q_{0}}+\frac{k_{1}^{*}}{m \omega_{0}^{2}} \frac{q^{*}}{q_{0}}+\frac{k_{3}^{*}}{m \omega_{0}^{2}} \frac{q^{* 3}}{q_{0}^{3}}$
$=\frac{\Omega_{z}^{*}}{\omega_{0}^{2}} \frac{\dot{q}^{*}}{q_{0}}+2 \frac{S^{*}}{\omega_{0}} \frac{\dot{q}^{*}}{q_{0}}+\frac{u^{*}}{m \omega_{0}^{2}} \frac{1}{q_{0}}$

where the parameters are defined as follows:

$q=\frac{q^{*}}{q_{0}}, d_{x y}=\frac{d_{x y}^{*}}{m \omega_{0}}, \Omega_{z}=\frac{\Omega_{z}^{*}}{\omega_{0}}, u_{x}=\frac{u_{x}^{*}}{m \omega_{0}^{2}} \frac{1}{q_{0}}, u_{y}=\frac{u_{y}^{*}}{m \omega_{0}^{2}} \frac{1}{q_{0}}$

$\omega_{x}=\sqrt{\frac{k_{x}}{m \omega_{0}^{2}}}, \omega_{y}=\sqrt{\frac{k_{y}}{m \omega_{0}^{2}}}, \omega_{x y}=\frac{k_{x y}}{m \omega_{0}^{2}}$,

$\delta_{x}=\frac{k_{x}^{3}}{m \omega_{0}^{2}} q_{0}^{2}, \delta_{y}=\frac{k_{y}^{3}}{m \omega_{0}^{2}} q_{0}^{2}$

Consequently, the non-dimensional representation becomes

$\ddot{q}+D \dot{q}+q k_{1}+k_{3} q^{3}=\Omega_{z}^{2} \dot{q}+2 S \dot{q}+u$

where

$q=\left[\begin{array}{l}x \\ y\end{array}\right], u=\left[\begin{array}{l}u_{x} \\ u_{y}\end{array}\right], D=\left[\begin{array}{cc}d_{x} & d_{x y} \\ d_{x y} & d_{y}\end{array}\right], k_{1}=\left[\begin{array}{cc}\omega_{x}^{2} & \omega_{x y} \\ \omega_{x y} & \omega_{y}^{2}\end{array}\right]$, $k_{3}=\left[\begin{array}{cc}\delta_{x} & 0 \\ 0 & \delta_{y}\end{array}\right], S=\left[\begin{array}{cc}0 & \Omega_{z} \\ -\Omega_{z} & 0\end{array}\right]$

Simulation is done to observe the influence of stiffness, damping and temperature on gyroscope performance to minimize time and cost of expensive trial and error with the actual fabrication cycle.

MATLAB Simulink [14] is used for simulation and computation. Spring stiffness, damping coefficient and temperature are known to have significant influence on the output performance of gyroscope. Thus, the objective of the simulation is to quantify the effects of these parameters. The spring stiffness and damping coefficient are varied from half to double of their nominal values. The temperature is varied from $0^{0} \mathrm{C}$ to $45^{0} \mathrm{C}$ to observe the temperature effect on stiffness, damping coefficient and on sensing magnitude. For the simulation model, the nominal values for the proof mass, stiffness, and damping are extracted from the literature reported in [12]. Proof mass, $\mathrm{m}=0.57 \mathrm{e}^{-8} \mathrm{~kg}$, damping coefficient along the drive-axis, $\mathrm{d}_{\mathrm{xx}}=0.429 \mathrm{e}^{-6} \mathrm{~N} \mathrm{~s} / \mathrm{m}$, damping error due to manufacturing imperfection, $\mathrm{d}_{\mathrm{xy}}=0.0429 \mathrm{e}^{-6} \mathrm{~N} \mathrm{~s} / \mathrm{m}$, damping coefficient along the sense-axis, $d_{y y}=0.687 \mathrm{e}^{-36} \mathrm{~N} \mathrm{~s} / \mathrm{m}$, spring stiffness along the drive-axis, $\mathrm{k}_{\mathrm{xx}}=80.98 \mathrm{~N} / \mathrm{m}$, stiffness error 
due to manufacturing imperfection, $\mathrm{k}_{\mathrm{xy}}=5 \mathrm{~N} / \mathrm{m}$, spring stiffness along the sense-axis, $\mathrm{k}_{\mathrm{yy}}=71.62 \mathrm{~N} / \mathrm{m}$, angular velocity, $\Omega_{\mathrm{z}}=5$ $\mathrm{rad} / \mathrm{s}$ are considered.

\section{MODEL RESULTS}

\section{A. Oscillation Frequency}

To identify the first resonance frequency, input frequency is varied, a sinusoidal electromagnetic force is introduced at the drive-axis and output is sensed both in drive and sense directions (Fig. 2). Usually the drive mode displacement is higher compared to sense mode due to the energy transfer.

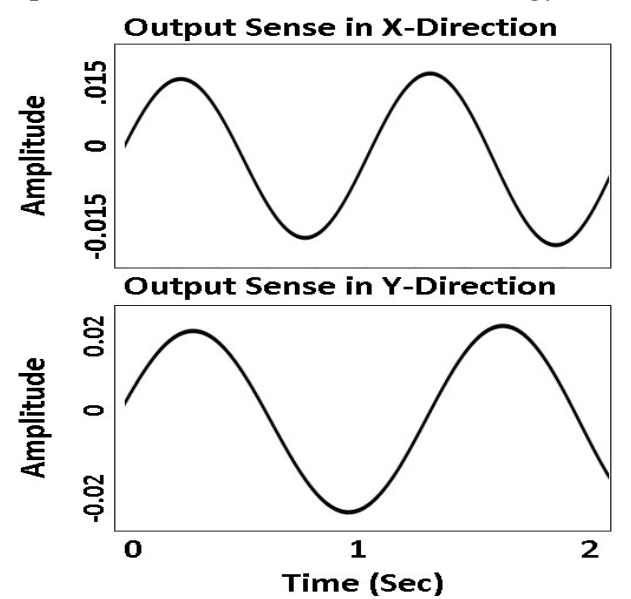

Figure 2. Displacement in drive mode (x) and sense mode (y) direction.

After sweeping the simulation with the frequency range of 1 $\mathrm{kHz}$ to $60 \mathrm{kHz}$ (Fig. 3), the resonance frequency is found at around $9.4 \mathrm{kHz}$. So, for better focusing, the frequency range is restricted from $10 \mathrm{kHz}-30 \mathrm{kHz}$ for other comparisons.

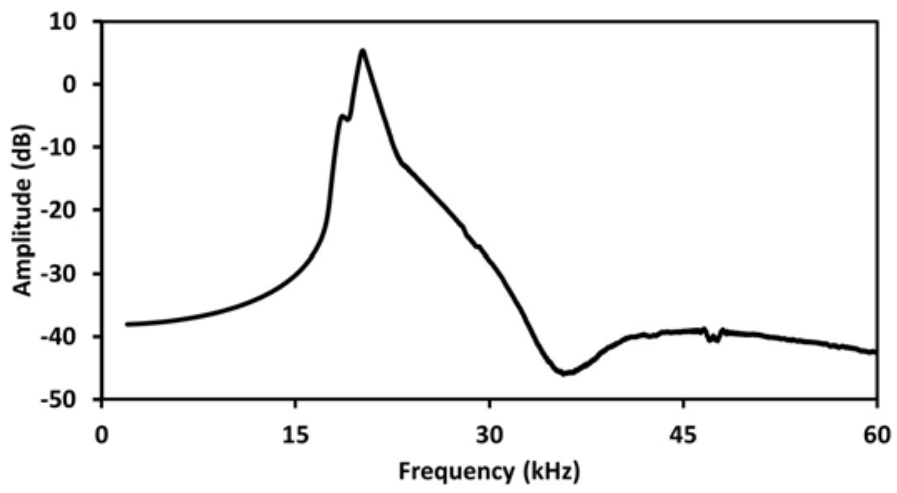

Figure 3. Frequency versus magnitude graph.

\section{B. Variation in Stiffness}

Suspension beam along drive and sense direction provides the stiffness necessary in each direction. Keeping the $\mathrm{k}_{\mathrm{yy}}$ value constant at $71.62 \mathrm{~N} / \mathrm{m}, \mathrm{k}_{\mathrm{xx}}$ is varied from $50 \%$ (normalized value 0.5 ) to $150 \%$ (normalized value 1.5 ). The normalized value of tuned reference stiffness $(80.98 \mathrm{~N} / \mathrm{m})$ is considered as 1. It is observed that magnitudes at resonance frequencies show linear decreasing trend (Fig. 4) and amplitudes decrease (Fig. 5) as stiffness increases. However, decreasing the stiffness creates phase lag and distortion in output response.

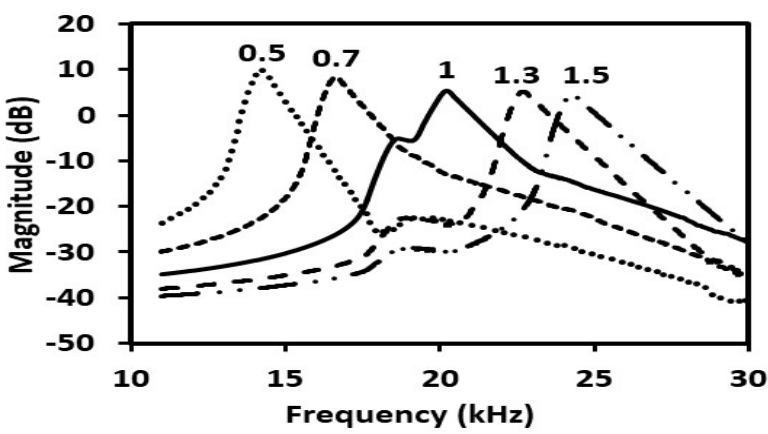

Figure 4. Effect of drive mode stiffness on resonance frequency and amplitude.

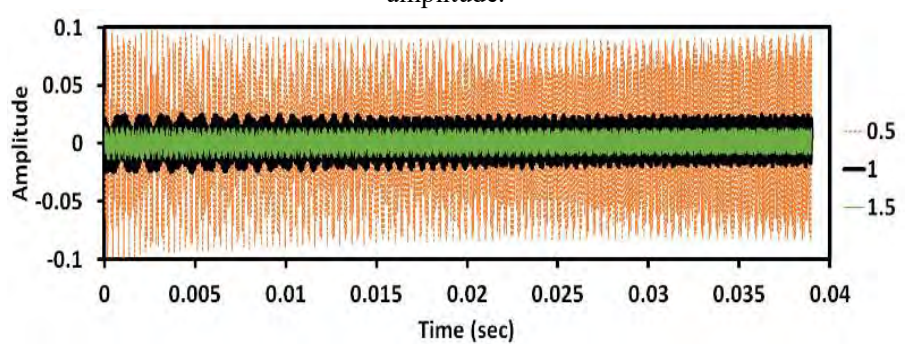

Figure 5. Effect of drive mode stiffness on output sense (sense mode stiffness is constant)

Keeping the $\mathrm{k}_{\mathrm{xx}}$ value constant, $\mathrm{k}_{\mathrm{yy}}$ is varied. It is observed that shift in resonance frequency is non-significant (Fig. 6).

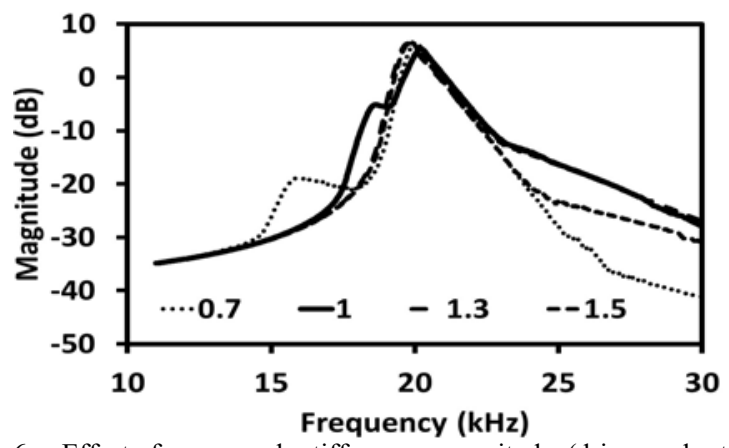

Figure 6. Effect of sense mode stiffness on magnitude (drive mode stiffness is constant).

Variation in stiffness $\left(\mathrm{k}_{\mathrm{xx}}\right.$ and $\left.\mathrm{k}_{\mathrm{yy}}\right)$ along both axes decreases magnitudes at resonance frequencies and resonance frequencies shift towards higher frequencies (Fig. 7) and amplitudes also decreases as stiffness increases (Fig. 8).

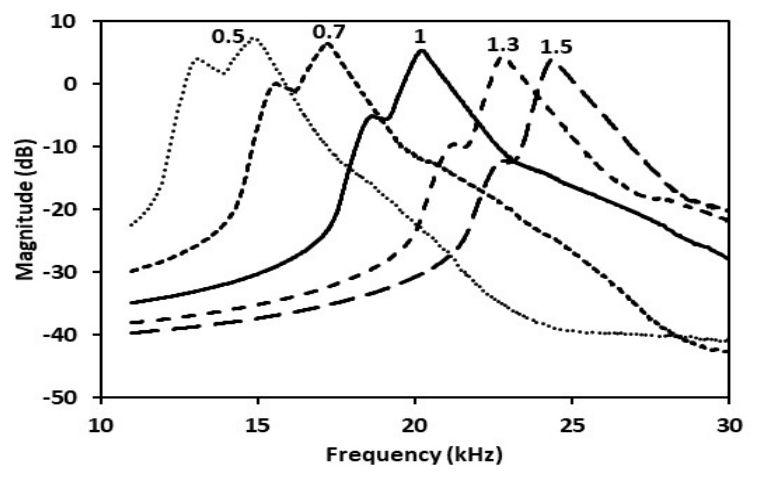

Figure 7. Combined effect of drive mode stiffness and sense mode stiffness on magnitude. 


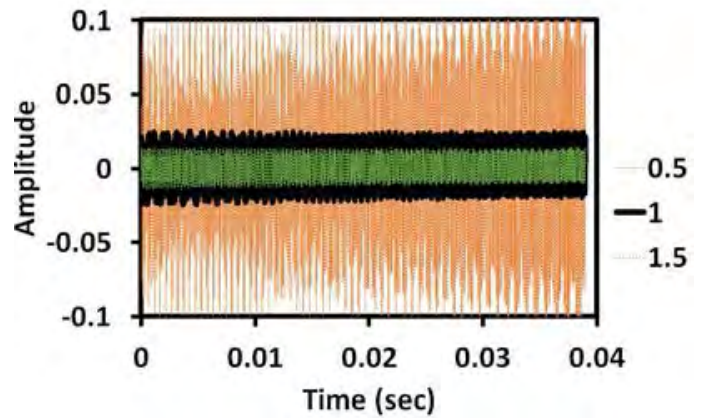

Figure 8. Effect of drive mode stiffness and sense mode stiffness on output sense.

\section{Variation in Damping}

Here, damping along drive axis is varied (Fig. 9). It is obvious from the figure that the resonance frequencies do not change that much but slope steepness decreases after resonance frequencies.

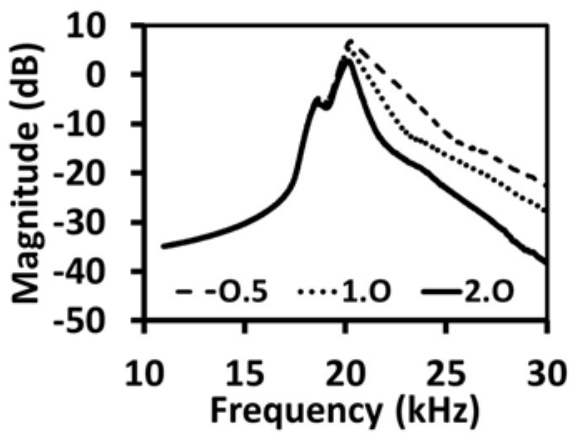

Figure 9. Effect of drive mode damping on magnitude.

\section{Temperature Variation}

One key disadvantage of MEMS gyrosocpe is its high thermal sensitivity. The frequency of oscillation drifts with temperture. Temperature variation mainly affects the stiffness and damping of the supporting beams. Here investigation on the temperature sensitivity for stiffness and damping is done. The variation of stiffness with temperature can be modeled using simplified linear relationship of [7] $K(T)=K_{0}(1-k \Delta T)$, where $K_{0}$ is the stiffness coefficient at reference temperature $300 \mathrm{~K}$ and $k=70$ ppm.

Variation of damping coefficient with temperature can be modeled as [7] $d(T)=d_{0} \cdot 1.28^{\ln \left(\frac{T}{300}\right)}$, where $\mathrm{T}$ is temperature in $\mathrm{K}, d_{0}$ is demping coefficient at reference temperature of 300 $K$. Magnitude shows positive increasing trend with the increase of temperature when stiffness and temperature relationship shows linear behaviour. The damping coefficient within industrial temperature range shows decreasing trend as temperature increases (Fig. 10, 11).

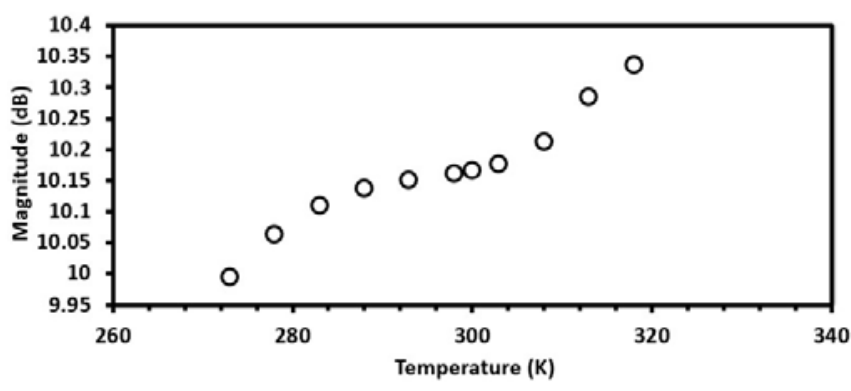

Figure 10. Effect of temperature on linear drive mode stiffness and magnitude

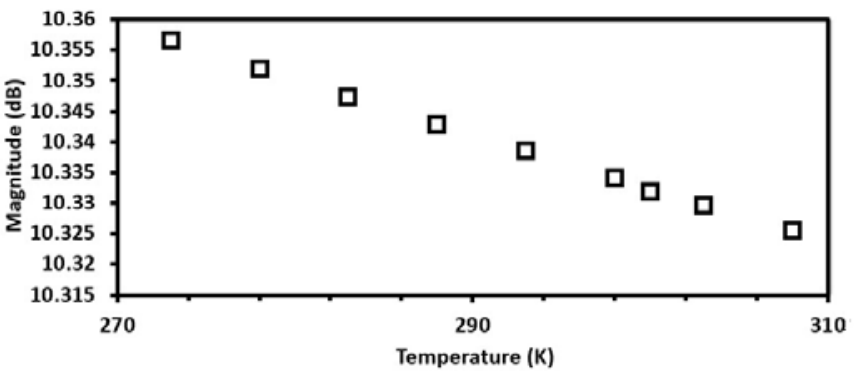

Figure 11. Effect of temperature on non-linear damping and magnitude

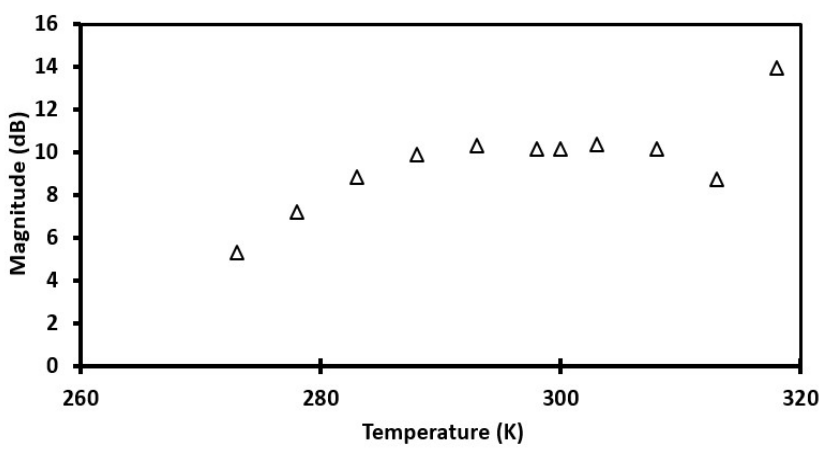

Figure 12. Effect of temperature on non-linear drive mode stiffness and magnitude.

The spring stiffness and temperature relationship shows nonliner behavior, the magnitude increases non-linearly up to a certain point before decreasing. At the end of the industrial temperature range, the magnitude increases drastically (Fig. 12)

\section{CONCLUSION AND FUTURE WORK}

This paper investigates the effect of variation of suspending stiffness coefficient, damping coefficient and temperature on the performance of MEMS gyroscope. Simulation results show how these parameters affect the error i.e. mismatch between the input and sense signals. This will help to design appropriate system parameters and controllers to increase gyroscope accuracy. Simulation results also show that stiffness is the dominant gyroscope parameter. The temperature can negatively impact resonance frequency and sense magnitude. In the future, we plan to extend this by including experimental validation of our results with MEMS gyroscopes and implementing a controller to minimize these effects and maximize the gyroscope performance. 


\section{ACKNOWLEDGEMENT}

The authors would like to thank the Natural Sciences and Engineering Research Council of Canada (NSERC) and University of Windsor for financial support.

\section{REFERENCES}

[1] M. J. Ahamed, D. Senkal, S. Member, A. A. Trusov, and A. M. Shkel, "Study of High Aspect Ratio NLD Plasma Etching and Postprocessing of Fused Silica and Borosilicate Glass," J. Microelectromechanical Syst., vol. 24, no. 4, pp. 790-800, 2015.

[2] J. Liu, J. Jaekel, D. Ramdani, N. Khan, D. S.-K. Ting, and M. J. Ahamed, "Effect of Geometric and Material Properties on Thermoelastic Damping (TED) of 3D Hemispherical Inertial Resonator," in ASME International Mechanical Engineering Congress and Exposition, 2016, vol. 10, pp. 1-6. doi: 10.1115/IMECE2016-66277.

[3] A. M. Shkel, M. Ahamed, and D. Senkal, "Method of fabricating micro-glassblown gyroscopes," US 9,702,728 B2, 2017.

[4] D. Senkal, M. J. Ahamed, M. H. A. Ardakani, S. Askari, and A. M. Shkel, "Demonstration of 1 million Q -factor on microglassblown wineglass resonators with out-of-plane electrostatic transduction," $J$. Microelectromechanical Syst., vol. 24, no. 1, pp. 29-37, 2015.

doi: 10.1109/JMEMS.2014.2365113.

[5] F. Braghin, F. Resta, E. Leo, and G. Spinolab, "Nonlinear dynamics of vibrating MEMS," Sensors Actuators A Phys., vol. 134, no. 1, pp. 98-108, 2007. doi: 10.1016/j.sna.2006.10.041

[6] J. Fei and C. Batur, "A novel adaptive sliding mode control with application to MEMS gyroscope," ISA Trans., vol. 48, no. 1, pp. 73-78, 2009. doi: $10.1016 /$ j.isatra.2008.10.008.

[7] M. Wen et al., "Modeling and analysis of temperature effect on MEMS gyroscope," Electron. Components Technol. Conf., pp. 20482052, 2014. doi: 10.1109/ECTC.2014.6897584

[8] J. Wang, S. Ban, and Y. Yang, "A Differential Self-Integration DDot Voltage Sensor and Experimental Research," IEEE Sens. J., vol. 15, no. 7, pp. 3846-3852, 2015. doi: 10.1109/JSEN.2015.2399413.

[9] W. T. Sung, S. Sung, J. G. Lee, and T. Kang, "Design and performance test of a MEMS vibratory gyroscope with a novel AGC force rebalance control," J. Micromechanics Microengineering, vol. 17, no. 10, pp. 1939-1948, 2007. doi: 10.1088/0960-1317/17/10/003.

[10] W. Juan and J. Fei, "Adaptive fuzzy approach for non-linearity compensation in MEMS gyroscope," Trans. Inst. Meas. Control, vol. 35, no. 8, pp. 1008-1015, 2013. doi: 10.1177/0142331212472224.

[11] R. G. Albrecht Bertram, Solid Mechanics: Theory, Modeling, and Problems, First Edit. Sachsen-Anhalt, Germany: Springer, 2013.

[12] J. Fei, W. Dai, M. Hua, and Y. Xue, "System dynamics and adaptive control of MEMS gyroscope sensor," IFAC Proc., vol. 44, no. 1, pp. 3551-3556, 2011. doi: 10.3182/20110828-6-IT-1002.00034.

[13] M. R. Moghanni, J. Keighobadi, and A. Ghanbari, "Fuzzy adaptive sliding mode controller for MEMS vibratory rate gyroscope," IFAC Proc., vol. 44, no. 1, pp. 4192-4197, 2011. doi: 10.3182/20110828-6-IT-1002.03569.

[14] Simulink, "Mathworks," 2018. [Online]. Available: https://www.mathworks.com/products/simulink.html. [Accessed: 26Mar-2018]. 


\title{
Lagrangian, Hamiltonian, and Energy-based Control for Space Tethered System
}

\author{
Junjie Kang and Zheng H. Zhu \\ York University \\ Toronto, Canada \\ kangjunjie1989@gmail.com; gzhu@yorku.ca
}

\begin{abstract}
A typical and useful way to derive the dynamics equation of the tethered systems is by means of the Lagrange's equations and various dynamic models of different tethered missions are established by the Lagrangian formulation. The Hamiltonian formulation is also widely used in the mechanical systems for its well-known symplectic structure property. With this in mind, the dynamics equation of the tethered system's motion are deduced by Hamilton's equations in this research. The relation between the Lagrangian and Hamiltonian is shown by Legendre transformation. The goodness of the Hamiltonian formulation is intuitive to reveal the Energy balance property that corresponds to the passivity property. Furthermore, the Hamiltonian energy function of tethered system is employed for facilitating the controller design. In order to bring the system into operations, the energy based control is to achieve the tethered system for precise positioning. Simulations are used to demonstrate the effectiveness of the designed controller.
\end{abstract}

Keywords-Tethered satellite system; Passivity; Energy-based control.

\section{INTRODUCTION}

Space tethered systems have been drawn great attention on its significant potential for the space missions utilization during the past decades. The tethered satellite system consists of two or more satellites which are connected by a long cable. The key challenge to bring the tethered space systems into operation is to successfully deploy the tether to the desired position in a stable and fast manner. However, the dynamics of the tethered systems are quite complex for the overall flexibility, then toward getting insight into the dynamics and control of the deployment for tethered systems, the simplified dumbbell model of the tethered systems are usually taken [1]. The mother and daughter satellites are considered as point mass connected by an inextensible massless tether. Thus, the important aspects of the dynamic behavior of the tethered system can be investigated by the simplified model without the involving the complicated mathematical equations.
In the past researches, the dynamics and control of tethered satellite system are well studied. The dynamic model of tethered system is usually derived by the Lagrangian formulation because it is easy to apply and solve the equations of motion for such two-body problem. A lot of research achievement of tethered systems are gained space applications such as the debris towed by tether, tethered satellite formation, tethered robot, etc. Among these applications, space tether deployment is the key issue, which is still a hot topic. The deployment control of the tethered system is not easy because it is prone to swing or liberating due to the coriolis force induced by deployment. Sometimes, the fast deployment speed may cause too large swing amplitude, which should be avoided to proceed space tethered operations. Many researchers are focusing on the problem how to achieve the tether deployment fast and stable with suppressing the libration [2-7]. For example, a tension control law in terms of the tether length and its velocity was firstly raised by Rupp for tether deployment [2]. Then, Fujii proposed a mission function control for the tether deployment based on the Lyapunov function [3]. A linear tension control for tether deployment was presented by Pradeep through the linearization [4]. Sun and Zhu developed a fractional order control law to eliminate the overshooting [5]. Further, Wen proposed a saturated controller by imposing a special saturation function to deal with tension constraints [6]. Kang and Zhu design a fractional order sliding mode tension control to counter the perturbations or uncertainties [7].

In this study, we proposed a natural controller design approach which is closely related to the dynamics of the tethered system. The energy-based control is used for precise positioning and effective suppressing the libration. Firstly, the dynamic equations are derived by the Lagrange's equations. The obtained dynamic equations of the tethered system are further represented in the compact form of Euler-Lagrange. The important passivity of the inherent aspect of tethered system's dynamics is revealed with specific matrix. In addition, Hamiltonian formulation is used to derive the dynamic equations of motion, which is an equivalent way of Lagrangian. Hamiltonian formulation is also widely used in the mechanical systems for its well-known symplectic structure property. Thus, energy balance property of the tethered system equivalent to passivity is given. The property is generally founded by 
Hamiltonian formulation rather than specifying the expressions of the matrix. Finally, the energy-based control is developed and asymptotic stability of the closed-loop system is proved to ensure the precise positioning.

\section{MODELING FORMULATION}

The widely used model of tethered space system control is the dumbbell model that is a long tether connecting two satellites at its ends, as shown in Fig.1 [8]. The system is moving in the orbit of the earth. The orbital motion coordinate is located as $o-x y z$. The $y$-axis directs toward the Earth center. The $x$-axis is perpendicular to the $y$-axis in the orbital plane and pointing at the velocity's direction of the orbit. The $z$-axis complies with the right-hand rule. The mass of the Main satellite and Subsatellite is $m_{1}$ and $m_{2}$, respectively. To bring insight of space tether system, following assumptions are made usually.

a) The field of the earth is central gravitational field and the Main and Sub satellites are particles.

b) The mass ratio of the Subsatellite over Main satellite is sufficiently small such that coordinate $o-x y z$ always remains in its nominal orbit.

c) The tether is massless and its flexibility is ignored.

d) The tether length $l$ is very short compared to the distance $R$ form coordinate $o-x y z$ to the center of earth.

e) External perturbation force is ignored. The orbit is circular and only motion in the orbital plane is considered.

f) Tether tension is the only control force acts along the tether. The tether is deployed/retrieved through the tension control, i.e., the required tension is produced by the motor driving the tether reel in/out system.

Based upon the assumptions, the TSS motion can be decomposed into the orbital motion of the system's center of mass (CM) and the local libration motion $\theta$ of tether about the $\mathrm{CM}$ in the orbital plane, as shown in Fig.1.

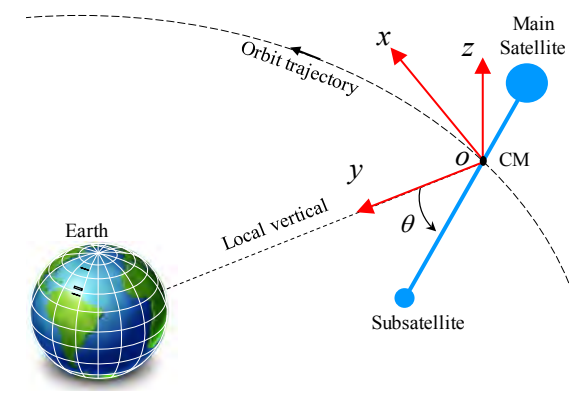

Figure 1. Sketch of the space tether system

Accordingly, the kinetic energy of the tethered system reads $K_{0}=0.5\left(m_{1}+m_{2}\right) R^{2} \Omega^{2}+0.5 m_{0} l^{2}\left(\theta^{\prime}+\Omega\right)^{2}+0.5 m_{0}\left(l^{\prime}\right)^{2}$, where the prime ( $)^{\prime}$ denotes the derivative with respect to time $t$, and $\Omega$ is the orbital angular velocity. $m_{0}=m_{1} m_{2} /\left(m_{1}+m_{2}\right)$ is the equivalent mass. Due to $l<<R$, the tethered system's potential energy can be approximated $U_{0}=-\left(m_{1}+m_{2}\right) R^{2} \Omega^{2}+0.5 m_{0} l^{2} \Omega^{2}\left(1-3 \cos ^{2} \theta\right)$.

\section{A. Lagrangian Formulation}

Take the Lagrangian function as $L=K-U$, the dynamic equations of the tethered system can be straightly obtained according to the Lagrange's Equation,

$$
\frac{d}{d t} \frac{\partial L}{\partial \dot{s}}-\frac{\partial L}{\partial s}=Q
$$

where $s=\{l, \theta\}$ is the generalized coordinates and the nonconservative force $Q_{l}=-\bar{T}$ and $Q_{\theta}=0 . \bar{T}$ is the tether tension. Hence, the equations of motion are,

$$
\begin{aligned}
& l^{\prime \prime}-l\left[\left(\theta^{\prime}+\Omega\right)^{2}+\Omega^{2}\left(3 \cos ^{2} \theta-1\right)\right]=-\frac{\bar{T}}{m_{e}} \\
& l^{2} \theta^{\prime \prime}+2 l l^{\prime}\left(\Omega+\theta^{\prime}\right)+\frac{3}{2} l^{2} \Omega^{2} \sin 2 \theta=0
\end{aligned}
$$

For sake of simplicity, the dimensionless variables are introduced to normalize the equations of motion $[6,7]$, such as,

$$
\lambda=l / l_{n} \quad T=\bar{T} /\left(m_{0} l_{n} \Omega^{2}\right) \quad \tau=\Omega t \quad \dot{s}=\mathrm{d} s / \mathrm{d} \tau
$$

where $l_{n}$ is a nominal constant of tether length. $T$ is the dimensionless tension that is to regulate the tether deployment, $\tau$ is the dimensionless time which equals to the true anomaly. Then, the dimensionless model of tethered system is presented as,

$$
\begin{aligned}
& \ddot{\lambda}-\lambda\left[(1+\dot{\theta})^{2}-1+3 \cos ^{2} \theta\right]=-T \\
& \lambda^{2} \ddot{\theta}+2 \lambda \dot{\lambda}(1+\dot{\theta})+3 \lambda^{2} \sin \theta \cos \theta=0
\end{aligned}
$$

It should be noted here, $\lambda_{\max } \geq \lambda \geq \lambda_{\min }>0$ to satisfy the physical real situation and avoid the singularity of second equation of the Eq.(3).

Further, to show the properties of tethered system, (3) is represented as the general form of Euler-Lagrange mechanical system,

$$
M(q) \ddot{q}+C(q, \dot{q}) \dot{q}+G(q)=u
$$

where $q=\operatorname{col}(\lambda, \theta)$ is the general coordinates, $M(q)$ is the mass matrix, $C(q, \dot{q})$ is the Coriolis and centrifugal force terms, $G(q)$ is the gravity term, and $u=\operatorname{col}(-T, 0)$ is the generalized force.

$$
M(q)=\left[\begin{array}{cc}
1 & 0 \\
0 & \lambda^{2}
\end{array}\right] \quad C(q, \dot{q})=\left[\begin{array}{cc}
0 & -\lambda(\dot{\theta}+2) \\
\lambda(\dot{\theta}+2) & \lambda \dot{\lambda}
\end{array}\right] \quad G(q)=\left[\begin{array}{l}
-3 \lambda \cos ^{2} \theta \\
\frac{3}{2} \lambda^{2} \sin 2 \theta
\end{array}\right]
$$

From the dimensionless equations (4), a dimensionless Lagrangian $L(q, \dot{q})$ could be obtained as follows,

$$
L(q, \dot{q})=K(q, \dot{q})-U(q)=\frac{1}{2} \dot{\lambda}^{2}+\frac{1}{2} \lambda^{2}\left[(\dot{\theta}+1)^{2}-1+3 \cos ^{2} \theta\right]
$$


where $K(q, \dot{q})=\frac{1}{2} \dot{\lambda}^{2}+\frac{1}{2} \lambda^{2}\left[(\dot{\theta}+1)^{2}-1\right]$ and $U(q)=-\frac{3}{2} \lambda^{2} \cos ^{2} \theta$ are the dimensionless kinetic energy and potential energy, respectively.

Property 1. The inertia matrix $M(q)$ is positively bounded, $\zeta_{\min } \leq\|M(q)\| \leq \zeta_{\max } \cdot \zeta_{\min }$ and $\zeta_{\max }$ are the minimum and maximum eigenvalue of inertia matrix, respectively.

Property 2. There exists a positive constant $k_{g}$ satisfying $\|\partial G(q) / \partial q\| \leq k_{g}$.

Property 3. The time derivative of the mass matrix and the matrix of coriolis and centrifugal force terms are dependent with each other. Such that, $0.5 \dot{M}(q)-C(q, \dot{q})$ is a skewsymmetry matrix.

$$
\dot{q}^{T}[0.5 \dot{M}(q)-C(q, \dot{q})] \dot{q}=0
$$

Define an energy function $E$ satisfying the transformation $E=\dot{q}^{T} \frac{\partial L}{\partial \dot{q}}-L$, then one has,

$$
E=\frac{1}{2} \dot{\lambda}^{2}+\frac{1}{2} \lambda^{2} \dot{\theta}^{2}-\frac{3}{2} \lambda^{2} \cos ^{2} \theta=\frac{1}{2} \dot{q}^{T} M(q) \dot{q}+U(q)
$$

The derivative of the energy function can be directly obtained, such that,

$$
\begin{aligned}
\dot{E} & =\dot{q}^{T} M \ddot{q}+\frac{1}{2} \dot{q}^{T} \dot{M} \dot{q}+\dot{U} \\
& =\dot{q}^{T}\left(\frac{1}{2} \dot{M}-C\right) \dot{q}+\dot{q}^{T} u+\dot{q}^{T} G-\dot{q}^{T} G \\
& =\dot{q}^{T} u=-\dot{\lambda} T
\end{aligned}
$$

The energy function is $E$ bounded from below, if $\exists P \geq-\infty$, such that the potential energy function $U(q) \geq P$. Then, system satisfies passivity property as follows,

Property 4. Define the function $S=E-P \geq 0$ as the storage function, then the system (4) with inputs $u$ and outputs $\dot{q}$ is passive in general, such that,

$$
S(t)-S(0) \leq \int_{0}^{t} \dot{q}^{T} u \mathrm{dt}
$$

Assumption 1. The libration angle of the payload always remains in $(-\pi / 2, \pi / 2),|\theta|<\pi / 2$.

\section{B. Hamiltonian Formulation}

In Hamiltonian mechanics, the canonical coordinates $(q, p)$ is used to describe the physical system's motion. Related to the Lagrangian formulation, the Hamiltonian is a function of $(q, p)$ instead of $(q, \dot{q})$ in Lagrangian. It is understood that $\dot{q}$ is replaced by $q$ and $p$, thus $\dot{q}$ is in terms of $(q, p) \cdot p=\partial L / \partial \dot{q}$ is the generalized momentum.

The generalized momentum $p=\operatorname{col}\left(p_{\lambda}, p_{\theta}\right)$ is obtained as,

$$
\begin{gathered}
p_{\lambda}=\frac{\partial L}{\partial \dot{\lambda}}=\dot{\lambda} \\
p_{\theta}=\frac{\partial L}{\partial \dot{\theta}}=\lambda^{2}(\dot{\theta}+1)
\end{gathered}
$$

Invoking the Legendre transformation, the Hamiltonian function can be derived by,

$$
H(q, p)=\dot{q}^{T} p-L(q, \dot{q}(q, p))
$$

The Hamiltonian of the tethered satellite system is obtained, such that

$$
\begin{aligned}
H(q, p) & =\frac{1}{2} \dot{\lambda}^{2}+\frac{1}{2} \lambda^{2} \dot{\theta}^{2}-\frac{3}{2} \lambda^{2} \cos ^{2} \theta \\
& =\frac{1}{2} p_{\lambda}^{2}+\frac{\lambda^{2}}{2}\left(\frac{p_{\theta}}{\lambda^{2}}-1\right)^{2}+U(q)
\end{aligned}
$$

Hamilton Equations used for mechanical system is presented as follows,

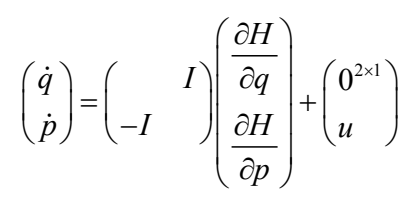

The motivation to use Hamiltonian formulation instead of Lagrangian formulation is the symplectic structure of Hamilton equations in (14). Then the equations of motion of the tethered system can be expressed as,

$$
\begin{aligned}
& \ddot{\lambda}=-\lambda \dot{\theta}^{2}+\lambda(\dot{\theta}+1)+3 \lambda \cos ^{2} \theta-T \\
& \lambda^{2} \ddot{\theta}+2 \lambda \dot{\lambda}(\dot{\theta}+1)=-3 \lambda^{2} \sin \theta \cos \theta
\end{aligned}
$$

It is easy to find that (15) is equivalent to (3) which is derived by the Lagrangian formulation.

Further, the time derivative of $H$ could be directly calculated as,

$$
\begin{aligned}
& \dot{H}(q, p)=\frac{\partial^{T} H}{\partial q} \dot{q}+\frac{\partial^{T} H}{\partial p} \dot{p} \\
& =\left(\frac{\partial^{T} H}{\partial q}, \frac{\partial^{T} H}{\partial p}\right)\left(\begin{array}{ll}
I &
\end{array}\right)\left(\frac{\partial^{T} H}{\partial q}, \frac{\partial^{T} H}{\partial p}\right)^{T}+\frac{\partial^{T} H}{\partial p} u \\
& =-\frac{\partial H}{\partial p_{\lambda}} T=-\dot{\lambda} T
\end{aligned}
$$

Equation (16) indicates the energy balance property of tethered system. The increase in energy (Hamiltonian quantity) of the system equals to the power supply. The relation shown in (16) is actually equivalent with passivity (9), Moreover, it is quite straight to observe the intrinsic property of tethered system (3) by the Hamiltonian formulation.

\section{ENERGY-BASED CONTROL}

The aim of controller design is to deploy the tethered system to its desired position. As well known, the stable equilibrium position of the tethered system lies in local vertical. Thus, the desired states of tethered system is usually set at the 
downward equilibrium $(\lambda, \dot{\lambda}, \theta, \dot{\theta})=\left(\lambda_{d}, 0,0,0\right) . \lambda_{d}$ is the desired tether length and tether angle is zero. By virtue of the passive property of the tethered system, the controller design for tethered system deployment is posed based on the energy modified Lyapunov function in this section.

From the Hamiltonian formulation, the quantity of Hamiltonian energy function obtained by the Legendre transformation is lower bounded when the potential energy function $U(q)-P \geq 0$, then one can take a function $P=\bar{U}(q)=\left.U(q)\right|_{\theta=0}$ to shift the Hamiltonian function to be positive such that,

$$
V_{1}=H-P=\frac{1}{2} \dot{\lambda}^{2}+\frac{1}{2} \lambda^{2} \dot{\theta}^{2}-\frac{3}{2} \lambda^{2} \cos ^{2} \theta+\frac{3}{2} \lambda^{2}
$$

It is obvious that (17) is always positive. Thus, one can select the function $V_{1}$ as the Lyapunov function candidate, and then controller is designed to make the closed-loop system stable.

Take the derivative of the function $V_{1}$,

$$
\dot{V}_{1}=\dot{H}-\dot{P}=-T \dot{\lambda}+3 \lambda \dot{\lambda}
$$

Thus, the closed-loop tethered system is passive if the one take output $\dot{\lambda}$ and input $T$ as follows,

$$
T=3 \lambda+k_{v} \dot{\lambda}, k_{v}>0
$$

where $k_{v}$ is an strictly positive constant.

Then, substituting (19) into (18), which yields,

$$
\dot{V}_{1}=\dot{H}-\dot{P}=-T \dot{\lambda}+3 \lambda \dot{\lambda}=-k_{v} \dot{\lambda}^{2}
$$

It is straightforward to know that the closed-loop tethered system is Lyapunov stable under the controller (19). However, the final tether length is not certain since $V_{1}$ is not convex at $\lambda=\lambda_{d}, \theta=0$. It is easy to check with the Hessian Matrix of $V_{1}$, at the desired position, such that,

$$
\left.\frac{\partial^{2} V_{1}}{\partial q^{2}}\right|_{\substack{\lambda=\lambda_{d} \\
\theta=0}}=\left[\begin{array}{cc}
3 \sin ^{2} \theta & 3 \lambda \sin (2 \theta) \\
3 \lambda \sin (2 \theta) & 3 \lambda^{2} \cos (2 \theta)
\end{array}\right]_{\substack{\lambda=\lambda_{d} \\
\theta=0}}=\left[\begin{array}{cc}
0 & 0 \\
0 & 3 \lambda_{d}^{2}
\end{array}\right]
$$

It is obvious that the Hessian Matrix $\left.\frac{\partial^{2} V_{1}}{\partial q^{2}}\right|_{\substack{\lambda=\lambda_{d} \\ \theta=0}}$ is not positive definite. Thus, even if the tether may arrive to the desired length but it will not be able to stay there.

To achieve the desired final length along the vertical, the energy Lyapunov function should be modified so that it is convex at the desired position. For the purpose of this, we construct a Lyapunov function as follows,

$$
V_{2}=V_{1}+\frac{1}{2} k_{p}\left(\lambda-\lambda_{d}\right)^{2}
$$

where $k_{p}$ is an strictly positive constant. It is obvious to find that $V_{2} \geq 0$ and compared with $V_{1}$, a positive term $\frac{1}{2} k_{p}\left(\lambda-\lambda_{d}\right)$ is added, which has the unique minimum at desired tether length. Then, at the desired equilibrium we have,

$$
\begin{gathered}
\left.\frac{\partial V_{2}}{\partial q}\right|_{\substack{\lambda=\lambda_{d} \\
\theta=0}}=\left[\begin{array}{c}
3 \lambda \sin ^{2} \theta+k_{p}\left(\lambda-\lambda_{d}\right) \\
\frac{3}{2} \lambda^{2} \sin (2 \theta)
\end{array}\right]_{\substack{\lambda=\lambda_{d} \\
\theta=0}}=0 \\
\left.\frac{\partial^{2} V_{2}}{\partial q^{2}}\right|_{\substack{\lambda=\lambda_{d} \\
\theta=0}}=\left[\begin{array}{cc}
3 \sin ^{2} \theta+k_{p} & 3 \lambda \sin (2 \theta) \\
3 \lambda \sin (2 \theta) & 3 \lambda^{2} \cos (2 \theta)
\end{array}\right]_{\substack{\lambda=\lambda_{d} \\
\theta=0}}=\left[\begin{array}{cc}
k_{p} & 0 \\
0 & 3 \lambda_{d}^{2}
\end{array}\right]
\end{gathered}
$$

Thus, it indicates that $V_{2}$ is convex, then it is stable to stay at the desired equilibrium. Then, the controller (19) should be modified accordingly. The control law becomes,

$$
T=3 \lambda+k_{p}\left(\lambda-\lambda_{d}\right)+k_{v} \dot{\lambda}, k_{p}>0, k_{v}>0
$$

Theorem 1: Under the controller (25) and Assumption 1, the deployment control of the closed-loop tethered system is asymptotically stable at desired downward equilibrium.

Proof. Select the Lyapunov function candidate as $V_{2}$ and take the derivative of it, yields that,

$$
\dot{V}_{2}=-T \dot{\lambda}+3 \lambda \dot{\lambda}+k_{p}\left(\lambda-\lambda_{d}\right)
$$

Recall (25), one has,

$$
\dot{V}_{2}=-k_{v} \dot{\lambda}^{2} \leq 0
$$

From (27), the Lyapunov function $V_{2}$ is non-increasing, then one has $V_{2} \leq V_{2}(0)$. The trajectory of all the states will converge to the largest invariant set $W=\left\{q \in \mathbb{R} \mid \dot{V}_{2}=0\right\}$ as the time approaches to infinity. The set $W$ indicates $\dot{\lambda}=0$, which further means the tether length keeps constant $\lambda=$ constant . Then, recall (13) and (16), one can have,

$$
\frac{1}{2} \lambda^{2} \dot{\theta}^{2}-\frac{3}{2} \lambda^{2} \cos ^{2} \theta=\text { constant }
$$

Combine (28) with first equation of (15), we can know that there are at most two isolate solutions of $\dot{\theta}$ for $\dot{\theta} \in \mathbb{R}$. Then, one can have that,

$$
\ddot{\theta}=0
$$

Substitute (29) into the second equation of (15), yields,

$$
\sin \theta \cos \theta=0
$$

Further, the solutions of tether angle can be obtained under the Assumption 1, we have $\theta=0$. Combine with (25) and (15), we have,

$$
k_{p}\left(\lambda-\lambda_{d}\right)=0
$$

Then, one can get the unique solution for tether length $\lambda=\lambda_{d}$. Therefore, the deployment of tethered system has unique equilibrium at $(\lambda, \dot{\lambda}, \theta, \dot{\theta})=\left(\lambda_{d}, 0,0,0\right)$ under the controller (25) with the Assumption 1. The equilibrium 
$(\lambda, \dot{\lambda}, \theta, \dot{\theta})=\left(\lambda_{d}, 0,0,0\right)$ is concluded to be asymptotically stable, which completes the proof.

Remark 1: In the proof, the LaSalle's invariance theory is used to prove the asymptotic stability of the closed-loop system. However, it should be noted that it actually can't be directly applied because of unboundedness of tether angle. But it can be circumvented by variable transform as in $[6,10]$, which is omitted in the proof because it has no effect on the conclusion of asymptotic stability.

Remark 2: The convex of the function in (24) actually implies the asymptotically stable of the closed-loop system. Because the convex of the function at desired equilibrium means that any state of motion in the small domain near desired equilibrium has the minimum at desired equilibrium. That is the reason we construct the function to make the energy Lyapunov function have minimum at the desired equilibrium.

\section{SimUltion AND DISCUSSION}

In this section, numerical experiments are carried on to verify the effectiveness of the proposed controller. The simulations are conducted in MATLAB R2017a using the numerical integrator ODE45. The tethered satellite system is assumed to orbit circularly with an altitude of $220 \mathrm{~km}$ and the orbital velocity is $1.18 \times 10^{-3} \mathrm{rad} / \mathrm{s}$. The maximum tether length is $100 \mathrm{~km}$. The corresponding initial and final parameters of the tether system are selected as $\left(\lambda_{0}, \dot{\lambda}_{0}, \theta_{0}, \dot{\theta}_{0}\right)=(0.01,0.5,0,0)$ and the desired states $\left(\lambda_{d}, \dot{\lambda}_{d}, \theta_{d}, \dot{\theta}_{d}\right)=(1,0,0,0)$, respectively.

Define two potential energy functions $U_{1}=U_{0}+P$ and $U_{2}=U_{1}+\frac{1}{2} k_{p}\left(\lambda-\lambda_{d}\right)^{2}$ according to the Lyapunov function $V_{1}$ and $V_{2}$. Parameters in these functions are set as $k_{p}=4$ and $\lambda_{d}=1$. Then, sketch the graph of the function in configuration space of $\lambda-\theta$, see Fig. 2. As shown in the graph of first subplot, the function $U_{1}$ has the minimum if the tether angle is zero but it is not depending on tether length. It indicates that the tethered system is able to deploy to the vertical direction but the deployed tether length is not certain maybe any value. However, seen from the second subplot, the function $U_{2}$ has the unique minimum at desired position. It can be observed that the function $U_{2}$ is convex around the small neighborhood of the desired equilibrium.

Further, the control laws (19) and (25) are used to clarify the stability of the closed-loop tethered system. The control gains are chosen as $k_{v}=1$ in (19) and $k_{p}=2, k_{v}=4$ in (25). The profiles of the position states and tension in tether are presented in Figs.3-5. Seen in Fig. 3, the length profiles show that both two proposed controller can achieve a stable tether deployment. The controller (25) fulfill the tether deployment to desired length successfully, however the controller (19) does not. It can be found that the tether angle profiles converge to zero as we want for both two controllers, see Fig. 4. The tension in tether for two controllers are shown in Fig.5, tension is positive and finally equal to the static balance force. In conclusion, the simulation results agree with the theoretical analysis given in Section III and the proposed controller (25) can perform the asymptotic stability for deployment control of the tethered system.
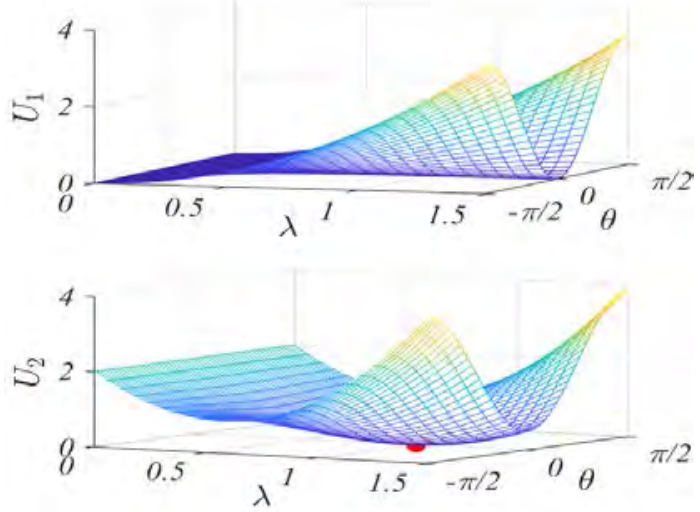

Figure 2. $U_{1}$ and $U_{2}$ in configuration space $\lambda-\theta$

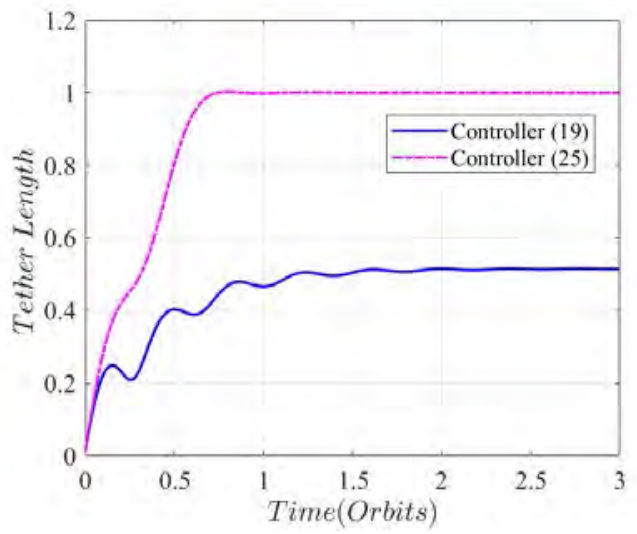

Figure 3. The deployed dimensionless tether length

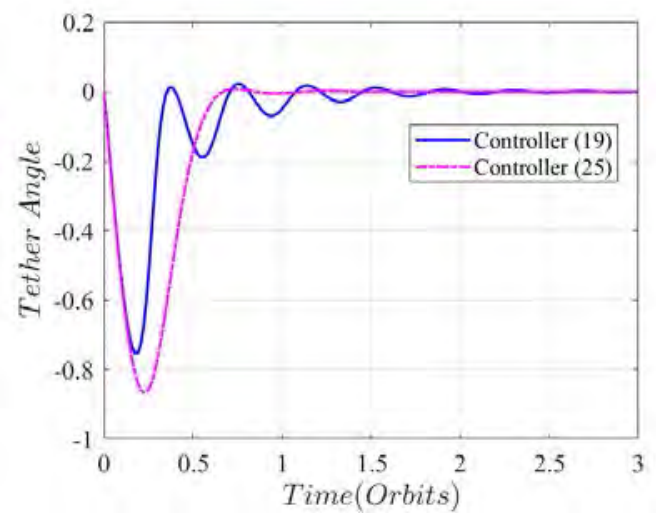

Figure 4. The tether angle during tether deployment 


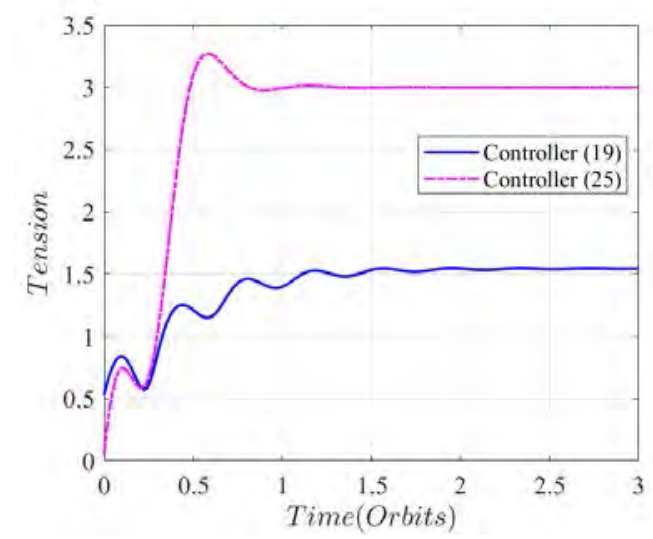

Figure 5. Tether tension during tether deployment

\section{CONCLUSTION}

In this study, we focus on the dynamics and control of space tethered system's deployment in two dimensional space. In order to get insight of the dynamic behavior of the tethered system, the dynamic model is formulated by the way of Lagrangian and Hamiltonian, respectively. In Lagrangian formulation, the dynamic equation is written into the EulerLagrange form and the passivity property is further presented by the specifically chosen matrix of coriolis/centrifugal force. In alternative way, the equivalent property of energy balance in the tethered system is performed by Hamiltonian formulation, which is much more intuitively due to the symplectic structure. This property is actually in general rather than depending on specified coriolis/centrifugal matrix. By virtue of the Hamilton formulation, the deployment control of the tethered system is studied based on Energy-based control methodology. Controllers are proposed for stabilizing tether deployment. The Lyapunov stability and asymptotical stability are analyzed for each controller, respectively. Finally, the results are verified by the numerical simulations.

\section{REFERENCES}

[1] K. D. Kumar, "Review on Dynamics and Control of Nonelectrodynamic Tethered Satellite Systems," Journal of Spacecraft and Rockets, vol. 43, no. 4, pp. 705-720, July, 2006. doi: 10.2514/1.5479.

[2] C. C. Rupp, A tether tension control law for tethered subsatellites deployed along local vertical, Marshall Space Flight Center, Huntsville, AL, United States, September, 1975.

[3] H. Fujii, and S. Ishijima, "Mission function control for deployment and retrieval of a subsatellite," Journal of Guidance, Control, and Dynamics, vol. 12, no. 2, pp. 243-247, March, 1989. doi: 10.2514/3.20397.

[4] S. Pradeep, "A new tension control law for deployment of tethered satellites," Mechanics Research Communications, vol. 24, no. 3, pp. 247-254, Feburary, 1997.

[5] G. Sun, and Z. H. Zhu, "Fractional-Order Tension Control Law for Deployment of Space Tether System," Journal Of Guidance Control And Dynamics, vol. 37, no. 6, pp. 2062-2066, Nov, 2014. doi: 10.2514/1.G000496.

[6] H. Wen, Z. H. Zhu, D. Jin, and H. Hu, "Space Tether Deployment Control with Explicit Tension Constraint and Saturation Function," Journal of Guidance, Control, and Dynamics, vol. 39, no. 4, pp. 916-921, April, 2015. doi: 10.2514/1.G001356.

[7] J. Kang, Z. H. Zhu, W. Wang, A. Li, and C. Wang, "Fractional order sliding mode control for tethered satellite deployment with disturbances," Advances in Space Research, vol. 59, no. 1, pp. 263-273, January, 2017. doi: 10.1016/j.asr.2016.10.006.

[8] M. P. Cartmell, and D. J. McKenzie, "A review of space tether research," Progress in Aerospace Sciences, vol. 44, no. 1, pp. 1-21, January, 2008. doi: 10.1016/j.paerosci.2007.08.002.

[9] J. Kang, Z. H. Zhu, W. Wang, C. Wang, and A. Li, "Energy-based output feedback tension control for space tether deployment under physical constraints", in 2018 American Control Conference, 2018 (in press). doi: 10.23919/ACC.2018.8431257.

[10] R. Lozano, I. Fantoni, and D. J. Block, "Stabilization of the inverted pendulum around its homoclinic orbit," Systems \& Control Letters, vol. 40, no. 3, pp. 197-204, 2000. doi: 10.1016/S0167-6911(00)00025-6 . 


\title{
Direct Switching Position Control Algorithms for Pneumatic Actuators using On/Off Solenoid Valves
}

\author{
Yile Zhang and Gary M. Bone* \\ Department of Mechanical Engineering \\ McMaster University \\ Hamilton, Canada \\ *Corresponding author. E-mail: gary@ mcmaster.ca
}

\begin{abstract}
Pneumatic actuators are advantageous in terms of cost, power to weight ratio and inherent safety. However, their dynamics makes precise closed-loop position control very difficult in practice. Two sliding-mode control algorithms for controlling the position of a pneumatic cylinder by directly switching four on/off solenoid valves are proposed in this paper. The solenoid valves are much less expensive than the commonly used servo or proportional valves. The proposed algorithms are compared to two state of the art position control algorithms. Based on experiments on a high friction cylinder with various payloads, the proposed controllers provide superior performance in terms of valve switches per second, steady state error, settling time and overshoot. The achieved number of valve switches per second is also about one tenth of the number required by the pulse-width modulation method that is commonly used with on/off valves. This should result in prolonged valve lifetimes and reduced maintenance costs.
\end{abstract}

Keywords-actuator; pneumatic; position control; sliding-mode control; solenoid valve.

\section{INTRODUCTION}

Pneumatic actuators are widely used in automation because they provide several advantages. They are low cost, clean and provide a high power to weight ratio. They are also inherently safe due to their natural compliance. However, the closed-loop control of their position for servoing applications is very difficult and remains an active research topic (e.g. [1]-[5]). Our interest is on the closed-loop position of pneumatic cylinders. The majority of previous papers employed proportional or servo valves to control the flow of air to/from the cylinder's two chambers. In this paper, on/off solenoid valves will be used since they are much less expensive than proportional/servo valves (e.g., US $\$ 40$ vs. US\$800). This advantage is offset by the disadvantage that their flow behaviour is discontinuous, making smooth and precise position control more difficult to achieve. While pulse-width modulation (PWM) may be used with on/off valves to approximate a proportional valve in position control applications (e.g.,[6]-[8]) it also causes frequent valve switching leading to shorter valve life. In

Research sponsored by the Natural Sciences and Engineering Research

Council of Canada (NSERC) through a Discovery Grant. contrast, systems employing the direct switching method only switch the valves when it is necessary for the desired closedloop performance. This reduced switching frequency prolongs the valve life, leading to reduced repair and replacement costs. In spite of its advantages, relatively few researchers have investigated the direct switching approach.

In [9], a double acting cylinder was controlled using four two-way on/off solenoid valves (i.e. two valves per cylinder chamber) and an optical encoder for position feedback. Their sliding-mode control (SMC) algorithm used three operating modes for the valves, and was the first to consider the problem of excessive valve switching. This algorithm will be further described in section III. For a $2 \mathrm{~kg}$ payload and a $40 \mathrm{~mm}$ step input, the steady state error (SSE) was $0.1 \mathrm{~mm}$ and the overshoot (OS) was less than $10 \mathrm{~mm}$. They did not report any robustness results. A more sophisticated SMC algorithm with seven valve operating modes was proposed in [10]. They used the same valve configuration as in [9]. The introduction of the four additional modes and two additional tuning parameters was shown to improve both the tracking errors and valve switching frequency. For a $0.9 \mathrm{~kg}$ payload and $40 \mathrm{~mm}$ step input the 7-mode controller reduced the positive OS from 7.2 $\mathrm{mm}$ to $0.76 \mathrm{~mm}$ (i.e., $89 \%$ reduction) compared with the 3mode controller from [9]. For the same payload and a multiple sine wave reference input the switching frequency with the 7mode controller was $48 \%$ less than with the 3-mode controller, while the tracking errors were similar. Nonlinear model predictive control algorithms were used to directly switch the valves in [11] and [12]. While these algorithms have the potential to outperform those from [9] and [10] they require a system model and greater computing power to implement.

In this paper we propose modified versions of the SMC algorithms from [9] and [10], and compare their experimental position control performance with the original algorithms. The system structure is described in section II. Next, the designs of the existing and proposed control algorithms are presented. Experimental results, including robustness tests, are presented in section IV. Finally, conclusions are drawn in section V. 


\section{SYSTEM STRUCTURE}

A schematic of the system structure is shown in Fig. 1. As in [9] and [10], two two-way on/off valves (also termed 2/2 valves) are connected to each chamber of the cylinder. This has the advantage of allowing the pressure of each chamber to be independently controlled. The cylinder drives a payload mass horizontally. The position and cylinder pressures are fed back to PC-based control system. The PC switches the valves via optocouplers.

\section{CONTROLLER DESIGN}

\section{A. Three-mode Sliding Mode Control Algorithm}

In this section, the three-mode sliding mode controller (abbreviated as SMC3) proposed by Nguyen et al. [9] is briefly summarized. The three operating modes are defined as follows:

Mode 1: Both chambers' valves are closed

$\left(u_{1}=0, u_{2}=0, u_{3}=0\right.$ and $\left.u_{4}=0\right)$

Mode 2: Chamber A charges and chamber B discharges

$\left(u_{1}=1, u_{2}=0, u_{3}=0\right.$ and $\left.u_{4}=1\right)$

Mode 3: Chamber A discharges and chamber B charges $\left(u_{1}=0, u_{2}=1, u_{3}=1\right.$ and $\left.u_{4}=0\right)$

Mode 2 is used to move the piston in the positive direction and mode 3 is used to move it in the negative direction. Mode 1 is used to reduce the chattering when the tracking error is small enough and to save energy.

They use the second-order sliding surface:

$$
s=\frac{\ddot{e}}{\omega^{2}}+\frac{2 \zeta \dot{e}}{\omega}+e
$$

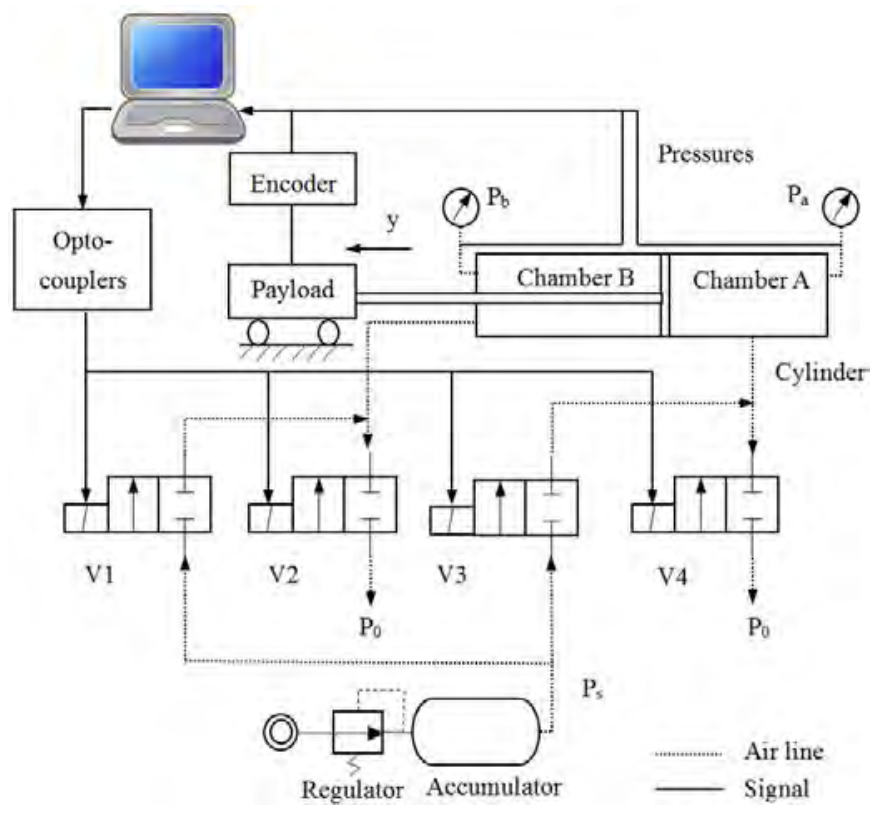

Figure 1. System schematic diagram. where $e=y-y_{d}$ is the position error, $y$ is the actual position, $y_{d}$ is the desired position, and $\zeta$ and $\omega$ are constant and positive numbers. The value of $s$ determines the mode according to:

$$
\begin{cases}s>\varepsilon, & \text { mode } 3 \\ s<-\varepsilon, & \text { mode } 2 \\ -\varepsilon \leq s \leq \varepsilon, & \text { mode } 1\end{cases}
$$

where $\mathcal{E}$ is the mode 1 boundary limit. It is used to reduce the valve switching caused by control chattering. To limit the tracking error it is desirable to choose $\varepsilon$ as small as possible. However, $\varepsilon$ also needs to be large enough to reduce chattering.

\section{B. Seven-mode Sliding Mode Control Algorithm}

The seven-mode sliding mode controller (abbreviated as SMC7) proposed by Hodgson et al. [10] is briefly summarized in this section. They extended SMC3 from three to seven operating modes with the goal of reducing the number of valve switches per second. The valve states for the seven modes are defined in Table 1. Modes 1, 6 and 7 are the same as SMC3 modes 1, 2 and 3, respectively. Modes 2-5 are new.

As with SMC3 the sliding surface is defined by (1). They extended the three regions of $s$ used with SMC3 (see (2)) to five regions. The regions, corresponding modes and qualitative forces are listed in Table 2 . In addition to deadband $\mathcal{E}$, they introduce a larger boundary limit $\beta$. Examining Table 2, regions $s \leq-\beta,-\varepsilon<s \leq \varepsilon$ and $s>\beta$ map to unique modes. Regions $-\beta<s \leq-\varepsilon$ and $\varepsilon<s \leq \beta$ map to two modes each, and additional logic based on the chamber pressures are required to identify the unique mode to apply. The principle behind the mode selection is the mode producing the larger acceleration should be chosen since it will reduce the tracking error faster.

For region $-\beta<s \leq-\varepsilon$, they deduced that the steady state payload acceleration with mode 2 is roughly proportional to $P_{s}-P_{b}$ where $P_{s}$ is the supply pressure and $P_{b}$ is the pressure inside chamber B. Similarly, with mode 4 the steady state acceleration is roughly proportional to $P_{a}-P_{0}$ where $P_{a}$ is the pressure inside chamber $\mathrm{A}$ and $P_{0}$ is atmospheric pressure. They then defined

$$
E_{1}=\left(P_{s}-P_{b}\right)-\left(P_{a}-P_{0}\right)
$$

where if $E_{1}$ is positive mode 2 should produce a larger acceleration than mode 4 . So the following switching logic is

TABLE I. VALVE STATES FOR THE SEVEN OPERATING MODES USED BY THE SMC7 CONTROLLER

\begin{tabular}{|c|c|c|c|c|c|c|c|}
\hline \multirow{2}{*}{ Valve } & \multicolumn{7}{|c|}{ Mode } \\
\cline { 2 - 8 } & $\mathbf{1}$ & $\mathbf{2}$ & $\mathbf{3}$ & $\mathbf{4}$ & $\mathbf{5}$ & $\boldsymbol{6}$ & $\mathbf{7}$ \\
\hline$u_{1}$ & 0 & 1 & 0 & 0 & 0 & 1 & 0 \\
\hline$u_{2}$ & 0 & 0 & 1 & 0 & 0 & 0 & 1 \\
\hline$u_{3}$ & 0 & 0 & 0 & 0 & 1 & 0 & 1 \\
\hline$u_{4}$ & 0 & 0 & 0 & 1 & 0 & 1 & 0 \\
\hline
\end{tabular}


applied in the region $-\beta<s \leq-\varepsilon$ :

$$
\begin{cases}E_{1}>0, & \text { mode } 2 \\ E_{1} \leq 0, & \text { mode } 4\end{cases}
$$

For region $\varepsilon<s \leq \beta$, a similar approach is employed. Note that the steady state acceleration will be negative. They defined

$$
E_{2}=\left(P_{s}-P_{a}\right)-\left(P_{b}-P_{0}\right)
$$

where if $E_{2}$ is positive mode 5 should produce a larger acceleration than mode 3 . So the following switching logic is applied in the region $-\beta<s \leq-\varepsilon$ :

$$
\begin{cases}E_{2}>0, & \text { mode } 5 \\ E_{2} \leq 0, & \text { mode } 3\end{cases}
$$

In order to reduce the number of switches between modes when (5) and (6) are applied, they introduced a timeout parameter $\tau$. Mode 2, 3, 4 or 5 is maintained for a period of $\tau$ or longer. A larger $\tau$ therefore reduces the switching but may also lead to larger tracking errors.

\section{Proposed Sliding Mode Control Algorithms}

We propose two SMC algorithms in this section. Both are modifications of the existing algorithms, and are based on the idea that adding integral action can reduce settling time and SSE if it is properly applied.

In order to add integral action, the sliding surface is based on $\int_{0}^{t} e d t$ rather than $e$. Thus, we define the new sliding surface as:

$$
s=\frac{\ddot{e}}{\omega^{3}}+\frac{3 \zeta \dot{e}}{\omega^{2}}+\frac{3 \zeta^{2} e}{\omega}+\int_{0}^{t} e d t
$$

where $\int_{0}^{t} e d t$ provides the integral action. Integral windup may occur when there is a large change in the desired position or a large disturbance, and the integral term accumulates a significant error during the transient. The time required to reduce this large integral term can lead to overshoot and a longer settling time. Anti-windup is implemented by bounding the integral action as follows:

$$
\left|\int_{0}^{t} e d t\right| \leq e_{\text {limit }}
$$

The first proposed algorithm, termed integral SMC3

TABLE II. FIVE REGIONS OF $S$ USED BY THE SMC7 CONTROLLER

\begin{tabular}{|c|c|c|}
\hline Region & Corresponding Modes & $\begin{array}{c}\text { Qualitative Pneumatic } \\
\text { Force }\end{array}$ \\
\hline$s \leq-\beta$ & 6 & Large positive \\
\hline$-\beta<s \leq-\varepsilon$ & 2 or 4 & Small positive \\
\hline$-\varepsilon<s \leq \varepsilon$ & 1 & Zero \\
\hline$\varepsilon<s \leq \beta$ & 3 or 5 & Small negative \\
\hline$s>\beta$ & 7 & Large negative \\
\hline
\end{tabular}

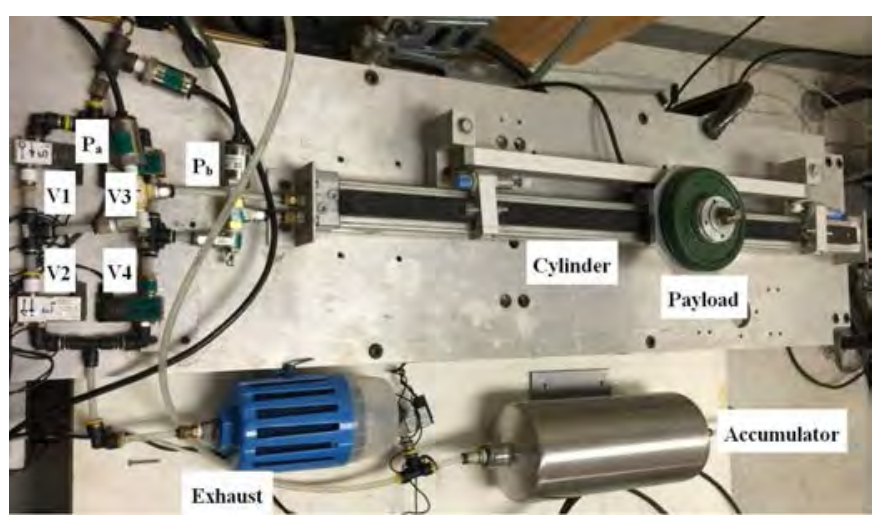

Figure 2. System hardware (PC and optocouplers are not shown).

(ISMC3), is derived from SMC3 by replacing (1) with (7) and (8). The second proposed algorithm, termed integral SMC7 (ISMC7), is similarly derived from SMC7 by replacing (1) with (7) and (8).

\section{EXPERIMENTS}

\section{A. Hardware}

The hardware implementation of the system structure presented in section II is shown in Fig. 2. The cylinder (Festo, model number DGPL-25-600) is rodless; and has a $600 \mathrm{~mm}$ stroke and $25 \mathrm{~mm}$ bore. The four on/off valves are made by MAC, model number 34B-AAA-GDFB-1BA. The linear encoder has a resolution of $0.01 \mathrm{~mm}$. The pressure sensors are SSI Technologies, model number P51-100-A-B-I36-5V-000000 . The supply pressure is regulated at $0.6 \mathrm{MPa}$. All of the sensor signals and control signals are interfaced with the PC using a National Instruments PCIe-6365 card. The PC runs 64bit Windows 7 with a $3.10 \mathrm{GHz}$ Intel i5-2400 processor and 8.00 GB RAM. This data acquisition and control system is programmed in $\mathrm{C}$ and operates at a $1 \mathrm{kHz}$ sampling frequency.

\section{B. Experimental Results and Discussion}

To test both the transient and steady state performance, the desired position trajectory consists of a series of step changes. The step heights are $200 \mathrm{~mm},-200 \mathrm{~mm}, 100 \mathrm{~mm}$ and $10 \mathrm{~mm}$. The total duration is $4 \mathrm{~s}$. The controller parameters were tuned for a nominal payload of $2.14 \mathrm{~kg}$.

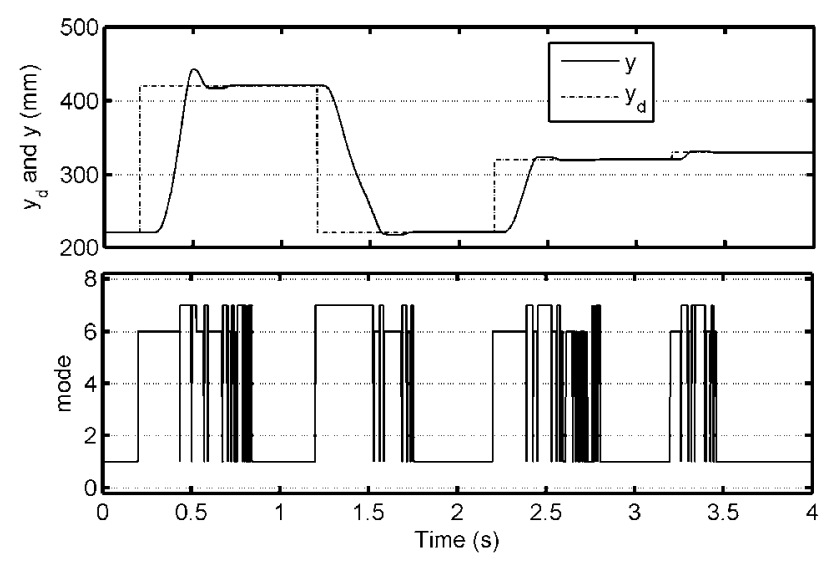

Figure 3. Experimental result for SMC 3 with the nominal payload. 

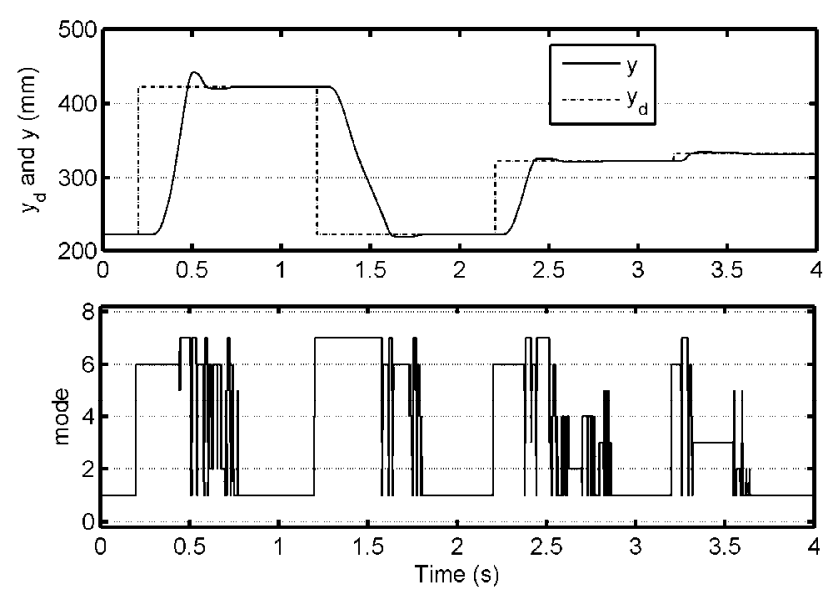

Figure 4. Experimental result for SMC7 with the nominal payload.

Sample responses for the four controllers with the nominal payload are plotted in Figs. 3-6. In these figures SMC3 and ISMC3 modes 2 and 3 are plotted as 6 and 7 (which are equivalent in terms of the valve states) to allow the 3-mode and 7-mode plots to be more easily compared.

Comparing Figs. 3 and 4 the responses obtained with SMC3 and SMC7 appear to be quite similar in terms of their position tracking and number of mode switches. In contrast, the performance of ISMC3 (Fig. 5) is clearly better than SMC3 (Fig. 3). ISMC3 exhibits lower OS and longer periods were no mode switches occur. ISMC7 (Fig. 6) demonstrates similar improvements over SMC7 (Fig. 4). As with SMC3 and SMC7, the benefits of ISMC7 relative to ISMC3 are not obvious from these plots. A quantitative comparison based on numerous experiments will be discussed next.

In addition to tests with the nominal payload, tests were performed with a decreased payload $(0.95 \mathrm{~kg})$ and an increased payload $(3.24 \mathrm{~kg})$ to quantify each controller's robustness to payload mismatch. The performance metrics include the SSE, OS, and the settling time $t_{s}$, which is defined here as the time for the response to settle within $1 \mathrm{~mm}$ of the desired steady state position. These were averaged over the four step changes. The final metric is the average number of times each valve is switched per second (SPS). Five tests were

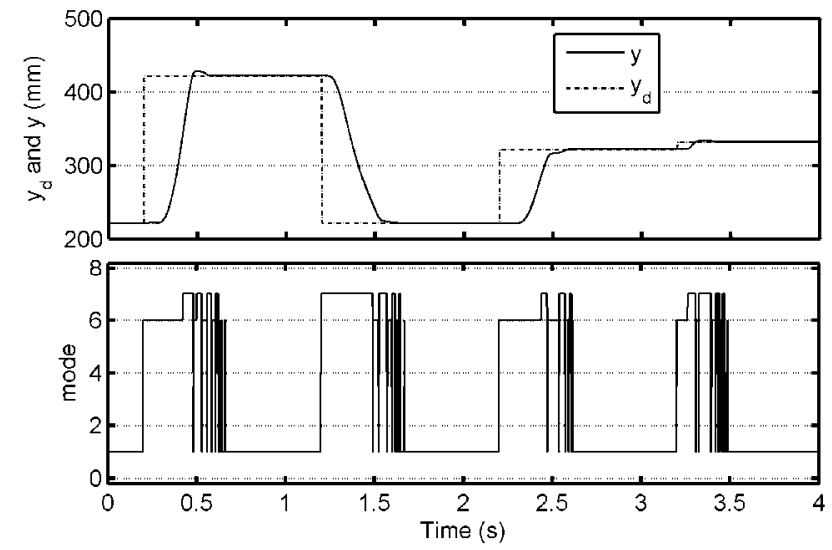

Figure 5. Experimental result for ISMC3 with the nominal payload.
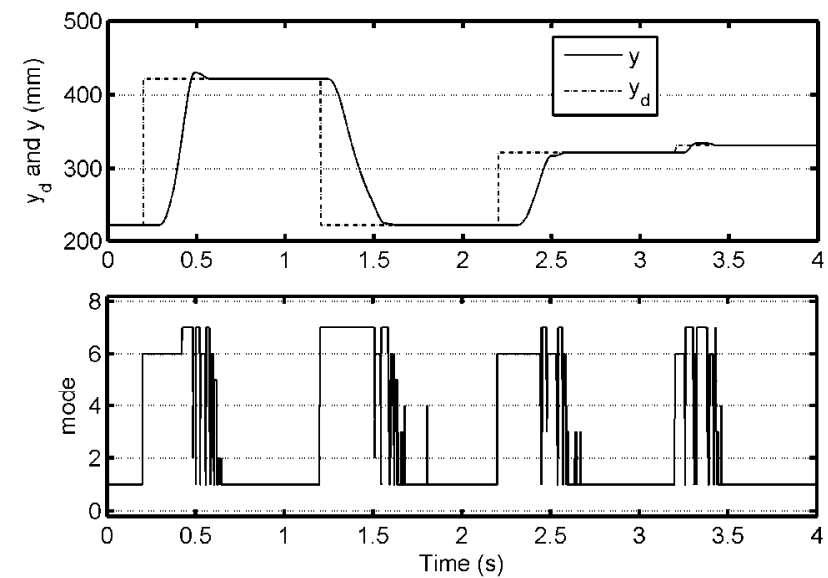

Figure 6. Experimental result for ISMC7 with the nominal payload. performed with each controller and payload combination. The averaged results of these tests are reported in Table III.

Examining the Table III results for the nominal payload, the SPS of SMC7 is $18 \%$ smaller than that of SMC3. For SSE, $t_{s}$ and OS the differences are $-6 \%,+10 \%$ and $-22 \%$, respectively. While SMC7 demonstrates improved performance vs. SMC3, the improvement is modest compared to the results reported in [10]. It is hypothesized that the much larger static friction due to the seals of the Festo DGPL cylinder used in our experiments $(\sim 80 \mathrm{~N}$ vs. $\sim 0.3 \mathrm{~N}$ for the Airpel M16 cylinder used in [10]) is the reason for the modest improvements observed here for SMC7.

The tabulated results for both ISMC3 and ISMC7 are consistently superior to the SMC3 and SMC7 results. For example, with the nominal payload the reductions in SPS, SSE, $t_{s}$ and OS for ISMC3 vs. SMC3 were 37\%, 57\%, 15\% and $65 \%$, respectively. The improvements with the altered payloads were smaller. For example, with the increased payload, ISMC3 reduced SPS, SSE, $t_{s}$ and OS by $43 \%, 19 \%$, $16 \%$ and $47 \%$ respectively, compared with SMC3. The improvements in SSE and $t_{s}$ were expected due to the integral

TABLE III. AVERAGED EXPERIMENTAL RESULTS

\begin{tabular}{|c|c|c|c|c|c|}
\hline Controller & Payload & SPS & $\begin{array}{c}\text { SSE } \\
(\mathbf{m m})\end{array}$ & $\begin{array}{c}\mathbf{t}_{\mathbf{s}} \\
(\mathbf{s})\end{array}$ & $\begin{array}{c}\text { OS } \\
(\mathbf{m m})\end{array}$ \\
\hline SMC3 & Nominal & 17.85 & 0.35 & 0.40 & 7.99 \\
\hline SMC3 & Decreased & 19.50 & 0.21 & 0.34 & 2.87 \\
\hline SMC3 & Increased & 20.35 & 0.26 & 0.45 & 12.53 \\
\hline SMC7 & Nominal & 14.60 & 0.33 & 0.44 & 6.20 \\
\hline SMC7 & Decreased & 16.03 & 0.28 & 0.42 & 2.43 \\
\hline SMC7 & Increased & 18.60 & 0.40 & 0.47 & 12.77 \\
\hline SMC13 & Nominal & 11.20 & 0.15 & 0.34 & 2.78 \\
\hline SMC13 & Decreased & 14.05 & 0.17 & 0.35 & 0.78 \\
\hline SMC13 & Increased & 11.65 & 0.21 & 0.38 & 6.61 \\
\hline SMCI7 & Nominal & 9.65 & 0.19 & 0.34 & 3.28 \\
\hline SMCI7 & Decreased & 11.88 & 0.20 & 0.35 & 1.14 \\
\hline SMCI7 & Increased & 8.55 & 0.17 & 0.38 & 6.60 \\
\hline
\end{tabular}


action included in ISMC3, but the improvements in the other two metrics were not. The results also show that the performances of ISMC7 and ISMC3 are similar, except that ISMC7 lowered the SPS. For example, with the increased payload the SPS was $27 \%$ smaller with ISMC7. Lastly, the results with the altered payloads demonstrate the SPS, SSE and $t_{s}$ metrics were robust to changing the payload, but the OS was not.

\section{CONCLUSION}

We have proposed two control algorithms, ISMC3 and ISMC7, for controlling the position of a pneumatic cylinder by directly switching the on/off solenoid valves. The proposed algorithms experimentally outperform the existing algorithms in terms of the SPS, SSE, $t_{s}$ and OS performance metrics. The proposed algorithms also switch the valves much less than if PWM was used. For example, the PWM period used in [7] produces an SPS of 100 (assuming the duty cycle is not equal to 0 or $100 \%$ ). This is 10 times larger than the SPS achieved by ISMC7 in our experiments. This reduced switching should prolong the life of the valves, and lower maintenance costs. Since the performances of ISMC3 and ISMC7 are similar, it is recommended that ISMC3 be used since it does not require any pressure sensing.

\section{REFERENCES}

[1] R. A. Rahman, L. He and N. Sepehri, "Design and experimental study of a dynamical adaptive backstepping-sliding mode control scheme for position tracking and regulating of a low-cost pneumatic cylinder," Int.J. Robust Nonlinear Control, vol. 26, pp. 853-875, 2016. doi: 10.1002/rnc.3341.

[2] V. Nazari and B. Surgenor, "Improved position tracking performance of a pneumatic actuator using a fuzzy logic controller with velocity, system lag and friction compensation," Int. J. Control Autom., vol. 14, pp. 1376-1388, 2016. doi: 10.1007/s12555-015-0202-0.

[3] R. A. Rahman and N. Sepehri, "Pneusimulator: A simulation software for studying performance of controllers in pneumatic actuating systems," Int. J. Fluid Power, vol. 18, pp. 70-72, 2017.

doi: 10.1080/14399776.2017.1264670.
[4] L. Zhao, Y. Xia, Y. Yang and Z. Liu, "Multicontroller Positioning Strategy for a Pneumatic Servo System Via Pressure Feedback," IEEE Trans. Ind. Electron., vol. 64, pp. 4800-4809, 2017. doi: 10.1109/TIE.2017.2674605

[5] F. Soleymani, S. M. Rezaei, M. Zareinejad, K. Baghestan and A.Rahimi, "Position control of a servo-pneumatic actuator with mismatched uncertainty using multiple-surface sliding mode controller and high-gain observer," Trans. Inst. Meas. Control, vol. 39, pp. 1497-1508, 2017. doi: $10.1177 / 0142331216640870$.

[6] R. B. van Varseveld and G. M. Bone, "Accurate position control of a pneumatic actuator using on/off solenoid valves", IEEE/ASME Trans. Mechatronics, vol. 2, no. 3, pp. 195-204, 1997. doi: $10.1109 / 3516.622972$.

[7] K. Ahn and S. Yokota, "Intelligent switching control of pneumatic actuator using on/off solenoid valves," Mechatronics, vol. 15, pp. 683702, 2005. doi: 10.1016/j.mechatronics.2005.01.001.

[8] X. Shen, J. Zhang, E. Barth, and M. Goldfarb, "Nonlinear model-based control of pulse width modulated pneumatic servo systems", ASME J. Dyn. Syst. Meas. Control, vol. 128, pp. 663-669, 2006. doi: $10.1115 / 1.2232689$.

[9] T. Nguyen, J. Leavitt, F. Jabbari, and J. E. Bobrow, "Accurate sliding mode control of pneumatic systems using low-cost solenoid valves," IEEE/ASME Trans. Mechatronics, vol. 12, no. 2, pp. 216-219, 2007. doi: 10.1109/TMECH.2007.892821.

[10] S. Hodgson, M. Q. Le, M. Tavakoli and M. T. Pham, "Improved tracking and switching performance of an electro-pneumatic positioning system", Mechatronics, vol. 22, no. 1, pp. 1-12, 2012. doi: 10.1016/j.mechatronics.2011.10.007.

[11] A. Grancharova and T. Johansen, "Design and comparison of explicit model predictive controllers for an electropneumatic clutch actuator using on/off valves," IEEE/ASME Trans. Mechatronics, pp. 665673, 2011. doi: 10.1109/TMECH.2010.2049365.

[12] G. M. Bone, M. Xue and J. Flett, "Position control of hybrid pneumaticelectric actuators using discrete-valued model-predictive control," Mechatronics, vol. 25, pp. 1-10, 2015.

doi: 10.1016/j.mechatronics.2014.10.009. 


\section{Design and Simulation of the First Joint for a Prospective 5 Degrees of Freedom Manipulator with MR Actuators*}

\author{
Sergey Pisetskiy \\ Department of Electrical and Computer Engineering \\ The University of Western Ontario \\ London, ON, Canada \\ Email: spisetsk@uwo.ca
}

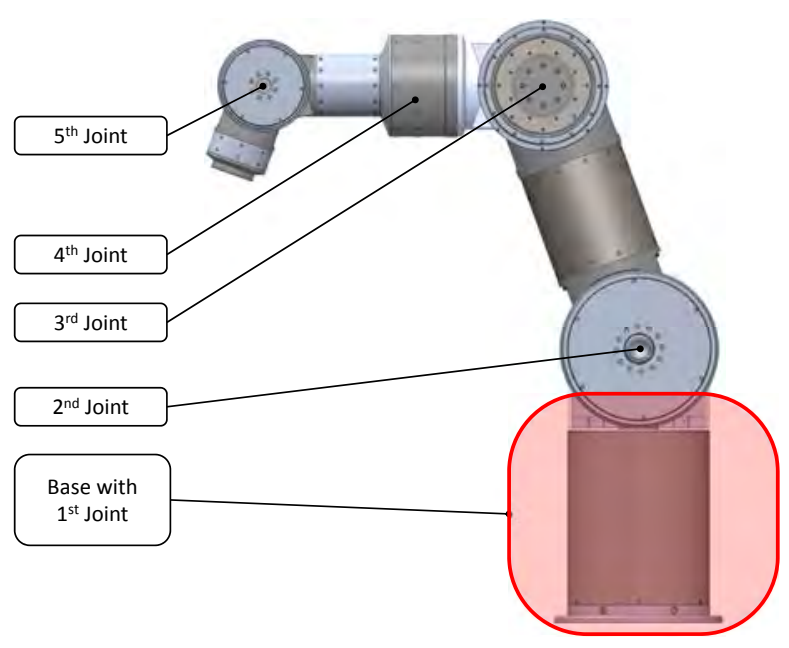

Fig. 1. Visualisation of the prospective 5-DOF robot.

\begin{abstract}
This paper presents the mechanical design and mathematical modeling for the first joint of a five degrees of freedom collaborative manipulator. The robot is actuated using Magneto-Rheological (MR) clutches in order to ensure full backdrivability and intrinsic torque control for safe human-friendly operation. One of the unique features of this design is the usage of a single motor for powering all five joints of the robot simultaneously and independently. After a brief description of the MR clutch principles, the details of the mechanical design and a state-space representation of the first joint are discussed. The paper will conclude with some preliminary simulation results.
\end{abstract}

\section{INTRODUCTION}

In recent years, the need for a human-friendly robot that can safely operate in close proximity to humans is driving progress in robotic technologies. Many research groups [4] [5] [6] [9] [10] achieved remarkable results in the development of automated systems that can interact with the person without posing a significant threat of injury.

Among all sources of danger, the risk of injury caused by a collision between a human and a robot is one of the biggest. Direct interactions make collisions unavoidable, and mitigating impacts from such collisions is one of the top priorities for research and development.

A number of approaches address the safety of robotic systems through development of compliant actuators. Possible solutions include variable stiffness transmission approach, elastic actuation, MR brakes and MR clutches, joint torque control, Distributed MacroMini Actuation, etc.

In this paper the Distributed Active Semi Active (DASA) approach [7] is briefly discussed, and the first steps in the development of an innovative five degrees of freedom (5-DOF) manipulator are presented.

DASA approach is intended to address the safety at the level of transmission, while the majority of commercially available

*This work was supported in part by Canad Foundation for Innovation (CFI) and Natural Sciences and Engineering Research Council (NSERC) of Canada under grant No.25031 and RGPIN-346166.

\author{
Mehrdad R. Kermani \\ Department of Electrical and Computer Engineering \\ The University of Western Ontario \\ London, ON, Canada \\ Email: mkermani@eng.uwo.ca
}

robotics platforms (KUKA LBR iiwa, Kinova JACO, Franka Emika) utilize the joint torque control methodology, that could be subject to software errors or control system malfunction.

Rigorous studies regarding the safety of MR clutch for direct interactions with humans were presented previously [7]. This paper does not discuss and validate the safe characteristics of MR clutches for human-robot interactions.

The contributions of this work are as follows:

- An innovative mechanical design of the base of the intended 5-DOF manipulator is introduced. The base comprises a single motor that actuates all joints of the robot, a Harmonic Drive (HD) gearbox, and a pair of MR clutches for the first joint.

- $\quad$ The derivation of a state-space model of the first joint transmission is presented.

- $\quad$ The simulation results for a simple control algorithm and the developed mathematical model are discussed. 


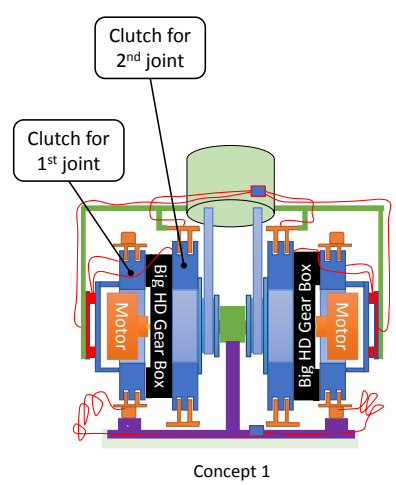

Concept 1

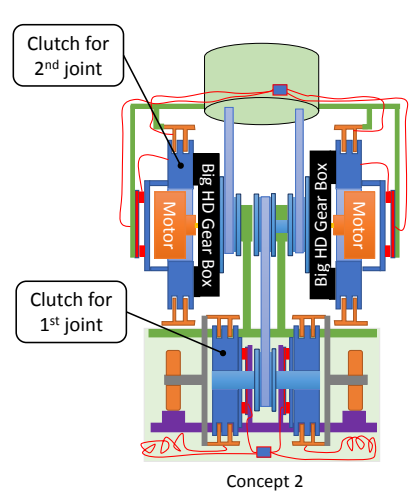

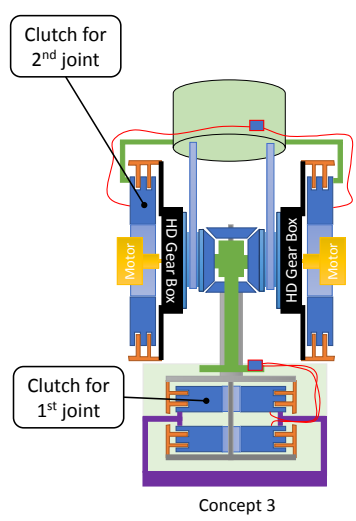

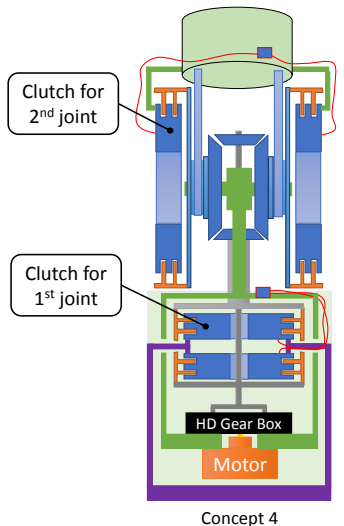

Fig. 2. Robot base design concepts

\section{OVERVIEW}

\section{A. Design Objective}

The main objective of the project is to develop a robotic manipulator using Magneto-Rheological actuators that address both safety and performance requirements of a collaborative robot. The manipulator includes five degrees of freedom that when combined with the degrees of freedom of the endeffector provide full kinematic redundancy. All joints of the intended manipulator are powered with the single motor located at the base of the robot. The targeted payload for the manipulator is $10 \mathrm{~kg}$ at full extension of the robotic arm.

Each joint of the manipulator is actuated using a pair of MR clutches coupled in an antagonistic configuration. The manipulator in total includes $10 \mathrm{MR}$ clutches and the torque is directly delivered by the clutches to the corresponding joint without gear reduction mechanism.

\section{B. Working Principles of MR clutches}

The utilization of a pair of MR clutches at each joint of the manipulator is a key feature of the proposed design. The main function of the MR clutch is the continual transmission of the actuation torque from the motor side to the load side. By changing the viscosity of the MR fluid in the clutch the exact amount of transmission torque between moving parts is regulated. The viscosity of MR fluid can be precisely controlled by applying a magnetic field in accordance with the requirement of the load transfer. The details of MR clutch construction and its features can be found in [2] [3] [8].

A pair of MR clutches can be configured to provide antagonistic actuation of a robot joint. In this case, the rotors of the MR clutches are rotated in opposite directions and the stators of both MR clutches are attached to a common shaft. Depending on the electromagnetic activation of each clutch, the torque can be applied by one or both MR clutches in either direction.

A complete comparison of various MR clutch configurations can be found in [1][2].

\section{Design of the Manipulator Base}

\section{A. Early design concepts}

At the early stage of this work, multiple design concepts for the base of the manipulator were considered and analyzed. In order to reduce the mass of the robot base, a lightweight high-speed motor with a harmonic drive gear box was decided to be used. Low weight along with a high gear ratio became the main reasons for choosing an expensive HD transmission for gear reduction.

Four concepts that were studied in details are shown in Fig. 2.

- The first concept comprises two motors and two pairs of clutches mounted co-axially on the wide moving frame. The two MR clutches of the 1st joint run on a circular rail. The motors are located inside the 1 st joint clutches, two harmonic drive gear boxes mounted between the 1 st and 2 nd joint clutches.

- The second concept is similar to the one discussed previously with the exception that the $2^{\text {nd }}$ joint clutches and the two motors and two harmonic drives are positioned above the $1^{\text {st }}$ joint clutches in order to reduce the diameter of the base.

- The third concept has a set of miter gears to change the orientation of the axis of rotation in order to mount $1^{\text {st }}$ joint clutches coaxial in the base. A pair of $2^{\text {nd }}$ joint clutches with a pair of motors and harmonic drives are positioned above the base.

- The fourth concept utilizes only one motor and one harmonic drive gear box. The pair of $1^{\text {st }}$ joint clutches is mounted coaxially to the vertical axis of rotation. The pair of $2^{\text {nd }}$ joint clutches is positioned above the base. A set of bevel gears distribute the motion between the clutches of the first and second joints.

The main disadvantage of the first concept is the unacceptable large size of the base and the need to use slip rings to actuate the magnetic coil and receive measurements from Hall sensors inside the MR clutches. Slip rings add additional 
resistance to the electrical circuit, bring more friction to the moving joint and may corrupt the signals of the sensors.

The second concept improves the base's footprint, but still requires slip rings to energize MR clutches and connect to Hall sensors.

The third and fourth concepts both have a small footprint in the base and do not require slip rings for MR clutches. But the third design requires two motors and two harmonic drives to ensure antagonistic actuation of the joints, while the last concept can use a single motor with a single HD gear box.

Taking into account the features described above, concept 4 was selected for further development.

\section{B. Transmission and control}

In selected configuration the single motor at the bottom of the robot base provides motion to the upper joints through a serial transmission that consists of several shafts and belts. As seen in Fig. 3, rotation speed of the motor shaft is reduced by the harmonic drive gear box.
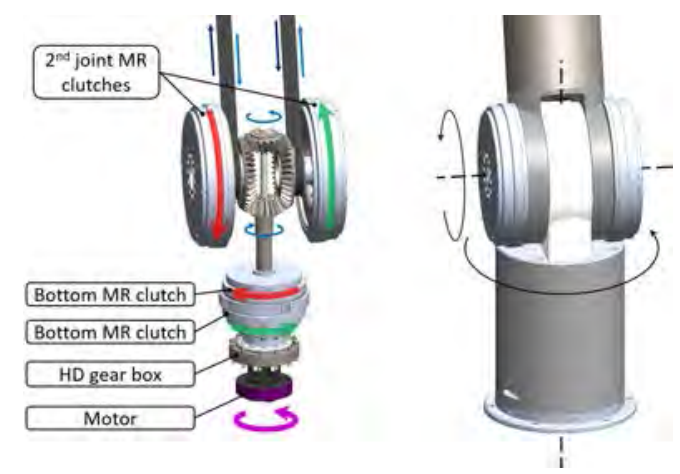

Fig. 3. Transmission of the 1-st and 2-nd joints of the manipulator.

The set of bevel gears located at the 2nd joint produce rotation in two opposite directions. Both clockwise and counterclockwise rotation is delivered to the pair of clutches at each joint through the belt transmission. Two belts moving in opposite directions provide antagonistic motion for the rotors in each pair of MR clutches.

\section{Design of the robot base (1-st joint)}

Manipulator base is designed to be properly mounted on the horizontal surface. The base comprises a pair of MR clutches that actuate the first joint responsible for the rotation of the second link around the vertical axis, as shown in Fig. 4. The single motor that drives all 5 upper joints is located at the very bottom in the base; it is fixed on the movable motor frame that rotates together with the upper links around the vertical axis of the base.

The wave generator of the harmonic drive gear box is directly connected to the motor shaft. The circular spline of the harmonic drive is mounted on the movable motor frame, while the flexspline is connected to the inner shaft and to the rotor of the bottom MR clutch via cylindrical adapters. The harmonic drive has high reduction ratio that allows utilization of the light but powerful high-speed electric motor.

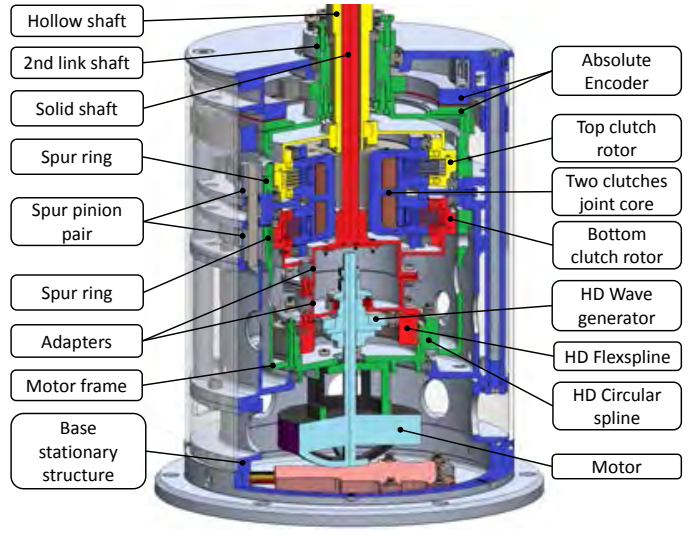

Fig. 4. Robot base zonal section view.

In order to compensate for the reaction moment, the torque from the motor and harmonic drive is transmitted from the motor frame to the upper links spur gears arrangement. The large spur gear - in the shape of a ring (spur gear ring) is located on the top of the motor frame. It is in mesh with six pinion pairs mounted on the stationary fixed cylindrical support. Through these pinion pairs, the rotation of the motor frame spur gear ring is mechanically transmitted to the similar spur gear ring that is mounted on the cylindrical frame of the second link. This construction allows to mechanically connect the motor frame with the second link frame, and at the same time provide fixation of the MR clutches to the stationary part of the base.

In the center of the manipulator base, there is a pair of MR clutches responsible for the rotation of the second robot link along the vertical axis (first joint). In order to save weight and space, both MR clutches share a joint magnetic core with two separate windings. Each clutch designed to generate torque up to $15 \mathrm{~N} \cdot \mathrm{m}$.

While the rotor of the bottom MR clutch is actuated directly by the circular spline of the harmonic drive, the rotor of the top clutch is rotated in opposite direction by the hollow shaft coming from the set of bevel gears located in the second link on top of the robot base.

In order to measure the angular position of the second joint, there is an absolute encoder mounted at the top plate of the manipulator base.

\section{Design of the 2nd joint}

The frame of the second link is mounted on the hollow shaft that spins around the vertical axis of the robot base. The link comprises a set of bevel gears to produce rotation in two opposite directions for all the upper joints of the robot. The transmission of the motion to the upper joints realized through the two belts as shown in Fig. 3.

From the harmonic drive located in the base, the rotation of the solid inner shaft is coming to the small bevel gear on the top of the link. Two big bevel gears in mesh with the small gear spin in opposite directions to each other with reduced speed 1:2. The bottom small bevel gear in mesh with big 
bevels rotates in an opposite direction relative to the top one. The hollow shaft coming from the bottom gear transmits the rotation to the rotor of the top MR clutch in the base.

Spinning in clockwise (CW) and counter-clockwise (CCW) directions big bevel gears rotate the rotors of two MR clutches of the second joint. The clutches should be able to produce up to $200 \mathrm{~N} \cdot \mathrm{m}$ torque each in order to actuate the third link of the robot.

\section{Modelling in MATLAB/Simulink}

\section{A. State-Space Model of the Joint}

In order to develop a state-space model, the simplified scheme of the manipulator first joint was considered, as shown in Fig. 5.

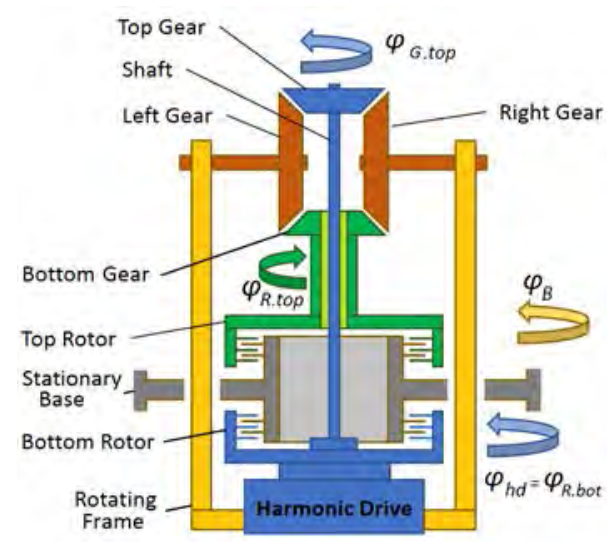

Fig. 5. Manipulator First Joint Scheme

In this figure, four separate groups of parts with common motions are identified with different colors, namely, HD Drive, bottom rotor, shaft, and top gear shown in blue; bottom gear, hollow shaft, and top rotor in green; rotating frame in yellow; and finally, left and right gears in red.

Assuming that the resulting angles, angular velocities, and angular accelerations of all parts of the assembly in the first group (blue color) are in the counterclockwise direction (looking from the top), then it can be shown that,

$T_{h d}-T_{R . b t m . i n e r t}-T_{R . b t m . f r}-T_{R . b t m . m r}-T_{\text {Sh.elast }}=0$

where $T_{h d}$ is the torque on Harmonic Drive, $T_{R \text {.btm.inert }}$ is the torque due to the inertia of the bottom rotor, $T_{R . b t m . f r}$ is the torque on clutch bottom rotor due to friction, $T_{R . b t m . m r}$ is the torque on bottom rotor due to MR effect, and $T_{\text {Sh.elast }}$ is the torque due to the elasticity of the shaft.

Harmonic drive output torque is a function of time,

$$
\begin{aligned}
T_{h d} & =T_{h d}(t) \\
T_{R . b t m . i n e r t} & =I_{R . b t m} \ddot{\phi}_{R . b t m}
\end{aligned}
$$

where $I_{R . b t m}$ and $\phi_{R . b t m}$ are the moment of inertia and the angle of the rotor of the bottom clutch respectively.

$$
T_{R . b t m . f r}=T_{R . b t m . v i s c}+T_{R . c o n s t . f r}
$$

where $T_{R . b t m . v i s c}$ is the torque on bottom rotor due to viscosity, $T_{R \text {.const.fr }}$ is the torque due to constant friction.

$$
T_{R . b t m . v i s c}=b_{R . v i s c} \dot{\phi}_{R . b t m}
$$

where $b_{\text {R.visc }}$ is the coefficient of viscous friction.

$$
\phi_{R . b t m}=\phi_{h d}
$$

where $\phi_{h d}$ is the angle of Harmonic Drive.

The torque due to the elasticity of the shaft can be calculated,

$$
T_{\text {Sh.elast }}=k_{S h} \Delta \phi=k_{s h}\left(\phi_{h d}-\phi_{G . t o p}\right)
$$

where $k_{s h}$ is the stiffness of the shaft, $\phi_{G . t o p}$ is the angle of the top bevel gear (top of the shaft).

For the second group of parts (green color), it is assumed that resulting angles, angular velocities, and angular accelerations of all parts of the assembly have clockwise direction (looking from the top), then it can be shown that,

$T_{G . b t m}-T_{R . t o p . i n e r t}-T_{R . t o p . f r}-T_{R . t o p . m r}-\frac{1}{2} T_{b . g . r e s u l t}=0$

where $T_{G . b t m}$ is the torque acting on the bottom bevel gear, $T_{R . t o p . i n e r t}$ is the torque due to the inertia of the clutch top rotor, $T_{R . t o p . f r}$ is the torque on clutch top rotor due to friction, $T_{R . t o p . m r}$ is the torque on clutch top rotor due to MR effect, $T_{\text {b.g.result }}$ is the resulting torque from bevel gears.

Due to mechanical differential properties, the torque acting on bottom gear is always equal to the top gear torque $T_{G \text {.top }}$,

$$
\begin{gathered}
T_{G . b t m}=T_{G . t o p}=T_{\text {Sh.elast }} \\
T_{\text {R.top.inert }}=I_{\text {R.top }} \ddot{\phi}_{\text {R.top }}
\end{gathered}
$$

where $I_{R . t o p}$ and $\phi_{R . t o p}$ are the moment of inertia and the angle of the rotor of the top clutch respectively.

$$
T_{R . t o p . f r}=T_{R . t o p . v i s c}+T_{R . c o n s t . f r}
$$

where $T_{R . t o p . v i s c}$ is the torque on bottom rotor due to viscosity. The top rotor rotates in opposite direction relative to the rotation of the stationary base.

$$
T_{\text {R.top.visc }}=b_{\text {R.visc }} \dot{\phi}_{R . t o p}
$$

where $\phi_{G . b t m}$ is the angle of the bottom bevel gear.

$$
\begin{gathered}
\phi_{\text {R.top }}=\phi_{G . b t m} \\
T_{\text {R.top.visc }}=b_{\text {R.visc }} \dot{\phi}_{G . b t m}
\end{gathered}
$$

In the third group (yellow color) the HD drive with the Motor is fixed on the rotating frame, so the reaction from the generated torque $T_{h d}$ is directly transmitted to the frame. Assuming that resulting angles, angular velocities, and angular accelerations of all parts of the frame assembly have counterclockwise direction (looking from the top),

$$
T_{\text {Sh.elast }}-T_{h d}+T_{G . b t m}-T_{F . \text { inert }}+T_{F}-T_{b . g . r e s u l t}=0
$$

where $T_{F \text {.inert }}$ is the torque due to the inertia of the rotating frame, $T_{F}$ is the torque acting on rotating frame from upper 
links (external). In this case torques $T_{\text {Sh.elast }}, T_{G . b t m}$, and $T_{F}$ have the same (counterclockwise) direction, while torques $T_{h d}$ and $T_{F . \text { inert }}$ have opposite direction.

$$
\begin{gathered}
T_{G . b t m}=T_{G . t o p}=T_{\text {Sh.elast }} \\
T_{\text {F.inert }}=I_{F} \ddot{\phi}_{F}
\end{gathered}
$$

where $I_{F}$ and $\phi_{F}$ are the moment of inertia and the angle of the rotating frame respectively.

In the fourth group (red color) the left and right bevel gears comprise the mechanical differential as well as transmit the torque from the top and bottom gears to the rotating frame.

According to design, both bevel gears have the same gear ratio $R_{b . g}$ in mesh with the top and with the bottom bevel gears. It is assumed that external torques applied to the left and right bevel gears have counterclockwise direction when looking from left and from right side respectively, so their resulting torque is:

$$
T_{b . g . r e s u l t}=R_{b . g} T_{G . L}+R_{b . g} T_{G . R}
$$

where $T_{G . L}$ and $T_{G . R}$ are the external torques acting on the left and right bevel gear respectively.

Angles of the mechanical differential in the rotating frame are governed by the following relation:

$$
\begin{gathered}
\phi_{G . t o p}+\left(-\phi_{G . b t m}\right)-2 \phi_{F}=0 \\
\phi_{G . b t m}=\phi_{G . t o p}-2 \phi_{F}
\end{gathered}
$$

In case of the motionless frame assembly, the angle $\phi_{G . b t m}$ has opposite direction relative to $\phi_{G \text {.top }}$ due to the property of mechanical differential.

For angular velocities and accelerations,

$$
\begin{aligned}
& \dot{\phi}_{G . b t m}=\dot{\phi}_{G . t o p}-2 \dot{\phi}_{F} \\
& \ddot{\phi}_{G . b t m}=\ddot{\phi}_{G . t o p}-2 \ddot{\phi}_{F}
\end{aligned}
$$

\begin{tabular}{|c|c|}
\hline $\begin{array}{l}x_{1}=\phi_{h d}(t), \\
x_{2}=\dot{\phi}_{h d}(t), \\
x_{3}=\phi_{G . t o p}(t), \\
x_{4}=\dot{\phi}_{G . t o p}(t), \\
x_{5}=\phi_{F}(t), \\
x_{6}=\dot{\phi}_{F}(t), \\
T_{1}=T_{h d}(t), \\
T_{2}=T_{R . b t m . m r}(t), \\
T_{3}=T_{R . t o p . m r}(t), \\
T_{4}=T_{G . L}(t),\end{array}$ & $\begin{array}{l}T_{5}=T_{G . R}(t), \\
T_{6}=T_{F}(t), \\
T_{7}=T_{R . c o n s t . f r}, \\
I_{1}=I_{R . b t m}, \\
I_{2}=I_{R . t o p}, \\
I_{3}=I_{F}, \\
b=b_{R . v i s c}, \\
k=k_{s h}, \\
R=R_{b . g} .\end{array}$ \\
\hline
\end{tabular}

Using relations described above, the following system with state equations was derived:

$$
\begin{aligned}
& {\left[\begin{array}{c}
\dot{x}_{1} \\
\dot{x}_{2} \\
\dot{x}_{3} \\
\dot{x}_{4} \\
\dot{x}_{5} \\
\dot{x}_{6}
\end{array}\right]=\left[\begin{array}{cccccc}
0 & 1 & 0 & 0 & 0 & 0 \\
-\frac{k}{I_{1}} & -\frac{b}{I_{1}} & \frac{k}{I_{1}} & 0 & 0 & 0 \\
0 & 0 & 0 & 1 & 0 & 0 \\
\frac{k\left(4 I_{2}+I_{3}\right)}{I_{2} I_{3}} & 0 & -\frac{k\left(4 I_{2}+I_{3}\right)}{I_{2} I_{3}} & -\frac{b}{I_{2}} & 0 & \frac{2 b}{I_{2}} \\
0 & 0 & 0 & 0 & 0 & 1 \\
\frac{2 k}{I_{3}} & 0 & -\frac{2 k}{I_{3}} & 0 & 0 & 0
\end{array}\right]\left[\begin{array}{l}
x_{1} \\
x_{2} \\
x_{3} \\
x_{4} \\
x_{5} \\
x_{6}
\end{array}\right]} \\
& +\left[\begin{array}{ccccccc}
0 & 0 & 0 & 0 & 0 & 0 & 0 \\
\frac{1}{I_{1}} & -\frac{1}{I_{1}} & 0 & 0 & 0 & 0 & -\frac{1}{I_{1}} \\
0 & 0 & 0 & 0 & 0 & 0 & 0 \\
-\frac{2}{I_{3}} & 0 & -\frac{1}{I_{2}} & -\frac{3 R}{I_{3}} & -\frac{3 R}{I_{3}} & \frac{2}{I_{3}} & -\frac{1}{I_{2}} \\
0 & 0 & 0 & 0 & 0 & 0 & 0 \\
-\frac{1}{I_{3}} & 0 & 0 & -\frac{R}{I_{3}} & -\frac{R}{I_{3}} & \frac{1}{I_{3}} & 0
\end{array}\right]\left[\begin{array}{c}
T_{1} \\
T_{2} \\
T_{3} \\
T_{4} \\
T_{5} \\
T_{6} \\
T_{7}
\end{array}\right]
\end{aligned}
$$

where variables are:

\section{B. Verification of the model in MATLAB/Simulink software}

Based on the state-space system above, the MATLAB/Simulink model was developed and analyzed. Based on the preliminary SolidWorks model analysis, the following values of variables were used in simulation:

$k=550[\mathrm{~N} \cdot \mathrm{m} / \mathrm{rad}]$ - Elastic coefficient of the shaft,

$b=0.5[\mathrm{~N} \cdot \mathrm{m} \cdot \mathrm{sec} / \mathrm{rad}]$ - Viscous friction coefficient,

$I_{1}=0.012\left[\mathrm{~kg} \cdot \mathrm{m}^{2}\right]$ - Inertia moment of bottom rotor,

$I_{2}=0.010\left[\mathrm{~kg} \cdot \mathrm{m}^{2}\right]-$ Inertia moment of top rotor,

$I_{3}=0.064\left[\mathrm{~kg} \cdot \mathrm{m}^{2}\right]$ - Inertia moment of rotating frame.

To build a discrete time model, a minimum required sampling rate was estimated (Nyquist criterion) and the sampling period $T s=0.01 \mathrm{sec}$ was selected.

In order to verify the developed model, the HD drive torque, continuous friction torque, and top clutch MR torque were applied in a form of a step-function:

- $\quad$ At the time $t=5 \mathrm{sec}$ the HD drive started to generate torque $T_{h d}=1 \mathrm{~N} \cdot \mathrm{m}$, the constant friction torque $T_{R . b t m . f r}=0.1 \mathrm{~N} \cdot \mathrm{m}$ appeared in the bottom clutch, and the constant friction torque $T_{R . t o p . f r}=0.1 \mathrm{~N} \cdot \mathrm{m}$ appeared in the top clutch;

- $\quad$ At the time $t=150 \mathrm{sec}$ the top MR clutch started to generate torque $T_{R . t o p . m r}=0.1 \mathrm{~N} \cdot \mathrm{m}$.

Resulting states representing angle and angular velocity of the bottom rotor, top bevel gear, rotating base, and derived parameters for top rotor are shown in Fig. 6 and Fig. 7.
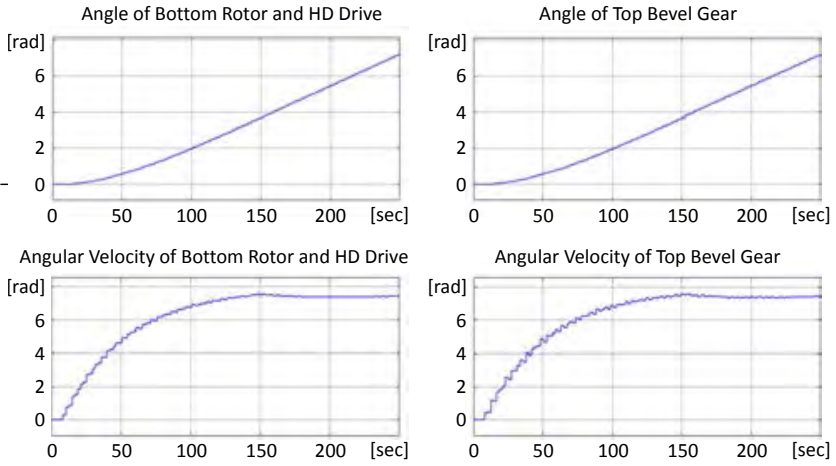

Fig. 6. Graphs of output angles and angular velocities for bottom rotor and top bevel gear

It can be observed on the graphs that at the time $=5 \mathrm{sec}$ when the harmonic drive started to generate torque $T_{h d}=1$ $N \cdot m$, angular velocities of the bottom rotor, top bevel gear, 

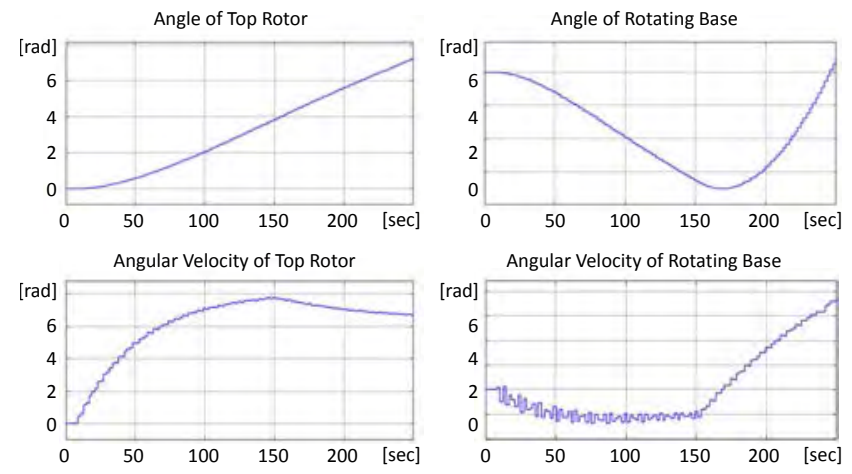

Fig. 7. Graphs of output angles and angular velocities for top rotor and rotating base gear

top rotor, and rotating base started to rise towards the certain values where the viscous friction of the parts equalizes generated torque. The rotating base started to turn in a clockwise direction and reached about $-0.07 \mathrm{rad}$ angle at the time $=150$ sec. On the angular velocity graphs for the top bevel gear and rotating base, one can clearly see the oscillations due to the flexibility of the shaft. Oscillations of the top and bottom rotor are not so visible but still can be noted.

When the top MR clutch was activated at $t=150 \mathrm{sec}$ and started to generate torque, the angular velocity of the top rotor started to decline, both bottom rotor and top bevel gear speeds showed a minor reduction, the angular velocity of the base changed its direction to counter-clockwise and the angle started to rise.

Shown on the graphs, the behavior of the parts corresponded very well with the expected motions of the actual hardware. Additional analogous simulations were performed to ensure the correct behavior of the developed system.

\section{Simple control design in MATLAB/Simulink software}

For further analysis, the simple input transducer is designed to process the desired reference trajectory of the rotating base into the controlling signals for clutches and HD drive. The required torques are calculated proportional to the derivative of the desired angle with respect to the time. The desired reference trajectory designed in MS Excel is shown with red color in Fig. 9.

The graphs of the torques applied to the system to follow desired trajectory of the rotating base are shown in Fig.8.

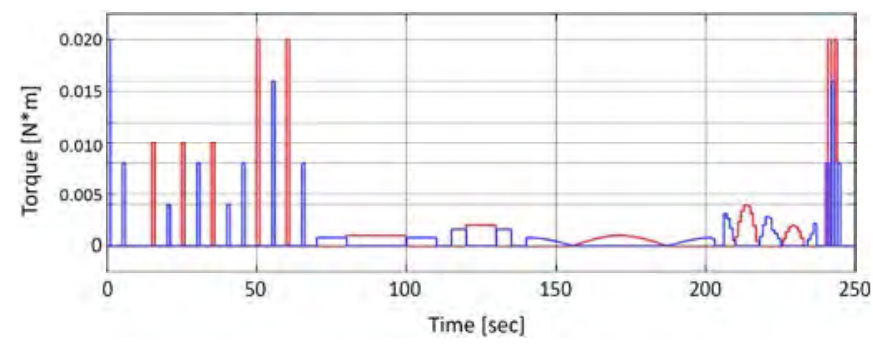

Fig. 8. Graph of the torque applied to the top (blue) and bottom (red) clutch in order the rotating base to follow the desired trajectory
It can be seen on the presented graphs, that in order to ensure the base is following the desired trajectory, the bottom MR clutch torque $T_{2}$ fluctuates from 0 up to $0.020 \mathrm{~N} \cdot \mathrm{m}$. The top MR clutch torque $T_{3}$ reaches $0.025 \mathrm{~N} \cdot \mathrm{m}$. Both clutches cannot produce negative torque due to physical limitations, so the minimum torque is $0 N \cdot m$ as shown on the graphs.

The rotation base follows the desired trajectory close enough. The graph of the simulated angle is shown in Fig.9.

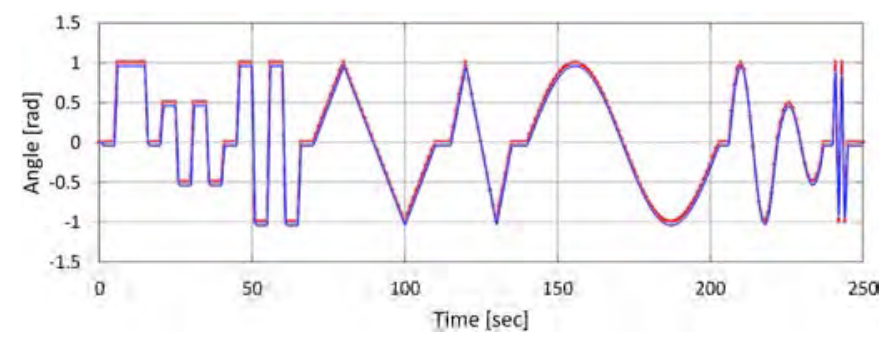

Fig. 9. Graph of the desired (red) and resulting (blue) trajectory of the rotating base.

The profile on the graph (blue) is almost identical to the profile of the desired trajectory (red) shown in Fig. 9. The error between the guiding profile and the actual angle of the base stays within +/- $0.04 \mathrm{rad}$.

\section{CONCLUSIONS}

Design and simulation results for the first joint of the prospective 5-DOF manipulator, presented in this paper, demonstrate the possibility of building the complex robot that can be actuated by a single motor at the base. The use of MR clutches in the joints of the manipulator brings power and safety to the system.

Further work is planned to model and simulate the rest of the joints in the robot. Future steps include parts manufacturing, control system development, assembling and testing the manipulator.

\section{REFERENCES}

[1] A. S. Shafer and M. R. Kermani, "Design and validation of a magnetorheological clutch for practical control applications in humanfriendly manipulation," in IEEE International Conference on Robotics and Automation, pp. 4266-4271, 2011. doi: 10.1109/ICRA.2011.5980258.

[2] W. Li, P. Yadmellat, and M. R. Kermani, "Design optimization and comparison of magneto-rheological actuators," in 2014 IEEE International Conference on Robotics and Automation (ICRA), 2014. doi: 10.1109/ICRA.2014.6907599.

[3] M. Moghani and M. R. Kermani, "Design and development of a hybrid Magneto-Rheological clutch for safe robotic applications," in 2016 IEEE International Conference on Robotics and Automation (ICRA), 2016. doi: 10.1109/ICRA.2016.7487474.

[4] M. Zinn, O. Khatib, and B. Roth, "A new actuation approach for human friendly robot design," in IEEE International Conference on Robotics and Automation, 2004. doi: 10.1109/ROBOT.2004.1307159.

[5] Dongjun Shin, I. Sardellitti, and O. Khatib, "A hybrid actuation approach for human-friendly robot design," in 2008 IEEE International Conference on Robotics and Automation (ICRA), pp. 1747-1752, 2008. doi: 10.1109/ROBOT.2008.4543453. 
[6] K. Kong, J. Bae, and M. Tomizuka, "Control of rotary series elastic actuator for ideal force-mode actuation in human-robot interaction applications," IEEE/ASME Trans. Mechatronics, vol. 14, no. 1, pp. 105-118, 2009. doi: 10.1109/TMECH.2008.2004561.

[7] A. S. Shafer and M. R. Kermani, "On the feasibility and suitability of mr fluid clutches in human-friendly manipulators," IEEE/ASME Trans. Mechatronics, vol. 16, no. 6, pp. 1073-1082, 2011.

doi: 10.1109/TMECH.2010.2074210.

[8] P. Fauteux, M. Lauria, B. Heintz, and F. Michaud, "DualDifferential Rheological Actuator for High-Performance Physical Robotic Interaction," IEEE Transactions on Robotics, vol. 26, no. 4 pp. 607-618, Aug. 2010. doi: 10.1109/TRO.2010.2052880.

[9] S. Diller, C. Majidi, and S. Collins, "Exoskeleton walking with a lightweight, low power electroadhesive clutch and spring," in 2016 IEEE International Conference on Robotics and Automation (ICRA), pp. $682-689,2016$.

[10] A. S. Shafer and M. R. Kermani, "Development of high performance intrinsically safe 3-DOF robot," in 2014 IEEE

International Conference on Robotics and Automation (ICRA), 2014. doi: 10.1109/ICRA.2014.6906919.

[11] M. S. Santina, A. R. Stubberud, and G. H. Hostetter, Digital Control System Design, 2nd ed. Orlando, United States of America: Saunders College Publishing, a Harcourt Brace College Publisher, 1994. 


\section{Comparative Analysis of OptiTrack Motion Capture Systems}

\author{
Joshua S. Furtado, Hugh H. T. Liu \\ University of Toronto Institute for Aerospace Studies \\ University of Toronto \\ Toronto, Canada \\ joshua.furtado@mail.utoronto.ca
}

\author{
Gilbert Lai, Herve Lacheray, Jason Desouza-Coelho \\ Quanser \\ Markham, Canada \\ gilbert.lai@quanser.com
}

\begin{abstract}
A comparative analysis of four different OptiTrack motion capture systems is provided in this paper. Tracking accuracy, workspace volume, marker size and camera range are the main criteria used for comparison. The tracking accuracy is tested using Quanser's linear motion platform by comparing the measured position of the cart with the position obtained using the encoder. The workspace volume limits are obtained by flying a LiteHawk Neon drone in circles of increasing radius at different heights until tracking fails. The relationship between marker size and camera range is obtained through theoretical calculations. Experimental as well as theoretical results are presented, illustrating the performance of these four systems. These will serve as a baseline to select the right motion capture system for any particular application.
\end{abstract}

Keywords- Motion capture; OptiTrack; Optical-Passive

\section{INTRODUCTION}

Motion capture (mocap) is the process of recording the movement of objects or people. It involves measuring the position as well as orientation of the objects or people in physical space [1]. The technology was originally developed for gait analysis in the life science market but is now used in a wide variety of other fields. Some of these include computer graphics animation, robotics and military use.

Over the past few decades, a lot of advancements have been made in motion capture technology resulting in many different approaches such as:

- Optical-passive: Retroreflective markers are tracked by a set of infrared cameras. It is the most practical and commonly used method in the industry [2]. The motion capture systems selected for comparative analysis in this paper are all optical-passive.

- Optical-active: Infrared LED markers are tracked by a set of infrared cameras. Note that a suitable power supply source is required to power active markers.

- Video: Software is used to track the object motion in each frame. Depending on the object detection and tracking algorithm used, the accuracy of the results may vary. This technique is less accurate than the marker-based solutions.

- Inertial: Independent of cameras, this technique uses inertial measurement sensors on the subject. Short term accuracy is a major drawback.

Optical-passive motion capture systems are widely used for validation and control of computer vision and robotics in an indoor laboratory setting. They have great practical value in indoor mobile robotics applications. Hence, choosing the right motion capture system for a particular application is extremely important. In this paper, a series of systematic tests are performed using four different OptiTrack motion capture systems to characterize their performance. The results obtained from these tests will provide a baseline for users to choose the right motion capture system for any computer vision or robotics application based on tracking accuracy, size of workspace, marker size and camera range.

This paper is organized as follows. In Section II, the four different OptiTrack motion capture systems selected for evaluation are presented. In Section III, the experimental setup used to test the performance of these motion capture systems is described and the test procedure is explained in detail. The results obtained by performing the test using the four different systems are showcased in Section IV. Finally, the conclusions drawn from this work are presented in Section V.

\section{MOTION CAPTURE SYSTEMS}

OptiTrack makes high-performance motion capture systems at an affordable price. A single OptiTrack motion capture system may track the 6 degrees of freedom (DOF) pose of one or more objects in the workspace. Objects tracked in space need to be defined as rigid bodies. Each rigid body is a cluster of reflective markers arranged in a unique configuration. Fig. 1 shows two quadrotors (a LiteHawk Neon and LiteHawk Highroller) defined as rigid bodies, one using $1 / 2$-inch by $1 / 2$ inch square markers and the other using 5/8-inch diameter spherical markers. OptiTrack manufactures spherical markers with diameters ranging from $1 / 4$-inch to $3 / 4$-inch. Additionally, OptiTrack also does fabricate reflective 1/3-inch diameter dots and $1 / 2$-inch by $1 / 2$-inch squares. Depending on the size and limitations of the rigid body, appropriate size markers may be 


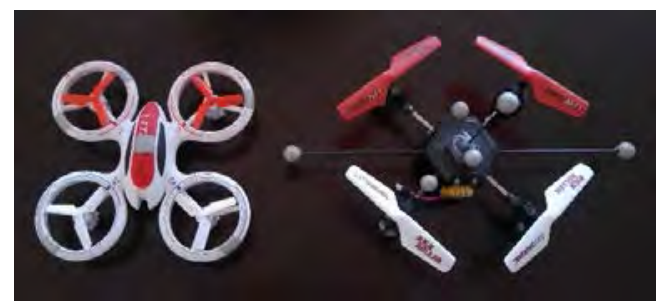

Figure 1. LiteHawk Neon and Highroller quadrotors tagged with retroreflective markers

chosen. For example, we use squares on the LiteHawk Neon due its payload capacity and spherical markers on the LiteHawk Highroller because of its fuselage design.

In this paper, four different OptiTrack systems are chosen for comparative analysis, comprising: Flex 3 (x12), Flex 13 (x6), Prime 13 (x6) and Prime 17W (x6). Table 1 provides detailed specifications for the cameras used in each of these systems. The specifications provided are obtained directly from the manufacturer's website [3]. The number of cameras for each of the systems are chosen to cover roughly the same workspace area and to be able to track rigid bodies using square markers. Note that the dot and square markers expose less retroreflective surface area compared to the spherical markers. The Flex 3 cameras have a much lower resolution compared to the rest. The ethernet connectivity of the Prime series allows for a faster frame rate and lower latency compared to the Flex series. All cameras use wide angle lenses. The system cost is the number of cameras times the cost per camera. However, this does not include the costs of the hubs, proprietary software and the ground control station as they are much smaller compared to the cost of the cameras.

TABLE I. MOTION CAPTURE SYSTEMS CHOSEN FOR COMPARATIVE ANALYSIS

\begin{tabular}{|c|c|c|c|c|}
\hline & $\begin{array}{c}\text { OptiTrack } \\
\text { Flex } 3(\times 12)\end{array}$ & $\begin{array}{c}\text { OptiTrack } \\
\text { Flex } 13(\times 6)\end{array}$ & $\begin{array}{c}\text { OptiTrack } \\
\text { Prime } 13 \\
(\times 6)\end{array}$ & $\begin{array}{c}\text { OptiTrack } \\
\text { Prime } \\
17 \mathrm{~W}(\times 6)\end{array}$ \\
\hline Resolution & $\begin{array}{c}640 \times 480(0.3 \\
\mathrm{MP}) \\
\end{array}$ & $\begin{array}{c}1280 \times 1024 \\
(1.3 \mathrm{MP})\end{array}$ & $\begin{array}{c}1280 \times 1024 \\
(1.3 \mathrm{MP})\end{array}$ & $\begin{array}{c}1664 \times 1088 \\
(1.7 \mathrm{MP})\end{array}$ \\
\hline Frame rate & $100 \mathrm{FPS}$ & $120 \mathrm{FPS}$ & $240 \mathrm{FPS}$ & $360 \mathrm{FPS}$ \\
\hline Lens & $4.5 \mathrm{~mm}$ & $5.5 \mathrm{~mm}$ & $5.5 \mathrm{~mm}$ & $6 \mathrm{~mm}$ \\
\hline $\begin{array}{l}\text { Horizontal } \\
\text { FOV }\end{array}$ & $46.2^{\circ}$ & $56^{\circ}$ & $56^{\circ}$ & $70^{\circ}$ \\
\hline Vertical FOV & $34.7^{\circ}$ & $46^{\circ}$ & $46^{\circ}$ & $49^{\circ}$ \\
\hline Connectivity & USB 2.0 & USB 2.0 & Ethernet & Ethernet \\
\hline Latency & $10 \mathrm{~ms}$ & $8.3 \mathrm{~ms}$ & $4.2 \mathrm{~ms}$ & $2.8 \mathrm{~ms}$ \\
\hline $\begin{array}{l}\text { Cost per } \\
\text { camera }\end{array}$ & 599 USD & 999 USD & 1999 USD & 3499 USD \\
\hline System cost & 7188 USD & 5994 USD & 11994 USD & 20994 USD \\
\hline
\end{tabular}

\section{EXPERIMENTAL SETUP AND TEST PROCEDURE}

The four different motion capture systems are compared using tracking accuracy, workspace volume, marker size and camera range. The tracking accuracy and workspace volume are obtained experimentally while the relationship between marker size and camera range is derived geometrically based on the pinhole camera model.

\section{A. Tracking Accuracy}

Fig. 2 shows the experimental setup used to test for rigid body tracking accuracy. Quanser's linear motion platform is used where the cart and the two ends of the track are defined as rigid bodies, tracked by the motion capture system. The positions of the rigid bodies are attained using the motion capture system. The cart is driven on the track by a DC motor via a rack and pinion mechanism that ensures consistent and continuous traction [4]. It has an encoder with a resolution of 4096 counts/rev to measure its position. To start, the cart is placed at one of the ends and using the encoder feedback, a PID position controller is used to move the cart with a fixed amplitude at increasing frequency. The position of the cart on the track is obtained using its initial position, the track unit vector (defined using the positions of the two ends of the track) and the encoder count. The calculated position is then compared with the cart position obtained from the motion capture system (to test the accuracy of the four different motion capture systems). For each camera system, three sets of tests are done in three different table orientations and for each orientation, data for five experimental runs are logged.

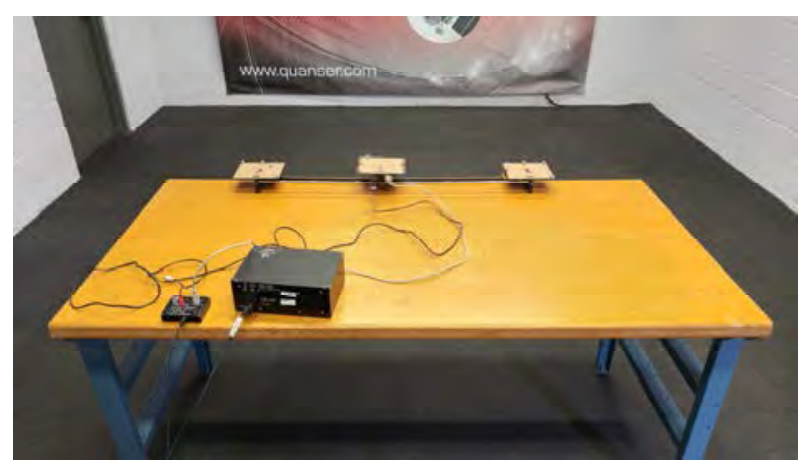

Figure 2. Experimental setup to test tracking accuracy

Let $P_{C C}$ and $P_{C E}$ denote the positions of the cart measured using the motion capture system and calculated based on the encoder count respectively. Let $P_{E 1}$ and $P_{E 2}$ denote the positions of the two ends of the cart obtained using the motion capture system.

$$
\hat{t}=\frac{P_{E 1}-P_{E 2}}{\left\|P_{E 1}-P_{E 2}\right\|_{2}}
$$

where $\hat{t}$ is the track unit vector. The displacement of the cart from its initial position, $d$ can be calculated using the encoder count, $c$. The effective linear tracking resolution, $k=2.2749 \times$ $10^{-5} \mathrm{~m}$ is based on the gear ratio, pitch and encoder resolution.

$$
d=k c
$$

The position of the cart at any time, $t$ can then be calculated as follows: 


$$
P_{C E}(t)=P_{C C}\left(t_{0}\right)+\hat{t} d
$$

The tracking error, $e$ at every time instant, $t$ is then given by

$$
e(t)=P_{C E}(t)-P_{C C}(t) .
$$

Fig. 3 shows the experimental data for a single run using the OptiTrack Prime 13 motion capture system. The position controller moves the cart back and forth with a fixed amplitude of $0.15 \mathrm{~m}$ at increasing frequency. The $\mathrm{x}, \mathrm{y}$ and $\mathrm{z}$ positions of the cart measured using the motion capture system (shown in blue) are compared with those obtained using the encoder count (shown in orange). Fig. 4 shows the errors in position along the $\mathrm{x}, \mathrm{y}$ and $\mathrm{z}$ direction for the same run.
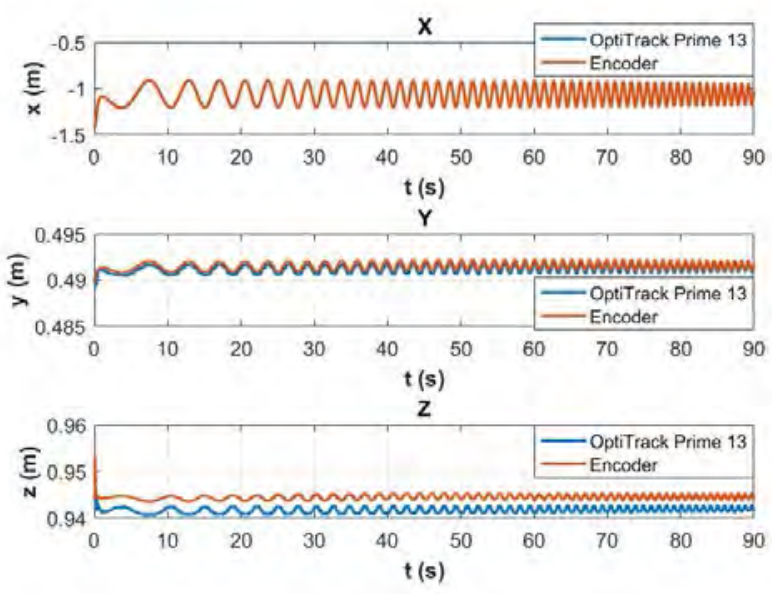

Figure 3. Measured (OptiTrack Prime 13) vs. encoder position of cart
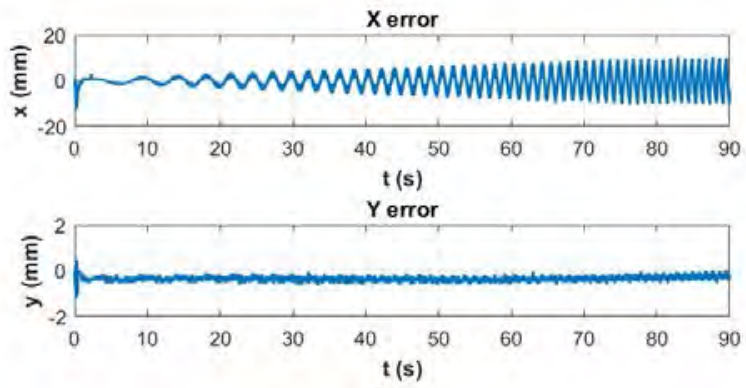

Z error

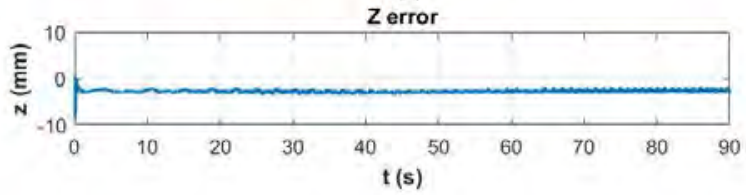

Figure 4. Tracking error (OptiTrack Prime 13)

It is important to note the tracking accuracy of the motion capture system for different cart velocities, accelerations and jerks. The velocity of the cart, $V_{C C}$ is obtained using a fourth order forward finite difference approximation [5] of the cart position given by

$$
V_{C C}(t)=\frac{\left(\begin{array}{c}
-25 P_{C C}(t)+48 P_{C C}(t+h)-36 P_{C C}(t+2 h) \\
+16 P_{C C}(t+3 h)-3 P_{C C}(t+4 h)
\end{array}\right)}{12 h}
$$

where $h$ is the sample time. A sample time of $0.001 \mathrm{~s}$ is used in all experimental runs. Similarly, acceleration and jerk are obtained by differentiating the approximated velocity and acceleration respectively.

\section{B. Workspace Volume}

The workspace of each motion capture system is the volume in which a rigid body can be successfully tracked. To estimate the workspace volumes of the four different motion capture systems, a LiteHawk Neon drone is flown around in the workspace to test its limits. A PID controller is used to control the position of the drone in the workspace using measurements from the motion capture system as feedback. The drone is flown around in circular trajectories of increasing radius starting at an altitude of $z=0.25 \mathrm{~m}$ until tracking is lost. This is then continued for altitudes all the way up to $z=3 \mathrm{~m}$ with increments of $\Delta z=0.25 \mathrm{~m}$. By logging the position of the drone continuously during the experiment, the workspace volume can be estimated.

\section{Marker Size and Camera Range}

OptiTrack manufactures spherical markers of sizes ranging from 1/4-inch to 3/4-inch in diameter. Limited by camera resolution and Field Of View (FOV), there is a maximum range to see markers of a particular size. Based on camera imaging geometry, the number of pixels seen in a marker of known size at a distance can be calculated. Knowing the marker diameter, $d$, the maximum cross-sectional area for each marker can be calculated using

$$
A_{C S}=\frac{\pi}{4} d^{2}
$$

If $r=a \times b$ pixels is the camera resolution, the aspect ratio is

$$
A R=\frac{a}{b}
$$

Given the horizontal FOV $\left(h_{F O V}\right)$, vertical FOV $\left(v_{F O V}\right)$ and focal length of the camera $(f)$, the diagonal FOV can be calculated as follows

$$
F O V=2 \operatorname{atan} 2\left(\frac{f \sqrt{\tan ^{2}\left(\frac{v_{F O V}}{2}\right)+\tan ^{2}\left(\frac{h_{F O V}}{2}\right)}}{2}, f\right)
$$

The area seen by the camera at a distance, $h$ is

$$
S=\frac{\left(2 h \tan \left(\frac{F O V}{2}\right)\right)^{2}}{A R+\frac{1}{A R}} .
$$


The number of pixels seen in a marker at a distance, $h$ is

$$
N=\frac{a b A_{C S}}{S} .
$$

\section{RESUlTS}

The experimental and theoretical results for the four different motion capture systems are presented in this section. Average error for different frequencies, velocities, accelerations and jerks of the cart are provided in the tracking accuracy subsection. Top and sectional views of the workspace volumes are shown in the workspace volume subsection. In the last subsection, the number of pixels seen in a marker by a single camera is related to its range based on the calculations in the previous section. Fig. 5 shows a scatter plot of the average error plotted against system cost. Note that the Flex 3 motion capture system is comprised of twelve cameras (twice as many as the number of cameras in the other motion capture systems). The Flex 3 cameras are mounted at a lower height to be able to track the $1 / 2$-inch by $1 / 2$-inch squares given their lower resolution compared to the rest of the cameras. The area seen by the cameras is thus much lower. Hence, twelve cameras are used to cover roughly the same workspace volume. The system costs reflect important characteristics of the cameras such as resolution, frame rate and latency based on the average tracking error of the four systems.

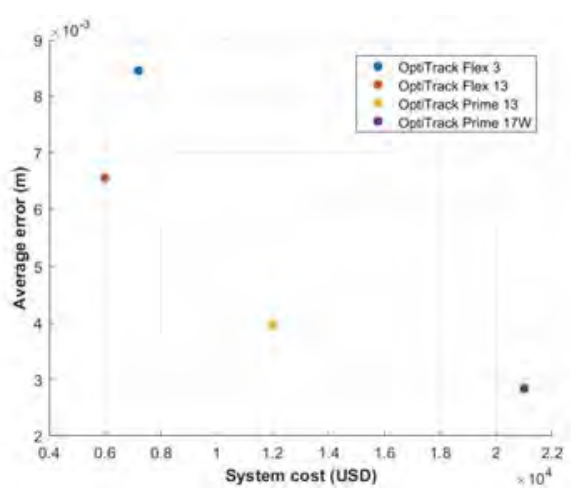

Figure 5. Average error vs. system cost

\section{A. Tracking Accuracy}

Fig. 6 shows the average error plotted against the frequency at which the cart moves back and forth along the track with an amplitude of $0.15 \mathrm{~m}$. The rise in average error with an increase in frequency follows a linear trend. The rate at which error increases with increase in motion frequency is a function of the frame rate and latency of the camera system. Figs. 7, 8 and 9 show plots of the average error vs. cart velocity, acceleration and jerk respectively. Note that the cart velocity, acceleration and jerk are derived using fourth order forward finite difference approximation of the cart position measured using the motion capture system. This is only an approximation and is subject to error. Straight lines were used to fit the data best from scatter plots of the average error vs. cart velocity, acceleration and jerk. The average error increases linearly with increase in cart velocity, acceleration and jerk. Once again, it can be noted that
Prime series have a lower tracking error compared to the Flex series because of higher frame rate and lower latency.

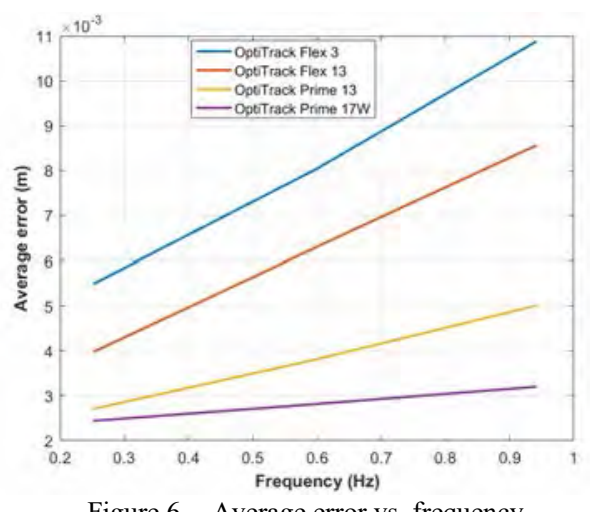

Figure 6. Average error vs. frequency

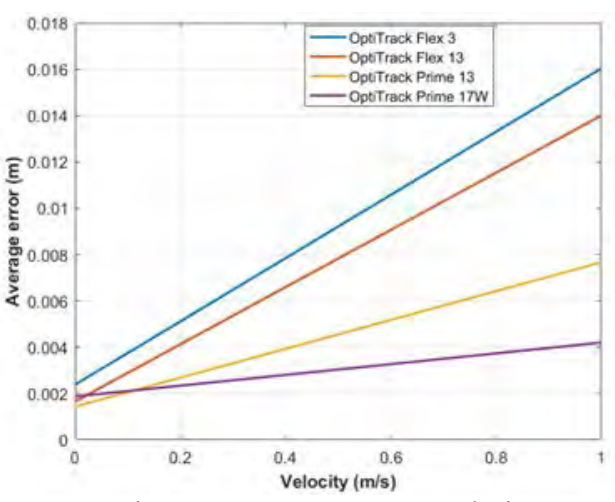

Figure 7. Average error vs. velocity

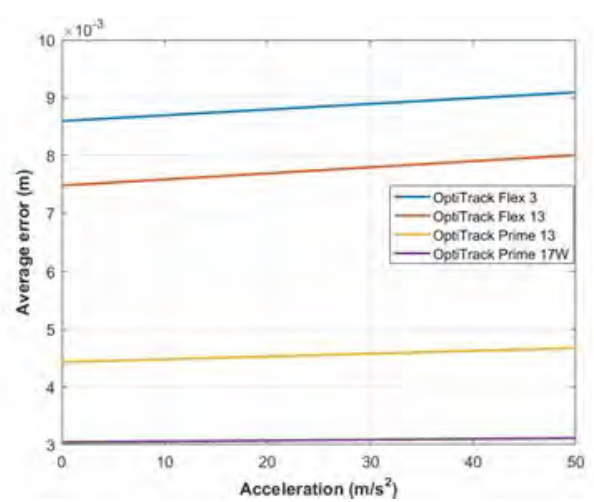

Figure 8. Average error vs. acceleration

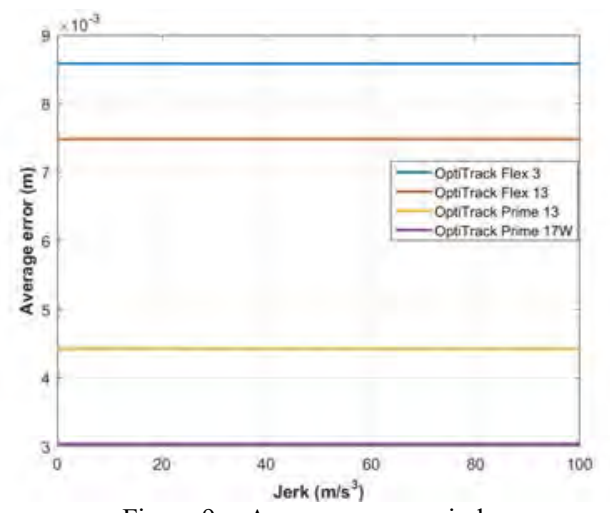

Figure 9. Average error vs. jerk 


\section{B. Workspace Volume}

The limits of the workspace volume for each of the four motion capture systems were tested by flying the LiteHawk Neon drone in circular trajectories of increasing radius at different heights. Fig. 10 shows the top and sectional views for each of the four different motion capture systems. The shape of the workspaces roughly resembles that of a dome. The top views show the circles at different heights within which the drone can fly while being tracked successfully. It can be noted that the flying radius in each transverse plane decreases with increase in height. The workspace volume depends on many factors such as the positioning of the cameras, resolution, focal length and FOV.

\section{Marker Size and Camera Range}

Fig. 11 shows the relationship between marker sizes and range for the different camera systems. In the first plot, the number of pixels as a function of distance is seen for different marker sizes using a Flex 13 camera. The larger the marker, the more pixels are seen by the camera and hence can be seen from a longer range. The number of marker pixels seen by a camera decreases exponentially with increase in range. In the second plot, comparing the four different cameras using the same size marker at a fixed distance, the Flex 13 and Prime 13 capture the most number of pixels closely followed by the Prime $17 \mathrm{~W}$ leaving the Flex 3 with the least. For a marker to be detected, at least 4 pixels are needed to be seen by a camera. The black line in the plots indicates this threshold.

\section{CONCLUSIONS AND RECOMMENDATIONS}

The Prime series cameras have a lower tracking error for aggressive rigid body manoeuvres due to higher frame rate and lower latency. High frame rate and low latency are useful in estimating rigid body velocity, acceleration and jerk more accurately. The OptiTrack Prime $17 \mathrm{~W}$ cameras offer maximum workspace volume due to wider angle lens with bigger FOV. Using a camera with a larger FOV will result in a bigger workspace volume. The OptiTrack Flex 13, Prime13 and Prime $17 \mathrm{~W}$ cameras offer longer marker range due to higher resolution and longer focal length. The camera resolution is important based on the type of markers used to identify rigid bodies in space. It will determine the minimum separation distance between the markers to be identified uniquely, and the minimum size of marker needed at a particular distance from the camera.

\section{REFERENCES}

[1] Vicon Motion Systems Ltd., "What is motion capture," VICON. [Online]. Available: https://www.vicon.com/what-is-motion-capture [Accessed: Feb. 13, 2018].

[2] P. Nogueira, "Motion Capture Fundamentals: A Critical and Comparative Analysis on Real-World Applications," Faculdade Eng. Univ. Porto, Nov. 2011. [3] NaturalPoint, Inc. DBA OptiTrack, "Compare Cameras," OptiTrack. [Online]. Available: https://optitrack.com/hardware/compare/ [Accessed Feb. 13, 2018]. [4] Quanser, "Linear Motion Platform Product Categories," Quanser. [Online]. Available: https://www.quanser.com/products_category/linear-motion-platform/ [Accessed Feb. 13, 2018].

[5] B. Fornberg, "Generation of Finite Difference Formulas on Arbitrarily Spaced Grids," Math. Comp., vol. 51, no. 184, pp. 699-706, 1988.

doi: 10.1090/S0025-5718-1988-0935077-0.
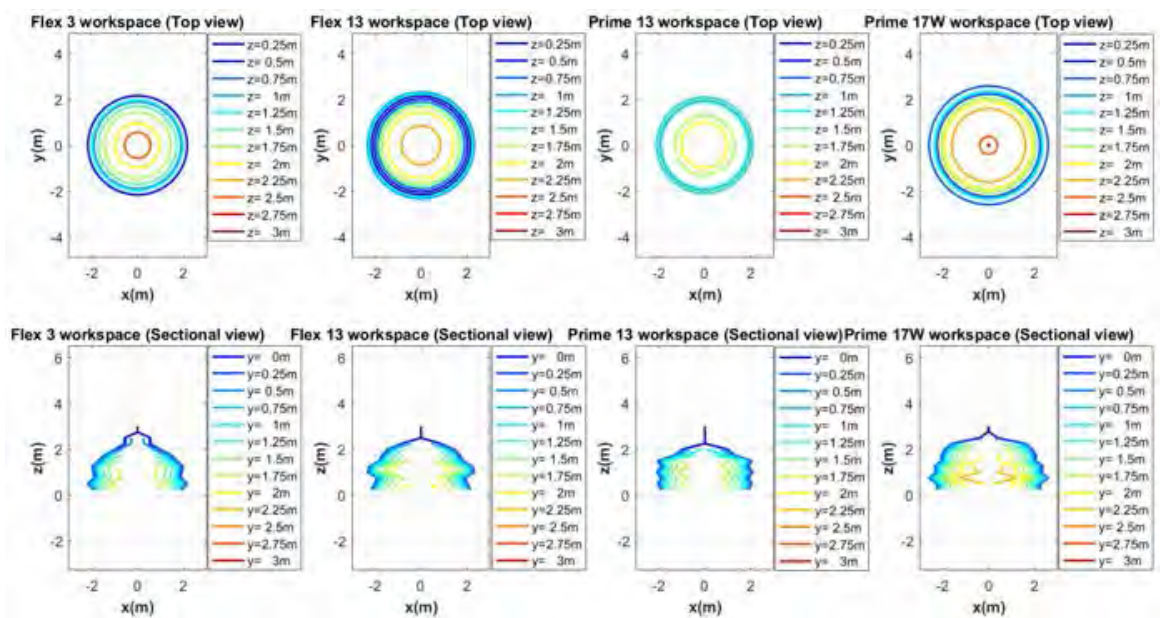

Figure 10. Workspace volume
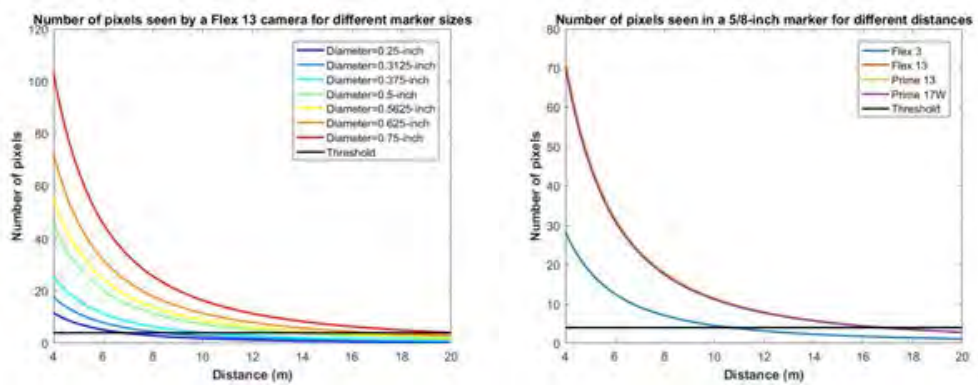

Figure 11. Number of pixels vs. distance 


\title{
Impact Force Reduction Strategies to Achieve Safer Human-robot Collisions
}

\author{
Behrad Rouzbeh and Gary M. Bone* \\ Department of Mechanical Engineering \\ McMaster University \\ Hamilton, Canada \\ *Corresponding author. E-mail: gary@mcmaster.ca
}

\begin{abstract}
The increasing use of robots operating close to people has made human-robot collisions more likely. In this paper, strategies intended to reduce the impact force to a safe level, without sacrificing the robot's performance, are investigated. The strategies can be applied to a robot arm without modifying its internal hardware. They include the existing strategies: lowering the actuator controller's stiffness; actuator switched off upon impact detection; withdrawing the arm upon impact detection; and adding a compliant cover. We also propose the novel strategy of limiting the controller's feedback term. The collision scenario studied is a robot arm colliding with a person's constrained head. An improved lumped parameter model of the constrained impact is proposed. Simulation results are included for a UR5 collaborative robot. Sixteen combinations of the impact force reduction strategies are compared. The results show that using a high stiffness controller with a feedback limit and compliant cover reduces the impact force to a safe level, and achieves precise trajectory tracking.
\end{abstract}

Keywords-collaborative robot; compliant covering; humanrobot collision; impact force; robot control; robot safety

\section{INTRODUCTION}

In emerging applications, such as collaborative robots and service robots, robot arms (also termed "manipulators") may operate in close proximity with people. This proximity makes human-robot collisions much more likely so the ability to reduce the human-robot impact force to a safe level is a critical requirement for these robots. Research on the design and control of safe collaborative robots (also termed "humanfriendly robots" in the literature) aims to address this safety requirement without sacrificing the robot's performance and functionality.

Previous researchers have proposed a wide variety of approaches for reducing the human-robot impact force. In [1]

Research sponsored by the Natural Sciences and Engineering Research Council of Canada (NSERC) through a Discovery Grant. the impact force is reduced by applying a time-varying limit to the actuator torques based on a dynamic model of the robot. While this can work in certain situations, an undesirable reduction in the precision of the robot's trajectory tracking may also occur when the actuator torques are limited. Reducing the inertia of the robot is very effective at reducing the impact force. The robot developed by DLR [2] is a prime example of this approach whose special design and use of lightweight materials, such as carbon fiber, produced a very low inertia arm. However, this is a costly solution and obviously cannot be used to improve the safety of conventional robots. Reducing the stiffness of the transmission between the actuators and links is another approach for improving the collision safety, e.g. [3][4]. With conventional robots the transmission between each actuator and link is very stiff so both of their inertias contribute to the severity of the collision. The objective of variablestiffness actuation (VSA) is to dynamically decouple the actuator's rotor inertia from the link's inertia. Since the contact occurs between the human and the link, and the link's inertia is lower than the combined inertia, the impact force will be reduced by the VSA's decoupling effect. The lower the stiffness the lower the force, however low transmission stiffness also tends to lower the precision of the robot's position control. Similarly, a lower arm velocity also reduces the impact force, but clearly sacrifices the robot's performance. The VSA mitigates these two problems by lowering the stiffness when the velocity is high, and increasing the stiffness when the velocity is low. This addresses the safety aspect, but will also cause the tracking precision to deteriorate during the higher velocity sections of its motion trajectories. Furthermore, using VSAs for the robot's six joints will increase the complexity, cost and inertia of the arm. Adding an electromechanical clutch between the link and actuator is an alternate approach to decouple the actuator's and link's inertias [5][6]. This behaves like a VSA with two stiffnesses and is termed a "series clutch actuator" (SCA). When clutch is engaged the transmission's stiffness is very high, and when it is disengaged the stiffness drops to zero. Unlike a VSA, the SCA should not affect the robot's performance during normal conditions since the clutch should only disengage when a collision happens. The clutch's torque threshold can also be 
varied to satisfy both the safety and performance requirements [5]. Unfortunately, using SCAs will increase the complexity, cost and inertia of the robot arm.

While the approaches in [2]-[6] all have merit, we are interested in methods for reducing the impact force that can be applied to existing robot arms with little or no modifications to the arm's hardware. The actuator torque limiting method in [1] is such an approach, but has serious side effects as previously discussed. A better approach is to add a collision detection and reaction strategy to the robot's control software, e.g. [7]-[9]. The detection can use either the robot's existing sensors [7][8] or externally mounted sensors [9]. The former approach requires a dynamic model of the robot while the latter does not. Using either approach detection times of a few ms can be achieved [7]-[9]. Various reaction strategies may be executed after contact detection to reduce the impact force, such as: braking to a stop [7][9], switching the actuator off [8], or having the arm reflexively withdrawn from the location of the impact [8]. Another method for increasing the safety of an existing robot is to add a compliant cover over its surface, e.g. [7][10][11]. The cover's stiffness can be chosen to satisfy a safety constraint without being excessively thick [11]. The compliant cover cannot be used with the end effector if it interferes with its function. If this is the case, quick release mechanisms, such as the QuickSTOP collision device (Applied Robotics Inc.), can be used to increase the safety of collisions with the end effector.

In this paper we will investigate strategies intended to reduce the impact force when a robot collides with a human's head, while maintaining the robot's performance during normal conditions. These can be applied to an existing robot without modifying its internal hardware. They include the previously applied strategies: lowering the actuator controller's stiffness, actuator switched off by controller upon impact detection, withdrawing the arm as fast as possible upon impact detection, and adding a compliant cover. We also propose the novel strategy of limiting the magnitude of the feedback term used with the controller.

The collision scenario is described in section II. In section III the impact model for this scenario is derived. Section IV covers the controller design. Simulation results are presented and discussed in section $\mathrm{V}$ and conclusions are drawn in section VI.

\section{COLLISION SCENARIO}

Although the human-robot impact could occur almost anywhere on the human's body we will investigate impact with the human head since it is the most likely to result in serious injuries. The collision scenarios being studied are illustrated in Fig. 1. We assume that the impact takes place at the tip of the forearm link when the robot arm is fully extended as shown. This contact location and elbow angle maximizes the moment of inertia of the arm which will produce the maximum impact force, all other parameters being equal. We also assume the impact direction and human's surroundings do not allow the head to move - this is termed a "constrained impact" and produces much larger forces than the unconstrained case where the head is allowed to move [11].

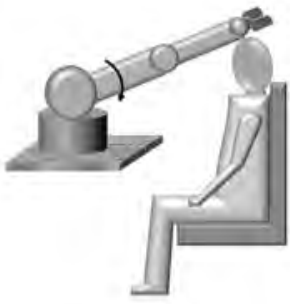

(a)

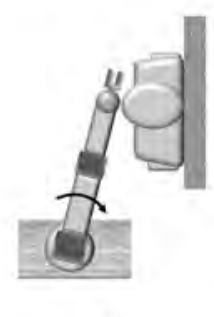

(b)
Figure 1. Illustration of the collision scenarios being studied. a) Head impact caused by robot's shoulder joint movement. b) Head impact caused by robot's waist joint movement.

The robot is assumed to be a standard articulated arm with six or seven motorized rotary joints. The impact may be caused by either the rotation of the robot's shoulder joint (Fig. 1a) or its waste joint (Fig. 1b). The elbow joint is assumed to be infinitely stiff which is a conservative assumption.

\section{HUMAN-ROBOT IMPACT MODELING}

As is common practice in the literature [3][4][10][11][12], the human-robot impact dynamics in the normal direction are modelled by a linear one dimensional lumped parameter model consisting mainly of masses and springs. Fig. 2 shows the schematic for the linear robot-head impact model. The actuator force (commanded by the controller), equivalent masses of the actuator and link are included. The mass of the head is shown but not included in our equations since it is constrained. Knowing the elastic modulus and contact area of the compliant material, the compliant cover can be modeled as a spring acting between the link and head. The human head's stiffness is also modelled as a spring. Its value for robot-head contact is included in the international technical specification for collaborative robots [12]. Including this spring makes the model more realistic than previous models that assumed the skull is perfectly rigid. Displacement due to transmission deflection adds one degree of freedom to the model which is shown as spring acting between the actuator position and the robotic arm position. Most existing robots use a low-backlash high-precision transmission at each joint which has a high stiffness compared to the controller's stiffness (due to feedback). Thus the transmission spring can be reasonably neglected, making the actuator and links act as a single mass. Fig. 3 shows the simplified model that is employed in the remainder of this paper. is:

The conventional position controller equation for the joint

$$
\tau_{\text {cont }}=K_{p}\left(\theta_{d}-\theta_{r}\right)+K_{d}\left(\dot{\theta}_{d}-\dot{\theta}_{r}\right)+I_{r} \ddot{\theta}_{d}
$$

subject to:

$$
\left|\tau_{\text {cont }}\right| \leq \tau_{\max }
$$

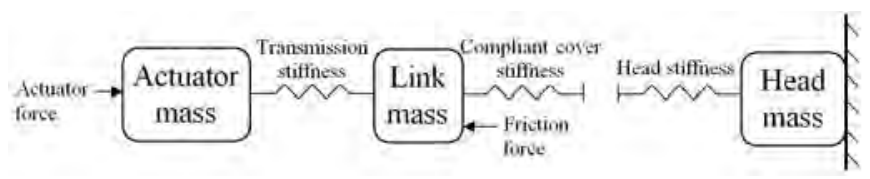

Figure 2. Linear robot link-head blunt constrained impact model. 


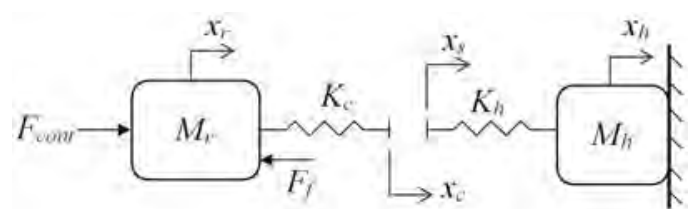

Figure 3. Simplified model for robot link-head blunt constrained impact.

where $\tau_{\text {cont }}$ is the joint torque to be applied by the actuator; $\tau_{\max }$ is the maximum actuator torque; $\theta_{r}$ is the joint angle; $\theta_{d}$ is the desired angle; $K_{p}$ and $K_{d}$ are the proportional and derivative feedback gains respectively; $I_{r}$ is the combined inertias of the actuator and links; and $I_{r} \ddot{\theta}_{d}$ is the feedforward term used to compensate the inertial torque. Assuming the post-impact deflections are small, the equivalent controller force in the normal direction at the point of contact is:

$$
F_{\text {cont }}=K_{\text {cont }}\left(x_{d}-x_{r}\right)+C_{\text {cont }}\left(\dot{x}_{d}-\dot{x}_{r}\right)+M_{r} \ddot{x}_{d}
$$

where $K_{\text {cont }}=K_{p} / L^{2}, C_{\text {cont }}=K_{d} / L^{2}, M_{r}=I_{r} / L^{2}, x_{d}=\theta_{d} L$, $x_{r}=\theta_{r} L$ and $L$ is the distance from the impact point to the joint. If all displacements are relative to $x_{h}$, the robot's equation of motion is:

$$
M_{r} \ddot{x}_{r}=F_{c o n t}-F_{f}-F_{i}
$$

where $F_{f}$ is the friction force and $F_{i}$ is the impact force. The impact force is given by:

$$
F_{i}=\left\{\begin{array}{cc}
K_{h c} x_{r} & x_{r}>0 \\
0 & x_{r} \leq 0
\end{array}\right.
$$

where:

$$
K_{h c}=K_{h} K_{c} /\left(K_{h}+K_{c}\right)
$$

Substituting (3) and (5) into (4) and rearranging gives:

$$
M_{r} \ddot{x}_{r}+C_{r} \dot{x}_{r}+K_{r} x_{r}=M_{r} \ddot{x}_{d}+C_{\text {cont }} \dot{x}_{d}+K_{\text {cont }} x_{d}-F_{f}
$$

where:

$$
\begin{gathered}
C_{r}=C_{c o n t} \\
K_{r}=\left\{\begin{array}{ll}
K_{h c}+K_{c o n t} & x_{r}>0 \\
K_{c o n t} & x_{r} \leq 0
\end{array}\right. \text { and }
\end{gathered}
$$

The friction force is modelled as the sum of dry and viscous components as follows:

$$
F_{f}=\left\{\begin{array}{c}
f_{k} \operatorname{sign}\left(\dot{x}_{r}\right)+k_{v} \dot{x}_{r} \quad \dot{x}_{r} \neq 0 \\
F_{\text {cont }}-F_{i} \quad \dot{x}_{r}=0 \wedge\left|F_{\text {cont }}-F_{i}\right|<f_{s} \\
f_{s} \operatorname{sign}\left(F_{\text {cont }}-F_{i}\right) \quad \text { otherwise. }
\end{array}\right.
$$

where $f_{k}$ is the kinetic friction force, $f_{s}$ is the static friction force and $k_{v}$ is the coefficient of viscous friction.

Lastly, the deflections of the compliant cover and head may be calculated using (11) and (12), respectively.

$$
\begin{gathered}
\Delta x_{c}=x_{c}-x_{r}=F_{i} / K_{c} \text { and } \\
x_{s}=F_{i} / K_{h}
\end{gathered}
$$

\section{CONTROLLER DESIGN}

The position controller can be designed for trajectory tracking by specifying the desired closed-loop bandwidth, $f_{b w}$, and damping ratio, $\zeta$. The corresponding controller gains are given by:

$$
\begin{gathered}
K_{p}=I_{r} \omega_{n}{ }^{2} \\
K_{d}=2 \zeta \sqrt{K_{p} I_{r}}
\end{gathered}
$$

where:

$$
\omega_{n}=2 \pi f_{b w} /\left(\zeta+\sqrt{1+\zeta^{2}}\right)
$$

The proposed feedback limited (FBL) controller is obtained by replacing (1) with:

$$
\begin{gathered}
\tau_{\text {cont }}=\tau_{f b}+\tau_{f f} \\
\tau_{f b}=K_{p}\left(\theta_{d}-\theta_{r}\right)+K_{d}\left(\dot{\theta}_{d}-\dot{\theta}_{r}\right) \text { and } \\
\tau_{f f}=I_{r} \ddot{\theta}_{d}
\end{gathered}
$$

subject to (2) and:

$$
\left|\tau_{f b}\right| \leq \tau_{\text {lim }}
$$

where $\tau_{\text {lim }}$ is the feedback torque limit.

\section{Simulations}

Simulations were created to compare the position control performance and impact forces produced under a variety of conditions. The simulated robot is a Universal Robot model UR5 which is one of the most common collaborative robots in use today. The robot's commanded trajectory is defined such that it collides with the head at a velocity of $0.25 \mathrm{~m} / \mathrm{s}$ which is the highest speed permitted by the Canadian [13] and international safety standards [14] when a human is within reach of a robot arm. Two different sets of controller gains $K_{p}$ and $K_{d}$ were used, one for a high bandwidth, high stiffness controller (HSC) and the other for a lower stiffness, lower bandwidth controller (LSC). The two bandwidths for the HSC and LSC are $50 \mathrm{~Hz}$ and $5 \mathrm{~Hz}$, respectively. The corresponding stiffnesses are $355 \mathrm{kN} / \mathrm{m}$ and $3.55 \mathrm{kN} / \mathrm{m}$, respectively. The other simulation parameters are listed in Table I.

Several impact conditions have been simulated and comparisons made. The HSC and HSC were simulated with and without compliant cover (CC). The other impact force reduction strategies simulated are: limiting the controller feedback force applied to the link (FBL); switching off the actuator when the impact is detected (SOA); and implementing an arm withdrawal reflex (AWR) by applying the maximum actuator torque in the opposite direction. The impact detection 
TABLE I. SIMULATION PARAMETERS

\begin{tabular}{|c|c|c|}
\hline Parameter & Value & Description \\
\hline$d t$ & $0.01 \mathrm{~ms}$ & Integration timestep \\
\hline$f_{k}$ & $19.4 \mathrm{~N}$ & Kinetic friction force ${ }^{a}$ \\
\hline$f_{s}$ & $19.4 \mathrm{~N}$ & Static friction force ${ }^{\mathrm{a}}$ \\
\hline$K_{c}$ & $25 \mathrm{kN} / \mathrm{m}$ & Compliant cover stiffness [11] \\
\hline$K_{h}$ & $150 \mathrm{kN} / \mathrm{m}$ & Head stiffness [12] \\
\hline$k_{v}$ & $0.554 \mathrm{Ns} / \mathrm{m}$ & Coefficient of viscous friction \\
\hline$L$ & $0.85 \mathrm{~m}$ & $\begin{array}{l}\text { Distance from joint to impact } \\
\text { location on the robot }[16\end{array}$ \\
\hline$M_{r}$ & $9.57 \mathrm{~kg}$ & Equivalent robot mass ${ }^{\mathrm{c}}$ \\
\hline$T_{d}$ & $5 \mathrm{~ms}$ & Impact detection delay \\
\hline$T_{s}$ & $1 \mathrm{~ms}$ & Controller sampling period \\
\hline$\zeta$ & 0.7 & Controller damping ratio \\
\hline$\tau_{\text {lim }}$ & $20 \mathrm{Nm}$ & Controller feedback limit \\
\hline$\tau_{\max }$ & $150 \mathrm{Nm}$ & Maximum joint torque [15] \\
\hline
\end{tabular}

delay for the AWR was assumed to be $5 \mathrm{~ms}$. The robot was simulated tracking a trapezoidal velocity trajectory before collision and the tracking root-mean-squared-error (RMSE) values were computed. To make the tracking more realistic the friction coefficients were subjected to a $5 \%$ random variation from the nominal. Table II lists the results for the cases simulated.

The International Organization for Standardization (ISO) specifies $130 \mathrm{~N}$ as the maximum permissible force for the skull and forehead. Studying the results in the table, it can be observed that the robot with HSC and no CC, FBL or AWR exerted an impact force of $410 \mathrm{~N}$ (case 1), well beyond the ISO safety limit. This $410 \mathrm{~N}$ value is used as the benchmark for comparison. Using the LSC reduced the impact force $24 \%$ to $313 \mathrm{~N}$ (case 9), while adding a carefully selected limit with the
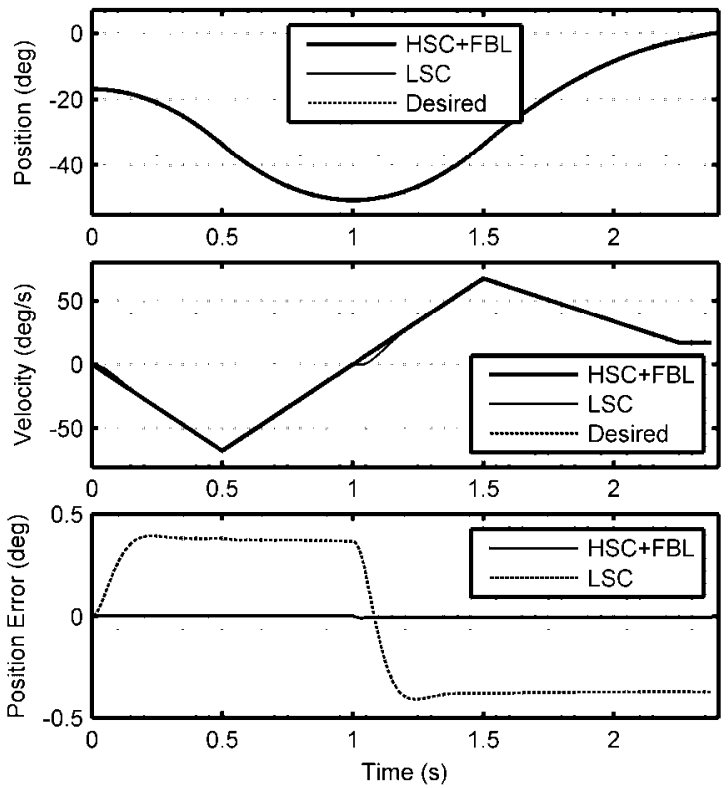

Figure 4. Comparison of the joint's motion tracking performances before impact for higher stiffness controller with feedback limit (HSC+FBL) and the lower stiffness controller (LSC).
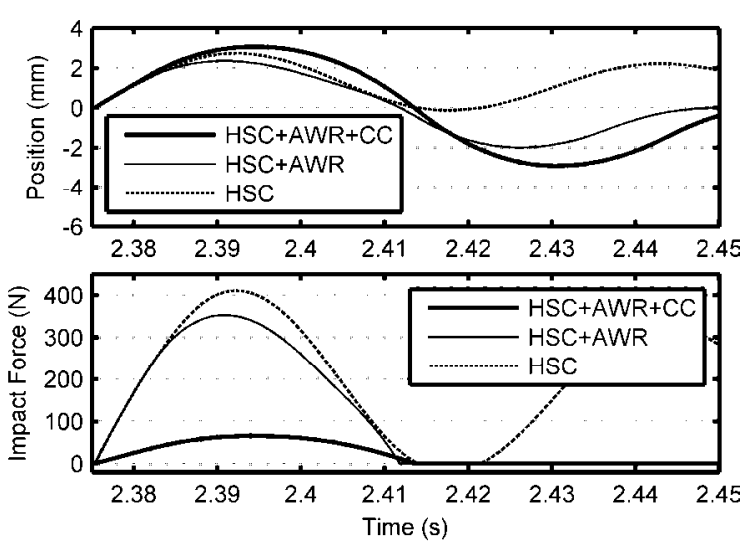

Figure 5. Post-impact link tip position and impact force for the high stiffness controller with different features: with no impact reduction feature (HSC), with arm withdrawal reflex (HSC+AWR), and with arm withdrawal reflex and compliant cover (HSC+AWR+CC).

FBL strategy reduced the force $26 \%$ to $303 \mathrm{~N}$ (case 2). Although these two strategies have a similar effect on the impact force, the RMSE before the impact is $99 \%$ smaller when FBL is used (case 2 vs. case 9). Fig. 4 compares the tracking performance for these two cases. The larger position and velocity errors with LSC are obvious in this figure. Adding $\mathrm{CC}$ to the robot with HSC reduced the impact force $36 \%$ (case 5) and when combined with FBL, the impact force reduction is $72 \%$ (case 6) and the force of $117 \mathrm{~N}$ is less than the ISO limit.

For the uncovered robot with HSC, SOA (case 3) and AWR (case 4) produced similar forces to FBL (case 2) since the impact force rises so fast that it is near its peak before the impact is detected by the SOA and AWR strategies. However when $\mathrm{CC}$ is used with HSC the force rises more slowly, allowing SOA (case 7) and AWR (case 8) to reduce the impact force by $75 \%$ and $84 \%$, respectively, to values well within the ISO safety limit. Fig. 5 compares the effectiveness of AWR strategy with and without $\mathrm{CC}$ to the benchmark case (without any force reduction method) for the HSC. With the benchmark case, in addition to large forces it can be observed that repeated impacts occur.

Regarding the LSC, cases 9-16 show a similar pattern to the corresponding HSC cases 1-8. Fig. 6 shows the results for the
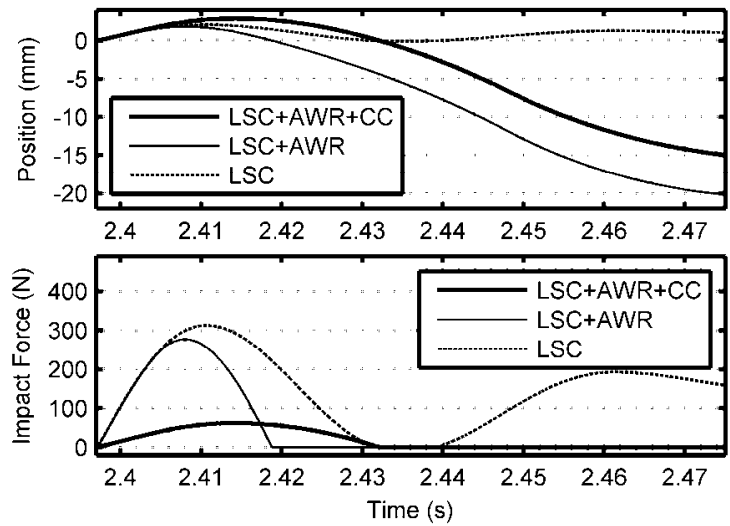

Figure 6. Post-impact link tip position and impact force for the low stiffness controller with different features: with no impact reduction feature (LSC), with arm withdrawal reflex (LSC+AWR), and with arm withdrawal reflex and compliant cover (LSC+AWR+CC). 
LSC and the same combinations of AWR and CC as in Fig. 5. Compared with Fig. 5, the same improvement can be observed when $\mathrm{CC}$ is added, with the main difference being the arm is withdrawn much further back after the impact when using the LSC. From these figures and the tabulated results it can be observed that the LSC provides no significant safety advantage when $\mathrm{CC}$ and AWR are used together.

\section{CONCLUSION}

Several strategies for reducing the impact force caused by the collision of a robot arm with a constrained human head were investigated. The effect of the strategies on the robot's trajectory tracking precision during normal operation was also studied. An improved lumped parameter model for the constrained impact was proposed for simulating the collisions. Sixteen combinations of impact reduction strategies were simulated and compared for a UR5 collaborative robot. Compared to the uncovered robot with the HSC, the LSC (with $1 \%$ of the HSC's stiffness) only made the impact force slightly smaller, and made the tracking errors much larger. The SOA and AWR strategies were effective when CC was used, but have the disadvantage of requiring rapid impact detection. Combining the proposed FBL strategy with HSC and $\mathrm{CC}$ reduced the impact force to less than the ISO safety limit and achieved precise trajectory tracking. This combination also does not require any impact detection.

\section{REFERENCES}

[1] J. Heinzmann and A. Zelinsky, "Quantitative safety guarantees for physical human-robot interaction," Int. J. Rob. Res., vol. 22, no. 7-8, pp.479-504, 2003. doi: $10.1177 / 02783649030227004$.

[2] G. Hirzinger, A. Albu-Schäffer, M. Hähnle, I. Schaefer, and N. Sporer, "On a new generation of torque controlled light-weight robots," in Proc. IEEE Int. Conf. Robot. Automat., 2001, pp. 3356-3363. doi: 10.1109/ROBOT.2001.933136.

[3] A. Bicchi and G. Tonietti, "Fast and 'Soft-Arm' Tactics," IEEE Robot. Autom. Mag., vol. 11, no. 2, pp. 22-33, Jun. 2004. doi: 10.1109/MRA.2004.1310939.

[4] D. Hyun, H. S. Yang, J. Park, and Y. Shim, "Variable stiffness mechanism for human-friendly robots," Mech. Mach. Theory, vol. 45, no. 6, pp. 880-897, 2010. doi: 10.1016/j.mechmachtheory.2010.01.001.

[5] N. Lauzier and C. Gosselin, "Performance indices for collaborative serial robots with optimally adjusted series clutch actuators," J. Mech. Robot., vol. 4, no. 2, p. 21002, 2012. doi: 10.1115/1.4005723.

[6] M. Moghani and M. R. Kermani, "Design and development of a hybrid magneto-rheological clutch for safe robotic applications," in Proc. IEEE Int. Conf. Robot. Automat., 2016, pp. 3083-3088. doi: 10.1109/ICRA.2016.7487474.

[7] K. Suita, Y. Yamada, N. Tsuchida, K. Imai, H. Ikeda, and N. Sugimoto, "A failure-to-safety' Kyozon' system with simple contact detection and stop capabilities for safe human-autonomous robot coexistence," in Proc. IEEE Int. Conf. Robot. Automat., 1995, vol. 3, pp. 3089-3096. doi: 10.1109/ROBOT.1995.525724.

TABLE II. SIMULATION RESULTS

\begin{tabular}{|c|c|c|c|c|c|c|c|c|}
\hline $\begin{array}{c}\text { Case } \\
\text { Number }\end{array}$ & $\begin{array}{c}\text { Controller Stiffness, } \\
K_{\text {cont }}(\mathrm{kN} / \mathrm{m}) \\
\end{array}$ & $\begin{array}{c}\text { Controller } \\
\text { Bandwidth }(\mathrm{Hz})\end{array}$ & $\begin{array}{c}\text { Switch Off } \\
\text { Actuator }\end{array}$ & $\begin{array}{c}\text { Arm } \\
\text { Drawback }\end{array}$ & $\begin{array}{c}\text { Compliant } \\
\text { Cover }^{\mathrm{a}}\end{array}$ & $\begin{array}{l}\text { Limit on } \\
\text { Feedback } \\
\end{array}$ & $\begin{array}{c}\text { Tracking } \\
\text { RMSE (deg) }\end{array}$ & $\begin{array}{c}\text { Peak Impact } \\
\text { Force }(\mathrm{N})\end{array}$ \\
\hline 1 & 355 & 50 & No & No & No & No & $3.73 \mathrm{E}-3$ & 410 \\
\hline 2 & 355 & 50 & No & No & No & Yes & $3.79 \mathrm{E}-3$ & 303 \\
\hline 3 & 355 & 50 & Yes & No & No & No & $3.73 \mathrm{E}-3$ & 329 \\
\hline 4 & 355 & 50 & No & Yes & No & No & $3.73 \mathrm{E}-3$ & 353 \\
\hline 5 & 355 & 50 & No & No & Yes $(10.5 \mathrm{~mm})$ & No & $3.73 \mathrm{E}-3$ & 264 \\
\hline 6 & 355 & 50 & No & No & Yes $(4.7 \mathrm{~mm})$ & Yes & $3.79 \mathrm{E}-3$ & 117 \\
\hline 7 & 355 & 50 & Yes & No & Yes $(4.1 \mathrm{~mm})$ & No & $3.73 \mathrm{E}-3$ & 103 \\
\hline 8 & 355 & 50 & No & Yes & Yes $(2.6 \mathrm{~mm})$ & No & $3.73 \mathrm{E}-3$ & 66 \\
\hline 9 & 3.55 & 5 & No & No & No & No & 0.362 & 313 \\
\hline 10 & 3.55 & 5 & No & $\mathrm{No}$ & No & Yes & 0.405 & 303 \\
\hline 11 & 3.55 & 5 & Yes & No & No & No & 0.362 & 299 \\
\hline 12 & 3.55 & 5 & No & Yes & No & No & 0.362 & 277 \\
\hline 13 & 3.55 & 5 & No & No & Yes $(5.3 \mathrm{~mm})$ & No & 0.362 & 132 \\
\hline 14 & 3.55 & 5 & No & No & Yes $(4.7 \mathrm{~mm})$ & Yes & 0.405 & 116 \\
\hline 15 & 3.55 & 5 & Yes & No & Yes $(4.1 \mathrm{~mm})$ & No & 0.362 & 102 \\
\hline 16 & 3.55 & 5 & No & Yes & Yes $(2.5 \mathrm{~mm})$ & No & 0.362 & 62 \\
\hline
\end{tabular}

a. Maximum compliant cover deflections during impact are shown in brackets. 


\section{REFERENCES}

[8] A. De Luca, A. Albu-Schaffer, S. Haddadin, and G. Hirzinger, "Collision detection and safe reaction with the DLR-III lightweight manipulator arm," in Proc. IEEE/RSJ Int. Conf. on Intell. Robots and Syst., 2006, pp. 1623-1630.

[9] S. Jeong and T. Takahashi, "Impact force reduction of manipulators using a dynamic acceleration polytope and flexible collision detection sensor," Adv. Robot., vol. 23, no. 3, pp. 367-383, 2009.

doi: $10.1163 / 156855308 \times 397578$.

[10] S. Haddadin, T. Laue, U. Frese, S. Wolf, A. Albu-Schäffer, and G.Hirzinger, "Kick it like a safe robot: Requirements for 2050," Robot. Auton. Syst., vol. 57, pp. 761-775, 2009. doi: 10.1016/j.robot.2009.03.004.

[11] L. Zeng and G. M. Bone, "Design of elastomeric foam-covered robotic manipulators to enhance human safety," Mech. Mach. Theory, vol. 60, pp. 127, 2013. doi: 10.1016/j.mechmachtheory.2012.09.010.
[12] ISO/TS 15066:2016, Robots and robotic devices - Collaborative robots. International organization for standardization, 2016.

[13] CAN/CSA-Z434-14 - Industrial robots and robot systems. Canadian Standards Association, 2014.

[14] ISO 10218-1:2011, Robots for industrial environments - Safety requirements - Part I: Robot. International organization for standardization,2011.

[15] K. Kufieta, "Force Estimation in Robotic Manipulators: Modeling, Simulation and Experiments, UR5 as a case study," Diploma Thesis, Norwegian University of Science and Technology, Department of Engineering Cybernetics, Norway, Jan. 29, 2014.

[16] P. M. Kebria, S. Al-wais, H. Abdi and S. Nahavandi, "Kinematic and dynamic modelling of UR5 manipulator," in Proc. IEEE Int. Conf. Syst., Man, and Cyber., 2016, pp. 4239-4234. doi: 10.1109/SMC.2016.7844896. 


\title{
Estimation and Control of a Double-Inverted Pendulum
}

\author{
Bowen $\mathrm{Xu}^{*}$, You Lyu, and S. A. Gadsden \\ College of Engineering and Physical Sciences, \\ University of Guelph, Ontario, Canada \\ *bxu04@uoguelph.ca
}

\begin{abstract}
This paper studies estimation and control methods applied on a double-inverted pendulum. The most popular estimation strategy, referred to as the Kalman filter (KF), was programmed and implemented on a double-inverted pendulum built by Quanser (Markham, Ontario). A linear quadratic regulator (LQR) was used to control the actuator joints of the double-inverted pendulum to keep it vertically balanced under different conditions. The application of the KF improved the tracking performance. The results of the paper are discussed, and future work is considered.
\end{abstract}

Keywords-double inverted pendulum; control system; Kalman filter; estimation.

\section{INTRODUCTION}

The double-inverted pendulum is one of the most popular mechatronic research systems used to study control and estimation strategies. The system itself is relatively easy to build, and is naturally unstable which makes it quite popular to study. The complex dynamics of a double-inverted pendulum offers a system suitable for the development and examination of new control and estimation methodologies. The findings can be applied to problems in current research, such as holding the balance of a robot or rotor-based aircraft [1]. In order to improve the control performance of the system, estimation strategies can be used to provide better knowledge of the states [1].

State and parameter estimation theory is an important field in mechanical and electrical engineering. The strategies are used to predict, estimate, or smooth out important system state and parameters [2,3]. The Kalman filter (KF) is the most popular estimation strategy, and yields a statistically optimal solution to the linear estimation problem [4]. The goal of the KF is to minimize the state error covariance, which is a measure of the estimation accuracy and is defined as the expectation of the state error squared [5]. A popular nonlinear form of the Kalman filter is the unscented Kalman filter (UKF). It utilizes statistics to approximate the nonlinear probability density function (PDF) [6]. These filters can be applied on mechanical or electrical systems to improve the overall control tracking performance [4].

The brief paper is organized as follows. In Section II, the linear-quadratic regulator (LQR) controller, the standard KF, and the MATLAB simulation is summarized. The application results are provided and discussed in Section III. Conclusions and future work are considered in the final section of the paper.

\section{METHODOLOGY}

\section{A. Model and Linear-quadratic regulator (LQR) control}

The double-inverted pendulum has a complex dynamic system [7]. In order to improve the control performance (e.g., balancing the system dynamics), a linear-quadratic regulator control strategy is applied in this paper. Quanser (Markham, Ontario) built the double-inverted pendulum system used in this paper [7]. The results of this paper were built upon this platform and the provided software. The first step of applying the LQR control strategy is to obtain the state-space model of the system. The motion equations may be obtained by using the following Euler-Lagrange equation, as follows [7]:

$$
Q_{i}=\frac{\partial L}{\partial t \partial_{\dot{q} 1}}-\frac{\partial L}{\partial q_{i}}
$$

The generalized coordinate $q_{i}$ may be found as follows [7]:

$$
\begin{aligned}
& q(t)^{T}=\left[\begin{array}{lll}
x_{c}(t) & \alpha(t) & \theta(t)
\end{array}\right] \\
& \dot{q}(t)^{T}=\left[\begin{array}{lll}
\frac{\partial x_{c}(t)}{\partial t} & \frac{\partial \alpha(t)}{\partial t} & \frac{\partial \theta(t)}{\partial t}
\end{array}\right]
\end{aligned}
$$

where the above parameters are defined as follows [7]:

$$
\begin{aligned}
& Q_{1}=\frac{\partial^{2} L}{\partial t \partial \dot{x}_{C}}-\frac{\partial L}{\partial x_{c}} \\
& Q_{2}=\frac{\partial^{2} L}{\partial t \partial \dot{\alpha}}-\frac{\partial L}{\partial \alpha} \\
& Q_{3}=\frac{\partial^{2} L}{\partial t \partial \dot{\theta}}-\frac{\partial L}{\partial \theta}
\end{aligned}
$$

The Lagrange (or energy) equation for the double-inverted pendulum system is defined by:

$$
L=T-V
$$

In equation (7), $T$ refers to the kinetic energy of the doubleinverted pendulum system, $V$ is the total potential energy of the double-inverted pendulum system. According to equations (4) through (7), the generalized forces of the system may be found as follows [7]:

$$
\begin{gathered}
Q_{1}=F_{c}-B_{e q} \dot{x_{c}} \\
Q_{2}=-B_{p 1} \dot{\alpha} \\
Q_{3}=-B_{p 2} \dot{\theta}
\end{gathered}
$$


After obtaining the nonlinear motion equations, a Jacobian matrix is calculated and the linear state-space model may be found as follow:

$$
\begin{aligned}
& \dot{x}=A x+B u \\
& y=C x+D u
\end{aligned}
$$

where $x$ refers to states, $u$ is the input from the motor, $A$ is the system matrix, $B$ is the input matrix, $C$ is the measurement matrix, and $D$ is the feedforward matrix. The state and output equations are respectively as follows:

$$
\begin{gathered}
x^{T}=\left[\begin{array}{llllll}
x_{c} & \alpha & \beta & \dot{x}_{c} & \dot{\alpha} & \dot{\theta}
\end{array}\right] \\
y^{T}=\left[\begin{array}{lllll}
x_{1} & & x_{2} & & x_{3}
\end{array}\right]
\end{gathered}
$$

After obtaining the linear state-space matrices, the LQR controller can be implemented. The LQR control strategy can provide full feedback control of the system [4]. The LQR equation is defined by [7]:

$$
J=\int_{0}^{\infty} x(t)^{\prime} Q x(t)+u(t)^{\prime} R u(t) d t
$$

In equation (15), $Q$ and $R$ are system and measurement noise covariances, respectively:

$$
Q=\left[\begin{array}{ccccccc}
10 & 0 & 0 & 0 & 0 & 0 & 0 \\
0 & 50 & 0 & 0 & 0 & 0 & 0 \\
0 & 0 & 50 & 0 & 0 & 0 & 0 \\
0 & 0 & 0 & 0 & 0 & 0 & 0 \\
0 & 0 & 0 & 0 & 0.9 & 0 & 0 \\
0 & 0 & 0 & 0 & 0 & 0.9 & 0 \\
0 & 0 & 0 & 0 & 0 & 0 & 1
\end{array}\right]
$$

In order to reduce regulation error of the linear cart, an integral term is added:

$$
\begin{aligned}
& \eta=\left[\begin{array}{cc}
A & 0 \\
1 & 0
\end{array}\right] \eta+\left[\begin{array}{c}
B \\
0
\end{array}\right] u \\
& {\left[\begin{array}{lllllll}
x_{c}-x_{c, d} & \alpha & \theta & \dot{x}_{c} & \dot{\alpha} & \dot{\theta} & \int\left(x_{c}-x_{c, d}\right) d t
\end{array}\right]}
\end{aligned}
$$

According to the control law, the equation of control feedback of this double-inverted pendulum may be found as follows:

$$
u=-K(\eta)
$$

There are three important model characterizations for control of the double-inverted pendulum: observability, controllability, and stability. Observability of a system states can be observed from the system output [8]. In this paper, the system is observable but not completely observable because the measurement matrix is a non-square matrix. The dynamic system is said to be controllable which means for any desired final state, the system can be driven by the input [8]. In this paper, by calculating the rank of the controllability matrix, the controllability can be proven. The system is stable if every bounded input produces a bounded output (principle of BIBO stability). In this paper, the stability was calculated using Maple, because some poles are positive, the system is considered to be unstable [9].

\section{B. Kalman Filter and Matlab Simulink}

For the control of the double-inverted pendulum, the cart position, and both pendulum angles are measured. According to the measurements, a voltage is determined that is used by the control system to actuate the cart's motor. As a result, the cart balances the double inverted pendulum and keeps the upright, vertical position.

For the software, the programs Matlab and Simulink were used. For this system, the controller could only be implemented in Simulink, whereas the programming environment of Matlab offers more possibilities. The workstation provides specialized Simulink blocks to communicate with the pendulum control. In real-time, the measurements of the position and angles are processed to control the motor by setting the voltage (as per the Figure 1). Simulink was used as the interface with the workstation, and Matlab as the data processor and background algorithms. The goal of this setup was to go beyond the setup provided commercially by Quanser in [7], and create a setting that enables real-time signal processing and the capability to add features over the time without losing the overview.

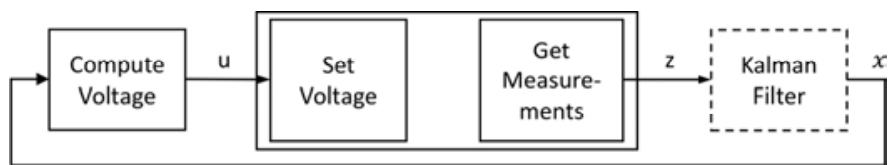

Figure 1. Control loop of the double-inverted pendulum.

\section{Implementation of the Kalman Filter}

For the Kalman filter, a linear, discrete-time, and timeinvariant system model is used [10]:

$$
\begin{gathered}
x_{k+1}=A x_{k}+B u_{k}+w_{k} \\
z_{k+1}=C x_{k+1}+v_{k+1}
\end{gathered}
$$

The Kalman filter is based on two stages: prediction and update. In each stage, the state vector and its covariance matrix are calculated. To simplify the notation, we write for the expected value of the a-priori density of the prediction as follows:

$$
E\left\{x_{k+1} \mid z_{k}\right\}=\dot{x}_{k+1}
$$

For the expected value of the a-posteriori density of the update as follows:

$$
E\left\{x_{k+1} \mid z_{k}\right\}=\hat{x}_{k+1}
$$

The estimation criterion is the minimization of the square norm if the estimation error:

$$
E\left\{\left|\hat{x}(n)-x(n)^{2}\right| z(n)\right\} \rightarrow \min
$$

With the linearized matrices of the dynamic coefficient matrix $A$, the coupling matrix $B$, and the measurement matrix $C$, we can use the extended Kalman filter (EKF) to decrease our measurement deviation. For the computation, the equations are shown below [11]:

Prediction stage:

$$
\begin{gathered}
\hat{x}_{k+1 \mid k}=A \hat{x}_{k \mid k}+B u_{k} \\
P_{k+1 \mid k}=A P_{k \mid k} A^{T}+Q_{k}
\end{gathered}
$$


Update stage:

$$
\begin{gathered}
K_{k+1}=P_{k+1 \mid k} C^{T}\left(C P_{k+1 \mid k} C^{T}+R_{k+1}\right)^{-1} \\
\hat{x}_{k+1 \mid k+1}=\hat{x}_{k+1 \mid k}+K_{k+1}\left[z_{k+1 \mid k}-C \hat{x}_{k+1 \mid k}\right] \\
P_{k+1 \mid k+1}=\left(I-K_{k+1} C\right) P_{k+1 \mid k}
\end{gathered}
$$

The unscented Kalman filter (UKF) belongs to the group of sigma point Kalman filter, which uses a weighted statistical linear regression strategy that approximates the nonlinear model statistically [6].

\section{Implementation of the Developed Interface}

The Kalman filters are implemented in a Matlab block in Simulink. The advantage is its simplicity. The block has to be created, the function to be written and the connection of inputs and outputs to be made. The drawbacks are the computation time that might be slower in comparison with the usage of Simulinkonly state space blocks. Nevertheless, the Matlab function block can be used. The value of the gain 'FilterChoice' can be modified using the user interface. With the if-operation and the modification of the choice input, different code sections can be executed, performing different KF options. The voltage input for the KF is provided by a 'Data Store Read' block. If we would connect the input $u$ with the output of the voltage determination step, we would create an algebraic loop. Due to the discrete state space model, the computation has to be executed in a finite time step. By calling itself, an infinite circle is created making a discrete state space computation impossible. Due to this, instead of initiating the execution of the KF function by the arrival of the voltage input, we write the input $u$ in a 'Data Store Write' block and the value can be read out if the measurements initiate the execution of the block.

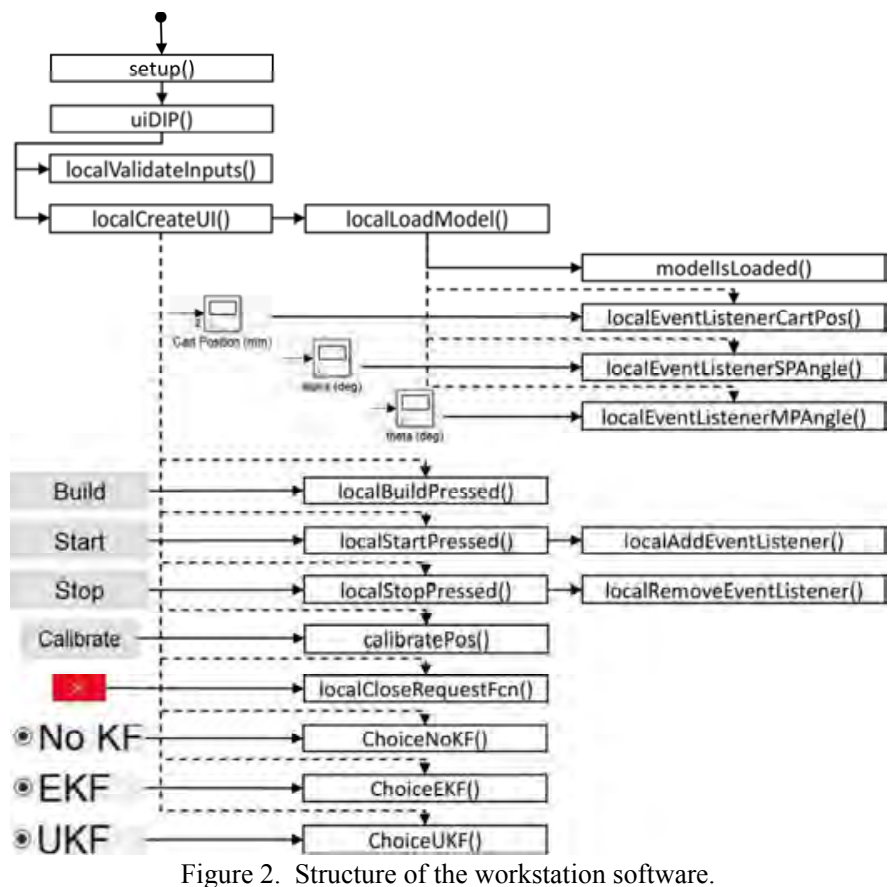

Matlab can modify the Simulink model and the user interface during the simulation by changing the parameters in Matlab's data structure. In the opposite way, Matlab obtains the data from
Simulink over event-listeners and from the user interface over callbacks. In order to accomplish this, the event listeners and callback functions have to be implemented first. The function 'localCreateUI' creates the figure of the user interface with all components and adds callback functions that are executed, each time the buttons are pressed. Additionally, this function also executes the function 'localLoadModel', which implements the event-listeners for the measurements of the cart position and the angles of the short and medium pendulum. Each time, a value arrives at one of the event-listener blocks, the event-listener function in Matlab is executed. By this approach, a bidirectional connection between the Simulink model and the user interface over the model is possible.

\section{SIMULATION RESULTS}

\section{A. Software Simulation}

The software only simulation is based on Matlab and Simulink. It provides software-based system running simulation for this double inverted pendulum system. All of the important parameters of the system such as output voltage, cart position, and pendulum angles are visible by real time graphs. Software only simulations can be roughly divided into ideal and non-ideal cases. Ideal types do not have system and measurement noises included in the simulation. While the non-ideal cases take all these factors in count and the results are closer to real system. In this paper, we test system performance for all these situations.

The system is built in Simulink and all components are represented by functional blocks. There are two key parts in the system design, namely LQR balance controller and the pendulum system. The pendulum system itself can be simulated in several ways. Regardless, using any method to simulate the pendulum system, there is only one input (motor voltage) for this system. The three output states are cart position and angles of the two pendulums.

\section{1) Ideal Case}

This specific simulation employs an ideal system model, which contains no noise interference. The results should be ideal inputs and output which will be considerably different from the hardware-connected case. However, this insight-view provides a convenient way to become familiar with the system and can benefit the configuration and optimization of the physical system. Simulink is the platform to perform this work. All of the parameters will be monitored in Simulink scopes. The Matlab file provides all the matrices and parameters of the system. The operation of the Simulink model is based on content loaded into Matlab's workspace. The pendulum system itself is represented by the state space model. Data flow for this target mainly contains the input, which is the voltage applied to the cart motor. The output are the three states of the system, cart position, angle of the first pendulum and the angle of the second pendulum, respectively. LQR balance control is the selected controller in this paper; however, the popular PID controller could also be used. The LQR part sends the calculated voltage values to control the motor motions. The simulation performed is set to contain two position values: the cart is supposed to stay at one place for ten seconds, then move to the second position and stay there for the next ten seconds, before returning to the original position. The simulation results are shown next. 


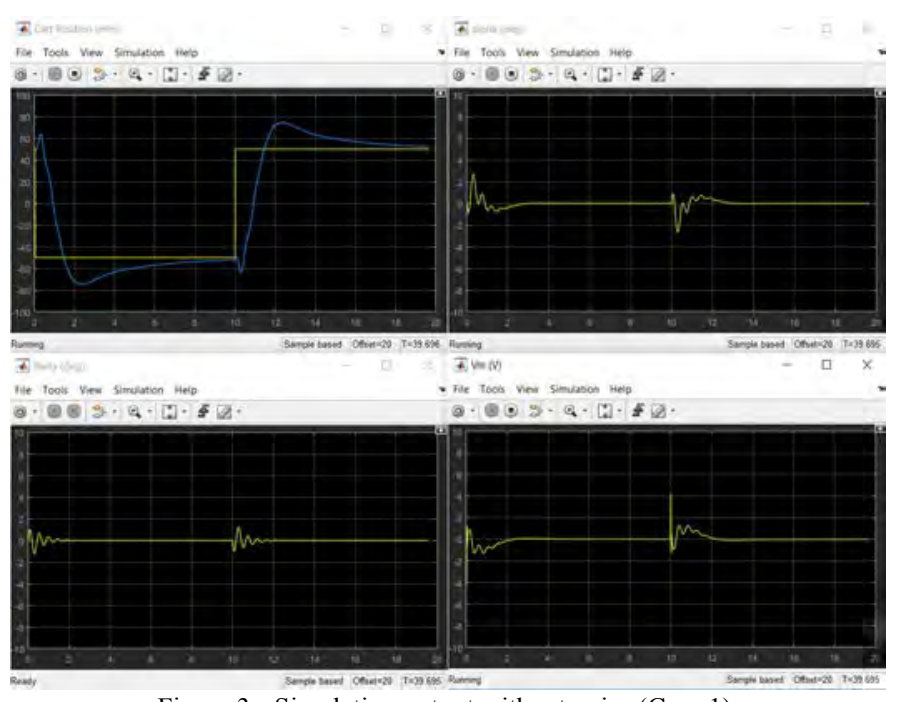

Figure 3. Simulation output without noise (Case 1).

In Figure 3(top-left), the yellow curve is the position setting and the blue curve is the cart output. The simulated cart moves in a rather smooth manner to reach the settling points. Compared with the yellow curve, the blue curve shows reasonable overshoot and low steady state error. The joint angles and voltage show small fluctuations, only occurs when the cart moves from one location to another.

\section{2) Noisy Measurement Case}

In this case, noisy measurements are considered and added to the simulation. In order to improve the control results, the Kalman filter was added to estimate the states. The noisy signal with filtered results are shown in Figure 4.

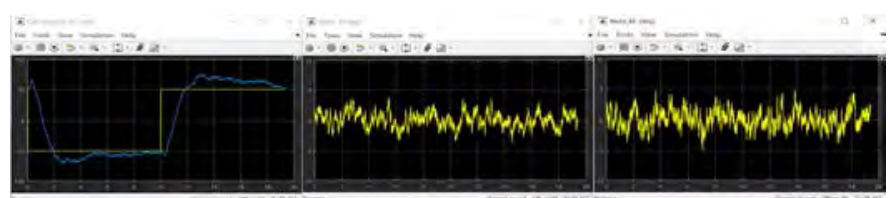

Figure 4. Simulation output with the KF (Case 2).

The results contain small fluctuations during the stabilization process of the cart (after quick position movements). The simulation shows that the steady-state error stays approximately at the same level compared with the ideal case. In contrast, the angles were more unstable. However, unlike the ideal case, the fluctuations never settle.

\section{3) System with Process and Measurement Noise}

In this case, the simulation contains both system and measurement noise. The cart position results without the addition of the KF shows significantly more fluctuations compared to the ideal case. The angles reveal the same trend as the cart position. In contrast, the addition of the KF shows more fluctuations which means the cart requires additional movements to keep the system balanced.

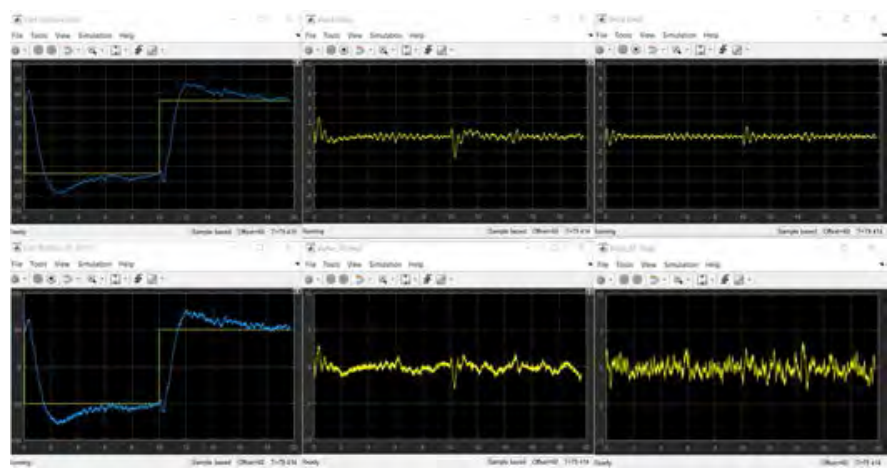

Figure 5. Simulation output with the KF (Case 3).

\section{B. Experimental System and Software Simulation}

This section focuses on the simulation with the pendulum. The motion and status of the real system is considerably different from the simulated type. The goal is to achieve and demonstrate the successful control of the double-inverted pendulum system.

\section{1) Normal Case}

In case, consider the system noise covariance matrix as per equation (16) and the measurement uncertainty covariance matrix as per equation (17). The normal case results are shown in the following figure.

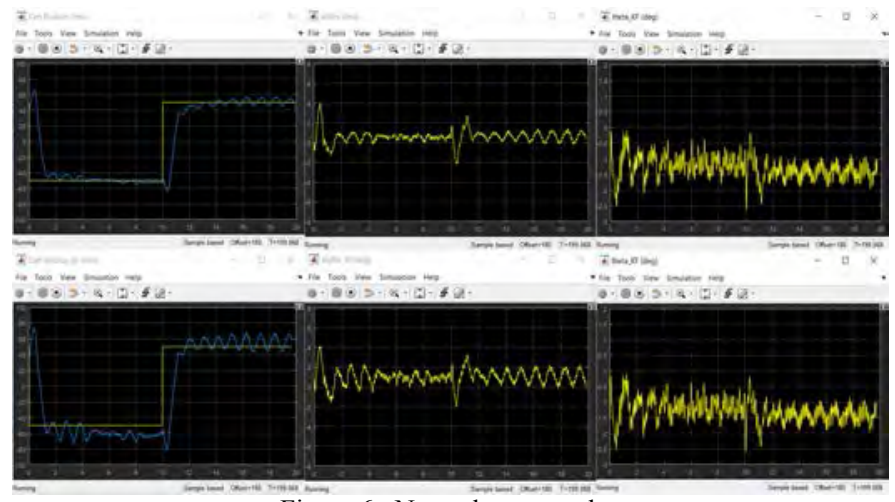

Figure 6. Normal case results.

The movement of the cart is fast and steady. The cart moves forward and backward in small scales to keep the balance of both pendulums. The angle values fluctuate as the cart moves to keep the whole system balanced. The addition of the KF improved the overall steady-state error of the system. Note also that the two angle states were quite similar in these two cases.

\section{2) Noisy Case}

The noisy system cases were simulated by increasing the noise covariance. The results are shown as per Figure 7. The system noise covariance matrix was changed as follows:

$$
Q=\left[\begin{array}{ccccccc}
30 & 0 & 0 & 0 & 0 & 0 & 0 \\
0 & 350 & 0 & 0 & 0 & 0 & 0 \\
0 & 0 & 100 & 0 & 0 & 0 & 0 \\
0 & 0 & 0 & 0 & 0 & 0 & 0 \\
0 & 0 & 0 & 0 & 0.5 & 0 & 0 \\
0 & 0 & 0 & 0 & 0 & 0.5 & 0 \\
0 & 0 & 0 & 0 & 0 & 0 & 1
\end{array}\right]
$$



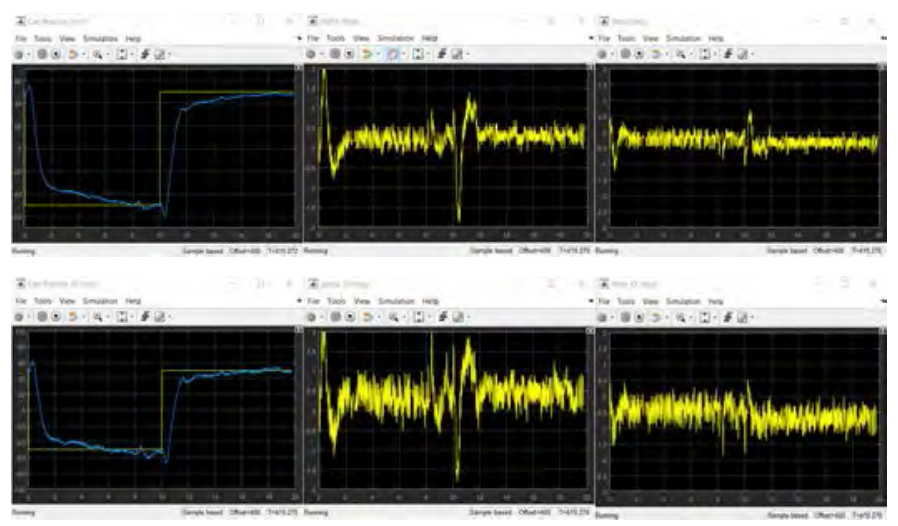

Figure 7. Noisy case results.

The cart moves quickly but in small movements in this case. The addition of the KF to the system improves the system performance, and the required settling time was decreased. It is important to note that both the LQR and the LQR with the KF methods were able to balance the double-inverted pendulum system. However, the LQR with KF method had improved response and faster settling time.

\section{CONCLUSIONS AND FUTURE WORK}

In this brief paper, the results of applying a controller and estimation strategy on a double-inverted pendulum were summarized and discussed. With well-defined parameters and a well-tuned KF, the system is able to overcome instabilities and external forces. The results demonstrate that the KF results are closer to the true values, which means that the LQR control strategy with the KF provides a better performance for the double-inverted pendulum system. Future work will look at implementing other control strategies, such as an adaptive PID, and comparing the results.

\section{REFERENCES}

[1] H. Wang and S. Jiang, "LQR control of single inverted pendulum based on square root filter," Adv. Mater. Res., vols. 433-440, pp. 6999-7003, 2012. doi: 10.4028/www.scientific.net/AMR.433-440.6999.

[2] B. D. O. Anderson and J. B. Moore, Optimal Filtering. Englewood Cliffs: Prentice-Hall, 1979.

[3] Y. Bar-Shalom, X. Rong Li, and T. Kirubarajan, Estimation with Applications to Tracking and Navigation. New York: John Wiley and Sons, Inc., 2001. doi: 10.1002/0471221279.

[4] S. A. Gadsden, "Smooth variable structure filtering: Theory and applications," Ph.D. Dissertation, McMaster Univ., Hamilton, Ontario, 2011.

[5] M. S. Grewal and A. P. Andrews, Kalman Filtering: Theory and Practice Using MATLAB, 3rd ed. New York: John Wiley and Sons, Inc., 2008. doi: 10.1002/9780470377819.

[6] S. A. Gadsden and A. S. Lee, "Advances of the smooth variable structure filter: Square-root and two-pass formulations," J. Appl. Remote Sens., vol. 11, no. 1, pp. 1-19, Mar. 2017. doi: 10.1117/1.JRS.11.015018.

\section{[7] Linear Motion Servo Plants: IP012 DBLP Laboratory}

Manual, Markham, Ontario: Quanser, 2017.

[8] B. Moore, "Principal component analysis in linear Systems: Controllability, observability, and model reduction," IEEE Trans. Autom. Control, vol. 26, no. 1, pp. 17-32, Feb. 1981. doi: 10.1109/TAC.1981.1102568.

[9] N. Nise, Control Systems Engineering, 4 ed. New York: John Wiley and Sons, Inc., 2004.

[10] S. A. Gadsden and S. R. Habibi, "A new form of the smooth variable structure filter with a covariance derivation," in 49th IEEE Conf. Decision and Control, Atlanta, GA, 2010, pp. 7389-7394. doi: 10.1109/CDC.2010.5717397.

[11] S. A. Gadsden and S. R. Habibi, "A new robust filtering strategy for linear systems," J. Dyn. Sys., Meas., Control, vol. 135, no. 1, p. 014503-014512, Oct. 2012. doi: 10.1115/1.4006628. 


\section{Comparison of Sliding Mode Controller and Linear Quadratic Regulator (LQR) on a Two-Wheel Inverted Pendulum Robot: Design and Experiments}

\author{
Mikail S. Arani \\ Department of Mechanical, \\ Industrial \& Aerospace \\ Engineering Concordia \\ University \\ Montreal, QC, Canada \\ m_shapoo@encs.concordia.
}

ca

\author{
Hamid Ebrahimi Orimi \\ Department of Mechanical, \\ Industrial \& Aerospace \\ Engineering Concordia \\ University \\ Montreal, QC, Canada \\ Ha_ebrah@encs.concordia.ca
}

\author{
Wen-Fang Xie \\ Department of Mechanical, \\ Industrial \& Aerospace \\ Engineering \\ Concordia University \\ Montreal, QC, Canada \\ wfxie@encs.concordia.ca
}

\author{
Henry Hong \\ Department of Mechanical, \\ Industrial \& Aerospace \\ Engineering \\ Concordia University \\ Montreal, QC, Canada \\ henry.hong@concordia.ca
}

\begin{abstract}
The current paper investigates two control methods for stabilizing a two-wheel inverted pendulum (TWIP) robot. It is well-known that the controller in the mobile robot plays a critical role in self-balancing and stabilizing. The TWIP robot has two DC gear motors with a high-resolution encoder and zero backlash, but with friction. It is a highly nonlinear and unstable system, which poses challenges for controller design. In this paper, a mathematical dynamic model is built using Lagrangian function method. Furthermore, a sliding mode controller (SMC) is proposed for auto-balancing and yaw rotation. A comparison of the proposed SMC controller and linear quadratic regulator (LQR) has been conducted. The experimental test results demonstrate the SMC controller outperforms (LQR) in terms of transient performance and disturbance rejection ability.
\end{abstract}

Keywords- Linear Quadratic Regulator (LQR), Sensor data fusion, Sliding mode control, Two-wheel inverted pendulum (TWIP) robot

\section{INTRODUCTION}

In recent years, self-balancing robots have attracted increasing attention in both industry and academia, since designing and testing control algorithms become more achievable with the rapid development of microcontrollers. Two-wheeled inverted pendulum (TWIP) robots have become more and more popular due to its light weight, small footprint, rapid rotation and high maneuverability. One of the applications of using these robots can be a service robot platform like Segway. However, the inherent instability and nonlinearity of the TWIP requires a sophisticated control scheme.

PID is a commonly used non-model based control method for self-balance robot, as there is no need to build the mathematics model. The three parameters can be tuned by trial and error or by experience. Although PID owns some robustness to disturbance and uncertainties to a certain extent, it cannot handle the sudden, large disturbance and modeling error [1]. Moreover, the control input cannot perfectly be optimized by using PID control. It could be targeted by having state feedback control (SFC) with optimized linear quadratic regulator (LQR) [2]. However, the optimized SFC can only work in the certain operating range and cannot react to system uncertainty. When the operating point is changed, the control gains have to be re-tuned in order to stabilize the robot, which is not very practical in the operation. Recently, the soft computing techniques such as fuzzy logic and neural networks have been used to control TWIP robot [3]. Two fuzzy controllers based on Mamdani and Takagi-Sugeno are designed for an inverted pendulum subjected to disturbance. Moreover, artificial neural network-based real-time switching dynamic controller is designed to solve balancing problem on various loose surfaces such as sand, pebble and soil [4]. A visual robot control interface is developed in $\mathrm{C}++$ software development environment so that robot controller parameters can be changed as desired.

Recently, the model-based control of TWIP has been proposed to deal with the nonlinearity and disturbance. In [6], a linear SFC has been designed based on the linearized model, which needs very accurate dynamic models of the robots. One of the typical model-based control is SMC method. SMC is known to have a good robustness to the model uncertainties and disturbance. It can deal with the nonlinear unstable system with easy implementation, insensitivity to parameters variation and quick response independence of external disturbances. SMC has been successfully used for controlling the TWIP robot. In [5], a back-stepping slide model control has been proposed for balancing and trajectory tracking of a twowheeled robot. The balancing and angular velocity controllers are implemented in a cascade way.

In this paper, a dynamic model of a customer built TWIP robot is derived by using Lagrangian function method. The robot is composed of two gear DC motors driving two wheels, a structure, battery, sensors and controller. It is a highly nonlinear and unstable system. Two controllers, i.e. LQR and SMC have been designed for balancing and stabilizing the robot. Both controllers have been implemented on the robot and experimental tests have been carried out to compare performances of two controllers. The results demonstrate the 
superiority of SMC controller in terms of transient performance and disturbance rejection capability.

The paper is organized as follows. The paper begins with an introduction and the next section provides a dynamic of the model of the TWIP robot built in our lab. In Section III, the LQR and SMC are designed and the simulation results of both controllers are presented. Section IV presents the experiment results of both controllers. The conclusion is drawn in Section $\mathrm{V}$.

\section{DYNAMIC MODELLING}

The TWIP robot is built in our lab to serve as test platform for various controllers. In order to control this type of unstable robot effectively, the first step is to model the robots dynamic behavior in the yaw and pitch motions. The schematic diagram of the TWIP robot is shown in Figure 1 and the parameters' description has been provided in TABLE 1 .

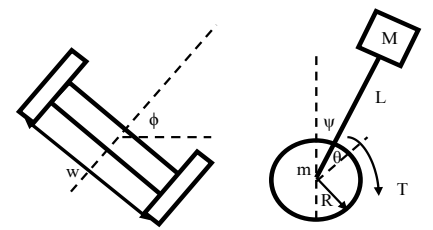

Figure. 1 Schematic diagram of the TWIP robot.

The dynamic model of the robot is built based on the Lagrangian function method [6]. Equation (1) presents Lagrangian where $\mathrm{L}_{1}, \mathrm{~L}_{2}$ and $\mathrm{B}$ are translation kinematic energy, rotational kinematic energy and potential energy, respectively.

$$
L_{a}=L_{1}+L_{2}+B
$$

It is assumed that the robot has 3 degree of freedoms (DOFs). The generalized variables of the robot are angle of the wheel $(\theta)$, pitch angle $(\phi)$ and yaw angle $(\psi)$ [7]. According to the robot dynamics, the translational and rotational kinetic energies are described as follows:

$$
\begin{gathered}
L_{1}=\frac{1}{2} m\left(\dot{x}_{l}^{2}+\dot{y}_{l}{ }_{l}^{2}\right)+\frac{1}{2} m\left(\dot{x}_{r}^{2}+\dot{y}_{r}^{2}\right)+\frac{1}{2} M\left(\dot{x}_{b}{ }^{2}+\dot{y}_{b}{ }^{2}+\dot{z}_{b}{ }^{2}\right) \\
L_{2}=\frac{1}{2} J_{w} \dot{\theta}^{2}{ }_{l}+\frac{1}{2} J_{w} \dot{\theta}^{2}{ }_{r}+\frac{1}{2} J_{\psi} \dot{\psi}^{2}+\frac{1}{2} J_{\phi} \dot{\phi}^{2}{ }_{l}+\frac{1}{2} n^{2} J_{m}\left(\dot{\theta}_{l}-\dot{\psi}\right)^{2}+\frac{1}{2} n^{2} J_{m}\left(\dot{\theta}_{r}-\psi\right)^{2} \\
B=M g z_{b}
\end{gathered}
$$

The Lagrangian equations are:

$$
\begin{aligned}
& \frac{d}{d l}\left(\frac{\partial L_{a}}{\dot{\theta}}\right)-\frac{\partial a}{\partial \theta}=F_{l}+F_{r} \\
& \frac{d}{d l}\left(\frac{\partial L_{a}}{\dot{\psi}}\right)-\frac{\partial a}{\partial \psi}=-F_{l}-F_{r} \\
& \frac{d}{d l}\left(\frac{\partial L_{a}}{\partial \dot{\phi}}\right)-\frac{\partial a}{\partial \phi}=\frac{W}{2 R}\left(F_{r}-F_{l}\right)
\end{aligned}
$$

\begin{tabular}{|c|c|c|}
\hline Parameters & Unit & Description \\
\hline $\mathrm{W}=0.242$ & $m$ & Body Width \\
\hline $\mathrm{M}=1.047$ & $\mathrm{~kg}$ & Body Mass \\
\hline $\mathrm{m}=0.118$ & $\mathrm{~kg}$ & Wheels mass \\
\hline $\mathrm{R}=0.060$ & $m$ & Diameter of wheel \\
\hline $\mathrm{g}=9.810$ & $\mathrm{~m} / \mathrm{s}^{2}$ & Gravity acceleration \\
\hline $\mathrm{L}=0.030$ & $m$ & $\begin{array}{l}\text { Distance of the center of } \\
\text { the mass from the Wheel } \\
\text { axle }\end{array}$ \\
\hline $\mathrm{D}=0.050$ & $m$ & Body Depth \\
\hline $\mathrm{n}=64: 1$ & \multicolumn{2}{|c|}{ Gear ratio } \\
\hline $\mathrm{J}_{\mathrm{W}}=\mathrm{mR}^{2} / 2$ & $\mathrm{kgm}^{2}$ & Wheel inertia moment \\
\hline $\mathrm{J}_{\Psi}=\mathrm{ML}^{2} / 2$ & $\mathrm{kgm}^{2}$ & Body pitch inertia moment \\
\hline $\mathrm{J}_{\phi}=\mathrm{M}\left(\mathrm{W}^{2}+\mathrm{D}^{2}\right) / 12$ & $\mathrm{kgm}^{2}$ & Body yaw inertia moment \\
\hline $\mathrm{R}_{\mathrm{m}}=1.900$ & $\Omega$ & DC motor resistance \\
\hline $\mathrm{K}_{\mathrm{t}}=13.400$ & $m N m / A$ & DC motor torque constant \\
\hline $\mathrm{K}_{\mathrm{b}}=1.400$ & $m v / r p m$ & $\begin{array}{l}\text { DC motor back E.M.F } \\
\text { constant }\end{array}$ \\
\hline $\mathrm{fm}=0.0022$ & \multicolumn{2}{|c|}{$\begin{array}{l}\text { Friction coefficient between body } \\
\text { and DC motor }\end{array}$} \\
\hline$\theta, \psi, \phi$ & \multicolumn{2}{|c|}{$\begin{array}{l}\text { Rotary angle of the wheel, pitch angle } \\
\text { and yaw angle of the robot }\end{array}$} \\
\hline $1, \mathrm{r}, \mathrm{b}$ & \multicolumn{2}{|c|}{$\begin{array}{l}\text { Subscripts indicating left or right wheels } \\
\text { and the robot body, respectively }\end{array}$} \\
\hline
\end{tabular}

where, $F_{l}$ and $F_{r}$ are the torques on the left and right wheels, respectively. Moreover, they could be defined as follows:

$$
\begin{aligned}
& F_{l}=n K_{t} i_{l}+T_{s}+\sigma_{m}\left(\dot{\psi}-\dot{\theta}_{l}\right. \\
& F_{l}=n K_{t} i_{r}+T_{s}+\sigma_{m}\left(\dot{\psi}-\dot{\theta}_{r}\right.
\end{aligned}
$$

TABLE 1. Parameters of Two Wheeled Inverted Pendulum

By substituting the kinetic and potential energies in the Lagrangian equations:

$$
\begin{aligned}
& \left((2 m+M) R^{2}+2 J_{w}+2 n^{2} J_{m}\right) \ddot{\theta}+ \\
& \left(M R L \cos \psi-2 n^{2} J_{m}\right) \ddot{\psi}-M L R \psi^{2} \sin \psi=F_{\theta} \\
& \left(M R L \cos \psi-2 n^{2} J_{m}\right) \ddot{\theta}+\left(M L 2+J_{\psi}+2 n^{2} J_{m}\right) \ddot{\psi} \\
& -M g L \sin \psi-M L^{2} \dot{\phi}^{2} \sin \psi \cos \psi=F_{\psi} \\
& \left(0.5 m W^{2}+J J_{\phi}+\frac{W^{2}}{2 R^{2}}\left(J_{w}+n^{2} J_{m}\right)+M L^{2} \sin ^{2} \psi\right) \ddot{\phi} \\
& +2 M L^{2} \dot{\psi} \dot{\phi} \sin \psi \cos \psi=F_{\phi}
\end{aligned}
$$

The aforementioned equations can be transformed to nonlinear state space, by defining:

as the state variables:

$$
\theta, \dot{\theta}, \psi, \dot{\psi}, \phi, \dot{\phi}
$$

$$
X=\left[x_{1}, x_{2}, x_{3}, x_{4}, x_{5}, x_{6}\right]=[\theta, \dot{\theta}, \psi, \dot{\psi}, \phi, \dot{\phi}] .
$$

Hence, the general form of the state space is given as following equations:

$$
M(x) \dot{x}=f(x)+u
$$

where:

$$
M(x)=\left[\begin{array}{cccccc}
1 & 0 & 0 & 0 & 0 & 0 \\
M_{21} & M_{22} & -M_{24} & M_{24} & 0 & 0 \\
0 & 0 & 1 & 0 & 0 & 0 \\
-M_{21} & M_{24} & M_{21} & M_{44} & 0 & 0 \\
0 & 0 & 0 & 0 & 1 & 0 \\
0 & 0 & 0 & 0 & M_{65} & M_{06}
\end{array}\right]
$$




$$
\begin{gathered}
f(x)=\left[\begin{array}{l}
f_{1} \\
f_{2} \\
f_{3} \\
f_{4} \\
f_{5} \\
f_{6}
\end{array}\right]=\left[\begin{array}{c}
x \\
M L R x_{4}^{2} \sin x_{3}+2 T_{s} \\
x \\
M g L \sin x_{3}+M L^{2} x_{6}^{2} \sin x_{3} \cos x_{3} \\
x \\
-2 M L^{2} x_{4} x_{6} \sin x_{3} \cos x_{3}
\end{array}\right] \\
\mathbf{u}=\left[\begin{array}{c}
0 \\
u_{2} \\
0 \\
-u_{2} \\
0 \\
u_{6}
\end{array}\right]=\left[\begin{array}{cc}
0 & 0 \\
\frac{n k_{t}}{R_{m}} & \frac{n k_{t}}{R_{m}} \\
0 & 0 \\
\frac{n k_{t}}{R_{m}} & -\frac{n k_{t}}{R_{m}} \\
0 & 0 \\
\mid \frac{n k_{1} w}{2 R R_{m}} & -\frac{n k_{1} w}{2 R R_{m}}
\end{array}\right]\left[\begin{array}{l}
v_{r} \\
v_{l}
\end{array}\right]
\end{gathered}
$$

$\mathrm{u}$ is the control input which is generated by the controllers in this study.

\section{CONTROLLER DESIGN AND SIMULATION}

\section{A. State feedback control having $L Q R$}

To stabilize the two-wheel inverted pendulum, the optimal SFC have been designed using LQR. Following is the linear quadratic regulator objective function:

$J=\int_{0}^{\infty} x^{T}(t) Q x(t)+u^{T}(t) R u(t) d t$

The optimal control input to satisfy the above mentioned equation is:

$$
u(t)=-K x(t) \text {, where } K=R^{-1} B^{T} P
$$

$\mathrm{P}$ can be calculated by solving Riccati equation:

$$
A^{T} P+P A-P B R^{-1} B^{T} P+C^{T} C=0
$$

Where A, B and C are state space matrices. Two optimal LQRs have been designed for left and right DC motors.

There are two measurements of the angle from two different sources. The measurement from accelerometer gets affected by sudden horizontal movements (it could be used to measure the $\psi$ ) and the measurement from gyroscope gradually drifts away from actual value (it could be used to measure $\theta$ ). In other words, the accelerometer reading gets affected by short duration signals and the gyroscope reading is affected by long duration signals. To stabilize the robot, two state feedback controllers work simultaneously to control the states of DC motors.

The state feedback controller has been applied to the simulated model which has been presented in the Eq. (15). Figure 2 is the block diagram of closed-loop states control for the TWIP. The gains details are given as in TABLE 2.

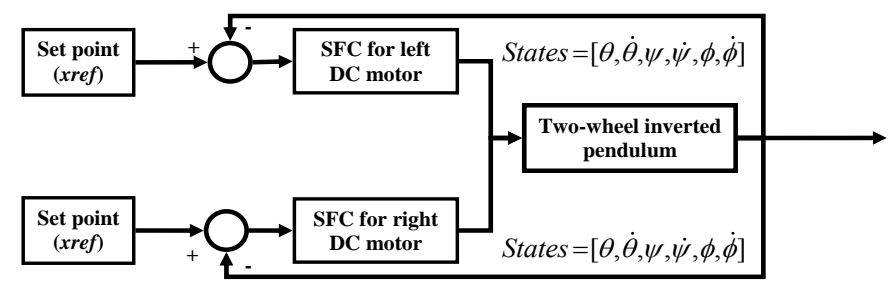

Figure 2. TWIP closed loop block diagram with LQR s $x r e f:$ It represents the desired states, in this case to stabilize the TWIP the $x r e f=0 \mathrm{rad}$ (Figure 2).

\begin{tabular}{|c|cccccc|}
\hline & \multicolumn{5}{|c|}{$\left[k_{1}, k_{2}, k_{3}, k_{4}, k_{5}, k_{6}\right]$} \\
\hline $\begin{array}{c}\text { Left } \\
\text { motor }\end{array}$ & {$\left[\begin{array}{lllllll}-0.7071 & -0.3078 & -11.3966 & -1.6791 & 0.0000 & 0.2118\end{array}\right]$} \\
\hline $\begin{array}{c}\text { Right } \\
\text { motor }\end{array}$ & {$\left[\begin{array}{llllll}-0.7071 & -0.3078 & -11.3966 & -1.6791 & 0.0000 & -0.2118\end{array}\right]$} \\
\hline
\end{tabular}

The simulation of the closed-loop SFC has been done in SIMULINK using ode45 method with variable time step. As shown in Figure 3, two different initial pinch angles have been provided to evaluate the performance of LQR.
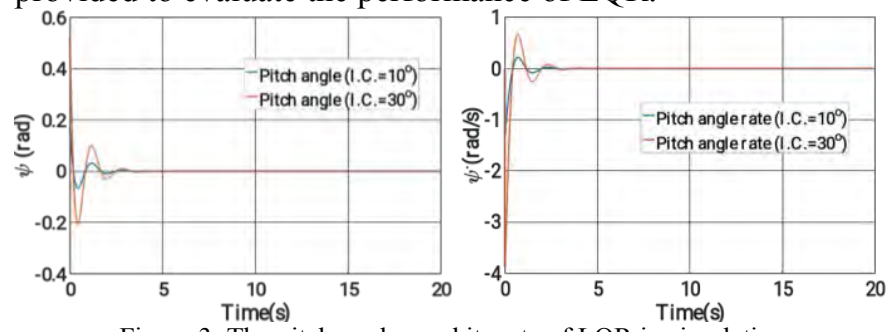

Figure 3. The pitch angle cand its rate of $L Q R$ in simulation

\section{A. Sliding mode controller}

As the yaw motion and self-balancing of the robot need to be controlled properly, a sliding mode control is proposed and designed to achieve self-balancing and stabilizing. Due to the system's nonlinearity and uncertainty, LQR could not precisely balance the pendulum and reject the large disturbance within short time period. Hence, the sliding mode controller is designed to handle the nonlinearity of the system using the sliding surface approach.

The design of the wheel angle sliding mode controller is presented as follows:

$$
\begin{aligned}
& \mathrm{s}_{1}=\mathrm{c}_{1} \psi+\mathrm{c}_{2} \dot{\psi} \\
& \dot{\mathrm{s}}_{1}=-\varepsilon_{1} \operatorname{sgn}\left(s_{1}\right) \\
& \mathrm{u}_{2}=\frac{c_{1}\left(M_{24}^{2}-M_{22} M_{44}\right) \dot{\psi}+\left(M_{24}^{2}+\mathrm{M}_{22} M_{44}\right) \varepsilon_{1} \operatorname{sgn}\left(s_{1}\right)}{c_{2}\left(-M_{24}-M_{22}\right.}+ \\
& \frac{C_{2} M_{24} f_{2}-C_{2} M_{22} f_{4}}{C_{2}\left(-M_{24}-M_{22}\right.}+ \\
& \frac{c_{2} M_{12}\left(-M_{24}+M_{22}\right)-\mathrm{C}_{2} M_{21}\left(M_{24}+\mathrm{M}_{22}\right) \dot{\psi}}{n}
\end{aligned}
$$

The design of the yaw motion sliding mode controller is presented as follows:

$$
\mathrm{s}_{2}=\mathrm{c}_{3} \phi+\mathrm{c}_{4} \dot{\phi}
$$

By substituting the equations to the robot dynamic, the second input can be calculated as:

$$
\mathrm{u}_{6}=\frac{M_{66} \varepsilon_{2} \operatorname{sgn}(s)}{-c_{4}}-f_{6}+\dot{\phi}\left(M_{65}-C_{3} M_{66}\right)
$$

where:

$$
\begin{aligned}
& \mathrm{f}_{2}=\operatorname{MLRx}_{4}^{2} \sin \mathrm{x}_{3}+2 \mathrm{~T}_{\mathrm{s}} \\
& f_{4}=M g L \sin x_{3}+M L^{2} x_{6}^{2} \sin x_{3} \cos x_{3}
\end{aligned}
$$




$$
f_{6}=-2 M L^{2} x_{4} x_{6} \sin x_{3} \cos x_{3}
$$

From Eq. 26 and Eq. 27, the inputs of left and right wheeels are:

$$
\begin{aligned}
& \mathrm{v}_{\mathrm{r}} \frac{\mathrm{RR}_{\mathrm{m}}}{\mathrm{nk}_{\mathrm{t}} \mathrm{W}} \mathrm{u}_{6}-\frac{\mathrm{R}_{\mathrm{m}}}{2 \mathrm{nk}_{\mathrm{t}}} \mathrm{u}_{2} \\
& \mathrm{v}_{\mathrm{l}}=-\frac{\mathrm{RR}_{\mathrm{m}}}{\mathrm{nk}_{\mathrm{t}} \mathrm{W}} \mathrm{u}_{6}-\frac{\mathrm{R}_{\mathrm{m}}}{2 \mathrm{nk}_{\mathrm{t}}} \mathrm{u}_{2}
\end{aligned}
$$

Figure 4 depicts the block diagram of closed look position control with sliding mode controller for the TWIP.

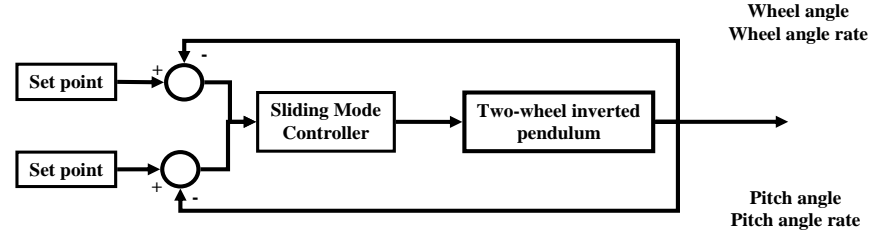

Figure 4. SMC block diagram for TWIP

The angular reaction of the wheels is presented in Figure 5:
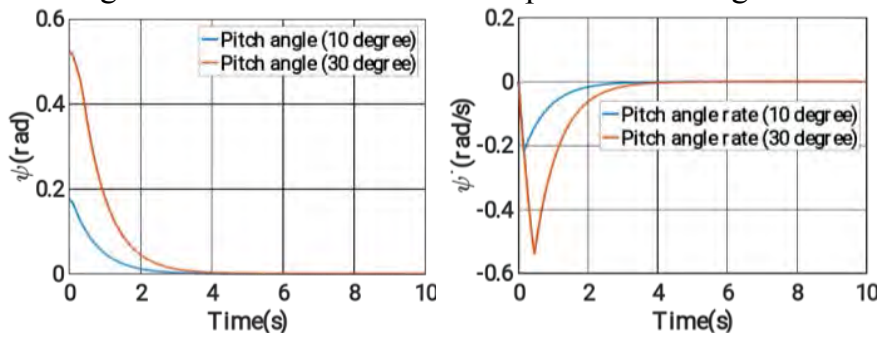

Figure 5. The pitch angle and its rate of SMC in simulation

TABLE 3. TRANSIENT PERFORMANCE OF SFC AND SMC IN SIMULATION

\begin{tabular}{|c|c|c|c|c|}
\hline & \multicolumn{2}{|c|}{ LQR } & \multicolumn{2}{c|}{ SMC } \\
\hline Initial condition & $10^{\circ}$ & $30^{\circ}$ & $10^{\circ}$ & $30^{\circ}$ \\
\hline Rise Time (s) & 0.181 & 0.166 & 3.144 & 4.374 \\
\hline $\begin{array}{c}\text { Settling Time (s) } \\
\begin{array}{c}\text { Percent of overshoot } \\
(\%)\end{array}\end{array}$ & 3.25 & 3.04 & 1.638 & 2.581 \\
\hline
\end{tabular}

The simulation results are summarized in TABLE 3. From this table, one can see that the SMC has better transient performance than LQR does in terms of settling time and percent of overshoot.

\section{EXPERIMENT}

This TWIP robot is built with four main parts: controller and sensors, gear DC motors, battery, and structure. Arduino Uno is the controller of the TWIP and allows the driver shield to drive gear DC motors. The gear DC motors of the robot could robustly keep the robot stable. The driver shield is L298, which is a dual full-bridge driver. It can transform the real time data from Arduino board to the DC motors. To record the angular position like pitch, yaw and wheel angles, Arduino has been connected to two different sensors. (i.e., IPU 6050 which has accelerometer sensors, gyroscope). As it contains 16-bits analog to digital conversion hardware for each channel, it could be more precise.

In addition, using $\mathrm{X}$-bee shield can wirelessly provide the data transmission to computer. The gear-DC-motors made by Faulhaber with maximum revolution of $350 \mathrm{rpm}$. Moreover, the power is supplied by Li-Po battery/4000 mA. The diameter of wheels is $120 \mathrm{~mm}$. the center mass of robot is located in the middle of the wheels' axis.

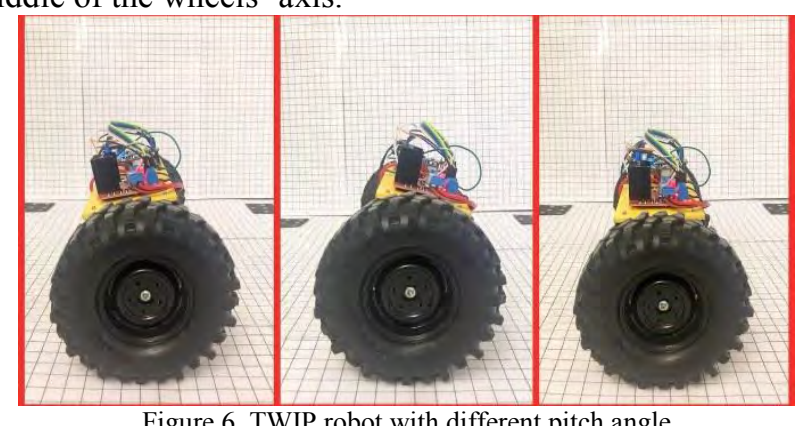

Figure 6. TWIP robot with different pitch angle

The experimental results of pitch angle and its rate of SFC controller are presented in Figure 7.
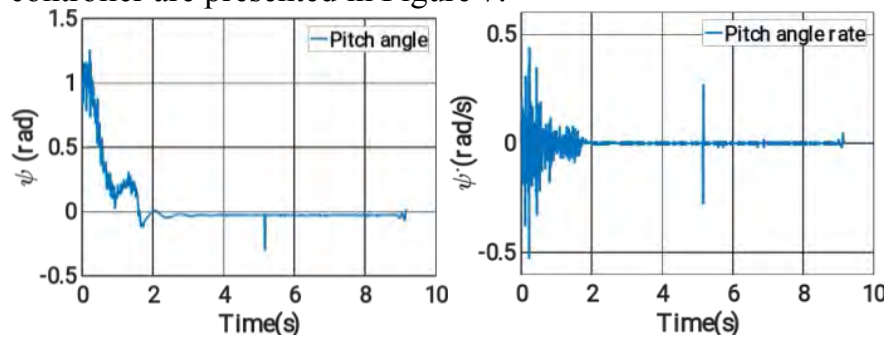

Figure 7. The experiment results of pitch angle and its rate of LQR In the next, to evaluate the performance of the sliding mode control, the pitch angle and its rate have been illustrated in Figure 8 .
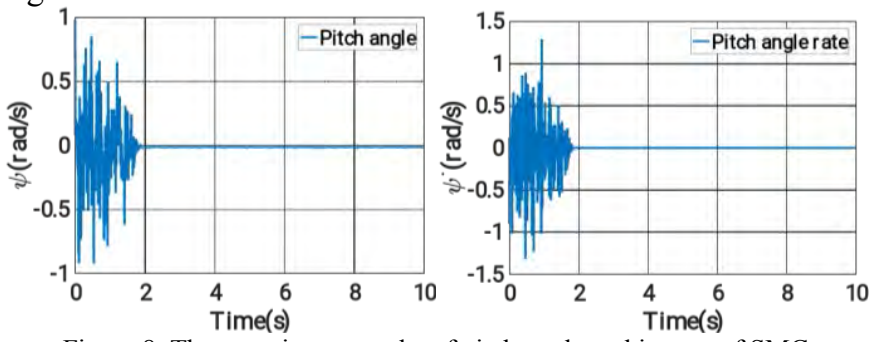

Figure 8. The experiment results of pitch angle and its rate of SMC

In both experimental tests, a big push has been applied to the TWIP robot around 1 second to test the disturbance rejection ability of both controllers. From Figs. 7 and 8, one can see that both SFC and SMC can recover from the push and achieve self-balancing. However, it takes SMC less than 2 seconds to settle in the zero angles position while SFC takes more than 2 seconds to reach the self-balancing state. The video of the experiments is uploaded in Youtube. https://youtu.be/a6w5zxU8IBU. The experimental tests demonstrate that SMC outperforms the LQR.

\section{CONCLUSION}

In this paper, a customer designed TWIP robot and it's modeling and control are presented. The ribot is an inherent 
unstable and nonlinear system, which poses challenge for control design. A SMC for balancing and steering movement is designed based on the dynamic model derived by the Lagrangian function method. From simulation results of the LQR and SMC for the TWIP robot, it can be concluded that the SMC has better transient performance in stabilizing the TWIP robot. To further evaluate the SMC and LQR performances, experimental tests have been conducted and validated the effectiveness of the designed controllers. As it is presented in the aforementioned test results, the performances of SMC is superior to that of LQR in terms of settling time and robustness. The future work includes the further improvement of control performances considering the actuation constraints.

\section{REFERENCES}

[1] A. N. K. Nasir, M. A. Ahmad, and R. M. T. Raja Ismail, "The control of a highly nonlinear two-wheels balancing robot: A comparative assessment between LQR and PID-PID control schemes," World Academy of Science, Eng. Technol., vol. 70, pp. 227-232, 2010.

[2] J. Villacres et al., "Controllers comparison to stabilize a two-wheeled inverted pendulum: Pid, lqr and sliding mode control," Int. J. Control Syst. Rob., vol. 1, pp. 29-36, 2016.

[3] H. Marzi, "Fuzzy control of an inverted pendulum using AC induction motor actuator," in Proc. IEEE Int. Conf. Computational Intelligence for Measurement Systems and Applications, La Coruna, Spain, 2006, pp. 109-114. doi: 10.1109/CIMSA.2006.250767.

[4] A. Unluturk and O. Aydogdu, "Adaptive control of two-wheeled mobile balance robot capable to adapt different surfaces using a novel artificial neural network-based real-time switching dynamic controller," Int. J. Adv. Rob. Syst., vol. 14, no. 2, pp. 1-9, Apr. 2017. doi: $10.1177 / 1729881417700893$.

[5] M. Velazquez et al., "Velocity and motion control of a self-balancing vehicle based on a cascade control strategy," Int. J. Adv. Rob. Syst., vol. 13, no. 3, p. 106, Jan. 2016. doi: 10.5772/63933.

[6] N. R. Gans and S. A. Hutchinson, "Visual servo velocity and pose control of a wheeled inverted pendulum through partial-feedback linearization," in Proc. IEEE/RSJ Int. Conf. on Intelligent Robots and Systems, Beijing, China, 2006, pp. 3823-3828. doi:10.1109/IROS.2006.281788.

[7] F. Dai et al., "A two-wheeled inverted pendulum robot with friction compensation," Mechatronics, vol. 30, pp. 116-125, Sept. 2015.

doi: 10.1016/j.mechatronics.2015.06.011. 


\section{Robust Cooperative Close Formation Flight Control of Multiple Unmanned Aerial Vehicles}

\author{
Qingrui Zhang \\ The Institute for Aerospace Studies University of Toronto \\ (UTIAS) \\ 4925 Dufferin St. Toronto, Canada M3H 5T6 \\ qingrui.zhang@mail.utoronto.ca
}

\begin{abstract}
This paper investigates the close formation control problem of multiple unmanned aerial vehicles (UAVs). A robust cooperative control algorithm is proposed in light of the uncertainty and disturbance estimation technique. In the proposed design, each UAV is assigned a virtual leader which defines the desired position for the corresponding UAV in close formation. Bidirectional communication topology is assumed for UAVs in close formation, based on which a cooperative control law is thereafter established on each UAV. Model uncertainties and formation aerodynamic disturbances are efficiently estimated and compensated using a uncertainty and disturbance estimator. Eventually, the efficacy of the proposed design will be validated via the close formation simulation of five aircraft.
\end{abstract}

Keywords-component; close formation control; cooperative control; Multi-UAVs; uncertainty and disturbacne estimation

\section{INTRODUCTION}

Close formation flight stems from the formation flight of migrating birds [1]. It has received extensive research attention in past decades [2, 3]. The research interest in close formation flight is motivated by its potential in drag reductions and fuel savings. When an aircraft is flying in air, trailing vorticescircular patterns of rotating airs-will be left behind it. The trailing vortices are divided into downwash wake and upwash wake. If a follower aircraft is managed to fly at certain proper relative position to a leader aircraft, the upwash wake could be used to reduce the drag of the follower aircraft, thereby decreasing energy expenditure. According to flight tests by NASA, close formation flight can generate 13\%-20\% drag reductions and $14 \%-18 \%$ fuel savings on a follower aircraft [4]. Wind-tunnel experimental studies by Cho et al indicate $12 \%$ increase in the lift-to-drag ratio of a follower aircraft in close formation against solo flight [5].

However, close formation flight is always a challenging problem for unmanned aerial vehicles. Highly efficient and accurate formation control algorithms are indispensable, as close formation flight only makes sense when a follower is keeping a certain optimal position to its leader. The theoretical

\author{
Hugh H. T. Liu \\ The Institute for Aerospace Studies University of Toronto \\ (UTIAS) \\ 4925 Dufferin St. Toronto, Canada M3H 5T6 \\ liu@uitas.utoronto.ca
}

analysis by the authors shows more $30 \%$ drag reduction by close formation flight could be lost, if a follower fails to track the optimal relative position by $10 \%$ wing span [6]. In addition, the trailing vortices, working like wind gusts, will induce other adverse aerodynamic disturbances on the follower aircraft, which increases the difficulty of the control design [6].

So far, the close formation control problem has been studied using different methods, such as PI controller [7], sliding mode control [8], LQR control [9], MPC control [10], adaptive control [11], and robust control [12]. However, all of them were developed under the leader-follower architecture, and there was no cooperation between two UAVs. The efficacy of the existing methods can only be guaranteed for close formation flight of two or three aircraft. Increase of formation size (number of UAVs) will result in dramatic loss of efficiency and accuracy for the existing methods. To deal with the deficiency of the existing methods in close formation flight of more than three UAVs, a cooperative control method is proposed in this paper. A bidirectional communication topology is employed. UAVs in close formation are required to communicate with some of their neighbors. To enhance the robustness against model uncertainties and formation aerodynamic disturbances, the uncertainty and disturbance estimation technique is employed and combined with the proposed cooperative formation controller. The efficiency of the proposed control algorithm is verified via the close formation simulation of five aircraft.

The paper is organized as follows. In section II, a nonlinear UAV model is introduced together with the close formation aerodynamics. Section III presents the robust cooperative control methods. The numerical simulations are given in Section IV. Eventually, some conclusion remarks are provided in Section V.

\section{PRELIMINARIES}

\section{A. Aircraft model}

The proposed cooperative control law will perform as an outer-loop formation tracking controller. Hence, a 6-state nonlinear UAV model is presented for the control design. 


$$
\left\{\begin{array}{l}
\dot{x}_{i}=v_{i} \cos \chi_{i}, \dot{y}_{i}=v_{i} \sin \chi_{i}, \dot{h}_{i}=\eta_{i} \\
\dot{v}_{i}=\left(T_{i}-D_{i}-\Delta D_{i}\right) / m_{i} \\
\dot{\chi}_{i}=\left(L_{i}+\Delta L_{i}\right) \sin \mu_{i} /\left(m_{i} v_{i}\right) \\
\dot{\eta}_{i}=\left(L_{i}+\Delta L_{i}\right) \cos \mu_{i} / m_{i}-g
\end{array}\right.
$$

where $x_{i}, y_{i}$, and $h_{i}$ denote the position of the $\mathrm{i}$-th UAV in the inertial frame, $v_{i}$ is the ground speed projection on a horizontal plane, $\eta_{i}$ is the climbing rate, $\chi_{i}$ is the heading angle, $\mu_{i}$ is the bank angle, $L_{i}$ is the lift, $D_{i}$ is the drag, $T_{i}$ is the thrust, $g$ is the gravity acceleration, $\Delta D_{i}$ and $\Delta L_{i}$ aerodynamic forces induced by the trailing vortices of leader aircraft. The control inputs are chosen to be $T_{i}, \mu_{i}$, and $L_{i}$. The vortex-induced forces $\Delta D_{i}$ and $\Delta L_{i}$ are given by

$$
\begin{aligned}
& \Delta L_{i}=\int_{-\frac{b}{2}}^{\frac{b}{2}} q_{\infty} c(s) C_{l_{\alpha}} \Delta \alpha(s) d s \\
& \Delta D_{i}=L_{i}^{\prime} \sin (\overline{\Delta \alpha})=\left(L_{i}+\Delta L_{i}\right) \sin (\overline{\Delta \alpha})
\end{aligned}
$$

where $\Delta \alpha$ is the vortex-induced change of the angle of attack along the wing span as shown in Figure 1 , while $\overline{\Delta \alpha}$ is the effective average change of the angle of attack. Notice that $\Delta L_{i}$ and $\Delta D_{i}$ are actually functions of the relative positions between a follower aircraft and its leader. Shown in Figure 2 and 3 are the relationships between non-dimensional lift and drag coefficients with respect to the lateral and vertical separations. More detailed results can be found at [6].

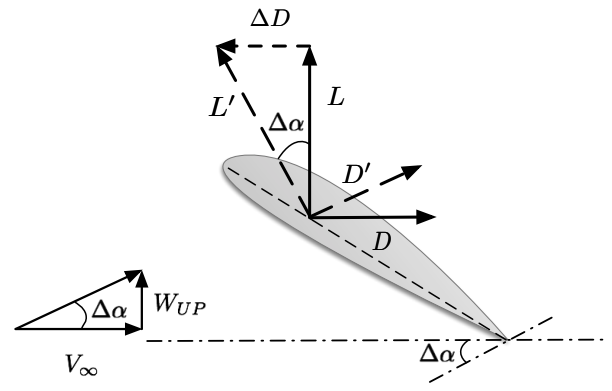

Figure 1: Lift rotation due to the upwash wake

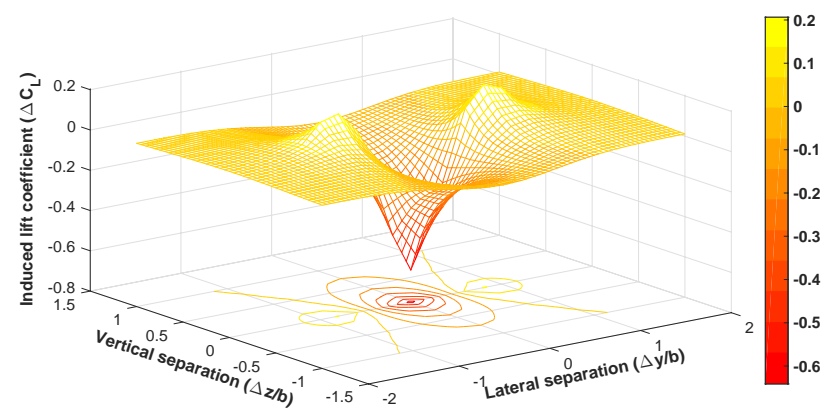

Figure 2: Vortex-induced lift coefficient variation

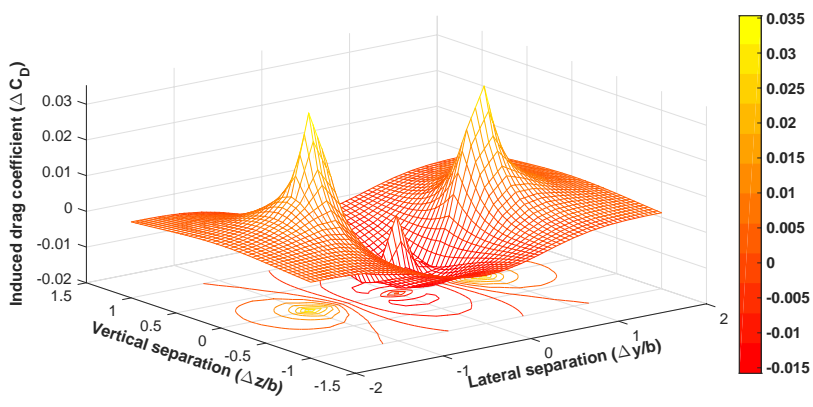

Figure 3: Vortex-induced drag coefficient variation

\section{B. Control conversion}

In the control design, $D_{i}$ is taken as part of the model uncertainties. Differentiating the kinematic equations of Model (1) will yield

$$
\left\{\begin{array}{c}
\ddot{x}_{i}=\dot{v}_{i} \cos \chi_{i}-v_{i} \dot{\chi}_{i} \sin \chi_{i} \\
\ddot{y}_{i}=\dot{v}_{i} \sin \chi_{i}+v_{i} \dot{\chi}_{i} \cos \chi_{i} \\
\ddot{h}_{i}=\dot{\eta}_{i}
\end{array}\right.
$$

Define

$u_{x i}=\frac{T_{i}}{m_{i}} \cos \chi_{i}-\frac{L_{i}}{m_{i}} \sin \mu_{i} \sin \chi_{i}$

$u_{y i}=\frac{T_{i}}{m_{i}} \sin \chi_{i}+\frac{L_{i}}{m_{i}} \sin \mu_{i} \cos \chi_{i}$

$u_{z i}=\frac{L_{i}}{m_{i}} \cos \mu_{i}-g$

$d_{x i}=-\frac{D_{i}+\Delta D_{i}}{m_{i}} \cos \chi_{i}-\frac{\Delta L_{i}}{m_{i}} \sin \mu_{i} \sin \chi_{i}$

$d_{y i}=-\frac{D_{i}+\Delta D_{i}}{m_{i}} \sin \chi_{i}+\frac{\Delta L_{i}}{m_{i}} \sin \mu_{i} \cos \chi_{i}$

$d_{z i}=\frac{\Delta L_{i}}{m_{i}} \cos \mu_{i}$

Eventually, the nonlinear model is transformed into a standard $2^{\text {nd }}$-oder integrator model as shown in (2).

$$
\ddot{\rho}_{i}=u_{\rho i}+d_{\rho i} \quad\left(\rho \in\left\{\begin{array}{lll}
x & y & z
\end{array}\right\}\right)
$$

\section{ROBUST COOPERATIVE CONTROL}

The entire control law is

$$
u_{\rho i}=u_{\rho 0 i}-\hat{d}_{\rho i} \quad\left(\rho \in\left\{\begin{array}{lll}
x & y & z
\end{array}\right\}\right)
$$


where $u_{\rho 0 i}$ is the baseline cooperative control, $\hat{d}_{\rho i}$ is the estimation of the uncertainties and disturbances.

\section{A. Baseline cooperative control}

In this subsection, a cooperative baseline control law is proposed for close formation flight of $\mathrm{N}$ UAVs where $\mathrm{N}>2$. The major expectation is to increase the scalability, efficiency, and robustness of close formation flight by introducing certain cooperative mechanism. In the cooperative control method, a virtual leader together is introduced to generate the reference signals for each vehicle. All the virtual leaders together defines the optimal formation shape (usually, "V-shape") for a group of $\mathrm{N}$ UAVs, as shown in Figure 4. A bidirectional communication topology is employed.

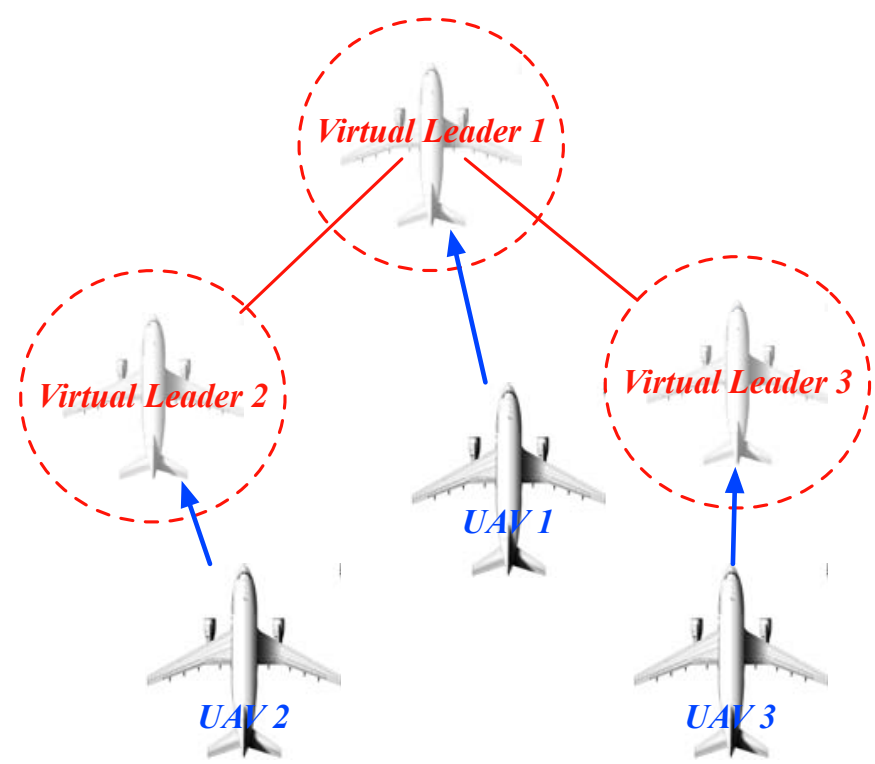

Figure 4 : Close formation organization

Define the position of each virtual leader as $\vec{r}_{i}=\left[\begin{array}{lll}r_{x i} & r_{y i} & r_{z i}\end{array}\right]^{T}$. Assume the $1^{\text {st }}$ and $2^{\text {nd }}$ of $r_{x i}, r_{y i}$, and $r_{z i}$ are available. Define the position tracking error as $e_{\rho i}=\rho_{i}-r_{\rho i}$. The baseline cooperative control law for i-th $\mathrm{UAV}$ is

$$
\begin{array}{lc}
u_{\rho 0 i}= & \ddot{r}_{\rho i}-K_{P \rho} e_{\rho i}-K_{V \rho} \dot{e}_{\rho i} \\
& -\sum_{j \in \mathbb{N}_{i}}\left[C_{P \rho}\left(e_{\rho i}-e_{\rho j}\right)+C_{V \rho}\left(\dot{e}_{\rho i}-\dot{e}_{\rho j}\right)\right]
\end{array}
$$

where $\rho \in\left\{\begin{array}{lll}x & y & z\end{array}\right\}, \quad K_{P \rho}, K_{V \rho}, C_{P \rho}, C_{V \rho}>0$, $\mathbb{N}_{i}$ denotes the set of neighbors of $i$-th aircraft.

\section{B. Uncertainty and disturbance estimator}

The uncertainty and disturbance estimation term $\hat{d}_{\rho i}$ is designed by applying (4) to (3).

$$
\ddot{\rho}_{i}=u_{\rho 0 i}-\hat{d}_{\rho i}+d_{\rho i} \quad\left(\rho \in\left\{\begin{array}{lll}
x & y & z
\end{array}\right\}\right)
$$

In terms of results in [], $d_{\rho i}$ is estimated using a stable lowpass first-order filter $G_{\rho i}(s)$, where

$$
G_{\rho i}(s)=\frac{1}{T_{\rho i} s+1}
$$

Hence, $\hat{d}_{\rho i}$ is given by

$$
\hat{d}_{\rho i}=\frac{1}{T_{\rho} s+1} d_{\rho i}
$$

where $T_{\rho}$ is the time constant, $d_{\rho i}$ is usually unavailable. However, $d_{\rho i}$ is equal to $\ddot{\rho}_{i}-u_{\rho 0 i}+\hat{d}_{\rho i}$ according to Eq. (5). Replace $d_{\rho i}$ by $\ddot{\rho}_{i}-u_{\rho 0 i}+\hat{d}_{\rho i}$ in (6). Via basic mathematical derivations, we can get

$$
\hat{d}_{\rho i}=\frac{1}{T_{\rho} S}\left(\ddot{\rho}_{i}-u_{\rho 0 i}\right)
$$

Specifying (7) in the time-domain will yield

$$
\hat{d}_{\rho i}=\frac{1}{T_{\rho}}\left(\dot{\rho}_{i}(t)-\dot{\rho}_{i}(0)-\int_{0}^{t} u_{\rho 0 i} d \tau\right)
$$

where $\dot{\rho}_{i}(t)$ is a measurable speed signal which makes (8) always practically applicable.

\section{NumericAl SimUlations}

In the numerical simulations, close formation flight of 5 UAVs is considered. Among the $5 \mathrm{UAVs}$, one UAV performs as the very leader for the entire formation. This formation leader will be in charge of tracking the trajectory defined for the entire formation. The virtual leaders are defined according to the optimal relative position requirements to the formation leader. In the simulation results, the leader UAV for the entire formation is labelled to be "Leader", while the other UAVs are labelled to be "Follower 1", "Follower 2", "Follower 3", and "Follower 4", respectively. The control gains are

$$
\begin{array}{ll}
K_{P x}=K_{P y}=K_{P z}=1.5, & K_{V x}=K_{V y}=K_{V z}=3 \\
C_{P x}=C_{P y}=C_{P z}=1, & C_{V x}=C_{V y}=C_{V z}=2
\end{array}
$$




$$
T_{x}=T_{y}=T_{z}=0.1
$$

The communication topology of the five UAVs are shown in Figure 5. The formation trajectory tracking performance is shown in Figure 6 in which the 3-D trajectory is projected on a horizontal plane. Detailed tracking error responses are shown in Figure 7.

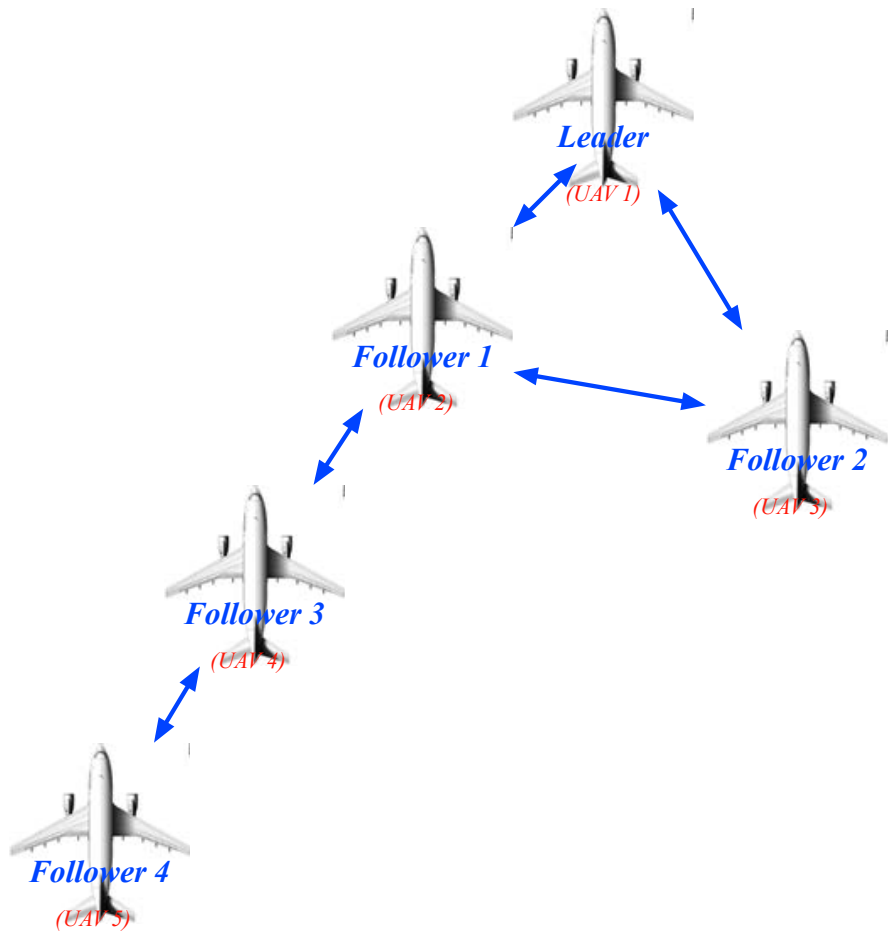

Figure 5 : Communication Topology

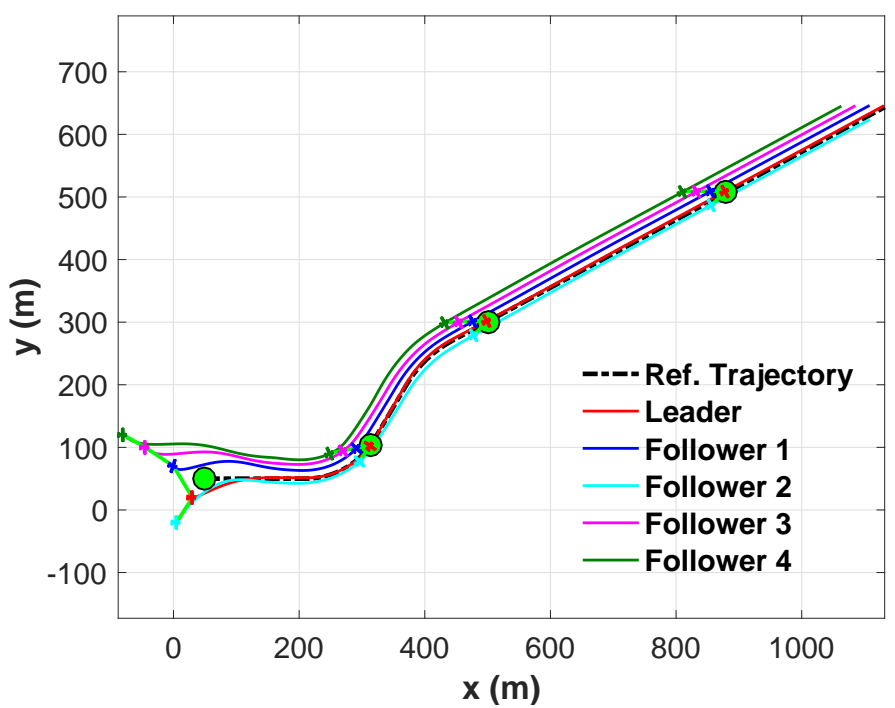

Figure 6: Formation trajectory projection on a 2-D horizontal plane
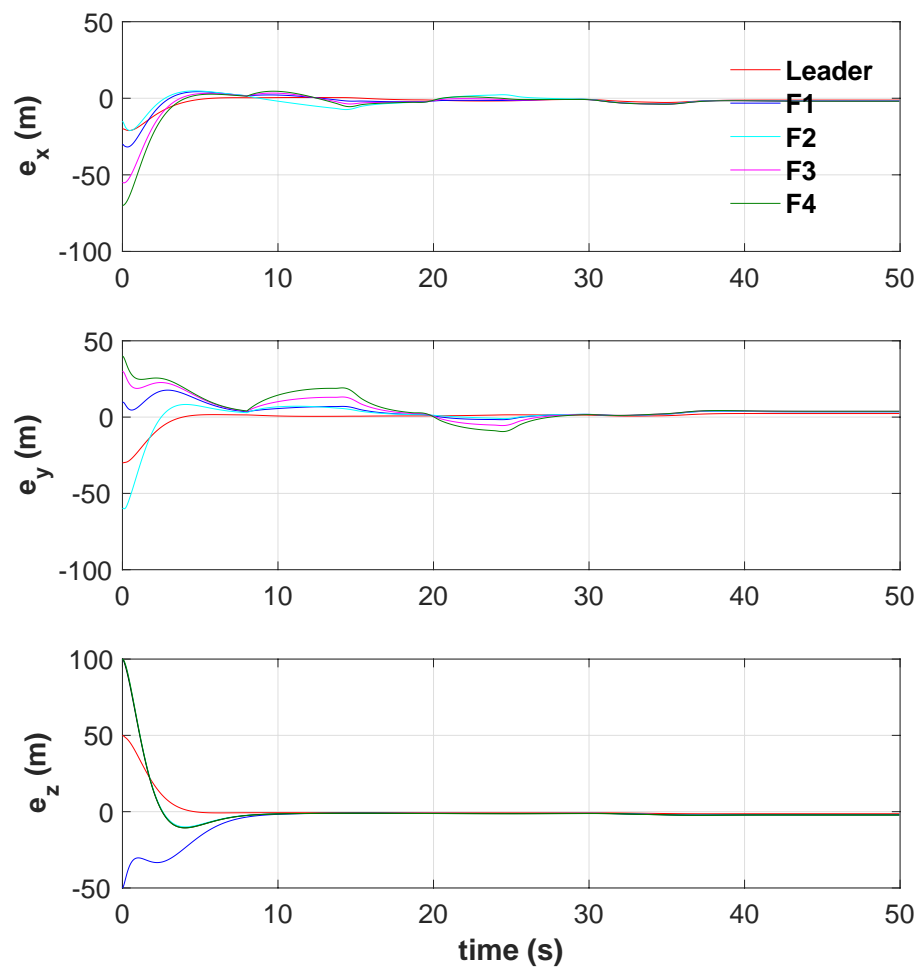

Figure 7: Formation tracking error responses

\section{CONCLUSION}

This paper presents an efficient robust cooperative close formation control algorithms. Based on the proposed design, close formation flight can be easily extended to the case of more than three UAVs. The efficacy of the proposed design is verified via a close formation simulation of five UAVs.

\section{REFERENCES}

[1] S. J. Portugal et al., "Upwash exploitation and downwash avoidance by flap phasing in ibis formation flight," Nature, vol. 505, no. 7483, pp. 399402, Jan. 2014. doi: 10.1038/nature12939.

[2] J. M. Fowler and R. D'Andrea, "Distributed control of close formation flight," Proceedings of the 41st IEEE Conf. on Decision and Control, IEEE, Las Vegas, Nevada USA, 2002. doi: 10.1109/CDC.2002.1184308.

[3] M. Brodecki and K. Subbarao, "Autonomous Formation Flight Control System Using In-Flight Sweet-Spot Estimation," J. of Guidance, Control, and Dynamics, vol. 38, no. 6, pp. 1083-1096, 2015. doi: 10.2514/1.G000220.

[4] R. Ray, B. Cobleigh, M. J. Vachon and C. S. John, "Flight Test Techniques Used to Evaluate Performance Benefits During Formation Flight," Proc. AIAA Atmospheric Flight Mechanics Conf. and Exhibit, AIAA, Monterey, California, AIAA 2002-4492, 2002. doi: 10.2514/6.2002-4492.

[5] H. Cho, S. Lee, S. and C. Han, "Experimental study on the aerodynamic characteristics of a fighter-type aircraft model in close formation flight," $J$. Mechanical Science and Technology, vol. 28, no. 8, pp. 3059-3065, 2014. doi: 10.1007/s12206-014-0713-2.

[6] Q. Zhang and H. H. T. Liu, "Aerodynamics modeling and analysis of close formation flight," J. Aircr., vol. 54, no. 6, pp. 2192-2204, 2017. doi: 10.2514/1.C034271.

[7] M. Pachter, J. J. D. Azzo, and A. W. Proud, "Tight formation flightcontrol," J. Guidance, Control, and Dynamics, vol. 24, no. 2, pp. 246 254, 2001. doi: 10.2514/2.4735. 
[8] S. Singh, M. Pachter, P. Chandler, S. Banda, S. Rasmussen and C. Schumacher, "Input-output invertibility and sliding mode control for close formation flying," Int. J. Robust Nonlinear Control, vol. 10, no. 10, pp. 779-797, 2000.

doi: 10.1002/1099-1239(200008)10:10<779::AID-RNC513>3.0.CO;2-6.

[11] Q. Zhang and H. H.-T. Liu, "Integrator-augmented robust adaptive control design for close formation flight," in Proc. 2017 AIAA Guidance, Navigation, and Control Conf. Grapevine, Texas: AIAA, 2017. doi: org/10.2514/6.2017-1255.

[12] Q. Zhang and H. H.-T. Liu, "Robust design of close formation flight control via uncertainty and disturbance estimator," in Proc. 2016 AIAA Guidance, Navigation, and Control Conf. San Diego, California, USA: AIAA, 2016, AIAA 2016-2102. doi: $10.2514 / 6.2016-2102$.

[13] Q. Zhang and H. H. T. Liu, "UDE-Based Robust Command Filtered Backstepping Control for Close Formation Flight," IEEE Trans. Ind. Electron., vol. 65 , no. 11 , pp. 8818-8827, 2018, published online, doi: 10.1109/TIE.2018.2811367. 


\section{Propeller Performance in Presence of Freestream}

\author{
Mojtaba Hedayatpour, Mehran Mehrandezh \\ Faculty of Engineering and Applied Science \\ University of Regina \\ Regina, Canada \\ \{hedayatm,mehran.mehrandezh\}@,uregina.ca
}

\author{
Farrokh Janabi-Sharifi \\ Department of Mechanical and Industrial Engineering \\ Ryerson University \\ Toronto, Canada \\ fsharifi@,ryerson.ca
}

\begin{abstract}
This paper presents mathematical modeling for thrust force and moments generated by a propeller. In particular, the effects of freestream on propeller's performance are investigated. We introduce some of the applications of the proposed model in modeling multi-rotor UAVs which helps to increase stability or maneuverability of the vehicle. In the end, simulation results for thrust force and moments of an example propeller in presence of a uniform freestream are presented.
\end{abstract}

Keywords-component; propeller; aerodynamics; thrust force; unmanned aerial vehicles, freestream

\section{INTRODUCTION (HEADING 1)}

Multi-rotors have gained significant attention in recent years. Due to their simplicity and maneuverability, they have been used in a broad spectrum of applications such as bioengineering [1], agronomy [2], sports [3] and inspection of infrastructures [4].

In modeling of UAVs, aerodynamic model of propellers plays an important role as it determines the majority of forces and moments in the system. Therefore, an accurate model for the propellers is crucial in analyzing such a system. In the literature, typically, the thrust force and moment generated by the propeller is assumed to be proportional to the square of its angular velocity and the effects of freestream on its performance is usually neglected by assuming small freestream velocities [5], [6], [7]. However, this model is not valid in high speed flight and its accuracy deteriorates as flight speed increases [8], [9].

The freestream may affect propeller's performance depending on its direction and magnitude. Generally, these effects could change propeller's performance in two ways: i) changing the effective angle of attack of the blades; and ii) changing the local airflow velocity over the blades. The former effect is caused when there is a freestream with its velocity vector parallel to the angular velocity vector of the propeller while the latter is caused by any freestream with its velocity vector perpendicular to the angular velocity vector of the propeller. Studying these effects not only helps us to derive a more realistic mathematical model for multi-rotors but also helps to find more stable and power-optimal configurations for such vehicles. In this paper we use Blade Element Theory (BET) to calculate the thrust force and moment of each blade element.

The paper is organized as follows: Section II presents the effects of freestream on propeller's performance along with simulation results for thrust force and moments of an example propeller. Finally, the paper concludes in Section III followed by presenting some of the applications of the proposed model in multi-rotor UAV research.

\section{EFFECTS OF FREESTREAM ON PROPELLER'S PERFORMANCE}

\section{A. Notation}

The term ${ }^{I} \boldsymbol{\omega}_{p}$ denotes that $\boldsymbol{\omega}$ belongs to $p$ and is expressed in frame $I$. Angular velocity vector of the vehicle is represented by $\boldsymbol{\omega}_{B}=(p, q, \mathrm{r})^{T}$ where $p, q$ and $r$ are roll, pitch and yaw rates respectively. Also, 2-Norm of $\boldsymbol{\omega}$ is represented by $\|\omega\|$ and absolute value of $s$ is shown by $|s|$.

\section{B. Effects of Freestream with Its Velocity Vector}

Perpendicular to Angular Velocity Vector of the Propeller

Suppose we have a propeller turning at angular velocity $\boldsymbol{\omega}_{p}$ as expressed in a frame attached to its COM as shown in Fig. 1. The propeller has two blades of radius $R_{b}$ and is assumed to have constant chord $c$. For simplicity, first, we assume there is an almost uniform freestream with velocity vector $V_{\infty_{1}}$, as shown in blue in Fig. 1, which is parallel to the y-axis.

Consider a blade element (small hashed area in Fig. 2) of length $c$ and differential width $d r_{b}$ where $r_{b}$ is the distance of the blade element from the root of the blade. As shown in Fig. 2 , the rotation of the blade generates relative air flow velocity with magnitude $r_{b}\left\|\omega_{p}\right\|$, over each blade element. As the propeller is turning, the relative air flow velocity over the blade element could either be increased or decreased depending on the azimuth angle of the blade and direction of the freestream velocity. The azimuth angle $\psi_{p}$ is defined as the angle between 
the blade and the direction of $V_{\infty_{1}}$. Therefore, the resultant relative air flow velocity over each blade element can be written as:

$$
v=r_{b}\left\|\omega_{p}\right\|+\left\|V_{\infty_{1}}\right\| \sin \quad p
$$

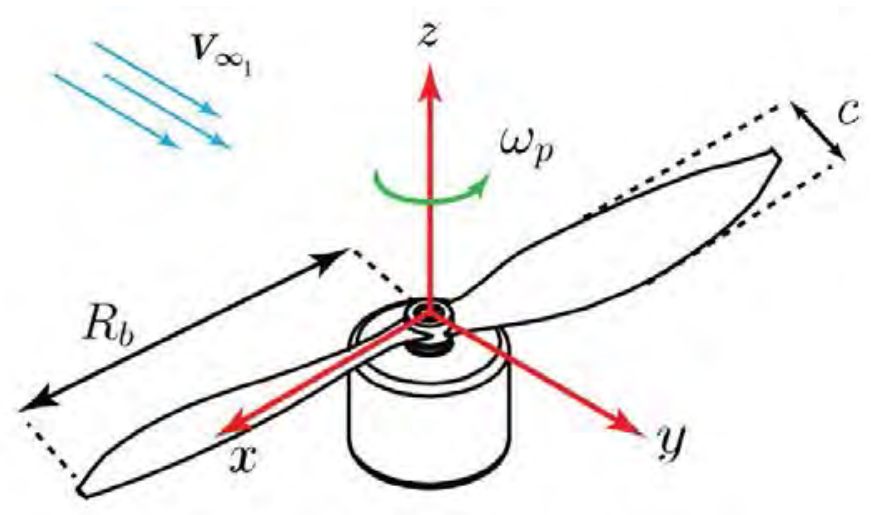

Figure 1: Schematic of a propeller with a frame attached to its COM.

It can be seen in Fig. 2, for the advancing blade $\left(0 \leq \psi_{p} \leq \pi\right)$, freestream velocity increases the relative air flow velocity over the blade and for the retreating blade ( $\left.\pi \leq \omega_{p} \leq 2 \pi\right)$, it decreases the relative air flow velocity. The changes in the relative air flow velocity with azimuth angle affects the overall thrust of the propeller and it generates a moment in the direction of the freestream velocity as shown in blue.

Therefore, using (1) and according to Blade Element Theory, thrust force and moments of each blade element can be calculated as follows:

$$
\begin{gathered}
d f_{p}=\frac{1}{2} \rho_{a} c C_{L} v^{2} d r_{b} \\
d \tau_{d p}=\frac{1}{2} \rho_{a} c C_{D} v^{2} r_{b} d r_{b} \\
d \tau_{p}=\frac{1}{2} \rho_{a} c C_{L} v^{2} r_{b} \sin \psi_{p} d r_{b}
\end{gathered}
$$

where $\rho_{a}$ represents air density and $C_{L}$ and $C_{D}$ are the lift and drag coefficient of the airfoil of the blade respectively. Also, $f_{p}$ represents thrust force of the blade element, $\tau_{d_{p}}$ represents the moment due to drag force of the blade element and $\tau_{p}$ represents the moments due to change in thrust force with respect to the azimuth angle of the blade.
By integrating (2), (3) and (4) over blade radius $r_{b}$ and azimuth angle $\psi_{p}$, average thrust force and the average moments for one blade as functions of freestream velocity and angular velocity of the propeller can be calculated as follows:

$$
\begin{gathered}
f_{p}=\frac{1}{2} \rho_{a} c C_{L}\left(\frac{2 R_{b}^{3}}{3}\left\|\omega_{p}\right\|^{2}+\left\|V \infty_{1}\right\|^{2} R_{b}\right) \\
\tau_{d_{p}}=\frac{1}{4} \rho_{a} c C_{D}\left(R_{b}\left\|\omega_{p}\right\|^{2}+\left\|V \infty_{1}\right\|^{2} R_{b}^{2}\right) \\
\tau_{p}=\frac{1}{2} \rho_{a} c C_{L} R_{b}^{3}\left\|\omega_{p}\right\|\left\|V_{\infty_{1}}\right\|
\end{gathered}
$$

Note that by assuming zero freestream velocity, equations (5), (6) and (7) yield the simplified model for thrust force and moments of a propeller which is widely used in the literature (i.e., [3], [10], [11], [12]).

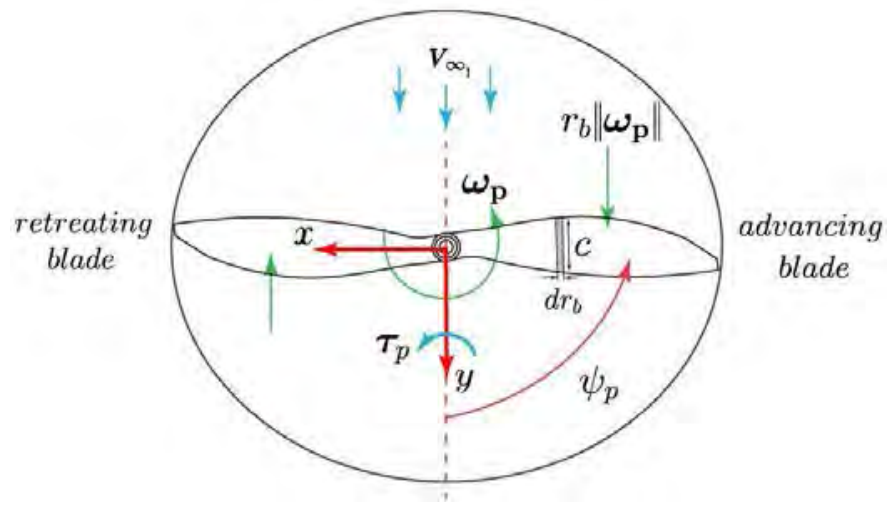

Figure 2: A propeller in presence of freestream.

Using the proposed model, simulation results for two complete turns of a propeller with angular velocity $\left\|\omega_{p}\right\|=900 \mathrm{rad} / \mathrm{s}$ in presence of freestream velocity is presented in Fig. 3. Note that the direction of rotation and freestream velocity are the same as those in Fig. 2. The remaining parameters of the simulations are as follows: $c=0.03 \mathrm{~m}$, $C_{L}=1.022, C_{D}=0.01, R_{b}=0.08 \mathrm{~m}$ and $\rho_{a}=1.225 \mathrm{~kg} / \mathrm{m}^{3}$. In Fig. 3, the top plot presents variations of thrust force with respect to blade azimuth. The red color represents the thrust force when $\left\|V_{\infty_{1}}\right\|=0$, which is constant, meaning that the relative air flow velocity over the blade element is constant for all azimuth angles. The blue color, represents thrust force of the propeller as a function of azimuth angle when freestream velocity is nonzero, $\left\|V_{\infty_{1}}\right\|=10 \mathrm{~m} / \mathrm{s}$. Comparing both scenarios, it can be seen that for nonzero freestream velocity and for $0 \leq \psi_{p} \leq \pi$ thrust force is increased while for $\pi \leq \omega_{p} \leq 2 \pi$ it is decreased which is due to higher relative air flow velocities on the advancing blade than that over the retreating blade. The 
yellow color shows the average of thrust force of the propeller when $\left\|V_{\infty_{1}}\right\|=10 \mathrm{~m} / \mathrm{s}$. Results show that in presence of nonzero freestream velocity the average thrust force of the propeller increases.
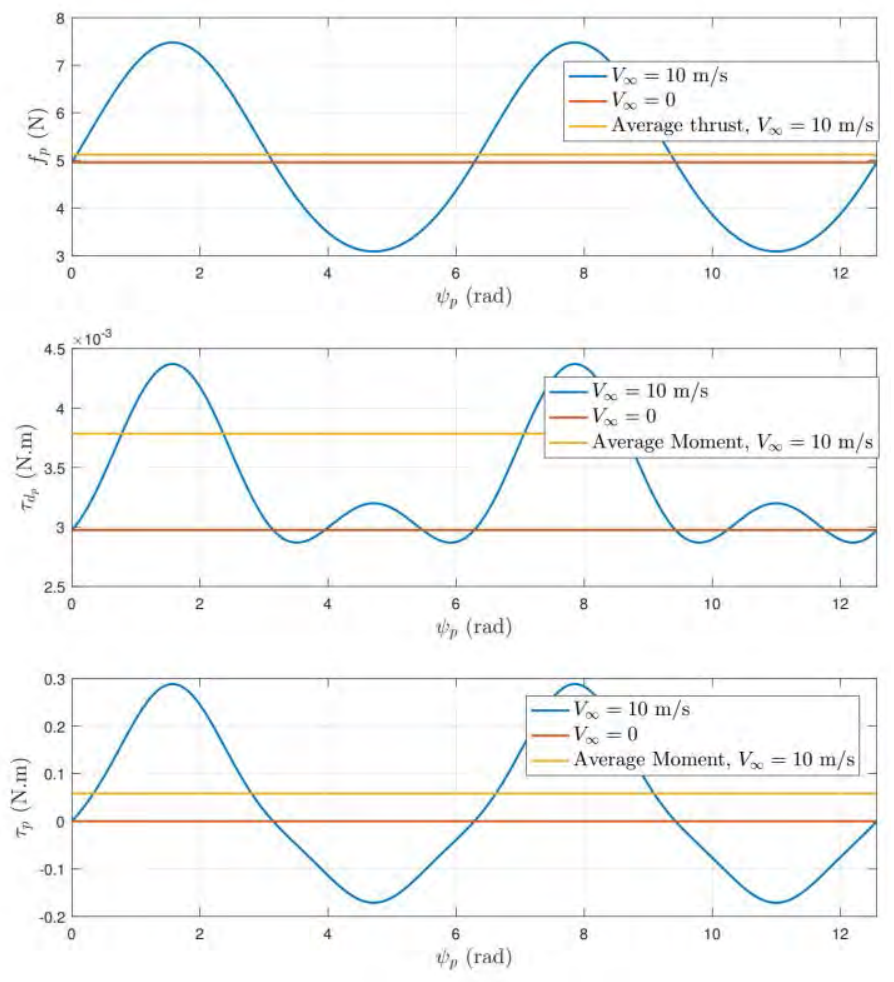

Figure 3: Simulation results for thrust force and moments of a propeller in presence of freestream.

The middle and bottom plots in Fig. 3 present variations of the moments due to drag and freestream $\tau_{d_{p}}$ and $\tau_{p}$ ) versus azimuth angle respectively. The red color represents the moment when $\left\|V_{\infty_{1}}\right\|=0 \mathrm{~m} / \mathrm{s}$, blue color represents variations of moment in presence of freestream velocity $\left\|V_{\infty_{1}}\right\|=10 \mathrm{~m} / \mathrm{s}$ and the yellow color represents the average moment of the propeller when $\left\|V_{\infty_{1}}\right\|=10 \mathrm{~m} / \mathrm{s}$.

\section{Effects of Freestream with Its Velocity Vector in Parallel with Angular Velocity Vector of the Propeller}

We continue investigating the effects of freestream on propeller's performance. However, as shown in Fig. 4, this time we assume the freestream velocity vector, $V_{\infty_{2}}$, is parallel to the angular velocity vector of the propeller, $\omega_{p}$, as expressed in the frame attached to it.

Suppose the propeller is turning at angular velocity $\omega_{p}$ as shown in Fig. 5. In absence of freestream, there will be an airflow velocity vector $r_{b} \omega_{p}$ over each blade element as shown in green. Also, for each blade element, the angle of attack $(\mathrm{AOA}) \Theta$ is defined as the angle between the chord of the blade element and the local airflow velocity vector $r_{b} \omega_{p}$.

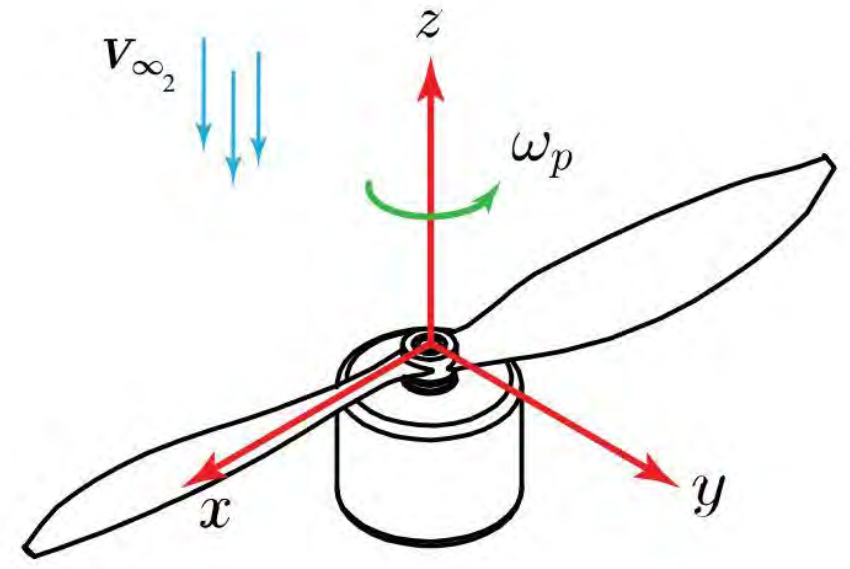

Figure 4: Propeller in presence of freestream. The freestream velocity vector $V_{\infty_{2}}$ is assumed to be parallel with the angular velocity vector of the propeller $\omega_{p}$.

Now, consider an almost uniform freestream with velocity vector $V_{\alpha_{2}}$ in the positive direction of z-axis as shown in blue in Fig. 5 top. It can be seen that such a freestream changes the direction and magnitude of the resultant airflow velocity over the blade element as shown in red in Fig. 5 top. Therefore, the new angle of attack $\Theta^{\prime}$, in presence of freestream is greater than that in absence of freestream $\left(\Theta^{\prime} \geq \Theta\right)$.

However, if the freestream velocity vector is in the negative direction of z-axis (see Fig. 5 bottom), it changes the direction and magnitude of the resultant airflow velocity vector such that it decreases the effective angle of attack $\left(\Theta^{\prime} \leq \Theta\right)$.
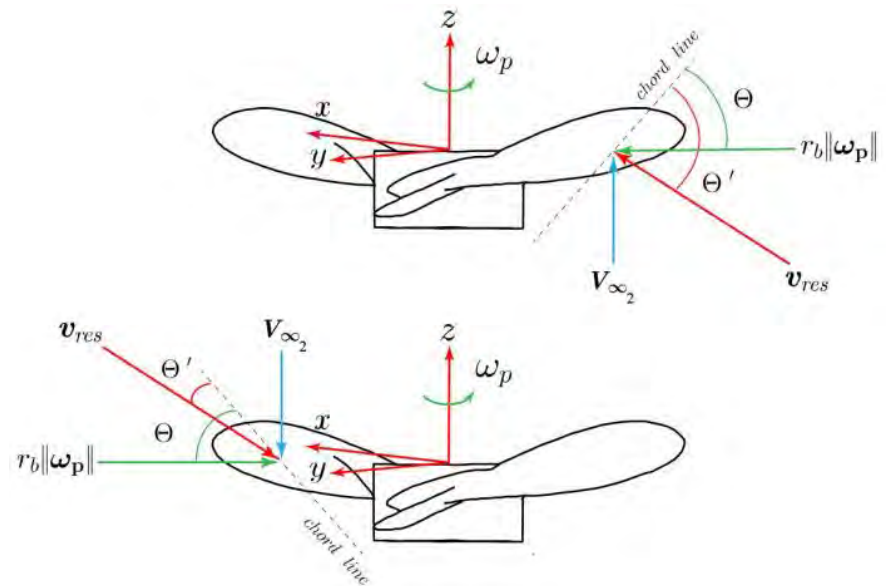

Figure 5: Propeller in presence of freestream. The freestream velocity vector $V_{\infty_{2}}$ is assumed to be parallel with $\omega_{p}$. On top, $V_{\infty_{2}}$ is the positive direction of $\mathrm{z}$-axis and in bottom it is assumed to be in the opposite direction.

The importance of studying AOA is because it directly affects the lift coefficient of the blade element and consequently affects the thrust force generated by the propeller. At low speed flight (subsonic) and assuming small angles, the lift coefficient $C_{L}$ changes almost linearly with AOA which can be written as follows [13]: 


$$
\frac{\Delta C_{L}}{\Delta \Theta}=\sigma
$$

where $\sigma$ is a constant which is determined through experiments in wind tunnel. From (2), we know that any increase (decrease) in $C_{L}$ increases (decrease) the thrust force of the blade element. Therefore, one can summarize the results as follows:

- Any freestream with positive (negative) z-component velocity (expressed in the propeller's frame) increases (decreases) the AOA which increases (decreases) the thrust force.

Furthermore, to formulate the changes in thrust force of the propeller, first we can write the changes in AOA of each blade element as follows:

$$
\Delta \Theta=\Theta-\Theta^{\prime}=\arctan \frac{\left\|V_{\infty_{2}}\right\|}{\left\|r_{b} \omega_{p}\right\|}
$$

Finally, using (2), (8) and (9), the changes in thrust force of the propeller can be written as follows:

$$
\Delta f_{p}=\frac{1}{4} \rho_{a} c \sigma R_{b}^{2}\left\|V_{\infty_{2}}\right\|\left\|\omega_{p}\right\|
$$

From (10), it can be seen that the changes in thrust force is proportional to the magnitude of the freestream velocity vector $V_{\infty_{2}}$.

Table I presents the parameters involved in the modeling.

TABLE I. Propeller Parameters

\begin{tabular}{|c|c|}
\hline$\omega_{p}$ & angular velocity vector of the propeller \\
\hline$\Theta$ & angle of attack \\
\hline$C_{L}$ and $C_{D}$ & lift and drag coefficients of the airfoil \\
\hline$V_{\infty}$ & freestream velocity vector \\
\hline$\sigma$ & slope of $C_{L}$ vs $C_{D}$ curve for the airfoil \\
\hline$R_{b}$ & blade radius of the propeller \\
\hline$c$ & chord of the blade \\
\hline$\rho_{a}$ & air density \\
\hline
\end{tabular}

\section{CONCLUSIONS}

In summary, we presented the significance of the effects of freestream on performance of propellers. We formulated these effects as functions of propeller parameters and also the parameters of the freestream. Table I, presents all parameters involved in the proposed propeller model.
The proposed propeller model may be used in modeling multi-rotor UAVs. Such a model is used in quadcopters where it is shown that by tilting the rotors by a small angle as shown in Fig. 6, more stable or maneuverable configurations can be found [8]. The model is also used in modeling spinning-type multi-rotor UAVs to find optimal-power hover solutions. Fig. 7, presents a monospinner UAV with a single propeller where the spinning fuselage generates a freestream over the propeller [9].

For the future work, we plan to compare the proposed model with experimental results from experiments in wind tunnel for a particular propeller and investigate the accuracy and shortcomings of the model.

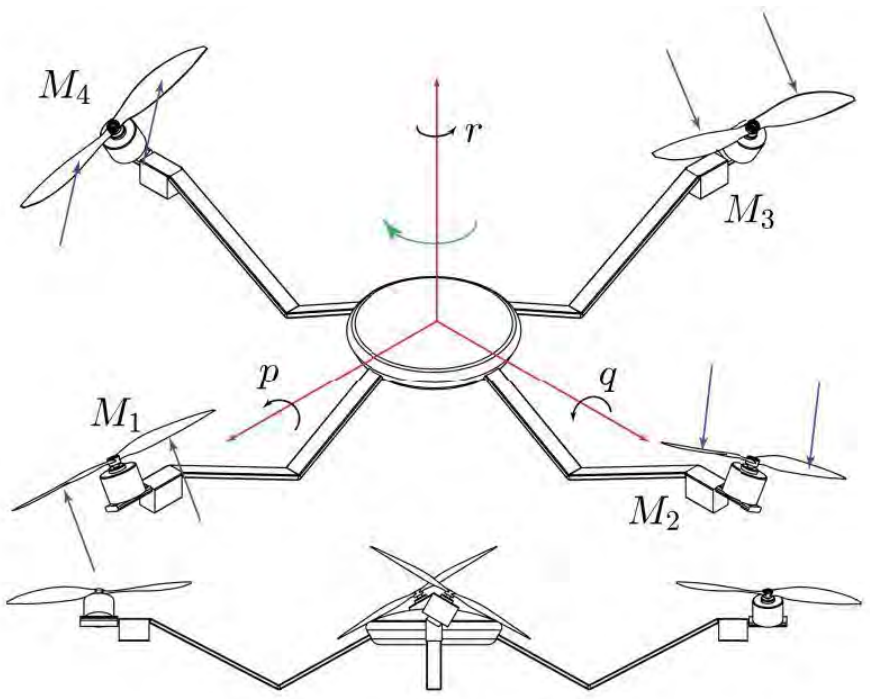

Figure 6: A quadcopter with tilted rotors. The blue arrows shows the direction of freestream velocity on each propeller as expressed in the body frame (red).

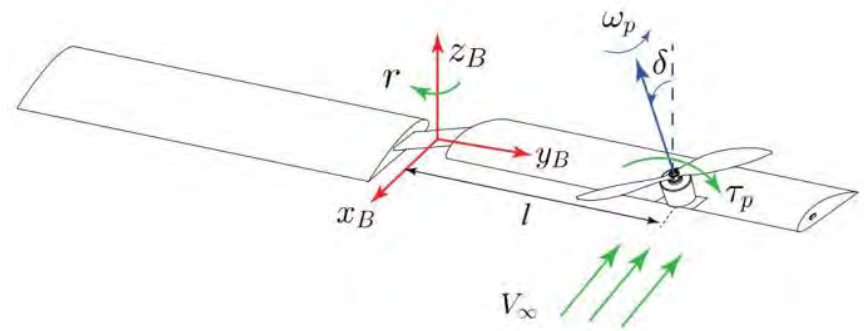

Figure 7: A monospinner UAV with tilted rotor. As the fuselage is spinning with yaw rate $r$, the propeller experiences a freestream, as shown in green.

\section{REFERENCES}

[1] J. Thomas et al., "Avian-inspired grasping for quadcopter micro uavs,” Bioinspir. Biomim., vol. 9, pp. 25010-25019, May 2014. doi: $10.1088 / 1748-3182 / 9 / 2 / 025010$.

[2] J. Rasmussen et al., "Potential uses of small unmanned aircraft systems (uas) in weed research," Weed Res., vol. 4, no. 53, pp. 242-248, 2013.

[3] M. Muller, S. Lupashin, and R. D'Andrea, "Quadrocopter ball juggling," in 2017 IEEE/RSJ Int. Conf. on IROS, pp. 5113-5120. 
[4] L. F. Luque-Vega et al., "Power line inspection via an unmanned aerial system based on the quadrotor helicopter," in MELECON 2014 - 17th IEEE Mediterranean Electrotechnical Conf., pp. 393-397. doi: 10.1109/MELCON.2014.6820566.

[5] M. Y. Amir and V. Abbass, "Modeling of quadrotor helicopter dynamics," in Int. Conf. on Smart Manufacturing Application, 2008, pp. 100-105.

doi: 10.1109/ICSMA.2008.4505621.

[6] D. Mellinger, N. Michael, and V. Kumar, "Trajectory generation and control for precise aggressive maneuvers with quadrotors," Int. J. Rob. Res., vol. 31, no. 5, pp. 664-674, Apr. 2012. doi: $10.1177 / 0278364911434236$.

[7] J. Wang et al., "Attitude free position control of a quadcopter using dynamic inversion," in AIAAInfotech@Aerospace, 2011, pp.29-31.

[8] M. Hedayatpour, M. Mehrandezh, and F. Janabi-Sharifi, "A unified approach to configuration-based dynamic analysis of quadcopters for optimal stability," in 2017 IEEE/RSJ Int. Conf. on IROS, pp. 5116-5121. doi: 10.1109/IROS.2017.8206397.

[9] M. Hedayatpour, M. Mehrandezh, and F. Janabi-Sharifi, "Revised propeller dynamics and energy-optimal hovering in a monospinner," in the 4th Int. Conf. of Control, Dynamic Systems, and Robotics, Aug. 2017, pp. 1-8. doi: 10.11159/cdsr17.135

[10] M. W. Mueller and R. D'Andrea, "Relaxed hover solutions for multicopters: application to algorithmic redundancy and novel vehicles," Int. J. of Rob. Res., vol. 35, no. 8, pp. 873-889, July 2016. doi: 10.1177/0278364915596233.

[11] M. W. Mueller and R. D'Andrea, "Stability and control of a quadrocopter despite the complete loss of one, two, or three propellers," in 2014 IEEE Int. Conf. on Robotics and Automation (ICRA), p. 4552. doi: 10.1109/ICRA.2014.6906588.

[12] M. Hehn and R. D'Andrea, "Quadrocopter trajectory generation and control," in 18th IFAC World Congr, Milano, 2011, pp. 1485-1491. doi:10.3182/20110828-6-IT-1002.03178.

[13] J. D. Anderson, Fundamentals of Aerodynamics, 5th ed. New York: McGraw-Hill, 2016. 


\section{ADVANCED ENERGY SYSTEMS}




\section{Temperature Measurements of $\mathbf{n}$-Heptane Injection and Compustion in the Ignition Quality Test (IQT $\left.{ }^{\mathrm{TM}}\right)$}

\author{
Osama Hmood and Edgar Matida \\ Department of Mechanical and Aerospace Engineering \\ Carleton University \\ Ottawa-Ontario, Canada \\ e-mail: osama.hmood@carleton.ca
}

\author{
Dave Gardiner, Luc Menard, Omar Ramadan, \\ Aaron Wilcox, and Gary Webster \\ Advanced Engine Technology Ltd. \\ Ottawa-Ontario, Canada \\ e-mail: gary@aet.ca
}

\begin{abstract}
An experimental work has been conducted to investigate the ignition of $n$-heptane in the ignition quality tester $\left(\mathrm{IQT}^{\mathrm{TM}}\right)$ by using fine gage thermocouples. A set of eight buttwelded exposed junction thermocouples (TCs) were inserted inside the chamber of $\mathrm{IQT}^{\mathrm{TM}}$ at different preselected locations to get the temperature variation spatially and temporally in the period prior to the fuel injection up to the mixture ignition. The data were recorded by using a high frequency data acquisition system with a sampling rate of $10 \mathrm{kHz}$ per channel. The experimental data were characterized at set point temperature $584{ }^{\circ} \mathrm{C}$ and pressure 21.3 bar. For reproducibility purposes, 32 injections have been averaged and presented. A drop in temperature due to fuel spray cooling effects has been recorded by TCs at the chamber centerline whereas TCs near the chamber walls did not show a significant drop in temperature. Thus, there was no evidence that the fuel spray reached the chamber walls. The measurements showed that the combustion occurs in the main part of the chamber and the region near the fuel injector nozzle did not show a significant rise in temperature.
\end{abstract}

Keywords- heptane; combustion; injection; pressure; spray; thermocouple; temperature.

\section{INTRODUCTION}

Diesel engines are more economical than gasoline engines but generate different toxic emissions (particulate matter, PM, in addition to elevated NOx, as well as $\mathrm{CO}$, and hydrocarbons) in their exhaust gases, which present major risks on public health and environment. Extensive work has been conducted on combustion of diesel fuel and its influence on the performance of compression ignition engines [1]. Most studies discuss the measurement of the time interval between the start of fuel injection until the start of combustion which is called ignition delay (ID) period.

The ignition quality of diesel-like fuels is rated through their cetane number $(\mathrm{CN})$ which is inversely proportional to the ID period. The Cooperative Fuel Research (CFR) test engine under designation number ASMT D613 [2] is commonly used to determine the $\mathrm{CN}$ of diesel fuels. Techniques which use constant volume combustion chambers to analyze diesel ignition have been developed and the results have shown an excellent agreement with engines [3]. Therefore, three standard test methods [4] using constant volume combustion chambers have been developed to test diesel fuels and obtain their Ignition Delay (ID) or Derived Cetane Number (DCN). The Ignition Quality Tester (IQT ${ }^{\mathrm{TM}}$ ), the most precise device used to rate diesel fuels in the present [5], is one of these standards with designation number ASTM D6890.

Most of the experimental data involving the $\mathrm{IQT}^{\mathrm{TM}}$ are related to pressure measurements. There seems to be a void in temperature measurements, although temperature distributions inside the IQT ${ }^{\mathrm{TM}}$ during operation conditions have been obtained numerically $[6,7]$. The present work aims to analyze $\mathrm{n}$-heptane spray behavior and combustion in the IQT ${ }^{\mathrm{TM}}$ through temperature measurements by using multiple fine gage thermocouples located throughout the combustion chamber. These measurements have the potential to identify the regions within the chamber where fuel vaporization takes place and combustion is initiated.

\section{EXPERIMENTAL SETUP}

\section{A. Ignition Quality Tester (IQT $\left.{ }^{T M}\right)$ Overview and Setting}

The IQT ${ }^{\mathrm{TM}}$ has been developed and automated by Advanced Engine Technology Ltd. (AET) [8] to make it a precise and accurate system for oil industry, research and regulatory applications $[9,10]$. It is a bench-scale device which is mainly utilized to determine the ignition quality of diesel and alternative diesel fuels. It is comprised of a constant volume combustion chamber with intake/exhaust systems, a fuel injection system, temperature/pressure sensors and a dataacquisition system. Detailed information on the IQT ${ }^{\mathrm{TM}}$ can be found in [4].

There are six ports and holes on the walls of the IQT ${ }^{\mathrm{TM}}$ chamber as shown in Figure 1: nozzle body port on one end of the chamber, pressure transducer port on the other end, air inlet valve, exhaust outlet valve, and front \& back thermocouple 


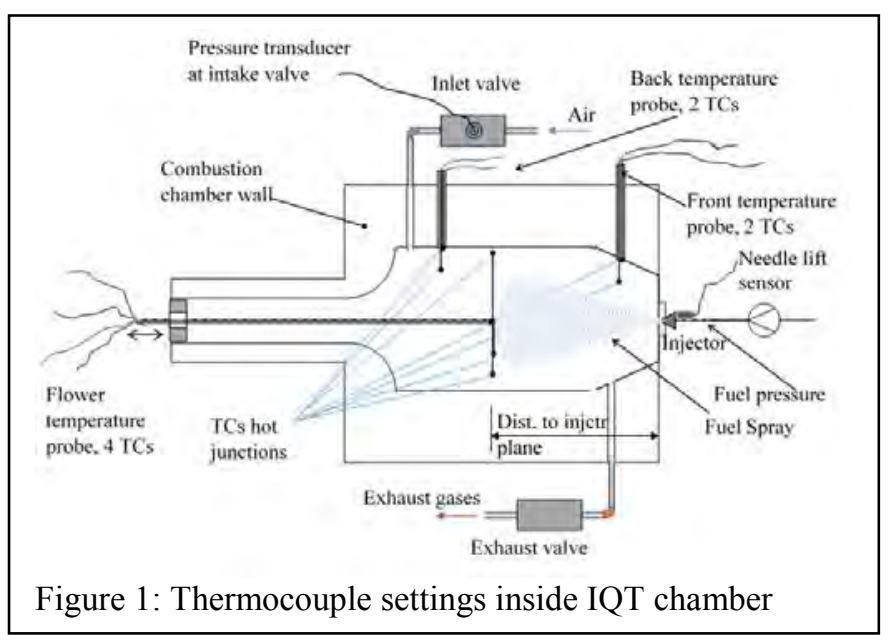

holes. Nine electrical heaters of cartridge-type are embedded in the chamber walls and are used to heat chamber walls up to approximately $600^{\circ} \mathrm{C}$. The combustion chamber is a cavity of cylindrical and conical shapes with total volume of $0.213 \pm 0.002$ liters. The charge-air gains the heat from the hot walls of the chamber. Both ends of the $\mathrm{IQT}^{\mathrm{TM}}$ chamber are maintained at a lower temperature compared to the temperature of main portion of the chamber to protect sensors located at the extremities.

The fuel is injected into the chamber as a hollow spray cone from an inward opening single-hole pintle-type nozzle. The spray characteristics from this nozzle design and others are well studied [11]. The nozzle needle starts to lift and the fuel is injected when the pressure of the liquid fuel line behind the nozzle reaches $17.92 \mathrm{MPa}$. The main injection duration lasts for approximately $2 \mathrm{~ms}$ [12].

\section{B. Temperature Measurement Settings}

Only three out of the six ports on chamber wall could be used to install temperature probes that would protrude into the chamber: front and back TC ports and pressure transducer port. An 1/8 inch probe with two TCs could be used with either front or back TC ports because those ports are already fitted for $1 / 8$ inch temperature probes and they did not require any modifications.

Slight modifications have been made on the IQT ${ }^{\mathrm{TM}}$ chamber to allow adjustment of the axial temperature probe. The port of the pressure transducer was used to insert a probe with $4 \mathrm{TCs}$, which was named "flower" temperature probe (FTP). The coolant adapter of the pressure sensor was modified to accommodate 3/16 inch diameter probe. The pressure sensor was moved with its housing and fitted to the intake valve port. The data from the pressure sensor at the new location was verified against the standard location, showing adequate fidelity. Thus, a total of 8 TCs ( 2 at the front port, 2 at the back port, and 4 through the pressure transducer port) were fitted inside and near the wall of the combustion chamber as shown in Figure 1.

\section{Thermocouple Selection and Temperature Probe Fabrication.}

A robust temperature probe was required in order to withstand the harsh combustion environment presented by the high temperature, pressure and turbulence, and to track and record the instantaneous temperature variation. Thermocouples (TC) were selected for the present measurements due to their relative robustness, response time, and wide temperature range of operation $[13,14]$ compared to other temperature sensors.

Thermocouple of type $\mathrm{K}$ has been selected to be used in all experiments due to its high sensitivity $(41 \mu \mathrm{V} / \mathrm{C})$ and availability [13].

Three sizes of TC wires (0.003", 0.005 ", and 0.010 " of a butt welded hot junction end) were tested to achieve a compromise between response and durability. Although they exhibited good transient response, the 0.003 " and 0.005 " TCs did not withstand the harsh environment. They were damaged during warming-up time of the combustion chamber or at mid combustion. The 0.010 " TC had a very good transient response to the temperature variations especially at discriminatory points (Start Of Injection (SOI) and Start Of Combustion (SOC)). Thus, 0.010 " butt welded TC wires of type K from Omega were adopted for temperature measurements.

Based on the dimensions of the front and back TC ports and pressure transducer port, two sizes (1/8" and 3/16") of stainless steel sheath tubes were used to encase the temperature probes. An alumina ceramic insulator rod was used around the TCs before insertion of the probe into the sheath tubes [15]. The $1 / 8$ " probe contains two thermocouples and the $3 / 16$ " probe contains four thermocouples. Each thermocouple required two holes in the ceramic insulator rod. A maximum of 4 holes (2 TCs) and 8 holes could be created on the face of a $2.5 \mathrm{~mm}$ and $3.2 \mathrm{~mm}$ diameter ceramic rods, respectively.

\section{Connections and Data logging}

Tests were conducted in the laboratory at the AET facilities. All sensors were fitted to the chamber and connected to the data logger before the test as shown in Figure 2. Two temperature probes with $2 \mathrm{TCs}$ each were fitted in the front and back TC ports. The flower temperature probe (FTP) with 4 TCs was inserted into the chamber from the pressure transducer port through a bored coolant adapter. All the TC wires of the hot junction side of the FTP were kept straight in the axial direction at first. Then, they were bent inside the chamber by $90^{\circ}$ to be distributed radially (see Figure 1). TCs wires were bent manually by getting access to them from the injector nozzle side. For sealing purposes, a reusable Teflon ferrule was used with the stainless steel compression fittings to allow the FTP to be traversed inside the chamber.

All the sensors are connected to a laptop through a data acquisition system from Omega of model OMB-DAQ-3005. DAQ software (Daq view) settings are adjusted to synchronize the feedback from the sensors. The data were collected for the period beginning $10 \mathrm{~ms}$ before needle lift until the end of combustion, with a sampling interval of $0.1 \mathrm{~ms}$. The recorded 


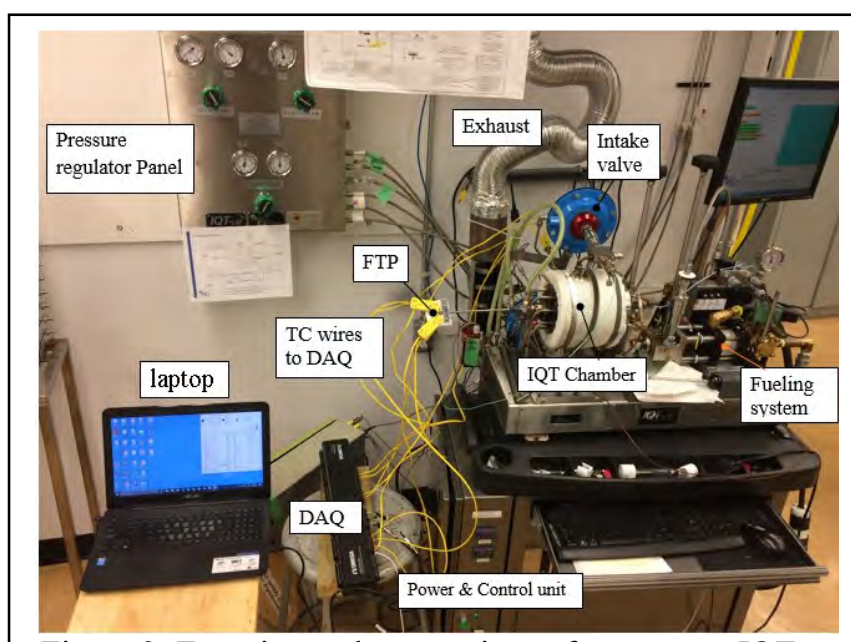

Figure 2: Experimental connections of sensors on IQT to the DAQ

data sets for all injections were saved automatically into the preselected file names. A Matlab code was created to analyze and present the collected data. The data of 32 test injections were low-pass filtered first and then the mean values were calculated for temperatures, pressure and needle lift as shown in the following figures below.

\section{E. Points of Temperatre Measurements}

Temperature variations were captured at 20 different locations inside the IQT ${ }^{\mathrm{TM}}$ chamber as shown in Figure 3. The front or back TCs locations are: first TC was at the wall chamber plane, and the second TC was $8 \mathrm{~mm}$ from the wall in a radial direction. The 4 TCs of the FTP were distributed radially as follows: the first $\mathrm{TC}$ was at chamber centerline $(\mathrm{r}=0 \mathrm{~mm})$, the second TC was $12 \mathrm{~mm}$ from center, the third was $18 \mathrm{~mm}$ from center, and the fourth was $25 \mathrm{~mm}$ from the center which is almost on the wall.

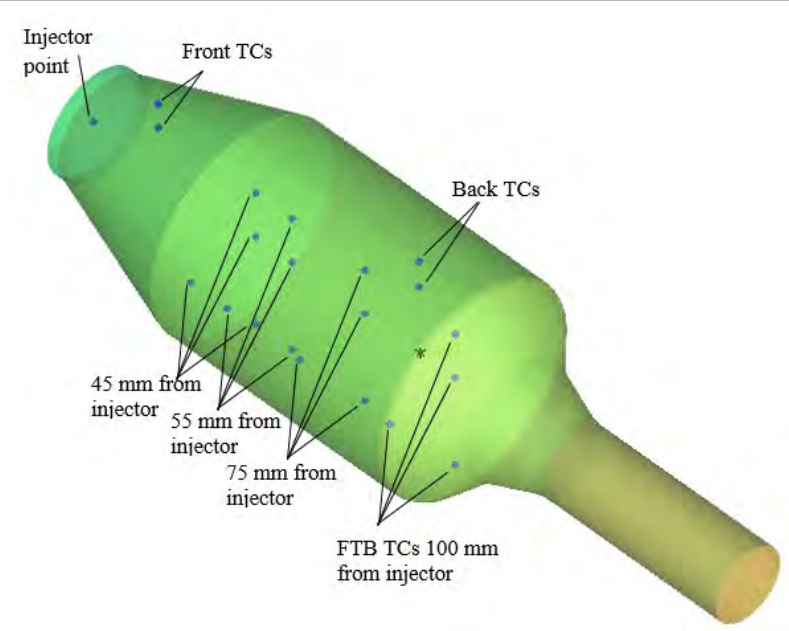

Figure 3: Temperature measurement locations in the IQT chamber

\section{RESULTS AND DISCUSSION}

The IQT ${ }^{\mathrm{TM}}$ was calibrated first and the test conditions were $584^{\circ} \mathrm{C}$ for set point, $310 \mathrm{psi}$ (21.3 bar) for charge air pressure and 0.8231 grams per 10 injections of heptane. Four runs on heptane were conducted at four different axial locations of the FTP, where the distances from the probe plane to the injector were 100, 75, 55, and $45 \mathrm{~mm}$ as shown in Figure 3. The three main parameters: nozzle needle position, combustion chamber pressure and spatial gas temperature are collected from needle lift sensor, pressure transducer (mounted in a new position) and TC probes, respectively.

\section{A. Needle lift and Chamber Pressure}

The nozzle needle lift position and the chamber pressure traces for 32 individual injections, along with their mean value trace (thick line) are presented in Figures 4 \& 5, respectively. The ignition delay period begins when the needle lift signal begins to rise. All injections have the same starting point and the peak of needle lift is around $0.6 \mathrm{~mm}$. The main injection duration lasts in about $2 \mathrm{~ms}$.

The chamber pressure (Figure 5) initially drops slightly due to cooling effects of evaporating spray. The required time for atomization, vaporization, mixing and heating of the injected fuel is called physical ignition delay period. During this period, the pressure and temperature inside the chamber decrease. This period is followed by a sudden increase in pressure and temperature due to heat released from the chemical reaction.

\section{B. Temperature measurement results}

The temperature variation at two locations were selected to be presented in this section: chamber centerline at a plane 55 $\mathrm{mm}$ from injector, and back port at chamber wall.

The time history of the gas temperature measured by FTP for 32 injections is given in Figure 6. The temperature drops initially due to the evaporation of the spray (as mentioned earlier), then the combustion will take place. The maximum temperature measured by FTP was around $1300{ }^{\circ} \mathrm{C}$. Notice the strong scatter of the temperature measurements from 25 to 500 $\mathrm{ms}$, indicating significantly different combustion processes for each injection during this interval. Nevertheless, the onset of

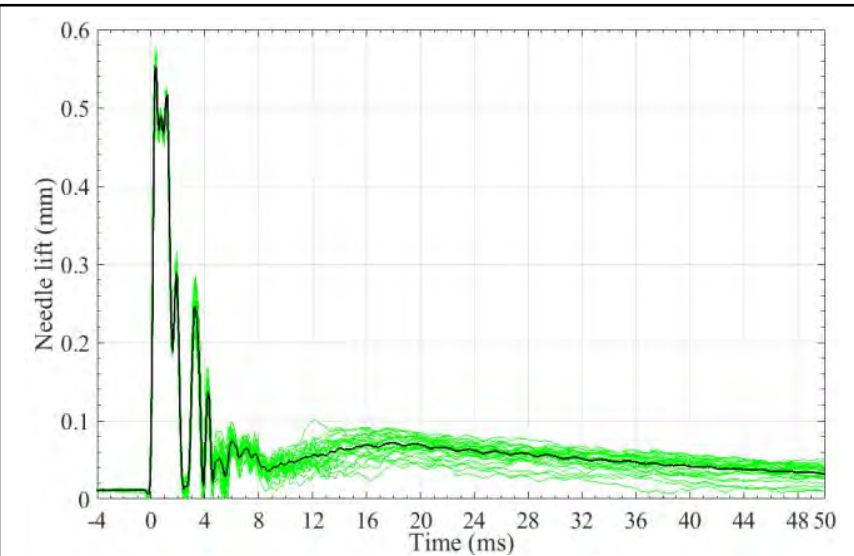

Figure 4: Needle lift trace of 32 injections with mean 


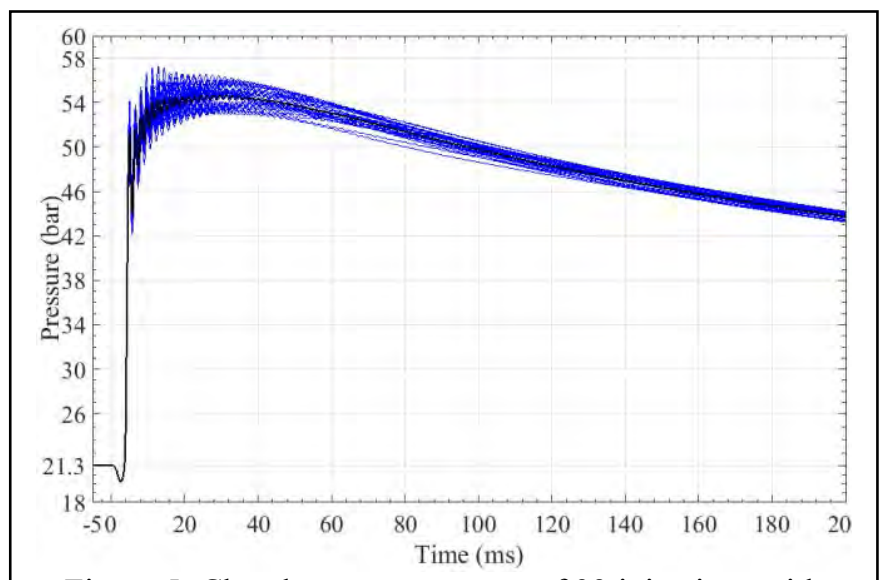

Figure 5: Chamber pressure trace of 32 injections with mean

combustion (the region of interest from 0 to $5 \mathrm{~ms}$ ) shows high reproducibility.

Temperature profiles at back port of chamber (Figure 7) are slightly different to that at the main part of the chamber (Figure 6). There was no drop in temperature recorded after fuel injection and before the sudden increase in temperature. In this case, a maximum temperature around $850{ }^{\circ} \mathrm{C}$ was recorded. The peak temperature at chamber wall, (Figure 7) continues for a very short period of time and then drops.

\section{Mean Temperature, Pressure and Needle lift Results at ID Period}

To observe clearly temperature variation with pressure during the ID period, the mean values of 32 low pass filtered test injections are presented for the time prior to the fuel injection up to the fuel's IOC as shown in Figures 8 and 9. Three parameters (temperature, pressure and needle lift) are shown on the each figure.

Figure 8 gives temperature variations in four locations along the chamber centerline. The temperature of pressurized air prior to the fuel injection at locations 3 and 4 is higher than that at locations 1 and 2 by almost $10^{\circ} \mathrm{C}$ and it drops when the released

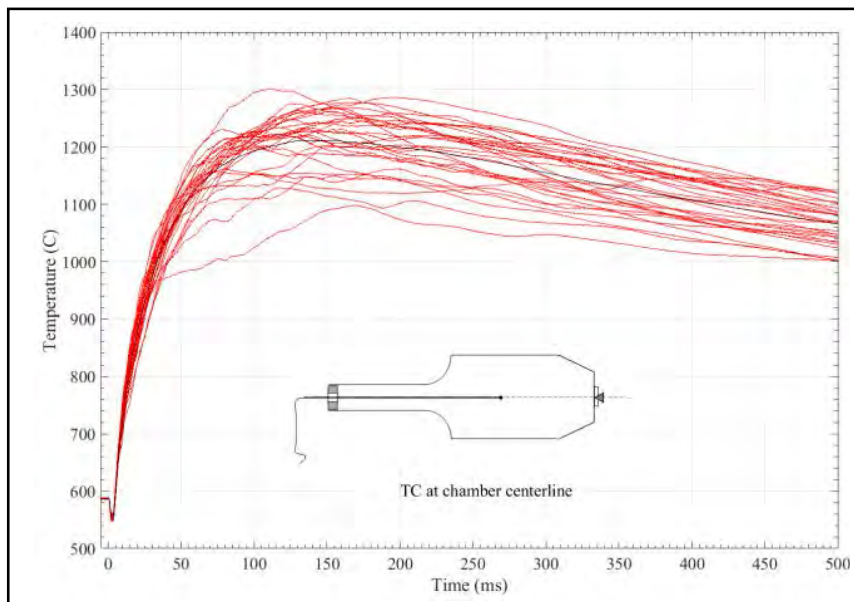

Figure 6: Temperature variation of 32 test injections with mean value from FTP

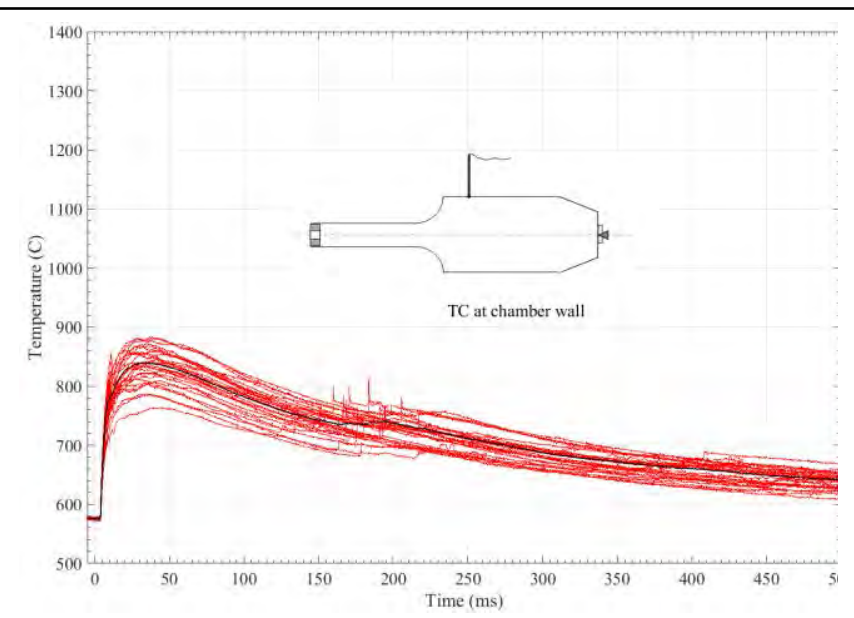

Figure 7: Temperature variation of 32 test injections with mean value at chamber wall, back port

spray from injector nozzle reaches the thermocouples. All four curves have shown a drop in temperature for various periods and are followed by that a sudden increase in temperature. The drop in temperature was due to the high vaporization rate of the fuel, which absorbs heat during that time. The starting point of that drop in temperature depends on the location of the thermocouple. Thus, the closest TC (line 4) to the injector nozzle sensed the spray first after SOI by $0.5 \mathrm{~ms}$ whereas the farthest one (line 1) sensed the spray after almost $1.25 \mathrm{~ms}$ from SOI. The lowest temperature was measured at location 2 . The rate of change in temperature $(\mathrm{dT} / \mathrm{dt})$ in the rear part of the chamber (locations 1 and 2) was close to zero (temperature does not change with time) and remains at lowest point for a while $(0.5-0.8 \mathrm{~ms})$ before the sharp rapid increase which gives an indication of the IOC at that location. After that, the rate of increase in $\mathrm{dT} / \mathrm{dt}$ is almost the same for all locations.

The results from the standard front and back TC locations of the IQT ${ }^{\mathrm{TM}}$ (at the wall and $8 \mathrm{~mm}$ from the wall) are presented

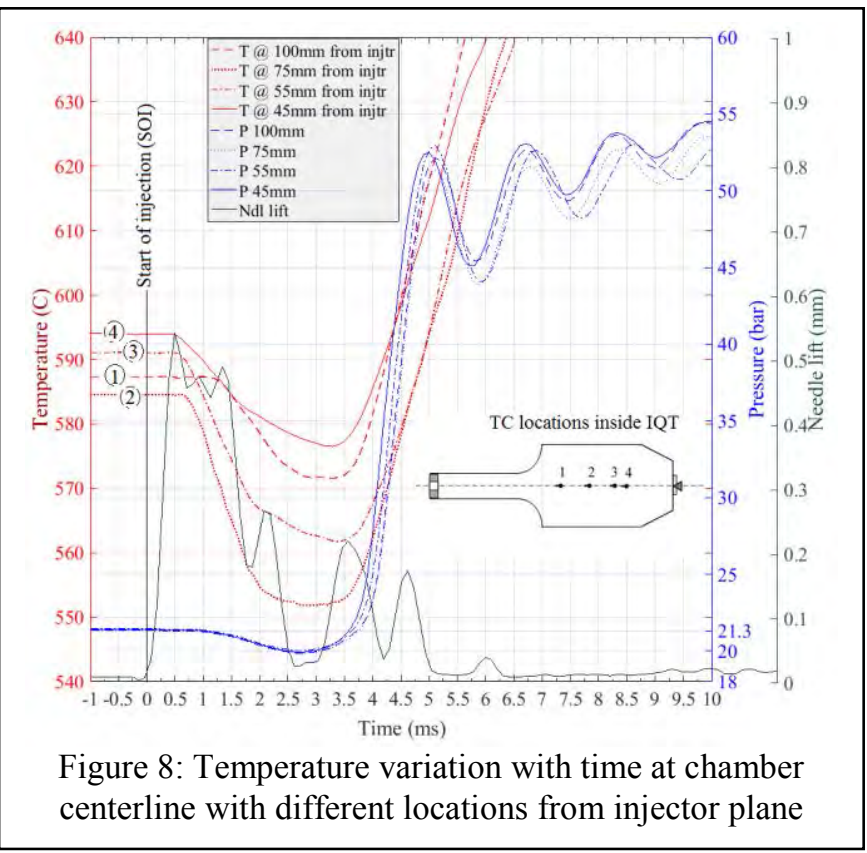




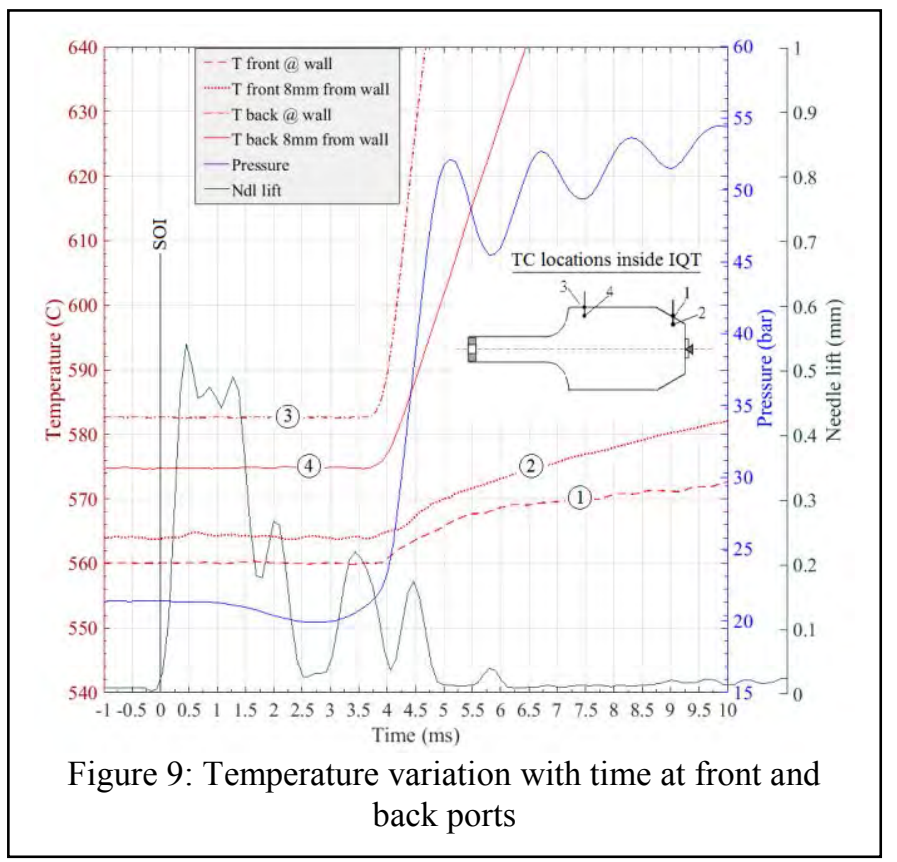

in the Figure 9. None of these TC locations recorded a drop in temperature during the period between SOI and SOC. The temperatures at the front $\mathrm{TC}$ location showed only a minor increase after IOC whereas a sudden increase in temperature was measured from front port TCs. For the sake of conciseness, additional data at different locations are not shown here.

\section{CONCLUSIONS}

Fine gage, exposed junction thermocouples were successfully used to trace temperature variations at twenty different locations in the combustion chamber of an ignition quality tester. The significant drop (about $30^{\circ} \mathrm{C}$ ) in temperature due to an evaporative cooling effect of the fuel spray was recorded at the region of chamber centerline. On the other hand, no evidence of cooling by fuel spray droplets was observed in measurements at and near the chamber walls, where only sudden rise in temperature (due to combustion) was recorded. These results indicate that fuel spray vaporization in the IQT ${ }^{\mathrm{TM}}$ takes place in the compressed, heated air within the combustion chamber rather than through contact with the hot chamber walls.

\section{REFERENCES}

[1] P. A. Lakshminarayanan and Y. V. Aghav, Modelling Diesel Combustion. Dordrecht; New York: Springer, 2010. doi: 10.1007/978-90-481-3885-2.

[2] A. I. Standards, "Standard test method for cetane number of diesel fuel oil," in Annual Book of ASTM Standards, vol. 5.05, pp. 3-19, ASTM International, Feb. 2015.

[3] T. W. Ryan and T. J. Callahan, "Engine and Constant Volume Bomb Studies of Diesel ignition and Combustion," in SAE Technical Paper Series, 1988. doi: $10.4271 / 881626$.

[4] A. I. Standards, "Standard test method for determination of derived cetane number (den) of diesel fuel oils- ignition delay and combustion delay using a constant volume combustion chamber method," in Annual Book of ASTM Standards, vol. 5.05, pp. 158-187, ASTM International, Feb. 2015.

[5] J. Yanowitz, M. A. Ratcliff, R. L. McCormick, J. D. Taylor, and M. J. Murphy, "Compendium of Experimental Cetane Numbers," Office of Scientific and Technical Information (OSTI), Feb. 2017. doi: 10.2172/1345058.

[6] G. E. Bogin Jr. et al., "Numerical and Experimental Investigation ofnHeptane Autoignition in the Ignition Quality Tester (IQT)," Energy \& Fuels, vol. 25, no. 12, pp. 5562-5572, Dec. 2011. doi: 10.1021/ef201079g.

[7] H.-L. Tsai, J.-Y. Chen, and G. T. Chin, "Validation of a Newly Developed n-Heptane Reduced Chemistry and Its Application to Simulations of Ignition Quality Tester, Diesel, and HCCI Combustion," Journal of Engineering for Gas Turbines and Power, vol. 136, no. 12, p. 121505, Jul. 2014. doi: $10.1115 / 1.4027891$.

[8] "Ignition quality tester (IQT)," 2017. [Online]. Accessed on: June 15, 2017.

[9] L. N. Allard, et al., "Diesel fuel ignition quality as determined in the ignition Quality Tester (IQT)-part II," Tech. Rep., SAE Technical Paper 971636, 1997. doi: 10.4271/971636.

[10] L. N. Allard, et al., "Diesel fuel ignition quality as determined in the Ignition Quality Tester (IQT) - part IV," in SAE Technical Paper, SAE International, 2001-01-3527, 2001. doi: 10.4271/2001-01-3527.

[11] S. G. Daviault, "Characterization of the fuel injection process within the ignition quality tester (IQT)," Master's thesis, Carleton University, 2011.

[12] A. E. T. Ltd., IQT ${ }^{\mathrm{TM}}$ User Manual, 2009.

[13] A. Tong, "Improving the accuracy of temperature measurements," Sensor Review, vol. 21, no. 3, pp. 193-198, 2001. doi: 10.1108/02602280110398044.

[14] C. S. McEnally, Ü. Ö. Köylü, L. D. Pfefferle, and D. E. Rosner, "Soot volume fraction and temperature measurements in laminar nonpremixed flames using thermocouples," Combustion and Flame, vol. 109, no. 4, pp. 701-720, Jun. 1997. doi: 10.1016/S0010-2180(97)00054-0.

[15] P. Auerkari, Mechanical and physical properties of engineering alumina ceramics, Technical Research Centre of Finland Espoo, 1996. 


\title{
A new mechanical band pass filter design for energy harvesting
}

\author{
Alireza Keshmiri, Nan Wu \\ Department of Mechanical Engineering \\ University of Manitoba \\ Winnipeg, Canada \\ keshmira@myumanitoba.ca, Nan.Wu@umanitoba.ca
}

\begin{abstract}
This work presents an analytical methodology for development of a mechanical band pass filter for energy scavenging. The model is based on Adomian decomposition method to derive the dynamic response of the non-uniform smart structure with strain rate damping to external environmental excitations and efficiently harvest the subsequent vibrational energy. The steady state response of the nonlinearly tapered cantilever beam subjected to a harmonic base motion is obtained and electromechanical outputs in open circuit condition are analytically derived. In other to design a band pass filter, an array of cantilever beams with nonlinear geometry and surface bonded piezoelectric layers are considered. Therefore, the filter can choose one harvester to resonate at one particular ambient vibration frequency and reach the maximum electromechanical output. Compared with uniform beam design, it is also demonstrated that the proposed non-uniform configuration can generate higher electrical output over a wide frequency range.
\end{abstract}

Keywords - Band pass filter; Piezoelectric energy harvesting; Non-uniform structure; Adomian decomposition method.

\section{INTRODUCTION}

Energy harvesting from ambient energy sources has become a dynamic research field and received so much attention in recent years because of huge number of applications. With decreasing electrical power requirements in wireless electronics and increasing utilization of sensors in different structures, energy harvesting to power sensors at the location without batteries limitations is appealing for various engineering automation and monitoring systems. Therefore, energy harvesters are designed to convert the environment energies into usable power form to supply the small wireless sensor systems throughout the sensor lifetime.

Alternatively, energy scavenging based on vibration is becoming more attractive. Conversion of mechanical vibration energy into electrical energy can be achieved through three conversion mechanisms: electrostatic, electromagnetic, and piezoelectric [1]. The small size, custom shape fabrication ability and high power conversion potential are the key and fundamental advantages of piezoelectric approach for vibration energy harvesting [2]. Even a small external excitation leads to considerable amount of generated voltage without requiring any low-efficient initiation mechanical mechanism [3].

The most common energy harvester design is the cantilever beam with surface bonded piezoelectric layers transversely vibrating because of the base excitation. Although it is not essentially the best design in terms of output energy, it is wellknown because of its simple and cost-efficient configuration. Furthermore, most of piezoelectric energy harvesters work on the resonance mechanism to raise the strain distribution in the piezoelectric layer and eventually increase the output electrical power. Due to the small size factor, resonance frequency of the harvester increases and makes it undesirable for low frequency vibrations. The common response to this problem is adding a proof mass at the free end $[4,5]$. But, this optimal resonance condition is not given in most of the environmental cases with variable vibration conditions. Given this, having a single harvester design capable of operating over a range of vibration frequencies would be desirable. Additionally, refining the geometry of the scavenger is itself a major improvement area. A trapezoidal profile can distribute the strain evenly such that maximum stain is obtained at every point in the piezoelectric energy harvester. A harvester with trapezoidal geometry can generate more than twice energy than a harvester with rectangular shape [6].

One of the biggest disadvantages of common vibration energy harvesters is the very limited practical bandwidths over which energy can be scavenged. In order to increase the frequency range of vibration energy harvesters, two approaches of tuning the resonant frequency and widening the bandwidth are suggested in the literature [7]. Based on the widen bandwidth methodology, the operational frequency range of the vibration energy generator can be extended by using an array of harvesters with different natural frequencies. In other words, the piezoelectric energy harvester should have appropriate bandwidth in the range of peak-power frequencies of the ambient vibrations. This frequency range can be easily measured in different environments. Such a device with this particular characteristic can be called a piezoelectric band pass filter [5].

The work presented in this research endeavors to challenge previous energy harvesting designs and fabricate a novel and advanced energy harvester system that possesses enough bandwidth in the range of ambient vibration frequencies. The model developed in this paper has included nonlinear geometry 
rather than a simple uniform pattern. The dynamic steady state solution of the harmonic base excitation problem for lateral vibration of the non-uniform smart structure with strain rate damping is presented and electromechanical outputs of the piezoelectric patches in open circuit condition are analytically derived. The mathematical process is based on Adomian decomposition method (ADM) and the mechanical model is applicable to any slender beam which allows neglecting shear deformation and rotatory inertia effects. Finally, it is demonstrated that such an arrangement of non-uniform cantilever piezoelectric coupled beams can be made into a band pass filter when the dimensions are selected properly.

\section{THEORETICAL MODEL}

The proposed bimorph piezoelectric energy harvester configuration consists of a nonlinearly tapered cantilever beam with piezoelectric patches bonded on the top and bottom surfaces, Fig. 1. The geometry of the structure is described by $L$ length of the beam, $2 b_{0}$ width of the beam at the fixed end, $2 h_{0}$ thickness of the beam at the fixed end, $L_{p}$ the piezoelectric patch length and $h_{p}$ thickness of the piezoelectric layer. The energy harvesting efficiency of the piezoelectric coupled cantilever beam can be investigated by setting the mechanical vibration input for the system in the form of $f(t)=Y e^{j \varphi t}$ where $Y$ is the amplitude of the base displacement, $\varphi$ is the angular frequency of the vibration and $j$ is the unit imaginary number. The differential equation that describes the forced lateral vibration of a smart beam with non-uniform geometry and structural strain rate damping can be expressed by,

$$
\left.\rho A(x) \frac{\partial^{2} w(x, t)}{\partial t^{2}}+\frac{\partial^{2}}{\partial x^{2}} E I(x) \frac{\partial^{2} w(x, t)}{\partial x^{2}}+C_{s} I(x) \frac{\partial^{3} w(x, t)}{\partial x^{2} \partial t}\right]=-\rho A(x) \phi^{2} Y e^{j p t}
$$

where $x$ is position variable along length of the beam, $t$ is time variable, $\rho$ is the equivalent mass density, $A(x)$ is crosssectional area, $w(x, t)$ is the deflection function, $E$ is the equivalent elasticity modulus, $I(x)$ is the second moment of area and $C_{s}$ is the strain-rate damping coefficient. Moreover, boundary conditions for the non-uniform cantilever beam are defined as,

$$
\begin{aligned}
& {[w(x, t)]_{x=0} \quad 0,\left[\frac{\partial w(x, t)}{\partial x}\right]_{x=0}=0,} \\
& {\left[E I(x) \frac{\partial^{2} w(x, t)}{\partial x^{2}} C_{s} I(x) \frac{w(x, t)}{\partial x \partial t}=0,\right.} \\
& {\left[\frac { \partial } { \partial x } \left(E I(x) \frac{\partial^{2} w(x, t)}{\partial x^{2}} C_{s} I(x) \frac{\partial w(x, t)}{\partial x \partial t}=0 .\right.\right.}
\end{aligned}
$$

Using the normal mode approach, the solution of Eq. (1) is assumed to be a linear combination of the normal modes of the beam denoted by a convergent series as,

$$
w(x, t)=\sum_{i=1}^{\infty} X_{i}(x) T(t),
$$

where $X_{i}(x)$ are mass normalized modes and $T_{i}(t)$ are modal participation coefficients for the $i$ th vibration mode. Since the system is proportionally damped, eigenfunctions represented by $X_{i}(x)$ are normal mode eigenfunctions of the corresponding undamped free vibration problem $[8,9]$.

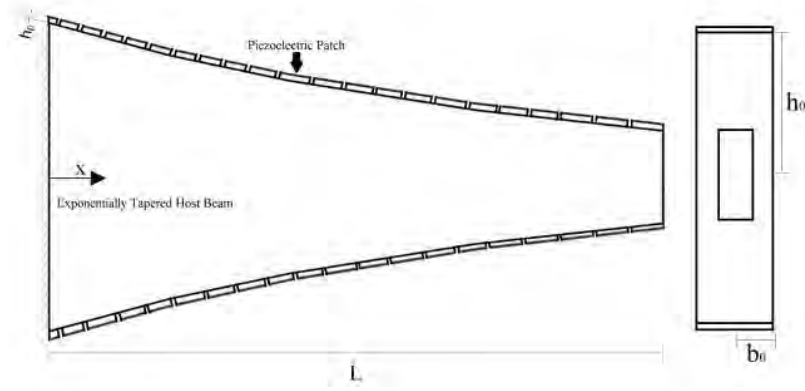

Figure 1. A nonlinearly tapered cantilever beam with piezoelectric patches mounted on its surface, side and front views.

Consequently, normal modes can be calculated by solving,

$$
E I(x) \frac{d^{4} X_{i}(x)}{d x^{4}}+2 E \frac{d I(x)}{d x} \frac{d^{3} X_{i}(x)}{d x^{3}}+E \frac{d^{2} I(x)}{d x^{2}} \frac{d^{2} X_{i}(x)}{d x^{2}}-\omega_{i}^{2} \rho A(x) X_{i}(x)=0
$$

where $\omega_{\mathrm{i}}^{2}$ are the undamped natural frequencies of the structure.

In order to solve the Eq. (4), ADM is used. The solution obtained by ADM is an infinite series; an $n$-term approximation usually serves as a practical solution. Since the methodology itself is not the main focus of this research and it is perfectly addressed in $[10,11]$, more details will not be discussed here. Hence, by applying ADM, solution of Eq. (4) which is the mode shape function in the recurrence format can be expressed as,

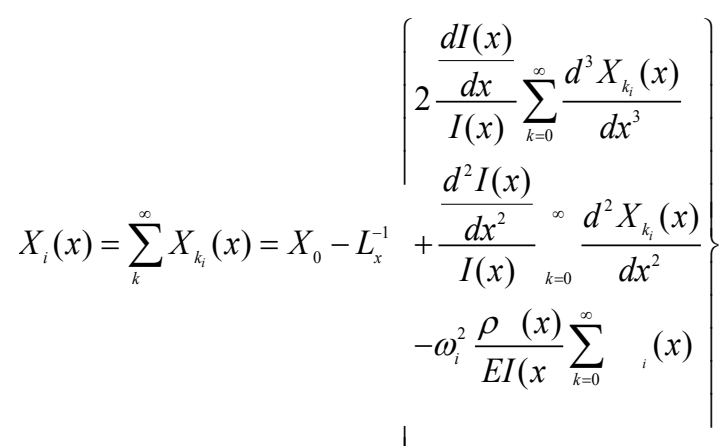

where the inverse operator $L_{x}^{-1}$ is a four fold integration with respect to $\mathrm{x}$ Additionally, $X_{0}=C_{1}+C_{2} x+C_{3} x^{2} / 2 !+C_{4} x^{3} / 3$ ! and $C_{1}$ to $C_{4}$ are constants which can be evaluated from the system boundary conditions. Thus, all $X_{k}$ components are evaluated and the mode shape function is obtained. As many terms as required to obtain a more accurate shape function can be considered.

Clearly, normal mode eigenfunctions satisfy the orthogonality conditions,

$$
\begin{aligned}
& \int_{x=0}^{L} \rho A(x) X_{m}(x) X_{n}(x) d x=\left\{\begin{array}{l}
1 \rightarrow m=n \\
0 \rightarrow m \neq
\end{array},\right. \\
& \int_{x=0}^{L} \frac{d^{2}}{d x^{2}}\left[E I(x) \frac{d^{2} X_{m}(x)}{d x} X_{n}(x) d x=\left\{\begin{array}{c}
\omega_{m}^{2} \rightarrow m=n \\
0 \rightarrow m \neq
\end{array} .\right.\right.
\end{aligned}
$$

Therefore, the modal participation coefficients $T_{i}(t)$ are the solution of the ordinary differential equation, 


$$
\begin{aligned}
& \frac{d^{2} T_{i}(t)}{d t^{2}}+2 \xi_{i} \omega_{i} \frac{d T(t)}{d t}+\omega_{i}^{2} T_{i}(t)= \\
& Q_{i}(t)=\rho \phi^{2} Y\left[\int_{0}^{L} X(x) A(x) d x\right\rceil e^{j \phi t},
\end{aligned}
$$

where $2 \xi_{\mathrm{j}} \omega_{\mathrm{i}}=C_{s} \omega_{\mathrm{i}}^{2} / E$ and $\xi_{j}$ is the structural strain-rate damping ratio of $i$ th vibration mode. Finally, the modal response can be obtained by Duhamel integral,

$$
T_{i}(t)=\frac{1}{\omega_{d i}} \int_{0}^{t} Q_{i}(\tau) e^{-\xi_{i j}(t-\tau)} \sin \omega_{d i}(t \quad \tau) d \tau,
$$

where $\omega_{\mathrm{di}}=\omega_{\mathrm{i}}\left[1-\xi_{\mathrm{i}}^{2}\right]^{0.5}$ is the $i$ th mode damped natural frequency. However, the steady state solution of Eq. (7) can be expressed as,

$$
T_{i}(t)=\frac{\rho \phi^{2} Y \int_{0}^{L} X_{i}(x) A(x) d x}{\omega_{i}^{2}-\phi^{2} \quad j 2 \xi_{i} \omega_{i} \phi} e^{j \phi t} .
$$

As a result, normal mode eigenfunctions given by Eq. (5) and modal participation coefficients obtained by Eq. (9) can be used in Eq. (3) to represent the dynamic steady state response of the smart structure relative to its base as,

$$
w(x, t) \quad{ }_{i 1}^{\infty} X_{i}(x) T_{i}(t)=\sum_{i=}^{\infty} X_{i}(x) \frac{\rho \phi^{2} Y \int_{0}^{L} X_{i}(x) A(x) d x}{\omega_{i}^{2}-\phi+j 2 \xi_{i} \omega_{i} \phi} e^{j \phi t} .
$$

In order to obtain the electrical outputs of the piezoelectric energy harvester, the following piezoelectric constitutive equation is considered,

$$
D_{3}(x, t) \quad d_{31} \sigma_{1}(x, t)+e_{33}^{\sigma} E_{3}(t),
$$

where $D_{3}$ is the electrical displacement, $d_{31}$ (Coulomb/ $\mathrm{N}$ or $\mathrm{m} / \mathrm{V}$ ) is the piezoelectric coefficient with polarization in direction 3 due to the external stress in direction $1, \sigma_{l}$ is the axial stress along 1-axis, $e_{33^{\sigma}}$ is the permittivity at constant stress and $E_{3}$ is the electrical field along 3-axis [12]. It should be noted that 1,2 and 3 directions are aligned with $x, y$ and $z$ axes, respectively.

By replacing the axial stress $\sigma_{1}$ with bending strain $\varepsilon_{1}$ and knowing that there is no applied electric field on the piezoelectric patch $E_{3}(t)=0$, Eq. (11) can be rewritten as,

$$
D_{3}(x, t)=d_{31} E_{p} \varepsilon_{1}(x, t),
$$

where $E_{p}$ is the piezoelectric elasticity modulus and $V(t)$ is the electric voltage over the piezoelectric area. Furthermore, the longitudinal bending strain $\varepsilon_{l}(x, t)$ in the structure can be calculated as,

$$
\varepsilon_{1}(x, t)=-h_{c} \frac{w(x, t)}{=-} h(x)+\frac{h_{p}}{2} \mid \frac{\partial^{2} w(x, t)}{\partial x^{2}},
$$

where $h_{c}$ is the distance between center of the piezoelectric layer and the beam's neutral axis and $\delta^{2} w(x, t) / \delta x^{2}$ is the curvature of the structure. Consequently, the electric displacement becomes,

$$
D_{3}(x, t)=-d_{31} E_{p}\left(h(x)+\frac{h_{p}}{2}\right) \frac{\partial^{2} w(x, t)}{\partial x^{2}} .
$$

The electric displacement $D_{3}(x, t)$ is related to the output electric charge $q(t)$ by integration over the electrode area. Since the vibration mode of the energy harvester is not restricted to the first mode, segmented piezoelectric patches and electrodes in order to harvest the maximum output charge are utilized. This is due to the fact that using continuous electrodes results in phase difference in the strain distribution which leads to charge cancellation [8].

$$
\left.q(t)=-\sum_{k=1}^{N_{p}} \int_{x=(k-1) L_{p}}^{k L_{p}} d_{31} E_{p} b(x)\left(h(x)+\frac{h_{p}}{2}\right) \frac{\partial^{2} w(x, t)}{\partial x^{2}} d x\right\},
$$

where $N_{p}$ is the total number of piezoelectric patches mounted on the surface of the beam and obviously $N_{p} L_{p}=L$.

The generated voltage $v(t)$ can be calculated by dividing the produced electric charge to the capacitance as,

$$
v(t)=\frac{q(t)}{C_{p}}=-\frac{d_{31} E_{p}}{C_{p}} \sum_{k=1}^{N_{p}}\left\{\int_{(k-1) p_{p}}^{k L_{p}} b(x)\left(h(x)+\frac{h_{p}}{2}\right) \frac{\partial^{2} w(x, t)}{\partial x^{2}} d x\right\},
$$
layer.

where $C_{p}=e_{33}{ }^{\varepsilon} L b_{0} / h_{p}$ is the capacitance of the piezoelectric

The goal of this research is to estimate the open circuit voltage across the piezoelectric layers. So the backward coupling in the relations is ignored. Finally, steady state voltage can be obtained by using Eq. (10) in Eq. (16) as,

$$
v(t)=-\frac{d_{31} E \rho \phi^{2} Y}{C_{p}} \sum_{1}^{\infty}\left\{\sum_{k=}^{N_{p}} \int_{(k-1)}^{k_{p}} p(x)\left(h(x)+\frac{h_{p}}{p} \int \frac{d^{2} X_{i}(x)}{d x^{2}} d x\right] \frac{\int_{0}^{L} X_{i}(x) A(x) d x}{\omega_{i}^{2}-\phi^{2}+j 2 \xi_{i} \omega_{i} \phi}\right\} e^{j i t} .
$$

In order to provide a more general form of steady state response and not considering specific $Y$ and $\varphi$ values, frequency response functions (FRFs) of the electromechanical outputs are obtained and presented. Firstly, the voltage FRF is defined as the ratio of the steady state voltage output to the base acceleration. Hence, voltage FRF is described as,

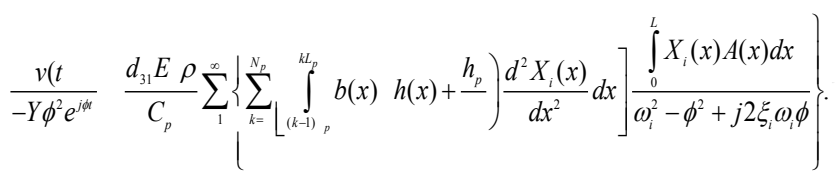

Secondly, the relative tip motion FRF is presented as ratio of the beam vibration amplitude at the tip to the amplitude of the base excitation. It is worth mentioning that the presented model is not limited to the motion at the tip and can be applied to any point along the beam. By using Eq. (10), tip motion FRF is defined as,

$$
\frac{w(L, t)}{Y e^{j \phi t}}=\rho \phi^{2} \sum_{i=1}^{\infty}{ }_{i}(L) \frac{\int_{0}^{L} X_{i}(x) A(x) d x}{\omega_{i}^{2}-\phi \quad j 2 \xi_{i} \omega_{i} \phi} .
$$

When the excitation frequency is equal to the natural frequency of the $i$ th mode $\left(\varphi=\omega_{i}\right)$, all the terms in the FRF 
relations other than the mode of interest can be ignored. As a result, the amplitude of voltage output and tip motion FRFs are rewritten as,

$$
\begin{gathered}
\left.\| \frac{(t)}{-Y \phi^{2} e^{j \phi t} \|} \frac{d_{31} E_{p} \rho}{\xi_{i} \omega_{i}^{2} C_{p}}\left\{\sum_{k=1}^{N_{p}} \mid \int_{(k-1) L_{p}}^{k L_{p}} b(x)\left(h(x)+\frac{h_{p}}{2}\right) \frac{d^{2} X_{i}(x}{d x^{2}} d x\right]\right\}\left[\int_{0}^{L} X_{i}(x) A(x) d x\right\rfloor . \\
\left\|\frac{w(L, t)}{Y e^{j \phi t}}\right\|=\frac{\rho \phi^{2}}{2 \xi_{i}} X_{i}(L) \int_{0}^{L} X_{i}(x) A(x) d x .
\end{gathered}
$$

\section{MECHANICAL BAND PASS FILTER}

In this section, the probability of designing a mechanical band pass filter from an array of non-uniform beams is investigated. The material properties and electromechanical coefficients are listed in Table 1. The excitation of the piezoelectric energy harvester is due to the harmonic base translation and the steady state dynamic behavior of the system is of interest. Furthermore, structural strain-rate damping ratios for the first and second vibration modes are assumed to be $\xi_{1}=\xi_{2}=0.03$. Finally, two steady state electromechanical FRFs, voltage output and beam tip motion, are examined.

TABLE I. GEOMETRY AND MATERIAL PROPERTIES OF THE

\begin{tabular}{|c|c|c|c|c|}
\hline \multicolumn{5}{|c|}{ Host beam } \\
\hline$L(m)$ & $b_{0}(m)$ & $h_{0}(m)$ & $E(G P a)$ & $\rho\left(\mathrm{kg} / \mathrm{m}^{3}\right)$ \\
\hline 0.3 & 0.05 & 0.005 & 210 & 7800 \\
\hline \multicolumn{5}{|c|}{ PZT } \\
\hline $\bar{h}(m)$ & $E_{p}(G P a)$ & $\rho\left(\mathrm{kg} / \mathrm{m}^{3}\right)$ & $d_{31}(\mathrm{pm} / \mathrm{V})$ & $\mathrm{e}_{33^{\varepsilon}}(n F / m)$ \\
\hline 0.0004 & 66 & 7800 & -190 & 15.93 \\
\hline
\end{tabular}
PIEZOELECTRIC ENERGY HARVESTER.

In order to design a mechanical band pass filter, an array of non-uniform beams is considered. The idea of an ensemble of beam-mass system working as a band pass filter is borrowed from [5] and further developed in here. The system consists of several nonlinearly tapered piezoelectric coupled cantilever beams, each of which has different geometrical taper ratio and hence different resonance frequencies. Consequently, the assembled system has a wide operational frequency range and the filter can automatically choose one harvester to resonate at one particular ambient vibration frequency. When the natural frequency of the energy harvester matches the peak power frequency of the source, the output power is the most efficient. Additionally, to partially overcome the stability and size drawbacks, all the harvesters in the system have similar length, volume and mass. As a result, the entire system can work as a mechanical band pass filter depending on geometrical dimensions and taper ratios. Although the non-uniformity function in the presented model is not constrained to any specific type of function and is a general definition, here an exponential variation function $g(x)=g_{0} e^{-m x / L}$ is considered where $g_{0}$ is the initial value, $m$ is the taper ratio and $0 \leq x \leq L$.

Fig. 2 represents the nonlinear geometry effect on the electric voltage output in frequency domain. As can be seen, the amplitude of the voltage output drastically increases when the excitation frequency is close to the fundamental natural frequency of the harvester. It is worthwhile to mention that for every excitation frequency, maximum electrical output is gained when the piezoelectric energy harvester is working in open circuit condition [13]. As shown, higher nonlinearity in geometry of the harvester results in substantial increase of the voltage amplitude. For instance, by increasing the geometry taper ratio from 0 to 0.8 , the voltage output for the first two vibration modes show up to 15.46 times higher outputs, Fig. 2. Therefore, a system comprising only five non-uniform energy harvesters with different taper ratios and same volume/mass can function like a band pass filter over a wide frequency band $\left[\omega_{\min }-\omega_{\max }\right]=[290-550] \mathrm{rad} / \mathrm{s} \approx[45-90] \mathrm{Hz}$. Besides, the proposed non-uniform design substantially increases the voltage output of the system compared to the uniform one [5].

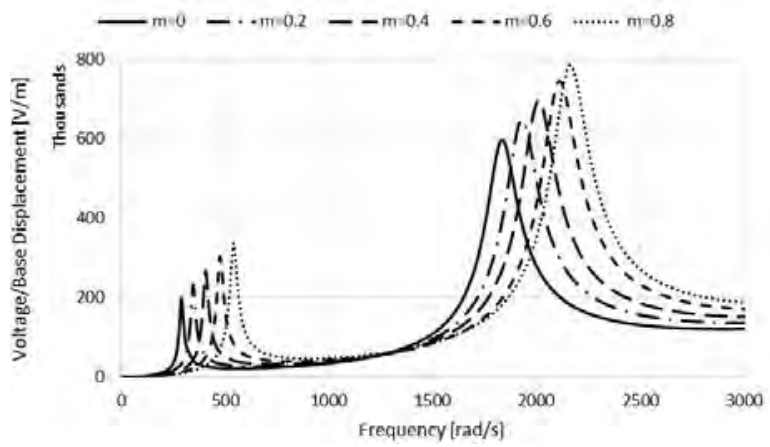

Figure 2. Voltage output FRF for an array of non-uniform piezoelectric energy harvesters with five different geometrical taper ratios.

Finally, the tip displacement in frequency domain for various nonlinear geometries is shown in Fig. 3. Likewise, increasing the nonlinearity in the geometry of the harvesters leads to higher natural frequencies and tip displacements. As mentioned earlier, trapezoidal profile can distribute the strain evenly such that maximum strain is obtained at every point in the piezoelectric energy harvester.

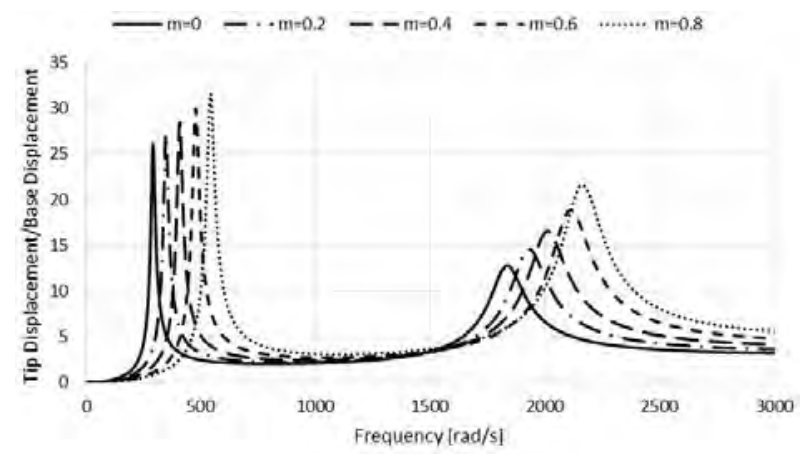

Figure 3. Beam tip motion FRF for an array of non-uniform piezoelectric energy harvesters with five different geometrical taper ratios.

\section{CONCLUSION}

In this paper, an analytic electromechanical model for a nonlinearly tapered cantilever piezoelectric energy harvester is presented. The non-uniform piezoelectric harvester is assumed to be excited by harmonic base motion in the transverse direction. By using ADM, the steady state dynamic behavior of the smart structure with non-uniform geometry and structural strain-rate damping is obtained and closed-form expressions for the electromechanical parameters are derived. Analytically 
obtained equations are used to determine the electric charge, voltage output and tip motion FRFs to be able to see the frequency response of the non-uniform harvesters. The electromechanical FRFs are plotted against frequency in open circuit condition which leads to the maximum output. Finally, a mechanical band pass filter including an array of nonlinearly tapered piezoelectric bimorph cantilever beams, each of which has different geometrical taper ratio and hence different resonance frequencies is designed and presented.

In summary, a new approach to increase the frequency range and electrical outputs of vibration energy harvesters by widening the bandwidth of the generator and introducing nonlinear structural geometry design is developed and presented. This is achieved through employing an array of nonuniform harvesters with different resonance frequencies. The new nonlinear geometry design has demonstrated to be promising to considerably increase the electromechanical outputs of the band pass filter in a wide frequency domain. This research challenges previous energy harvesting designs and formulates an innovative harvester configuration which functions optimally and efficiently on extensive range of ambient vibration sources.

\section{ACKNOWLEDGMENT}

This research was undertaken, in part, thanks to funding supports from University of Manitoba, Research Manitoba and Natural Sciences and Engineering Research Council of Canada (NSERC).

\section{REFERENCES}

[1] S. Roundy, P. K. Wright and J. Rabaey, "A study of low level vibrations as a power source for wireless sensor nodes," Computer Communications, vol. 26, no. 11, pp. 1131-1144, 2003. doi: 10.1016/S0140-3664(02)00248-7.

[2] N. G. Elvin, A. A. Elvin and M. Spector, "A self-powered mechanical strain energy sensor," Smart Mater. Struct., vol. 10, pp. 293-299, 2001. doi: 10.1088/0964-1726/10/2/314.

[3] T. Hehn and Y. Manoli, CMOS Circuits for piezoelectric energy harvesters, Netherlands: Springer, 2015. doi: 10.1007/978-94-017-9288-2.

[4] A. Erturk and D. J. Inman, "Mechanical considerations for modeling of vibration-based energy harvesters," in Proceedings of the ASME IDETC 21st Biennial Conference on Mechanical Vibration and Noise, Las Vegas, NV, 2007. doi: 10.1115/DETC2007-35440.

[5] S. Shahruz, "Design of mechanical band-pass filters for energy scavenging," Journal of Sound and Vibration, vol. 292, issues 3-5, pp. 987-998, 2006. doi: 10.1016/j.jsv.2005.08.018.

[6] S. Roundy, E. S. Leland, J. Baker, E. Carleton, E. Reilly, E. Lai, B. Otis, J. M. Rabaey and P. K. Wright, "Improving power output for vibrationbased energy scanvengers," Pervasive Computing, vol. 4, no. 1, pp. 28-36, 2005. doi: 10.1109/MPRV.2005.14.

[7] D. Zhu, M. J. Tudor and S. P. Beeby, "Strategies for increasing the operating frequency range of vibration energy harvesters: a review," Meas. Sci. Technol., vol. 21, pp. 1-29, 2010. doi: 10.1088/0957-0233/21/2/022001.
[8] A. Erturk and D. J. Inman, "On mechanical modeling of cantilevered piezoelectric vibration energy harvesters," J. Intell. Mater. Syst. Struct., vol. 19, no. 11, pp. 1311-1325, 2008. doi: 10.1177/1045389X07085639.

[9] T. K. Caughey and M. E. O'Kelly, "Classical normal modes in amped linear dynamic systems," J. Appl. Mech., vol. 32, pp. 583-588, 1965. doi: $10.1115 / 1.3627262$.

10. G. Adomian, "A Review of the Decomposition Method in Applied Mathematics," Journal of Mathematical Analysis and Applications, vol. 135, issue 2, pp. 501-544, 1988. doi: 10.1016/0022-247X(88)90170-9.

[11] A. Keshmiri, N. Wu and Q. Wang, "Free vibration analysis of a nonlinearly tapered cone beam by Adomian decomposition method," International Journal of Structural Stability and Dynamics, 2018. doi: 10.1142/S0219455418501018.

[12] IEEE Standard on Piezoelectricity, New York: IEEE, 1987.

[13] A. Erturk and D. J. Inman, "A distributed parameter electromechanical model for cantlievered piezoelectric energy harvesters," J. Vib. Acoust, vol. 130, pp. 1-14, 2008. doi:10.1115/1.2890402. 


\title{
Analysis of Thermal Losses in Air-Water Interfacial Solar Heating Systems
}

\author{
Shuzhe Wang, Paul G. O'Brien \\ Department of Mechanical Engineering \\ Lassonde School of Engineering, York University \\ Toronto, Canada \\ szwang94212@gmail.com
}

\begin{abstract}
This paper reports a numerical analysis of solardriven seawater desalination systems with two configurations: a floating photo-thermal film at the air-water interface with and without an insulation layer located beneath its surface. Heat transfer processes from the film including conduction, convection and radiation have been evaluated at different film temperatures to determine the relative contributions of different heat loss mechanisms from the film. Convective heat transferred to the bulk seawater is found to be the dominant heat loss path, and can be reduced from $6.95 \times 10^{7} \mathrm{~J} / \mathrm{m}^{2} \cdot \mathrm{h}$ to $8.77 \times$ $10^{5} \mathrm{~J} / \mathrm{m}^{2} \cdot \mathrm{h}$, a difference of almost two orders of magnitude, by inserting an insulation layer beneath the photo-thermal film. Thus, the addition of an insulating layer is suggested as an important design component to effectively minimize heat losses in air-water interfacial solar heating systems.
\end{abstract}

Keywords-solar interfacial heating; desalination; heat transfer

\section{INTRODUCTION}

Worldwide freshwater scarcity has been a longstanding issue due to the demands of an ever-growing population. In addition to environmental conservation, freshwater production is an important solution for meeting this urgent need. Seawater covers most of the earth's surface, and is considered to be a sustainable source of continuous freshwater supply. However, the predominant methods used to desalinate seawater, such as multistage flash distillation (MSF) and reverse osmosis (RO), typically consume $5 \sim 26$ times as much energy as the latent heat of water (about $2400 \mathrm{~kJ} / \mathrm{kg}$ ) [1], [2]. A promising alternative approach is solar-driven seawater desalination, whereby heat generated from the solar irradiance is used to evaporate water, which minimizes the need for fossil fuels and electric power. However, traditional solar-driven water desalination systems exhibit low efficiencies (typically $34-40 \%$ for a single basin solar still [3]) due to large heat losses to the bulk water reservoir.

To improve solar distillation systems, numerous photothermal materials with different morphologies and surface properties have been investigated for their performance of absorbing solar irradiance and producing heat to enhance evaporation. In this regard, plasmon resonance phenomena in metallic materials, such as $\mathrm{Au}$ [4], [5], $\mathrm{Ag} \mathrm{[6]} \mathrm{and} \mathrm{Cu}$ [7], [8], have been investigated in terms of light absorption and localized heat generation for accelerated water evaporation. For example, Jin et al. achieved a the solar-thermal conversion efficiency (the ratio of the thermal energy used for evaporation and the total incident solar irradiance) of $80.3 \%$ using $\mathrm{Au}$ nanoparticles dispersions under 220 sun illumination $\left(1\right.$ sun $\left.\approx 1 \mathrm{~kW} / \mathrm{m}^{2}\right)$ [4]. Besides, carbon-based materials such as graphene and reduced graphene oxide have gained popularity for solar-driven distillation applications in the past few decades on account of their excellent photo-thermal properties. Y. Ito et al. found that nitrogen doped nano-porous graphene sheets floating on the airwater interface were heated to $100{ }^{\circ} \mathrm{C}$ under concentrated solar irradiation while the bulk water remained at $35^{\circ} \mathrm{C}$, reaching an $80 \%$ conversion efficiency of received solar radiation to the latent heat of water [9]. This strategy of using a photo-thermal film located at the air-water interface has promising potential to increase the efficiency of solar desalination systems because heat generated from the absorbed solar energy is localized at the air-water interface where the evaporation process occurs.

To date, simulations and mathematical analysis of heat and mass transfer in traditional solar distillation plants [10]-[13] and molecular dynamic modeling for general evaporation [14], [15] have been reported in the literature. However, few studies have investigated heat loss mechanisms in photo-thermal films used for air-water interfacial solar heating from a heat transfer perspective to seek quantitative explanations for their superior performance. Herein, numerical analysis is performed to investigate the performance of two air-water interfacial solar heating configurations, wherein 1) a thin film of black photothermal material (e.g. graphene and its derivatives) floats directly on the air-water interface, and 2) the film structure is similar to the first configuration but with a thermal-insulation layer inserted between the photo-thermal film and the bulk water. Simplified models are used to study the convective, conductive and radiative heat transfer losses from the photothermal film to its surrounding environment. Comparisons between these heat losses and the energy contributed to evaporate water are made in order to provide recommendations for optimizing the design of solar-driven water desalination systems. 


\section{MATHEMATICAL MODEL AND ANALYSIS}

\section{A. General Assumptions}

Herein we model two air-water interfacial solar heating configurations, namely 1) floating photo-thermal film without an insulation layer and 2) floating photo-thermal film with an insulation layer. In practice, the photo-thermal film has a thickness at the micron scale and exhibits high absorptivity towards solar irradiance such that its temperature is sufficiently increased to provide thermal energy to facilitate the water evaporation process. In this work, to simplify the heat transfer analysis, it is assumed that the solar irradiance heats the film isothermally to a constant temperature $T_{f}$, which is reasonable considering the high thermal conductivity and small thickness of the film. Furthermore, in practice the photo-thermal film would be porous to allow for vapour to escape from the reservoir. However, an assumption is made that the photo-thermal film is flat on both its upper and lower surfaces and has zero thickness for simplification. Also, small turbulence induced by mass transfer (replenishment) from the bulk to the heat-up zone below the film is not considered. Moreover, the analysis presented herein assumes a one-dimensional, quasi-steady state case, and horizontal heat diffusion along the planar direction of the film is not considered. It is also assumed that the thermal properties of the film are independent of temperature and its heat capacity is negligible. The air is quiescent and remains at constant temperature, $T_{a}$, at distances far above the photo-thermal film. Both the air and water vapor behave as ideal gases. It is assumed that sidewalls of the tank are thermal-insulated without edge effects and the temperature of the water far beneath the air-water interface is at a constant value, $T_{w 0}$. The parameters considered in the analysis as well as the dimensions and related physical properties are provided in Table I.

\section{B. Floating Photo-Thermal Film without an Insulation Layer}

In this model the photo-thermal film floats on the air-water interface and is in direct contact with the water (as shown in Fig. 1 (a) and Fig. 1 (b)). A thermal resistance network for the heat transferred from the photo-thermal film is provided in Fig. 1 (c). This thermal resistance network is consistent with the assumption that evaporation takes place in an ultra-thin layer at the air-water interface where the porous photo-thermal film (with zero thickness) resides. Except for evaporating water, heat is lost from the photo-thermal film due to convection to the air, $Q_{v a}$, radiation to the air, $Q_{r a}$, convection to the bulk water, $Q_{v w}$, and radiation to the bulk water $Q_{r w}$. The surface area of the film, $\mathrm{A}$, is assumed to be $0.3 \mathrm{~m} \times 0.3 \mathrm{~m}$.

Convective heat losses are through natural convection mainly, and are calculated using Newton's Law of cooling:

$$
\begin{gathered}
Q_{v a}=A \overline{h_{a}}\left(T_{f}-T_{a}\right) \\
Q_{v w}=A \overline{h_{w}}\left(T_{f}-T_{w 0}\right)
\end{gathered}
$$

where $\overline{h_{a}}$ and $\overline{h_{w}}$ are the heat transfer coefficients for convection to the air and water, respectively, and are determined by the following empirical correlations:

$$
\begin{gathered}
R a_{L}=\frac{g \beta \Delta T L_{C}{ }^{3}}{\alpha \gamma} \\
\overline{N u_{L}}=\left\{\begin{array}{l}
0.54 R a_{L}^{1 / 4}\left(\text { upper surface } 10^{7} \leq R a_{L} \leq 10^{11}\right) \\
0.27 R a_{L}{ }^{1 / 4}\left(\text { lower surface } 10^{5} \leq R a_{L} \leq 10^{10}\right)
\end{array}\right. \\
\bar{h}=\frac{k \overline{N u_{L}}}{L_{C}}
\end{gathered}
$$

where $\Delta T$ is the relevant temperature difference.

Radiative heat transfer losses are determined using the Stefan-Boltzmann Law:

$$
\begin{gathered}
Q_{r a}=A \varepsilon \sigma\left(T_{f}^{4}-T_{a}^{4}\right) \\
Q_{r w}=A \varepsilon \sigma\left(T_{f}^{4}-T_{w 0}^{4}\right)
\end{gathered}
$$

Thermal energy transferred from the film for evaporation consists of both the sensible and latent heat of water, and is given by the following formula:

$$
Q_{\text {evap }}=A \dot{m}\left[\Delta H+C\left(T_{f}-T_{w 0}\right)\right]
$$

The temperature of the vapor is assumed to be the same as that of the film, so the sensible heat for a rise of water temperature from the initial temperature $T_{w 0}$ to $T_{f}$ is included in the calculation for $Q_{\text {evap }}$. Methods used to determine the evaporation rate, $\dot{m}$, are discussed in Section III with reference to Fig. 3.
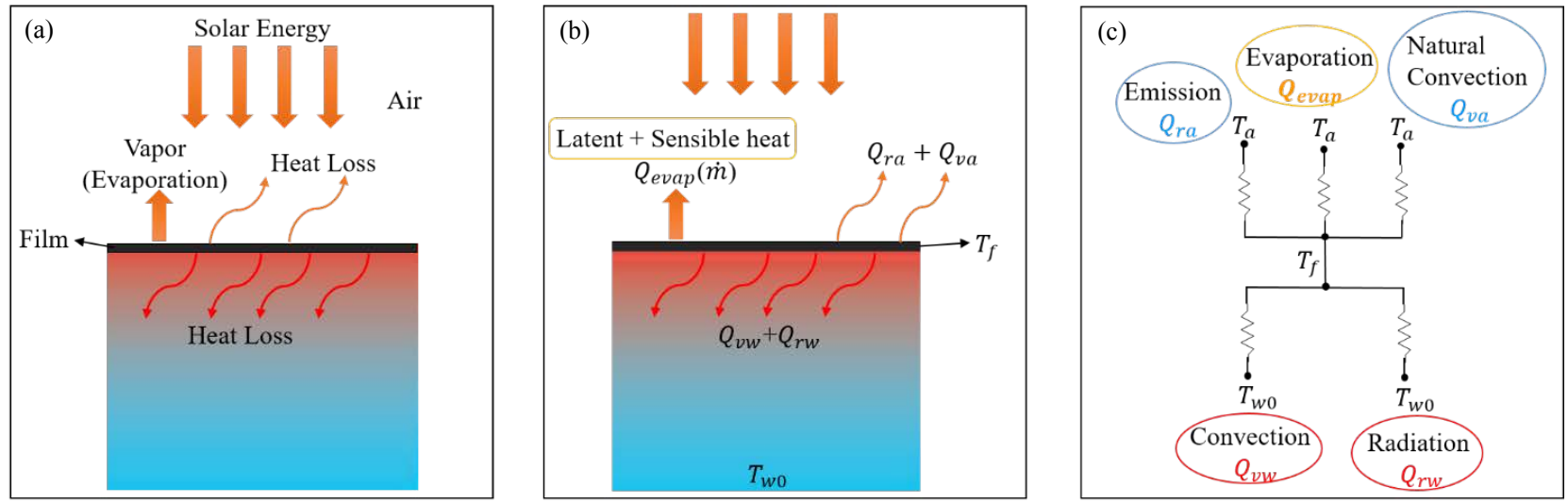

Figure 1. (a) (b) Simplified heat transfer model for a solar interfacial heating model using a floating photo-thermal film. (c) Thermal resistance network for the floating photo-thermal film. 

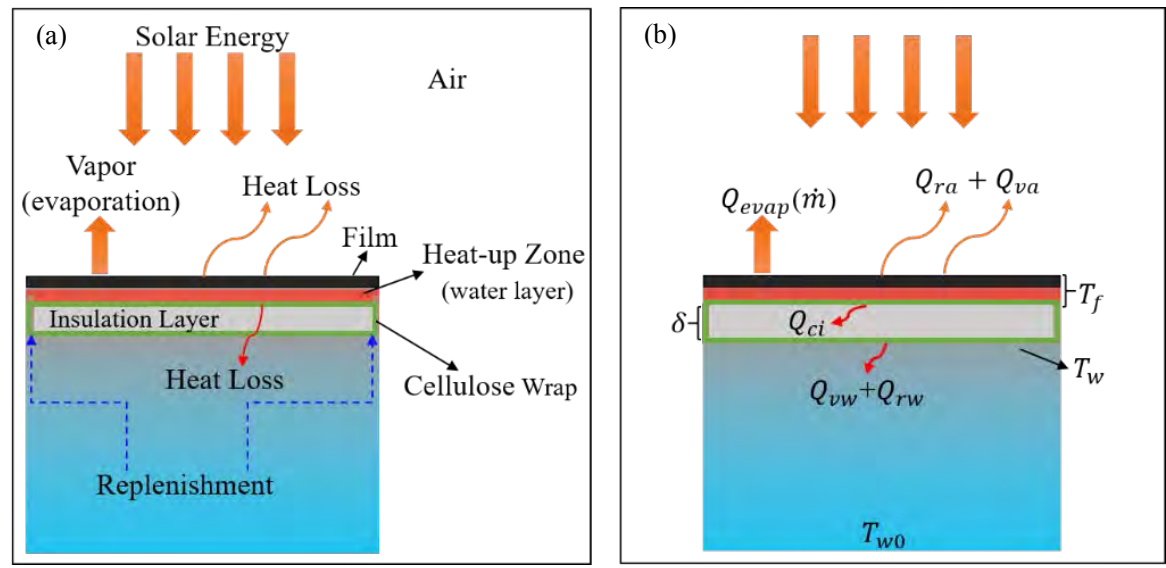

Figure 2. (a) (b) Simplified heat transfer model for solar interfacial heating model using photo-thermal film with an insulation layer underneath.

\section{Floating Photo-Thermal Film with an Insulation Layer}

In the second air-water interfacial solar heating configuration, an insulation layer (such as cellular glass or polystyrene foam with a low thermal conductivity of about 0.05 $\mathrm{W} / \mathrm{m} \cdot \mathrm{K}$ ) with a thickness of $\delta$ is inserted below the photothermal film to reduce convective loss to the bulk water (Fig. 2 (a) and Fig. 2 (b)).

In this configuration, a structure similar to that of a cellulose wrap [16] is applied for replenishment during evaporation, i.e. water is driven by capillary forces from the bulk. A thin water layer (with a thickness of $\sim 0.1 \mathrm{~mm}$ ), herein referred to as the "heat-up zone" in Fig. 2 (a), is thus created and resides on top of the insulating layer, right below the photo-thermal film. It is assumed that the temperature of the heat-up zone is equal to that of the film, $T_{f}$. The amount of thermal energy transferred from the film downwards through the insulator is equal to the sum of the convective and radiative heat losses from the bottom of the insulation layer to the bulk water:

$$
Q_{c i}=Q_{v w}+Q_{r w}
$$

Thus, it is assumed that the total heat loss from the photo-thermal film is the sum of three heat loss mechanisms: radiation and convection to the air, and conduction to the insulator. Equation

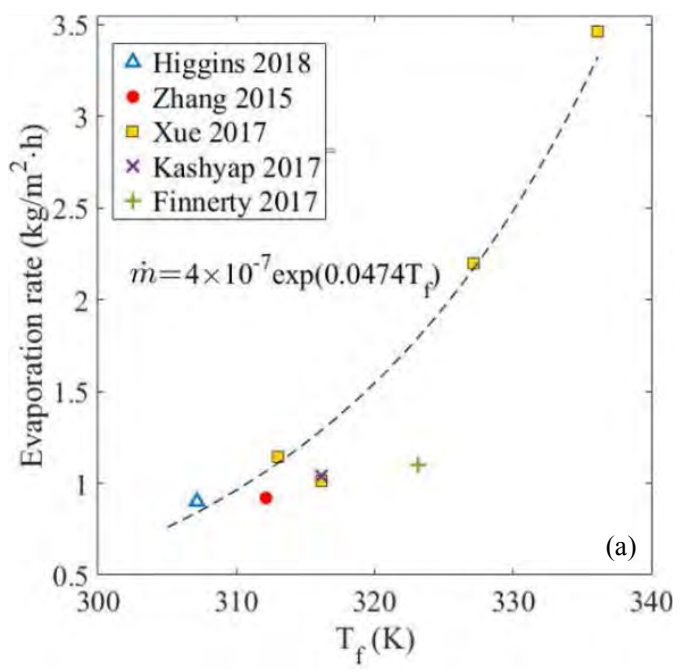

(1) - (7) are used again to determine the heat losses in the second configuration, except the temperature at the insulating layerbulk water interface, $T_{w}$, is used to calculate the radiative and convective heat losses to the water. $T_{w}$ is determined using (9). Conduction through the insulator is given by the following equation:

$$
Q_{c i}=\frac{\mathrm{A} k_{i}\left(T_{f}-T_{w}\right)}{\delta}
$$

where $k_{i}$ is the thermal conductivity of the insulator.

\section{RESULTS AND DISCUSSION}

To determine the evaporation rate at the air-water interface as a function of the photo-thermal film temperature, $T_{f}$, an empirical relationship has been determined using experimental data reported in the literature [17]-[21]. That is, based on evaporation rates reported for air-water interfacial solar heating employing various photo-thermal sheet-like carbon materials such as graphene, rGO (reduced graphene oxide) and graphite, an exponential curve fitting for the evaporation rate has been applied and the results are shown in Fig. 3 (a). The evaporation rates achieved are between $0.92 \mathrm{~kg} / \mathrm{m}^{2} \cdot \mathrm{h}$ and $3.5 \mathrm{~kg} / \mathrm{m}^{2} \cdot \mathrm{h}$ in most studies.

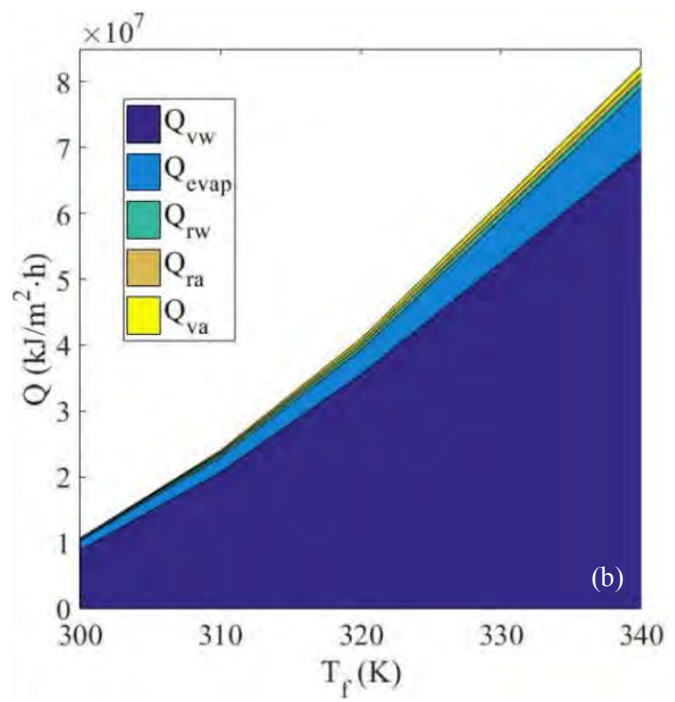

Figure 3. (a) Exponential fitting of the data from previous work $\left(R^{2}=0.8415\right)$ [17]-[21]. (b) Thermal energy transferred from the photo-thermal film due to water evaporation, convection and radiation to the water, and convection and radiation to ambient air in the floating film without an insulation layer configuration. 
For the first configuration, floating photo-thermal film without an insulation layer, thermal energy transferred through the different paths are plotted in Fig. 3 (b). Every heat transfer pathway steadily increases with increasing $T_{f}$, as the temperature difference between the photo-thermal film and its surrounding environment increases. When the photo-thermal film is at a temperature of $340 \mathrm{~K}$, the amount of energy used to evaporate water $\left(Q_{\text {evap }}\right)$ is $\sim 9.6 \times 10^{6} \mathrm{~J} / \mathrm{m}^{2} \cdot \mathrm{h}$, whereas the convective loss to the bulk water, $Q_{v w}$, is almost 7 times larger than $Q_{\text {evap }}$, and dominates over other heat transfer processes. Thus, natural convection to the bulk water is the major heat loss mechanism, limiting the efficiency of air-water interfacial solar heating evaporation systems. It can be noted that the sum of the heat lost to the air $\left(Q_{r a}\right.$ and $\left.Q_{v a}\right)$ and radiation to the bulk water $\left(Q_{r w}\right)$ is only $4 \%$.

The effectiveness of placing an insulation layer beneath the photo-thermal film to mitigate convective heat losses from the film to the bulk water is analyzed in the second configuration, floating photo-thermal film with an insulation layer. The results show the addition of an insulating layer significantly alters the thermal energy loss distribution. As shown in Fig. 4 (a), for an insulating layer with a thickness of $0.001 \mathrm{~m}$, the convective heat $Q_{v w}$ is reduced dramatically because the thermal insulation layer reduces the heat transfer lost from the photo-thermal film to the underlying bulk water. For example, for a photo-thermal film temperature of $T_{f}=340 \mathrm{~K}, Q_{v w}=8.77 \times 10^{5} \mathrm{~J} / \mathrm{m}^{2} \cdot \mathrm{h}$, which is just $1.26 \%$ of its former value of $6.95 \times 10^{7} \mathrm{~J} / \mathrm{m}^{2} \cdot \mathrm{h}$ for the configuration where the insulating layer is absent. Consequently, the percentage of heat used in evaporating water, $Q_{\text {evap }}$, increases from $11 \%$ to $76 \%$ of the total energy transferred from the photo-thermal film. Moreover, the influence of the insulator layer thickness on $Q_{v w}$ is also shown in Fig. 4 (b). As the thickness of the insulating layer increases the convective heat transfer losses to the bulk water are further decreased. For the thickest insulator layer considered in this study, $\delta=0.1 \mathrm{~m}$, convective loss to the bulk water decreases below $9000 \mathrm{~J} / \mathrm{m}^{2} \cdot \mathrm{h}$, which is even lower than $Q_{r a}$ and $Q_{v a}$ when $300 \mathrm{~K}<T_{f}<340$ $\mathrm{K}$. Thermal energy dissipations to the air, namely $Q_{r a}$ and $Q_{v a}$,

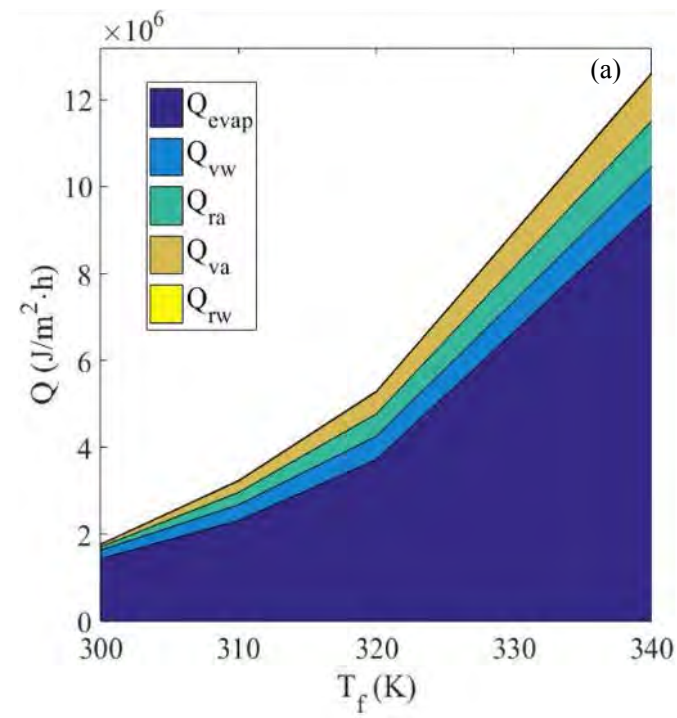

stay unchanged because they only depend on $T_{f}$ and $T_{a}$, regardless of the presence of an insulation layer under the film.

In conclusion, numerical analysis of the thermal energy losses in air-water interfacial solar heating systems shows that convection to the bulk water is the dominant loss mechanism, wasting up to over $80 \%$ of the available thermal energy. To improve the overall performance of interfacial air-water solar desalination systems, the application of an insulation layer beneath the photo-thermal film is recommended to effectively supress heat losses to the bulk of the reservoir.

TABLE I. PARAmeters AND PHOTO-THERMAL FILM DimENSIONS

\begin{tabular}{|c|l|l|l|}
\hline \multicolumn{5}{|c|}{ Nomenclature } \\
\hline$T_{f}$ & $\begin{array}{l}\text { Temperature of the photo- } \\
\text { thermal film }\end{array}$ & $\sigma$ & $\begin{array}{l}\text { Stefan-Boltzmann } \\
\text { constant }\left(\mathrm{W} / \mathrm{m}^{2} \cdot \mathrm{K}^{4}\right)\end{array}$ \\
\hline$T_{a}$ & $\begin{array}{l}\text { Temperature of the air }(296 \\
\mathrm{K})\end{array}$ & $g$ & $\begin{array}{l}\text { Acceleration of gravity } \\
\left(\mathrm{N} / \mathrm{m}^{2} \cdot \mathrm{s}\right)\end{array}$ \\
\hline$T_{w 0}$ & $\begin{array}{l}\text { Initial temperature of the } \\
\text { water }(288 \mathrm{~K})\end{array}$ & $\overline{N u_{L}}$ & Nusselt number \\
\hline$Q_{v a}$ & $\begin{array}{l}\text { Convective heat transfer to } \\
\text { the air }\left(\mathrm{J} / \mathrm{m}^{2} \cdot \mathrm{s}\right)\end{array}$ & $h_{f g}$ & $\begin{array}{l}\text { Latent heat of water } \\
(\mathrm{kJ} / \mathrm{kg})\end{array}$ \\
\hline$Q_{v w}$ & $\begin{array}{l}\text { Convective heat transfer to } \\
\text { the bulk water }\left(\mathrm{J} / \mathrm{m}^{2} \cdot \mathrm{s}\right)\end{array}$ & $\varepsilon$ & $\begin{array}{l}\text { Emissivity of the film }(\approx \\
0.9)\end{array}$ \\
\hline$Q_{r a}$ & $\begin{array}{l}\text { Radiative heat transfer to the } \\
\text { air }\left(\mathrm{J} / \mathrm{m}^{2} \cdot \mathrm{s}\right)\end{array}$ & $\beta$ & $\begin{array}{l}\text { Volume thermal } \\
\text { expansion coefficient } \\
(/ \mathrm{K})\end{array}$ \\
\hline$Q_{r w}$ & $\begin{array}{l}\text { Radiative heat transfer to the } \\
\text { bulk water }\left(\mathrm{J} / \mathrm{m}^{2} \cdot \mathrm{s}\right)\end{array}$ & $\alpha$ & $\begin{array}{l}\text { Thermal diffusivity } \\
\left(\mathrm{m}^{2} / \mathrm{s}\right)\end{array}$ \\
\hline$Q_{c i}$ & $\begin{array}{l}\text { Conductive heat transfer to } \\
\text { the insulation layer }\left(\mathrm{J} / \mathrm{m}^{2} \cdot \mathrm{s}\right)\end{array}$ & $\gamma$ & Kinetic viscosity $\left(\mathrm{m}^{2} / \mathrm{s}\right)$ \\
\hline$\delta$ & $\begin{array}{l}\text { Thickness of the insulation } \\
\text { layer }(\mathrm{m})\end{array}$ & $\dot{m}$ & $\begin{array}{l}\text { Evaporation rate or vapor } \\
\text { flux close to film surface } \\
\text { in the air }\left(\mathrm{kg} / \mathrm{m}^{2} \cdot \mathrm{h}\right)\end{array}$ \\
\hline$L_{c}$ & $\begin{array}{l}\text { Characteristic length of the } \\
\text { film }(\mathrm{A} / \text { Perimeter }=0.075 \mathrm{~m})\end{array}$ & $\mathrm{k}$ & $\begin{array}{l}\text { Thermal conductivity } \\
\text { (W/m } \cdot \mathrm{K})\end{array}$ \\
\hline$R a_{L}$ & Rayleigh number & $C$ & $\begin{array}{l}\text { Specific heat capacity of } \\
\text { water }(\mathrm{kJ} / \mathrm{kg} \cdot \mathrm{K})\end{array}$ \\
\hline
\end{tabular}

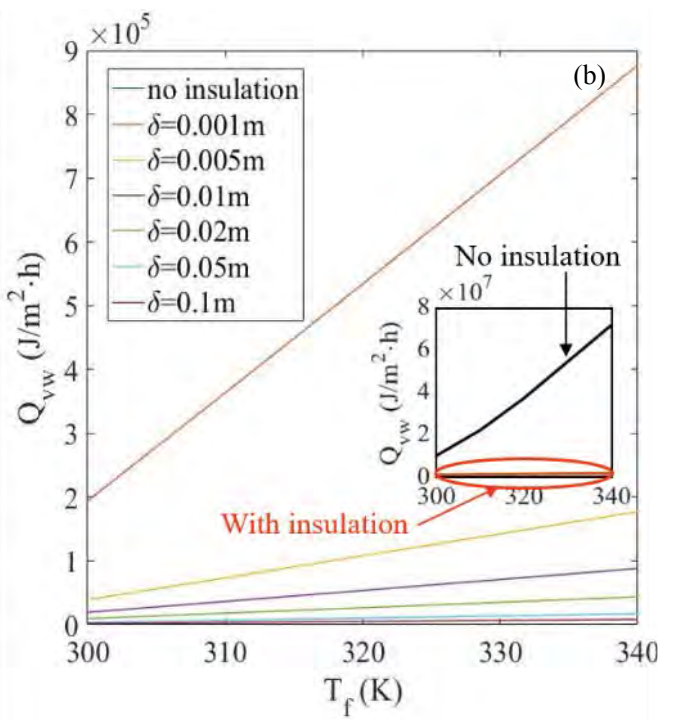

Figure 4. (a) Thermal energy transfer from the photo-thermal film as a function of its temperature due to water evaporation, convection and radiation to the water, and convection and radiation to the ambient air for the configuration with an insulating layer ( $\delta=0.001 \mathrm{~m}$ ). (b) Convective heat losses to the bulk water as a function of the photo-thermal film temperature for different thicknesses, $\delta$, of the insulating layer situated beneath the film. The inset shows that convective losses to the bulk water for the configuration with an insulation layer is almost two orders of magnitude less than that of the configuration without an insulation layer. 


\section{ACKNOWLEDGMENT}

This research was supported by the Natural Sciences and Engineering Research Council of Canada.

\section{REFERENCES}

[1] N. P. Cheremisinoff, Handbook of water and wastewater treatment technologies, Boston: Butterworth-Heinemann, 2002.

[2] A. Al-Karaghouli and L. L. Kazmerski, "Energy consumption and water production cost of conventional and renewable-energy-powered desalination processes," Renew. Sustain. Energy Rev., vol. 24, pp. 343-356, Aug. 2013. doi: 10.1016/j.rser.2012.12.064.

[3] C. L. Ong et al., "A novel concept of energy reuse from high concentration photovoltaic thermal (HCPVT) system for desalination," Desalination, vol. 295, pp. 70-81, June 2012. doi: 10.1016/j.desal.2012.04.005.

[4] H. Jin et al., "Steam generation in a nanoparticle-based solar receiver," Nano Energy, vol. 28, pp. 397-406, Oct. 2016. doi: 10.1016/j.nanoen.2016.08.011.

[5] S. Yu et al., "The impact of surface chemistry on the performance of localized solar-driven evaporation system," Sci. Rep., vol. 5, no. 1, p. 13600, Sept. 2015. doi: 10.1038/srep13600.

[6] G. M. Akselrod et al., "Large-area metasurface perfect absorbers from visible to near-infrared," $A d v$. Mater., vol. 27, no. 48, pp. 8028-8034, Dec. 2015. doi: 10.1002/adma.201503281.

[7] C. Zhang et al., "Shape-controlled synthesis of high-quality Cu7S4 nanocrystals for efficient light-induced water evaporation," Small, no. 38, pp. 5320-5328, Oct. 2016. doi: 10.1002/smll.201601723.

[8] P. Fan et al., "Large-scale cauliflower-shaped hierarchical copper nanostructures for efficient photothermal conversion," Nanoscale, vol. 8, no. 30, pp. 14617-14624, July 2016. doi: 10.1039/C6NR03662G.

[9] Y. Ito et al., "Multifunctional porous graphene for high-efficiency steam generation by heat localization," Adv. Mater., vol. 27, no. 29, pp. 43024307, Aug. 2015. doi: 10.1002/adma.201501832.

[10] G. N. Tiwari, H. N. Singh, and R. Tripathi, "Present status of solar distillation," Sol. Energy, vol. 75, no. 5, pp. 367-373, Nov. 2003. doi: 10.1016/j.solener.2003.07.005.

[11] Y. H. Zurigat and M. K. Abu-Arabi, "Modelling and performance analysis of a regenerative solar desalination unit," Appl. Therm. Eng., vol. 24, no. 7, pp. 1061-1072, May 2004.

doi: 10.1016/j.applthermaleng.2003.11.010.

[12] M. K. Phadatare and S. K. Verma, "Influence of water depth on internal heat and mass transfer in a plastic solar still," Desalination, vol. 217, no. 1-3, pp. 267-275, Nov. 2007. doi: 10.1016/j.desal.2007.03.006.

[13] R. Tripathi and G. N. Tiwari, "Desalination effect of water depth on intemal heat and mass transfer for active solar distillation," Desalination, vol. 173, pp. 187-200, Mar. 2005.

doi: 10.1016/j.desal.2004.08.032.

[14] G. Nagayama et al., "On the evaporation rate of ultra-thin liquid film at the nanostructured surface: A molecular dynamics study," Int. J. Therm. Sci., vol. 49, no. 1, pp. 59-66, Jan. 2010.

doi: 10.1016/j.ijthermalsci.2009.06.001.

[15] H. R. Seyf and Y. Zhang, "Effect of nanotextured array of conical features on explosive boiling over a flat substrate: A nonequilibrium molecular dynamics study," Int. J. Heat Mass Transf., vol. 66, pp. 613-624, Nov. 2013. doi: 10.1016/j.ijheatmasstransfer.2013.07.025.
[16] X. Li et al., "Graphene oxide-based efficient and scalable solar desalination under one sun with a confined 2D water path," Proc. Natl. Acad. Sci., vol. 113, no. 49, pp. 13953-13958, Dec. 2016. doi: 10.1073/pnas.1613031113.

[17] M. W. Higgins et al., "Carbon fabric based solar steam generation for waste water treatment," Sol. Energy, vol. 159, pp. 800-810, Jan. 2018. doi: 10.1016/j.solener.2017.11.055.

[18] L. Zhang et al., "Hydrophobic light-to-heat conversion membranes with self-healing ability for interfacial solar heating," Adv. Mater., vol. 27, no. 33, pp. 4889-4894, Sept. 2015. doi: 10.1002/adma.201502362.

[19] G. Xue et al., "Robust and low-cost flame-treated wood for highperformance solar steam generation," ACS Appl. Mater. Interfaces, vol. 9, no. 17, pp. 15052-15057, Apr. 2017. doi: 10.1021/acsami.7b01992.

[20] V. Kashyap et al., "Flexible anti-clogging graphite film for scalable solar desalination by heat localization," J. Mater. Chem. A, pp. 1522715234, June 2017. doi: 10.1039/C7TA03977H.

[21] C. Finnerty et al., "Synthetic graphene oxide leaf for solar desalination with zero liquid discharge," Environ. Sci. Technol., vol. 51, no. 20, pp. 11701-11709, Sept. 2017.

doi: 10.1021/acs.est.7b03040. 


\title{
Inlet Channel Effects on Archimedes Screw Generators
}

\author{
Adil Khan, Scott Simmons, Murray Lyons, William Lubitz ${ }^{1}$ \\ School of Engineering \\ University of Guelph \\ Guelph, Ontario, Canada \\ 1'wlubitz@uoguelph.ca
}

\begin{abstract}
The Archimedes screw is one of the oldest machines in the world, and has been used for various applications including hydroelectric power generation. This project investigated the effect of inlet conditions on a laboratory-scale Archimedes screw as a generator. The performance of the Archimedes screw was measured with both a narrow inlet channel and a wider inlet basin. The required inlet water depth was experimentally determined for both the channel and the basin, for a range of operating flow rates and screw rotation speeds. The tests with and without the channel were conducted at 9 different speeds and 9 different flows. The data was analyzed and plotted for efficiency, head, inlet depth, nondimensional inlet depth with the respective flow and speed associated to each data point. The experimental data was used to develop an inlet depth model. The data was analyzed and plotted for efficiency, head, inlet depth, non-dimensional inlet depth with the respective flow and speed associated to each data point. It was found that there was a notable efficiency difference between the basin and channel inlets only at low volume flow rates and high rotation speeds.
\end{abstract}

Keywords-Archimedes screw; inlet channel; efficiency; head

\section{INTRODUCTION}

The Archimedes screw is an ancient machine that has been used continually for millennia. Initially the Archimedes screw was used for drainage and irrigation purposes [1]. Applications of the Archimedes screw today include, but are not limited to, wastewater treatment plant pumps, low-lying land pumping stations, irrigation systems, fish conveyors, and generators [2].

An Archimedes screw consists of a set of helical planes, or flights, which are connected to a cylindrical central shaft, and supported on bearings that allow it turn within a close-fitting cylindrical trough or enclosure [3]. The design of the Archimedes screw is such that when the screw is tilted, the blades of the screw trap water discrete volumes of water called buckets [1]. The rotation of the screw allows the water to travel within the blades. The Archimedes screw can be used either as a pump or a generator. When water is moving from the bottom to the top, work is done turning the screw and it is being used as a pump. When water is moving from the top to the bottom, work is done by the rotating Archimedes screw and it can be used to turn a generator to produce electricity.
In the 1930's there were over 300 Archimedes screws in the Netherlands being used as drainage pumps in low-lying regions [4]. Use of the Archimedes screw for power generation began with experimental tests conducted at the University of Prague between 1993 and 1995 by Brada [2]. These experiments demonstrated that an Archimedes screw used for power generation could have more than $80 \%$ efficient converting hydraulic energy into mechanical energy [2]. It has been shown and proven that better conversion efficiencies can be attained by using larger screws as per Hellmann and Kleemann [2].

Currently over 400 Archimedes screw generators (ASGs) have been installed in Europe, with the first being installed only in 1993 [5]. Archimedes screw generators remain rare outside Europe. The first connection of an Archimedes screw generator to a power grid in North America was in 2013 by Greenbug Energy near Waterford, Ontario, Canada [5]. Unlike most hydro turbines, fish can pass through an operating Archimedes screw unharmed [5]. Additional advantages of Archimedes screws include straightforward design, good efficiency at non-optimum flows, ease of use, and low environmental impacts [6].

Water for an ASG is typically supplied by an inlet channel that conveys water from a reservoir or other source to the inlet of the screw. During design of an ASG installation, it is essential to ensure that the water depth that can be supplied at the inlet to the screw will be sufficient to provide the desired flow rate and amount of filling once the water enters the screw. If the inlet basin level is too low, for example, it will not be possible to fill the buckets in the operating screw.

The required inlet water level will be a function of both the screw rotation speed and the volume flow rate of water to be supplied. For example, if the screw is turning very quickly, there will be insufficient time to fill each bucket of the operating screw, and the screw will be unable to produce the desired power.

The goal of this project was to experimentally investigate the impact of volume flow rate and screw rotation speed on the inlet basin water depth. Two cases were tested to check the effect of inlet channel geometry on this relationship: one with a narrow inlet channel and the other with a much wider

A range of cross-sections can be used in an inlet channel. The channel used for this experiment had a rectangular cross-section, with a flat bottom and vertical, parallel side walls. 
The project involved the use of an inlet channel for the testing to develop a relationship of a non-dimensional model which can predict head, flow, and speed. The outlet channel losses were held constant for all tests during the experiment because the depth of the water at the screw outlet was always below the screw in the lower basin. The inlet channel losses depend upon the depth of water entering the screw and leaving the screw. It should be noted that the Borda-Carnot entrance loss technique can be used for calculating the inlet channel head loss and has been done by [2]. Kozyn found that the actual power loss at the entrance was small relative to other losses in an ASG.

This project involved the construction of a rectangular cross section channel which was connected to the Archimedes screw generator. This connecting channel was used to develop relationships with respect to the flow, head, torque, and speed of an Archimedes screw generator. The objective of the project was to determine the required water depth to achieve a specific volume flow rate through the Archimedes screw generator over a range of screw speeds within the operating range of the Archimedes screw generator.

\section{EXPERIMENTAL Methodology}

Experiments were conducted at the University of Guelph Archimedes screw testing facility with, and without the rectangular inlet channel installed within the upper supply basin to the Archimedes screw. Tests were conducted on a threebladed screw with a $31.6 \mathrm{~cm}$ outer diameter, $16.8 \mathrm{~cm}$ inner cylinder diameter, $31.8 \mathrm{~cm}$ pitch and $122 \mathrm{~cm}$ flighted length

The setup of the testing facility involved an upper basin that fed the Archimedes screw with water and that water would output to a lower basin (Fig. 1). The water from the lower basin was fed to the upper basin through a pipe. There was a pump which allowed for the water to flow through the pipe to get to the upper basin. Upon completion of the experiments with just the Archimedes screw, a rectangular cross-section inlet channel was designed with width of $31.8 \mathrm{~cm}$, length of $122 \mathrm{~cm}$ from upstream channel entrance to screw entrance. The sides of the channel entrance were designed with a radius of $2.5 \mathrm{~cm}$ to reduce channel entrance losses. The floor of the channel was aligned with the bottom-most edge of the screw inlet. This channel was machined by a local machining company in Guelph and installed in the Archimedes screw testing facility. The channel was installed and then tests were conducted at the 9 different flows and 9 different rotation speeds. The flows and speeds that were tested were the same with and without the channel in place.

The screw was operated at an inclination angle $\beta$ of $24.5^{\circ}$, at rotation speeds of RPM, 25 RPM, 30 RPM, 35 RPM, 40 RPM, 50 RPM, 60 RPM, 70 RPM, and 80 RPM. At each rotation speed, torque and inlet basin depth were recorded for flows of 6 $\mathrm{L} / \mathrm{s}$ to $14 \mathrm{~L} / \mathrm{s}$ in one $\mathrm{L} / \mathrm{s}$ increments. These ranges of speed and flow were chosen to provide a range of results across the performance envelope of the screw.

The Archimedes screw was operated in generator mode and the instruments which were available in the University of Guelph Archimedes screw testing facility were used for the measurements of flow, head, torque, and speed $[3,8]$.

The shaft power generated by the screw $P$ was calculated from the angular rotation speed of the screw $\omega$ and torque $T$ :

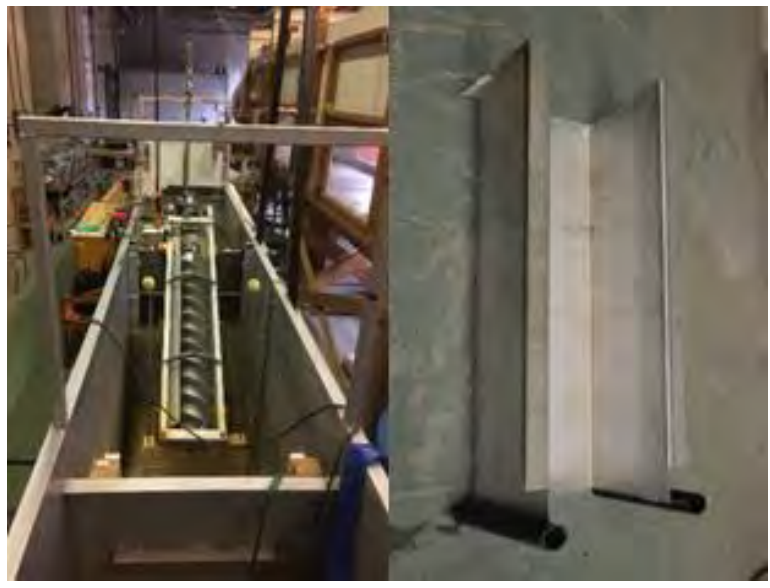

Figure 1. Archimedes screw (left) and inlet channel (right).

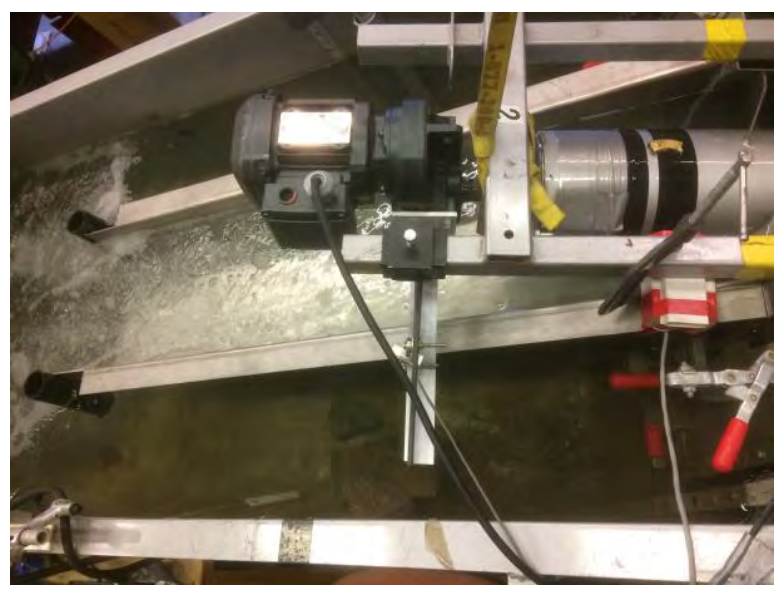

Figure 2. Upper basin with channel installed, with top of screw frame including gear motor and torque arm.

$$
P=\omega T
$$

The rotational speed of the screw was measured with a magnetic switch which was positioned just above the shaft at the inlet of the screw. A magnet secured on the screw shaft caused the magnetic switch to close once revolution [3]. The switch state was recorded by a National Instruments (NI) USB-6009 Data Acquisition (DAQ) unit connected to a laptop computer running NI LabVIEW. The magnetic switch data was recorded at a frequency of $1000 \mathrm{~Hz}$.

It should be noted that ASGs rotate relatively slowly. In practice, most full-scale ASGs used for power generator rotate at a speed close to

$$
\omega_{m}=\frac{5 \pi}{3 D_{o}^{2 / 3}}
$$

where $D_{0}$ is the screw outer diameter in meters and $\omega_{m}$ is rotation speed in radians per second.

Screw torque was measured by an Omegadyne LC703-25 load cell with one end fixed to an arm attached to a variablefrequency drive that regulated rotation speed, and the other end fixed to a stationary point on the support frame of the screw [8]. 
Efficiency vs Speed at $6 \mathrm{~L} / \mathrm{s}$

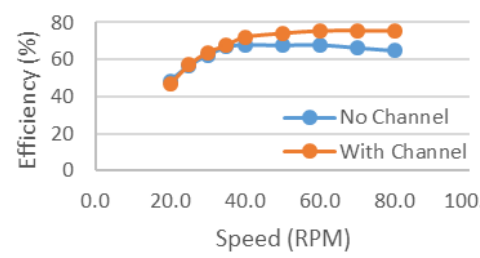

Efficiency vs Speed at $9 \mathrm{~L} / \mathrm{s}$

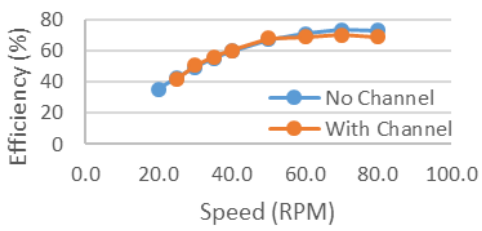

Efficiency vs Speed at $12 \mathrm{~L} / \mathrm{s}$

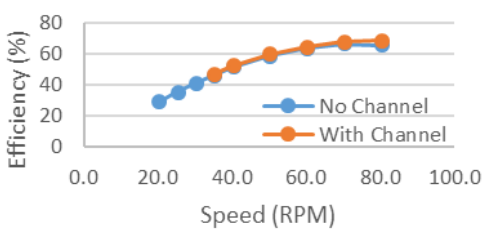

Efficiency vs Speed at $7 \mathrm{~L} / \mathrm{s}$

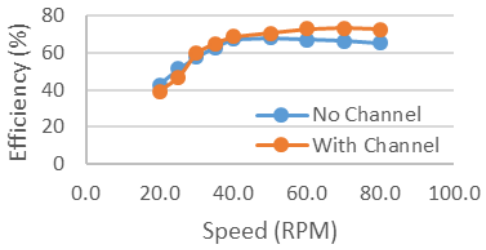

Efficiency vs Speed at $10 \mathrm{~L} / \mathrm{s}$

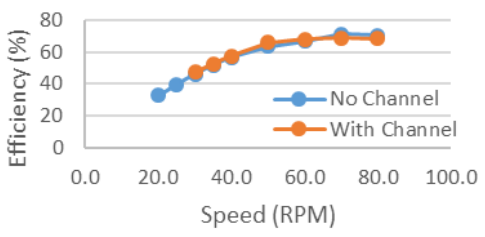

Efficiency vs Speed at $13 \mathrm{~L} / \mathrm{s}$

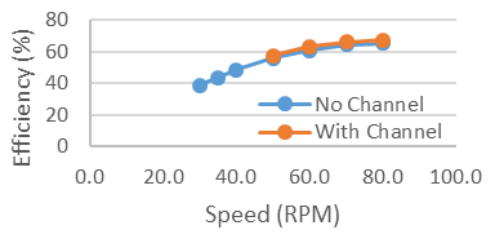

Efficiency vs Speed at $8 \mathrm{~L} / \mathrm{s}$

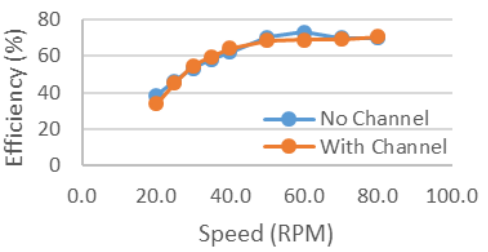

Efficiency vs Speed at $11 \mathrm{~L} / \mathrm{s}$

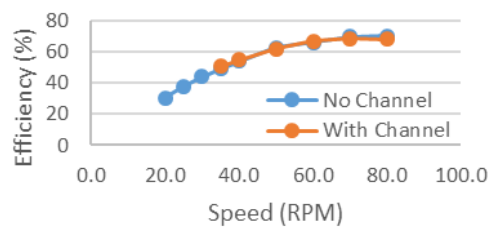

Efficiency vs Speed at $14 \mathrm{~L} / \mathrm{s}$

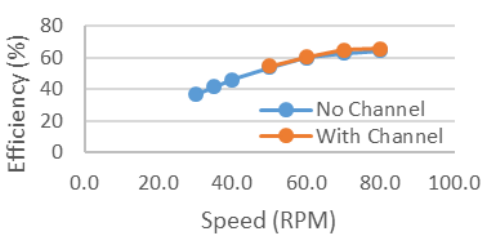

Figure 4. Efficiency vs Speed at Specified Flows

The load cell was installed so that the line of action through the cell was $26.5 \mathrm{~cm}$ from the screw axis of rotation.

The maximum power available to the screw is

$$
P_{\max }=\rho g h Q
$$

where $\rho$ is water density, $g$ is the gravitational constant $(9.81$ $\left.\mathrm{m} / \mathrm{s}^{2}\right), h$ is the height difference between the free surfaces in the upper and lower basin, and $Q$ is volume flow rate of water. The screw was operated with the receiving basin water level below the outlet of the screw to ensure consistence, so $h$ was taken as the vertical distance from the inlet basin free surface, to the lowermost point on the screw outlet. The heights of the screw as tested are shown in Fig. 3. The efficiency of the screw is then

$$
\eta=\frac{P}{P_{\max }}
$$

The water level in the inlet basin was measured using a Keller Valueline depth gauge and visually confirmed. Volume flow rate was measured with an Omega FTB740 flow meter

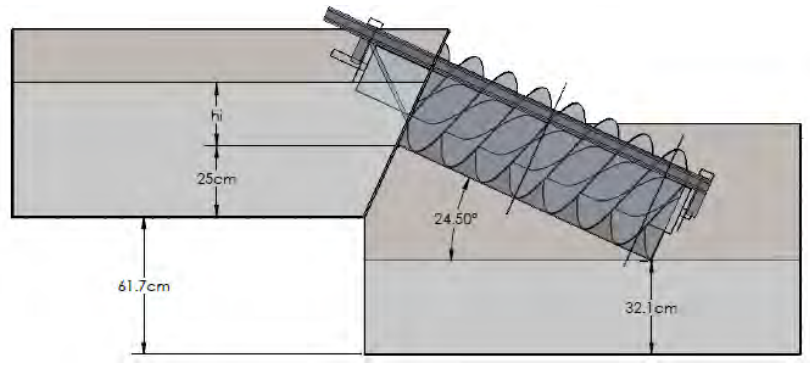

Figure 3. Heads and vertical distances as tested. installed in the return pipe that through which water is pumped from the lower basin back to the inlet basin. All flow through the system also passes through the screw, so this measurement also reflects the flow rate through the screw.

Non-dimensional inlet depth was calculated as

$$
\zeta=\frac{h_{i}}{D_{o} \cos \beta}
$$

where $h_{i}$ is vertical distance between the inlet basin free surface, and the lowest point of the screw inlet opening (Fig. 3).

\section{ERROR ANALYSIS}

An error analysis was performed in which observed sensor errors and uncertainties were propagated through the calculations to determine the uncertainty of calculated efficiency and power. Power generated by the screw is the product of torque and angular velocity. The magnetic switch observing rotation was recorded at a frequency of $1000 \mathrm{~Hz}$, and rotation rate was based on the precise time between the series of full rotations within the one minute sampling period. This allowed for the uncertainty in angular velocity to be negligible relative to the torque measurement [3]. The uncertainty for the torque measurements was previously found to be $0.22 \mathrm{Nm}$ [8]. The overall result was that under nominal conditions, the uncertainty of the calculated power was $\pm 1.0 \mathrm{~W}$ and efficiency uncertainty at nominal conditions is \pm 0.015 .

\section{ResUlts AND DisSUCSION}

The effect of supplying water to the screw through a narrow inlet channel, rather than directly from a wide supply basin, was 
relatively minor. Figs. 4 and 5 show that across much of the tested range, the efficiency of the screw was effectively the same whether it was operating with a narrow inlet channel or the wider inlet basin. The effect of the inlet channel only becomes apparent at low volume flow rates and higher rotational speeds. Above 40 RPM the efficiency of the screw is higher with the channel then without the channel for $6 \mathrm{~L} / \mathrm{s}$. A similar trend occurred at $7 \mathrm{~L} / \mathrm{s}$, while at all higher flow rates there were no significant differences in efficiency given the uncertainty in the calculated efficiency.

Interestingly, in low flow, high rotation conditions, the efficiency of the screw was higher with the narrow channel in place than it was without the channel. This was not expected: it was anticipated that the presence of the channel would result in decreased overall efficiency. The overall head across the screw is measured in the upstream area of the basin, and so any channel losses with the inlet channel would be expected to produce additional head loss, reducing system efficiency relative to the case of operating with the screw being supplied directly from the wide inlet basin.

It was determined that the efficiency difference was due to entrance effects when water enters the screw. Figs. 6 to 9 show the water surface at the entrance to the operating Archimedes screw. With the channel in place there is a relatively linear surface, and flow is relatively uniform into the screw. Without the channel, water enters the screw from the wide basin from both the front and sides of the inlet. Fig. 10 shows a notably variation in water level at the entrance, due to the flow from the basin sides turning around the sharp edged inlet and into the screw entrance. This was seen for both the $6 \mathrm{~L} / \mathrm{s}$ and $7 \mathrm{~L} / \mathrm{s}$ flows. This additional entrance loss without the channel in place results in an increased head loss. At higher flow rates, there was little apparent difference in the water surface at the entrance with and without the channel in place.

Note that the data point with $8 \mathrm{~L} / \mathrm{s}$ and 60 RPM may be subject to experimental error. It is believed that some air was drawn by the pump into the return pipe while this data point was taken, causing the flow meter to erroneously measure a slightly lower-than-actual flow.

Considering inlet depth generally (with or without the narrow inlet channel present), it was found that higher inlet basin depth was required in order to increase flow rates or decrease rotation speeds. Fig. 5 graphically shows this relationship.

Simple models of inlet depth were developed by first noting that screw rotation speed and volume flow rate would be the dominant dependent variables:

$$
\begin{aligned}
h_{i} & =f(\omega, Q) \\
\zeta & =f\left(\frac{\omega}{\omega_{m}}, \frac{Q}{Q_{m}}\right)
\end{aligned}
$$

where

$$
Q_{m}=\left[\frac{\pi}{4}\left(D_{o}{ }^{2}-D_{i}{ }^{2}\right)\right]\left[\frac{S \omega}{2 \pi}\right]
$$

is a theoretical rate at which material would pass through the full cylindrical volume of the screw (excluding the inner cylinder) due to screw rotation only.
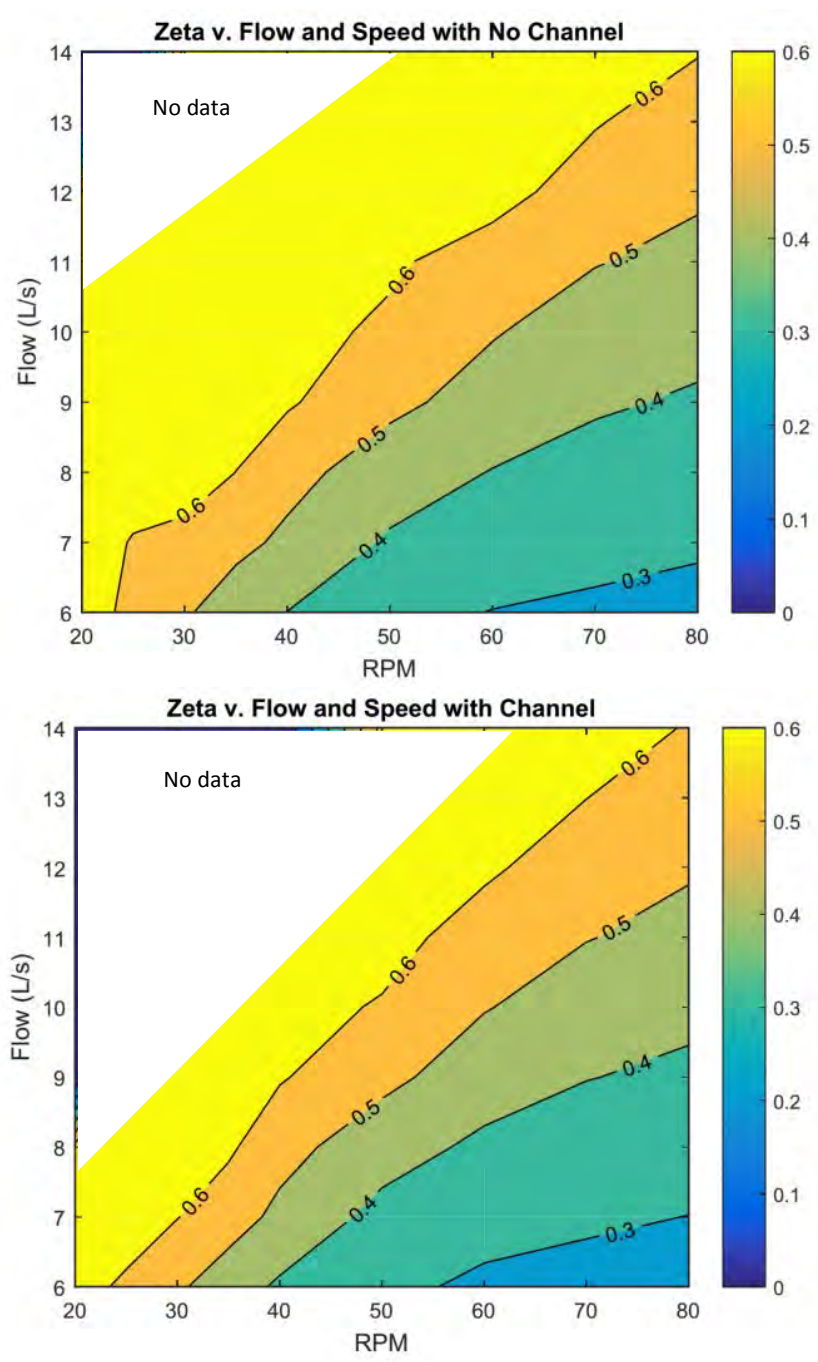

Figure 5. Contours of non-dimensional inlet depth vs. flow and rotation speed without channel installed (top) and with channel in place (bottom)

Simple predictive models were then developed by fitting the measured data (e.g. Fig. 4) to Eqns. 6 and 7. The resulting model for the inlet depth in dimensional form is

$$
h_{i}=\frac{Q}{\left[15.51 \mathrm{~L}^{1} \mathrm{~m}^{-1} \mathrm{rad}^{-1}\right] \times \omega}
$$

This equation gives a fairly accurate representation of the inlet depth with a mean absolute error of $3.3 \%$. Similarly, a nondimensionalized relationship was found as

$$
\zeta=\frac{\omega Q}{6.84\left(\omega_{m} Q_{m}\right)}
$$

The equation developed for the non-dimensional inlet depth with respect to the flow and speed was found by trial and error. This equation gives a representation of the non-dimensional inlet depth with a mean absolute error of $9.4 \%$.

It should be noted that scaling between lab ASGs and full scale ASGs has been identified as an issue in need of further study [3]. While Eqn. 10 is cast non-dimensionally, it should be noted that entrance Reynolds number and Froude number will 


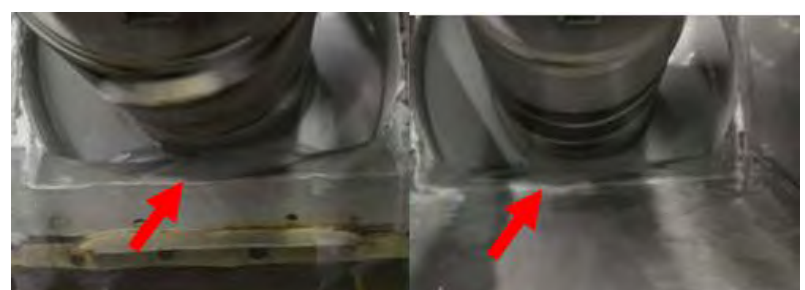

Figure 6. Flow of $6 \mathrm{~L} / \mathrm{s}$ with no channel (left) and channel (right) at 80 RPM at the inlet of the screw

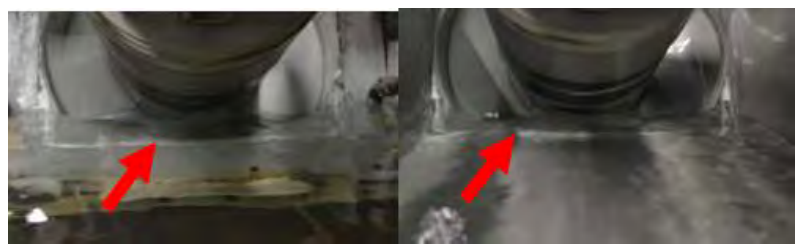

Figure 7. Flow of $7 \mathrm{~L} / \mathrm{s}$ with no channel (left) and channel (right) at 80 RPM at the inlet of the screw

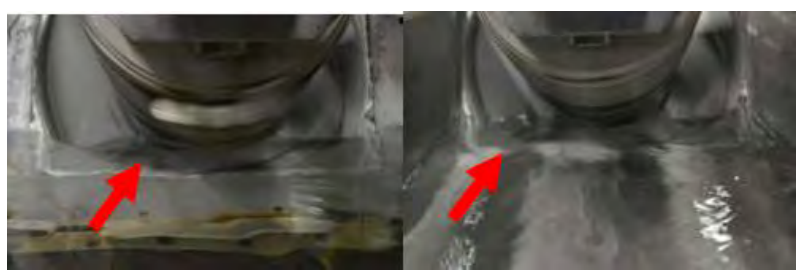

Figure 8. Flow of $8 \mathrm{~L} / \mathrm{s}$ with no channel (left) and channel (right) at 80 RPM at the inlet of the screw

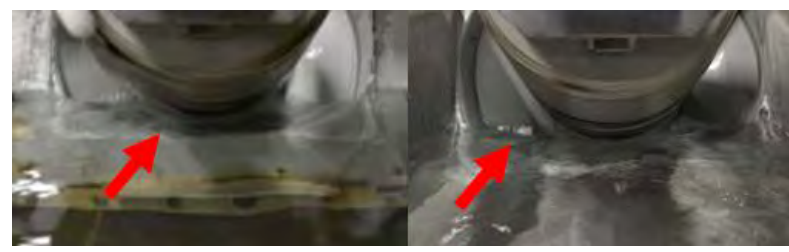

Figure 9. Flow of $9 \mathrm{~L} / \mathrm{s}$ with no channel (left) and channel (right) at 80 RPM at the inlet of the screw

change with ASG scale, and effects such as the criticality of the entrance flow are not included in this simple model. Therefore, Eqn. 10 should be used with caution in differing situations.

\section{CONCLUSION}

The Archimedes screw is a practical device that has been used for many years with various applications. There are still many things to learn about the Archimedes screw. This project investigated the inlet water depth to required achieve a specific volume flow rate through the Archimedes screw generator over a range of screw speeds. Tests were conducted with both a narrow inlet channel and wider inlet basin at the entrance to the screw. The measured efficiency was higher with the channel than without the channel for the $6 \mathrm{~L} / \mathrm{s}$ and $7 \mathrm{~L} / \mathrm{s}$ cases above 40 RPM. Entrance effects at the screw were identified as a possible cause of the reduced efficiency without channel present. There can be other reasons which would require further research into this topic. Relationships were developed for both the dimensional and non-dimensional inlet depth required to provide a specified volume flow rate across a range of rotational speeds. Additional testing of these relationships with data from other Archimedes screws is needed to verify prediction accuracy and determine if the relationships are broadly applicable to all Archimedes screws.

\section{ACKNOWLEDGMENTS}

The work documented in this study was completed as part of a much larger project financially supported by Greenbug Energy Inc. and the Natural Sciences and Engineering Research Council (NSERC) of Canada through the Collaborative Research and Development (CRD) program.

\section{REFERENCES}

[1] C. Rorres, "The turn of the screw: Optimal design of an Archimedes screw". Journal of Hydraulic Engineering, 126(1), pp 72-80, 2000. doi: 10.1061/(ASCE)0733-9429(2000)126:1(72).

[2] D. M. Nuernbergk and C. Rorres, "Analytical Model for Water Inflow of an Archimedes Screw Used in Hydropower Generation", Journal of Hydraulic Engineering, vol. 139, no. 2, pp 213-220, 2012. doi: 10.1061/(ASCE)HY.1943-7900.0000661.

[3] A. Kozyn and W. D. Lubitz, "A power loss model for Archimedes screw generators," Renewable Energy, vol. 108, pp. 260-273, Aug. 2017. doi: 10.1016/j.renene.2017.02.062.

[4] G. Nagel, Archimedean Screw Pump Handbook, Schwäbisch Gmünd RITZ Pumpenfabrik OHG, pp 10-11, 1968

[5] W. D. Lubitz, M. Lyons, and S. Simmons, "Performance model of archimedes screw hydro turbines with variable fill level", Journal of Hydraulic Engineering, vol. 140, no. 10, p. 4014050, Oct. 2014. doi:10.1061/(ASCE)HY.1943-7900.0000922

[6] Greenbug Energy Inc, "What are the benefits of using Archimedes Screws over other technologies? - GreenBug Energy - micro hydro", 2017, GreenBug Energy - micro hydro. Retrieved 21 May 2017. [Online]. Available: http://greenbugenergy.com/sp_faq/what-are-thebenefits-of-usingarchimedes-screws-over-other-technologies.

[7] D. A. Chin, Water-resources engineering (Third ed.), Pearson Education, pp. 140-144, 2013.

[8] K. Songin, "Experimental Analysis of Archimedes Screw Turbines", Masters Thesis, University of Guelph. August 2017. [Online]. Available: https://atrium.lib.uoguelph.ca/xmlui/bitstream/handle/10214/11481/Song in_Kathleen_201708_MASc.pdf?sequence $=1$. 


\title{
Investigation of Short-Side-Chain lonomer and Membrane for Proton Exchange Membrane Fuel Cells
}

\author{
Samaneh Shahgaldi \\ Department of Mechanical and Mechatronics Engineering, \\ University of Waterloo, 200 University Avenue West, \\ Waterloo, ON N2L 3G1, Canada \\ samaneh.shahgaldi@uwaterloo.ca
}

\author{
Adnan Ozden \\ Department of Mechanical and Mechatronics Engineering, \\ University of Waterloo, 200 University Avenue West, \\ Waterloo, ON N2L 3G1, Canada \\ aozden@uwaterloo.ca
}

\author{
Xianguo Li \\ Department of Mechanical and Mechatronics Engineering, University of Waterloo, \\ 200 University Avenue West, Waterloo, ON N2L 3G1, Canada \\ xianguo.li@uwaterloo.ca
}

\begin{abstract}
Proton exchange membrane (PEM) fuel cells have been progressively designed to become suitable for hightemperature operation to achieve further performance improvements. However, the current state-of-the-art fuel cell materials, such as long-side-chain (LSC) ionomers and membranes, are not suitable for high-temperature operation, requiring development and investigation of alternative materials. In this study, short-side-chain (SSC) membrane and ionomer are considered as potential materials, and performance of a membrane-electrode assembly (MEA) manufactured with the SSC ionomer and membrane is experimentally investigated in a scaled-up fuel cell $\left(45 \mathrm{~cm}^{2}\right)$. Comparison is made with an MEA based on the LSC ionomer and membrane under identical preparation and testing conditions. The catalyst layers (CLs) made of either SSC or LSC ionomer are characterized through scanning electron microscopy (SEM) to understand their surface morphology and microstructure. Results show that the SSC ionomer embedded in the CL provides much more uniform surface morphology and well-proportioned microstructural characteristics than its LSC counterpart. Further, the MEA based on SSC ionomer and membrane demonstrates considerable performance superiorities under all the applied operating conditions. Furthermore, the performance of the MEA based on the SSC ionomer and membrane is found to be less sensitive to changes in operating conditions.
\end{abstract}

Keywords- Proton exchange membrane fuel cell; Short-sidechain ionomer; Short-side-chain membrane; High-temperature fuel cell operation

\section{INTRODUCTION}

Proton exchange membrane (PEM) fuel cells are an energy-conversion device that converts the chemical energy of hydrogen and oxygen into electricity through electrochemical reactions. In recent years, PEM fuel cell technology has perceivably advanced and reached the early stage of commercialization, but along with remaining technical challenges to be overcome for further improvements in performance [1-3]. To overcome many of these challenges, fuel cells have been gradually designed to provide trouble-free performance at high operating temperatures.

Fuel cells adopted for low-temperature operation are highly susceptible to carbon monoxide (CO) poisoning, since the adsorption of $\mathrm{CO}$ on the catalyst nanoparticles is favored at low temperatures [4]. Thus, high-temperature operation would certainly increase the $\mathrm{CO}$ tolerance, which can in turn eliminate prohibitive technological costs through catalyst loading reduction. Further, a fuel cell generates a considerable amount of heat due to electrochemical reactions and associated irreversibilities that needs to be effectively removed to maintain the operating temperature, requiring the development of effective cooling strategies. Thus, if the cell is adopted for high-temperature operation, there will be no necessity for well-planned cooling strategies. Besides, the overall system efficiency can be substantially boosted through recovering of excess heat as steam for direct heating or pressurized conditions. High-temperature operation can also substantially minimize the damage caused by fuel impurities [5], as it does not require high-level of humidification for pressurization, unlike low-temperature operation, and hence the problems associated with water management can also be mitigated. High-temperature operation can also simultaneously increase the diffusion rate of the reactants and simplify detachment of water molecules from the catalyst layer (CL) - which would consequently facilitate the diffusion of the reactants into the three-phase boundary.

Although high-temperature operation provides the aforementioned promises, today's fuel cells perform only well at low temperatures, typically $60-80^{\circ} \mathrm{C}$ [6]. This restriction originates from unsuitableness of the archetypal membranes and ionomers for high-temperature operation (i.e., long-side chain (LSC) membranes and ionomers), since they are 
typically made of perfluorosulfonic acid. Dupont's Nafion ${ }^{\circledR}$ can be given as a good example of these ionomers and membranes, in which proton transport is governed by "Vehicular" and "Grotthus" mechanisms [7,8] - the mechanisms function only well in the presence of adequate water, unlikely to be seen at high temperatures [9]. Recently, several alternatives, known as short-side chain (SSC) ionomers and membranes, have been introduced into the fuel cell market to make fuel cells viable for high-temperature operation (see [10], for example). The SSC ionomers and membranes can provide favorable thermal transition temperature and ion exchange capacity (EIC), without facing any swelling and/or dissolution [11]. These characteristics also enable them to absorb and retain water even at elevated temperatures. Even though the SSC ionomers and membranes are popular owing to their suitability for high-temperature operation, their cell performance characteristics are still under vehement investigations.

The objective of the present work is therefore to investigate cell performances of the SSC ionomers and membranes under different cell conditions: varied cell temperatures, varied anode and cathode flow rates, and varied anode and cathode relative humidities (RHs). To understand the morphological and microstructural differences between the SSC and LSC ionomers, scanning electron microscopy (SEM) analyses of the CLs made of these ionomers are performed. For comparison, cell performances of the membrane-electrode assemblies (MEAs) with the SSC ionomer-based CLs and membranes are evaluated. Cell performances are compared with those of the MEAs based on the LSC-based ionomers and membranes under the same conditions.

\section{EXPERIMENTAL}

The catalyst inks are prepared by using carbon-supported platinum (50\% Pt to C ratio, Tanaka Kikinzoku Kogyo K.K.) as a catalyst, either short-side-chain ionomer (Aquivion ${ }^{\circledR}$ with equivalent weight of $790 \mathrm{~g} \mathrm{eq}^{-1}$ ) or long side chain ionomer ( Nafion (EW) $1100 \mathrm{~g} \mathrm{eq}^{-1}$ ) as a binding agent, and 2-proponal (IPA, 99.9\%, Sigma ${ }^{\circledR}$-Aldrich) as a solvent. Proper amount of these materials is mixed in a $20 \mathrm{ml}$ vial, followed by mechanical mixing in an ultrasonic bath for $1 \mathrm{~h}$. The PtC/ionomer ratio in the electrodes is kept constant as 3.0/1.0. The ink prepared is deposited onto the selected membrane (either Aquivion E87-05S with equivalent weight of $890 \mathrm{~g} \mathrm{eq}^{-1}$ or Nafion 211) via catalyst-coated membrane (CCM) method. The CCM is then sandwiched between the anode and cathode gas diffusion layers (GDLs, Avcarb GDS 3250) to obtain the MEA. The electrode active areas of the MEAs are $45 \mathrm{~cm}^{2}$, and the Pt loadings in the anode and cathode electrodes are kept constant as $0.10 \mathrm{mg} / \mathrm{cm}^{2}$ and $0.40 \mathrm{mg} / \mathrm{cm}^{2}$, respectively. Cell performances of the MEAs based on different ionomers and membranes are obtained under the same conditions: a varied cell temperature, varied anode and cathode flow rates, and varied anode and cathode relative humidities. The polarization curves are obtained by potentiostatically reducing the cell voltage and recording the corresponding current values. Each polarization curve is the average of three polarization curves obtained on three different days, with a standard deviation no more than $5 \%$.

\section{RESULTS AND DISCUSSION}

\section{A. Morphological and Microstructural Characteristics of the Catalyst Layers}

Figs. 1 (a) and (b) present the top view of the CLs made of the SSC and LSC ionomers. Both the CLs have similar compact and porous surface characteristics - such characteristics are quite common for the CLs made of Pt-C catalyst. Since the CLs are subjected to gold coating prior to SEM analyses, the cracks apparent on the surface could be associated with the gold deposition. Darker particles in the images show the Pt-C particles, while black holes represent the pores existing in the regions near the surface. Ionomer surrounding the Pt-C particles is shown by lighter contrast in the images. Clearly, both the CLs have homogeneous surface characteristics, but the SSC ionomer-based CL seems to have relatively more open pores, along with virtually no ionomer agglomeration (see Fig. 1(a)), clearly indicating the SSC ionomer's compatibility with the Pt-C particles. Such compatibility could be related to inherent binding characteristics of the SSC ionomer. In contrast, in the LSC ionomer-based $\mathrm{CL}$, the ionomer films are more prone to accumulate and build up bigger ionomer-ionomer agglomerates (see Fig. 1(b)), which is not so desirable, because such agglomerates shrink the available pores that are available for mass transport. Therefore, compared to the LSC ionomer-based CL, the SSC ionomer-based one has relatively more open pores. The pore characteristics of the electrodes based on these CLs are investigated by the Method of Standard Porosimetry (MSP) under identical testing conditions, and results are in good agreement with the SEM images. It is seen that the SSC ionomer-based CL has relatively a relatively higher porosity $(73.10 \%)$ than the LSC ionomer-based one $(68.80 \%)$.

\section{B. Fuel Cell Performance Testing}

Scaled-up single cell performance tests are conducted to provide practical insights into the differences between the CLs and membranes made of the SSC and LSC ionomers, under various operating conditions, i.e., different cell temperatures and different flow rates, and relative humidities (RHs) of air and hydrogen. For the virtue of repeatability, each polarization curve is obtained from measurements conducted on three different days under identical conditions. Figs. 2 (a) and (c) compare the polarization and power density curves of the MEAs with the CLs made of two different ionomers - SSC and LSC - as polymeric binders and membranes as a function of varying air and hydrogen flow rates. The other testing parameters are held constant, such that the cell temperature is set as $75^{\circ} \mathrm{C}$, while the back pressures and RHs of the hydrogen and air streams are set as $35 \mathrm{kPag}$ and $100 \%$, respectively. Two different flow rates of hydrogen and air are applied. Fixed anode and cathode flow rates of 4.45 and $9.001 / \mathrm{min}$ applied is named as "high flow", while the anode and cathode stoichiometries of $1.20 / 2.00$ is named as "low flow", throughout the rest of this paper. As evident from Fig. 2 (a), the MEA with the SSC-ionomer based CLs and membrane is relatively less sensitive to flow rates of the reactants. The open circuit voltages (OCVs) obtained from this MEA at the highand low-flow rates are almost the same, while there is a slight 
difference between the current densities when the cell voltage is decreased, such that the maximum power densities obtained under high- and low-flow conditions are $0.96 \mathrm{~W} / \mathrm{cm}^{2}$ and 0.94 $\mathrm{W} / \mathrm{cm}^{2}$, respectively. On the other hand, the MEA with the LSC-based CLs and membrane shows almost the same performance at low current densities under both the high- and low-flow conditions, as seen in Fig. 2 (b). However, a noticeable reduction in cell performance is observed, as the cell is operated at high current densities, and the reactants are supplied under low-flow conditions. This trend indicates that the LSC-based CL and membrane provide conditiondependent performance, further suggesting that the LSC-based components are highly sensitive to changes in operating conditions. This sensitivity probably originates from the dimensional changes in the LSC ionomer; the LSC ionomer potentially experiences severe swelling at high current densities, blocking the pores available for the mass transport. In contrast, the MEA with the SSC ionomer-based CLs and membrane shows almost the same performance, regardless of flow conditions, indicating the stability of this ionomer, which potentially experiences less swelling, as the current density is increased. Such behaviour probably preserves the porosity of the electrode, thus almost the same mass transport capability is

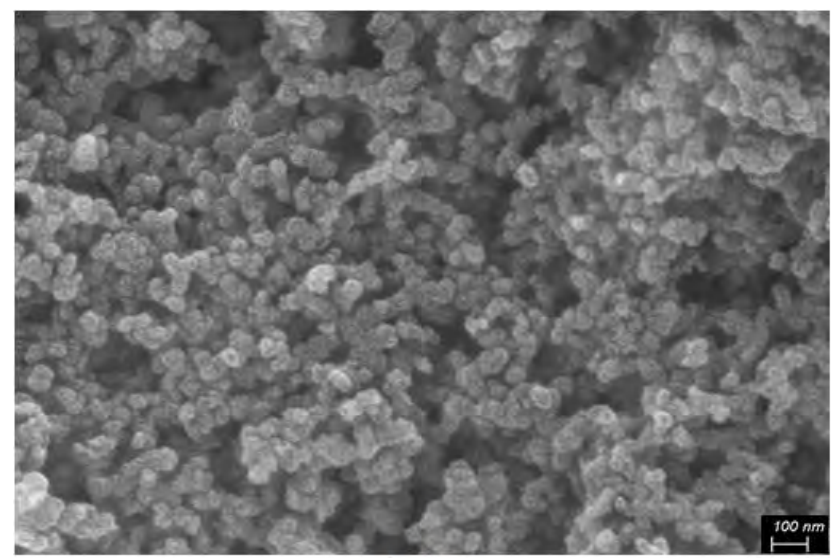

(a)

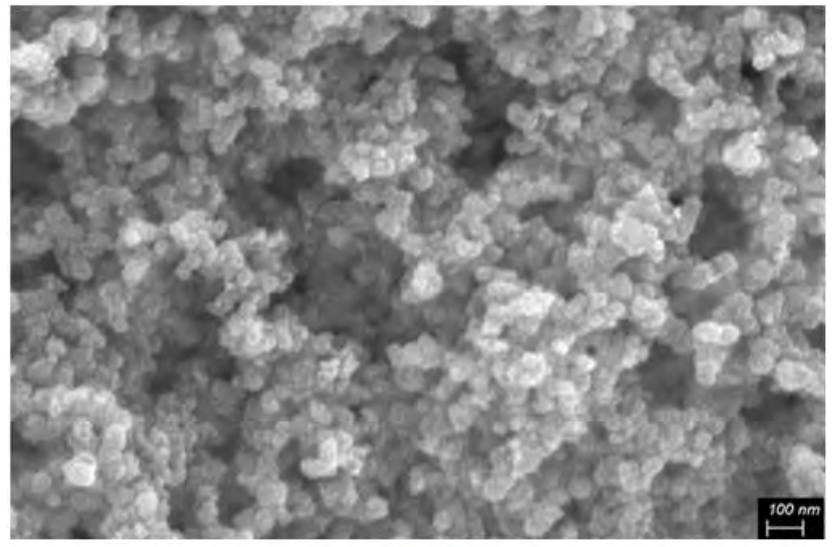

(b)

Figure 1. Scanning electron microscopy (SEM) images of the catalyst layers (CLs) made of (a) short-side chain (SSC) ionomer and (b) long-side-chain (LSC) ionomer.
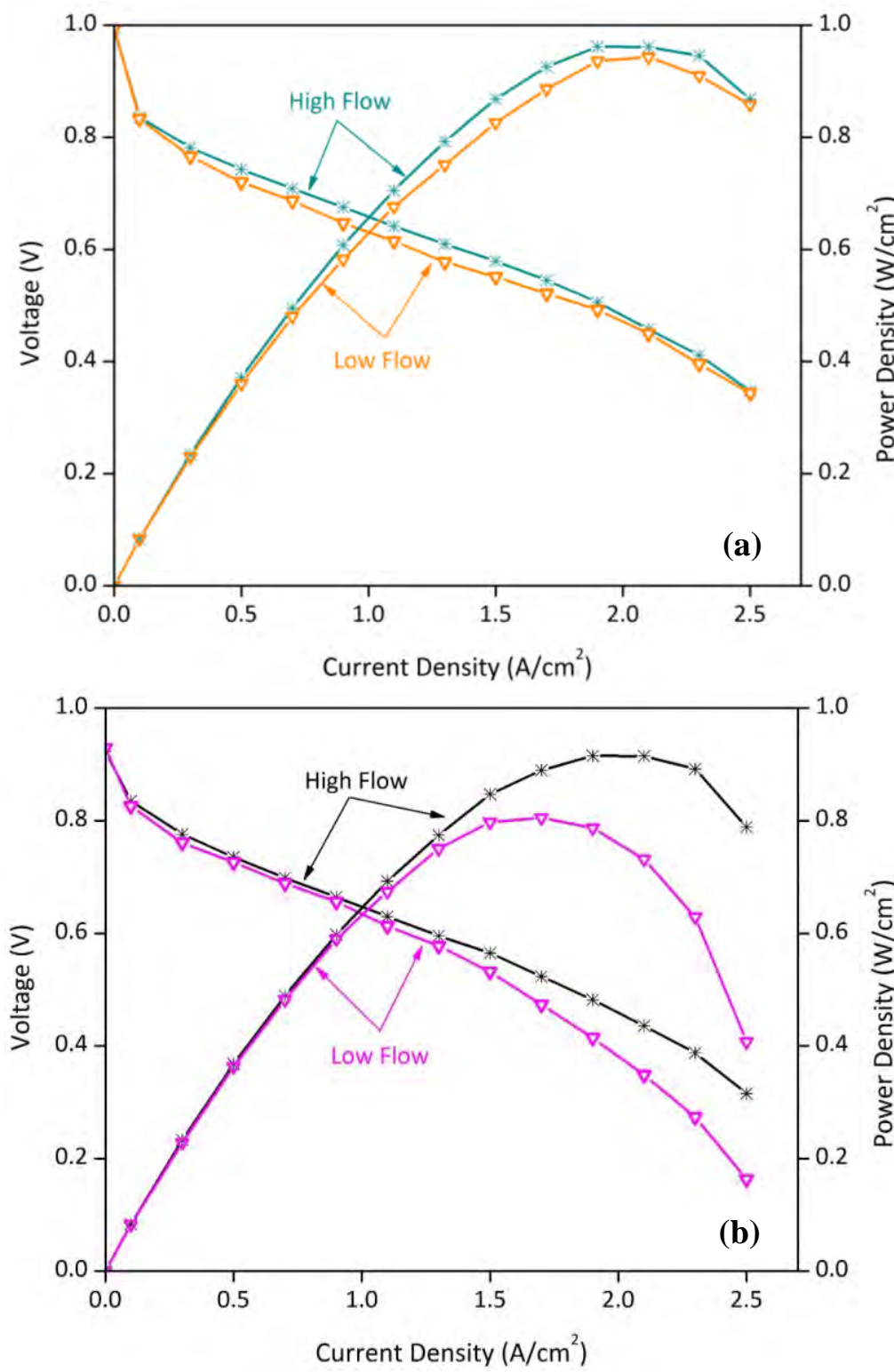

Figure 2. Polarization and power curves of the MEAs with the catalyst layers (CLs) and membranes made of either (a) SSC or (b) LSC ionomers under low and high flow conditions.

maintained, regardless of the flow conditions. By comparing Figs. 2 (a) and 2 (b), it can be concluded that the MEA with the SSC ionomer-based CLs and membrane yields better cell performance under high- and low-flow conditions than its LSC ionomer-based counterpart; such a performance superiority may be attributed to the SSC-based components' higher stability, crystallinity, and proton-transport capability [11].

Cell temperature is another critical parameter affecting the capability of the constituents in dealing with mass, heat, and electron transport. Thus, performances of the MEAs with the CLs and membranes based on either SSC or LSC ionomers are investigated at two different cell temperatures, namely, at $75^{\circ} \mathrm{C}$ and $95^{\circ} \mathrm{C}$. These temperatures are specifically chosen, because the LSC-ionomers and membranes demonstrate the best performance at the cell temperature of $75^{\circ} \mathrm{C}$; meanwhile, it is also known that their effective operation is limited to $100^{\circ} \mathrm{C}$. Hence, temperatures chosen are expected to provide a better understanding of the differences between the SSC and 

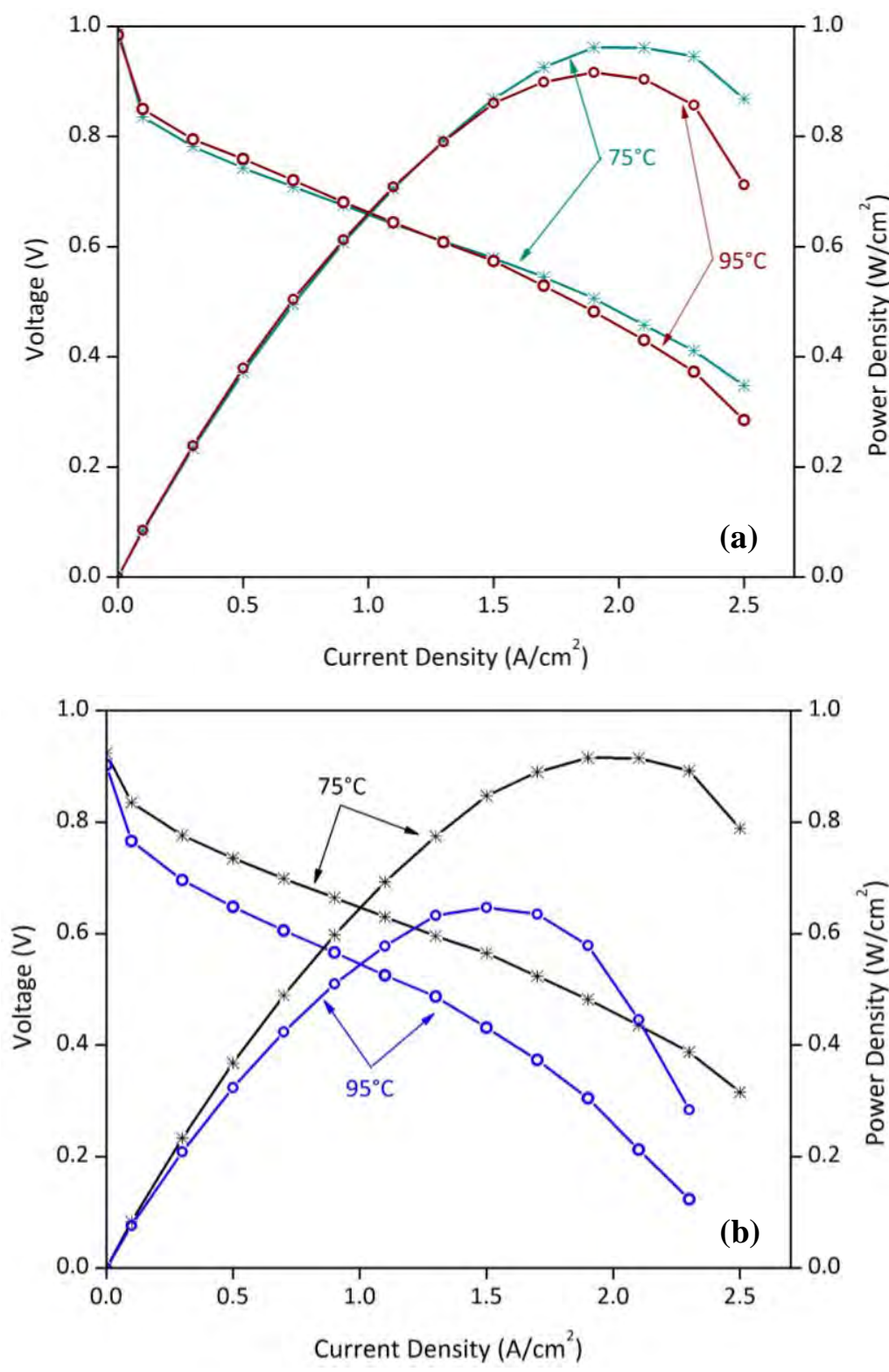

Figure 3. Polarization and power curves of the MEAs with the catalyst layers (CLs) and membranes made of either (a) SSC or (b) $\mathrm{LSC}$ ionomers at $75^{\circ}$ and $95^{\circ} \mathrm{C}$.

LSC ionomers and membranes. For a proper comparison, the other operating parameters are held constant during performance testing, such that the flow rates, back pressures, and RHs of the air and hydrogen streams are set to 4.45 and $9.00 \mathrm{l} / \mathrm{min}, 35 \mathrm{kPag}$, and $100 \%$, respectively. As seen from Fig. 3, the MEA with the SSC ionomer-based CLs and membrane shows virtually the same performance in activation-polarization and ohmic-polarization dominant regions, as the operating temperature is increased from $75^{\circ} \mathrm{C}$ to $95^{\circ} \mathrm{C}$, while a slight drop in cell voltage is detected at high current densities. For example, at the constant current density of $2.50 \mathrm{~A} / \mathrm{cm}^{2}$, the performance of the MEA with the SSCbased ionomer and membrane drops almost $17 \%$, as the cell temperature is increased from $75^{\circ} \mathrm{C}$ to $95^{\circ} \mathrm{C}$. However, the MEA with the LSC ionomer-based CLs and membrane performs quite differently in activation-, ohmic-, and concentration-polarization dominant regions with increasing cell temperature (see Fig. 3(b)). The drop in cell voltage is even more substantial at high current densities, such that at the current density of $2.30 \mathrm{~A} / \mathrm{cm}^{2}$, a voltage drop of about $68 \%$ is detected. The performance differences between the two MEAs may be better understood by taking a closer look at the maximum power densities obtained at the cell temperatures of $75^{\circ} \mathrm{C}$ and $95^{\circ} \mathrm{C}$. The MEA with the SSC ionomer-based CLs and membrane yields the maximum power densities of 0.96 $\mathrm{W} / \mathrm{cm}^{2}$ and $0.92 \mathrm{~W} / \mathrm{cm}^{2}$ at the cell temperatures of $75^{\circ} \mathrm{C}$ and $95^{\circ} \mathrm{C}$, respectively. However, the maximum power densities obtained at the cell temperatures of $75^{\circ} \mathrm{C}$ and $95^{\circ} \mathrm{C}$ from the MEA with the LSC ionomer-based CLs and membrane are $0.92 \mathrm{~W} / \mathrm{cm}^{2}$ and $0.65 \mathrm{~W} / \mathrm{cm}^{2}$, respectively. Clearly, increasing cell temperature results in severe deterioration in performance of the MEA with the LSC ionomer-based CLs and membrane, but only slight deterioration in performance of the MEA with the SSC ionomer-based CLs and membrane - a finding indicating that the SSC ionomer-based cell components are far less sensitive to changes in the operating temperature; or alternatively, the proton transport in the SSC ionomer and membrane is effectively maintained at elevated temperatures. This behaviour could be related to the high crystallinity and better proton transport capability of the SSC ionomer at elevated temperatures. Further, owing to their higher crystallinity and lower equivalent weight, the SSC ionomer and membrane experience less swelling, which is not only useful for preserving the interfacial characteristics at the threephase boundary but also for maintaining the electrode microstructural characteristics over a wide range of cell temperature. Furthermore, due to their lower equivalent weight, the SSC ionomer and membrane probably retain adequate water, which in turn enables them not to suffer from membrane dehydration, hence function well even at elevated temperatures. In the case of the MEA with the LSC ionomerbased CLs and membrane, high-temperature operation probably leads to degradation in the LSC-ionomer based components, i.e., excessive swelling, and thus both the interfacial and microstructural characteristics may not be maintained at high cell temperatures. The joint effect of these changes in turn leads to significant deterioration in the cell performance in both ohmic- and concentration-polarization dominant regions (see Fig. 3).

The effect of the RHs of the air and hydrogen streams on performances of the MEAs with the CLs and membranes made of either SSC or LSC ionomers are also investigated. Fig. 4 shows the power and polarization curves of the MEAs under the RHs of $55 \%$ and $100 \%$. Clearly, a decrease in the RHs of the air and hydrogen streams negatively impacts performances of both the MEAs. However, the MEA with the SSC ionomer-based CLs and membrane seems to experience less performance drop with decreasing RHs, compared to the one with the LSC ionomer-based CLs and membrane. For example, the MEA with the SSC ionomer-based CLs and membrane experiences a cell voltage drop of $13 \%$ at the constant current density of $1.50 \mathrm{~A} / \mathrm{cm}^{2}$, as the RHs are decreased from $100 \%$ to $55 \%$, while drop in cell voltage of the MEA with the LSC-based CLs and membrane is almost $46 \%$. The maximum power densities obtained for the SSC- and LSC-based MEAs at $55 \% \mathrm{RH}$ are $0.76 \mathrm{~W} / \mathrm{cm}^{2}$ and 0.55 $\mathrm{W} / \mathrm{cm}^{2}$, respectively. A noticeably better cell performance of the SSC-based MEA is probably due to the higher waterretention capability of the SSC ionomer and membrane, 

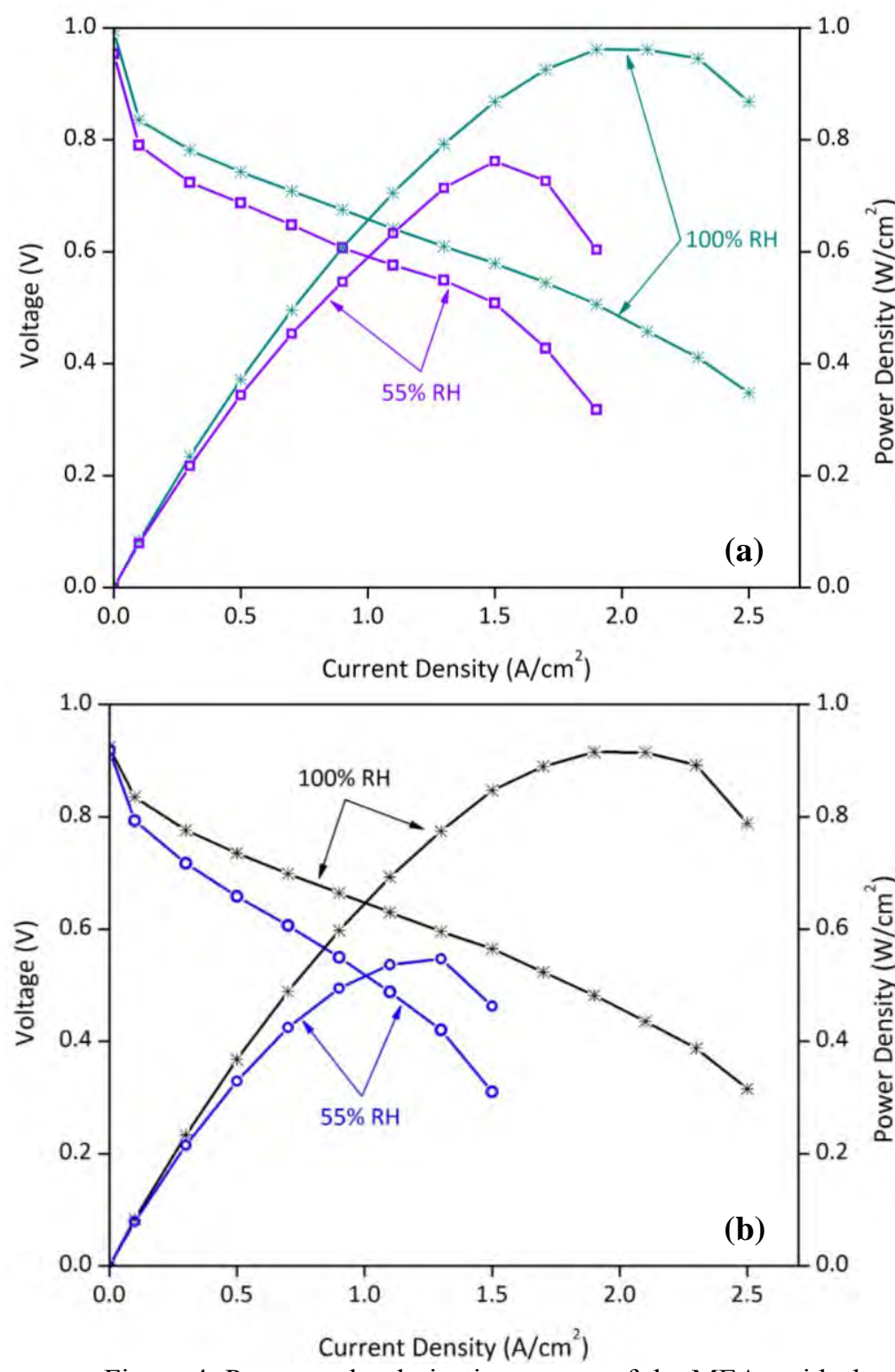

Figure 4. Power and polarization curves of the MEAs with the catalyst layers (CLs) and membranes made of either (a) SSC or (b) LSC ionomer under different RHs of air and hydrogen streams.

compared to their LSC counterparts, since being able to operate well under low-humidity conditions requires effective proton-transport capability. This advanced proton-transport capability is probably due to its lower equivalent weight than the LSC ionomer, which provides relatively higher number of hydrophilic sulfonic acid groups. The presence of more sulfonic acid groups inside both the ionomer and membrane yields effective proton transport even when the cell is operated under low RHs, thus the cell suffers less from ohmic polarization and performs much better.

\section{CONCLUSIONS}

In this study, the effect of ionomer structure in the membrane and catalyst layer (CL) on the performance of proton exchange membrane (PEM) fuel cells is experimentally investigated in a scaled-up cell $\left(45 \mathrm{~cm}^{2}\right)$. Short-side-chain (SSC) ionomer and membrane are applied in a membrane-electrode assembly
(MEA), and cell performance of this MEA is investigated over a wide range of operating conditions: different cell temperatures $\left(75^{\circ} \mathrm{C}\right.$ and $\left.95^{\circ} \mathrm{C}\right)$, air and hydrogen flow rates (low- and high-flow conditions), and air and hydrogen relative humidities $(100 \% \mathrm{RH}$ and $55 \% \mathrm{RH})$. Comparison is made with an MEA based on long-side-chain (LSC) ionomer-based CLs and membrane. The morphological and microstructural characteristics of the SSC ionomer-based CLs are investigated, and comparison is made with the LSC ionomer-based CLs. The pore characteristics of the electrodes with these CLs are also investigated. Results indicate that the SSC ionomer not only offers more uniform surface morphology and microstructure, but also provides desirable coverage characteristics; adequate Pt-C coverage without blocking the pores. Thus, the SSC ionomer-based electrode has a relatively higher porosity $(73.10 \%)$ than the LSC ionomer-based electrode $(68.80 \%)$. The performance test indicates that the MEA with the SSC ionomer-based CLs and membrane shows superior performance than the one with the LSC ionomerbased CLs and membrane under all the studied conditions. In particular, the SSC ionomer and membrane are found to be quite suitable for high-temperature operation, due to their favorable water-retention capability (hence proton-transport capability) at high temperatures. Lastly, the MEA based on SSC-ionomer and membrane is found to be less sensitive to variations in operating conditions.

\section{REFERENCES}

[1] A. Ozden, I. E. Alaefour, S. Shahgaldi, X. Li, C. O. Colpan, F. Hamdullahpur, "Chapter 40 - Gas Diffusion Layers for PEM Fuel Cells: Ex- and In-Situ Characterization," In Exergetic, Energetic and Environmental Dimensions, eds. Ibrahim Dincer, Can Ozgur Colpan, Onder Kizilkan, Academic Press, 2017.

[2] S Shahgaldi, J. Hamelin, "Stability study of ultra-low Pt thin film on TiO2-C coreshell structure and TiO2encapsulated in carbon nanospheres as cathode catalyst in PEMFC," Fuel, vol. 150, pp. 645-655, 2015. doi:10.1016/j.fuel.2015.02.002.

[3] A. Ozden, S. Shahgaldi, J. Zhao, X. Li, F. Hamdullahpur, "Assessment of graphene as an alternative microporous layer material for proton exchange membrane fuel cells," Fuel, vol. 215, pp. 726-734, 2018. doi:10.1016/j.fuel.2017.11.109.

[4] M. Ercelik, A. Ozden, E. Seker, C. O. Colpan, "Characterization and performance evaluation of PtRu/CTiO2 anode electrocatalyst for DMFC applications," Int. J. Hydrogen Energy, vol. 2, pp. 1-12, 2016. doi:10.1016/j.ijhydene.2016.12.020.

[5] S. Bose, T. Kuila, T. X. H. Nguyen, N. H. Kim, K. T. Lau, J. H. Lee, "Polymer membranes for high temperature proton exchange membrane fuel cell: Recent advances and challenges," Prog. Polym. Sci., vol. 36, pp. 813-843, 2011. doi:10.1016/j.progpolymsci.2011.01.003.

[6] S. Shahgaldi, J. Hamelin, "Improved carbon nanostructures as a novel catalyst support in the cathode side of PEMFC: A critical review" Carbon N. Y., vol. 94, pp. 705-728, 2015. doi:10.1016/j.carbon.2015.07.055.

[7] A. Ozden, M. Ercelik, Y. Ozdemir, Y. Devrim, C. O. Colpan, "Enhancement of direct methanol fuel cell performance through the inclusion of zirconium phosphate," Int. J .Hydrogen Energy, vo. 42, pp.21501-21517, 2017. doi:10.1016/j.ijhydene.2017.01.188.

[8] A. Ozden, M. Ercelik, Y. Devrim, C. O. Colpan, F. Hamdullahpur, "Evaluation of sulfonated polysulfone/zirconium hydrogen phosphate composite membranes for direct methanol fuel cells," Electrochim Acta, vol. 256, pp. 196-210, 2017. doi:10.1016/j.electacta.2017.10.002. 
[9] M. Ercelik, A. Ozden, Y. Devrim, C. O. Colpan, "Investigation of Nafion based composite membranes on the performance of DMFCs," Int. J. Hydrogen Energy, vol. 42, pp. 2658-2668, 2016. doi:10.1016/j.ijhydene.2016.06.215.

[10] S. Shahgaldi, I. Alaefour, X. Li, "Influence of ionomer structures and ratios on performance and degradation of PEM fuel cells," vol. 83, no. 1, pp. 71-78, 2018. 2018;83:71-8. doi: 10.1149/08301.0071ecst.

[11] C. Lei, D. Bessarabov, S. Ye, Z. Xie,S. Holdcroft, T. Navessin, "Low equivalent weight short-side-chain perfluorosulfonic acid ionomers in fuel cell cathode catalyst layers," J. Power Sources, vol. 196, pp. 6168-6176, 2011. doi:10.1016/j.jpowsour.2011.03.024. 


\title{
Thermal Management for Efficient Fast Refill of Compressed Natural Gas
}

\author{
Aiqing Lan, Guoyu Zhang, Ri Li*, Joshua Brinkerhoff \\ School of Engineering \\ The University of British Columbia, Okanagan campus \\ Kelowna, Canada
}

\begin{abstract}
As an alternative fuel, compressed natural gas (CNG) plays an important role fueling transportation. Like driving other types of vehicles, drivers of CNG-powered vehicles prefer to refill on-board CNG tanks in a short time, which is referred to as fast refill. Accompanied by a fast pressure increase in the cylinder, temperature will also rise significantly due to re-compression work with limited time for the heat to dissipate. This phenomenon ends with a common problem referred to as under-refill, which means less mass filled than the standards. This article presents a summary of the research works to solve this problem and improve fill efficiency by thermal management. Twodimensional axisymmetric CFD (computational fluid dynamics) simulations of unsteady, compressible, turbulent flow in fast refill process are conducted. Three different types of thermal management concepts are discussed, including active cooling, pre-chilling and real-time chilling. The results show that thermal management is a promising way to mitigate the under-refill problem with a maximum of $7 \%$ increase of fill efficiency achieved by active cooling. These provide guidelines for future research on improving fill efficiency through cooling strategies in which real-time chilling should be firstly focused on.
\end{abstract}

Keywords- Compressed natural gas; Fast refill; Thermal management; Computational fluid dynamics

\section{INTRODUCTION}

Natural gas [1] has become an important alternative fuel for transportation. The number of natural gas vehicles (NGVs) has escalated speedily at an annual rate of $24 \%$ since 2005 [2] and now it powers roughly 22.4 million vehicles around the world [3]. Due to the comparatively low density, natural gas is carried either as liquefied low-temperature LNG (liquefied natural gas) or as high-pressure CNG (compressed natural gas) with the latter currently having higher market share [4].

For CNG fuelled vehicles, a common problem is underrefill when the on-car gas cylinder is fast filled ( $<5$ mins) at CNG station. It is caused by temperature rise in the filling process followed by earlier reaching the standard pressure. This means less mass of CNG filled as the temperature inside

*Corresponding author: sunny.li@ubc.ca finally recovers to ambient temperature and the pressure inside drops. The temperature rise is a result of the heat generated by recompression work and inadequate time for the heat to be removed out naturally through cylinder wall with material of low conductivity. It is at least about $10^{\circ} \mathrm{C}$ above the ambient temperature at the end of fast refill [5]. Fill efficiency is thus typically only $80 \%$ for ambient temperature at $20{ }^{\circ} \mathrm{C}$ when the efficiency is defined as the ratio of the actual filled mass to the capacity at the rated pressure and ambient temperature [6].

To mitigate the under-refill problem, the simplest idea is to over-pressurise the cylinder when refilling it, which has a risk of safety issues. The second way that has been considered is to develop good strategy with modified feeding conditions to minimize the re-compression heat. FarzanehGord et al. [7] showed that existing procedure proposed [8] requires appropriate controlling facilities, limiting the applicability. Compared to the above two methods, thermal management is a more promising way to achieve higher filling efficiency, in which cooling technique and strategies are introduced to lower the CNG cylinder's temperature before or during the refilling process. Zhang et al. [9] numerically investigated an active cooling concept and achieved a $7 \%$ increase of fill efficiency at the expense of utilizing a simple cooling loop, which shows good prospect for thermal management in improving the fill efficiency of CNG.

There are very few previous studies focusing on thermal management of efficient fast refill of CNGs. The article presents a summary of the work done on thermal management to improve fill efficiency of CNG cylinder, involving three types of thermal management method: active cooling, pre-chilling and real-time chilling. The effects of these methods on thermal behaviour and fill efficiency of the CNG fast refill process are presented and discussed.

\section{NMERICAL MODEL AND THERMAL BEHAVIOUR OF THE FAST REFILL PROCESS}

To quantify the temperature rise and its effect on the fill efficiency, a 2-D CFD model is built for the fast fill process, employing ANSYS Fluent ${ }^{\circledR}$, a commercial CFD package. Gas inside the cylinder is modelled as an axisymmetric flow. For simplicity, the working fluid is pure methane with real 
gas properties determined using the Redlich-Kwong equation of state [10]. The standard $\mathrm{k}-\varepsilon$ turbulence model with enhanced wall treatment is applied with modified coefficients $\left(\mathrm{C}_{1 \varepsilon}=1.52, \mathrm{C}_{1 \varepsilon}=1.92, \mathrm{C}_{\mu}=0.09\right)$ [11]. As shown in Fig 1, model geometry is based on a type-III cylinder which is one of the four types of cylinder commonly used for natural gas vehicles. The type-III cylinder wall consists of the aluminum liner and the carbon fiber wrap. The dimensions of the cylinder follow the model used by Nahavandi [12]. The gas enters the cylinder through a converging nozzle that has an entrance diameter of $0.01 \mathrm{~m}$ and an exit diameter of $0.005 \mathrm{~m}$. Figure 2 shows the unstructured mesh applied in conventional fast filling case. Mesh is refined near the inlet region where there expect big gas property changes and gradients.

A convection boundary condition is imposed on the outer wall of the cylinder with a constant heat transfer coefficient of $10 \mathrm{~W} / \mathrm{m}^{2} \cdot \mathrm{K}$, and the ambient air temperature is kept constant at $300 \mathrm{~K}$ throughout the filling process. At the inner wall, a no-slip boundary condition is imposed. The initial pressure of the gas inside the cylinder is $2 \mathrm{MPa}$. A constant total enthalpy boundary condition is applied corresponding to a total temperature of $300 \mathrm{~K}$. A total pressure that linearly ramps from 4 to $20.6 \mathrm{MPa}$ in the first 3 seconds of the fill process and then is maintained at 20.6 MPa for the remainder of the fill. The refill is considered to end at time $t$ when the average gas pressure $\mathrm{p}_{\mathrm{g}}(\mathrm{t})$ satisfies the following equation:

$$
\frac{p_{g}(t)-p_{i}}{p_{s}-p_{i}}=95 \%
$$

where $p_{s}$ and $p_{i}$ are the total supply pressure and initial pressure, respectively.

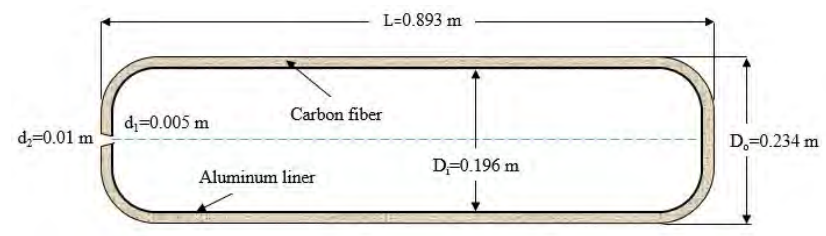

Figure 1. The computational domain for conventional fast filling process

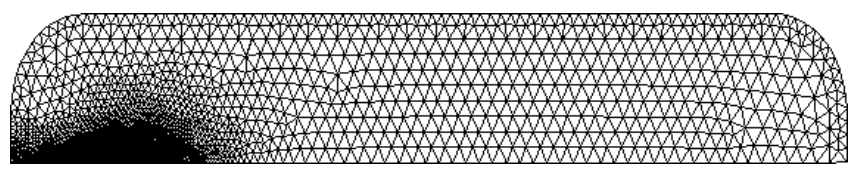

Figure 2. The computational mesh used for fast refill process without cooling

Figures 3-4 present the temporal variation of the average temperature and mass filled, respectively, within the cylinder during conventional fast refill process. The temperature rises quickly in the first 5 seconds, and after that, the temperature increase slows down and ends at a final temperature $29 \mathrm{~K}$ higher than the ambient temperature. Totally, $3.35 \mathrm{~kg}$ natural gas is filled into the cylinder. Potential of reducing the final temperature and increasing the final mass can be seen related to different thermal management concepts.

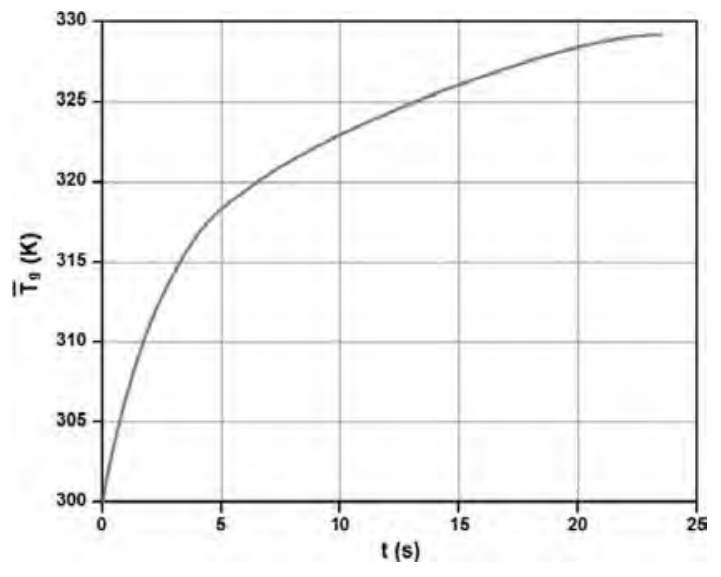

Figure 3. Dynamic change in gas average temperature

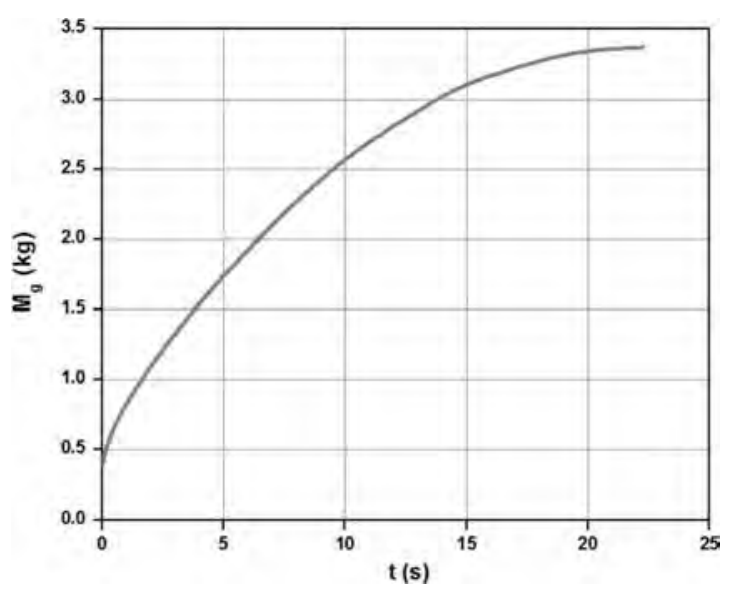

Figure 4. Dynamic change in mass filled

\section{IMPROVING FILL EFFICIENCY BY ACTIVE COOLING}

In the concept of active cooling, the natural gas is filled and cooled simultaneously in the filling process with coolant loop used to help remove heat to the ambient environment. There are two important features of the active cooling. The first is that the feeding flow rate of coolant can be adjusted so it can actively adjust the target temperature of the cooling, which is different from some passive heat removal device as heat pipes. The second feature is that the ultimate low temperature it can reach is the ambient temperature.

The heat removal performance of active cooling is investigated by using 2-D axisymmetric CFD simulation on a simplified model with location effects considered. The cooling system is expected to be composed of cooling coils installed inside the cylinder (Fig 5) which are connected to a coolant pump and a heat exchanger, which are not displayed in Fig 5. For simplicity, only the inside cooling coils are considered in the model (Fig 6) and they are placed in the front or back part of the cylinder. A uniform and constant temperature $300 \mathrm{~K}$ is applied at the coil surface. It represents 
the ideal extreme temperature that the cooling loop can reach assuming an ambient temperature of $300 \mathrm{~K}$.

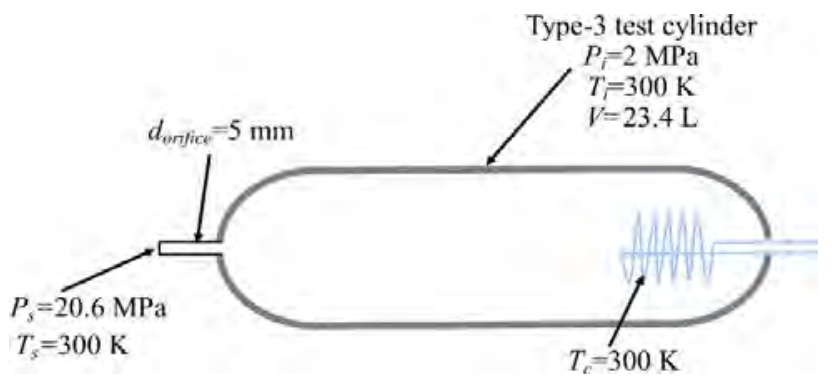

Figure 5. Schematics on the active cooling concept

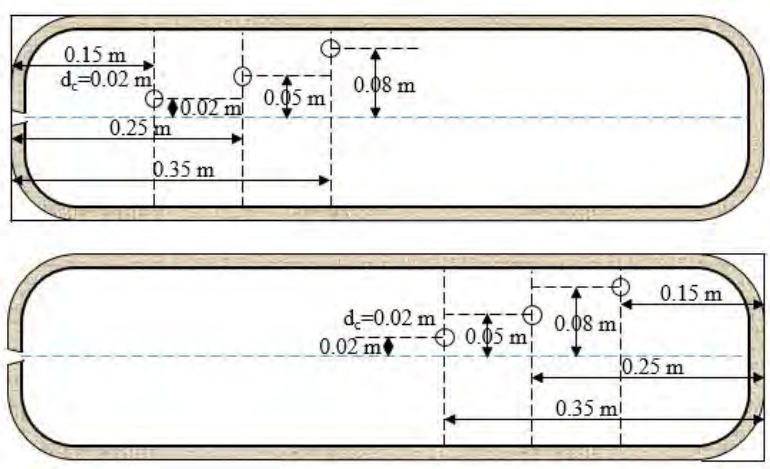

Figure 6. The computational domain for filling process with active cooling in the front and back

Figures 7-8 present the temporal variation of the average gas temperature and mass within the cylinder during the fill, respectively, with active heat removal via the cooling coils in the front and the back. Comparison has been made with conventional fast refuelling without cooling. As shown in Fig 7 , different from the monotonic increase of the temperature for the case without cooling, the temperature for the active cooling cases starts to go down after the first 5 seconds' increase. This results from a stronger effect of cooling than that of recompression heating. The maximum average temperatures are $313.3 \mathrm{~K}$ and $310 \mathrm{~K}$, respectively, for front cooling and back cooling, while the maximum value could be as high as $329 \mathrm{~K}$ for no cooling. Through active cooling, $7 \%$ more mass of gas is filled into a cylinder when cooling happens at the back part of the gas cylinder, while the fill efficiency only increases by $4.5 \%$ when the cooling coils are placed at the front. Apparently, fast refill is more efficient when active cooling is conducted in the back of the cylinder. These improvements are accompanied by longer fill time, which, however, is still comparable to that of conventional liquid fuel.

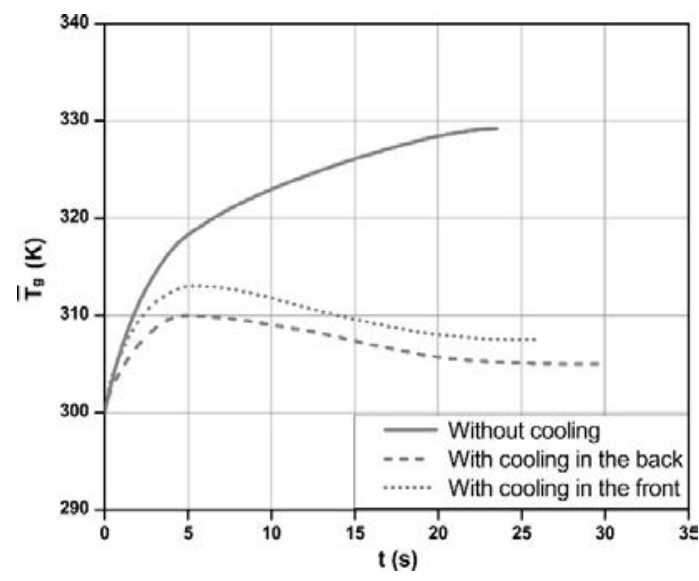

Figure 7. Dynamic change in gas average temperature

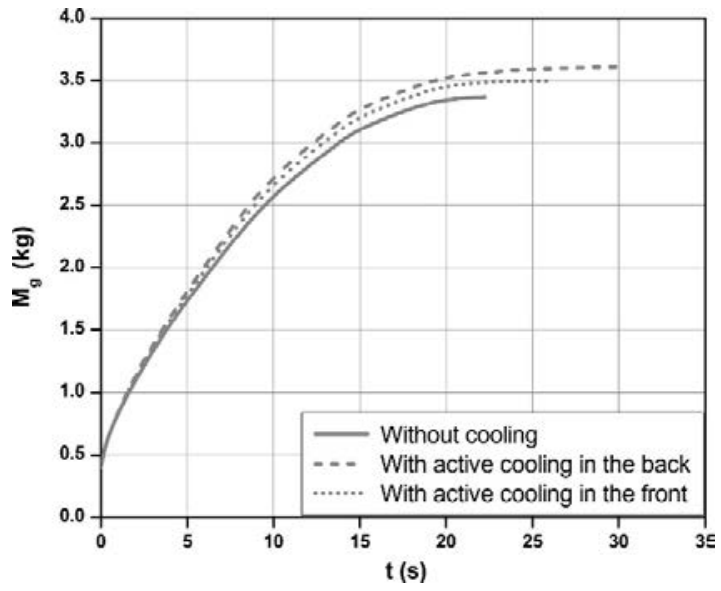

Figure 8. Dynamic change in the mass of gas filled

\section{IMPROVING FILL EFFICIENCY BY PRE-CHILLING}

Although active cooling can help improve the fill efficiency by $7 \%$, there are still two limitations for this method. Firstly, the coolant temperature is no lower than the ambient temperature, leading to limited maximum cooling ability. Secondly, excessively high pressure difference across the coil wall requires material of high mechanical strength and possible leakage will reduce the fuel quality. A new concept named pre-chilling is then considered in this section. As displayed by Fig 9, a pre-chilled mass block made of aluminium alloy is designed to be placed in the back side of the cylinder. There is a coolant circulation to chill the mass to a temperature below the ambient temperature prior to the beginning of gas filling. The chilled mass is then responsible for cooling the gas filled in the filling process. The liquid refrigerant (R134a) from truck air conditioning system [13] can be used to cool down the aluminium mass prior to the filling process. 


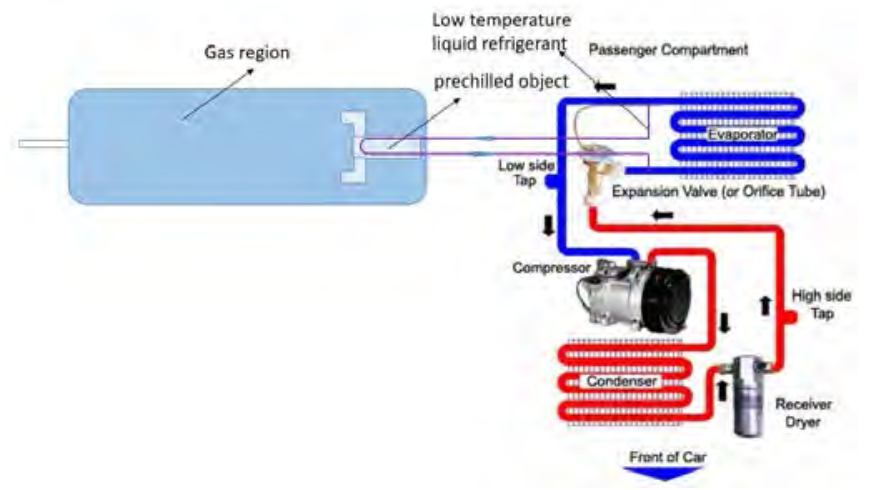

Figure 9. Schematic diagram of pre-chilling achieved by refrigerant circulation

The geometry of model used in simulation is designed with fins, as displayed in Fig 10. The pre-chilled mass takes $5 \%$ of the gas volume. Saturated liquid refrigerant flow with temperature of $-1.2^{\circ} \mathrm{C}$ and pressure of $275.79 \mathrm{kPa}$ [14] is used to chill the mass with 4 different refrigerant mass flow rates tested ranging from $0.0035 \mathrm{~kg} / \mathrm{s}$ to $0.014 \mathrm{~kg} / \mathrm{s}$. For comparison, another case is also simulated with a constant temperature boundary condition $\left(-1.2^{\circ} \mathrm{C}\right)$ applied at the wall of the channel, which corresponds to infinite flow rate.

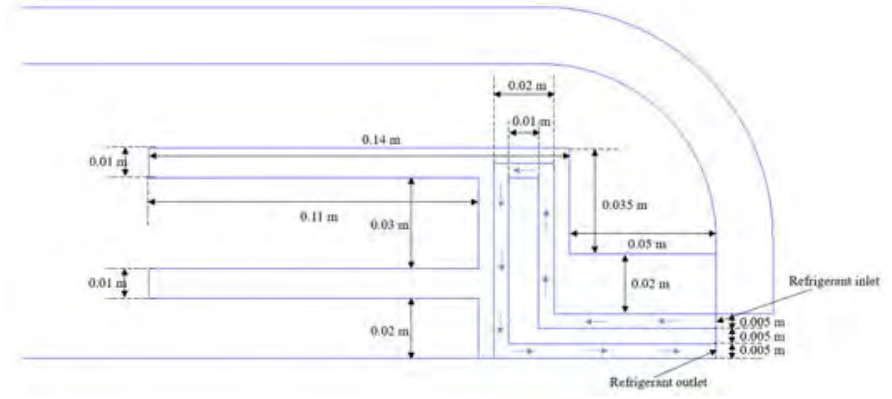

Figure 10. Dimension and location of the redesigned pre-chilled device

The first step in simulation is to pre-chill the mass attached to the bottom before starting gas filling. The change of average temperature of the pre-chilled mass is plotted in Fig 11. The duration of the chilling is 30 minutes and increasing the refrigerant flow rate means lower temperature within a fixed time. For this ideal case with infinite flow rate, the heat sink can be chilled to $0^{\circ} \mathrm{C}$ in only 5 minutes, this is the shortest time that pre-chilling takes with current inlet conditions in the model.

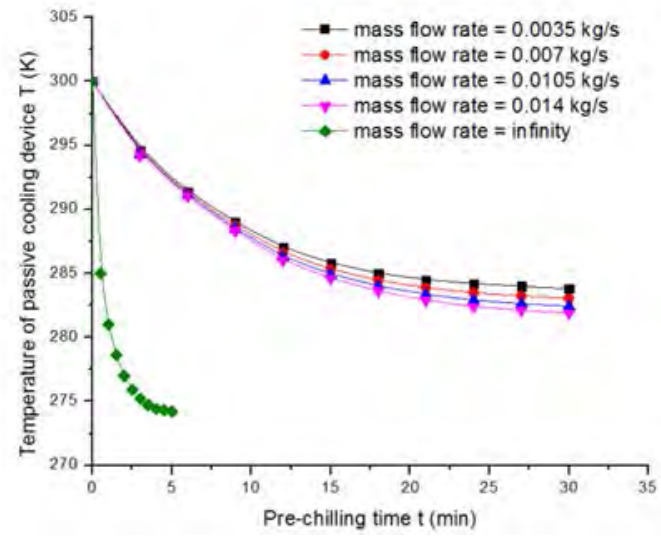

Figure 11. Average temperature of the pre-chilled device vs. pre-chilling time

Second step is to fill the CNG into the cylinder with refrigerant loop stopped. Two pre-chilled cases are presented in Fig. 12. In case (a), the heatsink has been pre-chilled by a refrigerant mass flow rate of $0.0035 \mathrm{~kg} / \mathrm{s}$ for 30 minutes. Case (b) is pre-chilled by constant boundary temperature for 5 minutes. At the end of the refill, compared with the case without cooling, pre-chilling achieved with constant boundary temperature increases the filled mass by $6.6 \%$, while the number drops to $5 \%$ for case (a), for which a refrigerant flow rate of $0.0035 \mathrm{~kg} / \mathrm{s}$ is applied. This indicates that pre-chilling is also an effective way to improve fill efficiency.

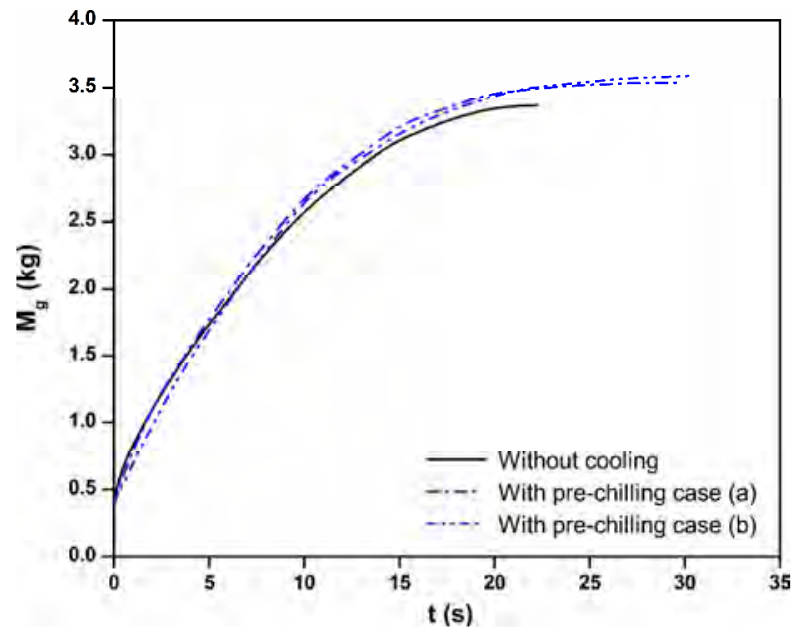

Figure 12. Dynamic change in the mass of gas filled

\section{IMPROVING FILL EFFICIENCY WITH REAL-TIME CHILLING}

One disadvantage for pre-chilling is that the total filling time exceeds the fast refill regime. Increasing mass flow rate to a high enough value is a possible way to reduce the time, but mass flow rate can also be restricted by the power of coolant supplying system. A new concept called real-time chilling is proposed to avoid the long pre-chilling time. While the gas inside the cylinder is heated by compression 
work done by the incoming gas flow, it is possible to utilize the expansion (the Joule-Thomson effect) of another flow of CNG outside the cylinder to cool the gas inside the cylinder. As shown in Fig. 13, there is cavity at the outside of the rear heat sink. Another flow of natural gas only for chilling expands with gas temperature lowered. The low temperature expansion flow removes heat from the gas inside the cylinder actively. This chilling flow starts simultaneously with the filling flow into the cylinder. The low pressure chilling flow coming out of the cavity can be transported to other natural gas systems or re-compressed to the gas reservoir. This concept and related model still need to be investigated as a main future work to verify its effect on efficient fast refill.

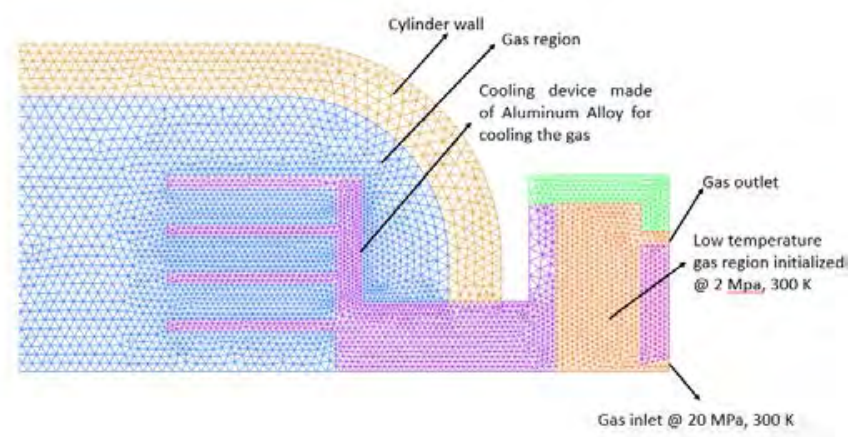

Figure 13. The rear part of the cylinder model for real-time chilling

\section{CONCLUSION}

In this paper, in terms of improving the fill efficiency of CNG fast refill, three different thermal management methods are presented with some research results displayed, giving a whole map of cooling strategies including the effects on the thermal behaviour and fill efficiency of the CNG fast refill process.

Some conclusions can be drawn from the results of research on active cooling and pre-chilling. Both methods can effectively increase the fill efficiency of the fast refill process for CNG. For active cooling, the best improvement that can be achieved with the current design is $7 \%$, which is higher than $4.5 \%$ with active cooling conducted at the front part of the cylinder. This location effect indicates that it is better to apply cooling to the rear part of the cylinder. For pre-chilling, time spent and results from this method are dependent on the mass flow rate of supplying refrigerant flow when inlet conditions are fixed. For the ideal case with infinite flow rate, the pre-chilling can achieve a maximum improvement on fill efficiency by $6.6 \%$, which is slightly lower than that of active cooling. Pre-chilling also takes much more time to cool the thermal mass before filling the cylinder, which is less time-economic than active cooling.

Compared with the first two methods researched, the real-time chilling concept can balance the time efficiency and cooling effects. It presents good prospect and need further work to verify this idea. Among the three thermal management concepts discussed in this paper, each of them has its advantages and disadvantages. Proper thermal management method should be chosen based on comprehensive assessment of the compatibility and practicality of each method in a specific scenario.

Current research has provided important guidelines for thermal management on efficient fast refill combining the advantage of acceptable computation time by using 2-D axisymmetric model. In the future, real-time chilling concept will be firstly examined based on current model. After that, as the 2-D model may cause abnormal shape of the coolant loop and the fins of the heat sink, a 3-D model will be built for better realization of the cooling strategies and better understanding of the thermal and flow field. Also, basic experiment will be done to verify certain thermal management concepts.

\section{REFERENCES}

[1] E. J. Moniz, et al., "The future of natural gas: an interdisciplinary MIT study," Cambridge, MA: Massachusetts Institute of Technology, 2011, pp. $1-308$.

[2] M. I. Khan, T. Yasmin, and A. Shakoor, "Technical overview of compressed natural gas (CNG) as a transportation fuel," Renew. Sustain. Energy Rev., vol. 15, pp. 785-797, Nov. 2015. doi: $10.1016 /$ j.rser.2015.06.053.

[3] NGV. (2014). Worldwide NGV statistics [Online]. Available: http:// www.ngvjournal.com/worldwide-ngv-statistics/. Accessed: March 22, 2018.

[4] M. J. Economides, K. Sun, and G. Subero, "Compressed natural gas (CNG): an alternative to liquefied natural gas (LNG)," SPE Prod. Oper., vol. 21, no. 02, pp. 318-324, May 2006. doi: 10.2118/92047-PA.

[5] K. J. Kountz, "Modeling the fast fill process in natural gas vehicle storage cylinders," in American Chemical Society (No.

CONF-940301--42), San Diego, CA, 1994. [Online]. Available: https:// www.osti.gov/servlets/purl/10177364.

[6] K. J. Kountz, "Natural gas storage conditions as a function of operating environment," Inst. Gas Technol., Chicago, IL, Rep. PB-96-191077/XAB, 1996.

[7] K. Kountz, W. Liss, and C. Blazek, "A new natural gas dispenser control system," presented at the 1998 Int. Gas Res. Conf., San Diego, CA.

[8] M. Farzaneh-Gord, M. Deymi-DashteBayaz, and H. R. Rahbari, "Effects of gas types and models on optimized gas fuelling station reservoir's pressure," Braz. J. Chem. Eng., vol. 30, no. 2, pp. 399-411, June 2013. doi: $10.1590 / \mathrm{S} 0104-66322013000200017$.

[9] G. Zhang et al., "Analytical and numerical study on the fast refill of compressed natural gas with active heat removal," J. Nat. Gas Sci.Eng., vol. 45, pp. 552-564, Sept. 2017. doi: 10.1016/j.jngse.2017.06.023.

[10] Q. Li et al., "Effects of geometry and inconstant mass flow rate on temperatures within a pressurized hydrogen cylinder during refueling," Int. J. Hydrogen Energy, vol. 37, no. 7, pp. 6043-6052, Apr. 2012. doi: 10.1016/j.ijhydene.2011.12.020.

[11] P. Ouellette and P. G. Hill, "Turbulent transient gas injections," $J$. Fluids Engineering, vol. 122, no. 4, pp. 743-752, 2000. doi: $10.1115 / 1.1319845$.

[12] N. N. Nahavandi and M. Farzaneh-Gord, "Numerical simulation of filling process of natural gas onboard vehicle cylinder," J. Braz. Soc. Mech. Sci. Eng., vol. 35, no. 3, pp. 247-256, Oct. 2013.

[13] J. Stepnicka. (2010, May 15). A basic description of Automotive AC Systems and how they work [Online]. Available: http:// www.autorepairinstructions.com/archives/ac-system-how.

[14] F. Bagheri et al., "Theoretical and experimental investigation into antiidling A/C systemsfor trucks," Energy Convers. Manage., vol. 98, no. 1, pp. 173-183, Jul. 2015. doi: 10.1016/j.enconman.2015.03.114. 


\title{
Energy Benefits of Integrating Transportation Energy with a Net Zero Energy Solar Building Using Captured Waste Hydrogen from Electrochemical Plants and Biogas from Various Bio Gasification Processes
}

\author{
Shahryar Garmsiri, Marc A. Rosen \\ Faculty of Engineering and Applied Science \\ University of Ontario Institute of Technology \\ Oshawa, Ontario, Canada
}

\begin{abstract}
When considering a net zero energy building, the transportation energy sector is often viewed as a separate entity. Currently, hydrocarbons are the main source of energy used in the transportation sector. In an effort to reduce greenhouse gas emissions, more automotive manufacturers are moving towards alternatively fueled vehicles that are electric or fuel cell powered. Electrochemical and other process industries frequently vent or flare hydrogen into the atmosphere and most community municipal waste is buried underground (landfill) or burned to generate electricity resulting in greenhouse gas emissions. Energy benefits and the methods of achieving net zero energy status for a solar building and reduction of greenhouse gas emissions through the integration of these waste streams and transportation energy are discussed.
\end{abstract}

Keywords- Waste hydrogen, biogas, biodigestion, chlor-alkali, fuel cell, transportation energy integration, net zero energy building, building integrated photovoltaic/thermal, waste energy recovery

\section{INTRODUCTION}

Hydrocarbon fuels that are responsible for a relatively large proportion of the overall greenhouse gas (GHG) emissions are the main source of energy used in the transportation sector. An overview of 2015 reported emissions by sector was published in 2017 by Environment and Climate Change Canada [1]. Emissions reported in 2014 by sector in the City of Toronto [2] indicate that the transportation sector contributes $35 \%$ of overall emissions while $53 \%$ of the overall emissions are produced by buildings. The City of Toronto has committed to reducing GHG emissions by $80 \%$ of 1990 levels by 2050 , through increases of renewable and district power generation [2]. To this end the City of Toronto has developed a zero emissions building framework, which targets building performance to near-zero emissions level by the year 2030 [2].

A net zero energy building (NZEB) is a building that offsets all of its energy consumption by using renewable energy sources within the building's built environment [3]. Most net zero energy buildings use the electrical grid as a buffer to avoid the need for energy storage. The building's energy demand is typically generated on-site through a

National Sciences and Engineering Research Council (NSERC) renewable energy source such as solar. A net zero energy building's performance is increased through efficiency measures and waste energy recovery so that the balance of energy needed within the building, both thermal and electrical, is usually met by the renewable source of energy. However, solar energy is intermittent due to weather conditions and the balance is not met through the renewable source of energy; hence the building relies on the electrical grid for the energy demand.

Climate change has become an important topic world-wide, as have reduction targets for GHG emissions. The transport sector is identified as one of the key sectors contributing to climate change $[4,5]$. Along with the growing gap between oil supply and demand globally, and the need to reduce GHG emissions, the use of alternative fuels and energy efficiency improvements are receiving increased attention [6].

In an effort to meet emission targets set out by each country, alternative methods of energy production must be considered such as solar power, wind energy, bio gasification, and waste energy recovery techniques. Although hydrogen energy technology has received much attention in the last decade with significant progress in performance, reliability, cost and efficiency, producing hydrogen from fossil fuels is not a clean, sustainable approach. There are also economic, intermittency and efficiency implications of hydrogen production from renewable energy sources. One method of addressing these implications is through the recovery of waste energy in a community.

Electrochemical and other process industries such as chloralkali plants frequently vent or flare hydrogen process gas into the atmosphere. The sodium chlorate used as a reactant for water purification and paper bleaching processes in a chloralkali plant produces hydrogen as a by-product. This waste hydrogen can be captured and purified for use in a hydrogen proton exchange membrane (PEM) fuel cell for transportation or stationary power generation.

A study of the economics of hydrogen production, storage and delivery was conducted by International Energy Agency (IEA) [7] for transportation use. The environmental benefits were outlined, with key points demonstrating the potential benefits of renewable hydrogen production, and utilization for 
various modes of transportation. The Integrated Waste Hydrogen Utilization Project (IWHUP), the world's first wastehydrogen capture system for fuel cell applications, was installed at a chemical plant in North Vancouver, British Columbia [8]. Additional waste-hydrogen capture systems are in development.

When considering a net zero energy building or community, the transportation energy sector is often viewed as independent from the building sector of the community. In this paper, the integration of transportation energy with a net zero energy building utilizing captured waste hydrogen from chloralkali plants and biogas from various bio gasification processes is examined.

Energy benefits and the methods of achieving net zero energy status for a solar building, and the reduction of greenhouse gas emissions through the integration of transportation energy are described. The objective of this paper is to demonstrate usable waste gas reduction and community waste energy recovery for use in the building and transportation sectors while utilizing a fuel cell vehicle's energy storage and production capabilities.

\section{BACKGROUND}

\section{A. Net zero energy building}

A net zero energy building (NZEB) is a building that offsets all of its energy consumption by using renewable energy sources within the building's built environment [3]. Net zero energy performance may be achieved by a combination of reducing building energy demand and generating electricity through the increased use of renewables, efficiency measures, waste energy recovery methods and integration of transportation energy.

Most net zero energy buildings use the electrical grid as a buffer to avoid the need for energy storage. The building's energy demand is typically generated on-site through a renewable energy source such as solar. Because solar energy remains intermittent due to the weather conditions, it may not be considered as a dependable source of energy to meet the building's energy demands.

\section{B. Solar energy}

In a photovoltaic (PV) module, a portion of the incident solar energy is converted into useful electricity, while the rest is either reflected or dissipated as heat. There are two methods of installing PV modules on a building. A building added photovoltaic (BAPV) system has the PV modules installed as an addition to the building envelope. A building integrated photovoltaic (BIPV) system has the PV modules forming part of the building installation. When adding a heat recovery system for module cooling purposes and to capture generated waste heat by the BIPV solar module, the system is referred to as a building integrated photovoltaic/thermal (BIPV/T) system. The BIPV/T system not only generates electricity, but also provides useful heat that allows for a reduction in the building heating loads $[9,10]$.

\section{Electrochemical chlor-alkali industry plants}

In a chlor-alkali facility, chlorine $\left(\mathrm{Cl}_{2}\right)$ and sodium hydroxide $(\mathrm{NaOH})$ are manufactured by electrolysis of brine, a salt solution [11]. The main technologies applied for chloralkali production are mercury cell, diaphragm and membrane cell electrolysis, mainly using sodium chloride $(\mathrm{NaCl})$ as feed [11]. Each of these processes represents a different method of keeping the chlorine that is produced at the anode separate from the caustic soda and hydrogen that are produced at the cathode.

The inputs are primarily salt and water; acids and chemical precipitants are used to remove impurities and output chlorine and caustic solution [11]. The main pollutant outputs are chlorine gas emissions to air, free oxidants to water, spent acids, cooling agents, and impurities removed from the input brine salt and hydrogen gas.

\section{Waste hydrogen gas}

The chlor-alkali industry produces chlorine and caustic solution (sodium or potassium hydroxide) simultaneously through decomposition of salt in water. The basic principle in the electrolysis of a sodium chloride solution occurs at the anode and cathode. At the anode, chloride ions are oxidized and chlorine $\left(\mathrm{Cl}_{2}\right)$ is formed. At the cathode, hydrogen $\left(\mathrm{H}_{2}\right)$ and hydroxide ions $\left(\mathrm{OH}^{-}\right)$are formed by the reaction of the sodium in the amalgam with water.

During chlorine gas compression and cooling, most of the chlorine gas is condensed. However, by diluting the remaining chlorine gas with air, the concentration of non-condensable gases such as hydrogen can be kept below the explosion limit. The remaining gases after liquefaction have to be purged from the system and are led to a chlorine absorption unit. The remaining impurities in the gases in the chlorine absorption unit is absorbed and the remaining gas is $97.5-99.9 \%$ pure hydrogen [12].

This pure hydrogen gas can be used in hydrogen proton exchange membrane (PEM) fuel cells for stationary or vehicle use applications. Often times the gas is either sold to merchants or vent/flared into the atmosphere. In this study, the focus is made on the vent/flared hydrogen gas into the atmosphere.

In a chlor-alkali electrochemical plant, hydrogen is produced in a fixed ratio of $28 \mathrm{~kg}$ per tonne of chlorine used or approximately $1200 \mathrm{~kg}$ per day ranging up to approximately $2700 \mathrm{~kg}$ per day [11].

\section{E. Biogasification processes}

There are a variety of ways to use wastes and to convert them into useful energy. Some of these include gasification processes that uses wood or coal, and anaerobic digesters that use biodegradable material such as municipal solid waste and sewage sludge to create a biogas that is useful for combustion to generate power. This useful gas can be further processed and converted to hydrogen to be used in fuel cells.

Gasification is a process of converting organic materials into carbon monoxide $(\mathrm{CO})$, hydrogen $\left(\mathrm{H}_{2}\right)$, and carbon dioxide $\left(\mathrm{CO}_{2}\right)$. The process involves burning of solid fuels like wood or coal to complete combustion with inadequate air supply, 
creating an output gas called synthesis gas with potential for combustion. The power produced from gasification and combustion of the synthesis gas obtained from biomass is considered to be a source of renewable energy $[13,14,15,16]$. This synthesis gas can further be processed in a steam reformer for subsequent conversion to hydrogen.

Some examples of demonstration projects include the 20 MW synthesis gas from forest residues by GoBiGas project in Gothenburg, Sweden [18], a 2 MW electricity production facility in Gussing, Austria by the Renewable Energy Network Austria (RENA) [19] and the US Air Force in Hurlburt Field, Florida [17].

\section{F. Integration of transportation and renewable sources of energy}

Several studies concerning the possibilities of integrating renewable sources of energy with the transportation sector have been pursued. Some of these studies include electricity produced by wind turbines in combination to plug-in hybrid vehicles (PHEVs) [20], leading to the potential of using electric vehicle battery storage as a medium for excess energy produced by wind power [21]. Similarly, solar photovoltaic (PV) systems have received attention in tandem with electric vehicles (EVs). A study conducted in Germany [22] demonstrated the potential of $\mathrm{CO}_{2}$ emissions reduction in the transportation sector when using EV's paired with solar PV's.

The method of integrating transportation energy with a building is referred to as vehicle-2-grid (V2G) [23,24]. Although most of the research is based on EVs, attention is required for fuel cell vehicles (FCV) as well. A FCV can generate power using its onboard stored hydrogen as compared to an EV that can be seen simply as a storage medium that cannot produce electricity. An advantage to a FCV over an EV is the potential of storing a greater amount of energy in compressed gas form and generating electricity using the onboard fuel cell. FCVs can also be refueled much faster than EVs.

There are several benefits when integrating transportation energy to achieve a net zero energy balance for a building. The integration using a FCV helps to eliminate the need for centralized large power generators, and provides a vector for the utilization of industrial waste hydrogen gas, synthesis gas and biogas from gasification and anaerobic digester processes converted to hydrogen during a steam reformation process.

\section{G. Fuel cell systems}

There are several types of fuel cells that can be used for stationary and vehicle applications. The proton exchange membrane fuel cell (PEMFC) capitalizes on the essential simplicity of the fuel cell. Most biogases have low heating values, due to the high levels of carbon oxides and nitrogen, which are not attractive for gas engines. The molten carbonate fuel cell (MCFC), solid oxide fuel cell (SOFC), and phosphoric acid fuel cell (PAFC) are able to handle very high concentrations of carbon oxides [25].

Although there are several fuel cell types that can be used for creating electrical power, the PEM fuel cell is considered more advantageous than MCFC, SOFC and PAFC types. This is due to the limitations MCFC, SOFC and PAFC have over the PEMFC. The limitations are that MCFC, SOFC and PAFC operate at higher temperatures and are not ideal for stop and go applications. But a PEMFC is not limited to the high operating temperatures and has the capability of stop and go operation, making it an advantageous fuel cell for vehicle application [25].

\section{ANALYSIS AND METHODOLOGY}

Modelling community and transportation energy use can present several challenges, such as acquiring and generating appropriate input data based on highly variable energy usage patterns. How and when occupants exercise their energy needs is a key consideration for all green building planning, design, operations and decision-making.

Fuel cell vehicles can be used as a new power generation source, supplying electricity to homes, and to the grid. The V2G mode helps implement this connection and uses the hydrogen fuel cell powertrain and the stored hydrogen on board the vehicle as a new power generation source supplying electricity.

\section{A. House electrical consupmtion}

A bungalow style house is chosen for this study, however the model can be applied to any size and type of house. It is assumed that all the appliances in the house are electrical including the domestic hot water tank. A list of common appliances and devices that use electricity are outlined in Table 1 . 
TABLE I. ELECTRICAL APPLIANCES IN THE HOUSE

\begin{tabular}{|c|c|c|c|}
\hline \multirow{2}{*}{$\begin{array}{c}\text { Appliance and } \\
\text { Device }\end{array}$} & \multicolumn{3}{|c|}{ Description } \\
\hline & Spot measurement $(W)$ & Model & $C O P$ \\
\hline Electric furnace & 4,600 & NT14A018A & 0.83 \\
\hline Air conditioner & 10,000 & 21ECM10 & 1.00 \\
\hline $\begin{array}{l}\text { Electric domestic } \\
\text { hot water }\end{array}$ & 3,500 & RTEX-04 & \\
\hline Fridge & 57.20 & $\begin{array}{c}\text { LG } \\
\text { LTCS24223 }\end{array}$ & \\
\hline Clothes washer & 13.70 & $\begin{array}{c}\mathrm{LG} \\
\mathrm{WM}^{2} 900 \mathrm{H}^{*} \\
\mathrm{~A}\end{array}$ & \\
\hline Clothes dryer & 7,200 & $\begin{array}{c}\text { DLEX7600 } \\
\text { VE }\end{array}$ & \\
\hline LED television & 70 & LGUH6100 & \\
\hline Electric stove & 3,800 & $\begin{array}{c}\text { YWEE510S } \\
\text { 0FS }\end{array}$ & \\
\hline Dishwasher & 27.30 & $\begin{array}{c}\text { Samsung } \\
\text { DW80K704 } \\
9\end{array}$ & \\
\hline Microwave & $2.20-10$ & & \\
\hline $\begin{array}{l}\text { Lighting (Candle } \\
\text { light, A19, T8, } \\
\text { MR16, PAR30) }\end{array}$ & $5,10,18,5,14$ & LED & \\
\hline Coffee maker & $70-1,000$ & & \\
\hline $\mathrm{PC}$ and monitor & $6.4-75$ & & \\
\hline Laptop & $2-25$ & & \\
\hline Stereo & 30 & & \\
\hline DVD player & $3.8-17$ & & \\
\hline $\begin{array}{ll}\text { Video game } \\
\text { consoles }\end{array}$ & $3.6-36$ & & \\
\hline Chargers & $4-13$ & & \\
\hline $\begin{array}{l}\text { All in one printer } \\
\text { and scanner }\end{array}$ & 4.40 & & \\
\hline Router & 8 & & \\
\hline Phone & 2.80 & & \\
\hline
\end{tabular}

The heating and cooling electrical consumption from the electric heater and air conditioner used for this house depends on the wall insulation and the assumption of steady state heat loss and heat gain in the house. The heat loss and heat gain for the house is calculated as follows:

$$
\text { Q }=\square A \Delta T
$$

where $\mathrm{Q}$ is the heat loss during winter and heat gain during summer, $U$ is the measure of the effectiveness of the total insulation, $\mathrm{A}$ is the area of the house, and $\Delta \mathrm{T}$ is the outside air and house internal temperature. The comfortable indoor air temperature for winter according to a study is between $20^{\circ} \mathrm{C}$ and $23^{\circ} \mathrm{C}$ [26], while the comfortable indoor air temperature for summer is between $23^{\circ} \mathrm{C}$ and $25^{\circ} \mathrm{C}$ [26].
The effectiveness of the insulation of the house is calculated using the R-value of the insulating materials. The $\mathrm{R}$ value is the capacity of an insulating material that resists heat flow, and the higher the R-value, the greater the insulation for the application.

The $U_{\text {total }}$ the measure of the effectiveness of an insulator for the house, is calculated from the total $U$ values of the windows, doors, walls, floors, ceiling, and roof that comprises of the components of a house. The lower the $U$ value, the better the material for heat insulation. The $\mathrm{U}_{\text {total }}$ can be calculated as follows:

$$
\bigsqcup_{\text {total }}=\bigsqcup_{\text {window }}+\bigsqcup_{\text {door }}+\bigsqcup_{\text {wall }}+\bigsqcup_{\text {floor }}+\bigsqcup_{\text {ceiling }}+\bigsqcup_{\text {roof }}
$$

The electric consumption of the electric heater and air conditioner is calculated for each hour of the year. Similarly the electrical usage of appliances, lighting apparatuses and other appliances in the house are quantified as follows:

$$
E_{\text {House_total }}=E_{\text {Appliances }}+E_{\text {lighting }}+E_{\text {Devices }}
$$

where $\mathrm{E}_{\text {appliances }}$ is the total electricity used by the appliances for the duration of an hour, $E_{\text {lighting }}$ is the total electricity used by the lights in the house for an hour, and $E_{\text {Devices }}$ is the total electricity used by devices in the house that use electricity for an hour. The appliances, lighting and devices used for this study are listed in Table 1.

\section{B. Solar power generation}

The number of solar panels that can be installed onto the rooftop of the house can be written as:

$$
N_{p v}=\left(A_{\text {roof }} / 4\right)^{*}(1 / \text { Apv })
$$

where $N_{P V}$ is the number of solar panels, $A_{\text {roof }}$ is the area of the roof and APV is the area of the solar panel. The solar array area can be calculated as:

$$
A_{\text {array }}=A_{p v}{ }^{*} \mathrm{Npv}
$$

The maximum power capacity of the total solar PVs installed on the rooftop can be calculated as follows:

$$
\text { PV } V_{\text {total }}=A_{\text {array }}{ }^{*} \eta_{\text {module }}
$$

where $\eta_{\text {module }}$ is the solar module efficiency.

By varying the area of the roof and calculating the maximum power capacity, the total solar PVs can also vary and this is can be used for various house sizes. Using the information calculated the electricity produced per hour (EPV) can be obtained from the NREL website [27].

\section{Fuel cell vehicle fuel usage}

There are several examples of development of hydrogen vehicles in the past decade. The automotive sector is a good 
candidate for using hydrogen to reduce $\mathrm{CO}_{2}$ emissions, as the quantity of vehicles exceeds the numbers of other modes of transportation. On board hydrogen storage is in the range of approximately 5 to $13 \mathrm{~kg}$, a requirement to enable a driving range of more than $480 \mathrm{~km}$ (300 miles) for the full platform of light-duty automotive vehicles using fuel cells [28].

The fuel cell hydrogen usage can be made comparable to the house electricity usage and the solar panel power generation by using (7) to convert $1 \mathrm{~kg}$ of hydrogen to $\mathrm{kWh}$.

$$
33.22 \mathrm{kWh} / \mathrm{I} \mathrm{kghydrogen}{ }^{*} \eta \text { PeM fuel cell }=\text { Ehydrogen }
$$

where the PEM fuel cell efficiency is assumed to be $60 \%$ and $E_{\text {hydrogen }}$ is the useable $\mathrm{kWh}$ per $\mathrm{kg}$ of hydrogen. Equation (8) can be used to determine the $\mathrm{kWh}$ of energy on board a fuel cell vehicle:

$$
\text { Ehydragen } * \text { Shydrogen storage }=\text { EFCV }
$$

where $\mathrm{S}_{\text {hydrogen storage }}$ is the fuel tank size in $\mathrm{kg}$, and $\mathrm{E}_{\mathrm{FCV}}$ is the amount of energy on board measured in $\mathrm{kWh}$.

The electricity usage rate per kilometer is based on an assumption of combined city and highway driving. In this study it is assumed that the driving distance is constant with no road traffic or diversions of the path traveled between the workplace and the house.

\section{RESULTS AND DISCUSSION}

The data for the electrical consumption of the house, the power generated by the solar PV and the hydrogen usage by the FCV (and hence the amount potentially available for building operations) can be determined on an hourly basis for the duration of a year.

In this case with the current solar panel installation, the sum of the electricity produced and the electricity used by the house makes this house a net negative energy building. The calculations show that a total of $17,650 \mathrm{kWh}$ of electricity is used from the grid annually as shown in Figure 1.

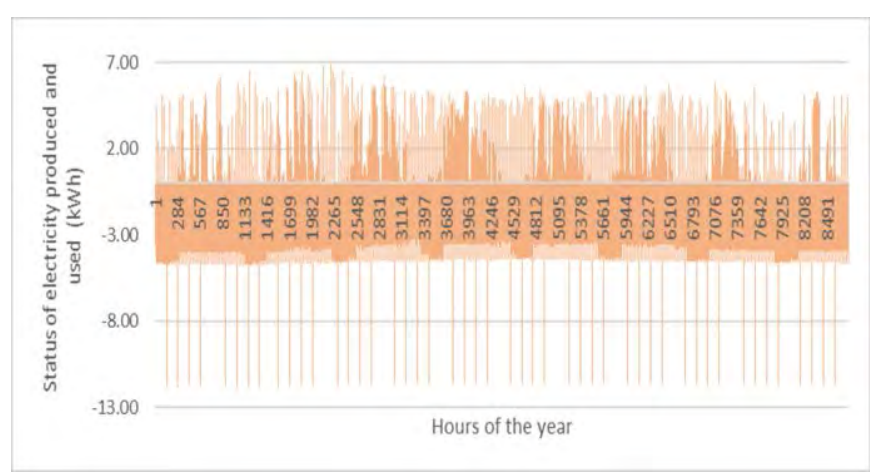

Figure 1. Total summed electricity produced by the solar PV and the electricity used by the house $(\mathrm{kWh})$

From this study it is observed that the electricity produced by the solar PV is not fully utilized during the day, while the occupants are away at work and most of the electricity consumption is during peak times when they have arrived back at the house from the workplace. To demonstrate this, day 1 is extracted from Figure 1 and shown in Figure 2 below.

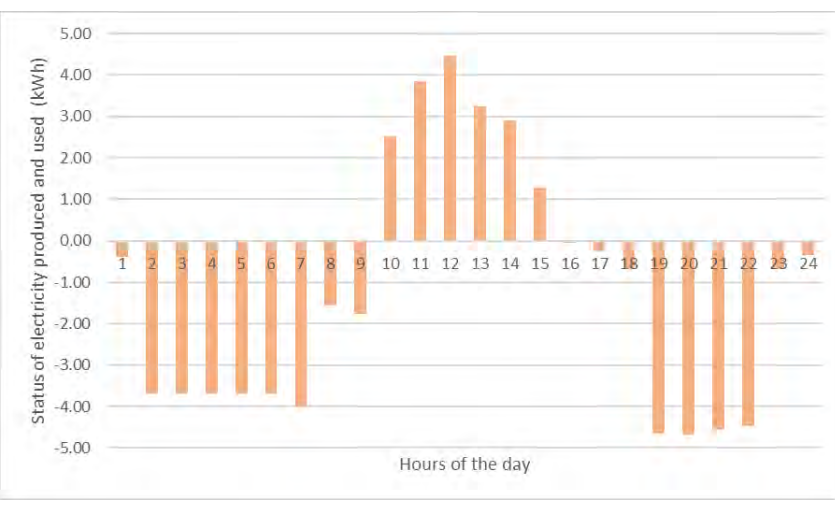

Figure 2. Sum of electricity produced and electricity used for Day $1(\mathrm{kWh})$

The FCV can be used to offset the net shortfall of the electricity produced by the solar PV throughout the day by using the hydrogen fuel cell on board the vehicle during high peak hours. The surplus electricity generated between 10am and $3 \mathrm{pm}$ is sent to the grid but is subsequently offset, during high peak times through the integration of FCV to the house. The integration of the FCV to the house for Day 1 can be observed in Figure 3, where the orange bar represents the sum of the electricity produced by the solar PV and the electricity used by the house compared to the green lines representing when electricity from the FCV was used. The house becomes a net positive energy building annually with $3,321 \mathrm{kWh}$ provided by the integration of FCV. This indicates that approximately $21,000 \mathrm{kWh}$ of solar PV energy that was not fully used by the house may be sent to the grid. The integration of the FCV powered by waste gas streams indicates that the house is fully powered from zero emission sources maintaining its net zero energy status.

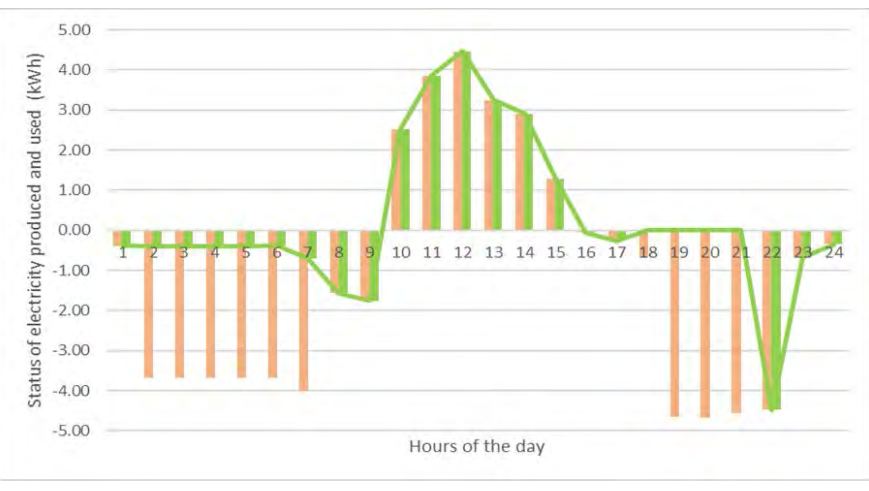

Figure 3. FCV integration to the zero energy building (kWh)

\section{CONCLUSIONS}

The integration of the FCV with a net zero energy building can be beneficial. This is due to the solar PV energy not being fully used by the unoccupied house during the day. The fuel recovered from the waste streams, such as hydrogen from electrochemical plants, synthesis gas converted to hydrogen 
using steam reformation from bio gasification and anaerobic digestion processes, is used by the FCVs. The FCV's power generation capability allows the house to remain fully powered from zero emission sources and to utilize waste streams from the community.

\section{REFERENCES}

[1] [1] Environment and climate change Canada. Overview of 2015 reported emissions. Gatineau, Quebec. [Online] Available: https:// www.ec.gc.ca/ges-ghg/82BA1E22-9653-45F1-8EC2-9BF8A2151555/ ECCC_GHGRP_OverviewOfReported2015Emissions.pdf . Accessed on 1 February, 2018.

[2] City planning division. The City of Toronto zero emissions building framework. Toronto, Ontario. [Online] Available: https://web.toronto.ca/wp-content/uploads/2017/11/9875-Zero-EmissionsBuildings-Framework-Report.pdf. Accessed on January 29, 2018.

[3] Pless, S. D., Torcellini, P.A., "Zero energy buildings: A classification system based on renewable energy supply options,", National Renewable Energy Laboratory, Golden, CO, Report NREL/TP-550-44586, 2010.

[4] Commission of Integrated Transport. Transport and climate change, advice to government from the commission for integrated transport. London: CFIT-Publications; 2007. [Online]. Available:

http://www.cambridgeenergy.com/archive/ 2007-02-08/commissioninteg-trans.pdf. Accessed on January 5, 2015.

[5] Stanley J, Loader C. Road transport and climate change: stepping off the greenhouse gas. Report for the bus industry confederation, Victoria. 2008. [Online]. Available: http://www. ozebus.com. Accessed on November 5, 2014.

[6] P. Kumar, R. Britter, and N. Gupta, "Hydrogen Fuel: Opportunities and Barriers," J. Fuel Cell Sci. Technol., vol. 6, no. 2, p. 21009, 2009. doi: 10.1115/1.3005384.

[7] IEA (International Energy Agency)/OECD (Organisation for Economic Co-operation and Development). Transport energy and CO2. Paris: IEAPublications; 2009. [Online]. Available: http://www.iea.org/publications/ freepublications/publication/transport2009.pdf . Accessed on: January 19, 2018.

[8] "Dynetek hydrogen trailer system for IWHUP," Fuel Cells Bulletin, vol. 2007, no. 12, pp. 8-9, Dec. 2007. doi: 10.1016/S1464-2859(07)70426-1.

[9] T. T. Chow, J. W. Hand, and P. A. Strachan, "Building-integrated photovoltaic and thermal applications in a subtropical hotel building," Appl. Therm. Eng., vol. 23, no. 16, pp. 2035-2049, Nov. 2003.

doi: 10.1016/S1359-4311(03)00183-2.

[10] O. Zogou and H. Stapountzis, "Energy analysis of an improved concept of integrated PV panels in an office building in central Greece," App. Energy, vol. 88, no. 3, pp. 853-866, Mar. 2011. doi: 10.1016/j.apenergy.2010.08.023.

[11] IPPC (Integrated Pollution Prevention and Control), Reference document on best available techniques in the chlor-alkali manufacturing industry. 2000. [Online] Available: http://www.lanuv.nrw.de/bref/Chlor\% 20Alkali.pdf. Accessed on: February 6, 2018.

[12] Metcon Sales and Engineering. Gas scrubber system. 2015. [Online]. Available: http://www.metconeng.ca/uploads/1/5/3/5/15354030/stsscrubber.pdf. Accessed on: January 18, 2018.

[13] National Non-Food Crops Centre. "Review of Technologies for Gasification of Biomass and Wastes, NNFCC project 09/008" (PDF). York YO10 5DG, UK.
[14] The Clean and Renewable Energy Source. www.biomass.uk.com. [Online]. Available: https://web.archive.org/web/20110910155945/http:// www.biomass.uk.com/. Accessed on: 29 December 2017.

[15] Thermal Gasification of Biomass, International Energy Agency Task 33, www.gastechnology.org. [Online]. Available: http://web.archive.org/ web/20170509160354/http://gastechnology.org/. Accessed on: December 16, 2017.

[16] Plasma gasification: Clean renewable fuel through vaporization of waste. [Online]. Available:www.waste-management-world.com. January 7 , 2009. Accessed on: January 9, 2018.

[17] A. M. Wright, "AFSOC makes 'green' history while investing in future," US Air Force Special Operations Command, April 28, 2011. [Online]. Available: https://www.afsoc.af.mil/News/Article-Display/ Article/162788/afsoc-makes-green-history-while-investing-in-future/. Accessed on: January 28, 2018.

[18] Göteborg Energi's biogas plant GoBiGas is now fully operational GoBiGas. [Online]. Available: gobigas.goteborgenergi.se. Accessed on: January 9, 2018.

[19] Gussing Biomass Power Plant. [Online]. Available: www.clarkeenergy.com. Accessed 29 January, 2018.

[20] K. F. Valentine, W. G. Temple, and K. M. Zhang, "Electric vehicle charging and wind power integration: Coupled or decoupled electricity market resources?," in 2012 IEEE Power and Energy Society General Meeting, 2012. doi: 10.1109/PESGM.2012.6344885.

[21] J. R. Pillai, S. Huang, P. Thogersen, J. Moller, and B. Bak-Jensen, "Electric vehicles in low voltage residential grid: A Danish case study," in 2012 3rd IEEE PES Innovative Smart Grid Technologies Europe (ISGT Europe), 2012. doi: 10.1109/ISGTEurope.2012.6465804.

[22] L.-M. Ritte, S. Mischinger, K. Strunz, and J. Eckstein, “Modeling photovoltaic optimized charging of electric vehicles," in 2012 3rd IEEE PES Innovative Smart Grid Technologies Europe (ISGT Europe), 2012. doi: 10.1109/ISGTEurope.2012.6465792.

[23] H. Lund and W. Kempton, "Integration of renewable energy into the transport and electricity sectors through V2G," Energy Policy, vol. 36, no. 9, pp. 3578-3587, Sep. 2008. doi: 10.1016/j.enpol.2008.06.007.

[24] M. V. Romeri, "Considering Hydrogen Fuel Cells Powertrain as Power Generation Plant," World Electric Vehicle Journal, vol. 4, no. 4, pp. 933-938, Dec. 2010. doi: 10.3390/wevj4040933.

[25] J. Larminie and A. Dicks, "Fuel Cell Systems Analysed," in Fuel Cell Systems Explained, John Wiley \& Sons, Ltd., pp. 369-389, 2013. doi: 10.1002/9781118878330.ch11.

[26] Direct energy. What's the best temperature for my thermostat in winter? Toronto, Ontario. [Online]. Available: http://www.directenergy.com/blog/ best-temperature-heater-winter/. Accessed on January 3, 2018.

[27] Natural renewable energy laboratory (NREL). Solar resource data PVWatts calculator. Golden, Colorado. [Online]. Available: http://pvwatts.nrel.gov/pvwatts.php. Accessed on 19 December, 2017.

[28] Hydrogen storage technologies. Energy efficiency and renewbale energy. US department of Energy. [Online]. Available: http:// www1.eere.energy.gove/hydrogenandfuelcells/storage/ current_technology.html, 2009. 


\section{Predicting the Consumption Speed of a Premixed Flame Subjected to an Unsteady Stretch Rate}

\author{
Meysam Sahafzadeh, Seth B. Dworkin \\ Department of Mechanical and Industrial Engineering \\ Ryerson University \\ Toronto, Canada \\ seth.dworkin@ryerson.ca
}

\author{
Larry W. Kostiuk \\ Department of Mechanical Engineering \\ University of Alberta \\ Edmonton, Canada \\ lkostiuk@ualberta.ca
}

\begin{abstract}
The stretched laminar flame model provides a convenient approach to embed realistic chemical kinetics when simulating turbulent premixed flames. When positiveonly periodic strain rates are applied to a laminar flame there is a notable phase lag and diminished amplitude in heat release rate. Similar results have been observed with respect to the other component of stretch rate, namely the unsteady motion of a curved flame front when the stretch rates are periodic about zero. Both cases showed that the heat release rate or consumption speed of these laminar premixed flames can vary significantly from the quasi-steady flamelet model. Deviation from quasi-steady behaviour increases for conditions further from stoichiometric such that unsteady time scales of the flow are of the same magnitude as the chemistry. A challenge remains in how to use such results predictively for local and instantaneous consumption speed for small segments of turbulent flames where their stretch history is not periodic. This paper uses a frequency response analysis as a characterization tool to simplify the complex non-linear behaviour of premixed methane air flames for equivalence ratios from 1.0 down to 0.7 , and frequencies from quasi-steady up to $2000 \mathrm{~Hz}$ using flame transfer functions. Various linear and nonlinear models were studied to identify appropriate flame transfer functions for low and higher frequency regimes, as well as to extend the predictive capabilities of these models. Linear models were only able to accurately predict the flame behaviour below a threshold of when the fluid and chemistry time scales are the same order of magnitude.
\end{abstract}

Keywords: Laminar Premixed Flames, Transient Response, Linear and Nonlinear Systems, Frequency Response Analysis

\section{INTRODUCTION}

Understanding turbulent combustion is important due to industrial applications such as internal combustion engines and furnaces. The challenges related to predicting turbulent combustion behavior have been discussed extensively in terms of their time-dependent and multidimensional nature, as well as having a large range of hydrodynamic time and length scales interacting with the time and length scales of the chemistry [1]. In this regard,, the formulation of these problems is well-posed by a system of partial differential equations and algebraic constraints that represent the conservation of mass and energy, as well as considerations of momentum and individual species while being subjected to convection, diffusion, and chemical reaction. All these phenomena are captured in direct numerical simulations with appropriate boundary conditions, but their solutions, due to these equations being highly nonlinear and strongly coupled, come at a significant computational cost [2], and thereby creates the need for model-level understandings.

A conceptual modeling approached that allows for estimation of local burning rates with pre-calculated complex chemistry is based on flamelets, which can collectively represent a turbulent flame. In their classical premixed form, flamelets provide a local consumption speed of the flame (referenced to their reactant state), and are generally assumed to be local planar structures subjected to a specified steady strain rate and of fixed reactant composition. This consumption speed, $S_{L}$, is the rate mass is converted from reactants to equilibrium products per unit area of flame and divided by the density of the reactants, and can readily be converted to the local heat release rate per unit flame area knowing the enthalpy of reaction. The validity of these assumptions has been challenged in terms of the response of laminar flames to changing positive strain rates [3], or mixture composition fluctuations [4]. Key observations for these planar flames included the flame responding in a quasi-steady manner at low frequencies, but at high frequencies the burning rate response becoming significantly attenuated, as well as having a phase lag.

Sahafzadeh et al. [5] showed the same results occur when flame stretch was created through the motion of a curved flame where both positive and negative transient stretch rates were applied. As a result, within an unsteady flow, classical flamelet models would predict the wrong magnitude of local burning and any expected maxima or minima events would occur at the wrong time. Lastly, an alternate approach to reduce the 
computational load of complex chemistry is to use reduced chemistry and simple transport properties. It has been found that the transient response of a stretched flame is not necessarily captured accurately due to the change in the internal flame structure [6].

In the current work, the approach is to keep the full chemistry and then estimate the instantaneous local consumption speed by a frequency response analysis in the form of a flame transfer function using the local stretch history as the input.

The focus of this paper is on premixed combustion in the Damköhler and Karlovitz regimes where reaction occurs in identifiable flame structures that are locally subjected to unsteadiness in the flow. The objective of this paper is to further develop a model of a flame for the practical purpose of capturing the impact of unsteadiness on the rates of conversion from reactants to products. To be explicit, no consideration is given here to spatial variations along a flame, which is another important characteristic of turbulent flames.

\section{FREQUENCY RESPONSE ANALYSIS}

To create a useful transfer function from a frequency response analysis, consideration needs to be given to its input and output. Within the context of premixed turbulent flame modeling in the flamelet regime, a goal is often to represent the flame as an interface with burning rates that vary over the surface depending on local conditions. For example, basic flamelet libraries are used to assign a consumption speed to an element of the flame depending on its equivalence ratio and stretch rate. Since one of the goals of the current work is to better account for the unsteadiness in turbulent flames, which includes unsteadiness in stretch rate, the input to the transfer function was chosen to be the varying stretch rate and the output is the varying local consumption speed. By choosing stretch rate, the transfer function could be seen as independent of the geometry that creates it, and therefore applicable to flamelets of a turbulent flame.

The numerically modeled flame used to produce the results for this frequency response analysis has been previously reported in the combustion literature [5], and is therefore only briefly described here. Reactants of methane and air of a specifiable equivalence ratio flow radially outward from the inlet boundary $(\mathrm{r}=5 \mathrm{~cm})$ and through the outlet boundary $(\mathrm{r}=$ $11 \mathrm{~cm}$ ). The mean and fluctuating components of the inlet mass flow rate were specified so that all the chemical reactions occur within the domain and are well removed from the boundaries. The model is based on a finite volume approach for solving the discretized equations for mass, momentum, energy, and species. The thermodynamic and transport properties come from CHEMKIN, and the reaction mechanism is based on GRI 3.0 [7] involving 36 species and 219 reactions.

For the simulations, the mass flow rate was changed sinusoidally with time (Eq. 1) at the inlet boundary.

$\dot{m}(t)=\dot{m}_{0}+B \operatorname{Sin}(\omega t)$
In Eq. $1, \dot{m}$ is the mass flow rate at the inlet boundary, $\dot{m}_{0}$ the mean mass flow rate at steady state, $B$ represents the amplitude of the oscillation, and $\omega$ is the angular frequency. The frequency was varied between $20 \mathrm{~Hz}$ and $2000 \mathrm{~Hz}$. The reason to consider frequencies up to $2000 \mathrm{~Hz}$ is for the relevance to turbulent flows. For example, in the middle of a premixed turbulent duct flame stabilized by a backward facing step, mean frequency of the flame motion across a fixed point was observed to be in this range [8]. In the present study, the equivalence ratio was varied from 1.0 to 0.7 in order to compare the stoichiometric condition to lean flames, which are usually of interest due to their applicability to low-emission combustion systems.

The geometry studied herein has unique features of flame dynamics relative to the counter-flow configuration, which is usually used to generate flamelet libraries. The steady flame is unstretched due to the balance of curvature and flow divergence (i.e., strain). However, when the flame is exposed to a flow field fluctuation, the expanding flame is subjected to positive stretch, whereas the contracting flame generates negative stretch, which has generally been ignored in the literature. The magnitude of the stretch rate could be changed by varying mean flame curvature, amplitude and frequency of oscillations, equivalence ratio, and fuel type. The choice of the marker, usually associated with a scalar field, to convert a flame with internal structure spread over space to an interface affects the magnitude of the stretch rate. To reduce this sensitivity, it is common to choose a marker that is on the trailing edge of the flame structure [9]. The flame marker used in this work is an iso-therm based on the temperature field with a value of $90 \%$ of the maximum temperature. Once calculated, this changing stretch rate becomes the input for the response analysis to create the transfer function.

The output for the response analysis is related to the total heat release, which is the chemical energy source term integrated for all species and in all control volumes in the domain, and is defined by Eq. 2 .

$\dot{E}_{b}=\sum_{i=1}^{N_{c v}} \sum_{k=1}^{N_{s p}} h_{k} \dot{\omega}_{k} \mathbb{V}(i)$

In Eq. $2, \dot{E}_{b}$ is referred to here as the rate of enthalpy conversion from chemical to sensible sources for the whole flame, otherwise known as the burning rate, $\mathbb{V}(i)$ represents the volume of cell $i, h_{k}$ is the molar specific enthalpy of species $k$, and $\dot{\omega}_{k}$ is the molar rate of creation of species $k$ per unit volume.

Fig. 1 shows this instantaneous consumption speed as a phase plot with respect to the corresponding instantaneous stretch rate. The quasi-steady response is a point (not shown in the figure) located on $\kappa=0$ and $S_{L}=0.2$, which is essentially the steady state value and would be referred to as $S_{L}^{O}$. At very low frequencies $(20 \mathrm{~Hz})$, the relationship between local flame speed and stretch rate is almost linear. By further increasing the frequency, the line becomes an ellipse. The slope of the ellipses are negative which has been shown in other works for 
methane-air flames in the same range of equivalence ratio [1]. The sensitivity of the local flame speed to the stretch rate first increases (i.e., the vertical amplitude of the ellipse) in low the frequency regime and then decreases in the high frequency regime. For the case of $\varphi=0.8$, this changing behaviour is seen in the variation from 100 to $200 \mathrm{~Hz}$ where the consumption speed increases, and from 200 to $500 \mathrm{~Hz}$ where the consumption speed decreases. Hence, there seems to be separate low and high frequency responses.

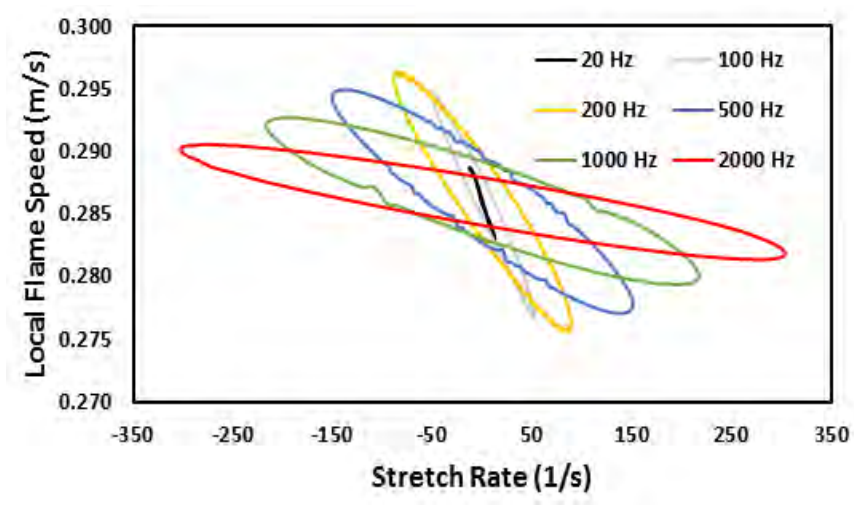

Figure 1. Local flame speed versus stretch rate for the equivalence ratio of $\varphi=0.8$ and quasi-steady response for the frequencies between $20 \mathrm{~Hz}$ and 2000 $\mathrm{Hz}$

From this analysis, it is obvious that the flame response to a changing stretch rate depends on parameters such as equivalence ratio and the frequency of oscillation. Therefore, it would seem desirable to find a describing function to replace the cumbersome complex chemistry computations of transient response of turbulent and laminar premixed flames.

\section{LINEAR HIGHER ORDER ANALYSIS}

In this section of the paper, in order to include flame behavior to a flow change upstream of the flame front, one needs to simulate each case for a wide range of frequencies and various input functions such as step, ramp, pulse, etc. This time consuming process can be replaced by one or more transfer functions to define the overall behavior of the flame. With regard to the application of this study, the input to the transfer functions are converted to stretch rate. The calculation of stretch rate is adopted from [5]. Therefore, the relationship between the stretch rate and local flame speed, which has been ignored in the current approach to flamelet modeling, reveals how flamelets respond to the time-dependent motion of a curved flame front. In order to have a better prediction of the transfer functions, a Polynomial Discrete-Time ARX (Autoregressive Exogenous) Model [10], which is a generalized transfer function, has been used. Equation (5) shows the general form of linear (due to the linear relationship between input and output) higher order (more than one term) transfer functions.

$$
C\left(z^{-1}\right) y(t)=\sum_{i=1}^{n u} D_{i}\left(z^{-1}\right) u_{i}\left(t-n k_{i}\right)
$$

In this equation, $u(t)$ is the input, $y(t)$ is the output, $\mathrm{C}$ and $\mathrm{D}$ are polynomials expressed in the time-shift operator $z^{-1}, u_{i}$ is the $i^{\text {th }}$ input, $n u$, the total number of inputs, and $n k_{i}$, the $i^{t h}$ input delay that characterizes the transport delay. In the present study, the system is SISO (single input/single output), therefore the transfer function takes a more familiar form wherein $i=1$. A Z-transform (Time-Discrete Fourier Transformation) can be used to convert the discrete-time form of the transfer function (Eq. 3) into a complex frequency domain representation.

This approach was first used to estimate the transfer function of the flame (with equivalence ratio of 0.8 ) response at $20 \mathrm{~Hz}$ fluctuations in mass flow rate. Then, the flame response to higher frequencies has been predicted using this transfer function. It has been observed that the accuracy of predictions decreases with increasing frequency. Increasing error in prediction of the transient response in the low frequency regime confirms that nonlinearity of the system increases with increasing frequency at each equivalence ratio. However, it could be considered insignificant until the oscillation reaches the high frequency regime, where the intrinsic structure of the flame may be changed locally. In the next step, the whole range of low frequencies (frequencies less than $200 \mathrm{~Hz}$, which is the cut-off frequency for equivalence ratio of 0.8 ) has been used to generate the transfer function (Eq. 6). Figure 5 shows that the average accuracy of the predictions is $\sim 88 \%$ for frequencies up to $200 \mathrm{~Hz}$; however, the flame response experiences a significant drop in accuracy after $200 \mathrm{~Hz}$ (not shown in the figure).

$$
\begin{aligned}
& C(z)=1+0.9623 z^{-1}+0.636 z^{-2}+0.2826 z^{-3}+ \\
& 0.122 z^{-4} \\
& D(z)=-0.6376 z^{-1}+0.6915 z^{-2}+0.5377 z^{-3}- \\
& 0.5923 z^{-4}
\end{aligned}
$$

$\mathrm{C}(\mathrm{z})$ and $\mathrm{D}(\mathrm{z})$ are the expressions represented in Eq. 3. The fourth order transfer function results indicate that a minimum of the first four terms on each side of Eq. 3 is required to have the most accurate predictions. It should be noted that $\mathrm{C}(\mathrm{z})$ and $\mathrm{D}(\mathrm{z})$ are usually referred to as the poles and zeros of a transfer function in the literature.

Based on the higher order linear model, a transfer function (Eq. 5) is generated for predicting the high frequency regime transient response. Figure 3 illustrates the accuracy of this model for frequencies higher than $200 \mathrm{~Hz}$.

$$
\begin{aligned}
& C(z)=1+0.2641 z^{-1}-0.2513 z^{-2}-0.3054 z^{-3}- \\
& 0.2756 z^{-4} \\
& D(z)=0.0012 z^{-1}-0.0044 z^{-2}+0.0051 z^{-3}- \\
& 0.0019 z^{-4}
\end{aligned}
$$

In order to study the effect of the amplitude on flame behavior and the transfer function, the ratio of the oscillations to the mean value was doubled. The results depict a fourth order transfer function similar to the previous cases, which 
predicts the high frequency responses $(70 \%$ for $500 \mathrm{~Hz}, 84 \%$ for $1000 \mathrm{~Hz}$, and $91 \%$ for $2000 \mathrm{~Hz}$ ), with different coefficients.

The fourth order equation is the effective transfer function for various equivalence ratios. For example, a stoichiometric flame was tested with different flame curvature and amplitude. The optimum transfer function has the same format as it does with the other cases. The accuracies for the high range of frequencies is $83 \%$ for $500 \mathrm{~Hz}, 93 \%$ for $1000 \mathrm{~Hz}$, and $94 \%$ for $2000 \mathrm{~Hz}$. The higher accuracy at the same frequencies was expected due to the faster burning rate at the stoichiometric condition compared to the lean flames.

As can be concluded from the recent results in this section, the whole range of frequencies is divided in two regimes based on the cut-off frequency obtained from numerical simulations of transient response of laminar premixed flames for each equivalence ratio, and a separate transfer function is defined for each section. Although the accuracies are in an acceptable range, there may be considerable improvement using nonlinear models to predict the output. Therefore, in order to study the capability of nonlinear models to predict the flame response more accurately, different nonlinear transfer functions were analyzed in the next section.

\section{NONLINEAR SYSTEM ANALYSIS}

As shown in the previous section, although linear transfer functions can achieve accurate prediction in the low frequency range of flame response to changes in stretch rate, they are not able to predict the response at some high frequencies. As mentioned in the introduction, in analyzing flame instabilities, flow velocity perturbations (as the input to the system) change the heat release rate (as the output) by influencing the flame surface area. Therefore, flame transfer functions are studied using the flame response to velocity fluctuations. The heat release rate modulations result in acoustic pressure perturbations, and could be linked to inlet flow velocity oscillations. Therefore, in order to capture the nonlinearities in the thermos-acoustic coupling, the flame transfer function has been replaced by the flame-describing function [11]. It has been reported that in premixed combustion systems, the relationship between velocity and heat release makes the system nonlinear, whereas gas dynamic processes generally stay in the linear regime [12].

Among these predictive models, using NARX (Nonlinear Autoregressive Exogenous) led to better results in estimating the transient response of the flame for the whole range of frequencies, including both low and high frequency regimes. Figure 3 illustrates the NARX predictions in comparison to the complex chemistry numerical results for the flame with an equivalence ratio of 0.8 at different frequencies. NARX models (which are an extension of ARX in polynomial transfer functions) are flexible nonlinear functions that are able to capture the complex behavior in nonlinear systems such as the current data set. Eq. (6) illustrates the structure of a linear Single Input/Single Output ARX model:

$y(t)+a_{1} y(t-1)+a_{2} y(t-2)+\cdots+a_{n a} y(t-n a)=$ $b_{1} u(t)+b_{2} u(t-1)+\cdots+b_{n b} u(t-n b+1)+e(t)$
Where $u, y$ and $e$ are the input, output, and noise, respectively. This structure implies that the output $y(t)$ is predicted as a weighted sum of past output values and current and past input values. na is the number of past output terms, and $n b$ is the number of past input terms used to predict the current output. It has been shown that the surface wrinkling in turbulent flames at one location, in addition to the local velocity perturbation, also depends on the flame surface fluctuations at previous times upstream of the flame front [13]. Therefore, an ARX model could capture this memory effect occurring in turbulent flames by modifying the laminar flamelet response to a change in upstream flowrate.

After applying the nonlinear ARX model to predict the flame response, the results need to be validated by comparing the estimations with time series datasets obtained from complex chemistry numerical simulations. The nonlinear ARX model has been applied to three different datasets including, the low frequency regime (nonlinear ARX 2 in Fig. 2), the high frequency regime (nonlinear ARX 2 in Fig. 3), and the whole range of frequencies (nonlinear ARX 1 in Fig. 2 and 3). Therefore, three different nonlinear transfer functions were generated and tested against the numerical computations. As can be concluded from Figs. 2 and 3, nonlinear ARX model 2 based on two different frequency regimes, shows accurate predictions for each frequency compared to the linear higher order ARX model and nonlinear ARX model 1. It should be noted that these models are all capable of predicting the transient responses due to their storing of time histories in their structures. However, because of the nonlinear relationship between the input and output at high frequencies, nonlinear models are more accurate in general.
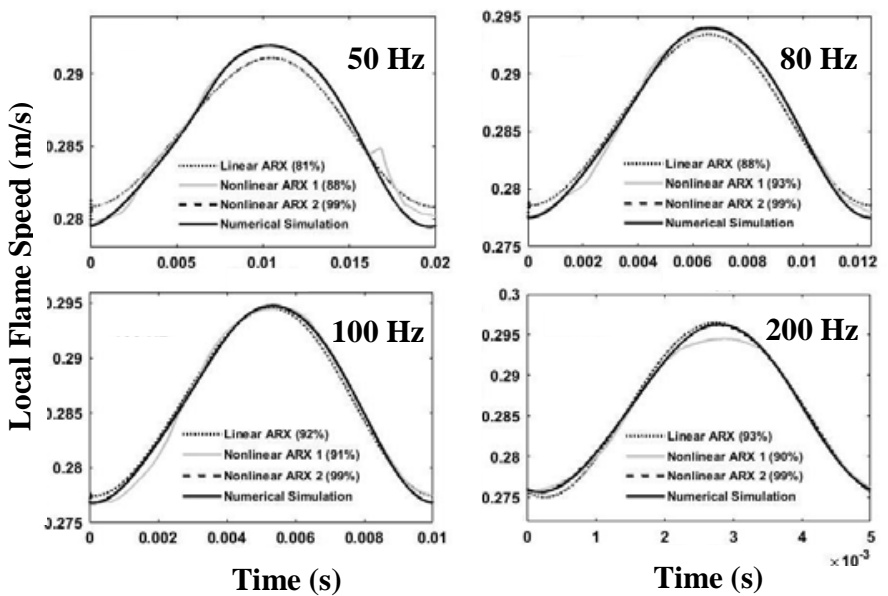

Figure 2. Estimation of transient response of a laminar premixed flame for $\varphi=0.8$ using a higher order linear ARX model and a nonlinear ARX model in the low frequency regime 


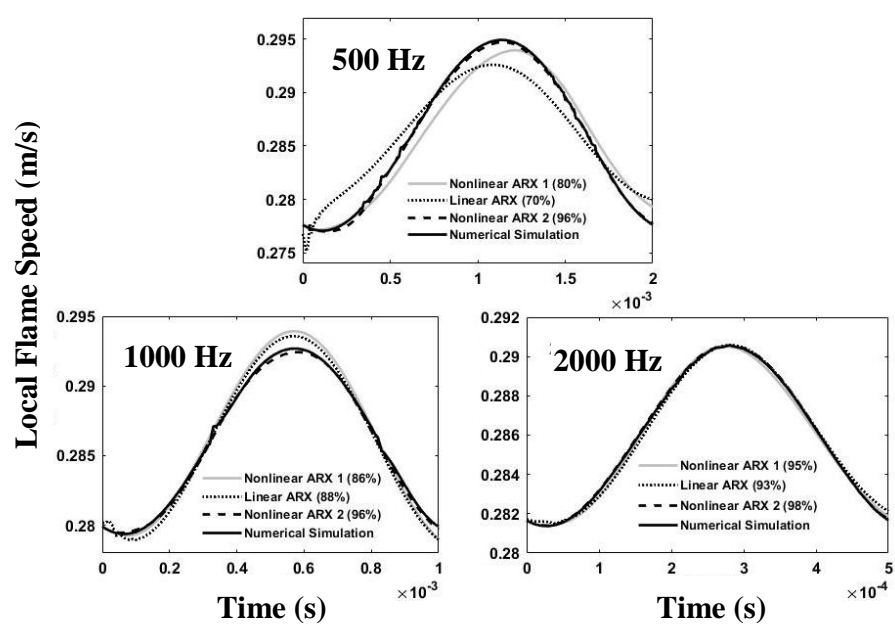

Figure 3. Estimation of transient response of a laminar premixed flame for $\varphi=0.8$ using the higher order linear ARX model and nonlinear ARX model in the high frequency regime

\section{CONCLUSIONS}

In this paper, the transient response data on the rate of conversion of chemical to sensible enthalpy, and the local flame speed for a laminar premixed flame that was exposed to periodic flow conditions, was analyzed in order to estimate a transfer function between these inputs and outputs. The study of the transient response analysis of a curved flame could be a potential modification to the quasi-steady assumption used in laminar flamelet libraries in order to improve premixed turbulent combustion modeling. The transient response dataset was taken from a previous study, which was a complex chemistry simulation of an inwardly burning laminar premixed (lean methane/air) flame in a cylindrically-symmetric geometry for different equivalence ratios $(0.7-1.0)$ with cyclic exposure to positive and negative stretch rates at different frequencies $(0$ $-2000 \mathrm{~Hz}$ ). In this paper, the higher order linear and nonlinear analyses have been performed for equivalence ratio of 0.8 only, to show the capability of transfer functions to capture the flame behavior when exposed to a flow perturbation upstream of the flame front.

A linear first order model was applied to the data and it fit the low frequency results for each flame. However, the flame behavior deviates from that of a linear first order system as the frequency increased and approached conditions where the chemical and flow time scales were similar. Applying higher order linear transfer functions improved the predictions (except for in the high frequency regime) due to the capability of the transfer function to store the time histories in its structure.

Nonlinear models, such as the nonlinear ARX model, could correctly predict the transient local flame speed over the whole range of frequencies and changing flow conditions. This transfer function could also reconstruct the local flame speed response for any arbitrary flow input, which enables the model to capture the transient effects of a flame in any conditions.
Therefore, estimating a flame transfer function for laminar premixed flames could yield a better understanding of flame dynamics and could be used in order to modify the quasisteady assumption in laminar flamelet models of turbulent combustion. Using the generated transfer functions based on the simulation datasets, the transient complex chemistry results were predicted with an accuracy over $70 \%$.

\section{ACKNOWLEDGMENTS}

The authors would like to thank the Natural Sciences and Engineering Research Council of Canada (NSERC) for financial support. This research was undertaken, in part, thanks to funding from the Canada Research Chairs program.

\section{References}

[1] J. Driscoll, "Turbulent premixed combustion: Flamelet structure and its effect on turbulent burning velocities," Prog. Energy Combust. Sci., vol. 34, no. 1, pp. 91-134, 2008. doi:10.1016/j.pecs.2007.04.002.

[2] H. Wang, E. R. Hawkes, J. H. Chen, "Turbulence-flame interactions in DNS of a laboratory high Karlovitz premixed turbulent jet flame," Phys Fluids, vol. 28, no. 9, 2016. doi:10.1063/1.4962501.

[3] A. F.Ghoniem, C. A. Petrov, "The transient response of strained laminarpremixed flames," Combust. Flame, vol. 102, pp. 401-417, 1995. doi:10.1016/0010-2180(95)00032-2.

[4] R. Lauvergne, F. N. Egolfopoulos, "Unsteady response of C3H8/air laminar premixed flames submitted to mixture composition oscillations," Proc. Combust. Inst., vol. 28, p. 1841-1850, 2000. doi:10.1016/S0082-0784(00)80587-1.

[5] M. Sahafzadeh, L. W. Kostiuk, S. B. Dworkin, "Transient response of a laminar premixed flame to a radially diverging/converging flow," Combust. Flame, vol. 179, pp. 51-62, 2017. doi:10.1016/j.combustflame.2017.01.015.

[6] T. Poinsot, D. Veynante, Theoretical and numerical combustion, Philadelphia: Edwards Inc., 2005.

[7] G. P. Smith, D. M. Golden, M. Frenklach, N. W. Moriarty, B. Eiteneer, M. Goldenberg, C. T. Bowman, R. K. Hanson, S. Song, W. C. Gardiner, Jr., V. V. Lissianski, Z. Qin, "http://www.me.berkeley.edu/gri_mec," [Online].

[8] I. G. Shepherd, J. B. Moss, "Characteristic Scales for Density Fluctuations in a Turbulent Premixed Flame," Combust. Sci. Technol., vol. 33, no. 5-6, pp. 231-243, 1983. doi:10.1080/00102208308923678.

[9] G.K. Giannakopoulos, M. Matalon, C.E. Frouzakis, A.G. Tomboulides, "The curvature Markstein length and the definition of flame displacement speed for stationary spherical flames," Proc. Combust. Inst., vol. 35, pp. 737-743, 2015. doi:10.1016/j.proci.2014.07.049.

[10] R. Vidal, "Recursive identification of switched ARX systems," Automatica, vol. 44, no. 9, pp. 2274-2287, 2008. doi:10.1016/j.automatica.2008.01.025.

[11] N. Noiray, D. Durox, T. Schuller, S. Candel, "A unified framework for nonlinear combustion instability analysis based on the flame describing function," J. Fluid Mech., vol. 615, p. 139-167, 2008. doi:10.1017/S0022112008003613.

[12] A. P. Dowling, "Nonlinear Self-Excited Oscillations of a Ducted Flame," $J$. Fluid Mech, vol. 346, p. 271-290, 1997. doi:10.1017/S0022112097006484.

[13] S. Hemchandra, T. Lieuwen, "Local consumption speed of turbulent premixed flames - An analysis of "memory effects"," Combust. Flame, vol. 157, no. 5, p. 955965, 2010. doi:10.1016/j.combustflame.2009.10.007. 


\title{
Wind Turbine Wake Effect Visualization and LiDAR Measurement Techniques
}

\author{
Michael McKinnon, David A. Johnson \\ Mechanical and Mechatronics Engineering \\ University of Waterloo \\ Waterloo, Canada \\ mmckinno@uwaterloo.ca
}

\begin{abstract}
The expansion of wind energy development has resulted in larger wind farms and closer placement of turbines to utilize the space available. Each turbine produces a wake that affects downstream turbines, which causes issues such as production loss, blade loading, and fluctuating electrical output. In order to minimize this impact, the wake produced by turbines must first be understood. Experimental methods were used in the exploration of the wake effects of wind turbines. Smoke visualization inside a controlled wind facility was used to determine the helical vortex wake distribution behind a $3.3 \mathrm{~m}$ diameter turbine as well as the tip vortex shedding on the blade. Wind Doppler Light Detection and Ranging (LiDAR) measurement devices are used to measure the velocity of aerosols to determine the wind velocity. This technique can be used to measure the airspeed in the wake of a wind turbine. The University of Waterloo Wind Energy Group has a ZephIR z150 LiDAR to use in this study. The LiDAR was verified for accuracy against a cup and vane anemometer. The results determined that the LiDAR was suitable to provide accurate measurements in further turbine wake experiments. The University of Waterloo's facilities provide the advantage to study wake effects under controlled wind conditions as well as under realistic open-air conditions.
\end{abstract}

Keywords-wind energy; wake effects; LiDAR; wind farm layout

\section{INTRODUCTION}

Increasing energy demands and the need for cleaner electricity sources have fueled the development of renewable energy, especially wind energy [1]. More wind farms are being developed with increased density of turbine spacing to utilize areas with high wind conditions. Therefore, wind turbine interaction is becoming a greater concern when developing and operating wind farms.

Wakes are produced by the wind turbines which affect other downstream turbines. This can cause blade loading problems, affect rotor performance, cause fluctuating electrical output, and reduce production [2]. It is important to study the wake effects of wind turbines to be able to design wind farms to reduce the effects on downstream turbines.
Several methods have been explored to measure and simulate the behaviour of the wakes. Some key behaviour characteristics include wake recovery, meandering, and expansion [2]. Experimental methods include wind tunnel tests and field measurements. The University of Waterloo's Wind Energy Group has the capability to test small-scale wind turbines in both wind tunnel and field conditions. The issue with most wind tunnel experiments is obtaining accurate results while scaling wind turbines to fit the wind tunnel specifications. The University of Waterloo's Controlled Wind Facility is able to house a $3 . .3 \mathrm{~m}$ diameter wind turbine with the benefit of obtaining controlled wind conditions up to $13 \mathrm{~m} / \mathrm{s}$. Additionally, the University has a $10 \mathrm{~m}$ wind turbine located in the external environment to test actual field conditions that would be experienced by commercial wind turbines.

With these two wind turbines, the characteristics of wind turbine wakes can be studied and compared to get an understanding of the theoretical and actual behaviour of the wake. Experimental analysis using smoke visualization gives a visual of the wake profile, while a Light Detection and Ranging (LiDAR) measurement device measures the air speed in the wake. Both methods are used to determine the wake effects of the turbines in controlled and external conditions.

\section{EXPERIMENTAL FACILITIES}

Experiments to determine the behaviour of wind turbine wakes were tested inside the University of Waterloo Controlled Wind Facility. The large-scale wind turbine testing facility uses six $75 \mathrm{~kW}$ variable speed fans to obtain consistent wind conditions up to $13 \mathrm{~m} / \mathrm{s}$ in the test area [3]. Fig. 1 shows the

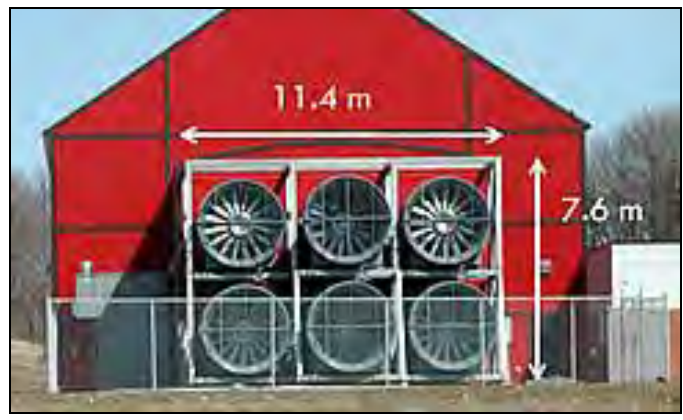

Figure 1. Controlled Wind Facility fans 
fans on the outside of the facility.

A $3.3 \mathrm{~m}$ diameter wind turbine, shown in Fig. 2(a), was developed by the UW Wind Energy Group to operate inside the controlled wind facility. The wind turbine, called the UW WEG turbine, has an output of $3 \mathrm{~kW}$ under $11 \mathrm{~m} / \mathrm{s}$ wind conditions.

A small-scale, grid connected wind turbine is located in the Region of Waterloo and is used by the Wind Energy Group for open-air testing. The turbine, manufactured by Wenvor Technologies, has two blades with passive variable pitching and a diameter of $10 \mathrm{~m}$. The hub height is $30 \mathrm{~m}$ and has a power rating of $35 \mathrm{~kW}$. The Wenvor turbine is shown in Fig. 2(b).

\section{FLOW VISUALIZATION}

Smoke visualization was used inside the Controlled Wind Facility to gain a visual understanding of the wake behaviour as well as provide quantitative measurements. Two smoke visualization techniques exist to study the wake behaviour of turbines [4]. Each method was explored in the experiments. The first method attached smoke emitters to the tip of the one of the blades. This method was used to analyze the wake behind the turbine. The second method ignited smoke in front of the turbine directed at the tip of the blade sweep to analyze the tip vortices.

Both experiments were performed at a wind speed of $6 \mathrm{~m} / \mathrm{s}$ and constant rotational speed of 100 RPM with the UW WEG turbine, resulting in a tip speed ratio of approximately 3.9. The behaviour of the smoke was captured at various views.

A view of the progression from the tip ejection experiment using method 1 can be seen in Fig. 3. As the smoke propagates downstream, a helical pattern can be seen. This shows a typical helical vortex wake distribution. As vortex cylinders are shed from the blades of the turbine, they follow a helical path due to the rotation of the blade. The wake expansion can also be

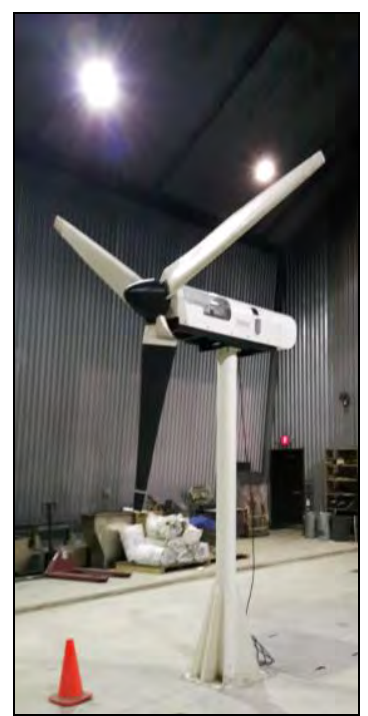

(a)

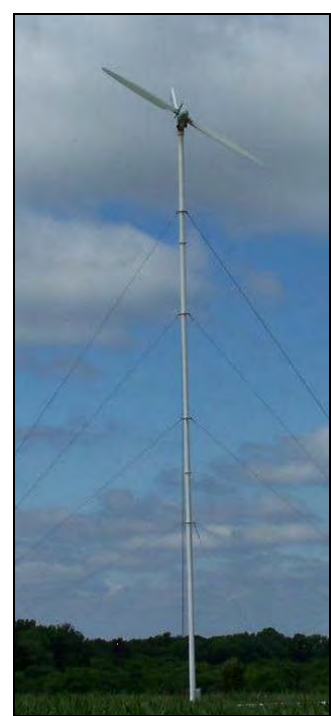

(b)
Figure 2. University of Waterloo's wind turbines: (a) UW WEG Turbine and (b) Wenvor Turbine

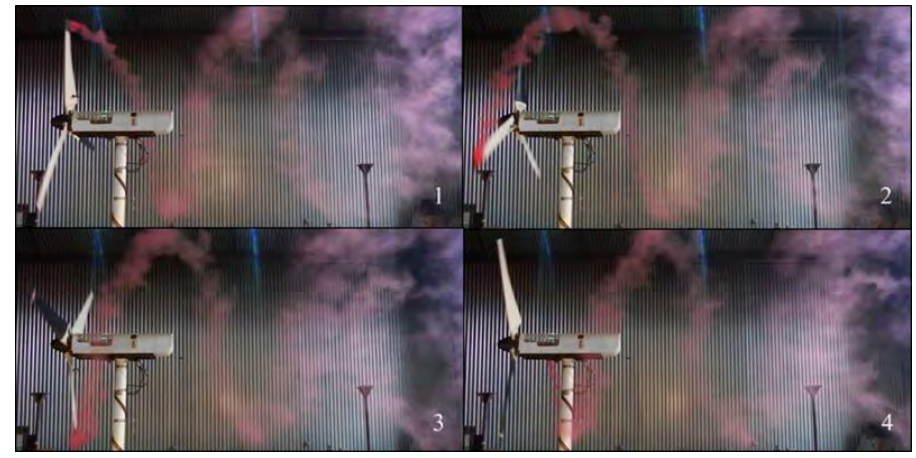

Figure 3. Tip smoke visualization progression of the UW WEG turbine for $6 \mathrm{~m} / \mathrm{s}$ wind speed and a blade rotaion of $100 \mathrm{RPM}$

determined from the smoke visualization experiment. As the smoke moves downstream, the diameter increases due to this expansion.

A close up of the tip of the blade in Fig. 4 shows the angle at which the smoke leaves the blade tip. This is known as the helix angle, $\phi$, or the flow angle at the blade tip [5]. This angle is used to determine the direction of the vortex cylinder parallel to the axis of rotation. As a result, the induced velocity at the rotor plan can be calculated using Biot-Savart law if the vorticity strength in known. The experiment resulted in a helix angle of $25^{\circ}$ from the setup parameters.

The second smoke emission method released smoke upstream of the turbine to visualize the vortices being shed from the blade tips. A tip vortex forms as the blade passes through the smoke. The vortex moves downstream and another is formed as the next blade passes. This effect is seen in the results of the experiment in Fig. 5, which shows a tip vortex being formed and vortex that has moved downstream with the flow. The distance between these vortices is related to the helical path of the vortex cylinders that was seen in the previous experiment.

\section{LIDAR MEASUREMENTS}

Wind Doppler LiDARs have the capability to measure the velocity of particulates in the air, which are used as seeding material to determine the wind speed [6]. The LiDAR achieves

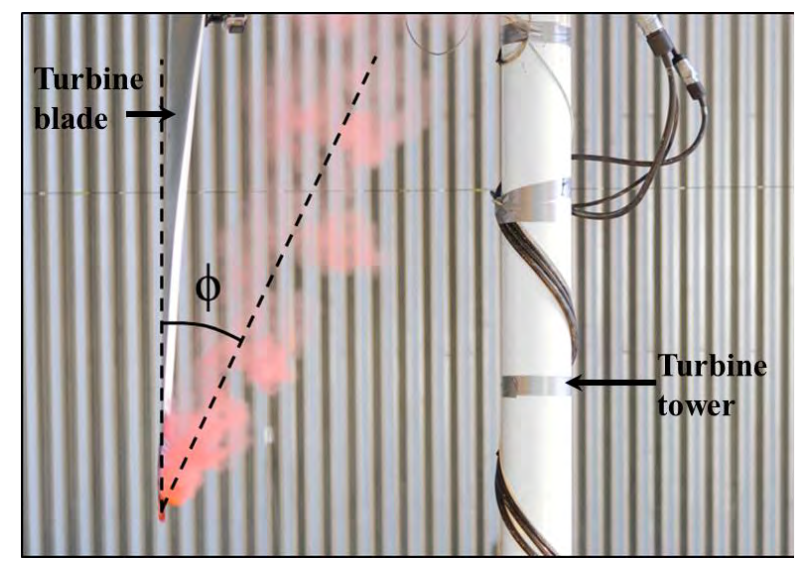

Figure 4. Helix Angle of the UW WEG turbine for $6 \mathrm{~m} / \mathrm{s}$ wind speed and a blade rotaion of 100 RPM 


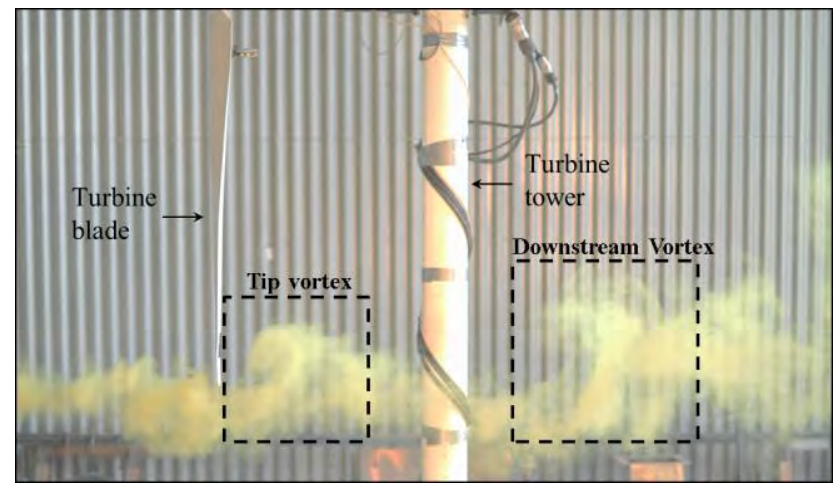

Figure 5. Upstream smoke visualization of the UW WEG turbine $6 \mathrm{~m} / \mathrm{s}$ wind speed and a blade rotaion of 100 RPM

this by the use of a laser, which uses the Doppler Effect from the scattered light reflected from the particulate to calculate the speed of that particle.

Therefore, to measure the velocity of the air in the wake of the turbine, a ZephIR z150 LiDAR, shown in Fig. 6, is used. The LiDAR has the ability to measure various heights and uses a conical sweeping pattern to calculate the direction of the wind. Therefore, it can be used to get a representation of the velocity profile in the wake as it progresses downstream.

Before using the LiDAR for wake measurements, it was first verified for accuracy [8]. A test was conducted to compare the LiDAR against a cup and vane anemometer, each at different heights in atmospheric conditions. The anemometers were attached to the Wenvor wind turbine tower and located at a vertical height of $10 \mathrm{~m}$ from ground level and a height of 20 $\mathrm{m}$ for the cup and vane anemometers respectively.

Due to constraints on the LiDAR, a minimum height of 12 $\mathrm{m}$ was achieved to compare against the cup anemometer. The vane anemometer was used to verify the wind direction. The LiDAR was located $18 \mathrm{~m}$ away from the Wenvor tower to avoid interference due to the conical sweeping of the laser. The LiDAR was placed at a similar distance away from a nearby tree line and ensured that the ground level was consistent with the tower. A view of the setup is shown in Fig. 7. Data was

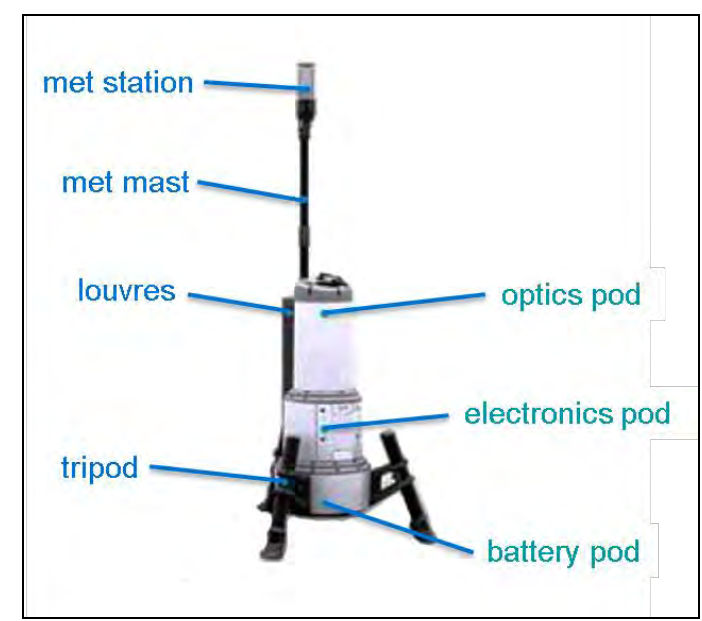

Figure 6. ZephIR z150 LiDAR [7] collected in 10-minute averages over the span of one hour.

The result of the velocity comparison for the LiDAR and cup anemometer is shown in Fig. 8. Due to the limitations of the LiDAR minimum measurable height, the measurements were scaled based on the power law to get equivalent values at $10 \mathrm{~m}$. Both measurements followed the same trend and were within the error of measurements. However, the data was skewed, which could be the result of the scaling or a bias in the cup anemometer measurements.

Similar to the velocity comparison, the direction measurements also showed similar trends as seen in Fig. 9. Again, the comparison shows the measurements were with error. Therefore, it was concluded that the LiDAR measurements were within reasonable accuracy to use for wake measurements.

In order to take measurements in the wake of a turbine, the LiDAR needs to be modified to convert the conical sweep of the laser to a single point line-of-sight laser [9]. This ensures that the measurement position can be placed at a single point and then traversed to gain a profile of the wake.

\section{CONCLUSION}

Understanding wake behaviour of wind turbines is important for the development of new wind farms and design of wind turbines. Understanding can be achieved through experiments using flow visualization and LiDAR measurements.

The University of Waterloo Wind Energy Group uses two wind turbines to contribute to the study of wakes. The two turbines have the benefit to compare controlled wind versus open-air conditions. A controlled wind facility allows for the testing of a $3.3 \mathrm{~m}$ diameter wind turbine under steady wind conditions up to $13 \mathrm{~m} / \mathrm{s}$, while an external $10 \mathrm{~m}$ diameter turbine experiences realistic variable wind effects.

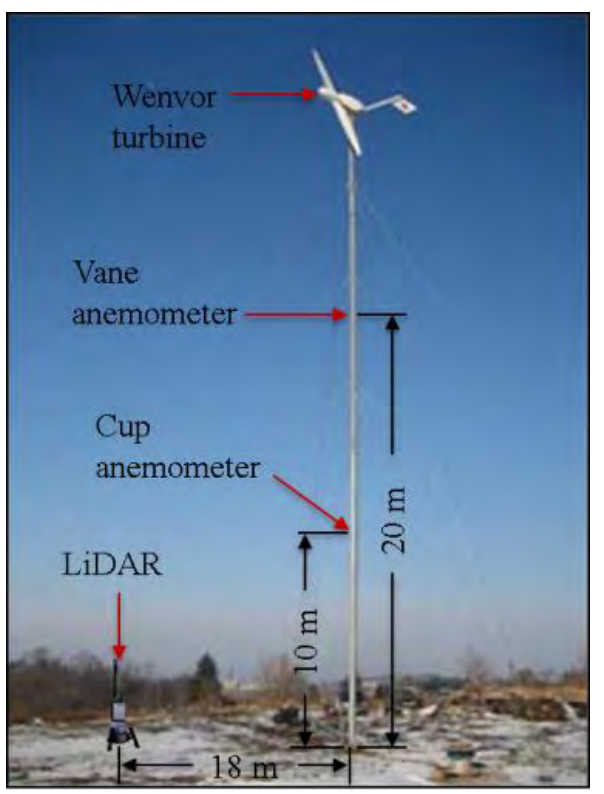

Figure 7. LiDAR verification test setup 


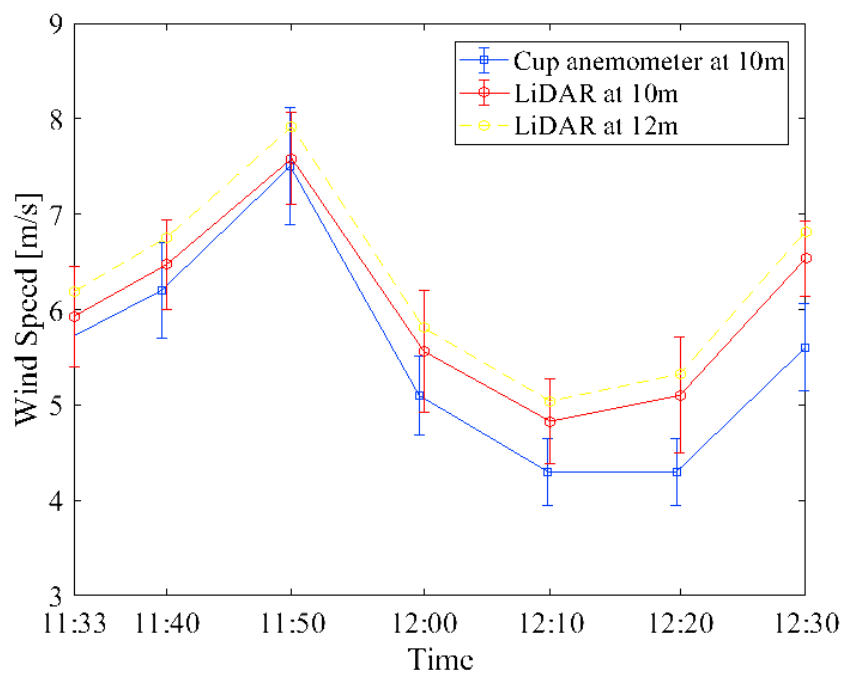

Figure 8. Cup anemometer and LiDAR velocity comparison

Smoke visualization was used to determine the wake characteristics such as a helical vortex distribution, tip vortex shedding, and wake expansion inside the Controlled Wind Facility. This was completed with two smoke visualization techniques that emit smoke from the tip of a turbine blade and another to emit smoke upstream of the turbine directed at the sweeping blade tips. In the first method, the helix angle was able to be measured with a value of $25^{\circ}$. The second method showed the formation and downstream propagation of the tip vortices.

Wind Doppler LiDAR measures the speed of aerosols in the wind to calculate the wind speed. A ZephIR z150 LiDAR was measured against a cup anemometer at a height of $10 \mathrm{~m}$ to verify the wind velocity measurements. The LiDAR was also measured against a vane anemometer with a $20 \mathrm{~m}$ height to verify wind direction measurements. The comparison between the LiDAR and both anemometers showed the same trend and were within error, resulting in the conclusion that the LiDAR had sufficient accuracy to use in the measurement of air speeds. Therefore, the LiDAR is able to be used to measure the

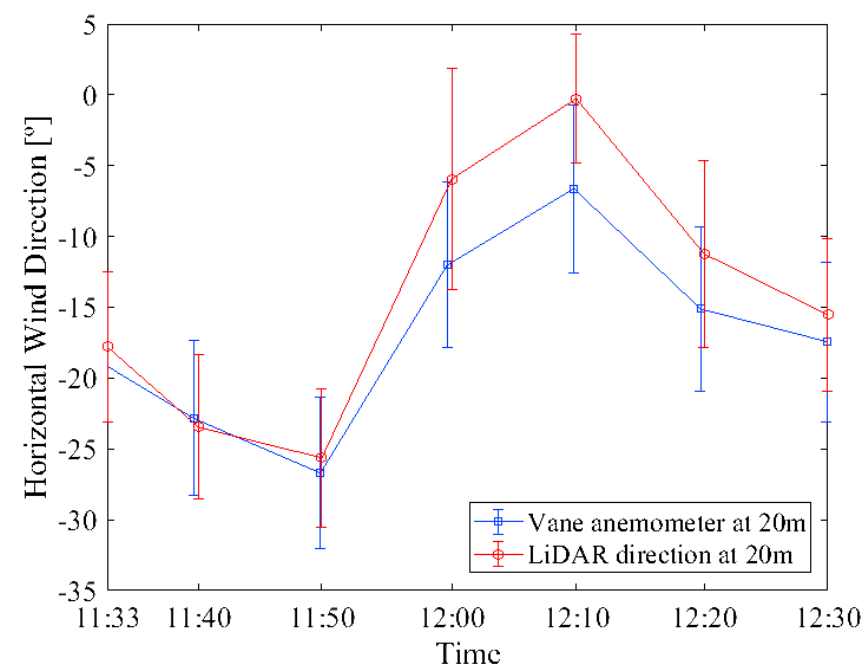

Figure 9. Vane anemometer and LiDAR wind direction comparison velocity profile in the wakes of the turbines.

The comparison between controlled and external conditions will allow for a better relation between the theoretical understanding of wake effects and the actual behaviour of a wind turbine. With this, better optimization of wind farms can lead to increased density while reducing production losses and turbine maintenance.

\section{ACKNOWLEDGMENT}

The authors acknowledge the help and support of the University of Waterloo Wind Energy Group and technicians in the aid of experiments and contribution to the research topic.

\section{REFERENCES}

[1] Government of Canada National Energy Board, "Canada's Energy Future 2017," Calgary, AB, 2017.

[2] R. Stevens and C. Meneveau, "Flow structure and turbulence in wind farms," Annu. Rev. Fluid Mech., vol. 49, pp. 311-339, 2017. doi: 10.1146/annurev-fluid-010816-060206.

[3] T. E. Gallantand D. A. Johnson, "In-blade angle of attack measurement and comparison with models," J. Phys. : Conf. Ser., 2016. doi: 10.1088/1742-6596/753/7/072007.

[4] L. J. Vermeer, J. N. Sørensen, and A. Crespo, "Wind turbine wake aerodynamics," Prog. Aerosp. Sci., vol. 39, pp. 467-510, 2003. doi: 10.1016/S0376-0421(03)00078-2.

[5] T. Burton et al., Wind Energy Handbook. Chester, UK: John Wiley \& Sons Ltd., 2001. doi: 10.1002/0470846062.

[6] C. Werner, "Doppler Wind Lidar" in Lidar, C. Weitkamp, Ed. New York: Springer, 2005, pp. 325-354.

[7] Natural Power, "ZephIR Operations and Maintenance Manual," ZephIR LiDAR, Malvern, Worcs, 2009.

[8] M. Cayla, "Comparison of ZephIR measurements against cup anemometry and power curve assessment," Natural Power, Strasbourg, France, 2010.

[9] F. Bingöl, "Adapting a Doppler laser anemometer to wind energy," M. Sc. thesis, Fluid Mech. Section, Technical University of Denmark, Lymgby, Denmark, 2005 . 


\title{
Polymeric Triboelectric Nanogenerator: Effects of Polymer Type, Geometry, and Porosity on Triboelectrification
}

\author{
Hossein Abdoli and Siu N. Leung \\ Department of Mechanical Engineering \\ York University \\ Toronto, ON, Canada \\ sunny.leung@lassonde.yorku.ca
}

\begin{abstract}
Triboelectric nanogenerator (TENGs), based on the principles of triboelectrification and electrostatic induction, have recently been viewed as a promising approach to harvest mechanical energy, which would otherwise be wasted through dissipation to surrounding. Large contact area and large surface area have been identified as the basic design principles to promote the performances of TENGs. In this study, particulate leaching method was used to introduce and tailor the open-cell morphology of negative triboelectric layers in TENGs. The effects of material type, material geometry, open-cell morphology on the level of triboelectrification induced by mechanical motions were also investigated. Experimental results revealed that polyvinylidene fluoride (PVDF) performed better than high density polyethylene (HDPE) as the negative triboelectric layer of the TENG. Furthermore, increasing the contact area, reducing the thickness, and introducing fine pores would enhance the performances of TENGs.
\end{abstract}

Keywords-energy harvesting; geometry; open-cell morphology; polymer; triboelectricification

\section{INTRODUCTION}

Triboelectrification, in conjunction with electrostatic induction, has been developed into an innovative energy harvesting technology - triboelectric nanogenerators (TENGs) [1]. Although different types of technologies based on electrostatic, electromagnetism, and piezoelectricity exist to convert mechanical energy into electrical energy [2], TENGs have recently been drawing attentions. It is considered as a promising solution to harvest mechanical energy, which will otherwise be wasted, into useful energy. In addition, TENGs are also capable of powering sensors, portable electrics, and biomedical microsystems $[1,3]$.

There are several different modes of TENGs. Among them, the contact-separation mode is one of the simplest ones. In this mode, two friction materials with different triboelectric properties contact and separate periodically. Each of them is attached to an electrode. During contact, electrons transfer from one material to the other because of their abilities to attract electrons. As a result, the material loses electrons would become positively charged while the other gains electrons would be negatively charged. Subsequently, the two oppositely charged materials separate and a potential difference will be generated between them. This will electrostatically induce a current flow along an external circuit connected to the two electrodes adhered by the two materials in order to compensate the potential difference. When the two materials are pressed again, the potential difference will decrease and a reverse current flow will be induced. This periodic action will in turn generate an alternating current [3].

Although triboelectrification is a well-known phenomenon, its fundamental is not fully understood yet. Various parameters can affect the degree of triboelectrification of materials, and thereby the performances of TENGs. These factors include humidity, external force applied to induce the periodic contact and separation of friction materials, chemical composition of the friction layers, geometrical parameters (e.g., thickness and contact area), and morphological parameters (e.g., shape and surface area).

Recently, researchers have conducted studies to investigate the effects of these governing factors on the degree of triboelectrification in order to optimize the performances of TENGs [1-3]. Wang revealed that microstructuring the surface of PDMS, which was used as the negative side of the friction materials, could influence the charge density accumulated on the material surfaces [4]. It is believed that the creation of pyramidbased micro-patterns is effective to enhance the contact area and potentially the triboelectrification. Lee et al. suggested that the introduction of a film-covered pillar structure on PDMS would enhance the conformable contact and adhesion energy, which would in turn increase triboelectrication [5]. Another recent work fabricated a hydrophobic polydimethylsiloxane (PDMS) sponge to study the effect of pore size and humidity on TENG parameters [2]. They observed that TENG voltage and current density greatly increased when PDMS pore size reduced from $10 \mu \mathrm{m}$ to $0.5 \mu \mathrm{m}$. Furthermore, the developed voltage only slightly decreased when relative humidity increased from 20 to $80 \%$, which showed reasonable stability of hydrophobic structure under different humidity conditions. Chen et al. posed that matrix of high dielectric nanoparticles like strontium titanate $\left(\mathrm{SrTiO}_{3}\right)$ in PDMS can enhance charge density of TENG and improve the power output of the device by five times [6]. 
This paper studied the effects of material type and material geometry (e.g., thickness and contact area) on the electrification of polymer-based TENGs. After identifying the appropriate material type, particulate leaching was used to introduce and tailor the open-cell structures of negative triboelectric layers. Parametric studies were conduct to elucidate how the open-cell morphology of the triboelectric layer influenced the output performances of TENGs. This work enhanced the understanding of material design on their triboelectriciation and provided new and effective strategy to enhance the performances of TENGs.

\section{EXPERIMENTAL}

\section{A. Materials}

Polyvinylidene fluoride (PVDF, Kynar 741, Arkema) and high density polyethylene (HDPE, Sclair ${ }^{\circledR}$ 2710, NOVA Chemicals) were used to fabricate the negative triboelectric layers of TENGs. Thermoplastic polyurethane (TPU, Estane ${ }^{\circledR}$ 2103-70A, Lubrizol) was used to fabricate the positive triboelectric layers of TENGs. Table I summarizes the physical properties of PVDF, HDPE, and TPU. Sodium chloride $(\mathrm{NaCl})$ was used as the leaching agent to fabricate porous negative triboelectric PVDF layers. The electrodes of TENGs were made by copper tapes with conductive adhesive. All materials were used as received without any modification.

TABLE I. PhysicAl PROPERTIES OF PVDF, HDPE, AND TPU

\begin{tabular}{|l|c|c|}
\hline \multirow{2}{*}{ Polymer } & \multicolumn{2}{|c|}{ Physical Properties } \\
\cline { 2 - 3 } & Density & Young's Modulus \\
\hline HDPE & $951 \mathrm{~kg} / \mathrm{m}^{3}$ & $1000 \mathrm{MPa}$ \\
\hline PVDF & $1780 \mathrm{~kg} / \mathrm{m}^{3}$ & $1700 \mathrm{MPa}$ \\
\hline TPU & $1060 \mathrm{~kg} / \mathrm{m}^{3}$ & $2.1-5.2 \mathrm{MPa}$ \\
\hline
\end{tabular}

\section{B. Sample Preparation}

Solid triboelectric negative layers were fabricated by compression molding (Craver Press, $4386 \mathrm{CH}$ ) PVDF powders or HDPE pellets at $185^{\circ} \mathrm{C}$ and $140^{\circ} \mathrm{C}$, respectively, into samples with various thicknesses and areas. In the first step, the heat platens of the compression molding machine were brought into contact with the mold, which loaded with polymer powders or pellets, for 5 minutes to ensure the complete melting of the materials. Subsequently, the molding pressure was ramped up to $27 \mathrm{MPa}$ and was held at the set pressure for 5 minutes to fabricate the disc samples. Similar procedures were used to fabricate the solid TPU triboelectric positive layers, which were $0.5 \mathrm{~mm}$ thick.

Porous PVDF triboelectric negative layers were fabricated by compression molding and particulate leaching process using the procedures indicated below:

STEP 1. $\mathrm{NaCl}$ powders were sieved into different sizes (i.e., $<53$ $\mu \mathrm{m}$ and $53-250 \mu \mathrm{m}$,).

STEP 2. PVDF powders were dry-blended with $80 \mathrm{wt} \% \%$ sieved $\mathrm{NaCl}$ powders.

STEP 3. PVDF-NaCl mixtures were molded by a compression molding machine at $185^{\circ} \mathrm{C}$ and $27 \mathrm{MPa}$ into disc samples of desired thicknesses and areas. All porous samples are $0.50 \mathrm{~mm}$ in their thicknesses and $3.14 \mathrm{~cm}^{2}$ in their areas.

STEP 4. Molded samples were submerged under water at room temperature (i.e., $23^{\circ} \mathrm{C}$ ) for 72 hours to leach out the $\mathrm{NaCl}$ crystals. Water was changed regularly to avoid the saturation of $\mathrm{NaCl}$.

STEP 5. The open-cell PVDF samples were then dried in an oven at $50^{\circ} \mathrm{C}$ for 24 hours.

Table II summarizes the key parameters being studied in this work to elucidate the effects of material type, material geometry, and open-cell morphology of the negative triboelectric layer on the triboelectrification of the TENG.

TABLE II. PhYSICAL PROPERTIES OF PVDF, HDPE, AND TPU

\begin{tabular}{|l|c|c|}
\hline \multicolumn{1}{|c|}{ Parameter } & Value & Unit \\
\hline Material Type & PVDF and HDPE & - \\
\hline Thickness & $0.15,0.22$, and 0.50 & $\mathrm{~mm}$ \\
\hline Area & $2.0,4.0,6.0$, and 8.0 & $\mathrm{~cm}^{2}$ \\
\hline Pore Size & $<53$ and $53-250$ & $\mu \mathrm{m}$ \\
\hline
\end{tabular}

\section{Sample Characterization}

The morphology and structure of the samples were characterized by a scanning electron microscope (SEM) (FEI Company Quanta 3D FEG. The cross-sections of porous PVDF samples were exposed by cryo-fracturing the samples under liquid nitrogen. The fractured surfaces were then sputter-coated with gold (Denton Vacuum, Desk V Sputter Coater).

Open-cell contents of porous PVDF samples were measured by a gas pycnometer (Quantachrome, Ultrapyc 1200e). Nitrogen was used to determine to total volume of the solid part and the closed-cell $\left(V_{t}\right)$ by employing Archimedes' principle of fluid displacement, and Boyle's Law of gas expansion. Then, the open-cell content $\left(O_{c}\right)$ can be determined by Equation (1).

$$
O_{c}=\frac{V_{g}-V_{t}}{V_{g}} \times 100 \%
$$

where $V_{g}$ is the geometric volume of the open-cell foam sample.

A simple contact-mode TENG, as illustrated in Figure 1, was constructed by using two pieces of cast acrylic plates as the substrates. Copper electrodes with conductive adhesive were adhered to the two plates. The positive and negative triboelectric layers were adhered to the two electrodes. Conducting wires were used to connect the two electrodes to an oscilloscope (Tektronix, TBS1052B-EDU) to measure the output voltage of the TENG during its operation. The measurement was obtained by averaging the output voltages generated by the mechanical vibration as a result of dropping the TENG's top plate, which was attached with a $195 \mathrm{~g}$ mass, onto its bottom plate for at least 25 times from a height of $3 \mathrm{~cm}$. All data points were obtained from the characterization of three samples and the error bars present the standard deviations of the data values. 


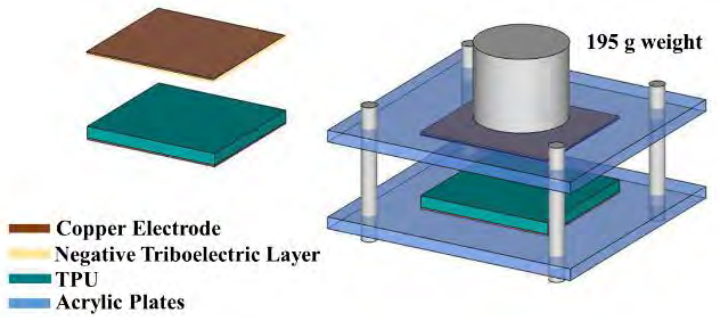

Figure 1. Schematic of Triboelectric Nanogenerator Setup

\section{RESULTS AND DISCUSSION}

The performance of TENG was predominantly depending on the degrees of triboelectrification in both the positive and the negative triboelectric layer. In this context, parametric studies were conducted to study the effects of polymer type, triboelectric layer's geometry, as well as foam morphology on the degrees of triboelectrification of these friction layers.

\section{A. Effect of Polymer Type on Triboelectricification}

The tendencies of materials to gain, lose, or hold electrons when contacting other materials depend on their chemical compositions. Literatures have reported different triboelectric series to organize materials according their ability to gain electrons. These data tables indicated that both PVDF and HDPE have higher tendencies than TPU to gain electrons. Therefore, when they are pairing up with TPU in a TENG, PVDF and HDPE will serve as the negative triboelectric layers while TPU will serve as the positive triboelectric layer. To compare their abilities to draw electrons away from TPU upon periodic contact and separation, solid PVDF and HDPE samples with the same contact area (i.e., $4 \mathrm{~cm}^{2}$ ) and varying thicknesses were used as the negative triboelectric layers of the TENG setup. Figure 2 illustrate the average peak voltages generated by TENG consisted of PVDF and HDPE as the negative triboelectric layers. It can be observed that, regardless of the layer's thickness, the peak voltages generated in the TENGs with PVDF negative triboelectric layers were $\sim 2$ times of those generated in the TENGs with HDPE negative triboelectric layers.

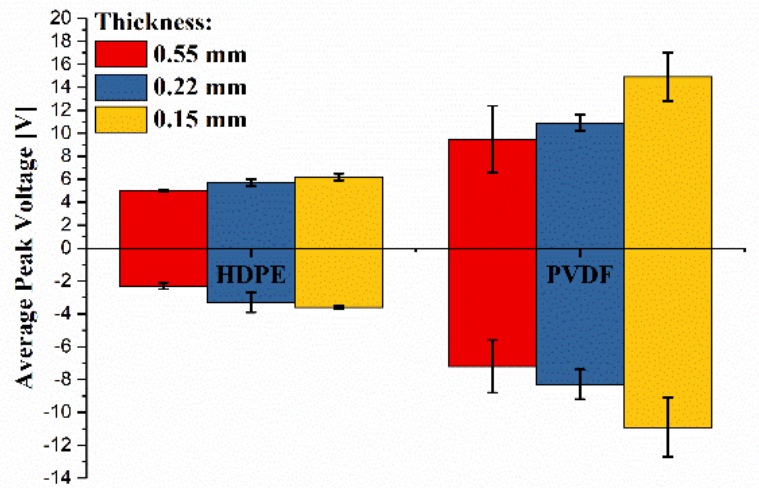

Film Type

Figure 2. Comparison of PVDF and HDPE's Performances on Triboelectrification for Contact Area of $4 \mathrm{~cm}^{2}$

\section{B. Effects of Sample Geometry on Triboelectricification}

The effects of triboelectric layer's thicknesses and contact area on the degree of triboelectrification were investigated by measuring the changes in vibration-induced voltage generated as these two geometric factors of the negative triboelectric layer (i.e., PVDF and HDPE) were varied. Figures 3 and 4 illustrate the effects of the thickness and contact area of the PVDF layer and the HDPE layer, respectively, on the peak voltages generated during periodic contact and separation of the TENG. Likewise, regardless of the thickness and the contact area, the uses of PVDF as the negative triboelectric layer consistently resulted in higher degrees of triboelectrification than the uses of HDPE.

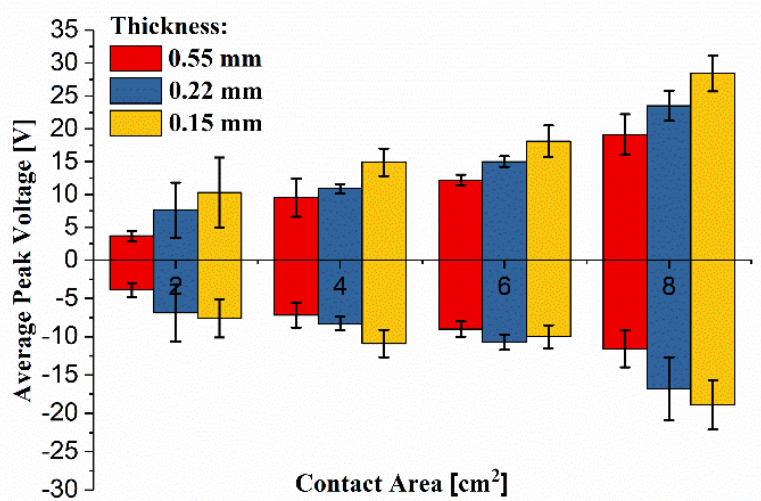

Figure 3. Effects of PVDF Layer's Geometry (i.e., Thickness and Contact Area) on Triboelectrification

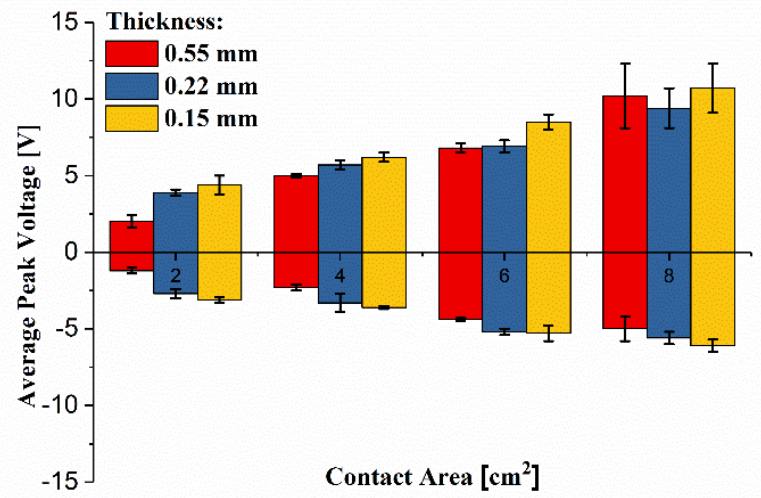

Figure 4. Effects of HDPE Layer's Geometry (i.e., Thickness and Contact Area) on Triboelectrification

For both PVDF and HDPE, it can also be observed that the peak output voltages of the TENG increased by increasing the triboelectric layer's contact area or decreasing the triboelectric layer's thickness. The effect of contact area on the degree of triboelectrification was intuitive. Increasing the contact area would result in the accumulation of more charges between the 
opposite triboelectric layers, leading to a higher degree of electrification during the periodic contact and seperation between the two layers. In constrast, decreasing the thickness of the PVDF or HDPE layer increased the peak output voltage. This could be attribute to the higher degree of electrostatic induction by the charged triboelectric layers and the electrodes. Experimental results confirmed the analytical model of the induced charge density (i.e., Equation (2)) derived by Wang et al. [7].

$$
\sigma^{\prime}=\frac{\sigma d_{3} \varepsilon_{r 1} \varepsilon_{r 2}}{d_{1} \varepsilon_{r 1}+d_{3} \varepsilon_{r 1} \varepsilon_{r 2}+d_{2} \varepsilon_{r 2}} \times 100 \%
$$

where $\sigma^{\prime}$ is the induced charge density; $\sigma$ is the triboelectric charge density $\varepsilon_{r 1}$ and $\varepsilon_{r 2}$ are the relative permittivity of the positive triboelectric layer and negative triboelectric layer, respectively; $d_{1}, d_{2}$, and $d_{3}$ are the thicknesses of the positive triboelectric layer, negative triboelectric layer, and the air gap, respectively.

\section{Effects of Open-Cell Morphology on Triboelectricification}

Figure 5 illustrates an SEM micrograph of a representative open-cell PVDF sample prepared by using $80 \mathrm{wt} \%$ of $\mathrm{NaCl}$ with particle size of $53 \mu \mathrm{m}-250 \mu \mathrm{m}$. The individual cells resembled the cubic shape of $\mathrm{NaCl}$ crystals. It can be observed that the cubic pores were uniformly distributed and interconnected throughout the PVDF matrices. Figure 6 revealed the effect of $\mathrm{NaCl}$ particle size (i.e., pore size after the particulate leaching process) on the open-cell contents of porous PVDF foams fabricated by using $80 \mathrm{wt} \% \mathrm{NaCl}$.

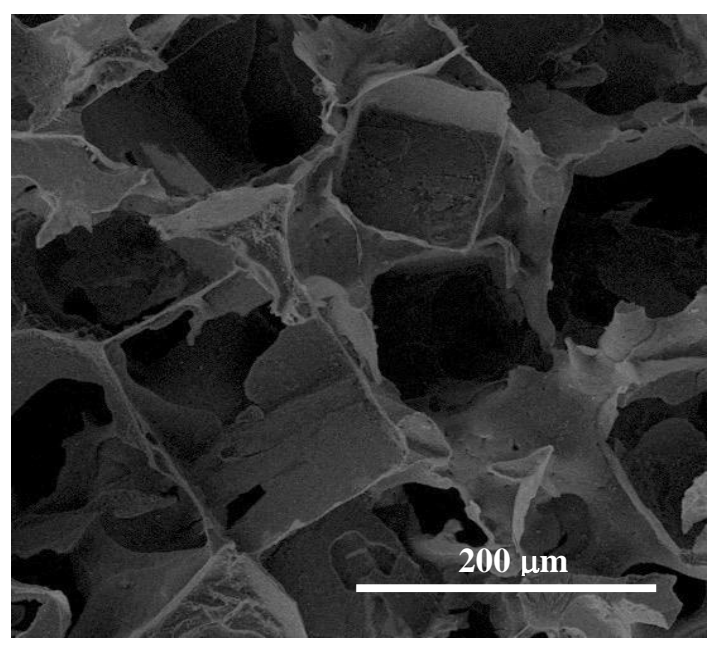

Figure 5. SEM Micrograph of Porous PVDF Negative Triboelectric Layer

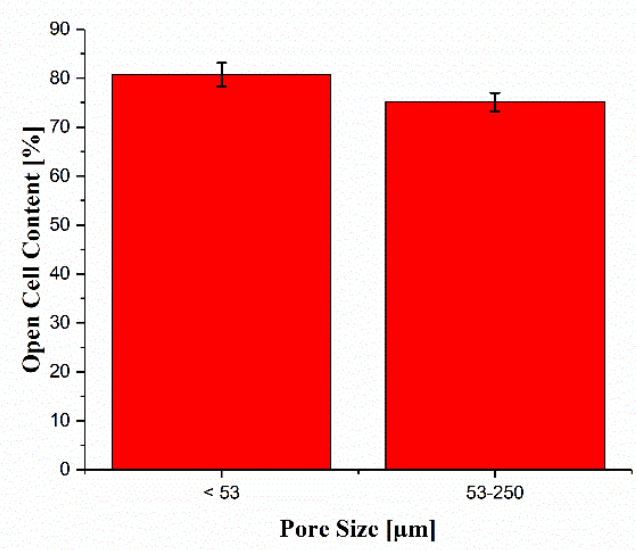

Figure 6. Effect of $\mathrm{NaCl}$ Particle Size on the Open-Cell Content of the Porous PVDF Layer

It can be observed that smaller $\mathrm{NaCl}$ particle sizes would yielded porous PVDF foams with higher open-cell contents. The negative correlation between these two parameters could be attributed to the higher packing efficiency of smaller particles. Figure 6 show that smaller $\mathrm{NaCl}$ particle size would increase the open-cell content of the fabricated PVDF foams after the complete leaching of $\mathrm{NaCl}$. The higher packing efficiency of smaller $\mathrm{NaCl}$ particles would promote the interconnectivity among the particles, increasing the opening among adjacent pores after the leaching process.

Figure 7 revealed that open-cell PVDF layer led to a higher degree of triboelectrification than solid PVDF layer. Furthermore, the promoted performance became more significant as the pore size decreased. It is believed that the presence of pores in the PVDF layer would enhance the degree of triboelectrification in three-fold. On the one hand, the ominpresence of pores throughout the PVDF layer significantly increased the surface area of the triboelectric layer. This would increase the accumulation of charges during the periodic contact and seperation of the opposite layers. On the other hand, this also reduced the local thickness of the triboelectric layer. It is speculated that this would enhance the electrostatic induction and thereby increased the peak voltage generated. Finally, the promoted conformabiity of the contact surfaces introduced by the PVDF foam structures would enhance adhension energy, which would then promote the triboelectric output [5]. 


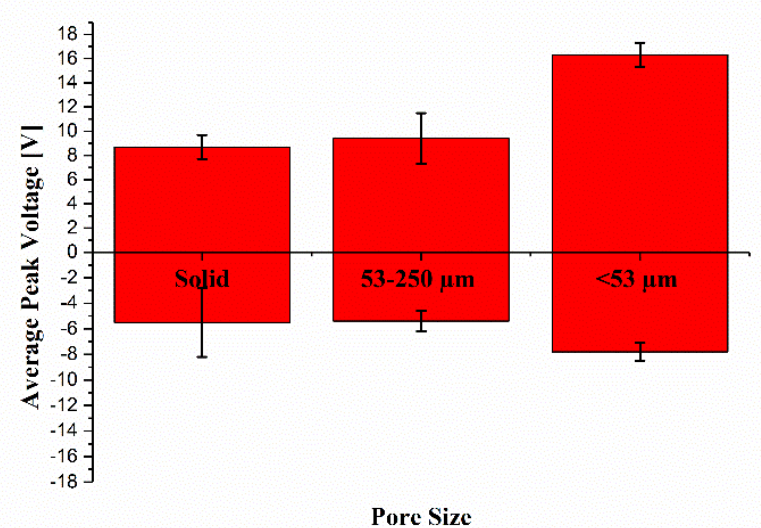

Figure 7. Effects of PVDF Layer's Morohology (Porosity) on Triboelectrification

\section{CONCLUSION}

The study has investigated the importance of polymer type, contact area, triboelectric layer thickness, and porosity on the triboelectric output voltage of TENG. Experimental results revealed that PVDF was a more efficient negative triboelectric material than HDPE, which increased the peak voltage by $\sim 2$ times. Furthermore, increasing the contact area and decreasing the triboelectric layer thickness positively influenced the degree of triboelectrification and/or electrostatic induction. Finally, particulate leaching, using $\mathrm{NaCl}$ as the leaching agent, could introduce open-cell structures within the PVDF layer. The presence of pores and the reduction in pore size were found to be effective strategies to promote the triboelectric output of TENG.

\section{ACKNOWLEDGMENT}

The authors are grateful about the financial support by the Natural Sciences and Engineering Research Council of Canada.

\section{REFERENCES}

[1] H. Peng, X. Sun, and X. Fang, "5 - Energy Harvesting Based on Polymer," in Polymer Materials for Energy and Electronic Applications. London, UK Elsevier, 2017, pp. 151-196. doi: 10.1016/B978-0-12-811091-1.00005-7.

[2] K. Y. Lee et al., "Hydrophobic sponge structure-based triboelectric nanogenerator," Adv. Mater., vol. 26, pp. 5037-5042, May 2014. doi: 10.1002/adma.201401184.

[3] Z. L. Wang et al. Triboelectric Nanogenerators, 1st ed. Switzerland: Springer, 2016, pp. 1-46. doi: 10.1007/978-3-319-40039-6.

[4] Z. L. Wang, "Triboelectric nanogenerators as new energy technology for self-powered systems and as active mechanical and chemical sensors," ACS Nano., vol. 7, pp. 9533-9557, Nov. 2013. doi: 10.1021/nn404614z.

[5] J. H. Lee et al., "Remarkable increase in triboelectrification by enhancing the conformable contact and adhesion energy with a film-covered pillar structure," Nano Energy, vol. 34, pp. 233-241, Jan. 2017. doi: 10.1016/j.nanoen.2017.02.032.

[6] J. Chen et al., "Enhancing Performance of Triboelectric Nanogenerator by Filling High Dielectric Nanoparticles into Sponge PDMS Film," ACS Appl. Mater. Interfaces, vol. 8, pp. 736-744, 2016. doi: 10.1021/acsami.5b09907.

[7] J. A. Cross, Electrostatics: Principles, Problems and Applications. Bristol, UK: CRC Press, 1987, pp. 91-144. 


\section{Effects of Steam Injection on the Performance of Natural Gas Combined Cycle Power Generation System}

\author{
Nitin Varia \\ Department of Automotive, Mechanical and \\ Manufacturing Engineering \\ University of Ontario Institute of Technology \\ Oshawa, L1H 7 K4, Canada \\ Bale V. Reddy \\ Department of Automotive, Mechanical and \\ Manufacturing Engineering \\ University of Ontario Institute of Technology \\ Oshawa, L1H 7 K4, Canada \\ bale.reddy@uoit.ca
}

\author{
Dipal Patel \\ Department of Automotive, Mechanical and \\ Manufacturing Engineering \\ University of Ontario Institute of Technology \\ Oshawa, L1H 7 K4, Canada \\ dipal.patel@uoit.ca
}

T. Srinivas

School of Mechanical Sciences

Vellore Institute of Technology

Vellore, India

lsriivast@vit.ac.in

\begin{abstract}
In the present work, combined cycle power generation configuration studies with natural gas as a primary fuel. Steam is injected in main combustion chamber and reheat combustion chamber individually and simultaneously to understand the performance of combined cycle work output and greenhouse gas emissions. The effect of pressure ratio, gas turbine inlet temperature on combined cycle work output, thermal efficiency and exergy efficiency carried out with and without steam injection. It is observed that the steam injection increases gas cycle efficiency and decreases the steam cycle efficiency. Ideal pressure ratio found to be 25 in all different combined cycle power generation system configurations. Maximum $\mathrm{CO}_{2}$ emission reduction (7.2\%) occurs when steam injected in reheater combustion chamber.
\end{abstract}

Keywords - combined cycle; steam; turbines; energy efficiency; exergy efficiency

\section{INTRODUCTION}

This Research investigations are conducted to study the combined cycle power generation systems with various options to increase efficiency and to reduce carbon dioxide emissions. Srinivas et. al. [1] conducted parametric simulation of steam injected gas turbine combined cycle. Waldyr [2] compared the humid air turbine (HAT) cycle with simple gas turbine cycle, steam injected gas turbine and also with the combined cycle. Nishida et. al. [3] investigated the performance characteristics of the regenerative steam injected gas turbine system. Shukla and Singh [4] conducted thermodynamic analysis of steam injected gas turbine cycle power plant with inlet air cooling. Bahrami et. al., [5] conducted performance comparison between steam injected gas turbine and combined cycle during frequency drops. Korakianititis et. al. [6] conducted performance investigations for combine-cogeneration power plants with performance enhancements. In the present work the effect of steam injection in main and reheat gas turbine combustion chambers and the role of operating variables for different natural gas fired combined cycle power generation systems are investigated.

\section{Confriguration And Methodology}

\section{A. Natural gas combined cycle configuration}

Natural gas fired combined power generation systems are gaining popularity due to their higher combustion efficiency and reduced emission. Different combined cycle power generation configurations are considered in the present work and are listed in Table 1. Fig.ure 1 shows the schematic diagram of these configurations.

The topping cycle consists of air compressor $(C 1)$ followed by an intercooler $(I C)$. Air is further compressed in second air compressor (C2). Compressed air is burned with methane in combustion chamber (CC1). In case of configuration \#2 and \#3 from Table 1, a fraction of a steam $(\zeta)$ is injected in the first combustion chamber. Products of first combustion chamber enters main gas turbine (GT1) and produced work output. Exhaust gas from main gas turbine further burned with methane in second combustion chamber (CC2). In case of configuration $\# 3$, a fraction of steam $(\omega)$ is injected in reheater combustion chamber. Product of reheater combustion chamber enters the reheater gas turbine (GT2) to produce work output. Exhaust gas from gas turbine enters heat recovery steam generator (HRSG) which produces steam in the bottoming cycle. The Steam then enters the steam turbine $(S T)$ and produces work output. Fraction of steam $(\zeta$ and $\omega)$ is taken out at particular pressure and used in topping cycle for power augmentation. Water vapor from steam turbine is then condensed in condenser and recirculates in the 
bottoming cycle through pump. It is assumed that system is operating at steam state steady flow conditions.

TABLE I. CONFIGURATIONS

\begin{tabular}{|c|l|}
\hline Configuration & \multicolumn{1}{c|}{ Description of Study } \\
\hline 1 & $\begin{array}{l}\text { Combustion of air and methane without stream } \\
\text { injection }\end{array}$ \\
\hline 2 & $\begin{array}{l}\text { Combustion of air and methane with steam } \\
\text { injection in main combustion chamber }\end{array}$ \\
\hline 3 & $\begin{array}{l}\text { Combustion of air and methane with steam } \\
\text { injection in main combustion chamber and reheater } \\
\text { combustion chamber }\end{array}$ \\
\hline
\end{tabular}

Figure 1 shows the generalized schematic diagram for all configuration listed in Table 1. Air is compressed from ambient condition into air compressor $(C 1)$ and $(C 2)$. An intercooler $(I C)$ is used between $C 1$ and $C 2$ to bring the air temperature down and to reduce overall compressor work. Compressed air $\left[\phi \mathrm{O}_{2}+\right.$ $(\theta \times 3.76) N_{2}$ ] enters combustion chamber 1 (CC1) and burned with methane fuel $(\alpha)$ at constant pressure. Combusted gas has high thermal energy and expanded partially into Gas Turbine 1 (GT1) to obtain shaft work $\left(\dot{W}_{G T 1}\right)$. Partially expanded gas enters combustion chamber $2(C C 2)$ and burned with methane fuel $(\beta)$ to elevate the thermal energy of the gas. Gas coming out of $C C 2$ is expanded in Gas Turbine 2 (GT2) to obtain shaft work $\left(\dot{W}_{G T 2}\right)$. Gas coming out of GT2 is passed through Heat Recovery Steam Generator (HRSG) to generate more steam which passes through Steam Turbine $(S T)$ to obtain shaft work $\left(\dot{W}_{S T}\right)$. Saturated water flows through pump $(\mathrm{P})$ in order to increase pressure and then passed through HRSG, which then completes the Rankine cycle.

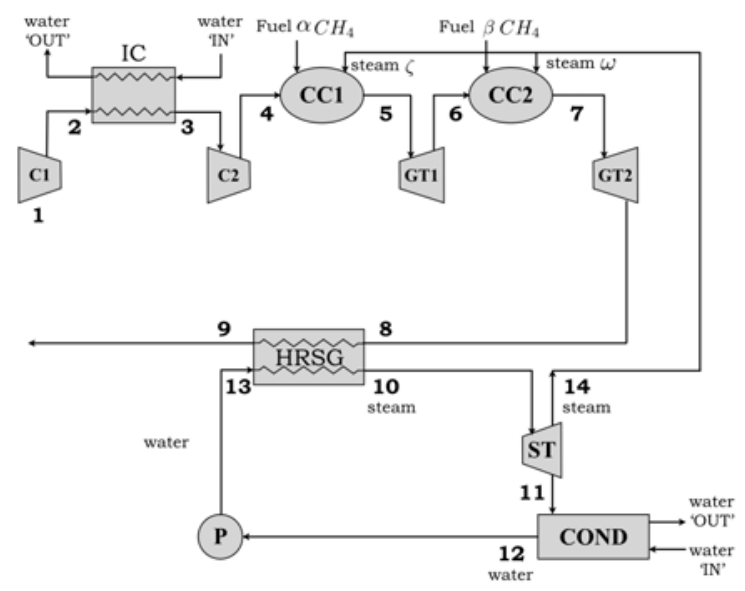

Figure 1. Schematic diagram of configuration 1,2 and 3 (Listen in Table-1)

The configuration \#2 has basic schematic same as configuration \#1, except a fraction of steam $(\zeta)$ is extracted from $S T$ and injected inside combustion chamber $C C 1$ with air and fuel. The fraction of steam $\zeta$ is percentage of air mass flow which enters combustion chamber $C C 1$ at state 4 . The steam $\zeta$ is then taken out from steam turbine at the stage where steam pressure is $5 \%$ higher than pressure of combustion chamber 1 (CC1). The configuration \#3 is an extension from configuration
$\# 2$, where fraction of steam $(\omega)$ is injected inside of reheater combustion chamber (CC2). Steam is taken out of steam turbine at $5 \%$ higher pressure present inside combustion chamber 2 (CC2).

TABLE II. FUEL BALANCE ON $C C 1$ AND $C C 2$ FOR CONFIGURATION \#1.

\begin{tabular}{|c|c|c|c|c|c|c|c|c|c|c|}
\hline 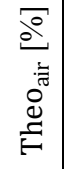 & $\frac{\sqrt{0}}{8}$ & $\frac{\sqrt{2}}{\sim}$ & $\begin{array}{l}\Xi \\
0 \\
\Xi \\
\Xi\end{array}$ & $\frac{\sqrt{2}}{a}$ & $\frac{2}{3}$ & 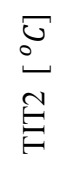 & $\frac{2}{d^{\circ}}$ & $\frac{\sum^{u}}{3^{u}}$ & $\frac{\sum_{\Delta}}{\Delta}$ & $\underset{3}{\stackrel{\vec{d}}{=}}$ \\
\hline 200 & 40 & 0 & 1000 & 60 & 0 & 1222 & 43.38 & 250363 & 146469 & 348051 \\
\hline 200 & 2.22 & 0 & 1074 & 57.78 & 0 & 1217 & 44.01 & 250363 & 145701 & 353104 \\
\hline 200 & 44.44 & 0 & 1095 & 55.56 & 0 & 1213 & 44.64 & 250363 & 144921 & 358139 \\
\hline 200 & 46.67 & 0 & 1116 & 53.33 & 0 & 1208 & 45.26 & 250363 & 144128 & 363155 \\
\hline 200 & 48.89 & 0 & 1137 & 51.11 & 0 & 1204 & 45.89 & 250363 & 143321 & 368153 \\
\hline 200 & 51.11 & 0 & 1157 & 48.89 & 0 & 1199 & 46.51 & 250363 & 142502 & 373130 \\
\hline 200 & 53.33 & 0 & 1178 & 46.67 & 0 & 1194 & 47.13 & 250363 & 141670 & 378088 \\
\hline 200 & 55.56 & 0 & 1198 & 44.44 & 0 & 1189 & 47.74 & 250363 & 140824 & 383024 \\
\hline 200 & 57.78 & 0 & 1218 & 42.22 & 0 & 1184 & 48.35 & 250363 & 139966 & 38793 \\
\hline 200 & 60 & 0 & 1238 & 40 & 0 & 1179 & 48.96 & 250363 & 139094 & 392832 \\
\hline
\end{tabular}

\section{B. Thermodynamic Analysis and Methodology}

For the considered configurations energy and exergy analyses are conducted with steam injection in gas turbine combustion chambers. Equations are developed for the configurations and the role of steam injection and effect of operating variables are simulated on performance and carbon dioxide emissions..

TABLE III. FUEL BALANCE ON CC1 AND CC2 FOR CONFIGURATION \#3.

\begin{tabular}{|c|c|c|c|c|c|c|c|c|c|}
\hline 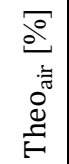 & $\frac{\sqrt{2}}{d^{\circ}}$ & $\begin{array}{l}\Xi \\
0 \\
E \\
E \\
E\end{array}$ & $\frac{\sqrt{0}}{3}$ & 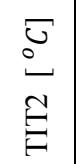 & $\frac{\sqrt{2}}{d^{2}}$ & $\begin{array}{l}\frac{\sum}{2} \\
3^{0}\end{array}$ & $\underset{3}{3}$ & 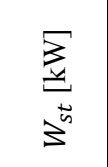 & 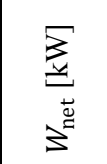 \\
\hline 200 & 1 & 173 & 1 & 169 & 46.93 & 250363 & 03 & 136722 & 376533 \\
\hline 200 & 2 & 1162 & 2 & 1146 & 46.52 & 250363 & 492837 & 132014 & 373246 \\
\hline 200 & 3 & 1152 & 3 & 1123 & 46.08 & 250363 & 493950 & 127296 & 369729 \\
\hline 200 & 4 & 1142 & 4 & 1102 & 45.62 & 250363 & 494858 & 122566 & 365996 \\
\hline 200 & 5 & 132 & 5 & 1081 & 45.13 & 250363 & 495575 & 117824 & 362060 \\
\hline 200 & 6 & 1122 & 6 & 1061 & 44.61 & 250363 & 496115 & 113068 & 357934 \\
\hline 200 & 7 & 1112 & 7 & 1042 & 44.08 & 250363 & 496490 & 108297 & 353627 \\
\hline 200 & 8 & 1103 & 8 & 1023 & 43.52 & 250363 & 496710 & 103510 & 349152 \\
\hline 200 & 9 & 1094 & 9 & 1005 & 42.94 & 250363 & 496787 & 98707 & 344516 \\
\hline 200 & 10 & 1085 & 10 & 987.5 & 42.34 & 250363 & 496729 & 93886 & 339730 \\
\hline
\end{tabular}

All simulations have been conducted using Engineering Equation Solver (EES). 


\section{RESULTS AND DisCUSSIONS}

\section{A. Effects of pressure ratio on combined performance with fraction of steam injection}

The effects of pressure ratio on combined cycle for both thermal and exergy efficiency is shown in Fig 2. It can be seen from Fig. 2 that there is a sharp increase in thermal efficiency for initial pressure ratio range from 5 to 25 . For configuration $\# 1$, there is a reduction in thermal efficiency corresponding to pressure ratio of 25 to 40 whereas for configurations \#2 and \#3, there is no such noticeable reduction in thermal efficiency after optimum pressure ratio has reached. Furthermore it can be seen from Fig. 2 that there is an increase of $1.57 \%$ exergy efficiency corresponding to $5 \%$ steam injection in $C C 1$ and $C C 2$.

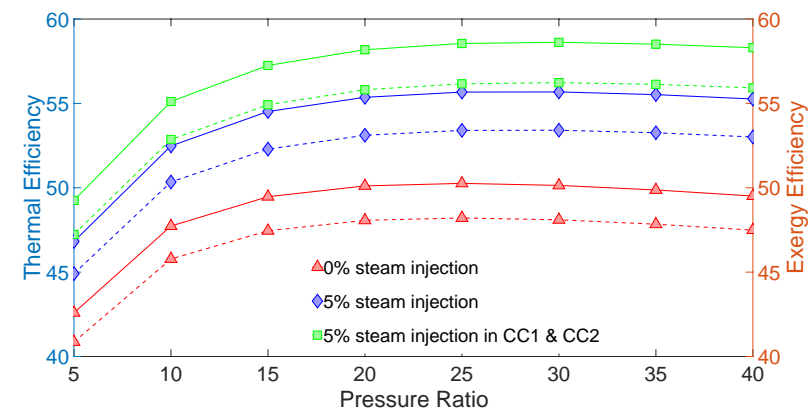

Figure 2. Effects of pressure ratio on combined cycle for both thermal (solid line) and exergy efficiency (broken line)

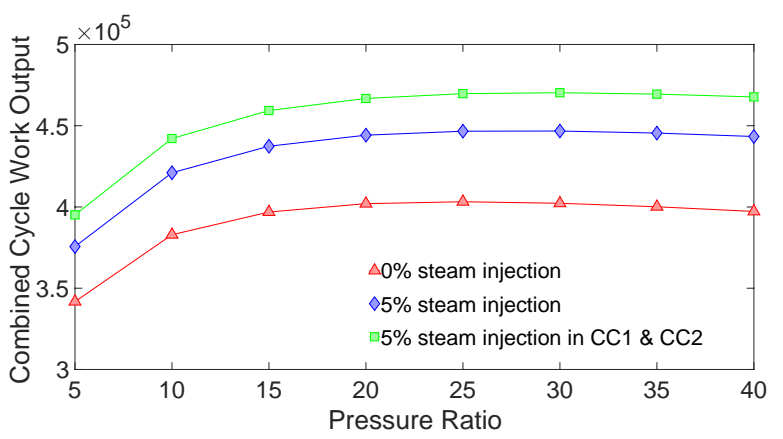

Figure 3. Effects of pressure ratio on the combined cycle work output

\section{B. Effects on $\mathrm{CO}_{2}$ emission with fraction of steam injection}

Amount of fuel reduction with steam injection has direct impact on $\mathrm{CO}_{2}$ emissions. The operating parameters of the combined system are set as pressure ratio $=25$, Ambient temperature $=25^{\circ} \mathrm{C}$, Ambient pressure $=1$ bar, Steam temperature $=500{ }^{\circ} \mathrm{C}$ and Steam pressure $=100$ bar. The effects of steam injection on $\mathrm{CO}_{2}$ emission is shown in Fig. 4. When steam $\zeta$ is injected to $C C 1$, a constant TIT1 is maintained through reduction on air mass flow, thus reduction in compressor work. At this flow rate, combined cycle net work output increase by $3.2 \%$, because of increase in mass flow of the steam from $0 \%$ to $10 \%$ in $G T 1$. In order to maintain constant combined cycle work output, fuel $(\alpha)$ consumption reduces. And when steam $\omega$ is injected in CC2 only, mass flow rate affects only GT2 and through reduction of fuel $\beta$ in $\mathrm{CC} 2,7.2 \%$ of $\mathrm{CO}_{2}$ emission reduction observed. However, when steam $\zeta$ and $\omega$ were injected in $C C 1$ and $C C 2$ together, only $0.9 \%$ of $C_{2}$ emission was observed. The results indicates that injecting steam in $C C 2$ alone has greater effects on reduction of $\mathrm{CO}_{2}$ emissions.

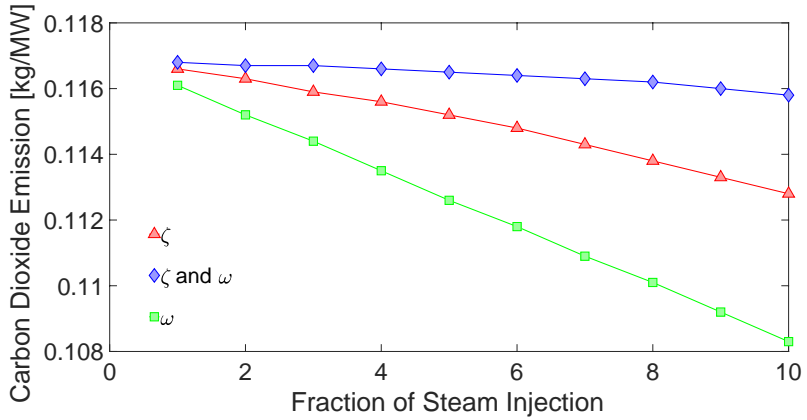

Figure 4. Effects of steam injection in $C C 1$ and $C C 2$ on Carbon Dioxide Emission.

\section{Performance analysis on fuel ratio for combined cycle with fraction of steam injection}

Table II shows fuel supply ratio between $C C 1$ and $C C 2$.At pressure ratio of 25 and Theo $_{\text {air }}$ of $200 \%$, ideal fuel supply is $\alpha=54 \%$ and $\beta=46 \%$. At this fuel supply TIT 1 and TIT 2 are very close to each other, which then becomes and important factor to gas turbine efficiency. Furthermore, Table III shows steam $\zeta$ and $\omega$ injected at $C C 1$ and $C C 2$ respectively. As steam $\zeta$ injected in $C C 1$, there is a reduction in TIT1 due to increased mass from the steam, which further resulting in reduction of flame temperature. There is a sharp reduction in TIT1 of $88^{\circ} \mathrm{C}$ with $10 \%$ steam injection in CC1. Further addition of steam $\omega$ in $C C 2$ resulting further reduction of TIT2. Work output of the gas turbines are increased about $5 \mathrm{MW}$ but sharp decline in the net work output (37MW) due to work output lost from the steam turbine. Furthermore Table III shows ideal theoretical air input at different steam injection to maintain the same net work output. Theoretical air is reduced by $32 \%$. It is assumed that complete combustion takes place in both $C C 1$ and $C C 2$. Steam injection decreases the amount of excess air in the combustion chamber, which also supports in controlling the temperature. The amount of steam injection has a limit depending on the air quality on the compressor. As a result of decreased fuel mass, the flue gas from the combustion chamber decreases resulting reduction in $\mathrm{CO}_{2}$ and $\mathrm{NO}_{2}$ emission (Fig. 4).

\section{Exergy destruction in combined cycle system}

Figure 6 shows exergy destruction in each individual component in combined cycle compared to overall exergy destruction in the cycle. These results corresponds to fixed turbine inlet temperature of $1200{ }^{\circ} \mathrm{C}$ and pressure ratio of 25 . The main source of exergy destruction in the combined cycle unit are the main combustion chamber $(C C 1)$, reheat combustor 
(CC2) and heat recovery steam generator (HRSG) which are responsible for $37 \%, 20 \%$ and $16 \%$ respectively of the total exergy destruction. It can be seen from the Fig. 6 that the combustors of topping cycle have the highest exergy destruction. Moreover, reducing the destruction in the combustors of topping cycle will lead to a significant improvement in the exergetic efficiency and also reduced destruction in the combined cycle.

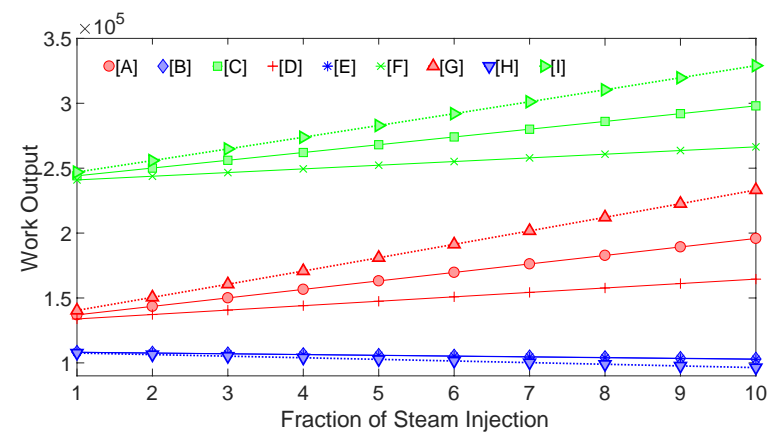

Figure 5. Effects o work output with steam injection for Configuration \#1, \#2 and $\# 3$.

TABLE IV. EFFECTS OF WORK OUTPUT WITH STEAM INJECTION FOR CONFIGURATION \#1, \#2 AND \#3 (FIG. 5).

\begin{tabular}{|c|l|c|}
\hline & \multicolumn{1}{|c|}{ Work Output } & Steam injection in: \\
\hline$[\mathrm{A}]$ & Gas Cycle Work Output & $C C 1$ \\
\hline$[\mathrm{B}]$ & Steam Cycle Work Output & $C C 1$ \\
\hline$[\mathrm{C}]$ & Combined Cycle Work Output & $C C 1$ \\
\hline$[\mathrm{D}]$ & Gas Cycle Work Output & $C C 2$ \\
\hline$[\mathrm{E}]$ & Steam Cycle Work Output & $C C 2$ \\
\hline$[\mathrm{F}]$ & Combined Cycle Work Output & $C C 2$ \\
\hline$[\mathrm{G}]$ & Gas Cycle Work Output & $C C 1$ and $C C 2$ \\
\hline$[\mathrm{H}]$ & Steam Cycle Work Output & $C C 1$ and $C C 2$ \\
\hline$[\mathrm{I}]$ & Combined Cycle Work Output & $C C 1$ and $C C 2$ \\
\hline
\end{tabular}

The exergy destruction in the combustion chamber is related to chemical reaction that occurs in combustion process. The exergy destruction ratios that associated with both turbines are less than $10 \%$ of total exergy destruction of the power plant. Although the rejected heat in the condenser is considered as tremendous amount from first law of thermodynamics perspective, the exergy destruction ratio associated with the condenser unit is low because the steam at condenser condition does not have potential power to produce useful work. As the fraction of steam (5\%) injected in $C C 1$ and $C C 2$, there is a drop of exergy destruction in $C C 1$ and $C C 2$ by $2.3 \%$ and $2 \%$ respectively. Steam injection adds more useful work and lowers the requirement of fuel amount in combustion chamber, that further reduces the destruction.

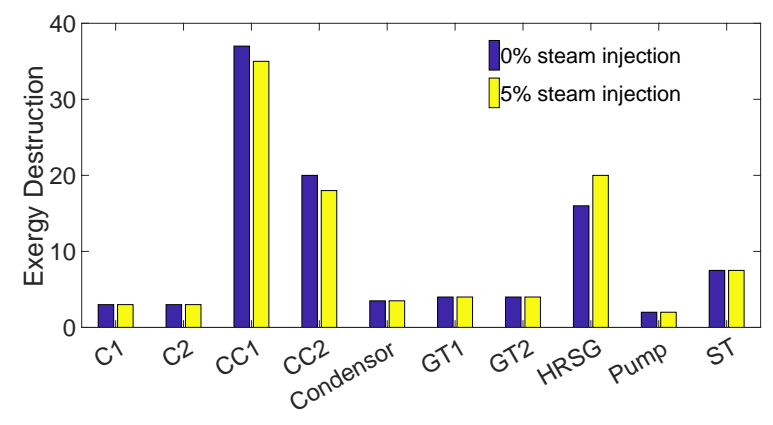

Figure 6. Percentage of exergy destruction in each component as compared to overall destruction on combined cycle with steam injection.

\section{CONCLUSIONS}

It is observed that the steam injection increases gas cycle efficiency and decreases the steam cycle efficiency. Ideal pressure ratio found to be 25 for considered combined cycle power generation configurations. Steam injection in main combustion chamber and reheat combustion chamber individually has more benefit than steam injection in both combustion chambers together. Maximum $\mathrm{CO}_{2}$ emission reduction $(7.2 \%)$ occurs when steam injected in reheat combustion chamber Thermal efficiency of combined cycle system increased by $8.2 \%$ when $10 \%$ steam injection in both combustion chambers. The present results and trends on steam injection effect on work output and efficiency are in agreement with results reported in the literature.

\section{REFERENCES}

[1] T. Srinivas, A.V.S.S.K.S. Gupta and B.V. Reddy, "Parametric simulation of steam injected gas turbine combined cycle," Proc. Inst. Mech. Eng., Part A, vol. 221, pp. 873-883, Jan. 2007. doi: 10.1243/09576509JPE418.

[2] L. R. G. Waldyr, "Comparison between the HAT cycle and other gas turbine based cycles, efficiency, specific power and water consumption," Energy Convers. Manage., vol. 38, nos. 15-17, pp. 1595-1604, Oct. - Nov. 1997. doi: 10.1016/S0196-8904(96)00220-8.

[3] K. Nishida, T. Takagi, and K. Shinichi, "Regenerative steam injection gas turbine systems," J. App. Energy, vol. 81, no. 3, pp. 231-246, Jul. 2005. doi: 10.1016/j.apenergy.2004.08.002.

[4] A. K. Shukla and O. Singh, "Thermodynamic analysis of steam injected gas turbine cycle power plant with inlet air cooling," Int. J. Ambient Energy, vol. 38, no. 6, pp. 556-566, Feb. 2017. doi: 10.1080/01430750.2016.1155495.

[5] S. Bahrami, A. Ghaffari, M. Genrup, and M. Thern, "Performance comparison between steam injected gas turbine and combined cycle during frequency drops," Energies, vol. 8, no. 8, pp. 7582-7592, Jul. 2015. doi: $10.3390 / \mathrm{en} 8087582$.

[6] T. Korakianitis, J. Grantstrom, P. Wassingbo, and A. F. Massardo, "Parametric performance of combined-cogeneration power plants with various power and efficiency enhancements," J. Eng. Gas Turbines and Power, vol. 127, no. 1, pp. 65-72, Feb. 2005. doi: 10.1115/1.1808427. 


\title{
Numerical Simulation of Gas-Liquid Phenomena in the Cathode of a Stirred Tank Reactor Proton Exchange Membrane Fuel Cell
}

\author{
Xichen Wang, Biao Zhou ${ }^{1}$, Mengcheng Jiang \\ Department of Mechanical, Automotive and Materials Engineering \\ University of Windsor \\ Windsor, ON, Canada, N9B 3P4
}

\begin{abstract}
Liquid water management is still a critical issue in the improvement of proton exchange membrane fuel cell (PEMFC) performance. In this work, for the first time, the liquid water behavior and transport inside the cathode of a PEMFC with a stirred tank reactor (STR) design, rather than the conventional PEMFC flow channel design, are numerically studied. The volume of fluid (VOF) method is employed in the simulation to track the gas-liquid interface. The capability of STR-PEMFC to reduce the liquid water flooding is also investigated and verified from the simulation, showing the potential of this channel-less type fuel cell in the further development.
\end{abstract}

Keywords-proton exchange membrane fuel cell; water management; volume of fluid; stirred tank reactor.

\section{INTRODUCTION}

Generally, fuel cells can be classified into five major types according to the electrolyte used: the phosphoric acid fuel cell (PAFC), the solid-oxide fuel cell (SOFC), the alkaline fuel cell (AFC), the molten carbonate fuel cell (MCFC), and the proton exchange membrane fuel cell (PEMFC) [1]. Among these types of fuel cells, PEMFC has been considered as one of the best potential candidates for vehicle and distributed power applications, mainly because of their low operating temperature, high power density, and good start-stop cycling durability. However, PEMFCs suffer from a water management problem that may cause a decrease in power output. Because the operating temperature of a PEMFC is under $100^{\circ} \mathrm{C}$ (usually $50-100^{\circ} \mathrm{C}$ ), water generated from the electrochemical reaction easily condenses into liquid water and accumulates in the cathode.

Channels in the bipolar plate of the PEMFC can distribute the gas flow and remove excess liquid water, which is an effective way to improve the water management [2]. Many different types of flow field channel designs have been developed so far and most of them can be classified into three categories: parallel, serpentine, and interdigitated. In 2004, an innovative PEMFC with a porous layer connected to a stirred tank reactor (STR) rather than conventional gas flow channels was developed by Benziger et al. [3]. This STR PEMFC provides ideally suitable approaches to examine the fuel cell dynamics in the reaction and transport processes. The further experiments and analysis of the operating STR fuel cell were also reported by Hogarth and Benziger [4]. The results showed that compared to the conventional PEMFC with serpentine channels under humidified feeds or dry feeds, the STR fuel cell with dry feeds can provide higher power density and voltage at high current density. The features of this channel-less PEMFC include "dry-feeding, auto-humidification and self-draining" [4]. These research works by Benziger et al. [3, 4] not only revealed a simplified method for the PEMFC design, operation and control, but also indicated the remarkable advantages of auto-humidification in the commercialization of PEMFCs. However, no research to date has investigated the liquid water behavior and transport inside the STR PEMFC.

Comparing with other numerical models applied in the simulation of water management in PEMFCs such as the twofluid model [5-7] and the mixture model [8-10], the volume of fluid (VOF) model [11] has the advantage of simulating the liquid water removal process by tracking the interface between the liquid and gaseous phases. In recent years, VOF method has been recognized as the most popular approach in the simulation of gas-liquid two-phase flow in PEMFCs [12] and a series of numerical studies have been reported in this area [1319].

In this study, a three-dimensional, two-phase flow model is developed to study the gas-liquid dynamics in the cathode of a PEMFC with STR flow field design. The corresponding simulations are conducted by using the VOF method. The liquid water general transport and emerging process are presented and discussed.

\section{NUMERICAL MODEL DESCRIPTION}

\section{A. Computational Domain and Boundary Conditions}

The numerical simulation domain (Fig. 1) is an STR PEMFC cathode, having similar dimensions to Hogarth and Benziger's model [4], consisting of a porous layer connected to a stirred tank reactor rather than conventional gas flow channels.

The porous layer is $0.019 \mathrm{~m} \times 0.019 \mathrm{~m} \times 0.0003 \mathrm{~m}$ (in the $\mathrm{X}, \mathrm{Y}$ and $\mathrm{Z}$ direction respectively). The attached STR is 0.015 $\mathrm{m} \times 0.015 \mathrm{~m} \times 0.0017 \mathrm{~m}$ and has an open rectangular plenum

${ }^{1}$ Corresponding Author and Principal Investigator: Dr. Biao Zhou, bzhou@uwindsor.ca, 1-519-253-3000 ext. 2630 
as the gas flow field, minus four distributed pillars at intervals of $0.003 \mathrm{~m}$ in the $\mathrm{X}-\mathrm{Y}$ plane. The dimension of these pillars is $0.003 \mathrm{~m} \times 0.003 \mathrm{~m} \times 0.0017 \mathrm{~m}$. A gas inlet channel and an outlet channel with the same dimensions as the pillars are connected to the STR and distributed on the diagonal corners. Gravity acts along the diagonal of the plenum connecting the gas inlet and outlet channels and is directed toward the outlet. The porosity of the porous layer is 0.3 .

The no-slip boundary condition is applied to the walls in STR model. The liquid mass flow rate is set as $1.331 \times 10^{-4} \mathrm{kgs}^{-1}$ and the gas mass flow rate is set as $6.615 \times 10^{-5} \mathrm{kgs}^{-1}$. The pressure outlet boundary condition is applied at the outlet. The contact angle for the side walls of plenum, upper walls of plenum and walls of porous layer are $53^{\circ}, 43^{\circ}$ and $130^{\circ}$ respectively.

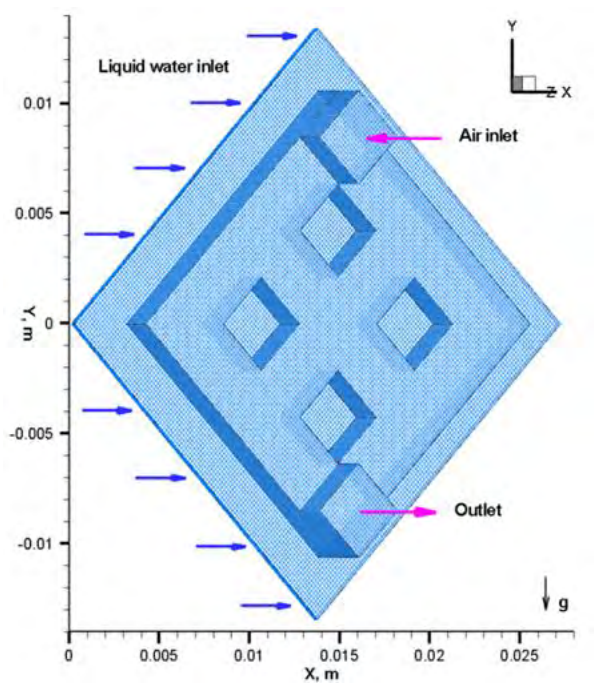

Figure 1. Computational domain of STR model.

\section{B. Computational Methodology}

The VOF method is employed in the present study to track the gas-liquid flow interface. Air is considered as the gaseous phase and liquid water is considered as the liquid phase. The fluid flow is laminar because of the low flow velocity and the small size of the channels. Heat transfer and phase changes are not considered in this study to reduce the computation time. The simulations are conducted using the commercial software package ANSYS Fluent. The governing equations for VOF method are the same as those employed in our previous work by Wang and Zhou [15].

\section{Grid Independency}

The computational domain of the STR model is meshed by 167,400 cells with a total volume of $4.602 \times 10^{-7} \mathrm{~m}^{3}$. The cell size is approximately $1.67 \times 10^{-4} \mathrm{~m}$ in the $\mathrm{X}$ and $\mathrm{Y}$ directions, whereas the dimensions in the Z-direction are $1.4 \times 10^{-4} \mathrm{~m}$ in the plenum domain and $5 \times 10^{-5} \mathrm{~m}$ in the porous layer domain. This grid size has been validated for PEMFC-related simulations by Le et al. [14], and the grid independency check is achieved by increasing and decreasing certain percentages of grid cells.

\section{RESULTS AND DISCUSSION}

A. General Process of Liquid Water Transport in STR Model

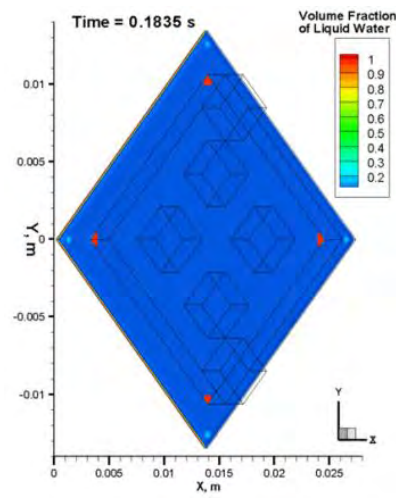

(a)

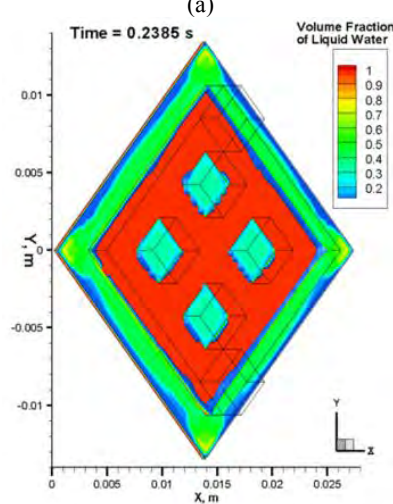

(c)

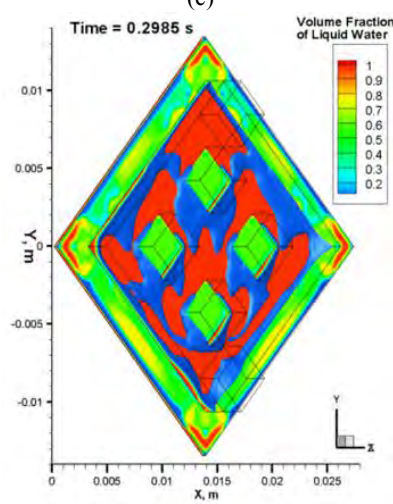

(e)

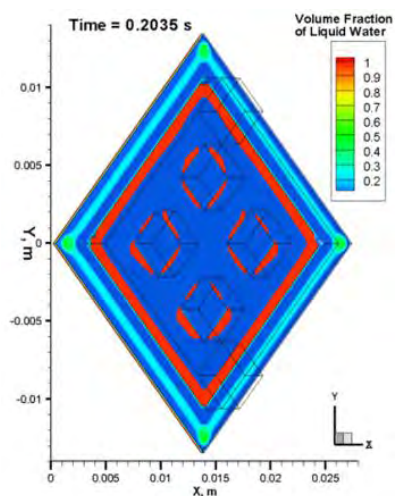

(b)

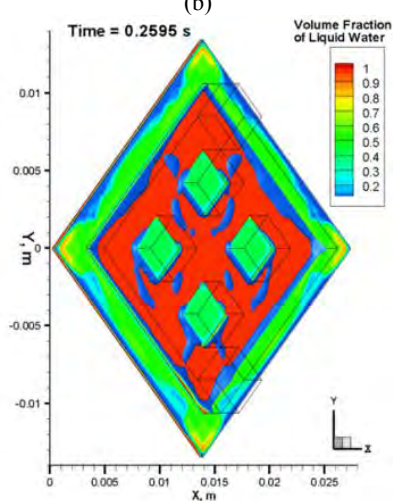

(d)

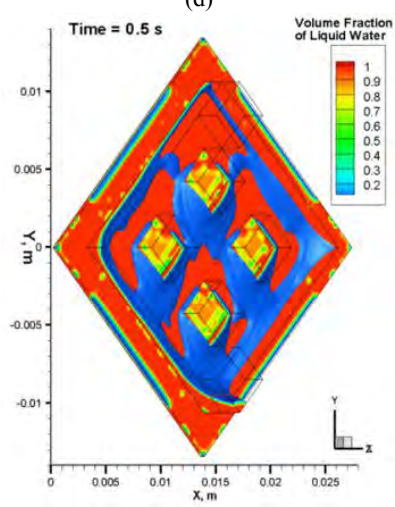

(f)
Figure 2. General liquid water transport in the STR model.

Fig. 2 shows the general process of liquid water transport over time at several selected time instants, which can be divided into the following sub-processes:

1) The liquid water generated from the electrochemical reaction is supplied continuously from the liquid inlet boundary, i.e., the back surface of the porous layer.

2) The liquid water migrating through the porous layer reaches the interface between the porous layer and the plenum, first at the four corners (Fig. 2(a)), then at the peripheral edges of the plenum domain and the edges of four pillars (Fig. 2(b)). 
3) Liquid water passes through the interface and emerges along the sidewalls of the plenum domain and also at the sidewalls of each pillar (Fig. 2(c)). Some liquid water emerges from the circumjacent areas near the pillars as well (Fig. 2(d)).

4) The liquid water emerging from the peripheral edges of the plenum domain and from the edges of the pillars merge together with the liquid water from the circumjacent areas near the pillars, forming a "tooth shape" (Fig. 2(e)).

5) As more water enters the plenum, the areas below the pillars become flooded. With the force of gravity and the gas flow, the liquid water drains out of the computational domain through the outlet channel (Fig. 2(f)).

\section{B. Liquid Water Distribution in Plenum}

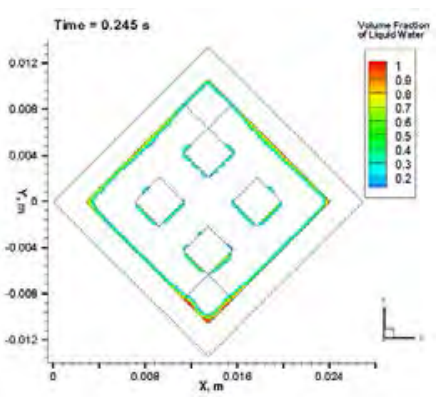

(a)

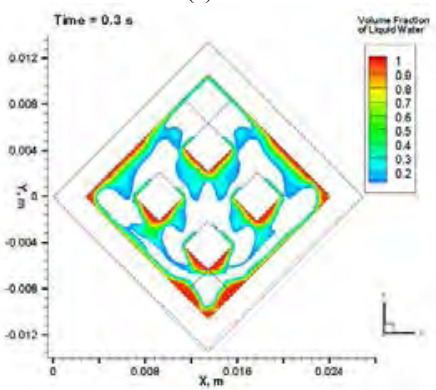

(c)

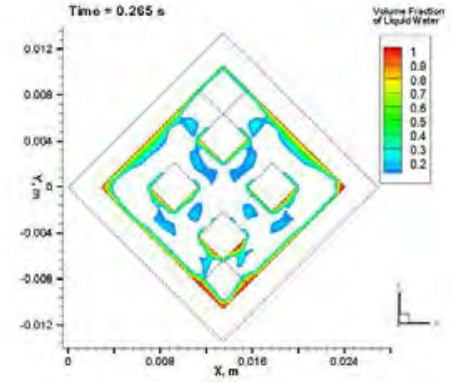

(b)

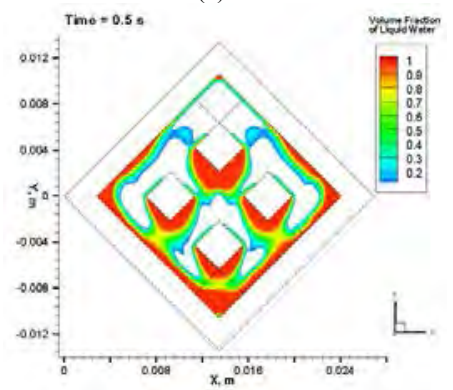

(d)
Figure 3. Liquid water distribution on plenum plane near interface $(Z=$ $0.00031 \mathrm{~m}$ ) in the STR model.

Fig. 3 shows the liquid water distribution on the $X-Y$ plane extracted from the plenum domain at $Z=0.00031 \mathrm{~m}$, which is very close to the interface of the porous layer. At first, liquid water emerges from the peripheral edges of the plenum domain and the edges of pillars (Fig. 3(a)), especially the lower edges. Subsequently, water emerges from the circumjacent areas near the lower sides of the four pillars and also the upper sides of the top pillar and gradually merges with the water from the edges of plenum (Fig. 3(b)), forming tooth-like shapes (Fig. 3(c)). Finally, the lower areas near the pillars become flooded, as well as the areas near the peripheral edges of the plenum (Fig. 4(d)). It can be observed that the liquid water appears mainly in the lower part of the plenum, which is caused by the direction of gravity and the locations of the inlet and outlet channels.

\section{CONCLUSIONS}

In this study, the gas-liquid behavior inside an STR PEMFC is numerically investigated through the VOF method. The liquid water general transport process and liquid water distribution in the Plenum are presented and discussed. It is found that a PEMFC cathode with the STR design has a promising capability for water drainage, so that the fluid flow field in the STR model rarely becomes blocked or fully flooded, which weakens the reduction of the power density of fuel cell. These numerical results verify that self-draining liquid water is one of the merits of the STR PEMFC, which has been noted by Hogarth and Benziger [4].

\section{ACKNOWLEDGMENT}

The authors are grateful for the support from the Natural Sciences and Engineering Research Council of Canada (NSERC) and the University of Windsor.

\section{REFERENCES}

[1] R. O'Hayre et al., Fuel cell fundamentals. Hoboken, N.J.: John Wiley \& Sons, 2016.

[2] X. Li and I. Sabir, "Review of bipolar plates in PEM fuel cells: Flow-field designs," Int. J. Hydrogen Energy, vol. 30, no. 4, pp. 359-371, Mar. 2005. doi: 10.1016/j.ijhydene.2004.09.019.

[3] J. Benziger et al., "The stirred tank reactor polymer electrolyte membrane fuel cell," AIChE J., vol. 50, no. 8, pp. 1889-1900, Aug. 2004. doi: 10.1002/aic.10158.

[4] W. H. J. Hogarth and J. B. Benziger, "Operation of polymer electrolyte membrane fuel cells with dry feeds: Design and operating strategies," $J$. Power Sources, vol. 159, no. 2, pp. 968-978, Sept. 2006. doi: 10.1016/j.jpowsour.2005.11.079.

[5] Q. Ye and T. V. Nguyen, "Three-dimensional simulation of liquid water distribution in a PEMFC with experimentally measured capillary functions," J. Electrochem. Soc., vol. 154, no. 12, pp. B1242-B1251, Dec. 2007. doi: $10.1149 / 1.2783775$.

[6] P. C. Sui, S. Kumar, and N. Djilali, "Advanced computational tools for PEM fuel cell design: Part 1. Development and base case simulations," $J$. Power Sources, vol. 180, no. 1, pp. 410-422, May 2008. doi: 10.1016/j.jpowsour.2008.02.007.

[7] H. Wu, P. Berg, and X. Li, "Steady and unsteady 3D non-isothermal modeling of PEM fuel cells with the effect of non-equilibrium phase transfer," Appl. Energy, vol. 87, no. 9, pp. 2778-2784, Sept. 2010. doi: 10.1016/j.apenergy.2009.06.024.

[8] H. Meng and C.-Y. Wang, "Model of two-phase flow and flooding dynamics in polymer electrolyte fuel cells," J. Electrochem. Soc., vol. 152, no. 9, pp. A1733-A1741, Sept. 2005. doi: 10.1149/1.1955007.

[9] S. Basu, J. Li, and C.-Y. Wang, "Two-phase flow and maldistribution in gas channels of a polymer electrolyte fuel cell," J. Power Sources, vol. 187, no. 2, pp. 431-443, Feb. 2009. doi: 10.1016/j.jpowsour.2008.11.039.

[10] P. P. Mukherjee, Q. Kang, and C.-Y. Wang, "Pore-scale modeling of two-phase transport in polymer electrolyte fuel cells - progress and perspective," Energy Environ. Sci., vol. 4, no. 2, pp. 346-369, Feb. 2011. doi: 10.1039/B926077C. 
[11] P. Quan et al., "Water behavior in serpentine micro-channel for proton exchange membrane fuel cell cathode," J. Power Sources, vol. 152, pp. 131-145, Dec. 2005. doi: 10.1016/j.jpowsour.2005.02.075.

[12] R. B. Ferreira et al., "Numerical simulations of two-phase flow in proton exchange membrane fuel cells using the volume of fluid method-A review," J. Power Sources, vol. 277, pp. 329-342, Mar. 2015.

doi: 10.1016/j.jpowsour.2014.11.124.

[13] K. Jiao and B. Zhou, "Accelerated numerical test of liquid behavior across gas diffusion layer in proton exchange membrane fuel cell cathode," J. Fuel Cell Sci. Technol., vol. 5, no. 4, p. 041011, Nov. 2008. doi: 10.1115/1.2971020.

[14] A. D. Le et al., "Numerical simulation and experimental validation of liquid water behaviors in a proton exchange membrane fuel cell cathode with serpentine channels," J. Power Sources, vol. 195, no. 21, pp. 7302-7315, Nov. 2010. doi: 10.1016/j.jpowsour.2010.05.045.

[15] X. Wang and B. Zhou, "Liquid water flooding process in proton exchange membrane fuel cell cathode with straight parallel channels and porous layer," J. Power Sources, vol. 196, no. 4, pp. 1776-1794, Feb. 2011. doi: 10.1016/j.jpowsour.2010.09.092.
[16] S. Kang and B. Zhou, "Numerical study of bubble generation and transport in a serpentine channel with a T-junction," Int. J. Hydrogen Energy, vol. 39, no. 5, pp. 2325-2333, Feb. 2014.

doi: 10.1016/j.ijhydene.2013.11.115.

[17] S. Kang, B. Zhou, and M. Jiang, "Bubble behaviors in direct methanol fuel cell anode with parallel design," Int. J. Hydrogen Energy, vol. 42, no. 31, pp. 20201-20215, Aug. 2017. doi: 10.1016/j.ijhydene.2017.06.149.

[18] R. B. Ferreira, D. S. Falcão, V. B. Oliveira, and A. M. F. R. Pinto, "Numerical simulations of two-phase flow in an anode gas channel of a proton exchange membrane fuel cell," Energy, vol. 82, pp. 619-628, Mar. 2015. doi:10.1016/j.energy.2015.01.071.

[19] R. B. Ferreira et al., "1D+3D two-phase flow numerical model of a proton exchange membrane fuel cell," Appl. Energy, vol. 203, pp. 474-495, Oct. 2017. doi: 10.1016/j.apenergy.2017.06.048. 


\title{
Gas-Liquid Phenomena with Dynamic Contact Angles in the Cathode of a Parallel Proton Exchange Membrane Fuel Cell
}

\author{
Xichen Wang, Biao Zhou ${ }^{1}$, Mengcheng Jiang \\ Department of Mechanical, Automotive and Materials Engineering \\ University of Windsor \\ Windsor, ON, Canada, N9B 3P4
}

\begin{abstract}
Among the numerical studies on liquid water transport in Proton Exchange Membrane Fuel Cells (PEMFCs), the static contact angle (SCA) model is generally used, while the dynamic contact angle (DCA) model is only applied on the simulation of droplet behaviors with simple geometries like a single microchannel. In this study, the DCA model is employed to study the gas-liquid phenomena inside the cathode of PEMFC with parallel flow field design, i.e., Parallel-DCA model. The water emerging and transport processes are simulated based on this Parallel-DCA model using the volume of fluid (VOF) method. The numerical results are also compared to those from the Parallel-SCA model with the same computational domain and operating conditions.
\end{abstract}

Keywords-proton exchange membrane fuel cell; volume of fluid; dynamic contact angle; parallel.

\section{INTRODUCTION}

As the main product in the cathode, water plays an important role in the performance of a proton exchange membrane fuel cell (PEMFC). Too much or too little liquid water will result in an increase of voltage loss, which is considered to be a key point affecting the performance of PEMFCs. Therefore, water management, particularly liquid water management, is a significant technical issue in the optimization of the PEMFC performance, which has received further attention in this research area.

Comparing with existing numerical models employed in the simulation of gas-liquid dynamics in PEMFCs, the volume of fluid (VOF) model, which was proposed by Quan et al. [1], has the advantage of tracking the interface between the liquid and the gas so that the liquid water distribution and transport can be described in detail. Recently, for the numerical studies on twophase flow in PEMFCs, the VOF method has been widely used and recognized as an effective tool in this area and a series of research works have been reported [2-9].

However, all the aforementioned numerical studies only considered static contact angle (SCA) as wall boundary condition. It is known that the geometric dimensions of PEMFCs are quite small, namely from a microscopic view, wall adhesion and surface tension significantly affect the

${ }^{1}$ Corresponding Author and Principal Investigator: Dr. Biao Zhou, bzhou@uwindsor.ca, 1-519-253-3000 ext. 2630 gas/liquid distribution and transport, thus the contact angle is unlikely to stay at equilibrium. Djilali et al. $[10,11]$ noted that liquid water behavior on the GDL surface is affected by the contact angle when water emerges from a pore. Consequently, in the numerical models of PEMFCs (unsteady, two-phase flow model), it is not reliable to use static contact angle (SCA) as the wall boundary condition. Sikalo et al. [12] conducted experimental and numerical studies for droplet impact on horizontal surface, and it is revealed that there are obvious limitations using SCA as one of the boundary conditions and the DCA should be considered. The Hoffman function [13], an empirical correlation for DCA (also known as Kistler's Law), was employed in [12] to predict the contact angle values:

$$
\begin{gathered}
\theta_{d}=f_{\text {Hoff }}\left[C a+f_{\text {Hoff }}^{-1}\left(\theta_{s}\right)\right] \\
f_{\text {Hoff }}(x)=\arccos \left\{1-2 \tanh \left[5.16\left(\frac{x}{1+1.31 x^{0.99}}\right)^{0.706}\right]\right\}
\end{gathered}
$$

In (1), $C a$ is the capillary number and $f_{\text {Hoff }}^{-1}\left(\theta_{s}\right)$ is the shift factor obtained from the inverse of the Hoffman function under SCA [14]. Hoffman function is considered as one of the promising formulae in DCA simulations [15]. However, so far, nearly all the numerical models with DCAs are for simple geometries and use the DCA only for a single wall boundary [16-18]. Recently, Jiang et al. [15] found that the AR-DCA model, which is implemented with Hoffman function and considers both advancing and receding dynamic contact angles, can well simulate droplet behaviors and dynamic wetting behaviors. This AR-DCA model is validated against a series of experiments for droplet impact on horizontal and inclined surfaces from Sikalo et al. [19, 20], showing its potential to be applied in the simulation of gas-liquid phenomena in PEMFCs.

In this study, we extend our previous work [4] by applying DCAs at multiple wall boundaries to investigate the gas-liquid phenomena inside the cathode of a parallel PEMFC, i.e., Parallel-DCA model. The general liquid water transport processes are presented and discussed, and also compared to those from Parallel-SCA model. 


\section{NUMERICAL MODEL DESCRIPTION}

\section{A. Numerical Simulation Domain}

The numerical simulation domain for the parallel model represents the geometry of a full-scale cathode side of a single PEMFC, as shown in Fig. 1. The detailed dimensions and descriptions of this domain can be found in [4].

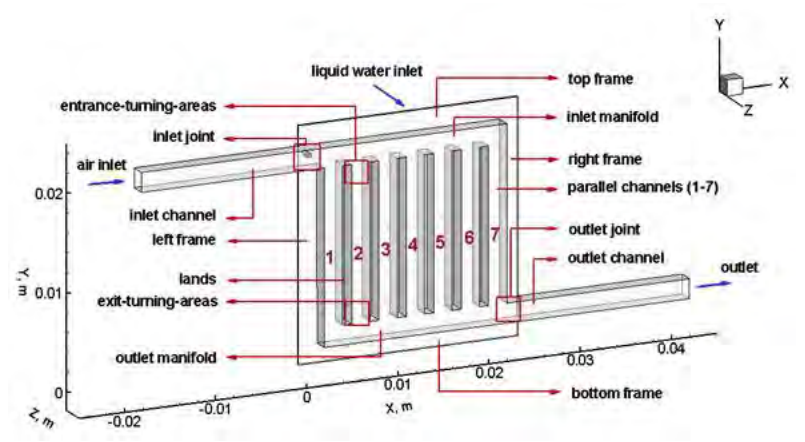

Figure 1. Computational domain of Parallel model.

\section{B. Computational Methodology}

A three-dimensional PEMFC cathode model is developed in this study to investigate gas-liquid phenomena and water transport processes. Air is modeled in the gaseous phase and liquid water is modeled in the liquid phase; these two-phases are assumed to be immiscible. The VOF method is applied to track the liquid-gas flow interface. The governing equations and corresponding computational methodology were reported in our previous work by Wang and Zhou [4]. The method to implement DCA model was reported by Jiang and Zhou [15]. More details and descriptions can be found in [4] and [15].

\section{Boundary Conditions and Grid Independency}

The accelerated model [3] is employed in this study by adjusting the mass flow rate of air and liquid water, which can significantly save the computation time. The mass flow rate for the liquid water inlet and gas inlet is set as $1.7 \times 10^{-4} \mathrm{kgs}^{-1}$ and $2.0 \times 10^{-5} \mathrm{kgs}^{-1}$ respectively. The initial contact angle for the side walls of channels, upper walls of channels and walls of porous layer are $40^{\circ}, 43^{\circ}$ and $140^{\circ}$ respectively. In ParallelSCA model, the contact angles are constant for the wall boundaries, whereas in the Parallel-DCA model, the contact angles vary with the change of the flow field and can be expressed as parameters associated with time and location.

The Parallel-DCA model and Parallel-SCA model [4] share the same computational domain and the mesh set up and independency has also been reported in [4].

\section{RESULTS AND DISCUSSION}

\section{A. General Process of Liquid Water Transport in Parallel- DCA Model}

The general process of liquid water transport in the ParallelDCA model, as shown in Fig. 2, can be summarized into several sub-processes:

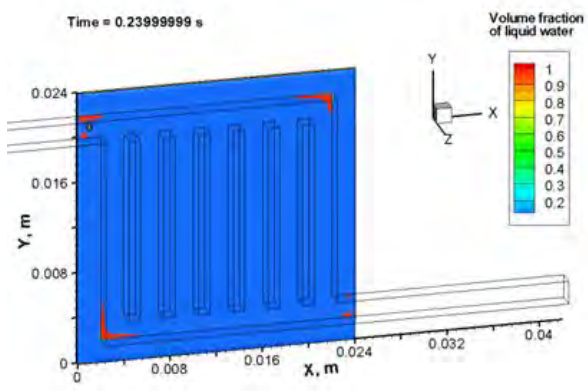

(a)

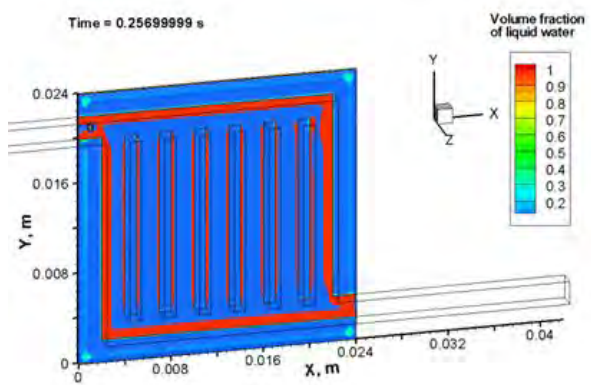

(b)

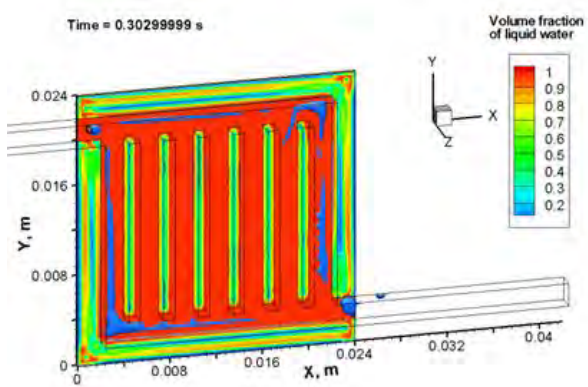

(c)

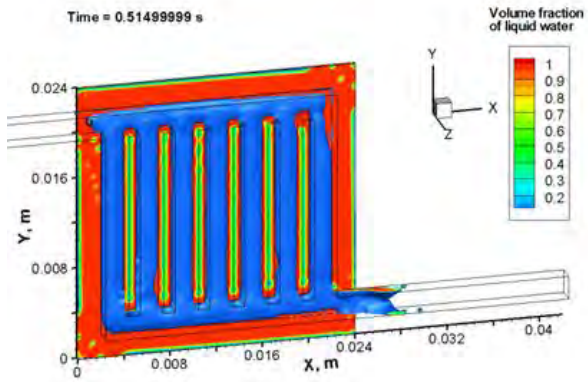

(d)

Figure 2. General liquid water transport in the Parallel-DCA model.

1) Liquid water is supplied from the back surface of the porous layer with a constant mass flow rate to simulate the liquid water generation through the electrochemical reaction.

2) The liquid water reaches the top surface of the porous layer, first accumulates at the four corners of the interface under the channels domain (Fig. 2(a)), and then the frame and the edges of each parallel channel (Fig. 2(b)).

3) Liquid water from the porous layer emerges into the gas flow channels first from the interface under the frame of the channels domain, in the middle area of the channels (Fig. 3(c)). 
4) More liquid water emerges into the channels. With time, channels become flooded and liquid water starts to drain out through the outlet channel, as shown in Fig. 2(d).

In summary, the general process of water transport and removal in the Parallel-DCA model are similar to those of the Parallel-SCA model [4] in the early stage (Fig. 2(a) and (b)). However, some different phenomena can be observed: for the Parallel-DCA model, the liquid water emerges into the gas channels first from the middle area of the interface under the channels, whereas for the Parallel-SCA model, the liquid water emerges into the gas channels from the peripheral edges of the lands and the peripheral edges of the channels domain. The detailed comparisons are provided and illustrated in the following sections.

\section{B. Comparison of Emerging Process}

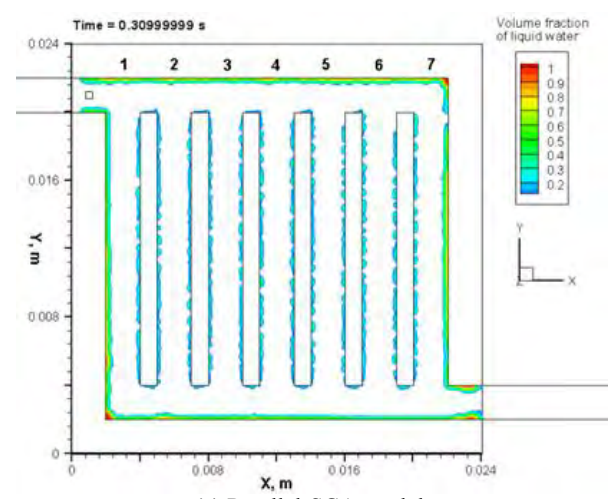

(a) Parallel-SCA model.

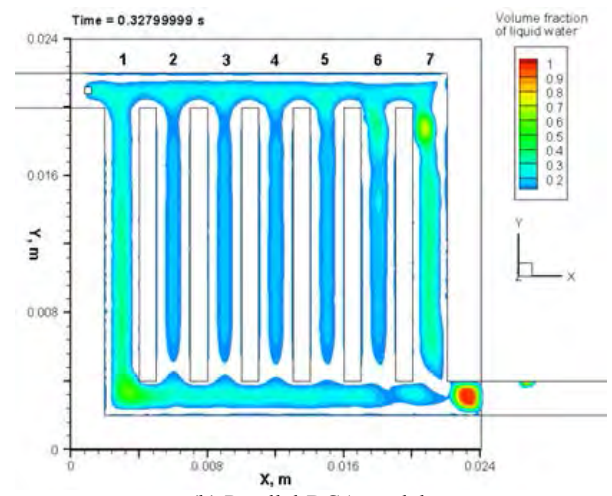

(b) Parallel-DCA model.

Figure 3. Comparison of the liquid water distribution near interface at $\mathrm{Z}=$ $0.00031 \mathrm{~m}$.

Fig. 3 (a) and (b) show the liquid water emerging pattern in the gas flow channels from Parallel-SCA and Parallel-DCA models respectively, located at the plane extracted near the interface $(Z=0.00031 \mathrm{~m})$ from the channel domain. It can be further observed that for Parallel-SCA model, the liquid water enters into the gas flow channels from the peripheral edges of the channel domain and emerges from the land edges (Fig. 3(a)); whereas for Parallel-DCA model, the liquid water mainly passes through the interface under the middle area, with only a small amount of water emerging from the edges of the lands and moving along the sidewalls (Fig. 3(b)).
The detailed emergence and growth of liquid water from the porous layer into the channels is presented by Fig. 4, which shows the liquid water volume fraction with different reference velocity vectors located at the No. 7 parallel channel on the plane (extracted from the computational domain at $\mathrm{Y}=0.012$ $\mathrm{m}$ as illustrated in Fig. 4(a)). For Parallel-SCA model, due to the uniform water generation rate on the back surface of the porous layer, the liquid water from the porous layer enters into the channels from the land edges; it can also be observed that there is a pair of vortices - an upper vortex and a lower one in the cross-section of the parallel channels, referred to as Dean vortices, which normally occur in curved channels or pipes [21]. For Parallel-DCA model, the liquid water emerges from the porous layer enters into the channel at the middle area, and the pair of vortices has a left-right distribution.

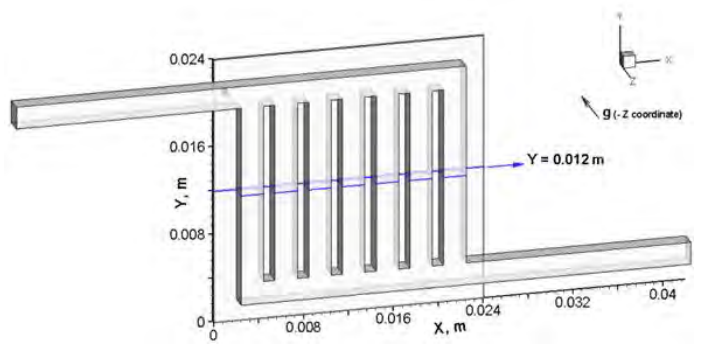

(a) Schematic of extracted plane.

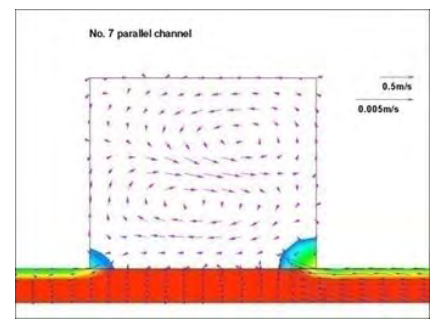

(b) Parallel-SCA model.

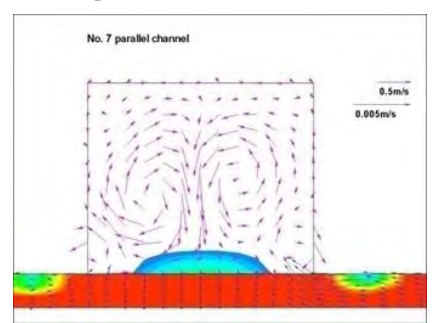

(c) Parallel-DCA model.
Figure 4. Comparison of liquid water emerging on plane at $\mathrm{Y}=0.012 \mathrm{~m}$ (enlarged plane with velocity vectors).

\section{Comparison of Flow Pattern in the Draining Process}

The draining process in the Parallel-SCA and ParallelDCA models can be explained by Fig. 5, which shows the deformation of the draining liquid water in the outlet channel. For the Parallel-SCA model, valley-shaped water near the outlet joint will deform into a top-bottom stratified flow pattern while moving along the outlet channel to drain out, as shown in Fig. 5(a); whereas for the Parallel-DCA model, the deformation of the liquid water in the outlet channel is in a stratified-slug pattern, as shown in Fig. 5(b). Also, it can be observed that there are several splashed, small droplets in the outlet channel adhered to the sidewalls of the outlet channel, because their inertial force is not strong enough to overcome the friction or wall adhesion. 


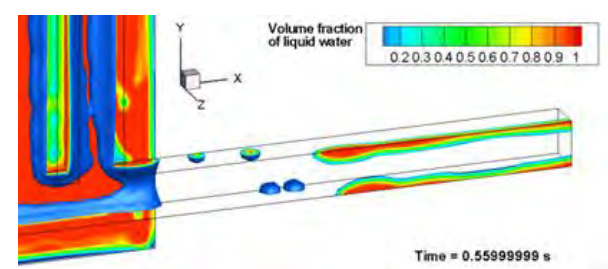

(a) Parallel-SCA model

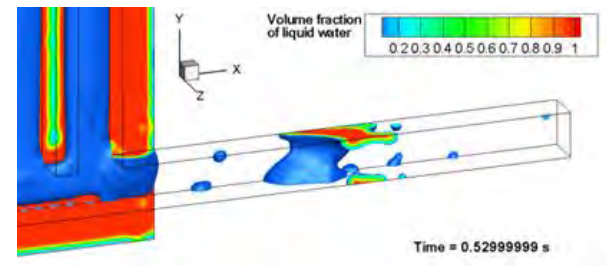

(b) Parallel-DCA model.

Figure 5. Comparison of the flow pattern in the draining process.

\section{CONCLUSIONS}

In this study, the Parallel-DCA model is proposed by applying DCA at multiple wall boundaries in a PEMFC cathode. The gas-liquid phenomena and water transport processes are simulated and investigated using the ParallelDCA model and VOF method. The numerical results are compared to those from the Parallel-SCA model [4]. It is indicated that although the general water transport processes from these two models are similar, there are several differences observed from the phenomena that can be summarized as follows:

(1) Emerging pattern (SCA model: liquid water first emerges from the edges of the interface under the channels; DCA model: liquid water first emerges from the middle of the interface under the channels).

(2) Dean vortices evolution (SCA model: upper-lower distribution; DCA model: left-right distribution).

(3) Draining deformation (SCA model: top-bottom stratified flow; DCA model: stratified-slug flow).

In the future, we will further investigate the potential of the DCA model to be applied in the simulation of gas-liquid phenomena in PEMFCs with other complex flow field designs.

\section{ACKNOWLEDGMENT}

The authors are grateful for the support from the Natural Sciences and Engineering Research Council of Canada (NSERC) and the University of Windsor.

\section{REFERENCES}

[1] P. Quan et al., "Water behavior in serpentine micro-channel for proton exchange membrane fuel cell cathode," J. Power Sources, vol. 152, pp. 131-145, Dec. 2005. doi: 10.1016/j.jpowsour.2005.02.075.

[2] A. D. Le and B. Zhou, "A numerical investigation on multi-phase transport phenomena in a proton exchange membrane fuel cell stack," J. Power Sources, vol. 195, no. 16, pp. 5278-5291, Aug. 2010. doi: 10.1016/j.jpowsour.2010.03.014.
[3] K. Jiao and B. Zhou, "Accelerated numerical test of liquid behavior across gas diffusion layer in proton exchange membrane fuel cell cathode," J. Fuel Cell Sci. Techol., vol. 5, no. 4, p. 041011-041011-10, Sept. 2008. doi: $10.1115 / 1.2971020$.

[4] X. Wang and B. Zhou, "Liquid water flooding process in proton exchange membrane fuel cell cathode with straight parallel channels and porous layer," J. Power Sources, vol. 196, no. 4, pp. 1776-1794, Feb. 2011 doi: 10.1016/j.jpowsour.2010.09.092.

[5] Y. Ding et al., "Simulations of two-phase flow distribution in communicating parallel channels for a PEM fuel cell," Int. J. Multiphase Flow, vol. 52, pp. 35-45, June 2013. doi: 10.1016/j.ijmultiphaseflow.2012.12.001.

[6] J. H. Jo and W. T. Kim, "Numerical simulation of water droplet dynamics in a right angle gas channel of a polymer electrolyte membrane fuel cell," Int. J. Hydrogen Energy, vol. 40, no. 26, pp. 8368-8383, July 2015. doi: 10.1016/j.ijhydene.2015.04.122.

[7] Y. Qin et al., "Effective removal and transport of water in a PEM fuel cell flow channel having a hydrophilic plate," Appl. Energy, vol. 113, pp. 116-126, Jan. 2014. doi: 10.1016/j.apenergy.2013.06.053.

[8] S. Kang and B. Zhou, "Numerical study of bubble generation and transport in a serpentine channel with a T-junction," Int. J. Hydrogen Energy, vol. 39, no. 5, pp. 2325-2333, Feb. 2014. doi: 10.1016/j.ijhydene.2013.11.115.

[9] S. Kang, B. Zhou, and M. Jiang, "Bubble behaviors in direct methanol fuel cell anode with parallel design," Int. J. Hydrogen Energy, vol. 42, no. 31, pp. 20201-20215, Aug. 2017. doi: 10.1016/j.ijhydene.2017.06.149.

[10] X. Zhu, P. C. Sui, and N. Djilali, "Numerical simulation of emergence of a water droplet from a pore into a microchannel gas stream," Microfluid. Nanofluid., vol. 4, no. 6, pp. 543-555, June 2008. doi: 10.1007/s10404-007-0209-9.

[11] X. Zhu, P. C. Sui, and N. Djilali, "Three-dimensional numerical simulations of water droplet dynamics in a PEMFC gas channel," J. Power Sources, vol. 181, no. 1, pp. 101-115, June 2008. doi: 10.1016/j.jpowsour.2008.03.005

[12] Š. Šikalo et al., "Dynamic contact angle of spreading droplets: Experiments and simulations, "Phys. Fluids, vol. 17, no. 6, p. 062103, June 2005. doi: 10.1063/1.1928828.

[13] S.F. Kistler, "Hydrodynamics of wetting," in Wettability, vol. 6, Surfactant Science Series, J. C. Berg, Ed., New York: Marcel Dekker, Inc., 1993, pp. 311-430.

[14] R. L. Hoffman, "A study of the advancing interface. I. Interface shape in liquid-gas systems," J. Colloid Interface Sci., vol. 50, no. 2, pp. 228-241, Feb. 1975. doi: 10.1016/0021-9797(75)90225-8.

[15] M. Jiang, B. Zhou, and X. Wang, "Comparisons and validations of contact angle models," Int. J. Hydrogen Energy, vol. 43, no. 12, pp. 6364-6378, Mar. 2018. doi: 10.1016/j.ijhydene.2018.02.016.

[16] C. Miller, "Liquid water dynamics in a model polymer electrolyte fuel cell flow channel," M.A.Sc. Thesis, Univ. of Victoria, Victoria, B.C., 2009.

[17] C. Fang et al., "3-D numerical simulation of contact angle hysteresis for microscale two phase flow," Int. J. Multiphase Flow, vol. 34, no. 7, pp. 690-705, July 2008. doi: 10.1016/j.ijmultiphaseflow.2007.08.008.

[18] M. Jiang and B. Zhou, "Numerical study of droplet impact on inclined surface: Viscosity effects," ECS Trans., vol. 83, no. 1, pp. 127-136, Jan. 2018. 2018. doi: 10.1149/08301.0127ecst.

[19] Š. Šikalo, C. Tropea, and E. N. Ganic, "Impact of droplets onto inclined surfaces," J. Colloid Interface Sci., vol. 286, no. 2, pp. 661-669, June 2005. doi: 10.1016/j.jcis.2005.01.050.

[20] Š. Šikalo and E. N. Ganic, "Phenomena of droplet-surface interactions," Exp. Therm. Fluid Sci., vol. 31, no. 2, pp. 97-110, Nov. 2006. doi: 10.1016/j.expthermflusci.2006.03.028.

[21] W. R. Dean, "XVI. Note on the motion of fluid in a curved pipe," The Lon., Edin., Dub. Phil. Mag. and J. Sci., vol. 4, no. 20, pp. 208-223, July 1927. doi: $10.1080 / 14786440708564324$. 


\section{Synthesis of Diamond-like Carbon by PECVD on Different Substrates for Fuel Cell Applications}

\author{
Ibrahim Alaefour \\ Department of Mechanical and Mechatronics Engineering \\ University of Waterloo, Waterloo, Ontario N2L 3G1 \\ Ibrahim.Alaefour@uwaterloo.ca
}

\author{
Jian Zhao \\ Department of Mechanical and Mechatronics Engineering \\ University of Waterloo, Waterloo, Ontario N2L 3G1 \\ jian.zhao@uwaterloo.ca
}

\author{
Samaneh Shahgaldi \\ Department of Mechanical and Mechatronics Engineering \\ University of Waterloo, Waterloo, Ontario N2L 3G1 \\ samaneh.shahgaldi@uwaterloo.ca \\ Xianguo Li \\ Department of Mechanical and Mechatronics Engineering \\ University of Waterloo, Waterloo, Ontario N2L 3G1 \\ xianguo.li@uwaterloo.ca
}

\begin{abstract}
Lower cost, lighter weight, and higher electrical conductivity are among many advantages of using metallic bipolar plates (BPs) over the conventional graphitic materials in PEM fuel cell applications. In this study, diamond-like carbon (DLC) coatings are deposited on top surfaces of stainless steel (SS316) and Aluminum (A15052) substrates via Plasma Enhanced Carbon Vapor Deposition (PECVD) in order to increase the durability. Further, the effect of different plasma power intensities and type of precursor gases, including methane $\left(\mathrm{CH}_{4}\right)$ and acetylene $\left(\mathrm{C}_{2} \mathrm{H}_{2}\right)$, on the growth of DLC films is studied. Various ex-situ characterization techniques have been used to examine the strength of adhesion, morphologies, structures/compositions, and wettability of the DLC coatings. It is found that coatings produced from $\mathrm{CH}_{4}$ gas show superior adhesion in comparison to those obtained from the $\mathrm{C}_{2} \mathrm{H}_{2}$ gas. Results indicate that higher deposition power intensity can produce more $\mathrm{sp}^{3}$ bonds than $\mathrm{sp}^{2}$ bonds. It is also determined that the type of the metallic substrates has predominant roles on the morphology of the DLC coatings. In addition, a moderate power intensity of $250 \mathrm{~W}$ results in desirable properties in DLC, including adhesion and wettability, compared to higher and lower plasma power intensities.
\end{abstract}

Keywords- Proton exchange membrane fuel cell; Bipolar plates; Diamond-like carbon.

\section{INTRODUCTION}

Bipolar plates (BPs) are one of the crucial components in polymer electrolyte membrane (PEM) fuel cells. They conduct electrons between adjoining cells, distribute reactants, facilitate water and heat management, and provide mechanical support. To accomplish these functions, bipolar plates must exhibit a number of desirable mechanical and surface properties. However, most commercial bipolar plates are made of heavy- graphite and are considered to be the bulkiest and the second most-expensive component in the manufacture of PEM fuel cell stacks [1]. Due to the technical and cost requirements of BPs [1-3], metallic bipolar plates have received considerable attention to replace conventional graphite materials, but their durability inside the acidic PEM fuel cell environment is a challenging issue. Various surface modifications and protective coatings have been investigated, including [4]: noble metals such as gold [5], metal nitrides, and metal carbides. In the last decade, diamond-like carbon (DLC) has been considered an innovative candidate as a coating material for metallic BPs in PEM Fuel Cells [6]. In general, DLC can be formed as a thin film at relatively low deposition temperatures on metallic substrates. One of the most suitable DLC deposition techniques is Plasma Enhanced Chemical Vapor Deposition (PECVD) [79]. Carbon-based layer is deposited on stainless steel (SS304) via PECVD, and the coating sample presents the lower interfacial contact resistance in comparison to the SS304 without coating [10]. In other work, a $1 \mu \mathrm{m}$-thin layer of nickel is applied between the SS304 and carbon-base coating to improve adhesion [11]. The electrical conductivity of DLC film under different voltages is also reported [12]. Due to the potential advantages of using aluminum, different carboncomposite coatings for aluminum-base bipolar plates have also been investigated [13] [14]. However, information about DLC coating on the aluminum surfaces is limited. Therefore, the objective of this work is to investigate the effect of deposition parameters on the DLC properties for metallic-base substrates (stainless steel SS316 and aluminum A15052).

\section{EXPERIMENTAL AND CHARACTERIZATIONS}

The DLC coating is deposited on A15052 and SS316 substrates by using the fully automated INTLVAC's AUTOSYS control system. $\mathrm{CH}_{4}$ and $\mathrm{C}_{2} \mathrm{H}_{2}$ are separately used as a precursor gas, and the carrier gas is argon. Different plasma power intensities of 70,250, and $1000 \mathrm{~W}$ are applied in 
order to investigate its impact of plasma power on the various characteristics of DLC coatings, whereas the argon gas percentage is fixed at $30 \%$ and the deposition time is a constant of $74 \mathrm{~min}$. Various ex-situ characterization methods are employed to investigate the properties of the deposited films. Scanning electron microscopy is used to study the morphology of the prepared samples. Since the adhesion of the coating DLC films is one of the most challenging tasks of surface engineering, tape pull-off tests are used to check the coatings' adhesion to the metallic substrates. The morphology of the coated film is investigated by field emission-scanning electron microscopy (FE-SEM). Further, X-ray photoelectron (XPS) spectroscopy is commonly used for quantitative comparison between the molecule compositions existing on the coated surface. The DLC surface wettability indicates the hydrophobicity behavior of the BP coatings in order to determine whether they are suitable for PEM Fuel cell applications. The surface wettabilities of the DLC coatings on different A15052 and SS316 samples are evaluated by measuring the static contact angle $(\theta)$, a characterization technique that uses a Ramé-Hart contact angle apparatus.

\section{RESULTS}

\section{A. Adhesion}

Table 1 summarizes the results of the adhesion tests between the DLC coatings on SS316 and Al5052 substrates, under the classifications as passed, failed, and partially failed. It shows the impact of varying the precursor gas between acetylene $\left(\mathrm{C}_{2} \mathrm{H}_{2}\right)$ and methane $\left(\mathrm{CH}_{4}\right)$, and the power intensities between $70 \mathrm{~W}$ and $250 \mathrm{~W}$, then $1000 \mathrm{~W}$ on the adhesion of the DLC coatings with the constant carrier gas (argon) percentage of $30 \%$ and deposition time of $74 \mathrm{~min}$.

TABLE 1. ADHESION TESTS OF DLC COATINGS ON SS316 AND AL5052 SUBSTRATES:

\begin{tabular}{|c|c|c|c|c|c|}
\hline \multicolumn{4}{|c|}{ Deposition Parameters } & $\begin{array}{l}\text { Substrate } \\
\text { Materials }\end{array}$ & $\begin{array}{c}\text { Substrate } \\
\text { Materials Al5052 }\end{array}$ \\
\hline $\begin{array}{c}\text { Power } \\
(W)\end{array}$ & $\begin{array}{l}\mathrm{H} / \mathrm{C} \\
\text { gas }\end{array}$ & $\begin{array}{l}A r \\
(\%)\end{array}$ & $\begin{array}{l}\text { Time } \\
\text { (min) }\end{array}$ & Pass/Failed & Pass/Failed \\
\hline 70 & $\mathrm{C}_{2} \mathrm{H}_{2}$ & 30 & 74 & Failed & Failed \\
\hline 250 & $\mathrm{CH}_{4}$ & 30 & 74 & Passed & Passed \\
\hline 1000 & $\mathrm{CH}_{4}$ & 30 & 74 & Failed & Passed \\
\hline
\end{tabular}

It is observed that employing $\mathrm{CH}_{4}$ gas can result in higher adhesion than $\mathrm{C}_{2} \mathrm{H}_{2}$ gas, especially with a moderate plasma power intensity of 250 for both A15052 and SS316 substrates. This adhesion enhancement can be attributed to the different growth mechanisms of carbon composites between $\mathrm{CH}_{4}$ gas and those produced from $\mathrm{C}_{2} \mathrm{H}_{2}$. This difference occurs because DLC generated by using $\mathrm{CH}_{4}$ produces more hydrogen in reaction, hence the etching reaction is developed during the deposition processes [15]. It is also noticed that increasing the plasma power intensity has a negative effect on the adhesion between the SS316 substrate and the coating. That is, a higher power intensity of $1000 \mathrm{~W}$ can result in poor DLC adhesion in comparison to $250 \mathrm{~W}$. The poor adhesion at higher plasma power intensity is associated with a fast deposition rate as well as the growth of thicker DLC films. Consequently, the residual stress is higher inside the films due to the noticeable increase in $\mathrm{sp}^{3}$ bonding fraction, as will be discussed by the XPS analysis. On the other hand, varying the plasma power intensity has a negligible impact on the adhesion of the DLC coating on Al 5052 substrates. These results signify the roles of the metallic substrate on the adhesion property.

\section{B. Morphology}

Figure 1 (a-d) shows the surface morphologies of two different DLC coatings deposited on SS316 and Al5052 substrates at two different power intensities of $250 \mathrm{~W}$ and 1000 $\mathrm{W}$. These images are taken from the top surface without using any gold coating. Figure 1 ( $a$ and $b$ ) are obtained using SS316, while $30 \%$ of carrier gas is applied. These images show homogenous coverage, with the presence of big island-shaped regions (see the images with high magnification on top of the SEM images). Both surfaces are covered with dark and light particles, and this contrast can be attributed to the accumulation of particles with different electrical-conductivity properties. The DLC structure is basically amorphous carbon coordinated with $\mathrm{sp}^{2}$ and $\mathrm{sp}^{3}$ structures with different conductivity. It is expected that the $\mathrm{sp}^{3}$ bond is responsible for the mechanical properties, while $\mathrm{sp}^{2}$ bond presents the electronic properties. It can be seen that Figure 1 (a) is covered with a higher amount of darker particles, in comparison to Figure 1 (b). These results may suggest that using higher power in the PECVD process leads to a higher $\mathrm{sp}^{3}$ structure. Further, Figure 1 (c and d) demonstrates the top surface of the coating on the Al5052 substrate under identical deposition conditions as those for Figure 1 ( $a$ and $b$ ). It can be observed that the two images have uniform and homogenous coverages with a stripe-like structure, which is considerably different than island-shaped structure in the SS316 substrate. These images clearly suggest that the types of substrate are a predominant factor in the morphology of the DLC coating. However, the deposition conditions also play important roles. For instance, Figure 1 (a) and (c) are prepared under identical conditions using different substrates, but the morphology of the samples is completely different (island versus stripe).
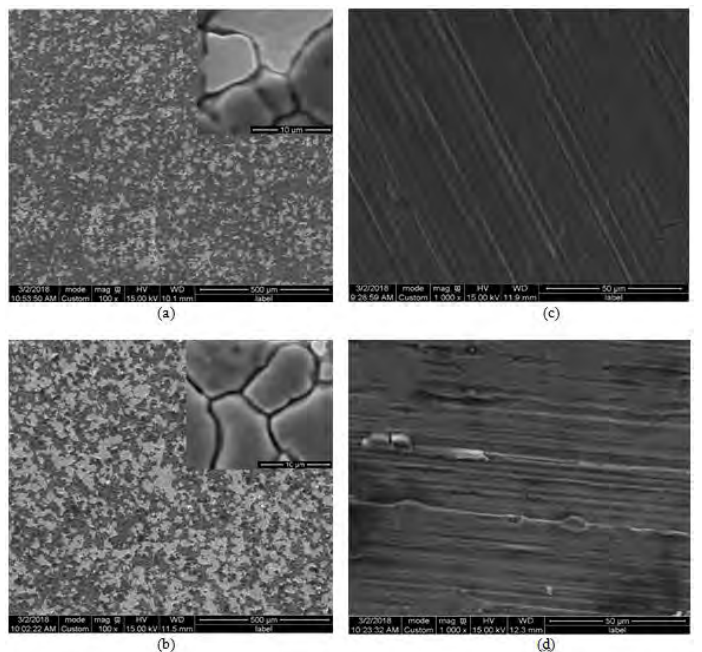

Figure 1. SEM images of coated films of DLC deposited SS316 substrates with plasma power intensity of (a) $250 \mathrm{~W}$ and (b) $1000 \mathrm{~W}$, whereas (c) and (d) for coated films of DLC deposited on A15052 substrates with plasma intensities of 250 and $1000 \mathrm{~W}$, respectively 


\section{X-ray Analysis}

Figures 1 (a) and (b) show the XPS spectra of DLC films deposited on SS316 substrates for plasma power intensities of $250 \mathrm{~W}$ and $1000 \mathrm{~W}$, respectively, with a fixed carrier gas (argon) ratio of $30 \%$. The coating films are obtained under a deposition time of $74 \mathrm{~min}$. Since the surface properties of the DLC films are strongly influenced by the ratios between the $\mathrm{sp}^{3}$ and $\mathrm{sp}^{2}$ of the carbon bonds, it can be expected that DLC with higher $\mathrm{sp}^{2}$ carbon bonds will show graphene-like properties. It can be observed from Figure 1 (a-d) that the curve peak of DLC film deposited is deconvoluted into two components, which indicate the existences of different carbon bonds including $\mathrm{sp}^{2}$ and $\mathrm{sp}^{3}$. It can be observed from Figure 1 (a) that about $81 \%$ of the DLC coated film deposited on the SS316 is $\mathrm{sp}^{2}$ carbon bonds. However, increasing the deposition power intensity to $1000 \mathrm{~W}$ lowered the amount of graphene-like bonds in the coating films by $15 \%$. Further, figure 1 (c) shows curve peaks at 284 corresponds to the $\mathrm{sp}^{2}$ binding energy on its spectrums, while the peak of DLC deposited with $1000 \mathrm{~W}$ has been shifted to the higher binding energy of $285 \mathrm{eV}$. It can be seen that the percentage of $\mathrm{sp}^{2}$ is $70.9 \%$ in the coated samples produced with $250 \mathrm{~W}$, while the DLC coatings obtained with $1000 \mathrm{~W}$ contain $58.7 \% \mathrm{sp}^{3}$ bonds. On the other hand, the second peak components occur at $286 \mathrm{eV}$, indicating the $\mathrm{C}=\mathrm{O}$ bond in the coating film due to contamination. This suggests that a higher power intensity of $1000 \mathrm{~W}$ can result in incensing of the $\mathrm{sp}^{3}$ bond in the DLC films. Further, these results may suggest that the metallic-substrate material and the depositionpower intensity can impact the deposited-film properties.

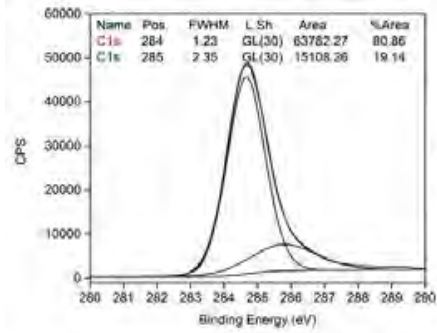

(a)

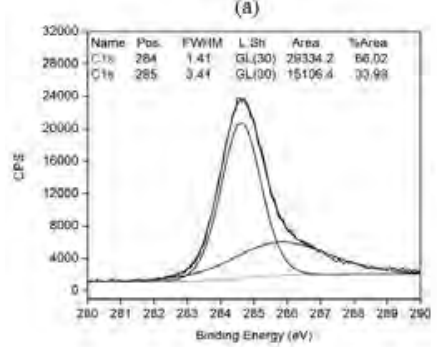

(b)

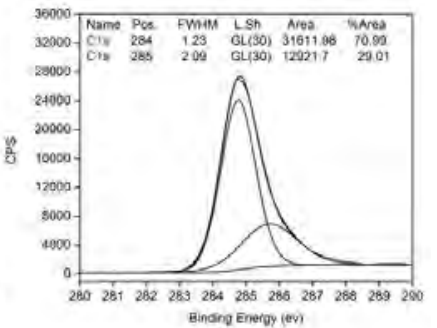

(c)

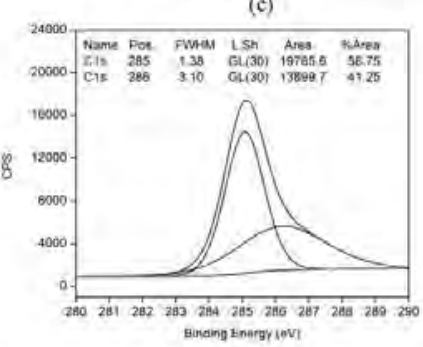

(d)
Figure 2. XPS analysis spectra of coating films of DLC with different power intensities (a) $250 \mathrm{~W}$ and (b) $1000 \mathrm{~W}$ deposited on SS316 substrates. Whereas coatings deposited on A15052 substrates with power intensity of (c) $250 \mathrm{~W}$ and (d) $1000 \mathrm{~W}$. Deposition conditions: $\mathrm{CH}_{4}$ is a precursor gas, argon gas percentage of $30 \%$, time of $74 \mathrm{~min}$, and voltage $120 \mathrm{~V}$.

\section{Wettability}

Water management is a crucial issue inside operating PEM Fuel cells. The wettability and water repellency of the bipolar plates are important surface properties for water removal [16].
Therefore, the DLC coatings of the bipolar plate coatings surface must exhibit hydrophobicity behavior and water repellency. The surface wettabilities of the DLC coatings on different A15052 and SS316 substrates are evaluated as in Table 2, showing the average values of the water contact angles $\left(\theta^{\circ}\right)$ for the coated surface of SS316 and A15052 substrates. The angles vary from $80^{\circ}$ to $82^{\circ}$ for the SS316 substrate, and from $76^{\circ}$ to $91^{\circ}$ for Al5052 under different plasma power intensities. Table 2 may suggest that the substrate material has a strong impact on the surface wettability characteristics. It demonstrates that increasing the deposition power intensity results in lowering the contact-angle values due to the diamond-like carbon structure [17]. This observation is found to be in good agreement with the XPS analysis discussed above, which showed that a higher percentage of $\mathrm{sp}^{3}$ bonds in the coating with a higher power intensity of $1000 \mathrm{~W}$ in comparison to $250 \mathrm{~W}$. Further, it is seen that coated films deposited with a lower power intensity of $250 \mathrm{~W}$ results in a higher contact angle of $91^{\circ}$.

TABLE 2: STATIC CONTACT ANGLE $(\Theta)$ OF DLC DEPOSITED ON AL5052 AND SS316 SUBSTRATES

\begin{tabular}{|c|l|c|c|c|c|}
\hline \multicolumn{4}{|c|}{ Deposition Parameters } & $\begin{array}{c}\text { Substrate } \\
\text { Materials } \\
\text { SS316 }\end{array}$ & $\begin{array}{c}\text { Substrate } \\
\text { Materials Al5052 }\end{array}$ \\
\hline $\begin{array}{c}\text { Power } \\
(\text { W) }\end{array}$ & $\begin{array}{l}\text { H/C } \\
\text { gas }\end{array}$ & $\begin{array}{l}\text { Ar } \\
(\%)\end{array}$ & $\begin{array}{c}\text { Time } \\
(\text { min })\end{array}$ & $\begin{array}{c}\text { Contact angle } \\
\left({ }^{\circ}\right.\end{array}$ & $\begin{array}{c}\text { Contact angle } \\
\left({ }^{\circ}\right)\end{array}$ \\
\hline 250 & $\mathrm{CH} 4$ & 30 & 74 & 82 & 91 \\
\hline 1000 & $\mathrm{CH} 4$ & 30 & 74 & 80 & 76 \\
\hline
\end{tabular}

\section{CONCLUSIONS}

The effect of power intensity on the carbon composite coatings deposited on aluminum (A15052) and stainless steel (SS316) substrates has been investigated. Adhesion, SEM, XPS, and wettability measurements have been conducted to understand the characteristics and the compositions of the deposited coatings. It has been observed that coatings generated from $\mathrm{CH}_{4}$ provide better adhesion than those coatings produced from $\mathrm{C}_{2} \mathrm{H}_{2}$ gas. Results suggest that the ratio between $\mathrm{sp}^{2}$ and $\mathrm{sp}^{3}$ bonds in the DLC coatings is strongly affected by the plasma power intensity. It has been found that a large percentage $\mathrm{sp}^{3}$ (diamond-like) in the coating is produced at a high power density at the cost of the $\mathrm{sp}^{2}$ bond (graphenelike). Therefore, the surface morphology and composition of the DLC coatings are strongly influenced by the deposition parameters and material of the metallic substrates. The coatings generated under power intensity of $250 \mathrm{~W}$ shows stronger adhesion with the metallic A15052 and SS316 substrates than those produced higher power of $1000 \mathrm{~W}$. Further, the DLC coatings deposited on metallic substrates demonstration hydrophobicity properties with a contact angle of between $80^{\circ}$ of $90^{\circ}$.

\section{REFERENCES}

[1] J. a. M. Spendelow, "Fuel Cell System Cost-2013”, DOE Fuel and Cell Technologies Office Record, Record \# 13012.

[2] S. Chunhui, P. Mu, Y. Qin, and Y. Runzhang, "Studies on Preparation and Performance of Sodium Silicate/Graphite Conductive Composites," J. Comp. Mat., vol. 40, pp. 839-848, May 1, 2006.

doi: $10.1177 / 0021998306061296$. 
[3] D. J. L. Brett and N. P. Brandon, "Review of Materials and Characterization Methods for Polymer Electrolyte Fuel Cell Flow-Field Plates," J. Fuel Cell Sci. Technol., vol. 4, pp. 29-44, Feb. 2006. doi: $10.1115 / 1.2393303$.

[4] S. Karimi, N. Fraser, B. Roberts, and F. Foulkes, "A Review of Metallic Bipolar Plates for Proton Exchange Membrane Fuel Cells: Materials and Fabrication Methods," Adv. Mater. Sci. Eng., vol. 2012, p. 22, 2012. doi: $10.1155 / 2012 / 828070$.

[5] R. L. Borup and N. E. Vanderborgh, "Design and Testing Criteria for Bipolar Plate Materials for Pem Fuel Cell Applications," MRS Proceedings, vol. 393, p. 151, 1995. doi: 10.1557/PROC-393-151.

[6] C. C. Wang, "Novel Structured Metal Bipolar Plates for Low Cost Manufacturing," Project ID\# : FC105 2014.

[7] R. Paul, S. Hussain, S Majumder, S Varma, and A. K. Pal, "Surface plasmon characteristics of nanocrystalline gold/DLC composite films prepared by plasma CVD technique," Mater. Sci. Eng. B: Solid, vol. 164, pp. 156-164, 2009. doi: 10.1016/j.mseb.2009.09.009.

[8] G. A. Viana, et al., "Diamond-like carbon deposited by plasma technique as a function of methane flow rate," Diamond Relat. Mater., vol. 19, nos. 7-9, pp. 756-759, Jul. - Sept. 2010. doi: 10.1016/j.diamond.2010.01.043.

[9] D. Caschera, F Federici, S Kaciulis, L Pandolfi, A Cusmà, and G. Padeletti, "Deposition of Ti-containing diamond-like carbon (DLC) films by PECVD technique," Mater. Sci. Eng. C, vol. 27, nos. 5-8. pp. 1328-1330, Sept. 2007. doi: 10.1016/j.msec.2006.06.027.

[10] T. Fukutsuka, T. Yamaguchi, S.-I. Miyano, Y. Matsuo, Y. Sugie, and Z. Ogumi, "Carbon-coated stainless steel as PEFC bipolar plate material," $J$. Power Sources, vol. 174, no. 1, pp. 199-205, Nov. 2007.

doi: 10.1016/j.jpowsour.2007.08.096.
[11] C.-Y. Chung, S.-K. Chen, P.J. Chiu, M.-H. Chang, T.-T. Hung, and T.H. Ko, "Carbon film-coated 304 stainless steel as PEMFC bipolar plate," $J$. Power Sources, vol. 176, no. 1, pp. 276-281, Jan. 2008. doi: 10.1016/j.jpowsour.2007.10.022.

[12] Md. K. Hassan, B.K. Pramanik and A. Hatta., "Electrical Resistivities of the Diamond-like carbon films fabricated from methane and acetylen using RF plasma," New Diamond and Frontier Carbon Technology, vol 16, no. 4, pp. 211-219. 2006.

[13] J. R. Mawdsley, J. D. Carter, X. Wang, S Hiyogi, C. Q. Fan, R. Koc, and G. Osterhout, "Composite-coated aluminum bipolar plates for PEM fuel cells," J. Power Sources, vol. 231, no. 1,pp. 106-112, Jun. 2013. doi: 10.1016/j.jpowsour.2012.12.074.

[14] S.-J. Lee, C.H. Huang and Y.P. Chen., "Investigation of PVD coating on corrosion resistance of metallic bipolar plates in PEM fuel cell," J. Mater. Process. Technol., vol. 140, nos. 1-3, pp. 688-693, Sept. 2003. doi: 10.1016/S0924-0136(03)00743-X.

[15] M. Aksak S. Kir and Y. Selamet, "Effect of the growth temperature on carbon nanotubes grown by thermal chemical vapor deposition method." $J$. Optoelectron. Adv. Mater. Symp., vol. 1 no. 3, pp. 281-284, 2009.

[16] S. A. Shakhshira, Y. Wang, I. E. Alaefour, and X. Li, "The Influence of Channel Wettability on Two-Phase Flow and Polymer Electrolyte Membrane Fuel Cell Performance," ECS Transactions, vol. 42, pp. 109-115, 2012. doi: $10.1149 / 1.4705486$.

[17] J. Martí-González and E. Bertran, "Mechanical and Surface Characterization of Diamond-Like Carbon Coatings onto Polymeric Substrate," arXiv preprint .arXiv:1509.08512, 2015. 


\section{Effects of Oxyfuel Combustion on the Performance of Natural Gas Combined Cycle Power Generation System}

\author{
Nitin Varia \\ Department of Automotive, Mechanical and \\ Manufacturing Engineering \\ University of Ontario Institute of Technology \\ Oshawa, L1H 7 K4, Canada \\ Bale V. Reddy \\ Department of Automotive, Mechanical and \\ Manufacturing Engineering \\ University of Ontario Institute of Technology \\ Oshawa, L1H 7 K4, Canada \\ bale.reddy@uoit.ca
}

\author{
Dipal Patel \\ Department of Automotive, Mechanical and \\ Manufacturing Engineering \\ University of Ontario Institute of Technology \\ Oshawa, L1H 7 K4, Canada \\ dipal.patel@uoit.ca \\ T. Srinivas \\ School of Mechanical Sciences \\ Vellore Institute of Technology \\ Vellore, India \\ 1sriivast@vit.ac.in
}

\begin{abstract}
In the present work, natural gas combined cycle power generation configurations are investigated with oxyfuel combustion. Steam is also injected in main combustion chamber and reheat combustion chamber to understand the performance of combined cycle work output and greenhouse gas emission. It is observed that the steam injection increases gas cycle efficiency and decreases the steam cycle efficiency. $\mathrm{CO}_{2}$ emission reduction of $3.2 \%$ when steam injection in both combustion chambers for oxyfuel cycle. In oxyfuel combustion, higher ratio of recycle flue gas brings higher thermal efficiency and highest thermal efficiency is achieved when steam is injected in gas turbine main combustion chamber only.
\end{abstract}

Keywords - combined cycle; oxyfuel; turbines; energy efficiency; exergy efficiency

\section{INTRODUCTION}

There is growing research investigations conducted on natural gas combined cycle power generation system with oxyfuel combustion approach due to the ability to capture carbon dioxide emissions and for carbon capture and storage. Mietzko et. al. [1] reported comparison of natural gas combined cycle power plants with post combustion and oxyfuel technology at different $\mathrm{CO}_{2}$ capture rates. Stanger et. al. [2] presented details on oxyfuel combustion for $\mathrm{CO}_{2}$ capture in power plants. They reported an overview of the current state of the art technology on the development of oxyfuel combustion systems for $\mathrm{CO}_{2}$ capture. Sammak et. al., [3] reported conceptual mean-line design of single and two-shaft oxyfel gas turbine in a semi closed oxyfuel combustion combined cycle. Thorbergsson and Gronstedt [4] conducted thermodynamic analysis of two competing mid-sized oxyfuel combustion combined cycles.
Jericha et. al., [5] presented gas turbine with $\mathrm{CO}_{2}$ retention for 400MW oxyfuel system Graz Cycle. Woollatt and Franco [6] reported details on conceptual aerodynamic design of turbomachinery components for natural gas oxyfuel cycles. Mathieu and Bolland [7] presented results on comparison of costs for natural gas power generation with $\mathrm{CO}_{2}$ capture. In the present work, natural gas combined cycle power generation configurations are investigated with with oxy fuel combustion. Steam is also injected in main combustion chamber and reheat combustion chambers to understand the performance of combined cycle work output and greenhouse gas emissions.

\section{Confriguration And Methodology}

\section{A. Natural gas combined cycle configuration}

Natural gas fired oxyfuel combustion combined power generation systems gaining popularity for carbon capture and storage. In the present work different oxyfuel combined cycle power generation configurations as listed in Table 1 are considered. Figure 1 shows the schematic diagram of these configurations.

The topping cycle consists of air compressor $(C 1)$ followed by an intercooler $(I C)$. Air is further compressed in second air compressor $(C 2)$. Compressed air is burned with methane in combustion chamber (CC1). In case of configuration \#2 and \#3 from Table 1, a fraction of a steam $(\zeta)$ is injected in the first combustion chamber. Products of first combustion chamber enters main gas turbine (GT1) and produced work output. Exhaust gas from main gas turbine further burned with methane in second combustion chamber (CC2). In case of configuration $\# 3$, a fraction of steam $(\omega)$ is injected in reheater combustion chamber. Product of reheater combustion chamber enters the 
reheater gas turbine (GT2) to produce work output. Exhaust gas from gas turbine enters heat recovery steam generator $(H R S G)$ which produces steam in the bottoming cycle. The Steam then enters the steam turbine $(S T)$ and produces work output. Fraction of steam $(\zeta$ and $\omega)$ is taken out at particular pressure and used in topping cycle for power augmentation. Water vapor from steam turbine is then condensed in condenser and recirculates in the bottoming cycle through pump. It is assumed that system is operating at steam state steady flow conditions.

In configuration $\# 1$, pure oxygen $(\phi)$ obtained from air separator unit $(A S U)$ is used as oxidizing agent and burned in $C C 1$ with main combustion chamber fuel $\alpha$ and in $C C 2$ with fuel supply $\beta$. Expanded gas after GT2 is passed through $H R S G$ and steam is generated to operate bottoming Rankin cycle. Exhaust gas after $H R S G$ contains only $\mathrm{CO}_{2}$ and water vapor. Water vapor is condensed through water separator $(W S)$ and removed from exhaust gas.

Combustion of methane and pure oxygen produces very high flame temperature, which is not suitable for turbine operation at this stage. A fraction of $\mathrm{CO}_{2}$, defined as $\lambda$ is compressed in three compressors $(C 3, C 4, C 5)$ coupled with intercooler between each compression. Highly compressed $\mathrm{\lambda CO}_{2}$ is removed from the cycle for sequestration. Remaining part of $\mathrm{CO}_{2}$ defined as $(1-\lambda)$ is compressed in $C 1$ and $C 2$ and recycled back to $C C 1$ to bring the flame temperature down to suitable operative condition.

TABLE I. CONFIGURATIONS

\begin{tabular}{|c|l|}
\hline Configuration & \multicolumn{1}{c|}{ Description of Study } \\
\hline$\# 1$ & $\begin{array}{l}\text { Combustion of oxygen and methane without stream } \\
\text { injection }\end{array}$ \\
\hline$\# 2$ & $\begin{array}{l}\text { Combustion of oxygen and methane with steam } \\
\text { injection in main combustion chamber }\end{array}$ \\
\hline$\# 3$ & $\begin{array}{l}\text { Combustion of oxygen and methane with steam } \\
\text { injection in main combustion chamber and re- } \\
\text { heater combustion chamber }\end{array}$ \\
\hline
\end{tabular}

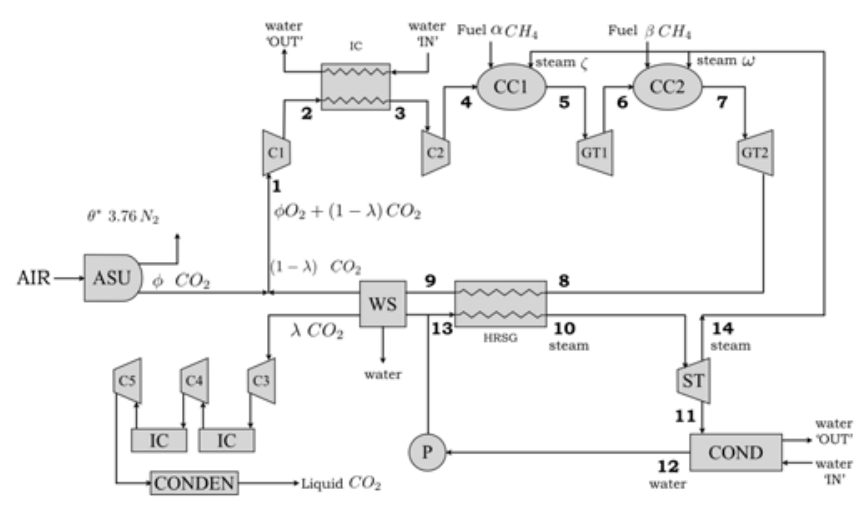

Figure 1. Schematic diagram of configuration 1, 2 and 3 (Listen in Table-1)

Similar to configuration \#1, in configuration \#2, a fraction of steam $\zeta$ is extracted from $S T$ and injected inside $C C 1$ with pure

Identify applicable sponsor/s here. (sponsors) oxygen $\phi$ and fuel $\alpha$. The $C C 2$ is not injected with steam, where steam is taken from steam turbine at $5 \%$ higher pressure than pressure present inside the CC1. As an extension of configuration $\# 2$, in configuration $\# 3$, additional steam $\omega$ is taken from steam turbine and injected inside of $C C 2$. Pressure at which $\omega$ is extracted from steam turbine is $5 \%$ higher than pressure present at $C C 2$.

TABLE II. EFFECTS OF FLUE GAS RECYCLE ON TURBINE INLET TEMPERATURE IN COMBINED CYCLE STEAM - CONFIGURATION \#1.

\begin{tabular}{|c|c|c|c|c|c|c|}
\hline$\lambda[\%]$ & 90 & 80 & 60 & 40 & 20 & 0 \\
\hline TIT1 $\left[{ }^{\circ} \mathrm{C}\right]$ & 1552 & 1643 & 1871 & 2194 & 2687 & 3545 \\
\hline TIT2 $\left[{ }^{\circ} \mathrm{C}\right]$ & 1619 & 1697 & 1880 & 2105 & 2383 & 2710 \\
\hline
\end{tabular}

\section{B. Thermodynamic Analysis and Methodology}

For the considered natural gas oxyfuel combined cycle power generation systems energy and exergy analyses are conducted. Equations are developed and simulations are conducted to investigate the role of flue gas recycle ratio and steam injection on thermal efficiency and $\mathrm{CO}_{2}$ reduction.

All simulations have been conducted using Engineering Equation Solver (EES).

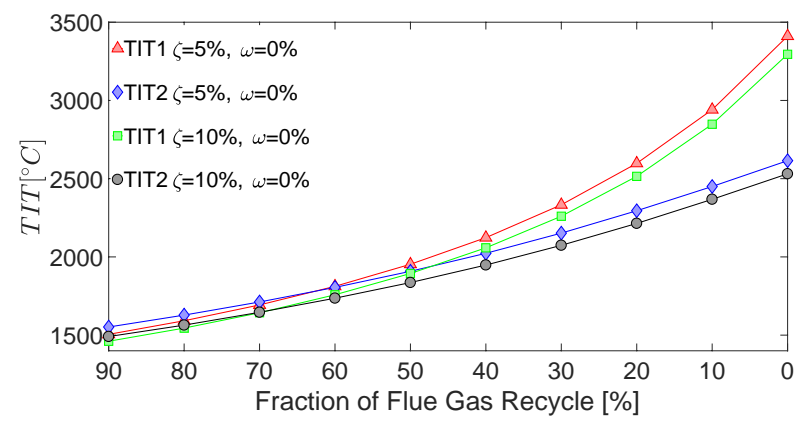

Figure 2. Effects of steam injection on TIT with fraction of flue gas recycle (Configuration \#2)

\section{RESULTS AND DisCUSSIONS}

\section{A. Effects of flue gas recycle on turbine inlet temperatures}

When fuel is burned with pure oxygen, it produces very high TIT (Turbine Inlet Temperature) which is not desirable for current operational turbine blades.

To bring TIT to operable level, the flue gas $\lambda$ is recycled to the combustion chamber once all water vapor is isolated. Table 2 shows the effects of $\lambda$ on TIT1 and TIT2 when fuel supply is maintained at $\alpha=54 \%$ and $\beta=46 \%$. 


\section{B. Effects of steam injection on TIT with fraction of flue gas recycle}

Figure 2 shows the effects of steam injection (with 5\% and $10 \%$ respectively) only in the $C C 1$. Steam addition helps to lower the TIT as it adds mass of steam in combustion chamber. As more steam in injected in $C C 1$, additional mass flow from steam reduces the TIT to the operational level of $1000^{\circ} \mathrm{C}-$ $1400^{\circ} \mathrm{C}$ (i.e. configuration $\# 3$, figure not shown here). Furthermore fig. 3 shows combined cycle efficiency if maximum when steam injected only in $C C 1$ and minimum when steam is injected in $C C 1$ and $C C 2$ together. Higher efficiency can be obtained from configuration \#2 and \#3.

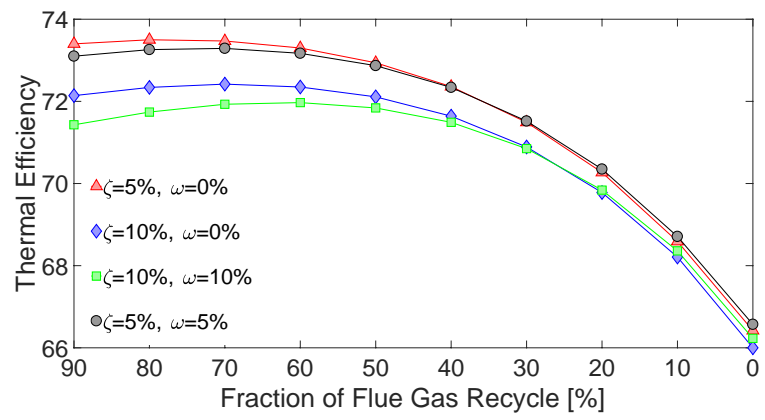

Figure 3. Effects of steam injection on combined cycle thermal efficiency with fractioin of flue gas recycle (Configuration \#2 and \#3)

\section{Effects of pressure ratio on combined cycle work output, efficiencies and $\mathrm{CO}_{2}$ emission}

Various pressure ratio applied to the oxyfuel combustion system at fixed TIT 1 and $T I T 2$ of $1200^{\circ} \mathrm{C}$. Figure 4 shows the effects of pressure ratio on both the topping cycle work output and combined cycle work output for all configuration listed in Table 1.

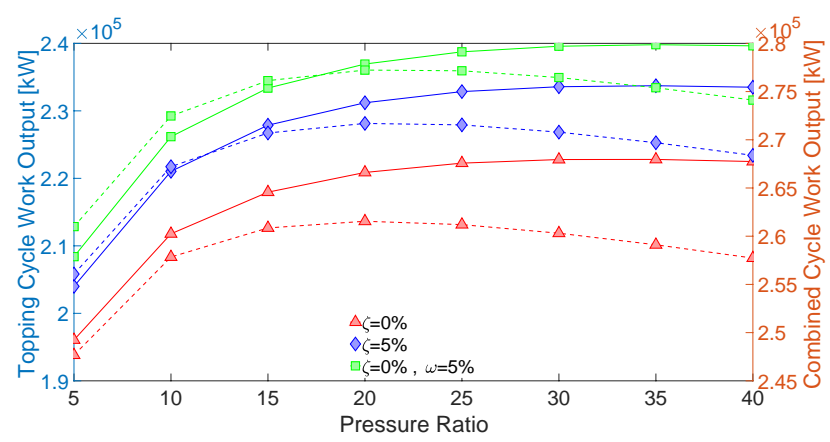

Figure 4. Effects of pressure ratio on topping cycle work output (left axis) and on combined cycle work output (right axis dotted line) for Configuration $\# 1$, \#2 and \#3.

It can be seen from Fig 4 that topping cycle increases work output up to pressure ratio of 25 and then remains constant at higher pressure ratio. Adding 5\% steam injection at $C C 1$ further increases the topping work output and 5\% steam injection at
$C C 1$ and $C C 2$ brings topping cycle work slightly higher. There is an increase of $2.3 \%$ work output when steam injected in $C C 1$ only and $0.3 \%$ further increase when steam is injected in both combustion chambers.

Figure 5 shows the $\mathrm{CO}_{2}$ emission for all configuration considered here. It can be observed from Fig 5 that, the lowest emission accounts in configuration \#3 with corresponding pressure ratio of 25 and steam injection of $5 \%$.

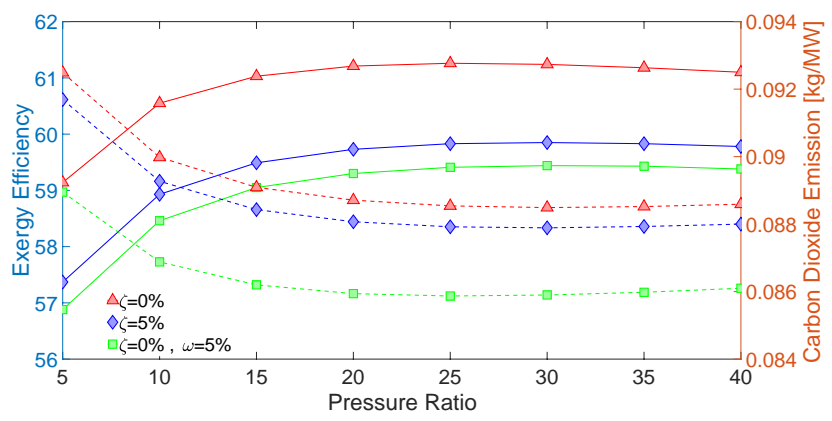

Figure 5. Effects of pressure ratio on exergy efficiency (left axis) and on $\mathrm{CO}_{2}$ emission (right axis dotted line) for Configuration \#1, \#2 and \#3

\section{CONCLUSIONS}

$\mathrm{CO}_{2}$ emission reduction of $3.2 \%$ when steam injection in both combustion chambers for oxyfuel cycle. In oxyfuel combustion, higher ratio of recycle flue gas brings higher thermal efficiency and highest thermal efficiency is achieved when steam is injected in main combustion chamber only. More than $10 \%$ steam in combustion chamber brings combined cycle thermal efficiency down. The present results and trends on oxyfuel combustion work are in agreement with results reported in the literature.

\section{REFERENCES}

[1] J. Mietzko, S. Ehlers, A. Kather, "Comparison of natural gas combined cycle power plants with post combustion and oxyfuel technology at different CO2 capture rates," Energy Procedia, vol. 86, pp. 2-11, 2016. doi: 10.1016/j.egypro.2016.01.001

[2] R. Stanger et al., "Oxyfuel combustion for $\mathrm{CO} 2$ capture in power plants," Int. J. Greenhouse Gas Control, vol. 125, pp. 40-55, Sept. 2015. doi: 10.1016/j.ijggc.2015.06.010

[3] M. E. Sammak, T. Thorbergsson., M. Gronstedt., M. Genrup, "Conceptual Mean-Line design of sngle and two-shaft oxyfuel gas turbine in a semiclosed oxyfuel combustion combined cycle," J. Eng. Gas Turbine Power, vol. 135, no. 8, Aug. 2013. doi: 10.1115/1.4023886

[4] E. Thorbergsson and T. Gronstedt, "A thermodynamic analysis of two competing mid-sized oxyfuel combustion combined cycles," J. Energy, vol. 2016. 2438431. Dec. 2015. doi: 10.1155/2016/2438431

[5] H. Jericha, W. Sanz and E. Goettlich, "Gas turbine with CO2 retention-400 MW oxyfuel system Graz cycle," in Proc. CIMAC World Congress on Combustion Energy Technology, Vienna, Austria, May, 2007.

[6] G. Woollatt and F. Franco, "Natural gas oxyfuel cycles -Part I: Conceptual aerodynamic design of turbo-machinery components," Energy Procedia, vol.1, no. 1, pp. 573-580, Feb. 2009.

doi: 10.1016/j.egypro.2009.01.076.

[7] P. Mathieu and O. Bolland, "Comparison of costs for natural gas power generation with CO2 capture," Energy Procedia, vol. 37, pp. 2406-2419, 2013. doi: 10.1016/j.egypro.2013.06.122. 


\section{Advanced Exergy Analysis of $\mathrm{LiCl}-\mathrm{H}_{2} \mathrm{O}$ Absorption Air Conditioning System}

\author{
Julia Aman \\ Turbulence \& Energy Lab \\ Centre for Engineering Innovation \\ University of Windsor \\ Windsor, Ontario \\ email: amanj@uwindsor.ca
}

\author{
Paul Henshaw \\ Turbulence \& Energy Lab \\ Centre for Engineering Innovation \\ University of Windsor \\ Windsor, Ontario \\ email: henshaw@uwindsor.ca
}

\author{
David S-K Ting \\ Turbulence \& Energy Lab \\ Centre for Engineering Innovation \\ University of Windsor \\ Windsor, Ontario \\ email: dting@uwindsor.ca
}

\begin{abstract}
Increasing energy demand for air conditioning due to climate change is posing a continuous threat to the environment. Absorption air-conditioning systems driven by solar thermal or waste heat energy are an alternative for providing cooling comfort in a sustainable manner. The crystallization problem of high performance $\mathrm{LiBr}-\mathrm{H}_{2} \mathrm{O}$ absorption cooling system hinders its small-scale applications. In this study, the potential of a $10 \mathrm{~kW} \mathrm{LiCl}-\mathrm{H}_{2} \mathrm{O}$ absorption refrigeration system is discussed and analyzed. The new concept of advanced exergy analysis is coupled with conventional thermodynamic analyses, which provides the available potential of each component for overall system performance improvement. The analyses uncovered that only $45 \%$ of the total exergy loss is due to each component's own internal irreversibilities, whereas the remaining is through the interaction of the irreversibilities of other components in the system. The analyses also reveal that $43 \%$ of the total exergy loss is unavoidable and $57 \%$ can be reduced by improving the overall system efficiency.
\end{abstract}

Keywords- $\mathrm{LiCl}-\mathrm{H}_{2} \mathrm{O}$; vapor absorption refrigeration; exergy analysis; advanced exergy analysis

\section{INTRODUCTION}

Global warming poses a continuous threat to the environment. At the same time, air-conditioning demand is increasing significantly on a global scale as a result of climate change, population growth, standard of living conditions and industrialization. Presently, air conditioning alone accounts for $40 \%$ to $55 \%$ of the total building energy consumption [1], which is a major sector of the total world energy consumption and carbon emissions [2]. The traditional vapor compression air-conditioning system is usually used for providing this cooling comfort, but consumes a huge amount of electricity, triggering the construction of new fossil fuel power plants worldwide. Therefore, it is imperative to employ renewable energy technologies in order to minimize climate change while meeting the increased energy demand. A vapor absorption air conditioning system, driven by solar thermal energy, can replace the traditional vapor compressor by a thermally-driven one by using an environmentally friendly refrigerant, without utilizing grid electricity.

The most common absorbent-refrigerant working pairs for this cooling system are $\mathrm{LiBr}-\mathrm{H}_{2} \mathrm{O}$ and $\mathrm{NH}_{3}-\mathrm{H}_{2} \mathrm{O}$. The higher efficiency $\mathrm{LiBr}-\mathrm{H}_{2} \mathrm{O}$ absorption system dominates in large scale air-conditioning applications. However, due to the crystallization problem, the $\mathrm{LiBr}-\mathrm{H}_{2} \mathrm{O}$ absorption system cannot be scaled down for small residential applications. Meanwhile, the thermophysical properties of $\mathrm{LiCl}-\mathrm{H}_{2} \mathrm{O}$ shows promise for small residential-scale applications. Many researchers have shown that $\mathrm{LiCl}-\mathrm{H}_{2} \mathrm{O}$ has advantages over a $\mathrm{LiBr}-\mathrm{H}_{2} \mathrm{O}$ system in terms of cycle performance as well as low energy consumption [3, 4-8]. Hence, it is important to perform a detailed thermodynamic analysis of a $\mathrm{LiCl}-\mathrm{H}_{2} \mathrm{O}$ vapor absorption refrigeration system (VARS) in order to improve the cycle performance.

To quantify the potential of useful energy in any system, exergy analysis identifies the irreversibilities of the system components through the exergy destruction and losses. This process determines the true inefficiencies in the system and where the losses are taking place. Exergy analysis measures the actual improvement potential of the system. Exergy signifies the maximum work potential of a system that can be produced when the system proceeds from initial state to final state in relation to the surrounding environment. In this study, the exergy analysis was performed for each component and the total exergy destruction of each component was divided into endogenous, exogenous, unavoidable, and avoidable exergies. This relatively new exergy analysis is known as advanced exergy analysis. Finally, a comparison of conventional and advanced exergy analyses was performed on the component(s) that need to be improved via reducing the exergy losses. 


\section{SYSTEM DESCIPTION}

The schematic of a single-effect $\mathrm{LiCl}-\mathrm{H}_{2} \mathrm{O}$ absorption cooling system is shown in Fig. 1. The core components of this cooling system are the absorber, generator, condenser and evaporator. Two expansion (throttle) valves are used for reducing the pressure. A solution heat exchanger is considered to preheat the weak solution of $\mathrm{LiCl}-\mathrm{H}_{2} \mathrm{O}$ leaving the absorber with the heat of the high temperature strong solution that flows back from the generator to the absorber.

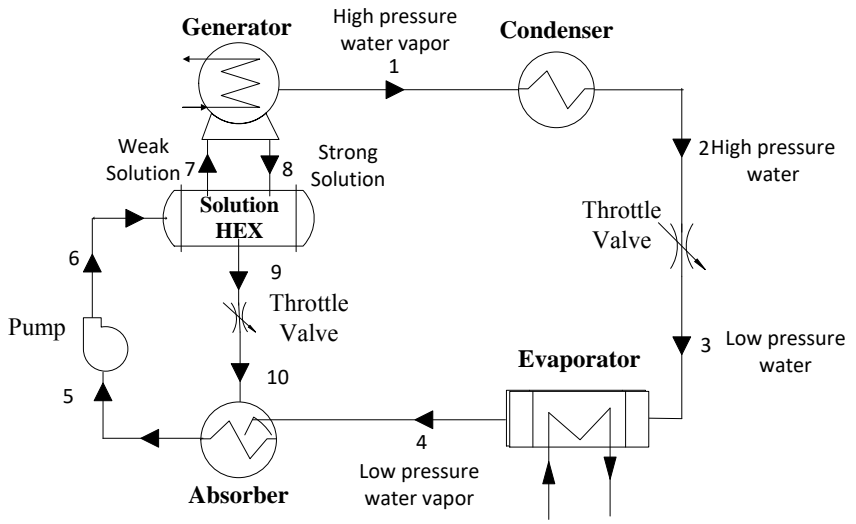

Figure 1: $\mathrm{LiCl}-\mathrm{H}_{2} \mathrm{O}$ Vapor Absorption Refrigeration System (VARS)

\section{THERMODYNAMIC ANALYSIS}

For a thermodynamic model of a vapor absorption refrigeration cycle, the principles of mass and energy conservation, and exergy balances was applied for each component of the system. The main components: generator, condenser, evaporator, absorber, and solution heat exchanger have been considered. To analyze the thermodynamic cycle, a control volume was applied to each component. All analyses were performed by considering the system under steady-state conditions.

\section{A. Conventional Exergy Analysis}

The mass and the energy balance of each component of the $\mathrm{LiCl}-\mathrm{H}_{2} \mathrm{O}$ refrigeration cycle in a control volume can be written as

Mass Conservation:

$$
\sum \dot{m}_{i n}-\sum \dot{m}_{\text {out }}=0
$$

Energy Conservation:

$$
\sum \dot{Q}=\sum \dot{m}_{\text {out }} h_{\text {out }}-\sum \dot{m}_{\text {in }} h_{\text {in }}+\dot{W}
$$

where $\dot{m}$ is the mass flow rate $(\mathrm{kg} / \mathrm{s}), \dot{Q}$ is the heat transfer rate $(\mathrm{kW}), h$ is the specific enthalpy $(\mathrm{kJ} / \mathrm{kg})$ and $\dot{W}$ is the rate of work leaving the control volume $(\mathrm{kW})$.

The exergy balance in a control volume can be determined by [9]

$$
\dot{E}_{D}=\sum(\dot{m} e)_{\text {in }}-\sum(\dot{m} e)_{\text {out }}+\sum \dot{E}_{Q_{\text {in }}}-\sum \dot{E}_{Q_{\text {out }}}+\sum \dot{W}
$$

where $\dot{E}_{D}$ represents the rate of exergy destruction (loss) in the system. (me $)_{i n}$ is the rate of exergy entering the control volume, $(\dot{m} e)_{o u t}$ is the rate of exergy leaving the control volume, $\dot{E}_{Q}$ represents the rate of exergy destruction in terms of heat transfer, to/from the component, and $\dot{W}$ is the mechanical work done by or on the control volume.

At any constant temperature $(\mathrm{T})$,

$$
E_{Q}=\phi\left(1-\frac{T_{r e f}}{T}\right)
$$

The overall exergy per unit mass of a fluid can be calculated by [10]

$$
e=\left(h-h_{0}\right)-T_{\text {ref }}\left(s-s_{0}\right)
$$

where $e$ is the specific exergy, $h$ is the specific enthalpy and $s$ is the entropy at a given temperature $T$, and $h_{o}$ is the enthalpy, and $s_{o}$ is the entropy at the environmental temperature $T_{r e f}$. The reference temperature and pressure are $\mathrm{T}_{\text {ref }}=273.15 \mathrm{~K}$ and $\mathrm{P}_{\text {ref }}$ $=101.325 \mathrm{kPa}$, respectively. The specific exergy, specific enthalpy and entropy of a $\mathrm{LiCl}-\mathrm{H}_{2} \mathrm{O}$ solution at a given temperature and pressure were calculated by using EES software [11]. In conventional exergy analysis, the exergy destruction [12] and the exergy destruction ratio [13] can be written as

$$
\begin{gathered}
\dot{E}_{D, k}=\left(\dot{E}_{k}\right)_{\text {in }}-\left(\dot{E}_{k}\right)_{\text {out }} \\
y_{k}=\frac{\dot{E}_{D, k}}{\dot{E}_{D, \text { total }}}
\end{gathered}
$$

In Equations (6) \& (7), $\dot{E}_{D, k}$ is the exergy destruction rate of $\mathrm{k}^{\text {th }}$ component, $\left(\dot{E}_{k}\right)_{\text {in }}$ and $\left(\dot{E}_{k}\right)_{\text {nut }}$ are the exergy entering/leaving to/from the $\mathrm{k}^{\text {th }}$ component, $y_{k}$ is the exergy destruction ratio of the $\mathrm{k}^{\text {th }}$ component over the total exergy destruction of the system. The exergy destruction provides the thermodynamic inefficiency of each component of the system [14]. The energy and the exergy equations of each component of the $\mathrm{LiCl}-\mathrm{H}_{2} \mathrm{O}$ absorption refrigeration system are shown in Table 1.

\section{B. Advanced Exergy Analysis}

The exergy destruction of each component can be split into endogenous/exogenous and unavoidable/avoidable exergy destruction parts [15]. Dividing exergy destruction of a component into endogenous and exogenous parts provides the actual exergy destruction caused by the component itself and the exergy destruction of that component caused by the remaining components of the system. As a result, it will provide information as to whether the focus to improve the system performance should be in the component itself or on the remaining components of the system. Alternatively, when the component operates at actual (real) and theoretical conditions, dividing the exergy destruction into unavoidable and avoidable parts determines the exergy destruction that cannot be eliminated. Table 2 shows the different operating conditions that were applied for this advanced exergy analysis. 
TABLE 1: ENERGY AND EXERGY BALANCE EQUATIONS

\begin{tabular}{|c|c|c|}
\hline $\begin{array}{l}\text { Cycle } \\
\text { Components }\end{array}$ & Energy balance equation & Exergy balance equation \\
\hline Generator & $\begin{array}{l}\dot{Q}_{g e n}=\dot{m}_{1} h_{1}+\dot{m}_{7} h_{7}-\dot{m}_{6} h_{6} \\
X_{6} \dot{m}_{6}=X_{7} \dot{m}_{7}\end{array}$ & $\dot{E}_{D, g e n}=\dot{m}_{6} e_{6}-\dot{m}_{1} e_{1}-\dot{m}_{7} e_{7}+\dot{Q}_{g e n}\left(1-\frac{T_{o}}{T_{g e n}}\right)$ \\
\hline Condenser & $\dot{Q}_{c o n}=\dot{m}_{1}\left(h_{1}-h_{2}\right)$ & $\dot{E}_{D, \text { con }}=\dot{m}_{1}\left(e_{1}-e_{2}\right)-\dot{Q}_{\text {con }}\left(1-\frac{T_{o}}{T_{c o n}}\right)$ \\
\hline Evaporator & $\dot{Q}_{\text {eva }}=\dot{m}_{4} h_{4}-\dot{m}_{3} h_{3}$ & $\dot{E}_{D, e v a}=\dot{m}_{1}\left(e_{3}-e_{4}\right)+\dot{Q}_{e v a}\left(1-\frac{T_{o}}{T_{e v a}}\right)$ \\
\hline Absorber & $\dot{Q}_{a b s}=\dot{m}_{8} h_{8}+\dot{m}_{4} h_{4}-\dot{m}_{5} h_{5}$ & $\dot{E}_{D, a b s}=\dot{m}_{4} e_{4}+\dot{m}_{8} e_{8}-\dot{m}_{5} e_{5}-\dot{Q}_{a b s}\left(1-\frac{T_{o}}{T_{a b s}}\right)$ \\
\hline \multirow[t]{2}{*}{ Solution HEX } & $\begin{array}{l}\mathrm{T}_{6}=\frac{\dot{m}_{7}}{\dot{m}_{5}} \eta_{\text {HEX }} \mathrm{T}_{7}+\left(1-\frac{\dot{m}_{7}}{\dot{m}_{5}} \eta_{\text {HEX }}\right) \mathrm{T}_{5} \\
\mathrm{~h}_{\mathrm{g}}=\mathrm{h}_{7}-\frac{\dot{m}_{5}}{\dot{m}_{7}}\left(\mathrm{~h}_{6}-\mathrm{h}_{5}\right)\end{array}$ & $\dot{E}_{D, H E X}=\dot{m}_{7} e_{7}+\dot{m}_{5} e_{5}-\dot{m}_{8} e_{8}-\dot{m}_{6} e_{6}$ \\
\hline & & $\dot{E}_{D, \text { total }}=\dot{E}_{D, \text { gen }}+\dot{E}_{D, \text { con }}+\dot{E}_{D, \text { eva }}+\dot{E}_{D, a b s}$ \\
\hline
\end{tabular}

TABLE 2: ALGORITHM AND ASSUMPTION OF PARAMETERS FOR DIFFERENT OPERATING CONDITIONS

\begin{tabular}{|c|c|c|c|c|}
\hline $\begin{array}{l}\text { Cycle } \\
\text { Components }\end{array}$ & Algorithm & Ideal cycle & Real cycle" & $\begin{array}{l}\text { Enavoidable } \\
\text { cycle* }^{*}\end{array}$ \\
\hline Generator & $\begin{array}{l}P_{g e n}=P_{\text {cond }}\left(1-\Delta P_{g e n}\right) \\
T_{g e n}=\left(T_{g e n}-\Delta T_{g e n}\right)\end{array}$ & $\begin{array}{l}\Delta P_{g e n}=0 \\
\Delta T_{g e n}=0\end{array}$ & $\begin{array}{l}\Delta P_{g e n}=2 \% \\
\Delta T_{g e n}=5{ }^{\circ} \mathrm{C}\end{array}$ & $\begin{aligned} \Delta P_{g e n} & =0.2 \% \\
\Delta T_{g e n} & =0.5{ }^{\circ} \mathrm{C}\end{aligned}$ \\
\hline Condenser & $\begin{array}{l}T_{\text {cond }}=\left(T_{\text {cand }}+\Delta T_{\text {eond }}\right) \\
T_{\text {eond }} \rightarrow P_{\text {cond }}\end{array}$ & $\Delta T_{\text {cond }}=0$ & $\Delta T_{\text {eand }}=5^{\circ} \mathrm{C}$ & $\Delta T_{\text {cond }}=0.5^{\circ} \mathrm{C}$ \\
\hline Evaporator & $\begin{array}{l}T_{e v a}=T_{e v a}+\Delta T_{e v a} \\
T_{e v a} \rightarrow P_{e v a}\end{array}$ & $\Delta T_{e v a}=0$ & $\Delta T_{e v a}=2^{\circ} \mathrm{C}$ & $\Delta T_{\text {eva }}=0.2^{\circ} \mathrm{C}$ \\
\hline Absorber & $\begin{array}{l}T_{a b s}=T_{a b s}+\Delta T_{a b s} \\
P_{a b s}=P_{e v a}\left(1-\Delta P_{a b s}\right)\end{array}$ & $\begin{array}{l}\Delta T_{a b s}=0 \\
\Delta P_{a b s}=0\end{array}$ & $\begin{array}{l}\Delta T_{a b s}=5^{\circ} \mathrm{C} \\
\Delta P_{a b s}=2 \%\end{array}$ & $\begin{array}{l}\Delta T_{a b s}=0.5^{\circ} \mathrm{C} \\
\Delta P_{a b s}=0.2 \%\end{array}$ \\
\hline Solution HEX & $\Delta T_{H E X}=T_{7}-T_{\mathrm{g}}$ & $\begin{array}{c}\Delta T_{\text {HEXX }}=0 \\
\eta_{M E X X}=100 \%\end{array}$ & $\begin{array}{l}\Delta T_{\text {HEXX }}=5^{\circ} \mathrm{C} \\
\eta_{H E X}=80 \%\end{array}$ & $\begin{aligned} \Delta T_{H E X} & =0.5{ }^{\circ} \mathrm{C} \\
\eta_{H E X} & =95 \%\end{aligned}$ \\
\hline $\begin{array}{l}\text { Solution } \\
\text { concentration }\end{array}$ & $X_{\text {strong }}=\varphi\left(T_{g e n}, P_{g e n}\right)$ & & & \\
\hline
\end{tabular}

\section{Endogenous/Exogenous Exergy Destruction}

The total exergy destruction $\left(E_{D, k}^{r e a l}\right)$ of a component $(\mathrm{k})$ can be divided into endogenous $\left(E_{D, k}^{E N}\right)$ and exogenous $\left(E_{D, k}^{E X}\right)$ parts and can be written as [9]

$$
\dot{E}_{D, k}^{\text {real }}=\dot{E}_{D, k}^{E N}+\dot{E}_{D, k}^{E X}
$$

The endogenous exergy destruction of a component is associated with the internal irreversibilities of that component only when the other components are without irreversibilities. This can be determined by considering the case where this component will work on real operating conditions while the other components of the system will work in ideal (theoretical) operating conditions. Therefore, the endogenous exergy destruction is only due to the inefficiency of the $\mathrm{k}$ component. The remaining exergy destruction is the exogenous exergy destruction in that component. This exergy destruction represents the inefficiency of the $\mathrm{k}$ component and the inefficiencies of the remaining components of the system.

\section{Unavoidable/Avoidable Exergy Destruction}

In real operating conditions, every component of a system works with some limitations such as technical and design limitations including material availability or cost of the materials etc. Therefore, some part of the total exergy destruction of a component cannot be reduced. This is called the unavoidable exergy destruction $\left({ }^{E_{D, k}^{U N}}\right)$. The rest of the total exergy destruction of the component is avoidable $\left({ }^{E_{D, k}^{A V}}\right)$, which is represented by Equation (9). The unavoidable exergy destruction of a component is calculated assuming that the component is isolated from the system. The most reasonable operating conditions for calculating the unavoidable exergy destruction are the lowest temperature difference and pressure losses [15-17]. The unavoidable conditions are determined based on the maximum improvement that can be achieved for the component.

$$
\dot{E}_{D, k}^{\text {real }}=\dot{E}_{D, k}^{U N}+\dot{E}_{D, k}^{A V}
$$

\section{Combination of Two Dimensions of Exergy Destruction}

The unavoidable exergy destruction of a component can be split again into endogenous unavoidable exergy destruction and exogenous unavoidable exergy destruction of that component. The exogenous unavoidable parts of a component cannot be reduced any more due to the technical limitations of the other components of the system for a given design and structure [17]. For a component, it can be determined by subtracting the endogenous unavoidable exergy destruction from the total unavoidable exergy destruction of that component and is defined by

$$
\dot{E}_{D, k}^{U N}=\dot{E}_{D, k}^{E N-U N}+\dot{E}_{D, k}^{E X-U N}
$$

The endogenous avoidable part of the exergy destruction of a component can be reduced by improving the efficiency of that component. It can be can be determined by

$$
\dot{E}_{D, k}^{E N}=\dot{E}_{D, k}^{E N-U N}+\dot{E}_{D, k}^{E N-A V}
$$

Likewise, the exogenous avoidable exergy destruction of a component can be reduced by the improving the design and structure of the overall system and also by improving the efficiency of the remaining components of the system. It is defined by

$$
\dot{E}_{D, k}^{E X}=\dot{E}_{D, k}^{E X-U N}+\dot{E}_{D, k}^{E X-A V}
$$

Therefore, the effect of the exergy destruction of each component on the overall system performance can be determined through this advanced exergy analysis. The total exergy destruction of a component is

$$
\dot{E}_{D, k}^{r e a l}=\dot{E}_{D, k}^{E N-U N}+\dot{E}_{D, k}^{E N-A V}+\dot{E}_{D, k}^{E X-U N}+\dot{E}_{D, k}^{E X-A V}
$$

\section{Theoretical Considerations}

The following assumptions have been used for analyzing the thermodynamic cycle in this study:

1. The system is operating under steady state conditions.

2. $\mathrm{LiCl}-\mathrm{H}_{2} \mathrm{O}$ solutions are assumed to be in equilibrium in the generator and in the absorber at their corresponding pressures and temperatures.

3. The throttle valve is a constant enthalpy adiabatic process.

4. The vapor leaving the generator is $100 \%$ water vapor.

5. The refrigerant leaving the condenser is saturated water, and leaves the evaporator as water vapor. 
6. The air cooling is considered for cooling of the condenser and the absorber at an atmospheric temperature of $25^{\circ} \mathrm{C}$.

\section{RESULTS AND ANALYSIS}

The thermodynamic values of different states of a 10

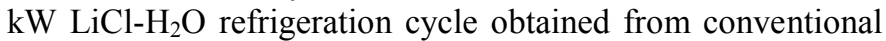
exergy analysis under ideal (theoretical) and real operating conditions are summarized in Table 3 . The exergy destruction $\left(E_{D, K}\right)$ and exergy destruction ratio $\left(y_{D, K}\right)$ of each component under ideal, real, and unavoidable operating conditions are shown in Table 4. Table 4 shows that the highest exergy destruction is in the absorber followed by the condenser, the generator and the evaporator for ideal and unavoidable operating conditions. However, the solution heat exchanger has the second highest exergy destruction after the absorber in real operating condition because of the heat exchanger effectiveness. These losses are due to high irreversibilities occurring in the absorber and the generator. This is because of the high temperature difference across the heat exchanger in the absorber, mass transfer between the two differently concentrated solutions and the mixing losses in the absorber and the generator [18]. Furthermore, the refrigerant (water) vapor is superheated when it leaves the generator/separator. As a result, a higher temperature is required under the same operating conditions, and this leads to higher thermodynamic losses in the generator. In addition, the condenser requires more cooling in order to cool the superheated water vapor that comes from the generator and this generates more exergy destruction in the condenser. This conventional exergy analysis suggests that the exergy loss in the absorber needs to be reduced first to improve the overall system efficiency. However, the irreversibilities of the other components in the system are partly responsible for the generator and condenser exergy destructions, and the extent of responsibility can be determined by advanced exergy analysis.

In advanced exergy analysis, the exergy destruction of each component is split into four parts. The analyses have been performed based on the operating conditions in Table 2. The results of this analysis are shown in Table 5.

TABLE 4: RESUlts OF CONVENTIONAL EXERGY ANALYSIS OF A $10 \mathrm{KW}$ LiCl- $\mathrm{H}_{2} \mathrm{O}$ ABSORPTION REFRIGERATION CYCLE UNDER DIFFERENT OPERATING CONDITIONS

\begin{tabular}{lcccccc}
\hline $\begin{array}{l}\text { Cycle } \\
\text { Components }\end{array}$ & \multicolumn{2}{c}{ Ideal } & \multicolumn{2}{c}{ Real } & \multicolumn{2}{c}{ Unavoidable } \\
\cline { 2 - 7 } & $\begin{array}{c}E_{D, k} \\
(k W)\end{array}$ & $\begin{array}{c}y_{D, k} \\
(\%)\end{array}$ & $\begin{array}{c}E_{D, k} \\
(k W)\end{array}$ & $\begin{array}{c}y_{D, k} \\
(\%)\end{array}$ & $\begin{array}{c}E_{D, k} \\
(k W)\end{array}$ & $\begin{array}{c}y_{D, k} \\
(\%)\end{array}$ \\
\hline Generator & 6.13 & 18.8 & 10.31 & 13.6 & 6.18 & 18.8 \\
Condenser & 7.54 & 23.1 & 7.70 & 10.1 & 7.51 & 22.9 \\
Evaporator & 6.13 & 18.8 & 7.53 & 9.7 & 6.11 & 18.6 \\
Absorber & 10.3 & 31.6 & 33.04 & 42.7 & 10.47 & 31.9 \\
Solution HEX & 2.53 & 7.8 & 18.76 & 24.3 & 2.60 & 7.9 \\
\hline
\end{tabular}

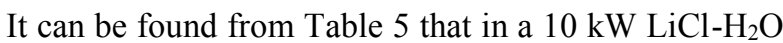
absorption air conditioning system, the exogenous exergy destruction $\left(\dot{E}_{D, k}^{E X}\right)$ of the absorber and the solution heat exchanger is higher than the endogenous exergy destruction ( $\dot{E}_{D, k}^{E N}$ ). This is opposite for generator, condenser and evaporator. This demonstrates that the other components' irreversibilities have high impact on the absorber and the solution HEX performance. Whereas, the internal irreversibility of the generator, the condenser and the evaporator itself needs to be reduced. Therefore, to improve the overall system efficiency, the components themselves should be examined for design and performance improvement.

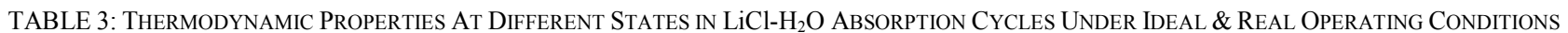

\begin{tabular}{|c|c|c|c|c|c|c|c|}
\hline State & $\begin{array}{c}\text { Temperature } \\
\left({ }^{\circ} \mathrm{C}\right) \\
\text { Ideal/Real } \\
\end{array}$ & $\begin{array}{c}\text { Pressure } \\
(\mathbf{k P a}) \\
\text { Ideal/Real } \\
\end{array}$ & $\begin{array}{c}\text { Mass flow } \\
(\mathrm{g} / \mathrm{s}) \\
\text { Ideal/Real } \\
\end{array}$ & $\begin{array}{c}\% \\
\text { Concentration } \\
\text { Ideal/Real } \\
\end{array}$ & $\begin{array}{c}\text { Enthalpy } \\
(\mathbf{k J} / \mathbf{k g}) \\
\text { Ideal/Real } \\
\end{array}$ & $\begin{array}{c}\text { Entropy } \\
\text { (kJ/kgK) } \\
\text { Ideal/Real } \\
\end{array}$ & $\begin{array}{c}\text { Exergy, e } \\
\text { (kJ/kg) } \\
\text { Ideal/Real } \\
\end{array}$ \\
\hline $\begin{array}{l}\text { Bubble pump generator } \\
\text { refg exit (1) }\end{array}$ & $78 / 75$ & $5.63 / 7.2$ & $4.2 / 4.3$ & 100 & $2646 / 2640$ & $8.6 / 8.5$ & $87 / 120$ \\
\hline Condenser refg exit (2) & $35 / 40$ & $5.63 / 7.2$ & $4.2 / 4.3$ & 100 & $147 / 168$ & $0.51 / 0.57$ & $0.59 / 1.43$ \\
\hline Evaporator refg inlet (3) & $7 / 9$ & $1 / 1.15$ & $4.2 / 4.3$ & 100 & $147 / 168$ & $0.51 / 0.57$ & $0.59 / 1.43$ \\
\hline Evaporator refg exit (4) & $7 / 9$ & $1 / 1.15$ & $4.2 / 4.3$ & 100 & $2513 / 2517$ & $8.97 / 8.92$ & $-157 /-139$ \\
\hline Absorber sol exit (5) & $35 / 40$ & $1 / 1.15$ & $25 / 45.9$ & $41.07 / 42.95$ & $155 / 182$ & $0.31 / 0.35$ & $-72 /-2.3$ \\
\hline Sol HEX inlet (6) & $35 / 40$ & $5.63 / 7.2$ & $25 / 45.9$ & $41.07 / 42.95$ & $155 / 182$ & $0.31 / 0.35$ & $-72 /-2.3$ \\
\hline Generator sol inlet (7) & $78 / 68$ & $5.63 / 7.2$ & $25 / 465.9$ & $41.07 / 42.95$ & $268 / 254$ & $0.65 / 0.57$ & $78 / 89$ \\
\hline Generator sol exit (8) & $78 / 75$ & $5.63 / 7.2$ & $21 / 45.5$ & $49.41 / 43.35$ & $327 / 275$ & 0.210 .62 & $150 / 95$ \\
\hline Sol HEX exit (9) & $35 / 47$ & $5.63 / 7.2$ & $21 / 45.5$ & $49.41 / 43.35$ & $190 / 202$ & $0.16 / .039$ & $91 / 44$ \\
\hline Absorber sol inlet (10) & $35 / 47$ & $1 / 1.15$ & $21 / 45.5$ & $49.41 / 43.35$ & $190 / 202$ & $0.16 / 0.39$ & $91 / 44$ \\
\hline
\end{tabular}

refg $=$ refrigerant; sol $=$ solution 
The percentage of exogenous exergy destruction rate of the solution heat exchanger $(87 \%)$, the absorber $(63 \%)$, and the generator $(42 \%)$ are higher than that of the evaporator $(17 \%)$ and the condenser $(2 \%)$.

TABLE 5: Results OF ADVANCED EXERGy ANALYSIS OF A LiCl- $\mathrm{H}_{2} \mathrm{O}$ ABSORPTION REFRIGERATION CYCLE

\begin{tabular}{|c|c|c|c|c|c|c|c|c|c|}
\hline \multirow[t]{2}{*}{ Components } & \multirow{2}{*}{$\begin{array}{l}E_{D, k}^{\text {real }} \\
(k W)\end{array}$} & \multirow{2}{*}{$\begin{array}{l}E_{D, F}^{F N} \\
(k W)\end{array}$} & \multirow{2}{*}{$\begin{array}{c}E_{D W}^{E X} \\
(k W)\end{array}$} & \multirow{2}{*}{$\begin{array}{c}E_{D k}^{\| N} \\
(k W)\end{array}$} & \multirow{2}{*}{$\begin{array}{l}E_{b .}^{A D V} \\
(k W)\end{array}$} & \multicolumn{2}{|c|}{$U N$} & \multicolumn{2}{|c|}{$A V$} \\
\hline & & & & & & $\begin{array}{c}E_{D N}^{Z N-U N} \\
(k W)\end{array}$ & $\begin{array}{c}E_{D Y}^{E X X N} \\
(\mathrm{~kW})\end{array}$ & $\begin{array}{c}E_{B .}^{E N-A V} \\
(\mathrm{~kW})\end{array}$ & $\begin{array}{c}E_{b 1}^{E X-A V} \\
(k W)\end{array}$ \\
\hline \multirow[t]{2}{*}{ Generator } & 10.31 & 603 & 428 & 6,18 & 4.13 & 60 & 018 & 0.03 & 41 \\
\hline & & $58 \%$ & $42 \%$ & $60 \%$ & $40 \%$ & $58 \%$ & $2 \%$ & $0 \%$ & $40 \%$ \\
\hline \multirow[t]{2}{*}{ Condenser } & 7.70 & 7.54 & 0.16 & 7.5 & 0.2 & 7.45 & 0.05 & 0.09 & 0.11 \\
\hline & & $98 \%$ & $2 \%$ & $97 \%$ & $3 \%$ & $97 \%$ & $1 \%$ & $1 \%$ & $1 \%$ \\
\hline \multirow[t]{2}{*}{ Evaporator } & 7.53 & 622 & 131 & 614 & 1.39 & 611 & 0.03 & 0.11 & 128 \\
\hline & & $83 \%$ & $17 \%$ & $82 \%$ & $18 \%$ & $81 \%$ & $0 \%$ & $1 \%$ & $17 \%$ \\
\hline \multirow[t]{2}{*}{ Absorber } & 3304 & 12,38 & 20,66 & 10.47 & 2257 & 10.43 & 0.04 & 195 & 20.62 \\
\hline & & $37 \%$ & $63 \%$ & $32 \%$ & $68 \%$ & $32 \%$ & $0 \%$ & $6 \%$ & $62 \%$ \\
\hline \multirow[t]{2}{*}{ Solution HEX } & 18.76 & 2.53 & 16.23 & 2.6 & 16.16 & 2.48 & 0.12 & 0.05 & 16.11 \\
\hline & & $13 \%$ & $87 \%$ & $14 \%$ & $86 \%$ & $13 \%$ & $1 \%$ & $0 \%$ & $86 \%$ \\
\hline \multirow[t]{2}{*}{ Total } & 77 & 34.7 & 42.6 & 32.9 & 44.5 & 32.5 & 0.4 & 2.2 & 42.2 \\
\hline & & $45 \%$ & $55 \%$ & $43 \%$ & $57 \%$ & $42 \%$ & $0.5 \%$ & $3 \%$ & $54.5 \%$ \\
\hline
\end{tabular}

It is also noted from Table 5 that the total unavoidable

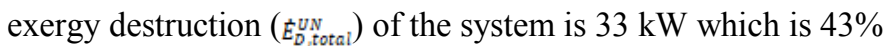
of the total exergy destruction rate. While $E_{D}^{E N-\text { total }}=32 \mathrm{~kW}$ $(42 \%)$ is due to the components themselves. Therefore, the minimum exergy destruction rate of the system is $33 \mathrm{~kW}$ and this cannot be reduced further. In practice, only the avoidable part of the total exergy destruction can be reduced. It is noticed that the avoidable part of the exergy destruction is $57 \%$ (44 $\mathrm{kW})$ of the total exergy destruction rate $\left(E_{D \text {, total }}^{\text {real }}=77 \mathrm{~kW}\right)$. However, only $3 \%(2.2 \mathrm{~kW})$ of the total exergy destruction can be eliminated by improving the components' own efficiencies, and $54.55 \%(42 \mathrm{~kW})$ will be lowered by improving overall system efficiency. The value of $\dot{E}_{D, a b s}^{E N-A V}$ in Table 5 indicates only $6 \%$ of the exergy destruction of the absorber can be reduced by technological improvement. It is also noticed that the exogenous avoidable is much higher than the endogenous avoidable exergy destruction ( $\dot{E}_{D}^{E X-A V}>>\dot{E}_{D}^{E N-A V}$ ) for all components. Therefore, the improvement of the efficiencies of other components plays a significant role in enhancing the overall system efficiency.

To evaluate the overall system performance in advanced exergy analysis, the avoidable exergy destruction is the focus as this has the potential for improvement. However, it is shown in Fig. 2 that the endogenous exergy destruction of the overall system is $45 \%$, out of which, $42 \%$ is unavoidable. Whereas, almost all of $55 \%$ of the exogenous exergy destruction is avoidable as shown in Fig 2. These results show the interaction between the components and how the internal irreversibilities of each component influence the overall system efficiencies. In this analysis, the higher unavoidable exergy destruction rate indicates that the system efficiency can be optimized by optimizing the operating conditions. The technological improvement of the system component, the size of the component, and the high heat and mass transfer mechanism can also have the positive impact to reduce the unavoidable exergy destruction rate.

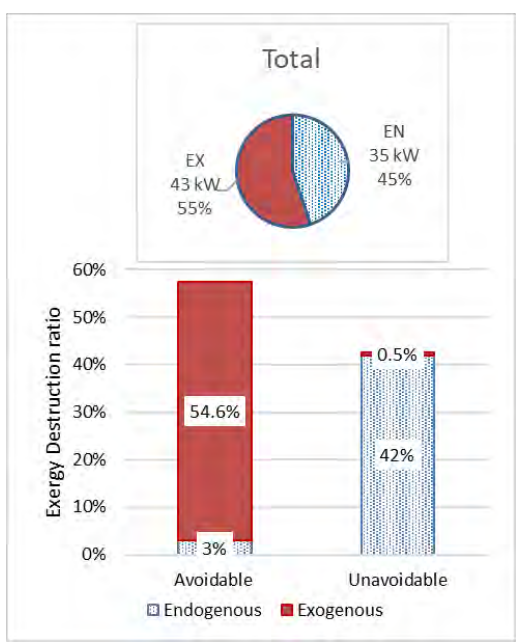

Figure 2: Exergy destruction ratio of the overall VAR system based on advanced exergy analysis.

Furthermore, based on the conventional and advanced exergy analyses, the influence of each component on the overall exergy destruction is shown in Fig. 3. Conventional exergy analysis concludes that the highest potential for improvement is the absorber, followed by the solution HEX and the generator, in order to improve the overall system performance. However, the advanced exergy analysis suggests that $32 \%(10.47 \mathrm{~kW})$ of the total exergy destruction in the absorber is unavoidable, and $6 \%$ is available by improving the absorber itself. Whereas, all avoidable exergy destruction of the generator $(40 \%)$, the solution HEX $(86 \%)$, and the evaporator $(17 \%)$ are exogenous avoidable. This clearly indicates that the irreversibilities of the overall system should be reduced for the system performance improvement. Furthermore, it is also noticed that $98 \%$ of the condenser exergy destruction is due to its internal irreversibilities, of which, $97 \%$ are unavoidable and $1 \%$ can be improved by improving other components efficiency.

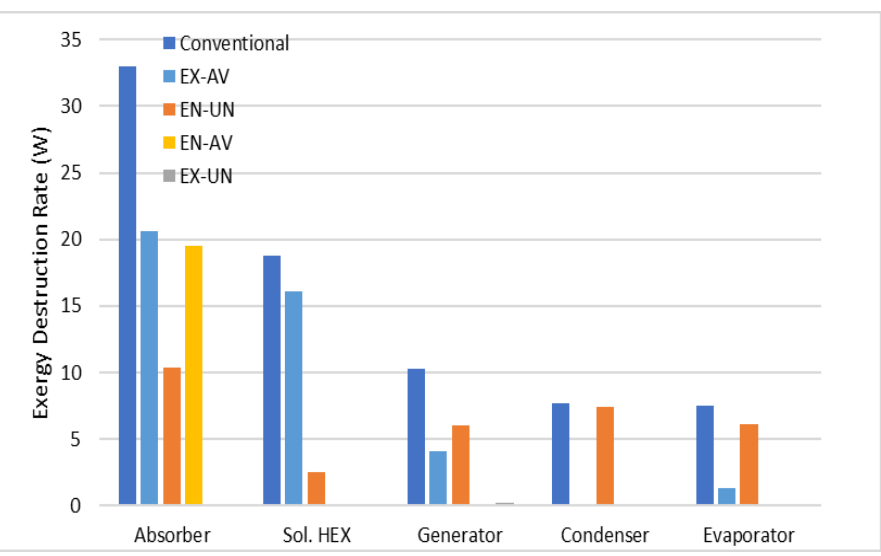

Figure 3: Exergy destruction rate of each component of a $\mathrm{LiCl}-\mathrm{H}_{2} \mathrm{O}$ absorption refrigeration cycle based on conventional exergy and advanced exergy analyses. 


\section{CONCLUSIONS}

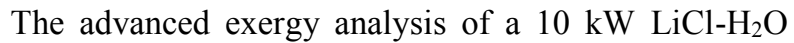
absorption air-conditioning system offers useful information in identifying the component that has the most potential for the overall system improvement. This information cannot be provided by a conventional exergy analysis. The avoidable exergy destruction identifies the potential of each component that can be improved by technological improvement. The endogenous avoidable and exogenous avoidable exergy destruction relate the interactions between the components' irreversibilities and quantify the importance of the relative improvement of each component and the overall system.

Finally, the analysis in this study is a very effective tool for the exergoeconomic optimization of energy conversion systems. It can provide useful information to the designer for investment in order to reduce the endogenous avoidable and exogenous avoidable parts of exergy destruction and to measure the potential improvement of the overall system structure.

\section{ACKNOWLEDGMENT}

This work is made possible by the Natural Sciences and Engineering Research Council of Canada.

\section{REFERENCES}

[1] W. Su and X. Zhang, "Thermodynamic analysis of a compressionabsorption refrigeration air-conditioning system coupled with liquid desiccant dehumidification," Appl. Therm. Eng., vol. 115, pp. 575-585, Mar. 2017.doi: 10.1016/j.applthermaleng.2016.12.071.

[2] Z. Liu, A. Li, Q. Wang, Y. Chi, and L. Zhang, "Performance study of a quasi grid-connected photovoltaic powered DC air conditioner in a hot summer zone," Appl. Therm. Eng., vol. 121, pp. 1102-1110, Jul. 2017. doi: 10.1016/j.applthermaleng.2017.03.132.

[3] J. Aman, P. Henshaw, and D. S.-K. Ting, "Bubble-pump-driven LiBr-H 2 $\mathrm{O}$ and LiCl-H $2 \mathrm{O}$ absorption air-conditioning systems," Ther. Sci. Eng. Prog., vol. 6, pp. 316-322, Jun. 2018. doi: 10.1016/j.tsep.2017.10.022.

[4] G. S. Grover, M. A. R. Eisa, and F. A. Holland, "Thermodynamic design data for absorption heat pump systems operating on water-lithium chloridePart one. Cooling," Heat Recovery Syst. and CHP, vol. 8, no. 1, pp. 33-41, Jan. 1988. doi: 10.1016/0890-4332(88)90039-7

[5] V. H. F. Flores, J. C. Román, and G. M. Alpírez, "Performance analysis of different working fluids for an absorption refrigeration cycle", Am. J. Environ. Eng., vol. 4, pp. 1-10, 2014. doi: 10.5923/s.ajee.201401.01.

[6] X. She, Y. Yin, M. Xu, and X. Zhang, "A novel low-grade heat-driven absorption refrigeration system with $\mathrm{LiCl}-\mathrm{H} 2 \mathrm{O}$ and $\mathrm{LiBr}-\mathrm{H} 2 \mathrm{O}$ working pairs," Int. J. Refrig., vol. 58, pp. 219-234, Oct. 2015.

doi: 10.1016/j.ijrefrig.2015.06.016.

[7] T. K. Gogoi and D. Konwar, "Exergy analysis of a H 2 O-LiCl absorption refrigeration system with operating temperatures estimated through inverse analysis," Energy Convers. Manage., vol. 110, pp. 436-447, Feb. 2016. doi: 10.1016/j.enconman.2015.12.037.

[8] A. Bejan, G. Tsatsaronis, M. Moran, "Thermal design and optimization", New York: Wiley, 1996.
[9] S. Kelly, G. Tsatsaronis, and T. Morosuk, "Advanced exergetic analysis: Approaches for splitting the exergy destruction into endogenous and exogenous parts," Energy, vol. 34, no. 3, pp. 384-391, Mar. 2009. doi: 10.1016/j.energy.2008.12.007.

[10] L. Zhu and J. Gu, "Second law-based thermodynamic analysis of ammonia/ sodium thiocyanate absorption system," Renewable Energy, vol. 35, no. 9, pp. 1940-1946, Sep. 2010. doi: 10.1016/j.renene.2010.01.022.

[11] EES (Engineering Equation Solver). F-Chart software, Academic Commercial V9. (2015), 941(1992-2017). [Online]. Available: www.fchart.com.

[12] I. Janghorban Esfahani, S. Lee, and C. Yoo, "Evaluation and optimization of a multi-effect evaporation-absorption heat pump desalination based conventional and advanced exergy and exergoeconomic analyses," Desalination, vol. 359, pp. 92-107, Mar. 2015. doi: 10.1016/j.desal.2014.12.030.

[13] T. Morosuk, G. Tsatsaronis, A. Boyano, and C. Gantiva, "Advanced exergybased analyses applied to a system including LNG regasification and electricity generation," Int. J. Energy and Environ. Eng., vol. 3, no. 1, p. 1, 2012. doi: 10.1186/2251-6832-3-1.

[14] A. Vidal, R. Best, R. Rivero, and J. Cervantes, "Analysis of a combined power and refrigeration cycle by the exergy method," Energy, vol. 31, no. 15, pp. 3401-3414, Dec. 2006. doi: 10.1016/j.energy.2006.03.001.

[15] T. Morosuk, G. Tsatsaronis, "Comparative evaluation of LNG-based cogeneration systems using advanced exergetic analysis", Energy, vol. 36, no. 6, pp. 3771-3778, Jun. 2011. doi: 10.1016/j.energy.2010.07.035.

[16] T. Morosuk and G. Tsatsaronis, "Comparative evaluation of LNG - based cogeneration systems using advanced exergetic analysis," Energy, vol. 36, no. 6, pp. 3771-3778, Jun. 2011. doi: 10.1016/j.energy.2007.09.012.

[17] S. E. May, I. Boukholda, and A. Bellagi, "Energetic and exergetic analysis of a commercial ammonia water absorption chiller," Int. J. Exergy, vol. 8, no. 1, p. 33, 2011. doi: 10.1504/IJEX.2011.037213.

[18] F. Cziesla, G. Tsatsaronis, and Z. Gao, "Avoidable thermodynamic inefficiencies and costs in an externally fired combined cycle power plant," Energy, vol. 31, no. 10-11, pp. 1472-1489, Aug. 2006. doi: 10.1016/j.energy.2005.08.001. 


\section{State of Charge and Parameter Estimation of Electric Vehicle Batteries}

\author{
Richard Bustos*, Abu Raihan Mohammad Siddique, \\ and Taranjit Cheema \\ College of Engineering and Physical Sciences, \\ University of Guelph, Ontario, Canada \\ *rbustos@uoguelph.ca
}

\begin{abstract}
Due to rising global environmental issues, electric vehicles (EV) are growing in popularity and will eventually replace vehicles that use internal combustion engines (ICE). EVs draw their power from batteries. Batteries are highly nonlinear storage elements used in a constantly changing environment making them highly dynamic and mathematically complex. In order to approximate the driving range of an EV, the state of charge (SOC) of the battery, which cannot be directly measured, has to be estimated accurately. SOC is highly dependent on the following parameters: internal resistance, temperature, and open circuit voltage. In this paper, two battery equivalent circuit models (ECM) are analyzed in conjunction with a thermal model to track the inner temperature of the battery. The states of the battery are estimated using the popular Kalman filter (KF) and unscented Kalman filter (UKF), and the results are discussed.
\end{abstract}

Keywords-electric vehicles; equivalent circuit models; Kalman filter; interactive multiple model; state of charge.

\section{INTRODUCTION}

The effects of technology, and in particular automobiles, on the environment are a growing concern in society. Automobile technology is rapidly moving towards a relatively eco-friendlier solution in an attempt to minimize the output of harmful greenhouse gasses: electric vehicles (EV) [1]. When compared to petroleum-based vehicles using the internal combustion engine (ICE), EVs have a smaller footprint, of course depending on the energy source [2].

In an EV, batteries store the electrical energy in an electrochemical reaction for later use. There are several types of batteries in the industry. The most popular are lead-acid, nickel, alkaline and lithium-ion [1]. Lithium has become very popular because it is the lightest of all metals, has the greatest electrochemical potential and provides the largest specific energy per weight [1]. Current lithium-ion battery technology allows EV to cover about $180-350 \mathrm{~km}$ per battery charge $[1,2]$. Unfortunately, batteries' full load capacity degrades over time as they are subject to charging cycles, resulting in a lower driving range throughout its lifetime [3]. In addition, the state of charge (SOC) and voltage measurement from the cell's terminal is crucial information to determine the available energy in the battery, which can be used to determine the

\author{
S. Andrew Gadsden ${ }^{\psi}$ and Shohel Mahmud \\ College of Engineering and Physical Sciences, \\ University of Guelph, Ontario, Canada \\ *gadsden@uoguelph.ca
}

available driving range of the EV. It is not possible to have direct measurements of the SOC [2]. The main difficulties are as follows:

- The battery packs of EVs have hundreds of cells connected in series; the different accumulated potential of each cell voltage is different to each other making it hard to have unified compensation or elimination methods.

- Voltage measurements require high precision. Other parameters are estimated according to voltage measurements. Required voltage precision is around 1 $\mathrm{mV}$ to have low carried \% errors [3].

The SOC of a battery can help estimate current driving range and prevent the battery pack from over charge and over discharge [3]. SOC is not easy to calculate for, as it would demand extensive computing times, expensive instrumentation or have the vehicle stopped preventing its application in real time [1, 4]. A solution is to generate estimates of the parameters, which requires a quality model, and good estimation strategy for the various scenarios: temperature, power demands, and state of function $[3,5]$.

In this paper, two battery electrical equivalent circuit models (ECM) are analyzed in conjunction with a thermal model. The states of the battery are estimated using the Kalman filter (KF) and unscented Kalman filter (UKF) [6, 7]. In addition, an interacting multiple model (IMM) is implemented for fault detection. Section II presents different filter techniques for the state of charge estimation process. Section III shows equivalent model of thermal, Rint, and Thevenin model. A numerical study and the simulated results are illustrated in Section IV. The paper is concluded in Section V.

\section{State OF Charge Estimation}

Three different estimation strategies, the Kalman filter (KF), unscented Kalman filter (UKF) and the interacting multiple model (IMM) method, are used to estimate the SOC, terminal voltage, and temperature behaviour of the battery. The following section briefly summarizes the filters and their algorithms. 


\section{A. Kalman Filter $(K F)$}

The Kalman filter (KF) was first introduced by R.E. Kalman in 1960 [8]. The KF provides the optimal solution, in terms of state estimation error, for known linear systems in the presence of white, Gaussian noise [8]. The system dynamic model and measurement model is described by the following two equations, respectively [6-8]:

$$
\begin{gathered}
x_{k+1}=A_{k} x_{k}+B_{k} u_{k}+w_{k} \\
z_{k+1}=C_{k+1} x_{k+1}+v_{k+1}
\end{gathered}
$$

where $A$ is the system matrix, $B$ is the input matrix, $C$ is the output matrix, $x$ is the system states, $z$ is the measurement output, $u$ is the input, $w$ is the system noise, and $v$ is the measurement noise. The following equations represent the KF estimation process [6].

Prediction stage:

$$
\begin{aligned}
& \hat{x}_{k+1 \mid k}=A_{k} \hat{x}_{k \mid k}+B_{k} u_{k} \\
& P_{k+1 \mid k}=A_{k} P_{k \mid k} A_{k}^{T}+Q_{k}
\end{aligned}
$$

Update stage:

$$
\begin{gathered}
K_{k+1}=P_{k+1 \mid k} C_{k+1}^{T}\left[C_{k+1} P_{k+1 \mid k} C_{k+1}^{T}+R_{k+1}\right]^{-1} \\
\hat{x}_{k+1 \mid k+1}=\hat{x}_{k+1 \mid k}+K_{k+1}\left[z_{k+1}-C_{k+1} \hat{x}_{k+1 \mid k}\right] \\
P_{k+1 \mid k+1}=\left[1-K_{k+1} C_{k+1}\right] P_{k+1 \mid k}
\end{gathered}
$$

\section{B. Unscented Kalman Filter (UKF)}

The UKF is a form of sigma point Kalman filter (SPKF) which is based on a statistical linear regression strategy which linearizes the nonlinear model statistically [7]. In simple steps, SPKF techniques generate a certain number of points referred as 'sigma points' from the projected probability distribution of the states. These points are then projected using the nonlinear system model, to obtain the 'a posteriori' estimate for probability distribution. The main difference characteristic of the SPKF is that it does not calculate the Jacobian matrices of the system in an attempt to linearize the system often yielding better results than other forms of the KF such as the Extended Kalman Filter (EKF). In the literature, one of the most popular type of SPKF is the UKF.

The following is a summary of the UKF algorithm [8]:

Intialization:

$$
\begin{gathered}
X_{0, k \mid k}=\hat{x}_{k \mid k} \\
W_{0}=\frac{k}{(n+k)} \\
\hat{x}_{k \mid k}+\left(\sqrt{(n+k) P_{k \mid k}}\right)_{i} \\
W_{i}=\frac{1}{[2(n+k)]} \\
X_{i+n, k \mid k}=\hat{x}_{k \mid k}-\left(\sqrt{(n+k) P_{k \mid k}}\right)_{i}
\end{gathered}
$$

Prediction:

$$
W_{i}=\frac{1}{[2(n+k)]}
$$

$$
\begin{aligned}
\hat{X}_{i, k+1 \mid k} & =f\left(X_{i}, u_{k}, w_{k}\right) \\
\hat{x}_{k+1 \mid k} & =\sum_{i=0}^{2 n} W_{i, k \mid k}, u_{k} \\
P_{k+1 \mid k}=\sum_{i=0}^{2 n} W_{i}\left(\hat{X}_{i, k+1 \mid k}\right. & \left.-\hat{x}_{k+1 \mid k}\right)\left(\hat{X}_{i, k+1 \mid k}-\hat{x}_{k+1 \mid k}\right)^{T} \\
\hat{Z}_{i, k+1 \mid k} & =h\left(\hat{X}_{i, k+1 \mid k}, u_{k}\right) \\
\hat{Z}_{k+1 \mid k} & =\sum_{i=0}^{2 n} W_{i} Z_{i, k+1 \mid k}
\end{aligned}
$$

Update:

$$
\begin{gathered}
P_{z z . k+1 \mid k}=\sum_{i=0}^{2 n} W_{i}\left(\hat{Z}_{i, k+1 \mid k}-\hat{z}_{k+1 \mid k}\right)\left(\hat{Z}_{i, k+1 \mid k}-\hat{z}_{k+1 \mid k}\right)^{T} \\
P_{x z . k+1 \mid k}=\sum_{i=0}^{2 n} W_{i}\left(\hat{X}_{i, k+1 \mid k}-\hat{x}_{k+1 \mid k}\right)\left(\hat{Z}_{i, k+1 \mid k}-\hat{z}_{k+1 \mid k}\right)^{T} \\
K_{k+1}=P_{x z . k+1 \mid k} P_{z z . k \mid k}^{-1} \\
\hat{x}_{k+1 \mid k+1}=\hat{x}_{k+1 \mid k}+K_{k+1}\left(z_{k+1}-\hat{z}_{k+1 \mid k}\right) \\
P_{k+1 \mid k+1}=P_{k+1 \mid k}-K_{k+1} P_{z z, k+1 \mid k} K_{k+1}^{T}
\end{gathered}
$$

\section{Interacting Multiple Mode (IMM)}

The interacting multiple model (IMM) strategy is useful when a system behaves according to more than one mode of operation or regime. Most engineering systems perform according to a number of different models. The IMM utilizes a finite number of models that calculates likelihood values based on the state estimates and state error covariance. These likelihood values are used to determine operating mode probabilities, and can be used for fault detection $[6,9]$.

A summary of the IMM algorithm broken down into 5 steps is shown below [8]:

Calculation of the mixing probablities:

$$
\begin{gathered}
\mu_{i|j, k| k}=\frac{1}{\overline{c_{j}}} p_{i j} \mu_{i, k} \\
\overline{c_{j}}=\sum_{i=1}^{r} p_{i j} \mu_{i, k}
\end{gathered}
$$

Mixing stage:

$$
\begin{gathered}
\hat{x}_{0 j, k \mid k}=\sum_{i=1}^{r} \hat{x}_{i, k \mid k} \mu_{i|j, k| k} \\
P_{0 j, k \mid k}=\sum_{i=1}^{r} \mu_{i|j, k| k}\left\{P_{i, k \mid k}+\left(\hat{x}_{i, k \mid k}-\hat{x}_{0 j, k \mid k}\right)\left(\hat{x}_{i, k \mid k}-\hat{x}_{0 j, k \mid k}\right)^{T}\right\} \\
\text { Mode }- \text { matched filtering: }_{\Lambda_{j, k+1}=\mathcal{N}\left(z_{k+1} ; \hat{z}_{j, k+1 \mid k}, S_{j, k+1}\right)} \\
\Lambda_{j, k+1}=\frac{1}{\sqrt{\left|2 \pi S_{j, k+1}\right|_{A b s}}} \exp \left(\frac{-\frac{1}{2} e_{j, z, k+1}^{T} e_{j, z, k+1 \mid k}}{S_{j, k+1}}\right)
\end{gathered}
$$

Mode probability update:

$$
\mu_{j, k}=\frac{1}{c} \Lambda_{j, k+1} \sum_{i=1}^{r} p_{i j} \mu_{i, k}
$$




$$
c=\sum_{j=1}^{r} \Lambda_{j, k+1} \sum_{i=1}^{r} p_{i j} \mu_{i, k}
$$

State estimate and covariance combination:

$$
\begin{aligned}
\hat{x}_{k+1 \mid k+1} & =\sum_{j=1}^{r} \mu_{j, k+1} \hat{x}_{j, k+1 \mid k+1} \\
P_{k+1 \mid k+1}=\sum_{j=1}^{r} \mu_{j, k+1} & \left\{P_{j, k+1 \mid k+1}\right. \\
+ & \left(\hat{x}_{j, k+1 \mid k+1}\right. \\
- & \left.\left.\hat{x}_{k+1 \mid k+1}\right)\left(\hat{x}_{j, k+1 \mid k+1}-\hat{x}_{k+1 \mid k+1}\right)^{T}\right\}
\end{aligned}
$$

III. Electrical Equivalent Circuit Models

ECM are models based on electrical components such as ideal voltage sources, resistors, and capacitors to simulate the behaviour of the battery. The vast majority of ECM models are semi-empirical models where the process of calculating your final outputs is based on two distinctive activities. First, the OCV with respect to SOC must be known beforehand by performing laboratory experiments where the battery is put through discharge and resting cycles. Second, the ECM parameters such as resistors and capacitors must be calibrated through $\mathrm{I} / \mathrm{O}$ data using parameter identification techniques such as least square methods. ECM model design may be easily achieved, but depending on the output and accuracy, they can vary greatly [1]. Moreover, computational power and the simulation time needed to simulate the battery is low. These characteristics make ECM models more suitable for use and implementation in a battery management system (BMS) [12].

\section{A. Thermal Model}

Battery thermal management is critical for tracking and estimating parameters, as well as avoiding severe consequences. The thermal model used for determining the core temperature, $T_{c}$, and the surface temperature of the cell, $T_{S}$, is shown in Figure 1, where the core and the surface of the cell are modeled as RC branches [13]. The core's resistance, $R_{c}$, models the amount of heat flux leaving or entering the core and the capacitor, $C_{c}$, models the core's ability to store heat. The surface of the battery was modeled similarly, yielding four parameters. The two inputs to the model are demanded/supplied current, $I_{s}$, and forced convection to the skin of the cell, $T_{f}$.

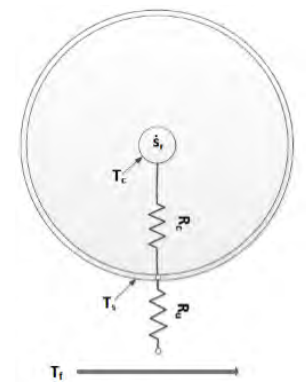

Figure 1. Schematic diagram of the battery thermal model [13].

The two differential equations for a cylinder cell were previously analyzed by [14] and [4]:

$$
\dot{T}_{c}=\frac{1}{C_{c}}\left(\dot{S}_{c}+\frac{\dot{T}_{s}-T_{c}}{R_{c}}\right)
$$

$$
\dot{T}_{c}=\frac{1}{C_{s}}\left(\frac{T_{f}-T_{s}}{R_{u}}-\frac{T_{s}-T_{c}}{R_{c}}\right)
$$

where $\dot{s}_{r}=\left(O C V-V_{T}\right) I_{s}, \mathrm{OCV}$ is the open circuit voltage, $V_{T}$ is the terminal voltage. The following is the state space representation of the two differential equations:

$$
\begin{gathered}
A=\left(\begin{array}{cc}
-\frac{1}{R_{c} C_{c}} & \frac{1}{R_{c} C_{c}} \\
-\frac{1}{R_{c} C_{s}} & -\frac{1}{C_{s}}\left(\frac{1}{R_{c}}+\frac{1}{R_{s}}\right)
\end{array}\right) \\
B=\left(\begin{array}{cc}
\frac{O C V-V_{t}}{C_{c}} & 0 \\
0 & -\frac{1}{C_{s} R_{s}}
\end{array}\right) \\
u=\left[\begin{array}{ll}
I_{s} & T_{f}
\end{array}\right]^{\prime}
\end{gathered}
$$

This thermal model was used across all simulated ECMs. Furthermore, a value of $25^{\circ} \mathrm{C}$ was used for $T_{f}$ to simulate the battery inside a laboratory with the air conditioner on. The values for the model's parameters were taken from [13] and used to generate the simulation results.

\section{B. Rint Model}

The most basic and commonly used battery model is the Rint model. It consists of an ideal battery with an open-circuit voltage $U_{O C}$ and a constant equivalent internal series resistance $R_{O} . U_{L}$ is the terminal voltage of the battery and $I_{L}$ is the load current with a negative value at charging and a positive value at discharging:

$$
U_{L}=U_{O C}-R_{O}
$$

The equations for the state space representation of the Rint model are as follows:

$$
\begin{gathered}
x=\left[S O C, T_{\text {core }}, T_{\text {surface }}\right]^{\prime} \\
A=\left(\begin{array}{cc}
1 & 0 \\
0 & A_{\text {thermal }}
\end{array}\right) \\
B=\left(\begin{array}{ll}
\frac{1}{C_{\text {battery }}} & 0 \\
0 & B_{\text {thermal }}
\end{array}\right) \\
u=\left[\begin{array}{ll}
I_{s} & T_{f}
\end{array}\right]^{\prime}
\end{gathered}
$$


The state space representation of the system was discretized for implementation purposes. Once the SOC was derived based on the state space, the algorithm would update the parameter values for that SOC level and calculate the corresponding $V_{O C}$. The terminal voltage $\left(V_{T}\right)$ was derived using equation (13). The following section describes the corresponding output of the model based on Normal Conditions.

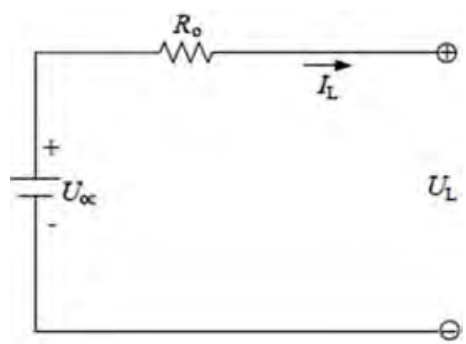

Figure 2. Schematic circuit diagram of Rint Model [7].

\section{Thevenin Model}

The Thevenin model is often referred to as a first order battery model. It consists of a parallel RC circuit in series with a resistance. As shown in Figure 3, it is mainly composed of three parts: open-circuit voltage source $\left(U_{O C}\right)$, internal resistance, and equivalent capacitances. The internal resistances include the ohmic resistance $\left(R_{O}\right)$ connected in series and the polarization resistance $\left(R_{T h}\right)$ connected in parallel. Polarization resistance is used so that the battery does not provide energy (or 'drain') right away to the system. The equivalent capacitance $\left(C_{T h}\right)$ is used to describe the transient response during charging and discharging. $\left(U_{T h}\right)$ is the Thevenin voltage across the $\left(C_{T h}\right),\left(I_{T h}\right)$ is the outflow current of $\left(C_{T h}\right)$ and $\left(U_{T h}\right)$ is the terminal voltage. The electrical behavior (dynamics) of the Thevenin model can be expressed by Eq. 18 [11].

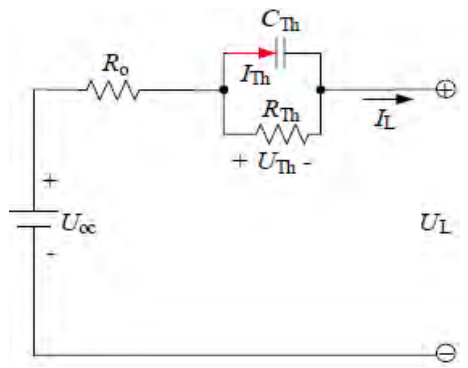

Figure 3. Schematic circuit diagram of Thevenin Model [11].

$$
\begin{gathered}
\dot{U}_{T h}=-\frac{U_{T h}}{R_{T h} C_{T h}}+\frac{I_{L}}{C_{T h}} \\
U_{L}=U_{O C}-U_{T h}-I_{L} R_{o}
\end{gathered}
$$

\section{NUMERICAL SimUlation AND RESUltS}

Figure 4 depicts the supplied current used across the different models in this numerical study. Positive current was assumed to demand from the battery, thus discharging the battery. The algorithm to simulate the battery was developed in MATLAB ${ }^{R}$. The SOC was estimated based on the following equation:

$$
S O C=S O C_{o}+\frac{1}{C_{\text {battery }}} \int_{0}^{t} I_{s} d t
$$

where the $C_{\text {battery }}$ refers to the nominal cell or battery capacity.

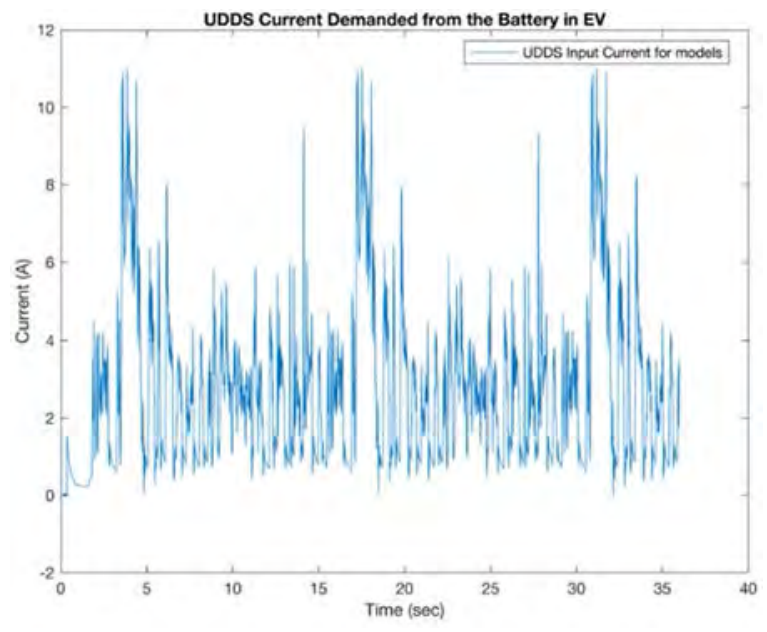

Figure 4. UDDS current profile.

As mentioned earlier, due to their dependence on SOC levels, model parameters curves are often derived offline experimentally using constant current (CC), and constant voltage $(\mathrm{CV})$ charge and discharge cycles under constant temperature by using a thermal chamber. Similar experiments are performed at different temperatures and lookup tables are created for the models. Afterwards, the data is processed using software such as MATLAB to match parameters to a desired transfer function [15]. Other parameter identification techniques use genetic algorithms (GA) [14].

In this paper, the MATLAB ${ }^{\circ}$ Simulink model was obtained from [17] and modified for the corresponding models to optimize the values of the model parameters: $U_{O C}, R_{O}, R_{T h}$, and $C_{T h}$. Furthermore, the input current and output voltage shown on Figure 5 were used for optimizing the parameter values.
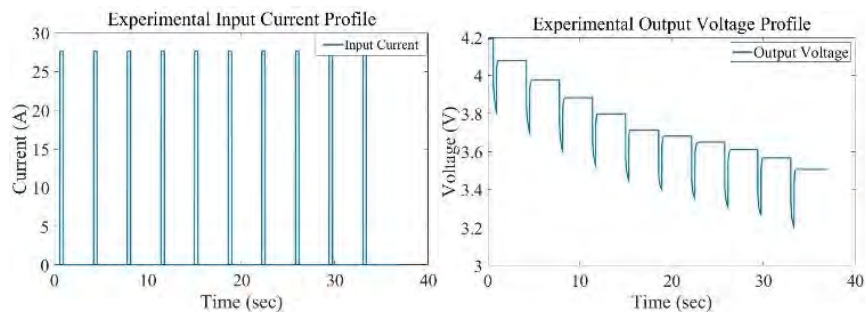

Figure 5. Input current Profile used across all models.

Figure 6 illustrates the resulting VOC-SOC curve from the optimization process. The VOC gradually increases with increasing SOC and reaches maximum at $4.2 \mathrm{~V}$. This VOCSOC curve was used for both models. Figure 7 shows the resulting capacitance values for the Thevenin capacitance, $C_{t h}$ for all levels of SOC. The nonlinearity of $C_{t h}$ is inherited by the model; making it nonlinear and thus, resulting in the implementation of the UKF method. Figure 8 depicts the calibration results of the Simulink model using the inputoutput data shown in Figure 5. 
Lastly, the system and measurement noise covariance matrices were set to $Q=10^{-9}$ eye(4) and $R=$ $\operatorname{diag}\left(\left[\begin{array}{lll}10^{-4} & 0.00172 & 10^{-4}\end{array}\right]\right)$. The initial state error covariance was set to $P=10 Q$ and the $C_{\text {battery }}$ was 27.625 Ah.

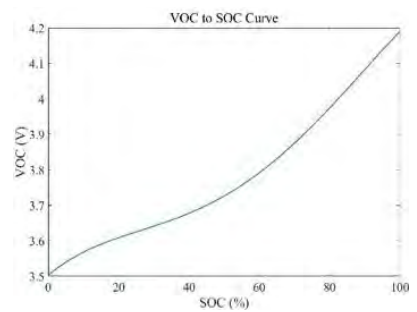

Figure 6. Open circuit voltage with respect to percentage of SOC for Rint model.

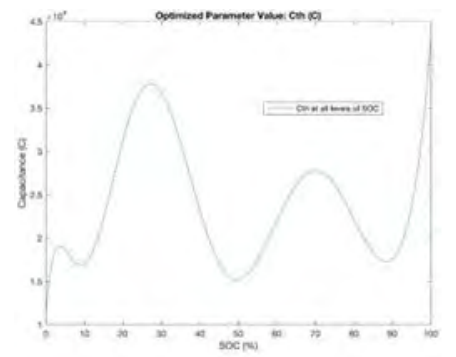

Figure 7. $C_{t h}$ profile with respect to percentage of SOC for Thevenin model.
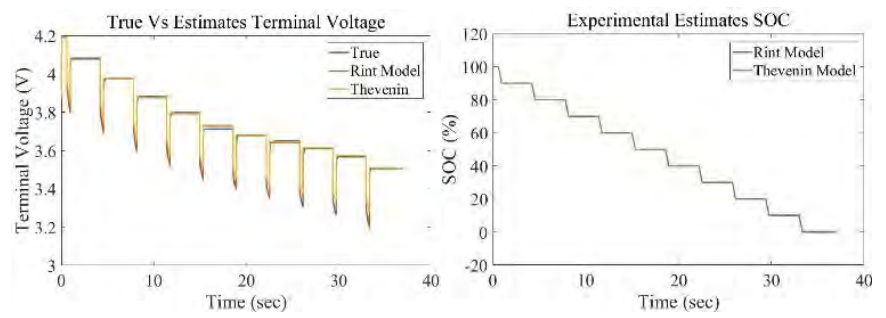

Figure 8. Calibrated Results of (a) terminal volatge and (b) SOC between Rint and Thevenin model.

\section{A. Rint Model}

The terminal voltage and the percentage of SOC for KF and IMM-KF of Rint model is presented in Fig. 10(a) and 10(b), respectively. Both terminal voltage and percentage of SOC decrease as time passes and $\mathrm{KF}$ almost follows the true value. However, as before, the IMM-KF tries to follow the trajectory but is slightly off from the true value. From Fig. 10(b), the KF follows the true SOC value and the IMM-KF is relatively close.

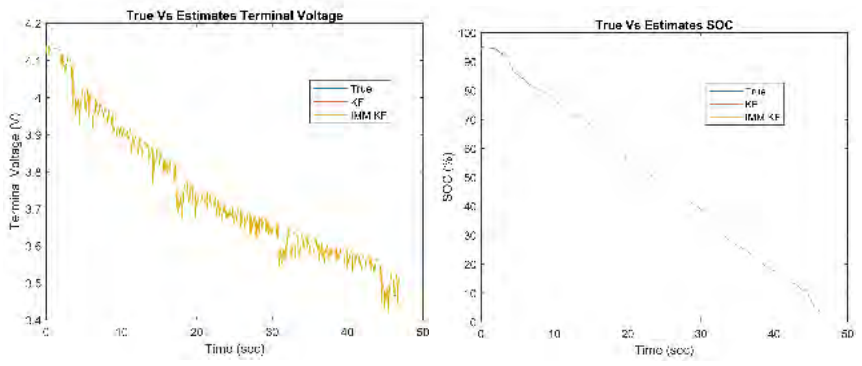

Figure 9. Simulated results of Rint model. (a) Terminal volatge and (b) SOC along with time for KF and IMM KF.
Using Thermal model, the temperature effect for Rint model is also analyzed. Figure 11(a) and 11(b) show the core and surface temperature behavior with respect to time of Rint model, respectively. Both temperatures decrease with time. Moreover, the surface temperature exponentially deceases and approaches $6{ }^{\circ} \mathrm{C}$ whereas the core temperature goes to nearly $23^{\circ} \mathrm{C}$. This is due to the thermal resistance between the core and surface, which is higher than the resistance between the surface and the air (e.g., natural cooling effect).
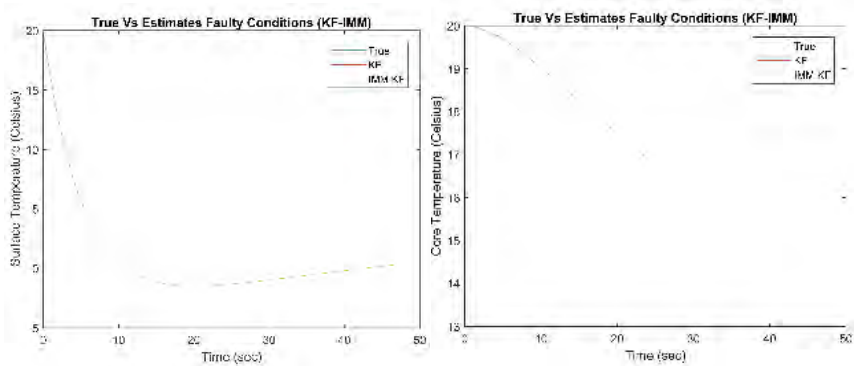

Figure 10. Simulated results of (a) core and (b) surface temperature for the KF and IMM-KF (Rint model).

\section{B. Thevenin Model}

The terminal voltage and SOC as a function of time are illustrated in Fig. 9(a) and 9(b), respectively. Terminal voltage shows the comparison between true values, UKF and IMMUKF. It can be clearly seen from Fig. 9(a) that with time, the terminal voltage decreases and UKF follows the true value. Whereas the IMM-UKF is a slightly off from the true value. In this scenario, the UKF obtains the best result. A similar trend can be identified from the SOC curve in Fig. 9(b).
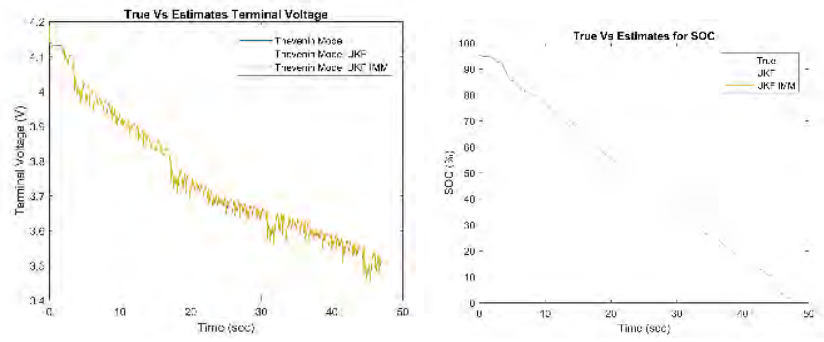

Figure 11. Simulated results of Thevenin model. (a) Terminal volatge and (b) SOC for the KF and IMM-KF.

\section{CONCLUSIONS}

An analysis on two ECM battery models, as well as a popular thermal model, was presented in this paper. Moreover, states such as $S O C, V_{T}, T_{c}$, and $T_{S}$ of the battery were estimated using the KF, UKF and IMM-KF estimation strategies. Parameter identification was performed using a modified MATLAB ${ }^{\circledR}$ Simulink model to calibrate battery models to a corresponding set of input-output data. Finally, the IMM was implemented for successful fault detection were the battery was aged and the thermal core resistance parameter was increased. Future work involves the development of an experimental testbed and implementation and comparison of other nonlinear battery models. 


\section{REFERENCES}

[1] A. Davide, Battery Management System for large Lithium-Ion Battery Packs, Norwood: Artech House, 2010

[2] Z. Y. Hou, P. Y. Lou and C. C. Wang, "State of Charge, State of Health, and State of Function Monitoring for EV BMS," in IEEE Int. Conf. Consumer Electronics, 2017. doi: 10.1109/ICCE.2017.7889332.

[3] L. Lu, X. Han, J. Li, J. Hua and M. Ouyang, "A review on the key issues for lithium-ion battery management in electric vehicles," J. Power Sources, vol. 226, pp. 272-288, 2013. doi: 10.1016/j.jpowsour.2012.10.060.

[4] Y. Masoudi, "Real-time Optimal Battery Thermal Management System Controller for Electric and Plug-in Hybrid electric Vehicles," University of Waterloo, Waterloo, 2017

[5] A. Millner, "Modeling Lithium Ion battery degradation in electric vehicles," in IEEE Conf. on Innovative Technol. Effic. Reliab. Electr. Supply, 2010. doi: 10.1109/CITRES.2010.5619782.

[6] S. A. Gadsden, "Smooth Variable Structure Filtering: Theory and Applications," Ph.D. Thesis, McMaster University, Hamilton, Ontario, 2011.

[7] E. A. Wan and R. Van Der Merwe, "The unscented Kalman filter for nonlinear estimation," in Proceedings of the IEEE 2000 Adaptive Systems for Signal Processing, Communications, and Control Symposium (Cat. No.00EX373). doi: 10.1109/ASSPCC.2000.882463.

[8] S. A. Gadsden, "Smooth Variable Structure Filtering: Theory and Applications," Hamilton, 2011.

[9] S. A. Gadsden and A. S. Lee, "Advances of the Smooth Variable Structure Filter: Square-Root and Two-Pass Formulations," J. Appl. Remote Sens., vol. 11, no. 1, pp. 1-19, 2017. doi: 10.1117/1.JRS.11.015018.

[10] M. Farag, "Lithium-Ion Batteries Modelling and State of Charge Estimation", Hamilton: McMaster University, 2013.
[11] H. He, R. Xiong and J. Fan, "Evaluation of Lithium-Ion Battery Equivalent Circuit Models for state of charge estimation by an experimental Approach," Energies, vol. 4, pp. 582 - 598, 2011 doi: 10.3390/en4040582.

[12] A. Nikolian et al., "Classsification of Electric Modelling and Characterization Methods of Lithium-ion Mierlo," in EUR.ELCTR.VEH.CONGR, 2014.

[13] I. Arasaratnam, J. Tjong, R. Ahmed, M. El-Sayed, and S. Habibi, "Adaptive temperature monitoring for battery thermal management," in 2013 IEEE Transp. Electrif. Conf. Expo (ITEC), 2013. doi: 10.1109/ITEC.2013.6574504.

[14] C. Park and A. K. Jaura, "Dynamic Thermal Model of Li-Ion Battery for Predictive Behavior in Hybrid and Fuel Cell Vehicles," in SAE Tech. Pap. Ser., 2003. doi: 10.4271/2003-01-2286.

[15] S. Zhao and D. A. Howey, "Global Sensitivity Analysis of Battery Equivalent Circuit Model Parameters," in 2016 IEEE Veh. Power Propul. Conf. (VPPC), 2016. doi: 10.1109/VPPC.2016.7791696.

[16] V. Sangwan, A. Sharma, R. Kumar, and A. K. Rathore, "Estimation of optimal li-ion battery parameters considering c-rate, SOC and temperature," in 2016 7th India Int. Conf. Power Electron. (IICPE), 2016. doi: 10.1109/IICPE.2016.8079484.

[17] J. Gazzarri, "MathWorks" 1008 2017.[Online]. Available: https://www.mathworks.com/matlabcentral/fileexchange/36019lithiumbattery-model-simscape-language-and-simulink-design-optimization. Accessed: February 6, 2018 


\section{Optimal Charging of Li-lon Batteries Based on an Electrolyte Enhanced Single Particle Model}

\author{
Xianke Lin \\ Department of Automotive, Mechanical and \\ Manufacturing Engineering, \\ University of Ontario Institute of Technology, \\ Oshawa, Canada \\ Xiaoguang Hao \\ Department of Mechanical Engineering, \\ University of Michigan, Ann Arbor, USA
}

\begin{abstract}
Lithium ion batteries play important roles as energy storage solutions in electric vehicle, portable devices, and renewable energy systems. There are many issues facing lithium ion batteries. One of them is the long charging time due to the slow electrochemical dynamics. Fast charging is one of the most difficult techniques that affect the acceptance of the electric vehicles. This paper presents a single particle battery model for charging optimization. The single particle model is enhanced with electrolyte dynamics. An optimal charging problem is formulated based on the electrolyte enhanced single particle model. Safety constraints are identified and imposed on the optimal charging problem. Multiple techniques have been developed to reduce the computational load. The fast charging strategy is developed. The results show that the fast charging strategy includes multiple phases and is able to reduce the charge time significantly.
\end{abstract}

Keywords-fast charging; single particle model; Li-ion battery

\section{INTRODUCTION}

Limited fossil fuel reserve and global warming provide a strong driving force for transportation electrification and clean energy technology. Lithium ion batteries play an important role as the most widely used energy storage system, and therefore are essential to the sustainable development of our society. There are still many issues facing lithium ion batteries. One of them is the long charging time due to the slow electrochemical dynamics. Compared with conventional vehicle which refuels in couple minutes, electric vehicle takes several hours to recharge. Battery recharge also requires meticulous control due to complex electrochemical reactions, immeasurable internal states, and serious safety concerns. It is thus important and also the focus of this article to develop a systematic approach solving for the optimal charging strategy while ensuring the safety during the charging process.

\author{
Zhenyu Liu \\ Department of Mechanical Engineering, \\ Zhejiang University, Hangzhou, China \\ Weiqiang Jia \\ Department of Mechanical Engineering, \\ Zhejiang University, Hangzhou, China
}

There are many charging protocols in the literature, such as constant-current / constant-voltage $(\mathrm{CC} / \mathrm{CV})$, pulse current charging, multi-stage charging [4], constant power charging, pulse voltage charging [1], [2], [3], neural networks[5], and fuzzy logic $[6,7]$. The most widely used charging protocol is the constant-current / constant-voltage (CC/CV). These approaches use predefined charging profiles with fixed current, voltage, or power constraints. Therefore, they are simple to implement. Most of them are based on the basic knowledge or experience of battery dynamics. These charging methods don't provide the best achievable performance determined by the electrochemistry.

This paper aims to develop a systematical approach for solving the optimal charging strategy based on a single particle model with electrolyte dynamics. An optimal control problem is mathematically formulated. The fast charging strategy is investigated. Multiple techniques, including time to SOC domain conversion and electrolyte dynamics approximation, have been developed in this paper to reduce the computational burden while maintaining the accuracy.

The rest of the paper is structured as follows. In section II, the physics-based single particle model is presented, and the electrolyte dynamics is simplified and added to the single particle model. The model is validated against experimental data. In section III, the optimal charging problem is formulated, and the fast charging strategy is then derived and investigated. In section IV, the optimized fast charging strategy is discussed in details. Section V concludes the presented work.

\section{EElectrolyte EnHanced Single Particle Model}

In the following we describe in detail the single particle model with electrolyte dynamics.

A graphite $/ \mathrm{LiFePO} 4$ cell with $400 \mathrm{mAh}$ is considered in this study. The single particle approach is used here. In the SP model, electrodes are assumed to consist of spherical intercalation particles with identical size. The surface of the spherical particle is scaled to that of the porous electrode. The key assumption in the single particle model is that the current 
distribution is taken to be uniform along the thickness of the electrode. Figure 1 provides a schematic of the SP model with electrolyte dynamics.

The mass balance of lithium ions in an intercalation particle of electrode active material is described by Fick's second law in a spherical coordinate system. Here the diffusion equation is simplified by using two-term polynomial approximation [8-10] as follows:

Cathode:

$$
\begin{aligned}
& \frac{d x_{p, a v g}}{d t}=\frac{-3 J_{p}}{F R_{p} c_{p, \max }}, J_{p}=\frac{I_{a p p}}{S_{p}} \\
& x_{p, \text { surf }}-x_{p, \text { avg }}=\frac{-J_{p} R_{p}}{5 F D_{s, p} c_{p, \max }}
\end{aligned}
$$

Anode:

$$
\begin{aligned}
& \frac{d x_{n, \text { avg }}}{d t}=\frac{-3 J_{n}}{F R_{n} c_{n, \max }}, J_{n}=\frac{I_{a p p}}{S_{n}} \\
& x_{n, \text { surf }}-x_{n, \text { avg }}=\frac{-J_{n} R_{n}}{5 F D_{s, n} c_{n, \max }}
\end{aligned}
$$

where $x_{i, a v g}$, electrode's SOC, is the ratio of the solid average concentration to the maximum solid concentration $c_{i, \max }$ for each electrode. $J_{i}$ is the current density for each electrode. $S_{i}$ is the electroactive surface area for each electrode. $I_{\text {app }}$ is the applied current. $R_{i}$ is the particle radius. $x_{i, \text { surf }}$ is the ratio of the solid surface concentration to the maximum solid concentration. $\mathrm{D}_{\mathrm{s}, \mathrm{i}}$ is the solid phase diffusion coefficient. $\mathrm{F}$ is the Faraday constant.

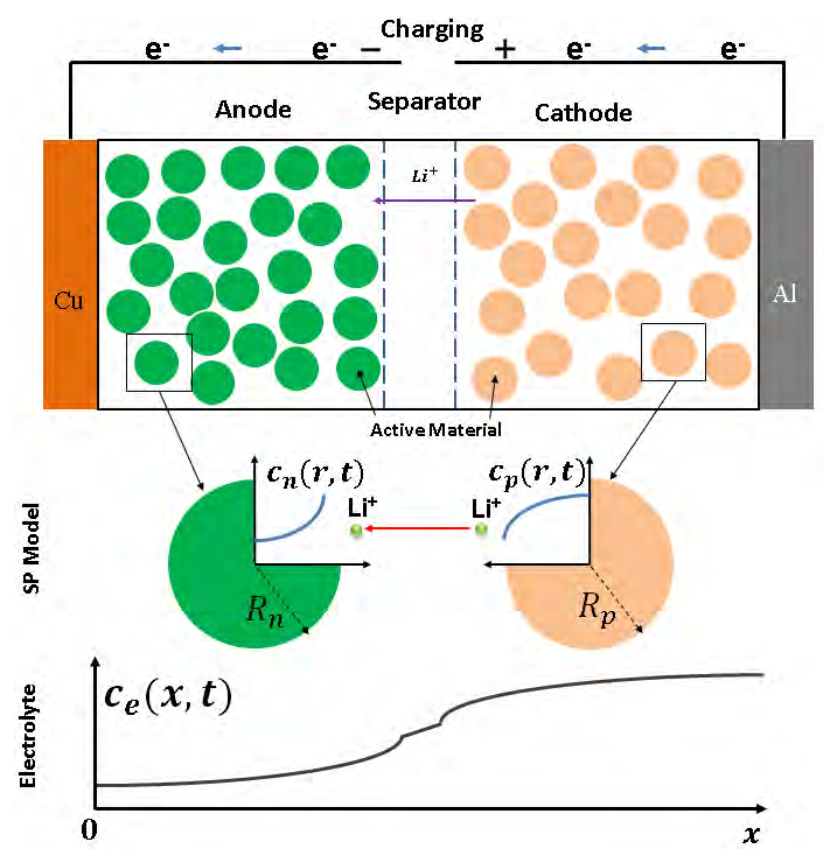

Figure 1. Electrolyte enhanced single particle model

The rate of lithium intercalation and deintercalation reactions is related to the surface SOC and over potential, and is expressed through the Butler-Volmer equation as follows.

$$
J_{i}=k_{i}\left[\exp \left(\frac{\alpha_{a, i} F}{R T} \eta_{i}\right)-\exp \left(\frac{-\alpha_{c, i} F}{R T} \eta_{i}\right)\right]
$$

where $\eta_{i}$ is the over-potential for lithium ion deintercalation/intercalation reactions. $\alpha_{\mathrm{a}, \mathrm{i}}$ and $\alpha_{\mathrm{c}, \mathrm{i}}$ are the anodic transfer coefficient and cathodic transfer coefficient respectively. $\mathrm{R}$ is the gas constant. $\mathrm{T}$ is the temperature. $\mathrm{k}_{\mathrm{i}}$ is the reaction rate coefficient.

$\eta_{\mathrm{p}}$ and $\eta_{\mathrm{n}}$ can be estimated as[11]

$$
\eta_{i}=\frac{R T}{\alpha_{a}} \ln \left(\xi_{i}+\sqrt{\xi_{i}^{2}+1}\right)
$$

where

$$
\xi_{i}=\frac{J_{i}}{2 k_{i}}
$$

The over-potentials are calculated by the following equations

$$
\begin{aligned}
& \eta_{p}=\phi_{\mathrm{p}}-\mathrm{U}_{p}^{\theta} \\
& \eta_{n}=\phi_{n}-\mathrm{U}_{n}^{\theta}
\end{aligned}
$$

where $\phi_{i}$ is the electrode's potential. $U_{i}^{\theta}$ is the open circuit potential in the appendix.

The cell voltage equals to the difference of solid phase potential between the positive and negative electrodes plus the voltage loss due to the internal resistance.

$$
V_{\text {cell }}=\phi_{\mathrm{p}}-\phi_{\mathrm{n}}+I_{\text {app }} R_{\text {cell }}
$$

where $R_{\text {cell }}$ is the internal resistance.

The material balance for the binary electrolyte in the liquid phase is given by [12]

$$
\begin{gathered}
\frac{\partial\left(\varepsilon_{2} c_{e}\right)}{\partial t}=\frac{\partial}{\partial x}\left(D_{e}^{e f f} \frac{\partial}{\partial x} c_{e}\right)+\frac{1-t_{+}^{0}}{F} J_{i} \\
\left.\frac{\partial c_{e}}{\partial x}\right|_{x=0}=\left.\frac{\partial c_{e}}{\partial x}\right|_{x=L}=0
\end{gathered}
$$

where $c_{e}$ is the lithium ion concentration, $\varepsilon_{2}$ is the electrolyte phase volume fraction, $D_{e}^{e f f}$ is the effective electrolyte phase Li diffusion coefficient. $t_{+}^{0}$ is the transference number.
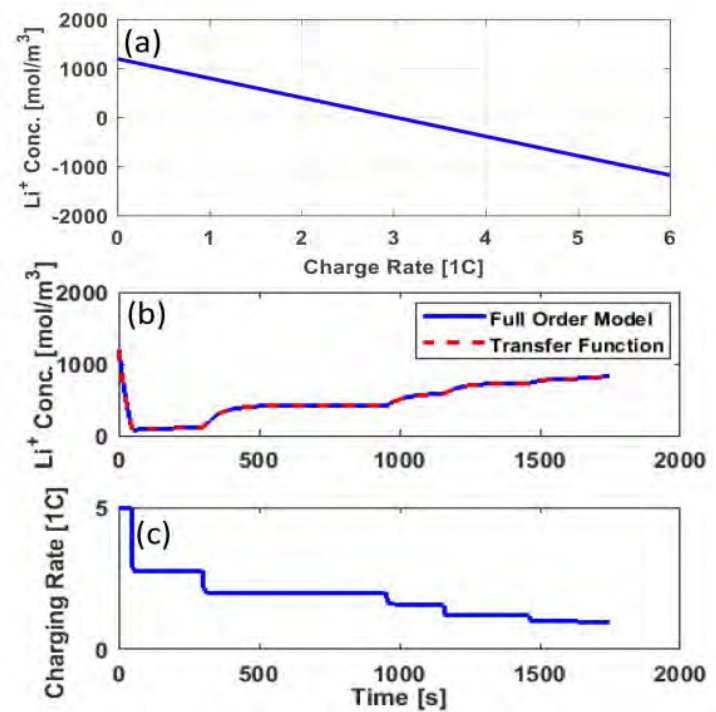

Figure 2. (a) Equilibrium $\mathrm{Li}^{+}$concentration at different charge rates. (b) Comparison of $\mathrm{Li}^{+}$concentration responses between full order model and transfer function. (c) Input current profile to both full order model and transfer function. 
During charging, the longer the diffusion distance of the $\mathrm{Li}^{+}$ ions, the greater the decrease of the $\mathrm{Li}^{+}$concentration in the electrolyte. The lowest electrolyte phase lithium ion concentration occurs near the anode current collector (the diffusion distance is longest from the cathode). Therefore, the lithium depletion could occur near the anode current collector. Based on the full-order model in our previous work [12], a static map is generated to predict the equilibrium $\mathrm{Li}^{+}$ concentration at the anode current collector surface (here the diffusion distance is longest) at different charging rates as shown in Figure 2. The $\mathrm{Li}^{+}$concentration decreases at the anode current collector as the charge rate increases. It reaches 0 $\mathrm{mol} / \mathrm{m}$ when charge rate reaches about $2.9 \mathrm{C}$ as shown in Figure 2. When the charge current continues to increase beyond $2.9 \mathrm{C}$, the equilibrium $\mathrm{Li}^{+}$concentration decreases to negative values. This is impossible and meaningless in reality. However, it's possible in the simulation, because the concentration is just a continuous variable. Although the negative equilibrium $\mathrm{Li}^{+}$concentration beyond $2.9 \mathrm{C}$ is meaningless in reality, it is actually very useful for predicting concentration decreasing rate at high charge rates. The lower the negative equilibrium lithium concentration at a charging rate, the greater the decreasing rate of the current lithium concentration at that charging rate.

TABLE I. BATtERY MODEL PARAMTERS

\begin{tabular}{|l|c|c|c|}
\hline \multicolumn{1}{|c|}{ Parameter } & $\begin{array}{c}\text { Negative } \\
\text { electrode }\end{array}$ & Separator & $\begin{array}{c}\text { Positive } \\
\text { electrode }\end{array}$ \\
\hline Electrode thickness $(\mathrm{m})$ & $70 \times 10^{-6}$ & $20 \times 10^{-6}$ & $90 \times 10^{-6}$ \\
\hline Particle radius $R_{s}(\mathrm{~m})$ & $3 \times 10^{-6}$ & & $0.2 \times 10^{-6}$ \\
\hline Electroactive surface area $S_{i}\left(\mathrm{~m}^{2}\right)$ & 0.8458 & & 12.6641 \\
\hline Active material volume fraction $\varepsilon_{1}$ & 0.5384 & & 0.4179 \\
\hline Filler volume fraction $\varepsilon$ & 0.172 & & 0.172 \\
\hline electrolyte phase volume fraction $\varepsilon_{2}$ & 0.2896 & 1 & 0.4101 \\
\hline Max. concentration $c_{i, m a x}\left(\mathrm{~mol} \mathrm{~m}^{-3}\right)$ & 26390 & & 22806 \\
\hline Initial electrode's SOC, $x_{i, a v g}$ & 0.017 & & 0.7862 \\
\hline Li diffusion coefficient $D_{s, i}\left(\mathrm{~m}^{2} \mathrm{~s}^{-1}\right)$ & $3.9 \times 10^{-14}$ & & $2 \times 10^{-17 \mathrm{ad}}$ \\
\hline Initial electrolyte concentration $\left(\mathrm{mol} \mathrm{m}^{-3}\right)$ & 1200 & 1200 & 1200 \\
\hline Charge transfers coefficient $\alpha_{a}, \alpha_{c}$ & 0.5 & & 0.5 \\
\hline Reaction rate coefficient $k_{i}\left(\mathrm{Am}^{-2}\right)$ & $9.77 \times 10^{-2}$ & & $1.19 \times 10^{-2}$ \\
\hline $\begin{array}{l}\text { Time constant for diffusion dynamics } \Delta T \\
\text { (s) }\end{array}$ & 60 & 60 & 60 \\
\hline
\end{tabular}

ad: adjusted

The nonlinear electrolyte diffusion equation has high fidelity. However, it's very computationally costly due to its PDE nature. Therefore, a first-order transfer function is used to approximate the $\mathrm{Li}$ concentration dynamics at anode current collector. The first-transfer function is given as follows.

$$
G(s)=\frac{c_{e}(s)}{c_{e_{-} e q}(s)}=\frac{1}{\Delta T s+1}
$$

where $c_{\mathrm{e}}(\mathrm{s})$ is the instantaneous lithium concentration (in Laplace domain) at anode current collector. $\mathrm{c}_{\mathrm{e}_{-} \mathrm{eq}}(\mathrm{s})$ is the equilibrium lithium concentration at a given charge rate. $\Delta \mathrm{T}$ is the time constant for the diffusion dynamics.

The transfer function in Laplace domain can be converted into time domain as follows

$$
\frac{d c_{e}}{d t}=\frac{1}{\Delta T}\left(c_{e_{-} e q}-c_{e}\right)
$$

The above first-order equation is able to accurately predict the lithium concentration evolution at the anode current collector. In this equation, lower negative $\mathrm{c}_{\mathrm{e}_{-} \text {eq }}$ will lead to lower negative value of $\frac{\mathrm{dc}_{\mathrm{e}}}{\mathrm{dt}}$, which means greater lithium ion decreasing rate. Therefore, a negative value beyond $2.9 \mathrm{C}$ in Figure 2 indicates a high decreasing rate of $\mathrm{c}_{\mathrm{e}}$. A comparison between transfer function prediction and the full-order model[12] prediction is shown in Figure 2. A charging current profile is input into both transfer function and full-order model. And the resultant $\mathrm{Li}^{+}$concentration profiles at anode current collector are compared. As shown in Figure 2, the transfer function has a very high accuracy (almost 100\%) of predicting the $\mathrm{Li}^{+}$concentration compared with full-order model. Therefore the diffusion dynamics of electrolyte can be simplified by using a static map of equilibrium lithium concentration at different charge rates and a first-order transfer function. This technique developed in this paper significantly reduces the computational cost, and also the states needed in the optimal charging problem.

The battery model parameters are summarized in Table I and more details can be found in [12-14].

The single particle model with electrolyte dynamics is validated against experiment data. The battery cells used are graphite $/ \mathrm{LiFePO}_{4}$ cells with $400 \mathrm{mAh}$ nominal capacity. As shown in Figure 3, a current profile is applied to both the single particle model and the real battery cell. Voltage responses from both simulation and experiment are compared. The RMSE of voltage is about $41 \mathrm{mV}$.

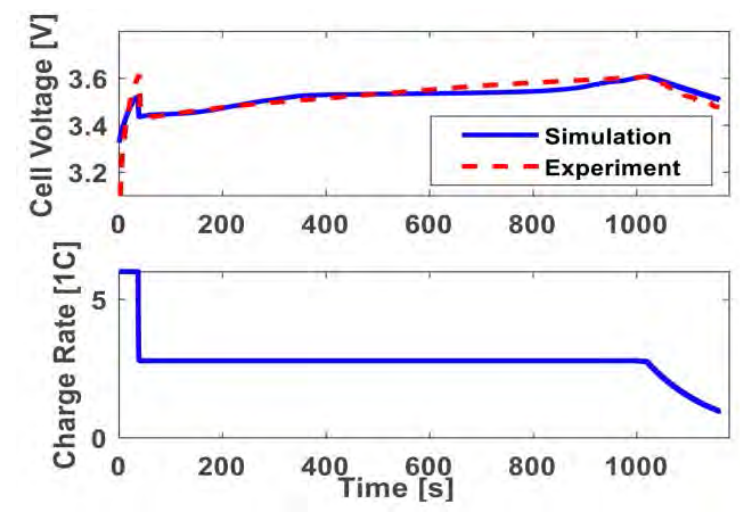

Figure 3. Experiment results for fast charging strategy.

\section{CHARGING STRATEGY OPTIMIZATION}

Based on the validated single particle model, an optimal charging problem is formulated with the aim to reduce the charge time while ensuring the safety constraints. The cost function is given by 


$$
\min _{(t), t_{f}} \int_{t_{0}}^{t_{f}} 1 \cdot d t
$$

where $\left(t_{f}-t_{0}\right)$ is the charge time. The optimization variables are the input current $I(t)$ and final time $t_{f}$, with the state variables, $x_{i, a v g}$ (electrode's SOC) and $c_{2}$ (electrolyte concentration).

The above optimal charging problem is formulated over time domain. The final time is a variable. To further reduce the state variables and optimization variables, the problem is converted from time domain into SOC domain. In this paper, the anode electrode SOC, $\mathrm{x}_{\mathrm{n}, \mathrm{avg}}$, is used to represent the SOC for the battery cell. When the battery is being charged, the anode SOC starts at a very low level (close to 0), and increases to a target high level. Therefore, we can formulate the cost function over anode SOC domain instead of time domain. Anode SOC is first divided in to many small segments $\triangle \mathrm{SOC}$. Over each $\triangle \mathrm{SOC}$ segment, the charging time can be calculated by the following equation.

$$
t_{\text {char }}=\frac{\Delta S O C \cdot S_{n} \cdot F \cdot R_{n} \cdot C_{n, \max }}{\cdot I(S O C)}
$$

where $t_{\text {char }}$ is the charge time for the segment $\triangle \mathrm{SOC}$. Therefore, the new cost function in anode SOC domain becomes

$$
\min _{(S O C)} \int_{S O C_{l o}}^{S O C_{h i}} t_{c h a r}
$$

where anode electrode $\mathrm{SOC}_{\mathrm{lo}}=0.017$ (fully discharged) and $\mathrm{SOC}_{\mathrm{hi}}=0.61$ (fully charged). After the time to SOC conversion, the state variable SOC is eliminated, and optimization variable $t_{f}$ is also eliminated. The optimization variable is now the input current I(SOC) only. The only state variable is the electrolyte concentration $\mathrm{c}_{\mathrm{e}}$ at anode current collector. The above time to SOC conversion technique simplifies the optimal control problem, and reduces the states and control inputs.

The optimal control problem has the following constraints for model dynamics, inputs, and states.

$$
\begin{gathered}
0<\mathrm{I}(\mathrm{t}) \leq \mathrm{I}_{\max } \\
\quad c_{e}>0 \\
0 \leq x_{n, \text { surf }} \leq 1 \\
0 \leq x_{p, \text { surf }} \leq 1 \\
V_{\text {cell }}<4 V
\end{gathered}
$$

These constrains protect the active particle from overcharge (saturation on anode and depletion on cathode), electrolyte from depletion and cell voltage from overvoltage.

Dynamic Programming (DP) is a very powerful, general tool for solving sequential decision making problems. DP as an extremely powerful approach has been extensively applied to real-world optimal control problems, including hybrid vehicle control system[15], path planning for autonomous vehicles[16], hydroelectric generation scheduling[17], etc...

\section{OPTIMIZATION RESULTS}

In this section, the optimal charging problem is solved by dynamic programming. The optimized charging strategy is studied and discussed in details.

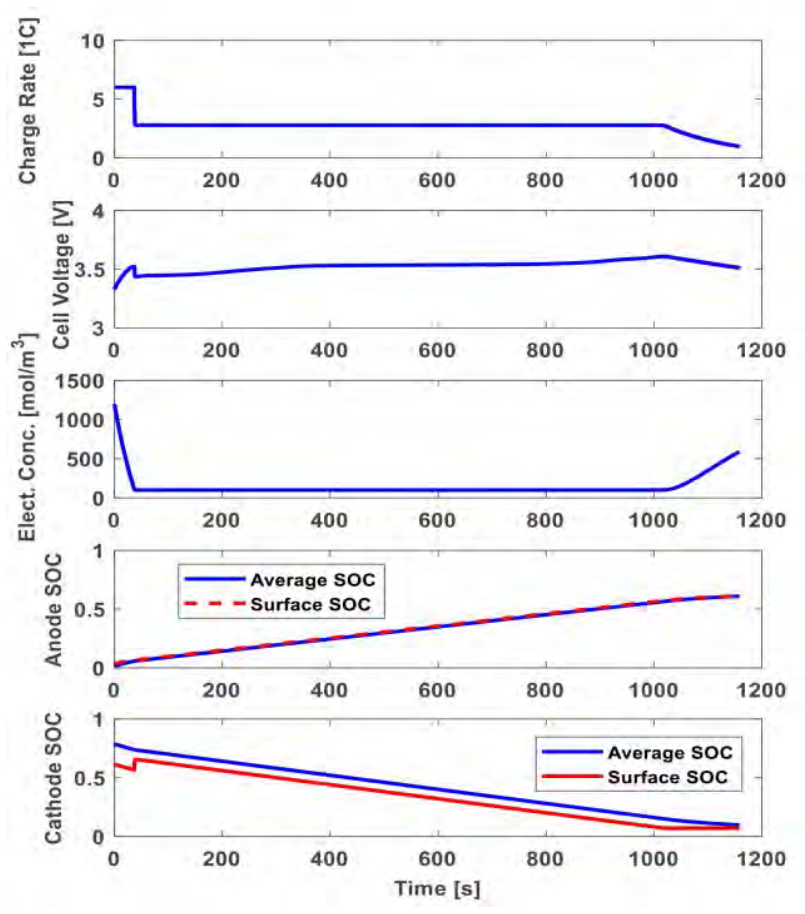

Figure 4. Optimization results for fast charging strategy.

The charging strategy pursued by this work aims to charge a battery as fast as possible. Dynamic programming is applied to find the optimal charging strategy which achieves the minimum charging time. The optimized charging current trajectory is shown in Figure 4. It takes about 19 minutes 18 seconds to achieve a target anode SOC of $61 \%$ (fully charged) from an initial anode SOC 1.7\% (fully discharged).

As shown in Figure 4, the optimized charging process can be divided into three stages. In the first stage, a high maximum current is applied until the $\mathrm{Li}^{+}$concentration in the electrolyte at the anode current collector depletes. In the second stage, the current is lowered to a level to avoid lithium depletion in the electrolyte and maintain a constant and small $\mathrm{Li}^{+}$concentration in the electrolyte at the anode current collector. In the third stage (close to the end of charge), the lithium concentration on the cathode surface decreases and gets close to zero, the charge current is gradually lowered to avoid the lithium depletion on the cathode surface. The whole charging process follows constant-current / constant-electrolyte-concentration / constantcathode-concentration $(\mathrm{CC} / \mathrm{CEC} / \mathrm{CCC})$.

\section{CONCLUSION}

This paper enhances the single particle model with electrolyte dynamics. Based on the enhanced model, the fast charging strategy is investigated. An optimal charging problem is formulated to solve for the fast charging strategy that achieves the minimum charging time. Dynamic programming as an extremely powerful approach has been used to find the optimal fast charging strategy. Multiple techniques, including electrolyte dynamics approximation and time to SOC domain 
conversion, have been used in this paper to reduce the computational cost and dimensions of the problem for the dynamic programming. Fast charging strategy is investigated and discussed in details. This charging strategy takes the shortest time to charge the battery, about 19 minutes 18 seconds in this study. The fast charging strategy can be divided into three stages and follows constant-current / constantelectrolyte-concentration / constant-cathode-concentration (CC/CEC/CCC).

\section{ACKNOWLEDGMENT}

This works was supported by the NSERC Discovery Program.

\section{APPENDIX}

The equilibrium potentials are obtained by $\mathrm{C} / 50$ charge and discharge, as shown in Figure A1.
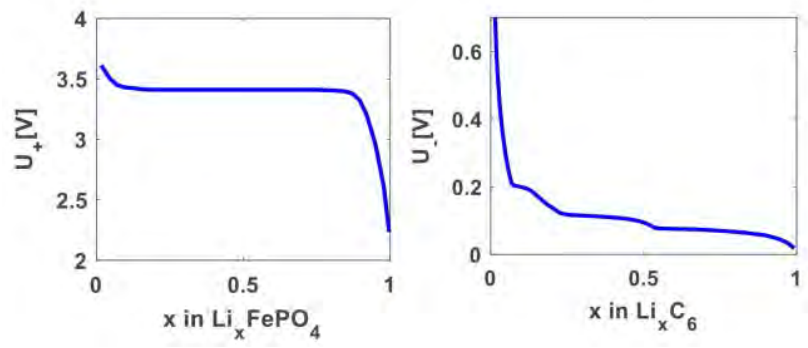

Figure A1. Equilibrium potentials for anode and cathode

\section{REFERENCES}

[1] P. H. L. Notten, J. H. G. Op het Veld, and J. R. G. Van Beek, "Boostcharging Li-ion batteries: A challenging new charging concept," J. Power Sources, vol. 145, no. 1, pp. 89-94, July 2005. doi: 10.1016/j.jpowsour.2004.12.038.

[2] B. Purushothaman and U. Landau, "Rapid charging of lithium-ion batteries using pulsed currents a theoretical analysis," J. Electrochem. Soc., vol. 153, no. 3, pp. A533-A542, 2006. doi: 10.1149/1.2161580.

[3] K. A. Smith, "Electrochemical control of lithium-ion batteries [applications of control]," IEEE Control Systems Mag., vol. 30, no. 2, pp. 18-25, Apr. 2010. doi: 10.1109/MCS.2010.935882.

[4] D. Anseán, et al., "Fast charging technique for high power lithium iron phosphate batteries: A cycle life analysis," J. Power Sources, vol. 239, pp. 9-15, Oct. 2013. doi: 10.1016/j.jpowsour.2013.03.044.

[5] Z. Ullah, B. Burford, and S. Dillip, "Fast intelligent battery charging: neural-fuzzy approach," IEEE Aerosp. Electron. Syst. Mag., vol. 11, no. 6, pp. 26-34, June 1996. doi: 10.1109/62.500207.

[6] H. Surmann, "Genetic optimization of a fuzzy system for charging batteries," IEEE Trans. Ind. Electron., vol. 43, no. 5, pp. 541-548, Oct. 1996. doi: 10.1109/41.538611.

[7] Y. H. Liu and Y. F. Luo, "Search for an optimal rapid-charging pattern for Li-ion batteries using the Taguchi approach," IEEE Trans. Ind. Electron., vol. 57, no. 2, pp. 3963-3971, Dec. 2010.

doi: 10.1109/TIE.2009.2036020.

[8] S. K. Rahimian, S. Rayman, and R.E. White, "State of charge and loss of active material estimation of a lithium ion cell under low earth orbit condition using Kalman filtering approaches," J. Electrochem. Soc., vol. 159, no. 6, pp. A860-A872, 2012. doi: 10.1149/2.098206jes.
[9] C. Wang, W. Gu, and B. Liaw, "Micro-Macroscopic Coupled Modeling of Batteries and Fuel Cells I. Model Development," J. Electrochem. Soc., vol. 145, no. 10, pp. 3407-3417, 1998. doi: $10.1149 / 1.1838820$.

[10] P. Ramadass, P., et al., "Development of first principles capacity fade model for Li-ion cells," J. Electrochem. Soc., vol. 151, no. 2, pp. A196-A203, 2004. doi: 10.1149/1.1634273.

[11] D. Di Domenico, G. Fiengo, and A. Stefanopoulou, "Lithium-ion battery state of charge estimation with a Kalman filter based on a electrochemical model," in Proc. IEEE Int. Conf. Control Appl., San Antonio, TX, 2008, pp. 702-707.

[12] X. Lin, et al., "A comprehensive capacity fade model and analysis for Li-ion batteries," J. Electrochem. Soc., vol. 160, no. 10, pp. A1701-A1710, 2013. doi: 10.1149/2.040310jes.

[13] P. Arora, M. Doyle, and R.E. White, "Mathematical Modeling of the Lithium Deposition Overcharge Reaction in Lithium-Ion Batteries Using Carbon-Based Negative Electrodes," J. Electrochem. Soc., vol. 146, no. 10, pp. 3543-3553, 1999. doi: 10.1149/1.1392512.

[14] M. Safari, et al., "Multimodal physics-based aging model for life prediction of Li-ion batteries," J. Electrochem. Soc., vol. 156, no. 3, pp. A145-A153, 2009. doi: 10.1149/1.3043429.

[15] C.C. Lin, et al., "Power management strategy for a parallel hybrid electric truck," IEEE Trans. Control Syst. Technol., vol. 11, no. 6, pp. 839-849, Nov. 2003. doi: 10.1109/TCST.2003.815606.

[16] M. Flint, M. Polycarpou, and E. Fernandez-Gaucherand, "Cooperative path-planning for autonomous vehicles using dynamic programming," IFAC Proc. Vol., vol. 35, no. 1, pp. 481-486, 2002. doi:10.3182/20020721-6-ES-1901.01305.

[17] S C. Chang, et al., "Hydroelectric generation scheduling with an effective differential dynamic programming algorithm, "IEEE Trans. Power Syst., vol. 5, no. 3, pp. 737-743, Aug. 1990.

doi: $10.1109 / 59.65900$. 


\section{D numerical study of Hill mounted VAWT}

\author{
Belabes Belkacem \\ Centre de Développement des Energies Renouvelables, \\ CDER, BP 62 Route de l'Observatoire, Bouzaréah, 16340 \\ Algiers, Algeria \\ belabes.belkacem@gmail.com
}

\author{
Marius Paraschivoiu \\ Concordia University, Montréal, H3G1M8, Québec, \\ Canada \\ marius.paraschivoiu@concordia.ca
}

\begin{abstract}
The ground topography effect on the wind flow is significant. The knowledge of the flow behavior near ground is crucial in the development of wind power, especially in the choice of suitable sites and for estimation of energy production. In this paper, the numerical prediction of the flow over a three-dimensional hill model and the analysis of placement of Savonius turbines on top of the hill are presented. The numerical analysis is based on the finite volume method implemented in the ANSYS CFX 15 Software using the Shear-Stress Transport (SST) turbulence model. The numerical results for a conventional Savonius rotor and a vertical-axis spiral wind rotor are both satisfactory compared with experimental data. The performances of these turbines, installed on the hilltop, are studied for different height positions. Furthermore, the influence of the hill size on the extracted power is investigated. At $\mathrm{TSR}=1$, the power coefficient of a conventional rotor is increased from 0.15 to 0.32 when the rotor is installed at a height of $0.25 \mathrm{~m}$ above the top of the hill, while it reaches 0.40 when the hill is two to three times higher. The helical Savonius rotor tested gives even higher power coefficient of 0.44 .
\end{abstract}

Keywords-component; Savonius wind turbine; 3D Computational Fluid Dynamics; SST turbulence model; Power Coefficient; hill mounted turbine

\section{INTRODUCTION}

The ground topography affects the velocity, direction and turbulence intensity of the wind and can be used advantageously to increase energy extraction from wind. In this work, a hill topology is considered. Flow separation at the lee side of a hill is still an unresolved issue where the field measurements show a rapid decrease in speed-up ratio. Different explanations are available regarding the issue; for instance: [1] conjectured that this three dimensional flow separation at the lee slope is due to the blockage effect of neighboring hills, but according to [2] the presence of the nearby topography does not affect the flow neither at the top nor at the downstream of the hill. For [2], hills have a significant influence on the wind speed. A smooth and not too steep hill causes acceleration of the wind and makes the wind accelerate when flowing towards the hilltop. The resultant increase in energy content is called hill impact. [2] and [3], find a good agreement between the experimental results and the computational results both at windward slope and at the lee side of the hill. [4] Investigate two-dimensional numerical simulations of flow over two common types of topographic features (a cliff and a hill) and three types of turbulence models. The standard k- $-\varepsilon$, Durbin's model and Shin's model are tested for predicting flow over this topographic. In this study, Shin's model gave better results compared to other models.

The wind flow over terrains can provide an important advantage when installing wind turbines. An appropriate selection of a suitable land for wind turbines can lead to an increased output of energy. However, understanding the many factors to be taken into account and the interaction with the turbine is needed. In this study, the interaction of the flow over the hill and the helical Savonius vertical axial wind turbine is analysed to increase the energy extraction.

The main objective of this paper is to analysis the improvement of the power output when the helical Savonius wind rotor developed by [5] and [6] are placed on top of a hill. The paper will show that a significant improvement of the power coefficient $(C p)$ can be achieved. In this study, the Savonius rotor is placed at different vertical locations on top of the hill and for different heights of the hill.

\section{HILL AND SAVONIUS WIND TURBINE GEOMETRIES}

The hill geometry employed in this work is shown in Fig. 1. It is a three-dimensional axisymmetric defined by [7] as follows:

$$
\mathrm{z}(\mathrm{x}, \mathrm{y})=\frac{\mathrm{H} \cos ^{2} \pi\left(\mathrm{x}^{2}+\mathrm{y}^{2}\right)^{\frac{1}{2}}}{2 \mathrm{~S}}
$$


The specific height used in the comparison with experimental data is $\mathrm{H}=40 \mathrm{~mm}$ and length $\mathrm{S}=100 \mathrm{~mm}$. It gives a maximum slope of about $32^{\circ}$. For the turbine mounted simulations, $\mathrm{H}=2 \mathrm{~m}$ and $\mathrm{S}=5 \mathrm{~m}$. Fig. 1 shows the details of the side view of the three dimensional hill and the coordinate system.

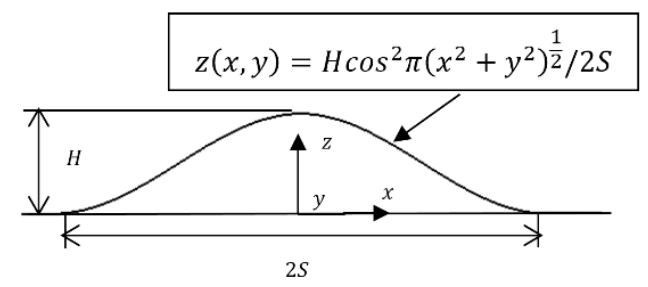

Figure 1. Topographic features [7]

The placement of turbines in complex land topology is becoming more and more common, although these areas are not always ideal sites, due to the high shear and turbulence levels in the wind flow. In this context, for a systematic analysis, the Savonius rotor is placed at a distance (L) above the hill. The Savonius rotor has an "S-shaped" cross-section constructed by two semicircular buckets. [6] conducted experimental investigations on a single stage helical Savonius rotor placed between end plates but without shaft. The helical rotor has a $90^{\circ}$ twist angle of the two blades evenly distributed around the vertical axis. The three-dimensional geometrical model of each blade can be approximately created by sweeping a base profile at $90^{\circ}$ along a spirally ascending curve, as shown in Fig. 2 and Table 1.
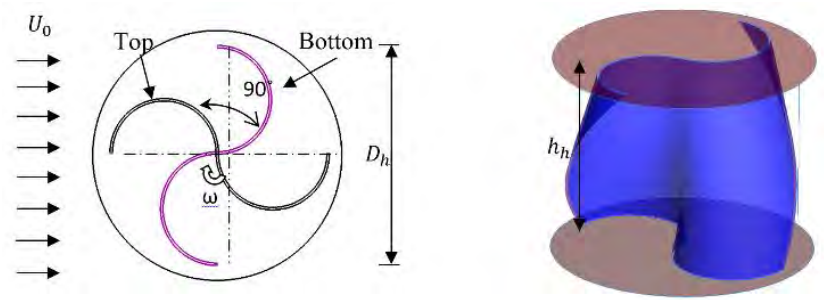

Figure 2. Schematic representations for a helical Savonius rotor .

TABLE I. BASIC PARAMETERS OF THE CONVENTIONAL SAVONIUS ROTOR [5] [6].

\begin{tabular}{|c|c|}
\hline Parameter & Value $(\mathrm{m})$ \\
\hline $\mathrm{D}$ & 0.33 \\
\hline $\mathrm{d}$ & 0.184 \\
\hline $\mathrm{e}$ & 0.038 \\
\hline $\mathrm{h}_{\mathrm{r}}$ & 0.23 \\
\hline $\mathrm{D}_{\mathrm{h}}$ & 0.23 \\
\hline $\mathrm{h}_{\mathrm{h}}$ & 0.202 \\
\hline
\end{tabular}

\section{ANALYSIS PARAMETERS}

Savonius turbines are mainly drag-driven devices made up of two or multiple blades. The difference in the drag forces acting on the rotor blades causes the Savonius turbine to rotate. The performance is defined by [5] as the power coefficient that is a function of power $\mathrm{P}$, rotor swept area $A s$, and free stream speed $U_{0}$ as follows:

$C_{P}=\frac{P}{\frac{1}{2} \rho A_{S} U_{0}^{3}}$

The moment coefficient $(\mathrm{Cm})$ of a Savonius turbine is determined as a function of torque $T$. It is defined by [5] as follows:

$C_{m}=\frac{T}{\frac{1}{4} \rho A_{s} D U_{0}^{2}}$

The Tip Speed Ratio TSR or $\lambda$ is the ratio between the rotational speed at the tip of the blade and the velocity of the wind. It is defined by [5] as follows:

$\lambda=\frac{\omega D}{2 U_{0}}$

The Reynolds number, $R e$, based on the rotor diameter is calculated as follows:

$R_{e}=\frac{\rho U_{0} D}{\mu}$

where $\mu$ is the dynamic viscosity of atmospheric air.

\section{NUMERICAL SIMULATION}

A hexahedral mesh is used for the stationary domain as shown in Fig. 3, and a tetrahedral mesh is used for the rotating domain containing the turbine as shown in Fig. 4. The total number of grid cells equals to 1.5106 . Note that the number of cells for both the conventional and helical rotors is almost the same. The meshes are generated using the ICEM CFD code. The grid node density in the rotating domain is higher than in the stationary domain. The prism layer of grid elements were extruded from the edges of the blade and the surface of the hill to improve the grid quality and to capture with sufficient precision the boundary layer flow. The height of the first prism layer above the surface was set such that the $y+$ value for the first layer of elements from the wall is below 2, depending on the rotation velocity of the rotor. There were nine layers in total, and a layer growth rate of 1.2 was chosen. 


\section{GRID AND TIME STEP INDEPENDENCE ANALYSIS}

Figure 3. The grid mesh around the hill Schematic.

Figure 4. The grid mesh around the blades.

Fig. 5 shows the boundary conditions and the computational domain. The inlet boundary condition is applied on to three faces [8]: the front and sides. The inlet boundary condition uses an atmospheric boundary layer profile as presented in equation (6) [9], with a horizontal velocity of 9 $\mathrm{m} / \mathrm{s}$ value at the height of the hilltop. The atmospheric boundary layer profile is defined as follows:

$U(z)=\frac{u_{*}}{k} \ln \left(\frac{z}{z_{0}}\right)$

where:

$\mathrm{u}_{*}$ : Frictional velocity.

$\mathrm{k}$ : Von Karman coefficient $(\mathrm{k}=0.4)$.

$\mathrm{z}_{0}$ : Roughness parameter $(\mathrm{Z} 0=0.01 \mathrm{~mm})$.

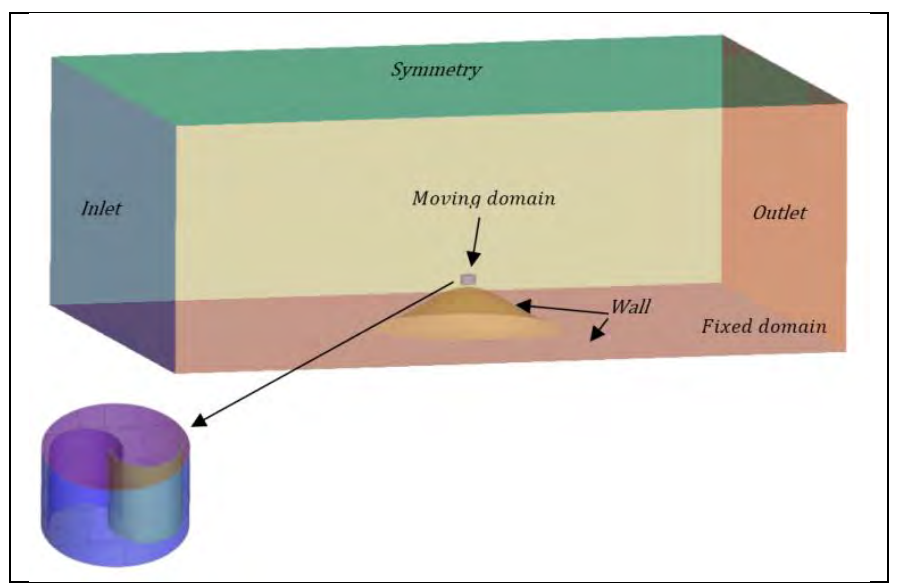

Figure 5. Computational Domain and Boundary Condition.
A mesh independence test is conducted in order to select the mesh needed to be in the converged spatial discretization regime for the moment coefficient value. The computational domain meshes are progressively refined until the observed physical quantities are stabilized. The grid refinement is performed using three types of meshes consisting of 1.0, 1.5, and 2 million grids. The convergence of each case needed more than 6 cycles. Fig. 6 shows the moment coefficient during the last two rotations of the conventional turbine rotor versus the total number of cells used. The computed moment coefficient does not show a significant difference when the total number of cells used is more than 1 million. The difference in moment coefficients between the grids of 1.0 and 1.5 million is in the order of $4 \%$, and this difference decrease to $0.4 \%$ between grid of 1.5 and 2 million grids. Based on these results, the following computations were carried out using a mesh consisting of 1.5 million cells.

Several studies also present a temporal discretization analysis: [10] uses 1 degrees/step, [11] reports 0.25 degree/step, while [12] uses 0.12 degrees/step, and [13] uses $0.02 \mathrm{~s}$. Based in these results, a verification study was performed using time steps ranging from 0.5 degrees/step to 1.5 degrees/step at a tip speed ratio of 1.0 and a free stream speed of $9 \mathrm{~m} / \mathrm{s}$. Periodic convergence of the rotor torque output was then evaluated by monitoring the dynamic torque for the selected time steps over the last two revolutions. Fig. 6 demonstrates that time step independence is achieved with a time step of 1 degree/step as for 0.5 degrees/step only a slight change in the torque is observed. The maximum difference is $3.13 \%$. Therefore, to reduce the time of calculation, the conservative choice in this study is to select a time step of 1 degree/step.

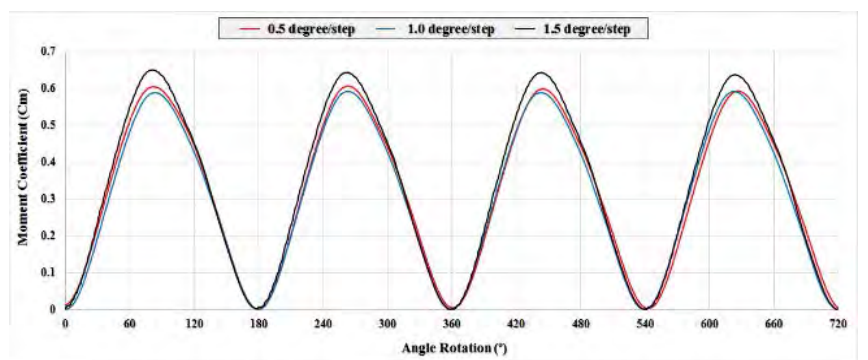

Figure 6. Torque variation during the last two revolutions at $\lambda=1.0$ and 9 $\mathrm{m} / \mathrm{s}$ for different time steps (conventional rotor).

\section{VALIDATIONS OF THE NUMERICAL METHODOLOGY}

In this part, two validations are performed to evaluate the proposed numerical methodology. The simulation results of the flow around a helical wind turbine Savonius with two blades using the SST turbulence model, were compared with the experimental results from [6]. Fig. 7 shows the comparison of the predicted average power coefficients with the measured 
data for various tip speed ratios (TSRs). A maximum power coefficient for conventional rotor $C_{P \max }$ of 0.155 is computed for the tip speed ratio of 0.8 , and a maximum power coefficient for the helical rotor $C_{P \max }$ of 0.169 was found at around 0.70 tip speed ratio. The computational results have a good agreement with experimental data for both cases.

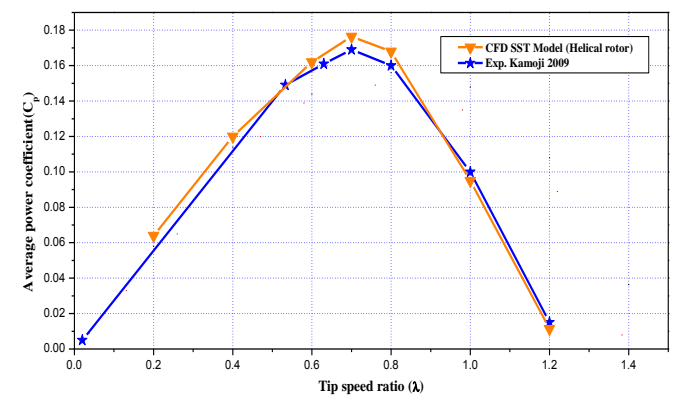

Figure 7. Comparison of the power coefficients from numerical and experimental results.

The second validation is related to the numerical simulation of the flow over the three-dimensional hill. The experimental data is obtained from a wind tunnel investigation by [13]. Fig. 10 shows the good agreement of the vertical profiles of the normalized velocity component of $(\mathrm{U})$ at various positions on the centerline of the hill.

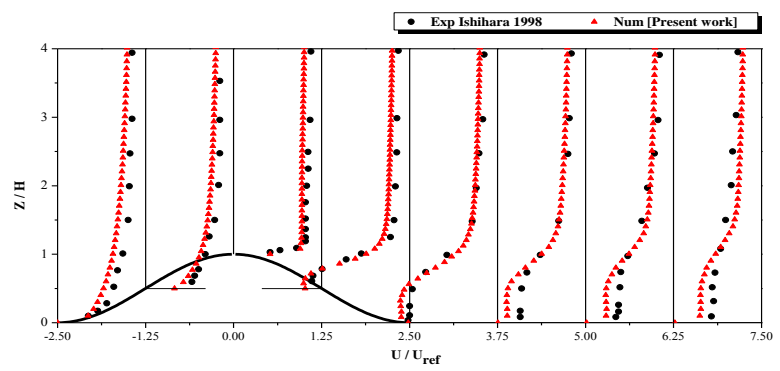

Figure 8. Comparison of the vertical profiles of mean velocity component $(U$ $/ U_{\text {ref }) \text {. }}$

\section{RESULTS AND DISCUSSION}

\section{A. $\quad$ Effect of different height positions (L)}

Numerical simulations of the flow over the hill mounted wind turbine were conducted for different placement height $\mathrm{L}$ : $\mathrm{L}=0.25 \mathrm{~m}, \mathrm{~L}=0.5 \mathrm{~m}$ and $\mathrm{L}=1 \mathrm{~m}$ above the hill. The results of the average power coefficient extracted from both wind turbines, as a function of the tip speed ratio, for a wind velocity inlet of $9 \mathrm{~m} / \mathrm{s}$ are presented in Fig. 9.

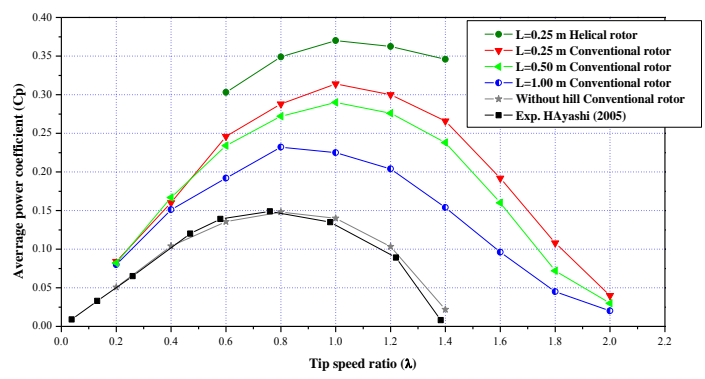

Figure 9. Average power coefficient at different vertical locations of the rotor.

In order to evaluate the power coefficient obtained from an isolated wind turbine both experimental and numerical cases are also reported. One can observe that the maximum values of the average power coefficient is obtained at a height of $\mathrm{L}=0.25$ $\mathrm{m}$ from the top of the hill. The position closet to the hill provides the higher power coefficient as the velocity is highest when measured close to the surface of the hill. The increase in maximum $\mathrm{Cp}$ is more than double than for an undisturbed flow. The maximum also occurs at a higher TSR as the effective wind reaching the turbine is higher. In fact, the ratio of maximum velocities squared $(12.372 / 92=1.89)$ is almost double and is close to the observed $\mathrm{Cp}$ increase. Taking this best position ( $\mathrm{L}=0.25$ ) obtained for the conventional rotor, and calculating the $\mathrm{Cp}$ for the vertical-axis helical wind rotor gives an even higher maximum $\mathrm{Cp}$. The maximum value of power coefficient for helical rotor is approximately 0.37 while, it is 0.32 for the conventional rotor in the tip speed ratio of 1 . This increase in power coefficient is due to the generation of swirling flow generated inside the wind turbine, these observations are also reported by [6] and [14]. This increase is also about twice the difference between the two turbines in an unperturbed flow.

\section{B. Effect of hill height}

In this section, the effect of the hill size on the power production is analyzed for the best position of the turbine rotor found previously. Numerical simulations were performed for the turbine position at $\mathrm{L}=0.25 \mathrm{~m}$ for different hill heights. Three cases are studied: hill height $=1 \mathrm{H}$, hill height $=2 \mathrm{H}$ and hill height $=3 \mathrm{H}$. Note that $\mathrm{H}$ is the standard height of the hill used in the previous section. The atmospheric boundary layer is set such that it has $9 \mathrm{~m} / \mathrm{s}$ velocity at the respective height, i.e. $\mathrm{H}, 2 \mathrm{H}$ or $3 \mathrm{H}$. A very important increase of the power coefficient was obtained, as shown in Fig. 10. 


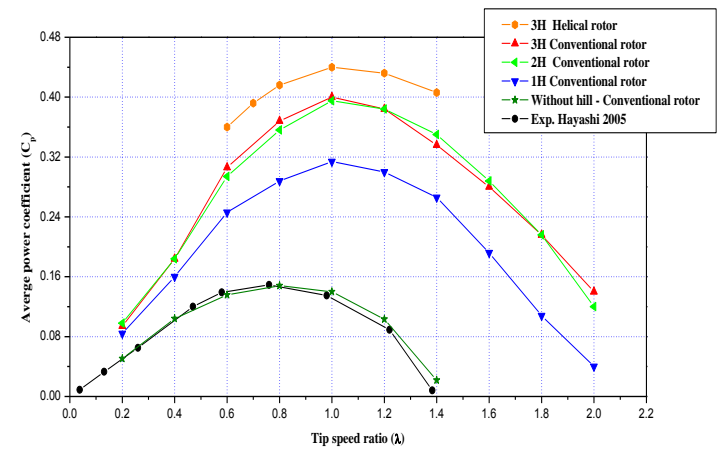

Figure 10. Variation of average power coefficient at different hill heights.

This figure illustrates the influence of the hill height on the performances up to a certain height. The cases of hill height $=2 \mathrm{H}$ and $3 \mathrm{H}$ give approximately the same maximum power coefficient which is close to 0.4 . The same boundary conditions and the hill height are applied to simulate the helical rotor. Again, the power coefficient of this newer design is higher than that obtained by the conventional rotor at the same conditions. A $\mathrm{Cp}=0.44$ is reached which is very high for a Savonius turbine. The difference between the three cases is quite clear in Fig. 11; the velocity in the case of $1 \mathrm{H}$ arrives at $18 \mathrm{~m} / \mathrm{s}$, while it is reaches $25 \mathrm{~m} / \mathrm{s}$ in the case of $2 \mathrm{H}$ and $3 \mathrm{H}$, which explain that the velocity is growing by $25 \%$ when the height is doubled.
Figure 11. Velocity and pressure contours at different plane with different hill heights (Conventional rotor). a) Longitudinal plan (XZ), b) Transversal plan (YZ).

These planes are vertical to the rotor axis to display the turbulent features around the helical rotor as indicated in
Fig.12. The pressure distribution and contours of the velocity at the maximum power coefficient are presented and compared in Fig. 13 for different sections. The flow fields at each horizontal section are not identical due to its helical geometry along the rotor axis. At this angle, plan 4 and 5 report that this part of the blade contributes significantly to the creation of clockwise torque. These results show that the wind did not directly influence the concave surface of the blade. Instead, it impacted on the convex surface of blade 2, and then hit the concave surface of blade 1

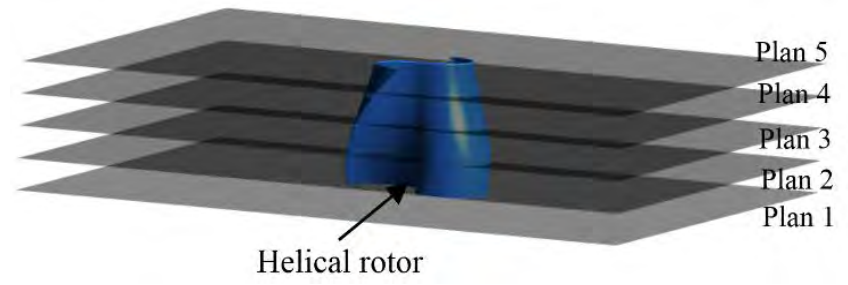

Figure 12. Fig. 18. Vertical sections of blades.

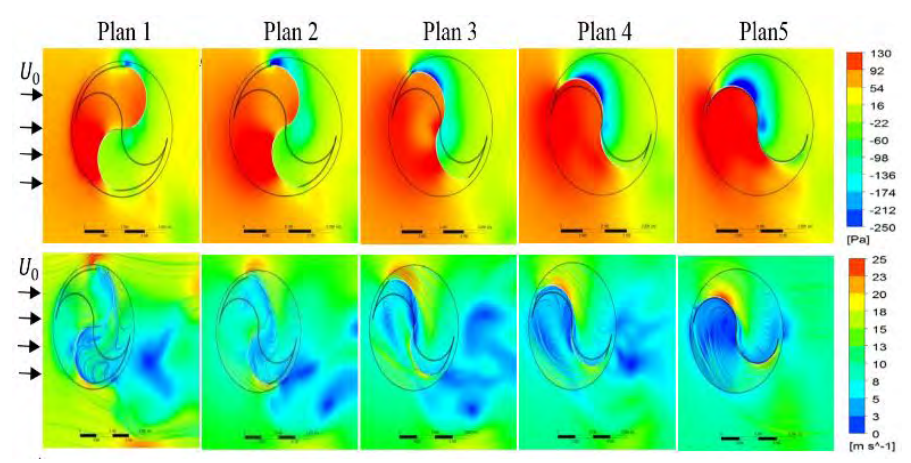

Figure 13. Velocity and pressure contours at different section vertical of helical rotor.

\section{C. $\quad$ Influence of wind velocity}

The main factor affecting the power output of wind turbine is the incoming wind velocity. The importance of wind speed is that the power is directly proportional to the cube of wind speed according to equation 2 . The power coefficient 
represents the aerodynamic efficiency of the wind turbine, which differs from one turbine to another. Three simulations are performed for different incoming wind velocities using the same computational domain and boundaries conditions as well as the best position from the previous cases $(\mathrm{L}=0.25 \mathrm{~m}$ and hill height $=3 \mathrm{H}$ ). The objective is to analyze the effect of the velocity on the coefficient of power.

Fig. 14 shows the numerical results of the average power coefficients at different Reynolds numbers calculated by equation (5) for three speeds $(6,9,12,15 \mathrm{~m} / \mathrm{s})$. The difference between the three cases is clearly illustrated in this figure, there is a proportional relation between wind velocity and power coefficient, it can be noted that the average power coefficient increases slightly with increasing wind velocity. The maximum value of the power coefficient appears in the range of the tip speed ratio of $(\lambda=0.8$ to $\lambda=1.2)$, which agree with the previous studies [5].

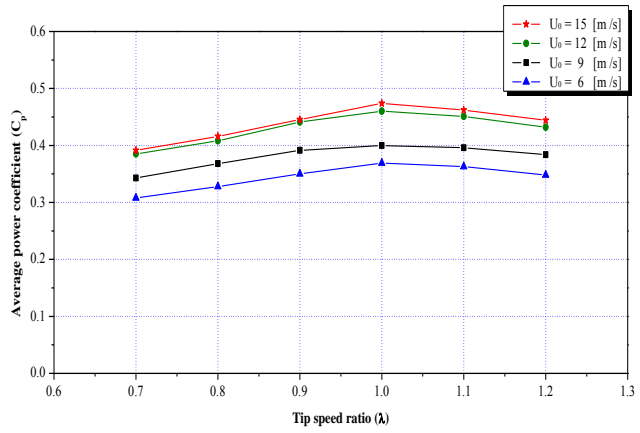

Figure 14. Power coefficient as a function of tip speed ratio at different wind velocity.

\section{CONCLUSION}

In this study, the flow analysis over a three-dimensional hill with a one-stage Savonius turbine is carried out numerically. Results obtained by the SST turbulence model are analyzed and compared with experimental data. The proposed methodology indicates that the numerical predictions of the flow field as well as power coefficient calculations are in reasonable agreement with experiment data. The flow around a three dimensional hill is characterized by an acceleration of the wind towards the hilltop and deceleration on the leeward side. It is demonstrated that it is possible to improve the low performance of the Savonius turbine by placing them at the top of a hill, which is clearly the best position. The extracted power increases significantly, more than doubles, when placed on top of a hill. The study of the aspect ratio of the Savonius turbine indicates that the optimum aspect ratio is different when the turbine is placed on the hill. The paper also shows that a Savonius rotor with a helical geometry always performs better than the conventional Savonius turbine.

\section{REFERENCES}

[1] H. G. Kim and V. Patel,"Test of turbulence models for wind flow over terrain with separation and recirculation," Boundary-Layer Meteorology, vol. 94, no.1, pp. 5-21, 2000. doi: 10.1023/A:1002450414410.

[2] F. A. Castro, et al. (2003). "Simulation of the Askervein Flow. Part 1: Reynolds Averaged Navier-Stokes Equations (k_ $\in$ Turbulence Model)," Boundary-Layer Meteorology, vol. 107, no.3, pp. 501-530, 2003. doi: 10.1023/A:1022818327584.

[3] N. N. Sørensen, General-purpose flow solver applied to flow over hills, Technical University of Denmark Danmarks Tekniske Universitet, Risø National Laboratory for Sustainable Energy, Risø Nationallaboratoriet for Bæredygtig Energi, Wind Energy Division Afdelingen for Vindenergi, Aeroelastic Design Aeroelastisk Design, 1995.

[4] Y.F. Lun, et al., "Numerical simulation of flow over topographic features by revised $\mathrm{k}-\varepsilon$ models," Journal of Wind Engineering and Industrial Aerodynamics, vol. 91, no. 1, pp. 231-245, 2003.

doi: 10.1016/S0167-6105(02)00348-3.

[5] T. Hayashi et al.,"Wind tunnel tests on a different phase threestage Savonius rotor," JSME International Journal Series B Fluids and Thermal Engineering, vol. 48, no. 1, pp. 9-16, 2005. doi: 10.1299/jsmeb.48.9.

[6] M. Kamoji, et al., "Performance tests on helical Savonius rotors," Renewable Energy, vol. 34, no. 3, pp. 521-529, 2009. doi:10.1016/j.renene.2008.06.002.

[7] Z. Liu, et al., "LES study of turbulent flow fields over a smooth 3-D hill and a smooth 2-D ridge," Journal of Wind Engineering and Industrial Aerodynamics vol. 153, pp. 1-12, 2016. doi: 10.1016/j.jweia.2016.03.001.

[8] P. Larin, et al., "CFD based synergistic analysis of wind turbines for roof mounted integration." Journal of Wind Engineering and Industrial Aerodynamics, vol. 156. pp. 1-13, 2016. doi: 10.1016/j.jweia.2016.06.007.

[9] T. Ishihara and K. Hibi, "Numerical study of turbulent wake flow behind a three-dimensional steep hill." Wind and Structures, vol. 5, no. 2-4, pp. 317-328, 2002.

[10] M. Shaheen, et al., "Numerical study of two-bucket Savonius wind turbine cluster," Journal of Wind Engineering and Industrial Aerodynamics, vol. 137, pp. 78-89, 2015. doi: 10.1016/j.jweia.2014.12.002.

[11] S. McTavish et al.,"Steady and rotating computational fluid dynamics simulations of a novel vertical axis wind turbine for smallscale power generation." Renewable Energy, vol. 41, pp. 171-179, 2012. doi: 10.1016/j.renene.2011.10.018.

[12] Y. F. Wang, and M.-S. Zhan, "Effect of barchan dune guide blades on the performance of a lotus-shaped micro-wind turbine," Journal of Wind Engineering and Industrial Aerodynamics, vol. 136, pp. 34-43, 2015. doi:10.1016/j.jweia.2014.10.014.

[13] A. Krishnan, and M. Paraschivoiu, "3D analysis of building mounted VAWT with diffuser shaped shroud," Sustainable Cities and Society, vol. 27, pp. 160-199, 2015. doi: 10.1016/j.scs.2015.06.006.

[14] Ishihara, T. and K. Hibi, "An experimental study of turbulent boundary layer over a steep hill," in Proceedings of the 15th National Symposium on Wind Engineering, 1998.

[15] J.V. Akwa, et al."Discussion on the verification of the overlap ratio influence on performance coefficients of a Savonius wind rotor using computational fluid dynamics," Renewable Energy, vol. 38, no. 1, p. 141149, 2012. doi: 10.1016/j.renene.2011.07.013. 
MANUFACTURING 


\section{The Effect of Nanoparticle Concentration on MQL Performance when Machining Ti-6Al-4V titanium alloy}

\author{
Abdelkrem A. Eltaggaz \\ Advanced Manufacturing Lab (AML) \\ School of Engineering, University of Guelph \\ Guelph, Canada \\ aeltagga@uoguelph.ca
}

\author{
Ibrahim M. Deiab \\ Advanced Manufacturing Lab (AML) \\ School of Engineering, University of Guelph \\ Guelph, Canada \\ ideiab@uoguelph.ca
}

\begin{abstract}
The main purpose of using cutting fluid during machining processes is to reduce the cutting temperature and friction, and to wash away chips from the cutting zone. However, excessive use of conventional cutting fluid negatively influences human health and environment. Therefore, much research has attempted to improve cutting fluid performance with superior tribological and thermal properties, and to reduce the amount of cutting fluid to minimize machining cost and impact on environment. Recently, Minimum Quantity Lubrication (MQL) technique has been widely investigated as a good alternative to flood coolant. Although MQL improves machining results, its removal heat capability still needs to improve. In this paper, in order to enhance the thermal conductive and viscosity of MQL, nanoparticles were dispersed to make nanofluid coolant. Nanofluids have attracted the attention of investigators due to their good high thermal conductivity and ability to remove heat. In this study, the effect of the cutting speed, feed rate, and nanoparticle concentrations on machining titanium Ti-Al6-V4 alloy were investigated by performing ANOVA analysis. The nanofluid coolant was prepared by adding Aluminum Oxide $\left(\mathrm{Al}_{2} \mathrm{O}_{3}\right)$ nanoparticles to base fluid (vegetable oil) at different weight concentrations $(0,2$, and $4 \%$ wt).

The ANOVA analysis found that the nanoparticle concentrations and feed rate had a significant influence on surface roughness. The tool wear was also observed to be affected by nanoparticle concentration significantly. The nanoparticles concentration had a significant impact on the flank wear as it could improve the thermal conductivity and lubrication properties of the cutting fluid and reduce the coefficient of friction between the tool- workpiece and toolchip. In terms of the effect of nanoparticle concentrations, $4 \%$ wt provided better improvements in both surface roughness and tool wear compared to $2 \%$ wt concentration and the pure MQL.
\end{abstract}

Keywords-component; Titanium Ti-Al6-V4 alloy, Tool wear, Surface roughness, Minimum Quantity Lubrication (MQL), Nanocutting fluid

\section{INTRODUCTION}

The effectiveness of machining processes is greatly dependent on the use of cutting fluid to reduce cutting forces and cutting temperature. However, such cutting fluids have negative effects on environment and health. In an effort to eliminate these contrary impacts, many experiments have been conducted using minimum quantity lubrication (MQL) as the main cooling technique. MQL can be also identified as neardry machining (NDM) or micro-lubrication $[1,2]$. Klocke et al. [3] identified the MQL as a cooling technique that uses only a small amount of lubricant, about 3 to 4 orders of magnitude less than flood coolant, at the flow rate of 50 to $500 \mathrm{ml} / \mathrm{h}$. As a result of the lubricant penetration ability into the chip/tool interface, MQL reduces cutting temperature and friction, and extends tool life. Therefore, MQL improves the surface finish of the product. Several investigations have revealed that the MQL technique is a viable alternative to the flood coolant under similar performance parameters $[4,5,6]$. In addition, from the environmental perspective, the MQL method has been shown to be suitable for green machining.

Spraying cutting fluid during machining difficult-to-cut materials, such as titanium, is crucial to reduce the high generated temperature at the cutting zone, thus extending tool life. The MQL technique provides relatively effective machining results, but enhancing its heat removal capacity is still required. MQL with nanoparticles has been suggested to be an effective method for reducing environmental and machine operator hazards due to its good tribological property and antibacterial feature $[7,8]$.

At present, efforts have been made to develop advanced machining processes utilizing less lubrication [10]. For further enhancement, adding nanoparticles into MQL (better known as MQL-nanofluid) may provide much better product quality due to the rolling action of nanoparticles, which could remarkably minimize cutting forces. Also, dispersing nanoparticles into base fluid of MQL would enhance the thermal conductivity and viscosity of the coolant $[10,11]$. Nano-cutting fluids make an important contribution toward meeting the heat dissipation challenge during machining processes as they provide a high practical thermal conductivity value compared to the base fluid [12]. This value can be attributed to the outstanding properties 
of the added nanoparticles (e.g. high heat convection coefficient and thermal conductivity). Moreover, the appropriate dispersion of nanoparticles into the base fluid provides a promising advantage in cutting processes as it helps to reduce the coefficient of friction in the tool-chip interface zone [13]. It has also been revealed that nano-cutting fluids have superior cooling properties due to their effective heat extraction properties [14].

Generally, most research agrees that nanofluids provide higher thermal conductivity in comparison with base fluid; however, determining the appropriate concentration of the nanoparticles is a big challenge. The addition of excessive nanoparticles could result in nanoparticle agglomeration which reduces the lubrication property. Lee et al. [7] stated that the physical parameters such as size and shape of nanoparticles affect their tribological properties. The study was found that determining the appropriate concentration of nanoparticles in base fluid is important to achieve better surface quality and lubrication. Sharma et al. [15] have also observed that an increase of nanoparticle concentration improves the thermal conductivity and viscosity of the nanofluid. Zhang et al. [16] carried out grinding experiments on 45 steel under the jet MQL nanofluid (soybean oil $+\operatorname{MoS} 2$ ) with different mass fraction concentrations $(2,4,6,8$, and $10 \%)$ of $\mathrm{MoS}_{2}$ nanoparticles. It was noted that increasing mass fraction nanoparticle concentration from $2 \%$ to $6 \%$ noticeably reduces the specific grinding energy, grinding forces, and friction coefficient. With further increases in nanoparticle concentration (above than $6 \%$ ), grinding forces, specific grinding energy, and friction coefficient increased. This increase can be attributed to the agglomeration of nanoparticles which cannot lubricate the grinding zone, thus negatively affecting the surface finish. It was concluded that $6 \%$ mass fraction concentration of nanoparticles provided the lowest specific grinding energy, tangential grinding forces, and friction coefficient. Vasu et al. [17] performed turning machining on Inconel 600 alloy under MQL with different volume fractions of $\mathrm{Al}_{2} \mathrm{O}_{3}$ (aluminum oxide) and regular MQL vegetable oil. It was reported that the MQL with 6\% nanoparticle concentration resulted considerable improvement in the surface roughness, tool wear, cutting forces, and cutting temperature compared to MQL with $4 \%$ volume fraction and regular MQL.

\section{EXPERIMENTATION AND METHODOLOGY}

Experiments were conducted by turning titanium Ti-Al6V4 alloy in order to investigate the effects of cutting speed, feed rate, and concentration of nanofluid on tool wear and surface roughness. The tests were performed on a CNC lathe machine using standard coated carbide turning inserts (CNMG120416MR) with a $1.6 \mathrm{~mm}$ nose radius. The machining tests were carried out using MQL nanofluid with different concentrations of volume fraction $(0,2$, and $4 \mathrm{wt} \%)$, at different levels of cutting speed $(120,170$, and $220 \mathrm{~m} / \mathrm{min})$, and feed rate $(0.1,0.15$, and $0.2 \mathrm{~mm} / \mathrm{rev})$ as shown in Table 1 . The depth of cut for each cutting pass was $0.5 \mathrm{~mm}$ with cutting length of $50 \mathrm{~mm}$. Each test was repeated twice for more reliable data, and findings were recorded for maximum flank wear (VB) and surface finish (Ra).

The air-oil mixture of the MQL system was supplied by the stand-alone booster system (Eco-Lubric) with a nominal oil flow rate of $80 \mathrm{ml} / \mathrm{min}$ and air pressure of $0.5 \mathrm{MPa}$. In addition, the vegetable oil ECOLUBRIC E200 was used as a base cutting fluid. This fluid, rapeseed, is environmentally harmless, suitable for industrial application, and has a biodegradability of $90 \%$ in 28 days [18]. The position and the angle of the MQL nozzle were adjusted by experimental observation to avoid being blocked by chips.

For the preparations of the nano-cutting fluid, Aluminum Oxide gamma nanoparticles $\left(\mathrm{Al}_{2} \mathrm{O}_{3}\right)$ were used as nanoadditives. $\mathrm{Al}_{2} \mathrm{O}_{3}$ nanoparticles were selected because of their superior tribological property and anti-toxic aspect. It has 20 $\mathrm{nm}$ average diameter, 95\% purity, and $138 \mathrm{~m} 2 / \mathrm{g}$ specific surface areas. Dispersion of nanoparticles into the base fluid vegetable oil is an important aspect, which effects on thermal conductivity and viscosity of resultant nano-cutting fluid. An ultrasonic machine (AQUASONIC-50HT) was used for 3 hours at $60^{\circ} \mathrm{C}$ to disperse nanoparticles into the base cutting fluid. However, the dispersion of nanoparticles into the base fluid is considered as challenge, due to the strong van der Waals interactions which result in nanoparticle agglomeration, clogging, and sedimentation. Thus, using a physical or chemical treatment such as surfactants is recommended to ensure sufficient dispersion of nanoparticles [19, 20]. Sodium Dodecyl Sulfate (SDS) was used as a surfactant (i.e. $0.2 \mathrm{gm}$ ). Surfactants are believed to make the nanoparticles performance more hydrophilic, and to increase the nanoparticles surface charges, thereby increasing the repulsive forces between the nanoparticles.

In this work, experiments and analysis of variance (ANOVA) analysis was conducted for each of the design parameters and labelled as A (cutting speed), B (feed rate), and $\mathrm{C}$ (nanoparticles concentration) as in Table 2. MINTAB 17 software was used to do design of ANOVA, which was performed to investigate the effect of design parameters on tool wear and machined surface quality. Using the ANOVA, the importance of cutting parameters and nanoparticle concentration with respect to surface roughness and tool wear was studied in order to find out the optimum combination of cutting parameters. All ANOVA analysis were conducted with confidence level of $95 \%(\alpha=0.5)$.

TABLE 1. SCHEME OF EXPERIMENT

\begin{tabular}{|c|c|c|c|}
\hline $\begin{array}{c}\text { Experiment } \\
\text { No. }\end{array}$ & $\begin{array}{c}\text { Speed } \\
(\mathbf{m} / \mathbf{m i n})\end{array}$ & $\begin{array}{c}\text { Feed rate } \\
(\mathbf{m m} / \mathbf{r e v})\end{array}$ & $\begin{array}{c}\text { Nanoparticles } \\
(\mathbf{w t} \%)\end{array}$ \\
\hline 1 & 120 & 0.1 & 0 \\
\hline 2 & 120 & 0.15 & 2 \\
\hline 3 & 120 & 0.2 & 4 \\
\hline 4 & 170 & 0.1 & 2 \\
\hline 5 & 170 & 0.15 & 4 \\
\hline 6 & 170 & 0.2 & 0 \\
\hline 7 & 220 & 0.1 & 4 \\
\hline 8 & 220 & 0.15 & 0 \\
\hline 9 & 220 & 0.2 & 2 \\
\hline
\end{tabular}




\section{RESULTS AND DISCUSSION}

In the current paper, the findings were recorded for max flank tool wear (VB) and surface roughness (Ra). Table 2 describes the max flank wear and surface roughness determined on the cutting tool and machined surface of ADI respectively. The flank wear varied from 0.162 to $0.721 \mathrm{~mm}$, and surface roughness varied from 0.512 to $2.81 \mu \mathrm{m}$. The main effects plots for $\mathrm{Ra}$ and VB are shown in Figures 1 and 2 respectively. Based on the analysis, low surface roughness was obtained at a feed rate of $0.1 \mathrm{~mm} / \mathrm{rev}$, a cutting speed of 170 $\mathrm{m} / \mathrm{min}$, and a nanoparticle concentration of $2 \mathrm{wt} \%$. Feed rate and nanoparticles wt $\%$ were found to have the greatest impact on surface roughness quality (Figure 1), contributing $46.55 \%$ and $40.75 \%$ respectively (Table 3 ). As known in machining process, the feed rate has a significant effect on the surface roughness. In addition, the impact of the increase in nanoparticle concentration can be attributed to the increase in nanoparticles which can penetrate in the chip and workpiece surface and settle in any micro-grooves or slits in the workpiece surface.

TABLE 2. THE MACHINING PARAMETER LEVELS AND THE OUTPUT MEASUREMENTS

\begin{tabular}{|c|c|c|c|c|c|}
\hline $\begin{array}{c}\text { Experiment } \\
\text { No. }\end{array}$ & A & B & C & $\begin{array}{c}\text { Surface } \\
\text { roughness } \\
(\boldsymbol{\mu m})\end{array}$ & $\begin{array}{c}\text { Maximum } \\
\text { flank } \\
\text { wear } \\
(\mathbf{m m})\end{array}$ \\
\hline 1 & 1 & 1 & 1 & 0.891 & 0.481 \\
\hline 2 & 1 & 2 & 2 & 0.852 & 0.241 \\
\hline 3 & 1 & 3 & 3 & 1.692 & 0.162 \\
\hline 4 & 2 & 1 & 2 & 0.512 & 0.192 \\
\hline 5 & 2 & 2 & 3 & 1.421 & 0.171 \\
\hline 6 & 2 & 3 & 1 & 2.812 & 0.721 \\
\hline 7 & 3 & 1 & 3 & 0.563 & 0.211 \\
\hline 8 & 3 & 2 & 1 & 1.890 & 0.561 \\
\hline 9 & 3 & 3 & 2 & 0.951 & 0.242 \\
\hline
\end{tabular}

In terms of tool wear, the lowest max flank wear (VB) was obtained at a cutting speed of $120 \mathrm{~m} / \mathrm{min}$, a feed of $0.2 \mathrm{~mm} / \mathrm{rev}$, and a nanoparticle concentration of $4 \mathrm{wt} \%$. Generally, the cutting speed significantly affects the tool wear due to the high heat generation at the cutting zone. However, in Figure 2, it can be seen that nanoparticle concentration was found to be the most significant factor, at $90 \%$ contribution (Table 4), with regard to the tool wear. This effect on tool wear behavior was likely because of the good thermal conductivity of the nanoparticle and its ability to penetrate into the chip/workpiece zone, which improve the machining performance, viscous property, and heat removal ability of the nanofluid.

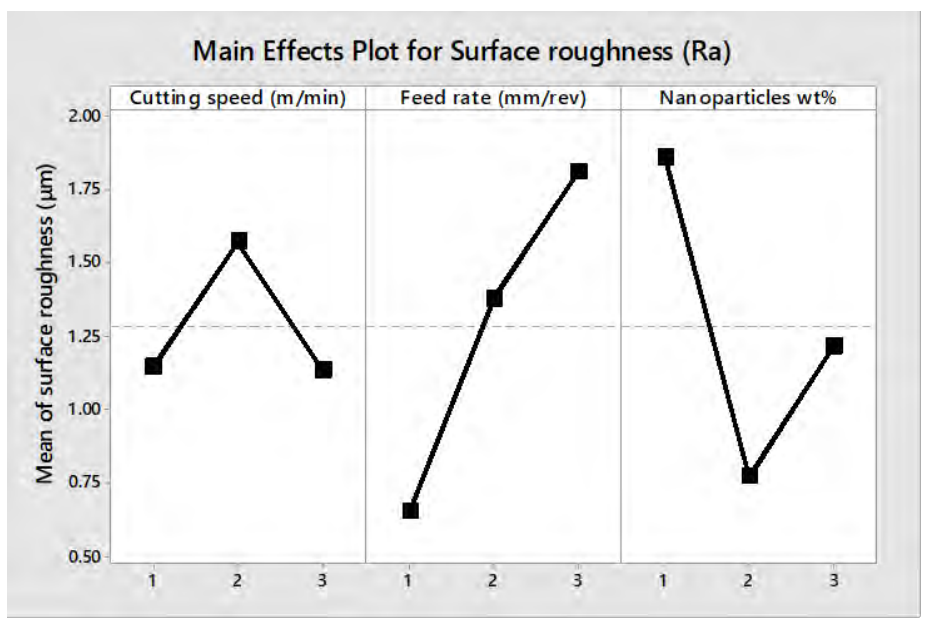

Figure 1. The main effects plot for surface roughness $(R a)$

TABLE 3 ANALYSIS OF VARIANCE FOR SURFACE ROUGHNESS.

\begin{tabular}{|c|c|c|c|c|}
\hline Source & $\begin{array}{c}\text { Degrees } \\
\text { of } \\
\text { freedom }\end{array}$ & $\begin{array}{c}\text { Sum of } \\
\text { squares }\end{array}$ & $\begin{array}{c}\text { Mean of } \\
\text { squares }\end{array}$ & $\begin{array}{c}\text { \% of } \\
\text { contribution }\end{array}$ \\
\hline Speed & 2 & 0.373 & 0.186 & 8.45 \\
\hline Feed & 2 & 2.0551 & 1.027 & 46.55 \\
\hline $\begin{array}{c}\text { Nanoparticles } \\
\%\end{array}$ & 2 & 1.799 & 0.899 & 40.75 \\
\hline Error & 2 & 0.187 & 0.093 & 4.24 \\
\hline Total & 8 & 4.414 & & 100 \\
\hline
\end{tabular}

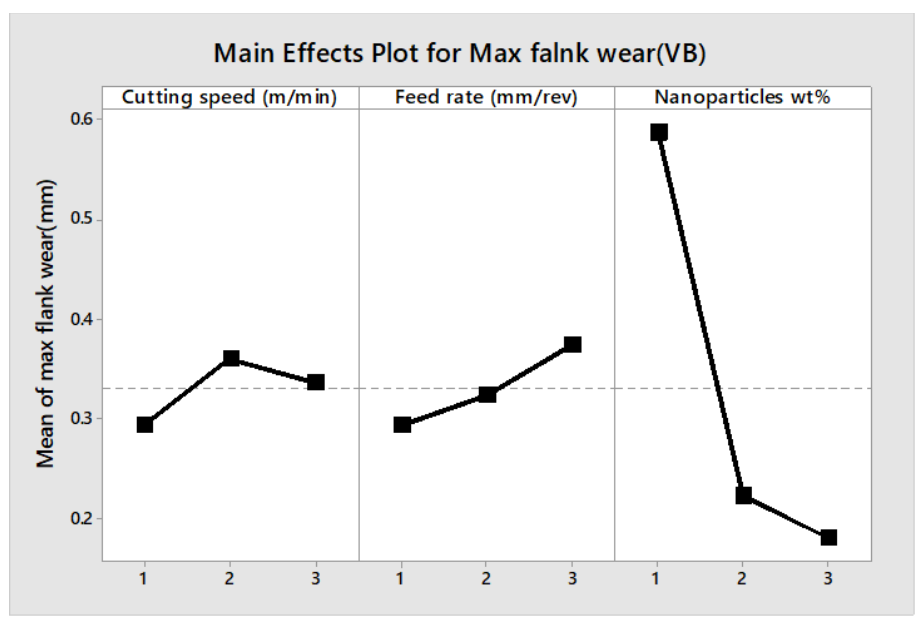

Figure 2. The main effects for the max flank wear (VB) 
TABLE 4. ANALYSIS OF VARIANCE FOR MAX FLANK WEAR

\begin{tabular}{|c|c|c|c|c|}
\hline Source & $\begin{array}{c}\text { Degrees } \\
\text { of } \\
\text { freedom }\end{array}$ & $\begin{array}{c}\text { Sum of } \\
\text { squares }\end{array}$ & $\begin{array}{c}\text { Mean of } \\
\text { squares }\end{array}$ & $\begin{array}{c}\text { \% of } \\
\text { contribution }\end{array}$ \\
\hline Speed & 2 & 0.006 & 0.003 & 2.04 \\
\hline Feed & 2 & 0.009 & 0.004 & 3.00 \\
\hline $\begin{array}{c}\text { Nanoparticles } \\
\%\end{array}$ & 2 & 0.298 & 0.149 & 90.0 \\
\hline $\begin{array}{c}\text { Error } \\
\text { Total }\end{array}$ & 2 & 0.016 & 0.008 & 4.85 \\
\hline & 8 & 0.331 & & 100 \\
\hline
\end{tabular}

\section{CONCLUSION}

In this study, cutting speed, feed rate, and different nanoparticle concentrations (wt \%) in MQL were selected as cutting parameters. According to ANOVA, the feed rate ( 40.75 $\%$ contribution) and nanoparticle concentration (46.55\% contribution) were the most significant parameters that determined the surface roughness on the titanium Ti- $6 \mathrm{Al}-4 \mathrm{~V}$ alloy. The ANOVA analysis also showed that the nano-fluid at 2 and $4 \mathrm{wt} \%$ concertation of $\mathrm{Al}_{2} \mathrm{O}_{3}$ provided better results than regular MQL in determining surface roughness. The tool wear was also found to be affected by nanoparticle concentration. The analysis indicated that the nanoparticles concentration had a significant influence on the flank wear, at $90 \%$ contribution, as it could enhance the thermal conductivity and lubrication properties of the cutting fluid and minimize the coefficient of friction between the tool- workpiece and tool-chip. Ultimately, it can be concluded that the nano-fluid improves the surface finish and tool life as a coolant during machining titanium TiAl6-V4 alloy. Furthermore, $\mathrm{Al}_{2} \mathrm{O}_{3}$ nanoparticles are improved the machining performance of MQL. However, determining the appropriate nanoparticle concentration needs more investigations.

\section{ACKNOWLEDGMENT}

The authors acknowledge the support of the Natural Sciences and Engineering Research Council of Canada (NSERC) and Ontario centers of Excellence (OCE).

\section{REFERENCES}

[1] B. Boswell et al., "A review identifying the effectiveness of minimum quantity lubrication (MQL) during conventional machining," Int. J. Adv. Manuf. Techno., vol. 92, no. 1-4, pp. 321-340, Feb. 2017.

doi:10.1007/s00170-017-0142-3.

[2] J. S. Dureja et al., "Performance evaluation of coated carbide tool in machining of stainless steel (AISI 202) under minimum quantity lubrication (MQL)," Inter. J. Preci. Eng. Manufacturing-Green Techno., vol. 2, no. 2, pp. 123-129, Apr. 2015. doi: 10.1007/s40684-015-0016-9.

[3] F. Klocke and G. Eisenblätter, "Dry Cutting," CIRP Annals, vol. 46, no. 2, pp. 519-526, 1997. doi: 10.1016/S0007-8506(07)60877-4.

[4] A. Eltaggaz et al, "Coolant strategy influence on tool life and surface roughness when machining ADI," Int. J. Adv. Manuf. Techno., vol. 94, no. 9-12, pp. 3875-3887, Sep. 2017. doi: 10.1007/s00170-017-1088-1.

[5] A. K. Sharma, A. K. Tiwari, and A. R. Dixit, "Effects of Minimum Quantity Lubrication (MQL) in machining processes using conventional and nanofluid based cutting fluids: A comprehensive review," J. Cleaner Prod., vol. 127, pp. 1-18, Jul. 2016. doi: 10.1016/j.jclepro.2016.03.146.
[6] I. Deiab, S. W. Raza, and S. Pervaiz, "Analysis of Lubrication Strategies for Sustainable Machining during Turning of Titanium Ti-6Al-4V Alloy," Procedia CIRP, vol. 17, pp. 766-771, 2014. doi:10.1016/j.procir.2014.01.112.

[7] P.-H. Lee et al., "An experimental study on micro-grinding process with nanofluid minimum quantity lubrication (MQL)," Inter. J. Precis. Eng. Manu., vol. 13, no. 3, pp. 331-338, Mar. 2012. doi: $10.1007 / \mathrm{s} 12541-012-0042-2$.

[8] P.-H. Lee et al., "Environmentally-Friendly Nano-fluid Minimum Quantity Lubrication (MQL) Meso-scale Grinding Process Using Nanodiamond Particles," in 2010 Inter. Conf. on Manufacturing Automation, 2010. doi: 10.1109/ICMA.2010.27.

[9] P. Sreejith and B. K. . Ngoi, "Dry machining: Machining of the future," J. Mater. Proc. Techno., vol. 101, no. 1-3, pp. 287-291, Apr. 2000. doi: 10.1016/S0924-0136(00)00445-3.

[10] A. K. Sharma, A. K. Tiwari, and A. R. Dixit, "Progress of Nanofluid Application in Machining: A Review," Mater. Manuf. Processes, vol. 30, no. 7, pp. 813-828, Oct. 2014. doi: 10.1080/10426914.2014.973583.

[11] R. Saidur, K. Y. Leong, and H. A. Mohammad, "A review on applications and challenges of nanofluids," Renewable Sustainable Energy Rev., vol. 15, no. 3, pp. 1646-1668, Apr. 2011.

doi: 10.1016/j.rser.2010.11.035.

[12] S.-S. Hsieh, H.-H. Liu, and Y.-F. Yeh, "Nanofluids spray heat transfer enhancement," Int. J. Heat Mass Transfer, vol. 94, pp. 104-118, Mar. 2016. doi: 10.1016/j.jjheatmasstransfer.2015.11.061.

[13] M. Alberts, K. Kalaitzidou, and S. Melkote, "An investigation of graphite nanoplatelets as lubricant in grinding," Inter. J. Mach. Tools and Manu., vol. 49, no. 12-13, pp. 966-970, Oct. 2009. doi: 10.1016/j.ijmachtools.2009.06.005.

[14] R. R. Srikant et al., "Applicability of cutting fluids with nanoparticle inclusion as coolants in machining," Proc. Inst. Mech. Eng., Part J, vol. 223, no. 2, pp. 221-225, Dec. 2008.

doi:10.1016/10.1243/13506501JET463.

[15] A. K. Sharma et al., "Characterization and experimental investigation of A12O3 nanoparticle based cutting fluid in turning of AISI 1040 steel under minimum quantity lubrication (MQL)," Mater. Today: Proc., vol. 3, no. 6, pp. 1899-1906, 2016. doi: https://doi.org/10.1016/j.matpr.2016.04.090.

[16] Y. Zhang et al., "Experimental evaluation of MoS2 nanoparticles in jet MQL grinding with different types of vegetable oil as base oil," J. Cleaner Prod., vol. 87, pp. 930-940, Jan. 2015. doi: 10.1016/j.jclepro.2014.10.027.

[17] V. Vasu and G. Pradeep Kumar Reddy, "Effect of minimum quantity lubrication with $\mathrm{Al} 2 \mathrm{O} 3$ nanoparticles on surface roughness, tool wear and temperature dissipation in machining Inconel 600 alloy," Proc. Inst. Mech. Eng., Part N, vol. 225, no. 1, pp. 3-16, Mar. 2011. doi: $10.1177 / 1740349911427520$.

[18] I. Deiab, S. W. Raza, and S. Pervaiz, "Analysis of Lubrication Strategies for Sustainable Machining during Turning of Titanium Ti-6Al-4V Alloy," Procedia CIRP, vol. 17, pp. 766-771, 2014.

doi: 10.1016/j.procir.2014.01.112.

[19] Y. Hwang et al., "Thermal conductivity and lubrication characteristics of nanofluids," Curr. Appl. Physics, vol. 6, pp. e67-e71, Aug. 2006. doi: 10.1016/j.cap.2006.01.014.

[20] H. Zhu et al., "Preparation and thermal conductivity of suspensions of graphite nanoparticles," Carbon, vol. 45, no. 1, pp. 226-228, Jan. 2007. doi:10.1016/j.carbon.2006.07.005. 


\section{Multi-objective Optimization During Machining Ti-6Al-4V Using Nano-Fluids}

\author{
H. Hegab*, W. Abdelfattah, A. Mohany, H. Kishawy \\ Department of Automotive, Mechanical and \\ Manufacturing Engineering \\ University of Ontario Institute of Technology \\ Oshawa, Canada
}

\author{
S. Rahnamayan \\ Department of Electrical, Computer and Software \\ Engineering \\ University of Ontario Institute of Technology \\ Oshawa, Ontario
}

\begin{abstract}
Several properties make titanium and its alloy the primary candidate to attain weight and functional advantages because of its promising properties such as high strength to weight ratio, high corrosion resistivity, and high yield stress. Although titanium alloys have superior properties, some inherent characteristics such as high chemical reactivity and low thermal conductivity lead to poor machinability and result in premature tool failure and shortened tool life. In order to overcome the heat dissipation challenge during machining of titanium alloys, nano-cutting fluids are utilized as they offer higher observed thermal conductivity values compared to the base oil. Thus, in the current work, multi-walled-carbon nanotubes (MWCNTs) cutting fluids along with minimum quantity lubrication (MQL) have been employed during machining Ti-6Al-4V. On the other hand, developing a multiobjective optimization model for machining titanium alloys is a promising step in order to minimize machining cost, achieve excellent surface quality, and increase the cutting tool life by selecting the optimal cutting conditions (i.e. cutting speed, feed rate, depth of cut). In this study, response surface methodology (RSM), and genetic algorithm (GA) are employed to model and optimize three main machining responses: tool wear, surface quality, and power consumption. Three main independent processes parameters are considered when machining titanium alloys, namely; cutting speed, feed rate, and percentage of added nano-additives.
\end{abstract}

Keywords-machining; optimization; modeling; response surface methodology (RSM); nano-cutting fluid; genetic algorithm (GA), NSGA-II

\section{INTRODUCTION}

Titanium alloys are broadly used in various industrial applications in military, aerospace, power generation, automotive, and other fields due to their promising mechanical, physical, and chemical characteristics; such as, high yield strength, high strength to weight ratio, high toughness and high creep, corrosion, and wear resistance [1]. These materials also retain their hardness and strength at high temperatures [2] which make them one of the primary candidates for aerospace, nuclear, power generation, and automotive applications.
However, despite the above-mentioned superior characteristics, titanium alloys are inherently difficult to cut due to their high stresses and high cutting temperatures generated when they are being machined. This is mainly attributed to low thermal conductivity of titanium which adversely affects the tool life and can lead to a premature tool failure. Because of their low thermal conductivity, the generated heat during machining titanium alloys is mainly dissipated through the cutting tool and cooling media other than the workpiece or chip. In addition, titanium alloys become chemically reactive at a high cutting temperature and react with some tool materials which also deteriorate the cutting tool and accelerate the tool failure. Moreover, the resultant chip shape in titanium machining is serrated or saw-toothed as localized adiabatic shear bending and intense shear strain rate exist in the primary shear zone due to the high temperature at the chip-tool interface. Some other properties, which make titanium alloys difficult to cut and impose barriers towards their widespread applications, are low elastic modulus, strain hardening, tendency to adhesion, and forming built-up edges $[3,4]$.

A promising technology in the application of cutting fluid is known as minimum quantity lubricant (MQL). MQL is a cooling/lubrication method in which an optimal amount of cutting fluid is forced to penetrate into the cutting zone by means of compressed air. Among the available lubrication techniques, the MQL technique with vegetable oil offers the best environmental friendly solution. Not only does this technique provide the optimum amount of lubricant, utilizing vegetable oil presents a promising alternative to overcoming harmful environmental impacts of commercial cutting fluids, such as mineral oil. The major important factor to improve the cooling and lubricant functions in the MQL method is utilizing certain cutting fluids such nano-fluids which can develop its wettability aspects $[5,6]$. Carbon nanotubes (CNTs) are among the nanoadditives that have superior properties. The diameters of these nano-additives vary from few nanometers to hundreds of nanometers, and their lengths range between tens of nanometers to several centimeters [7]. Proposing new nano-cutting fluids is contributing to overcome the heat dissipation challenge during the cutting processes as it offers a highly observed thermal conductivity value in comparison with the base lubricants. 
Additionally, it is shown that nano-cutting fluids have superior cooling properties due to its good heat extraction capabilities [8]. Nano-cutting fluids have shown promising effects on the cutting performance characteristics through different cutting operations such as turning, milling and grinding as has been presented in some previous studies [9-14, 22-26]. Also, a previous work introduced a detailed model to assess the sustainability aspects (including cutting fluids application) for machining processes [27]. The proper dispersion of nano-additives into the base cutting fluid shows a promising advantage in cutting processes as it helps to reduce the induced friction between the workpiece and cutting tool [15]. Also, nano-cutting fluids can contribute in facing the heat dissipation challenge during cutting processes. The main objective of this paper is to model and optimize the machining process of Ti-6Al-4V with MQL-nano-fluids using RSM and multi-objective evolution algorithm (i.e., NSGA-II). Three main machining responses are tool wear, surface quality, and power consumption, while three main independent process parameters, as decision variables, are considered during machining titanium alloys as follows: cutting speed, feed rate, and percentage of added nano-additives. The experimental design and methodology will be discussed in the next section, while section 3 will discuss the experimental results, machining responses modeling and multi-objective optimization results. Finally, section 4 will discuss the study conclusions.

\section{EXPERIMENTATION}

In order to investigate the effects of dispersed MWCNTsnano-fluid on the cutting quality performance, experiments are carried out by turning of Ti-6Al-4V. Ti-6Al-4V (UNS R56400) is utilized as the experiments workpiece. The tests are performed on $\mathrm{CNC}$ lathe machine using standard carbide turning inserts CNMG 432MMH13A (ANSI standard). Cutting tests are performed under MQL strategy, using different levels of cutting speed, feed rate, and weight percentages of added nanoadditives. The depth of cut for each cutting pass is $0.2 \mathrm{~mm}$. The cutting tool information is listed in the Table I. Regarding the MQL system, the air-oil mixture was supplied by the stand-alone booster system (Eco-Lubric) installed on the machine tool with a nominal oil flow rate of $40 \mathrm{ml} / \mathrm{hr}$ and air pressure of $0.5 \mathrm{MPa}$. The average surface roughness parameter has been used to evaluate the machined surface quality using a surface roughness tester (i.e., Mitutoyo SJ.201). Through the use of a toolmaker's microscope, flank wear was measured subsequent to each cutting pass. In addition, a Power Sight Manager was employed throughout the machining process in effort to assist with logging power consumption.

TABLE I. THE USED CUTTING INSERT AND TOOL HOLDER DURING MACHINING Ti-6Al-4V

\begin{tabular}{|c|c|}
\hline & $\begin{array}{c}\text { Cutting tool information during machining } \\
\text { Ti-6Al-4V }\end{array}$ \\
\hline Cutting tool & Coated carbide insert (CNMG 120416MR (ISO)) \\
\hline Tool holder & $\begin{array}{c}\text { SANDVICK SCLCR-2525M12 with clearance angle 5o and } \\
\text { rake angle 0o, nose radius of } 1.58 \mathrm{~mm}\end{array}$ \\
\hline
\end{tabular}

Multi-walled carbon nanotubes (MWCNTs) with 13-20 nm average diameter, $95 \%$ purity, $10-30 \mu \mathrm{m}$ length, and $110 \mathrm{~m}^{2} / \mathrm{g}$ specific surface area were used for nano-cutting fluid preparations. Dispersion of MWCNTs into the base cutting fluid is an important factor which affects the resultant nano-cutting fluid thermal conductivity and viscosity. The dispersion of MWCNTs into the base cutting fluid is performed using an ultrasonic device (AQUASONIC-50HT) over the course of 3 hours at $60^{\circ} \mathrm{C}$, which is then followed by a step that offers thorough mixture, using a magnetic stirrer (Hot Plate Stirrer3073-21) for 30 minutes to ensure full dispersion of MWCNTs into the resultant nano-cutting fluid.

In this work, three design variables (i.e., cutting speed, cutting feed, and added nano-additives percentage) are used at three levels each. Table II indicates the design variables studied and the assignment of the corresponding levels. L9 orthogonal array (L9OA) based on the Taguchi method is employed in Table III [16]. Tests are replicated three times and average response values are calculated. The full factorial array in this work is L27OA $\left(3^{3}\right)$; however, fractional factorial array L9OA based on the design of experiments methodology has been employed to save time and cost.

TABLE II. THE LEVELS ASSIGNMENT TO DESIGN VARIABLE

\begin{tabular}{|c|c|c|c|c|}
\hline Design variables & Symbol & Level 1 & Level 2 & Level 3 \\
\hline $\begin{array}{c}\text { Cutting speed } \\
\text { (m/min) }\end{array}$ & A & 120 & 170 & 220 \\
\hline $\begin{array}{c}\text { Feed Rate } \\
\text { (mm/rev) }\end{array}$ & B & 0.1 & 0.15 & 0.2 \\
\hline $\begin{array}{c}\text { Nano-additives } \\
\text { (wt. \%) }\end{array}$ & C & $0 \%$ & $2 \%$ & $4 \%$ \\
\hline
\end{tabular}

TABLE III. THE STUDIED PARAMETER LEVELS AND THE PLAN OF EXPERIMENTS

\begin{tabular}{|c|c|c|c|}
\hline \multirow{2}{*}{$\begin{array}{c}\text { Cutting } \\
\text { test \# }\end{array}$} & $\begin{array}{c}\text { A: Cutting speed } \\
\text { (m/min) }\end{array}$ & $\begin{array}{c}\text { B: Feed rate } \\
(\mathrm{mm} / \text { rev })\end{array}$ & $\begin{array}{c}\text { C: Added } \\
\text { MWCNT } \\
\text { (wt. \%) }\end{array}$ \\
\hline 1 & 120 & 0.1 & 0 \\
\hline 2 & 120 & 0.15 & 2 \\
\hline 3 & 120 & 0.2 & 4 \\
\hline 4 & 170 & 0.1 & 2 \\
\hline 5 & 170 & 0.15 & 4 \\
\hline 6 & 170 & 0.2 & 0 \\
\hline 7 & 220 & 0.1 & 4 \\
\hline 8 & 220 & 0.15 & 0 \\
\hline 9 & 220 & 0.2 & 2 \\
\hline
\end{tabular}

\section{RESULTS AND DISCUSSIONS}

\section{A. Cutting tests results}

The flank wear, surface roughness, and power consumption results are listed as shown in Table IV. Regarding the flank wear results, a maximum flank wear of $0.4 \mathrm{~mm}$ [17] is used as the tool life criteria otherwise, in case of not reaching the end of tool life 
criteria, the cutting stopped after nine cutting passes. Test 3 showed the lowest flank wear value. The cutting conditions at test 3 are cutting speed of $120 \mathrm{~m} / \mathrm{min}$, feed rate of $0.15 \mathrm{~mm} / \mathrm{rev}$ and 4 wt. $\%$ of added MWCNTs. The highest wear value was found after test 8 (after 6 cutting passes) and after test 6 (after 5 cutting passes). Both of cutting tests 8 and 6 have been performed without using nano-additives.

When 2 wt. \% of added MWCNs is utilized, the results showed that cutting test 2 offered the lowest power consumption. The cutting conditions at this test are cutting speed of $120 \mathrm{~m} / \mathrm{min}$ and feed rate of $0.15 \mathrm{~mm} / \mathrm{rev}$. While the highest power consumption value has been obtained at cutting test 8 which has been performed using classical MQL without nano-additives. In general, MWCNTs nano-fluid has shown better performance in reducing the power consumption compared to the tests performed without any nano-additives. For surface roughness results, it is clear that MWCNTs nano-fluid showed promising results to improve the induced surface quality; however, cutting tests 3 and 9 didn't show effective results compared to the other tests performed using nano-additives as these tests have been performed at the higher value of feed rate level (i.e. $0.4 \mathrm{~mm} / \mathrm{rev}$ ). Cutting test 4 showed the best surface quality. The cutting conditions of this test include cutting speed of $170 \mathrm{~m} / \mathrm{min}$, feed rate of $0.1 \mathrm{~mm} / \mathrm{rev}$ and $2 \mathrm{wt}$. \% of added MWCNTs. The obtained results will be used in the next step to build three different mathematical models for the studied machining responses.

TABLE IV. THE MACHINING OUTPUTS RESULTS

\begin{tabular}{|c|c|c|c|}
\hline \multirow{2}{*}{$\begin{array}{c}\text { Cutting } \\
\text { test \# }\end{array}$} & $\begin{array}{c}|c| \\
\mathrm{F}_{1}: \text { Flank wear } \\
(\mathrm{mm})\end{array}$ & $\begin{array}{c}\mathrm{F}_{2} \text { : Surface } \\
\text { roughness } \\
(\mu \mathrm{m})\end{array}$ & $\begin{array}{c}\mathrm{F}_{3} \text { : Power } \\
\text { consumption } \\
(\text { Watt. } \mathrm{h})\end{array}$ \\
\hline 1 & 0.44 & 0.89 & 2325 \\
\hline 2 & 0.18 & 0.85 & 2084 \\
\hline 3 & 0.16 & 1.85 & 2192 \\
\hline 4 & 0.29 & 0.51 & 2138 \\
\hline 5 & 0.21 & 1.063 & 2230 \\
\hline 6 & 0.62 & 2.8 & 2454 \\
\hline 7 & 0.45 & 0.55 & 2610 \\
\hline 8 & 0.56 & 1.89 & 2344 \\
\hline 9 & 0.42 & 1.43 & 2634 \\
\hline
\end{tabular}

\section{B. Cutting processes modeling}

The modeling of the studied cutting processes is implemented using response surface methodology (RSM) to study the effects of the studied design variables on the measured responses. RSM has been employed in previous studies to optimize the machining parameters effects on different machining characteristics [18]. The developed models are used to express; the generated power consumption, surface roughness, and flank wear. It should be stated that all studied responses have "lower-the-better" criteria. The purpose of mathematical models relates the process responses to ease the optimization of the process. The model's validation is demonstrated using the average percentage of deviation $(\Delta)$ which can be calculated as shown in equations (1-2) where Rm is the measured response, $R_{p}$ is the predicted response, $n$ is the number of runs, and $\Delta_{\mathrm{i}}$ is the percentage of deviation per run (i).

The developed models using MWCNTs nano-fluid for flank wear, power consumption, and surface roughness are provided as shown in equations (3-5), respectively. The design variables quadratic and interaction effects are used to express the developed model as all studied design variables have three levels ( $2^{\text {nd }}$ degree of freedom). The average model accuracy for the flank wear under using MWCNTs nano-fluid is about $89.31 \%$, while $98.6 \%$ average model accuracy is calculated for the power consumption model, and the surface roughness model provides an average model accuracy of $93.71 \%$. It should be stated that all mathematical models are valid within the studied the range for the independent process parameters

$$
\begin{gathered}
\Delta_{\mathrm{I}}=\operatorname{ABS}\left(\frac{\mathrm{R}_{\mathrm{M}}-\mathrm{R}_{\mathrm{P}}}{\mathrm{R}_{\mathrm{M}}}\right) * 100 \% \\
\Delta=\frac{\sum_{\mathrm{I}=1}^{\mathrm{N}} \Delta_{\mathrm{I}}}{\mathrm{N}}
\end{gathered}
$$

$$
\begin{aligned}
\mathrm{VB}_{1}= & 7.2 * 10^{-6} \mathrm{~A}^{2}+10.96 \mathrm{~B}^{2}+188.6 \mathrm{C}^{2}-7 * \\
& 10^{-3} \mathrm{AB}-2.2 * 10^{-3} \mathrm{AC}-94 \mathrm{BC}+0.22 \\
\mathrm{PC}_{1}= & 0.01 \mathrm{~A}^{2}-2579.76 \mathrm{~B}^{2}+399783 \mathrm{C}^{2}- \\
& 0.35 \mathrm{AB}-75.26 \mathrm{~A}-52343 \mathrm{BC}+2071.63 \\
\mathrm{RA}_{1}= & 8.6 * 10^{-6} \mathrm{~A}^{2}+58.32 \mathrm{~B}^{2}+1480.65 \mathrm{C}^{2}- \\
& 0.002 \mathrm{AB}-0.21 \mathrm{AC}-268.55 \mathrm{BC}+0.27
\end{aligned}
$$

Subjected to the following constraints:

$$
\begin{gathered}
120 \leq \mathrm{A} \leq 220 \\
0.1 \leq \mathrm{B} \leq 0.2 \\
0 \leq \mathrm{C} \leq 0.04 \\
\mathrm{~A}, \mathrm{~B}, \mathrm{C} \geq 0
\end{gathered}
$$




\section{Cutting processes optimization}

In the current study, the non-dominated sorting genetic algorithm (NSGA-II) has been utilized to solve the current multi-objective problem. The NSGA-II $[19,20]$ is one of the popularly used evolutionary multi-objective optimization algorithm which attempt to find optimal Pareto-front solutions. This technique is mainly based on using an elitist principle, and explicit diversity preserving mechanism, called crowding distance. The evolutionary operators of the NSGA-II are based on genetic algorithm's, which are namely cross-over, mutation, and selection. The three mathematical models developed using RSM have been used in the optimization stage.

A constant population size of 50 has been used in this work, while the cross-over rate was 0.8 , and the mutation probability was 0.01 . Tournament selection scheme has been used. Also, an example for the solution converges stages for objective functions 1 and 2 is provided as shown in Figure 2, and The Pareto-front for the three objectives with same GA parameters is provided as shown in Figure 3. The Pareto-front progress is provided as shown in Figure 1. In addition, different values for population number $(\mathrm{Np})$, and cross-over probability $(\mathrm{Cp})$ have been applied to check the results sensitivity, and no significant changes have been observed. Also, a partial consistency between the Paretofront results using different values of population size, and crossover probability with the experimental data has been noticed. The chart for the corresponding design variables with its Paretofront values for each case has been created, and the last case with $\mathrm{Np}=200$, and $\mathrm{Cp}=0.6$ has been presented as shown in Figure 1 and Table V. Depending on the literature review of selecting the optimal machining parameters, and the obtained results from the multi-objective optimization algorithm (i.e., NSGA-II), the optimal machining settings have been chosen.

The optimal levels for cutting speed, feed rate, and the percentage of added nano-additives are obtained at index \#13 (see Table V), which were; $136.5 \mathrm{~m} / \mathrm{min}, 0.123 \mathrm{~mm} / \mathrm{rev}$, and 2.82 wt.\%. Regarding the optimal levels of the nano-additives concertation, it has been found that lowest flank wear has been observed when using 4wt. \% MWCNTs, while the lowest power consumption and surface roughness have been obtained when using 2 wt. $\%$. On the other hand, the multi-objective algorithm (NSG-II) results showed that the optimal nano-additives concertation was 2.82 wt. \%. The selected optimal level (nanoadditives weight percentage) obtained from optimization procedure provides more physical sense as the highest percentage of nano-additive (4 wt. \%) could cause more deteriorations to the cutting processes because of the highest occurrence possibility of the drastic ploughing effects which could increase the induced nano-additives wear and accordingly increase the overall tool wear. When there is an abundance of nano-additives in the resultant nano-fluid, they collide with and are impeded by the asperities on the work surface and hence stronger cutting forces are generated. As a result, the nanoadditive induced wear is increased with increasing the nanoadditives concretion as similarly discussed in a previous work [21] (see Figure 3). On the other hand, decreasing the nanoadditives concentration would decrease the resultant nano-fluid thermal conductivity and would affect the heat dissipation performance. In addition, decreasing the nano-additives concentration means less nano-additives in the workpieceinterface area, and therefore the induced friction would increase as can be seen in Figure 3. Based on the above explanations, it can be concluded that $2.82 \mathrm{wt}$ \% MWCNTs provides more physical sense as it strikes a balance between achieving reasonable heat transfer performance, and avoiding the drastic ploughing effects.

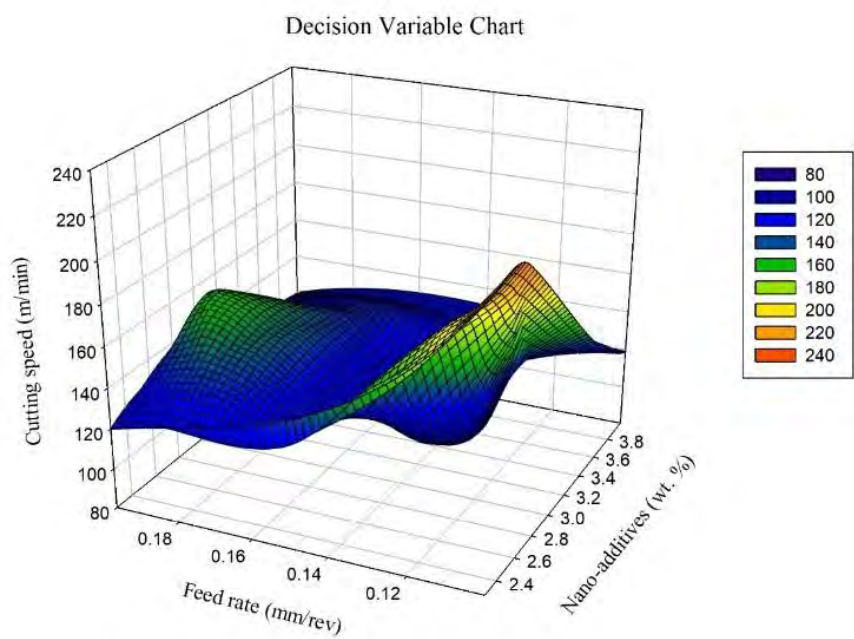

Figure 1. Design variables crosspdoing to the Pareto-front values at $\mathrm{Np}=200$, and $\mathrm{Cp}=0.6$

TABLE V. THE PARETO-FRONT OPTIMAL SOLUTIONS (OPTIMAL SOLUTION VALUES AND CORRESPONDING OPTIMAL DECISION VARIABLES)

\begin{tabular}{|c|c|c|c|c|c|c|}
\hline$\#$ & $\mathbf{F}_{\mathbf{1}}$ & $\mathbf{F}_{\mathbf{2}}$ & $\mathbf{F}_{\mathbf{3}}$ & $\mathbf{A}$ & $\mathbf{B}$ & $\mathbf{C}$ \\
\hline 1 & 0.2538 & 1.609 & 1872.583 & 120.0165 & 0.1976 & 2.28 \\
\hline 2 & 0.1343 & 1.8509 & 1972.339 & 120.0091 & 0.1969 & 3.99 \\
\hline 3 & 0.3865 & 0.3354 & 2220.757 & 216.428 & 0.1007 & 2.33 \\
\hline 4 & 0.3306 & 0.4136 & 2162.84 & 195.3334 & 0.1055 & 2.71 \\
\hline 5 & 0.2014 & 1.3933 & 1915.211 & 156.6597 & 0.1933 & 3.1 \\
\hline 6 & 0.3014 & 0.4721 & 2122.888 & 183.7243 & 0.1146 & 2.34 \\
\hline 7 & 0.1437 & 1.7749 & 1943.891 & 120.0743 & 0.1976 & 3.76 \\
\hline 8 & 0.3664 & 0.3601 & 2197.254 & 209.7226 & 0.1024 & 2.64 \\
\hline 9 & 0.2089 & 1.1313 & 2147.599 & 125.4769 & 0.1254 & 3.77 \\
\hline 10 & 0.1767 & 1.1828 & 2023.023 & 137.7389 & 0.1599 & 3.51 \\
\hline 11 & 0.2645 & 0.5666 & 2090.814 & 169.2304 & 0.1203 & 2.69 \\
\hline 12 & 0.16 & 1.6703 & 1916.766 & 120.6147 & 0.1967 & 3.45 \\
\hline $\mathbf{1 3}$ & $\mathbf{0 . 2 1 7 8}$ & $\mathbf{0 . 7 1 7 1}$ & $\mathbf{2 0 5 9 . 0 1}$ & $\mathbf{1 3 6 . 5 1 1 8}$ & $\mathbf{0 . 1 2 3 2}$ & $\mathbf{2 . 8 2}$ \\
\hline 14 & 0.2149 & 0.9247 & 1981.948 & 130.0624 & 0.1505 & 2.41 \\
\hline 15 & 0.225 & 1.4476 & 1895.653 & 141.7001 & 0.1936 & 2.65 \\
\hline 16 & 0.227 & 1.2574 & 1926.464 & 139.7971 & 0.1798 & 2.5 \\
\hline 17 & 0.2214 & 0.8655 & 1992.583 & 133.2566 & 0.146 & 2.32 \\
\hline 18 & 0.1343 & 1.8509 & 1972.339 & 120.0091 & 0.1969 & 3.99 \\
\hline
\end{tabular}



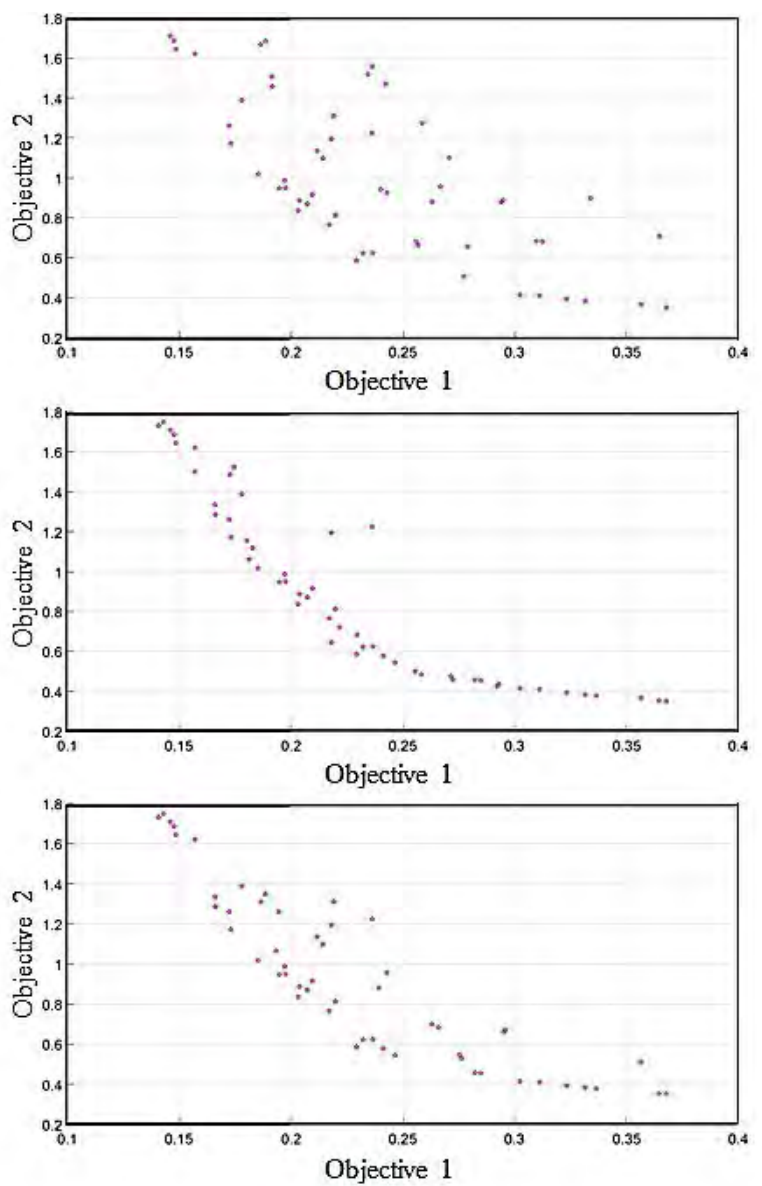

Figure 2. The solution converges stages for objective functions 1 and 2

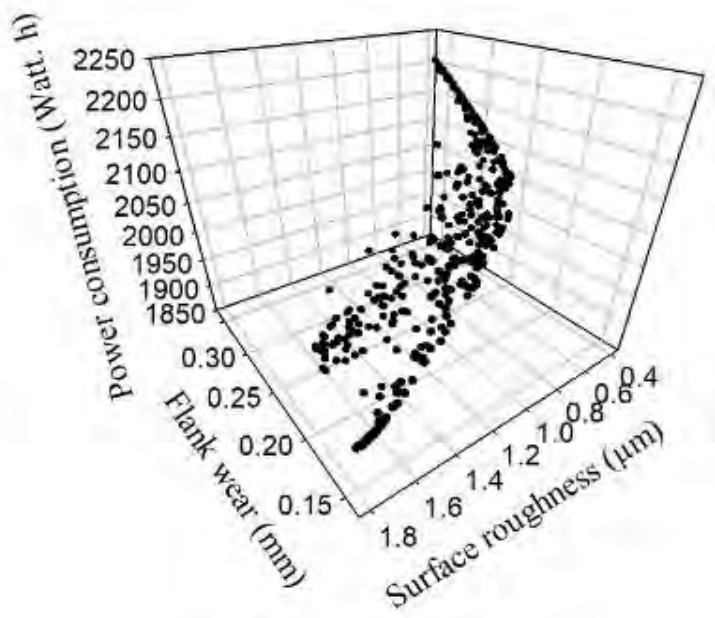

Figure 3. The Pareto-front for the three studied objectives

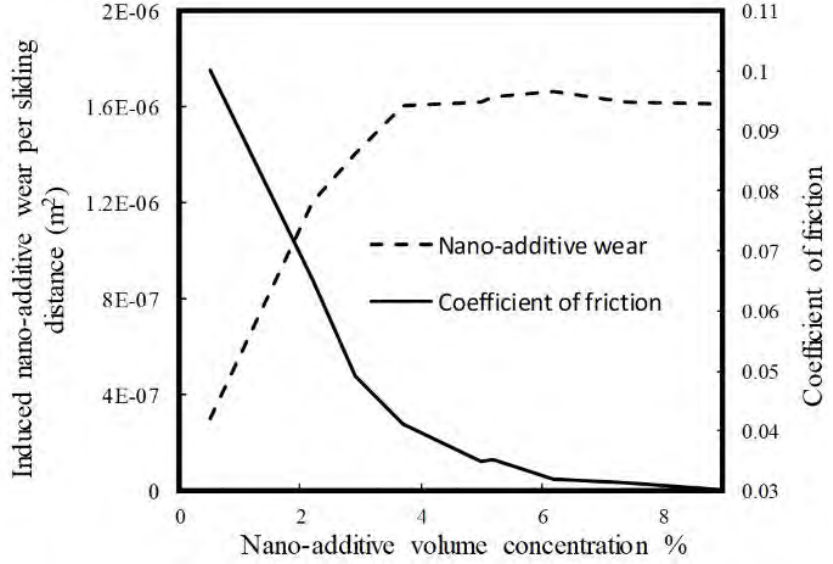

Figure 4. The induced nano-additives wear versus nano-additives concentration [21]

\section{CONCLUSIONS}

In the current work, multi-walled-carbon nanotubes (MWCNTs) cutting fluids along with minimum quantity lubrication (MQL) have been employed during machining Ti$6 \mathrm{Al}-4 \mathrm{~V}$. On the other hand, a multi-objective optimization model has been utilized for machining titanium alloys, which is a promising step in order to minimize machining cost, achieve excellent surface quality, and increase the cutting tool life by selecting the optimal cutting conditions such as cutting speed, feed rate, depth of cut. In this work, MWCNTs nano-fluid showed promising results in enhancing the machining performance during cutting Ti-6Al-4V. It is mainly attributed to the improved heat transfer and tribological characteristics of the employed oil. Also, response surface methodology (RSM), and multi-objective genetic algorithm (NSGA-II) are employed to model and optimize three main machining responses, namely, tool wear, surface quality, and power consumption. Different values for population number, and cross-over probability have been applied to check the results sensitivity; however, no significant changes have been observed. Also, the results showed a partial agreement between Pareto-front solutions using different values of population size, and cross-over probability with the experimental data. Depending on the literature review of selecting the optimal machining parameters, and the obtained results from the multi-objective algorithm, the optimal machining settings have been chosen, as selected solution form Pareto-front set. The optimal levels for cutting speed, feed rate, and the percentage of added nano-additives are obtained at index \#13, which are $136.5 \mathrm{~m} / \mathrm{min}, 0.123 \mathrm{~mm} / \mathrm{rev}$, and $2.82 \mathrm{wt} \%$, respectively. The selected optimal level (nano- additives weight percentage) obtained from optimization procedure provides more physical sense as it strikes a balance between achieving reasonable heat transfer performance, and avoiding the drastic ploughing effects. Finally, studying and analyzing the MQLnano-fluid tribological and heat transfer mechanisms is still required in order physically obtain the nano- additives size and percentage effects on the cutting processes performance. 


\section{ACKNOWLEDGMENT}

The authors acknowledge the support of the Natural Sciences and Engineering Research Council of Canada (NSERC).

\section{REFERENCES}

[1] A. Choragudi, M.A. Kuttolamadom, J.J. Jones, M. L. Mears, T. Kurfess, "Investigation of the machining of titanium components in lightweight vehicles," In SAE International Congress, 2010.

[2] S. Jaffery and P. Mativenga, "Assessment of the machinability of Ti-6Al-4V alloy using the wear map approach," The International Journal of Advanced Manufacturing Technology, vol. 40, issues 4-8, pp. 687-696, 2009, doi: 10.1007/s00170-008-1393-9.

[3] E. Ezugwu, and Z. Wang, "Titanium alloys and their machinability-a review," J. Mater. Process. Technol., vol. 68, issue 3, pp. 262-274, 1997. doi: 10.1016/S0924-0136(96)00030-1.

[4] A. Shokrani,V. Dhokia, and S.T. Newman, "Environmentally conscious machining of difficult-to-machine materials with regard to cutting fluids," Int. J. Mach. Tool. Мanu., vol. 57, pp. 83-101, 2012.

doi: 10.1016/j.ijmachtools.2012.02.002.

[5] M. Amrita, R. Srikant, and A. Sitaramaraju, "Performance evaluation of nanographite-based cutting fluid in machining process," Mater. Manuf. Processes, vol. 29, issue 5, pp. 600-605, 2014. doi: $10.1080 / 10426914.2014 .893060$.

[6] S. Khandekar, M.R. Sankar, V. Agnihotri, J. Ramkumar, "Nano-cutting fluid for enhancement of metal cutting performance," Mater. Manuf. Processes, vol. 27, issue 9, pp. 963-967, 2012. doi: 10.1080/10426914.2011.610078.

[7] Nanomaterials, U.R., US Research Nanomaterials, Inc, The Advanced Nanomaterials Provider, US Research Nanomaterials.

[8] R.R. Srikant, D. N. Rao, M.S. Subrahmanyam, V. P. Krishna, "Applicability of cutting fluids with nanoparticle inclusion as coolants in machining," Proc. Inst. Mech. Eng., Part J, vol. 223, issue 2, pp. 221-225, 2008. doi: 10.1243/13506501JET463.

[9] A. K. Sharma, A. K. Tiwari, and A.R. Dixit, "Progress of nanofluid application in machining: a review," Mater. Manuf. Processes, vol. 30, issue 7, pp. 813-828, 2015, doi: 10.1080/10426914.2014.973583.

[10] T. Rajmohan, S. Sathishkumar, and K. Palanikumar, "Effect of a nanoparticle-filled lubricant in turning of AISI 316L stainless steel (SS)," Part. Sci. Technol., vol. 35, issue 2, pp. 201-208, 2017, doi: 10.1080/02726351.2016.1146812.

[11] R.K. Singh, A.K. Sharma, A.R. Dixit, A.K. Tiwari, A. Pramanik, A. Mandal, "Performance evaluation of alumina-graphene hybrid nano-cutting fluid in hard turning.,"J. Cleaner Prod., vol. 162, pp. 830-845, 2017. doi: 10.1016/j.jclepro.2017.06.104.

[12] T. Bakalova, L. Svobodová, P. Rosická, K. Borůvková, L. Voleský, L., P. Louda, "The application potential of $\mathrm{SiO} 2, \mathrm{TiO} 2$ or Ag nanoparticles as fillers in machining process fluids," J. Cleaner Prod., vol. 142, pp. 2237-2243, 2017. doi: 10.1016/j.jclepro.2016.11.054.

[13] S. Paul, A.K. Singh, and A. Ghosh, "Grinding of Ti-6Al-4V Under Small Quantity Cooling Lubrication Environment Using Alumina and MWCNT Nanofluids," Mater. Manuf. Processes, vol. 32, issue 6, pp. 608-615, 2017. doi: 10.1080/10426914.2016.1257797.

[14] G. Liu, C. Li, Y. Zhang, M. Yang, D. Jia, X. Zhang, H. Zhai, "Process parameter optimization and experimental evaluation for nanofluid MQL in grinding Ti-6Al-4V based on grey relational analysis," Mater. Manuf.

Processes, vol. 33, issue 9, pp. 950-963, 2008.

doi: $10.1080 / 10426914.2017 .1388522$

[15] M. Alberts, K. Kalaitzidou, and S. Melkote, "An investigation of graphite nanoplatelets as lubricant in grinding," Int. J. Mach. Tool. Manu.,vol. 49, issue 12, pp. 966-970, 2009. doi: 10.1016/j.ijmachtools.2009.06.005.
[16] D.M. Byrne, "The Taguchi approach to parameter design," Quality Progress, vol. 20, no.12, pp. 19-26, 1987.

[17] E. O. Ezugwu, R.B. Da Silva, J. Bonney, A.R. Machado, "Evaluation of the performance of CBN tools when turning Ti-6Al-4V alloy with high pressure coolant supplies," Int. J. Mach. Tool. Manu., vol. 45, no. 9, pp. 1009-1014, 2005. doi: 10.1016/j.ijmachtools.2004.11.027.

[18] S.A. Bagaber, and A.R. Yusoff, "Multi-objective optimization of cutting parameters to minimize power consumption in dry turning of stainless steel 316," J. Cleaner Prod., vol. 157, pp. 30-46, 2017. doi: 10.1016/j.jclepro.2017.03.231.

[19] K. Deb, "Multi-objective optimisation using evolutionary algorithms: an introduction," in Multi-objective evolutionary optimisation for product design and manufacturing, L. Wang, A.H.C. Ng, K. Deb, Eds., New York: Springer, 2011, pp. 3-34. doi: 10.1007/978-0-85729-652-8_1.

[20] K. Deb, A. Pratap, S. Agarwal, T.A.M.T. Meyarivan, "A fast and elitist multiobjective genetic algorithm: NSGA-II," IEEE Trans. Evol. Comput.,, vol. 6, issue 2, pp.182-197, 2002, doi: 10.1109/4235.996017.

[21] H. Ghaednia, and R.L. Jackson, "The effect of nanoparticles on the real area of contact, friction, and wear," J. Tribol. vol. 135, no 4, p. 041603, 2013, doi: $10.1115 / 1.4024297$

[22] H. Hegab, U. Umer, M. Soliman, H.A. Kishawy, "Effects of nano-cutting fluids on tool performance and chip morphology during machining Inconel 718," The International Journal of Advanced Manufacturing Technology, vol. 96, issue 9-12, pp., 3449-3458, 2018. doi: 10.1007/s00170-018-1825-0.

[23] H.Hegab, U. Umer, I. Deiab, H. Kishawy, "Performance evaluation of Ti$6 \mathrm{Al}-4 \mathrm{~V}$ machining using nano-cutting fluids under minimum quantity lubrication," The International Journal of Advanced Manufacturing Technology, vol. 95, issue 1-12, pp. 4229-4241, 2018. doi: $10.1007 / \mathrm{s} 00170-017-1527-z$.

[24] A, Eltaggaz, P. Zawada, H. A. Hegab, I. Deiab, H.A. Kishawy, "Coolant strategy influence on tool life and surface roughness when machining ADI," The International Journal of Advanced Manufacturing Technology, vol. 94, no. 9-12, pp. 3875-3887, 2017, doi: 10.1007/s00170-017-1088-1.

[25] H. Hegab, H.A. Kishawy, M.H. Gadallah, U. Umer, I. Deiab, "On machining of Ti-6Al-4V using multi-walled carbon nanotubes-based nanofluid under minimum quantity lubrication," The International Journal of Advanced Manufacturing Technology, vol. 97, issue 5-8, pp. 1593-1603, 2018. doi: 10.1007/s00170-018-2028-4.

[26] A. Eltaggaz, H. Hegab, I. Deiab, H.A. Kishawy, "Hybrid nano-fluidminimum quantity lubrication strategy for machining austempered ductile iron (ADI)," International Journal on Interactive Design and Manufacturing (IJIDeM), vol. 12, issue 4, pp. 1273-1281, 2018. doi: 10.1007/s12008-018-0491-7.

[27] H. A. Hegab, B. Darras, H.A. Kishawy, "Towards sustainability assessment of machining processes," J. Cleaner Prod., vol. 170, pp.694-703, 2018. doi: 10.1016/j.jclepro.2017.09.197.

[28] H. Hegab, H.A. Kishawy, M.H. Gadallah, U. Umer, I. Deiab, "On machining of Ti-6Al-4V using multi-walled carbon nanotubes-based nanofluid under minimum quantity lubrication," The International Journal of Advanced Manufacturing Technology, vol. 97, no. 5-8, pp. 1593-1603, 2018. doi: 10.1007/s00170-018-2028-4.

[29] A. Eltaggaz, H. Hegab, I. Deiab, H.A. Kishawy, "Hybrid nano-fluidminimum quantity lubrication strategy for machining austempered ductile iron (ADI)," International Journal on Interactive Design and Manufacturing (IJIDeM), vol. 12, no. 4, pp. 1273-1281, 2018. doi: 10.1007/s12008-018-0491-7.

[30] H. A. Hegab, B. Darras, H.A. Kishawy, "Towards sustainability assessment of machining processes," J. Cleaner Prod., vol. 170, pp. 694-703, 2018. doi: 10.1016/j.jclepro.2017.09.197. 


\section{Comparison of WC-10Co-4Cr coatings properties produced by HVOF and HVOLF processes for application in petrochemical industry}

\author{
Anas Mahmoud Atieh \\ Department of Industrial Engineering \\ German Jordanian University \\ Amman, Jordan \\ Anas.atieh@gju.edu.jo
}

\author{
Abdulaziz AlHazaa \\ Physics \& Astronomy Department \\ King Saud University \\ Riyadh, KSA
}

\author{
Rohit Upadhyaya \\ Research and Development Department \\ Metallizing Equipment Co. Pvt. Ltd \\ Jodhpur, India
}

\begin{abstract}
This research work presents the results of a comparative study conducted to compare the coatings properties of $\mathrm{WC}-10 \mathrm{Co}-4 \mathrm{Cr}$ coats produced by two different Oxy-fuel coating process; high velocity oxy-gas fuel (HVOF) and high velocity oxy-liquid fuel (HVOLF) thermal spraying techniques. The coats were deposited directly on low carbon steel substrate without bonding coats. Scanning electron microscopy (SEM) was performed to study microstructural analysis and to quantify the porosity and cross-sectional coat thickness. Furthermore, the mechanical properties of both coating processes were defined in terms of bond strength and micro hardness. The results show that the liquid fuel sprayed coatings (HVOLF) produced higher adhesion strength coats $(\sim$ $73 \mathrm{MPa}$ ) compared to $(\sim 68 \mathrm{MPa})$ for HVOF. Similar results observed for micro-hardness of $1255 \mathrm{VHN}$ and $1032 \mathrm{VHN}$, respectively. The surface roughness and porosity were less for HVOLF $4.32 \mu \mathrm{m} / 0.85 \%$ compared to HVOF results of $5.26 \mu \mathrm{m} / 1.29 \%$ porosity. This superior result in coats properties of HVOLF compared to HVOF was attributed due to less decarburization in HVOLF and hence less production of hard secondary phases of $\mathrm{W}_{2} \mathrm{C}$.
\end{abstract}

Keywords-component; HVOF; HVOLF, WC-10Co-4Cr; Coating properties

\section{INTRODUCTION}

Engineering components in service under sever operational conditions i.e. erosive, corrosive and wear requires continuous maintenance using coated alloy materials [1,2].
Thermal spraying techniques such as HVOF and HVOLF can be utilized to produce a variety of wear/erosion resistance coatings onto a wide range of substrate material with numerous possibility of coating powders [3]. Semi-molten material state is deposited onto the substrate through the acceleration of powder particles to high velocity through the coating nozzle while physical interlocking occurred at substrate surface and splat deformation occurred due to high kinetic energy hence the process produces dense coat with superior wear and corrosion resistance [3-7].

Ceremet coating of WC-CoCr composed of hard WC phase and relatively softer $\mathrm{Co}$ and $\mathrm{Cr}$ have been developed and studied extensively by many researchers [8] and resulted in dense, good toughness and high corrosive resistance coatings $[9,10]$.

In this paper, the effect of fuel type either gaseous (propane) in HVOF compared to liquids aviation turbine fuel (ATF) in HVOLF is studied on the produced coats in terms of hardness, adhesion strength, porosity, thickness and microstructure.

\section{Methodology}

Commercial feedstock powder of WC-10Co-4Cr was sprayed by two techniques HVOF and HVOLF, Fig.1 shows the SEM micrograph of feedstock powder. As shown in the figure the shape and morphology of the powder particles are not uniformed for the $\mathrm{WC}-10 \mathrm{Co}-4 \mathrm{Cr}$ powder. 


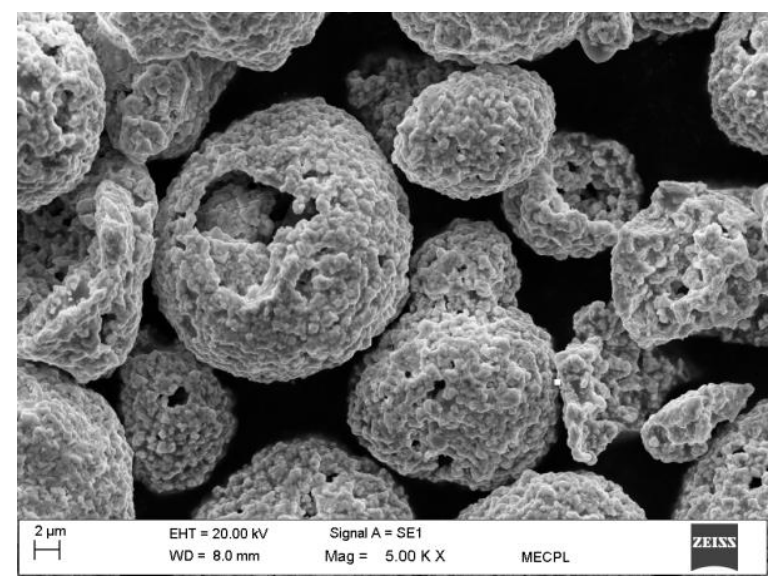

\section{Figure 1. SEM micrograph of WC-10Co-4Cr feedstock powder}

The grain size of the powder was measured as $15-45 \mu \mathrm{m}$ of agglomerated grains. Round specimen of $30 \mathrm{~mm}$ diameter and $10 \mathrm{~mm}$ high were prepared by blasting with alumina powder $\left(\mathrm{Al}_{2} \mathrm{O}_{3}\right)$ and air blown for cleaning. The HVOF and HVOLF spraying process was carried out at the laboratories of Metallizing Equipment Company Pvt. Ltd. a Nobal accredited laboratory (CERT. No. T-3514). Adhesion test was carried out at universal testing machine UTM Instron USA - Model 5969 (50 kN Capacity) according to ASTM C-633:2013 standards. A Shimadzu HVM-G Vickers micro-hardness tester was used to measure micro hardness according to ASTM E384-2011 where five measurements are taken for each sample with test force of HV0.3 and 8 seconds dwell time. Carl-Ziess (UK) scanning electron microscopy (SEM) model EVO-18 was utilized to measure porosity as per ASTM E-2109:2007 standards cross sectional thickness as per EN ISO 1463:2003 standards, and for microstructural analysis.

\section{RESULTS AND DISCUSSION}

\section{A. Microstructural and surface analysis}

The microstructure of the coatings were examined using the SEM, and the micrographs of the WC-10Co-4Cr HVOF and HVOLF are shown in Fig.2 a \& b, respectively.

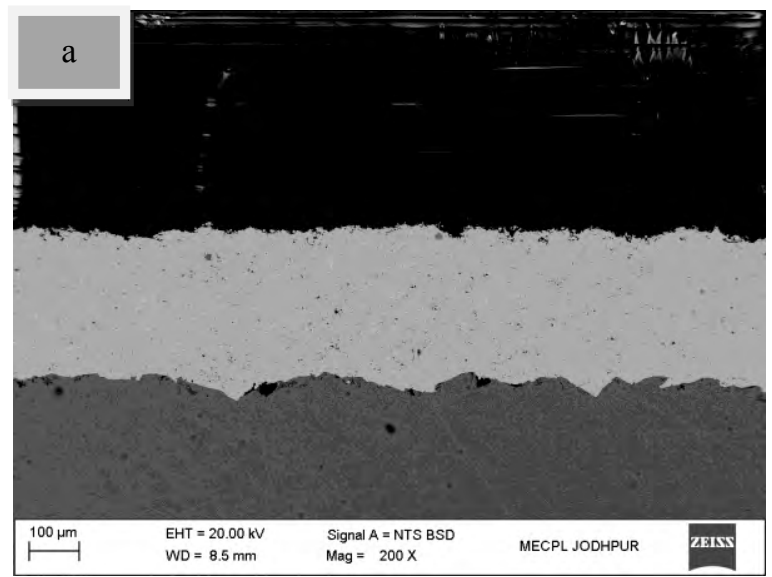

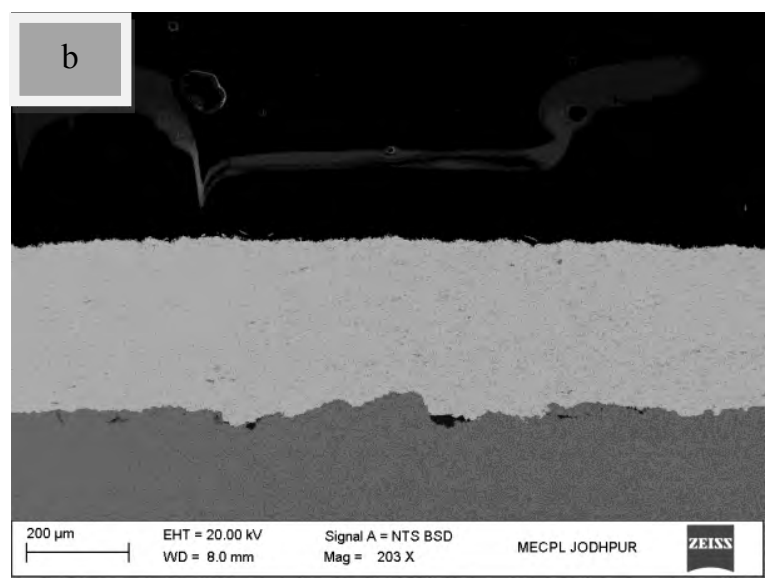

Figure 2. SEM micrograph for WC-10Co-4Cr (a) HVOF and (b) HVOLF sprayed coating

It can be seen that the coatings produced by both processes HVOF and HVOLF resulted in a homogenous coats. The microstructural analysis showed that HVOF coating contained higher percentage porosity of $1.29 \%$ at a total magnification of $200 \mathrm{X}$ compared to $0.85 \%$ for HVOLF coating under same total magnification.

Furthermore, the produced coating thickness were higher for HVOLF coatings compared to HVOF under same spraying conditions for both processes. The Fig. 3 below shows the average coating thickness measured on a diagonal of the cross section for both samples.

The surface roughness of the as sprayed coatings $(\mathrm{Ra})$ were lower for HVOLF compared to HVOF, which indicates smoother surface finish produced by HVOLF, Fig. 4 shows the average surface finish for both processes in the as sprayed condition.

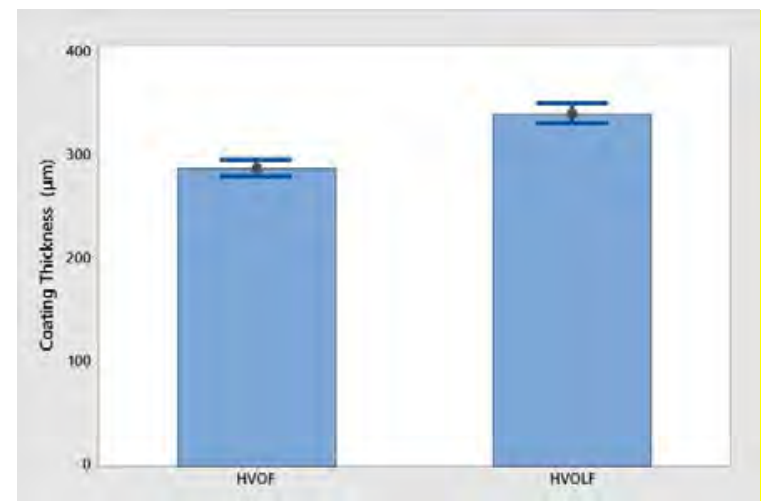

Figure 3. Average coat thickness measured on the cross section for HVOF and HVOLF 


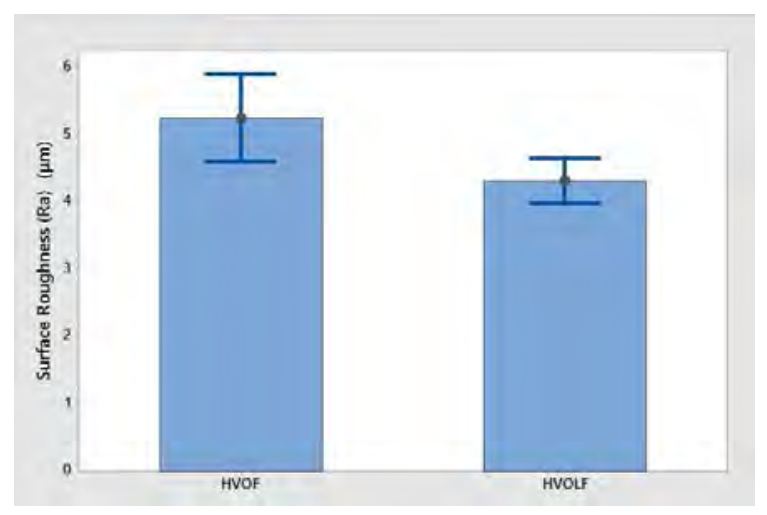

Figure 4. Average surface roughness measured for HVOF and HVOLF

\section{B. Microhardness measurments}

The average microhardness value for the HVOF was 1032 VHN and a higher average hardness value of $1255 \mathrm{VHN}$ was obtained for the HVOLF process. The coatings in both processes showed a uniform hardness profile by the five hardness measurements taken at different locations at the coatings through the entire thickness of the coatings confirming the homogeneity of the produced coatings.

\section{Table 1. Microhardness measurements at coatings} produced by HVOF and HVOLF

\begin{tabular}{|l|c|c|c|c|c|}
\hline & $\mathbf{1}$ & $\mathbf{2}$ & $\mathbf{3}$ & $\mathbf{4}$ & $\mathbf{5}$ \\
\hline HVOF & 955 & 1026 & 989 & 1087 & 1102 \\
\hline HVOLF & 1286 & 1324 & 1166 & 1206 & 1292 \\
\hline
\end{tabular}

The higher coating density in HVOLF using the liquid fuel can explain this increase in microhardness.

\section{Adhesion strength}

The strength tests were performed in order to compare the bondability and adhesion of the coatings to substrate. In both HVOF and HVOLF a failure in glue were observed, Fig. 5 shows the bond strength results of both coatings in $\mathrm{MPa}$.

In thermal spray, processing of $\mathrm{WC}$ a decarburization occurs during the spraying producing reaction products $\mathrm{W}_{2} \mathrm{C}$, $\mathrm{W}$ and $\mathrm{C}$. It was reported by Saha et al. that $\mathrm{W}$ and $\mathrm{C}$ may dissolve in the Co matrix and promote the formation of amorphous Co matrix [11]. In the current research work, it is expected that less decarburization occurred in case of HVOLF. Less formation of hard phases $\mathrm{W}_{2} \mathrm{C}$ may explain the higher bond strength of HVOLF compared to HVOF as $\mathrm{W}_{2} \mathrm{C}$ may work as a stress raiser within the coating matrix and resulted in coat failure.

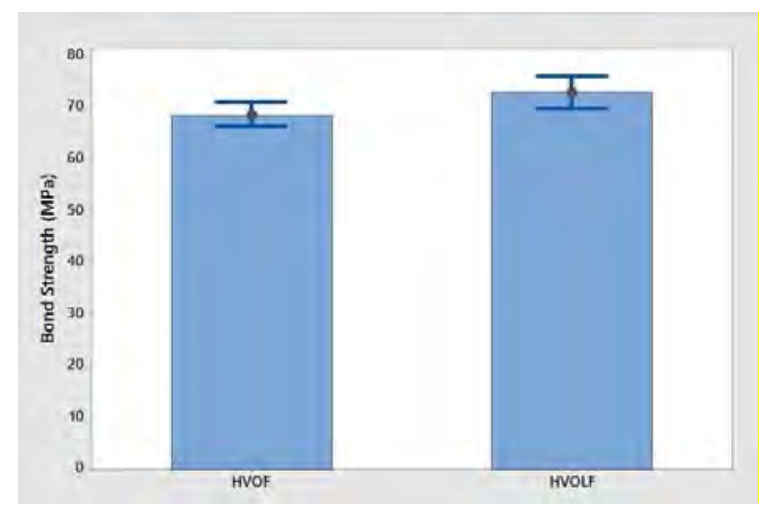

Figure 5. Average bond strength for HVOF and HVOLF

\section{CONCLUSIONS}

In this study, two thermal spraying processes of HVOF and HVOLF deposited WC-10Co-4Cr successfully. The results showed that liquid fuel process (HVOLF) sprayed coatings have less porosity $(0.85 \%$ compared to $1.29 \%$ in HVOF), higher coating thickness $(341 \mu \mathrm{m}$ compared to $288 \mu \mathrm{m})$, higher microhardness of $1255 \mathrm{VHN}$, and higher bond strength with 73 $\mathrm{MPa}$ in HVOLF compared to $68 \mathrm{MPa}$ in HVOF).

The increase in the mechanical properties of the coatings and therefore the performance was attributed to the formation of less hard phases due to less decarburization effect of WC and formation of amorphous Co matrix.

\section{ACKNOWLEDGMENT}

The authors gratefully acknowledge the financial support provided by German Jordanian University, Grant number SATS 12/2015. Samples preparations and characterization as provided by Metallizing Equipment Co. Pvt. Ltd (MEC) Jodhpur, India are also acknowledged.

\section{REFERENCES}

[1] Spadlo, S., Depczynski, W., and P. Mlynarczyk, "Selected Properties Of High Velocity Oxy Liquid Fuel (HVOLF) - Sprayed Nanocrystalline WC-Co Infralloytm S7412 Coatings Modified By High Energy Electric Pulse," Metalurgija, vol. 56, no. 3-4, pp. 412-414, 2017. [Online]. Available: https://hrcak.srce.hr/180998.

[2] J. R. Davies, Handbook of Thermal Spray Technology, ASM International, 2004.

[3] A. Galloway et al., "Performance evaluation of HVOF deposited cermet coatings under dry and slurry erosion," Surf. Coat. Technol., vol. 300, pp. 118-127, Aug. 2016. doi: 10.1016/j.surfcoat.2016.05.039. 
[4] T. A. Ben Mahmud, A. M. Atieh, and T. I. Khan, "The Wear Behavior of HVOF Sprayed Near-Nanostructured WC-17\%Ni(80/20) Cr Coatings in Dry and Slurry Wear Conditions," J. Mater. Eng. Perform., vol. 26, no. 7, pp. 3507-3515, Jul. 2017. doi: 10.1007/s11665-017-2739-y.

[5] G. Bolellia, L.Lusvarghia, and M. Barlettab, "HVOF-Sprayed WCCoCr Coatings on Al Alloy: Effect of the Coating Thickness on the Tribological Properties," Wear, vol. 267, no. 5-8, pp. 944-953, Jun. 2009. doi:10.1016/j.wear.2008.12.066.

[6] J. R. García et al., "Fatigue effect of WC coatings thermal sprayed by HVOF and laser treated, on medium carbon steel," Eng. Failure Anal., vol. 18 , no. 7 , pp. 1750-1760, Oct. 2011. doi: 10.1016/j.engfailanal.2011.03.026.

[7] J. G. La Barbera-Sosa et al., "Fatigue behavior of a structural steel coated with a WC-10Co- $4 \mathrm{Cr} /$ Colmonoy 88 deposit by HVOF thermal spraying," Surf. Coat. Technol., vol. 220, pp. 248-256, Apr.2013. doi: 10.1016/j.surfcoat.2012.05.098.

[8] A. C. Karaoglanli et al., "Comparison of tribological properties of HVOF sprayed coatings with different composition," Surf. Coat. Technol., vol. 318, pp. 299-308, May 2017. doi: 10.1016/j.surfcoat.2017.02.021.

[9] L. Thakur et. al., "An investigation on erosion behavior of HVOF sprayed WC-CoCr coatings," Appl. Surf. Sci., vol. 258, no. 3, pp. 12251234, Nov. 2011. doi: 10.1016/j.apsusc.2011.09.079.

[10] M. Barletta et al., "Wear and Corrosion Behavior of HVOF-Sprayed WC-CoCr Coatings on Al Alloys," J. Therm. Spray Technol., vol. 19, no. 1-2, pp. 358-367, Sep. 2009. doi: 10.1007/s11666-009-9387-1.

[11] G. C. Saha, T. I. Khan, and L. B. Glenesk, "Development of Wear Resistant Nanostructured Duplex Coatings by High Velocity Oxy-Fuel Process for Use in Oil Sands Industry," J. Nanosci. Nanotechnol., vol. 9, no. 7, pp. 4316-4323, Jul. 2009. doi: 10.1166/jnn.2009.M52. 


\section{Compressive Instabilities in Metal-Coated Polymer Microtrusses}

\author{
Bharat Bhaga \\ University of Toronto Institute for Aerospace Studies \\ Toronto, Ontario \\ bharat.bhaga@mail.utoronto.ca
}

Abstract-Lightweight structures directly contribute to the sus-
tainability of aviation, as their use reduces the structural weight
of aircraft which in turn reduces fuel burned during flight. One
family of lightweight structures are metal-coated polymers. Hybrid
nanocrystalline microtrusses are a member of this family. These
structures are fabricated by 3D printing complex truss-like structures
out of polymer material, and electrodepositing nanocrystalline metal
onto the polymer. Recent work has shown that buckling instabilities
govern the strength of these systems. Hence this study focuses on
modelling local shell buckling, one of the critical buckling mecha-
nisms. This paper briefly reviews existing models for filled-shell local
shell buckling, and outlines the development of an improved model.
Index Terms-compression; buckling; filled-shell; microtruss;
nanocrystalline; instability

\section{INTRODUCTION}

Electrodepositing nanocrystalline metals onto 3D printed polymers creates hybrid materials with exceptional properties, including high strength and stiffness-to-density as well as nearly limitless geometric control. These hybrid materials can be used to reduce the weight of aircraft and other transportation vehicles, directly decreasing fuel consumption, cost and environmental impact. To achieve this, accurate models of the mechanical behaviour of hybrid nanometal-polymer materials and structures must be developed. This paper will explore the compressive properties of polymer struts enhanced with nanocrystalline metal coatings.

A nanometal polymer hybrid structure is produced by creating a polymer preform through 3D printing and subsequently coating the preform with nanocrystalline metal [1], as seen in Fig. 1. The reduced grain sizes of nanocrystalline metals relative to conventional metals allow for increased strength as per the Hall-Petch effect [2]. While other properties of nanocrystalline metals, such as wear resistance, also benefit by reducing grain size [3], ductility and modulus tend to decrease. Truss-like structures are very appealing because of their high ratio of stiffness-to-mass. These structures exhibit mass-efficiency and low-weight while combining the highstrength benefits of nanocrystalline metals.

The production methods of nanometal-polymer hybrids also support the goals of sustainability. 3D printing of polymer structures yields less material waste than typical subtractive techniques, while also allowing for the manufacturing of complex geometries with ease. Furthermore, the use of electrodeposition techniques is cost-efficient and is relatively benign environmentally [3]. Elecrotrodeposition also allows for controlling the deposited nanometal crystal sizes $[3,4]$. Together

\author{
Craig A. Steeves \\ University of Toronto Institute for Aerospace Studies \\ Toronto, Ontario \\ csteeves@utias.utoronto.ca
}

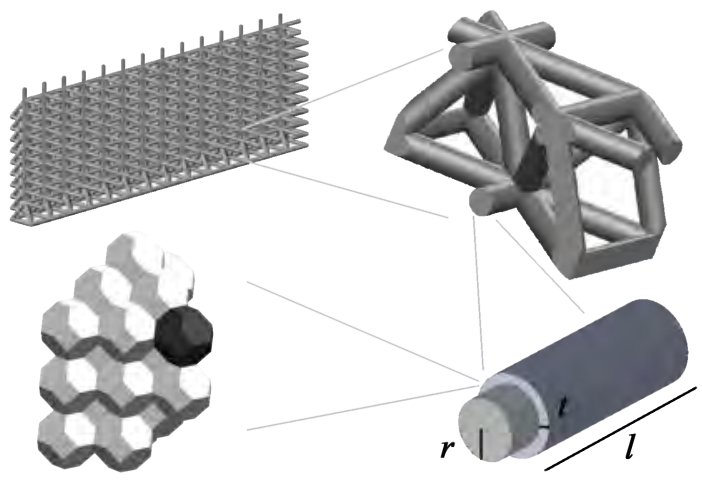

Fig. 1: Various scales of a nanocoated microtruss structure. Clockwise from upper left: coated microtrus structure, truss unit cells, individual struts, atoms at the material level [image modified from [4]]

with control over the geometry of the struts $(r, l$ and $t$ in Fig 1), this provides four length scales over which the behaviour of the nanocoated microtruss structure can be controlled.

Previous studies on nanocoated microtrusses have shown that their failure mechanisms are driven by compressive instabilities of the struts [4]. As the truss elements of nanocoated microtrusses are metal-coated polymer cylinders (as seen in Fig. 1), the present research explores the compressive instabilities associated with metal coated polymer cylinders.

\section{Literature REVIEW}

The two compressive instabilities of interest for metalcoated polymer cylinders are local shell buckling and global (or Euler) buckling, as seen in Fig. 2. Local shell buckling is characterized by the appearance of waves on the surface of the cylindrical shell $[5,6]$, while global buckling results in the lateral deflection of a column relative to its original undeformed axis.

For a nanometal-polymer hybrid, the nanometal coating provides all of the structural strength, and hence failure of the hybrid is governed by failure of the nanometal coating. Past investigations of metal-coated microtrusses assumed that the failure mechanisms of the struts only depended on the geometry and material properties of the metal shell [4], and that the polymer core provided no structural contribution. However, for thinly coated microtruss structures, where the cross-sectional area of the polymer is much more than that of the shell, the polymer contributes to the load-bearing capacity 


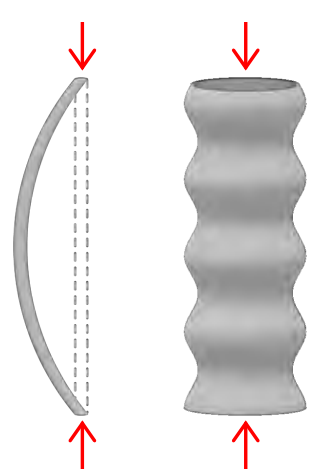

Fig. 2: Appearance of global buckling (left) and local shell buckling (right)

of the struts, while increasing their resistance to buckling [79].

\section{A. Hollow Shell Buckling}

Local shell buckling of filled cylindrical shells - or simply filled-shell buckling - is closely related to local buckling of hollow shells. The local shell buckling load for a thin cylindrical shell in axial compression was found independently [10] by Lorenz [11], Timoshenko [6], and von Karman and Tsien [12]. At the inception of buckling, the strain energy of the shell consists only of the direct compressive stress and strain. Buckling occurs when it is more energy efficient to deform the shell in sinusoidal waves than to continue to deform the shell by direct compression. For a thin cylindrical shell in axial compression, the buckling stress is found to be $[6,11,13-15]$ :

$$
\sigma=\frac{E}{\sqrt{3\left(1-\nu^{2}\right)}} \frac{t}{r}
$$

where $E$ is the Young's modulus of the shell's material, $\nu$ is its Poisson's ratio, and $t$ and $r$ are the thickness and inner radius of the shell, respectively.

Various derivations of (1) have shown that the buckled shape of the shell - be it axisymmetric or non-axisymmetric - is not a factor in determining its buckling load [6, 12]. In addition, the end conditions also do not affect the buckling load provided that the cylinder is sufficiently long $[6,12,13,15]$.

Experimental investigations of hollow-shell buckling have shown that buckling loads in practice are strongly affected by geometric imperfections, material imperfections, and loading asymmetry $[13,16]$. This was confirmed through the experiments of Tennyson [17], who manufactured nearly imperfection-free cylinders and ensured careful axial load distributions around the circumferences of the cylinders.

\section{B. Filled shell buckling}

Filled-shell buckling models utilize separate treatments of the shell and core behaviours. Most models for filled-shell buckling are intended for foam-filled metal or polymer tubes and experiments to validate them have used these material combinations.

The problem of local shell buckling of filled cylindrical shells was driven by the need to understand buckling of solid propellant rockets under axial loads $[7,8,18]$. The earliest attempt at a filled-shell buckling model by Myint [8] emulated the approach used by von Karman and Tsien [12]. Uniquely, the Myint model assumed that a shear interaction between the shell and core was important. Later studies showed that the shear interactions between the shell and the core were negligible [7, 9]. More recently, Karam and Gibson [7] utilized a foundation model derived by Gough et al. [16], which was originally intended for buckling of face sheets on sandwich panels subject to axial compression.

A variety of experimental investigations were also undertaken to investigate the phenomena of filled shell buckling. The majority of the experiments were performed using metal tubes filled with polymer or polymer-like foams [18, 19], intended to mimic the structure of solid propellant rockets or natural materials like plant stems. Through these experiments, it was reported that the filler material prevented or hindered the formation of some buckling deformations. It was also found that a better agreement between theory and experiment occurred with shells when the thickness-to-radius ratios were large $[18,19]$, due to the fact that buckling loads for thick shells are less prone to imperfection sensitivity.

While the Karam and Gibson model is currently the best available model for filled-shell buckling, there are several drawbacks. The Karam and Gibson model utilizes a stress function originally intended for buckling of face sheets on composite sandwich beams $[16,20]$, and is thus not axisymmetric. Moreover, their model becomes less accurate when the coating thickness is large [20] as the core shear stresses become too large to properly capture using their model.

\section{CORE MOdel}

The development of a core model requires an understanding of the behaviour of a solid polymer cylinder. During local shell buckling of a filled cylinder under an axial load, the core is subject to radial sinusoidal displacements which are the consequence of buckling, as shown in Fig. 3. The following assumptions are utilized:

- the shell and core are perfectly bonded;

- both the shell and core materials behave elastically;

- the buckling displacements are sinusoidal;

- there are many waves that form when the shell buckles;

- the core conforms to the buckled shape of the shell; and

- the shell is very thin compared to the radius (i.e. $t / r<0.1)$.

The Southwell model employs stress functions to calculate the stress field in an axysymmetrically-loaded isotropic cylinder [21]. This describes the stress state of a thick hollow or solid cylinder, depending upon the boundary conditions. Fig. 4 shows the generalized geometry and appropriate boundary conditions for which the Southwell model is valid. The solution to this domain using the Southwell model provides the complete stress state of the axisymmetric section, and hence the entire cylinder. 


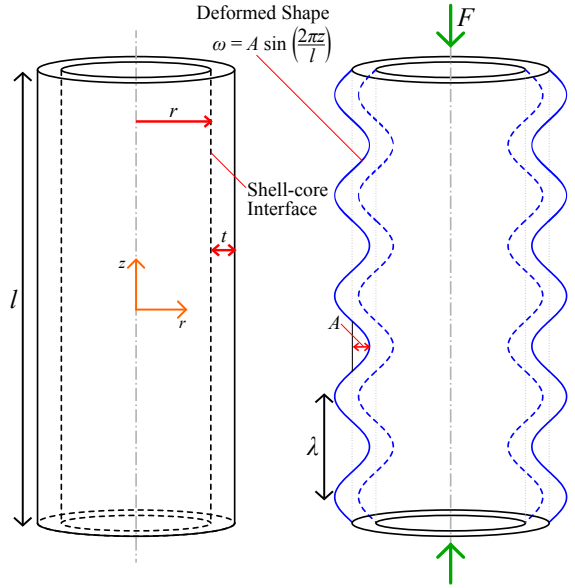

Fig. 3: A metal-coated polymer cylinder prior to buckling deformations (left) and at after buckling (right). The cylinder has radius $r$, length $l$ and shell thickness $t$ while the buckle wavelengths have amplitude $A$ and wavelength $\lambda$ caused by the axial load $F$

While commercial finite element packages are effective in determining the stress state of many geometries quite readily, the use of a custom-written program developed solely for analysing this geometry is a more efficient solution. The solution of the Southwell model is tailored to represent the specific stress states of an axisymmetric domain, allowing the use of coarser meshes to achieve an accurate solution. The governing equations for the Southwell model are [21]:

$$
\begin{gathered}
\frac{\partial^{2} \phi}{\partial r^{2}}-\frac{1}{r} \frac{\partial \phi}{\partial r}+\frac{\partial^{2} \phi}{\partial z^{2}}=0 \\
\frac{\partial^{2} \psi}{\partial r^{2}}-\frac{1}{r} \frac{\partial \psi}{\partial r}+\frac{\partial^{2} \psi}{\partial z^{2}}=\frac{\partial^{2} \phi}{\partial z^{2}},
\end{gathered}
$$

where $r$ is the radial ordinate direction, $z$ is the axial ordinate direction, and $\phi$ and $\psi$ are stress functions which must be determined. Once $\phi$ and $\psi$ are obtained, the individual stress components can be calculated using their equations written in terms of $\phi$ and $\psi[21]$ :

$$
\begin{aligned}
\sigma_{r} & =\frac{1}{r}\left(\frac{\partial \phi}{\partial r}+\frac{\partial \psi}{\partial r}\right)-\frac{1}{r^{2}}[\psi+(1-\nu) \phi] \\
\sigma_{\theta} & =\frac{\nu}{r} \frac{\partial \phi}{\partial r}+\frac{1}{r^{2}}[\psi+(1-\nu) \phi] \\
\sigma_{z} & =-\frac{\nu}{r} \frac{\partial \psi}{\partial r} \\
\tau_{z r} & =\frac{1}{r} \frac{\partial \phi}{\partial z},
\end{aligned}
$$

where $\sigma_{r}$ is the radial stress, $\sigma_{\theta}$ is the circumferential stress, $\sigma_{z}$ is the axial stress and $\tau_{z r}$ is the shear stress as seen in Fig. 4. It is to be noted that the Karam and Gibson model does not account for the circumferential stress [7, 20].

A general analytical solution is not obtainable for (2). A finite differencing solution was developed by Allen et al. [21] which utilizes second-order central differencing throughout the domain. Since the stress components are written in terms of $\phi$ and $\psi$ (i.e. (3)) the governing equations of these stress functions (i.e. (2)) are discretized. Details of the discretization can be found in Allen [21]. With the stress distributions known

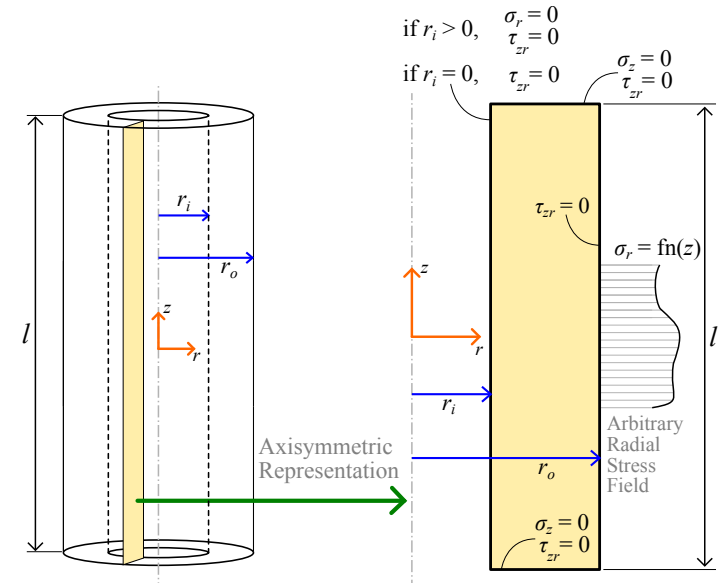

Fig. 4: Cylinder geometry (left) and axisymmetric domain (right) for Southwell model. The cylinder has length $l$, inner radius $r_{i}$ and outer radius $r_{o}$. Boundary conditions for the domain extremeties are given in terms of the values of the shear stress $\tau_{z r}$, axial stress $\sigma_{z}$ and radial stress $\sigma_{r}$

for the axisymmetric domain of Fig. 4, the internal energy of the cylinder can be readily calculated.

A modified domain is used in conjunction with the Southwell model in order to determine the stress state in the core of a filled cylindrical shell undergoing buckling deformations. A sinosoidal stress loading is utilized because buckling deformations are sinusoidal [6-9, 12, 14, 15, 19], and these lead to sinusoidal stress fields. A section of the core undergoing buckling deflections is analysed using the Southwell model, as shown in Fig. 5. Comparing Fig. 4 and Fig. 5, the new domain makes use of periodic boundary conditions, while a sinosoidal radial stress loading acts on the cylinder and the axisymmetric section. The periodic boundary conditions ensure that a calculation done for one buckle wavelength $\lambda$ can be reused when many waves are present $[6,7,12]$. The sinusoidal radial stress field is of the form:

$$
\sigma_{r}=\sigma_{0} \cos \left(\frac{m \pi z}{l}\right)
$$

where $\sigma_{0}$ is a given load magnitude, $m$ is the number of half waves, and $l$ is the length of the cylinder. Since the loading configuration represents one full wavelength, $m$ is set to 2 . The application of the radial stress field (i.e. (4)) leads to sinusoidal radial deflections with a magnitude of $A$ as seen in Fig 3 .

Analyses using the Southwell model, as well as finite element analyses, reveals that for small wavelengths to radius ratios (i.e. small $\lambda / r$ ) the value of the internal energy, $U$, is insensitive to the buckle wavelength $\lambda$. This implies that one calculation using the Southwell model for a given core material will provide the fitting constant $K$ using:

$$
K=\frac{2 U}{m A^{2} \pi r},
$$

where $K$ is the fitting constant. Once $K$ is determined, the expression for the core energy $U_{p}$ is found for any core radius $r$ and number of buckle wavelengths $m / 2$ :

$$
U=U_{p}=\frac{m}{2} K A^{2} \pi r
$$




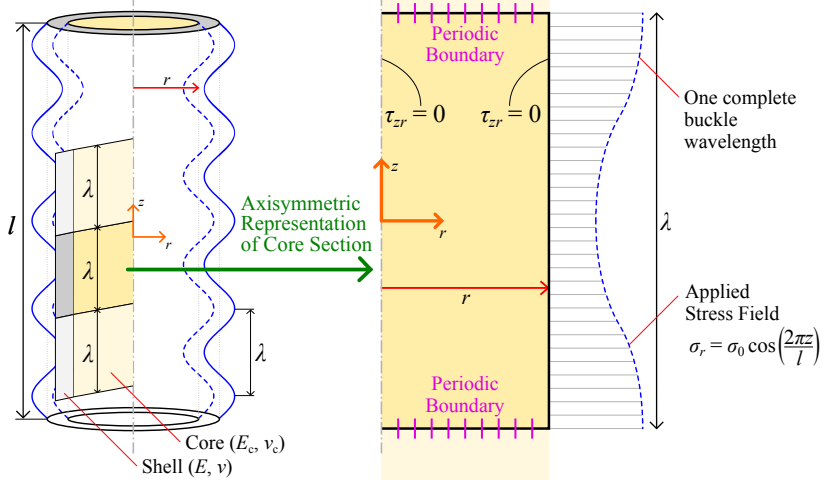

Fig. 5: Geometry, boundary conditions, and load for modified Southwell domain corresponding to buckling deflections of a filled cylindrical shell

This surrogate model is required for the core behaviour so that the core energy can be calculated for various core geometries, while also providing a differentiable function for the internal energy. The value of $K$ is dependant only on the material properties of the core, and must be recalculated when a new core material is used. The core energy expression (Eqn. 6) is used together with the shell energy terms to determine the buckling load for the filled shell system.

\section{BUCKLING LOAD DERIVATION}

While undergoing buckling, the energy sinks of a hollow cylindrical shell include the bending energy $\left(U_{b}\right)$ and the circumferential stretching energy $\left(U_{c}\right)$. The geometry and relevant material properties of a filled shell system are shown in Fig. 3 and Fig. 5.

The shell bending energy refers to the energy associated with the bending action of the shell sections as they undergo sinusoidal buckling deformations. It takes the form [6]:

$$
\begin{aligned}
U_{b} & =\int_{0}^{l}\left[\frac{A^{2} m^{4} \pi^{4}}{l^{2}} \sin ^{2}\left(\frac{m \pi x}{l}\right)\right]\left[\frac{\pi r E t^{3}}{6\left(1-\nu^{2}\right)}\right] \mathrm{d} x \\
& =\frac{\pi E r t^{3}}{24\left(1-\nu^{2}\right)} \frac{m^{4} \pi^{4}}{l^{4}} A^{2} l,
\end{aligned}
$$

where $t$ is the shell thickness, $E$ is the Young's Modulus of the shell material, $l$ is the length of the shell, $r$ is the shell's radius, and $\nu$ is the shell material Poisson's ratio.

The energy resulting from the circumferential tension or compression of the shell hoop sections is defined as [6]:

$$
\begin{aligned}
U_{c} & =\int_{0}^{2 \pi} \int_{r}^{r+t} \int_{0}^{l} \int_{0}^{\epsilon_{r c}} \frac{E}{r} A \sin \left(\frac{m \pi z}{l}\right) r \mathrm{~d} \epsilon \mathrm{d} z \mathrm{~d} r \mathrm{~d} \theta \\
& =\frac{\pi E t A^{2} l}{2 r} .
\end{aligned}
$$

During buckling, the total internal energy $\left(U_{i}\right)$ of a filled cylinder is the sum of the shell bending energy (7), the shell circumferential stretching energy (8), and the core energy (6). The external energy $\left(U_{e}\right)$ arises due to the applied axial force, $F$ (see Fig. 3). These two energy terms, respectively, are:

$$
U_{i}=U_{b}+U_{c}+U_{p}
$$

and

$$
U_{e}=\frac{F m^{2} \pi^{2} A^{2}}{4 l}
$$

At the inception of buckling, the following differential relation holds:

$$
\frac{\partial U_{e}}{\partial A}=\frac{\partial U_{i}}{\partial A}
$$

This is known as the bifurcation point: a further increase in $F$ will either lead to buckling or continued axial loading without buckling. While in perfect systems buckling will not take place, imperfections will always cause real systems to buckle [13].

Equating (9) and (10) and applying the operation as in (11), the elastic buckling load of a metal-coated polymer cylinder in axial compression is found. Adding on the load-bearing capacity of the core gives an expression of the form:

$$
F=\left(1+\frac{E_{c}}{E} \frac{r}{2 t}\right)\left[\frac{2 \pi^{3} r E t^{3}}{3\left(1-\nu^{2}\right)} \frac{1}{\lambda^{2}}+\frac{E t}{2 r \pi} \lambda^{2}+\frac{K r}{\pi} \lambda\right] \text {. }
$$

Equation (12) applies for any $\lambda$, but only one critical $\lambda$ value exists for a given cylinder with known material properties. This critical $\lambda$ value is found by finding the positive real root of the polynomial expression $\partial F / \partial \lambda=0$. Once found, the critical $\lambda$ value is substituted into (12) to give the critical load for the filled shell system of interest.

\section{Finite Element Verification}

Buckling analysis was undertaken in Abaqus to verify the predicted loads. This was done for ranges of cylinder lengthto-radius $(l / r)$ ratios and thickness-to-radius $(t / r)$ ratios. Many $l / r$ ratios were used to ensure that the buckling load was insensitive to cylinder length. All geometries were represented axisymmetrically, and the subspace algorithm was used to solve for the eigenvalues and corresponding buckled shapes. A perturbation was applied in the form of an axial displacement at one end of each cylinder, while keeping the opposing end fixed and allowing both ends to expand radially. There were at least 5 elements through the coating thicknesses after meshing was carried out. The lowest eigenvalue calculated by the solver was taken to be the buckling load.

The $t / r$ ratios studied ranged between 0.005 and 0.1 . Ratios below 0.005 indicate very low thicknesses which are not practical for nanocoated microtruss structures, while those above 0.1 correspond to very thick coatings which are outside of the range of thin-shell buckling assumptions.

Fig. 6 shows a graph of the non-dimensional buckling loads as predicted by Abaqus along with the theoretical buckling loads predicted by both the new elastic theory (i.e. (12)) and the Karam and Gibson theory [7]. The theoretical curves and Abaqus results were found for $E_{c} / E=0.01$, this value being typical for metal-coated polymer cylinders where the polymer material is ABS or a similar 3D printed material. The buckling loads for different $l / r$ values (overlapping points at each $t / r$ value in Fig. 6) are not identical due to slight differences in mesh characteristics. However, the are sufficiently close 


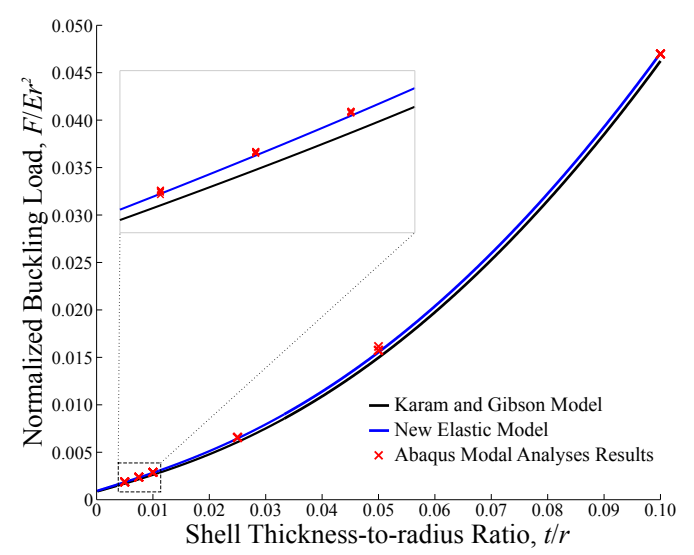

Fig. 6: Non-dimensional loads calculated for the new elastic model and the Karam and Gibson model, with Abaqus modal analyses results included $\left(E_{c} / E=0.01, \nu=0.3\right.$ and $\left.\nu_{c}=0.35\right)$

to indicate no dependence of the buckling load on cylinder length.

As seen in Fig. 6, both the Karam and Gibson theory and the new elastic theory predict lower loads than the Abaqus results. The Karam and Gibson theory predicts loads that are up to $10 \%$ lower than those predicted by Abaqus, while the new theory predicts loads that are no less than $3 \%$ of the values. The agreement between the Abaqus results and the new model theory improves at higher $t / r$ values.

\section{A. Discussion}

The improved predictions of the new elastic model are in accordance with the increased energy that the this model predicts. The circumferential stress component found using the Southwell model is not accounted for in the Karam and Gibson model, leading to lower energy predictions and lower predicted buckling loads. While the Southwell model is a more complete description of the stress state of a core subject to buckling deformations, the Karam and Gibson theory is simpler to implement in practice.

Though the Southwell model involves more steps to find the behaviour of the elastic core (i.e. finding the fitting, $K$ ), the loading configuration can be changed for several other situations involving filled shell buckling. This can include hollowing out the core or assuming no adhesion between the shell and the core. These cases have not been studied in the existing literature.

While the present model predicts the buckling strength of metal-coated polymer cylinders, adapting it for use with hybrid microtruss structures will require topological information of a given microtruss.

\section{CONCLUding REMARKS}

A new model has been developed that accurately accounts for the energy state in the polymer core of a metal-coated polymer cylinder as the cylinder undergoes sinusoidal buckling deformations. The model utilizes the Southwell stress model, which describes the stress state in cylindrical geometries under axisymmetric loads. This model has been used to represent the core behaviour of a filled-shell system undergoing buckling, and agrees more closely with finite element results. Although the model is more complex to implement than existing theories, such as the Karam and Gibson method, it is more adaptable to different cylinder geometries or adverse shellcore adhesion situations.

\section{REFERENCES}

[1] L. Gordon, B. Bouwhuis, M. Suralvo, J. Mccrea, G. Palumbo, and G. Hibbard, "Micro-truss nanocrystalline Ni hybrids," Acta Materialia, vol. 57, no. 3, pp. 932-939, Feb. 2009. doi: 0.1016/j.actamat.2008.10.038.

[2] K. Kumar, H. Van Swygenhoven, and S. Suresh, "Mechanical behavior of nanocrystalline metals and alloys," Acta Materialia, vol. 51, no. 19, pp. 5743-5774, Nov. 2003. doi: 10.1016/j.actamat.2003.08.032.

[3] U. Erb, "Electrodeposited nanocrystals: Synthesis, properties and industrial applications," Nanostruct. Mater., vol. 6, no. 5-8, pp. 533-538, 1995. doi: 10.1016/0965-9773(95)00114-X.

[4] A. Lausic, C. Steeves, and G. Hibbard, "Effect of grain size on the optimal architecture of electrodeposited metal/polymer microtrusses," J. Sandwich Struct. Mater., vol. 16, no. 3, pp. 251-271, Apr. 2014. doi: $10.1177 / 1099636213515507$.

[5] K. R. F. Andrews, G. L. England, and E. Ghani, "Classification of the axial collapse of cylindrical tubes under quasi-static loading," Int. J. Mech. Sci., vol. 25, no. 9-10, pp. 687-696, 1983. doi: 10.1016/0020-7403(83)90076-0.

[6] S. P. Timoshenko and J. M. Gere, Theory of Elastic Stability. New York: McGraw-Hill, 1961.

[7] G. N. Karam and L. J. Gibson, "Elastic buckling of cylindrical shells with elastic cores - Part I: Analysis," Int. J. Solids and Struct., vol. 32, no. 8-9, pp. 1259-1283, Apr.-May 1995. doi: 10.1016/0020-7683(94)00147-O.

[8] T. Myint-U, "Stability of axially compressed core-filled cylinders," $A I A A$ Journal, vol. 4, no. 3, pp. 552-553, 1965. doi: 10.2514/3.3481.

[9] P. Seide, "The stability under axial compression and lateral pressure of circuluar-cylindrical shells with a soft elastic core," J. Aeronaut. Sci., vol. 29, no. 7, pp. 851-862, 1962.

[10] J. Teng and J. Rotter, Buckling of Thin Metal Shells. London: CRC Press, 2004. doi: 10.4324/9780203301609.

[11] R. Lorenz, "Achsensymmetrische verzerrungen in dunnwandingen hohlzzylindern," VDI Z. (1857-1968), vol. 52, no. 43, pp. 1706-1713, 1908.

[12] T. von Karman and H.-S. Tsien, "The buckling of thin cylindrical shells under axial compression," J. Aeronaut. Sci., vol. 8, no. 8, pp. 303-312, June 1941. doi: $10.2514 / 8.10722$

[13] D. Bushnell, "Buckling of shells - pitfall for designers," AIAA Journal, vol. 19, no. 9, pp. 1183-1226, Sept. 1981. doi: 10.2514/3.60058.

[14] L. Donnell, "A new theory for the buckling of thin cylinders under axial compression and bending," Trans. ASME, Aeronaut. Eng., vol. AER-56-12, pp. 795-806, 1935.

[15] S. B. Batdorf, "A simplified method of elastic stability analysis for thin cylindrical shells: Part I - Donnell's equation," NACA TN 1341, 1947.

[16] G. Gough, C. Elam, and N. de Bruyne, "The stabilization of a thin sheet by a continuous supporting medium," J. R. Aeronaut. Soc., vol. 44, pp. 1243, Jan. 1940. doi: 10.1017/S036839310010495X.

[17] R. Tennyson, "An experimental investigation of the buckling of circular cylindrical shells in axial compression using the photoelastic technique," UTIAS Report No. 102, 1964. 
[18] G. N. Karam and L. J. Gibson, "Elastic buckling of cylindrical shells with elastic cores - Part 2: Experiments," Int. J. Solids Struct., vol. 32, no. 8-9, pp. 1285-1306, Apr.-May 1995. doi: 10.1016/0020-7683(94)00148-P.

[19] W. S. Goree and W. A. Nash, "Elastic stability of circular cylindrical shells stabilized by a soft elastic core," Exp. Mech., vol. 2, no. 5, pp. 142$149,1962$.

[20] H. G. Allen, Analysis and design of structural sandwich panels. Hungary: Pergamon Press, 1969.

[21] D. N. De G. Allen, Relaxation methods in engineering and science. New York: McGraw-Hill, 1954. 


\title{
Simulating Layup Defects During Tow Steering in Automated Fiber Placement
}

\author{
Nima Bakhshi and Mehdi Hojjati \\ Concordia Center for Composites (CONCOM), \\ Concordia University, \\ Montreal, Canada.
}

\begin{abstract}
Automated Fiber Placement (AFP) offers valuable advantages which make this technology a suitable candidate for producing high-quality parts in the aerospace industry. However, there are limitations: number of defects may arise during the fiber placement including wrinkles at the inside edge, and blisters in the middle of the prepreg tows. These defects have severe effects on the layup quality and consequently, on the performance and quality of the final part. Therefore, efforts shall be pointed to avoid them. A deeper understanding of the defect formation processes as well as tools and techniques for modeling them is indispensable for fully harnessing the potential of AFP technology. In the present study, a physics-based modeling approach is presented for the global modeling of defects in AFP. The application of this approach for detecting and modeling the blisters and outof-plane wrinkles that appear during fiber steering, is discussed, although it should be noted that the application is not limited to the case of fiber steering and can be expanded to different scenarios. Preliminary results of the simulations are presented. AFP trials are performed to validate the model. The trends and patterns of both wrinkles and blisters are found to be in good agreement with experimental results.
\end{abstract}

Keywords - Automated Fiber Placement; layup defect; simulation; manufacturing.

\section{INTRODUCTION}

Automated Fiber Placement has been increasingly used in the aerospace industry to manufacture high-quality aerospace parts. Manufacturing objectives including increasing productivity and reproducibility as well as decreasing labor intensity and minimizing the number of parts can be accomplished using this technology. One of the unique features of AFP is its ability to deliver up to 32 prepreg tows in a course with different speeds. This feature namely, the differential tow pay-out is what enables the AFP machines to laminate complex geometries and Variable Stiffness Composites (VSC). In complex geometries, steering the fiber path away from the geodesic path of the mold to maintain the desired fiber orientation is inescapable. Moreover, differential tow pay-out allows engineers to design a family of composites with curvilinear fiber paths, called VSCs. A more efficient load path between supports and load points is formed by curvilinear fibers which allows exploiting the full potential of the directionality of composite laminae [1].

Despite its numerous advantages and capabilities, AFP comes with its own limitations. The productivity of the lamination process and the quality of final parts are significantly influenced by the complexity of the part, as well as the process parameters such as lay-up temperature and speed, compaction force and fiber tension. Several different defects may appear in AFP (especially during fiber steering) such as out-of-plane wrinkles at the inside edge of the tow, blisters (circular delaminations in form of a blister) in the middle of the tow, tow pull ups and shearing effects. These defects will cause lower part quality with reduced reproducibility by deviating the fibers from the original prescribed path, increasing resin rich areas, etc. Consequently, efforts must be aimed at avoiding them $[1,2]$.

The key mechanism resisting against the formation of most layup defects is prepreg tack [3-5]. Tack is a complex systemic property of prepreg-tool interface that can be reasonably controlled by process parameters. Experimental trials performed during this study as well as a review of the literature demonstrated that tack levels as a function of process parameters can be generally understood considering the formation of strong Intimate Contact (IC), stress relaxation effects in the prepreg resin and the resin's cohesive strength. Perhaps layup temperature is the most sensitive process parameter that affects the prepreg tack and layup quality. By increasing the temperature, the resin viscosity decreases and a better IC is achieved, resulting in higher tack. Experimental observations, however, have reported a peak in the tack of prepregs and pressure sensitive adhesives as a function of temperature. This is due to the fact that reduction of resin viscosity (with increasing temperatures) is accompanied by the decline of resin strength. Consequently, at very high temperatures, a good contact is formed, whereas the lower strength of the resin results in cohesive failure within the prepreg (as opposed to interfacial failure at the prepreg-tool interface for lower temperatures). Layup speed in the AFP is inversely proportional to the contact time. As such, the lower speed means more time for IC formation, lower strain rates and more time for relaxation of stresses in the prepreg resin, all of which result in higher tack levels. Increasing compaction force, 
again, helps to establish a good IC and increases the prepreg tack. A peak is also observed for the tack as a function of compaction force, since the further increase of the force will result in redistribution of resin content and a contraction after the loading is released. Both these effects help the prepreg to detach from the tool surface (lower tack). Tack also has a peak around moderate values of relative humidity (50-60\%), which is generally attributed to the changes in resin viscosity and strength. Another important factor is the age of prepreg. As the material ages, crosslinking reaction occurs in the resin and the degree of cure increases. This will result in a stiffer material with lower tack. Using fresh prepregs within their shelf life helps in having a better quality layup. Other parameters such as tool's surface roughness, and, resin and tool surface energies also play a role in both establishing the IC and the amount of work of adhesion, however, their macroscopic effects on tack is not very well understood at the moment and further investigation is required.

The formation of wrinkles at the inside edge of the prepreg tow has been studied from both experimental [7-10] and modeling [11-13] perspectives. These approaches usually state a steering limit as the minimum radius that results in a defectfree layup. Experimental approaches are expensive, timeconsuming and increase material wastage since they are based on trial and error. On the other hand, modeling approaches take advantage of the incremental deposition of prepreg tows in the AFP, and assume that wrinkles are formed individually, in isolation from each other [12]. It has been noted that the assumption employed in the local modeling approach does not always follow the experimental observations [11]. Although these methods have gained some success, they both have limitations as stated. Recently, Hörmann [11] has recommended changing the modeling strategy for the future work by considering the global behavior of the prepreg tow, instead of using the local approach's assumption.

In the present paper, a framework for simulating the layup defects in the AFP is presented in the finite element software ABAQUS. The application of this approach for modeling the out-of-plane wrinkles at the inside edge of the steered prepreg tows is presented. The cohesive zone modeling technique with a bilinear traction-separation law is used to represent the prepreg tack at the prepreg-tool interface. In addition, it is found that the location of blister sites can be found using the simulations, which can be used to further explore the causes behind the blister formation. Preliminary results of the simulations are presented. Automated Fiber Placement trials are performed to validate the model. The trends and patterns of both wrinkles and blisters are found to be in very good agreement with experimental results.

\section{MODELING STRATEGY}

\section{A. Prepreg feeding and steering mechanisms}

The ABAQUS [14] model constitutes five parts: A rigid tool, a rigid bar which simulates the steering path, a compaction roller, a guide for the movement of prepreg tow, and finally the prepreg tow. The assembled model with the used boundary conditions are presented in Fig. 1. Appropriate boundary conditions and kinematic coupling constraints are

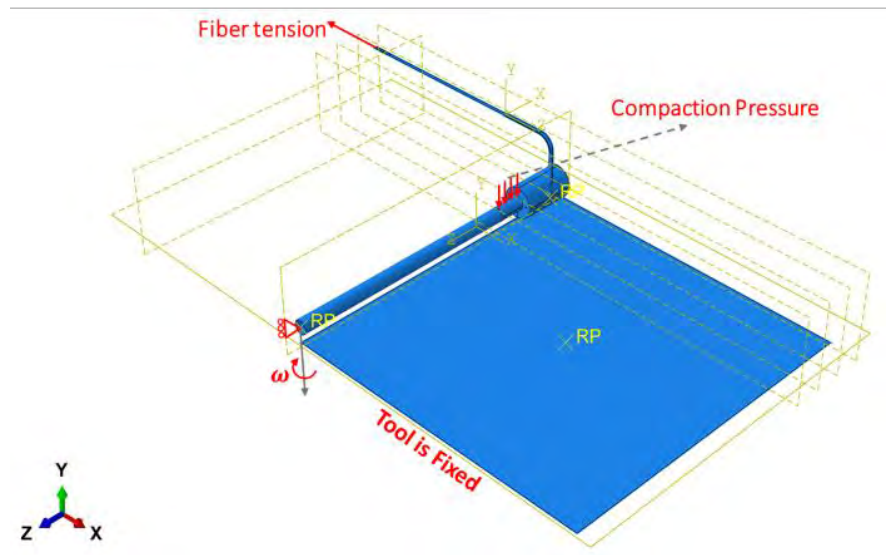

Figure 1. The assembled model.

created between the free end of the prepreg tow and the guide, to simulate the prepreg feeding mechanism. A light pressure simulating the compaction force is applied on top of the rigid bar, and a light force is applied to the free end of the tow to simulate the fiber tension which keeps the tows straight. A constant angular speed is applied to the free end of the rigid bar, such that it simulates the linear speed of the AFP head during the experiments.

\section{B. Prepreg tack}

Several different approaches are available for modeling fracture and delamination including continuum mechanics, fracture mechanics, XFEM and damage mechanics. For modeling the prepreg tack, an especial approach in the damage mechanics, called Cohesive Zone Modelling (CZM) is utilized [15]. Tack is merely intended to be modeled as an interfacial property; the delamination surface is known in the AFP process (at the prepreg-tool interface); and finally, since the location of out-of-plane wrinkles (and more generally, layup defects) are not known, the approaches requiring specification of an initial crack cannot be employed. Since CZM complies with all of the modeling requirements of prepreg tack, it has been chosen for the present study. CZM is well established in commercial finite element codes and more importantly, effective methods are available for characterizing the cohesive zone properties. They can be introduced into the ABAQUS model with the surfacebased cohesive zone modeling technique as a contact property. The cohesive contact is defined such that any node on the prepreg that comes into contact with the tool surface experiences the cohesive interactions. This modeling technique employs a bilinear traction-separation law to define the traction between the surfaces as a function of the separation between the surfaces. It is worthwhile to mention that in addition to the aforementioned desirable properties, viscoelastic material properties can be defined for the first part of the tractionseparation law which in conjunction with an exponential softening (damage evolution) can account for the ratedependent viscoelastic properties of prepreg tack.

Here, a bilinear traction-separation law for the CZM is employed which constitutes two linear sections (Fig. 2). The first section (elastic section) is defined through the slope of the line while the second part, corresponding to the damage evolution, requires a damage initiation criterion and a second 


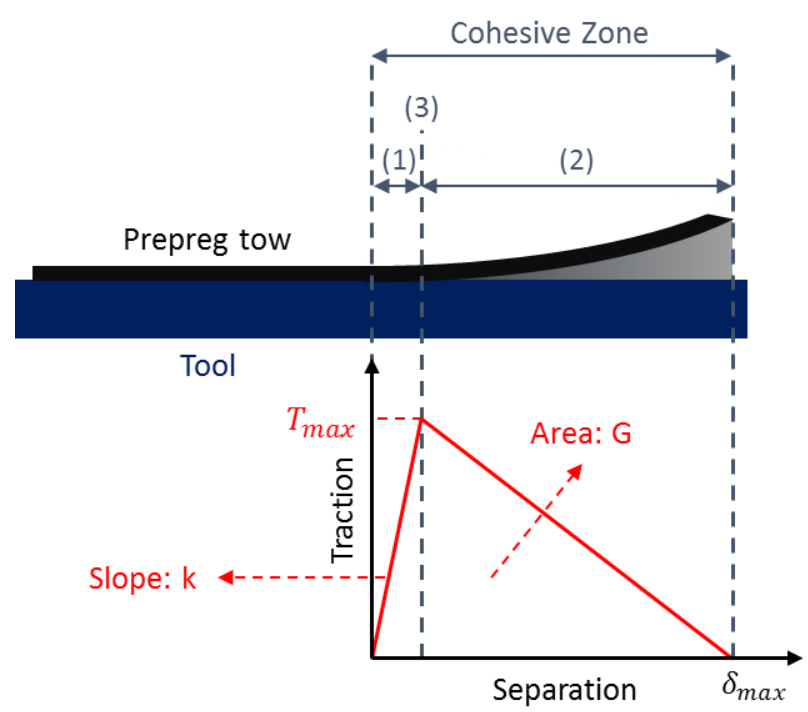

Figure 2. Bilinear traction separation law:

(1) Damage initiation (elastic), (2) Damage propagation (softening),

(3) Damage initiation criterion.

measure, for controlling the damage. Maximum nominal stress is chosen to establish the damage initiation and total fracture energy with linear softening is used to define the damage evolution.

From the fact that the maximum separation is, obviously, bigger than the separation at which the damage initiates, a simple relation should hold between the fracture energy (i.e. work of adhesion/tack), $G$, the maximum traction, $T_{\max }$, and stiffness (or the slope of the damage initiation part), $k$ :

$$
k>T_{\max }^{2} / 2 G
$$

\section{Solution method}

Several models with different mesh sizes were studied to find the appropriate trade-off between the computation time and resolution of the models. Abaqus / Explicit solver with full geometric nonlinearities was used to solve the model. The explicit solver can handle the nonlinearities that arise as a result of large deformations in the prepreg tow and material degradation in the traction-separation law, very well. It should be noted that on the contrary to the Standard solver, the value of stiffness, $k$, does matter in the Explicit solver. It is known that in order to avoid convergence problems, Standard solver treats the stiffness of the $\mathrm{CZ}$ in a manner similar to the penalty method, therefore the stiffness value should be large and it does not affect the final results significantly [14].

TABLE I.

PREPREG MECHANICAL PROPERTIES

\begin{tabular}{cccc}
\hline$E_{1}(\mathbf{G P a})$ & $\boldsymbol{E}_{2}(\mathbf{M P a})$ & $\boldsymbol{G}_{12}(\mathbf{M P a})$ & $\boldsymbol{v}_{12}$ \\
\hline 31 & 0.046 & 3.025 & 0.2 \\
\hline
\end{tabular}

\section{MATERIAL CHARACTERIZATION}

The mechanical properties (except for the transverse modulus) of the uncured prepreg are experimentally measured in the previous work [13], using the tensile tests and bias extension tests. The transverse modulus was calculated using Puck's formula. These values are used in the present study since the same material was used in the AFP experimental trials. The material properties are summarized in table 1.

A commonly used method for measuring the unknown parameters required to fully characterize the prepreg tack using bilinear traction-separation laws is probe tack test method [4, 6]. This method was initially introduced in the Pressure Sensitive Adhesives industry to quantitatively measure the tackiness of adhesives and later was adopted to be used for measuring prepreg tack. In this testing method usually, a circular probe comes into contact with the prepreg at a controlled temperature and relative humidity, until a specified force is reached. Then it remains in contact with the prepreg for a given amount of time (dwell time), and subsequently, it is pulled away. During the retraction stage, force and displacement data are recorded to be further analyzed. Generally, the maximum recorded stress or the work of adhesion can be used to represent the quantitative value for tack.

Fig. 3 shows a typical normalized stress-displacement data obtained from the probe tack test (figure is reproduced from previous work [13]). As can be seen in this figure, the stiffness and maximum traction can be directly measured using probe test. The fracture energy (i.e. work of adhesion) can also be easily obtained by calculating the area under the traction data. These three parameters are sufficient for fully defining the bilinear CZ traction-separation law.

It should be noted that, although probe tack test provides a straightforward method for finding the required information for the model, its results might be hard to be related to the actual process parameters. The correlation between the AFP head's speed, on one hand, and the dwell time and probe speed (during both compression and retraction stages of probe test), on the other hand, is not clear. Moreover, the effects of temperature on the prepreg tack that is measured through probe test might be contrary to what actually occurs during the AFP. Increasing

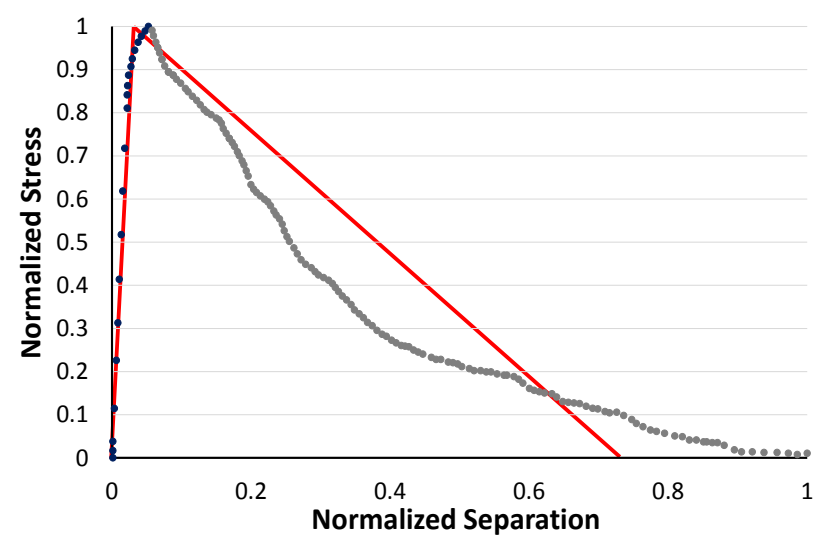

Figure 3. Typical data from probe tack test (reproduced from [13]), Data points: experimentally measured data, Red line: fitted bilinear traction-separation law. 


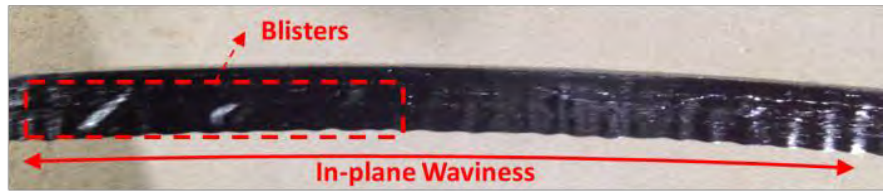

Figure 4. Blisters and in-plane waviness are appeared in the steered tows.

the temperature (which results in lowering the resin viscosity) in the probe test generally causes the resin to flows away from the probe surface. Consequently, lower tack results are usually recorded for increasing temperatures, while increasing temperature in the AFP clearly strengthens the interface (at least in the practical process range). Developing a more realistic approach for measuring prepreg tack with regards to AFP will remain for the future investigation. Here, available data in the literature $[6,13]$ that are in a close range of the experimental process parameters are used.

\section{RESULTS AND DISCUSSION}

AFP trials were performed using the $0.2 \mathrm{~mm}$-thick and 6.35 $\mathrm{mm}$-wide prepreg tows with the material properties presented in table 1. Tows are steered with the radius of $558.8 \mathrm{~mm}$ (22"). Results of the experiments and simulations are presented in this section.

In addition to the in-plane fiber waviness (Fig. 4) which is the most primitive result of length discrepancies between the inside and outside edges of a steered tow, a very commonly observed defects were blisters in the middle of the prepreg tows (Fig. 4 and 5). Initially, it was postulated that the blisters are occurring due to the high flow rate of the hot Nitrogen gas (heating system of the AFP). However, decreasing the flow rate proved to be ineffective in preventing their formation. During the simulations, it was found that in configurations similar to those in which blisters have appeared, several small zones are formed that have reached the damage initiation criterion but the damage has not progressed much. The results of the corresponding simulation are presented in Fig. 6 . The red color represents the areas where the damage initiation criterion of the traction-separation law is met. Those areas that have not completely buckled yet (i.e. the damage has not evolved $100 \%$ and consequently, wrinkles have not been formed) correspond to the blister zones and are marked with yellow circles. It can

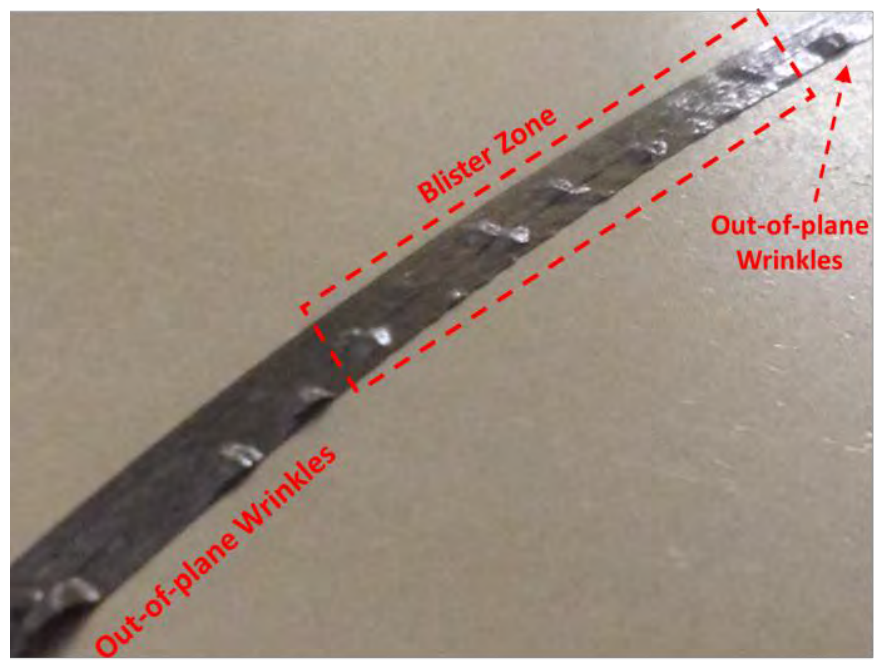

Figure 5. Blisters and out-of-plane wrinkles are appeared in the steered tows.

be observed from this figure that the wrinkles are clearly distinguished by the large deformation of the tow while the blister zones look intact (although they have a damaged interface which causes the blisters to appear in practice).

A qualitative comparison of Fig. 6 with the experimental results (Fig. 5) shows an excellent agreement, meaning that blisters are in fact mainly originating from the stress distribution resulted from fiber steering, and interface failure (i.e. not sufficient levels of tack) rather than other physical factors. Moreover, the pattern and frequency of appearance of out-of-plane wrinkles are also in good agreement between the simulation and experiments.

A commonplace result of the simulations is presented in Fig. 7. As it is demonstrated in this figure, the outside edge of the prepreg tow is under sever tensile stress. This tensile stress has two origins: the fiber tension force that is exerted on the free end of the tow which partly helps in keeping the loose tow straight (in reality this load is indeed induced in the prepreg tow in the delivery system), and the tensile stress that is induced as a result of fiber steering (bending stress). In the viewpoint of Fig. 7, two wrinkles are appeared. It is evident that compressive stress is built up at the inside edge of the tow

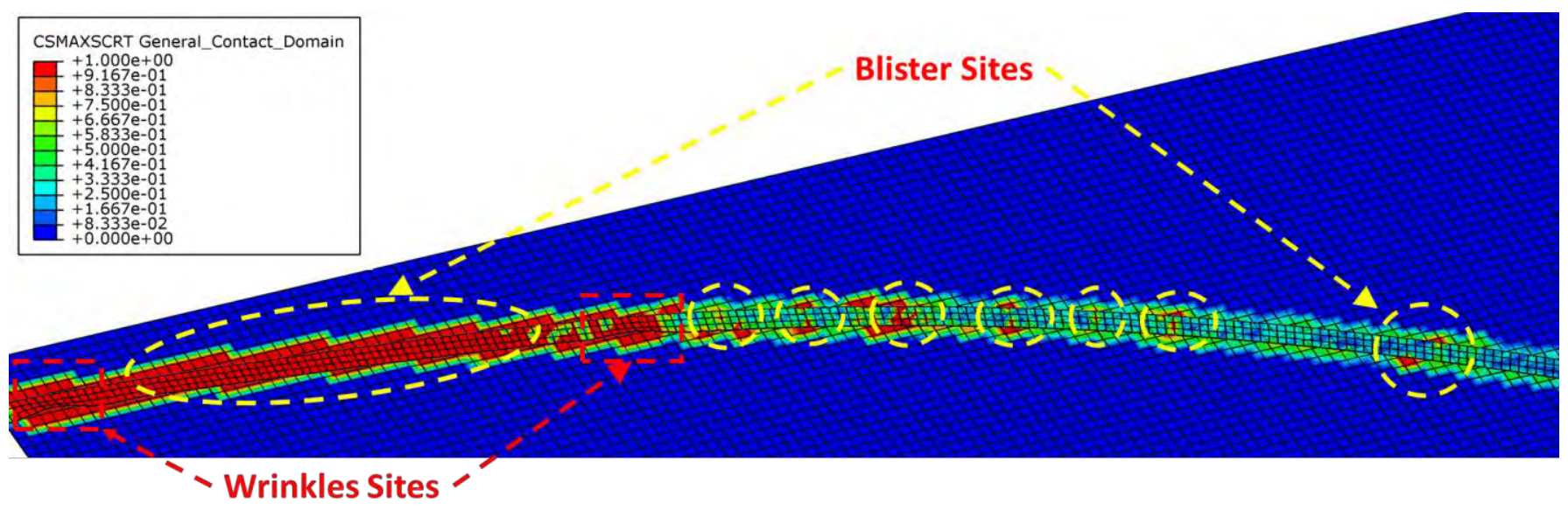

Figure 6. Simluation results: the damage initiation criteria. 


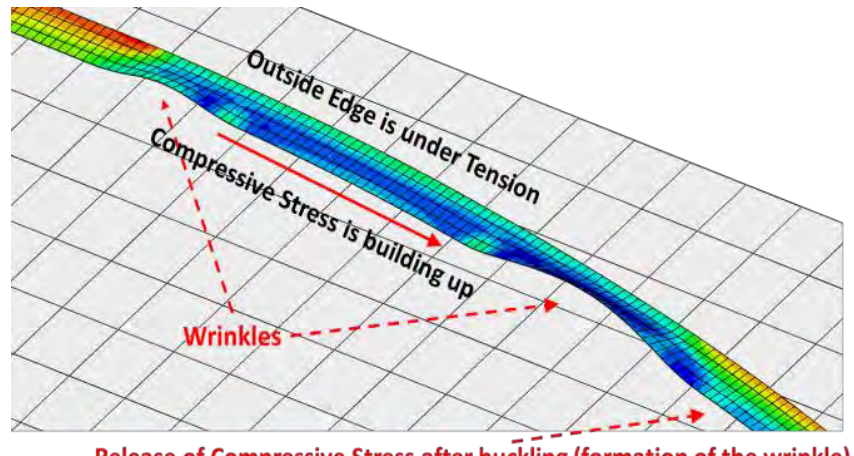

Release of Compressive Stress after buckling (formation of the wrinkle)

Figure 7. A commonplace result of the simulations.

between the two wrinkles. More importantly, small low-stress zones are visible in the vicinity of both wrinkles indicating that wrinkles are indeed acting as a stress relief mechanism.

\section{CONCLUSION}

A new global defect modelling approach for the layup process of Automated Fiber Placement is presented in the present work. A simple mechanism is created to simulate the feeding mechanism of the prepreg tow. The surface-based cohesive zone modelling technique using a bilinear tractionseparation law is used to model the prepreg tack. Application of the proposed approach for the challenging and commonplace case of fiber steering which is one of the major defective layup scenarios in AFP is discussed. Experimental AFP trials were performed and a very good agreement between the simulations and experimental results is found. It is shown that using the index representing the damage initiation criterion and the deformed shape of the prepreg tow, two of the major defects occurring during the tow steering namely, out-of-plane wrinkles and blisters, can be predicted and identified.

\section{ACKNOWLEDGMENT}

The authors would like to acknowledge the financial support from the Natural Sciences and Engineering Research Council of Canada (NSERC). Also, we would like to thank Mr. Jeffrey Fortin-Simpson of CONCOM's AFP laboratory for his help and assistance in sample preparations.

\section{REFERENCES}

[1] D. H.-J. A. Lukaszewicz, C. Ward, and K. D. Potter, “The engineering aspects of automated prepreg layup: History, present and future," Compos. B., vol. 43, no. 3, pp. 997-1009, Apr. 2012. doi: 10.1016/j.compositesb.2011.12.003.

[2] G. G. Lozano, A. Tiwari, C. Turner, and S. Astwood, "A review on design for manufacture of variable stiffness composite laminates," Proc. - Inst. Mech. Eng., Part B, vol. 230, no. 6, pp. 981-992, Sep. 2015. doi: $10.1177 / 0954405415600012$.

[3] K. J. Ahn, J. C. Seferis, T. Pelton, and M. Wilhelm, "Analysis and characterization of prepreg tack," Polym. Compos., vol. 13, no. 3, pp. $197-$ 206, Jun. 1992. doi: 10.1002/pc.750130308.

[4] O. Dubois, J.-B. Le Cam, and A. Béakou, "Experimental Analysis of Prepreg Tack," Exp. Mech., vol. 50, no. 5, pp. 599-606, Mar. 2009. doi: 10.1007/s11340-009-9236-7.

[5] R. J. Crossley, P. J. Schubel, and N. A. Warrior, "The experimental determination of prepreg tack and dynamic stiffness," Compos. A, vol. 43, no. 3, pp. 423-434, Mar. 2012. doi: 10.1016/.compositesa.2011.10.014.

[6] Wohl, C., et al., Tack Measurements of Prepreg Tape at Variable Temperature and Humidity, 2017.

[7] S. Nagendra, S. Kodiyalam, J. Davis, and V. Parthasarathy, "Optimization of tow fiber paths for composite design," in 36th Struct. Dyn., Mater. Conf., 1995. doi: $10.2514 / 6.1995-1275$.

[8] M. Wiehn, M. and R. Hale, Low cost robotic fabrication methods for tow placement, in 47 the International SAMPE Symposium and Exhibition 2002.

[9] J. Chen, T. Chen-Keat, M. Hojjati, A. Vallee, M.-A. Octeau, and A. Yousefpour, "Impact of layup rate on the quality of fiber steering/cut-restart in automated fiber placement processes," Sci. Eng. Compos. Mater., vol. 22, no. 2, Jan. 2015. doi: 10.1515/secm-2013-0257.

[10] R. Smith, Z. Qureshi, R. Scaife, and H. El-Dessouky, "Limitations of processing carbon fibre reinforced plastic/polymer material using automated fibre placement technology," J. Reinf. Plast. Compos., vol. 35, no. 21, pp. 1527-1542, Aug. 2016. doi: 10.1177/0731684416659544.

[11] P. Hörmann, Thermoset Automated Fibre Placement-on Steering Effects and Their Prediction, Verlag Dr. Hut., 2016.

[12] M. Y. Matveev, P. J. Schubel, A. C. Long, and I. A. Jones, "Understanding the buckling behaviour of steered tows in Automated Dry Fibre Placement (ADFP)," Compos., A, vol. 90, pp. 451-456, Nov. 2016. doi: 10.1016/j.compositesa.2016.08.014.

[13] M. Belhaj and M. Hojjati, "Wrinkle formation during steering in automated fiber placement: Modeling and experimental verification," J. Reinf. Plast. Compos., vol. 37, no. 6, pp. 396-409, Jan. 2018. doi: $10.1177 / 0731684417752872$.

[14] F. Abaqus, F., ABAQUS 6.14 Documentation. Dassault Systemes, Providence, RI, USA, 2014.

[15] R. A. Sauer, "A Survey of Computational Models for Adhesion," $J$. Adhes., vol. 92, no. 2, pp. 81-120, Oct. 2015.

doi: 10.1080/00218464.2014.1003210. 


\title{
Computational Modelling of Radiofrequency Cardiac Ablation to Study the Effect of Cooling on Lesion Parameters
}

\author{
Carolyn Berkmortel, Hamed Avari, Eric Savory \\ Department of Mechanical and Materials Engineering \\ University of Western Ontario \\ London, Ontario \\ cberkmor@uwo.ca
}

\begin{abstract}
Radiofrequency ablation (RFA) is a technique used to treat cardiac arrhythmias. It creates lesions in the heart by creating thermal damage. Due to limitations associated with in vivo as well as in vitro studies, computational methods assist in further analysis of the problem by allowing for quicker and more diverse parametric studies and hence, a more thorough understanding of the physics involved. These computational models have been proven to be good representations of the process by accurately modelling the catheter with simplified geometry and boundary conditions. Although these studies have inconsistencies in material properties (due to the variation of thermal and mechanical properties in biological tissue) as well as different methods of creating the geometry and applying the boundary conditions, overall they are quite similar. The effects of esophageal cooling were investigated to understand its effect on the process. It was determined that using the standard model found within the literature, the esophageal cooling changed the lesion depth by less than $18 \%$, while changing the maximum tissue temperature by as much as $13.4 \%$.
\end{abstract}

Keywords: Biomedical, simulation, computational fluid dynamics

\section{INTRODUCTION}

Radiofrequency ablation (RFA) is used in patients to treat cardiac arrhythmias (a cardiovascular disorder involving irregular heart rhythm). Atrial fibrillation, the most common type of cardiac arrhythmia, is where the atrial electrical signal is chaotic. This condition can be life threatening and must be treated [1]. For the past decade RFA has been the most common and effective method for treating atrial fibrillation that is resistant to drug treatment [2]. In this procedure, the tissue surrounding the pulmonary arteries is heated by a radiofrequency current (causing joule heating in the tissue) until the tissue is permanently damaged and a lesion is formed. This process isolates the incorrect electrical signals that originate there, so normal heart electrical function can resume [3,4]. One of the risks of this process is the development of an atrialesophageal fistula. This is a rare (it only occurs in less than $1 \%$ of patients after RF procedures) but serious condition that can potentially result in a patient's death [5]. Atrial-esophageal fistula is caused by thermal damage to the esophagus; however, the exact mechanism of injury is not yet well understood [6]. Studies have been conducted, both experimentally and computationally, to further understand the heat transfer in RF ablation, but there are still many unknowns regarding how thermal damage to the esophagus occurs and how it can be prevented.

\section{LITERATURE REVIEW}

The first RFA study [7] employed a 2-D axially symmetric mathematical model but no computational simulations were conducted. The first models with a geometric domain that discretized the equations for voltage and temperature distribution (that were solved by a computer program) were based on 2-D geometry [8-10]. Experiments were conducted in these studies to determine convective heat transfer coefficients in the heart as well as the heart tissue properties [8-10]. Much of the subsequent research drew heavily on this initial work for geometry, tissue properties and convection coefficients for blood flow in the heart, notably Berjano and co-workers [4, 1114]. A proven computational model strategy has emerged from the research and several aspects of that model will be considered in detail here.

\section{A. Geometry}

The geometry of these models varies, as each study considers a different situation, or models a specific experiment. Most studies model the catheter as a cylinder with a hemispherical tip. The catheter diameter is specified in French (where $1 \mathrm{Fr}=1 / 3 \mathrm{~mm}$ ), ranging from 6-8 Fr, whilst the length ranges from $3-5 \mathrm{~mm}$. The catheter is modelled partially embedded into the tissue in almost every scenario, with only one study accounting for tissue deformation [15]. Above the tissue is the blood, which is sometimes modelled and sometimes excluded (see blood modeling section for more information). The overall size of the model is determined through a Cauchy independence test which involves seeking the smallest domain possible without affecting the results. For most studies, the overall size of the domain is less than a cube with $80 \mathrm{~mm}$ sides. Studies often use axial symmetry or face symmetry. Axial 
symmetry cases cannot model blood flow and must use convection coefficients, but computation time is reduced significantly.

Most studies consider the orientation of the catheter to be perpendicular or parallel to the tissue. One study has considered the effect of catheter angle on lesion size and found that the parallel orientation produces smaller lesions [16], and so, for the purposes of finding the maximal lesion size, the perpendicular orientation is recommended. When the catheter is perpendicular to the tissue, the depth of the catheter into the tissue must be considered. In most studies, the depth used comes from previous research or is obtained from experimental measurements of ablated tissue. Currently, there is one experimental study relating catheter force to tissue depth [17].

The final parameter that varies in the model geometries is tissue thickness. In humans, the myocardium thickness in the posterior wall of left atrium is not uniform and varies from patient to patient. Therefore, it would be expected to vary in models as well. Many studies use very thick and unrealistic myocardial thicknesses to ensure that the bottom boundary condition does not affect the lesion size. For example, one recent study uses $20 \mathrm{~mm}$ thickness [4] in place of modelling multiple layers of tissue which would complicate the model. Some studies do include other layers, such as fat and esophageal layers, instead of using an unrealistically thick myocardium thickness $[5,18]$.

\section{B. Governing Equations}

The governing equations are consistent across all of the literature. The Bioheat equation is used in conjunction with Laplace's equation to take into account joule heating in the tissue and to give accurate temperature distributions. Fluid flow (if included) is governed by the continuity equation and the laminar, incompressible Navier-Stokes equations. For several studies, the vaporization of tissue is included. To account for the vaporization, the latent heat is included as the latent heat of water and there are different properties for the liquid and gaseous forms of tissue. It has been noted by some researchers that this method is inaccurate and should not be used. Instead, the focus should be on keeping the temperatures below the vaporization temperature of water, $100^{\circ} \mathrm{C}$ [19]. For most studies heat loss caused by blood perfusion and the metabolic heat generation terms in the Bioheat equation are negligible and, hence, neglected [7].

\section{Catheter Boundary Conditions}

The boundary conditions on the tip of the electrode vary in the present models. The types of boundary conditions used are: constant temperature, constant temperature through voltage regulation, constant voltage, constant power through voltage regulation, and constant maximum tissue temperature through voltage regulation. In addition to these five boundary conditions, the catheter may be modeled with or without saline irrigation (depending on the type of catheter modelled). The irrigated catheters slightly change the geometry of the model but leave most parameters and boundary conditions unchanged as they simply add another inlet to the blood flow. Irrigated catheters are more clinically relevant at the present time [4].
Applying a constant voltage boundary condition to model a constant power ablation is shown to be an acceptable approximation with a relatively high accuracy. However, if constant voltage is used, then other parameters are in a state of flux which affects how the lesion develops over time. For this reason, a constant voltage boundary condition is not always used. The next most common boundary condition is using voltage regulation to hold either temperature or power constant. In order to model these boundary conditions, the voltage applied at the active electrode must be regulated through a control feedback loop. These changing boundary conditions are implemented through the use of software modifications such as MATLAB integration into the program ANSYS, user defined functions (udfs) within the program, or other program macros [4].

\section{Modelling The Effect of Blood}

Most of the modelling research uses experimental measurement to determine the convective coefficient in the myocardium. Then, they apply a convective boundary condition to the tissue surface rather than modelling actual blood flow. The main methods to model blood flow in RFA include: using convection coefficients excluding the blood from the model; using convective coefficients with the blood included in the model (stagnant); the same as the previous method but with temperature-dependent blood conductivity; and, finally, modelling the blood flow itself. Interestingly, no study has yet included pulsating or turbulent blood flow (or both) in their model. The studies that use convection coefficients do take into account these parameters, often averaging the high and low turbulent blood flow conditions [8]. Most papers use a similar method. In future work, it is recommended to investigate a more accurate representation of the actual blood flow in the heart by studying the effects of pulsatile flow and turbulence.

\section{E. Other Boundary Conditions}

For all models, the boundary conditions other than the electrode and blood boundary condition are the same. There is a specified temperature at all surfaces far away from the electrode, which varies from $37.5^{\circ} \mathrm{C}$ to $36^{\circ} \mathrm{C}$. There, zero electrical flux is specified on all the walls and surfaces. The bottom surface is the only one that is different with a specified temperature and a specified voltage of $0 \mathrm{~V}$ to mimic the effect of the grounding pad. All of the studies for RFA in the heart that were reviewed here, used these boundary conditions except for one, which studied the effect of using a cooling balloon in the esophagus to prevent thermal injury [5]. This study applied a convective boundary condition to the bottom face (where the esophagus is located) rather than a prescribed temperature. This is the only study of that kind and is a potential method of preventing atrial-esophageal fistula. The aspect of esophageal cooling is one that needs further investigation. Overall, one may trust that these standard boundary conditions are justified in their use and produce accurate lesion sizes.

\section{F. Material Properties}

The material properties, like density, viscosity and thermal conductivity, used in these models represent one of the greatest differences between the different models [20]. Tissue properties are very variable and inconsistent and so any material property 
introduces error in the results. There have been several studies examining the effect of material properties on the results obtained [21-25], including changing thermal and electrical conductivity as well as determining how those conductivities should be changed as temperature changes in the model. Material properties, such as myocardial density, and blood properties, including density, viscosity, electrical conductivity, and thermal conductivity, are important, but they can be taken from previous studies or reliable sources as they do not greatly affect lesion size [22]. Some models have also included tissue vaporization while others have ignored it, or limited the tissue temperatures to below the vaporization point of $100^{\circ} \mathrm{C}$. In two recent cardiac studies $[4,26]$ the vaporization is taken into account with the following equation.

$$
\frac{\partial(\rho h)}{\partial t}=\frac{\partial T}{\partial t} \cdot\left\{\begin{array}{cr}
\rho_{l} c_{l}, & 0 \leq T \leq 99^{\circ} \mathrm{C} \\
H_{f g} C, & 99<T \leq 100^{\circ} \mathrm{C} \\
\rho_{g} c_{g}, & T>100^{\circ} \mathrm{C}
\end{array}\right.
$$

$\rho_{\mathrm{i}}=$ density of tissue phase $\left(\mathrm{kgm}^{-3}\right)$

$\mathrm{c}_{\mathrm{i}}=$ specific heat $\left(\mathrm{Jkg}^{-1} \mathrm{~K}^{-1}\right)$

$\mathrm{H}_{\mathrm{fg}}=$ latent heat $\left(\mathrm{Jkg}^{-1}\right)$

$\mathrm{C}=$ tissue water content $(\%)$

$\mathrm{i}=l$ corresponds to the liquid tissue phase

$\mathrm{i}=g$ corresponds to the gaseous tissue phase

It was found that the equations chosen to model material properties do not affect lesion diameter significantly [22]. To achieve accurate results the thermal and electrical tissue conductivities must be temperature-dependent, following one of the equations in the in Table 1 in [22]. The tissue properties vary across studies, but the effect on the results appears to be minimal so long as the tissue properties are made temperaturedependent in some way [22]. A 3.5\% maximum difference in final lesion size was found between identical models with different material properties [22].

\section{G. Lesion Size Measurement}

In the beginning of RFA modelling the Arrhenius thermal damage equation was used to calculate the lesion size [7,9,27]. This equation suggests, for heart tissue, a thermal damage temperature of greater than $48.9^{\circ} \mathrm{C}$ [28]. After this general temperature limit had been established and confirmed [7,9,27], most papers merely used an arbitrarily selected isotherm to measure lesion size because of its simplicity. The majority of studies use a $50^{\circ} \mathrm{C}$ isotherm. The $50^{\circ} \mathrm{C}$ isotherm has been tested many times and is shown to be a consistent and accurate measurement for the lesion size created.

Having reviewed the literature concerning RFA modelling, the present work seeks to use the best available strategy to examine the influence of key parameters on the performance of a non-irrigated RFA catheter and investigate the effect of esophageal cooling.

\section{Non-IRrigated CATHETER Ablation Model}

In the present work an improved model was created to examine effect of esophageal cooling. The geometry of González-Suárez and Berjano [26] was replicated, the same governing equations and material properties were used and, at first, all the same boundary conditions were implemented so

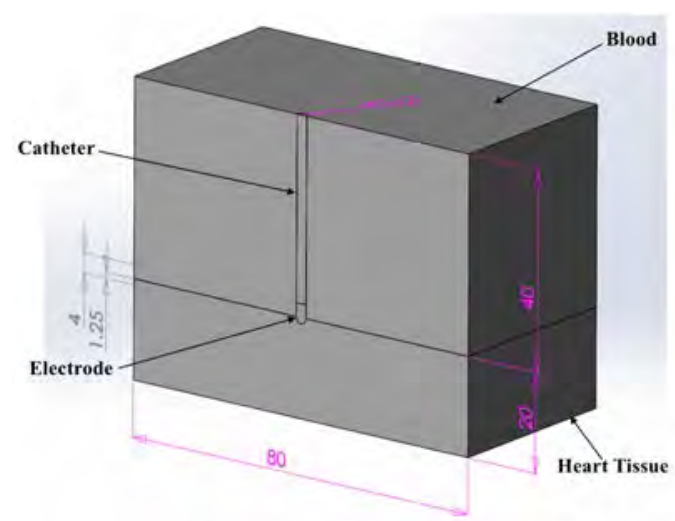

Figure 1. Geometry for non-irrigated catheter model. Dimension not shown is the depth which is $40 \mathrm{~mm}$.

that the present model could be validated. Following this the effect of convective cooling was studied on the bottom face. This study was then repeated with subsequent models that had the same geometry as the first, except for a smaller myocardial thickness to better represent the real-life situation.

\section{A. Geometry}

The geometry, shown in Fig. 1, consists of a $7 \mathrm{Fr}-4 \mathrm{~mm}$ (d $=2.33 \mathrm{~mm}, \mathrm{~L}=4 \mathrm{~mm}$ ) cylindrical catheter with a hemispherical tip embedded $1.25 \mathrm{~mm}$ into myocardial tissue whose thickness, $\mathrm{H}$, was varied from $20 \mathrm{~mm}$ to $10 \mathrm{~mm}$ and then $5 \mathrm{~mm}$. The overall domain of the model was the same as González-Suárez and Berjano's [26] who obtained those dimensions by means of a convergence test. Symmetry was used to reduce computation time. The model by González-Suárez and Berjano [26] included a thermistor embedded in the catheter to measure the overall temperature of the catheter and regulate the voltage to maintain constant temperature, but this was excluded from the present model as it was an unnecessary complication.

\section{B. Governing Equations and Material Properties}

The governing equations for this model are as discussed above and the material properties in Table I were used. For this tissue, $\mathrm{H}_{\mathrm{fg}}=2.162 \mathrm{Jm}^{-3}, \mathrm{C}=75 \%$, and $\rho_{\mathrm{l}}=958 \mathrm{kgm}^{-3}$ (at $100^{\circ} \mathrm{C}$ ) [26], so the vaporization latent heat is $2257 \mathrm{kJkg}^{-1}$. The equations were implemented in ANSYS Fluent v18.2 by udfs. The viscosity of blood was set to $0.0021 \mathrm{kgm}^{-1} \mathrm{~s}^{-1}$.

TABLE I. MATERIAL PROPERTIES USED IN NON-IRRIGATED MODEL

\begin{tabular}{|c|c|c|c|c|}
\hline & $\begin{array}{c}\text { Density, } \rho \\
\left(\mathrm{kgm}^{-3}\right)\end{array}$ & $\begin{array}{c}\text { Specific } \\
\text { Heat, c } \\
\left(\mathrm{Jkg}^{-1} \mathbf{K}^{-1}\right) \\
\end{array}$ & $\begin{array}{c}\text { Thermal } \\
\text { Cond., k } \\
\left(\mathbf{W m}^{-1} \mathbf{K}^{-1}\right)\end{array}$ & $\begin{array}{c}\text { Elec. } \\
\text { Cond., } \sigma \\
\left(\mathrm{Sm}^{-1}\right) \\
\end{array}$ \\
\hline Catheter & 70 & 1045 & 0.026 & $1 \times 10^{-5}$ \\
\hline Electrode & 21500 & 132 & 71 & $4.6 \times 10^{6}$ \\
\hline Blood & 1000 & 4180 & 0.541 & 0.667 \\
\hline Tissue & $\begin{array}{c}l-1060 \\
g-370.44\end{array}$ & $\begin{array}{c}l-3111 \\
g-2155.92\end{array}$ & $\begin{array}{c}\text { See } \\
\text { Equation } 2\end{array}$ & $\begin{array}{c}\text { See } \\
\text { Equation } 3\end{array}$ \\
\hline
\end{tabular}

$$
\begin{gathered}
k(T)=\left\{\begin{array}{cc}
0.531+0.0012(T-37), & 0<T \leq 100^{\circ} \mathrm{C} \\
0.606, & T>100{ }^{\circ} \mathrm{C}
\end{array}\right. \\
\sigma(T)=\left\{\begin{array}{cc}
0.541 e^{0.015(T-37),} & 0<T \leq 100^{\circ} \mathrm{C} \\
1.3-0.274(T-100), & 100<T \leq 105^{\circ} \mathrm{C} \\
1.371 \times 10^{-4}, & T>105^{\circ} \mathrm{C}
\end{array}\right.
\end{gathered}
$$


TABLE II. COMPARISON OF RESULTS OBTAINED WITH VARIOUS BOTTOM BOUNDARY CONDITIONS

\begin{tabular}{|c|c|c|c|c|c|}
\hline $\begin{array}{c}\text { Boundary } \\
\text { Condition On } \\
\text { Bottom Face }\end{array}$ & $\begin{array}{l}\text { Lesion } \\
\text { Depth } \\
(\mathrm{mm})\end{array}$ & $\begin{array}{l}\text { Lesion } \\
\text { Width } \\
(\mathrm{mm})\end{array}$ & $\begin{array}{l}\text { Max } \\
\text { Blood } \\
\text { Temp } \\
\left({ }^{\circ} \mathbf{C}\right) \\
\end{array}$ & $\begin{array}{c}\text { Max } \\
\text { Tissue } \\
\text { Temp } \\
\left({ }^{\circ} \mathbf{C}\right) \\
\end{array}$ & $\begin{array}{c}\text { Bottom } \\
\text { Boundary } \\
\text { Temp } \\
\left({ }^{\circ} \mathrm{C}\right) \\
\end{array}$ \\
\hline \multicolumn{6}{|c|}{$20 \mathrm{~mm}$ Thick Tissue } \\
\hline$T=37^{\circ} \mathrm{C}$ & 7.53 & 11.18 & 65.65 & 99.85 & 37.00 \\
\hline $\begin{array}{c}T=15^{\circ} \mathrm{C}, h=1000 \\
\mathrm{Wm}^{-2} \mathrm{~K}^{-1}\end{array}$ & 7.47 & 11.10 & 68.55 & 99.95 & 17.37 \\
\hline $\begin{array}{c}T=15^{\circ} \mathrm{C} \\
h=2000 \mathrm{Wm}^{-2} \mathrm{~K}^{-1}\end{array}$ & 7.44 & 11.11 & 64.15 & 99.95 & 16.28 \\
\hline \multicolumn{6}{|c|}{$10 \mathrm{~mm}$ Thick Tissue } \\
\hline $\mathrm{T}=37^{\circ} \mathrm{C}$ & 8.012 & 12.01 & 65.35 & 104.45 & 37.00 \\
\hline $\begin{array}{c}T=15^{\circ} \mathrm{C}, h=1000 \\
W^{-2} K^{-1}\end{array}$ & 6.767 & 11.24 & 64.25 & 101.95 & 20.38 \\
\hline $\begin{array}{c}T=15^{\circ} \mathrm{C} \\
h=2000 \mathrm{Wm}^{-2} \mathrm{~K}^{-1}\end{array}$ & 6.665 & 11.15 & 64.15 & 101.75 & 17.93 \\
\hline \multicolumn{6}{|c|}{$5 \mathrm{~mm}$ Thick Tissue } \\
\hline$T=37^{\circ} \mathrm{C}$ & 4.54 & 10.20 & 66.95 & 112.35 & 37.00 \\
\hline $\begin{array}{c}T=5^{\circ} \mathrm{C} \\
h=2000 \mathrm{Wm}^{-2} \mathrm{~K}^{-1}\end{array}$ & 4.07 & 8.54 & 65.35 & 116.35 & 16.78 \\
\hline $\begin{array}{c}T=5^{\circ} \mathrm{C}, \\
h=20,000 \mathrm{Wm}^{-2} \mathrm{~K}^{-1}\end{array}$ & 3.85 & 8.29 & 65.75 & 127.55 & 6.95 \\
\hline $\begin{array}{c}T=-5^{\circ} \mathrm{C} \\
h=2000 \mathrm{Wm}^{-2} \mathrm{~K}^{-1}\end{array}$ & 3.99 & 8.10 & 60.15 & 116.85 & 8.85 \\
\hline $\begin{array}{c}T=-5^{\circ} \mathrm{C}, \\
h=20,000 \mathrm{Wm}^{-2} K^{-1}\end{array}$ & 3.91 & 7.23 & 55.95 & 98.25 & -2.04 \\
\hline
\end{tabular}

TABLE III. COMPARING PRESENT RESUltS TO PREVIOUS EXPERIMENTAL WORK BY GONZÁLEZ-SUÁREZ AND BERJANO [26], 20 MM THICK TISSUE

\begin{tabular}{|c|c|c|c|}
\hline & $\begin{array}{c}\text { Previous } \\
\text { Work [26] }\end{array}$ & $\begin{array}{c}\text { Present } \\
\text { Results }\end{array}$ & $\begin{array}{c}\text { Percent } \\
\text { Difference (\%) }\end{array}$ \\
\hline Depth (mm) & 7.53 & 7.53 & 0 \\
\hline Width (mm) & 11.92 & 11.18 & 6.41 \\
\hline $\mathbf{T}_{\text {max,tissue }}\left({ }^{\circ} \mathbf{C}\right)$ & 102.4 & 99.85 & 2.52 \\
\hline $\mathbf{T}_{\text {max,blood }}\left({ }^{\circ} \mathbf{C}\right)$ & 79.9 & 65.65 & 19.58 \\
\hline
\end{tabular}

\section{Boundary Conditions}

The voltage boundary condition on the catheter was set to $25 \mathrm{~V}$ for a RF duration of $60 \mathrm{~s}$. The bottom face had a specified voltage of $0 \mathrm{~V}$. All remaining walls and interfaces were specified with zero electrical flux. Thermal boundary conditions were only specified on outside walls and on the bottom face. All outside walls were set at $37^{\circ} \mathrm{C}$. The bottom face was tested with a variety of boundary conditions including a constant temperature of $37^{\circ} \mathrm{C}$ and then various convective boundary conditions. All walls for the fluid domain were noslip and coupled with the adjoining material. The blood had a perpendicular laminar flow with an inlet velocity of $8.5 \mathrm{~cm} \cdot \mathrm{s}^{-1}$, and a zero-pressure outlet.

\section{Results}

Mesh convergence and time convergence were performed until the results were independent of both. The final step size was $0.05 \mathrm{~s}$ for $60 \mathrm{~s}$ and the final mesh had 419,362 elements with maximum and minimum face sizes of $1 \mathrm{~mm}$ and $4.99 \times 10^{-2} \mathrm{~mm}$, respectively. The lesion size was calculated using the $50^{\circ} \mathrm{C}$ isotherm for all models. Lesion depth is the maximum depth from the top of the tissue reached by the lesion and lesion width is the width of the isotherm at its widest point. All studies were run for sixty seconds. The key results can be seen in Table II and Fig. 2.

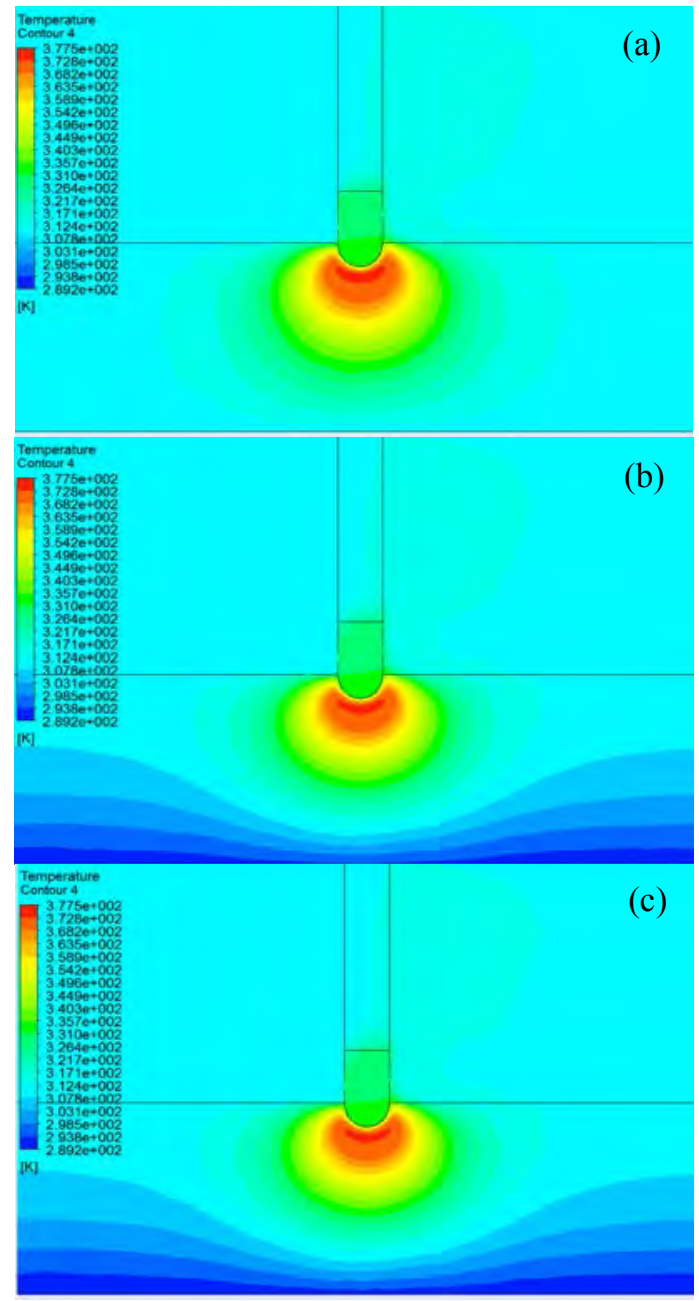

Figure 2. Results for $10 \mathrm{~mm}$ thick tissue. (a) $\mathrm{T}=37^{\circ} \mathrm{C}$ (b) $\mathrm{T}=15^{\circ} \mathrm{C}, \mathrm{h}=$ $1000 \mathrm{Wm}^{-2} \mathrm{~K}^{-1}$ (c) $\mathrm{T}=15^{\circ} \mathrm{C}, \mathrm{h}=2000 \mathrm{Wm}^{-2} \mathrm{~K}^{-1}$

For the $20 \mathrm{~mm}$ thick tissue the cooling had very little impact on lesion size due to the thickness of the tissue. The $37^{\circ} \mathrm{C}$ case was compared with that in González-Suárez and Berjano [26] for validation purposes in Table III. Maximal blood temperature is the only value that is significantly different, which is most likely due to a coarser mesh in the blood domain in the present model. The $10 \mathrm{~mm}$ thick tissue allowed for cooling to be much more effective in reducing lesion size. The lesion depth was reduced by a maximum of $18.4 \%$, the maximum tissue temperature was reduced by about $3^{\circ} \mathrm{C}$, and the bottom boundary temperature was reduced by $69.4 \%$. The $5 \mathrm{~mm}$ tissue was affected the most by cooling as the lesion size was decreased by as much as $16.4 \%$.

\section{COnclusions And Summary}

Previous models are, for the most part, experimentally validated; however, they do have some shortcomings. Most notably, the force applied to the catheter and the depth are only related in one published study. Another shortcoming is the lack of accurate blood flow modelling. To obtain an accurate representation of the real system, blood flow should be modelled as pulsatile and turbulent, and not by constant laminar flow or a convection coefficient. 
The computational results match previous data which have been verified using experimental data. The $5 \mathrm{~mm}$ thickness is representative of the total distance from catheter tip to inner esophageal wall [18] meaning the esophagus tissue and heart tissue layers were combined in this model. In future studies these two layers could be separated and have distinct properties as has been done in previous models [5]. The non-irrigated model demonstrates that regardless of tissue thickness cooling the esophageal wall has less than a $19 \%$ effect on the depth of the lesion, and as much as a $13.4 \%$ lowering of the temperature on the esophageal wall. This means that cooling on the esophageal wall can be used to effectively lower the damage to the esophagus without adversely affecting the procedure when a non-irrigated catheter is used. This model has temperatures that are too cool and would damage the esophagus, but the temperature of the cooling fluid or the convection coefficient can be easily changed. These results are in agreement with Berjano and Hornero [5], who also examined the effect of esophageal cooling. The present study demonstrates that cooling can be effectively added to the esophagus without comprising the RFA procedure.

\section{ACKNOWLEDGMENT}

This work was funded by Baylis Medical, the Natural Sciences and Engineering Research Council of Canada and MITACS. The guidance and support of Gareth Davies and Amanda Hartley of Baylis Medical is greatly appreciated.

\section{REFERENCES}

[1] Heart \& Stroke Foundation, "Atrial fibrilation." [Online] Available: http://www.heartandstroke.com/site/c.ikIQLcMWJtE/b.5052135/ k.2C86/Heart_disease_Atrial_fibrillation.htm. Accessed: Dec. 1, 2017. [2] S. Mohanty et. al, "Outcomes of atrioesophageal fistula following catheter ablation of atrial fibrillation treated with surgical repair versus esophageal stenting," J. Cardio. Electrophysiology, vol. 25, no. 6, pp. 579-584, 2014.

[3] C. Townsend, D. Beauchamp, M. Evers, and L. Kenneth, Sabiston Textbook of Surgery: The Biological Basis of Modern Surgical Practice. Elsevier Saunders, Philadelphia, pp. 236, 2012.

[4] A. González-Suárez, E. Berjano, J. Guerra, and L. GerardoGiorda, "Computational modeling of open-irrigated electrodes for radiofrequency cardiac ablation including blood motion-saline flow interaction," in PLoS ONE, vol. 11(3), pp. 1-18, 2016.

[5] E. Berjano, and F. Hornero, "A cooled intraesophageal balloon to prevent thermal injury during endocardial surgical radiofrequency ablation of the left Atrium: a finite element study," in Physics in Medicine and Biology, vol. 50(20), pp. N269-N279, 2005.

[6] J. Pérez, A. D’Avila, A. Aryana, and E. Berjano, "Electrical and thermal effects of esophageal temperature probes on radiofrequency catheter ablation of atrial fibrillation: results from a computational modeling study," J. Cardio. Electrophysiology, vol. 26, no.5, pp. 556-564, 2015.

[7] D. Haines, and D. Watson, "Tissue heating during radiofrequency catheter ablation: a thermodynamic model and observations in isolated perfused and superfused canine right ventricular free wall," Pacing and Clinical Electrophysiology, vol. 12, no.6, pp. 962-976, 1989.

[8] S. Labonté, "A theoretical study of radio-frequency ablation of the myocardium," Doctoral Thesis. University of Ottawa, 1992.

[9] S. Labonté, "A computer simulation of radio-frequency ablation of the endocardium," in IEEE Trans. Biomed. Eng., vol. 41(9), pp. 883-890, 1994

[10] S. Labonté, and S. Stuchly, "Radio-frequency ablation of the myocardium: a numerical model," from the Annual International Conference of the IEEE Eng. Medicine and Biology Society, vol. 13, no.2, pp. 619-620, 1991.
[11] J. Lequerica, E. Berjano, M. Herrero, and F. Hornero, "Reliability assessment of a cooled intraesophageal balloon to prevent thermal injury during RF cardiac ablation: an agar phantom study," J. Cardiovascular Electrophysiology, vol. 19, no.11, pp. 1188-1193, 2008.

[12] F. Burdío et. al, "Research and development of a new RF-assisted device for bloodless rapid transection of the liver: computational modeling and in vivo experiments," Biomed. Eng. Online, vol. 8, no. 6, 2009.

[13] J. Lopez, M. Rivera, and E. Berjano, "Analytical transient-time solution for temperature in non perfused tissue during radiofrequency ablation," Applied Mathematical Modelling, vol. 42, pp. 618-635, 2017.

[14] A. Qadri, N. Chia, and E. Ooi, "Effects of saline volume on lesion formation during saline-infused radiofrequency ablation," Applied Mathematical Modelling, vol. 43, pp. 360-371, 2017.

[15] H. Cao, M. Speidel, J. Tsai, M. Van Lysel, V. Vorperian, and J. Webster "FEM analysis of predicting electrode-myocardium contact from RF cardiac catheter ablation system impedance," in IEEE Trans. on Biomedical Eng., vol. 49, no.6, pp. 520-526, 2002.

[16] M. Wood et. al, "Effect of electrode orientation on lesion sizes produced by irrigated radiofrequency ablation catheters," J. of Cardio.

Electrophysiology, vol. 20(11), pp. 1262-1268, 2009.

[17] R. Francés et. al, "Characterization of the impact of catheter-tissue contact force in lesion formation during cavo-tricuspid isthmus ablation in an experimental swine model," Europace, vol. 16, no.11, pp. 1679-1683, 2014.

[18] F. Hornero, and E. Berjano, "Esophageal temperature during radiofrequency-catheter ablation of left atrium: a three-dimensional computer modeling study," J. Cardio. Electrophysiology, vol. 17(4), pp. 405-410, 2006.

[19] I. Chang, "Considerations for thermal injury analysis for RF ablation devices," Open Biomedical Eng. J., vol. 4, no.2, pp. 3-12, 2010.

[20] E. Berjano, "Theoretical modeling for radiofrequency ablation: state-ofthe-art and challenges for the future," Biomed. Eng. Online, vol. 5, no. 24, 2006.

[21] D. Panescu, and J. Webster, "Effects of changes in electrical and thermal conductivities on radiofrequency lesion dimensions," from Proceedings of the IEEE International Conference of the Eng. in Medicine and Biology Society, vol. 154, no. 1, pp. 154-156, 1997.

[22] M. Trujillo, and E. Berjano, "Review of the mathematical functions used to model the temperature dependence of electrical and thermal conductivities of biological tissue in radiofrequency ablation," in International $J$. of Hyperthermia, vol. 29(6), pp. 590-597, 2013.

[23] S. Lobo et. al, "RF tumour ablation: computer simulation and mathematical modelling of the effects of electrical and thermal conductivity," International J. of Hyperthermia, vol. 21(3), pp. 199-213, 2005.

[24] S. Quallich, K. Kriege, and P. Iaizzo, "The effects of radiofrequency or cryothermal ablation on biomechanical properties of isolated human or swine cardiac tissues," in IEEE J. of Translational Eng. in Health and Medicine, vol. 4, pp. 1-5, 2015.

[25] S. Tungjitkusolmun, E. Woo, H. Cao, J. Tsai, V. Vorperian, and J. Webster, "Thermal-electrical finite element modelling for radio frequency cardiac ablation: effects of changes in myocardial properties," Medical \& Biological Eng. \& Computing, vol. 38, pp. 562-568, 2000.

[26] A. González-Suárez, and E. Berjano, "Comparative analysis of different methods of modeling the thermal effect of circulating blood flow during RF cardiac ablation," in IEEE Transactions on Biomed. Eng., vol. 63, no. 2, pp. 250-259, 2016.

[27] J. Pearce, and S. Thomen, "Numerical models of RF ablation in myocardium," from IEEE International Conference of the Eng. in Medicine and Biology Society, and Canadian Medical and Biological Eng. Conference, vol. 7, pp. 269-270, 1995.

[28] D. Haines, D. Watson, and F. Verow, "Electrode radius predicts lesion radius during radiofrequency energy heating: validation of a proposed thermodynamic model," Circulation Research, vol. 67(1), pp. 124129, 1990. 


\section{FEM Assisted Observations of Cutting Forces, Temperature and Chip Curling in Oblique Machining}

\author{
Salman Pervaiz \\ Department of Mechanical and Industrial Engineering \\ Rochester Institute of Technology - Dubai Campus \\ P.O. Box 341055 Dubai, United Arab Emirates \\ sxpcad@rit.edu
}

\author{
Sathish Kannan \\ Department of Mechanical Engineering \\ American University of Sharjah \\ P.O. Box 26666 Sharjah, United Arab Emirates \\ skannan@aus.edu
}

\begin{abstract}
It is easier to numerically model the machining process assuming the orthogonal cutting conditions, however the realistic industrial machining arrangements are closer to the oblique machining conditions. In oblique machining the cutting edge is located at a certain inclination angle resulting in a third radial force component. The inclination angle in oblique machining arrangement has a controlling influence over the chip curling and resulting plastic deformation is more complex as compared to the orthogonal machining arrangements. The current study has incorporated a threedimensional finite element model to predict the machining of Inconel 718 using SIALON cutting tool materials under oblique machining conditions. The study incorporates different cutting edge-based inclination angles and resulting simulated machining performance has been reported. The machining performance has been evaluated by observing the simulated cutting forces, cutting temperature, and chip curling.
\end{abstract}

Keywords-component; chip curling, oblique machining, FEM

\section{INTRODUCTION}

As compared to the orthogonal machining setup, oblique machining provides more realistic cutting scenario with respect to the practical application in the metal cutting sector. The predictions about the machining performance under oblique machining arrangement is more reliable and closer to the practical application. Machining performance of a cutting process can be predicted using either analytical or numerical approaches. Numerical approaches are easier to deal with complex geometries and boundary conditions but at the expense of higher computational cost and time. When it comes to the oblique machining performance prediction most of the literature is based on the analytical modelling and very limited studies are available using numerical modeling approaches especially for difficult-to-machine materials such as Inconel 718.

Several researchers have focused their research work to utilize numerical modeling techniques for the prediction of different machining performance indicators. Shaw et al. [1] initially introduced the concept about the normal rake angle. The concept was further evolved in the work conducted by researchers such as Stabler [2,3], Brown and Armarego [4], and Russell and Brown [5] etc. Luk [6] investigated the chip flow under oblique machining setup. The study was conducted using analytical solution method considering mechanistic approach. Lin et al. [7] explored the orthogonal plane strain machining theory to predict the cutting forces under oblique machining conditions. The study revealed that cutting forces can be predicted using the combined knowledge of cutting parameters, materials flow stress and thermal behavior. Arsecularatne et al. [8] investigated the tool life under oblique cutting. The study utilized the theory of tool life using cutting temperature and investigated the tool life for oblique conditions. The experimental validation of predicted results was performed using the plain carbon steel with different tool geometries and cutting conditions.

Lazoglu and Islam [9] utilized finite difference based numerical method to explore the cutting temperature related 3D simulations under oblique cutting arrangements. The predicted results were found to be in good agreement with the experimental findings. Komanduri et al. [10] investigated the influence of both normal rake and inclination angles for the oblique machining of single crystal aluminum using 3D molecular dynamics simulations. The study measured cutting force components, cutting force ratio and specific energy. The study revealed that normal rake angle has controlling influence over the cutting force components and specific cutting energy. Feng and Zang [11] studied oblique machining process using a thermo-elastic-plastic coupled finite element (FE) model. The study was conducted using high speed steel (H11) and ANSI1045 steel as cutting tool and workpiece materials respectively. The study monitored cutting forces, stresses and cutting temperatures at the cutting interface and chips. The study revealed that role of inclination angle in decreasing cutting forces is very minor.

Song et al. [12] developed a model to predict the cutting forces using the vector transformation method and basic mechanistic approach towards the friction at tool - chip interface. The study considered that friction at interface is same in the orthogonal cutting as well as with different inclination angles. The simulated results were found to be in good agreement with experimental data. Grzesik and Zak [13] investigated the surface integrity of different iron-based 
materials such as carbon, low-alloyed, stainless steel and spheroidal iron under oblique machining conditions. The study has incorporated carbide and $\mathrm{A} 12 \mathrm{O} 3-\mathrm{TiC}$ ceramic cutting tools using inclination angle of $55^{\circ}$. The study revealed the potential of oblique machining to replace conventional finish turning and grinding operations as at higher feed rates oblique turning provides higher surface finish. The study also revealed that maximum value of compressive stress was produced closer to the machined surface, and sensitive to the strain hardening exponent of the workpiece material.

Moufki et al. [14] conducted a study to develop a thermomechanical modelling approach for the oblique machining and simulated results were validated experimentally as well. The study utilized an analytical method based on materials strain rate sensitivity, strain hardening and thermal softening. The model provided predictions for the cutting forces and chip flow. The model was developed for the continuous chip conditions. The model provided predictions in close agreement with the experimental readings. Abdellaoui and Bouzid [15] developed a thermo-mechanical methodology to model the oblique machining. The study developed a computational algorithm and controlled the oblique machining parameters using a ratio of tool-chip contact length and chip ratio. The predicted results were found in good agreement with the experimental findings.

Grzesik and Zak [16] in another study performed friction quantification under oblique machining arrangements using CBN chamfered cutting tools. The study developed an analytical-empirical model of friction. The study incorporated the effect of equivalent rake face and associated equivalent rake angle. The study was conducted on spheroidal cast iron. The study revealed that at large negative rake angles, friction was highly influenced by the inclination angles. Llanos et al. [17] conducted a study where machining performance of AISI4140 steel was investigated using uncoated cemented carbide cutting tools. The study utilized finite element (FE) based model using Arbitrary Lagrangian Eulerian (ALE) formulation. The study revealed that accurate chip formation is linked with the proper identification of frictional behavior at the tool - chip interface. Pawade et al. [18] conducted an analytical study to predict the specific shear energy for Inconel 718 under oblique machining conditions. The study incorporated the Inconel 718 material behavior using Johnson-Cook (JC) material model. JC model can incorporate strain hardening, strain rate sensitivity and thermal softening. The study revealed that shear band spacing linearly increases by increasing the feed rate. The specific shear energy model also revealed that it is more sensitive to thermal softening, strain hardening and shearing strain. The predictions were found in very good agreement with the experimental findings.

It is well understood by the metal cutting community that oblique machining arrangements are more practical than the orthogonal cutting arrangements. However, it has been observed that there is very limited finite element based numerical studies available in literature when comes to the oblique machining. It is even more rare to find in literature that such numerical models are developed for difficult-to-cut materials using modern cutting tool materials. The aim of this study is to utilize a finite element (FE) based numerical approach to predict the machining performance of Inconel 718 under oblique machining conditions.

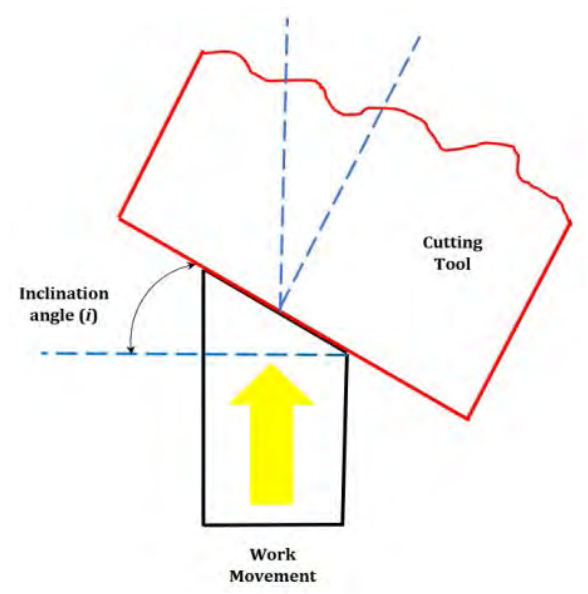

(a)

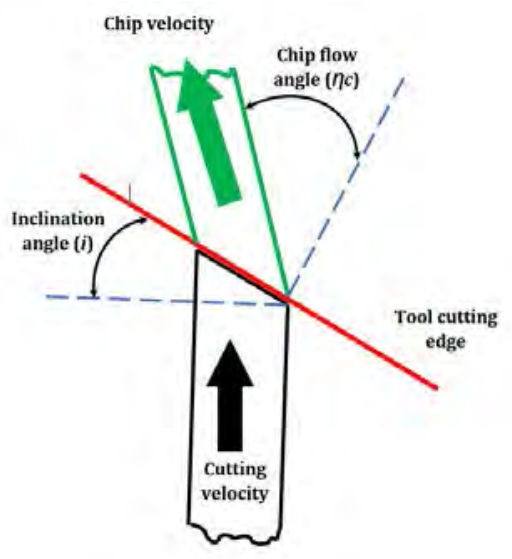

(b)

Figure 1. Schematic illustration of oblique machining (a) Inclination angle (i), and (b) Chip flow angle (Пc) (redrawn from [10], [19])

\section{OBLIQUE MACHINING VIEW}

In literature it is very frequent to find the numerical models for orthogonal cutting (2D) arrangements. Oblique cutting (3D) is different to orthogonal cutting due to the presence of inclination angle $(i)$ as shown in Fig. 1a and $1 b$.

Inclination angle $(i)$ is the angle that tool cutting edge makes with the normal to the cutting velocity vector. Due to the presence of inclination angle, chip flow is also at an angle other than the right angle as seen in orthogonal cutting case.

\section{FINITE ELEMENT MODELING}

Finite element (FE) model was prepared by using a customized software for machining application known as Thirdwave AdvantEdge [20]. In Thirdwave AdvantEdge software, chip formation is simulated by utilizing dynamic explicit lagrangian formulation. The software can execute coupled thermo-mechanical transient analysis and adaptive remeshing techniques. The software was first presented by Marusich and Ortiz [21]. Thirdwave AdvantEdge has built in 
material library for the cutting tools and workpieces. Fig. 2 represents the schematic illustration from Thirdwave AdvantEdge.

To utilize different engineering materials for different applications, there is a built-in material library in the software package. These material models can mimic the materials behavior by simulating flow stresses, strain rate sensitivity and temperature-based effects (thermal softening). The current study was based on the oblique machining (3D) setup, where cutting tool material was selected to be SIALON and workpiece material was selected to be Inconel 718. By default, AdvantEdge software utilizes power law to model the constitutive behavior of the Inconel 718. The constitutive power law can incorporate the effects related to strain hardening, strain rate sensitivity and thermal softening. The power law is mentioned in Eq. 1 .

$$
\sigma\left(\varepsilon^{P}, \dot{\varepsilon}, T\right)=g\left(\varepsilon^{P}\right) \Gamma(\dot{\varepsilon}) \Theta(T)
$$

In Eq. $1, g\left(\varepsilon^{P}\right), \Gamma(\dot{\varepsilon})$ and $\Theta(T)$ represent strain hardening, strain rate sensitivity and thermal softening respectively, and the functions are further elaborated in equations 2, 3 and 4 [22].

$$
\begin{aligned}
& \left(\varepsilon^{P}\right)=\sigma_{0}\left(1+\frac{\varepsilon^{P}}{\varepsilon_{0}^{P}}\right)^{1 / \mathrm{n}} \\
& \Gamma(\dot{\varepsilon})=\sigma_{0}\left(1+\frac{\dot{\varepsilon}}{\dot{\varepsilon}_{0}}\right)^{1 / \mathrm{m}} \\
& \Theta(T)=C_{0}+C_{1} T^{1}+\cdots C_{5} T^{5}
\end{aligned}
$$

In Eqs. 2, 3 and 4, $\sigma_{0}$ represents initial yield stress, $\varepsilon^{P}$ represents plastic strain, $\varepsilon_{0}^{P}$ represents reference plastic strain, $\dot{\varepsilon}$ represents strain rate, $\dot{\varepsilon}_{0}$ is reference plastic strain rate, $\mathrm{C} 0-\mathrm{C} 5$ represents coefficients for the polynomial fit, $\mathrm{T}$ is temperature, $\mathrm{n}$ represents strain hardening exponent, and $\mathrm{m}$ represents strain rate sensitivity coefficient [20].

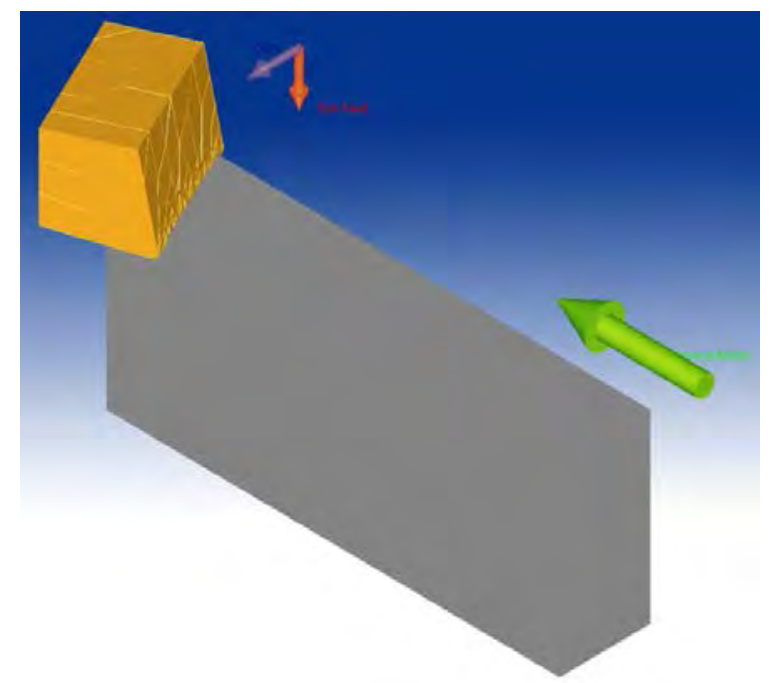

Figure 2. Schematic illustration of oblique machining in Thirdwave AdvantEdge
TABLE 1. PARAMETERS USED FOR NUMERICAL SIMULATIONS.

\begin{tabular}{|lc|}
\hline Simulation Parameters & Values \\
\hline Cutting Tools & SAILON \\
Workpiece Materials & Inconel 718 \\
Normal Rake angle $\left(^{\circ}\right)$ & 10 \\
Cutting Speed $(\mathrm{m} / \mathrm{min})$ & 300 \\
Feed rate $(\mathrm{mm} / \mathrm{rev})$ & 0.15 \\
Depth of Cut $(\mathrm{mm})$ & 1 \\
Length of Cut $(\mathrm{mm})$ & 3 \\
Friction Co-efficient & 0.5 \\
Lead angle $\left({ }^{\circ}\right)$ & 0 \\
Initial Temperature $\left(^{\circ}\right)$ & 20 \\
Relief Angle $\left({ }^{\circ}\right)$ & 5 \\
Inclination Angle $\left(^{\circ}\right)$ & $0,5,10,15,20,25$ \\
Cutting Environment & Dry \\
\hline
\end{tabular}

Like other FE codes, AdvantEdge software employs a damage model to integrate damage into the material model. The damage function D is stated in Eq. 5 [20].

$$
D=\sum_{i} \frac{\Delta \varepsilon_{i}^{p}}{\varepsilon_{f_{i}}^{p}}
$$

To have a precise cutting simulation, it is very important that proper friction should be modelled at the cutting tool-chip interface. In Thirdwave AdvantEdge friction at tool-chip interface is simulated using Coulomb law as represented in the Eq. 6 [20]. Coulomb law associates shear stresses by taking them proportional to the normal stresses and constant of proportionality is frictional coefficient $(\mu)$.

$$
\tau=\mu \sigma_{n}
$$

Selection of frictional constant is a difficult task because of complex sliding behavior at tool-chip interface. It is also reported that chip hardness is different and generally harder than the workpiece hardness, and hardness variation can lead to variation in friction coefficient [23,24]. Table 1 provides the information about the cutting parameters utilized for numerical simulations. In the current study the numerical simulations were limited to five different values of inclination angles ranging from $0-25^{\circ}$. The $0^{\circ}$ inclination angle leads to the orthogonal setup and acts like a reference for other values of inclination angles.

\section{SimUlated RESUlts AND Discussion}

The 3D simulated outcomes were studied for the different machining performance indicators such as cutting, thrust and oblique force components, cutting temperature and chip flow.

\section{A. Simulated Cutting Forces}

Cutting force components are very important machining performance indicators. These components have very significant role as they reveal the power consumption and vibrational effects in the machining process. They also play an important role towards the machine tool construction and fixture designing/ requirements. In this sub-section, simulated cutting forces at different inclination angles ranging from $0^{\circ}-$ $25^{\circ}$ were compared. The sample cutting force signals from the Thirdwave AdvantEdge softwares have been reported in Figures $3 \mathrm{a}$ and $3 \mathrm{~b}$. 


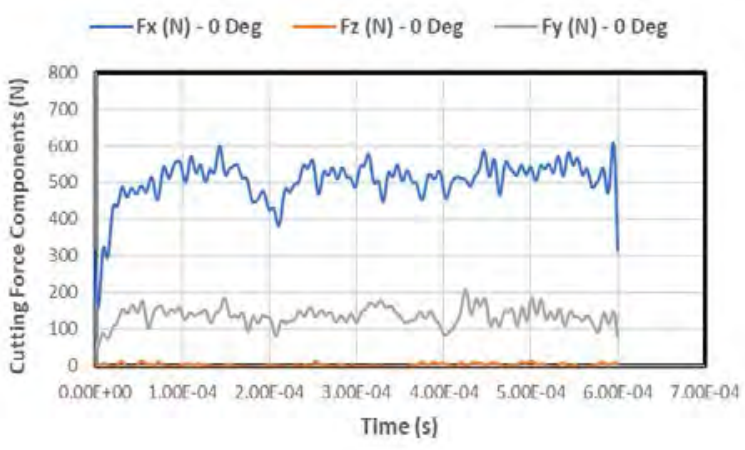

(a)

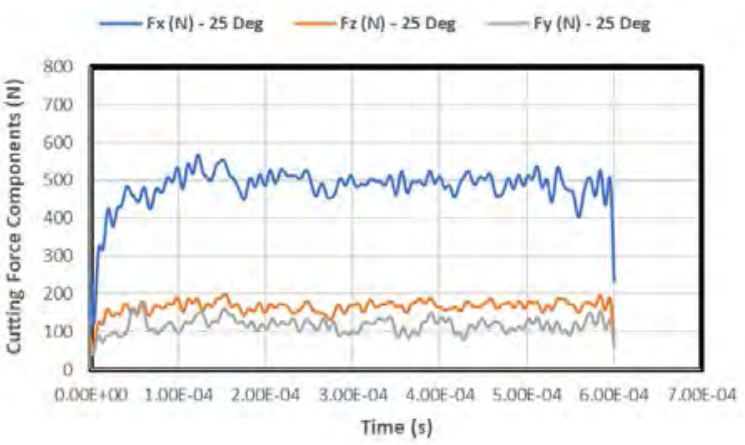

(b)

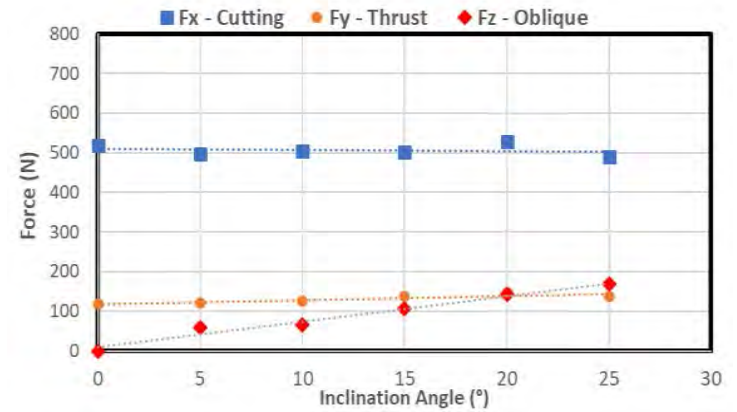

(c)

Figure 3. Simulated cutting force components (a) $0^{\circ}$ Inclination Angle, (b) $25^{\circ}$ Inclination Angle, (c) Simulated average cutting force components, $0^{\circ}-25^{\circ}$ inclination angles

The cutting force trends for all three cutting force components at all inclination angles have been reported in Figure $3 \mathrm{c}$. It can be observed that minor fluctuation has been reported for the cutting and thrust force components. The Fzoblique force component was majorly affected by the inclination angle.

\section{B. Simulated Cutting Temperature}

Cutting temperature has been attributed as one of the major machining performance indicators in the metal cutting sector. It is the deciding factor towards the cutting tool life, and many wear mechanisms such as diffusion and adhesion are linked with the cutting temperature. The sample cutting temperature signals from the Thirdwave AdvantEdge softwares have been reported in Figures $4 \mathrm{a}$ and $4 \mathrm{~b}$.
The average cutting temperature trends for all inclination angles has been reported in Figure 4c. The trend reveals that cutting temperature decreases slightly by increasing inclination angle.

\section{Simulated Chip Flow}

Simulated chip flow is another important machining parameter that has an important role in overall machining performance. Simulated results of chip flow for different inclination angles $0^{\circ}-25^{\circ}$ have been reported in Figures 5 and 6. Simulated chip flow observations revealed that increasing the inclination angles results in increasing the chip flow angle $\left(\prod_{\mathrm{c}}\right)$ as shown in Figure 6.

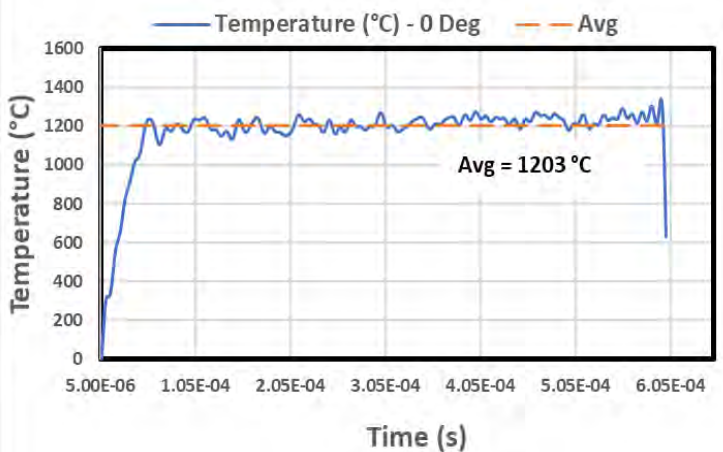

(a)

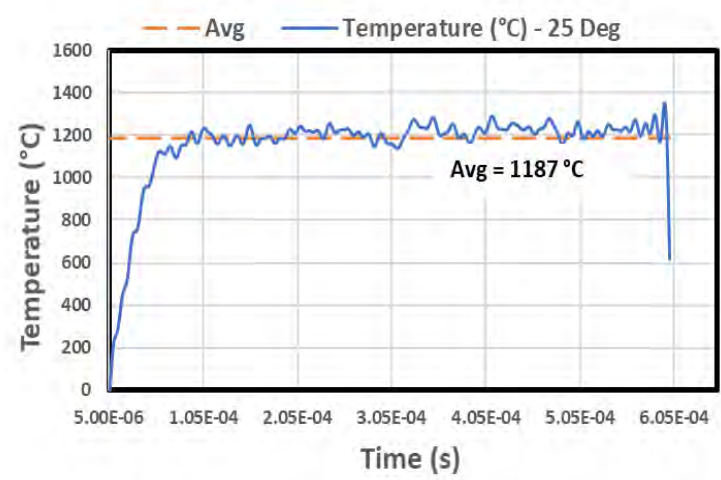

(b)

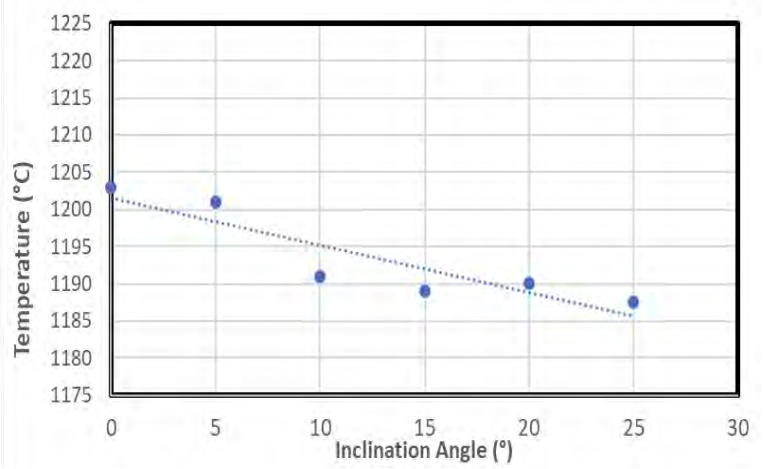

Figure 4. Simulated cutting temperatures (a) $0^{\circ}$ Inclination Angle, (b) $25^{\circ}$ Inclination Angle, (c) Simulated average cutting temperatures $0^{\circ}-25^{\circ}$ inclination angles 


\section{CONCLUSIONS}

The conclusions drawn from this study have been reported as under.

Inclination angle has negligible influence over the cutting force component which represents that it has negligible role towards power consumption. Thrust force component was found little sensitive to the inclination angle. Oblique force component was highly influenced by the inclination angle. The results were found in agreement with experimental and analytical studies present in the available literature. The cutting temperature slightly decreased by the introduction of inclination angles.

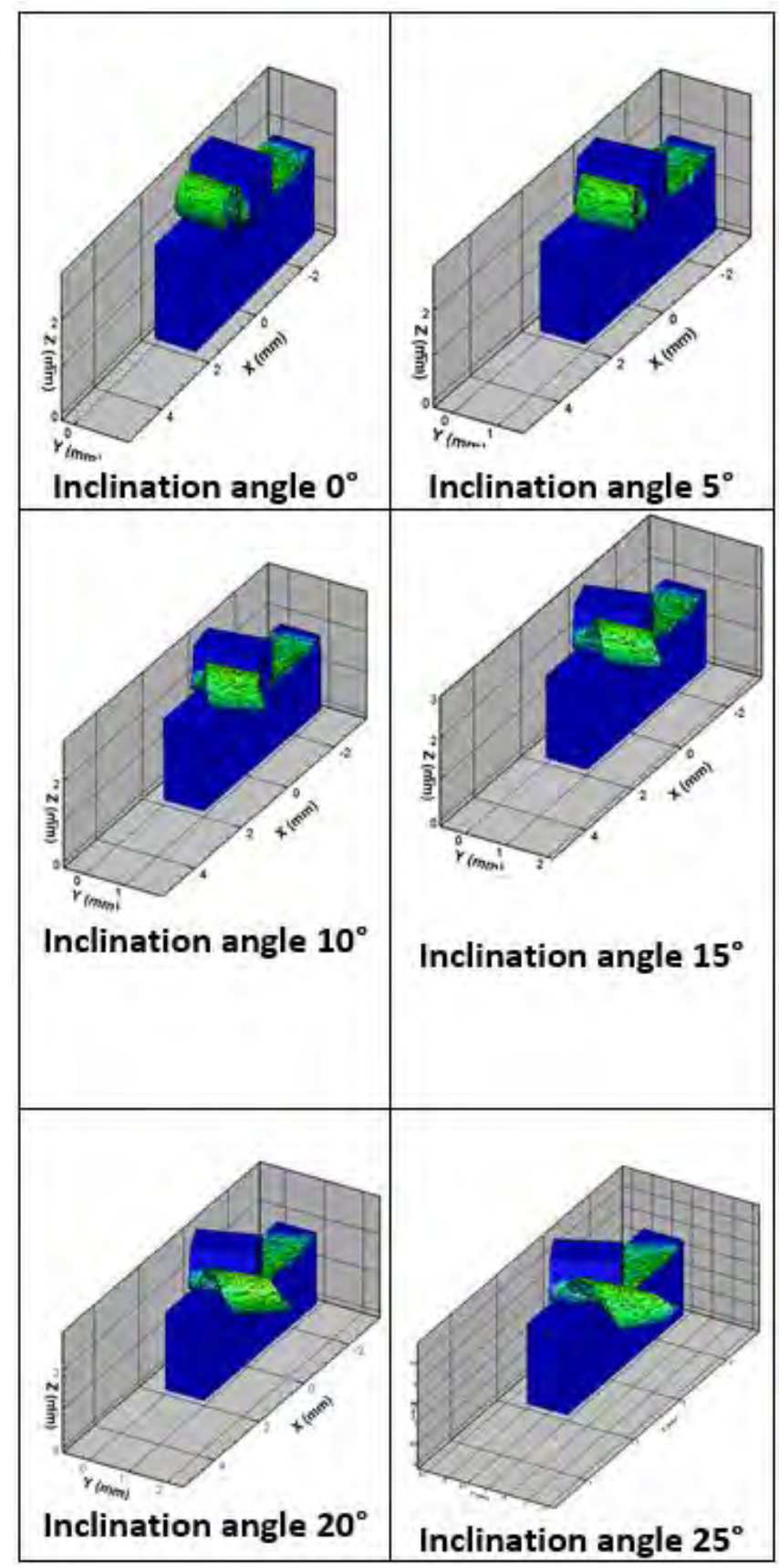

Figure 5. Simulations of chip flow, $0^{\circ}-25^{\circ}$ inclination angles

\section{ACKNOWLEDGEMENTS}

Authors sincerely acknowledge the financial support provided by Dubai Silicon Oasis Authority (DSOA) and Rochester Institute of Technology - Dubai (RIT-D).

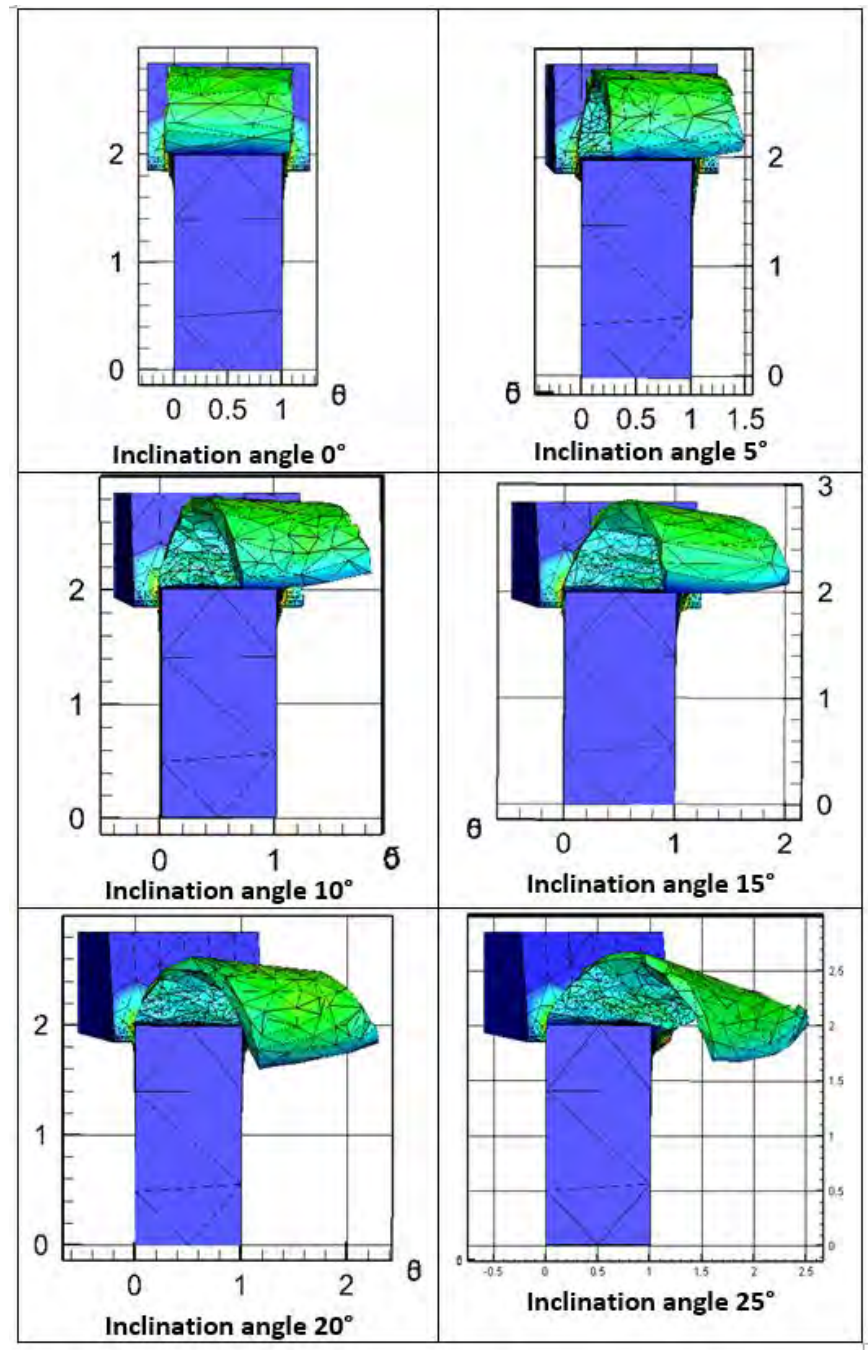

Figure 6 . Front view of chip flow, $0^{\circ}-25^{\circ}$ inclination angles

\section{REFERENCES}

[1] M. C. Shaw, N. H. Cook, and P. A. Smith, "The mechanics of three dimensional cutting operations," Trans. ASME, vol. 74, pp. 1055-1064, 1952.

[2] G. V. Stabler, "The fundamental geometry of cutting tools," Proc. Inst. Mech. Eng., vol. 165, pp. 14-21, 1951. doi: 10.1243/PIME_PROC_1951_165_008_02.

[3] G. V. Stabler, "The basic nomenclature of cutting tools," Inst. Prod. Eng. J., vol. 34, pp. 264-279, 1955. doi: 10.1049/ipej.1955.0030.

[4] R. H. Brown and E. J. A. Armarego, "Oblique machining with a single cutting edge,” Int. J. Mach. Tool Des. Res., vol. 4, pp. 9-25, 1964. doi: 10.1016/0020-7357(64)90006-X.

[5] J. K. Russell and R. H. Brown, "The measurement of chip flow direction," Int. J. Mach. Tool Des. Res., vol. 6, pp. 129-138, 1966. doi: 10.1016/0020-7357(66)90018-7.

[6] W. K. Luk, "The direction of chip flow in oblique cutting," Int. J. Prod. Res., vol. 10, no. 1, pp. 67-76, 1972. doi: 10.1080/00207547208929906.

[7] G. C. Lin, P. Mathew, P. L. B. Oxley, and A. R. Watson, "Predicting Cutting Forces for Oblique Machining Conditions," Proc. Inst. Mech. Eng., vol. 196, pp. 141-148, 1982. doi:. 10.1243/PIME_PROC_1982_196_015_02. 
[8] J. Arsecularatne, "Prediction of tool life in oblique machining with nose radius tools," Wear, vol. 198, no. 1-2, pp. 220-228, 1996. doi: 10.1016/0043-1648(96)06975-X.

[9] I. Lazoglu and C. Islam, "Modeling of 3D temperature fields for oblique machining," CIRP Ann. - Manuf. Technol., vol. 61, no. 1, pp. 127-130, 2012. doi: $10.1016 /$ j.cirp.2012.03.074.

[10] R. Komanduri, M. Lee, and L. M. Raff, "The significance of normal rake in oblique machining," Int. J. Mach. Tools Manuf., vol. 44, no. 10, pp. 1115-1124, 2004. doi: 10.1016/j.ijmachtools.2004.02.015.

[11] G. Fang and P. Zeng, "Three-dimensional thermo-elastic-plastic coupled FEM simulations for metal oblique cutting processes," J. Mater. Process. Technol., vol. 168, no. 1, pp. 42-48, 2005. doi: 10.1016/j.jmatprotec.2004.10.013.

[12] G. Song, S. Sui, and L. Tang, "Precision prediction of cutting force in oblique cutting operation," Int. J. Adv. Manuf. Technol., vol. 81, no. 14, pp. 553-562, 2015. https://doi.org/10.1007/s00170-015-7206-Z

[13] W. Grzesik and K. Zak, "Surface integrity generated by oblique machining of steel and iron parts," J. Mater. Process. Technol., vol. 212, no. 12 , pp. $2586-2596,2012$. doi: 10.1016/j.jmatprotec.2012.07.019.

[14] A. Moufki, A. Devillez, D. Dudzinski, and A. Molinari, "Thermomechanical modelling of oblique cutting and experimental validation," Int. J. Mach. Tools Manuf., vol. 44, no. 9, pp. 971-989, 2004. doi: 10.1016/j.ijmachtools.2004.01.018.

[15] L. Abdellaoui and W. Bouzid, "Thermomechanical approach for the modeling of oblique machining with a single cutting edge," Mach. Sci. Technol., vol. 20, no. 4, pp. 655-680, 2016. doi: $10.1080 / 10910344.2016 .1224020$.

[16] W. Grzesik and K. Zak, "Friction quantification in the oblique cutting with CBN chamfered tools." Wear. vol. 304, no. 1-2, pp. 36-42, 2013. doi: 10.1016/j.wear.2013.04.020.

[17] I. Llanos, J. A. Villar, I. Urresti, and P. J. Arrazola, "Finite element modeling of oblique machining using an arbitrary lagrangian-eulerian formulation," Mach. Sci. Technol., vol. 13, no. 3, pp. 385-406, 2009. doi: 10.1080/10910340903237921.

[18] R. S. Pawade, H. A. Sonawane, and S. S. Joshi, "An analytical model to predict specific shear energy in high-speed turning of Inconel 718," Int. J. Mach. Tools Manuf., vol. 49, no. 12-13, pp. 979-990, 2009. doi: 10.1016/j.ijmachtools.2009.06.007.

[19] M. C. Shaw, Metal Cutting Principles. Oxford University Press, Oxford, 1986.

[20] ThirdWaveSystems, Third Wave AdvantEdge ${ }^{\mathrm{TM}}$ User's Manual Version 7.3. 2017.

[21] T.D., Marusich and O. M., "Modeling and Simulation of High Speed Machining," Int. J. Numer. Methods Eng., vol. 38, no. 21, p. 3675 3694, 1995.

[22] ThirdWaveSystems, "Third Wave AdvantEdge ${ }^{\mathrm{TM}}$ User's Manual Version 7.0," p. 378, 2015.

[23] K. W. Kim, W. Y. Lee, and H. Sin, "A finite element analysis for the characteristics of temperature and stress in micro-machining considering the size effect," Int. J. Mach. Tools Manuf., vol. 39, no. 9, pp. 15071524, 1999. doi: 10.1016/S0890-6955(98)00071-6.

[24] A. Davoudinejad, P. Parenti, and M. Annoni, "3D finite element prediction of chip flow, burr formation, and cutting forces in micro endmilling of aluminum 6061-T6," Front. Mech. Eng., vol. 12, no. 2, pp. 203-214, 2017. doi: 10.1007/s11465-017-0421-6. 


\section{D Printing of Metallic Structures from a Green Ink}

\author{
Chao Xu, Louis Laberge Lebel, Daniel Therriault* \\ Mechanical engineering department \\ Polytechnique Montreal \\ Montreal, Canada \\ Daniel.therriault@polymtl.ca
}

\author{
Arslane Bouchemit, Gilles L'Espérance \\ Metallurgy engineering department \\ Polytechnique Montreal \\ Montreal, Canada
}

\begin{abstract}
A green metallic ink is developed for 3D printing of metallic structures featuring high mechanical and electrical performances. The metallic ink consists of steel micro powders and a water-based chitosan/acetic acid polymer solution which replaces the previously used toxic polylactic acid (PLA)/dichloromethane (DCM) polymer solution. The optimized ink is printed at room temperature to build a metal/polymer hybrid structure. While printing, a fan is used to blow air over the ink filament upon extrusion to accelerate the solvent evaporation and shorten the solidification time, which significantly reduces the sagging and deformation. After a drying period at ambient conditions, the as-printed structure is then thermally treated using a furnace. The polymer binder is decomposed and the metal powders are sintered, resulting in a strong metallic structure. Melted copper is infiltrated into the sintered structure to achieve a fully dense metal/metal hybrid structure. The sintered structure exhibits high stiffness $(205 \mathrm{GPa})$, electrical conductivity $\left(9 \times 10^{5} \mathrm{~S} / \mathrm{m}\right)$ and low filament porosity (7\%).
\end{abstract}

Keywords: 3D printing, metallic ink, environment-friendly, metallic structures, secondary metal infiltration

\section{INTRODUCTION}

Recently, 3D printing approaches have been developed to fabricate diverse metallic structures. ${ }^{[1-3]}$ They offer many advantages such as more design flexibility, time efficient and less material waste over traditional metal manufacturing methods (e.g., machining). 3D printed metallic structures are applied in many fields such as medical ${ }^{[4-5]}$, energy storage ${ }^{[6-7]}$, sensors ${ }^{[8-9]}$, and microelectronics ${ }^{[10-11]}$. Powder-bed approaches like selective laser sintering/melting (SLS/SLM) are mature, commercialized and progressively more widely used. These approaches are based on fine metal micro powders that are evenly spread out in a powder bed. A high-intensity laser is used to selectively fuse them to build layer-by-layer 3D structures ${ }^{[12]}$ However, the intense laser system is very costly and is a potential safety issue for the operator. In addition, the repeated scanning of the laser beam results in excessive material powder oxidation, loss of alloying elements and dramatical temperature change (which causes residual stresses and defects). ${ }^{[13-14]}$
To overcome these shortcomings, solvent-cast 3D printing (SC-3DP) is proposed, which combines room-temperature 3D printing and subsequent thermal treatments. This extrusionbased printing method employs an viscous metallic suspension consisting of metal powders, polymer binder and volatile solvent, referred as the ink. ${ }^{[15]}$ Under applied pressure, the metallic ink is extruded from a fine nozzle and deposited on a substrate in a layer-by-layer fashion. Post the filament extrusion, the volatile solvent quickly evaporates and the ink turns into solid state. The as-printed object is a metal/polymer hybrid structure which is then thermally treated to burn the polymer binder and to sinter the metal powders, resulting in a fully metallic structure. ${ }^{[15]}$ Many researchers adapted this approach to fabricate various 3D metallic structures featuring complex 3D geometries and high mechanical and electrical performances. ${ }^{[15-17]}$ Although this type of process is simple and practical, it generally uses a volatile and toxic solvent (e.g., dichloromethane (DCM)), as the polymer binder solvent. We developed a nontoxic metallic ink consisting of chitosan, acetic acid, deionized water and metal powders. Chitosan is a biocompatible polymer which is dissolved in acetic acid aqueous solution as a viscous hydrogel. The metal powders are evenly dispersed in the hydrogel, resulting in a metallic suspension (Fig.1a). The metallic ink is extruded under a certain pressure and deposited on a substrate using a 3-axis deposition robot. After extrusion, the solvent (acetic acid and water) evaporates, whereas the chitosan and metal powders are left in the ink filament. Fig. 1b shows the schematic of subsequent thermal treatments. The as-printed sample is first sintered at $1165^{\circ} \mathrm{C}$ in order to thermally decompose the chitosan and sinter the metal powder. The sintered sample is a slightly porous metallic structure. To obtain a fully dense metallic structure, the sintered sample can be heated at $1120^{\circ} \mathrm{C}$ with a piece of copper placed on top of it. At this temperature, the melted copper can infiltrate the sintered structure to achieve a metal $/$ metal hybrid structure.

\section{RESULTS AND DISCUSSION}

Fig. 2 shows an optical image and SEM images of the asprinted, sintered and copper infiltrated scaffolds printed with a tapered nozzle (tip inner diameter $=50 \mu \mathrm{m}$ ) at a printing speed 

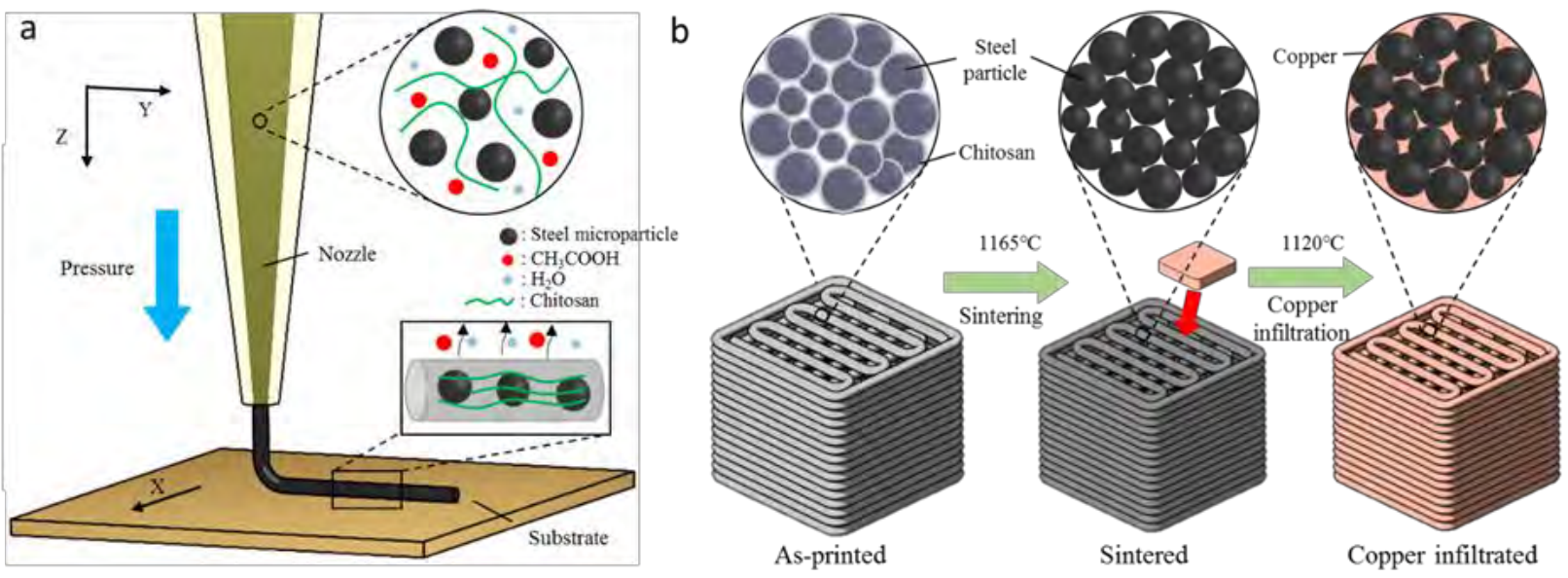

Fig. 1. Schematic of the fabrication process of a 3D metallic scaffold combining the following steps. (a) Solvent-cast 3D printing of a metallic ink consisting of steel microparticles and chitosan/acetic acid polymer solution. The metallic ink is loaded in a syringe and extruded through a micro nozzle under a certain pressure at room temperature. The solvent content evaporates upon extrusion assisted by an airflow. (b) Subsequent thermal treatments: the dried as-printed sample is thermally treated. The polymer is decomposed and the steel particles are sintered. Melted copper is infiltrated into the sintered structures to achieve a fully dense metallic structure.

of $7 \mathrm{~mm} / \mathrm{s}$. The three types of scaffolds are placed on a Canadian dollar. The as-printed scaffold has a dark gray color because the steel powders are covered by chitosan. After sintering, chitosan is removed and steel powders are brought closer. The sintered scaffold turns into light grey and shrinks in size. The copper infiltrated scaffold turns to brown red due to the appearance of copper. There are no significant shape distortion or oxidation occurred to the sintered and copper infiltrated scaffolds. The SEM images of the as-printed scaffold shows that steel powders are covered and bound by the polymer binder, chitosan (Fig. 2a). Before sintering, a debinding step is performed to remove the chitosan within the structure. After debinding, the structure integrity is held by the friction forces between the steel powders. The steel powders are sintered at a temperature slightly lower than their melting point. The powders are connected through the appearance of necks between them. The size and amount of the necks increase gradually, which densifies the sintered structure and reduces the pores. In the sintered scaffold (Fig. 2b), the chitosan is completely removed and the steel powders are directly connected with each other through the necks. For the copper infiltration, a piece of copper is placed on top of the sintered structures inside the oven. The sample is heated at $1120{ }^{\circ} \mathrm{C}$. This temperature is higher than the melting point of copper $\left(1085^{\circ} \mathrm{C}\right)$, but lower than the previously used sintering temperature. Mainly driven by capillary forces, the melted copper fills the pores within the filaments of the structure (Fig. $2 \mathrm{c})$.
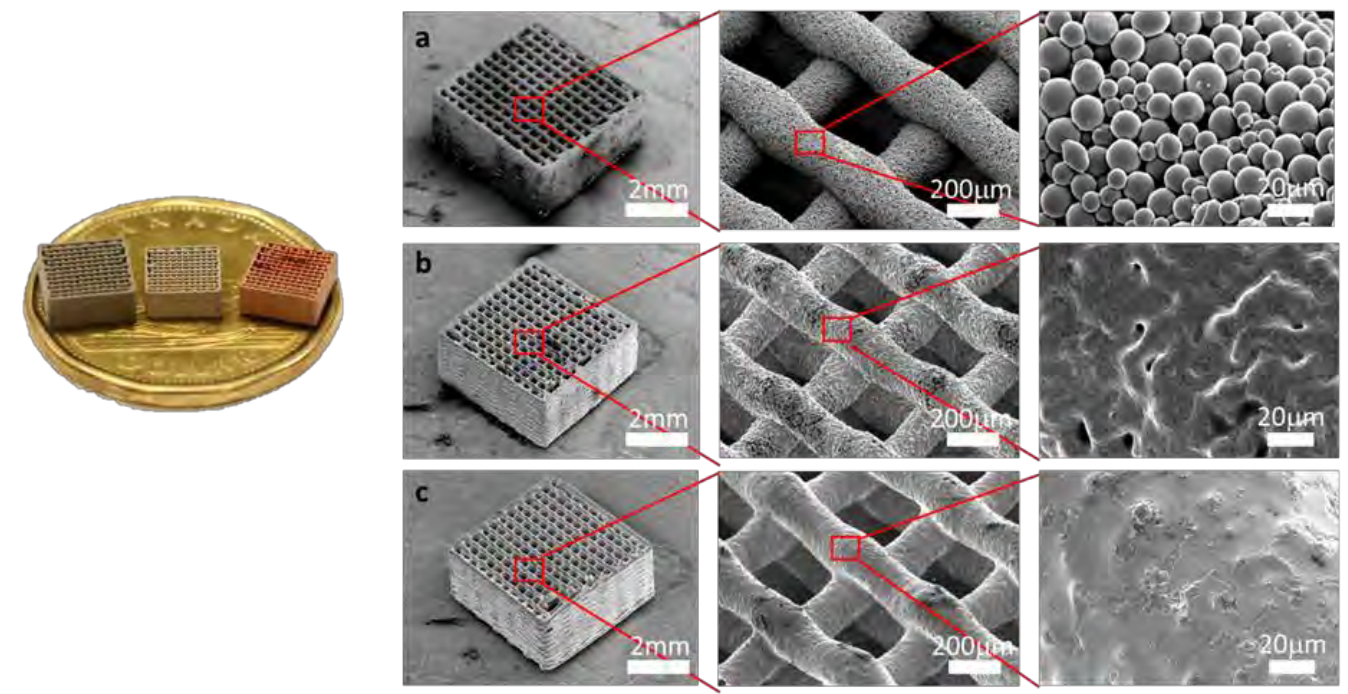

Fig. 2. Optical and SEM images of the scaffolds printed using a tapered nozzle (tip inner diameter $=50 \mu \mathrm{m})$ at a printing speed of $7 \mathrm{~mm} / \mathrm{s}$, (a) as-printed, (b) sintered, (c) copper infiltrated, and their close-up views. 
The linear size reduction, filament porosity, electrical conductivity and mechanical properties of as-printed, sintered and copper infiltrated samples are characterized (Fig. 3). The samples have size variations in every stage compared to the designed size. It is caused by the swelling upon extrusion, solvent evaporation, debinding, sintering and copper infiltration. The linear size reduction is studied and the result is shown in Fig. 3a. The extruded ink filament swells due to the pressure drop and then shrinks as a result of the solvent evaporation. The dried as-printed sample has similar size as the designed one ( $>1 \%$ linear expansion). After debinding and sintering, the sintered structure shrinks by around $12 \%$ compared to designed size. The copper infiltrated sample exhibits less size reduction ( $\sim 9 \%$ linear reduction) than the sintered one. The filament porosity of sintered samples is around $7 \%$ (Fig. 3b). After copper infiltration, the majority of the pores are filled by melted copper. The filament porosity of copper infiltrated samples is lower than $1 \%$. The conductivity of sintered samples is around $9 \times 10^{5} \mathrm{~S} / \mathrm{m}$, which is $64 \%$ of that of the bulk material (Fig. 3c). As copper is more conductive than steel, the copper infiltrated sample presents an even higher conductivity of $\sim 1.3 \times 10^{6} \mathrm{~S} / \mathrm{m}$. Fully dense sintered and copper infiltrated tensile bars, of which the cross section of the narrow part is $\approx 3.6 \times 1.6 \mathrm{~mm}$, are prepared for tensile test. It is carried out on a MTS Insight machine with a $50 \mathrm{kN}$ load cell (MTS 569332-01) at a crosshead speed of 1 $\mathrm{mm} / \mathrm{min}$ and using an extensometer (gauge length $=8 \mathrm{~mm}$, MTS 632.26, C-20). Five specimens for each sample type are tested. The Young's modulus of sintered samples is around $205 \mathrm{GPa}$, which equals $95 \%$ of that of bulk material (Fig. 3d). After copper infiltration, the stiffness is slightly decreased, but the ductility and the ultimate tensile strength are significantly improved.

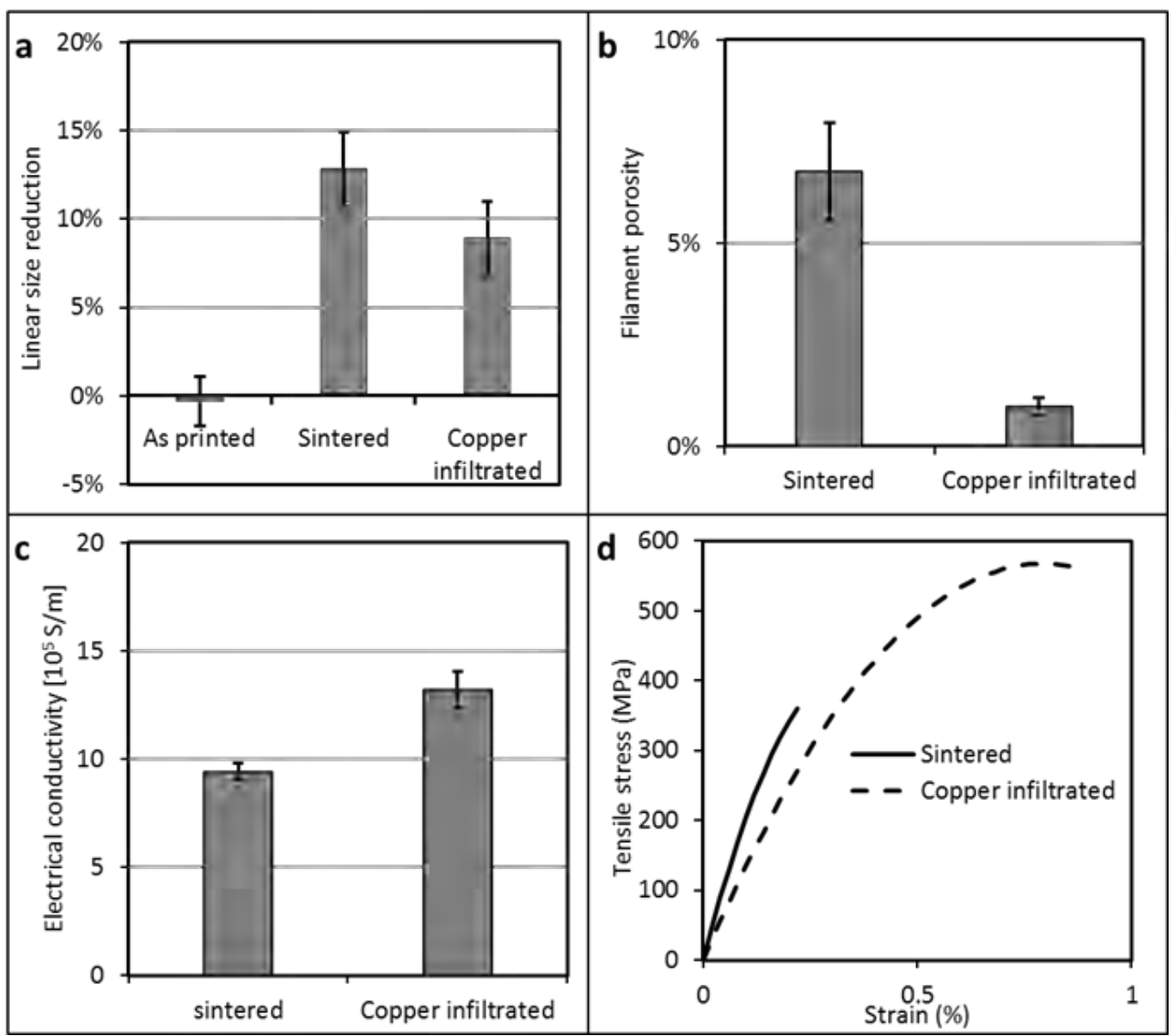

Fig. 3. (a) Linear size reduction, (b) filament porosity, (c) electrical conductivity and (d) mechanical properties of the as-printed, sintered and copper infiltrated samples

\section{CONCLUSION}

We developed an environmental metallic ink for SC-3D printing of highly dense metallic structures. The ink contains metal powders, biocompatible polymer binder and a nontoxic volatile solvent. Metallic and metal/metal hybrid structures are fabricated by subsequent thermal treatments. The linear size reduction, filament porosity, electrical conductivity and mechanical properties of the fabricated structures are characterized. The sintered structure has a Young's modulus close to that of the bulk material. The copper infiltrated structure exhibits less filament porosity, higher electrical conductivity and better ductility. The reported ink can replace the commonly used toxic ink to create $3 \mathrm{D}$ metallic structures for higher performances, lower environmental impact and more effective utilization of metallic materials. 


\section{ACKNOWLEDGMENT}

The authors acknowledge the financial support from NSERC (Natural Sciences and Engineering Research Council of Canada, grant number: RGPIN 312568-2013) and Auto 21 of the Canadian Network of Centers of Excellence (NCE) program. A scholarship for $\mathrm{Mr}$. Xu was also provided by the China scholarship Council (CSC) and the Fonds de recherche du Quebec - Nature et technologies (FRQNT).

\section{REFERENCES}

[1] J. H. Martin, B. D. Yahata, J. M. Hundley, J. A. Mayer, T. A. Schaedler, and T. M. Pollock, "3D printing of high-strength aluminium alloys," Nature, vol. 549, no. 7672, pp. 365-369, Sep. 2017. doi: 10.1038/nature23894.

[2] I. Todd, "Metallurgy: No more tears for metal 3D printing." Nature, vol. 549, no. 7672, pp. 342-343, Sep. 2017. doi: 10.1038/549342a.

[3] D. D. Gu, W. Meiners, K. Wissenbach, and R. Poprawe "Laser additive manufacturing of metallic components: Materials, processes and mechanisms," Int. Mater. Rev., vol. 57, no. 3, pp. 133-164, May 2012. doi: 10.1179/1743280411Y.0000000014.

[4] M. H. Elahinia, M. Hashemi, M. Tabesh, and S. B. Bhaduri, "Manufacturing and processing of NiTi implants: A review," Prog. Mater. Sci., vol. 57, no. 5, pp. 911-946, June 2012. doi:10.1016/j.pmatsci.2011.11.001

[5] X. Wang, et al. "Topological design and additive manufacturing of porous metals for bone scaffolds and orthopaedic implants: A review." Biomater., vol. 83, pp. 127-141, Mar. 2016. doi: 10.1016/j.biomaterials.2016.01.012.

[6] B. Mendoza-Sánchez and Y. Gogotsi, "Synthesis of two-dimensional materials for capacitive energy storage," Adv. Mater., vol. 28, no. 29, pp. 6104-6135, Jun. 2016. doi: 10.1002/adma.201506133.

[7] A. E. Jakus, S. L. Taylor, N. R. Geisendorfer, D. C. Dunand, and R. N. Shah, "Metallic architectures from 3D-printed powder-based liquid inks," Adv. Funct. Mater., vol. 25, no. 45, pp. 6985-6995, Nov. 2015. doi: 10.1002/adfm.201503921.
[8] J. H. Song et al., "Surface-embedded stretchable electrodes by direct printing and their uses to fabricate ultrathin vibration sensors and circuits for 3D structures," Adv. Mater., vol. 29, no. 43, p. 1702625 , Oct. 2017. doi: 10.1002/adma.201702625.

[9] Y. S. Rim, S.-H. Bae, H. Chen, N. De Marco, and Y. Yang, "Recent progress in materials and devices toward printable and flexible sensors," Adv. Mater., vol. 28, no. 22, pp. 4415-4440, Feb. 2016 doi: 10.1002/adma.201505118.

[10] J. W. Boley, E. L. White, G. T.-C. Chiu, and R. K. Kramer, "Direct writing of gallium-indium alloy for stretchable electronics," Adv. Funct. Mater., vol. 24, no. 23, pp. 3501-3507, Feb. 2014. doi: $10.1002 / \mathrm{adfm} .201303220$.

[11] M. D. Dickey, "Stretchable and soft electronics using liquid metals," Adv. Mater., vol. 29, no. 27, p. 1606425, Apr. 2017. doi: 10.1002/adma.201606425.

[12] R. D. Farahani, M. Dubé, and D. Therriault, "Three-dimensional printing of multifunctional nanocomposites: Manufacturing techniques and applications," Adv. Mater., vol. 28, no. 28, pp. 5794-5821, May 2016. doi: 10.1002/adma.201506215.

[13] H. Gong, K. Rafi, H. Gu, G. D. Janaki Ram, T. Starr, and B. Stucker, "Influence of defects on mechanical properties of Ti-6Al-4V components produced by selective laser melting and electron beam melting," Mater. Des., vol. 86, pp. 545-554, Dec. 2015. doi: 10.1016/j.matdes.2015.07.147.

[14] E. O. Olakanmi, R. F. Cochrane, and K. W. Dalgarno, "A review on selective laser sintering/melting (SLS/SLM) of aluminium alloy powders: Processing, microstructure, and properties," Prog. Mater. Sci., vol. 74, pp. 401-477, Oct. 2015. doi: 10.1016/j.pmatsci.2015.03.002.

[15] B. Y. Ahn, D. Shoji, C. J. Hansen, E. Hong, D. C. Dunand, and J. A. Lewis, "Printed origami structures," Adv. Mater., vol. 22, no. 20, pp. 2251-2254, May 2010. doi: 10.1002/adma.200904232.

[16] C. Xu, A. Bouchemit, G. L'Espérance, L. Laberge Lebel, and D. Therriault, "Solvent-cast based metal 3D printing and secondary metallic infiltration," J. Mater. Chem. C., vol. 5, no. 40, pp. 10448-10455, 2017. doi: 10.1039/C7TC02884A.

[17] E. Peng, X. Wei, T. S. Herng, U. Garbe, D. Yu, and J. Ding, "Ferrite -based soft and hard magnetic structures by extrusion free-forming," RSC Advances, vol. 7, no. 43, pp. 27128-27138, 2017. doi: 10.1039/C7RA03251J. 


\section{A Study of the Thermal and Mechanical Properties of Recycled Crosslinked HDPE}

\author{
Jardel Nobrega dos Santos \\ Department of Mechanical Engineering \\ University of Manitoba \\ Winnipeg, Canada \\ nobregaj@myumanitoba.ca
}

\author{
Raghavan Jayaraman, $\mathrm{PhD}$ \\ Department of Mechanical Engineering \\ University of Manitoba \\ Winnipeg, Canada \\ raghavan.jayaraman@umanitoba.ca
}

\begin{abstract}
Crosslinked high-density polyethylene (XHDPE) is used to manufacture large and rigid products, such as fuel tanks, using a rotomolding process. Currently, defective and post-consumer parts are not recycled and sent to landfills. Hence, reuse of this material, through compression molding is the objective of this study. Thermal characterization indicated that the recycled XHDPE, in pulverized powder form, could melt although the heat of fusion was less than that of virgin material. However, while the virgin XHDPE could be compression molded at the melting temperature, the recycled XHDPE required higher temperature. The recycled XHDPE started to degrade at lower temperature than virgin XHDPE. Based on this data, optimal compression molding process conditions were chosen to prepare dense tensile test coupons. Both materials exhibited similar tensile properties demonstrating the recyclability of XHDPE.
\end{abstract}

Rotomolding; compression molding; recycled XHDPE.

\section{INTRODUCTION}

Rotomolding is a common manufacturing process used to produce large and strong parts with a uniform thickness. One of the main plastics used is the High-Density polyethylene (HDPE). For some applications, the HDPE must be crosslinked to make the parts stronger and increase the barrier resistance

HDPE is a thermoplastic with a density of $0.940-$ $0.965 \mathrm{~g} / \mathrm{cm}^{3}[1]$ and melting between $128^{\circ} \mathrm{C}$ and $138^{\circ} \mathrm{C}$ [2]. These properties are slightly changed after crosslinking. The crosslinking process results in bonding of the chain to itself and to other chains [3].

The crosslinked polyethylene is prepared using either a crosslinking agent (peroxide or silane) or electron beam radiation. During rotomolding, the crosslinking caused by the peroxide initiator increases the gel content $(>80 \%$ as claimed by rotomolder using this materials), which reduces the flow of the material when heated above the melting temperature.

Due to this characteristic, XHDPE cannot be recycled and the industrial and post-consumer waste is currently sent to the landfill. Published studies on recycling this material include energy recovery [4], use of the ground material as a filler [5], degradation to low molecular weight polyethylene using solid state shearing [6], molten state shearing [7], ultrasonic process [8], and supercritical process [9]. The degraded material was subsequently blended with other thermoplastic polymers to manufacture parts.

This research is focused on reuse of this material, without blends, through compression molding. The thermal behavior of the recycled XDPE is characterized and compared with the virgin XDPE to understand the issues in reusing this material. Based on this, the compression molding conditions are chosen to mold tensile specimens. The tensile properties of recycled XHDPE are compared with virgin XHDPE to evaluate former's recyclability. Preliminary results are presented and discussed here.

\section{EXPERIMENTAL}

\section{A. Materials and Methods}

Exxon Mobil's Paxton 7000 Series XHDPE was used in this study. Pulverized powder of the virgin and recycled material was supplied by a rotomolder in Manitoba. The melting characteristics of these two materials were studied using TA Instruments' Q2000 DSC (Differential Scanning Calorimeter). Samples were ramped at a rate of $10^{\circ} \mathrm{C} / \mathrm{min}$ from $-50^{\circ} \mathrm{C}$ to $250^{\circ} \mathrm{C}$ under nitrogen atmosphere. The degradation characteristics was studied using TA Instruments' Q500 TGA (Thermogravimetric Analyzer). Samples were ramped at a rate of $10^{\circ} \mathrm{C} / \mathrm{min}$ from $25^{\circ} \mathrm{C}$ to $550^{\circ} \mathrm{C}$ using nitrogen and air as the purge gas. Based on these test results, compression molding temperature was chosen. Molding pressure was chosen based on preliminary trials. ASTM standard tensile test coupons were molded using these conditions and tested as per ASTM 638 using MTS Insight test machine.

\section{RESULTS AND DISCUSSION}

\section{A. Differential Scanning Calorimeter Results}

The results from DSC experiments, shown in Fig. 1, indicate that both materials melt around $130^{\circ} \mathrm{C}$. A completely crosslinked material would not melt. The results suggest that XHDPE either did not completely crosslink during the rotomolding process or was de-crosslinked by the pulverization process. 


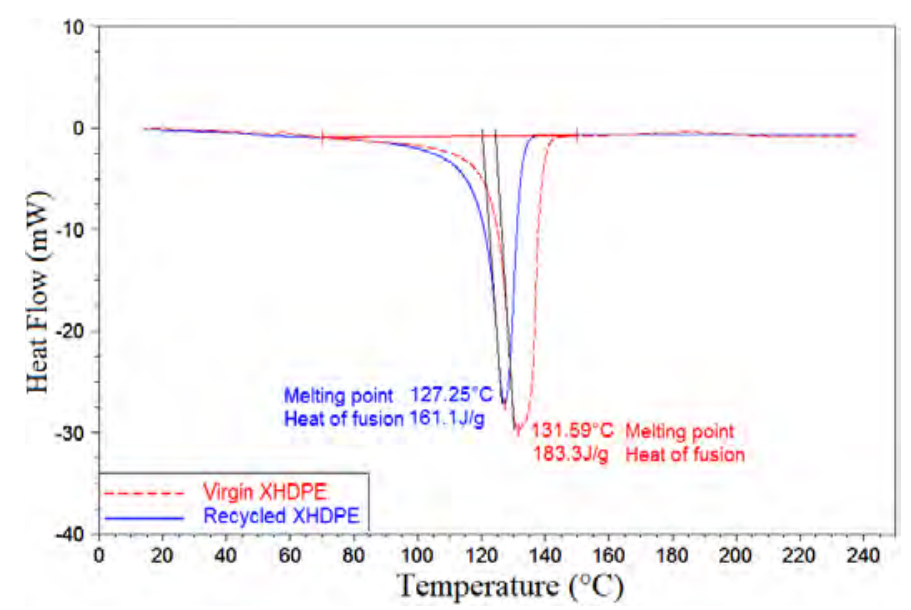

Figure 1. DSC test results for virgin and recycled XHDPE

The lower heat of fusion for recycled XHDPE than the virgin XHDPE suggests that there is residual crosslinking in the pulverized powders of recycled XHDPE. This result confirms that the recycled XHDPE, in pulverized powder form, can melt and hence is recyclable

\section{B. Thermogravimetric Analysis}

The results from TGA experiments shown in the Fig. 2 (purge gas - nitrogen) indicate that both the virgin and the recycled XDPE degrade similarly.

The decomposition initiation and completion temperatures as well as the weight loss are similar for both materials. These temperatures are much higher than the molding temperatures.

Depending of the molding conditions, the XHDPE may or may not come in contact with air. Hence, TGA test was repeated using air as a purge gas (Fig. 3).

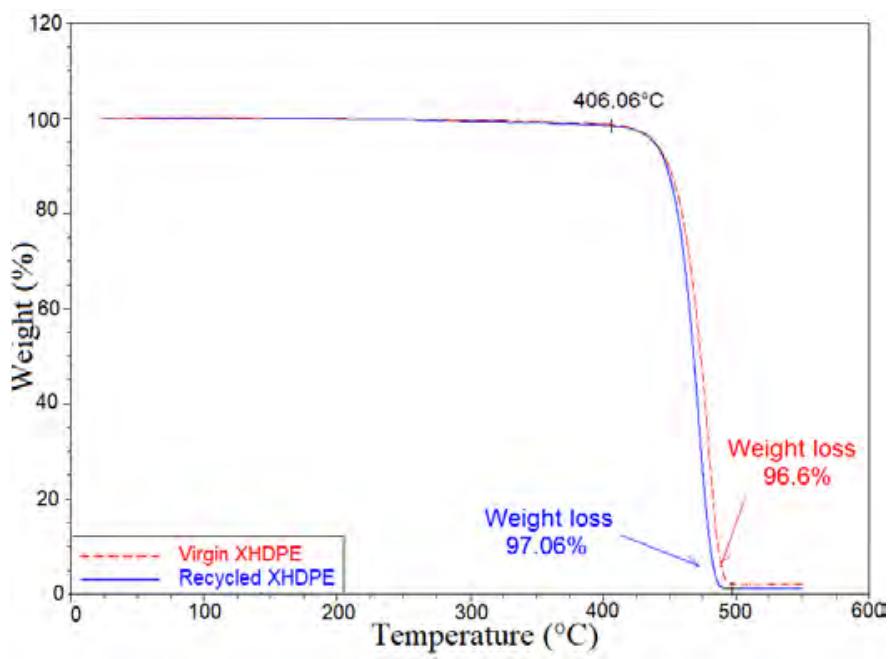

Figure 2. TGA test results for virgin and recycled XHDPE (Nitrogen)

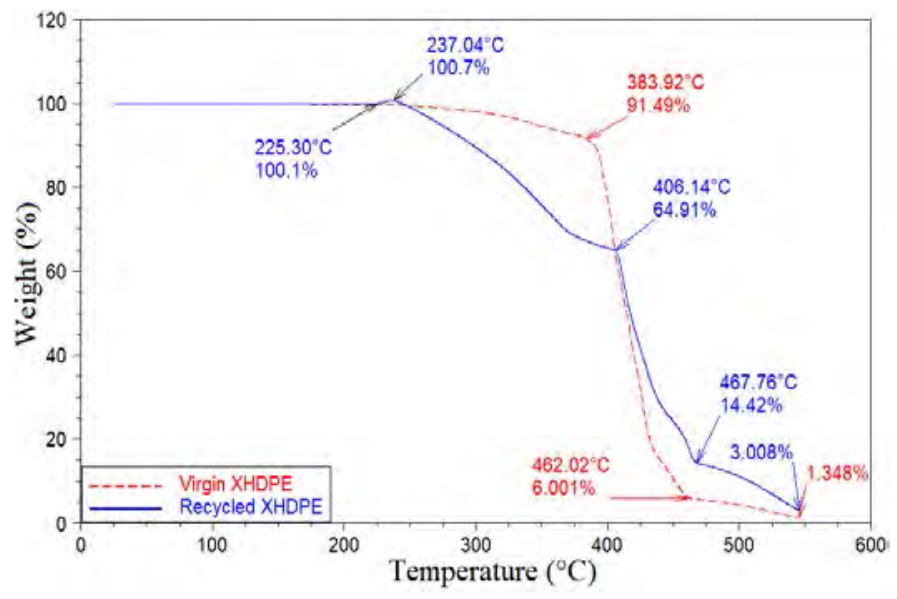

Figure 3. TGA test results for virgin and recycled XHDPE (Air)

The TGA results in Fig. 3 indicate that the recycled XHDPE is affected by the presence of air resulting in the formation of different products, suggested by the various slopes of the curve. Hence, the molding temperature was limited to $250^{\circ} \mathrm{C}$.

\section{Compression molding}

Initial trials indicated that while virgin XHDPE could be melted and molded at the melting temperature, recycled XHDPE had to be molded at a considerably higher temperature and pressure. Tab. I presents the final compression molding conditions that resulted in a dense product without defects.

\section{Mechanical properties}

The tensile test results for both materials are presented in Fig. 4. The results show that the recycled XHDPE has a very similar stress versus strain curve when compared with the virgin material.

The tensile strengths of the virgin and recycled XHDPE are $20.25 \mathrm{MPa}$ and $20.5 \mathrm{MPa}$ respectively. The fracture strain of the virgin and recycled material are $344.5 \%$ and $273.6 \%$ respectively. While there is a slight decrease in the strain to failure of the recycled XHDPE, the tensile strength and modulus for both materials were similar.

TABLE I. COMPRESSION MOLDING SETTINGS

\begin{tabular}{|l|c|c|c|}
\hline \multirow{2}{*}{\multicolumn{1}{|c|}{ Material }} & \multicolumn{3}{|c|}{ Parameters } \\
\cline { 2 - 4 } & Temperature & Pressure & Time \\
\hline Virgin XHDPE & $150^{\circ} \mathrm{C}$ & $0.286 \mathrm{MPa}$ & $30 \mathrm{~min}$ \\
\hline Recycled XHDPE & $230^{\circ} \mathrm{C}$ & $2.86 \mathrm{MPa}$ & $30 \mathrm{~min}$ \\
\hline
\end{tabular}




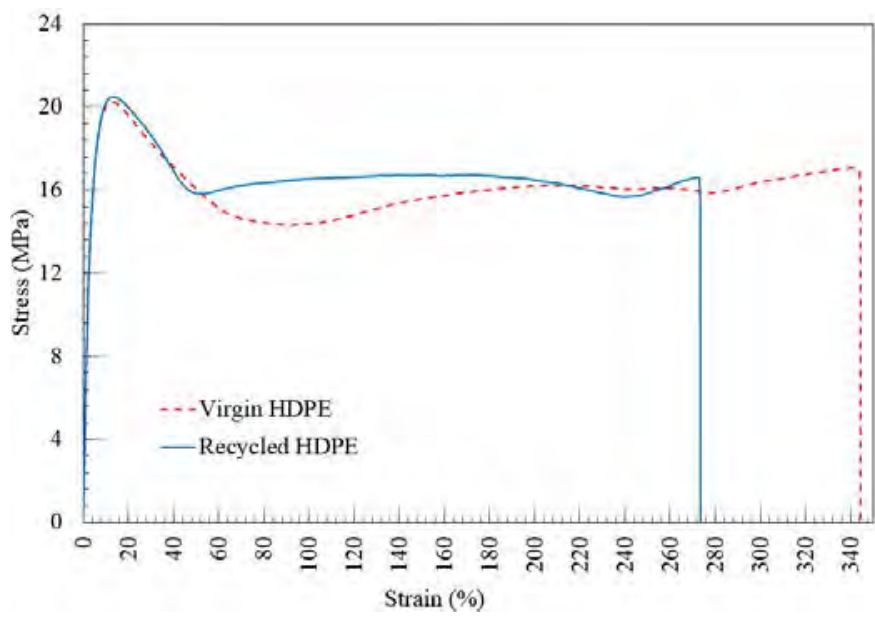

Figure 4. Tensile test results for virgin and recycled XHDPE

\section{SUMMARY}

The thermal properties of Virgin and recycled XHDPE were investigated using DSC and TGA. Both materials exhibited melting around $130^{\circ} \mathrm{C}$, although the heat of fusion for recycled material was less than that for the virgin material. The recycled material was successfully compression molded at a temperature and pressure higher than that used for the virgin material. Both materials exhibited similar tensile properties with the exception of strain to failure, which was slightly less for the recycled material. These results suggest that XHDPE can be reused through compression molding. Further studies are underway to study the mechanism of fusion of recycled XHDPE powder and the effect of pulverization on its recyclability.

\section{ACKNOWLEDGEMENTS}

The authors would like to gratefully acknowledge the support from Mr. Cameron Hay and Mr. Ramon Timchuk of Acrylon Plastics, $\mathrm{MB}$ in providing the material and in discussions.

\section{REFERENCES}

[1] A. B. Strong, Plastics: Materials and Processing, 3rd ed. London: Pearson, 2005.

[2] W. D. J. Callister, Materials Science and Engineering: An Introduction., 4th ed. New York, Wiley, 1996.

[3] M. Yamaguchi and K. I. Suzuki, "Rheological Properties and Foam Processability for Blends of Linear and Crosslinked Polyethylenes," J. Polym. Sci. Part B: Polym. Phys., vol. 39, no. 18, pp. 2159-2167, 2001. doi: 10.1002/polb.1189.

[4] A. Boss et al., "New Technology for Recycling of Plastics from Cable Waste" in 8th International Conference on Insulated Power Cables., Versailles., Yvelines, 2011, pp.1.

[5] J. Navratil et al., "Recycling of Irradiated High-Density Polyethylene," Radiat. Phys. Chem., vol. 106, pp. 68-72, Jan. 2015. doi: 10.1016/j.radphyschem.2014.06.025.

[6] H. Wu, M. Liang, and C. Lu, "Non-Isothermal Crystallization Kinetics of Peroxide-Crosslinked Polyethylene: Effect of Solid State Mechanochemical Milling," Thermochim. Acta, vol. 545, pp. 148156, Oct. 2012. doi: 10.1016/j.tca.2012.07.008.

[7] Y. Sekiguchi, N. Ohkawa, H. Nojo, and S. Hashimoto, "Development of Recycling Technology of XLPE," in 8th International Conference on Insulated Power Cables., Versailles., Yvelines, 2011.

[8] K. Huang, A. I. Isayev, and J. Zhong, "Ultrasonic Decrosslinking of Crosslinked High-Density Polyethylene: Effect of Screw Design," J. Appl. Polym. Sci., vol. 131, no. 17, pp. 8506-8521, 2014. doi: 10.1002/app.40680.

[9] B. K. Baek et al., "Continuous Supercritical Decrosslinking Extrusion Process for Recycling of Crosslinked Polyethylene Waste," J. Appl. Polym. Sci., vol. 132, no. 6, 2015. doi: 10.1002/app.41442. 


\section{Non-Contact Auxillary Fixture for Better Machining of Thin Flexible Workpiece Using Eddy Currents}

\author{
Robinderpal Singh \\ Advanced Manufacturing Lab (AML) \\ School of Engineering \\ University of Guelph \\ Guelph, Ontario, Canada. \\ robinder@uoguelph.ca
}

\author{
Ibrahim M. Deiab \\ Advanced Manufacturing Lab (AML) \\ School of Engineering \\ University of Guelph \\ Guelph, Ontario, Canada. \\ ideiab@uoguelph.ca
}

\begin{abstract}
Thin flexible parts are widely used in the aerospace industry and require a more precise surface finish. The main problem in achieving high-quality product is the vibration induced during machining of thin-walled parts. Clamping these thin parts or using rigid supports for parts, such as for propeller blades, lead to distortion. This issue may be solved using a noncontact auxiliary fixture. The new fixture developed in this research is an eddy current damper for passive control of vibrations in electrically conducting workpieces, using neodymium permanent magnets without any external power source. Two thin aluminum beams with different cross-section and same length were used to test the developed fixture. To increase the overall damping, conducting sheets were attached at the free end of beams. Impact and machining tests were conducted to validate the new fixture. The results showed higher damping and better machining stability leading to enhanced surface quality of the workpiece.
\end{abstract}

Keywords- Thin-flexible parts; vibration; fixture; eddy current damper; passive damping

\section{INTRODUCTION}

Machining of thin-walled workpieces is required in several industries, most notably the aerospace industry. During machining of thin parts, vibration is the main factor affecting the surface finish and tolerance. Milling is one of the most common processes used for thin workpieces. To improve the stability of the workpiece, i.e. to damp the vibrations, various methods are applied including choosing optimized machining parameters or using primary or auxiliary contacting or non-contacting fixtures [1]. In recent studies, distinct theoretical models have been created, and systems have been analyzed for damping of vibration, mainly in the cantilever beams. This research analyzes and controls the vibrations during machining of cantilever beamlike workpieces for better stability and surface finish.

In the aerospace industry, accuracy and precision of the machining process and the manufactured parts, along with the production rate and the tool life, are the primary areas for research. The parts that are more flexible, such as, the impellers, propeller blades etc., need much higher accuracy and a smooth surface finish. Chatter is widely studied in the literature. Lai et al. [2] studied chatter characteristics of a cylindrical thin-walled workpiece. The relation of the wall thickness and the inner diameter was analyzed to study machining vibration characteristics. Numerical and experimental methods were used to investigate the dynamic characteristics of the workpiece. Campa et al. [3] came up with a method to damp the vibrations in thin-floor milling using a bull-nose end mill. A mathematical model was created to predict the behavior of workpiece during the milling process and to generate a stability lobe diagram for selecting the optimal spindle speed for better stability during machining. Junjin et al. [4] worked on the use of magnetorheological (MR) fluid, which can be tuned using a magnetic field by changing its state from liquid to solid by aligning the magnetic particles in a single direction, to damp the vibrations in a thin cantilever beam and found a considerable change in the vibrations.

\section{EDDY CURRENT DAMPER}

Damping of vibrations in thin-walled electrically conducting workpieces can be achieved using magnetism. The use of eddy currents to oppose the motion of the workpiece utilizes the basic principle of using a magnetic field for vibration damping.

When a conductor moves in a magnetic field, electrical currents are induced in it, known as eddy currents. These currents generate a counter magnetic field with an opposing force, known as the Lorentz force. This Lorentz force (F) is directly opposite to the direction of movement of the conductor moving with a velocity (v) with respect to the magnetic field (B), as shown in Figure 1. When the movement of the conductor is perpendicular to the polar axis of the magnet, the radial component of magnetic field does not contribute to the damping effect due to lack of much interaction with the conductor. On the other hand, when the conductor moves parallel to the axis of poles of magnet, only the radial component of magnetic flux is responsible for inducing eddy currents and creating damping forces. 


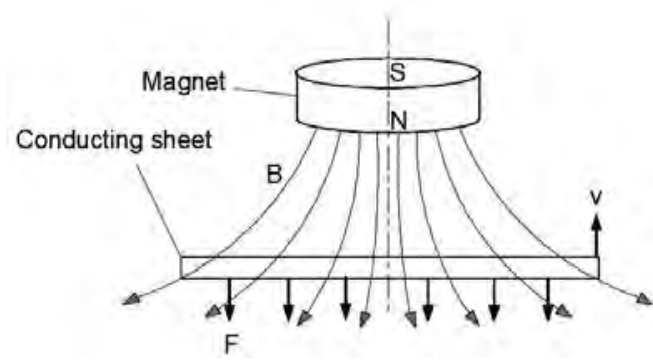

(a)

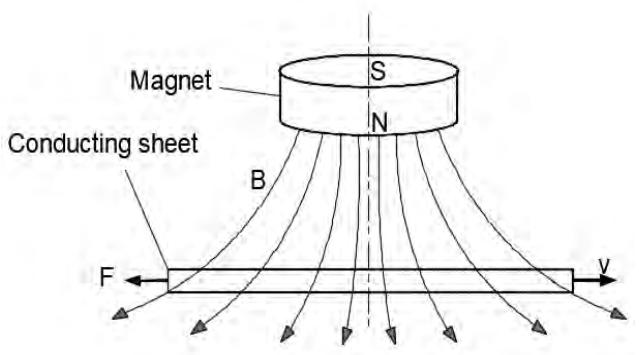

(b)

Figure 1: (a) Damping force using radial magnetic flux, and (b) Damping using axial magnetic flux

The induced current density $(\vec{J})$ can be obtained as,

$$
\vec{J}=\sigma(\vec{v} \times \vec{B})
$$

In the above equation, the conductor has conductivity $\sigma$, velocity $\vec{v}$ w.r.t the magnetic field and magnetic flux density $\vec{B}$.

The damping force of the system due to the magnetic field can be determined analytically. The damping force depends on the magnetic flux density $(B)$, the relative velocity of the vibrations $(v)$, and the properties of the conductor used, which includes a thickness $(\delta)$ and conductivity $(\sigma)$.

From the relation given, the damping force due to axial magnetic field lines, as used by Bae et al., [5], is given as

$$
F=-\left(\sigma \delta B^{2} S\left(\alpha_{1}+\alpha_{2}\right)\right) v
$$

Where $\mathrm{S}$ is the area of pole projection, and $\alpha_{1}$ and $\alpha_{2}$ are the conducting sheet's effect on damping force.

The case of a radial magnetic flux, as studied by Sodano et al. [6] is given as:

$$
F=\int_{v} J \times B d v=-2 k \pi \sigma \delta \int_{0}^{r_{c}} y B_{y}^{2}\left(y, l_{g}\right) d y
$$

With $\mathrm{k}$ as the spring coefficient of the system, $r_{c}$ as the equivalent radius of conducting sheet, and $l_{g}$ as the air gap between the magnet and the conducting sheet.

This eddy current creates an opposing field and force which opposes the motion of the conductor. This damping effect can be used for a thin-walled cantilever beam using a strong magnetic field around it such that the oscillation of the beam in the magnetic field induces a Lorentz force or damping force. For better results and more control, electromagnets can be used in this vibration damping methods [7]. The eddy current damping process is contact-less, wear-free, fast and safe. Also, with the electromagnets, the magnetic field or the force can be controlled using different input current or coil turns, making it more adjustable for different work conditions [8].

Sodano with Jae-Sung Bae [6] developed a passive eddy current damper which used radial components of magnetic flux for vibration damping, using permanent magnets. The damper showed an increase in damping ratio up to 150 times for a thin cantilever beam, when fixed at the free end. The above study was continued by Sodano et al. [9] to change the passive current damper to an active eddy current damper by using electromagnets instead of permanent magnets. With this active system, the magnetic field strength can be varied actively so as to induce eddy currents even without much movement of the conductor. This system is effective when the movement of the conductor in a magnetic field is not enough to induce significant eddy currents.

Another study was performed by Cheng et al. [10] using a permanent magnet for damping of vibrations. In their work, a conduction coil was attached to the cantilever beam to vary the resistance during induction of eddy currents using a permanent magnet. Ebrahimi et al. [11] worked on passive eddy current damper and developed an analytical model to select the permanent magnets of optimum strength and size.

\section{EXPERIMENTAL SETUP AND METHODOLOGY}

This research was conducted to develop a non-contacting auxiliary fixture, as shown in Figure 2, for machining stability of cantilever-like workpieces, such as propeller and impeller blades, to suppress machining vibrations and enhance the surface finish of the end-product. The fixture developed has an aluminum frame to limit interaction with the magnetic field. The 'magnet holder' holds the neodymium magnet and can be moved to change the gap between the magnet and the workpiece. Allen bolts are used to lock the 'magnet holders' at any point. Use of permanent magnets on either side of the workpiece is an ideal option when the workpiece moves perpendicular to the polaraxis of the magnets for maximum interaction of magnetic field lines.

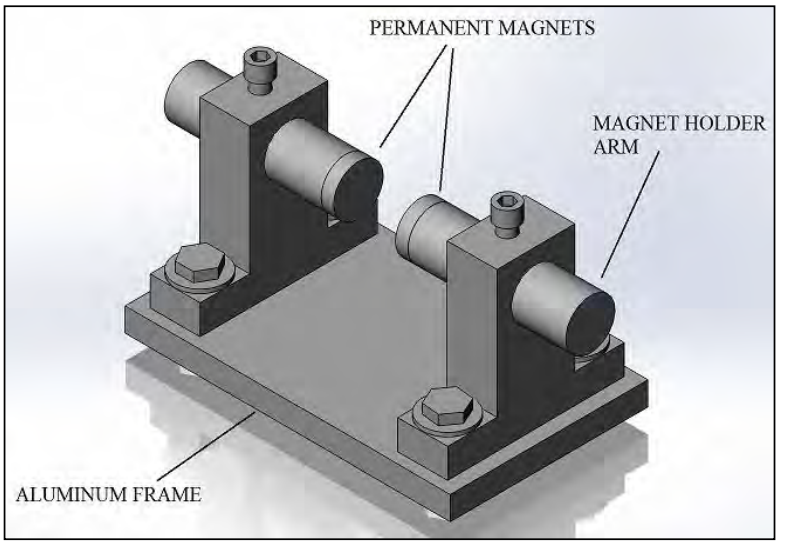

Figure 2: Developed experimental setup 
Two Aluminum beams (6061-T6), one of dimensions 170 $\mathrm{mm} \times 25 \mathrm{~mm} \times 6 \mathrm{~mm}$ (referred as 'thick beam') and the other of dimensions $170 \mathrm{~mm} \times 38.5 \mathrm{~mm} \times 3.58 \mathrm{~mm}$ (referred as 'thin beam'), were selected as the workpiece because of resemblance to a propeller blade. Permanent magnets (Neodymium N-48), of diameter $25.4 \mathrm{~mm}$ and thickness $6.35 \mathrm{~mm}$ each, were used to induce the eddy currents in the workpiece to produce the damping effect whenever there is a motion in the workpiece due to induced vibrations. The beams were secured on one end in a vise and the eddy current fixture was placed at the free end, as shown in Figure 3. Aluminum sheets $(50 \mathrm{~mm} \times 37 \mathrm{~mm} \times 1.5$ $\mathrm{mm}$ ) were glued at the free ends of both beams (as in Figure 3(b) and 3(c)).

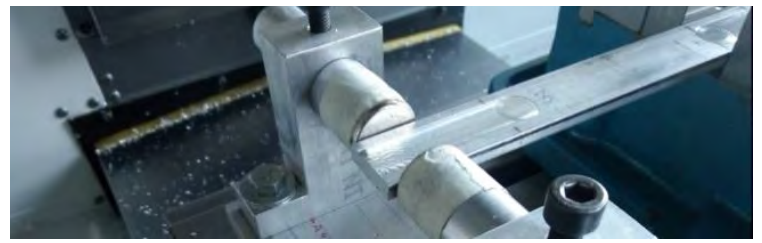

(a)

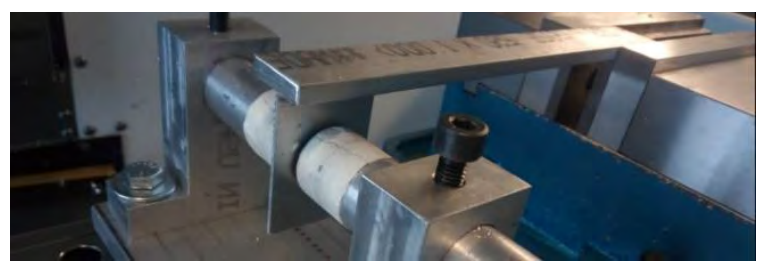

(b)

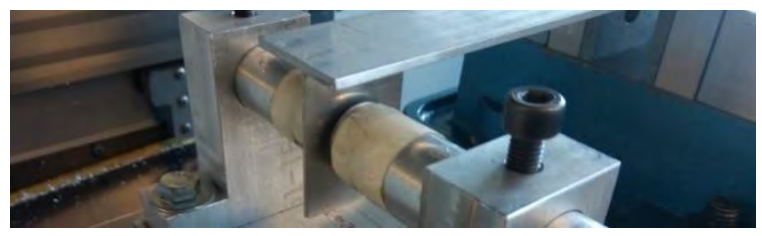

(c)

Figure 3. Different setups of the test workpiece (a) Beam 1 (without additional conducting element), (b) Beam 2 (thick beam with aluminum sheet), and (c) Beam 3 (thin beam with aluminum sheet element)

The three beam arrangements (Beam-1 i.e. thick beam without the additional aluminum sheet, Beam-2 i.e. thick beam with the aluminum sheet glued and Beam-3 i.e. thin beam with the aluminum sheet glued) used are given in Figure 3, with a constant gap of $0.8 \mathrm{~mm}$ between the magnet and the beam for each arrangement. The natural frequencies and the modes of each beam, with and without the fixture, were obtained using impact hammer test through LMS Test.lab by Siemens (as shown in Figure 4). The frequency response functions were then obtained from the LMS Test-lab software to get the average damping ratios and damping ratios for each mode for all the beams. The results of Test-lab, for natural frequencies and modes, were confirmed using ANSYS modal analysis. ANSYS Maxwell was used to simulate the magnets to see the maximum magnetic flux on the beam.

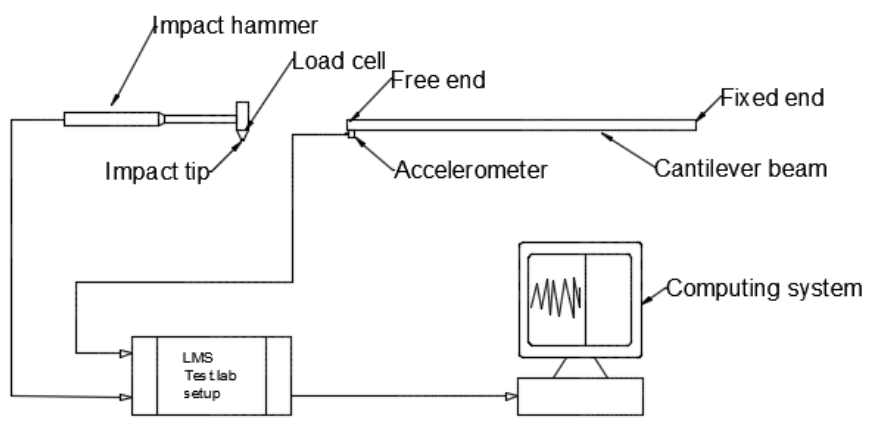

Figure 4: Impact test setup schematic

Observing the damping effect, Beam-2 and Beam-3 were machined in HAAS 3-axis milling machine at $2400 \mathrm{rpm}$ of spindle speed with a feed rate of 15 inches per minute to measure the acceleration during machining and to observe the surface finish of the machined workpiece. A 4 flute 3/8-inch HSS flat end mill tool was used for machining of the workpiece to create a 3-inch slot of 20 thou depth from the free end inwards, at the center of beams with aluminum sheets attached. Siemens Test.lab 'Signature' module was used to measure the online acceleration signals. A profilometer was used to measure the surface roughness of the workpiece.

\section{RESULTS AND DISCUSSION}

The permanent magnets were simulated using the ANSYS Maxwell software and it was observed that the magnetic flux density increases as the distance between the magnets decreases. Thus, as per the magnetic flux density results, the air gap between the cantilever beam and the magnets on either side was kept constant at $0.8 \mathrm{~mm}$. The damping effect can be clearly seen in the frequency response function plots in Figure 5.

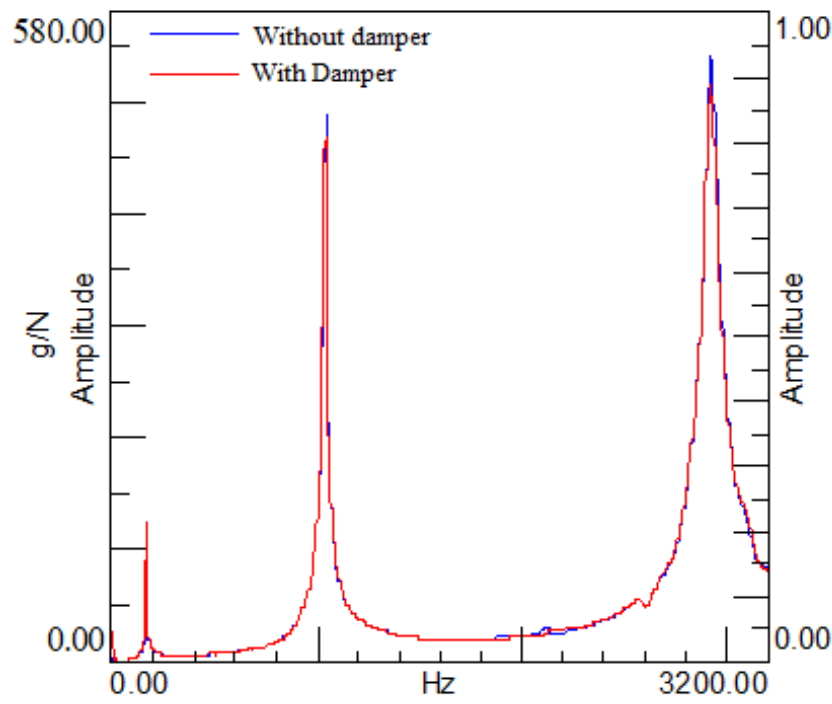

(a) 


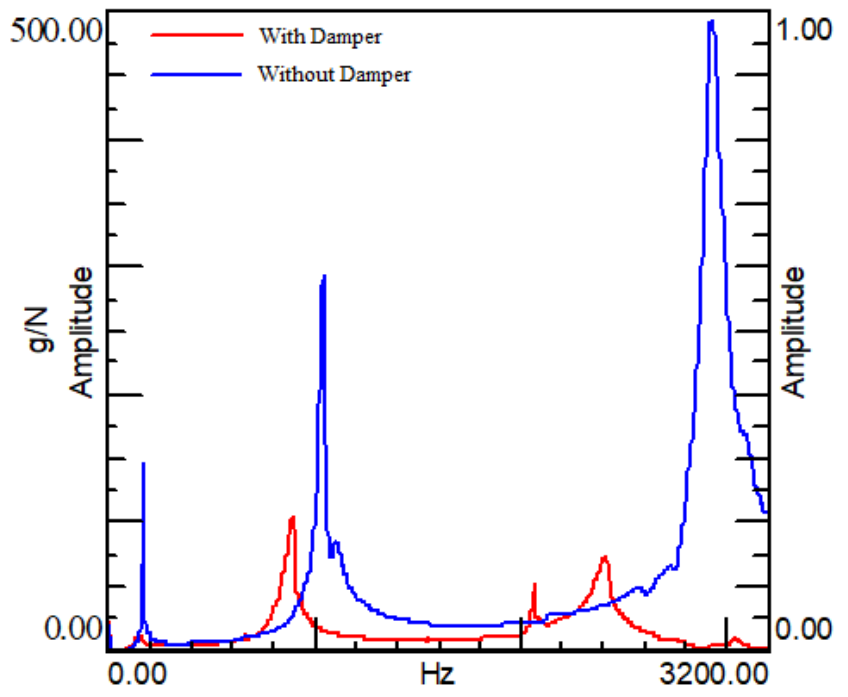

(b)

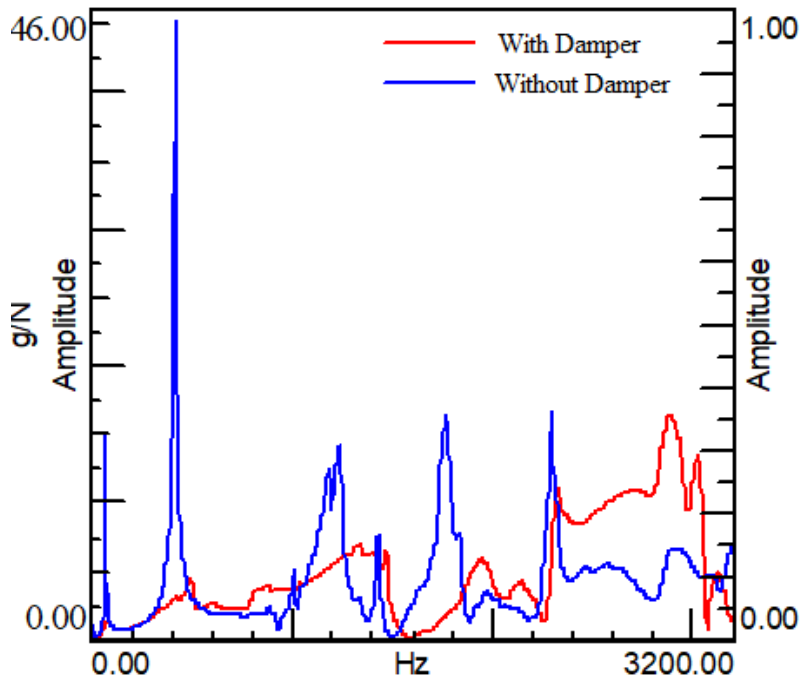

(c)

Figure 5: Frequency response function plots for (a) Beam-1, (b) Beam-2, and (c) Beam-3.

\section{IV.I. MODAL ANALYSIS}

The frequency response function (FRF) plots for the three cases of the beams (with and without aluminum sheet) were obtained from LMS Test.lab software, given in Figure 5. For Beam-1, as from the FRF plot in Figure 5(a), there is not much effect of the damper on the amplitude and damping ratio by the use of damper.

The average damping ratio ( $\% \%)$ for Beam-1 increased from $2.95 \%$ to $3.41 \%$ with the use of damper. However, for Beam-2, it changed from $3.51 \%$ to $12.26 \%$ and for Beam-3, it moved from $6.66 \%$ to $40.38 \%$. Table- 1 and Table- 2 shows the change in natural frequency, amplitude and damping ratio for the first three modes of Beam-2 and Beam-3, which were considered for this research. The maximum damping ratio and minimum amplitude for Beam-2 and Beam-3 were observed at mode 1 of each beam arrangement where the damping ratio increased more than 7 times for the Beam-2 and 12 times for Beam-3 and the amplitude decreased by more than 10 times, when the damper was placed at the free end of the beams.

The Beam-2 and Beam-3, with a conducting sheet attached to the free end, experienced the maximum damping because of use of the axial component of the magnetic field and the closeproximity of the magnets, providing a higher magnetic flux for more eddy currents. The closely packed magnetic field lines passing through the beam had the maximum interaction with the beam, even with minimum movements. The thicker beam with conducting sheet has higher stiffness making it less affected by the eddy currents than the thin beam. The damping force also depends on the thickness of the conducting sheet attached. Making the sheet thicker will increase the eddy current's density but can also make the system unstable.

TABLE 1: Modal testing results for Beam-2

\begin{tabular}{|c|c|c|c|c|c|c|}
\hline Parameter & & Without Damper & & & With Damper & \\
\hline Mode 1 & 165.6 & 146.72 & 1.06 & 146.8 & 11.87 & 7.79 \\
\hline Mode 2 & 1040.2 & 293.22 & 1.00 & 893.5 & 104.88 & 2.25 \\
\hline
\end{tabular}

TABLE 2: Modal testing results for Beam-3

\begin{tabular}{|c|c|c|c|c|c|c|}
\hline Parameter & \multicolumn{3}{|c|}{ Without Damper } & \multicolumn{3}{|c|}{ With Damper } \\
\hline Modes & $\begin{array}{l}\text { Natural frequency } \\
(\mathrm{Hz})\end{array}$ & Amplitude $(\mathrm{g} / \mathrm{N})$ & Damping ratio $(\zeta \%)$ & Natural frequency $(\mathrm{Hz})$ & Amplitude (g/N) & Damping ratio $(\zeta \%)$ \\
\hline Mode 1 & 68.7 & 15.27 & 1.73 & 71.8 & 1.47 & 21.53 \\
\hline
\end{tabular}




\section{IV.II. MACHINING TEST}

The machining tests were performed for Beam-2 and Beam3 only, because of the significant damping observed during modal analysis. The machining tests showed a reduction in the acceleration of both the beams during machining. For Beam-2, the maximum positive acceleration reduced from 415 to $180 \mathrm{~g}$. For thin beam with aluminum sheet, the maximum acceleration dropped from $368 \mathrm{~g}$ to $164 \mathrm{~g}$, as shown in the Figure 6. This change in the acceleration can be related to the damping shown in the modal testing. However, Beam-3 has lower stiffness which causes it to bend a bit when the cutting tool makes a contact, making the beam in less contact with the tool, as can be seen in the machined surface without the damping fixture. This causes undercutting of the beam. When the damping fixture was applied at the end of the beam, the damping force resisted the motion of the beam, making a better contact with the cutting tool. Also, the irregular pattern of the acceleration was caused by continuously changing of overhung mass when the cutting tool moves toward the clamped end of the beam.
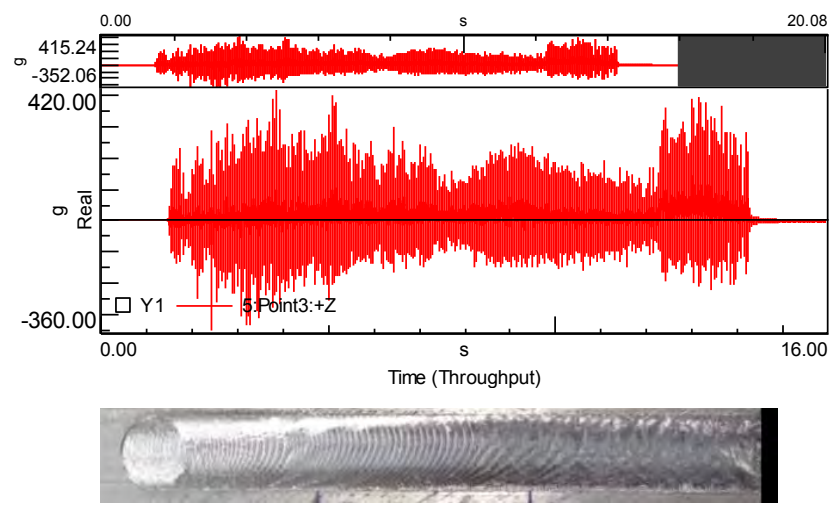

(a)
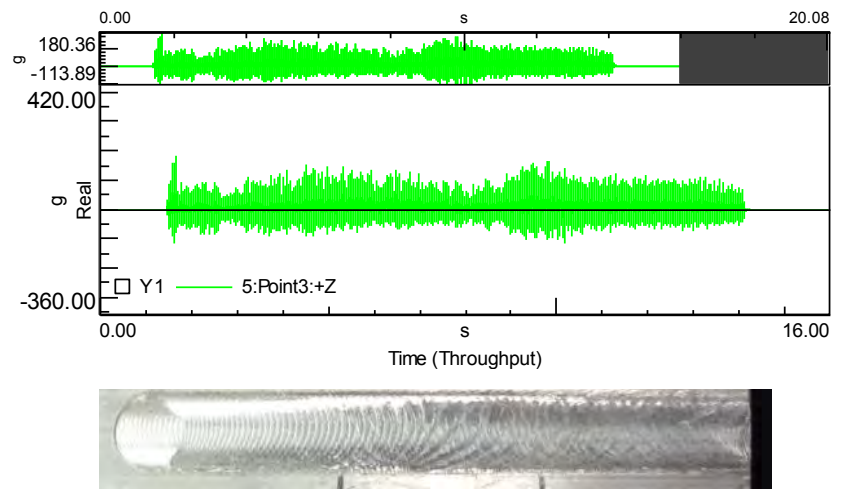

(b)

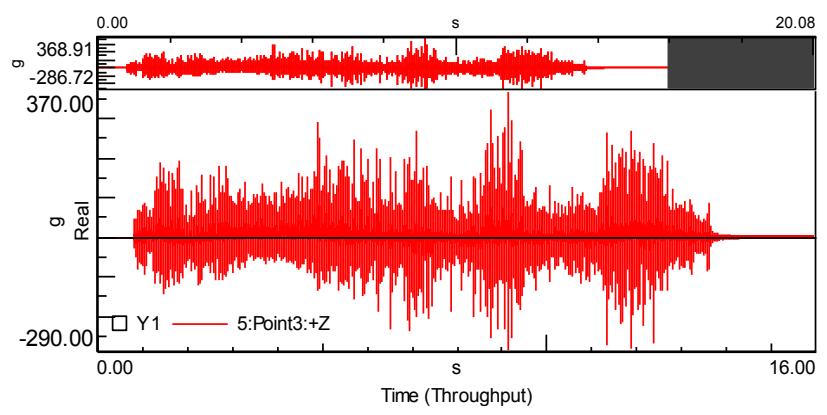

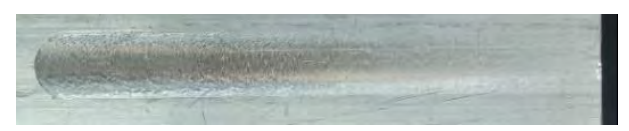

(c)

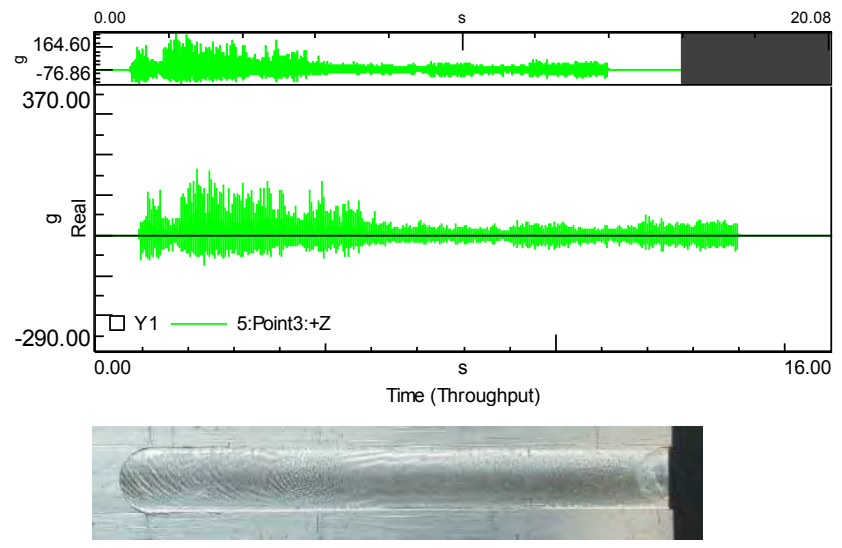

(d)

Figure 6: Machining results at 2400 rpm spindle speed, 15 inches per minute of feed and 20 thousandth-of-an-inch cutting depth for (a) Beam-2 without damper, (b) Beam-2 with damper, (c) Beam-3 without damper, and (d) Beam-3 with damper.

The average surface roughness of the beams was measured using a profilometer and Beam-2 showed a better surface finish, with average roughness values $\left(\mathrm{R}_{\mathrm{a}}\right)$ changing from $3.52 \mu \mathrm{m}$ to $2.17 \mu \mathrm{m}$, while for Beam-3 the initial impact causes deflection of the beam making it difficult to compare the surface roughness, but a higher material removal rate was observed (undercutting was prevented by the damper).

\section{CONCLUSIONS}

Machining of thin-walled parts is considered a challenge because of the chatter induced due to lower stiffness of workpieces. To counter this issue, an auxiliary fixture, based on eddy currents, was developed to damp the vibrations in a cantilever beam and to improve the surface finish of the product. The fixture developed is a passive eddy current damper which is simple to use and does not need a power supply for its operation.

The damping force (Lorentz force) applied by the eddy current damper reduced the vibrations in the workpiece when there is an interaction of a conductor with the magnetic field. The damping force was increased by attaching a conducting sheet to the workpiece to increase the magnetic flux density with respect to the conductor sheet. The damping force was observed as maximum when the cantilever beam workpiece has low stiffness, a conducting sheet is attached to the free end and the gap between the magnets is kept small. The fixture proposed highly damps the first three modes of the tested beams.

The modal testing and the machining tests validated the usability of eddy current dampers for better machining stability, by suppressing chatter. Better surface finish and high material removal rate was observed by using the proposed auxiliary fixture. Future work will focus on optimizing the thickness of the conducting sheet attached and also on using it as a tuned mass damper. 


\section{ACKNOWNLEDGEMENT}

This work was supported by the Natural Sciences and Engineering Research Council of Canada (NSERC).

\section{REFERENCES}

[1] G. Quintana and J. Ciurana, "Chatter in machining processes: A review," Int. J. Mach. Tools Manuf., vol. 51, no. 5, pp. 363-376, 2011. doi:10.1016/j.ijmachtools.2011.01.001.

[2] J. Y. Chang, G. J. Lai, and M. F. Chen, "A study on the chatter characteristics of the thin wall cylindrical workpiece," Int. J. Mach. Tools Manuf., vol. 34, no. 4, pp. 489-498, 1994. doi:10.1016/0890-6955(94)90080-9.

[3] F. J. Campa, L. N. Lopez De Lacalle, and A. Celaya, "Chatter avoidance in the milling of thin floors with bull-nose end mills: Model and stability diagrams," Int. J. Mach. Tools Manuf., vol. 51, no. 1, pp. 43-53, 2011. doi:10.1016/j.ijmachtools.2010.09.008.

[4] J. Ma, D. Zhang, B. Wu, M. Luo, and B. Chen, "Vibration suppression of thin-walled workpiece machining considering external damping properties based on magnetorheological fluids flexible fixture," Chinese J. Aeronaut., vol. 29, no. 4, pp. 1074-1083, 2016. doi:10.1016/j.cja.2016.04.017.

[5] D. Bae, Jae-Sung;kwak,Moon k.;Inman, "Vibration suppression of a cantilever beam using eddy current damper," J. Sound Vib., vol. 284, pp. 805-824, 2004. doi:10.1016/j.jsv.2004.07.031.

[6] H. A. Sodano, J. S. Bae, D. J. Inman, and W. Keith Belvin, "Concept and model of eddy current damper for vibration suppression of a beam," J. Sound Vib., vol. 288, no. 4-5, pp. 1177-1196, 2005. doi:10.1016/j.jsv.2005.01.016.

[7] H. D. Wiederick, N. Gauthier, D. A. Campbell, and P. Rochon, "Magnetic braking: Simple theory and experiment," Am. J. Phys., vol. 55, no. 6, pp. 500-503, 1987. doi:10.1119/1.15103.

[8] O. Rodrigues, O. Taskar, S. Sawardekar, H. Clemente, and G. Dalvi, "Design \& Fabrication of Eddy Current Braking System," Int. Res. J. Eng. Technol., vol. 3, no. 4, pp. 809-815, 2016.

[9] H. A. Sodano and D. J. Inman, "Non-contact vibration control system employing an active eddy current damper," J. Sound Vib., vol. 305, no. 4-5, pp. 596-613, 2007. doi:10.1016/j.jsv.2007.04.050.

[10] T. Cheng and I. Oh, "Vibration Suppression of Flexible Beam Using Electromagnetic Shunt Damper," IEEE Trans. Magn., vol. 45, no. 6, pp. 2758-2761, 2009. doi:10.1109/TMAG.2009.2020549.

[11] B. Ebrahimi, M. B. Khamesee, and F. Golnaraghi, "Permanent magnet configuration in design of an eddy current damper," Microsyst. Technol., vol. 16, no. 1-2, pp. 19-24, 2010.

doi:10.1007/s00542-008-0731-z. 


\section{Shaker Vibration Test of Epoxy Composites Reinforced with Pristine and Functionalized Carbon Nanotubes}

\author{
M. Rafiee*, M. Labrosse \\ Department of Mechanical Engineering, University of \\ Ottawa \\ Ottawa, ON K1N 6N5, Canada \\ mrafiee20@gmail.com,mrafiee@uottawa.ca
}

\author{
F. Nitzsche \\ Department of Mechanical and Aerospace Engineering, \\ Carleton University, 1125 Colonel By Drive, \\ Ottawa, ON K1S 5B6, Canada
}

\begin{abstract}
The vibration and damping characteristics of epoxy composites reinforced with pristine and functionalized multi-walled carbon nanotubes (MWCNTs) were investigated experimentally with potential use as integral passive damping elements in structural composite applications. The MWCNTs were introduced into the acetone solvent and then mixed with epoxy resin through a sonication process and mechanical stirring. The solvent was evaporated by means of magnetic hot plate and the hardener was added to the mixture once it was cooled down to room temperature. The MWCMTs/epoxy mixture was then injected into a mold to form the nanocomposite specimen. Nanocomposite specimens were fabricated for six different MWCNT loadings $(0.02,0.041$, $0.061,0.123,0.25$ and $0.37 \mathrm{wt} \%)$. Microstructural analysis, tensile and bending tests were carried out to examine the effects of pristine multi-walled carbon nanotubes (pMWCNTs) and functionalized multi-walled carbon nanotubes (f-MWCNTs). The frequency response functions (FRFs), coherence and phase diagrams of nanocomposites were measured using a shaker vibration technique. The periodic upchirp signal was generated by a shaker to excite the cantilever nanocomposite specimen at the base. The damped natural frequencies and damping ratios were obtained for different loadings of MWCNTs. The experimental results indicated that the damped natural frequencies of p-MWCNTs/epoxy and fMWCNTs/epoxy composites increased by adding MWCNTs up to 0.12 wt. $\%$ and, after that, decreased with higher MWCNT content. Another finding was that $\mathrm{p}-\mathrm{MWCNT}$ sere beneficial to improve the damping ratio of nanocomposites. While the damping ratios of f-MWCNTs with loadings of 0.02-0.06 wt.\% were higher than p-MWCNTs ones, they did not increase at higher CNTs contents for the first mode of vibration.
\end{abstract}

Keywords-experimental vibration, carbon nanotubes, polymer composite; vibration damping; functionalize CNTs; epoxy nanocomposite.

\section{INTRODUCTION}

Carbon nanotubes-reinforced polymers have attracted considerable interest due to their potential to exhibit physicochemical properties, such as mechanical, electrical, and thermal properties, that are improved compared to those of their hosting polymers[1-4].

In addition, the investigation of the vibration and damping behavior of composites is an important issue in mechanical and aerospace engineering. Many researchers have studied them both experimentally [5-7] and theoretically [1,7]. A review of state-of-the-art in vibration of composite beams and blades was presented by Rafiee et al. [2]. Studies in the recent years have shown that carbon nanotubes can introduce damping to attenuate the vibrations and noise of two-phase and three-phase epoxy nanocomposites by dissipating substantial amounts of vibration energy [5-7]. However, no study has been reported on the vibration and damping characteristics of functionalized MWCNTs-reinforced epoxy composites.

The present research aimed to understand the practical aspects related to the vibration and damping behavior of pristine and functionalized MWCNTs/epoxy composites with potential use as integral passive damping elements in structural composite applications. In addition, th study also focused on evaluating the effect of MWCNT functionalization on mechanical properties of two-phase epoxy resin composites.

\section{MATERIALS, PROCESSING AND FABRICATION}

\section{A. Materials}

The pristine multi-wall carbon nanotube (NC7000) and amine functionalized multi-wall carbon nanotube (NC7000NH2) were received from Nanocyl S.A., Belgium, with an average diameter and length of $9.5 \mathrm{~nm}$ and $1.5 \mu \mathrm{m}$, respectively. MWCNTs were produced via the Catalytic Chemical Vapor Deposition (CCVD) process with carbon purity of $90 \%$. Low viscosity diglycidyl ether of bisphenol-A (DGEBA) epoxy resin PT-2712 Part A and diethyltoluenediamine (DETDA) curing agent PT-2712 Part B1 produced by PTM\&W Industries, Inc., USA were used. Epoxy 
resin Part A and B mixing weight ratio was 100/22 as per manufacturer recommendation.

\section{B. Processing and fabrication of MWCNTs/polymer nanocomposites}

The two-phase polymer matrix composites reinforced by pristine multi-wall carbon nanotube (p-MWCNTs) or functionalized multi-wall carbon nanotube (f-MWCNTs) were prepared with nanotube loadings of $0.025,0.050,0.075,0.15$, 0.3 and 0.45 of DGEBA $w \mathrm{t} \%$. The equivalent nanotube loadings with respect to total weight (DGEBA+DETDA) were $0.020,0.041,0.061,0.123,0.246$ and $0.369 \mathrm{wt} \%$, respectively. Figure 1 shows a schematic of the method for the preparation of the MWCNTs/epoxy nanocomposites.
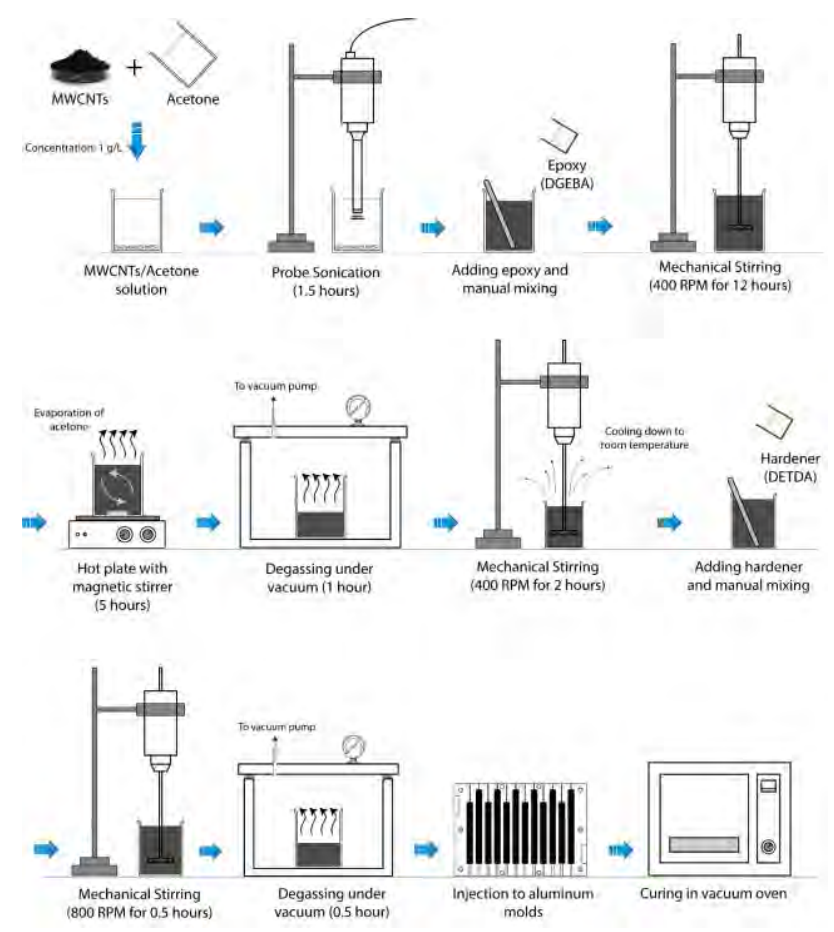

Figure 1. Fabrication process of MWCNTs/epoxy two-phase composites.

The samples were cured at room temperature for 15 hours followed by a post cure of $1{ }^{\circ} \mathrm{C} / \mathrm{min}$ ramp rate, held every $20^{\circ} \mathrm{C}$ for an hour, then continued until a final hold of three hours at $100^{\circ} \mathrm{C}$ to remove the possible thermal history.

\section{EXPERIMENTAL SETUP}

The shaker vibration test was used to measure the natural frequencies and damping factors of nanocomposites specimens in the form of cantilever beams. The clamped-free beam was excited by a vibration shaker (Brüel \& Kjær Sound \& Vibration Measurement A/S, Denmark) at the clamped end, and the response of the beam was tracked by means of accelerometers. The computed frequency response functions (FRFs) gave information about the natural frequencies of the composite beam.

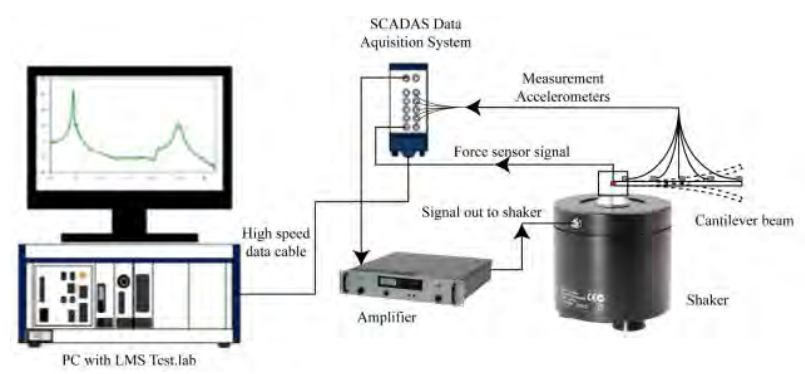

Fig. 2. Schematic diagram of the experimental procedure for vibration testing of MWCNTs- reinforced composites.

Rectangular specimens $12.7 \mathrm{~mm}$ (0.5 in) wide, $3.175 \mathrm{~mm}$ thick (1/8 in) and $152.4 \mathrm{~mm}$ long (6 in) were prepared for vibration tests according to ASTM E756. The free beam length is defined as the distance between the clamp and the specimen tip and was measured as $130 \mathrm{~mm}$. Figure 2 illustrates the instrumentation used for the vibration test. Acceleration was measured at five locations by means of miniature lightweight accelerometers. A piezoelectric crystal force transducer (Quartz ICP $\AA$ Force Ring, PCB $\AA$ Model 201B03, measurement range of $2.224 \mathrm{kN}$ ) was used to measure the excitation force exerted by the electrodynamic shaker. A power amplifier was required to drive the shaker. Periodic chirp excitation signals were used with a frequency bandwidth of $0-1024 \mathrm{~Hz}$ to guarantee the highest quality measurements. The tests were performed using the LMS Test.Lab by Siemens ${ }^{\circledR}$, which is an integrated solution for experimental vibrational analysis including tools for data acquisition (LMS SCADAS III with eight measurement channels and two output channels), test execution and data processing (LMS Testing Solution Software). The LMS SCADAS III was used to acquire the signals from the force sensor and the piezoelectric accelerometers and to control the amplifier connected to the shaker, by means of a digital-analog converter output. Spectral Testing module of LMS Testing Solution Software was used for shaker test. The Modal Analysis module was then used to analyze the power spectral density (PSD) of the excitation signal, the FRFs and the coherence functions, and to calculate FRF sum functions (i.e. the sum of the five FRFs). Finally, the PolyMAX frequency domain identification method [27], which is based on a least squares fit, was used to identify the natural frequencies, damping ratios and vibration modes of MWCNTs/epoxy nanocomposites. All measurements were acquired in the frequency range 10-1024 Hz, with a resolution of $0.125 \mathrm{~Hz}$. Each measurement was averaged over 20 acquisitions.

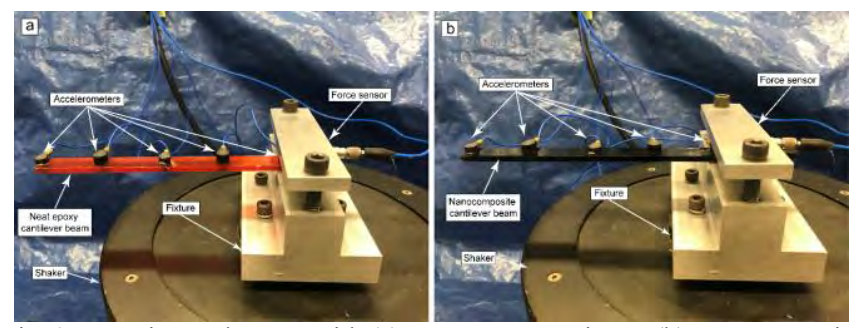

Fig. 3. Experimental set-up with (a) neat epoxy specimen, (b) nanocomposite specimen. 


\section{RESULTS AND DISCUSSION}

Damped natural frequencies, frequency-response functions (FRFs), phase diagram, coherence, and damping ratios about the first three vibration modes were obtained and shown in Figures 4 through 7.
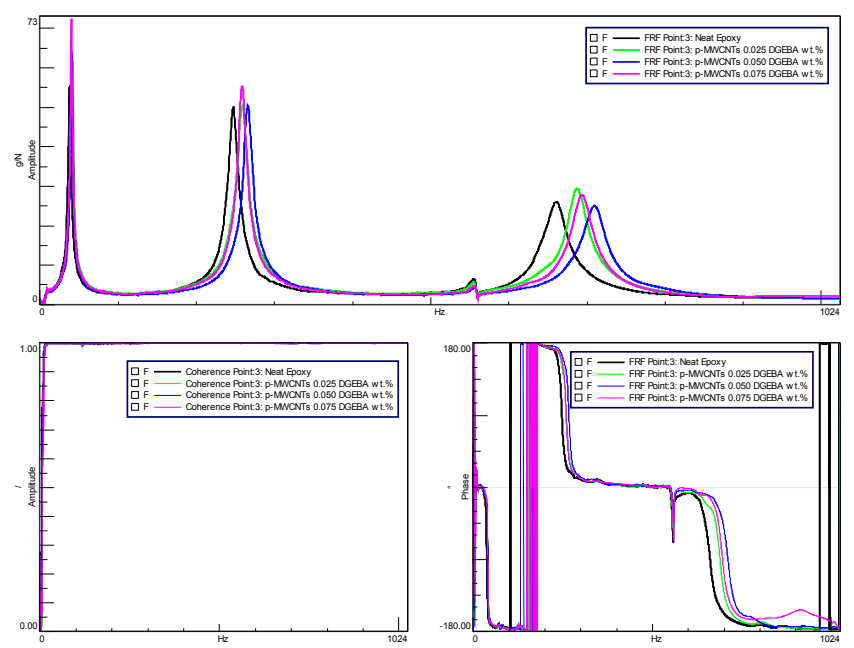

Fig. 4. FRFs, coherence and phase diagrams for neat epoxy and p-MWCNTs $(0.025,0.050$ and 0.075 DGEBA wt.\%) reinforced composites

As can be seen, the damped natural frequencies of $\mathrm{p}$ MWCNTs/epoxy and f-MWCNTs/epoxy composites increased by adding CNTs up to $0.12 \mathrm{wt.} \%$, and decreased after that with higher MWCNTs content. The maximum damping improvement of $9.4 \%$ happened at $0.02 \mathrm{wt} \%$ of $\mathrm{f}$ MWCNTs/epoxy composites while it was $7.2 \%$ with the equivalent pristine MWCNTs. The improvement in damping of the fundamental natural frequency was more significant than that at higher natural frequencies. For instance, addition of $0.02 \%$ of f-MWCNTs improved the damping of the fundamental, second and third natural frequencies by $9.4 \%$, $7.5 \%$ and $6.3 \%$, respectively.
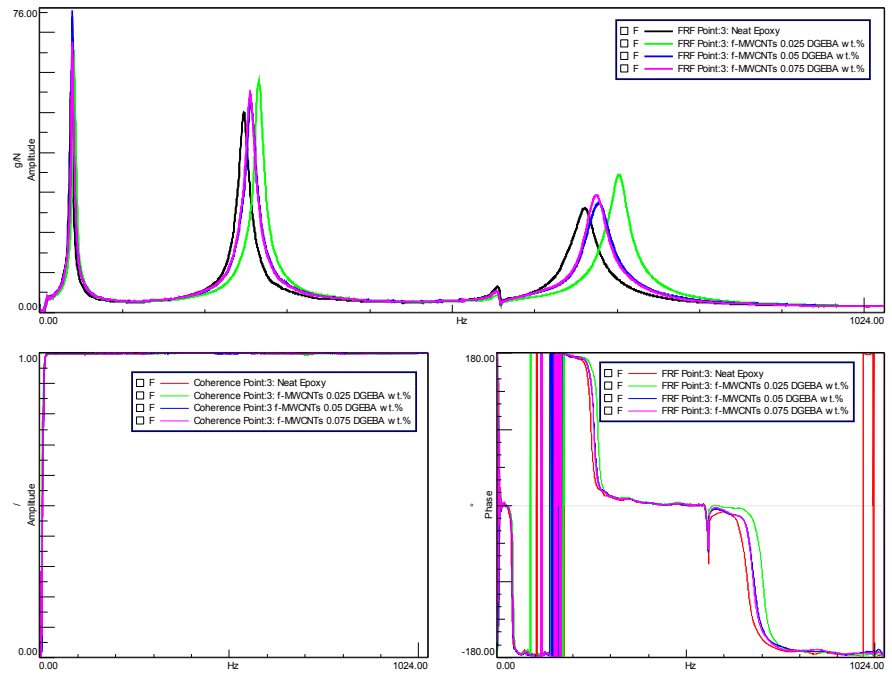

Fig. 5. FRFs, coherence and phase diagrams for neat epoxy and f-MWCNTs $(0.025,0.050$ and 0.075 DGEBA wt.\%) reinforced composites
The relative effects of pristine vs. functionalized carbon nanotubes on the damping ratios of epoxy-based composite beams were investigated and are depicted in Fig. 6 and 7, respectively. As one can observe from Fig. 6, the damping ratios dropped by $18 \%$ till loading of 0.06 wt. $\%$ and then improved by adding $\mathrm{p}-\mathrm{MWCNTs}$ in the composites for the first vibration mode. A $46 \%$ improvement in damping ratio of $p-$ MWCNTs/epoxy composite was observed with a loading of 0.37 wt.\%. For f-MWCNTs/epoxy composites, a decrease in damping ratio can be observed over the whole range of loadings While the damping ratios with f-MWCNTs at loadings of 0.02-0.06 wt.\% were still higher than those with pMWCNTs, they did not increase with higher CNT contents for the first mode of vibration.

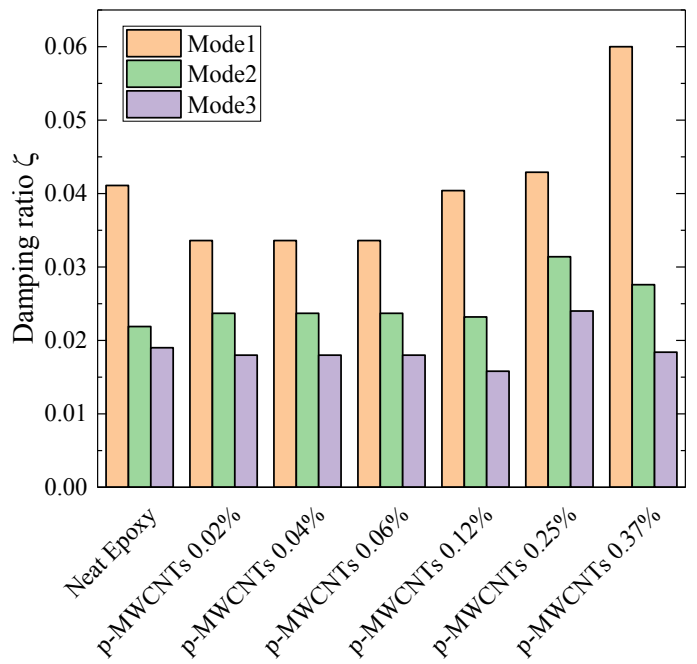

Fig. 6. Damping ratio of p-MWCNTs-reinforced composites with different loadings.

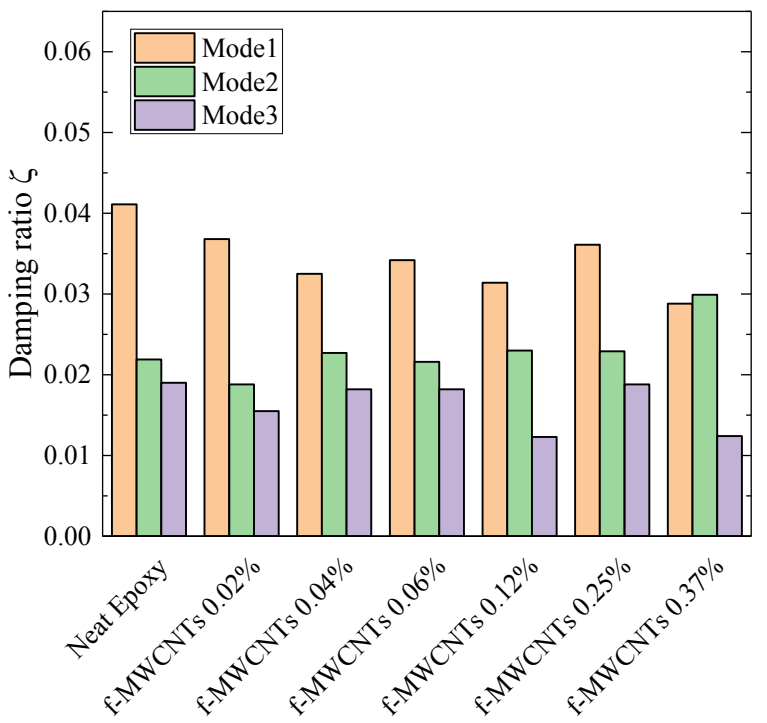

Fig. 7. Damping ratio of f-MWCNTs-reinforced composites with different loadings.

For the second mode of vibration, both pristine and functionalized multiwalled carbon nanotubes improved the damping characteristics of nanocomposite beams. There was 
over $40 \%$ improvement in damping ratio in p-MWCNTs/epoxy composites at $0.25 \mathrm{wt} . \%$ loading. The maximum improvement in damping ratio in f-MWCNTs/epoxy composites was observed at the 0.37 wt.\% loading and reached $36.5 \%$. Damping ratio results for the third mode of vibration showed no significant improvements.
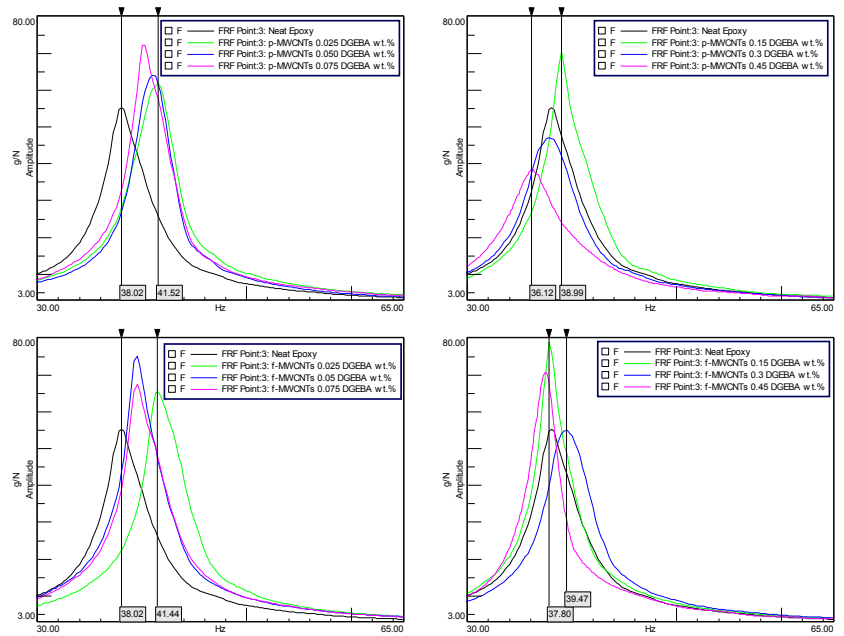

Fig. 8. Magnification of FRFs, coherence and phase diagrams around fundamental resonance peak of MWCNTs/epoxy.
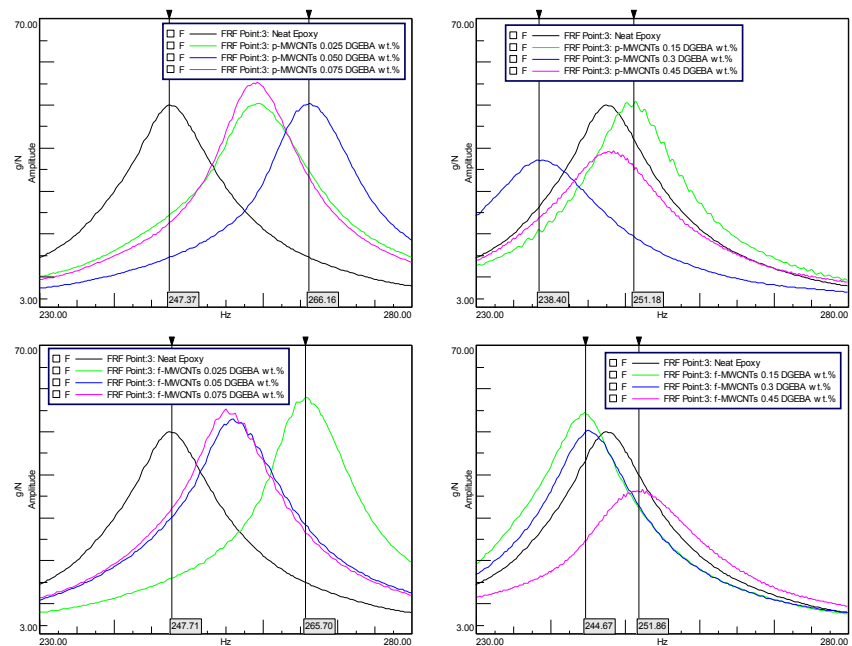

Fig. 9. Magnification of FRFs, coherence and phase diagrams around second resonance peak of MWCNTs/epoxy.

\section{CONCLUSION}

The experimental vibration and damping analyses of $\mathrm{p}$ MWCNTs/epoxy and f-MWCNTs/epoxy nanocomposites were completed. Nanocomposite specimens were fabricated for six different MWCNT loadings (0.02, 0.041, 0.061, 0.123, 0.25 and $0.37 \mathrm{wt} \%$ ). Microstructural analyses and tensile and bending tests were carried out to examine the effects of $p$ MWCNTs and f-MWCNTs. The frequency response functions, coherence and phase diagrams of nanocomposites were obtained using a periodic up-chirp forced vibration technique. The damped natural frequencies and damping ratios were obtained for different loadings of MWCNTs. The experimental results indicated that the damped natural frequencies of $\mathrm{p}$ MWCNTs/epoxy and f-MWCNTs/epoxy composites increased by adding CNTs up to 0.12 wt.\% and decreased with higher MWCNTs content. Another finding was that the improvement in fundamental natural frequency was more significant compared than that at higher natural frequencies. The forced vibration test also confirmed the beneficial effect of $p$ MWCNTs in improving the damping ratio of nanocomposites for the first and second vibration modes. However, a decrease in damping ratio was observed in f-MWCNTs/epoxy composites. For instance, a $46 \%$ improvement in damping ratio associated with fundamental vibration mode was observed at loadings of $0.37 \mathrm{wt} \%$ in p-MWCNTs/epoxy composites. While the damping ratios with f-MWCNTs at loadings of $0.02-$ 0.06 wt. $\%$ were still higher than with $\mathrm{p}$-MWCNTs, they were not increasing at higher CNTs contents for the first mode of vibration.

\section{REFERENCES}

[1] M. Rafiee, X. Q. He, S. Mareishi, and K. M. Liew, "Modeling and stress analysis of smart CNTs/fiber/polymer multiscale composite plates," Int. J. Appl. Mech., vol. 6, no. 3, p. 1450025, 2014.

doi: $10.1142 / \mathrm{S} 1758825114500252$.

[2] M. Rafiee, F. Nitzsche, and M. Labrosse, "Dynamics, vibration and control of rotating composite beams and blades: A critical review," ThinWalled Struct., vol. 119, pp. 795-819, 2017.

doi: 10.1016/j.tws.2017.06.018.

[3] I. Rafique, A. Kausar, and B. Muhammad, "Epoxy Resin Composite Reinforced with Carbon Fiber and Inorganic Filler: Overview on Preparation and Properties," Polym. Plast. Technol. Eng., vol. 55, no. 15, pp. 1653-1672, 2016. doi: 10.1080/03602559.2016.1163597.

[4] G. Mittal, V. Dhand, K. Y. Rhee, S.-J. Park, and W. R. Lee, "A review on carbon nanotubes and graphene as fillers in reinforced polymer nanocomposites," J. Ind. Eng. Chem., vol. 21, pp. 11-25, 2015. doi: 10.1016/j.jiec.2014.03.022.

[5] Fig. 11. Magnification of FRFs, coherence and phase diagrams around second resonance peak of MWCNTs/epoxy.

[6] H. Rajoria and N. Jalili, "Passive vibration damping enhancement using carbon nanotube-epoxy reinforced composites," Compos. Sci. Technol., vol. 65, no. 14, pp. 2079-2093, 2005. doi: 10.1016/j.compscitech.2005.05.015.

[7] S. C. Her and S. W. Yeh, "Fabrication and Characterization of the Composites Reinforced with Multi-Walled Carbon Nanotubes," J. Nanosci. Nanotechnol., vol. 12, no. 10, pp. 8110-8115, 2012.

doi: 10.1166/jnn.2012.4518.

[8] K. Mehar, S. K. Panda, and T. R. Mahapatra, "Theoretical and experimental investigation of vibration characteristic of carbon nanotube reinforced polymer composite structure," Int. J. Mech. Sci., vol. 133, pp. 319-329, 2017. doi: 10.1016/j.ijmecsci.2017.08.057. 


\section{The Effect of High Strain Rate on the Piezoresistance of Polydimethylsiloxane with Carbon Nanotubes}

\author{
Farouk Wahsh \\ Mechanical Engineering Department \\ York University \\ Toronto, Canada \\ faroukw@my.yorku.ca
}

\author{
Aleksander Czekanski \\ Mechanical Engineering Department \\ York University \\ Toronto, Canada \\ alex.czekanski@lassonde.yorku.ca
}

\begin{abstract}
This paper describes the piezoresistive properties of a nanocomposite made of polydimethylsiloxane and multiwalled carbon nanotubes that is subjected to high strain rate. We highlight the production process of the composite, the testing protocol, and the results obtained.
\end{abstract}

Keywords: piezoresistance; nanocomposite; high strain rate; polydimethylsiloxane; carbon nanotubes; Kolsky bar

\section{INTRODUCTION}

Over the past decade, smart materials have become increasingly popular owing to their intrinsic functional properties that allow for fast, easy, and inexpensive sensing and actuating.

Composites based on nanoscale fillers such as carbon nanotubes (CNTs) are considered the new generation of smart materials. Since their discovery in 1991, CNTs have had a significant impact on the field of material science. They are cylindrical in shape and consist of carbon molecules covalently bonded together to form a tube-shaped lattice, lending them their unique properties - superior electrical and magnetic conductivity, lightness, and strength. Polydimethylsiloxane (PDMS) is the simplest member of the silicone polymer family and is characterized by its attractive mechanical properties and translucency.

Much work has been done on developing highly sensitive strain sensors made from CNT-polymer nanocomposites. This paper investigates the potential of PDMS mixed with CNTs in the development of high strain rate (impact) sensors.

\section{Methodology}

\section{A. Composite Production Process}

The material in question is an elastomeric composite made from PDMS as the matrix and multi-walled carbon nanotubes (MWCNTs) as the phase. The production of this composite was a complex, time-consuming, and dangerous process. First, there is the issue of avoiding exposure, since CNTs are considered hazardous and not enough research has been done on the risks they pose to human health. Second, the dispersion of CNTs in PDMS was difficult to achieve. The reason for that is the tendency of CNTs to agglomerate over time when mixed in liquids. To prevent these agglomerations, chloroform was used as a solvent to aid the dispersion [1], and the material was sonicated repeatedly throughout the process. Third, the curing time of PDMS at room temperature is unfavorably long at 48 to $72 \mathrm{~h}$. This creates a wide window in which CNTs can agglomerate. To remedy this problem, the PDMS was cured at $110^{\circ} \mathrm{C}$, which reduced curing time to $30 \mathrm{~min}$. Last, to cure the material at high temperature, a custom press-casting heated mold was developed.

The mold was made from two blocks of aluminum cut using computer numerical control (CNC). The bottom part (drag) contained the PDMS, while the top part (cope) had an emboss that forced the PDMS on the surface out of the cast area and into channels on all four sides of the mold that contained the excess material. The cope was hinged to the drag of the mold and closed with a mechanism resembling that of a waffle maker. This design prevents imperfections caused by air pockets and bubbles that would otherwise get trapped during assembly below the cope and inside the cast. Moreover, three though-all holes were drilled in the cope below the surface of the cast to house two cartridge heaters and one thermocouple. The heaters were connected to a relay, a thermocouple, and a PID controller in a circuit that controls the temperature inside the mold according to the user's selection.

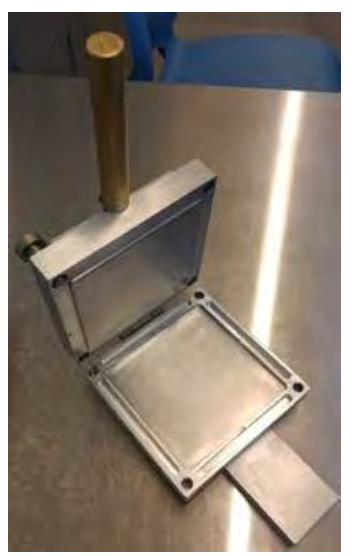

Figure 1. Heated Press-casting Mold 


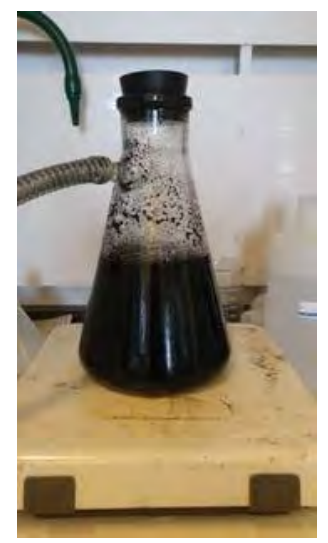

Figure 2. PDMS/CNT Solution - Chloroform Expulsion

Our primary objective was to study the effect of CNT concentration in PDMS on the electrical resistivity when the material was subjected to high strain rates. Therefore, three different CNT concentrations were studied, ranging from $1 \mathrm{wt}$. $\%$ to 2 wt. $\%$, with 0.5 wt. $\%$ increments.

Dispersion is a spatial quality that represents how evenly distributed CNTs are in the PDMS matrix. Manually mixing CNTs in PDMS does not generate enough shear forces to overcome the inter-tube Van der Waals forces and separate the tubes from their aggregations [1]. On the other hand, more advanced techniques such as mechanical stirring and sonication were found to deliver better results in dispersing nanoparticles in solutions. However, the efficiency of these methods is greatly reduced with the use of highly viscous liquids such as PDMS. Therefore, a solvent was used to dissolve the PDMS, thereby reducing its viscosity. Chloroform was chosen for two reasons. One, CNTs were found to disperse well in chloroform compared with their dispersion in other reagents like toluene [1]. Two, chloroform dissolved PDMS without chemically reacting with it, unlike other reagents that might provide better dispersibility. Once the dispersion process was complete, the solution was heated to evaporate the chloroform and expel it from the solution. To speed up the process, a vacuum was introduced to encourage the chloroform's evaporation as shown in Fig. 2. Finally, the solution was cast in the mold to produce a $3 \mathrm{~mm} \times 120 \mathrm{~mm} \times 120 \mathrm{~mm}$ sheet of the composite.

The production process ${ }^{1}$ of the material is summarized in the following eight steps:

1. Chloroform was mixed with MWCNTs at a ratio of 1/15 g.mL $\mathrm{mL}^{-1}$ (MWCNT: $\mathrm{CHCl}_{3}$ ) in a beaker and sonicated for $1 \mathrm{~h}$.

2. Chloroform was mixed with PDMS part A at a ratio of 2/3 g. $\mathrm{mL}^{-1}\left(\mathrm{PDMS} \mathrm{CHCl}_{3}\right)$.

3. The solutions from steps 1 and 2 were mixed and sonicated for $3 \mathrm{~h}$.

4. The solution from step 3 was heated in an Erlenmeyer flask under vacuum at $62{ }^{\circ} \mathrm{C}$ while mechanically stirring at $1200 \mathrm{r} . \mathrm{min}^{-1}$ for $3 \mathrm{~h}$ to expel the chloroform.

${ }^{1}$ Applicable to 1 wt. $\%$ of CNTs. For 1.5 wt. $\%$ and 2 wt. \%, the concentration of CNTs in PDMS was adjusted accordingly.

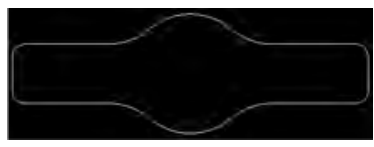

Figure 3. Sample Shape

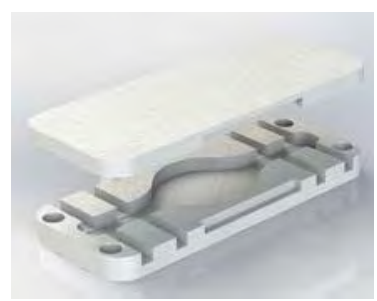

Figure 4. Probe Insertion Guide Housing

5. Part B was added to the solution from step 4, and this solution was manually mixed for $10 \mathrm{~min}$.

6. The solution from step 5 was put in a vacuum chamber to remove any air bubbles trapped under the surface.

7. After degassing, the solution was carefully cast in the mold at a controlled temperature of $110^{\circ} \mathrm{C}$ for $30 \mathrm{~min}$.

8. The mold was carefully opened, and the sheet casting was removed from it.

To prepare the material for testing, samples were laser cut from the sheets produced using an Epilog Laser Fusion M2 cutter in the shape shown in Fig. 3.

To measure the electrical resistance of the material, a fourwire connection [2] was used to control current through the sample and measure the voltage change along the sample. The distance between the probes was controlled for all samples, since it impacts measured potential significantly. A fixed distance was achieved by the design and fused deposition modelling of a small housing for the samples that guide the insertion of the probes, as shown in Fig. 4.

\section{B. Testing}

Testing the material's electrical resistance response to high strain rate was performed using a Kolsky (Split-Hopkinson) (Fig. 5). The Kolsky bar was manufactured in house [3] and was used to subject the material to high strain rates. MATLAB was used for system control, and a National Instruments USB6351 DAQ device was used for data acquisition.

After the probes were inserted in the sample and connected to current and voltage source meters following the four-wire connection, the sample was sandwiched between the incident and transmitted bars of the Kolsky bar, as shown in Fig. 4.

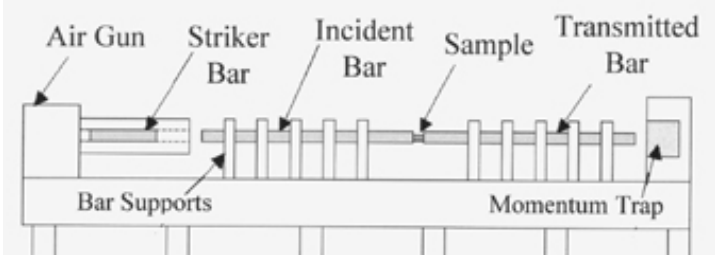

Figure 5. Kolsky (Split-Hopkinson) Bar Schematic 


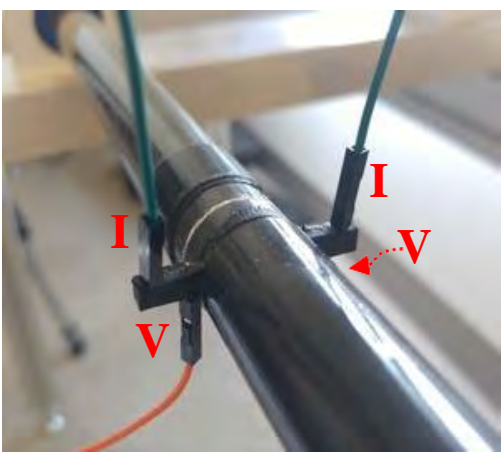

Figure 6. Sample Setup on Kolsky Apparatus

The air-gun pressure was kept constant at 100 psi for all tests to approach reproducibility of results. The DAQ was synced with the trigger through a MATLAB code such that it started collecting data from the strain gauges and the sample the moment the trigger on the Kolsky bar was pulled. The data sampling went on for $5 \mathrm{~s}$ from the moment the trigger was pulled.

\section{RESULTS AND OBSERVATIONS}

\section{A. Composite Production}

The sheets of composite produced were checked for quality to ensure samples were as uniform as possible. First, the surface of the sheet was inspected for cavities caused by trapped air. Second, the sheets were cut in different areas, and the cross-section was inspected for cavities as well. Since the material is black, imperfections within each sample were hard to detect nondestructively. However, a method used to test the uniformity of the sample ensured that the initial resistance of the samples was within the same range. In that way, samples with significant imperfections were eliminated.

We faced several challenges during the production process. First, the chloroform had to be completely evaporated from the material before it was cast. Any remaining chloroform would threaten the chemical purity of the composite, leading to incorrect results. Excess chloroform also affects the physical structure of the final composite. If chloroform exists in the material casted, it will quickly evaporate inside the mold because of the high curing temperature. This creates cavities at the surface of the casting and affects its physical structure, as shown in Fig. 7(a).

Achieving good results depended on trial and error. It took three attempts to finally arrive at a reliable process that produced good-quality sheets of PDMS with CNTs. The sheets produced were free from surface and body imperfections according to our visual inspections. Fig. 7(b) shows a successfully produced sample. Samples were laser cut from these sheets for testing.
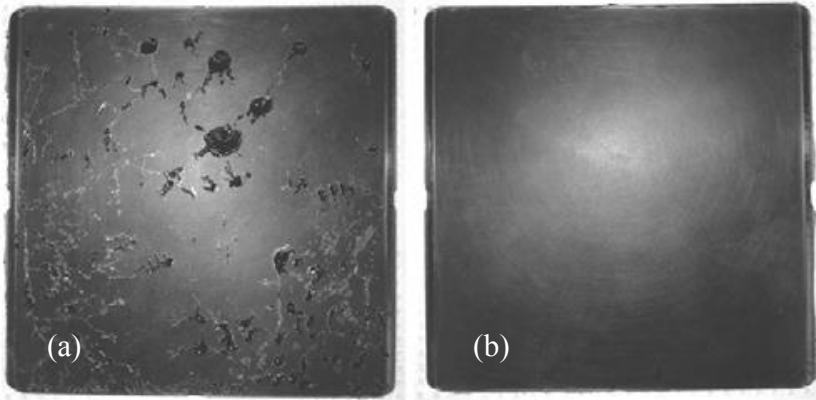

Figure 7. (a) Incomplete Chloroform Evaporation. (b) Complete Chloroform Evaporation.

\section{B. Test Results and Observations}

Dynamic tests were conducted on five samples for each concentration of CNTs in PDMS. The results obtained were found to be consistent and exhibited similar trends. However, only data from one representative set of samples are presented here.

Fig. 8 shows the voltage measured from the sample during the initial moments of impact. The normalized voltage from the strain gauges on the incident and transmitted bars is also shown and represents the stress waves propagating through the system. Early observation of the CNT-PDMS plot shows a spike in voltage reaching up to 2.5 times the initial voltage. Moreover, the voltage was found to rise in steps that are synced with the propagating stress waves in the system. Furthermore, the sample voltage was observed to lag half a period behind the incident wave and was in phase with the transmitted wave.

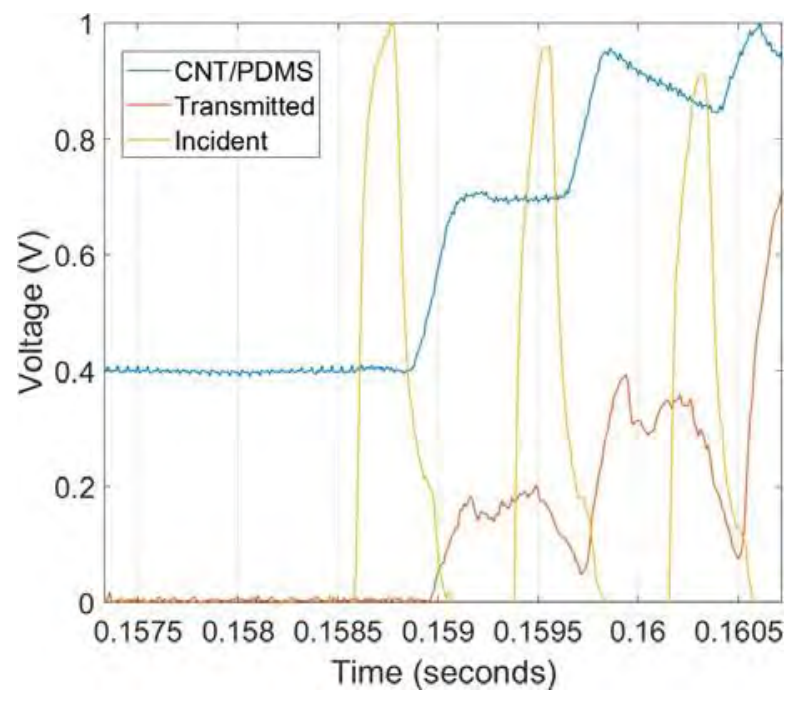

Figure 8. Transmitted and incident voltage output. (1wt. \% sample)

Fig. 9 shows the resistance change within the sample plotted against the strain. The stress-strain curve of the material is also plotted on the graph to emphasize the observed correlation. The change in resistance aligned with the stress/strain curve of the sample, indicating a strong correlation between electrical resistance and applied stress on the material. 


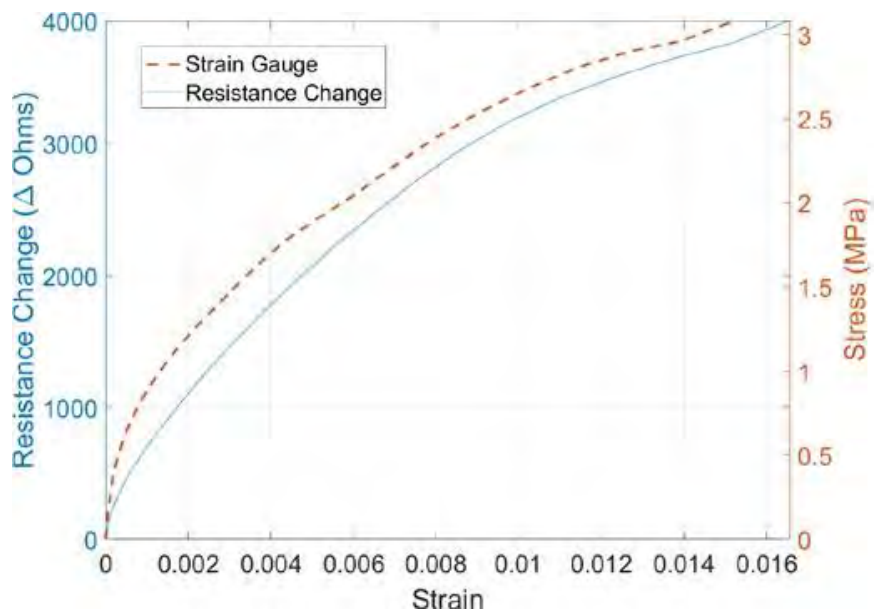

Figure 9. Strain Gauge and Resistance Change vs. Strain at Initial Contact

Fig. 10 shows the change in resistance within samples of different CNT concentration plotted against time. The observed rise in resistance was consistent at all three concentrations. However, we observed that increasing the CNT concentration in samples led to smaller resistance changes within them. The rate at which the resistance of the composite changed decreased when CNT concentration in the PDMS increased.

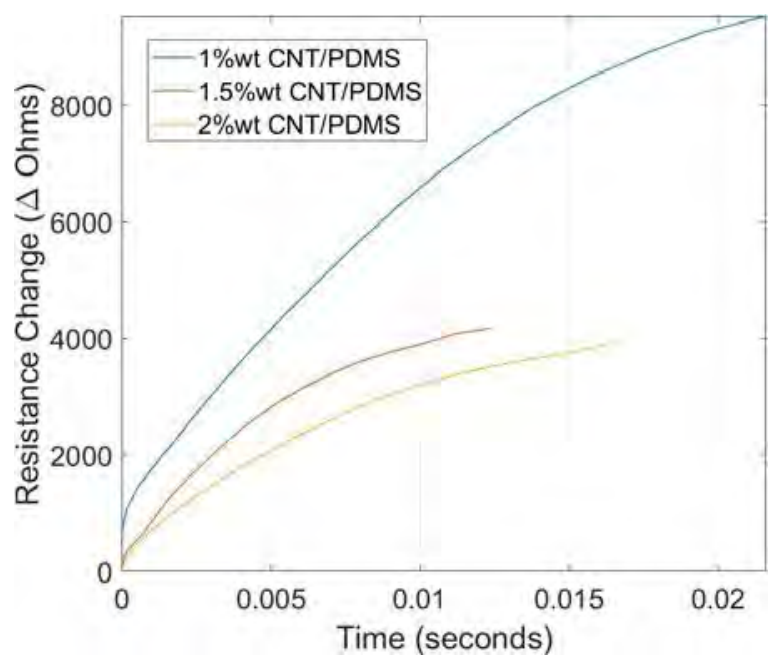

Figure 10. Resistance Change with respect to time for different CNT/PDMS weight percentage.

\section{CONCLUSION}

The effect of high strain rate on the piezoresistive properties of elastomeric composites shows promise in the potential development of high strain rate sensors. The results show a strong correlation between high strain rate and electrical resistance, indicating piezoresistive behavior in the material.

The elastomeric composite was prepared using Sylgard 184 PDMS and MWCNTs. The material was sonicated to break down the CNT chains and agglomerations. Our results show that chloroform functioned well as a solvent for the purposes of dispersing CNTs in PDMS. A custom press casting mold was developed to efficiently cast sheets of the material that were then laser cut to produce the samples for dynamic testing using the Kolsky bar. During tests, the current through the material was controlled, and the voltage and strain were measured.

The electrical resistance was found to spike upon loading with a lag of half a period relative to the incident wave and inphase relative to the transmitted wave. The slow recovery of voltage indicates viscoelastic behavior in the material [4]. The magnitude of the peak resistance was found to be 2.5 times the initial resistance, which represents the signal to be detected. The peak value was found to be directly related to the strain rate (as further research will show). By detecting this peak, we hope to determine the strain rate through means of statistical analysis. This research has proven the potential of CNTimpregnated elastomers for the development of impact force sensors. Future work includes further testing on the material to confirm preliminary results and gather enough data to build a robust statistical model. The goal of the model is to generate stress values from the input, the measured electrical resistance across the material.

\section{ACKNOWLEDGMENT}

The authors gratefully acknowledge the generous financial support of Lassonde School of Engineering (York University) and NSERC-Quanser Chair in Design Engineering.

\section{REFERENCES}

[1] C. Liu and J. Choi, "Improved Dispersion of Carbon Nanotubes in Polymers at High Concentrations," Nanomaterials, vol. 2, no. 4, pp. 329-347, 2012. doi: 10.3390/nano2040329.

[2] M. Heaney, "Ch. 7. Electrical Conductivity and Resistivity," in Electrical Measurement, Signal Processing, and Displays. John G.Webster, Ed. Boca Raton: CRC Press, 2003. doi: 10.1201/9780203009406.

[3] M. S. Chaudhry, "On the Characterization of Engineered Elastomers At High Strain Rates," (unpub.MA thesis) York Univ., [Online]. Available: https://yorkspace.library.yorku.ca/xmlui/handle/10315/33410. Accessed on August 5, 2017.

[4] Y. Zezin and E. Lomakin, "Study of Viscoelastic Properties of Elastomers Reinforced by Nanoparticles," Mech. Solids, vol. 50, no. 2, pp. 117-126, May 2015. doi: 10.3103/S0025654415020016. 


\title{
Design of Hard Compaction Rollers for Automated Fiber Placement on Complex Mandrel Geometries
}

\author{
Tom T. Zacchia, Farjad Shadmehri, Jeffrey Fortin-Simpson, Suong V. Hoa \\ Concordia Center for Composites (CONCOM), Concordia University \\ Montreal, Canada \\ Email: tomzacchia@live.com
}

\begin{abstract}
This paper presents a methodology for designing hard compaction rollers for an automated fiber placement (AFP) head with a hot gas torch heating system for laying thermoplastic carbon fiber reinforced plastic (CFRP) tapes. Some manufacturing defects and alternative designs are reviewed to highlight the importance of AFP compaction roller conformity and surface adhesion. The formulation of a general parametric description of the design challenge is derived and adequately addresses the problem on a tow-to-tow basis.
\end{abstract} roller

Keywords: Automated Fiber Placement; CFRP; compaction

\section{INTRODUCTION}

Manufacturers of aircrafts widely use CFRP to produce large parts, such as airfoil covers and fuselages. An example of such is the use of automated fiber placement manufacturing to produce Boeing's 787 Dreamliner's fuselage [3]. AFP is a highly flexible and effective process for manufacturing parts at a high rate of production. However, with parts of increasing geometrical complexity, there are difficulties for a single roller with fixed geometry to provide uniform pressure to compact the tape onto the surface of the part. It is necessary either to have a conformable roller that can adjust its geometry to fit the geometry of the surface of the part, or to have multiple rollers with different geometries that correspond to the geometries at different locations of the part. The objective of this paper is to introduce a methodology for designing hard AFP compaction rollers.

\section{THERMOPLASTICS MANUFACTURING DEFECTS AND LITERAUTRE SURVEY}

The quality of the final product is highly dependent on a number of manufacturing parameters, such as temperature, pressure and lay-up speed (see Fig. 1). Within the context of this study, pressure and consolidation during the manufacturing process are the primary concerns.

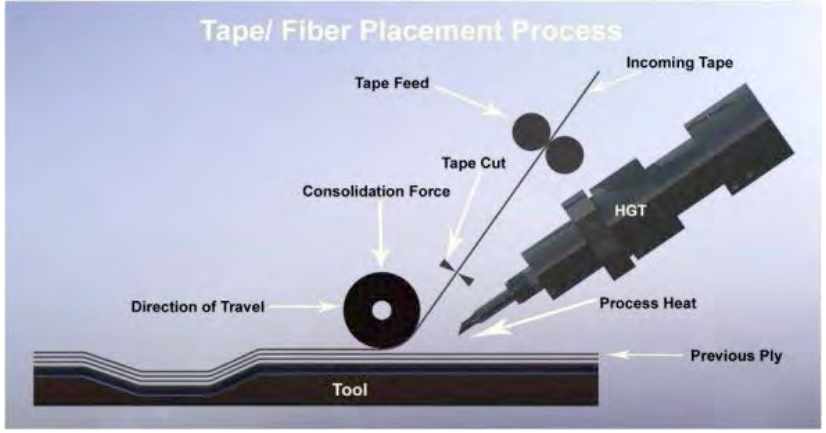

Figure 1. Fiber Placement Illustration (photo courtesy of Automated Dynamics)

During the manufacturing process pressure is applied after temperature is increased. The sustained pressure during the solidification phase of the process prevents the fiber network from springing back [1]. Under adequate pressure, suppressing fiber spring back minimizes voids that exist between individual layers. Pressure also plays a critical role in the development of intimate contact between plies, which in turn is an important parameter for autohesion. In the most severe case, inadequate conformity between the AFP compaction roller and the mandrel's surface can dramatically reduce pressure applied to the CFRP tape and localize the force (see Fig. 2).

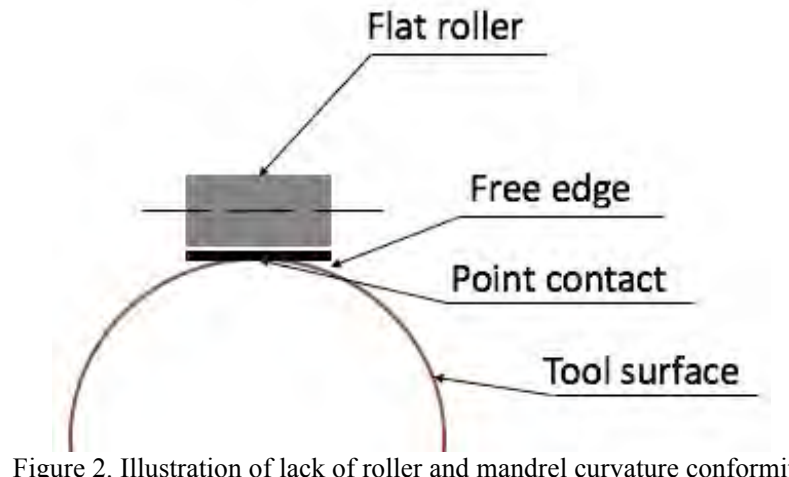

Figure 2. Illustration of lack of roller and mandrel curvature conformity 
Denkena, Schmidt, and Weber introduced a modular AFP laying head in 2016, which includes a unique set of height adjustable compaction rollers that are pneumatically controlled, thus increasing the flexibility of the design (see Fig. 3) [2]. The compaction rollers used are made of a flexible material, as it is best suited for thermoset CFRP manufacturing, and heating is done with a laser. Qualitatively, experiments conducted have validated the effectiveness of the compaction unit both on flat and contoured surfaces. For thermoplastic manufacturing that employs a hot gas torch heating element, hard rollers are better suited to perform the task.

NASA's Phase II SBIR program led to the development of a number of innovative conformable compactors [4]. Research published by Lamontia, Gruber, Waibel, Cope, and Hulcher included a study on defects caused by poor compactor to surface conformity as well as the design of a highly conformable compaction system. This study highlights that when compactors lack sufficient conformity with the tool surface, the time/temperature/pressure requirements for high-quality laminates are not fulfilled due to the concentration of forces exerted on high points of the tape [4]. An example of one of the high conformity compactor designs can be seen in Figure 4. This design features 76 heated segments that are covered in shims, which protect the fibers during manufacturing. The compactors were constructed and tested, however the authors did not report the results of those experiments in their study.

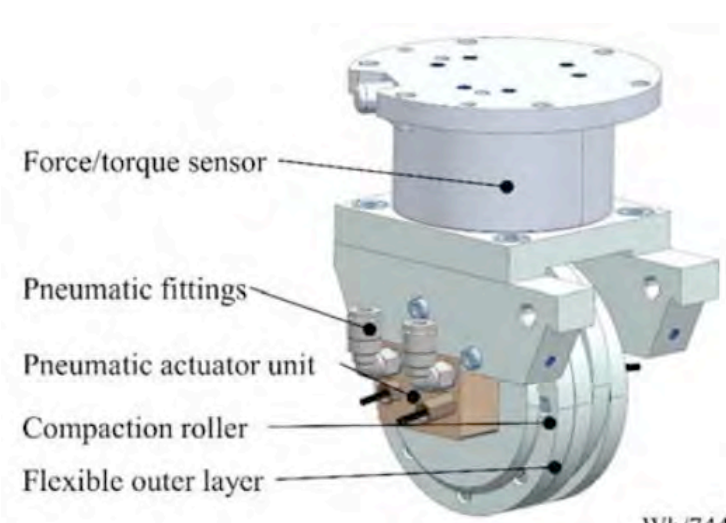

$\mathrm{Wb} / 74419 \bigcirc \mathrm{IFW}$

Figure 3. Modular Compaction Unit [2]

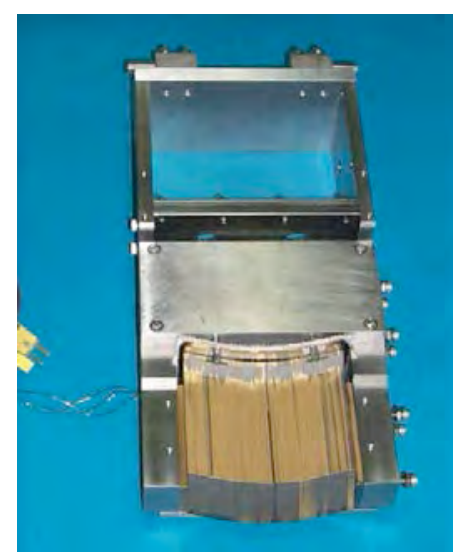

Figure 4. Hot line compactor featuring 76 heated segments [4]

To the best of the author's knowledge there exists very little research describing an approach to designing hard compaction rollers for thermoplastic CFRP manufacturing; this is the motivation behind the study.

\section{Methodology}

A cylindrical mandrel with a radius of Rs and a compaction roller with a profile radius of $\mathrm{Ri}$ was used to formulate a general equation to describe the adherence of the two components: with $\mathrm{Ri}>\mathrm{Rs}$. A local coordinate system was positioned at the center of the cylinder. However, in Fig. 5 the axes are translated onto the surface of the tape to better describe their directions: $\mathrm{x}$ (normal to the surface), $\mathrm{y}$ (transverse to the layup direction) and $\mathrm{z}$ (in the layup direction).

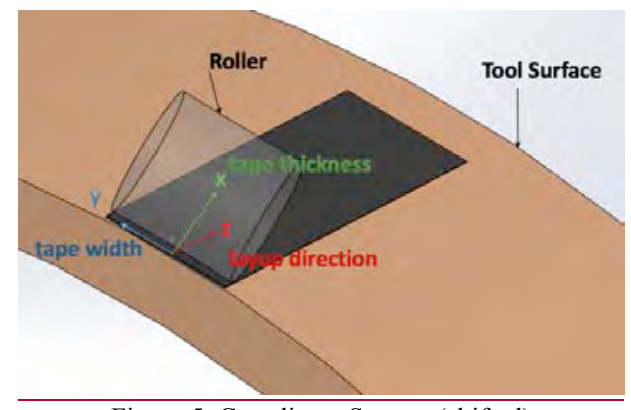

Figure 5. Coordinate System (shifted)

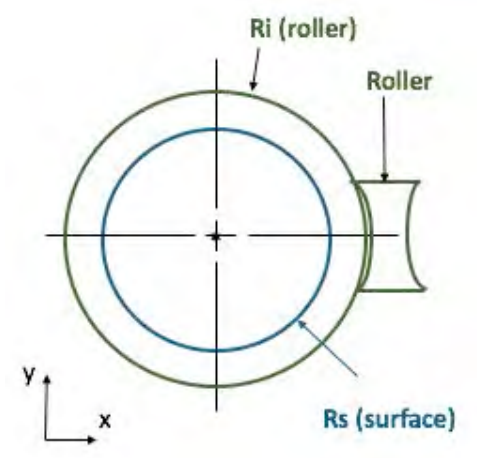

Figure 6. Concentric circles in design space 
In the XY plane, the roller's profile and instantaneous curvature at the tool's surface was represented by two concentric circles centered at the origin (see Fig. 6). Compression of the tape during manufacturing can be modeled by translating the circle of smaller curvature, Rs, by $\Delta X$. The value of $\Delta \mathrm{X}$ is such that the tape is at its minimum thickness under compression, $C$. The aforementioned variable can be determined experimentally.

$$
\Delta X=(R i-R s)-C
$$

With reference to Fig. 7, a number of variables are needed in order to fully describe the distance of the gap that lies between the roller and the mandrel, at the edge of the tape. These are given in Table 1 . The angle $\theta$ (either for the roller or for the surface) is given by,

$$
\theta=\sin ^{-1} \frac{w}{2 R}
$$

The coordinates of the contact point between the roller and the edge of the tape can be described as,

$$
\left[\begin{array}{l}
x_{i} \\
y_{i}
\end{array}\right]=R_{i}\left[\begin{array}{c}
\cos \left(\theta_{i}\right) \\
\frac{w}{2 \cdot R_{i}}
\end{array}\right]
$$

The coordinates of the contact point between the mandrel surface and the edge of the tape are,

$$
\left[\begin{array}{l}
x_{s} \\
y_{s}
\end{array}\right]=R_{s}\left[\begin{array}{c}
\cos \left(\theta_{s}\right) \\
\frac{w}{2 \cdot R_{S}}
\end{array}\right]
$$

As previously mentioned $\mathrm{Rs}$ has to be translated by $\Delta \mathrm{X}$, the result is a new set of variables $\left(\mathrm{x}_{\mathrm{s}}\right.$ ' and $\left.\mathrm{y}_{\mathrm{s}}{ }^{\prime}\right)$.

$$
\left[\begin{array}{l}
x_{s}^{\prime} \\
y_{s}^{\prime}
\end{array}\right]=\left[\begin{array}{l}
x_{s} \\
y_{s}
\end{array}\right]+\left[\begin{array}{c}
\Delta X \\
0
\end{array}\right]
$$

The gap between the roller and the surface of the mandrel at the edge of the tape is,

$$
\begin{gathered}
t=x_{i}-x_{s}{ }^{\prime} \\
t=R_{i} \cos \left(\theta_{i}\right)-\left[R_{s} \cos \left(\theta_{s}\right)+(R i-R s-C)\right] \\
t-C=R_{s}\left(1-\cos \theta_{s}\right)-R_{i}\left(1-\cos \theta_{i}\right)
\end{gathered}
$$

The quantity (t-C) represents the lack of desired compaction. The condition $\mathrm{t} / \mathrm{C}=1$ represents optimal compaction, while $\mathrm{t} / \mathrm{h}=1$ represents no compaction. If an allowable minimum compaction (say $\mathrm{t} / \mathrm{h}=\alpha$ ) is specified, then the profile of the roller can be designed such that $\mathrm{C}<\mathrm{t}<$ $\alpha \cdot h$.

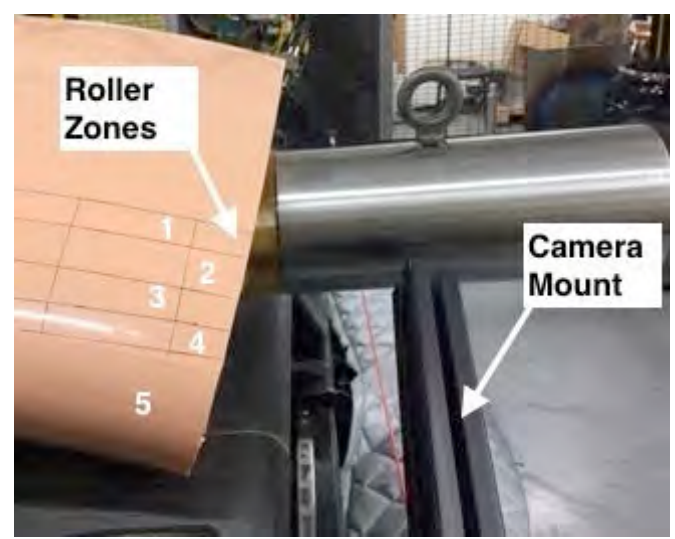

Figure 7. Boundary conditions of roller curvature

\section{EXPERIMENTAL INVESTIGATION}

The set up used for preliminary experimental investigations is seen in Fig. 7. The mandrel's surface is divided into 5 segments, each with a unique range of surface curvature, and 6 compaction rollers were manufactured. An extruded $\mathrm{T}$ slot bar was fixed onto a tripod and the assembly was positioned such that the camera's lens is parallel to the subject being captured.

Images were then processed using ImageJ. Fig. 8 depicts an area of 16,362 pixels which serves as a reference when calculating the percentage of the total area occupied by the gap, if any exits. The aforementioned gap area was calculated by drawing a perimeter encapsulating the gap using ImageJ's polygon tool, see Fig. 9.

The results recorded in table II highlights the successful application of equation 6 , since the rollers conform well within their intended range of surface curvatures, e.g. roller 1 conforms to segment 1 . In addition, it was observed that even in the least severe case, when the gap area was only $1.5 \%$ of the reference area, the gap distance, $t$, measured in ImageJ exceeded the thickness of the CFRP tape $(0.127 \mathrm{~mm})$.

\section{CONCLUSION}

A methodology for calculating an appropriate hard compaction roller profile curvature for a given tool surface curvature, used during thermoplastic AFP processing, has been presented. Equation 6 can be used to guide the design of compaction rollers for geometries of varying curvatures.

Initial experimental investigations validate equation 6 based on the conformity study that was performed in section IV. Future investigations include using the rollers to manufacture a CFRP component on the same mandrel and study manufacturing defects. The manufacturing process will consist of stopping the AFP head when a particular roller no longer adequately conforms to the tool surface and changing it for a more appropriate compaction roller. 


\section{REFERENCES}

[1] S. V. Hoa, Principles of the manufacturing of composite materials, 2nd ed. Lancaster: DEStech Publications, Inc., 2007, pp. 329-380

[2] B. Denkena, C. Schmidt, and P. Weber, "Automated fiber placement head for manufacturing of innovative aerospace stiffening structure,"

Procedia Manuf., vol. 6, pp. 96-104, 2016.

doi: 10.1016/j.promfg.2016.11.013.

[3] J. Sloan, Composite World (2008, June 30). ATL and AFP: Defining the megatrends in composite aerostructures [Article Post]. Available: https://www.compositesworld.com/articles/atl-and-afp-defining-themegatrends-in-composite-aerostructures.

[4] M. Lamontia et al., "Conformable compaction system used in automated fiber placement of large composite aerospace structures," in Proc. 23rd Int. SAMPE Europe Conf., Paris, France, 2002, pp. 745-756.

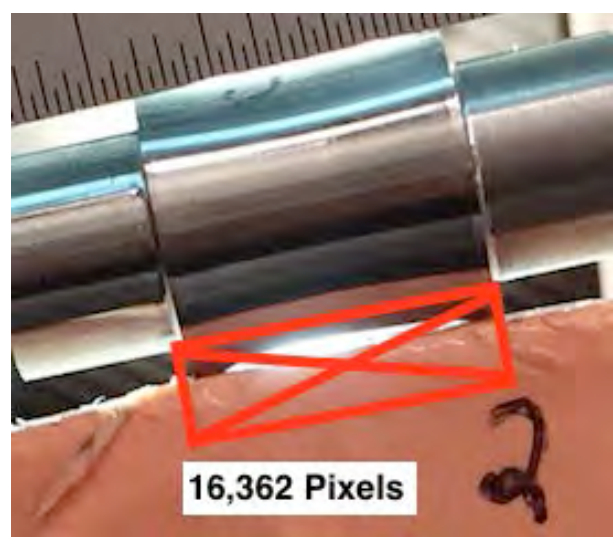

Figure 8. Reference Area

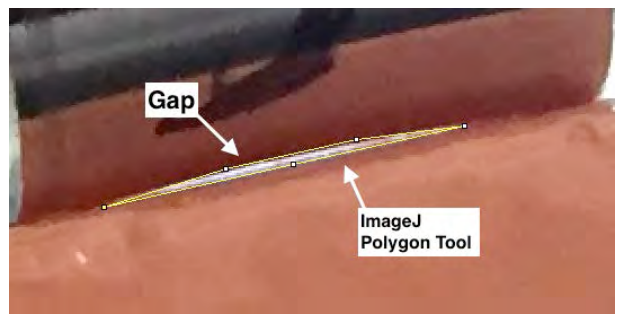

Figure 9. Calculating Gap Area (yellow polygon)

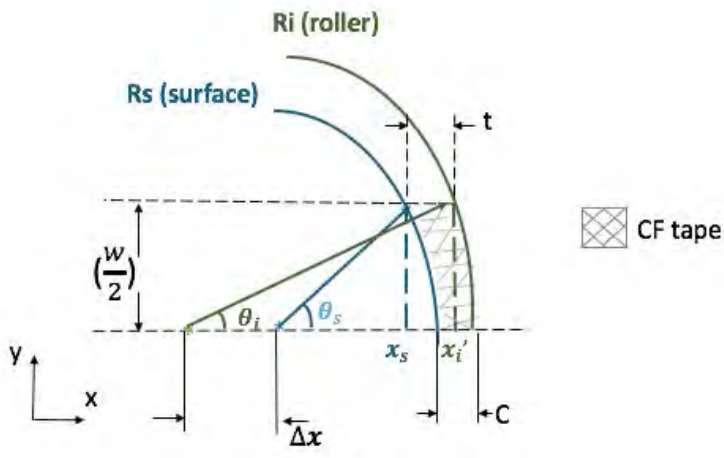

Figure10. Boundary conditions of roller curvature
TABLE I. VARIABLES USED

\begin{tabular}{|l|l|}
\hline \multicolumn{1}{|c|}{ Variable } & \multicolumn{1}{c|}{ Description } \\
\hline$C$ & Minimum allowable thickness under compression \\
\hline$w$ & Width of one tow \\
\hline$t$ & Gap between roller and mandrel surface et edge of tape \\
\hline$h$ & Thickness of one tow / tape \\
\hline $\mathrm{Rs}$ & Radius of curvature of surface \\
\hline $\mathrm{Ri}$ & Radius of curvature of compaction roller \\
\hline $\mathrm{X}_{\mathrm{s}}$ & x coordinate corresponding to position on surface at $w / 2$ \\
\hline $\mathrm{x}_{\mathrm{i}}$ & $\mathrm{x}$ coordinate corresponding to position on roller at $w / 2$ \\
\hline
\end{tabular}

TABLE II. PERCENTAGE AREA OCCUPIED BY GAP

\begin{tabular}{|c|c|c|c|c|c|c|c|}
\cline { 3 - 8 } \multicolumn{2}{c|}{} & \multicolumn{6}{|c|}{ Roller I.D } \\
\cline { 2 - 8 } \multicolumn{2}{c|}{} & $\mathbf{1}$ & $\mathbf{2}$ & $\mathbf{3}$ & $\mathbf{4}$ & $\mathbf{5}$ & $\mathbf{6}$ \\
\hline \multirow{3}{*}{ Zone } & $\mathbf{1}$ & $\mathbf{0 . 0} \%$ & $\mathbf{0 . 0} \%$ & $5.2 \%$ & $2.5 \%$ & $3.2 \%$ & $20.7 \%$ \\
\cline { 2 - 9 } & $\mathbf{2}$ & $\mathbf{0 . 0 \%}$ & $\mathbf{0 . 0} \%$ & $3.2 \%$ & $6.6 \%$ & $52.4 \%$ & $34.3 \%$ \\
\cline { 2 - 9 } & $\mathbf{3}$ & $3.1 \%$ & $1.5 \%$ & $\mathbf{0 . 0} \%$ & $7.1 \%$ & $40.5 \%$ & $38.9 \%$ \\
\cline { 2 - 9 } & $\mathbf{4}$ & $7.3 \%$ & $2.8 \%$ & $\mathbf{0 . 0} \%$ & $\mathbf{0 . 0} \%$ & $25.0 \%$ & $33.9 \%$ \\
\cline { 2 - 9 } & $\mathbf{5}$ & $8.0 \%$ & $3.6 \%$ & $5.6 \%$ & $\mathbf{0 . 0} \%$ & $\mathbf{0 . 0} \%$ & $\mathbf{0 . 0} \%$ \\
\hline
\end{tabular}




\section{The Effect of Minimum Quantity Lubrication on the the FSW Process Performance}

\author{
Wisam Al-Wajidi \\ School of Engineering \\ University of Guelph \\ Guelph, Ontario, Canada \\ walwajid@uoguelph.ca
}

\author{
Ibrahim Deiab \\ School of Engineering \\ University of Guelph \\ Guelph, Ontario, Canada \\ ideiab@uoguelph.ca
}

\author{
Fantahun M. Defersha \\ School of Engineering \\ University of Guelph \\ Guelph, Ontario, Canada \\ fdefersh@uoguelph.ca
}

\begin{abstract}
The effect of Minimum Quantity Lubrication (MQL) on performance of Friction Stir Welding (FSW) process for Aluminum Alloy 6061-T651 plates was investigated. MQL was used as a cooling and lubrication medium. Five different levels of rotational speed and three feed speed ranges with and without MQL were tested. The effect of MQL on the mechanical properties of the weld joints was studied throughout means of Ultimate Tensile Strength (UTS) tests. Statistical analyses were run to study the relationship between certain process parameters and response. The results showed that the average UTS of the welds is improved when MQL was applied to most of the process variables. The highest UTS was achieved at spindle speed with $1600 \mathrm{rpm}$ and with feed rate $180 \mathrm{~mm} / \mathrm{min}$ due to improvement of the grain growth.
\end{abstract}

Keywords-component; FSW; friction stir welding; MQL; Aluminum Alloys; AA6061-T651; statistical analysis

\section{INTRODUCTION}

Over the past decade, manufacturing companies have been forced to consider the environmental and social effects of their products instead of only focusing on economic benefits. Sustainability implies dealing with more than simply analyzing and modifying the performance of manufacturing processes as related to the environment. Aluminum is promising for the automotive industry because of its high strength/weight ratio, emissions, safety, corrosion resistance, and sustainability [1]. Unfortunately, high thermal and electrical conductivity of aluminum alloys are problematic in fusion and resistance welding [2]. The researchers have investigated relevant aspects, such as FSW method mechanics, metallurgical evolution of the material, the material flow, and bonding conditions. They have focused on finding effective welding variables for various materials to optimize the performance of the joints [3].

The friction stir welding process (FSW) avoids the usual problems that occur when using conventional welding to join low melting point materials or dissimilar materials. In FSW, metals are welded without attaining the melting temperature of the metals. During the FSW processes, the rotating probe slowly moves into a butt joint line between two rigidly clamped plates or sheet metals as shown in Figure 1.

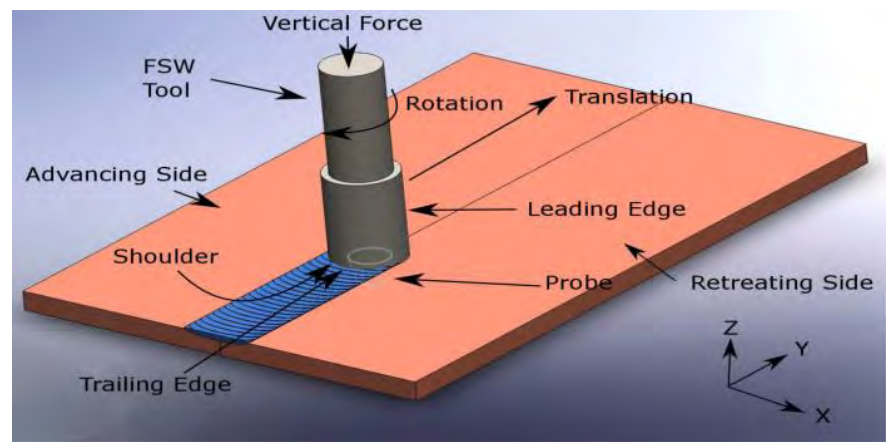

FIGURE. 1: SCHEMATIC ILLUSTRATION OF FRICTION STIR WELDING

FSW processes were first applied on aluminum alloys because of their low melting temperatures, and were found to be an effective technique. Improvement of the strength of common friction stir welded joints is possible through careful control of temperature. In several solid-state welding techniques, external liquid cooling has been used to improve weld performance. Frictional contact between the workpiece and the tool shoulder, while the tool is traveling, will generate heat; usually, the temperature is about 80 to $90 \%$ of the melting temperature of the materials [4]. 
There are several parameters in FSW that can be employed to control the process. These include spindle speed, feed speed, tool geometry, and tilting angle of the tool. Cooling strategies are one of several possible techniques to enhance the properties of the friction stir welding process. Several studies have investigated the effect of cooling strategies on the properties of friction stirring tools and the FSW joints.

As a new and sustainable method of lubrication and cooling, MQL optimizes the use of metalworking fluids [5]. The MQL process replaces regular lubrication methods, resulting in more sustainable manufacturing processes, thus acting as an achievement-oriented technology. This process has been widely verified as a near-dry process with respect to human health, cost, and environmental friendliness. The MQL process uses a small quantity of oil and sprays of compressed air. As a cooling system it is used for many manufacturing processes, but has not previously been used for FSW. Because MQL uses small amounts of highly biodegradable and environmentally friendly oils, it has promise as a sustainable manufacturing process.

The main objective of this research is to study the effect of the MQL scheme on the FSW process and the joints of AA 6061-T651 within appropriate process parameters.

\section{MethodOLOGY}

\section{A. Experimental Design}

The present study uses a full factorial experimental design (FFD) to achieve the response measurements. A statistical analysis of variance (ANOVA) test is conducted to determine the corresponding importance value of the input variables. Straightforwardly, the objective of the ANOVA test in this study is to distinguish which process variables are significant, and to determine the contribution of each variable. To compare pairs of treatment means and to find which process variable has a significant effect, the Fisher Least Significant Difference (LSD) method is used with $95 \%$ confidence. For analyzing the results, Minitab software was utilized to investigate the statistical analysis. Five different levels of rotational speed and three feed speed ranges are applied in this study, in addition to the coolant lubrication strategies in two levels as shown in Table (1).

TABLE I. : FSW PROCESS CONDITIONS

\begin{tabular}{|c|c|c|c|c|c|}
\hline Spindle Speed (rpm): & 1000 & 1300 & 1600 & 1900 & 2200 \\
\hline Feed rate $(\mathrm{mm} / \mathrm{min})$ : & 100 & 180 & 260 & & \\
\hline
\end{tabular}

\section{B. Experimental Setup}

The FSW processes in this study will use $3.175 \mathrm{~mm}$ plate sample thickness of AA 6061-T651 cut into $65.5 \times 56.5 \mathrm{~mm}$ workpieces. Table 2 shows the typical mechanical properties of AA 6061-T651.
TABle 2: The MechaniCAL Properties of AA 6061-T6 [6]

\begin{tabular}{|c|c|c|c|}
\hline $\begin{array}{c}\text { Ultimate Tensile } \\
\text { strength }(\mathbf{M P a})\end{array}$ & $\begin{array}{c}\text { Tensile strength } \\
(\mathbf{M P a})\end{array}$ & $\begin{array}{c}\text { Yield strength } \\
(\mathbf{M P a})\end{array}$ & $\begin{array}{c}\text { Elongation } \\
(\boldsymbol{\%})\end{array}$ \\
\hline $260-310$ & $228-283$ & $193-262$ & $10-11.62$ \\
\hline
\end{tabular}

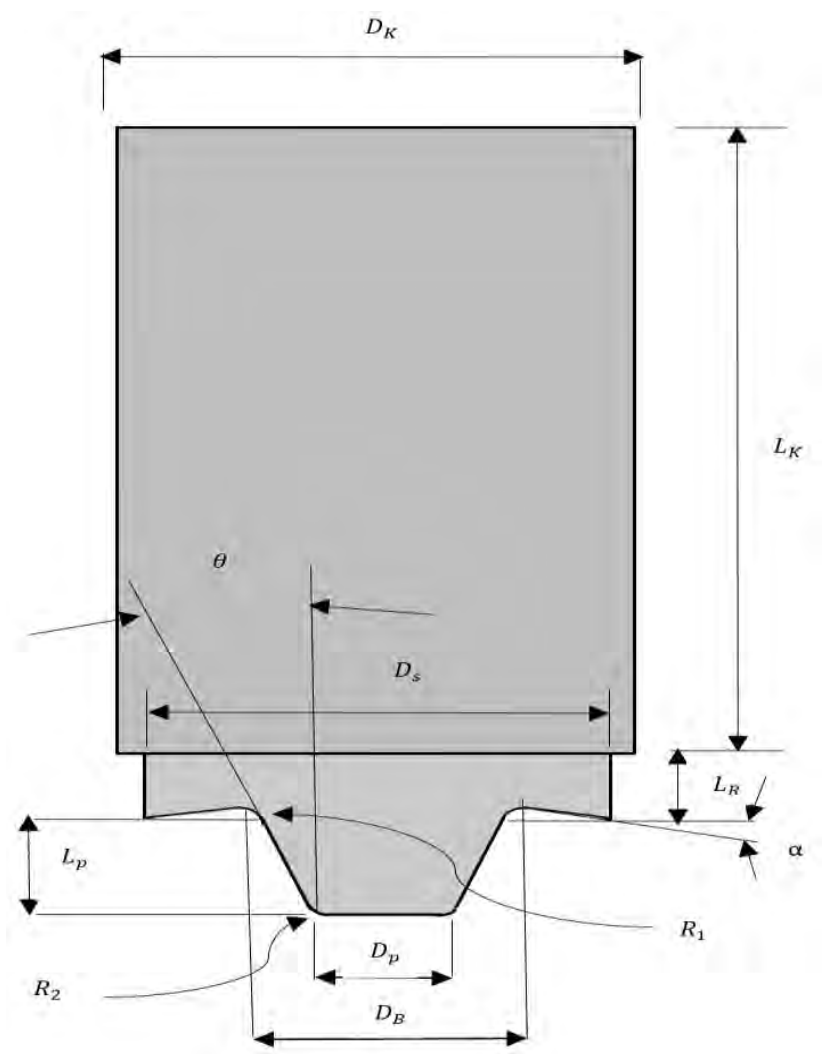

FIGURE (2) DEMONSTRATES THE GEOMETRICAL DIMENSIONS OF THIS TOOL.

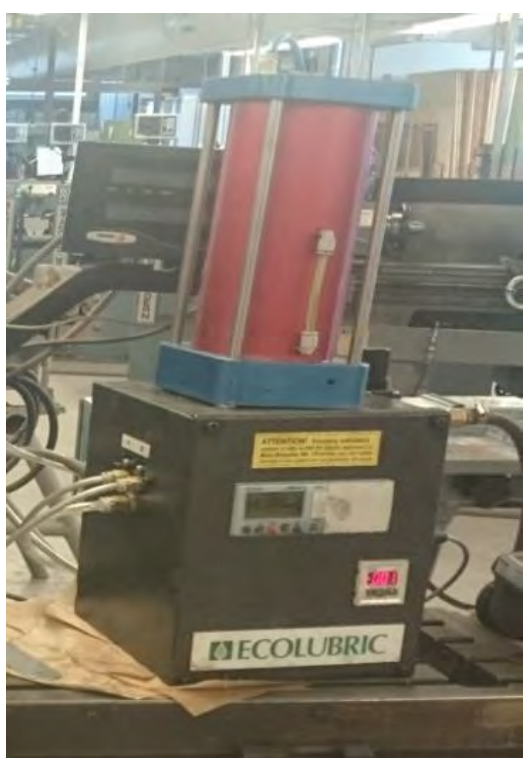

FIG. 3: MQL SYSTEM 
In this study, the tools are manufactured from A2 tool steel due to its resistance to thermal fatigue, cracking, and high toughness. The pin is a smooth trapezoidal cylinder, containing no threads, tapers, or other welding enhancement designs as shown in Figure (2).

To enhance the FSW process characteristics, the MQL process is applied in this study. Figure (3) explains the micro lubricating MQL system that is used with different variables. This system using a micro-pump which deliver too small amounts of oil. The vegetable oil that will be used is rapeseed oil, and ECOLUBRIC E200.

The specimens are machined from each welded plate subjected to tensile testing with geometry according to ASTM standards, using a dog bone shape in a sample of $32 \mathrm{~mm}$ gauge length. Tensile testing is applied to each weld at room temperature and perpendicular to the weld direction using an Instron-type tensile testing machine. Three samples are machined from each welded plate. For each sample prepared, ultimate tensile strength and efficiency are calculated, and the results are averaged.

\section{DATA ANALYSIS}

Table (6) shows the ANOVA test for the UTS of the welded metals. This analysis is conducted at a confidence interval of $95 \%$. As the P-value is zero for the spindle speed and the feed rate factors, they have significant effects on UTS results. As shown in the table, the lubricant factor is not independently significant. This might be because it has only two levels, while it is observed that the interaction of the lubricant factor with the spindle speed factor and feed rate factor has a significant effect. This reflects the importance of the types of the lubricant on the spindle speed and feed rate. Additionally, there is a significant effect of the triple interaction between the spindle speed, feed rate, and the lubricant.

\begin{tabular}{lcccll}
\multicolumn{5}{c}{ TABLE (6): ANOVA TEST RESULTS FOR TENSILE STRENGTH } \\
\hline Source & DF & Adj SS & AdjMS & F-value & P-Value \\
\hline Spindle Speed & 4 & 110916 & 27729 & 601.09 & 0 \\
Feed Rate & 2 & 9524 & 4762.1 & 103.23 & 0 \\
$\begin{array}{l}\text { Lubricant } \\
\text { Spindle }\end{array}$ & 1 & 18 & 17.7 & 0.38 & 0.537 \\
$\begin{array}{l}\text { Speed*Feed rate } \\
\text { Spindle }\end{array}$ & 8 & 7993 & 999.1 & 21.66 & 0 \\
$\begin{array}{l}\text { Speed*Lubricant } \\
\text { Feed }\end{array}$ & 4 & 3902 & 975.5 & 21.15 & 0 \\
$\begin{array}{l}\text { Rate*Lubricant } \\
\text { Spindle Speed }\end{array}$ & 2 & 2209 & 1104.4 & 23.94 & 0 \\
*Feed & & & & & \\
Rate*Lubricant & 8 & 4609 & 576.1 & 12.49 & 0 \\
Error & 90 & 4152 & 46.1 & & \\
Total & 119 & 143322 & & & \\
\hline
\end{tabular}

Note: DF: The total Degree of Freedom, Adj SS: Adjusted sums of squares, Adj MS: Adjusted mean squares.

Table (7) shows the Fisher pairwise comparisons of the effect of the spindle speed levels on UTS. The spindle speed with $1600 \mathrm{rpm}$ records the highest UTS, which significantly differs from 1900, 2200, and 1000 rpm respectively; while there is no significant difference between 1600 and $1300 \mathrm{rpm}$. Also, the levels of 1300 and $1900 \mathrm{rpm}$ are significantly different from 2200 and $1000 \mathrm{rpm}$. As tool spindle speed increases more than $1600 \mathrm{rpm}$, the resulting higher temperature cause to wider Heat Affected Zone, that leading to reduction in tensile strength at the jointed area. Also, the friction produces progressively more intensive stirring and mixing of the metal workpiece from lower to upper surface, thus decreasing the tensile strength. This means that any future study can rely on these levels for optimization or economic concept.

TABLE (7): FISHER TEST RESUlTS FOR SPINDLE SPEED OF UTS

\begin{tabular}{cccc}
\hline Levels $($ rpm $)$ & $\boldsymbol{N}$ & Mean & Grouping \\
\hline $\mathbf{1 6 0 0}$ & 24 & 205.19 & $\mathrm{~A}$ \\
$\mathbf{1 3 0 0}$ & 24 & 196.85 & $\mathrm{AB}$ \\
$\mathbf{1 9 0 0}$ & 24 & 187.38 & $\mathrm{~B}$ \\
$\mathbf{2 2 0 0}$ & 24 & 160.34 & $\mathrm{C}$ \\
$\mathbf{1 0 0 0}$ & 24 & 121.46 & $\mathrm{D}$
\end{tabular}

The feed rate factor has recorded the highest effect on the UTS at $180 \mathrm{~mm} / \mathrm{min}$ as shown in Table (8), which presents the Fisher pairwise comparisons of the feed rate levels on UTS. As Fisher test of the feed rate factor, $180 \mathrm{~mm} / \mathrm{min}$ has a significant effect on the tensile strength. The heat generated is reduced when the feed rate becomes higher because the interaction time between the material and FSW tool is decreased; that the reduced heat generation might produce the formation of a tunnel defect inside the insufficient metal flow of the bottom region. Thus, when the feed rate is increased, correspondingly, the tensile strength properties also increase to a maximum at $180 \mathrm{~mm} / \mathrm{min}$.

TABLE (8): FISHER TEST RESULTS FOR FEED RATES OF UTS

\begin{tabular}{cccc}
\hline Levels $(\mathbf{m m} / \mathbf{m i n})$ & $\boldsymbol{N}$ & Mean & Grouping \\
\hline 180 & 40 & 186.60 & P-Yalue \\
100 & 40 & 170.18 & B \\
260 & 40 & 165.94 & B \\
& & & 0.537
\end{tabular}

IV. CONCLUSIONS: 0

This study investigated the effect of MQLOon the welding properties of AA 6061-T651. Statistical analysis is conducted to achieve the influence of controlled factors on the tensile strength. According to the ANOVA results, the following conclusions may be drawn: 0

1. The controlled parameters, spindle speed and the feed rate, have a significant effect on weld joint strength.

2. The interaction of lubricant factor has a significant effect with the spindle speed factor and feed rate factor, which means that the lubricant depends on the value of these factors.

3.The spindle speed with $1600 \mathrm{rpm}$ records the highest UTS, it might be because of finer grain size produced at this spindle speed. 
4. The feed rate factor has recorded the highest effect on the UTS at $180 \mathrm{~mm} / \mathrm{min}$.

\section{ACKNOWLEDGMENT}

The authors acknowledge the support of the Natural Sciences and Engineering Research Council of Canada (NSERC) and Ontario centers of Excellence (OCE). The first author acknowledges the Iraqi Ministry of Higher Education and Scientific research for their support of this research.

\section{REFERENCES}

[1] C. A. Ungureanu, "Design for sustainability: product life-cycle analysis in aluminum auto body applications," M. Thesis, Univ. Kentucky, 2007. [Online]. Available: https://uknowledge.ukyedu/gradschool theses/449.

[2] G. Mathers, The Welding of Aluminium and its Alloys, Woodhead Publishing, 2002.

[3] L. Fratini, G. Buffa, and R. Shivpuri, "Influence of material characteristics on plastomechanics of the FSW process for T-joints." Mater. Des., vol. 30, no. 7, pp. 2435-2445, Aug. 2009.

doi: $10.1016 /$ j.matdes.2008.10.014.

[4] N. Dialami, M. Chiumenti, M. Cervera, and C. Agelet De Saracibar. "Challenges in thermo-mechanical analysis of friction stir welding processes." Arch. Comput. Methods Eng., vol. 24, no. 1, pp. 189-225, Jan. 2017. doi: 10.1007/s11831-015-9163-y.

[5] A. K. Sharma, A. K. Tiwari and A. R. Dixit. "Effects of Minimum Quantity Lubrication (MQL) in machining processes using conventional and nanofluid based cutting fluids: A comprehensive review." J. Cleaner Prod., vol. 127, no. 20, pp. 1-18, Jul. 2016. doi:10.1016/j.jclepro.2016.03.146.

[6] J. R. Davis, Alloying: Understanding the Basics. ASM International, 2013. 


\title{
Review Paper on Digital Manufacturing \& Design
}

\author{
Mukhtiar Singh \\ Department of Mechanical Engineering, \\ Lovely Professional University \\ Jalandhar - Delhi G.T. Road, Phagwara, Punjab 144411 \\ mukhtiarts@gmail.com
}

\begin{abstract}
The transformation is happening through digital manufacturing and design (DM\&D) - a shift from paper-based processes to digital processes in the manufacturing industry.as technology is having a huge impact on the ways products or services are designed \& manufactured. Ever increasing customer demands for newer products with better features is transforming the dynamic of manufacturing towards embracing digital technology. Mass customization of products and services is preferred over mass production. There is an increased pressure upon businesses to serve an individual customer at a competitive cost comparable to the mass production cost, with shortest possible development time and production by focusing on levels of customization, quality and performance along with competitive price and value. With the advancements made in Information Technology, Material Science, Production Technologies and Supply Chain Strategies for the past 50 years' manufacturers are well positioned to challenge the traditional methods the products are designed and manufactured. Digital manufacturing and design encompasses visualization, manufacturing simulation, ergonomic and human factor analyses, holistic view of product and process design, development and product design sensitive to the process constraints and capabilities. The next stages of the new era and next Industrial Revolution termed as INDUSTRY 4.0. This article is all about defining a transformational shift from conventional methods of manufacturing to Digital Manufacturing adopting factors like Digital Thread, Advanced Analysis, Intelligent Machining, Security in Manufacturing, Model based system Engineering and reconfigurability in manufacturing systems etc
\end{abstract}

Keywords- Digital Manufacturing \& Design, Digital Thread, Intelligent Machining, Digital Manufacturing Security, Cyber Security, Model Based System Engineering

\section{INTRODUCTION}

The manufacturing environment radically changed over the last decades as manufacturing companies strived to improve their performance in a globalized, interconnected and volatile market environment. Manufacturing companies in the 21 st century face increasingly frequent and unpredictable market changes driven by global competition, including the rapid introduction of new products and constantly varying product demand. Traditional approaches of dedicated production lines, although still in use/practice, are becoming irrelevant with changing market requirements such as; increases in product demand, product changes, such as the introduction of a new product in the line, and system failures, such as a machine breakdown, in a cost effective or timely manner. In a highly competitive market and high volume manufacturing, such as automotive, the introduction of automation and information and communication technologies (ICT) on the shop floor, supported by digital manufacturing tools, has led to more flexible production systems which are capable of dealing with the volatile market demands and having a mixed product flow Digital Manufacturing \& Design is a platform for exploring new methods for manufacturing product. Digital manufacturing evolved from manufacturing practices such as design for manufacturing(DFM), flexible manufacturing (FM), lean manufacturing (LM) and computer-integrated manufacturing (CIM) which requires the collaboration across process and product design. The first Industrial Revolution, began in mid-1800s represented the transition from manual production to mechanical production, empowered by outfitting of water and steam power control [1]. The Second Industrial Revolution came at the beginning of the $20^{\text {th }}$ century with the advancement of methodologies that empowered large scale manufacturing, including the mechanical production system [2]. The Third Industrial Revolution, otherwise called Digital Revolution, represented the advances made conceivable via robotization as mechanical and simple advances offered approach to computerized gadgets between the 1950s and the 1970s. The Fourth Industrial Revolution which is also called Industry 4.0 is the succeeding stage in the development of manufacturing.

The cyber capabilities resulting from advances in computing combined with physical systems to have enabled a highly intelligent, SMART (Sustainable Manufacturing and Advanced Robotics Technologies) machines that integrates design, and produces robotized manufacturing ecosystem [3]. The Formal definition of Manufacturing is the way towards changing over the crude materials, segments, or parts into completed products that meet a client's desires. Manufacturing is the most tangible part of the product lifecycle because it results in a clear outcome [4]. 
In recent years, industrial nations around the globe have invested heavily in new technologies, software, and services to advance digital design and manufacturing using cyber-physical systems, data analytics, and highperformance computing for optimum product and process design. In product life cycle stages, design begins in an abstract fashion with conceptual design and concludes with detail design, where final detailed part specifications are created. The subsequent step of the process is to take the product from a design and develop it. This stage includes defining the items that need to be manufactured and how they will be created and assembled. In product design and development process, production prototypes are created and checked to see if they meet performance and cost requirements. As well as long term production capabilities are established [5]. Next stage is support stage where once produced and purchased by the customer, the product must still be supported. The support stage of the lifecycle is where you provide the customer with information to assist in the effective use of the product and maintain the operational performance of the product. Product's lifecycle ends with disposal and recycling. The dispose state of the lifecycle draws on information from the design and build phases to understand the composition and what portions of a product can be recycled into future applications [6]. The dispose stage may also play a crucial role in iterating the design to further develop a product that meets performance expectations and that can be disassembled at the end of life. With the possibilities of many iterations, multiple opportunities for improvement have been identified for traditional design and manufacturing processes over the time. In a product's lifecycle, many iterations may be required before customer approval is received.

There are various challenges associated with traditional paper bases processes as in paper based processes, information sharing can consume valuable time and delay the development process. In traditional processes, there is also a lag during and in- between stages in the lifecycle as necessary approvals are sought [7]. Sometimes, this can even result in losing first-tomarket competitive advantage. Another challenge in paper based processes is that maintaining a single source of true information for product and process definitions is very difficult and can limit the capabilities for data analysis and evidence based decision-making. Frequent communication gaps can result when planned changes are made during design and development process, as well as after the production launch. To address all these issues a new approach called digital manufacturing is most appropriate. The concept of digital manufacturing is an integrated suite of tools that work with product definition data to support tool design, manufacturing process design, visualization, modelling and simulation, data analytics, and other analyses necessary to optimize the manufacturing process as this approach uses advanced computing power to optimize the product lifecycle. [8]. Digital manufacturing allows you to more efficiently and commandingly share information in the design process.
The term Digital thread refers to the communication framework that allows a connected data flow and integrated view of the asset's data throughout its lifecycle across traditionally siloed functional perspectives can be used to create a computer based digital twin which is an integrated system of data, models, and analyses that can be used in design, manufacturing, support, and disposal. The concept of digital thread is very useful in designing smart manufacturing which strives to orchestrate and optimize business, digital and physical processes across factories and the entire product value chain. The digital thread also provides a formal framework for controlled interplay of authoritative technical and as-built data with the ability to access, integrate, transform and analyze data from disparate systems throughout the product lifecycle into actionable information. This whole idea is called as the Fourth Industrial Revolution, Industry 4.0 [9].

\section{PRODUCT LIFECYCLE \& CHANGE TRANSITIONED}

Product lifecycle refers to all the stages of a product concept design to disposal and recovery. Product lifecycle can be seen from different points of view. All in all, item lifecycle covers three primary stages: start of life, including outline and assembling; center of life, including use, administration, and upkeep; at last the end of life, these products are re-collected, arranged, dismantled, re-manufactured, reused, \& disposed [10]. Different organization have different motivations to collect product lifecycle data. A couple of them are transparency, business assessment, change, and estimating. Full transparency empowers both the purchasers and producers to settle on better educated choices [11]. Cases of transparency incorporate the arrival of the nourishment data on sustenance items and the arrival of natural effects data on products to customers. With a specific end goal to have transparency, we have to gather and offer information of various parts of an item or venture. Another motivation to collect product lifecycle data is to use data for business evaluation and improvement [12]. Continuous improvement requires continuous evaluation of the business process from different aspects. Continuous evaluations require continuous collection of data to evaluate [13]. By collecting data, the enterprise management is able to forecast valuable information. An example of this is when an enterprise collects the market trends to predict future market demands. Another is when an enterprise collects the energy usage of an industrial machine to predict its failure. As we just learned, the product life-cycle covers three main phases: beginning of life, middle of life, and end of life. The information flow during the beginning of life is quite complete and is collected using different information management systems, such as computer-aided design, or CAD models, computer-aided manufacturing, or CAM systems, and product data management or PDM systems [14]. The information flow becomes less complete after the beginning of life cycle. In fact, the information flow is stopped after product is delivered to a consumer for the majority of today's consumer products such as household 
appliances and vehicles. This is because after products are delivered to consumers, the ownership of product is no longer with companies and is transferred to customers, therefore, it is not easy for companies to track product usage data generated by consumers. As a result, decision makers involved in each phase of a product lifecycle make decision based on an incomplete and inaccurate product lifecycle data from other phases, which in many cases result into inefficient decisions. Product lifecycle data can be categorized under static data and dynamic data [15]. Static data is mainly generated at the beginning of life phase and rarely changes during the lifetime of the product. Examples of static data are bill of materials, material content, take back information, disassembly instruction, return policies, and recycling information. Static data is fairly complete and can be collected through existing data management systems. The dynamic information incorporates mainly the information created during usage phase. Cases of progression information which utilize designs, natural conditions, and adjusting activities [16]. Dynamic information is regularly lost and is hard to acquire during product lifecycle. Product lifecycle have numerous applications. Assume that organizations can track and follow product utilization information produced by buyers \& consumers [17]. Companies could gain substantial business advantages if they use product usage data to improve their products and optimize relevant operations [18]. For example, lifecycle acquisition can be used to enable productrelated services. Product lifecycle data is valuable and can be used to facilitate the service delivery such as maintenance, repair, and after-sales services. It is particularly important in the aviation industry. In aviation industry, capital equipment, and products have long service lives and complex configurations [19]. The productivity of the business is not just from the offers of capital gear and flying machines, yet from keeping up them for an expected 30 or more-year life expectancy. Along these lines, upkeep and repair organizations mean to limit support cost and turnaround time to boost income. Because of the many-sided quality of the framework, robotized data recovery and item structure data can help organizations effortlessly recognize their potential disappointments and acquire the data essentially for part repair and tooling plan. Gathering item lifecycle information is a spine for an incorporated enterprise.

Data standards are very useful to establish a relationship between two different datasets to create information or connect machines from different manufacturers. They specify a consistent data format so that the data can be shared and transformed into usable information. Some examples are, STEP and IGES, are data standards for sharing product design data like geometry [20]. MT Connect is a standard that supports the capture and sharing of information from machining equipment. Mil Standard 31000A and A SME 1441 define ways to share product model information using a technical data package to support the product life cycle.

\section{DIGITAL THREAD}

From the initial concept, products evolve through a regular sequence of plan, design, build, support, and dispose. During each of these stages, a colossal measure of information is produced. Although traditionally, much of it has not been captured, it can be processed into very valuable information that can impact the product life cycle in many different ways. A name's been given to describe all of the data generated in every part of a product's life cycle- the digital thread. The digital thread concept seamlessly integrates information through the value chain from requirements gathering through feasibility reviews, design, manufacturing, testing, and on to final sustainment and disposal phases. This means specialists, throughout the process, can work on the product, and process definitions simultaneously to inform decisions, through the life of a system or product. While promising, adoption of the digital thread was initially limited, but has continued to gain momentum. Like any paradigm shift, the digital thread approach is potentially disruptive, especially to organizations that do not have in house information technology resources [21]. For example, the information technology foundation for the digital thread has typically been viewed as overhead, simply a cost of doing business that needs to be shortened.

The digital thread paradigm views the IT infrastructure as an investment that will generate a return in increased effectiveness and responsiveness. A second challenge, facing the digital thread, is the transitioning profile of manufacturing jobs from skilled to smart or information workers that combine their expertise with physical systems, and the ability to work with the data generated in the production process [22]. This change will more tightly link production staff with the design process. Giving them more opportunities to impact the design of a product that they create. A third challenge facing the digital thread implementation is natural. How do we keep the information secure? Traditional processes used physical documents that could be controlled and locked up. There's a natural resistance to putting valuable intellectual property in digital form, especially as retailers seemingly report intrusions on a regular basis. Information security is a key component of the digital thread, and as we all see, is incorporated from the beginning [23]. Sharing data and information between different functions and systems of the product life cycle can add significant value to the entire development process. Some examples are, faster product launches, clearer communication between suppliers and manufacturers, resulting in fewer errors, reduced rework and scrap. Reduced inventory in the supply chain. Reduced unit product cost which increases profit margin. And even the expansion to additional markets.All around, the techniques by which products are composed and fabricated are progressing at a quick pace just directly in front of us. Traditionally processes, checked and controlled by people, are presently being controlled and observed by brilliant sensors and actuators in this new era of manufacturing [24]. 
Intelligence of a system can be perceived as the capability of the system to achieve a goal or to sustain desired behavior in business of uncertain conditions. In context of manufacturing, intelligent machining is a criterion in which machine tools can identify their own states and the environment in which they are operating, and can execute start, control, and terminate machine activities [25]. In other words, intelligent machines are self-worth and can make decisions related to assembling processes. The intelligent machining worldview is empowered to the coordination of keen sensors and controls. Advances in sensor technologies and automated data systems, provide new opportunities for real time collection of valuable measurements that can be converted into actionable data to make smart decisions [26].

The data acquisition and management module is comprised of the data aggregator component, a data post processor, and a data buffer component. The data aggregator component collects time synchronized raw data from external sensors. Since the data generated from different sensors can be in different formats, they need to be transformed into single format to enable it to be processed into information. Data aggregators allow for this interoperability [27]. Empty connect, is an example of industry standard data aggregation agent that allows for data aggregation. Data processor processes the collected data into suitable data formats that can be used by machine learning algorithms to make smarter decisions. Whereas, data buffer allows for data buffering so that the unwanted data can be erased at this point. The data buffer stores only relevant data that could be used by machine learning algorithms to generate actionable intelligence. Data buffers act where in between the data processing and machine learning algorithms and allows for efficient storage [28]. The data dividend machine learning and knowledge discovery module, uses the processed monitoring data to construct a data dividend prediction model for the target machine. The prediction model is used in conjunction with the data from past experiences to monitor and control the machining tool.

\section{ADVANCED MANUFACTURING ENTERPRISE}

According to the National Institute of Standards and Technology, advanced manufacturing is an entity that makes extensive use of computer, high precision and data innovation incorporated with a superior workforce in a creation framework equipped for outfitting a heterogeneous mixture of products in little or huge volumes with both the productivity of large scale manufacturing and the adaptability of custom assembling so as to react quickly to client demands. Anderson Economy Group has defined has defined advanced manufacturing as, operations that create advanced products, use innovative techniques in their manufacturing, and are inventing new processes and technologies for future manufacturing [29]. Advanced Manufacturing is not quite recently the work of robots and cutting-edge forms yet in addition incorporates the assembling of imaginative items and utilization of innovative standards. Components of cutting edge producing are arranged under two primary classes, tangible and intangible components. Tangible elements of manufacturing systems include manufacturing processes, industrial control systems, industrial robotics, assembly systems, material transportation systems and storage systems. Intangible elements of assembling frameworks incorporate all arranging and administration part of the assembling firm, cases of cutting edge innovations incorporate Nano-fabricating, semiconductor, additive manufacturing and bioproducing.

\section{DIGITAL MANUFACTURING SECURITY}

Digital manufacturing via the digital thread requires the consideration of security in all domains including network, desktop, hardware, and software applications. Security is, in fact, an integral part of the overall digital manufacturing design since it involves various valuable intellectual property resources, which we call IP throughout this course [30]. And should not be confused with IP, an acronym used in computer networks to mean Internet protocols. These IP resources are mostly information such as hardware designs, machine control instructions, payroll transactions, or business deals. The leak of IP information can cause huge loss to your company. And therefore, IP needs primary attention while designing system security. Threats to IP, come from various sources that includes business competitors and state affiliated actors. The biggest threats to IP is the insider threat. Since insiders are the people with legitimate access to important information and hence, such threats are hard to detect. Therefore, in DMD security, we need different levels of security to provide restricted access to the information. Such access control will allow only certain authorities in the organization to access the IP information. Recent developments in digital manufacturing may be categorized into two major groups. The developments of the first group have followed a bottom-up approach considering digital manufacturing, and extending its concepts, within a wider framework e.g. the digital factory or enterprise. The developments of the second group have followed a top- down approach considering the technologies in sup- port of individual aspects of digital manufacturing, e.g. e-collaboration and simulation. The digital factory includes models, methods, and tools for the sustainable support of factory planning and factory operations. It includes processes based on linked digital models connected with the product model. At a theoretical level, several researchers have contributed to the definition of the digital factory vision and suggested how this vision could be implemented in reality. From a technological point of view, new frameworks for distributed digital manufacturing have appeared on the scene. Recent developments focus on a new generation of decentralized factory control algorithms known as 'agent based'. A software agent, first, is a self-directed object, second, has its own value systems and a means of communicating with other such objects, and, third, continuously acts on 
its own initiative. A system of such agents, called a multi-agent system, consists of a group of identical or complementary agents that act together. Agentbased systems encompassing real-time and decentralized manufacturing decision-making capabilities have been reported.

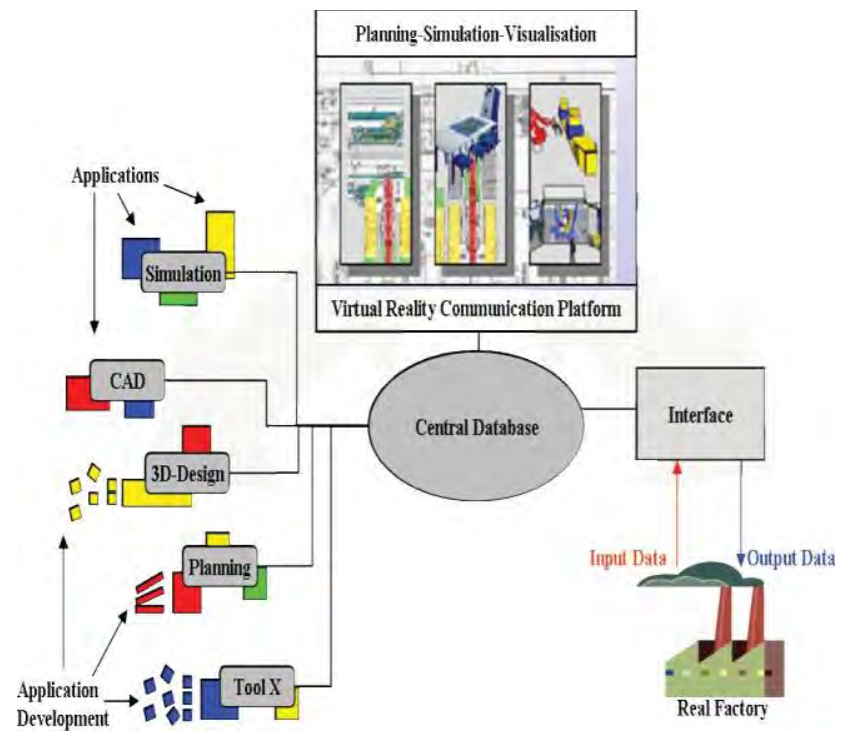

The vision of the digital factory

\section{MODEL BASED SYSTEM ENGINEERING}

The INCOSE systems engineering handbook points to three meaningful definitions of systems engineering [31]. A complete definition - Systems Engineering is an integrative approach which intends to empower the recognition of acknowledged systems. It focuses on characterizing client needs and required outputs, in the development cycle. Documenting stipulations and then proceeding with design composites and system demonstration while considering the complete problem [32]. Operations, cost and schedule, performance, training and support, test, manufacturing and disposal [33]. It accommodates all the disciplines and specialty groups into a team effort framing a structure advancement process that proceeds from idea, to production, to operation. It additionally considers both the business and industrial needs of all clients with the goal providing a quality product that meets the consumer needs.

Systems engineering is a reiterative process of top down synthesis, reinforcement, and operation of a realworld system that satisfies, in near optimal manner, the full range of requirements for the system. From an operational perspective, System engineering is a development engineering that scrutinize on the design and operation of the entire framework as particular from the parts.

\section{A. System Engineering \& Lifecycle}

A system progresses through its life cycle as the result of actions. Performed and managed by people in organizations, using processes for execution of their actions. The life cycle stages used the standard are concept, development, creation, usage, support and retirement. Different models have emerged for managing the processes within and across life cycle stages [34]. The first life cycle model for systems engineering that is the waterfall model. The waterfall model is a specified, sequential process that follows a predetermined sequence. Waterfall is easily converted into schedules with deliverables, where the process flows downwards through all the stages that are required, concept, development, production, utilization, support and retirement. By its nature, in waterfall there is an embedded assumption that requirements are well understood and not likely to change during the execution of a project. The second approach is actually a class of methods. Incremental and iterative methods. Incremental and iterative methods have proven to be successful for small, simpler systems or elements of a system due to their focus on flexibility and judicious risk taking.

\section{B. Model-Based Definition}

According to INCOSE, model-based systems engineering, also known as MBSE, is the formalized application of modeling to support system requirements, design, investigation, confirmation, and approval exercises starting in the reasonable outline stage and proceeding through advancement and later life cycle stages. Model-based systems engineering is still in a dynamic state of development and is a vast topic to fully address. As a result, this course is offering a broad perspective with connections throughout the course that give you the opportunity to learn more about a topic of interest. A key component of model-based systems engineering is the model-based definition, or MBD. Model-based definition embodies the concept of moving away from paper-based documentation and drawings to digital, 3D CAD representation, manufacturing data, and performance models. An example of the dynamic state of MBSE, the definition of MBD is still evolving and converging. Standards, like ASME Y 14.41, ISO 16792 , and MIL-STD-31000, can provide guidance on how to annotate drawings. But multiple perspectives exist in regards to what makes up a model. The framework to use a model-based system a framework is required provides the guiding structure to implement analysis throughout the lifecycle of the product. An analysis framework connects a variety of simulation tools -- for example, Ansys, Nastran, MATLAB, and tools built in-house-in such a way that outputs of one analysis provide the input for subsequent analyses without human intervention. The Model-Based Systems Engineering (MBSE) framework is Siemens offering HEEDs. HEEDs provide a means of computationally exploring possible design alternatives in search of specific performance goals.

VII. Cloud-based Design and Manufacturing 
Cloud-based design and manufacturing (CBDM) refers to a service-oriented networked product development model in which service consumers are able to configure products or services and reconfigure manufacturing systems through Infrastructure-as-a-Service (IaaS), Platform-as-a-Service (PaaS), Hardware-as-a-Service (HaaS), and Software-as-aService (SaaS) [35]. In the IaaS model, cloud service providers offer on-demand access to computing resources such as virtual machines and cloud storage. In the PaaS model, cloud service providers deliver computing platforms such as social collaboration platforms, programming and execution 4 environments for cloud computing. In the HaaS model, cloud service providers and consumers are enabled to rent and lease manufacturing equipment such as milling machines and 3D printers. In the SaaS model, cloud service consumers are enabled to run computationally expensive application software such as AutoCAD remotely without installing and running the software on their local computers [36]. Cloud-Based Design (CBD) refers to a networked design model that leverages cloud computing, service-oriented architecture (SOA), Web 2.0 (e.g., social network sites), and semantic web technologies to support cloud-based engineering design services in distributed and collaborative environments. Cloud-Based Manufacturing (CBM) refers to a networked manufacturing model that exploits on-demand access to a shared collection of diversified and distributed manufacturing resources to form temporary, reconfigurable production lines which enhance efficiency, reduce product lifecycle costs, and allow for optimal resource allocation in response to variable-demand customer generated tasking

\section{CONCLUSIONS}

Digital Manufacturing \& Design creates a product at speed beyond human imagination. The DMD domain is inspired by a detailed thread of product, handled information and data, which brings about significant protected innovation. Industries can reduce their paper based processes to digital process which at last will lead in increase in productivity. When development of product was made initially in 8 to 9 months now had been reduced to 20 to 30 days and this is digital manufacturing. DMC will become a platform used by millions of people daily. Users can easily add their own manufacturing recipes and examine their works in their own web browsers. The digital thread is networked at several functions and levels in order to enhance efficiency and effectiveness.

DMD reduces the effects on the supply chain by transparently sharing efficiency information, whether man, machine, materials, or methods, throughout the supply chain. Increasing supply chain confidence between tiers, reducing excess inventory that's contained within the supply chain, and allowing for realizing more efficient and agile supply chain models. Data analytics ideas have taken into account the development of new assembling ideal models including the smart manufacturing, social assembling and cloud based manufacturing. Digital manufacturing incorporates technologies for the virtual representation of factories, buildings, resources, machine systems equipment, labor staff and their skills, as well as for the closer integration of product and process development through modelling and simulation Closing the gap between the product definition and the actual manufacturing production activities within the enterprise, fully transforming tacit manufacturing knowledge into tangible, and, finally, digital knowledge, optimizing data management, and developing standard models are some key priorities.

\section{REFERENCES}

[1] T. Brugo, R. Palazzetti, S. Ciric-Kostic, X.T. Yan, G. Minak, and A. Zucchelli, "Fracture mechanics of laser sintered cracked polyamide for a new method to induce cracks by additive manufacturing," Polym. Test., vol. 50, pp. 301-308, Apr. 2016. doi: 10.1016/j.polymertesting.2016.01.024.

[2] A. J. Capel, S. Edmondson, S. D. Christie, R. D. Goodridge, R. J. Bibb, and M. Thurstans, "Design and additive manufacture for flow chemistry," Lab Chip, vol. 13, no. 23, pp. 4583- 4590, 2013. doi:10.1039/c31c50844g.

[3] S. Choi, C. Jun, W. B. Zhao, and S. D. Noh, "Digital manufacturing in smart manufacturing systems," in Advances in Production Management Systems Conf. Proc., Tokyo, Jpn., pt. II, pp. 21-29, 2015. doi: 10.1007/978-3-319-22759-7_3.

[4] S. Choi, B. Kim, and S. Noh, "A diagnosis and evaluation method for strategic planning and systematic design of a virtual factory in smart manufacturing systems," Int. J. Precis. Eng. Manuf., vol. 16, no. 6, pp. 1107-1115, 2015.

doi: 10.1007/s12541-015-0143-9.

[5] M. Cotteleer, J. Holdowsky, and M. Mahto, The 3D opportunity primer, DU Press, 2014.

[6] J. Coykendall, M. Cotteleer, J. Holdowsky, and M. Mahto, "3D opportunity in aerospace and defense: Additive manufacturing takes flight," Deloitte Insights, June 2, 2014. [Online]. Available: https://www2.deloitte.com/insights/us/en/focus/3dopportunity/additive-manufacturing-3d-opportunity-in-aerospace.html. L. Ding L, D. Davies, C. A. McMahon, "The integration of lightweight representation and annotation for collaborative design representation," Res. Eng. Des., vol. 20, no. 3 , pp. 185-200, 2009. doi: 10.1007/s00163-009-0077-2 .

[7] R. R. Dehoff, C. Tallman, C.E. Duty, W. H. Peter, Y. Yamamoto, W. Chen, and C. A. Blue, "Case study: additive manufacturing of aerospace brackets.,"Advanced Materials and Processes, vol. 171, no. 3, 2013.

[8] P. Delaporte and A. P. Alloncle, "[INVITED] Laser-induced forward transfer: A high resolution additive manufacturing technology," Optics \& Laser Technology, vol. 78, part A, pp. 33-41, 2016 doi: 10.1016/j.optlastec.2015.09.022.

[9] I. Foster, Y. Zhao, I. Raicu, S. Lu, Cloud computing and grid computing 360degree compared. In: Grid computing environments workshop, Austin. 2008.

[10] V. Jovanovic and N.W. Hartman, "Web-based virtual learning for digital manufacturing fundamentals for automotive workforce training," International Journal of Continuing Engineering Education and Life Long Learning XIV, vol. 23, no. 3-4, pp. 300-310, 2013. doi: 10.1504/IJCEELL.2013.055403.

[11] L. Keating, "You can now print edible pizza with a 3D 'Foodini' printer," TECH TIMES, November 6, 2014. [Online]. Available: http://

www.techtimes.com/articles/19630/20141106/startup-serves-up-edible- pizzaburgers-3dfoodini-printer.htm.

[12] H. Kim, J.-K. Lee, J.-H. Park, B. -J. Park, and D.-S. Jang, "Applying digital manufacturing technology to ship production and the maritime environment," Integrated Manufacturing Systems, 13(5), 295-305, 2002. doi: 10.1108/09576060210429748.

[13] Kim Porter, Jarrod Phipps, Adam Szepkouski, \& S. Abidi, "3D opportunity serves it up: Additive manufacturing and food," June 18, 2015, Deloitte Insights. [Online] Available: https://www2.deloitte.com/insights/us/en/focus/3dopportunity/3d-printing-in-the-food-industry.html. 
[14] P. J. Kitson, S. Glatzel, W, Chen, C. G. Lin, Y.F. Song, and L. Cronin, "3D printing of versatile reaction ware for chemical synthesis," Nature Protocols, vol. 11, no. 5, pp. 920-936, 2016. doi: 10.1038/nprot.2016.041.

[15] R. Kurzrock, and D. J. Stewart, "Click chemistry, 3D-printing, and omics: the future of drug development," Oncotarget, vol. 7, no. 3, pp. 2155-2158, 2016. doi:10.18632/oncotarget.6787.

[16] B. H. Li, L. Zhang, S. L. Wang, F. Tao, J. W. Cao, X. D. Jiang, et al, "Cloud manufacturing: a new service-oriented networked manufacturing model," Computer Integrated Manufacturing Systems, vol. 16, no. 1, pp. 1 -7, 2010.

[17] L. Monostori, J. Váncza, S. R. Kumara, "Agent-based systems for manufacturing," CIRP Ann, vol. 55, no. 3, pp. 697-720, 2006. doi: 10.1016/j.cirp.2006.10.004.

[18] F. Piller, A. Vossen, C. Ihl, "From social media to social product development: the impact of social media on co-creation of innovation," Unternehmung, vol 66, no. 1, pp. 7-27, 2012. doi:10.5771/0042-059X-2012-1-7.

[19] E. Red, D. French, G. Jensen, S. S. Walker, P. Madsen, "Emerging design methods and tools in collaborative product development.," J. Comput. Inf. Sci. Eng. vol. 13, no. 3, 031001, 2013. doi: 10.1115/1.4023917.

[20] E. Red, V. Holyoak, C. G. Jensen, F. Marshall, J. Ryskamp, Y. Xu, "?-CAx: a research agenda for collaborative computer-aided applications," Computer Aided Design and Applications, vol. 7, no. 3, pp. 387-404, 2010.

doi: 10.3722/cadaps.2010.387-404.

[21] D. Schaefer, Ed, Product development in the socio-sphere: game changing paradigms for 21st century breakthrough product development and innovation, London, UK: Springer; 2014. p. 235. doi:10.1007/978-3-319-07404-7.

[22] Shen W, Hao Q, Yoon HJ, Norrie DH. Applications of agent-based systems in intelligent manufacturing: an updated review. Adv Eng Inform, vol. 20, no. 4, pp. 415-431, 2006. doi:10.1016/j.aei.2006.05.004.

[23] I.E. Sutherland, "Sketch pad a man-machine graphical communication system,". In: Proceedings of the SHARE design automation workshop, ACM; 1964. p. 6-329. doi: $10.1145 / 800265.810742$.

[24] T. Tolio, D. Ceglarek, H. A. ElMaraghy, A. Fischer, S. J. Hu, L. Laperrière, et al,."SPECIES - co- evolution of products, processes and production systems," CIRP Ann., vol. 59, no. 2, pp. 672-693, 2010. doi: 10.1016/j.cirp.2010.05.008.

[25] K.T. Ulrich Kand S. D. Eppinger, Product design and development, vol. 384. New York: McGraw- Hill; 1995.

[26] D. Wu, J.L. Thames, D. W. Rosen, D. Schaefer, "Enhancing the product realization process with cloud-based design and manufacturing systems," Trans ASME J. Comput. Inform. Sci. Eng., vol. 13, no. 4, 041004-041004-14, 2013. doi: $10.1115 / 1.4025257$.

[27] L. Wang, W. Shen, S. Lang, "Wise-ShopFloor: a web-based and sensor-driven eshop floor," Trans. ASME J. Comput. Inform. Sci. Eng., vol. 4, no. 1, pp. 56-60, 2004. doi: 10.1115/1.1647122.

[28] D. Wu, D. W. Rosen, D. Schaefer, "Cloud-based design and manufacturing: status and promise," In: Cloud-based design and manufacturing: a service-oriented product development paradigm for the 21st century, D. Schaefer, Ed., London, UK: Springer; 2014. p. 282. doi: 10.1007/978-3-319-07398-9_1.

[29] D. Wu, M. J. Greer, D. W. Rosen, D. Schaefer, "Cloud manufacturing: strategic vision and state-of- the-art," J. Manuf. Syst., vol. 32, no. 4, 2pp. 564-579, 2013. doi: 10.1016/j.jmsy.2013.04.008.

[30] L. Wang, W. Shen, H. Xie, J. Neelamkavil, A. Pardasani, "Collaborative conceptual design — state of the art and future trends," Computer Aided Des., vol. 34, no. 13, pp. 981-996, 2002. doi:10.1016/S0010-4485(01)00157-9.

[31] L.Wang, W. Shen, "DPP: an agent-based approach for distributed process planning," J. Intell. Manuf., vol. 14, no. 5, pp. 429-439, 2003.

doi: 10.1023/A:1025797124367.
[32] D. Wu, D. W. Rosen, D. Schaefer, "Modeling and analysing the material flow of crowdsourcing processes in loud-based manufacturing systems using stochastic petri nets," in Proceedings of the ASME 2014 international manufacturing science and engineering conference (MSEC14), Paper Number: MSEC2014-3907, Detroit, Michigan, US, 2014. [Online]. Available: http:// www.compositesworld.com/cdn/cms/beyond-big-data-harnessing-the-industrialinternet-for-wind-power-5-638.jpg.

[33] S. Yim, D. W. Rosen, "Build time and cost models for additive manufacturing process selection," in Proceedings of the ASME 2012 international design engineering technical conference \& computers and information in engineering conference, IDETC/CIE12, Paper Number: DETC2012-70940, Chicago, US, 2012. doi:10.1115/DETC2012-70940.

[34] C. Giffi, B. Gangula, P. Illinda, "3D opportunity for the automotive industry: Additive manufacturing hits the road," Deloitte Insights, May 19, 2014. [Online]. Available: https://www2.deloitte.com/insights/us/en/focus/3d-opportunity/ additive-manufacturing-3d-opportunity-in-automotive.html.

[35] J. Manyika, M. Chui, J. Bughin, R. Dobbs, P. Bisson, and A. Marrs, "Disruptive technologies: advances that will transform life, business, and the global economy," McKinsey Global Institute San Francisco, CA, 2013.

[36] D. Wu, D. W. Rosen, D. Schaefer, "Cloud-based design and manufacturing: status and promise," in Cloud-based design and manufacturing (CBDM): a service-oriented product development paradigm for the 21st century, D. Schaefer, Ed., Springer, London, UK: Springer, pp. 1-24, 2014. doi:10.1007/978-3-319-07398-9_1. 
COMPUTATIONAL MECHANICS 


\section{A Novel Finite Strain Visco-hyperelasticity Based Constitutive Model for Elastomers}

\author{
Rohan Thakkar \\ Department of Mechanical Engineering \\ York University \\ Toronto, Ontario, Canada \\ Email: rohan33@yorku.ca
}

\author{
Aleksander Czekanski \\ Department of Mechanical Engineering \\ York University \\ Toronto, Ontario, Canada \\ Email: alex.czekanski@lassonde.yorku.ca
}

\begin{abstract}
A novel three-dimensional finite strain viscohyperelastic constitutive model is proposed to capture the strain rate dependency of rubber-like materials. The overall material behavior is defined by cumulative description of hyperelasticity and nonlinear viscoelasticity. The hyperelastic part is based on exponential logarithmic Hart-Smith strain energy function and the viscous part comprises of a fading integral which links the current stresses to the applied strain history. The derived analytical framework is verified with respect to experimental data. The potential of the proposed model has been constituted by an excellent fit between proposed model and considered test data.
\end{abstract}

Keywords-Rubber-like materials, Strain rate, Visco-hyperelasticity, Constitutive modeling

\section{INTRODUCTION}

Rubber-like materials undergo large deformations at relatively low stresses and recover their initial shape upon removal of the load. This exceptional ability makes them suitable and without a doubt irreplaceable materials in vital engineering applications. This uncommon behavior is caused by the underlying coiled long chain polymer molecules. These chains straighten when the material is stretched and recoil upon removal of the load. This substantial aspect contributes towards their extraordinary mechanical behavior and makes rubbers a strain rate dependent material. This rate dependency is associated with the rearrangement of the molecular chains [1].

Rubber viscoelasticity can be described by two distinct theories: a) Linear viscoelasticity b) Non-linear viscoelasticity. To summaries, linear viscoelasticity models are constituted by considering parallel and/or series arrangements of elastic springs and linear viscous dash-pots. These rheological analogies are superimposed by Boltzman's principle and the relaxation function is approximated with a prony series formulation[2], [3], [4]. Whereas, the non-linear viscoelastic constitutive relations are formulated by employing generalized fading history integral function [5], [6]. In this approach, an approximation of matrix stress functional defines the strain history on stresses.
In order to prescribe time dependent response of the elastomers, quasi-linear viscoelastic frame work requires to be represented by 4-6 term prony series approximations. As a result, large number of material constants are needed to be determined [7], [8], [9]. In contrast, the number of material constants can be reduced by articulating nonlinear viscoelasticity. Moreover, numerical implementation of such formulations is a straightforward procedure and the material parameter identification is comparatively less time consuming task. For that reason, a novel power-exponential strain history functional has been proposed in the nonlinear finite strain visco-hyperelastic framework to capture rate dependency in various types of elastomers. For the purpose of validation, derived constitutive relations are compared with the literature based high strain rate experimental data for polyurea [10].

\section{Constitutive Model Development}

\section{A. Hyperelasticity}

Hyperelastic constitutive laws are derived from strain energy density $W$. It expresses the stored elastic strain energy in material per unit reference volume as a function of the principle strain or stretch invariants.

$$
W=f\left(I_{1}, I_{2}, I_{3}\right),
$$

Where $I_{1}, I_{2}$ and $I_{3}$ are the invariants of left Cauchy-Green tensor $\left(B=F F^{T}\right)$ defined as:

$$
\begin{gathered}
I_{1}=\operatorname{tr}(B)=\lambda_{1}^{2}+\lambda_{2}^{2}+\lambda_{3}^{2} \\
I_{2}=(\operatorname{tr} B)^{2}-\operatorname{tr} B^{2}=\lambda_{1}^{2} \lambda_{2}^{2}+\lambda_{2}^{2} \lambda_{3}^{2}+\lambda_{3}^{2} \lambda_{1}^{2} \\
I_{3}=J=\sqrt{\operatorname{det}(F)}=\sqrt{\left(\lambda_{1}^{2} \lambda_{2}^{2} \lambda_{3}^{2}\right)}
\end{gathered}
$$

Cauchy stress in an incompressible hyperelastic continua is given by[11]:

$$
\sigma^{e}=-p^{e} I+2\left[\left(\frac{\partial W}{\partial I_{1}}+I_{1} \frac{\partial W}{\partial I_{2}}\right) B-\frac{\partial W}{\partial I_{2}} B^{2}\right]
$$

where where $p^{e}$ is a hydrostatic pressure term which needs to be determined from boundary conditions and $I$ is an identity 
tensor. Time independent behavior of elastomers is characterized using Hart-Smith strain energy [12] as it provides distinctive advantages in modeling finite strain hyperelastic materials due to its exponential form[13], [14], [15], [16].

$$
W_{H S}=C_{1} \int \exp \left(C_{3}\left(I_{1}-3\right)^{2}\right) d I_{1}+3 C_{2} \ln \frac{I_{2}}{3}
$$

Here $C_{1}, C_{2}$ and $C_{3}$ are model parameters and need to be determined from experiments.

Let us consider an in-compressible \{i.e. $J=1$ in eq.(3) material deformation under uniaxial extension mode. If the stretch in the loading direction is denoted by $\lambda$ then the deformation gradient $F$ for this loading becomes:

$$
F=\left[\begin{array}{ccc}
\lambda & 0 & 0 \\
0 & \lambda^{-0.5} & 0 \\
0 & 0 & \lambda^{-0.5}
\end{array}\right]
$$

Consequently the invariants are depicted as:

$$
I_{1}=\lambda^{2}+2 \lambda^{-1}, I_{2}=\lambda^{-2}+2 \lambda
$$

From eq. (3) and (4), we arrived to uniaxial stress-stretch relation as:

$$
\sigma_{11}^{e}=-p^{e}+2 \lambda^{2}\left(\frac{I_{1} C 2}{I_{2}}+C 1 \exp ^{C 3\left(I_{1}-3\right)^{2}}-\frac{C 2 \lambda^{2}}{I_{2}}\right)
$$

Then after, the hydrostatic pressure term $p^{e}$ is calculated by applying uniaxial loading condition $\sigma_{22}^{e}=\sigma_{33}^{e}=0$

$$
\sigma_{22}^{e}=0=-p^{e}+\frac{2}{\lambda}\left(\frac{I_{1} C 2}{I_{2}}+C 1 \exp ^{C 3\left(I_{1}-3\right)^{2}}-\frac{C 2}{\lambda I_{2}}\right)
$$

Finally, by substituting the expression for $p^{e}$ into eq(7); the we derived incompressible uniaxial hart-smith stress-strain law as:

$$
\begin{gathered}
\sigma_{11}^{e}=2\left(\lambda^{2}-\lambda^{-1}\right)\left[\frac{I_{1} C 2}{I_{2}}+C 1 \exp ^{C 3\left(I_{1}-3\right)^{2}}\right. \\
\left.-\frac{\left(\lambda^{2}+\lambda^{-1}\right) C 2}{\lambda I_{2}}\right]
\end{gathered}
$$

\section{B. Viscoelasticity}

The generalized constitutive relation for non-linear incompressible viscoelastic material behavior is given by[17]:

$$
\sigma^{v}=-p_{v} I+F(t) \cdot \prod_{\tau=-\infty}^{t}\{\mathrm{C}(\tau)\} \cdot F(t)^{t}
$$

where $\sigma^{v}$ is time dependent cauchy stress tensor, $p$ is the hydro-static pressure term also called Lagrange Multiplier and $\prod$ is a strain history function given by:

$$
\prod_{\tau=-\infty}^{t}\{\mathrm{C}(\tau)\}=\int_{-\infty}^{t} \Phi\left(I_{1}, I_{2}\right) m(t-\tau) \dot{E(\tau)} d \tau
$$

In equation (11), $\Phi\left(I_{1}, I_{2}\right)$ is the function depending on invariants of strain tensor $C$ and the strain rate $E \dot{E}(\tau)$ is defined as:

$$
\dot{E}=\frac{1}{2}\left(\dot{F}^{T} F+F^{T} \dot{F}\right)
$$

$m(t)$ is a relaxation function which is represented by a series of exponential series functions as

$$
m(t)=\sum_{i=1}^{N} \exp \left(-\frac{t-\tau}{\theta_{i}}\right)
$$

where $\theta_{i}$ is the relaxation time. Several researchers have assigned $N$ values greater than/equal to 2 in their work[18]. In order to reduce the number of material constants with single relaxation time scheme i.e. $N=1$, we are proposing a novel suitable representation of the function $\Phi\left(I_{1}, I_{2}\right)$ as:

$$
\Phi\left(I_{1}, I_{2}\right)=E \dot{E}(\tau){ }^{A_{1}} \cdot A_{2} \cdot \exp \left(I_{2}-3\right)^{A_{3}}
$$

The starting point for the time integration is at the instant when loading commences. It is assumed that the effect of deformation history for $\tau<0$ on the stress at time $t>0$ is negligible. Thus, the period of deformation history which is considered to effect the stress response and hence the limits of integration in the second term on the right-hand side of Eq.11 becomes $[0, t]$ rather than $[-\infty, t]$.

Substituting Eqs. 14 and 13 with $\mathrm{N}=1$ into Eq. 11 results in the following proposed integral approximation for $\prod$

$$
\prod=\int_{0}^{t} \dot{E}^{A_{1}} \cdot A_{2} \cdot \exp \left(I_{2}-3\right)^{A_{3}} \exp \left(-\frac{t-\tau}{\theta_{1}}\right) \dot{E} d \tau
$$

Substitution of eq.(15) into eq.(10) yields a finite strain viscoelastic model for incompressible materials as

$$
\begin{aligned}
& \sigma^{v}=-p_{v} I+F \cdot\left[\int_{0}^{t} \dot{E}^{A_{1}} \cdot A_{2} \cdot \exp \left(I_{2}-3\right)^{A_{3}} .\right. \\
& \left.\exp \left(-\frac{t-\tau}{\theta_{1}}\right) \dot{E(\tau)} d \tau\right] \cdot F^{t}
\end{aligned}
$$

\section{Visco-hyperelasticity}

Visco-hyperelastic behavior is generally introduced by aggregating hyperelasticity and viscocity i.e. $\sigma=\sigma^{e}+\sigma^{v}[18]$, [19].

$$
\begin{gathered}
\sigma=-p I+2\left[\left(\frac{\partial W}{\partial I_{1}}+I_{1} \frac{\partial W}{\partial I_{2}}\right) B-\frac{\partial W}{\partial I_{2}} B^{2}\right]+F \\
{\left[\int_{0}^{t} \dot{E}^{A_{1}} \cdot A_{2} \cdot \exp \left(I_{2}-3\right)^{A_{3}} \cdot \exp \left(-\frac{t-\tau}{\theta_{1}}\right) E \dot{E}(\tau) d \tau\right] \cdot F^{t}}
\end{gathered}
$$

where $\sigma$ is the total stress and $p$ is the total hydrostatic pressure incorporating static and viscoelastic components. Inserting $\dot{E}_{11}=\lambda \dot{\lambda}$ into eq.(17) concludes the uniaxial stress as:

$$
\begin{gathered}
\sigma_{11}=-p^{v}+\sigma_{11}^{e}+ \\
\lambda^{3} \int_{0}^{t}(\lambda \dot{\lambda})^{A_{1}} \cdot A_{2} \cdot \exp \left(I_{2}-3\right)^{A_{3}} \cdot \exp \left(-\frac{t-\tau}{\theta_{1}}\right) \dot{\lambda} d \tau
\end{gathered}
$$


By applying uniaxial loading condition that $\sigma_{11}=0$ into eq.(18), $p^{v}$ is formulated as:

$$
\begin{gathered}
p^{v}=-\frac{\lambda^{-1}}{2}\left[\int_{0}^{t} \lambda^{-2}(\lambda \dot{\lambda})^{A_{1}} \cdot A_{2} \cdot \exp \left(I_{2}-3\right)^{A_{3}} .\right. \\
\left.\exp \left(-\frac{t-\tau}{\theta_{1}}\right) \dot{\lambda} d \tau\right]
\end{gathered}
$$

Replacing $p^{v}$ in eq(18), we derived the uniaxial stressdeformation expression as:

$$
\begin{gathered}
\sigma_{11}=\sigma_{11}^{e}+\lambda^{3} \int_{0}^{t}(\lambda \dot{\lambda})^{A_{1}} \cdot A_{2} \cdot \exp \left(I_{2}-3\right)^{A_{3} .} \\
\exp \left(-\frac{t-\tau}{\theta_{1}}\right) \dot{\lambda} d \tau+-\frac{\lambda^{-1}}{2}\left[\int_{0}^{t} \lambda^{-2}(\lambda \dot{\lambda})^{A_{1}} \cdot A_{2} .\right. \\
\left.\exp \left(I_{2}-3\right)^{A_{3}} \cdot \exp \left(-\frac{t-\tau}{\theta_{1}}\right) \dot{\lambda} d \tau\right]
\end{gathered}
$$

\section{VERIFICATION OF PROPOSED MODEL}

We have considered experimental data reported by Roland et. al [10] for verifying derived analytical results. They have performed uniaxial tensile test on polyurea from moderate to high strain rates using drop weight apparatus. The hyperelastic

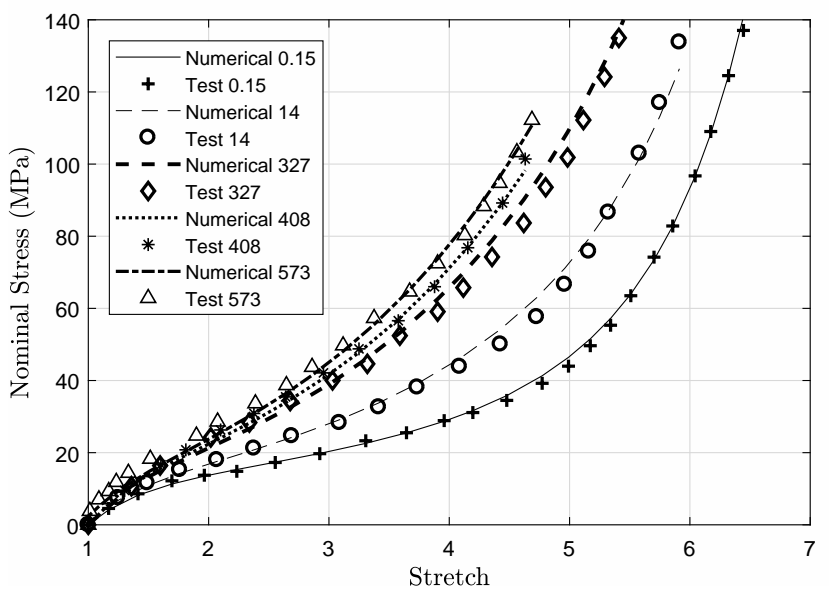

Fig. 1: Comparison of Roland et. al [10] data with proposed model

Hart-Smith material parameters are determined with respect to quasi-static (in this case $0.15 \mathrm{~S}^{-1}$ ) experimental data. The detailed procedure for parameter identification is described in [13]. Where as the material parameters for the viscoelastic part has been derived by considering test data for the strain rates of $14 \mathrm{~S}^{-1}$ and $573 \mathrm{~S}^{-1}$. These required material constants have been optimized using non-linear least square function "Fmincon" in MATLAB (see Table I). Fig. (??) demonstrates an excellent agreement between the proposed model results and the experimental data. Notably, the model is adequate to predict the material response for the rates of $327 \mathrm{~S}^{-1}$ and $408 \mathrm{~S}^{-1}$ accurately even though the relevant experimental data have not been considered for the material parameters identification.
TABLE I: Optimized material parameters

\begin{tabular}{|c|c|c|c|c|c|c|}
\hline \multicolumn{3}{|c|}{ Hyperelastic model } & \multicolumn{4}{|c|}{ Viscoelastic model } \\
\hline \hline$C_{1}$ & $C_{2}$ & $C_{3}$ & $\theta_{1}$ & $A_{1}$ & $A_{2}$ & $A_{3}$ \\
\hline 0.4474 & 12.79 & $8.1 \mathrm{E}-4$ & $5.4 \mathrm{E}-3$ & -0.7355 & 259.89 & 0.05 \\
\hline
\end{tabular}

\section{CONCLUSION AND FUTURE WORK}

To summarize, a novel visco-hyperelastc material law has been proposed to capture rate dependency in elastomers by adopting generalized non-linear viscoelasticity. Analytical stress-strain relations are derived for uniaxial tension mode. An excellent agreement between numerical results and experimental data reported by Roland et. al [10] has been obtained. Also, the predictive capability of the model has been demonstrated. Following the promising findings, the conducted work will be extended by considering broader spectrum of experimental data. The developed model will be implemented in commercial finite element code via user defined subroutine to analyze complex geometries and ladings.

\section{ACKNOWLEDGMENT}

The authors would like to acknowledge Dr.Taisiya Sigaeva for her assistance in the implementation of hyperelasticity. The grants provided by Lassonde School of Engineering and NSERC Quanser Chair in Design Engineering are thankfully acknowledged.

\section{REFERENCES}

[1] J.D. Ferry,Viscoelastic properties of polymers, John Wiley \& Sons, 1980.

[2] J.C. Simo, "On a fully three-dimensional finite-strain viscoelastic damage model: formulation and computational aspects," Computer methods in applied mechanics and engineering, vol. 60, issue 2, pp. 153-173, 1987. doi: 10.1016/0045-7825(87)90107-1.

[3] H.T. Banks, S. Hu, and Z.R. Kenz, "A brief review of elasticity and viscoelasticity for solids," Advances in Applied Mathematics and Mechanics, vol. 3, issue 1, pp. 1-51, 2011. doi: 10.4208/aamm.10-m1030.

[4] A.D. Drozdov, "A constitutive model for nonlinear viscoelastic media," Int. J. Solids Struct., vol. 34, issue 21, pp. 2685-2707, 1997. doi: 10.1016/S0020-7683(96)00178-3.

[5] C. Truesdell, W. Noll, A. Pipkin, "The non-linear field theories of mechanics," J. Appl. Mech., vol. 33, issue 4, p. 958, 1966. doi: $10.1115 / 1.3625229$.

[6] B. Bernstein, E. Kearsley, L. Zapas, "A study of stress relaxation with finite strain," Trans. Soc. Rheol., vol. 7, issue 1, pp. 391-410, 1963. doi: $10.1122 / 1.548963$.

[7] M. Pawlikowski, "Non-linear approach in visco-hyperelastic constitutive modelling of polyurethane nanocomposite," Mech. Time-Depend. Mater., vol. 18, issue 1, pp. 1-20, 2014. doi: 10.1007/s11043-013-9208-2.

[8] J. Shim, D. Mohr, "Rate dependent finite strain constitutive model of polyurea," Int. J. Plast., vol. 27, issue 6, pp. 868- 886, 2011. doi: 10.1016/j.jplas.2010.10.001.

[9] S. Goh, M. Charalambides, J. Williams, "Determination of the constitutive constants of non-linear viscoelastic materials," Mech. TimeDepend. Mater., vol. 8, issue 3, pp. 255-268, 2004. doi: 10.1023/B:MTDM.0000046750.65395.fe. 
[10] C. Roland, J. Twigg, Y. Vu, P. Mott, "High strain rate mechanical behavior of polyurea," Polymer, vol. 48, issue 2, pp. 574-578, 2007. doi:10.1016/j.polymer.2006.11.051.

[11] R. S. Rivlin, D. Saunders, "Large elastic deformations of isotropic materials. vii. experiments on the deformation of rubber," Philos. Trans. $R$. Soc., A, vol. 243, issue 865, pp. 251-288, 1951. doi: 10.1098/rsta.1951.0004.

[12] L. Hart-Smith,"Elasticity parameters for finite deformations of rubberlike materials,"Zeitschrift für Angewandte Mathematik und Physik (ZAMP), vol. 17, issue 5, pp. 608-626, 1966. doi: 10.1007/BF01597242.

[13] R. Thakkar, and A. Czekanski, "A comparative finite element analysis of hart-smith hyperelastic model under uniaxial, planar and equi-biaxial tension". In ASME 2017 International Mechanical Engineering Congress and Exposition, Tampa, Florida, 2017. doi: 10.1115/IMECE2017-72138.

[14] G. Chagnon, G. Marckmann, E. Verron, "A comparison of the hart-smith model with arruda-boyce and gent formulations for rubber elasticity," Rubber Chem. Technol., vol. 77, issue 4, pp. 724- 735, 2004. doi:10.5254/1.3547847.

[15] L. Hoss, R.J. Marczak, "A new constitutive model for rubber-like materials," Mecánica Computacional, vol. 23, pp. 2759-2773, 2010.

[16] M. Hossain, P. Steinmann, "More hyperelastic models for rubber-like materials: consistent tangent operators and comparative study," J. Mech. Behav. Mater., vol. 22, issue 1-2, pp. 27-50. 2013. doi: $10.1515 / \mathrm{jmbm}-2012-0007$.

[17] F.J. Lockett, Nonlinear viscoelastic solids. London: Academic Press, 1972.

[18] L. Yang, V. Shim, C. Lim, C., "A visco-hyperelastic approach to modelling the constitutive behaviour of rubber," International Journal of Impact Engineering, vol. 24, issue 6, pp. 545-560, 2000. doi: 10.1016/S0734-743X(99)00044-5.

[19] H. Khajehsaeid, J.Arghavani, R. Naghdabadi, S. Sohrabpour, 2014. "A visco-hyperelastic constitutive model for rubber-like materials: A ratedependent relaxation time scheme," Int. J. Eng. Sci. (Oxford, U. K.), vol. 79, pp. 44-58, 2014. doi: 10.1016/j.ijengsci.2014.03.001. 


\section{Modal Participation Factors and Their Potential Applications in Aerospace: A Review}

\author{
Michelle Guzman Nieto*, Mostafa S.A. ElSayed ${ }^{* *}$ \\ Dept. of Mechanical and Aerospace Engineering \\ Carleton University \\ Ottawa, Ontario, Canada \\ *Michelle.guzman@carleton.ca \\ **Mostafa.elsayed@carleton.ca
}

\author{
Denis Walch \\ Bombardier Aerospace \\ St-Laurent, Quebec, Canada \\ Denis.Walch@aero.bombardier.com
}

\begin{abstract}
In this paper we review different definitions of Modal Participation Factor (MPF) available in the literature and propose a simple classification and unified concept for their application with particular focus in aerospace engineering. A case study is presented to aid the reader understand under what conditions each definition of MPF applies, its physical interpretation and which definitions have the potential to act as response tracking mechanisms during design optimization.
\end{abstract}

Keywords: modal participation factor, modal contribution factors, dynamic analysis, mode tracking

\section{INTRODUCTION}

In practice, complex structures must be analyzed under a large set of dynamic operating conditions to ensure that the design will meet prescribed functional requirement.

During design optimization, a relatively small number of such operating conditions are selected as critical and are used to minimize or maximize the objective function. However, the dynamic response is strongly dependent on the physical characteristics of the structure and thus the use of a deterministic set of critical operating conditions may lead to unexpected behaviors. To monitor inexpensively the behavior of the design space during optimization, a response tracking mechanism based on MPFs could lead to large computational savings and improve the optimization process by considering the variations of the response of the design space as the design evolves.

MPFs have been used in the past as response tracking mechanisms in several engineering disciplines. For instance, Keong et al. [1] tracked a targeted mode during the design optimization of a head suspension assembly by defining a participation factor that resembled the most to the mode of interest. In the field of civil engineering, MPFs have been widely used to approximate the dynamic response of buildings without performing any dynamic analysis [2-13]; this procedure was later on applied in aerospace for the case of a cantilever wing model [14].

The concept of MPF, however, seems to be ambiguous. A simple search of the words "modal participation factor" in the open literature quickly reveals that several variants of this concept exist. For instance, in electrical engineering [15-18] the term MPF refers to the product between the left and the right eigenvectors; other researchers [19-22] used simple definitions for MPF such as the same term to refer to the components of the eigenvector matrix. On the other hand, [23-28] defined different convenient mathematical forms as modal participation factor suitable to their specific applications.

This significant variation in the definitions of MPFs is also conveys into a wide range of applications. For instance, Wallrapp \&Wiedemann [29] computed a quasi-static modal solution and a corresponding participation factor to assess the importance of each mode retained in the solution; Chung-Band and Young Bahng [30] utilized a participation factor based on modal strain energy to identify dominant modes; Van Lagenhove \&Brughmans [31] reviewed three kinds of MPFs as methods to identify the best location for sensors to correlate a Finite Element Model (FEM) with experimental results. Similarly, other researchers [32,33] used different definitions of MPFs to correlate experimental with numerical results.

In this paper, we propose a unified concept and a simple classification of the different mathematical definitions of modal participation factors found in the literature. A case study is presented to aid the reader to understand under what conditions each definition applies, its physical interpretation and which definitions have the potential to act as response tracking mechanisms during design optimization.

\section{MODAL CONTRIBUTION FACTORS}

The complex behavior of a dynamic structure can be decomposed into a set of simple harmonic motions $\mathrm{q}_{\mathrm{i}}(\mathrm{t})$, also known as modal responses, linearly coupled with amplitude ratios. This transformation is achieved by finding the so-called natural frequencies, $\omega_{\mathrm{r}}$ and mode shapes, $\Phi_{\mathrm{I}}$ that satisfy the characteristic equation (i.e., the undamped free vibration problem) and applying the following variable change:

$$
\mathrm{u}(\mathrm{t})=\Phi \mathrm{q}(\mathrm{t})
$$

Where $u(t)$ is the nodal vector of physical displacements, and $\Phi$ is the matrix of eigenvectors. Expanding (1) in a summation form we obtain: 


$$
\mathrm{u}_{\mathrm{n}}(\mathrm{t})=\phi_{\mathrm{n}, 1} \mathrm{q}_{1}(\mathrm{t})+\ldots+\phi_{\mathrm{n}, \mathrm{i}} \mathrm{q}_{\mathrm{i}}(\mathrm{t})
$$

From (2), the modal contribution can be defined as the quantity of movement that each mode contributes to the total nodal response [34-37]. In other words, it is the product between the modal response $\mathrm{q}_{\mathrm{i}}(\mathrm{t})$ and the amplitude ratio $\phi_{\mathrm{n}, \mathrm{i}}$, denoted here with the Greek letter gamma $(\gamma)$.

$$
\gamma_{n, i}(t)=\phi_{n, i} q_{i}(t)
$$

All definitions of modal participation factors aim to rank the relative importance of $\gamma_{\mathrm{n}, \mathrm{i}}$, nevertheless, these definitions can be subdivided in two classes. The first class (Class I) is derived from the equations of motion and it's commonly known as static modal participation factors (SMPF). SMPFs have the potential to be used as a reanalysis tool since they predict the magnitude of $\mathrm{q}_{\mathrm{i}}$ [42-51]. The second class (Class II) is intended solely to assess the relative importance of a mode within the solution of a system response.

We should make emphasis on the term "relative importance of a mode", since the interpretation of the results obtained from the second class of participation factors is dependent on its mathematical definition, as discussed below in section IV.

\section{CLASS I}

The amplitude of a modal response can be expressed as the product of a constant called modal participation factor $\Gamma_{i}$ and a time dependent term $\mathrm{D}_{\mathrm{i}}(\mathrm{t})[7,14,36,37]$.

$$
\mathrm{q}_{\mathrm{i}}(\mathrm{t})=\Gamma_{\mathrm{i}} \mathrm{D}_{\mathrm{i}}(\mathrm{t})
$$

Though in general the time history of the response may be of interest, it is the value of the maximum displacement that will be used to designing or optimizing a structure. The maximum value of the response $\mathrm{u}^{0}$ is directly proportional to the maximum dynamic response $\mathrm{D}^{0}$.

$$
\mathrm{u}^{0}=\Phi \Gamma \mathrm{D}^{0}
$$

Where $\mathrm{u}^{0}$ is the vector of maximum nodal displacements, $\mathrm{D}^{0}$ is the dynamic amplification vector and $\Gamma$ is the diagonal matrix of modal participation factors. To find appropriate expressions for $\Gamma$ and $\mathrm{D}^{0}$ we resort to the definition of the SMPF.

We begin by decomposing the vector of external forces into two components: a time invariant vector $f$ and a time dependent expression $\mathrm{p}(\mathrm{t})$.

$$
\mathrm{F}(\mathrm{t})=f \mathrm{p}(\mathrm{t})
$$

The vector $f$ can be expanded into a matrix of static forces $f^{s t}$ acting at node $\mathrm{j}$ in the mode $\mathrm{i}$; where the sum of the columns of $f^{s t}$ is equal to the magnitude of the $\mathrm{j}$-th component of the vector $f$ $[14,37]$.

$$
f_{\mathrm{j}, \mathrm{i}}^{\mathrm{st}}=\Gamma_{\mathrm{i}, \mathrm{i}} \phi_{\mathrm{j}, \mathrm{i}} \quad\left\{\begin{array}{l}
\text { for } \mathrm{i}=1,2, \ldots, \mathrm{N} \\
\text { for } \mathrm{j}=1,2, \ldots, \mathrm{n}
\end{array}\right.
$$

where:

$$
\Gamma=\operatorname{diag}\left(\Phi^{\mathrm{T}} \tilde{f}\right)
$$

When the eigenvectors are mass normalized, the vector of total static displacement can be found by the use of Hooke's law.

$$
\mathrm{u}_{\mathrm{j}}^{\mathrm{st}}=\sum_{\mathrm{i}=1}^{\mathrm{N}} \frac{\Gamma_{\mathrm{i}, \mathrm{i}}}{\omega_{\mathrm{i}}^{2}} \phi_{\mathrm{j}, \mathrm{i}} \quad \text { for } \mathrm{i}=1,2, \ldots, \mathrm{N}
$$

The vector of total static displacement can then be related to the vector of total dynamic displacements by introducing (9) into (2).

$$
u_{j}^{o}=u_{j}^{s t} \omega_{i}^{2} D_{i}^{o}
$$

Depending on the form of the excitation force $p(t)$, a particular method can be selected to find an analytical solution for $\mathrm{D}^{0}$, as explained below.

\section{A. Steady-state}

From [36], a dynamic amplification factor $\mathrm{R}_{\mathrm{i}}$ is defined as the ratio between the amplitude of the dynamic response $\mathrm{D}_{\mathrm{i}}^{\mathrm{o}}$ and the amplitude of the static response $\mathrm{D}_{\mathrm{i}}^{\mathrm{st}}$.

$$
\mathrm{R}_{\mathrm{i}}=\frac{\mathrm{D}_{\mathrm{i}}^{\mathrm{o}}}{\mathrm{D}_{\mathrm{i}}^{\mathrm{st}}}
$$

Where the amplitude of the static response can be found by neglecting the contribution of the acceleration and the velocity terms in the well-known mass-normalized modal equations of motion.

$$
\mathrm{D}_{\mathrm{i}}^{\mathrm{st}}=\frac{1}{\omega_{i}^{2}}
$$

And the dynamic amplification factor for an undamped single degree of freedom (SDOF) system is given by (13), when the unitary periodic function $\mathrm{p}(\mathrm{t})$ is equal to 1 .

$$
\mathrm{R}_{\mathrm{i}}=\frac{1}{\left(\omega_{\mathrm{i}}^{2}-\omega^{2}\right)}
$$

By introducing (11-13) into (10) we find an inexpensive expression that allows to estimate the maximum amplitude of the nodal physical displacements during steady-state vibration.

$$
\mathrm{u}^{\mathrm{o}}=\mathrm{u}^{\mathrm{st}} \mathrm{R}
$$

To compare the output of this method with respect to others, a steady-state modal participation fraction $\mathrm{L}_{\mathrm{i}}^{\text {st }}$ is defined in (15). This fractional number allows to compare the relative amplitudes of the modal responses $\mathrm{q}_{\mathrm{i}}$ for a given design and loading condition.

$$
\mathrm{L}_{\mathrm{i}}^{\mathrm{st}}=\frac{\left|\Gamma_{\mathrm{i}} \mathrm{D}_{\mathrm{i}}^{\mathrm{o}}\right|}{\sum_{\mathrm{i}}^{\mathrm{N}}\left|\Gamma_{\mathrm{i}} \mathrm{D}_{\mathrm{i}}^{\mathrm{o}}\right|}=\frac{\left|\Gamma_{\mathrm{i}} /\left(\omega_{\mathrm{i}}^{2}\left(\omega_{\mathrm{i}}^{2}-\omega^{2}\right)\right)\right|}{\sum_{\mathrm{i}}^{\mathrm{N}}|| \Gamma_{\mathrm{i}} /\left(\omega_{\mathrm{i}}^{2}\left(\omega_{\mathrm{i}}^{2}-\omega^{2}\right)\right) \mid}
$$

\section{B. Transient}

The steady participation factors neglect the contribution of the transient components of the time domain solution. When the global maximum is of particular interest, we aim to estimate the maximum dynamic amplitude response.

$$
\mathrm{D}_{\mathrm{i}}^{\max }=\max (\mathrm{D}(\mathrm{t}))
$$

The transient participation fraction $L_{i}^{t s}$ can then be defined similarly to the steady participation fraction as the ratio between the maximum amplitude of the harmonic solution and the sum of the maximum amplitude of all the modes retained.

$$
\mathrm{L}_{\mathrm{i}}^{\mathrm{ts}}=\frac{\left|\Gamma_{\mathrm{i}} \mathrm{D}_{\mathrm{i}}^{\max }\right|}{\sum_{\mathrm{i}}^{\mathrm{N}}\left|\Gamma_{\mathrm{i}} \mathrm{D}_{\mathrm{i}}^{\max }\right|}
$$

\section{Internal loads Participation Factor}

For point loads, the element internal force is computed as the product of the element stiffness matrix and the corresponding 
displacement vector of the nodes that conform the element in the global coordinate system.

When the element and global coordinate systems do not coincide a rotation matrix, $\mathrm{C}^{\mathrm{T}}$, is necessary to ensure that the internal forces computed, $\mathrm{N}_{\mathrm{e}}$, are expressed in the element coordinate system.

$$
\mathrm{N}_{\mathrm{e}}(\mathrm{t})=\mathrm{C}^{\mathrm{T}} \mathrm{K}_{\mathrm{e}} \mathrm{U}_{\mathrm{e}}(\mathrm{t})
$$

Where $\mathrm{K}_{\mathrm{e}}$ and $\mathrm{U}_{\mathrm{e}}(\mathrm{t})$ are the element stiffness matrix and the vector of nodal displacements in the global coordinate system, respectively.

We can expand (18) into its modal components as:

$$
\mathrm{N}_{\mathrm{e}}(\mathrm{t})=\mathrm{C}^{\mathrm{T}} \mathrm{K}_{\mathrm{e}} \Phi_{\mathrm{e}} \mathrm{q}(\mathrm{t})=\mathrm{C}^{\mathrm{T}} \mathrm{K}_{\mathrm{e}} \Phi_{\mathrm{e}} \Gamma \mathrm{D}(\mathrm{t})
$$

The magnitude of $\Gamma \mathrm{D}(\mathrm{t})$, determines the dominance of a mode in the solution, whereas the product $\Sigma_{\mathrm{e}}=\mathrm{C}^{\mathrm{T}} \mathrm{K}_{\mathrm{e}} \Phi_{\mathrm{e}}$ determines the impact of such mode in the $\mathrm{r}$ component of the element load where $r \in\{1: 6\}$. A participation factor can then be defined as:

$$
\mathrm{L}_{\mathrm{r}, \mathrm{i}}^{\text {load }}=\frac{\mid \Sigma_{\mathrm{e}_{\mathrm{r}, \mathrm{i}} \Gamma_{\mathrm{i}} \mathrm{D}_{\mathrm{i}} \mid}}{\sum_{\mathrm{i}=1}^{\mathrm{N}}\left|\Sigma_{\mathrm{e}_{\mathrm{r}, \mathrm{i}}} \Gamma_{\mathrm{i}} \mathrm{D}_{\mathrm{i}}\right|}
$$

\section{CLASS II}

The following definitions intend to assess inexpensively the relative importance of a mode and to ensure that a sufficient number of eigenvalues are retained to recover the solution in the physical domain. The most widely used definition is that of the modal mass participation factor, in practice modes with negligible modal effective mass are removed from the equations of motion, since its participation to the overall response is small $[20,38]$.

However other definitions have been proposed, for instance, Wilson [25] used a mode participation factor similar to (8) to estimate which modes should be retained in a subsequent mode superposition analysis. Carlbom et al. [26] presented four criteria to select important modes during the dynamic analysis of rail vehicles, and recently, Kammer, et.al. [39] proposed a new definition to select important elastic modes in free-free systems. In the following subsections we present most common definitions found in literature relevant to structural analysis.

\section{A. Modal Mass Participation Factor}

The modal effective mass was initially derived for a single degree of freedom system subject to an enforced motion through the base. The reaction force at the base $F_{\text {base }}$ due to the enforced acceleration $\ddot{U}_{0}$ in the frequency domain is given by:

$$
F_{\text {base }}(\omega)=m\left[1+\left(\frac{\omega}{\omega_{n}}\right)^{2} H\left(\frac{\omega}{\omega_{n}}\right)\right] \ddot{U}_{o}(\omega)
$$

In this context the effective mass of the system is $\mathrm{m}$ and it is directly proportional to the reaction force at the base. Thus, a large modal effective mass indicates a large inertial force.

The above analysis has been generalized to unconstrained multi-degree of freedom systems (MDOF) through a CraigBrampton transformation matrix $[9,38]$, and the resulting equation is (22), where $M$ is the mass matrix. This equation, relates the rigid-body (or unconstrained) modes $\Phi^{\mathrm{r}}$ with the elastic modes $\Phi^{\mathrm{e}}$.

$$
\Gamma^{\mathrm{mm} \mathrm{T}}=\Phi^{\mathrm{rT}} \mathrm{M} \Phi^{\mathrm{e}}
$$

We can prove that the product of the modal mass participation factors $\Gamma^{\mathrm{mm}}$ divided by the modal mass $M_{q}$ is identical to the mass matrix of unconstrained degrees of freedom. This, expressed in component form is the so called modal effective mass $\mathrm{M}^{\text {eff }}$.

$$
\mathrm{M}_{\mathrm{p}, \mathrm{i}}^{\mathrm{eff}}=\frac{\Gamma_{\mathrm{p}, \mathrm{i}}^{\mathrm{mm} \mathrm{T}} \Gamma_{\mathrm{i}, \mathrm{p}}^{\mathrm{mm}}}{\mathrm{M}_{\mathrm{i}, \mathrm{q}}}
$$

Where the subscript $p$ indicates the number of unconstrained elastic degrees of freedom (DoF) and $i$ is the number of modes retained in the analysis.

Though the definition of the modal effective mass has been derived using a free-free MDOF system, an important point should be kept in mind: the modal characteristics of the elastic degrees of freedom must be computed with respect to a boundary DoF, which should be constrained for the analysis $[6,8,38]$. Otherwise, the results extracted using the method described above are ill-founded and the participation of the elastic DoF's will tend to be zero.

Finally, for comparison purposes with other methods we can define an effective modal mass fraction $\mathrm{L}_{\mathrm{p}, \mathrm{i}}^{\mathrm{mm}}$ as the ratio between the effective modal mass and the total mass of the system:

$$
\mathrm{L}_{\mathrm{p}, \mathrm{i}}^{\mathrm{mm}}=\frac{\mathrm{M}_{\mathrm{p}, \mathrm{i}}^{\mathrm{eff}}}{\sum_{\mathrm{j}=1}^{\mathrm{n}} \mathrm{M}_{\mathrm{j}}}
$$

\section{B. Free-Free Participation Factor}

Recently [39], presented a new definition to estimate the relative importance of each mode for a free-free system, based on the concept of Effective Interface Mass (EIM), commonly used in the dynamic analysis of subsystems that will be attached to larger assemblies.

The equations of motion for a dynamic free-free system in matrix form are described as:

$$
\ddot{\mathrm{q}}(\mathrm{t})+\omega_{i}^{2} \mathrm{q}(\mathrm{t})=\Phi^{\mathrm{T}} f \mathrm{p}(\mathrm{t})
$$

Using equation (1) to recover the physical displacements from (25) and solving for the physical acceleration, we obtain:

$$
\ddot{\mathrm{u}}(\mathrm{t})=\Phi \ddot{\mathrm{q}}(\mathrm{t})=\Phi \Phi^{\mathrm{T}} f p(\mathrm{t})-\Phi \omega_{\mathrm{i}}^{2} \mathrm{q}(\mathrm{t})
$$

In (26), the modes that are strongly excited by the external force vector are, in consequence, expected to contribute strongly to the physical acceleration at the nodes. Thus, we can define a free-free modal participation factor $\Gamma_{i}^{f r e e}$ as the trace of the product between two eigenvectors.

$$
\Gamma_{\mathrm{i}}^{\mathrm{free}}=\operatorname{tr}\left(\Phi_{\mathrm{i}} \Phi_{\mathrm{i}}^{\mathrm{T}}\right)
$$

Since our interest lies in evaluating the relative importance of the elastic modes, the free-free participation factor $\mathrm{L}_{i}^{\text {free }}$ should be compared against the elastic degrees of freedom only.

$$
\Gamma^{\text {elas }}=\operatorname{tr}\left(\Phi^{\text {elas }} \Phi^{\text {elas }}{ }^{\mathrm{T}}\right)
$$


Thus, the free effective mass is defined as the fraction between the free-free modal participation factors and the trace of the product of the matrix of elastic eigenvectors.

$$
\mathrm{L}_{\mathrm{i}}^{\text {free }}=\frac{\Gamma_{\mathrm{i}}^{\text {free }}}{\Gamma^{\text {elas }}}
$$

\section{Modal Strain Energy Participation Factor}

Several authors [40-41] have proposed the use of the element modal strain energy $\mathrm{MSE}_{\mathrm{ei}}$ as assessment criteria. The element modal strain energy is evaluated at each element stiffness submatrix $\mathrm{K}_{\mathrm{e}}$ in the global coordinate system.

$$
\mathrm{MSE}_{\mathrm{ei}}=\frac{1}{2} \Phi_{\mathrm{e}, \mathrm{i}}^{\mathrm{T}} \mathrm{K}_{\mathrm{e}} \Phi_{\mathrm{e}, \mathrm{i}}
$$

Furthermore, the authors in [40] defined a modal strain participation fraction as the ratio between the strain energy and the kinetic energy of the system in free vibration.

$$
\mathrm{L}_{\mathrm{ei}}^{\mathrm{MSE}}=\frac{\Phi_{\mathrm{i}}^{\mathrm{T}} \mathrm{K}_{\mathrm{e}} \Phi_{\mathrm{i}}}{\Phi_{\mathrm{i}}^{\mathrm{T}} \mathrm{M} \Phi_{\mathrm{i}} \omega_{\mathrm{i}}^{2}}
$$

A large modal strain fraction at the element $\mathrm{e}$, at the $i$-th mode indicates that the element is a major load carrying component for such mode. Thus, any structural modifications in that element will produce significant changes in the modal characteristics of the structure and vice versa.

\section{CASE STUdy}

A simple 5-degree of freedom model was created to assess the performance of each of the methods described above. As shown in figure (1), the model consists of a five DOFs cantilever beam. Each beam element has a stiffness of $8700[\mathrm{~N} / \mathrm{m}]$ and a $5 \%$ modal critical damping is also considered.

The model is excited by a force $f$ of magnitude $100 \mathrm{~N}$ applied at the $5^{\text {th }}$ node with a periodic function and an excitation frequency of $5 \mathrm{rad} / \mathrm{s}$ as shown in (32). This analysis can be seen as a simplified aircraft wing under windmilling incident.

The modal equation of motion is solved in the time domain using Runge-Kutta's numerical method [52] and the result is presented in figure 2 .

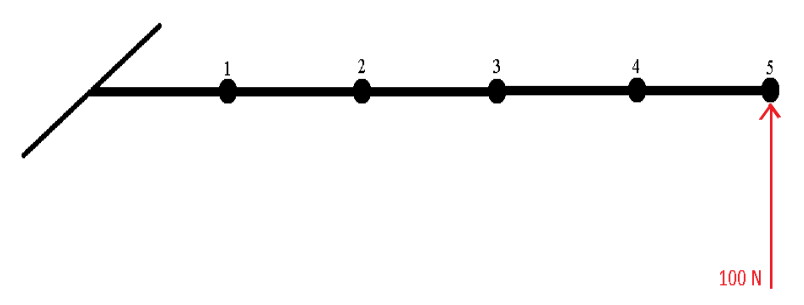

Figure 1. Case study: cantinlever beam

$$
\mathrm{p}(\mathrm{t})=\sin (\omega \mathrm{t})
$$

The modal steady-state $\mathrm{L}_{\mathrm{i}}^{\text {st }}$, transition $\mathrm{L}_{i}^{t s}$, modal effective mass $\mathrm{L}_{i}^{m m}$ and free-free $\mathrm{L}_{i}^{f f}$ participation fractions are compute using equations (15), (17), (24) and (29), respectively, and the results are shown in figure 3 . This figure gives an idea of the difference in magnitude obtained with each method, however the results should be interpreted independently.
Both, the steady-state $\mathrm{L}_{\mathrm{i}}^{\text {st }}$ and the transition $\mathrm{L}_{i}^{t s}$ participation fractions are estimates of the amplitude of the modal response, therefore the percentage of participation is well in agreement with figure 2: the amplitude of the first mode is very large in comparison to that of the fifth mode for the given excitation frequency.

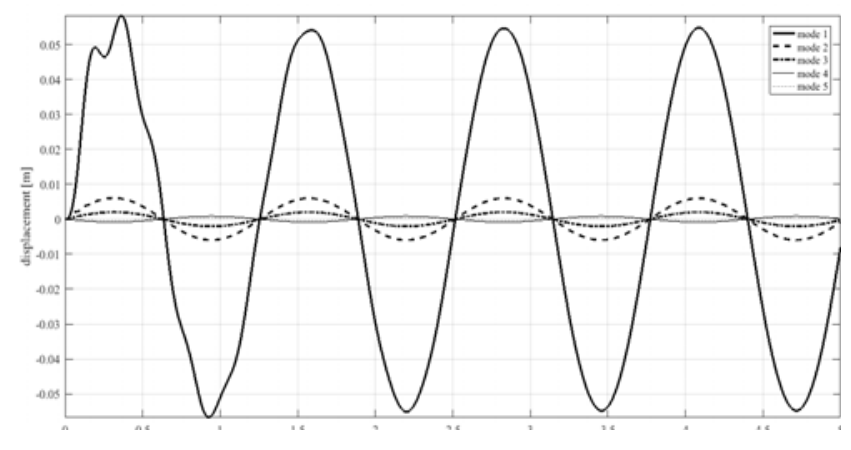

Figure 2. Modal response.

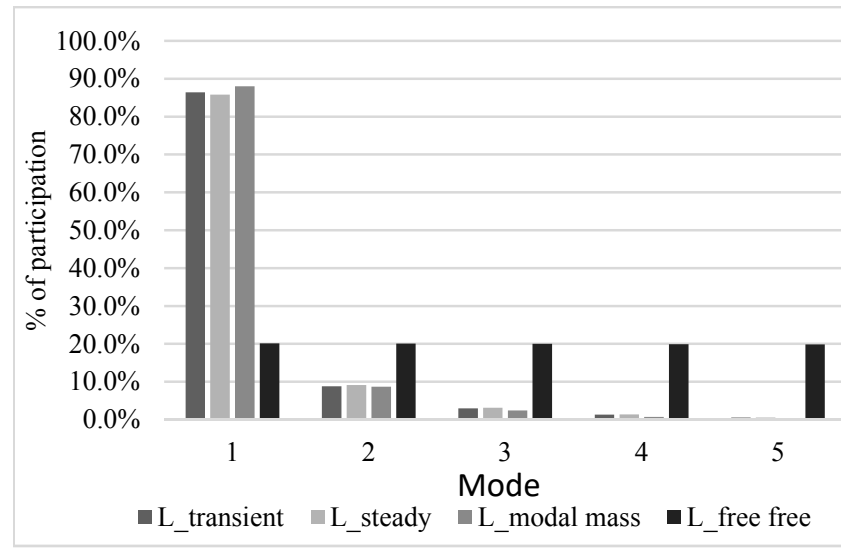

Figure 3. Modal participation factors.

The modal effective mass participation factor is also in agreement with figure 2, a large modal effective mass indicates that a particular mode has a large modal inertial component, and thus it is easier to excite.

The estimated free-free participation fractions are mostly in disagreement with the rest of the participation fractions computed, since its magnitude reveals that the participation of all modes is almost identical. Note that the definition of $L_{i}^{f f}$ is simply the trace of the modal residues, i.e. how the node $\mathrm{i}$-th responds to an excitation applied at the $\mathrm{j}$-th node.

Using both, the steady-state and transition modal response participation factors, the contribution of each mode to the internal load was estimated using equation (20). The modal strain energy was also computed using equation (31). In order to compare the three sets of results, the values of each matrix were normalized with respect to the component with the largest magnitude and the results are displayed in figure 4.

Take for example figure 6 (d), according to the modal strain energy, it appears that the second mode becomes more relevant to the response of element 4 , thus, any structural modification 
that could impact the behavior of the second mode would also have a major impact in the magnitude of the load experienced by element 4 . However, the results obtained using equation (32) are in disagreement with those of the modal strain energy, which indicates that the internal load is dominated by the first elastic mode.
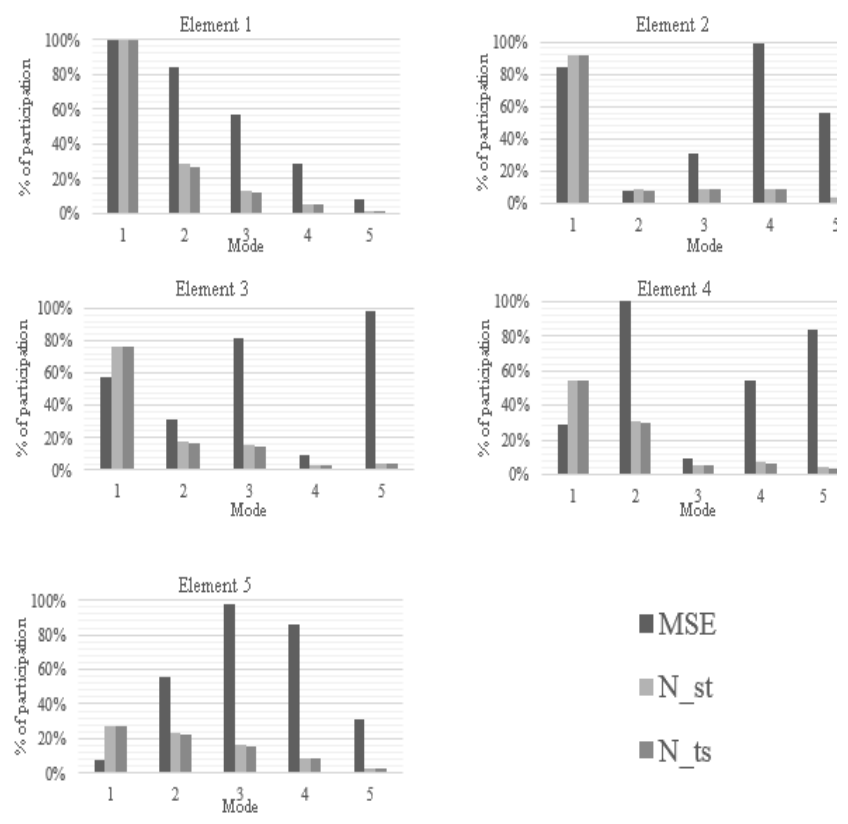

Figure 4. Internal load modal participation factors.

Using the steady and the transient modal response participation factors, the peak in plane shear force was recovered using equation (19) with (15) and (17), respectively. Table I shows a comparison and percentage error between the recovered load and the actual peak load computed using MSC NASTRAN [53].

TABLE I. PEAK SHEAR FORCE PER ELEMENT

\begin{tabular}{|c|c|c|c|c|c|}
\hline \multicolumn{7}{|c|}{ Peak shear force per element } \\
\hline \multirow{2}{*}{ Element } & $\begin{array}{c}\text { Actual } \\
\text { Peak } \\
\text { Load }\end{array}$ & $\begin{array}{c}\text { Load } \\
\Gamma_{i} D_{i}{ }^{s t}\end{array}$ & \%error & $\begin{array}{c}\text { Load } \\
\Gamma_{i} D_{i}{ }^{t s}\end{array}$ & \%error \\
\hline 1 & 55.8422 & 51.9211 & $7.02 \%$ & 60.3657 & $8.10 \%$ \\
\hline 2 & 55.2437 & 51.7904 & $6.25 \%$ & 47.0600 & $-14.81 \%$ \\
\hline 3 & 54.1775 & 51.5293 & $4.89 \%$ & 52.3115 & $-3.44 \%$ \\
\hline 4 & 52.6880 & 51.1385 & $2.94 \%$ & 67.5420 & $28.19 \%$ \\
\hline 5 & 50.8545 & 50.6189 & $0.46 \%$ & 37.18662 & $-26.88 \%$ \\
\hline
\end{tabular}

The percentage error of the steady-state modal response participation factors $\mathrm{L}_{\mathrm{i}}^{\text {st }}$ is due to the omission of the transient terms. Since the peak load occurs during the transition period, the load recovered corresponds in fact to the actual steady-state in-plane shear force.

On the other hand, error of the peak load recovered using the transient modal response participation factor $\mathrm{L}_{i}^{t s}$ was found to be significantly larger. This is due to an implicit assumption in (16): the peak of all the modal responses are in phase, thus the method tends to either over or underestimate the value of the peak load.

\section{CONCLUSIONS}

The first class of modal response participation factors have the potential to be used as reanalysis tools. When the excitation force is periodic and the interest lies in solving for the steadystate response, the $\mathrm{L}_{\mathrm{i}}^{\text {st }}$ fraction provides accurate results.

The second class of modal response participation factors are independent of the excitation and depend solely on the mass and stiffness distribution, in this sense, this type of factors should not be used as a measure of the relative amplitude of the modal responses. Instead, their application should be restricted, for example, in the case of the modal effective mass fraction, to determine the number of modes to be retained in the analysis (except when the model has an enforced acceleration through the base $[2,3])$. Such methodology can serve as an inexpensive response tracking mechanism in complex structures during design optimization.

The modal strain energy, on the other hand, is an inexpensive expression that relates the mass and stiffness distribution of the structure to its modal characteristics. This expression can be useful to visualize and correlate how modifying the physical properties at element level can impact the behavior of a specific mode.

\section{REFERENCES}

[1] G. K. Lau and H. Du, "Topology optimization of head suspension assemblies using modal participation factor for mode tracking," Microsyst. Technol., vol. 11, no. 12, pp. 1243-1251, June 2005. doi: 10.1007/s00542-005-0607-4.

[2] E. Miranda, "Approximate seismic lateral deformation demands in multistory buildings," J. Struct. Eng., vol. 125, no. 4, pp. 417-425, Apr. 1999. doi: 10.1061/(ASCE)0733-9445(1999)125:4(417).

[3] B. K. Oh, M. S. Kim, Y. Kim, T. Cho, and H. S. Park, "Model updating technique based on modal participation factors for beam structures," Comput.-Aided Civ. Infrastruct. Eng., vol. 30, no. 9, pp. 733-747, Apr. 2015. doi: 10.1111/mice.12139.

[4] T. Kobori, N. Koshika, K. Yamada, and Y. Ikeda, "Seismic-responsecontrolled structure with active mass driver system. Part 1: Design," Earthquake Eng. Struct. Dyn., vol. 20, no. 2, pp. 133-149, 1991. doi: 10.1002/eqe.4290200204.

[5] H. G. Park, T. Eom, and H. Lee, "Factored modal combination for evaluation of earthquake load profiles," J. Struct. Eng., vol. 133, no. 7, pp. 956-968, July 2007. doi: 10.1061/(ASCE)0733-9445(2007)133:7(956).

[6] A. K. Chopra and C. Chintanapakdee, "Drift spectrum vs. modal analysis of structural response to near-fault ground motions," Earthquake Spectra, vol. 17, no. 2, pp. 221-234, May 2001. doi: 10.1193/1.1586173.

[7] M. Palermo, S. Silvestri, G. Gasparini, and T. Trombetti, "Seismic modal contribution factors," Bull. Earthquake Eng., vol. 13, no. 10, pp. 2867-2891, Apr. 2015. doi: 10.1007/s10518-015-9757-7.

[8] S. F. Ghahari, F. Abazarsa, M. A. Ghannad, and E. Taciroglu, "Reponseonly modal identification of structures using strong motion data," Earthquake Eng. Struct. Dyn., vol. 42, no. 8, pp. 1221-1242, Nov. 2012. doi: 10.1002/eqe.2268.

[9] A. Girard and N. A. Roy, "Modal effective parameters in structural dynamics," Rev. Euro. Eléments Finis, vol. 6, no. 2, pp. 233-254, Jan. 1997. doi: 10.1080/12506559.1997.10511267.

[10] T. Igusa, A. D. Kiureghian, and J. L. Sackman, "Modal decomposition method for stationary response of non-classically damped systems," Earthquake Eng. Struct. Dyn. vol. 12, no. 1, pp. 121-136, 1984. doi: 10.1002/eqe.4290120109. 
[11] A. S. Whittaker, M. C. Constantinou, O. M. Ramirez, M. W. Johnson, and C. Z. Chrysostomou, "Equivalent lateral force and modal analysis procedures of the 2000 NEHRP Provisions for buildings with damping systems," Earthquake Spectra, vol. 19, no. 4, pp. 959-980, Nov. 2003. doi: 10.1193/1.1622391.

[12] E. Miranda and S. Taghavi, "Approximate floor acceleration demands in multistory buildings. I: Formulation," J. Struct. Eng., vol. 131, no. 2, pp. 203-211, Feb. 2005. doi: 10.1061/(ASCE)0733-9445(2005)131:2(203).

[13] E. L. Wilson, A. Der Kiureghian, and E. P. Bayo, "A replacement for the srss method in seismic analysis," Earthquake Eng. Struct. Dyn., vol. 9, no. 2, pp. 187-192, 1981. doi: 10.1002/eqe.4290090207.

[14] L. Romera and S. Hernandez, "An improved technique for modal contribution factors of dynamic responses," presented at the 40th Structures, Structural Dynamics, and Materials Conference and Exhibit, St. Louis, MO, 1999. doi: 10.2514/6.1999-1242.

[15] A. Arief, M. B. Nappu, A. Nizar, and Z. Y. Dong, "Determination of DG allocation with modal participation factor to enhance voltage stability," presented at the 8th International Conference on Advances in Power System Control, Operation and Management, Hong Kong, 2009. doi: 10.1049/cp.2009.1850.

[16] A. Gebreselassie and J. H. Chow, "Investigation of the effects of load models and generator voltage regulators on voltage stability," Int. J. Electr. Power Energy Syst., vol. 16, no. 2, pp. 83-89, Apr. 1994. doi: 10.1016/0142-0615(94)90043-4.

[17] A. Arief, M. B. Nappu, Z. Y. Dong, and M. Arief, "Under voltage load shedding incorporating bus participation factor," in 2010 Conference Proceedings IPEC, 2010, pp. 561-566. doi:10.1109/IPECON.2010.5697058

[18] W. A. Hashlamoun, M. A. Hassouneh, and E. H. Abed, "New results on modal participation factors: Revealing a previously unknown dichotomy," IEEE Trans. Autom. Control, vol. 54, no. 7, pp. 1439-1449, Jul. 2009. doi: 10.1109/TAC.2009.2019796.

[19] R. MacNeal, The NASTRAN Theoretical Manual (level 15.5): Msr-40, MacNeal-Schwendler Corp., Los Angeles, CA, 1972.

[20] T. Irvine. (2013, March 28). Effective modal mass and modal participation factors [Online]. Available: http://citeseerx.ist.psu.edu/viewdoc/summary?doi=10.1.1.366.1903

[21] A. Girard and N. A. Roy, "Modal effective parameters in structural dynamics," Rev. Euro. élém. finis, vol. 6, no. 2, 233-254, Jan. 1997. doi: 10.1080/12506559.1997.10511267.

[22] E. J. Kuhar and C. V. Stahle, "Dynamic transformation method for modal synthesis," AIAA J., vol. 12, no. 5, pp. 672-678, May 1974. doi: 10.2514/3.49318.

[23] J. T. Chen, K. H. Chen, I. L. Chen, and L. W. Liu, "A new concept of modal participation factor for numerical instability in the dual BEM for exterior acoustics," Mech. Res. Commun., vol. 30, no. 2, pp. 161-174, Mar. 2003. doi: 10.1016/S0093-6413(02)00351-8.

[24] J. T. Chen, H.-K. Hong, and C. S. Yeh, "Modal reaction method for modal participation factors in support motion problems," Commun. Numer. Methods Eng., vol. 11, no. 6, pp. 479-490, Jun. 1995. doi: 10.1002/cnm.1640110602.

[25] E. L. Wilson, M.-W. Yuan, and J. M. Dickens, "Dynamic analysis by direct superposition of Ritz vectors," Earthquake Eng. Struct. Dyn., vol. 10, no. 6, pp. 813-821, Nov./Dec. 1982. doi: $10.1002 /$ eqe.4290100606.

[26] P. F. Carlbom, "Combining MBS with FEM for rail vehicle dynamics analysis," Multibody Syst. Dyn., vol. 6, no. 3, pp. 291-300, Oct. 2001. doi: 10.1023/A:1012072405882.
[27] A. J. Salmonte, "Considerations on the residual contribution in modal analysis," Earthquake Eng. Struct. Dyn., vol. 10, no. 2, pp. 295-304, Mar. 1982. doi: 10.1002/eqe.4290100210.

[28] D. . Photiadis, B. . Houston, X. Liu, J. . Bucaro, and M. . Marcus, "Thermoelastic loss observed in a high Q mechanical oscillator," Phys. $B$ (Amsterdam, Neth.), vol. 316-317, pp. 408-410, May 2002. doi: 10.1016/S0921-4526(02)00528-8.

[29] O. Wallrapp and S. Wiedemann, Simulation of deployment of a flexible solar array," Multibody Syst. Dyn., vol. 7, no. 1, pp. 101-125, Feb. 2002. doi: 10.1023/A:1015295720991.

[30] C. B. Yun and E. Y. Bahng, "Substructural identification using neural networks," Comput. Struct., vol. 77, no. 1, pp. 41-52, Jun. 2000 doi: 10.1016/S0045-7949(99)00199-6.

[31] T. Van Lagenhove and M. Brughmans, "Using MSC/NASTRAN and LMS/PRETEST to find an optimal sensor placement for modal identification and correlation of aerospace structures," in 2nd MSC Aerospace Conference, Long Beach, CA, 1999, pp. 7-11.

[32] J. C. Asmussen and R. Brincker, "Modal analysis based on the random decrement technique: Application to civil engineering structures," in Dynamics of Structures 1993-1997: Description of the Projects in the Research Programme. Aalborg, DK: Dept. of Building Technology and Structural Engineering, Aalborg Univ., 1997, pp. 39-55.

[33] S. M. Fang and J. M. Niedzwecki, "Comparison of airfoil and ribbon fairings for suppression of flow-induced vibrations," Int. J. Comput. Methods Exp. Meas., vol. 2, no. 1, pp. 30-45, Mar. 2014. doi: 10.2495/CMEM-V2-N1-30-45

[34] A. Przekop, S. A. Rizzi, and D. S. Groen, "Nonlinear acoustic response of an aircraft fuselage sidewall structure by a reducedorder analysis," presented at the IX International Conference if Recent Advances in Structural Dynamics, Southampton, UK, July 2006

[35] L. Zhang, R. Brincker, and P. Andersen, "Modal indicators for operational modal identification," presented at the 19th International Modal Analysis Conference (IMAC), Kissimmee, FL, Feb. 2001.

[36] A.K.Chopra, Dynamics of Structures: Theory and Applications, 2nd ed. Upper Saddle River, NJ: Prentice Hall, 2001

[37] A. K. Chopra, "Modal analysis of linear dynamic systems: Physical interpretation," J. Struct. Eng., vol. 122, no. 5, pp. 517-527, May 1996. doi: 10.1061/(ASCE)0733-9445(1996)122:5(517).

[38] J. J. Wijker, Spacecraft Structures. Heidelberg, DE: Springer-Verlag, 2008. doi: 10.1007/978-3-540-75553-1.

[39] D. C. Kammer, J. Cessna, and A. Kostuch, "An effective mass measure for selecting free-free target modes," presented at the $23 \mathrm{rd}$ International Modal Analysis Conference, Orlando, FL, Jan. 2005.

[40] H. Li, H. Yang, and S. L. J. Hu, "Modal strain energy decomposition method for damage localization in 3D frame structures," J. Eng. Mech., vol. 132, no. 9, pp. 941-951, Sep. 2006. doi: 10.1061/(ASCE)0733-9399(2006)132:9(941).

[41] L. Li, Y. Hu, and X. Wang, "Numerical methods for evaluating the sensitivity of element modal strain energy," Finite Elem. Anal. Des., vol. 64, pp. 13-23, Feb. 2013. doi: 10.1016/j.finel.2012.09.006.

[42] X.-K. Zou and C.-M. Chan, "An optimal resizing technique for seismic drift design of concrete buildings subjected to response spectrum and time history loadings," Comput. Struct., vol. 83, no. 19-20, pp. 1689-1704, July 2005. doi: 10.1016/j.compstruc.2004.10.002.

[43] H. P. Chen, "Efficient methods for determining modal parameters of dynamic structures with large modifications," J. Sound Vib., vol. 298, no. 1-2, pp. 462-470, Nov. 2006. doi: 10.1016/j.jsv.2006.06.002 
[44] M. Lou and G. Chen, "Modal perturbation method and its applications in structural systems," J. Eng. Mech., vol. 129, no. 8, pp. 935-943, Aug. 2003. doi: 10.1061/(ASCE)0733-9399(2003)129:8(935).

[45] W. M. To and D. J. Ewins, "Non-linear sensitivity analysis of mechanical structures using modal data," Proc. Inst. Mech. Eng., Part C, vol. 205, no. 1, pp. 67-75, Jan. 1991. doi: 10.1243/PIME_PROC_1991_205_092_02.

[46] F. Massa, B. Lallemand, and T. Tison, "Multi-level homotopy perturbation and projection techniques for the reanalysis of quadratic eigenvalue problems: The application of stability analysis," Mech. Sys. Signal Process., vol. 52-53, pp. 88-104, Feb. 2015. doi:10.1016/j.ymssp.2014.07.013.

[47] K. Ki-Ook and D. V. Wallerstein, "Modal design sensitivities for multiple eigenvalues," Comput. Struct., vol. 29, no. 5, pp. 755-762, Jan. 1988. doi: 10.1016/0045-7949(88)90343-4.

[48] A. Saito, H. Suzuki, M. Kuroishi, and H. Nakai, "Efficient forced vibration reanalysis method for rotating electric machines," $J$. Sound Vib., vol. 334, pp. 388-403, Jan. 2015. doi: 10.1016/j.jsv.2014.09.004.

[49] A. Dutta and C. V. Ramakrishnan, "Error estimation in finite element transient dynamic analysis using modal superposition," Eng. Comput., vol. 14, no. 1, pp. 135-158, Feb. 1997. doi: $10.1108 / 02644409710157668$.

[50] M. D. Grissom, A. D. Belegundu, and G. H. Koopmann, "A reduced eigenvalue method for broadband analysis of a structure with vibration absorbers possessing rotatory inertia," J. Sound Vib., vol. 281, no. 3-5, pp. 869-886, Mar. 2005. doi: 10.1016/j.jsv.2004.02.040.

[51] E. W. Constans, A. D. Belegundu, and G. H. Koopmann, "Optimally designed shell enclosures with tuned absorbers for minimizing sound power," Optim. Eng., vol. 1, no. 1, pp. 67-86, 2000. doi: 10.1023/A:1010046506443.

[52] J. C. Butcher, The Numerical Analysis of Ordinary Differential Equations: Runge-Kutta and General Linear Methods. New York: Wiley-Interscience, 1987.

[53] Quick Reference Guide. MSC Nastran, Santa Ana, CA, 2004. 


\section{Meso-Scale Computational Modeling of Hypervelocity Impact Damage in Advanced Materials}

\author{
Aleksandr Cherniaev \\ Department of Mechanical, Automotive and Materials \\ Engineering \\ University of Windsor \\ Windsor, Canada
}

\author{
Igor Telichev \\ Department of Mechanical Engineering \\ University of Manitoba \\ Winnipeg, Canada
}

\begin{abstract}
Modeling of hypervelocity impact (HVI) on materials and structures is often associated with high computational expenses, especially when inhomogeneous materials are involved. To reduce computational cost, complex materials are often represented in modeling as homogeneous substances with the effective properties similar to those of the real materials. Although this approach has been successfully used in modeling of HVI on different materials with complex architecture, there are applications where it may not be applicable due to significant influence of materials' mesoscale features on resulting HVI damage. Two of such applications are considered in this study, and include simulation of HVI on sandwich panels with metallic foamcores, and composites fabricated by filament winding. In the former case, adequate modeling of the multi-shock action of the foam ligaments on hypervelocity fragment cloud propagating through the foam core requires an explicit representation of the foam geometry in numerical model. In the latter case, the meso-scale modeling is required due to experimentally observed dependence of HVI damage of the composite on the particular filament winding pattern used in its fabrication. The study presents numerical models developed for both of these applications and compares numerical results with obtained experimental data.
\end{abstract}

\section{Hypervelocity impact, orbital debris, advanced materials}

\section{INTRODUCTION (HEADING 1)}

Models for the simulation of impact behavior of different inhomogeneous materials have been described in the literature and implemented in commercial software packages, such as Autodyn, LS-Dyna etc. These models are often based on the homogenization of advanced materials with complex microand meso-structure, and their representation in the modeling as a macroscopically homogeneous media with effective properties equivalent to those of the real materials. Although computationally efficient, this approach may be too simplistic for some materials with meso-scale inhomogeneities. This paper considers two problems, where simulation of damage of inhomogeneous materials required meso-scale representation of the materials' architecture. These examples are modeling of hypervelocity impact on a foam-core sandwich panel and simulation of HVI-induced damage in CFRP composites fabricated by filament winding.

Open-cell foam-core sandwich panels are considered by many researchers as a promising alternative to honeycomb-core panels in MMOD protection applications. For instance, in an experimental study presented by NASA [1], foam-core sandwich panels (FCSPs) with $25-30 \%$ lower areal density than HCSPs demonstrated better ballistic performance. In [2], it was determined that, depending on impact conditions, doublelayer foam shields provided from 3 to $15 \%$ improvement in critical projectile diameter as compared to double-layer honeycomb shields of similar weight. The performance improvement in case of using the foams was not explained only as a result of the simple absence of cells that "channel" the fragment cloud. Rather, the radial expansion of fragment cloud and repeated impact of fragments on individual foam cell ligaments induced further fragmentation, melt, and vaporization of fragments [3].

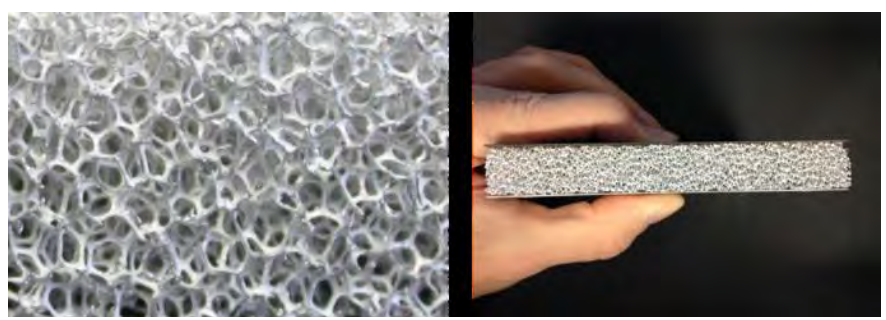

Figure 1. Open-cell aluminum foam (left) and a fragment of a foam-core sandwich panel (right)

Material models implemented in commercial hydrocodes and recommended for simulation of impact on foams are represented, for example, by the LS-DYNA's *MAT_057 (*MAT_LOW_DENSITY_FOAM), $\quad$ *MAT_063 (*MAT_CRUSHABLE_FŌAM), and a set of equations of state for porous materials in AUTODYN (Crushable foam EOS, Compaction EOS, P-Alpha EOS). These and other available material models treat the foams as homogeneous materials and, therefore, a priori unable to represent multi- 
shock interactions of foam ligaments with fragments of debris cloud propagating through the foam.

Composite fabricated by filament winding is another example of a material with the meso-scale inhomogeneity. This feature results from multiple interweavings of filament bands forming a filament-wound composite part. In space, these materials can be used in spacecraft propellant tanks (so-called composite overwrapped pressure vessels) and remote manipulator systems (e.g., Canadarm). Behavior of composites under HVI has been studied experimentally and numerically by many researchers, e.g. [4, 5]. However, most of the reported work was confined to the standard laminated composites and homogenization-based simulation techniques, whereas less attention has been paid to the filament-wound materials.

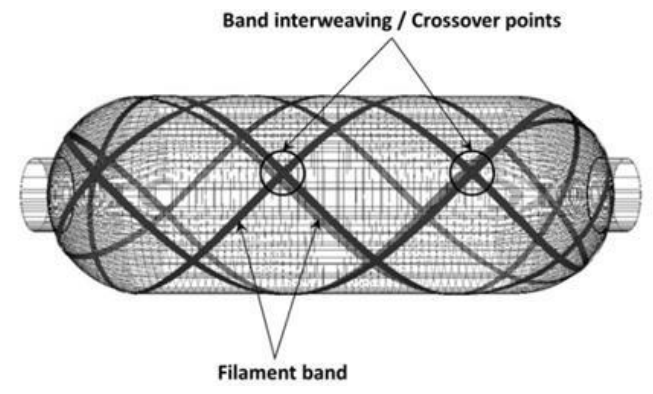

Figure 2. Filament winding

In filament winding, the carriage unit of a winding machine moves back and forth relative to the rotating mandrel and lays down on it the resin-impregnated fibers (towpreg) at a specific angle. Fibers are placed onto a mandrel in the form of filament bands, each containing few thousands of fibers. Fig. 2 illustrates the initial stages of filament winding. At each circuit (one back and forth pass of the carriage unit along a mandrel), filament bands are laid on a mandrel at "+" (forward motion) and "-" (back motion) angle. Each circuit results in crossovers between filament bands of "+" and "-" angle. The presence of crossovers is intrinsic to filament winding and forms its well recognizable patterns. The choice of a particular winding pattern is often up to a manufacturing engineer, as different patterns can be easily programmed using software supplied with the filament winding equipment (Fig. 3).
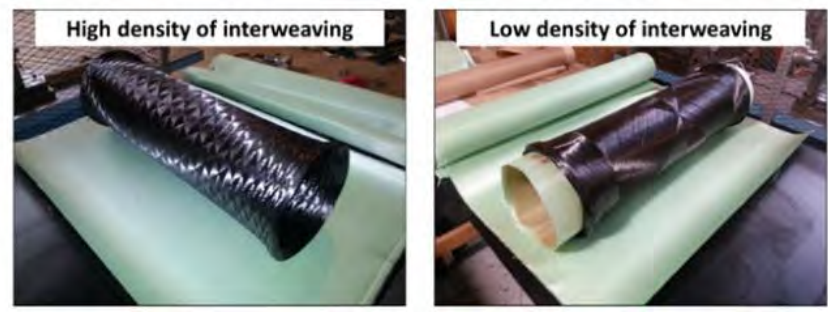

Figure 3. CFRP weaves of different winding patterns

Also, filament-wound composites contain two types of voids: voids internal to filament bands, and voids at the filament bands' crossover regions (Fig. 4). Voids of the latter type represent additional meso-scale feature of filament-wound composites. It is believed that they may result in stress concentration at crossovers leading to formation of cracks and preliminary disintegration of the filament wound composite when subjected to static or impact loading.
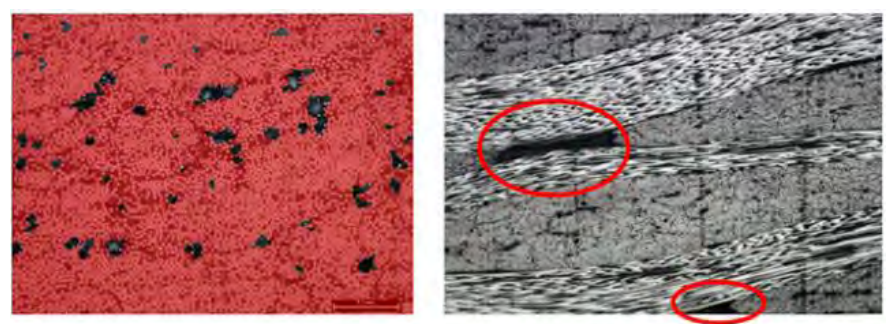

Figure 4. Voids in the filament-wound composite: intra-band voids (left) and voids at the crossovers (right)

\section{MODELING OF OPEN-CELL FOAM}

A mesoscale approach to the modeling of open-cell foam geometry must be used in the HVI simulations to represent multi-shock interactions of the foam ligaments and hypervelocity fragment cloud propagating through the foam. The original structure that forms metallic foam is a threedimensional array of bubbles having a maximum volume for the minimal surface area and surface energy. During the fabrication process, membranes of the bubbles are being removed, leaving an interconnected network of solid struts. A common approach to geometric modeling of open-cell foams is based on Wearie-Phelan idealization [6]. It represents a structure consisting of equal-volume bubbles of two different shapes: namely, the 14-sided tetrakaidecahedron (Fig. 5a) and the 12-sided dodecahedron (Fig. 5b). It is believed that Wearie-Phelan packing provides a minimal surface area for a given volume of cells/bubbles. The periodic domain (translation unit) of the Wearie-Phelan tessellation is shown in Fig. 5c.

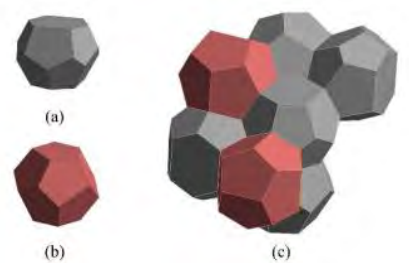

Figure 5. Wearie-Phelan packing

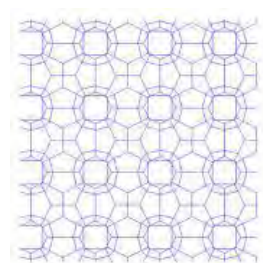

(a)

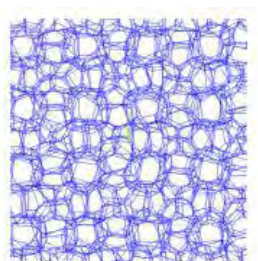

(b)

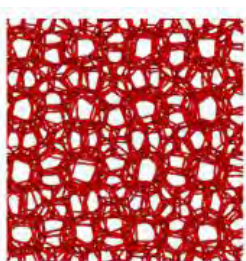

(c)
Figure 6. Mesoscale geometric modeling of aluminum foam: from structured frame to randomized solid model

It should be noted that the use of Wearie-Phelan idealization suggests a regular array of bubbles; however, real foams are random structures. To increase the realism of the modeling, therefore, a foam randomization mechanism is implemented. The overall algorithm for the geometric modeling can be described as follows: 1) generation of the representative volume element of aluminum foam with the specified dimensions based on Wearie-Phelan idealization [at 
this stage, the geometric model consists of multiple lines connected with each other at nodal points (see Fig. 6a)]; 2) randomization of the foam using node perturbation (see Fig. $6 \mathrm{~b}$ ); and 3) development of a solid geometric model using the randomized line-based geometry (see Fig. 6c).

The foam modeling algorithm has been implemented in the form of a script written in ANSYS Parametric Design Language. The SPH model of the foam-core sandwich panel is depicted in Fig. 7. It consists of approximately two million SPH particles. To represent thin ligaments of aluminum foam with a satisfactory accuracy, a smoothing length of $0.015 \mathrm{~mm}$ has been used. The model exploited the idea of quarter-symmetry. Although aluminum foam has a random structure and is not exactly symmetrical relative to any cutting plane, isotropy of its effective properties allows for such an assumption. The foam in the model is present only in the central region of the panel, where the most energetic fragments with the greatest damage potential will propagate. Additional lateral extension of the foam model was found to be impractical, as it was associated with high computational expenses.
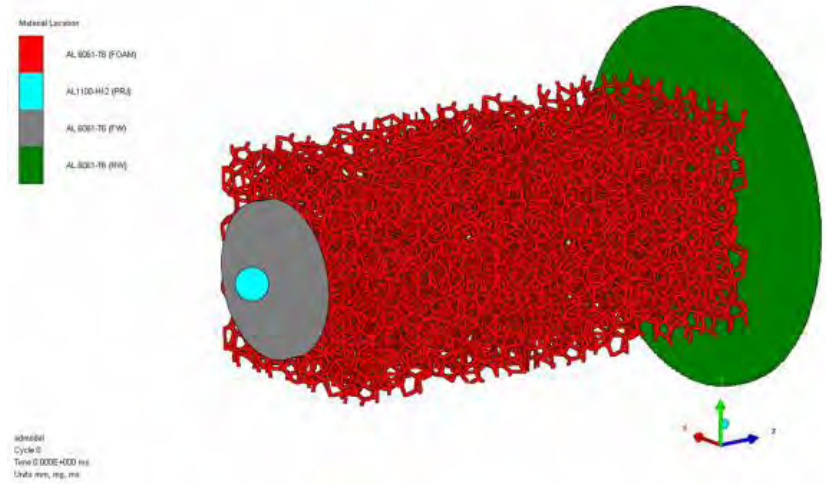

Figure 7. Numerical model of the open cell foam-core sandwich panel

Results of the conducted simulation are presented in Fig. 8, which demonstrates three consequent moments of time when the debris cloud propagates through the aluminum foam. It can be seen in the figure that multi-shock action of the foam effectively breaks up fragments of the projectile and the front bumper, converting them into a cloud of small, disperse particles. No perforation of the rear wall was detected during the numerical experiment.

In view of the novelty of the foam modeling approach (i.e., its mesoscale representation in HVI analysis), the numerical prediction was verified by means of a physical experiment. The experiment was carried out using a two-stage light gas gun at the HIT Dynamics, Ltd., impact testing facility (Canada). The tested panel consisted of two Al6061-T6 facesheets and 16mm-thick 3\% 40 ppi aluminum foam from ERG Aerospace. It should be noted that the tested panel had a slightly lower facesheet thickness $(0.4 \mathrm{~mm}$ instead of $0.5 \mathrm{~mm}$, as used in the numerical tests.
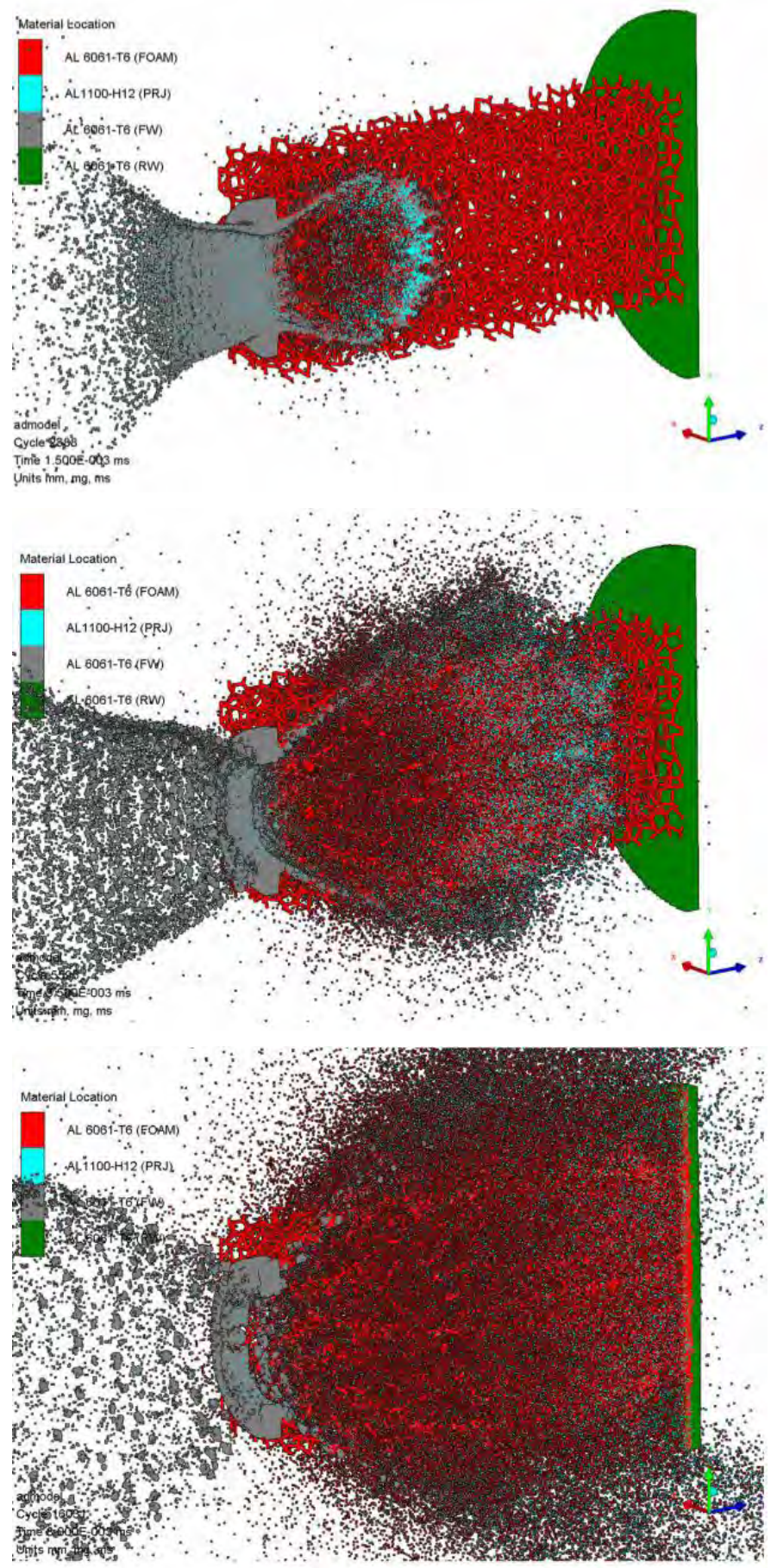

Figure 8. Results for the foam-core sandwich panel: a) $t=1.5$ $\mu s$; b) $t=3.5 \mu \mathrm{s}$; and c) $t=8.5 \mu \mathrm{s}$

The panel was impacted by a $1 \mathrm{~mm}$ aluminum projectile at $6.965 \mathrm{~km} / \mathrm{s}$, and the resulting damage to the panel is shown in Fig. 9. Rear-wall damage was represented by discoloration and barely noticeable bulging. No perforation of the rear wall was detected, which supported the results of the numerical simulation. 

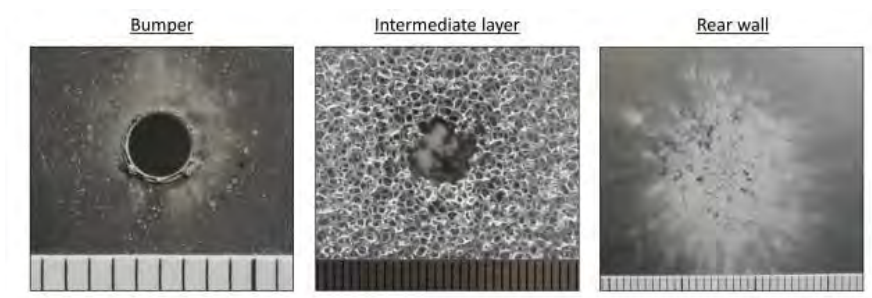

Figure 9. Experimental results for the foam-core sandwich panel
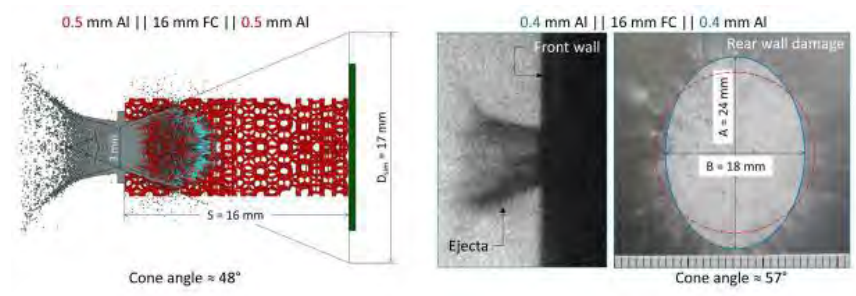

Figure 10. Shapes of ejecta clouds and cone angle measurements

Also, debris expansion angles (also referred to as "cone angles") were procured for both cases. For the model, this angle could be measured directly, as shown in Fig. 10, and this was found to be equal to $48 \mathrm{deg}$. To measure the experimental value of this angle, it was assumed that the debris cloud expanded within a cone, with one base having the diameter of the perforation hole in the front facesheet $(\sim 3 \mathrm{~mm})$ and the second base with the diameter of the damaged region on the rear wall. As the damaged area on the rear facesheet had a slightly elliptical shape (possibly because of the randomness of the foam; see Fig. 10), the corresponding cone base was represented in the calculations by a circle with the same area as the ellipse (indicated by the dashed line in Fig. 10). With this approximation, the debris expansion angle in the experiment was equal to $57 \mathrm{deg}$. The observed 9 deg difference was most likely the result of the different facesheet thicknesses of the modeled panel $(0.5 \mathrm{~mm})$ and the panel used in the experiment $(0.4 \mathrm{~mm})$. The model, therefore, was deemed to provide a good approximation of the physical phenomenon.

\section{MODELING OF FILAMENT-WOUND COMPOSITES}

Numerical techniques for the modeling of composite behavior under hypervelocity impact loading have been described in the literature $[5,7]$ and implemented in commercial software packages. These techniques are designed for the standard laminated composites. They are based on the homogenization of a composite laminate, which is represented in the modeling as a macroscopically homogeneous orthotropic media with effective properties equivalent to those of the real material.

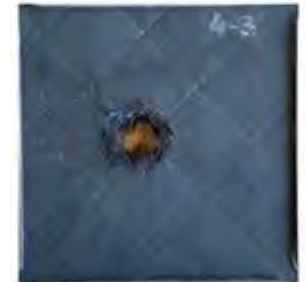

Front side

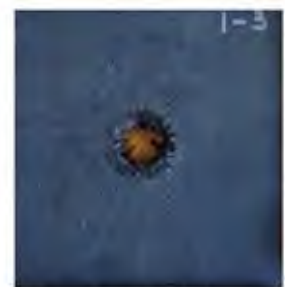

Front side

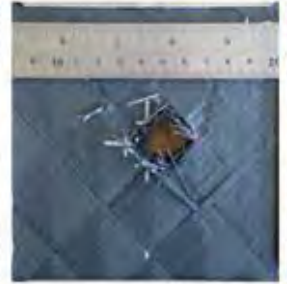

Rear side

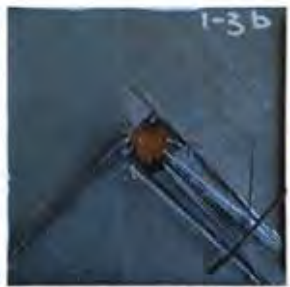

Rear side

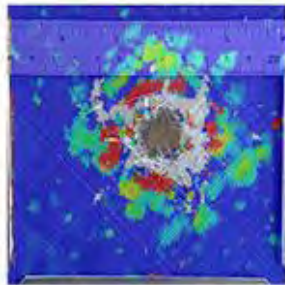

Ultrasound

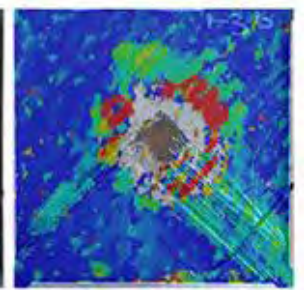

Ultrasound
Figure 11. HVI damage to a HDI composite (top) and a LDI composite material (bottom)

[impact conditions: $7 \mathrm{~km} / \mathrm{s} ; 4.76 \mathrm{~mm}$ aluminum projectile; 0.8 mm-thick aluminum bumper in front of the composite]

Conducted physical experiments revealed dependence of HVI damage of filament-wound composites on the particular winding pattern used in fabrication, as shown in Fig.11 In the case of pattern with the high density of interweaving (HDI) of filament bands, a specimen inspection revealed a "containment" of the impact damage within the region restricted by $2 \times 2$ weave units adjacent to the perforated hole. In case of low density of interweaving (LDI), the specimen damage propagates outwards along the direction of the fibers, especially in the bottom left and bottom right directions. Correspondingly, the homogenization-based techniques are inapplicable to this class of materials. Instead, in this study, a meso-scale modeling approach was used to represent the material structure in HVI simulations.

The meso-scale representation of the composite materials included explicit modeling of fiber-reinforced filament bands with ultrathin resin-rich regions between them. The following assumptions regarding the behavior of the materials of filament bands and resin-rich regions were made:

1. The material of a filament band is homogeneous, orthotropic, and linearly elastic until failure. The intra-band failure is assumed to be brittle, which is the case for most CFRP systems.

2. The material of the resin-rich regions is homogeneous, isotropic, and linearly elastic until failure. The linear elasticity assumption fits well for the thermoset-resin composites, as thermosets typically do not undergo a significant amount of plastic deformation prior to an initiation of failure.

3. Assumingly, the inter-band damage of the composite, such as delamination, is completely confined to the resin-rich region. The behavior in the resin-rich regions after a failure initiation is determined by such macro-scale parameters as fracture toughness, which allows for the consideration of both adhesive and cohesive types of inter-band fracture. 
4. Fracture toughness of thermoset-resin composites does not reveal strain-rate dependence, which is supported by several investigations by previous researchers [8 - 9].

As materials of filament bands and resin rich-regions are assumed to be linearly elastic until failure, their strength models only require the input of elastic constants. Pressure volume response was modeled using nonlinear EOS for composite filament bands, and the Gruneisen EOS for isotropic resin-rich layers. Failure within the filament bands and resin reach layers was modeled using a continuum damage mechanics model available in AUTODYN.

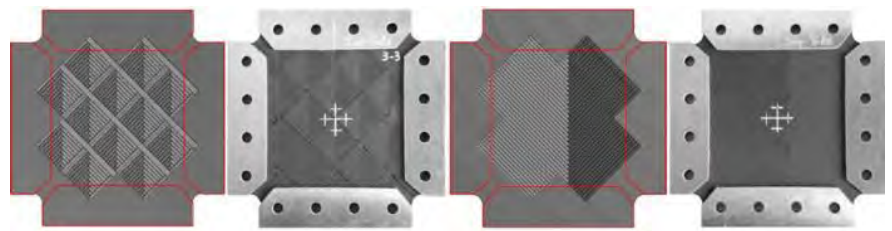

Figure 12. Geometrical modeling of HDI (left) and LDI (right) composites

The modeling approach employed in this study is based on simultaneous utilization of both SPH and FEM in each numerical simulation. The SPH method was used to represent the behavior of parts that exhibited fragmentation, namely aluminum projectile projectiles and bumpers. At the same time, composite panels with a meso-scale representation of filament winding patterns, which contained multiple interfaces between filament bands and resin-rich regions, were modeled using FEM in the Lagrangian formulation combined with the erosion mechanism. The use of erosion helped to avoid excessive mesh distortions and tangling at high deformations.

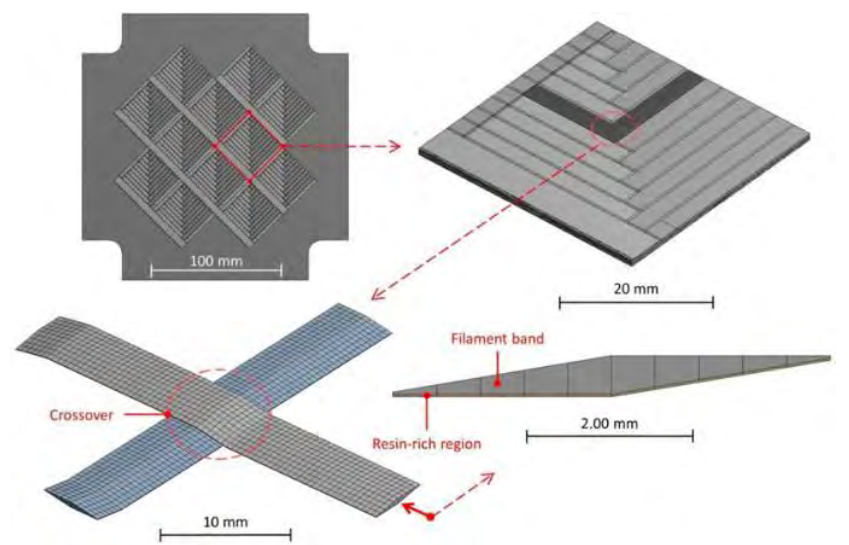

Figure 13. Meso-scale modeling of the filament-wound composite

The detailed meso-scale representation of filament winding was used only in the region of interest to minimize the computational cost. Around that region, the composite was represented by a homogeneous orthotropic material with effective properties equivalent to those of the real material. The geometrical models of the HDI and LDI panels compared with the real specimens are represented in Fig. 12.
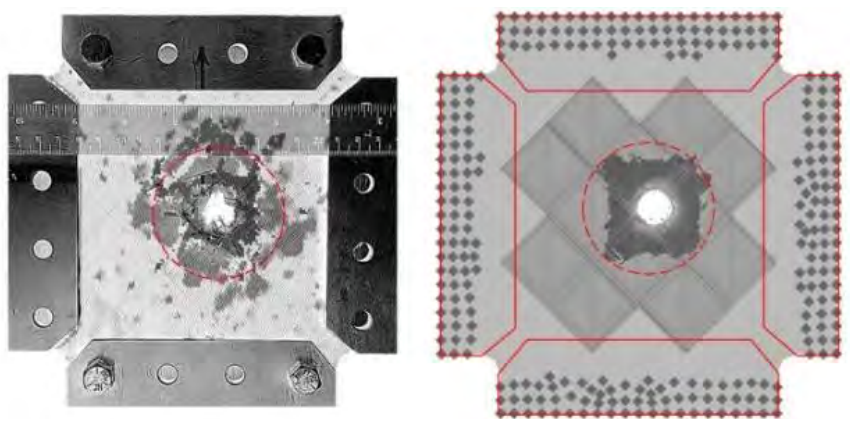

HDI: superposition of all damaged surfaces
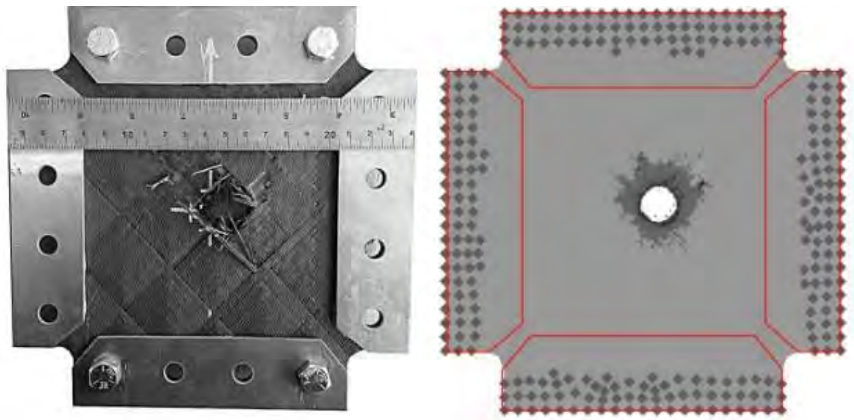

HDI: rear surface damage only
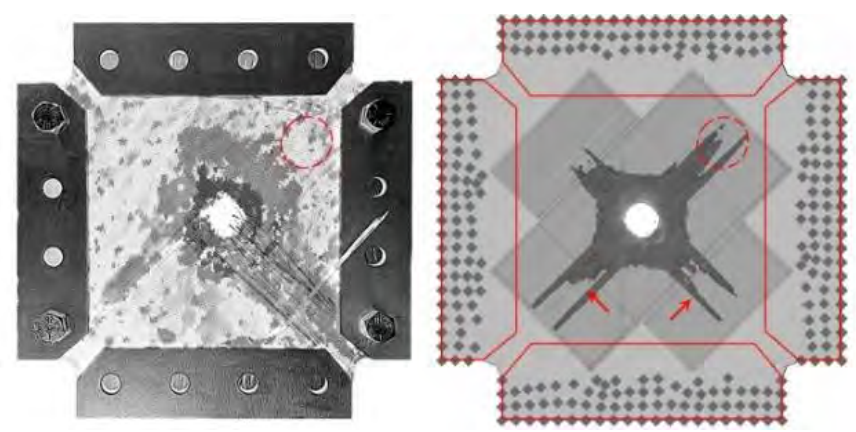

LDI: superposition of all damaged surfaces
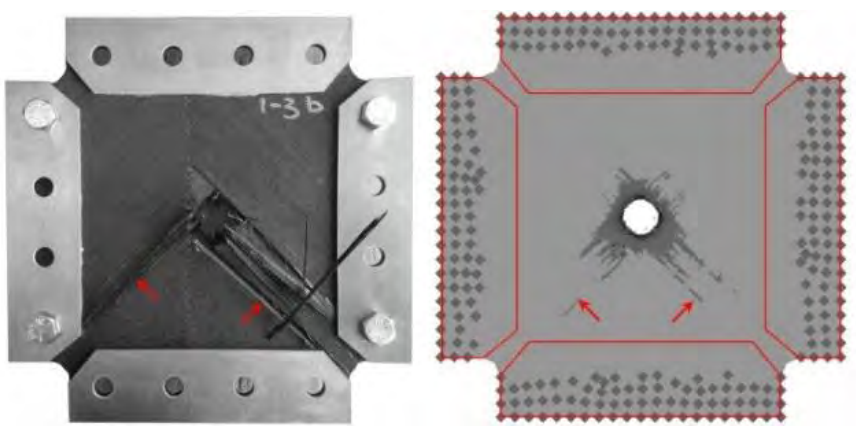

LDI: rear surface damage only

Figure 14. Composite damage: comparison of experimental results and numerical predictions

Fig. 13 represents the main features of the numerical models, including: a macro-scale appearance of the specimen model; a translational unit of the filament winding pattern model; a pair of interweaving filament bands; and a crosssection of a filament band with an adjacent resin-rich region. 
Voids at crossover points were represented explicitly in the meso-scale model.

Fig. 14 represents comparison of experimental and modeling results in terms of the damage to composite panels. It reveals the following:

- Simulations tend to somewhat underpredict the damaged area as compared to the damage detected by C-Scan. The exact reason for the underprediction is unknown, but may be attributed to the presence of a strain-rate dependence of the composite fracture toughness that was ignored in the modeling.

- For the HDI specimen, the simulation predicts a "containment" of the HVI damage within the region restricted by $2 \times 2$ HDI units adjacent to the perforated hole, which is the same damage pattern observed in the experiment.

- For the LDI specimen, the simulation predicts further propagation of the damage outwards along the fiber direction, which is also consistent with the experimental results. It should be noted, however, that the simulation also predicts the propagation of damage in the top-right corner of the specimen (encircled in Fig. 14) that was not observed on the C-Scan image.

- Damage patterns on the rear surface predicted by simulations for both HDI and LDI specimens are qualitatively in very good agreement with the experiment, as shown in Fig. 14.

\section{CONCLUSIONS}

For the foam-core sandwich panels, the meso-scale representation of the foam geometry is essential in HVI simulations, as it accounts for multi-shock interactions of the foam ligaments and hypervelocity fragment cloud propagating through the foam. The modelling approach considered in this study can be used in simulations estimating ballistic limit of foam core sandwich panels under hypervelocity impact.
Hypervelocity impact damage of a filament-wound composite is significantly dependent on the filament winding pattern used in its fabrication. In the presence of the winding pattern dependence, adequate simulation of HVI damage requires the utilization of a relatively high level of detail in representing a filament-wound composite. In this regard, the introduced meso-scale modeling approach was found to be suitable for capturing the main features of the HVI damage in the composites with different winding patterns. This approach can be used in the design of spacecraft filament-wound components, in order to determine the critical level of damage that is sustained due to orbital debris impacts.

\section{REFERENCES}

[1] J. Yasensky and E. Christiansen, "Hypervelocity Impact Evaluation of Metal Foam Core Sandwich Structures," NASA Johnson Space Center; Houston, TX, United States, 2007, JSC63945.

[2] S. Ryan, T. Hedman and E. I. Christiansen, "Honeycomb vs. foam: Evaluating potential upgrades to ISS module shielding," Acta Astronaut., vol. 67, 818-825, 2010. doi: 10.1016/j.actaastro.2010.05.021.

[3] S. Ryan and E. L. Christiansen, "Hypervelocity impact testing of advanced materials and structures for micrometeoroid and orbital debris shielding," Acta Astronaut., vol. 83, 216-231, 2013. doi: 10.1016/j.actaastro.2012.09.012.

[4] C. H. Yew and B. K. Rodney, "A study of damage in composite panels produced by hypervelocity impact," Int. J. Impact Eng., vol. 5, 729-38, 1987, 2008. doi: 10.1016/0734-743X(87)90087-X.

[5] M. Wicklein, S. Ryan, D. M. White and R. A. Clegg, "Hypervelocity impact on CFRP: testing, material modelling, and numerical simulation," Int. J. Impact Eng., vol. 35 (1), 1861-1869, 2008. doi: 10.1016/j.ijimpeng.2008.07.015.

[6] D. Weaire and R. Phelan, "A counter-example to Kelvin's conjecture on minimal surfaces," Philos. Mag. Lett., vol. 69 (2), pp. 107-110, 1994. doi: 10.1080/09500839408241577.

[7] D. M. White, E. A. Tylor, and R. A. Clegg R.A, "Numerical simulation and experimental characterization of direct hypervelocity impact on spacecraft hybrid carbon fibre/Kevlar composite structure," Int. J. Impact Eng., vol 29, no.1, pp. 779-790, 2003. doi: 10.1016/j.ijimpeng.2003.10.024.

[8] C. T. Sun and C. Han. "A method for testing interlaminar dynamic fracture toughness of polymeric composites," Compos. Part. B. Eng., vol. 35, no. 6-8, pp. 647-55, 2004. doi: 10.1016/j.compositesb.2004.04.006.

[9] J. L. Tsai, C. Guo, and C. T. Sun, "Dynamic delamination fracture toughness in unidirectional polymeric composites," Compos. Sci. Technol., vol. 61, no. 1, pp. 87-94, 2001. doi: 10.1016/S0266-3538(00)00197-4. 


\title{
Design, Fabrication, and Calibration of a MEMS based Sensing Rosette for Quantifying the Influence of Strain Engineering on the Piezoresistive Coefficients
}

\author{
Amr A. Balbola and Walied A. Moussa \\ Mechanical Engineering \\ University of Alberta \\ Edmonton, Canada \\ amr.balbola@ualberta.ca
}

\begin{abstract}
Strain has been used extensively in enhancing the electron mobility for high speed and low power transistors. As stretching the silicon atoms away beyond their normal atomic space has a significant influence on the carrier mobility. In this paper, to study the effect of strained silicon on piezoresistivity, the strained silicon will be integrated into a ten element sensing rosette, that utilizes the unique properties of crystalline silicon over the (111) plane to fully extract the six stress components in fully temperature compensated manner. Two chips were designed and fabricated, where the pre-strain state was induced onto the silicon substrate during microfabrication. In the first design, a highly compressive film (stressor layer) was utilized to globally produce a tensile strain at the front side of the substrate where the sensing elements were fabricated. While in the second design, the stressor layer was patterned in a way allowing for inducing both local tensile and compressive transverse uniaxial pre-strain onto the substrate. In another word, stressor strip with intrinsic compressive stress will cause a tensile pre-strain underneath it and compressive stress on both sides. This allows for applying different local strain using the same stressor rather than using nitride capping for tensile or silicon germanium for compressive as used on strained based transistors. To evaluate the effect of the pre-strain on the piezoresistive coefficients, uniaxial, thermal, and hydrostatic loading will be utilized to calibrate both designs.
\end{abstract}

Keywords-component; Strain Engineering; MEMS; Piezoresistivity, local stressor, biaxial pre-strain, uniaxial prestrain, intrinsic stress.

\section{INTRODUCTION}

STRAIN ENGINEERING is a cutting edge technology that is being employed mainly to enhance the performance of many CMOS devices [1] as shown in Figure 1. To continue Moore's law without scaling, strain technology has been utilized in many high volume production, such as Intel [2], [3] , IBM [4], Freescale [5], and Texas Instruments [6]. For decades, thermal mismatches [7], lattice mismatches [8], and non-equilibrium deposition [9] have been utilized extensively in producing prestrain state globally and locally. For instance, stress accompanying with silicide and nitride layers were used on integrating strain with the CMOS devices as pictured in Figure 1. Since 1992, biaxial [10] [11] [12], uniaxial [13] [14], and three dimensional stress [15] were induced onto different transistors to allow for higher performance and speed without going smaller. Many techniques have been employed to apply different controlled strain, such as silicide [16], nitride capping [17], dual stress linear [18], shallow trenches isolation [15], stress memorization technique [13], and selective epitaxial SiGe/Si-C layer [3] [19] [20].

As mobility is directly related to the piezoresistive coefficients, these coefficients were utilized to evaluate the influence of strained silicon on the electron and hole mobility. Accordingly, strain engineering has high influence on the piezoresistive coefficients. Hence, strain would have a tremendous impact on the piezoresistive based sensing rosette. For instance, strain engineering could improve the sensitivity of piezoresistive based stress sensors by 30 percent [21]. In this work, a biaxial and transverse strain will be produced and integrated with a piezoresistive sensing rosette, which will allow for quantifying the effect of strain on the piezoresistive coefficients. Tensile and compressive transverse local strains are applied using the same stressing layer rather than using nitride capping for tensile or silicon germanium for compressive strain [2], [3]. Unlike other type of stress, transverse uniaxial stress has the same effect on both electron and hole mobility [16]. 


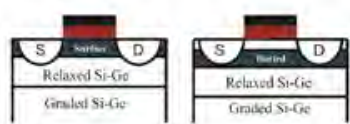

(1992)

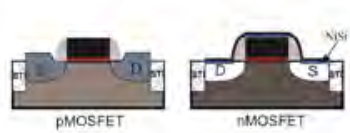

(2004)
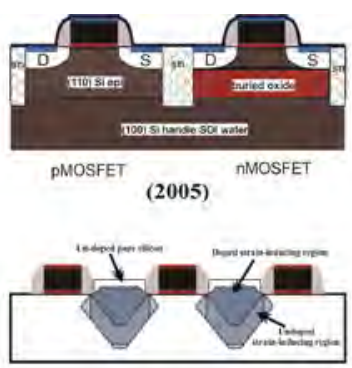

(2014)

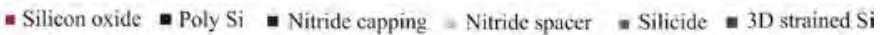
- Tensile biaxial strained $\mathrm{Si}$ = Tensile unaxial strained $\mathrm{Si}$ \# Compressive unaxial strained $\mathrm{Si}$

Figure 1. MOSFET structure changes in the past 30 years [2]

\section{STRESSOR DESIGN}

A ten-elements sensing rosette, which was developed by the author's group, was utilized to study both global biaxial and local uniaxial strain. This chip was fabricated on (111) silicon to develop a set of independent linear equations that yield the six stress components with full-temperature compensation [22], [23]. The strained silicon technology has been integrated during microfabrication via deposition of highly compressive plasma enhanced chemical vapor deposition (PECVD) nitride film that bends the substrate as plotted in Figure 2. This will produce a tensile biaxial strain at the top side of the substrate, where the sensing rosette is located. Considering that the inplane stresses are equal on all directions and the (111) plane has isotropic elastic properties [24], the biaxial elastic modulus can be utilized to calculate the pre-stain. A compressive nitride film stress of $600 \mathrm{MPa}$ was measured via the wafer curvature, however the finite element analysis for the stress distribution shows large stress losses up to $70 \%$ between the film and substrate.

The same stressing layer was patterned in a way allowing for producing uniaxial local strain onto the silicon substrate as shown in Figure 3. Both tensile and compressive strains were applied using the same layer. Also the shallow trenches technique was used to maximize the strain produced at the piezoresistors' area. The shallow trenches were etched on both sides of the piezoresistors using reactive ion etching. The effect of the stressors and the trenches was numerically studied extensively via ANSYS finite element software package. The result shows that nitride stressor would generate uniaxial tensile and compressive transverse stress around $50 \mathrm{MPa}$. The simulation indicates a dominant influence of film thickness on the stress transmitted to the silicon, which can reach up to 150 and -200 for tensile and compressive stress respectively, as presented in Figure 4. While the shallow trenches introduce more strain up to 200 and $-250 \mathrm{MPa}$ for tensile and compressive stress respectively.

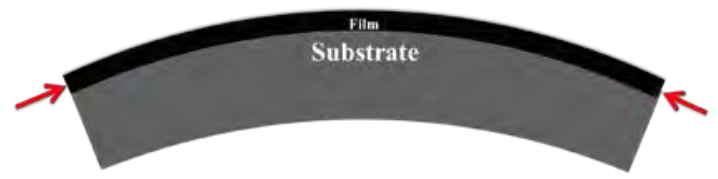

Figure 2. Curvature caused by highly compressive film

Canadian Microsystems Corporation (CMC).

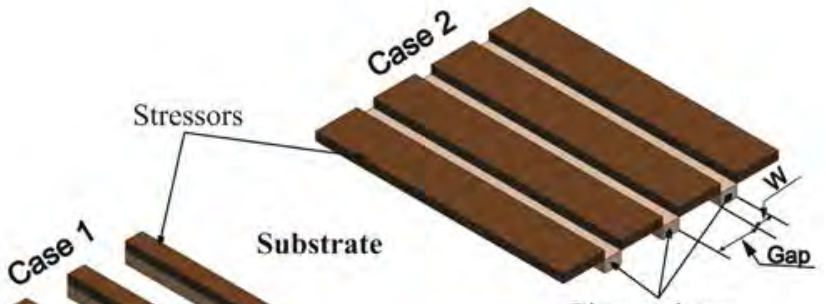

Piezoresistors under compressive stress

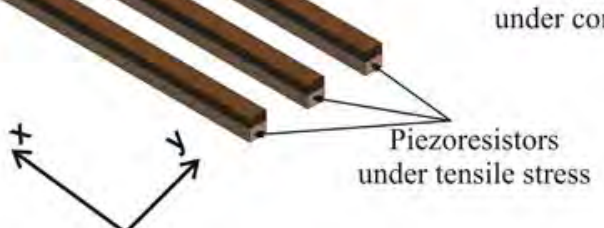

Figure 3. The mechanism of producing tensile and compressive pre-strain

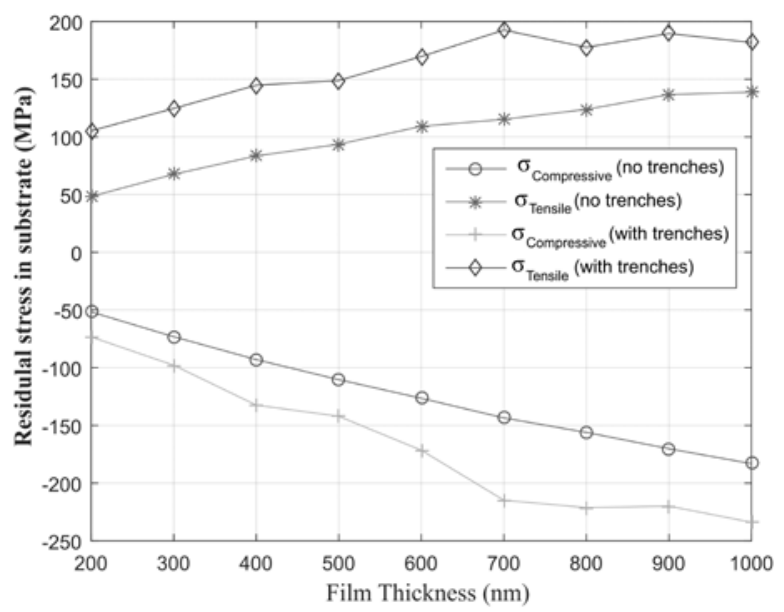

Figure 4. Stress transferred to the silicon versus the film thickness

\section{MICROFABRICATION}

Two different designs have been fabricated in the nanoFAB and the MEMS/NEMS Advanced Design Laboratory (ADL) at the University of Alberta. In the first design, a biaxial tensile pre-strain was induced at the front side of the substrate. While both tensile and compressive uniaxial stresses were integrated in the second chip. Both recipes were fabricated on a p-type (111) prime silicon wafers which were initially cleaned using piranha and buffered-oxide etches (BOE). The main fabrication steps are creating the piezoresistive sensing elements and applying the pre-stress state as represented in Figure 5. A highly compressive layer of PECVD silicon nitride was locally deposited to induce tensile and compressive strain onto the sensing elements rather than straining the whole wafer. Finally, prior to the metallization, an additional predeposition diffusion step was carried out to create $\mathrm{n}+$ region at the contact vias to obtain ohmic contact behavior between $\mathrm{Al}$ and Si. The fabricated chips were experimentally characterized to assess the piezoresistors functionality. The concentration profile was measured using a time-of-flight secondary ion mass spectrometry (ToF-SIMS). Photomicrographs of both designs are shown in Figure 6 and Figure 7. 
(1)

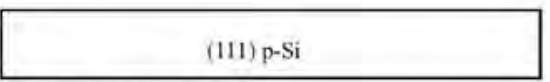

Start with p-type (111) silicon wafer Wafer Cleaning using Piranha and BOF:

(2)

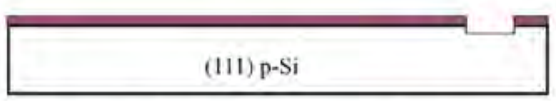

Optical Photolithography to pattern the aliganient marks Silicon DRIE to etch the alignment marks through the silicon

(3)

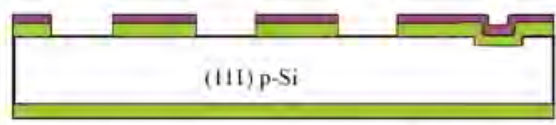

Thermal Wet Oxidation to grow oxide layer as diffusion mask

Optical Photolithography then BOE to pattern the doping window through the oxide

4)

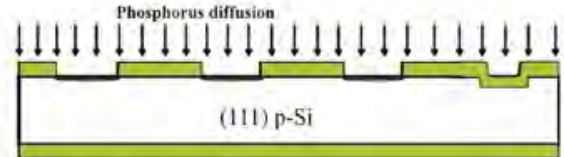

Phosphorus pre-deposition

(5)

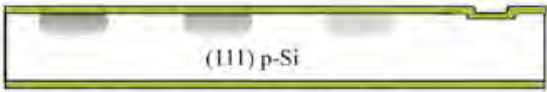

Drive-in to diffuse the surface concentrated dopants Thermal oxidation to grow oxide layer
(6)

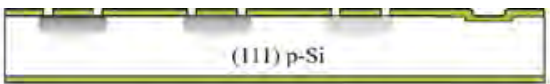

Optical Photolithography then BOE to pattern the contact vias

(7)

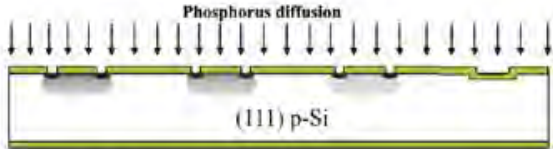

Phosphorus Diffusion to create $n+$ contacts

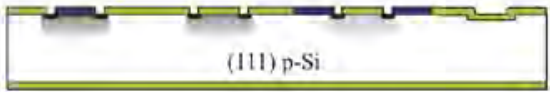

Optical Photolithography then BOE to pattern the stressors area PECVD to grow a layer of PECVD silicon nitride

Optical Photolithography then Plasma RIE to etch the stressors and the contact vias

(9)

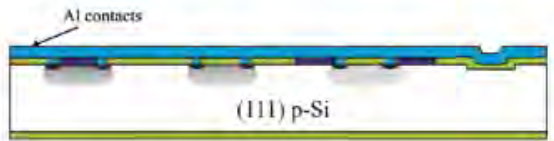

Sputtering of Aluminum layer for metal contacts

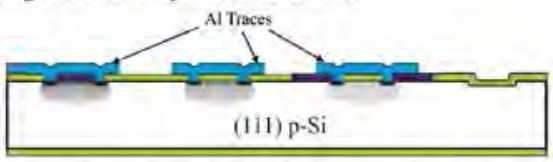

Optical Photolithography then Al etching to pattern the metal contact:

\section{Silicon \\ Photoresist \\ Wet thermal oxide \\ PECVD nitride \\ Al contacts}

Figure 5. Microfabrication process flow of strained ten-element sensing rosette

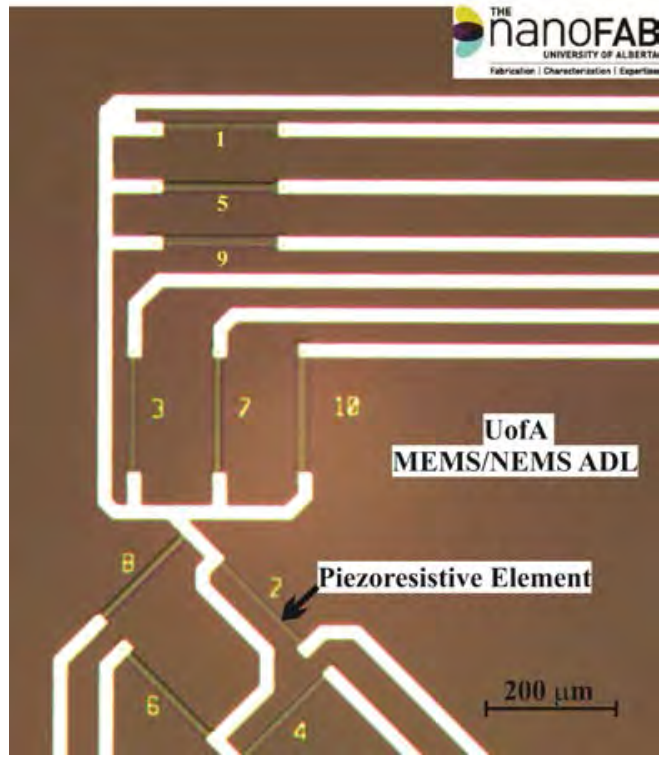

Figure 6. Photomicrograph of the microfabrication biaxial strained based sensing rosette

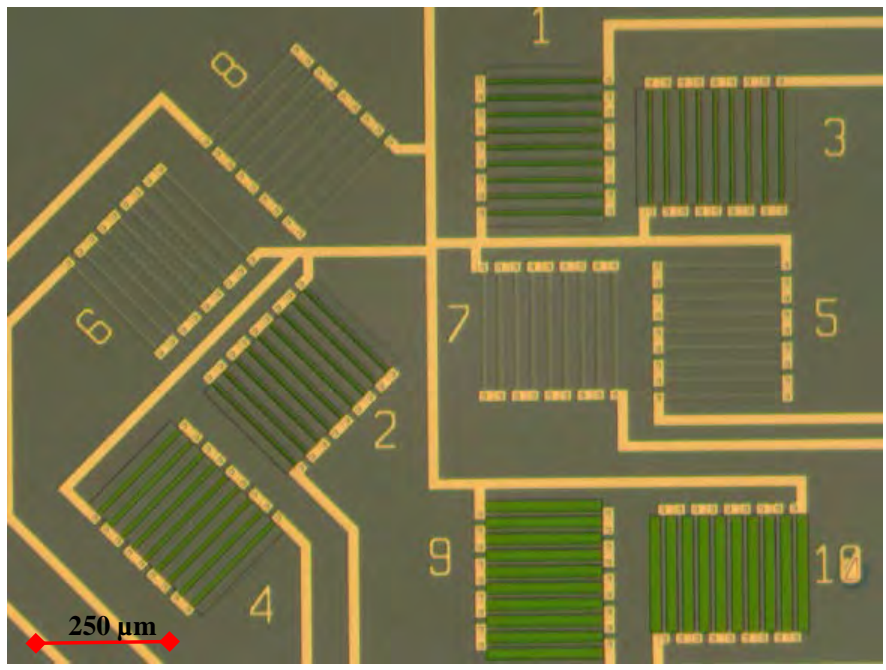

Figure 7. Photomicrograph of the microfabrication uniaxial strained based sensing rosette 


\section{CALIBRATION}

A full calibration of the piezoresistive coefficients over (111) $\left(B_{1}, B_{2}, B_{3}\right)$ were carried out to quantify the influence of strained silicon on piezoresistive coefficients. The typical calibration results would be used to calculate the piezoresistive coefficients. This test was carried out using a four-point bending (4PB) setup, environmental chamber, and hydrostatic test [21]. Applying known uniaxial stress on the current fabricated sensing chip will give $B_{1}$ and $B_{2}$ directly, while hydrostatic load will measure $B_{3}$. This calibration setup does not require packaging of the sensing die, where the calibration die is a part of a rectangular beam cut from the wafer, and is connected to a zero-insertion force (ZIF) connector using aluminum traces as shown in Figure 8 . These aluminum traces are connected to voltmeter to provide six bias voltages which are enough for fully calibrating the piezoresistive coefficients. The resistance changes from the $0^{\circ}$ sensing elements $\left(R_{1}, R_{5}\right.$, and $R_{9}$ ) are used to determine the $B_{1}$ parameters, while $B_{2}$ was calibrated via the sensing elements oriented at $90^{\circ}\left(R_{3}, R_{7}\right.$, and $R_{10}$ ) as shown in (1). Unlike the $B_{1}$ and $B_{2}, B_{3}$ can be calculated from $0^{\circ}$ or $90^{\circ}$ sensing elements using the hydrostatic test.

$$
B_{1}=\frac{\partial}{\partial \sigma}\left(\frac{\Delta R_{0}}{R_{0}}\right) \quad \text { and } \quad B_{2}=\frac{\partial}{\partial \sigma}\left(\frac{\Delta R_{s 0}}{R_{s 0}}\right)
$$

The test procedure started with measuring the nominal resistance of the six piezoresistors at no load. Then the load was increased incrementally and measured. At each load increment, the resistances were measured again to calculate the change in resistance. Figures 9-11 show the typical results came from the calibration, where the slopes represent the $B_{1}, B_{2}$ and $B_{3}$.

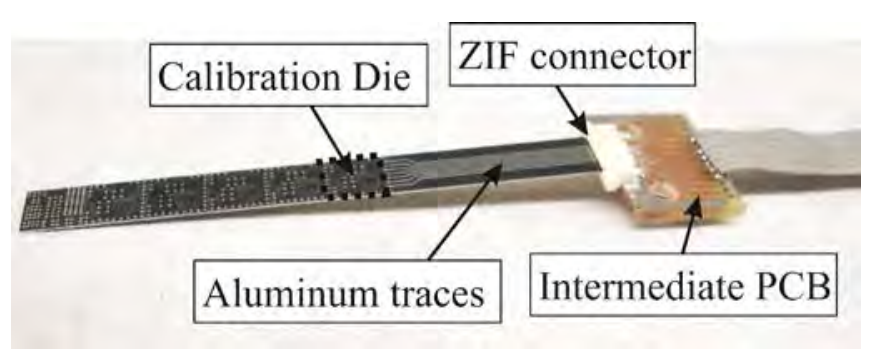

Figure 8 . The silicon beam that used in calibration

\section{CONCLUSION}

In the frame of employing strain engineering on enhancing the performance of a MEMS 3D stress sensor, a strained based piezoresistive sensing rosette was developed for studying the effect of strain on the piezoresistive coefficients. The intrinsic stress accompanied with PECVD silicon nitride was utilized to induce a pre-strain into the silicon substrate during microfabrication. Both biaxial global and uniaxial transverse local strain was produced using the same stressing layer. A full study including design, fabrication, and calibration was carried out to build a chip that can be used to quantify the strain effect.

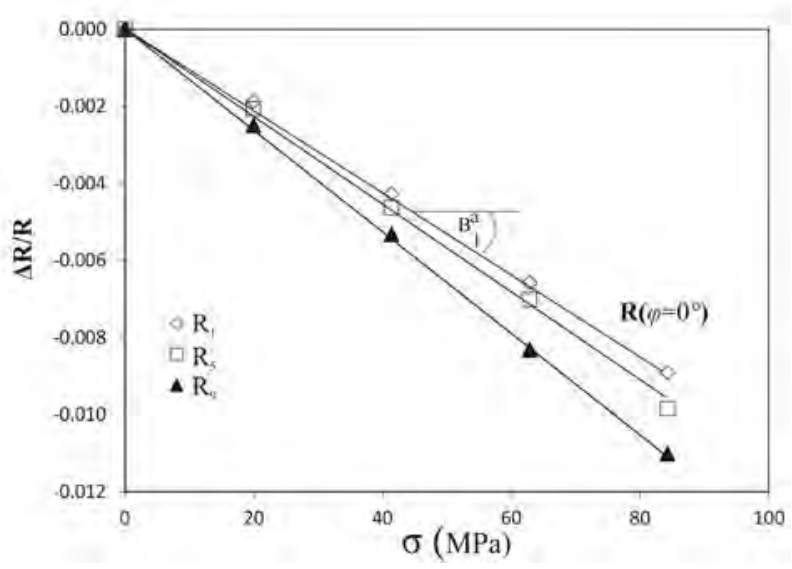

Figure 9. Typical 4PB results for R1, R5, and R9

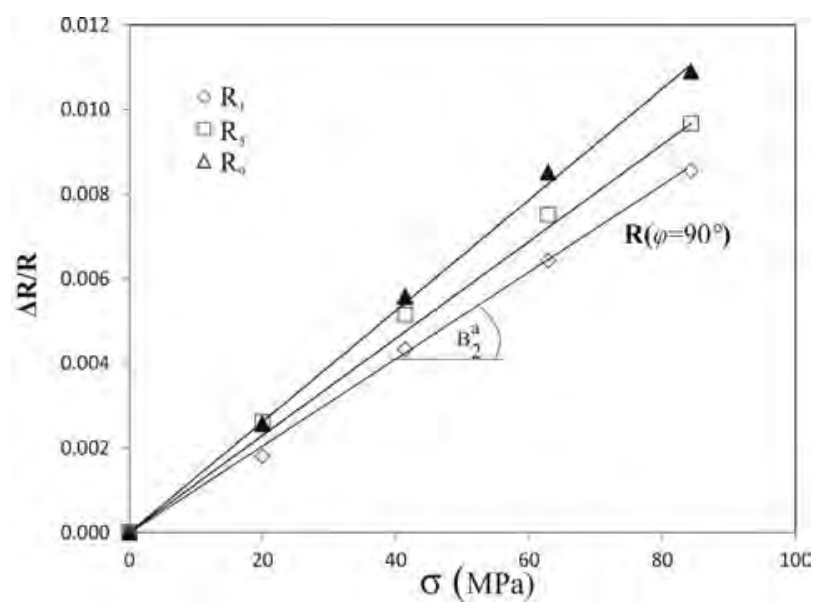

Figure 10. Typical 4PB results for R3, R7, and R10

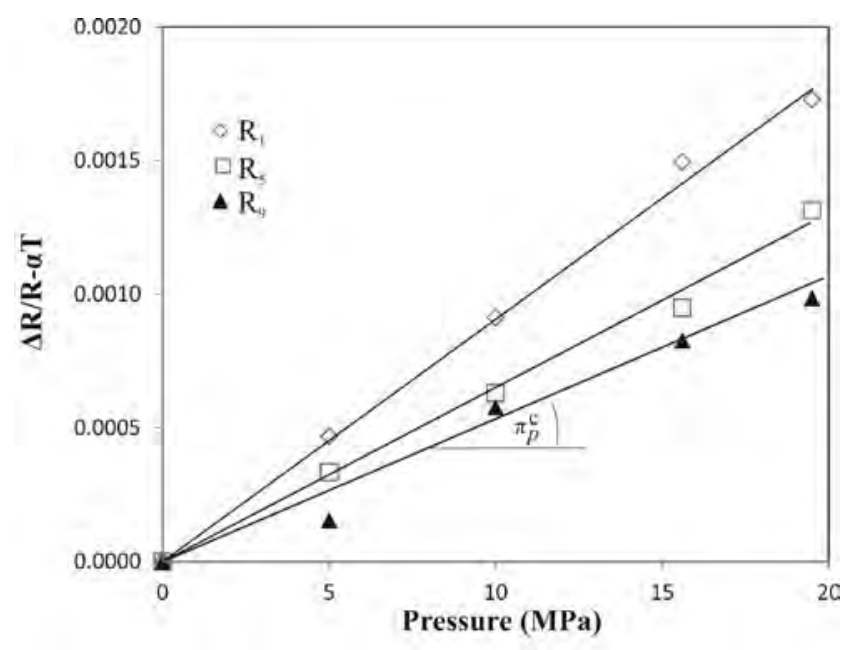

Figure 11. Adjusted hydrostatic calibration data 


\section{ACKNOWLEDGMENT}

This work was partially funded by the Canadian Microsystems Corporation (CMC). The authors would like to thank this organization for their support.

\section{REFERENCES}

[1] E. Parton and P. Verheyen, "Strained silicon - the key to sub-45 nm CMOS," III-Vs Rev Adv Semicond Mag, vol. 19, no. 3, pp. 28-31, 2006. doi: 10.1016/S0961-1290(06)71590-3.

[2] S. E. Thompson, M. Armstrong, and C. Auth, "A 90-nm logic technology featuring strained-silicon," IEEE Trans. Electron. Devices, vol. 51, no. 11, pp. 1790-1797, 2004. doi: 10.1109/TED.2004.836648.

[3] S. E. Thompson et al., "A logic nanotechnology featuring strainedsilicon," IEEE Electron. Device Lett., vol. 25, no. 4, pp. 191-193, 2004. doi: 10.1109/LED.2004.825195.

[4] Q. Ouyang et al., "Investigation of CMOS devices with embedded SiGe source/drain on hybrid orientation substrates," in Digest of Technical Papers.2005 Symposium on VLSI Technology, 2005, vol. 2005, pp. 2829. doi: $10.1109 / .2005 .1469199$.

[5] Y. G. G. Wang et al., "Effects of uniaxial mechanical stress on drive current of 0.13 um MOSFETs," IEEE Trans Electron Devices, vol. 50, no. 2, pp. 529-531, Feb. 2003. doi: 10.1109/TED.2002.808450.

[6] P. R. R. Chidambaram, C. Bowen, S. Chakravarthi, C. Machala, and R. Wise, "Fundamentals of silicon material properties for successful exploitation of strain engineering in modern CMOS manufacturing," IEEE Trans Electron Devices, vol. 53, no. 5, pp. 944-964, May 2006. doi: 10.1109/TED.2006.872912.

[7] A. F. Bower and L. B. Freund, "Analysis of stress-induced void growth mechanisms in passivated interconnect lines," J. Appl. Phys., vol. 74, no. 6, p. 3855 , 1993. doi: $10.1063 / 1.354480$.

[8] A. L. Roitburd, "Equilibrium structure of epitaxial layers," Phys Status Solidi, vol. 37, no. 1, pp. 329-339, 1976. doi: 10.1002/pssa.2210370141.

[9] M. P. Besland, M. Lapeyrade, F. Delmotte, and G. Hollinger, "Interpretation of stress variation in silicon nitride films deposited by electron cyclotron resonance plasma," J. Vac Sci Technol A Vacuum, Surfaces, Film, vol. 22, no. 5, p. 1962, 2004. doi: 10.1116/1.1776179.

[10] J. Welser, J. L. Hoyt, and J. F. Gibbons, "NMOS and PMOS transistors fabricated in strained silicon/relaxed silicon-germanium structures," Electron Devices Meet 1992 IEDM '92 Tech Dig Int, pp. 1000-1002, 1992. doi: org/10.1109/IEDM.1992.307527.

[11] J. Welser, J. L. Hoyt, and J. F. Gibbons, "Electron mobility enhancement in strained-Si n-type metal-oxide-semiconductor field-effect transistors," IEEE Electron Device Lett., vol. 15, no. 3, pp. 100-102, 1994. doi: $10.1109 / 55.285389$.

[12] J. Welser, J. L. Hoyt, S. Takagi, and J. F. Gibbons, "Strain dependence of the performance enhancement in strained-Sin-MOSFETs," Proc. 1994 IEEE Int. Electron. Devices Meet., pp. 373-376, 1994. doi: 10.1109/IEDM.1994.383389.
[13] Chien-Hao Chen et al., "Stress memorization technique (SMT) by selectively strained-nitride capping for sub-65nm high-performance strained-Si device application," in Digest of Technical Papers 2004 Symposium on VLSI Technology, 2004., pp. 56-57.

[14] H. S. Yang et al., "Dual stress liner for high performance sub-45nm gate length SOI CMOS manufacturing," in IEDM Technical Digest. IEEE International Electron Devices Meeting, 2004, 2004, pp. 1075-1077. doi: 10.1109/IEDM.2004.1419385.

[15] C.-H. Ge et al., "Process-strained Si (PSS) CMOS technology featuring 3D strain engineering," in IEEE International Electron Devices Meeting 2003, pp. 73-76. doi: 10.1109/IEDM.2003.1269169.

[16] A. Steegen, M. Stucchi, A. Lauwers, K. Maex, and K. U. Leuven, "Silicide induced pattern density and orientation dependent transconductance in MOS transistors," in Electron Devices Meeting, 1999. IEDM '99. Technical Digest. International, 1999, pp. 497-500. doi: 10.1109/IEDM.1999.824201.

[17] S. Ito et al., "Mechanical stress effect of etch-stop nitride and its impact on deep submicron transistor design," in International Electron Devices Meeting 2000. Technical Digest. IEDM (Cat. No.00CH37138), 2000, pp. 247-250. doi: 10.1109/IEDM.2000.904303.

[18] A. Shimizu et al., "Local mechanical-stress control (LMC): a new technique for CMOS-performance enhancement," in Electron Devices Meeting, 2001. IEDM '01. Technical Digest. International, 2001, pp. 433-436. doi: 10.1109/IEDM.2001.979529.

[19] K. W. A. K. W. Ang et al., "Enhanced performance in $50 \mathrm{~nm} \mathrm{~N}$ MOSFETs with silicon-carbon source/drain regions," in IEDM Technical Digest. IEEE International Electron Devices Meeting, 2004., 2004, pp. 1069-1071. doi: 10.1109/IEDM.2004.1419383.

[20] K.-J. Chui, K.-W. Ang, N. Balasubramanian, M.-F. Li, G. S. Samudra, and Y.-C. Yeo, "n-MOSFET with silicon-carbon source/drain for enhancement of carrier transport," IEEE Trans. Electron. Devices, vol. 54, no. 2, pp. 249-256, Feb. 2007. doi: 10.1109/TED.2006.888629.

[21] A. A. Balbola, M. O. Kayed, and W. A. Moussa, "Studying the influence of n-type strained (111) silicon on the piezoresistive coefficients," IEEE Sens. J., vol. 17, no. 2, pp. 302-310, Jan. 2017. doi: 10.1109/JSEN.2016.2616759.

[22] H. H. Gharib and W. A. Moussa, "On the feasibility of a new approach for developing a piezoresistive 3D stress sensing rosette," IEEE Sens J., vol. 11, no. 9, pp. 1861-1871, Sep. 2011. doi: 10.1109/JSEN.2010.2102350.

[23] H. H. Gharib and W. A. Moussa, "Microfabrication and calibration of a single-polarity piezoresistive three-dimensional stress sensing chip," $J$. Micromechanics Microengineering, vol. 23, no. 3, p. 35019, Mar. 2013. doi: 10.1088/0960-1317/23/3/035019.

[24] M. J. Madou, Fundamentals of Microfabrication: The Science of Miniaturization, Second Edition. CRC Press, 2002. doi: $10.1201 / 9781482274004$. 


\section{Heat Conduction in a Layerd Structure with an Interface Crack Using the Dual Phase Lag Model}

\author{
Zengtao Chen \\ Department of Mechanical Engineering \\ University of Alberta \\ Edmonton, AB, Canada \\ Zengtao.chen@ualberta.ca
}

\author{
Keqiang $\mathrm{Hu}$ \\ Department of Chemical and Materials Engineering \\ University of Alberta \\ Edmonton, AB, Canada \\ Keqiang.hu@gmail.com
}

\begin{abstract}
In this paper, the transient heat conduction in a layered composite with an insulated interface crack parallel to the boundaries is investigated by using the dual phase lag (DPL) model. Fourier and Laplace transforms are applied and the mixed boundary value problem for the cracked structure under temperature impact is reduced to solving a singular integral equation. The temperature field in time domain is obtained and the intensity factor of temperature gradient is defined. Numerical studies show that overshoot phenomenon may occur due to the combined effect of the insulated crack and application of the DPL heat conduction model. The thermal conductivity and the phase lag parameters have strong influence on the dynamic intensity factor of temperature gradients. The results obtained by the dual phase lag model can be reduced to that by the hyperbolic model and that by the parabolic model.
\end{abstract}

Keywords- transient heat conduction; dual-phase lag model; non-Fourier heat conduction; Singular integral equation

\section{INTRODUCTION}

High-rate heat transfer has become a major concern in modern industries and accurate heat conduction analysis is of great importance for the material and structural integrity. For applications involving high power density, extremely short times or cryogenic temperatures, the classical parabolic heat diffusion theory as stipulated by Fourier's law of heat conduction becomes ineffective [1]. A unified heat conduction model that accounts for spatial and temporal effects in macroand micro-scale heat transfer in a one temperature formulation has been proposed and was experimentally supported [2], namely the dual-phase-lag (DPL) model.

Inherent defects in materials such as dislocations and cracks may disturb the temperature distribution when thermal loading is applied to the material, and singularities may be developed in the neighborhood of discontinuities. Heat conduction problems of cracked materials using the classical Fourier heat conduction model have been investigated by some researchers [3, 4]. Some investigations on crack problems in thermo-elastic materials have been made using the hyperbolic heat conduction model. Transient temperature field around a thermally insulated crack in a substrate bonded to a coating has been obtained by Chen and $\mathrm{Hu}$ [5] using the hyperbolic heat conduction model; and based on the same theory, $\mathrm{Hu}$ and Chen [6] obtained the transient temperature and thermal stress distributions around a partially insulated crack in a thermo-elastic strip. The problem of a finite crack in a material layer under the theory of non-Fourier heat conduction has been investigated by Wang and Han [7]. By applying the DPL model to a cracked half-plane under transient thermal loading, the dynamic temperature field around a partially insulated crack has been obtained [8].

To the author's knowledge, the transient interface crack problem in a layered composite under thermal loading by applying the dual phase lag model has not yet been reported in the literature. In this paper, we analyze the transient temperature field around an insulated interface crack in a layered composite under temperature impact using the dual phase lag model. The effect of the parameters of the dual phase lag model and the geometric size of the cracked body on the temperature disturbance field are investigated.

\section{PROBLEM DESCRIPTION}

Consider a thermo-elastic, double-layered structure containing an interface crack of length $2 c$ parallel to the boundaries of the structure, as shown in Fig. 1. The thicknesses of the upper and lower layers are $h_{1}$ and $h_{2}$, respectively. The layered structure is initially at the uniform temperature of zero, and is suddenly heated to a temperature, $T_{1}$ and $T_{2}$ on the upper and the lower boundaries, respectively, and $H(t)$ denotes the Heaviside step function. The crack surfaces are assumed to be thermally insulated, which indicates that the thermal transfer across the crack faces vanishes.

In order to account the effects of finite heat propagation and micro-structural interaction, the Fourier's law of heat conduction is modified to the DPL model [2],

$$
q+\tau_{q} \frac{\partial q}{\partial t}+\frac{\tau_{q}^{2}}{2} \frac{\partial^{2} q}{\partial t^{2}}=-k \cdot\left(\nabla T+\tau_{T} \frac{\partial}{\partial t} \nabla T\right)
$$


where $q$ is the heat flux, $T$ is the temperature, $k$ is the thermal conductivity of the material, $\nabla$ is the spatial gradient operator, $t$ is the physical time at which observation on heat transport is made, $\tau_{q}$ and $\tau_{T}$ are the phase lags of the heat flux and temperature gradient, respectively, which are two intrinsic thermal properties of the material. The heat flux precedes the temperature gradient for $\tau_{q}<\tau_{T}$, and the temperature gradient precedes the heat flux for $\tau_{q}>\tau_{T}$.

Conservation of the local energy with vanishing heat source applies [9]:

$$
-\nabla q=\rho C_{p} \cdot \frac{\partial T}{\partial t}
$$

where $\rho$ and $C_{p}$ are the mass density and the specific heat capacity, respectively.

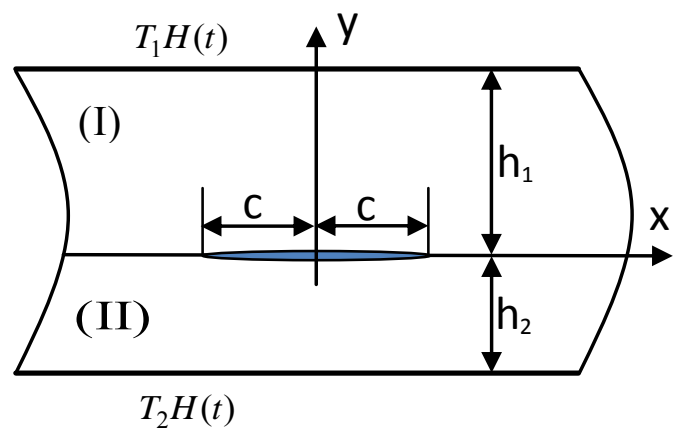

Figure 1. Geometry of cracked double-layered structure under transient thermal impact on the upper and lower surfaces

Incorporating (1) with (2) leads to the heat conduction equations in the following form

$$
\begin{gathered}
\nabla^{2} T^{(1)}+R_{1} \frac{\partial}{\partial t}\left(\nabla^{2} T^{(1)}\right)=a^{2}\left[\frac{\partial T^{(1)}}{\partial t}+\frac{\partial^{2} T^{(1)}}{\partial t^{2}}+\frac{1}{2} \frac{\partial^{3} T^{(1)}}{\partial t^{3}}\right] \\
\nabla^{2} T^{(2)}+R_{2} \frac{\partial\left(\nabla^{2} T^{(2)}\right)}{\partial t}=\frac{a^{2}}{r_{\alpha}}\left[\begin{array}{c}
\frac{\partial T^{(2)}}{\partial t}+r_{\tau} \frac{\partial^{2} T^{(2)}}{\partial t^{2}} \\
+\frac{r_{\tau}^{2}}{2} \frac{\partial^{3} T^{(2)}}{\partial t^{3}}
\end{array}\right] . \\
R_{1}=\tau_{T}^{(I)} / \tau_{q}^{(I)}, R_{2}=\tau_{T}^{(I I)} / \tau_{q}^{(I)} \\
r_{\alpha}=\alpha^{(I I)} / \alpha^{(I)}, r_{\tau}=\tau_{q}^{(I I)} / \tau_{q}^{(I)}
\end{gathered}
$$

where $a=c / L$ is a characteristic length parameter of the material, the superscript "(1), (2)" represent the variables in the upper layer and the lower layer, respectively. It is noted that the following dimensionless variables have been used for simplicity:

$$
T^{(1)}=T^{(I)} / T_{1}, T^{(2)}=T^{(I I)} / T_{1}, L=\sqrt{\alpha^{(I)} \tau_{q}^{(I)}}
$$

and the time have been normalized as $t / \tau_{q}^{(I)}$.
The heat conduction equations are subjected to the following boundary and initial conditions in the dimensionless form as

$$
\begin{gathered}
T^{(1)}\left(x, h_{1}, t\right)=1, \quad(|x|<\infty, t>0) . \\
T^{(2)}\left(x,-h_{2}, t\right)=T_{2} / T_{1}, \quad(|x|<\infty, t>0) . \\
q^{(1)}\left(x, 0^{+}, t\right)=q^{(2)}\left(x, 0^{-}, t\right), \quad(|x| \geq c) . \\
T^{(1)}\left(x, 0^{+}, t\right)=T^{(2)}\left(x, 0^{-}, t\right), \quad(|x| \geq c) . \\
\frac{\partial T^{(1)}\left(x, 0^{+}, t\right)}{\partial y}=\frac{\partial T^{(2)}\left(x, 0^{-}, t\right)}{\partial y}=0, \quad(|x|<c) . \\
T^{(j)}=0, \frac{\partial T^{(j)}}{\partial t}=0, \quad \frac{\partial^{2} T^{(j)}}{\partial t^{2}}=0, \quad(t=0, j=1,2) .
\end{gathered}
$$

\section{METHOD OF THE PROBLEM}

Application of Laplace transforms to $(3,4)$ leads to:

$$
\begin{aligned}
& T^{*}(x, y, p)=\int_{0}^{\infty} T(x, y, t) \exp (-p t) d t \\
& T(x, y, t)=\frac{1}{2 \pi i} \int_{B r} T^{*}(x, y, p) \exp (p t) d p
\end{aligned}
$$

where the superscript "**" denotes the quantities in the Laplace domain and $\mathrm{Br}$ stands for the Bromwich path of integration.

By considering the initial conditions and boundary conditions, we can obtain the appropriate temperature field in the Laplace domain by using Fourier transforms as

$$
\begin{aligned}
& T^{*(1)}(x, y, p)=\int_{-\infty}^{\infty}\left[\begin{array}{l}
D_{1}(\xi, p) \exp (m y) \\
+D_{2}(\xi, p) \exp (-m y)
\end{array}\right] \exp (-i x \xi) d \xi \quad\left(0 \leq y \leq h_{1}\right) \quad \\
&+ W_{1}(y, p) \\
& T^{*(2)}(x, y, p)=\int_{-\infty}^{\infty}\left[\begin{array}{l}
E_{1}(\xi, p) \exp (n y) \\
+E_{2}(\xi, p) \exp (-n y)
\end{array}\right] \exp (-i x \xi) d \xi \\
&+W_{2}(y, p) \quad\left(-h_{2} \leq y \leq 0\right)
\end{aligned}
$$

where $D_{j}(\xi, p), E_{j}(\xi, p)(j=1,2)$ are unknowns to be determined and $m, n, W_{1}(y, p), W_{2}(y, p)$ are known functions.

Introduce the temperature density function as

$$
\phi(x, p)=\frac{\partial T^{*(1)}\left(x, 0^{+}, p\right)}{\partial x}-\frac{\partial T^{*(2)}\left(x, 0^{-}, p\right)}{\partial x} .
$$

It is clear from the boundary conditions (10) that

$$
\int_{-c}^{c} \phi(t, p) d t=0 \text {. }
$$

The satisfaction of the mixed boundary value problem on the crack face plane leads to the singular integral equation for $\phi(x, p)$ as follows 


$$
\int_{-c}^{c} \phi(t, p)\left[\frac{1}{t-x}+H(x, t, p)\right] d t=2 \pi u\left(b_{2}-b_{1}\right) .
$$

where $H(x, t, p)$ is the kernel function and its expression is omitted here.

The solution of the singular integral equation (18) under the single-valuedness condition (17) may be expressed as [10]:

$$
\phi(c x, p)=\Phi(x, p) / \sqrt{1-x^{2}} .
$$

where $\Phi(x, p)$ is bounded and continuous on the interval $[-1,1]$. From the properties of symmetry and the condition (17), it is seen that $\Phi(x, p)$ is an odd function of $x$, i.e., $\Phi(-x, p)=-\Phi(x, p)$.

By using the numerical method of Erdogan [10], Singular integral equations (17) and (18) can be solved at discrete points as

$$
\begin{gathered}
\sum_{k=1}^{N} \frac{1}{N} \Phi\left(t_{k}, p\right)\left[\frac{1}{t_{k}-x_{r}}+H\left(x_{r}, t_{k}, p\right)\right]=2 u\left(b_{2}-b_{1}\right) \\
\quad(r=1,2, \ldots, N-1) \\
\sum_{k=1}^{N} \frac{\pi}{N} \Phi\left(t_{k}, p\right)=0 . \\
t_{k}=\cos [(2 k-1) \pi / 2 N], \quad(k=1,2, \ldots, N) . \\
x_{r}=\cos (r \pi / N), \quad(r=1,2, \ldots, N-1)
\end{gathered}
$$

Once the function $\Phi(t, p)$ is obtained, the function $D_{2}(\xi, p)$ can be obtained by applying the Chebyshev quadrature for integration as

$$
\begin{gathered}
D_{2}(\xi, p) \cong-\frac{1}{2 \pi \xi G(\xi, p)} \sum_{m=1}^{N} w_{m} \Phi\left(x_{m}, p\right) \sin \left(x_{m} \xi\right) \\
x_{m}=\cos \left(\frac{2 m-1}{2 N} \pi\right), \quad(m=1,2, \ldots, N) \\
w_{m}=\pi / N
\end{gathered}
$$

The functions $D_{1}(\xi, p), E_{j}(\xi, p)(j=1,2)$ can be expressed in terms of $D_{2}(\xi, p)$ as

$$
\begin{aligned}
& D_{1}(\xi, p)=-D_{2}(\xi, p) \exp \left(-2 m h_{1}\right) \\
& E_{1}(\xi, p)=-\frac{m K_{1}(p)\left[1+\exp \left(-2 m h_{1}\right)\right]}{n K_{2}(p)\left[1+\exp \left(-2 n h_{2}\right)\right]} D_{2}(\xi, p) . \\
& E_{2}(\xi, p)=\frac{m K_{1}(p)\left[1+\exp \left(-2 m h_{1}\right)\right]}{n K_{2}(p)\left[1+\exp \left(2 n h_{2}\right)\right]} D_{2}(\xi, p)
\end{aligned}
$$

\section{TEMPERATURE FIELD}

The substitution of $(21,22)$ into $(14,15)$ can give the temperature in the Laplace domain, and the temperature in time domain can be obtained by applying the Laplace inverse transform.

Of particular interest are the temperature gradients in the cracked media under thermal loadings. A temperature gradient is a physical quantity that describes in which direction and at how much rate the temperature changes the most rapidly around a particular location. The temperature gradients in the Laplace domain can be obtained as

The singular temperature gradients near the right crack tip in the Laplace domain can be obtained as

$$
\begin{gathered}
T_{, y}^{*(1)}(r, \theta, p)=-\frac{\Phi(1, p) \sqrt{c}}{2 \sqrt{2 r}} \cos \left(\frac{\theta}{2}\right) . \\
T_{, y}^{*(2)}(r, \theta, p)=-\frac{K_{1}(p)}{K_{2}(p)} \frac{\Phi(1, p) \sqrt{c}}{2 \sqrt{2 r}} \cos \left(\frac{\theta}{2}\right) . \\
T_{, x}^{*(1)}(r, \theta, p)=\frac{\Phi(1, p) \sqrt{c}}{2 \sqrt{2 r}} \sin \left(\frac{\theta}{2}\right) . \\
T_{, x}^{*(2)}(r, \theta, p)=\frac{K_{1}(p)}{K_{2}(p)} \frac{\Phi(1, p) \sqrt{c}}{2 \sqrt{2 r}} \sin \left(\frac{\theta}{2}\right) .
\end{gathered}
$$

The temperature gradients near the crack tip in the radial direction in the Laplace domain can be obtained as

$$
\begin{gathered}
T_{, r}^{*(1)}(r, \theta, p)=-\frac{\Phi(1, p) \sqrt{c}}{2 \sqrt{2 r}} \sin \left(\frac{\theta}{2}\right) . \\
T_{, r}^{*(2)}(r, \theta, p)=-\frac{K_{1}(p)}{K_{2}(p)} \frac{\Phi(1, p) \sqrt{c}}{2 \sqrt{2 r}} \sin \left(\frac{\theta}{2}\right) .
\end{gathered}
$$

where the subscript ",$j$ " $(j=x, y, r)$ denote the temperature gradient in $x, y$ and radial direction, respectively; $(r, \theta)$ are the polar coordinates measured from the crack tip defined by

$$
r^{2}=(x-c)^{2}+y^{2}, \quad \tan (\theta)=y /(x-c) .
$$

In front of the crack tip at $\theta=-\pi$, the temperature gradient reaches the maximum value and the intensity factor of the temperature gradient (IFTG) near the crack tip can be defined as [11],

$$
\begin{gathered}
K_{T}^{*(1)}(p)=\left.\lim _{r \rightarrow 0} 2 \sqrt{r} T_{, r}^{*(1)}(r, \theta, p)\right|_{\theta=-\pi}=\frac{\Phi(1, p) \sqrt{c}}{\sqrt{2}} \\
K_{T}^{*(2)}(p)=\left.\lim _{r \rightarrow 0} 2 \sqrt{r} T_{, r}^{*(2)}(r, \theta, p)\right|_{\theta=-\pi}=\frac{K_{1}(p)}{K_{2}(p)} \frac{\Phi(1, p) \sqrt{c}}{\sqrt{2}}
\end{gathered}
$$

and the radial intensity factor of the temperature gradient (RIFTG) near the crack tip can be defined as

$$
\begin{aligned}
& K_{r}^{*(1)}(\theta, p)=\lim _{r \rightarrow 0} 2 \sqrt{r} T_{, r}^{*(1)}(r, \theta, p)=-\frac{\Phi(1, p) \sqrt{c}}{\sqrt{2}} \sin \left(\frac{\theta}{2}\right) \cdot(29) \\
& K_{r}^{*(2)}(\theta, p)=\lim _{r \rightarrow 0} 2 \sqrt{r} T_{, r}^{*(2)}(r, \theta, p)=-\frac{K_{1}(p)}{K_{2}(p)} \frac{\Phi(1, p) \sqrt{c}}{\sqrt{2}} \sin \left(\frac{\theta}{2}\right)
\end{aligned}
$$

By applying the inverse Laplace transform, the near cracktip temperature gradients in time domain can be obtained as

$$
T_{, y}^{(1)}(r, \theta, t)=-\frac{K_{T}^{(1)}(t)}{2 \sqrt{r}} \cos \left(\frac{\theta}{2}\right) .
$$




$$
\begin{gathered}
T_{, y}^{(2)}(r, \theta, t)=-\frac{K_{T}^{(2)}(t)}{2 \sqrt{r}} \cos \left(\frac{\theta}{2}\right) . \\
T_{, x}^{(1)}(r, \theta, t)=\frac{K_{T}^{(1)}(t)}{2 \sqrt{r}} \sin \left(\frac{\theta}{2}\right) . \\
T_{, x}^{(2)}(r, \theta, t)=\frac{K_{T}^{(2)}(t)}{2 \sqrt{r}} \sin \left(\frac{\theta}{2}\right) . \\
T_{, r}^{(1)}(r, \theta, t)=-\frac{K_{T}^{(1)}(t)}{2 \sqrt{r}} \sin \left(\frac{\theta}{2}\right) . \\
T_{, r}^{(2)}(r, \theta, t)=-\frac{K_{T}^{(2)}(t)}{2 \sqrt{r}} \sin \left(\frac{\theta}{2}\right) .
\end{gathered}
$$

where the intensity factor of temperature gradient (IFTG) in the time domain, $K_{T}^{(j)}(t)(j=1,2)$ is given as

$$
\begin{gathered}
K_{T}^{(1)}(t)=\frac{\sqrt{c}}{2 \sqrt{2} \pi i} \int_{B r} \Phi(1, p) \exp (p t) d p . \\
K_{T}^{(1)}(t)=\frac{\sqrt{c}}{2 \sqrt{2} \pi i} \int_{B r} \frac{K_{1}(p)}{K_{2}(p)} \Phi(1, p) \exp (p t) d p .
\end{gathered}
$$

and the radial intensity factor of temperature gradient (RIFTG) near the crack tip in time domain is

$$
\begin{aligned}
K_{r}^{(1)}(\theta, t) & =-\frac{\sqrt{c}}{2 \sqrt{2} \pi i} \int_{B r} \Phi(1, p) \sin \left(\frac{\theta}{2}\right) \exp (p t) d p \\
& =-K_{T}^{(1)}(t) \sin \left(\frac{\theta}{2}\right) \\
& K_{r}^{(2)}(\theta, t)=-K_{T}^{(2)}(t) \sin \left(\frac{\theta}{2}\right) .
\end{aligned}
$$

It can be seen from (33) that the dynamic temperature gradients possess a $r^{-1 / 2}$ singularity near the crack tip, which is in agreement with the corresponding static thermal crack problem $[3,11]$. The dynamic effect is merely introduced by the IFTGs, which are time-dependent as shown in $(34,35)$. It can be observed that the maximum temperature gradient appears at the angle $\theta=\mu \pi$, which corresponds to the lower and the upper crack surfaces near the crack tip; this conclusion is in agreement of the physical intuition that abrupt temperature changes occur near the crack tip. The effect of the geometric size $h_{1}$ and $h_{2}$ on the radial intensity factor of temperature gradient (RIFTG) is through the function $\Phi(1, p)$, and the geometric size does not affect the angular function of the temperature gradient.

\section{NUMERICAL RESULTS AND DISCUSSION}

The temperature field in the time domain can be obtained by applying the numerical inversion of Laplace transform, as detailed in Miller and Guy [12]. The geometric size of the composite is chosen as $h_{1} / c=1, h_{2} / c=2, L=1$, without loss of generality.

The temperature distribution in the cracked layers is shown in Figs. 2 and Figs. 3 for the cases of $k_{1} / k_{2}=0.5$ and $k_{1} / k_{2}=2$, respectively. Other related material parameters of the two layers are assumed to be $\tau_{q}^{(1)}=\tau_{q}^{(2)}=10^{-9} s$, $\tau_{T}^{(1)}=2.0 \times 10^{-9} s \quad, \quad \tau_{T}^{(2)}=0.5 \times 10^{-9} s \quad$. The normalized temperature applied on the boundaries of the layers are assumed to be $T_{1}=1, T_{2}=2$.

For the case of $k_{1} / k_{2}=0.5$, the disturbance of the thermally insulated crack on the temperature field can be observed from the iso-temperature lines in Fig. 2, and there is a temperature jump across the crack faces. The interference of the insulated crack results in the higher temperature in the inner region of the heat conduction medium than that on the boundary, this is called temperature overshooting phenomenon, as shown in Fig. 2, which corresponds to the normalized time $t=2.0$. This temperature overshooting phenomenon is of great importance in thermal engineering applications such as safety design of the electronic or mechanical devices under severe thermal loadings [13]. Fig. 3 display the temperature distribution in the cracked layers for the case of $k_{1} / k_{2}=2$, temperature overshooting phenomenon is observed and there is a temperature gap across the crack. Comparing to Fig. 2, it can be seen that the temperature intensification around the crack varies as the thermal conductivity parameters changes.

Of much interest is the temperature distribution on the crack faces as the insulated crack interrupt the temperature in the cracked composite. The temperature on the crack faces and crack face extended lines is shown in Fig. 4 for different time points. It is observed that there is temperature jump across the insulated crack on the interface of the bonded layers, while outside the crack region the temperature on the interface is continuous. As the time changes, the temperature on crack faces and the extended lines changes accordingly. It can be seen that the temperature on part of the upper face may exceed the temperature on the boundary.

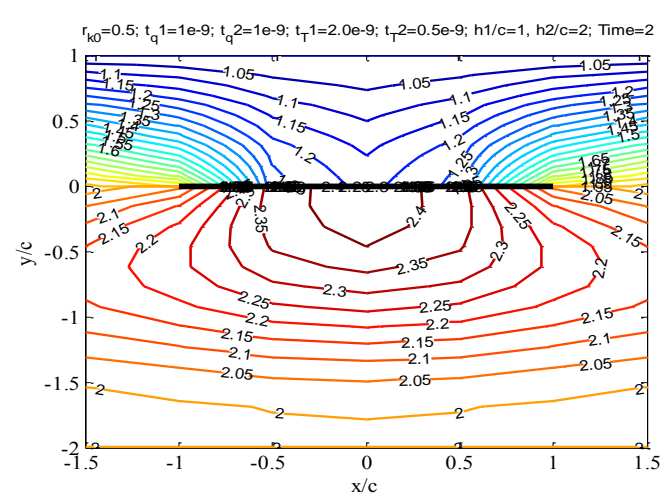

Figure 2. Temperature distribution in cracked layers when $k_{1} / k_{2}=0.5$ at $t=2.0$. 


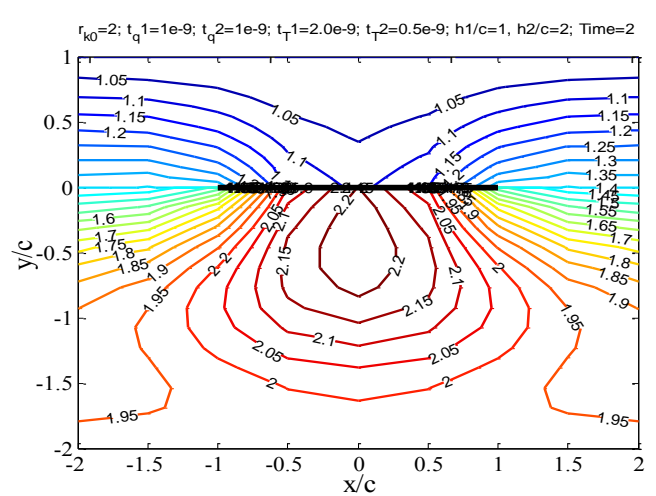

Figure 3. Temperature distribution in cracked layers when $k_{1} / k_{2}=2$ at

$$
t=2.0 \text {. }
$$

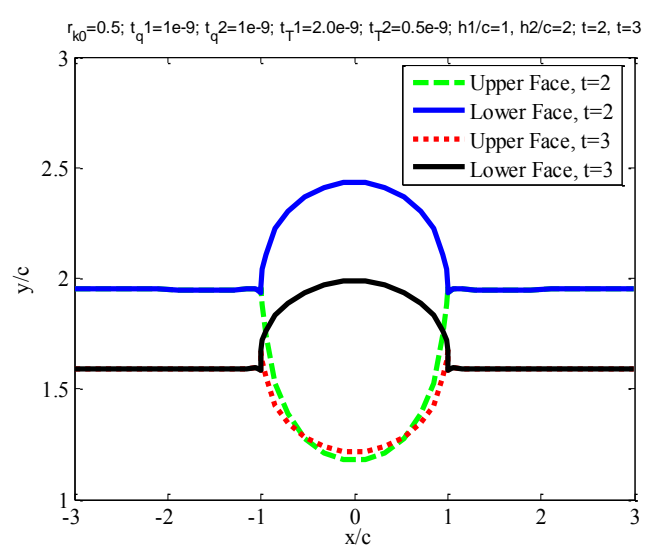

Figure 4. Temperature distribution on crack faces and extended lines when

$$
k_{1} / k_{2}=0.5 \text {. }
$$

\section{CONCLUSIONS}

The transient temperature distribution in a layered composite with an interface crack under temperature impact loading has been studied using the dual phase lag model. The crack lies parallel to the boundary and is assumed to be thermally insulated. Fourier and Laplace transforms are applied to solve the temperature field, and the mixed boundary problem is reduced to solving a singular integral equation. An asymptotic analysis and inverse Laplace transform are applied to obtain the temperature field in the time domain and the intensity factor of temperature gradient is defined. Numerical studies show that overshoot phenomenon may occur due to the combined effect of the insulated crack and application of the DPL heat conduction model. The thermal conductivity and the phase lag parameters have strong influence on the dynamic intensity factor of temperature gradients.

\section{REFERENCES}

[1] D. S. Chandrasekharaiah, "Hyperbolic Thermoelasticity: A Review of Recent Literature," Appl. Mech. Rev., vol. 51, no. 12, pp. 705729, Dec. 1998. doi: 10.1115/1.3098984.

[2] D. Y. Tzou, Macro- to Micro-scale Heat Transfer: The Lagging Behavior, Washington, DC: Taylor \& Francis, 1997.

[3] G. C. Sih, "Heat Conduction in the Infinite Medium with Lines of Discontinuities," J. Heat Transfer, vol. 87, no. 2, pp. 293-298, May. 1965. doi: 10.1115/1.3689092.

[4] C. Y. Chang and C. C. Ma, "Transient Thermal Conduction Analysis of a Rectangular Plate with Multiple Insulated Cracks by the Alternating Method," Int. J. Heat Mass Transfer, vol. 44, no. 13, pp. 2423-2437, Jul. 2001. doi: 10.1016/S0017-9310(00)00292-1.

[5] Z. T. Chen and K. Q. Hu, "Hyperbolic Heat Conduction in a Cracked Thermoelastic Half-Plane Bonded to a Coating," Int. J. Thermophys., vol. 33, no. 5, pp. 895-912, May. 2012. doi: $10.1007 / \mathrm{s} 10765-012-1190-4$.

[6] K. Hu and Z. Chen, "Thermoelastic Analysis of a Partially Insulated Crack in a Strip Under Thermal Impact Loading Using the Hyperbolic Heat Conduction Theory," Int. J. Eng. Sci., vol. 51, pp. 144-160, Feb. 2012. doi: 10.1016/j.ijengsci.2011.10.009.

[7] B. Wang and J. Han, "Fracture Mechanics Associated with NonClassical Heat Conduction in Thermoelastic Media," Sci. China Phys. Mech. Astron., vol. 55, no. 3, pp. 493-504, Mar. 2012. doi: $10.1007 / \mathrm{s} 11433-012-4637-5$.

[8] K. Q. Hu and Z. T. Chen, "Transient Heat Conduction Analysis of a Cracked Half-Plane Using Dual-Phase-Lag Theory," Int. J. Heat Mass Transfer, vol. 62, pp. 445-451, 2013. doi: 10.1016/j.ijheatmasstransfer.2013.03.032.

[9] H.S. Carslaw and J.C. Jaeger, Conduction of heat in solids, Oxford, England: Oxford Science Publications, Clarendon Press, 1990.

[10] A. C. Eringen, "Complex function technique" in Continuum Physics, vol. 2, New York: Academic Press, 1975, pp. 523-603.

[11] D. Y. Tzou, "The Singular Behavior of the Temperature Gradient in the Vicinity of a Macrocrack Tip," Int. J. Heat Mass Transfer, vol. 33, no. 12, pp. 2625-2630, Dec. 1990. doi: 10.1016/0017-9310(90)90198-4.

[12] M. K. Miller and W. T. Guy, "Numerical Inversion of the Laplace Transform by Use of Jacobi Polynomials," SIAM J. Numer. Anal., vol. 3, no. 4, pp. 624-635, Dec. 1966. doi: $10.1137 / 0703055$.

[13] M. Xu, J. Guo, L. Wang, and L. Cheng, "Thermal Wave Interferenceas the Origin of the Overshooting Phenomenon in Dual-Phase-Lagging Heat Conduction," Int. J. Therm. Sci., vol. 50, no. 5, pp. 825-830, May. 2011. doi: 10.1016/j.ijthermalsci.2010.12.006. 


\section{Scaling and Machine Learning Analysis of Turbulent Fluxes of Momentum and Heath in the Microclimate of an Urban Canyon}

\author{
Mohsen Moradi ${ }^{1}$, William D. Lubitz ${ }^{2}$, Amir A. \\ Aliabadi* \\ School of Engineering \\ University of Guelph \\ Guelph, Canada \\ ${ }^{1}$ moradim@uoguelph.ca, ${ }^{2}$ wlubitz@uoguelph.ca \\ * Corresponding Author: aliabadi@uoguelph.ca
}

\author{
E. Scott Krayenhoff \\ School of Environmental Sciences \\ University of Guelph \\ Guelph, Canada \\ skrayenh@uoguelph.ca
}

\begin{abstract}
Turbulent flow inside the urban roughness sublayer, despite its complexities, plays a crucial role in the microclimate of the built environment. The parameterization of flow in the urban roughness sublayer provides a better understanding of turbulent exchange process leading to accurate weather forecasting. This study focused on developing relationships between turbulent quantities, including momentum and heat fluxes, and mean quantities such as mean wind speeds. Field data, including wind directions, wind speeds, and thermal stability conditions, were collected from an urban canopy in Guelph, Ontario, Canada during the summer 2017. Comparative data was obtained from a nearby rural station. A systematic scaling analysis was performed to identify a range of quantities highly related to turbulent fluxes. All combinations of quantities leading to dimensionless groups were evaluated. Linear and nonlinear correlation coefficients between different groups of variables identified when mean and turbulent quantities were related. Significant improvement in correlation coefficients was observed using high order polynomial regression, revealing the challenge of developing a robust model for predicting nonlinear behavior of turbulence. This study also used artificial neural networks (ANNs) to find nonlinear relationships between turbulent and mean quantities. As used here, an ANN is a multivariable function which attempts to approach the exact value of turbulent flux based on independent variables, properly chosen from dimensionless groups. Results showed that these approaches can successfully relate most, but not all, turbulent quantities to mean quantities.
\end{abstract}

Keywords-component; microclimate, urban canopy; turbulence; artificial neural network; scaling

\section{INTRODUCTION}

Flow interactions between near-surface regions and the atmosphere in built-up areas cause spatial and temporal flow complexity leading to phenomena such as channeling flow, secondary vortices, complex three-dimensional patterns and turbulence. Within the lower level of the urban atmosphere, which is the region from the surface up to the top of the atmospheric roughness sublayer, net transport of energy and pollutants is greatly influenced by the turbulent flow. Therefore, a general understanding of turbulent exchange processes in the lower urban atmosphere can give insight into underlying flow features. Numerous studies have investigated flow characteristics in mesoscale models that characterize large spatial and temporal scales. Three-dimensional effects, which are significant up to two or more times building heights, and the methods used for approximating the atmospheric roughness sublayer, can considerably change modeled flow and dispersion processes. Another important feature of flow is the formation of shear layers at roof level, which oscillate at dominant frequencies due to instabilities. Turbulence transport into the canopy is driven by these shear layers and turbulence characteristics vary by any change in the upwind conditions. More flow complexity arises in canopies with unequal building heights. Side separation layers, jetting flow from the windward face of downstream buildings, and channeling flow in a finite street length, can all cause additional difficulties when investigating in-canyon flows in these environments [1-4]. Analytical and numerical studies focused on the UCL are not developed enough to capture all of the important features of flow in these regions. It has been suggested that empirical relationships can be developed for the variables of interest. Turbulent exchange processes are usually investigated by measuring momentum and heat fluxes. Similarity theory is used to parameterize variables and guide the design of experiments to record the most desirable information [5]. Successful use of similarity theory requires guessing relevant variables that can be determined from measureable parameters, and ensuring these are relevant to predicting the unknown variables of interest. Numerous studies have employed Monin-Obukhov similarity theory to estimate wind, temperature, and turbulence statistics profiles as functions of friction velocity and length scales [6]. Additionally, accurate and reliable measurements of meteorological quantities, which can be obtained from field measurements or approximated from wind tunnel studies, are required to relate turbulent fluxes to other known quantities in the flow such as mean velocity or temperature. There have been many attempts to parametrize and describe turbulent transport in rough but horizontally homogenous environments. However, turbulence characteristics are still not well understood in 
horizontally inhomogeneous urban areas and further investigations are required. The pioneering efforts of Roth and Oke [7], followed by many others, have shown how to parametrize turbulent fluxes in horizontally inhomogeneous built areas. Most of these studies attempted to find or modify correlations between turbulent quantities and Monin-Obukhov length over the canopy. It has been found that there can be strong nonlinear relationships between different quantities within the canyon [8].

\section{OBJECTIVE}

This study investigated the relationships among different turbulent and mean quantities within and above an urban canyon under all wind directions, wind speeds, and thermal stability conditions. Scaling analyses were completed to identify relationships between variables. The study used measured microclimate data collected around an urban canyon in Guelph, Ontario, Canada during the summer 2018. The canyon has an aspect ratio of unity and is located on the University of Guelph campus. Strong nonlinear relationships between turbulent and mean quantities were observed. These were investigated by fitting high order polynomial equations to the data set. Artificial neural networks (ANNs) were also employed to find how turbulent fluxes can be related to groups of known variables to allow investigation of non-linear relationships. The approach presented here can be extended to turbulent flow in other canopies with different morphologies and climates.

\section{OBSERVATION SITE}

Data was collected from an urban microclimate field campaign held at the University of Guelph, Guelph, Ontario, Canada from 13th to 25th of August 2017 (Fig. 1). Meteorological conditions, including fluctuating velocity, surface and atmospheric temperature values, were measured within the urban canyon and on the roof-top of the building situated at the southwest canyon wall, using RM Young 81000 three-dimensional sonic anemometers recording at 4 and $32 \mathrm{~Hz}$. Data was also collected from the Guelph Turfgrass Institute, an open-field station representative of rural conditions located 1.9 $\mathrm{km}$ northeast of the canyon site [9]. Since local wind direction can significantly affect turbulence quantities and flow patterns within and above the urban canopy, the field data were collected and analyzed under all wind directions and thermal stability conditions.

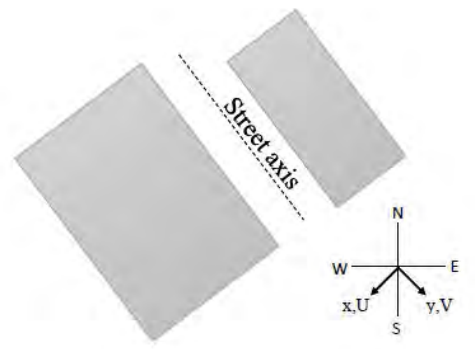

Figure 1: Top view of urban site

\section{METHODOLOGY}

Most variables will be considered to have mean and fluctuating components: $X=\bar{X}+x$ where $x$ is the turbulent fluctuating component and the overbar notation signifies a 30 minute time average of a variable. Lowercases are used to expresses variances and fluxes, e.g. $\overline{u^{2}}$ represents velocity variance in the $\mathrm{x}$ direction. Subscripts $R, S$ and $t$ denote rooflevel, street-level, and rural area measurements. ANNs, which can discover nonlinear relationships between various parameters, were employed to relate turbulent quantities

TABLE I. SCALING OF TURBULENT FLUXXS AT STREET (LEFT) AND ROOF (RIGHT) LEVELS

\begin{tabular}{|c|c|c|c|c|c|c|}
\hline \multirow{2}{*}{ 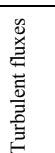 } & \multicolumn{6}{|c|}{$\begin{array}{c}\text { Urban Flow Variables } \\
\text { Correlation Coefficient }(\mathrm{Co})\end{array}$} \\
\hline & \multicolumn{2}{|c|}{ Street level } & \multicolumn{4}{|c|}{ Roof level } \\
\hline$\overline{u v}$ & $\begin{array}{c}\overline{v^{2}} s \\
\mathrm{Co}=0.90\end{array}$ & $\begin{array}{c}\overline{v w}_{S} \\
\text { Co }=0.96\end{array}$ & $\begin{array}{l}{\overline{V_{S}}}^{2},{\overline{W_{R}}}^{2}, u_{* R}{ }^{2} \\
0.2<\text { Co }<0.4 \\
w^{2}{ }_{R}, \bar{S}_{R}{ }^{2}\end{array}$ & \multicolumn{3}{|c|}{${\overline{S_{t}}}^{2}, \bar{u}_{R}, \overline{v^{2}}{ }_{R}, T K E_{R}$} \\
\hline$\overline{u w}$ & $\begin{array}{c}\bar{U}_{S}^{2}, \bar{S}_{S}^{2} \\
0.8<\text { Co }<0 .\end{array}$ & $\begin{array}{c}{\overline{W_{S}}}^{2}, u_{* S}^{2}, \overline{u^{2}} s \\
w_{S}^{2}, T K E_{S} \\
0.9<\text { Co }<1.0\end{array}$ & $\begin{array}{c}{\overline{U_{R}}}^{2},{\overline{V_{R}}}^{2},{\overline{S_{t}}}^{2} \\
{\overline{U_{S}}}^{2},{\overline{S_{S}}}^{2},{\overline{v^{2}}}_{S} \\
0.2<\text { Co }<0.4\end{array}$ & $\begin{array}{c}{\overline{V_{S}}}^{2},{\overline{v^{2}}}_{R}, T K E_{R} \\
0.4<\text { Co }<0.5\end{array}$ & $\begin{array}{l}u_{* R}^{2}, \\
0.5<\end{array}$ & $\begin{array}{l}{ }_{R}, \overline{w^{2}}{ }_{R} \\
<0.6\end{array}$ \\
\hline$\overline{v w}$ & & $\begin{array}{l}\overline{i v}_{S} \\
<1.0\end{array}$ & $\begin{array}{l}{\overline{v W_{R}}},{\overline{S_{S}}}^{2},{\overline{v^{2}}}_{S} \\
0.2<\text { Co }<0.4\end{array}$ & $\begin{array}{c}\bar{V}_{R}^{2}, \bar{W}_{R}^{2} \\
{\overline{u^{2}}}_{R},{\overline{w^{2}}}_{R} \\
0.5<\text { Co }<0.6\end{array}$ & $\begin{array}{c}{\overline{S_{R}}}^{2}, \bar{S}_{t}^{2} \\
{\overline{v^{2}}}_{R}, T K E_{R} \\
0.6<\text { Co }<0.7\end{array}$ & $\begin{array}{c}u_{* R}^{2} \\
0.7<\mathrm{Co}<0.8\end{array}$ \\
\hline$\overline{u t}$ & $\begin{array}{c}\overline{v t}_{R} \\
\text { Co }=-0.21\end{array}$ & $\begin{array}{c}\overline{w t}_{S} \\
\text { Co }=-0.99\end{array}$ & & $\mathrm{Co}=$ & & \\
\hline$\overline{v t}$ & & & & $\begin{array}{l}\overline{w t}_{R}, \bar{u} \\
0.2<\mathrm{C}\end{array}$ & $\begin{array}{l}, \overline{w t}_{S} \\
<0.4\end{array}$ & \\
\hline$\overline{w t}$ & $\begin{array}{c}\overline{v t}_{R} \\
\text { Co }=0.21\end{array}$ & $\begin{array}{c}\overline{u t}_{S} \\
\text { Co }=-0.99\end{array}$ & & $\begin{array}{r}\overline{v t}_{R} \\
0.2<\mathrm{C}\end{array}$ & $\begin{array}{l}\bar{l}_{R} \\
<0.4\end{array}$ & \\
\hline
\end{tabular}


to mean variables or those that can be measured conveniently. Properly scaled mean quantities form the input layers and turbulent fluxes are predicted at the output layer. Multilayer feedforward backpropagation network was used to find relationships between inputs and outputs while minimizing errors. The backpropagation algorithm is an iterative procedure that tries to modify weights based upon errors determined for all layers. Problems such as overfitting may arise in training a network, resulting in a poor generalization [10]. To avoid this, a simple optimization procedure was used to find a suitable network architecture containing an optimum number of hidden layers and neurons. It was speculated that in cases where the underlying relationships between variables are relatively simple, linear regression can outperform neural networks for relating turbulent and mean quantities. Therefore, it was necessary to understand the strength of linear or nonlinear correlations between different quantities before seeking complex relationships. Before creating a neural network, a systematic dimensional analysis was performed. All possible dimensionless groups containing two or three variables were built. Then, this analysis was used to distinguish between existing linear and nonlinear relationships. Moreover, high order polynomial functions were fit to the measured data to understand the severity of nonlinear relationships. This procedure provided us with a selection of quantities with known strong linear or nonlinear relationships.

\section{RESUlTS AND DisCUSSION}

\section{A. Two-variable Diemsionless Groups}

A systematic dimensional analysis identified all of the relevant two-variable dimensionless groups in table I. Those groups with linear correlation coefficients higher than 0.2 between turbulent fluxes and other quantities are shown. Some strong linear correlations were found at street level that were mostly related to other turbulent quantities such as variances along the street axis, $\overline{v^{2}}$. Significant linear correlation coefficients between different turbulent fluxes at street level, e.g. $\overline{u v}_{S}$ and $\overline{v w}_{S}$, provide an opportunity to find turbulent fluxes of interest by knowing the value of other fluxes, which may be accessible to determine. Among all momentum and heat fluxes, those with components in the vertical direction, i.e. $\overline{u w}$ and $\overline{w t}$, play important roles in the correct representation of the urban boundary layer. It is evident that there is a significant correlation between the momentum flux in the vertical direction, $\overline{u w}_{S}$, and other mean quantities including $\bar{U}_{S}{ }^{2}$ and $\bar{W}_{S}{ }^{2}$. Unlike at street level, the roof level turbulent fluxes did not exhibit high linear correlations with other variables. It is worth noting that there are still some linear relationships between vertical momentum fluxes, $\overline{v w}_{R}$ and $\overline{u w}_{R}$, and mean quantities with correlation coefficients of 0.4 to 0.6 . In comparison with flow within the canyon, wind speed and wind direction at roof-level are more likely to follow the flow patterns in the rural area. Linear correlations between rural wind speed, $\bar{S}_{t}{ }^{2}$, and momentum fluxes at roof level are shown in table I. Some other important correlations were also found, including relationships between $\overline{u v}_{R}, \overline{u w}_{R}$ and $\overline{v w}_{R}$, and squared mean velocity components within the canyon, $\bar{V}_{S}{ }^{2}$ and $\bar{U}_{S}{ }^{2}$. It is revealed that turbulent quantities at street level can be determined indirectly through mean quantities at the roof level and the rural site. Among all two-variable dimensionless groups, heat fluxes at roof-level only correlated with other heat fluxes at roof and street level. Higher order polynomial fits were also used to attempt to correlate the measured data for each two-variable dimensionless group. An example of the corresponding correlation coefficients is shown in Fig. 3. Comparing the value of correlation coefficients of linear and higher order polynomial functions, particularly groups containing $\bar{U}_{R}{ }^{2}, \bar{U}_{S}{ }^{2}$ and $\bar{W}_{S}{ }^{2}$, shows the existence of strong nonlinear relationships. For example, using a second order polynomial function to relate $\overline{u w}_{R}$ to $\bar{U}_{R}{ }^{2}$ increases the correlation coefficient by 178 percent. Fig. 4 shows how using the second order polynomial can improve the prediction of the relationship between $\overline{u w}_{R}$ and $\bar{U}_{R}{ }^{2}$. However, there was no significant change in correlation coefficients for groups containing $\bar{V}_{S}^{2}$ or $\bar{V}_{R}^{2}$. Likewise, using nonlinear functions for other vertical turbulent fluxes did not produce noticeable changes in correlation coefficient (not shown here). It can be concluded that nonlinear regression can significantly improve relationships between turbulent and some mean quantities. This highlights the importance of developing a proper nonlinear function which can describe nonlinear behavior of turbulent fluxes.

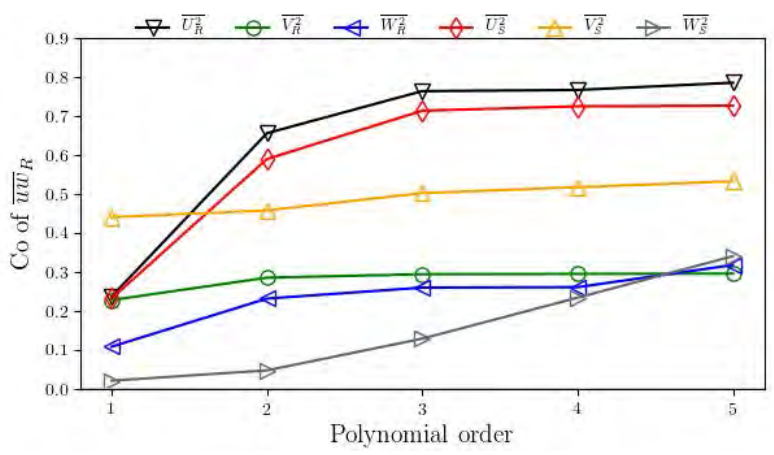

Figure 3: Correlation coefficient (Co) for different order polynomial regressions of the momentum flux

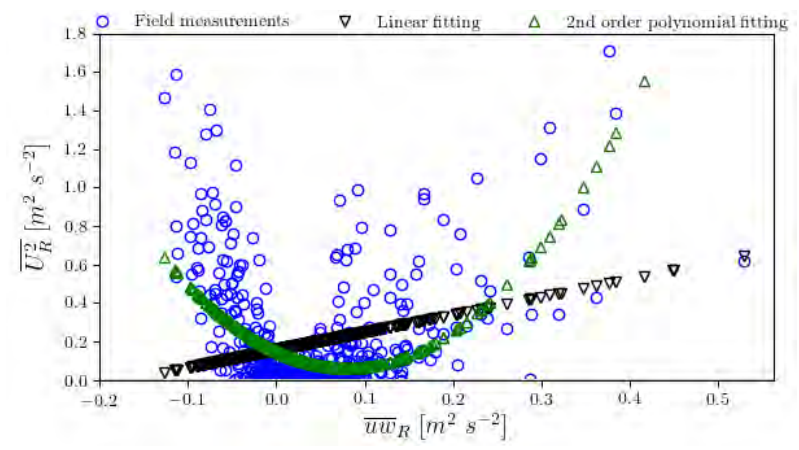

Figure 4: Comparison of linear and high order polynomial regression of momentum of flux

\section{B. Three-variable Diemsionless Group}

The same algorithm used above was also applied to threevariable dimensionless groups. Fig. 5 shows how higher order polynomial regressions can modify correlation coefficients. Vertical heat flux at roof level, $\overrightarrow{w t}_{R}$, had a strong nonlinear relationship with temperature differences between surface and adjacent atmosphere multiplied by vertical velocity (solid line). There are poor linear and nonlinear relationships between other heat flux (horizontal heat fluxes are not shown) and mean 
quantities. In this figure, $\bar{T}_{s r R}$ and $\bar{T}_{s r s}$ represents surface temperature at roof and street levels.

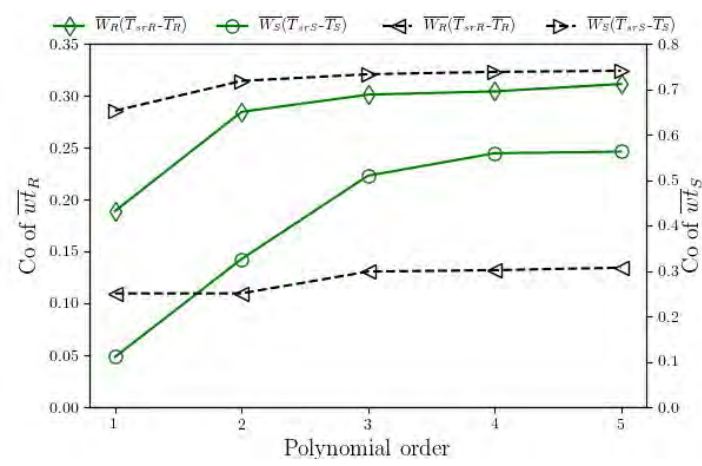

Figure 5: Correlation coefficient ( $\mathrm{Co}$ ) for different polynomial regressions of vertical heat flux; primary axis (solid lines) and secondary axis (dash lines)

\section{Developing a Multivariable Function}

The scaling studies provided insight into what mean quantities can be related to turbulent quantities. The unsteady and, to some extent, unpredictable nature of turbulent flows, particularly within a canyon, make turbulence modeling difficult and it seems unreliable to try to predict most features of turbulence using only one quantity. It has been suggested that multivariable functions can limit duplicity of solutions for a turbulence problem [11]. Thus, multivariable functions were developed using ANNs. Among all two and three-variable groups, those which exhibited high linear correlation coefficients between turbulent and mean quantities were chosen as candidates in the search for multivariable functions. The capability of an ANN to restrict and predict turbulent fluxes was evaluated by performing statistical

TABLE II. COMPARISON OF MULTIVARIABLE FUNCTIONS WITH SINGLE-VARIBLE FUNCTION OF TURBULENT FLUXES WITHIN CANYON

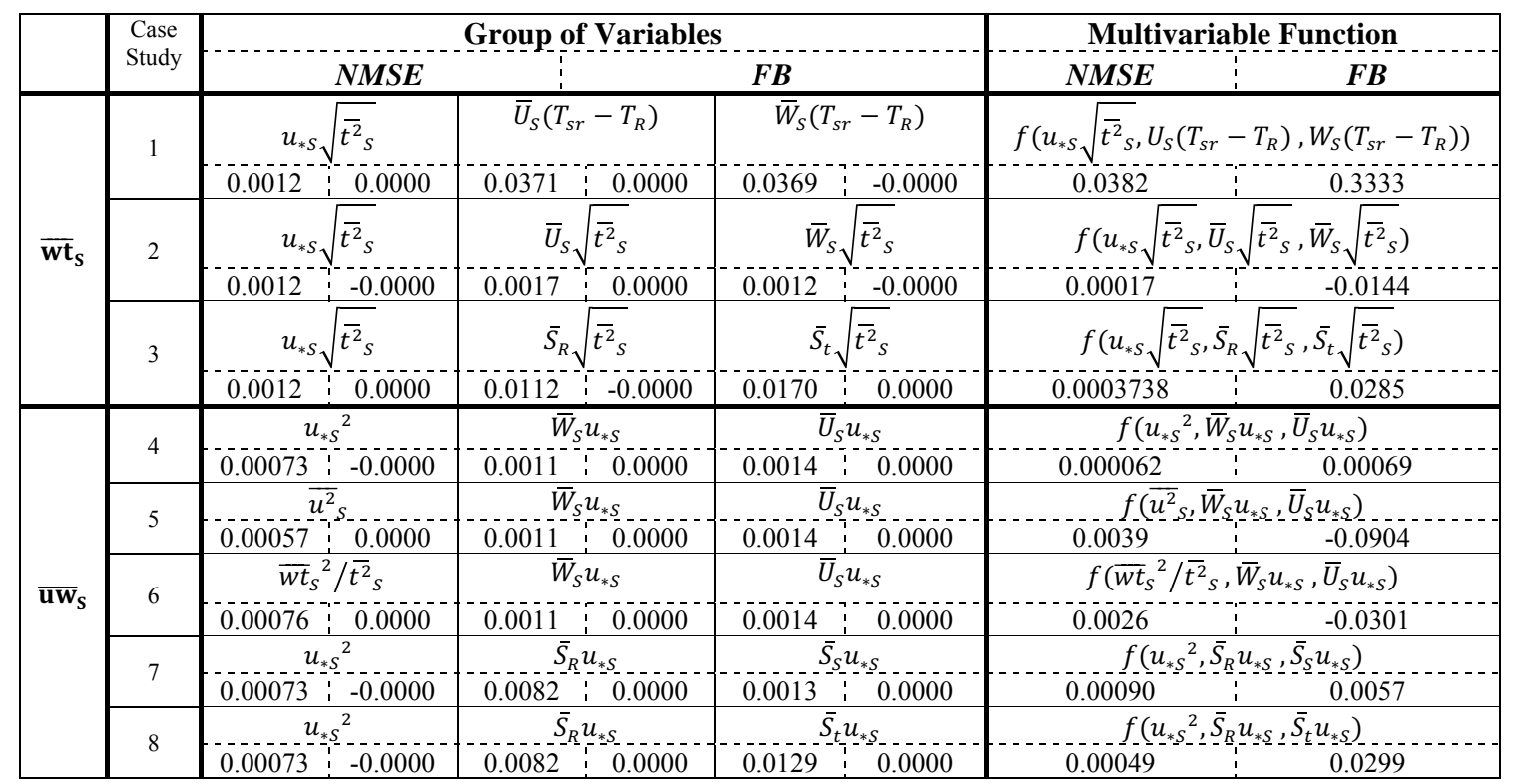

TABLE III. COMPARISON OF MULTIVARIABLE FUNCTIONS WITH SINGLE-VARIBLE FUNCTION OF TURBULENT FLUXE AT ROOF-LEVEL

\begin{tabular}{|c|c|c|c|c|c|c|}
\hline & \multirow{2}{*}{$\begin{array}{l}\text { Case } \\
\text { Study }\end{array}$} & \multicolumn{3}{|c|}{ Group of Variables } & \multicolumn{2}{|c|}{ Multivariable Function } \\
\hline & & $N M S E$ & 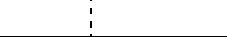 & $\boldsymbol{F B}$ & $N M S E$ & $F B$ \\
\hline \multirow{4}{*}{$\overline{\mathbf{u w}}_{\mathbf{R}}$} & \multirow[b]{2}{*}{9} & $u_{* R}^{2}$ & $\bar{U}_{R} u_{* R}$ & $\bar{U}_{S} u_{* R}$ & \multicolumn{2}{|c|}{$f\left(u_{* R}{ }^{2}, \bar{U}_{R} u_{* R}, \bar{U}_{S} u_{* R}\right)$} \\
\hline & & $\begin{array}{l:l}0.0081 & -0.0000\end{array}$ & $0.0022,0.0000$ & $0.0054: 0.0000$ & & -0.0121 \\
\hline & \multirow{2}{*}{10} & $\overline{w t}_{R}^{2} / \bar{t}^{2}$ & $\overline{u t}_{R}^{2} / \bar{t}^{2}$ & $\bar{U}_{R} u_{* R}$ & \multicolumn{2}{|c|}{$f\left(\overline{w t}_{R}^{2} / \bar{t}_{R}^{2}, \overline{u t}_{R}^{2} / \bar{t}_{R}^{2}, \bar{U}_{R} u_{* R}\right.$} \\
\hline & & $\begin{array}{l:l}0.0082 & -0.0000\end{array}$ & $\begin{array}{l:l}0.0082 & 0.0000\end{array}$ & 0.0022 & 0.0011 & 0.0400 \\
\hline
\end{tabular}

analysis. Two metrics fractional bias (FB) and normalized mean square error (NMSE) were employed [12]. NMSE represents the overall scatter between observed and predicted quantities while FB quantifies the overall under- or over-estimation of measured quantities. Tables II and III show how efficiently and precisely ANNs can predict vertical turbulent fluxes. FB and NMSE were calculated for single-variable functions obtained from linear regressions of each induvial group of variables, and multivariable functions generated by ANN containing each group as an independent variable. Friction velocity, $u_{* S}=$ $\left(\overline{u w}_{S}^{2}+\overline{v w}_{S}^{2}\right)^{1 / 4}$, played an important role in scaling mean quantities leading to proper prediction of the turbulent fluxes. In comparison with linear regressions for the same case, an ANN often reduced NMSE by one order of magnitude. An ANN with input variables of $u_{* S} \sqrt{\overline{t^{2}}}{ }_{S}, \bar{S}_{R} \sqrt{\overline{t^{2}}}, \bar{S}_{t} \sqrt{\overline{t^{2}}}{ }_{S}$ showed 68 to 98 percent improvement in prediction of vertical heat flux at street level, $\overline{w t}_{s}$ compared to linear correlations. Employing ANNs also provided an opportunity to directly relate turbulent fluxes to horizontal rural velocity, $S_{t}$, with an acceptable accuracy. 
Notable improvements also occurred in ANN predictions of $\overline{u w}_{S}$ and $\overline{u w}_{R}$ with $f\left(u_{* S}{ }^{2}, \bar{W}_{S} u_{* S}, \bar{U}_{S} u_{* S}\right)$ and $f\left(u_{* R}{ }^{2}, \bar{U}_{R} u_{* R}, \bar{U}_{S} u_{* R}\right)$ as multivariable functions, respectively. On the other hand, FB deteriorated when an ANN was used to predict fluxes, but the results were still considered acceptable, meeting the range of acceptance criteria [13]. Figs. 6 and 7 compare predictions of hourly time series of turbulent fluxes using ANN and field measurements. These figures are based on case studies 3 and 9 , as referred to in Tables II and III, which represented significant improvement in prediction of relevant turbulent fluxes. Field data were collected at all wind speeds and directions, so no clear trend was expected for hourly flux time series. The level of agreement between observations and the ANN predictions is remarkable. This suggests that a well-trained ANN, such as built herein, can ingest, the inherently chaotic flow behavior within and above the canopy. However, ANNs failed to predict other turbulent fluxes, such as $\overline{u v}_{S}$ and $\overline{w t}_{R}$, using mean quantities. Additionally, as depicted in Tables II and III, linear regressions in cases 1 and 5 outperformed nonlinear multivariable functions.

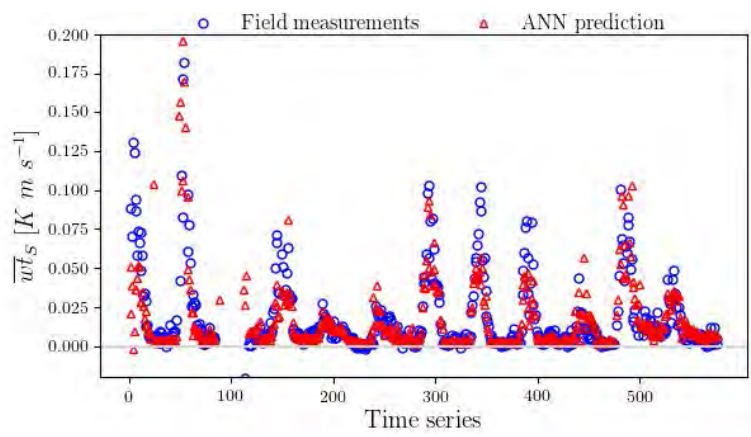

Figure 6: Comaprison of ANN results with measurement data of vertical heat flux within the canyon

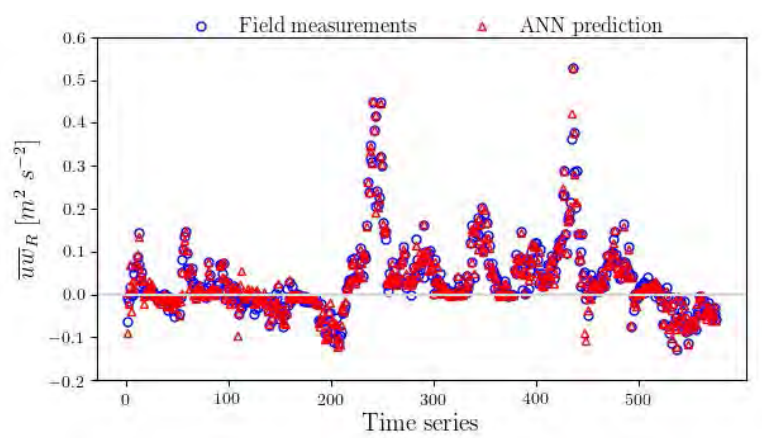

Figure 7: Comaprison of ANN results with measurement data of vertical momentum flux at roof-level

\section{CONCLUSIONS}

Systematic scaling analysis was performed to find relationships between turbulent and mean quantities within and above the urban canopy. Field data was collected under all wind directions, wind speeds, and thermal stability conditions from a street canyon within an urban canopy, and also a rural area, in Guelph, Ontario, Canada. Several approaches were used to establish relationships between turbulent and mean quantities. The significance of nonlinear behavior and relationships between quantities was evaluated by creating dimensionless groups of variables containing two or three quantities. High linear correlations for vertical momentum flux at both roof and street levels were found with mean wind speeds. This linear behavior is much more profound at street-level. Applying higher order polynomial functions to the field data revealed some strong nonlinear relationships. It was observed that using nonlinear functions can improve correlation coefficients significantly, for example, enhancing correlation coefficient by 178 percent for the relationship between $\overline{u w}_{R}$ and $\bar{U}_{R}{ }^{2}$. Similar results were found for the vertical heat flux. Additionally, it was found that artificial neural networks (ANNs) can be generated to predict such strong nonlinear relationships. The predictive capability of ANNs was evaluated for the current dataset by comparing NMSE and FB calculated by ANN and linear regressions. It was found that a well-trained ANN accurately predicted vertical momentum and heat fluxes within and above the canyon. However, in some cases linear regression outperformed ANN predictions. The different approaches are complementary to one another, and can be used in combination to further develop robust optimization algorithms for predicting turbulence quantities from mean meteorological values.

\section{ACKNOWLEDGEMENTS}

The lead author acknowledges the financial support of the NSERC Discovery Grant program. All authors thank the following individuals who helped with the urban microclimate field campaign: Denis Clement, Steve Nyman, Chris Duiker, Manuela Racki, Jeffrey Dafoe, Joanne Ryks, Ryan Smith, Stephen Stajkowski, and Baharam Gharabaghi.

\section{REFERENCES}

[1][1] A. Martilli, J. L. Santiago, and F. Salamanca, "On the representation of urban heterogeneities in mesoscale models," Environ. Fluid Mech., vol. 15, no. 2, pp. 305-328, Nov. 2013. doi: 10.1007/s10652-013-9321-4.

[2] R. E. Britter and S. R. Hanna, "Flow and dispersion in urban areas," Аnпи. Rev. Fluid Mech., vol. 35, no. 1, pp. 469-496, Jan. 2003.-496. doi: 10.1146/annurev.fluid.35.101101.161147.

[3] S. E. Belcher, "Mixing and transport in urban areas," Philosophical Transactions of the Royal Society A: Mathematical, Physical and Engineering Sciences, vol. 363, no. 1837, pp. 2947-2968, Dec. 2005. doi: $10.1098 /$ rsta.2005.1673.

[4] D. Zajic, H. J. S. Fernando, R. Calhoun, M. Princevac, M. J. Brown, and E. R. Pardyjak, "Flow and Turbulence in an Urban Canyon," J. Applied Meteorology and Climatology, vol. 50, no. 1, pp. 203-223, Jan. 2011. doi: 10.1175/2010JAMC2525.1.

[5] R. B. Stull, Ed., An Introduction to Boundary Layer Meteorology, Springer Netherlands, 1988. doi: 10.1007/978-94-009-3027-8.

[6] A. Venkatram and M. Princevac, "Using measurements in urban areas to estimate turbulent velocities for modeling dispersion," Atmos. Environ., vol. 42, no. 16, pp. 3833-3841, May 2008. doi: 10.1016/j.atmosenv.2007.12.061.

[7] M. Roth and T. Oke, "Turbulent transfert: relationships over an urban surface. I: spectral characteristics," Quart. J. Roy. Meteorol. Soc., vol. 119, 1071-1104, 1993

[8] L. Quan and F. Hu, "Relationship between turbulent flux and variance in the urban canopy," Meteorology and Atmospheric Physics, vol. 104, no. 1-2, pp. 29-36, Nov. 2008. doi: 10.1007/s00703-008-0012-5. 
[9] A. Aliabadi, M. Moradi, D. Clement, W. D. Lubitz and B. Gharabaghi, "Flow and temperature dynamics in an urban canyon under all wind directions, wind speeds, and thermal stability conditions," Environ. Fluid Mech., (submitted).

[10] G. Dreyfus, Neural networks: methodology and applications, Springer, Berlin, Heidelberg, 2005

[11] S. J. Linz and J. C. Sprott, "Elementary chaotic flow," Phys. Let. A, vol. 259, no. 3-4, pp. 240-245, Aug. 1999. doi: 10.1016/S0375-9601(99)00450-8

[12] A. A. Aliabadi et al., "Effects of Roof-Edge Roughness on Air Temperature and Pollutant Concentration in Urban Canyons," Bound-Layer Meteorol., vol. 164, no. 2, pp. 249-279, Apr. 2017.

doi: 10.1007/s10546-017-0246-1.

[13] J. C. Chang and S. R. Hanna, "Technical descriptions and users guide for the boot statistical model evaluation software package," version 2.0, 2005. 


\section{Mechanical Analysis of Multi-Directional Functionally Graded Cellular Plates}

\author{
H. Niknam \\ Department of Bioresource Engineering \\ McGill University \\ Montreal, Canada \\ D. Therriault \\ Department of Mechanical Engineering \\ Polytechnique Montreal \\ Montreal, Canada
}

\author{
A. H. Akbarzadeh \\ Department of Bioresource Engineering \& \\ Department of Mechanical Engineering \\ McGill University \\ Montreal, Canada \\ D. Rodrigue \\ Department of Chemical Engineering \\ Université Laval \\ Quebec, Canada
}

\begin{abstract}
In this study, the concept of multi-directional functionally graded cellular material (FGCM) is introduced. FGCMs consist of two spatially-varying engineered phases: solid and void. Different parameters, such as relative density, cell topology, cell orientation, and cell elongation, can be tailored in multiple directions to optimize their mechanical performance. We implement a homogenization technique to evaluate the structural response of plates made by advanced cellular solids. The homogenized effective properties are used in a third-order shear deformation theory (TSDT) formulation. The governing differential equations are solved by a finite element method to predict the mechanical response of FGCM plates. The numerical results reveal that it is possible to increase the buckling load as much as $115 \%$ and decrease the maximum deflection about $60 \%$ by using an FGC structure.
\end{abstract}

Keywords - Architected advanced materials, Functionally graded cellular materials, Homogenization, FDM 3D prining

\section{INTRODUCTION}

Over the last 50 years, several investigations revealed that cellular materials may replace fully dense solids in many different applications [1,2]. Opposed to conventional materials which gain their properties merely from their material composition, cellular solids gain their properties mainly from their underlying architectures [3]. Several investigations have been performed to study the mechanical behavior of cellular materials. The majority of these early investigations on the mechanical properties of advanced porous materials was summarized by Gibson and Ashby in their textbook on "Cellular Solids, Structures and Properties" [1]. Furthermore, application of cellular materials as mechanical elements was first introduced in the design of structural sandwich panels in 1969 when periodic honeycomb sheets were used as the core of sandwich panels [4]. These studies and many others [5-9], shed some light on the properties and possible applications of cellular materials.
The characteristic properties of the representative cell (relative density and void (pore) topology), were uniform across the lightweight structure in all the initial studies mentioned above. However, recent technical developments in advanced manufacturing techniques, like additive manufacturing [10] and powder metallurgy [11], lead to new opportunities to design and manufacture architected cellular structures, in which the geometrical features and material composition of their constituent unit cells can vary in a specified direction. The cellular structures in which the cell' relative density and topology vary across the lightweight structures with a predefined distribution function are called "Functionally Graded Cellular (FGC)" structures. This idea is inspired by natural and biological materials such as bamboo, plants and Humboldt beak.

A few studies are available in the literature implementing the idea of graded cellular properties in the structural mechanical design. One of the earliest research in the field of FGC structures was performed to apply the idea of graded honeycomb structures to obtain a Poisson-curving structure; i.e. a structure which experiences a significant change in its thickness as a result of a prescribed curvature [12]. Afterwards, a finite element based micromechanical model was proposed to predict the fracture toughness of FGC foams [13]. Moreover, FGC structures were shown to be suitable for practical applications such as orthopedic hip implants [14] and cores of aero-engine fan blade [15].

In the present article, we perform a comprehensive study on the architected multi-directional FGCMs with a focus on architected cellular plates. The cell topology is modelled by a superellipse function [16], and the effective properties are obtained by using standard mechanics homogenization [17]. A generalized power-law function is proposed to model the variation of cell characteristics in multiple directions. The governing equations for the structural analysis of FGC plates are obtained by using Reddy's third-order shear deformation theory [18], and solved by a finite element model. The advantages of FGC structures over regular cellular structures are illustrated 
through various examples and the effect of different distribution functions are investigated.

\section{Modelling of FunCtionally GRAded CELLULAR Plates}

\section{A. Formulation of Cell Geometry}

In this work, an FGC plate with length $L_{t o t}$, width $W_{t o t}$, and thickness $T_{\text {tot }}$ is considered in the $x y-$ plane as shown in Fig. 1. The plate consists of $N_{x}$ cells in $x$-direction, $N_{y}$ cells in $y$-direction, and $N_{z}$ cells in $z$-direction, where $(x, y, z)$ refer to the global Cartesian coordinate system also shown in Fig. 1.

The properties of cellular materials depend on their relative density and void topology. Here, a focus is made on extruded two-dimensional square cells in which the void topology of each cell is introduced according to the following superellipse function [16]:

$$
\left[\begin{array}{l}
\tilde{x} \\
\tilde{y}
\end{array}\right]=\left[\begin{array}{l}
r_{x} \cos ^{n} \emptyset \\
r_{y} \sin ^{n} \emptyset
\end{array}\right] R(\theta)
$$

where $0 \leq \emptyset \leq 2 \pi, \tilde{x}$ and $\tilde{y}$ are the local coordinate system whose origin is at the center of the unit cell. Three parameters $\left(r_{x}, r_{y}, n\right)$ have been introduced to control and optimize the void size and topology. Equation (1) simplifies to a circular cell with radius $r_{0}$ for the case of $n=1$ and $r_{x}=r_{y}=r_{0}$. Moreover, $R(\theta)=\left[\begin{array}{cc}\cos \theta & -\sin \theta \\ \sin \theta & \cos \theta\end{array}\right]$ is the rotation matrix enabling to rotate the void topology within each cell. To allow a systematic study for the effect of void topology and void orientation on the mechanical responses of FGC structures, four independent parameters are considered: shape parameter $(n)$, aspect ratio $\left(A . R .=\frac{r_{y}}{r_{x}}\right)$, relative density $\left(\rho_{\text {rel }}\right)$, and orientation angle $(\theta)$. In this study, these four parameters are assumed to vary in the range of $[0.01,3],[1,3],[0.01,1]$ and $[0, \pi]$, respectively. Fig. 2 presents the effect of $n$ and $A$. $R$. on the void topology of periodic cellular materials. It should be mentioned that cell topologies for which the void geometry intersect with cell boundaries has been marked as "Geometrically Inadmissible" in Fig. 2.

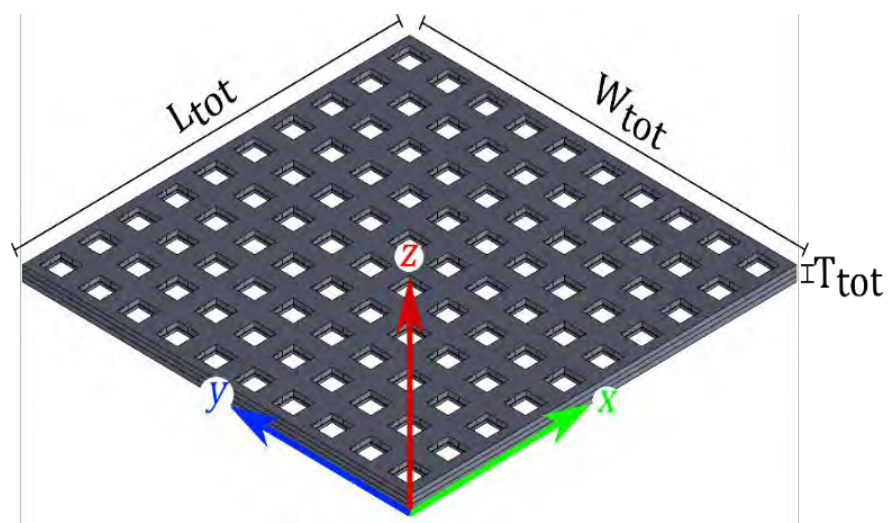

Fig. 1- Homogenous cellular plate

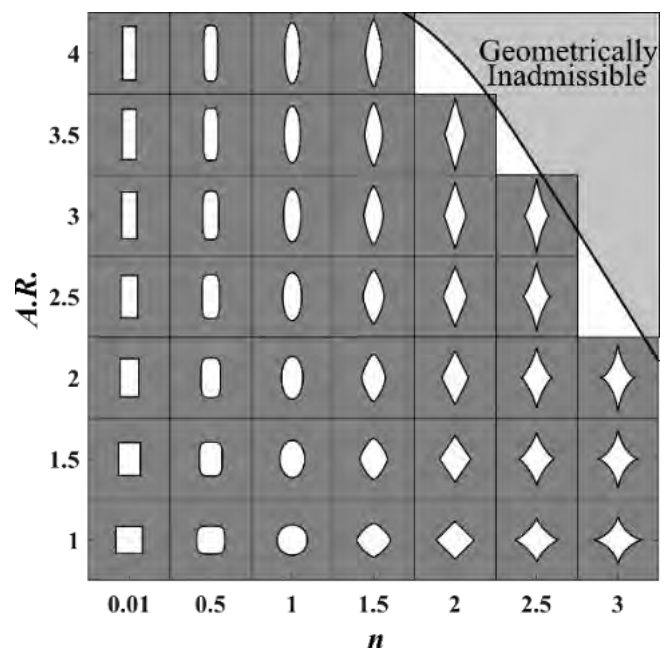

Fig. 2- Achievable cell topologies by a $2 \mathrm{D}$ superellipse function as a function of the shape parameter $(n)$ for $\boldsymbol{\rho}_{\text {rel }}=\mathbf{0 . 9}$ and different aspect ratios (A.R.)

\section{B. Formulation of Cell Topology Variation in FGCMs}

A general power-law function is introduced here to represent any property distribution throughout the length, width and thickness of the material as:

$P(\bar{x}, \bar{y}, \bar{z})=P_{1}+\left(P_{0}-P_{1}\right)\left(1-\frac{\bar{x}}{\bar{x}_{0 P}}\right)^{m x_{P}}\left(1-\frac{\bar{y}}{\bar{y}_{0 P}}\right)^{m y_{P}}\left(1-\frac{\bar{z}}{\bar{z}_{0 P}}\right)^{m z_{P}}$

where $P$ represents any of the four void topology parameters ( $n$, $A . R ., \rho_{\text {rel }}$, and $\left.\theta\right), P_{0}$ is its value at the origin $(x=0, y=0$, $z=0)$ and $P_{1}$ is its extreme value at $x=\bar{x}_{0 P}, y=\bar{y}_{0 P}$ and $z=$ $\bar{z}_{0 P}$. In all cases, an overbar indicates dimensionless coordinates defined as:

$$
\bar{x}=\frac{x}{L_{t o t}}, \bar{y}=\frac{y}{W_{t o t}}, \bar{z}=\frac{z}{T_{t o t}}
$$

Moreover, $m x_{P}, m y_{P}$ and $m z_{P}$, introduced in Eq. (3), are even integers indicating the distribution profile function. If $m x_{P}$, $m y_{P}$ or $m z_{P}$ equals zero, the property in the $x, y$ or $z$ direction results in a constant value, respectively; i.e. a homogenous distribution of cellular materials in the respective direction. If $P_{0}=P_{1}$, the property has a constant value $P_{0}$ all over the domain.

\section{Formulation of Functionally Graded Cellular Plates}

In this study, the effective elastic properties of all the cells modelled by the superellipse formulation are obtained by standard mechanics homogenization [17]. The material property chart, or so-called Ashby chart [1], is an effective presentation giving an overview of solid materials properties lying in a certain characteristic range. As a result, Fig. 3 presents the Ashby chart for well-known solid materials along with 2D extruder cellular materials with superellipse topologies. Fig. 3 shows that the range of Young's modulus for cellular materials can be extended to regions which cannot be achieved by fully dense solid materials.

To develop a methodology which can be implemented for thin and relativity-thick cellular plates, Reddy's third-order shear deformation theory (TSDT) [18] was used. According to TSDT, the transverse shear stresses are presented as a quadratic 
function through the plate thickness. Therefore, unlike the firstorder shear deformation theory (FSDT), it is not necessary to introduce any shear correction factor in the formulation.

Based on the abovementioned assumptions and by applying Hamilton's approach [18], the governing equations for the present problem, in which the cell topology and subsequently elastic properties are varying in multiple directions, will be obtained as:

$$
\begin{aligned}
& N_{x x, x}+N_{x y, y}=I_{0} \ddot{u}_{0}+J_{1} \ddot{\varphi}_{x}-c_{1} I_{3} \ddot{w}_{0, x} \\
& N_{y y, y}+N_{x y, x}=I_{0} \ddot{v}_{0}+J_{1} \ddot{\varphi}_{y}-c_{1} I_{3} \ddot{w}_{0, y} \\
& c_{1} P_{x x, x x}+2 c_{1} P_{x y, x y}+c_{1} P_{y y, y y}+Q_{x, x}+Q_{y, y}- \\
& 3 c_{1}\left(R_{x, x}+R_{y, y}\right)+q=c_{1}\left(I_{3} \ddot{u}_{0}+J_{4} \ddot{\varphi}_{x}-\right. \\
& \left.c_{1} I_{6} \ddot{w}_{0, x}\right)_{, x}+c_{1}\left(I_{3} \ddot{v}_{0}+J_{4} \ddot{\varphi}_{y}-c_{1} I_{6} \ddot{w}_{0, y}\right)_{, y}+I_{0} \ddot{w}_{0} \\
& M_{x x, x}+M_{x y, y}-c_{1}\left(P_{x x, x}+P_{x y, y}\right)-Q_{x}+3 c_{1} R_{x}= \\
& J_{1} \ddot{u}_{0}+J_{2} \ddot{\varphi}_{x}-c_{1} J_{4}\left(\ddot{\varphi}_{x}+\ddot{w}_{0, x}\right) \\
& M_{y y, y}+M_{x y, x}-c_{1}\left(P_{y y, y}+P_{x y, x}\right)-Q_{y}+3 c_{1} R_{y}= \\
& J_{1} \dot{v}_{0}+J_{2} \ddot{\varphi}_{y}-c_{1} J_{4}\left(\ddot{\varphi}_{y}+\ddot{w}_{0, y}\right)
\end{aligned}
$$

where comma represents partial differentiation with respect to $x$ or $y$, dots denotes differentiation with respect to time and $u_{0}$, $v_{0}, w_{0}, \varphi_{x}$ and $\varphi_{y}$ are the displacements and rotations of the transverse normal on the plane $z=0$. Moreover, $c_{1}=\frac{4}{3 T_{\text {tot }}^{2}}$ and $N_{\alpha \beta}, M_{\alpha \beta}, P_{\alpha \beta}, Q_{\alpha \beta}$ and $R_{\alpha \beta}$ are stress resultants, $I_{i}$ and $J_{i}$ are moments of inertia and $q$ is the transverse mechanical load.

Equations 4-8 are discretized and solved using rectangular conforming element combined with Lagrangian and Hermitian interpolation functions as presented in reference [18].

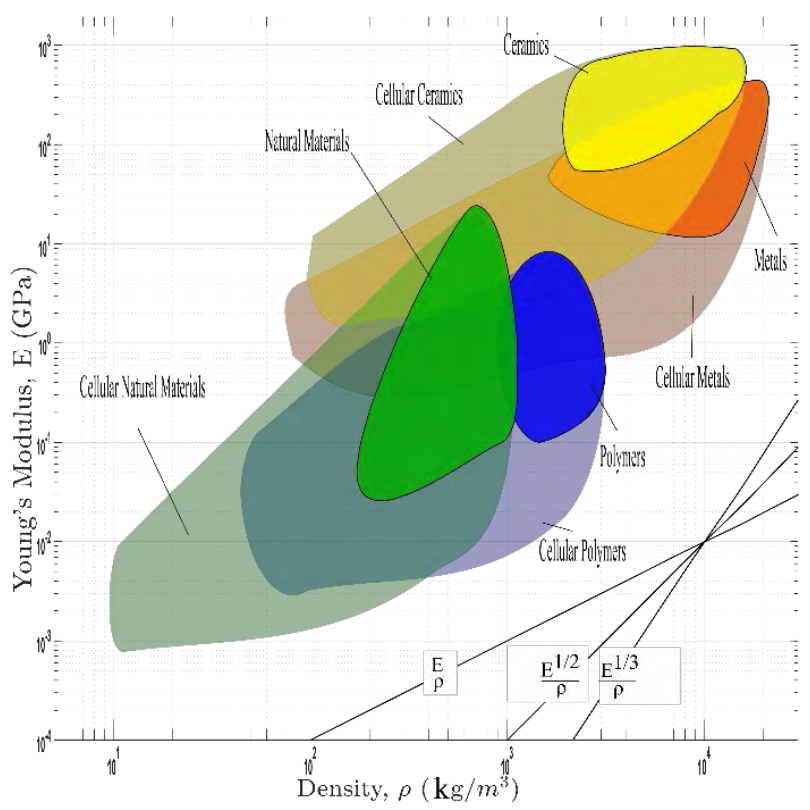

Fig. 3- Ashby chart for cellular materials of superellipse cell topology

\section{RESULTS AND DISCUSSION}

In this section, the numerical results are presented for homogenous and functionally graded cellular materials. The non-dimensional parameters used to represent the mechanical responses of FGC plates are defined as:

$$
\begin{aligned}
& \bar{W}_{\text {max }}=100 W_{\text {max }} \frac{E_{s} W_{\text {tot }}^{3}}{F L_{\text {tot }}^{2}} \\
& \bar{\Omega}=\Omega \frac{L_{\text {tot }}^{2}}{W_{\text {tot }}} \sqrt{\frac{\rho_{s}}{E_{S}}} \\
& \bar{N}_{c r}^{x x}=\frac{10^{4} \widehat{N}_{x x} \lambda L_{\text {tot }}^{2}}{W_{\text {tot }}^{3}}
\end{aligned}
$$

where $F$ is the resultant of the uniform transverse load $(F=$ $\left.q A_{\text {solid }}\right)$, while $W_{\text {max }}, \Omega$ and $\widehat{N}_{x x}$ are the maximum deflection of the plate, natural frequency and in-plane compressive load, respectively. Moreover, the size of each constituent unit cell is considered to be the same. Therefore, the total relative density of the cellular plate can be written as:

$$
\rho_{\text {rel,plate }}=\frac{\sum_{i=1}^{N} \rho_{\text {rel }, i}}{N}
$$

where $\rho_{\text {rel,i }}$ is the relative density of $i^{\text {th }}$ cell and $N$ is the total number of cells in a cellular plate.

\section{A. Structural Response of Homogenouos Cellular Plates}

An optimized structural design requires lightweight but stiff structural elements with minimum deflection, maximum mechanical buckling load, and maximum fundamental frequency. The variation of maximum bending deflection as a function of relative density is shown in Fig. 4. The highest stiffness among all the topologies studied in this article is associated with square void. Consequently, it is observed that the maximum deflection of a plate made of square shape voids is the lowest.

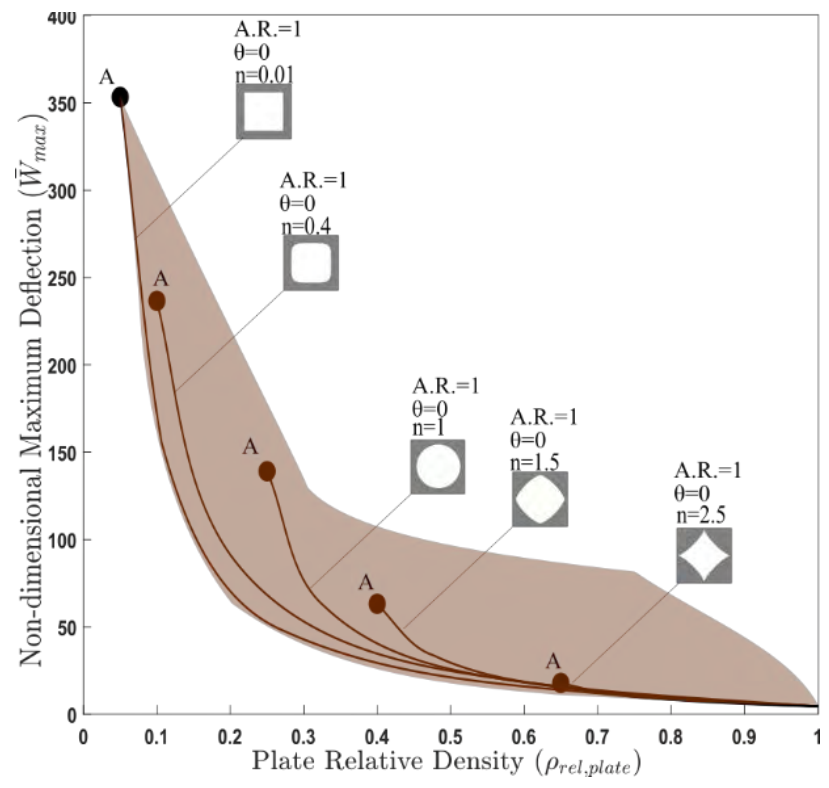

Fig. 4- Non-dimensional maximum deflection of a SSSS cellular plate with $\frac{L_{t o t}}{T_{t o t}}=\mathbf{1 0}$ made of $\mathbf{1 0} \times \mathbf{1 0}$ cells of super ellipsoidal void 


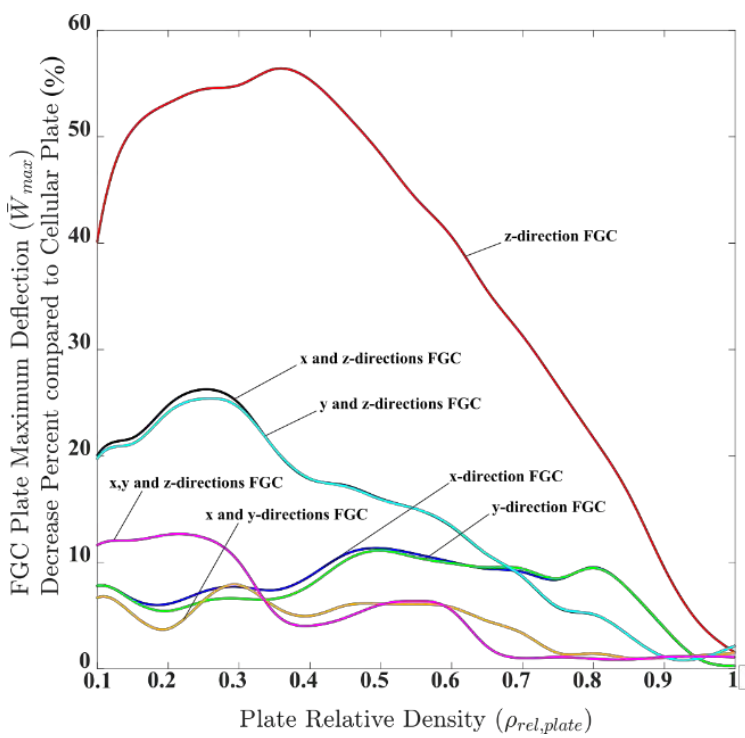

Fig. 5- Decrease of the maximum deflection of alternative multi-directional FGC square plates made by square void shape as a function of the relative density for SSSS plate with $\frac{L_{t o t}}{T_{\text {tot }}}=\mathbf{1 0}$

\section{B. Structureal Response of Multi-Directional FGC Plates}

The effect of density variation in different directions on the stiffening of FGC plates subjected to a uniformly distributed load is presented in Fig. 5. It can be observed that density gradient in the z-direction has the most dominant effect on the maximum deflection variation, while directional density variation in the $x$ - and $z$-directions can decrease the maximum deflection by $26 \%$, the maximum improvement attainable by one directional density variation in the $z$-direction can be up to $56 \%$ for the maximum deflection of architected FGC plates. It is important to note that our conclusion here is only limited to the proposed variation formula defined in Eq. (2) for multidirectional graded cellular materials and for SSSS plates.

To gain insight into the most effective density gradient, the percentage of increase in the critical buckling load is plotted in Fig. 6 as a function of $\left(\rho_{\text {rel } 1}-\rho_{\text {rel0 }}\right)$, where $\rho_{\text {rel } 1}$ is the relative density at $\bar{x}_{0 \rho}=\bar{y}_{0 \rho}=\bar{z}_{0 \rho}=0.5$ and $\rho_{\text {relo }}$ represents the relative density at the origin. Figure 9 shows that for a density gradient in the $z$-direction, the maximum improvement occurs when $\left(\rho_{\text {rel } 1}-\rho_{\text {rel0 }}\right)<0$; i.e. when the center of the FGC plate is more porous than the origin at the FGC plate edges. When the density varies in the $x$-direction or $y$-direction, the conclusion is reversed. Fig. 6 implies that a $z$-directional FGC plate can have a maximum buckling load when the density at the center of the thickness (mid-plane) is higher at the top and bottom of the plate. For the $x$-directional and $y$-directional FGC plates, higher relative densities at the sides than in the center is more desirable for increasing the critical buckling load. It is also concluded that for all one-directional FGC plates, a higher difference between the side and center density $\left|\rho_{\text {rel1 }}-\rho_{\text {relo }}\right|$, usually leads to a higher critical buckling load increase. It must be noted that the effect of local buckling is not taken into account in the buckling results.

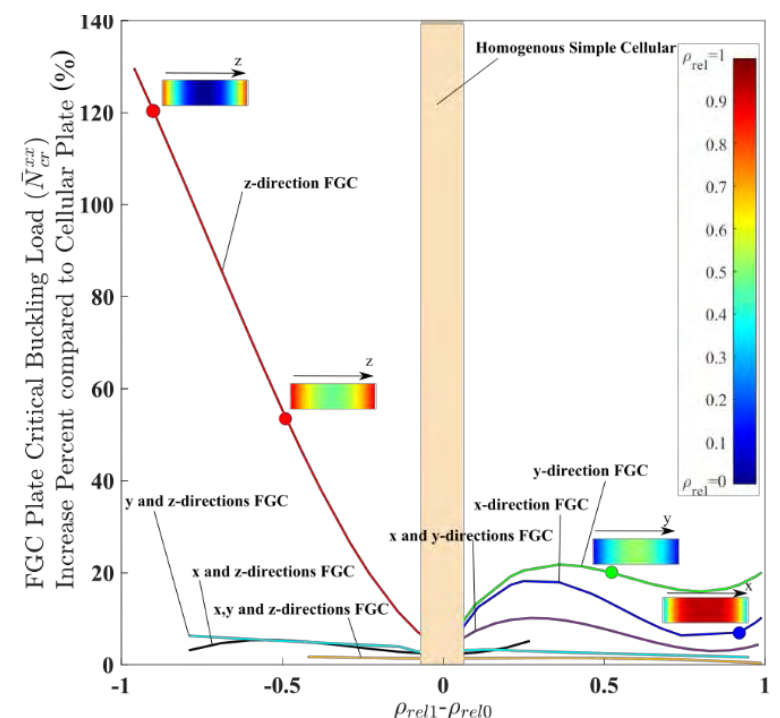

Fig. 6- Effect of the difference between the extreme values of relative density gradient $\left(\boldsymbol{\rho}_{\text {rel1 } 1}-\boldsymbol{\rho}_{\text {rel2 }}\right)$ on the critical buckling load ( $x$-direction) achievable by FGC design for SSSS plate with $\frac{L_{t o t}}{T_{\text {tot }}}=\mathbf{1 0}$

\section{CONCLUSION}

In this work, the concept of multi-directional functionally graded cellular materials was used to produce architected advanced materials. In particular, the mechanical and structural performance of FGCMs were investigated to be used as constitutive elements for lightweight plates. The results showed their important advantages compared to homogenous cellular materials. The superellipse formula was implemented to model the topology of the architected cells and a general power-law function was introduced to control the cell distribution attributes in three different orthogonal directions. The effective cells properties were predicted by standard mechanics homogenization and the functionally graded cellular plate was modeled based on TSDT. The governing equations were solved by using a finite element method. The results clearly showed that architected cellular materials, in the form of superellipsoidal voids, can significantly expand the range of elastic and structural properties leading to lightweight but stiff solutions for advanced materials and structures. Moreover, it was shown that tailoring the topological features of cellular materials through the graded cellular plates thickness more significantly affected their structural response than changing the corresponding features in planar directions.

\section{ACKNOWLEGEMENT}

A.H. Akbarzadeh acknowledges the financial support by McGill University and Natural Sciences and Engineering Research Council of Canada (NSERC) through NSERC Discovery Grant RGPIN-2016-0471. The authors also acknowledge financial support by the Research Center for High Performance Polymer and Composite Systems (CREPEC). 


\section{REFERENCES}

[1] L. J. Gibson and M. F. Ashby, Cellular Solids, Cambridge University Press, 1997. doi: 10.1017/CBO9781139878326.

[2] Robert, F., "An isotropic three-dimensional structure with Poisson's ratio $=$ ?1," J. Elasticity, vol. 15, no. 4, pp. 427-430, 1985. doi: 10.1007/BF00042531.

[3] A. Akbarzadeh, S. Rankohi, "Multifunctional and multiphysics materials as load bearing structural components," in Resilient Infrastructure, London, 2016.

[4] H. G. Allen, "Analysis of sandwich beams and struts by strain energy methods," in Analysis and Design of Structural Sandwich Panels: Chapter 4, Pergamon, pp. 57-75, 1969. doi: 10.1016/B978-0-08-012870-2.50008-0.

[5] M. Avalle, G. Belingardi, and R. Montanini, "Characterization of polymeric structural foams under compressive impact loading by means of energy-absorption diagram," Inter. J. Impact Eng., vol. 25, no. 5, pp. 455-472, May 2001. doi: 10.1016/S0734-743X(00)00060-9.

[6] A. H. Akbarzadeh, J. W. Fu, Z. T. Chen, and L. F. Qian, "Dynamic eigenstrain behavior of magnetoelastic functionally graded cellular cylinders," Compos. Struct., vol. 116, pp. 404-413, Sep. 2014. doi: 10.1016/j.compstruct.2014.05.036.

[7] A. Vigliotti and D. Pasini, "Analysis and design of lattice materials for large cord and curvature variations in skin panels of morphing wings," Smart Mater. Struct., vol. 24, no. 3, p. 37006, Feb. 2015. doi: 10.1088/0964-1726/24/3/037006.

[8] J. T. B. Overvelde, S. Shan, and K. Bertoldi, "Compaction Through Buckling in 2D Periodic, Soft and Porous Structures: Effect of Pore Shape," Adv. Mater., vol. 24, no. 17, pp. 2337-2342, Mar. 2012. doi: 10.1002/adma.201104395.

[9] S. Janbaz, H. Weinans, and A. A. Zadpoor, "Geometry-based control of instability patterns in cellular soft matter," RSC Advances, vol. 6, no. 24, pp. 20431-20436, 2016. doi: 10.1039/C6RA00295A.

[10] D. W. Rosen, "Computer-Aided Design for Additive Manufacturing of Cellular Structures," Computer-Aided Design and Applications, vol. 4, no. 5, pp. 585-594, Jan. 2007.

doi: 10.1080/16864360.2007.10738493.

[11] B. H. Smith, S. Szyniszewski, J. F. Hajjar, B. W. Schafer, and S. R. Arwade, "Steel foam for structures: A review of applications, manufacturing and material properties," J. Constructional Steel Res., vol. 71, pp. 1-10, Apr. 2012. doi: 10.1016/j.jcsr.2011.10.028.

[12] T. C. Lim, "Functionally graded beam for attaining Poisson-curving," J. Mater. Sci. Lett., vol. 21, no. 24, pp. 1899-1901, 2002.

doi: 10.1023/A:1021688009461.
[13] S.-J. Lee, J. Wang, and B. V. Sankar, "A micromechanical model for predicting the fracture toughness of functionally graded foams," Int. J. Solids Struct., vol. 44, no. 11-12, pp. 4053-4067, Jun. 2007. doi: 10.1016/j.ijsolstr.2006.11.007.

[14] S. Arabnejad Khanoki and D. Pasini, "Multiscale Design and Multiobjective Optimization of Orthopedic Hip Implants with Functionally Graded Cellular Material," J. Biomech. Eng., vol. 134, no. 3, p. 31004, 2012. doi: 10.1115/1.4006115.

[15] C. Lira, F. Scarpa, and R. Rajasekaran, "A Gradient Cellular Core for Aeroengine Fan Blades Based on Auxetic Configurations," J. Intell. Mater. Syst. Struct., vol. 22, no. 9, pp. 907-917, Jun. 2011. doi: $10.1177 / 1045389 X 11414226$.

[16] N. T. Gridgeman, "Lame Ovals," The Mathematical Gazette, vol. 54, no. 387, p. 31, Feb. 1970. doi: 10.2307/3613154.

[17] B. Hassani and E. Hinton, "A review of homogenization and topology opimization II - analytical and numerical solution of homogenization equations," Comput. Struct., vol. 69, no. 6, pp. 719-738, Dec. 1998. doi: 10.1016/S0045-7949(98)00132-1.

[18] J. N. Reddy, "Analysis of functionally graded plates," Inter. J.Numer. Meth. Eng., vol. 47, no. 1-3, pp. 663-684, Jan. 2000. doi: 10.1002/(SICI)1097-0207(20000110/30)47:1/3<663::AIDNME787>3.0.CO;2-8 


\title{
Gradient Elasticity Modelling for the Analysis of Fiber Composites with Fiber Resistant to Flexure
}

\author{
Mahdi Zeidi, Chun IL Kim \\ Mechanical Engineering Department \\ University of Alberta \\ Edmonton, AB, Canada \\ e-mails: zeidi@ualbert.ca, cikim@ualberta.ca
}

\begin{abstract}
A model for the deformation of an elastic solid reinforced by embedded fibers is presented in which elastic resistance of the fibers to bending is incorporated. We developed the equilibrium equations and necessary boundary conditions, Within the framework of strain-gradient elasticity, which describe the finite plane deformations of fiber-reinforced composite materials. The resulting nonlinear coupled $4^{\text {th }}$ order partial differential equations are numerically solved by using the FEM. Within the limitation of superposed incremental deformations, a complete analytical solution is also obtained.
\end{abstract}

Finite elasticity; strain gradient theory; fiber-reinforced materials; flexure; superposed incremental deformations; finite element method; finite plane deformations

\section{INTRODUCTION}

The mechanics of fiber-reinforced materials is a wellestablished subject [1-4] that has significantly advanced our knowledge on and practices in the development of composite materials. One way of addressing this problem is to examine the local behavior of an individual microstructure-matrix system including the interfacial region. Such investigations are essential to extracting the mechanical properties of those materials, yet rather inefficient at predicting the general behavior of the composite materials under prescribed forces and/or displacements. Within this prescription, much theoretical work has been developed based on the simple concept of an anisotropic material where the material response function depends on the classical deformation gradient with the augmented constraints of bulk incompressibility and fiber inextensibility. A general theory for an elastic solid with fibers resistant to flexure, stretch and twist is presented in [4].

In the present work, we develop a continuum model in which the fibers accommodate elastic resistance to flexure. The fibers are treated as continuously distributed spatial rods of the Kirchhoff type where the kinematics are based on their position field and a director field. We seek a complete model describing the finite plane deformation of fiber composites which offers fiber resistance to flexure loading. The basic kinematics and constitutive framework are presented in Section II. Via the computation of variational derivatives and the virtual-work statement, in Section II the corresponding equilibrium equation is derived in which the bulk incompressibility condition is augmented in a weak sense. In section III, we also consider an example in the case of Neo-Hookean materials with necessary boundary conditions. In section IV, a set of numerical solutions is obtained via a finite element analysis. In Section V, a complete analytical solution of the linearized system is obtained for the case when the fiber composite is subjected to uniform bending moment at its edge. It is good to mention that the presented model can serve as an alternative 2D Cosserat theory of nonlinear elasticity.

\section{KINEMATICS AND EQUILIBRIUM EQUATIONS}

We propose that the mechanical response of the fiber material is governed by the following strain energy function:

$$
\begin{gathered}
\mathrm{W}(\mathbf{F}, \mathbf{G})=\widehat{\mathrm{W}}(\mathbf{F})+\mathrm{W}(\mathbf{G}), \\
\mathrm{W}(\mathbf{G})=\frac{1}{2} \mathrm{C}(\mathbf{F})|\mathrm{g}|^{2} .
\end{gathered}
$$

where $\mathbf{F}$ and $\mathbf{G}$ are the gradient of the deformation and the second gradient of the deformation, respectively. Further, C refers to the material property of fibers which, in general, independent of the deformation gradient. The orientation of a particular fiber is given by

$$
\begin{aligned}
& \lambda=|\mathbf{d}|, \\
& \tau=\lambda^{-1} \mathbf{d} .
\end{aligned}
$$

Where

$$
\mathbf{d}=\mathbf{F D}
$$

in which $\mathbf{D}$ is the unit tangent to the fiber trajectory in the reference configuration. Equation (3) can be derived by taking the derivative of $r(s)=\chi(X(s))$, upon making the identifications $\mathrm{D}=\mathrm{X}^{\prime}(\mathrm{s})$ and $\mathrm{d}=r^{\prime}(\mathrm{s})$. The expression for geodesic curvature of an arc (r (s)) is then obtained from (3) as

$$
\mathbf{g}=\mathbf{r}^{\prime \prime}=\frac{\mathrm{d}\left(\mathbf{r}^{\prime}\right)}{\mathrm{ds}}=\frac{\partial(\mathbf{F} . \mathbf{D})}{\partial \mathbf{X}} \frac{\mathrm{d} \mathbf{X}}{\mathrm{ds}}=\nabla|\mathbf{F D}| \mathbf{D}
$$

To accommodate the bulk incompressibility condition, we consider the following energy functional

\footnotetext{
NSERC of Canada via Grant \#RGPIN 04742 and U of A start-up grant.
} 


$$
\begin{gathered}
\mathrm{E}=\int_{\mathrm{w}} \mathrm{U}(\mathbf{F}, \mathbf{G}, p) \mathrm{dA}, \\
\mathrm{U}(\mathbf{F}, \mathbf{G}, p)=\mathrm{W}(\mathbf{F}, \mathbf{G})-p(J-l) .
\end{gathered}
$$

where $J$ is determinant of $\mathbf{F}$ and $p$ is a Lagrange-multiplier field. The induced variation of the energy is then evaluated as

$$
\dot{\mathrm{E}}=\int_{\mathrm{w}} \dot{\mathrm{W}}(\mathbf{F}, \mathbf{G}) \mathrm{dA}
$$

Where

$$
\dot{U}(\mathbf{F}, \mathbf{G}, p)=W_{\mathbf{F}} \cdot \dot{\mathbf{F}}-W_{\mathbf{G}} \cdot \dot{\mathbf{G}}-p \dot{J}
$$

So we have

$$
P_{i A}=\frac{\partial W}{\partial F_{i A}}-p F_{i A}^{*}-C g_{i, B} D_{B} D_{A}
$$

Therefore, the corresponding Euler equation can be obtained as

$$
P_{i A, A}=0 \text { or } \operatorname{Div}(\mathbf{P})=0
$$

\section{NEO-HOOKEAN MATERIALS}

In the case of incompressible Neo-Hookean materials, the energy density function is given by

$$
\begin{gathered}
\widehat{\mathrm{W}}(\mathbf{F})=\frac{\mu}{2} \operatorname{tr}(\mathbf{C})=\frac{\mu}{2} \operatorname{tr}\left(\mathbf{F}^{\mathrm{T}} \mathbf{F}\right)=\frac{\mu}{2} \mathbf{F} . \mathbf{F} \\
\mathrm{W}(\mathbf{F}, \mathbf{G})=\frac{\mu}{2} \mathbf{F} . \mathbf{F}+\frac{1}{2} \mathrm{C}|\mathrm{g}|^{2}
\end{gathered}
$$

where $\mu$ and $\mathrm{C}$ are the material constant of the matrix and fiber, respectively. Accordingly, the corresponding Euler equation can be obtained as

$$
P_{i A, A}=\mu F_{i A, A}-p_{, A} F_{i A}^{*}-C g_{i, A B} D_{A} D_{B}=0
$$

where $\varepsilon_{\mathrm{ij}}$ is the 2-D permutation; $\varepsilon_{12}=-\varepsilon_{21}=1, \varepsilon_{11}=-\varepsilon_{22}=0$. Equation (12) together with the incompressibility condition furnishes a coupled PDE system for $\chi_{1}, \chi_{2}$ and $p$. i.e.

$$
\begin{gathered}
\mu\left(\chi_{1,11}+\chi_{1,22}\right)-p_{, 1} \chi_{2,2}+p_{, 2} \chi_{2,1}-\mathrm{C} \chi_{1,1111}=0 \\
\mu\left(\chi_{2,11}+\chi_{2,22}\right)+p_{, 1} \chi_{1,2}-p_{, 2} \chi_{1,1}-\mathrm{C} \chi_{2,1111}=0 \\
\chi_{1,1} \chi_{2,2}-\chi_{2,1}=1 .
\end{gathered}
$$

When the fiber's directions are either normal or tangential to the boundary, we have

$$
\begin{gathered}
t=P_{i A} N_{A}, \\
m_{i}=C g_{i} D_{A} N_{A} D_{B} N_{B},
\end{gathered}
$$

$$
f_{i}=0
$$

where

$$
\begin{gathered}
P_{i A}=\mu F_{i A}-p F_{i A}^{*}-g_{i, B} D_{A} D_{B}, \\
g_{i}=F_{i A, B} D_{A} D_{B} .
\end{gathered}
$$

\section{FINITE ELEMENT ANALYSIS OF THE 4TH ORDER COUPLED PDE}

It is not trivial to demonstrate numerical analysis procedures for coupled PDE systems, especially for those with high order terms, since the piece wise linear function adopted in FE analysis has limited differentiability up to second order. For preprocessing, Equation (13) can be recast as

$$
\begin{gathered}
\mu\left(\mathrm{R}+\chi_{1,22}\right)-p_{, 1} \chi_{2,2}+p_{, 2} \chi_{2,1}-\mathrm{C} \chi_{1,1111}=0 \\
\mu\left(\mathrm{Q}+\chi_{2,22}\right)+p_{, 1} \chi_{1,2}-p_{, 2} \chi_{1,1}-\mathrm{C} \chi_{2,1111}=0 \\
\mathrm{Q}-\chi_{1,11}=0 \\
\mathrm{R}-\chi_{2,11}=0, \\
\mathrm{~A}-\mu\left(\chi_{1,11}+\chi_{1,22}\right)-\mathrm{CR}_{, 11}=0 \\
\mathrm{~A}-\mu\left(\chi_{2,11}+\chi_{2,22}\right)-\mathrm{CQ}_{, 11}=0 .
\end{gathered}
$$

where $\mathrm{Q}=\chi_{1,11}$ and $\mathrm{R}=\chi_{2,11}$. By employing the Picard iterative process, the nonlinear terms in the above can be treated as

$$
\begin{gathered}
-\mathrm{A}_{\text {initial }} \chi_{2,2}^{\text {initial }}+\mathrm{B}_{\text {initial }} \chi_{2,1}^{\text {initial }} \Longrightarrow-\mathrm{A}_{0} \chi_{2,2}^{0}+\mathrm{B}_{0} \chi_{2,1}^{0} \\
\mathrm{~A}_{\text {initial }} \chi_{1,2}^{\text {initial }}-\mathrm{B}_{\text {initial }} \chi_{1,1}^{\text {initial }} \Longrightarrow \mathrm{A}_{0} \chi_{1,2}^{0}-\mathrm{B}_{0} \chi_{1,1}^{0}
\end{gathered}
$$

Thus, we write

$$
\begin{gathered}
-\mathrm{A}_{\mathrm{N}-1} \chi_{2,2}^{\mathrm{N}-1}+\mathrm{B}_{\mathrm{N}-1} \chi_{2,1}^{\mathrm{N}-1} \Longrightarrow-\mathrm{A}_{\mathrm{N}} \chi_{2,2}^{\mathrm{N}}+\mathrm{B}_{\mathrm{N}} \chi_{2,1}^{\mathrm{N}} \\
\mathrm{A}_{\mathrm{N}-1} \chi_{1,2}^{\mathrm{N}-1}-\mathrm{B}_{\mathrm{N}-1} \chi_{1,1}^{\mathrm{N}-1} \Longrightarrow \mathrm{A}_{\mathrm{N}} \chi_{1,2}^{\mathrm{N}}-\mathrm{B}_{\mathrm{N}} \chi_{1,1}^{\mathrm{N}}
\end{gathered}
$$

where the values of $A$ and $B$ continue to be refreshed based on their previous estimations as iteration progresses. The weak form of Eq. (16)1 is given by

$$
0=\int_{\Omega} \mathrm{w}_{1}\left(\mu\left(\mathrm{R}+\chi_{1,22}\right)-\mathrm{A} \chi_{2,2}+\mathrm{B} \chi_{2,1}-\mathrm{CR}_{, 11}\right) \mathrm{d} \Omega
$$

where $\Omega, \partial \Gamma$ and $\mathrm{N}$ are the domain of interest, the associated boundary, and the rightward unit normal to the boundary $\partial \Gamma$ in the sense of the Green-Stoke's theorem, respectively. The unknowns, $\chi_{1}, \chi_{2}, \mathrm{Q}, \mathrm{R}, \mathrm{A}$ and $\mathrm{B}$ can be written in the form of Lagrangean polynomial. Finally, we assemble the local stiffness matrices and obtain the following systems of equations in the Global form. 
For demonstration purpose, we consider a rectangular fiber composite where one end is fixed and the other end is subjected to uniform bending in order to examine fibers' reinforcing effects against to flexure. We also note here that data are obtained under the normalized setting. The convergence criteria are set for both nonlinear terms (i.e. A and B) and the deformed profiles at $\mathrm{y}=0$.

$$
\begin{gathered}
\left|\mathrm{A}_{\mathrm{n}+1}-\mathrm{A}_{\mathrm{n}}\right|=\mathrm{e}_{1} \leq \varepsilon, \\
\left|\mathrm{B}_{\mathrm{n}+1}-\mathrm{B}_{\mathrm{n}}\right|=\mathrm{e}_{2} \leq \varepsilon,
\end{gathered}
$$$$
\varepsilon=\text { Maximum error. }
$$

It is clear from Fig. 1 that the adopted numerical method demonstrates fast convergence within 20 iterations. The deformation profile and contour show smooth transitions as they approach the boundary (Figs. 2, 3).

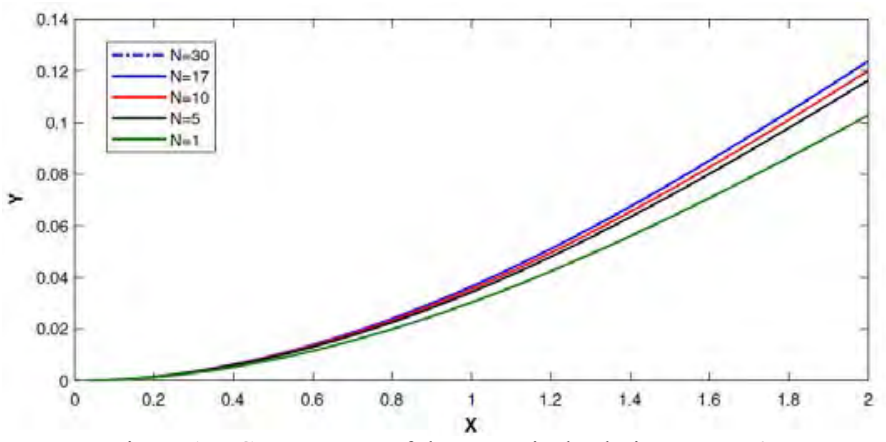

Figure 1. Convergence of the numerical solutions at $y=0$

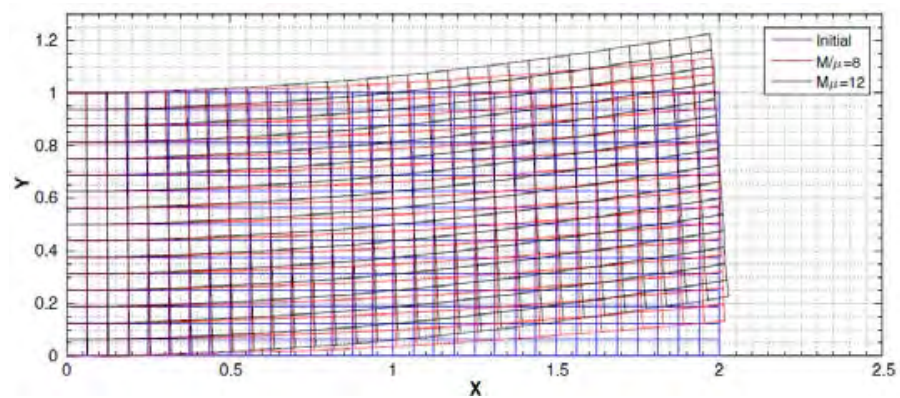

Figure 2. Deformed configurations with respect to $M / \mu$ when $C / \mu=150$

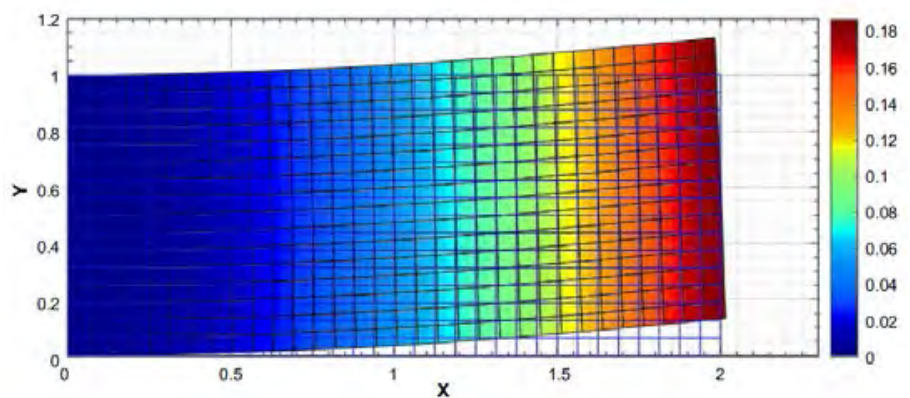

Figure 3. Deformation contour when $\mathrm{C} / \mu=150$ and $\mathrm{M} / \mu=8$

\section{SOLUTION TO THE LINEARIZED PROBLEM}

We introduce scalar field $\phi$ as

$$
\begin{gathered}
\mathbf{u}=\mathbf{k} \times \nabla \phi, \mathbf{k}(\text { unit normal), } \\
u_{i}=\varepsilon_{\lambda i} \phi_{, \lambda},
\end{gathered}
$$

The linearized Euler equation can be rewritten as

$$
\dot{P}_{, i}=\mu \varepsilon_{\lambda i}\left(\phi_{, \lambda 11}+\phi_{, \lambda 22}\right)-C \varepsilon_{\lambda i} \phi_{, \lambda 1111}
$$

So the final equation will be

$$
\begin{gathered}
\Delta H-\alpha H_{, 1111}=0, \\
H=\Delta \phi \text { and } \alpha=\frac{C}{\mu}>0 .
\end{gathered}
$$

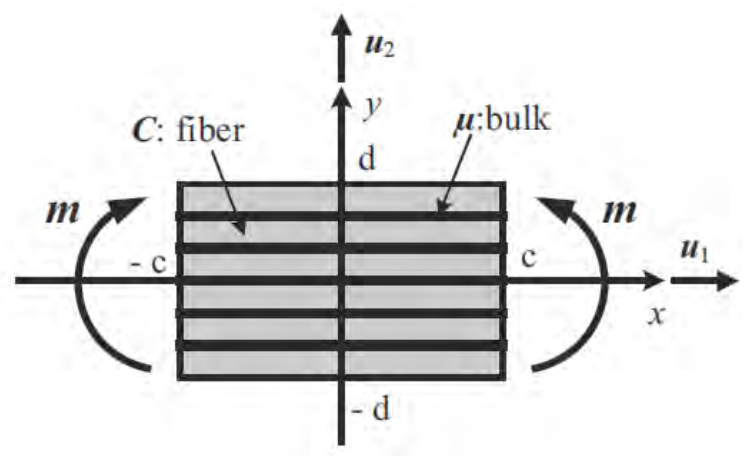

Figure 4. Schematic of problem

The general solution for the above equation can be found as

$$
\phi=\sum_{m=1}^{\infty}\left[\begin{array}{c}
\left(e^{a_{m} x}\left(A_{m} \cos b_{m} x+B_{m} \sin b_{m} x\right)+\right. \\
e^{-a_{m} x}\left(C_{m} \cos b_{m} x+D_{m} \sin b_{m} x\right) \\
\times\left(E_{m} \cos m y+F_{m} \sin m y\right)
\end{array}\right\}+K
$$

where $\mathrm{K}$ is a solution of Laplace's equation given by

$$
K=\sum_{n=1}^{\infty}\left[\left(G_{n} \cosh n x+H_{n} \sinh n x\right)\left(I_{n} \cos n y+J_{n} \sin n y\right)\right]
$$

In above equations $\mathrm{a}_{\mathrm{m}}$ and $\mathrm{b}_{\mathrm{m}}$ are

$$
a_{m}=\frac{\sqrt{2 m \sqrt{\alpha}+1}}{2 \sqrt{\alpha}}, b_{m}=\frac{\sqrt{2 m \sqrt{\alpha}-1}}{2 \sqrt{\alpha}} .
$$

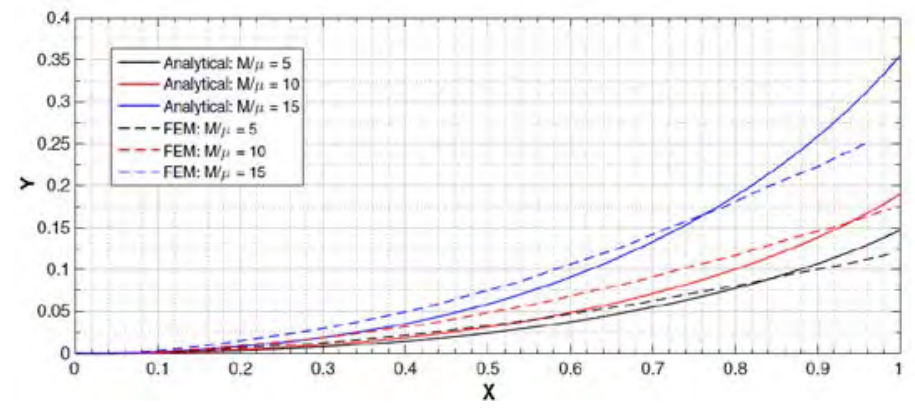


Figure 5. Solutions of the bending problem with $C / \mu=150$. Nonlinear solution (dashed line), linear solution (solid line)

The analytical (linear) solution shows good agreement with nonlinear solution (FEM) for the small deformation regime, while larger values of $\mathrm{M}$ induce a significant discrepancy between the linear and nonlinear solution (see, Fig. 5). As a result, the obtained analytical solution has limitations in large deformation analysis. However, it can still be used in the design and analysis of fiber composites, particularly for CNCreinforced composites, where the deformations of the systems are expected to be relatively 'small'.

\section{CONCLUSION}

A continuum-based model is developed in finite plane elastostatics in which fibers resistant to flexure is taken into account. The fibers are regarded as continuously distributed spatial rods of the Kirchhoff type in which the kinematics are based on a position field and a director field. The equilibrium equation of the fiber-reinforced composite materials is derived by the variational computation and method of virtual work.

These constitute a highly nonlinear coupled PDE system which is treated numerically via the FEM. Within the prescription of superposed incremental deformations, a complete linear theory is developed through which an analytical solution of the corresponding linearized PDE system is obtained. The obtained analytical solution demonstrates good agreement with nonlinear solution for the small deformation regime, yet has limited predictions for large deformation analysis.

\section{ACKNOWLEDGMENT}

The author would like to thank Dr. Steigmann for stimulating his interest in this subject. This work was supported by the Natural Sciences and Engineering Research Council of Canada via Grant \#RGPIN 04742 and the University of Alberta through a start-up grant.

\section{REFERENCES}

[1] J. F. Mulhern, T. G. Rogers, and A. J. M. Spencer, "A continuum theory of a plastic-elastic fibre-reinforced material," Inter. J. Eng. Sci., vol. 7, no. 2, pp. 129-152, Feb. 1969. doi: 10.1016/0020-7225(69)90053-6.

[2] A. C. Pipkin and T. G. Rogers, "Plane Deformations of Incompressible Fiber-Reinforced Materials," J. Appl. Mech., vol. 38, no. 3, p. 634, 1971. doi: $10.1115 / 1.3408866$.

[3] A. J. M. Spencer and K. P. Soldatos, "Finite deformations of fibrereinforced elastic solids with fibre bending stiffness," Int. J. Nonlinear Mech., vol. 42, no. 2, pp. 355-368, Mar. 2007. doi: 10.1016/j.ijnonlinmec.2007.02.015.

[4] P. Neff, "A finite-strain elastic-plastic Cosserat theory for polycrystals with grain rotations," Int. J. Eng. Sci., vol. 44, no. 8-9, pp. 574-594, May 2006. doi: 10.1016/j.ijengsci.2006.04.002.

[5] D. J. Steigmann, "Theory of elastic solids reinforced with fibers resistant to extension, flexure and twist," Int. J. Nonlinear Mech., vol. 47, no. 7, pp. 734-742, Sep. 2012. doi: 0.1016/j.jijnonlinmec.2012.04.007.

[6] M. Zeidi and C. I. Kim, "Mechanics of fiber composites with fibers resistant to extension and flexure," Math. Mech. Solids, vol. 24, no. 1, pp. 3-17, Sep. 2017. doi: 10.1177/1081286517728543.

[7] F. dell'Isola and D. Steigmann, "A Two-Dimensional Gradient-Elasticity Theory for Woven Fabrics," J. Elasticity, vol. 118, no. 1, pp. 113-125, May 2014. doi: 10.1007/s10659-014-9478-1.

[8] C. Truesdell., W. Noll, "The non-linear field theories of mechanics," in: Handbuch der Physik, vol. III/3, S. Flugge, Ed., Springer, Berlin, 1965.

[9] M. Zeidi and C. I. Kim, "Finite plane deformations of elastic solids reinforced with fibers resistant to flexure: complete solution," Arch. Appl. Mech., vol. 88, no. 5, pp. 819-835, Jan. 2018. doi: 10.1007/s00419-018-1344-3.

[10] N. F. Dow, "Study of stresses near a discontinuity in a filamentreinforced composite material," Gen. Elect. Co. Report number R63SD-61, 1963. 


\section{D Dynamical Model for Liquid Sloshing Simulation in a Partially Filled Elliptical Tank}

\author{
O. Noui \\ Department of Applied Science \\ University of Quebec at Chicoutimi \\ Chicoutimi, Qc, Canada \\ omar.noui@uqac.ca
}

\author{
M. Bouazara \\ Department of Applied Science \\ University of Quebec at Chicoutimi \\ Chicoutimi, Qc, Canada \\ mbouazar@uqac.ca
}

\author{
M. J. Richard \\ Department of Mechanical Engineering \\ Laval University \\ Quebec, Qc, Canada \\ mrichard@gmc.ulaval.ca
}

\begin{abstract}
Many of 2D mechanical models have been developed to simulate liquid sloshing of a partially filled tank with different shapes. However, those models didn't represent properly the complex liquid motion, especially in the case of portable tanks. Indeed, forces exerted on the liquid can be lateral, longitudinal and vertical. Then, liquid displacement and pressure forces applied to the tank walls are undervalued and may cause design flaws. In this case, 2D mechanical models are ineffective for liquid motion simulation. In previous studies, a $3 \mathrm{D}$ equivalent mechanical model has been developed. This dynamical model is used to simulate different liquid motion in a partially filled tank that consider any sort of excitement forces and get more accurate results in terms of displacements and pressure forces. In this study, a brief description of the new dynamical model is given, including the liquid discretization process, stiffness and damping coefficients computing method and equations of motion. Afterward, the model is applied to an elliptical cross section tank to obtain displacement and pressure forces of the liquid. Finally, the results are compared to the literature.
\end{abstract}

Keywords: liquid sloshing, tank truck, equivalent model, elliptical tank, 3D simulation.

\section{INTRODUCTION}

There are several equivalent mechanical models that simulate 2D liquid movement in a partially filled tank with different shapes such as spring-mass and simple pendulum models [1-4]. The use of such models in the liquid simulation provides convincing results when it comes to lateral sloshing. However, they didn't represent the global liquid motion in a tank. On the other hand, 3D mechanical models as spring-mass lattice are used for modeling complex motions of deformable bodies. Especially in computer graphical animation, springmass systems are often used for their simplicity and rapid implementation [5-7]. Moreover, the use of these models in the simulation of deformable bodies provides both geometrical and physical aspects. In a previous study, a new equivalent 3D dynamical model was developed to simulate the global motion of the liquid in a horizontal tank [8]. A mathematical method for liquid discretization showed that liquid splits in multiple particles along each axis. Then, the masse and coordinates of the center of mass of each particle are computed. The movement of each particle is simulated by the displacement of its center of mass. All centers of mass constitute the nodes of the mesh. Each two adjacent nodes are linked by flexible edges having a parallel spring and damper. To adapt the model to all used forms of tanks, the generic tank cross-section, developed by Kang [9], was employed. In another study [10], we showed how to obtain stiffness and damping coefficient depending on the liquid and the tank design. Afterward, equations of motion were obtained and solved using a computation software program, namely Maple 16 [11]. In this study, the 3D dynamical model is applied to simulate liquid motion and pressure forces in a partially filled tank with an elliptical cross-section. Indeed, this tank shape is one of the most used shapes. Many researches have been dedicated to study this tank geometry. It allows a lower center of mass comparing to a cylindrical tank. We show a brief description of the dynamical model. Afterward, some results are generated and compared to the literature.

\section{3D DYNAMICAL MODEL}

\section{A. Liquid discratization}

In this study, the tank lateral walls are assumed to be straight without any camber. The liquid discretization is made by dividing the length $X$ of the tank by $M$ parts, the height $Z_{h}$ of the free surface by $P$ parts and each portion between two heights $k . Z_{h} / P$ and $(k-1) \cdot Z_{h} / P$ by $N$ parts in each direction of the $\overrightarrow{O Y}$ axis (left side and right side of the $\overrightarrow{O Z}$ axis), with $1 \leq k \leq$ $P$ and $M, N, P \in \mathbb{N}^{*}$. Thus, we obtain $M .2 N$. $P$ particles, noted $p_{i, j, k}$ with $1 \leq i \leq M, 1 \leq j \leq 2 N$ and $1 \leq k \leq P$. Note that the positions of each couple of particles $p_{i, N-(l-1), k}$ and $p_{i,(N+1)+(l-1), k}$ are symmetric with respect to the $\overrightarrow{O Z}$ axis $(1 \leq$ $l \leq N)$. Each particle is represented by its center of mass, noted $G_{i, j, k}$, which are the nodes of the model. Mass $m_{i, j, k}$ and coordinates $\vec{p}_{i, j, k}$ of each node are computed depending on its location [8]. The following figures show an example of the liquid discretization for a half full elliptical tank with $M=12$, $N=4$ and $P=5$ : 


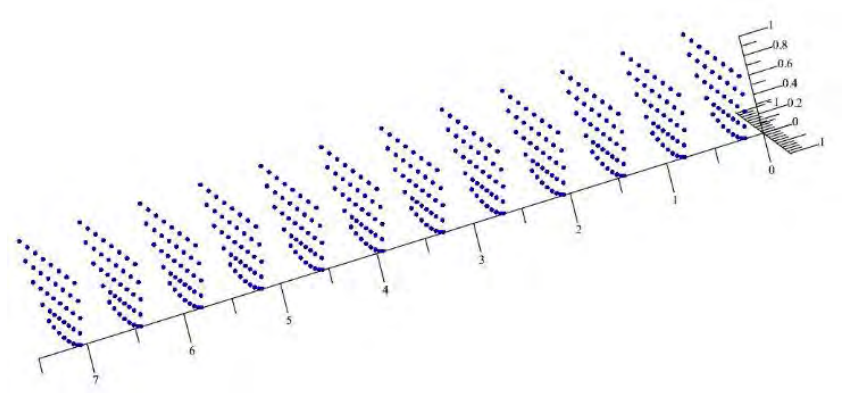

Figure 1: Example of liquid discretization in 3D

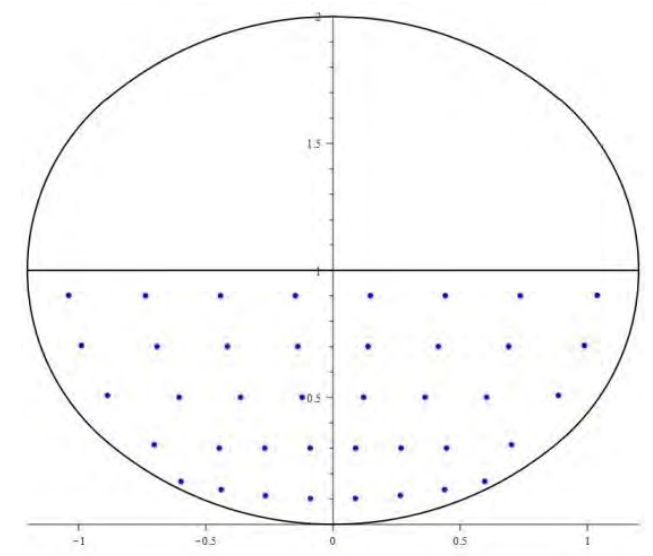

Figure $2: 2 \mathrm{D}$ view of the liquid discretization.

In this example, the model contains of 480 nodes. Then, we will obtain 1440 equations of motion, three equations for each node.

\section{B. Stiffness and damping coefficient}

Each node $G_{i, j, k}$ is linked to six links: two links $A_{1, i, j, k}$ and $A_{1, i+1, j, k}$ along the $\overrightarrow{O X}$ axis, two links $A_{2, i, j, k}$ and $A_{2, i, j+1, k}$ along the $\overrightarrow{O Y}$ axis and two links $A_{3, i, j, k}$ and $A_{3, i, j, k+1}$ along the $\overrightarrow{O Z}$ axis as shown in the following scheme:

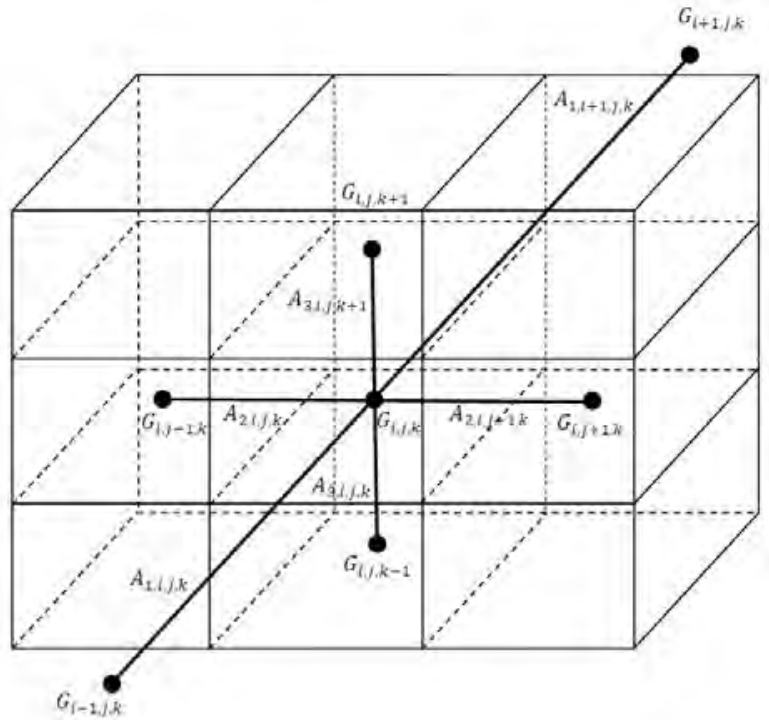

Figure 3: Links between a node $\boldsymbol{G}_{\boldsymbol{i}, \boldsymbol{j}, \boldsymbol{k}}$ and its adjacent nodes.
Each link $A_{q, i, j, k}$, with $q=1,2,3$, contains a parallel linear spring with a stiffness $K_{q, i, j, k}$ and linear damper with a damping coefficient $c_{q, i, j, k}$ as shown in the figure below:

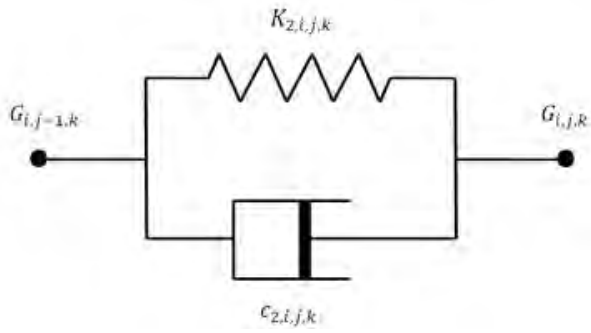

Figure 4: composition of the flexible link.

The nodes contacting the tank walls $G_{1, j, k}, G_{M, j, k}, G_{i, 1, k}$, $G_{i, N, k}$ and $G_{i, j, 1}$ are attached to the structure by the same type of the links. Another special case of the nodes $G_{i, j, P}$, that represent the free surface of the liquid, are linked by only 5 links because they have no contact with the tank structure. The methodology used to compute stiffness and damping coefficient of the model is discussed as follows:

Firstly, stiffness $K_{1, i, j, k}$ along the $\overrightarrow{O X}$ axis is computed. It is supposed that the liquid is divided along the $\overrightarrow{O Z}$ axis. Each part of the liquid between two heights $Z_{k-1}$ and $Z_{k}$ represents a vibration mode. The following formula is used to compute the natural frequency $\omega_{n}$ of each vibration mode $n$ of the liquid in rectangular tank [12]:

$$
\omega_{n}{ }^{2}=\pi(2 n-1) \frac{g}{a} \tanh \left[\pi(2 n-1)\left(\frac{h}{a}\right)\right]
$$

Where $a$ is the width of the tank, $h$ is the height of the liquid and $g=9.81 \mathrm{~m} . \mathrm{s}^{-2}$ is the gravity acceleration. This formula is adapted to our model and becomes:

$$
\omega_{x k}^{2}=\pi(2 n-1) \frac{g}{L} \tanh \left[\pi(2 n-1)\left(\frac{Z_{h}}{L}\right)\right]
$$

Where $n=P-k+1$. Indeed, when the cylinder is placed horizontally, the tank may be considered as rectangular [1]. Then, stiffness $K_{x k}$ of each spring that simulate each mode $k$ is computed by:

$$
K_{x k}=m_{x k} \cdot \omega_{y k}^{2}
$$

Where $m_{x k}$ is the mass of the liquid between two heights $Z_{k-1}$ and $Z_{k}$. Afterward, it is supposed that each spring $K_{x k}$ is a set of $M+1$ springs in series with equal stiffness $K_{x k, i}$ computed by the following formula:

$$
K_{x k, i}=(M+1) \cdot K_{x k}
$$

Then, it is supposed that each spring $K_{x k, i}$ is a set of parallel springs with the same stiffness $K_{1, i, j, k}$ such as:

$$
K_{1, i, j, k}=\frac{K_{x k, i}}{2 N} \text { with } 1 \leq j \leq 2 N
$$

Finally, proceeding by the same way for each part of the liquid between two heights $Z_{k-1}$ and $Z_{k}$, all stiffness along the $\overrightarrow{O X}$ axis are obtained.

Secondly, stiffness $K_{2, i, j, k}$, along the $\overrightarrow{O Y}$, axis are computed. The method is identical to the one used previously for stiffness along the $\overrightarrow{O X}$ axis. It is supposed that the liquid is divided along the $\overrightarrow{O Z}$ axis. The formula (1) that compute natural 
frequencies of each part of the liquid between $Z_{k-1}$ and $Z_{k}$ is adapted as follows:

$$
\omega_{y k}^{2}=\pi(2 n-1) \frac{g}{Y_{k-1}+Y_{k}} \tanh \left[\pi(2 n-1)\left(\frac{Z_{h}}{Y_{k-1}+Y_{k}}\right)\right](6)
$$

Where $Y_{k}$ is the half width of the tank corresponding to the height $Z_{k}$. In fact, the width of the tank along the $\overrightarrow{O Y}$ axis varies depending on the height of the studied liquid part. Therefore, the value of width $a$ in the formula (1) is replaced by the average width between to the heights $Z_{k-1}$ and $Z_{k}$. The stiffness $K_{y k}$ describing each mode is computed by:

$$
K_{y k}=m_{y k} \cdot \omega_{y k}^{2}
$$

Where $m_{y k}$ is the mass of the liquid between $Z_{k-1}$ and $Z_{k}$. It is supposed that the spring $K_{y k}$ is a set of $M$ parallel springs with the same stiffness:

$$
K_{y k, i}=\frac{K_{y k}}{M} \text { with } 1 \leq i \leq M
$$

Afterward, we split each stiffness $K_{y k, i}$ in $2 N+1$ springs in series with equal stiffness, noted $K_{2, i, j, k}$, with:

$$
K_{2, i, j, k}=(2 N+1) \cdot K_{y k, i}
$$

All stiffness $K_{2, i, j, k}$ values along the $\overrightarrow{O Y}$ axis are then obtained by the proposed computational procedures.

Thirdly, the stiffness $K_{3, i, j, k}$ of springs along the $\overrightarrow{O Z}$ axis is computed. In this case, there is no formula in the literature that can be used to compute natural frequencies of vertical sloshing. For this, an important assumption is considered. Indeed, the vertical displacement of the liquid is usually limited. For conventional shapes of the tank, vertical displacement $\Delta z$ of the liquid center of mass is $0.04 \mathrm{~m}$ on average [9]. Considering this assumption, first, it is supposed that there is only one spring, namely $K_{z}$, attached to the center of mass of the liquid. The maximum stretch of this spring should be $\Delta z$. Assuming that the mass of the center of mass is the total liquid mass $m_{t}$, then, stiffness $K_{Z}$ is computed as follows:

$$
K_{z}=\frac{m_{t} \cdot g}{\Delta z}
$$

Then, the spring $K_{z}$ is considered as a set of $P$ springs in series with equal stiffness, noted $K_{z k}$ with $1 \leq k \leq P$. Each stiffness $K_{z k}$ to be calculated by the formula:

$$
K_{z k}=P . K_{z z}
$$

Afterwards, each spring $K_{z k}$ is considered to be a set of $2 M N$ parallel springs $K_{3, i, j, k}$ computed as follows:

$$
K_{3, i, j, k}=K_{z k} / 2 M N
$$

Stiffness of each spring $K_{3, i, j, k}$ along the $\overrightarrow{O Z}$ axis is then obtained.

Finally, the damping coefficients $c_{q, i, j, k}$ with $q=1,2,3$ are assumed to be equal to 100 times the dynamical viscosity coefficient $\eta$ of the liquid multiplied by the corresponding link length. For example, the damping coefficient of a link between two nodes $G_{i, j, k}$ and $G_{i+1, j, k}$ along the $\overrightarrow{O X}$ axis

$$
c_{1, i, j, k}=100 . \eta \cdot\left\|\vec{p}_{i, j, k}-\vec{p}_{i-1, j, k}\right\|
$$

Where $\|\vec{x}\|$ is the Euclidian norm of the vector $\vec{x}$. To obtain consistent results, a calibration factor is added to the model such that all the values of stiffness along $\overrightarrow{O X}$ axis and $\overrightarrow{O Y}$ axis to be multiplied by this factor. The value of this factor is used depending on the number of nodes in the model. Virtually, as the number of nodes decreases extremely, the calibration factor increases. This factor can be explained by the lack of precision when the mesh is less refined. In this case, parameters of the links are underestimated and should be corrected.

\section{Equations of motion}

Displacement, velocity and acceleration vectors of a node $G_{i, j, k}$ are noted respectively $\vec{r}_{i, j, k}(t), \vec{v}_{i, j, k}(t)$ and $\vec{a}_{i, j, k}(t)$. The forces applied on each node are: stress force $\vec{T}_{i, j, k}$ of the springs attached to the nodes, damping force $\vec{A}_{i, j, k}$ and input force $\vec{F}_{i, j, k}$. Stress force $\vec{T}_{i, j, k}$ is calculated by the following formula:

$$
\begin{gathered}
\vec{T}_{i, j, k}=\vec{T}_{1, i, j, k}+\vec{T}_{1, i+1, j, k}+\vec{T}_{2, i, j, k}+\vec{T}_{2, i, j+1, k}+\vec{T}_{3, i, j, k} \\
+\vec{T}_{3, i, j, k+1}
\end{gathered}
$$

Where the stress force of each spring attached to the node is computed by the Hook law. For example:

$$
\begin{aligned}
\vec{T}_{1, i, j, k}=-K_{1, i, j, k} & \left(\left\|\vec{r}_{i, j, k}-\vec{r}_{i-1, j, k}\right\|\right. \\
& \left.-\left\|\vec{p}_{i, j, k}-\vec{p}_{i-1, j, k}\right\|\right) \cdot \frac{\vec{r}_{i, j, k}-\vec{r}_{i-1, j, k}}{\left\|\vec{r}_{i, j, k}-\vec{r}_{i-1, j, k}\right\|}
\end{aligned}
$$

Damping force $\vec{A}_{i, j, k}$ of each node is computed by:

$$
\begin{gathered}
\vec{A}_{i, j, k}=\vec{A}_{1, i, j, k}+\vec{A}_{1, i+1, j, k}+\vec{A}_{2, i, j, k}+\vec{A}_{2, i, j+1, k} \\
+\vec{A}_{3, i, j, k}+\vec{A}_{3, i, j, k+1}
\end{gathered}
$$

Where the damping forces applied by each attached damper is computed by the viscosity law. For example:

$$
\vec{A}_{1, i, j, k}=-c_{1, i, j, k} \cdot\left(\vec{v}_{i, j, k}-\vec{v}_{i-1, j, k}\right)
$$

The input force $\vec{F}_{i, j, k}$ is calculated by the formula below:

$$
\vec{F}_{i, j, k}=m_{i, j, k}\left[\begin{array}{lll}
a_{x} & a_{y} & a_{z}
\end{array}\right]^{t}
$$

Where $a_{x}, a_{y}$ and $a_{z}$ are respectively the longitudinal, lateral and vertical accelerations applied on the tank. Using the Newton second law, we obtain for each node $G_{i, j, k}$ the following equations of motion:

$$
\vec{T}_{i, j, k}+\vec{A}_{i, j, k}+\vec{F}_{i, j, k}=m_{i, j, k} \cdot \vec{a}_{i, j, k}
$$

To obtain the global system of motion equations, the global displacement vector is computed by $\vec{r}$, the global velocity vector by $\vec{v}=\overrightarrow{\dot{r}}$ and the global acceleration vector by $\vec{a}=\overrightarrow{\dot{r}}$. These 3 vectors contain $3 M N P$ components. Moreover, note by $[\mathcal{M}]$ the $3 M N P \times 3 M N P$ diagonal mass matrix such as each mass $m_{i, j, k}$ is repeated 3 times. The global input forces vector is then given by:

$$
\vec{F}=[\mathcal{M}] \cdot \overrightarrow{a_{\text {ext }}}
$$

where $\overrightarrow{a_{\text {ext }}}$ is given by:

$$
\overrightarrow{a_{\text {ext }}}=\left[\begin{array}{lllllll}
a_{x} & a_{y} & a_{z} & \ldots & a_{x} & a_{y} & a_{z}
\end{array}\right]^{t}
$$

The global stress forces $3 M N P$-vector $\vec{T}$ is equal to:

$$
\vec{T}=\left[\begin{array}{lllllll}
T_{x, 1,1,1} & T_{y, 1,1,1} & T_{z, 1,1,1} & \ldots & T_{x, M, N, P} & T_{y, M, N, P} & T_{z, M, N, P}
\end{array}\right]^{t}
$$

Similarly, global damping forces $3 M N P$-vector $\vec{A}$ is obtained by the same manner. Finally, the global system of equations of motion is given as follows:

$$
[\mathcal{M}]\left(\overrightarrow{\ddot{r}}-\overrightarrow{a_{e x t}}\right)+\vec{A}-\vec{T}=0
$$




\section{RESULTS AND DISCUSSION}

In this section, the new $3 \mathrm{D}$ dynamical model is used to simulate liquid sloshing in a partially filled elliptical tank that is one of the most used geometry of portable tanks. The purpose of this simulation is to compare liquid displacement and pressure forces obtained by this model to the results obtained in the literature by two different methods [13]. The first model is an analytical model considering the liquid as a rigid body. Liquid motion is simulated by the movement of center of mass of the body. The second method is obtained by using a numerical simulation software. We compare maximum, both lateral and vertical, displacements of the center of mass of the liquid and maximum pressure forces generated by liquid sloshing.

To obtain lateral displacement $Y_{g}$ and vertical displacement $Z_{g}$ of the center of mass of the liquid using the 3D model developed in this study, the following formulas are used:

$$
\begin{aligned}
& Y_{g}=\frac{1}{m_{T}} \sum_{\substack{i=1, j=1, k=1 \\
M, 2 N, P}}^{M, 2 N, P} m_{i, j, k} \cdot y_{i, j, k} \\
& Z_{g}=\frac{1}{m_{T}} \sum_{i=1, j=1, k=1}^{M, 2 N, P} m_{i, j, k} \cdot z_{i, j, k}
\end{aligned}
$$

Where $m_{T}$ is the total mass of the liquid, $y_{i, j, k}$ and $z_{i, j, k}$ are respectively lateral and vertical displacement of the node $G_{i, j, k}$. Using the forth order numerical derivation, lateral acceleration is computed to obtain lateral pressure force $F_{y}$.

A $50 \%$ filled elliptical tank is considered in this simulation with $X=7.5 \mathrm{~m}, a=1.2 \mathrm{~m}$ and $b=1 \mathrm{~m}$. The liquid is supposed to be a domestic oil with a density $\rho=966 \mathrm{~kg} \cdot \mathrm{m}^{-3}$ and a dynamic viscosity $\eta=0.048 \mathrm{~kg} \cdot \mathrm{m}^{-1} \cdot \mathrm{s}^{-1}$. The selected values for the discretizing model are $M=12, N=4$ and $P=$ 5 . Therefore, the dynamical model consists of 480 nodes and 585 links (Figure 1). Firstly, a vehicle engagement in a curve maneuver is used as an input force with maximum lateral acceleration $A=3 \mathrm{~m} \cdot \mathrm{s}^{-2}$. The following table shows maximum displacements and lateral pressure forces obtained by three different models.

TABLE I. COMPARING RESULTS OF LIQUID DISPLACEMENTS AND PRESSURE FORCES ACCORDING TO THE NEW 3D MODEL AND THE LITERATURE.

\begin{tabular}{|c|c|c|c|}
\cline { 2 - 3 } \multicolumn{1}{c|}{} & \multicolumn{2}{c|}{ Literature } & \multirow{2}{*}{ New 3D } \\
\cline { 2 - 3 } & $\begin{array}{c}\text { Analytical } \\
\text { Model }\end{array}$ & $\begin{array}{c}\text { Numerical } \\
\text { Model }\end{array}$ & \\
\hline Lateral displacement $(m)$ & 0.18 & 0.27 & 0.246 \\
\hline Vertical displacement $(m)$ & 0.023 & 0.073 & 0.111 \\
\hline Lateral pressure force $(\mathrm{KN})$ & 40.5 & 52 & 51.05 \\
\hline
\end{tabular}

The table above shows accurate results in term of displacements and lateral pressure forces. Indeed, the numerical model using the simulation software is supposed to be the most accurate with respect to real displacements and lateral pressure forces. Note that the results obtained by the new 3D model are closer to the numerical model than the analytical model of the literature [13]. We can also notice that the lateral pressure forces are undervalued by the analytical model while the new 3D model developed in this study is more accurate. Thus, these results validate the new $3 \mathrm{D}$ model and it is possible to use it for tank design and for the study of vehicle stability. Note also that for the simulation of the center of mass motion for a duration of 12 seconds, the computer required approximately one hour of computation time. Which is much shorter than the computation time of a numerical simulation software which requires hours, or even days of computation. Then we can show the real results obtained by the new model by evaluating displacement of each node of the mesh. The figure below shows the displacement of the liquid after one second of motion in a curve:

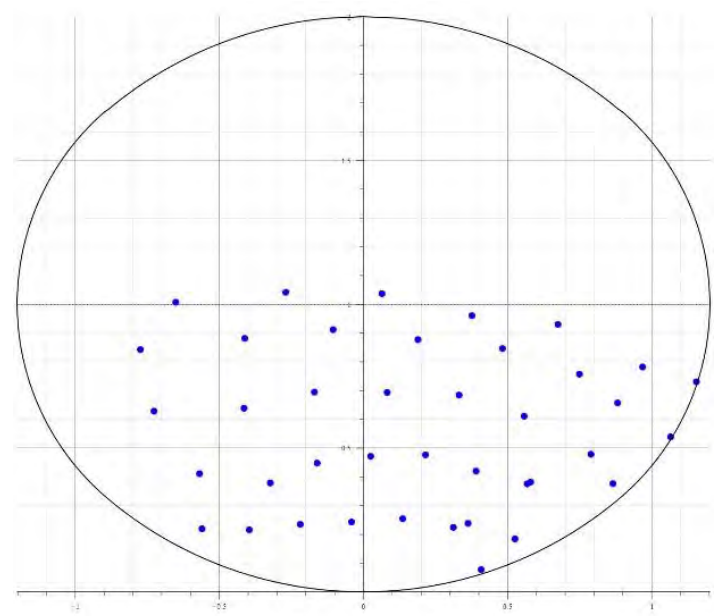

Figure 5: liquid displacement after $1 \mathrm{~s}$ of the vehicle engagement in a curve.

Figure 6 shows displacement of each node representing the liquid after two seconds of the vehicle movement in a curve:

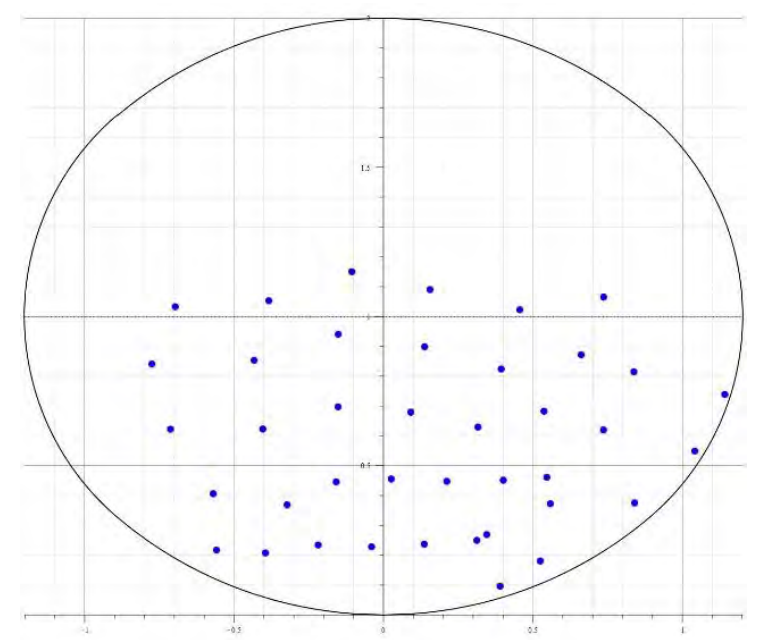

Figure 6: liquid displacement after $2 \mathrm{~s}$ of the vehicle engagement in a curve.

The two figures above show that not only does the new model provide accurate values of displacement of the center of mass and pressure forces generated by the liquid motion, but it is also possible to visualize the global liquid motion by evaluating displacement of each node. In addition, it is also possible to find an approximation of the shape of the liquid free surface by linking the adjacent nodes that represent the free surface. 


\section{CONCLUSION}

In this study, a new 3D-dynamical model is described and used to simulate liquid sloshing in an elliptical tank. Firstly, we showed a brief description of the dynamical model. This model is based on the idea of modeling the liquid as a deformable body using a spring-mass lattice. A special method is used for liquid discretization. Each mass of the liquid is simulated by a node of the mesh that represents its center of mass. The adjacent nodes are linked by a flexible link containing a parallel spring and damper. Afterwards, stiffness and damping coefficient of each link are computed using some assumptions. The equations of motion of each node are then calculated and compiled to obtain the global system of equations of the $3 \mathrm{D}$ model. All formulas and equations are programmed and solved using Maple 16 software. To compare the results of the model with the literature, identical dimensions of an elliptical tank are used as well as the filling rate, the external force applied and the liquid properties. Comparing the results with the literature allows to conclude that the new model provide results closer to the numerical models that require a longer computation time. Moreover, the new model allows a visualization of the global movement of the liquid by evaluating displacement of each node of the mesh. Future works will focus on generating more results for other tank geometries, filling rates and other vehicle maneuver. It will be possible to compare results generated by this new model with other researches.

\section{ACKNOWLEDGMENT}

The work reported in this article is financed by the natural sciences and engineering research council of Canada (NSERC) and the university of Quebec at Chicoutimi.

\section{REFERENCES}

[1] H. N. Abramson, "The Dynamic Behavior of Liquids in Moving Containers," NASA SP-106, 1966.

[2] R. Ranganathan, Y. Ying, and J. B. Miles, "Analysis of fluid slosh in partially filled tanks and their impact on the directional response of tank vehicles," in SAE Technical Paper Series, 1993. doi: 10.4271/932942.

[3] A. A. El Damatty and A. M. I. Sweedan, "Equivalent mechanical analog for dynamic analysis of pure conical tanks," Thin-Walled Structures, vol. 44, no. 4, pp. 429-440, Apr. 2006. doi: 10.1016/j.tws.2006.03.016.

[4] O. Noui, "Étude et modélisation des citernes en aluminium pour véhicule routier," Master's thesis, University Of Quebec at Chicoutimi, 2012.

[5] X. Provot, "Deformation constraints in a Mass-Spring Model to Describe Rigid Cloth Behavior," National Institute for Research in Computer Science and Control, France, 1995.

[6] M. Balci and H. Foroosh, "Real-time 3D Fire Simulation Using a SpringMass Model," in 2006 12th International Multi-Media Modelling Conference, Jan. 2006. doi: 10.1109/MMMC.2006.1651309.
[7] J. Mesit, R. Guha, and S. Chaudhry, "3D Soft Body Simulation Using Mass-spring System with Internal Pressure Force and Simplified Implicit Integration," J. Computers, vol. 2, no. 8, Oct. 2007. doi: $10.4304 /$ jcp.2.8.34-43.

[8] Bouazara, O. Noui, and M. J. Richard, "3D Dynamical Model for Liquid Motion Simulation in a Partially Filled Tank," in Volume 4A: Dynamics, Vibration, and Control, 2014. doi: 10.1115/IMECE2014-36211.

[9] X. Kang,"Optimal tank design and directional dynamic analysis of liquid cargo vehicles under steering and braking," Ph.D. Thesis, Concordia University Canada, Montreal, 2001.

[10] O. Noui, M. Bouazara, and M. J. Richard, "Study of Liquid Motion and Pressure Forces Applied on the Walls of Partially Filled Moving Tank," in Volume 4A: Dynamics, Vibration, and Control, 2016. doi: 10.1115/IMECE2016-65170.

[11] Maple 16, www.maplesoft.com. MapleSoft, Waterloo, ON, Canada.

[12] F.T. Dodge, "The new dynamic behaviour of liquids in moving containers," Southwest Research Institute (SRI), San Antonio, Texas, 2008.

[13] M. Toumi, M. Bouazara, and M. J. Richard, "Impact of liquid sloshing on the behaviour of vehicles carrying liquid cargo," Euro. J. Mech. A., vol. 28 , no. 5, pp. 1026-1034, Sep. 2009.

doi: 10.1016/j.euromechsol.2009.04.004. 


\author{
I \\ a C n i i $\mathbf{n ~ F a}$ \\ na i $S$ \\ Michelle Guzman Nieto ${ }^{*}$, Mostafa S.A. ElSayed ${ }^{* *}$ \\ Dept. of Mechanical and Aerospace Engineering \\ Carleton University \\ Ottawa, Ontario, Canada \\ *Michelle.guzman@carleton.ca \\ **Mostafa.elsayed@carleton.ca
}

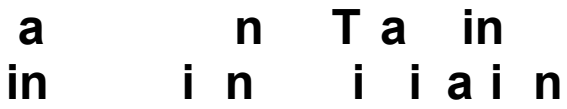

\author{
Denis Walch \\ Bombardier Aerospace \\ St-Laurent, Quebec, Canada \\ Denis.Walch@aero.bombardier.com
}

\begin{abstract}
As an industrial practice, complex structures are analyzed under a large set of dynamic operating conditions from which design load cases are selected. During design optimization, response tracking mechanisms based on modal participation factors could lead to large computational savings. In this paper we review the use of static modal participation factors as an inexpensive method to approximate the modal response of a dynamic system and extend the analysis to estimate the peak modal response when the structure is subject to a shock or pulse disturbances. A case study is presented as a proof of concept where the derived approximate peak loads are also estimated.
\end{abstract}

Keywords: modal contribution factor, modal contribution factors, dynamic analysis, static modal decomposition

\section{INTRODUCTION}

In practice, complex structures must be analyzed under a large set of dynamic operating conditions to ensure that the design will meet prescribed requirement.

During optimization, a relatively small number of such operating conditions are selected as critical and are used to minimize or maximize the objective function. However, the dynamic response is strongly dependent on the physical characteristics of the structure and thus the use of a deterministic set may lead to unexpected dynamic behaviors during different stages of design optimization.

To alleviate some of this issues, constraints such as frequency range, displacement and stresses were sometimes imposed in the optimization algorithms to tailor the structures dynamic response [1-3]; other approach was to specify a certain amount of material to attain a specific natural frequency [4]. These approaches relay heavily on a deterministic set of dynamic responses and could often lead to conservative and even off-optimal-performance designs.

Alternatively, several researchers [5-7] considered the inclusion of the dynamic behavior within the optimization problem through equivalent static load (ESL) sets that were computed using the solution of the transient analysis or approximated using modal analysis.

In the same spirit as [5-7], the dynamic behavior of any multi-degree of freedom system can be approximated through static modal participation factors (SMPF) as shown in [8]. The SMPF are inexpensive to compute and allow to assess the importance of a mode in the solution. Changes in modes with large participation factors, will then, induce large changes in the dynamic response. Thus, a response tracking mechanism based on static modal participation factors could lead to large computational savings and improve the optimization process by monitoring the dynamic response and the internal peak load of elements.

In this paper, an improved method based on SMPF is presented to approximate the modal peak responses when the structure is subjected to a shock or pulse disturbances. A case study is presented as a proof of concept, where the approximated peak out of plane bending moment at the wing root of an aircraft is approximated using SMPF.

\section{MODAL CONTRIBTION FACTORS}

The complex response of a dynamic structural system can be deconstructed into a set of simple harmonic motions $\mathrm{q}_{\mathrm{i}}(\mathrm{t})$, also known as modal responses, linearly coupled with amplitude ratios. This transformation is achieved by finding the system modal pairs in the form of natural frequencies $\omega_{i}$ and mode shapes $\Phi_{\mathrm{i}}$ that satisfy the characteristic equation of undamped free vibration system which are employed to find the system response as the following variable change:

$$
\mathrm{u}(\mathrm{t})=\Phi \mathrm{q}(\mathrm{t})
$$

Where $u(t)$ is the nodal vector of physical displacements, and $\Phi$ is the modal matrix of column concatenated eigenvectors.

Expanding (1) in a summation form we obtain:

$$
\mathrm{u}_{\mathrm{n}}(\mathrm{t})=\phi_{\mathrm{n}, 1} \mathrm{q}_{1}(\mathrm{t})+\ldots+\phi_{\mathrm{n}, \mathrm{i}} \mathrm{q}_{\mathrm{i}}(\mathrm{t})
$$

From (2), the modal contribution can be defined as the quantity of movement that each mode contributes to the total nodal response $[9,10]$. In other words, it is the product between 
the modal response $\mathrm{q}_{\mathrm{i}}(\mathrm{t})$ and the amplitude ratio $\phi_{\mathrm{n}, \mathrm{i}}$, denoted here with the Greek letter gamma $(\gamma)$ as:

$$
\gamma_{n, i}(t)=\phi_{n, i} q_{i}(t)
$$

The amplitude of each modal response can be expressed as the product of a constant called modal participation factor $\Gamma_{\mathrm{i}}$ and a time dependent term $\mathrm{D}_{\mathrm{i}}(\mathrm{t})$ [9-11] which is expressed as:

$$
\mathrm{q}_{\mathrm{i}}(\mathrm{t})=\Gamma_{\mathrm{i}} \mathrm{D}_{\mathrm{i}}(\mathrm{t})
$$

Though in general the time history of the response may be of interest, it is the value of the maximum displacement that will be used for the sizing or the design optimization of a structure. Thus, from (5), the maximum value of the response is directly proportional to the maximum dynamic response which is expressed as:

$$
\mathrm{u}^{0}=\Phi \Gamma \mathrm{D}^{0}
$$

Where $\mathrm{u}^{0}$ is the vector of maximum nodal displacements, $\mathrm{D}^{0}$ is the dynamic modal response vector subjected to a unitary load and $\Gamma$ is the diagonal matrix of modal participation factors.

Appropriate expressions for $\Gamma$ and $\mathrm{D}^{0}$ can be found through static modal decomposition as addressed in the next section.

\section{STATIC MODAL PARTICIPATION FACTORS}

The distribution of external forces acting on the structure can be expressed as a time invariant vector $f$ and a time dependent expression $\mathrm{p}(\mathrm{t})$.

$$
\mathrm{F}(\mathrm{t})=f \mathrm{p}(\mathrm{t})
$$

Since the vector $f$ is independent of time we can try to estimate a static modal deflection caused by the distribution of the forces by expanding $f$ into a matrix of static forces $f^{s t}$ acting at each node $\mathrm{j}$ and within each mode $\mathrm{i}$; where the sum of the columns of $f^{\beta t}$ is equal to the magnitude of the $\mathrm{j}$-th component of the vector $f$ [8-11] which is expressed as:

$$
f_{\mathrm{j}, \mathrm{i}}^{\mathrm{st}}=\Gamma_{\mathrm{i}, \mathrm{i}} \phi_{\mathrm{j}, \mathrm{i}} \quad\left\{\begin{array}{l}
\text { for } \mathrm{i}=1,2, \ldots, \mathrm{N} \\
\text { for } \mathrm{j}=1,2, \ldots, \mathrm{n}
\end{array}\right.
$$

Where $\mathrm{n}$ and $\mathrm{N}$ are the number of nodes and number of modes retained in the solution, respectively

$$
\Gamma=\operatorname{diag}\left(\Phi^{\mathrm{T}} \tilde{f}\right)
$$

When the eigenvectors are normalized with respect to mass, the vector of total static displacement can be found by the use of Hooke's law, which yields to:

$$
\mathrm{u}_{\mathrm{j}}^{\mathrm{st}}=\sum_{\mathrm{i}=1}^{\mathrm{N}} \frac{\Gamma_{\mathrm{i}, \mathrm{i}}}{\omega_{\mathrm{i}}^{2}} \phi_{\mathrm{j}, \mathrm{i}} \quad \text { for } \mathrm{i}=1,2, \ldots, \mathrm{N}
$$

The vector of total static displacement can then be related to the vector of total dynamic displacements by introducing (9) into (5) which results in:

$$
u_{j}^{o}=u_{j}^{s t} \omega_{i}^{2} D_{i}^{o}
$$

To derive an appropriate expression for $\mathrm{D}^{0}$, we begin by recalling the classical equation of motion in the modal coordinates, where the eigenvectors have been massnormalized:

$$
\ddot{\mathrm{q}}(\mathrm{t})+\omega_{\mathrm{i}}^{2} \mathrm{q}(\mathrm{t})=\Phi^{\mathrm{T}} f \mathrm{p}(\mathrm{t})
$$

Where the operator (*) denotes the second derivative with respect to time.

By introducing (4) and (8) into (11), we obtain (12), where the term $\Gamma$ is factored out of the resulting expression resulting in:

$$
\ddot{D}(\mathrm{t})+\omega_{i}^{2} D(t)=p(t)
$$

Equation (12) is simply the modal equations of motion subjected to a unit dynamic load; thus, depending on the form of the excitation force $\mathrm{p}(\mathrm{t})$, a particular method can be selected to find an analytical solution to (12) and consequently $\mathrm{D}^{0}$.

\section{A. Steady-state Response}

When $\mathrm{p}(\mathrm{t})$ is a periodic function, a dynamic amplification factor $R_{i}$ can be defined as the ratio between the amplitudes of the dynamic response $\mathrm{D}_{i}^{o}$ and the static response $\mathrm{D}_{i}^{\text {st }}$ [9] expressed as:

$$
\mathrm{R}_{\mathrm{i}}=\frac{\mathrm{D}_{\mathrm{i}}^{\mathrm{o}}}{\mathrm{D}_{\mathrm{i}}^{\mathrm{st}}}
$$

The amplitude of the static response is found by neglecting the contribution of the acceleration term in the well-known mass-normalized modal equations of motion, yielding to (14).

$$
\mathrm{D}_{\mathrm{i}}^{\mathrm{st}}=\frac{1}{\omega_{\mathrm{i}}^{2}}
$$

The dynamic amplification factor is the steady-state amplitude of an undamped system subjected to a unit periodic function which is expressed as:

$$
\mathrm{R}_{\mathrm{i}}=\frac{1}{\left(\omega_{\mathrm{i}}^{2}-\omega^{2}\right)}
$$

By introducing (13-15) into (10), we find the inexpensive expression, i.e. (16) that allows to estimate the maximum amplitude of the nodal physical displacements during steadystate vibration, which is expressed as:

$$
\mathrm{u}^{\mathrm{o}}=\mathrm{u}^{\mathrm{st}} \mathrm{R}
$$

To compare the output of this method with respect to the proposed methodology, a steady-state modal participation fraction $\mathrm{L}_{\mathrm{i}}^{\mathrm{st}}$ is defined in (17). This fractional number allows to compare the relative amplitudes of the modal responses $\mathrm{q}_{\mathrm{i}}$ for a given design and loading condition.

$$
\mathrm{L}_{\mathrm{i}}^{\mathrm{st}}=\frac{\left|\Gamma_{\mathrm{i}} \mathrm{D}_{\mathrm{i}}^{\mathrm{o}}\right|}{\sum_{\mathrm{i}}^{\mathrm{N}}\left|\Gamma_{\mathrm{i}} \mathrm{D}_{\mathrm{i}}^{\mathrm{o}}\right|}=\frac{\left|\Gamma_{\mathrm{i}} /\left(\omega_{\mathrm{i}}^{2}\left(\omega_{\mathrm{i}}^{2}-\omega^{2}\right)\right)\right|}{\sum_{\mathrm{i}}^{\mathrm{N}}|| \Gamma_{\mathrm{i}} /\left(\omega_{\mathrm{i}}^{2}\left(\omega_{\mathrm{i}}^{2}-\omega^{2}\right)\right) \mid}
$$

\section{B. Transient Response I}

The steady participation factors neglect the contribution of the transient components of the time domain solution. When the global maximum is of particular interest, we aim here to estimate the maximum dynamic amplitude of system response computed as:

$$
\mathrm{D}_{\mathrm{i}}^{\max }=\max (\mathrm{D}(\mathrm{t}))
$$

The transient participation fraction $L_{i}^{t s}$ can then be defined similarly to the steady participation fraction as the ratio between the maximum amplitude of the harmonic solution and the sum of the maximum amplitude of all the modes retained in the system which is expressed as: 


$$
\mathrm{L}_{\mathrm{i}}^{\mathrm{ts}}=\frac{\left|\Gamma_{\mathrm{i}} \mathrm{D}_{\mathrm{i}}^{\max }\right|}{\sum_{\mathrm{i}}^{\mathrm{N}}\left|\Gamma_{\mathrm{i}} \mathrm{D}_{\mathrm{i}}^{\max }\right|}
$$

\section{Transient Response II - Analytical solutions}

To obtain (19) the solution of the modal equations of motion, necessary for (18) calculations, is required a priori. As shown in [12] the solution of (18) was approximated using a quasi-static solution. Here we introduce a new participation factor based on the closed form solution of a Single Degree of Freedom System (SDOF).

Since this method requires the analytical solution of the modal equations of motion in the time domain, we use here the definition given in (20) as an example of an excitation signal which represent the analytical model of Tuned Discreet Gust (TDG) of an aero structure. However the methodology is applicable for any forcing function where a closed form solution exists.

$$
p(t)=1-\cos (\omega t)
$$

where $\omega$ is the forcing frequency.

The analytical solution in the time domain for an undamped SDOF system subjected to a unitary pulse, as shown in (20), is given by:

$$
D_{i}=\left\{\begin{array}{cr}
\frac{1}{\omega_{\mathrm{i}}^{2}}\left(1-\cos \left(\omega_{\mathrm{i}} \mathrm{t}\right)\right)-\frac{2}{\omega^{2}-\omega_{\mathrm{i}}^{2}} \sin \left(\frac{\left(\omega_{\mathrm{i}}+\omega\right) \mathrm{t}}{2}\right) \sin \left(\frac{\left(\omega_{\mathrm{i}}-\omega\right) \mathrm{t}}{2}\right) & 0 \leq \mathrm{t} \leq \tau \\
\frac{1}{\omega_{\mathrm{i}}^{2}}\left[\cos \left(\omega_{\mathrm{i}}(\mathrm{t}-\tau)\right)-\cos \left(\omega_{\mathrm{i}} \mathrm{t}\right)\right]+\frac{1}{2 \omega_{\mathrm{i}}\left(\omega-\omega_{\mathrm{i}}\right)}\left[\cos \left(\left(\omega-\omega_{\mathrm{i}}\right) \tau+\omega_{\mathrm{i}} \mathrm{t}\right)-\cos \left(\omega_{\mathrm{i}} \mathrm{t}\right)\right]- \\
\cdots \frac{1}{2 \omega_{\mathrm{i}}\left(\omega+\omega_{\mathrm{i}}\right)}\left[\cos \left(\left(\omega+\omega_{\mathrm{i}}\right) \tau-\omega_{\mathrm{i}} \mathrm{t}\right)-\cos \left(\omega_{\mathrm{i}} \mathrm{t}\right)\right] & \tau \leq \mathrm{t}
\end{array}\right.
$$

Where $\tau$ is the pulse length. From the classical theory of shock, the maximum response will occur during the application of the pulse whenever the forcing frequency is smaller than twice the natural frequency of the system [13]. Thus, the solution for the maximum amplitude of the dominant elastic mode and the time $t_{1}^{\max }$ at which it occurs can be estimated by differentiating (21) with respect to time during the application of the pulse and finding the roots of the derivative; alternatively, computing the complete time response of the dominant mode using (21) and extracting the peak values.

Then, the rest of the dynamic responses can be estimated by computing (20) at $t_{1}^{\max }$, that is:

$$
D_{i}^{\max }=D_{i}\left(t_{1}^{\max }\right)
$$

The transient response II participation fraction is defined similar to equations (17) and (19) as:

$$
\mathrm{L}_{\mathrm{i}}^{\mathrm{tsII}}=\frac{\left|\Gamma_{\mathrm{i}} \mathrm{D}_{\mathrm{i}}^{\max }\right|}{\sum_{\mathrm{i}}^{\mathrm{N}}\left|\Gamma_{\mathrm{i}} \mathrm{D}_{\mathrm{i}}^{\max }\right|}
$$

\section{Internal load Participation Factor}

For point loads, the element internal forces are computed as the product of the element stiffness matrix $\mathrm{K}_{\mathrm{e}}$ and the corresponding displacement vector $\mathrm{U}_{\mathrm{e}}(\mathrm{t})$ of the nodes that conform the element in the global coordinate system.

When the element and global coordinate systems do not coincide a rotation matrix $\mathrm{C}^{\mathrm{T}}$ is necessary to ensure that the internal forces computed, $\mathrm{N}_{\mathrm{e}}(\mathrm{t})$, are expressed in the element coordinate system as:

$$
\mathrm{N}_{\mathrm{e}}(\mathrm{t})=\mathrm{C}^{\mathrm{T}} \mathrm{K}_{\mathrm{e}} \mathrm{U}_{\mathrm{e}}(\mathrm{t})
$$

Where $\mathrm{K}_{\mathrm{e}}$ and $\mathrm{U}_{\mathrm{e}}(\mathrm{t})$ are the element stiffness matrix and the vector of nodal displacements in the global coordinate system., respectively.

We can expand equation (24) into its modal components as:

$$
\mathrm{N}_{\mathrm{e}}(\mathrm{t})=\mathrm{C}^{\mathrm{T}} \mathrm{K}_{\mathrm{e}} \Phi_{\mathrm{e}} \mathrm{q}(\mathrm{t})=\mathrm{C}^{\mathrm{T}} \mathrm{K}_{\mathrm{e}} \Phi_{\mathrm{e}} \Gamma \mathrm{D}(\mathrm{t})
$$

Equation (23) gives an important insight into the variables that affect the internal loads sustained by each element. The term $\Gamma$ is constant for a given loading conditions and its magnitude depends on the matrix of eigenvectors. On the other hand, the magnitude of $\mathrm{D}(\mathrm{t})$ is strongly dependent on the initial conditions and the ration between the forcing and the natural frequencies of the system. The magnitude of $\Gamma \mathrm{D}(\mathrm{t})$, thus, determines the dominance of a mode in the solution, whereas the product $\Sigma_{\mathrm{e}}=\mathrm{C}^{\mathrm{T}} \mathrm{K}_{\mathrm{e}} \Phi_{\mathrm{e}}$ determines the impact of such mode in the $\mathrm{r}$ component of the element load where $r \in\{1: 6\}$. A participation factor can then be defined as:

$$
\mathrm{L}_{\mathrm{r}, \mathrm{i}}^{\text {load }}=\frac{\left|\Sigma_{\mathrm{e}_{\mathrm{r}, \mathrm{i}}} \Gamma_{\mathrm{i}} \mathrm{D}_{\mathrm{i}}\right|}{\sum_{\mathrm{i}=1}^{\mathrm{N}}\left|\Sigma_{\mathrm{e}_{\mathrm{r}, \mathrm{i}}} \Gamma_{\mathrm{i}} \mathrm{D}_{\mathrm{i}}\right|}
$$

\section{CASE STUDY}

An aircraft stick model based on a Bombardier Aircraft platform was created to assess the performance of each of the methods described herein. The model is excited by a forcing vector $f$ with a linear distribution of a $1000 \mathrm{~N}$ on the wing root and $480 \mathrm{~N}$ on the wing tip of the form given in equation (20). The modal responses were calculated in MSC Nastran [14] and the normalized results are displayed in Figure 2.

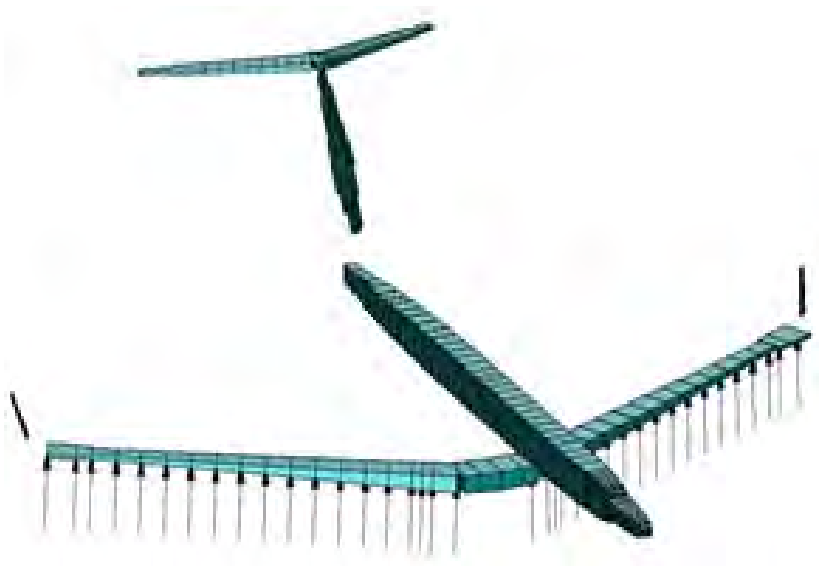

Figure 1. Case study: airplane stick model. 


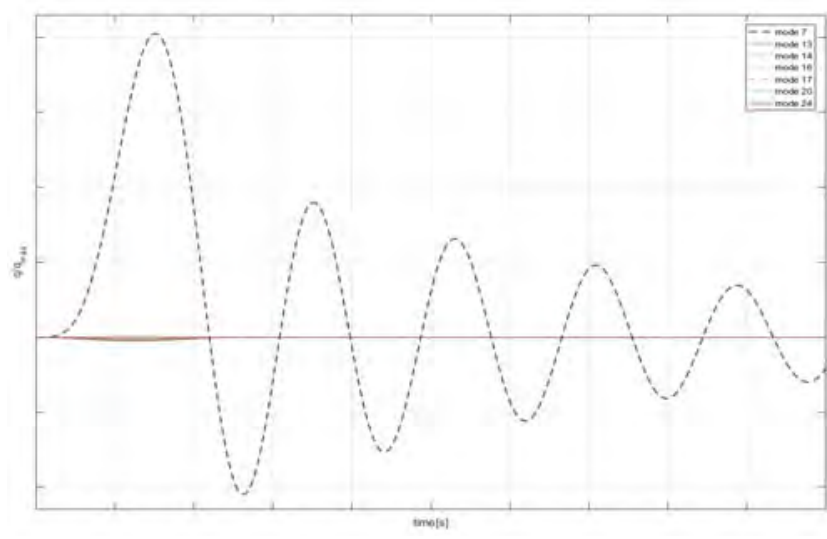

Figure 2. Normalized modal responses.

The modal participation factors of the free-free airplane model were computed using (17), (19) and (23) and the results are presented in Table I.

The results are well in agreement with the transient response of Figure 1; the first elastic mode dominates entirely the response, followed by the elastic modes 7,8 and 10 . The peak out of plane bending moment of the wing root was estimated also in MSC Nastran, and approximated using (25) with (17), (19) and (23). The percentage of error is shown in figure 3.

TABLE I. MODAL PARTICIPATION FACTORS

\begin{tabular}{|c|c|c|c|}
\hline \multicolumn{4}{|c|}{ Modal Participation Fractions } \\
\hline Elastic mode & $L_{i}^{t s}$ & $\mathrm{~L}_{\mathrm{i}}^{\mathrm{tsI}}$ & $\mathrm{L}_{\mathrm{i}}^{\mathrm{st}}$ \\
\hline 1 & $94.67 \%$ & $95.41 \%$ & $95.93 \%$ \\
\hline 2 & $0.00 \%$ & $0.00 \%$ & $0.00 \%$ \\
\hline 3 & $0.00 \%$ & $0.00 \%$ & $0.00 \%$ \\
\hline 4 & $0.00 \%$ & $0.00 \%$ & $0.00 \%$ \\
\hline 5 & $0.00 \%$ & $0.00 \%$ & $0.00 \%$ \\
\hline 6 & $0.00 \%$ & $0.00 \%$ & $0.00 \%$ \\
\hline 7 & $0.21 \%$ & $0.18 \%$ & $0.16 \%$ \\
\hline 8 & $0.27 \%$ & $0.22 \%$ & $0.20 \%$ \\
\hline 9 & $0.00 \%$ & $0.00 \%$ & $0.00 \%$ \\
\hline 10 & $0.31 \%$ & $0.27 \%$ & $0.24 \%$ \\
\hline
\end{tabular}

Both of the transient approximations present better results than that of the steady-state participation factor, which has an error of $32 \%$. This result is expected since the modal amplitude computed using (17) treats the excitation as a periodic function.

That is, even though the participation factor obtained using $L_{i}^{\text {st }}$ is well in agreement with Figure 1, the actual magnitude of the modal response is not. This large variation is reflected in the large error of the estimated out of plane bending moment.

The static modal participation factor $L_{i}^{t s}$ was computed using the time domain solution of the modal response extracted from
Nastran; its associated error is due an implicit assumption of equation (18): the modal responses are considered to be in phase. This means that the recovered amplitudes are not time consistent, thus the method tends to either over or underestimate the value of the internal load, even when the exact solution is known.

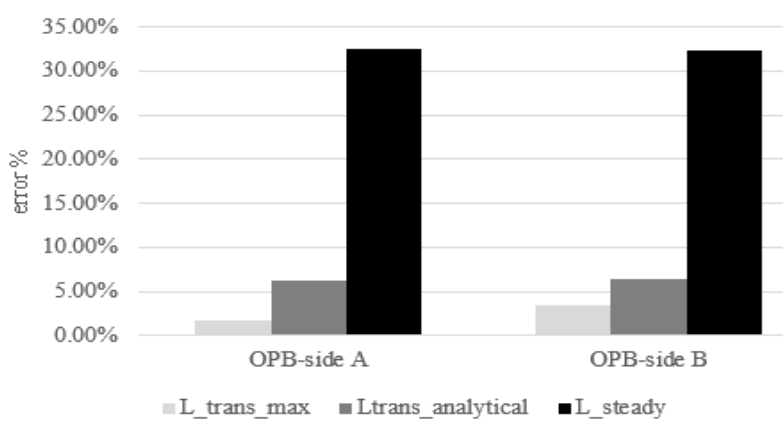

Figure 3. Normalized modal responses.

In the case of $\mathrm{L}_{\mathrm{i}}^{\text {tsII }}$ the approximation could be improve by considering modal damping in the derivation of the analytical solution (23).

Finally, the modal participation fraction of the out of plane bending moment at the wing root were computed using (26) with (17), (19) and (23). The results are shown and compared in Table II.

TABLE II. ELEMENT INTERNAL LOAD MODAL PARTICIPATION FACTORS

\begin{tabular}{|c|c|c|c|}
\hline \multicolumn{4}{|c|}{ Out of Plane Bending Moment at wing root contribution fractions } \\
\hline Elastic mode & $L_{i}^{t s}$ & $\mathrm{~L}_{\mathrm{i}}^{\mathrm{tsI}}$ & $\mathrm{L}_{\mathrm{i}}^{\text {st }}$ \\
\hline 1 & $92.449 \%$ & $93.444 \%$ & $94.263 \%$ \\
\hline 2 & $0.000 \%$ & $0.000 \%$ & $0.000 \%$ \\
\hline 3 & $0.000 \%$ & $0.000 \%$ & $0.000 \%$ \\
\hline 4 & $0.000 \%$ & $0.000 \%$ & $0.000 \%$ \\
\hline 5 & $0.000 \%$ & $0.000 \%$ & $0.000 \%$ \\
\hline 6 & $0.000 \%$ & $0.000 \%$ & $0.000 \%$ \\
\hline 7 & $0.196 \%$ & $0.168 \%$ & $0.152 \%$ \\
\hline 8 & $0.195 \%$ & $0.159 \%$ & $0.150 \%$ \\
\hline 9 & $0.000 \%$ & $0.000 \%$ & $0.000 \%$ \\
\hline 10 & $0.056 \%$ & $0.049 \%$ & $0.043 \%$ \\
\hline
\end{tabular}

Once again, the first elastic mode dominates entirely the response. However, it is noticed that the participation of the modes shown in Table II decrease. This means that term $\Sigma_{\mathrm{e}}$ from equation (25) increased the participation of higher order modes.

\section{CONCLUSIONS AND FUTURE WORK}

Static modal participation factors have the potential to be used as response tracking mechanisms during structural design optimization, since they are inexpensive to compute and allow 
to estimate the peak internal load of the elements. Thus, large design spaces could be easily updated without the need to run any dynamic analysis.

The methodology presented herein is applicable for systems with concentrated nodal forces, however, in aerospace applications we mostly encounter distributed loads such as aerodynamic and inertia loads. The extension of this methodology for aeroelastic response analysis is of particular interest and will be addressed in our future work.

\section{REFERENCES}

[1] R. Grandhi, "Structural optimization with frequency constraints: A review," AIAA J., vol. 31, no. 12, pp. 2296-2303, Dec. 1993. doi: $10.2514 / 3.11928$.

[2] S. S. Rao, "Optimization of airplane wing structures under gust loads," Comput. Struct., vol. 21, no. 4, pp. 741-749, Jan. 1985. doi: 10.1016/0045-7949(85)90150-6.

[3] V. R. Rao, N. G. R. Iyengar, and S. S. Rao, "Optimization of wing structures to satisfy strength and frequency requirements," Comput. Struct., vol. 10, no. 4, pp. 669-674, Aug. 1979. doi: 10.1016/0045-7949(79)90010-5.

[4] G. N. Vanderplaats, "Structural optimization-Past, present, and future," AIAA J., vol. 20, no. 7, pp. 992-1000, Jul. 1982. doi: 10.2514/3.51158.

[5] W. S. Choi and G. J. Park, "Transformation of dynamic loads into equivalent static loads based on modal analysis," Int. J. Numer. Methods Eng., vol. 46 , no. 1 , pp. $29-43$, Sep. 1999 doi: 10.1002/(SICI)1097-0207(19990910)46:1<29::AID-NME661>3.0.CO;2-D.

[6] W. Choi and G. Park, "Structural optimization using equivalent static loads at all time intervals," Comput. Methods Appl. Mech. Eng., vol. 191, no. 19-20, pp. 2105-2122, Mar. 2002. doi: 10.1016/S0045-7825(01)00373-5.

[7] W.-S. Choi, K. B. Park, and G. J. Park, "Calculation of equivalent static loads and its application," Nucl. Eng. Des., vol. 235, no. 22, pp. 2337-2348, Nov. 2005. doi: 10.1016/j.nucengdes.2005.05.030.

[8] L. Romera and S. Hernandez, "An improved technique for modal contribution factors of dynamic responses," in 40th Structures, Structural Dynamics, and Materials Conference and Exhibit, St. Louis, MO, 1999. doi: 10.2514/6.1999-1242.

[9] A. K. Chopra, "Modal analysis of linear dynamic systems: Physical interpretation," J. Struct. Eng., vol. 122, no. 5, pp. 517-527, May 1996. doi: 10.1061/(ASCE)0733-9445(1996)122:5(517).

[10] A. K. Chopra, Dynamics of Structures: Theory and Applications to Earthquake Engineering, 2nd ed., Upper Saddle River, NJ: Prentice Hall, 2001.

[11] M. Palermo, S. Silvestri, G. Gasparini, and T. Trombetti, "Seismic modal contribution factors," Bull. Earthquake Eng., vol. 13, no. 10, pp. 2867-2891, Apr. 2015. doi: 10.1007/s10518-015-9757-7.

[12] O. Wallrapp and S. Wiedemann, "Simulation of deployment of a flexible solar array, Multibody Sys. Dyn., vol. 7, no. 1, pp. 101-125, Feb. 2002. doi: 10.1023/A:1015295720991.

[13] C. W. De Silva, Ed., Vibration and Shock Handbook. Boca Raton, FL: CRC Press, 2005.

[14] R. H. MacNeal, Ed., The NASTRAN Theoretical Manual (Vol. 221). Hampton, VA: Scientific and Technical Information Office, NASA, 1970. 


\section{A Higher Order Theory of Beams and its Application to the MEMS/NEMS Analysis and Simulations}

\author{
A. Czenkanski \\ Department of Mechanical Engineering \\ York University \\ Toronto, Canada \\ alex.czekanski@lassonde.yorku.ca
}

\author{
V.V. Zozulya \\ Composite Materials Department \\ Centro de Investigacion Cientifica de Yucatan A.C. \\ Merida, Mexico \\ zozulya@cicy.mx
}

\begin{abstract}
A higher order theory for beams based on expansion of the two dimensional (2-D) equations of elasticity into Legendre's polynomials series has been developed. The 2-D equations of elasticity have been expanded into Legendre's polynomials series in terms of a thickness coordinate.. Cases of the first and second approximations have been considered in details. For obtained boundary-value problems, a finite element method (FEM) has been used and numerical calculations have been done with MATLAB. Developed theory has been applied for study stress-strain state of the electrostatically and thermally actuated MEMS/NEM.
\end{abstract}

\section{Keywords- higher order beam theory; MEMS; NEMS; actuators}

\section{INTRODUCTION}

Micro-Electro-Mechanical Systems (MEMS) are microscale devices or miniature embedded systems involving one or more components that enable higher-level functionality. MEMS have a characteristic length scale between $1 \mathrm{~mm}$ and 1 $\mu \mathrm{m}$. Similarly Nano-Electro-Mechanical Systems (NEMS) nanoscale devices. NEMS have a characteristic length scale between $1 \mathrm{~nm}$ and $100 \mu \mathrm{m}$ [3]. The MEMS and NEMS are widely used in engineering industries, communications, defense systems, health care, information technology, environmental monitoring, etc. [3]. Fabrication of the MEMS and NEMS rapidly increase from year to year. Therefore modeling, simulation and mathematical analysis are very important for optimizing process of their fabrication and further safety exploration.

Many MEMS and NEMS structures and devices can be considered as thin-walled structures that are exposed to mechanical loading, high temperature and electromagnetic fields and are in mechanical and thermal contact with other structural elements and massive bases through thin heat conducting layers. Often in the analysis and simulation of such devices classic models based in Euler-Bernoulli or Timoshenko hypothesis give an inaccurate result [1]. For accurate analysis and simulation of such structures and devices higher order theories may be more preferable. These theories are based on expansion of the stress-strain and temperature field components into polynomial Legendre's series in term of thickness [5]. Such an approach has significant advantages because of Legendre's polynomials are orthogonal and as result obtained equations are simpler. This approach was extended and applied to thermally actuated MEMS and NEMS in $[6,7]$.

In this presentation high order, models of beams based on nonlocal theory of elasticity have been developed and applied for MEMS and NEMS modeling and computer simulations.

\section{2-D NONLOCAL THEORY OF ELASTICITY}

We consider a curved elastic rod in a 2-D Euclidian space, which occupies the domain $V=\Omega \times[-h, h]$ with a smooth boundary $\partial V$. Here $2 h$ is thickness, $\Omega=[-l, l]$ is the middle line of the rod and $2 l$ is its length. The boundary of the rod $\partial V$ can be presented in the form $\partial V=S \cup \Omega^{+} \cup \Omega^{-}$, where $\Omega^{+}$and $\Omega^{-}$are the upper and lower sides and $S$ denotes lateral sides.

According to the theory of nonlocal elasticity [2], the basic equations for linear, homogeneous, isotropic, nonlocal elastic solid are given in the form of the following system of differential equations.

The equations of motion have the form

$$
\nabla \cdot \boldsymbol{\sigma}+\mathbf{b}=\rho \ddot{\mathbf{u}}
$$

Here $\mathbf{b}$ is a vector of body forces, $\rho$ is a density of material, $\ddot{\mathbf{u}}$ is the acceleration vector.

Kinematic relations simplify and have the form

$$
\boldsymbol{\varepsilon}=\left(\nabla \mathbf{u}+(\nabla \mathbf{u})^{T}\right)
$$

Here $\mathbf{u}$ is a displacements vector and $\boldsymbol{\varepsilon}$ is a tensor of small deformations.

According to Eringen's nonlocal elasticity theory [1] the stress at a point $\mathbf{X}$ in a body is functional of the strain field at every point of the body. In differential form nonlocal constitutive relations are presented as 


$$
\left(1-\left(e_{0} a\right)^{2} \nabla^{2}\right) \boldsymbol{\sigma}=\bar{\lambda}(t r \boldsymbol{\varepsilon}) \mathbf{I} \delta_{i j}+2 \mu \boldsymbol{\varepsilon}
$$

Here $\bar{\lambda}=\lambda \frac{2 \mu}{\lambda+2 \mu}, \lambda$ and $\mu$ are Lamè constants of classical elasticity, $e_{0}$ is the constant of material, $a$ is a parameter describes internal characteristic length.

The differential equations of motion in the form of displacements can be represented as the following

$$
\mathbf{L}_{u} \cdot \mathbf{u}+\left(1-\left(e_{0} a\right)^{2} \nabla^{2}\right)\left(\mathbf{b}-\rho \mathbf{I}_{u} \cdot \ddot{\mathbf{i}}\right)=0
$$

where $\mathbf{I}_{u}$ is the matrix operator that has on the main diagonal elements equal to one and

$$
\mathbf{L}_{u} \cdot \mathbf{u}=\mu \nabla^{2} \mathbf{u}+(\bar{\lambda}+\mu) \nabla(\nabla \cdot \mathbf{u})
$$

Differential operator of Hamilton and its combina-tions in orthogonal system of coordinates related to the middle line of the beam are presented in [5].

\section{1-D FORMULATION}

In order to reduce the 2-D problem for the couple stress theory of elastic curved beams to a 1-D one, we expand the physical parameters, that describe the stress-strain state of the beam into the Legendre polynomials series along the coordinate $x_{2}$. Then any continuous function $f(p)$ can be represented by Legendre's polynomial series according to formula

$$
f\left(x_{2}\right)=\sum_{k=0}^{\infty} a_{k} P_{k}(\varpi), a_{n}=\frac{2 k+1}{2} \int_{-h}^{h} f(\varpi) P_{k}(\varpi) d x_{2}
$$

where $\varpi=x_{2} / h$.

By expanding all functions contained in the equations (1)(3) in the Legendre's polynomial series one can obtain differential equations of motion for Legendre's polynomial series coefficients of the displacements in the form (4).

For the first order approximation theory we have

$$
\begin{aligned}
& u_{\alpha}\left(x_{1}, x_{2}\right)=u_{\alpha}^{0}\left(x_{1}\right) P_{0}(\omega)+u_{\alpha}^{1}\left(x_{1}\right) P_{1}(\omega), \\
& \varepsilon_{\alpha \beta}\left(x_{1}, x_{2}\right)=\varepsilon_{\alpha \beta}^{0}\left(x_{1}\right) P_{0}(\omega)+\varepsilon_{\alpha \beta}^{1}\left(x_{1}\right) P_{1}(\omega) \\
& \sigma_{\alpha \beta}\left(x_{1}, x_{2}\right)=\sigma_{\alpha \beta}^{0}\left(x_{1}\right) P_{0}(\omega)+\sigma_{\alpha \beta}^{1}\left(x_{1}\right) P_{1}(\omega)
\end{aligned}
$$

The matrix differential operator $\mathbf{L}_{u}$ vectors of displacements and body forces have the form

$$
\mathbf{L}_{u}=\left|\begin{array}{llll}
L_{11}^{00} & L_{12}^{00} & L_{11}^{01} & L_{12}^{01} \\
L_{21}^{00} & L_{22}^{00} & L_{12}^{01} & L_{22}^{01} \\
L_{11}^{10} & L_{12}^{01} & L_{11}^{11} & L_{12}^{11} \\
L_{21}^{10} & L_{22}^{10} & L_{21}^{11} & L_{22}^{11}
\end{array}\right|, \quad \mathbf{u}=\left|\begin{array}{c}
u_{1}^{0} \\
u_{2}^{0} \\
u_{1}^{1} \\
u_{2}^{1}
\end{array}\right|, \quad \mathbf{b}=\left|\begin{array}{c}
b_{1}^{0} \\
b_{2}^{0} \\
b_{1}^{1} \\
b_{2}^{1}
\end{array}\right|
$$

For the second order approximation theory we have

$$
\begin{aligned}
& u_{\alpha}\left(x_{1}, x_{2}\right)=u_{\alpha}^{0}\left(x_{1}\right) P_{0}(\omega)+u_{\alpha}^{1}\left(x_{1}\right) P_{1}(\omega)+u_{\alpha}^{2}\left(x_{1}\right) P_{2}(\omega), \\
& \varepsilon_{\alpha \beta}\left(x_{1}, x_{2}\right)=\varepsilon_{\alpha \beta}^{0}\left(x_{1}\right) P_{0}(\omega)+\varepsilon_{\alpha \beta}^{1}\left(x_{1}\right) P_{1}(\omega)+\varepsilon_{\alpha \beta}^{2}\left(x_{1}\right) P_{2}(\omega), \\
& \sigma_{\alpha \beta}\left(x_{1}, x_{2}\right)=\sigma_{\alpha \beta}^{0}\left(x_{1}\right) P_{0}(\omega)+\sigma_{\alpha \beta}^{1}\left(x_{1}\right) P_{1}(\omega)+\sigma_{\alpha \beta}^{2}\left(x_{1}\right) P_{2}(\omega) .
\end{aligned}
$$

The matrix differential operator $\mathbf{L}_{u}$ vectors of displacements and body forces have the form

$$
\mathbf{L}_{u}=\left|\begin{array}{llllll}
L_{11}^{00} & L_{12}^{00} & L_{11}^{01} & L_{12}^{01} & L_{11}^{02} & L_{12}^{02} \\
L_{21}^{00} & L_{22}^{00} & L_{21}^{11} & L_{22}^{01} & L_{21}^{02} & L_{22}^{02} \\
L_{11}^{10} & L_{12}^{10} & L_{11}^{11} & L_{12}^{11} & L_{11}^{12} & L_{12}^{12} \\
L_{21}^{10} & L_{22}^{10} & L_{21}^{11} & L_{22}^{11} & L_{12}^{12} & L_{22}^{12} \\
L_{11}^{20} & L_{12}^{20} & L_{11}^{21} & L_{12}^{21} & L_{11}^{22} & L_{12}^{22} \\
L_{21}^{20} & L_{22}^{20} & L_{21}^{21} & L_{21}^{12} & L_{21}^{22} & L_{22}^{22}
\end{array}\right|,\left|\begin{array}{l}
u_{1}^{0} \\
u_{2}^{0} \\
u_{1}^{1} \\
u_{2}^{1} \\
u_{1}^{2} \\
u_{2}^{2}
\end{array}\right|, \mathbf{f}=\cdot\left|\begin{array}{l}
f_{1}^{0} \\
f_{2}^{0} \\
f_{1}^{1} \\
f_{2}^{1} \\
f_{1}^{2} \\
f_{2}^{2}
\end{array}\right|
$$

Explicit expressions for differential operators in the matrix differential operators (8) for the beams are presented in [3].

The equations presented here can be used for modeling and stress-strain calculations of the beams by considering nonlocal effects at micro- and nanoscales

\section{APPLICATION TO MODELING OF THE ELECTROSTATICALLY ACTUATED MICRO AND NANO BEAMS}

Let us consider an elastic beam of the length $l$, width $b$ and thickness $2 h$ which is suspended above the rigid foundation with an initial gap $h_{0}$ in the electromagnetic field. The beam and foundation are perfect conductors and separated by a dielectric medium of permittivity $\varepsilon_{0} \varepsilon_{r}$, where $\varepsilon_{0}=8.854 \cdot 10^{-12} \mathrm{~F} / \mathrm{m}$ is the vacuum permittivity and $\varepsilon_{r}$ is the relative permittivity. A positive potential difference $V$ between the two conductors causes the beam to electrostatically deflect downwards. We assume that the gap $h_{0}$ is commensurable with the beam displacements which are assumed to be small. There is an electro conducting medium in the gap between the foundation and the beam, which does not resist the beam deformation.

From an electrical point of view, the system behaves as a variable gap capacitor. We do not consider fringing fields in the present work. Therefore a distributed force on the deformable beam due to the electric field depends on the potential difference between the two conductors and on their geometries. Because of we consider that $h_{0} / l \square 1$, it is reasonable to assume that at every point the electrostatic force per unit length depends only on the local deflection and equals the force per unit length acting on an infinitely long straight beam separated by a distance $h_{0}+u_{2}\left(x_{1}\right)$ from a ground plane. Then the magnitude of the electrostatic force $F_{e}$ acting on the deformable electrode along its normal is given by

$$
F_{e}=\frac{\varepsilon_{0} V^{2}}{2\left(h_{0}-u_{2}\right)^{2}}
$$

Thus the expression for the electrostatic force at a point on the plate depends only on the local gap $h_{0}$ and the validity of the analysis is limited to those variable gap capacitors which are locally parallel to each other. 
For convenience we introduce the nondimensional parameters of the beam deformation $\hat{u}_{\alpha}^{k}=u_{\alpha}^{k} / h_{0}$ and the nondimensional coordinate $\hat{x}_{1}=x_{1} / l$. Then the electrostatic force (11) can be presented in the form

$$
F_{e}=\frac{\alpha V^{2}}{\left(1-\hat{u}_{2}\right)^{2}}, \quad \alpha=\frac{3 \varepsilon_{0} l^{2}}{4 \mu b h^{2} h_{0}^{2}}
$$

An applied direct current (DC) voltage $V$ between the beam and foundation results in the deflection of the beam and a consequent change in the system capacitance. The applied voltage has an upper limit beyond which the electrostatic force is not balanced by the elastic restoring force in the deformable conductor. Beyond this critical voltage the deformable conductor snaps and touches the lower rigid foundation. As it was mentioned in introduction, this phenomenon is called pullin instability. We study static response of beam till pull-in instability using high order models developed here for fixedfixed and simply supported beams.

For solution of the system of differential equation (4) with operators (8) we used simple iterative algorithm as it have been proposed in [4]. Algorithm consists in the following. We start from the initial value of $\mathbf{u}^{0}\left(x_{1}\right)$, for example and calculate next value form the equation

$$
\mathbf{u}^{n+1}=\mathbf{T} \cdot \mathbf{u}^{n}
$$

where is the operator that act in the Banach space, which properties depend on used model. In our case it is the operator inverse to (8).

Algorithm (13) is not always convergent, and for its convergence operator $\mathbf{T}$ has to be a contraction in corresponding Banach space. In that case $\mathbf{T}$ has a fixed point which is a solution of the system of differential equations (4) and can be obtained from the initial value $\mathbf{u}^{0}\left(x_{1}\right)$ using iterative algorithm (13), as the limit of the iterative sequence. We do not study here conditions for convergence of the algorithm, it can be done mathematically using methods of functional analysis.

This approach we explore for investigation of the pull-in instability of the beams using first order and second order theories developed here. On each step of iteration we calculate displacements using differential equations (4) with differential operators (8)for first and second order theories respectively. Corresponding differential equations we solve numerically using finite element method (FEM). All calculations and post processing analysis have been done using commercial software MATLAB. We performed finite element analysis with MATLAB. We used PDE mode with coefficient form impute module. The differential equations (4)are presented in the form convenient for MATLAB input. In 1-D PDE coefficient module are used finite elements of Lagrange type from linear to quantic order. Also in MATLAB there is option for mesh refinement. For details we refer to the corresponding software manuals. Our numerical experiments show that for the problem under consideration FEM has good convergence and use quadratic elements with one mesh refinement gives accurate results.

We calculated dimensionless vertical displacements of the beam using first order and second order and Euler-Bernoulli theories. Calculations have been done using iteration algorithm (13) defined above. The algorithm demonstrates good convergence for $V<V_{i n}$, where $V_{\text {in }}$ is the critical value of the applied voltage $V$, that corresponds to pull-in instability of the beam and called pull-in voltage. In the vicinity of $V_{\text {in }}$ convergence of the algorithm becomes worse and worse and finally is broken down for value of $V$ close to $V_{i n}$. We define approximate value of $V_{i n}$ using that convergence property of the algorithm. For fixed-fixed beam all considered theories give the same result $V_{\text {in }} \approx 81 \mathrm{~V}$. Results of calculation of the displacements at the middle point $x_{1}=l / 2$ for various values of the applied voltage $V$, up to point of pull-in instability are presented. The results obtained using different theories are in a good correspondence.

\section{APPLICATION TO MODELING OF THE THERMALLY ACTUATED MICRO AND NANO BEAMS}

Let us consider an elastic beam of the length $l$, width $b$ and thickness $2 h$, which is settled above the rigid foundation with an initial gap $h_{0}$ in the thermal field. There is a heat-conducting medium in the gap between the foundation and the beam. The medium does not resist the beam deformation, and heat exchange between the foundation and the beam is due to the thermal conductivity of the medium. We assume that the gap $h_{0}$ is commensurable with the beam displacements which are assumed to be small.

With taking into account temperature field the 1-D differential equations of thermoelasticity and head conductivity of the beam can be presented in the form

$$
\begin{aligned}
& \mathbf{L}_{u} \cdot \mathbf{u}+\mathbf{L}_{\theta} \cdot \boldsymbol{\theta}+\mathbf{f}=0, \\
& \mathbf{L}_{\theta \theta} \cdot \boldsymbol{\theta}+\mathbf{Q}+\boldsymbol{\chi}=0
\end{aligned}
$$

The matrix differential operator $\mathbf{L}_{u}$ and vectors $\mathbf{u}$ and $\mathbf{f}$ have the forms (8) and (10) for first and second order approximations, respectively. The differential operators $\mathbf{L}_{\theta \theta}$ and $\mathbf{L}_{\theta}$ and vectors $\boldsymbol{\theta}, \mathbf{Q}$, and $\boldsymbol{\chi}$ can be presented in the following forms.

For the first order approximation theory

$$
\begin{aligned}
& \theta_{i}(\boldsymbol{x})=\theta_{i}^{0}\left(x_{1}\right) P_{0}(\omega)+\theta_{i}^{1}\left(x_{1}\right) P_{1}(\omega), \\
& \chi_{i}(\boldsymbol{x})=\chi_{i}^{0}\left(x_{1}\right) P_{0}(\omega)+\chi_{i}^{1}\left(x_{1}\right) P_{1}(\omega)
\end{aligned}
$$




$$
\mathbf{L}_{\theta}=\mathbf{E} \cdot\left|\begin{array}{c}
L_{1}^{0} \\
L_{2}^{0} \\
L_{1}^{1} \\
L_{2}^{1}
\end{array}\right|, \boldsymbol{\theta}=\left|\begin{array}{c}
\theta_{1}^{0} \\
\theta_{2}^{0} \\
\theta_{1}^{1} \\
\theta_{2}^{1}
\end{array}\right|, \quad \mathbf{L}_{\theta \theta}=\left|\begin{array}{cc}
L^{0} & 0 \\
0 & L^{1}
\end{array}\right|, \mathbf{Q}=\left|\begin{array}{c}
Q_{2}^{0} \\
Q_{2}^{1}
\end{array}\right|, \boldsymbol{\chi}=\left|\begin{array}{c}
\bar{\chi}^{0} \\
\bar{\chi}^{1}
\end{array}\right|
$$

For the second order approximation theory

$$
\begin{aligned}
& \theta_{i}(\boldsymbol{x})=\theta_{i}^{0}\left(x_{1}\right) P_{0}(\omega)+\theta_{i}^{1}\left(x_{1}\right) P_{1}(\omega)+\theta_{i}^{2}\left(x_{1}\right) P_{2}(\omega), \\
& \chi_{i}(\boldsymbol{x})=\chi_{i}^{0}\left(x_{1}\right) P_{0}(\omega)+\chi_{i}^{1}\left(x_{1}\right) P_{1}(\omega)+\chi_{i}^{2}\left(x_{1}\right) P_{2}(\omega)
\end{aligned}
$$

$$
\mathbf{L}_{\theta}=\mathbf{E} \cdot\left|\begin{array}{c}
L_{1}^{0} \\
L_{2}^{0} \\
L_{1}^{1} \\
L_{2}^{1} \\
L_{1}^{2} \\
L_{2}^{2}
\end{array}\right|, \boldsymbol{\theta}=\left|\begin{array}{c}
\theta_{1}^{0} \\
\theta_{2}^{0} \\
\theta_{1}^{1} \\
\theta_{2}^{1} \\
\theta_{1}^{2} \\
\theta_{2}^{2}
\end{array}\right|, \quad \mathbf{L}_{\theta \theta}=\left|\begin{array}{ccc}
L^{0} & 0 & 0 \\
0 & L^{1} & 0 \\
0 & 0 & L^{2}
\end{array}\right|, \mathbf{Q}=\left|\begin{array}{c}
Q_{2}^{0} \\
Q_{2}^{1} \\
Q_{2}^{2}
\end{array}\right|, \boldsymbol{\chi}=\left|\begin{array}{c}
\bar{\chi}^{0} \\
\bar{\chi}^{1} \\
\bar{\chi}^{2}
\end{array}\right|(18)
$$

In some cases under action of mechanical load and temperature filed lower side $\Omega^{-}$of the beam can be in unilateral mechanical contact with the rigid foundation. In this case the area of close mechanical contact $\partial V_{e}$ and contact forces are not known in advance. Therefore unilateral mechanical contact conditions have the form of inequalities

$$
u_{n}=\geq h_{0}, q_{n} \geq 0,\left(u_{n}-h_{0}\right) q_{n}=0, \quad \forall \mathbf{x} \in \partial V_{e}
$$

For the case if temperature set on upper side of the beam $\theta^{+}\left(x_{1}\right)$ and on the foundation $\theta^{-}\left(x_{1}\right)$ respectively, then contact conditions through heat conduction layer we have

$$
T_{k}=\frac{\lambda_{T}\left(h_{0}-u_{2}\right)\left(3 \theta^{+}+6 \theta^{0}-10 \theta^{1}\right)+\lambda_{T}^{*} h \theta^{-}}{9 \lambda_{T}\left(h_{0}-u_{2}\right)+\lambda_{T}^{*} h}
$$

Here $u_{2}\left(x_{1}\right)$ is calculated using representation (7) and (9) for the first and second order approximation theories, respectively.

It is important to mention, that systems of differential equations of thermoelasticity an heat conductivity (14) are coupled. Their connectedness is not the one usually related to dynamical thermoelasticity. We consider stationary problem and here the connectedness of the corresponding equations caused by change of the heat conducting conditions during the microbeam deformations. One can see that in the equations of heat is presented function $u_{2}\left(x_{1}\right)$ that is deflection of the beam. Presence of the function $u_{2}\left(x_{1}\right)$ in the equation (20) turns the problem into nonlinear one.

For solution of the problem we use iterative algorism developed in $[51,53]$. In the first step of iteration we assume that deflection of the beam $u_{2}\left(x_{1}\right)=0$. In this case we have traditional uncoupled problem of thermoelasticity and heat conductivity. For that uncoupled problem any analytical or numerical method can be used and corresponding equations of thermoelasticity and heat conductivity can be solved independently. We refer below to this case as uncoupled or traditional formulation. In the next steps of iterations we substitute in (20) deflection $u_{2}\left(x_{1}\right)$ obtained from the solution of the problem in previous step of iteration. Our previous and fulfilled here researches show that in the problems under consideration algorithm is convergent and convergence is fast enough.

In this study the differential equations of thermoelasticity and heat conductivity (14) we solve numerically using finite element method (FEM). All calculations and post processing analysis have been done using commercial software MATLAB and COMSOL Multiphysics. We performed finite element analysis with COMSOL Multiphysics. We used PDE mode with coefficient form impute module. The differential equations (14) are presented in the form convenient for MATLAB input. In 1-D PDE coefficient module are used finite elements of Lagrange type from linear to quantic order. Also in MATLAB there is option for mesh refinement. For details we refer to the corresponding software manuals. Our numerical experiments show that for the problem under consideration FEM has good convergence and use quadratic elements with one mesh refinement gives accurate results. Obtained with MATLAB results of the finite element analysis then have been further analyzed and compared with results obtained by Euler-Bernoulli theory.

\section{ACKNOWLEDGMENT}

This work was partly supported by the Committee of Science and Technology of Mexico (CONACYT) by the Research Grants (Ciencia Basica, Reference No 256458), which is gratefully acknowledged. The grants provided by Lassonde School of Engineering and NSERC Quanser Chair in Design Engineering are also acknowledged.

\section{REFERENCES}

[1] H. Altenbach and V.A. Eremeev, Shell Like Structures. Non Classical Theories and Applications, New York: Springer, 2011, 761 p.

[2] A.C. Eringen, Microcontinuum Field Theories I. Foundations and Solids, New York: Springer, 1999, $336 \mathrm{p}$.

[3] S.E.Lyshevski. Nano- and Micro-Electromechanical Systems. Fundamentals of Nano- and Microengineering, 2nd edition. Boca Raton: CRC Press LLC, 2005, 744 p. doi: 10.1201/9781315219288.

[4] J.A. Pelesko and D.H. Bernstein, Modeling of MEMS and NEMS, Chapman \& Hall, 2002, 364 p.

[5] I.N. Vekua Shell Theory, General Methods of Construction, Boston: Pitman Advanced Pub. Program, 1986, 302 p.

[6] V.V. Zozulya and A. Saez, "A high order theory of a thermo elastic beams and its application to the MEMS/NEMS analysis and simulations," Archive of Applied Mechanics, vol. 86, no. 7, pp. 1255-1272, 2016. doi:10.1007/s00419-015-1090-8.

[7] V.V. Zozulya and A. Saez, "A high order theory of a thermo elastic beams and its application to the MEMS/NEMS analysis and simulations," Archive of Applied Mechanics, vol. 86, no.7, pp. 1255-1272, 2015. doi: 10.1007/s00419-015-1090-8.

[8] A. Czekanski, S. A. Meguid, "Solution of dynamic frictional contact problems using nondifferentiable optimization," International Journal ofMechanical Sciences, vol. 43, pp. 1369-1386, 2001. doi: 10.1016/S0020-7403(00)00095-3. 


\section{Coupled Orbital-Attitude Dynamics of Flexible Electric Solar Wind Sail}

\author{
Gangqiang Li \\ Department of Earth Science and Space Engineering \\ York University \\ Toronto, Canada \\ lgq1984@yorku.ca
}

\author{
Zhenghong Zhu \\ Department of Mechanical Engineering \\ York University \\ Toronto, Canada \\ gzhu@yorku.ca
}

\begin{abstract}
This paper studies the dynamic characteristics of an electric solar wind propulsion system. To study the coupled interaction of the orbital and self-spinning motions of an electric solar wind propulsion system, a high-fidelity model is built up by using the nodal position finite element method, where the axial elastic and transverse dynamic motions of tether with the electric effects are considered. The coupling effects between the orbital and self-spinning motions are identified and explained, its results show that they have a significant impact on the system dynamics.
\end{abstract}

Keywords-Dynamic characteristic; Electric solar wind sail; High-fidelity; Nodal position finite element method; Coupling

\section{INTRODUCTION}

The electric solar wind sail (E-sail) is an innovative propulsion system to extract momentum from the high-speed plasma streams in the solar wind $[1,2]$. The E-sail consists of a main spacecraft connected with a multiple of long and thin conductive tethers that are positively charged by a solarpowered electron gun on the spacecraft. Each tether contains a remote unit at its end, which comprises a small gas or ion thruster to control the main tether's angular velocity $[3,4]$.

The precise dynamics of E-sail requires a high-fidelity model of the tethers. Since the length of main tethers is several orders of magnitude larger than dimension of the main spacecraft/remote units, the attitude dynamics of the main spacecraft and remote the units can be safely omitted. Thus, the E-sail model consists of inter-connected tethers with lumped masses at the center of E-sail and the tips of main tethers. Many efforts have been devoted to the modelling of Esail [3]. Initially, the E-sail was simplified as a spherical rigid pendulum model to investigate dynamic behavior of the sailplane attitude dynamics subjected to the tether voltage [5, 6]. The complicated dynamic behavior of E-sail caused by the tether dynamics is completely omitted. Then, the initial study was expanded by modeling each main tether as a rigid bar to study the coupled orbital-attitude dynamics of E-sail in a heliocentric transfer mission [6-8]. However, the rigid bar model ignores the influence of spring effect of tether on the configuration and attitude of E-sail, which is critical for its application in space exploration. To address the challenge, the main tethers are modeled by elastic catenary theory, and the shape of the main tether is parameterized by the ratios of the E-sail force over the centrifugal force and the spin plane orientation over the solar wind direction [9]. Although effective, both the rigid bar and elastic catenary models cannot describe the slack phenomenon of tethers that may happen dynamically. Furthermore, the analytical solution of elastic catenary model exists in very limited cases, which is problem specific. To address the problem, the discretized model of tether has been proposed. A typical approach is to discretize tethers into a series of lumped mass connected by massless springs and dampers $[4,10]$. It transforms the nonlinear partial differential equations of a tether into a set of ordinary differential equations, and it is actually a special case of the finite element method [11]. The finite element method is probably the most appealing technique among all numerical methods. It discretizes the continuous tether into a finite number of elements to approximate the solution within each element. The main advantage of the finite element method over the lumped method is its capability to handle the complex geometries with different tether properties along the tether length in an algorithmic fashion $[11,12]$.

Once the high-fidelity model of the E-sail is developed, the coupled dynamics of the E-sail should be investigated, such as the coupling effect between the orbital motion and the selfspinning motion around the principal axis of the E-sail $[9,10]$

\section{FINITE ELEMENT FormULATION OF ELECTRIC SOLAR WIND SAIL}

\section{A. Coordinate Systems}

Consider an E-sail as shown in Fig. 1, where the main and auxiliary tethers are divided into finite number of elements. Each element is assumed to resist tensile load only. Both main spacecraft and remote units are modeled as lumped masses without attitude dynamics. The dynamic motion of the E-sail can be described by three generalized coordinate systems: the global heliocentric-ecliptic inertial coordinate system 
$\left(O_{g} X_{g} Y_{g} Z_{g}\right)$ and the orbital coordinate system $\left(O_{o} X_{o} Y_{o} Z_{o}\right)$ as shown in Fig.1. The origin of the heliocentric-ecliptic inertial coordinate system $O_{g}$ is at the center of the Sun, where the positive $X_{g}$-axis points in the vernal equinox direction, the positive $Y_{g}$-axis is along the normal direction of the ecliptic plane, and the positive $Z_{g}$-axis completes a right-hand coordinate system. The origin of the orbital coordinate system $O_{o}$ is at the center of mass (CM) of the E-sail, where the positive $Z_{o}$-axis points from the Sun to the $\mathrm{CM}$ of E-sail, the positive $X_{o}$-axis is perpendicular to the $Z_{g}$-axis and $Z_{o}$-axis, and the positive $Y_{o}$-axis completes a right-hand coordinate system.

\section{B. Modeling of E-sail by the nodal position coordinates formulation}

The nodal position finite element method is applied to model the complex E-sail due to its advantages in handling large rigid-body motion coupled with small elastic deformation in a simple and accurate formulation.

Considering a two-node straight element in the global heliocentric-ecliptic coordinate system. Taking the $k$-th element as an example, an arbitrary position inside the element can be expressed as,

$$
\boldsymbol{X}=\boldsymbol{N}_{k} \cdot \boldsymbol{X}_{e, k}
$$

where $\boldsymbol{X}=(X, Y, Z)^{T}$ is the position vector inside the element in the global inertial coordinate system, and $\boldsymbol{X}_{e, k}=\left(X_{k}, Y_{k}, Z_{k}, X_{k+1}, Y_{k+1}, Z_{k+1}\right)^{T}$ is the nodal coordinate vector with subscripts referring to the node numbers of the $k$ th element, $\boldsymbol{N}_{k}$ is the shape function matrix defined in [11,12]

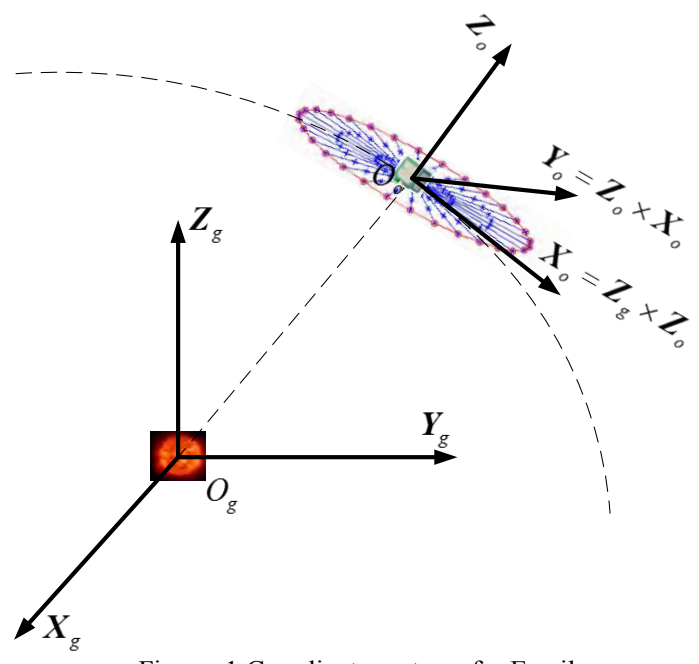

Figure. 1 Coordinate systems for E-sail

From the Hooke's law, the elastic stress of $k$-th element can be written as,

$$
\sigma_{k}=E_{k} \varepsilon_{l, k}=E_{k}\left(\frac{L_{e, k}-L_{e 0, k}}{L_{e 0, k}}\right)=E_{k} \boldsymbol{B}_{e, k} \boldsymbol{X}_{e, k}-E_{k}
$$

where the subscript $k$ denotes $k$-th element, $\varepsilon_{l, k}$ is the longitudinal strain of element, $L_{e 0, k}$ is the unstretched length of the element. $E_{k}$ is the Young's modulus of tether material, and $\boldsymbol{B}_{e, k}$ is the element strain matrix defined in $[11,12]$.

The element mass matrix $\boldsymbol{M}_{e, k}$ can be derived from the kinetic energy, such as,

$$
T_{e, k}=\frac{1}{2} \int_{0}^{L_{e, k}} \rho_{k} A_{k} \dot{\boldsymbol{X}}_{e, k}{ }^{T} \dot{\boldsymbol{X}}_{e, k} d s=\frac{1}{2} \dot{\boldsymbol{X}}_{e, k}^{T} \boldsymbol{M}_{e, k} \dot{\boldsymbol{X}}_{e, k}
$$

where $\rho_{k}$ and $A_{k}$ are the material density and cross-section area of the tether with the subscript $k$ indicating the $k$-th element.

The symbol ( ) denotes the first order derivative with respect to time, and element mass matrix $\boldsymbol{M}_{e, k}$ is given in. It should be noted that the mass matrix is constant in the global inertial coordinate system.

The strain energy due to the longitudinal deformation can be written as,

$$
\begin{aligned}
U_{e, k}= & \frac{1}{2} \int_{0}^{L_{e, k}} E_{k} A_{k} \varepsilon_{l, k}^{2} d s=\frac{1}{2} \boldsymbol{X}_{e, k}^{T} \boldsymbol{K}_{e, k} \boldsymbol{X}_{e, k}-\boldsymbol{X}_{e, k}^{T} \boldsymbol{F}_{k, k}+ \\
& \frac{1}{2} E_{k} A_{k} L_{e, k}
\end{aligned}
$$

where $\boldsymbol{K}_{e, k}$ is the nonlinear element stiffness matrix and $\boldsymbol{F}_{k, k}$ is the generalized nodal force vector resulting from the elasticity of element in the global inertial coordinate system[11,12].

The virtual work done by external forces including the gravitational and Coulomb forces can be written as,

$$
\begin{gathered}
\delta W_{g, k}=\int_{0}^{L_{e, k}} \delta \boldsymbol{X}^{T} \rho_{k} \boldsymbol{g}_{c} A_{k} d s=\delta \boldsymbol{X}_{e, k}^{T} \boldsymbol{F}_{g, k} \\
\delta W_{c, k}=\int_{0}^{L_{e, k}} \delta \boldsymbol{X}^{T} \boldsymbol{f}_{c} d s=\delta \boldsymbol{X}_{e, k}^{T} \boldsymbol{F}_{c, k}
\end{gathered}
$$

where $\boldsymbol{F}_{g, k}$ and $\boldsymbol{F}_{c, k}$ are the vectors of equivalent nodal gravitational and Coulomb forces, respectively.

Accordingly, the Hamilton's principle for the E-sail from $t_{1}$ to $t_{2}$ can be obtained as,

$$
\delta \Pi=\int_{t_{1}}^{t_{2}}\left(\delta T_{e, k}-\delta U_{e, k}+\delta W_{g, k}+\delta W_{c, k}\right) d t=0
$$

Equation (7) yields the dynamic equation as, 


$$
\frac{d}{d t}\left(\frac{\partial T_{e, k}}{\partial \dot{\boldsymbol{X}}_{e, k}^{T}}\right)-\frac{\partial T_{e, k}}{\partial \boldsymbol{X}_{e, k}^{T}}+\frac{\partial U_{e, k}}{\partial \boldsymbol{X}_{e, k}^{T}}=\boldsymbol{F}_{g, k}+\boldsymbol{F}_{c, k}
$$

Substituting Eqs. (3) and (4) into Eq. (8) and rearranging them into the matrix form yield the following dynamic equation for the $k$-th element,

$$
\boldsymbol{M}_{e, k} \ddot{X}_{e, k}+\boldsymbol{K}_{e, k} \boldsymbol{X}_{e, k}=\boldsymbol{F}_{k, k}+\boldsymbol{F}_{g, k}+\boldsymbol{F}_{c, k}
$$

The dynamic equations of E-sail can be obtained by assembling Eq. (9) with the standard assembly procedure in the finite element method $[12,13]$. It is worth pointing out that the primary variables in the NPFEM are the nodal position coordinates instead of the nodal displacements in the conventional finite element method. It should be noted the internal damping of the tether is not considered in the flight dynamics due to the lack of experimental data in space. Nonetheless, the damping effect generally stabilizes the disturbance to the E-sail system. Thus, the neglect of damping will not affect the validation of current investigation.

\section{RESULTS AND DISCUSSIONLI}

In the current paper, the E-sail is assumed fully deployed and spinning. The dynamic equations of the E-sail are solved numerically by an implicit 4th order Runge-Kutta GaussianLegendre scheme with a Symplectic property that is suitable for the long-term numerical integration [12]. The maximum iteration number and error tolerance of each iteration step are set to 10 and 10-9, respectively. The physical properties of the E-sail are listed in Table. I.

TABLE I. PHYSICAL PROPERTIES OF E-SAIL

\begin{tabular}{|l|c|}
\hline Parameters & Values \\
\hline Mass of main satellite $(\mathrm{kg})$ & 100 \\
\hline Number of main tethers (auxiliary tethers) & 10 \\
\hline Length of each main tether $(\mathrm{km})$ & 10 \\
\hline Material type of main/auxiliary tether & Aluminum/Kapton \\
\hline Material density of main tether $\left(\mathrm{kg} / \mathrm{m}^{3}\right)$ & 2700 \\
\hline Material density of auxiliary tether $\left(\mathrm{kg} / \mathrm{m}^{3}\right)$ & 1420 \\
\hline Elastic modulus of main tether $\left(10^{9} \mathrm{~N} / \mathrm{m}^{2}\right)$ & 70 \\
\hline Diameter of main tether $(\mathrm{m})$ & $7.3810^{-5}$ \\
\hline Elastic modulus of the auxiliary tether $\left(10^{9} \mathrm{~N} / \mathrm{m}^{2}\right)$ & 2.5 \\
\hline Width of auxiliary tether $(\mathrm{m})$ & 0.03 \\
\hline
\end{tabular}

\section{A. The steady state of self-spinning E-sail}

The initial equilibrium conditions, such as the tension of tether, for the given initial position and velocity of E-sail are unknown. Consequently, a special start-up procedure is developed with very small numerical damping to damp out the initial transient oscillation until the steady state of spinning Esail is obtained. In this case, the main and auxiliary tethers of E-sail are modeled by one truss element respectively. The tethers are assumed spinning and subjected to a central gravitational field, but the center of E-sail is stationary in the space. The numerical damping is removed after $500 \mathrm{~s}$ and then the simulation continued for another 5,900 s. The results of the steady state of a spinning E-sail are shown in Fig. 5. As shown in Fig. 2(a), the numerical damping successfully damps out the transient oscillation in the first $500 \mathrm{~s}$. Then, the system state maintains constant as expected. The same phenomenon can be observed in the tensions of the main and auxiliary tethers, and the angular velocities of the remote units, seen in Figs. 2(b)2(c).
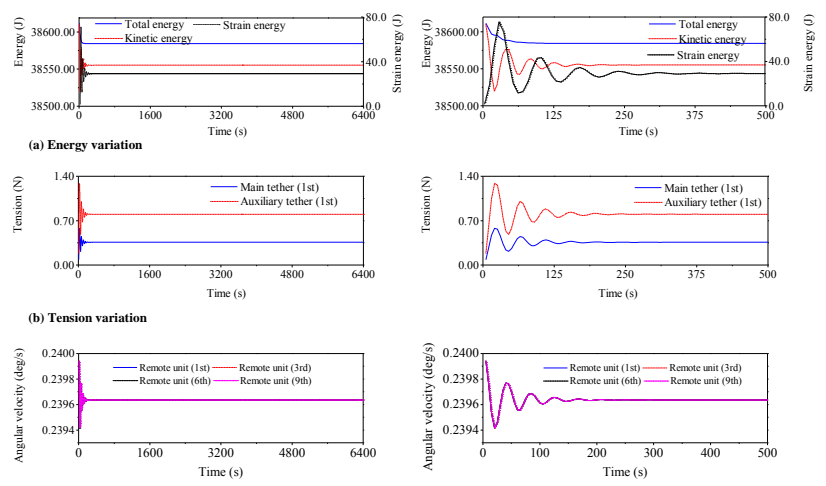

(c) Angular velocity variation

Figure. 2. Steady state of a self-spinning E-sail (a) energy (b) tension in main and auxiliary tethers (c) angular velocity of remote unit.

\section{B. The coupled orbital and attitude dynamics of E-sail}

The coupling effect between the orbital and spinning motions of E-sail is investigated. The E-sail is temporally assumed to move in a circular orbit with a radius of $R=1 \mathrm{AU}$. Accordingly, the orbital angular velocity $\Omega$ of E-sail can be calculated by $\Omega=\sqrt{\mu_{s} / R^{3}}$, where $\mu_{s}$ and $R$ are the gravitational constant and orbital radius, respectively. In this case, the main and auxiliary tethers are modeled with one truss element respectively. The initial spin rate $\omega$ and the time step in the simulation are set as $0.24 \mathrm{deg} / \mathrm{s}$ and $0.01 \mathrm{~s}$, respectively.

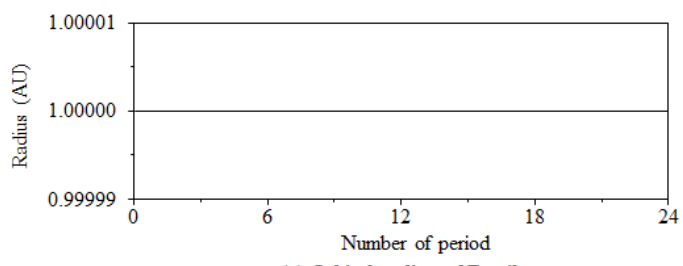

(a) Orbital radius of E-sail

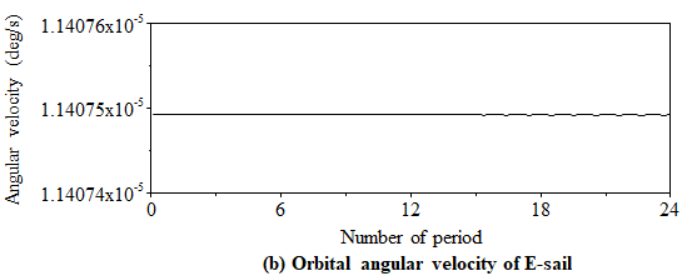

Figure.3. The results of the orbital dynamic motion of E-sail

The characteristics of the coupled motion between the orbital and spinning motions of E-sail is analyzed for a period of 36,000 s. The simulation results are shown in Figs. 3 to 4, where the time scale is changed from seconds to the number of 
period of self-spinning, $\omega_{0} t /(2 \pi)$. First, it can be found in Fig. 3 that the orbital radius and angular velocity of E-sail subject to the gravity are constant as expected. It shows that the assumption of constant orbital radius $R$ and orbital angular velocity $\Omega$ is reasonable. Second, Fig. 4 shows that the spin rate, the length of main tether, and the tension in the main and auxiliary tethers change periodically at the frequency of spin rate of E-sail, which is corresponding to the term of $(\boldsymbol{L} \cdot \boldsymbol{\Omega}) \boldsymbol{\omega}$ for the spin rate. Fig. 4(a-b) shows the variations of main tether length and its rate vs the time. They are all very small. Thus, the induced Coriolis effects $2 \boldsymbol{\omega} \times \dot{\boldsymbol{L}}^{\prime}$ and $2 \boldsymbol{\Omega} \times \dot{\boldsymbol{L}}^{\prime}$ are negligible in the short term. Fig. 4(c) shows the spin rate changes from the maximum to minimum in every period. This phenomenon is caused by the coupling between the orbital and spinning motions of E-sail as presented in section 2.3. Moreover, it is seen that the tension variation in the auxiliary tether is in phase with the spin rate, while it is out of phase with the tension in the main tether, see Fig. 4(d). The reason is that the periodic motion of the spin rate induces the periodic motion of the auxiliary tether and remote unit within one period. Furthermore, the centrifugal force $(\boldsymbol{\omega} \cdot \boldsymbol{\omega}) \boldsymbol{L}$ causes periodical variation of tension in the main tether. Finally, three conclusions can be drawn from the above results: (i) the coupling effect between the orbital and spinning motions of Esail will induce a periodic oscillation of the spin rate. Then, the oscillation of spin rate induces the oscillation of tension in the main and auxiliary tethers. This phenomenon is not observed in previous references $[5,10]$. (ii) for the short-term analysis, the assumption of constant value of orbital radius and angular velocity is reasonable. (iii) the Coriolis effects $2 \boldsymbol{\omega} \times \dot{\boldsymbol{L}}^{\prime}$ and $2 \boldsymbol{\Omega} \times \dot{\boldsymbol{L}}^{\prime}$ are the long-term effect [5].
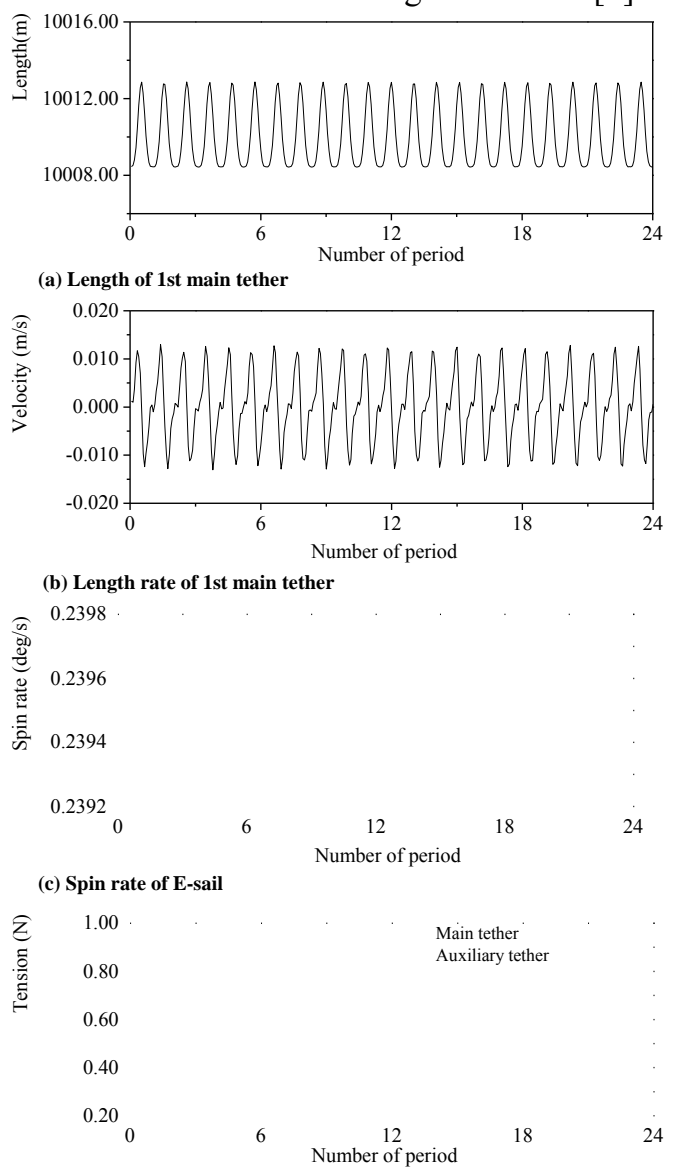

(d) Tension in the main and auxilliary tethers

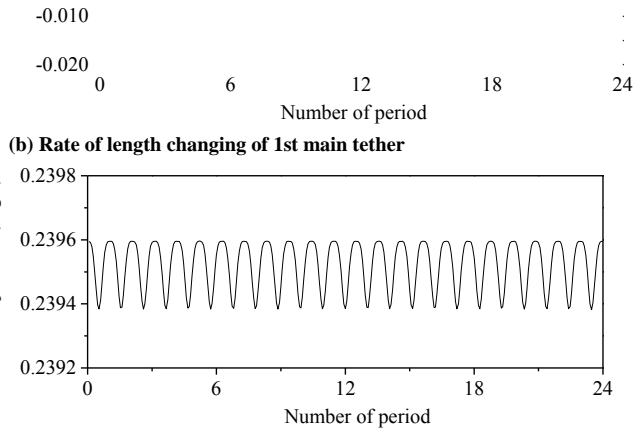

(c) Spin rate of E-sail

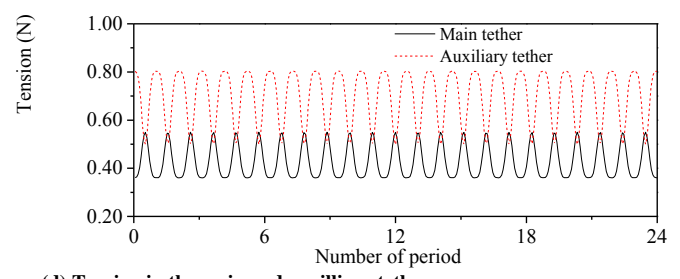

(d) Tension in the main and auxilliary tethers

Figure.4. The results of spinning of E-sail. (a) Spin rate (b)-(c) Tether length and rate of length changing of Main tether (1st) (d) Tension in the main (1st) and auxiliary tethers (1st).

\section{CONCLUSION}

A high-fidelity model of an electric solar wind sail is developed by using the nodal position finite element method. The coupling effects between the orbital and self-spinning motion are analyzed when the system subjects to the gravitational force only, and major coupling terms are identified and examined. The numerical simulations show that the coupling effect between the orbital and self-spinning motions causes a periodic variation of the spin rate and tension in the tethers.

\section{ACKNOWLEDGMENT}

This work is supported by the Discovery Grant of Natural Sciences and Engineering Research Council of Canada and Canadian Space Agency

\section{REFERENCES}

[1] P. Janhunen, "Electric sail for spacecraft propulsion," J. Propul. Power vol. 20, no. 4, pp. 763-764, Jul. 2004. doi: 10.2514/1.8580.

[2] P. Janhunen and A. Sandroos, "Simulation study of solar wind push on a charged wire: Basis of solar wind electric sail propulsion, Ann. Geophys., vol. 25, no. 3, pp. 755-767, Mar. 2007. doi: 10.5194/angeo-25-755-2007.

[3] G. Mengali, A.A. Quarta and P. Janhunen, "Electric sail performance analysis," J. Spacecr. Rockets, vol. 45, no. 1, pp. 122-129, Jan. 2008. doi: $10.2514 / 1.31769$.

[4] P. Toivanen and P. Janhunen, "Electric solar wind sail: Deployment, long-term dynamics, and control hardware requirements," in: Advances in Solar Sailing, M. Macdonald, Ed. Berlin: Springer-Verlag, ch. 58, pp. 977-987. doi: 10.1007/978-3-642-34907-2_58.

[5] P.K. Toivanen and P. Janhunen, "Spin plane control and thrust vectoring of electric solar wind sail," J. Propul. Power, vol. 29, no. 1, pp. 178-185, Jan. 2013. doi: 10.2514/1.B34330.

[6] P. Toivanen, P. Janhunen and J. Envall, "Electric sail control mode for amplified transverse thrust," Acta Astronaut., vol. 106, pp. 111-119, Jan. 2015. doi: 10.1016/j.actaastro.2014.10.031.

[7] M. Huo, J. Zhao, S. Xie and N. Qi, "Coupled attitude-orbit dynamics and control for an electric sail in a heliocentric transfer mission," PLOS ONE, vol. 10, no. 5, p. e0125901, May 2015. doi: 10.1371/journal.pone.0125901. 
[8] M. Huo, G. Mengali and A. A. Quarta, "Electric sail thrust model from geometrical perspective," J. Guid. Control Dyn., vol. 41, no. 3, pp. 735-741, Mar. 2018. doi: 10.2514/1.G003169.

[9] P. Toivanen and P. Janhunen, "Thrust vectoring of an electric solar wind sail with a realistic sail shape, Acta Astronaut., vol. 131, pp. 145-151, Feb. 2017. doi: 10.1016/j.actaastro.2016.11.027.

[10] P. Janhunen and P. Toivanen, "TI tether rig for solving secular spinrate change problem of electric sail," arXiv:1603.05563, Mar. 2016.

[11] Z. H. Zhu and S. A. Meguid, "Elastodynamic analysis of aerial refueling hose using curved beam element," AIAA J., vol. 44, no. 6, pp. 1317-1324, Jun. 2006. doi: 10.2514/1.17311.

[12] G.Q. Li and Z.H. Zhu, "Long-term dynamic modeling of tethered spacecraft using nodal position finite element method and symplectic integration," Celest. Mech. Dyn. Astr., vol. 123, no. 4, pp. 363-386, Aug. 2015. doi: 10.1007/s10569-015-9640-5.

[13] F. J. Sun, Z. H. Zhu, and M. LaRosa, "Dynamic modeling of cable towed body using nodal position finite element method," Ocean Eng., vol. 38, no. 4, pp. 529-540, Mar. 2011. doi: 10.1016/j.oceaneng.2010.11.016. 


\title{
Buckling and Post Buckling Behavior for Unsymmetrical Laminates Part I: Curing Cycle
}

\author{
A. Y. Elruby ${ }^{1}$, T. Ahmed ${ }^{2}$, S. Nakhla ${ }^{3 *}$ \\ ${ }^{1} \mathrm{Ph} . \mathrm{D}$. Candidate. ${ }^{2}$ Graduate student and ${ }^{3 *}$ Assistant Professor \\ Dept. of Mechanical Engineering, Faculty of Engineering and Applied Science \\ Memorial University of Newfoundland \\ St. John's, Canada \\ E-mail*: satnakhla@mun.ca
}

\begin{abstract}
This is the first of two companion papers that examine the elastic buckling and post-buckling behavior of thin unsymmetric cross-ply laminates. When cured in a flat mold these panels possess two cylindrical equilibrium configurations. From stability perspective this problem is identified as a bifurcation buckling problem. As explained in literature thermal mismatch between plies and existing geometric imperfections triggered this behavior. Therefore, and according to Koiter it is necessary to measure and account for these geometric imperfections to accurately predict the cured shapes. Since imperfection measurement is challenging and cannot be possible in the stage of design. This work applies a unified finite element methodology based on Koiter's theory to predict the cured shapes. This methodology is consistently applied in commonly used finite element computer codes, ABAQUS, ANSYS and LS-DYNA, and their predictions are compared.
\end{abstract}

Keywords- bistable, unsymmetric, cross-ply, curing, buckling, post-buckling.

\section{INTRODUCTION}

When thin unsymmetric composite laminate is cured in a press or an autoclave, it will develop out-of-plane deformations at room temperature. These deformations are due to mismatch in thermal expansion behavior and elastic properties of laminae within the laminate. Hyer [1] reported the shapes of several families of unsymmetric laminates and observed that the roomtemperature shapes do not always conform to the predictions of classical lamination theory. To justify this mismatch Hamamoto and Hyer [2] measured the cured laminate and reported its lack of thickness uniformity, i.e., thicker in the middle than it is near the edges. The reason for such non-uniform thickness was explained to be caused by resin flow during curing resulting in differences in fiber volume faction from one location to the next within the laminate. Some other factors were also suggested to be responsible for geometric imperfections, such as the handlayup technique in which it is impossible to precisely orient or stack different laminae.

Several solutions reported in literature focused on predicting the cured shapes of unsymmetric cross-ply laminates [3]. The first solution among these was developed by Hyer [4] presenting the extended classical lamination (ECLT) theory based on the Principal of Minimum Total Potential Energy (PMTPE). Predictions obtained from ECLT was limited to unsymmetric square laminates with straight edges. The first finite element solution was originally proposed by Schlecht and Schulte [5] in which they added a subroutine in finite element (FE) code MARC applying so-called backward method to obtain the cured shapes of unsymmetric square cross-ply laminates. Their finite element analysis (FEA) predictions agreed with Hyer's measurements and analysis [1, 4]. The first analytically correct FEA methodology using Abaqus finite element code was initially presented then published by Tawfik et al. $[6,7]$. Their methodology was based on Koiter's theory [8] and their predictions of room temperature cured shapes were in good agreement with Hyer's measurements and analysis [1, 4]. Giddings et al. [8] developed an improved FE model in ANSYS V11.0 to predict the cured shapes of thin unsymmetric laminates. They developed two FE models, the first model was based on 'idealised' nominal ply-thickness which required a temporary force during the initial curing simulation to allow convergence. While the second 'improved model' used optical microscopy, data obtained post curing to account for resin rich areas in the FE simulation. Giddings et al. [8] reported excellent predictions using the 'improved model' and acceptable predictions by the model based on idealised ply-thickness. In summary, to enable FEA to accurately predict the cured shapes of thin unsymmetric laminates one must account for deviations in geometry such as resin rich areas. Meanwhile measuring the specific to certain laminate deviation may not be possible prior to manufacturing. Therefore, an analytical solution based on Koiter's theory provides the potential for accurate predictions prior to manufacturing, e.g. during the design stages.

To this end the current work aimed at providing a general applicability of the proposed methodology of Tawfik et al. [6, 7]. Their proposed methodology which they developed in Abaqus was consistently applied in Ansys and LS-Dyna. Cured shapes of thin unsymmetric laminated as predicted by all $\mathrm{FE}$ codes, ABAQUS, ANSYS and LS-DYNA were compared, and conclusions were provided. 
TABLE II. ELEMENT TYPES IN DIFFERENT FE CODES

\section{MethodOlgy}

\section{A. Problem definition}

Tawfik [10] and Tawfik et al. [6, 7, 11] were pioneers in investigating the shapes of unsymmetric cross-ply laminate under thermal curing stresses using FEA. Based Koiter's theory [8], considering the effect of the geometric imperfections, they developed a FE based methodology for predicting such cured shapes.

Tawfik et al. $[6,7,11]$ implemented their FE methodology in ABAQUS and proved that without geometric imperfection cured shape of an unsymmertic laminate cannot be predicted by FEA accurately. They did an extensive study regarding shape prediction using their FE methodology and finally came up with two distinct useful relations which they named as the temperature-curvature relationship and the design curve.

This current work is focused on presenting a generalized FE based methodology to predict the shapes of unsymmetric crossply laminates under thermal curing load by replicating Tawfik's ABAQUS model in two other commercial FE codes, ANSYS and LS-DYNA, and finally comparing the ANSYS and LSDYNA results with the ABAQUS results.

\section{B. Description of basic FEA model}

The cross-ply unsymmetric laminate used in this model has square geometric configuration and it is a $\left[0_{n} / 90_{n}\right]$ laminate of Hexcel IM7/8551-7 graphite/epoxy prepreg with mechanical properties provided in Table I.

Tawfik [10] conducted the convergence study with all the shell elements available in Abaqus element library and ended with the selection of S4R as the element type and $20 \times 20$ as the mesh size for optimal modeling of this problem in ABAQUS.

For the present work uses shell elements and $20 \times 20$ mesh for modeling. Table II documents the details about the element types used in three different FE codes.

The centre point of the laminate geometry is fixed in all degrees of freedom during the curing cycle. The applied load during curing cycle is a temperature drop with a magnitude of $156^{\circ} \mathrm{C}$. Fig. 1 shows the basic ABAQUS model used for this curing cycle problem.

TABLE I. MECHANICAL PROPERTIES OF MATERIAL AS REPORTED BY TAWFIK [10]

\begin{tabular}{|c|c|c|c|c|c|c|c|}
\hline Material & $\begin{array}{c}\boldsymbol{E}_{\mathbf{1 1}} \\
(\mathbf{G P a})\end{array}$ & $\begin{array}{c}\boldsymbol{E}_{22} \\
(\mathbf{G P a})\end{array}$ & $\begin{array}{c}\boldsymbol{G}_{\mathbf{1 2}} \\
(\mathbf{G P a})\end{array}$ & $\boldsymbol{v}_{\mathbf{1 2}}$ & $\begin{array}{c}\boldsymbol{\alpha}_{\mathbf{1}} \\
\left(\mathbf{1 0}^{-\mathbf{6}} /{ }^{\mathbf{0}} \mathbf{C}\right)\end{array}$ & $\begin{array}{c}\boldsymbol{\alpha}_{\mathbf{2}} \\
\left(\mathbf{1 0}^{-\mathbf{6}} /{ }^{\mathbf{}} \mathbf{C}\right)\end{array}$ & $\begin{array}{c}\boldsymbol{t} \\
(\boldsymbol{\mu m})\end{array}$ \\
\hline $\begin{array}{c}\text { IM7/8551-7 } \\
\text { graphite/epo } \\
\text { xy prepreg }\end{array}$ & 141.18 & 7.20 & 4.45 & 0.30 & 0.30 & 30.98 & 138.75 \\
\hline
\end{tabular}

\begin{tabular}{|c|c|c|c|c|}
\hline FE code & $\begin{array}{c}\text { Element } \\
\text { name }\end{array}$ & $\begin{array}{c}\text { Element } \\
\text { type }\end{array}$ & $\begin{array}{c}\text { Number } \\
\text { of DOF* }\end{array}$ & Description \\
\hline $\begin{array}{c}\text { ABAQUS } \\
{[12]}\end{array}$ & S4R & $\begin{array}{c}\text { Shell } \\
\text { element }\end{array}$ & 6 & $\begin{array}{c}\text { A four-node general- } \\
\text { purpose finite-membrane- } \\
\text { strain shell with reduced } \\
\text { integration for hourglass } \\
\text { control }\end{array}$ \\
\hline $\begin{array}{c}\text { ANSYS } \\
{[13]}\end{array}$ & Shell 181 & $\begin{array}{c}\text { Shell } \\
\text { element }\end{array}$ & 6 & $\begin{array}{c}\text { A four-node thin to } \\
\text { moderately-thick shell } \\
\text { with both full and reduced } \\
\text { integration schemes }\end{array}$ \\
\hline $\begin{array}{c}\text { LS-DYNA } \\
{[14]}\end{array}$ & Type 16 & $\begin{array}{c}\text { Shell } \\
\text { element }\end{array}$ & 6 & $\begin{array}{c}\text { A four-node fully } \\
\text { integrated thin shell with } \\
\text { assumed strain } \\
\text { interpolation and available } \\
\text { hourglass control option }\end{array}$ \\
\hline
\end{tabular}

*Degrees of freedom
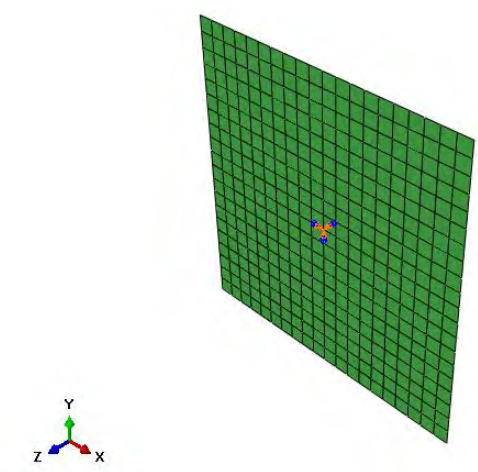

Figure 1. Image of basic model from ABAQUS

\section{Equation and curves presented by Tawfik and Tawfik et al.}

1) The temperature-curvature relationship

Utilizing the concept of geometric imperfection, Tawfik et al. $[6,7,11]$ used their ABAQUS model to predict the bifurcation point or the tendency of retaining cylindrical-like. Two curvatures of two adjacent sides of the square unsymmetric cross-ply laminate ware plotted in a temperature vs. curvature graph, keeping the cure cycle relative temperature along the abscissas and curvatures along the ordinates. The point from which the two curvatures start deviating from each other is the bifurcation point and the graph was named as temperaturecurvature relationship. This deviation of two curvatures of two adjacent sides indicates the tendency of a unsymmetric crossply laminate to retain two different curvatures with bistable behavior while curing. Their ABAQUS analysis predicted the bifurcation point to occur at a relative temperature of $140.60{ }^{\circ} \mathrm{C}$ with a laminate curvature of $0.290 \mathrm{~m}^{-1}$. Curvatures were approximate using (1).

$$
\kappa=\frac{1}{R} \cong \frac{8 d}{C^{2}+4 d^{2}}
$$

where $\kappa$ is the approximated curvature, $R$ is the radius, $d$ is the out-of-plane displacement and $C$ is the chord length as shown in Fig. 2. 


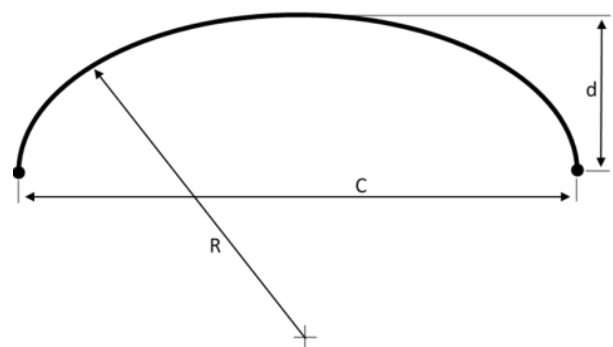

Figure 2. Curvature of laminate

\section{2) The design curve}

Tawfik [10] and Tawfik et al. [6, 7, 11] predicted the out-ofplane deformation and expressed it as the effect of laminate side length-to-thickness ratio. This relationship was named as the design curve while keeping the non-dimensional curvature along the abscissas and non-dimensional side length along the ordinates. This design curve shows that the relative side lengthto-thickness ratio is one of the parameters controlling the panel's bistable behavior and there is a critical value of side length in units of thickness that makes the laminate to have cylindrical-like shape. This non-dimensional critical side length for square $\left[0_{n} / 90_{n}\right]$ laminate was documented as $L_{C}^{*}=82.8$, below which the cured panel assumes a saddle shape and above it the panel is bistable with cylindrical-like equilibrium shapes. They defined the non-dimensional side length and nondimensional curvature as respectively (2) and (3).

$$
\begin{gathered}
L^{*}=\frac{L}{t} \\
\kappa^{*}=\kappa \times L \times \frac{180}{\pi}
\end{gathered}
$$

where $L^{*}$ and $\kappa^{*}$ are non-dimensional side length and nondimensional curvature respectively. While $L, t$ and $\kappa$ are the side length, thickness and curvature of the laminate respectively.

\section{Modelling curing cycle in ABAQUS}

Tawfik [10] and Tawfik et al. [6, 7, 11] presented, in ABAQUS the cylindrical-like cured shapes of an unsymmetric laminate can be easily obtained through three major steps as follows

- Eigenvalue buckling analysis of the perfect geometry using the *BUCKLE option which is also known as buckling analysis.

- Introducing geometric imperfections as a linear superposition of buckling eigenmodes of the perfect geometry using *IMPERFECTION option.

- A geometrically non-linear load-displacement analysis of the imperfect structure using the *STATIC, STABILIZE method which is one kind of post buckling analysis.
Tawfik [10] and Tawfik et al. $[6,7,11]$ stated, one of the major step of their methodology is accounting for geometric imperfection. So, they studied the influence of imperfection size on predicting the bifurcation point and figured out that imperfection in a must for predicting cylindrical-like cured shape and the most suitable imperfection size for this problem is one tenth of the laminate thickness. This current work will also use the same size of imperfection.

Tawfik [10] mentioned about two different procedures available in ABAQUS for solving non-linear systems of equations and among those two, the one which can introduce an artificial damping into the solution is more suitable for problems with local instability and hence enables to capture the cylindrical shape during the curing cycle and in ABAQUS it is available in the name of STABILIZE.

Further for modeling the curing cycle in LS-DYNA and ANSYS Tawfik's three-step methodology will be followed as guideline.

\section{E. Modelling curing cycle in LS-DYNA}

With LS-DYNA cylindrical-like cured shapes of an unsymmetric laminate can be predicted as follows.

- Eigenvalue buckling analysis of the perfect geometry using the *CONTROL_IMPLICIT_EIGENVALUE [14] option.

- Introducing geometric imperfection as a linear superposition of buckling eigenmodes of the perfect geometry using *PERTURBATION_NODE [14] option.

- A geometrically non-linear load-displacement analysis of the imperfect structure using the *CONTROL_IMPLICIT_STABILIZATION [14] option.

ABAQUS creates the buckling results in normalized form but in LS-DYNA eigen buckling analysis results are not normalized. So, this should be taken into consideration while selecting the value of the scale factor.

\section{F. Modelling curing cycle in ANSYS}

Unlike ABAQUS and LS-DYNA solving this current problem in ANSYS requires following four steps:

- Static analysis step to get an initial deformation that is going to be used in the Eigen buckling analysis.

- Eigen buckling analysis step is used to generate the Eigen modes for the model based on the applied thermal load in the static analysis step using any available eigen solver i.e.; SUBOPT [13].

- Based on extracted Eigen mode shapes the geometry of the model is to be perturbed using a required imperfection value. The command UPCOORD [13] is used by multiplying a scale factor of imperfections to a specified step results.

- The last step is to solve a non-linear analysis for the imperfect geometry obtained from the previous step using STABILIZE [13] command. 
Like LS-DYNA eigen buckling analysis results in ANSYS are not normalized by default. So, this should be taken into consideration while selecting the value of the scale factor.

\section{G. A unified Finite Element Analysis methodology}

Curing of a laminate is a locally instable nonlinear problem which is highly imperfection sensitive. So, modeling the curing cycle in FEA requires solving a linear perturbation associated with geometric imperfection and a nonlinear load-displacement analysis in a row [10]. Implementation of modeling technique proposed by Tawfik in these three FE codes can be summarized as:

Modeling a curing cycle in any FEA code requires three available options.

- An eigenvalue solver for buckling analysis to get the eigenmodes.

- An option to introduce a geometric imperfection into the structure.

- An implicit non-linear solver that can introduce an artificial damping into the solution to make this locally instable problem to capture the cylindrical-like shape.

Tawfik [10] first came up with this three-step Finite Element Analysis methodology and can be used as a unified guideline for modelling curing cycle in any FEA as LS-DYNA and ANSYS.

\section{RESULTS AND DISCUSSION}

Tawfik [10] did an extensive study to figure out the effect of geometric imperfection on predicting the cured shapes of unsymmetric laminates and proved using ABAQUS that without any imposed geometric imperfection FEA cannot capture the cylindrical-like shape and predicts the saddle shape. Fig. 3 is the predicted saddle shape by ABAQUS in absence of imperfection and Fig. 4 is the cylindrical-like shape in the presence of imperfection.

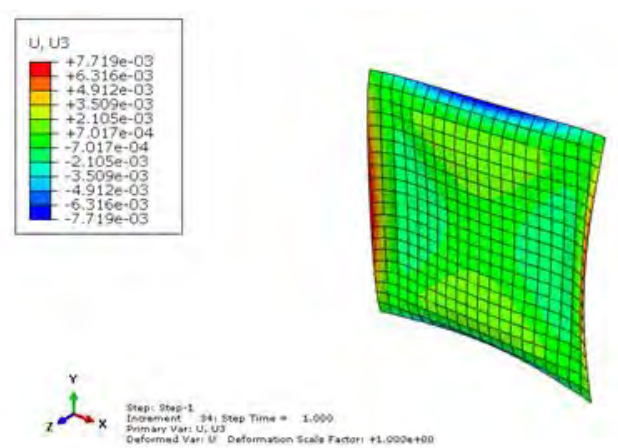

Figure 3. Saddle shape predicted by ABAQUS in absence of geometric imperfection

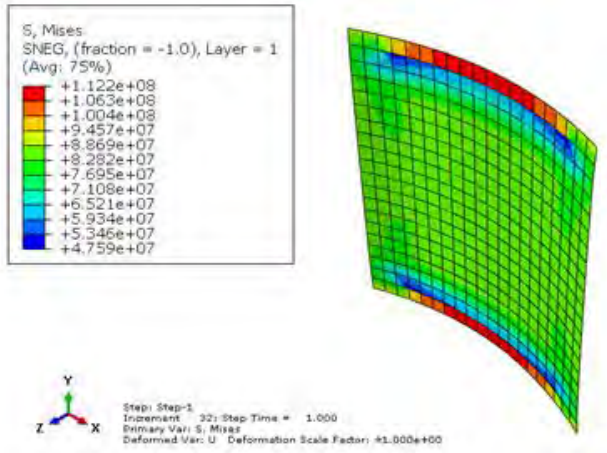

Figure 4. Cylindrical-like shape predicted by ABAQUS in presence of geometric imperfection

With the same size of imperfection suggested by Tawfik [10] and without imperfection LS-DYNA and ANSYS both predict cylindrical-like and saddle shape respectively as ABAQUS does. Fig. 5 is the predicted saddle shape by LSDYNA in absence of imperfection and Fig. 6 is the predicted cylindrical-like shape in presence of imperfection. Fig. 7 and Fig. 8 respectively represents the saddle and cylindrical-like shape predicted by ANSYS.

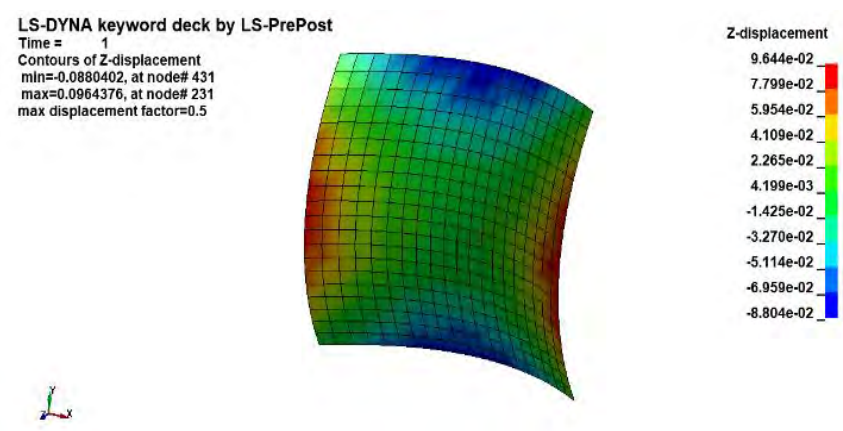

Figure 5. Saddle shape predicted by LS-DYNA in absence of geometric imperfection

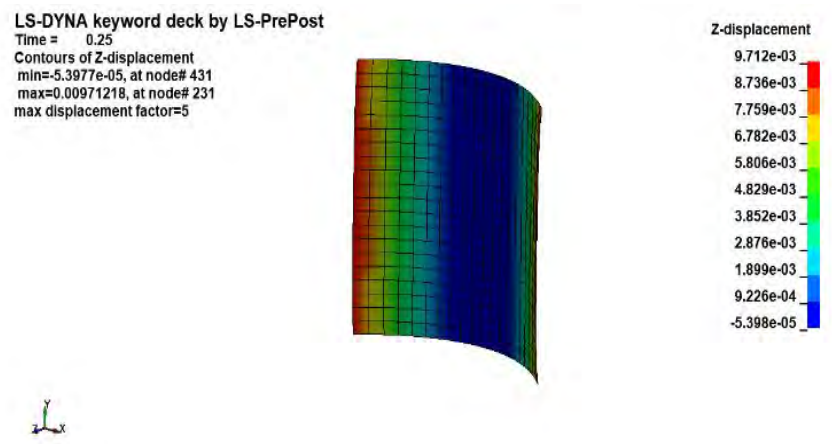

Figure 6. Cylindrical-like shape predicted by LS-DYNA in presence of geometric imperfection 


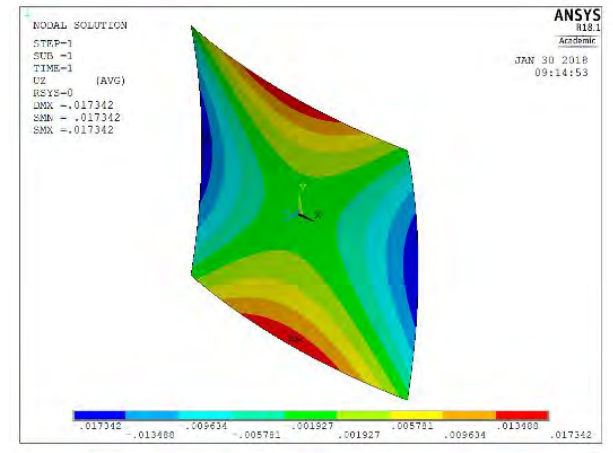

Figure 7. Saddle shape predicted by ANSYS in absence of geometric imperfection

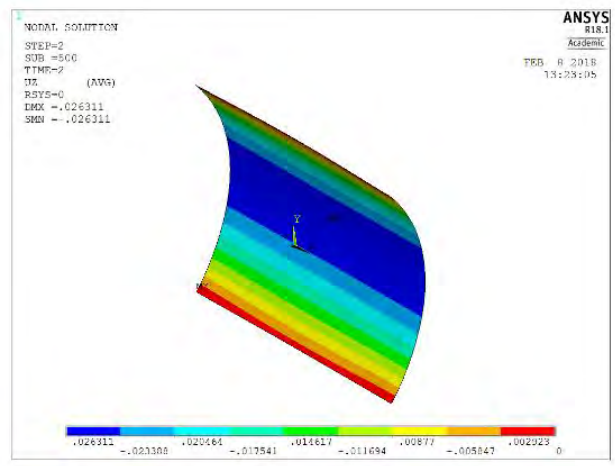

Figure 8. Cylindrical-like shape predicted by ANSYS in presence of geometric imperfection

To compare the ABAQUS, ANSYS and LS-DYNA results, both the temperature-curvature relationship and the design curve from these three codes have been plotted and compared with each other.

To plot the temperature-curvature relation Tawfik's [10] results in ABAQUS were reiterated in ABAQUS, ANSYS and LS-DYNA while recording curvatures of both sides and thermal load at every time increment. Fig. 9 shows the temperature curvature relation from ABAQUS, ANSYS and LS-DYNA for square $\left[0_{\mathrm{n}} / 90_{\mathrm{n}}\right]_{\mathrm{T}}$ laminate of Hexcel IM7/8551-7 graphite/epoxy prepreg in the same graph. The figure also shows the results from ABAQUS, ANSYS and LS-DYNA are in an excellent agreement and the bifurcation temperature prediction from these three FE codes is almost the same.

To plot the design curve the side length of the square $\left[0_{n} / 90_{n}\right]_{T}$ laminates was varied from $0.01 \mathrm{~m}$ to $0.15 \mathrm{~m}$ keeping the laminate thickness and layup unchanged while recording the corresponding curvatures of the two sides of the laminate at the end of the curing cycle. Fig. 9 Shows the design curve from ABAQUS, ANSYS and LS-DYNA for square $\left[0_{n} / 90_{n}\right]_{T}$ laminate of Hexcel IM7/8551-7 graphite/epoxy prepreg in the same graph and it also shows the results from ABAQUS, ANSYS and LS-DYNA are in an excellent agreement and the critical side length prediction from these three FEA codes is almost the same.

A good agreement of ANSYS and LS-DYNA results with the ABAQUS result makes Tawfik's three-step FE based methodology unified.

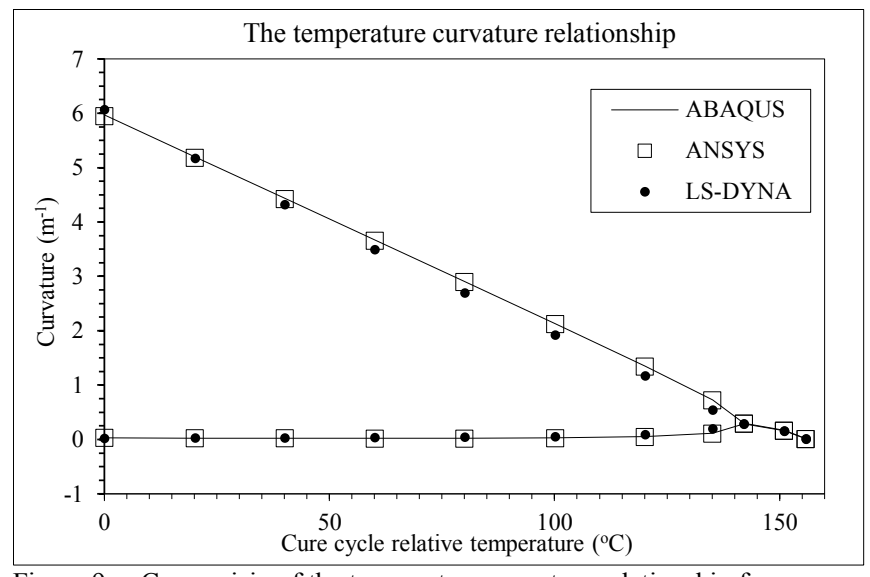

Figure 9. Comparisin of the temperature curvature relationship for square $\left[0_{n} / 90_{n}\right]_{T}$ laminate of Hexcel IM7/8551-7 graphite/epoxy prepreg from ABAQUS, ANSYS and LS-DYNA.

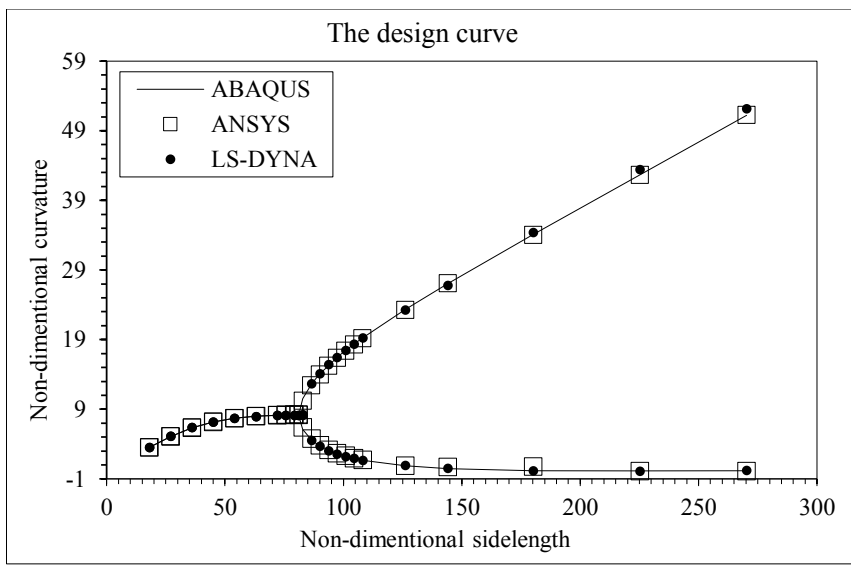

Figure 10.Comparison of the design curve for square $\left[0_{n} / 90_{n}\right]_{T}$ laminate from ABAQUS, ANSYS and LS-DYNA

\section{CONCLUSION}

This work addressed the buckling behavior of thin unsymmetric laminates and their out-of-plane cured shapes. An analytically correct methodology is obtained from literature and applied in commonly used finite element computer codes, Abaqus, Ansys and LS-Dyna. Predicted out-of-plane cured shapes of thin unsymmetric cross-ply laminated were compared and results from all FE codes were found in excellent agreement. Therefore, the currently used analytically correct methodology proved its generality and applicability in commonly used FE software codes.

\section{REFERENCES}

[1] M. W. Hyer, "Some Observations on the Cured Shape of Thin Unsymmetric Laminates," J. Compos. Mater., vol. 15, no. 2, pp. 175-194, Mar. 1981. doi: $10.1177 / 002199838101500207$.

[2] H. Akira and M. W. Hyer, "Non-linear temperature-curvature relationships for unsymmetric graphite-epoxy laminates," Inter. J. Sol. Struct., vol. 23, no. 7, pp. 919-935, 1987. doi: 10.1016/0020-7683(87)90087-4. 
[3] S. A. Emam and D. J. Inman, "A Review on Bistable Composite Laminates for Morphing and Energy Harvesting," App. Mech Rev., vol. 67, no. 6, p. 60803, Dec. 2015. doi: 10.1115/1.4032037.

[4] M. W. Hyer, "Calculations of the Room-Temperature Shapes of Unsymmetric Laminatestwo," J. Compos. Mater., vol. 15, no. 4, pp. 296-310, Jul. 1981. doi: 10.1177/002199838101500401.

[5] M. Schlecht and K. Schulte, "Advanced Calculation of the RoomTemperature Shapes of Unsymmetric Laminates," J. Compos. Mater., vol. 33, no. 16, pp. 1472-1490, Aug. 1999. doi: 10.1177/002199839903301601.

[6] S. Tawfik, X. Tan, S. Ozbay and E. Armanios, "Modeling of anticlastic stability in elastically tailored composites," in Proceedings of 20th annual technical ASC conference, Drexel University, Philadelphia, Pennsylvania, 2005.

[7] S. Tawfik, X. Tan, S. Ozbay and E. Armanios, "Anticlastic Stability Modeling for Cross-ply Composites," J. Compos. Mater., vol. 41, no. 11, pp. 1325-1338, 2006. doi: 10.1177/0021998306068073.

[8] Koiter WT. On the stability of elastic equilibrium, Ph.D. Thesis, 1945, Polytechnic Institute Delft, NASA TTF-10833; 1967.

[9] P. F. Giddings, C. R. Bowen, A. I. T. Salo, H. A. Kim, and A. Ive, "Bistable composite laminates: Effects of laminate composition on cured shape and response to thermal load," Compos. Struct., vol. 92, no. 9, pp. 2220-2225, Aug. 2010. doi: 10.1016/j.compstruct.2009.08.043.

[10] S. Tawfik, "Stability and morphing characteristics of bistable composite laminates, $\mathrm{PhD}$ dissertation, School of Aerospace Engineering, Georgia Institute of Technology, Atl., Georg., 2008. [Online].

Available: https://smartech.gatech.edu/bitstream/handle/1853/24702/ tawfik_samer_a_200808_phd.pdf.

[11] S. Tawfik, D. S. Dancila and E. Armanios, "Planform Effects Upon the Bistable Response of Cross-Ply Composite Shells," Composites, Part A, vol. 42, no. 7, pp. 825-833, 2011. doi: 10.1016/j.compositesa.2011.03.012.

[12] Simulia, D. S. (2014). Abaqus 6.14 user manual. Providence, RI, USA: DS SIMULIA Corp.

[13] ANSYS Inc. (2015). ANSYS, U. S. Version 16.2, Canonsburg, USA.

[14] LS-DYNA Manual: Volume I, Version 971, 7374. Livermore Software Technol. Corp., Livermore, CA, $2013 .$. 


\title{
Buckling and Post Buckling Behavior for Unsymmetrical Laminates Part II: Stability Characteristics
}

\author{
T. Ahmed ${ }^{1}$, A. Y. Elruby ${ }^{2}$, S. Nakhla ${ }^{3 *}$ \\ ${ }^{1}$ Graduate student, ${ }^{2} \mathrm{Ph}$.D. Candidate and ${ }^{3 *}$ Assistant Professor \\ Dept. of Mechanical Engineering, Faculty of Engineering and Applied Science \\ Memorial University of Newfoundland \\ St. John's, Canada \\ E-mail*: satnakhla@mun.ca
}

\begin{abstract}
This is the second of two companion papers that examine the elastic buckling and post buckling behavior of unsymmetric cross-ply laminates. The existence of geometric imperfection and thermal mismatch between plies result in these panels to retain two equilibrium configurations despite being cured in a flat mold. These equilibrium configurations conform to cylindrical shapes of orthogonal and opposite curvatures. Under externally applied load a panel undergoes snap-through behavior from one equilibrium shape to the other. The unified finite element methodology based on Koiter's theory and presented in the first companion paper is extended to predict the required force responsible for snap-through behavior. Accordingly, ABAQUS, ANSYS and LS-DYNA finite element codes are used to predict the critical snap-through forces and their predictions are compared.
\end{abstract}

Keywords-stability; bistable; snap-through; snap-back; finite element; post buckling

\section{INTRODUCTION}

It was observed that a cured thin unsymmetric cross-ply laminate will possess two equilibrium configurations. These equilibrium configurations conform to two cylindrical shapes of equal and opposite curvatures which was initially reported by Hyer [1]. The cured panel when triggered it undergoes snapthrough behavior from one equilibrium shape to the other, hence called bistable. A panel exhibit this post buckling response if it originally possessed large out-of-plane at room temperature caused by thermal stresses in its layers. The effect of thermal stresses and the existence was originally explained and documented by Hamamoto and Hyer [2].

Bistable panels found many applications in morphing and energy harvesting as discussed in Emam and Inman [3]. It is credited to Hyer to present the first analytical solution to predict the out of plane deformations of these panels [4]. Based on the Principal of Minimum Total Potential Energy PMTPE, Hyer developed the extended classical lamination theory (ECLT). Later Hyer's colleagues Dano [5] and Schultz [6] extended the ECLT to predict the requirements to trigger snap-through behavior using shape memory alloys (SMA) and piezoelectric actuators, respectively. Tawfik [7] extended his ABAQUS finite element (FE) methodology to predict the force required to trigger snap-through behavior in panels with various planforms. This methodology was based on Koiter's theory [8] in accounting for geometric imperfections and originally proposed by Tawfik et al. $[9,10]$. ABAQUS based FE predictions of snap-through loads and test results reported in [7] were in good agreement. Therefore, the proposed ABAQUS based FE methodology proved to be successful predicting post buckling behavior. As reported by Emam and Inman [3] many researchers worked on the same problem, i.e. triggering snapthrough behavior in bistable panels. Meanwhile, the ABAQUS FE methodology proposed by Tawfik [7] proved successful application in predicting mechanical force or piezoelectric actuators requirements. Moreover, this methodology is analytically correct and do not require obtaining any measurement of actual imperfection in the manufactured panel. Therefore, this methodology possesses potential to be used during the design stages of morphing applications.

The current work targeted generalizing the proposed methodology of Tawfik [7] and investigating the applicability in wide variety of FE computer codes. Therefore, this proposed methodology was consistently applied in commonly used FE codes ANSYS and LS-DYNA. The force required to trigger snap-through behavior in thin unsymmetric cross-ply laminates was predicted by ABAQUS, ANSYS and LS-DYNA. Predictions obtained from FE analysis were compared with test values and proved to be consistent and in good agreement.

\section{Methodolgy}

\section{A. Problem definition}

Tawfik [7] and Tawfik et al. [9, 10,11] were the pioneer in investigating the stability of unsymmetric cross-ply laminates using Finite Element Analysis (FEA). Utilizing two back to back non-linear load-displacement analysis they developed a FE based methodology to examine the bistable characteristics of thin unsymmetric cross-ply laminates using ABAQUS, a commercial FE code, and presented their analysis outcome as a load-displacement curve to show that a cured unsymmetric 
cross-ply laminate is stable in its both equilibrium positions which is termed as the bistable behavior. For different geometries, forces required to trigger snap-through of such bistable laminates were also predicted and compared with test measurements.

This current work is focused on presenting a generalized FE based methodology to capture the bistable characteristics of a unsymmetric cross-ply laminate under thermal load by replicating Tawfik's ABAQUS [9, 10] work in two other commercial FE codes, ANSYS and LS-DYNA, and finally comparing their results with Tawfik's ABAQUS and test results.

\section{B. Description of basic FEA model}

The unsymmetric cross-ply laminate used in this problem has a square geometric configuration and it is a thin $\left[0_{2} / 90_{2}\right]_{\mathrm{T}}$ laminate of Hexcel IM7/8551-7 graphite/epoxy prepreg with mechanical properties provided in Table I. Tawfik [7] conducted the convergence study with all the shell elements available in ABAQUS element library and ended with the selection of S4R as the element type and $20 \times 20$ as the mesh size for optimal modeling of this problem in ABAQUS. This present work will use the same mesh size and the similar types of elements in two other FE codes. Table II documents the details about the element types used in three different FE codes.

Tawfik [7] mention that investigation of stability characteristics is a subsequent analysis of curing and in FEA bistable behavior of a thermally cured unsymmetric cross-ply laminate can easily be captured by performing an extra nonlinear load-displacement analysis right after the curing cycle. Only the boundary conditions and loading need to be changed during this extra step. During the first step, the curing cycle, center point of the laminate geometry is kept fixed in all degrees of freedom and the applied load is a temperature drop with a magnitude of $156{ }^{\circ} \mathrm{C}$. During the second step, for analyzing stability characteristics, temperature of the cured shape is kept constant at $-156{ }^{\circ} \mathrm{C}$ and the center point of the cured shape is kept free only in z-direction while the four corners are kept free in all degrees other than the z-direction. A concentrated force, $P$, with a value higher than the snap-through load is applied along the z-direction at the center point as displayed in Fig. 1.

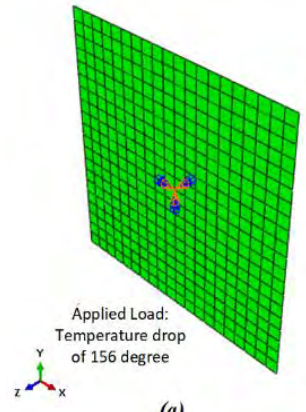

(a)

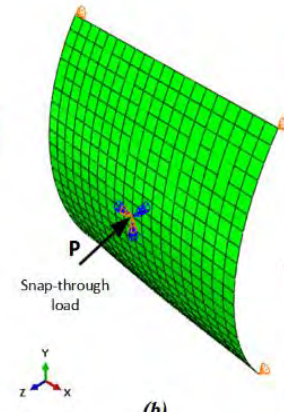

(b)

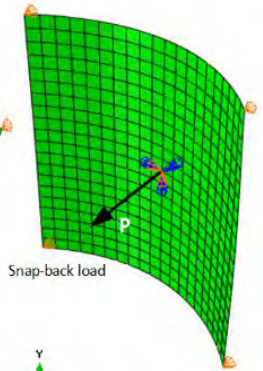

(c)
Figure 1. ABAQUS model at different stages of analysis showing the corresponding shapes, boundary conditions and applied load; a) at the beginning of first step $b$ ) at the beginning of the second step c) at the end of the second step.
TABLE I. MECHANICAL PROPERTIES OF MATERIAL AS REPORTED IN KIM [12] AND ALSO USED BY TAWFIK [7].

\begin{tabular}{|c|c|c|c|c|c|c|c|}
\hline Material & $\begin{array}{c}\boldsymbol{E}_{11} \\
(\mathbf{G P a})\end{array}$ & $\begin{array}{c}\boldsymbol{E}_{22} \\
(\mathbf{G P a})\end{array}$ & $\begin{array}{c}\boldsymbol{G}_{12} \\
(\mathbf{G P a})\end{array}$ & $\boldsymbol{v}_{12}$ & $\begin{array}{c}\boldsymbol{\alpha}_{1} \\
\left(\mathbf{1 0}^{-\mathbf{6}} /{ }^{\mathbf{0}} \mathbf{C}\right)\end{array}$ & $\begin{array}{c}\boldsymbol{\alpha}_{2} \\
\left(\mathbf{1 0}^{-\mathbf{6}} /{ }^{\mathbf{0}} \mathbf{C}\right)\end{array}$ & $\begin{array}{c}\boldsymbol{t} \\
(\boldsymbol{\mu m})\end{array}$ \\
\hline $\begin{array}{c}\text { IM7/8551-7 } \\
\text { graphite/epoxy } \\
\text { prepreg }\end{array}$ & $\begin{array}{c}146.1 \\
4\end{array}$ & 8.472 & 3.879 & 0.341 & 0.30 & 30.98 & 138.75 \\
\hline
\end{tabular}

TABLE II. ELEMENT TYPES IN FE CODES.

\begin{tabular}{|c|c|c|c|c|}
\hline FEA code & $\begin{array}{c}\text { Element } \\
\text { name }\end{array}$ & $\begin{array}{c}\text { Element } \\
\text { type }\end{array}$ & $\begin{array}{c}\text { Number } \\
\text { of DOF* }\end{array}$ & Description \\
\hline $\begin{array}{c}\text { ABAQUS } \\
{[13]}\end{array}$ & S4R & $\begin{array}{c}\text { Shell } \\
\text { element }\end{array}$ & 6 & $\begin{array}{c}\text { A four-node general- } \\
\text { purpose finite-membrane- } \\
\text { strain shell with reduced } \\
\text { integration for hourglass } \\
\text { control }\end{array}$ \\
\hline $\begin{array}{c}\text { ANSYS } \\
{[14]}\end{array}$ & Shell 181 & $\begin{array}{c}\text { Shell } \\
\text { element }\end{array}$ & 6 & $\begin{array}{c}\text { A four-node thin to } \\
\text { moderately-thick shell } \\
\text { with both full and reduced } \\
\text { integration schemes }\end{array}$ \\
\hline $\begin{array}{c}\text { LS-DYNA } \\
{[15]}\end{array}$ & Type 16 & $\begin{array}{c}\text { Shell } \\
\text { element }\end{array}$ & 6 & $\begin{array}{c}\text { A four-node fully } \\
\text { integrated thin shell with } \\
\text { assumed strain } \\
\text { interpolation and available } \\
\text { hourglass control option }\end{array}$ \\
\hline
\end{tabular}

*Degrees of freedom

Fig. 1 shows the ABAQUS model and its geometric shapes at different stages of the stability analysis. It also illustrates the corresponding boundary conditions and applied loads associated with those stages.

\section{Notations ued by Tawfik and Tawfik et al.}

Tawfik [7] and Tawfik et al. [11] used some important notations to describe the stability characteristics of a thermally cured bistable unsymmetric cross-ply laminate.

- First equilibrium shape: Laminate's shape when the larger curvature is along the x-axis, Fig. $2 \mathrm{a}$.

- Second equilibrium shape: Laminate's shape when the larger curvature is along the y-axis, Fig. $2 b$.

- Snap-through: Forcing a laminate to snap from first equilibrium shape to the second equilibrium shape, Fig. 2a.

- Snap-back: Forcing a laminate to snap from the second equilibrium shape to the first equilibrium shape, Fig. $2 \mathrm{~b}$.

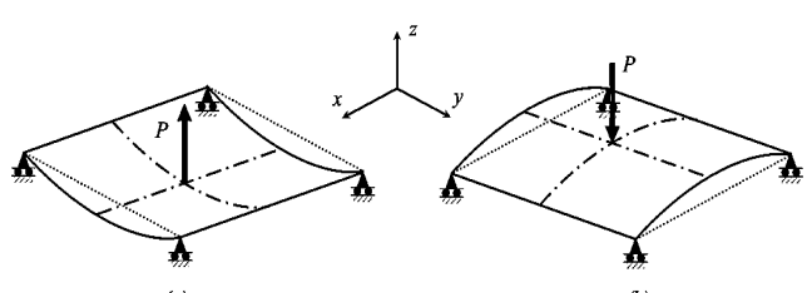

(a)

(b)

Figure 2. Triggering snap-through in cylindrical panel via concentrated force $[7,11]$. 


\section{Snap-through and snap-back analysis with ABAQUS}

Tawfik [7] and Tawfik et al. [9, 10] showed that snapthrough and snap-back can be captured in ABAQUS using following steps

- $\quad$ Eigenvalue buckling analysis of the perfect geometry using the *BUCKLE keyword to get the eigenmodes.

- Introduce imperfections into the geometry using *IMPERFECTION keyword.

- Non-linear load-displacement analysis of the imperfect geometry using the *STATIC, STABILIZE keyword to get the cured shape.

- A second non-linear load displacement analysis of the cured shape using *STATIC, STABILIZE keyword to capture the snap-through and snap-back.

Among these four steps, first three are for obtaining the cured shape while the fourth step is for snap-through and snapback of that cured shape $[7,9,10]$.

The second non-linear load-displacement analysis in Tawfik's [7, 9] two-step FE methodology was pursued by performing the following sub steps

- Boundary condition of the cured shape was reset such that the center point of the panel was free only in zdirection and the four corner points were free in all degrees other than the z-direction.

- A concentrated force, $P$, higher than the snap-through load was applied at the center point along the $\mathrm{z}$ direction such that it tended to switch the curved laminate to its second equilibrium shape.

- As the laminate switched to its second equilibrium shape the applied load was removed to make sure that the retained shape was stable.

- After the second equilibrium position was found force, $\mathrm{P}$, was applied again along the $\mathrm{z}$-direction such that it tended to switch the panel to its first equilibrium shape. Boundary conditions were kept unchanged.

- As the laminate switched back to its first equilibrium shape the load was removed once again to make sure that this retained shape was stable.

Further, for modeling of the snap behavior in LS-DYNA and ANSYS Tawfik's two-step methodology will be followed as a guideline.

\section{E. Snap-through and snap-back analysis with LS-DYNA}

With LS-DYNA snap behavior of cured bistable unsymmetric laminate can easily be captured as follows

- $\quad$ Eigenvalue buckling analysis of the perfect geometry using the *CONTROL_INPLICIT_EIGENVALUE [15] keyword.

- Introduce imperfections into the geometry using *PERTURBATION_NODE [15] keyword.

- Non-linear load-displacement analysis of the imperfect geometry to capture the cured shape using the *CONTROL_IMPLICIT_STABILIZATION [15] keyword.
- A second non-linear load displacement analysis of the cured shape to capture the snap-through and snap-back using *CONTROL_IMPLICIT_STABILIZATION keyword.

Like ABAQUS the first three steps are for obtaining the cured shape and the fourth one is for snap-through and snapback of that cured shape.

\section{F. Snap-through and snap-back analysis with ANSYS}

Unlike ABAQUS and LS-DYNA solving snap-through and snap-back in ANSYS require following steps

- Static analysis step to get an initial deformation that is going to be used in the Eigen buckling analysis.

- Following is the eigen buckling analysis to generate the $1^{\text {st }}$ eigenmode under thermal loading using any available eigensolver.

- Based on extracted eigenmode shape, the geometry of the model is to be perturbed using a required imperfection size. The command UPCOORD [14] is used to introduce the imperfection.

- The next step is to solve a non-linear analysis of the imperfect geometry using STABILIZE [14] command to get the cured shape.

- A second non-linear load displacement analysis of that cured shape using STABILIZE [14] command.

First four steps are for obtaining the cured shape and the fifth one is for snap-through and snap-back of that cured shape.

\section{G. A unified Finite Element Analysis methodology}

Successful implementation of Tawfik's ABAQUS based FE methodology in ANSYS and LS-DYNA can be summarized as follows

Modeling snap-through and snap-back to investigate the stability characteristics of a bistable unsymmetric cross-ply laminate in any FE code requires two back to back analysis

- $1^{\text {st }}$ analysis is for obtaining the cured shape of that unsymmetric cross-ply laminate.

- $2^{\text {nd }}$ analysis is the non-linear load displacement analysis of that cured shape to obtain the snap-through and snap-back.

Tawfik [7] and Tawfik et al. [9, 10,11] first came up with this two-step FE methodology and can be used as a unified guideline for studying stability characteristics of a bistable unsymmetric cross-ply laminate in any FE code, like LS-DYNA or ANSYS.

\section{RESUlTS AND DisCUSSION}

Fig. 3 shows the comparison of load-displacement curves extracted from ABAQUS, ANSYS and LS-DYNA illustrating that they are in good agreement.

Fig. 4 shows the two equilibrium shapes of a bistable laminate extracted from ABAQUS, while Fig. 5 and Fig. 6 display the equilibrium shapes solved by ANSYS and LSDYNA respectively. 


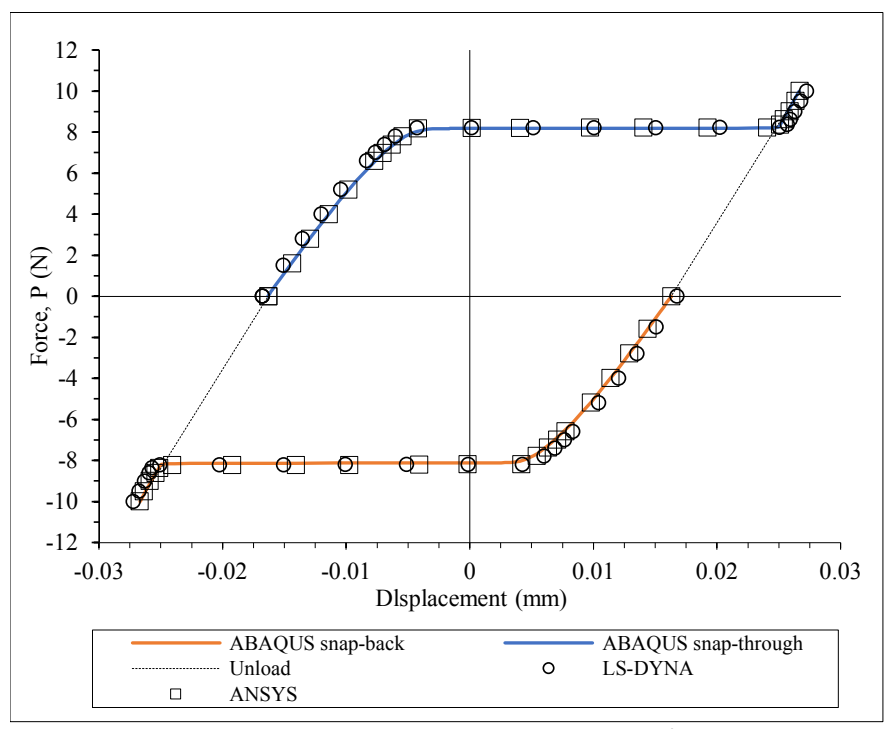

Figure 3. Load-displacement curve for a $150 \times 150 \mathrm{~mm}^{2}$ cured laminate made of Hexcel IM7/8551-7 graphite/epoxy prepreg with $\left[0_{2} / 90_{2}\right]_{\mathrm{T}}$ stacking.

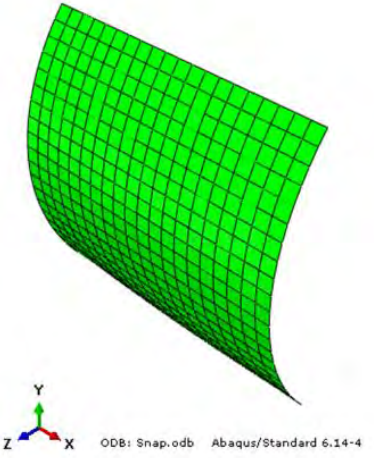

(a)

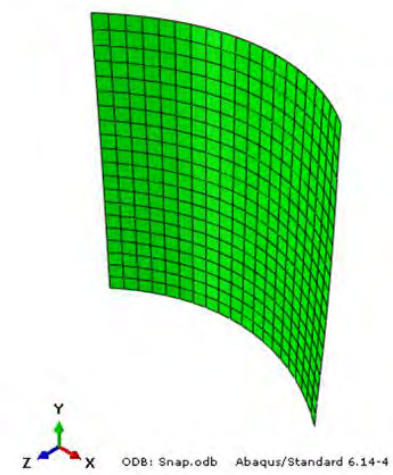

(b)
Figure 4. a) First equilibrium shape and b) Second equilibrium shape extracted from ABAQUS.

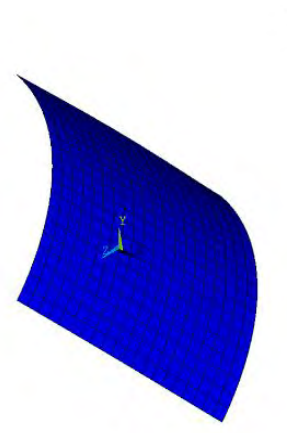

(a)

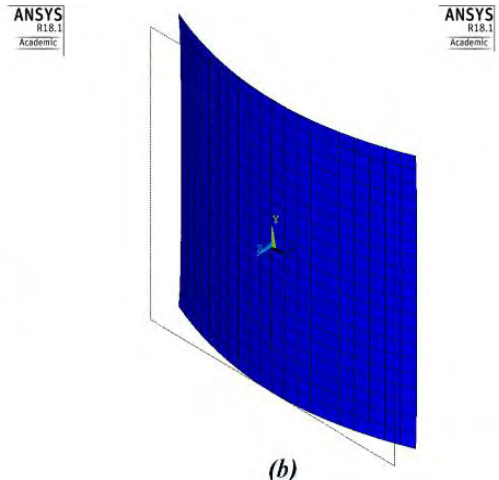

(b)
Figure 5. a) First equilibrium shape and b) Second equilibrium shape extracted from ANSYS.

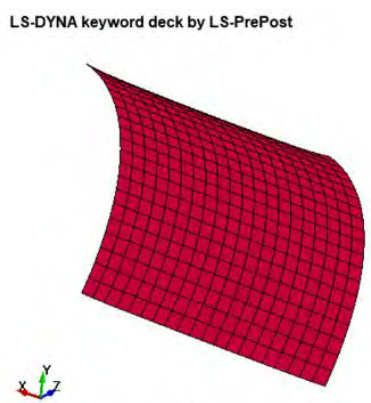

(a)

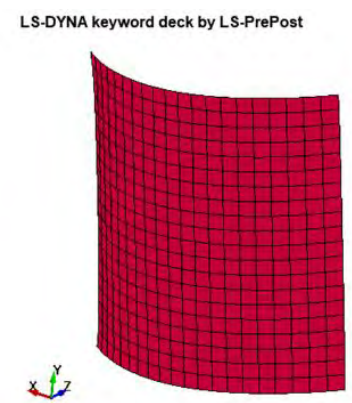

(b)
Figure 6. a) First equlibrium shape and b) Second equilibrium shape extracted from LS-DYNA.

TABLE III. EXPERIMENTAL AND FE SNAP-THROUGH LOADS OF SQUARE SPECIMENS

\begin{tabular}{|c|c|c|c|c|c|c|c|}
\hline \multirow{2}{*}{$\begin{array}{l}\text { Sidelength [7] } \\
(\mathrm{mm} \times \mathbf{m m})\end{array}$} & \multirow{2}{*}{$\begin{array}{c}\text { Test [7] } \\
\mathbf{P}(\mathbf{N})\end{array}$} & \multicolumn{2}{|c|}{ ABAQUS [7] } & \multicolumn{2}{|c|}{ LS-DYNA } & \multicolumn{2}{|c|}{ ANSYS } \\
\hline & & $\mathbf{P}(\mathbf{N})$ & $\%$ & $\mathbf{P}(\mathbf{N})$ & $\%$ & $\mathbf{P}(\mathbf{N})$ & $\%$ \\
\hline $151 \times 150$ & 9.61 & 8.72 & -9.29 & 8.69 & -9.50 & 8.76 & -8.84 \\
\hline $123 \times 125.5$ & 8.90 & 8.15 & -8.46 & 8.19 & -7.87 & 8.06 & -9.44 \\
\hline $100 \times 101$ & 8.09 & 7.54 & -6.76 & 7.49 & -7.29 & 7.20 & -11.00 \\
\hline $87 \times 88$ & 5.11 & 4.93 & -3.46 & 5.09 & -0.21 & 4.82 & -5.68 \\
\hline $67 \times 67$ & 2.75 & 2.7 & -1.81 & 2.69 & -1.84 & 2.68 & -2.55 \\
\hline
\end{tabular}

TABLE IV. EXPERIMENTAL AND FE SNAP-BACK LOADS OF SQUARE SPECIMENS

\begin{tabular}{|c|c|c|c|c|c|c|c|}
\hline Sidelength [7] & Test [7] & \multicolumn{2}{|c|}{ ABAQUS [7] } & \multicolumn{2}{c|}{ LS-DYNA } & \multicolumn{2}{c|}{ ANSYS } \\
\cline { 3 - 8 }$(\mathbf{m m} \times \mathbf{m m})$ & $\mathbf{P}(\mathbf{N})$ & $\mathbf{P}(\mathbf{N})$ & $\mathbf{\%}$ & $\mathbf{P}(\mathbf{N})$ & $\mathbf{\%}$ & $\mathbf{P}(\mathbf{N})$ & $\%$ \\
\hline $151 \times 150$ & 10.39 & 9.42 & -9.36 & 9.49 & -8.63 & 9.48 & -8.76 \\
\hline $123 \times 125.5$ & 10.06 & 9.25 & -8.02 & 9.29 & -7.61 & 9.50 & -5.57 \\
\hline $100 \times 101$ & 8.27 & 7.75 & -6.35 & 7.69 & -6.89 & 7.68 & -7.13 \\
\hline $87 \times 88$ & 6.12 & 5.37 & -12.27 & 5.53 & -9.61 & 5.22 & -14.71 \\
\hline $67 \times 67$ & 4.45 & 2.97 & -33.26 & 3.05 & -31.37 & 2.78 & -37.53 \\
\hline
\end{tabular}

Tawfik [7] also studied the effect of geometry on the stability characteristics by changing the sidelength of thin $\left[0_{2} / 90_{2}\right]_{\mathrm{T}}$ square laminate and documented the snap-through and snapback loads comparing the ABAQUS predictions with the test measurements. Table III shows the snap-through loads predicted in this present work by LS-DYNA and ANSYS while table IV shows the snap-back loads. These two tables also illustrate the percentage differences in predictions from these three FE codes while compared with the test measurements, where all the test measurements and ABAQUS results are from Tawfik [7]. ABAQUS, ANSYS and LS-DYNA all are in good agreement with the test measurements which proves the generality of that two-step FE methodology developed by Tawfik [7] and Tawfik et al. [9, 10, 11].

\section{CONCLUSION}

The current work addressed post buckling behavior in thin unsymmetric cross-ply laminates and stability characteristics. An analytically correct methodology is obtained from literature and applied in commonly used finite element computer codes, ABAQUS, ANSYS and LS-DYNA. Critical forces required to trigger snap-through behaviour in thin unsymmetric cross-ply laminated were compared with test measurement and found to be in good agreement. Therefore, the adopted analytically 
correct methodology proved generality and recommended for analysis and design of unsymmetric composites morphing applications.

\section{REFERENCES}

[1] M. W. Hyer, "Some observations on the cured shapes of thin unsymmetric laminates," J. Compos. Mater., vol. 15, no. 2, pp. 175-194, 1981. doi: 10.1177/002199838101500207.

[2] A. Hamamoto and M. W. Hyer, "Non-linear temperature-curvature relationships for unsymmetric graphite-epoxy laminates," Int. J. Solids Struct., vol. 23, no. 7, pp. 919-935, 1987. doi:10.1016/0020-7683(87)90087-4.

[3] S. A. Emam and D. J. Inman, "A review on bistable composite laminates for morphing and energy harvesting," Appl. Mech. Rev., vol. 67, no. 6, pp. 060803, Dec. 2015. doi: 10.1115/1.4032037.

[4] M.W. Hyer, "Calculations of the room-temperature shapes of unsymmetric laminates," J. Compos. Mater., vol. 15, no. 4, pp. 296-310, July 1981. doi: 10.1177/002199838101500401.

[5] M. Dano, "SMA-induced deformations in general unsymmetric laminates," Ph.D. dissertation, Eng. Sci. Mech., Virginia Polytechnic Univ., Blacksburg, Virginia, 1997.

[6] M. R. Schultz, "Use of piezoelectric actuators to effect snap-through behavior of unsymmetric composite laminates," Ph.D. dissertation, Eng. Sci. Mech., Virginia Polytechnic Univ., Blacksburg, Virginia, 2003.

[7] S. Tawfik, "Stability and morphing characteristics of bi-stable laminates," Ph.D. dissertation, Georgia Technol., Aerosp. Eng., Atlanta, Georgia, 2008.
[8] W. T. Koiter, "On the stability of elastic equilibrium," Ph.D. dissertation, Polytechnic Institute Delft, Delft, Netherlands, NASA TTF-10833, 1945.

[9] S. Tawfik et al., "Modeling of anticlastic stability in elastically tailored composites," in Proc. 20th Annu. Tech. ASC Conf., Drexel Univ., Philadelphia, PA, 2005.

[10] S. Tawfik et al., "Anticlastic stability modeling for cross-ply composites," J. Compos. Mater., vol. 41, no. 11, pp. 1325-1338, 2006. doi: 10.1177/0021998306068073.

[11] S. Tawfik, D. S. Dancila, and E. Armanios, "Planform effects upon the bistable response of cross-ply composite shells," Composites, Part A, vol. 42, no. 7, pp. 825-833, 2011. doi: 10.1016/j.compositesa.2011.03.012.

[12] I. Kim, "Development and analysis of elastically tailored composite star shapedbeam sections," Ph.D. dissertation, Aerosp. Eng., Georgia Technol., Atlanta, Georgia, 2003

[13] Abaqus 6.14 user manual. DS SIMULIA Corp., Providence, RI, 2004.

[14] ANSYS manual, Version 16.2, ANSYS Inc., Canonsburg, USA, 2015.

[15] LS-DYNA Manual: Volume I, Version 971, 7374. Livermore Software Technol. Corp., Livermore, CA, 2013. 


\title{
Inclusion of Aerodynamic Effects in Multibody Dynamics
}

\author{
BP Minaker ${ }^{\dagger}$ PhD PEng, M Sun, J Defoe PhD \\ Department of Mechanical, Automotive, \& Materials Engineering \\ University of Windsor \\ Windsor, Ontario, Canada \\ bminaker@uwindsor.ca
}

\begin{abstract}
The Equations of Motion (EoM) software, developed by University of Windsor Vehicle Dynamics and Control Research Group, can be used to generate linear or linearized equations of motion for mechanical systems, and is particularly well suited to vehicle dynamics. This paper describes an effort to extend its capability to include the effects of wings on the motion of multibody systems.
\end{abstract}

Keywords- multibody dynamics; aerodynamics; equations of motion; stability; eigenvalues; open source software

\section{INTRODUCTION}

This paper discusses the Equations of Motion (EoM) multibody dynamics software developed by University of Windsor Vehicle Dynamics and Control Research Group, and an attempt to extend its capability to include the effects of wings on the motion of multibody systems.

The EoM software can be used to generate linear or linearized equations of motion for multibody mechanical systems. The software is entirely open source and runs within the MATLAB $^{\circledR}$ framework, or in the syntax compatible open source alternative, Octave. It has also recently been ported to Julia, a new open source high performance programming language optimized for numerical computing. It is freely available online at the software hosting site www.github.com.

The input of the software is a simple function file describing the system in question, and the output is the state space form of the equations, and optionally, a report tabulating the results of a linear analysis in a .pdf format, and animations of the mode shapes that can be viewed using any virtual reality modelling language (VRML) viewer.

When analyzing a multibody system, EoM will first read the information from the input data, and build the necessary stiffness and constraint Jacobian matrices required to find all the preload and constraint forces. Once these are known, the stiffness matrices are updated with the tangent stiffness terms. The kinematic differential equations relating position and velocity

\footnotetext{
$\dagger$ corresponding author
}

are then combined with the Newton-Euler equations of motion, reduced to a minimal coordinate set, and cast in the following form:

$$
\left[\begin{array}{cc}
\mathbf{E} & \mathbf{0} \\
\mathbf{0} & \mathbf{I}
\end{array}\right]\left\{\begin{array}{l}
\dot{x} \\
y
\end{array}\right\}=\left[\begin{array}{ll}
\mathbf{A} & \mathbf{B} \\
\mathbf{C} & \mathrm{D}
\end{array}\right]\left\{\begin{array}{l}
\boldsymbol{x} \\
\boldsymbol{u}
\end{array}\right\}
$$

A careful examination will show that this system, known as the descriptor form of the state space, can be further reduced to standard state space form, if the $\mathbf{E}$ matrix is non-singular. If it is singular, then the system becomes a set of differentialalgebraic equations. Nevertheless, a singular value decomposition approach can be used to reduce the system to an equivalent lower dimensional standard state space form. From this state matrix form, EoM will perform a number of linear analyses, e.g., calculate the eigenvalue of each mode to determine the stability, compute the frequency response between any inputs or outputs that have been defined, and determine the minimal realization (the minimum dimensional system with equivalent input-output behaviour).

\section{EXtension to InClude AERodynamic EFFECTS}

The intention of the work described in this paper is to extend the capabilities of the EoM software to include aerodynamic effects. The EoM software is primarily used to model vehicle dynamics; there are many cases where aerodynamic effects could have significant influence on vehicle behaviour. The addition of aerodynamic items to EoM would enable automated construction of multibody dynamic models of a number of systems, e.g, a vehicle ride (bounce-pitch) model with front and rear wings, a motorcycle with an aerodynamic fairing, or an aircraft with discrete flexible model elements (e.g. a torsionally flexible fuselage). More elaborate examples might include an aircraft during lift-off or landing, where both the tires and the aero surfaces are contributing to the motion, or an aircraft towing a second aircraft with a cable, as is commonly used when launching gliders.

\section{A. Basic wing example}

To illustrate the inclusion of aero effects on a dynamic vibrating system, consider the equations of motion for the 
spring mass system, with a single wing attached, as depicted in Fig. 1. The development begins with the breakdown of the net aerodynamic force into lift and drag. The equations for lift force $L$ and drag force $D$ are written as a function of the dynamic pressure $q$, where $\rho$ represents the air density, $V$ the airstream velocity, and $S$ the wing area.

$$
\begin{aligned}
q & =\frac{1}{2} \rho V^{2} \\
L & =q S C_{\mathrm{L}} \\
D & =q S C_{\mathrm{D}}
\end{aligned}
$$

The nondimensional coefficients of lift and drag are $C_{\mathrm{L}}$ and $C_{\mathrm{D}}$, respectively.

Note that the lift and drag forces are assumed to remain parallel and perpendicular to the airflow direction respectively, measured in a reference frame that moves with the wing. If the wing is moving vertically downward, the airflow appears to have an upward velocity component, quantified by the angle of attack. The angle of attack can be written in terms of the vertical speed of the wing, and simplified by assuming small angles.

$$
\alpha \approx \alpha_{0}-\frac{\dot{z}}{V}
$$

In effect, the directions of the aero forces as defined above change relative to the ground fixed reference frame. Taking the vertical component of the aero forces gives:

$$
Z=L \cos \left(\alpha-\alpha_{0}\right)+D \sin \left(\alpha-\alpha_{0}\right)
$$

To linearize, a truncated Taylor series is used.

$$
Z \approx Z_{0}+\left.\frac{\partial Z}{\partial \alpha}\right|_{\alpha=\alpha_{0}}\left(\alpha-\alpha_{0}\right)
$$

The lift and drag coefficients are assumed to be functions of the angle of attack.

$$
\begin{array}{r}
\frac{\partial Z}{\partial \alpha}=\frac{\partial L}{\partial \alpha} \cos \left(\alpha-\alpha_{0}\right)-L \sin \left(\alpha-\alpha_{0}\right) \\
+\frac{\partial D}{\partial \alpha} \sin \left(\alpha-\alpha_{0}\right)+D \cos \left(\alpha-\alpha_{0}\right) \\
\left.\frac{\partial Z}{\partial \alpha}\right|_{\alpha=\alpha_{0}}=\frac{\partial L}{\partial \alpha}+D=q S\left(\frac{\partial c_{\mathrm{L}}}{\partial \alpha}+c_{\mathrm{D}}\right)
\end{array}
$$

As a result:

$$
Z=Z_{0}+q S\left(\frac{\partial c_{\mathrm{L}}}{\partial \alpha}+c_{\mathrm{D}}\right)\left(\alpha-\alpha_{0}\right)
$$

Noting that $\alpha-\alpha_{0}=-\dot{z} / V$

$$
Z=Z_{0}-\frac{q S}{V}\left(\frac{\partial c_{\mathrm{L}}}{\partial \alpha}+c_{\mathrm{D}}\right) \dot{z}
$$

Combining the sum of the aerodynamic and spring forces, and equating to mass times acceleration gives the familiar second order linear differential equation of motion. Note that the deflection $z$ is measured from equilibrium, where any static lift forces $Z_{0}$ are offset by the spring preload $k z_{0}$, (weight force is neglected, but would have no effect in this example).

$$
m \ddot{z}=Z-k\left(z+z_{0}\right)=Z_{0}-\frac{1}{V} \frac{\partial Z}{\partial \alpha} \dot{z}-k\left(z+z_{0}\right)
$$

Simplifying:

$$
m \ddot{z}+\frac{q S}{V}\left(c_{L \alpha}+c_{\mathrm{D}}\right) \dot{z}+k z=0
$$

The dynamic pressure $q$ is parabolic in $V$, so the wing behaves effectively as a damper whose coefficient increases linearly with airspeed. This is noteworthy in a vehicle dynamics context, as it is in contrast to a typical slip-based tire model, where the tire is effectively a damper whose coefficient varies as the inverse of forward speed.

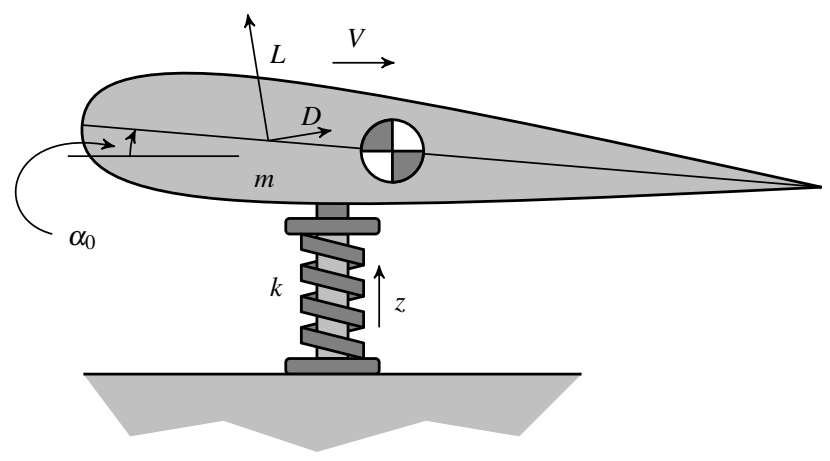

Figure 1. Spring mass wing model. Note that the lift and drag forces are assumed to remain parallel and perpendicular to the airflow direction respectively, measured in a reference frame that moves with the wing.

While the example illustrates the development of the equation of motion for a simple system, in fully three dimensional motion, the effective damping term becomes a full $6 \times 6$ matrix, where the three forces and three moments are written as linear functions of linear and angular speed. A typical vehicle based coordinate system is used, where the $\mathrm{x}$ axis is the vehicle's direction of travel, and the $\mathrm{z}$ axis is vertical. Symmetry in the $\mathrm{xz}$ plane is assumed; the effect is that fully half of the 36 damping terms go to zero. In this notation, the components of force in the three coordinate axes are $X, Y, Z$, while $L, M, N$ are the corresponding moments. The linear and angular velocities are represented as $u, v, w$, and $p, q, r$, respectively. The wingspan is denoted as $b$, and $c$ is the chord (i.e., $S=b c$ for a rectangular planform wing). These $6 \times 6$ matrices can be easily incorporated into the EoM generated equations of motion.

The full equation is written as: 


$$
\left\{\begin{array}{c}
X \\
Y \\
Z \\
L / b \\
M / c \\
N / b
\end{array}\right\}=-\frac{q S}{V}\left[\begin{array}{cccccc}
C_{X u} & 0 & C_{X w} & 0 & C_{X q} & 0 \\
0 & C_{Y v} & 0 & C_{Y p} & 0 & C_{Y r} \\
C_{Z u} & 0 & C_{Z w} & 0 & C_{Z q} & 0 \\
0 & C_{L v} & 0 & C_{L p} & 0 & C_{L r} \\
C_{M u} & 0 & C_{M w} & 0 & C_{M q} & 0 \\
0 & C_{N v} & 0 & C_{N p} & 0 & C_{N r}
\end{array}\right]\left\{\begin{array}{c}
u \\
v \\
w \\
\frac{b}{2} p \\
\frac{c}{2} q \\
\frac{b}{2} r
\end{array}\right\}
$$

or:

$$
\left\{\begin{array}{c}
X \\
Y \\
Z \\
L \\
M \\
N
\end{array}\right\}=[\mathbf{L}]\left\{\begin{array}{c}
u \\
v \\
w \\
p \\
q \\
r
\end{array}\right\}
$$

where:

$$
\mathbf{L}=-\frac{q S}{V}\left[\begin{array}{cccccc}
C_{X u} & 0 & C_{X w} & 0 & \frac{1}{2} c C_{X q} & 0 \\
0 & C_{Y v} & 0 & \frac{1}{2} b C_{Y p} & 0 & \frac{1}{2} b C_{Y r} \\
C_{Z u} & 0 & C_{Z w} & 0 & \frac{1}{2} c C_{Z q} & 0 \\
0 & b C_{L v} & 0 & \frac{1}{2} b^{2} C_{L p} & 0 & \frac{1}{2} b^{2} C_{L r} \\
c C_{M u} & 0 & c C_{M w} & 0 & \frac{1}{2} c^{2} C_{M q} & 0 \\
0 & b C_{N v} & 0 & \frac{1}{2} b^{2} C_{N p} & 0 & \frac{1}{2} b^{2} C_{N r}
\end{array}\right]
$$

\section{B. EoM system elements}

In EoM, each component of a system is defined as an 'item'. The most basic type of item is a 'body', which defines a rigid body. The other types of items define either rigid or flexible connectors that are attached to one or more rigid bodies. All

\begin{tabular}{|c|c|}
\hline Type of item & Definition \\
\hline body & a rigid body \\
\hline spring & $\begin{array}{l}\text { a two point elastic spring, with linear or torsional stiffness } \\
\text { and damping, non-zero free length }\end{array}$ \\
\hline link & a two point massless rigid link \\
\hline rigid_point & $\begin{array}{l}\text { a generic point constraint with a variable number of } \\
\text { constraint forces and moments }\end{array}$ \\
\hline flex_point & $\begin{array}{l}\text { a point spring with translational and/or rotational } \\
\text { stiffness and damping }\end{array}$ \\
\hline nh_point & $\begin{array}{l}\text { a non-holonomic constraint to prevent velocity but not } \\
\text { displacement }\end{array}$ \\
\hline beam & $\begin{array}{l}\text { a zero mass beam spring with bi-directional bending } \\
\text { and shear stiffness }\end{array}$ \\
\hline $\begin{array}{l}\text { load } \\
\text { actuator } \\
\text { sensor }\end{array}$ & $\begin{array}{l}\text { constant forces or moments applied to the system } \\
\text { applied force or moment, proportional to an input signal } \\
\text { used to measure displacement, velocity, or acceleration }\end{array}$ \\
\hline
\end{tabular}
the available types of items are listed in Table I.

TABLE I. Types of items in EoM

A typical vehicle model in EoM will consist of rigid bodies, both rigid and flexible connectors, and other items like static loads (to include the effect of preloads), actuators to apply time varying forces, and sensors to measure the resulting motion. Joints constrain bodies together while allowing certain degrees of freedom; ball joints (spherical joints) and hinges (revolute joints) are both typical. Both are modelled in EoM as 'rigid_point' type items, where the appropriate number of constraints can be specified in each case, along with an axis to define the associated directions. Suspension springs and shock absorbers are modelled as uni-directional two-point springs, and tires as 'flex_point' items, i.e., springs with zero free length.

As the aerodynamic forces described above are expressed using a linear dependency on the velocities, they are included as a special case of the 'flex_point' item, where the full $6 \times 6$ matrix is supplied, as opposed to a typical point spring damper where a single coefficient and a direction vector suffice. It is noteworthy that the terms generated by traditional mechanical damper will always result in a symmetric damping matrix. Because the wing behaviour requires a potentially asymmetric matrix, there is no possible combination of dampers that would provide an equivalent motion response. In order to properly capture the wing behaviour, the full matrix must be supplied.

\section{Formulation of THE EQUATIONS OF MOtion}

The linearized equations of motion of the model are automatically generated by EoM. A detailed description of the method is given in Minaker and Rieveley[1], and expanded in Minaker[2]. The set of differential equations are written as

$$
\left[\begin{array}{ccc}
\mathbf{I} & \mathbf{0} & \mathbf{0} \\
\mathbf{0} & \mathbf{M} & -\mathbf{G} \\
\mathbf{0} & \mathbf{0} & \mathbf{0}
\end{array}\right]\left\{\begin{array}{l}
\dot{p} \\
\dot{w} \\
\dot{\boldsymbol{u}}
\end{array}\right\}+\left[\begin{array}{ccc}
\mathbf{V} & -\mathbf{I} & \mathbf{0} \\
\mathbf{K} & \mathbf{L} & -\mathbf{F} \\
\mathbf{0} & \mathbf{0} & \mathbf{I}
\end{array}\right]\left\{\begin{array}{l}
p \\
w \\
u
\end{array}\right\}=\left\{\begin{array}{c}
\mathbf{0} \\
f_{\mathrm{c}} \\
\boldsymbol{u}
\end{array}\right\}
$$

where the mass matrix $\mathbf{M}$ results from the Newton-Euler equations, and is tri-diagonal as is typical. The stiffness matrix $\mathbf{K}$ is the sum of terms resulting from deflection of elastic elements, and additional tangent stiffness matrix terms resulting from preload in the connectors. The $\mathbf{L}$ matrix contains the traditional viscous damping matrix, plus terms due to the inertia forces, i.e., centripetal forces and gyroscopic moments. It is in this $\mathbf{L}$ matrix that the aero terms are included. The $\mathbf{V}$ matrix results from the linearization of the kinematic differential equations, and is nonzero only in the event of a non-zero velocity reference condition.

The $\mathbf{F}$ and $\mathbf{G}$ matrices are used to allow the inclusion of rate dependency in the input forces, i.e., the applied forces are written as

$$
\boldsymbol{f}_{\mathrm{a}}=\mathbf{F} \boldsymbol{u}+\mathbf{G} \dot{u}
$$

where $\boldsymbol{u}$ is the input. The $\boldsymbol{p}$ vector represents the global locations and small angle orientations, and the $\boldsymbol{w}$ vector represents the body fixed linear and angular velocities. The constraint forces $\boldsymbol{f}_{\mathrm{c}}$ are still present in the intermediate formulation, but are eliminated through a coordinate reduction process. The linearized constraint equations are written as

$$
\left[\begin{array}{ccc}
\mathbf{J}_{\mathrm{h}} & \mathbf{0} & \mathbf{0} \\
-\mathbf{J}_{\mathrm{h}} \mathbf{V} & \mathbf{J}_{\mathrm{h}} & \mathbf{0} \\
\mathbf{0} & \mathbf{J}_{\mathrm{nh}} & \mathbf{0}
\end{array}\right]\left[\begin{array}{cc}
\dot{\boldsymbol{p}} & \boldsymbol{p} \\
\dot{\boldsymbol{w}} & \boldsymbol{w} \\
\dot{\boldsymbol{u}} & \boldsymbol{u}
\end{array}\right]=\left[\begin{array}{cc}
\mathbf{0} & \mathbf{0} \\
\mathbf{0} & \mathbf{0} \\
\mathbf{0} & \mathbf{0}
\end{array}\right]
$$

where the $\mathbf{J}_{\mathrm{h}}$ and $\mathbf{J}_{\mathrm{nh}}$ matrices represent holonomic and nonholonomic constraint equations, respectively. An orthogonal 
complement of the Jacobian is used to reduce the number of coordinates.

\section{WING ANALYSIS}

In order to generate the wing properties used to fill the associated damping matrix, a numerical approach is required. Luckily, a well-developed solution already exists.

\section{A. Athena Vortex Lattice}

Athena Vortex Lattice (AVL) is an open source software tool developed at MIT by Mark Drela and Harold Youngren[3]. It has numerous capabilities related to the flight-dynamic analysis of rigid aircraft of arbitrary configuration. While AVL can already produce a linearized aerodynamic model to predict aircraft stability, its models are limited to treating the aircraft as a single rigid body.

However, individual lifting and control surfaces can also be modelled in AVL (rather than full aircraft), and the full aerodynamic properties can be output in a format suitable for inclusion in other codes, such as EoM.

The relevant functionality of AVL for this paper is its ability to produce stability derivatives (the elements of the system matrix relating state derivatives to the state values) at any flight condition. By definition, vortex lattice codes work in three dimensions; it is not possible to carry out two-dimensional analysis using AVL. AVL can be expected to produce accurate results when the lifting surfaces are thin and angles of attack (and sideslip) are small ( $\ll 1 \mathrm{rad}$ ). In a vortex lattice code, the surfaces and the wakes that trail them downstream are represented with vortex sheets of infinitesimal thickness. By discretizing the aerodynamic surface(s) of interest with horseshoe vortices distributed along the span and chord, a numerical solution for the aerodynamic forces and moments along the surface(s) can be obtained. A full description of vortex lattice methods is beyond the scope of this paper, but see for example Lan[4], Lamar and Gloss[5], and Miranda, Elliot, and Baker[6] for the foundational work that underpins AVL. An important limitation of AVL is the assumption of quasi-steady flow[3]. This means that oscillatory motions must be sufficiently slow so that the period of oscillation is long compared to the time it takes for the flow to travel the length of the wing chord. This can be quantified using the reduced frequency $\beta$, where

$$
\beta=\frac{\omega c}{V} \ll 1
$$

For typical aircraft in flight this is almost always true, but in alternative applications of AVL for use in EoM such as ground vehicles, this condition should be checked. Finally, the angular velocities (roll, pitch, and yaw rates) must be slow enough to ensure that small angle assumptions continue to hold.
Quantitatively, this means:

$$
\begin{aligned}
& |p b / 2 V|<0.10 \\
& |q c / 2 V|<0.03 \\
& |r b / 2 V|<0.25
\end{aligned}
$$

Again, while this is normally not an issue for aircraft, if AVL analysis is used for other applications, these should be checked to ensure solution validity.

\section{EXAMPLE PROBLEM}

In order to demonstrate the capabilities of the combined EoM/AVL software tool, a test model was developed. It is an expansion of the swivel wheel problem, as presented by Schwab and Meijaard[7], and before them by Den Hartog[8]. The model is used to predict the shimmy phenomenon, as sometimes observed in aircraft nose wheel landing gear, or more commonly on the front wheels of a grocery cart. The model consists of a single rigid body, constrained to planar motion, and travelling at a fixed forward speed. The motion of the body is reacted by a lateral spring placed at the front, to represent the lateral stiffness of the wheel carrier or support, and a no-slip tire (modelled as a nonholonomic constraint) at the rear. The wheel shimmy problem is noteworthy, as it can predict unstable behaviour. The response is strongly dependent on the location of the mass centre; when it is near the midway point between the tire and the lateral spring, the system is stable, while locations near either end result in an unstable motion. The instability is oscillatory; the amplitude grows with time.

In this example, the shimmy problem is repeated with an aerodynamic surface is added to the model. A symmetric generic thin surface model is used for the airfoil. The reference point of the airfoil is taken at one quarter of the chord from the leading edge. The wing is fixed such that its reference point is located at the front of the body at the same location where the lateral stiffness acts. It is noteworthy that shifting the longitudinal location of the wing does have an effect on its influence. As the wing is moved closer to the zero-slip tire, its angle of attack is reduced. In fact, the tire constraint forces the angle of attack to be zero at that point, minimizing the influence of the wing on the overall motion. A schematic diagram of the problem is shown in Figure 2.

In order to establish a baseline result, the model is run first with no wing present. The default properties of the mechanical system are: $m=5.0 \mathrm{~kg}, l=1.0 \mathrm{~m}, I_{\mathrm{z}}=1.05 \mathrm{~kg} \mathrm{~m}^{2}, k=1.5 \mathrm{~N} / \mathrm{m}$, and $u=10.0 \mathrm{~m} / \mathrm{s}$. The location of the mass centre is varied from the front to the rear of the model. The results are then repeated with the wing present. The resulting aerodynamic properties are: $L_{2,2}=7.59895 \mathrm{Ns} / \mathrm{m}, L_{2,6}=-2.05293 \mathrm{Ns} / \mathrm{rad}$, $L_{6,2}=0.153319 \mathrm{Ns}, L_{6,6}=0.224167 \mathrm{Nms} / \mathrm{rad}, b=1.0 \mathrm{~m}$, and $c=0.5 \mathrm{~m}$. 


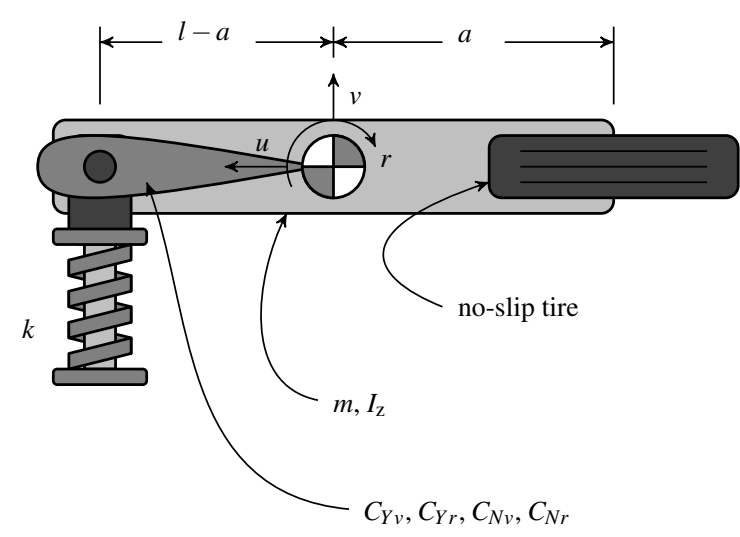

Figure 2. Swivel wheel example problem, with added aerodynamic effect. The swivel wheel exhibits the shimmy phenomenon, with potential instability, as a function of the location of the mass centre.

\section{A. Results}

Once the EoM software has generated the equations of motion, a number of linear analyses are automatically conducted. By analyzing the eigenvalues, the stability and natural frequencies of the motion can be found. The resulting eigenvalues govern the form of the motion. The eigenvalue can be expressed in the form of $s=a \pm b$ i, i.e., it is frequently a complex number. If the real part is positive, the motion will be unstable; if the real part is negative, the motion is stable as it gradually approaches zero with time. The imaginary part controls the oscillatory frequency of the motion. If the imaginary part is equal to zero, there will be no oscillation in the unforced motion.

The results are plotted in Figures 3 and 4. Figure 3 shows both eigenvalues. Note that the slow eigenvalue is complex, while the fast eigenvalue, i.e., the more negative, is real. Figure 4 shows the real part of the slow eigenvalue, which is unstable in some cases, with a much tighter zoom, to better illustrate the zero crossings. Note that if the centre of mass is located in the range $0.3 l<a<0.7 l$, then the system without the wing is stable. When the wing is added, the stability range increases to $0.12 l<a<0.88 l$.

\section{CONCLUSIONS}

The paper outlines the development of an open source multibody code for the analysis of the dynamics of mechanical systems, including aerodynamic effects. An example illustrates how mechanical system behaviour can be influenced by the addition of aerodynamic effects. The example shown is relatively simple in scope when compared to the type of problems that could be analyzed using the combined EoM and AVL software, but serves to illustrate the concept. Future work will expand this approach to more extensive applications, such as ground vehicles with multiple aerodynamic surfaces or flexible aircraft.

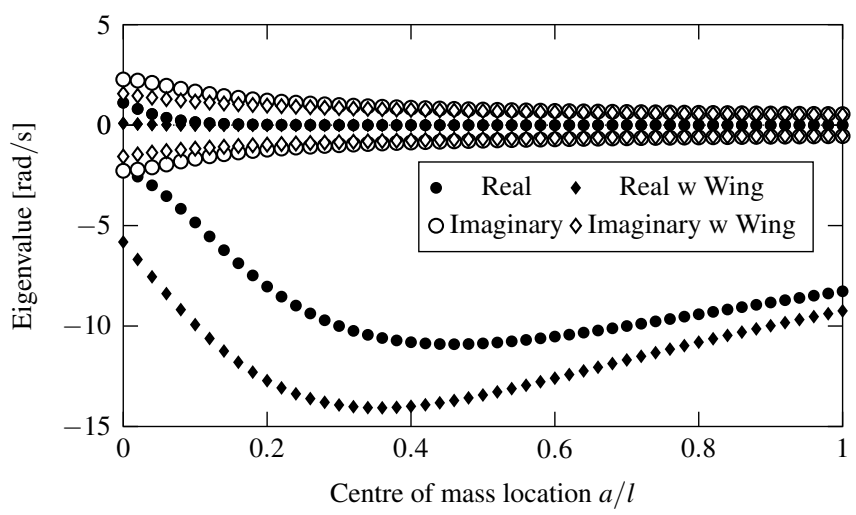

Figure 3. Eigenvalues vs centre of mass location. The effect of the addition of the wing on the eigenvalues is a significant increase in stability, and decrease in the shimmy frequency in the cases where the centre of mass is shifted rearward.

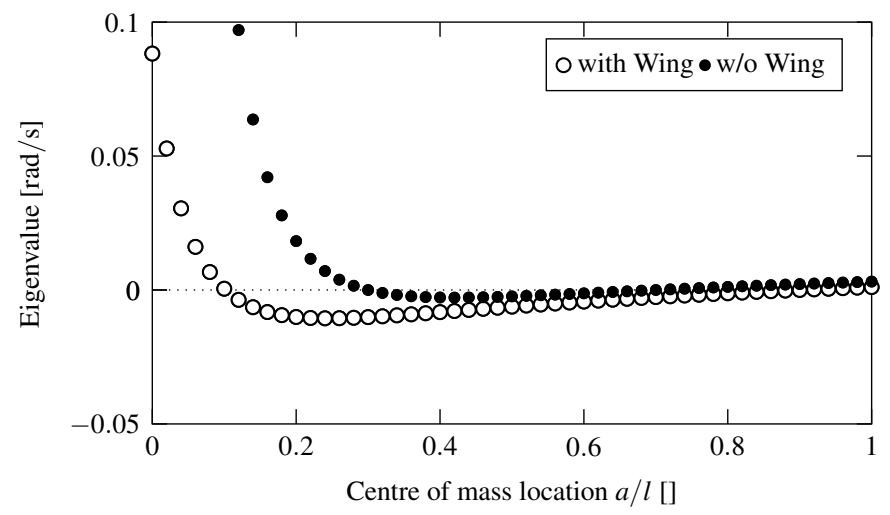

Figure 4. Slow eigenvalues vs centre of mass location, zoomed. Note that if the centre of mass is located in the range $0.3 l<a<0.7 l$ then the system without the wing is stable. When the wing is added the range is expanded.

\section{REFERENCES}

[1] B. P. Minaker and R. J. Rieveley, "Automatic generation of the non-holonomic equations of motion for vehicle stability analysis," Veh. Sys. Dyn., vol. 48, no. 9, pp. 1043-1063, Sep. 2010. doi: 10.1080/00423110903248702.

[2] B. P. Minaker, "The tangent stiffness matrix in rigid multibody vehicle dynamics," Mathematical and Computer Modelling of Dynamical Systems, vol. 21, no. 3, pp. 288-310, Sep. 2014. doi: 10.1080/13873954.2014.953549.

[3] M. Drela and H. Youngren, Athena vortex lattice, AVL. [Online]. Available: http://web.mit.edu/drela/Public/web/avl, Accessed on: Jan.10, 2018.

[4] C. E. Lan, "A Quasi-Vortex-Lattice Method in Thin Wing Theory," $J$. Aircr., vol. 11, no. 9, pp. 518-527, Sep. 1974. doi: 10.2514/3.60381.

[5] J.E. Lamar and B.B. Gloss, "Subsonic aerodynamic characteristics of interacting lifting surfaces with separated flow around sharp edges predicted by a vortex-lattice method", NASA Technical Report NASA-TN-D-7921, 1975.

[6] L.R. Miranda, R.D. Elliot, and W.M. Baker, "A generalized vortex lattice method for subsonic and supersonic flow applications", NASA Technical Report NASACR-2865, 1977.

[7] A.L. Schwab and J.P. Meijaard, "Dynamics of Flexible Multibody Systems with Non-Holonomic Constraints: A Finite Element Approach", Multibody System Dynamics vol. 0, pp. 107-123, 2003. doi: 10.1023/A:1024575707338.

[8] J.P. Den Hartog, Mechanical Vibrations, fourth edition, McGraw-Hill, New York, 1956. 


\title{
Numerical Study of Inlet Gas Velocity Effects on Droplet Behaviors in Microchannel
}

\author{
Mengcheng Jiang, Biao Zhou ${ }^{1}$ \\ Department of Mechanical, Automotive and Materials Engineering \\ University of Windsor \\ Windsor, ON, Canada, N9B 3P4
}

\begin{abstract}
In this study, the numerical simulation of liquid water behaviors inside a single straight microchannel is conducted using Volume of fluid (VOF) method and dynamic contact angle (DCA) model. Two different gas inlet velocities are considered in the simulation to investigate the gas velocity effects on the droplet behaviors. The general process of the liquid water evolvement inside the channel are presented and discussed. The results indicate that the water droplet will form into long slug flow under lower gas inlet velocity and thin film flow under higher gas inlet velocity.
\end{abstract}

Keywords-droplet behaviors; microchannel; volume of fluid method; dynamic contact angle.

\section{INTRODUCTION}

Proton exchange membrane fuel cells (PEMFCs) are energy conversion devices that can produce electrical power from hydrogen and air, releasing only water and heat. PEMFC is one of the promising energy power sources for nextgeneration vehicles and distributed power applications, mainly because of their advantages such as zero-emission, low operation temperature, high power density and quietness.

However, water management is still one of the most critical challenges for fuel cell commercialization. Thoroughly understanding the gas-liquid dynamics inside PEMFCs will help researchers to optimize the PEMFC designs. Numerical simulation using volume of fluid (VOF) method has been recognized as an effective approach to investigate the liquid water behaviors inside the gas channels. So far, for PEMFCrelated simulations, the static contact angle (SCA) model is generally used [1-7] while the dynamic contact angle (SCA) model is still under development. Recently, several researchers have applied DCA model to simulate the droplet dynamics and evolvement [8-11] and it is indicated that DCA model is more applicable than SCA model in the prediction of droplet behaviors. Zhou's group proposed the AR-DCA model [12] implemented with Hoffman function [13], which is able to simulate both advancing and receding dynamic contact angles. This AR-DCA model has been successfully validated against a series of experiments for droplet impact on horizontal and inclined surfaces from Sikalo et al. [14, 15], showing its potential to be applied in the simulation of gas-liquid twophase in microchannels.

${ }^{1}$ Corresponding Author and Principal Investigator: Dr. Biao Zhou, bzhou@uwindsor.ca, 1-519-253-3000 ext.2630
However, only a few of the researchers reported the simulations of droplet behaviors in microchannels using DCA model: Fang et al. [16] employed a contact angle hysteresis model to simulate gas-liquid flows and the results showed that the contact angle distribution in the microchannel will significantly affect the slug elongation and instability; Miller [17] and $\mathrm{Wu}$ [18] implemented DCA model with Hoffman function to simulate the liquid water transport and behaviors in gas channel, and it is indicated that the dynamic contact line treatment is very crucial in the simulation of gas-liquid dynamics.

In this study, as one part of the progress for the DCA model development, we further extend our research to the simulation of droplet behaviors in a single straight microchannel. The numerical results under different gas inlet velocity will be presented and discussed.

\section{NUMERICAL MODEL DESCRIPTION}

\section{A. Computational Domain}

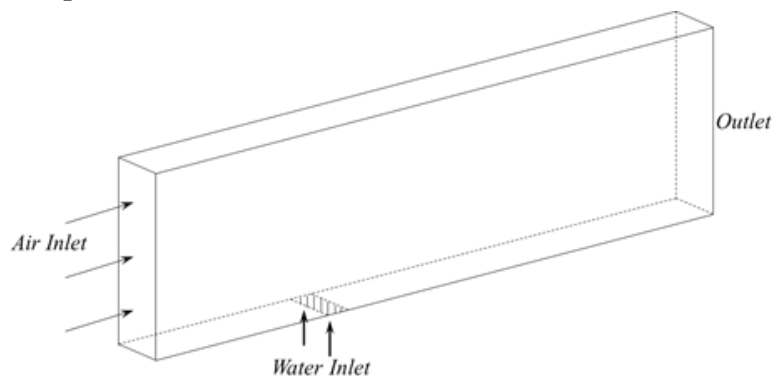

Figure 1. Schematic of computational domain in this study.

In this study, a three-dimensional single straight channel with rectangular cross section is built up as the computational domain. The dimensions of the channel are $0.05 \mathrm{~mm}$ in depth, $0.5 \mathrm{~mm}$ in width and $5 \mathrm{~mm}$ in length. The liquid water enters into the channel through a $0.02 \mathrm{~mm}$ rectangular slot on the bottom wall, which is located at $1.65 \mathrm{~mm}$ away from the inlet boundary.

\section{B. Computational Methodology}

In the simulation, the VOF method is used to track the gasliquid flow interface. The governing equations are as follows: 
The mass conservation equation:

$$
\frac{\partial(\rho)}{\partial t}+\nabla \cdot(\rho \vec{u})=0
$$

The mixture density and viscosity in each computational cell can be calculated by:

$$
\begin{gathered}
\rho=s_{l} \rho_{l}+s_{g} \rho_{g} \\
\mu=s_{l} \mu_{l}+s_{g} \mu_{g}
\end{gathered}
$$

where $s_{l}$ and $s_{g}$ are the liquid and gas volume fraction respectively, and the sum of the volume fraction is:

$$
s_{l}+s_{g}=1
$$

Then the continuity equation for the liquid phase can be expressed as the following form:

$$
\frac{\partial\left(s_{l} \rho_{l}\right)}{\partial t}+\nabla \cdot\left(s_{l} \rho_{l} \vec{u}\right)=0
$$

The momentum equation is:

$$
\frac{\partial}{\partial t}(\rho \vec{u})+\nabla \cdot(\rho \vec{u} \vec{u})=-\nabla p+\nabla \cdot\left[\mu\left(\nabla \vec{u}+\nabla \vec{u}^{\mathrm{T}}\right)\right]+S_{m}
$$

The surface normal $\hat{n}$ is determined by:

$$
\hat{n}=\hat{n}_{w} \cos \theta_{d}+\hat{t}_{w} \sin \theta_{d}
$$

where $\hat{n}_{w}$ and $\hat{t}_{w}$ represent the unit vectors normal and tangential to the wall respectively. Dynamic contact angle $\theta_{d}$ is applied at the wall boundaries through a user defined function (UDF) code. The methodology to calculate and implement $\theta_{d}$ has been reported in our previous work [12] and more details can be found in [12].

\section{Boundary Conditions and Mesh Set Up}

In the numerical simulation, the no-slip boundary condition is applied at channel walls. The DCA is considered on both side wall and bottom wall. The initial contact angle (i.e., SCA) for all the boundary walls is $108^{\circ}$. The volume flow rate of liquid water is set as $50 \mu L / \mathrm{min}$; the gas inlet velocities $V_{\text {inlet }}$ are set as $4.8 \mathrm{~m} / \mathrm{s}$ and $21.9 \mathrm{~m} / \mathrm{s}$ respectively to investigate the effects of different gas inlet velocities on the droplet behaviors and evolvement.

The whole computational domain is meshed by approximately 125500 cells, with minimum cell volume of $2 \times 10^{-7} \mathrm{~mm}^{3}$ and maximum cell volume of $1.4 \times 10^{-6} \mathrm{~mm}^{3}$. The grid size is approximately $0.01 \mathrm{~mm}$ in $\mathrm{X}-$, $\mathrm{Y}$ - and Z- direction with a grid refinement applied near the bottom wall.

\section{RESULTS AND DISCUSSION}

Fig. 2 presents the water droplet evolvement process in the single straight microchannel under relative low velocity $\left(V_{\text {inlet }}=\right.$ $4.8 \mathrm{~m} / \mathrm{s}$ ), with the liquid water volume fraction contours. The dark blue area represents the gas phase while the red area represents the liquid water. From this series of figure under selected time instances, the main process of droplet evolvement can be described as follows:
1) At the very beginning, the water enters into the channel with a constant volume flow rate, as shown in Fig. 2(a).

2) The liquid water continues to emerge and form the droplet with time (from $t=0.2 \mathrm{~ms}$ to $10 \mathrm{~ms}$, as shown in Fig. 2(b-c)).

3) When it comes to about $16.4 \mathrm{~ms}$, the droplet almost blocks the channel (Fig. 2(d)) and reaches a critical point; then the droplet is blown away towards the outlet direction due to the force exerted at the windward side from the inlet gas, as shown in Fig. 2(e).

4) From $t=16.6 \mathrm{~ms}$ to $18.0 \mathrm{~ms}$ (Fig. 2(e-g)), the droplet significantly deforms from "tall-standing" shaped slug to "long-lying" shaped slug, mainly due to the continuous pressure from the inlet gas and the interactions among the pressure, surface tension and shear stress.

5) After $18.0 \mathrm{~ms}$, the deformation and evolvement of the water slug become stable, as shown in Fig. 2(g-h).
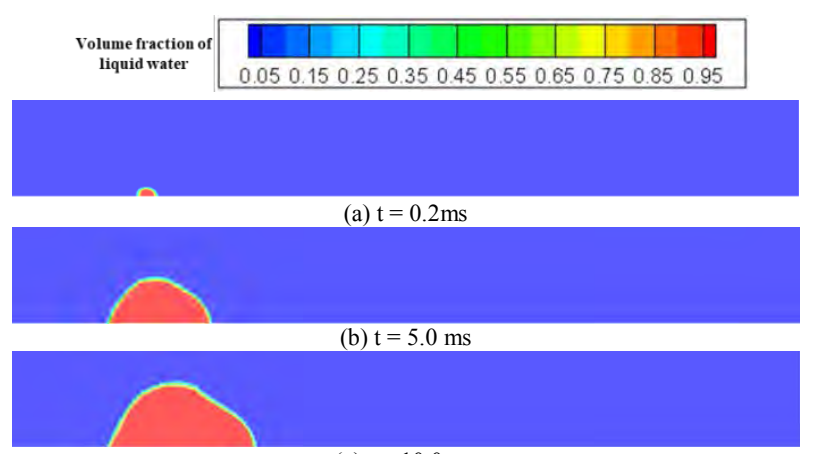

(c) $\mathrm{t}=10.0 \mathrm{~ms}$

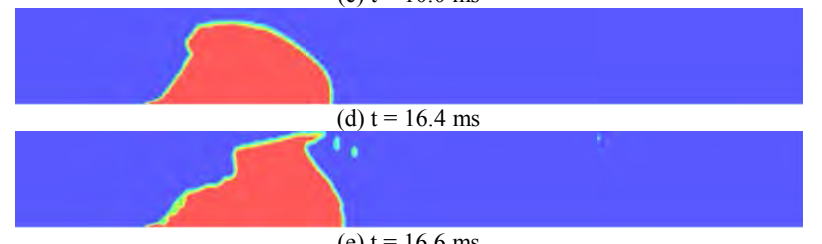

(e) $\mathrm{t}=16.6 \mathrm{~ms}$

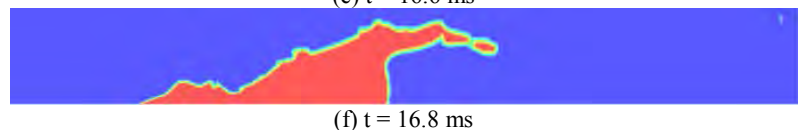

(f) $\mathrm{t}=16.8 \mathrm{~ms}$

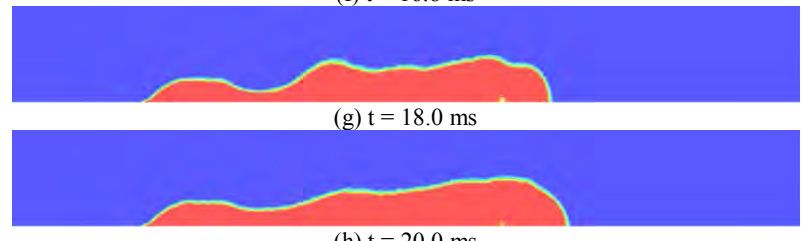

(h) $\mathrm{t}=20.0 \mathrm{~ms}$

Figure 2. Liquid water droplet evolvement under $V_{\text {inlet }}=4.8 \mathrm{~m} / \mathrm{s}$.

When the air inlet velocity is increased to $21.9 \mathrm{~m} / \mathrm{s}$, the remarkably different phenomena for the droplet behaviors and evolvement can be observed, as shown in Fig. 3.

1) At the beginning of the simulation, the water enters into the channel with a constant volume flow rate, as shown in Fig. 3(a). 
2) From $t=2.0 \mathrm{~ms}$ to $4.4 \mathrm{~ms}$, the droplet continues to grow in the microchannel. Due to the higher gas inlet velocity, the droplet starts to deform at the early stage and tends to form small liquid film at the trailing side, as shown in Fig. 3(c).

3) From $t=4.4 \mathrm{~ms}$, when the deformation reaches a critical point, the upper part of the droplet is blown away, as shown in Fig. 3(d-e).

4) After $4.6 \mathrm{~ms}$, the water droplet deforms into film flow and moves along the bottom wall. Some small splashed water droplet can also be observed in the channel, as shown in Fig. 3(f). This is mainly caused by the high pressure and strong air flow between the upper wall and the top side of droplet.

5) With time, the water accumulates at the leading side and forms a new droplet, as shown in Fig. 3(g), and the rest remains in the regime of film flow.

6) As shown in Fig. 3(h), the droplet further elongates and forms a long, thin and continuous film flow with waves. The liquid water will also drain out through the outlet.
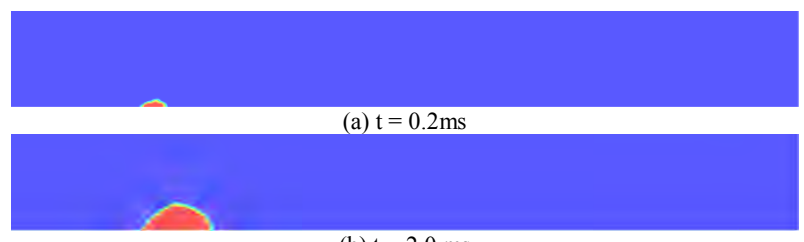

(b) $\mathrm{t}=2.0 \mathrm{~ms}$

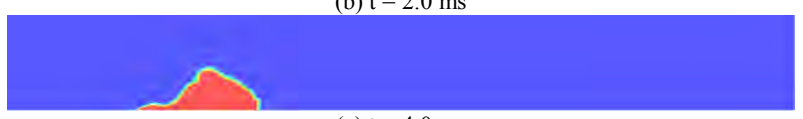

(c) $\mathrm{t}=4.0 \mathrm{~ms}$

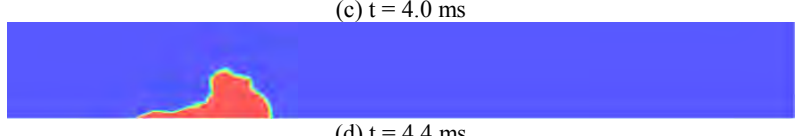

(d) $\mathrm{t}=4.4 \mathrm{~ms}$

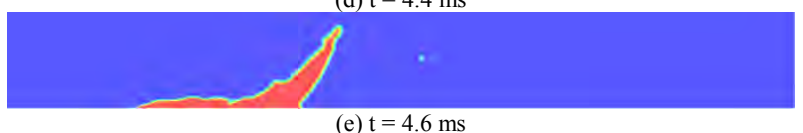

(e) $\mathrm{t}=4.6 \mathrm{~ms}$

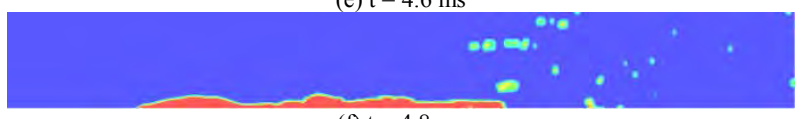

(f) $\mathrm{t}=4.8 \mathrm{~ms}$

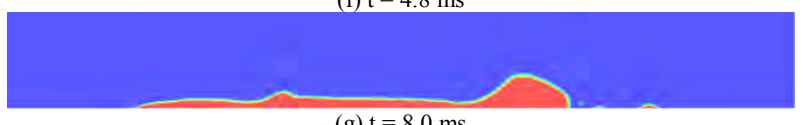

(g) $\mathrm{t}=8.0 \mathrm{~ms}$

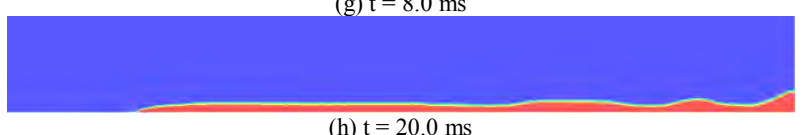

(h) $\mathrm{t}=20.0 \mathrm{~ms}$

Figure 3. Liquid water droplet evolvement under $V_{\text {inlet }}=21.9 \mathrm{~m} / \mathrm{s}$.

\section{CONCLUSIONS}

This paper presents the numerical study of droplet behavior and evolvement in a single straight microchannel. The effects of gas inlet velocity are investigated by considering two different conditions $(4.8 \mathrm{~m} / \mathrm{s}$ and $21.9 \mathrm{~m} / \mathrm{s})$.
Dynamic contact angle is considered as one of the wall boundary conditions instead of static contact angle.

From the numerical results, it can be found that the two cases in this study (one with lower gas inlet velocity and another with higher velocity) share some similar droplet evolvement phenomena: the droplet first emerge and grow inside the channel near the liquid inlet area; with time, the droplet will reach a critical point and then be blown away towards the channel outlet side.

Also, several significantly different phenomena can also be captured: under lower gas inlet velocity $(4.8 \mathrm{~m} / \mathrm{s})$, the liquid water can remain in a droplet much longer than that of the higher inlet velocity condition $(21.9 \mathrm{~m} / \mathrm{s})$; within the same time period $(20 \mathrm{~ms})$, the liquid water will form into long slug flow under lower inlet velocity, while the continuous long film flow can be observed under higher inlet velocity. It can be concluded that the gas inlet velocity has remarkable effects on the droplet deformation and evolvement in the microchannel, mainly reflected in the flow regime.

\section{ACKNOWLEDGMENT}

The authors are grateful for the support from the Natural Sciences and Engineering Research Council of Canada (NSERC) and the University of Windsor. The authors also would like to thank Compute Canada and Sharcnet for the support of computing resource.

\section{REFERENCES}

[1] K. Jiao and B. Zhou, "Accelerated numerical test of liquid behavior across gas diffusion layer in proton exchange membrane fuel cell cathode," J. Fuel Cell Sci. Technol., vol. 5, no. 4, pp. 041011, Nov. 2008. doi: 10.1115/1.2971020.

[2] A. D. Le and B. Zhou, "Fundamental understanding of liquid water effects on the performance of a PEMFC with serpentine-parallel channels," Electrochim. Acta, vol. 54, no. 8, pp. 2137-54, Mar. 2009. doi: $10.1016 /$ j.electacta.2008.10.029.

[3] S. Kang et al., "Liquid water flooding in a proton exchange membrane fuel cell cathode with an interdigitated design," Int. J. Energy Res.. vol, 35 , no. 15 , pp. 1292-311, Dec. 2011. doi: 10.1002/er.1858.

[4] X. Wang and B. Zhou, "Liquid water flooding process in proton exchange membrane fuel cell cathode with straight parallel channels and porous layer," J. Power Sources, vol. 196, no. 4, pp. 1776-94, Feb. 2011. doi: 10.1016/j.jpowsour.2010.09.092.

[5] Y. Ding Y, X. T. Bi, and D. P. Wilkinson, "Numerical investigation of the impact of two-phase flow maldistribution on PEM fuel cell performance," Int. J. Hydrogen Energy, vol. 39, no. 1, pp. 469-80, Jan. 2014. doi: $10.1016 /$ j.ijhydene.2013.10.047.

[6] M. Ashrafi et al., "Simulation and experimental validation of droplet dynamics in microchannels of PEM fuel cells," Heat Mass Transfer, vol. 52, no. 12, pp. 2671-86, Dec. 2016. doi: 10.1007/s00231-016-1771-z.

[7] R. B. Ferreira et al., "1D+ 3D two-phase flow numerical model of a proton exchange membrane fuel cell," Appl. Energy, vol. 203, pp. 474-95, Oct. 2017. doi: 10.1016/j.apenergy.2017.06.048. 
[8] Š. Šikalo et al., "Dynamic contact angle of spreading droplets: Experiments and simulations," Phys. Fluids, vol. 17, no. 6, pp. 062103, June 2005. doi: 10.1063/1.1928828.

[9] S. F. Lunkad, V. V. Buwa, and K. D. Nigam, "Numerical simulations of drop impact and spreading on horizontal and inclined surfaces," Chem. Eng. Sci., vol. 62, no. 24, pp. 7214-24, Dec. 2007. doi: 10.1016/j.ces.2007.07.036.

[10] D. Legendre and M. Maglio, "Numerical simulation of spreading drops," Colloids Surf., A., vol. 432, pp. 29-37, Sept. 2013.

doi: 10.1016/j.colsurfa.2013.04.046.

[11] M. Jiang and B. Zhou, "Numerical study of droplet impact on inclined surface: viscosity effects," ECS Trans., vol. 83, no. 1, pp. 127-36, Jan. 2018. doi: 10.1149/08301.0127ecst.

[12] M. Jiang, B. Zhou, and X. Wang, "Comparisons and validations of contact angle models," Int. J. Hydrogen Energy, vol. 43, no. 12, pp. 6364-6378, Mar. 2018. doi: 10.1016/j.ijhydene.2018.02.016.

[13] S. F. Kistler, "Hydrodynamics of wetting," in Wettability, J. C. Berg, Ed., New York: Marcel Dekker Inc., 1993, pp. 311-430.

[14] Š. Šikalo, C. Tropea, and E. N. Ganić, "Impact of droplets onto inclined surfaces," J. Colloid Interface Sci., vol. 286, no. 2, pp. 661-669, June 2005. doi: 10.1016/j.jcis.2005.01.050.

[15] Š. Šikalo and E. N. Ganić, "Phenomena of droplet-surface interactions," Exp. Therm. Fluid Sci., vol. 31, no. 2, pp. 97-110, Nov. 2006. doi: 10.1016/j.expthermflusci.2006.03.028

[16] C. Fang et al., "3-D numerical simulation of contact angle hysteresis for microscale two phase flow," Int. J. Multiphase Flow, vol. 34, no. 7, pp. 690-705, Jul. 2008.

doi: 10.1016/j.jimultiphaseflow.2007.08.008

[17] C. Miller, "Liquid water dynamics in a model polymer electrolyte fuel cell flow channel, " M.A.Sc. thesis, Dept. Mech. Eng., Univ.

Victoria, Victoria, B.C., 2009.

[18] T. C. Wu, "Two-phase flow in microchannels with application to PEM fuel cells," Ph.D. dissertation, Dept. Mech. Eng., Univ.

Victoria, Victoria, B.C., 2015. 


\title{
Using Visco-Plastic Lubrication for Suspension Transportation
}

\author{
Saeed Garmeh, Ali Dolatabadi and Ida Karimfazli \\ Department of Mechanical, Industrial and Aerospace Engineering \\ Concordia University \\ Montreal, Canada
}

\begin{abstract}
This study focuses on a common problem in suspension plasma spraying called clogging. Different mechanisms causing that issue are described and a technique called visco-plastic lubrication is introduced as a solution to this problem. Numerical modelling has been done using mentioned technique and effect of viscosity ratio of the fluids on the flow are investigated.
\end{abstract}

Keywords; clogging; suspension plasma spraying; visco-plastic lubrication; numerical modelling

\section{INTRODUCTION AND BACKGROUND}

Coating plays an important role in surface engineering industries. The aim of coating is to enhance physical and mechanical properties of the material surface [1]. Among different spraying processes, suspension plasma spraying (SPS) is common and popular. In SPS, suspension which carries the material particles that we desire to coat them on top of a substrate, is injected into a plasma torch. These particles melt and stick to the substrate and then they are solidified and adhered to the surface.

Typical suspensions used in SPS have concentration of about $20 \%$ wt. which means only $20 \%$ of the mass of the suspension is composed of particles. This relatively low concentration consumes a lot of energy and time in spraying. One way to reduce the spraying time is to increase the concentration of the particles. For instance, if we use $50 \%$ wt. suspension instead of $20 \%$ wt., we save time and energy with a factor of 2.5 which is significant. However, the chance of agglomeration and clogging that is a common issue in SPS is also raised.

Different ways of clogging occurrence are investigated and a technique called visco-plastic lubrication is introduced and modelled numerically as a solution to the clogging issue. In suspensions, when the concentration of particles is increased, the interaction between particles and the tube wall grows. There are two general ways that cause clogging. (a)

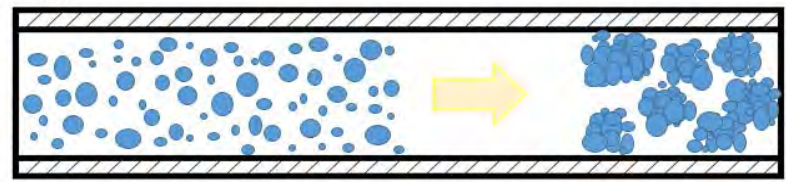

(b)

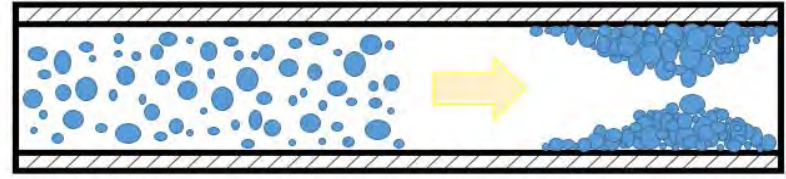

Fig. 1 Schematic of (a) Particle-Particle interactions and (b) Particle-Wall interactions for high concentration suspension inside the tube

Firstly, when the solid particles in the suspension interact with each other, they may adhere together and make bigger agglomerates. These agglomerated parts can be trapped in the carrying tube or injector and cause clogging. A visual representation of this phenomena is shown in Fig. 1(a).

Secondly, when these particles interact with the tube wall and injector, they can be attached to the tube and decrease the passing area gradually to a point that suspension couldn't flow throw the tube and clogging would be the result. Fig. 1(b) shows this phenomena schematically.

For clarifying the problem, possibility of clogging due to the particle-particle interaction was focused. Suspensions of Titanium Dioxide (TiO2) with different concentrations of 20, 30,40 and $50 \%$ wt. were made and analyzed using laser diffraction system (Malvern Spraytec, UK). Measurement tests presented in Fig. 3 and Table 1 show no considerable difference. If the suspension is prepared properly, for concentration up to $50 \%$, there will be no significant change in particle size distribution. The test indicates the main reason behind the clogging is interaction of particles with the tube wall which is shown in Fig 1(b). This conclusion motivates us to implement a technique for preventing contact of suspension with the tube wall. 
(a)

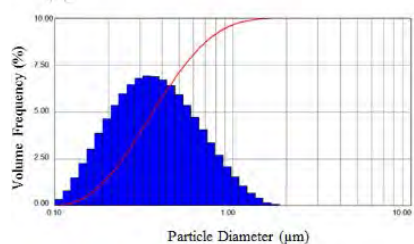

Particle Diameter $(\mu \mathrm{m})$

(c)

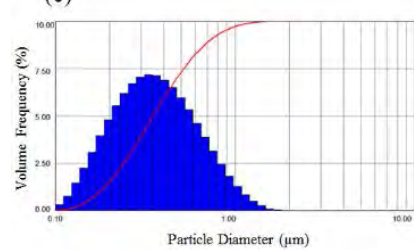

(b)

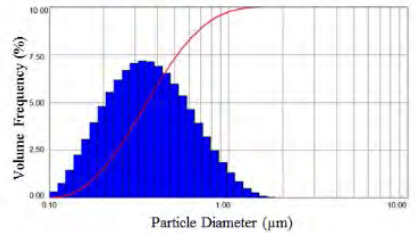

(d)

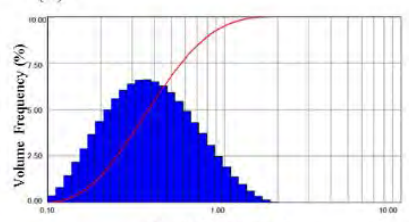

Particle Diameter $(\mu \mathrm{m})$
Fig. 2 Particle size distribution for TiO2 suspensions with concentrations of (a) $20 \%$ wt. (b) $30 \%$ wt. (c) $40 \%$ wt. and (d) $50 \%$ wt.

Table 1. Distribution parameters of suspended particles

\begin{tabular}{cccc}
\hline & $\begin{array}{c}\mathbf{D}(\mathbf{1 0}) \\
\boldsymbol{\mu m}\end{array}$ & $\begin{array}{c}\mathbf{D}(\mathbf{5 0}) \\
\boldsymbol{\mu m}\end{array}$ & $\begin{array}{c}\mathbf{D}(\mathbf{9 0}) \\
\boldsymbol{\mu m}\end{array}$ \\
$\mathbf{2 0 \%}$ & 0.1793 & 0.3549 & 0.7391 \\
$\mathbf{3 0 \%}$ & 0.1784 & 0.3512 & 0.7266 \\
$\mathbf{4 0 \%}$ & 0.1782 & 0.3503 & 0.7243 \\
$\mathbf{5 0 \%}$ & 0.1798 & 0.3736 & 0.8196 \\
\hline
\end{tabular}

Proposed solution to this problem is visco-plastic lubrication. In this technique, we have a core annular flow where the core fluid is high concentration suspension and it is lubricated with a yield stress fluid. The outstanding character of yield stress fluids is that they are able to flow only if they are submitted to a stress above a critical value called yield stress of the material. Otherwise they deform like solids [2]. Utilizing yield stress fluid prevents the core fluid to get in contact with the tube wall by preserving an unyielded plug around the core fluid [3]. Fig. 3 shows a schematic of visco-plastic lubrication.

(a)

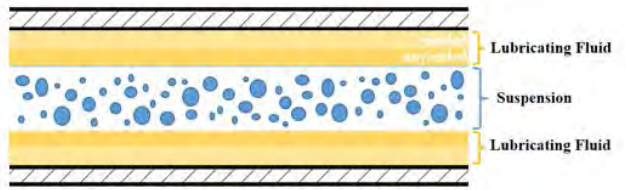

(b)

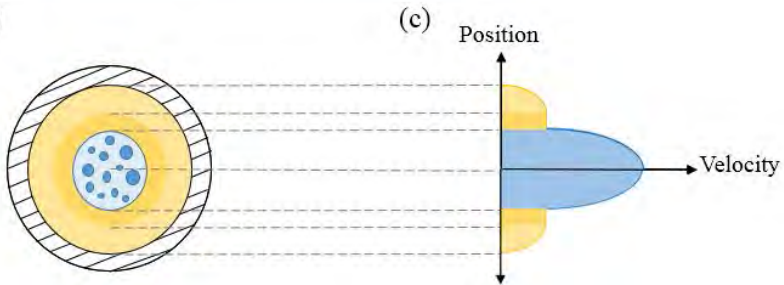

Fig. 3 Schematic of visco-plastic lubrication of suspension in (a) side view, (b) front view and (c) velocity profile of the flow
In this research, $0.3 \%$ Carbomer gel used as lubricating fluid which is a yield stress fluid. Result of the rheology test on the gel and the fitted curves are shown in Fig. 4. The curves generated using MATLAB Curve Fitting tool and the equations of the curves are as follows:

Herschel-Bulkley Model:

$$
\tau=44.18+13.84 \times \dot{\gamma}^{0.2986}
$$

Bingham Model:

$$
\tau=55.12+0.80 \times \dot{\gamma}
$$

Where $\tau$ is the stress and $\dot{\gamma}$ is strain rate. According to (1) and (2), the yield stresses predicted by Herschel-Bulkley and Bingham models are $44.18 \mathrm{~Pa}$ and $55.12 \mathrm{~Pa}$, respectively. As it can be understood from Fig. 4, the Herschel-Bulkley model can predict the behavior of the yield stress fluid more accurately. For calculation of effective viscosity, slope of predicted curve by Bingham model is used when the flow begins [4]. Therefore, $0.80 \mathrm{~Pa}$.s considered as the effective viscosity of the $0.3 \%$ Carbomer Gel.

\section{MECTHODOLOGY:}

\section{A. Numerical model}

All simulations were performed by ANSYS Fluent package. For modelling the multiphase flow, the mixture model was used. This model can be used to model multiphase flows where the phases move at different velocities. The mixture model can model $\mathrm{n}$ phases by solving the momentum, continuity, volume fraction equations for the secondary phases, and algebraic expressions for the relative velocities [5]. The governing equations are as follow:

\section{1) Continuity Equation}

$$
\frac{\partial\left(\rho_{m}\right)}{\partial t}+\nabla \cdot\left(\rho_{m} \vec{v}_{m}\right)=0
$$

Where $\rho_{m}$ is the mixture density according to (4) and $\vec{v}_{\mathrm{m}}$ is the mass-averaged velocity according to (5):

$$
\begin{array}{r}
\rho_{m}=\sum_{k=1}^{n} \alpha_{k} \rho_{k} \\
\vec{v}_{m}=\frac{\sum_{k=1}^{n} \alpha_{k} \rho_{k} \vec{v}_{k}}{\rho_{m}}
\end{array}
$$

Where $\mathrm{n}$ is number of phases. Current numerical study is for a flow of 2 phases. $\alpha_{k}, \rho_{k}$ and $\vec{v}_{k}$ are volume fraction, density and velocity of phase $\mathrm{k}$, respectively. $\mathrm{k}=1$ is representing the lubricating fluid and $\mathrm{k}=2$ is the core fluid.

\section{2) Momentum Equation}

$$
\begin{gathered}
\frac{\partial}{\partial t}\left(\rho_{m} \vec{v}_{m}\right)+\nabla \cdot\left(\rho_{m} \vec{v}_{m} \vec{v}_{m}\right)=-\nabla P+\nabla \cdot\left[\mu_{m}\left(\nabla \vec{v}_{m}+\overrightarrow{v_{m}^{T}}\right)\right] \\
+\rho_{m} \vec{g}+\vec{F}+\nabla \cdot\left(\sum_{k=1}^{n} \alpha_{k} \rho_{k} \vec{v}_{d r, k} \vec{v}_{d r, k}\right)
\end{gathered}
$$




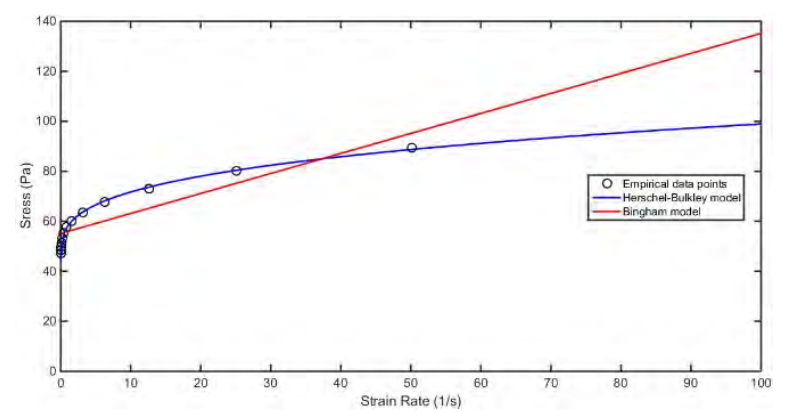

Fig. 4 Flow curve of $0.3 \%$ Carbomer Gel and fitted curves of Bingham and Herschel-Bulkley models

Where $\vec{F}$ is the body force, and $\mu_{m}$ is the viscosity of the mixture:

$$
\mu_{m}=\sum_{k=1}^{n} \alpha_{k} \mu_{k}
$$

And $\vec{v}_{d r, k}$ is the drift velocity for secondary phase $k$ :

$$
\vec{v}_{d r, k}=\vec{v}_{k}-\vec{v}_{m}
$$

\section{3) Volume Fraction Equation for the Secondary Phase}

From the continuity equation for secondary phase $(k=2)$, the volume fraction equation for secondary phase 2 can be obtained:

$$
\begin{gathered}
\frac{\partial}{\partial t}\left(\alpha_{2} \rho_{2}\right)+\nabla \cdot\left(\alpha_{2} \rho_{2} \vec{v}_{m}\right) \\
=-\nabla \cdot\left(\alpha_{2} \rho_{2} \vec{v}_{d r, 2}\right)+\left(\dot{m}_{12}-\dot{m}_{21}\right)
\end{gathered}
$$

Where $\dot{m}_{12}$ is the mass transfer from phase 1 to phase 2 and $\dot{m}_{21}$ is the mass transfer from phase 2 to phase 1 .

\section{B. Dimensional analysis}

According to The Buckingham Pi theorem, groups of dimensionless numbers are presented in Table 2. This study concentrates on investigating the effect of viscosity ratio of core fluid to lubricating fluid on the flow.

Core fluid and lubricating fluid have viscosities of $\mu_{\text {core }}$ and $\mu_{\text {lub }}$, densities of $\rho_{\text {core }}$ and $\rho_{\text {lub }}$ and average velocities of $V_{\text {core }}$ and $V_{l u b}$, respectively. $L$ and $D$ are the length and diameter of the tube and $d_{i}$ is the interface diameter of developed flow. $\tau_{y}$ is the yield stress for lubricating fluid and $\Delta P$ is the pressure drop alongside the tube.

Table 2. Dimensionless groups

\begin{tabular}{|c|c|c|c|}
\hline$\Pi_{1}=\frac{\rho_{\text {core }} V_{\text {core }} d_{i}}{\mu_{\text {core }}}$ & $\Pi_{2}=\frac{\tau_{y} D}{V_{\text {lub }} \mu_{\text {lub }}}$ & $\Pi_{3}=\frac{\Delta P}{\rho_{\text {core }} V_{\text {core }}^{2}}$ & $\Pi_{4}=\frac{\mu_{\text {core }}}{\mu_{\text {lub }}}$ \\
\hline$\Pi_{5}=\frac{V_{\text {core }}}{V_{\text {lub }}}$ & $\Pi_{6}=\frac{\rho_{\text {core }}}{\rho_{\text {lub }}}$ & $\Pi_{7}=\frac{L}{D}$ & $\Pi_{8}=\frac{d_{i}}{D}$ \\
\hline
\end{tabular}

According to table $2, \Pi_{1}$ is the Reynolds number based on the core fluid properties. $\Pi_{2}$ is the Bingham number for lubricating fluid which is the ratio of yield stress to a typical viscous stress of the flow [4]. $\Pi_{3}$ is dimensionless pressure drop based on the core fluid properties, $\Pi_{4}, \Pi_{5}$ and $\Pi_{6}$ are the ratios of core fluid to lubricating fluid properties. $\Pi_{7}$ and $\Pi_{8}$ are groups of the normalized length and interface diameter.

\section{Geometry and computational domain}

The domain inside the tube that is modeled is a cylinder with diameter of $D$ and length of $L$. The grid is made up of quadrilateral cells. ICEM CFD 14.0 was used for generating the mesh. Number of cells used in this study is 211,167 and a grid dependency study has been done to ensure the results are independent from the mesh.

\section{Boundary Conditions:}

This study aims to reveal the relation of viscosity ratio to the flow parameters. Both fluids are injected co-axially to the domain. The velocity inlet boundary condition is assigned for inlets of core and lubricating fluids. Core fluid is injected from the center of cylinder. Pressure outlet used as the boundary condition at the exit of the flow. The wall boundary condition with no-slip is assigned to the tube wall.

In the modelling, the flow rate of each fluid kept at a constant value which results a stable core annular flow. Then, different values were set to the viscosity of the core fluid. By keeping the lubricating fluid parameters constant in all the simulations and just changing the viscosity of the core fluid, different viscosity ratios obtained and simulation repeated for each of those ratios.

\section{E. Simulation results}

Effects of changing the viscosity ratio over dimensionless groups are demonstrated in Fig. 6. Velocity profiles of the flow are also presented in Fig. 7. Note that the velocity and position are normalized by average velocity of lubricating fluid $\left(V_{l u b}\right)$ and diameter of the pipe $(D)$, respectively.

Contours of volume fraction of core fluid in a cut plane alongside the modelled tube for different viscosity ratios are shown in Fig. 8. The tube is long enough to ensure the flow is fully developed.

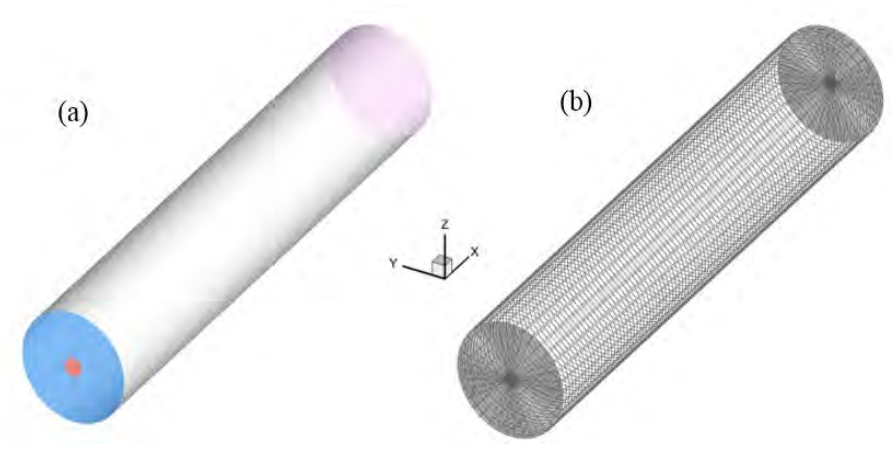

Fig. 5 (a) Geometry and (b) Generated grid 


\section{SUMMARY AND CONCLUSION}

The simulation results showed that using the visco-plastic lubrication technique can prevent suspension to get in contact with the tube wall by forming a solid-like annulus around the core fluid which in fact it can solve the clogging issue. Investigating different viscosity ratios of core fluid to lubricating fluid showed us as the ratio increases, the interface diameter rises which means there is more core fluid in each cross section of the tube. (a)

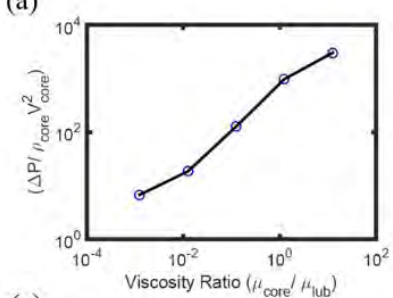

(c)

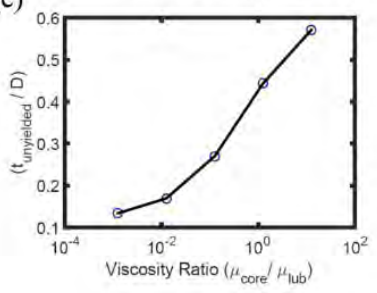

(b)

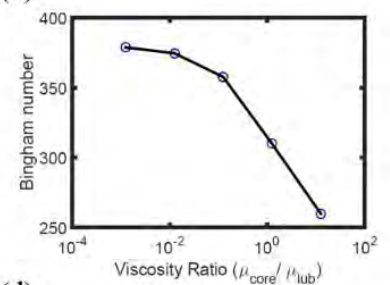

(d)

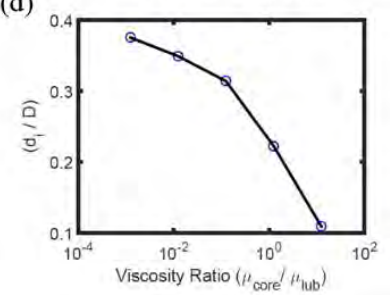

Fig. 6 Effects of changing the viscosity ratio on (a) dimensionless pressure drop (b) Bingham number, (c) normalized thickness of unyielded plug $\left(t_{\text {unyielded }} / D\right)$ around the core fluid and (d) normalized interface diameter $\left(d_{i} / D\right)$ for $L=200$ $\mathrm{mm}, D=38.1 \mathrm{~mm}$ and Reynolds number of $1392,110,6.9,0.41,0.03$ for viscosity ratio of $0.00125,0.0125,0.125,1.25$ and 12.5 , respectively.

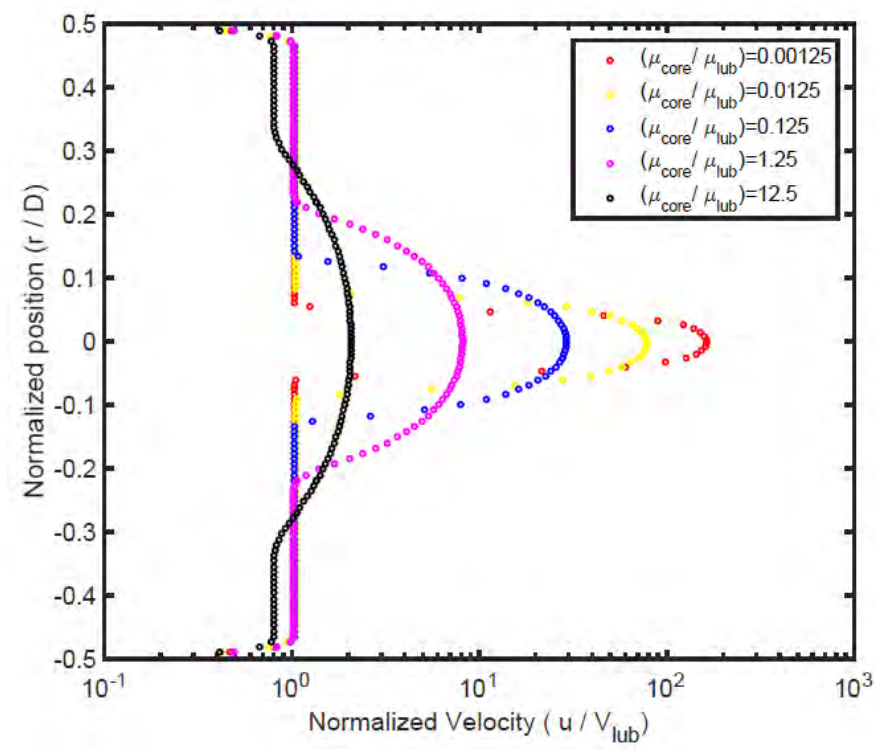

Fig. 7 Velocity profiles for different viscosity ratios

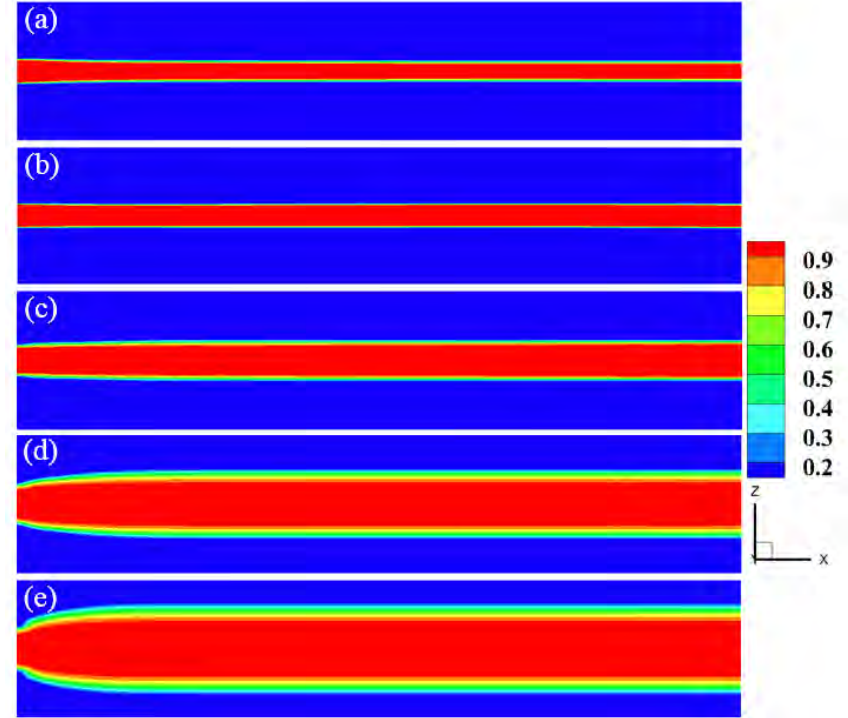

Fig. 8 Contours of volume fraction of core fluid for viscosity ratio of (a) 0.00125 , (b) 0.0125 , (c) 0.125 , (d) 1.25 , (e) 12.5

In all the cases, an unyielded plug was formed around the core suspension. When the viscosity ratio increased, reduction in the thickness of the plug was observed. As long as there is an annulus plug around the core, the interfacial instabilities cannot grow which means the flow is stable. Similar to our expectation, increasing the viscosity of the core fluid resulted in increasing the dimensionless pressure drop $\left(\Pi_{3}\right)$. In addition, increasing the viscosity ratio caused reduction of Bingham number.

Since we are interested in maximizing the flow rate ratio of core fluid to lubricating fluid, the future work of this study will focus on assigning different flow rates for each fluid in order to obtain maximum possible flow rate ratio. In addition, performing experiment in parallel is planned to confirm the simulation results.

\section{ACKNOWLEDGMENT}

The authors acknowledge the financial support from Natural Sciences and Engineering Research Council (NSERC) of Canada.

\section{REFERENCES}

[1] P. Fauchais, A. Joulia, S. Goutier, C. Chazelas, M. Vardelle, A. Vardelle and S. Rossignol, "Suspension and solution plasma spraying,"Journal of Physics D: Applied Physics, vol. 46, 224015, 2013. doi: 10.1088/0022-3727/46/22/224015.

[2] P. Coussot, "Yield stress fluid flows: A review of experimental data. Journal of Non-Newtonian Fluid Mechanics, vol. 211, pp. 31-49, 2014. doi: 10.1016/j.jnnfm.2014.05.006.

[3] S. Hormozi, D. Martinez and I. Frigaard, "Stable core-annular flows of viscoelastic fluids using the visco-plastic lubrication technique," Journal of NonNewtonian Fluid Mechanics, vol. 166, pp. 1356-1368, 2011. doi: 10.1016/j.jnnfm.2011.08.013.

[4] S. Hormozi, K. Wielage-Burchard and I. Frigaard (2011). Entry, start up and stability effects in visco-plastically lubricated pipe flows. Journal of Fluid Mechanics, vol. 673, pp. 432-467, 2011. doi: 10.1017/S0022112010006348.

[5] ANSYS Fluent (2011). Fluent 14 documentation. User's guide 


\title{
Vortex-Induced Vibrations of a Low-tension Cable-Sheave System Modeled Using Nonlinear Finite Elements
}

\author{
Cassidy Westin, Rishad A. Irani \\ Department of Mechanical and Aerospace Engineering \\ Carleton University \\ Ottawa, Canada \\ cassidy.westin@carleton.ca; rishad.irani@carleton.ca
}

\begin{abstract}
This work presents a finite element model of a cablesheave system constructed to model cable vibrations due to vortex shedding in low-tension cases. The study assesses the potential for vortex shedding to lead to detachment of the cable from the sheave. The absolute nodal coordinate formulation is utilized to define the cable structural dynamics. Vortex shedding forces are incorporated by coupling the cable's equations of motion to a Van der Pol equation, also known as a wakeoscillator and the cable-sheave interaction is described using a contact penalty method. The study examines the contribution of vortex shedding occurring at the cable's natural frequency to the cable motion. For the cases studied the model demonstrates that vortex shedding alone results in very small vibration amplitudes and thus is unlikely to result in cable detachment.
\end{abstract}

Keywords-finite element method; absolute nodal coordinate formulation; cable-sheave interaction; vortex-induced vibration

\section{INTRODUCTION}

Cable-sheave systems are commonly used in marine applications for towing and lifting. The cable is subjected to highly dynamic loading due to the ship motion, hydrodynamic forces and wind loading which can result in the cable falling off the sheave. Cables exposed to external fluid flow experience vortex induced vibrations (VIV) resulting from the periodic shedding of vortices in the cables wake. The vortex shedding produces oscillating lifting and drag forces on the cable, which can excite a resonance response in the cable if the shedding frequency matches the fundamental frequency of the cable. Excessive cable vibrations can result in wear of both the cable and the sheave and possible detachment of the cable from a sheave. It is thus useful to quantify the vibration amplitudes due to wind loading using a dynamic model and assess the potential for vortex shedding to lead to cable detachment.

Gabbai and Benaraoya [1] give an overview of the various modeling approaches used to predict the response of cylinders to the vortex shedding forces. One modelling approach known as a wake-oscillator model utilizes a second order differential equation, such as the Van der Pol equation, to represent the flow in the wake. The oscillator is coupled with the structural dynamics of the cable using forcing terms in both equations. This modelling approach does not require numerical modeling of the flow field and can be easily incorporated into existing dynamic cable models. Existing studies of cable vibrations using wake oscillator models typically utilize continuous equations to represent the cable structural dynamics. The contribution of the current research is the coupling of a wake-oscillator model and a finite element cable model, which has not been extensively studied.

This paper presents the construction of a finite element model using the absolute nodal coordinate formulation (ANCF). The model, used for a planar cable-pulley system in the researchers previous work [2], has been extended to include three dimension contact with the sheave grooves. Additionally, oscillating lift and drag forces using an existing wake-oscillator model [3] have been incorporated. Cases of low cable tension are considered as cable detachment is more likely to occur when the cable is slack. High sustained wind velocities are neglected in the study and only the contribution of vortex shedding at the cable's natural frequency is considered. Section 2 of the paper describes the cable model and the formulation of the external forces. Case studies examining the potential for VIV to lead to cable detachment are presented in Section 3. The paper ends with concluding remarks in Section 4.

\section{Finite Element And External LoAd Model}

The finite element model is comprised of $N$ two node cable elements. Each node has 6 degrees of freedom consisting of a position vector and a vector tangent to the cable centerline. All degrees of freedom are defined in the absolute coordinate frame. An additional parameter $p \in[0, l]$ is used to interpolate 
between nodes. The absolute coordinates $\mathbf{r}$ of a point on the cable is defined using a vector of generalized coordinate $\mathbf{q}$ and a cubic shape function $\mathbf{S}(p)$ which interpolates between the nodes of each element:

$$
\mathbf{r}(p)=\mathbf{S}(p) \mathbf{q}=\left[\begin{array}{lll}
x & y & z
\end{array}\right]^{T} .
$$

For a fully parameterized ANCF beam element of unstretched length $l$, the generalized coordinates consist of the Cartesian coordinates $\mathbf{r}$ and the parametric slopes $\partial \mathbf{r} / \partial p$ at each node:

$$
\mathbf{q}=\left[\begin{array}{llll}
\mathbf{r}_{1}{ }^{T} & (\partial \mathbf{r} / \partial p)_{1}{ }^{T} & \mathbf{r}_{2}{ }^{T} & (\partial \mathbf{r} / \partial p)_{2}{ }^{T}
\end{array}\right]^{T}
$$

where subscripts 1 and 2 represent the respective nodes of the element or parameter values of $p=0$ and $p=l$, respectively. The shape function representing a cubic spline is

$$
\mathbf{S}(p)=\left[\begin{array}{c}
\left(1-3(p / l)^{2}+2(p / l)^{3}\right) \mathbf{I} \\
\left((p / l)-2(p / l)^{2}+(p / l)^{3}\right) \mathbf{I} \\
\left(3(p / l)^{2}-2(p / l)^{3}\right) \mathbf{I} \\
\left((p / l)^{2}-(p / l)^{3}\right) \mathbf{I}
\end{array}\right]^{T}
$$

where $\mathbf{I}$ is a $3 \times 3$ identity matrix.

The standard form of the Newton-Euler equations are given for a single element as

$$
\mathbf{M} \ddot{\mathbf{q}}+\mathbf{K q}+\mathbf{C} \dot{\mathbf{q}}-\mathbf{Q}=0,
$$

where $\mathbf{M}$ is the mass matrix, $\mathbf{K}$ is the elastic stiffness matrix, $\mathbf{C}$ is the damping matrix, $\mathbf{Q}$ is an external force vector, and $\mathbf{q}$ is the vector of generalized coordinates defined in Equation 2. The equations of motion for the full system are formulated from Equation 4 using the embedding technique described in [4]. The Newton-Euler equations are solved using a numerical ODE integrator to determine the cable motion over time. The matrices $\mathbf{M}, \mathbf{K}$ and $\mathbf{C}$ and force vector $\mathbf{Q}$ will be defined in the following sections.

\section{A. Mass Matrix}

Using a variational mass lumping approach the mass matrix is derived directly from the element kinetic energy [5] and is given by

$$
\mathbf{M}=\frac{\partial^{2} E_{T}}{\partial \dot{\mathbf{q}} \partial \dot{\mathbf{q}}}=\int_{0}^{l} \rho A \mathbf{S}(\mathbf{p})^{T} \mathbf{S}(\mathbf{p}) d p
$$

where $E_{T}$ is the kinetic energy of the element, $\rho$ is the cable density and $A$ is the cable cross-sectional area.

\section{B. Stiffness}

Similarly, the stiffness matrix is derived from the strain energy $E_{S}$ of the element given by [6]:

$$
E_{S}=\frac{1}{2} \int_{0}^{l}\left[E A \epsilon_{l}^{2}(p)+E I \kappa^{2}(p)\right] d p
$$

where $E$ is the Young's modulus of the cable material, $A$ is the cross-sectional area, $I$ is the second moment of area, $\epsilon_{l}$ is the longitudinal strain, and $\kappa$ is the curvature of the element. The internal stiffness force is then given by the derivative of the strain energy with respect to the coordinate vector $\mathbf{q}$. The force vector can by separated into longitudinal and transverse components, $\mathbf{Q}_{l}$ and $\mathbf{Q}_{t}$. In order to simplify the computation of these forces it is assumed that the longitudinal strain $\epsilon_{l}$ is small and also constant throughout the element. The forces can then be derived as shown by Berzeri et al. [6]:

$$
\mathbf{Q}_{\mathbf{l}}=\left[E A \epsilon_{l} \int_{0}^{l} \mathbf{S}^{\prime T} \mathbf{S}^{\prime} d p\right] \mathbf{q}
$$

where $\mathbf{S}^{\prime}$ is the derivative of $\mathbf{S}$ with respect to the parameter $p$. The longitudinal strain is determined from the arc length $s$ of the element [2]:

$$
\epsilon_{l}(\mathbf{q})=\frac{s(\mathbf{q})-l}{l}
$$

where $s$ is the arc length and $l$ is the unstretched element length. The arc length is defined by integrating the norm of $\partial \mathbf{r} / \partial p$ with respect to $p$ over the length of the element [7]. The arc length $s$ is approximated using a numerical quadrature

$$
s(\mathbf{q})=\sum_{i=1}^{N_{I}} w_{i} \sqrt{\mathbf{r}^{\prime}\left(p_{i}\right)^{T} \mathbf{r}^{\prime}\left(p_{i}\right)} \frac{l}{N_{I}}
$$

where $i$ denotes the integration point, $N_{I}$ is the total number of integration points, $w_{i}$ is the quadrature weight. The trapezoidal rule is used with quadrature weights defined

$$
w_{i}= \begin{cases}0.5, & i=1, N_{I} \\ 1, & i=2,3, \ldots, N_{I}-1\end{cases}
$$

The transverse stiffness force $\mathbf{Q}_{t}$ is defined [6]

$$
\mathbf{Q}_{t}=\left[\int_{0}^{l} E I \mathbf{S}^{\prime \prime T} \mathbf{S}^{\prime \prime} d p\right] \mathbf{q}
$$

where $I$ is the second moment of area. The general stiffness matrix $\mathbf{K}$ from Equation 4 is then given by

$$
\mathbf{K}=\int_{0}^{l} E I \mathbf{S}^{\prime \prime} \mathbf{S}^{\prime \prime} d p+\epsilon_{l}(\mathbf{q}) E A \int_{0}^{l} \mathbf{S}^{\prime T} \mathbf{S}^{\prime} d p .
$$

\section{Damping}

The damping matrix $\mathbf{C}$ from Equation 4 serves both to include energy dissipation and attenuate high frequency vibrational modes. The cable model uses the Rayleigh-damping approach [8], wherein the damping matrix is defined as a linear combination of the mass and stiffness matrices:

$$
\mathbf{C}=\alpha \mathbf{M}+\beta \mathbf{K}
$$

where $\alpha$ and $\beta$ are scalar damping coefficients. The massproportional damping term represents external viscous damping while the stiffness-proportional damping term represents internal frictional damping. Since the external fluid damping 
can be incorporated into the aerodynamic drag force, the massproportional term will be neglected by setting $\alpha$ equal to zero.

Given a specified damping ratio $\xi$, the corresponding value of the remaining coefficient $\beta$ is given by [9]

$$
\beta=2 \xi \frac{1}{\omega_{n}}
$$

where $\omega_{n}$ is the natural frequency of the cable.

\section{External Forces}

The vector of external forces $\mathbf{Q}$ includes the gravitational body force, the normal contact forces along the sheave surfaces and the aerodynamic loading. The formulation of the forces is provided in the following sections.

1) Contact Force: In order to model the cable-sheave interaction, a contact penalty is used. The cable is allowed to "penetrate" the sheave surface and the normal force os defined as a function of the of the relative penetration $\delta$. The normal force per unit length $\mathbf{f}_{N}$ acting at a single point on the element is defined using the Hunt-Crossley model [10], which represents the surface as a non-linear spring-damper:

$$
\mathbf{f}_{N}=k_{N} \delta^{n}(1+D \dot{\delta}) \mathbf{u}_{n}
$$

where $\mathbf{u}_{n}$ is the unit vector normal to the sheave surface at the point of contact, $k_{N}$ is the contact stiffness, $\delta$ is the relative "penetration" of the node into the surface, $D$ is a damping coefficient and $n$ is a positive constant with a value between 1 and 1.5 from the Hertz contact theory [11]. In the present analysis, a contact stiffness of $1.5 \times 10^{7} \mathrm{~N} / \mathrm{m}$ and a value of $n$ of 1.5 is used based on the values used by Bulin et al [12] and the researchers' previous work [2].

The radius of curvature of the groove is assumed to be equal to the radius of the cable. Two surfaces are defined that are parallel to the straight walls of the sheave groove and intersect at the center of curvature of the groove. In the cross-section shown in Figure 1, the two surfaces are straight solid lines and the sheave groove is a dotted line. If the cable centerline lies below either of the solid lines, a penalty force is produced proportional to the penetration of the cable node into the surface. If the cable node lies above both lines, no normal force is applied. The contact penalty is illustrated in Figure 1. The normal force per unit length is evaluated at $N_{I}$ discrete points points per element. The distributed force in the element coordinates is then calculated from the discrete force distribution that will be discussed in Section II-D4.

2) Drag Force: The drag force per unit length acting at a given point on the cable is given by [13]

$$
\mathbf{f}_{D}=-\frac{1}{2} C_{D} \rho_{w} d\left|\mathbf{V}_{n}\right| \mathbf{V}_{n}
$$

where $C_{D}$ is the drag coefficient, $d$ is the cable diameter, $\mathbf{V}_{n}$ is the normal component of the relative velocity $V$, and $\mu$ is the dynamic viscosity of the fluid. The relative velocity and its normal component are calculated as follows:

$$
\mathbf{V}=\dot{\mathbf{r}}-\mathbf{U}
$$

$$
\begin{gathered}
\mathbf{V}_{t}=\left(\mathbf{V} \cdot \mathbf{u}_{t}\right) \mathbf{u}_{t} \\
\mathbf{V}_{n}=\mathbf{V}-\mathbf{V}_{t}
\end{gathered}
$$

where $\mathbf{U}$ is the velocity of the free stream, $\dot{\mathbf{r}}$ is the velocity of the point on cable centerline. The unit vector tangent to the cable centerline $\mathbf{u}_{t}$ is equivalent to $\frac{\mathbf{r}^{\prime}}{\left|\mathbf{r}^{\prime}\right|}$. The drag coefficient $C_{D}$ is given by

$$
C_{D}=1.1+4 R e^{0.8}, \quad 30<\operatorname{Re}<10^{5}
$$

where $R e$ is the Reynolds number.

3) Wake Oscillator Model: A two-degree of freedom wakeoscillator developed by Bai and Qin [3] is used to describe the vortex shedding forces on the cable. The variable $w(t)$ is introduced, with the function $d^{2} \dot{w}(t)$, where $d$ is the cable diameter, corresponding to the vortex strength of the wake. The evolution of $w$ over time satisfies a van der Pol equation of the form

$$
\ddot{w}+a_{1} \omega_{s t}\left[1-\frac{a_{2}(\dot{w})^{2}}{\omega_{s t}^{2}}\right] \dot{w}+\omega_{s t} w=\frac{a_{3} \omega_{s t}}{d} \dot{Y}
$$

where $a_{1}, a_{2}$ and $a_{3}$ are empirical parameters, $\omega_{s t}$ is the Strouhal frequency in rad/s, and $Y$ is the displacement of the cable in the cross-flow direction. Typical values for $a_{1}, a_{2}$ and $a_{3}$, proposed by Facchinetti [14] and Bai and Qin [3] are 0.3, 0.2 and 12 , respectively.

The forces acting on the structure due to the vortex shedding are then evaluated:

$$
\begin{gathered}
f_{S W}=-\frac{C_{D 0} \rho d^{4}}{32 \pi^{3} S t^{3} U} \dot{w} \ddot{w} \\
f_{C F}=\frac{C_{L 0} \rho U d^{2}}{8 \pi S t} \dot{w}
\end{gathered}
$$

where $f_{S W}$ is the force per unit length acting in the streamwise direction, $f_{C F}$ is the force per unit length acting in the cross-flow direction, $C_{D 0}$ and $C_{L 0}$ are the drag and lift coefficients associated with the vortex shedding, with assumed values of 0.2 and 0.3 , respectively. In the absolute coordinate frame, the cross-flow direction corresponds to the y-axis, while the stream-wise direction corresponds to the z-axis.

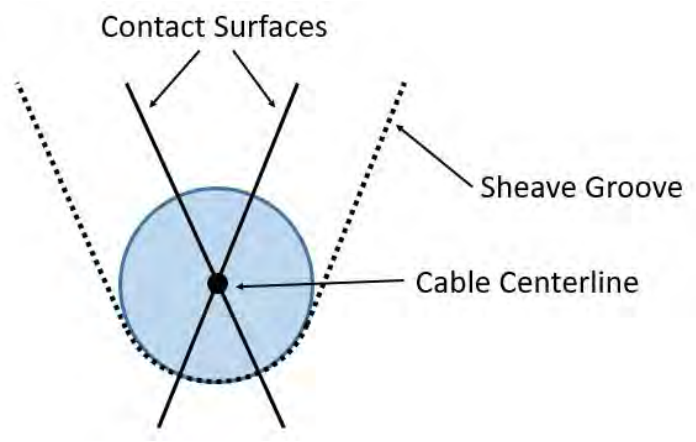

Fig. 1: Sheave Groove Cross-Section 
For each node on the cable exposed to the wind, a degree of freedom representing the wake variable $w$ is added to the system equations of motion. The wake coefficients are interpolated linearly to provide the discrete force distributions along each element.

4) Total Distributed Force: The force distributions determined for the normal contact, stationary drag and oscillating drag and lift are added together to give the total force distribution $\mathbf{f}$. The force acting on the element in the element coordinates $\mathbf{Q}$ is then found by integrating over the length of the cable element:

$$
\mathbf{Q}=\int_{0}^{l} \mathbf{S}(p)^{T} \mathbf{f} d p .
$$

Again, a numerical quadrature is used to approximate the integral:

$$
\mathbf{Q}=\sum_{i=1}^{N_{I}} w_{i} \mathbf{S}(p)^{T} \mathbf{f}_{i} \frac{l}{N_{I}}
$$

where $i$ denotes the integration point, $N_{I}$ is the total number of integration points and $w_{i}$ is the quadrature weight. Ten integration points per element were used for all simulations described in this report.

The gravitational body forces are defined similarly with $\mathbf{f}=$ $\left[\begin{array}{lll}0 & -\rho A g & 0\end{array}\right]^{T}$ where $\rho$ is the cable density and $g$ is the acceleration due to gravity. Since the gravitational force is constant, the integration can be performed symbolically using Equation 21 prior to the simulation.

\section{CASE Studies}

\section{A. Model Parameters}

The system consists of a cable suspended by two identical sheaves. Each cable end is attached to a point mass as shown in Fig. 2. The wind vector is aligned with the z-axis. Static drag and oscillating lift and drag are applied only to the portion of the cable suspended between the two sheaves. A number of case studies were performed. The system properties used for the simulations are listed in Table I. The cable properties used are for a wire rope with $6 \times 37$ construction, commonly used for marine lifting and hoisting applications [15]. The sheave diameters are calculated using the minimum recommended sheave-to-rope diameter ratio of 18 [15].

Values for the cable bending stiffness $E I$ and damping ratio $\xi$ are not readily available, thus conservative values were used. A minimum bending stiffness value can be calculated by neglecting the friction between individual wires and assuming the total bending stiffness is the sum of the bending stiffnesses of the individual wires [16]. Using the average diameter $d_{a v g}$ and assuming the lay angle $\theta$ is small, the bending stiffness becomes

$$
E I=E \sum \frac{\pi d_{w}{ }^{4}}{64} \cos \theta=E N_{w} \frac{\pi d_{a v g}{ }^{4}}{64}
$$

where $N_{w}$ is the number of wires. Based on the parameters listed in Table I, the bending stiffness is estimated to be 0.73
TABLE I: Model parameters.

\begin{tabular}{|l|c|}
\hline Cable diameter, $d$ & $20 \mathrm{~mm}$ \\
Cable mass per unit length, $\mu$ & $1.38 \mathrm{~kg} / \mathrm{m}$ \\
Damping ratio, $\xi$ & 0.10 \\
Cable elastic modulus, $E$ & $75.8 \mathrm{GPa}$ \\
Cable bending stiffness, $E I$ & $0.73 \mathrm{Nm}^{2}$ \\
Cable metallic area, $A$ & $1.64 \times 10^{-4} \mathrm{~m}^{2}$ \\
Sheave radius at root, $R$ & $0.18 \mathrm{~m}$ \\
Sheave groove angle & 30 degrees \\
Sheave groove depth & $30 \mathrm{~mm}$ \\
\hline
\end{tabular}

TABLE II: Case study parameters.

\begin{tabular}{|c|c|c|c|c|}
\hline Case & 1 & 2 & 3 & 4 \\
\hline Span length (m) & 2 & 5 & 5 & 5 \\
Cable Tension (N) & 200 & 200 & 500 & 1000 \\
Natural frequency (rad/s) & 19.9 & 7.57 & 12.0 & 16.9 \\
Wind velocity (m/s) & 0.302 & 0.121 & 0.190 & 0.269 \\
\hline
\end{tabular}

$\mathrm{Nm}^{2}$. Damping ratios for wire ropes undergoing bending vary with cable tension and have been shown to be as high as $37 \%$ for slack cables [17]. A conservative damping ratio of $10 \%$ was used for the simulations.

The natural frequency of the catenary is estimated by treating the cable as a thin Euler-Bernoulli beam pinned at both ends and is given by [18]

$$
\omega_{n}=\left(\frac{\pi}{L}\right)^{2} \sqrt{\frac{E I}{m}\left[1+\frac{T}{E I}\left(\frac{L}{\pi}\right)^{2}\right]}
$$

where $L$ is the beam length, $m$ is the mass per unit length, $T$ is the axial tension, and $E I$ is the bending stiffness. The vortex shedding frequency can be calculated using the Strouhal relation [3]:

$$
f_{S t}=\frac{S t U}{d}=\frac{\omega_{S t}}{2 \pi}
$$

where $S t$ is the Strouhal number, assumed to have a value of 0.2 [14], $U$ is the free stream velocity and $d$ is the cable diameter.

Simulations were performed for three cases; the model parameters specific to each case are given in Table II. In each case, the wind velocity was chosen such that the shedding frequency matches the estimated natural frequency of the cable.

\section{B. Mesh Convergence}

Fig. 2 shows how the cable was divided into five segments: the two free cable ends with point masses, the two segments in contact with the sheaves and the catenary suspended between the sheaves. The wind load is applied only to the catenary, segment 3. Each segment was further divided into elements. A smaller element length is desirable for the elements in contact with the sheave as the curvature is greater. A mesh convergence was performed by allowing the system to reach 


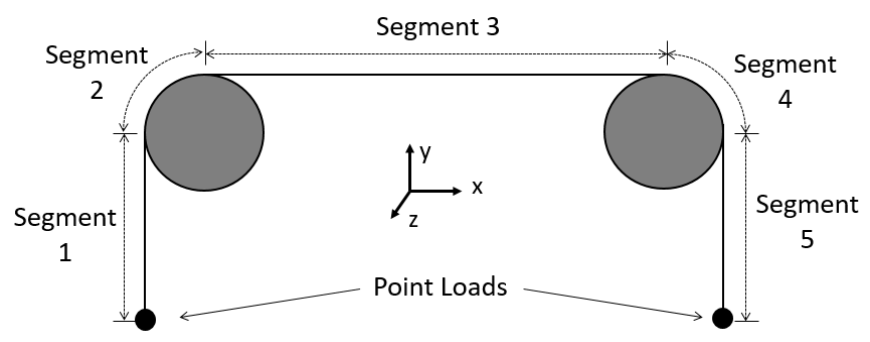

Fig. 2: Diagram of cable-sheave system. Air flow is along the $\mathrm{z}$-axis.

equilibrium with no wind load applied and iteratively reducing the length of the elements in the contact arc and comparing the contact force at the top of the sheave. The number of elements was found to have a minimal effect on the contact force.

For segment 3, the convergence is determined by simulating 20 seconds of motion with the wind load applied. The maximum amplitudes of the motion at the mid-point of the span were compared. Convergence was considered to occur when the change in the output was less than $1 \%$. Fig. 3 plots the mesh convergence for the vertical amplitudes of the 2 and $5 \mathrm{~m}$ spans. For the final simulations, 5 elements were used for each of the contact segments while 12 and 16 elements were used for the 2 and $5 \mathrm{~m}$ cable spans. The free cable ends were assumed not to undergo significant transverse motion, thus only 2 elements were used for each.

\section{Results}

For each case in Table II, the motion was simulated until a steady oscillation was obtained. Fig. 4 displays the vertical motion at midpoint of the span the for Case 4. The results for each test case are shown in Table III. The maximum amplitude at the midpoint of the span was $4.5 \times 10^{-6} \mathrm{~m}$ for Case 4 . At the sheave, the maximum amplitude was $3.3 \times 10^{-6} \mathrm{~m}$. The amplitude of the motion is much smaller than the $30 \mathrm{~mm}$ sheave groove depth.

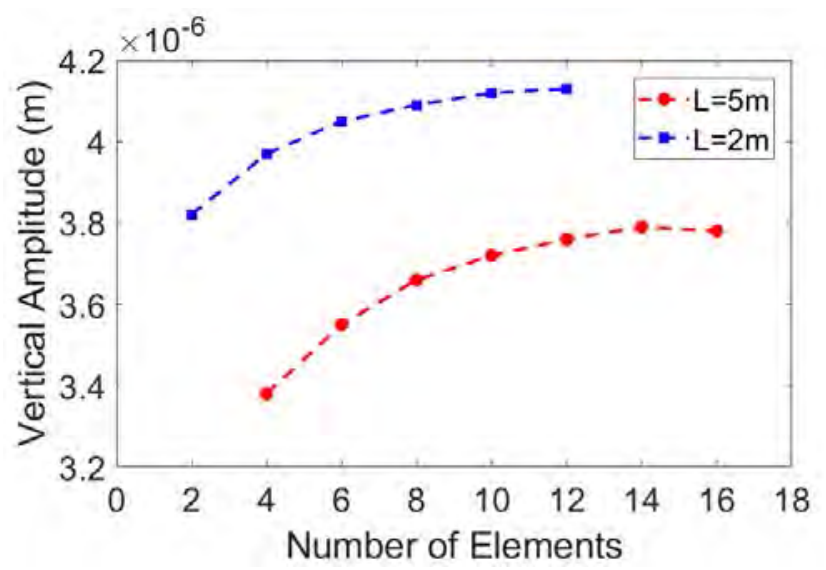

Fig. 3: Convergence curves for segment 3
TABLE III: Simulated vibration amplitudes.

\begin{tabular}{|c|c|c|c|c|}
\hline Case & 1 & 2 & 3 & 4 \\
\hline Vertical, Mid-span $\left(10^{-6} \mathrm{~m}\right)$ & 4.1 & 3.8 & 4.4 & 4.5 \\
Streamwise, Mid-span $\left(10^{-6} \mathrm{~m}\right)$ & 0.30 & 0.35 & 0.30 & 0.30 \\
Vertical, Sheave $\left(10^{-6} \mathrm{~m}\right)$ & 0.48 & 1.38 & 3.3 & 2.3 \\
\hline
\end{tabular}

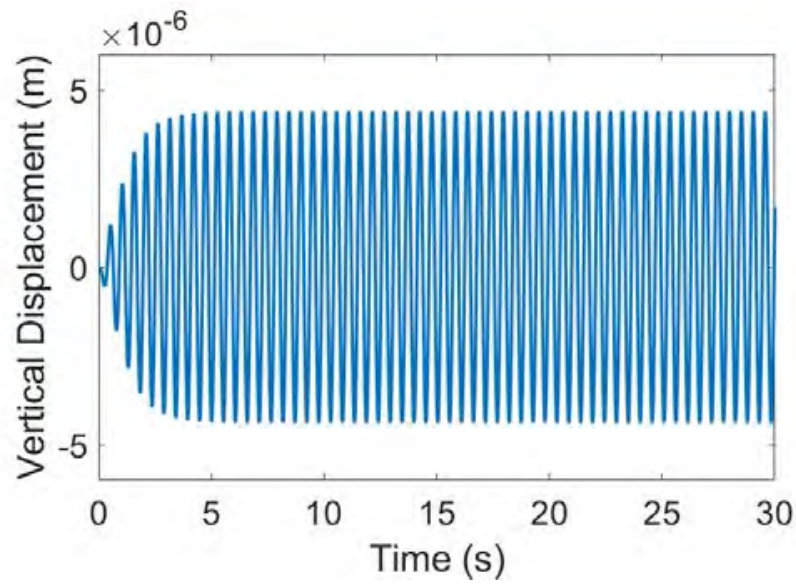

Fig. 4: Mid-span vertical displacement for Case 4

\section{CONCLUSION}

In this paper a dynamic finite element model of a lowtension cable sheave system was used to simulate the vortexinduced vibration of the cable due to wind loading and assess the potential for wind loading to lead to detachment of the cable from the sheave. The model, constructed using the absolute nodal coordinate formulation, utilized a wake-oscillator model to describe the vortex shedding forces. Also, three-dimensional contact with the sheave grooves was introduced. Several case studies were performed for systems of varying span length and cable tension with the wind velocity selected to excite the fundamental mode of the cable. The vibration amplitudes were determined to be small - the maximum amplitude observed at the sheave was only $3.3 \times 10^{-6} \mathrm{~m}$ - thus wind loading alone is unlikely to lead to cable detachment. In future work, the wake-oscillator parameters can be tuned based on wind tunnel experiments. Scenarios where more severe vibrations are likely, such as cables with ice accretion, can also be examined in this manner. Further analysis can be performed to assess the effects of ship motion and higher sustained wind speeds on the response.

\section{ACKNOWLEDGMENT}

The authors would like to thank the Natural Sciences and Engineering Research Council of Canada (NSERC), Kraken Robotic Systems Inc., Rolls-Royce Canada Limited and Carleton University for their support of this research.

\section{REFERENCES}

[1] R. Gabbai and H. Benaroya, "An overview of modeling and experiments of vortex-induced vibration of circular cylinders," J. Sound Vib., vol. 282, no. 3, pp. 575-616, 2005. doi: 10.1016/j.jsv.2004.04.017. 
[2] C. Westin and R. A. Irani, "Cable-pulley interaction with dynamic wrap angle using the absolute nodal coordinate formulation," in Proceedings of the 4th Inter. Conf. Contr. Dyn. Sys. and Robotics, 2017.

doi: $10.11159 /$ cdsr17.133.

[3] X. Bai and W. Qin, "Using vortex strength wake oscillator in modelling of vortex induced vibrations in two degrees of freedom," Euro. $J$.

Mechanics-B/Fluids, vol. 48, pp. 165-173, 2014.

doi:10.1016/j.euromechflu.2014.05.002.

[4] A. A. Shabana, "Forms of the dynamic equations," Computational Dynamics, Third Edition, pp. 177-210. doi: 10.1002/9780470686850.ch4.

[5] A. A. Shabana, "Flexible multibody dynamics: review of past and recentdevelopments," Multibody system dynamics, vol. 1, no. 2, pp. 189-222, 1997. doi: 10.1023/A:1009773505418.

[6] M. Berzeri and A. A. Shabana, "Development of simple models for the elastic forces in the absolute nodal co-ordinate formulation," J. Sound Vib. , vol. 235, no. 4, pp. 539-565, 2000. doi: 10.1006/jsvi.1999.2935.

[7] M. Ciftci, Flexible multibody analysis using Absolute Nodal Coordinate Formulation. PhD thesis, MS Thesis, Middle East Technical Univ., Ankara, Turkey, 2014.

[8] L. K"ubler, P. Eberhard, and J. Geisler, "Flexible multibody systems with large deformations and nonlinear structural damping using absolute nodal coordinates," Nonlinear Dyn., vol. 34, no. 1-2, pp. 31-52, 2003. doi: 10.1023/B:NODY.0000014551.75105.b4.

[9] I. Chowdhury and S. P. Dasgupta, "Computation of rayleigh damping coefficients for large systems," Electron. J. Geotech. Eng., vol. 8, no. 0, pp. $1-11,2003$

[10] U. Lugr'1s, J. Escalona, D. Dopico, and J. Cuadrado, "Efficient and accurate simulation of the cable-pulley interaction in weight-lifting machines," in 1st Joint International Conference on Multibody System Dynamics, 2010.

[11] P. Flores, J. Ambrósio, J. C. P. Claro, and H. M. Lankarani, "Influence of the contact-impact force model on the dynamic response of multi-body systems," Proceedings of the Institution of Mechanical Engineers, Part K: J. Multi-body Dyn., vol. 220, no. 1, pp. 21-34, Mar. 2006.

doi: 10.1243/146441906X77722.

[12] R. Bul'ın, M. Haj`zman, and P. Polach, "Nonlinear dynamics of a cable-pulley system using the absolute nodal coordinate formulation," Mech. Res. Commun., vol. 82, pp. 21-28, 2017. doi: 10.1016/j.mechrescom.2017.01.001.

[13] S. Takehara, Y. Terumichi, and K. Sogabe, "Motion of a submerged tether subject to large deformations and displacements," J. Sys. Des. Dyn., vol. 5, no. 2, pp. 296-305, 2011. doi: 1 0.1299/jsdd.5.296.

[14] M. L. Facchinetti, E. De Langre, and F. Biolley, "Coupling of structure and wake oscillators in vortex-induced vibrations," J. Fluids Struct., vol. 19, no. 2, pp. 123-140, 2004. doi: 10.1016/j.jfluidstructs.2003.12.004.

[15] "U.S. Navy wire-rope handbook volume 1," Tech. Rep. ADA955305, Naval Sea Systems Command, 1976.

[16] K. Spak, G. Agnes, and D. Inman, "Cable parameters for homogenous cable-beam models for space structures," in Dynamics of Civil Structures, vol. 4, pp. 7-18, Springer, 2014.

[17] Z. Zhu and S. Meguid, "Nonlinear fe-based investigation of flexural damping of slacking wire cables," Int. J. Solids Struct., vol. 44, no. 16, pp. 5122-5132, 2007. doi: 10.1016/j.ijsolstr.2006.12.024.

[18] N. Al-Raheimy, "Free vibration of simply supported beam subjected to axial force," J Babylon Univ Eng Sci, vol. 20, no. 1, pp. 301-315, 2012. 


\title{
Convergence Control for Topology Optimization
}

\author{
Dylan Bender and Ahmad Barari \\ Faculty of Engineering and Applied Science \\ The University of Ontario Institute of Technology \\ Oshawa, Canada \\ Ahmad.Barari@uoit.ca
}

\begin{abstract}
Proportional-Integral-Derivative (PID) control theory is applied to the evolutionary rate of the Bi-Directional Evolutionary Structural Optimization (BESO) method to control aspects of the convergence such as the rise time, stability, and other convergence characteristics. When the PID controller is applied to the BESO topology optimization method, its behavior resembles that of a second order linear system and its response depends on whether it is an overdamped, critically damped or underdamped system. The new algorithm replaces the evolutionary rate control parameter with the three gain values of the controller, namely, the proportional gain, the integral gain and the derivative gain for further control the structure's evolution.
\end{abstract}

Keywords - topology optimization; adaptive optimization; Proportional-Integral-Derivative; Bi-Directional Evolutionary Structural Optimization ; PID control

\section{INTRODUCTION}

Structural optimization consists of three main categories, which are size, shape and topology optimization. Size optimization aims to find an optimal design by optimizing variables such as cross-sectional dimensions or thicknesses. Shape optimization achieves structural optimization by changing pre-determined boundaries. Topology optimization, the concern of this paper, aims to determine the best locations and geometries of cavities within the design domain [1]. Fig. 1 shows a sample problem definition and resulting optimal structure of the Bi-Directional Evolutionary Structural Optimization method of topology optimization (BESO).. Although the resulting structure has much less material and weight, but it maintain the required mechanical stiffness for the given loading and boundary condition. Reducing weigh of the components is highly desired in various applications, particularly in design and manufacturing of automotive and aerospace products.

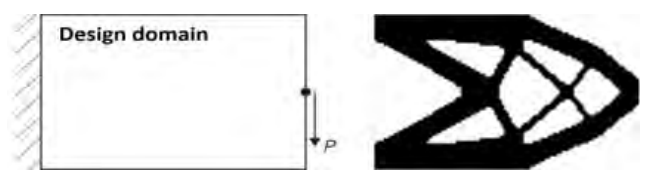

Figure 1. Sample topology optimization problem definition (left) and resulting structure (right)

Significant developments have been seen in topology optimization methodologies within the last two decades [2], the majority of which are based of Finite Element Analysis. However, applications of the developed topology optimization methods have been very limited due (i) the limitations in manufacturing and inspection of the complex topologies, and (ii) the computational complexity of the topology optimization algorithms. A successful design process needs to be conducted by considering the details of the manufacturing and inspection requirement [3]. Although the additive manufacturing processes are still not able to produce high surface qualities [4], they are highly flexible in producing the complex geometries resulting by the topology optimization methodologies [5]. Also. The advances in coordinate metrology algorithms allow relatively fast inspection of the complex topologies resulting by the topologically optimized designs [6].

The computational complexity is another limiting factor in implementation of the topology optimization algorithms. There is not that much control on the convergence of these algorithms and their computational efficiencies are not addressed well. This paper will focus on the convergence control of a typical topology optimization algorithm, Bi-directional Evolutionary Structural Optimization. BESO first introduced by [7]. The BESO method contains several control parameters including target volume fraction, penalty, minimum density, filter radius, mesh resolution, and the focus of this paper, the evolutionary rate. Traditionally, the evolutionary rate is taken to be a constant value, usually $1 \%, 2 \%$ or even as high as $4 \%$, but there exists potential to use the evolutionary rate as a parameter of adaptive optimization in the form of a Proportional-IntegralDerivative controller in conjunction with closed-loop feedback.

Feedback is a very powerful idea. Its use has often had revolutionary consequences with drastic improvements in performance [8]. The usefulness of PID controls lies in their 
general applicability to most control systems. In particular, when the mathematical model of the plant is not known and therefore analytical design methods cannot be used, PID controls prove to be most useful [9]. For this paper, the volumetric error signal is used to control the evolutionary rate of the BESO algorithm. The volumetric error is calculated as the difference in the targeted volume fraction and the current iterations volume fraction which can then be integrated and differentiated to produce the three inputs of the PID controller.

\section{BACKGROUND}

\section{A. Bi-directional Evolutionary Structural Optimization}

In the Bi-Directional Evolutionary Structural Optimization (BESO) method, the optimal topology is determined according to the relative ranking of the elemental sensitivity numbers. The sensitivity numbers of the elements are conceptualized to be the increase in the mean compliance, $\mathrm{C}$, of a structure as a result of the removal of the ith element is equal to its elemental strain energy [1]. By this definition, the optimization problem is to find the most effective way to remove elements so that the increase in the compliance is minimal as shown in (1).

Minimize:

$$
C=\frac{1}{2} f^{T} u
$$

Where $\mathbf{f}$ is the nodal DOF force vector and $\mathbf{u}$ is the nodal displacement vector, both of which has the size $2 *($ nely +1$) *($ nel $x+1)$ where nelx and nely are the number of elements in the $\mathrm{x}$ - and $\mathrm{y}$-dimensions respectively of the finite element mesh.

The study on the effect of element removal on the stiffness is referred to as the sensitivity analysis. Sensitivity numbers, which indicate the change in the overall stiffness (strain energy), have been formulated using information available from a static finite element analysis [10]. The sensitivity number of the ith element is calculated as:

$$
\frac{\partial C}{\partial x_{i}}=-\frac{1}{2} p x_{i}^{p-1} u_{i}^{T} K_{i}^{0} u_{i}
$$

Each iteration of the topology optimization problem is subject to equation (3) and (4) where $V^{*}$ is the prescribed target volume, $\mathrm{V}_{\mathrm{i}}$ and $\mathrm{x}_{\mathrm{i}}$ are the volume and density of each element respectively and $\mathrm{N}$ is the total number of elements.

$$
\begin{gathered}
V^{*}-\sum_{i=1}^{N} V_{i} x_{i}=0 \\
x_{i}=x_{\min } \text { or } 1
\end{gathered}
$$

The target volume for the subsequent iteration $\left(\mathrm{V}_{\mathrm{i}+1}\right)$ or $\left(\mathrm{V}^{*}\right)$ is determined based on the current structural volume, $\mathrm{V}_{\mathrm{i}}$ the target structural volume, $\mathrm{V}_{\mathrm{c}}$, and the main focus of this paper, the evolutionary rate, ER. The subsequent target volume is calculated as follows:

$$
V_{i+1}=\left\{\begin{array}{l}
\max \left(V_{i}(1-E R)_{0} V_{c}\right) \text { when } V_{i}>V_{c} \\
\max \left(V_{i}(1+E R)_{0} V_{c}\right) \text { when } V_{i} \leq V_{c}
\end{array}\right.
$$

Convergence Criterion:

$$
\text { error }=\frac{\left|\sum_{i=1}^{W} C_{k-i+1}-\sum_{i=1}^{W} C_{k-N-i+1}\right|}{\sum_{i=1}^{W} C_{k-1+1}} \leq \tau
$$

The soft-kill BESO MATLAB code downloaded from the website www.isg.rmit.edu.au is used as a test platform for the methods proposed in this paper. The design domain is assumed to be rectangular and discretized using four node plane stress elements. Below, in fig. 2, a short cantilever is taken as an example of the resulting structure of the unmodified code (the graph was generated by the authors of this paper) with the input data being: nelx $=120$, nely $=60$, volfrac $=0.5, E R=0.02$, and $\operatorname{rmin}=3$.

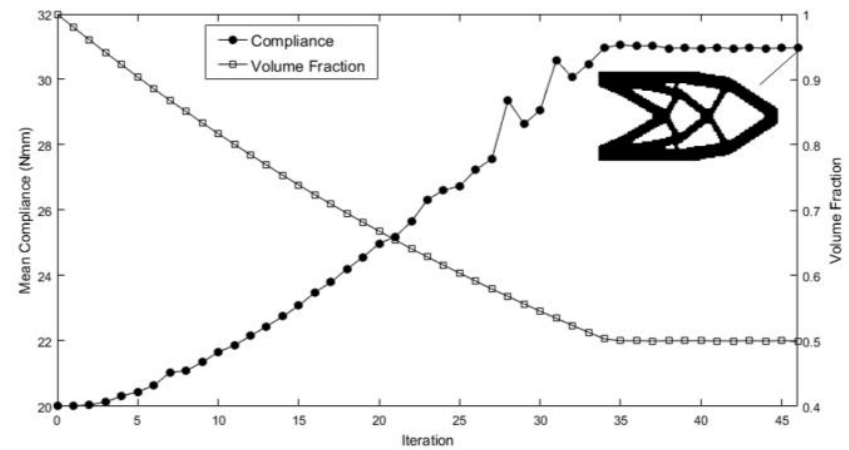

Figure 2: Results of a topology optimized short cantilever with optimization parameters: nelx $=120$, nely $=60$, volfrac $=0.5, \mathrm{ER}=0.02$, rmin $=3, \mathrm{p}=3$. Resulting compliance: $30.96 \mathrm{Nmm}$.

\section{B. PID Control of a Second Order Linear System}

A standard Proportional-Integral-Derivative controller, or PID controller for short is a form of a "three-term" controller whose transfer function is wrtten as:

$$
G(s)=K_{p}+K_{I} \frac{1}{s}+K_{D} s
$$

The "three-term" functionalities are the proportianl term, the integral term, and the derivative term. The proportional term provides an overall control action proportional to ther error signal through the proportional gain factor. The integral term reduces steady-state errors through low-frequency compensation by an integrator and the derivative term improves the transient response through high-frequency compensation via a differentiator [8]. The effects of increasing these terms can be seen below in Table 1. Rise time refers to the number of iterations to reach the target volume for the first time, overshoot is the maximum peak value of the response curve (volumetric error curve) measured from target volume. Settling time refers the number of iterations for the response curve to reach and stay within a range of the final volume fraction. Steady state error refers to the difference between the final equallibrium and the target volume of the response curve. 
Table 1. Effects of independent P, I, and D tuning [7]

\begin{tabular}{|l|l|l|l|l|l|}
\hline $\begin{array}{l}\text { Closed- } \\
\text { Loop } \\
\text { Response: }\end{array}$ & $\begin{array}{l}\text { Rise } \\
\text { Time }\end{array}$ & Overshoot & $\begin{array}{l}\text { Settling } \\
\text { Time }\end{array}$ & $\begin{array}{l}\text { Steady } \\
\text { State } \\
\text { Error }\end{array}$ & Stability \\
\hline $\begin{array}{l}\text { Increasing } \\
\mathrm{K}_{\mathrm{P}}\end{array}$ & Decrease & Increase & $\begin{array}{l}\text { Small } \\
\text { Increase }\end{array}$ & Decrease & Degrade \\
\hline $\begin{array}{l}\text { Increasing } \\
\mathrm{K}_{\mathrm{I}}\end{array}$ & $\begin{array}{l}\text { Small } \\
\text { Decrease }\end{array}$ & Increase & Increase & $\begin{array}{l}\text { Large } \\
\text { Decrease }\end{array}$ & Degrade \\
\hline $\begin{array}{l}\text { Increasing } \\
\mathrm{K}_{\mathrm{D}}\end{array}$ & $\begin{array}{l}\text { Small } \\
\text { Decrease }\end{array}$ & Decrease & Decrease & $\begin{array}{l}\text { Minor } \\
\text { Change }\end{array}$ & Improve \\
\hline
\end{tabular}

A family of unit-step response curves with various values of damping coefficients, $\zeta$ is shown in fig. 3 . In control theory, a system with a damping coefficient of $\zeta=0$ is considered marginally stable and results in a sinusoidal error signal. A damping coefficient of $0<\zeta<1$, results in a system known as underdamped where the error signal exhibits a damped sunusoidal oscillation and at steady state, no error exists between input (target volume) and output (actual volume). A damping coefficient of $\zeta=1$ is considered critically damped (often the ideal situation) which exhibits the fastest response. A coefficient of $\zeta>1$ is known as an overdamped system and is sluggish in responding to any inputs.

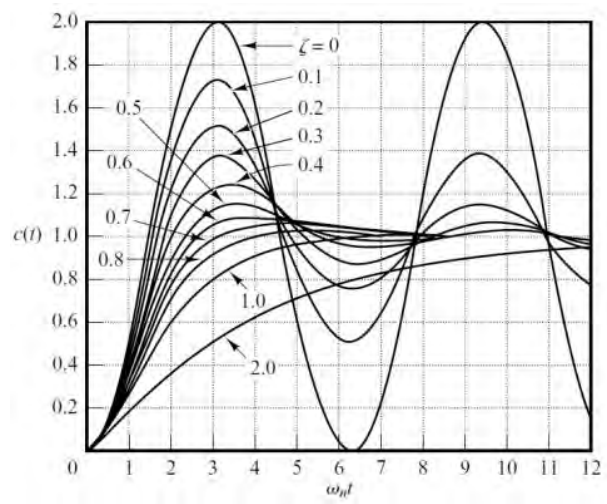

Figure 3. Unit-step response curves for a second order system with varying damping ratios [9]

\section{Methodology}

It is expected that, the smaller the value of the element removal ratio used, the more accurate is the final design, at the expense of larger computation time [10]. This philosophy is based on elemental removal ratio strategy considered with respect to the current number of elements. The motivation behind the applied control theory is to allow for large amounts of material to be removed at the beginning of the optimization when there exists a lot of inefficient material and for small amounts of material to be removed towards then end of the optimization as the volumetric error approaches zero.

The plot of volume versus iteration number can be generalized as a nonlinear function which starts at some arbitrary initial volume, then approaches and eventually reaches the prescribed target volume. Note that the traditional constant evolutionary rate results in a volume graph that resembles a piecewise linear function as shown in fig. 2 but the adaptive evolutionary rate results in a nonlinear graph as presented as seen in fig. 6 .

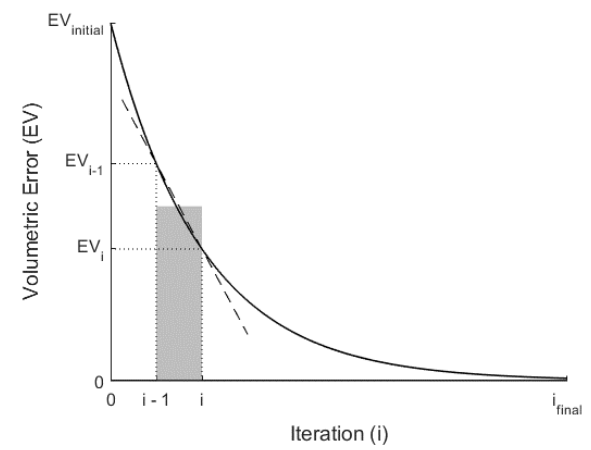

Figure 4. The volumetric error plot with a visualization of the volumetric error integral calculation (shaded grey box) and the volumetric error derivative (dashed line)

The volumetric error is calculated as the difference in the current structural volume and the target structural volume and demonstrated convergence is graphed in fig. 4. Volumetric Error $\left(E_{\mathrm{v}}\right)$ is calculated as:

$$
E_{V_{i}}=V_{\text {target }}-V_{i}
$$

The volumetric integral error is caluclated to be the integral of the volumetric error signal which may be visualized as the area under the volumetric error graph as demonstrated in fig. 4. Volumetric Integral Error $\left(\int E_{D}\right)$ is calculated as:

$$
\int_{0}^{i} E_{v} \approx \int_{0}^{i-1} E_{v}+\frac{E_{v_{i}-1}+E_{v}}{2}(i-(i-1))
$$

Where $i$ denotes the current iteration number. Note that the (i - (i - 1))term of (8) evaluates to 1, and therefore may be omitted during implementation.

The volumetric error derivative is calculated as the rate of change of the volumetric error. This can be simplified to represent the slope of the line passing through two adjacent points of the volumetric error graph as shown in the right graph of fig. 4. Volumetric Error Derivative $\left(E_{v}{ }^{\prime \prime}\right)$ is calculated as:

$$
\frac{d E_{V_{i}}}{d i} \approx \frac{E_{v_{i}}-E_{v_{i-1}}}{i-(i-1)}
$$

Similar to (9), the (i - (i - 1)) term of equation (8) evaluates to 1 , and therefore may be omitted during implementation.

Evolutionary Rate (ER):

$$
E R_{\mathrm{i}}=K_{P} E_{v_{\mathrm{i}}}+K_{I} \int_{0}^{\mathrm{i}} E_{\mathrm{V}}+K_{D} E_{v_{\mathrm{i}}}^{v}
$$

An alternative formula may be used for the next iterations target volume in lieu of (5). This method allows for the underdamped cases where the evolution's volume overshoots the target volume and returns to an equilibrium at the target volume. 


$$
V_{\mathrm{i}+1}=\min \left(\max \left(V_{\mathrm{i}}-E R_{v} 0\right), 1\right)
$$

Tuning a proportional-integral-derivative (PID) controller appears to be conceptually intuitive, but can be hard in practice, if multiple (and often conflicting) objectives such as short transient and high stability are to be achieved [11]. There exist many suggested methods for tuning a PID controller but the majority of them require increasing the proportional gain until the system becomes unstable or marginally unstable which breaks down the topology optimization algorithm when implemented.

For tuning the PID controller, the authors suggest starting with a derivative gain of zero and a very small value of the integral gain $\left(0.0001<\mathrm{k}_{\mathrm{I}}<0.001\right)$ slowly increasing the proportional gain until just after the algorithm has reached similar performance (e.g. similar compliance and settling time) as the standard algorithm. Then, the user should start increasing the derivative gain to make the volume convergence plot resemble that of a critically damped system. If the system seems to take too long to converge, the user should increase the integral gain slightly. The authors have find that the controller exhibits similar performance characteristics for varying mesh resolutions so it is suggested that the tuning be carried out on a low-resolution mesh then applied to the applicable high-resolution mesh.

\section{RESULTS AND DISCUSSION}

The first sample topology optimization with the PID controller presented in fig. 5 is to demonstrate how closely its volumetric convergence resembles that of the response of a second order linear dynamic system. With the gain tuning values of $K_{P}=0.2, K_{D}=0.3$ and $K_{I}=0.04$, it can be seen that at first the algorithm overshoots its target volume of $50 \%$ and reaches a volume fraction of $29 \%$, then overshoots the target again on its way back up and reaches a volume fraction of $55 \%$ before eventually reaching its target and settling down. This behaviour resembles that of a system with a damping coefficient of 0.3 .

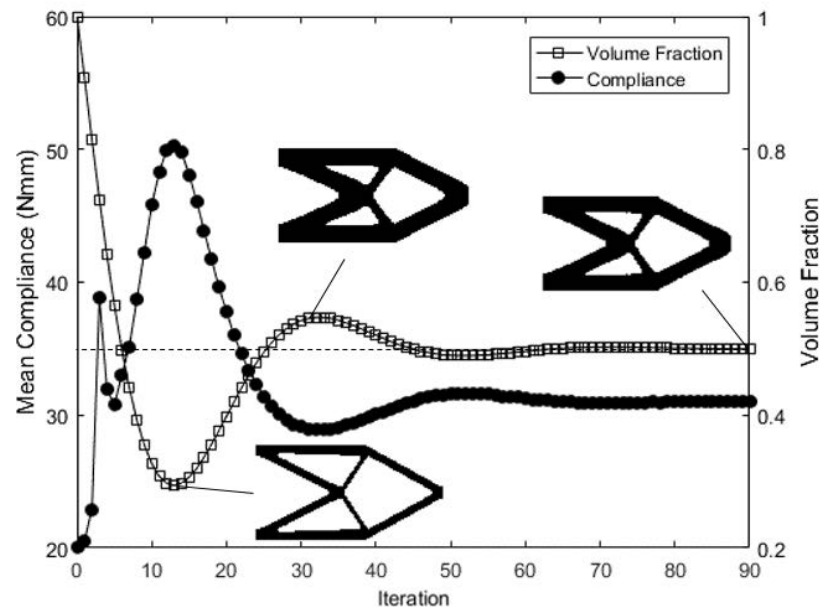

Figure 5. Topology optimization result with a PID tuning of $\mathrm{k}_{\mathrm{P}}=0.2, \mathrm{k}_{\mathrm{D}}=0.3$, $\mathrm{k}_{\mathrm{I}}=0.04$, resulting in a final compliance of $31.06 \mathrm{Nmm}$
The second sample optimization run aims to demonstrate similar performance to that of the standard BESO algorithm but with improved convergence characteristics. The example also uses the standard volume calculation of (5) instead of the new proposed volume calculation of (12) like the previous example. It can be seen that with a controller tuning of $\mathrm{k}_{\mathrm{P}}=0.2, \mathrm{k}_{\mathrm{D}}=0.3, \mathrm{k}_{\mathrm{I}}=0.04$, the optimization reaches a similar compliance within the same number of iterations. The volume convergence and incidentally the compliance make big changes at first when the volumetric error is the highest and small, gradual changes towards the end when the volumetric error is near zero.

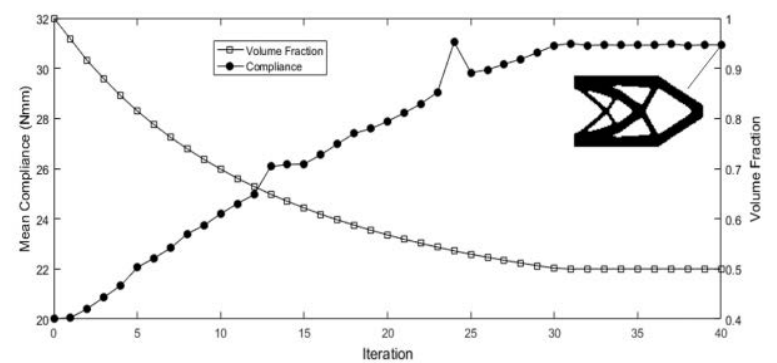

Figure 6. Topology optimization result with a PID tuning of: $\mathrm{k}_{\mathrm{P}}=0.125, \mathrm{k}_{\mathrm{D}}=$ $0.35, \mathrm{k}_{\mathrm{I}}=0.002$, resulting in a final compliance of $30.93 \mathrm{Nmm}$

The addition of the PID controller to the evolutionary rate of the BESO algorithm uses very little resources but provides the user with increased control on the algorithm's convergence as well as other surprising characteristics. The authors have found tunings that can achieve better compliance in less iterations than the standard BESO algorithm but the convergence plot is highly unstable. The authors have also found tunings that seem to provide length scale invariance regardless of the mesh resolution chosen. These intriguing cases will explored further in a future publication as the goal of this paper was to just introduce the topic.

Additionally, the evolutionary rate plays the similar role as the move limit in mathematical programming and the step size in optimality criteria methods [10]. This fact could be used to extend the PID control of the evolutionary rate of the BESO method to the move limit of the SIMP or MMA methods and will also be explored in future publications.

\section{CONCLUSION}

This paper presents an approach to control the convergence of Bi-Directional Evolutionary Structural Optimization algorithm. It is shown the evolutionary rate is an effective control parameter for a closed-loop Proportional-IntegralDerivative controller in the Bi-Directional Evolutionary Structural Optimization method. The developed methodology provides the user with effective control of various convergence characteristics of the volumetric evolution during the topology optimization process. 


\section{ACKNOWLEDGMENT}

The research support provided by the Natural Science and Engineering Research Council of Canada (NSERC) is greatly appreciated.

\section{REFERENCES}

[1] X. Huang and Y. Xie, Evolutionary topology optimization of continuum structures. Chichester, West Sussex, U.K: Wiley, 2010. doi: 10.1002/9780470689486.

[2] G. I. N. Rozvany, "A critical review of established methods of structural topology optimization," Struct. Multidiscip. Optim., vol. 37, no. 3, pp. 217-237, Jan. 2008. doi: 10.1007/s00158-007-0217-0.

[3] F. Kaji and A. Barari, "Evaluation of the surface roughness of additive manufacturing parts based on the modelling of cusp geometry," IFACPapersOnLine, vol. 48, no. 3, pp. 658-663, 2015. doi: 10.1016/j.ifacol.2015.06.157.

[4] A. Barari, "Inspection of the machined surfaces using manufacturing data," J. Manuf. Syst., vol. 32, no. 1, pp. 107-113, Jan. 2013. doi: 10.1016/j.jmsy.2012.07.011.

[5] N. Siraskar, R. Paul, and S. Anand, "Adaptive slicing in additive manufacturing process using a modified boundary octree data structure," J. Manuf. Sci. Eng., vol. 137, no. 1, p. 011007, 2014. doi: $10.1115 / 1.4028579$.

[6] H. Gohari and A. Barari, "A quick deviation zone fitting in coordinate metrology of NURBS surfaces using principle component analysis," Meas., vol. 92, pp. 352-364, 2016.

doi: 10.1016/j.measurement.2016.05.050.

[7] O. Querin, G. Steven, and Y. Xie, "Evolutionary structural optimisation (ESO) using a bidirectional algorithm," Eng. Comput., vol. 15, no. 8, pp. 1031-1048, 1998. doi: 10.1108/02644409810244129.

[8] K. Åström and T. Hägglund, "The future of PID control," Control Eng. Pract., vol. 9, no. 11, pp. 1163-1175, 2001.

doi: 10.1016/S0967-0661(01)00062-4.

[9] K. Ogata, Modern control engineering, 5th ed. Delhi: Pearson, 2016.

[10] D. Nha Chu et al., "On various aspects of evolutionary structural optimization for problems with stiffness constraints," Finite Elem. Anal. Des., vol. 24, no. 4, pp. 197-212, 1997. doi: $10.1016 / \mathrm{S} 0168-874 \mathrm{X}(96) 00049-2$.

[11] K. H. Ang, G. Chong, and Yun Li, "PID control system analysis, design, and technology," IEEE Trans. Control Syst. Technol., vol. 13, no. 4, pp. 559-576, 2005. doi: 10.1109/TCST.2005.847331. 


\title{
Incorporating symmetric ardening ehavior in odeling the Cold Expansion rocess of AZ31B Sheet
}

\author{
Sasan Faghih, Seyed Behzad Behravesh, Hamid Jahed \\ Mechanical and Mechatronics Engineering Department, University of Waterloo \\ Waterloo, Ontario
}

\begin{abstract}
The Variable Material Properties (VMP) method of elastoplastic analysis for axisymmetric problems is extended to the applications of the cold expansion problem for magnesium sheets exhibiting asymmetric hardening behavior. To investigate the effect of hardening asymmetry in the stressstrain results, a detailed 3D finite element (FE) model with symmetric material properties was also generated for the cold expansion problem. Comparison between the results of the VMP and the FE model revealed the significant effect of hardening asymmetry.
\end{abstract}

Keywords- Asymmetric hardening; VMP; Cold expansion; Finite element

\section{INTRODUCTION}

Lightweighting, as an effective approach to improve fuel efficiency and reduce greenhouse gasses emissions, has been a major strategy in the transportation industry [1]. Magnesium, as the lightest structural metal, has been a candidate for replacing the existing heavier materials in this industry. Magnesium application in the aerospace industry goes back to early aircraft development in 1930s, mostly for non-load bearing components, such as gearbox casing [2]. Recently, it has been considered also for structural components [3].

In aerospace structures, fastened joints are the most common assembly technique [4]. Rivet joints, causing stress concentration in structures, are highly susceptible to fatigue failure [5]. One of the approaches to improve the fatigue life is to create compressive residual stress around the hole by plastic expansion. There are several ways of expanding the hole, such as hole edge expansion, ball expansion and tapered mandrel. Due to the surface damage caused by these methods [4], they have been replaced by a more advanced method called split sleeve cold expansion. This method, which consists of pulling a tapered mandrel covered with split sleeve through the hole [6], is now widely used in aerospace industry as the fatigue life improvement technique [7]. From the design point of view, determination of the residual stress due to cold expansion is key to estimation of fatigue life. Such stresses have been shown to strictly depend on the stress-strain material model employed in the calculation/simulation [8]. Current calculation/simulation models for cold expansion process, either finite element models [9], [10] or analytical models [11], [12] are based on simple material models, which can only consider symmetric yield and hardening behaviors. The variable material property (VMP), with capability of considering actual material behavior [13][15], is proven to be able to consider the asymmetric behavior ([16]).

Wrought magnesium alloys, show asymmetric behavior, which is defined as different yield strength and hardening behavior under tension and compression. Such asymmetry is arising from twinning deformation [17] and is different from hydrostatic stresses-associated strength-differential effect seen in high strength steels [18]. In addition, wrought magnesium alloys exhibit anisotropy, i.e., the material behavior varies in different orientations. It has been shown that in rolled AZ31B, asymmetric behavior is dominant, and anisotropy is not significant [19].

In this paper, a simple numerical method based on the VMP approach [20], with the capability of considering the asymmetric hardening behavior, is implemented to model the cold expansion process. A detailed 3D finite element model assuming symmetric hardening behavior is developed for the same problem. A comparison is made between the asymmetric solution from the VMP method and the symmetric solution from the FE analysis, to investigate the effect of material symmetry.

\section{MATERIAL AND MODELING}

\section{A. Split sleeve cold expansion process}

In split sleeve cold expansion process, a tapered mandrel surrounded with a split sleeve, is pulled through the hole. Since the summation of the maximum diameter of the mandrel and split sleeve thickness is greater than the initial hole diameter, the hole expands. Upon removal of the mandrel elastic region around the hole springs back, which creates compressive residual stress around the hole. Schematic illustration of the split sleeve cold expansion process is shown in Fig. 1. Details of the geometry of different parts that are used in this study is given in Table 1. Sheet material is AZ31B with properties given in [21]. 


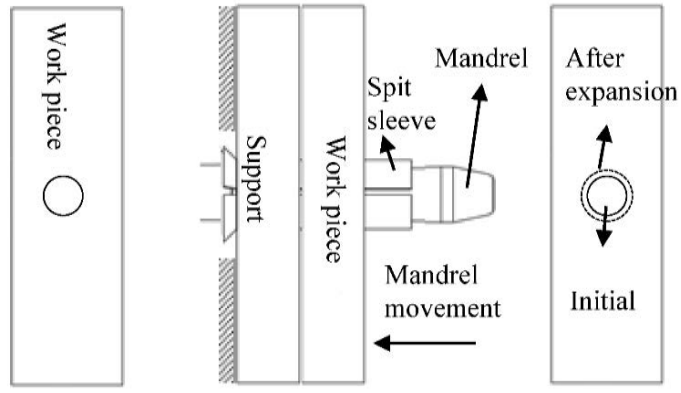

Figure 1. Schematic illustration of the split sleeve cold expansion process

Table 1. Geometry of different parts used in the cold expansion model

\begin{tabular}{|c|c|c|}
\hline Part & dimension & size $(\mathbf{m m})$ \\
\hline Mandrel & Major diameter & 5.84 \\
\hline Split sleeve & Thickness & 0.20 \\
\hline Workpiece & Thickness & 4.00 \\
\hline Workpiece & Initial hole diameter & 6.00 \\
\hline
\end{tabular}

\section{B. $V M P$ approach}

First, a brief review of the VMP method is presented here. Total strain in a body subjected to general force can be written as:

$$
\varepsilon_{i j}=\varepsilon_{i j}^{e}+\varepsilon_{i j}^{p}
$$

in which $\varepsilon, \varepsilon^{\mathrm{e}}$, and $\varepsilon^{\mathrm{p}}$ stand for total strain, elastic strain, and plastic strain, respectively. According to Hook's law:

$$
\varepsilon_{i j}{ }^{e}=\frac{1+v}{v} \sigma_{i j}-\frac{v}{E} \sigma_{k k} \delta_{i j}
$$

where $v, E, \delta, \sigma$ are Poisson's ratio, Young's modulus, Kronecker delta, and stress components. According to the Hencky's total deformation theory:

$$
\begin{gathered}
\varepsilon_{i j}^{p}=\phi S_{i j} \\
S_{i j}=\sigma_{i j}-\frac{1}{3} \sigma_{k k} \delta_{i j} \\
\phi=\frac{3}{2} \frac{\varepsilon_{e q}^{p}}{\sigma_{e q}}
\end{gathered}
$$

where $\mathrm{S}$ stands for deviatoric stress and $\phi$ is a scalar value, obtained from uniaxial stress-strain curve. Combining "(3)"and “(2)" with “(1)" results in:

$$
\begin{gathered}
\varepsilon_{i j}=\left(\frac{1+v}{E}+\phi\right) \sigma_{i j}-\left(\frac{v}{E}+\frac{\phi}{3}\right) \sigma_{k k} \delta_{i j} \\
\varepsilon_{i j}=\frac{1+v_{e f f}}{E_{e f f}} \sigma_{i j}-\frac{v_{e f f}}{E_{e f f}} \sigma_{k k} \delta_{i j} \\
E_{e f f}=\frac{3 E}{3+2 E \phi} \\
v_{e f f}=\frac{3 v+E \phi}{3+2 E \phi}
\end{gathered}
$$

In which, the "eff" subscript stands for the effective value.

To solve an axisymmetric boundary value problem, such as a cylinder subjected to an internal pressure, Fig. 2, the body is discretized into a finite number of annuli. The VMP method, being an iterative solution, starts with a fully elastic solution in the first iteration. Then, the equivalent stress is calculated for all the elements. For the elements where the equivalent stress is higher than the yield strength of the material, the values of $v_{\text {eff, }} E_{\text {eff }}$ are updated according to Equation (4), which are used in the next solution iteration. The effective Young's modulus at each element is found from the projection method, illustrated in Fig. 2(b). According to the projection method, the effective Young's modulus is the secant modulus at the strain value obtained in the previous iteration, point $b^{\prime}$ in Fig. 2(b). Eliminating $\phi$ in Equation (4) gives the effective Poisson's ratio. Then, the elastic solution in each iteration is obtained from the well-known Lame's solution in the plane stress condition [20]. This procedure is repeated until the equivalent stress and strain values for all the elements match the uniaxial stress-strain curve. In case of the cold expansion problem, the process is modeled as a cylinder, with inside diameter equal to the hole diameter and outside diameter which is five times the hole diameter, subjected to radial displacement at inner radius.

As mentioned earlier, in magnesium alloys uniaxial tension and compression curves are not the same. To consider the asymmetric behavior, the Cazacu-Barlat yield criterion [22] was implemented to calculate the equivalent stress in the VMP method iterations. This criterion is given by:

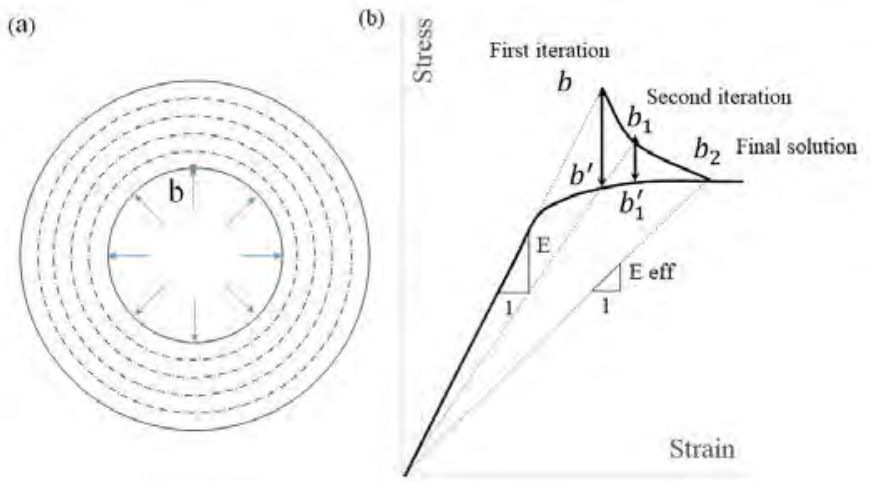

Figure 2. (a) Pressurized thick-walled cylinder discretized into finite number of annuli, (b) demonstraion of the projection method

$$
\begin{gathered}
\sigma_{e q}=A\left(J_{2}^{\frac{3}{2}}-c J_{3}\right)^{\frac{1}{3}} \\
c=\frac{3 \sqrt{3}}{2}\left(\frac{\sigma_{y t}^{3}-\sigma_{y c}^{3}}{\sigma_{y t}^{3}+\sigma_{y c}^{3}}\right) \\
A=3\left(3^{\frac{3}{2}}-2 c\right)^{\frac{-1}{3}}
\end{gathered}
$$


In which, $\sigma_{\mathrm{yt}}, \sigma_{\mathrm{yc}}, \mathrm{J}_{2}$ and $\mathrm{J}_{3}$ are tension and compression yield strenghts, second and third invariants of deviatoric stress tensor, respectively. For the case of symmetric materials, i.e. $\sigma_{\mathrm{yt}}=\sigma_{\mathrm{yc}}$, this yield criterion reduces to the Von-Mises criterion. The asymmetry parameter, c, determines the shape of the yield locus. The parameter $\mathrm{c}$ at a given plastic strain is obtained from the ratio of stresses on the uniaxial tension and compression curves (Fig. 3) in that plastic strain. Therefore, the shape of the yield locus for the Cazacu-Barlat criterion evolves during the plastic deformation, while it is fixed for the Von-Mises criterion. It is noteworthy that for both the symmetric (Von-Mises criterion) and asymmetric (Cazacu-Barlat criterion) solutions, only the uniaxial tension curve is used in the projection method. For the asymmetric solution, the evolution of the parameter $\mathrm{c}$ accounts for the compression hardening curve being different than the tension curve.

To obtain the residual stress field created by cold expansion two steps of loading and unloading are required. The unloading solution is similar to the loading part, but now each element has a specific unloading behavior defined by the level of loading strain. For the unloading phase, a pressure with the same value as the pressure at the end of the loading step with an opposite sign is applied to the inner radius of the cylinder. The negative pressure in the unloading step simulates the removal of the mandrel. Since in the Cazacu-Barlat yield criteria, tension and compression curves need to be determined. For the unloading solution, second part of the tension-compression curve (Fig. 4b) is considered as tensile curve. Likewise, the second part of the compression-tension (Fig. 4a) is considered as compression curve as the input for the VMP method. Simple interpolation method was used to generate the curves that are in between unloading curves shown in Fig. 4. After solving the unloading part, the residual stress field was obtained by:

$$
\begin{gathered}
\sigma_{\text {Radial }}^{\text {Res }}=\sigma_{\text {Radial }}^{\text {load }}+\sigma_{\text {Radial }}^{\text {Unload }} \\
\sigma_{\text {Hoop }}^{\text {Res }}=\sigma_{\text {Hoop }}^{\text {load }}+\sigma_{\text {Hoop }}^{\text {Unload }}
\end{gathered}
$$

In which, $\sigma^{\text {Res }}, \sigma^{\text {Load }}, \sigma^{\text {Unload }}$ are residual stress, stress in the loading step, and the stress in the unloading step, respectively.

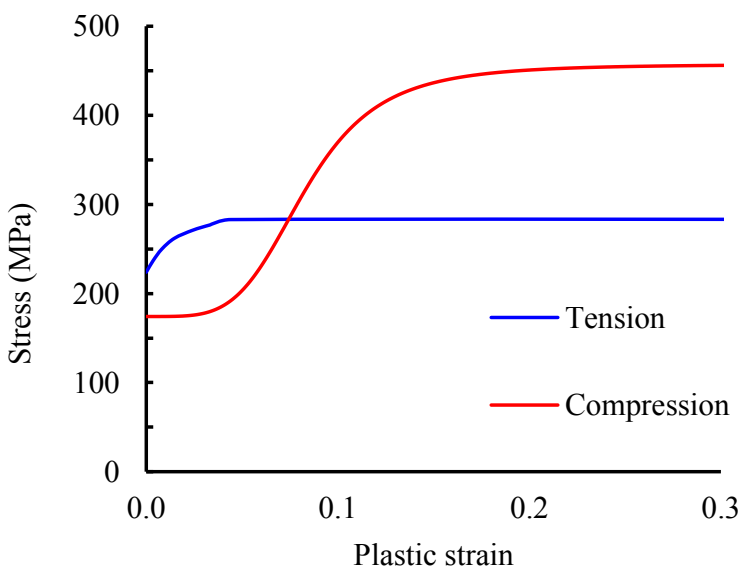

Figure 3. Uniaxial tension and compression behavior of rolled AZ31B [21]
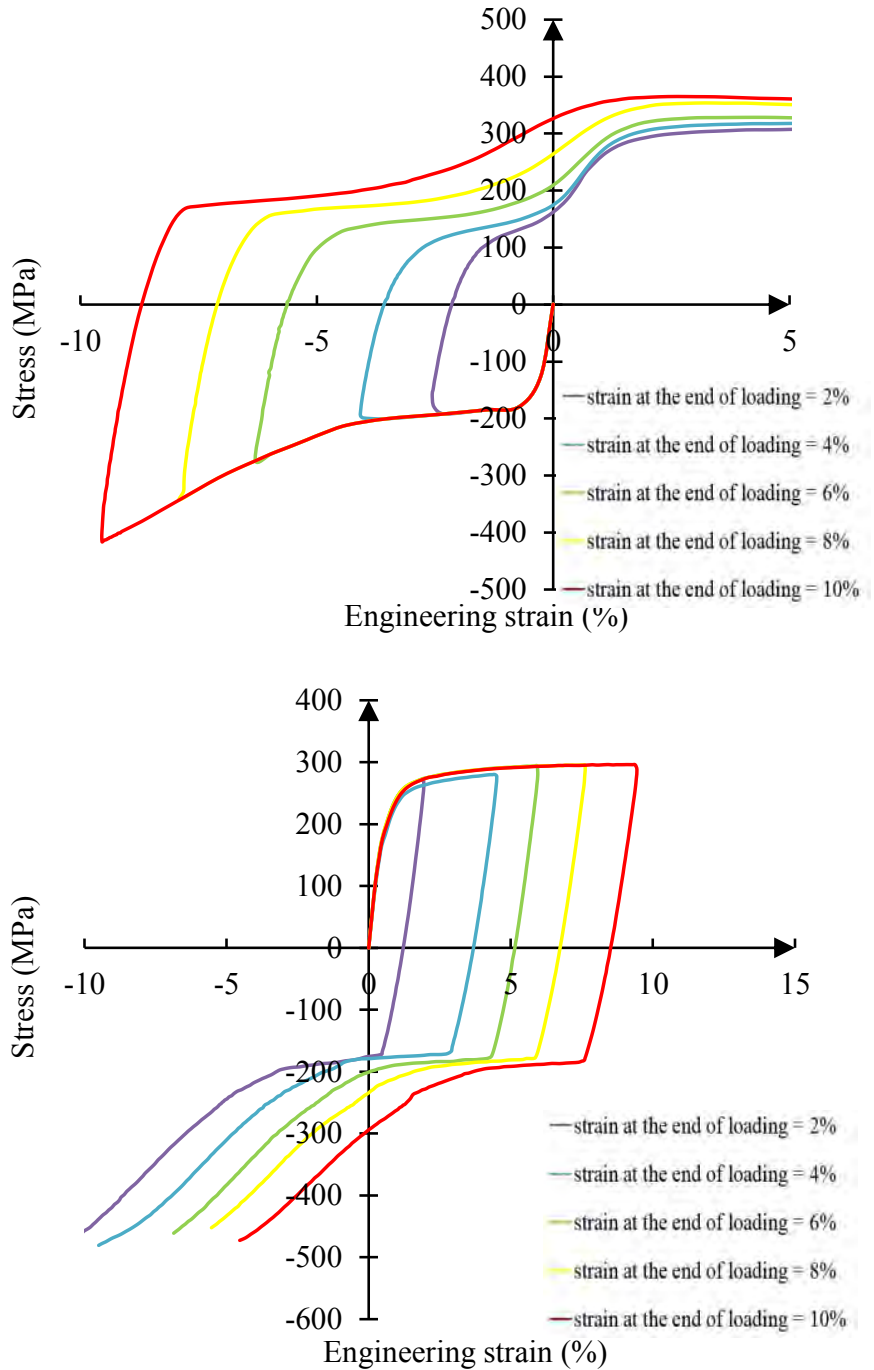

Figure 4. (a) Compression-tension and (b) tension-compression behavior for rolled AZ31B ([21],[23])

\section{Finite element}

ABAQUS finite element software was used in this study to simulate the cold expansion process (Fig. 5). In order to apply a more realistic boundary condition, the support of the workpiece was also included in the model. Owing to the symmetry, only half of the structure was modeled. Boundary conditions of the model are shown in Fig. 6. On the X-Y plane, movement of the external edges of the workpiece and the support were constrained. Mandrel was considered rigid, while the split sleeve and steel support were linear elastic material with the Young's modulus of $200 \mathrm{GPa}$ and Poison's ratio of 0.3 . The workpiece followed the tensile curve (Fig. 3) for the loading step, and the hardening behavior was assumed to be isotropic for the unloading step. Three surface-to-surface contacts were applied to the model: between mandrel and split sleeve, split sleeve and workpiece, and workpiece and support. They all were considered as frictionless contact. Hexahedral linear brick elements with 8 nodes and one integration point, C3D8R, were used to construct the workpiece. Because the most important region of the workpiece is the area close to the hole, the mesh size was biased towards the hole. 


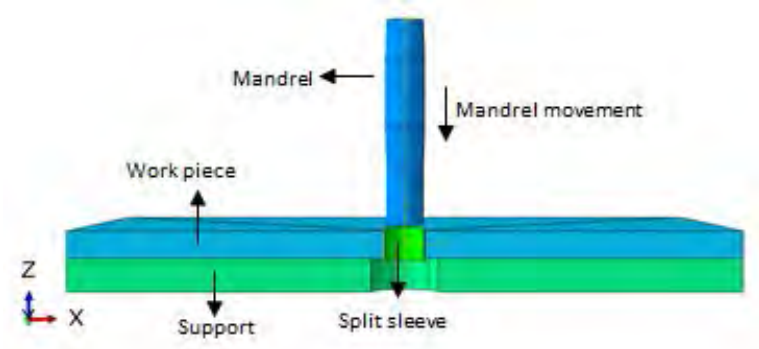

Figure 5. Assembly view of the 3D model

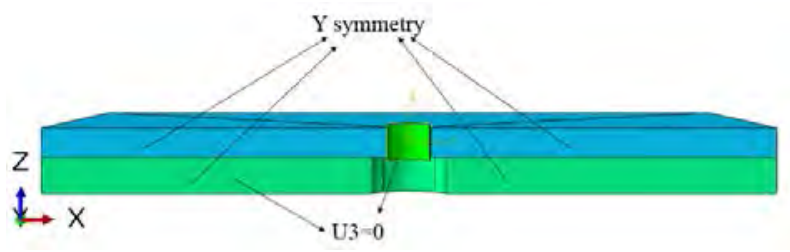

Figure 6. Boundary condition of the model

III. RESULT AND DISCUSSION

To validate the results of the VMP solution, a comparison between the VMP and the ABAQUS results using only the tension curve and assuming isotropic hardening in both VMP and ABAQUS was made. Fig. 7 and Fig. 8 show the loading and residual stress distributions, respectively. The results from ABAQUS is for the mid-plane of the workpiece and far from the position of split. As expected, the results are in good agreement. The difference can be attributed to the fact that ABAQUS is a 3D model that considers more detail such as effect of support for workpiece, contact between mandrel and split sleeve and workpiece, while the VMP models the process as a 2D uniform expansion. Fig. 9 and Fig. 10 show the effect of considering asymmetric behavior in loading and unloading in stress distribution.

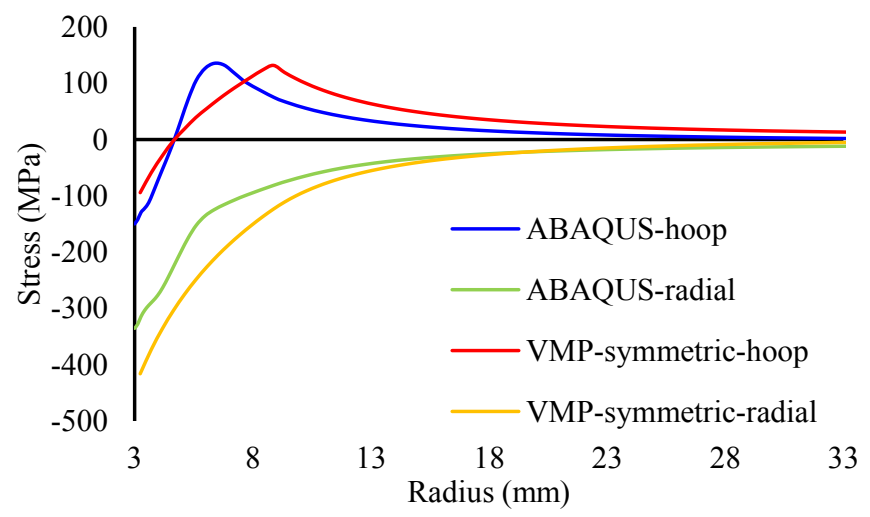

Figure 7. Hoop and radial stress distribution at the end of loading assuming symmetric hardening behavior

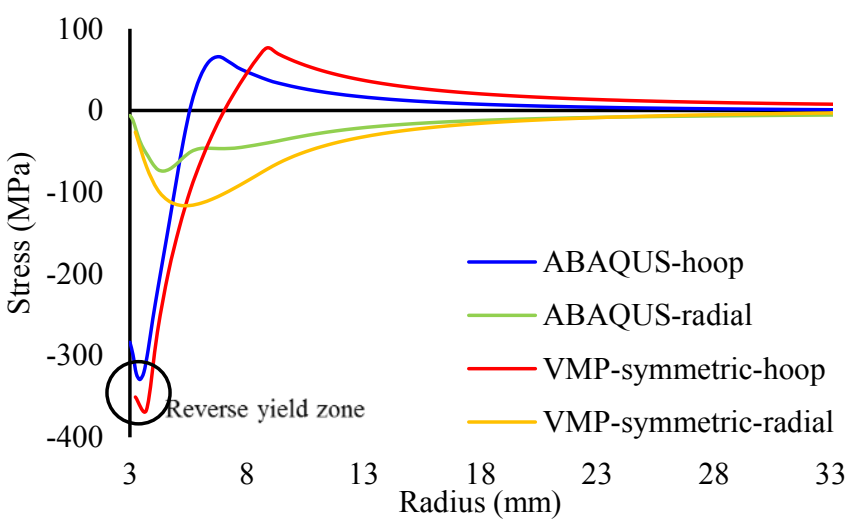

Figure 8. Residual hoop and radial stress distribution assuming symmetric hardening behavior

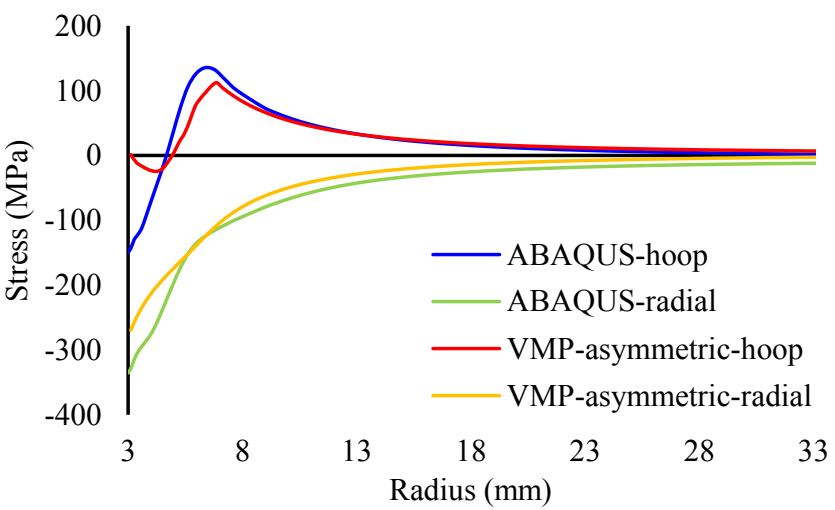

Figure 9. Hoop and radial stress distribution at the end of loading assuming symmetric hardening in the ABAQUS and asymmetric hardening in the VMP

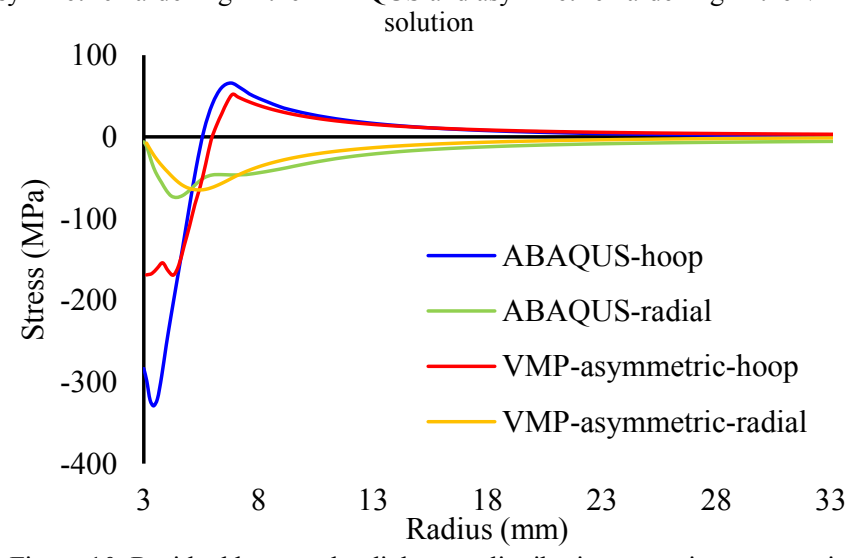

Figure 10. Residual hoop and radial stress distribution assuming symmetric hardening in the ABAQUS and asymmetric hardening in the VMP solution

In Fig. 9 and Fig. 10, the results of VMP is obtained with asymmetric material model, while in ABAQUS, symmetric behavior was assumed.

Hoop stress distributions close to the hole in loading and unloading steps were significantly different between the symmetric ABAQUS and asymmetric VMP solutions. Close to hole, which is the area of interest for the cold expansion problem, the VMP predicts the value of hoop stress to be close to zero, Fig. 9. Likewise, the residual hoop stress distribution is meaningfully different. ABAQUS predicts the reverse yield zone to be close to the hole, while Asymmetric VMP model predicts the reverse yield zone to be larger, i.e. farther from the hole (Fig. 10). This can be explained by different unloading 
curves used in ABAQUS and VMP. VMP considers actual material unloading behavior (Fig. 4), in which reverse yield in the unloading part, happens at lower stress, while in ABAQUS model, the unloading curve is constructed using the isotropic hardening assumption. Moreover, considering the actual unloading behavior of the material by VMP and assuming isotropic hardening rule by ABAQUS, also contributed to the difference between the calculated residual stress field. The difference in residual stress distribution is also due to difference in the hoop stress distribution in the loading step. The plastic strain obtained from VMP around the hole during the loading step is shown in Fig. 11, for symmetric and asymmetric behavior. The larger plastic strain in the asymmetric solution is due to compression behavior accounted for in this solution. Because the yield stress in compression is smaller than that in tension (Fig. 3), it is expected that the plastic strain close to hole to be higher in the asymmetric model compared to the symmetric model.

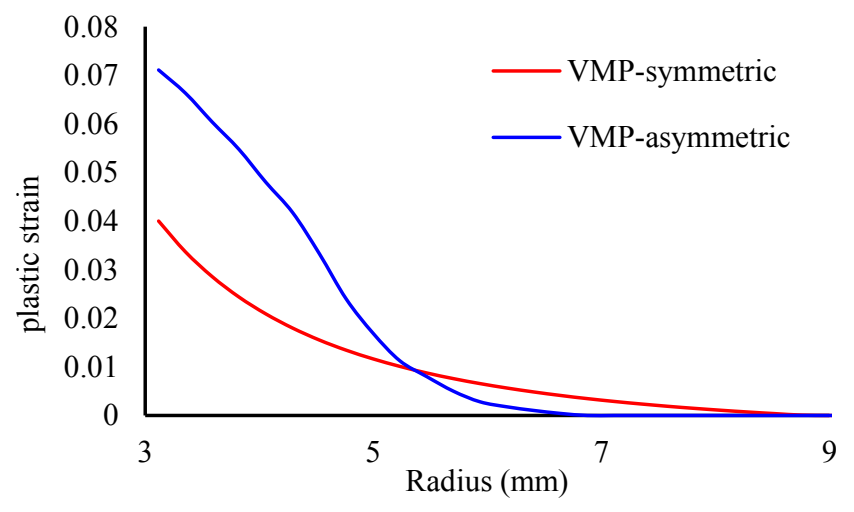

Figure 11. Plastic strain around the hole at the end of loading

\section{CONCLUSTION}

The cold expansion process in AZ31B wrought magnesium alloy has been modeled using the finite element and the VMP methods. The asymmetric hardening behavior of AZ31B was captured by the VMP, but the finite element method did not account for the asymmetric hardening behavior. Following conclusions can be drawn from this study:

- The VMP method is capable of modeling the cold expansion process. For symmetric materials, the results of this simple 2D model are in agreement with predictions of the 3D finite element model in the mid-plane.

- Asymmetric hardening behavior of materials can significantly affect the stress distribution in loading and unloading steps of the cold expansion process.

- Hoop stress distribution is significantly different in the asymmetric model, while the radial stress is almost not affected.

\section{REFERENCES}

[1] J. P. Immarigeon, R. T. Holt, A. K. Koul, L. Zhao, W. Wallace, and J. C. Beddoes, "Lightweight materials for aircraft applications," Mater. Charact., vol. 35, no. 1, pp. 41-67, 1995. doi: 10.1016/1044-5803(95)00066-6.

[2] L. Ostrovsky and Y. Henn, "Present state and future of magnesium application in aerospace industry," Int. Conf. New Challenges Aeronaut. ASTEC 07, pp. 1-5, 2007.
[3] G. Song and a. Atrens, "Understanding Magnesium Corrosion-A Framework for Improved Alloy Performance," Adv. Eng. Mater., vol. 5, no. 12, pp. 837-858, 2003. doi: 10.1002/adem.200310405.

[4] Y. Fu, E. Ge, H. Su, J. Xu, and R. Li, "Cold expansion technology of connection holes in aircraft structures: A review and prospect," Chinese J. Aeronaut., vol. 28, no. 4, pp. 961-973, 2015.

doi: 10.1016/j.cja.2015.05.006.

[5] G. L. Kulak, J. W. Fisher, and J. H. A. Struik, Guide to design criteria for bolted and riveted joints, vol. 15, no. 1. 1988.

[6] D. A. Clark and W. S. Johnson, "Temperature effects on fatigue performance of cold expanded holes in 7050-T7451 aluminum alloy," Int. J. Fatigue, vol. 25, no. 2, pp. 159-165, 2003.

do: 10.1016/S0142-1123(02)00070-1.

[7] V. Achard, A. Daidie, M. Paredes, and C. Chirol, "Optimization of the Cold Expansion Process for Titanium Holes," Adv. Eng. Mater., vol. 19, no. 6, pp. 1-13, 2017. doi: 10.1002/adem.201500626.

[8] A. A. Garcia-Granada, V. Lacarac, D. J. Smith, M. J. Pavier, R. Cook, and P. Holdway, "3D residual stresses around cold expanded holes in a new creep resistant aluminium alloy," Trans. Eng. Sci., vol. 25, pp. 103-116, 1999.

[9] V. Nigrelli and S. Pasta, "Finite-element simulation of residual stress induced by split-sleeve cold-expansion process of holes," J. Mater. Process. Technol., vol. 205, no. 1-3, pp. 290-296, 2008. doi: 10.1016/j.jmatprotec.2007.11.207.

[10] S. J. Houghton and S. K. Campbell, "Identifying the residual stress field developed by hole cold expansion using finite element analysis," Fatigue Fract. Eng. Mater. Struct., vol. 35, no. 1, pp. 74-83, 2012. doi: 10.1111/j.1460-2695.2011.01616.x.

[11] Rich and Impellizzeri, "Fatigue analysis of cold-worked and interference fit fastener holes," ASTM Spec Tech Publ 637, pp. 153 $175,1977$.

[12] G. S. Wang, "An elastic-plastic solution for a normally loaded center hole in a finite circular body," Int. J. Press. Vessel. Pip., vol. 33, no. 4, pp. 269-284, 1988. doi: 10.1016/0308-0161(88)90075-0.

[13] H. Jahed, S. B. Lambert, and R. N. Dubey, "Variable material property method in the analysis of cold-worked fastener holes," J. Strain Anal. Eng. Des., vol. 35, no. 2, pp. 137-142, 2000. doi: 10.1243/0309324001514080.

[14] H. Jahed, B. A. Moghadam, and M. Shambooli, "Re-Autofrettage," J. Press. Vessel Technol., vol. 128, no. 2, p. 223, 2006. doi: $10.1115 / 1.2172619$.

[15] S. Faghih, H. Jahed, and S. B. Behravesh, "Variable Material Properties (VMP) Approach: A Review on Twenty Years of Progress," J. Press. Vessel Technol., 2018. doi: 10.1115/1.4039068.

[16] M. A. Khayamian, B. Behravesh, and H. Jahed, "Incorporation ofasymmetric yield and hardening behaviour in axisymmetric elastoplastic problems," Mater. Des., vol. 99, pp. 490-499, 2016. doi: 10.1016/j.matdes.2016.03.089.

[17] J. Albinmousa, H. Jahed, and S. Lambert, "Cyclic axial and cyclic torsional behaviour of extruded AZ31B magnesium alloy," Int. J. Fatigue, vol. 33, no. 11, pp. 1403-1416, 2011. doi: 10.1016/j.ijfatigue.2011.04.012.

[18] J. Casey and H. Jahedmotlagh, "The strength-differential effect in plasticity," Int. J. Solids Struct., vol. 20, no. 4, pp. 377-393, 1984. doi: 10.1016/0020-7683(84)90047-7.

[19] S. B. Behravesh, "Fatigue Characterization and Cyclic Plasticity Modeling of Magnesium Spot-Welds," University of Waterloo, 2013. 
[20] H. Jahed and R. N. Dubey, "An axisymmetric method of elasticplastic analysis capable of predicting residual stress field," $J$.

Press.Vessel Technol., vol. 119, no. 3, pp. 264-273, 1997.

doi: $10.1115 / 1.2842303$.

[21] S. B. Behravesh, H. Jahed, and S. Lambert, "Characterization of magnesium spot welds under tensile and cyclic loadings," Mater.

Des., vol. 32, no. 10, pp. 4890-4900, 2011.

doi: 10.1016/j.matdes.2011.06.001.

[22] O. Cazacu and F. Barlat, "A criterion for description of anisotropy and yield differential effects in pressure-insensitive metals," Int. J.

Plast., vol. 20, no. 11 SPEC. ISS., pp. 2027-2045, 2004.

[23] A. Yazdanmehr and H. Jahed, "Large strain compression-tension behavior of AZ31B rolled sheet in the rolling direction,"

UNPUBLISHED. 


\section{Effect of Additives on Cutting Temperature of Glass Fibers Reinforced Polymers}

\author{
H. Kuate-Togue, J-F. Chatelain \\ Mechanical engineering department \\ École de technologie supérieure \\ Montréal, Canada \\ jean-francois.chatelain@etsmtl.ca
}

\author{
C. Ouellet-Plamondon \\ Construction engineering department \\ École de technologie supérieure \\ Montréal, Canada \\ claudiane.ouellet-plamondon@etsmtl.ca
}

\begin{abstract}
Composites are abrasive materials that generate heat during machining operations. This heat generates thermal damage locally and may severely alter the mechanical properties of the components if it exceeds the glass transition temperature of the matrix. This research studies the influence of additives, mixed to the epoxy matrix of Glass Fiber Reinforced Polymer (GFRP), on the cutting temperature. The results show that wax together with clay and a wetting agent, contribute to reduce the maximum cutting temperature, on a $300 \mathrm{~mm}$ length of cut, a value up to $28 \%$ as compared to a plain epoxy matrix.
\end{abstract}

Machining; temperature; GFRP; composite; additives

\section{INTRODUCTION}

The advanced industries such as the automotive, aerospace and wind industries are constantly innovating to improve the performance in terms of the weight and mechanical performance of their products. Traditionally used materials are metals that provide good mechanical performances. Metallic materials not only have good mechanical properties but are also relatively easy to machine. On the other hand, metals have a fairly high density, which implies high energy consumption by vehicles and mobile structures built with these materials. In order to reduce energy consumption, industries have introduced composite materials into the design and manufacture of mechanical parts. However, although these materials are mechanically efficient with a density that leads to reduced energy consumption, these materials have an abrasiveness that influences the temperature during machining operations.

The composite materials generally consist of a thermoset or thermoplastic matrix, and fibers as reinforcement elements. The most used and widespread fibers are carbon and glass mainly because of their mechanical and thermal properties. The use of composite materials in the manufacturing of parts or structures often requires cutting operations, like drilling, trimming or milling, before the assembly process [1]. However, these machining operations pose difficulties and quality issues because of their heterogeneity and anisotropic behavior as well as the abrasiveness of the fibers $[2,3]$.
The abrasiveness of the fibers alone is a cause of wear for the cutting tools. This wear is the source of a temperature increase which in turn provokes thermal damage on the cut surfaces and on the mechanical properties, specifically if the glass transition temperature ( $\mathrm{Tg}$ ) of the matrix is exceeded [46]. Previous studies showed that cutting temperature may reach values up to $450^{\circ} \mathrm{C}[7,8]$ during machining if tool wear is not properly controlled.

The tool wear do not only affect the temperature, but also the cutting forces. In fact, both phenomena are related, so that monitoring the forces may help to diagnose a worn tool $[6,8$, 9].

This research proposes to introduce additives or chemical components within the matrix as a solution to improve the machining process. The research aims to find additives that would allow a better interaction in the matrix-fiber contact surface in order to maintain good or improved mechanical performances [10-12], while reducing the temperature of cut which lead to better quality and lesser tool wear. The introduction of additives as a solution to reduce the cutting temperature was initially explored by Lasseur [5] study showing the performance of three additives. This paper will consider the study of five additives over a length of cut equal to $300 \mathrm{~mm}$.

\section{METHODOLOGY}

\section{A. Materials}

The material with which the experiments were made consists of long glass fibers with modified epoxy resin as matrix. The characteristics of the fibers and the resin are summarized in Table I.

TABLE I. CHARACTERISTICS OF THE FIBERS AND EPOXY RESIN

\begin{tabular}{|l|l|l|l|l|l|}
\hline Materials & Name & Company & $\begin{array}{c}\text { Density } \\
{\left[\mathbf{g} / \mathbf{c m}^{3}\right]}\end{array}$ & $\begin{array}{c}\text { Surface } \\
\mathbf{d e n s i t y} \\
{\left[\mathbf{g} / \mathbf{m}^{2}\right]}\end{array}$ & $\begin{array}{c}\text { Resin } \\
\text { viscosity } \\
{[\mathbf{P a . s}]}\end{array}$ \\
\hline Fibers & E-Glass & TEXONIC & 1,239 & 440 & - \\
\hline Resin & $\begin{array}{l}\text { Marine } \\
820\end{array}$ & ADTECH & 1,106 & - & 1,2 \\
\hline
\end{tabular}


Different laminate of $300 \mathrm{~mm}$ by $300 \mathrm{~mm}$ size were manufactured using the infusion method. A total of 16 unidirectional plies were stacked with a $45^{\circ}$ angle in order to have a trimming direction with respect to the fiber orientation of $-45^{\circ}$. This configuration is the worst one in terms of surface quality resulting from trimming operations. In fact, previous studies proved that such fiber orientation generates more delamination (uncut fibers and fiber pullout) and worst surface roughness than any other orientation with respect to the cutting direction [2, 13-16]. The thickness of all plaques manufactured with the various epoxy mixtures was controlled to be of $4.7 \mathrm{~mm}$, to ensure no variability in the results due to the fiber content. Five mixtures of epoxy were considered in this study considering three different additives with the following concentrations:

- Unmodified resin (RE)

- $\operatorname{Resin}+1 \%$ wt wetting agent (WA)

- $\operatorname{Resin}+1 \%$ wt WA $+1.5 \%$ wt clay (CL)

- $\operatorname{Resin}+1 \%$ wt WA + 2\% wt wax (WAX)

- $\operatorname{Resin}+1 \%$ wt WA $+1 \%$ wt WAX $+1 \%$ wt CL.

All mixtures were made according to the protocol proposed by Quaresimin, et al. [10], and Withers, et al. [11]. The characteristics and tradenames of the additives are summarized in table II. Based on these studies, as well as on the supplier technical documentation, these additives should play the following roles:

- The wetting agent, which is an antistatic, should allow diffusion, homogenization and stabilization of the additives in the mixture with the resin.

- The clay particles, which are particles having storage stability and sag resistance should improve the mechanical properties of the composite.

- The wax particles which have a low transition temperature $288.15 \mathrm{~K}\left(115^{\circ} \mathrm{C}\right)$ and low viscosity should therefore melt at the cutting surface during high temperature machining. It is expected to act as a lubricant to the cutting surface thus reducing the cutting temperature.

TABLE II. CHARACTERISTICS OF THE ADDITIVES

\begin{tabular}{|l|l|}
\hline \multicolumn{1}{|c|}{ Additives } & \multicolumn{1}{c|}{$\begin{array}{c}\text { Characteristics and } \\
\text { Supplier }\end{array}$} \\
\hline Wetting agent (WA) & BYK-W 972, BYK \\
\hline Clay particles (CL) & $\begin{array}{l}\text { Ømax }=59 \mu \mathrm{m} \text { GARAMITE- } \\
1958, \mathrm{BYK}\end{array}$ \\
\hline Wax particles (WAX) & $\begin{array}{l}\text { Ømax }=11 \mu \mathrm{m}, \mathrm{CERAFLOUR} \\
996, \mathrm{BYK}\end{array}$ \\
\hline
\end{tabular}

\section{B. Cutting temperature acquisition}

The temperature acquisition was done through two thermocouples welded symmetrically on diametrically opposed teeth of the cutting tool. The thermocouples are of type $\mathrm{K}$ having a diameter of $0.076 \mathrm{~mm}(0.003 ")$. The welded tip and the stripped thermocouples were covered with OmegaBond400 cement which has high thermal conductivity. Finally, the tip is covered with a J-B weld epoxy resin to reinforce the shear strength and prevent the weld from easily tearing during machining. In addition, it was determined experimentally that the distance between the cutting zone and the welded tip on the tool is $1.7 \mathrm{~mm}$ in order to avoid a detachment of the thermocouples due to the chip flow and temperature variation [5, 7]. In addition, the closer are the thermocouples to the cutting zone, the more accurate will be the measurements [2, 9]. The cutting tool type is a CVD (Chemical Vapor Deposition) diamond coated having a $9.5 \mathrm{~mm}$ diameter with following characteristics:

TABLE III. CHARACTERISTICS OF THE CUTTING TOOL

\begin{tabular}{|l|l|l|}
\hline \multirow{4}{*}{ Machining Tool } & Material & Tungsten Carbide \\
\cline { 2 - 3 } & Coating material & Diamond \\
\cline { 2 - 3 } & Coating process & $\begin{array}{l}\text { Chemical Vapor Deposition } \\
\text { (CVD) }\end{array}$ \\
\cline { 2 - 3 } & Diameter & $9.5 \mathrm{~mm}\left(3 / 8^{\prime \prime}\right)$ \\
\cline { 2 - 3 } & Number of teeth & 6 \\
\cline { 2 - 3 } & Helix angle & $10^{\circ}$ \\
\hline
\end{tabular}

The tool instrumented with thermocouples is mounted in a holder equipped with a wireless transmission system on which the red-AL (aluminum) and yellow-CR (chromium) pairs of wires are connected to transmit the signal during the cutting process (fig. 1). The cutting operations were performed using a 3-axis Huron K2X10 CNC machine having a maximum speed of $28000 \mathrm{RPM}$ and power of $40 \mathrm{~kW}$. Not only the temperature data was recorded, but also the cutting forces through a Kistler 9255B dynamometric table, as shown in figure 2 . The forces data will help to understand the first contact between the tool and the laminate.

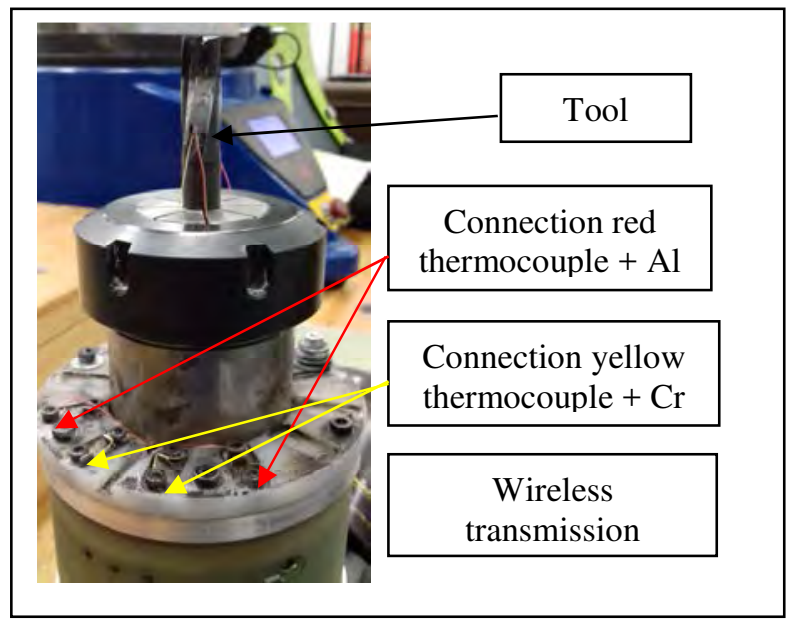

Figure 1. Tool mounting on the spindle 


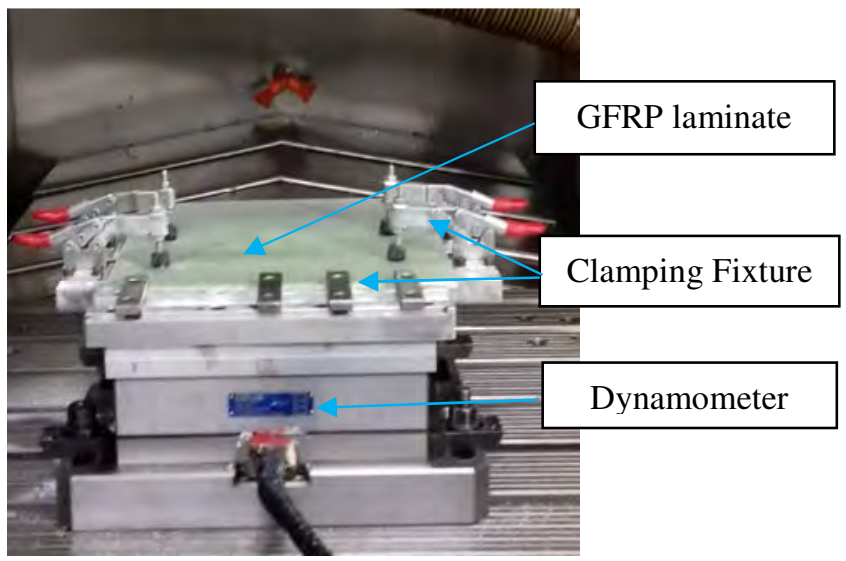

Figure 2. Experimental cutting configuration

With the objective of observing the influence of additives on the cutting temperature, the cutting parameters were fixed constant. The maximum allowed rotational speed of the wireless telemetry system used for temperature acquisition being $10000 \mathrm{RPM}$, the cutting speed of the laminates was fixed to $300 \mathrm{~m} / \mathrm{min}$. Regarding the feed rate, based on previous experiments, it was fixed to $1140 \mathrm{~mm} / \mathrm{min}(0.0190 \mathrm{~mm} /$ tooth $)$. The cutting operation was carried out over a distance of 300 $\mathrm{mm}$.

\section{RESULTS AND DISCUSSION}

The cutting operations for the different laminates started at room temperature, which corresponds $20{ }^{\circ} \mathrm{C}$. The figure 3 shows the temperature along a cutting distance of $300 \mathrm{~mm}$ for all types of laminate. It can be noted that the laminates containing the WA, the WA-WAX, and the WA-WAX-CL have a lower cutting temperature than the reference laminate containing only the epoxy resin (RE). In contrast, only the cutting temperature for the WA-CL laminate is higher than that of the reference laminate. Focusing on the laminates containing respectively WA-CL, WA, WA-WAX, one could validate how the wax plays a beneficial role of lubricating after a certain cutting temperature. The curves start almost from the same point and they are close to each other over a distance of $20 \mathrm{~mm}$. Afterwards, we observe that the WA-CL and WA-WAX-CL curves are visibly off the other three curves. From this result, one may conclude of a combinatory phenomenon between the clay and the wax which favors a reduction of the cutting temperature. However, more study is further required to confirm this hypothesis, since the standard deviation of the temperature differences remains large for some mixtures as shown in Figure 4.

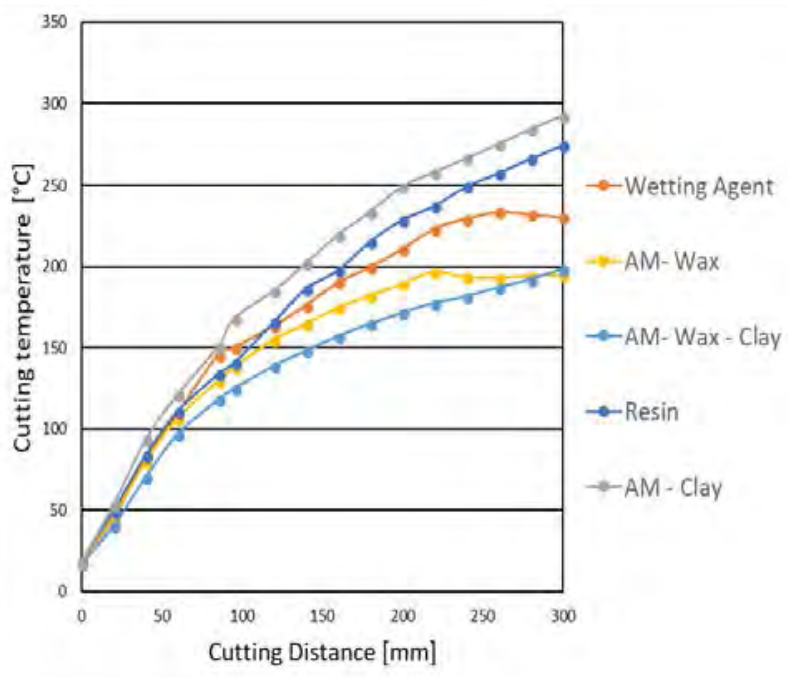

Figure 3. Cutting temperature over a distance of $300 \mathrm{~mm}$.

Evaluating the average temperature differences (Figure 4) relative to the reference temperature which is that of the unmodified resin, it can be noted as expected that the average temperature difference is positive only for the laminate WACL. However, the values of the average temperature difference are significant over a cutting distance of $300 \mathrm{~mm}$, unlike those obtained by Lasseur [5] over a reduced distance of $100 \mathrm{~m}$. The negative values of the average temperature differences observed for the WA, WA-WAX, WA-WAX-CL laminates reflect a reduction in cutting temperature compared to the reference (Resin). Figure 4 also shows standard deviations of the temperature differences. It is observed, however, that the range of standard deviations obtained with the WA-WAX and WA-WAX-CL laminates does not intercept the reference axis as opposed to the gap obtained with the WA laminate. Hence, the wetting agent (WA), alone, would not be appropriate to reduce the cutting temperature. The addition of the clay to the wetting agent would involve an increase in cutting temperature. The figure 5 shows the maximum temperature values achieved for each laminate. The graph reflects the analysis mentioned above. In addition, from this figure, we observe that the laminates containing WA-WAX and WA-WAX-CL are those that actually lead to a reduction of the cutting temperature by taking into account the standard deviations of the temperature differences. Thus, there is a decrease of respectively $28.7 \%$ and $26.5 \%$ in the cutting temperature. 


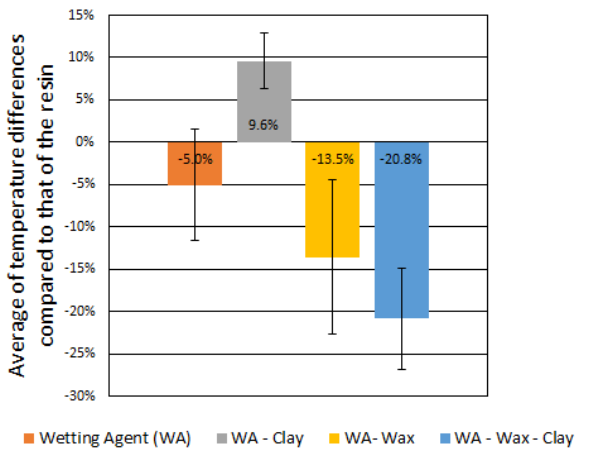

Figure 4. Average of temperature differences compared to that of the resin over a distance of $300 \mathrm{~mm}$.

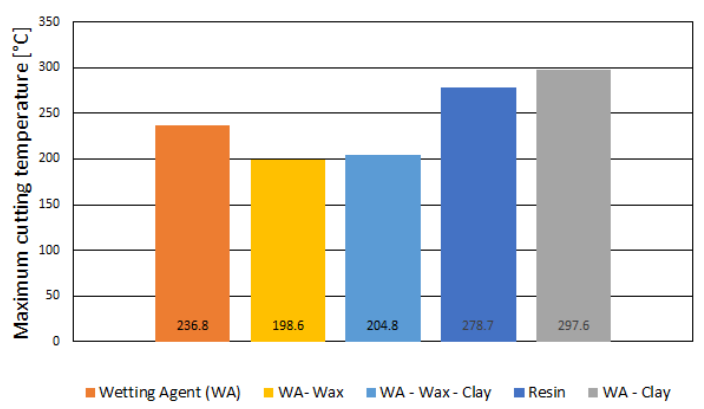

Figure 5. Maximum cutting temperature over a distance of $300 \mathrm{~mm}$.

\section{CONCLUSION}

The objective of this work was to study the influence of additives on the cutting temperature of GFRP laminates. It is apparent that the cutting temperature of the laminates made from WA-CL additives to the epoxy generates higher cutting temperature. Addition of wax to the epoxy clearly lowers the cutting temperature. The best mixture found was the WAWAX-CL with a maximum temperature decrease up to $28 \%$. Further development will focus on the effect of these mixtures on the mechanical properties of the GFRP composite.

\section{REFERENCES}

[1] B. L. B. Furet, Benoit, "Milling and drilling of composite materials for the aeronautics: feature aeronautics," JEC Magazine \#18, June-July 2005.

[2] M. H. El-Hofy, S. L. Soo, D. K. Aspinwall, W. M. Sim, D. Pearson, and P. Harden, "Factors Affecting Workpiece Surface Integrity in Slotting of CFRP," Procedia Eng., vol. 19, pp. 94-99, 2011. doi:10.1016/j.proeng.2011.11.085.

[3] J. P. Davim and P. Reis, "Damage and dimensional precision on milling carbon fiber-reinforced plastics using design experiments," J. Mater. Process. Technol., vol. 160, no. 2, pp. 160-167, Mar. 2005. doi:10.1016/j.jmatprotec.2004.06.003.

[4] K.-M. Li, C. Wang, and W.-Y. Chu, "An improved remote sensing technique for estimating tool-chip interface temperatures in turning," $J$. Mater. Process. Technol., vol. 213, no. 10, pp. 1772-1781, Oct. 2013. doi:10.1016/j.jmatprotec.2013.04.014.
[5] B. Lasseur, "Étude de l'effet d'additifs sur la qualité de la coupe et les performances mécaniques d'un matériau composite," École de technologie supérieure, 2017.

[6] J. Delahaigue, J.-F. Chatelain, and G. Lebrun, "Influence of Cutting Temperature on the Tensile Strength of a Carbon Fiber-Reinforced Polymer," Fibers, vol. 5, no. p. 46, Dec. 2017. doi:10.3390/fib50400464,

[7] G. Mullier and J. F. Chatelain, "Influence of Thermal Damage on the Mechanical Strength of Trimmed CFRP," Inter. J. Mech. Aerospace, Indus., Mechatronic and Manu. Eng., vol. 9, no. 9, pp. 1558-1566, 2015.

[8] H. Hamedanianpour, Effect to tool wear on quality of carbon fiber reinforced polymer laminate during edge trimming (Mgm, no. 2013:21]). Montréal: École de technologie supérieure, 2013, pp. 1 ressource en ligne (xviii, 143 pages).

[9] R. Teti, K. Jemielniak, G. O'Donnell, and D. Dornfeld, "Advanced monitoring of machining operations," CIRP Annals, vol. 59, no. 2, pp. 717-739, 2010. doi:10.1016/j.cirp.2010.05.010.

[10] M. Quaresimin, M. Salviato, and M. Zappalorto, "Fracture and interlaminar properties of clay-modified epoxies and their glass reinforced laminates," Eng. Fract. Mech., vol. 81, pp. 80-93, Feb. 2012. doi:10.1016/j.engfracmech.2011.10.004.

[11] G. Withers, J. Souza, Y. Yu, L. Cercone, V. Khabashesku, and D. Davis, "Improved mechanical properties of a water-activated polyurethane-glass fiber composite reinforced with amino-functionalized carbon nanofibers," $J$. Compos. Mater., vol. 50, no. 6, pp. 783-793, Apr. 2015. doi:10.1177/0021998315581510.

[12] S. Gurusideswar and R. Velmurugan, "Strain rate sensitivity of glass/epoxy composites with nanofillers," Mater. Des., vol. 60, pp. 468-478, Aug. 2014. doi:10.1016/j.matdes.2014.03.065.

[13] O. Pecat, R. Rentsch, and E. Brinksmeier, "Influence of Milling Process Parameters on the Surface Integrity of CFRP," Procedia CIRP, vol. 1, pp. 466470, 2012. doi:10.1016/j.procir.2012.04.083.

[14] T. Rajasekaran, B. K. Vinayagam, K. Palanikumar, and S. Prakash, "Influence of machining parameters on surface roughness and material removal rate in machining carbon fiber reinforced polymer material," in Frontiers in Automobile and Mechanical Engineering -2010, 2010. doi:10.1109/FAME.2010.5714801.

[15] M. Ramulu, "Machining and surface integrity of fibre-reinforced plastic composites," Sadhana, vol. 22, no. 3, pp. 449-472, Jun. 1997. doi:10.1007/BF02744483.

[16] J. F. Chatelain, I. Zaghbani, and J. Monier, "Effect of Ply Orientation on Roughness for the Trimming Process of CFRP Laminates," Inter. J. Mech. Aerospace, Indus. Mechatronic and Manu. Eng., vol. 6, no. 8, pp. 1515-1522, 2012. doi:10.5281/zenodo.1329697. 


\section{Energy Transfer Mechanisms in the Ranque-Hilsch Vortex Tube}

\author{
Nolan Dyck \\ Department of Mechanical Engineering \\ The University of Western Ontario \\ London, Ontario, Canada \\ Email: ndyck@uwo.ca
}

\author{
Anthony Straatman \\ Department of Mechanical Engineering \\ The University of Western Ontario \\ London, Ontario, Canada \\ Email: astraatman@eng.uwo.ca
}

\begin{abstract}
The Ranque-Hilsch Vortex Tube (RHVT) is a small tube without any moving parts receiving compressed air from a tangential inlet and exhausting cold air from a small hole at one end of the tube while exhausting hot air from an annular exit at the opposite end. Nearly a century has passed since the RHVT was first patented, and the mechanism responsible for this socalled "temperature separation" remains unclear. The present work tests the hypothesis that kinetic energy is transferred from the stream of air leaving the cold exit to the air stream leaving the hot exit.

To test the hypothesis, a parametric study using an axisymmetric model of the RHVT has been carried out using CFD software, wherein the pressure at the hot exit was varied. The study has been validated against previous experimental and computational models. The results show that the dominant mode of energy transfer between the two streams is work transfer, which supports the hypothesis.
\end{abstract}

Index Terms-Ranque-Hilsch Vortex Tube, Compressible flow, Computational Fluid Dynamics

\section{INTRODUCTION}

Consider the schematic shown in figure 1. A compressible fluid is supplied at the entrance shown at some pressure $p_{\text {in }}$. Provided $p_{\text {in }}$ exceeds $p_{\text {cold }}$ and $p_{\text {hot }}$, some fraction of the inlet mass $\mu_{\mathrm{c}}=\dot{m}_{\text {cold }} / \dot{m}_{\text {in }}$ leaves via the cold exit, while the remaining fraction $1-\mu_{c}$ is exhausted at the hot exit. While this observation is rather unremarkable, many researchers [1], [2], [3], [4] have observed physical experiments in which the static temperature of cold exit stream was significantly lower than that of the inlet stream, while the static temperature of the hot exit stream was significantly higher than that of the inlet stream. This "temperature separation" was first noticed by George J. Ranque [5] in 1922, and is often referred to as the Ranque effect. In a later investigation Hilsch [1] discussed the effects of varying the geometric constraints of the flow boundaries. The vortex tube is now commonly referred to as the Ranque-Hilsch Vortex Tube (RHVT) after the aforementioned researchers. Sometimes the abbreviation is shortened to VT.

While vortex tubes are easy to construct and operate, the mechanism(s) responsible for the temperature separation phenomenon are still unknown, as noted in a recent review by Thakare et al. [6]. Many researchers have conducted studies and proposed theories of operation over the past century, and while much data is available, the complex interaction between fluid mechanics and thermodynamics remains unclear.

Ranque [5] originally suggested that the temperature separation could be explained using the isentropic ideal gas relation, wherein the sudden expansion at the inlet causes a decrease in density and a sharp drop in temperature. Experiments by Gao et al. [7] and Xue et al. [8] indicate this theory significantly over-predicts the temperature drop at the cold exit. Furthermore this does not explain the corresponding temperature increase at the hot exit.

Some numerical studies have revealed the presence of recirculation region(s) at various points within the VT. Both Ahlborn and Groves [2], and Xue and others [9] have suggested multiple re-circulation zones exist, each one behaving as a heat pump to move energy away from the core and towards the periphery. While earlier papers have provided mediocre evidence for this theory, more recent papers reporting the results of steady and unsteady, 3D, RANS and LES results have included streamline plots depicting multiple recirculation zones occurring within short vortex tubes [10], [11], [12], [13]. The re-circulation zones appear to be mobile and sensitive to geometry and boundary conditions.

A third theory states that cold stream transfers kinetic energy to the hot stream through viscous shear. This work transfer theory was first proposed by Hilsch [1], who suggested that 'internal friction' was the mode by which energy is transferred from the axis to the periphery. Aljuwayhel et al. [14] have since analyzed the results of an axisymmetric CFD model and provided quantitative evidence that work transfer from the cold stream to the hot stream is dominant mechanism of energy transfer in the RHVT. More recently, Polihronov et al. [15] and Tlili El May et al. [16] have observed wall shear stress spikes in 3D simulations of the VT, suggesting the cold stream is transferring kinetic energy to the hot stream.

In the present work we aim to quantify the energy transfer between the cold stream and the hot stream, testing our hypothesis that shear work is the primary mechanism of temperature separation. Section II provides the governing equations applicable to the current analyses, section III details the CFD model used, section IV reports the results of our simulations, and section $\mathrm{V}$ analyzes the energy transfer 


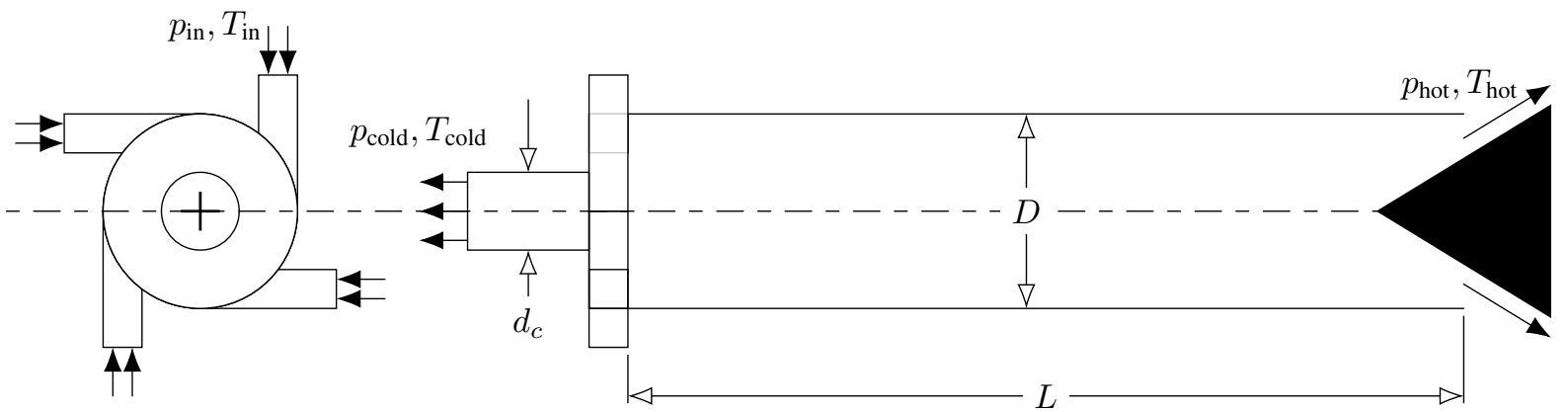

Fig. 1. A schematic of a typical Ranque-Hilsch Vortex Tube.

between the cold and hot streams.

\section{Governing EQUATIONS}

The conservation of mass is

$$
\frac{\partial \rho}{\partial t}+\nabla \cdot(\rho \mathbf{u})=0
$$

where $\mathbf{u}$ is the velocity, $\rho$ is the density, $t$ is time, and $\boldsymbol{\nabla}$ is the gradient operator. The conservation of momentum for a Newtonian fluid where Stokes' hypothesis has been invoked is

$$
\frac{\partial \mathbf{u}}{\partial t}+(\mathbf{u} \cdot \boldsymbol{\nabla}) \mathbf{u}=-\frac{\boldsymbol{\nabla} p}{\rho}+\frac{\mu}{\rho}\left(\nabla^{2} \mathbf{u}+\frac{1}{3} \boldsymbol{\nabla}(\boldsymbol{\nabla} \cdot \mathbf{u})\right),
$$

where $p$ is the thermodynamic pressure and $\mu$ is the dynamic viscosity. The conservation of internal energy is

$$
\rho\left(\frac{\partial \varepsilon}{\partial t}+\mathbf{u} \cdot \boldsymbol{\nabla} \varepsilon\right)=-p(\boldsymbol{\nabla} \cdot \mathbf{u})-\boldsymbol{\nabla} \cdot \mathbf{q},
$$

where $\varepsilon$ is the specific internal energy and $\mathbf{q}$ is the heat flux vector.

\section{A. Auxillary equations}

All fluids analyzed in this work are assumed to obey the ideal gas equation of state:

$$
p=\rho R_{s} T,
$$

where $R_{s}$ is the specific ideal gas constant, and $T$ is the static, absolute temperature.

We will further assume a constant heat capacity for all fluids analyzed in this work, so that the internal energy and enthalpy may be respectively written as

$$
\begin{aligned}
& \varepsilon=c_{v} T, \\
& h=c_{p} T,
\end{aligned}
$$

where $c_{v}$ is the volumetric heat capacity and $c_{p}$ is the isobaric heat capacity. Fluids which obey the ideal gas law and have constant heat capacities are called perfect gases [17].

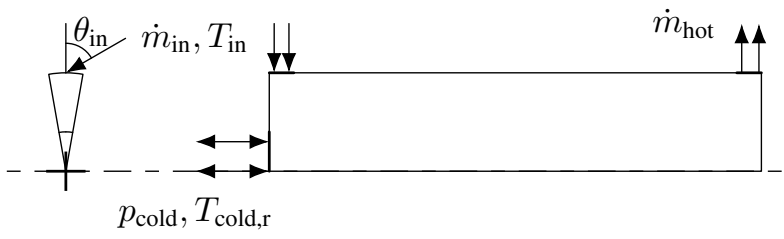

Fig. 2. Simplified VT geometry for CFD study

TABLE I

PARAMETERS OF THE PRESENT CFD STUDY

\begin{tabular}{||l|l||}
\hline Measurement & Value \\
\hline Working tube length [mm] & 106 \\
Working tube inner diameter $[\mathrm{mm}]$ & 11.4 \\
Nozzle total inlet area $\left[\mathrm{mm}^{2}\right]$ & 8.2 \\
Cold exit diameter $[\mathrm{mm}]$ & 6.2 \\
Cold exit area $\left[\mathrm{mm}^{2}\right]$ & 30.3 \\
Hot exit area $\left[\mathrm{mm}^{2}\right]$ & 95 \\
Nozzle angle $\left[{ }^{\circ}\right]$ & 75.48 \\
\hline Inlet conditions & \\
\hline mass flow rate $\left[\mathrm{g} \mathrm{s}^{-1}\right]$ & 8.34 \\
Total Temperature $\left[{ }^{\circ} \mathrm{C}\right]$ & 21.21 \\
\hline
\end{tabular}

\section{CFD STUDY}

To analyze the energy transfer between the hot and cold streams we have performed a CFD analysis of the commercial vortex tube studied by Skye et al. [4]. The simplified vortex tube geometry is visible in Fig. 2, and the relevant geometric parameters are given in Table I.

For each simulation, the mass flow rate at the inlet is $\dot{m}_{\text {in }}=$ $8.34 \mathrm{~g} \mathrm{~s}^{-1}$, the inlet velocity angle is $\theta_{\text {in }}=75.48^{\circ}$, and the inlet total temperature is $T_{\mathrm{in}}=21.21^{\circ} \mathrm{C}$. Skye et al. notes the presence of reverse flow at the cold exit for small cold mass fractions, so an opening boundary condition is applied at the cold exit, where the total temperature of the recirculating air, $T_{\text {cold, },}$, is set to the bulk mean total temperature of the leaving air, $T_{\text {cold }}$. To replicate the experiment conditions of Skye et al. the cold exit pressure $p_{\text {cold }}$ has been set to the experimentally measured value for each cold mass fraction.

Two non-orthogonal structured grids have been generated 


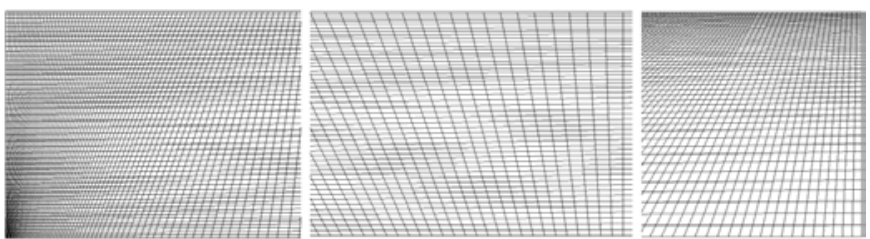

Fig. 3. Sample views of the fine mesh at inlet, middle, and hot outlet end.

using ANSYS Workbench ${ }^{\circledR}$ Meshing Software [18]: a relatively course grid containing 1890 nodes and a fine grid containing 41778 nodes. Sample images of the fine grid are provided in Fig. 3. The $\mu_{\mathrm{c}}=0.208$ case was run using each mesh and the results were compared. The cold exit temperature differed by $0.22 \%$ and the hot exit temperature differed by $0.017 \%$, indicating our results are grid-independent. The fine grid has been used for the remainder of the simulations reported on in this work.

In each simulation convergence was achieved when the rms residuals for the mass, momentum, and turbulent equations were fell below $10^{-6}$, while the energy equation rms residuals fell below $10^{-5}$.

ANSYS-CFX ${ }^{\circ} 16$ [19] has been used to setup and solve each of the simulations. The standard $k-\varepsilon$ turbulence model has been used.

\section{Results AND Discussion}

The measured temperature separation values at the cold and hot exits are defined by

$$
\begin{gathered}
T_{\mathrm{s}, \mathrm{c}}=T_{\text {in }}-T_{\text {cold }} \\
T_{\mathrm{s}, \mathrm{h}}=T_{\text {hot }}-T_{\text {in }}
\end{gathered}
$$

where the total temperatures have been used. The cold and hot temperature separation values have been plotted in Figs. 4 and 5 alongside data obtained from experimental and computational results. The cold exit temperature separation appears to peak at a cold mass fraction of $\mu_{\mathrm{c}} \approx 0.35$, while the hot exit temperature separation steadily increases with cold mass fraction. The 3D, unsteady Large Eddy Simulations conducted by Farouk and Farouk [20] predict the cold stream temperature separation more accurately than the present model.

Despite our less accurate predictions, the present model still closely captures the trend of the experimental data at both the hot and cold exits. A possible explanation for the discrepancy between the present model and Farouk and Farouk's is that the mechanism present in the axisymmetric models is augmented or supplemented by unsteady circumferential variations. In either case, the results of the axisymmetric model can be further analyzed to gain more insight into at least one temperature separation mechanism.

Fig. 6 shows a typical streamline plot of the axisymmetric VT, where several interesting features are present. First, the hot stream occupies only a narrow band around the tube

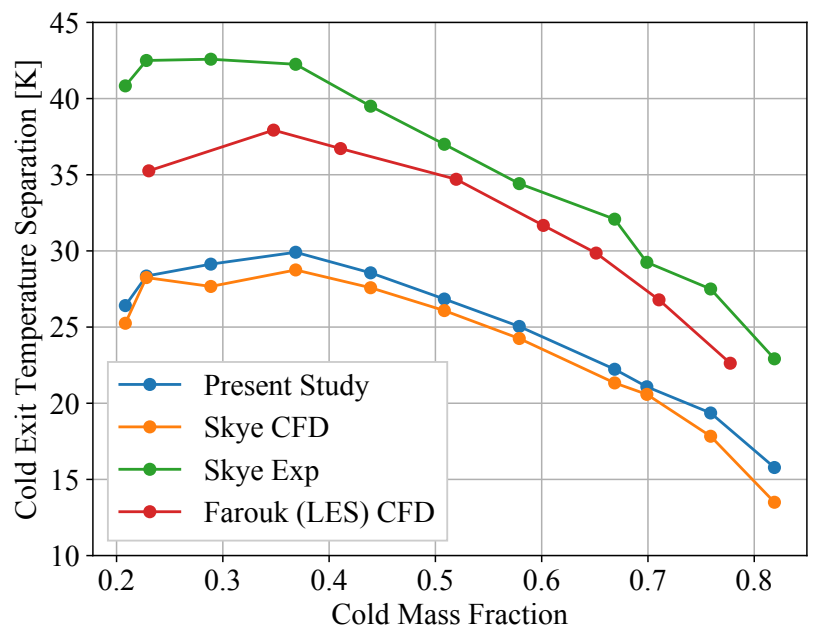

Fig. 4. Cold exit temperature separation.

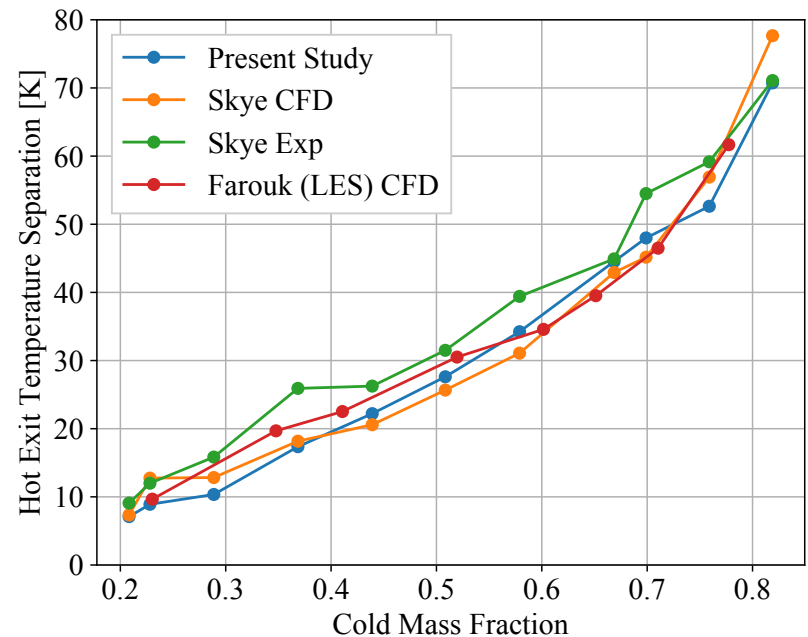

Fig. 5. Hot exit temperature separation.

wall, while the rest of the domain is occupied by the slender reverse flow region of the cold stream. Furthermore there is a recirculation region present in at the cold outlet, caused by the strong radial pressure variation. This recirculation region disappears for cold mass fractions greater than 0.4. Finally, a stagnation streamline connects the inlet to a zero velocity region near the hot outlet. When the stagnation streamline is revolved about the tube axis, it represents a stream surface separating the hot stream from the cold stream.

\section{Energy Transfer Modes}

With the converged solution fields at hand, the energy transfer modes across the stagnation streamline may be computed. We follow the same procedure as Aljuwayhel et al. [14], by computing the various energy transfers across the stagnation streamline. The differential energy transfers associated with conduction heat transfer, circumferential shear work, and axial shear work are given by 


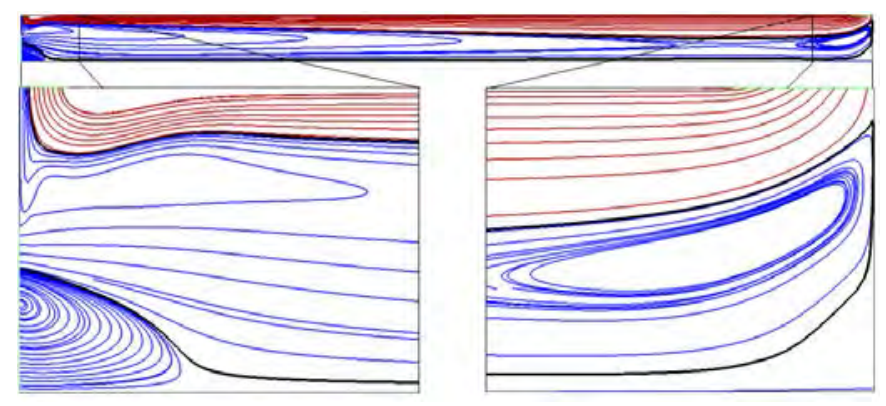

Fig. 6. Streamline plot of axisymmetric CFD results, with a cold mass fraction of 0.208 . Red streamlines proceed from the inlet to the hot exit, blue streamlines terminate at the cold exit, and two stagnation streamlines are represented by thick black lines. The stagnation streamline of interest divides the hot and cold streams.

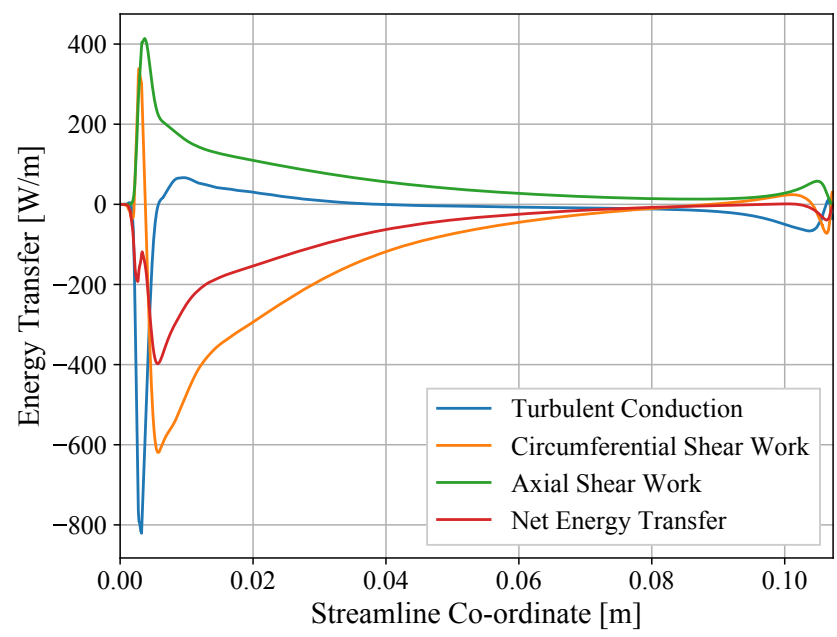

Fig. 7. Energy transfer across the stagnation streamline for the case $\mu_{\mathrm{c}}=$ 0.208

$$
\begin{aligned}
\frac{\partial \dot{Q}}{\partial s} & =-2 \pi k_{\mathrm{eff}} r \frac{\partial T}{\partial n} \\
\frac{\partial \dot{W}_{\theta}}{\partial s} & =-2 \pi \mu_{\mathrm{eff}} r v_{\theta} r \frac{\partial}{\partial n}\left(\frac{v_{\theta}}{r}\right) \\
\frac{\partial \dot{W}_{z}}{\partial s} & =-2 \pi \mu_{\mathrm{eff}} v_{z} r \frac{\partial v_{z}}{\partial n}
\end{aligned}
$$

where $s$ is a streamline co-ordinate, and $n$ is the normal vector pointing towards the hot stream. The contributions from each of these have plotted as a function of the streamline co-ordinate for two cold mass fractions Figs. 7 and 8. In the case of a low cold mass fraction, the majority of the energy transfer between the streams takes place near the inlet, diminishes as the streamsurface contracts towards the axis, and recovers near the hot exit. The circumferential shear work transfer is the dominant energy transfer mechanism. For a cold mass fraction of 0.819 , the majority of the energy transfer takes place near the hot exit.

The total energy transferred via each mechanism can be found by integrating over the stream line, and the results

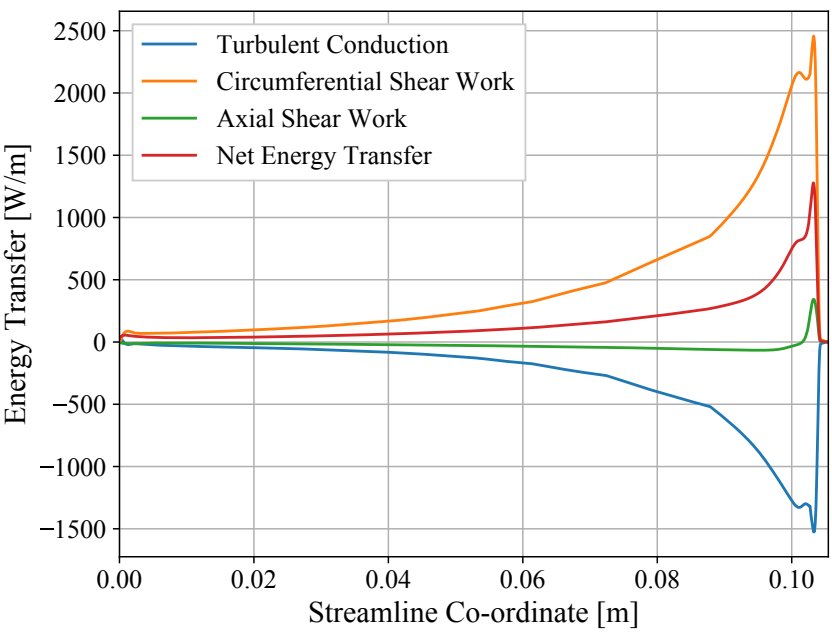

Fig. 8. Energy transfer across the stagnation streamline for the case $\mu_{\mathrm{c}}=$ 0.819

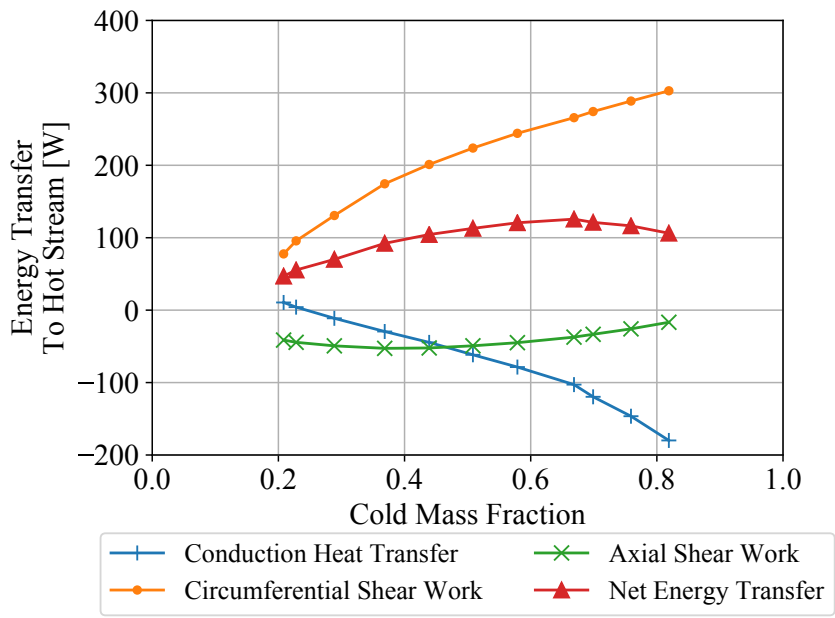

Fig. 9. Total Energy transfer across the stagnation streamlines.

have been plotted as a function of cold mass fraction in Fig. 9. As the cold mass fraction increases, the energy transferred through circumferential shear work and conduction heat transfer increase. Since the energy is transferred in opposite directions, the net effect is only a modest increase in energy transfer.

To validate our findings, we have compared the energy increase of the flow leaving the hot exit with the net energy transfer to the hot stream across the stagnation streamline. The hot stream energy increase has been computed using

$$
\Delta E_{\mathrm{hot}}=\left(1-\mu_{\mathrm{c}}\right) \dot{m} c_{p} T_{\mathrm{s}, \mathrm{h}}
$$

The percent differences between the energy transfer across the stagnation streamline and equation 12 have been plotted in Fig. 10. Overall the discrepancies are modest. It is likely there is a small amount of energy transferred via radial shear at smaller mass fractions since the stagnation streamlines have 


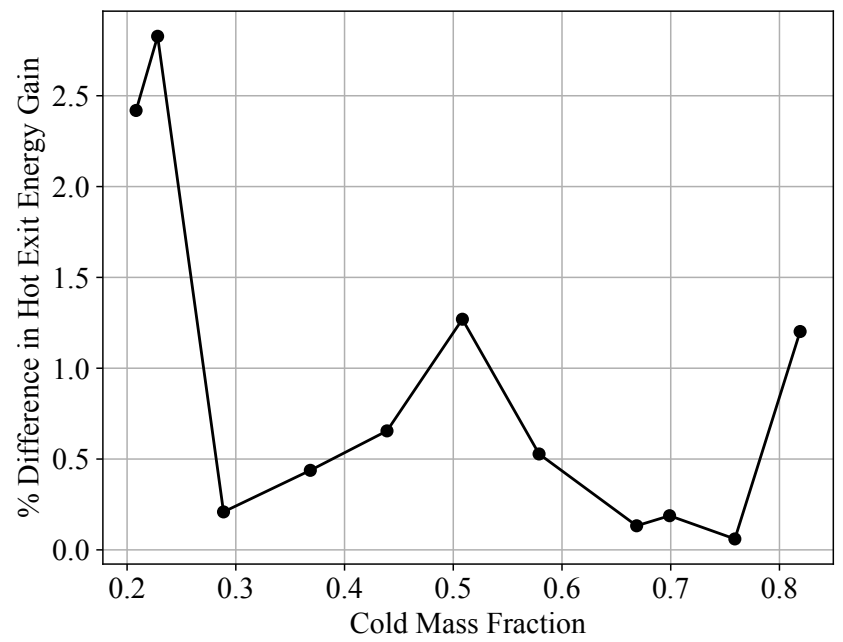

Fig. 10. Percent difference between energy increase computed based on the difference between the inlet and hot exit and the total energy transferred across the stagnation streamline.

the most significant radial variation, which could account for the greater discrepancies at low mass fractions.

\section{CONCLUSION}

In this work we have re-analyzed the vortex tube studied by Skye et al. [4] using an axisymmetric CFD model. It was observed that the trends in temperature separation matched those seen in experiments, though they are less accurate than 3D unsteady CFD models. We have also computed the transfers of kinetic and thermal energies across the stagnation streamline, demonstrating that circumferential shear work transfer is the dominant energy transfer mechanism.

Although the hypothesis has been supported, result is unsatisfying as the study fails to answer the deeper questions about the mechanism behind temperature separation; a complete picture of the energy movement in the system is still elusive, and there aren't any clear indications of which parameters are most important in vortex tube design. Furthermore, recirculation regions near the boundaries are present and cast doubt on the correctness of the velocity field. Future work will focus on better matching the boundary conditions between experiments and computational results.

\section{ACKNOWLEDGMENT}

The authors gratefully acknowledge the financial support from the Natural Sciences and Engineering Research Council of Canada (NSERC).

\section{REFERENCES}

[1] R. Hilsch, "The use of the expansion of gases in a centrifugal field ascooling process," Rev. Sci. Instrum., vol. 18, no. 2, pp.108-113, 1947. doi: 10.1063/1.1740893.
[2] B. Ahlborn and S. Groves, "Secondary flow in a vortex tube," Fluid Dyn Res., vol. 21, no. i, pp. 73-86, 1997. doi:10.1016/S0169-5983(97)00003-8.

[3] K. Dincer et al., "Experimental investigation of the performance of a Ranque-Hilsch vortex tube with regard to a plug located at the hot outlet," Int. J. Refrig., vol. 32, no. 1, pp. 87-94, Jan. 2009. doi: 10.1016/j.ijrefrig.2008.06.002.

[4] H. M. Skye, G. F. Nellis, and S. A. Klein, "Comparison of CFD analysis to empirical data in a commercial vortex tube," Int. J. Refrig., vol. 29, no. 1, pp. 71-80, Jan. 2006. doi: 10.1016/j.ijrefrig.2005.05.004.

[5] G. J. Ranque, "Experiments on expansion in a vortex with simultaneous exhaust of hot air and cold air," J. Phys. Radium, vol. 4, no. 7, pp. 112- 114, 1933.

[6] H. R. Thakare, A. Monde, and A. D. Parekh, "Experimental, computational and optimization studies of temperature separation and flow physics of vortex tube: A review," Renewable Sustainable Energy Rev., vol. 52, pp. 1043-1071, Dec. 2015. doi: 10.1016/j.rser.2015.07.198.

[7] C. M. Gao et al., "Experimental study on a simple Ranque-Hilsch vortex tube," Cryogenics, vol. 45, no. 3, pp. 173-183, Mar. 2005. doi: 10.1016/j.cryogenics.2004.09.004

[8] Y. Xue and M. Arjomandi, "The effect of vortex angle on the efficiency of the Ranque-Hilsch vortex tube," Exp. Therm. Fluid Sci., vol. 33, no. 1, pp. 54-57, Oct. 2008. doi: 10.1016/j.expthermflusci.2008.07.001.

[9] Y. Xue, M. Arjomandi, and R. Kelso, "The working principle of a vortex tube," Int. J. Refrig., vol. 36, no. 6, pp. 1730-1740, Sept. 2013. doi:10.1016/j.ijrefrig.2013.04.016.

[10] R. Liew et al., "Maxwell's demon in the ranque-hilsch vortex tube," Phys. Rev. Lett., vol. 109, no. 5, pp. 3-6, Aug. 2012. doi: 10.1103/PhysRevLett.109.054503.

[11] H. R. Thakare and A. D. Parekh, "Computational analysis of energy separation in counter-flow vortex tube," Energy, vol. 85, pp. 62-77, June 2015. doi: 10.1016/j.energy.2015.03.058.

[12] S. E. Rafiee and M. M. Sadeghiazad, "Experimental study and 3D CFD analysis on the optimization of throttle angle for a convergent vortex tube," J. Mar. Sci. Appl., vol. 15, no. 4, pp. 388-404, Dec. 2016.

[13] V. Bianco et al., "A comparison of the application of RSM and LES turbulence models in the numerical simulation of thermal and flow patterns in a double-circuit Ranque-Hilsch vortex tube," Appl. Therm. Eng., vol. 106, pp. 1244-1256, Aug. 2016.

doi: 10.1016/j.applthermaleng.2016.06.095.

[14] N. F. Aljuwayhel, G. F. Nellis, and S. A. Klein, "Parametric and internal study of the vortex tube using a CFD model," Int. J. Refrig., vol. 28, no. 3, pp. 442-450, May 2005. doi: 10.1016/j.ijrefrig.2004.04.004.

[15] J. G. Polihronov, A. G. Straatman, and C. T. DeGroot, "On the Thermodynamics of Angular Propulsion," in 10th Int. Conf. on Heat Transfer, Fluid Mechanics and Thermodynamics, Orlando, FL, 2014.

[16] O. Tlili El May et al., "CFD investigation of a vortex tube : Effect of the cold end orifice in the temperature separation mechanism," Sci. Acad. Trans. Renewable Energy Syst. Eng. Technol., vol. 1, no. 3, pp. 84-89, 2011.

[17] K. Masatsuka, I do like CFD, Vol.1: Governing equations and exact solutions, 2nd ed., 2013. [Online] Available: Software Cradle.

[18] Workbench User's Guide. ANSYS Inc, 2015, pp. 724-746. [Online].

[19] ANSYS CFX-Pre User's Guide. ANSYS Inc., 2011, pp. 724-746. [Online].

[20] T. Farouk and B. Farouk, "Large eddy simulations of the flow field and temperature separation in the Ranque-Hilsch vortex tube," Int. J. Heat Mass Transfer, vol. 50, no. 23-24, pp. 4724-4735, Nov. 2007. doi: 10.1016/j.ijheatmasstransfer.2007.03.048. 


\section{Thermal Conductivity of Advanced Architected Cellular Materials}

\author{
A. Mirabolghasemi \\ Department of Bioresource Engineering \\ McGill University \\ Montreal, Canada \\ D. Therriault \\ Department of Mechanical Engineering \\ Polytechnique Montreal \\ Montreal, Canada
}

\begin{abstract}
Architected cellular materials, as a novel class of low density materials, gain their unprecedented multifunctional performance mainly from their underlying architecture. In this paper, we focus on thermal conductivity of cellular materials. Standard mechanics homogenization with periodic boundary conditions is used to determine the thermal conductivity of cells with supershape pores. The computational results confirm that a wide range of possible anisotropic behaviour for thermal conductivity is achievable for cellular materials. Effective thermal conductivity of shellular materials based on three triply periodic minimal surfaces are also compared with those of cells with supershape pores. It is found that unlike the shellular materials, which only cover a narrow portion of thermal conductivity vs. relative density chart, cellular materials with anisotropic effective thermal conductivity could be engineered by employing supershape pores in cells.
\end{abstract}

\begin{tabular}{l}
\multicolumn{1}{c}{ Keywords - Architected } \\
conductivity; Homogenization; \\
materials
\end{tabular}

\section{INTRODUCTION}

Limited material and energy resources, economical restrictions, and concerns over the prospect of global climate changes, promote the design and manufacturing of lightweight materials with tunable multifunctional properties. Architected cellular materials are one of the cutting-edge alternative lightweight and optimized materials which can simultaneously satisfy multiple functionalities, from structural stiffness to thermal insulation and heat exchanging [1].

Cellular materials are divided into two categories: foams, where a gaseous phase is randomly dispersed in a continuous solid medium, and periodic lattices, which consist of a periodic architected cell [2]. The concept of using cellular metals emerged in early 1970s, by inspiration from the excellent

\author{
A. H. Akbarzadeh \\ Department of Bioresource Engineering \& \\ Department of Mechanical Engineering \\ McGill University \\ Montreal, Canada \\ Email: hamid.akbarzaeh@mcgill.ca
}

\author{
D. Rodrigue \\ Department of Chemical Engineering \\ Université Laval \\ Quebec, Canada
}

properties of natural materials like bone, wood and cork. Hexagonal honeycomb structures in sandwich cores, used in aviation industry, can be named as one of the first applications of periodic cellular materials [3]. Driven by the high performance of these cellular materials and recent development of additive manufacturing, advanced polymeric and metallic cellular materials are being widely fabricated. A non-exhaustive list of applications of cellular materials found in the literature includes: lightweight structural elements in aircrafts and highspeed trains, energy-absorbing elements in automotive industry, thermal insulation, thermal energy storage devices, hydrogen storage tanks, and scaffold for tissue engineering [2-11].

To exploit multifunctional potentials of cellular materials, a mathematical predictive model, capable of accurately predicting their effective properties, is of crucial importance. Early efforts on this subject range from the simple volumetric averaging of properties of constituent cellular materials, so-called as 'rule of mixtures method', to several empirical equations for predicting the multiphysical properties of cellular materials [2, 12]. Investigations have clearly shown that cell architecture, in addition to the properties of the constituents' materials, plays a significant role in the emergence of their outstanding properties $[13,14]$. In this regards, as thermal conductivity, electrical conductivity and magnetic permeability are all mathematically described by the Laplace equation, existing approaches used in the electric and magnetic fields have been also applied for thermal properties of cellular materials $[15,16]$. The introduction of thermal-circuit method, based on the analogy between the electrical and thermal conductivities, could be considered as a turning point in theoretical modelling of thermal conductivity of cellular materials [16]. It is worth mentioning that most of the analytical models have been mainly developed for random pore distribution [17], or simple pore topologies, e.g. cubic, circular, cylindrical, and spherical [18, 19], leaving more complex periodic architectures with potential superior multifunctional properties unexplored. Although analytical upper and lower bounds, e.g. those bounds presented by Hashin 
and Strikman [20] or thermal-circuit method, are valuable for estimating the effective thermal conductivity with a minimum knowledge about the actual heat flow and temperature profile in cellular materials, advanced computational models, e.g. Latticebased Monte Carlo approach [21], micropoloar modelling [22], and standard mechanics [23] and asymptotic homogenizations [24], have been developed to precisely take into account the effect of microarchitecture of cellular materials to accurately predict their effective thermal properties. The physical properties and application of cellular materials have been summarize in several textbooks, among which the book by Gibson and Ashby [2] provide the most comprehensive overview.

In this paper, we present two methodologies for predicting the effective thermal conductivity of architected cellular materials: (1) Theoretical modelling using the thermal-circuit method [25] and (2) Computational modeling based on standard mechanics homogenization. While the theoretical model is used to provide a narrow bound for thermal conductivity of cellular materials with simple 2D microarchitecture, standard mechanics homogenization is used to determine the exact thermal conductivity of cellular materials with complex 2D microarchitecture. To highlight the effect of cell topology on the thermal conductivity of periodic cellular materials, modified supershape formula [26] has been employed to create 2D pore shapes on square cells. Moreover, to address the ever growing interests on the lightweight 3D cellular materials, shellular materials based on three triply-periodic minimum surfaces have been analyzed and compared with the rest of selected cellularbased metamaterials.

\section{CAD Design Of ARChitected Cellular Materials}

To apply computational standard mechanics homogenization to explore the effect of topology and relative density on the effective thermal conductivity of cellular materials, 2D square unit cell with one supershape pore and 3D cubic shellular representative volume element (RVE) are selected.

\section{A. 2D RVEs}

As a powerful formula for creating a variety of pore shapes, superformula (1) is selected and modified to generate void geometries while scaling and rotation ( $\beta$ ) parameters are considered to increase the possibilities of pore topologies.

$$
\begin{array}{r}
{\left[\begin{array}{l}
\mathrm{x} \\
\mathrm{y}
\end{array}\right]=[|\cos (\mathrm{m} \phi / 4)|+|\sin (\mathrm{m} \phi / 4)|]^{\mathrm{n}}\left[\begin{array}{l}
\cos (\phi) \\
\sin (\phi)
\end{array}\right]} \\
(-\pi \leq \phi \leq \pi, \mathrm{m}=1 \sim 8, n=-5 \sim 5)
\end{array}
$$

Fig 1 shows sample 2D RVEs for different topological parameters. The pore shape corresponding to each set of geometrical parameters, introduced in (1), is directly modeled inside ANSYS APDL (Fig. 2) by creating several keypoints and using them to make spline curves. The area surrounded by the spline curves is formed, scaled, rotated and moved to the center of the RVE to be subtracted from the RVE and to finalize the 2D RVE.

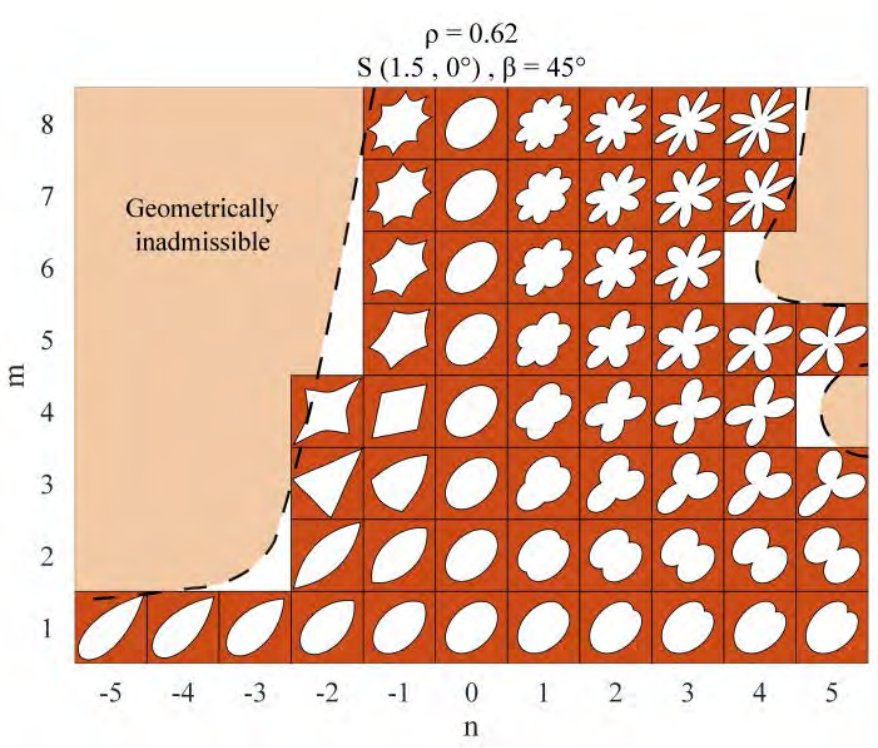

Figure 1. Sample 2D RVEs for different parameters: $\rho=0.62$, scaled by 1.5 in horizontal direction, and rotated by $45^{\circ}$

\section{B. $3 D$ shellular RVEs}

A Triply Periodic Minimal Surface (TPMS) is a non-selfintersecting surface in $\mathrm{R}^{3}$, which has a crystalline structure repeated in three independent directions and has zero mean curvature at each point [27]. The presence of TPMS in natural structures, like biological membranes [28] and crystals [27], has inspired researchers to consider TPMS architectures in tissue engineering, and biomimetic material design [29-32]. It is worth mentioning that "Shellular" term has recently been used in the literature to represent thin TPMS cellular shells. Among many known TPMSs, Schwarz's Primitive (P) and Diamond (D) and Schoen's Gyroid $(\mathrm{G})$ are selected. These surfaces can be trigonometrically approximated using the following level surface equations [33]:

$$
\begin{aligned}
& \text { G: } \cos x \cdot \sin y+\cos y \cdot \sin z+\cos z \cdot \sin x=f \quad(f=0) \\
& \text { P: } \cos \mathrm{x}+\cos \mathrm{y}+\cos \mathrm{z}=f \quad(f=0,0.4,0.8) \\
& \text { D: } \sin x \cdot \sin y \cdot \sin z+\sin x \cdot \cos y \cdot \cos z+ \\
& \cos x \cdot \sin y \cdot \cos z+\cos x \cdot \cos y \cdot \sin z=f \\
& (f=0)
\end{aligned}
$$

We develop a MATLAB code to solve the level surface equations to determine the coordinates of several points on the surface. These points together with multiple cross section curves satisfying the surface equations are firstly created inside Solidworks using a Visual Basic code and then are used to create a smooth surface. This part of the surface is then patterned to create the mid-surface of the shellular RVE and subsequently thickened in order to add the desired thickness (as illustrated in Fig. 3). To focus more on the lightweight structures, five thicknesses $\left(t_{r e l}\right)$ between 2 to 10 percent of RVE's length are considered. Fig. 4 demonstrates some of the selected shellular architectures. 

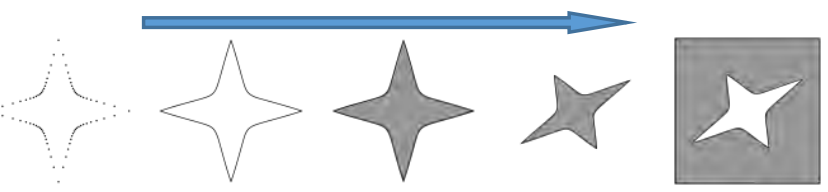

Figure 2. Different steps in modeling of 2D RVEs

\section{PREDICTIVE METHODS}

Fourier's law assumes the following linear relation between heat flux $(\overrightarrow{\mathrm{q}})$ and temperature gradient $(\vec{\nabla} \mathrm{T})$ through a symmetric thermal conductivity tensor $\left(\mathrm{K}_{\mathrm{eff}}\right)$ :

$$
\vec{q}=-K_{e f f} \vec{\nabla} T
$$

For a thermally isotropic homogenous solid material, $K_{\text {eff }}$ tensor reduces to $\mathrm{KI}$, where $\mathrm{I}$ is the identity tensor and $\mathrm{K}$ is called isotropic thermal conductivity. However, in the case of a cellular material, overall thermal conductivity is generally anisotropic and depends on microarchitectural parameters of the cells [1].

Since the simplistic volumetric averaging approach is indifferent to cell architecture, it is unable to capture the anisotropic thermal properties of cellular materials and its prediction can significantly overestimate the effective thermal conductivity. To address these shortcomings, several analytical and computational methods have been developed, among which thermal-circuit method (or Resistor approach) has been widely used for the theoretical upper and lower bounds of thermal conductivity for a given cell geometry [4]. More advanced methods, e.g. computational homogenization, are required to exactly model the microarchitecture of cellular materials.

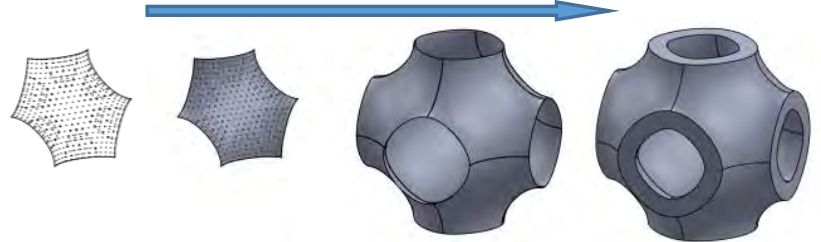

Figure 3. Steps for 3D modeling of a 3D shellular RVE

It is worth mentioning that the contribution of heat transfer mechanisms other than conduction, i.e. radiation and convection, is assumed to be small and consequently disregarded. For a cellular material made by a highly conductive matrix and an empty void or a gaseous inclusion, this assumption is valid for small pore sizes working at temperature ranges close to the ambient [34-37].

\section{A. Analytical model}

Using the analogy between thermal and electric fields, the thermal circuit method with parallel and series configurations is used to derive closed-from expressions for the upper and lower bounds of thermal conductivity of cellular materials with rectangular or elliptic inclusion/pore. In this approach, thermal gradient is analogous to electric voltage, the heat flow represents electric current and thermal resistance, being equal to the reciprocal of thermal conductivity for a unit cell, corresponds to electric resistance [25]. To establish this model, as presented in Fig. 5, the unit cell is divided into rectangular elements acting as thermal resistors, while the heat flux is considered to be along " $y$ " direction and perfect thermal contact is assumed between the matrix (solid cell walls) and the filler material (such as air for cellular materials).

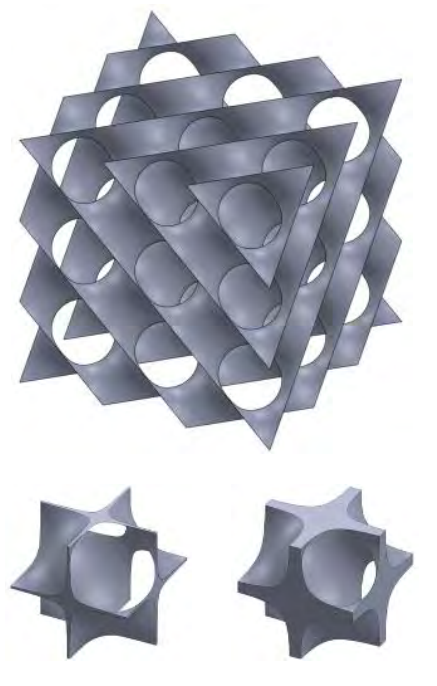

(a)

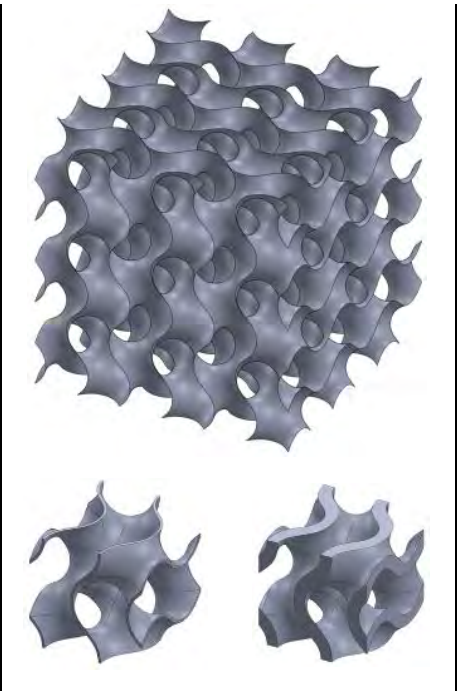

(b)

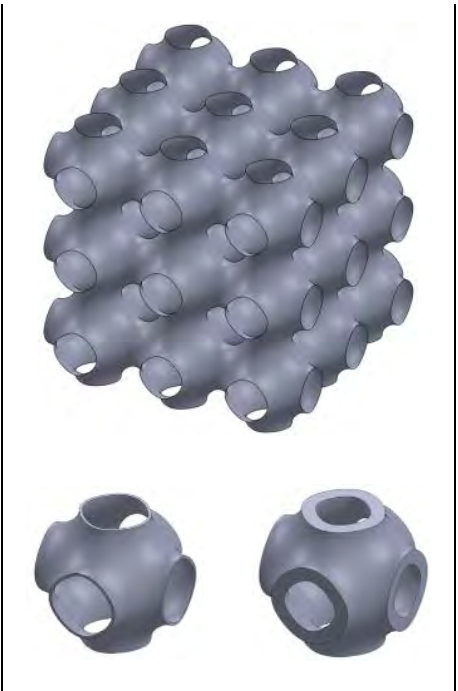

(c)
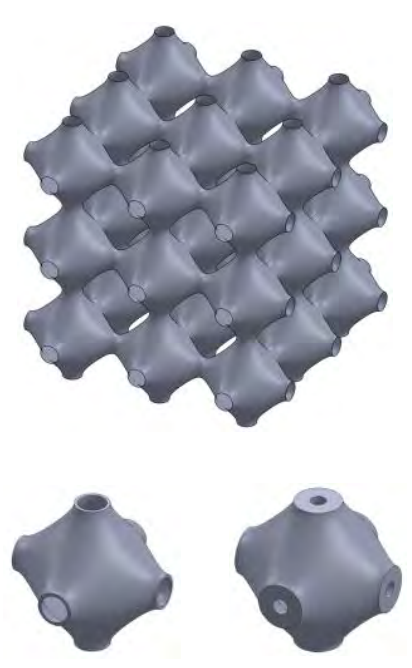

(d)

Figure 4. $3 \times 3 \times 3$ TPMS cells together with two shellular RVEs at 0.02 and 0.10 relative thicknesses: (a) Schwarz D, (b) Gyroid, (c) Schwarz P with $f=0$, and (d) Schwarz $P$ with $f=0.8$ 


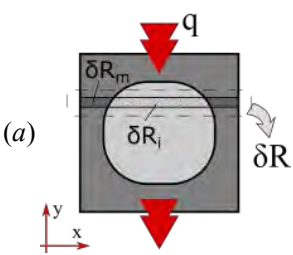

$$
\begin{gathered}
\delta R^{y}=\left(\frac{1}{\delta R_{m}^{y}}+\frac{1}{\delta R_{i}^{y}}\right)^{-1} \\
R^{y}=\sum \delta R^{y}
\end{gathered}
$$

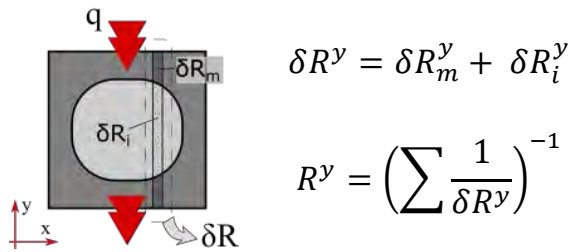

Figure 5. Schematic view of the thermal resistant elements for: (a) Horizontal iso-thermal lines and (b) Vertical adiabatic lines

Closed-form thermal conductivity formulations are presented in Table I, where $k_{m}$ and $k_{i}$ are the thermal conductivity of the matrix and inclusion respectively, $\lambda_{a}=a / l \quad, \quad \lambda_{b}=b / l \quad, \quad \lambda_{k}=k_{i} / k_{m}(<1)$, $\theta=\operatorname{atan}\left(\sqrt{\left(\left(1-\lambda_{k}\right) \lambda_{a}\right)^{-2}-1}\right.$ and $A=\lambda_{b}\left(1 / \lambda_{k}-1\right)$. It can be found that 'Vertical adiabatic lines' expressions for both rectangular and elliptic geometries reduce to $k_{\text {eff }}^{y y} / k_{m}=1-\lambda_{a}$ when $k_{i}=0$, which corresponds to cellular materials with empty pores. The relative density of the matrix can be calculated as $\left(\rho_{m}\right)_{r e l}=\left(l^{2}-a b\right) / l^{2}=1-\lambda_{a} \lambda_{b}$ and $\left(\rho_{m}\right)_{\text {rel }}=\left(l^{2}-\pi a b / 4\right) / l^{2}=1-\pi \lambda_{a} \lambda_{b} / 4$, for cells with rectangular and elliptic inclusion/pore, respectively. As the symmetry in the selected architectures of matrix and inclusion/pore dictates, the off-diagonal terms of the thermal conductivity tensor are zero $\left(K_{\text {eff }}^{x y}=0\right)$ and thermal conductivity in " $x$ " and " $y$ " are equal.

\section{B. Computational standard mechanics homogenization}

Under the assumption that an RVE of a periodic cellular material is repeated in all three directions and the RVE is far from the boundaries, the following periodic boundary conditions (4) together with independent unit thermal gradients (5) on the cell boundaries are adopted [23]:
Periodicity in $x$-direction:

$$
T\left(x_{0}, y, z\right)-T\left(x_{0}+l_{x}, y, z\right)=l_{x}\left(\frac{\partial T}{\partial x}\right)_{i}
$$

Periodicity in $y$-direction:

$$
T\left(x, y_{0}, z\right)-T\left(x, y_{0}+l_{y}, z\right)=l_{y}\left(\frac{\partial T}{\partial y}\right)_{i}
$$

Periodicity in $z$-direction:

$$
T\left(x, y, z_{0}\right)-T\left(x, y, z_{0}+l_{z}\right)=l_{z}\left(\frac{\partial T}{\partial z}\right)_{i}
$$

Independent unit thermal gradients:

$$
(\overline{\nabla T})_{i}=\left(\frac{\partial T}{\partial x}, \frac{\overline{\partial T}}{\partial y}, \overline{\partial T}\right)_{i z}= \begin{cases}(1,0,0), & i=1 \\ (0,1,0), & i=2 \\ (0,0,1), & i=3\end{cases}
$$

where $i$ is the thermal loading case number and $\mathrm{x}_{0}, \mathrm{y}_{0}$ and $\mathrm{z}_{0}$ are the locations of the three faces of the cubic RVE, while $\mathrm{l}_{\mathrm{x}}, \mathrm{l}_{\mathrm{y}}$ and $\mathrm{l}_{\mathrm{z}}$ represent RVE's dimensions along " $x$ ", " $y$ " and " $z$ " axis, and $\overline{\nabla T}$ is the average macroscopic thermal gradient applied to the RVE's boundaries in order to calculate microscopic thermal gradients inside the cell. Since the RVE is

TABLE I. CLOSED-FORM EXPRESSIONS FOR $k_{e f f}^{y y} / k_{m}$ OF CELLS MADE BY THERMALLY ISOTROPIC AND HOMOGENEOUS MATRIX AND FILLER MATERIALS, HAVING RECTANGULAR AND ELLIPTIC INCLUSION/PORE SHAPES, UNDER THE ASSUMPTIONS OF 'HORIZONTAL ISO-THERMAL LINES' OR 'VERTICAL ADIABATIC LINES'

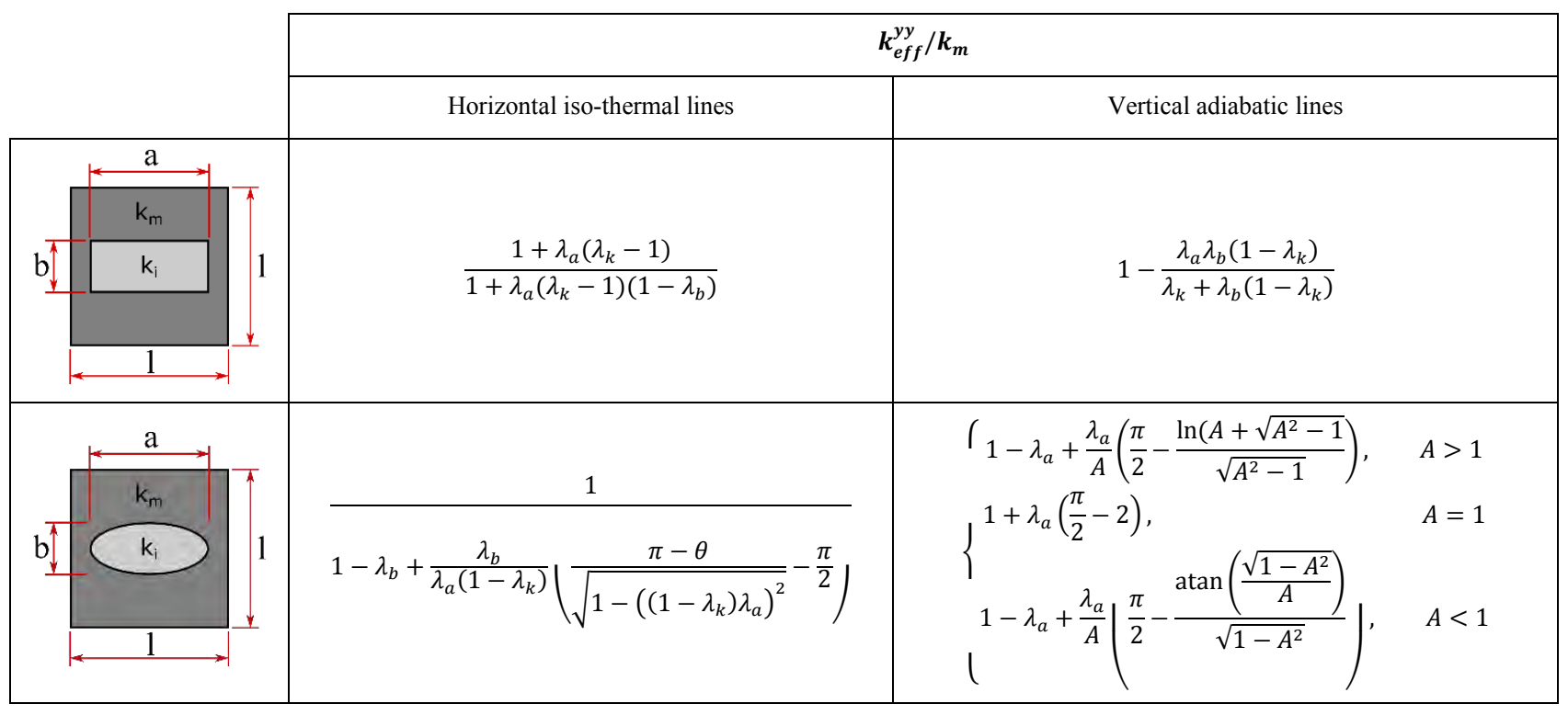


a unit square in two-dimensional (2D) analysis and a unit cube in three-dimensional (3D) analysis of this research, Eqs. (4) and (5) can be further simplified to:

$$
\left(\begin{array}{l}
\mathrm{T}\left(\mathrm{x}_{0}, \mathrm{y}, \mathrm{z}\right)-\mathrm{T}\left(\mathrm{x}_{0}+1, \mathrm{y}, \mathrm{z}\right) \\
\mathrm{T}\left(\mathrm{x}, \mathrm{y}_{0}, \mathrm{z}\right)-\mathrm{T}\left(\mathrm{x}, \mathrm{y}_{0}+1, \mathrm{z}\right) \\
\mathrm{T}\left(\mathrm{x}, \mathrm{y}, \mathrm{z}_{0}\right)-\mathrm{T}\left(\mathrm{x}, \mathrm{y}, \mathrm{z}_{0}+1\right)
\end{array}\right)_{\mathrm{i}}=\left(\begin{array}{l}
1 \\
0 \\
0
\end{array}\right)_{\mathrm{i}=1},\left(\begin{array}{l}
0 \\
1 \\
0
\end{array}\right)_{\mathrm{i}=2},\left(\begin{array}{l}
0 \\
0 \\
1
\end{array}\right)_{\mathrm{i}=3}
$$

In 2D analysis, " $z$ " dimension diminishes leading to two independent thermal loading cases. Energy balance equation, i.e. energy equation combined with Fourier heat conduction over the RVE, is solved using finite element method (FEM). The effective thermal conductivity of the cell can then be calculated by the volumetric averaging of the resultant heat flux based on standard mechanics homogenization:

$$
\bar{K}_{i j}=\frac{1}{V_{R V E}} \int K_{i k} M_{k j}^{T} d V_{R V E} \quad(i, j, k=1,2,3)
$$

where $\overline{\mathrm{K}}_{\mathrm{ij}}$ is the effective thermal conductivity tensor, $\mathrm{V}_{\mathrm{RVE}}$ represents the volume of the RVE, $K_{i k}$ is the local thermal conductivity tensor, and the matrix of $\mathrm{M}^{\mathrm{T}}$ relates the average and the local temperature gradients by $\nabla T=M^{T} \overline{\nabla T}$.

\section{RESULTS AND DISCUSSION}

To show the effect of filler material on the overall thermal conductivity and to examine the validity of neglecting the thermal conductivity of the gas inside the cellular materials, the homogenized thermal conductivity of a square cell with an empty square pore is benchmarked against the effective thermal conductivity of the same cell infilled with air at room temperature. As shown in Fig. 6, even for relative densities as low as $10 \%$, air's thermal conductivity can be neglected without affecting the effective thermal conductivity when the thermal conductivity of the matrix material is several orders of magnitude higher than the air, such as in metallic cellular materials.

Under the validated assumption of neglecting the air, derived closed form expression for the cells with rectangular and elliptical pore topologies are compared with the results obtained by standard mechanics homogenization. As illustrated in Fig. 7, for all aspect ratios of pores $(1,2$ and 3 in the horizontal direction), distances between higher and lower bounds of the effective thermal conductivity of the cells with elliptical pores are larger than those for the cells with rectangular pores. Moreover, by increasing the relative density, the gap between the theoretical bounds increases. In this case, although the pore geometry is accounted for deriving the theoretical upper and lower bounds, the simplifying assumptions made in deriving the closed form formula based on the thermal-circuit method make it impossible to accurately predict the effective thermal conductivity.

Close to the smallest possible relative density for each aspect ratio of pore, the effective thermal conductivity of cells with elliptical pores reveals sharper drops in comparison with cells with rectangular pores. This is partly because of the higher rate of decreasing of the minimum wall thickness with respect to decreasing in the relative density for the former topologies.

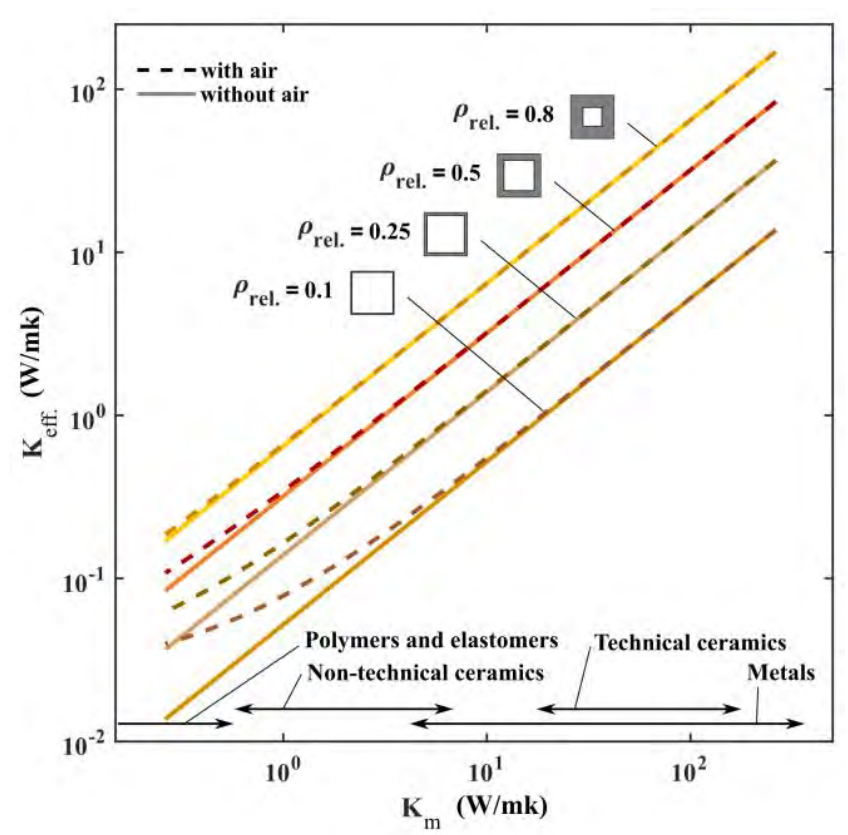

Figure 6. Effective thermal conductivity of air-filled ( $k_{\text {air }}=0.0263 \mathrm{~W} / \mathrm{m} . \mathrm{K}$ ) and empty $\left(k_{i}=0\right)$ cellular materials for a square pore shape versus thermal conductivity of the solid matrix (plotted in a log-log scale)

The standard mechanics homogenization is applied to cells with supershape pore topologies, while different aspect ratios (1 to 3 , with 0.5 increments) and rotations (generally from 0 to $90^{\circ}$ ) of the pore has been taken into account. The predicted in-plane effective thermal conductivities are generally anisotropic, therefore instead of $K_{x x}, K_{y y}$ and $K_{x y}$, the effective principal thermal conductivities are presented in Fig. 8. Sample relative densities are highlighted with different colors to show the achievable range of $K_{l}$ and $K_{2}$ by the chosen pore topologies. The large area of $K_{l}$ versus $K_{2}$ for each relative density allows the engineering of architected cellular materials, while keeping the weight constant.

Unlike most of the considered 2D pore geometries, the computational analysis shows that $\mathrm{P}, \mathrm{D}$ and $\mathrm{G}$ types of shellular materials are thermally isotropic and thus only one value is reported in Fig. 9 as the thermal conductivity for each microarchitecture. The $\mathrm{G}, \mathrm{D}$ and $\mathrm{P}$ shellular materials (when $f=$ 0 ) are found to have almost equal thermal conductivity at each relative density, which is in agreement with the findings in [38]; however, by increasing the value of $f$ for the $\mathrm{P}$ shellular materials, the effective thermal conductivity decreases.

\section{CONCLUDING REMARKS}

Theoretical and computational methodologies have been introduced in this paper for obtaining the effective thermal conductivity of architected cellular materials. We first examine the validity of neglecting the thermal conductivity of the air inside the pores of cellular materials when we determine the effective thermal conductivity of cellular materials. We then derive closed-form expressions for the upper and lower bounds of the in-plane effective thermal conductivity of cellular materials with rectangular and elliptic pores. The computational predictions of standard mechanics homogenization are also used 


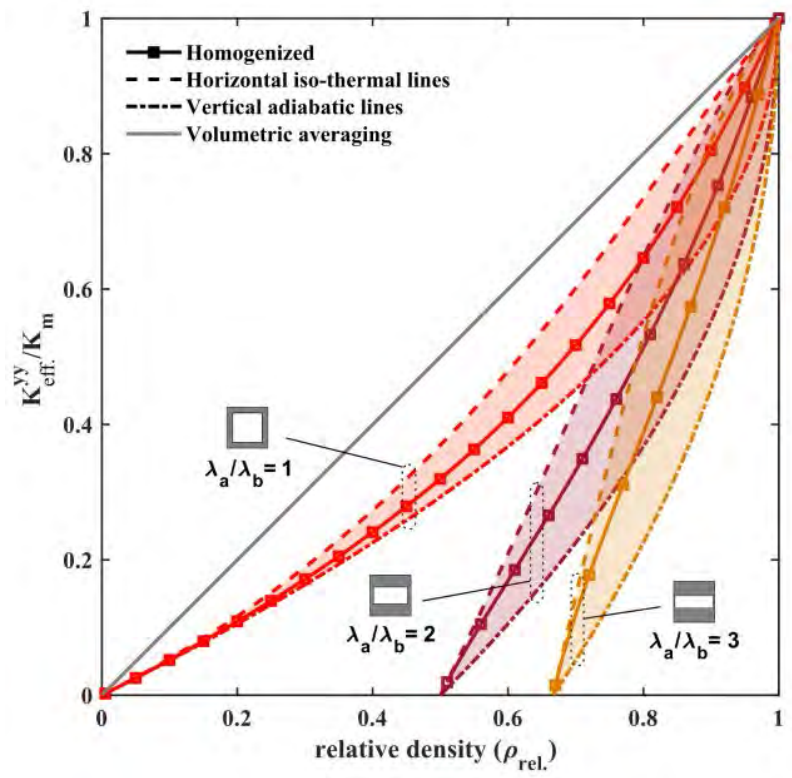

(a)

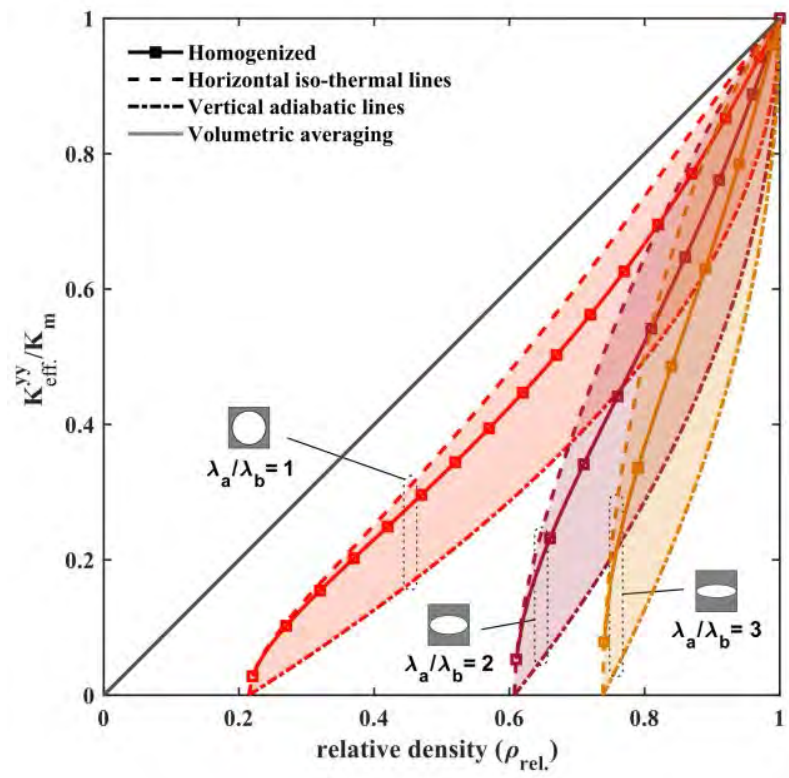

(b)

Figure 7. Theoretical upper and lower bounds along with computational homogenized values of effective thermal conductivity in " $y$ " direction, normalized by the thermal conductivity of the solid matrix: (a) Rectangular pore and (b) elliptic pore

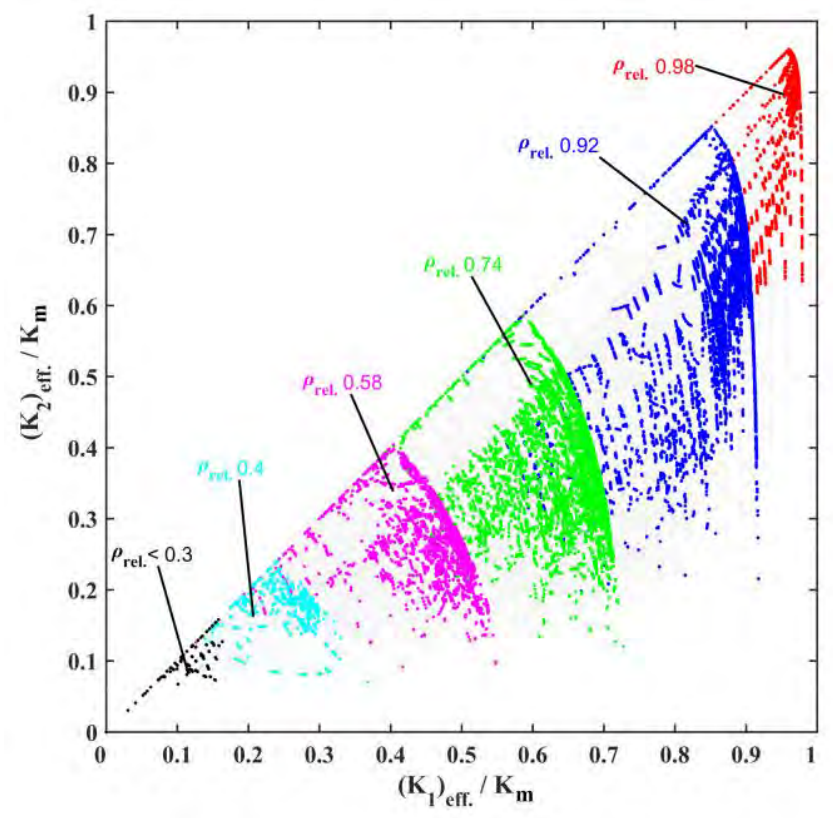

Figure 8. Normalized effective principal thermal conductivities, obtained using standard mechanics homogenization (for 2D supershape voids)

as benchmark for analyzing the effect of cell microarchitecture on effective thermal conductivity. The predictions show that increasing aspect ratio of the pores, widens the gap between the theoretical upper and lower bounds of thermal conductivity. Computational homogenized predictions are found to be close to the theoretical upper bound for small relative densities. The results present a wide range of achievable anisotropic effective thermal conductivity for different relative densities. Different TPMSs are also selected to create 3D shellular materials. For the considered range of relative density $\left(\rho_{\text {rel }}<25 \%\right), \mathrm{G}, \mathrm{D}$ and $\mathrm{P}$

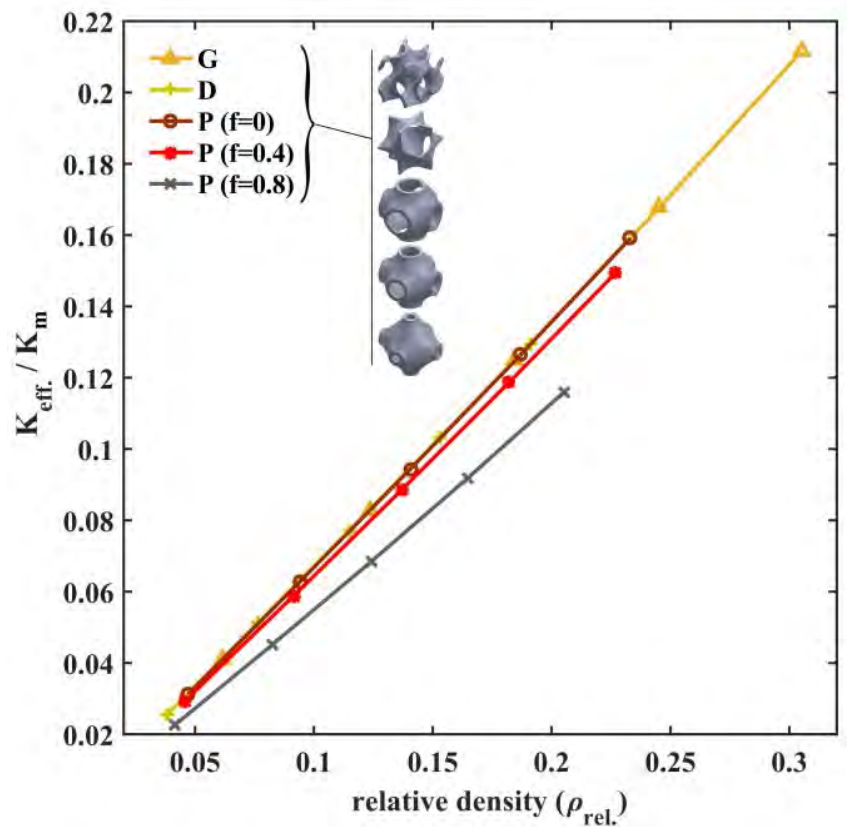

Figure 9. Effective thermal conductivity of shellular materials, normalized by thermal conductivity of the solid matrix

shellular materials with $f=0$, are found to have equal homogenized thermal conductivity. The methodology presented in this paper sheds light on the thermal application of lightweight and mechanically robust advanced cellular materials manufacturable by additive manufacturing technology.

\section{ACKNOWLEDGMENT}

A.H. Akbarzadeh acknowledges the financial support by McGill University and Natural Sciences and Engineering 
Research Council of Canada (NSERC) through NSERC Discovery Grant RGPIN-2016- 29 0471. The authors also acknowledge financial support by the Research Center for High Performance Polymer and Composite Systems (CREPEC).

\section{REFERENCES}

[1] A. Shafaroudi and S. Rankohi, "Multifunctional and Multiphysics Materials As Load-Bearing Structural Components," CSCE Annu. Conf., London, Jun. 2016. pp. 1-9. [Online]. Available: https://ir.lib.uwo.ca/ csce2016/London/Structural/35/.

[2] "L.J. Gibson and M.F. Ashby, Cellular solids: Structure \& properties, Oxford: Pergamon Press, ISBN: 0-08-036607-4, 1988, $357+$ ix pages, $\$ 35.00$," book review in Adv. Polym. Technol., vol. 9, no. 2, pp. 165-166, 1989. doi: 10.1002/adv.1989.060090207.

[3] A. Öchsner and T. Fiedler, "Effective Thermal Properties of HollowSphere Structures: A Finite Element Approach," in Cellular and Porous Materials: Thermal Properties Simulation and Prediction, Wiley-VCH Verlag GmbH \& Co. KGaA, pp. 31-71, Jan. 2008. doi: 10.1002/9783527621408.ch2.

[4] D. Baillis and R.M. Coquard, "Radiative and Conductive Thermal Properties of Foams,"in Cellular and Porous Materials: Thermal Properties Simulation and Prediction, Wiley-VCH Verlag GmbH \& Co. KGaA, pp. 343-384, Jan. 2008. doi: 10.1002/9783527621408.ch11.

[5] I.V. Belova and G.E. Murch, "Thermal Properties of Composite Materials and Porous Media: Lattice-Based Monte Carlo Approaches," in Cellular and Porous Materials: Thermal Properties Simulation and Prediction, WileyVCH Verlag GmbH \& Co. KGaA, pp. 73-95, Jan. 2008. doi: 10.1002/9783527621408.ch3.

[6] Belova, I.V., C. Vehyhl, T Fiedler, and G. E. Murch, "Analysis of anisotropic behaviour of thermal conductivity in cellular metals," Scr. Mater., vol. 65, no. 5, pp. 436-439, Sept. 2011.

doi: 10.1016/j.scriptamat.2011.05.029.

[7] T. Fiedler et al., "Determination of the thermal conductivity of periodic APM foam models," Int. J. Heat Mass Transfer, vol. 73, pp. 826-833, Jun. 2014. doi: 10.1016/j.ijheatmasstransfer.2014.02.056.

[8] Gasia, J., L. Miró and L.F. Cabeza, "Materials and system requirements of high temperature thermal energy storage systems: A review. Part 2: Thermal conductivity enhancement techniques," Renewable Sustainable Energy Rev., vol. 60, pp. 1584-1601, Jul. 2016.

doi: 10.1016/j.rser.2016.03.019.

[9] P. Kumar, F. Topin and J. Vicente, "Determination of effective thermal conductivity from geometrical properties: Application to open cell foams," Int. J. Therm. Sci., vol. 81, pp. 13-28, Jul. 2014.

doi: 10.1016/j.ijthermalsci.2014.02.005.

[10] H. Montazerian, E. Davoodi, M. Asadi-Eydivand, J. Kadkhodapour, M. Solati-Hashjin,"Porous scaffold internal architecture design based on minimal surfaces: A compromise between permeability and elastic properties," Materials \& Design, vol. 126, pp. 98-114, 2017. doi: 10.1016/j.matdes.2017.04.009.

[11] W. Wang, X. Yang, B. Han, Q. Zhang, X. Wang, T. Lu, "Analytical design of effective thermal conductivity for fluid-saturated prismatic cellular metal honeycombs," Theor. Appl. Mech. Lett., vol. 6, no. 2, pp. 69-75, Mar. 2016. doi: 10.1016/j.taml.2016.01.003.

[12] P .Ranut, "On the effective thermal conductivity of aluminum metal foams: Review and improvement of the available empirical and analytical models," Applied Thermal Engineering, vol. 101, no. 25, pp. 496-524, May 2016. doi: 10.1016/j.applthermaleng.2015.09.094.
[13] A. H. Akbarzadehab, J. W. Fu, L. Liu, Z. T. Chen, and D. Pasini, "Electrically conducting sandwich cylinder with a planar lattice core under prescribed eigenstrain and magnetic field," Composite Structures, vol. 153, pp. 632-644, Oct. 2016. doi: 10.1016/j.compstruct.2016.06.058.

[14] Z. Lu, Q. Wang, X. Li, and Z. Yang, "Elastic properties of two novel auxetic 3D cellular structures," Int. J. Solids Struct., vol. 24, pp. 46-56, 2017. doi: 10.1016/j.jijsolstr.2017.05.031.

[15] R. Courant and D. Hilbert, Methods of Mathematical Physics, WileyVCH Verlag GmbH., 2008, pp. I-XXII.

[16] R Singh, "Thermal Conduction Through Porous Systems," in Cellular and Porous Materials: Thermal Properties Simulation and Prediction, Wiley-VCH Verlag GmbH \& Co. KGaA, pp. 199-238, Jan. 2008. doi: 10.1002/9783527621408.ch7.

[17] T. H. Bauer, "A general analytical approach toward the thermal conductivity of porous media," Int. J. Heat Mass Transfer, vol.36, no. 17, pp. 4181-4191, Nov. 1993.doi: 10.1016/0017-9310(93)90080-P.

[18] D. Baillis and R. Coquard, "Radiative and Conductive Thermal Properties of Foams," in Cellular and Porous Materials: Thermal Properties Simulation and Prediction, Wiley-VCH Verlag GmbH \& Co. KGaA, pp. 343-384, Jan. 2008. doi: 10.1002/9783527621408.ch11

[19] L. Gong, Y. Wang, X. Cheng, R. Zhang, and H. Zhang, "A novel effective medium theory for modelling the thermal conductivity of porous materials," Int. J. Heat Mass Transfer, vol. 68, pp. 295-298, Jan. 2014. doi: 10.1016/j.ijheatmasstransfer.2013.09.043.

[20] Z.Hashin and S. Shtrikman, "A Variational Approach to the Theory of the Effective Magnetic Permeability of Multiphase Materials," J. App. Physics, vol. 44, no. 10, pp. 3125-3131, Jun. 2004.

doi: $10.1063 / 1.1728579$.

[21] T. Fiedler, I. V. Belova, A Rawson, and G. E. Murch, "Optimized Lattice Monte Carlo for thermal analysis of composites," Computational Mater. Sci., vol. 95, pp. 207-212, Dec. 2014. doi: 10.1016/j.commatsci.2014.07.029.

[22] A. T. Karttunen, J. N. Reddy and J. Romanoff, "Micropolar modeling approach for periodic sandwich beams," Compos. Struct., vol. 185, pp. 656-664, Feb. 2018. doi: 10.1016/j.compustruct.2017.11.064.

[23] S. J. Hollister and N. Kikuchi, "A comparison of homogenization and standard mechanics analyses for periodic porous composites," Comp. Mech., vol 10, no. 2, pp. 73-95, Mar. 1992. doi: $10.1007 / \mathrm{BF} 00369853$.

[24] N. S. Bakhvalov and G. Panasenko, Homogenisation: Averaging Processes in Periodic Media, Springer Science \& Business Media, 2012.

[25] F. P. Incropera, Fundamentals of Heat and Mass Transfer, John Wiley \& Sons, 2016.

[26] M. Matsuura, "Gielis' superformula and regular polygons," J. Geom., vol. 106, no. 2, pp. 383-403, 2015. doi: 10.1007/s00022-015-0269-z.

[27] A. L. Mackay, "Periodic minimal surfaces," Nature, vol. 314, p. 604, 1985. doi: 10.1038/314604a0.

[28] Y. Deng and M. Mieczkowski, "Three-dimensional periodic cubic membrane structure in the mitochondria of amoebaeChaos carolinensis," Protoplasma, vol. 203, no. 1, pp. 16-25, 1998. doi: $10.1007 / \mathrm{BF} 01280583$. 
[29] Z. Gan, M. D. Turner and M. Gu, "Biomimetic gyroid nanostructures exceeding their natural origins," $S c i$. $A d v$., vol. 2, no. 5, May 2016. doi: 10.1126/sciadv.1600084.

[30] J. Shin, S. Kim, D. Jeong, H. G. Lee, D. Lee, J Y. Lim, and J. Kim "Finite Element Analysis of Schwarz P Surface Pore Geometries for TissueEngineered Scaffolds," Math. Prop. Eng., vol. 2012. doi: $10.1155 / 2012 / 694194$

[31] S. C. Kapfer, S. T. Hyde, K. Mecke, C. H. Arns, andG E. SchröderTurk, "Minimal surface scaffold designs for tissue engineering," Biomater. vol. 43, no. 29, pp. 6875-6882, Oct. 2011.

doi: 10.1016/j.biomaterials.2011.06.012.

[32] J. Lei and Z. Liu, "The structural and mechanical properties of graphene aerogels based on Schwarz-surface-like graphene models." Carbon, vol. 130, pp. 741-748, 2018.

doi: 10.1016/j.carbon.2018.01.061.

[33] I. Maskery et al., "Insights into the mechanical properties of several triply periodic minimal surface lattice structures made by polymer additive manufacturing," Polymer, vol. 152, pp. 62-71, 2017.

doi: 10.1016/j.polymer.2017.11.049.

[34] P. G. Collishaw and J. R. G. Evans, "An Assessment of Expressions for the Apparent Thermal-Conductivity of Cellular Materials," J. Mater. Sci., vol. 29, no. 9, pp. 2261-2273, 1994. doi: 10.1007/BF00363413.

[35] T. Fiedler, I. V. Belova and G. E. Murch, "Theoretical and Lattice Monte Carlo analyses on thermal conduction in cellular metals," Compu. Mater. Sci., vol. 50, no. 2, pp. 503-509, 2010.

doi: 10.1016/j.commatsci.2010.09.011.

[36] L. Boldrin, F. Scarpa and R. Rajasekaran, "Thermal conductivities of iso-volume centre-symmetric honeycombs," Comp. Struct., vol. 113, no. 1, pp. 498-506, 2014. doi: 10.1016/j.compstruct.2014.03.013.

[37] W. Van De Walle and H. Janssen, "Validation of a 3D pore scale prediction model for the thermal conductivity of porous building materials," Energy Procedia, vol. 132, pp. 225-230, 2017.

doi: 10.1016/j.egypro.2017.09.759.

[38] D. W. Abueidda, R. K Abu Al-Rub, A. S. Dalaq, D.-W. Lee, K. A. Khan, and I. Jasiuk, "Effective conductivities and elastic moduli of novel foams with triply periodic minimal surfaces," Mech. Maters., vol. 95, pp. 102-115, Apr. 2016. doi: 10.1016/j.mechmat.2016.01.004. 


\title{
Displacement Flows of Viscoplastic Fluids in Near-Horizontal Channels: Numerical and Analytical Simulations
}

\author{
Ali Eslami, Roozbeh Mollaabbasi, Seyed Mohammad Taghavi \\ Department of Chemical Engineering, Laval University, Quebec, QC, Canada \\ Seyed-Mohammad.Taghavi@gch.ulaval.ca
}

\begin{abstract}
The miscible displacement flow of a viscoplastic fluid by a Newtonian fluid is studied numerically and analytically in a near-horizontal 2D channel. We focus on a density-unstable flow configuration where the displacing fluid is heavier than the displaced one with a laminar imposed flow. Using numerical and analytical approaches, we demonstrate that the channel inclination has the significant impacts on the viscoplastic flow morphology and front height. In addition, we study the yield stress impacts on the viscoplastic flow features.
\end{abstract}

Keywords-Displacement flow; viscoplastic fluid; inclination impacts, numerical and analytical approaches

\section{Introduction}

Many industrial processes require the cleaning and removal of a gelled-like material (i.e., typically viscoplastic) from a duct. For example, it can be mentioned oil \& gas well cementing, waxy crude oil pipeline restarts, gasassisted injection molding, biomedical applications (mucus, biofilms), cleaning of equipment and environmental surfaces, and food processing [2]. An import issue in these processes is the existence of the residual deposits. The aforementioned processes may have different fluid properties (e.g., different densities/viscosities and miscible/immiscible liquids) and flow geometries (e.g., pipe, annulus, channel). In this study, we numerically and analytically consider buoyant miscible, iso-viscous displacement flows of a viscoplastic fluid (displaced fluid) by a Newtonian one (displacing fluid) in a plane channel where the imposed flow is laminar.

Previously, we have investigated the impacts of three dimensionless numbers (i.e., the Bingham number, the densimetric Froude number and the Reynolds number) on the displacement of a viscoplastic fluid by a Newtonian fluid of higher density numerically [1] and analytically [4]; which covered various buoyancy effects from very small to very large Froude numbers. For brevity, we refer to these papers for a more detailed introduction and review of previous works. The main finding in the previous works was that the yield stress can lead to appear various flow regimes and have a significant effect on the displacing fluid front profile and residual layer thickness. The novelty of the current work is in studying impacts of channel inclination on the viscoplastic displacement flows at very small Froude number, through numerical and analytical approaches, which have not been studied so far.

\section{Governing Equations}

In this study the miscible displacement flow of a Bingham fluid (light fluid) by a Newtonian one (heavy fluid) along a channel is studied. These fluids have the same viscosity whereas their density are different. We choose Cartesian coordinates $(\hat{x}, \hat{y})$ with $\hat{x}$ representing the streamwise direction. ${ }^{1}$ The flow geometry and notation used in the current study is represented in Fig. 1. The governing dimensionless equations of motion are:

$$
[1+\phi A t] R e\left[\frac{\partial}{\partial t} \mathbf{u}+(\mathbf{u} . \nabla) \mathbf{u}\right]=
$$

\footnotetext{
${ }^{1}$ In this paper, we adopt the convention of denoting dimensional quantities with the symbol and dimensionless quantities without.
} 


$$
\begin{aligned}
-\nabla p+\nabla \cdot \tau & +\frac{\phi R e}{F r^{2}} \mathbf{e}_{g}, \\
\nabla \cdot \mathbf{u} & =0, \\
C_{t}+\mathbf{u} \cdot \nabla C & =\frac{1}{P e} \nabla^{2} C,
\end{aligned}
$$

where $p, \mathbf{u}$ and $\tau$ are the pressure, the velocity and the deviatoric stress. Here $\mathbf{e}_{g}=(\cos \beta,-\sin \beta)$ and the function $\phi(C)=2 C-1$ interpolates linearly between -1 and 1 for $C \in[0,1]$. No slip conditions are satisfied at the walls, the heavy fluid enters fully developed (plane Poiseuille profile) at $x=-L / 4$ (inlet) and outflow conditions are applied at $x=3 L / 4$ (outlet).

Five dimensionless numbers appear in (1-3) including: the Reynolds number $R e=\frac{\hat{V}_{0} \hat{D}}{\hat{\nu}}$ (here $\hat{D}$ is the channel width), Péclet number $P e=\frac{\hat{V}_{0} \hat{D}}{\hat{D}_{m}}$ (here $\hat{D}_{m}$ is the molecular diffusivity), densimetric Froude number $\mathrm{Fr}=$ $\frac{\hat{V}_{0}}{\sqrt{A t \hat{g} \hat{D}}}$, channel inclination $\beta$, Atwood number $A t=$ $\frac{\left(\hat{\rho}_{H}-\hat{\rho}_{L}\right)}{\left(\hat{\rho}_{H}+\hat{\rho}_{L}\right)}$ which represents a dimensionless density difference, where $\hat{\rho}_{H}$ and $\hat{\rho}_{L}$ are the density of Newtonian fluid and Bingham fluid, respectively. Since $\hat{\rho}_{H}>\hat{\rho}_{L}$ (i.e., the heavy fluid displaces the light fluid), $A t>0$ in this paper. It should be mentioned that displacement flows with small density differences were considered, for which the Boussinesq approximation is implemented. The density differences can remarkably affect buoyancy effects, captured by the densimetric Froude number. Finally, $P e \gg 1$ which means that on the timescale of interest, $\hat{D}_{m}$ does not play a major role in the flows studied.

The constitutive laws for Newtonian fluid (displacing fluid) is $\tau(\mathbf{u})=\dot{\gamma}(\mathbf{u})$ with $\dot{\gamma}=\nabla \mathbf{u}+(\nabla \mathbf{u})^{T}$ and for the Bingham fluid (displaced fluid) which includes a yield stress is:

$$
\begin{array}{r}
\tau_{2}(\mathbf{u})=\left[1+\frac{B n}{\dot{\gamma}(\mathbf{u})}\right] \dot{\gamma}(\mathbf{u}) \Leftrightarrow \tau_{2}(\mathbf{u})>B n, \\
\dot{\gamma}(\mathbf{u})=0 \Leftrightarrow \tau_{2}(\mathbf{u}) \leq B n,
\end{array}
$$

where the second invariants $(\dot{\gamma}(\mathbf{u}))$ and $\tau_{2}(\mathbf{u})$, are defined by:

$$
\begin{gathered}
\dot{\gamma}(\mathbf{u})=\left[\frac{1}{2} \sum_{i, j=1}^{2}\left[\dot{\gamma}_{i j}(\mathbf{u})\right]^{2}\right]^{1 / 2}, \\
\tau_{2}(\mathbf{u})=\left[\frac{1}{2} \sum_{i, j=1}^{2}\left[\tau_{2, i j}(\mathbf{u})\right]^{2}\right]^{1 / 2},
\end{gathered}
$$

where $B n$ is the Bingham number: $B n \equiv \frac{\hat{\tau}_{Y}}{\hat{\mu}_{1}\left[\hat{V}_{0} / \hat{D}_{0}\right]}$.

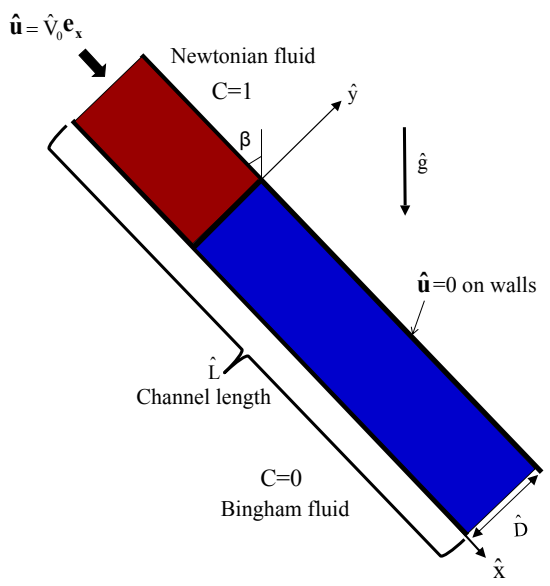

Figure 1: Schematic view of the numerical domain and initial flow configuration.

\section{Computational Method}

The equations of motion was discretized using a mixed finite element-finite volume method, using the classical augmented Lagrangian method of [3] to resolve unyielded zones. More details of the numerical approach are described in [5]. The present numerical algorithm was implemented in $\mathrm{C}++$ and solved using an open source platform, PELICANS. More details about employing this platform for numerical simulation of displacement flow are given in [6].

In the current simulation study, 63,000 mesh cells $(1500 \times$ 42) were used for all simulations while the meshes (regular rectangular) in the $y$-direction were refined slightly towards the channel walls. The initial interface between the two fluids was placed at distance $L / 4$ from the channel inlet in the computational domain which means that at $t=0$, the two fluids are separated by an imaginary gate valve located at $x=0$. It is worth noting that the dependency of results on the mesh size have been examined and also the results are validated which shows a great agreement with the previous model [1]. Note that the computational cost is high.

\section{Analytical Method}

In this part, a lubrication approximation model is developed to simplify the governing displacement flow equations and propose a semi-analytical solution for the heavy and light fluid flux functions to reduce the computational cost. Therefore, we scale our equations using a dimensionless length-scale, $\delta^{-1} \gg 1$, over which the interface elongates. We assume that $\delta$ has a size of $\hat{D} / \hat{L}$, in which $\hat{L}$ denotes the characteristic spreading length of the interface. Defining $\delta x=X, \delta t=T, \delta p=P, v=\delta V$ and following standard methods, we can now re-scale our equations to (assume that 
$\delta \rightarrow 0$ with fixed $R e$ ):

$$
\begin{aligned}
0 & =-\frac{\partial P}{\partial X}+\frac{\partial}{\partial y} \tau_{k, X y}+\frac{\partial}{\partial z} \tau_{k, X z} \pm \frac{\chi}{2}, \\
0 & =-\frac{\partial P}{\partial y} \mp \delta \frac{\chi}{2} \tan \beta, \\
0 & =-\frac{\partial P}{\partial z}
\end{aligned}
$$

where $\chi=\frac{2 R e \cos \beta}{F r^{2}}$. The interface is advected via a kinematic condition furnishing the dependence of the velocity on space and time:

$$
\frac{\partial h}{\partial T}+u \frac{\partial h}{\partial X}=V .
$$

Combining the incompressibility condition with the kinematic condition results in

$$
\frac{\partial h}{\partial T}+\frac{\partial q}{\partial X}=0,
$$

where $q$ is defined as:

$$
q=\int_{0}^{h} u d y .
$$

\section{Results}

In this section the computational results are presented. In a typical simulation, as time progresses, due to the imposed velocity, the heavy fluid layer penetrates into the light fluid layer and displaces it. Through the displacement flow process, different interesting flow patterns are formed at the interface between fluids.

Figure 2 shows the concentration maps of the numerical simulation as time evolves, run for $R e=400$ and $F r=$ 0.9 . The yield stress in the horizontal channel $\left(\beta=90^{\circ}\right)$ for the Fig. $2 \mathrm{a}$ and Fig. $2 \mathrm{~b}$ is different such that the yield stress for Fig. 2a is zero ( $B n=0$, Newtonian fluid) while for the Fig. $2 \mathrm{~b}$ is considerable ( $B n=0$, Bingham fluid). A nearly symmetric displacement flow is observed in Fig. 2a and the displacing finger is similar to a Poiseuille-like profile. At $B n=10$, the displacement flow remains symmetric and the front shape is similar to a plug-like profile (see Fig. 2b). The only difference between the Fig. 2b (horizontal channel) and Fig. $2 \mathrm{c}\left(\beta=88^{\circ}\right)$ is that the channel is more inclined with respect to vertical in the latter. Figure $2 \mathrm{~b}$ shows a qualitatively stable flow while in the Fig. $2 \mathrm{c}$ the yield stress of the displaced fluid has not been able to damp interfacial instabilities due to channel inclination.

Figure 3 presents examples of our simulations, for $\beta=$ $80^{\circ}, B n=50, R e=400$ and $F r=0.9$ at given time. Initially at $x=0$, the Newtonian fluid and Bingham one are completely separated. However, as time progresses, impacts of the imposed flow and the density ratio, leads to penetration of the heavy fluid through the light fluid and a)

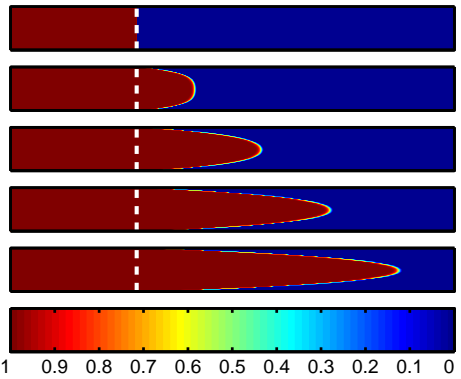

b)

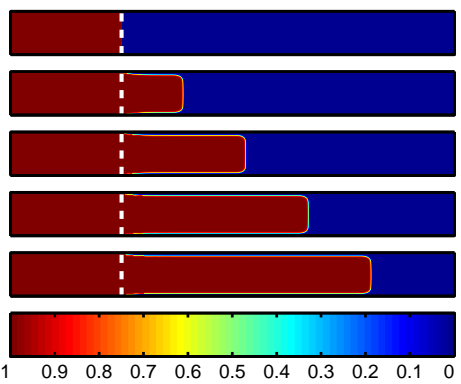

c)

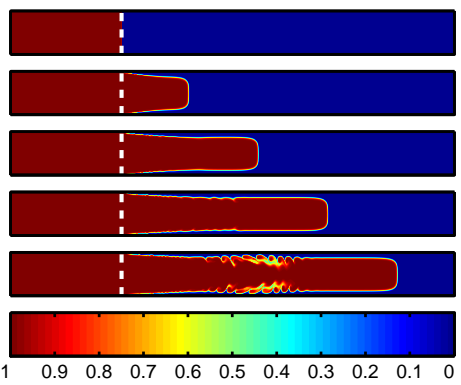

Figure 2: Concentration colormaps of the displacement flow at times $t=[0,8.75, \ldots, 35]$ for $R e=400$ and $F r=0.9$; a) $B n=0$ and $\beta=90^{\circ}$. b) $B n=10$ and $\beta=90^{\circ}$. c) $B n=10$ and $\beta=88^{\circ}$. The last image at the bottom of each subfigure is the colorbar of the concentration values. The white broken lines display the position of the initial interface $x=0$ (gate valve). The domain size shown is $1 \times 100$.

attempts to sweeps it. Figure. 3a depicts the concentration colormap of the displacement flow such that at longer spatial position with respect to the initial gate valve, the displaced layers adjacent to the walls become apparently static. Furthermore, it can be observed that the displacement flow is stable over a long time. The shear stress colormaps at the same time as the colormap of concentration is plotted in Fig. 3b, confirming that the stress field asymmetry results in asymmetric static layers on the top/bottom walls. Figure 3c displays the speed contours, $V=\sqrt{V_{x}^{2}+V_{y}^{2}}$, at the same simulation parameters as in Fig. 3a, where $V_{x}$ and $V_{y}$ are the stream-wise and depthwise velocity components, respectively. A Poiseuille profile is observed before 


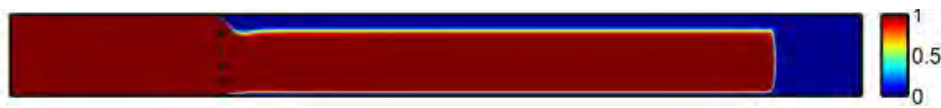

b)

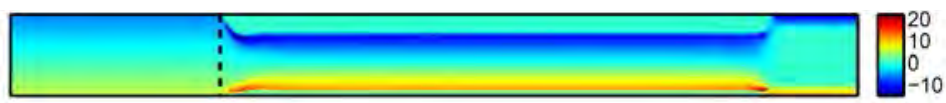

c)

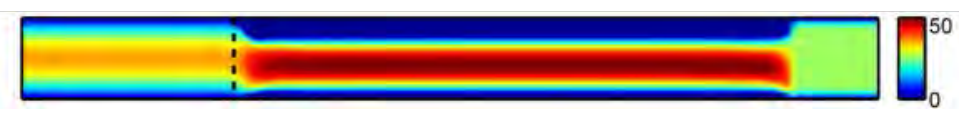

d)

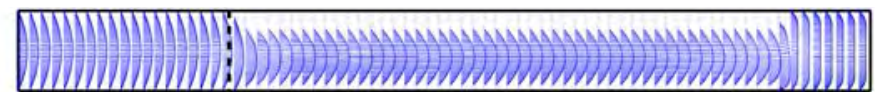

Figure 3: Computational results for $B n=50, \operatorname{Re}=400$ and $F r=0.9$ and $\beta=80^{\circ}$ at time $t=34$ : a) Concentration colormap; b) Shear stress colormap; c) Speed contours: $V=\sqrt{V_{x}^{2}+V_{y}^{2}}$. d) Velocity vectors. The black broken lines display the position of the initial interface $x=0$.

a)

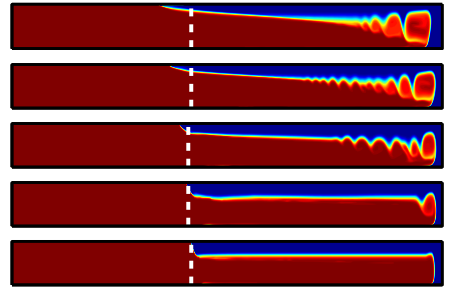

b)

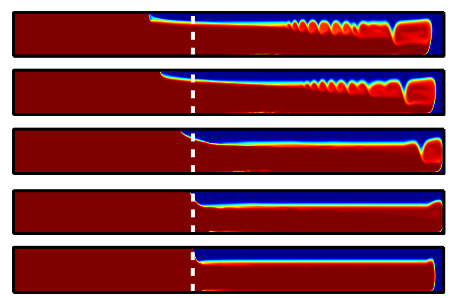

c)

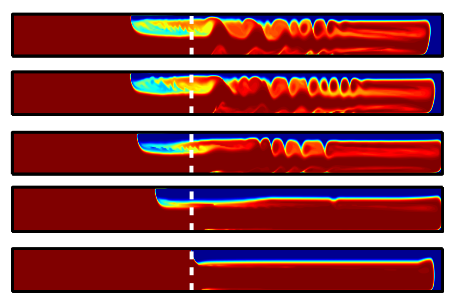

Figure 4: Panorama of concentration colormaps at $t=16, F r=0.5$ and $R e=400$ for a) $\beta=90^{\circ}$, b) $\beta=88^{\circ}$ and c) $\beta=82^{\circ}$. The rows from top to bottom show $B n=0, B n=5, B n=20, B n=50, B n=100$. The domain size shown is $1 \times 60$.

the gate valve location such that the high-speed regions remain towards the channel center. However, the speed contours are zero within the displaced fluid layers adjacent the upper and lower walls after the gate valve, which means that the remaining displaced layers are completely static. Figure $3 \mathrm{~d}$ shows the velocity vector fields along the channel length. This graph confirms that the flow is nearly stable and that the displaced fluid layer close to top wall is entirely static. It can be observed that the fluid within the displaced layer has a zero velocity.

The effects of yield stress value and channel inclination for $R e=400$ and $F r=0.5$ are presented in Fig. 4. Figure $4 \mathrm{a}, \mathrm{b}$ and $\mathrm{c}$ show panoramas of the concentration colormaps at $t=16$ for $\beta=90^{\circ}, \beta=88^{\circ}$ and $\beta=82^{\circ}$, respectively. As seen, for a given channel inclination, by increasing the dimensionless yield stress, the flow morphology changes remarkably. For example, at $\beta=88^{\circ}$, the interfacial instabilities decrease by increasing the Bingham number such that the yield stress can damp the interfacial waves. Moreover, the trailing front has an indirect relation with $B n$. In other words, at small yield stress the trailing front moves upwards, while at larger Bingham number, the moving of the trailing front completely stops. It also can be seen, at fixed $B n$, by increasing the channel inclination the flow becomes more unstable and trailing front shows different behavior. Finally, it can be conclude that from Fig. 4 and Fig. 2, at large Froude number, the displacement flow is nearly symmetric which means that the displacing fluid moves more or less along the center of the channel. However, at small $F r$, the displacement flow is asymmetric; displacing finger advances approximately near the lower wall of the channel.

The analytical results are presented in Fig. 5 and Fig. 6. Figure 5 shows the dependency of the displacing front height to yield stress. By increasing the yield stress of displacing fluid the front hight decreases and the static layer increases.

Figure 6 shows the effect of increasing the buoyancy force $(\chi)$ in the heavy-light fluids displacement. Increasing the inclination of the channel reinforces the buoyancy force therefore, the buoyancy overcomes the yield stress and the back flow is observed in Fig. 6c. Our results show that the analytical approach is also generally able to predict the variation of flow morphology with yield stress and channel inclination in the viscoplastic fluid displacement with low computational cost. 

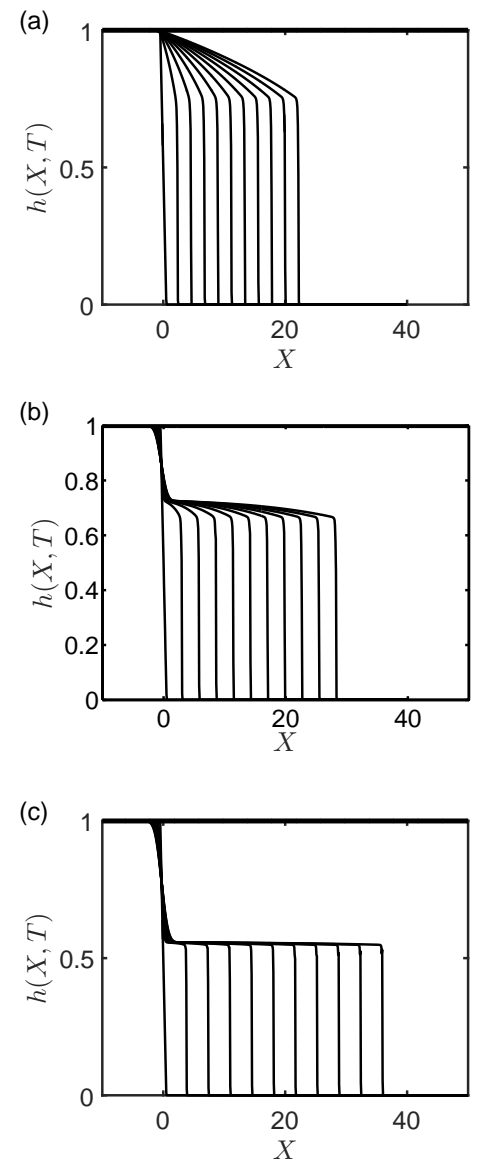

Figure 5: Interface evolution in time, $T=0,2, \ldots ., 18,20 . \beta$, $\mathrm{Fr}$ and $\operatorname{Re}$ are $90^{\circ}, 0.5$ and 400 respectively in all figures. $B n$ number is 0,5 and 50 from up to down. The displacing fluid is Newtonian.

\section{Conclusion}

In this study, we have numerically and analytically considered a fascinating displacement flow where the heavier displacing fluid (Newtonian fluid) pushes the lighter displaced fluid (viscoplastic fluid) along a 2D plane channel. During the miscible displacement flow, the results demonstrate that the variation of channel inclination and yield stress has the remarkable impacts on the flow morphology. Also the results show that the yield stress (at larger Bingham number) damps the interfacial instabilities. The viscoplastic displacement flows, at given $B n$, for more channel inclination can be more unstable.

\section{References}

[1] A. Eslami, I. A. Frigaard, and S. M. Taghavi, "Viscoplastic fluid displacement flows in horizontal channels: Numerical simulations," J. non-Newt. Fluid Mech., vol. 249, pp. 79-96, Nov. 2017. doi: 10.1016/j.jnnfm.2017.10.001.
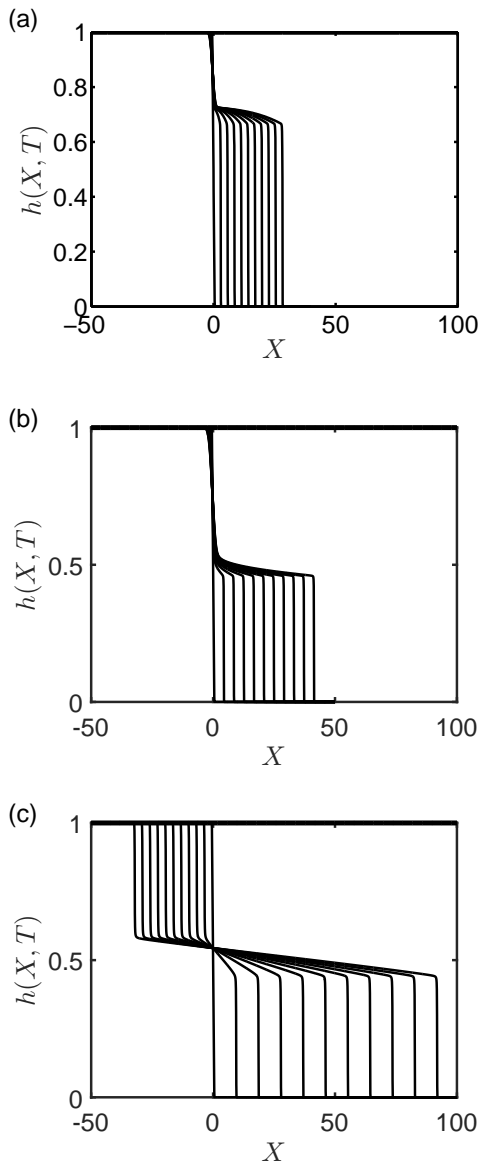

Figure 6: Interface evolution in time, $T=0,2, \ldots ., 18,20$. $B n, F r$ and $R e$ are 20, 0.5 and 400 respectively in all figures. $\beta$ is $90^{\circ}, 88^{\circ}$ and $82^{\circ}$ from up to down. The displacing fluid is Newtonian.

[2] A. Eslami and S. M. Taghavi, "Viscous fingering regimes in elasto-visco-plastic fluids," J. non-Newt. Fluid Mech., vol. 243, pp. 79-94, May 2017. doi: 10.1016/j.jnnfm.2017.03.007.

[3] R. Glowinski and P. Le Tallec, "Augmented Lagrangian and operator-splitting methods in nonlinear mechanics," Phys. Fluids, vol. 19, p. 084106, 2007. doi: 10.1137/1.9781611970838.

[4] R. Mollaabbasi and S. M. Taghavi, "Buoyant displacement flows in slightly non-uniform channels,"J. Fluid Mech., vol. 795. pp. 876913, Apr. 2016. doi: 10.1017/jfm.2016.232.

[5] A. Roustaei, "Yield stress fluid flows in uneven geometries: applications to the oil and gas industry," PhD dissertation, University of British Columbia, Vancouver, BC, 2016.

[6] S. M. Taghavi et al., "Stationary residual layers in buoyant Newtonian displacement flows," Phys. Fluids, vol. 23, no. 4, p. 044105, Apr. 2011. doi: 10.1063/1.3581063. 


\section{Development and Validation of a Numerical Model for Vibration of Power Lines}

\author{
Mohammad Hadi Jalali \\ Faculty of Engineering and Applied Science \\ Memorial University \\ St. John's, Canada \\ mjalalinodou@mun.ca
}

\author{
Geoff Rideout \\ Faculty of Engineering and Applied Science \\ Memorial University \\ St. John's, Canada \\ gdrideout@mun.ca
}

\begin{abstract}
Modal testing is being investigated as a means of non-destructive evaluation of wooden utility pole strength. In order to understand the effects of conductors on the dynamics of the poles, a numerically efficient model based on lumped segments for the conductor has been developed and experimentally validated. The cable is modeled as number of lumped segments jointed with axial and torsional springs and dampers representing the cable's compliance and damping. In order to validate the models, an experimental set up for vibration testing of the cable has been built. Time response measurement and modal testing are performed and the comparison of the experimental results with the numerical results show that the lumped segment model has the fidelity to capture the dynamics of the cables efficiently and accurately.
\end{abstract}

Keywords; Cable dynamics; Lumped segment model; Modal testing; Bond graph model

\section{INTRODUCTION}

Wooden utility poles are extensively used in North America for electrical power transmission and distribution. These wooden poles are subject to deterioration due to aging, rot, woodpecker damage, and fungal attack. Developing a nondestructive pole evaluation method based on modal testing requires numerical modeling of the poles and attached cables (conductors). Because cables are attached to the utility poles, vibrations of the cables affect the measurement of the modal properties of the poles. Therefore, a numerically efficient model should be developed to capture the dynamics of the cables.

Barry et. al. [1] investigated the vibrations of a single transmission line conductor with attached Stockbridge damper. They modeled the system as a double-beam system and derived the equations of motion of the system using Hamilton's principle. They validated the analytical results with experimental results and investigated the effect of damper location and characteristics on the conductor natural frequencies. Ricciardi et al. [2] developed a continuous model for vibration analysis of cables with sag considering the bending stiffness. They derived the vibration equation of motion of a sagged cable as an Euler-Bernoulli beam and solved the equation by an exact method. They performed a parametric study to investigate the effect of sag and bending stiffness on the cable natural frequencies and validated the results using finite element (FE) and finite difference methods. The exact method that they used could not be used when the cable is attached to a pole or moving support structure. Barbieri et. al. [3] used linear and nonlinear finite element models for analyzing the dynamic behavior of three different transmission line cables. They validated the numerical results by experimental tests and investigated the effect of inclusion of Stockbridge damper and linearity of the FE model on the natural frequencies. They concluded that linear finite element models provide good results for short cables only. Li et. al [4] and Wang et. al [5] presented a simplified computational model of a high-voltage transmission tower-line system under out-ofplane and in-plane vibrations due to seismic excitations. The transmission cables and their supporting towers were modeled as a lumped mass system. They did not consider the bending stiffness of conductors and their model was a simplified model for obtaining the response to seismic excitations. They obtained the mass and stiffness matrices of the coupled system and derived the linear equations of motion and validated the theoretical results with experiments. Papailiou [6] for the first time proposed a model for conductors that took into account the interlayer friction and the interlayer slip in the conductor during bending, obtaining a bending stiffness that changes with the bending displacement and the tension applied to the conductor. Spak et. al. [7] reviewed helical cable models with a focus on cable damping modeling. They described the work done in the literature on the inclusion of damping through frictional effects, variable bending stiffness, and internal friction. Spak et. al. [8, 9] developed the distributed transfer function method to model cables and simple cabled structures. They included shear effects, tension, and hysteretic damping for modeling of helical stranded cables and investigated the effect of cables on the dynamics of cabled structures using the developed model and by experimental tests. Pinto et. al [10] developed a bond graph model for a wooden pole with a cable attached to the free end. In their paper, the cable was modeled as a series of point masses connected by translational springs and the pole was represented 
by a modal expansion based on separation of variables. They obtained the modal parameters of the cable and pole-cable systems numerically and experimentally. They concluded that their model should be expanded and improved from point masses to rigid bodies with rotational inertia, connected with bending stiffness and damping elements as well as axial ones.

The bond graph formalism, details of which can be found in [11] is chosen because of the ease with which it allows expansion of the model by adding segments in a modular way. Bond graphs, which use a small set of generalized elements to model multi-domain systems, facilitate connection of the cable model with other subsystem models such as poles, regardless of whether the poles are modeled using lumped segments or modal expansion.

This paper consists of two main sections. In Section II, a bond graph lumped segment model of the cable is presented. In this model, the cable is modeled as number of segments that are attached to each other by springs and dampers representing the axial and bending compliances and damping of the cable, respectively. Section III describes the experimental set up and procedure that is used for performing time series measurement and modal testing of the stranded cable. The modeling assumptions and the results are explained in Section IV.

\section{LUMPED SEGMENT MODEL}

\section{A. Theoretical Development}

In this paper, the cable is modeled as a pinned-pinned beam divided into number of segments. A numerical solution is obtained by a lumped segment approximation that captures axial and bending motions. To the purpose of modeling, two types of coordinate systems are used in the model. The first coordinate system is the inertial coordinate system. A body-fixed coordinate system is attached to each segment, the origin of which is located at the center of gravity and the local $x$ axis of which is in the axial direction. The advantages of using body coordinate system are that axial springs between the segments can be modeled simply along the local coordinates, and that orientation angles and inertia properties are more easily handled if the model is expanded to three dimensions. Figure 1 shows representative segments of the cable, and local and global coordinate systems.

The cable is represented by a series of rigid cylinders of equal length and cross section; each rigid body has the same properties as the corresponding portion of the cable. As the number of segments approaches infinity, the behavior of the lumped segment model will converge to that of the continuous cable. The length of each segment is defined by the number of rigid bodies, $n$. For each segment, three points are defined: $B$ in the left end of the segment, $A$ in the right end and $G$ in the center of mass. Point $A$ of element $i$ and point $B$ of element $i+1$ are linked by one torsional and two linear springs. The torsional spring represents the bending compliance or flexural rigidity of the beam in the $x-y$ plane, and the linear springs in $x$ and $y$ local directions represent the axial and shear compliance respectively. Each spring is complemented with a damper acting in parallel to it that acts as the cable material damping. Figure 1 shows the connection of each segment to the next and previous segments using torsional and linear springs, also illustrates the body-fixed coordinate system. For simplicity purposes, the dampers are not shown in the figure.

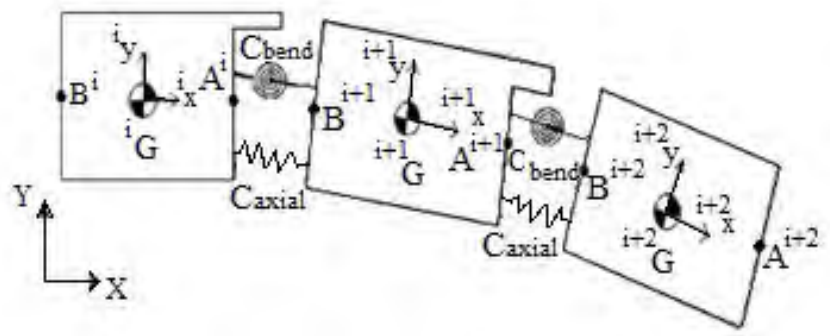

Figure 1. Multibody dynamic model

\section{Cable Compliance:}

Equations (1)-(2) present the axial and bending compliance of the cable, respectively [12].

$$
\begin{aligned}
C_{\text {axial }} & =\frac{E A}{l} \\
C_{\text {bend }} & =\frac{E I}{l}
\end{aligned}
$$

where:

$E=$ Young's modulus of beam

$A=$ Cross sectional area of the cable

$l=$ Length of the cable segment

$I=$ Area moment of inertia of the cross section

\section{Cable Inertia:}

The translational inertia of the lumped segments is equal to their mass. The rotational inertia of a cylinder about the axis perpendicular to the body-fixed coordinate plane, through the center of mass $G$, is as follows.

$$
J_{G}=1 / 12 m\left(3 / 4 d^{2}+l^{2}\right)
$$

Where $m$ is mass of a segment, $d$ is diameter of cable cross section, and $l$ is segment length.

\section{Cable Damping:}

Damping in the cable is due to the material damping associated with the hysteresis energy losses in the material and interlayer wire slip. Because the stranded cable consists of number of layers, the layers can slip on each other during vibration and this interlayer friction increases the damping in the stranded cables. In this paper, axial and bending dampers are placed in parallel with the springs in the model. The damper values are tuned to give decay of the response and natural frequencies close to experimental results. Further work should be done in the future to model the damping in the numerical model more accurately. 


\section{B. Kinematics of Rigid Bodies}

In the bond graph model, we need to develop the velocity relations between points $G$ and $A$ and $B$ in Figure 1. Eq'ns (4)(5) represent the velocity of end points $A$ and $B$ in the local coordinate of the segment, respectively.

$$
\begin{gathered}
{ }^{i} \vec{V}_{A}=\left[\begin{array}{c}
V_{A x} \\
V_{A y}
\end{array}\right]={ }^{i} \vec{V}_{G}+{ }^{i} \vec{V}_{A / G}=\left[\begin{array}{c}
V_{G x} \\
V_{G y}
\end{array}\right]+\left[\begin{array}{c}
0 \\
A G \omega
\end{array}\right] \\
{ }^{i} \vec{V}_{B}={ }^{i}\left[\begin{array}{c}
V_{B x} \\
V_{B y}
\end{array}\right]={ }^{i} \vec{V}_{G}+{ }^{i} \vec{V}_{B / G}={ }^{i}\left[\begin{array}{c}
V_{G x} \\
V_{G y}
\end{array}\right]+\left[\begin{array}{c}
0 \\
-B G \omega
\end{array}\right]
\end{gathered}
$$

where

${ }^{i} \vec{V}_{A / G},{ }^{i} \vec{V}_{B / G}=$ relative velocity of points A and B with respect to $G$

$\omega=$ angular velocity of segment

Positive rotational speed of the segment is considered counterclockwise. Having defined the endpoint velocities, their relative motions are constrained by axial and bending springs and dampers. The velocity of center of mass $(G)$ of each segment, while defined in the local coordinate system, must be transformed to the inertial coordinate system in order to apply gravity, and to allow system initialization through a static vertical displacement of a point on the cable, which is then released to create free vibration. The coordinate transformation for velocity of point $G$ is as follows.

$$
{ }^{I} \vec{V}_{G}=\left[\begin{array}{c}
V_{G x} \\
V_{G y}
\end{array}\right]=\left[\begin{array}{cc}
\cos \theta & -\sin \theta \\
\sin \theta & \cos \theta
\end{array}\right]{ }^{i} \vec{V}_{G}
$$

where $\theta=$ angle of rotation of segment $i$.

Also, because the end point velocities of each segment need to be related, both end point velocities should be expressed in the same frame, in this case the local coordinate of the segment to the right. Eq. (7) represents the coordinate transformation relation between two connected sections.

$$
{ }^{i+1}\left[\begin{array}{l}
V_{A x} \\
V_{A y}
\end{array}\right]=\left[\begin{array}{cc}
\cos \left(\theta_{i+1}-\theta_{i}\right) & \sin \left(\theta_{i+1}-\theta_{i}\right) \\
-\sin \left(\theta_{i+1}-\theta_{i}\right) & \cos \left(\theta_{i+1}-\theta_{i}\right)
\end{array}\right]^{i}\left[\begin{array}{l}
V_{A x} \\
V_{A y}
\end{array}\right]
$$

Eq. (8) represents Newton's Law for the segment in local coordinates.

$$
{ }^{i} \sum \vec{F}=\frac{d}{d t}{ }^{i} \vec{P}={ }^{i}\left[\begin{array}{l}
m \dot{v}_{x} \\
m \dot{v}_{y}
\end{array}\right]+\vec{\omega} \times\left[\begin{array}{l}
m v_{x} \\
m v_{y}
\end{array}\right]=\left[\begin{array}{l}
m \dot{v}_{x} \\
m \dot{v}_{y}
\end{array}\right]+\left[\begin{array}{c}
-m \omega v_{y} \\
m \omega v_{x}
\end{array}\right]
$$

The first term on the right-hand side is modeled with generalized inertias in the bond graph, and the second term using modulated gyrators.

\section{Bond Graph Model}

The bond graph model of the cable consists of 30 segments, which is enough to produce the first few frequencies with reasonable accuracy. Each segment is connected to the next segment with a connection sub-model and each connection sub -model contains the compliance and damping bond graph elements. Figure 2 shows the bond graph model of a segment of the cable. A bond graph 1-junction represents a generalized Kirchoff loop law, with all bonded elements having the same flow, and efforts algebraically summing to zero. A bond graph 0 -junction represents a generalized node law, with all bonded elements having the same effort. The 0 -junction also sums velocities of bonded elements to zero, making it the element used to enforce velocity constraints. As can be seen from Figure 2 , there are two modulated transformers (MTF), one for each row of Eq. (6), two MTF's for defining Eq'ns (4) and (5) and one modulated gyrator (MGY) for defining the Euler's Equations inner product terms of Eq. (8). The power bonds (with half-arrows) contain both force and velocity information. In Figure 2 sample junction equations are given. The 1-junction representing Newton's Law in the $x$ direction sums forces, including constraint forces from adjoining elements that are on the same bonds that communicate velocity of points A and B to adjoining elements. Left superscripts indicate the local $(i)$ or inertial (0) frame. Figure 3 shows the bond graph of one connection sub-model between two adjoining segments. As can be seen in Figure 3, there are four MTF's for defining coordinate transformation Eq. (7). The top row of the bond graph represents the velocities and elements in the $x$ and $y$ directions and the most bottom row represents the bending compliance.

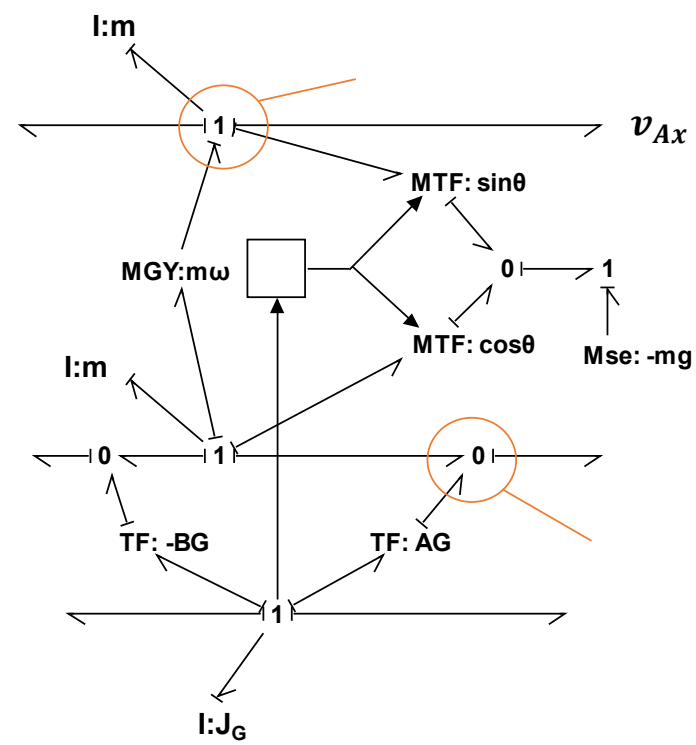

Figure 2. Bond Graph Model of a Segment of the Cable 
TABle 1. MAterial Properties of CABLE

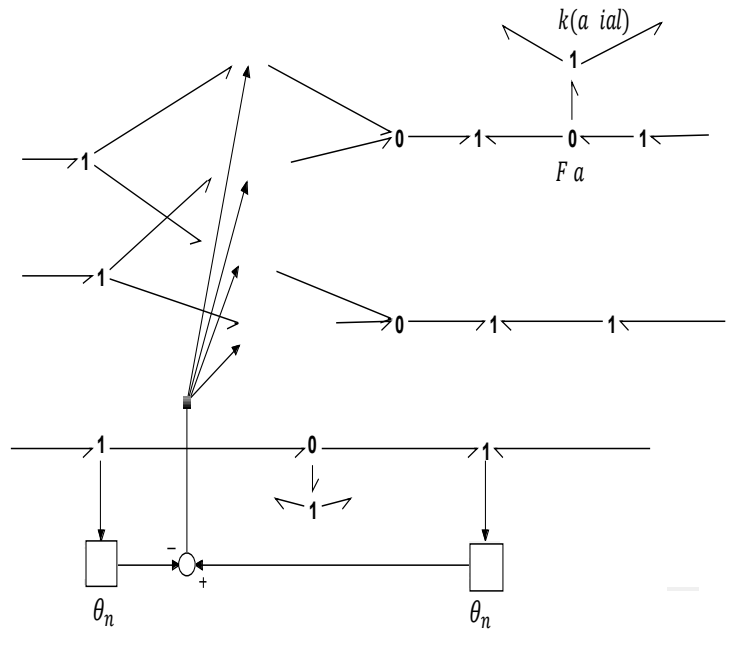

Figure 3. Bond graph model of complaint connection between consequent segments

In Figure 3 , the 0 -junction $F_{\text {axial }}$ defines the velocity $v_{k \text { (axial) }}$ as the difference between $v_{B x}$ on segment $i+1$ and $v_{A x}$ on segment $i$ defined in the $i+1$ coordinate system. The 1junction equates the velocities of the parallel axial spring and damper. The bending spring/damper velocity is defined similarly. The block diagram elements define the relative angle between segments for the velocity transformation.

Point B of the left-most segment and Point A of the rightmost segment are attached to the wall and therefore have zero velocity. Thus, two zero flow sources are used at each point in the $x$ and $y$ directions. In order to create the desired initial tension in the cable, the model is given zero initial conditions and then stretched until the desired tension is achieved. In this paper, four values of tension are considered for the analysis. For applying the stretching displacement to the right end of the cable in bond graph model, a flow source was temporarily applied to the 1-junction associated with the velocity of the right end of the cable in local $x$ direction. After creating the desired tension, the zero flow source was reinstated at the right end. The cable was then excited, with results of the bond graph model compared to experimental results.

\section{EXPERIMENTAL VIBRATION TESTING OF THE CABLE}

\section{A. Cable Bending Stiffness Measurement}

The material properties of the conductor used for testing are tabulated in Table 1. The bending stiffness $(E I)$ of the cable should be measured as it is stranded cable and its bending stiffness is not equal to the bending stiffness of solid cable with the same diameter [6]. Figure. 4 shows the setup used for measuring the bending stiffness $(E I)$ and consequently area moment of inertia $(I)$ of the cable.

\begin{tabular}{|c|c|c|c|c|}
\hline Material & $\begin{array}{c}\text { Length } \\
{[\mathrm{m}]}\end{array}$ & $\begin{array}{c}\text { Density } \\
{\left[\mathrm{kg} / \mathrm{m}^{3}\right]}\end{array}$ & $E[\mathrm{GPa}]$ & $\begin{array}{c}\text { Area } \\
{\left[\mathrm{m}^{2}\right]}\end{array}$ \\
\hline $\begin{array}{c}\text { Stainless } \\
\text { Steel 7× } \\
7 \text { wires }\end{array}$ & 3.6 & 9537 & 195 & $2.78 \times 10^{-5}$ \\
\hline
\end{tabular}

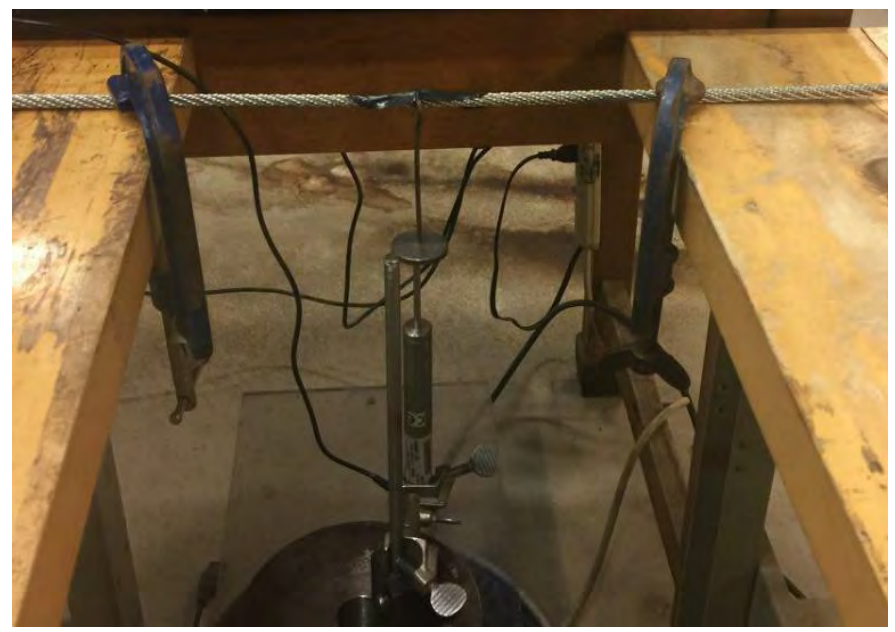

Figure 4. Set up for Bending Stiffness Measurement

A section of the cable is clamped between two ends and a fixedfixed configuration is made. Various weights are hung in the middle of the cable section and a LDVT is used to measure the deflection of the cable at midspan. By using standard forcedeflection formulae for a fixed-fixed beam with force applied at midspan, the bending stiffness of the cable is obtained [13]. It should be noted that the value of measured $E I$ from bending test is between theoretical minimum and maximum bending stiffness bounds for the stranded cables. $E I_{\min }$ corresponds to when all the layers of the cable slip on each other and $E I_{\max }$ is for all the wires are bonded and make a solid cable. The details of the theoretical bounds for bending stiffness can be found in [6]. Bending of the cable in a cantilever configuration was also performed with different lengths of the cable and the results were the same, which shows that the bending stiffness of the cable is not sensitive to length, over the range of segment lengths considered in this work. A measured constant value for $E I$ is directly used in the bond graph model in numerical analyses. Table 2 presents the value of $E I$ for three different lengths of cable sections.

TABLE 2. VALUE OF MEASURED EI WITH DIFFERENT LENGTHS

\begin{tabular}{|c|c|}
\hline Length $(\mathrm{mm})$ & $E I\left(\mathrm{Nm}^{2}\right)$ \\
\hline 205 & 0.555 \\
\hline 364 & 0.444 \\
\hline 400 & 0.547 \\
\hline
\end{tabular}




\section{B. Experimental Modal Testing}

Figure 5 shows the set up used for modal impact testing of the cable.

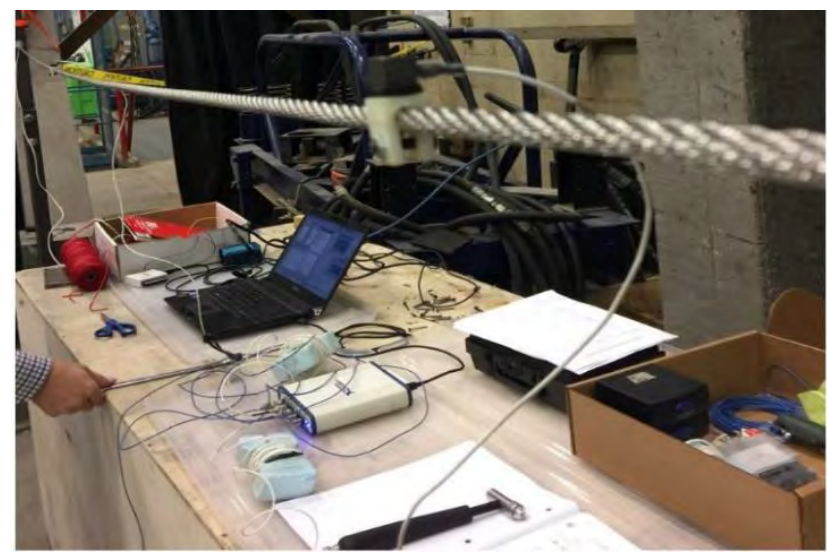

Figure 5. Cable Vibration Test Set up

Figure 6 presents a schematic illustrating two set-ups used for testing the cable. Two pin support structures hold the cable. The right end of the cable is attached to a threaded rod to adjust the static tension of the cable. A load cell is at the left end of the cable to measure the longitudinal tension of the cable. The load cell is directly inline with the cable to measure the longitudinal tension of the cable. The rectangular solid shapes and the black arrows represent respectively the position of the accelerometers and the hammer hits along the cable.

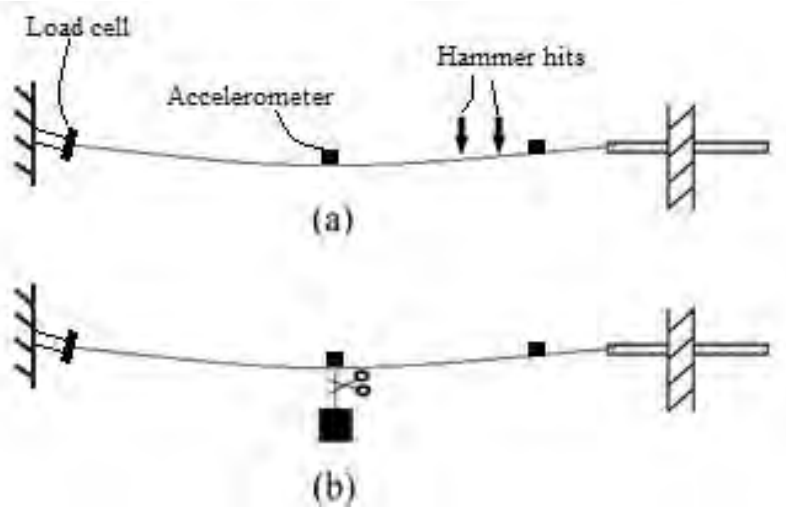

Figure 6. Schematic view of cable vibration testing

Modal tests are performed with a Bruel \& Kjaer 8205-002 impact hammer, 4507 B 004 70-g accelerometers, and a National Instruments NI USB-4432 power supply and signal conditioner. Cable tension is measured with a load cell. Also, the software ModalView [14] is used to analyze the data. All modal tests averaged 5 hits.

Figure 6 (a) illustrates the set up used to perform hammer modal testing over the cable, experiment " $\mathrm{A}$ ". With accelerometers positioned at 0.3 and $1.8 \mathrm{~m}$ from the right side, the measurements taken hitting the hammer in two different positions, at $1 / 4$ and $1 / 5$ of the cable span. The modal test was performed with different tensions which are tabulated in Results section.
Another experiment, "B", is performed as illustrated by Figure 6 (b). The cable is supported the same way as before, however now there is a mass attached to the middle of the cable through a string. Cutting the string creates a force excitation that can be replicated in simulation.

\section{Results AND Discussions}

The Frequency Domain Toolbox in the $20 \operatorname{sim}^{\circledR}$ bond graph simulation environment numerically generates a system transfer function from which eigenvalues are calculated. Table 3 presents the experimental and numerical results of the natural frequencies and damping ratios of the tensioned cable. In the simulation, an impulse force is applied at the quarter of the cable span which is in the 7th cable segment and the time response is obtained at the midspan of the cable. The result of experiment (b) in Figure 6 and corresponding simulation results are illustrated in Figure 7 and Figure 8. The measured and simulated acceleration time response of the midspan can be compared from these figures. Figures 7-8 show accelerations of similar magnitude. Discrepancies are primarily attributed to inaccurate high-frequency modes inherent in a lumped-segment representation. Figure 9 presents the stabilization diagram obtained from ModalView to extract the natural frequencies of the cable with $350 \mathrm{~N}$ tension. The peaks in the stabilization diagram correspond to the natural frequencies of the cable.

\begin{tabular}{|c|c|c|c|c|}
\hline \multirow{2}{*}{$\begin{array}{c}\text { Tension } \\
(\mathrm{N})\end{array}$} & \multicolumn{2}{|c|}{ Modal Testing } & \multicolumn{2}{|c|}{ Simulation } \\
\hline & $\begin{array}{l}\text { Freq } \\
(\mathrm{Hz})\end{array}$ & $\begin{array}{c}\text { Damp } \\
\text { (\%) }\end{array}$ & $\begin{array}{l}\text { Freq } \\
(\mathrm{Hz})\end{array}$ & $\begin{array}{c}\text { Damp } \\
\text { (\%) }\end{array}$ \\
\hline \multirow[t]{3}{*}{91.4} & 2.37 & 4.025 & 2.41 & 1.612 \\
\hline & 4.52 & 16.4 & 4.77 & 11.511 \\
\hline & 6.88 & 3.880 & - & - \\
\hline \multirow[t]{3}{*}{140.6} & 4.53 & 0.645 & 4.21 & 5.672 \\
\hline & 6.53 & 0.939 & - & - \\
\hline & 10.15 & 1.18 & 13.03 & 2.014 \\
\hline \multirow[t]{3}{*}{230.8} & 4.96 & 1.074 & 5.19 & 4.223 \\
\hline & 8.19 & 0.699 & 8.32 & 1.770 \\
\hline & 12.39 & 0.900 & 13.31 & 54.881 \\
\hline \multirow[t]{3}{*}{300.7} & 5.14 & 0.913 & 5.11 & 32.85 \\
\hline & 9.46 & 0.357 & 9.19 & 91.908 \\
\hline & 14.22 & 0.436 & 14.8 & 2.741 \\
\hline \multirow[t]{3}{*}{350} & 5.35 & 0.878 & 5.02 & 1.918 \\
\hline & 10.18 & 0.258 & 10.34 & - \\
\hline & 15.27 & 0.343 & - & - \\
\hline \multirow[t]{3}{*}{400} & 5.58 & 0.807 & 5.07 & 17.997 \\
\hline & 10.87 & 0.209 & 10.3 & 5.509 \\
\hline & 16.27 & 0.487 & 15.23 & 0.778 \\
\hline
\end{tabular}




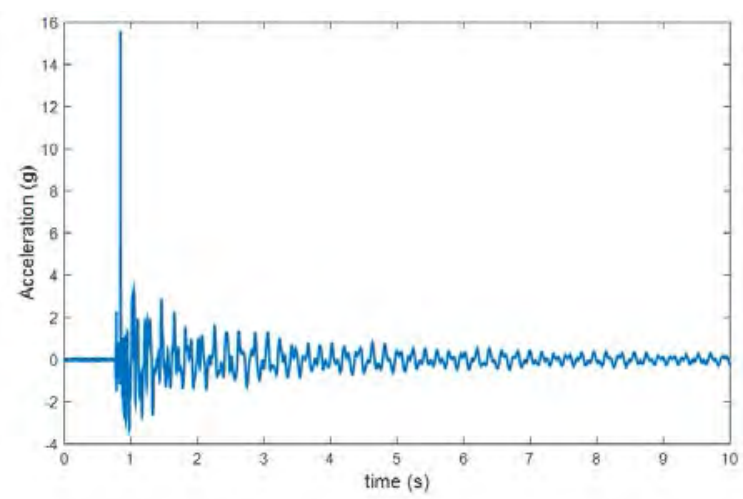

Figure 7. Acceleration time series of the midspan of the cable- Experimental result

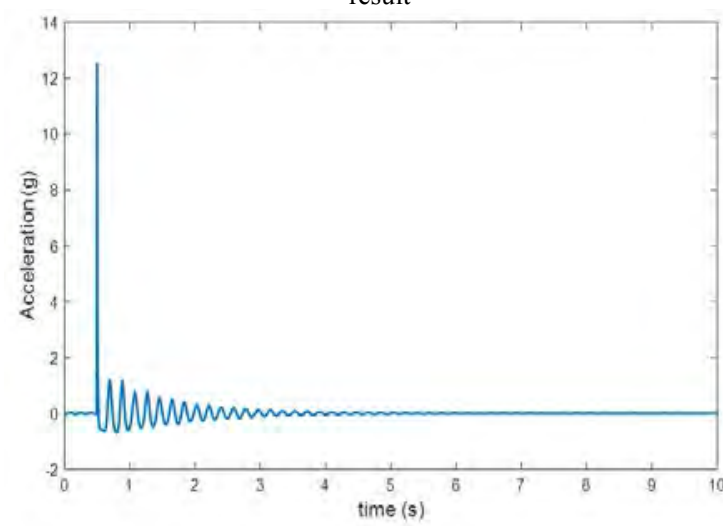

Figure 8. Acceleration time series of the midspan of the cable- Simulation result

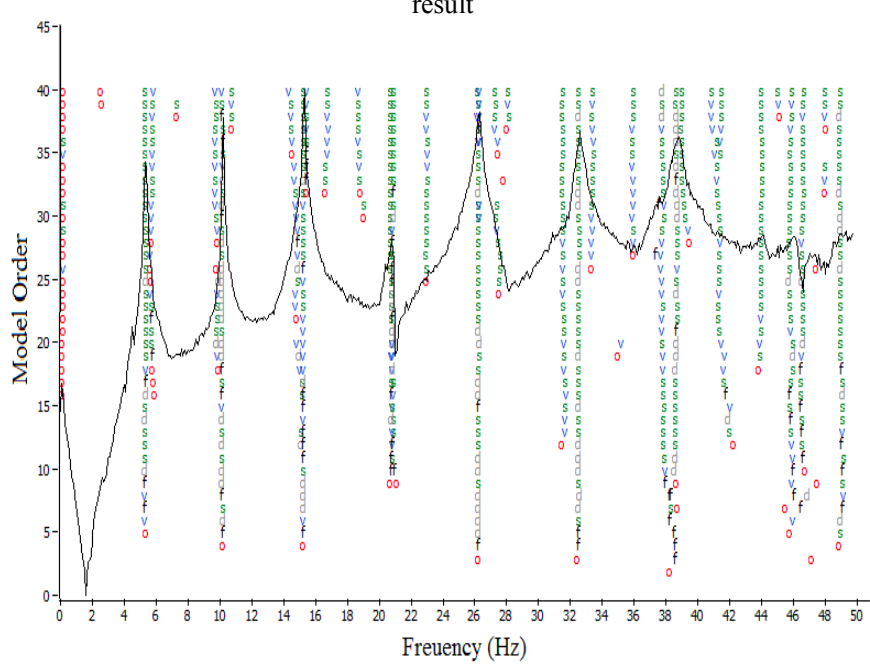

Figure 9. Experimental stabilization diagram

\section{CONCLUSIONS AND FUTURE WORK}

A bond graph lumped segment model was developed to obtain the modal data of a conductor. The natural frequencies and damping ratios from modal testing of the physical setup were compared to those from simulation, along with time responses. The overall system frequencies were predicted with the simulation model for the range investigated. Discrepancies in damping ratios, and in time response, are attributed to the use of simple linear viscous damper elements. Developing and tuning a more accurate damping model remains an open research problem. In addition, as the overall goal of the research is to capture the effects of cable on dynamics of poles, cable numerical models should be combined with the pole models to investigate the effects of cables in a line of pole-cables in the future work.

\section{REFERENCES}

[1] O. Barry, J. Zu, and D. Oguamanam, “Analytical and experimental investigation of overhead transmission line vibration," J. Vibration and Control, vol. 21, no. 14, pp. 2825-2837, Jan. 2014. doi: $10.1177 / 1077546313517589$.

[2] G. Ricciardi and F. Saitta, "A continuous vibration analysis model for cables with sag and bending stiffness," Eng. Struct., vol. 30, no. 5, pp. 1459-1472, May 2008. doi: 10.1016/j.engstruct.2007.08.008.

[3] N. Barbieri, M. K. Tenório Calado, M. J. Mannala, K. F. de Lima, and G. de Sant'Anna Vitor Barbieri, "Dynamical analysis of various transmission line cables," Procedia Eng., vol. 199, pp. 516-521, 2017. doi: 10.1016/j.proeng.2017.09.143.

[4] H.-N. Li, W.-L. Shi, G.-X. Wang, and L.-G. Jia, "Simplified models and experimental verification for coupled transmission tower-line system to seismic excitations," J. Sound and Vibration, vol. 286, no. 3, pp. 569585, Sep. 2005. doi: 10.1016/j.jsv.2004.10.009.

[5] M.L. Q. Wang, H.N. Li, "Aseismic calculation of transmission towerconductor system," Earthquake Engineering and Engineering Vibration, vol. 9, pp. 73-87, 1989.

[6] K. O. Papailiou, "On the bending stiffness of transmission line conductors," IEEE Trans. on Power Delivery, vol. 12, no. 4, pp. 1576-1588, 1997. doi: 10.1109/61.634178.

[7] K. Spak, G. Agnes, and D. Inman, "Cable Modeling and Internal Damping Developments," Appl. Mech. Rev., vol. 65, no. 1, p. 10801, Mar. 2013. doi: 10.1115/1.4023489.

[8] K. S. Spak, G. S. Agnes, and D. J. Inman, "Modeling vibration response and damping of cables and cabled structures," J. Sound and Vibration, vol. 336, pp. 240-256, Feb. 2015. doi: $10.1016 /$ j.jsv.2014.10.009.

[9] K. S. Spak, G. S. Agnes, and D. Inman, "Towards Modeling of CableHarnessed Structures: Cable Damping Experiments," in 54th AIAA/ ASME/ASCE/AHS/ASC Structures, Structural Dynamics, and Materials Conference, 2013. doi: 10.2514/6.2013-1889.

[10] P. Ferreira Pinto and G. Rideout, "Development and Validation of an In-Situ Utility Pole Simulation Model for Virtual Modal Testing," in Volume 13: Acoustics, Vibration, and Wave Propagation, 2016. doi: 10.1115/IMECE2016-67463.

[11] D. C. Karnopp, D. L. Margolis, and R. C. Rosenberg, System Dynamics. John Wiley \& Sons, Inc., 2012 doi: $10.1002 / 9781118152812$

[12] L. Meirovitch, Fundamentals of Vibrations, McGraw Hill, 2000.

[13] R.C. Hibbeler, Mechanics of Materials, Pearson, 2011.

[14] ModalVIEW, ABSignal b.v., 2012. 


\section{On The Finite Element Modeling Of Turbo Machinery Rotors In Rotor Dynamic Analysis}

\author{
Mohammad hadi Jalali \\ Faculty of Engineering and Applied \\ Science \\ Memorial University \\ St. John's, Canada
}

\author{
Nima Nouri \\ Isfahan University of Technology \\ Department of Mechanical \\ Engineering \\ Isfahan, Iran
}

\author{
Saeed Ziaei-Rad \\ Isfahan University of Technology \\ Department of Mechanical \\ Engineering \\ Isfahan, Iran
}

\begin{abstract}
In this study, a program based on finite element method is developed for rotor dynamic analysis of gas turbine rotors. In the FE model of the rotors, various minor and major parts of the rotor are modeled using the cylindrical and tapered Timoshenko beam elements and the lateral vibration behavior of the rotor is evaluated. In the paper, the lateral vibration behavior of a certain gas turbine rotor is analyzed using the developed finite element program and coupled lateral-torsional vibration behavior of the rotor is analyzed using $3 \mathrm{D}$ finite element model. A good agreement exists between the results obtained from two FE models. Two design models are used for the rotor one of which has 2 bearings and the other one has 4 bearings with specific locations. The effects of the number of the bearings on the critical speeds, operational deflection shapes and unbalance response of the rotor is investigated. It is found that the number of the bearings has significant effect on the first critical speed but slight effect on the second and third critical speeds. It is demonstrated that the number of the bearings can be used as one of the system design parameters.
\end{abstract}

Keywords; Timoshenko beam elements, Dynamic analysis, Campbell diagram, Unbalance response, Operational deflection shape

\section{INTRODUCTION}

Rotor dynamics is a branch of systems dynamics dealing with mechanical systems in which at least one part, usually defined as rotor, rotates with significant angular momentum [14]. It is vital to consider the dynamics of the rotating machines in the design stage to avoid catastrophic failures that may occur because of resonance condition in their operation. Dynamic analysis of rotating machines and turbo machinery rotors has been performed by many researchers and finite element method has been of interest of many researchers recently as a numerically efficient method to analyze the dynamics of rotating machines and mechanical structures [5-10].

Chiang et al. [11] developed finite element models to investigate the dynamic characteristics of single- and dualrotor-bearing turbomachinery systems. The models were analyzed to predict the natural frequencies, produce critical speed maps, and estimate the bearing stiffness. They demonstrated that the speed ratio of the high speed to lowspeed shaft in dual-rotors could be used as one of the dual-rotor system design parameters. Jeon et al. [12] performed a full rotor dynamic analysis for a high thrust liquid rocket engine fuel turbo pump using finite element method. They found out that the rear bearing stiffness was the most important parameter for the critical speed and instability, because the first vibration mode of the rotor was turbine side shaft bending mode. Jeon et al. [13] used 1D and three-dimensional finite element methods to perform critical speed analysis of a 30-ton thrust turbo pump while considering the casing structural flexibility. They also analyzed the effect of loads on the bearings on the dynamic behavior of the rotor. Jalali et al. $[14,15]$ predicted the dynamic behavior of a rotor-bearing system with a 1D finite element model, a 3D finite element model and experimental modal test. They obtained natural frequencies and mode shapes of the rotor at rest under free-free boundary condition using beam model, 3D model and modal test. Also, they performed a full rotor dynamic analysis for the rotor using both FE models. Brusa and Zolfini [16] investigated the dynamic behavior of the Galileo Galilei Ground (GGG) test facility through numerical and experimental analyses. They used one dimensional beam finite element model to model the system and They compared the results with experimental results. Creci et al. [17] performed a full rotor dynamic analysis for a $5-\mathrm{KN}$ thrust gas turbine using a 1D finite element model considering bearing stiffness and damping dynamics. They obtained mode shapes and operational deflection shapes of the rotor and they performed a transient analysis to simulate the transition of the system through resonance.

In this paper, a finite element model based on cylindrical and tapered Timoshenko beam elements, by considering the rotary inertia, gyroscopic effect and shear deformations, is used to study the dynamic behavior of a gas turbine rotor. The difference of this beam FE model with the models in the literature is that the various minor and major parts of the rotor are modeled with beam elements in this model, therefore dynamics of the rotor can be evaluated exactly. A 3D finite element model based on solid elements is also constructed in 
Ansys software to validate the beam FE results. A parameter study is carried out to examine the effects of the number of the bearings on the lateral vibration behavior of the rotor. The mode shapes, Campbell diagram, critical speeds, operational deflection shapes (ODS) and unbalance response of the rotor are obtained in both cases with 2 bearings and 4 bearings with specific locations to compare the dynamics of the designed rotors and to evaluate which design would be better. It is found that the number of the bearings can be a design parameter, because it influences the vibration behavior of the rotor significantly.

\section{THEORETICAL FORMULATION}

The rotor system can be divided into rigid disks, rotor segments, and linear bearing supports. The vector of the nodal displacements, i.e., of the generalized coordinates of a Timoshenko beam element used in this study is:

$$
q=\left[\begin{array}{llllllll}
x_{1} & y_{1} & \theta_{x 1} & \theta_{y 1} & x_{2} & y_{2} & \theta_{x 2} & \theta_{y 2}
\end{array}\right]
$$

Where $x_{1}, y_{1}, \theta_{x 1}$ and $\theta_{y 1}$ represent node 1 's displacements and rotations about the nodal axes and $x_{2}, y_{2}, \theta_{x 2}$ and $\theta_{y 2}$ represent node 2 's displacements and rotations about the nodal axes. The value of displacements through the elements can be obtained by shape functions and the nodal displacements from the following equation.

$$
\begin{aligned}
& \left\{\begin{array}{c}
x \\
\theta_{y}
\end{array}\right\}=\left[\begin{array}{llll}
N_{11} & N_{12} & N_{13} & N_{14} \\
N_{21} & N_{22} & N_{23} & N_{24}
\end{array}\right]\left\{\begin{array}{c}
x_{1} \\
\theta_{y 1} \\
x_{2} \\
\theta_{y 2}
\end{array}\right\} \\
& \left\{\begin{array}{c}
y \\
\theta_{x}
\end{array}\right\}=\left[\begin{array}{llll}
N_{11} & N_{12} & N_{13} & N_{14} \\
N_{21} & N_{22} & N_{23} & N_{24}
\end{array}\right]\left\{\begin{array}{c}
y_{1} \\
\theta_{x 1} \\
y_{2} \\
\theta_{x 2}
\end{array}\right\}
\end{aligned}
$$

Where $N_{i j}(i, j=1,2)$ are the shape functions of Timoshenko beam element which can be found in [18]. The motion equation of a complete rotor-bearing system that is axially symmetrical around its spin axis and rotates at a constant spin speed $\Omega$, is in the following general form:

$$
\mathbf{M} \ddot{\mathbf{q}}(\mathrm{t})+(\mathbf{G}) \dot{\mathbf{q}}(\mathrm{t})+(\mathbf{K}) \mathbf{q}(\mathrm{t})=\mathbf{f}(\mathrm{t})
$$

Where $q(t)$ is a vector containing the generalized coordinates, referred to an inertial frame and the other matrices are the assembled matrices for the system. $\mathbf{M}$ is the symmetric mass matrix, $\mathbf{G}$ is the skew-symmetric gyroscopic matrix, $\mathbf{K}$ is the symmetric stiffness matrix, and $\mathbf{f}(\mathrm{t})$ is a time-dependent vector in which all forcing functions are listed. The gyroscopic matrix contains inertial and hence conservative terms that, in the case of rotor dynamics, are strictly linked with the gyroscopic moments acting on the rotating parts of the machine. When dealing with rotating systems, one of the forcing functions is usually that caused by the residual unbalance that, although small, cannot nevertheless be neglected. Unbalance forces are harmonic functions of time, with an amplitude proportional to $\Omega^{2}$ and a frequency equal to $\Omega$.

Most flexible rotors can be considered as beam-like structures. Under fairly wide assumptions, the lateral behavior of a beam can be considered as uncoupled from its axial and torsional behavior [1, 18]. The same uncoupling is usually assumed in rotor dynamics, with the difference that no further uncoupling between bending in the principal planes is possible. When the flexural behavior can be uncoupled from the axial and torsional ones, Equation (3) holds for the first one.

In order to solve the eigenvalue problem of the Equation (3) (with $\mathbf{f}(\mathrm{t})=0$ ), the second-order homogeneous Equation (3) should be reduced into $2 \mathrm{n}$ first-order differential equations. A 2n-column vector $\mathrm{X}=\left\{\begin{array}{l}\dot{\boldsymbol{q}} \\ \boldsymbol{q}\end{array}\right\}$ is used so that Equation (3) with zero force can be expressed as:

$$
\left[\begin{array}{cc}
\mathbf{0} & \mathbf{M} \\
\mathbf{M} & \mathbf{G}
\end{array}\right]\left[\begin{array}{c}
\ddot{\mathbf{q}} \\
\dot{\mathbf{q}}
\end{array}\right]+\left[\begin{array}{cc}
-\mathbf{M} & \mathbf{0} \\
\mathbf{0} & \mathbf{K}
\end{array}\right]\left\{\begin{array}{l}
\dot{\mathbf{q}} \\
\mathbf{q}
\end{array}\right\}=\left\{\begin{array}{l}
\mathbf{0} \\
\mathbf{0}
\end{array}\right\}
$$

Equation (4) can be simplified into:

$$
\mathbf{A} \dot{\mathbf{X}}+\mathbf{B X}=\mathbf{0}
$$

By assuming $\mathbf{X}=\{\boldsymbol{\psi}\} e^{\lambda t}$, Equation (5) can be expressed as:

$$
\lambda \mathbf{A}+\mathbf{B}\{\boldsymbol{\psi}\}=\mathbf{0}
$$

The eigenvalues of the Equation (6) is $\lambda_{k}=\sigma_{k}+i \omega_{k}$ where $\sigma_{k}$ is the decay rate of the kth natural frequency and $\omega_{k}$ is the kth natural frequency. When the natural frequencies of the rotor at various rotor speeds are calculated, the Campbell diagram can be plotted. The natural frequencies of the rotor at various rotor speeds can be calculated by solving the eigenvalue problem Equation (6). In addition, the unbalance response of the rotor can be calculated by obtaining the solution of the Equation (7) which is derived when in Equation (3), $\mathbf{f}(\mathrm{t})$ is a harmonic function of time, with amplitude proportional to $\Omega^{2}$ and a frequency equal to $\Omega$ and by assuming the response to be $\mathbf{q}(\mathrm{t})=\mathbf{q}_{0} e^{i \omega t}$.

$$
\left(-\Omega^{2} \mathbf{M}+\mathrm{i} \Omega \mathbf{G}+\mathbf{K}\right) \mathbf{q}_{0}=\Omega^{2} \mathbf{f}_{r}
$$

\section{FINITE ELEMENT MODELS}

\section{A. Beam Finite element Model}

The studied rotor is a single spool gas turbine rotor and consists of two fan stages, six compressor stages and two turbine stages. The compressors and fans are made of aluminum alloy by the density of $4437 \mathrm{~kg} / \mathrm{m}^{3}$, Young modulus of $113.8 \mathrm{GPa}$ and the Poisson ratio of 0.342 . The material of the other parts of the rotor is assumed to be steel by the density 
of $7700 \mathrm{~kg} / \mathrm{m}^{3}$, Young modulus of $215 \mathrm{GPa}$ and the Poisson ratio of 0.3 . The total length of the shaft is $70 \mathrm{~cm}$. Figure 1 and Figure 2 show the finite element design model of the rotor with 2 and 4 bearings, respectively. In these Figures, disks D1 and D2 are the fan stages, D3-D8 are the compressor stages, D9 and D10 are the turbine stages and D11 is the turbine starter. The model of the rotor in both Figures is the same and only the number of the bearings is different. The model consists of 60 nodes and 78 Timoshenko beam elements. A mesh convergence analysis is performed to explain why this number of nodes and elements is used in the beam model, but the results are not presented in the paper for brevity. The parts with the same colors in Figs. 1-2 have the same materials. The effects of rotary inertia, gyroscopic moments and shear deformations are included in the formulations and the damping is neglected. The dimensions of the various parts of the rotor are exactly equal to those in the 3D FE model. Every node used in the system has 4 degrees of freedom. These include translations in the nodal directions and rotations about nodal axes. In order to consider the inertial properties of fans, compressors and turbines and turbine starter, concentrated masses are used at the place of each one. Table 1 shows the geometric properties of the discs. Inertial properties of the disks can be calculated from the dimensions given in Table 1 . Cylindrical Timoshenko beam elements are used to model the various parts of the shaft and the generator. It should be noted that, in order to model the rotor more accurately, some of the elements have equal nodes. It means that they are on top of each other with the same nodes. Tapered Timoshenko beam elements are used to model the minor parts such as the gaps between different stages of the fans, compressors and turbines. Springs are used to model the bearings at the place of bearings. Table 2 shows the stiffness coefficients of the bearings. The stiffness and damping cross-coupled terms for the bearings are considered null.

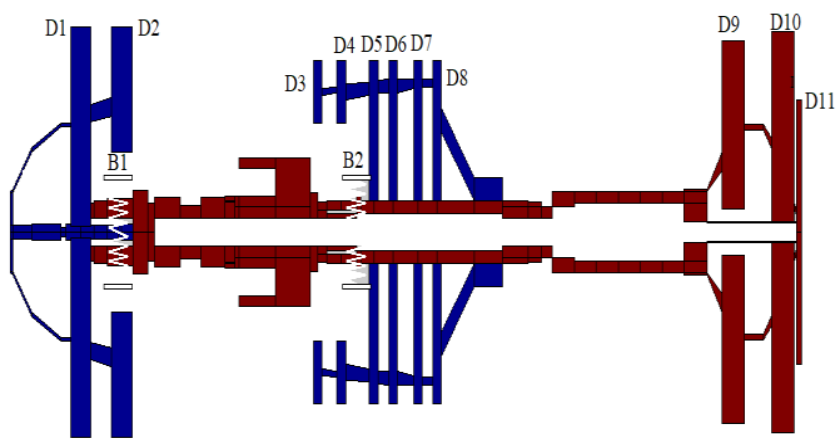

Figure 1. FE model of rotor with 2 bearings

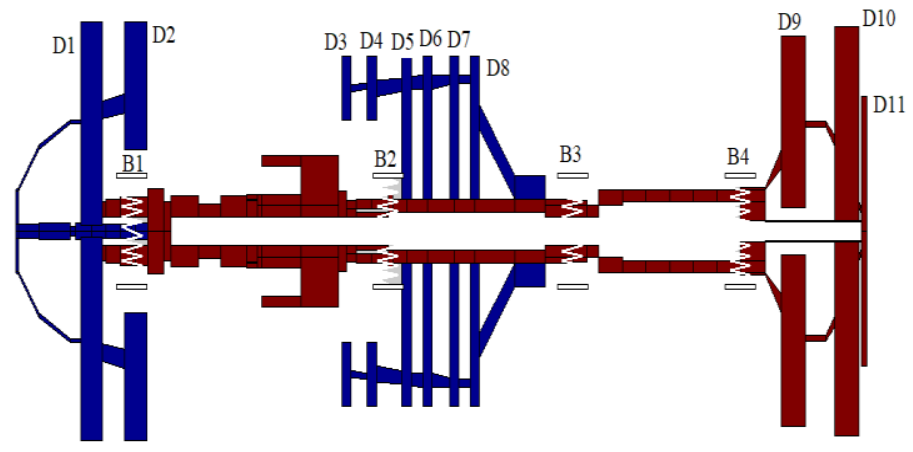

Figure 2. FE model of rotor with 4 bearings

TABLE 1. GEOMETRIC PROPERTIES OF DISCS

\begin{tabular}{|c|c|c|c|c|c|c|c|}
\hline Discs & D1 & D2 & $\begin{array}{c}\text { D3- } \\
\text { D4 }\end{array}$ & $\begin{array}{c}\text { D5- } \\
\text { D8 }\end{array}$ & D9 & D10 & D11 \\
\hline $\begin{array}{c}\text { Outer } \\
\text { diameter } \\
(\mathrm{mm})\end{array}$ & 262 & 262 & 219 & 219 & 244 & 257 & 169 \\
\hline $\begin{array}{c}\text { Inner } \\
\text { diameter } \\
(\mathrm{mm})\end{array}$ & 7.06 & 102 & 140 & 40 & 30.1 & 13.31 & 0 \\
\hline $\begin{array}{c}\text { Width } \\
(\mathrm{mm})\end{array}$ & 17.5 & 17.98 & 7.62 & 7.62 & 20 & 20 & 5 \\
\hline
\end{tabular}

TABLE 2. STIFFNESS COEFFICIENTS OF THE BEARINGS

\begin{tabular}{|c|c|}
\hline Bearing Number & Stiffness (N/m) \\
\hline B1 & $1.57 \mathrm{e}+07$ \\
\hline B2 & $1.77 \mathrm{e}+07$ \\
\hline B3 & $3.91 \mathrm{e}+06$ \\
\hline B4 & $7.04 \mathrm{e}+06$ \\
\hline
\end{tabular}

B. 3D Finite Element Model

The coupled lateral-torsional vibration analysis of the rotor is carried out using 3D FEM. The Ansys software is used to construct the 3D finite element model with 10 node tetrahedral solid elements. Figure 3 shows the 3D finite element model of the rotor. The model consists of 50809 nodes and 27982 solid elements. All the parts are considered elastic and the material and geometric properties of this rotor model are exactly equal to that of the beam finite element model. Spring elements in the two lateral directions are used at the place of the bearings to model the bearings for 2 and 4 bearing cases. 


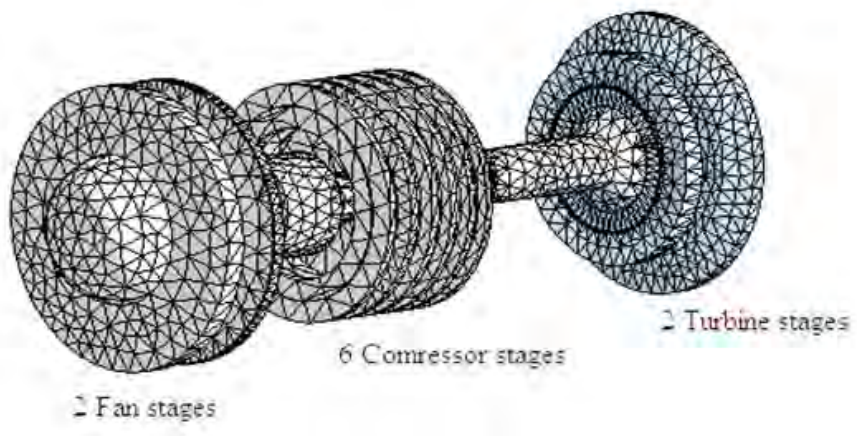

Figure 3. 3D Finite Element Model

\section{RESUlts}

\section{A. Dynamic Analysis of the Rotor with 2 Bearings}

In order to investigate the dynamic behavior of the rotor at operational speeds, critical speeds and the Campbell diagram are obtained using the beam FE model. The speed range considered is 0 to $36000 \mathrm{rpm}$. The Campbell diagram of the rotor obtained from beam FEM excluding the rigid body modes is illustrated in Figure 4. The three first bending critical speeds of the rotor corresponding to the forward whirling obtained from beam FE model are $2099.97 \mathrm{rpm}, 10479 \mathrm{rpm}$ and 15556 rpm, respectively. Also, the Campbell diagram obtained from 3D finite element model excluding the rigid body modes is shown in Figure 5. As can be seen, the gyroscopic softening/stiffening of some of the modes have minor error between two Figs. which is expected because of the difference in modeling methods of the inertial properties of the disks in two FE models.

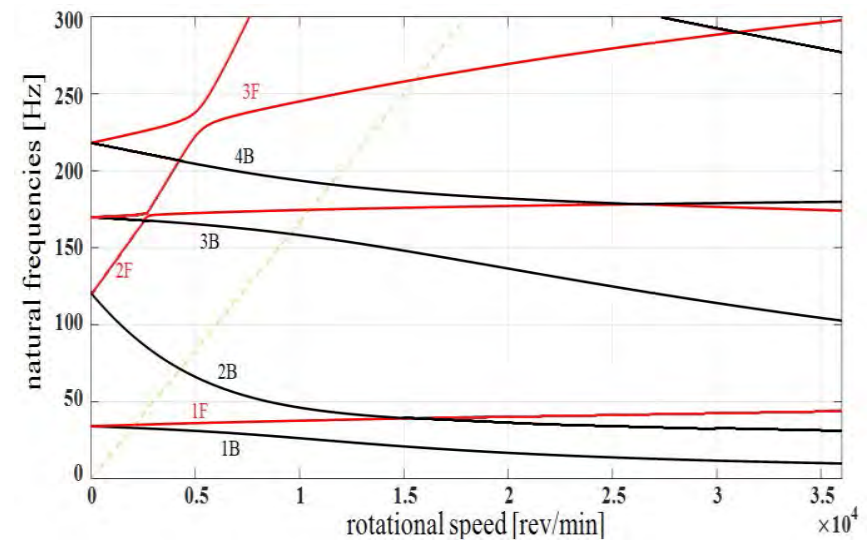

Figure 4. Campbell diagram of the rotor with 2 bearings (Beam FE model)

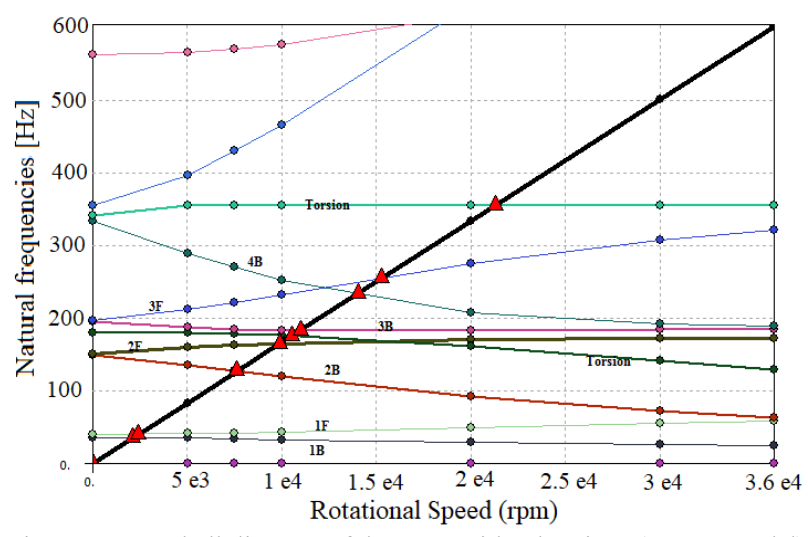

Figure 5. Campbell diagram of the rotor with 2 bearings (3D FE model)

The critical speeds obtained from two models are presented in Table 3. It should be noted that from the beam finite element model, only the bending critical speeds of the rotor are calculated but from 3D finite element model, the torsional and bending critical speeds are calculated.

\begin{tabular}{|c|c|c|c|c|c|}
\hline \multicolumn{1}{|c|}{ TABLE 3. CRITICAL SPEEDS OF THE ROTOR WITH 2 BEARINGS } \\
\begin{tabular}{|c|c|c|c|} 
Critical \\
Speed
\end{tabular} & $\begin{array}{c}\text { First } \\
\text { bending }\end{array}$ & $\begin{array}{c}\text { Second } \\
\text { bending }\end{array}$ & $\begin{array}{c}\text { First } \\
\text { torsional }\end{array}$ & $\begin{array}{c}\text { Third } \\
\text { bending }\end{array}$ & $\begin{array}{c}\text { Second } \\
\text { torsional }\end{array}$ \\
\hline Beam & 2099.97 & 10479 & N/A & 15556 & N/A \\
FE & (rpm) & & & & \\
\hline 3D FE & 2416.2 & 9869.1 & 10503 & 15252 & 21250 \\
\hline (rpm) & & & & & \\
\hline Error & 13.0 & 6.1 & N/A & 1.9 & N/A \\
\hline
\end{tabular}

In order to investigate the dynamic behavior of the rotor more practically and to verify the critical speed obtained from the Campbell diagrams, an imbalance of $1 \mu \mathrm{m}$ is considered at the gravity center of all of the discs which have inner diameter equal to the outer diameter of the shaft where they are located, to simulate the worst unbalancing condition. The imbalance is considered at the gravity center of disks D5, D6, D7, D8 and D10 (Table 1). The operational deflection shapes (ODS) at two bending critical speeds obtained from the Campbell diagrams are shown in Figure 6. 

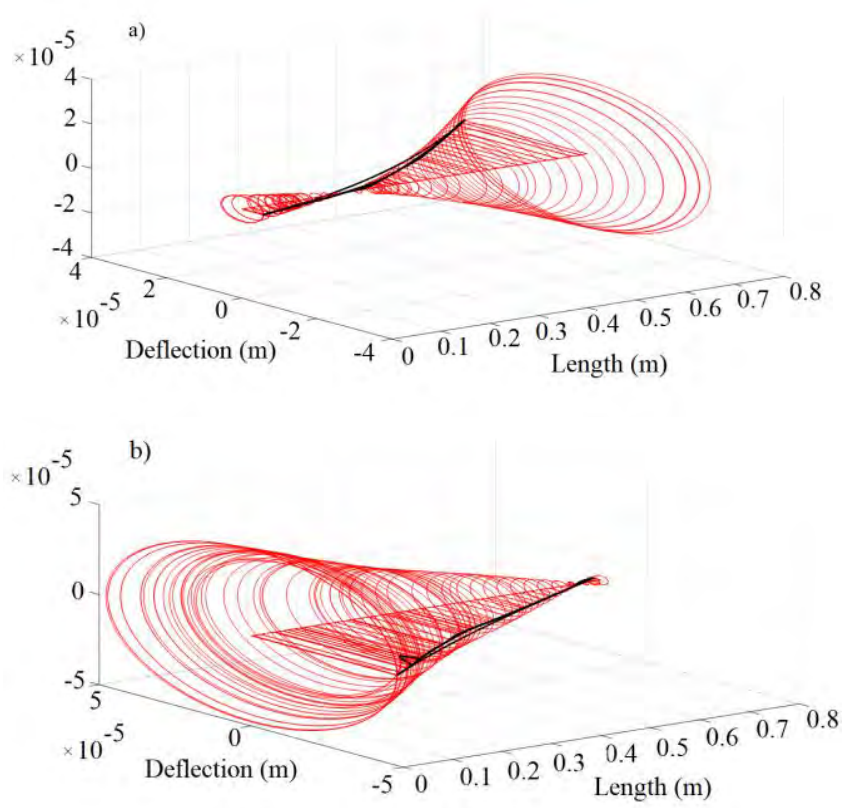

Figure 6. Operational deflection shape of the rotor with 2 bearings at a) first b) second critical speed (Beam model)

Figure 7. shows the unbalance response of the rotor evaluated the location of concentrated masses of disks D5, D6, D7, D8 and D10 at which the imbalance is considered. Nodes 27, 28, $29,30,45$ are the location of concentrated masses of disks D5, D6, D7, D8 and D10, respectively. It can be seen that displacement peaks at the critical speeds points which were previously calculated.

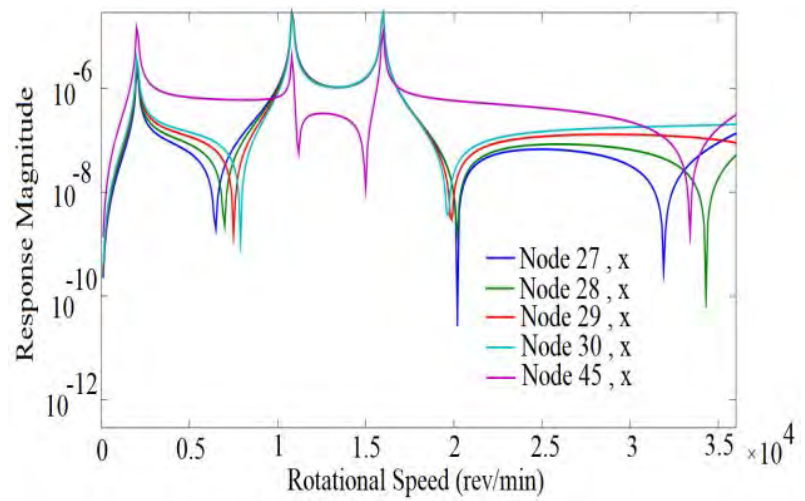

Figure 7. Unbalance response of the rotor with 2 bearings (Beam model)

\section{B. Dynamic Analysis of the Rotor with 4 Bearings}

The Campbell diagram, operational deflection shapes and unbalance response to the worst unbalancing condition is obtained also in this case. The considered speed range is again 0 to $36000 \mathrm{rpm}$. The Campbell diagrams, excluding rigid body modes, obtained from beam FE model and Ansys are shown in the Figure 8 and Figure 9, respectively. The critical speeds obtained from two models are compared with each other in Table 4.

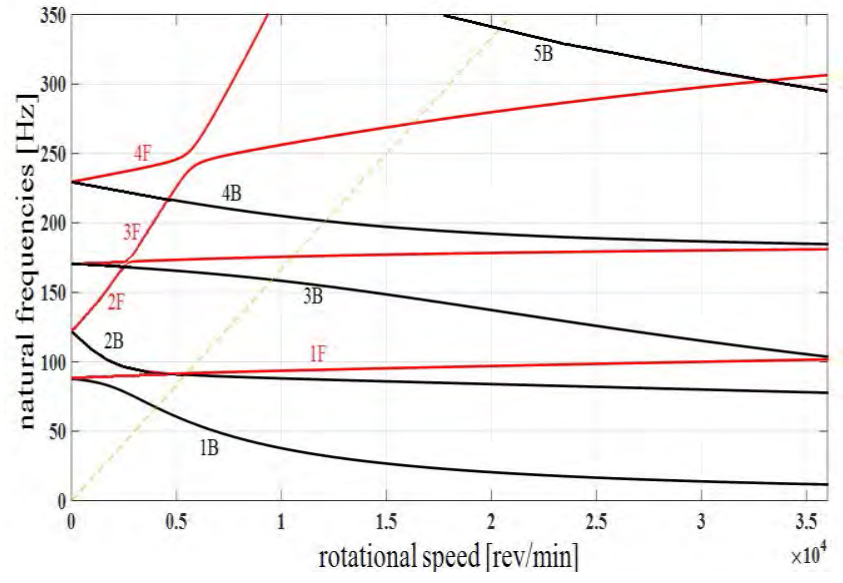

Figure 8. Campbell diagram of the rotor with 4 bearings (Beam FE model)

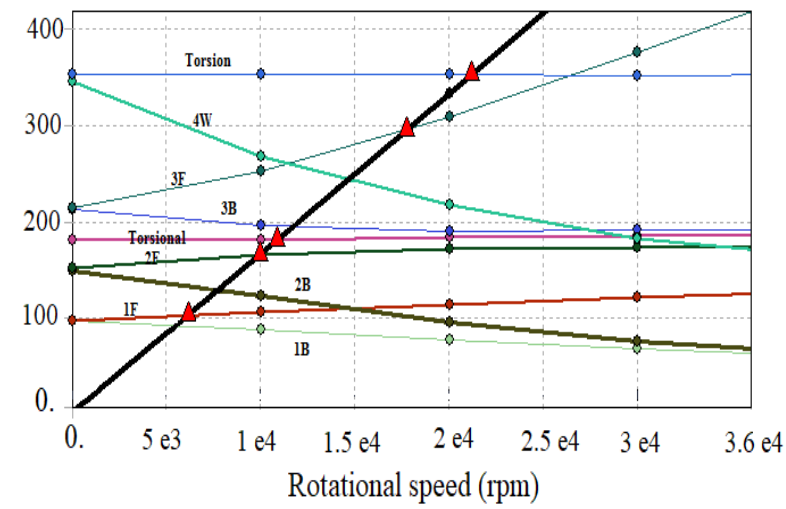

Figure 9. Campbell diagram of the rotor with 4 bearings (3D FE model)

TABLE 4. CRITICAL SPEEDS OF THE ROTOR WITH 4 BEARINGS

\begin{tabular}{|c|c|c|c|c|c|}
\hline $\begin{array}{l}\text { Critical } \\
\text { Speed }\end{array}$ & $\begin{array}{c}\text { First } \\
\text { bending }\end{array}$ & $\begin{array}{l}\text { Second } \\
\text { bending }\end{array}$ & $\begin{array}{c}\text { First } \\
\text { torsional }\end{array}$ & $\begin{array}{l}\text { Third } \\
\text { bending }\end{array}$ & $\begin{array}{l}\text { Second } \\
\text { torsional }\end{array}$ \\
\hline $\begin{array}{c}\text { Beam } \\
\text { FE } \\
\text { (rpm) }\end{array}$ & 5507.26 & 10549 & $\mathrm{~N} / \mathrm{A}$ & 16308 & $\mathrm{~N} / \mathrm{A}$ \\
\hline $\begin{array}{l}\text { 3D FE } \\
(\mathrm{rpm})\end{array}$ & 5446.9 & 9894.2 & 10868 & 17697 & 21180 \\
\hline $\begin{array}{l}\text { Error } \\
(\%)\end{array}$ & 1.1 & 6.6 & $\mathrm{~N} / \mathrm{A}$ & 7.8 & $\mathrm{~N} / \mathrm{A}$ \\
\hline
\end{tabular}

An imbalance same as which was considered in the previous section is considered also in this case and the operational deflection shapes (ODS) at two bending critical speeds obtained from the Campbell diagrams and the unbalance response are calculated. The operational deflection shapes at two bending critical speeds are shown in Figure 10 
and the unbalance response evaluated at the location of disks D5, D6, D7, D8 and D10 is shown in Figure 11.
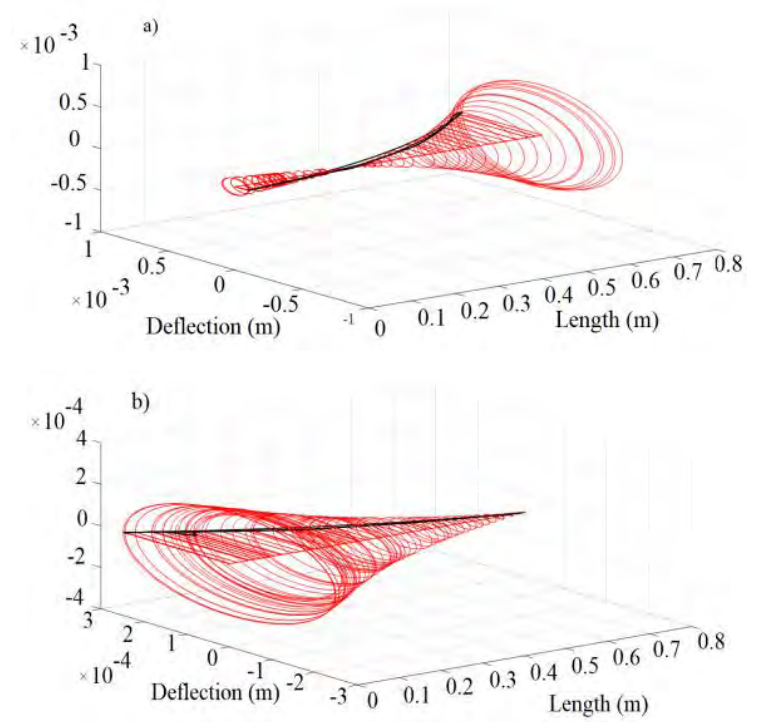

Figure 10. Operational deflection shape of the rotor with 4 bearings at a) first b) second critical speed (Beam model)

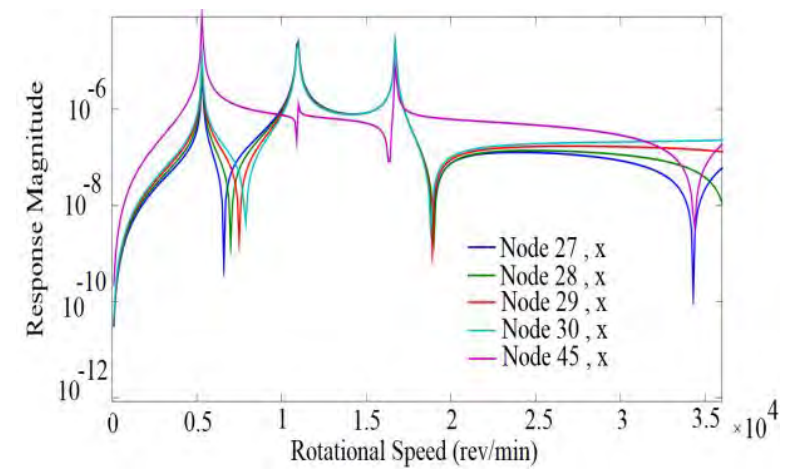

Figure 11. Unbalance response of the rotor with 4 bearings (Beam model)

By comparing the critical speeds of the rotor with 2 and 4 bearings from Tables 3 and 4, it is obvious that the number of the bearings has significant effect on the value of the first critical speed but slight effect on the value of the second and third critical speeds.

Also, by comparing the operational deflection shapes of the rotor with 2 and 4 bearings from Figure 6 and Figure 10, respectively, it is found that the effect of the number of the bearings on the overall deflection of the rotor at the first critical speed is much more than this effect on the overall deflection of the rotor at the second critical speed. In addition, the overall deflection of the rotor with 2 bearings at the critical speeds is much lower than the overall deflection of the rotor with 4 bearings at the critical speeds. It should be noted that in this paper, the effects of the number of the bearings on the dynamics of this rotor is evaluated but the locations of the bearings also can affect the dynamics of the rotor.

\section{CONCLUSIONS}

A finite element model based on Timoshenko beam elements is presented. In this model, various minor and major parts of a gas turbine rotor are modeled using cylindrical and tapered beam elements. A full rotor dynamic analysis is carried out to evaluate the dynamic behavior of the rotor at operational speeds and to avoid vibration problems. Also, a 3D finite element model is constructed, and the lateraltorsional vibration behavior of the rotor is predicted. The comparison of the results shows good agreement between the results obtained from two FE models. The effect of the number of the bearings on the dynamic behavior of the rotor is also studied from the beam finite element model. It is found that the overall deflection of the rotor with 2 bearings (at specific locations) at its first two bending critical speeds is much lower than the overall deflection of the rotor with 4 bearings (at specific locations) at its first two bending critical speed. In addition, it is found that the effect of the number of the bearings on the overall deflection of the rotor at the first critical speed is much more than this effect on the overall deflection of the rotor at the second critical speed.

\section{References}

[1] G. Genta, Dynamics of Rotating Systems, Springer2005. doi: 10.1007/0-387-28687-X.

[2] H. Cao, L. Niu, S. Xi, X. Chen, "Mechanical model development of rolling bearing-rotor systems: A review," Mech. Systems and Signal Processing, vol. 102, pp. 37-58. Mar. 2018

doi: $10.1016 /$ j.ymssp.2017.09.023.

[3] B. Shahriari, M.H. Jalali and M. R. Karamooz Ravari, "Vibration analysis of a rotating variable thickness bladed disk for aircraft gas turbine engine using generalized differential quadrature method," Proc. Inst. Mech. Eng., Part G, vol. 231, no. 14, pp/ 2739-2749, Jan. 2017. doi: $10.1177 / 0954410016684360$.

[4] M. H. Jalali, B. Shahriari, "Elastic Stress Analysis of Rotating Functionally Graded Annular Disk of Variable Thickness Using Finite Difference Method," Math. Probl. Eng., vol. 2018, pp. 7, ID 1971674. doi: 10.1155/2018/1871674.

[5] S. Nabavi, L. Zhang," Design and Optimization of Wideband Multimode Piezoelectric MEMS Vibration Energy Harvesters," Proc. Eurosensors 2017, vol. 1, no. 4. p. 596. Aug. 2017. doi: 10.3390 /proceedings 1040586 .

[6] M. Rafiee, F. Nitzsche and M. Labrosse, "Dynamics, vibration and control of rotating composite beams and blades: A critical review," ThinWalled Structures, vol. 119, pp. 795-819., 2017.

doi: 10.1016/j.tws.2017.06.018.

[7] M. H. Jalali, B. Shahriari, M. Ghayour, S. Ziaei-Rad, and S. Yousefi, "Evaluation of Dynamic Behavior of a Rotor-Bearing System in Operating Conditions," Conf. World Academy of Science, Engineering and

Technology, International Journal of Mechanical, Aerospace, Industrial and Mechatronics Engineering, vol. 8, no. 10. pp.1675-1679, 2014.

[8] S. Nabavi and L. Zhang, "Design and Optimization of Piezoelectric MEMS Vibration Energy Harvesters Based on Genetic Algorithm," IEEE Sens. J., vol. 17, no. 22, pp. 372-7382, 2017. doi: 10.1109/JSEN.2017.2756921.

[9] M.H. Jalali, B. Shahriari, O. Zargar, M. Baghani, and M. Baniassadi, "Free Vibration Analysis of Rotating Functionally Graded Annular Disc of Variable Thickness Using Generalized Differential Quadrature Method," Scientia Iranica, vol. 25, no. 2, pp. 728-740, Mar. \& Apr. 2018. 
[10] B. Parsi, M. Bahrami, A.M. Esfahani, B.S. Sany, "Calibration verification of a low-cost method for MEMS accelerometers," Trans. Inst. Meas. Control, vol. 36, no. 5, pp. 579-587, Dec. 2013.

doi: $10.1177 / 0142331213513607$.

[11] H.-W. Chiang, C.-N. Hsu, S.-H. Tu, "Rotor-Bearing Analysis for Turbomachinery Single- And Dual-Rotor Systems," $J$.

Propul. Power, vol. 20, no. 20, pp. 1096-1104, 2004.

doi: $10.2514 / 1.3133$.

[12] S.M. Jeon, H.D. Kwak, S.H. Yoon, J. Kim, "Rotordynamic analysis of a high thrust liquid rocket engine fuel (Kerosene) turbopump," Aerosp. Sci. Technol., vol. 26, no. 1, pp. 169-175, May-Apr. 2013.

doi: 10.1016/j.ast.2012.03.005.

[13] S.-M. Jeon, H.D. Kwak, S. Hwan Yoon, J. Kim, "Rotordynamic Analysis of a Turbopump with the Casing Structural Flexibility," J.

Propul. Power, vol. 24, no. 23, pp. 422-436, 2008.

doi: 10.2514/1.33551.

[14] M.H. Jalali, M. Ghayour, S. Ziaei-Rad, B. Shahriari, "Dynamic analysis of a high speed rotor-bearing system," Meas., vol. 53, pp. 1-9, 2014 doi: 10.1016/j.measurement.2014.03.010.

[15] M.H. Jalali, M. Ghayour, S. Ziaei-Rad, B. Shahriari, S. Yousefi, "Rotordynamic Analysis of a Small Rotor-Bearing System," 4th Int. Conf. Acous. Vibr. Tehran, Iran, 2014.

[16] E. Brusa, G. Zolfini, "Dynamics of Multi-Body Rotors: Numerical and Experimental FEM Analysis of the Scientific Earth Experiment Galileo Galilei Ground," Meccanica, vol. 37, no. 3, pp. 239-254, 2002. doi: 10.1023/A:1020147020815

[17] G. Creci, J. Carlos Menezes, J. Barbosa, J. Aparecido Corra, "Rotordynamic Analysis of a 5-Kilonewton Thrust Gas Turbine by Considering Bearing Dynamics," J. Propul. Power, vol. 27, no. 2, pp. 330-336, 2011. doi: 10.2514/1.B34104.

[18] J.S. Rao, "Finite Element Methods for Rotor Dynamics," in History of Rotating Machinery Dynamics, Springer Netherlands, Dordrecht, 2011, pp. 269-297. doi: 10.1007/978-94-007-1165-5_16. 


\section{STUDENT PAPER COMPETITION}




\title{
Assessment of Aliphatic Based Soot Inception in Laminar Diffusion Flames
}

\author{
Nemanja Ceranic, Seth B. Dworkin \\ Department of Mechanical and Industrial Engineering \\ Ryerson University \\ Toronto, Canada
}

\begin{abstract}
Soot models are key components of computation fluid dynamic combustion codes that attempt to prescribe how soot is formed. However, due to the complex nature of soot formation, not all pathways may have been fully characterized. This work investigates numerically the influence that an aliphatic-collision (open-chain hydrocarbon) based soot inception model has on soot formation for coflow ethylene/air and methane/air laminar diffusion flames. In the literature, prediction of the soot volume fraction along the centerline of coflow ethylene flames is lacking in accuracy. Similarly for methane flames, soot formation on the wings are under predicted by many models. A new collision based inception model has been developed for specific aliphatics, and applied using an existing framework for molecular collision, in conjunction with pyrene based inception. The purpose of this model is not to be completely fundamental in nature, but more so a proof of concept in that by using physically realistic values for surface reactivity and collision efficiency, this collision mechanism can account for soot formation deficiencies that exist with just polycyclic aromatic hydrocarbon (PAH) based inception. Using this new model, the peak soot volume fraction along the centerline of an ethylene flame can be increased while the peak soot volume fraction along the wings remains unchanged, showing potential to significantly improve the model's predicative capability. Applying this model to a methane flame has resulted in an increase in the soot volume fraction in both the centerline and the wings, again improving predictive capability.
\end{abstract}

\section{INTRODUCTION}

Any form of hydrocarbon combustion leads to the generation of soot. These particles are hazardous to human health $[1,2]$ as well as dangerous for the environment [3-5]. Thus it is necessary that the characterization of soot formation be well understood in order to design more environmentally friendly combustion devices. Numerical combustion models require extensive knowledge of soot formation in order to obtain meaningful results. One of the advantages that numerical modelling can provide is that it allows researchers to test new theories at virtually no cost compared to experimental techniques.

Soot formation is a complex process consisting of polycyclic aromatic hydrocarbon (PAH) gas-phase growth, particle nucleation, surface growth via surface reaction and $\mathrm{PAH}$ condensation, surface oxidation, particle coalescence, particle coagulation and fragmentation, gas-phase scrubbing, and radiation [6]. These pathways are widely considered by the scientific community to be the accepted routes of soot formation, however, due to its complex nature, other pathways may exist that have yet to be fully characterized.

Soot models have been the subject of discussion for several decades and continue to improve to this day. The three classifications of models are empirical, semi-empirical, and predominantly fundamental models. Semi-empirical soot models [7, 8] may have a foundation in the physics behind soot formation, but lack the fundamental physics behind the problem as they rely on tunable parameters. Although these models may not accurately portray what is occurring inside of the flame, they do give insight as to which parameters may be correlated. They are an essential milestone towards the creation of a fundamental model.

One such model is the Hydrogen-Abstraction-CarbonAddition (HACA) growth mechanism introduced by Frenklach and Wang [9]. This model traditionally uses surface reactivity $(\alpha)$ as a tunable constant or function for matching numerical peak soot predictions to experimental peaks [9]. Therein lies the issue that current soot models face, which is, although peak soot may be correctly predicted, other regions of the flame may not be correctly predicted through the same tuning of $\alpha$. It has been postulated that the centerline of coflow laminar diffusion flames are dominated by inception whereas the wings are dominated by surface growth through the HACA mechanism [10]. By tuning $\alpha$ to experimental peaks, which occur along the wings, the prediction in the wings region may be corrected. However, many models do not have sufficient reaction chemistry or appropriate sub mechanisms to accurately capture the trends along the centerline, and tend to under predict soot formation in that region [11-14].

Based on recent literature, soot is able to undergo mass growth in the absence of gas phase hydrogen atoms and nascent soot can be rich in aliphatic molecules in premixed flames [15]. This observation indicates that the HACA mechanism is not likely to be responsible for this growth. The HACA mechanism removes hydrogen from the surface of molecules meaning that gas phase hydrogen atoms would be present 
whenever this mechanism is active. If the atoms are not present, another mechanism is responsible for this growth. It is proposed that aliphatic-collision based inception may have some influence on this soot mass growth. A semi-empirical model has been generated to investigate the impact that additional aliphatic collision based inception in the soot formation model would have on a variety of coflow laminar diffusion flames. More details on the physics and assumptions of the model will be explained in the following sections. The model is applied to both pure ethylene/air and methane/air laminar diffusion flames that use the same burner dimensions. Two parameters are key to this model which are the surface reactivity, $\alpha$, and the collision efficiency, $\beta$.

\section{BURNER AND NUMERICAL MODEL}

CoFlame is the in-house FORTRAN code that this research implements, which was recently formalized and published in Eaves et al. [16]. The code has been parallelized to reduce computational cost and has been validated against ethylene/air and methane/air combustion [16]. The first flame that is simulated is a coflow ethylene/air laminar diffusion flame that was originally studied by Santoro et al. [17, 18]. In those works, Santoro and coworkers had conducted experiments for several flames, four of which were pure ethylene/air diffusion flames. Of those flames, the one of particular interest is the second non-smoking flame (NSII) due to its prevalence in the literature and abundance of experimental data [19-22]. The NSII has a fuel velocity of $3.98 \mathrm{~cm} / \mathrm{s}$ and an air velocity of 8.9 $\mathrm{cm} / \mathrm{s}$ [18]. The burner consists of an $11.1 \mathrm{~mm}$ diameter inner fuel passage with a wall thickness of $1 \mathrm{~mm}$ surrounded by an outer air passage with a diameter of $101.6 \mathrm{~mm}$ [18]. The second flame that is modelled follows the work of Lee et al. [23], which uses the same burner as the Santoro flame, but methane instead of ethylene as the fuel. For the methane flame, the fuel velocity is $10.24 \mathrm{~cm} / \mathrm{s}$ while the air velocity is $11.94 \mathrm{~cm} / \mathrm{s}$ [24]. The computational domain consists of a nonuniform axisymmetric mesh of $384 \mathrm{CVs}$ in the axial direction and $150 \mathrm{CVs}$ in the radial direction for both of the modelled flames as the burner dimensions are the same.

\section{ALIPHATIC COLLISION MECHANISM}

For the mechanism, it is assumed that aliphatic molecules collide together and under the right conditions may stick together. These conditions are accounted for in the collision efficiency that is prescribed in the CoFlame code. This mechanism was developed to function in a similar manner to particle coalescence, which has been implemented in the CoFlame code and validated. The primary difference between this mechanism and particle coalescence being the specific molecules that are colliding and contributing to soot inception. Particle coalescence uses large PAHs for inception, such as Benzo-a-pyrene, whereas this mechanism uses specific aliphatic species for the same means. A value is given to the collision efficiency to indicate that only $\mathrm{X}$ in every $\mathrm{Y}$ collisions will result in effective sticking. For example, if the collision efficiency is set 0.01 , this indicates that 1 in every 100 collisions will result in sticking, and the other 99 will result in rebound. Once the molecules stick together, they are treated as an incipient soot particle. This in effect means that this mechanism is contributing to the inception of soot, in addition to traditional PAH routes. Furthermore, this collision efficiency is highly dependent on molecular dynamics. Each of the aliphatic molecules considered has a radius and a concentration. The size and concentration inherently have an influence on the soot mass growth as the number of collisions increases with an increase in either one of those two parameters. It is important to note that the purpose of this mechanism is to simply link the aliphatics in the gas phase to the incipient solid soot phase, so as to assess their potential impact on soot mass growth and spatial distribution. The rate of inception is calculated according to kinetic theory:

$$
\partial \mathrm{N} / \partial \mathrm{t}=\beta \sqrt{ }\left[\left(8 \pi \mathrm{k}_{\mathrm{B}} \mathrm{T}\right) / \mu_{\mathrm{AB}}\right]\left(\mathrm{r}_{\mathrm{A}}+\mathrm{r}_{\mathrm{B}}\right)^{2} \mathrm{~A}_{\mathrm{V}}^{2}[\mathrm{~A}][\mathrm{B}]
$$

where $\beta$ is the collision efficiency, $\mathrm{k}_{\mathrm{B}}$ is the Boltzmann constant, $A_{V}$ is Avogadro's number, $\mu_{A B}$ is the collisional reduced mass for the two colliding aliphatics, $r_{A}$ and $r_{B}$ are the radii of the two colliding aliphatics, and [A] and [B] are the concentrations of the two colliding aliphatics [25].

The present study implements a 94 species mechanism, which models ethylene or methane combustion and PAH growth [16]. Some of the aliphatic species have been filtered out of use in the inception model based on their concentrations and carbon mass. Extremely low concentrations with low carbon mass such as $\mathrm{CH}_{2}$ have been removed from the proposed mechanism. The results of this analysis have determined that only 6 species of aliphatics are of particular interest in the present study. Those species are: $\mathrm{CH}_{4}$ (methane), $\mathrm{C}_{2} \mathrm{H}_{2}$ (acetylene), $\mathrm{C}_{2} \mathrm{H}_{6}$ (ethane), $\mathrm{C}_{3} \mathrm{H}_{6}$ (propene), $\mathrm{C}_{4} \mathrm{H}_{6}$ (butyne), and lastly $\mathrm{C}_{3} \mathrm{H}_{8}$ (propane).

While the present model does not represent the complete physics behind the problem, it can be used to determine the potential for mass transfer from the aliphatic gas phase to the soot solid phase. This strategy allows for analysis to be carried out, such that relationships can be made between the aliphatic species and soot mass growth. Adjusting the collision efficiency of aliphatics, and further adjustment to the soot surface reactivity parameter, as was done in [11] is a key component to this exercise. The present analysis has shown that the soot volume fraction, with respect to the proposed mechanism, can be tuned through the collision efficiency. Increasing the efficiency, leads to more soot growth whereas decreasing it has the opposite effect. Through this adjustment, it is hypothesized that the centerline soot volume fraction can be modified to better predict experimental values along the centerline of laminar diffusion flames.

The surface reactivity is a value that ranges from 0 to 1 and is representative of the portion of a soot particle's surface area that is available for chemical reaction. CoFlame allows for this surface reactivity to be modelled as either a constant or a function of temperature history. In the present study, it is taken as a constant value. This parameter is a factor in determining 
HACA growth and oxidation. Decreasing the surface reactivity normally leads to a decrease in the soot volume fraction as the HACA mechanism's contribution to surface growth decreases. Further adjustment of the surface reactivity, beyond what is already provided in literature $[11,26]$ comes in when a new growth mechanism is introduced into the numerical model as is the case in the present study.

\section{RESULTS AND DISCUSSION}

The generation of the model was predicated on being able to observe a positive influence that aliphatic-collision based inception would have on soot formation along the centerline of a laminar diffusion flame. It was not clear a priori if drawing aliphatics out of the gas phase for soot inception would reduce the amount of carbon available for PAHs or HACA growth thereby reducing soot concentrations in some locations of the flame. In order for the model to have some significance it had to be able to increase the soot volume fraction along the centerline while the wings remained close to the experimental peaks for the NSII flame. If that condition could not have been met then the model would not have a positive correlation between aliphatic collision based inception and soot distribution.

Figure 1 shows the predicted soot volume fraction along the pathline of maximum soot of the NSII flame as a function of height above the burner for varying collision efficiencies. The data are compared to a model without aliphatic inception and to experimental results. The solid horizontal line and gray band in Figure 1 at $9.7 \mathrm{ppm}$ indicates the experimentally measured peak value and associated uncertainty. It can be seen from Figure 1 that the predictive capability along the wings of the flame is generally unaffected by the addition of aliphatic inception. Furthermore, only collision efficiencies less than 1e-10 can lead to reasonable results. Adding aliphatic inception, however, shifts the location of peak soot formation to higher heights above the burner.

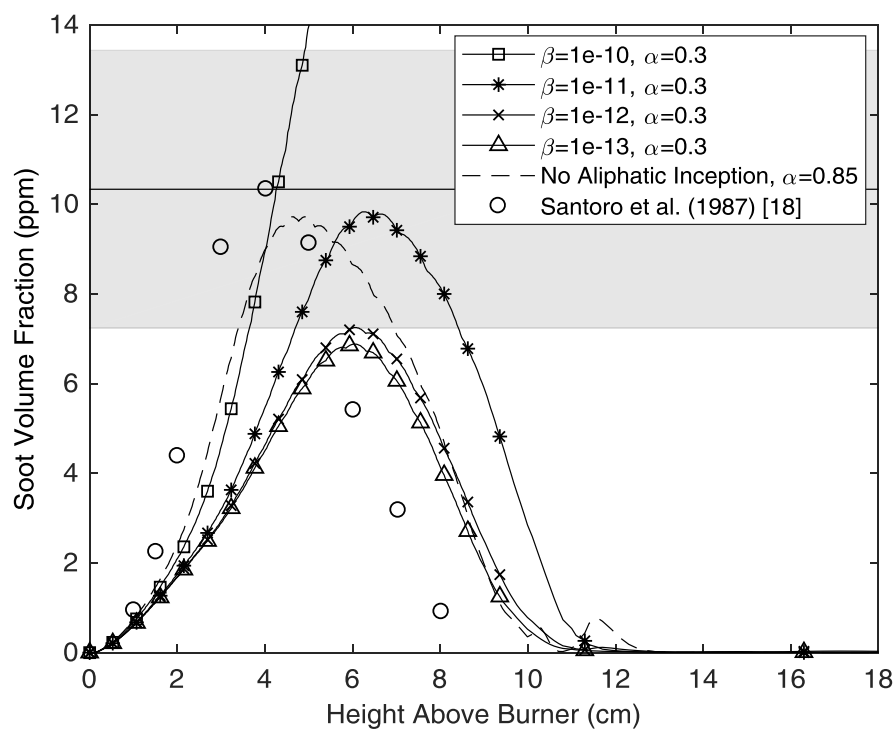

Figure 1. Predicted soot volume fraction as a function of height above the burner for varying collision efficiencies from 1e-10 to 1e-13 with constant surface reactivity of 0.3 along the wings of the NSII flame. The results of a model without aliphatic inception with $\alpha=0.85$ is shown alongside experimental results from [18]. The horizontal black line and gray band denote the experimentally measured peak.

Figure 2 shows the predicted soot volume fraction along the centerline of the NSII flame as a function of height above the burner for the same varying collision efficiencies. Here too the data are compared to a model without aliphatic inception and to experimental results. Again, only collision efficiencies less than 1e-10 lead to physically realistic results. Otherwise, implementing aliphatic based inception results in only a modest increase of soot volume fraction along the flame centerline. These figures illustrate that when $\alpha$ is set to 0.3 and $\beta$ is set to $1 \mathrm{e}-11$ there is an increase in the soot volume fraction along the centerline while the wings remain close to the experimental peaks. The same upward peak shifting phenomenon can be observed along the centerline in Figure 2. Also, the results of varying the collision efficiency and surface reactivity show that for the NSII flame, $\alpha$ can be lowered to a more physically realistic [27] value in order to correctly predict the wings peak soot volume fraction. In order to obtain results close to the experimental peaks for the NSII flame using CoFlame without an aliphatic collision based inception model, $\alpha$ had to be set to 0.85 .

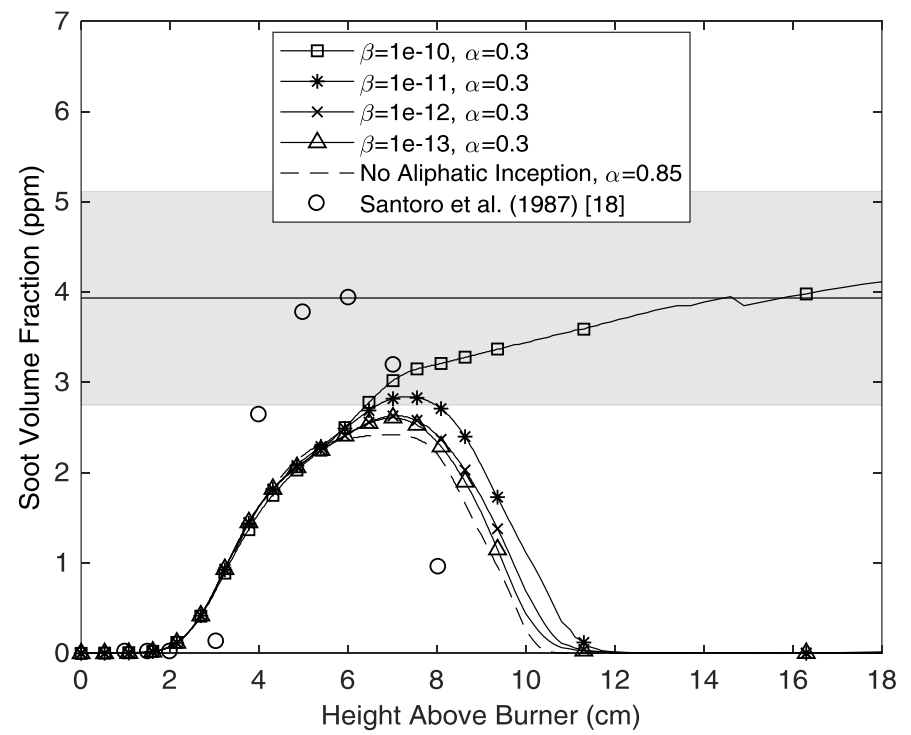

Figure 2. Predicted soot volume fraction as a function of height above the burner for varying collision efficiencies from 1e-10 to $1 \mathrm{e}-13$ with a constant surface reactivity of 0.3 along the centerline of the NSII flame. The results of a model without aliphatic inception with $\alpha=0.85$ is shown alongside experimental results from [18]. The horizontal black line and gray band denote the experimentally measured peak.

Similarly, the predicted soot volume fraction of the methane flame as a function of height above the burner for varying collision efficiencies and surface reactivity compared to a model without aliphatic inception and experimental results is shown in Figures 3 and 4 . The results along the wings are shown in Figure 3 of the methane flame while Figure 4 shows the data along the centerline. The methane flame serves as a good benchmark to test the aliphatic collision based inception model for several reasons. The first and foremost reason is that 
methane flames are known to be dominated by inception over surface growth through the HACA mechanism as the concentration of acetylene throughout the flame is lower compared to ethylene combustion [12]. Since methane is a single carbon fuel, much less acetylene is formed when it is burned, leaving less opportunity to achieve accurate model predictions by refining HACA rates. Secondly, current soot models typically under predict the soot volume fraction along both the centerline and the wings in methane flames [12]. Using the same value for $\alpha$ and $\beta$ as for the NSII flame that achieved the aforementioned condition, 0.3 and $1 \mathrm{e}-11$ respectively, resulted in the under prediction of the soot volume fraction in both the centerline and wings. In ethylene flames, the surface reactivity can compensate for deficiencies in the chemical kinetic mechanism [12]. However, since methane flames are less influenced by HACA growth, the deficiencies in the reaction scheme remain prevalent. The latest version of CoFlame has added reactions specifically for methane flames as detailed by Chernov et al. [12]. Although there is an improvement in the model's predictive capability, increasing soot concentrations, even when the surface reactivity is set to its theoretical limit of unity, both regions of the flame remain under predicted. When the aliphatic collision based inception is applied with the same $\beta$ of $1 \mathrm{e}-11$ as before and $\alpha$ is increased to 1 , the theoretical maximum, there is an increase in the soot volume fraction along both the centerline and wings. Once again, the same peak shifting phenomenon can be observed. The aliphatic collision based inception model is able to improve the comparisons to experimental data, but not rectify all remaining inaccuracies in the methane flame.

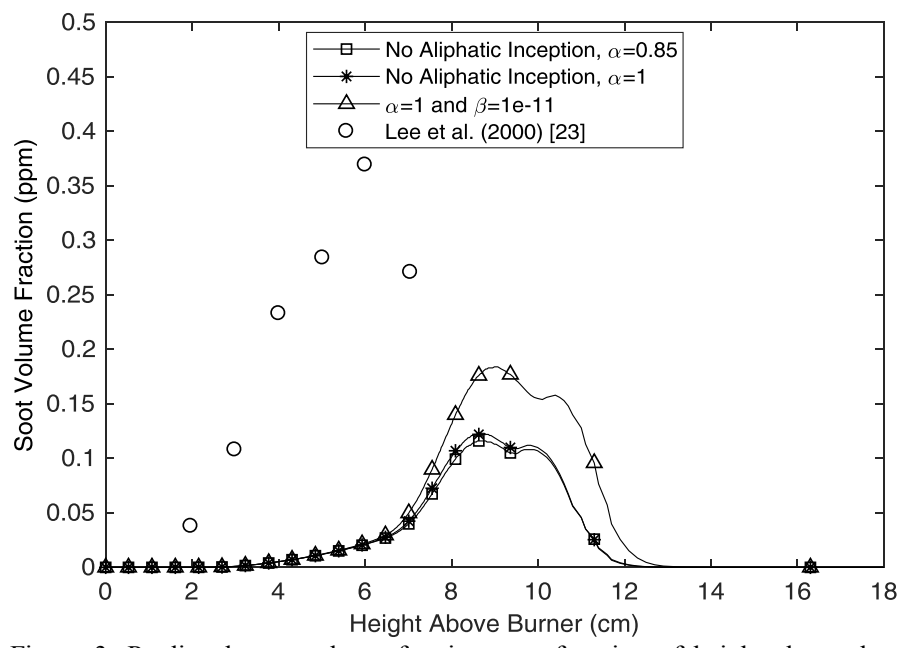

Figure 3. Predicted soot volume fraction as a function of height above the burner for a model without aliphatic inception using $\alpha$ of 0.85 and 1.0 along the wings of the methane flame. The aliphatic inception model is shown using the same $\beta$ as for the NSII, 1e-11. The experimental work of [20] is plotted.

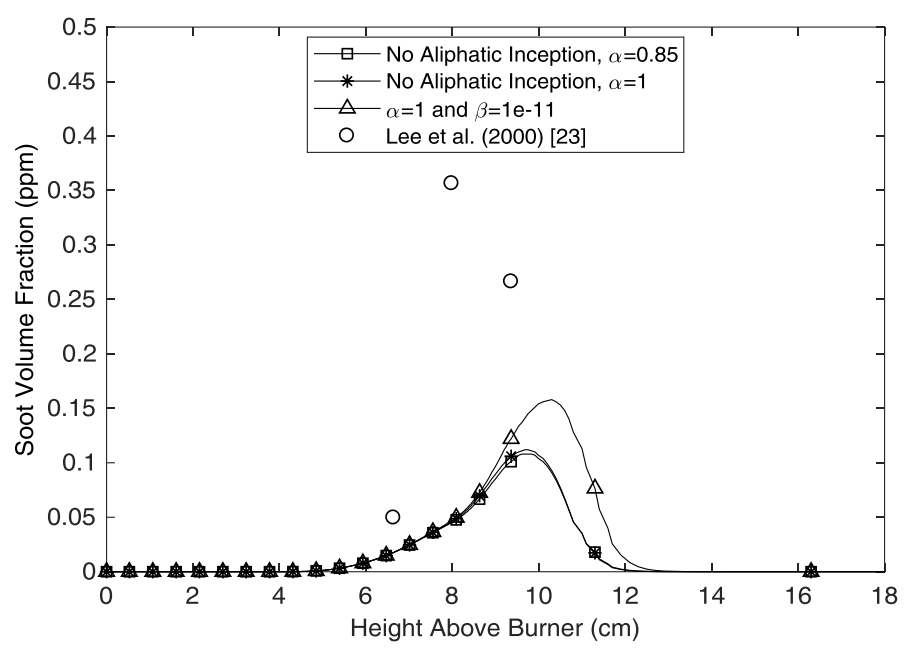

Figure 4. Predicted soot volume fraction as a function of height above the burner for a model without aliphatic inception using $\alpha$ of 0.85 and 1.0 along the centerline of the methane flame. The aliphatic inception model is shown using the same $\beta$ as for the NSII, 1e-11. The experimental work of [20] is plotted.

\section{SUMMARY AND CONCLUSIONS}

A new collision based inception mechanism was developed using an existing framework for larger PAHs but applied to aliphatics. The aliphatic inception mechanism was combined with PAH inception and implemented in the CoFlame code. The influence that an aliphatic-collision based inception model would have on the soot volume fraction distribution for coflow ethylene/air and methane/air laminar diffusion flames was investigated. It was found that for the ethylene flame, a surface reactivity, $\alpha$ of 0.3 and a collision efficiency, $\beta$ of $1 \mathrm{e}-11$ resulted in an increase in the peak soot volume fraction along the centerline, better predicting experimental values, while the predicted peak soot volume fraction along the wings was not degraded. For the methane flame, using the same $\beta$ of $1 \mathrm{e}-11$ resulted in an increase in both the centerline and wings of the flame as compared to using the same $\alpha$ in a soot model without aliphatic-collision based inception. Future work remains to test the applicability of the model to other combustion systems, in particular those for which model predictions do not completely explain or characterize experimental data.

To test the validity of the model further, the results will be expanded to other flame systems in order to ensure applicability to a wide variety of flames. The results of the mechanism need to be applied to the other pure ethylene/air diffusion flames studied by Santoro et al. $[17,18]$ to observe the effects this mechanism would have in those systems. The other ethylene flames suffer from the same predictive discrepancies as the NSII flame. A potential pathway of interest may also be to consider aliphatic molecule condensation and determine if it plays a significant role in soot formation. The current model acts as a proof of concept in that transfer of carbon mass from the aliphatic gas phase to soot particles seems to significantly improve the model's predictive capability in the centerline region of the flames studied, without degrading the HACA growth dominated wings. 


\section{ACKNOWLEDGEMENTS}

The authors thank Dr. Nadezhda Slavinskaya and Prof. Uwe Riedel of the German Aerospace Center (DLR) for providing the chemical reaction mechanism, thermodynamic data, and transport data for ethylene combustion and $\mathrm{PAH}$ formation. The authors acknowledge the Natural Sciences and Engineering Research Council of Canada (NSERC) for financial support. This research was enabled in part by support provided by Sharcnet of Compute Canada.

\section{REFERENCES}

[1] N. A. Janssen et al. Health Effects of Black Carbon, World Health Organization, 2012.

[2] N. A. H. Janssen et al., "Black Carbon as an Additional Indicator of the Adverse Health Effects of Airborne Particles Compared with PM 10 and PM 2.5," Environ. Health Perspect., vol. 119, no. 12, pp. 1691-1699, Dec. 2011. doi: 10.1289/ehp.1003369.

[3] S. Vedal, "Ambient Particles and Health: Lines that Divide," J. Air Waste Manage. Assoc., vol. 47, no. 5, pp. 551-581, May 1997.

doi: 10.1080/10473289.1997.10463922.

[4] J. Hansen and L. Nazarenko, "Soot climate forcing via snow and ice albedos," Proc. Natl. Acad. Sci., vol. 101, no. 2, pp. 423-428, Dec. 2003. doi: 10.1073/pnas.2237157100.

[5] J. Peng et al., "Markedly enhanced absorption and direct radiative forcing of black carbon under polluted urban environments," Proc. Natl. Acad. Sci., vol. 113, no. 16, pp. 4266-4271, Mar. 2016. doi: 10.1073/pnas.1602310113.

[6] B. S. Haynes and H. G. Wagner, "Soot formation," Prog. Energy Combust. Sci., vol. 7, no. 4, pp. 229-273, Jan. 1981.

doi: 10.1016/0360-1285(81)90001-0.

[7] M. Fairweather, W. P. Jones, H. S. Ledin, and R. P. Lindstedt, "Predictions of soot formation in turbulent, non-premixed propane flames," Symp. (Int.) Combust., vol. 24, no. 1, pp. 1067-1074, Jan. 1992. doi: 10.1016/S0082-0784(06)80126-8.

[8] I. M. Kennedy, "Models of soot formation and oxidation," Prog. Energy Combust. Sci., vol. 23, no. 2, pp. 95-132, Jan. 1997. doi: 10.1016/S0360-1285(97)00007-5.

[9] M. Frenklach and H. Wang, "Detailed modeling of soot particle nucleation and growth," Symp. (Int.) Combust., vol. 23, no. 1, pp. 1559-1566, Jan. 1991. doi: 10.1016/S0082-0784(06)80426-1.

[10] V. Chernov, Q. Zhang, M. J. Thomson, and S. B. Dworkin, "Numerical investigation of soot formation mechanisms in partially-premixed ethylene-air co-flow flames," Combust. Flame, vol. 159, no. 9, pp. 2789-2798, Sep. 2012. doi: 10.1016/j.combustflame.2012.02.023.

[11] S. B. Dworkin, Q. Zhang, M. J. Thomson, N. A. Slavinskaya, and U. Riedel, "Application of an enhanced PAH growth model to soot formation in a laminar coflow ethylene/air diffusion flame," Combust. Flame, vol. 158, no. 9, pp. 1682-1695, Sep. 2011. doi: 10.1016/j.combustflame.2011.01.013.

[12] V. Chernov, M. J. Thomson, S. B. Dworkin, N. A. Slavinskaya, and U. Riedel, "Soot formation with $\mathrm{C} 1$ and $\mathrm{C} 2$ fuels using an improved chemical mechanism for PAH growth," Combust. Flame, vol. 161, no. 2, pp. 592-601, Feb. 2014. doi: 10.1016/j.combustflame.2013.09.017.

[13] J. Appel, H. Bockhorn, and M. Frenklach, "Kinetic modeling of soot formation with detailed chemistry and physics: laminar premixed flames of $\mathrm{C} 2$ hydrocarbons," Combust. Flame, vol. 121, no. 1-2, pp. 122-136, Apr. 2000. doi: 10.1016/S0010-2180(99)00135-2.

[14] N. M. Marinov et al., "Aromatic and Polycyclic Aromatic Hydrocarbon Formation in a Laminar Premixed n-Butane Flame," Combust. Flame, vol. 114, no. 1-2, pp. 192-213, Jul. 1998. doi: 10.1016/S0010-2180(97)00275-7.
[15] H. Wang, "Formation of nascent soot and other condensed-phase materials in flames," Proc. Combust. Inst., vol. 33, no. 1, pp. 41-67, 2011. doi: 10.1016/j.proci.2010.09.009.

[16] N. A. Eaves, Q. Zhang, F. Liu, H. Guo, S. B. Dworkin, and M. J. Thomson, "CoFlame: A refined and validated numerical algorithm for modeling sooting laminar coflow diffusion flames," Comput. Phys. Commun., vol. 207, pp. 464-477, Oct. 2016. doi: 10.1016/j.cpc.2016.06.016.

[17] R. J. Santoro, H. G. Semerjian, and R. A. Dobbins, "Soot particle measurements in diffusion flames," Combust. Flame, vol. 51, pp. 203218, Jan. 1983. doi: 10.1016/0010-2180(83)90099-8.

[18] R. J. Santoro, T. T. Yeh, J. J. Horvath, and H. G. Semerjian, "The Transport and Growth of Soot Particles in Laminar Diffusion Flames," Combust. Sci. Technol., vol. 53, no. 2-3, pp. 89-115, Jun. 1987. doi: $10.1080 / 00102208708947022$.

[19] N. A. Eaves, A. Veshkini, C. Riese, Q. Zhang, S. B. Dworkin, and M. J. Thomson, "A numerical study of high pressure, laminar, sooting, ethane-air coflow diffusion flames," Combust. Flame, vol. 159, no. 10, pp. 3179-3190, Oct. 2012. doi: 10.1016/j.combustflame.2012.03.017.

[20] F. Liu, H. Guo, G. Smallwood, and omer Gulder, "Numerical modelling of soot formation and oxidation in laminar coflow nonsmoking and smoking ethylene diffusion flames," Combust. Theory Modell., vol. 7, no. 2, pp. 301-315, Jun. 2003.

doi: $10.1088 / 1364-7830 / 7 / 2 / 305$.

[21] C. M. Megaridis and R. A. Dobbins, "Soot aerosol dynamics in a laminar ethylene diffusion flame," Symp. (Int.) Combust., vol. 22, no. 1, pp. 353-362, Jan. 1989. doi: 10.1016/S0082-0784(89)80041-4.

[22] M. M. Constantine and A. D. Richard, "Comparison of Soot Growth and Oxidation in Smoking and Non-Smoking Ethylene Diffusion Flames," Combust. Sci. Technol., vol. 66, no. 1-3, pp. 1-16, Jul. 1989. doi: $10.1080 / 00102208908947136$.

[23] K.-O. Lee et al., "Soot formation effects of oxygen concentration in the oxidizer stream of laminar coannular nonpremixed methane/air flames," Combust. Flame, vol. 121, no. 1-2, pp. 323-333, Apr. 2000. doi: 10.1016/S0010-2180(99)00131-5.

[24] A. Khosousi and S. B. Dworkin, "Detailed modelling of soot oxidation by $\mathrm{O} 2$ and $\mathrm{OH}$ in laminar diffusion flames," Proc.

Combust. Inst., vol. 35, no. 2, pp. 1903-1910, 2015.

doi: 10.1016/j.proci.2014.05.152.

[25] M. Saffaripour, A. Veshkini, M. Kholghy, and M. J. Thomson, "Experimental investigation and detailed modeling of soot aggregate formation and size distribution in laminar coflow diffusion flames of Jet A-1, a synthetic kerosene, and n-decane," Combust. Flame, vol. 161, no. 3, pp. 848-863, Mar. 2014. doi: 10.1016/j.combustflame.2013.10.016.

[26] A. Veshkini, S. B. Dworkin, and M. J. Thomson, "Understanding soot particle size evolution in laminar ethylene/air diffusion flames using novel soot coalescence models," Combust. Theory Modell., vol. 20, no. 4, pp. 707-734, Jun. 2016. doi: 10.1080/13647830.2016.1169319.

[27] A. Veshkini, S. B. Dworkin, and M. J. Thomson, "A soot particle surface reactivity model applied to a wide range of laminar ethylene/air flames," Combust. Flame, vol. 161, no. 12, pp. 3191-3200, Dec. 2014. doi: 10.1016/j.combustflame.2014.05.024. 


\title{
Glass Micro-cutting by Spark Assisted Chemical Engraving
}

\author{
Lucas A. Hof, Rolf Wüthrich \\ Department of Mechanical \& Industrial Engineering \\ Concordia University \\ Montreal, Canada \\ lucas.hof@concordia.ca,rolf.wuthrich@concordia.ca
}

\begin{abstract}
Glass remains a difficult to machine material. Its brittleness, chemical resistance and relatively high thermal conductivity challenges available technologies. An interesting approach is to the use of hybrid technologies such as Spark Assisted Chemical Engraving (SACE) which is a thermochemical process. Micro-drilling and micro-milling were intensively characterised and developed. SACE can however as well be used to micro-cut glass. This aspect was so far never discussed in the literature and no systematic data about its performance are available. As a first step, basic parameters such as depth of cut and tool feed rate must be determined. This communication aims to report systematic experiments on micro-cutting glass by SACE technology and demonstrates how using available data from SACE microdrilling the maximal depth-of-cut and tool feed can be determined. Relations for depth-of-cut and tool feedrate are proposed to use as guidance for glass micro-cutting.
\end{abstract}

Keywords - micro-machining; glass; spark assisted chemical engraving; micro-cutting; advanced manufacturing

\section{INTRODUCTION}

The application of glass science to the improvement of industrial tools occurred only in the past century, with a few exceptions. Glass has been employed in many forms to fabricate glazing and containers for centuries while it is now entering new applications that are appearing in micro and even nanotechnology like fibers, displays and Micro-ElectroMechanical-System (MEMS) devices [1]. Many qualities make glass attractive since it is transparent, chemically inert, environmentally friendly and its mechanical strength and thermal properties. In fact, no other materials being massproduced have shown such qualities over so many centuries. Nowadays glass offers recycling opportunities and allows for tailoring new and dedicated applications. Moreover, glass is radio frequency (RF) transparent, making it an excellent material for sensor and energy transmission devices. Another advantage of using glass in microfluidic MEMS devices [2] is its relatively high heat resistance, which makes these devices suitable for high temperature microfluidic systems [3] and sterilization by autoclaving.
However, glass remains a difficult to machine material. Its brittleness, chemical resistance and relatively high thermal conductivity challenges available technologies. An interesting approach is to the use of hybrid technologies such as Spark Assisted Chemical Engraving (SACE) [4] which is a thermochemical process (Fig. 1).

In SACE process, a voltage is applied between tool- and counter-electrode dipped in an alkaline solution - typical $\mathrm{NaOH}$ or $\mathrm{KOH}$ (Fig. 1). At high voltages (around $30 \mathrm{~V}$ ), the bubbles evolving around the tool electrode coalesce into a gas film and discharges occur from the tool to the electrolyte through it. Glass machining becomes possible due to thermally promoted etching (breaking of the Si-O-Si bond) [4].
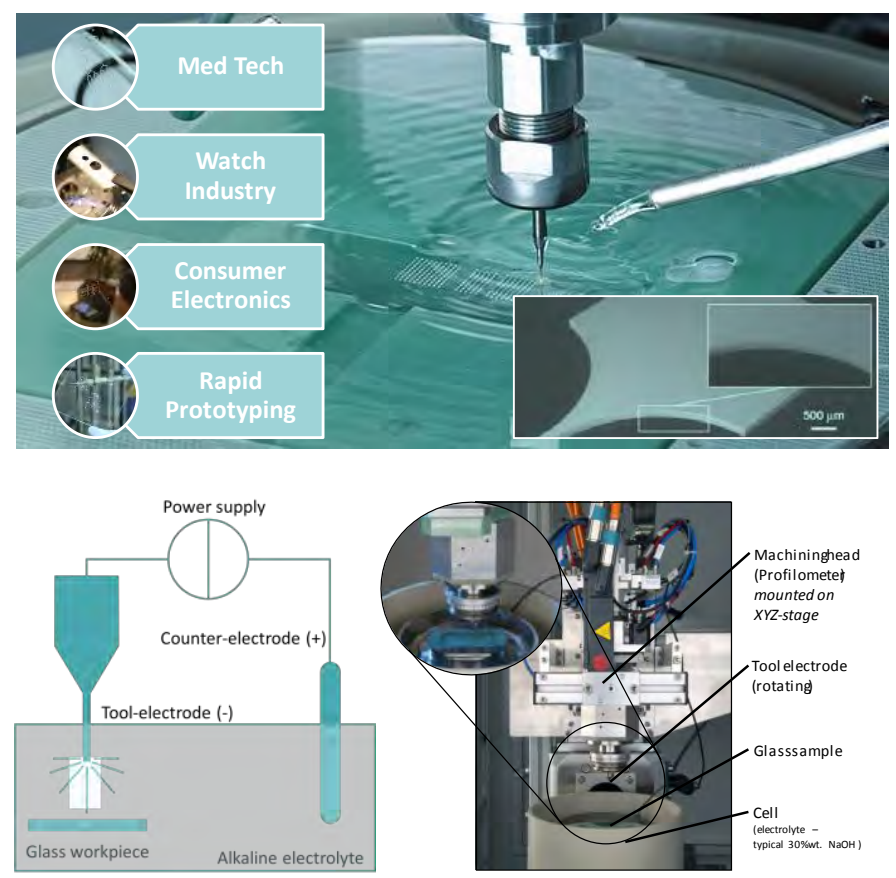

Figure 1. Above left: Overview of targeted applications/markets for SACE glass machining. Above right: SACE machined micro-hinge (scale bar $=500$ $\mu \mathrm{m}$ - width of 'hinge' $=30 \mu \mathrm{m}$ - thickness glass $=100 \mu \mathrm{m}$ ). Below left: Principles of SACE machining. Below right: SACE machining head. 
Developed since the sixties in last century in academia [5], SACE found recently its entrance in the industrial world. Micro-drilling and micro-milling were intensively characterised and developed. The introduction of pulsed voltage machining did made a further significant step forward in term of quality of machining. SACE can however as well be used to micro-cut glass. This aspect was so far never discussed in the literature and no systematic data about its performance are available. As a first step, basic parameters such as depth of cut and tool feed rate must be determined. This is the aim of the present communication which reports systematic experiments on micro-cutting glass by SACE and demonstrates how using available data from SACE micro-drilling maximal depth-of-cut and tool feed can be determined.

\section{MATERIALS AND METHODS}

Micro-cutting of pyrex glass (microscope slides) was performed on a Posalux FP1-SACE machine (Fig. 2). The machine incorporates a force sensor in the machining head that allows online measurement of the axial force (down to $1 \mathrm{mN}$ ) exerted on the tool-electrode. As electrolyte $30 \mathrm{wt} \%$ Potassium Hydroxide $(\mathrm{KOH})$, prepared from de-ionised water, was used. Low viscous electrolyte is chosen to reduce the chance that flushing with fresh electrolyte at the machining zone becomes the limiting factor for machining/cutting.

Cylindrical tool electrodes, made of tungsten carbide, 100 $\mu \mathrm{m}$ diameter with a bevel of $45^{\circ}$ where used. The spindle rotated at $1000 \mathrm{rpm}$. Cuts were performed over an equivalent length of at least two minutes of micro-cutting, adapted at the operating cutting speed.

After cutting, images with an optical microscope (Keyence VHX 5000) were acquired in order to evaluate precisely the realized depth-of-cut and to evaluate the cut qualitatively.

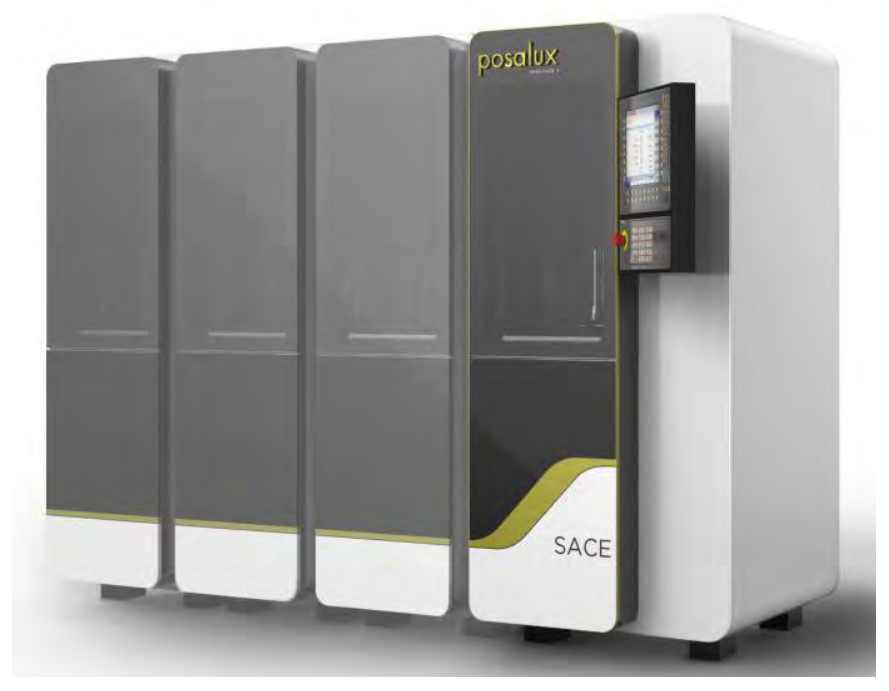

Figure 2. Posalux FP1-SACE machine (modular system)

\section{THEORY}

Material removal rate in SACE machining is limited, in case flushing of the machining zone happens easily, by heat propagation in the glass workpiece [4]. In the case of microcutting, electrolyte can access freely to the machining zone by appropriate flushing, using side tubes for electrolyte supply in the machining cell (Fig. 1). Further machined material is removed readily from it through the cut. Consequently, as a first approximation, one can discuss the relation between the axial depth of cut $p$ and horizontal feed rate $F$ (see Fig. 3 for a schematic) in terms of time needed for the heat to propagate inside the workpiece: one has to give enough time to the workpiece to heat up while one moves the tool.

Consider a tool of radius $r$. In the time interval

$$
t_{F}=\frac{2 r}{F}
$$

the tool will move over its own size. If heat propagates at the rate $h_{r}$ inside the workpiece, then the time $t_{h}$ needed to heat a distance $p$ is given by

$$
t_{h}=\frac{p}{h_{r}}
$$

If heat propagation is the rate limiting process, the tool cannot move faster than the feed rate $F$ at an axial depth of cut $p$ such that ( $t_{F}$ and $t_{h}$ must be of the same order of magnitude):

$$
F \cdot p=2 r \cdot h_{r}
$$

Equation (3) shows that, under the stated hypothesis that machining is only limited by heat propagation, the maximal value of the product $F \cdot p$ is, for a given tool and workpiece, a constant value. Note that, as the time $t$ needed to cut a path of length $L$ and depth $d$ is given by

$$
t=L \cdot d \frac{1}{F \cdot p}
$$

in SACE cutting, the time needed to perform a given cut is essentially constant regardless of the values of $F$ and $p$ chosen.

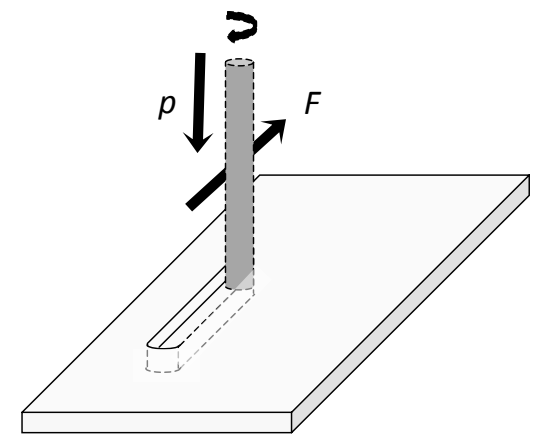

Figure 3. Schematic representation of micro-cuting by SACE process with a rotating tool-electrode (radius $r$ ) moving horizontally with feedrate $F$ at an axial depth of cut $p$. 
The heat propagation rate $h_{r}$ is function of the geometry of the problem and the energy put into the system (i.e. energy produced by the spark activity around the tool electrode). Estimations of the heat propagation rate in case of drilling for cylindrical tools are reported in the literature [4] and can be used as a first input.

Explicit calculation of the product $F \cdot p$ using equation (5.23) from [4] is found to be:

$$
F \cdot p=4 a \cdot \frac{\kappa-1}{\kappa}\left[1-\exp \left(-\pi\left(\frac{\kappa-1}{\kappa}\right)^{2}\right)\right]
$$

with $a$ the thermal diffusity of the workpiece $\left(a \sim 6 \cdot 10^{-7} \mathrm{~m}^{2} / \mathrm{s}\right.$ for Pyrex ${ }^{\circledR}$ glass). Relations between the dimensionless number $\kappa$ and voltage $U$ are empirically derived in section 5.2 of [4]:

$$
\kappa=0.018 \cdot U+0.615
$$

Equation (5) and (6) allows to establish a direct relationship between the machining voltage $U$ and the product $F \cdot p$ which can be used to define the material removal rate (MRR) when multiplying with the tool diameter.

\section{RESULTS AND DISCUSSION}

To determine experimentally the maximal depth-of-cut $p$ at a given tool feed rate $\mathrm{F}$ a series of cuttings of $0.4 \mathrm{~mm}$ thick glass slides $\left(\right.$ Pyrex $\left.^{\circledR}\right)$ were performed. The cut was considered to be successful if

a) The tool didn't break, and

b) The measured depth of the cut was equal or higher than the imposed depth of cut during machining.

From a practical point of view, thanks to the force sensor inside the tool-electrode holder, it was possible to detect none successful cuts due to an increased force acting on the tool (a few $\mathrm{mN})$. Pulsed voltage with $2.5 \mathrm{~ms}$ pulse high time $\left(t_{\text {high }}\right)$ and $0.1 \mathrm{~ms}$ pulse low time $\left(t_{\text {low }}\right)$ was used. In such conditions the gas film shows high stability and very few bubbles are observed around the tool-electrode. Cutting experiments were performed at three pulsed machining voltages (high, intermediate and low) as denoted in table 1 .

The average input voltage $U_{\text {avg }}$ can be calculated according:

$$
U_{\text {avg }}=\left(t_{\text {high }}-t_{\text {low }}\right) /\left(t_{\text {high }}+t_{\text {low }}\right) \Delta U_{\text {pulse }}+U_{\text {lowlevel }}
$$

TABLE I. SACE MACHINING SETTINGS

\begin{tabular}{lcccc}
\hline \multicolumn{4}{c}{ Pulsed Voltage Input } \\
\hline & High Level & Low Level & Period & Duty Cycle \\
\hline Low & $30 \mathrm{~V}$ & $17.5 \mathrm{~V}$ & $2.6 \mathrm{~ms}$ & $96.15 \%$ \\
Intermediate & $33 \mathrm{~V}$ & $17.5 \mathrm{~V}$ & $2.6 \mathrm{~ms}$ & $96.15 \%$ \\
High & $35 \mathrm{~V}$ & $17.5 \mathrm{~V}$ & $2.6 \mathrm{~ms}$ & $96.15 \%$ \\
\hline
\end{tabular}

TABLE II. HEAT PROPAGATION IN GLASS WORKPIECE

\begin{tabular}{ll}
\hline Machining voltage $[\mathbf{V}]$ & $\mathbf{F} \cdot \mathbf{p}\left[\mathrm{mm}^{2} / \mathbf{m i n}\right]$ \\
\hline 30 & 1.0 \\
33 & 1.6 \\
35 & 2.2 \\
\hline
\end{tabular}

Using these machining voltages (table 1 and equation (7)) as input in equations (5) and (6) we estimate the values for $F$. $p$ as outlined in table 2 .

As shown in Fig. 4, the maximal depth of cut for a given tool feed follows equation (3). The dashed lines were obtained by least square fits.

The quality of the cut is assessed by optical microscopy for the different machining voltages (low - intermediate - high voltage) and at varying feed rate and depth-of-cut (Fig. 5).

It can be clearly observed that quality of the glass cut generally increases when the machining voltage reduces (indents of $\sim 75 \mu \mathrm{m}-60 \mu \mathrm{m}-50 \mu \mathrm{m}$ for respectively $35 \mathrm{~V}-$ $33 \mathrm{~V}-30 \mathrm{~V}$ at maximum cutting depth for each voltage and feedrate $=5 \mathrm{~mm} / \mathrm{min}$.)). Decreasing the depth of cut results generally also in higher machining quality. In the extremes, when machining at same feedrate $-F=40 \mathrm{~mm} / \mathrm{min}$ - and low depth of cut, the machining quality is around the same for all applied machining voltages.

Reasonable quality of cut in glass by SACE technology can be obtained using the derived tool feed vs. depth of cut relations. It should be noted that this model is not optimized for quality of cut surface and other strategies, such as 'SACE polishing' by approaching the surface with small lateral increments of the tool-electrode at very low voltage, can be followed to achieve surface qualities in the order of a few microns.

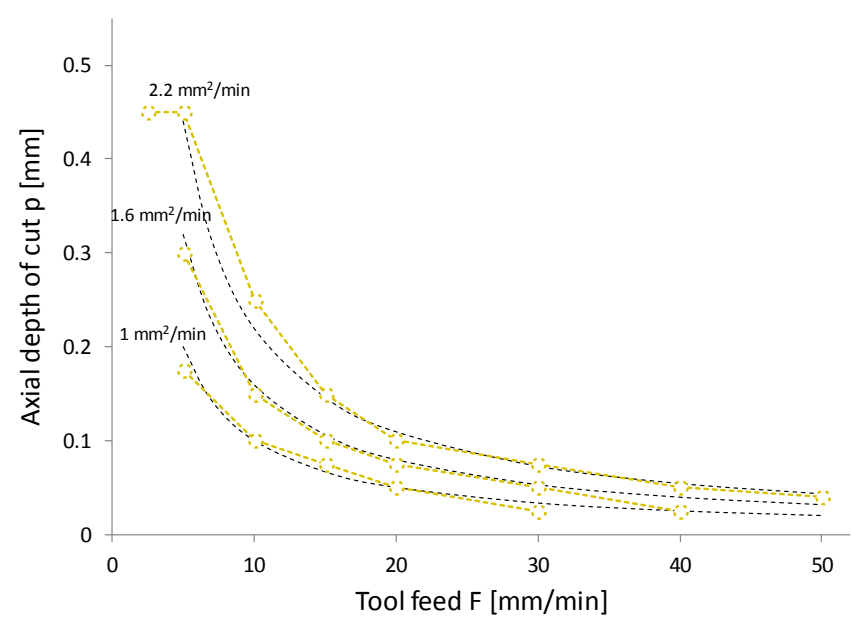

Figure 4. Yellow: Empirical values for maximal depth-of-cut $p$ [mm] versus tool feedrate $F[\mathrm{~mm} / \mathrm{min}]$ for tungsten carbide $100 \mu \mathrm{m}$ diameter tools. Grey dashed lines: Theoretical model values. 
Machining voltage $-35 \mathrm{~V}: 17.5 \mathrm{~V} / 2.5 \mathrm{~ms}: 0.1 \mathrm{~ms}$

Glass thickness: $400 \mu \mathrm{m}$

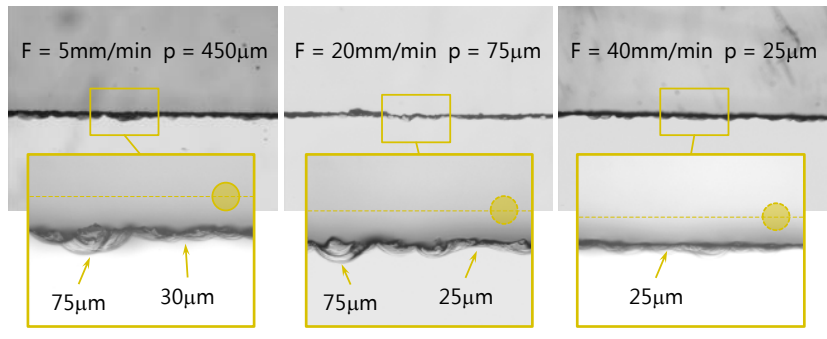

Machining voltage $-33 \mathrm{~V}: 17.5 \mathrm{~V} / 2.5 \mathrm{~ms}: 0.1 \mathrm{~ms}$

Glass thickness: $400 \mu \mathrm{m}$

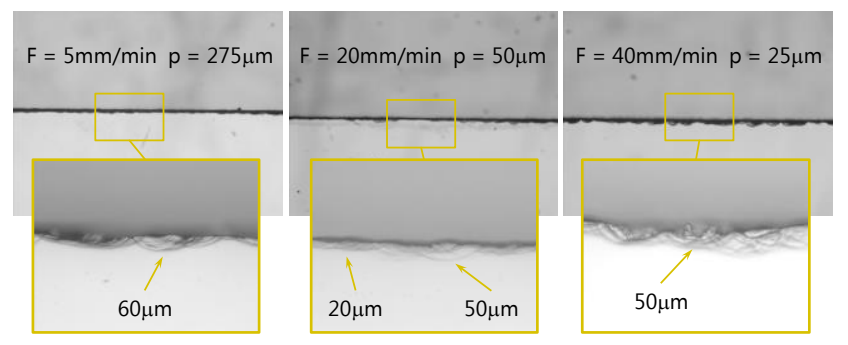

Machining voltage $-30 \mathrm{~V}: 17.5 \mathrm{~V} / 2.5 \mathrm{~ms}: 0.1 \mathrm{~ms}$

Glass thickness: $400 \mu \mathrm{m}$

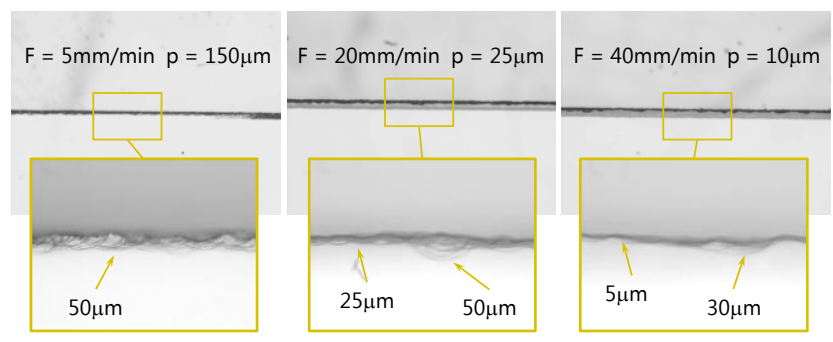

Figure 5. Microscopy (Keyence VHX 5000) analysis of cut quality at different machining voltage, feedrate and depth-of-cut.

\section{CONCLUSIONS}

- Cutting parameters for tungsten carbide $100 \mu \mathrm{m}$ diameter cylindrical tools (with $45^{\circ}$ bevel) were determined;

- A simple model relating the lateral tool feedrate $F$ to the maximum depth-of-cut $p$ based on the assumption that machining is only limited by heat tranfer in the workpiece was presented and matches well the measured data;

- Cut quality was evaluated by optical micro-graphs for different machining voltages (high, medium and low voltage).

The developed model allows to predict the maximum depth of cut for a given tool feedrate, which eliminates timeconsuming 'trial runs' before machining to determine the best cutting parameters. Hence, this study contributes to further optimization of SACE technology for industrial use.

\section{ACKNOWLEDGMENT}

This work was supported by the Natural Sciences and Engineering Research Council of Canada (NSERC). L.A.H. would like to thank the Quebec MEESR for the 'bourse d'excellence pour étudiants étrangers' (Doctoral students V1) and acknowledge Posalux SA for the fruitful discussions and work performed on their FP1-SACE machine.

\section{REFERENCES}

[1] E. Le Bourhis, Glass, Mechanics and Technology. Wiley-VCH, 2014.

[2] G. M. Whitesides, "The origins and the future of microfluidics.," Nature, vol. 442, no. 7101, pp. 368-373, 2006. doi: 10.1038/nature05058.

[3] D. Sinton, "Energy: the microfluidic frontier.," Lab Chip, vol. 14, no. 17, 2014.doi: 10.1039/C4LC00267A.

[4] R. Wüthrich and J. D. Abou Ziki, Micromachining Using Electrochemical Discharge Phenomenon. Elsevier, 2015.

[5] H. Kurafuji and K. Suda "Electrical discharge drilling of glass" Annals of the CIRP, 16, pp. $415-419,1968$. 


\section{A Finite Element Model to Study the Effect of Porosity Location on the Elastic Modulus of a Cantilever Beam}

\author{
Stephen Handrigan \\ Department of Mechanical Engineering \\ Memorial University of Newfoundland \\ St. John's, Canada
}

\author{
Sam Nakhla \\ Department of Mechanical Engineering \\ Memorial University of Newfoundland \\ St. John's, Canada
}

\begin{abstract}
The effect of the location of porosity concentration on elastic modulus of a cantilever beam is investigated. First, two-dimensional investigation with beam theory, EulerBernoulli and Timoshenko, was performed to estimate the modulus based on load-deflection curve. Second, threedimensional finite element model (FEM) in Abaqus was developed to identify the effect of porosity concentration. The use of macro-models such as beam theory and threedimensional FEM enabled enhanced understanding of the effect of porosity on modulus.
\end{abstract}

Keywords-porosity, elastic modulus, FEA, micro-mechanical, cantilever

\section{INTRODUCTION}

\section{A. Background}

It is known that porosity affects the mechanical properties of metals. In many materials, increases in macroscale pore sizes have shown to decrease ultimate strength, yield stress, and fatigue life [1]. However, due to advancements in manufacturing, pores in metals tend to be on the microscale instead of macroscale. This presents new concerns since the effect on a material's mechanical properties due to this microporosity is unknown [2]. This is critical because without understanding how microporosity affects a material, the ability to predict behaviour due to loading throughout its life cycle is difficult. Throughout the life cycle of a structure, exposure to various environmental conditions, sometimes harsh, is possible. Due to these environmental conditions, it is possible that porosity can be increased in the material. As such, structures may be affected in various locations, and in differing amounts, depending on the exposure to the environment. It is critical to understand if the location of porosity has an effect on a structure.

\section{B. Purpose of Study}

It is known that porosity has an effect on elastic modulus. The work of Morrissey and Nakhla [3] presented a literature review on existing models available in literature. These models, mostly empirical, describe the effect of porosity on elastic modulus. Morrissey and Nakhla developed a two-dimensional finite element model (FEM) that successfully captured the effect of porosity on elastic modulus in tension.

In the current work, a three-dimensional FEM is developed to investigate the effect of porosity on modulus. The effects of uniform distribution or concentrated zones of porosity was investigated. All FEM results were compared to test data reported in literature.

\section{PROCEDURES}

\section{A. Understanding Experimental Setup and Data}

The first step in this study was to examine experimental load versus deflection data for micro-cantilevers. For this study, the work by Gong [4] was first analyzed to understand the correct beam theory to apply for determining elastic modulus, as well as to develop the three-dimensional FEM. It is reported in [4], for beam 5, an experimental elastic modulus of $147 \mathrm{GPa}$. As well, their three-dimensional FE model captured the trend of porosity reduction with an average error in prediction of $38 \%$ compared to experimental.

It is reported in [4] that samples were heat treated such that an average grain size of $8-10 \mu \mathrm{m}$ was obtained. From these samples, the micro-cantilevers were produced at the University of California, Berkeley (UCB) using a focused ion beam (FIB). The FIB was used to cut three trenches using a 7-15 nA beam current - forming a U-shaped trench that had a width of 20-30 $\mu \mathrm{m}$ and a depth of $10 \mu \mathrm{m}$. Then using a 1-3 nA beam current, the outline of the beam was refined. Lastly, the sample was rotated $45^{\circ}$ both clockwise and counter-clockwise around the length of the beam to allow for cutting of the triangular bottom of the beam. Using a MicroMaterials nanoindenter with a square tip, UCB was able to obtain the load and deflection data for the micro-cantilevers. The depth of indentation into bulk material was removed from the experimental deflection to ensure only the displacement due to bending is accounted. Lastly, the load was applied with a displacement rate of 10 $\mathrm{nm} / \mathrm{s}$ to the tip of the micro-cantilever until fracture.

The micro-cantilever is approximately $7.5 \mu \mathrm{m}$ tall, $4 \mu \mathrm{m}$ wide, and $28 \mu \mathrm{m}$ long - load is applied at approximately $27 \mu \mathrm{m}$ from the root, this will be taken to be the length since deflection is also measured at this location. The cross-section is pentagonal shaped, proposed in [5]. The beam is considered to be short and stubby with a length-to-height ratio of less than 
four. The beam is not undergoing uniform bending (concentrated load introduces shear forces) and the beam is not rigidly connected to the support. Due to these factors, shear effects may be highly pronounced at the root. Therefore, both Euler-Bernoulli and Timoshenko beam theories were used in the current study.

Using the load-deflection curve reported in [4], the data was extracted and the slope for the linear section was determined to be approximately $1932 \mathrm{~N} / \mathrm{m}$. Next, the modulus was calculated using Euler-Bernoulli and Timoshenko beam theory, resulting in $148 \mathrm{GPa}$ and $154 \mathrm{GPa}$, respectively. This is compared to the reported modulus in [4] in Table I below. Due to the beam geometry, boundary conditions, and the manner at which the load is applied, it is most likely that Timoshenko beam theory is more accurate because the effect due to shear is included. As such, Timoshenko beam theory will be the method of calculating the elastic modulus within this study.

TABLE I. COMParison OF CAlculated Modulus

\begin{tabular}{|c|c|c|}
\hline Beam Theory & This Study & Reported in [4] \\
\hline Euler-Bernoulli & $148 \mathrm{GPa}$ & $147 \mathrm{GPa}$ \\
\hline Timoshenko & $154 \mathrm{GPa}$ & - \\
\hline
\end{tabular}

For this study, several assumptions are made. Uranium Dioxide is highly anisotropic [6-7]; however, it is assumed that the material acts as an isotropic material since the microcantilevers are ideally contained within a single crystal-grain. It is reported in [4] that not all micro-cantilevers are within a single grain; however, without additional information on number of grains and grain orientation, the assumption will remain. The FEM assumes the beam is solid, homogeneous and has a constant cross-section. Lastly, it is assumed that the effect on Poisson's ratio for porosities less than $5 \%$ is negligible [89]. The FEM assumes uniform porosity distribution across the cross-section.

\section{B. Finite Element Model - Three-Dimensional Beam}

To build the FEM, a three-dimensional, deformable solid part was created in Abaqus. The substrate was sketched and extruded to create a cube. From the front face, the geometry was sketched and extruded to create the beam. The actual beam from [4] and the currently developed FEM is shown in Fig.1. The beam and substrate were then partitioned to allow for separate modification of material properties and mesh development. The beam was further partitioned into three segments of equal length, shown in Fig. 2. This allows for different material properties to be applied to each segment. The next step was to develop the mesh. The mesh was refined differently within the beam than the substrate. The beam had 11,088 3D Stress Hex Quadratic (Reduced Integration) elements, while the substrate had 4,464 3D Stress Hex Quadratic (Reduced Integration) elements.

The next step was to apply boundary conditions to the FEM. The side, rear, and bottom faces of the substrate have fixed boundary condition, while the top and front faces, as well as the beam, are free surfaces, as shown in Fig. 3 (lighter colours indicate free while dark indicate fixed). Next, a tip load was applied to the beam. The deflection is measured from the bottom side, directly under the location of the applied load, to ensure indentation into the top surface of the beam due to the load was not accounted, thus skewing the deflection data.

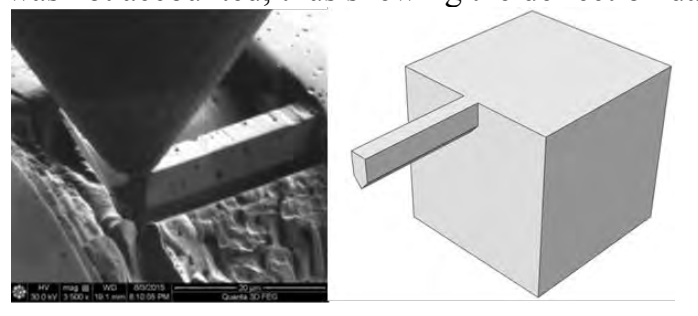

Figure 1. SEM Picture of Experimental Setup [4] (left), Abaqus FEM this study (right)

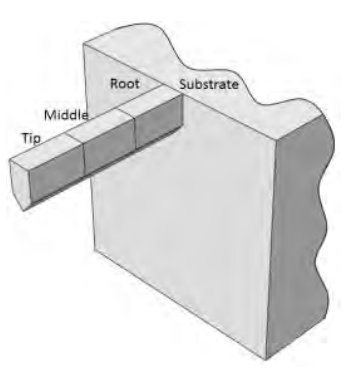

Figure 2. Beam Sections (From left to right: Tip, Middle, Root, Substrate).

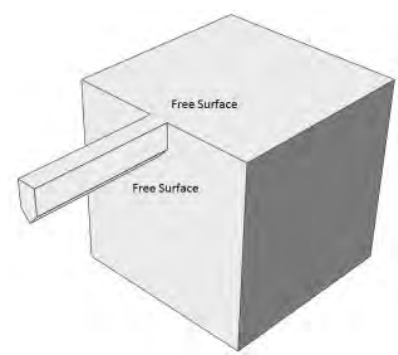

Figure 3. Boundary Conditions (Top, Front, and Beam are free surfaces; all other sides fixed).

Once the FEM was developed, the first test was to determine the calculated modulus of a perfect beam with nominal modulus and zero porosity. This was to provide a baseline for the predicted modulus due to the boundary conditions alone. However, it was unsure if the size of the substrate would have an effect on the results and as such, the FEM was first tested with various substrate sizes.

The substrate was constrained to be a solid cube in each case. Four substrate sizes were considered and the dimensions were scaled due to the beam's largest dimension - height. Sizes were labelled A through D, with A being the smallest and D the largest. Substrate size A was approximately the same size as the height of the beam. Substrate sizes B, C, and D were approximately one-and-a-half times, three-times, and six-times larger than the beam height, respectively. A comparison of the moduli obtained for the four cases is shown below in Table II. As can be seen, the size of the substrate does affect the response of the beam; however, as long as the substrate is at least three-times the height of the beam, the effect is 
insignificant. This is expected since when the substrate is small, the fixed boundary condition has a greater effect on the rigidity of the root of the beam, thus, stiffening the beam and over-predicting the modulus. As the substrate size is increased, this effect is reduced to a point such that the modulus is unchanged since the fixed boundary condition is sufficiently far enough away from the root of the beam. For this study, Substrate size D was chosen for the FEM to ensure the fixed boundary condition did not influence the results.

TABLE II. COMPARISON OF SUBSTRATE SIZE

\begin{tabular}{|l|l|}
\hline Substrate Size & Modulus Calculated (GPa) \\
\hline A & 186.7 \\
\hline B & 182 \\
\hline C & 180 \\
\hline D & 180 \\
\hline
\end{tabular}

With the size of the substrate determined, the modulus obtained due to the boundary conditions alone was $18 \%$ less than the nominal value. This will be considered the base case value and the effect on modulus due to porosity amount and location will be compared.

\section{Finite Element Model-Reduction in Modulus due to Porosity under Bending}

Before an analysis can be completed, a FEM must first be developed to determine the percent reduction in elastic modulus versus the position of porosity concentration along the beam, similar to [3]. A two-dimensional FEM was developed to determine this reduction. The FEM was a long, slender beam, with uniform cross-section, and rigidly supported at the root.

The FEM is assumed to have a nominal elastic modulus with zero pores to establish a base case. Next, the elastic modulus is calculated from load and deflection data at the tip. Using this calculated modulus, it was normalized with the nominal value to provide a percent reduction in modulus. This was repeated with various pore locations and pore sizes to simulate various porosities and porosity concentration locations. The results of this FEM are shown below in Fig. 4.

As the porosity concentration moves further away from the root, the percent reduction in elastic modulus decreases. These percent reduction values were applied to the nominal elastic modulus for Uranium Dioxide and new moduli were calculated for when porosity is concentrated within different sections of the beam. A similar process was completed for when the porosity is uniformly distributed over the entire length of the beam. In this case, the percent reduction was approximately equal to the reduction experienced when porosity was concentrated at the tip for both porosities.

Next, these reduced moduli were imported into the threedimensional FEM for the various setups. These moduli were applied to specific sections of the beam where the porosity was to be concentrated while the remaining sections of the FEM, including the substrate, were considered to be equal to the nominal modulus of $219 \mathrm{GPa}$ for Uranium Dioxide [10].

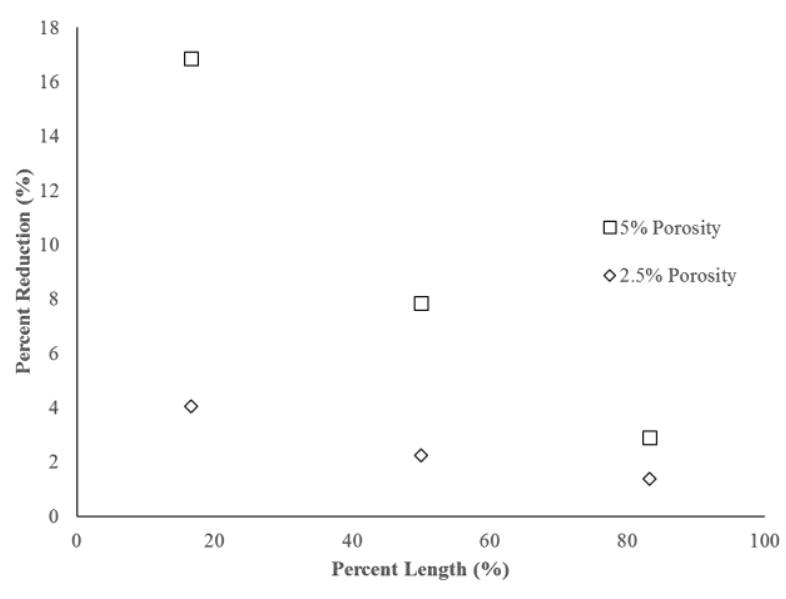

Figure 4. Percent reductions in modulus due to porosity concentration along percent length of the beam.

\section{RESULTS AND DISCUSSION}

The results for moving the porosity concentration from the root to the tip is shown in Table III. When the porosity is concentrated at the root, the reduction in modulus is the greatest, while moving towards the tip this reduction decreases. This trend is further supported by the Reduction in Modulus due to Porosity under bending FEM. Furthermore, Gong has successfully demonstrated in [4] that the location of pore concentration does affect the modulus, with the largest effect at the root and decreasing away from the root.

When comparing the percent reductions determined from the two-dimensional and three-dimensional FE models in this study, the values differ. There are several reasons for these discrepancies: 1) different applied boundary conditions, 2) different cross-section, thus different second moments of area three-dimensional FEM is pentagonal whereas twodimensional represents rectangular, and 3) general errors converting from two-dimensional to three-dimensional FEM.

When comparing to Gong's results, there is a large difference in the values. There are several reasons for these discrepancies: 1) pore location relative to neutral plane in Single Pore FEM [4] is unknown - pores further away will have a larger effect on reducing modulus, 2) pore distribution is not uniform across cross-section in Cluster Pore FEM [4] pores constrained within rectangular-portion of the beam (no pores located in the triangular section), and 3) uncertain boundary conditions - Gong initially completed a substratesize sensitivity-analysis, but for the FEM of the reconstructed beam, the substrate appears to be approximately the same size as the beam which would greatly influence the results due to the boundary conditions stiffening the beam.

In Fig. 5, the Abaqus FE models completed for this study are compared with experimental load versus deflection data reported in [4]. From this, it is evident that the FE models capture the trend of the experimental results from [4] with average error of $14.8 \%$. However, there is some error which can be contributed to the assumptions made in this study. The FE models completed in this study assumed a uniform, constant cross-section, free of imperfections, which is not the 
case when observing SEM images of the beam in [4]. As well, the FE models were isotropic due to the single-grain assumption, but as reported in [4] this was not true. Lastly, the porosity concentration in the FE models completed in this study do not include the effect of pores away from the neutral plane - it is assumed the porosity is concentrated uniformly across the cross-section with no bias away from the neutral plane.

In Fig. 6, all FE models by this study and by Gong are compared with the experimental data. It is evident that Gong's Single Pore Root FEM has the largest effect, comparable to this study's FEM for 5\% porosity concentrated at the root. The placement of this single pore from the neutral plane is unknown and as such it is difficult to compare to the FE models completed in this study.

In Fig. 7, all 2.5\% porosity models are compared with the experimental data. Again, Gong's Single Pore Root FEM has the largest effect on the load-deflection response. However, when comparing the Cluster Pore FE models from [4] to the FE models completed in this study, it is evident that the Cluster Pore FE models are over-predicting the elastic modulus. This may be due to the orientation chosen by Gong to model the cluster of pores. This orientation differs from the assumed uniform porosity concentration for the FE models completed in this study, hence the lower reductions.

In Fig. 8, a comparison is shown between Gong's experimental data and FE models. Gong's FE models show a much better agreement to the experimental data than is reported in [4] with an average error of $21.8 \%$ with experimental.

The FE models completed in this study, which assume uniform porosity distribution across the cross-section, show with certainty that porosities of $5 \%$ have a large effect on the behaviour of the beam when concentrated close to the root. However, if these large porosities are uniformly distributed over the length, or concentrated in a location away from the root - at or beyond half the beam length - the effect decreases drastically. Whereas, for porosities of $2.5 \%$ and lower, it can be concluded that there is minimal effect on the beam's modulus regardless of distribution and concentration throughout the length.

\section{CONCLUSIONS}

This study has proven that the amount of porosity and the location of said porosity has an effect on the elastic modulus. Several cases were analyzed and it was determined that porosities concentrated at the root have the largest effect on the elastic modulus of a cantilever, while porosities uniformly distributed over the length, or concentrated away from the root, have minimal effect on elastic modulus.

\section{ACKNOWLEDGEMENTS}

The authors are grateful for the financial support from Suncor Inc. The first author is grateful to Mr. Liam Morrissey for assisting in developing the Reduction in Modulus due to Porosity under bending FEM.

\section{REFERENCES}

[1] N. I. Romanova, G. S. Kreimer, and V. I. Tumanov, "Effects of residual porosity on the properties of tungsten carbide-cobalt hard alloys," Soviet Powder Metall Met Ceram, vol. 13, pp. 670-673, August 1974. doi:10.1007/BF00798346.

[2] R. A. Hardin and C. Beckermann, "Effect of porosity on the stifness of cast steel," Metall and Mat Trans A, vol. 38, pp. 2992-3006, December 2007. doi: 10.1007/s11661-007-9390-4.

[3] L. S. Morrissey and S. Nakhla, "A finite element model to predict the effect of porosity on elastic modulus in low porosity materials," Metall and Mat Trans $A$, in press.

[4] B. Gong, Finite Element Analysis of Micro-Cantilever Beam Experiments in UO2, November 2015.

[5] D. Di Maio and S. G. Roberts, "Measuring fracture toughness of coatings using focused-ion-beam-machined microbeams," Mat Res, vol.20, pp. 299-302, February 2005. doi: 10.1557/JMR.2005.0048.

[6] K. Gofryk et al., "Anisotropic thermal conductivity in uranium dioxide," Nat. Commun., August 2014. doi:10.1038/ncomms5551.

[7] F. Gupta, A. Pasturel, and G. Brillant, "Diffusion of oxygen in uranium dioxide: A first-principles investigation," Ohys. Rev. B, vol. 81, January 2010

[8] M. Asmani, C. Kermel, A. Leriche, and M. Ourak, "Influence of porosity on young's modulus and poisson's ratio in alumina ceramics," Euro Cera Soci, vol. 21, pp. 1081-1086, August 2001. doi: 10.1016/S0955-2219(00)00314-9.

[9] C. Yu, S. Ji, and Q. Li, "Effects of porosity on seismic velocities, elastic moduli and poisson's ratios of soli materials and rocks," Rock Mech and Geotech Eng, vol. 8, pp. 35-49, February 2016. doi: 10.1016/j.jrmge.2015.07.004.

[10] D. R. Olander, Fundamental Aspects of Nuclear Reactor Fuel Elements, p. 335 , 1976.doi: $10.2172 / 7343826$.

TABLE III. REDUCTION IN MODULUS DUE TO POROSITY

\begin{tabular}{|c|c|c|c|c|c|}
\hline \multirow{2}{*}{$\begin{array}{c}\text { Finite Element } \\
\text { Model }\end{array}$} & \multirow{2}{*}{ Porosity } & \multirow{2}{*}{$\begin{array}{c}\text { Reduction in Modulus } \\
\text { (2D FEM) }\end{array}$} & \multirow{2}{*}{$\begin{array}{c}\text { Reduction in Modulus } \\
\text { (3D FEM) }\end{array}$} & \multicolumn{2}{|c|}{ Reductions in Modulus Reported in [4] } \\
\hline & & & & Single Large Pore ${ }^{\mathrm{b}}$ & Cluster of Pores $^{\mathrm{c}}$ \\
\hline Root & $2.5 \%$ & $-3.0 \%$ & $-1.38 \%$ & $-20 \%$ & $-4.5 \%$ \\
\hline \multirow{2}{*}{ Middle } & $2.5 \%$ & $-1.2 \%$ & $-0.23 \%$ & $-8.6 \%$ & $-2.7 \%$ \\
\hline & $5.0 \%$ & $-6.8 \%$ & $-1.25 \%$ & - & - \\
\hline \multirow{2}{*}{ Entire } & $2.5 \%$ & $-0.3 \%$ & $-0.26 \%$ & - & - \\
\hline & $5.0 \%$ & $-1.1 \%$ & $-0.79 \%$ & - & - \\
\hline
\end{tabular}

b. Location of Pore relative to neutral axis unknown 


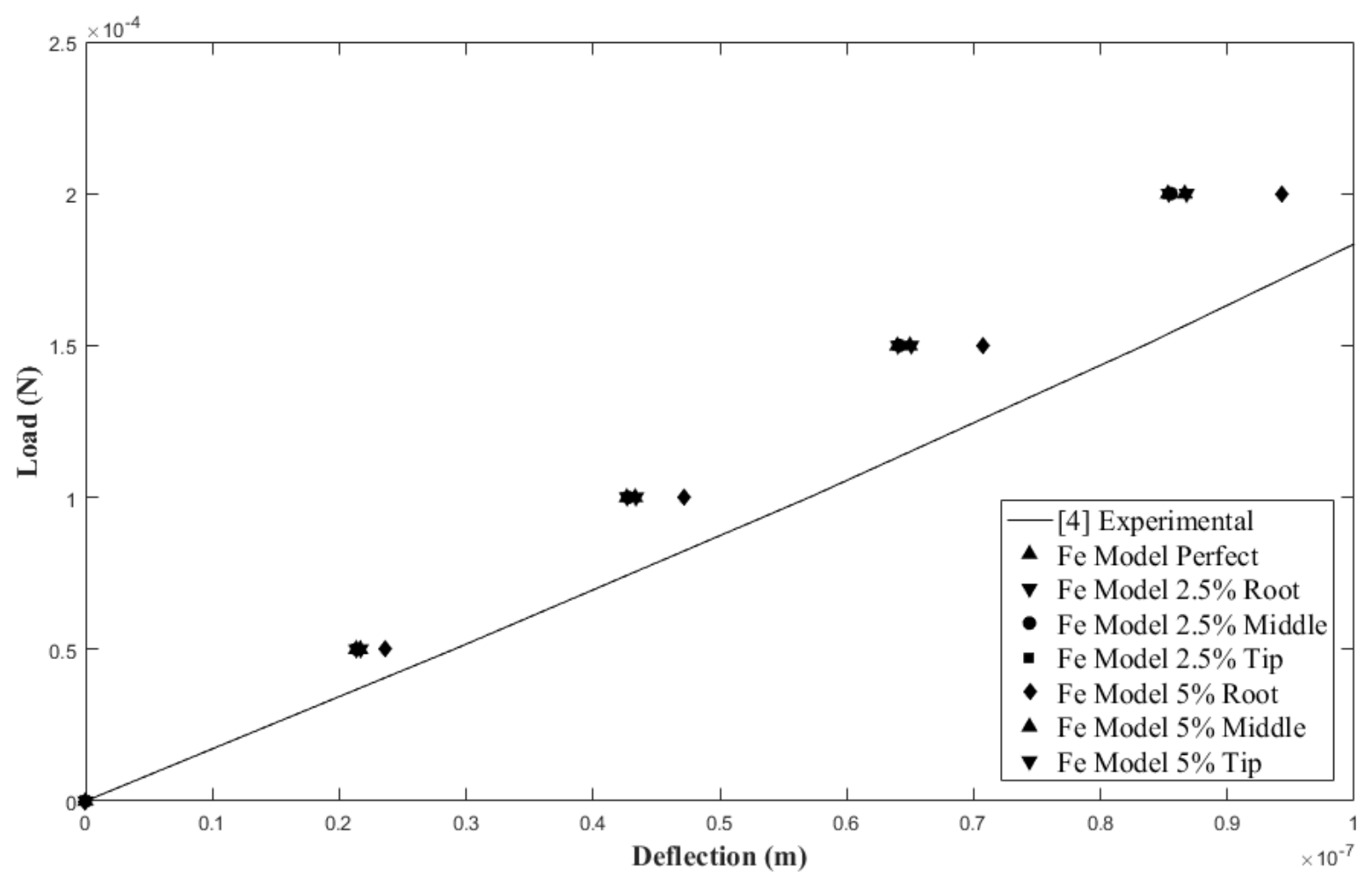

Figure 5. Load-Deflection Comparison of This Study with [4] Experimental.

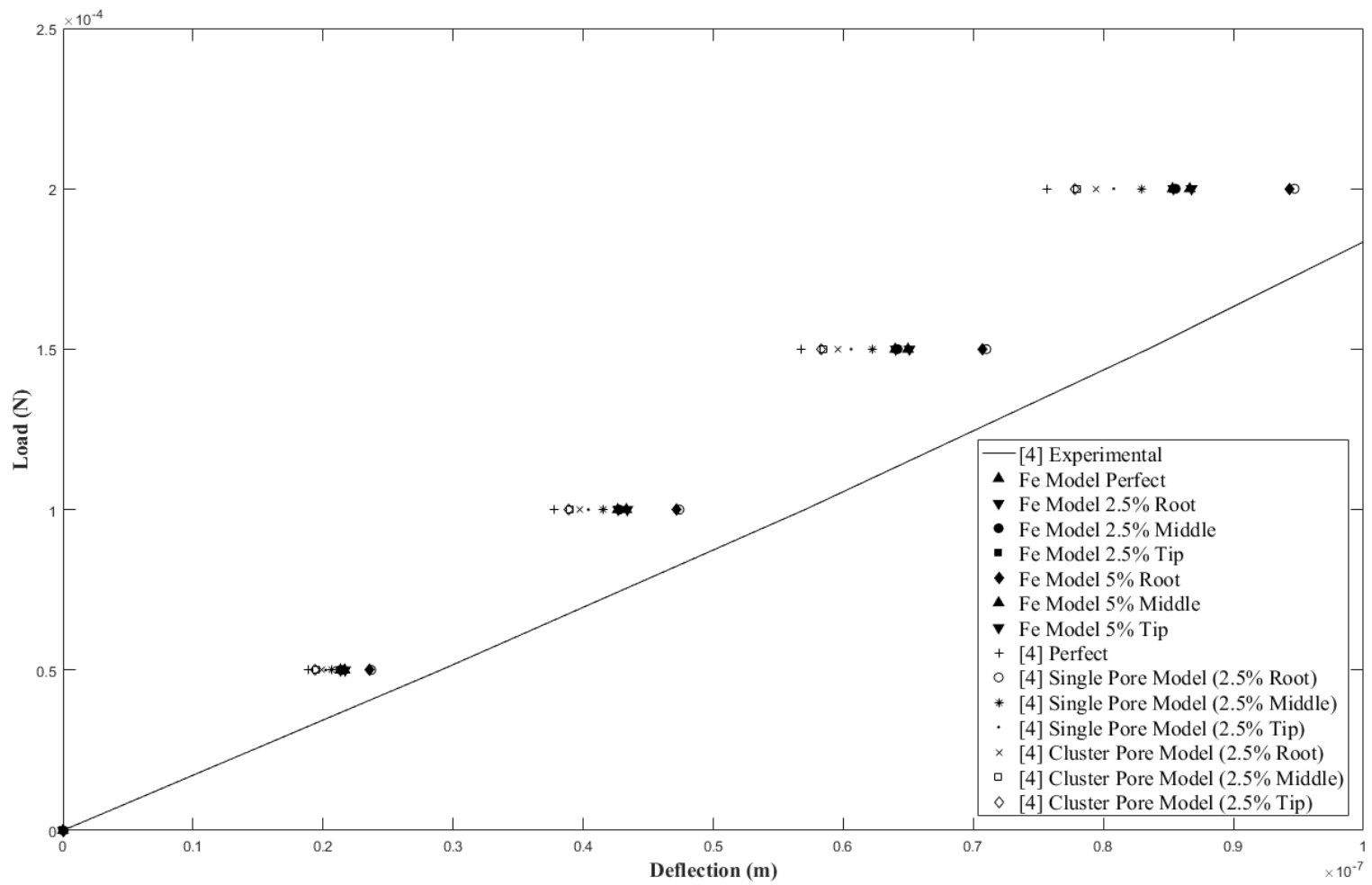

Figure 6. Load-Deflection Comparison of All Data. 


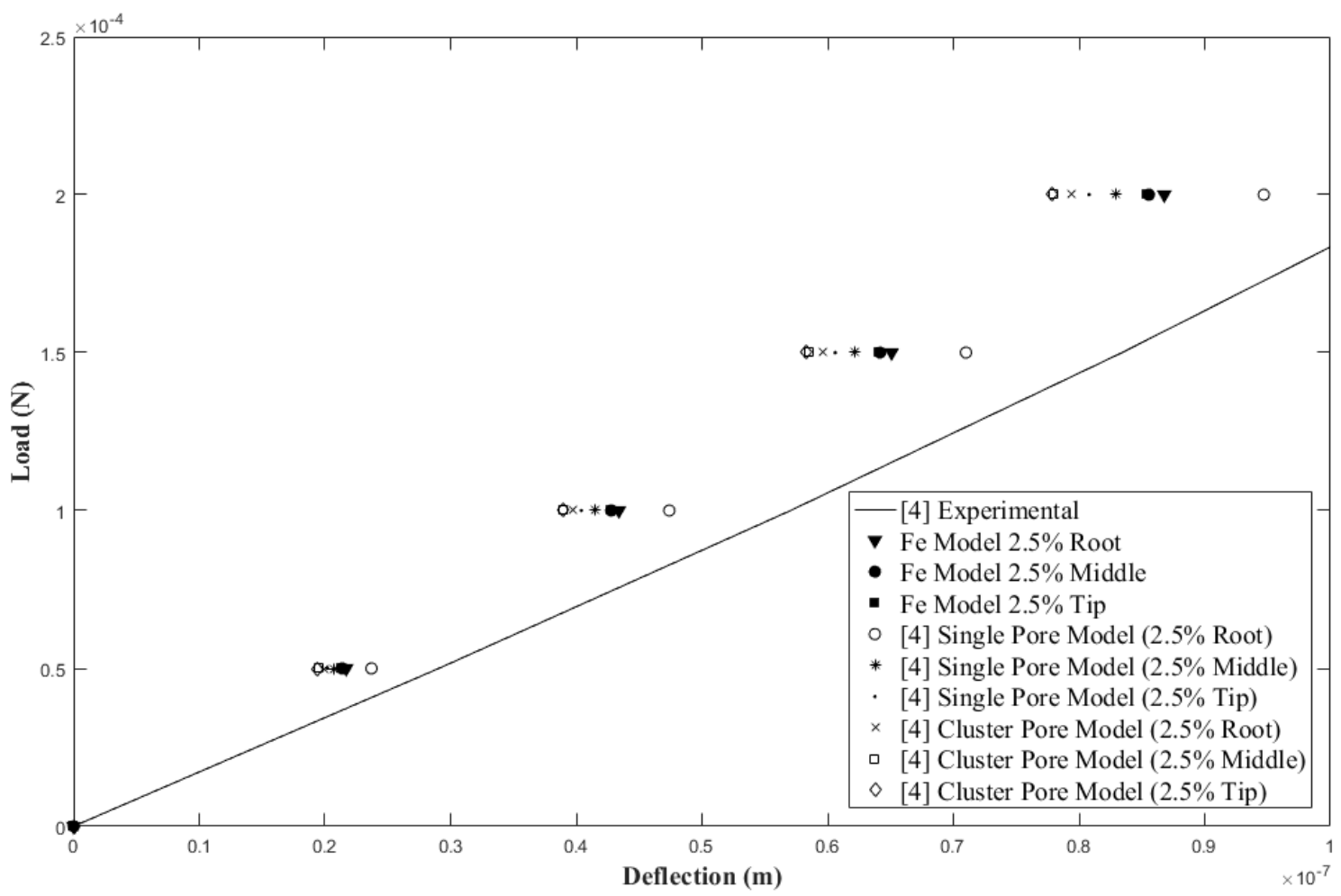

Figure 7. Load-Deflection Comparison of All 2.5\% Porosity FE Models with Experimental [4].

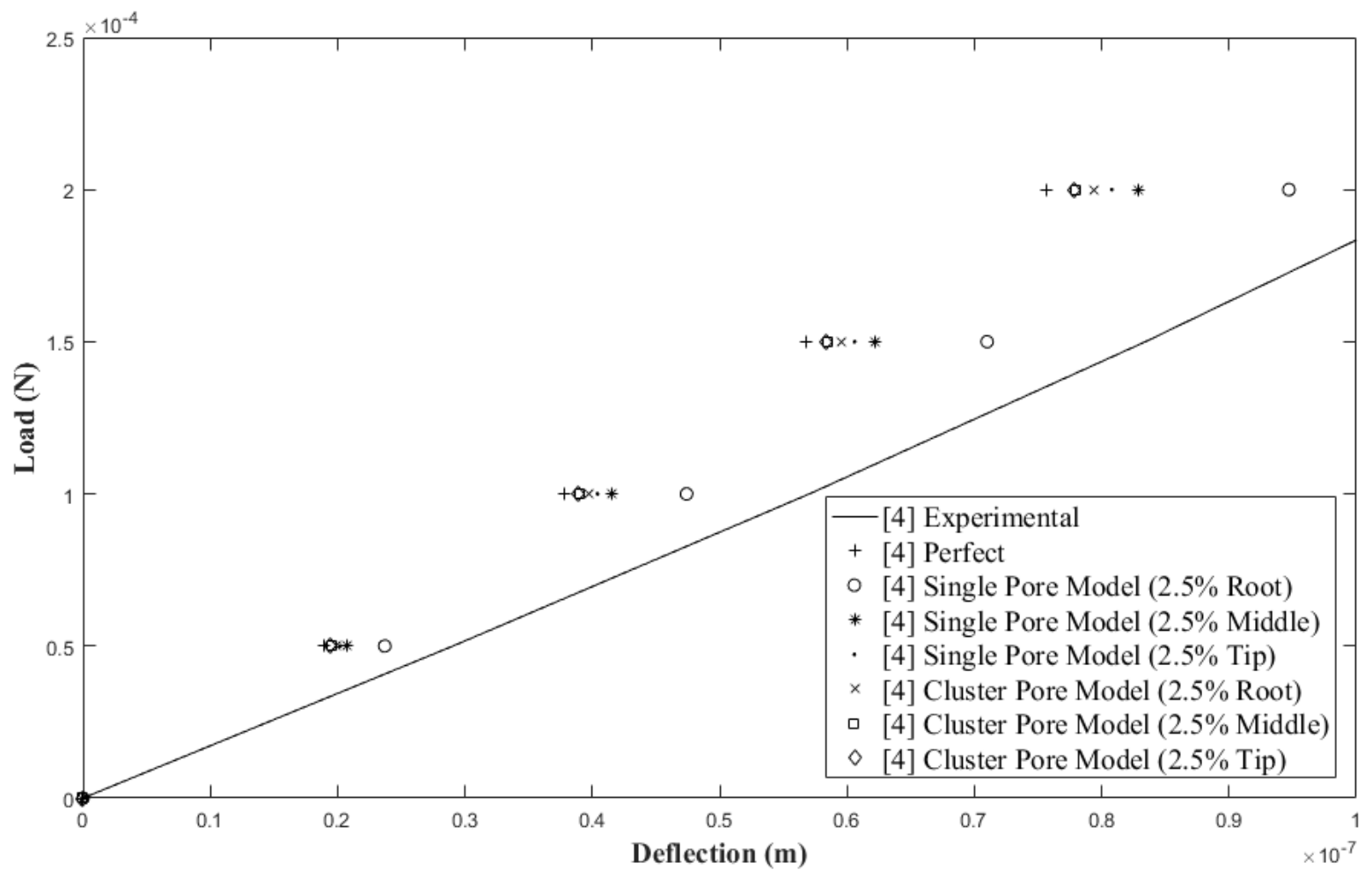

Figure 8. Load-Deflection Comparison of [4] FE Models and Experimental Data. 


\section{Microfluidic Method of Viscosity Measurement in Propane-Saturated Bitumen}

\author{
Mira Kim \\ Department of Mechanical Engineering \\ McGill University \\ Montreal, Canada
}

\author{
Nader Mosavat and David Sinton \\ Department of Mechanical Engineering \\ University of Toronto \\ Toronto, Canada
}

\begin{abstract}
In this paper, the viscosity propane-saturated bitumen was measured through a microfluidic chip. Property measurement of propane-saturated bitumen has been traditionally done on a heavy-duty PVT cell, and the application of microfluidics in high temperature and pressure conditions to measure the properties of bitumen is a novel technique. There are two steps in the viscosity measurement of propane-saturated bitumen. First, the propane saturated bitumen is prepared in a high-pressure piston cylinder through propane injection in bitumen at a pressure above its saturation pressure. This propane-bitumen sample is left to saturate till it there is no further pressure drop. Then the propane-saturated bitumen is pushed through the microfluidic chip and the viscosity of the bitumen is measured by the pressure differences and the imaging of the fluid end, as an application of the Poiseuille's law.
\end{abstract}

Keywords-Bitumen, Oil sands, Microfluidics, Propane, PVT, Viscosity, Diffusion

\section{INTRODUCTION}

Steam-assisted-gravity-drainage (SAGD) is a commercially successful oil recovery method for Canadian oil sands, also known as bitumen, by utilizing the heat transfer from steam condensation process to mobilize the highly temperature sensitive bitumen. Solvent-SAGD process is a modification of the SAGD process, where solvents are uses as additional components to reduce the viscosity of bitumen through mass transfer. [1] Vapor extraction technique (VAPEX) is an oil extraction process primarily using the effect of viscosity reduction through mass transfer by injecting light hydrocarbons as propane into bitumen. [2]

As the solvent saturation in bitumen is a crucial part of the VAPEX process, measurement studies to determine the saturation of solvents have been conducted. Pressure-decay method is a popular technique to measure the diffusivity in the gas-liquid systems. [3-7] The first step in the calculation of the diffusivity is to define the appropriate mass-transfer equations. [3] A simplified model of this is the unsteady one-dimensional mass diffusion equation, also known as the Fick's second law of diffusion. Fick's second law of diffusion can be written as below:

$$
\frac{\partial c}{\partial t}=D \frac{\delta^{2} c}{\partial z^{2}}
$$

where $\mathrm{c}$ is the solvent concentration in bitumen, $\mathrm{z}$ is the distance from the bottom of the diffusion cell, and D is the diffusion coefficient of the solvent in bitumen.

The application of Fick's law to the diffusion process in solvent-bitumen has been an interest to many scientists. Tharanivasan et al. [4] published a paper, applying three different boundary conditions to solve this equation. First is the equilibrium boundary condition, where the boundary condition assumes that the heavy oil-solvent interface is saturated with the solvent under the so-called equilibrium at all times. Second is the quasi-equilibrium boundary condition, where the heavy oil-solvent interface is assumed to be saturated with the solvent at the existing pressure in the solvent phase. Third is the nonequilibrium boundary condition, where the solvent mass-transfer flux across the interface is proportional to the difference between the solvent saturation concentration under the equilibrium pressure and the existing solvent concentration at the surface. According to Tharanivasan's paper, the mass transfer across the heavy oilpropane interface is best described by applying the quasiequilibrium boundary condition.

The study of solvent diffusion in bitumen has been conducted using experimental methods as well. Etminan et al. [5] published a paper on the constant pressure technique for gas diffusivity and solubility measurements in heavy oil and bitumen where the boundary conditions were set experimentally to an equilibrium state. This was accomplished by continuously injecting the required amount of gas into the gas cap from a secondary supply cell to maintain the pressure constant at the gas-liquid interface. Additionally, Behzadfar et al. [6] measured the diffusivity of carbon dioxide in bitumen in a pressure-decay method coupled with rheometer. Mixing due to shear imposed by a rheometer allows rapid direct measurement of the equilibrium pressure in the carbon dioxide-bitumen system.

Microfluidics methods of measuring diffusivity has been studied by Fadaei et al. [8] In Fadaei's paper, toluene was injected to a partially bitumen filled microfluidic chip, and one-dimensional diffusion of toluene and bitumen was quantified by through-plane visible-light transmission imaging. Then, by fitting the experimental data to the concentration profiles obtained using a numerical model, diffusion transport dynamics were quantified. The results showed that for intermediate toluene-mass fractions $(0.2-0.8)$, a constant diffusion coefficient of $2.0 \times 10^{-10} \mathrm{~m}^{2} / \mathrm{s}$ was measured. However, at low toluene mass fractions $(<0.2)$, 
significantly reduced diffusive transport was observed, and endpoint analysis indicated diffusion coefficients trending towards $4.3 \times 10^{-11} \mathrm{~m}^{2} / \mathrm{s}$. At high toluene mass fractions $(>0.8)$, the values trend towards $1.5 \times 10^{-10} \mathrm{~m}^{2} / \mathrm{s}$.

Fadeai et al. [9] also measured the diffusivity of bitumencarbon dioxide by using a cross-channel glass microfluidic chip. The device was initially filled with carbon dioxide at low pressure $(<1.0$ bar). A plug of bitumen was injected into the central $(50 \mu \mathrm{m}$ wide and $20 \mu \mathrm{m}$ deep) channel and subsequently exposed to high-pressure carbon dioxide on both ends. One-dimensional oil swelling in response to carbon dioxide diffusion was imaged over time. A simple mathematical approach was applied to calculate the diffusion coefficient based on the oil-swelling data. Measurement results are reported here at a range of pressures (1-5 MPa) and room temperature $\left(21{ }^{\circ} \mathrm{C}\right)$. The measured diffusion coefficients in this range are on the order of $10^{-10} \mathrm{~m}^{2} / \mathrm{s}$, in good agreement with the relevant published data using conventional methods. The biggest advantage of this method was that it required up to $10 \mathrm{~min}$ of time and a $1 \mathrm{~nL}$ plug of sample in comparison with conventional methods that require hours or days and up to $0.5 \mathrm{~L}$ of sample.

In this paper, measurement of bitumen viscosity with propane saturation was conducted using a microfluidic method. Though viscosity measurement through a microfluidic device has been previously done in biological research, [10-13] the property measurements of solvent saturated bitumen have traditionally been done in macroscale experimental apparatus. [14-22] The use of the glass microfluidic chip in this work allows experiments to have advantages in sample size reduction and miniaturization of system design, bringing the heavy-duty petroleum PVT research on lab-on-a-chip scale.

\section{EXPERIMENTAL METHOD}

The preparation of propane saturated bitumen was done on a high-pressure piston cylinder. Bitumen was filled in one end of the cylinder and water was filled on the other side. Then a certain volume quantity of liquefied propane was injected to the bitumen filled cylinder. Liquefied propane was used to quantify the amount of mass fraction in bitumen. Propane gas was liquified by drawing propane gas from the cylinder tank (Praxair) to a high-pressure syringe pump (ISCO 100D), and compressing the gas in the high-pressure syringe pump. Then the propane was injected to the bitumen filled side of the piston cylinder at a pressure quite above the saturation pressure, so that the propane saturation in bitumen could be achieved. Pressure was measured after the propane was injected in bitumen, and saturation was determined when the pressure drop was below the accuracy of the pressure gauges $( \pm 1 \mathrm{kPa})$. The pressure drop rate showed a gradual decrease until eventually there was no pressure further pressure drop. The theoretical saturation pressure values were derived from equality of fugacity between the liquid and vapor phases. If the vapor phase is pure propane, the equation for the saturation pressure $\mathrm{P}$ is

$$
\mathrm{P}=\mathrm{x}_{\mathrm{ca}} \gamma_{\mathrm{ca}} \mathrm{P} v_{\mathrm{ca}}
$$

where $\mathrm{Pv}_{\mathrm{ca}}$ is the vapor pressure of propane in $\mathrm{kPa}$, and $\gamma_{\mathrm{ca}}$ is the activity coefficient of propane in bitumen. According to literature, the best fit value of the activity coefficient was 1 . 158. This equation primarily indicates that higher pressure is needed to achieve a higher volume fraction of saturation. [2] Figure 1 shows the experimental setup to prepare the propanesaturated bitumen with the thought of connecting it to the microfluidic chip after the saturation. A high-pressure valve was used to close the system after the injection of propane to later connect it to the microfluidic chip.

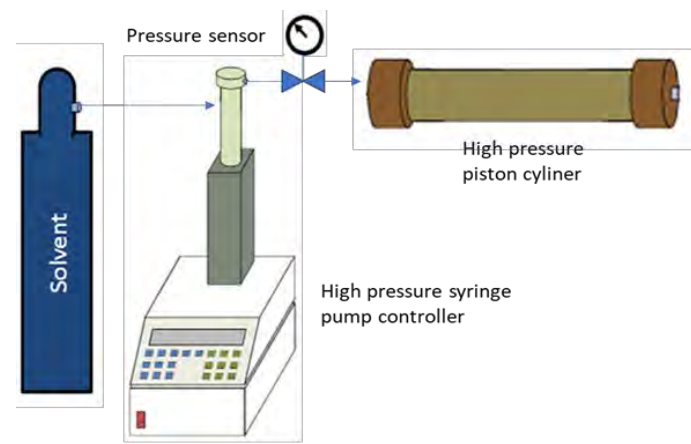

Fig. 1 Setup for propane saturation in bitumen

After the propane saturated bitumen sample was prepared, it was pushed to go through the microchannel in the microfluidic chip. The hydraulic diameter of the microfluidic chip was calibrated to be $76 \mu \mathrm{m}$ by reading the pressure and flowrate of a calibration fluid. The propane bitumen sample was pushed by applying a constant pressure on the other end of the cylinder by the ISCO pump. The pressures were 1.5 and 1.7 MPa, both values above saturation pressures. Poiseuille's law was used to measure the viscosity of the solvent-saturated bitumen through imaging the flow. Poiseuille's law is describe as below

$$
\frac{\mathrm{dx}}{\mathrm{dt}}=\frac{\Delta \mathrm{Pr}^{4}}{8 \mathrm{r}^{2} \mu \mathrm{x}}
$$

where $\mathrm{x}$ is the length of the fluid, $\mathrm{r}$ is the tube radius, $\mu$ is the viscosity of the fluid, and $\Delta \mathrm{P}$ is the total effective pressure. Figure 2 depicts the experimental setup for the viscosity measurement of propane saturated bitumen. 


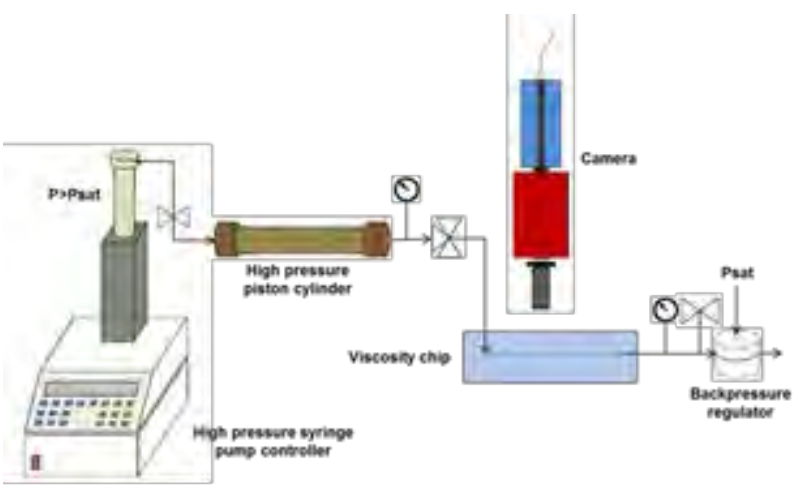

Fig. 2 Schematic for the viscosity measurement of the propane-saturated bitumen.

The experimental conditions of the two runs are summarized in Table 1. The propane fraction values were selected to compare a higher propane fraction ratio to a lower propane fraction ratio with the objective to demonstrate the effectiveness of solvent saturation in bitumen viscosity reduction.

Table 1 Experimental conditions and the measured viscosities for viscosity measurement of propane-saturated bitumen

\begin{tabular}{|c|c|c|c|c|c|}
\hline Run\# & $\begin{array}{c}\text { Bitumen } \\
\text { mass(g) }\end{array}$ & $\begin{array}{c}\text { Propane } \\
\text { mass } \\
\text { fraction }\end{array}$ & $\begin{array}{c}\text { Temperat } \\
\text { ure } \\
\left({ }^{\circ} \mathbf{C}\right)\end{array}$ & $\begin{array}{c}\text { Pressure } \\
(\mathbf{M P a})\end{array}$ & $\begin{array}{c}\text { Measured } \\
\text { Viscosity } \\
(\mathbf{c P})\end{array}$ \\
\hline $\mathbf{1}$ & 2.83 & 0.10 & 20 & 1.5 & 65,000 \\
\hline $\mathbf{2}$ & 2.83 & 0.25 & 20 & 1.7 & 565 \\
\hline
\end{tabular}

\section{RESULTS AND DISCUSSIONS}

In this section, two runs were done with different mass fractions of propane in bitumen. The experiments were captured using a video camera, and the video file was converted using a video to image converter. Figure 3 shows the movement of the bitumen in the first run by putting it into a sequence of frames in a time frame of frame per 10 seconds.
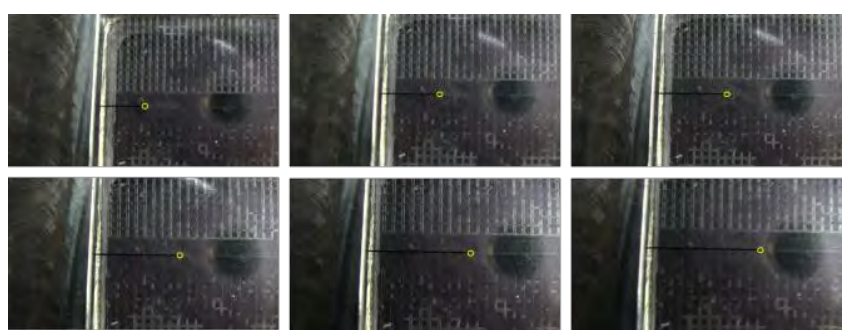

Fig. 3 Sequence of images of run \#1 in every 10 seconds.

Figure 4 is an image capture from the higher propane mass fraction component run. The images were extracted to be $1 / 3$ second per frame from the video to image converter.

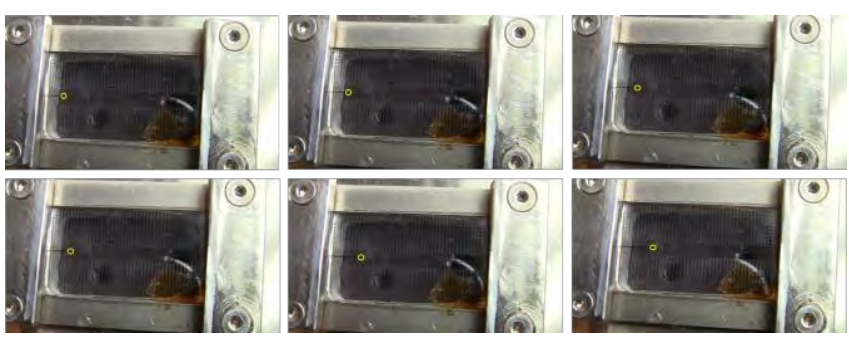

Fig. 4 Sequence of images of run $\# 2$ in every $1 / 3$ second.

Figure 5 shows a comparison of run $\# 1$ and run $\# 2$ in a fuller time frame. Run $\# 1$ had a significantly slower advancement in the flow, and it took 8-9 minutes for the propane-saturated bitumen sample to completely fill the channel. In run \#2, the propane saturated bitumen sample filled the channel in just 7 seconds, resulting in flow speed 2 order of magnitude higher than of run $\# 1$, indicating a significantly lower viscosity. a)

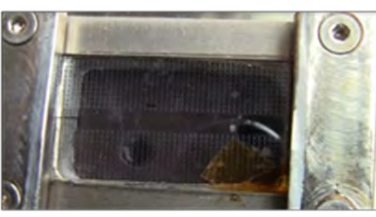

Time $=26 \mathrm{sec}$

b)

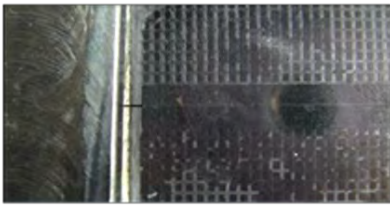

Time $=1 \mathrm{sec}$

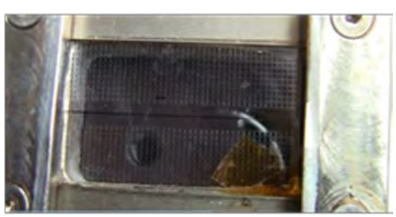

Time $=8 \operatorname{mins} 16 \mathrm{sec}$

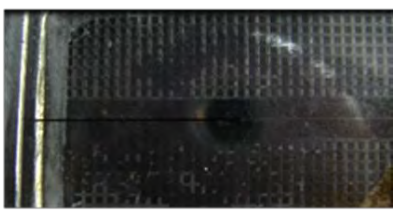

Time $=5 \mathrm{sec}$
Fig. 5 Images of propane-bitumen flow captured at different times during a) run $\# 1$ and b) run $\# 2$.

These images were converted manually into two datasets by calculating the distance of the bitumen front advancement. The grids in the microfluidic chip was a useful standard for this calculation, each grid providing a $1 \mathrm{~mm}$ scale. Then, an excel linear curve fitting function was used to calculate the slope of the plots, and through the calculated linear constant, the viscosity was calculated. Figure 6 shows the graphs depicting the location of the bitumen front in time.
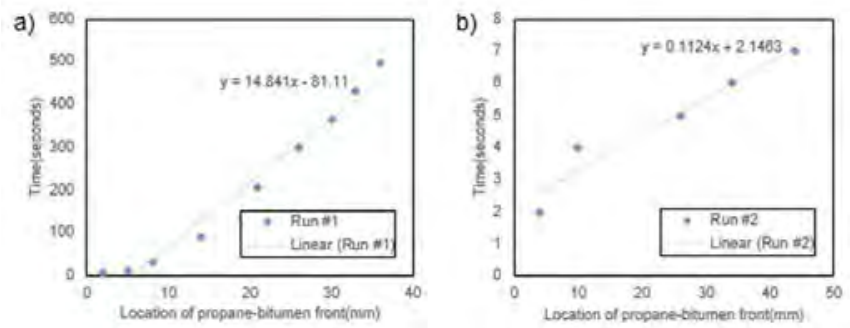

Fig. 6 Location of propane-bitumen flow front and time for a) run \#1, and b) run \#2. 
While the viscosity measurement of solvent-saturated bitumen is still under study, various numerical methods have been developed to estimate the viscosity of bitumen. Here, the measured viscosity was compared with the modified Shu correlation equation. The modified Shu equation for the mixture is:

$$
\begin{gathered}
\mu=\mu_{\text {Bit }}^{x_{\text {Bit }}} \times \mu_{g}^{x_{g}} \\
x_{\text {Bit }}=\frac{\alpha V_{\text {Bit }}}{\alpha V_{\text {Bit }}+V_{g}} \\
x_{g}=1-x_{\text {Bit }} \\
\alpha=\frac{0.000375 \Delta \rho^{-8.56653} \rho_{\text {Bit }}^{456001} \rho_{g}^{-5.27094}}{\ln \left(\mu_{\text {Bit }} / \mu_{g}\right)}
\end{gathered}
$$

where $\mu$ is the viscosity of the mixture, $V_{\text {Bit }}$ is the volume fraction of bitumen, $V_{g}$ is the volume fraction of solvent. The original equation for $\alpha$ is an empirical estimate based on 17 different crude oil and solvents. [22] The $\alpha$ for the Shu equation was modified for propane-saturated bitumen, as it was found that the original empirical estimate by Shu was not appropriate for liquified propane. [2] Furthermore, in this equation, the viscosity estimate was very parameter sensitive, and it is fair to say that an exact analytical solution for the viscosity of the propane-saturated bitumen does not exist, due to the multi-component structure of bitumen, and especially with its asphaltene components. Nevertheless, it is possible that some escape of propane gas and asphaltene deposition during the run could have resulted in a higher viscosity measurement than its actual viscosity.

Figure 7 depicts a graph of the Shu equation and the measured viscosity values. The line depicts the Shu equation and the red points are the measured values.

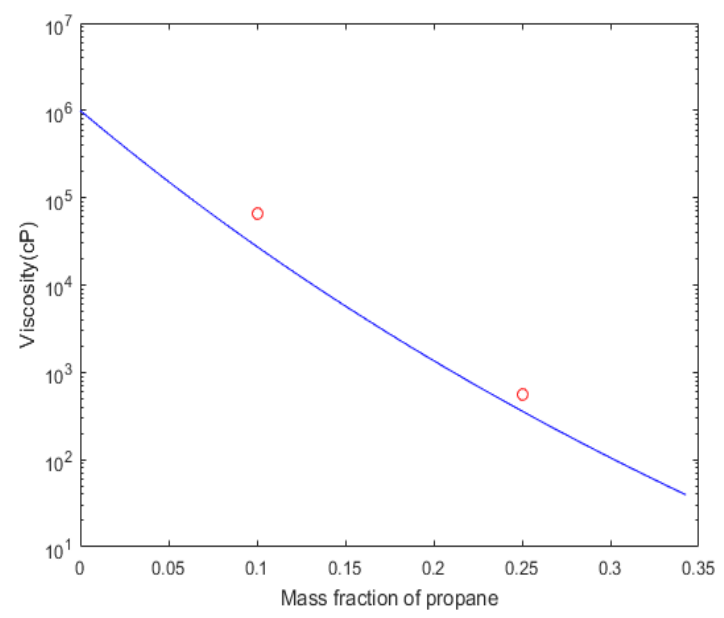

Fig. 7 Viscosity of propane-saturated bitumen through the Shu equation and experimental values. The line indicates the

Shu equation and the red points are the measured values.

\section{CONCLUSION}

In conclusion, viscosity measurements of propane-saturated bitumen showed a significant improvement in viscosity reduction. This indicates the effectiveness of the alternative method of viscosity reduction through mass transfer as a comparable method or combined method to viscosity reduction through heat transfer. Additionally, the application of microfluidics was important because it brought visualization of the sample flow, reduction of sample size, and miniaturization of the experimental setup.

Furthermore, this shows that microfluidics can be applied for PVT measurements in petroleum research. An important tool of the PVT measurements in petroleum research is a piston cylinder, and a development of a microfluidic piston cylinder that is capable of withstanding the conditions required for petroleum research, such as high temperature and pressure conditions, [23] and compatibility to harsh solvents would be the next possible direction to bring the heavy-duty petroleum research system to lab-on-chip scale.

\section{ACKNOWLEDGMENT}

Suncor Energy Services is gratefully acknowledged for providing financial support.

\section{REFERENCES}

[1] M. Kim, "In-situ visualization of steam assisted gravity drainage with chemical and solvent additives," Ph.D. dissertation, Dept. Mech. Ind. Eng., Univ. of Toronto, Toronto, ON, 2017.

[2] A. Badamchi-Zadeh, H. W. Yarranton, W. Y. Svrcek, and B. B. Maini, "Phase behaviour and physical property measurements for VAPEX solvents: Part I. Propane and Athabasca bitumen," J. Can. Pet. Technol. vol. 48, no. 1, pp. 54-61, Jan. 2009. doi: 10.2118/09-01-54.

[3] S. R. Upreti and A. K. Mehrotra, "Experimental measurement of gas diffusivity in bitumen: Results for carbon dioxide," Ind. Eng. Chem. Res., vol. 39, no. 4, pp. 1080-1087, Apr. 2000. doi: 10.1021/ie990635a

[4] A. K. Tharanivasan, C. Yang, and Y. Gu, "Measurements of molecular diffusion coefficients of carbon dioxide, methane, and propane in heavy oil under reservoir conditions," Energy Fuels, vol. 20, no. 6, pp. 2509-2517, Nov. 2006. doi: 10.1021/ef060080d.

[5] S. R. Etminan, B. B. Maini, Z. Chen, and H. Hassanzadeh, "Constantpressure technique for gas diffusivity and solubility measurements in heavy oil and bitumen," Energy Fuels, vol. 24, no. 1, pp. 533-549, Jan. 2010. doi: 10.1021/ef9008955.

[6] E. Behzadfar and S. G. Hatzikiriakos, 'Diffusivity of CO2 in bitumen: Pressure-decay measurements coupled with rheometry," Energy Fuels, vol. 28, no. 2, pp. 1304-1311, Jan. 2014. doi: 10.1021/ef402392r.

[7] S. K. Das and R. M. Butler, "Diffusion coefficients of propane and butane in Peace River bitumen," Can. J. Chem. Eng., vol. 74, no. 6, pp. 985-992, Dec. 1996. doi: 10.1002/cjce.5450740623.

[8] H. Fadaei, J. M. Shaw, and D. Sinton, "'Bitumen-toluene mutual diffusion coefficients using microfluidics," Energy Fuels, vol. 27, no. 4, pp. 2042-2048, Mar. 2013. doi: 10.1021/ef400027t.

[9] Fadaei, B. Scarff, and D. Sinton, "Rapid microfluidics-based measurement of CO2 diffusivity in bitumen," Energy Fuels, vol. 25, no. 10, pp. 4829-4835, Oct. 2011. doi: 10.1021/ef2009265.

[10] A. E. Kamholz, B. H. Weigl, B. A. Finlayson, and P. Yager, "Quantitative analysis of molecular interaction in a microfluidic channel: The t-sensor," Anal. Chem., vol. 71, no. 23, pp. 5340-5347, Dec. 1999. doi: 10.1021/ac990504j.

[11] Y. J. Kang, S. Y. Yoon, K.-H. Lee, and S. Yang, "A highly accurate and consistent microfluidic viscometer for continuous blood viscosity measurement," Artif. Organs, vol. 34, no. 11, pp. 944-949, Oct. 2010. doi: $10.1111 / \mathrm{j} .1525-1594.2010 .01078 . x$.

[12] Z. H. Silber-Li, Y. P. Tan, and P. F. Weng, "A microtube viscometer with a thermostat," Exp. Fluids, vol. 36, no. 4, pp. 586-592, Apr. 2004 doi: 10.1007/s00348-003-0730-x. 
[12] Z. H. Silber-Li, Y. P. Tan, and P. F. Weng, A microtube viscometer with a thermostat," Exp. Fluids, vol. 36, no. 4, pp. 586-592, Apr. 2004. doi: 10.1007/s00348-003-0730-x

[13] N. Srivastava and M. A. Burns, "Analysis of non-Newtonian liquids using a microfluidic capillary viscometer," Anal. Chem. vol. 78, no. 5, pp. 1690-1696, Mar. 2006. doi: 10.1021/ac0518046

[14] M. Kariznovi, H. Nourozieh, and J. Abedi, "Experimental apparatus for phase behavior study of solvent-bitumen systems: A critical review and design of a new apparatus," Fuel, vol. 90, no. 2, pp. 536-546, Feb. 2011. doi: 10.1016/j.fuel.2010.10.019

[15] H. Nourozieh, M. Kariznovi, and J. Abedi, "Physical properties and extraction measurements for the Athabasca bitumen + light hydrocarbon system: Evaluation of the pressure effect, solvent-to-bitumen ratio, and solvent type," J. Chem. Eng. Data, vol. 56, no. 11, pp. 4261-4267, Nov. 2011. doi: $10.1021 /$ je2008846

[16] C. T. Fu, V. R. Puttagunta, and G. Vilcsak, "Gas solubility of methane and ethane in Cold Lake bitumen at in situ conditions," J. Can. Pet. Tech., vol. 27, no. 4, Jul. 1988. doi: 10.2118/88-04-06

[17] F. A. Jacobs, J. K. Donnelly, and J. Stanislav, "Viscosity of gas saturated bitumen," Can. Pet. Sci. Tech., vol. 19, no. 4, pp. 46-50, Oct. 1980. doi: 10.2118/80-04-03

[18] W. Y. Svrcek and A. K. Mehrotra, "Properties of Peace River bitumen bitumen saturated with field gas mixtures," Can. Pet. Sci. Tech. vol. 28 , no. 2, pp. 50-56, Mar. 1989. doi: 10.2118/89-02-01

[19] S. G. Sayegh, D. N. Rao, S. Kokal, and J. Najman, "Phase behaviour and physical properties of heavy oil of linbergh heavy oil/CO2 mixtures," Can. Pet. Sci. Tech., vol. 29, no. 6, Nov. 1990. doi: 10.2118/90-06-02

[20] A. K. Mehrotra, R. R. Eastick, and W. Y. Svrcek, "Viscosity of Cold Lake bitumen and its fractions," Can. J. Chem. Eng., vol. 67, no. 6, pp. 1004-1009, Dec. 1989. doi: $10.1002 /$ cjee. 5450670620

[21] W. Y. Svrcek and A. K. Mehrotra, "Gas solubility, viscosity and density measurements for Athabasca bitumen," J. Can. Pet. Tech., vol. 21, no. 4, pp. 31-38, July 1982. doi: 10.2118/82-04-02

[22] W. R. Shu, "A viscosity correlation for mixtures of heavy oil, bitumen, and petroleum fractions," Soc. Pet. Eng. J., vol. 24, no. 3, pp. 277-282, Jun. 1984. doi: 10.2118/11280-PA

[23] E. F. Hasselbrink, T. J. Shepodd, and J. E. Rehm, "High-pressure microfluidic control in lab-on-a-chip devices using mobile polymer Monoliths," Anal. Chem., vol. 74, no. 19, pp. 4913-4918, Oct. 2002. doi: $10.1021 / \mathrm{ac} 025761 \mathrm{u}$ 


\section{Effect of Body Forces Due to Aircraft Acceleration on Ventilation Airflow and Contaminant Dispersion in Passenger Aircraft Cabins}

\author{
Hossam A. Elmaghraby \\ Mechanical Engineering Program, School of Engineering \\ University of Guelph \\ Guelph, Ontario N1G 2W1, Canada \\ helmaghr@uoguelph.ca
}

\author{
Yi Wai Chiang, Amir A. Aliabadi \\ Environmental Engineering Program, School of Engineering \\ University of Guelph \\ Guelph, Ontario N1G 2W1, Canada \\ chiange@uoguelph.ca \\ *Corresponding Author: aliabadi@uoguelph.ca
}

\begin{abstract}
Numerical simulations for the effect of body forces due to aircraft acceleration on the airflow and contaminant dispersion in a model for a passenger aircraft cabin are performed in this study. It was found that those body forces have a significant impact on the contaminant dispersion phenomena and concentrations, especially during the climb leg, where the concentration was almost triple its counterpart during the steady level flight case at the two monitoring locations. Air velocities, on the other side, increased noticeably during the climb and descent legs leading to evident changes in the airflow patterns, vorticity magnitudes, and at some locations, vorticity directions, as well.
\end{abstract}

Keywords-Aircraft ventilation; Air quality; Contaminants; Aircraft acceleration; Airflow vorticity; Numerical simulation; CFD

\section{INTRODUCTION}

Nowadays, billions of people every year prefer to use air travel rather than other means of transport because of the fast and reliable service the airliners provide. However, the environment inside a commercial aircraft cabin provides a fertile ground for deterioration of air quality and disease transmission among passengers if proper measures are not taken [1].

In the past two decades, numerous studies with different research approaches have investigated air quality in aircraft cabin environments using air distribution systems as a control measure. These approaches range from purely experimental [26] to entirely computational (numerical simulations) [7-9], or combinations of both [10-14].

Despite this large number of airflow and air quality studies in aircraft cabins, and although aircraft are moving with high speeds and accelerations accomplishing distinct flight mission legs (or intervals) with different dynamic conditions, such as takeoff, climb, cruise, descent, and landing [15], no study has considered the effect of the resulting body forces from these accelerations on the flow of ventilation air and contaminate dispersion inside aircraft cabins.
The main objective of this work is to fill the gap in the literature that always considered that the passenger aircraft are at rest or under cruise conditions, and that the ventilation airflow, buoyancy effects due to the occupants-generated thermal plumes, and contaminants dispersion within their cabins are only influenced by the gravitational acceleration. Moreover, the effect of the accelerations induced by the body forces acting on a typical modern passenger aircraft (Boeing 767-300), such as the lift and drag, in addition to the thrust of the jet engines and the weight of the aircraft itself, on the air distribution and gaseous contaminant dispersion inside an economy cabin section is to be investigated through computational fluid dynamics (CFD) simulations using the ANSYS FLUENT software. The mission legs, during which the simulations are performed. are climb, steady level cruise, and descent.

\section{METHODS}

\section{A. Simulated cabin geometry}

The aircraft cabin model used for the current study is adopted from the literature, and more specifically the experimental study in [4], and the numerical simulation followup work in [8].

The model resembles an actual size sectional economy-class cabin of a Boeing 767 passenger aircraft with 21 seats arranged in three rows (the seven abreast or 2-3-2 seat arrangement). The external dimensions of the cabin mockup are $4.9 \mathrm{~m}$ by $3.2 \mathrm{~m}$ by $2.1 \mathrm{~m}(\mathrm{~W}, \mathrm{~L}, \mathrm{H})$. The cabin mockup is located at the International Centre for Indoor Environment and Energy, Technical University of Denmark, Lyngby, Denmark. The cabin mockup appeared for the first time in the literature in [3] where more details about its configuration and control systems can be found. Fig. 1 shows a three-dimensional view for the created cabin model in the current study to the exact dimensions using the design software DesignModeler included in the commercial CFD package ANSYS 17.0, and a plan view for the seats.

\footnotetext{
*Address all correspondence to this author.
} 


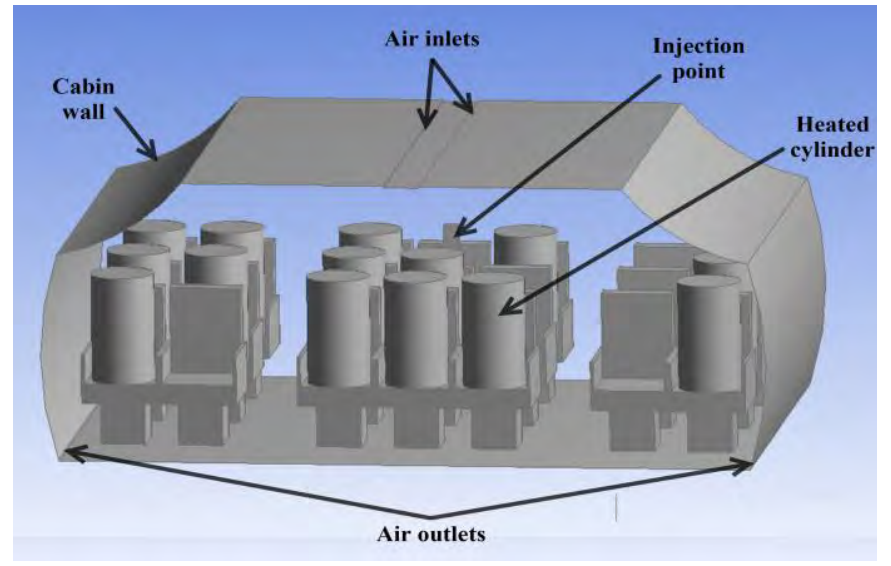

(a)

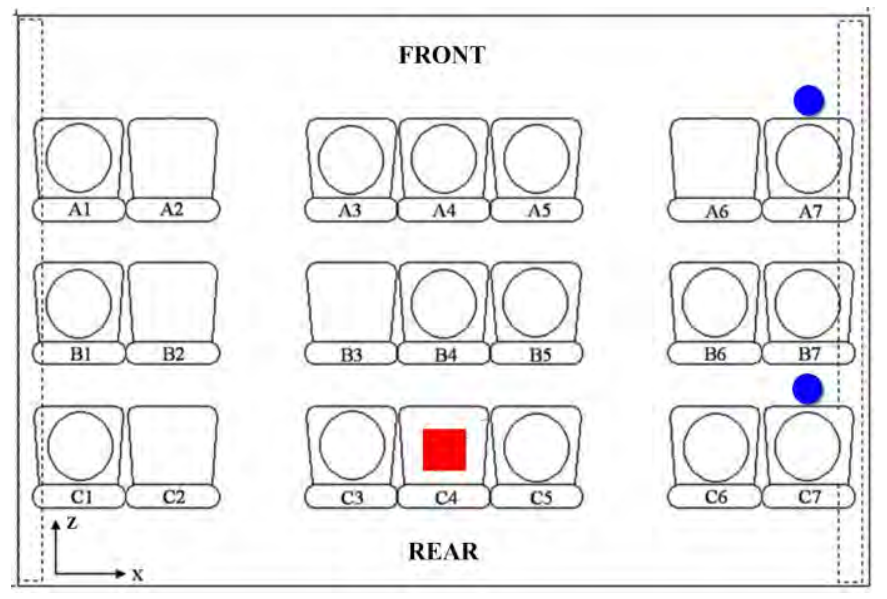

(b)

Figure 1. The computational cabin model used in the current study; (a) threedimensional view of the geometry built in ANSYS 17.0, and (b) plan view for the cabin with the cougher/injector position (red square), and the $\mathrm{SF}_{6}$ concentration monitoring points (blue circles)

\section{B. Model and boundary conditions}

The model's boundary conditions found in [4] and [8] were closely followed and implemented in the numerical solver FLUENT for the case of $200 \mathrm{~L} \mathrm{~s}^{-1}$ supply air flow rate, which was the only supply air flow rate considered. $\mathrm{SF}_{6}$ gas was released in the cabin to mimic the injection and transport of the cough's smallest size droplets (typically 1.6 to $3.0 \mu \mathrm{m}$ ), and which formed the largest number concentration of the injected droplet ensemble in the experiments. This was performed for the sake of model validation. Table I shows the boundary and inlet conditions for the current model.

The simulation time for the transient airflow part is $350 \mathrm{~s}$ at the start of which the cough $\left(\mathrm{SF}_{6}\right.$ release) was introduced for 1 $\mathrm{s}$ with released air volume of $0.4 \mathrm{l}$. Before this transient run, the airflow domain only was completely solved in the steady mode.
TABLE I. BOUNDARY AND INLET CONDITIONS FOR THE MODEL

\begin{tabular}{|l|l|}
\hline \multicolumn{1}{|c|}{ Boundary/inlet Conditions } & \multicolumn{1}{c|}{ Value } \\
\hline Supply air temperature & $24^{\circ} \mathrm{C}$ \\
\hline Supply air flow rate & $\begin{array}{l}200 \mathrm{~s}^{-1} \text { (corresponds to a supply } \\
\left.\text { velocity of } 2.61 \mathrm{~m} \mathrm{~s}^{-1}\right)\end{array}$ \\
\hline Supply air absolute humidity & $\begin{array}{l}0.92 \mathrm{~g} \mathrm{~kg}^{-1}(\text { corresponds to } 5 \% \mathrm{RH} \\
\text { at supply air temperature) }\end{array}$ \\
\hline Cabin wall temperature & $18{ }^{\circ} \mathrm{C}$ \\
\hline Heating cylinder heat release & $60 \mathrm{~W}$ per cylinder \\
\hline $\mathrm{SF}_{6}$ (cough) injection location & Seat $\mathrm{C} 4$ \\
\hline Air velocity at injection location & $10.6 \mathrm{~m} \mathrm{~s}^{-1}$ \\
\hline
\end{tabular}

The flow turbulence in the cabin was modeled using the renormalization group (RNG) k- $\varepsilon$ model of the Reynoldsaveraged Navier-Stokes (RANS) group based on recommendations for similar airflow simulation cases in closed spaces found in the literature [16-19].

\section{Grid independence test}

For defining the degree of independence of the obtained solution (airflow velocity and time-averaged $\mathrm{SF}_{6}$ concentration) from the grid size changes, a grid independence test is conducted. In the present work, three levels of grid fineness (sizes) were created, namely: coarse grid $(4,704,751$ elements), medium grid $(5,522,517$ elements), and fine grid $(7,375,800$ elements), in the order of grid element size decrease or fineness increase. The mesh refinement ratio (r) between each two consecutive grid levels was kept constant at 1.1.

Transient simulation runs were performed for airflow and $\mathrm{SF}_{6}$ dispersion in the cabin on each grid level with the identical boundary conditions mentioned previously in section B. Fig. 2 presents the normalized $\mathrm{SF}_{6}$ concentration time series (real-time concentration divided by time-averaged concentration) as they change with the simulation time for the three grid levels with respect to the experimental data (see section $\mathrm{D}$ ). The real-time $\mathrm{SF}_{6}$ concentration was monitored at the breathing level of the occupants (1.17 m) at seats A7 and C7 (blue circles in Fig.1).

In addition to the graphical comparison of solution on the three grid levels, the grid convergence index (GCI) is calculated to indicate the amount of asymptotic convergence that the solution achieves through determining the uncertainty in solution between two consecutive grid levels [20,21].

In the current study, the GCI is calculated using the following formula:

$$
G C I_{m n}=\frac{F_{s}\left|\epsilon_{m n}\right|}{r^{p}-1}
$$

where, $F_{s}$ is a factor of safety recommended to be 3.0 for comparisons of two meshes and 1.25 for comparison of three meshes (such as in the current model), $\epsilon_{m n}$ is relative error between the two solutions obtained on two consecutive grid levels, and $p$ is the order of convergence. For more information on the calculation procedure followed refer to [21,22]. 


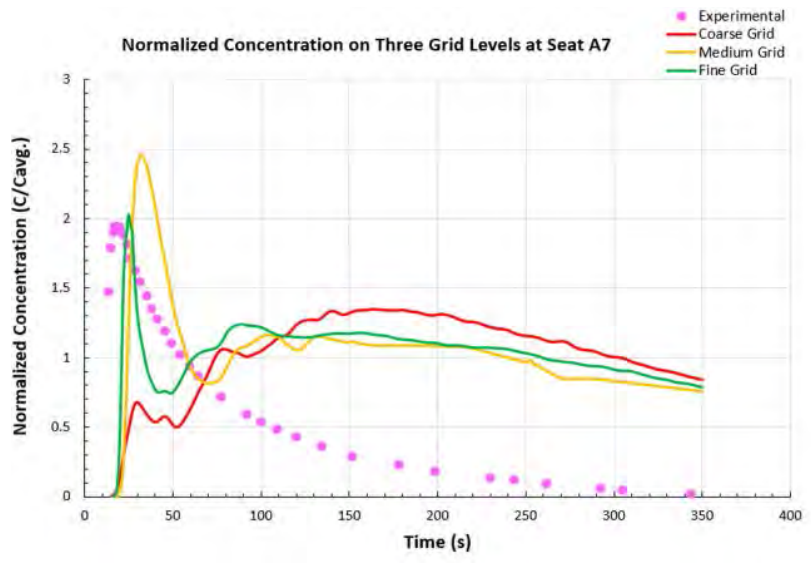

(a)

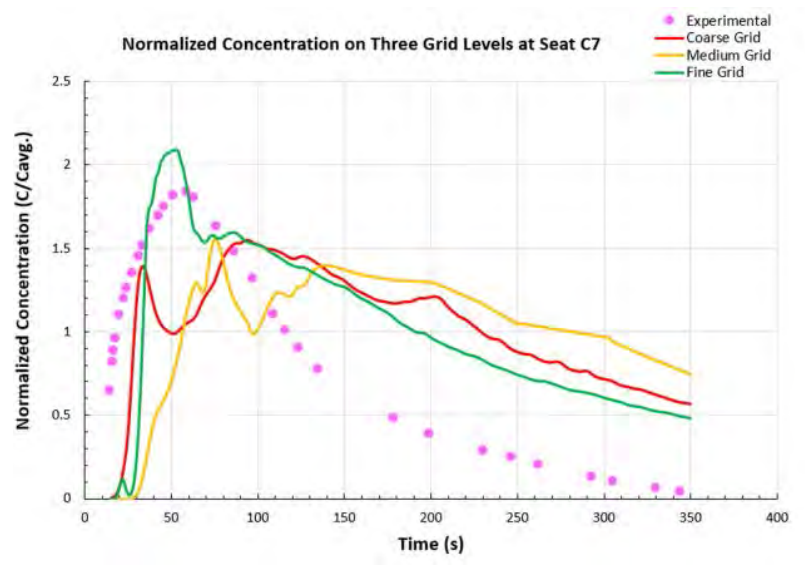

(b)

Figure 2. Normalized $\mathrm{SF}_{6}$ concentration change with the simulation time on the three grid levels; (a) at seat A7, and (b) at seat C7

The GCI for $\mathrm{SF}_{6}$ concentration calculations from the coarse to the medium grids was $3.13 \%$, and from the medium to the fine grids was $1.26 \%$ at seat A7. On the other hand, at seat C7, the GCI was $3.55 \%$ from the coarse to the medium grids, while it was equal to $1.41 \%$ between the medium and the fine grids. Based on these results, the fine grid level was found to exhibit enough grid independency of the solution, and therefore, will be used further.

\section{Model validation and error estimation}

The experimental data used for the aircraft cabin model validation was mainly extracted from the original study [4] considering the smallest particle size range $(1.6-3.0 \mu \mathrm{m})$ to be compared with the $\mathrm{SF}_{6}$ (passive tracer) concentration time series monitored at the two seats $\mathrm{A} 7$ and $\mathrm{C} 7$ over the simulation time. This is based on the findings of multiple studies in literature that indicate that the smallest size droplets ( 3 microns in diameter and less) behave like the gaseous substances (especially $\mathrm{SF}_{6}$ ) when dispersing in the cabin space $[5,10]$. Fig. 3 depicts the compared normalized concentration time series at seats $\mathrm{A} 7$ and $\mathrm{C} 7$ using the numerical solution obtained on the fine grid only.

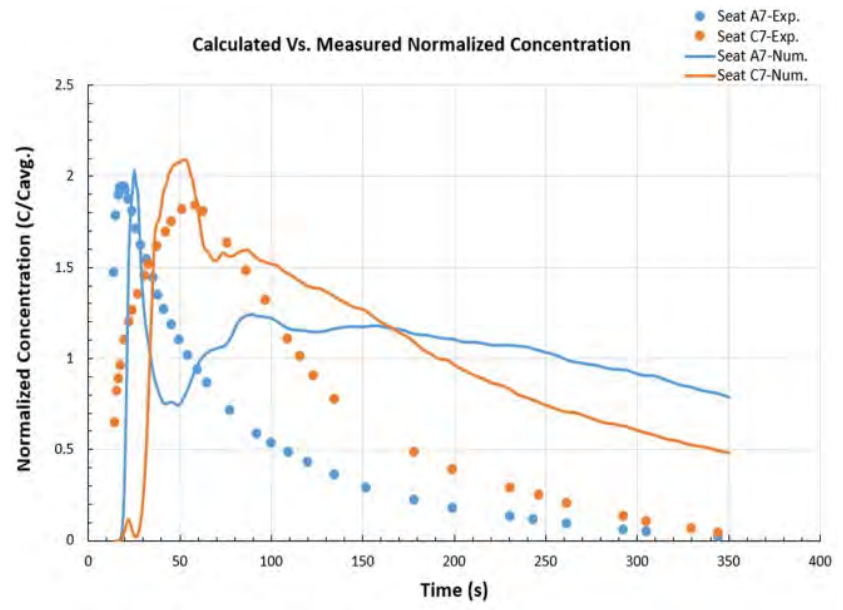

Figure 3. Comparison of the normalized concentration time series between the experiment and numerical simulation on the fine grid level

The error estimation in the current CFD model predictions for airflow velocity and concentration time series are calculated using a procedure which was first proposed by Steven Hanna in [23], and used by him and others later in multiple studies to express the error between the observed and predicted concentrations in atmospheric air quality models [24-26]. This procedure uses two performance measures to express the error, namely: the fractional mean bias (FB), and the normalized mean square error (NMSE), defined as follows:

$$
F B=\frac{2\left(\overline{C_{o}-C_{p}}\right)}{\left(\overline{C_{o}}+\overline{C_{p}}\right)},
$$

$$
N M S E=\frac{\left(\overline{\left(C_{o}-C_{p}\right)^{2}}\right)}{\left(\overline{C_{o}} * \overline{C_{p}}\right)},
$$

where, $C_{o}$ and $C_{p}$ are the observed and predicted concentrations, respectively.

Table II gives the FB and NMSE values calculated for the velocity magnitudes and normalized concentration time series between the experimental measurements and numerical predictions in the current study.

From Table II, it can be remarked that both error measures are noticeably lower for the air velocity magnitude than for the normalized concentration calculations. On the other hand, the NMSE values for normalized concentration at seat $\mathrm{C} 7$ are about $50 \%$ less than at seat $\mathrm{A} 7$, while $\mathrm{FB}$ values are almost the same at both seats. This shows that the shift between the observed and predicted concentration values is similar, but the spread of the predicted data with respect to the observed ones is two times higher at seat A7, which indicates less accurate predictions.

The decrease in prediction accuracy from seat $\mathrm{C} 7$ to seat $\mathrm{A} 7$ 
TABLE II.

FB AND NMSE VALUES FOR THE NUMERICAL PREDICTIONS

\begin{tabular}{|cc|cc|cc|}
\hline \multicolumn{2}{|c|}{ Air Velocity } & \multicolumn{4}{|c|}{ Normalized Concentration } \\
\hline \multirow{2}{*}{ FB } & NMSE & $\begin{array}{c}\text { FB } \\
(\text { Seat } \text { A7) }\end{array}$ & $\begin{array}{c}\text { NMSE } \\
\text { (Seat A7) }\end{array}$ & $\begin{array}{c}\text { FB } \\
\text { (Seat C7) }\end{array}$ & $\begin{array}{c}\text { NMSE } \\
\text { (Seat C7) }\end{array}$ \\
\hline 0.07977 & 0.02743 & 0.09705 & 0.96112 & 0.06922 & 0.42384 \\
\hline
\end{tabular}

can very well be attributed to the condition of airflow, and therefore that of the surrogate $\mathrm{SF}_{6}$ gas, from the emission source (at seat C4) to each seat. From C4 to C7 the flow is mainly lateral which is less susceptible to the bulk flow turbulence than the primarily longitudinal flow experienced from C4 to A7 (see Fig. 1).

Generally, the calculated FB and NMSE figures agree well with the graphical presentation for the measured and calculated concentration time series (Fig. 3).

\section{E. Calculation of aircraft body acceleration components}

The aircraft vertical acceleration $\left(\mathrm{a}_{\mathrm{v}}\right)$ and horizontal acceleration $\left(a_{h}\right)$ components were calculated during climb and descent legs using a basic approach adapted from different sources in aircraft dynamics literature [27] and online [28,29].

The calculation procedure relies on applying Newton's second law $\left(\sum \vec{F}=m \vec{a}\right)$ on two axes passing through the center of gravity of the aircraft; one is vertical and the other is horizontal. The forces in action are the lift (L) and drag (D) on the aircraft, in addition to the aircraft's weight (W) and the thrust of the jet engines (T). More information on the calculation procedure followed can be found in appendices A and $\mathrm{B}$ at the end of this paper.

\section{RESULTS AND DISCUSSION}

\section{A. Effect of aircraft body forces on contaminants dispersion}

The climb and descent simulations were run for the same time span of the steady level flight simulations (350 s), but with the new acceleration components, resulting from the climb and descent calculations, implemented in the numerical solver. Fig. 4 shows a comparison of the calculated concentration time series of $\mathrm{SF}_{6}$ during steady level flight, climb, and descent at the two set monitoring locations at seats A7 and C7.

It can be clearly noticed from Fig. 4 that the tracer gas concentration is significantly higher (up to $250 \%$ more) during the climb leg than the steady level flight case for most of the simulation time at the two locations. Consequently, the level of exposure of passengers sitting at any of the two seats to the contaminant, which can be interpreted from the area under the curves, significantly increases throughout the aircraft climb time. This poses greater infection risks on the health and wellbeing of most passengers in the cabin upon exposure to hazardous gaseous substances, in-cabin contaminants, or some infectious particulates during the aircraft climb leg that can take up from 20 to 30 minutes for some flights and aircraft models.

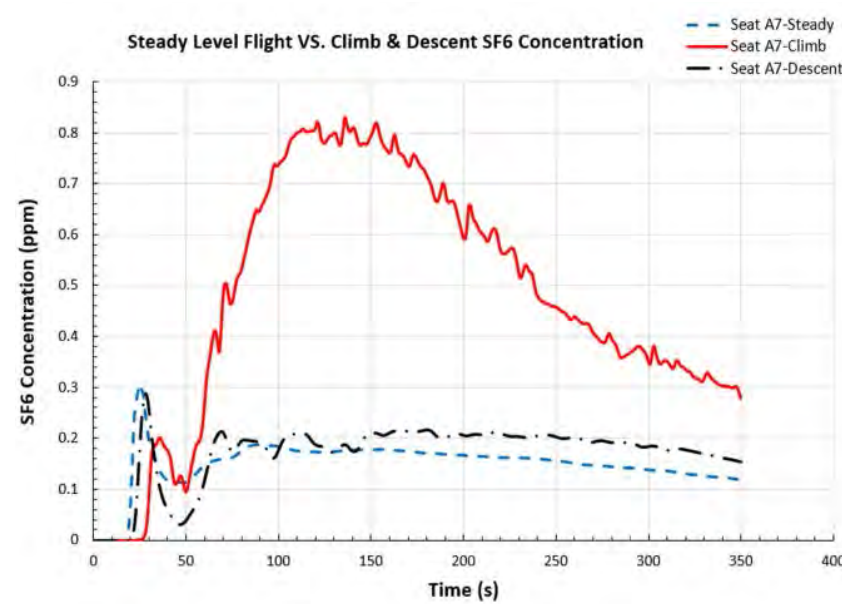

(a)

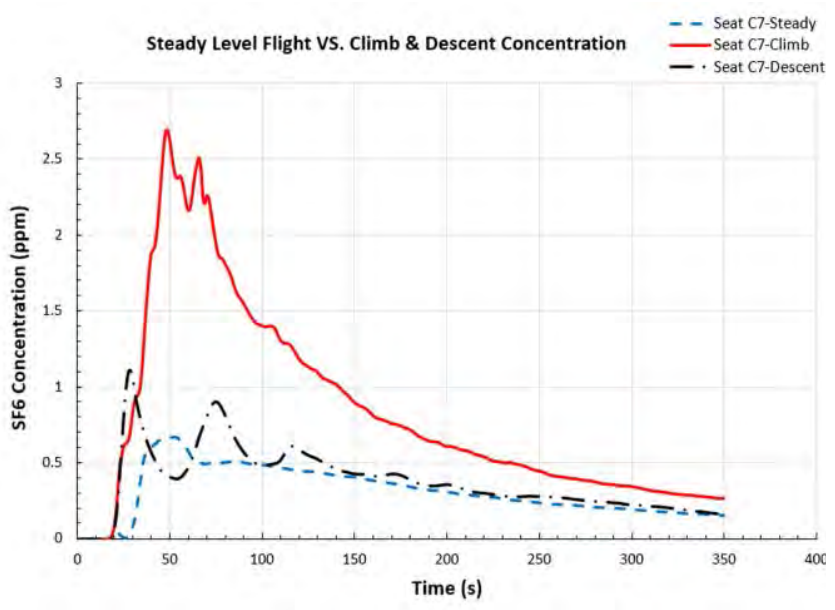

(b)

Figure 4. Comparison of the predicted $\mathrm{SF}_{6}$ concentration time series among steady level flight, climb, and descent scenarios; (a) at seat A7, and (b) at seat $\mathrm{C} 7$

On the other hand, the $\mathrm{SF}_{6}$ concentration time series during the descent leg does not noticeably differ from that for the steady level flight time, and the former can be seen increasing slightly over the latter at the beginning or near the end of the simulation run depending on the monitoring point location. This indicates that the level of passengers' exposure to contaminants is almost identical between the descent and the steady level flight scenarios at the breathing height. However, such similarity in the exposure between the two cases can be altered by changing the contaminant injection point or direction and/or varying the number of concentration monitoring sites and their locations, such factors is beyond the scope of the current study.

One factor that may have contributed to the considerable difference in the $\mathrm{SF}_{6}$ concentration time series between the climb and the descent legs is the difference between climb and descent rate of velocity change and the corresponding accelerations. The climb speed for most modern jet-powered passenger aircraft can reach up to $600 \mathrm{~km} \mathrm{hr}^{-1}$ (324 knots) or more, while the full-powered descent rate is limited to around 
$250 \mathrm{~km} \mathrm{hr}^{-1}$ (135 knots) only to ensure passengers' comfort. This large difference in aircraft speed between the two mission legs yield dissimilar aircraft accelerations, and therefore, distinct effect of the generated body forces on the contaminants dispersion rates and patterns inside the cabin.

Another factor in play is the difference between the climb and the descent (inclination) angles. During the take-off and climb legs, most jet-powered passenger aircraft adopt an inclination angle from $15^{\circ}$ to $20^{\circ}$, such an angle is much steeper than the small descent angle restricted mostly to $3^{\circ}$ to perform comfortable descents. As much as the rise in aircraft speed, the increase of the climb angle over the descent angle very well contribute in enhancing the dispersion rate of contaminants and changing their distribution contours within the cabin. This is because changing the flight path angles significantly alters the values of body force components acting on the aircraft in motion and the contained air on the vertical and horizontal lines, and therefore, the aircraft acceleration components in each of those two directions.

\section{B. Effect of aircraft body forces on airflow patterns and vorticity}

In addition to the effect the body forces have on the contaminant dispersion inside the cabin, they also affect the airflow velocity and patterns, which can be quantified using vorticities.

Airflow velocity magnitudes in all directions inside the cabin were in general greater during climb and descent than that throughout steady level flight. The monitored airflow velocity magnitudes for the simulated flight time increased anywhere between $1 \%$ and $45 \%$ during climb and between $6 \%$ and $42 \%$ during descent with respect to the steady level flight air velocity magnitudes. On the other hand, the air velocity components $\left(\mathrm{V}_{\mathrm{x}}\right.$, $\mathrm{V}_{\mathrm{y}}$, and $\mathrm{V}_{\mathrm{z}}$ ) exhibited different values of increase and decrease between climb and steady flight, and descent and steady flight, with no fixed trend.

The increase in airflow velocity magnitudes throughout the climb and descent legs changed the airflow patterns in the cabin to some degree. One form of this change is the increased air boundary-layer thickness adjacent to the cabin walls due to the tendency of the air to separate from the walls as it moves downward during climb and descent. In other words, the increased downward airflow velocities $\left(0.9 \mathrm{~m} \mathrm{~s}^{-1}\right.$ for climb and $0.8 \mathrm{~m} \mathrm{~s}^{-1}$ for descent compared to $0.65 \mathrm{~m} \mathrm{~s}^{-1}$ during the steady level flight) led to reduce the airflow attachment to the walls. Consequently, the strongest downward flow separation (thickest airflow boundary-layer) is noticed during climb.

Another effect the airflow velocity changes have on airflow patterns is the alteration of the vorticity magnitude and direction in the cabin. For rotational (non-zero vorticity) flows, such as the highly turbulent airflow in the cabin space, the vorticity vector $(\vec{\omega})$ is defined as the curl of the velocity as follows:

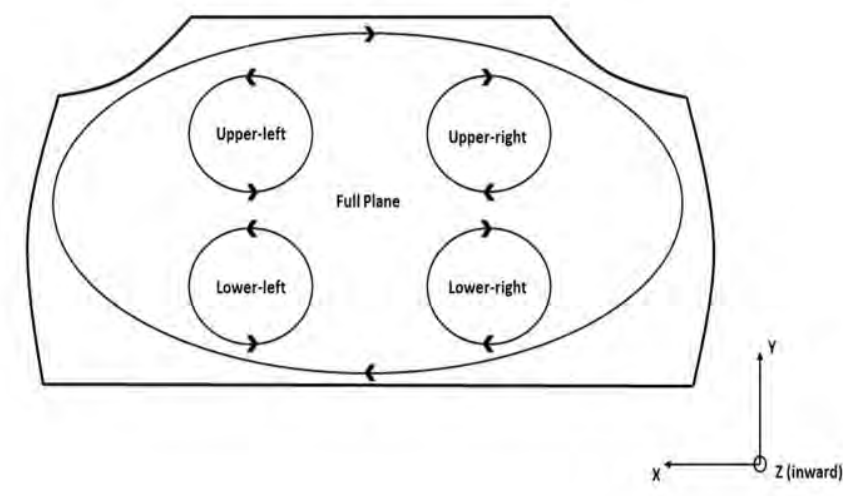

Figure 5. Example of the followed notation for estimating the z-component airflow vorticity $\left(\omega_{z}\right)$ on the full $x-y$ plane and its four side corners

$$
\vec{\omega}=\nabla \times \vec{V}=\omega_{x} \hat{\imath}+\omega_{y} \hat{\jmath}+\omega_{z} \hat{k},
$$

Fig. 5 shows an example of the airflow vorticity on the $x-y$ plane set at the middle of the cabin. From the figure, it can be clearly seen that the airflow vorticity is used to approximate circulation on the full planes, such as $x-y, x-z$, and $z-y$ (not shown in the figure, but corresponding to $\omega_{z}, \omega_{y}$, and $\omega_{x}$, respectively), and also at the four side corners of each plane, namely: the upper-left, upper-right, lower-left, and lower-right corners. This was made possible by calculating the average airflow velocities on separate line segments located at the top, bottom, right, left, and the center (horizontally and vertically) of each of the mentioned planes. Afterwards, the difference in magnitude between each pair of those velocities, and the distances between each two parallel lines on which they were calculated, are used to yield the vorticity vector components as follows:

$$
\begin{aligned}
& \omega_{x}=\frac{\Delta v_{z}}{\Delta y}-\frac{\Delta v_{y}}{\Delta z} \\
& \omega_{y}=\frac{\Delta v_{x}}{\Delta z}-\frac{\Delta v_{z}}{\Delta x} \\
& \omega_{z}=\frac{\Delta v_{y}}{\Delta x}-\frac{\Delta v_{x}}{\Delta y}
\end{aligned}
$$

Table III presents the calculated vorticity vector magnitudes and components on the full planes and the four side corners of each during the steady level flight, climb, and descent legs, and also, the percentage change of those values when each of the climb and descent legs is compared to the steady level flight case.

Studying Table III, it can be noticed that the vorticity vector magnitudes during climb and descent are always greater than those during steady level flight either on the full plane or any of 
TABLE III. AIRFLOW VORTICITY VALUES AND CHANGES BETWEEN STEADY LEVEL FLIGHT, CLIMB AND DESCENT LEGS

\begin{tabular}{|c|c|c|c|c|c|c|c|c|c|}
\hline \multirow{2}{*}{ Plane/side corner } & \multirow{2}{*}{ Flight Leg } & \multirow{2}{*}{$\begin{array}{c}\text { Vorticity }^{\mathrm{a}} \\
\text { Magnitude }\end{array}$} & \multicolumn{3}{|c|}{ Vorticity Components ${ }^{\mathrm{b}}$} & \multicolumn{4}{|c|}{ Change in Vorticity During Climb/Descent ${ }^{\mathrm{c}}(\%)$} \\
\hline & & & $\omega_{x}$ & $\omega_{y}$ & $\omega_{z}$ & $\begin{array}{c}\text { Vorticity } \\
\text { Magnitude }\end{array}$ & $\omega_{x}$ & $\omega_{y}$ & $\omega_{z}$ \\
\hline \multirow{3}{*}{ Full Plane } & Steady Flight & 0.009 & -0.004 & -0.0065 & 0.0046 & - & - & - & - \\
\hline & Climb & 0.014 & -0.01 & -0.0105 & 0.002 & 63.98 & 158.1 & 62.05 & -64.64 \\
\hline & Descent & 0.023 & 0.012 & -0.019 & 0.0043 & 163.6 & $221.1^{d}$ & 197.7 & -6.45 \\
\hline \multirow{3}{*}{ Lower-right Corner } & Steady Flight & 0.168 & 0.028 & -0.023 & 0.164 & - & - & - & - \\
\hline & Climb & 0.196 & -0.033 & -0.015 & 0.193 & 16.85 & $19.64^{\mathrm{d}}$ & -34.13 & 17.59 \\
\hline & Descent & 0.194 & 0.072 & -0.028 & 0.178 & 15.49 & 161.3 & 17.71 & 8.53 \\
\hline \multirow{3}{*}{ Lower-left Corner } & Steady Flight & 0.141 & 0.023 & -0.007 & -0.139 & - & - & - & - \\
\hline & Climb & 0.200 & 0.079 & -0.024 & -0.182 & 42.07 & 249.2 & 243.9 & 31.12 \\
\hline & Descent & 0.154 & -0.021 & -0.002 & -0.152 & 9.050 & $-9.83^{\mathrm{d}}$ & -70.06 & 9.64 \\
\hline \multirow{3}{*}{ Upper-right corner } & Steady Flight & 0.151 & -0.030 & -0.006 & 0.148 & - & - & - & - \\
\hline & Climb & 0.210 & -0.099 & 0.003 & 0.185 & 39.28 & 226.6 & $-57.81^{d}$ & 25.42 \\
\hline & Descent & 0.170 & 0.045 & -0.036 & 0.160 & 13.01 & $47.49^{\mathrm{d}}$ & 498.3 & 8.64 \\
\hline \multirow{3}{*}{ Upper-left corner } & Steady Flight & 0.159 & -0.035 & 0.0105 & -0.155 & - & - & - & - \\
\hline & Climb & 0.190 & 0.013 & -0.006 & -0.189 & 19.33 & $-61.66^{d}$ & $-47.39^{d}$ & 22.25 \\
\hline & Descent & 0.177 & -0.048 & -0.0109 & -0.169 & 10.82 & 35.94 & $3.99^{\mathrm{d}}$ & 9.41 \\
\hline
\end{tabular}

a. The unit for vorticity magnitude and components is $\mathrm{s}^{-1}$

b. $(+)$ is in clockwise direction and (-) is in counterclockwise direction

c. $(+)$ indicates an increase and (-) indicates a decrease from steady flight

d. Accompanied with a change in the direction of rotation

the four corners. This rise is mainly caused by the increased airflow velocity gradients everywhere in the cabin during climb and descent. This indicates stronger circulation of air in the cabin for each zone identified. Between climb and descent, the former has higher vorticity magnitudes at the corners, while this was not the case on the full plane only, where circulation patterns on each corner cancel each other out on the full plane. The greatest increase in vorticity magnitude, taking the steady level flight case as a reference, is seen on the full plane during descent with around $164 \%$ of increase. This was followed by the climb on the full plane, as well, with $64 \%$ increase, while, for the corners, the amounts of increase were relatively close in value and in the favor of climb, as previously mentioned.

On the other hand, the vorticity vector components exhibited both increases and decreases during climb and descent, with respect to the steady level flight case, with increases occurring slightly more frequently. However, those increases are not exclusive to one flight leg, and occur almost equally between climb and descent. One unique characteristic of vorticity components is that they can show the change in the vorticity direction between steady flight and climb, and steady flight and descent, in addition to the increase or decrease in magnitude. This is shown by a change in the sign of the vorticity component from positive (clockwise direction) to negative (counterclockwise direction), and vice versa. Those cases are also clearly demonstrated in Table 3 tagged by the footnote (d) to show that a change in the direction of airflow rotation occurs even if the vorticity intensity (magnitude) increases or decreases.

\section{CONCLUSIONS}

In this study, numerical simulations for the effect of body forces due to aircraft acceleration on the airflow and contaminant dispersion in a model for a passenger aircraft cabin are performed. The steady level flight leg which takes most of the flight time is taken as the reference case, to which the contaminant concentration and airflow changes during the climb and descent flight legs are compared. 
It was noticed that the concentration of the contaminant surrogate $\left(\mathrm{SF}_{6}\right)$ at the two set monitoring locations, and therefore the passengers' exposure, increased substantially during the climb leg from the steady level flight. However, this was not the case during the descent leg, throughout which the $\mathrm{SF}_{6}$ concentration did not considerably differ from that during the steady level flight time.

Airflow velocity magnitudes, on the other hand, increased everywhere in the simulated cabin during climb and descent from the steady flight case. But, this was not the case for the airflow velocity components $\left(\mathrm{V}_{\mathrm{x}}, \mathrm{V}_{\mathrm{y}}\right.$, and $\left.\mathrm{V}_{\mathrm{z}}\right)$ which showed different levels of increase and decrease with no fixed trend.

The change in airflow velocities had a significant effect on the airflow patterns and vorticity (approximation for circulation) in the cabin. Downward airflow coming from supply slots was less attached to the cabin walls during climb and descent than through the steady flight time. Additionally, airflow vorticity magnitudes always exhibited an increasing trend when the steady flight case was changed to either the climb or descent scenario, indicating greater air circulation in the cabin. This increasing trend, however, was not followed by the vorticity components on the full plane and its corners in each direction which showed various percentages of increase and decrease, and also displayed changes in the vorticity direction from steady flight to either climb or descent.

The findings indicate the potent effect the body forces have on the airflow behavior and contaminate dispersion inside the cabins of passenger aircraft and calls for more research attention to this topic to unveil some ventilation design remedies to the negative effects this may have on the health of aircraft occupants.

\section{ACKNOWLEDGMENT}

The authors thank the Government of Ontario, Canada, for providing the funding for this work in the form of the Ontario Trillium Scholarship (OTS) for the lead author. They also thank Christopher Y. H. Chao (Department of Mechanical Engineering, Hong Kong University of Science and Technology, Clear Water Bay, Hong Kong, China), and Man Pun Wan (Department of Mechanical \& Aerospace Engineering, Nanyang Technological University, Singapore) for providing their full research data. Finally, the authors recognize CMC Microsystems for providing the Academic Research license of ANSYS, and the Centre for Advanced Computing (CAC) at Queen's University (Kingston, ON, Canada) for providing the Frontenac Compute Cluster to run the simulations.

\section{REFERENCES}

H. A. Elmaghraby, Y. W. Chiang, and A. A. Aliabadi, "Ventilation strategies and air quality management in passenger aircraft cabins: A review of experimental approaches and numerical simulations," Sci. Technol. Built Environ., vol. 24, no. 2, pp. 160-175, 2018. doi: $10.1080 / 23744731.2017 .1387463$

[2] M. A. Waters, T. F. Bloom, B. Grajewski, and J. Deddens, "Measurements of Indoor Air Quality on Commercial Transport Aircraft," in The 9th International Conference on Indoor Air Quality and Climate, 2002, pp. 782-787.
P. Strøm-Tejsen, D. P. Wyon, L. Lagercrantz, and L. Fang, "Passenger evaluation of the optimum balance between fresh air supply and humidity from 7-h exposures in a simulated aircraft cabin," Indoor Air, vol. 17, no. 2, pp. 92-108, 2007. doi: $10.1111 / \mathrm{j} .1600-0668.2006 .00458 . x$.

[4] G. N. Sze To, M. P. Wan, C. Y. H. Chao, L. Fang, and A. Melikov, "Experimental study of dispersion and deposition of expiratory aerosols in aircraft cabins and impact on infectious disease transmission," Aerosol Sci. Technol., vol. 43, no. 5, pp. 466-485, 2009. doi: 10.1080/02786820902736658.

[5] F. Li, J. Liu, J. Pei, C. H. Lin, and Q. Chen, "Experimental study of gaseous and particulate contaminants distribution in an aircraft cabin," Atmos. Environ., vol. 85, pp. 223-233, 2014. doi: 10.1016/j.atmosenv.2013.11.049.

[6] Z. Fang, H. Liu, B. Li, A. Baldwin, J. Wang, and K. Xia, "Experimental investigation of personal air supply nozzle use in aircraft cabins," Appl. Ergon., vol. 47, pp. 193-202, 2015. doi:10.1016/j.apergo.2014.09.011.

[7] C.-H.- Lin, K. H. Dunn, R. H. Horstman, J. L. Topmiller, M. F. Ahlers, J. S. Bennett, L. M. Sedgwick, and S. Wirogo, "Numerical simulation of airflow and airborne pathogen transport in aircraft cabins--Part I: numerical simulation of the flow field.," ASHRAE Trans., vol. 111, no. 1, pp. 755-764, 2005.

[8] M. P. Wan, G. N. Sze To, C. Y. H. Chao, L. Fang, and A. Melikov, "Modeling the fate of xpiratory aerosols and the associated infection risk in an aircraft cabin environment," Aerosol Sci. Technol., vol. 43, no. 4, pp. 322-343, 2009. doi: $10.1080 / 02786820802641461$.

[9] M. Hassan, "Numerical Investigation of Improving Distribution Systems In Aircraft Passengers Cabins," Cairo University, Giza, Egypt, 2016.

[10] Z. Zhang, X. Chen, S. Mazumdar, T. Zhang, and Q. Chen, "Experimental and numerical investigation of airflow and contaminant transport in an airliner cabin mockup," Build. Environ., vol. 44, no. 1, pp. 85-94, 2009. doi: 10.1016/j.buildenv.2008.01.012.

[11] S. B. Poussou, S. Mazumdar, M. W. Plesniak, P. E. Sojka, and Q. Chen, "Flow and contaminant transport in an airliner cabin induced by a moving body: Model experiments and CFD predictions," Atmos. Environ., vol. 44, no. 24, pp. 2830-2839, 2010.doi: 10.1016/j.atmosenv.2010.04.053.

[12] T. T. Zhang, P. Li, and S. Wang, "A personal air distribution system with air terminals embedded in chair armrests on commercial airplanes," Build. Environ., vol. 47, no. 1, pp. 89-99, 2012. doi:10.1016/j.buildenv.2011.04.035.

[13] S. S. Isukapalli, S. Mazumdar, P. George, B. Wei, B. Jones, and C. P. Weisel, "Computational fluid dynamics modeling of transport and deposition of pesticides in an aircraft cabin," Atmos. Environ., vol. 68, pp. 198-207, 2013. doi: 10.1016/j.atmosenv.2012.11.019.

[14] F. Li, J. Liu, J. Ren, X. Cao, and Y. Zhu, "Numerical investigation of airborne contaminant transport under different vortex structures in the aircraft cabin," Int. J. Heat Mass Transf., vol. 96, pp. 287295, 2016. doi: 10.1016/j.ijheatmasstransfer.2016.01.004.

[15] D. G. Hull, Fundamentals of Airplane Flight Mechanics. New York: Springer Berlin Heidelberg, 2007.

[16] Z. J. Zhai, Z. Zhang, W. Zhang, and Q. (Yan) Chen, "Evaluation of various turbulence models in predicting airflow and turbulence in enclosed environments by CFD: Part 1 - summary of prevalent turbulence models," HVAC\&R Res., vol. 13, no. 6, pp. 853-870, 2007. doi: 10.1080/10789669.2007.10391459. 
[17] Z. Zhang, W. Zhang, Z. J. Zhai, and Q. Y. Chen, "Evaluation of Various Turbulence Models in Predicting Airflow and Turbulence in Enclosed Environments by CFD: Part 2-Comparison with Experimental Data from Literature," $H V A C \& R$ Res., vol. 13, no. 6, pp. 871-886, 2007.doi: 10.1080/10789669.2007.10391460.

[18] M. Wang and Q. Chen, "Assessment of various turbulence models for transitional flows in enclosed environment (RP-1271)," HVAC\&R Res., vol. 15, no. 6, pp. 1099-1119, 2009. doi: 10.1080/10789669.2009.10390881.

[19] W. Liu, J. Wen, C. H. Lin, J. Liu, Z. Long, and Q. Chen, "Evaluation of various categories of turbulence models for predicting air distribution in an airliner cabin," Build. Environ., vol. 65, pp. 118-131, 2013.10.1016/j.buildenv.2013.03.018.

[20] P. J. Roache, "Perspective: A Method for Uniform Reporting of Grid Refinement Studies," J. Fluids Eng., vol. 116, no. 3, pp. 405-413, 1994. doi: 10.1115/1.2910291

[21] A. A. Aliabadi, Theory and Applications of Turbulence: A Fundamental Approach for Scientists and Engineers. Guelph, ON, Canada: Amir Abbas Aliabadi Publications, 2018.

[22] A. A. Aliabadi, "Dispersion of Expiratory Airborne Droplets in a Model Single Patient Hospital Recovery Room with Stratified Ventilation," The University of British Columbia, Vancouver, Canada, 2013.

[23] S. R. Hanna, "Confidence limits for air quality model evaluations, as estimated by bootstrap and jackknife resampling methods," Atmos. Environ., vol. 23, no. 6, pp. 1385-1398, 1989. doi: 10.1016/0004-6981(89)90161-3.

[24] J. C. Chang and S. R. Hanna, "Air quality model performance evaluation," Meteorol. Atmos. Phys., vol. 87, no. 1-3, pp. 167196, 2004. doi: 10.1007/s00703-003-0070-7.

[25] S. Hanna and J. Chang, "Acceptance criteria for urban dispersion model evaluation," Meteorol. Atmos. Phys., vol. 116, no. 3-4, pp. 133-146, 2012. doi: 10.1007/s00703-011-0177-1.

[26] A. A. Aliabadi, E. S. Krayenhoff, N. Nazarian, L. W. Chew, P. R. Armstrong, A. Afshari, and L. K. Norford, "Effects of Roof-Edge Roughness on Air Temperature and Pollutant Concentration in Urban Canyons," Boundary-Layer Meteorol, vol. 164, no. 2, pp. 249-279, 2017. doi: 10.1007/s10546-017-0246-1.

[27] S. Gudmundsson, General Aviation Aircraft Design: Applied Methods and Procedures. Butterworth-Heinemann, 2013.

[28] National Aeronautics and Space Adminstration (NASA), "Forces in a climb," 2015. [Online]. Available: https://www.grc.nasa.gov/ www/k-12/airplane/climb.html\%0A. [Accessed: 10-Oct-2017]

[29] University of Southampton, "Weight, Geometry, Lift, Drag and Resources/Sydney aerodynamics for students/perf/perf_ac.html. http://www.southampton.ac.uk/ jps7/Aircraft Design Resources/ Sydney aerodynamics for students/perf/perf_ac.html. [Accessed: 10-May-2017]. 


\section{Appendix (A): Procedure FOR Determining THE AIRCRAFT ACCELERATION COMPONENTS DURING CLIMB}

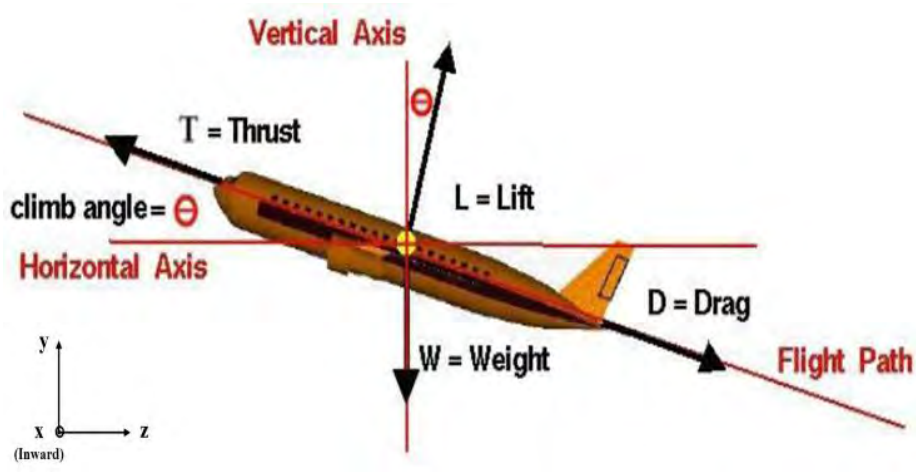

Figure 6. Forces on a passenger aircraft during climb with the two axes (horizontal and vertical) set for the calculation of acceleration components

Newton's second law is applied on the vertical and horizontal axes shown in the above figure,

$$
\sum \vec{F}=m \vec{a}
$$

On the vertical axis:

$$
\mathrm{T} \sin \theta-\mathrm{D} \sin \theta+\mathrm{L} \cos \theta-\mathrm{W}=\mathrm{m} \overrightarrow{\mathrm{a}_{\mathrm{v}}},
$$

and, on the horizontal axis:

$$
\mathrm{T} \cos \theta-\mathrm{D} \cos \theta+\mathrm{L} \sin \theta=\mathrm{m} \overrightarrow{\mathrm{a}_{\mathrm{h}}},
$$

where $\overrightarrow{\mathrm{a}_{\mathrm{v}}}$ and $\overrightarrow{\mathrm{a}_{\mathrm{h}}}$ are the vertical and horizontal acceleration components, respectively.

The unknowns $(\mathrm{T}, \theta, \mathrm{D}, \mathrm{L}, \mathrm{m})$ are estimated based on industrial specifications and dimensions for the Boeing 767300 aircraft.

$\mathbf{T}=462.6 \mathrm{KN}$ (for a twin-jet engine)

$\boldsymbol{\theta}=\mathbf{2 0}{ }^{\circ}$

$\mathbf{m}=\mathbf{1 5 9 , 2 1 0} \mathrm{kg}$ (max. takeoff weight)

$\mathrm{W}=\boldsymbol{m g}$

$$
\begin{aligned}
& \mathrm{D}=\mathrm{C}_{\mathrm{D}} * 0.5 \rho \mathrm{V}^{2} \mathrm{~A}, \\
& \mathrm{~L}=\mathrm{C}_{\mathrm{L}} * 0.5 \rho \mathrm{V}^{2} \mathrm{~A},
\end{aligned}
$$

where $C_{D}$ and $C_{L}$ are the drag and lift coefficients, respectively, $\rho$ is the air density, $V$ is the aircraft velocity (taken as $\mathbf{1 5 5} \mathbf{~ m ~ s}^{-1}$ ), and $A$ is the reference (wing) area.

$$
\mathrm{C}_{\mathrm{D}}=\mathrm{C}_{\mathrm{D}_{0}}+\mathrm{kC}_{\mathrm{L}}^{2}
$$

where $C_{D_{0}}$ is the part of drag coefficient due to friction and pressure on the aircraft body, and $k$ is a constant that incorporates the other part of the drag coefficient due to lift (lift induced drag).

$\mathbf{C}_{\mathbf{D}}=\mathbf{0 . 0 6}$ (approximation for the majority of aircraft aerofoils)

$$
\mathrm{k}=\frac{1}{\pi \mathrm{ARe}}
$$

where $e$ is a constant equal to 0.85 for twin engine wide-body aircraft, and $A R$ is the wing aspect ratio which is determined from:

$$
\mathrm{AR}=\frac{(\text { wing span })^{2}}{\text { wing area }}=\frac{(47.57)^{2}}{283.3}=7.987
$$

These yield $\mathbf{k}=\mathbf{0 . 0 4 6 8 8}$

Taking $C_{D_{0}}=0.017$ for twin engine wide-body, and substituting in equation 6 , yields $\mathbf{C}_{\mathbf{L}}=\mathbf{0 . 9 6}$

Substituting in equations 4 and 5, and assuming the density of air to be $1.2 \mathrm{~kg} \mathrm{~m}^{-3}$ (aircraft still close to sea level), the drag and lift forces on the aircraft during climb are determined to be:

$$
\mathrm{D}=229.473 \mathrm{KN} \text {, }
$$

and

$$
L=3671.568 \mathrm{KN}
$$

Substituting in equations 2 and 3 yields the vertical and horizontal components of the aircraft acceleration, respectively:

and

$$
\overrightarrow{\mathrm{a}_{\mathrm{v}}}=13.79 \mathrm{~ms}^{-2}=-1.4 \vec{g},
$$

$$
\overrightarrow{\mathbf{a}_{h}}=-7.14 \mathrm{~ms}^{-2}=0.73 \vec{g} .
$$

Lastly, the absolute acceleration components got are expressed in the form of relative acceleration components on the air inside the aircraft cabin before being implemented in Fluent. This is attained by reversing the sign of each acceleration component and superimposing it on any acceleration(s) that may exist in the same direction (e.g. gravity). This yield the relative acceleration components as follows:

$$
\begin{gathered}
\overrightarrow{\mathrm{a}_{\mathrm{y}}}=(-13.79-9.81)=-23.6 \mathrm{~ms}^{-2}=2.4 \vec{g}, \\
\left(\text { or } 23.6 \mathrm{~ms}^{-2} \text { acting downwards }\right)
\end{gathered}
$$

and

$$
\overrightarrow{\mathrm{a}_{\mathrm{z}}}=7.14 \mathrm{~ms}^{-2}=-0.73 \vec{g} .
$$

(or $7.14 \mathrm{~ms}^{-2}$ acting toward the tail of the aircraft) 


\section{ApPendix (B): Procedure FOR DETERMining THE AIRCRAFT ACCELERATION COMPONENTS DURING DESCENT}

For descent, the same procedure and parameters for climb apply, but the aircraft velocity $(\mathrm{V})$, descent angle $(\theta)$, and the density of air $(\rho)$, at the chosen descent elevation $(10000 \mathrm{ft}$. or $3050 \mathrm{~m}$ ), are adjusted to the new case.

$\mathbf{V}=\mathbf{7 0} \mathbf{m ~ s}^{-1}$ (full-powered descent)

$\boldsymbol{\theta}=\mathbf{3}^{\circ}$

$\rho=0.9 \mathrm{~kg} \mathrm{~m}^{-3}$

With the aircraft tilted downward, equations 2 and 3 in Appendix (A) are changed to:

On the vertical axis:

$$
\mathrm{L} \cos \theta+\mathrm{D} \sin \theta-\mathrm{T} \sin \theta-\mathrm{W}=\mathrm{m} \overrightarrow{\mathrm{a}_{\mathrm{v}}} \text {, }
$$

and, on the horizontal axis:

$$
\mathrm{L} \sin \theta+\mathrm{T} \cos \theta-\mathrm{D} \cos \theta=\mathrm{m} \overrightarrow{\mathrm{a}_{\mathrm{h}}}
$$

Substituting in equations 4 and 5 of Appendix (A) with the new velocity and air density, and using the same values for $\mathrm{C}_{\mathrm{D}}$ and $\mathrm{C}_{\mathrm{L}}$, yields:

and

$$
\mathrm{D}=37.481 \mathrm{KN}
$$

$$
\mathrm{L}=599.69 \mathrm{KN} \text {. }
$$

Substituting the D and $\mathrm{L}$ values got (keeping $\mathrm{T}$ and $\mathrm{m}$ the same) in equations 2 and 3 of Appendix (A) yields the vertical and horizontal components of aircraft acceleration during descent, respectively:

and

$$
\overrightarrow{a_{v}}=-6.1883 \mathrm{~ms}^{-2}=0.63 \vec{g}
$$

$$
\overrightarrow{a_{h}}=2.86 \mathrm{~ms}^{-2}=-0.29 \vec{g}
$$

Finally, the relative acceleration components are calculated for the descent leg using the same approach previously followed for the climb leg:

$$
\begin{gathered}
\overrightarrow{\mathrm{a}_{\mathrm{y}}}=(6.1883-9.81)=-3.622 \mathrm{~ms}^{-2}=0.37 \vec{g}, \\
\left(\text { or } 3.622 \mathrm{~ms}^{-2} \text { acting downwards }\right)
\end{gathered}
$$

and

$$
\overrightarrow{\mathrm{a}_{\mathrm{z}}}=-2.86 \mathrm{~ms}^{-2}=0.29 \overrightarrow{\mathrm{g}} \text {. }
$$

(or $2.86 \mathrm{~ms}^{-2}$ acting toward the head of the aircraft) 


\title{
Quantitative Natural Gas Discrimination For Pipeline Leak Detection Through Time-Series Analysis of an MOS Sensor Response
}

\author{
Matthew Barriault, Mahyar Mohaghegh Montazeri, Allen O’Brien, Homayoun Najjaran, Mina Hoorfar \\ School of Engineering \\ University of British Columbia \\ Kelowna, Canada
}

\begin{abstract}
In order to detect natural gas pipeline leaks, ethane in the natural gas must be discriminated from background methane emissions. Our gas detection apparatus is well-suited for this application due to its flexibility and low cost. We present a comparison of machine learning models for quantitative estimation of concentrations of both methane and ethane in a target gas sample, using a response over time from a single sensor in our apparatus. We also demonstrate that the use of synthetic data is very effective for training a model to discriminate between methane and ethane.
\end{abstract}

Keywords-machine learning; gas detection; microfluidic; diffusion simulation; pipeline leak detection

\section{INTRODUCTION}

Natural gas pipeline leak detection can be broadly categorized into "internal" and "external" methods, depending on whether the detector resides inside or outside the pipeline. Internal methods include acoustic measurement, pressure/flow monitoring, and statistical analysis. These methods also often make use of one of several mathematical modeling options to predict when a leak has occurred. External methods are more hardware-based, relying on, for example, acoustic, optical, soil monitoring, or vapor sampling sensors [1]. These hardware solutions can either be permanently installed in a fixed location, or used in conjunction with a mobile monitoring apparatus, such as a handheld detector or even a drone. We present here the application of a small, cost-effective, and highly flexible gas sensing apparatus that can be used in either permanent or mobile applications.

Leak detection requires differentiation between methane and ethane, since natural gas will typically contain $\sim 5 \%$ ethane and must be detected in the presence of background methane emission from, for example, nearby agriculture. However, the difference between the sensor's response to methane and ethane may not be immediately clear. We employ machine learning techniques to discover patterns that will enable this discrimination. To offset the requirements of some algorithms that a large dataset be provided, we also test the performance of our estimation models using synthetic data. Using simulations, we will be able to generate predictions of the sensor's responses to wide ranges of concentration, temperature, pressure and humidity. All of this can be used to train the pattern recognition system to be able to take into account the effect of these parameters and give more accurate results.

\section{SENSING APPARATUS AND DATA COLLECTION}

The sensing apparatus consists of a Figaro 2610 metal oxide semiconducting gas sensor embedded in a 3D-printed microchannel, which is coated with chromium, gold, and parylene-C to increase selectivity. Full fabrication details are given in [4]. This apparatus is quite flexible, and target gases can be altered simply by changing which MOS sensor is included. This is made easier by the fact that Figaro manufactures sensors with similar dimensions, but for a variety of target gases. Parallel work in our lab has demonstrated the suitability of this apparatus for nuisance sewer gas detection [2], wine identification [3], and breath analysis. Its small size enables it to be used for both stationary and mobile applications, which is extremely beneficial in the context of pipeline leak detection, where both types of devices may be needed, depending on the individual situation. Figure 1 displays a model of the microchannel sensing apparatus.

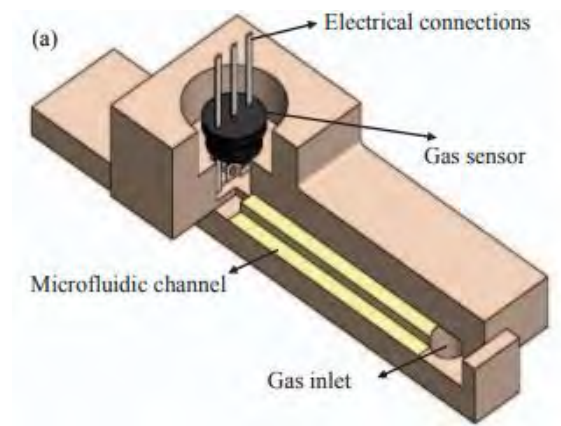

Figure 1. Gas sensing apparatus. Figure from [4]

Our dataset consists of time-series curves from our sensing apparatus in response to a variety of target gas concentrations. These targets are created through the use of a mass flow controller, and contain known concentrations of methane or ethane. We have not yet collected enough data with mixtures of both gases to make meaningful predictions, but this is the focus of ongoing work, primarily on using simulations to create synthetic mixture data to alleviate the time required to perform manual tests. Therefore, in the current work, the goal of the model is to not only determine which gas it has been exposed to, but also to estimate the concentration of that gas. 
The time-series curves are generated by exposing the sensor to the target gas for 40 seconds, then allowing the sensor to recover by placing it in fresh air for 150 seconds. An example curve is shown in Figure 2. The exposure and recovery phases are clearly distinguishable. Some of the curves' features are also shown, which will be discussed in Section IV-A.

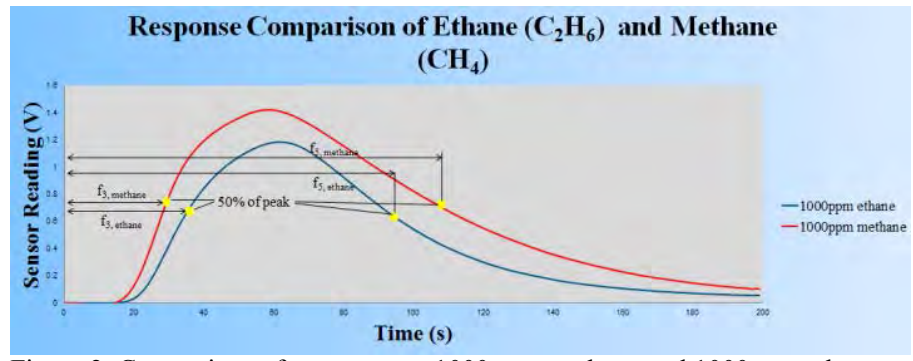

Figure 2. Comparison of responses to $1000 \mathrm{ppm}$ methane and 1000ppm ethane, with features \#3 and \#5 shown

\section{SIMULATION METHODOLOGY}

One of the main challenges of developing microfluidicbased gas detectors is calibration of the sensor, based on wide ranges of different parameters such as changes in the mixture concentration, temperature, pressure and humidity. This requires a vast number of experiments to generate enough data to be able to take into account the effect of each parameter accurately. Simulation of the gas sensor can help solve this problem as it reduces the number of experiments needed for calibrating the sensor, saves time, reduces human and instrument errors, and removes many limitations.

In the current study diffusion of a target gas inside the sensor's 3D printed micro-fluidic channel is studied. The simulation is done in a three dimensional model and the effect of gas adsorption to the channel walls is also applied to the simulation results.

This methodology does not take into account the individual differences between each sensor. To use the simulation data to train a model used to estimate real data, the simulations need to be tailored to account for the fact that the real sensor does not exactly match the theoretical model. However, we present here a proof of concept that synthetic data is well-suited to training a discriminative model such as those discussed in Section IV-B and IV-C. Such a calibration procedure that would allow the model to be trained on synthetic data and tested on real data is the focus of ongoing work.

The synthetic dataset was generated by simulating a target gas with concentrations from $100 \mathrm{ppm}$ to $1000 \mathrm{ppm}$ in increments of $100 \mathrm{ppm}$, with five repeats for each. To make our predictions more robust to day-to-day variations in the sensor, and to make our data more realistic, we introduce some randomness in the simulations. The actual simulated target concentrations were sampled from Gaussian distributions with means equal to the ideal target concentrations, and standard deviations of $10 \mathrm{ppm}$. Since this randomness is unknown to us in a real situation, the estimation targets for these tests are kept as the ideal targets i.e. $100 \mathrm{ppm}, 200 \mathrm{ppm}$, etc. The simulated curves for different concentrations of methane are shown in Figure 3.

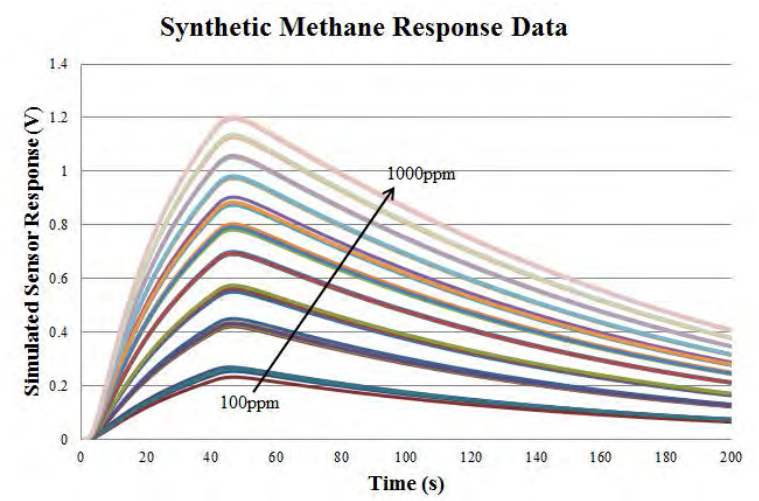

Figure 3. Simulation results for methane, with actual concentrations sampled from a Gaussian centered on the ideal concentration

\section{A. Diffusion model}

As in this study, diffusion of a mixture of gas into another is the governing transport phenomena. For this, the MaxwellStefan equation, which is an accurate model for multicomponent diffusion for low density gases, is chosen:

$$
\nabla x=-\sum_{\substack{\beta=1 \\ \alpha=1,2,3, \ldots, n}}^{n} \frac{}{C D_{a \beta}}\left(x_{\beta} N-x N_{\beta}\right),
$$

where $x_{i}$ is the mole fraction, $N_{i}$ the flux, $C$ the total concentration and $D_{i j}$ the diffusion coefficient of component $i$ in component $j$. It worth mentioning that, in a binary system, the well-known Fick's law can also be used to simplify the model. [5]

\section{B. Surface adsorption model}

As the gas diffuses inside the micro-fluidic channel, some of the molecules adsorb to or desorb from the channel walls which affects the transport phenomena rate. In this simulation the adsorption is taken into account using the Langmuir adsorption model, which considers the phenomena an equilibrium reaction and provides the adsorption and desorption rate as:

$$
\begin{aligned}
r_{a d} & =k_{a d} p_{A}[s] \\
r_{d} & =k_{d}\left[A_{a d}\right]
\end{aligned}
$$

Where $k_{a d}$ and $k_{d}$ are the adsorption (forward) and desorption (backward) reaction rates, $p_{A}$ partial pressure of $A$, $[S]$ empty sites concentration and $\left[A_{a d}\right]$ is the concentration of compound A molecules adsorbed on the surface. [6]

\section{Model assumptions and boundary conditions}

In this model we assume that there is no flow, and the diffusion is the governing transport phenomena. Also, there is no diffusion of gas molecules to the bulk of channel walls and the adsorption is occurring only on surface. The simulation model is shown in Figure 4. 
As the experimental test consists of two steps, exposure and recovery, the simulation is also designed in two different steps which have different initial and boundary conditions. In the exposure phase, there is no target gas inside the channel and it is filled with air. At time $=0$ s the sample concentration is introduced as the boundary condition at the opening of the channel and during this step the diffusion happens for 40s, at which point the recovery phase starts. The initial concentration is derived from the last time point of the previous step and the boundary condition will be set to zero concentration of the target gas.

It is worth mentioning that many other model assumptions are inherited from the diffusion and the surface adsorption models which can be found in the previously mentioned references.

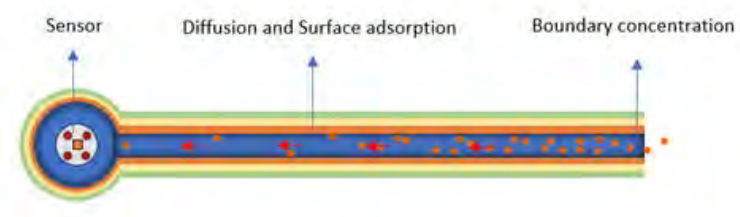

Figure 4. Diffusion simulation model

\section{CONCENTRATION ESTIMATION}

In this work, we evaluate the performance of both featurebased and feature-less classifiers. In the case of a featurebased model, the input to the classifier is a set of pre-designed features that are extracted from each of the time-series curves. In other words, the input is a set of training vectors $x_{i} \in$ $\mathbb{R}^{\boldsymbol{d}}, i=1 \ldots n$, where $n$ is the number of samples in the training dataset, and $d$ is the number of features extracted from each sample. For the feature-less models, the input is simply the raw data, so the training vectors are $x_{i} \in \mathbb{R}^{t}, i=1 \ldots n$, where $t$ is the length of each time-series sample. In both cases, the targets $y$ are the known concentrations of both methane and ethane: $y_{i} \in \mathbb{R}^{2}, i=1 \ldots n$. Feature-based methods have found extensive use with electronic noses, and a considerable effort has been made in past decades to design features that will produce good classification results [1]. However, it is desirable to use a classifier that does not require the handdesigning of such features. For this reason, we investigate the performance of a recurrent neural network, which can take the raw sensor data as input, without the need for any feature design.

Preliminary hyperparameter selection for each model (including model depth, width, and regularization parameters) was done using a common held-out test set consisting of $5 \%$ of the total data. Once hyperparameters were identified that maximized performance on the test set, the final performance of the model was evaluated by predicting the methane and ethane concentrations for each sample, using a leave-one-out method. Leave-one-out can be considered a special case of $\mathrm{k}$ fold validation with $k=n$, where $n$ is the total number of samples. This means that $n$ models were trained, with one sample excluded from each, giving the best possible prediction for each sample. The leave-one-out method becomes impractical for even moderately-sized datasets, for which standard k-fold validation should be used instead, with $k$ chosen such that the held-out test data in each case would be about $5 \%$ of the total dataset.

\section{A. Feature extraction}

In order to get an idea of which features will discriminate well between methane and ethane, we examine the comparison of the sensor's response to 1000ppm of each gas in Figure 2. The significant difference in speed of response, especially between 20 and 50 seconds, suggests that features such as the time at which the signal reaches $50 \%$ of its peak value might be useful. Along with good discrimination between gases, it is also important for the magnitudes of predicted concentrations to be accurate. For this reason, features such as the peak value and the area under the curve will also be useful because these features relate directly to the magnitude of the target gas concentration. Table 1 provides a full description of the features used.

Once the features have been extracted, they must be processed to ensure that the models can learn properly from them. This processing is to make the distributions of each of the features have a mean of zero, and a standard deviation of one. This is done so that one feature with a much larger magnitude than the others does not completely dwarf the contributions of the rest.

Table 1. Extracted features used for feature-based classifiers

\begin{tabular}{cc}
$\begin{array}{c}\text { Feature } \\
\text { Number }\end{array}$ & Feature Description \\
\hline 1 & Peak value \\
\hline 2 & Area under the curve \\
\hline 3 & $\begin{array}{c}\text { Time to } 50 \% \text { of peak value } \\
\text { (exposure phase) }\end{array}$ \\
\hline 5 & $\begin{array}{c}\text { Time to } 75 \% \text { of peak value } \\
\text { (exposure phase) }\end{array}$ \\
\hline 6 & $\begin{array}{c}\text { Time to } 50 \% \text { of peak value } \\
\text { (recovery phase) }\end{array}$ \\
& $\begin{array}{c}\text { Time to } 75 \% \text { of peak value } \\
\text { (recovery phase) }\end{array}$
\end{tabular}

\section{B. Feature-based models}

Many machine learning models perform poorly with timeseries data if the entire time-series is naively given to the model to use as training/testing data, due to their difficulty in learning temporal relationships. Multi-layer perceptrons (MLPs) and support vector machines (SVMs) are two such models. Therefore, we evaluate the performance of these model using extracted features.

A multilayer perceptron is a type of feed-forward neural network consisting of at least three layers, all but the first of which apply a nonlinear transform to a weighted sum of the previous layer's activations. The first layer is called the input layer, and it is where the features are input. The last layer is called the output layer, and it is where the network's predictions appear. Any layers in between these two are called 
hidden layers. Since a nonlinear transform is applied at every layer, the addition of more hidden layers means that the network can learn more complex functions [7]. The activation $z$ of layer $j$ is given by:

$$
z=\sigma\left(W_{i j} z_{i}+b\right)
$$

where $z_{j}$ is the activation vector of the previous layer, $b_{j}$ is the bias vector for layer $j, W_{i j}$ is the weight matrix between layers $i$ and $j$, and $\sigma$ is a nonlinear transform function, usually either the logistic sigmoid or the hyperbolic tangent function.

MLPs are supervised models, meaning that for the network to predict the correct output values, it must be allowed to learn on a training dataset for which the correct outputs are already known. The goal of this learning is for the network's predictions to be as close to the true outputs as possible. This is accomplished by altering the network's weights and biases ( $W_{i j}$ and $b_{j}$ for each layer) through a process known as backpropagation. It involves updating the weights and biases along a gradient that maximally decreases the error. The weights and biases are updated according to:

$$
\begin{gathered}
W_{i, \text { new }}=W_{i}-\delta \frac{\partial \varepsilon}{\partial W_{i}} \\
b_{, \text {new }}=b-\delta \frac{\partial \varepsilon}{\partial b}
\end{gathered}
$$

where $\delta$ is a constant called the learning rate, and $\varepsilon$ is the error function, such as the mean-squared loss function. The learning rate controls how large the weight and bias updates are. If it is too small, the network will train slowly, but if it is too large, the algorithm may not converge.

In our case, the network must have five input units, and two output units, since we are using five features to predict two concentrations. Preliminary testing indicates that three hidden layers with 50 units each performed best on our data.

A support vector machine is a binary classification model that can construct a very complex classification surface through the use of a kernel function. They are based around the idea of achieving the maximum margin separation between classes. They achieve non-linear classification by mapping the inputs into a higher-dimensional space, where linear classification may be able to be accomplished. This idea can also be extended to regression analysis by fitting a regression hyperplane to the training cases. This allows for a highly nonlinear regression surface. In our case, we actually have to fit two SVMs, one for each concentration target, since the standard SVM is applicable only to single targets [11].

\section{Feature-less models}

Recurrent neural networks (RNNs) differ from traditional neural networks in the fact that they incorporate memory. Each new data point in a time-series that is given to an RNN will produce not only an output, but also an update to the network's internal memory state. At each time step, the network's hidden units see not only the input data, but also the memory state. Using the training data, the network will learn how best to use this memory state throughout the duration of a single time series data vector [8]. A simple recurrent neural network is shown in Figure 5.

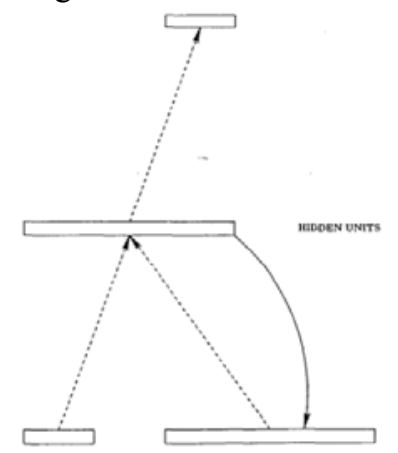

Figure 5. Simple recurrent neural network, with connections from the hidden units back to themselves. Figure from [8]

The hidden layer outputs for an RNN are similar to those of an MLP, except that the outputs are now indexed by time due to the fact that the output changes for every time point in each series. Also, there is an extra term in the expression, since the hidden layer's output at the previous time point influences the output at the current point:

$$
z_{, t}=\sigma\left(W_{i j} z_{i, t}+W_{h}{ }_{, t-1}+b\right) .
$$

In the above equation, $W_{h}$ represents the weight matrix from the previous hidden activation to the current hidden activation.

\section{RESULTS}

\section{A. Real data prediction}

As mentioned in Section IV, we are using a leave-one-out validation method for determining the quality of our models. Our quality metric is the mean-squared prediction error over our entire dataset. The error $\varepsilon$ is given by:

$$
\varepsilon=\sum_{i=0}^{n} \sum_{=0}^{d}\left(y_{i j}-y_{\text {pred }, i j}\right)^{2}
$$

where $y_{i j}$ refers to the $j^{\text {th }}$ concentration target for the $i^{\text {th }}$ data sample, $y_{\text {pred, } i j}$ is the network's prediction of $y_{i j}, n$ is the number of samples, and $d$ is the number of concentration targets per sample. In our case, $d$ is equal to two, since we are predicting methane and ethane. The error rates for the real data are presented in Table 2.

Table 2. Mean-squared error comparison for the models studied

\begin{tabular}{ccc} 
Model & $\begin{array}{c}\text { Mean-squared } \\
\text { Error }\end{array}$ & $\begin{array}{c}\text { Mean Error } \\
\text { (ppm) }\end{array}$ \\
\hline MLP & 1,845 & 43.0 \\
SVM & 3,079 & 55.5 \\
RNN (1 layer) & 22,781 & 150.9 \\
RNN (4 layers) & 8,276 & 91.0
\end{tabular}


While the RNN requires less effort in feature engineering, it did not perform as well as the more traditional feature-based models. This can be attributed to the fact that the RNN's performance increases significantly with a deep model (four layers vs one), and deep models tend to require much more training data than shallow models [9]. This makes the possibility of using synthetic data particularly attractive.

\section{B. Synthetic data prediction}

For the tests with synthetic data, there was no additional model parameter tuning; the same values were used on both the real and synthetic datasets. However, since we have 100 simulated examples, $\mathrm{k}$-fold validation with $k=20$ was used instead of the leave-one-out method. This significantly reduced the training time. The error rates for the synthetic dataset are presented in Table 3 .

Table 3. Mean-squared error rates for the sythetic dataset

\begin{tabular}{ccc} 
Model & $\begin{array}{c}\text { Mean-squared } \\
\text { Error }\end{array}$ & $\begin{array}{c}\text { Mean Error } \\
\text { (ppm) }\end{array}$ \\
\hline MLP & 74 & 8.6 \\
SVM & 288 & 17.0 \\
RNN (1 layer) & 4,120 & 64.2 \\
RNN (4 layers) & 6,025 & 77.6
\end{tabular}

As predicted, all of the models saw an improvement when using the synthetic dataset instead of the real data. This is likely due to the fact that the synthetic data is cleaner and contains less variance than the real data. In future tests, the randomness added to the synthetic data should be increased to match the distribution of the real data.

The deep RNN did not see as much of an improvement as the other models, which might mean that it requires additional training time since more data samples were used.

\section{CONCLUSION}

Even though the RNNs failed to outperform the feature-based methods, the overall results show that the quantification of methane and ethane with a single MOS sensor is very feasible, even when the identity of the target gas is unknown. Once a large volume of simulation data has been accumulated, the application of the models described in this work, along with the integration of our sensing apparatus with a stationary or mobile platform will be viable, low cost, and non-invasive method of detecting natural gas pipeline leaks.

\section{FUTURE WORK}

The estimation results presented here have been limited to the discrimination between two different gases, and concentration estimation of the target gas. Future work will include extension to estimating the concentration of both methane and ethane in an arbitrary mixture. To accomplish this, we anticipate the need for more information than a single time-series. For example, multiple different sensors can be used with one microchannel, and ideally one of them would be more sensitive to either methane or ethane than the other. Electronic noses typically do contain more than one type of sensor, but since methane and ethane are so similar, the approach of adding different types of sensors will not likely make a big improvement. The more promising approach is to use multiple identical sensors, each with a microchannel of a different length. This will accentuate the differences in diffusion between the two gases, rather than the difference in how they affect the sensor.

Future work will also include the extension of the algorithms described here to other projects in our lab, as described above [2][3]. In addition, we will be investigating a variant of the RNN called the long short-term memory (LSTM) network, which tends to learn longer-term dependencies better than regular RNNs [10].

\section{REFERENCES}

[1] J. Yan et al., "Electronic nose feature extraction methods: a review," Sensors, vol. 15, pp. 27804-27831, 2015. doi: 10.3390/s151127804.

[2] M. M. Montazeri et al., "A sensor for nuisance sewer gas monitoring," in IEEE Sens., Glasgow, 2017, pp. 1-3. doi: 10.1109/ICSENS.2017.8234327.

[3] M. Paknahad et al., "On-chip electronic nose for wine tasting: A digital microfluidic approach," IEEE Sens. J., vol. 17, no. 14, pp. 4322-4329, July 2017. doi: 10.1109/JSEN.2017.2707525.

[4] M. Paknahad et al., "Characterization of channel coating and dimensions of microfluidic-based gas detectors," Sens. Actuators, B, vol. 241, pp. 55-64, 2017. doi: 10.1016/j.snb.2016.10.048.

[5] R. B. Bird, W. E. Stewart, and E. N. Lightfoot, Transport Phenomena. New York, NY: J. Wiley, 2007.

[6] I. Langmuir, "The adsorption of gases on plane surfaces of glass, mica and platinum," J. Am. Chem. Soc., vol. 40, no. 9, pp. 1361-1403, 1918. doi: $10.1021 / \mathrm{ja} 02242 \mathrm{a} 004$.

[7] D. W Ruck, S. K Rogers, and M. Kabrinsky, "Feature selection using a multilayer perceptron," J. Neural Network Comput., vol. 2, no. 2, pp. 40-48, 1990.

[8] J. L. Elman, "Finding structure in time," Cognit. Sci., vol. 14, no. 2, pp. 179-211, Mar. 1990. doi: 10.1207/s15516709 $\operatorname{cog} 1402 \_1$.

[9] Z. Zhou and J. Feng, "Deep forest: Towards an alternative to deep neural networks," in Proc. 26th Int. Joint Conf. on Artif. Intell., Melbourne, 2017, pp. 3553-3559. doi: 10.24963/ijcai.2017/497.

[10] S. Hochreiter and J. Schmidhuber, "Long short-term memory," Neural Comput., vol. 9, pp. 1735-1780, 1997. doi: 10.1162/neco.1997.9.8.1735.

[11] S. R. Gunn, "Support vector machines for classification and regression," Faculty of Eng., Sci., and Math., Sch. of Electron. Comput. Sci., Univ. of Southampton, Tech. Rep., May 1998. 


\section{The Influence of Powder Size and Packing Density on the Temperature Distribution in Selective Laser Melting}

\author{
Diego Augusto de Moraes \\ Lassonde School of Engineering \\ York University \\ Toronto, Canada \\ moraes@yorku.ca
}

\author{
Aleksander Czekanski \\ Lassonde School of Engineering \\ York University \\ Toronto, Canada \\ alex.czekanski@lassonde.yorku.ca
}

\begin{abstract}
Metal powder properties in Selective Laser Melting (SLM) is among one of the most important factors when implementing new alloy developments for the equipment. In fact, not all commercially available metal powder alloys are ready to be implemented without a comprehensive set of tests. Besides the powder properties, we have a large number of building and environmental parameters that demands extensively research prior implementation. Although selected alloys are commercially available and documented to be used in SLM, including Ti6Al4V, SS316L and In718, the majority of it still not ready to be utilized in this system. The focus of this study is to use a thermal model in order to predict the thermal distribution of the process regarding different aspects of the powder properties, especially the thermal conductivity, when different powder packing densities and diameters are used. A Stainless Steel 304L will be utilized in this work, since it is not yet available to be commercially used. The main goal is to show the capabilities of the Finite Element Method in the pre-definition of optimal parameters for the process using a new alloy development. Our findings can be used as a pre-evaluation guideline when printing SS304L, since the comparison with similar experimental work in the field showed significant resemblance and outcomes. The temperature distributions show that the packing density has greater sensibility on the final temperature distributions, compared to the powder diameter variance. Two different power inputs are compiled and the temperature outcomes demonstrate that a power input of 100 Watts is recommended to use when printing SS304L, rather than 400 Watts that brings high temperature into the powder bed.
\end{abstract}

Keywords-component; Additive Manufacturing Modeling, Selective Laser Melting, Powder Compaction, Effective Thermal Conductivity, Finite Element Method

\section{INTRODUCTION}

Selective Laser Melting (SLM) is an Additive Manufacturing process that utilize metal powder in order to build full dense parts in a layer fashion. Currently, the technology is commercially distributed by few manufacturers

NSERC/Quanser Chair in Design Engineering program around the world, with a selection of alloys available, including Titanium, Stainless Steel, Aluminum, Cobalt-Chrome and Nickel-based alloys [1][2].

The fact that SLM uses a lower space, entitled bed, which is filled with fine metal powder, resulted in the association of this process with the Powder Bed Fusion (PBF) classification. Among them are Direct Laser Melting Sintering (DLMS), Electron Beam Melting (EBM) and Selective Laser Sintering (SLS). It is important to emphasize that all these processes were developed by different entities and timelines, and although some similarities, they have quite different set of parameters and mode of operations [3].

The main objective of this study is to demonstrate the high capability of FEM in this field, utilizing for that a low computational cost thermal model able to predict the temperature distributions when scanning a single layer and track of the powder bed. Some of the aspects of discrete method will be implemented, however only in the thermal conductivity implementation method. We start with the laser power input modeling, by using double-ellipsoidal as the heat flux input into the FEM model. Next the material is modeled, considering the state (solid, liquid, powder) and the temperature effects. Special attention is devoted to the thermal conductivity of the powder and for that a special effective thermal conductivity formulation is used to determine the variance in all conditions (packing density and powder diameter). Important devotion is given to the phase change and emissivity of powder as well. Finally, the model is built and compiled, leading to the results and analysis sessions. The powder diameter and the packing density are the main focuses here, in order to determine the impact on the temperature profile during two set of power input sources.

\section{EFFective Thermal Conductivity of POWDER BeD}

The contact of two adjacent spherical particles is essential when calculating the thermal conductivity, since the major heat exchange is done through conduction [4]. The respectively radius of contact is calculated by the Hertz theory applied for contact interaction of solid bodies [5]. This contact area is very small, especially in the present study, since no plasticity is 
considered and the only force acting among particles are gravity forces.

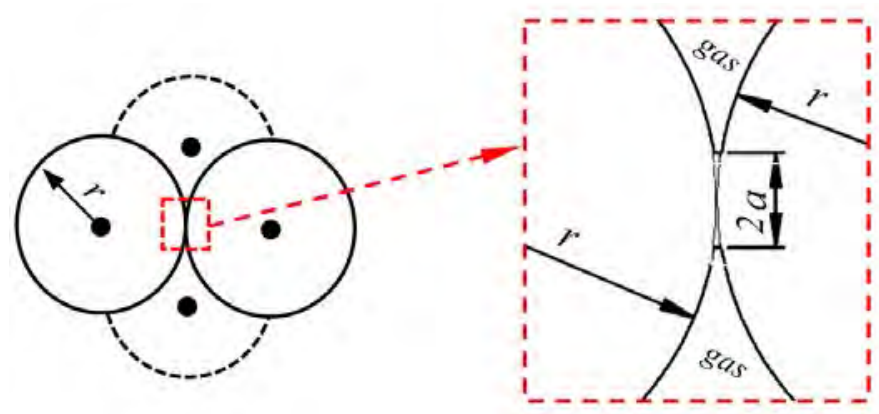

Figure 1 - Particles in contact (right) and the contact region (left)

The material used in the model is Stainless Steel 304L. This alloy has considerably low cost and yet still not available for the process in the industry. The data is extracted from Choong report, including Specific heat, density and the thermal conductivity, which are specified as temperature-dependent properties, essential when simulating high change in temperature distribution over small time increments [6]. To accommodate the phase transformation, the latent heat is included as well, which will modify the capacity heat during the powder melting [3].

The packing density affects the contact radius, consequentially influencing the emissivity and finally the final effective thermal conductivity of the powder bed. Below are the porosity-dependent emissivity for all packing densities and the final effective thermal conductivity of all cases from $300 \mathrm{~K}$ until $T_{\text {Solidus }}$ of SS304L [7].

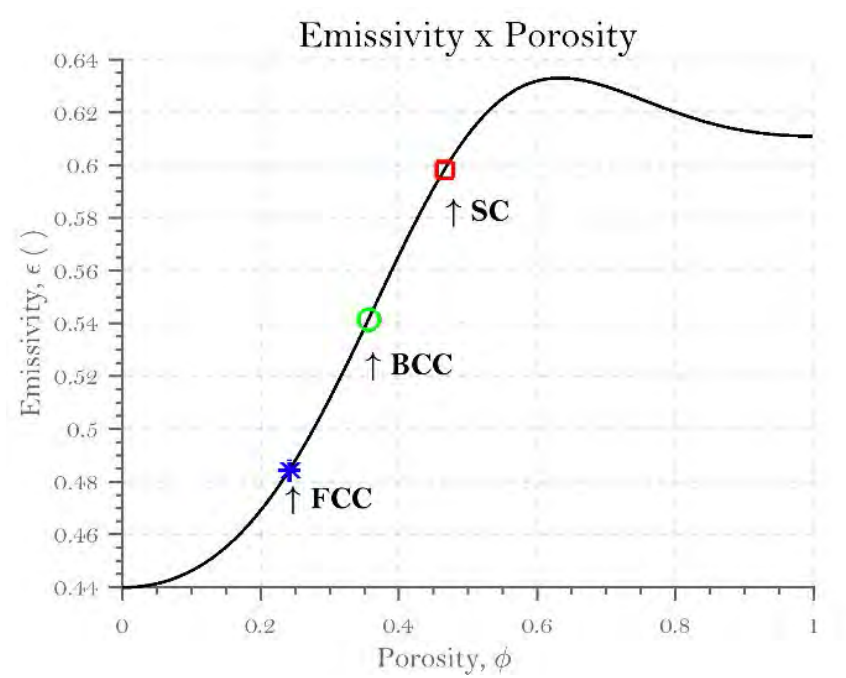

Figure 2 - Porosity-dependent emissivity of SC, BCC and FCC for SS304L [7]

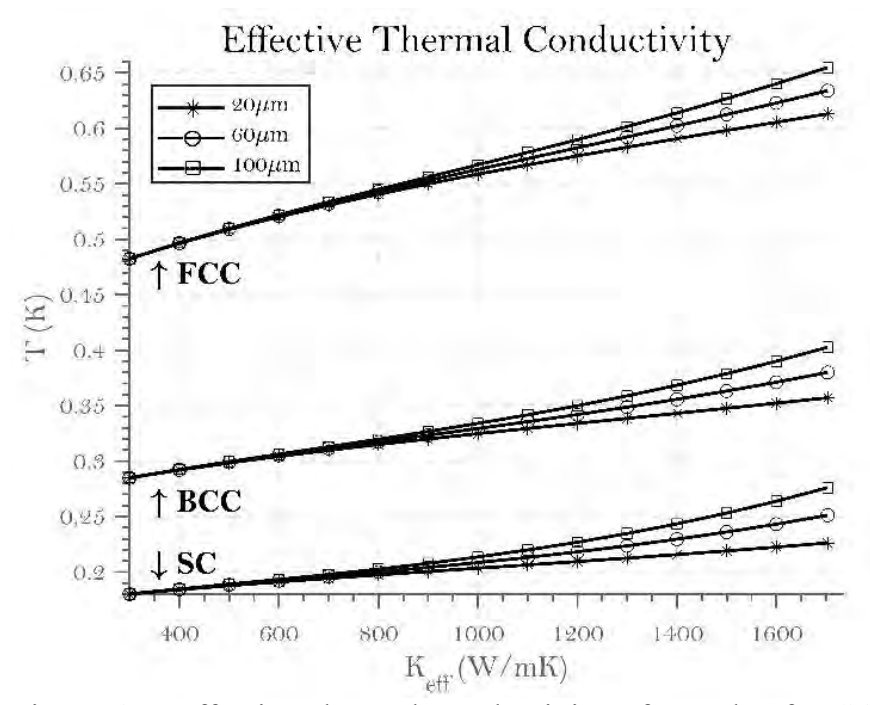

Figure 3 - Effective thermal conductivity of powder for SC, BCC and FCC with 20,60 and $100 \mu \mathrm{m}$ of powder diameter for SS304L [7]

In this study, it is considered three different packing densities, SC, BCC and FCC, with porosity of $0.2424,0.3571$ and 0.467 respectively, along with three powder diameters analysed, 20, 60 and $100 \mu \mathrm{m}$. On previously work the effects of powder packing in the bed was not analyzed and a general value of 0.400 in porosity was adopted [3]. The thermal radiation term is highly influenced by the powder diameter, temperature and emissivity of the powder bed. In the other hand, the thermal conductivity due the contact between particles will vary according to the contact ratio and the initial solid thermal conductivity, since the value of the fractional contact area, $\Lambda$, is less than $3 \times 10^{-4}[7]$.

It is noticeable that the powder diameter does not affect significantly the value of $k_{\text {eff }}$ for temperatures between $300 \mathrm{~K}$ and $800 \mathrm{~K}$. However, for higher temperatures, due the radiation term, there is a perceptible difference. For FCC, as example, there is around $7 \% k_{\text {eff }}$ increase when using $100 \mu \mathrm{m}$ instead $20 \mu \mathrm{m}$. The margin increases to $22 \%$ when doing the same comparison with SC, due the higher emissivity of SC compared to FCC. When comparing between the different packing densities, the $k_{\text {eff }}$ increase margin is even bigger, reaching up to $267 \%$.

\section{Thermal Modeling of SLM Heat Source}

The thermal model is defined in a way to handle different material phase (liquid, solid and powder state), as well for their variance during different temperatures. A three-dimensional model is established using DC3D8 hexahedron element with $25 \mu \mathrm{m}$ of size and a total domain of $1500 \times 1500 \times 75 \mu \mathrm{m}(1.5 \times 1.5 \mathrm{x}$ $0.075 \mathrm{~mm}$ ). A small domain is justifiable, given the small laser spot diameter, $50 \mu \mathrm{m}$, used in the model and the very low thermal conductivity present on the bed.

The material is modeled as temperature and state dependent, allowing to proper account more accurately the temperature 
distributions. There are some considerations that were made to preserve a low computational time/cost.

\section{Principal assumptions:}

- thermo-fluid effects were not considered, so Marangoni effect and fluid flow in the melt pool is not accounted;

- vaporization of the material is not implemented as well, but the phenomena only occur when utilizing considerably high laser power input $(P=400 \mathrm{~W})$;

- a single track of the laser is observed and the influence of the hatch distance is neglected in order to only focus on the effect of the packing structure and particle diameter in the temperature distributions.

- only average powder (particles) diameter is considered when calculating the effective thermal conductivity of powder state.

ABAQUS is the software used to perform the analysis, since its subroutines allow to model the moving heat source and keep track of the material state. The subroutines DFLUX and FILM were implemented in this study for Gaussian heat flux and convection/radiation definition respectively. The solution given is the temperature distribution of the process, described as $T(x, y, z, t)$. The problem is described by the three dimensional heat conduction equation (8), with the respective manufacturing and chamber environmental boundary conditions $[3,7]$.

\section{Results AND Discussion}

The numerical analysis was conducted for two laser power input (100 and 400 Watts), combined with three packing densities and three different powder diameters, giving 18 compiled result models that ran for 9,5 hours in total (average of approximately $30 \mathrm{~min}$ for each simulation). The temperature distribution was acquired in four central nodes through the thickness located in the middle of the powder bed.

The contact powder and substrate is neglected, however the pre-heat temperature from the substrate is entered as a boundary condition, with a defined pre-defined temperature of $643 \mathrm{~K}$. We have found that different powder diameters within the same packing density have no impact in the final temperature distributions on the model. However, between the three different packing densities the temperature differs. When using $\mathrm{P}=100 \mathrm{~W}$ (Figure 10), the maximum temperature achieved is around $1575 \mathrm{~K}$ at the surface, not enough to reach the melting point of the alloy utilized. However, it is essential to consider that powder can still melt in certain amount due necking between the small particles. The only concern here is at $-75 \mu \mathrm{m}$, which achieved a temperature of $1100 \mathrm{~K}$.

On the same temperature distribution plot, is noticeable that the temperature raises at the same rate for all the packing densities, but as soon as the temperature reaches the maximum amount and the laser scan moves forward, the temperature loss differs from each of the configurations. On the highlighted secondary plot in the same graph, it is shown that SC loses less heat than BCC and FCC. This is justifiable by the fact that SC has a smaller effective thermal conductivity among all, thus exchanging less heat. The biggest difference reaches up to $45 \mathrm{~K}$ between SC and FCC at the surface of the powder bed, representing $3 \%$.

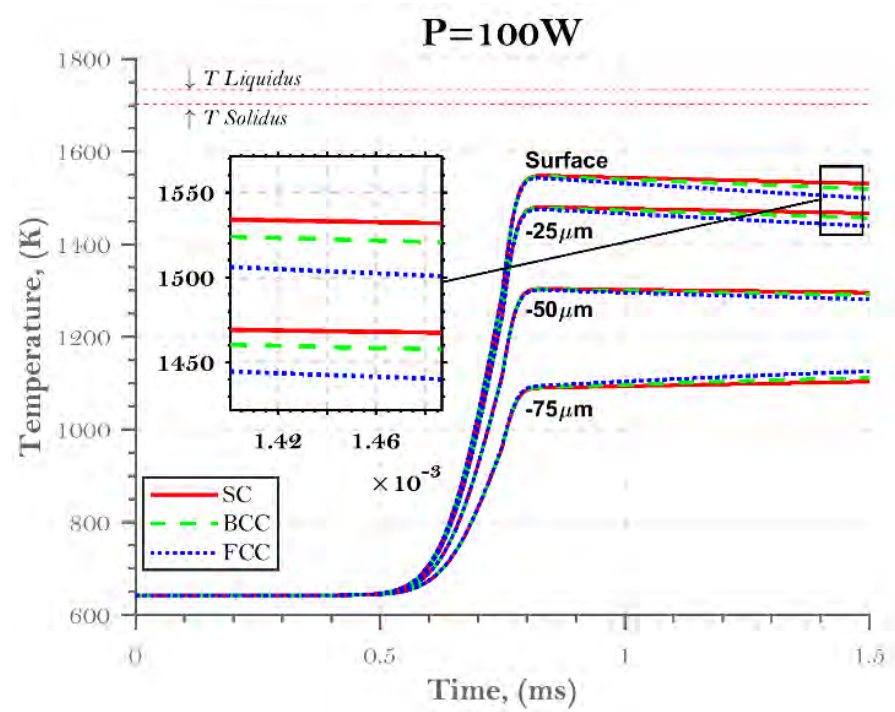

Figure 4 - Temperature distribution of Power input of 100W [7]

Another feature from the first analysis is the fact that the rate of change is very small after the temperature reaches its maximum. This happens because of the domain still on powder state, thus having low thermal conductivity overall. It is important to notice as well that the effective thermal conductivity, although not validated experimentally, followed the same trend experienced by the study of Alkahari et al [23]. In their study the thermal conductivity of SS316L was measured by laser flash technique and the outcomes show a tendency of superior thermal conductivity of $100 \mu \mathrm{m}$ particles when comparing to $20 \mu \mathrm{m}$, the same tendency happens when increasing the packing density.

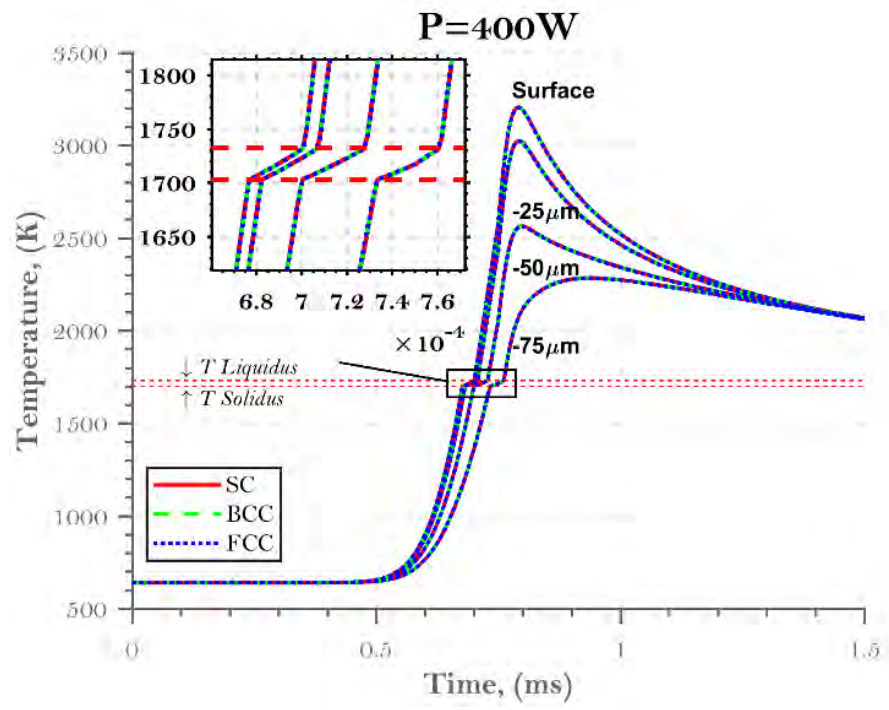

Figure 5 -Temperature distribution of Power input of $400 \mathrm{~W}$ [7]

The second power input $(\mathrm{P}=400 \mathrm{~W})$ is the one which the whole depth of the powder bed layer thickness reaches the 
melting point. However, the maximum temperature achieved at the surface is around $3250 \mathrm{~K}$, which is an undesirable condition that can lead to evaporation of the metal [8]. The bottom reaches around $2100 \mathrm{~K}$, which will most likely induce the melting of some area of the substrate or increase the heat affected area of the same. It is noteworthy here to observe that a gradient of around $1150 \mathrm{~K}$ between the top and bottom layer is induced, whereas at $\mathrm{P}=100 \mathrm{~W}$ the gradient is $475 \mathrm{~K}$. This fact alone is extremely important when analysing residual stresses during SLM. The higher temperature gradient and respectively the cooling rates induced by the process, higher is the residual stresses occurrence.

In the secondary plot of Figure 12, it is highlighted the melting point area between solidus and liquidus temperature (dashed red line). The change of rate of temperature during this interval proves the model is capable of interpreting the different properties states and the phase change by the latent heat of fusion, even with a small time incrementation used.

Furthermore, the results show a very close behaviour with the experimental studies using SS304L found in the literature. Abd-Elghany and Bourel had performed the closest testing to our set-up. They have conducted tensile specimens printing using a machine with $100 \mathrm{~W}$ and $30 / 50 / 70 \mu \mathrm{m}$ layer thickness, concluding that higher layer thickness produced low density and poor mechanical properties specimens [9]. This can be used as an reference to our set-up of $\mathrm{P}=100 \mathrm{~W}$ and layer thickness of $75 \mu \mathrm{m}$, which the laser input was not enough to bring the temperature to the melting point, thus consolidation between powder particles are poor.

\section{CONCLUSIONS}

In this research it is proposed a low cost FEM thermal model capable of measure temperature distributions through the powder bed and consequentially the temperature gradient, of a single layer and track of the laser scanning using SLM process. Although the packing density and powder diameter impact the final value of effective thermal conductivity of the powder bed, the only considerable effects in the temperature distribution was observed for lower power input $(\mathrm{P}=100 \mathrm{~W})$ and only for different packing densities. The reason why the powder diameter did not show major impact in the model is related to the minor effective thermal conductivity change regardless of the diameter and the fact that the model is based in the total volume occupied by the particles as a whole.

The significance of the results demonstrate that a proper compaction and density distribution of the powder bed is important to keep the cooling rates and temperature distributions within the desirable range, however these impacts are only perceivable outside the melt pool zone. In the other hand, by looking to the power input, we can see that $400 \mathrm{~W}$ with the current speed of $1 \mathrm{~m} / \mathrm{s}$ induce high temperature at the surface and through the layer thickness, very close to evaporation point.
This by itself brought a temperature gradient of around $1150 \mathrm{~K}$ through the layer thickness, which is the main cause of warping and residual stresses. For $\mathrm{P}=100 \mathrm{~W}$ the temperature did not reach the melting point at all, which could affect in the consolidation of the particles and in the final porosity of the built part.

Future research is necessary in order validate the model experimentally using different powder diameters and measure on which extent the powder bed is compacted or not.

\section{ACKNOWLEDGMENT}

This research is supported by Lassonde School of Engineering and NSERC/Quanser Chair in Design Engineering program.

\section{REFERENCES}

[1] "SLM Solutions Group AG" [Online]. Available: https://slm-solutions.com/. [Accessed: 30-Dec-2017].

[2] "Renishaw Manufacturing Systems" [Online]. Available: https:// renishaw.com/. [Accessed: 30-Dec-2017].

[3] D. A. de Moraes and A. Czekanski, "Thermal Modeling of 304L Stainless Steel for Selective Laser Melting: Laser Power Input Evaluation," in Int. Mech. Eng. Congr. Expo. Vol. 2: Adv. Manuf., 2017. doi: 10.1115/IMECE2017-72224.

[4] S. Sumin Sih and J. W. Barlow, "The Prediction of the Emissivity and Thermal Conductivity of Powder Beds," Part. Sci. Technol., vol. 22, no. 3, pp. 291-304, Jul. 2004. doi: 10.1080/02726350490501682a.

[5] K. L. Johnson, 1985, Contact Mechanics, Cambridge University Press, Cambridge. doi: 10.1017/CBO9781139171731.

[6] C. S. Kim, "Thermophysical properties of stainless steels," Office of Scientific and Technical Information (OSTI), Sep. 1975. doi: $10.2172 / 4152287$.

[7] D. de Moraes and A. Czekanski, "Parametric Thermal FE Analysis on the Laser Power Input and Powder Effective Thermal Conductivity during

Selective Laser Melting of SS304L," J. Manuf.and Mater. Process., vol. 2, no. 3, p. 47, Jul. 2018. doi: 10.3390/jmmp2030047.

[8] P. W. Fuerschbach, J. T. Norris, X. He, and T. Debroy, Understanding Metal Vaporization from Laser Welding, Albuquerque, 2003.

[9] K. Abd - Elghany and D. L. Bourell, "Property evaluation of 304L stainless steel fabricated by selective laser melting," Rapid Prototyping J., vol. 18, no. 5, pp. 420-428, Jul. 2012. doi: 10.1108/13552541211250418. 


\section{Experimental Results and Proposed Electromechanical Model for Vibrational Communication in Mining and Oil-Gas Drill Strings}

\author{
Md Shahriar Islam \\ Department of Mechanical \\ Engineering University of \\ Saskatchewan Saskatoon, SK, Canada \\ shahriar.islam@usask.ca \\ Nathan Peter \\ Development Engineering \& Manufacturing, Mining \\ and Minerals \\ Saskatchewan Research Council, Saskatoon, SK, \\ Canada Nathan.Peter@src.sk.ca
}

\author{
Travis Wiens \\ Department of Mechanical \\ Engineering University of \\ Saskatchewan Saskatoon, SK, Canada \\ t.wiens@usask.ca
}

\begin{abstract}
In borehole mining and oil and gas drilling, the drilling process is invisible from the surface, which is why a two-way communication method between the drill head and the surface is a must. The popular industrial instrumentation technique, widely known as measurement while drilling (MWD), has enabled industries to save costly employee hours and gain more control over the drill bit direction. There are four major methods used throughout history, where the mud pulse telemetry technique has become the state of the art because of its simplicity and convenience. However, like other methods, it has a major drawback of having a slow data transmission rate. Moreover, other methods have different issues which include large attenuation of the data that is transferred through drill pipes. Acoustic telemetry is the most recent method of transferring data through a solid medium, a technique using continuous wave propagation of forces in the wall of the pipe. A proper use of this new method is expected to become popular in the mining and oil-gas industries because of its faster data transmission rate and ability to ignore most attenuating parameters. This method is still in its optimization stage, and the use of a convenient tool to match the transducer with the parameters of the drill pipe and surroundings is still a challenge to overcome. This paper will propose a simulation model to simulate the transmission of data through drill pipes, and will show experimental results of such arrangement justifying the opportunities ahead in this field.
\end{abstract}

Keywords-acoustics; vibration; drill pipe; transmission line; communication; frequency response;

\section{INTRODUCTION}

Experts involved in surveying or extracting fossil fuel and minerals face a great challenge of drilling without seeing, which is why their usual methods include measuring real time data while drilling. This is commonly known as measurement while drilling (MWD) or logging while drilling (LWD). In addition, these operations involve large assemblies of equipment and inconveniences due to invisibility, complex well patterns, long operation times, high costs etc. The initial goal is to determine ore location, drilling orientation, properties of rock, dimensions of cavity and other parameters using sensor data assembled in the borehole assembly (BHA). This data is then transmitted to the surface from the wellbore (or the hole). Using basic trigonometry, a three-dimensional plot of the path of the well can be produced thus enabling an operator to measure the trajectory of the hole as it is drilled in real time. Therefore, it becomes easy to follow the preplanned direction of the drilled hole. Also, measurements to determine properties of the rock is done by taking natural gamma ray emissions from the rock [1]. Other information such as density, porosity, rock fluid pressures are taken by sensors based on sound or electricity [2]. All of this information helps to broadly determine what type of rock formation is being drilled, which in turn helps confirm the real-time location of the wellbore by comparing to seismic data. MWD downhole tools also allow the wellbore to be directed in a chosen direction, therefore known as directional drilling.

Historically there are four communication methods used, namely cable operated communication, mud pulse telemetry, vibration/pressure wave transmission and electromagnetic communication [3]. Among these, the most popular is mud pulse telemetry (MPT) which is a binary coding system and is the first of its kind to be used in the industry. It uses drilling fluid to transmit the pressure pulse created by a poppet type valve. The valve changes the drilling fluid's pressure thus the pressure wave propagates through the drilling fluid. At the surface the pressure differences are recorded and analyzed. This method, however, has its own issues such as 
non-homogeneity of the mud fluid and is only operational when the drilling fluid is running. Most importantly, due to signal attenuation in the fluid, its data transmission rate is slow on the scale of 1-10 bits per second. As a result, exploring other methods such as communication via structural vibration have become necessary [3].

Acoustic telemetry includes the use of piezo transducers to produce compression, torsion, or shear waves that propagate through the drill strings to the other end where they can be picked up using another piezo transducer or another type of sensor. The medium of pressure wave transmission is the solid steel pipe rather than viscous liquid as in mud pulse telemetry. The information picked up by different sensors is converted into digital data and is recorded into the memory and broadcast by the transducers as vibration or acoustic wave through the drill string. The transducer generates this vibration within the steel drill string by means of a high frequency ferroelectric ceramic piezoelectric material with the combination of two different masses [4]. The captured signal is transferred to a computer where a program decodes the digitized sensor signal and extracts the downhole information; this method can give faster data rates up to 100 bits per second [5]. The vibrational method and theories have been known from the beginning of the MWD, but because of the lack of an efficient transducer, the method did not flourish. Currently, because of advancements in piezoelectric ceramic technology, the method has resurfaced and is beginning to gain commercial popularity [6-9].

Acoustic telemetry uses the solid medium of the drill string itself and not the mud fluid which is why it has different attenuation. For typical oil and gas applications, the attenuation for acoustics systems varies from $2-10 \mathrm{~dB} / 1000$ feet and up to $30 \mathrm{~dB} / 1000$ feet if the pipe is badly worn [10-12]. Along with a number of patents, the work published in academic journals are found to focus on wave transmission of different types through mass jointed pipe strings [13-14]. Drumheller has done a considerable amount of work which included investigations of the basic theory of wave propagation, the hardware of communication tools, and collecting and explaining a history of industrial experimental results on attenuation. [15]. Carcione introduced a time-domain algorithm for the propagation of onedimensional axial, torsional, and flexural stress waves, which also includes transducer sources and sensors [16]. Based on this work some other works were modified and justified by experimental verification. Among the modifications include avoiding lateral vibration at the neutral point and a method to avoid them by a new approach of MPT and summarization of many more applications, such as wave loadings on offshore platforms, classical problems in wave propagation, and extensions to modern kinematic wave theory [17].

Most of the research work regarding drill bit communication focuses on communication through drill strings in the oil-gas industry but none address borehole mining, which has a number of important differences. In borehole mining, drill stems tend to be shorter, and larger in diameter and the whole pipeline can be within hundreds of meters, consisting of hundreds of pipes. Moreover, the impedance mismatch at each pipe joint is much less, because the pipe joints are screwed to each other with no extension in outside diameter as seen in the pipe joints of the oil-gas drill pipe. So, the associated reflection and resonances at the pipe joints are less.

In this paper a universal simulation model will be proposed that can account for any types of drill string along with the transmitter and receiver for acoustic wave propagation. The simulation model will be verified by experimental data, which will justify the model. A simulation model will be a convenient approach to understand the characteristics of the pipelines for different applications, which include the attenuation and frequency shifting of the system. Moreover, this will lead to the optimization and modification of the transducer and sensors to be used with any specific application. If the frequency response of the system is known then a particular communication scheme can be chosen beforehand which can employ different frequency contents for a faster data transmission. Future research works will be to optimize the transducer based on the application and convenience in the communication scheme and also to choose a particular communication scheme.

\section{EXPERIMENTAL MODELING}

An experimental apparatus was assembled for data transmission through drill strings as shown in Fig. 1. This experimental model includes Tonpilz-type piezoelectric transducers as both the transmitter and receiver. These transducers were designed for sonar applications. It is possible to use an accelerometer sensor at the receiving end, but in this experiment an identical transducer was used as the receiver. This configuration allows for two-way communication to occur if needed.

\section{A. Frequecny Response of the Transducer}

First, the experiment is performed attaching the transducers face to face with bolts. This experiment gives the frequency response of the two transducers shown in Fig. 2.

All control and data measurements are done by a National Instruments data acquisition system (NI-DAQ). A maximum length sequence (MLS) is a digital pseudorandom binary sequence that has a perfectly white, flat spectrum. This signal is used as an input signal into the driver transducer through an amplifier. The MLS is generated using linear feedback shift registers (LFSR) using Matlab ${ }^{\circledR}$ code created by Wiens [18] and

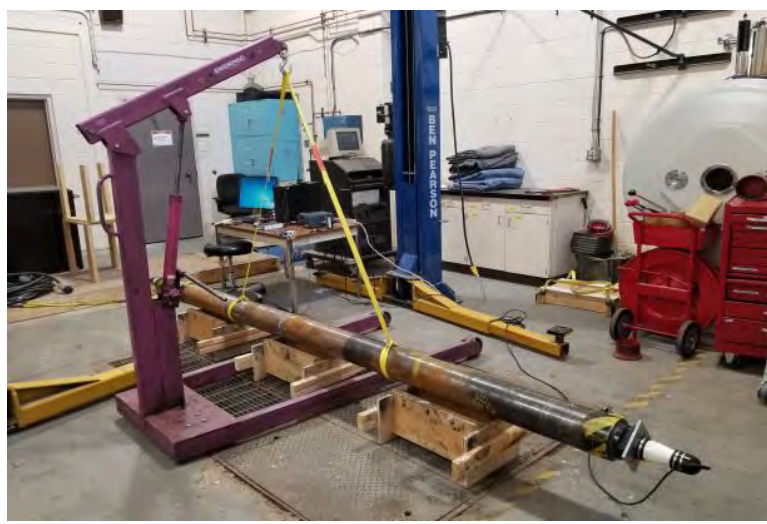

Figure 1. Arrangement for three pipes connected with transducers.

Saskatchewan Research Council. (sponsor) 


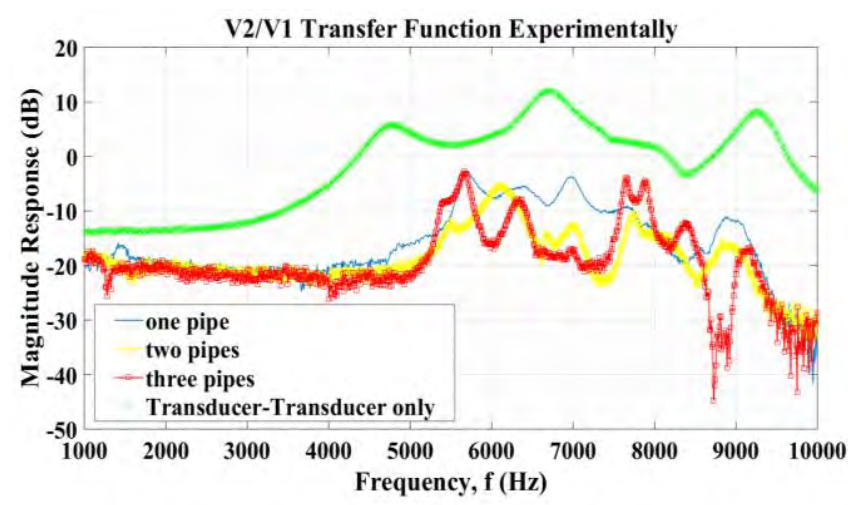

Figure 2. Frequecny Response of the transducers only connected face to face and transducers connected with one, two and three drill strings.

LSFR feedback values from Koopman [19]. The output is measured from the receiver transducer through a voltage divider which is recorded as voltage $\mathrm{v}_{2}$. The input voltage $\mathrm{v}_{1}$ is taken from the amplifier output through the voltage divider. The voltage divider is used here so that NI-DAQ device stays within the rated range of $\pm 10 \mathrm{~V}$, while the amplifier outputs $\mathrm{V}_{1}=100 \mathrm{~V}$. The MLS used has a sampling frequency of $20 \mathrm{kHz}$.

The measured data was then used to obtain transfer function

$$
\mathrm{ETFE}=\frac{\mathrm{v}_{2}}{\mathrm{~V}_{1}}
$$

where $V_{1}$ and $V_{2}$ are the fast Fourier transform (FFT) of the measured voltage $\left(\mathrm{v}_{1}\right.$ and $\left.\mathrm{v}_{2}\right)$ signals. This experimental method works well for pressure measurements of fluid transmission line [20] and is believed to be a good experimental approach for measuring vibration along any transmission line.

\section{B. One, Two and Three Drill Strings}

The transducers are connected with one drill string through the end caps. The drill string used for this experiment is collected from a mine site (in this application they are called jet rods). The transducers are then connected with two and three drill segment strings through end caps as shown in Fig. 1.

Fig. 2 shows the frequency response for different pipe arrangements with the transducers. It is clear from the figure that the frequency response range of the transducers is $4000 \mathrm{~Hz}$ to $10000 \mathrm{~Hz}$. For ease of understanding, the peak natural frequencies are extracted and Fig. 6 is formed to show that with the increase of pipes the natural frequencies of certain modes are reducing and more frequency contents become visible in the range.

\section{PRoposed Simulation Model}

A model shown in Fig. 3 is proposed in this section for simulation that can represent the experimental apparatus previously shown. A successful model that can account for a certain number of drill strings and comply with the experimental results must be able to account for a large number of drill strings connected together. The model includes a driver/transmitter transducer, then a block for a number of drill strings and a receiver transducer to act as a sensor.
To obtain an effective and more practical simulation model, the linearized one-dimensional piezoelectric ceramic equations found in Sherman [21] are used. Also, the transducer is considered as a simple mass-spring-damper model where piezoelectric stack materials are placed between the head mass and tail mass. The pipeline of a finite number of drill strings can be modeled using a number of transmission line models [22] connected together. This accounts for the input and output forces and displacements in terms of transfer functions that can calculate the system more in a computationally inexpensive way.

In Fig. 3, $v_{1}$ is the input forcing voltage at the driver transducer, $\mathrm{u}_{1}$ is the displacement at the driver transducer, $\mathrm{F}_{1}$ is the output mechanical force of driver transducer and also the input of the pipeline. $F_{2}$ is the input force at the outlet of transmission/pipeline and also the output from the receiver transducer. Similarly, $\mathrm{u}_{1}$ and $\mathrm{u}_{2}$ are the displacements at the inlet and outlet of the transmission line. $\mathrm{v}_{2}$ is the output voltage from the receiver transducer.

\section{A. Piezoelectirc Transducer}

A piezoelectric transducer is an electromechanical device that converts mechanical energy into electrical energy or vice versa. A lot of previous research has been performed on the design of an effective transducer, among them is a $50 \mathrm{kHz}$ Tonpilz type transducer developed by Baylis [23]. A detailed discussion on the basic design analysis of this type of transducer is made in the book by Sherman and Butler [21]. This particular transducer is comprised of a combination of two masses bolted together with piezoelectric ceramic placed in between.

The basic governing equations of piezoelectric ceramics are the combination of mechanical and electrical characteristics which are dependent on each other. The constitutive relations are usually called piezoelectric equations that apply the theory of piezoelectricity. The equation, describe the interaction effects of stress, strain, electric displacement, and the electric field. In the case where the strain and electric fields are approximately uniform, the mass of the piezoelectric material can be assumed negligible, and the amount of free charge is small, the behavior of the transducer can be modelled by the simplified constitutive equations given in [21]

$$
\begin{aligned}
& S=s^{E} T+d^{t} E \\
& D=d T+\varepsilon{ }^{T} E
\end{aligned}
$$

where $\mathrm{T}$ is the stress, $\mathrm{S}$ is the strain, $\mathrm{E}$ is the electric field, and $\mathrm{D}$ is the electric displacement. All of these variables are functions of position and time, where $\mathrm{T}$ and $\mathrm{S}$ are both symmetric second rank tensors. In these equations, $\mathrm{S}$ and $\mathrm{T}$ are $6 \times 1$ column matrices, $\mathrm{E}$ and $\mathrm{D}$ are $3 \times 1$ column matrices, $\mathrm{s}^{\mathrm{E}}$ is a

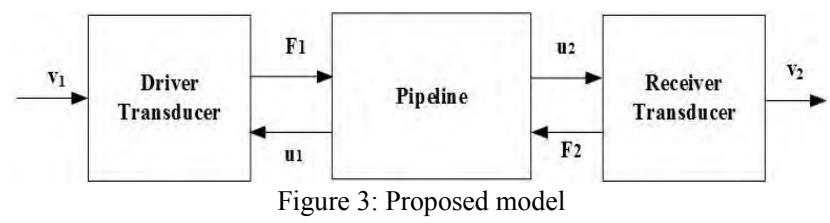


$6 \times 6$ matrix of elastic compliance coefficients, $d$ is a $6 \times 3$ matrix of piezoelectric coefficients ( $\mathrm{d}^{\mathrm{t}}$ is the transpose of $\mathrm{d}$ ), and $\varepsilon^{\mathrm{T}}$ is a $3 \times 3$ matrix of permittivity coefficients [24].

\section{B. Pipeline}

To model the drill string, a different approach is taken in this research by using a transmission line model which is often used in the hydraulic circuit design for fluid flow through a pipe. The transmission line model was initially developed by Krus [22] and is based on the assumption of a four pole equation and approximating the response with a number of transfer functions, as shown in Fig. 4. This model reduces to a compact transfer function model which only calculates the sates at inlet and outlet thus reducing the computational effort. There is other research done on the modification of this transmission line model in order to gain better accuracy and apply to other transmission line problems [25-27]. However, all of these are used in the application of fluid mechanics. For this research, pressure and flow are replaced with analogous force and velocity.

From analogy, in this model the pressure $\mathrm{P}$ and flow rate, $\mathrm{Q}$, can be replaced with force, $\mathrm{F}$, and velocity $\mathrm{u}$. They are related by the following equation [22]

$$
\begin{aligned}
& \mathrm{F}_{1}=\mathrm{C}_{1}+\mathrm{Z}_{\mathrm{c}} \mathrm{u}_{1} \\
& \mathrm{~F}_{2}=\mathrm{C}_{2}+\mathrm{Z}_{\mathrm{c}} \mathrm{u}_{2}
\end{aligned}
$$

where, $Z_{c}=A c \rho \quad$ is the characteristic impedance of the drill string, $C$ is the local speed of sound in the transmission line, $A$ is the cross-sectional area of the pipe, and $\rho$ is the material density of the drill string.

The transfer functions in Fig. 4 are as follows. They are calculated in the laplace domain, $s$.

$$
\begin{gathered}
\mathrm{H}_{1}(\mathrm{~s})=\frac{\mathrm{R}}{\mathrm{kTs}+1} \\
\mathrm{H}_{2}(\mathrm{~s})=\mathrm{Z}_{\mathrm{c}} \\
\mathrm{G}_{\mathrm{f}}(\mathrm{s})=\frac{\mathrm{kTe}^{\frac{-\mathrm{RA}}{2 \rho \mathrm{c}}} \mathrm{s}+1}{\mathrm{kTs}+1}
\end{gathered}
$$

where, $\mathrm{k}$ is an empirical factor. It was determined in [22] that $k=1.25$ is an acceptable value. The wave propagation time, $T=\frac{L}{c}$, is related to length of the pipe, $\mathrm{L}$, and local speed of sound, c. The distributed line resistance, $R=\frac{\mu \pi \mathrm{d}}{h} L$, assuming ideal laminar flow. This model neglects unsteady friction, and assumes a distributed resistance across the transmission line.

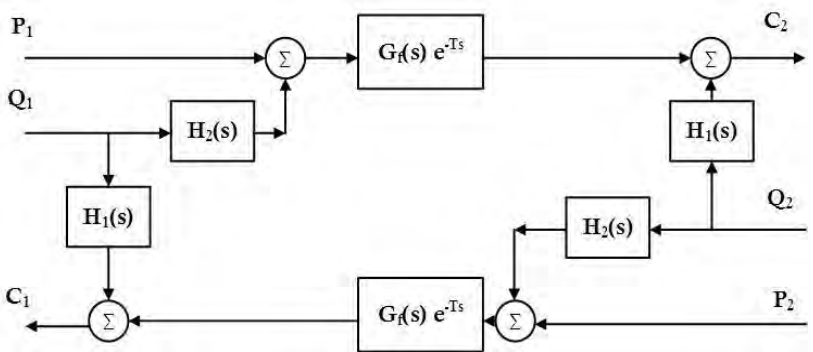

Figure 4. Block diagram of a fluid transmission line model [22].
The $e^{-T s}$ term with the $(s)$ transfer function is a time delay of time, $T$, simulating wave propagation.

\section{RESUlts OF SimUlATION MODEL AND COMPARISON}

The simulation model for the two transducers connected face to face show similar frequency response to that of experimental results except in the $1^{\text {st }}$ mode shown in Fig. 5, which is expected given the assumptions in the model. The assumptions are taken as the model consists of two mass spring damper system where the mass of the piezo ceramic is ignored and it is considered as a spring damper connection in between two masses [21]. Also, the actual transducer has masses bolted together at an unknown preload and the whole assembly is covered with neoprene type rubber material. So, these factors played an important role of moving the first mode of the experiment from the simulation model value.

Fig. 5 shows the frequency response of different pipe arrangements for the simulation model. The frequency response of different pipes have similar trend to that observed in the experimental result shown in Fig. 2.

Fig. 6 compares the resonant frequencies between the experimental apparatus and simulation model. The simulation model has a different range than the experimental model as shown in Fig. 5, which is why a different range is seen in the

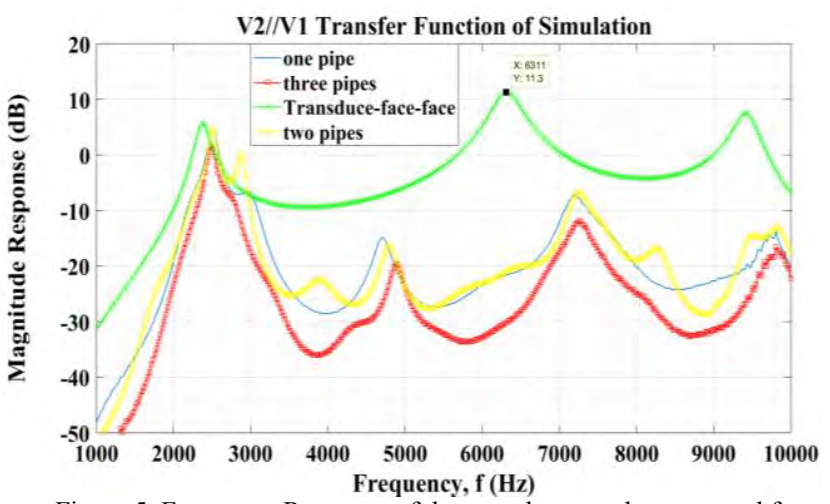

Figure 5. Frequecny Response of the transducers only connected face to face and transducers connected with one, two and three drill strings.

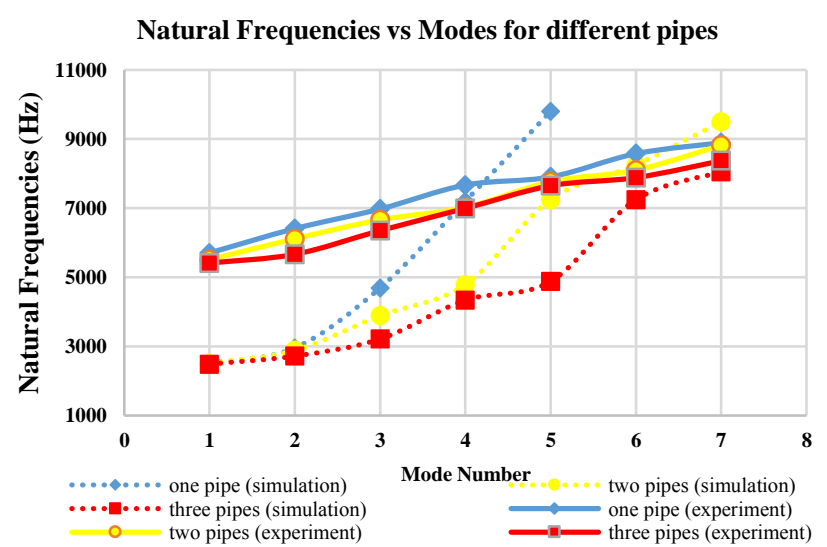

Figure 6. Comparison of Natural Frequencies of different Modes of different pipe arrangements of simulation model and experimental model. 
simulation model for the frequency response of different pipes than the experimental results. Also because the experimental apparatus is a drill string that has a construction of concentric inner and outer pipes supported by spacers of different materials, whereas in the simulation model a cylindrical type of pipe is considered with a similar mass matched with the experimental apparatus. However, a similar trend of decreasing natural frequencies of different modes with the increase of number of pipes is observed in both experimental and simulation model results. This is expected because the natural frequencies of a system change disproportionally with the square root of its mass.

\section{CONCLUSION}

The comparison of results between the simulation model and the experiment indicates that the simulation model is able to explain the wave propagation in long drill strings. Thus this can help with developing a communication scheme for data communication in drill string using methods that employ wide range of frequency content in a single transmission.

\section{ACKNOWLEDGMENT}

The authors would like to acknowledge the help of Mr. Douglas V. Bitner of University of Saskatchewan for his help with this research. The authors also acknowledge the support given by the Saskatchewan Research Council, SK, Canada for the experimental assembly and equipment, as well as financial support of this work.

\section{REFERENCES}

[1] J. J. Arps and J. L. Arps, "The subsurface telemetry problem - a practical solution," Journal of Petroleum Technology. Society of Petroleum Engineers, Vol. 16, Issue 05, May 1964. doi: doi.org/10.2118/710-PA.

[2] I. Dowell, A. Mills and M. Lora, "Chapter 15 - Drilling-Data Acquisition," In R. F. Mitchell. Petroleum Engineering Handbook. II - Drilling Engineering. Society of Petroleum Engineers. pp. 647-685, 2006. ISBN 978-1-55563-114-7.

[3] N. G. Franconi, A. P. Bunger, E. Sejdic and M. H. Mickle, "Wireless communication in oil and gas wells," Energy Technology, vol. 2, no. 12, pp. 996-1005, 2014. doi: 10.1002/ente.201402067.

[4] M. S. Islam, A. T. Dolovich, N. Peter and T. Wiens, "Acoustic drill pipe communications for mining," Proceeding of the Maintenance, Engineering and Reliability/Mine Operators Conference, Canadian Institute of Mining, Metallurgy and Petroleum (CIM), 2017. http://store.cim.org/en/acoustic-drillpipe-communications-for-mining

[5] D. S. Drumheller, S. S. Kuszmaul, "Acoustic Telemetry," Geothermal Research Department Sandia National Laboratories, Albuquerque, 2003. NM 87185-1 033. doi: 10.2172/918379.

[6] F. Dias, F. Marcancola, D. Wakabayashi and R. Piazza, "First real-time drill-stem test in deepwater using fully acoustic telemetry monitoring and control of the well," Abu Dhabi International Petroleum Exhibition and Conference. Society of Petroleum Engineers, 2014. doi: 10.2118/172111-MS

[7] J. R. Haddad, A. Salguero, C. Jaimes, "Application of telemetry technology in high-pressure wells to improve data accuracy in drill stem tests," SPE Oil and Gas India Conference and Exhibition. Society of Petroleum Engineers, 2017.

[8] E. Kreuzer, L. Krumm and M. A. Pick, "Investigation of the influence of screw connections in drill-strings on the propagation of torsional waves with respect to advanced stick-slip controllers," 33rd International Conference on Ocean, Offshore and Arctic Engineering. American Society of Mechanical Engineers, pp. V005T11A022-V005T11A022, 2014. doi: 10.1115/OMAE2014-24545.
[9] S. Fuchao, P. Xiaohan, Z. Lichen, W. Guoqing and J. Deli, "Research and validation of pipe acoustic transmission mechanism," International Journal of Multimedia and Ubiquitous Engineering, vol. 9, no. 11, pp. 161-170, 2014. doi: 10.14257/ijmue.2014.9.11.16.

[10] W. Cox and P. Chaney, 1981, "Telemetry system," US Patent 4,293,936. 1981.

[11] D. S. Drumheller, "Acoustic data transmission through a drillstring," US Patent 5,128,901, 1992.

[12] J. Orban and R. Squire, "Sonic vibration telemetering system," US Patent $5,373,481,1994$.

[13] T. G. Barnes and B. R. Kirkwood, "Passbands for acoustic transmission in an idealized drill string," The Journal of the Acoustical Society of America, vol. 51, no. 5B, pp. 1606-1608, 1972. doi: 10.1121/1.1913006.

[14] L. Brillouin, "Wave propagation in periodic structures: electric filters and crystal lattices," Courier Corporation, 2003.

[15] D. S. Drumheller, "Acoustical properties of drill strings," The Journal of the Acoustical Society of America, vol. 85, no. 3, pp. 1048-1064, 1989. doi: 10.1121/1.397488.

[16] J. M. Carcione and F. Poletto, "Simulation of stress waves in attenuating drill strings, including piezoelectric sources and sensors," The Journal of the Acoustical Society of America, vol. 108, no. 1, pp. 53-64, 2000. doi: $10.1121 / 1.429443$.

[17] W. C. Chin, "Wave Propagation in Drilling, Well Logging and Reservoir Applications," John Wiley \& Sons, 2014.

[18] T. Wiens, "Kasami sequences, m-sequences, linear feedback shift registers," 2009.[Online] Available: https://www.mathworks.com/matlabcentral/ fileexchange/22716.

[19] P. Koopman, "Maximal length lfsr feedback terms." [Online.] Available: https://users.ece.cmu.edu/ koopman/lfsr/index.html.

[20] J. ven der Buhs, "Investigation and Optimization of Hydraulic Step-down Switched Inertance Converters with Non-uniform Inertance Tubes", M.Sc. thesis, Department of Mechanical Engineering, University of Saskatchewan, Saskatoon, Canada, 2017.

[21] C. H. Sherman, J. L. Butler, "Transducers and Arrays for Underwater Sound," Springer, New York, 2007.doi: 10.1007/978-0-387-33139-3.

[22] P. Krus, K. Weddfelt and J. O. Palmberg, "Fast Pipeline Models for Simulation of Hydraulic Systems," Journal of Dynamic Systems Measurement and Control, 1994. doi:10.1115/1.2900667.

[23] C. Bayliss, "Application and Development of Finite Element Techniques for Transducer Design and Analysis," PhD Thesis, The University of Birmingham, Birmingham, UK, 1998.

[24] D. A. Berlincourt, D. R. Curran and H. Jaffe, "Piezoelectric and piezomagnetic materials and their function in transducers," Physical Acoustics, Vol. 1, Part A, ed. by W.P. Mason, Academic Press, New York, 1964. doi: 10.1016/B978-1-4832-2857-0.50009-5.

[25] N. Johnston, "The Transmission Line Method for Modelling laminar flow of Liquids in Pipelines," Journal of Systems and Control Engineering, pp. 586-597, 2012.

[26] N. Johnston, M. Pan and S. Kudzma, "An Enhanced Transmission Line Method for Modelling Laminar Flow of Liquid in Pipelines," Journal of Systems and Control Engineering, vol. 228, no. 4, pp. 193-206, 2013.

[27] J. ven der Buhs, T. Wiens, "Modelling Dynamic Response of Hydraulic Fluid Within Tapered Transmission Lines," Proceedings of the 15th Scandinavian International Conference on Fluid Power, Linköping, Sweden, 2017. 


\title{
Investigation into the Influence of Fused Deposition Modeling (FDM) Process Parameters on the Thermal Properties of 3D-Printed Parts
}

\author{
Ahmed Elkholy and Roger Kempers ${ }^{1}$ \\ Department of Mechanical Engineering, \\ York University, \\ Toronto, Canada \\ 1 Corresponding Author: Kempers@yorku.ca
}

\begin{abstract}
The mechanical properties of 3D-printed parts have been extensively studied and the effects of printing parameters on them have been investigated. However, there are limited reliable data for the thermal properties of the materials used for printing, which can impede the development of additively manufactured heat exchangers made from either pure polymers or composites. In the current study, the effect of the layer height and width have been investigated experimentally and numerically to explore the thermal anisotropic nature of unidirectional printed parts printed using fused deposition modelling (FDM). The results show that increasing the layer height and width causes deterioration in the thermal conductivity, which may reach $65 \%$ of reduction compared to the pure polymer.
\end{abstract}

Keywords: FDM, FFF, printing parameters, thermal conductivty, PLA, numerical modelling

\section{INTRODUCTION}

Polymers have many advantages - such as corrosion resistance, low weight, and smooth surfaces - which make the class a notable competitor to exotic metals in heat exchanger applications. Nevertheless, their low thermal conductivity narrows their application [1]. The addition of conductive fillers inside the polymer matrix is an effective remedy to this issue. Polymer composites are conventionally produced by an injection process [2]. However, controlling the injection process parameters, such as injection flow conditions, filler volume concentration, their distribution, and their orientation state inside the polymer matrix, is not practically achievable.

Additive manufacturing (AM) is an alternative approach to produce composite polymer components [3], [4]. It depends on laying the material layer by layer according to the designed 3D CAD model. Compared with subtractive methods, it has many advantages, such as shortening the production time cycle and reducing cost [5], [6]. AM has many techniques, such as stereo-lithography (SLA), fused deposition modeling (FDM), selective laser sintering (SLS), and laminated object manufacturing (LOM) [7].

Recent studies show the ability to produce a final prototype of polymer heat exchangers using either one of the previous methods. For example, Jia et al. [8] employed the FDM process to produce a heat sink made of thermally conductive graphite polymer composite for a 3D printer extruder. Their results showed that it achieved a similar energy dissipation effectiveness compared with the conventional aluminum one. In the same direction, Hymas et al. [9] established a new hybrid approach of FDM and embedded metallic strips to fabricate a composite polymer heat exchanger (CPHE). Kalsoom et al. [10] exploited the stereolithography process to produce an electronic heat sink from composite resin made of synthetic diamond fillers and acrylate polymer. In this context, the current work is dedicated to studying the effect of the FDM process parameters on the thermal properties.

FDM is the most commonly used process parameter due to its ability to produce low cost products with negligible waste, the wide availability of various plastic filaments, and because there is no need for chemical post-processing. The process theory is based on laying the material layer by layer on a heated bed using a continuous filament of thermoplastic that passes through a hot nozzle moving in the $\mathrm{X}-\mathrm{Y}$ plane. The nozzle motion is controlled according to the data generated by slicing software which is responsible for dividing the CAD model into separate layers. Once one layer is completed, the bed is lowered in order to begin the other one and so on until

a)

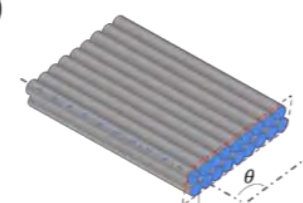

b)

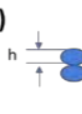

c)

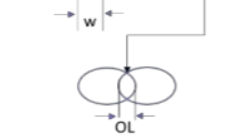

Figure 1: FDM process parameters; (a) raster angle, $\theta$; (b) layer height, $\mathrm{h}$

and width, w; (c) overlap, OL; (d) infill pattern.

d)
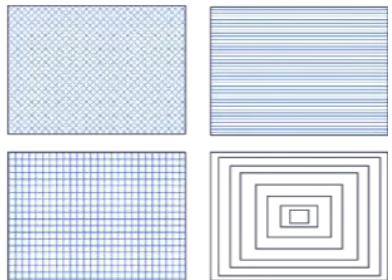
the whole product is built. This process has many parameters, such as layer height, layer width, overlap between layers, infill pattern, and raster angle, as shown in Fig. 1.

Due to the low mechanical and thermal properties of the FDM 3D-printed parts, many studies have focused on producing composite filament. However, this composite should have specific properties to be easily processed with FDM, such as viscosity, flexibility, stiffness, strength, and conductivity. Thus, for some cases where the filler concentration is high, additives - such as surfactants - are needed to produce homogeneous distribution of the filler inside the matrix. Some of these studies are presented here.

Nikzad et al. [11], [12] could increase the mechanical and thermal properties of Acrylonitrile Butadiene Styrene polymer (ABS) by including some metallic fillers-iron or copper-to produce a composite filament for FDM applications. They were able to successfully find the appropriate mixture percentage that meets the desired properties without facing processing issues during printing. They utilized the transient line source technique and the Differential Scanning Calorimetry (DSC) to measure thermal conductivity and the thermal capacity of the resulted mixture of composite polymer, respectively. Their results show the thermal conductivity achieved marked improvement above 30 vol \%. However, the thermal capacity deteriorated by incorporating the metallic fillers at any volume percentage. Furthermore, they examined the dynamic mechanical properties of the composite and demonstrated that high filler percentage reduced the material strength due to poor filler distribution, agglomeration, and the development of voids. Laureto et al. [13] used the guarded heat flow meter TCA300 to quantify the through-plane thermal conductivity of 3Dprinted parts made from the commercially available Polylactic acid (PLA) filament and its metal composites. Similar attempts were carried out, but with different metallic composites such as in the studies by Hwang et al. [14] and Masood et al. [15].

Ning et al. [2] examined experimentally the influence of process parameters such as nozzle temperature, raster angle, fill speed, and layer thickness on mechanical properties of carbon fiber reinforced polymers (CFRP). The adopted filament was made of an Acrylonitrile Butadiene Styrene (ABS) matrix with a volume concentration of 5\% of chopped carbon fiber. They concluded that the optimum parameters are $220{ }^{\circ} \mathrm{C},[0 / 90], 25 \mathrm{~mm} / \mathrm{s}$ and 0.15 respectively in terms of the tensile properties. However, regarding the ductility and toughness, the optimum values remain the same, except for the layer thickness and raster angle which have values of $0.25 \mathrm{~mm}$ and [45/45], respectively. Furthermore, they investigated the effect of adding these carbon fibers on the porosity of the FDM-printed parts using an SEM micrograph. Shemelya et al. [16] utilized the Transient Plane Source (TPS) to address the anisotropic thermal properties of the 3D-printed ABS composites filled with graphite, carbon fiber, and silver. Flaata et al. [17] developed a steady-state apparatus designed specifically to measure the thermal conductivity of 3D-printed composites and exploited it to test some feed stock materials available commercially for FDM such as PLA, ABS, brass PLA, bronze PLA, and stainless steel PLA. However, there is a large discrepancy between the values measured by Laureto [13], Shemelya et al. [16], and Flaata et al. [17]. For instance, ABS has a thermal conductivity of 0.35 according to [17] while it was tested by [16] and suggested to have a value ranging from 0.15 to 0.2 , depending on the direction of measurements; this represents a deviation of $57 \%-75 \%$. The same situation applies for PLA and its composites. Also, some studies addressed the manufacture of piezoelectric ceramics and ceramic composites via FDM [10], [18], [19].

In summary, the mechanical properties of 3D-printed parts have been extensively studied and the effects of printing parameters on them have been investigated. However, to the best of our knowledge, there are limited studies about their thermal properties. The achievement of creating a thermally efficient composite polymer heat exchanger (CPHE) hangs on investigation of the anisotropic nature of the printed parts to produce reliable thermal conductivity data and exploration of the effects of process variables on their performance. Therefore, in order to achieve this goal, two objectives must be met:

- Develop an accurate experimental facility for measuring the thermal conductivity of low conductive materials.

- Characterize the effect of the layer height and width on the thermal conductivity of unidirectional printed parts experimentally and numerically.

\section{MATERIALS AND METHODOLOGY}

\section{A. Preparation of Samples}

To examine the effect of the process variables on the thermal anisotropic nature of unidirectional printed parts, the specimens need to be fabricated in three different configurations of the same size, as shown in Fig. 2. The first one is for quantifying the thermal conductivity in z-direction $\left(\mathrm{k}_{\mathrm{zz}}\right)$, while second one is for $\mathrm{y}$-direction $\left(\mathrm{k}_{\mathrm{yy}}\right)$ and the last one is for $\mathrm{x}$-direction $\left(k_{x x}\right)$. However, only the first direction was printed and measured experimentally, while the other directions were investigated numerically and analytically. The experimental measurement was utilized as a validation step for the numerical model. The influence of the layer height was studied by printing the samples in these configurations with

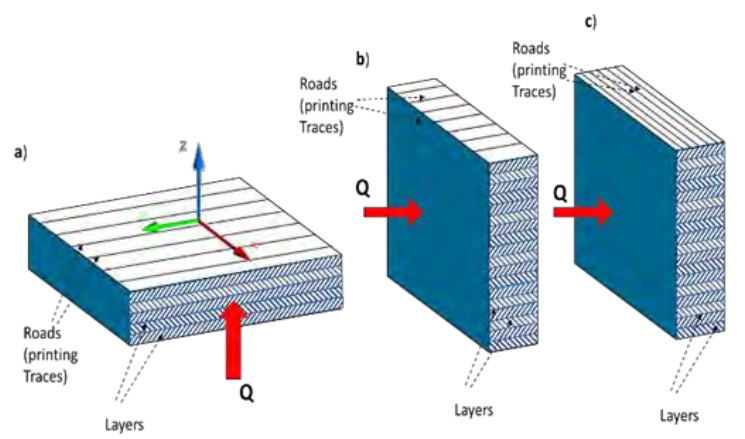

Figure 2: Different Configurations to measure the thermal conductivity in; (a) z-direction, $k_{z z}$, (b) y-direction, $k_{y y}$, (c) x-direction, $k_{x x}$. 
different values ranging from $0.05 \mathrm{~mm}$ to $0.3 \mathrm{~mm}$, while keeping the layer width and overlap constant at $0.4 \mathrm{~mm}$ and 0 $\mathrm{mm}$, respectively, which are the default printer setting. As for the layer width, its value was varied from $0.4 \mathrm{~mm}$ to $0.8 \mathrm{~mm}$, while maintaining the layer height and overlap at $0.15 \mathrm{~mm}$ and $0 \mathrm{~mm}$. The printing temperatures were $200{ }^{\circ} \mathrm{C}$ for the nozzle and $60{ }^{\circ} \mathrm{C}$ for the bed. All the samples were printed with line patterns with a raster angle of $90{ }^{\circ} \mathrm{C}$ in order to be unidirectional.

All samples were printed with $100 \%$ fill from PLA filament using the Ultimaker ${ }^{\circledR} 2$ printer. Each sample was printed to the size $40 \mathrm{~mm} \times 40 \mathrm{~mm} \times 3 \mathrm{~mm}$, which is the same cross section area of the test rig. An automatic ULTRAPOL polishing machine was utilized to ensure that the sample surfaces were parallel and its surface roughness was very small. The sample surface roughness was measured after polishing by means of a profile meter and its Ra value was about $0.462 \mu \mathrm{m}$.

\section{B. Microscopic Study}

Determining the thermal conductivity for the other directions numerically or analytically requires an investigation of the pattern of layers after printing, which was carried out using a microscopic study. Also, this study was used to obtain the air volume fraction inside the matrix. Fig. 3a depicts the effect of changing the layer height from $0.15 \mathrm{~mm}$ to $0.3 \mathrm{~mm}$ while keeping its width at the default value of $0.4 \mathrm{~mm}$. Similarly, Fig. 3b shows the layer width effect while keeping the layer height at $0.15 \mathrm{~mm}$. As can be seen from this figure, increasing the layer height increases the air volume fraction and the same effect happens for the layer width. Fig. 3 indicates that the pattern is homogeneous, except for some roads which are not connected to each other. This phenomenon is more probable to occur at small layer heights. This is because the melt flow sometimes finds low resistance in one direction more than the other when squeezed against the printer bed. Another important feature which appears in this figure is that the roads starts to completely disconnect when layer width is more than $0.5 \mathrm{~mm}$. This additional air gap between the roads most probably happens because the printer is not able to provide the correct amount of extrusion during the printing time which is controlled by the travelling speed. The printing speed was held constant at a value of $60 \mathrm{~mm} / \mathrm{s}$ for all the samples. Each photo was then converted into an 8-bit image type in order to perform thresholding. ImageJ software was employed to do the thresholding and calculate the air volume fraction. Another method was employed to measure the volume fraction of air which depends on weighting the sample while postulating that the air mass inside the gaps is negligible, and then applying the following equation:

$$
\begin{aligned}
& v_{f}=\frac{v_{s}-v_{p}}{v_{s}} \\
& v_{p}=\frac{m_{s}}{\rho}
\end{aligned}
$$

where $v_{f}, v_{p}, v_{s}$ are the volume fraction of air, the volume that the polymer occupies inside the printed part, and the printed part volume. $\rho$ is the polymer density which was measured for the PLA feed stock filament. Table 1 and 2 show a comparison between the air volume fraction resulting from the ImageJ method and weighting method. As can be seen from these tables, there is a large deviation between the two methods, especially for the layer height. The reason for this is that the tested sample under the microscope is broken only at one section and the photo captured part of this section which cannot be considered representative of the whole sample, especially in given the layers' disconnection problem. Therefore, the second method was assumed to be more accurate; however, the photos give a better insight into the pattern happening inside the printed part and will help to develop the numerical model.

TABLE. I. AIR VOLUME FRACTION AT DIFFERENT LAYER HEIGHTS AT CONSTANT LAYER WIDTH OF 0.4 MM

\begin{tabular}{|c|c|c|c|}
\hline \multirow{2}{*}{$\begin{array}{c}\text { Layer } \\
\text { Height}\end{array} \mathbf{h}$} & \multicolumn{3}{|c|}{ Air Volume Fraction \% } \\
\cline { 2 - 4 } & $v_{f} \%$ & $v_{f} \%$ & Weighting Method \\
\hline 0.1 & 4.1 & 8.2 & 0.14 \\
\hline 0.15 & 6.5 & 11.7 & 0.138 \\
\hline 0.2 & 9.8 & 13.5 & 0.13 \\
\hline 0.25 & 14.3 & 14.1 & 0.13 \\
\hline
\end{tabular}

TABLE. II. AIR VOLUME FRACTION AT DIFFERENT LAYER WIDTHS AT CONSTANT LAYER HEIGHT OF $0.15 \mathrm{MM}$

\begin{tabular}{|c|c|c|c|}
\hline \multirow{2}{*}{$\begin{array}{c}\text { Layer } \\
\text { WIDTH, }\end{array}$} & \multicolumn{3}{|c|}{ Air Volume Fraction \% } \\
\cline { 2 - 4 } W & ImageJ & \multicolumn{2}{|c|}{ Weighting Method } \\
\cline { 2 - 4 } & $v_{f} \%$ & $v_{f} \%$ & Uncertainty \% \\
\hline 0.4 & 4.1 & 11.7 & 0.137 \\
\hline
\end{tabular}

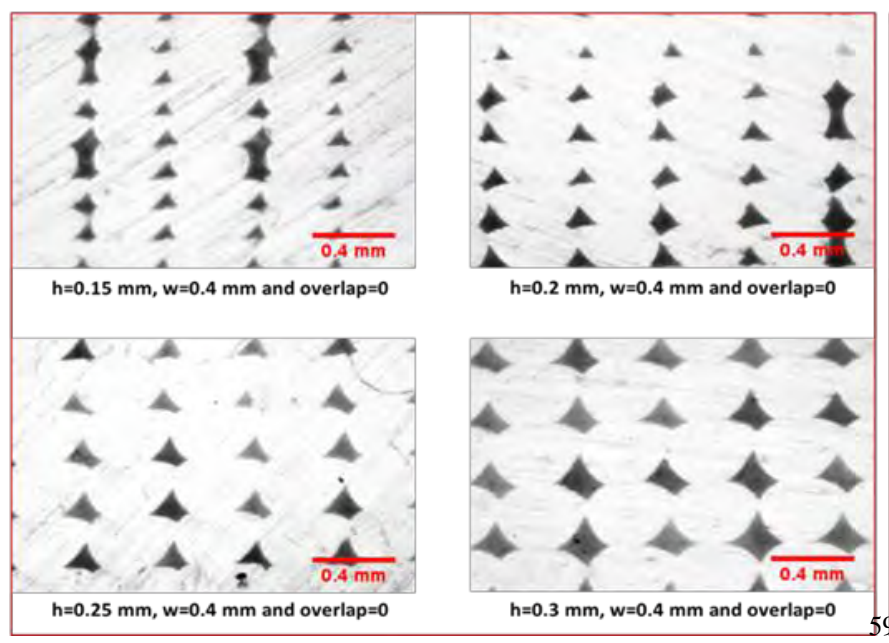

b)

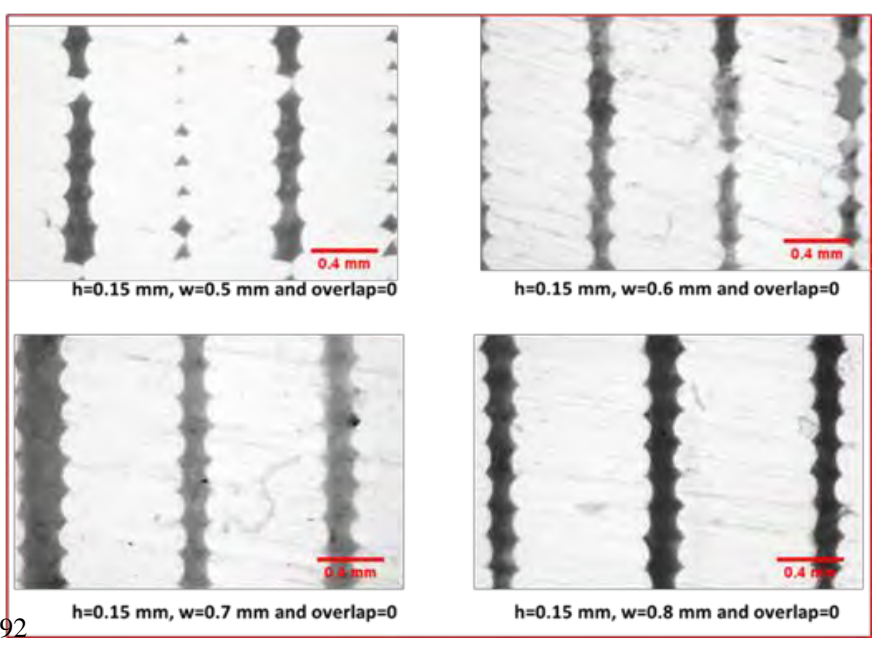




\begin{tabular}{|c|c|c|c|}
\hline \multirow{2}{*}{$\begin{array}{c}\text { Layer } \\
\text { WIDTH, }\end{array}$} & \multicolumn{3}{|c|}{ Air Volume Fraction \% } \\
\cline { 2 - 4 } & ImageJ & \multicolumn{2}{|c|}{ Weighting Method } \\
\hline 0.5 & 11.9 & 13.7 & 0.134 \\
\hline 0.6 & 13.7 & 15.3 & 0.131 \\
\hline 0.7 & 17.1 & 19 & 0.126 \\
\hline 0.8 & 18.1 & 24.5 & 0.117 \\
\hline
\end{tabular}

\section{Numerical Modeling}

The effective thermal conductivity in $\mathrm{z}$ or $\mathrm{x}$ directions can be predicted by simplifying the problem into a $2 \mathrm{D}$ model which resembles a unit cell that was extracted to represent the overall geometry. ANSYS fluent 18.2 software [20] was used to predict the heat flow by conduction through printed parts. The unit cell had a dimension of $h / 2$ in the z-direction and $w / 2$ in the x-direction, as shown in Fig. 4a. A temperature boundary condition was applied at the right and left boundaries of the domain, while insulated and symmetry conditions were adopted for the top and bottom boundaries, respectively, as shown in Fig. 4b. This configuration was employed to predict $k_{x x}$. As for the other direction, the boundary conditions were reversed, where the temperature gradient should be in the same direction as the thermal conductivity measurement direction. The energy equation solution results in the heat transfer which is used to calculate the effective thermal conductivity by exploiting Fourier's law of conduction. The simulation was repeated several times to find the layer height and width influence on the thermal properties. The same range stated in

a)

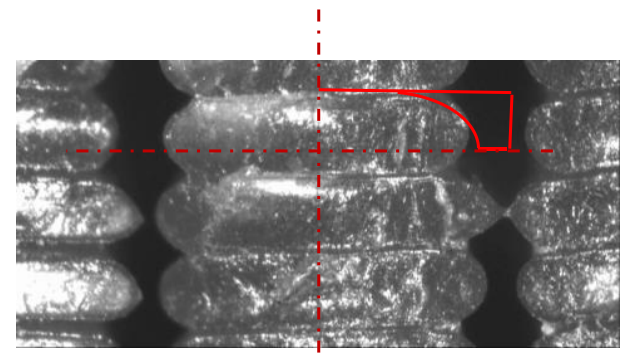

$\mathrm{h}=0.15 \mathrm{~mm}, \mathrm{w}=0.65 \mathrm{~mm}$ and $\mathrm{OL}=0$

b)

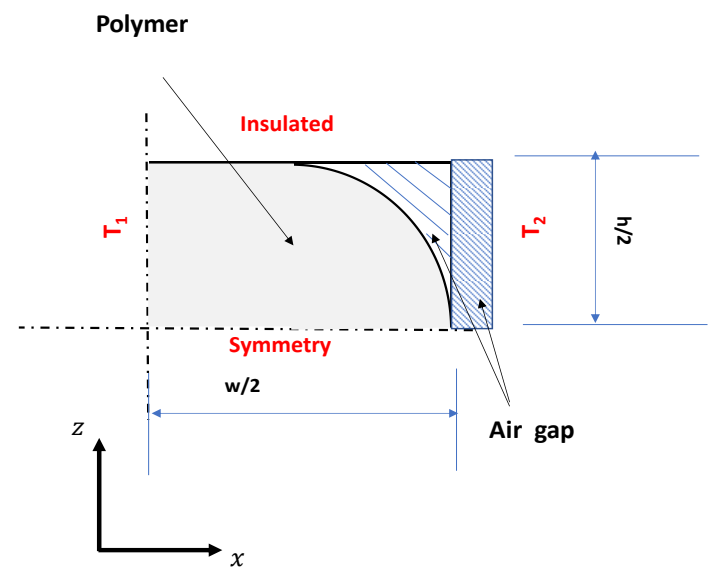

Figure 5: Numerical Model (a) unit Cell (b) domain.
Section A was used here for the numerical simulation. The employed temperature difference, $T_{1}-T_{2}$, was set equal to 1 for all cases. The volume fraction calculated by weighting the samples in the previous section was used to determine the air gap distance between the roads.

\section{Experimental Methodology}

Fig. 5 shows the suggested design which is mainly composed of two sides; one is serving as the heat source and the other one represents the heat sink, where the sample is sandwiched between them. The hot side is composed of two isothermal blocks manufactured from copper material, $\mathrm{k}=391.2 \mathrm{w} / \mathrm{m} \mathrm{k}$, with a small thickness to produce a uniform temperature field through them. The function of the first block is to supply the heat into the sample under testing. The secondary hot block was designed to surround the main one in order to guarantee that all the heat coming from the source flows into the sample only and there is no heat loss from the main block either from its sides or its top surface. The secondary block's power was controlled to ensure that the two blocks have the same temperature which was monitored at three different locations. It was assumed that the secondary power was adequately tuned when the temperature difference did not exceed $0.001{ }^{\circ} \mathrm{C}$ at steady state.

Similarly, the cold side consists of two blocks, which were also manufactured from copper material. Both have a $U$ channel which permits the chilled cooling water to flow through
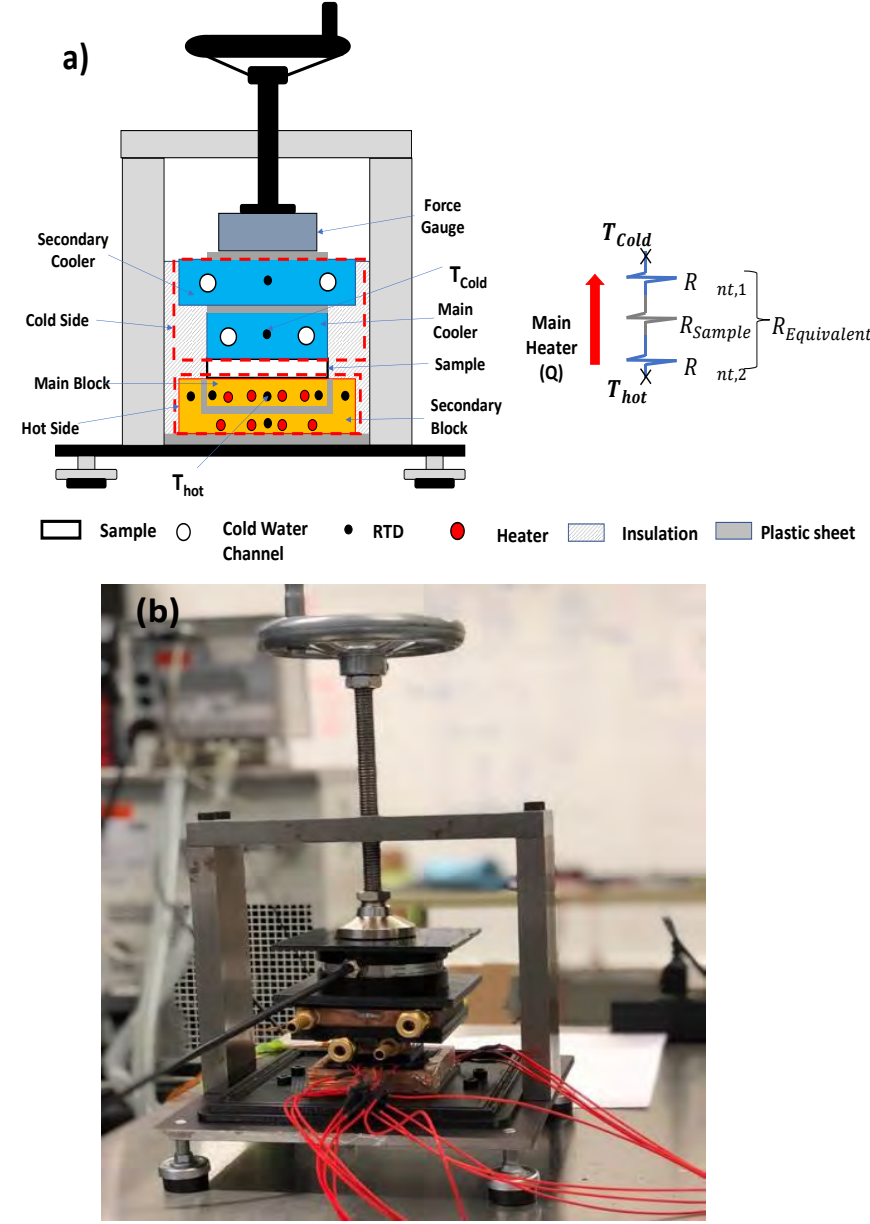

Figure 4: Experimental apparatus (a) Schematic (b) real rig. 
them. All the blocks and the sample were stacked above each other and forced against a force gauge using a clamping system. The secondary cold block function is to isolate the system from the clamping system base. All parts were confined into a large block filled with silica aerogel with a thermal conductivity of $0.014 \mathrm{w} / \mathrm{m} \mathrm{k}$.

The thermal conductivity can be easily calculated by measuring main block input power and temperature difference between RTD, as given by:

$$
k=\frac{Q \Delta t}{A_{c}\left(T_{\text {hot }}-T_{\text {cold }}\right)}
$$

where $k$ is the measured thermal conductivity, $\Delta t$ is the specimen thickness, $A_{c}$ is the sample cross section area and $\left(T_{h}-T_{c}\right)$ is the temperature difference between the main hot block and main cold one. Some aspects were considered while doing the testing to make sure that the contact resistance effect is negligible. Thermal interface material was applied at both sides of the sample and the clamping pressure was kept high at about 3 bars for all measurement. Moreover, the contact resistance value was calculated by testing the total thermal resistance for three samples, at the same operating conditions, with similar geometrical and printing parameters, except for the thickness which was different. The contact resistance value can be extracted by drawing its value against its thickness and performing a linear curve fitting. Its value was less than $2 \%$ of the total resistance, which can be discarded.

\section{RESULTS}

\section{A. The effect of layer height}

The thermal conductivity in z-direction $\left(k_{z z}\right)$ was measured experimentally and compared to the numerical model, as shown in Fig. 6. Each sample was measured three times and averaged to guarantee the reliability of the test rig. It is indicated that there is a good agreement between both of them which supports the possibility of using the numerical model to predict the thermal conductivity in $\mathrm{x}$-direction $\left(k_{x x}\right)$. The analytical parallel was employed to predict the thermal conductivity in the third direction $\left(k_{y y}\right)$ which is given by the following equation:

$$
K_{e}=\left(1-V_{f}\right) K_{m}+V_{f} K_{a}
$$

where $k_{e}$, is the effective thermal conductivity and $k_{m}$ and $k_{a}$ are the conductivities for the matrix and air respectively. $V_{f}$ is the air volume fraction inside the PLA matrix. The numerical model requires the thermal conductivity of pure polymer and this has been measured by printing a sample with a small layer height of $0.06 \mathrm{~mm}$ and a high percentage of overlap to ensure that there are no air gaps generated inside the part. Its value was $0.2207 \mathrm{w} / \mathrm{mk}$. The experimental results in Fig. 6 indicate that increasing the layer height reduces the thermal conductivity in $\mathrm{z}$-direction until a value of $0.133 \mathrm{w} / \mathrm{m} \mathrm{k}$ at layer height of $0.3 \mathrm{~mm}$ is reached, due to the increase of air volume fraction. This represents a percentage of reduction of about 40 $\%$ compared with pure PLA polymer. Figs. 6 and 7 illustrate that the effect of layer height is more significant in $\mathrm{z}$ and $\mathrm{x}$ directions, while it is nearly constant for $y$-direction. The thermal conductivities are higher in directions $\mathrm{z}$ and $\mathrm{y}$ than $\mathrm{x}$ direction. This is because the heat path in $\mathrm{x}$-direction is a series which increases the heat's overall resistance while it is parallel for the other directions. For $\mathrm{x}$-direction, the thermal conductivity decreases and then increases because when the layer height is small, the layers are not connected. In summary, the layer height increase causes a decrease in the thermal conductivity, which reaches values of reduction of $42 \%, 14 \%$, and $28 \%$ for $\mathrm{x}, \mathrm{y}$, and $\mathrm{z}$, respectively, compared with the pure polymer at a layer height of $0.3 \mathrm{~mm}$. The previous percentages were calculated based on the numerical model predictions.

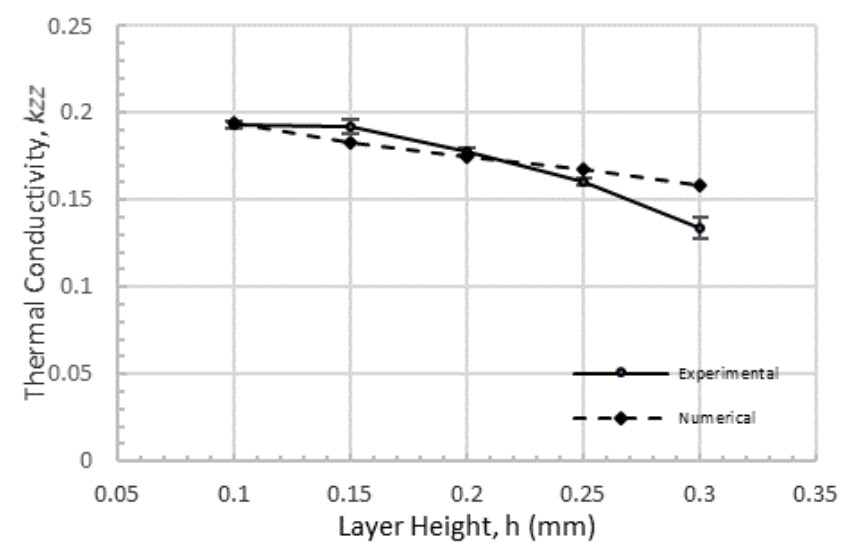

Figure 6: Comparison between the experimental results and numerical model for studying the effect of layer height on the thermal conductivity, $k_{z z}$ at constant layer width of $0.4 \mathrm{~mm}$.

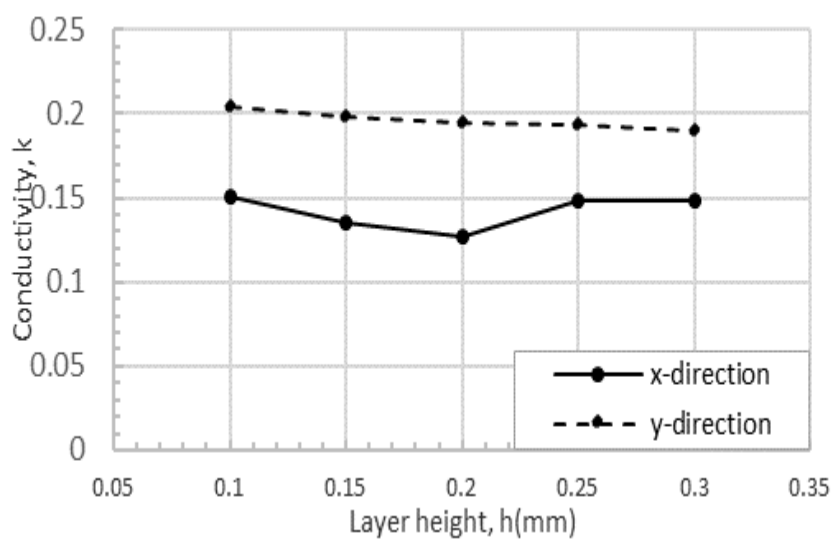

Figure 7: The effect of layer height on the thermal conductivity in y-direction $\left(k_{y y}\right)$ and $\mathrm{x}$-direction $\left(k_{x x}\right)$ at constant layer height of $0.15 \mathrm{~mm}$.

\section{B. The effect of layer width}

The influence of the layer width on thermal conductivity in $\mathrm{x}$ and $\mathrm{z}$ directions was predicted numerically while the parallel model was employed for y-direction, as shown in Fig. 8. Similar to the layer height, increasing the layer width causes 
deterioration for the thermal conductivity in all directions. Also, the thermal conductivity is higher for $\mathrm{y}$ and $\mathrm{z}$ directions than $\mathrm{x}$-direction, because of the same reason mentioned before. The layer width has a severe effect on the thermal conductivity in x-direction because the layers are completely disconnected in that direction.

The thermal conductivity in $\mathrm{x}$-direction reaches a low thermal conductivity value of $0.077 \mathrm{w} / \mathrm{mk}$ at a width of 0.8 $\mathrm{mm}$, which represents a $65 \%$ reduction compared with pure polymer, while the reduction is $28 \%$ and $25 \%$ for $\mathrm{y}$ and $\mathrm{z}$ directions, respectively. This highlights the importance of studying these parameters and demonstrates the possibility of tailoring the thermal properties of the printed parts, especially in the case of continuous fiber printing.

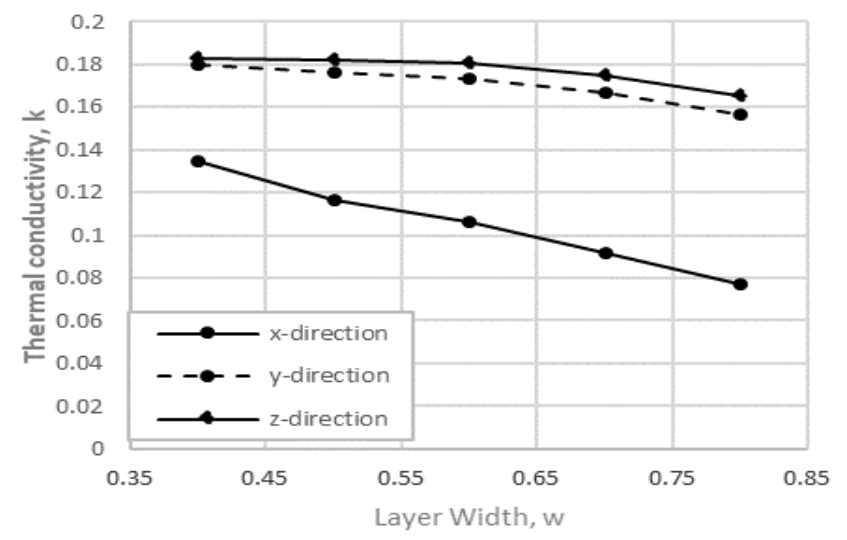

Figure 8: The effect of layer width on the thermal anisotropic nature of 3Dprinted parts.

\section{CONCLUSION}

This work examined the influence FDM process parameters on the anisotropic thermal properties of unidirectional 3D-printed parts. An experimental facility was developed to measure the effect of the layer height on the thermal conductivity in one direction, which was used as a validation step for the developed numerical model. The numerical model used in parallel with the analytical model was able to quantify the thermal conductivities in all directions. The main outcomes can be summarized into these points:

- Increasing either the layer height or width reduces thermal conductivity in all directions as a result of porosity generation.

- The effect of layer width is more significant than the layer height, especially when the layer disconnection problem exists.

- Increasing the layer height, while keeping the layer width constant at $0.4 \mathrm{~mm}$, results in a drop in the thermal conductivity which reaches a value of $42 \%$, $14 \%$, and $28 \%$ in $\mathrm{x}, \mathrm{y}$, and $\mathrm{z}$ directions, respectively, at a layer height of $0.3 \mathrm{~mm}$ compared with pure polymer.

- Increasing the layer width, at constant layer height of 0.15 , leads to a decline in thermal conductivity, reaching reduction percentages of $65 \%, 28 \%$, and
$25 \%$ in $\mathrm{x}, \mathrm{y}$, and $\mathrm{z}$ directions, respectively, at a layer width of $0.8 \mathrm{~mm}$.

\section{REFERENCES}

[1] I. L. Ngo, S. Jeon, and C. Byon, "Thermal conductivity of transparent and flexible polymers containing fillers: A literature review," Int. J. Heat Mass Transfer, vol. 98, pp. 219-226, 2016. doi: 10.1016/j.ijheatmasstransfer.2016.02.082.

[2] F. Ning, W. Cong, Y. Hu, and H. Wang, "Additive manufacturing of carbon fiberreinforced plastic composites using fused deposition modeling: Effects of process parameters on tensile properties," J. Compos. Mater., vol. 51, no. 4, pp. 451-462, 2017.doi:10.1177/0021998316646169.

[3] S. Kumar and J. P. Kruth, "Composites by rapid prototyping technology," Mater. Des., vol. 31, pp. 850-856, 2010. doi:10.1016/j.matdes.2009.07.045.

[4] A. C. De Leon, Q. Chen, N. B. Palaganas, J. O. Palaganas, J. Manapat, and R. C. Advincula, "High performance polymer nanocomposites for additive manufacturing applications," React. Funct. Polym., vol. 103, pp. 141-155, 2016. doi: 10.1016/j.reactfunctpolym.2016.04.010.

[5] B. Berman and F. G. Zarb, "3-D printing: The new industrial revolution," Bus. Horiz., vol. 55, pp. 155-162, 2011. doi:10.1016/j.bushor.2011.11.003.

[6] P. Deepa, "Fused Deposition Modeling - A Rapid Prototyping technique for Product Cycle Time Reduction cost effectively in Aerospace Applications," in Int. Conf. Adv. Eng. Technol., 2014, pp. 62-68.

[7] K. V. Wong and A. Hernandez, "A Review of Additive Manufacturing," ISRN Mech. Eng., vol. 2012, pp. 1-10, 2012.doi: 10.5402/2012/208760.

[8] Y. Jia, H. He, Y. Geng, B. Huang, and X. Peng, "High through-plane thermal conductivity of polymer based product with vertical alignment of graphite flakes achieved via 3D printing," Compos. Sci. Technol., vol. 145, pp. 55-61, 2017. doi:10.1016/j.compscitech.2017.03.035.

[9] D. M. Hymas, M. A. Arie, F. Singer, A. H. Shooshtari, and M. M. Ohadi, "Enhanced Air-Side Heat Transfer in an Additively Manufactured Polymer Composite Heat Exchanger," in IEEE Intersoc. Conf. Therm. Thermomech. Phenom. Electron. Syst., 2017.doi: 10.1109/ITHERM.2017.7992546.

[10] U. Kalsoom, A. Peristyy, P. N. Nesterenko, and B. Paull, "A 3D printable diamond polymer composite: a novel material for fabrication of low cost thermally conducting devices," RSC Adv., vol. 6, no. 44, pp. 38140-38147, 2016. doi: 10.1039/C6RA05261D.

[11] M. Nikzad, S. H. Masood, and I. Sbarski, "Thermo-mechanical properties of a highly filled polymeric composites for Fused Deposition Modeling," Mater. Des., vol. 32, no. 6, pp. 3448-3456, 2011.doi:10.1016/j.matdes.2011.01.056.

[12] M. Nikzad, S. H. Masood, I. Sbarski, and a Groth, "Thermo-Mechanical Properties of a Metal-filled Polymer Composite for Fused Deposition Modelling Applications," 5th Australas. Congr. Appl. Mech. ACAM 2007, pp. 319-324.

[13] J. Laureto, J. Tomasi, J. A. King, and J. M. Pearce, "Thermal properties of 3-D printed polylactic acid-metal composites," Prog. Addit. Manuf., vol. 2, no. 1-2, pp. 57-71, 2017. doi: 10.1007/s40964-017-0019-x.

[14] S. Hwang, E. I. Reyes, K. sik Moon, R. C. Rumpf, and N. S. Kim, "Thermomechanical Characterization of Metal/Polymer Composite Filaments and Printing Parameter Study for Fused Deposition Modeling in the 3D Printing Process," $J$. Electron. Mater., vol. 4, pp. 771-777, 2015. doi: 10.1007/s11664-014-3425-6.

[15] S. H. Masood and W. Q. Song, "Thermal characteristics of a new metal/polymer material for FDM rapid prototyping process," Assem. Autom., vol. 25, no. 4, pp. 309 315, 2005. doi: 10.1108/01445150510626451. 
[16] C. Shemelya, A. De La Rosa, A. R. Torrado, K. Yu, J. Domanowski, P. J.

Bonacuse, R. E. Martin, M. Juhasz, F. Hurwitz, R. B. Wicker, B. Conner, E.

MacDonald, and D. A. Roberson, "Anisotropy of thermal conductivity in 3D printed polymer matrix composites for space based cube satellites," Addit. Manuf., vol. 16, pp. 186-196, 2017. doi: 10.1016/j.addma.2017.05.012.

[17] T. Letcher, "Thermal Conductivity Testing Apparatus for 3D Printed Materials," in ASME Heat Transfer Summer Conf., Bellevue, WA, 2017, pp. 1-6. doi: 10.1115/HT2017-4856.

[18] M. K. Agarwala, R. Van Weeren, A. Bandyopadhyay, P. J. Whalen, A. Safari, and S. C. Danforth, "Fused deposition of ceramics and metals: an overview," in Proc. Solid Free. Fabr. Symp., 1996, pp. 385-392.

[19] A. Bandyopadhyay, R. K. Panda, T. F. McNulty, F. Mohammadi, S. C. Danforth, and A. Safari, "Piezoelectric ceramics and composites via rapid prototyping techniques," Rapid Prototyp. J., vol. 4, no. 1, pp. 37-49, 1998. doi: $10.1108 / 13552549810200285$.

[20] ANSYS, "ANSYS Fluent Theory Guide," ANSYS 16 Documentation, vol. 15317, no. July. p. 80, 2015. 


\title{
Sonification of flow instabilities in CFD aneurysm models
}

\author{
Daniel E. MacDonald ${ }^{1}$, Thangam Natarajan ${ }^{1}$, Richard C. Windeyer ${ }^{2}$, Peter W. Coppin ${ }^{2}$, David A. Steinman ${ }^{1}$ \\ ${ }^{1}$ Biomedical Simulation Laboratory \\ University of Toronto \\ Toronto, Canada \\ ${ }^{2}$ Perceptual Artifacts Laboratory \\ Ontario College of Art Design University \\ Toronto, Canada
}

\begin{abstract}
Recent investigations using image-based computational flow dynamics (CFD) have revealed turbulent-like blood flow instabilities (hemodynamics) within intracranial cerebral aneurysms and surrounding vasculature, which may contribute to aneurysm initiation, growth, and rupture. We describe a method derived from spectral decomposition of flow for inspecting and characterizing these "turbulent-like" hemodynamic structures in intracranial aneurysms by sonification of CFD generated data. Motivated by auscultation techniques, the current research seeks to address the limitations of conventional CFD animations by allowing the user to listen to complex flow patterns that are often difficult to discern visually. In the process, the proposed method of sonification is successfully applied to a series of datasets resulting from highfidelity numerical simulations of intracranial aneurysms. The resulting framework is shown to be highly efficient in performing parametric sonification in real time.
\end{abstract}

Keywords- CFD; biomedical; sonification; signal processing

\section{INTRODUCTION}

A cerebral aneurysm is the localized bulging of a weakened artery wall within the brain. Rupture occurs when the stresses exerted on the artery wall exceed the strength of the wall.

Blood flow instabilities (hemodynamics) are thought to contribute to the initiation, growth, and rupture of intracranial aneurysms [1], by stimulating degenerative remodeling (weakening) of the artery wall. The forces associated with these unstable flows are difficult to measure non-invasively using current technologies, thus, medical image-based CFD has been used to investigate these hemodynamic forces retroactively [1][2][3].

The initiation, growth, and rupture of these aneurysms is a complex, multifactorial process, but it is known that remodeling occurs according to flow conditions [4]. At Reynolds number of less than 500, turbulent-like flow has been observed in glass models of human intracranial aneurysms [5], starkly contrasting the commonly accepted threshold value of $\mathrm{Re}=2300$.

Using digitized patient-specific vasculature models extract-

This research was funded by the Canadian Natural Sciences and Engineering Research Council, the Social Sciences and Humanities Research Council, and the Heart \& Stroke Foundation. ed from clinical 3D angiography images, blood flow is simulated using advanced numerical methods. The results of such simulations are typically visualized as part of the process of data analysis.

These investigations have revealed turbulent-like flow instabilities within aneurysms or the vessels that host them, with implications for aneurysm rupture and initiation [1][2]. Such instabilities are typically represented through conventional visualization paradigms (glyphs, streamlines, isosurfaces), and though these can provide tangible and intuitive representations of the hemodynamics, visual differences among unstable laminar, transitional, and turbulent flow regimes in complex vascular geometries are often difficult to discern.

Auscultation refers to the established medical technique of listening to the sounds of the body for evaluation purposes [6]. For example, clinicians are trained to identify turbulent blood flows by recognizing auditory properties of duration, quality, and timing during examination of bruits in the carotid artery [6]. Doppler auscultation has been used in preliminary stages to listen to valvular movements and blood flow velocity sounds during cardiac examination, resolving sounds undetected by stethoscope [7], though to our knowledge, this technique has not been demonstrated on intracranial aneurysms.

Intracranial saccular aneurysms have been observed to emit acoustic signals (bruits) as a result of fluctuating hemodynamic flows thought to be characterized as turbulent-like, if not necessarily turbulent in the strict fluid mechanical sense [8][9][10]. These vibrations are difficult to measure in vivo due to difficulty differentiating these bruits from other bodily sounds, and as such are not a reliable source of diagnostic information [9].

Visually communicating spatiotemporal flow instabilities ignores the existing strengths of the human auditory system for resolving frequencies as compared to visual perception of spatial frequencies. For example, attempting to visually distinguish a difference of pitch between vibrating strings ignores the auditory ability to distinguish subtle pitch differences, of which [11] indicates can be as small as $0.2 \%$.

This lack of visual acuity is not only a problem of aneurysm visualization, but flow visualization in general. High frequency fluctuations require video or animation with an appropriately high frame rate (at least twice the fluctuation rate, in agreement with Nyquist's theorem) or greatly slowed video (thereby in- 
creasing time required to analyze data). This limitation becomes increasingly apparent as frequency of fluctuation increases.

Sonification is "the data-dependent generation of sound, if the transformation is systematic, objective and reproducible" [12]. The most common method of sonification, parameter mapping, involves careful mapping of data dimensions to auditory dimensions [13].

Sonification in the current work begins by decomposing the time domain values (velocity) in the frequency domain in order to discriminate regions of unstable flow. The current method allows inspection of individual frequency bands independently. Although point vector values remain the basis for our current work, the frequency domain processing between these vector values and the audio output distinguishes the current research from previous works. By sonifying the spectrogram, the envelope shape (percussive or smooth nature) is communicated along with spectral content.

In this context, a novel method originally inspired by Doppler ultrasound auscultation is presented for communicating the nature of the flow by augmenting conventional flow visualizations with data-driven sound (sonification) for the purposes of more easily identifying hemodynamic flow quality.

\section{METHOD}

High-fidelity CFD data sets produced using cases from the open-source Aneurisk database [14] serve as the basis of this methodology. These simulations were performed with a highresolution strategy where a high-fidelity Navier-Stokes solver was used in combination with fine spatial and temporal resolutions. Fully developed Womersley velocity profiles were applied at the inlet, and zero pressure was specified at the outlets.

The density of the fluid was $1.025 \mathrm{~g} / \mathrm{cm} 3$ and its corresponding viscosity 0.035 Poise was used. The computational mesh consisting of tetrahedral elements is generated using the open-source Vascular Modelling Toolkit (VMTK) [15] from segmented and preprocessed CT angiograms. A second-orderaccurate, minimally dissipative, and energy-preserving opensource CFD solver (Oasis) is used. Number of elements range from hundreds of thousands to millions of elements, with a time-step resolution on the order of $0.1 \mathrm{~ms}$ [16][17].

Within an interactive visualization environment (ParaView - an opensource scientific visualization tool), the average power spectral density of velocity-time traces is computed for a probed sample volume, defined by centre point and radius, in order to evaluate a local region of flow within the aneurysm sac. The probe radius is chosen based on two considerations: first, the real-time computation must support interactivity, and second, the probe should not be too spatially localized or too global. To define this balance of local and global volume, the upper bound of probe radius should neck diameter of the aneurysm, while the lower bound will be considered small enough to separate coherent Q-criterion structures. The frequency content and time-varying power of the resulting spectrogram are sent to a real-time audio server (SuperCollider sound server) to control the frequency and loudness of a bank of audio-rate sound generators. The summation of these generators results in a harmonically rich signal representative of the probed location.

This on-the-fly implementation is contained (though not constrained) within the ParaView Python shell and intended to run alongside a synchronized animation. Sound networking protocol Open Sound Control (OSC) is used to communicate the spectrogram data between the live ParaView environment and audio server. The audio presentation of the signal is highly configurable while maintaining audio-rate synthesis, allowing the user to pitch, slow down, or threshold by frequency band in real-time, synchronized to the visualization. Fig. 1 provides an overview of this process.

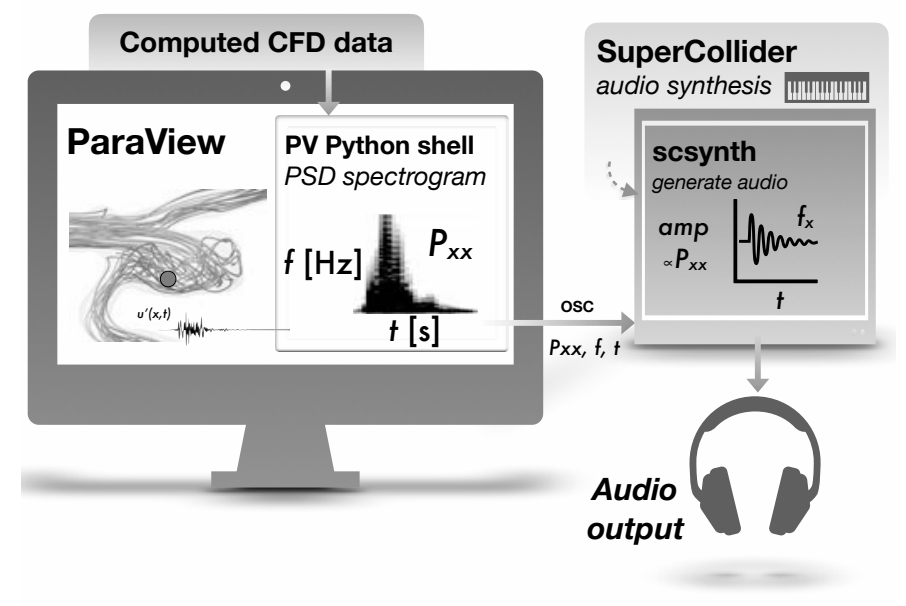

Figure 1: An overview of the method of implementation.

The structure of the sonification "instrument" was designed such that any aneurysm dataset (or any collection of spatiotemporal data) may be used as input with little manipulation (only requiring a transpose of velocity vectors). As the user probes the data, varying audio outputs are generated local to the probe. As such, different frequency content and amplitude envelopes are readily generated for each new sample of the dataset.

A guiding philosophy of sonic aesthetics was to caricature the existing physiological phenomena by generating sound inspired by fluid sounds existing in nature, and not necessarily to recreate the full acoustic field tied to the flow.

The basis of our parameter mapping was generated as simply as possible: the spectral content of velocity is mapped with auditory frequency values, while magnitude of spectral content is mapped with loudness. Use of spatial audio was considered to represent "space" of the mesh, but was ultimately found more useful for communicating the kinetic directionality of flow within the sample volume.

\section{A. Implementation}

Data is stored in binary data frames and accessed interactively from the Paraview render view. SuperCollider is used to generate the sonification from the flow data. The real-time selection, filtering, and spectral processing of flow velocity is described below. 
The chosen probe radius used is $0.25 \mathrm{~mm}$, containing approximately 50 discrete points within the volume. A list of point identifiers is generated for a 20 uniformly distributed random points within this local volume. The velocity values for these points through time will serve as the basis for the sonification, to be discussed in subsequent sections. This sonification is intended to accompany a synchronized visualization with time duration chosen by the user. As such, this pipeline is updated once for each period of flow. The data has temporal resolution of $0.004 \mathrm{~s}$ (2500 samples/second) and about 500000 points.

As shown in Fig 2, the velocity content for each point is extracted and filtered by criteria of spectral power index (SPI) to isolate flow instabilities.

SPI is a frequency domain decomposition method introduced by Khan et al as a means to quantifying the transient instabilities of turbulent-like pulsatile flows without cycle-tocycle variations [18]. This decomposition separates the pulsatile carrier signal from the turbulent-like flow instabilities. By using a high pass filter to remove the $99 \%$ most energycarrying frequencies (corresponding to a cutoff frequency of 25 $\mathrm{Hz}$ ), flow instabilities can be isolated. SPI is defined as the ratio of energy contained within the flow instabilities as a fraction of all signal energy.

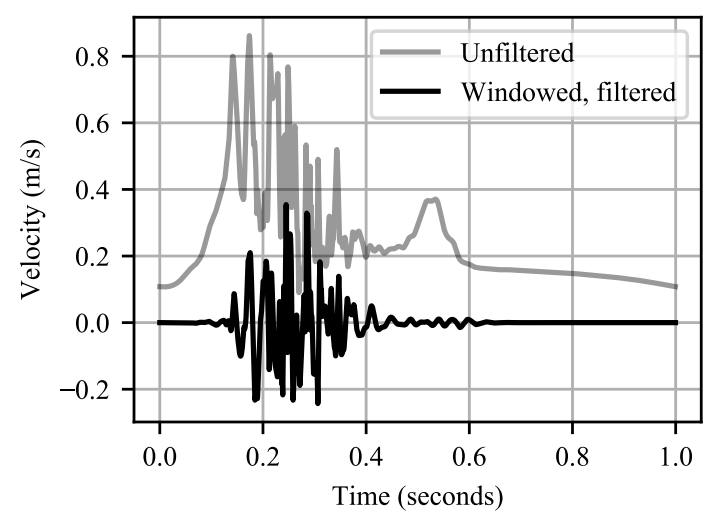

Figure 2. Isolation of velocity fluctuations by high-pass filtering the pulsatile signal according to SPI criteria. A Blackman window is used prior to filtering.

The local Power Spectral Density (PSD) spectrogram for the filtered velocity data associated with each point is calculated using a window length of 256 samples with 192 sample overlap, resulting in a smooth, temporally shifting view of the power content for each frequency bin. The average spectrogram for the sample volume is then computed. This matrix of power content corresponds to 128 frequency bins x 36 time bins.

A synthesizer template used for creating synth nodes (a synthdef) was generated in the SuperCollider environment and stored on scsynth (a real-time SuperCollider audio server). Using sound networking protocol Open Sound Control (OSC), the power matrix is sent to scsynth.

The frequency content and time-varying power of the computed spectrogram are fed to a bank of sound generators. Each frequency bin of the spectrogram corresponds to the frequency of a generator. The time-varying power values of the spectrogram are used to modulate the loudness (amplitude) of each generator in time.

The power associated with each bin corresponds to the temporal loudness (amplitude envelope) of the sound as well as a "complexity" parameter, while the frequency value corresponds to the resonant frequency of a constant skirt gain filter (Ringz generator) with noise input. The "complexity" parameter controls the cutoff frequency and modulation depth of a resonant high-pass filter. By pseudo-randomly shifting the cutoff frequency of the high pass filter near the resonant frequency of the Ringz generator, and with a shift depth proportional to the temporal power, sound textures resembling bubbles are generated to indicate a higher level of flow complexity.

The apparent directionality of flow is communicated by rotating an ambisonic B-format sound field around the user. Given the position of the aneurysm model onscreen, the directionality of flow relative to the user is determined and used to spatialize the sound; for flow visually moving left-to-right, the sound rotates correspondingly, and vice versa. The perceived rotation angle indicates the relative velocity orthogonal to the user.

This on-the-fly generation is intended to accompany a conventional flow visualization in ParaView. All computed data (signal filtering and extraction of PSD) is computed within the Paraview python shell.

\section{RESUlTS AND DISCUSSION}

A current example of the interactive implemented method can be found at [19].

The present framework of sonification results in a highly configurable audio representation allowing on-the-fly exploration of flow instabilities within CFD data. Frequency bands can be individually emphasized or extracted for closer inspection. Different sound textures, ranging from Doppler-inspired sounds to bubbly flow sounds, exist within the instrument framework. This sonification runs in real time next to a simplified conventional visualization on consumer grade hardware, allowing for flexible inspection of data.

Fig 3. illustrates a variety of flow conditions existing within an aneurysm. Velocity of flow is high entering the neck of the aneurysm, but contains minimal fluctuation. Entering the sac, it is observed that fluctuations increase substantially. The intensity of the spectral content is presented on a logarithmic color scheme and normalized for presentation. High-frequency fluctuations present within the aneurysm sac indicate disturbed flow, which may have implications for aneurysm initiation, growth, and rupture as described previously. Contours of Qcriterion for a time instant during the systole are shown for each point on a constant $y-z$ plane, where discrete locations of intense Q-criterion are observed.

The prototype has been tested with five aneurysm datasets, each of similar temporal and spatial resolution but varying flow complexity. Further exposure to new datasets will confirm the 


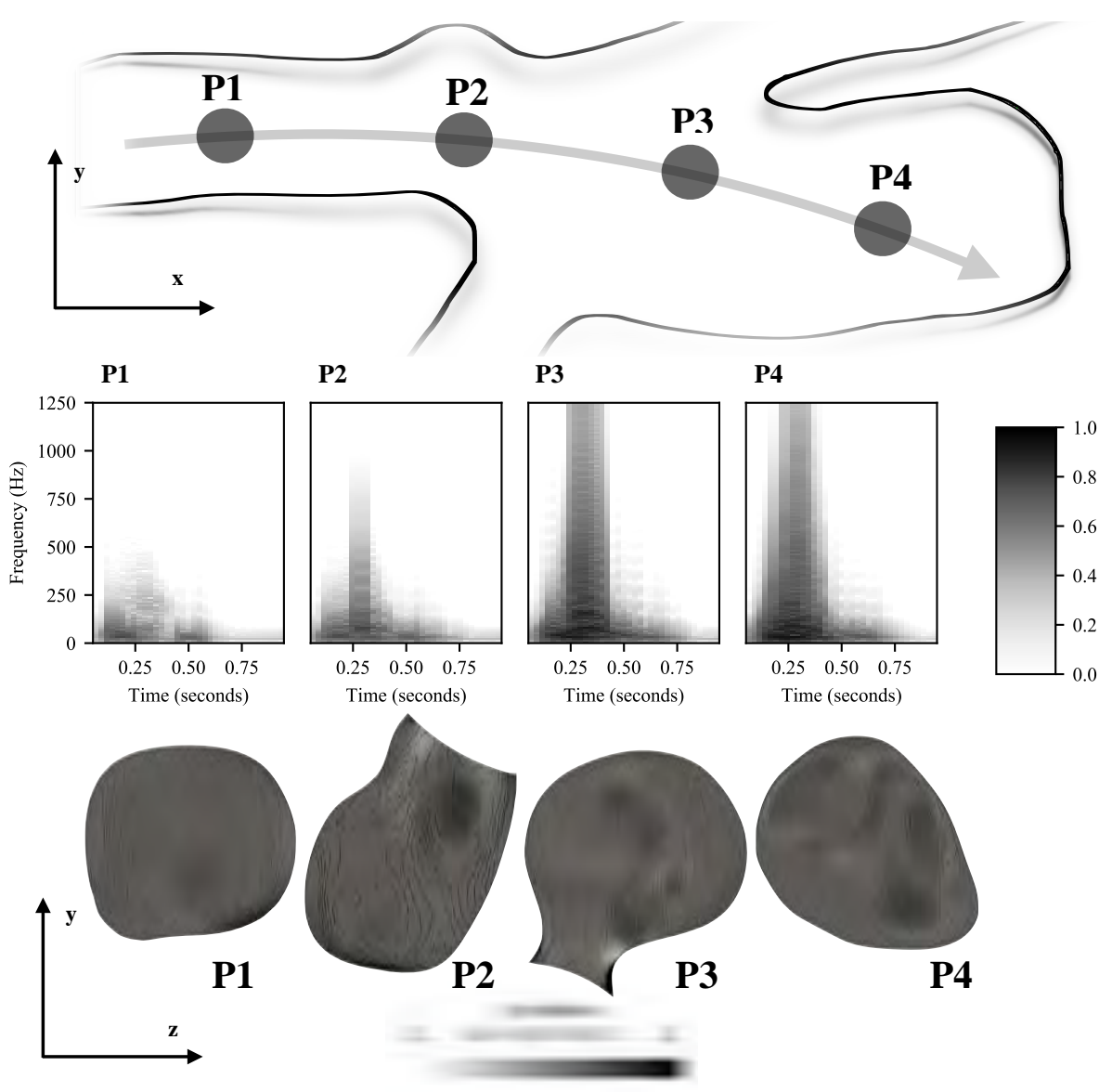

Figure 3. A comparison of spectral content and intensity of Q-criterion through the aneurysm neck and into the sac

existing method of psychological scaling, or suggest further refinement to accommodate a broader range of flow conditions.

The biological and clinical implications of high frequency content from a decomposed velocity signal are not in the scope of the current investigation; these effects are discussed in greater depth in CFD literature. The physiological presence of high frequency fluctuations, along with frequency-specific amplitude envelopes, are to be communicated to the user for interpretation. Other simplified strategies such as directly communicating Q-criterion to indicate turbulent flow were sonified, but in omitting so many dimensions of the underlying data, this simple feedback system was found too simplistic for anything other than locating regions of instability. The strength of this sonification lies in maintaining the nuance of the original signal.

The frequency resolution of the calculated PSD is limited by the temporal resolution of the data. By windowing the data, variation in time is gained at the expense of frequency resolution. This is a reasonable balance if the signals often approximate noise; broad temporal and spectral characteristics are communicated. From this balance, there are two potential dis-

advantages. First, the hard-set designation of frequency bands results in similar timbral properties between datasets. Secondly, and perhaps more importantly, if the signals have strong harmonic content, greater frequency resolution may be required to explore spectral relationships. Given the existing knowledge of spectral content in turbulent-like flow, the current chosen windowing parameters are acceptable.

The proposed method for interpreting cerebrovascular flows is not limited by application, and may be useful for other time-series spatial datasets containing instabilities. For example, we implemented a similar approach in the $3 \mathrm{D}$ creation suite Blender, for visualizing and sonifying flow around a vertical wind turbine, placing third in WestGrid's Visualize This! 2017 Challenge [20].

\section{CONCLUSION}

A novel method for sonifying CFD data for the purpose of better communicating high frequency fluctuations within simulated intracranial aneurysmal flows is described. This frequency-domain method is intended to augment conventional visualization in order to ease the interpretation of dense spatiotemporal datasets by allowing the user to visually concentrate on one field while listening to another. This method for data 
inspection builds on established analysis of intracranial hemodynamics with a focus on real-time inspection inspired by the existing field of auscultation.

\section{REFERENCES}

[1] K. Valen-Sendstad, K. A. Mardal, and D. A. Steinman, "High-resolution CFD detects high-frequency velocity fluctuations in bifurcation, but not sidewall, aneurysms," J. Biomech., vol. 46, no. 2, pp. 402-407, Jan. 2013. doi: 10.1016/j.jbiomech.2012.10.042.

[2] K. Valen-Sendstad, M. Piccinelli, and D. A. Steinman,"Highresolution computational fluid dynamics detects flow instabilities in the carotid siphon: Implications for aneurysm initiation and rupture?," $J$. Biomech., vol. 47, no. 12, pp. 3210-3216, Sept. 2014.

doi: 10.1016/j.jbiomech.2014.04.018.

[3] N. Varble et al., "Flow instability detected by high-resolution computational fluid dynamics in fifty-six middle cerebral artery aneurysms," J. Biomech. Eng., vol. 138, no. 6, p. 061009, May 2016. doi: 10.1115/1.4033477.

[4] S. Chien, "Mechanotransduction and endothelial cell homeostasis: The wisdom of the cell," Am. J. Physiol. Heart Circ. Physiol., vol. 292, no. 3, pp. H1209-H1224, Mar. 2007. doi: 10.1152/ajpheart.01047.2006.

[5] M. R. Roach, S. Scott, and G. G. Ferguson, "The hemodynamic importance of the geometry of bifurcations in the circle of Willis (Glass model studies)," Stroke: J. Cereb. Circ., vol. 3, no. 3, pp. 255-267, 1972. doi: 10.1161/01.STR.3.3.255.

[6] M. C. Kusko and K. Maselli, "Introduction to cardiac auscultation," in Learning Cardiac Auscultation, London, UK: Springer, 2015, pp. 3-14.

[7] M. J. Mc Loughlin and S. Mc Loughlin, "Cardiac auscultation: Preliminary findings of a pilot study using continuous wave doppler and comparison with classic auscultation," Int. J. Cardiol., vol. 167, no. 2 , pp. 590-591, Jul. 2013. doi: 10.1016/j.ijcard.2012.09.223.

[8] L. N. Sekhar et al., "Acoustic recordings from experimental saccular aneurysms in dogs." Stroke: J. Cereb. Circ., vol. 21, no. 8, pp. 12151221, Aug. 1990. doi: 10.1161/01.STR.21.8.1215.

[9] G. G. Ferguson, "Turbulence in human intracranial saccular aneurysms," J. Neurosurg., vol. 33, no. 5, pp. 485-97, 1970. doi: $10.3171 /$ jns. 1970.33 .5 .0485 .

[10] Y. Kurokawa, S. Abiko, and K. Watanabe, "Noninvasive detection of intracranial vascular lesions by recording blood flow sounds," Stroke: J. Cereb. Circ., vol. 25, no. 2, pp. 397-402, 1994.

doi: 10.1161/01.STR.25.2.397.

[11] B. C. J. Moore, "Frequency difference limens for short-duration tones." J. Acoust. Soc. Am., vol. 54, no. 3, pp. 610-619, 1973. doi: $10.1121 / 1.1913640$.

[12] T. Hermann, A. Hunt, and J. G. Neuhoff, The Sonification Handbook. Berlin: Logos Verlag, 2011.

[13] G. Dubus and R. Bresin, "A systematic review of mapping strategies for the sonification of physical quantities," PloS One, vol. 8, no. 12, p. e82491, 2013. doi: 10.1371/journal.pone.0082491.

[14] Emory University. (2013). AneuriskWeb: The aneurisk dataset repository [Online]. Available: http://ecm2.mathcs.emory.edu/ aneuriskweb

[15] S. Manini and L. Antiga. (2019). The vascular modeling toolkit [Online]. Available: http://www.vmtk.org

[16] M. O. Khan, K. Valen-Sendstad, and D. A. Steinman, "Narrowing the expertise gap for predicting intracranial aneurysm hemodynamics: Impact of solver numerics versus mesh and time-step resolution," Am. J.

Neuroradiol., vol. 36, no. 7, pp. 1310-16, 2015. doi: 10.3174/ajnr.A4263.

[17] M. Mortensen and K. Valen-Sendstad, "Oasis: A high- level/highperformance open source navier-stokes solver," Comput. Phys.

Commun., vol.188, pp. 177-188, Mar. 2015.

doi: 10.1016/j.cpc.2014.10.026.
[18] M. O. Khan et al., "On the quantification and visualization of transient periodic instabilities in pulsatile flows," J. Biomech., vol. 52, pp. 179-182, Feb. 2017. doi: 10.1016/j.jbiomech.2016.12.037.

[19] D. MacDonald. (2018, Feb. 27). Sonification of flow instabilities [Video file]. Available: https://www.youtube.com/watch?

$\mathrm{v}=\mathrm{UmDvnPjnpV4}$

[20] Compute Canada. (2017, Dec. 11). Interactive visualization and sonification of air flow [Video file]. Available: https://vimeo.com/246882338 
HEAT TRANSFER 


\title{
Droplet Impact, Spreading and Freezing on Metallic Surfaces of varying Wettability
}

\author{
Y. Pan, K. Shi, X. Duan, G. F. Naterer \\ Faculty of Engineering and Applied Science, Memorial University of Newfoundland, St. John's, NL, Canada \\ yp8367@mun.ca, kshi@mun.ca, xduan@mun.ca, gnaterer@mun.ca
}

\begin{abstract}
Ice formation and accumulation can lead to operational failure and risks for structures, including power transmission lines, aircraft, offshore platforms, marine vessels, and wind turbines. Liquid repellent and icephobic surfaces can reduce ice accretion and improve asset integrity and safety in harsh environments. There are significant needs to probe how wettability affects the droplet impact, ice nucleation and ice accretion processes on different kinds of micro-structured surfaces. This paper presents experimental results of droplet impact, icing delay time and ice accumulation on metallic surfaces with varying wettability. Several different designs of the hydrophobic surfaces are considered. A commercial hydrophobic coating is also used to enhance liquid repellent features and reduce ice accumulation. The results demonstrated that when the static contact angle increases, the total icing time increases, suggesting desirable icing delays. The total icing time decreases with lower surface temperature, higher impact velocity or smaller droplet diameter.
\end{abstract}

Keywords - superhydrophobic, anti-icing, wetting, freezing, icing-delay time

\section{INTRODUCTION}

In cold and harsh environments, the formation and accumulation of ice may cause significant hazards and hinder the field operations, for example, with power transmission lines, airplanes, offshore platforms, marine vessels, and wind turbine [1-4]. There are many ways to remove ice from structures, for example, with a high-frequency high-voltage short-circuit to melt the ice but it requires the operator to shut off power during de-icing operation [5-7]. Workers use hammers to remove the ice on wellhead platforms and chain bridges. These methods are inefficient, difficult, expensive, and often have safety and environmental issues [8]. In order to achieve a more effective way to reduce ice formation and adhesion, extensive research has been directed to develop hydrophobic metallic surfaces with water repellent and antiicing properties for applications in harsh environments [8, 9$10]$.

Superhydrophobic phenomena were first related to surface roughness by Dettre and Johnson [11]. Subsequently, the unique hydrophobic properties of lotus leaves have become an active research area because of the fundamental interests in wetting and directional flow of water [11]. The lotus effect has various advantages such as water repellent action, small flow resistance, and self-cleaning property from dirt. The static contact angle of a liquid droplet on a smooth surface is related to the following interfacial energies at the three phase contact line,

$$
\cos \theta=\frac{\gamma_{s v}-\gamma_{s l}}{\gamma_{l v}}
$$

where $\theta$ is the static contact angle. $\gamma_{s v}, \gamma_{l v}$, and $\gamma_{s l}$ represent the solid-vapor, liquid-vapor, and solid-liquid interfacial tensions, respectively. The contact angle hysteresis is also a key property to define the surface wettability. It is the difference between the advancing angle and receding angle when the solid surface is tilted, and the droplet starts to roll off [12-14]. The key properties of superhydrophobicity include the static contact angle larger than $150^{\circ}$ and small contact angle hysteresis (less than $10^{\circ}$ ).

For droplet impact dynamics, Ted Mao et al. [15] developed a semi empirical model to predict the maximum spread as a function of the Reynolds number $(\mathrm{Re})$, the Weber number (We), and the static contact angle. Ice nucleation submodel is also considered as heterogeneous ice nucleation at liquid-solid interface and the free-energy barrier $(\Delta G)$ is also estimated by N. H. Fletcher [16].

$$
T_{\text {interface }}=T_{\text {droplet }}+\left(T_{\text {substrate }}-T_{\text {droplet }}\right) \frac{\operatorname{erfc}\left(\frac{h}{2 \sqrt{a_{n s} t_{c}}}\right)}{\operatorname{erfc}\left(\frac{h}{2 \sqrt{a_{w} t_{c}}}\right)}
$$

where $a_{n s}$ and $a_{w}$ are thermal diffusivities of solid-air and water domain, respectively and $t_{c}$ is the total time that the droplet in contact with substrate.

In this paper, experimental studies are conducted to determine the effects of sample surface temperature, droplet impact speed, surface wettability, and droplet diameter on the total icing time of a single water droplet. 


\section{EXPERIMENTAL APPARATUS AND PROCEDURE}

An experimental apparatus was designed to investigate liquid droplet impact and icing on surfaces with varying wettabilities. Fig. 1 shows the experimental setup. It consists of a support stand (Fisher Scientific) with a tilt base (World Precision Instruments) of adjustable inclination angles, a cold plate (AAVID Thermally) that is mounted on the tilt base and controls the temperature of sample surfaces, and a thermal electric cooler (TE Technology). A small copper tube (SigmaAldrich, Stainless steel capillary tubing 1/16 in) is buried for generating the liquid droplets with controllable temperatures. To generate a small water droplet, several needles of different inner diameters were used at the bottom end of the droplet generator tubing. The liquid comes from a syringe pump (Harvard). The cold plate is connected to a thermal bath (Fisher Scientific) which provides control of the surface temperature of the material sample being tested.

With this setup, water droplets are emitted with controlled temperatures impacting on different surfaces under different temperatures. High speed imaging was conducted with a highspeed camera (Vision Research). The high-speed camera was connected to a laptop for video and photo. An LED light and a light diffuser (Edmund Optics) were used to provide light while capturing droplet impact and nucleation processes. The response of the oscillation time, droplet dynamics and total icing time were determined from the video taken by the highspeed camera. The uncertainty analysis of the equipment used is shown in table 1.

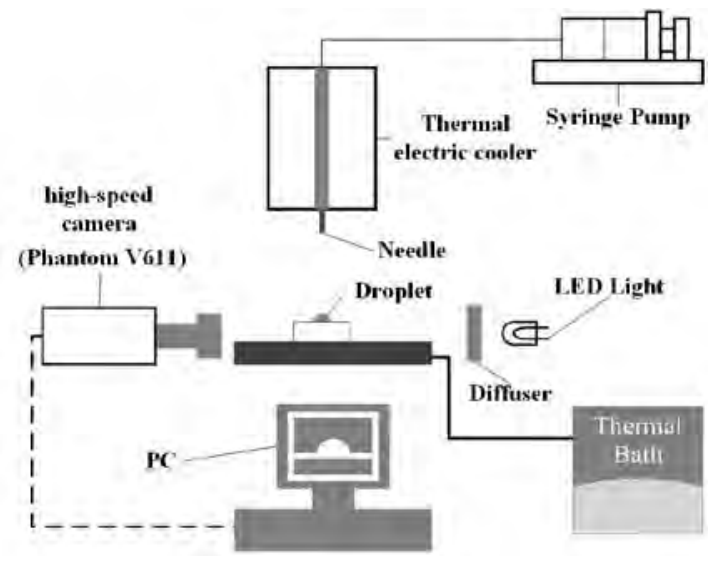

Fig. 1 Liquid droplet impact and icing experimental systems

Table 1. Experimental uncertainties of the experiments

\begin{tabular}{|c|c|c|}
\hline & Operating Range & $\begin{array}{c}\text { Uncertaint } \\
\mathrm{y}\end{array}$ \\
\hline Thermal Electric Cooler & -20 to $80^{\circ} \mathrm{C}$ & $\pm 0.1^{\circ} \mathrm{C}$ \\
\hline FLIR E60 Infrared Camera & -20 to $650^{\circ} \mathrm{C}$ & $\pm 2{ }^{\circ} \mathrm{C}$ \\
\hline $\begin{array}{c}\text { Isotemp Heated Bath } \\
\text { Circulators }\end{array}$ & -25 to $200^{\circ} \mathrm{C}$ & $\pm 0.01^{\circ} \mathrm{C}$ \\
\hline Measure tape & MAX $25^{\prime}$ & $\pm 0.5 \mathrm{~mm}$ \\
\hline Measure ruler & MAX $15 \mathrm{~cm}$ & $\pm 0.5 \mathrm{~mm}$ \\
\hline High Speed Camera & $\begin{array}{c}\text { MAX } 6242 \text { fps with full } \\
\text { resolution }\end{array}$ & $\pm 20 \mathrm{~ns}$ \\
\hline
\end{tabular}

Several machined and coated sample surfaces with various wettabilities were used in the experiment. The contact angles of various samples are between $77.2^{\circ}$ and $145.5^{\circ}$ including hydrophilic and hydrophobic surfaces. The surface material is stainless steel (17-4 PH) for all samples. The smooth sample is a normal stainless-steel surface with no mechanical machining and coating. Some samples have microscale surface structures fabricated by wired electrical discharge machining, laser maching [17], or sandblasting. Other samples are coated with low surface energy material to further increase the surface hydrophobicity. The coating used for the samples was a Metal Repellency Treatment coating from Aculon.

\section{RESULTS AND DISCUSSION}

The droplet dynamics and ice nucleation experiments on a flat surface were conducted under different conditions and captured by high-speed camera. The temperature of the sample surface was varied between $-10^{\circ} \mathrm{C}$ and $-13^{\circ} \mathrm{C}$. The droplet diameters were varied from $1.80 \mathrm{~mm}, 2.82 \mathrm{~mm}$, to $4.11 \mathrm{~mm}$. The droplet impact speeds were varied from $0.77 \mathrm{~m} / \mathrm{s}, 0.99$ $\mathrm{m} / \mathrm{s}$, to $1.17 \mathrm{~m} / \mathrm{s}$. The droplet temperature was kept at $5^{\circ} \mathrm{C}$. The surface wettability was changed from a hydrophilic smooth stainless-steel surface to a hydrophobic textured stainless-steel surface.

To better describe the dynamics of the droplet after impinging on the flat surface, a coordinate system is defined as shown in Fig. 2. The moment at which a water droplet first impacts the sample surface is named as the impact point. The $\mathrm{x}$-axis is parallel to the sample surface and the $\mathrm{y}$-axis is perpendicular to the sample surface, and it is positioned in the middle of the droplet. Fig. 3 clearly indicates a typical droplet impact and nucleation process on a flat smooth surface. Kinetic energy, surface tension, air drag, liquid viscosity, and surface structure play important roles during the droplet impact process. The surface temperature, droplet size, and surface wettability play important roles during the droplet nucleation process. In general, the water droplet goes to a dynamic phase after impacting the sample surface, and the ice nucleation phase (phase change) occurs after the dynamic phase. The dynamic phase contains: a spreading phase (Fig. 3 b-e); a retraction phase (Fig. $3 \mathrm{f}-\mathrm{i}$ ); and a relaxation phase which contains many cycles of spreading and retraction processes. The dynamic phase lasted $334 \mathrm{~ms}$ before moving into an ice nucleation phase.

The impact process of a water droplet $(\mathrm{D}=2.82 \mathrm{~mm})$ on the smooth sample surface at the temperature of $-10^{\circ} \mathrm{C}$ and impact speed at $0.77 \mathrm{~m} / \mathrm{s}$ is shown in Fig. 3. After hitting the sample surface, a lamella was formed from the base of the water droplet, and then a ring was formed. Most of the volume of the droplet stayed in the outside ring instead of inside the lamella (Fig. 3 b-e). At the end of the spreading phase, the water droplet reached its maximum contact diameter which is 5.31 $\mathrm{mm}$ (Fig. 3 e). The total spreading process lasted for $8 \mathrm{~ms}$. The retraction phase started right after the spreading phase (Fig. $3 \mathrm{f}$ i). During the retraction phase, the water droplet started to recoil from the outside ring to the inside lamella. After the relaxation phase which contained a number of cycles of spreading and retraction phases, the droplet became steady and 
reached its equilibrium state for about $334 \mathrm{~ms}$. The ice nucleation phase started after the dynamic phase, and the water droplet took about $12.9 \mathrm{~s}$ to freeze. The droplet freezing process initiated at the liquid-solid interface (Fig. 3 i), and it propagated to the top of the droplet (Fig. $3 \mathrm{k}$ ). A small tip was formed on the top of the droplet at the end of the ice nucleation phase (Fig. 3 1).

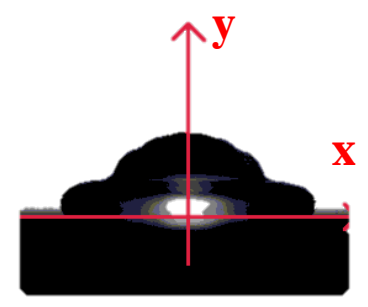

Fig. 2 Coordinate system of droplet impact and movement

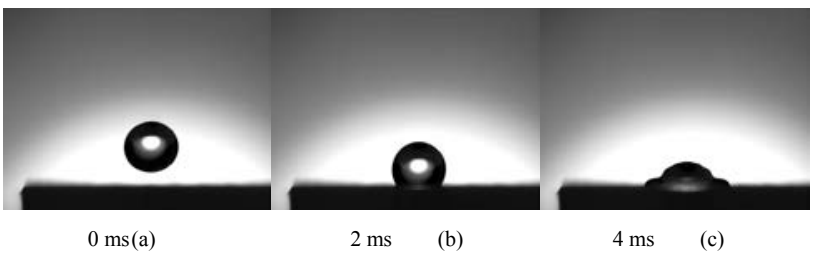

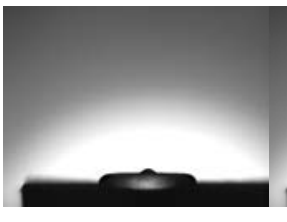

$6 \mathrm{~ms}$

(d)

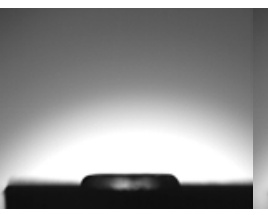

$8 \mathrm{~ms} \quad$ (e)

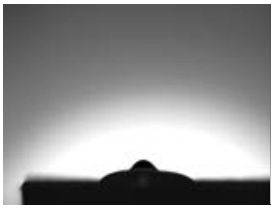

$12 \mathrm{~ms} \quad(\mathrm{~g})$

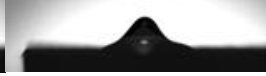

$14 \mathrm{~ms} \quad(\mathrm{~h})$
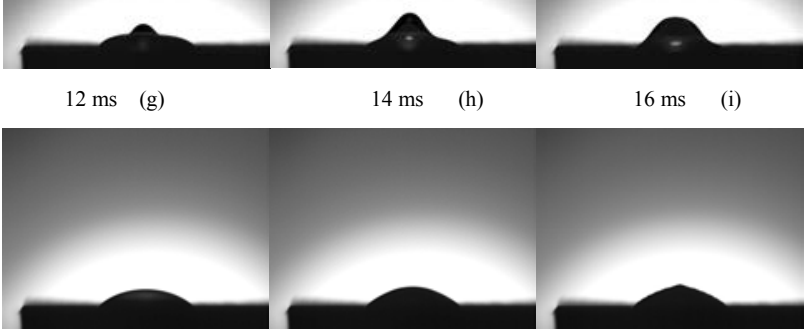

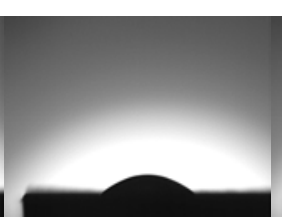

$8.774 \mathrm{~s}$

(k)
$334 \mathrm{~ms}$

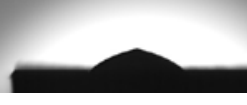

$12.902 \mathrm{~s} \quad(\mathrm{l})$

Fig. 3 Images of water droplet impact on a flat smooth surface $\left(D_{0}=2.82\right.$ $\mathrm{mm}, T_{s}=-10^{\circ} \mathrm{C}, v_{0}=0.77 \mathrm{~m} / \mathrm{s}$, and $T_{d}=5^{\circ} \mathrm{C}$ )

The impact process of a water droplet $(\mathrm{D}=2.82 \mathrm{~mm})$ on a varied channel textured surface [20] with Aculon coating at temperature of $-10^{\circ} \mathrm{C}$ is shown in Fig. 4. It was very similar to the smooth surface case with a lamella outside of the droplet formed right after impact, and then a ring formed. This means a large proportion of the droplet stays in the outside ring instead of the inside lamella (Fig. 4 d). The first spreading process lasted about $6 \mathrm{~ms}$, and the maximum spreading diameter was $4.28 \mathrm{~mm}$ (Fig. $4 \mathrm{~b}-\mathrm{d}$ ). The retraction process started at $8 \mathrm{~ms}$ (Fig. 4 e). Since the coated varied channel textured surface is hydrophobic, the height of the droplet is larger than a smooth surface during the retraction process (Fig. 4 e-h). The dynamic phase lasted about $532 \mathrm{~ms}$. Then the ice nucleation process is initiated and lasts for about $21.3 \mathrm{~s}$ (Fig. $4 \mathrm{i}-\mathrm{m}$ ). The droplet freezing process initiated at the liquid-solid interface (Fig. 4 i), and it propagated to the top of the droplet (Fig. 4 j-1). A small tip was formed on the top of the droplet at the end of the ice nucleation phase (Fig. 4 m). Compared with the total icing time on the smooth surface, the total icing time on this very hydrophobic surface is much longer under the same conditions.
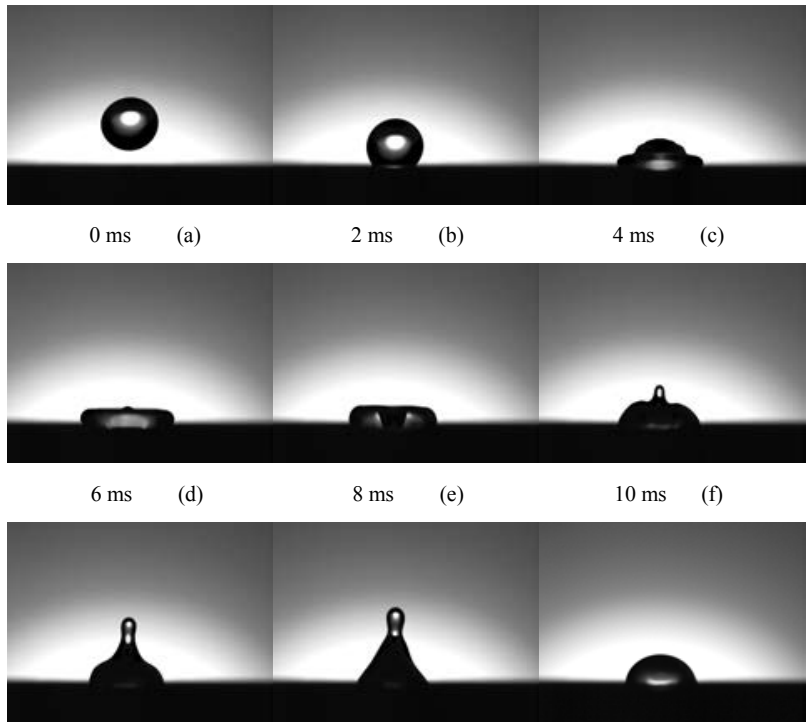
$12 \mathrm{~ms} \quad(\mathrm{~g})$
$14 \mathrm{~ms} \quad$ (h)
$532 \mathrm{~ms} \quad$ (i)

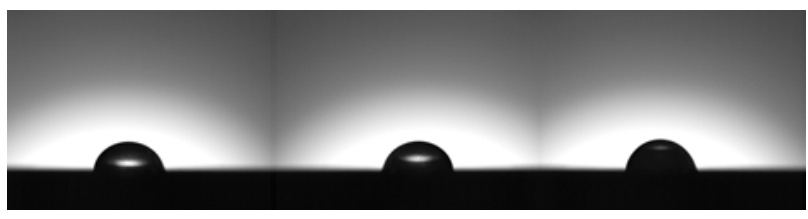

$3.376 \mathrm{~s} \quad$ (j)

$5.694 \mathrm{~s}$

(k)

$12.824 \mathrm{~s}$

(1)

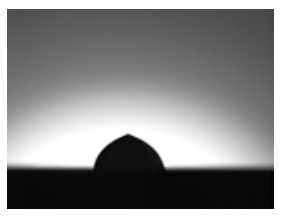

$21.324 \mathrm{~s} \quad(\mathrm{~m})$

Fig. 4: Images of water droplet impact on a flat coated varied channel surface $\left(D_{0}=2.82 \mathrm{~mm}, T_{s}=-10^{\circ} \mathrm{C}, v_{0}=0.77 \mathrm{~m} / \mathrm{s}\right.$, and $\left.T_{d}=5^{\circ} \mathrm{C}\right)$

The droplet nucleation experiment is performed with varying droplet impact speed, droplet diameter, sample surface temperature, and surface wettability. The experiments are performed to find out the relationship between different factors and droplet overall icing times. Five runs of experiments are conducted at each experimental condition. The following figures show the experimental result and analysis.

Fig. 5 indicates how the droplet icing time varies with respect to static contact angle and temperature. As the static contact angle increases, the icing time increases. The icing time decreases with decreasing temperature. Since the water droplet spreads less, oscillates longer, and partially sits on the hydrophobic surface with a low wettability, the contact area at 
solid-liquid interface is much smaller than a hydrophilic surface. The surface energy at the solid-liquid interface is lower when the contact angle is small, so the ice formation initiates earlier on a smooth surface than a hydrophobic surface. The total icing time is much longer on a hydrophobic surface due to a smaller solid-liquid contact area which means a smaller heat transfer rate.

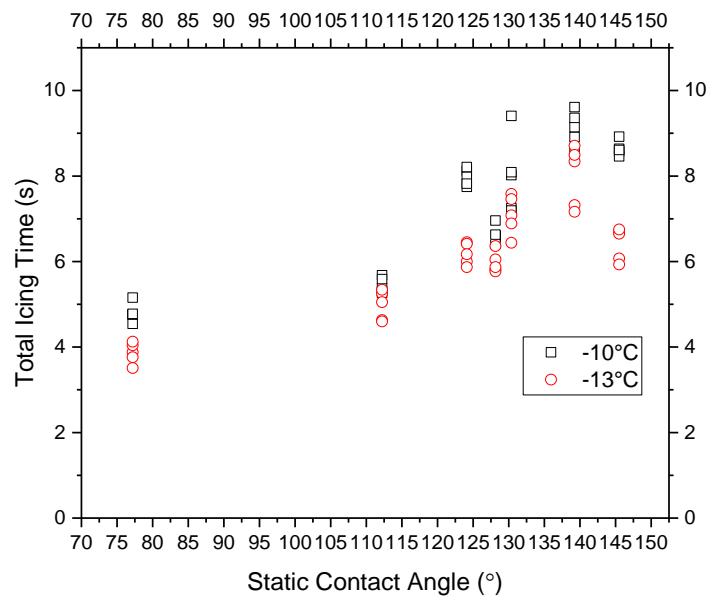

Fig. 5 Droplet icing time vs. Static contact angle at impact speed $=0.77 \mathrm{~m} / \mathrm{s}$ when the surface temperature is $-10{ }^{\circ} \mathrm{C}$ and $-13^{\circ} \mathrm{C}$.

Fig. 6 shows how the water droplet total icing time changes with respect to static contact angle at impact velocity of 0.77 $\mathrm{m} / \mathrm{s}, 0.99 \mathrm{~m} / \mathrm{s}$, and $1.17 \mathrm{~m} / \mathrm{s}$. From the figure, the total icing time decreases as the impact velocity increases. Since the impact velocity increases, the droplet spreads faster and the maximum contact area increases due to higher initial kinetic energy. Since the contact area and time during the dynamic phase increase while increasing the impact velocity, the heat transfer rate from droplet to the surface increases. The droplet may also penetrate into micro-grooves on the hydrophobic surface while increasing the impact velocity. In that case, the droplet penetration would also increase the heat transfer rate from droplet to the surface.

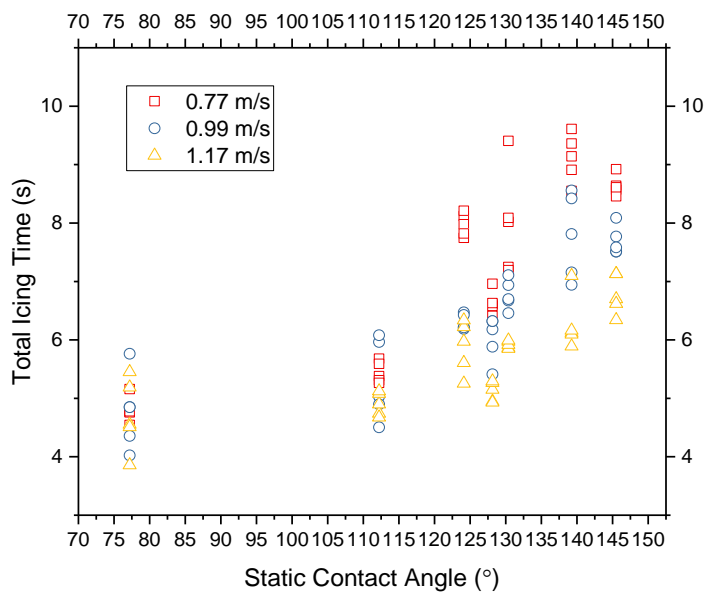

Fig. 6 Droplet icing time vs. Static contact angle at $-10{ }^{\circ} \mathrm{C}$ when the impact speed is $0.77 \mathrm{~m} / \mathrm{s}, 0.99 \mathrm{~m} / \mathrm{s}$, and $1.17 \mathrm{~m} / \mathrm{s}$.
Fig. 7 shows the relationship between the total icing time and droplet diameter which are $1.80 \mathrm{~mm}, 2.82 \mathrm{~mm}$, and 4.11 $\mathrm{mm}$. When increasing the droplet diameter and surface hydrophobicity, the droplet icing time increases. Since the volume of the droplet increases when increasing the diameter, more time and overall heat transfer are needed to fully freeze the droplet.

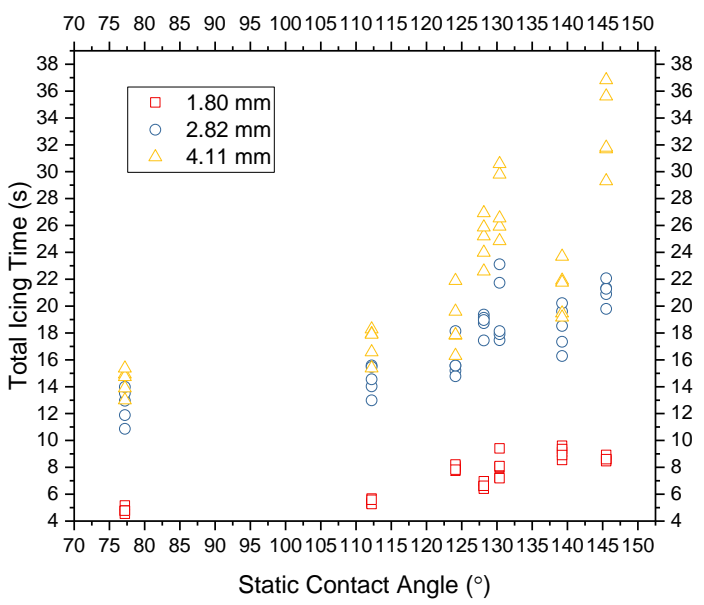

Fig. 7: Droplet icing time vs. static contact angle at $-10^{\circ} \mathrm{C}$ when the droplet diameter is $1.80 \mathrm{~mm}, 2.82 \mathrm{~mm}$, and $4.11 \mathrm{~mm}$.

\section{CONCLUSION}

This paper investigated the effects of surface wettability, droplet impact speed, surface temperature, and droplet diameter on the total icing time on surfaces. The experimental results demonstrated that when the surface wettability decreases, the total icing time increases. Since the water droplet spreads less, oscillates longer, and partially sits on surfaces with low wettability, the contact area at the solid-liquid interface is much smaller which means a lower heat transfer rate. The total icing time decreases with decreasing temperature, increasing impact velocity or decreasing droplet diameter. The water droplet spreads more and oscillates longer with increasing droplet size because the larger droplet contains more kinetic energy. Since the contact area and time during the dynamic phase increase while increasing the impact velocity, the heat transfer rate from droplet to the surface increases. So increasing droplet diameter, decreasing droplet impact speed, or increasing surface hydrophobicity can decrease or delay the droplet icing time.

\section{ACKNOWLEDGMENT}

Financial support for this research by Petroleum Research Newfoundland \& Labrador (PRNL) and the Natural Sciences and Engineering Research Council of Canada (NSERC) are gratefully appreciated.

\section{REFERENCES}

[1] Sojoudi, H., Wang, M., Boscher, N. D., Mckinley, G. H. and Gleason, K. K. "Durable and scalable icephobic surfaces: similarities and distinctions from superhydrophobic surfaces". Soft Matter, 2016 Vol 12, pp. 1938-1963. https://doi.org/10.1039/C5SM02295A 
[2] Bahadur, V., Mishchenko, L., Hatton, B., Taylor, A. J., Aizenberg, J., and Krupenkin. T.. 2011. "Predictive Model for Ice Formation on Superhydrophobic Surfaces" Langmuir 27, pp. 14143-14150. https:/ doi.org/10.1021/la200816f

[3] Yamada, Y., Ikuta, T., Nishiyama, T., Takahashi, K., and Takata, Y., "Droplet Nucleation on a Well-Defined Hydrophilic -Hydrophobic Surface of $10 \mathrm{~nm}$ Order Resolution", Langmuir 2014, Vol 30, pp. 14532-14537. https://doi.org/10.1021/la503615a

[4] Civil Aviation Authority. Aircraft Icing Handbook, 2000.

[5] Volat, C. and Farzaneh, M. "De-icing/Anti-icing Techniques for Power lines: Current Methods and Future Direction.” IWAIS XI, Montreal, June 2015 .

[6] Sullivan, C.R., Petrenko, V. F., McCurdy, J. D. and Kozliouk, V. "Breaking the ice: de-icing power transmission lines with highfrequency, high-voltage excitation". IEEE Industry Applications Magazine, Vol. 9, pp. 49-54. https://doi.org/10.1109, MIA.2003.1227872

[7] H. Liu, X. Gu, and W. Tang. "Icing and Anti-Icing of Railway Contact Wires". Reliability and Safety in Railway. 2012.

[8] Meuler, A. J., Smith, J. D., Varanasi, K. K., Mabry, J. M., Mckinley, G. H., and Cohen, R. E. "Relationship between Water Wettability and Ice Adhesion". ACS Appl. Mater. Interfaces, 2010, 2(11), pp. 3100-3110. https://doi.org/10.1021/am1006035

[9] Mishchenko, L., Hatton, B., Bahadur, V., Taylor, A., Krupenkin, T., and Aizenbery, J., 2010. "Design of Ice-free Nanostructured Surfaces Based on Repulsion of Impacting Water Droplets", ACS Nano 4(12), pp. 7699-7707.https://doi.org/10.1021/nn102557p
[10] Yoshimitsu, Z., Nakajima, A., Watanabe, T. and Hashimoto, K. "Effects of Surface Structure on the Hydrophobicity and Sliding Behavior of Water Droplets". Langmuir 2002, 18, 5818-5822. https:/ doi.org/10.1021/la020088p

[11] Johnson, R. E., Jr.; Dettre, R. H. "In Wetting of Low Energy Surfaces", Marcel Dekker: New York, NY, 1993; pp. 2-71.

[12] Wang, S., Liu, K., Yao, X. and Jiang, L. "Bioinspired Surfaces with Superwettability: New Insight on Theory, Design and Applications". American Chemical Society, 2015, 115, 8230-8293.

[13] Malavasi, I., Veronesi, F., Caldarelli, A., Zani, M., Raimondo, M. and Marengo, M. "Is a Knowledge of Surface Topology and Contact Angles Enough to Define the Droplet Impact Outcome?" Langmuir, 2016, 32, 6255-6262. https://doi.org/10.1021/acs.langmuir.6b01117

[14] Shi, Z., Liu, Z. Song, H. and Zhang, X. "Prediction of Contact Angle for Hydrophobic Surface Fabricated with Micro-Maching Based on Minimum Gibbs Free Energy". Applied Surface Science, 2016, 364 , 597-603. https://doi.org/10.1016/j.apsusc.2015.12.199

[15] Mao, T., Kuhn, D. C. S. and Tran, H. "Spread and Rebound of Liquid Droplets Upon Impact on Flat Surfaces". AICHE Journal, 1997, Vol. 43, No. 9. https://doi.org/10.1002/aic.690430903

[16] Hobbs, P. V. "Ice Physics", Oxford Classic Texts in the Physical Science.

[17] Cui, C., Collier, B., Duan, X., Poduska, K., "hydrophobic Steel Surfaces with Micron-scale and Sub-micron Structures from laser Fabrication", 2nd Thermal and Fluid Engineering Conference, April 2-5, 2017, Las Vegas, NV, US. https://doi.org/10.1615/TFEC2017.fnt.017613 


\title{
Preparation and Properties of Nanoparticle-enhanced Composite Phase Change Material with Ceramic Porous Media
}

\author{
Runfeng $\mathrm{Li}^{1,2}$, Yang Zhou ${ }^{2}$, Xili Duan ${ }^{1}$ \\ ${ }^{1}$ Faculty of Engineering and Applied Science, Memorial University of Newfoundland, St. John's, Canada \\ ${ }^{2}$ School of Mechanical, Electronic and Control Engineering. Beijing Jiaotong University, Beijing, China \\ runfengl@mun.ca, yzhou@bjtu.edu.cn, xduan@mun.ca
}

\begin{abstract}
Nanoparticle-enhanced tailing-paraffin composite phase change material (NCPCM) is fabricated by spontaneous melt infiltration. Industrial waste-iron tailing is used as raw material to prepare ceramic porous carrier with a foam-gel casting method. By adjusting the fabrication parameters, optimal NCPCM properties are obtained with paraffin content of $70 \% \sim 88 \%$ and thermal conductivity of $0.351 \sim 0.490$ $\mathrm{W} /(\mathrm{m} \cdot \mathrm{K})$, which is nearly $200 \%$ the thermal conductivity of paraffin wax. After 25 melting/solidification cycles, the nanoparticles remain well dispersion with overall stability in the composite phase change material, and the thermal conductivity slightly decreased from 0.349 to $0.317 \mathrm{~W} / \mathrm{m} \cdot \mathrm{K}$. With multiple melting and solidification cycles, a low weight loss of 2.3 7.8 wt.\% is demonstrated. The strength of ceramic frame is found to have a direct effect on the weight loss. Compared with exisiting nanoparticle-enhanced phase change material, the new NCPCM shows significantly improved thermal conductivity and better nanoparticle stability due to its ability to prevent nanoparticles from disposition.
\end{abstract}

Keywords: Nanoparticle; Phase Change Materials; Ceramic Porous Media; Thermophysical Properties; Dispersion.

\section{INTRODUCTION}

Energy storage plays an important role in meeting the growing global energy demand. Phase change materials (PCMs), with high storage density and isothermal process in phase change process, are often applied in latent heat energy storage systems where energy is stored in the form of latent heat during melting and recovered during solidification of the PCMs [1].

Paraffin wax is a reliable option for PCM in various engineering applications, such as storage of solar thermal energy, industrial waste heat recovery and insulation building material [2]. But the low thermal conductivity of paraffin causes low heat transfer rate during charging and discharging cycles [3]. Therefore, efforts have been made to enhance the rate of melting/freezing by utilizing different methods. An effective method of improving the melting/freezing rate is to add highly thermal conductive nanoparticles inside the PCM.
This nanoparticle-enhanced phase change material (NePCM) has been studied in a shell-and-tube thermal energy storage system [4]. Khodadadi and Hosseinizadeh [5] observed that NePCM exhibits higher heat release rate when compared to the conventional PCM partly due to the simultaneous increase of the thermal conductivity and reduction of the latent heat. Although nanoparticles can improve the thermal conductivity of the PCM, the stability issue presents a major challenge. It's very difficult to achieve long term uniform dispersion of nanoparticles in paraffin wax since nanoparticle sedimentation is almost unavoidable [1].

Another heat transfer enhancing technique involves composite phase materials (CPCM) incorporating porous ceramics. This technique can efficiently improve the thermal conductivity and maintain original shape of the PCM even when the wax is melted. Several authors reported the thermal enhancement of CPCMs. Zhao et al.[6] performed an experimental investigation to characterize the phase change processes (both melting and solidification) of paraffin wax inside copper metal foam. The porous materials, through capillary and surface tension forces, keep the PCM in stable shape at liquid phase [7]. Qian, et al. [8] fabricated shapestabilized composite phase change material (ss-CPCM) based on $\mathrm{SiO}_{2}$ materials. $\mathrm{SiO}_{2}$ acts as the carrier matrix to provide structural strength and prevent the leakage of melted PCM. Their results show that the use of porous medium enhances the melting/solidification process faster than pure PCM without a porous medium.

NCPCM is a new way that combines the advantages of NePCM and CPCM. Although the transport processes of nanofluids in a porous medium have been studied in recent years, the fabricating technology and thermal properties of NCPCM were barely reported. The key point is that the ceramic must have micron-sized pores and high porosity, good wettability and chemical stability with NePCM. Iron ore tailings (IOT) have been generated in steel production as an industrial waste all over the world, which caused economic, environmental and health-related problems [9]. Using IOT as a carrier to fabricate NCPCM is an efficient method for improving thermal properties, keeping the shape of meted paraffin, reducing industrial pollution and drastically 
decreasing the manufacturing cost. This paper presents the preparation and analyzes the thermal conductivity of NCPCM.

\section{MATERIALS AND PROCEDURE OF PREPARATION}

\section{A. Preparation of Tailing Porous Ceramic Media}

Tailing was obtained from iron mines of Miyun area in Beijing. Tailing porous ceramics were fabricated by a foam-gel casting method. First, iron tailing slurries with a solid loading of $45 \mathrm{wt} . \%$ were prepared by mixing ball-milling IOT powders, deionized water, Acrylamide, N,N'-Methylenebisacrylamide and ammonium polyacrylate for $15 \mathrm{~h}$. After that, Sodium dodecyl sulfate and lauryl alcohol were added into the slurries, followed by high-speed stirring to acquire foamed slurries. Tetraacetylethylenediamine and Ammonium persulfate were slowly added to the foamed slurries and mixed adequately. Then the slurries were poured into molds and gelled in the air for $10 \mathrm{~min}$ to get green porous bodies. Finally, the green bodies were dried and sintered at different temperatures of 1070, 1080, $1090,1100,1110$ and $1120^{\circ} \mathrm{C}$ for $7 \mathrm{~h}$.

\section{B. Preparation of NCPCM}

Technical grade paraffin wax with melting temperature 56 ${ }^{\circ} \mathrm{C}$ and graphene nanoplatelets were supplied by Sigma-Aldrich Canada. The porous ceramic and $99 \mathrm{~g}$ paraffin were heated to $150^{\circ} \mathrm{C}$ and $90^{\circ} \mathrm{C}$, respectively. Then $1 \mathrm{~g}$ of graphene nanoparticles were added into the melted paraffin and the mixture was stirred for $30 \mathrm{~min}$.

The mixture is under ultrasonic treatment for $5 \mathrm{~min}$ after stirring to prevent nanoparticles from aggregation. Then, the porous ceramics were put into a beaker full of melted paraffin to make sure that the melted paraffin covers the ceramics. The infiltration process needs $5 \mathrm{~min}$. After that, the beaker is let to cool down to $60{ }^{\circ} \mathrm{C}$ and the samples are taken out to dry in the air. Figure 1 shows pictures of samples of the porous ceramic, CPCM and NCPCM. Due to the black color of graphene nanoparticles, the color of NCPCM is much darker than the other samples.

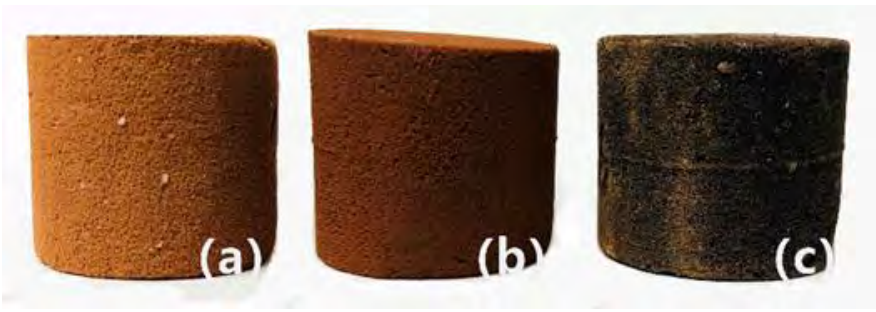

Figure 1. Pictures of porous medium (a), CPCM (b) and NCPCM (c)

\section{PROPERTIES OF POROUS CERAMIC AND NCPCM}

\section{A. Properties of Ceramic and Infiltration Result}

The porosity of porous ceramic is a key property to decide NePCM content in NCPCM which is measured by the Archimedes method in distilled water. Compressive strength was measured by a WDW-100E testing machine with a crosshead loading speed of $0.5 \mathrm{~mm} / \mathrm{min}$, using cylindrical specimens with a diameter of $20 \mathrm{~mm}$ and a height of $20 \mathrm{~mm}$. Phase composition of the sintered materials was identified by X-ray diffraction (D/MAX-III, Rigaku, Japan). Eight specimens were used to acquire average property. Pore size distribution was analyzed by the mercury intrusion method in an Auto Pore IV 9510 instrument.

Physical properties of the tailing porous ceramics sintered at different conditions are listed in Table 1, and the effects of sintering temperature on the compress strength are shown in Fig.2. Six samples were tested for each temperature point.

Table 1 Properties Of The Tailing Porous Ceramics

\begin{tabular}{|c|c|c|c|}
\hline $\begin{array}{c}\text { Sintering } \\
\text { temperature } \\
\left({ }^{\circ} \boldsymbol{C}\right)\end{array}$ & $\begin{array}{c}\text { Bulk density } \\
\left(\mathbf{g} / \text { cm }^{\mathbf{3}}\right)\end{array}$ & $\begin{array}{c}\text { Apparent } \\
\text { Porosity }(\%)\end{array}$ & Shrinkage (\%) \\
\hline 1070 & $0.39 \pm 0.01$ & $88.8 \pm 0.1$ & $3.81 \pm 0.43$ \\
\hline 1080 & $0.38 \pm 0.01$ & $87.4 \pm 0.4$ & $5.59 \pm 0.31$ \\
\hline 1090 & $0.36 \pm 0.01$ & $87.0 \pm 0.8$ & $8.20 \pm 1.42$ \\
\hline 1100 & $0.29 \pm 0.02$ & $85.5 \pm 0.1$ & $9.46 \pm 0.23$ \\
\hline 1110 & $0.48 \pm 0.01$ & $82.6 \pm 0.2$ & $10.74 \pm 0.31$ \\
\hline 1120 & $0.76 \pm 0.01$ & $71.6 \pm 0.4$ & $20.67 \pm 0.45$ \\
\hline
\end{tabular}

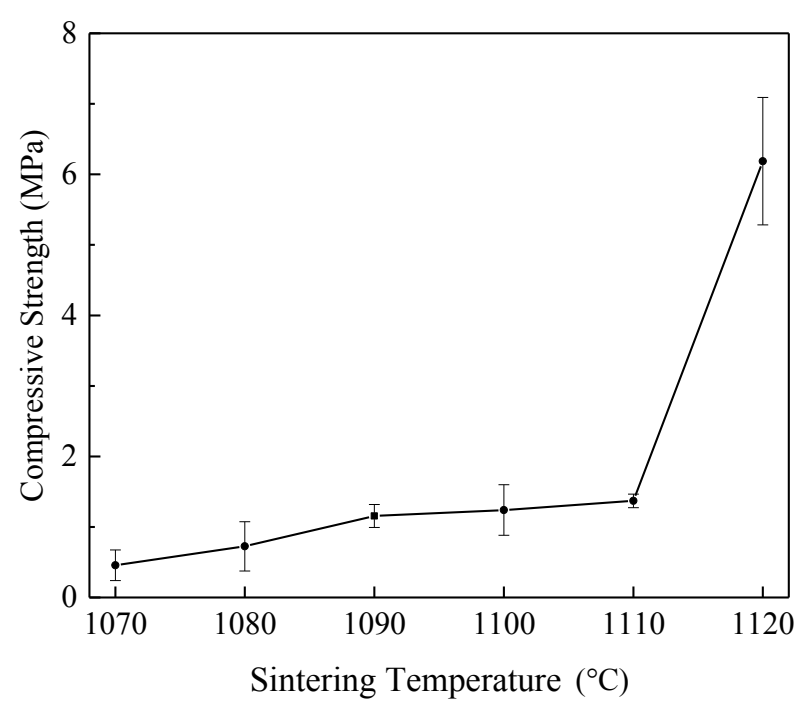

Figure 2. Relationship between sintering temperature and compressive strength

As temperature increased from 1070 to $1120{ }^{\circ} \mathrm{C}$, bulk density and shrinkage of ceramic increased from 0.39 to 0.76 $\mathrm{g} / \mathrm{cm}^{3}$ and 3.81 to $20.67 \%$ respectively, and the porosity decreased from $89 \%$ to $71 \%$. With the increase of sintering temperature, atomic diffusion kinetic energy increases, which enhances the sintering activity and makes the tailings particles closer. $1110{ }^{\circ} \mathrm{C}$ is likely a point where liquid phase is significantly generated, while higher temperatures lead to drastic shrinkage of the samples, resulting in increased porosity of the porous ceramics. Higher porosity of porous ceramics means bigger energy storage density of the NCPCM.

As we can see in Fig 2, with higher sintering temperatures, the compressive strength of porous ceramics increased due to the much stronger interconnection among quartz grains and framework of more glass phase generated in higher 
temperature. Based on the Gibson equation, the following relationship between porosity and strength was correlated from the experimental data:

$$
\sigma_{1}=129 \times(1-\Gamma)^{2.53}
$$

where $\Gamma$ is porosity, $\sigma_{1}$ is compression strength of porous ceramics.

When sintering temperature reached $1080{ }^{\circ} \mathrm{C}$ and held for 3 hours, porosity, bulk density and compression strength were $86.6 \%, 0.31 \mathrm{~g} / \mathrm{cm}^{3}$ and $0.75 \mathrm{MPa}$ respectively, which can satisfy the needs of NCPCM.

Figure 3 shows the phase composition of tailing porous ceramics sintered at $1080{ }^{\circ} \mathrm{C}$. The mineralogical composition of the porous samples determined by X-ray Diffraction (XRD) shows main crystalline phases like quartz $\left(\mathrm{SiO}_{2}\right)$, diopside $\left(\mathrm{CaMgSi}_{2} \mathrm{O}_{6}\right)$, augite $\left(\mathrm{Ca}(\mathrm{Mg}, \mathrm{Fe}, \mathrm{Al})(\mathrm{Si}, \mathrm{Al})_{2} \mathrm{O}_{6}\right)$. Anorthite $\left(\mathrm{CaAl}_{2} \mathrm{Si}_{2} \mathrm{O}_{8}\right)$ and hematite $\left(\mathrm{Fe}_{2} \mathrm{O}_{3}\right)$ were identified. This phase has good erosion resistance and forms the main mineral framework of the tailing porous ceramics, which are responsible for the physical properties and mechanical strength of porous ceramics.

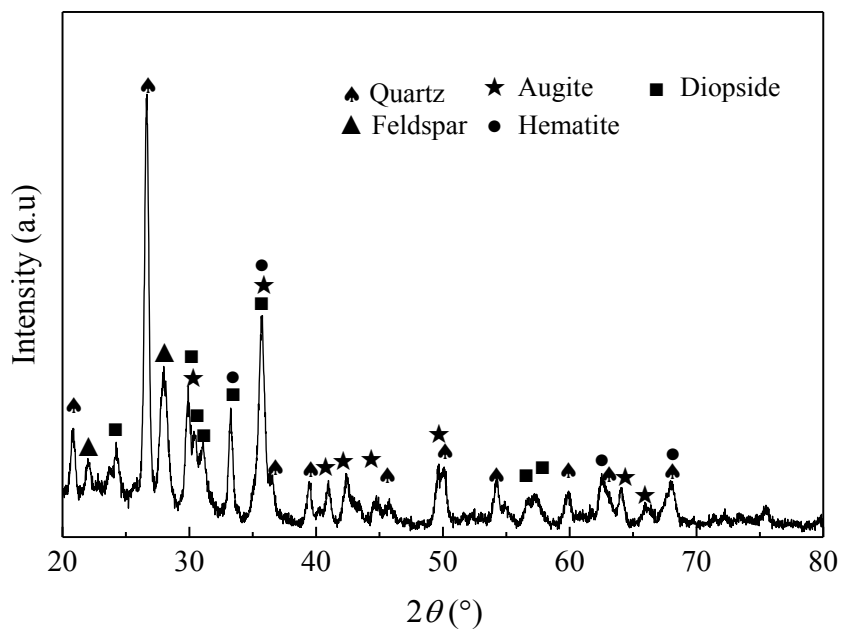

Figure 3. XRD patterns of porous ceramics sintered at $1080{ }^{\circ} \mathrm{C}$

Figure 4 shows the pore size distribution of porous tailing ceramics sintered at $1080{ }^{\circ} \mathrm{C}$ for 3 hours. It shows a bimodal distribution of the pore size of the porous tailing ceramic, with a weak peak at about $1.4 \mu \mathrm{m}$ and a strong peak at $45 \mu \mathrm{m}$, while the pore diameter was mainly in the range of $20-80 \mu \mathrm{m}$. According to previous work, when the radius of pores is smaller than $100 \mu \mathrm{m}$, the capillary force is larger than that from atmospheric pressure, which helps to eliminate air from the samples in the infiltration process. Also, when the pore radius is $3 \sim 35 \mu \mathrm{m}$, the differential pressure $\Delta \mathrm{P}$ changes from $6 \mathrm{MPa}$ to $1 \mathrm{MPa}$, which efficiently prevents the melted paraffin from leaking out of the porous medium.

Table 2 shows filling fractions of the prepared CPCM and NCPCM. The values indicate percentage of pores that are occupied by liquid paraffin (which has a density of $0.74 \mathrm{~g} / \mathrm{cm}^{3}$ ) and liquid nanoparticle enhanced paraffin (which has a density of $0.73 \mathrm{~g} / \mathrm{cm}^{3}$ due to the low density of graphene nanoparticles).
As we can see in Table 2, different sintering temperatures lead to different pore sizes of the samples, which causes variation in capillary forces and therefore changes in the infiltration efficient. Compared with the CPCM, adding graphene nanoparticles didn't seem to change the wettability of paraffin on the surface of the ceramics.

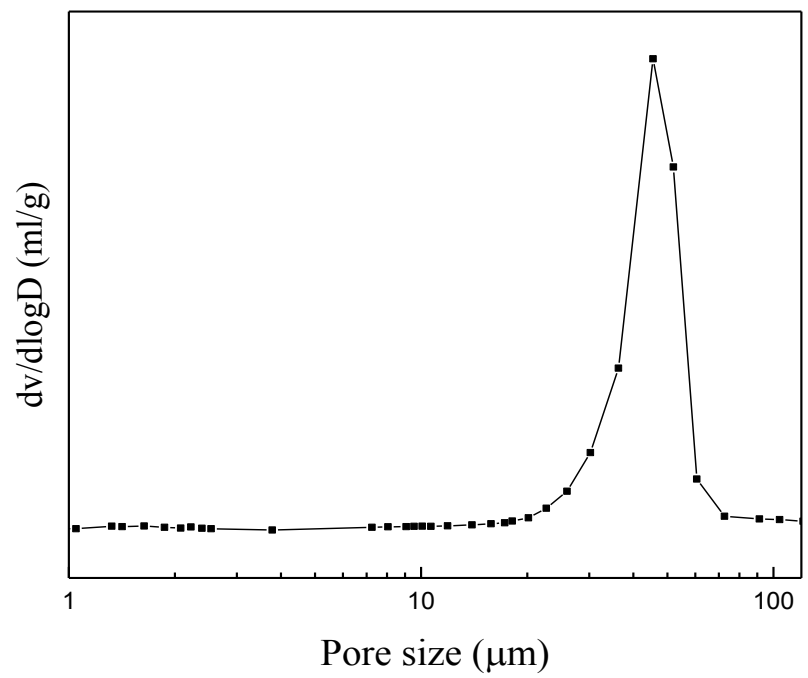

Figure 4. Pore size of sample sintered at $1080{ }^{\circ} \mathrm{C}$

Table 2 INFILTRATION OF CPCM AND NCPCM

\begin{tabular}{|c|c|c|c|}
\hline $\begin{array}{c}\text { Sintering } \\
\text { Temperature } \\
\left({ }^{\circ} \boldsymbol{C}\right)\end{array}$ & $\begin{array}{c}\text { Porosity of } \\
\text { Porous } \\
\text { Ceramic }(\%)\end{array}$ & $\begin{array}{c}\text { Filling Fraction of } \\
\text { CPCM }(\%)\end{array}$ & $\begin{array}{c}\text { Filling Fraction of } \\
\text { NCPCM }(\%)\end{array}$ \\
\hline 1070 & 89 & 98.08 & 99.21 \\
\hline 1090 & 86 & 98.91 & 99.08 \\
\hline 1110 & 82 & 98.13 & 97.30 \\
\hline
\end{tabular}

\section{B. Thermal Conductivity}

The thermal conductivities of iron ore tailing were measured on $5 \times 5 \times 3 \mathrm{~mm}^{3}$ machined specimens at room temperature, using the Thermal Transport Option (TTO) of Physical Properties Measurement System (PPMS, Model 6000, Quantum Design, USA). The NCPCMs, i.e., tailing ceramic/ NePCM composite materials, were measured with a KD2 Pro Thermal Properties Analyzer (Decagon Devices, USA). The thermal conductivity of the tailing ceramic and the NePCM is $1.41 \mathrm{~W} / \mathrm{m} \cdot \mathrm{K}$ and $0.282 \mathrm{~W} / \mathrm{m} \cdot \mathrm{K}$, respectively.

Figure 5 shows thermal conductivities of NCPCM, CPCM, $\mathrm{NePCM}$ and the base PCM (paraffin wax). With combined advantages of CPCM and NePCM, the new NCPCM shows a significant increase of thermal conductivity to $0.428 \mathrm{~W} / \mathrm{m} \cdot \mathrm{K}$, as compared to $0.229 \mathrm{~W} / \mathrm{m} \cdot \mathrm{K}$ of the base PCM. It was confirmed that the micron-sized pores can prevent nanoparticles from deposition. Also, the tailing ceramic has higher thermal conductivity than paraffin, which also helps improve the thermal properties.

\section{Multiple Melting Solidification Cycles}

Weight loss of samples was tested after 1, 5, 10, 15, 20 and 25 melting / solidification cycles. Figure 6 shows cross- 
sectional view of a sample after these cycles. As we can see in Fig. 6, there is no observable color change in the crosssectional view of the NCPCM, suggesting that the nanoparticles were still well dispersed in the PCM without deposition (otherwise there will be dramatic change of color gradient with dark colored area from nanoparticle deposition). These results demonstrate that the NCPCM technique can effectively prevent the nanoparticles from disposition.

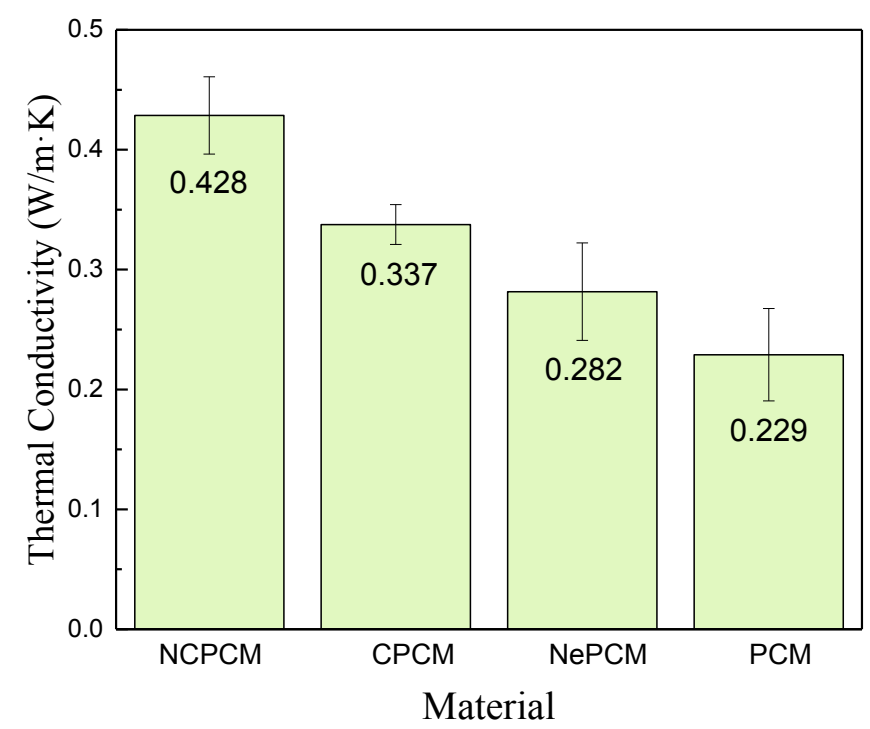

Figure 5. Thermal conductivities of NCPCM, CPCM, NePCM and pure PCM

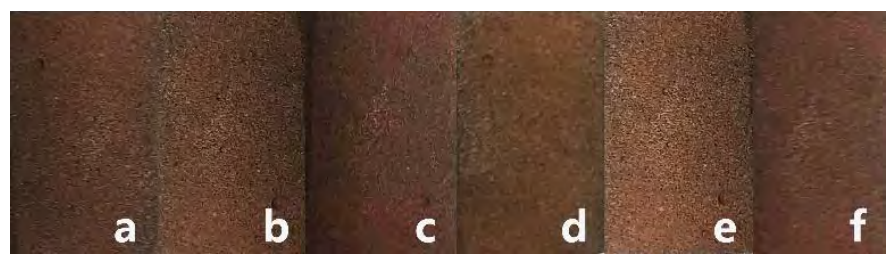

Figure 6. The section of sample after 1(a), 5(b), 10(c), 15(d), 20(e) and 25(f) times of melting / solidification cycles

Figure 7 shows weight losses of two NCPCM samples after $1,5,10,15,20$ and 25 melting / solidification cycles respectively.

As the number of melting/ solidification cycles increased, the mass of samples slightly decreased. With air fills into the sample after paraffin leaking, the thermal conductivity decreased from 0.349 to $0.317 \mathrm{~W} / \mathrm{m} \cdot \mathrm{K}$. This can be explained by evaporation and leak of paraffin. Comparing the two curves in the Figure 7, one can find that the leakage and weight loss are greatly affected by the porosity. As the porosity increases, more and more glass phase forms on internal walls of the ceramics promoting the strength of samples. Stronger wall of the pores can decrease the impact from liquid paraffin in melting and solidification and prevent the paraffin from leaking.

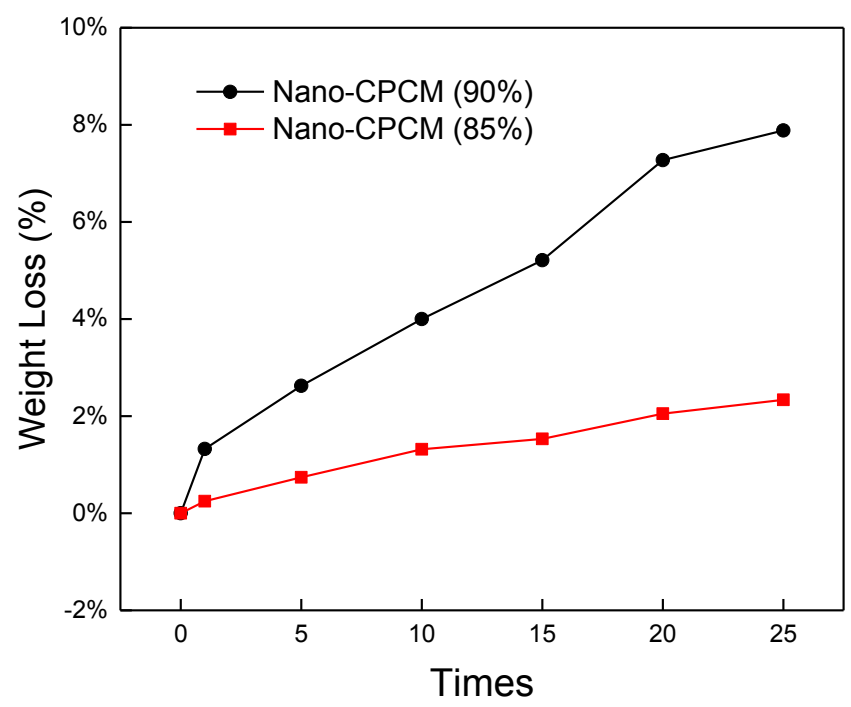

Figure 7. NCPCM Sample material weight loss with $90 \%$ and $85 \%$ porosity after multiple melting / solidification cycles

\section{CONCLUSIONS}

Nanoparticle enhanced tailing-paraffin composite phase change material (NCPCM) was successfully prepared using iron ore tailing, graphene nanoparticles and paraffin as raw materials with a foam-gel casting method and spontaneous infiltration technique. With higher sintering temperature, the porosity of ceramic decreased from $89 \%$ to $71 \%$ and the bond between particles become stronger as well. It was found that paraffin dispersed with graphene nanoparticles has good wettability with the tailing ceramic. It efficiently prevents leaking of the melted paraffin out from the porous medium. NCPCM significantly increases the thermal conductivity of $\mathrm{PCM}$ from $0.229 \mathrm{~W} / \mathrm{m} \cdot \mathrm{K}$ to $0.428 \mathrm{~W} / \mathrm{m} \cdot \mathrm{K}$. In addition, the porosity of the ceramic porous media has a significant effect on weight loss which was only $2.3 \mathrm{wt} . \%$ when porosity is smaller than $85 \%$. NCPCM with tailing porous ceramic demonstrates good mechanical integrity, with significantly improved and controllable thermal properties.

\section{ACKNOWLEDGMENT}

This work was supported by Beijing Jiaotong University, Memorial University of Newfoundland, and a Discovery Grant from the Natural Sciences and Engineering Research Council (NSERC) of Canada. The first author (R. Li) also gratefully acknowledges financial support from the China Scholarship Council.

\section{REFERENCES}

[1] R. Hossain, S. Mahmud, A. Dutta and I. Pop, "Energy storage system based on nanoparticle-enhanced phase change material inside porous medium," Int J Therm Sci., vol. 91, pp. 49-58, 2015. https:/ doi.org/10.1016/j.ijthermalsci.2014.12.023

[2] F. Chen and M. Wolcott, "Polyethylene/paraffin binary composites for phase change material energy storage in building: A morphology, thermal properties, and paraffin leakage study," Sol Energ Mat Sol C., vol. 137 , pp. 79-85, 2015. https://doi.org/10.1016/j.solmat.2015.01.010 
[3] S.F. Hosseinizadeh, A.A.R. Darzi and F.L. Tan, "Numerical investigations of unconstrained melting of nano-enhanced phase change material (NEPCM) inside a spherical container." Int J Therm Sci.. vol. 51, pp. 77-83, 2012. https://doi.org/10.1016/j.ijthermalsci.2011.08.006

[4] M. Parsazadeh and X. Duan, "Numerical and statistical study on melting of nanoparticle enhanced phase change material in a shell-and-tube thermal energy storage system." Applied Thermal Engineering, vol. 111 pp. 950-960, 2017. https://doi.org/10.1016/j.applthermaleng.2016.09.133

[5] J.M. Khodadadi and S.F. Hosseinizadeh, "Nanoparticle-enhanced phase change materials (NEPCM) with great potential for improved thermal energy storage," Int Commun Heat Mass. Transfer, vol. 34. pp. 534-543, 2007. https://doi.org/10.1016/j.icheatmasstransfer.2007.02.005

[6] C.Y. Zhao, W. Lu and Y. Tian, "Heat transfer enhancement for thermal energy storage using metal foams embedded within phase change materials (PCMs)," Solar Energy, vol. 84, pp. 1402-1412, 2010. https:/ doi.org/10.1016/j.solener.2010.04.022
[7] Y.L. Li, J.H. Li, W.W. Feng, X. Wang and H.G. Nian,, "Design and Preparation of the Phase Change Materials Paraffin/ Porous A12O3@Graphite Foams with Enhanced Heat Storage Capacity and Thermal Conductivity." Acs Sustain Chem Eng.. vol. 5 , pp. 7594-7603, 2012. https://doi.org/10.1021/acssuschemeng.7b00889'

[8] T.T. Qian, J.H. Li, H.W. Ma and J. Yang, "Numerical investigations of unconstrained melting of nano-enhanced phase change material (NEPCM) inside a spherical container." Int J Therm Sci.. vol. 51, pp. 77-83, 2012. https://doi.org/10.1016/j.ijthermalsci.2011.08.006

[9] M. Edraki, T. Baumgartl, E. Manlapig, D. Bradshaw, D.M. Franks and C.J. Moran, "Designing mine tailings for better environmental, social and economic outcomes: a review of alternative approaches," J Clean Prod., vol. 84, pp. 411-420, 2014. https://doi.org/10.1016

i.jclepro.2014.04.079 


\section{Effect of Dispersing Copper Oxide Nanoparticles to RT-35 Filled Circular Thermal Energy Storage System}

\author{
Soroush Ebadi*, Mohammadhossein Hajiyan, Syeda Tasnim \\ School of Engineering, University of Guelph, \\ Guelph, Canada \\ *sebadi@uoguelph.ca
}

\author{
Amir Abbas Aliabadi, Shohel Mahmud ${ }^{\psi}$ \\ School of Engineering, University of Guelph, \\ Guelph, Canada \\ *smahmud@uoguelph.ca
}

\begin{abstract}
Phase Change Materials (PCMs) have been widely used in Latent Heat Thermal Energy Storage Systems (LHTES) to store and release thermal energy. However, PCMs suffer from their low thermal conductivities, which leads to longer melting (charging) or solidification (discharging) time. Dispersing highly conductive nanoparticles into PCM is one of the methods to improve the thermal conductivity of the PCM. In this paper, we have experimentally and numerically investigated the effect of dispersing Copper Oxide nanoparticles into RT-35 filled a vertical cylindrical Thermal Energy Storage (TES) system. The TES system is consisted of two vertical cylindrical enclosures, which are attached to a thick acrylic plate. One of the enclosures is filled with pure PCM and the other one is filled with nano-PCM. The setup is insulated from the bottom and isothermally heated from its sides by using a constant temperature bath. To study more about the thermal and velocity fields, melting fraction and stored energy, a numerical simulation is conducted by using COMSOL Multiphysics software. It is concluded that although adding nanoparticles to PCM improves the melting rate, it is lowering the amount of energy stored inside the TES system.
\end{abstract}

\section{Keywords: Thermal energy storage (TES); Latent heat; Phase change material (PCM); nano- PCM}

\section{INTRODUCTION}

The increase in the amount of greenhouse gasses especially Carbon dioxide caused by burning fossil fuels has caused a serious environmental concern known as global warming. On the other hand, the increase in the worldwide population and industrial units have forced energy suppliers to search for alternate energy sources (i.e. renewable energy) and or use of energy efficiently. Thermal Energy Storage (TES) systems have developed with the aim of storing thermal energy effectively and release that energy in the demand time. TES systems are divided into three main groups including: (i) Sensible Heat TES system, (ii) Latent Heat TES system and, (iii) Thermo-Chemical TES system. However, among these three groups, LHTES system is more attractive. LHTES systems use PCMs to store or release thermal energy which offers two main advantages: (i) higher capacity of storing energy (i.e. 5-14 times higher than sensible heat TES) and (ii) smoother temperature fluctuations during the operation [1,2]. A wide variety of PCMs are available with different melting temperatures (Tm) which have been used for both cooling and heating purposes. Paraffin waxes, hydrated salts and organic compounds (e.g. Coconut oil) are the most available types of PCMs. Although PCMs seem to be a proper material for store/release of thermal energy, they suffer from a low thermal conductivity. This disadvantage leads to longer melting/solidification time, which affect the performance of the LHTES system [3]. Several methods have been proposed to enhance the thermal conductivity of PCMs such as (i) dispersing highly conductive nanoparticles into the PCM, which is the main purpose of this study, (ii) inserting metal fins, and (iii) the use of conductive porous medium [4]. Different weight fractions/volume fractions of nanoparticles can be dispersed in to the PCM to increasing its thermal conductivity. However, in practical, sedimentation is an issue caused by using high weight/volume fractions of nanoparticles $[5,6]$. Different configuration of PCM container such as rectangular, cylindrical (both horizontal/vertical), and spherical can be used. Cylindrical can be installed both vertically and horizontally according to a specific application/ occupied space [7].

In this paper, the main aim of study is to investigate the melting process of a nano-PCM filled in a vertical cylindrical enclosure. By surveying the literature, it can be found that a considerable number of researchers have studied the use of vertical cylindrical enclosures filled with nano-PCM. Wu et al. [8] performed a numerical study on the melting process of Paraffin enhanced with Copper nanoparticles filled in a cylindrical enclosure which was heated from the bottom. It was found that the melting rate was $13.1 \%$ improved by using $1 w t$. 
$\%$ of nanoparticles. Sciacovelli et al. [9] numerically studied the melting of paraffinic PCM with copper nanoparticles. In their investigation, the nano-PCM was isothermally heated by placing an inner tube at the center of cylindrical enclosure. It was reported that by using $4 \%$ volume fraction of nanoparticles, the melting time was decreased by $15 \%$. Das et al. [10] numerically studied the melting process of n-eicosane enhanced with carbon allotropes. The enclosure was isothermally heated placing an inner pipe at the center. It was concluded that by using $1 \%$ volume fraction of single-walled carbon nanotubes, the melting time melting time was decreased by $15 \%$. The melting time was decreased up to $25 \%$ with the use of $1 \%$ volume fraction of graphene. An experimental study on melting process of 1-dodeconal enhanced with carbon nanotubes was performed by Zeng et al. [11]. In their experiment, the cylindrical enclosure was insulated from the surroundings and heated from the bottom. It is reported that by dispersing the carbon nanotubes, melting rate was degraded due to the increase of viscosity and decrease in the natural convection. Fan et al. [12] experimentally investigated the effect of adding Graphene nanoplatelets in to tetradecanol on the melting rate. The cylindrical enclosure was isothermally heated from the bottom. It was reported that by using $3 \mathrm{wt} . \%$ of nanoparticles at the temperature of $55^{\circ} \mathrm{C}$, a $8 \%$ decrease in the melting time is observed.

According to the literature, the melting process of a nanoPCM inside a vertical cylindrical enclosure, insulated from the bottom, and isothermally heated from the sides has not been extensively investigated. In this work, an experimental and numerical investigation are performed to study the effect of adding Copper oxide nanoparticles into RT-35 with the melting temperature $\left(\mathrm{T}_{\mathrm{m}}\right)$ in a range of $34{ }^{\circ} \mathrm{C}-36{ }^{\circ} \mathrm{C}$. To do so, an experimental setup is built to visualize and compare the melting process of pure PCM and nano-PCM. Moreover, a COMSOL simulation is developed to compare the energy stored in PCM and nano-PCM.

\section{EXPERIMENTAL INVESTIGATION}

\section{A. Experimental setup}

The primary objective of the experimental work is to visualize and compare the melting process of PCM and nanoPCM. The experimental setup is consisted of two transparent acrylic pipe with the inner diameter of $4.4 \mathrm{~cm}$, a transparent water bath equipped with temperature regulator, a circulation pump, and a digital camera to capture the melting pattern periodically. Two cylindrical acrylic pipes are vertically attached to a thick plastic plate (thickness $=3.5 \mathrm{~cm}$ ) with the center-to-center distance of $13 \mathrm{~cm}$. The cylindrical pipes are thermally insulated from the bottom and to avoid any pressure at the top of PCM/nano-PCM, it is kept open from the top. A schematic of the experimental setup is shown in Fig.1.

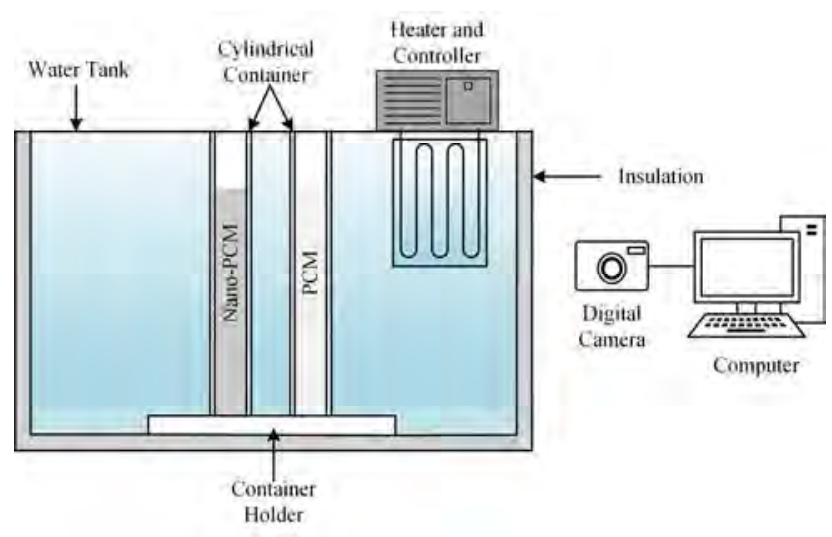

Fig.1. A schematic of the experimental setup

\section{B. Nano-PCM preparation}

One of the main challenges associated with the use of nano-PCM is the sedimentation of nanoparticles at the bottom of enclosure after several cycles of operation. Therefore, preparation of the nano-PCM is an important step for the experimental work. At the first step, the required amount of RT-35 and Copper oxide nanoparticles to have a $0.2 \mathrm{wt} . \%$ nano-PCM was weighed by a precise balance. It should be mentioned that dispersing Copper oxide nanoparticles into RT-35 results to a dark black fluid. Thus, to have a clear melting visualization, low amount of nanoparticles is used. Then, the mixture was heated up to $60{ }^{\circ} \mathrm{C}$ and stirred by using a magnetic stirrer for 24 hours. To ensure the homogeneity of mixture and release the trapped air inside the nano-PCM, an ultrasound sonicator was used for two hours. At the next step, the nano-PCM was kept in a bath with the temperature of $45^{\circ} \mathrm{C}$ for three hours (more than the time required for melting experiment) to see the amount of sedimentation. After ensuring the homogeneity of mixture, the nano-PCM was solidified gradually at the room temperature (i.e., $\mathrm{T}_{0=}$ $23{ }^{\circ} \mathrm{C}$ ). Solidification at the temperatures close to the $\mathrm{T}_{\mathrm{m}}$ requires a longer time, however, it provides the advantages of having less trapped air inside the nanoPCM and avoiding the sub-cooling effect. Table.1 shows the thermophysical properties of RT-35 and Copper Oxide nanoparticles.

Table 1. Properties of RT-35 and $\mathrm{CuO}$ nanoparticles

\begin{tabular}{|c|c|c|}
\hline Property & RT-35 [13] & Copper oxide[14] \\
\hline$\beta(1 / \mathrm{K})$ & $6 \times 10^{-4}$ & $85 \times 10^{-7}$ \\
\hline$h_{s f}(\mathrm{~kJ} / \mathrm{kg})$ & 170 & - \\
\hline$k(\mathrm{~W} / \mathrm{m} \mathrm{K})$ & 0.2 & 18 \\
\hline$\mu(\mathrm{Pa} . \mathrm{s})$ & 0.023 & - \\
\hline$c_{p}(\mathrm{~kJ} / \mathrm{kg} \mathrm{K})$ & 2 & 0.54 \\
\hline$\rho\left(\mathrm{kg} / \mathrm{m}^{3}\right)$ & 815 & 6500 \\
\hline
\end{tabular}




\section{Experimental procedure}

Several steps were taken to start the experiment. First, a predetermined amount of molten Pure PCM and nano-PCM was poured in cylindrical pipes at the same height $(\mathrm{H}=8.3$ $\mathrm{cm})$ representing Rayleigh number of $10^{7}$. Then, the $\mathrm{PCM} /$ nano-PCM were gradually solidified by keeping the setup at the room temperature. Next, the temperature of the constant bath was set to $45^{\circ} \mathrm{C}$. To ensure the accuracy of the thermal regulator, a T-type OMEGA thermocouple was placed inside the bath and monitored the temperature. The Cylindrical TES system enclosures were suddenly placed inside the bath to initiate the melting process. A digital camera was used to record the melting pattern periodically (i.e. every five minutes) during melting process.

\section{NUMERICAL INVESTIGATION}

\section{A. Numerical model}

To study the effect of dispersing nanoparticles to pure PCM on the thermal fields, melting fraction and energy stored, a numerical model with the same geometry as the experimental setup was developed by using COMSOL Multiphysics. The model is insulated from the bottom, isothermally heated from its sides, and filled with PCM/nanoPCM at the height of $\mathrm{H}$. The liquid phase of PCM/nano-PCM is assumed as a Newtonian incompressible fluid. A 2-D view of the numerical model is shown below.

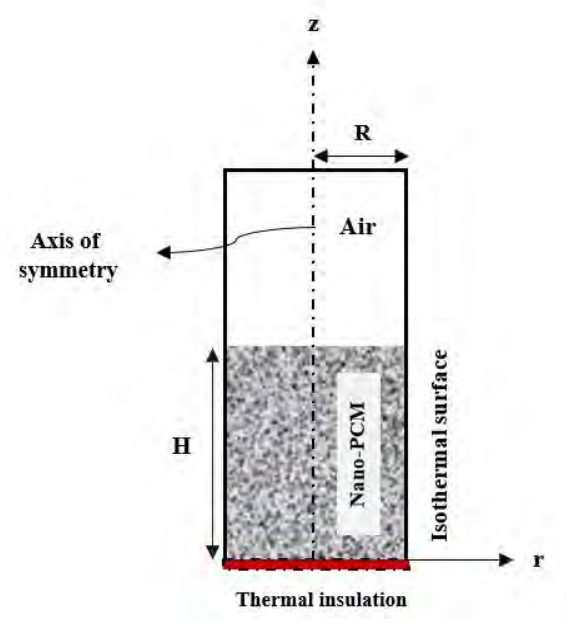

Fig. 2. A 2-D view of the numerical model

The governing equations representing the conservation of mass, momentum and transferred energy are presented below [15]:

$$
\text { Continuity equation : } \frac{\partial u}{\partial r}+\frac{u}{r}+\frac{\partial w}{\partial z}=0
$$

where $u$ and $w$ are the components of velocity in r-direction and $\mathrm{z}$ - direction, respectively.

Energy equation:

$$
\frac{\partial T}{\partial t}+u \frac{\partial T}{\partial r}+w \frac{\partial T}{\partial z}=\frac{k}{\rho C_{p}}\left[\frac{1}{r} \frac{\partial}{\partial r}\left(r \frac{\partial T}{\partial r}\right)+\frac{\partial^{2} T}{\partial z^{2}}\right]
$$

where $t, K, \rho$ and $C_{p}$ represent time, thermal conductivity, density and specific heat at constant pressure, respectively.

Momentum (z-direction):

$$
\frac{\partial w}{\partial t}+u \frac{\partial w}{\partial r}+w \frac{\partial w}{\partial z}=\frac{1}{\rho} \frac{\partial p}{\partial z}+\frac{\mu}{\rho}\left(\frac{\partial^{2} w}{\partial r^{2}}+\frac{1}{r} \frac{\partial w}{\partial r}+\frac{\partial^{2} w}{\partial z^{2}}\right)+g \beta\left(T-T_{m}\right)
$$

Momentum(r-direction):

$$
\frac{\partial u}{\partial t}+u \frac{\partial u}{\partial r}+w \frac{\partial u}{\partial z}=-\frac{1}{\rho} \frac{\partial p}{\partial r}+\frac{\mu}{\rho}\left(\frac{\partial^{2} u}{\partial r^{2}}+\frac{1}{r} \frac{\partial u}{\partial r}-\frac{u}{r^{2}}+\frac{\partial^{2} u}{\partial z^{2}}\right)
$$

where $\mu, g, \beta$ and $T$ represent viscosity, gravitational acceleration and coefficient of thermal expansion and temperature, respectively. The amount of stored energy is one of the main parameters for designing a LTES system which can be determined by Eq. 5 [16].

$$
E=\int_{0}^{t} \dot{Q}(t) d t
$$

In this equation, $\hat{Q}(t)$ is the transferred heat during the melting process which can be determined by Eq. 6 .

$$
\dot{Q}(t)=(\pi d) \int_{0}^{H}\left(-k \frac{\partial T}{\partial r}\right) d z+(2 \pi) \int_{0}^{R}\left(-k \frac{\partial T}{\partial z}\right) r d r
$$

Where $d$ and $k$ are the inner diameter of the circular pipe and thermal conductivity of the nano-PCM respectively.

Dispersion of nanoparticles to PCM results to new properties such as density, viscosity, thermal conductivity, and heat capacity. The thermophysical property relationships for the nano-PCM can be found in [17]. Moreover, as the change in the phase of the nano-PCM occurs within a transition stage, the properties of the nano-PCM are affected by a transition temperature range (i.e. $T=T_{m}-\Delta T$, and $T=T_{m}+\Delta T$ ). The thermophysical properties of the nano-PCM at the transition 
stage can be found in [18]. In addition, a Gaussian function is used to approximate the heat capacity during the phase change process [19].

\section{B. Grid size and time step independency test}

To test the mesh size and time intervals independency, simulations were carried out in different steps. First, the melting fraction was calculated by using three different mesh sizes including (i) 6965 elements, (ii) 15062 elements and (iii) 33905 elements. The result of this test is shown in Fig.3.

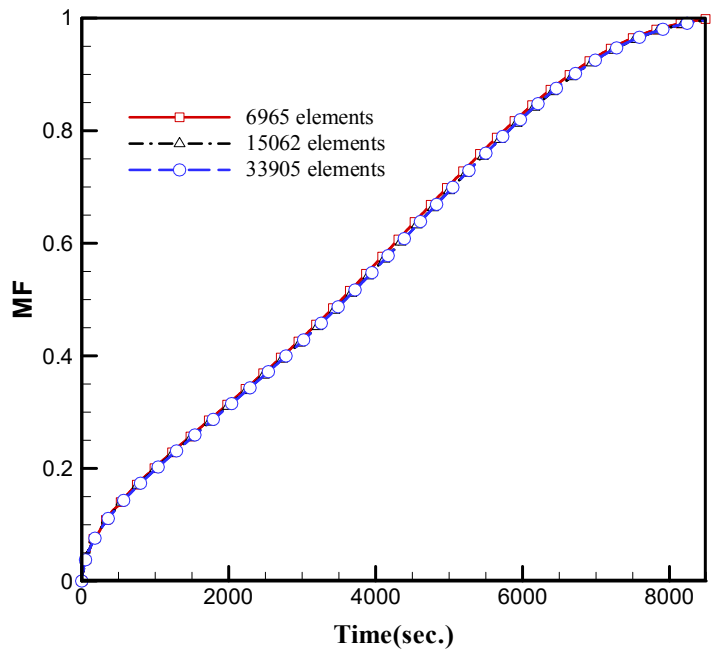

Fig. 3. Grid size independency test

As it can be seen from Fig. 3, the values of melting fraction for different meshes are almost identical. Hence, to obtain the proper accuracy and less simulation time, the mesh quality with 15062 elements was selected. At the next step, the time step independency is examined. In this test, melting fraction was calculated by using three different simulations with different time steps. Table 2 shows the different time steps used in these series of tests.

Table 2. Time intervals used to examine the time step independency

\begin{tabular}{|c|c|c|c|}
\hline \multirow{2}{*}{$\begin{array}{c}\text { Time zone } \\
\text { (s) }\end{array}$} & \multicolumn{3}{|c|}{ Time step } \\
& \multicolumn{3}{|c|}{$(\mathrm{s})$} \\
\hline & Test 1 & Test 2 & Test 3 \\
\hline 0 to 10 & 0.01 & 0.1 & 0.005 \\
\hline 10 to 100 & 0.1 & 1 & 0.05 \\
\hline 100 to 10000 & 10 & 100 & 5 \\
\hline
\end{tabular}

Fig.4 presents the result of these tests. As it can been seen, there is a slight difference in the values of melting fraction resulted by these three time steps. Therefore, to have both accuracy and less simulation time, the simulations were carried out by using the time interval in Test 2.

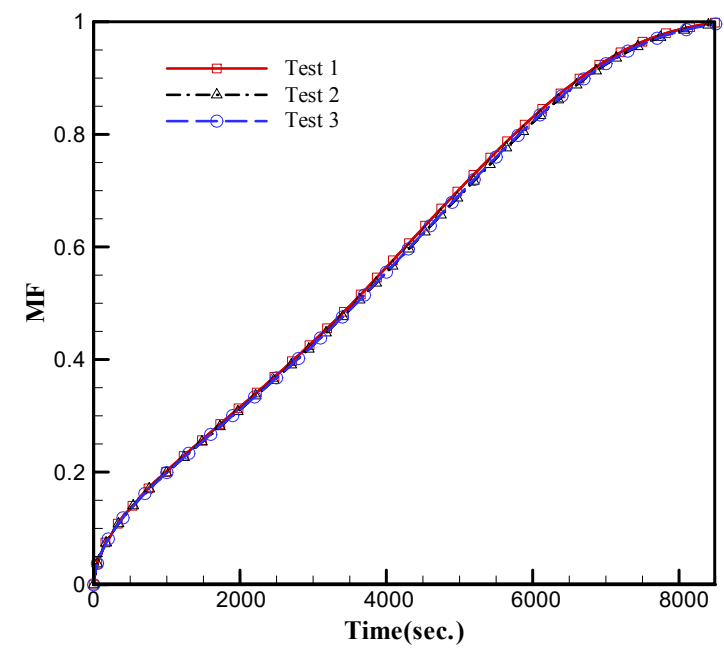

Fig. 4. Time step independency test

\section{Validation with experimental work}

Figure 5 presents a melting visualization comparison between numerical results and experimental work for 0.2 wt.\% of nano-PCM. To do so, some selected results obtained from numerical and experimental are compared. In general, a good agreement is achieved. However, there are some discrepancies in melting pattern, which may be due to the two main factors. First, the air trapped inside the nano-PCM in the experimental work was seen to cause a non-uniform melting at the times that trapped air was released. The other major factor is neglecting of the thermal resistance of the acrylic pipe in the numerical model. As a result, more heat could be transferred between hot wall and the nano-PCM during numerical simulation. 


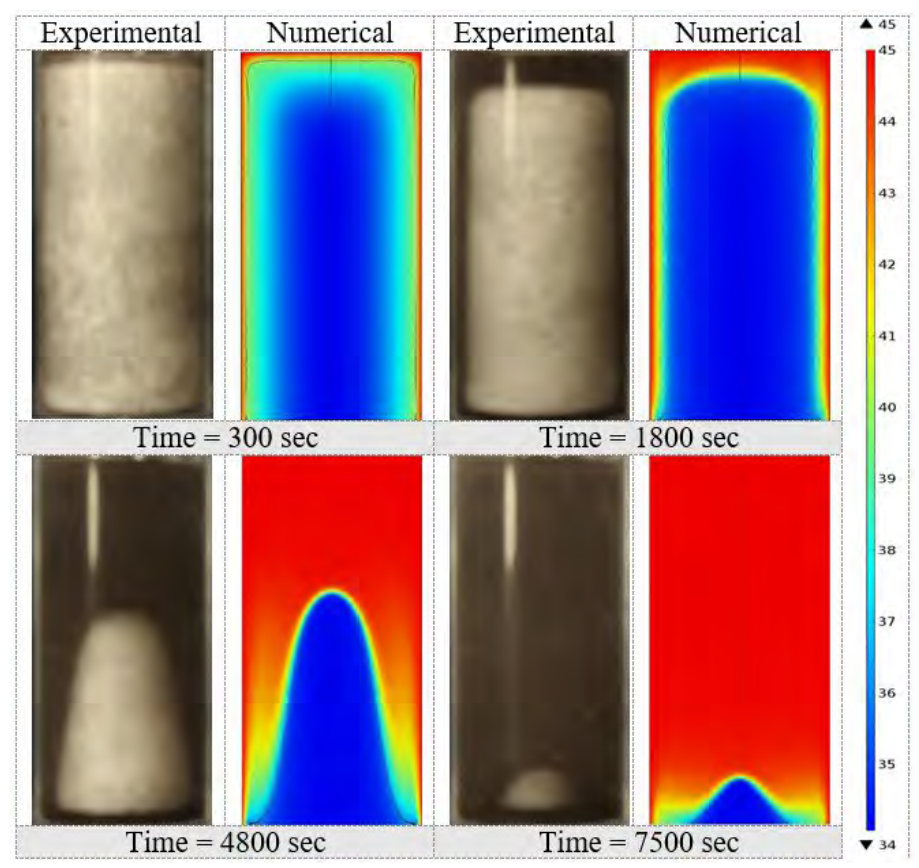

Fig. 5. Validation of numerical model with experimental work

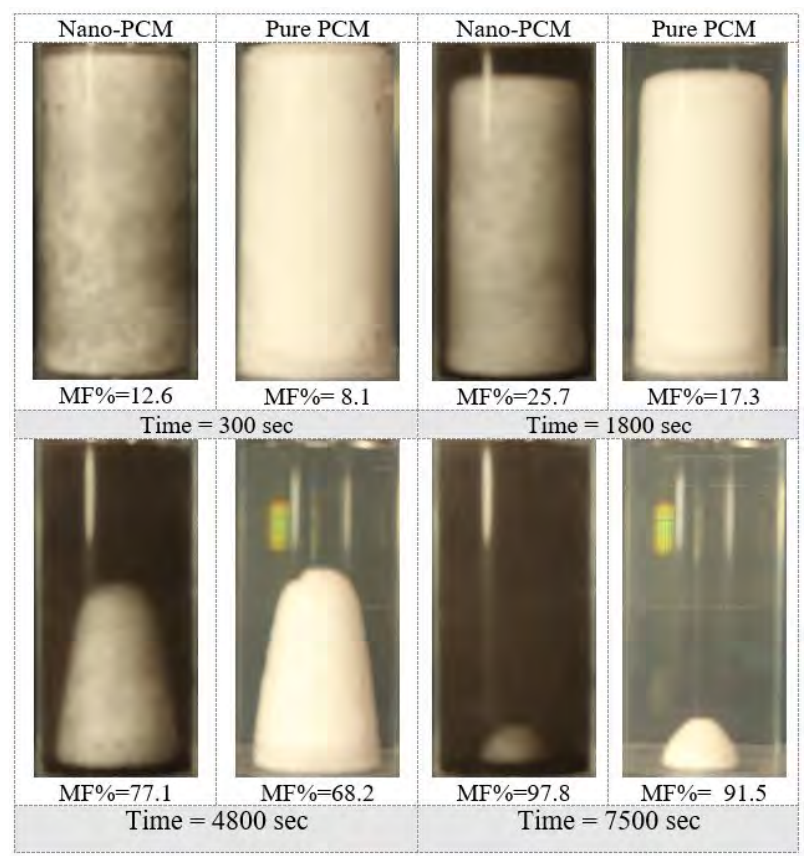

Fig. 6. Visualization of melting pattern for nano-PCM and pure PCM

\section{B. Energy Stored}

\section{A. Melting pattern visualization}

In this section a comparison of melting pattern between pure PCM and Nano-PCM is presented. Some selected results are presented in figure 6 . After a few minutes of initiating the experiment, a thin layer of molten PCM/nano-PCM appeared which lead to a solid-liquid interface. At the early stages of melting process (up to $300 \mathrm{~s}$ ), conduction was the dominant mode of heat transfer since the solid-liquid interface is parallel to the lateral walls. As time furthered, the buoyancy force started to dominate the viscous force and natural convection started to establish. This phenomenon can be clearly observed from the remaining shape of the solid PCM/nanoPCM. At the later stages of melting process (i.e. after 3600s), convection became the dominant mode of heat transfer which resulted to a dome shape solid $\mathrm{PCM} /$ nano-PCM. As it can be seen from the figure 6 , an improvement in the melting rate is observed by using 0.2 wt. \% nano-PCM. To assist the discussion, the melting fractions for both PCM and nano-PCM at different times was calculated from the digital photos by using a software called Grafula. Calculation of melting fraction from digital image was also used in other published works in the literature such as [20].

Figure 7 presents the stored energy during the melting process for both the nano-PCM and the pure PCM cases. At the beginning of melting process, the temperature difference between hot lateral walls of the circular enclosure and cold $\mathrm{PCM} /$ nano-PCM is more than the later stages of melting process. As a result, the slope of energy stored is higher at the early stages (i.e. up to 600s) than other times. From this point onward, the rate of energy-stored decreases until it reaches to zero by reaching to the thermal equilibrium. The amount of energy stored during the melting process mainly depends on the specific heat capacity and the latent heat capacity of nanoPCM/PCM. As it can be seen from the thermophysical relations of the nano-PCM presented in [21], adding nanoparticles to the PCM at the liquid phase increases the heat capacity, while, it decreases the latent heat capacity of the PCM. Therefore, it is expected to have a lower amount of energy stored in the nano-PCM which will be further degraded by adding higher weight/volume fractions of nano-particles. The amount of this decrease in the energy stored is not significant in this work due to the use of low weight fractions of nanoparticles in the experimental work. However, this phenomenon is more apparent in Groulx's numerical study [19]. 


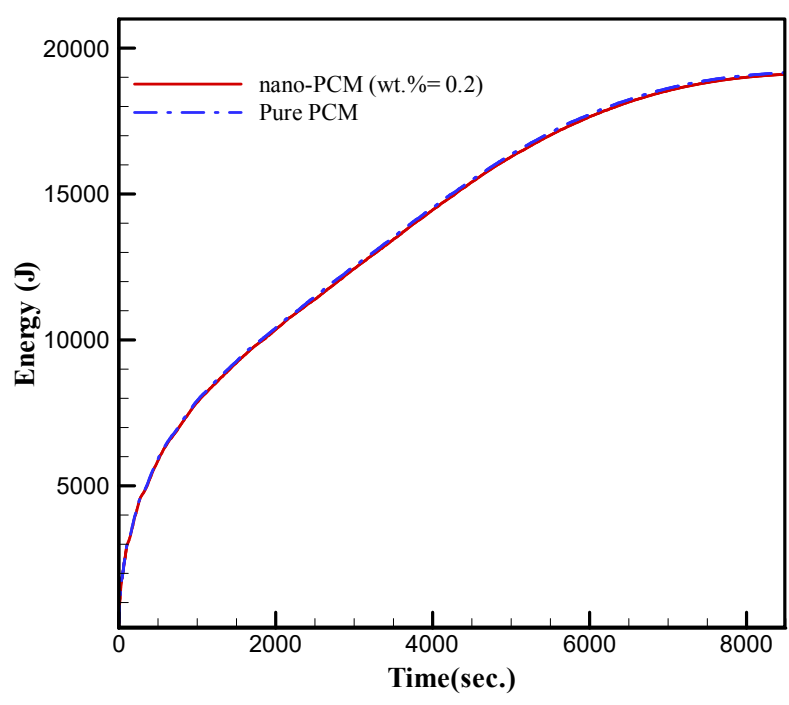

Fig.7. Stored energy

V. CONCLUSION

A numerical and experimental study were conducted to investigate the effect of dispersing copper oxide nanoparticles into the RT-35 as the base PCM on the melting rate and energy stored. The followings can be concluded from the results obtained from this work:

a) Dispersion of the Copper oxide nanoparticles in to the RT-35 increased the melting rate due to enhancing the thermal conductivity of base PCM. From the numerical simulations, it can be found that this improvement increases by using higher weight/volume fractions of the nanoparticles. However, it is worth mentioning that in practical, dispersion of high weight/volume fractions of nanoparticles results to the sedimentation issue.

b) The rate of energy stored is seen to be high at the beginning of melting process due to the high temperature gradient. This stored energy rate degraded to zero at the end of melting process due to the thermal equilibrium. In addition, nano-PCM showed a lower amount of energy stored.

c) Finally, as both melting time (charging) and the total energy stored are important for an optimal design of a LHTES system, it is recommended to maintain a balance between improvement in the thermal conductivity and decrease in the energy stored if use of nano-PCM is required.
REFERENCES

[1] A. Kumar and S. K. Shukla, "A Review on Thermal Energy Storage Unit for Solar Thermal Power Plant Application," Energy Procedia, vol. 74, pp. 462 469, Aug. 2015. doi: 10.1016/j.egypro.2015.07.728.

[2] B. Zalba, J. M. Marín, L. F. Cabeza, and H. Mehling, "Review on thermal energy storage with phase change: materials, heat transfer analysis and applications," Appl. Therm. Eng., vol. 23, no. 3, pp. 251-283, Feb. 2003. doi: 10.1016/S1359-4311(02)00192-8.

[3] G. Fang, H. Li, F. Yang, X. Liu, and S. Wu, "Preparation and characterization of nano-encapsulated n-tetradecane as phase change material for thermal energy storage," Chem. Eng. J., vol. 153, no. 1-3, pp. 217-221, Nov. 2009. doi: 10.1016/j.cej.2009.06.019.

[4] N. S. Dhaidan, "Nanostructures assisted melting of phase change materials in various cavities," Appl. Therm. Eng., vol. 111, pp. 193-212, Jan. 2017. doi: 10.1016/j.applthermaleng.2016.09.093.

[5] X. Zhang, X. Chen, Z. Han, and W. Xu, "Study on phase change interface for erythritol with nano-copper in spherical container during heat transport," Int. J. Heat Mass Transfer, vol. 92, pp. 490-496, Jan. 2016. doi: 10.1016/j.ijheatmasstransfer.2015.08.095.

[6] R. K. Sharma, P. Ganesan, V. V. Tyagi, H. S. C. Metselaar, and S. C. Sandaran, "Thermal properties and heat storage analysis of palmitic acid-TiO 2 composite as nano-enhanced organic phase change material (NEOPCM)," Appl. Therm. Eng., vol. 99, pp. 1254-1262, Apr. 2016. doi: 10.1016/j.applthermaleng.2016.01.130.

[7] N. Dhaidan and J. Khodadadi, "Melting and convection of phase change materials in different shape containers: A review," J. Renewable Sustainable Energy Rev., 43 (2015) 449-477. doi: 10.1016/j.rser.2014.11.017.

[8] S. Wu, H. Wang, S. Xiao, and D. Zhu, "Numerical Simulation on Thermal Energy Storage Behavior of Cu/paraffin nanofluids PCMs," Procedia Eng., vol. 31, pp. 240-244, 2012. doi: 10.1016/j.proeng.2012.01.1018.

[9] A. Sciacovelli, F. Colella, and V. Verda, "Melting of PCM in a thermal energy storage unit: Numerical investigation and effect of nanoparticle enhancement," Int. J. Energy Res., 37 (2013) 1610-1623.

doi: 10.1002/er.2974.

[10] N. Das, Y. Takata, M. Kohno, and S. Harish, "Effect of carbon nano inclusion dimensionality on the melting of phase change nanocomposites in vertical shell-tube thermal energy storage unit," Int. J. Heat Mass Transfer, vol. 113, pp. 423-431, Oct. 2017.

doi: 10.1016/j.ijheatmasstransfer.2017.05.101.

[11] Y. Zeng, L.-W. Fan, Y.-Q. Xiao, Z.-T. Yu, and K.-F. Cen, “An experimental investigation of melting of nanoparticle-enhanced phase change materials (NePCMs) in a bottom-heated vertical cylindrical cavity," Int. J. Heat Mass Transfer, vol. 66, pp. 111-117, Nov. 2013. doi: 10.1016/j.ijheatmasstransfer.2013.07.022.

[12] L.-W. Fan, Z.-Q. Zhu, Y. Zeng, Q. Lu, and Z.-T. Yu, "Heat transfer during melting of graphene-based composite phase change materials heated from below," Int. J. Heat Mass Transfer, vol. 79, pp. 94-104, Dec. 2014. doi: 10.1016/j.ijheatmasstransfer.2014.08.001.

[13] M. Esapour, M. J. Hosseini, A. A. Ranjbar, and R. Bahrampoury, "Numerical study on geometrical specifications and operational parameters of multi-tube heat storage systems," Appl. Therm. Eng., vol. 109, pp. 351-363, Oct. 2016. doi: 10.1016/j.applthermaleng.2016.08.083.

[14] S.-K. Choi, S.-O. Kim, and T.-H. Lee, "Computation of the Natural Convection of Nanofluid in a Square Cavity with Homogeneous and Nonhomogeneous Models," Numer. Heat Transfer, vol. 65, no. 4, pp. 287301, Nov. 2013. doi: 10.1080/10407782.2013.831695. 
[15] A. Bejan, Convection Heat Transfer, John Wiley \& Sons, Inc., 2013. doi: $10.1002 / 9781118671627$.

[16] C. J. Ho and J. Y. Gao, "An experimental study on melting heat transfer of paraffin dispersed with $\mathrm{Al} 2 \mathrm{O} 3$ nanoparticles in a vertical enclosure," Int. J. Heat Mass Transfer, vol. 62, pp. 2-8, Jul. 2013.

doi: 10.1016/j.ijheatmasstransfer.2013.02.065.

[17] J. M. Khodadadi and S. F. Hosseinizadeh, "Nanoparticle-enhanced phase change materials (NEPCM) with great potential for improved thermal energy storage," Int. Commun. Heat Mass Transfer, vol. 34, no. 5, pp. 534-543, May 2007. doi: 10.1016/j.icheatmasstransfer.2007.02.005.

[18] N. S. Dhaidan, J. M. Khodadadi, T. A. Al-Hattab, and S. M. Al-Mashat, "Experimental and numerical investigation of melting of phase change material/nanoparticle suspensions in a square container subjected to a constant heat flux," Int. J. Heat Mass Transfer, vol. 66, pp. 672-683, Nov. 2013.

doi: 10.1016/j.ijheatmasstransfer.2013.06.057.

[19] D. Groulx, "Numerical study of nano-enhanced PCMs: Are they worth it?" in Proceedings of the 1st Thermal and Fluid Engineering Summer Conference, New York City, USA, August 9-12, 2015.

[20] F. L. Tan, "Constrained and unconstrained melting inside a sphere," Int. Commun. Heat Mass Transfer, vol. 35, no. 4, pp. 466-475, Apr. 2008. doi: 10.1016/j.icheatmasstransfer.2007.09.008. 


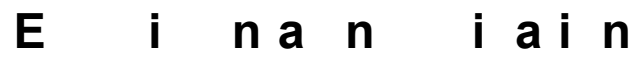

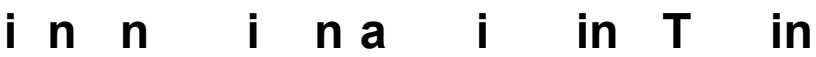

\author{
M.A. Elhajare, K. Pope, X. Duan \\ Department of Mechanical Engineering, \\ Memorial University of Newfoundland \\ St. John's, Canada
}

\begin{abstract}
In this paper, ice accretion on a wind turbine blade with a NACA 63415 airfoil is investigated with experimental techniques. Several different angles of attack, from $0^{\circ}$ to $90^{\circ}$, and wind velocities, $3 \mathrm{~m} / \mathrm{s}, 4 \mathrm{~m} / \mathrm{s}$ and $5 \mathrm{~m} / \mathrm{s}$ are studied. Tests are conducted in a climatic wind chamber with a fan and two spray nozzles. The largest quantity of ice accumulation on the blade was $7.2 \mathrm{~kg}$, at an angle of attack of $90^{\circ}$. The results of this paper provide valuable new experimental data for ice accretion on wind turbine blades.
\end{abstract}

\section{Keywords-Wind turbines; ice accretion; wind tunnel}

\section{INTRODUCTION}

In northern locations, ice is a significant obstacle for efficient operation of wind turbines. From measurements by Natural Resource's Canada, ice accretion can occur up to $20 \%$ of the time, between November and April [1]. Several problems for wind power are caused by ice accretion, including reduced power output due to disrupted aerodynamics, overload due to delayed stall, and increased fatigue of components due to imbalance of the ice load [2]. Ice accretion on a wind turbine occurs when super cooled liquid water droplets in fog, clouds or rain collide with a turbine and freeze on the surface.

To predict the annual power production for a wind turbine in northern locations, the wind and ice conditions must be considered [3]. Power output losses were estimated from $17 \%$ to $30 \%$ for wind turbine operating in locations that are affected by ice accumulation [4-6]. Ice accumulation changes with variations of the atmospheric temperature and water droplet size [2]. Better predictive techniques and experimental data are needed to predict ice accretion on wind turbine blades [7]. The external conditions at a cold climate site have several effects on wind turbine behaviour and on the efficiency of a wind farm.

Ice accumulation on a wind turbine blade causes distortion to the blades profile, which can impede the aerodynamic efficiency of an airfoil. The distortion frequently does not depend on the amount of ice accretion but on the location that accrues it; a small amount of ice accretion on the leading edge of a blade can significantly impair the aerodynamic performance. Additionally, ice accretion can cause wind turbine vibration, which can increase fatigue loads and cause structural failure [8].

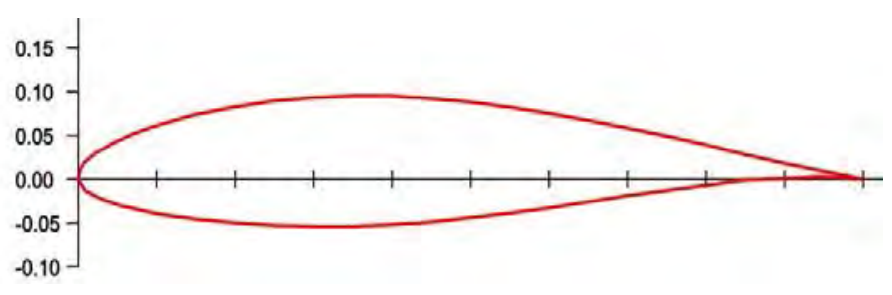

Figure 1. NACA 415-63 blade airfoil (NASA, 2014).

During icing conditions, a wind turbine is often shut-down to prevent damage caused by the ice accumulation. In this paper, experiments are conducted on a non-rotating wind turbine blade (NACA 415-63 airfoil (Fig. 1)). This research will focus on two important aspects of ice accumulation on non-rotating wind turbine blades, including (i) the quantity and (ii) location of ice accumulation.

\section{EXPERIMENTAL METHODOLGY}

In this section, details of the experimental setup used to study ice accretion on non-rotating wind turbine blades is presented. As illustrated in Fig. 2, a wind turbine blade of 0.93 $\mathrm{m}$ length, with a chord length of $19 \mathrm{~cm}$ at the root to $8.3 \mathrm{~cm}$ at the tip is manufactured for the experiments. The blade is made by carbon fiber. During the experiments, the thickness and chord of the blade are measured at four points (Tip, Mid1, Mid2, Root) six times (each $30 \mathrm{~min}$ ). A digital caliper is used to measure the thickness of ice and a digital scale to measure the mass of ice.

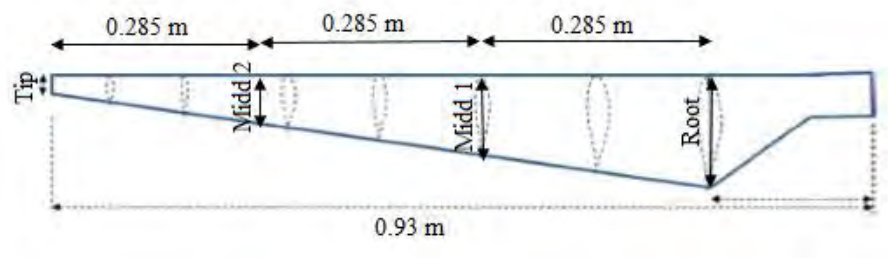

Figure 2. Schematic of wind turbine blade. 


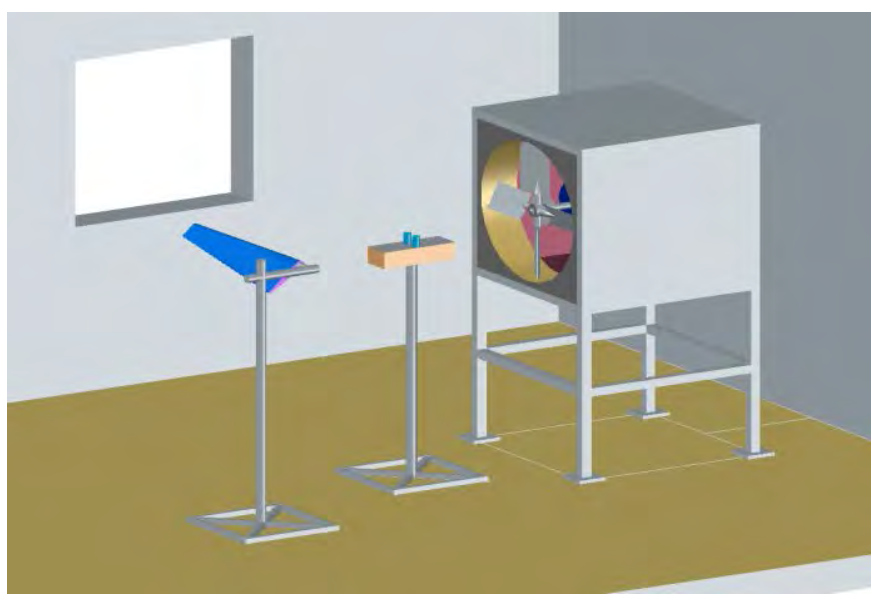

Figure 3. Schematic of experimental setup.

The experiments are conducted in a cold chamber with dimensions of $7.6 \mathrm{~m}, 4.6 \mathrm{~m}$, and $4.6 \mathrm{~m}$. The cold chamber is climatically controlled to allow sub-zero temperatures $\left(-10{ }^{\circ} \mathrm{C}\right.$ in this paper). As illustrated in Fig. 3, two FullJet spray nozzles are placed between the fan and the wind turbine blade. The wind velocity $(\mathrm{V})$ is maintained at $3.5 \mathrm{~m} / \mathrm{s}, 4 \mathrm{~m} / \mathrm{s}$ and $5 \mathrm{~m} / \mathrm{s}$. The positions of the blade and spray nozzle from the fan are $(1.5 \mathrm{~m}, 0.502 \mathrm{~m}, 0.835 \mathrm{~m})$ and $(0.833 \mathrm{~m}, 0.485 \mathrm{~m}, 0.321 \mathrm{~m})$, respectively. All coordinates are taken from the bottom left edge of the front side of the fan.

\section{RESULTS AND DISCUSSION}

In this section, new experimental results on wind turbine blade icing are presented. As presented in Table 1, 7 different experiments are conducted with different wind velocities and angle of attacks $(\alpha)$. Wind velocities from $3.5 \mathrm{~m} / \mathrm{s}$ to $5 \mathrm{~m} / \mathrm{s}$ are investigated for a $45^{\circ}$ angle of attack and angle of attacks from $0^{\circ}$ to $90^{\circ}$ are investigated for a wind velocity of $5 \mathrm{~m} / \mathrm{s}$. In all tests, the wind turbine blade has a horizontal angular position (Fig. 4). The liquid water content (LWC) is 0.6 and the mean volume diameter of the spray is $50 \mu \mathrm{m}$. All experiments are conducted for 3 hours.

\begin{tabular}{|c|c|} 
Table 1. Parameters of experiments \\
\begin{tabular}{|c|c|}
$\begin{array}{c}\text { Velocity } \\
(\mathrm{m} / \mathrm{s})\end{array}$ & $\begin{array}{c}\text { Angle of attack } \\
\left({ }^{\circ}\right)\end{array}$ \\
\hline 5 & $0^{\circ}$ \\
5 & $30^{\circ}$ \\
5 & $45^{\circ}$ \\
5 & $60^{\circ}$ \\
5 & $90^{\circ}$ \\
4 & $45^{\circ}$ \\
3.5 & $45^{\circ}$ \\
\hline
\end{tabular}
\end{tabular}

As illustrated in Fig. 4, the thickness of ice on the wind turbine blade is measured every 30 minutes during the 3 -hour test. Two different measurements (chord and width) are taken at four location of the blade span (Fig. 2). The chord length is measured before the tests, at each location, and then it is measured again with ice accumulation. The difference in the two measurements represents the thickness of ice on the leading and trailing edge (chord thickness of ice), which is plotted in Fig. 4a. As presented in Fig. 4, the majority of ice on the chord is at the leading edge. This is noteworthy as ice buildup on the leading edge can cause significant deterioration of aerodynamic performance [9]. The width ice thickness, represents the thickness of ice perpendicular to the chord and is plotted in Figure $4 \mathrm{~b}$. The results plotted in Figure 4 are for a wind velocity of $5 \mathrm{~m} / \mathrm{s}$ and an angle of attack of $45^{\circ}$. The transient results of other tests follow similar trends.

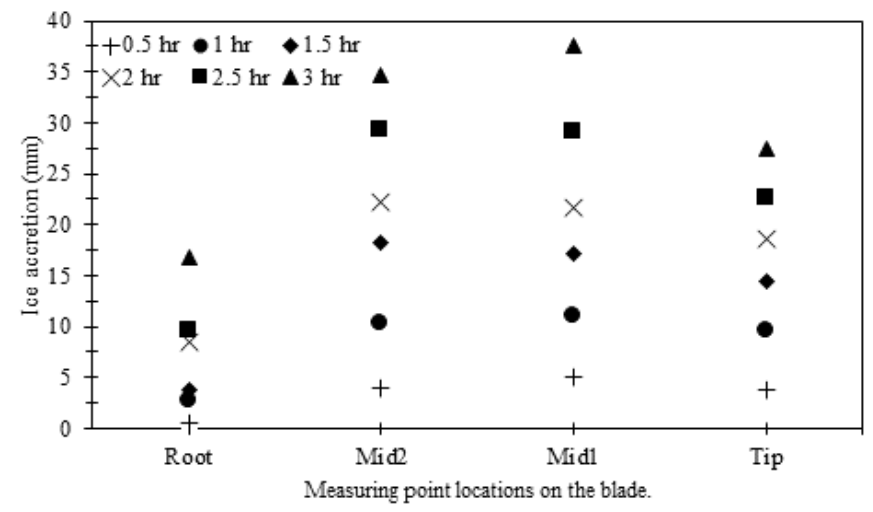

(a)

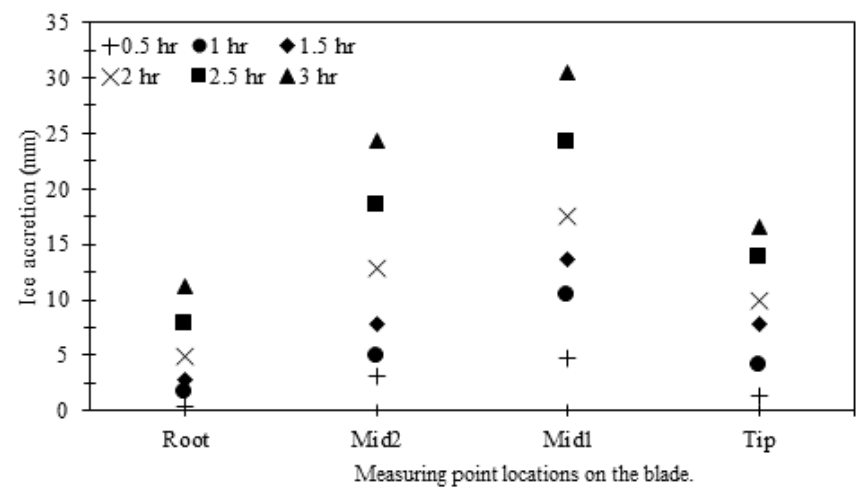

(b)

Figure 5. Transient ice accretion for $\mathrm{V}=5 \mathrm{~m} / \mathrm{s}$ and $\alpha=45^{\circ}$ on the (a) chord and (b) width of a wind turbine blade

As illustrated in Fig. 5, significant ice accretes on the leading edge of the wind turbine blade. Comparing Figs. 4 and $5 \mathrm{a}$, over $30 \mathrm{~mm}$ ice thickness is accreted on the leading edge, in the middle section. The root has the smallest thickness of ice, which is approximately half as thick as in the middle of the blade. The blade's tip also has significant leading edge ice accumulation, which is more than $25 \mathrm{~mm}$ thick.

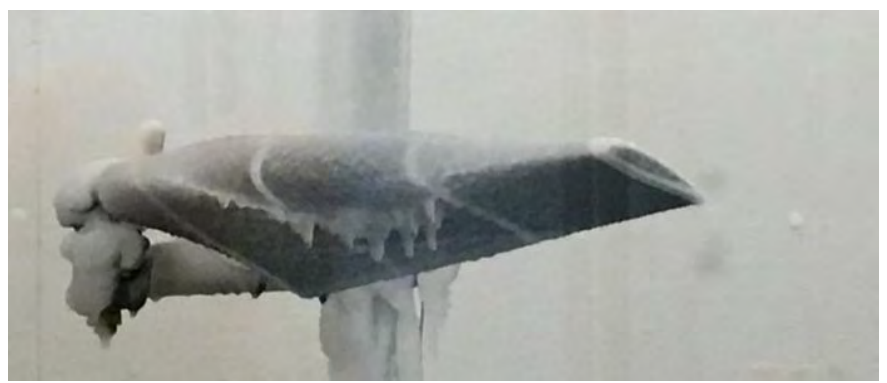

Figure 5. Ice accretion on a wind turbine blade at $V=5 \mathrm{~m} / \mathrm{s}$ and $\alpha=45^{\circ}$ after 3 hours of icing conditions. 


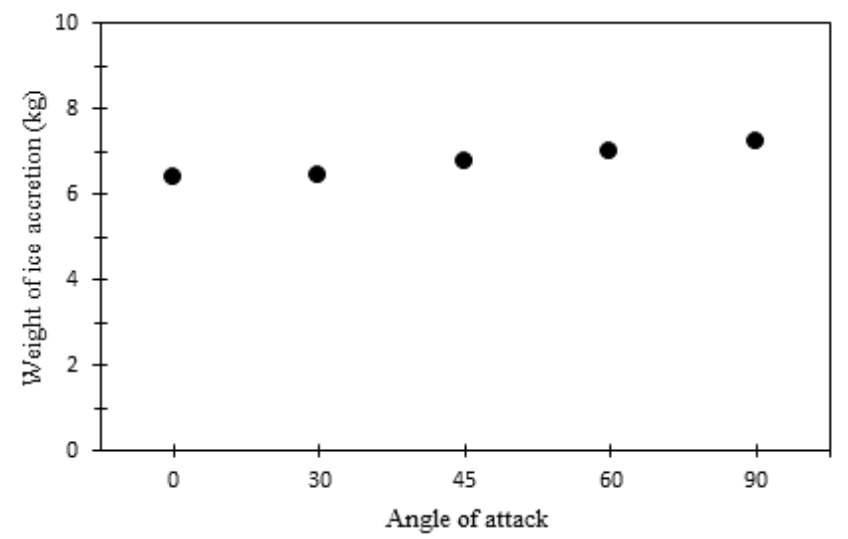

Figure 6. Weight of ice accreted on a wind turbine positioned at different angle of attacks for a wind velocity of $5 \mathrm{~m} / \mathrm{s}$.

As illustrated in Figure 6, the total quantity of ice that accretes on a non-rotating wind turbine blade is affected by the blade's angle of attack. At an angle of attack of $0^{\circ}$, the total ice accumulation is approximately $6.5 \mathrm{~kg}$, which increases to 7.2 $\mathrm{kg}$ at an angle of attack of $90^{\circ}$. The results of Fig. 6 suggest that angle of attack of a non-rotating wind turbine blade can affect the quantity of ice accretion. For conditions that are causing ice accretion, the position of the blade can be altered to help reduce the quantity of accumulated ice on the blade which can help reduce the amount of time the turbine is unable to be operated. However, when the angle of attack is low, the quantity of ice accumulation on the leading edge is higher than for a blade at $90^{\circ}$, despite more total ice accumulating on a blade at $90^{\circ}$.

In Figure 7, the quantity of ice accretion on the wind turbine blade for different wind velocities are presented. The increase in ice accretion is nearly linear with respect to wind speed. In the conditions of these experiments, the ice accretion quantity increased from approximately $3.45 \mathrm{~kg}$ at $3.5 \mathrm{~m} / \mathrm{s}$ to $4.92 \mathrm{~kg}$ at 5 $\mathrm{m} / \mathrm{s}$.

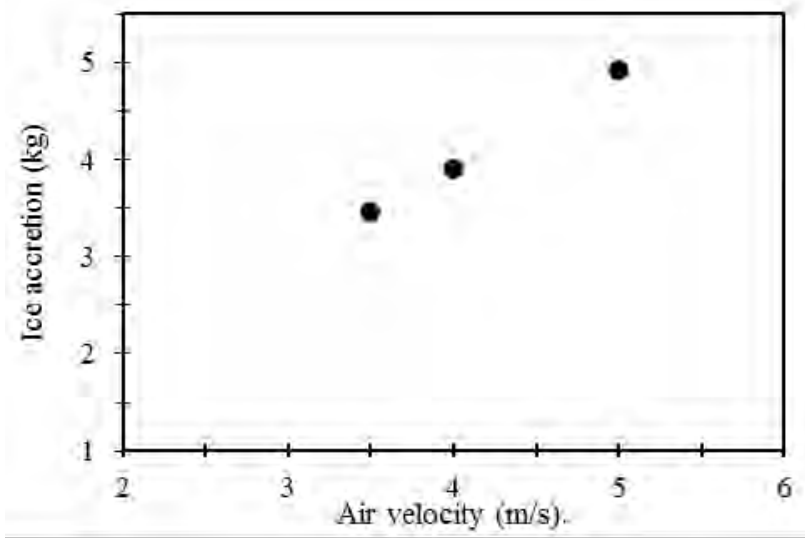

Figure 6. Weight of ice accreted on a wind turbine positioned at an angle of attack of $45^{\circ}$ in different wind velocities.

\section{CONCLUSIONS}

This paper studied the accumulation of ice on a wind turbine blade. The study was conducted for five different angles of attack $\left(0^{\circ}\right.$ to $\left.90^{\circ}\right)$ and three speeds of wind $(3.5 \mathrm{~m} / \mathrm{s}, 4$ $\mathrm{m} / \mathrm{s}$ and $5 \mathrm{~m} / \mathrm{s}$ ). The temperature was maintained at $-10{ }^{\circ} \mathrm{C}$ with a LWC of $0.6 \mathrm{~g} / \mathrm{m}^{3}$. The largest accumulation of ice was at 5 $\mathrm{m} / \mathrm{s}$, with the blade at $45^{\circ}$. More ice accretion occurred for higher angles of attack, however, more ice accumulated on the leading edge at lower angles of attack. The results also indicated a linear relationship between ice accumulation and wind velocity. The results of this paper provide new experimental data and insights for ice accretion on a wind turbine blade that has twist and varied chord length along the blade span.

\section{ACKNOWLEDGMENTS}

The authors gratefully acknowledge the financial support of the Research and Development Corporation of Newfoundland and Labrador and the Libyan Ministry of Higher Education and Scientific Research.

\section{REFERENCES}

[1] Canada Wind Energy Association. (2015). Wind energy market Installed capacity [Online]. Available: https://canwea.ca/wind-energy/installed-capacity/.

[2] M. C. Homola, M. S. Virk, T. Wallenius, P. J. Nicklasson, and P. A. Sundsbø, "Effect of Atmospheric Temperature and Droplet Size Variation on Ice Accretion of Wind Turbine Blades," J. Wind Eng. Ind. Aerod., vol. 98, no. 12, pp. 724-729, Dec. 2010 . doi: 10.1016/j.jweia.2010.06.007.

[3] B. Tammelin and H. Seifert, "Large Wind Turbines go into Cold Climate Regions," in European Wind Energy Association Conference., Copenhagen., 2001.

[4] S. Barber, Y. Wang, N. Chokani, and R. S. Abhari, "The Effect of Ice Shapes on Wind Turbine Performance," in 13th International Workshop on Atmospheric Icing, Andermatt, Switzerland, 2009.

[5] Ø. Byrkjedal, "Estimating Wind Power Production Loss due to Icing," in 13th International Workshop on Atmospheric Icing, Andermatt, Switzerland, 2009.

[6] M. C. Homola, G. Ronsten, and P. J. Nicklasson, "Energy Production Losses due to Iced Blades and Instruments at Nygardsfjell, Sveg and Aapua" in 13th International workshop on Atmospheric Icing, Andermatt, Switzerland, 2009.

[7] S. Fikke et al., "COST 727: Atmospheric Icing on Structures Measurements and data collection on icing: State of the Art," MeteoSwiss., 2006.

[8] A. S. Y. Alsabagh, W. Tiu, Y. Xu, M. S. Virk, "A Review of the Effects of Ice Accretion on the Structural Behavior of Wind Turbines," Wind Engineering., vol 37, no 1, pp 59-70, Feb. 2013. doi: 10.1260/0309-524X.37.1.59.

[9] G. M. Ibrahim, K. Pope, and Y. S. Muzychka, "Effects of Blade Design on Ice Accretion for Horizontal Axis Wind Turbines," J. Wind. Eng. Ind. Aerod., vol. 173, pp. 39-52, Feb. 2018. doi: 10.1016/j.jweia.2017.11.024. 


\author{
$T$ in an na $i \quad F$ in \\ Milad Rezvani Rad, André McDonald \\ Department of Mechanical Engineering \\ University of Alberta \\ Edmonton, AB, Canada \\ milad.rezvani@ualberta.ca \\ andre.mcdonald@ualberta.ca
}

\author{
Rick Marsden \\ Asset Integrity \\ Cenovus Energy \\ Calgary, AB, Canada \\ rick.marsden@cenovus.com
}

\begin{abstract}
Solidification of water in closed piping systems is a common phenomenon in both the industrial and residential sectors. In some cases, the resulting damage may be both costly and dangerous and thus warrants attention. Therefore, further study of the cooling and freezing behavior of stagnant water within closed pipes seems to be necessary. In this regard, several experiments were performed to investigate the freezing behavior of water in pipes. The inelastic deformation, work hardening, and fracture of the pipes that were caused by volume expansion due to the freezing of the water were also studied. Two conventional steel pipe materials, namely ASTM A333-6 and ASTM A106-B, were used in the freezing tests. The fracture surfaces of the pipes were analyzed by using both macroscale and microscale images of the surfaces. Fractography analysis of the fractured regions was also performed by using a scanning electron microscope. It was found that the damage caused during freezing to the A106-B pipe was considerably more than that in the A333-6 pipe. The results confirm that A333-6 should be used instead of A106-B in pressure equipment, such as pipes, that are exposed to low temperatures.
\end{abstract}

Keywords - Fracture analysis; ice accumulation; pipe bursting; pressure build-up; pressure vessel

\section{INTRODUCTION}

Failure of pressure equipment due to solidification of entrapped water in closed systems exposed to temperatures below freezing is very widespread and costly in both the industrial and residential sectors. According to the Insurance Information Institute (I.I.I.) [1], frozen pipes are one of the main problems that occur during winters and the financial losses because of ice, freezing, and related issues averaged $\$ 1.2$ billion annually in the United States alone over the last 20 years.

The failure of pipes in commercial and residential heating, ventilating, and air-conditioning (HVAC) systems in which pipes are located in low-temperature environments, is also common. The most conventional pipe materials that are used in HVAC systems are steel, copper, and chlorinated polyvinyl chloride (CPVC) [2]. In this regard, further study of freezing behavior of water within these pipes and the failure of such piping systems has been necessary. Gordon [3] conducted a series of experiments to study the freezing behavior of water within closed pipes and the bursting of pipes used for residential applications. Smith, et al. [4] performed freezing experiments with copper and steel pipes. In this study, it was shown that the ice front did not reach the rupture site and failure of the pipe occurred at a location in which water was entrapped. Edwards, et al. [5] studied bursting of three different plastic pipe materials. Given the noticeable inelastic deformation of the plastic pipes that could accommodate the expansion of freezing liquid, it was concluded that longer pipe lengths were required for the plastic pipes compared to the metallic pipes to cause over-pressurization and bursting.

Mathematical simulation of the cooling and freezing of water in pipes has been the subject of substantial research investigations [6 - 9]. In this regard, Sugawara, et al. [6] and Oiwake, et al. [7] performed both experimental and numerical studies to evaluate the internal pressure changes during the solidification of water. Sullivan and McDonald [8] developed a one-dimensional transient heat conduction mathematical model to estimate the cooling and freezing times of water within copper and polyvinyl chloride (PVC) pipes of various inner diameters. McDonald, et al. [9] compared the results obtained from experimental data with estimates from the mathematical model and concluded that the separation of variables method for a finite length-scale heat conduction problem is an acceptable method for use in developing a predictive model for the total cooling and freezing times of water in small diameter pipes.

The study of the current paper investigated the freezing behavior and the resulting failure in two conventional grades of carbon steel pipes. The objectives of this study were to: (1) investigate different stages of freezing in the closed pipes, (2) analyze the inelastic deformation and the resulting work hardening of the pipe materials, and (3) analyze the fracture surfaces to determine the fracture mechanism and failure mode for each rupture case. 


\section{Methodology}

Two 254-mm (10-inch) long, 51-mm (2-inch) diameter carbon steel pipes were used in the freezing experiments. The steel pipes were of two different materials namely ASTM A333-6 and ASTM A106-B. However, the wall thickness of both of the pipes was the same. Schedule 40 pipes, which are widely used in industry, were selected for the tests. The pipes were chosen to study the effects of pipe material on the resulting failure.

Each pipe assembly included a 254-mm (10-inch) long pipe with two A420 WPL6 butt-welded end caps, each including a 19-mm (3/4-inch) Class 3000 A350 LF2 threadolet. Welding consisted of a typical SMAW P1-P1 procedure using E6010 for the root and E7018 for the cap. The total length of the assembly that consisted of the pipe, the two end caps, and the two threadolets was approximately $381 \mathrm{~mm}$ (15 inches). A pipe assembly, which is related to a Schedule 40 ASTM A333 Grade 6 pipe, is shown in Fig. 1.

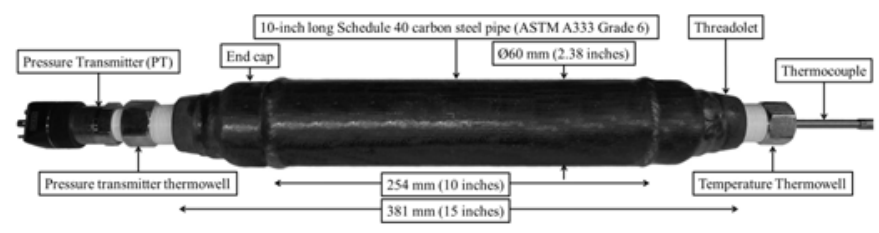

Figure 1. Pipe assembly, complete with the installed thermowells, thermocouple, and pressure transmitter

A thermowell (TW15, WIKA Instruments, Edmonton, AB, Canada) was installed into the threadolets at each end of the pipe. The thermowells were used for positioning the type $\mathrm{T}$ thermocouple (TC40, WIKA Instruments, Edmonton, AB, Canada) and the pressure transmitter (PT) (S-20, Klingenberg, Germany). The tip of the thermowell used for the PT was drilled through to enable pressure to be measured. A balloon filled with antifreeze was installed on the tip of the PT thermowell to ensure that no ice would form inside the pressure sensing pathway and to ensure that the peak pressure in the pipe, which occurs in the liquid phase, would be measured.

Temperature and pressure data were collected at regular time intervals from the thermocouples and the pressure transmitter by using two data acquisition systems. One data acquisition system (SCXI-1600, National Instruments, Austin, TX, USA) was used to measure the ambient and water/ice temperatures and a second data acquisition system (cDAQ9171, National Instruments, Austin, TX, USA) was utilized to collect and record pressure measurements. The NI MAX software was used to collect all the measurement readings. The rate at which data was collected from all the mentioned devices was one data point per second $(1 \mathrm{~Hz})$.

In order to assess the freezing behavior of water inside the closed pipes, several freezing tests were performed in an 18.2 $\mathrm{m}^{3}\left(640 \mathrm{ft}^{3}\right)$ cold room freezer (Foster Refrigerator USA, Kinderhook, NY, USA) that was equipped with a temperature controller. The experiments were all conducted with pipes that were oriented horizontally and under the same environmental ambient conditions. The pipes were placed in the cold room in advance of conducting the tests so that both the ambient and the water inside the pipe were at steady state. Care was taken to start all the tests at approximately the same temperature $\left(27^{\circ} \mathrm{C}\right)$. The cold room set-point temperature was $-25^{\circ} \mathrm{C}$ and the tests were stopped when the water inside the pipe reached $-15^{\circ} \mathrm{C}$.

All of the experiments involved leaving a small predetermined percentage of air space inside the pipe, rather than filling it completely with water. The reason for having air in the system was twofold. First, the first solidification plateau and ice cooling can only be seen when some air is entrapped in the closed system. Second, some air in the system prevents the pipe from yielding excessively and allows the pipe to be used for more freezing tests and, therefore, work hardening of the pipe material can be better observed and assessed. As a result, the amount of entrapped air in the pipe assemblies was set to a constant value of $5 \mathrm{vol} \%$ for all the tests so that the work hardening behavior of the pipe materials was studied without introducing the effect of variation in the air content.

Considering the volume expansion of the pipes due to inelastic deformation, the volume of the pipe assemblies was measured after each test. Then, the pipe assemblies were refilled with water so that the increase in the volume of the pipe assembly was filled with water and the volume percent of air could be maintained constant in all the freezing tests.

Eventually, after conducting repeated freezing tests, the piping assemblies failed. The fracture surfaces were cleaned in order to study the microscopic features of the fracture surfaces. The sectioned samples of the pipes were placed in an ultrasonic cleaner (Haver USC 200, HAVER \& BOECKER, Oelde, NRW, Germany) and cleaned with corrosion inhibited detergent solution (Citranox, Alconox, NY, USA) for 30 minutes to ensure that the oxide scale and deposits were removed from the fracture faces. The microstructure of the fracture surfaces was then examined by using a scanning electron microscope (Zeiss Sigma 300 VP-FE, Carl Zeiss Canada Ltd., Toronto, ON, Canada).

\section{RESULTS AND DISCUSSION}

\section{A. Freezing Behavior}

The temperature and pressure traces of water/ice for the first freezing test that was conducted on ASTM A333 Grade 6, hereafter referred to as A333-6, Schedule 40 steel pipe in which 5 vol.\% of air was entrapped in the pipe are shown in Fig. 2. The graph includes five stages, namely water cooling, the first solidification plateau, cooling of the mix of water and ice, the second solidification plateau, and the solid ice cooling. The first stage, which was related to the liquid cooling, began when the fans in the cold room started cycling (dropping the temperature in the room) and finished after a short supercooling period. The nucleation and growth of plate-like solid crystals known as dendritic ice occur at this stage and during the supercooling [10]. The length of the first solidification plateau in the second stage indicates the time needed for some of the liquid water in the pipe assembly to solidify so that a mixture of water and ice fills the entire volume of the pipe assembly. As the solidification proceeded through this stage, the annular ice formed inward and the dendritic ice was engulfed by solid ice [11]. At this point, solidification of water was paused due to the pressure increase. 
At the end of the first solidification plateau, all the water had not been transformed into solid ice and some water was still entrapped in the middle of the pipe assembly.

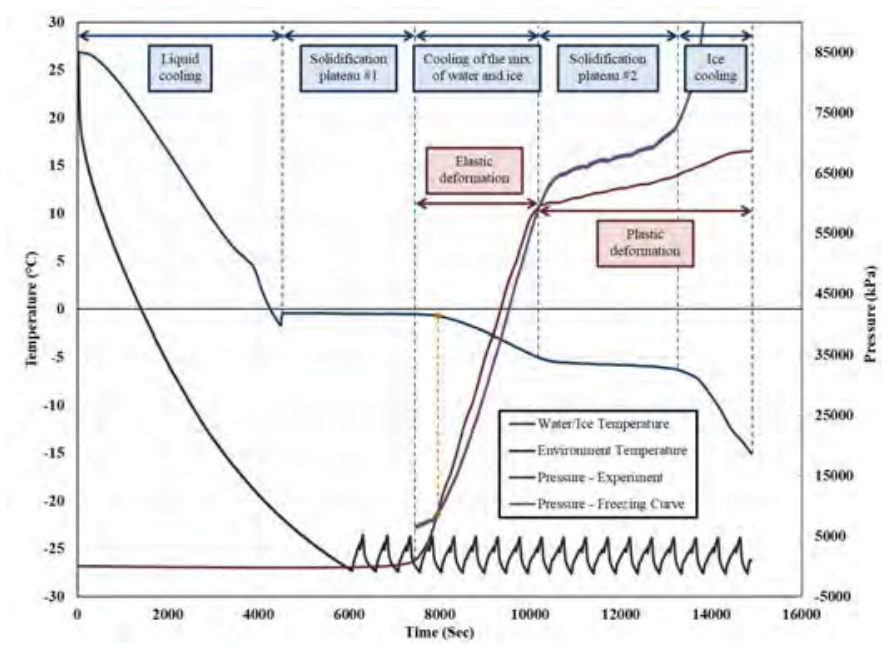

Figure 2. Curves of temperature and pressure versus time for water/ice in A333-6 Schedule 40 low carbon steel pipe

In the third stage and during the elastic deformation of the pipe, the pressure of water within the pipe increased sharply. As shown in Fig. 2, at the beginning of this stage, the average rate at which the water temperature decreased in this period was very low (order of $-0.02^{\circ} \mathrm{C} / \mathrm{min}$ ) because the pressure of the water was lower than the pressure that was calculated from the non-linear freezing relation for the hexagonal ice, which is given by Eq. (1) [12] as

$$
P_{\mathrm{f}}=6.11657 \times 10^{-4}-414.5 \times\left[\left(\frac{T_{\mathrm{f}}}{273.16}\right)^{8.38}-1\right]
$$

The values of pressure obtained from Eq. (1) were based on the transient temperature of the freezing water inside the pipe (see Fig. 2).

Further transformation of the water into ice caused an increase in the pressure of the freezing water such that the experimentally determined transient pressure curve intersected and crossed the transient pressure curve that was obtained from the non-linear freezing relation, which was representative of the magnitude of pressure that was required to maintain the freezing water in its liquid form. This point and its analogous point on the water temperature trace have been connected to each other with a dashed line (see Fig. 2) in order to highlight the interaction between water pressure and temperature. After this point, the high rate of increase in the pressure of the water caused a decrease in the rate of solidification of the rest of the pressurized water due to a continuous depression of the freezing point. Therefore, the heat loss in this period was mainly sensible as observed from the decrease in temperature of the ice-water mixture and consequently, the average rate of decrease of the water temperature changed noticeably from $0.02^{\circ} \mathrm{C} / \mathrm{min}$ before 7,960 seconds after the start of the experiment to $-0.12^{\circ} \mathrm{C} / \mathrm{min}$ after 7,960 seconds after the start of the experiment.

Once the carbon steel pipe entered the plastic deformation region, the pressure increased with time at a lower rate than that which occurred earlier in the elastic deformation region. As a result of further cooling of the freezing water when the pipe material was deforming plastically, the pressure on the water was not sufficiently high to maintain the freezing liquid in its liquid form and, therefore, the remaining water started transforming to solid ice. The average pressure that was obtained from the experiment during this period (solidification plateau \#2) was 5.2 $\mathrm{MPa}$ lower than the average pressure that was obtained from the freezing curve for the given temperatures (see Fig. 2). The transformation of water to ice, when the temperature trace plateaued again, was clearly observed at the time when the pipe material started deforming plastically.

The magnitude of freezing point depression correlates with the pressure of the freezing water, which itself is a function of the yield strength of the pipe and the pipe wall thickness according to the von Mises yield criterion and thick-walled pressure vessel theory relations, respectively. The yield strength of the pipe is not a constant value and varies due to work hardening from one test to another.

At the end of the second plateau, all the water in the pipe had been transformed into hexagonal ice. In the last stage, further cooling resulted only in temperature decrease of the solid ice. The increasing deviation of theoretical pressure values from the experimental values in this stage was likely due to a lack of pressurized water in the closed pipe. Therefore, comparison of pressure values between the non-linear freezing relation and the experiment is not valid in this stage.

\section{B. Inelastic Deformation and Work Hardening}

The inelastic deformation of the pipes that occurred during each freezing test was determined by measuring the diameter of the pipes by using a Pi-tape. The outer diameter of the pipes was measured before each test and after the failure of each pipe in the last test. The transverse strain values were calculated by comparing the current diameter of the pipe with its actual original value. As expected, the highest strains occurred near the middle of the pipe where the reinforcing effects of the circumferential welds had the lowest impact.

It was found that the maximum strain values that A106-B and A333-6 steel pipes could accommodate before rupture was $13.3 \%$ and $11.3 \%$, respectively. It should be noted that the minimum transverse elongation required before failure according to the standard respecting ASTM A106 Grade B steel, hereafter referred to as A106-B, is $16.5 \%$ [13]. Therefore, the material did not meet the criteria under the frozen pipe tests. However, the requirement of the minimum transverse elongation at failure for A333-6 steel is $11.4 \%$ [14], which is very close to the strain value at which the pipe failed.

The number of loading/unloading cycles had a profound impact on the pressure values that were required to promote plastic deformation of the pipe material in each freezing test. The work hardening of both A106-B and A333-6 Schedule 40 
steel pipes, which was caused by generation and movement of dislocations within the pipe material, can be observed clearly in Figs. 3 and 4, respectively.

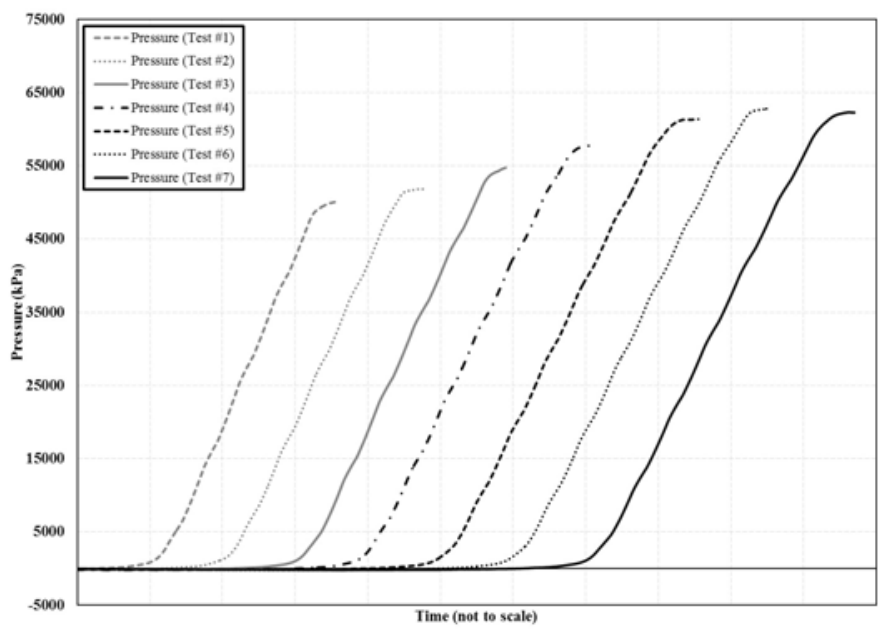

Figure 3. Effect of freezing/thawing cycles on yield pressure of A106-B Schedule 40 low carbon steel pipe

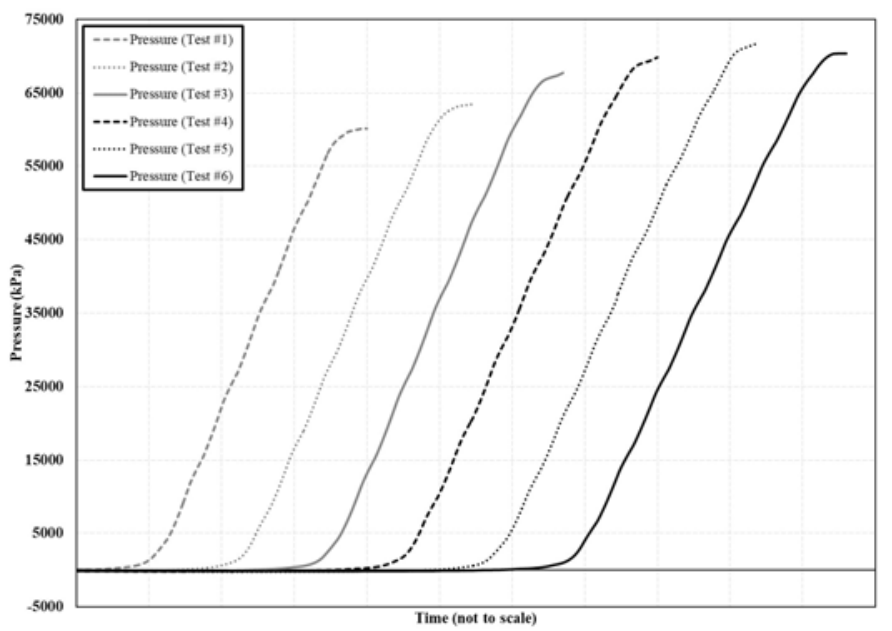

Figure 4. Effect of freezing/thawing cycles on yield pressure of A333-6 Schedule 40 low carbon steel pipe

A comparison of the peak pressures in the elastic region between the first and the last tests for both pipes shows approximately $23 \%$ and $17 \%$ increase in the pressure at which the pipe material started to deform plastically for A106-B and A333-6 Schedule 40 steel pipes, respectively (see Figs. 3 and 4). Therefore, the number of freezing/thawing (loading/unloading) cycles and the resulting work hardening of the pipe material had an impact on the increase of the peak pressures in the elastic region and, consequently, further depression of the freezing point of the remaining water in the closed system during the second solidification plateau according to Eq. (1).

A comparison of the temperature and pressure data of the first and the last tests that were performed with A333-6 steel pipe is shown in Fig. 5. The increase in the yield pressure and the decrease in the temperature at which temperature curve plateaued again can be clearly observed in Fig. 5 .

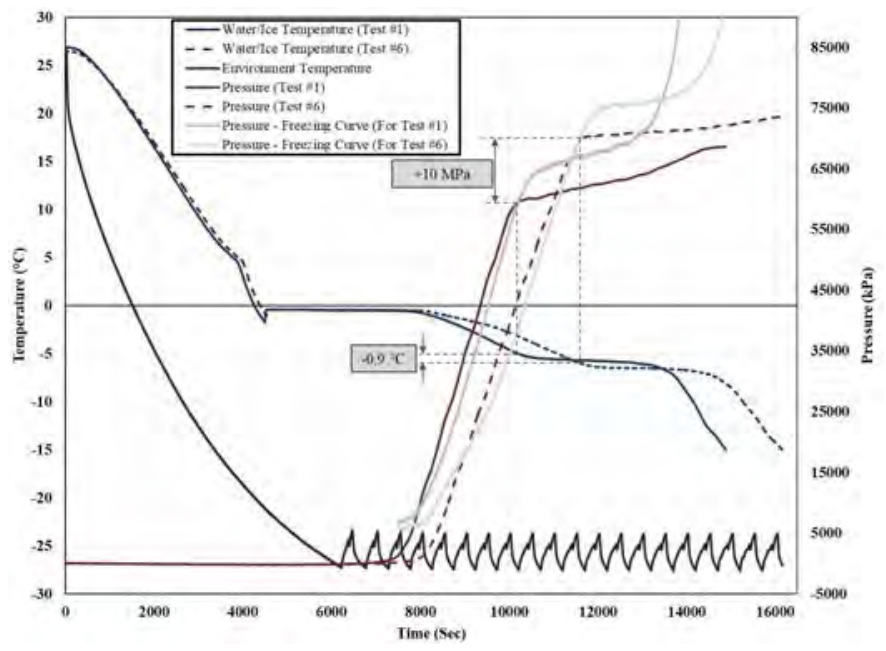

Figure 5. Effect of loading/unloading cycles on further depression of the freezing point at the beginning of the second solidification plateau

The relatively lower rate of temperature decrease in Test \#6 compared to that of Test \#1 at the beginning of the third stage (cooling of the mix of water and ice) was an indirect consequence of non-uniform bulging of the pipe assembly during the previous freezing tests, which resulted in the increase of the volume of the closed pipe from $649 \mathrm{ml}$ to 718 $\mathrm{ml}$. Therefore, more ice was required to be formed to compensate for the $10.6 \%$ increase in the volume of the pipe assembly and caused an increase in the water pressure to that which was required according to the freezing curve.

\section{Pipe rupture}

Failure of the pipes occurred after conducting several tests. The pattern of failure that is shown in Fig. 6 is very common in pipe rupture cases. As expected, both pipe assemblies fractured along the axial direction, which was perpendicular to the direction of the applied hoop stress where the maximum principal stress for the case of the cylindrical pressure vessel was located. Considering the geometry of the pipe assembly with thermowells installed, the center of the pipe is the part that froze last. It was the expansion of the ice plugs in the axial direction and the low compressibility of the entrapped water that caused freeze-induced pipe failure [4].

The gap at the failure site of the A333-6 steel pipe was remarkably narrower and shorter than that of the A106-B steel pipe. It is believed that the longer axial crack for the A106-B pipe compared to that of the A333-6 pipe was due to the decrease in the amount of energy that was absorbed by the pipe material during propagation of the crack, which was due to the lower toughness of A106-B material at low temperatures. While not dramatically obvious in these specific tests due to the low amount of stored energy released at failure, the improved toughness properties of A333-6 is expected to be much more relevant and evident in actual field conditions where large amounts of stored energy is released at failure. The higher toughness of A333-6 provides better resistance to a brittle 
fracture developing following a ductile overload. The dependency of the absorption energy in Charpy impact tests on temperature for an A106-B pipe can be found in literature [15].
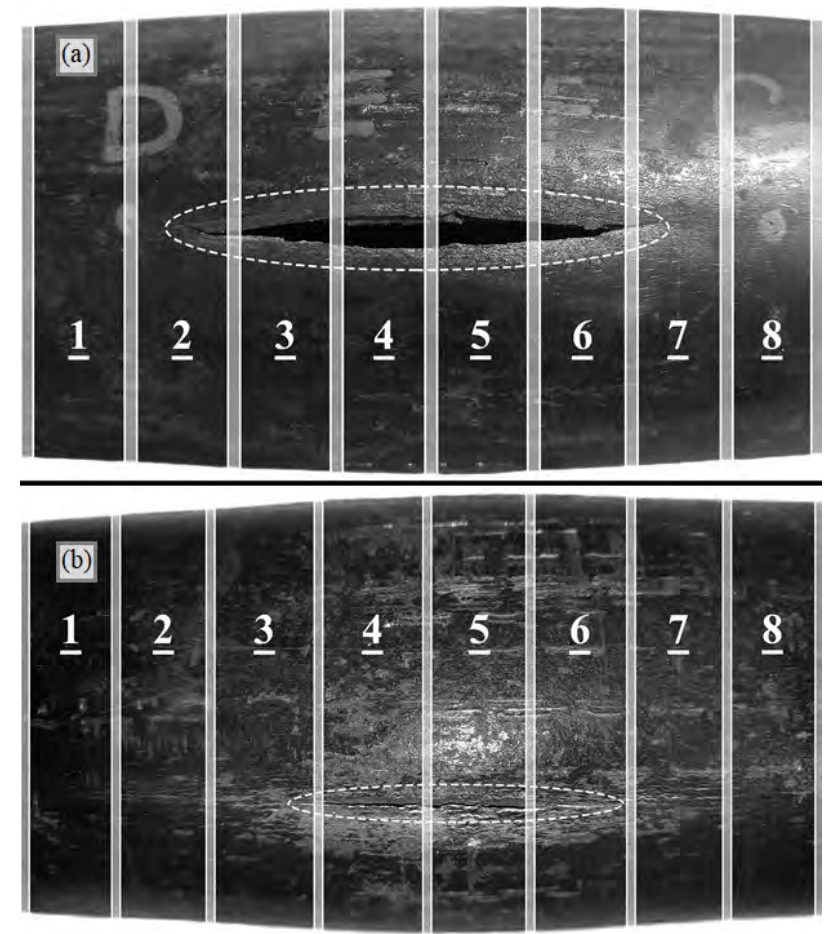

Figure 6. Failure of (a) A106-B and (b) A333-6 Schedule 40 low carbon steel pipe due to internal over-pressurization

The pipes were cut open after failure so that the fracture surfaces could be better observed. Eight rings, each one centimeter wide, were cut from the middle section of the pipe to study the fracture at different locations. The exact locations from which these eight rings were cut are shown in Fig. 6.

\section{Fracture Analysis}

In order to study the macroscopic features of the fracture surfaces, images of the sides of the fractured regions were taken, which can be seen in Figs. 7 and 8. The geometry and appearance of the fractured regions are the characteristics that may be used to determine the fracture mechanism and the failure mode of the pipe material. Both slant fracture and double-slant fracture features, which are typical of planes-stress loading conditions in ductile materials [16] and are common failure modes in pressure vessel and in the piping industry [17], can be observed in cross-sectional views of the sectioned rings from the fractured portions.

These types of fractures, which are common in tubes with low wall thicknesses due to their minimum through-thethickness stresses, are known to be ductile in nature [16]. It is well known that the tearing process is initiated by local thinning. The localization of two shear bands oriented at $45^{\circ}$ angles occurs inside the necked region [18 - 19]. The resulting slant failure and the necked regions can be observed in Figs. 7 and 8 , in the middle and in front of the axial cracks, respectively.

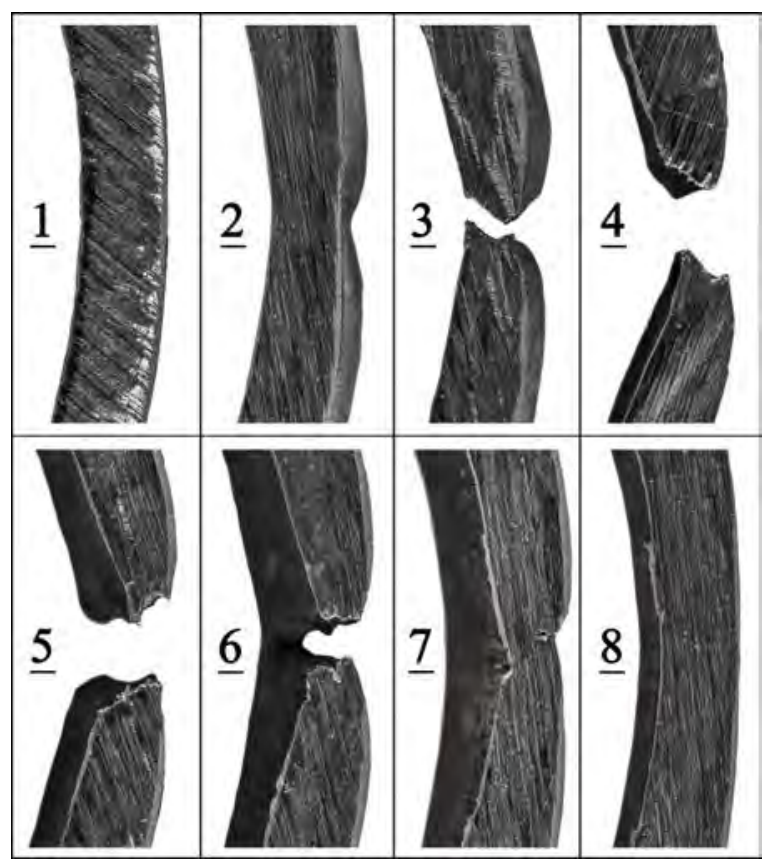

Figure 7. Cross-sectional views of the rings sectioned from A106-B Schedule 40 low carbon steel pipe

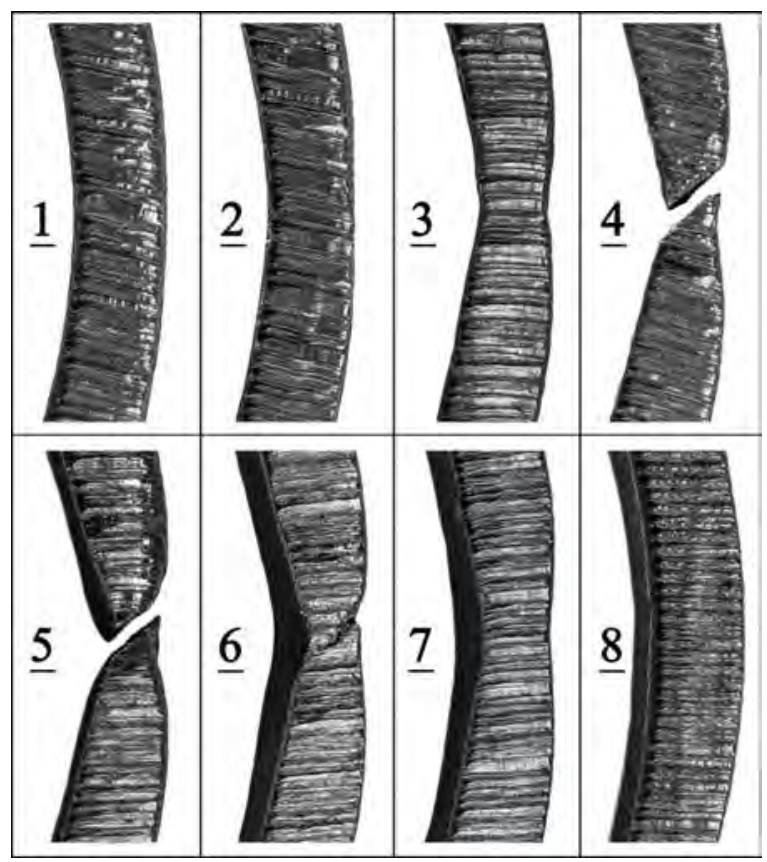

Figure 8. Cross-sectional views of the rings sectioned from A333-6 Schedule 40 low carbon steel pipe

\section{E. Fractography}

The higher-magnification fractographs show that the mode of failure was ductile tearing from void coalescence induced microcracks, which is characteristic of ductile fracture. This can be observed from coalescence of adjacent microvoids in the axial direction (see Fig. 9). The dull and fibrous surfaces of the fracture that contained many dimples are indicative of dimpled rupture fracture. 


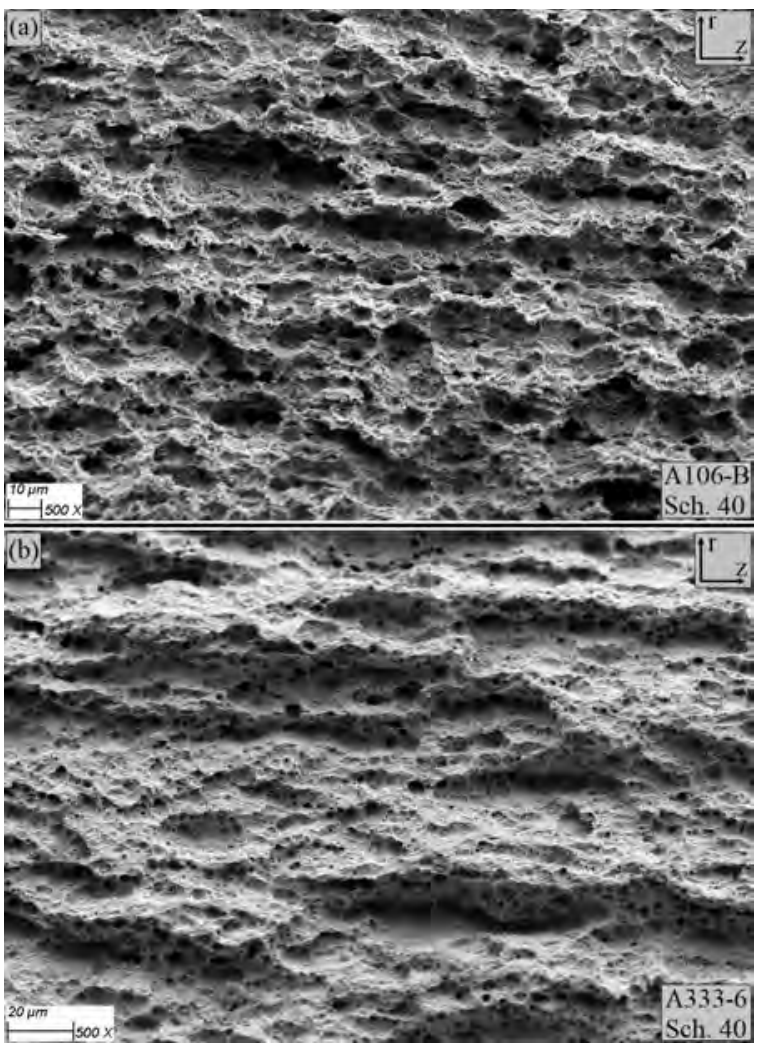

Figure 9. Micrograph of the fracture faces of (a) A106-B and (b)A333-6 Schedule 40 low carbon steel pipe (500X magnification)

\section{CONCLUSION}

Several freezing tests were performed on two steel pipe assemblies with the same dimensions, but with two different steel materials. By comparing the data, the following conclusions can be drawn.

- The freezing point depression of the water depended on the fluid pressure, which itself was a function of pipe material yield strength and wall thickness.

- The amount of work hardening mainly depended on the number of freezing/thawing cycles that the closed pipes had experienced. After performing several freezing tests, the pressure at which both A106-B and A333-6 steel pipes started deforming plastically was increased $23 \%$ and $17 \%$, respectively.

- Work hardening of the material caused depression of freezing point in the second solidification plateau. It was found that an increase in the yield pressure of A333-6 steel pipe material on the order of $10 \mathrm{MPa}$ $(1,450 \mathrm{psi})$ resulted in a $0.9^{\circ} \mathrm{C}$ decrease in the temperature at which the remaining entrapped water started to freeze to solid ice.

- $\quad$ Failure and bursting of the pipes occurred after several loading/unloading (freeze/thaw) cycles. Double-slant and slant fracture appearances were observed in the macroscopic examination of the cross-sectional views of the sectioned rings.
- Higher-magnification views of the fracture surfaces of both pipes confirmed dimpled rupture fracture, which was indicative of ductile fracture. It was observed that the axial cracks were formed by coalescence of adjacent microvoids in the axial direction.

- As expected, the resulting damage in A333-6 pipe was less noticeable than that in A106-B pipe due to decreased toughness of the A106-B material at low temperatures.

\section{REFERENCES}

[1] A. Carrns, "Avoiding Costly Home Damage from Winter's Cold", The New York Times, 02-Dec-2014.

[2] H.W. Stanford, HVAC Water Chillers and Cooling Towers: Fundamentals, Application, and Operation, CRC Press, New York, pp. 48. 2003. doi:10.1201/9780203912492.

[3] J. Gordon, An Investigation into Freezing and Bursting Water Pipes in Residential Construction, Research Report No. 96-1, Building Research Council, School of Architecture, University of Illinois at UrbanaChampaign, pp. 1-51. 2006.

[4] K.M. Smith, M.P. Van Bree, and J.F. Grzetic, "Analysis and Testing of Freezing Phenomena in Piping Systems", ASME International Mechanical Engineering Conference and Exposition, Boston, Massachusetts, 2008.doi: 10.1115/IMECE2008-69109.

[5] D.B. Edwards, K.M. Smith, D.E. Duvall, and J.F. Grzetic, "Analysis and Testing of Freezing Phenomena in Plastic Piping Systems", ASME International Mechanical Engineering Conference and Exposition, Lake Buena Vista, Florida, 2009. doi: 10.1115/IMECE2009-10071.

[6] M. Sugawara, N. Seki, and K. Kimoto, "Freezing limit of water in a closed circular tube", Wärme Stoffübertragung, vol. 17, pp. 187192, 1983.doi:10.1007/BF02570529.

[7] S. Oiwake, H. Saito, H. Inaba, and I. Tokura, "Study on dimensionless criterion of fracture of closed pipe due to freezing of water", Wärme Stoffübertragung, vol. 20, pp. 323-328, 1986. doi:10.1007/BF01002423.

[8]

E. Sullivan and A. McDonald, "Freezing time of water in small diameter tubes and pipes in residential and commercial HVAC applicactions", in Proceedings of The Canadian Society for Mechanical Engineering International Congress 2014, Toronto, Ontario, Canada, 2014.

[9] A. McDonald, B. Bschaden, E. Sullivan, and R. Marsden, "Mathematical simulation of the freezing time of water in small diameter pipes", Appl. Therm. Eng., vol. 73, pp. 142-153, 2014. doi: 10.1016/j.applthermaleng.2014.07.046.

[10] R. R. Gilpin, "The effects of dendritic ice formation in water pipes", Int. J. Heat Mass Transf., vol. 20, pp. 693-699. 1977. doi: 10.1016/0017-9310(77)90057-6.

[11] R.R. Gilpin, "The influence of natural convention on dendritic ice growth", J. Cryst. Growth, vol. 36, pp. 101-108, 1976. doi: 10.1016/0022-0248(76) $90220-7$

[12] M. Choukroun and O. Grasset, "Thermodynamic model for water and high-pressure ices up to $2.2 \mathrm{GPa}$ and down to the metastable domain", J. Chem. Phys., vol. 127, p. 124506. 2007. doi: 10.1063/1.2768957.

[13] ASTM A106/A106M-13, "Standard Specification for Seamless Carbon Steel Pipe for High-Temperature Service", ASTM International, West Conshohocken, PA, 2013, p. 3 .

[14] ASTM A333/A333M-11, "Standard Specification for Seamless and Welded Steel Pipe for Low-Temperature Service", ASTM International, West Conshohocken, PA, 2011, p. 3

[15] PVRC Recommendations on Toughness Requirements for Ferritic Materials, WRC Bulletin 175, Welding Research Council, 1972.

[16] W.T. Becker, Fracture Appearance and Mechanisms of Deformation and Fracture, Failure Analysis and Prevention, vol. 11, ASM Handbook, ASM International, Materials Park, Ohio, pp. 1162-1327, 2002.

[17] H. Huang and L. Xue, "Prediction of slant ductile fracture using damage plasticity theory", Int. J. Press. Vessels Pip., vol. 86, pp. 319-328, 2009. doi: 10.1016/j.ijpvp.2008.11.027.

[18] S.A. El-Naaman and K.L. Nielsen, "Observations on Mode I ductile tearing in sheet metals", Eur. J. Mech. A/Solid, vol. 42, 2013, pp. 5462.

[19] K.L. Nielsen and J.W. Hutchinson, "Cohesive traction-separation laws for tearing of ductile metal plates", Int. J. Impact Eng., vol. 48, 2012, pp. 628 15-23. doi: 10.1016/j.ijimpeng.2011.02.009. 


\section{$\mathbf{T}$}

\author{
Alireza Sarraf Shirazi \\ Department of Mechanical Engineering \\ University of British Columbia \\ Vancouver, BC, Canada \\ alireza.sarrafshirazi@gmail.com
}

\author{
Ian Frigaard \\ Department of Mechanical Engineering \\ University of British Columbia \\ Vancouver, BC, Canada \\ frigaard@math.ubc.ca
}

\begin{abstract}
A modified three-layer model for solid-liquid flow in horizontal pipes is developed, which overcomes the limitations of many previous models. The steady-state model predicts the pressure loss, critical velocity, concentration profile in the heterogeneous layer, mean heterogeneous layer and moving bed layer velocities, and bed layer heights for each set of parameters. We propose a new correlation for the turbulent solids diffusivity. This and the steady state model predictions show a good agreement with experimentally measured results in literature: for concentration profile in the heterogeneous layer and pressure loss, over a wide range of conditions [1]. In turbulent flow. the pressure loss vs mean velocity curve shows a characteristic minimum just before the critical velocity is attained.
\end{abstract}

Index Terms-three-layer model, solids transport, critical velocity, solids diffusivity, pressure gradient

\section{INTRODUCTION}

The flow of solid-liquid mixtures in the form of slurry occurs in a wide range of situations, e.g. river bed erosion and sedimentation, ocean-bed avalanches, transport of mined particulate, etc.. Pipe flows of water-sand slurry are commonly encountered in many applications such as mined slurry transport, hole cleaning, hydraulic fracturing and gravel packing in oil \& gas wells. There are numerous studies of the fundamental sub-processes of solids phase transport (erosion, dispersion, sedimentation, deposition, shear-migration) appearing over the past 20-30 years, and dating back to the 1950s.

Many different two-layer multi-phase hydraulics models have appeared in the slurry transport literature, e.g. the twolayer model of Shook and Bartosik [2], and many others, where a heterogeneous layer, and a sliding/stationary bed layer is predicted. Doron and Barnea [3] first introduced a three-layer slurry transport model. They identified the flow patterns of slurries flowing in horizontal pipes as the flow rate increases: (a) flow with a three-layer configuration, i.e. a stationary bed layer at the bottom, a sliding bed layer in the middle, and a heterogeneous layer at the top; (b) flow with a sliding bed and heterogeneous layer; (c) fully-suspended flow.

Transitions between the different observed flow regimes have historically formed one major axis of the research work on slurry transport. These are typically represented as transition velocities, one of the most important being the deposition, suspension or critical velocity: defining the onset of a bed at the bottom of the pipe. There are many correlations and models for predicting the deposition velocity in literature, e.g. that of Oroskar and Turian [4]. The empirical correlations and theoretical predictions are in qualitative agreement and what is interesting about the methodology in [4] is that it is based on modeling the physical balance between turbulent eddies suspending the particles and buoyancy driven settling.

Other critical velocity predictions in literature are based on a frictional pressure drop prediction, e.g. see [3]. Prediction of frictional pressure gradient is of key importance for industrial applications and has attracted many researchers since 1950's. For example, Turian and Yuan [5] developed correlations based on over 2800 data points, for the frictional pressure in each of the four different flow regimes. This is probably the most comprehensive empirical correlation developed to date.

Particle turbulent diffusivity prediction plays an important role in modeling the slurry flow and predicting the concentration distribution in the pipe. We mention here the work of Walton [6] who proposed a correlation to predict the mean particle diffusivity which consists of three empirical parameters, Based on which he derived an equation for the critical particle velocity. However, the correlation does not account for the dependence of the mean particle diffusivity on the solids concentration.

In the present paper we present a new three-layer model which is based on Doron and Barnea's concept [3], with the main change in defining the solids diffusivity. This affects the prediction of the concentration distribution in the hetero- 
geneous layer and with this the critical velocity. A number of the stress and force closure models in the bed layer were changed and the heterogeneous layer Reynolds number for fine particles was modified. Validation against experimental data is presented in [1]. In $\$[$ II we outline the model development and assumptions, and in \$III, we bring an example of reference outputs from the proposed model.

\section{Model DEVElopment}

In this study we outline a steady state three-layer model used to predict the transport of solids in horizontal wells, typically sand-water flows. These flows arise in many well operations and the model is developed to overcome limitations of existing three- and two-layer models. Conservation of mass equations for the two phases and momentum balance in each layer are combined in the model. A convection-diffusion equation equation models the solids distribution profile and average concentration in the heterogeneous suspension layer, above the bed. These 5 equations plus the imposed flow rate are solved iteratively to predict pressure drop, individual layer heights, mean layer velocities and solids distribution. Additional closure expressions are used to describe other effects.

\section{A. Dimensional analysis}

Steady state slurry flow along the pipe depends on many parameters such as the pipe diameter, $\hat{D}$, the liquid phase density, $\hat{\rho}_{l}$, the solids phase density, $\hat{\rho}_{s}$, the liquid phase viscosity, $\hat{\mu}_{l}$, the particle diameter in the solids phase, $\hat{d}_{p}$, Gravitational acceleration, $\hat{g}$, the flow rate of the slurry, $\hat{Q}$, measured positive in the downwards direction along the pipe, and the fraction of the flow rate due to the solids phase, $q$, (or alternatively, the mean volumetric concentration of solids in pipe cross section, say $C_{v}$ ). The last two mentioned parameters are dimensionless, but others are dimensional 1 Thus, the base flow is described by four dimensionless groups, plus the solids phase flux fraction, $q$, (or $C_{v}$ ). There are various equivalent choices for the four dimensionless groups, but we will adopt two geometric groups, which are the scaled diameter ratio, $\delta=\hat{d}_{p} / \hat{D}$, and the density ratio, $s=\hat{\rho}_{s} / \hat{\rho}_{l}$, and two others, the Froude number $(F r)$ and Reynolds number $(R e)$ :

$$
\begin{aligned}
& R e=\frac{\hat{\rho}_{l} \hat{D} \hat{U}_{s}}{\hat{\mu}_{l}}, \\
& F r=\frac{\hat{U}_{s}^{2}}{\hat{g} \hat{D}(s-1)},
\end{aligned}
$$

where $\hat{U}_{s}=4 \hat{Q} /\left(\pi \hat{D}^{2}\right)$. Other choices could have included a Richardson or Bagnold number.

The base set of five parameters $(R e, s, F r, \delta, q)$ is clearly not sufficient to fully describe all phenomena one is likely to encounter in a pipe flow. Characterising the particle distribution via a single parameter is considered as a simplification, apart from a size distribution particle shape can have significant effects and as soon bed formation and motion

\footnotetext{
${ }^{1}$ Throughout this paper we write all dimensional quantities with a $\hat{.}$ symbol and dimensionless parameters without.
}
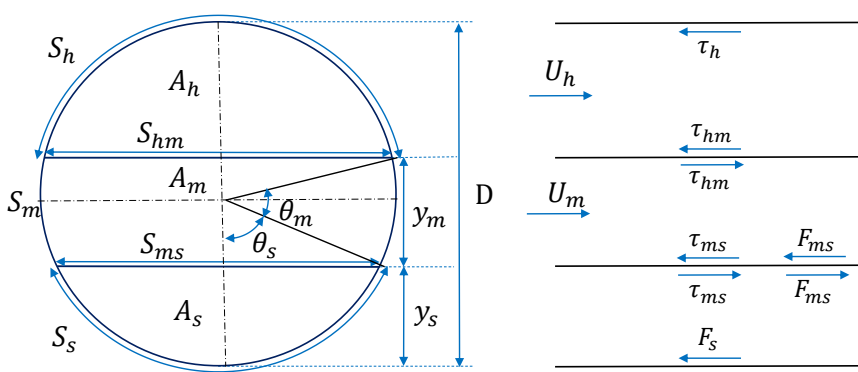

Fig. 1. Schematic of the proposed three-layer model, including geometrical parameters, moving and static bed layers positions, and stresses and forces on each layer

is considered other mechanical and geometric parameters become important, e.g. friction coefficient, maximal packing fraction, etc.

In order to render the model dimensionless we scale all lengths with $\hat{D}$, areas with $0.25 \pi \hat{D}^{2}$, velocities with $\hat{U}_{s}$. The stresses are scaled with $0.5 \hat{\rho}_{l} \hat{U}_{s}^{2}$, forces are scaled with $0.5 \hat{\rho}_{l} \hat{U}_{s}^{2} \hat{D}$ and the pressure gradient is scaled as follows:

$$
\frac{\partial \hat{p}}{\partial \hat{z}}=\left[\hat{\rho}_{l}\left(1-C_{m}\right)+\hat{\rho}_{s} C_{m}\right] \hat{g}+\frac{2 \hat{\rho}_{l} \hat{U}_{s}^{2}}{\pi \hat{D}} \frac{\partial p}{\partial z},
$$

where $0.25 \pi \hat{D}^{2} C_{v}=\hat{A}_{h} C_{h}+\hat{A}_{b} C_{\max }$, i.e. $C_{v}$ is the mean spatial solids concentration.

\section{B. Three-layer steady state model equations}

According to the flow parameters, the pipe cross-section may contain moving and/or stationary beds at the bottom of the pipe, of heights $\hat{y}_{m}$ and $\hat{y}_{s}$ respectively, see Fig. 1 . Above the bed the flow is assumed to consist of a heterogeneous slurry, in which the concentration (volume fraction) of solids varies with height $\hat{y}_{h}$. Within the bed the concentration is assumed to be at the maximal packing fraction, $C=C_{\max }$. The heterogeneous layer moves in the axial direction with mean speed $\hat{U}_{h}$ and the moving bed layer moves with mean speed $\hat{U}_{m}$.

Conservation of the total mass flow rate is represented by

$$
\hat{Q}=0.25 \pi \hat{D}^{2} \hat{U}_{s}=\hat{A}_{h} \hat{U}_{h}+\hat{A}_{m} \hat{U}_{m},
$$

and mass conservation of the solid and liquid phases is governed by:

$$
\begin{aligned}
\hat{Q} q & =C_{h} \hat{A}_{h} \hat{U}_{h}+C_{\max } \hat{A}_{m} \hat{U}_{m} \\
\hat{Q}(1-q) & =\left(1-C_{h}\right) \hat{A}_{h} \hat{U}_{h}+\left(1-C_{\max }\right) \hat{A}_{m} \hat{U}_{m}
\end{aligned}
$$

where $C_{h}$ is the mean solids concentration of the heterogeneous layer and the areas $\hat{A}_{h}$ and $\hat{A}_{m}$ are heterogeneous and moving bed cross section areas respectively. Evidently, one of Eqs. [3- 5] is redundant.

We select Cartesian coordinates such that $\hat{z}$ denotes axial distance along the pipe in the downwards direction, $\hat{y}$ measure distance perpendicularly upwards from the base of the pipe, 
viewed in a cross-section, and the $\hat{x}$ direction is orthogonal, within the plane of the cross-section. The axial momentum balance in heterogeneous and moving bed layers are:

$$
\begin{aligned}
& \hat{A}_{h} \frac{\partial \hat{p}}{\partial \hat{z}}=-\hat{S}_{h} \hat{\tau}_{h w}-\hat{S}_{h m} \hat{\tau}_{h m} \\
& \hat{A}_{m} \frac{\partial \hat{p}}{\partial \hat{z}}=-\hat{F}_{m w}-\hat{F}_{m s}-\hat{S}_{m s} \hat{\tau}_{m s}-\hat{S}_{m} \hat{\tau}_{m w}+\hat{S}_{h m} \hat{\tau}_{h m}
\end{aligned}
$$

where the perimeters $\hat{S}_{h}$ and $\hat{S}_{m}$ are illustrated in Fig. 1 The axial pressure gradient is denoted $\frac{\partial \hat{p}}{\partial \hat{z}}$. The mean shear stresses along $\hat{S}_{h w}$ and $\hat{S}_{m w}$ are denoted $\hat{\tau}_{h w}$ and $\hat{\tau}_{m w}$, respectively. $\hat{F}_{m s}$ is the dry friction force acting at the interface between the moving bed and stationary bed, $\hat{\tau}_{m s}$ is the hydrodynamic shear stress acting on the interface. $\hat{F}_{m w}$ is the dry friction force acting at the surface of contact of the moving bed with the pipe wall, $\hat{S}_{m w}$, and $\hat{\tau}_{m w}$ is the hydrodynamic shear stress acting on the pipe wall. Closure models for the stresses and forces are described in \$I-D

To obtain the velocity of the moving bed layer, which is a key feature of the three-layer model, we use the method suggested by [3], in which a moment balance equation is written on a solid particle in the lowermost stratum of the moving bed layer, which is at the verge of rolling (for more details, see [3]). Evaluating the moments exerted about the particle, just about to move, we get:

$$
\hat{U}_{m}=\sqrt{\frac{0.779\left(\hat{\rho}_{s}-\hat{\rho}_{l}\right) \hat{g} \hat{d}_{p}\left[C_{\max } \frac{\hat{y}_{m}}{\hat{d}_{p}}+\left(1-C_{\max }\right)\right]}{\hat{\rho}_{l} C_{D}}} .
$$

Within the heterogeneous layer, the solids distribution $C(\hat{y})$ is governed by a balance between sedimentation and turbulent resuspension:

$$
0=\frac{\partial}{\partial \hat{y}}\left[\hat{v}_{p} C+\hat{\epsilon}_{p} \frac{\partial C}{\partial \hat{y}}\right]
$$

where $\hat{v}_{p}$ is the sedimentation velocity and $\hat{\epsilon}_{p}$ is the turbulent particle diffusivity. At the interface between bed and heterogeneous layer we have $C=C_{\max }$, and at the top of the heterogeneous layer there is no flux of particles, so that:

$$
\hat{v}_{p} C+\hat{\epsilon}_{p} \frac{\partial C}{\partial \hat{y}}=0 .
$$

\section{Concentration distribution in the heterogeneous layer}

On scaling $\hat{y}$ with $\hat{D}$, and on integrating (9), the solids distribution within the heterogeneous layer $\left(y_{b}, 1\right]$ satisfies the following initial value problem:

$$
\frac{\partial C}{\partial y}+\frac{\hat{v}_{p} \hat{D}}{\hat{\epsilon}_{p}} C=0, \quad\left(y_{b}, 1\right], \quad C\left(y_{b}\right)=C_{0} .
$$

In the case that there is a bed, $\left(y_{b}>0\right)$, then the initial condition is given by $C_{0}=C_{\max }$. The differential equation
(11) is solved to give $C(y)$ and the area-averaged value of $C(y)$ is then computed as follows:

$$
\bar{C}\left(y_{b}\right)=\frac{\int_{y_{b}}^{1} C(y) \sqrt{1-(2 y-1)^{2}} \mathrm{~d} y}{\int_{y_{b}}^{1} \sqrt{1-(2 y-1)^{2}} \mathrm{~d} y},
$$

We note that the value of $\bar{C}$ depends also on the initial condition $C_{0}$, used in (11), and on any other dimensionless parameters in 11 . Thus, we write $\bar{C}=\bar{C}\left(y_{b}, C_{0}\right)$ and the height of the heterogeneous layer is determined from the equation:

$$
\bar{C}\left(y_{b}, C_{0}=C_{\max }\right)=C_{h} .
$$

In the case that there is no bed, $\left(y_{b}=0\right)$, then the initial condition is unknown but the average concentration must still equal $C_{h}$. Thus, $C(y)$ satisfies $\sqrt{11}$ and the initial condition is found from

$$
\bar{C}\left(y_{b}=0, C_{0}\right)=C_{h} .
$$

Evidently, the solution of (11) depends on the closure laws used for the sedimentation velocity, $\hat{v}_{p}$, and for the turbulent particle diffusivity, $\hat{\epsilon}_{p}$.

1) Sedimentation velocity, $\hat{v}_{p}$ : The sedimentation velocity is assumed to depend on the particle concentration according to Richardson and Zaki's hindered settling law [7]:

$$
\hat{v}_{p}=\hat{v}_{p 0}(1-C)^{n},
$$

where $\hat{v}_{p 0}$ is the settling velocity of a single particle and $n$ depends upon the particle Reynolds number, $R e_{p}$. The settling velocity of a single particle could be obtained by a force balance between the gravitational and drag forces:

$$
\hat{v}_{p 0}=\sqrt{\frac{4 \hat{g}(s-1) \hat{d}_{p} \sin \beta}{3 C_{D}\left(R e_{p}\right)}},
$$

where $C_{D}\left(R e_{p}\right)$ is the drag coefficient:

$$
C_{D}\left(R e_{p}\right)= \begin{cases}\frac{24}{R e_{p}} & R e_{p}<1.4 \\ \frac{A_{C D}}{R e_{p}^{0.625}} & 1.4 \leq R e_{p} \leq 500 \\ \frac{A_{C D}}{500^{0.625}} & 500<R e_{p}\end{cases}
$$

where $A_{C D}=24 / 1.4^{0.375}$, and

$$
R e_{p}=\frac{\hat{\rho}_{l} \hat{v}_{p 0} \hat{d}_{p}}{\hat{\mu}_{l}} .
$$

Using the groups $R e, F r$, and $\delta$, we can solve explicitly for $\hat{v}_{p 0}$, from which $R e_{p}$ is determined.

For very low particle Reynolds numbers, the exponent $n$ only depends on the diameter ratio $\delta$, whereas in the intermediate range of Reynolds number it depends on both $\delta$ and Reynolds number, and lastly, for high Reynolds numbers it is a constant. These closure expressions can be found in [7]. It is also worth mentioning that the range of $R e_{p}>500$ is unlikely to be attained. The values of $n$ are between 2.39 
for inertia dominated settling and 4.65 for viscous dominated settling, and also it is a strong function of $R e_{p}$ specially when $0.2 \leq R e_{p}<500$, while it is a weak function of $\delta$.

2) Turbulent particle diffusivity, $\hat{\epsilon}_{p}$ : For obtaining the solids diffusivity, we have adopted and modified Eskins model [8], which is based on an expression for the turbulent diffusivity in isotropic turbulence and is characterized by two empirical parameters that have been identified from the experimental data for solids concentration distribution available in the literature. Eskins model only covers a small range of diameter ratio $\left(\delta=3.636 e^{-4}\right.$ to $\left.9.320 e^{-3}\right)$, and also used the pressure drop model of Turian and Yuan [5] to obtain the pressure drop and the Fanning friction factor along the pipe. Here we have modified the solid diffusivity model taking into account a wider range of diameter ratio including much courser ones, using the pressure drop obtained by the present model. The modified solids diffusivity correlation is validated against the experimental data for solids concentration distribution and critical velocity in the literature; see [1]. The modified solid diffusivity correlation is as follows.

$$
\hat{\epsilon}_{p}=\alpha \delta^{\zeta}\left(\frac{\overline{\hat{\rho}}_{h}}{\hat{\rho}_{h}} \frac{d p}{d x}\right)^{\frac{2}{3}}\left(1+\frac{\tau_{s}}{T_{l}}\right) \hat{D} \hat{U}_{s},
$$

where $\overline{\hat{\rho}}_{h}$ is the mean slurry density in the heterogeneous layer, $\hat{\rho}_{h}$ is the slurry density which is a function of the local concentration, $\tau_{s}$ is the particle relaxation time, and $T_{l}$ is the Lagrangian time scale for the pipe flow. This model was fitted against the experimental concentration data using least square method and we obtained $\alpha=0.81$, and $\zeta$ was found to be dependent on the diameter ratio as

$$
\zeta=-333.3 \delta^{2}+26.37 \delta+1.355 .
$$

The particle relaxation time can be calculated as

$$
\tau_{s}=\tau_{s t} \frac{24}{R e_{p} C_{D}}
$$

where $\tau_{s t}$ is the Stokesian particle residence time in the flow.

\section{Closure equations for stresses and forces}

In the following subsections we define the various closures we have used for the stresses and forces in each layer. For friction factors we use the Fanning friction factor as opposed to the Darcy-Weisbach friction factor, i.e. the relation between a wall stress $\hat{\tau}_{w}$ and the friction factor $f$ is

$$
\hat{\tau}_{w}=0.5 f \hat{\rho} \hat{U}^{2},
$$

and typically $f=16 / R e$ in laminar flow. Explicit friction factor formulas will be defined in the following subsections for each layer.

1) Heterogeneous layer: The wall shear stress in the heterogeneous layer is defined by

$$
\hat{\tau}_{h w}=0.5\left[\hat{\rho}_{s} C_{h}+\hat{\rho}_{l}\left(1-C_{h}\right)\right] \hat{U}_{h}\left|\hat{U}_{h}\right| f\left(R e_{h}, 0\right),
$$

where $R e_{h}$ is the heterogeneous layer Reynolds number and $f_{C W}$ is the friction factor, based on the Colebrook-White relation for turbulent flows, as described below.
We base the heterogeneous layer Reynolds number $R e_{h}$ on the hydraulic diameter of the layer, on $\hat{U}_{h}$, on the mean density of the slurry and on an effective viscosity. Typically these flows are fully turbulent and the viscosity to use depends therefore on how viscous stresses are conveyed to the wall. In the case that the particles are larger than the viscous sub-layer in the flow, then the fluid effective viscosity is simply $\hat{\mu}_{l}$. In the case that the particle size is comparable to the thickness of the viscous sub-layer, we treat the slurry as a pseudo-fluid, with effective viscosity:

$$
\hat{\mu}_{s}=\hat{\mu}_{l}\left[1.0+2.5 C_{h}+10 C_{h}^{2}+0.0019 \mathrm{e}^{20 C_{h}}\right],
$$

see [9]. Eq. (23) is an extension of the Einstein-Thomas relation to higher concentrations and sand particles.

To estimate the size of the viscous sublayer $\hat{d}_{v}$ we use the estimate

$$
\hat{d}_{v} \approx 10 \frac{\hat{D}_{h}}{R e_{h} \sqrt{0.5 f\left(R e_{h}, 0\right)}}
$$

(i.e. the viscous layer is approximately $10 y^{+}$in turbulent duct flows). Here $\hat{D}_{h}$ is the hydraulic diameter of the heterogeneous layer and $f$ is the friction factor.

If the particle diameter $\hat{d}_{p}$ satisfies $\hat{d}_{p}<\hat{d}_{v}$ we assume 23. to be valid: $\hat{\mu}_{h}=\hat{\mu}_{s}$. If the particle diameter is significantly larger than $\hat{d}_{v}$, say $\hat{d}_{p}>10 \hat{d}_{v}$, we assume that $\hat{\mu}_{h}=\hat{\mu}_{l}$. For intermediate values of $\hat{d}_{p} / \hat{d}_{v}$ we interpolate between the above values. Finally, $R e_{h}$ could be obtained using the effective viscosity in the heterogeneous layer, $\hat{\mu}_{h}$. We note that the viscosity ratio $\hat{\mu}_{h} / \hat{\mu}_{l}$ and $R e_{h}$ depend on the 6 parameters $\left(R e, C_{h}, s, \delta, y_{b}, u_{h}\right)$.

The friction factor formula we use is based on the Colebrook-White equation for turbulent flow. The ColebrookWhite expression is:

$$
\sqrt{1 / f}=-2 \log \left(\frac{2.51}{R e \sqrt{f}+\frac{\epsilon_{r}}{3.71}}\right),
$$

which gives a Darcy-Weisbach friction factor $f\left(R e, \epsilon_{r}\right)$.

2) Interfacial stresses: The interfacial stresses are defined in essentially the same as the heterogeneous layer stresses, except that the velocity differences are used, and a roughness is assumed at each interface to determine the corresponding friction factors.

We assume that the "interface" between the heterogeneous and moving bed layers is rough, with the roughness $\epsilon_{r}=$ $\min \left\{1, \delta / d_{h}\right\}$ where $d_{h}$ is the dimensionless hydraulic diameter of the heterogeneous layer. We also assume the "surface" between heterogeneous layer and bed to be "rough", with roughness $\epsilon_{r}=\min \left\{1, \delta / d_{h}\right\}$. The interfacial stress is then defined as follows:

$$
\begin{aligned}
\hat{\tau}_{h m} & =0.5\left[\hat{\rho}_{s} C_{h}+\hat{\rho}_{l}\left(1-C_{h}\right)\right]\left(\hat{U}_{h}-\hat{U}_{m}\right) \\
& \times\left|\hat{U}_{h}-\hat{U}_{m}\right| f\left(R e_{h}, \min \left\{1, \delta / d_{h}\right\}\right) .
\end{aligned}
$$

Similarly, for the interface between the moving and stationary bed layers, we assume the roughness $\epsilon_{r}=\min \left\{1, \delta / d_{m}\right\}$, and accordingly, we define the dimensional interface stress as follows. 


$$
\begin{aligned}
\hat{\tau}_{m s} & =0.5\left[\hat{\rho}_{s} C_{\max }+\hat{\rho}_{l}\left(1-C_{\max }\right)\right] \hat{U}_{m}\left|\hat{U}_{m}\right| \\
& \times f\left(\operatorname{Re}_{h}, \min \left\{1, \delta / d_{m}\right\}\right),
\end{aligned}
$$

where $d_{m}$ is the dimensionless hydraulic diameter of the moving bed layer. In addition to the defined interfacial stresses in 26], we should consider the solid particle contribution to the friction force on the interface $S_{m s}$ due to the effect of the submerged weight of the particles in the moving bed layer. In the heterogeneous layer, it is assumed that the weight of the particles is entirely supported by turbulent suspension. In contrast, the submerged weight of the particles in the moving bed layer is supported by the wall and also the interface $S_{m s}$. The resulting dry friction force at the interface which is transmitted to the wall as a Coulomb friction term is defined as

$$
\hat{F}_{m s}=\eta\left(\hat{\rho_{s}}-\hat{\rho}_{l}\right) g C_{\max } \hat{y_{m}} \hat{S_{m s}} .
$$

3) moving bed layer: Our treatment of the moving bed layer wall shear stress is similar to that of the heterogeneous layer, which is defined as

$$
\begin{aligned}
\hat{\tau}_{m w} & =0.5\left[\hat{\rho}_{s} C_{\max }+\hat{\rho}_{l}\left(1-C_{\max }\right)\right] \hat{U}_{m}\left|\hat{U}_{m}\right| \\
& \times f\left(R e_{m}, 0\right) .
\end{aligned}
$$

Where $R e_{m}$ is the moving bed layer Reynolds number which could be determined as follows.

$$
R e_{m}=\operatorname{Re} d_{m}\left|u_{m}\right|\left(1+(s-1) C_{\max }\right) .
$$

As was discussed in \$II-D2 the submerged weight of the solid phase is partially transmitted through the bed to the wall as a Coulomb friction term. Thus, the dimensional term of the dry friction force at the wall is defined as follows.

$$
\begin{aligned}
& \hat{F}_{m w}=2 \eta\left(\hat{\rho}_{s}-\hat{\rho}_{l}\right) g C_{\max }\left(\frac{D}{2}\right)^{2} \\
\times & \left\{\left[2 \frac{\left(\hat{y}_{s}+\hat{y}_{m}\right)}{D}-1\right] \theta_{m}+\cos \left(\theta_{m}+\theta_{s}\right)-\cos \theta_{s}\right\} .
\end{aligned}
$$

4) Static bed layer, and transition to two-layer solution: The existence of a static bed is determined based on the momentum balance equation on the static bed layer. It should be checked whether the sum of driving forces on the static bed layer exceeds the maximal available resistance force on this layer. The driving forces on the static layer are pressure gradient, and the shear at the interface between the moving and static bed layers. The maximal available resistance is the dry friction force acting on the periphery of the static bed which is defined as follows.

$$
\begin{gathered}
\hat{F}_{m w}=2 \eta_{s}\left(\hat{\rho}_{s}-\hat{\rho}_{l}\right) g C_{\max }\left(\frac{D}{2}\right)^{2} \\
\times\left[\left(\frac{2 \hat{y}_{s}}{\hat{D}}-1\right)\left(\theta_{s}+\frac{\pi}{2}\right)+\cos \theta_{s}\right] .
\end{gathered}
$$

where $\eta_{s}$ is the dry static friction coefficient. if

$$
\hat{A}_{s} \frac{\partial \hat{p}}{\partial \hat{z}}+\hat{F}_{m s}+\hat{S}_{m s} \hat{\tau}_{m s} \leqslant \hat{F}_{s w},
$$

is satisfied, the static bed layer does not move, and it is indeed "static"; Otherwise, the static bed layer moves and we get a transition from three-layer model solution to the twolayer model solution, where we assume that there is one bed layer in the pipe which is moving. For solving the two-layer problem, we adopt the similar procedure to the three-layer problem. The only difference between the two problems is that in the two-layer model, five equations are numerically handled to get the five unknowns $\hat{U}_{h}, \hat{U}_{m}, \hat{y}_{m}, C_{h}, \frac{\partial \hat{p}}{\partial \hat{z}}$, whereas for three-layer model we deal with six equations to get six unknowns (aformentioned unknowns plus $\hat{y}_{s}$ ).

\section{RESULT AND DISCUSSION}

An example of reference outputs from the proposed model is given in Figs. 2(a-f). We present results for $C_{v}=$ $0.2,0.25,0.3,0.35$, over a wide range of mean superficial velocities, for the horizontal pipe diameter of $D=0.1 \mathrm{~m}$. For each example we present the solids phase flux fraction, frictional pressure drop, heterogeneous and moving bed layers velocities, and moving and static bed layers heights. Note that all of the outputs in Figs. 2(a-f) are dimensionless except for the pressure drop result (Fig. 2(f)). As mentioned before, the velocities are scaled with slurry mean superficial velocity $\hat{U}_{s}$, and the lengths are scaled with the pipe diameter $D$.

Eq. 11 suggests that the concentration distribution, and the bed height in the pipe depend on the competition between the sedimentation velocity and solid turbulent diffusivity. At low mean superficial velocities, the turbulent eddies are not strong enough to suspend the solid phase; As a result , a considerable portion of the pipe is covered with bed layers, and we get low mean delivered solids concentration (Figs. 2(a), 2( c), and2(e)). Also, the height of the static bed is larger than the moving bed (Figs. 2(c) and 2(e)). As a result, the mean heterogeneous layer velocity is at its maximum at low flow rates to satisfy the continuity equation (Fig. 2(b)).

As the mean superficial velocity increases, the turbulent eddies get more capable of suspending the solids; Thus, the mean delivered solids concentration increases as could be observed in Fig. 2(a). Furthermore, the height of moving bed increases as the height of static bed drops(Figs. 2(c) and 2 (e)). The total bed height also decreases with increasing the flow rate. From Eq. (8) it is obvious that the mean moving bed velocity $\hat{U}_{m}$ monotonically increases with the moving bed height as long as we get a three-layer configuration with a static bed, although Fig. 2(d) could be misleading as it shows the dimensionless mean moving bed velocity $u_{m}$ decreases with the flow rate increases.

As the flow rate increases, we reach a point where there is no static bed layer, and the pipe consists of the heterogeneous and the moving bed layers. As was discussed in \$II-D4, this is the transition from the three-layer to two-layer model solution, where the mean velocity and the height of the moving bed 
$\mathrm{g}$ bed

eneous

$m, 0)$,

(34a)
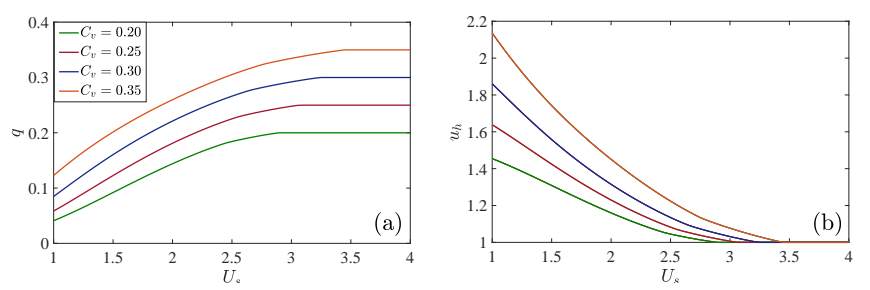

(34b)

which

ght of

to the

al and

all are

1] $\theta_{m}+\mathrm{cc}$

(36a)
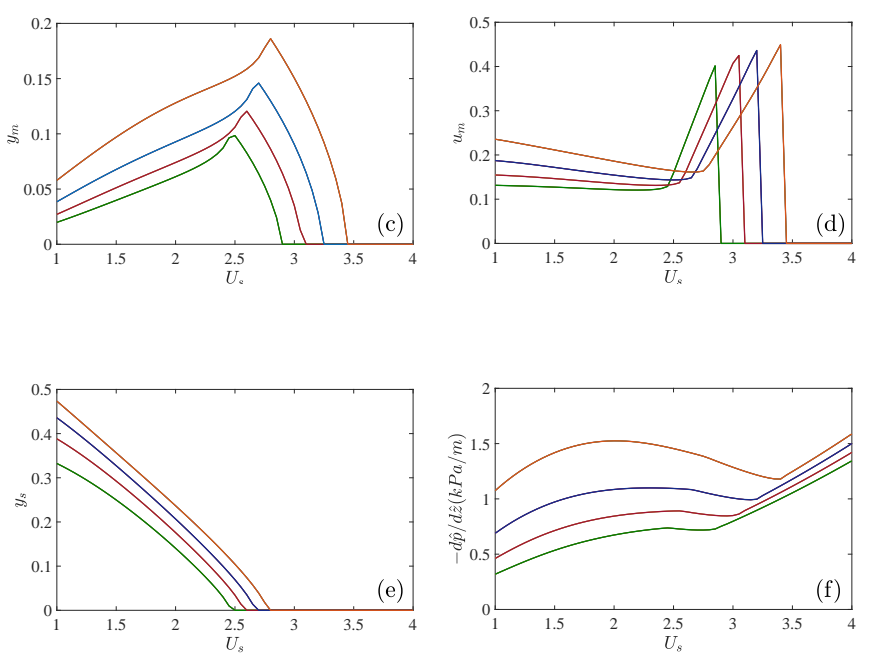

$\left.\theta_{s}\right)-\cos$

(36b)

Fig. 2. Example solutions from the proposed steady state three-layer model problem at various $C_{v}$, as a function og $\hat{U}_{s}$, for a sand-water mixture flowing in a horizontal pipe of diameter $\hat{D}=0.1 \mathrm{~m}$. Fixed parameters are $C_{\max }=$ $0.55, \hat{\rho}_{s}=2650 \mathrm{~kg} / \mathrm{m}^{3}, \hat{\rho}_{l}=1000 \mathrm{~kg} / \mathrm{m}^{3}, \hat{\mu}_{l}=9 \times 10^{-4}$ Pa.s, $\hat{d}_{p}=$ $7 \times 10^{-4} \mathrm{~m}, \eta=0.5$, and $\eta_{s}=0.7$.

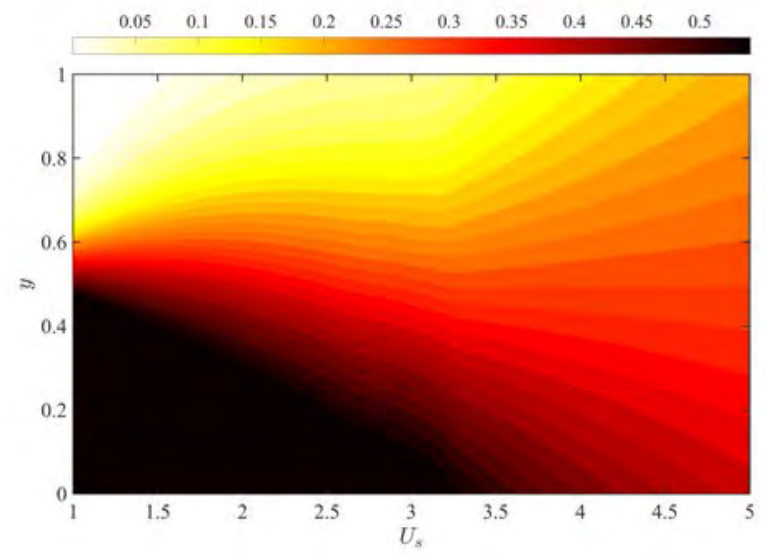

Fig. 3. colormap of concentration in $\left(\hat{U}_{s}, y\right)$ plane for $C_{v}=0.3$. Fixed parameters are $C_{\max }=0.55, \hat{\rho}_{s}=2650 \mathrm{~kg} / \mathrm{m}^{3}, \hat{\rho}_{l}=1000 \mathrm{~kg} / \mathrm{m}^{3}, \hat{\mu}_{l}=$ $9 \times 10^{-4}$ Pa.s, $\hat{d}_{p}=7 \times 10^{-4} \mathrm{~m}, \eta=0.5$, and $\eta_{s}=0.7$. layer reach their maximum values. As we further increase the flow rate, the height of moving bed decreases until we reach the deposition velocity where there is no sedimentation bed, and we get a single heterogeneous layer across the pipe, and evidently, the mean delivered solids concentration is equal to the mean spatial solids concentration (Fig. 22a)). As can be observed in Fig. 2(f), the pressure gradient reaches its minimum value at the deposition velocity. Furthermore, the deposition velocity increases with $C_{v}$. These findings are consistent with the published results of most of the researchers in the field, e.g. [2], [5].

Fig. 3 shows a colormap of solids concentration in the $\left(\hat{U}_{s}, y\right)$ plane for a constant value of $C_{v}=0.3$. At low flow rates, the bottom of the pipe is at maximum packing concentration $C_{\max }$ which indicates the existence of the sedimentation bed. Also, we observe that the concentration gradient is very large in the heterogeneous layer at low flow rates. As $\hat{U}_{s}$ increases, the total bed height decreases as was discussed before, and the concentration gradient drops as well. At very high velocities, the solids turbulent diffusivity becomes dominant and we get a pseudo-homogeneous slurry flow in the pipe.

\section{Conclusions}

A modified three-layer model for solid-liquid flow in horizontal pipes has been developed which predicts the pressure loss, critical velocity, concentration profile in the heterogeneous layer, mean heterogenous layer and moving bed layer velocities, and bed layer heights for each set of parameters. We have also proposed a new correlation for the turbulent solids diffusivity based on concentration profile and critical velocity comparison against the experimental data available in the literature. The pressure loss vs mean velocity curve shows a characteristic minimum just before the critical velocity is attained, in agreement with published research. 


\section{REFERENCES}

[1] A. Sarraf Shirazi and I. Frigaard, "A novel three-layer model for steadystate slurry flow in inclined pipes," unpublished.

[2] C. Shook and A. Bartosik, "Particlewall stresses in vertical slurry flows," Powder Technol., vol. 81, no. 2, pp. 117 - 124, 1994. doi: 10.1016/0032-5910(94)02877-X.

[3] P. Doron and D. Barnea, "A three-layer model for solid-liquid flow in horizontal pipes," Int. J. Multiphase Flow, vol. 19, no. 6, pp. 1029 1043, 1993. doi: 10.1016/0301-9322(93)90076-7.

[4] A. R. Oroskar and R. M. Turian, "The critical velocity in pipeline flow of slurries," AIChE J., vol. 26, no. 4, pp. 550-558, 1980. doi: 10.1002/aic.690260405.

[5] R. M. Turian and T.-F. Yuan, "Flow of slurries in pipelines," AIChE J., vol. 23, no. 3, pp. 232-243, 1977. doi: 10.1002/aic.690230305.

[6] I. Walton, "Eddy diffusivity of solid particles in a turbulent liquid flow in a horizontal pipe," AICHE J., vol. 41, no. 7, pp. 1815-1820, 1995. doi: 10.1002/aic.690410716.

[7] J. F. Richardson and W. N. Zaki, "Sedimentation and fluidisation: Part i," Chem. Eng. Res. Des., vol. 75, pp. S82-S100, 1997.

doi: 10.1016/S0263-8762(97)80006-8.

[8] D. Eskin, "A simple model of particle diffusivity in horizontal hydrotransport pipelines," Chem. Eng. Sci., vol. 82, pp. 84 - 94, 2012. doi: 10.1016/j.ces.2012.07.017.

[9] J. Schaan, R. J. Sumner, R. G. Gillies, and C. A. Shook, "The effect of particle shape on pipeline friction for newtonian slurries of fine particles," Can. J. Chem. Eng., vol. 78, no. 4, pp. 717-725, 2000.

doi: $10.1002 /$ cjce. 5450780414 . 


\title{
Adsorbent-Adsorbate Pairs for Solar Thermal Energy Storage in Residential Heating Applications: A Comparative Study
}

\author{
Kapil Narwal, R. Kempers, P. G. O’Brien \\ Lassonde School of Engineering \\ York University \\ Toronto, Canada \\ mr.k.narwal@gmail.com, kempers@yorku.ca, paul.obrien@lassonde.yorku.ca
}

\begin{abstract}
This paper investigates the feasibility of using different adsorbent-adsorbate pairs in a thermal energy storage cycle to store solar energy for residential heating applications in Canada. Silica gel, activated carbon, activated aluminum, zeolite-4A, zeolite-5A and zeolite-13X adsorbents paired with methanol and water adsorbates are considered. Calculations are made to determine the volume, mass and cost of the adsorbent-adsorbate pair required to heat a house with four occupants. Zeolite 4A-water and zeolite $13 \mathrm{X}$-water pairs are found to be the most economic (with an actual cost of 285 $\mathrm{CAD}$ and $374 \mathrm{CAD}$, respectively) and efficient (maximum heat of adsorption) adsorbent-adsorbate pairs with the minimum mass required, (290 kg and $226 \mathrm{~kg}$, respectively) to meet the spatial heating requirements of the house.
\end{abstract}

Keywords- Adsorption, adsorption pairs, thermal energy storage

\section{INTRODUCTION}

Policy changes incentivizing sustainable energy technologies have provided new opportunities for renewable sources of energy such as solar, wind, tidal and others. Investments in solar energy, which is abundantly available, has increased exponentially in recent years. However, solar energy is intermittent and should be coupled with energy storage technologies to reach its full potential. For example, one promising technology for utilizing solar energy in a Canadian climate is to store it in a thermal battery so it can be used at night to reduce building heating loads. As solar thermal energy storage costs are considerable, various technologies are being explored to optimize its capacity, operating temperature, and overall performance. Heat energy is stored at different temperatures depending on its intended application. Low temperature heat is useful for domestic air-conditioning applications whereas high temperature heat is extremely useful in industry. Furthermore, heat energy can be stored in various forms: sensible heat (solids, liquids, solid-liquid), latent heat (solid-solid, solid-liquid), chemical heat (reacting solids or liquids, gaseous compounds) and the heat of adsorption or absorption (physisorption, chemisorption, heat of solutions). Sensible heat storage technologies utilize high heat capacity materials, whereas latent heat energy storage technologies absorb/release heat when a material undergoes a phase change from solid to liquid or liquid to gas and vice-versa. On the other hand, chemical heat energy storage technologies utilize reversible thermo-chemical reactions (Rainer et al. 2012) to absorb and release heat. Sorption-based thermal storage technologies can be broadly categorized as absorption and adsorption processes, depending on whether the adsorbate atoms or molecules are dissolved in, or adhered to, the surface of the absorbent. Advantages of sorption-based thermal energy storage technologies are their minimal costs, and matured performance in heat pumping and solar refrigeration. In this article, adsorption-based thermal energy storage materials are investigated for their potential to store solar energy during the day to provide heat at night in a typical Canadian residential home.

\section{LITERATURE REVIEW}

Studies have been conducted to understand thermal energy storage technologies such as electric thermal storage heaters, salt hydrate technology, molten salt technologies and solar energy storage. (Bataineh and Taamneh 2016) conducted a feasibility analysis of both adsorption and absorption systems and concluded that extended adsorption and desorption times, poor exhibitions, and low coefficient of performance (COP) are hindering the widespread commercialization of these technologies. (Papadopoulos et al. 2003) reviewed existing solar energy based sorptive refrigeration technologies for applications in both residential and commercial spaces, and discussed the necessity of implementing energy policies to accelerate the flourishment of solar refrigeration technologies. (Cabeza et al. 2017) presented an exhaustive review on adsorption and absorption technologies in refrigeration and thermal heat storage and discussed research progression 
towards developing efficient working pairs with better storage capacities and optimal energy output. (Aliane et al. 2016) studied various solar energy technologies developed for adsorptive cooling. A comparative study of various experimental systems has been done to study the operative behaviors of individual components and whole systems. It was concluded that these technologies can potentially be extended to meet global energy demands by implementing some modest improvements. Further, (Alobaid et al. 2017) reviewed solar absorption cooling systems which were fifty percent more energy efficient than vapor compression based cooling systems. The COP and thermal collector efficiency of the solar based systems are found to vary from $0.1-0.91$ and $0.06-0.64$, respectively. It was also noted that solar collector costs comprise a significant portion of the entire system. (Zeyghami et al. 2015) discussed various concepts, designs, and experimental set-ups for thermomechanical solar adsorption systems while discussing performance enhancement approaches. (Goyal et al. 2016) presented the fundamentals of solar energy utilization in adsorption systems and provided a thorough discussion about technological and economic aspects, recognizing that poor heat and mass transfer performances, low COP, and higher costs are the major roadblocks preventing the development of competitive products in this technological field. It has also been observed that hybrid systems, which combine solar adsorption heating and cooling, enhance the system performances significantly. (Askalany et al. 2013) compared various adsorption cooling systems and presented a comparision based on adsorbentrefrigrent pairs on the basis of COP, specific cooling power, driving and evaporation temperatures. It has been observed that the systems employing silica gel-water and chloride composites-water pairs have the highest COP, while those employting zeolite-water pairs have the lowest COP. The lowest evaporation temperatures were found in a system that used metal hydride-water pairs and the lowest driving temperatures were observed for the case of silica-gel-methanol and chloride composites-methanol pairs, wheras the highest driving temperatures are found in zeolite-water pairs.

\section{ADSORPTION}

Adsorption involves the adhesion of adsorbate molecules on the adsorbent surface. The adsorbent is usually a porous material with a high surface area. Adsorbate molecules form a thin film on the surface of the adsorbent either by physisorption or chemisorption. Adsorption is referred to as physisorption if the adsorbate molecules are attached on the adsorbent surface by Vander Waals forces, and chemisorption if the adsorbate molecules are chemically attached at the adsorbent surface. Thermal heat storage, therefore, is a physiochemical phenomenon between the adsorbate and adsorbent molecules while condensation and evaporation facilitate the charging and release of adsorbate molecule onto and from the adsorbent, respectively.

\section{THERMAL ENERGY StORAGE CYCLE}

The thermal energy storage cycle for the application of residential heating in Canada, shown in Figure 1, comprises two main processes: desorption and adsorption.

Desorption process: During the day the solar irradiance provides the heat of adsorption required to drive the evaporation of adsorbate molecules from the adsorber. The average daily solar insolation available from November through March in Toronto, Canada is $7.8 \mathrm{MJ} / \mathrm{m}^{2}$ (October-9.3 $\mathrm{MJ} / \mathrm{m}^{2}$, November-5.1 MJ $/ \mathrm{m}^{2}$, December-4.3 MJ $/ \mathrm{m}^{2}$, January5.7 MJ $/ \mathrm{m}^{2}$, February-9.2 MJ $/ \mathrm{m}^{2}$ and March-13.2 MJ $/ \mathrm{m}^{2}$ ) [26]. Assuming the average size of a residential building with four occupants is $200 \mathrm{~m}^{2}$ [27] the net solar radiance available during these cold climatic conditions is $1.56 \mathrm{GJ}$ per day.

Adsorption process: During the night adsorbate molecules that have evaporated from the condenser/evaporator are adsorbed by the adsorbent, and the heat of vaporization is released to function as a heat supply for residential heating. The reaction that occurs during the desorption and adsorption processes can be written as follows:

Adsorbent $+n \cdot$ Adsorbate $\leftrightarrow$ Adsorbent $* n \cdot$ Adsorbate + Heat

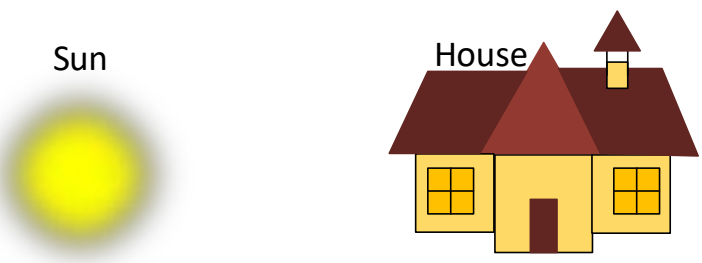

Heat of Vaporization Heat of Condensation
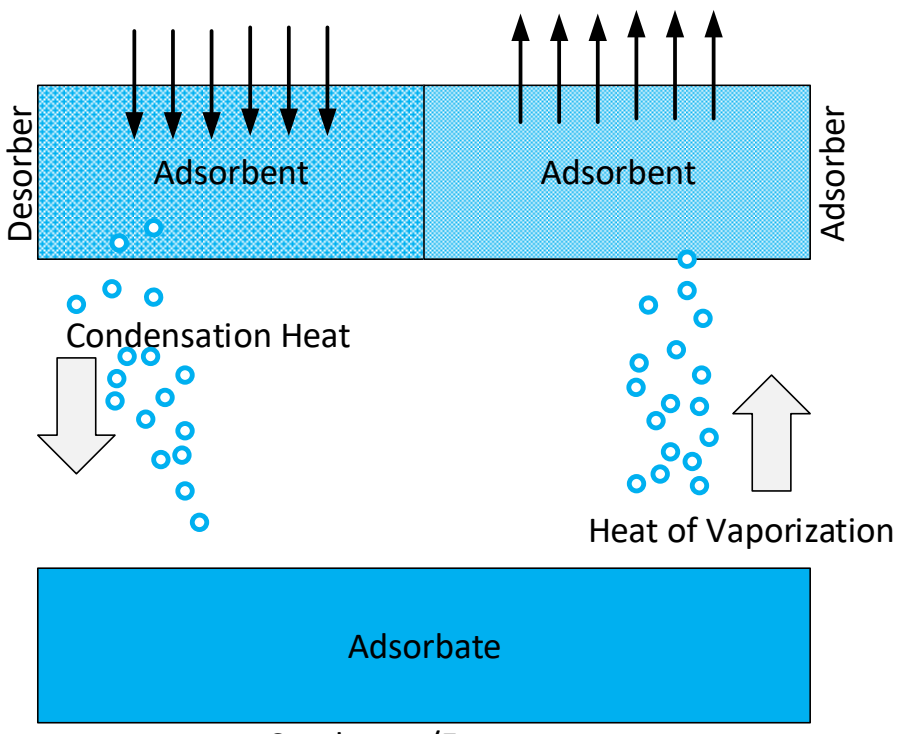

Condenser/Evaporator

Fig 1. Schematic diagram showing the desorption (left) and adsorption (right) processes in the thermal energy adsorption cycle. 


\section{ADSORPTION PAIRS}

The adsorbate and adsorbent form an essential component of the thermal energy storage cycle and various factors play a decisive role in selecting the right pair including the maximum adsorption capacity, heat of adsorption, energy capacity, specific heat of adsorbent, adsorbent density, critical pressure and critical temperature. The maximum adsorption capacity is the maximum amount of adsorbate an adsorbent can adsorb. The heat of adsorption is the heat released during the adsorption process, and can be determined by estimating the heat released in a calorimeter experiment or by adsorption isotherms. The energy capacity is the maximum energy an adsorbent can absorb or release. The specific heat of adsorbent is the heat required to raise the temperature of the adsorbent by one degree under constant pressure. At the critical temperature and critical pressure, the density of the adsorbate liquid and vapor is equal.

While many different adsorbates are available for various applications, the most commonly used are water, ammonia, methanol and ethanol. The latent heat of evaporation of water $(2258 \mathrm{~kJ} / \mathrm{kg})$ is very high compared to that of ammonia (1368 $\mathrm{kJ} / \mathrm{kg})$, methanol $(1100 \mathrm{~kJ} / \mathrm{kg}$ ) and ethanol (1100 kJ/kg). Thus, water is typically preferred, although other adsorbates can be used to produce sub-zero temperatures. Activated carbons, zeolites, silica gels, metal-organic frameworks, and potassium hydroxide $(\mathrm{KOH})$ are widely used adsorbents as they have a highly porous structure, which increases the adsorption capacity of the system. (Aristov 2007) presented selective water sorbents based on halides, sulphates and nitrates of alkaline and alkaline earth metals possessing an intermediate behavior between solid adsorbents, salt hydrates, and liquid absorbents. Also, an estimation was made on the thermodynamic equilibrium of these materials with water vapors. These sorbents are found to possess a better COP then silica gel-water and zeolite-water based systems. (Aristov 2013) reviewed existing literature on metalaluminophosphates, metal-organic frameworks, ordered porous solids, and porous carbons and composite frameworks. (Gordeeva and Aristov 2012) surveyed composite salts inside porous matrix (CSPM) sorbents. (Bhargav et al. 2017) studied the methanol-activated carbon fiber adsorption pair, and the adsorption capacity and desorption temperature were investigated under isobaric conditions using the Dubinin-Astakhov equation. (Brancato et al. 2015) tested various activated carbon fibers and composites of $\mathrm{LiBr}$ on silica gel adsorbents along with ethanol as a refrigerant. A thermo-physical analysis including nitrogen physisorption, specific heat and thermo-gravimetric equilibrium has been performed, and the Dubinin-Astakhov equation has been used to fit the equilibrium data. Furthermore, the thermodynamic performance was estimated by calculating the COP. (Erto et al. 2010) studied trichloroethylene adsorbates precipitated from water-based solutions on a set of 12 different activated carbon adsorbents. Results indicated that the adsorption capacity increases with an increase in Brunauer-Emmett-Teller (BET) surface area, micropore volume, and the percentage of carbon in the adsorbent. Further, the presence of sodium and tetrachloroethylene also increased the absorption capacity. (Meng and Park 2010) improved the $\mathrm{CO}_{2}$ adsorption capacity by inserting $\mathrm{KOH}$ in graphite nanofibers to increase its porosity under heat treatment at $700-1000^{\circ} \mathrm{C}$. The heat treatment temperature had a significant effect on the adsorption capacity and texture of graphite nanofibers, and a positive effect on surface area, total pore volume and micropore volume. (Saha et al. 2011) studied the adsorption of an ethanol-MIL101Cr pair theoretically and experimentally within a $0.1-0.9$ bar pressure range and a $30-70^{\circ} \mathrm{C}$ temperature range. One $\mathrm{kg}$ of MIL-101 $\mathrm{Cr}$ is found to adsorb up to $1.1 \mathrm{~kg}$ of ethanol at $30^{\circ} \mathrm{C}$. The Tóth equation has been used to fit experimental data with the goal of enhancing the adsorption capacity in the adsorption cooling cycle employing activated carbon fibers (ACF) as adsorbents. (Saha et al. 2015) studied various adsorbent-refrigerant pairs at a temperature of $77.3 \mathrm{~K}$. ACF (A-20), owing to its large surface area, MIL-101Cr was found to have $0.797 \mathrm{~kg} / \mathrm{kg}$ ethanol adsorption capacity. (Uddin et al. 2014) studied the highly porous Maxsorb III, $\mathrm{H}_{2}$ and $\mathrm{KOH}-\mathrm{H}_{2}$ surface treated Maxsorb III adsorbents for ethanol adsorption at $30-70^{\circ} \mathrm{C}$ and evaporation temperatures of $65^{\circ} \mathrm{C}$ experimentally. The Dubinin-Radushkevich and DubininAstakhov adsorption isotherm models have been used to obtain adsorption isotherms. Adsorption cycle performance studies were conducted using activated carbon-ethanol adsorption pairs. $\mathrm{H}_{2}$ surface treated Maxsorb III exhibits a COP of 0.51 with a specific cooling effect of $374 \mathrm{~kJ} / \mathrm{kg}$ at evaporation, heat source, and heat sink temperatures of $-5^{\circ} \mathrm{C}$, $100^{\circ} \mathrm{C}$ and $30^{\circ} \mathrm{C}$, respectively. (Zeng et al. 2017) studied the ammonia adsorption on four different kinds of activated carbons at $30^{\circ} \mathrm{C}$. MSC30 is found to possess a very high adsorption capacity for ammonia. A modified DubininAstakhov equation has been used for isothermal studies. (Dawoud et al. 2007) experimentally studied an adsorption system using a zeolite 13X-water adsorption pair. It was observed that an increase in the flow rates of the adsorber and desorber increase the heat discharge, while higher temperatures occured at lower flow rates. A radiation shield is found to be effective in minimizing radiation losses during the adsorption process. (Ansari et al. 2014) prepared nano-NaX zeolite using a hydrothermal process. The size and crystallinity of the $\mathrm{NaX}$ zeolites is found to increase up to 44 $\mathrm{nm}$ and $96 \%$, respectively, as the reaction time was increased from $1 \mathrm{~h}$ to $4 \mathrm{~h}$ and the reaction temperature increased from $90^{\circ} \mathrm{C}$ to $110^{\circ} \mathrm{C}$. (Tatler and Erdem-Şenatalar 2004) evaluated the effective diffusion coefficient of water in zeolite-4A coatings. It is observed that the evaluated results qualify the thermogravimetric analysis (TGA) and effective medium theory (EMT) based experimental results from the literature. (Solmuş et al. 2011) developed a zeolite-water pair based adsorption cooling system and investigated its performance at various evaporator temperatures. The COP of the system was about 0.25 at adsorption, desorption, and condenser temperatures of $45^{\circ} \mathrm{C}, 150^{\circ} \mathrm{C}$, and $30^{\circ} \mathrm{C}$, respectively, for evaporative temperatures ranging from $10^{\circ} \mathrm{C}$ to $22.5^{\circ} \mathrm{C}$. The mean volumetric specific cooling power density and the mean 
mass specific cooling power density were found to be 4.8 $\mathrm{kW} / \mathrm{m}^{3}$ and $6.4 \mathrm{~W} / \mathrm{kg}$, respectively. (Solmuş et al. 2010) used the Dubinin-Astakhov equation to determine a maximum adsorption capacity of $0.12 \mathrm{~kg}_{\mathrm{w}} / \mathrm{kg}_{\mathrm{ad}}$ for a natural zeolite. Furthermore, the isosteric heat was calculated and a correlation between adsorption and desorption processes was established.

\section{DISCUSSION}

Herein, different adsorbate-adsorbent pairs are investigated for their ability to store solar energy during the day to provide heating for a typical residential home in Canada. The average size of a residence with four occupants is around $200 \mathrm{~m}^{2}$ [26] with the overall energy requirements of 130 GJ yearly, or 0.36 GJ daily, by Statistics Canada [27], and it is assumed that 63\% of this energy is used for spatial heating [28]. Thus, it is assumed that $0.23 \mathrm{GJ}$ is required for spatial heating, which is much less than the average solar irradiance (1.6 GJ) estimated to be incident on a residential home in Toronto over the months from November to March (Table III).

Different adsorbate-adsorbent pairs comprising seven adsorbents (charcoal, silica gel, activated alumina, zeolie-4A, $5 \mathrm{~A},-13 \mathrm{X}$, and activated carbon) with water and methanol as

TABLE I. THERMOCHEMICAL PROPERTIES OF ADSORBENT-ADSORBATE PAIRS (RAINER ET AL. 2012)

\begin{tabular}{|c|c|c|c|c|c|c|c|c|c|c|}
\hline Material & Adsorbate & $\begin{array}{c}\text { Max. } \\
\text { adsorbate } \\
\text { capacity }\end{array}$ & $\begin{array}{c}\begin{array}{c}\text { Heat of } \\
\text { adsorption } \\
\text { (average) }\end{array} \\
\Delta h_{\text {ads }}\end{array}$ & $\begin{array}{c}\begin{array}{c}\text { Adsorbent } \\
\text { specific } \\
\text { heat }\end{array} \\
\text { Cadsorbent }\end{array}$ & $\begin{array}{l}\text { Net heat of } \\
\text { adsorption }\end{array}$ & $\begin{array}{l}\text { Net Heat of } \\
\text { adsorption per } \\
\text { unit volume of } \\
\text { adsorbent }\end{array}$ & $\begin{array}{l}\text { Volume of } \\
\text { Adsorbent } \\
\text { required for } \\
\text { residential heating }\end{array}$ & $\begin{array}{c}\text { Net } \\
\text { adsorbent } \\
\text { required }\end{array}$ & $\begin{array}{c}\text { Amount of } \\
\text { Adsorbate } \\
\text { required }\end{array}$ & $\begin{array}{c}\text { Total } \\
\text { mass of } \\
\text { system }\end{array}$ \\
\hline & & $\begin{array}{l}\text { kgadsorbate/ } \\
\text { kgadsorbent }\end{array}$ & $\begin{array}{c}\text { kJ/ } \\
\text { kgadsorbate }\end{array}$ & $\mathrm{kJ} \mathrm{kg}^{-1} \mathrm{~K}^{-1}$ & $\begin{array}{c}\text { kJ/ } \\
\text { kgadsorbent }\end{array}$ & $\mathrm{kJ} / \mathrm{m}^{3}$ & $\mathrm{~m}^{3}$ & kg & kg & kg \\
\hline Charcoal & Water & 0.4 & 2320 & 1.09 & 928 & 445440 & 0.52 & 247.84 & 99.14 & 346.98 \\
\hline Silica gel & Water & 0.37 & 2560 & 0.88 & 947.2 & 634624 & 0.36 & 242.82 & 89.84 & 332.66 \\
\hline Activated alumina & Water & 0.19 & 2480 & 1 & 471.2 & 461776 & 0.50 & 488.12 & 92.74 & 580.86 \\
\hline Zeolites 4A & Water & 0.22 & 4410 & 1.05 & 970.2 & 756756 & 0.30 & 237.06 & 52.15 & 289.22 \\
\hline Zeolites 5A & Water & 0.22 & 4180 & 1.05 & 919.6 & 625328 & 0.37 & 250.11 & 55.02 & 305.13 \\
\hline Zeolites $13 \mathrm{X}$ & Water & 0.3 & 4410 & 0.92 & 1323 & 793800 & 0.29 & 173.85 & 52.15 & 226.00 \\
\hline Active carbon & Methanol & 0.32 & 1400 & 0.9 & 448 & - & - & - & - & - \\
\hline Zeolites 4A & Methanol & 0.16 & 2300 & 1.05 & 368 & 287040 & 0.80 & 625.00 & 100.00 & 725.00 \\
\hline Zeolites 5A & Methanol & 0.17 & 2300 & 1.05 & 391 & 265880 & 0.87 & 588.24 & 100.00 & 688.24 \\
\hline Zeolites $13 \mathrm{X}$ & Methanol & 0.2 & 2400 & 0.92 & 480 & 288000 & 0.80 & 479.17 & 95.83 & 575.00 \\
\hline
\end{tabular}

TABLE II. THERMODYNAMIC PROPERTIES OF ADSORBATES FOR ADSORPTION COOLING AND HEATING [17]

\begin{tabular}{|c|c|c|c|c|c|}
\hline & Mol wt. & $\begin{array}{c}\text { Critical } \\
\text { temp. }\end{array}$ & $\begin{array}{c}\text { Critical } \\
\text { pressure }\end{array}$ & Density & Specific heat \\
\cline { 2 - 6 } & & $\mathbf{T}_{\mathrm{cr}}$ & $\mathbf{P}_{\mathrm{cr}}$ & $\mathbf{\rho}_{\mathrm{cr}}$ & $\mathbf{C}_{\mathrm{p} 0 \text {,cr }}$ \\
\hline Methanol & 32.042 & 512.64 & 8140 & 8547 & 0.061 \\
\hline Ethanol & 46.069 & 513.92 & 6120 & 5952 & 0.098 \\
\hline Ammonia & 17.031 & 405.65 & 11300 & 13889 & 0.038 \\
\hline Water & 18.015 & 647.3 & 22048 & 17857 & 0.037 \\
\hline 1-Propanol & 60.096 & 536.78 & 5120 & 4545 & 0.135 \\
\hline 2-Propanol & 60.096 & 508.3 & 4790 & 4525 & 0.133 \\
\hline
\end{tabular}

properties of adsorbate-adsorbent pairs are listed in Table I. The adsorbate capacity (figure 3 ) for the adsorbents considered herein varies from $0.16-0.37 \mathrm{~kg}$ adsorbate $/ \mathrm{kg}_{\text {adsorbent, }}$ with the silica gel-water having the maximum followed by active-carbon at $0.32 \mathrm{~kg}_{\text {adsorbate }} / \mathrm{kg}_{\text {adsorbent }}$ and zeolite- $13 \mathrm{X}$ at 0.3 $\mathrm{kg}_{\text {adsorbate }} / \mathrm{kg}_{\text {adsorbent }}$ whereas the heat of adsorption (figure 4) of zeolite is the largest with a value of $1400-4410 \mathrm{~kJ} / \mathrm{kg}_{\text {adsorbate }}$. Here the zeolite 4A-water and zeolite 13X-water pairs show high potential with energy capacities of 970 and 1320 $\mathrm{kJ} / \mathrm{kg}_{\text {adsorbent, }}$ respectively, which is the largest amongst all pairs considered. Thermodynamic properties viz. molecular weight, critical temperature, critical pressure, density and specific heat of some selective adsorbates for adsorption based cooling and heating are given in Table II. Further, Table I shows the net heat of adsorption (figure 8) per mass of adsorbent is the largest for zeolite 4A-water and zeolite 13X- 


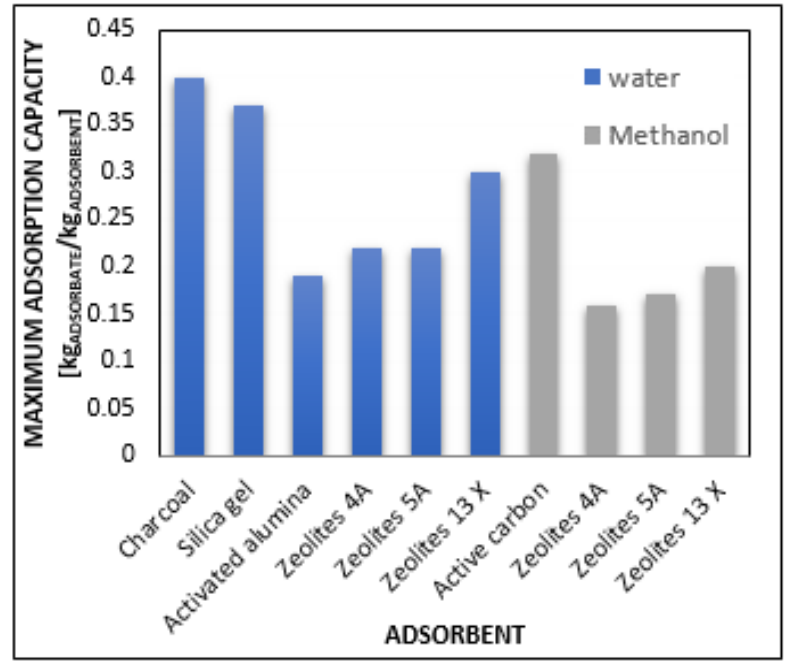

Fig 2. Maximum adsorption capacity of various adsorbents

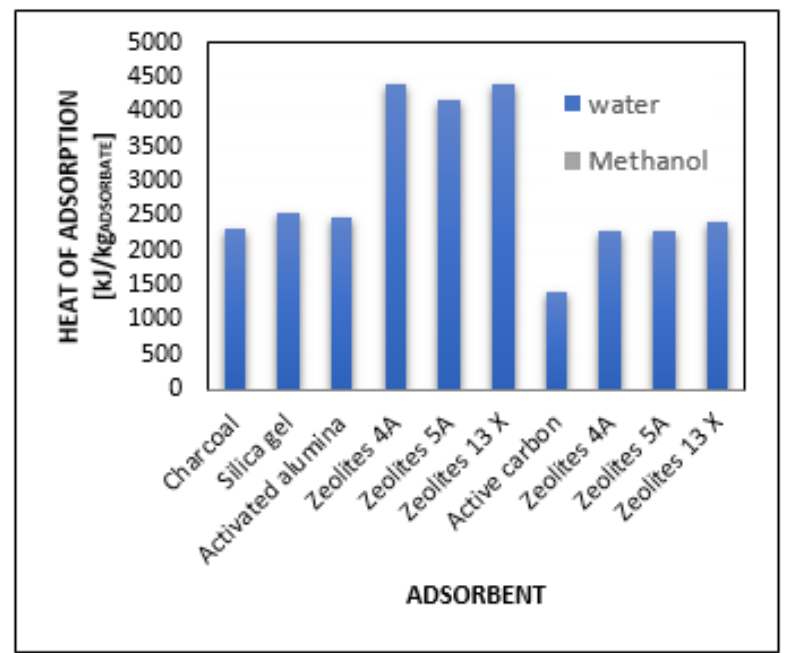

Fig 3. Heat of adsorption generated with one kilogram of various adsorbents

TABLE III. AVERAGE ENERGY REQUIREMENTS OF A HOUSE OF FOUR IN CANADA [SOURCE: STATISTICS CANADA]

\begin{tabular}{|l|c|}
\hline For a house of $\mathbf{4}$ occupants with an area $\mathbf{2 0 6 . 4} \mathbf{~ m}^{\mathbf{2}}$ & GJ \\
\hline Average energy requirement yearly & 130 \\
\hline Average energy requirement per Day & 0.36 \\
\hline $\begin{array}{l}\text { Average energy requirement for spatial heating per } \\
\text { Day }\end{array}$ & 0.23 \\
\hline $\begin{array}{l}\text { Average solar energy available per day in Toronto } \\
\text { (November-March) }\end{array}$ & 1.56 \\
\hline
\end{tabular}

water with values of 970 and $1323 \mathrm{~kJ} / \mathrm{kg}_{\text {adsorbent }}$, respectively. The total volume of adsorbent required to provide heating for a residential building with four occupants in Canada using a zeolite 4A-water pair is $0.30 \mathrm{~m}^{3}$, while the volume would be $0.29 \mathrm{~m}^{3}$ if a zeolite $13 \mathrm{X}$-water pair were to be used. If a zeolite

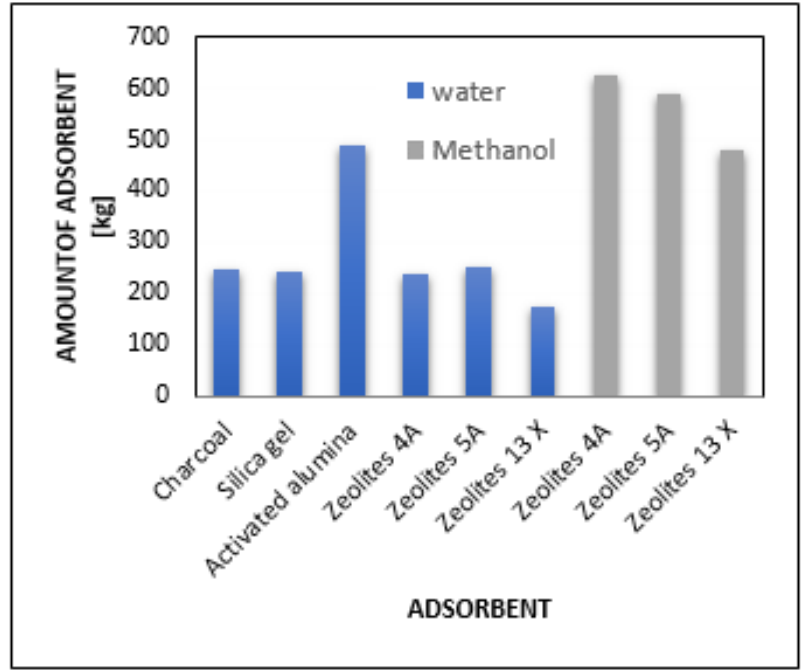

Fig 4. Amount of adsorbents needed to meet the energy requirements for a house with four occupants in Canada

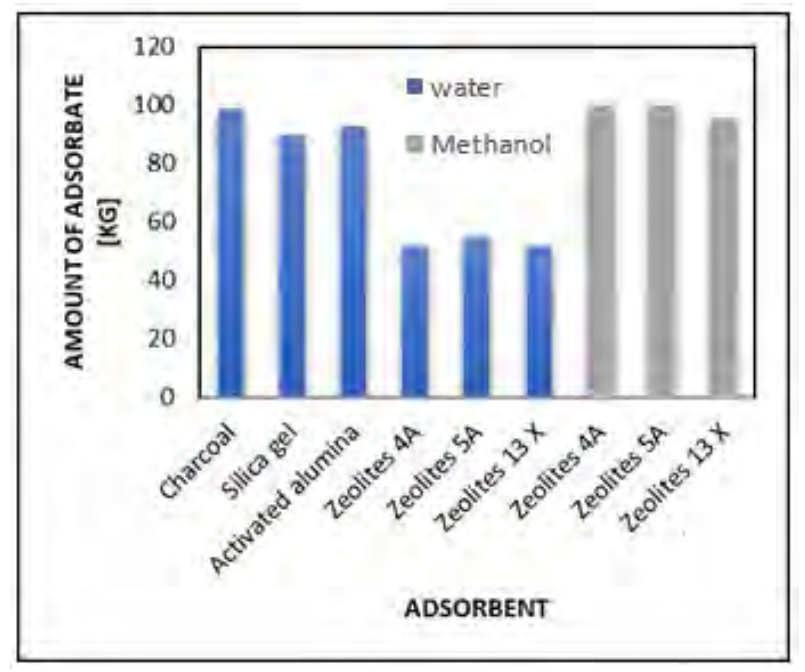

Fig 5. Amount of adsorbate needed to meet the energy requirements for a house with four occupants in Canada

5A-methanol pair were used as the adsorbent/adsorbate then the required volume would increase significantly to $0.87 \mathrm{~m}^{3}$. Also, zeolite 4A-water and zeolite 13X-water pairs have the minimum mass of adsorbent (figure 5) and adsorbate (figure 6) required. The total mass of the adsorption system including both adsorbent and adsorbate needed to meet the energy requirement of the house under consideration for zeolite 4Awater and zeolite 13X-water is $290 \mathrm{~kg}$ and $226 \mathrm{~kg}$, respectively. Now, if the adsorbent-absorbent pair is situated inside flat rectangular panels that are $1.7 \mathrm{~m}$ long, $1 \mathrm{~m}$ wide and $3 \mathrm{~cm}$ thick, it would take only 6 panels of zeolite 4A-water and 6 panels of zeolite 13X-water pairs to meet the spatial heating requirements. The total cost of the adsorbents in discussion is shown in Figure 9 and the cost of zeolite 4A and zeolite $13 \mathrm{X}$ adsorbents required to meet the energy for the house under consideration is $285 \mathrm{CAD}$ and $375 \mathrm{CAD}$, 


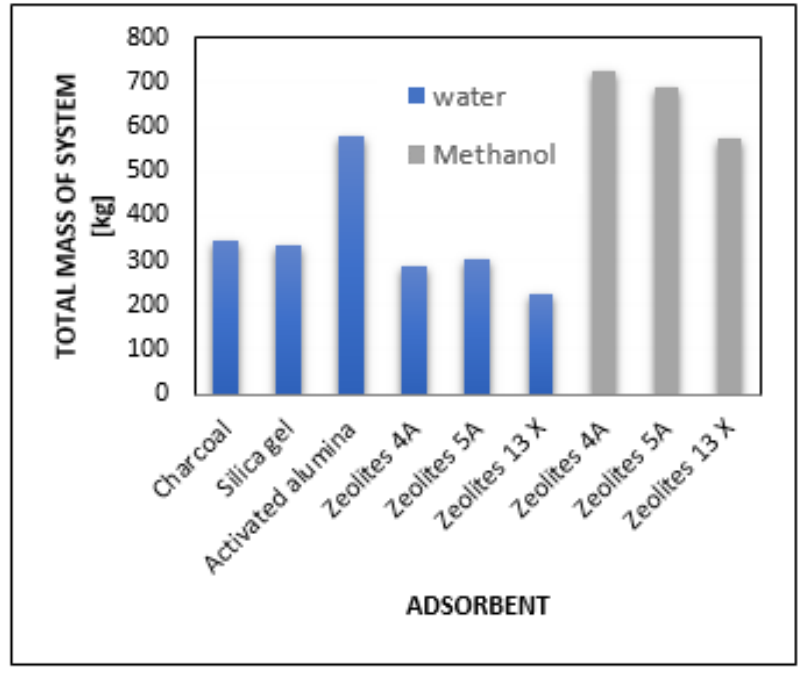

Fig 6. Total mass of the system (adsorbent and adsorbate) needed to meet the energy requirements for a house with four occupants in Canada

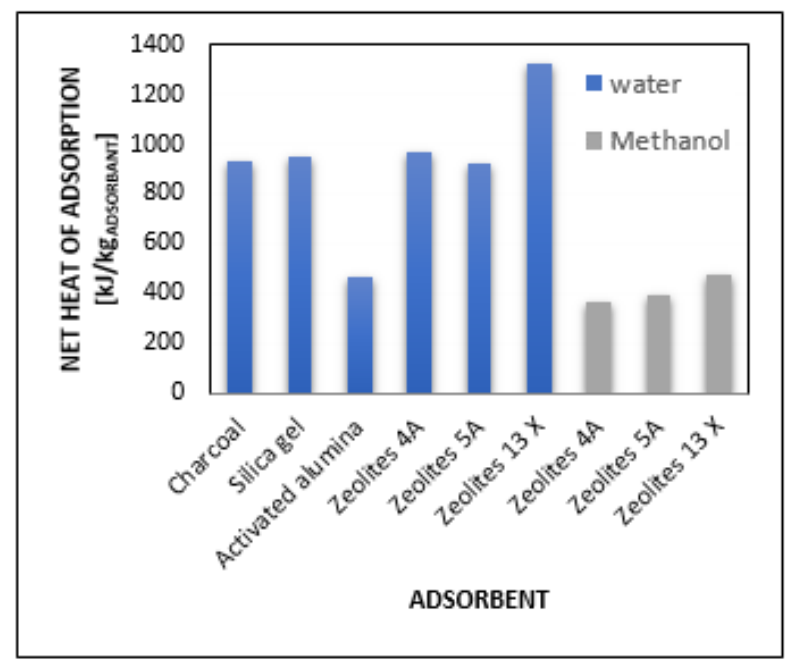

Fig 7. Net Heat of adsorption of various adsorbents

In comparison, an average of about $6.2 \mathrm{~m}^{3}$ [29] of natural gas per day is required to meet the spatial heating requirements for the residence under consideration, and the daily cost of using natural gas for spatial heating would be about 0.6 CAD. Further, assuming a carbon tax of 1 CAD per GJ [31] of energy produced, this cost would rise from $0.6 \mathrm{CAD}$ to 0.85 CAD daily $(\sim 19$ CAD monthly). Thus, the owner of the residence under consideration would be able to save $\sim 155$ $\mathrm{CAD}$ on their natural gas heating bill over the colder seasons of the year by using adsorptive technology to heat their home. Perhaps more importantly, considering $\mathrm{CO}_{2}$ emissions, $1 \mathrm{GJ}$ of energy generation from natural gas emits approximately $56 \mathrm{~kg}$ of $\mathrm{CO}_{2}$ [30]. For the residence with four occupants and a daily energy requirement of $0.23 \mathrm{GJ}, \sim 13 \mathrm{~kg}$ of $\mathrm{CO}_{2}$ is emitted daily to provide for heating. Thus, about 2.4 tonnes of $\mathrm{CO}_{2}$ emissions could potentially be prevented by heating a typical

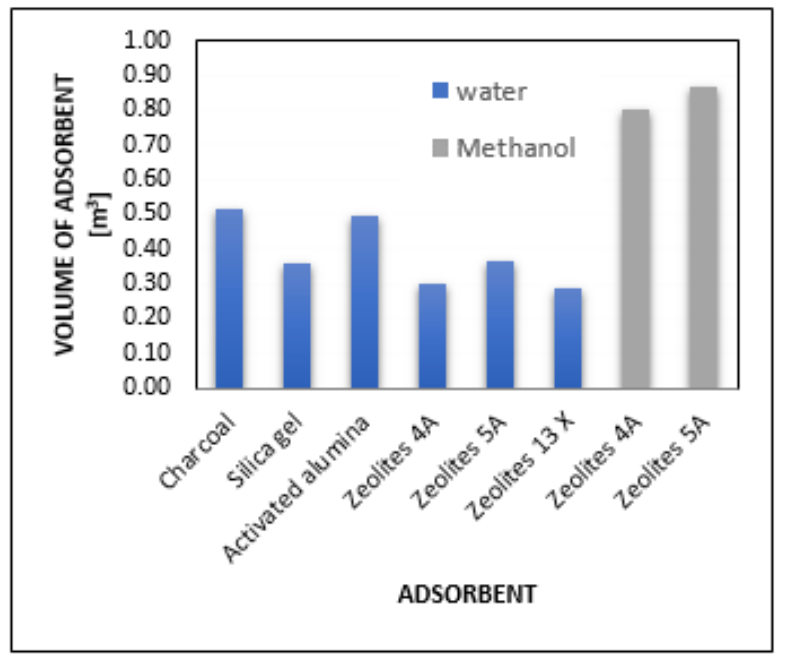

Fig 8. Volume of adsorbents needed to meet the energy requirements for a house with four occupants in Canada

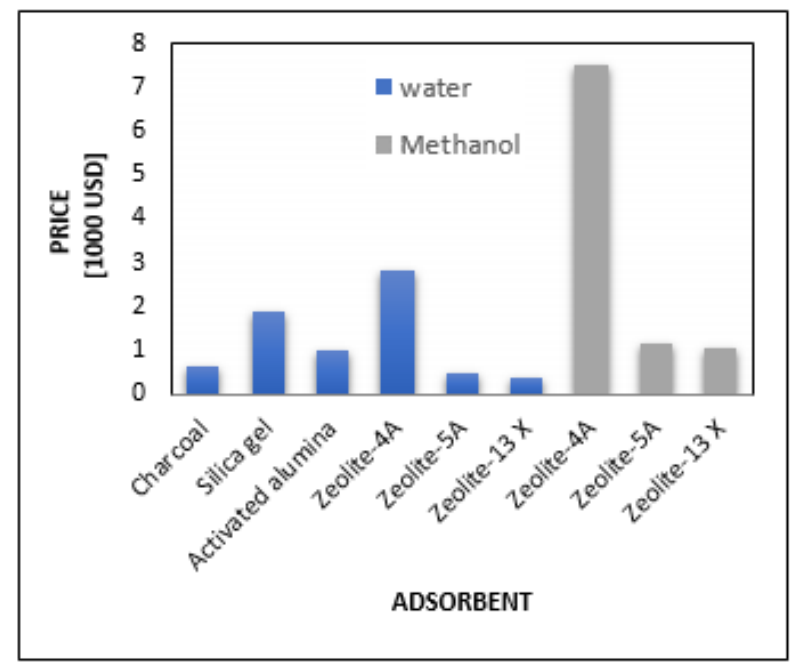

Fig 9. Costs of various adsorbents

Canadian residence using a thermal energy storage cycle based on adsorbate-adsorbent pairs instead of using natural gas. As $\mathrm{CO}_{2}$ emissions per capita in Ontario are $\sim 15$ tonnes, further research and development of sorption-based thermal energy storage cycles that store solar energy for residential heating applications is warranted.

\section{CONCLUSION}

Various adsorbent-adsorbate pairs have been investigated as the working pair in a thermal energy adsorption cycle that provides the energy required to heat a residence with four occupants in Canada. Amongst all pairs considered, the zeolite 4A-water and zeolite $13 \mathrm{X}$-water pairs have the maximum heat of adsorption and the minimum mass $(290 \mathrm{~kg}$ and $226 \mathrm{~kg}$, respectively) required to meet the heating demands. The net volume of zeolite $4 \mathrm{~A}$ and zeolite $13 \mathrm{X}$ required to meet the spatial heating requirements of the residence is $0.29 \mathrm{~m}^{2}$ and 
$0.30 \mathrm{~m}^{2}$, respectively. The cost of zeolite $4 \mathrm{~A}$ and zeolite $13 \mathrm{X}$ adsorbents for meeting the heating energy requirements of the residence is $285 \mathrm{CAD}$ and $374 \mathrm{CAD}$, respectively. Thus, zeolite 4A-water and zeolite $13 \mathrm{X}$-water pairs can potentially be used in a thermal energy adsorption cycle that stores solar energy so it can be used to provide heating for a residential home in Canada and further research in this area is warranted.

\section{ACKNOWLEDGEMENTS}

This research was supported by the Natural Sciences and Engineering Research Council of Canada

\section{REFERENCES}

[1] A. Aliane et al., "An illustrated review on solar absorption cooling experimental studies," Renew. Sustain. Energy Rev., vol. 65, pp. 443-458, Nov. 2016. doi: 10.1016/j.rser.2016.07.012.

[2] M. Alobaid et al., "A review of solar driven absorption cooling with photovoltaic thermal systems," Renew. Sustain. Energy Rev., vol. 76, pp. 728-742, Sept. 2017. doi: 10.1016/j.rser.2017.03.081.

[3] M. Ansari et al., "Preparation and characterization of nano-NaX zeolite by microwave assisted hydrothermal method," Adv. Powder Technol., vol. 25, no. 2, pp. 722-727, Mar. 2014. doi: 10.1016/j.apt.2013.10.021.

[4] Y. I. Aristov, "New family of solid sorbents for adsorptive cooling: Material scientist approach, " J. Eng. Thermophys., vol. 16, no. 2, pp. 63-72, June 2007. https://doi.org/10.1134/S1810232807020026.

[5] Y. I. Aristov, "Challenging offers of material science for adsorption heat transformation: A review," Appl. Therm. Eng., vol. 50, pp. 1610-1618, Feb. 2013. doi: 10.1016/j.applthermaleng.2011.09.003.

[6] A. A. Askalany et al., "An overview on adsorption pairs for cooling," Renew. Sustain. Energy Rev., vol. 19, pp. 565-572, Mar. 2013. doi: 10.1016/j.rser.2012.11.037.

[7] K. Bataineh and Y. Taamneh, "Review and recent improvements of solar sorption cooling systems," Energy Build., vol. 128, pp. 22-37, Sept. 2016. doi: 10.1016/j.enbuild.2016.06.075.

[8] H. Bhargav, B. M. Ramani, and V. Siva Reddy, "Experimental investigation on adsorption capacity of ACF-methanol pairs for cooling application," Int. J. Ambient Energy, vol. 750, pp. 1-4, May 2017. doi: 10.1080/01430750.2017.1318787.

[9] V. Brancato et al., "Ethanol adsorption onto carbonaceous and composite adsorbents for adsorptive cooling system," Energy, vol. 84, pp. 177-185, May 2015. doi: 10.1016/j.energy.2015.02.077.

[10] L. F. Cabeza, A. Solé, and C. Barreneche, "Review on sorption materials and technologies for heat pumps and thermal energy storage," Renew. Energy, vol. 110, pp. 3-39, Sept. 2017. doi: 10.1016/j.renene.2016.09.059.

[11] B. Dawoud, E. H. Amer, and D. M. Gross, "Experimental investigation of an adsorptive thermal energy storage," Int. J. Energy Res., vol. 31, pp. 135-147, Feb. 2007. doi: 10.1002/er.1235.

[12] A. Erto et al., "Factors affecting the adsorption of trichloroethylene onto activated carbons," Appl. Surf. Sci., vol. 256, no. 17, pp. 5237-5242, June 2010. doi: 10.1016/j.apsusc.2009.12.110.

[13] L. G. Gordeeva and Y. I. Aristov, "Composites 'salt inside porous matrix' for adsorption heat transformation: a current state-of-the-art and new trends," Int. J. Low-Carbon Technol., vol. 7, no. 4, pp. 288-302, Dec. 2012. doi: 10.1093/ijlct/cts050.

[14] P. Goyal et al., "Adsorption refrigeration technology - An overview of theory and its solar energy applications," Renew. Sustain. Energy. Rev., vol. 53, pp. 1389-1410, Jan. 2016. doi: 10.1016/j.rser.2015.09.027.
[15] L. Y. Meng and S. J. Park, "Effect of heat treatment on CO2 adsorption of KOH-activated graphite nanofibers," J. Colloid Interface Sci., vol. 352, no, 2, pp. 498-503, Dec. 2010. doi: 10.1016/j.jcis.2010.08.048.

[16] A. M. Papadopoulos, S. Oxizidis, and N. Kyriakis, "Perspectives of solar cooling in view of the developments in the air-conditioning sector," Renew. Sustain. Energy Rev., vol. 7, no. 5, pp. 419-438, Oct. 2003. doi: 10.1016/S1364-0321(03)00063-7.

[17] T. Rainer, B. Thomas, and H. Erich, "Heat Storage Media," in Ullmann's Encycl. Ind. Chem., vol. 17, Weinheim, Germany: Wiley-VCH, 2012, pp. 421-438.

[18] B. B. Saha et al., "Ethanol adsorption onto metal organic framework: Theory and experiments," Energy, vol. 79, pp. 363-370, Jan. 2015. doi: 10.1016/j.energy.2014.11.022.

[19] B. B. Saha et al., "Experiments for measuring adsorption characteristics of an activated carbon fiber, ethanol pair using a plate-fin heat exchanger," $H V A C R$ Res., vol. 12, pp. 767-782, Mar. 2011.

[20] I. Solmus et al., "Experimental investigation of a natural zeolite-water adsorption cooling unit," Appl. Energy, vol. 88, no. 11, pp. 4206-4213, Nov. 2011. doi: 10.1016/j.apenergy.2011.04.057.

[21] I. Solmus et al., "Adsorption properties of a natural zeolite-water pair for use in adsorption cooling cycles," Appl. Energy, vol. 87, no. 6, pp. 2062-2067, June 2010. doi: 10.1016/j.apenergy.2009.11.027.

[22] M. Tatler and A. Erdem-Senatalar, "Estimation of the effective diffusion coefficients in open zeolite coatings," Chem. Eng. J., vol. 102, no. 3, pp. 209 216, Sept. 2004. doi: 10.1016/j.cej.2004.04.001.

[23] K. Uddin et al., "Thermodynamic Analysis of Adsorption Refrigeration Cycles Using Parent and Surface Treated Maxsorb III / Ethanol Pairs," in Int. Refrigeration and Air Conditioning Conf., West Lafayette, IN, 2014, pp. 1-11.

[24] T. Zeng et al., "Performance of an Activated Carbon-Ammonia Adsorption Refrigeration System," Nat. Resour., vol. 8, no. 10, pp. 611-631, Oct. 2017. doi: 10.4236/nr.2017.810039.

[25] M. Zeyghami, D. Y. Goswami, and E. Stefanakos, "A review of solar thermomechanical refrigeration and cooling methods," Renew. Sustain. Energy Rev., vol. 51, pp. 1428-1445, Nov. 2015. doi: 10.1016/j.rser.2015.07.011.

[26] Government of Canada, "Photovoltaic potential and insolation dataset." March 20, 2017. Published by Natural Resources Canada. [Online]. Available: http:// www.nrcan.gc.ca/18366.

[27] Government of Canada, 2007 Households and the Environment: Energy Use (cat. no. 11-526-S.). Canada: Statistics Canada, 2010. [Online]. Available: https:// www.statcan.gc.ca/pub/11-526-s/11-526-s2010001-eng.pdf.

[28] Government of Canada, Energy Efficiency Trends in Canada, 1990-2009: December 2011 (cat. no. M141-1/2009E-PDF). Canada: Natural Resources Canada, 2012. [Online]. Available: http://oee.rncan.gc.ca/publications/statistics/trends11/pdf/ trends.pdf.

[29] T. Trombe and N. Rivers. (2017, Jan. 4). The cost of carbon pricing in Ontario and Alberta [Online]. Available: http://www.macleans.ca/economy/ economicanalysis/what-carbon-prices-in-alberta-and-ontario-will-cost-the-averagehousehold-and-why/.

[30] Government of Canada. (2015. Nov. 27). Natural Gas: A Primer [Online]. Available: https://www.nrcan.gc.ca/energy/natural-gas/5641.

[31] Government of Canada, Benchmarking Energy Intensity in the Canadian Steel Industry (cat. no. M144-125/2006E). Canada: Natural Resources Canada, 2007. Available: http://www.nrcan.gc.ca/energy/efficiency/industry/technical-info/ benchmarking/canadian-steel-industry/519. 


\section{Thermal Management of Electrical and Electronic Systems Using PCM}

\author{
Yazeed A. Alomair*, Muath A. Alomair, \\ School of Engineering, University of Guelph \\ Guelph, Ontario, Canada \\ *e-mail: yazeed@uoguelph.ca
}

\author{
Syeda Tasnim, , Hussein A. Abdullah, Shohel \\ Mahmud, ${ }^{\psi}$ \\ School of Engineering, University of Guelph \\ Guelph, Ontario, Canada \\ we-mail:smahmud@uoguelph.ca
}

\begin{abstract}
The present experimental research investigates transient thermal performance of a PCM-based thermal management system for cooling electronics components. The proposed system consists of a heatsink that is submerged into Rubitherm (RT-18) for melting experiments. The heat sink has a total length of $10 \mathrm{~cm}$, a width of $5 \mathrm{~cm}$ and a height of $13 \mathrm{~cm}$. The heat sink contains 4 vertical copper heat pipes and 34 horizontal aluminum fins. The proposed system is insulated from all sides except the front wall for periodic visualization pictures, and it is exposed to a constant heat flux boundary condition from the top with a 1-inch ${ }^{2}$ Omega heat flux heater with a wattage density of $10 \mathrm{~W} / \mathrm{in}^{2}$ at $100 \mathrm{~V}$. The PCM is heated from $0^{\circ} \mathrm{C}$ to about $51{ }^{\circ} \mathrm{C}$. The visualization results as well as the temperature distribution are discussed. Primarily, the results show that the melting process of the PCM manages the temperature of the heatsink and the base at which the heater is attached due to the high heat storage capacity of the PCM.

Keywords_latent heat thermal energy storage system; PCM, electronics thermal managemen; rectangular cavity; experimental measurement.
\end{abstract}

\section{INTRODUCTION}

Sensible heat Thermal Energy Storage (TES) systems are not as efficient as latent heat TES systems for extensive thermal discharge or high climate temperatures [1]. There are many advantages of incorporating PCM in TES systems. PCM-based TES systems can store energy for various periods of time (e.g., from minutes to seasons), which diversifies its applicability and scalability. PCM-based TES systems are used in many applications such as solar engineering, building energy consumption, greenhouse thermal control, food protection, occupant thermal comfort systems, and aircrafts thermal management. Latent heat storage is an efficient way of storing thermal energy for most applications since it can store high amount of energy at nearly constant temperature. Thus, PCM-based TES technologies have been used in electronics and electrical applications for thermal management. Electrical and electronics applications produce a massive amount of heat that minimizes the operation duration and the efficiency of the overall system. PCMs can maintain the optimal operation temperature limits of such delicate systems. However, such applications require $\mathrm{PCMs}$ with high thermal conductivity for more responsive thermal management. Unfortunately, PCMs have low thermal conductivities. In the literature, various methods have been utilized to increase the thermal conductivities of PCMs.

In automobile industry, for instance, a significant amount of carbon dioxide $\left(\mathrm{CO}_{2}\right)$ and other greenhouse gas emissions are produced by traditional transportation vehicles (by means of fossil fuel combustion process). Therefore, researchers have been investigating on how to overcome the major limitations and to grow the potential of electric vehicles in the automobile market. The electric energy storage system (electric battery) is a substantial component of an electric vehicle. Electric batteries necessitate an appropriate thermal management for more effective operation and longer life span at different operation conditions. The common key challenges with these electrical batteries are the small range of their optimal operational temperature, the intensive undesired power consumption associated with their excessive heat generation, the practicality in different climate conditions. Luckily, PCMbased thermal management technology addresses all these issues.

For instance, an improvement of $50 \%$ of an electric battery life span was accomplished for a small electric vehicle by combining TES technology and wax as a PCM with an electric scooter bike [2]. The wax was incorporated with graphite to enhance the thermal conductivity of the wax by up to 70 Wm-1K-1. This graphite-wax-based-TES system significantly increased the maximum driving distance of the small electric vehicle from $30 \mathrm{Km}$ to $55 \mathrm{Km}$ [2]. Moreover, Wang et al. performed an experimental study on electric automobiles battery safety and thermal management using heat pipes. Two battery cells producing $2.5-40 \mathrm{~W}$ of heat have been built and tested by a thermal camera at off-normal conditions. It was found that incorporating heat pipes helped to maintain the temperature below $40^{\circ} \mathrm{C}$ when the battery generates a maximum of $10 \mathrm{~W} / \mathrm{cell}$; when the battery cell generates 20-40 W/cell (uncommon thermal abuse), the proposed system could decrease the temperature of the battery to $70^{\circ} \mathrm{C}[3]$.

Heat generation in a great deal of engineering applications is nonuniform with time. This unstable behavior of heat generation is associated with undesired spikes of temperature 
increase [4]. The sudden increase of temperature could damage or decrease the efficiency of the electrical circuits. Thermal management systems that absorb the undesired heat by means of sensible and latent heat can maintain a nearly constant desired temperature. Number of researchers have investigated the improvements of thermal management performance considering many thermal enhancement methods. Hosseinizadeh et al. [5] conducted a numerical and experimental study on the performance of PCM incorporated with different configurations of fins for thermal management applications. This study considers different number, height, and thickness of fins at different power intensity. The outcomes show that the higher the number of fins and the higher the height of fins, the higher the overall thermal performance. However, increasing the fins thickness does not increase the overall thermal performance as significantly. Furthermore, Peleg et al. [6] conducted a numerical study to optimize the geometry, quantity the number of internal fins, and to optimize the amount of PCM for thermal management of electronics. The results show that at fewer fins, the temperature range increases, which decreases the PCM quantity to keep the electronics at operation temperature range. Rajesh, and Balaji [7] carried out an experimental study on the performance of PCM based thermal management storage systems using several numbers of pin fins $(0,33,27$, and 120 pin fins) for two different PCMs (n-eicosane and paraffin wax). The authors also performed an Artificial Neural Network and Genetic Algorithm optimization techniques to extend the operation time of the system to reach the set point and increase the thermal performance. The results show an improvement factor of 24 was achieved by using 72 pin fins combined with n-eicosane. $\mathrm{Li}-\mathrm{Wu}$ et al. [8] experimentally investigated the thermal performance of heatsink combined with high aspect-ratio carbon nanofillers at different loads. The results show that for heating cases, the use of high aspectratio carbon nanofillers is less effective in removing heat due to the weak natural convection to the environment. Thus, the authors have combined carbon nanotubes and graphene nanoplatelets, which increases the thermal recovery due to their high thermal conductivity. Yoram et al. [4] studied a combination of PCM and heat sink experimentally and numerically. The prototype is meant to absorb the undesired heat by the PCM (eicosane $\mathrm{C}_{2} \mathrm{OH}_{42}$ ) and to reject the heat to the surroundings by aluminum heat sinks with a fan. A twodimensional thermal model was developed and analyzed for estimation of thermal field. The experimental and numerical results of the base temperature and PCM melting behavior have some agreement. It was observed that as the heat input increases, the sensible-heat-based accumulation rate increases. Fok et al. [9] targeted cooling gadgets with power input of 3 to $5 \mathrm{~W}$ using PCM (neicosane) with and without fins. In this experimental study, the considered variables are the device orientation (vertical, horizontal, and $45^{\circ}$ incline), power input level $(3-5 \mathrm{~W})$, number of fins ( 3,6 fins), and the usage intensity (light to heavy). The main objective of this study is to investigate the thermal performance of small handheld devices when cooled using PCM with and without fins. It has been observed that cooling mobile devices using PCM with fins is feasible; however, an optimization on the device is required considering the amount of PCM used and all previously mentioned parameters.

In this experimental study, a PCM-based thermal management system is examined. The thermal management system contains of a heatsink with 34 horizontal aluminum fins integrated with 4 vertical copper heat pipes to increase the thermal conductivity of RT-18 PCM in a rectangular shaped cavity for electrical and electronics applications. The prototype is examined under transient thermal condition on the top side with maximum power input. All other walls of the enclosure are thermally insulated. This study investigates the thermal performance by means of visualization and the temperature distribution of the PCM during melting.

\section{Methodoligy}

\section{A. Components Details and Description}

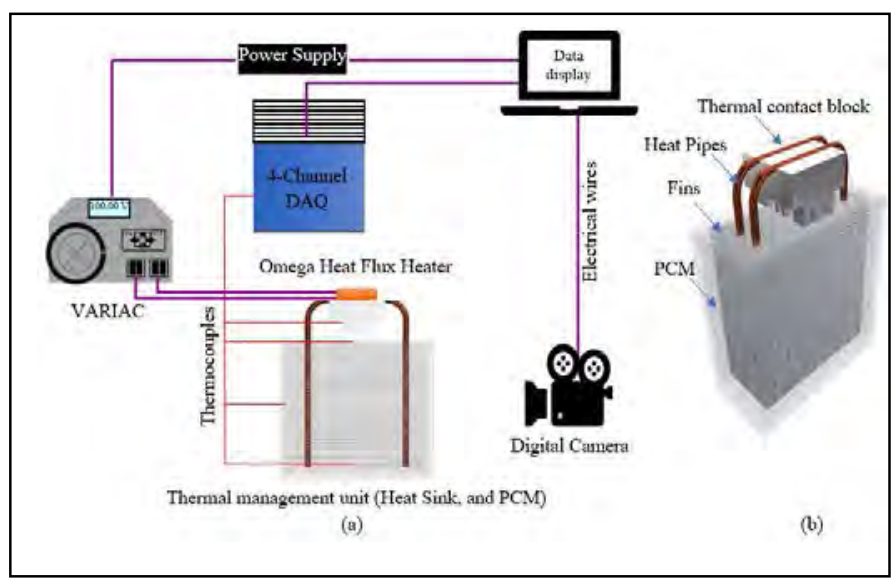

Figure 1: Schematic diagram of the (a) experimental setup and (b) components of thermal management system.

The components of the experimental setup are presented in Figure 1, (a), while Figure 1, (b) shows the components of thermal management system. The main components used in this experiment are: a variac, a DAQ system, an Omega flexible heater, a thermal management unit (heat sink, and PCM), 4 K-type thermocouples, a computer with LabVIEW software installed, and a digital camera. The variac feeds the omega heater with a series of different voltages that vary from $0-100 \mathrm{~V}$. 4 thermocouples are instrumented in the thermal management system at four locations (heat source, top fin, bottom fin, and PCM). The thermocouples are measuring the temperature across the heat sink using a DAQ system (NI USB-9162) from National Instruments. The LabVIEW software monitors and saves the thermocouples readings. The Omega heater (SRFG-101/10) is 1-inch ${ }^{2}$ with a wattage density of $10 \mathrm{~W} / \mathrm{in}^{2}$. The maximum operating temperature of the heater is $232^{\circ} \mathrm{C}$, and the maximum rated voltage is $115 \mathrm{VAC}$. The heater is in a direct contact with the heatsink from the top base as illustrated in Figure 1, (a). The heat sink has a total length of $10 \mathrm{~cm}$, a width of $5 \mathrm{~cm}$ and a height of 
$13 \mathrm{~cm}$. The heat sink contains four vertical copper heat pipes and 34 horizontal aluminum fins. The heat sink is submerged into a phase change material to assist the heat sink to dissipate heat coming from the electronic component (Omega heater). Therefore, this helps to maintain the operational average temperature for the electronic component. Rubitherm (RT-18) whose melting point ranges from $17-19^{\circ} \mathrm{C}$ was selected as thermal storage medium. The heat storage capacity of RT- 18 is $250 \mathrm{~kJ} / \mathrm{kg}$, and the maximum operation temperature is $48^{\circ} \mathrm{C}$. The container is made of a clear acrylic with a thickness of 0.24 -inch. The outer shell of the container is insulated by 1 inch thick Styrofoam. A high definition Canon camera "EOS Rebel T2i" is used to capture the images periodically during the melting process. The laptop monitor is used to display and save visualization pictures and the thermocouples readings.

\section{B. Experimental Procedure}

The experimental setup of the electronics thermal management system is presented in Figure 2. Initially, the thermal management system is placed in a refrigerator at about $\left(0^{\circ} \mathrm{C}\right)$ for 24 hours to ensure a uniform temperature across the PCM at a solid state. Then, the thermal management system is connected to the DAQ system and the heater is connected to the variac power supply. The digital camera starts taking pictures, and the LabVIEW software starts running to take the readings. The variac is turned on immediately at maximum voltage to feed omega heater with $(100 \mathrm{~V})$. Once the PCM is in a liquid form, the experiment is done.

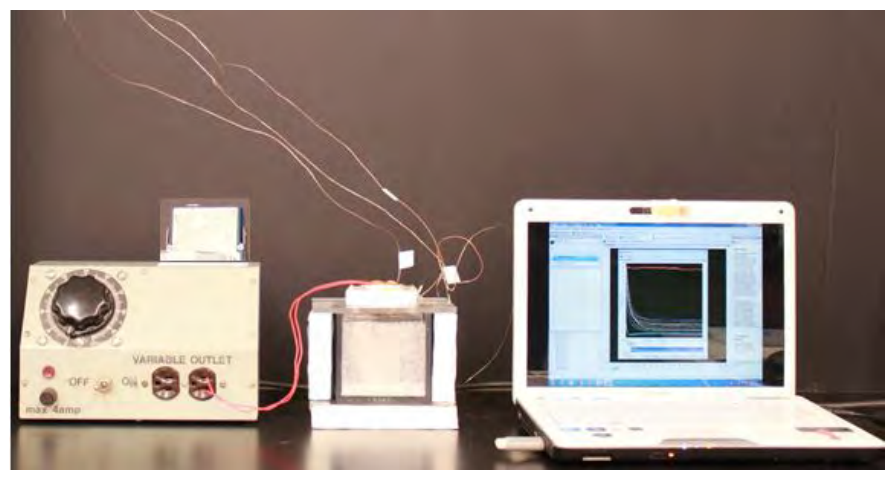

Figure 2: Experimental setup of the electronics thermal management system.

\section{RESULTS AND DISCUSSION}

\section{A. Experimental Temperature Distribution Analysis}

Figure 3 presents the experimental temperature profile in the thermal management system at $100 \mathrm{~V}$. The initial temperature of the $\mathrm{PCM}$ is $0^{\circ} \mathrm{C}$. The thermal management system is insulated from all sides except the front wall for visualization. The PCM was heated up to $34.7^{\circ} \mathrm{C}$. Four thermocouples are measuring temperatures at four locations in the thermal management system (heat spreader, top fin, bottom fin, and PCM). As can be seen from Figure 3 that once the heater is tuned on, most of the heat is absorbed by the aluminum heat spreader and top fin since they are close the heat source. It also can be seen from Figure 3 that once the heater is tuned on, the temperature of top fin increases rapidly from $0^{\circ} \mathrm{C}$ to $18^{\circ} \mathrm{C}$ by means of sensible heating. Then, the temperature of the top fin remains almost constant at the melting point temperature while the PCM was melting at the top. Once the melting of PCM is done at the top, the temperature of PCM increases due to sensible heating supplied by the heater. Note that temperature of PCM at the top and the heat source remains the same throughout the melting process. Further, it can be observed that the temperature of bottom fin increases less rapidly from $0^{\circ} \mathrm{C}$ to $15^{\circ} \mathrm{C}$ by means of sensible heating. The PCM temperature profile follows the same trend to that of the bottom fin. The melting of PCM occurs around $15^{\circ} \mathrm{C}$ and this is the reason the bottom fin and the PCM temperature remain nearly $15^{\circ} \mathrm{C}$ during the melting. The temperature of the PCM and the bottom fin increases due to sensible heating once melting of PCM is finished. Due to high thermal energy storage and low thermal conductivity of PCM, the temperature of PCM remains constant around melting point temperature for a longer period compared to bottom fin.

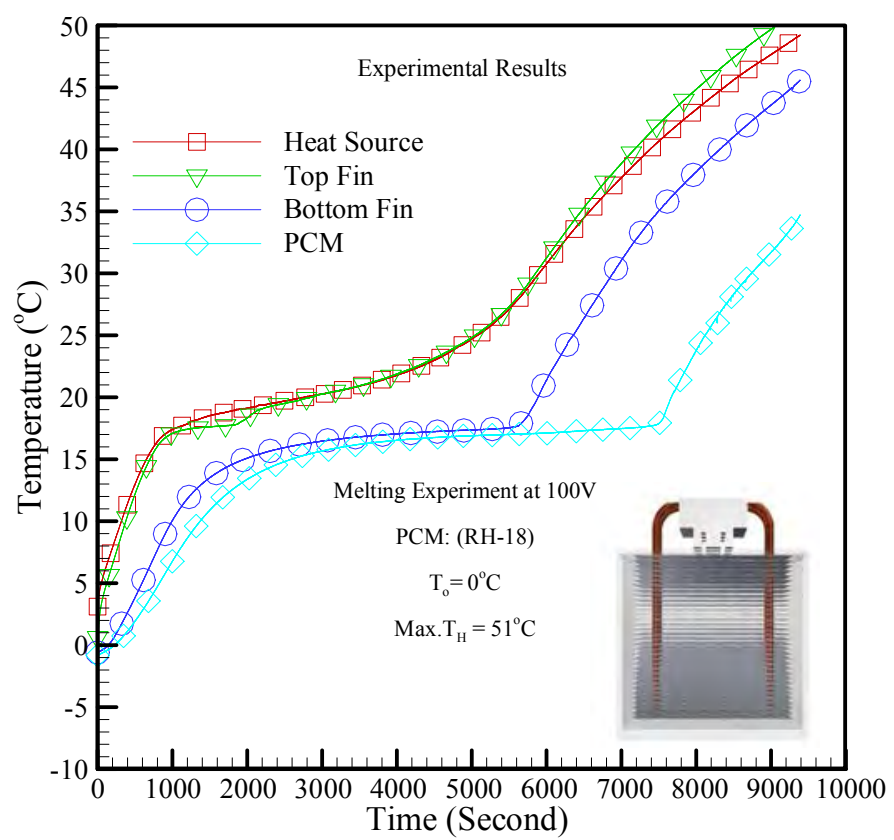

Figure 3: Temperature profile at different locations in the thermal management system at $100 \mathrm{~V}$.

\section{B. Experimental Visualization Results}

Since the heater is in direct contact with the top base of heat sink, the heat sink will absorb the heat through the base. Then, the heat will dissipate through the heat pipes and then to the fins. Therefore, the solid PCM starts absorbing the heat across the heatsink by conduction in a sensible form, as shown in Figure 4 (at time $=0-16$ minutes). The initial sensible heat storage takes about 1000 seconds (16 minutes). This sensible heat transfer keeps going until the PCM temperature reaches $17-19^{\circ} \mathrm{C}$ causing the PCM to start melting down, as shown in Figure 4 (at time $=25$ minutes). The melting starts from the right and left sides of the thermal storage system due to the vertical heat pipes that are transferring the heat from the top 
base. The melting process (latent heat storage process) takes about 4750 seconds (79 minutes) at nearly constant range of temperature $\left(17-19^{\circ} \mathrm{C}\right)$. During the melting process, heat will transfer to the liquid PCM by conviction in a latent form until all PCM become liquid. As the temperature increases above $19^{\circ} \mathrm{C}$, the heat will transfer by convection in a sensible form to the liquid PCM, as shown in Figure 4 (at time $=126$ minutes). This entire process helps to maintain the temperature of the attached electronic component at a desired temperature range.

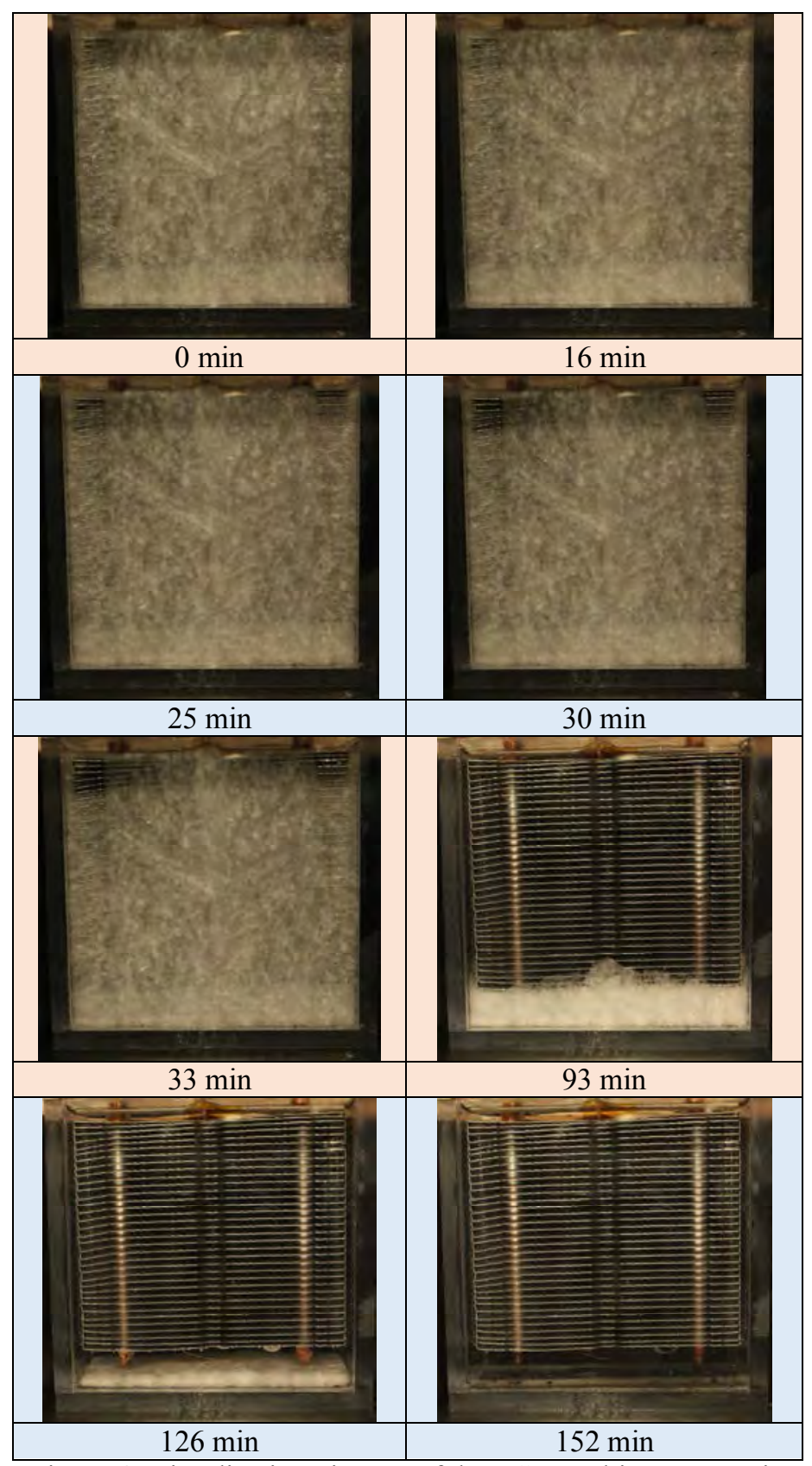

Figure 4: Visualization pictures of the PCM melting process in the thermal management system.

\section{CONCLUSION}

In this research, an experimental investigation has been carried out to examine the thermal performance of a PCMbased thermal management system for cooling of electrical and electronics components. The thermal management system consists of a heat sink that is submerged into Rubitherm (RT18) to assist the heat sink to dissipate the heat coming from the electronic component. The heat sink contains 34 horizontal aluminum fins and 4 vertical copper heat pipes. The thermal management system is insulated from all sides except the front wall for capturing periodic visualization pictures; the thermal management system is tested under a transient condition by a 1-inch ${ }^{2}$ Omega heat flux heater. The PCM was heated from $0^{\circ} \mathrm{C}$ to about $51{ }^{\circ} \mathrm{C}$. To assess and analyze the performance of the cooling system, the camera records the visualization of the melting process of PCM, while the DAQ system records the temperature profile in different locations of the thermal energy storage system. The visualization results as well as the temperature profile are discussed. Mainly, the results show that the melting process of the PCM manages the temperature of the heat spreader of the heatsink, at which, the heater is attached. This thermal control is associated with the high heat storage capacity of the PCM.

\section{REFERENCES}

[1] Z. Rao and S. Wang, " A review of power battery thermal energy management," Renewable Sustainable Energy Rev., vol. 15, no. 9, pp.4554-4571, Dec. 2011. doi: 10.1016/j.rser.2011.07.096.

[2] M. Malik, I. Dincer, and M. A. Rosen, "Review on use of phase change materials in battery thermal management for electric and hybrid electric vehicles," Int. J. Energy Res., vol. 40, no. 8, pp. 1011-1031, June 2016. doi: 10.1002/er.3496.

[3] Q. Wang et al., "Experimental investigation on EV battery cooling and heating by heat pipes," Appl. Therm. Eng., vol. 88, pp. 54-60, Sept. 2015. doi: 10.1016/j.applthermaleng.2014.09.083.

[4] Y. Kozak, B. Abramzon, and G. Ziskind, "Experimental and numerical investigation of a hybrid PCM-air heat sink," in Proc. ASME 2012 10th Int. Conf. Nanochannels Microchannels Minichannels, Rio Grande, Puerto Rico, pp. 767773.

[5] S. F. Hosseinizadeh, F. L. Tan, and S. M. Moosania, "Experimental and numerical studies on performance of PCM-based heat sink with different configurations of internal fins," Appl. Therm. Eng., vol. 31, no. 17-18, pp. 38273838, Dec. 2011. doi: 10.1016/j.applthermaleng.2011.07.031.

[6] P. P. Levin, A. Shitzer, and G. Hetsroni, "Numerical optimization of a PCMbased heat sink with internal fins," Int. J. Heat Mass Transf., vol. 61, pp. 638645, June 2013. doi: 10.1016/j.ijheatmasstransfer.2013.01.056.

[7] R. Baby and C. Balaji, "Thermal optimization of PCM based pin fin heat sinks: an experimental study," Appl. Therm. Eng., vol. 54, no. 1, pp. 65-77, May 2013. doi: 10.1016/j.applthermaleng.2012.10.056.

[8] L.-W. Fan et al., "Transient performance of a PCM based heat sink with high aspect-ratio carbon nanofillers," Appl.Therm. Eng., vol. 75, pp. 532-540, Jan. 2015. doi: 10.1016/j.applthermaleng.2014.10.050.

[9] S. C. Fok, W. Shen, and F. L. Tan, "Cooling of portable hand-held electronic devices using phase change materials in finned heat sinks," Int. J. Therm. Sci., vol. 49, no. 1, pp. 109-117, Jan. 2010. doi: 10.1016/j.ijthermalsci.2009.06.011. 


\title{
Investigation of Latent Heat Thermal Energy Storage System for Air-Conditioning Applications
}

\author{
Muath A. Alomair*, Yazeed A. Alomair,
School of Engineering, University of Guelph
Guelph, Ontario, Canada \\ Muath A. Alomair*, Yazeed A. Alomair,
School of Engineering, University of Guelph
Guelph, Ontario, Canada \\ Muath A. Alomair*, Yazeed A. Alomair,
School of Engineering, University of Guelph
Guelph, Ontario, Canada \\ *e-mail: alomairm@uoguelph.ca
}

\begin{abstract}
Around $40 \%$ of the total energy in USA is consumed by buildings, and about $\$ 370$ billion US dollar is spent each year to supply this energy and thus reduction in energy consumption is extremely important. One of the most effective and reliable ways to reduce energy consumption is the use of Phase Change Materials (PCMs) in Latent Heat Thermal Energy Storage (LHTES) systems. In this study an experimental setup was constructed to investigate the solidification process of PCM using two different types of heat exchangers: pipe heat exchanger and horizontal finned-pipe heat exchanger. The PCM used in this study is Rubitherm (RT-18) that has a melting point of $18^{\circ} \mathrm{C}$, and the heat transfer fluid (HTF) is water. To investigate the performance of pipe heat exchanger versus finned-pipe heat exchanger, two experiments were performed under identical initial, thermal and boundary conditions. The PCM is cooled down from $20^{\circ} \mathrm{C}$ to $5^{\circ} \mathrm{C}$ in both heat exchangers. Result of the temperature distribution as a function of time during the solidification process inside the TES system is presented and investigated. Visualization of the solidification process inside the TES system is also presented. Generally, the PCM is solid when its temperature is lower than the melting point, and it is liquid when its temperature is higher. PCM stores thermal energy during melting and releases heat as it solidifies. Both visualization and periodic temperature distribution results showed that as time progresses, the amount of the solid PCM increases in both heat exchangers. At a certain time, finnedpipe heat exchanger has higher amount of the solid PCM than the pipe heat exchanger. Also, the solidification time is reduced significantly when fins are added to the TES system. After 75,000 seconds of solidification, the average PCM temperature of pipe heat exchanger reached to $8.5^{\circ} \mathrm{C}$. Whereas, the average PCM temperature of the finned-pipe heat exchanger reached to $8.5^{\circ} \mathrm{C}$ after 19,000 seconds of solidification. Significant time reduction equivalent to 54,000 seconds is observed when the fins are added to the TES system. Employment of fin is preferred to improve heat transfer rate as the solidification process is enhanced due to the incorporation of fin.
\end{abstract}

\author{
Syeda Tasnim, Hussein A. Abdullah, Shohel Mahmud ${ }^{\psi}$, \\ School of Engineering, University of Guelph \\ Guelph, Ontario, Canada \\ w e-mail: smahmud@uoguelph.ca
}

Keywords- finned-pipe heat exchanger; pipe heat exchanger; phase change material; solidification; experimental investigation; solidificationv visualization; periodic temperature distribution

\section{INTROUCTION}

Buildings use approximately $40 \%$ of the total energy consumed in USA followed by industry and transportation [1]. Approximately $\$ 370$ billion US dollar is spent each year to supply this energy and thus reduction in energy consumption is extremely important [2]. One of the most efficient and reliable ways to reduce energy consumption is the use of PCMs in LHTES system [3]. Solid-liquid PCM have long been adopted as a thermal energy storage medium in LHTES system. Typically, a LHTES system consists of: (a) a PCM, (b) a container and (c) a thermal conductive surface for exchanging heat with the PCM $[4,5]$, a heat exchanger. In a typical LHTES system, a large amount of thermal energy is absorbed or released by the PCM during its nearly constant temperature phase change processes.

PCMs can be classified into (i) non-organic compounds (e.g., salt hydrate, metals, alloys) and (ii) organic compounds (e.g., wax). Organic PCMs can be further divided into (i) paraffinic PCMs (e.g., wax) and (ii) non-paraffinic PCMs (e.g., fatty acid). Paraffinic PCMs have extensively been used in LHTES system design because of their high latent heat of fusion, wide range of phase change temperatures, and good chemical stability [6]. Another advantage of paraffinic PCMs is that they have unlimited numbers of cycles [7]. However, PCMs have low thermal conductivity which results in reduction of the rate of melting and solidification of the PCMs [3]. Therefore, there are several ways to increase the performance of PCMs, such as using fins [8].

Bechiri and Mansouri [9] analyzed charging and discharging paraffin PCM in a shell and tube heat exchanger analytically. Anisur et al. [10] performed a 2-D analysis on a double wall circular tube heat exchanger with paraffin PCM for air cooling application. Heat Transfer Fluid (HTF) flow, pipes sizes and different radius of the PCM container were investigated analytically. Air was used as HTF, and the 
highest co-efficient of performance (COP) of the system was calculated to be 8.79 .

Anisur et al. [11] analytically and experimentally studied melting of PCM in a shell and tube heat exchanger for air cooling applications. They used Heptadecane PCM which had a melting point temperature of $22.33^{\circ} \mathrm{C}$. The analytical result was validated with the experimental result, and the $\mathrm{COP}$ of the system was calculated to be 4.16. Nithyanandam and Pitchumani [12] performed a numerical analysis on charging and discharging of PCM in finned heat pipes for concentrated solar power applications. The authors observed an enhancement of heat transfer by adding vertical fins, the larger the number of fins, the higher the heat transfer. Liu et al. [13] investigated melting characteristics of Paraffin PCM surrounding heat pipes. They found that when the inlet temperature of the heat pipe is increased, the total heat storage capacity and heat storage rates are increased. Also, the total heat storage capacity and heat storage rates are increased with decreasing the initial temperature of heat storage material. Khalifa et al. [14] performed an experimental and numerical study on PCM solidification around tube integrated with vertical fins versus a bare tube for solar thermal power generation application. Their findings illustrated that heat transfer performance was improved $24 \%$ compared to a bare tube.

Khalifa et al. [15] performed an experimental investigation on solidification of a high temperature PCM in a finned heat pipe duct. A significant improvement in the heat transfer rate was observed for the finned heat pipe case especially during later times of the solidification process. $\mathrm{Li}$ et al. [16] experimentally studied the melting and solidification of PCM in a flat heat exchanger with heat pipes. The system's performance showed a uniform temperature across the heat exchanger and an increase in the heat transfer inside the PCM. Rahimi et al. [8] performed an experimental analysis on melting and solidification of PCM inside a thermal energy storage system using copper pipes and aluminum fins. They found that when the aluminum fins were added to their system, the heat transfer in the PCM was increased. Other studies [17, 18] experimentally investigated melting performance of PCMs by using heat pipes. They studied the performance of their systems with increasing the heat pipe numbers and increasing the power input of the heat pipes. An enhancement of heat transfer was observed with increase in the numbers of heat pipes and input power of heat pipes.

Based on the literature review above, there is a lack of experimental studies that examined the effect of solidification (heat discharging) of PCMs with copper fins. The aim of this study is to compare the performance of a tube heat exchanger and finned-tube heat exchanger experimentally. RT-18 Rubitherm is used as PCM, and water is used as a heat transfer fluid (HTF). To compare the performance, transient temperatures are measured at different locations and visualization of the solidification process is performed. This information will enable us to assess the performance of heat exchanger with fins and help to able to understand the fundamental of liquid-solid phase transition and heat transfer during solidification of PCM.

\section{EXPERIMENTAL SETUP AND PROCEDURES}

\section{A. Description of experimental set-up}

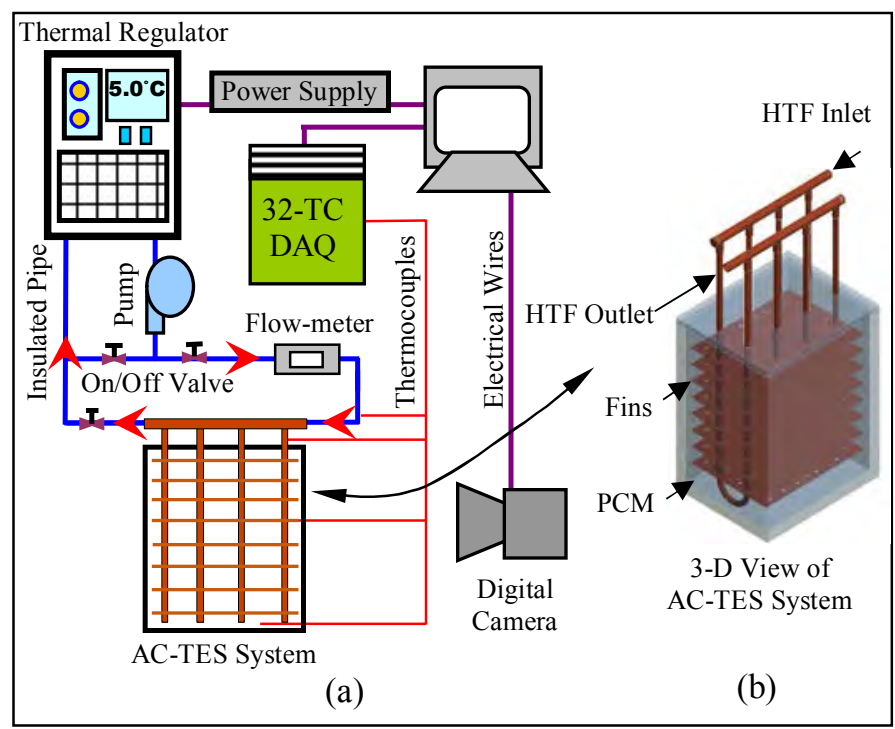

Figure 1: Schematic diagram of the (a) experimental setup and (b) components of thermal energy storage system (TES).

An experimental set-up was designed and built in order to visualize and investigate the solidification process of the PCM in LHTES system. Figure 1(a) shows a schematic diagram of the experimental set-up, and figure 1(b) shows the finned TES system. The experimental set-up in figure 1(a) consists of a (Polystat recirculator, model: 13042-01, supplier: Cole-Parmer) that controls water inlet temperature; a water pump (model: FP1, supplier: Cole Parmer), and a series of valves to regulate water flow rate; a digital flow meter (model: RT-375MI-LPM2, supplier: Blue-White) and the heat storage system. The set-up allows replacing readily the heat exchanger with no changes in the rest of equipment. $32 \mathrm{~K}$-type thermocouples (model: 5TCTT-K-30-36, supplier: Omega) were installed at different locations inside the TES system and the copper pipes to record temperatures at different times. These measurements were performed to ensure that the PCM was well distributed within all pipes and sheets. Temperatures were recorded as function of time in a computer via the DAQ system (model: NI9213, supplier: National Instruments) using LabView software (version: 15, supplier: National Instruments). A digital camera (model: EOS rebel T5, supplier: Canon) is connected to the computer to capture the images during the experiment periodically. The external surface of the TES system was totally covered with insulation foam to minimize heat losses. For both pipes and finned-pipes heat exchangers, the following temperatures measurements were taken: ambient temperature; water inlet and outlet temperatures of the TES system; water inlet and outlet temperatures for each U-shaped copper pipe; and halfway between water inlet and outlet of each U-shaped copper pipe (at the bottom point). The rest of the 17 thermocouples were distributed evenly through the PCM for 
the pipes heat exchanger. To measure the temperature distribution across the copper sheet wall for the finned-pipes heat exchanger, 5 thermocouples were distributed in the middle copper sheet (4 corners and 1 in the center). To measure the temperature distribution across the PCM, 12 thermocouples were evenly distributed in three elevations ( 4 corners for each elevation). The TES system in figure 1(b) consists of a transparent acrylic container filled with a PCM, copper pipes and copper sheets. The outer surface of the TES system was made of a $30-\mathrm{cm}$ height, $24.5-\mathrm{cm}$ width and $20-\mathrm{cm}$ depth. The thickness of the transparent acrylic wall is 1-cm (supplier: McMaster Carr). The used PCM is Rubitherm (RT-18) (supplier: Rubitherm) that has a solidifying range of $19^{\circ} \mathrm{C}-$ $17^{\circ} \mathrm{C}$, heat storage capacity of $250 \mathrm{~kJ} / \mathrm{kg}$, and a max operation temperature of $48^{\circ} \mathrm{C}$. The volume of PCM was $0.01 \mathrm{~m} 3$. Four U-shaped vertical copper pipes of a diameter of $0.9525 \mathrm{~cm}$

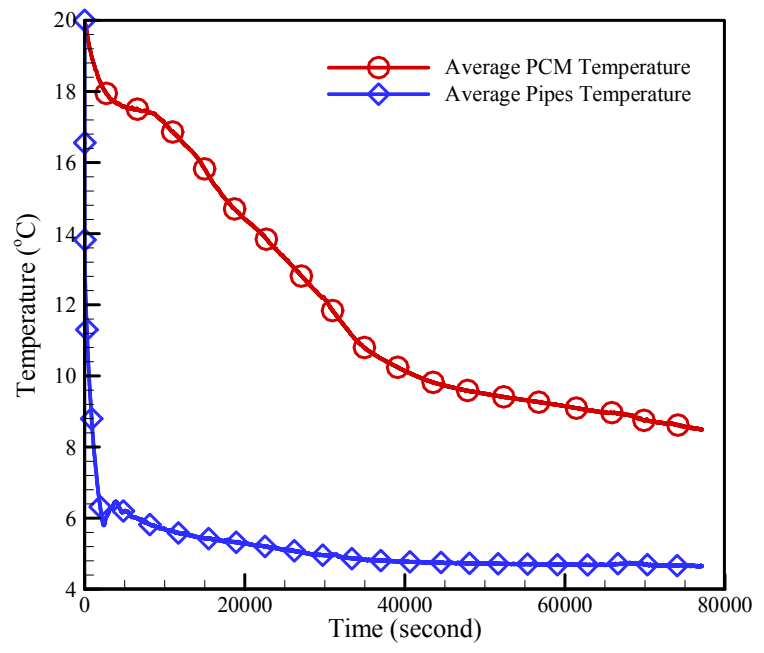

(a)

\section{RESULTS AND DISCUSSION}

\section{A. Temperature distribution of the solidification process}

Figures 2 (a) and (b) present periodic average temperature variation of the pipes, PCM and fins at different locations inside the TES system. The red curve represents the average PCM temperature while the average pipes temperature is presented in the blue curve. For the finned-pipes heat exchanger, the green curve represents the fins temperature. In general, the PCM is liquid when its temperature is higher than the melting point temperature $\left(18^{\circ} \mathrm{C}\right)$, and it solidifies when its temperature is lower. Initially, the PCM is liquid, and the temperature of the $\mathrm{PCM}$ is $20^{\circ} \mathrm{C}$. The solidification process is initiated when cold water at $5^{\circ} \mathrm{C}$ is bypassed through the copper pipes. The liquid PCM around the tube absorbs thermal

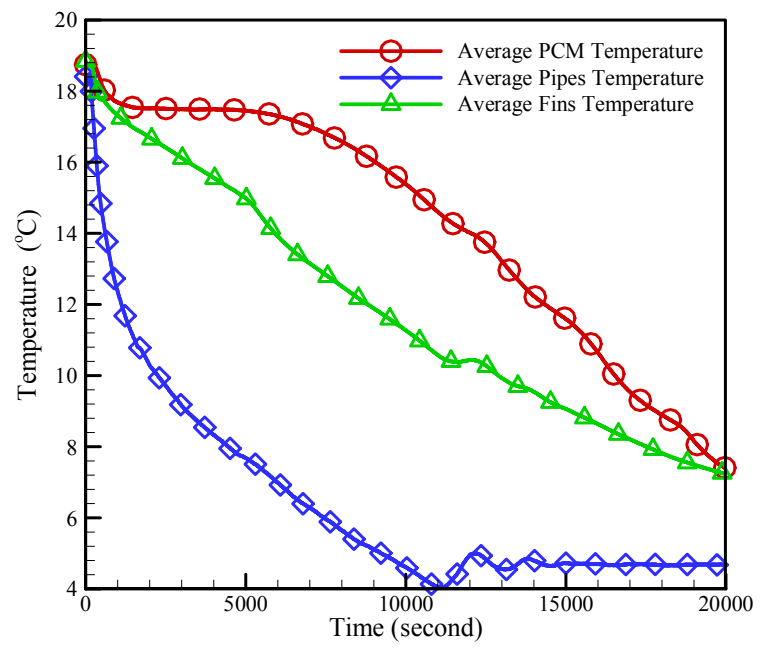

(b)

Figure 2: (a) Temperature distribution $\left({ }^{\circ} \mathrm{C}\right)$ versus time (second) for solidification process inside the pipe heat exchanger and (b) Temperature distribution $\left({ }^{\circ} \mathrm{C}\right.$ ) versus time (second) for solidification process inside the finned-pipe heat exchanger.

(0.375 inch) were passed through the center of the PCM. Pipes were distributed evenly from each side: 6-cm away from the front and back walls and 1-cm away from right and left side walls. These copper pipes carried water as the heat transfer fluid during the experiment. Eight horizontal copper sheets of $21.5-\mathrm{cm}$ width, $16.5-\mathrm{cm}$ depth, and 1-mm thickness are aligned on the base with $3-\mathrm{cm}$ spacing between them. The fins were soldered to the pipes to minimize the thermal contact resistance between the heat exchanger parts.

\section{B. Experimental Procedure}

The experiments were conducted in several steps. First, the TES system was filled with RT-18 PCM. Second, the temperature of the PCM was heated up to about $20^{\circ} \mathrm{C}$ (liquid phase). Third, the water of thermal regulator was cooled down to $5^{\circ} \mathrm{C}$ and supplied to the TES system through the copper pipes. Meanwhile, the temperatures of the water flow, copper sheets and PCM were recorded at each 10 second. Also, images of the solidification process were captured at each 10minute interval. energy from the cold water (HTF) via the tube surface, removes the sensible heat of the PCM and drops the temperature down to the freezing point. The PCM starts solidifying around the tube in the radial direction when the temperature drops below freezing point temperature. The thickness of the solid PCM grows with time around the tube. This solid PCM around the tube then behaves as an insulation material due to its low thermal conductivity compared to the copper tube material and slowed down the solidification process. Heat transfer through the solid PCM is dominated by conduction and as the thickness of the solid PCM increases around the tube, the heat transfer from the tube surface is further reduced. This reduction in heat transfer enhances the solidification time for a bare tube case. Adding horizontal copper fins decreases the thermal resistance during the solidification process and enhances the solidification time. The verage PCM temperature of pipe heat exchanger results shown in figure 2 (a) reached to $8.5^{\circ} \mathrm{C}$ after 75,000 seconds of cooling. The results of the finned-pipe heat exchanger in figure 2 (b) showed that the average PCM temperature reached 


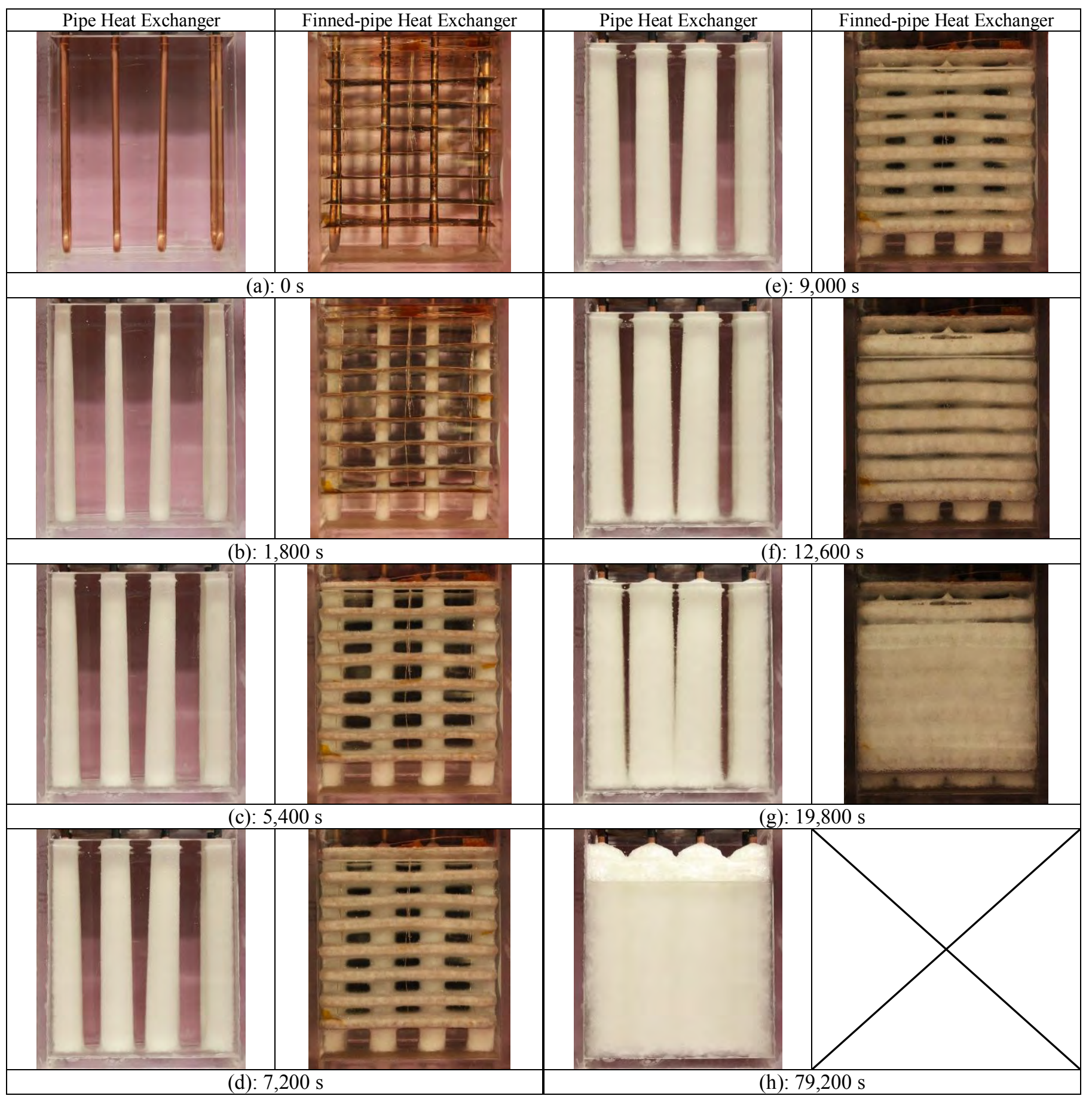

Figure 3: Different visualization captures for different times of the solidification experiments of the pipe heat exchanger versus finned-pipe heat exchanger. For the finned-pipe heat exchanger, the solidification process ends at 19,800 $\mathrm{s}$ and that is why no figure is provided to the last row $(\mathrm{h})$ in the right column.

to $8.5^{\circ} \mathrm{C}$ after 19,000 seconds of cooling. When the fins were added to the TES system, 54,000 seconds was reduced.

\section{B. Visualization of the solidification process}

Figure 3 shows visualization pictures captured of the solidification experiments of the pipe heat exchanger versus finned-pipe heat exchanger for different times from 0 second to 79,200 seconds (a-h). The left column represents the pipe heat exchanger experiment, and right column represents finned-pipe heat exchanger experiment. Each row represents a visualization comparison between pipe heat exchanger versus finned-pipe heat exchanger for the same boundary and thermal 
conditions at a certain time. In general, the PCM is liquid when its temperature is higher than the melting point temperature $\left(18^{\circ} \mathrm{C}\right)$, and it solidifies when its temperature is lower. At 0 second, the PCM is liquid, and the temperature of the PCM is $20^{\circ} \mathrm{C}$. The solidification process is initiated when cold water at $5^{\circ} \mathrm{C}$ is bypassed through the copper pipes any time after 0 second. It is observed that the amount of the solid PCM increases with time progression for both heat exchangers. Also, it is noticed that the amount of the solid PCM in finned-pipe heat exchanger is higher than the pipe heat exchanger.

\section{CONCLUSION}

An experimental set-up was constructed to investigate the solidification process of two heat exchangers (pipe heat exchanger versus finned-pipe heat exchanger). The PCM used in this study was RT-18 that has a melting point temperature of $18^{\circ} \mathrm{C}$. Water was used as heat transfer fluid. Two experiments were performed under identical initial, thermal and boundary conditions to investigate the performance of pipe heat exchanger versus finned-pipe heat exchanger. Periodic temperature distribution and visualization results of the solidification process inside the TES system were presented. Generally, the PCM is liquid when its temperature is higher than the melting point temperature $\left(18^{\circ} \mathrm{C}\right)$, and it solidifies when its temperature is lower. Also, PCM releases heat as it solidifies. Results for both heat exchangers showed that the amount of the solid PCM increases with time progression. Temperature distribution results indicated that the amount of the solid PCM in finned-pipe heat exchanger is higher than the pipe heat exchanger at a fixed time. To reach $8.5^{\circ} \mathrm{C}$ in the solidification process, the average PCM temperature of pipe heat exchanger needed 75,000 seconds and the average PCM temperature of finned-pipe heat exchanger needed 19,000 seconds. Therefore, 54,000 seconds is reduced once the fins were added to the TES system.

\section{REFERENCES}

[1] M. Collins (2017). Solar Thermal Research Laboratory (STRL) [Online]. Available: https://uwaterloo.ca/solar-thermal-research-laboratory/about.

[2] US DOE Building Windows and Envelope R\&D -Retrofit Focus EcoBuild December 10th, 2014.

[3] A. Sharma et al., "Review on thermal energy storage with phase change materials and applications," Renew. Sustain. Energy Rev., vol. 13, no. 2, pp. 3183-3145, Feb. 2009. doi: 10.1016/j.rser.2007.10.005.

[4] M. Lacroix, "Study of the heat transfer behavior of a latent heat thermal energy storage unit with finned tube." Int. J. Heat Mass Transfer, vol. 36, no. 8, pp. 2083-2092, 1993. doi: 10.1016/S0017-9310(05)80139-5.

[5] M. Lacroix, "Numerical simulation of a shell and tube latent heat thermal energy storage unit," Sol. Energy, vol. 50, no. 4, pp. 357-367, Apr. 1993. doi: 10.1016/0038-092X(93)90029-N.

[6] X. X. Han, Y. Tian, and C. Y. Zhao, "An effectiveness study of enhanced heat transfer in phase change materials (PCMs)," Int. J. Heat Mass Transfer, vol. 60, pp. 459-468, May 2013.

doi: 10.1016/j.ijheatmasstransfer.2013.01.013.

[7] Rubitherm (2017). Rubitherm Advantages [Online].

Available: https://www.rubitherm.eu/pcm.html

[8] M. Rahimi et al., "Analysis of geometrical and operational parameters of PCM in a fin and tube heat exchanger," Int. Commun. Heat Mass Transfer, vol. 53, pp. 109-115, Apr. 2014. doi: 10.1016/j.icheatmasstransfer.2014.02.025.
[9] M. Bechiri and K. Mansouri, "Analytical solution of heat transfer in a shell-andtube latent thermal energy storage system," Renewable Energy, vol. 74, pp. 825-838, Feb. 2015. doi: 10.1016/j.renene.2014.09.010.

[10] M. R. Anisur et al., "Analysis of a thermal energy storage system for air cooling-heating application through cylindrical tube," Energy Convers. Manage., vol. 76, pp. 732-737, Dec. 2013. doi: 10.1016/j.enconman.2013.08.031.

[11] M. R. Anisur et al., "Cooling of air using heptadecane phase change material in shell and tube arrangement: Analytical and experimental study," Energy and Buildings, vol. 85, pp. 98-106, Dec. 2014. doi: 10.1016/j.enbuild.2014.09.015.

[12] K. Nithyanandam and R. Pitchumani, "Computational studies on a latent thermal energy storage system with integral heat pipes for concentrating solar power," Appl. Energy, vol. 103, pp. 400-415, Mar. 2013. doi: 10.1016/j.apenergy.2012.09.056.

[13] X. Liu, G. Fang, and Z. Chen, "Dynamic charging characteristics modeling of heat storage device with heat pipe," Appl. Therm. Eng., vol. 31, no. 14-15, pp. 2902-2908, Oct. 2011. doi: 10.1016/j.applthermaleng.2011.05.018.

[14] A. Khalifa et al., "A numerical and experimental study of solidification around axially finned heat pipes for high temperature latent heat thermal energy storage units," Appl. Therm. Eng., vol. 70, no. 1, pp. 609-619, Sept. 2014. doi: 10.1016/j.applthermaleng.2014.05.080.

[15] A. Khalifa et al., "Performance of suspended finned heat pipes in hightemperature latent heat thermal energy storage, "Appl. Therm. Eng., vol. 81, pp. 242-252, Apr. 2015. doi: 10.1016/j.applthermaleng.2015.02.030.

[16] F. Li et al., "Experimental study on the thermal performance of a new type of thermal energy storage based on flat micro-heat pipe array," Energy Convers. Manage., vol. 112, pp. 395-403, Mar. 2016.

doi: 10.1016/j.enconman.2016.01.039

[17] J. Zhao et al., "Experimental investigation on thermal performance of phase change material coupled with closed-loop oscillating heat pipe (PCM/CLOHP) used in thermal management," Appl. Therm. Eng., vol. 93, pp. 90-100, Jan. 2016. doi: 10.1016/j.applthermaleng.2015.09.018.

[18] Q. Wang et al., "Thermal performance of phase change material/oscillating heat pipe-based battery thermal management system," Int. J. Therm. Sci., vol. 102, pp. 9-16, Apr. 2016. doi: 10.1016/j.ijthermalsci.2015.11.005. 


\section{Development of Absorbents with High Contact Area for Applications in Desiccant Coated Heat Exchanger}

\author{
Yunxi Li \\ Hisense Air Conditioner R\&D Centre \\ Hisense (Shandong) Air Conditioner Co. \\ Ltd. \\ Qingdao, China \\ liyunxi@hisense.com
}

\author{
Benwei Li \\ Hisense Air Conditioner R\&D Centre \\ Hisense (Shandong) Air Conditioner Co. \\ Ltd. \\ Qingdao, China \\ libenwei@hisense.com
}

\author{
Zhigang Wang \\ Hisense Air Conditioner R\&D Centre \\ Hisense (Shandong) Air Conditioner Co. \\ Ltd. \\ Qingdao, China \\ wangzhiganghh@hisense.com
}

\begin{abstract}
Desiccant coated heat exchanger (DCHE) is developed from the combination of desiccant absorbents and the conventional fin-tube heat exchanger. The performance of the DCHE is mainly due to the adsorption properties of the coated absorbents. Therefore, to improve the DCHE performance, we fabricated porous ion exchange resin (IER) particles which have highly increased contact areas to be used as the DCHE absorbents. The water uptake and adsorption ratio of the porous and non-porous absorbents were both measured and compared against each other. The results showed that the absorbents with porous structure possessed higher water capacity and adsorption rate than that of the nonporous IER.
\end{abstract}

Keywords: air-conditioner; exchanger; desiccant; porous; ion exchange resin

\section{INTRODUCTION}

Generally, the dehumidification and humidification are achieved via refrigerating to condense the moist air below the dew point or heating the liquid water above the vapor temperature. Both directions of the humidity control caused plenty of energy waste, which decreased the coefficient of performance (COP) as well ${ }^{[1-7]}$. Recently, to control the indoor humidity and improve the COP of the conventional airconditioning system, researches on adsorption refrigeration technology has increased due to many advantages such as utilizing solar and geothermal energies and wastes heat ${ }^{[6-12]}$. The desiccant coated fin-tube heat exchanger a kind of the DCHEs, developed from the classic fin-tube heat exchanger which is widely used in nowadays air-conditioning system [1420].

Absorbents such as active carbons, silica gel, zeolites or ion exchange resins are the key component of the desiccant coated fin-tube heat exchanger ${ }^{[2,8,11,13]}$. Great efforts have been dedicated to investigating the performance of these materials. For example, silica gel has been both analytically and experimentally studied ${ }^{[11-13]}$. Computer simulation was also applied to optimize the performance of the adsorption refrigeration ${ }^{[14-15]}$.

Compared to those inorganic porous materials, IER can directly attach to the fin surfaces instead of mixing with epoxy resin, which avoids the decreasing of the contact area of the absorbents. However, the IER swells while absorbing water and shrinks after evaporation. The frequent dimensional change of the resin caused the serious decline in durability. Besides, the adsorption properties are

In this work, we propose IER with aromatic chain structures to provide high thermal and mechanical stabilities, which improves the longevity of the absorbents. The aromatic IER was also fabricated with porous structures to increase contact surface areas. The test results showed improved humidity adsorption rate and capacity due to the porous surfaces.

\section{EXPERIMENTAL}

A. Sythesis of IER

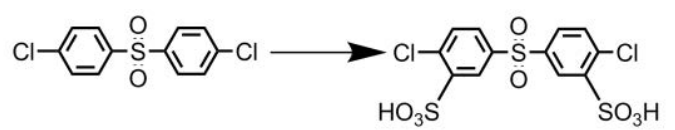

(a)

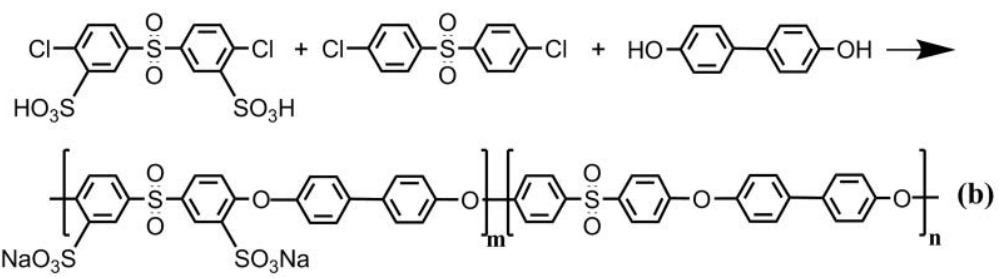




\section{Scheme 1. Preparation scheme of the sulphonated poly ether sulfone ion exchange resin (SPESIER).}

$B$.

\section{SPESIER}

Synthesis of the

The ion exchange resin was synthesized via two major steps as shown in Scheme 1. First, sulphonated bis (4-chlorophenyl) sulphone was obtained via a typical sulphonating procedure utilizing fuming sulfuric acid and bis (4-chlorophenyl) sulphone ${ }^{[21]}$. Then the sulphonated bis (4-chlorophenyl) sulphone was added into a three-necked, round-bottomed flask with $\mathrm{K}_{2} \mathrm{CO}_{3}$, biphenol and DMSO (solvent). The SPESIRE was polymerized via aromatic nucleophilic substitution polycondensation. The resulting polymer was washed with DI water and ethanol after polymerization.

C.

Prepareation of

porous absorbents particles.

The size of the common used absorbents particles are usually in the range of $60 \sim 100 \mu \mathrm{m}$. Therefore, to develop the absorbents with porous surfaces, the pore size should be much smaller than that of the particle.

In this work, the porous structure was fabricated by the solution casting method. First, the SPESIER was dissolved into NMP with the solid content of $10 \%$ and stirring for $12 \mathrm{~h}$ at room temperature to get a homogeneous solution. Secondly, 1Ethyl-3-methlimidazolium tetrafluoroborate $\left(\mathrm{EMMBF}_{4}\right)$ was separately added into the solution at concentrations of $50 \mathrm{wt} \%$ of the SPESIER. The mixtures were then stirred for another $12 \mathrm{~h}$ to ensure uniform dispersion. The casting solution was filtered before pouring onto a flat glass substrate. The glass plates with casting solution on top were first held in the oven at $60 \mathrm{C}$ for $12 \mathrm{~h}$ and then $80 \mathrm{C}$ in vacuum for another $24 \mathrm{~h}$ to remove solvents. The dried polymers were then removed from the glass plate and boiled in methanol until constant weight to remove the $\mathrm{EMMBF}_{4}$ and residuals. The porous polymers were powdered into small particles and the pore structure was observed under SEM.

$D$.

Water uptake

Water uptake was measured after completely drying of the samples at $120^{\circ} \mathrm{C}$ in vacuum. Then their weights $\left(\mathrm{W}_{\text {dry }}\right)$ were recorded before immersing into DI water. Tests were carried out at different temperatures $\left(20 \mathrm{C}, 40^{\circ} \mathrm{C}, 60^{\circ} \mathrm{C}, 80^{\circ} \mathrm{C}\right.$ and $\left.100{ }^{\circ} \mathrm{C}\right)$. The $\mathrm{W}_{\text {wet }}$ were measured after $10 \mathrm{mins}$ at each temperature. The water uptake of these membranes was calculated and compared as percentages by the following equation:

Water uptake $(\mathrm{wt} \%)=\left(\mathrm{W}_{\text {wet }}-\mathrm{W}_{\text {dry }}\right) / \mathrm{W}_{\mathrm{dry}} \times 100$

E.

Adsorption rate

The adsorption rate of the porous IER particles was measured and compared against the non-porous absorbents. The absorbent powder was first dried in the oven at the temperature of $120 \mathcal{C}$ until constant weight which was recorded as $\mathrm{W}_{\text {dry }}$. Then the absorbents were placed in a cube with the salt bath of $\mathrm{KCl}$ to create a constant relative humidity $(\mathrm{RH})$ atmosphere. The weight change of these absorbents was continuously measured every 100 seconds.

The adsorption ratio was evaluated by the following equation:

Adsorption ratio $(\mathrm{wt} \%)=\left(\mathrm{W}_{\text {wet }}-\mathrm{W}_{\text {dry }}\right) / \mathrm{W}_{\text {dry }} \times 100$

\section{RESULTS AND DISCUSSION}

A.

\section{Morphology}

$\mathrm{EMMBF}_{4}$ was used as the pore-forming agent due to its extraordinary compatibilities with polymers. $50 \mathrm{wt} \%$ of the $\mathrm{EMMBF}_{4}$ was added into the system and removed by methanol after membrane casting. The choice of solvent for membrane casting plays an important role in the manufacture of uniform pore size distribution. To lower the evaporation speed and avoid the split phase, NMP was used in the work. Figure 2 shows the field emission scanning electron microscopy (FESEM) images of the porous absorbents. The morphological structure exhibited uniform pore size distribution with an average diameter of $0.32 \mu \mathrm{m}$.

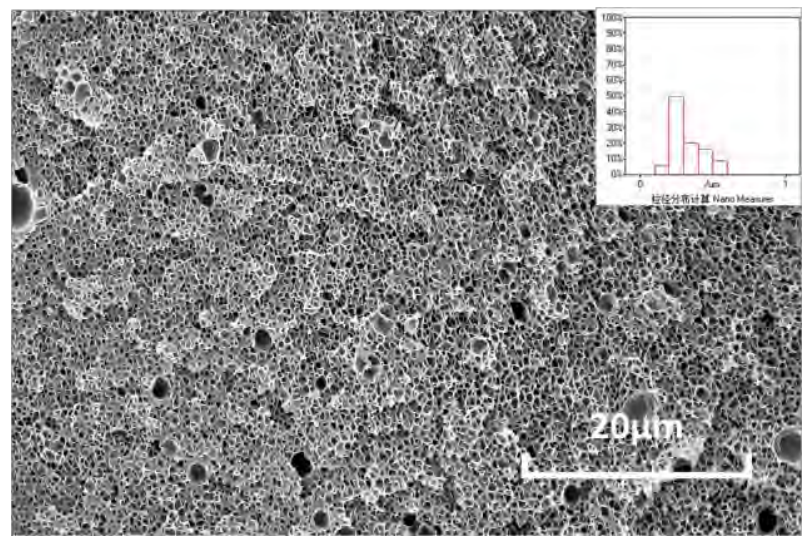

Figure 2. The SEM images of the porous IER.

$B$.

Water uptake

Water uptake at various temperatures could reveal the highest water capacity of the absorbents. As expected, both the porous and non-porous absorbents exhibited improved water uptake along with the increasing of temperature. Besides, as we can see in Fig. 3, the porous absorbents outperformed that of non-porous absorbents over the entire range of temperatures. The porous absorbent have a higher water uptake ratio of 5.9 $\mathrm{wt} \%$ than the non-porous particles $(5.6 \mathrm{wt} \%)$ after $10 \mathrm{mins}$ immersed into water at $20 \mathbb{C}$, (ih)dicating 2 the enhancement of the porous surfaces on water uptake. 


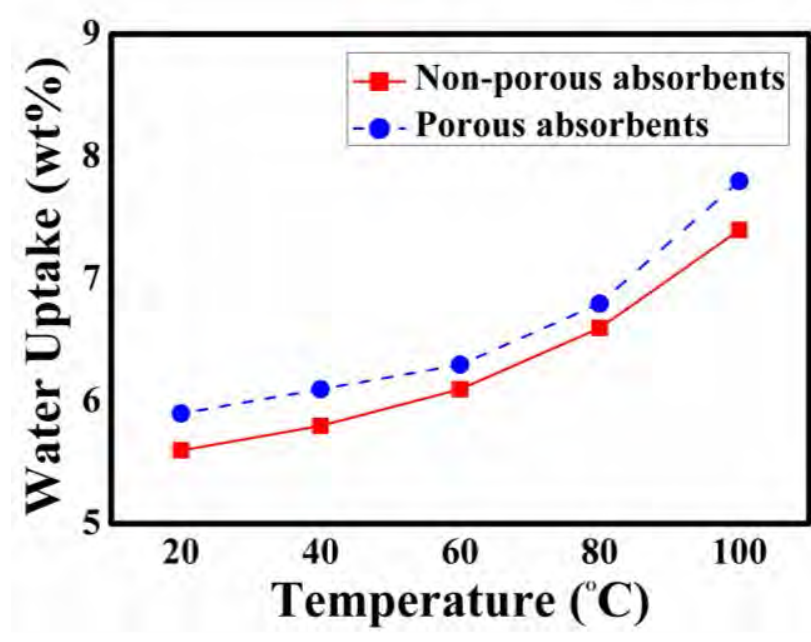

Figure 3. Water uptake of both porous and non-porous absorbents.

\section{Adsorption}

$\mathrm{KCl}$ salt bath was placed in a cube to create constant $\mathrm{RH}$ atmosphere. The RH was continuously measured until constant for $5 \mathrm{mins}(68.7 \% \mathrm{RH})$. Then the dried porous and non-porous absorbents were both placed in the oven and the weight change was recorded for comparison. Both samples exhibited high adsorption rate at first and slowed down after 300s. The porous absorbents showed higher adsorption rate than that of nonporous samples. As shown in figure 4, the porous absorbents also had higher adsorption ratio $(6.3 \mathrm{wt} \%)$ after fully absorbed.

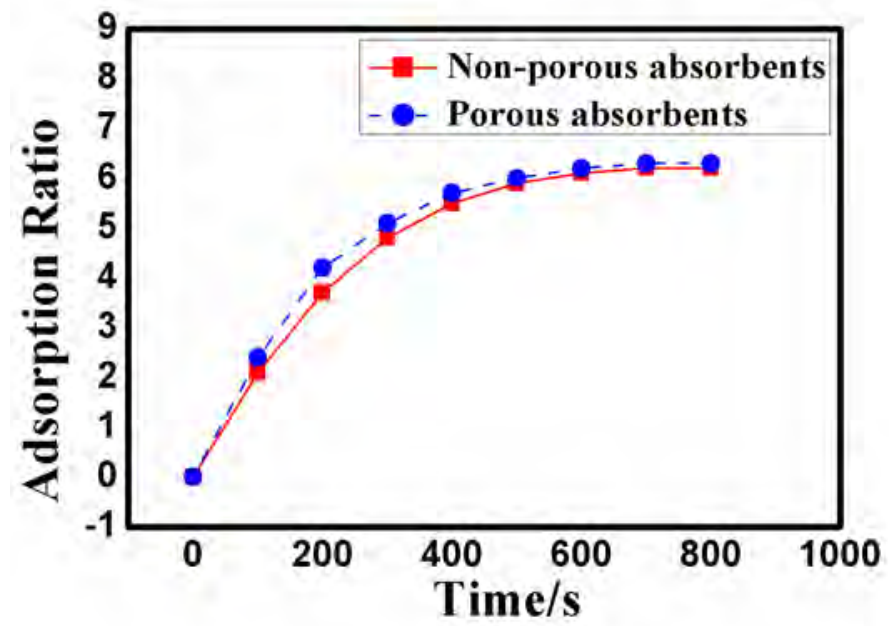

Figure 4. The adsorption ratio of both porous and nonporous absorbents.

\section{CONCLUSION}

The sulphonated poly ether sulfone ion exchange resin was successfully synthesized. To improve the adsorption capacity, the IER was fabricated with porous structure to increase the contact area with the moist air. Water uptake and adsorption ratio are investigated and compared with the non-porous absorbents. It turns out the porous absorbents exhibit both higher humidity adsorption rate and capacity due to the morphological changes. This works may provide a new method for developing high-performance desiccant coated heat exchangers.

\section{ACKNOWLEDGMENT}

We would like to thank the supporting of Hisense Air Conditioner R\&D Centre on this work.

\section{REFERENCES}

[1] M. A Mandegari and H. Pahlavanzadeh, "Introduction of a new definition for effectiveness of desiccant wheels," Energy, vol. 34, no. 6, pp. 797-803, June 2009. doi:10.1016/j.energy.2009.03.001.

[2] M. Tokarev, L. Gordeeva, and V. Romannikov, "New composite sorbent $\mathrm{CaCl} 2$ in mesopores for sorption cooling/heating," J. Therm. Sci., vol. 41, no. 5, pp. 470-474, Apr. 2002. doi:10.1016/S1290-0729(02)01339-X.

[3] N. Subramanyam, M. P. Maiya, and S. S. Murthy, "Application of desiccant wheel to control humidity in air-conditioning systems," J. Appl. Therm. Eng., vol. 24, no. 7-8, pp. 2777-2788, Dec. 2004. doi: 10.1016/j.applthermaleng.2004.04.008.

[4] I. Glaznev et al., "Composites CaCl2/SBA-15 for adsorptive transformationof low temperature heat: pore size effect," Int. J. Refrigeration, vol. 34, no. 5, pp. 1244-1250, Aug. 2011. doi: 10.1016/j.ijrefrig.2011.02.007.

[5] I. Ponomarenko et al., "Synthesis and water sorption properties of a new composite ' $\mathrm{CaCl} 2$ confined into SBA-15 pores'," Microporous Mesoporous Mater., vol. 129, no. 1-2, pp. 243-250, Apr. 2010. doi: 10.1016/j.micromeso.2009.09.023.

[6] L .Gordeeva and Y. I. Aristov, "Composites 'salt inside porous matrix' for adsorption heat transformation: a current state-of-the-art and new trends," Int. J. Low-Carbon Tech., vol. 7, no. 4, pp. 288-302, Dec. 2012.

doi: 10.1093/ijlct/cts050.

[7] T. S. Ge et al., "Simulation investigation on solar powered desiccant coated heat exchanger cooling system," Appl. Energ., vol. 93, pp. 532-540, May 2012. doi: 10.1016/j.apenergy.2011.11.089.

[8] T. S. Ge et al., "Experimental comparison and analysis on silica gel and polymer coated fintube heat exchangers," Energy, vol. 35, no. 7, pp. 2893-2900, July 2010. doi: 10.1016/j.energy.2010.03.020.

[9] R. K. Collier Jr., "Desiccant properties and their effect on cooling system performance," ASHRAE Trans., pp. 823-827, 1989.

[10] P. Stabat and D. Marchio, "Heat-and-mass transfers modelled for rotary desiccant dehumidifiers," Appl. Energ., vol. 85, no. 2-3, pp. 128-142, Feb.Mar. 2008. doi: 10.1016/j.apenergy.2007.06.017.

[11] X. Zheng et al., "Experimental study on silica gel-LiCl composite desiccants for desiccant coated heat exchanger," Int. J. Refrigeration, vol. 51, pp. 24 -32, Mar. 2015. doi: 10.1016/j.ijrefrig.2014.11.015.

[12] A. Frazzica et al., "Experimental and theoretical analysis of the kinetic performance of an adsorbent coating composition for use in adsorption chillers and heat pumps," Appl. Therm. Eng., vol. 73, no. 1, pp. 1022-1031, Dec. 2014. doi: 10.1016/j.applthermaleng.2014.09.004

[13] F. T. Ishugah et al., "Performance improvement of an adsorption chiller using composite adsorbent, silica gel impregnated with lithium chloride, paired with methanol as the adsorbate," Int. J. Air-Cond. Refrig., vol. 22, no. 2, pp. 1440003-1440017, Jan. 2014. doi: 10.1142/S2010132514400033. 
[14] Z.-S. Lu et al., "Experimental and theoretical analysis of the kinetic performance of an adsorbent coating composition for use in adsorption chillers and heat pumps," Appl. Therm. Eng., vol. 73, no. 1, pp. 1022-1031, Dec. 2014. doi:10.1016/j.applthermaleng.2014.09.004.

[15] A. Freni et al., "Simulation of water sorption dynamics in adsorption chillers: One, two and four layers of loose silica grains," Appl. Therm. Eng., vol. 44, pp. 69-77, Nov. 2012.

doi:10.1016/j.applthermaleng.2012.03.038.

[16] H. Demir, M. Mobedi, and S. Ulku, "The use of metal piece additives to enhance heat transfer rate through an unconsolidated adsorbent bed," Int. J. Refrig., vol. 33, no. 4, pp. 714-720, June 2010. doi: 10.1016/j.ijrefrig.2009.12.032.

[17] C.-H. Wu et al., "Enhancing the thermal conductivity of the heat exchanger in a noncompressive system as a means of energy efficiency improvement of the system," Int. J. Green Energy, vol. 6, no. 5, pp. 490-507, Oct. 2009. doi: 10.1080/15435070903228100.

[18] L.-W. Wang et al., "Thermal conductivity and permeability of consolidated expanded natural graphite treated with sulphuric acid," Carbon, 2011, vol. 49, no. 14, pp. 4812-4819, Nov. 2011.

doi: 10.1016/j.carbon.2011.06.093.

[19] Z. M. Khazimov et al., "Modeling of the motion of free convective drying agent in plastic helio dryer," J. Eng. Thermophys., vol. 23, no. 4, pp. 306-315, Oct. 2014.

doi: $10.1134 / \mathrm{S} 1810232814040080$

[20] M. A. Mandegari and H. Pahlavanzadeh, "Introduction of a new definition for effectiveness of desiccant wheels," Energy, vol. 34, no. 6, pp. 797-803, June 2009. doi: 10.1016/j.energy.2009.03.001.

[21] B. Liu, et al., "A comparative structure-property study of methylphenylated and fluoromethylphenylated poly(aryl ethers) and their gas permeabilities and permselectivities," Polym., vol. 46, no. 25, pp.

11279-11287, Nov. 2005. doi: 10.1016/j.polymer.2005.09.083. 


\title{
Effectiveness-NTU Relationships of Parallel-Plate Moving Bed Heat Exchangers
}

\author{
Pedro A. Isaza, Markus Bussmann* \\ Department of Mechanical and Industrial Engineering \\ University of Toronto \\ Toronto, Ontario, Canada, M5G-3S8
}

\begin{abstract}
Moving bed heat exchangers (MBHEs) are used in various industrial processes. Recently, analytical solutions to several MBHE heat transfer problems have been presented in the literature. In this work, the mathematical procedure by which these new solutions are used to construct effectivenessNTU relationships is presented, for parallel-plate configurations. Expressions for both co- and counter-current orientations are outlined. Effectiveness-NTU plots are then generated, and contrasted with those of fluid-fluid systems. As expected, a functionality with respect to the Number-ofTransfer-Units and the Capacity Ratio is observed. A novel dependency with respect to the Biot number is also demonstrated, whereby effectiveness decreases with increasing Biot number, due to the increasing resistance imparted by the diffusion of energy through the solids bulk. These effectiveness-NTU plots can serve as a design platform, which engineers can use to size and rate MBHEs.
\end{abstract}

Keywords-Effectiveness-NTU; Moving Bed Heat Exchangers; Analytical Solutions

\section{INTRODUCTION}

Describing energy transport into and out of beds of granular solids has been an area of active research for a number of years. Recently, research in the field has given increased attention to the mathematical modeling of heat transfer in moving bed heat exchangers. MBHEs typically consist of a bed of particulate solids flowing downwards by gravity, which exchanges thermal energy across a separating wall with a heating or cooling fluid. Compared with alternative technologies like fluidized beds, moving beds offer reduced investment costs, energy consumption and maintenance requirements $[1,2]$. These competitive advantages have prompted their use in processes ranging from nickel production [3, 4] and food sterilization [5], to flue gas abatement and biomass combustion [6, 7]. Most recently, MBHEs have found a use in solar power generation [8].

Moving bed heat exchangers can be organized in various configurations (i.e. parallel-plate, vertical pipe, etc.) and can accommodate different flow orientations (i.e. counter-current, co-current, or cross flow). Recent mathematical investigations into some of these heat transfer problems have identified analytical solutions for both parallel-plate and vertical pipe configurations [9-12]. The results outlined provide a sizing platform that engineers can now use to design and rate the thermal performance of these systems. One step that is absent in this new body of work, is the transformation of these expressions into effectiveness-NTU relationships that are commonly found in the heat exchanger literature.

The purpose of this paper is to detail the mathematical procedure by which effectiveness-NTU relationships can be established for parallel-plate MBHEs, using the recently presented analytical solutions. From the expressions, effectiveness-NTU curves are generated as a function of the dimensionless groups found in the fluid-fluid heat exchanger literature (i.e. Number-of-Transfer-Units and Capacity Ratio), as well as a new dimensionless group (i.e. Biot number).

\section{GOVERNING EQUATIONS}

The experimentally validated assumptions behind the energy model formulated for the parallel-plate MBHE, outlined by Isaza et al. $[9,10]$, include: The system operates under steady-state conditions, and the solids move with constant velocity under local thermal equilibrium [13-17]. The solids enter the exchanger at a constant temperature, and the thermo-physical properties are constant and isotropic. Heat conduction in the solids occurs in the lateral direction only [9] (i.e. negligible axial heat conduction - explored and validated in [12]), while convection occurs in the axial direction. Energy transfer in the cooling/heating fluid takes place via convection only (i.e. a convective coefficient quantifies transport by means of a SiederTate style correlation [18]). An overall heat transfer coefficient $\left(U_{o}\right)$ comprised of resistances in series due to contact (i.e. nearby wall effects) $[13-14,17,19-20]$, wall conduction and convection into the heating/cooling fluid describes energy transfer between the domains. Negligible axial heat conduction takes place along the exchanger wall [11]. Viscous energy dissipation, radiation effects, and sources of thermal energy are negligible. Figure 1 presents a schematic of the co- and countercurrent parallel-plate MBHEs under consideration.

Based on the Cartesian geometry in Fig. 1 and the above assumptions, the MBHE problems can be formulated as follows: 


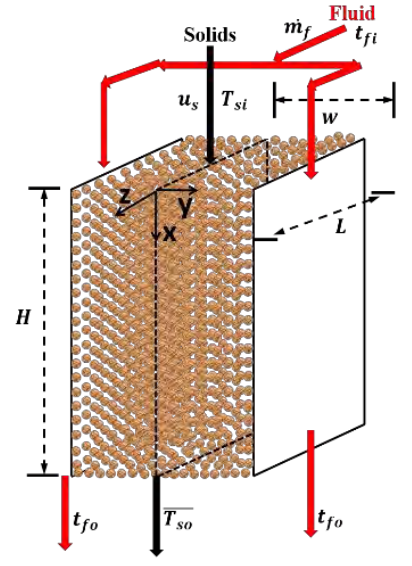

(a)

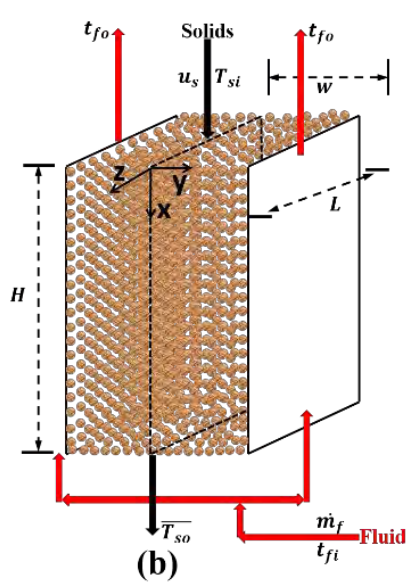

(b)
Figure 1 Co-current (a) and counter-current (b) parallel-plate MBHE schematics

$$
\begin{aligned}
\rho_{s} C_{p s} u_{s} \frac{\partial T_{s}(x, y)}{\partial x} & =k_{s} \frac{\partial^{2} T_{s}(x, y)}{\partial y^{2}} \quad x>0 ; 0<y<w \\
T_{s}(0, y)=T_{s i} \quad \text { at } \quad x=0 & \quad \text { at } y=0 \\
\left.\frac{\partial T_{s}(x, y)}{\partial y}\right|_{y=0}=\left.0 \quad k_{s} \frac{\partial T_{s}(x, y)}{\partial y}\right|_{y=w}= & U_{o}\left[T_{s}(x, w)-t_{f}(x)\right] \quad \text { at } \quad y=w
\end{aligned}
$$

where the complementary fluid problem is the following:

$$
\begin{array}{ccc}
U_{o} \cdot L\left[T_{s}(x, w)-t_{f}(x)\right]= \pm \frac{\dot{m}_{f}}{2} \cdot C_{p s} \frac{d t_{f}(x)}{d x} \quad & x>0 \\
t_{f}(0)=t_{f i}(+) & \text { or } \quad t_{f}(H)=t_{f i}(-)
\end{array}
$$

Note that the plus and minus signs correspond to the equations for the co-current [9] and counter-current [10] orientations respectively.

Like other heat transfer investigations, the analytical solutions to the above problems are presented subject to the following nondimensional variables $[9,10]$ :

$$
y^{*}=\frac{y}{w} ; \quad x^{*}=\frac{x}{H} ; \quad \theta_{s}=\frac{T_{s}-t_{f i}}{T_{s i}-t_{f i}} \quad \theta_{f}=\frac{t_{f}-t_{f i}}{T_{s i}-t_{f i}}
$$

Applying these to Eqs. (1) - (6) yields:

$$
\begin{gathered}
\frac{\partial^{2} \theta_{S}\left(x^{*}, y^{*}\right)}{\partial y^{* 2}}=\frac{B i}{N T U} \frac{\partial \theta_{S}}{\partial x^{*}} \\
\theta_{S}\left(0, y^{*}\right)=1 \quad \text { at } x^{*}=0 ; 0<y^{*}<1 \\
\left.\frac{\partial \theta_{S}\left(x^{*}, y^{*}\right)}{\partial y^{*}}\right|_{y^{*}=0}=0 \quad \text { at } \quad y^{*}=0 \\
\left.\frac{\partial \theta_{S}\left(x^{*}, y^{*}\right)}{\partial y}\right|_{y^{*}=1}=B i \cdot\left[\theta_{f}\left(x^{*}\right)-\theta_{S}\left(x^{*}, 1\right)\right] \quad \text { at } y^{*}=1 \\
\frac{d \theta_{f}\left(x^{*}\right)}{d x^{*}}=\mp N T U \cdot C \cdot\left[\theta_{f}\left(x^{*}\right)-\theta_{S}\left(x^{*}, 1\right)\right] \quad x^{*}>0 \\
\theta_{f}(0)=0(-) \quad \text { or } \quad \theta_{f}(1)=0(+)
\end{gathered}
$$

where the minus and plus correspond to the equations for the cocurrent [9] and counter-current [10] orientations. In Eqs. (8) (13) the Biot number $(B i)$, Number-of-Transfer-Units (NTU) and Capacity Ratio $(C)$ dimensionless groups are defined as:

$$
B i=\frac{U_{o} \cdot w}{k_{s}} ; \quad N T U=\frac{U_{o} \cdot H}{\rho_{s} C_{p s} u_{s} w} ; \quad C=\frac{\dot{m}_{s} \cdot C_{p s}}{\dot{m}_{f} \cdot C_{p f}}
$$

\section{ANALYTICAL SOLUTIONS}

Isaza et al. $[9,10]$ present the analytical solutions of the differential equations outlined by Eqs. (8) - (13). The method of solution begins with the application of a Laplace transformation. Once in the Laplace domain, the solid and fluid problems are decoupled, resulting in a well-posed second-order ordinary differential equation problem. Using standard techniques, the problems are solved and the Laplace inverse transform is identified by means of either the standard or the generalized expansion theorem detailed by Luikov [21]. As discussed in [10], to obtain a correct inverse transform for the solids temperature function in the counter-current case, the inverse transform needs to be examined separately for $C<1, C=1$ and $C>1$. This special analysis is driven by the expansion theorem, which requires the identification of the simple roots in the denominator function of the Laplace domain solution. Unlike co-current systems, in the counter-flow problem the roots are a function of $C$, which requires a case-by-case examination. Complete details of the methodology are presented by Isaza et al. [10].

\section{A. Co-Current Analytical Solution}

Following the methodology discussed, Isaza et al. [9] obtained the solution for the co-current problem. As will become evident later, for the purpose of developing effectiveness-NTU relations only the expression quantifying the solids average temperature at the exit is needed. That expression is given as:

$\overline{\theta_{s o}}=\frac{C}{1+C}+\sum_{n=1}^{\infty}\left[\frac{4 \sin \left(\lambda_{n}\right)}{\frac{4 C \sin ^{2}\left(\lambda_{n}\right)}{\lambda_{n}}+2 \lambda_{n}+\sin \left(2 \lambda_{n}\right)}\right] e^{-\frac{\lambda_{n}^{2} \cdot N T U}{B i}} \cdot \frac{\sin \left(\lambda_{n}\right)}{\lambda_{n}}$

which is subject to the transcendental equation:

$\frac{\lambda_{n} \cdot \sin \left(\lambda_{n}\right)}{\left[\frac{C}{\lambda_{n}} \sin \left(\lambda_{n}\right)+\cos \left(\lambda_{n}\right)\right]}=B i \quad$ where $\quad n=1,2,3 \ldots$

\section{B. Counter-Current Analytical Solution:}

For the counter-current configuration, the fluid outlet temperatures in [10] are required to build the effectiveness-NTU relationships. Recall that for counter-flow, the analytical solutions are a function of the capacity ratio, and the case-bycase expressions are presented below:

Fluid Outlet Temperature $-C<1$ :

$\theta_{f o}=\frac{\frac{C}{1-C}+C \cdot \sum_{n=1}^{\infty}\left[\frac{4 \sin \left(\lambda_{n}\right)}{\frac{4 C \sin ^{2}\left(\lambda_{n}\right)}{\lambda_{n}}-2 \lambda_{n}-\sin \left(2 \lambda_{n}\right)}\right] e^{-\frac{\lambda_{n}^{2} \cdot N T U}{B i} \cdot \frac{\sin \left(\lambda_{n}\right)}{\lambda_{n}}}}{\frac{1}{1-C}+C \cdot \sum_{n=1}^{\infty}\left[\frac{4 \sin \left(\lambda_{n}\right)}{\frac{4 C \sin ^{2}\left(\lambda_{n}\right)}{\lambda_{n}}-2 \lambda_{n}-\sin \left(2 \lambda_{n}\right)}\right] e^{-\frac{\lambda_{n}^{2} \cdot N T U}{B i} \cdot \frac{\sin \left(\lambda_{n}\right)}{\lambda_{n}}}}$ 
Fluid Outlet Temperature $-C=1$ :

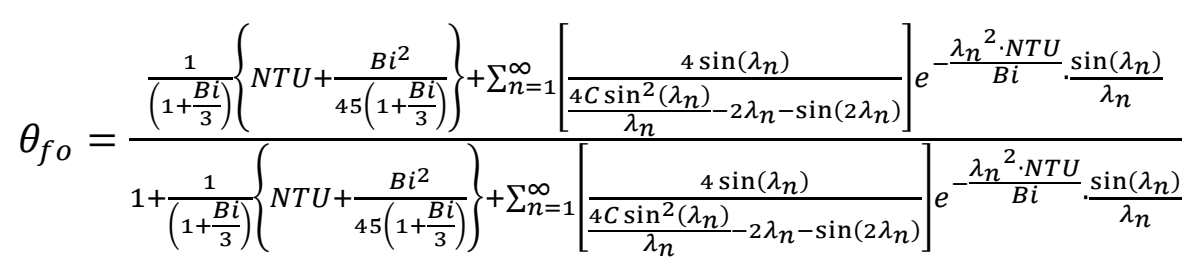

Fluid Outlet Temperature $-C>1$ :

$$
\theta_{f o}=\frac{\frac{C}{1-C}+C\left[\frac{4 \sinh (\mu)}{\frac{4 C \sinh ^{2}(\mu)}{\mu}-2 \mu-\sinh (2 \mu)}\right] \frac{\sinh (\mu)}{\mu} e^{\frac{\mu^{2} \cdot N T U}{B i}}+C \cdot \sum_{n=1}^{\infty}\left[\frac{4 \sin \left(\lambda_{n}\right)}{\frac{4 C \sin ^{2}\left(\lambda_{n}\right)}{\lambda_{n}}-2 \lambda_{n}-\sin \left(2 \lambda_{n}\right)}\right] e^{-\frac{\lambda_{n}^{2} \cdot N T U}{B i}} \cdot \frac{\sin \left(\lambda_{n}\right)}{\lambda_{n}}}{\frac{1}{1-C}+C\left[\frac{4 \sinh (\mu)}{\frac{4 C \sinh ^{2}(\mu)}{\mu}-2 \mu-\sinh (2 \mu)}\right] \frac{\sinh (\mu)}{\mu} e^{\frac{\mu^{2} \cdot N T U}{B i}}+C \cdot \sum_{n=1}^{\infty}\left[\frac{4 \sin \left(\lambda_{n}\right)}{\frac{4 C \sin ^{2}\left(\lambda_{n}\right)}{\lambda_{n}}-2 \lambda_{n}-\sin \left(2 \lambda_{n}\right)}\right] e^{-\frac{\lambda_{n}^{2} \cdot N T U}{B i}} \cdot \frac{\sin \left(\lambda_{n}\right)}{\lambda_{n}}}
$$

subject to the transcendental equations:

$\frac{\lambda_{n} \cdot \sin \left(\lambda_{n}\right)}{\left[\frac{-C}{\lambda_{n}} \sin \left(\lambda_{n}\right)+\cos \left(\lambda_{n}\right)\right]}=B i \quad$ where $\quad n=1,2,3 \ldots$

$\frac{\mu \cdot \sinh (\mu)}{\left[\frac{C}{\mu} \sinh (\mu)-\cosh (\mu)\right]}=B i$

\section{EFFECTIVENESS - NTU RELATIONS}

The effectiveness of a heat exchanger is defined as the ratio of the actual and the maximum heat transfer rate which is achievable in a given system [18, 22]. In the case of the MBHE, the actual heat transfer rate can be defined in terms of the energy lost or gained by the solids or the heating/cooling fluid. The maximum rate of energy transfer on the other hand, is established as the product of the largest temperature differential available in the system and the minimum capacity rate (i.e. the min capacity rate is defined as $c=\dot{m} \cdot C_{p}$ ). Mathematically, the effectiveness of the MBHE can then be defined as:
Equation (22), however, requires further examination as a function of $C$. In cases where $C$ is less than or equal to 1 , the fluid capacity rate exceeds that of the solids (i.e. $\dot{m}_{s} \cdot C_{p s}<$ $\dot{m}_{f} \cdot C_{p f}$ ) and the effectiveness expression adopts the form:

Effectiveness $-C \leq 1$ :

$$
\varepsilon=\frac{\dot{m}_{s} \cdot C_{p s} \cdot\left[\bar{T}_{s o}-T_{s i}\right]}{\dot{m}_{s} \cdot C_{p s} \cdot\left[t_{f i}-T_{s i}\right]}=\frac{\dot{m}_{f} \cdot C_{p f} \cdot\left[t_{f i}-t_{f o}\right]}{\dot{m}_{s} \cdot C_{p s} \cdot\left[t_{f i}-T_{s i}\right]}=1-\overline{\theta_{s o}}=\frac{\theta_{f o}}{C}
$$

Conversely, when $C>1$ the effectiveness expression becomes:

Effectiveness $-C \geq 1$ :

$$
\varepsilon=\frac{\dot{m}_{s} \cdot C_{p s} \cdot\left[\bar{T}_{s o}-T_{s i}\right]}{\dot{m}_{f} \cdot C_{p f} \cdot\left[t_{f i}-T_{s i}\right]}=\frac{\dot{m}_{f} \cdot C_{p f} \cdot\left[t_{f i}-t_{f o}\right]}{\dot{m}_{f} \cdot C_{p f} \cdot\left[t_{f i}-T_{s i}\right]}=\theta_{f o}=C\left[1-\overline{\theta_{s o}}\right]
$$

\section{A. Co-Current Effectiveness Relationships:}

Substituting Eq. (15) into Eqs. (23) and (24) the effectiveness-NTU relations for the co-current MBHE are found to be the following:

$$
\begin{aligned}
& \varepsilon=\frac{1}{1+C}-\sum_{n=1}^{\infty}\left[\frac{4 \sin \left(\lambda_{n}\right)}{\frac{4 C \sin ^{2}\left(\lambda_{n}\right)}{\lambda_{n}}+2 \lambda_{n}+\sin \left(2 \lambda_{n}\right)}\right] e^{-\frac{\lambda_{n}^{2} \cdot N T U}{B i} \cdot \frac{\sin \left(\lambda_{n}\right)}{\lambda_{n}} \quad \text { for } C \leq 1} \\
& \varepsilon=\frac{C}{1+C}-C \sum_{n=1}^{\infty}\left[\frac{4 \sin \left(\lambda_{n}\right)}{\frac{4 C \sin ^{2}\left(\lambda_{n}\right)}{\lambda_{n}}+2 \lambda_{n}+\sin \left(2 \lambda_{n}\right)}\right] e^{-\frac{\lambda_{n}^{2} \cdot N T U}{B i}} \cdot \frac{\sin \left(\lambda_{n}\right)}{\lambda_{n}} \text { for } C \geq 1
\end{aligned}
$$

$$
\varepsilon=\frac{\left[\dot{m} \cdot C_{p} \cdot \Delta T\right]_{s \text { or } f}}{\left[\dot{m} \cdot C_{p}\right]_{\min } \cdot \Delta T_{\max }}
$$

and $\left[\dot{m} \cdot C_{p}\right]_{\min }$ is associated with either the solids or the fluid depending on the conditions examined. Note that the definition applies regardless of the flow configuration.
Note that for the special case where $C=1$, Eq. (26) simplifies to Eq. (25) as expected.

\section{B. Counter-Current Effectiveness Relationships:}

Substituting Eqs. (17) - (19) into Eqs. (23) and (24), the effectiveness-NTU relations for a counter-current MBHE are found to be: 


$$
\begin{aligned}
& \left.\mathcal{E}=\frac{\frac{1}{1-C}+\sum_{n=1}^{\infty}\left[\frac{4 \sin \left(\lambda_{n}\right)}{\frac{1}{1-C}+C \cdot \sum_{n=1}^{\infty}\left[\frac{\sin ^{2}\left(\lambda_{n}\right)}{\lambda_{n}}-2 \lambda_{n}-\sin \left(2 \lambda_{n}\right)\right.}\right] e^{-\frac{\lambda_{n}^{2} \cdot N T U}{B i} \cdot \frac{\sin \left(\lambda_{n}\right)}{\lambda_{n}}}}{\frac{4 C \sin ^{2}\left(\lambda_{n}\right)}{\lambda_{n}}-2 \lambda_{n}-\sin \left(2 \lambda_{n}\right)}\right] e^{-\frac{\lambda_{n}^{2} \cdot N T U}{B i} \cdot \frac{\sin \left(\lambda_{n}\right)}{\lambda_{n}}} \quad C<1
\end{aligned}
$$

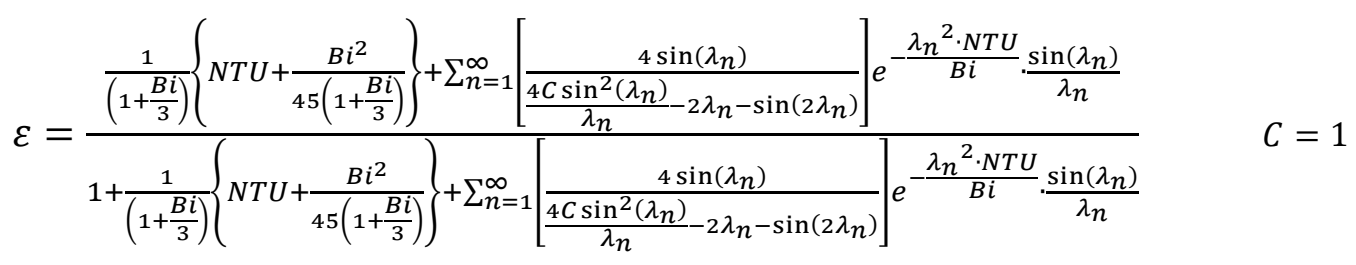

$$
\varepsilon=\frac{\frac{C}{1-C}+C\left[\frac{4 \sinh (\mu)}{\frac{4 C \sinh ^{2}(\mu)}{\mu}-2 \mu-\sinh (2 \mu)}\right] \frac{\sinh (\mu)}{\mu} e^{\frac{\mu^{2} \cdot N T U}{B i}}+C \cdot \sum_{n=1}^{\infty}\left[\frac{4 \sin \left(\lambda_{n}\right)}{\frac{4 C \sin ^{2}\left(\lambda_{n}\right)}{\lambda_{n}}-2 \lambda_{n}-\sin \left(2 \lambda_{n}\right)}\right] e^{-\frac{\lambda_{n}^{2} \cdot N T U}{B i} \cdot \frac{\sin \left(\lambda_{n}\right)}{\lambda_{n}}}}{\frac{1}{1-C}+C\left[\frac{4 \sinh (\mu)}{\frac{4 C \sinh ^{2}(\mu)}{\mu}-2 \mu-\sinh (2 \mu)}\right] \frac{\sinh (\mu)}{\mu} e^{\frac{\mu^{2} \cdot N T U}{B i}}+C \cdot \sum_{n=1}^{\infty}\left[\frac{4 \sin \left(\lambda_{n}\right)}{\frac{4 C \sin ^{2}\left(\lambda_{n}\right)}{\lambda_{n}}-2 \lambda_{n}-\sin \left(2 \lambda_{n}\right)}\right] e^{-\frac{\lambda_{n}^{2} \cdot N T U}{B i} \cdot \frac{\sin \left(\lambda_{n}\right)}{\lambda_{n}}} C 1} \quad C
$$

Several important observations can be made of Eqs. (25) (29). Like fluid-fluid systems [18, 22], the effectiveness of the MBHE is also a function of the Number-of-Transfer-Units $(N T U)$ and the Capacity Ratio $(C)$. This dependency exists regardless of the flow configuration. A new functionality of the MBHE effectiveness with respect to the Biot number is clear from Eqs. (25) - (29). As defined, the Biot number quantifies the magnitude of the resistance associated with lateral energy transfer through the particulate solids. In what follows, the effectiveness dependency on these dimensionless groups is explored graphically.

\section{EFFECTIVENESS - NTU GRAPHICAL ANALYSIS}

For ease of heat exchanger design, it is common for engineers to use the graphical representation of the effectiveness-NTU relations. This is particularly useful during rating exercises, where the area of the exchanger is known and the outlet temperatures need to be determined. As explained by Serth and Lestina [18], this calculation requires an iterative procedure which can be circumvented through the use of effectiveness-NTU plots. This ease of calculation is even more important for MBHEs, due to the presence of the infinite series in the above relations. For all of the plots presented below, the functions are evaluated with 600 terms. This provides sufficient accuracy for the range of dimensionless groups explored [9].

\section{A. Co-Current MBHEs: Effectiveness vs. NTU}

In fluid-fluid heat transfer, it is well known that the effectiveness depends only on $C$ and NTU [18, 22, 23]. Equations (25) and (26), however, clearly demonstrate that for MBHEs $\varepsilon$ also depends on $B i$. Figure 2 presents effectivenessNTU curves for the co-current configuration, as a function of $C$ and $B i$. For comparison purposes, the predictions of the fluidfluid expressions available in the literature are included in the plots.
From Fig. (2a) - (2c), like the fluid-fluid expressions, as the capacity ratio increases (i.e. from 0 to 1 ) the MBHE effectiveness decreases. This trend exists for all of the Biot numbers explored. Per the definition given by Eq. (22), an effectiveness of one describes a system where the maximum heat transfer rate is achieved. For a co-current system, this occurs when $C=0$, and outlines conditions where the heating/cooling fluid undergoes phase change (i.e. $\dot{m}_{f} \cdot C_{p f}$ tends to infinity). Similarly, when $C=0.5$ and $C=1$ (i.e. Figs. (2b) and (2c)) a maximum effectiveness of 0.67 and 0.5 is obtained. These values agree with the theoretical limits available under cocurrent conditions, whereby the effectiveness tends to $\frac{1}{1+C}$ as $N T U$ goes to infinity (i.e obtained by evaluating the fluid-fluid expressions and Eq. (25) as NTU $\rightarrow \infty$ ). At first glance, Figure (2d) would appear to deviate from this tendency; however, recall from Eqs. (24) and (26) that the effectiveness definition for the MBHE changes when $C$ is greater than one. Unlike fluid-fluid problems, the definition of $C$ here is static (i.e. $C$ is defined as the capacity rate of the solids divided by that of the fluid for all problems), rather than as the ratio of the minimum and maximum capacity rates. This requires the correction of multiplying by $C$, which exists between Eqs. (23) and (24). Having made this correction, Eq. (26) tends to the expected fluid-fluid effectiveness maxima of 0.6 for $C=1.5$. Furthermore, it can be readily shown that as $C$ increases beyond 1.5 , the effectiveness will again tend towards one.

Figure 2 also demonstrates the effect of the Biot number. Figures. (2a) - (2d) show that as the Biot number increases, from 0 (i.e. a "fluid -fluid" analog) to 100 , the $N T U$ required to reach the maximum effectiveness increases. In other words, as the Biot number increases, the area of heat transfer required to reach a given effectiveness also increases. This behavior is independent of the magnitude of $C$, and aligns with the physics of the problem considered. A large Biot number corresponds to a system where the diffusional resistance to lateral heat transfer within the solids is controlling. Under such conditions, the energy exchange 
(a)

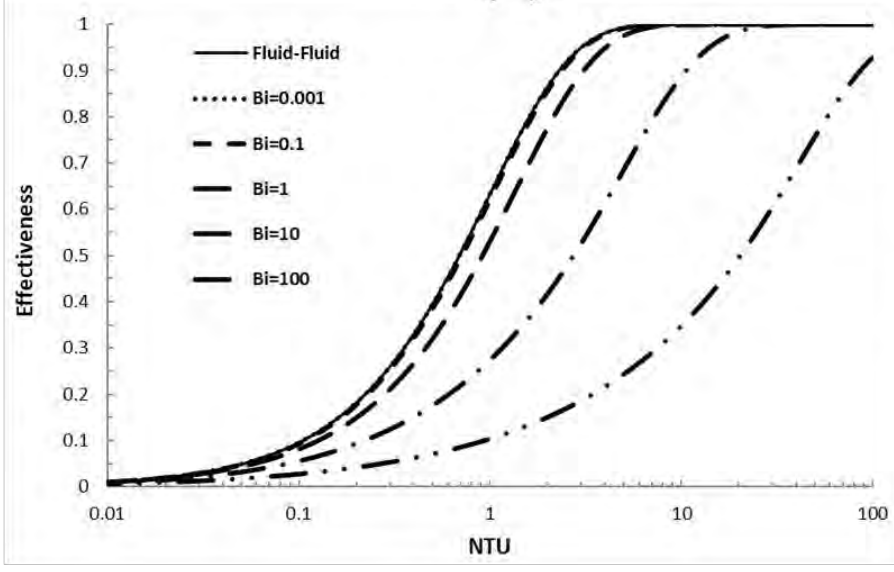

(c)

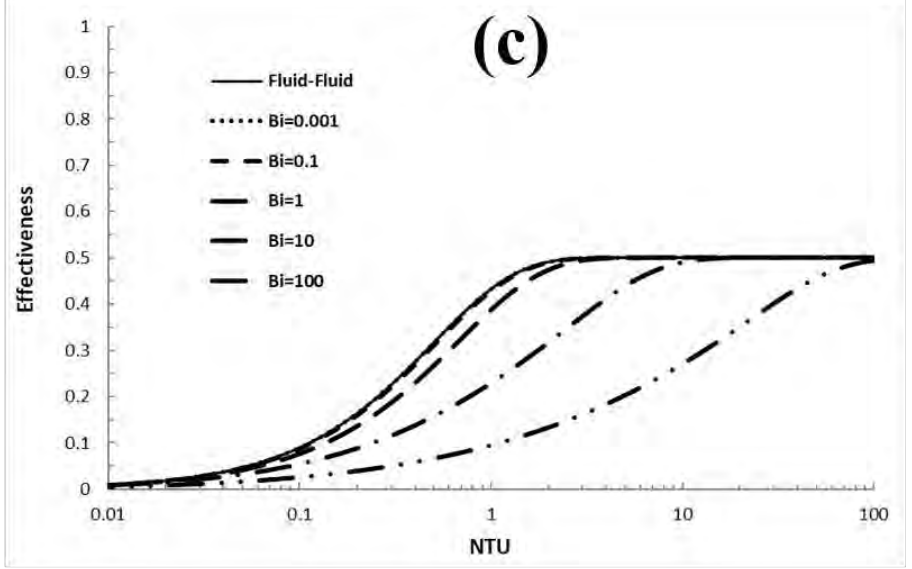

(b)
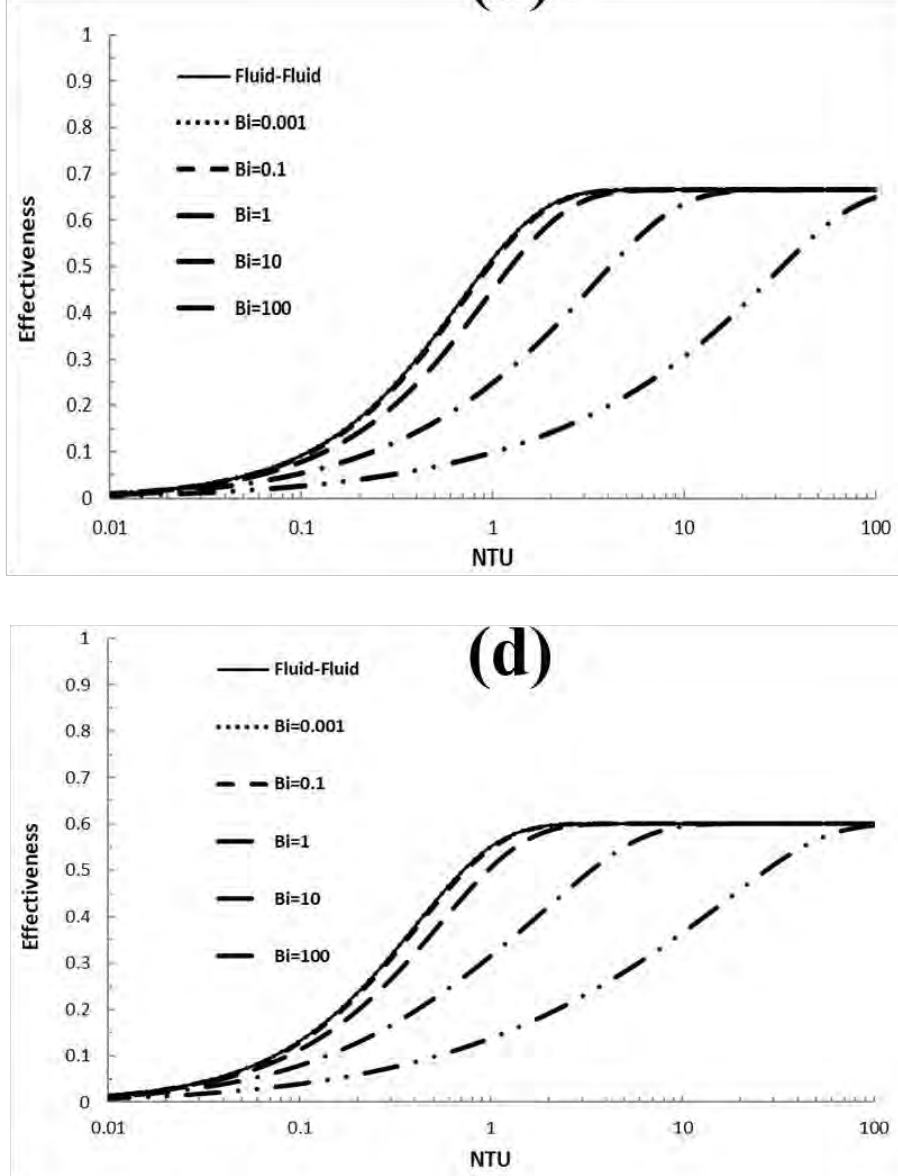

Figure 2. Effectiveness-NTU curves for co-current MBHEs with capacity ratios of (a) $C=0$, (b) $C=0.5$, (c) $C=1$, and (d) $C=1.5$

between the two domains is no longer controlled by the overall heat transfer coefficient (i.e. $U_{o}$ ) and heat transport is controlled by diffusion through the solids bulk. This behavior has been explained in detail by Isaza et al. [9].

\section{B. Counter-Current MBHEs: Effectiveness vs. NTU}

Now we consider the effectiveness-NTU behavior of the counter-current MBHE. As discussed in the literature, counterflow configurations can achieve higher heat transfer rates than co-current systems [24, 25]. As such, for a given amount of energy a smaller area is required. As discussed above, the effectiveness of counter-current fluid-fluid systems depends only on $C$ and $N T U$ [18, 22, 23]. Equations. (27) - (29), however, also demonstrate a Biot number dependency for counter-current MBHEs. Figure 3 presents the effectiveness curves for the counter-current configuration as a function of $N T U, C$ and $B i$. For comparative purposes, the predictions of the fluid-fluid expressions in the literature are included.

The results presented in Fig. 3, display some of the wellestablished characteristics of fluid-fluid systems. First, for all $C$ (i.e. Figs (3a) - (3d)) the effectiveness tends to one. This behavior arises from thermodynamic considerations. In a counter-flow system, given a sufficiently large heat transfer area (i.e. a sufficiently large $N T U$ ), the outlet temperature of the solids will tend to the fluid inlet temperature when $C<1$. From Eq. (23), if $\bar{T}_{s o} \sim t_{f i}$ the effectiveness of the unit tends to one, as per Figs. (3a) - (3c). A similar analysis when $C>1$ also demonstrates that given a sufficiently large area, the fluid outlet temperature will tend to the solids inlet temperature. From Eq. (24), one arrives at the expected maximum effectiveness of 1 observed in Fig. (3d). Secondly, for Figs. (3a) - (3c), as the capacity ratio increases, the $N T U$ required to achieve a given effectiveness also increases. This pattern is independent of the Biot number. For instance, for a Biot number of 1 , the NTU required to reach an effectiveness of 0.95 is $4.05,6.31$ and 25.35 for $C$ equal to $0,0.5$ and 1 respectively.

Figure 3 also depicts some of the Biot number dependencies discussed for the co-current system. For instance, when $C=0$ and $N T U=1$ (i.e. Fig (3a)), the effectiveness are 0.63, 0.62, 0.53, 0.27 and 0.1 for Biot numbers of $0.001,0.1,1,10$ and 100 respectively. Clearly, as the Biot number increases, the effectiveness decreases even under counter-current conditions. 
(a)
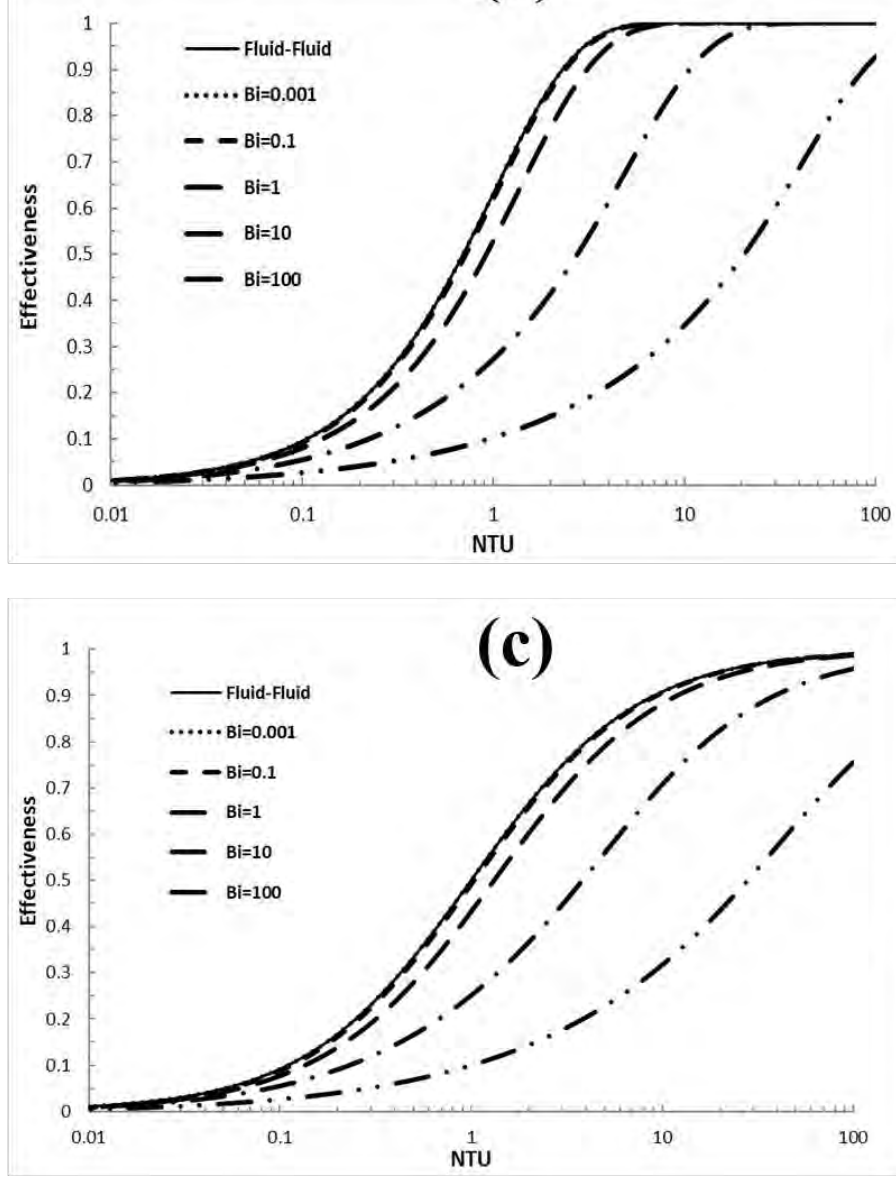

(b)
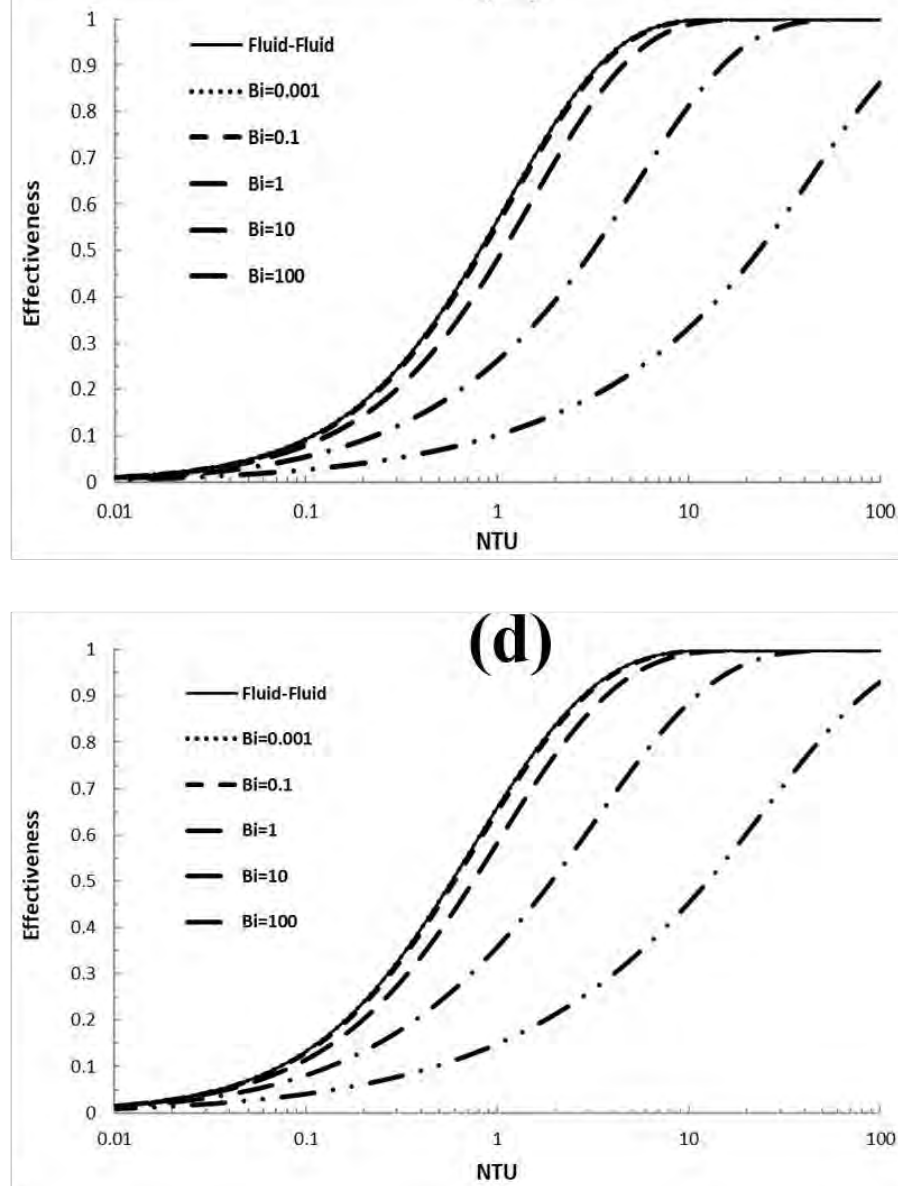

Figure 3. Effectiveness-NTU curves for counter-current MBHEs with capacity ratios of (a) $C=0,(\mathrm{~b}) C=0.5$, (c) $C=1$, and (d) $C=1.5$

By examining Figs. (3a) - (3d), it can be concluded that this dependency exists for all capacity ratios. In other words, regardless of the magnitude of $C$ the effectiveness of the MBHE will decrease with increasing Biot number. As discussed in Section V, large Biot numbers represent a system with a significant diffusional resistance in the solids. This results in the reduced effectiveness that's observed

\section{CONCLUSIONS}

This work presents a methodology for obtaining effectiveness-NTU relationships for parallel-plate MBHEs, based on new analytical solutions found in the literature. Relationships for both co- and counter-current systems are presented. Like fluid-fluid systems, the effectiveness-NTU expressions are found to be a function of the Number-ofTransfer-Units and the Capacity Ratio. Both orientations are shown to be in alignment with the thermodynamic expectations of the systems, and with the corresponding results for fluid-fluid exchangers. A novel dependency of the effectiveness with respect to the Biot number is observed. In particular, as the Biot number increases the effectiveness of the MBHE decreases. This is the case for both co-current and counter-current orientations. A large Biot number is associated with an increased diffusional resistance through the solids bulk, explaining the reduced effectiveness. The relationships and curves presented in this work can serve as a sizing and rating platform for design activities. 


\begin{tabular}{|c|c|}
\hline$\dot{m}_{f}$ & Fluid mass flow rate \\
\hline$\dot{m}_{s}$ & Solids mass flow rate \\
\hline$n$ & Integer number, positive \\
\hline$N T U$ & Number of transfer units, $=\frac{U_{o} \cdot A_{h x}}{\dot{m}_{s} \cdot C_{p s}}=\frac{U_{o} \cdot H}{\rho_{s} u_{s} w C_{p s}}$ \\
\hline$T_{S}$ & Solids temperature \\
\hline$T_{s i}$ & Solids entrance temperature \\
\hline$\overline{T_{s o}}$ & Solids average outlet temperature \\
\hline$t_{f}$ & Fluid temperature \\
\hline$t_{f i}$ & Fluid entrance temperature \\
\hline$t_{f o}$ & Fluid outlet temperature \\
\hline$U_{o}$ & Overall heat transfer coefficient \\
\hline$u_{s}$ & Solids velocity \\
\hline$w$ & Plate half width \\
\hline$x$ & Axial spatial coordinate \\
\hline$x^{*}$ & Dimensionless axial spatial coordinate, $=\frac{x}{H}$ \\
\hline$y$ & Lateral spatial coordinate \\
\hline$y^{*}$ & Dimensionless lateral spatial coordinate, $=\frac{y}{w}$ \\
\hline & Greek Letters \\
\hline$\varepsilon$ & Heat exchanger effectiveness, $=\frac{\left[\dot{m} \cdot C_{p} \cdot \Delta T\right]_{\text {orf }}}{\left[\dot{m} \cdot C_{p}\right]_{\min } \Delta T_{\max }}$ \\
\hline$\theta_{f}$ & Dimensionless fluid temperature function, $=\frac{t_{f}-t_{f i}}{T_{s i}-t_{f i}}$ \\
\hline$\theta_{f o}$ & Dimensionless fluid outlet temperature, $=\frac{t_{f o}-t_{f i}}{T_{s i}-t_{f i}}$ \\
\hline$\theta_{s}$ & Dimensionless solids temperature function, $=\frac{T_{s}-t_{f i}}{T_{s i}-t_{f i}}$ \\
\hline$\overline{\theta_{s o}}$ & Dimensionless solids average exit temperature \\
\hline$\lambda_{n}$ & $\mathrm{n}^{\text {th }}$ eigenvalue \\
\hline$\rho_{f}$ & Fluid density \\
\hline$\rho_{s}$ & Solids "effective" density \\
\hline
\end{tabular}

\section{REFERENCES}

[1] M. Colakyan, "Moving bed heat transfer and fluidized elutriation," Ph.D. dissertation, Dept. Chem. Eng., Oregon State Univ., Corvallis, OR, 1985.

[2] A. C. Pivem and M. J. S. de Lemos, "Laminar heat transfer in a moving porous bed reactor simulated with a macroscopic two-energy equation model," Int. J. of Heat Mass Transfer, vol. 55, no. 5, pp. 1922-1930, Mar. 2012. doi: 10.1016/j.ijheatmasstransfer.2011.11.047.

[3] M. H. I. Baird, N. V. Rama Rao, E. Tackie, and A. Vahed, "Heat transfer to a moving packed bed of nickel pellets," Can. J. Chem. Eng., vol. 86, no. 2, pp. 142-150, Mar. 2008. doi: 10.1002/cjce.20023

[4] R. Henda and D. J. Falcioni, "Modeling of heat transfer in a moving packed bed: Case of the preheater in nickel carbonyl process," J. Appl. Mech., vol. 73, no. 1, pp. 47-53, Apr. 2006. doi: 10.1115/1.1991862.
[5] J. J. Saastamoinen, "Heat exchange between two coupled moving beds by fluid flow," Int. J. of Heat and Mass Transfer, vol. 47, no. 6, pp. 1535-1547, Mar. 2004. doi: 10.1016/j.ijheatmasstransfer.2003.10.011.

[6] H. W. Pennline and J. S. Hoffmann, "Flue gas cleanup using the moving bed copper oxide process," Fuel Process. Technol., vol. 114, pp. 109- 117, Oct. 2013. doi: 10.1016/j.fuproc.2013.03.020.

[7] J. Yrjola and J. J. Saastamoinen, "Modelling and practical operation results of a dryer for wood chips," Drying Technol., vol. 20, no. 6, pp. 10771099, Dec. 2002. doi: 10.1081/DRT-120004041.

[8] T. Baumann and S. Zunft, "Theoretical and experimental investigation of a moving bed heat exchanger for solar central receiver power plants," J. Phys.: Conf. Ser., vol. 395, pp. 1-9, 2012. doi: $10.1088 / 1742-6596 / 395 / 1 / 012055$.

[9] P. A. Isaza, W. D. Warnica, and M. Bussmann. "Co-current parallel-plate moving bed heat exchanger: An analytical solution," Int. J. Heat and Mass Transfer, vol. 87, pp. 616-624, Aug. 2015. doi: 10.1016/j.ijheatmasstransfer.2015.02.079.

[10] P. A. Isaza, W. D. Warnica, and M. Bussmann. "Counter-current parallelplate moving bed heat exchanger: An analytical solution," Int. J. Heat and Mass Transfer, vol. 87, pp. 625-635, Aug. 2015. doi: 10.1016/j.ijheatmasstransfer.2015.02.056.

[11] P. A. Isaza, Y. Cai, W. D. Warnica, and M. Bussmann. "Co-current and counter-current vertical pipe moving bed heat exchangers: Analytical solutions," International Journal of Heat and Mass Transfer, vol. 95, pp. 1115-1128, 2015.

[12] P. A. Isaza, A. O'Brien, W. D. Warnica, and M. Bussmann. "Assessing axial heat conduction in moving bed heat exchangers," Int. $J$. Therm. Sci., vol. 120, pp. 303-313, Oct. 2017 doi: 10.1016/j.ijthermalsci.2017.06.004.

[13] M. Colakyan and O. Levenspiel, "Heat transfer between moving bed of solids and immersed cylinders," AIChE Symp. Ser., vol. 80, no. 241, pp. 156-168, Jan. 1984.

[14] W. N Sullivan and R. H. Sabersky, "Heat transfer to flowing granular media," Int. J. Heat Mass Transfer, vol. 18, no. 1, pp. 97-107, Jan. 1975. doi: 10.1016/0017-9310(75)90012-5.

[15] M.S. Brinn, S.J. Friedmen, F.A. Gluckert, and R.L. Pigford, "Heat transfer to granular materials," Ind. Eng. Chem, vol. 40, no. 6, pp. 1050-1061, 1948. doi: 10.1021/ie50462a016.

[16] N. K. Harakas and K. O. Beatty, "Moving bed heat transfer: Effect of interstitial gas with fine particles," Chem. Eng. Progr. Symp.Ser., vol. 59, pp.1050-1061, 1963.

[17] E. U. Schlünder, "Heat transfer to packed and stirred beds from the surface of immersed bodies," Chemical Engineering and Processing, vol. 18, no. 1, pp. 31- 53, 1984. doi: 10.1016/0255-2701(84)85007-2.

[18] R. W. Serth and T. G. Lestina, Process Heat Transfer: Principles Applications and Rules of Thumb, New York: Academic Press, 2014.

[19] T. Ohmori, M. Miyahara, M. Okazaki, and R. Toei, "Heat transfer in a conductive heating agitated dryer," Drying Technol., vol. 12, no. 1-2, pp. 299- 328, 1994. doi: 10.1080/07373939408959958.

[20] M. Kwapinska, G. Saage, and E. Tsotsas, "Continuous versus discrete modelling of heat transfer to agitated beds," Powder Technol., vol. 181, no. 3, pp. 331-342, Feb. 2008. doi: 10.1016/j.powtec.2007.05.025.

[21] A.V. Luikov, Analytical Heat Diffusion Theory. New York: Academic Press, 1968. doi: 10.1016/B978-0-12-459756-3.X5001-9.

[22] W. M. Kays, and A. L. London, Compact Heat Exchangers. New York: McGraw-Hill, 1984.

[23] J. P. Holman, Heat Transfer, 7th ed. New York: McGraw-Hill, 1990.

[24] A. C. Pivem and M. J. S. de Lemos, "Temperature distribution in a counterflow moving bed under a thermal nonequilibirum condition," Numer. Heat Transfer, Part A, vol. 61, no. 1, pp. 1-17, Jan. 2012. doi: 10.1080/10407782.2012.631384.

[25] S. Kakac, H. Liu, and A. Pramuanjaroenkij, Heat Exchangers: Selection, Rating and Thermal Design, 3rd ed. Boca Raton, FL: CRC Press, 2012. 


\title{
Numerical Simulation of Fluid Flow and Heat Transfer of the Supercritical Water in Different Fuel Rod Channels
}

\author{
Huirui Han, Chao Zhang \\ Mechanical \& Materials Engineering \\ Western University \\ London, Canada \\ czhang@eng.uwo.ca
}

\begin{abstract}
The supercritical water-cooled reactor was proposed as one of the Generation IV nuclear systems. Although many research works on the fluid flow and heat transfer of supercritical water in circular channels have been conducted, there is still lack of research on the fluid flow and heat transfer process in fuel bundles used in supercritical water-cooled nuclear reactors. Besides, fuel bundles have multiple fuel rods, the flow is an external flow, not internal flow as that in circle channels, which will cause the difference in the fluid flow phenomenon and heat transfer on the fuel rod cladding surface. In this work, the heat transfer and fluid flow characteristics of the supercritical water in the single-rod channel and the multi-rod channel are simulated numerically. The results show that there are secondary flows in both channels. The circumferential cladding surface temperature variation is large and should be considered in the future fuel rod design. With the same flow rate and heat flux input, the maximum cladding surface temperature in the multi-rod channel is much higher than that in the single-rod channel. Since the maximum cladding surface temperature is an important parameter for the safety of the nuclear reactor operation, it is recommended to use the multi-rod channel model to conduct numerical simulations for the fluid flow and heat transfer of the supercritical water in the Canadian SCWR.
\end{abstract}

Keywords- SCWR, heat transfer, single-rod, multi-rod, cladding surface temperature

\section{INTRODUCTION}

With the rapid growing population, the electricity generation amount is in high demand. Nuclear reactor power plants can provide higher power-to-sources rate, compared with thermal power plants using coals or natural gas. Canada has a long history in the development of the CANada Deuterium Uranium Pressurized Heavy Water Reactor (CANDU-PHWR), which has been operating for about half century. The Supercritical Water-Cooled Reactor (SCWR) is proposed as one of the six selected Generation IV reactor systems in the world since 2002 [1], which has unique advantages, such as higher thermal efficiency, lower coolant mass flow to the thermal power rate, and simpler components.

The studies for the flow in rod bundles have been conducted by several researchers. Kjellstrom [2] did the flow profile measurements of air flows in a triangular rod bundle. Trupp and Azad [3] changed the pitch-to-diameter ratios of hexagonal lattices between 1.2 and 1.5 and measured detailed turbulence profiles of the air flow. The eddy viscosity showed strong anisotropy. Carajilescov and Todreas [4] and Vonka [5] used Laser Doppler Anemometry to measure the water flow characteristics in triangular subchannels. They found that the secondary flow velocity was less than $1 \%$ of the mean flow velocity.

Many researchers have investigated the turbulent mixing rate of the fluids in channels. Jeong et al. [6] defined a new mixing factor and then evaluated all the experimental data from previous researchers on the turbulent mixing. It was found that the turbulent mixing of fluids depends strongly on the ratio of the distance between the center of two adjacent sub-channels and the hydraulic diameter of a sub-channel. Only very few experimental studies were carried out for the heat transfer and flow phenomenon of supercritical fluids because of the experiment environment restrictions. $\mathrm{Xi}$ et al. [7] did an investigation on the supercritical water flow between two heated parallel channels. Both inlet mass flow rate and outlet temperature oscillations were observed. Verma et al. [8] carried out the experiments using a scaled test facility of AHWR (Advanced Heavy Water Reactor) rod bundle. The effect of the spacer on the turbulent mixing rate in subchannels was investigated. The results showed that the turbulent mixing rate increased with the increase in the average Reynolds number.

The simulation results for the flow of the supercritical water in fuel bundles showed that the anisotropic turbulence models are more accurate. $\mathrm{Gu}$ et al. [9] simulated the supercritical water flow in a SCWR fuel bundle using the sub-channel method. The simulation results demonstrated that the turbulent mixing rate was sensitive to the asymmetric boundary condition. Mukohara et al. [10] conducted the sub-channel analysis in High Temperature Fast Supercritical Water-cooled Reactor (HTF-SCWR). It was found that the cladding surface 
temperature was sensitive to both the local power peak value and the sub-channel area. Yu et al. [11] developed a subchannel analysis code to analyze the thermo-hydraulic behavior of the CANDU-SCWR. The simulation results showed that this code can successfully simulate the steady state flows in subchannels.

The Canadian SCWR concept is based on CANDU. Because of the sharp variation of the supercritical water properties around the pseudo-critical point, it is important to use appropriate anisotropic turbulence models for the simulations of the supercritical water flow behaviors and heat transfer phenomenon in fuel bundles. Previous researchers Cheng et al. [12] and Zhang et al. [13] have proved that the anisotropic model RSM (Reynolds Stress Model) can give a better agreement with the experimental results for the supercritical water flow in channels compared with the isotropic two-equation turbulence models.

Previous numerical studies mainly focused on the flow and heat transfer phenomenon of the supercritical water in circle channels. However, the fuel bundle used in the SCWR has multiple fuel rods. It is time-consuming to simulate the fluid flow and heat transfer in the channel with multiple rods. Therefore, some researchers conducted simulations using simplified geometries, such as the work by Sun et al. [14], where the multiple fuel rod system was simplified as a singlerod system. Therefore, in this study, the CFD (Computational Fluid Dynamics) simulations are carried out for the fluid flow and heat transfer of the supercritical water in both the singlerod channel and multi-rod channel under the same operating conditions in order to compare the difference in the results between them. The CFD simulations are conducted with the RSM using ANSYS FLUENT 15.0.

\section{GOVERNING EQUATIONS AND NUMERICAL MODELS}

The governing equations for 3D steady flow and heat transfer are conservations of mass equation, momentum equation and energy equation, which is shown as follows in the Cartesian tensor [15]:

$$
\begin{gathered}
\frac{\partial}{\partial x_{i}}\left(\rho \overline{u_{i}}\right)=0 \\
\frac{\partial}{\partial x_{j}}\left(\rho \overline{u_{i} u_{j}}\right)=-\frac{\partial \bar{p}}{\partial x_{i}}+\frac{\partial}{\partial x_{j}}\left(\mu \frac{\partial u_{i}}{\partial x_{j}}-\rho_{u_{i} u_{j}}\right)+\rho g_{i} \\
\frac{\partial}{\partial x_{i}}\left(\overline{u_{i}} \rho_{c_{p}} T\right)=\frac{\partial}{\partial x_{i}}\left[\left(\lambda+\frac{c_{p} \mu_{t}}{P_{r_{t}}}\right) \frac{\partial T}{\partial x_{i}}\right]+\phi
\end{gathered}
$$

Here, $u$ is the velocity, $\mathrm{T}$ is the temperature, $\mu$ is the dynamic viscosity, $\rho$ is the density, $\lambda$ is the thermal conductivity, $c_{p}$ is the specific heat, $\mu_{t}$ is the turbulent viscosity, and $P_{r_{t}}$ is the turbulent Prandtl number. The RSM with the enhanced wall treatment is chosen in this study based on the recommendations from the previous studies [13]. ANSYS Fluent 15.0 is used to solve the governing equations. The SIMPLE scheme is selected for pressure correction, and QUICK method is used for the spatial discretization. The convergence criteria for continuum is $10^{-3}$, for the momentum and turbulence parameters are $10^{-5}$, and for the energy equations is $10^{-6}$.

\section{CONFIGURATIONS OF THE CHANNELS}

The cross-section views of the single-rod channel and the multi-rod channel are shown in Fig. 1 and Fig. 2, respectively. The working fluid is the supercritical water, and its properties are from Wagner [16]. The length of the channel is $1.5 \mathrm{~m}$. For the single-rod channel, the channel diameter is $9 \mathrm{~mm}$ and the rod diameter is $4 \mathrm{~mm}$. And for the multi-rod channel, the outer diameter is $9 \mathrm{~mm}$. There are 5 rods in the channel and their diameter is $1.788 \mathrm{~mm}$, so, the total cross-section areas of all 5 rods is equal to the cross-section area of the rod in the singlerod channel. The supercritical water flow in these channels is upward based on the configuration of the proposed SCWR [17]. The reference pressure is $25 \mathrm{MPa}$ [17].

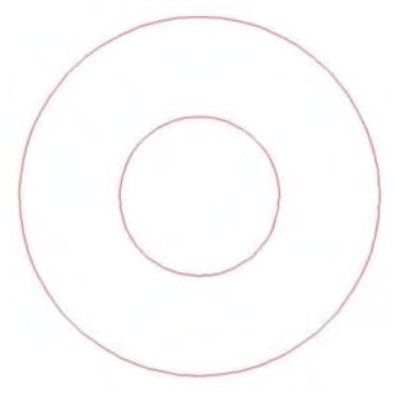

Figure 1. Cross-section view of the single-rod channel

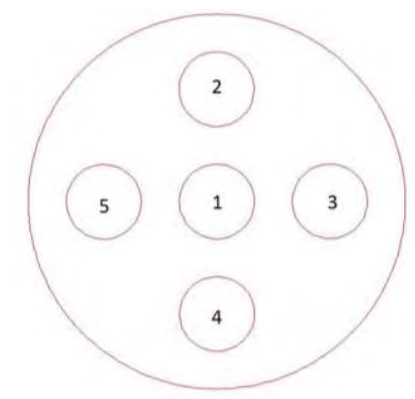

Figure 2. Cross-section view of the single-rod channel

Boundary conditions are as follows:

Inlet: The inlet velocity for each channel is $3 \mathrm{~m} / \mathrm{s}$, and the inlet temperature is $623.15 \mathrm{~K}$. Turbulence intensity is set as $5 \%$, and the hydraulic diameters are specified based on the geometrical shapes of the channels.

Outlet: Outflow is selected for each channel.

Walls: They are all smooth walls with the no-slip condition. The heat flux on the fuel rod surface is $10^{6} \mathrm{~W} / \mathrm{m}^{\wedge} 2$ based on the operating condition of the SCWR [17].

The cross-section views of the meshes for these two channels are shown in Fig. 3 and Fig. 4, respectively. The 
mesh refinement near the wall is performed so that the nondimensional distance to the wall is approximately 1 .

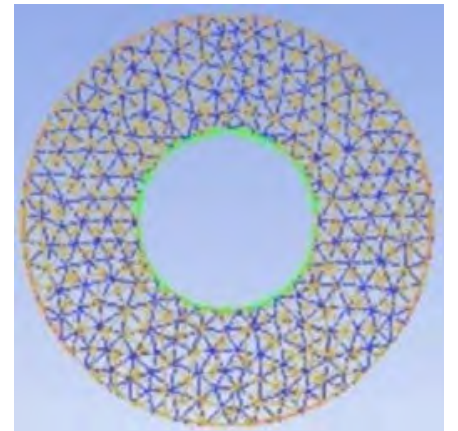

Figure 3. Cross-section view of the mesh for the single-rod channel

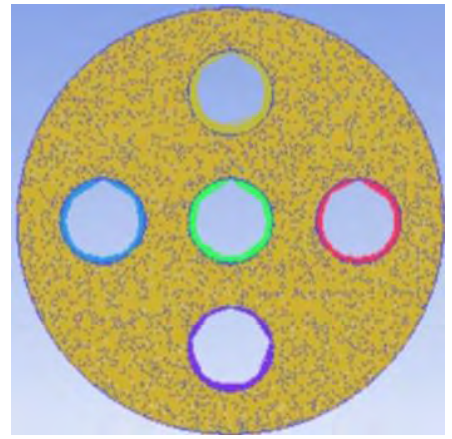

Figure 4. Cross-section view of the mesh for the single-rod channel

\section{RESUltS AND DisCUSSIONS}

Fig. 5 shows the outlet velocity vectors colored by the velocity magnitude of the supercritical water in the single-rod channel and the multi-rod channel. It is shown that there are secondary flows at the outlet plane in the single-rod channel and the multi-rod channel, especially near the fuel rod cladding surfaces. Fig. 6 shows the contours of the outlet velocity magnitudes in the single-rod channel and multi-rod channel. The velocity magnitudes at the outlets of these two channels are quite different. The velocity magnitude in the single-rod channel is much lower than that in the multi-rod channel. The maximum velocity at the outlet is $4.5 \mathrm{~m} / \mathrm{s}$ in the single-rod channel and $7 \mathrm{~m} / \mathrm{s}$ in the multi-rod channel. The velocity field will affect the heat transfer in the channel. Therefore, using a single-rod channel to replace the multi-rod channel used in the Canadian SCWR will cause inaccurate results.

The cladding surface temperature distribution in the singlerod channel is shown in Fig. 7. The difference in the cladding surface temperature along the circumference in the single-rod channel is less than $50 \mathrm{~K}$. Fig. 8 shows the cladding surface temperature distribution in the multi-rod channel. It can be seen that the cladding surface temperature difference along the circumference in the multi-rod channel can reach to about $150 \mathrm{~K}$.

Fig. 9 to Fig. 11 show the maximum and minimum cladding surface temperatures of each fuel rod at $\mathrm{z}=0.1 \mathrm{~m}$, $\mathrm{z}=0.8 \mathrm{~m}$, and $\mathrm{z}=1.5 \mathrm{~m}$ in the multi-rod channel. It can be seen that the maximum cladding surface temperature at these three planes all occur at the fuel rod \#4. And the minimum cladding surface temperature at these three planes occur at the fuel rods $\# 1$, \#5, \#5, respectively. The largest difference of maximum and minimum cladding surface temperatures occurs at the fuel rod\#4 at the outlet plane $\mathrm{z}=1.5 \mathrm{~m}$, which is $78.537 \mathrm{~K}$. The maximum cladding surface temperature is $670 \mathrm{~K}$ for the singlerod channel and $780 \mathrm{~K}$ for the multi-rod channel. The difference is $110 \mathrm{~K}$. Therefore, the multi-channel model should be used in the simulation to generate more accurate data used for the control system designs since the maximum cladding surface temperature is an important parameter for the safety of nuclear reactors.
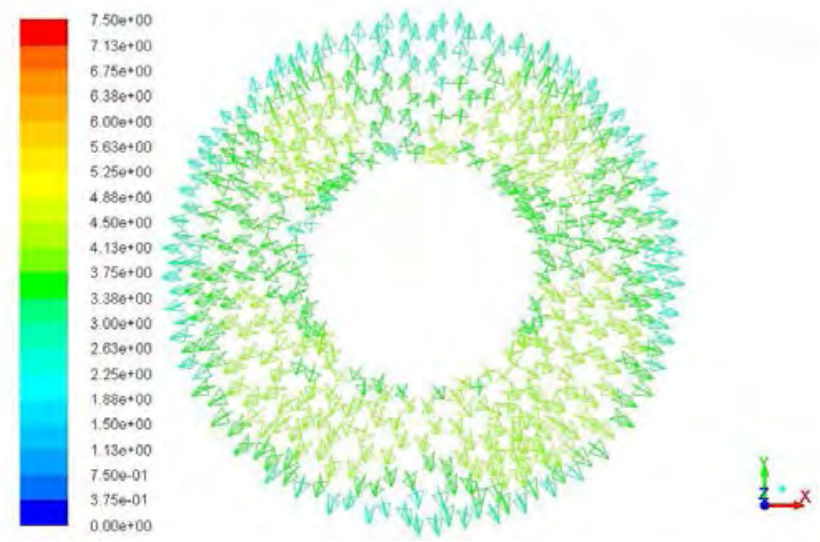

(a) Single-rod Channel
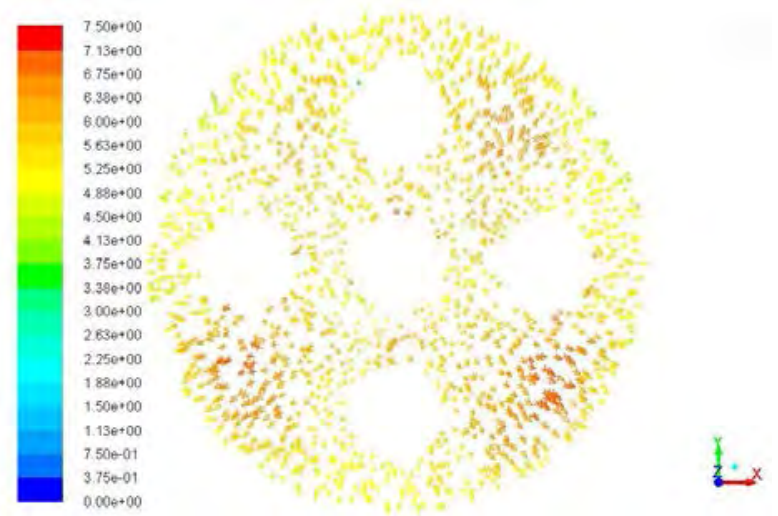

(b) Multi-rod Channel

Figure 5 . Outlet velocity vectors colored by the velocity magnitude $(\mathrm{m} / \mathrm{s})$ in the single-rod and multi-rod channels
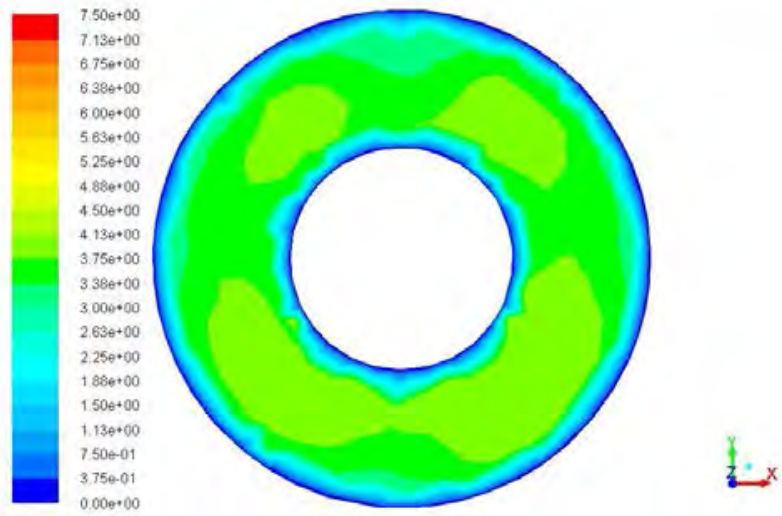

(a) Single-rod channel 


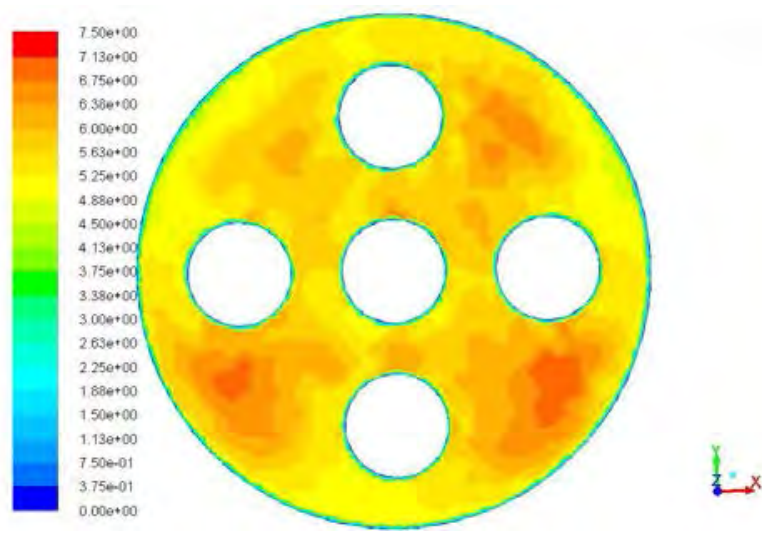

(b) Multi-rod channel

Figure 6 Outlet velocity magnitude $(\mathrm{m} / \mathrm{s})$ contours in the single-rod and multirod channels
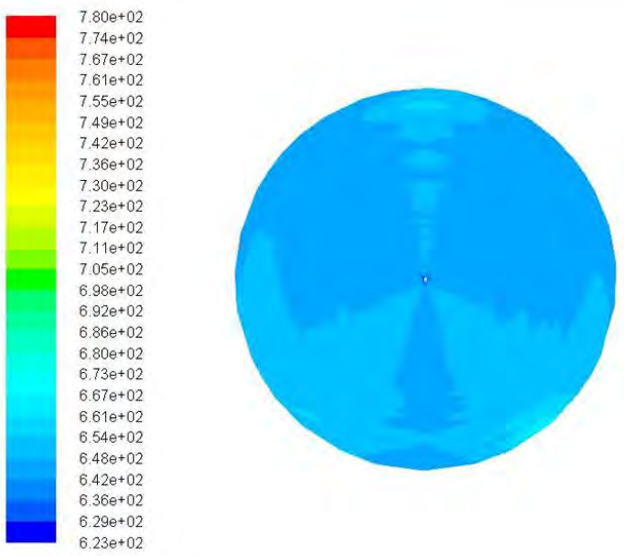

$6.23 e+02$

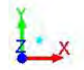

Figure 7 Cladding surface temperature (K) distribution in the single-rod channel
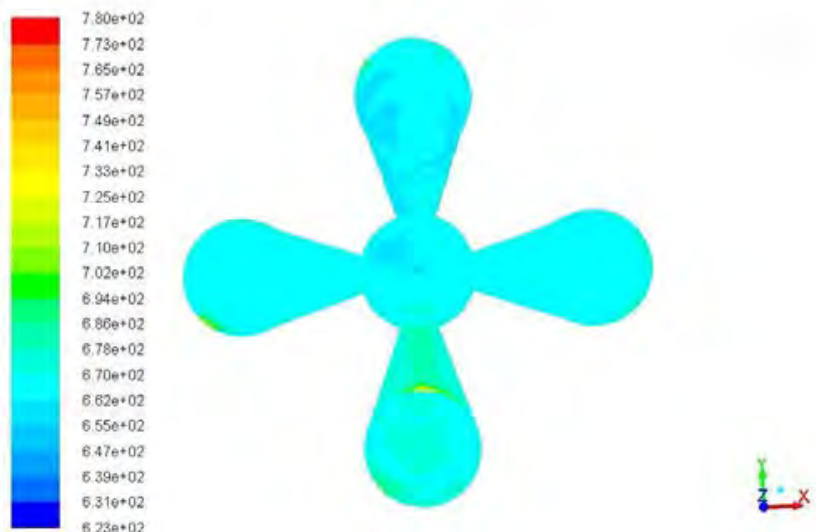

Figure 8 Cladding surface temperature $(\mathrm{K})$ distribution in the multi-rod channel

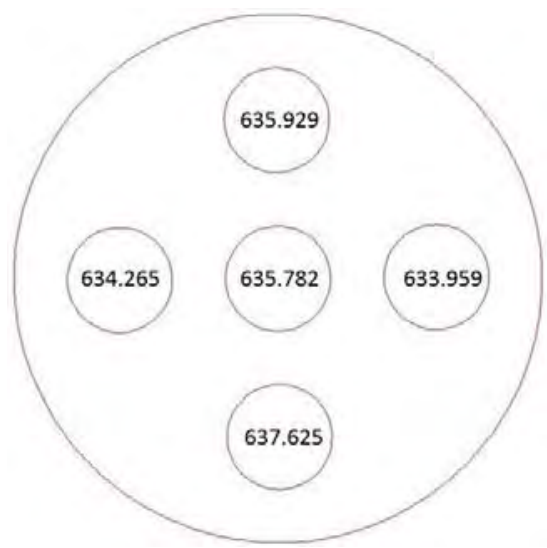

(a) Maximum temperature

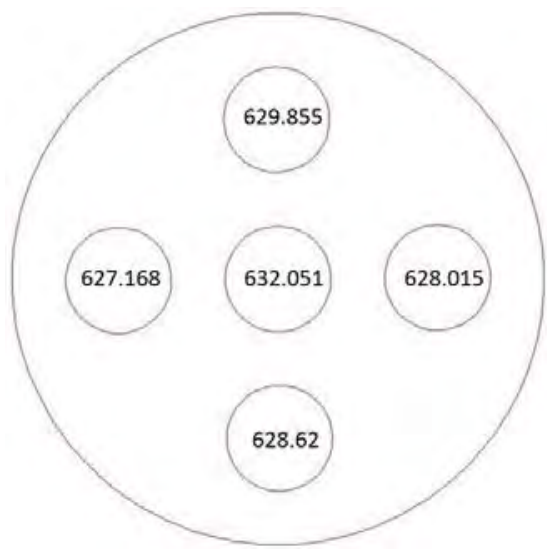

(b) Minimum temperature

Figure 9 Cladding surface temperatures $(\mathrm{K})$ at $\mathrm{z}=0.1 \mathrm{~m}$

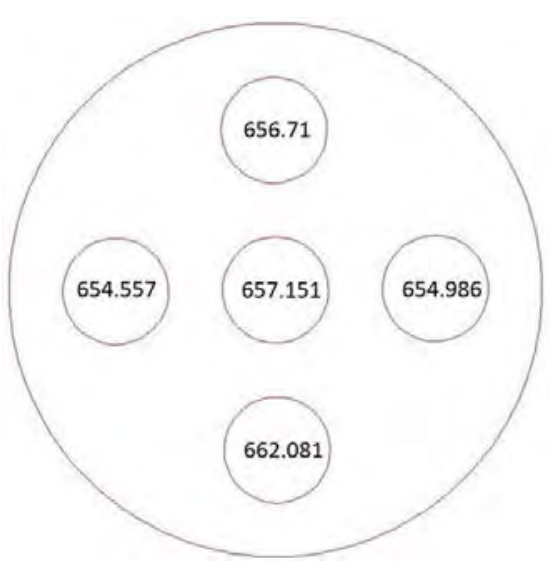

(a) Maximum temperature 


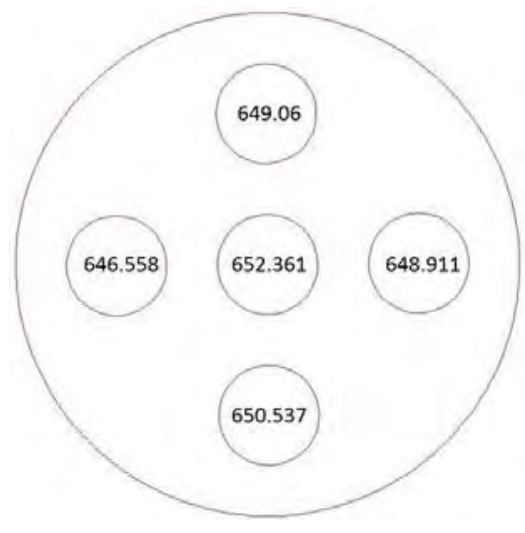

(b) Minimum temperature

Figure 10 Cladding surface temperatures $(K)$ at $z=0.8 m$

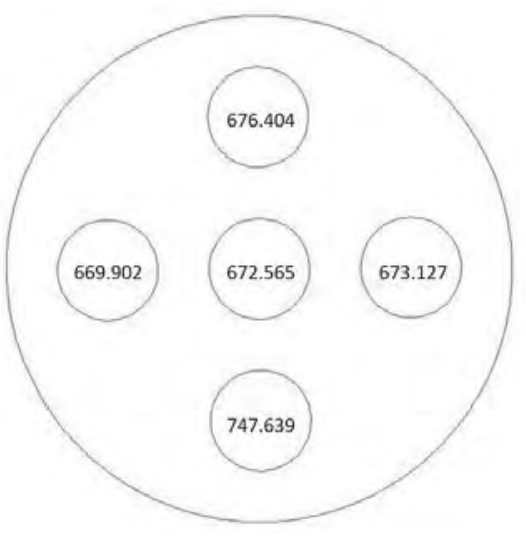

(a) Maximum temperature

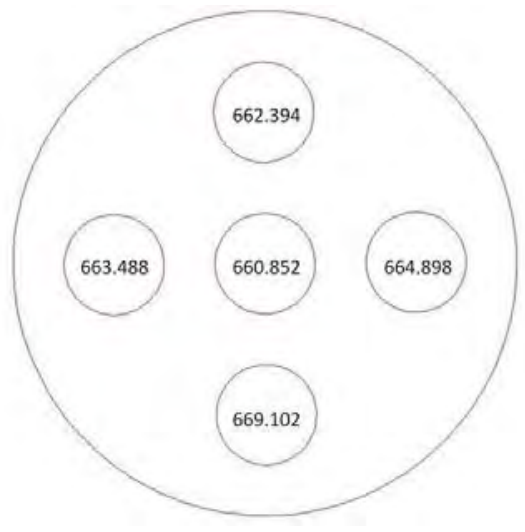

(b) Minimum temperature

Figure 11 Cladding surface temperatures $(K)$ at $z=1.5 \mathrm{~m}$

\section{CONCLUSIONS}

In this study, the fluid flow and heat transfer characteristics of the supercritical water in the single-rod channel and the multi-rod channel are compared. The results show that there are secondary flows in the single-rod channel. The maximum cladding surface temperature in the multi-rod channel is about $110 \mathrm{~K}$ higher than that in the single-rod channel. Besides, the cladding surface temperature distributions are also not same between the single-rod channel and multi-rod channel. The difference of the circumference cladding surface temperature for the multi-rod channel can be up to $78.537 \mathrm{~K}$. The large circumferential temperature difference should be considered in the Canadian SCWR fuel bundle design. Since the heat transfer characteristics for the single rod channel and the multi-rod channel are not similar, the numerical simulations of the Canadian SCWR should be performed for the multi-rod channel in order to obtain more accurate results.

\section{REFERENCES}

[1] USNERAC, A Technology Roadmap for Generation IV Nuclear Energy Systems, Retrieved from The U.S.DOE nuclear energy research advisory committee and the generation IV international forum, 2002.

[2] B. Kjellstrom, "Studies of turbulent flow parallel to a rod bundle of triangular array," Retrieved from AB Atomenergi, Sweden, vol.5, pp.190, May 1974.

[3] A.C. Trupp and R.S. Azad, "The structure of turbulent flow in triangular array rod bundles," Nucl. Eng. Des, vol.32, issue 1, pp. 47-84, 1975. doi: 10.1016/0029-5493(75)90090-4.

[4] P. Carajilescov and N.E. Todreas, "Experimental and analytical study of axial turbulent flows in an interior subchannel of a bare rod bundle," J. Heat Transfer, vol.2, pp. 262-268, 1976. doi: 10.1115/1.3450529.

[5] V. Vonka, "Measurement of secondary flow vortices in a rod bundle," Nucl. Eng. Des., vol.2, pp. 191-207, 1988. doi: 10.1016/0029-5493(88)90277-4.

[6] H.Y. Jeong, K.S. Ha,Y.M. Kwon, Y.B. Lee, and D. Hahn, "A dominant geometrical parameter affecting the turbulent mixing rate in rod bundles," Int. J. Heat Mass Transfer, vol.5, pp. 908-918, 2007. doi:10.1016/j.ijheatmasstransfer.2006.08.023.

[7] X. Xi, Z. Xiao, X. Yan, Y. Li, and Y. Huang, "An experimental investigation of flow instability between two heated parallel channels with supercritical water," Nucl. Eng. Des., vol. 278, pp. 171-181, 2014.

doi: 10.1016/j.nucengdes.2014.06.034.

[8] S.K. Verma, S.L. Sinha, and D.K. Chandraker, "Experimental investigation of effect of spacer on single phase turbulent mixing rate on simulated subchannel of Advanced Heavy Water Reactor," Ann. Nucl. Energy, pp. 186-195, 2017. doi: 10.1016/j.anucene.2017.06.020.

[9] H.Y. Gu, X. Cheng, and Y.H. Yang, "CFD analysis of thermal-hydraulic behavior of supercritical water in sub-channels," Nucl. Eng. Des., vol.240, issue 2, pp. 364-374, 2010. doi: 10.1016/j.nucengdes.2008.08.022.

[10] T. Mukohara, S. Koshizuka, and Y.Oka, "Subchannel analysis of a fast reactor cooled by supercritical light water," Prog. Nucl. Energy, vol.37, issues 1-4, pp. 197-204, 2000. doi: 10.1016/S0149-1970(00)00046-9.

[11] J.Y. Yu, S.T. Wang, and B.S. Jia, "Development of sub-channel analysis code for CANDU-SCWR," Prog. Nucl. Energy, vol.49, pp. 334-350, 2007.

[12] X. Cheng, B. Kuang, and Y.H. Yang, "Numerical analysis of heat transfer in supercritical water cooled flow channels," Nucl. Eng. Des., vol.237, issue 3, pp. 240-252, 2007. doi: 10.1016/j.nucengdes.2006.06.011.

[13] Y.N. Zhang, C. Zhang, \& J.Jiang, "Numerical simulation of heat transfer of supercritical fluids in circular tubes using different turbulence models," J. Nucl. Sci. Technol., vol. 48, issue 3, pp. 366-373, 2011.

doi: $10.1080 / 18811248.2011 .9711712$.

[14] P.W. Sun, "Dynamic model construction and control system design for Canadian supercritical water-cooled reactors," PhD dissertation, Dept. of Elect. Comp. Eng., University of Western Ontario, London, Canada, 2012. [Online]. Available: https://ir.lib.uwo.ca/etd/896.

[15] Ansys Fluent. Fluent 14.0 User's Guide, 2011.

[16] W.Wagner and A.Kruse, Properties of Water and Steam. New York: Springer, 1998.

66โ17] L.Leung, "Overview of SCWR Concepts," 2013. 


\author{
F a i i i s \\ Ian Mullett \\ Faculty of Engineering and Applied Science, Memorial \\ University of Newfoundland, St. John's, Canada \\ ihrm55@mun.ca \\ Xili Duan \\ Faculty of Engineering and Applied Science, Memorial \\ University of Newfoundland, St. John's, Canada \\ xduan@mun.ca
}

an i U

\author{
Zongming Wang \\ College of Chemical Engineering, China University of \\ Petroleum, Qingdao, Shandong, China \\ wzmcc@upc.edu.cn \\ Yishan Li \\ Shandong Special Equipment Inspection Institute Co. Ltd, \\ Jinan, Shandong, China \\ liyishan@sei.sd.cn
}

\begin{abstract}
Thermal oil heaters can be considered as an alternative to steam boilers for process heating use. Instead of boiling water, thermal oil heaters use heat transfer oils with high boiling points which allows operation at low pressures. To increase thermal oil heater efficiency, a nanofluid consisting of a common heat transfer oil, the synthetic TH66, and copper nanoparticles has been proposed. Based on existing correlations for nanofluids, a figure of merit (FOM) was created to evaluate heat transfer performance while factoring in pumping power increases. A maximum FOM increase of $13 \%$ was found for a thermal oil heater using the nanofluid when compared to one that uses the base fluid oil.
\end{abstract}

Keywords- Thermal Oil Heater; Nanofluid; Heat Transfer; Feasibility Study.

\section{INTRODUCTION}

Traditionally, water boilers are used to provide steam or heat for residential or industrial process applications. To reach the high temperatures required for process applications, high pressure steam is required. In lieu of using a steam boiler, a thermal oil heater is used to heat the oil. In general, the heaters are gas fired with a burner located in the center of a helical coil. The hot exhaust gases generally travel several passes over the coil to heat the thermal oil. Heat transfer fluids such as thermal oils, can be used as a substitute for steam and can reach much higher temperatures at minimal system pressures. The use of thermal oils also eliminates freezing and corrosion concerns, and no water treatment is necessary. Synthetic heat transfer oils provide higher stability at elevated temperatures, and offer higher thermal conductivity than other heat transfer fluids $(0.1$

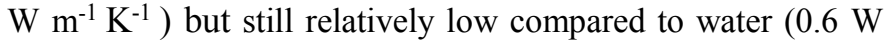
$\mathrm{m}^{-1} \mathrm{~K}^{-1}$ at room temperature).

In 1995, Choi published a paper showing the enhancement of thermal physical properties of a basefluid with dispersed nanoparticles, referred to as a nanofluid [1]. Since then, many papers have been published on the topic of nanofluids, mainly showing the thermal conductivity enhancement of water based nanofluids. Although the quantity of research is much smaller, significant enhancement has also been found for oil based nanofluids [2-6]. Thermal conductivity enhancement generally follows Maxwell's effective medium theory (EMT), but some studies have shown results that far surpass those predicted by EMT [7-8]. Several mechanisms have been proposed to explain the unusual enhancements, including Brownian motion, the interfacial layer model, and particle agglomeration, but no conclusive evidence has been found and the topic remains widely debated.

The present study will examine the feasibility of copper nanoparticles dispersed in a Therminol 66 (TH66) oil as base fluid for use in thermal oil heaters. TH 66 is a widely used and readily available, high temperature liquid phase heat transfer fluid. It is a modified terphenyl heat transfer oil with stability up to $345^{\circ} \mathrm{C}$ and provides excellent fouling resistance. Copper nanoparticles were chosen mainly due to their high thermal conductivity. Singh et al. showed that with the use of surfactants, copper nanoparticle suspensions in TH66 were stable [4]. Furthermore, Timofeeva et al. showed stable concentrations of up to 7\% for a TH66 nanofluid [3]. Published correlations and data will be used to evaluate any increase in heat transfer rates, but also any drawbacks, including increased pumping power and erosion.

\section{THERMOPHYSICAL PROPERTIES OF NANOFLUIDS}

In order to assess the improvements in convective heat transfer given by a nanofluid, several thermophysical properties must be determined. The effective density $\rho$ of a nanofluid with particle volume fraction $\phi$ is given by the rule of mixtures [9]:

$$
\rho_{n f}=\rho_{p} \phi+\rho_{b f}(1-\phi)
$$

where the subscripts $n f, p$, and $b f$ denote nanofluid, particle, and basefluid respectively. The aforementioned subscripts will be used throughout this paper. Similarly, the effective specific 
heat capacity $c$ of a nanofluid can also be given by the rule of mixtures [9]:

$$
c_{n f}=\frac{1}{\rho_{n f}}\left[\rho_{p} c_{p} \phi+\rho_{b f} c_{b f}(1-\phi)\right]
$$

Unlike density and specific heat, thermal conductivity is a much more complicated and widely debated topic. On way is to use Maxwell's effective medium theory (EMT) to estimate the thermal conductivity $k$ of spherical nanoparticles suspended in the base fluid based on the volume fraction as follows [10]:

$$
\frac{k_{n f}}{k_{b f}}=1+\frac{3\left(k_{p}-k_{b f}\right) \phi}{\left(k_{p}+2 k_{b f}\right)-\left(k_{p}-k_{b f}\right) \phi}
$$

Hamilton and Crosser [11] introduced a modification of Maxwell's EMT which included a shape factor that accounted for the sphericity of the particles. More recently, Nan et al. [12] introduced a further modification to Maxwell's theory which included the particle geometry and the interfacial heat flow resistance. For spherical particles and negligible interfacial resistance, both the Hamilton and Crosser and the Nan et al. models reduce to equation (3). Although some studies have shown abnormal enhancements of thermal conductivity [7-8], a review by Buongiorno et al. [13] showed that EMT accurately predicts the thermal conductivity of a nanofluid. The model proposed by Nan et al. was shown to be accurate for nanofluids within a narrow band of $\pm 10 \%$ when compared to a wide range of published data.

Finally, particle suspensions cause an increase in the viscosity of a fluid. An analytical solution for effective viscosity $\mu$ of a solid-fluid suspension is given by Einstein's expression [14]:

$$
\mu_{n f}=\mu_{b f}(1+2.5 \phi)
$$

At the low concentrations typical of nanofluids, equation (4) results in values very close to $\mu_{\mathrm{bf}}$ which contributes to an underestimation of the viscosity at low concentrations. Furthermore, experimental data suggests that the effective viscosity of a nanofluid is significantly higher than the base fluid, and higher than the results suggested by equation (4). Unfortunately, no general correlations exist for the viscosity of a nanofluid as they depend on the shape and material of nanoparticles. Empirical correlations exist but must be derived from a specific set of data pertaining to the base fluid and nanoparticle used. Aberoumand et al. [15] derived the following equation for the viscosity of a heat transfer oil based nanofluid using experimental data:

$$
\mu_{n f}=\mu_{b f}\left(1.15+1.061 \phi-0.5442 \phi^{2}+0.1181 \phi^{3}\right)
$$

It is important to note that equation (5) may provide an overestimation of the nanofluid viscosity. As the concentration goes to zero, $\mu_{\mathrm{nf}}$ will approach $1.15 \mu_{\mathrm{bf}}$. This may result in an overestimation of the viscosity at low concentrations. Due to the minimal research done on oil based nanofluids, and experimental results for a $\mathrm{Cu}$-TH66 nanofluid only existing up to $120^{\circ} \mathrm{C}$ [4], equation (5) will be used as an approximation. To not skew results, equation (5) will be used as an upper bound, and equation (4) will be used as a lower bound for viscosity.

\section{MATHEMATiCAl Model}

To assess the feasibility of nanofluids for use in a thermal oil heater, a mathematical model will be proposed in this section. Two main factors will be evaluated to determine if nanofluids are feasible for use in a thermal oil heater; the heat transfer coefficient (HTC) $h$ and the pumping power $P$. For single phase forced convection, the heat transfer coefficient will effectively show the heat transfer capability of the fluid. Although the nanofluid will have an increased thermal conductivity and specific heat capacity, which contribute to an increased HTC, a nanofluid will also have an increased viscosity, which will require an increase in pumping power to attain the same heat transfer. Therefore, both the HTC and pumping power required will need to be evaluated. There are two ways to compare single phase forced convection heat transfer; constant fluid velocity and constant pumping power. For the purposes of this work, constant fluid velocity will be used to simplify the model.

\section{A. Heat Transfer Coefficient}

A review by Prahbat et al. [17] concluded that nanofluids achieved no real enhancement of the HTC other than that caused by the augmentation of the thermal conductivity and the increase in viscosity. Prahbat et al. found that studies that showed an abnormal increase in HTC, defined their Reynolds number $R e$ in terms of the viscosity of the nanofluid at room temperature. When Reynolds number calculations were done using temperature dependent properties, the heat transfer coefficient matched those given by the Dittus Boelter correlations, within uncertainties.

Williams et al. [16] examined the turbulent convective heat transfer behavior of alumina and zirconia water based nanofluids. It was shown that if temperature dependent viscosities and thermal conductivities were used when calculating Reynolds, Prandtl, and Nusselt numbers, the Dittus Boelter correlations reproduced the convective HTC measured in the study. Therefore, the Dittus Boelter correlation for heating (equation (6)) will be used to evaluate the internal heat transfer coefficient of the thermal oil heater.

$$
h=0.023 \operatorname{Re}^{0.8} \operatorname{Pr}^{0.4}
$$

\section{B. Pumping Power}

Although the addition of nanoparticles will increase the thermal conductivity of a fluid, the added particles will also increase the viscosity of the fluid. If the viscosity increase is 
too great, the increase in pumping power may counteract the added heat transfer benefits of the nanofluid.

For a circular pipe of diameter $d$ and length $L$, and a turbulent internal flow with velocity $V$, the pumping power required is given by the following equation:

$$
P=Q \Delta p=\frac{\pi d^{4} V}{4} \frac{2 f V^{2} L}{d}
$$

where $f$ is the friction factor within the pipe. The above equation assumes that all pressure losses are given by major frictional losses. For heating, the friction factor is given by the Blasius equation:

$$
f=0.0791 R e^{-0.25}
$$

\section{Figure of Merit}

In order to assess the effectiveness of a nanofluid as a heat transfer fluid, a figure of merit (FOM) will be used to compare the performance of a nanofluid to the basefluid at various compositions and temperatures. The heat transfer coefficient (HTC) ratio will show the increase in $h$ for a nanofluid.

$$
\frac{h_{n f}}{h_{b f}}=\left(\frac{\rho_{n f}}{\rho_{b f}}\right)^{0.8}\left(\frac{c_{p n f}}{c_{p b f}}\right)^{0.4}\left(\frac{\mu_{n f}}{\mu_{b f}}\right)^{-0.4}\left(\frac{k_{n f}}{k_{b f}}\right)^{0.6}
$$

Although the HTC ratio will show an increase in heat transfer rate for a given fluid, the increased viscosity of the nanofluid can have adverse effects. The increased viscosity will increase the pumping power needed to maintain the same fluid velocity, thus decreasing the overall efficiency of the system. A simple figure of merit proposed by $\mathrm{Yu}$ et al. [18] compares the increase in heat transfer of a nanofluid to the basefluid, while accounting for pumping power.

$$
F O M=\left(\frac{h_{n f}}{h_{b f}}\right)\left(\frac{P_{b f}}{P_{n f}}\right)
$$

Substituting equations (6), (7), and (8) into equation (10) gives:

$$
F O M=\left(\frac{\rho_{n f}}{\rho_{b f}}\right)^{\frac{32}{55}}\left(\frac{c_{p n f}}{c_{p b f}}\right)^{\frac{2}{5}}\left(\frac{\mu_{n f}}{\mu_{b f}}\right)^{-\frac{26}{55}}\left(\frac{k_{n f}}{k_{b f}}\right)^{\frac{3}{5}}
$$

A FOM larger than 1 indicates a nanofluid that will have better overall heat transfer performance than the basefluid, and the higher the FOM, the better the nanofluid will perform as a heat transfer fluid.

\section{RESULTS AND DISCUSSION}

In order to assess the performance of a $\mathrm{Cu}$-TH66 as a nanofluid, two methods were considered while using the FOM presented in equation (11):

- Varying concentration phi at a constant temperature. A temperature of $300{ }^{\circ} \mathrm{C}$ was chosen as it gives a reasonable representation of a practical thermal oil heater operating temperature.

- Varying temperature at a constant concentration phi. A range of $0-6 \%$ was used to give a large cross section of data while staying within the stable range shown by Timofeeva et al. [3].

Thermophysical properties of TH66 were obtained from the manufacturer [19]. Figure 1 shows the increase in the FOM with increasing concentration. The higher viscosity estimated by equation (5) gives a FOM that is less than 1 for low concentrations. At $\phi \approx 0.02$, the FOM becomes greater than one showing an improvement from the base fluid. This is in alignment with published experimental data. Furthermore, as equation (5) most likely overestimates the viscosity at low concentrations, the point at which the nanofluid becomes beneficial lies between the curve using Einstein's viscosity relationship and thus, will occur at a lower concentration. A maximum FOM of 1.13 and 1.15 were found at $\phi=6 \%$ using the Aberoumand and Einstein models, respectively. The curves continue to trend upward, but the question of fluid stability must be asked at higher volume fractions. Assuming the real curve lies somewhere between the two curves, a significant increase in efficiency could be obtained from the use of the TH66 $\mathrm{Cu}$ nanofluid.

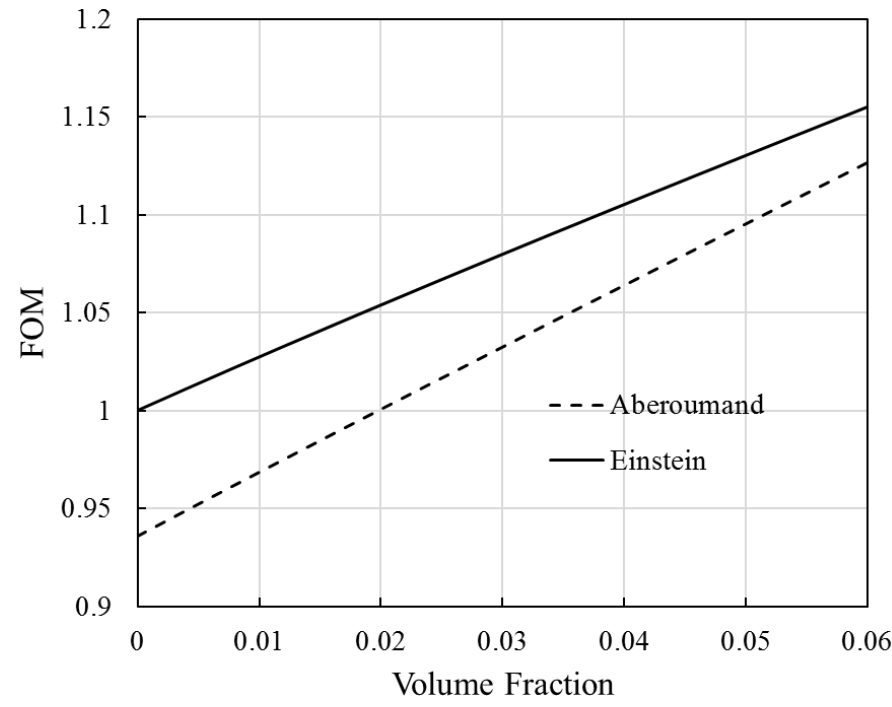

Figure 1. FOM of Cu-TH66 Nanofluid at $300^{\circ} \mathrm{C}$ with varying volume fraction

Figures 2 and 3 show the effects of increasing the temperature of the nanofluid at select volume fractions. These figures show that apart from the conservative values given by 
equation 5 for $\phi=0.01$, a FOM increase is observed for all reasonable temperatures. It is also important to note that a minimum FOM value is observed at $\mathrm{T} \approx 100-150^{\circ} \mathrm{C}$. This minimum efficiency may be important when considering inlet temperatures to a thermal oil heater.

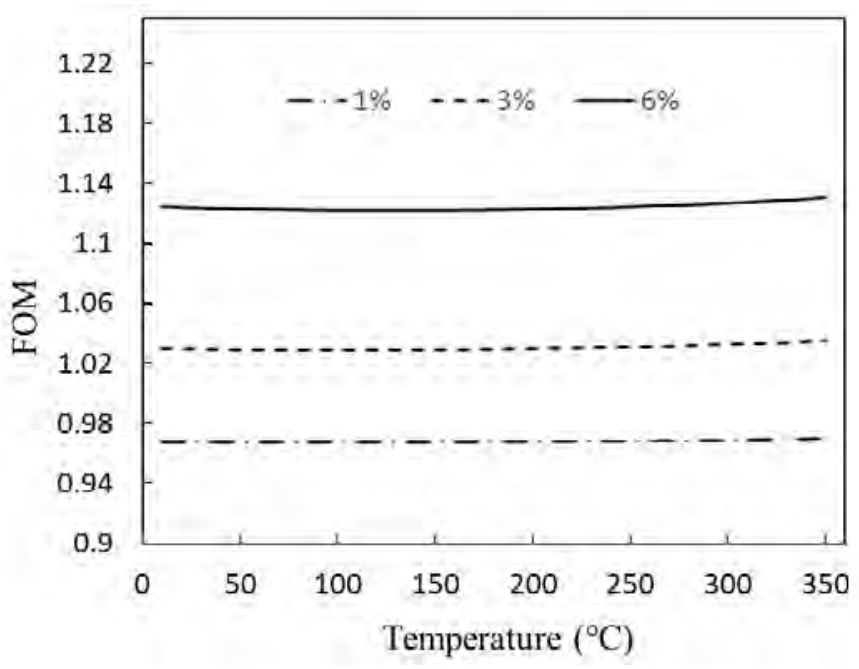

Figure 2. FOM using Aberoumand's viscosity correlation

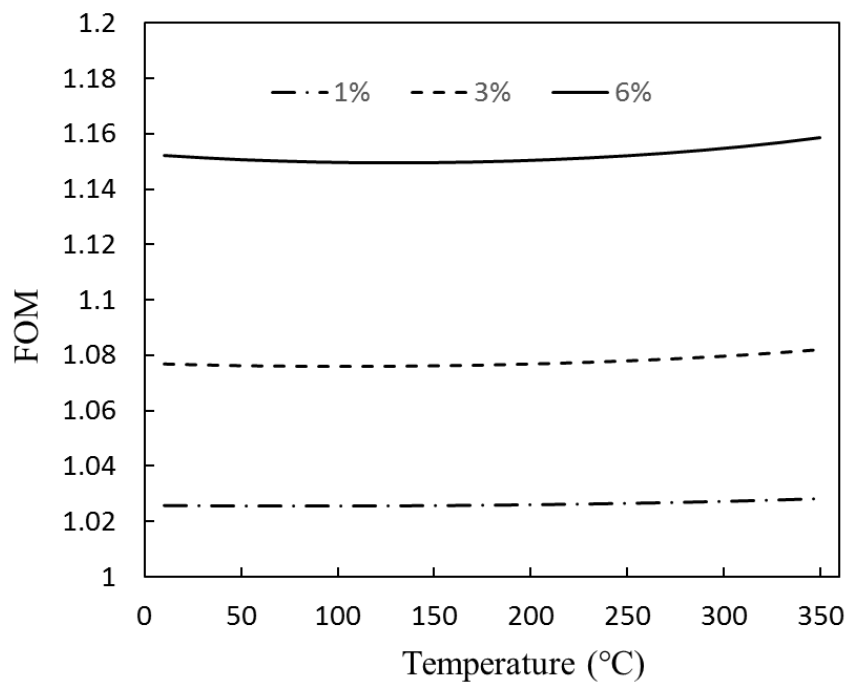

Figure 3. FOM using Einstein's viscosity correlation

In addition to the heat transfer performance increases, the nanofluid must be compatible with existing materials and not introduce unwanted corrosion or erosion effects. Molina et al. [20] tested the effects of an alumina ethylene glycol nanofluid on common cooling system materials, including aluminum and copper. Although little effects were observed on the copper specimens, there was a large surface roughness increase on the aluminum samples. Bubbico et al. [21] tested the effects of various nanofluids on many common process materials including copper, stainless steel, and aluminum. No effects were observed on the copper and stainless-steel specimens, but significant damage was caused to the aluminum specimens. It is important to note that the erosion observed was caused entirely by chemical corrosion due to the $\mathrm{pH}$ of the nanofluids rather than mechanical erosion.

It was also noted in the study that increased viscosity of the nanofluid caused pump failures. A separate test conducted by Routbort et al. [22] similarly showed effects on the pump system. In a process environment, it will be important to make sure that pump reliability will not be affected by the nanofluid. Increased pump capacity may also need to be considered to account for the increased nanofluid viscosity.

\section{RECOMMENDATIONS AND CONCLUSIONS}

From the above figures, it appears that it is feasible to use nanofluids in a thermal oil heater. Even with conservative values given by Aberoumand's viscosity correlation, the figure of merit (FOM) was found to be larger than 1 for all temperatures and $\phi>0.01$. In order to further assess the applicability, several further steps are needed to obtain a more precise answer:

- A more accurate viscosity relationship will be needed. Experimental viscosity data will be needed for a $\mathrm{Cu}$-TH66 nanofluid to give a relationship of the accepted form $\mu_{n f}=\mu_{b f}\left[1+A \phi+B \phi^{2}\right]$, which will allow for more accurate heat transfer performance calculations.

- The FOM given in equation (11) does not directly apply to a thermal oil heater. Although equation (11) gives a reasonable idea of the overall heat transfer performance of a nanofluid, it does not accurately model a thermal oil heater; rather heat transfer for turbulent flow in a pipe. A thermal oil heater model will be required to determine the exact performance increases, but that was not the scope of the present study.

- If an accurate thermal oil heater model can be formulated, an economic analysis can be performed using exact performance increases, upgrade costs, and fluid costs.

\section{REFERENCES}

[1] S. Choi and J. Eastman, "Enhancing thermal conductivity of fluid with nanoparticles," Proc. ASME Int. Mech. Eng. Congr. Expo. 2009, vol. 66, pp. 99-105, Jan. 1995.

[2] D. Li, W. Xie, and W. Fang, "Preparation and properties of copper- oilbased nanofluids," Nanoscale Res. Lett., vol. 6, no. 1, p. 373, 2011. doi: 10.1186/1556-276X-6-373.

[3] E. V. Timofeeva, M. R. Moravek, and D. Singh, "Improving the heat transfer efficiency of synthetic oil with silica nanoparticles," $J$. Colloid Interface Sci., vol. 364, no. 1, pp. 71-79, 2011. doi: 10.1016/j.jcis.2011.08.004.

[4] D. Singh, E. V. Timofeeva, M. R. Moravek, S. Cingarapu, W. Yu, T. Fischer, and S. Mathur, "Use of metallic nanoparticles to improve the thermophysical properties of organic heat transfer fluid used in concentrated solar power," Sol. En., vol. 105, pp. 468 - 478, 2014. doi: 10.1016/j.solener.2014.02.036.

[5] Y. Naresh, A. Dhivya, K. S. Suganthi, and K. S. Rajan, "Hightemperature thermo-physical properties of novel CuO-Therminol 55 nanofluid," Nanosci. Nanotechnol. Lett., vol. 4, no. 12, 2012. doi: 10.1166/nnl.2012.1454. 
[6] W. Yu, E. V. Timofeeva, D. Singh, D. M. France, and R. K. Smith, "Investigations of heat transfer of copper in Therminol 59 nanofluids," Int. J. Heat Mass Transfer, vol. 64, pp. 1196 - 1204, 2013. doi: 10.1016/j.ijheatmasstransfer.2013.03.023

[7] S. Murshed, K. Leong, and C. Yang, "Enhanced thermal conductivity of TiO 2-water based nanofluids," Int. J. Therm. Sci., vol. 44, no. 4, pp. 367 - 373, 2005. doi: 10.1016/j.ijthermalscie.2004.12.005.

[8] M. Chopkar, P. K. Das, and I. Manna, "Synthesis and characterization of nanofluids for advanced heat transfer applications," Scripta Materialia, vol. 55, no. 6, pp. 549 - 552, 2006. doi: 10.1016/j.sciptamat.2006.05.030.

[9] E. E. Michaelides, Nanofluidics. Cham: Springer International Publishing, 2014. doi: 10.1007/978-3-319-05621-0.

[10] J. C. Maxwell, A treatise on electricity and magnetism, vol. 1. Clarendon press, 1881.

[11] R. Hamilton and O. Crosser, "Thermal conductivity of heterogeneous two-component systems," in Industrial \& Engineering chemistry fundamentals, vol. 1, no. 3, pp. 187-191, 1962.

[12] Nan, R. Birringer, D. Clarke, and H. Gleiter, "Enhancing thermal conductivity of fluids with nanoparticles," J. App. Physics, vol. 364, no. 1, pp. 27-30, 2011.

[13] J. Buongiorno, et al, "A benchmark study on the thermal conductivity of nanofluids," J. App. Physics, vol. 106, no. 9, 2009. doi: $10.1063 / 1.3245330$.

[14] Einstein, "Berichtigung zu meiner arbeit:eine neue bestimmung der molekuuu"ldimensionen ," Annalen der Physik, vol. 339, no. 3, pp. 591592, 1911. doi: 10.1002/andp.19113390313.

[15] S. Aberoumand, A. Jafarimoghaddam, M. Moravej, H. Aberoumand, and K. Javaherdeh, "Experimental study on the rheological behavior of silver-heat transfer oil nanofluids and suggesting two empirical based correlations for thermal conductivity and viscosity of oil based nanofluids," App.Thermal Engineering, vol. 101, pp. 362-372, 2016. doi: 10.1016/j.applthermaleng.2016.01.148.
[16] W. Williams, J. Buongiorno, and L.-W. Hu, "Experimental investigation of turbulent convective heat transfer and pressure loss of alu- mina/water and zirconia/water nanoparticle colloids (nanofluids) in horizontal tubes," Journal of Heat Transfer, vol. 130, pp. 042412-042412-7, 03 2008.

[17] N. Prabhat, J. Buongiorno, and L.-w. Hu, "Convective Heat Trans- fer Enhancement in Nanofluids: Real Anomaly or Analysis Arti- fact?," ASME/JSME 2011 8th Thermal Engineering Joint Conf., pp. T10070T10070-10, 2011.

[18] W. Yu, D. M. France, E. V. Timofeeva, D. Singh, and J. L. Routbort, "Comparative review of turbulent heat transfer of nanofluids," Int. J. Therm. Sci., vol. 55, no. 21, pp. 5380 - 5396, 2012. doi: $10.1016 /$ j.ijheatmasstransfer.2012.06.034.

[19] Therminol, Therminol 66 - High Performance Highly Stable Heat Transfer Fluid, 1998.

[20] G. J. Molina, M. Hulett, V. Soloiu, and M. Rahman, "Erosion effects of nanofluids on selected cooling-system materials," in Tribo-Corrosion: Research, Testing, and Applications, pp. 47-65, ASTM International, Mar. 2013.

[21] R. Bubbico, G. P. Celata, F. DAnnibale, B. Mazzarotta, and C. Menale, "Experimental analysis of corrosion and erosion phenomena on metal surfaces by nanofluids," Chem. Eng. Res. Des., vol. 104, pp. 605 - 614, 2015. doi: 10.1016/j.cherd.2015.10.004.

[22] J. Routbort, D. Singh, E. Timofeeva, W. Yu, and R. Smith, "Erosion of radiator materials by nanofluids," Argonne National Laboratory, Vehicle Technologies-Annual Review, 2010. 


\title{
Effects of High Pressure Coolant on Machining Temperature and Machinability of AISI 304 Stainless Steel
}

\author{
Yassmin Seid Ahmed ${ }^{1}$, Stephen Clarence Veldhuis ${ }^{2}$ \\ McMaster Manufacturing Research Institute (MMRI), Department of Mechanical Engineering, McMaster University \\ Hamilton, Canada \\ 1 Seidahmy@mcmaster.ca \\ ²veldhu@mcmaster.ca
}

\begin{abstract}
Effective zones of high pressure coolant (HPC) have been identified in turning performing a wide range of machining operations on 304 stainless steel using uncoated carbide inserts. The effects of triple coolant jets on tool-chip interface temperature were studied. In addition, their performance are evaluated in terms of machining parameters by comparing with those of conventional coolant and dry cut. Chipping and catastrophic failure are the dominant factors of insert rejection for dry cut and conventional coolant, whereas progressive flank wear is observed for HPC within its effective zones at lower feed rate and higher cutting speed. It is found that the cutting force is reduced, surface finish is improved, chip width is narrowed and cooling effect is better with the use of HPC. The enhanced heat dissipation by triple jets is accredited as the primary reason for the reduction of cutting forces, surface roughness and tool wear.
\end{abstract}

Keywords-high pressure coolant; 304 stainless steel; tool wear; cutting forces; chip formation; surface roughness

\section{INTRODUCTION}

Machining is a manufacturing process where a sharp cutting tool penetrates the surface of a work material. The penetration causes shear deformation of the workpiece in order to remove material from the surface in the form of chips. During the formation of chips, the mechanical energy applied by the insert converts to heat energy [1]. Plastic deformation of the work material and friction between tool rake face, and flowing chips foster intense amount of heat at the cutting zone [2]. Machining of stainless steel and other difficult to cut materials requires instant heat transfer from the cutting edge of tool to aid tool life. Supply of high volume and HPC often provides the best answer. Conventional coolant does not reach the real cutting area near the cutting edge of the tool and vaporizes before it reaches the cutting area as the heat is very intense during machining [3]. 304 stainless steel is mainly used for Heat Exchangers, bushings, springs, bearings, gears, and shafts. Despite the increased usage of 304 stainless steel, they have relatively poor machinability characteristics compared to other metals, because of their poor thermal conductivity [4].
They are very difficult to machine and intense amount of heat generates during machining which must be reduced to enhance product quality and dimensional accuracy by proper application of coolant [5].

The concept of HPC may be a possible solution for high speed machining in achieving intimate chip-tool interaction, low cutting temperature and slow tool wear while maintaining cutting forces at reasonable levels. [6] Based on the necessity and versatility of HPC in machining, this study focuses on the machinability assessment of AISI 304 stainless steel by using uncoated carbide inserts at dry, conventional coolant, and triple jets HPC lubrication. The evaluation of HPC has been executed in two steps: firstly, based on the ability of HPC to reduce the cutting temperature, and secondly, the relevance of this temperature lowering with favorable curbing of the cutting force, tool wear, surface quality, and chip formation.

\section{EXPERIMENTAL PARAMETERS AND PROCEDURES}

In this research, a round bar $(120 \mathrm{~mm}$ diameter by $500 \mathrm{~mm}$ length) of an austenitic stainless steel AISI 304 was investigated during turning. The chemical composition of the material is shown in Table 1. To reveal the workpiece microstructure, a sample of the AISI 304 was prepared, polished and etched with Glycergia solution $(1 \mathrm{~mL}$ Glycerol + $20 \mathrm{~mL}$ hydrochloric Acid ( $\mathrm{HCl})+20 \mathrm{~mL} \mathrm{HNO} 3)$. The microstructure of the AISI 304 workpiece was characterized using a Nikon ECLIPSE IV 100 equipped with UC30 camera, see Figure 1. The turning process was performed using a Nakamura-Tome Sc-450 lathe. The cutting tool (Manufacturer: Kennametal) used for the experiments was an uncoated cemented carbide insert with $\mathrm{WC} / 6 \% \mathrm{Co}$. The designation of the insert is ISO WNMG432-SM with the following geometry characteristics: back rake angle, $\lambda 0=7^{\circ}$; clearance angle, $\alpha 0=0^{\circ}$; wedge angle, $\beta=82^{\circ}$; edge radius, $\mathrm{r}=34 \mu \mathrm{m}$ and nose radius, $\mathrm{R} \varepsilon=0.8 \mathrm{~mm}$. Tool-holder used for high pressure cooling was an ISO PWLNR 16-4DHP with three cooling nozzles used, supplied by Sandvik (Figure 2). The high pressure coolant was applied at a flow rate of 30 $\mathrm{L} / \mathrm{min}$ via three nozzles directed toward the rake face of the 
cutting tool at a pressure of 70 bar. The cutting fluid chosen was semi-synthetic coolant-CommCool тм 8800, manufactured by the Wallover Company (Harrow, ON, Canada), at a concentration of $7 \%$, typically used with stainless steel alloys.

Table 1: Chemical composition and mechanical properties of AISI 304.
\begin{tabular}{|c|c|c|c|c|c|c|c|}
\hline \multicolumn{1}{|c|}{ Chemical Composition \% } \\
\hline $\boldsymbol{C}$ & $\boldsymbol{S i}$ & $\boldsymbol{M n}$ & $\boldsymbol{p}$ & $\boldsymbol{S}$ & $\boldsymbol{C r}$ & $\boldsymbol{N i}$ & $\boldsymbol{N}$ \\
\hline 0.08 & 0.75 & 2.0 & 0.045 & 0.03 & 20 & 0.50 & 0.1 \\
\hline
\end{tabular}

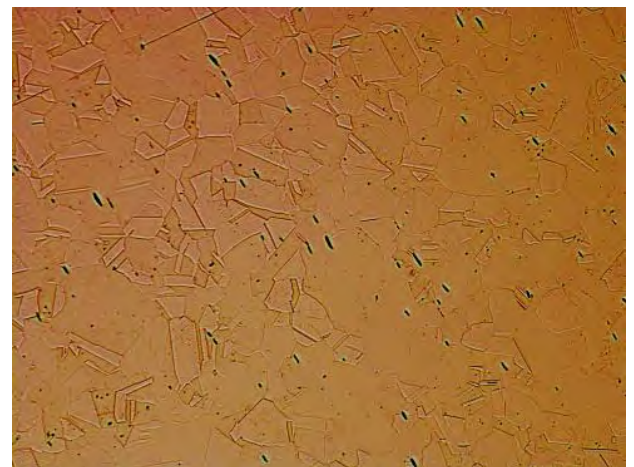

Figure 1. The microstructure of AISI 304 with an austenitic structure.

The investigations have been performed with respect to varied cutting speed $(60-100-150 \mathrm{~m} / \mathrm{min})$, feed rate $(0.1-0.2-$ $0.3 \mathrm{~mm} / \mathrm{rev}$ ) and cutting conditions (Dry-Convention coolant HPC). During turning operation the depth of cut was kept constant at $0.5 \mathrm{~mm}$ and a new tool insert was used for each run for all the machining runs.

The tool flank wear was measured using a KEYENCE VHX 5000 digital microscope, equipped with a CCD camera and image analyzer software. The tool life criterion was set to a flank wear of $0.3 \mathrm{~mm}$ according to the recommendation of the ISO 3685 Standard [8]. During the tests, the cutting tools were analyzed by SEM, using a Vega 3-TESCAN.

The chip compression ratio, the shear angle and the friction coefficient at the tool-chip interface were determined using standard methods [9]. The surface roughness of the machined workpiece was measured across the tool feed direction by means of an Alicona Infinite Focus-with the Profile roughness module. The procedures of surface roughness measurements were performed according to EN ISO standard 25178 [10]. Roughness measurements were taken with a cut-off wave length of $800 \mu \mathrm{m}$, a vertical resolution of $100 \mathrm{~nm}$ and a lateral resolution of $2 \mu \mathrm{m}$.

During the machining tests, the cutting force and chip-tool interface temperature measurements were performed with a $3 \mathrm{D}$ component tool holder Kistler dynamometer type with a data acquisition system and thermocouple sensors, respectively. The signals of the forces from the dynamometer were transmitted to a Kistler 5010 type amplifier, and then recorded on a computer using LABVIEW version 14.0 software. To measure chip-tool interface temperature, thermocouple placed just right side of insert through hole made at right side of shim. The signals of the temperature from the thermocouple were transmitted to analog K-type thermocouple amplifier, and then recorded on a computer using LABVIEW version 14.0 software.

(a) (b)

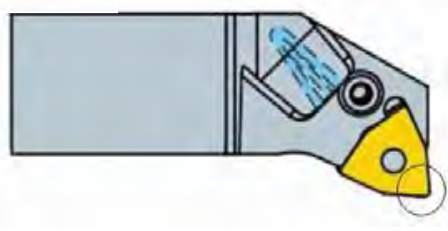

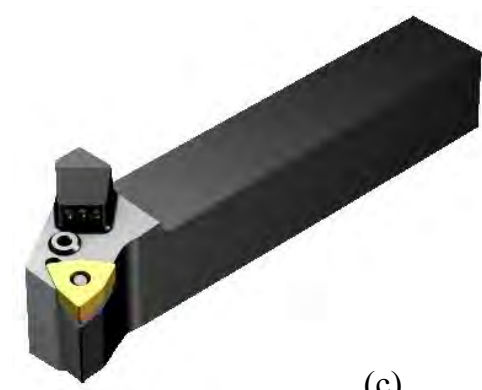

(c)

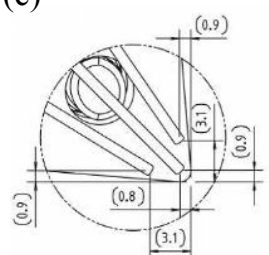

Figure 2.(a) High pressure coolant tool holder (b) concentrated flow cooling in and (b) destination of the flow cooling on the inserts [11].

\section{RESULS AND DISCUSSION}

\section{A. Effect of High Pressure coolant on Machining Temperature}

In this section, an investigation of effect of HPC on machining temperature are discussed. Figure 3 shows the interface of chip-tool and the direction of applied jets. Fluid jets are aimed at chip-tool interface in order to remove the originated heat [12]. The HPC when passed through a small diameter of jet opening produces high volumetric flow rate, which creates a thin boundary layer on impingement surface, and thus enhances heat transfer rate.

In this study, very high velocity of fluid jet induced by high pressure (70 bar) and small nozzle diameter $(1 \mathrm{~mm})$ enhance convective heat transfer rate [13]. Accordingly, three turbulent flows acting on the mutually perpendicular surfaces effectively control the cutting temperature by facilitating rapid removal of heat. The heat transfer rate can be enhanced if the jet impinges in the maximum temperature region of the cutting tool.

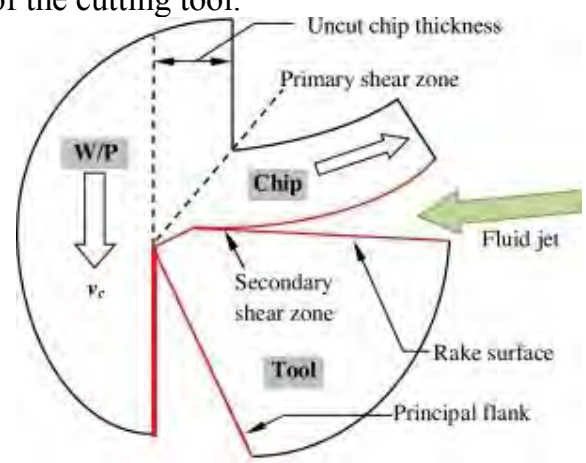

Figure 3: Chip-tool interface and the direction of applied jets [12].

Figure 4a exhibits the movement of chip-tool interface temperature with cutting speed at different feed rates. As it is shown, higher cutting speed is affiliated with elevated 
temperature which was expected owing to the dominant nature of endured friction by extended chip-tool contact area [14]. At the same time, the effect of higher feed rate is reflected by an increase in chip-tool interface temperature. This is endorsed to a higher rate of conversion of mechanical to thermal energy by the chip evolution by shearing of solid material [15]. Therefore, the recommended speed is $60 \mathrm{~m} / \mathrm{min}$ along with $0.1 \mathrm{~mm} / \mathrm{rev}$ feed rate to originate the minimum temperature of $22.5{ }^{\circ} \mathrm{C}$ under the employment of effective HPC jets.

Figure $4 \mathrm{~b}$ shows the typical temperature profiles of toolchip interface with machining time for dry cut, conventional coolant, and HPC at the recommended speed and feed. Temperature profiles for dry cut show gradual increase of temperature with machining time. Maximum temperature rise for dry cut is $140^{\circ} \mathrm{C}$, for conventional coolant is $36^{\circ} \mathrm{C}$, and for $\mathrm{HPC}$ is $22^{\circ} \mathrm{C}$ for the optimum cutting condition. HPC decreases the tool-chip temperature by $38 \%$ in comparison with conventional coolant and by $85 \%$ in comparison with dry cut indicating better cooling effect. Above results clearly indicate the effectiveness of HPC in reducing the temperature. This is endorsed to a higher rate of conversion of mechanical to thermal energy by the chip evolution by shearing of solid material [16].
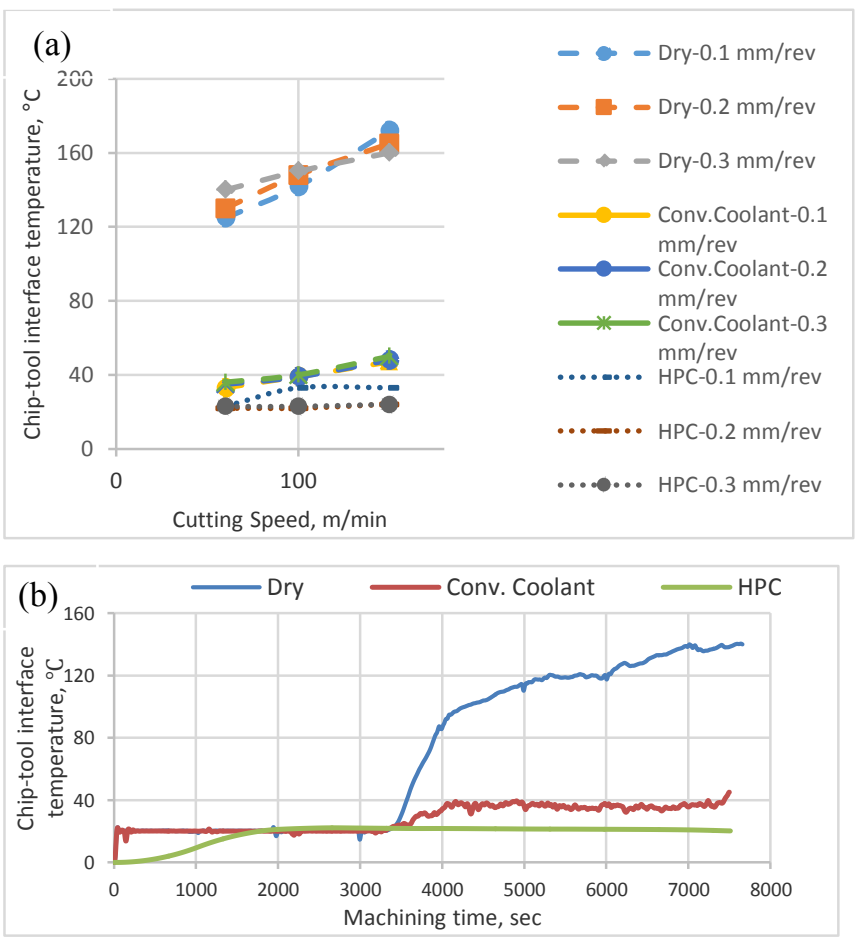

Figure 4: (a) chip-tool interface temperature values at different cutting conditions and corresponding chip-tool interface temperature profiles at optimum cutting conditions.

\section{B. Effect of High Pressure Coolant on Machining Performance}

Force components are almost always utilized to determine chip formation process of engineering materials. Although measuring forces directly help to determine power requirement for cutting the work material, it is also useful to interpret the whole cutting process [17]. The main cutting forces for the dry and two different cooling at optimum cuing conditions is depicted in Figure 5. As shown, main cutting forces are higher with dry and conventional coolant as compared to HPC. The reduction in the cutting force with HPC could be due to several reasons. HPC jet strikes the chip at a point very close to the cutting edge of insert, resulting in the reduction of the width of the chip. Consequently, the tool chip contact area on the rake face is reduced, which reduces the friction between insert and chip and eventually reduces cutting force. Another reason is that HPC is able to penetrate deeper into the cutting interface, thus providing more efficient cooling as well as lubrication [18-19]. The coolant water wedge created at the tool-chip interface reduces tool-chip contact length and forces, which can be also connected to benefits in friction conditions.

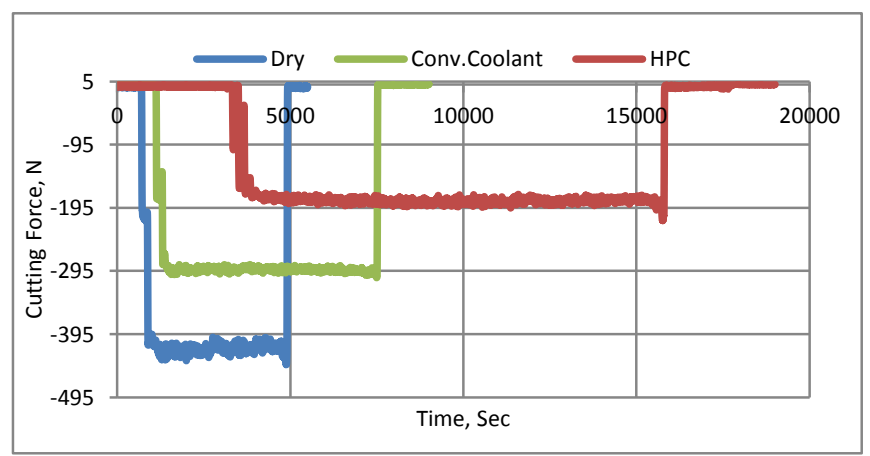

Figure 5: Main cutting forces for dry, conv. coolant and HPC at optimum cutting conditions.

The average surface roughness parameter $(\mathrm{Ra})$ has been studied and its behavior with the progression of cutting speed at different feed rates for dry, conventional coolant, and HPC is described in Figure 6a. It is evident from this figure that a reduced roughness is achieved at higher cutting speed. On the contrary, the sliding and adhesion of chips at low spindle speed are accounted for high surface roughness [20]. Also, the surface roughness is increased by the feed rate as a result of the straining effect of high feed rate [19].

In addition, in all the cases, best surface finish is obtained with HPC, followed by conventional coolant and then dry cut, see Figure $6 \mathrm{~b}$. When chipping occurs as it is shown in dry condition, the surface obtained is the worst and shows very high value of average surface roughness because of melting of chips on the workpiece surface. The improvement of surface finish with the use of HPC may be because of tool wear rate becomes slower. 
(a)

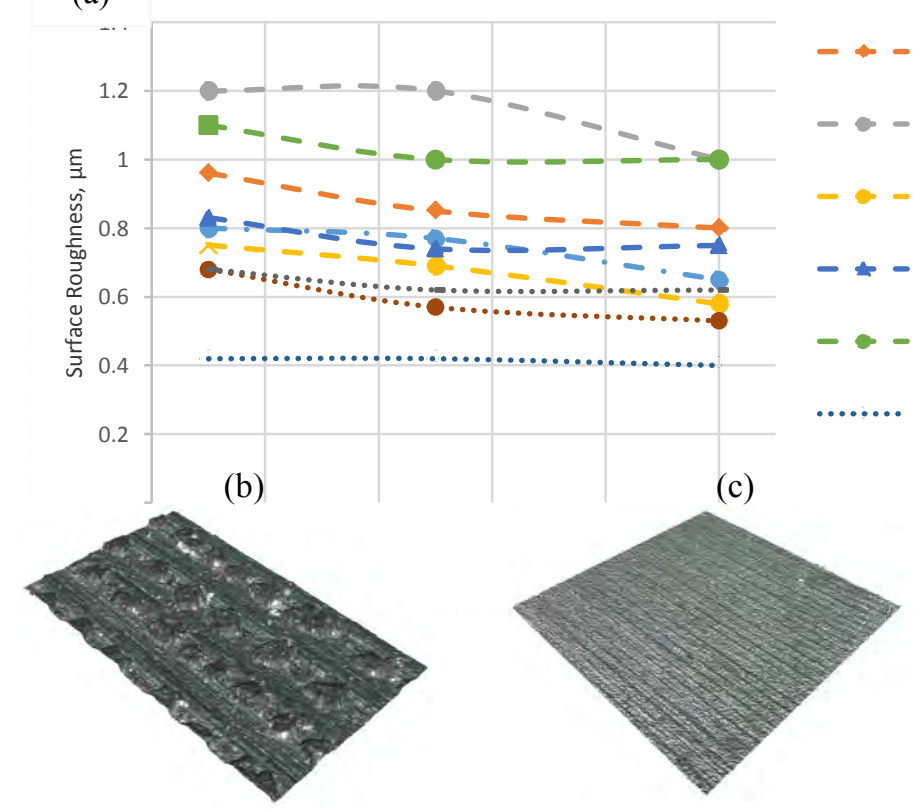

(d)

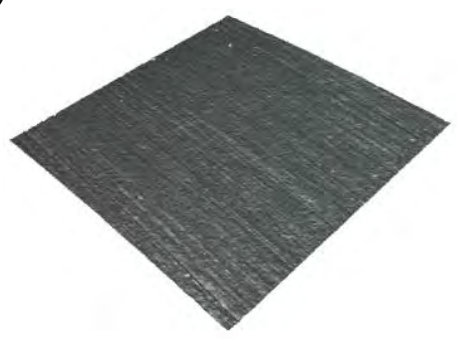

Figure 6: (a) average surface roughness at different cutting conditions and corresponding machined surface for (b) dry, (c) conv. coolant and (d) HPC at optimum cutting conditions.

To study the effectiveness of HPC, the chips were collected and presented in Figure 7. Although continuous and snarled chips are produced under dry and conventional coolant conditions, tubular chips are produced under HPC condition. HPC condition helps to break chips, in particular at lower feed rate by producing shorter tubular chips. In contrast, conventional coolant didn't show distinct effect on chip breakability. Under dry and conventional coolant, chip fracture is due to the negative bending moment created by an obstruction. However, effective chip breaking under HPC is due to the mechanical action of the high pressure jet. The 70 bar coolant pressure is equivalent to a force of

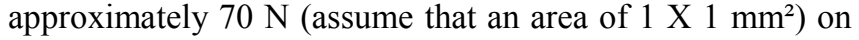
the chip which is large enough to fracture the chip by a positive bending moment, before making contact with an obstruction.

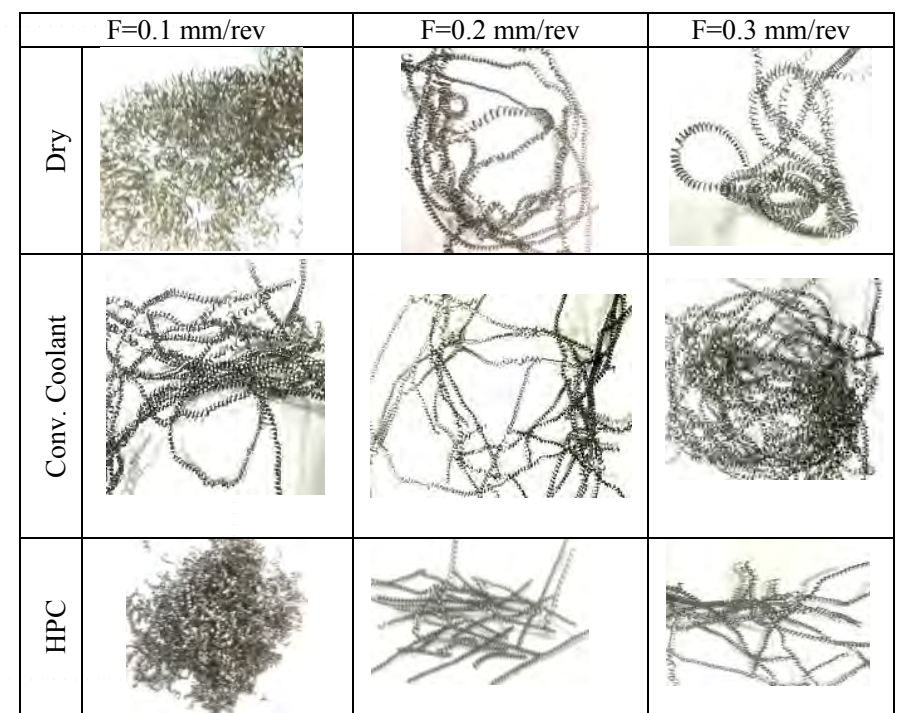

Figure 7: Chip breaking under various various cutting conditiond and feed rates.

Figure 8 shows tool life of carbide inserts with variation of cutting speed and feed rate, respectively. From these figures, HPC is found to be most effective for the cutting conditions of $\mathrm{f}=0.1 \mathrm{~mm} / \mathrm{rev}$ and $\mathrm{v}=150 \mathrm{~m} / \mathrm{min}$, which is considered as optimum cutting condition for this high pressure system. Experiments have been carried out by varying speed and feed rate, however one parameter is varied while other two are kept constant at optimum value and thus, effective zones of HPC are identified. At lower speed below $150 \mathrm{~m} / \mathrm{min}$, the use of HPC is not effective as high speed. At higher speeds, inserts fail due to chipping and excessive flank wear for dry cut and conventional coolant whereas only progressive flank wear is observed for HPC. HPC is also found to be very effective at lower feed rate $(0.1 \mathrm{~mm} / \mathrm{rev})$, reducing the chipping of inserts, which is observed for both dry cut and conventional coolant. In contrast at higher feed rate $(0.3 \mathrm{~mm} / \mathrm{rev})$, the use of HPC is found to be non-effective. The reasons for that is at lower feed rate, the generation of heat is low and the temperature rise in insert is also low and when the HPC is used, different modes of heat transfer take place. First film boiling starts and persists for very short time. Then vigorous nucleate boiling starts and then forced convection takes place [21]. At lower feed rate, pressure is high enough to remove the heat from the cutting zone. But when the feed rate is high, the temperature rise in the insert is high and film boiling persists for longer time and nucleate boiling may not take place [18]. As a result, no phase change takes place to reduce temperature. For this reason, the temperature rise causing chipping. 


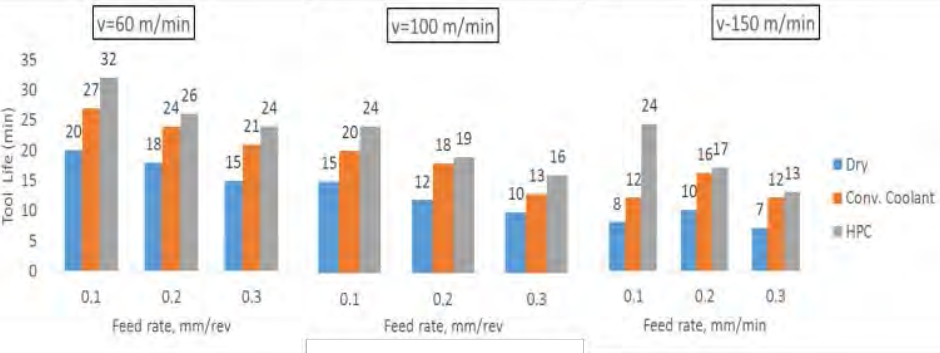

Figure 8. Recorded tool lives at various cutting conditions under dry, conv.coolant, and HPC.

Figure 9 shows SEM images of worn inserts at optimum cutting condition $(150 \mathrm{~m} / \mathrm{min}, 0.1 \mathrm{~mm} / \mathrm{rev})$. It is clear from the SEM pictures that inserts fail because of chipping for both dry cut and conventional coolant and largest wear zone is observed for dry cut, followed by conventional coolant. No chipping is observed for HPC and wear zone is also the smallest. Also, SEM analysis of the worn inserts show a smooth pattern in dry and conventional coolant conditions, indicating that diffusion wear mechanism is predominant. Diffusion wear is mostly dependent on the temperature at the interface. The HPC system has been to significantly reduce the temperature of the cutting tool which in turn reduces the diffusion wear rate (Figure 9c) and consequently increases tool life.
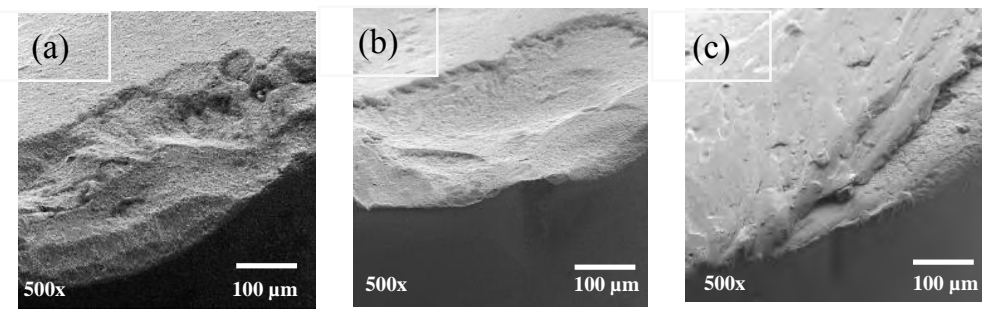

Figure 9. SEM images of worn inserts at optimum cutting conditions under (a) dry, (b) conv.coolant, and (c) HPC.

\section{ACKNOWLEDGMENT}

The authors would like to gratefully acknowledge the financial supported from the Natural Sciences and Engineering Research Council of Canada's (NSERC) Strategic Canadian Network for Research and Innovation in Machining Technology (CANRIMT).

\section{REFERENCES}

[1] M.B. Guemmour, A. Sahli, S. Kebdani and Sara Sahli, "Simulation of the chip formation and temperature distribution by the FEM," J. Appl. Sci., vol.15, pp.1138-1148, 2015. doi: 10.3923/jas.2015.1138.1148.

[2] W.Akhtar, J. Sun, P. Sun, W. Chen, and Z. Saleem, "Tool wear mechanisms in the machining of nickel based super-alloys: A review, " Frontiers of Mechanical Engineering, vol. 9. pp. 106-119, 2014. doi: 10.1007/s11465-014-0301-2.

[3] M. Kamruzzaman and N. R. Dhar, "The influence of high pressure coolant on temperature tool wear and surface finish in turning 17CrNiMo6 and 42CrMo4 steels," ARPN J. Eng. Appl. Sci., vol. 4, no. 6, 2009.
[4] R. A. Mahdavinejad and S. Saeedy, "Investigation of the influential parameters of machining of AISI 304 stainless steel," Sadhana, vol. 36, Part 6, pp. 963-970, 2011. doi: 10.1007/s12046-011-0055-z.

[5] H. Sharma and M. Sharma, "Influence of cutting fluids on quality and productivity of products in manufacturing industries," International Journal of Engineering, Management and Sciences, vol. 1, issue 11, pp.8-11, 2014.

[6] A.R Natasha, J. A. Ghani, C. H. Che Haron, and J. Syarif, "Temperature at the tool-chip interface in cryogenic and dry turning of Aisi 4340 using carbide tool," Int. J. Simul. Model., vol. 15, pp. 201-212, 2016. doi: 10.2507/IJSIMM15(2)1.314.

[7] ISO 15510-Stainless steels-Chemical composition: Annex $C$ : classification of grades, c.1: stainless steels, International Organization for Standardization, Geneva, Switzerland, 2014.

[8] ISO 3685-Tool Life Testing with Single-Point Turning Tools, 2nd ed., International Organization for Standardization, Geneva, Switzerland, 1993. (Revised in 2018).

[9] M. Shaw, Metal cutting principles, second ed., New York: Oxford University Press, 2005.

[10] ISO 25178-2 Geometrical Product Specifications (GPS) -Surface Texture: Area 1 Part 2: Terms, Definitions and Surface Texture Parameters, International Organization for Standardization, Geneva, Switzerland, 2012

[11] R. Drlička, V. Kročko, and M. Matúš, "Machinability improvement using high-pressure cooling in turning," Res. Agric. Eng. , vol. 60, pp.70-76, 2014. doi: 10.17221/38/2013-RAE.

[12] L. Liang, Y.Quan, and Z. Ke, "Investigation of tool-chip interface temperature in dry turning assisted by heat pipe cooling," Int. $J$. Adv. Manuf. Technol., vol. 54, pp.35-43, 2011.

[13] V. Katti, S. Prabhu, "Experimental study and theoretical analysis of local heat transfer distribution between smooth flat surface and impinging air jet from a circular straight pipe nozzle,". Int. J. Heat Mass Transfer, vol.51, pp.4480-4495, 2008. doi: 10.1016/j.ijheatmasstransfer.2007.12.024.

[14] E. Ezugwu, J. Bonney, "Finish machining of nickel-base Inconel 718 alloy with coated carbide tool under conventional and high-pressure coolant supplies," Tribol. Trans., vol.48, pp.76-81, 2005. doi: $10.1080 / 05698190590899958$.

[15] S. Cedergren, G. Petti, and G. Sjöberg, "On the influence of work material microstructure on chip formation, cutting forces and acoustic emission when machining Ti-6Al-4V," Procedia CIRP, vol.12, pp.55-60, 2013. doi:10.1016/j.procir.2013.09.011.

[16] T. Öpöz , X.Chen, "Chip Formation Mechanism Using Finite Element Simulation," Journal of Mechanical Engineering, vol. 62, pp. 11-22, 2016. doi: $10.5545 / \mathrm{sv}$-jme.2016.2523.

[17] M. Miaa, N. R. Dharb, "Effects of duplex jets high-pressure coolant on machining temperature and machinability of Ti-6Al-4V superalloy," Journal of Materials Processing Tech., vol. 252, 688-696, 2018. doi: 10.1016/j.jmatprotec.2017.10.040.

[18] o. Kumar, G. Singh , S.S Gill, "Impact of varying the nozzle stand off distance on cutting temperature in turning of EN-31 steel with minimum quantity lubrication," Int. J. Pure Appl. Res. Eng. Technol., vol.2, pp.931-936, 2013. doi: 10.15623/ijret.2013.0206003.

[19] M. Kamruzzamam and N.R. Dhar "The effect of high pressure coolan (HPC) jet in machining of 42CRMO4 steel by uncoated carbide inserts", Journal of Mechanical Engineering, vol. ME39, p.71-77, 2008.

[20] T. Bouchnak, A. Morel, and B. Furet, "Influence of high-pressure coolant assistance on the machinability of the titanium alloy Ti555-3," Machining Science and Technology, vol.19, pp.134-15, 2015. doi: 10.1080/10910344.2014.991029.

[21] A. Senthil Kumar, M. Rahman and S. L. Ng, "Effect of high-pressure coolant on machining performance," Int. J. Adv. Manuf. Technol., vol.20, pp.83-91, 2002. doi: 10.1007/s001700200128. 


\title{
Modeling of Structural and Environmental Effects on Microelectromechanical (MEMS) Vibratory Gyroscopes
}

\author{
Md. Imrul Kaes, D.S-K Ting, Mohammed Jalal Ahamed \\ Micro/Nano-Mechatronics Laboratory, Mechanical, Automotive \& Material Engineering \\ University of Windsor, Windsor, ON, Canada \\ Email: jahamed@uwindsor.ca
}

\begin{abstract}
-
In this paper we investigate the effects of stiffness, damping and temperature on the performance of a MEMS vibratory gyroscope. The stiffness and damping parameters are chosen because they can be appropriately designed to synchronize the drive and sense mode resonance to enhance the sensitivity and stability of MEMS gyroscope. Our results show that increasing the drive axis stiffness by $50 \%$ reduces the sense mode amplitude by $\sim 27 \%$ and augments the resonance frequency by $\sim 21 \%$. The stiffness and damping are mildly sensitive to typical variations in operating temperature. The stiffness increases by $1.25 \%$, while the damping decreases by $3.81 \%$, when the temperature is raised from $0^{\circ} \mathrm{C}$ to $45^{\circ} \mathrm{C}$. Doubling the damping reduces the oscillation amplitude by $80 \%$, but $\sim 1 \%$ change in the frequency. The predicted effects of stiffness, damping and temperature can be utilized to design a gyroscope for the desired operating condition.
\end{abstract}

Keywords- MEMS gyroscope, Resonator, Spring Stiffness Coefficient, Damping Coefficient, Temperature, Coriolis

\section{INTRODUCTION}

Microelectromechanical systems (MEMS) based inertial sensors such as accelerometers and gyroscopes are the most commercially successful MEMS sensors to date. With the rapid advancement of semi-conductor based mass manufacturing, the cost, size and power consumption are continuously decreasing, enabling new applications in automotive, aerospace, biomedical and consumer electronics. MEMS vibratory gyroscopes are physical sensors that can detect and measure the angular motion of an object relative to an inertial frame of reference. MEMS vibratory sensors show promising vibration characteristics. Fabrication accuracies are comparable to macro-scale high performance sensors [1]-[4]. A vibratory gyroscope uses a vibrating structure (proof mass) to determine the rate of rotation [5]. The vibrating proof mass tends to vibrate along the same plane even if its support rotates. The Coriolis Effect causes the object to exert a force on its support, and the rate of rotation can be determined from the exerted force. The energy is transferred from the vibrating drive axis to the sense axis through Coriolis force. The sense mode response detects the angular velocity. The sensing performance of the MEMS gyroscope can deteriorate because of the influence of time-varying parameters such as damping, cross stiffness, and environmental variations. They generate a frequency of oscillation mismatch between the two vibrating axes [6]. Due to fabrication imperfections and environmental effects, true values of resonant frequencies deviate from their nominal values (which is known as frequency mismatching). These lead to considerable reduction in the sensitivity of the gyroscope. Therefore, it is necessary to design proper stiffness and damping for achieving a robust gyroscope.

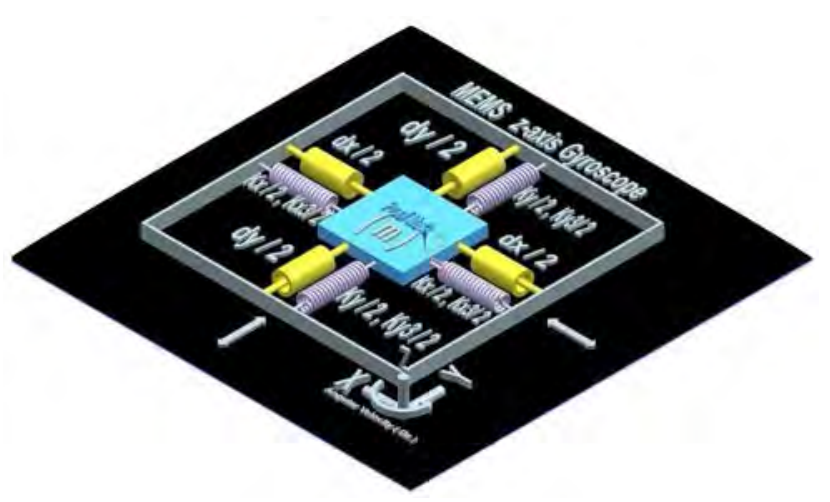

Figure 1. Schematic showing MEMS gyroscope model.

Optimization of time varying system parameters for better performance can be achieved by iterative modelling and simulation procedure. Various modeling techniques, including finite element analysis (FEA) modeling, analytical modeling and simplified lumped parameter modeling are employed [7]. FEA based modeling are robust, flexible and accurate in solving the complete multi-physics problem. Additionally, it can be coupled with controllers and electronics for selecting an appropriate controller [8].

Usually gyroscope displacement measurement varies under open loop scheme which can cause undesirable performance characteristics such as scale factor nonlinearity, limited dynamic range and narrow bandwidth [9]. In MEMS gyroscopes, 
manufacturing imperfection and noises often exist which negatively influence the resolution and performance. Errors due to noise are inevitable in actual MEMS gyroscopes, and thus, the controller has to be robust and effectively designed [10]. Postfabrication modeling of gyroscope using lumped-parameter techniques can shed light to the influence of system parameters, noise and environmental parameters on the gyro performance. This will allow iteration and design of a proper controller.

In this paper, a lumped-parameter model for predicting the gyroscope performance with the influence of various system parameters such as stiffness, damping and environmental condition (temperature) is analyzed. The results can be utilized to design a robust high performance gyroscope with efficient controller.

\section{MATHEMATICAL MODEL}

In a vibratory gyroscope the main sensing element is the proof mass, which is suspended above the substrate by several flexible beams. The overall dynamic system is usually modeled as a 2 degrees-of-freedom (2-DOF) spring-mass-damper system, as shown in Figure 1. The proof mass is suspended on the substrate using four springs (Figure 1), the other end of the springs are anchored to the substrate. An electrostatic actuation is used to give an oscillatory motion to the proof mass in the drive axis. When the proof mass is subject to an angular velocity, energy is transferred from drive axis to sense axis, causing it to oscillate. The mechanism for sensing position and velocity of the proof mass along the sense axis is present. Constant velocity of the proof mass and changing angular velocity of gyroscope about the $z$-axis are assumed. The MEMS vibratory gyroscope model includes proof mass $(m)$, drive axis displacement $(x)$, the angular velocity input $\left(\Omega_{z}\right)$, and sense axis displacement $(y)$.

According to Lagrange's [11] equation, the dynamics of the gyroscope can be described by the equations of motion below [12]:

$\mathrm{m}_{\ddot{x}}+\mathrm{d}_{\mathrm{xx}} \dot{x}+\mathrm{k}_{\mathrm{xx}} \mathrm{x}+\mathrm{k}_{\mathrm{xy}} \mathrm{y}+\mathrm{d}_{\mathrm{xy}} \dot{y}=\mathrm{u}_{\mathrm{x}}+2 \mathrm{~m} \Omega_{\mathrm{z}} \dot{y}$
$\mathrm{~m} \ddot{y}+\mathrm{d}_{\mathrm{yy}} \dot{y}+\mathrm{k}_{\mathrm{yy}} \mathrm{y}+\mathrm{k}_{\mathrm{xy}} \mathrm{x}+\mathrm{d}_{\mathrm{xy}} \dot{x}=\mathrm{u}_{\mathrm{y}}+2 \mathrm{~m} \Omega_{\mathrm{z}} \dot{x}$

Manufacturing imperfections are translated mainly to the asymmetric spring and damping terms, $\mathrm{k}_{\mathrm{xy}}$ and $\mathrm{d}_{\mathrm{xy}}$. The drive and sense axes spring and damping terms $\mathrm{k}_{\mathrm{xx}}, \mathrm{k}_{\mathrm{yy}}, \mathrm{d}_{\mathrm{xx}}$ and $\mathrm{d}_{\mathrm{yy}}$ are mostly known, but have small unknown variations from their nominal values. The proof mass can be determined accurately, and $\mathrm{u}_{\mathrm{x}}, \mathrm{u}_{\mathrm{y}}$ are the control forces in the $\mathrm{x}$ and $\mathrm{y}$ directions.

Dividing gyroscope dynamics by the reference mass, the following equation can be obtained [13]:

$q * *+\frac{D *}{m} q *+\frac{k 1 *}{m} \mathrm{q} *+\frac{k 3 *}{m}+\mathrm{q}^{* 3}$

$=\Omega^{*} \mathrm{q}^{*}+S^{*} * \mathrm{q}^{*}+2 \mathrm{~S}^{*} q^{*} *+\mathrm{u}^{*}$

where

$\mathrm{q}^{*}=\left[\begin{array}{l}x^{*} \\ y^{*}\end{array}\right], \mathrm{u}^{*}=\left[\begin{array}{l}u_{x^{*}} \\ u_{y^{*}}\end{array}\right], \mathrm{d}^{*}=\left[\begin{array}{cc}d_{x^{*}} & d_{x y^{*}} \\ d_{x y^{*}} & d_{y^{*}}\end{array}\right], \mathrm{k}_{1} *=\left[\begin{array}{cc}k_{x^{*}} & k_{x y^{*}} \\ k_{x y^{*}} & k_{y^{*}}\end{array}\right]$, $\mathrm{k}_{3} *=\left[\begin{array}{cc}k_{x^{3 *}} & 0 \\ 0 & k_{y^{3 *}}\end{array}\right], \mathrm{S} *=\left[\begin{array}{cc}0 & \Omega_{z^{*}} \\ -\Omega_{z^{*}} & 0\end{array}\right]$

Angular rate variation may be considered to be negligible. The equations of motion of the gyroscope are normalized to generalize the model. The final non-dimensional equation is derived in terms of normalized time, $t^{*}=\omega_{0} t$. With this, dividing both sides of the equation by the reference frequency and length gives [13]:

$\frac{\ddot{q}^{*}}{q_{0}}+\frac{d^{*}}{m \omega_{0}} \frac{\dot{q}^{*}}{q_{0}}+\frac{k_{1}^{*}}{m \omega_{0}^{2}} \frac{q^{*}}{q_{0}}+\frac{k_{3}^{*}}{m \omega_{0}^{2}} \frac{q^{* 3}}{q_{0}^{3}}$
$=\frac{\Omega_{z}^{*}}{\omega_{0}^{2}} \frac{\dot{q}^{*}}{q_{0}}+2 \frac{S^{*}}{\omega_{0}} \frac{\dot{q}^{*}}{q_{0}}+\frac{u^{*}}{m \omega_{0}^{2}} \frac{1}{q_{0}}$

where the parameters are defined as follows:

$q=\frac{q^{*}}{q_{0}}, d_{x y}=\frac{d_{x y}^{*}}{m \omega_{0}}, \Omega_{z}=\frac{\Omega_{z}^{*}}{\omega_{0}}, u_{x}=\frac{u_{x}^{*}}{m \omega_{0}^{2}} \frac{1}{q_{0}}, u_{y}=\frac{u_{y}^{*}}{m \omega_{0}^{2}} \frac{1}{q_{0}}$

$\omega_{x}=\sqrt{\frac{k_{x}}{m \omega_{0}^{2}}}, \omega_{y}=\sqrt{\frac{k_{y}}{m \omega_{0}^{2}}}, \omega_{x y}=\frac{k_{x y}}{m \omega_{0}^{2}}$,

$\delta_{x}=\frac{k_{x}^{3}}{m \omega_{0}^{2}} q_{0}^{2}, \delta_{y}=\frac{k_{y}^{3}}{m \omega_{0}^{2}} q_{0}^{2}$

Consequently, the non-dimensional representation becomes

$\ddot{q}+D \dot{q}+q k_{1}+k_{3} q^{3}=\Omega_{z}^{2} \dot{q}+2 S \dot{q}+u$

where

$q=\left[\begin{array}{l}x \\ y\end{array}\right], u=\left[\begin{array}{l}u_{x} \\ u_{y}\end{array}\right], D=\left[\begin{array}{cc}d_{x} & d_{x y} \\ d_{x y} & d_{y}\end{array}\right], k_{1}=\left[\begin{array}{cc}\omega_{x}^{2} & \omega_{x y} \\ \omega_{x y} & \omega_{y}^{2}\end{array}\right]$, $k_{3}=\left[\begin{array}{cc}\delta_{x} & 0 \\ 0 & \delta_{y}\end{array}\right], S=\left[\begin{array}{cc}0 & \Omega_{z} \\ -\Omega_{z} & 0\end{array}\right]$

Simulation is done to observe the influence of stiffness, damping and temperature on gyroscope performance to minimize time and cost of expensive trial and error with the actual fabrication cycle.

MATLAB Simulink [14] is used for simulation and computation. Spring stiffness, damping coefficient and temperature are known to have significant influence on the output performance of gyroscope. Thus, the objective of the simulation is to quantify the effects of these parameters. The spring stiffness and damping coefficient are varied from half to double of their nominal values. The temperature is varied from $0^{0} \mathrm{C}$ to $45^{0} \mathrm{C}$ to observe the temperature effect on stiffness, damping coefficient and on sensing magnitude. For the simulation model, the nominal values for the proof mass, stiffness, and damping are extracted from the literature reported in [12]. Proof mass, $\mathrm{m}=0.57 \mathrm{e}^{-8} \mathrm{~kg}$, damping coefficient along the drive-axis, $\mathrm{d}_{\mathrm{xx}}=0.429 \mathrm{e}^{-6} \mathrm{~N} \mathrm{~s} / \mathrm{m}$, damping error due to manufacturing imperfection, $\mathrm{d}_{\mathrm{xy}}=0.0429 \mathrm{e}^{-6} \mathrm{~N} \mathrm{~s} / \mathrm{m}$, damping coefficient along the sense-axis, $d_{y y}=0.687 \mathrm{e}^{-36} \mathrm{~N} \mathrm{~s} / \mathrm{m}$, spring stiffness along the drive-axis, $\mathrm{k}_{\mathrm{xx}}=80.98 \mathrm{~N} / \mathrm{m}$, stiffness error 
due to manufacturing imperfection, $\mathrm{k}_{\mathrm{xy}}=5 \mathrm{~N} / \mathrm{m}$, spring stiffness along the sense-axis, $\mathrm{k}_{\mathrm{yy}}=71.62 \mathrm{~N} / \mathrm{m}$, angular velocity, $\Omega_{\mathrm{z}}=5$ $\mathrm{rad} / \mathrm{s}$ are considered.

\section{MODEL RESULTS}

\section{A. Oscillation Frequency}

To identify the first resonance frequency, input frequency is varied, a sinusoidal electromagnetic force is introduced at the drive-axis and output is sensed both in drive and sense directions (Fig. 2). Usually the drive mode displacement is higher compared to sense mode due to the energy transfer.

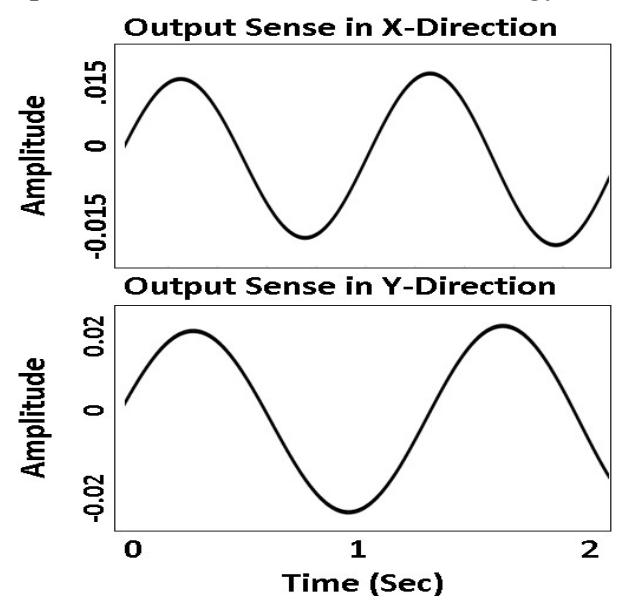

Figure 2. Displacement in drive mode (x) and sense mode (y) direction.

After sweeping the simulation with the frequency range of 1 $\mathrm{kHz}$ to $60 \mathrm{kHz}$ (Fig. 3), the resonance frequency is found at around $9.4 \mathrm{kHz}$. So, for better focusing, the frequency range is restricted from $10 \mathrm{kHz}-30 \mathrm{kHz}$ for other comparisons.

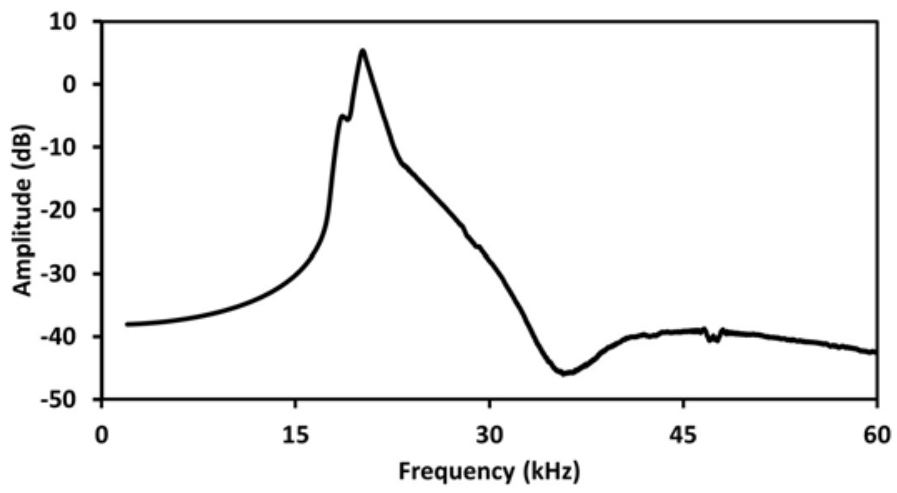

Figure 3. Frequency versus magnitude graph.

\section{B. Variation in Stiffness}

Suspension beam along drive and sense direction provides the stiffness necessary in each direction. Keeping the $\mathrm{k}_{\mathrm{yy}}$ value constant at $71.62 \mathrm{~N} / \mathrm{m}, \mathrm{k}_{\mathrm{xx}}$ is varied from $50 \%$ (normalized value 0.5 ) to $150 \%$ (normalized value 1.5 ). The normalized value of tuned reference stiffness $(80.98 \mathrm{~N} / \mathrm{m})$ is considered as 1. It is observed that magnitudes at resonance frequencies show linear decreasing trend (Fig. 4) and amplitudes decrease (Fig. 5) as stiffness increases. However, decreasing the stiffness creates phase lag and distortion in output response.

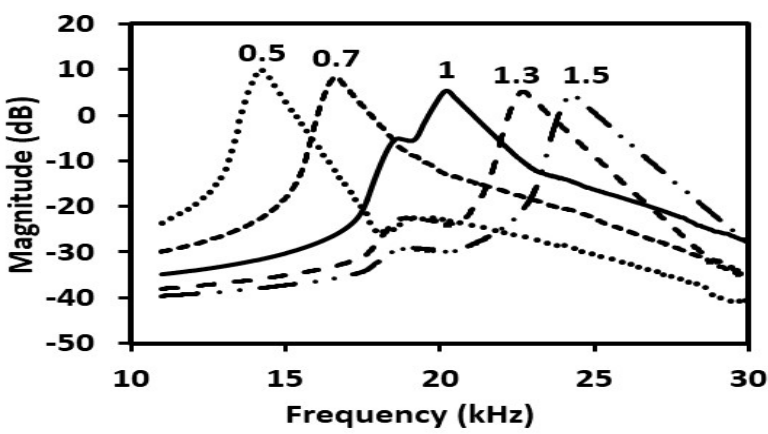

Figure 4. Effect of drive mode stiffness on resonance frequency and amplitude.

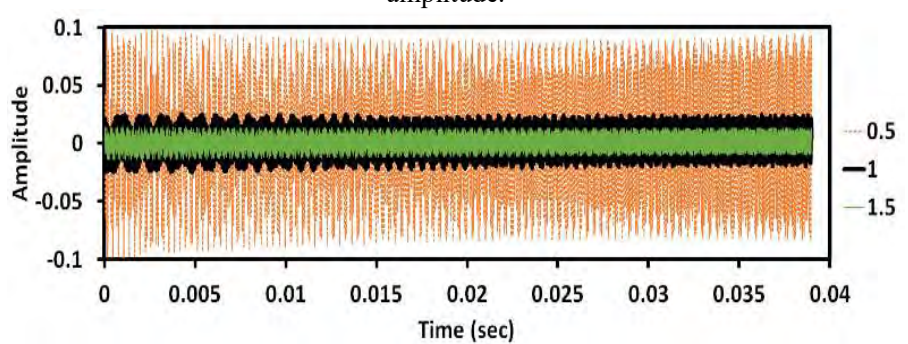

Figure 5. Effect of drive mode stiffness on output sense (sense mode stiffness is constant)

Keeping the $\mathrm{k}_{\mathrm{xx}}$ value constant, $\mathrm{k}_{\mathrm{yy}}$ is varied. It is observed that shift in resonance frequency is non-significant (Fig. 6).

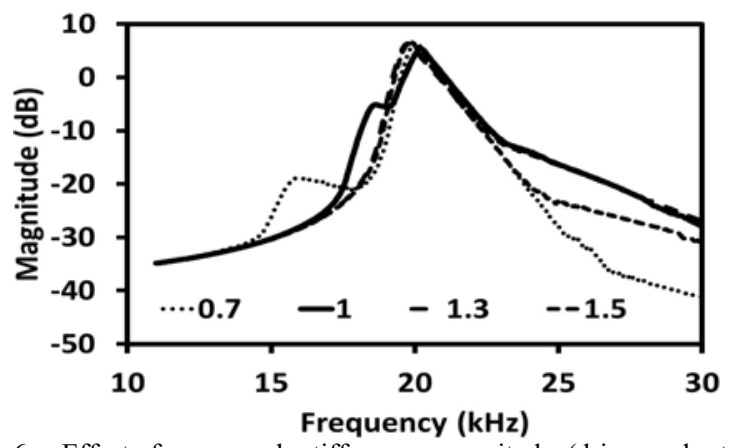

Figure 6. Effect of sense mode stiffness on magnitude (drive mode stiffness is constant).

Variation in stiffness $\left(\mathrm{k}_{\mathrm{xx}}\right.$ and $\left.\mathrm{k}_{\mathrm{yy}}\right)$ along both axes decreases magnitudes at resonance frequencies and resonance frequencies shift towards higher frequencies (Fig. 7) and amplitudes also decreases as stiffness increases (Fig. 8).

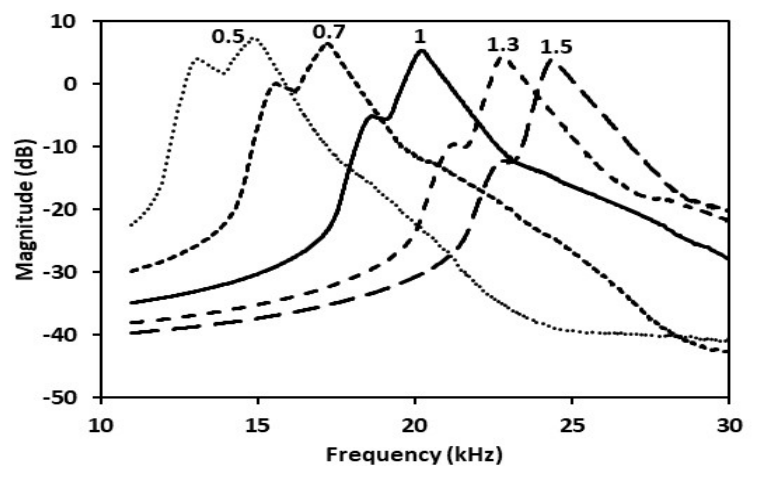

Figure 7. Combined effect of drive mode stiffness and sense mode stiffness on magnitude. 


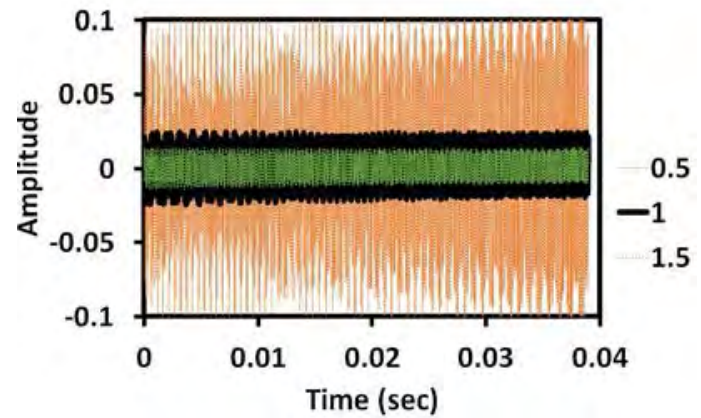

Figure 8. Effect of drive mode stiffness and sense mode stiffness on output sense.

\section{Variation in Damping}

Here, damping along drive axis is varied (Fig. 9). It is obvious from the figure that the resonance frequencies do not change that much but slope steepness decreases after resonance frequencies.

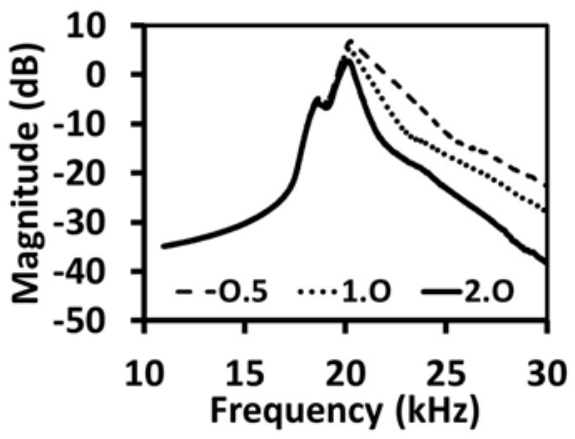

Figure 9. Effect of drive mode damping on magnitude.

\section{Temperature Variation}

One key disadvantage of MEMS gyrosocpe is its high thermal sensitivity. The frequency of oscillation drifts with temperture. Temperature variation mainly affects the stiffness and damping of the supporting beams. Here investigation on the temperature sensitivity for stiffness and damping is done. The variation of stiffness with temperature can be modeled using simplified linear relationship of [7] $K(T)=K_{0}(1-k \Delta T)$, where $K_{0}$ is the stiffness coefficient at reference temperature $300 \mathrm{~K}$ and $k=70$ ppm.

Variation of damping coefficient with temperature can be modeled as [7] $d(T)=d_{0} \cdot 1.28^{\ln \left(\frac{T}{300}\right)}$, where $\mathrm{T}$ is temperature in $\mathrm{K}, d_{0}$ is demping coefficient at reference temperature of 300 $K$. Magnitude shows positive increasing trend with the increase of temperature when stiffness and temperature relationship shows linear behaviour. The damping coefficient within industrial temperature range shows decreasing trend as temperature increases (Fig. 10, 11).

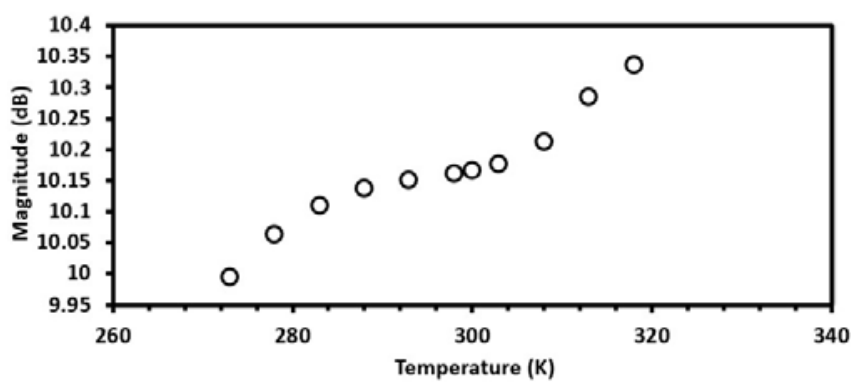

Figure 10. Effect of temperature on linear drive mode stiffness and magnitude

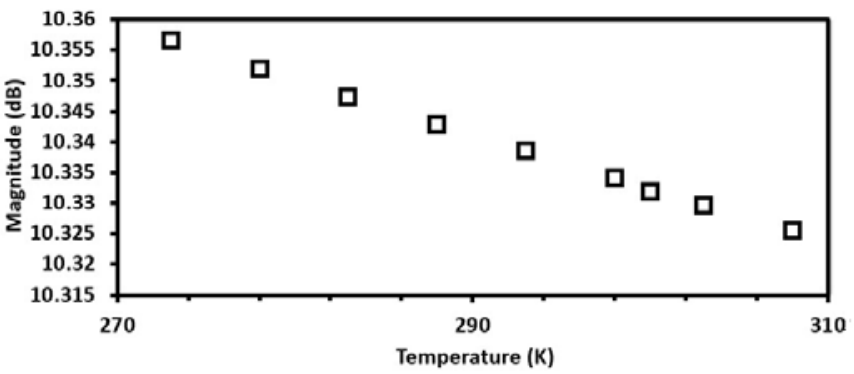

Figure 11. Effect of temperature on non-linear damping and magnitude

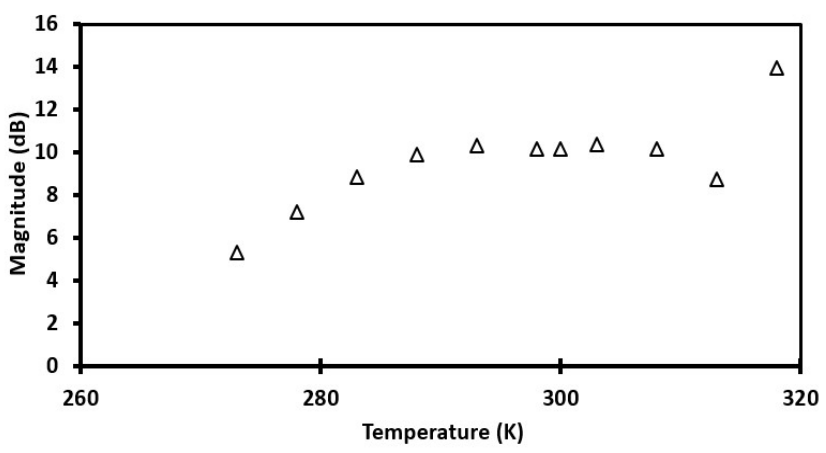

Figure 12. Effect of temperature on non-linear drive mode stiffness and magnitude.

The spring stiffness and temperature relationship shows nonliner behavior, the magnitude increases non-linearly up to a certain point before decreasing. At the end of the industrial temperature range, the magnitude increases drastically (Fig. 12)

\section{CONCLUSION AND FUTURE WORK}

This paper investigates the effect of variation of suspending stiffness coefficient, damping coefficient and temperature on the performance of MEMS gyroscope. Simulation results show how these parameters affect the error i.e. mismatch between the input and sense signals. This will help to design appropriate system parameters and controllers to increase gyroscope accuracy. Simulation results also show that stiffness is the dominant gyroscope parameter. The temperature can negatively impact resonance frequency and sense magnitude. In the future, we plan to extend this by including experimental validation of our results with MEMS gyroscopes and implementing a controller to minimize these effects and maximize the gyroscope performance. 


\section{ACKNOWLEDGEMENT}

The authors would like to thank the Natural Sciences and Engineering Research Council of Canada (NSERC) and University of Windsor for financial support.

\section{REFERENCES}

[1] M. J. Ahamed, D. Senkal, S. Member, A. A. Trusov, and A. M. Shkel, "Study of High Aspect Ratio NLD Plasma Etching and Postprocessing of Fused Silica and Borosilicate Glass," J. Microelectromechanical Syst., vol. 24, no. 4, pp. 790-800, 2015.

[2] J. Liu, J. Jaekel, D. Ramdani, N. Khan, D. S.-K. Ting, and M. J. Ahamed, "Effect of Geometric and Material Properties on Thermoelastic Damping (TED) of 3D Hemispherical Inertial Resonator," in ASME International Mechanical Engineering Congress and Exposition, 2016, vol. 10, pp. 1-6. doi: 10.1115/IMECE2016-66277.

[3] A. M. Shkel, M. Ahamed, and D. Senkal, "Method of fabricating micro-glassblown gyroscopes," US 9,702,728 B2, 2017.

[4] D. Senkal, M. J. Ahamed, M. H. A. Ardakani, S. Askari, and A. M. Shkel, "Demonstration of 1 million Q -factor on microglassblown wineglass resonators with out-of-plane electrostatic transduction," $J$. Microelectromechanical Syst., vol. 24, no. 1, pp. 29-37, 2015.

doi: 10.1109/JMEMS.2014.2365113.

[5] F. Braghin, F. Resta, E. Leo, and G. Spinolab, "Nonlinear dynamics of vibrating MEMS," Sensors Actuators A Phys., vol. 134, no. 1, pp. 98-108, 2007. doi: $10.1016 /$ j.sna.2006.10.041

[6] J. Fei and C. Batur, "A novel adaptive sliding mode control with application to MEMS gyroscope," ISA Trans., vol. 48, no. 1, pp. 73-78, 2009. doi: 10.1016/j.isatra.2008.10.008.

[7] M. Wen et al., "Modeling and analysis of temperature effect on MEMS gyroscope," Electron. Components Technol. Conf., pp. 20482052, 2014. doi: 10.1109/ECTC.2014.6897584.

[8] J. Wang, S. Ban, and Y. Yang, "A Differential Self-Integration DDot Voltage Sensor and Experimental Research," IEEE Sens. J., vol. 15, no. 7, pp. 3846-3852, 2015. doi: 10.1109/JSEN.2015.2399413.

[9] W. T. Sung, S. Sung, J. G. Lee, and T. Kang, "Design and performance test of a MEMS vibratory gyroscope with a novel AGC force rebalance control," J. Micromechanics Microengineering, vol. 17, no. 10, pp. 1939-1948, 2007. doi: 10.1088/0960-1317/17/10/003.

[10] W. Juan and J. Fei, "Adaptive fuzzy approach for non-linearity compensation in MEMS gyroscope," Trans. Inst. Meas. Control, vol. 35, no. 8, pp. 1008-1015, 2013. doi: 10.1177/0142331212472224.

[11] R. G. Albrecht Bertram, Solid Mechanics: Theory, Modeling, and Problems, First Ed. Sachsen-Anhalt, Germany: Springer, 2013.

[12] J. Fei, W. Dai, M. Hua, and Y. Xue, "System dynamics and adaptive control of MEMS gyroscope sensor," IFAC Proc., vol. 44, no. 1, pp. 3551-3556, 2011. doi: 10.3182/20110828-6-IT-1002.00034.

[13] M. R. Moghanni, J. Keighobadi, and A. Ghanbari, "Fuzzy adaptive sliding mode controller for MEMS vibratory rate gyroscope," IFAC Proc., vol. 44, no. 1, pp. 4192-4197, 2011.

doi: 10.3182/20110828-6-IT-1002.03569.

[14] Simulink, "Mathworks," 2018. [Online]. Available: https:// www.mathworks.com/products/simulink.html. Accessed: 26-Mar-2018. 


\title{
One-dimensional Fully Coupled Heat and Moisture Transfer Study Using the Finite Volume Method
}

\author{
Lam Dang, Wey H. Leong Alan S. Fung \\ Department of Mechanical and Industrial Engineering \\ Ryerson University \\ Toronto, Ontario \\ lam.dang@ryerson.ca, weyleong@ryerson.ca, alanfung@ryerson.ca
}

\begin{abstract}
In this paper, one-dimensional coupled heat and mass transfer is studied using the finite volume method. The simulated results are compared with the experimental results and show good agreements with the experimental data. Soil from Northern Victoria County (silt loam, a medium soil) is compacted in a cylinder with 5 heat pulse probes to measure the thermal and moisture responses. Moisture content at field capacity is used for the soil.
\end{abstract}

Keywords: Heat and mass transfer, soil, numerical analysis, finite volume method, heat pulse

\section{INTRODUCTION}

Global warming is currently causing serious issues around the world such as typhoons [1], more diseases [2] for humans, and habitat damages [3]. Consequently, many governments around the globe have devoted heavy efforts in reducing global warming which results from heavy consumption of energies (mostly fossil fuels) [3, 4]. Renewable energies have been encouraged to be used as they are more environmental friendly than the fossil fuels. A highly abundant and attractive renewable energy is the solar radiation from the Sun.

In order to store the thermal energy from solar radiation, thermal energy storages (TESs) are needed. A common and widely used TES form is the ground thermal energy storage (GTES). Storing at higher temperatures in the GTESs can better utilize the GTES. Consequently, it is better to store the solar heat in high-temperature (above $40^{\circ} \mathrm{C}$ ) ground because the heat can be readily retrieved for space heating without the use of a heat pump. A first-in-the-world example of successful application of using high temperature GTES is the Drake Landing Solar Community in Okotos, Alberta, Canada. The GTES can provide $90 \%$ space heating needs for the Community. The ground in Okotos contains mostly clay which has low moisture diffusivity, so the GTES in the Drake Landing Solar Community can store and retain most of the heat in the summer. However, not everywhere in the world has such type of soil. Therefore, understanding how heat and moisture transfer in various soil types at high temperatures is beneficial to the design of high-temperature GTES.

\section{GOVERNING EQUATIONS}

Modeling transport phenomena in the ground has been of great interests. The pioneers in modeling include Philip and de Vries [5] and Luikov [6]. Governing equations were developed based on the soil's hydraulic conductivity, temperature gradients, moisture potential, and volumetric moisture content. Heat conduction incorporating latent heat transfer by water vapor diffusion was considered and generalized with moisture and latent heat storage in vapor phase and sensible heat transfer by liquid migration in the soil.

Another early work was done by Demsey [7] who studied the coupled heat and moisture transfer and applied the finite difference method to numerically study the one-dimensional moisture in Lakeland fine sand (AASHO Classification A-3) under isothermal conditions (maximum $25^{\circ} \mathrm{C}$ ). Comparisons with laboratory experimental data showed good agreements between the numerical work and the lab data.

Rees et al. [8] and Janssen et al. [9] coupled heat and mass transfer equations to study how the moisture content affects the heat losses in underground structures. The researchers found that the coupled calculations give significantly higher heat losses than the calculations from simple heat and moisture transfer. In addition, the latent heat transfer by thermal vapor diffusion has a notable influence on the foundation heat loss.

Fully coupled heat and mass transfer equations can be formulated based on physical processes that occur in the soil can be described as follow [10]:

$$
\begin{gathered}
C_{\psi m} \frac{\partial \psi}{\partial t}+C_{T m} \frac{\partial T}{\partial t}=\nabla \cdot\left(D_{\psi m} \nabla \psi\right)+\nabla \cdot\left(D_{T m} \nabla T\right)+\frac{\partial K}{\partial z} \\
C_{\psi T} \frac{\partial \psi}{\partial t}+C_{T T} \frac{\partial T}{\partial t}=\nabla \cdot\left(D_{\psi T} \nabla \psi\right)+\nabla \cdot\left(k_{e f f} \nabla T\right)+C_{p w} \dot{\boldsymbol{m}}_{w} \cdot \nabla T \\
\text { where } C_{\psi m}=C_{\psi l}+C_{\psi v}, C_{T m}=C_{T l}+C_{T v}, C_{\psi T}=\rho_{l} \cdot h_{f g} \cdot C_{\psi v}, \\
C_{T T}=C_{s o i l}+\rho_{l} \cdot h_{f g} \cdot C_{T v}, C_{\psi l}=\left(\frac{\partial \theta_{l}}{\partial \psi}\right)_{T}, C_{T l}=\left(\frac{\partial \theta_{l}}{\partial T}\right)_{\psi}, \\
D_{\psi m}=K+D_{\psi v}, D_{T m}=D_{T v}, D_{\psi T}=\rho_{l} \cdot h_{f g} \cdot D_{\psi v},
\end{gathered}
$$




$$
\begin{gathered}
D_{\psi v}=f\left(\theta_{l}\right) D_{v a} \frac{\rho_{v s}}{\rho_{l}} \frac{\varphi g}{R_{w} T}, \dot{m}_{w}=\rho_{l} u_{l}, \varphi=\exp \left(\frac{\psi g}{R_{w} T}\right), \\
C_{\psi v}=\frac{\rho_{v s} \varphi}{\rho_{l}}\left[\frac{\left(\eta-\theta_{l}\right) g}{R_{w} T}-\left(\frac{\partial \theta_{l}}{\partial \psi}\right)_{T}\right], \quad C_{T v}=\frac{\rho_{v s} \varphi}{\rho_{l}}\left[\frac{\eta-\theta_{l}}{\rho_{v s}}\left(\frac{\partial \rho_{v s}}{\partial T}\right)-\left(\frac{\partial \theta_{l}}{\partial T}\right)_{\psi}\right], \\
D_{T v}=f\left(\theta_{l}\right) D_{v a} \varphi \frac{\rho_{v s}}{\rho_{l}}\left[\frac{1}{\rho_{v s}} \frac{\partial \rho_{v s}}{\partial T}-\frac{\psi g}{R_{w} T^{2}}+\frac{g}{R_{w} T}\left(\frac{\partial \psi}{\partial T}\right)_{\theta}\right] \frac{(\nabla T)_{p}}{\nabla T}, \\
K=K_{s a t} \frac{\left[1-|\alpha \psi|^{n-1}\left(1+|\alpha \psi|^{n}\right)^{-m}\right]^{2}}{\left(1+|\alpha \psi|^{n}\right)^{n / 2}}, \frac{(\nabla T)_{p}}{\nabla T}=\xi \cdot\left(\theta_{w}+\xi_{p} \theta_{p}+\sum_{i=1}^{n} \xi_{i} \theta_{i}\right)^{-1}
\end{gathered}
$$

and more definitions can be found in [10].

Eq. 1a is the moisture transfer equation in the soil and Eq. $1 b$ is the energy transfer equation in the soil. The terms on the left hand sides correspond to the stored mass and energy due to the temporal change in matric potential and temperature. The first two terms on right hand sides account for the mass transfer (Eq. 1a) and heat transfer (Eq. 1b) respectively due to moisture and temperature gradients. The last term in Eq. 1a represents the mass transfer by gravitational effects while that in Eq. $1 \mathrm{~b}$ is the sensible heat transfer by bulk liquid flow. The second term in square brackets of $D_{T v}$ is significant for $\psi<$ $10^{-4} \mathrm{~m}$ while the third term is needed for $\psi<-10^{-5} \mathrm{~m}$ [10].

As indicated by Deru [10], the last term on the right hand side (RHS) of Eq. $1 \mathrm{~b}$ is significant for only a short period after a large influx of moisture such as rainfall or irrigation. In the present experiment, there is no moisture addition into the soil during experimentation; therefore, the last term on the RHS of Eq. $1 \mathrm{~b}$ is ignored.

\section{EXPERIMENTAL SETUP}

The soil used is called Victoria soil (coded NB2 [11]) from Northern Victoria County in New Brunswick, Canada. It is a silt loam with solid density of $2540 \mathrm{~kg} / \mathrm{m}^{3}, 16.6 \%$ clay and $83.4 \%$ silt (by mass). The moist soil was prepared and packed carefully into a soil column.

In order to verify the governing equations (i.e., Eq. 1), a soil cell assembly is made as shown in Fig. 1. The heat pulse method is used to obtain soil temperature response due to a heat pulse, and a computer program developed by Knight $e t$ al. [11] is used to post-process the temperature response data in order to determine soil thermal properties and moisture content.

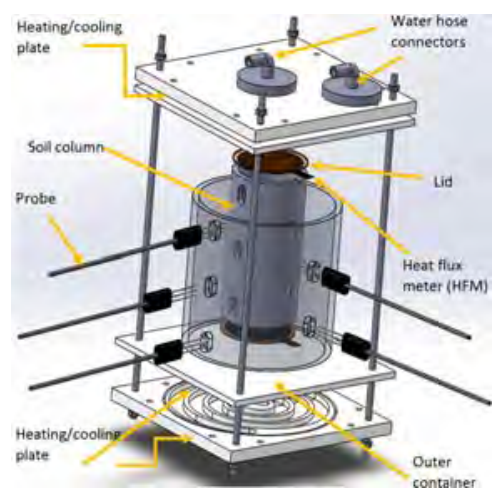

Figure 1. Soil cell assembly.
Fig. 2 shows a close-up view of a heat pulse probe. For ease of viewing the inner soil column in Fig. 1, the outer stainless-steel tube and lids are shown transparently. Water hose connectors are connected to thermally-controlled water baths (which provide the heating and cooling to the aluminum plates) through water hoses. The soil column is a stainlesssteel tube (63.5 $\mathrm{mm}$ ID, $76.2 \mathrm{~mm}$ OD, $147.9 \mathrm{~mm}$ length) that has five slots for inserting five heat pulse probes. The heating/cooling plates are made of aluminum. The soil cell assembly is connected to a data acquisition system that collects experimental data.

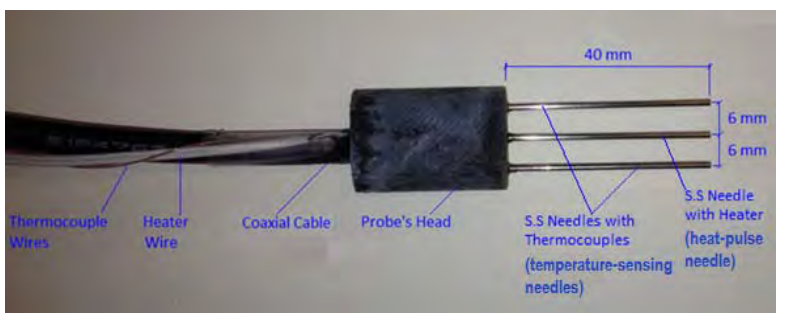

Figure 2. An in-house made heat pulse probe.

\section{NUMERICAL SOLUTION}

\section{A. One-dimensional Discretization Formulations - Finite Volume Method}

The one-dimensional finite volume formulations are developed according to the discretization schme shown in Fig. 3 with the following assumptions:

- The height of the soil column is $h$ (distance from node A to node B)

- There are $n$ control volumes (CVs) along $h$

- Each CV has length $\Delta z$ of $h / n$

- The distance from node A to node 1 is the same as that from $n$ to $\mathrm{B}$ and is equal to $\Delta z / 2$

- There are $n+2$ nodes

- The temperatures at nodes A and B are known at all times

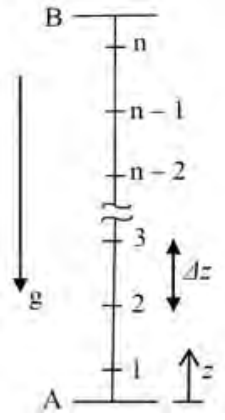

Figure 3. One-dimensional discretization scheme.

Discretizing Eq. 1 for nodes 2 to $n-1$ gives:

$$
\begin{aligned}
& a_{1} \psi_{P}+a_{2} T_{P}=a_{3} \psi_{N}+a_{4} T_{N}+a_{5} \psi_{S}+a_{6} T_{S}+G_{1} \\
& b_{1} \psi_{P}+b_{2} T_{P}=b_{3} \psi_{N}+b_{4} T_{N}+b_{5} \psi_{S}+b_{6} T_{S}+G_{2}
\end{aligned}
$$

where $a_{1}=C_{\psi m}+a_{3}+a_{5}, a_{2}=C_{T m}+a_{4}+a_{6}, b_{1}=C_{\psi T}+b_{3}+b_{5}$, $b_{2}=C_{T T}+b_{4}+b_{6}, \quad a_{3}=\frac{\beta \Delta t}{(\Delta z)^{2}}\left(D_{\psi m}\right)_{n}, a_{4}=\frac{\beta \Delta t}{(\Delta z)^{2}}\left(D_{T m}\right)_{n}, \quad \beta=0.5$ 


$$
\begin{gathered}
a_{5}=\frac{\beta \Delta t}{(\Delta z)^{2}}\left(D_{\psi m}\right)_{s} \quad a_{6}=\frac{\beta \Delta t}{(\Delta z)^{2}}\left(D_{T m}\right)_{s}, \quad b_{3}=\frac{\beta \Delta t}{(\Delta z)^{2}}\left(D_{\psi T}\right)_{n}, \\
b_{4}=\frac{\beta \Delta t}{(\Delta z)^{2}}\left(k_{e f f}\right)_{n}, \quad b_{5}=\frac{\beta \Delta t}{(\Delta z)^{2}}\left(D_{\psi T}\right)_{s}, \quad b_{6}=\frac{\beta \Delta t}{(\Delta z)^{2}}\left(k_{e f f}\right)_{s}, \\
G_{1}=\left\{C_{\psi m}-\frac{(1-\beta)}{\beta}\left[a_{3}^{o}+a_{5}^{o}\right]\right\} \psi_{P}^{o}+\left\{C_{T m}-\frac{(1-\beta)}{\beta}\left[a_{4}^{o}+a_{6}^{o}\right]\right\} T_{P}^{o} \\
+\frac{(1-\beta)}{\beta}\left\{a_{3}^{o} \psi_{N}^{o}+a_{5}^{o} \psi_{S}^{o}+a_{4}^{o} T_{N}^{o}+a_{6}^{o} T_{S}^{o}+\Delta z\left(K_{n}^{o}-K_{s}^{o}\right)\right\}+\frac{\beta \Delta t}{\Delta z}\left\{K_{n}-K_{s}\right\} \\
G_{2}=\left\{C_{\psi T}-\frac{(1-\beta)}{\beta}\left[b_{3}^{o}+b_{5}^{o}\right]\right\} \psi_{P}^{o}+\left\{C_{T T}-\frac{(1-\beta)}{\beta}\left[b_{4}^{o}+b_{6}^{o}\right]\right\} T_{P}^{o} \\
+\frac{(1-\beta)}{\beta}\left\{b_{3}^{o} \psi_{N}^{o}+b_{5}^{o} \psi_{S}^{o}+b_{4}^{o} T_{N}^{o}+b_{6}^{o} T_{S}^{o}\right\}
\end{gathered}
$$

where the superscript ${ }^{\circ}$ means the value at previous time step, $\mathrm{P}$ means the centroid of the current $\mathrm{CV}$ being considered, $\mathrm{N}$ means the north of the CV's centroid, $\mathrm{S}$ means the south of the CV's centroid.

The temperatures at nodes $\mathrm{A}$ and $\mathrm{B}\left(T_{A}\right.$ and $\left.T_{B}\right)$ are measured in the experiment and therefore they are used as prescribed boundary temperatures. The moisture balance at nodes $\mathrm{A}$ and $\mathrm{B}$, for impermeable surfaces due to the stainlesssteel lids, is [10]:

$$
0=-K \frac{\partial \Phi}{\partial n}-D_{\psi v} \frac{\partial \psi}{\partial n}-D_{T m} \frac{\partial T}{\partial n}
$$

where $\Phi=\psi+z$ with $z$ being positive upwards $(z=0$ at node A) and $\partial / \partial n=\partial / \partial z$.

As a result of Eq. 4, the matric potential gradients at nodes $\mathrm{A}$ and $\mathrm{B}$ respectively are:

$$
\begin{gathered}
\left.\frac{\partial \psi}{\partial z}\right|_{A}=\frac{-1}{K+D_{\psi v}}\left(\left.D_{T m} \frac{\partial T}{\partial z}\right|_{z=A}+K\right) \\
\left.\frac{\partial \psi}{\partial z}\right|_{B}=\frac{-1}{K+D_{\psi v}}\left(\left.D_{T m} \frac{\partial T}{\partial z}\right|_{z=B}+K\right)
\end{gathered}
$$

Applying Eq. 5a, the discretized governing equations at node 1 are:

$$
\begin{aligned}
& a_{1} \psi_{1}+a_{2} T_{1}=a_{3} \psi_{2}+a_{4} T_{2}+a_{5} \psi_{A}+a_{6} T_{A}+G_{1} \\
& b_{1} \psi_{1}+b_{2} T_{1}=b_{3} \psi_{2}+b_{4} T_{2}+b_{5} \psi_{A}+b_{6} T_{A}+G_{2}
\end{aligned}
$$

where $a_{1}=C_{\psi m}+a_{3}+a_{5}, \quad a_{2}=C_{T m}+a_{4}+a_{6}, \quad b_{1}=C_{\psi T}+b_{3}+b_{5}$, $b_{2}=C_{T T}+b_{4}+b_{6}, \quad a_{3}=\frac{\beta \Delta t}{(\Delta z)^{2}}\left(D_{\psi m}\right)_{2}, \quad a_{4}=\frac{\beta \Delta t}{(\Delta z)^{2}}\left(D_{T m}\right)_{2}, \quad a_{5}=0$,

$$
\begin{aligned}
& a_{6}=\frac{2 \beta \Delta t}{(\Delta z)^{2}}\left[\left(D_{T m}\right)_{1}+\left(\Lambda_{a}\right)_{1}\right], \quad b_{3}=\frac{\beta \Delta t}{(\Delta z)^{2}}\left(D_{\psi T}\right)_{2}, b_{4}=\frac{\beta \Delta t}{(\Delta z)^{2}}\left(k_{e f f}\right)_{2}, \\
& b_{5}=0, \quad b_{6}=\frac{2 \beta \Delta t}{(\Delta z)^{2}}\left[\left(k_{e f f}\right)_{1}+\left(\Lambda_{b}\right)_{1}\right], \quad \Lambda_{a}=\left(\frac{-D_{T m} \cdot D_{\psi m}}{K+D_{\psi v}}\right)_{1},
\end{aligned}
$$$$
\Lambda_{b}=\left(\frac{-D_{T m} \cdot D_{\psi T}}{K+D_{\psi v}}\right)_{1}, K_{1} \text { means } K \text { at node } 1 \text {, and }
$$

$\left(D_{x x}\right)_{1}$ and $\left(k_{\text {eff }}\right)_{1}$ mean $D_{x x}$ and $k_{\text {eff }}$ of CV 1 respectively

$$
\begin{aligned}
G_{1}= & \left\{C_{\psi m}-\frac{(1-\beta)}{\beta} a_{3}^{o}\right\} \psi_{1}^{o}+\left\{C_{T m}-\frac{(1-\beta)}{\beta}\left[a_{4}^{o}+a_{6}^{o}\right]\right\} T_{1}^{o} \\
& +\frac{\beta \Delta t}{\Delta z}\left\{K_{2}-K_{1}+\left(\frac{\Lambda_{a} K}{D_{T m}}\right)\right\} \\
& +\frac{(1-\beta)}{\beta}\left\{a_{3}^{o} \psi_{2}^{o}+a_{4}^{o} T_{2}^{o}+a_{6}^{o} T_{A}^{o}+\frac{\beta \Delta t}{\Delta z}\left(K_{2}^{o}-K_{1}^{o}+\left(\frac{\Lambda_{b} K}{D_{T m}}\right)_{1}^{o}\right)\right\} \\
G_{2}= & \left\{C_{\psi T}-\frac{(1-\beta)}{\beta} b_{3}^{o}\right\} \psi_{1}^{o}+\left\{C_{T T}-\frac{(1-\beta)}{\beta}\left[b_{4}^{o}+b_{6}^{o}\right]\right\}_{1}^{o} . \\
& +\frac{(1-\beta)}{\beta}\left\{b_{3}^{o} \psi_{2}^{o}+b_{4}^{o} T_{2}^{o}+b_{6}^{o} T_{A}^{o}\right\}
\end{aligned}
$$

Applying Eq. 5b, the discretized governing equations at node $n$ are:

$$
\begin{aligned}
& a_{1} \psi_{n}+a_{2} T_{n}=a_{3} \psi_{B}+a_{4} T_{B}+a_{5} \psi_{n-1}+a_{6} T_{n-1}+G_{1} \\
& b_{1} \psi_{n}+b_{2} T_{n}=b_{3} \psi_{B}+b_{4} T_{B}+b_{5} \psi_{n-1}+b_{6} T_{n-1}+G_{2}
\end{aligned}
$$

where $a_{1}=C_{\psi m}+a_{3}+a_{5}, a_{2}=C_{T m}+a_{4}+a_{6}, b_{1}=C_{\psi T}+b_{3}+b_{5}$, $b_{2}=C_{T T}+b_{4}+b_{6} \quad, \quad a_{3}=0 \quad, \quad a_{4}=\frac{\beta \Delta t}{(\Delta z)^{2}}\left(D_{T m}\right)_{n-1}$, $a_{5}=\frac{\beta \Delta t}{(\Delta z)^{2}}\left(D_{\psi m}\right)_{n-1} \quad, \quad a_{6}=\frac{2 \beta \Delta t}{(\Delta z)^{2}}\left[\left(D_{T m}\right)_{n}+\left(\Lambda_{c}\right)_{n}\right], \quad b_{3}=0$, $b_{4}=\frac{\beta \Delta t}{(\Delta z)^{2}}\left(k_{e f f}\right)_{n-1}, \quad b_{5}=\frac{\beta \Delta t}{(\Delta z)^{2}}\left(D_{\psi T}\right)_{n-1}, \quad b_{6}=\frac{2 \beta \Delta t}{(\Delta z)^{2}}\left[\left(k_{e f f}\right)_{n}+\left(\Lambda_{d}\right)_{n}\right]$, $G_{1}=\left\{C_{\psi m}-\frac{(1-\beta)}{\beta} a_{5}^{o}\right\} \psi_{n}^{o}+\left\{C_{T m}-\frac{(1-\beta)}{\beta}\left[a_{5}^{o}+a_{6}^{o}\right]\right\} T_{n}^{o}$

$$
\begin{aligned}
& +\frac{\beta \Delta t}{\Delta z}\left\{K_{n}-K_{n-1}+\left(\frac{\Lambda_{c} K}{D_{T m}}\right)_{n}\right\} \\
& +\frac{(1-\beta)}{\beta}\left\{a_{5}^{o} \psi_{n-1}^{o}+a_{4}^{o} T_{n-1}^{o}+a_{6}^{o} T_{B}^{o}+\frac{\beta \Delta t}{\Delta z}\left(K_{n}^{o}-K_{n-1}^{o}+\left(\frac{\Lambda_{c} K}{D_{T m}}\right)_{n}^{o}\right)\right\}
\end{aligned}
$$$$
\begin{aligned}
G_{2}= & \left\{C_{\psi T}-\frac{(1-\beta)}{\beta} b_{5}^{o}\right\} \psi_{n}^{o}+\left\{C_{T T}-\frac{(1-\beta) \Delta t}{(\Delta z)^{2}}\left[b_{4}^{o}+b_{6}^{o}\right]\right\} T_{n}^{o}, \\
& +\frac{(1-\beta)}{\beta}\left\{b_{5}^{o} \psi^{o}+b_{4}^{o} T^{o}+b_{6}^{o} T^{o}\right\}
\end{aligned}
$$

$$
\Lambda_{c}=\left(\frac{-D_{T m} \cdot D_{\psi m}}{K+D_{\psi v}}\right)_{n}, \quad \Lambda_{d}=\left(\frac{-D_{T m} \cdot D_{\psi T}}{K+D_{\psi v}}\right)_{n},
$$

where $K_{n}$ means $K$ at node $n$, and $\left(D_{x x}\right)_{n}$ and $\left(k_{\text {eff }}\right)_{n}$ mean $D_{x x}$ and $k_{\text {eff }}$ of CV $n$ respectively.

The initial conditions are as follows:

1. The temperatures at all nodes are at room temperature $\left(\sim 23^{\circ} \mathrm{C}\right)$

2. The relative humidity $(\varphi)$ of dry soil is the same as that of the lab room $(\sim 23 \%)$

3. The initial moisture content in wet soil can be used to calculate the initial matric potential using van Genuchten's method [12].

B. Solution Method 
For faster convergence, the partial elimination algorithm (PEA) [13] is applied to Eqs. 3, 6, and 7 as follow:

$$
\begin{gathered}
a_{1} \psi_{P}+a_{2} T_{P}=\sum a_{F}^{\psi} \psi_{F}+G_{3} \\
b_{1} \psi_{P}+b_{2} T_{P}=\sum a_{F}^{T} T_{F}+G_{4}
\end{gathered}
$$

where $\sum a_{F}^{\psi} \psi_{F}=a_{3} \psi_{N}+a_{5} \psi_{S}, \quad G_{3}=G_{1}+a_{4} T_{N}+a_{6} T_{S}$

$$
\sum a_{F}^{T} T_{F}=b_{3} T_{N}+b_{5} T_{S}, \quad G_{4}=G_{2}+b_{4} \psi_{N}+b_{6} \psi_{S}
$$

The expressions for $a_{\mathrm{i}}$ and $b_{\mathrm{i}}, G_{1}$ and $G_{2}$ can be derived from Eqs. 3, 6, and 7 for the nodes.

Multiplying Eq. 8a with $b_{2}$ and Eq. 8b with $a_{2}$ and isolating $\psi_{P}$ :

$$
a_{P}^{\psi} \psi_{P}=\sum a_{F}^{\psi} \psi_{F}+B_{P}^{\psi}
$$

where $a_{P}^{\psi}=\frac{a_{1} b_{2}-a_{2} b_{1}}{b_{2}}$ and $B_{P}^{\psi}=G_{3}-\frac{a_{2}}{b_{2}}\left(\sum a_{F}^{T} T_{F}+G_{4}\right)$

Similarly, multiplying Eq. 8a with $b_{1}$ and Eq. $8 \mathrm{~b}$ with $a_{1}$ and isolating $T_{P}$ :

$$
a_{P}^{T} T_{P}=\sum b_{F}^{T} T_{F}+B_{P}^{T}
$$

where $a_{P}^{T}=\frac{a_{1} b_{2}-a_{2} b_{1}}{a_{1}}$ and $B_{P}^{T}=G_{4}-\frac{b_{1}}{a_{1}}\left(\sum a_{F}^{\psi} \psi_{F}+G_{3}\right)$

Since the convection and diffusion coefficients are highly non-linear, an iterative approach is adopted from Moukalled and Saleh [13]:

1. Assume reasonable values for $\psi\left(\psi_{\text {guess }}\right)$ and $T\left(T_{\text {guess }}\right)$

2. Calculate the physical properties of soil using $\psi_{\text {guess }}$ and $T_{\text {guess }}$

3. Calculate the coefficients in Eqs. 3a, 6a, and 7a using $\psi_{\text {guess }}$ and $T_{\text {guess }}$

4. Obtain the coefficients in Eq. 9 using $\psi_{\text {guess }}$ and $T_{\text {guess }}$

5. Using tridiagonal matrix algorithm (TDMA), solve for nodal $\psi$ values $\left(\psi^{i}\right)$ where $i$ means current iteration and

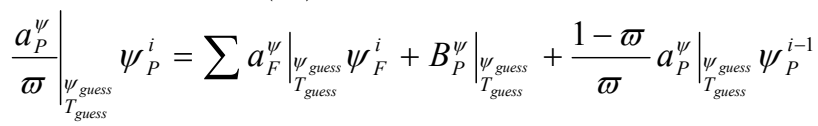

6. Calculate the coefficients in Eqs. $3 \mathrm{~b}, 6 \mathrm{~b}$, and $7 \mathrm{~b}$ using $\psi^{i}$ and $T_{\text {guess }}$

7. Calculate the coefficients in Eq. 10 using $\psi^{i}$ and $T_{\text {guess }}$

8. Using TDMA, solve for nodal $T$ values $\left(T^{i}\right)$ where $i$ means current iteration and

$\left.\frac{a_{P}^{T}}{\varpi}\right|_{\substack{\psi_{\text {guness }}^{i} \\ \text { ing }}} T_{P}^{i}=\left.\sum a_{F}^{T}\right|_{T_{\text {guess }}^{\psi^{i}}} T_{F}^{i}+\left.B_{P}^{T}\right|_{T_{\text {guess }}^{\psi^{i}}}+\left.\frac{1-\varpi}{\varpi} a_{P}^{T}\right|_{T_{\text {guess }}^{\psi^{i}}} T_{P}^{i-1}$

9. Compare $\psi^{i}$ and $T^{i}$ with the corresponding $\psi_{\text {guess }}$ and $T_{\text {guess }}$ as:

$$
\varepsilon_{\psi}=\frac{\psi^{i}-\psi_{\text {guess }}}{\psi_{\text {guess }}} \quad \text { and } \quad \varepsilon_{T}=\frac{T^{i}-T_{\text {guess }}}{T_{\text {guess }}}
$$

10. If $\varepsilon_{\psi}<0.001$ and $\varepsilon_{T}<0.001$, proceed to the next time step. Otherwise, repeat steps $1-9$ with $\psi_{\text {guess }}=\psi^{i}$ and $T_{\text {guess }}=T^{i}$ and $i$ in steps $5-9$ becomes $i+1$ and $i-1$ becomes $i$

\section{Matlab Code Validation}

The computer code is programmed in Matlab. First, it is necessary to perform a grid sensitivity study for deciding the optimum amount of nodes for subsequent numerical study. Figure 4 shows a graph of the percentage root-mean-square differences of temperature $(T)$ and moisture content $(\theta)$ vs. number of nodes at time of 30 minutes with a simulation timestep of $1 s$ in the case of heating top of the soil column to $90^{\circ} \mathrm{C}$ and cooling the bottom to $10^{\circ} \mathrm{C}$. The percentage root-meansquare difference (\% RMS Difference) is defined as:

$$
\frac{\% \text { RMS Difference }}{100 \%}=\sqrt{\sum_{i=1}^{n_{\text {ferer noldes }}}\left(\frac{\Xi_{2+3(i-1) \text {,more nodes }}-\Xi_{i, \text { fwer nodes }}}{n_{\text {fever nodes }}} \Xi_{i, \text { fewer nodes }}\right.}
$$

where $\Xi_{i}$ represents $T_{i}$ or $\theta_{i}$ at node $i$ and $n_{\text {fewer nodes }}$ is the total number of finite volumes or nodes in the case of fewer nodes. The successive increase of nodes is by tripling, i.e. more nodes $=3 \times($ fewer nodes $)$, so that the same height points between the case of fewer nodes and the case of more nodes can be compared directly, i.e., node number of $2+3 \times(i-1)$ in the case of more nodes has the same position as node number $i$ in the case of fewer nodes.

From Fig. 4, it can be seen that, as the number of nodes increases, the successive percentage RMS differences of $T$ and $\theta$ become smaller, which means that the values of $T$ and $\theta$ are converging toward an infinite-node solution. Due to limited computing power, memory and time constraint, it is decided to use 4437 finite volumes for the rest of the numerical study. For this number of finite volumes, the percentage RMS differences of $T$ and $\theta$ between 4000 and 6000 nodes are less than $1.1 \%$, and the size of each finite volume is about $33.3 \mu \mathrm{m}$.

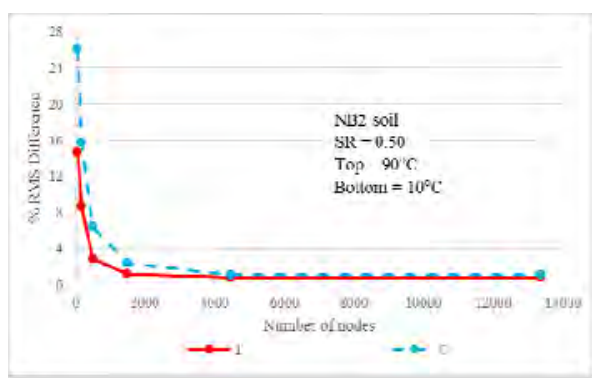

Figure 4. \% RMS differences of $T$ and $\theta v$ s. number of nodes.

The next step is to validate the Matlab code by simulating two cases, namely: only heat transfer and only moisture transfer. For the first case, a soil column (assumed to be semiinfinite) of height of $2 \mathrm{~m}$, density of $2,000 \mathrm{~kg} / \mathrm{m}^{3}$, thermal conductivity of $2.51 \mathrm{~W} / \mathrm{m} \cdot \mathrm{K}$, heat capacity of $837.2 \mathrm{~J} / \mathrm{kg} \cdot \mathrm{K}$, porosity of 0.50 , and initial uniform temperature $293 \mathrm{~K}$ are used. The top surface temperature of the soil is suddenly 
raised to $310 \mathrm{~K}$ (or $37^{\circ} \mathrm{C}$ ) at time $t=0$ and held constant. The numerical simulation is used to predict the temperature distribution in the soil after 1, 4, 9, 16, and 25 hours. The simulation time-step used is $1 \mathrm{~s}$. The results obtained are compared to the analytical solution of transient pure conduction in a semi-infinite wall [A64], assuming the heat flows in the vertical direction only. The derivation of the analytical solution assumes constant thermal properties, so Case 1 is limited to small temperature difference of $\Delta T=310$ $-293=17 \mathrm{~K}$ to reduce the effect of temperature on the thermal properties.

Fig. 5 shows the temperature distributions of the semiinfinite soil column at different times from the numerical model (i.e., Eq. 1) and the analytical solution. As shown in Fig. 5, the numerical solution from the Matlab code matches with the analytical solution.

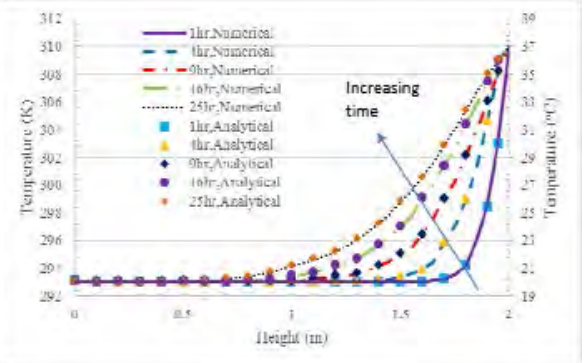

Figure 5. Temperature distributions of semi-infinite soil column at different times from numerical and analytical solutions.

For the second case, a column $(0.4 \mathrm{~m}$ high, assumed semiinfinite) of Yolo light clay soil is used to study the moisture behavior in the soil under isothermal condition at the room temperature. The soil initially is not fully saturated and subjected to an inflow of liquid water at the top surface such that the matric potential at the top surface (i.e., $z=40 \mathrm{~cm}$ ) is always constant at 0 or $-25 \mathrm{~cm}$. The matric potential at the bottom surface (i.e., $z=0 \mathrm{~cm}$ ) is always held constant at -600 $\mathrm{cm}$. Neglecting the vapor and thermal effects, the governing equation in one dimension can be written as [15]:

$$
\left(\frac{\partial \theta}{\partial \psi}\right) \frac{\partial \psi}{\partial t}=\frac{\partial}{\partial z}\left(K \frac{\partial \psi}{\partial z}\right)+\frac{\partial K}{\partial z}
$$

The initial and boundary conditions are: $\psi(0 s, z)=-6 m$, $\psi(t, 0.40 \mathrm{~m})=0 \mathrm{~m}$ or $-0.25 \mathrm{~m}$, and $\psi(t, 0 \mathrm{~m})=-6 \mathrm{~m}$.

Fig. 6 shows the numerical and analytical (from Philip [15]) solutions of moisture distributions at four different times of $10^{3} s(16.7 \mathrm{mins}), 10^{4} s(2.8 \mathrm{hrs}), 4 \times 10^{4} s(11.1 \mathrm{hrs})$ and $10^{5}$ $s(27.8 \mathrm{hrs})$ after moisture is first added at the top surface of the soil. As shown in the figure, when the top surface is fully saturated (i.e., $\psi$ is zero) or has a pool of water (i.e., $\psi$ is positive), more moisture can infiltrate into deeper places of the soil column. In addition, the water slowly infiltrates through the soil as clay is a fine soil which has low moisture permeability. Again, the numerical solution from the Matlab code matches very well with the analytical solution. After the two validations of the code, it is ready to simulate an actual experimental case and compare the results.
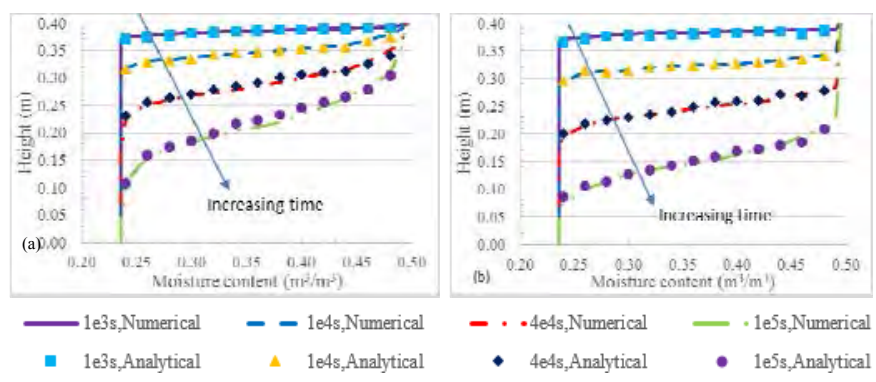

Figure 6. Moisture distributions in Yolo light clay soil column from numerical and analytical solutions for $(a) \psi(t, 40 \mathrm{~cm})=$ $0 \mathrm{~cm}$ and $(\mathrm{b}) \psi(t, 40 \mathrm{~cm})=-25 \mathrm{~cm}$.

\section{VERIFICATION OF EQ. 1}

A comparison between numerical and experimental results is made for a case of vertical soil column with the top of the soil cell heated to $90^{\circ} \mathrm{C}$ and the bottom cooled to $10^{\circ} \mathrm{C}$. The initial saturation ratio (SR) of the soil is 0.50 throughout the soil column. Each heat pulse probe can measure two positions of soil temperatures and moisture contents. Therefore, there are a total of ten positions from top to bottom of the soil column for the five probes; they are named N1 to N10, respectively. Fig. 7 shows the \% Errors (in terms of $T$ and $\theta$ ) for all ten positions over the experiment period of 18 hours. The \% Error is defined as follows:

$$
\% \text { Error }=100 \% \times \frac{(\operatorname{Tor} \theta)_{E q .1}-(T \text { or } \theta)_{\text {experiment }}}{(\operatorname{Tor} \theta)_{\text {experiment }}}
$$

From the figure, it can be seen that the theoretical model (Eq. 1) over-predicts the moisture contents by as much as $6.2 \%$ at $\mathrm{N} 1$ but under-predicts the temperatures by as much as $2.1 \%$ at N1. When the moisture content is over-predicted, the temperature becomes lower as there is more moisture around the region, resulting in higher thermal capacitance of the soil. The top near N1 is the most critical region because it experiences high temperatures and thermal gradients

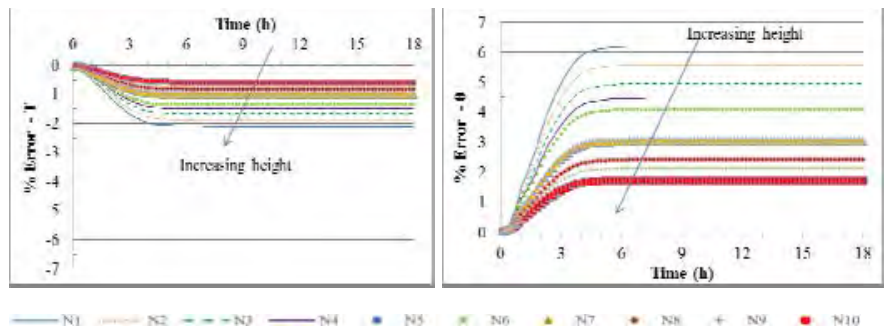

Figure 7. Percentage error of $T$ and $\theta$ (from Eq. 1) for NB2 soil $(S R \approx 0.50)$ and vertically heated from top.

\section{CONCLUSION}

The theoretical governing equations of fully coupled heat and mass transfer in soils by Deru [10] was used to study how the moisture and thermal responses behaved in a soil column containing a silt loam (NB2 soil) with initial moisture at field capacity, i.e. a saturation ratio of 0.5 , and temperature settings of $90^{\circ} \mathrm{C}$ and $10^{\circ} \mathrm{C}$ at top and bottom plates. The onedimensional discretization formulations by the finite volume 
method were developed to numerically solve the governing equations. The numerical analysis was programmed in Matlab and verified to agree well with the existing literature. Running the Matlab code, the results showed good comparisons with the experimental results. The highest percent error appeared to be $6.2 \%$. This is an encouraging preliminary result. Further experimental and numerical studies with different soils, temperature settings and initial moisture contents will be conducted to fully understand the coupled heat and moisture transfer in soils for high-temperature GTES.

\section{NOMENCLATURE}

$C$
$C_{p}$
$C_{T v}, C_{T m}$
$C_{\psi T}, C_{T T}$
$C_{\psi v}, C_{\psi m}$
$D_{v a}$
$D_{T}$
$D_{\theta}$
$D_{T v}, D_{T m}$
$D_{\psi v}, D_{\psi m}$
$D_{\psi T}$
$f(), f$
$g$
$h_{f g}$
$K$
$k$
$\dot{m}$
$P$
$P_{v}$
$r$
$R_{w}$
$S$
$t$
$u$
$T$
$z$

Volumetric heat capacity $\left(\mathrm{J} / \mathrm{m}^{3} \cdot \mathrm{K}\right)$

Specific heat capacity $(\mathrm{J} / \mathrm{kg} \cdot \mathrm{K})$

Thermal vapor and total moisture capacitances $\left(K^{-1}\right)$

Matric $\left(\mathrm{J} / \mathrm{m}^{4}\right)$ and thermal $\left(\mathrm{J} / \mathrm{m}^{3} \cdot \mathrm{K}\right)$ volumetric heat capacitances

Matric vapor and total moisture capacitance terms $\left(\mathrm{m}^{-1}\right)$

Molecular diffusivity of water vapor in air $\left(\mathrm{m}^{2} / \mathrm{s}\right)$

Thermal diffusivity $\left(\mathrm{m}^{2} / \mathrm{s}\right)$

Isothermal moisture diffusivity $\left(\mathrm{m}^{2} / \mathrm{s}\right)$

Thermal vapor and total moisture diffusivities $\left(\mathrm{m}^{2} / \mathrm{s} \cdot \mathrm{K}\right)$

Matric vapor and total moisture diffusivities $\left(\mathrm{m}^{2} / \mathrm{s}\right)$

Matric potential heat diffusivity $\left(\mathrm{W} / \mathrm{m}^{2}\right)$

Correction and interpolation factors

Gravitational acceleration $\left(=9.804 \mathrm{~m} / \mathrm{s}^{2}\right)$

Latent heat of vaporization $(\mathrm{J} / \mathrm{kg})$

Hydraulic conductivity of soil $(\mathrm{m} / \mathrm{s})$

Thermal conductivity $(\mathrm{W} / \mathrm{m} \cdot \mathrm{K})$

Mass flux $\left(\mathrm{kg} / \mathrm{m}^{2} \cdot \mathrm{s}\right)$

Total pressure in pore spaces $(\mathrm{Pa})$

Partial vapor pressure in pore spaces $(\mathrm{Pa})$

Radius $(m)$

Specific gas constant for water vapor $(=461.5 \mathrm{~J} / \mathrm{kg} \cdot \mathrm{K})$

Surface area $\left(m^{2}\right)$

Time $(s)$

Bulk velocity $(\mathrm{m} / \mathrm{s})$

Temperature $\left(K\right.$ or $\left.{ }^{\circ} \mathrm{C}\right)$

Vertical distance $(m)$

\section{Greek symbols}

$\nabla$

$\eta$

$\kappa$

$\mu$

$\psi$

$\rho$

$\sigma_{w a}$

$\tau$

$\theta$

$\Theta$

$\varphi$

$\xi$

$\Xi$

\section{Subscripts}

a

$c$

Convergence criteria

Soil porosity $\left(\mathrm{m}^{3}\right.$ of pore space per $\mathrm{m}^{3}$ of soil)

Permeability of soil $\left(\mathrm{m}^{2}\right)$

Dynamic viscosity $(\mathrm{kg} / \mathrm{m} \cdot \mathrm{s})$

Relaxation factor of iteration

Total soil matric potential for liquid flow $(m)$

Density $\left(\mathrm{kg} / \mathrm{m}^{3}\right)$

Surface tension of water in air $(\mathrm{N} / \mathrm{m})$

Empirical constant used in hydraulic conductivity equation

Volumetric moisture content/fraction $\left(\mathrm{m}^{3}\right.$ of water per $m^{3}$ of soil)

Degree of saturation

Relative humidity

Temperature gradient ratio $\left(=(\nabla T)_{p} /(\nabla T)\right)$

Dummy variable that represents another/other variable(s)

Air

Critical
$C V$
eff
$l$
$p$
$r e f$
$s$
$s a t$
$s v$
$T$
tot
$\psi$
$v$
$w$

\section{Superscripts}

$T$

$i$
Pertaining to control volume

Effective

Liquid

Pore

Reference

Solid

Saturation

Saturated vapor

Pertaining to thermal conditions

Total

Pertaining to moisture conditions

Vapor

Water

Transpose

Current iteration

\section{REFERENCES}

[1] I.-I. Lin, I.F. Pun, C.-C. Lien; "Category-6 Super Typhoon Haiyan in Global Warming Hiatus: Contribution from Subsurface Ocean Warming"; AGU Geophysical Research Letters, pp. 8547 - 8553; 2014.

[2] International Federation of Red Cross and Red Crescent Societies, World Disaster Report, [Ch. 2]; IFRC; 2004.

[3] The Earth Project, Are Fossil Fuels Really Bad for the Environment?, [Online]. Available: http://theearthproject.com/fossil-fuels-badenvironment/. Accessed: 12 Feb. 2018.

[4] Richard Matthews, How to End our Fossil Fuel Addiction by 2050? [Online]. Available: http://www.thegreenmarketoracle.com/2015/03/ infographic-how-to-endfossil- fuels-by.html. Accessed: 12 Feb. 2018.

[5] J. R. Philip, D. A. de Vries, "Moisture Movement in Porous Materials under Temperature Gradients", American Geophysical Union Transaction, vol. 38 (2), pp. $222-232,1957$. doi: 10.1029/TR038i002p00222.

[6] A. V. Luikov, Heat and Mass Transfer in Capillary-Porous Bodies, Advances in Heat Transfer, Ed. T. F. Irvine Jr. and J. P. Hartnett, pp. 123-184, 1964.

[7] B. J. Demsey, "A Mathematical Model for Predicting Coupled Heat and Water Movement in Unsaturated Soil", Int. J. Numer. Anal. Methods Geomech., vol. 2, pp. 19-34, 1978. doi: 10.1002/nag.1610020103.

Matric liquid (capillary) potential (pressure head) $(m)$
[8] S. W. Rees, Z. Zhou, H. R. Thomas, "The Influence of Soil Moisture Content Variations on Heat Losses from Earth-Contact Structures: An Initial Assessment", Building and Environment, vol. 36, pp. 157-165, 2001. doi: 10.1016/S0360-1323(99)00063-3.

[9] H. Janssen, J. Carmeliet, H. Hens, "The Influence of Soil Moisture in the Unsaturated Zone on the Heat Loss from Buildings via the Ground", J. Therm. Envelope Build. Sci. vol. 25, no. 4, pp. 275-298, 2002. doi: $10.1177 / 0075424202025004683$.

[10] M. Deru, "A Model for Ground-Coupled Heat and Moisture Transfer from Buildings," National Renewable Energy Laboratory, 1617 Cole Boulevard, Golden, Colorado, USA, Tech. Rep. NREL/ TP-550-33954, Contract No. DE-AC36-99-GO10337, 2003. 
[11] J. H. Knight, G. J. Kluitenberg, T. Kamai, J. W. Hopman,

"Semianalytical solution for dual-probe heat-pulse applications that accounts for probe radius and heat capacity", Vadose Zone J., vol. 11, no. 2, 2012. doi: 10.2136/vzj2011.0112.

[12] M. T. van Genuchten, "A Close-Form Equation for Predicting the Hydraulic Conductivity of Unsaturated Soils", Soil Sci. Soc. Am. J., vol. 44, pp. $892-898,1980$.

doi: $10.2136 /$ sssaj1980.03615995004400050002x.

[13] F. Moukalled, S. Saleh, "Heat and Mass Transfer in Moist Soil, Part I. Formulation and Testing", Numer. Heat Transfer, Part B, vol. 49, pp. 467-486, 2006. doi: 10.1080/10407790500510866.

[14] F. P. Incropera, D. P. DeWitt, Fundamentals of Heat and Mass Transfer, Wiley, New York, 2002.

[15] J. R. Philip, "The Theory of Infiltration: 1 . The infiltration equation and its solution", Soil Science, vol. 83, pp. 435 - 448; 1957. doi: 10.1097/00010694-195706000-00003. 


\title{
Thermal Management of PQ Transformer for a Passively Cooled Power Module
}

\author{
Anshuman Dey \\ School of Engineering, University of British Columbia \\ Kelowna, Canada \\ anshuman.dey@alumni.ubc.ca
}

\author{
Rahul Khandekar \\ Alpha Technologies Ltd \\ Burnaby, Canada \\ rahul.khandekar@alpha.ca
}

\author{
Navid Shafiei \\ Alpha Technologies Ltd \\ Burnaby, Canada \\ navid.shafiei@alpha.ca
}

\author{
Kevin Lau \\ Alpha Technologies Ltd \\ Burnaby, Canada \\ kevin.lau@alpha.ca
}

\author{
Wilson Eberle, Ri Li \\ School of Engineering, University of British Columbia, \\ Kelowna, Canada
}

\begin{abstract}
In this paper we study the thermal performance of a $5 \mathrm{~kW}$ rectifier module experimentally and numerically, with the aim of changing the cooling methodology from an active cooled (fan cooled) to a passively cooled system. Numerical model of the rectifier for fan cooled operation is developed and experimentally validated, following which the critical components in the system are identified. In this system, magnetic components like transformers were observed to have the poorest thermal performance. Given the lack of attention thermal management of magnetic components has received compared to switching components, we numerically study the thermal performance of a PQ 40/40 transformer in a passive (natural convection) scenario. Modifications to the transformer structure were studied and the heat transfer from the transformer was observed to be convection limited (large convection thermal resistance). Providing a minimum resistance conduction path from effective hot spot to ambient was observed to be the best practice. Further, providing a heat transfer path between the coil and core was observed to be crucial to transformer thermal performance.
\end{abstract}

Keywords - thermal management; CFD; passive cooling; $P Q$ 40/40 transformer;

\section{INTRODUCTION}

Power electronic systems play a vital role in telecommunication, aerospace and various other industries. Modern day power electronic systems generally run at high efficiencies due to the application of innovative conversion topologies. Despite this, a significant amount of heat is dissipated, especially for high power rated electronic systems.

The need for thermal management of electronic systems has been accentuated by both the tendency of components to fail at elevated temperatures and the trend of miniaturization of transistors in switching devices over the years. This is evidenced by the Arrhenius law [1], which states that device failure rates increase exponentially with operating temperature and by Moore's Law which states that the number of transistors in Integrated Circuits (ICs) approximately doubles every two years [2] respectively. This miniaturization of switching components has led to increased heat fluxes due to reduction of area available for heat transfer and increase in power dissipation. Modern day semiconductors are reported to dissipate around $1-100 \mathrm{~W} / \mathrm{cm}^{2}$ of heat [3].

Active cooling methods like fan cooling are the most commonly used cooling methodology for such electronic systems. Although this is a simple method and can handle thermal loads up to $0.04 \mathrm{~W} / \mathrm{cm}^{2}$ [4], it faces some drawbacks. Fan cooled systems generally consume parasitic power [5] and have low reliability. Further, fans produce a lot of noise and cannot be implemented in locations with strict acoustic regulations. The reliability and robustness offered by passive cooling systems has drawn attention, especially in the renewable energy sector for outdoor applications where access for maintenance is limited.

Irrespective of the cooling methodology, providing a system/module level cooling solution would require understanding of component level heat transfer before a foray is made into providing a system level cooling modification. The heat transfer characteristics of switching components like IC's and MOSFETS have been extensively studied by numerous authors [6]-[7] and the thermal bottlenecks have been identified. Magnetic components in such systems has received less attention. Biela and Kolar et al. [8] studied thermal performance of a transformer using a heat transfer component as an insert, Pavlovsky et al. [9] similarly used a conduction based methodology using a heat pipe for heat 
removal from hot spot. Apart from a few studies, most studies on transformer thermal management are limited to large scale oil cooled power transformers [10]-[11].

This study evaluates the thermal management of conventional PQ 40/40 transformers used in power conversion systems to achieve passive cooling.

\section{PROBLEM DEFINITION}

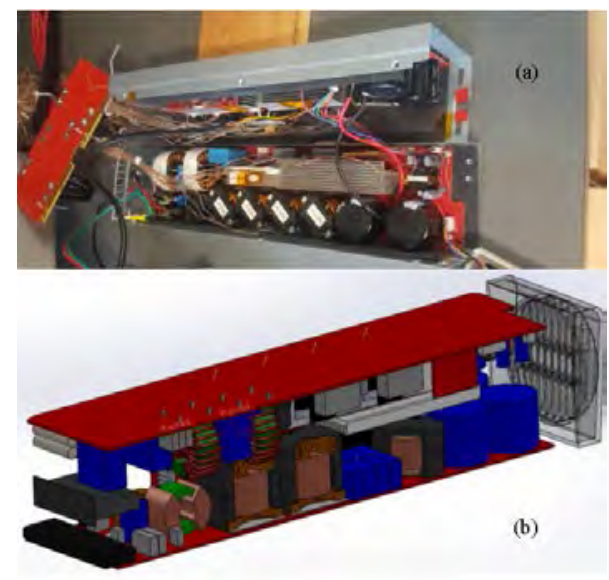

Figure 1. (a) Unbolted module under study. (b) CAD model of the module without chassis to highlight internal details

The objective of our research is to redesign a power module as shown in Fig. 1(a) from an active (fan cooled) to a purely passive design. The system in question is a $5 \mathrm{~kW}$ rated rectifier power module operating at $240 \mathrm{~V}$ AC input and providing $54 \mathrm{~V}$ DC output. The internal geometry of the power module can be visualized in Fig. 1(b) while the unbolted view of the module can be seen in Fig. 1(a).

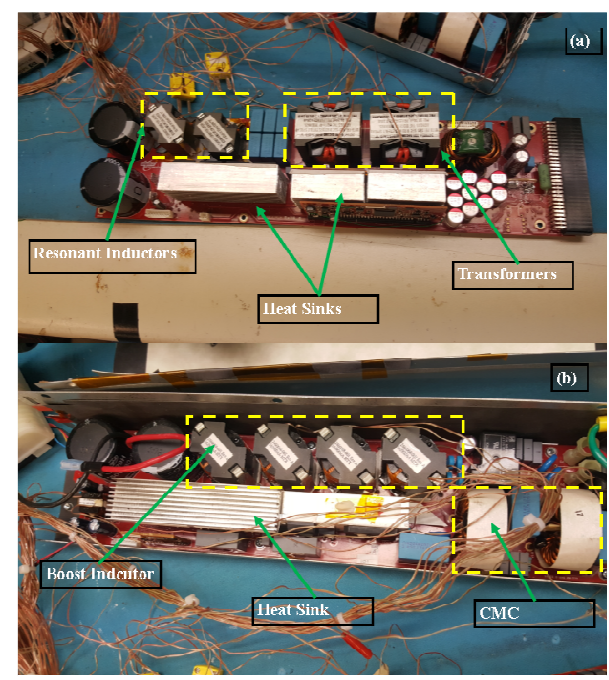

Figure 2. (a) DC-DC converter. (b) PFC converter

This power rectifier module utilizes two distinct topologies, the Power Factor Correction (PFC) converter and the DC-DC converter. The PFC converter provides a modulated signal to the DC-DC converter, which in turn provides the required DC output. The major heat dissipating components on the DC-DC side and PFC side can be seen in Fig. 2(a) and Fig. 2(b) respectively, with the switching components being mounted on the heat sinks

The two converters constituting the power conversion system are fixed facing each other as seen in Fig. 1 (b) with the PFC side on the top and DC-DC on the bottom. The system runs at an efficiency of $95.7 \%$ at full load and dissipates around $202 \mathrm{~W}$. This configuration has been designed for fan cooling, using a 12 VDC rated Delta model AFB0712SHCCM fan.

Now, to realize passive cooling of such a design, we follow the research methodology as described in Fig. 3.

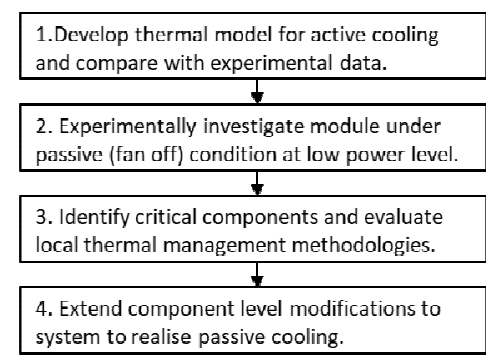

Figure 3: Research Methodology

The first step of our study is to develop a computational model for the current (fan cooled) operation of the system/module and compare with numerical results. This is to help validate our modelling methodology.

Once we have established confidence in our modeling methodology, the module is tested with its fan off at a low power level. This would help us characterize the challenge of passive cooling for such a design and help identify the critical components. Once the critical components are identified, the best practice is to study these components in isolation instead of analyzing their thermal performance in a system/module analysis, Further, if we can locally manage the heat being dissipated from these critical components, the system level thermal managment is greatly simplified and the local themal management methodology can be extended to the sytem level thermal mangement concept.

\section{EXPERIMENTAL SETUP}

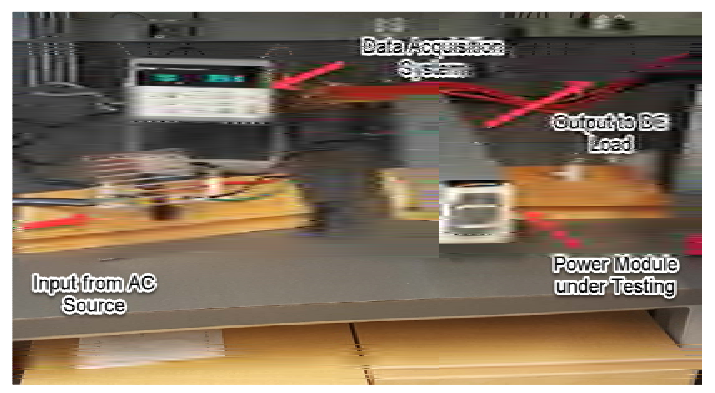

Figure 4: Experimental Setup for module under testing.

The experimental setup can be visualized in Fig. 4. Chroma model 6460 programmable AC source was used to provide a $240 \mathrm{~V}$ AC input to the unit under testing while a Chroma model 63204 DC electronic load was used as the output. The thermal performance of the module was evaluated by attaching $\mathrm{K}$ type 
thermocouples to components that dissipate heat. Keystone model 34970A data acquisition system was used to connect to the thermocouples for datalogging.

The fan cooled experimental analysis of the module was run until the temperature variation was less than $2{ }^{\circ} \mathrm{C}$ within one hour, which was considered as steady state. In this condition the module was run at multiple power levels. The passive (no fan) thermal testing on the other hand was done at a low power level of $0.5 \mathrm{~kW}(10 \%$ full load) as the module is designed for active (fan) cooling and the thermal performance can be expected to be poor. Further, the real time thermocouple data was closely monitored and the system was shut off when component temperatures were close to their safe operating threshold temperature.

\section{NUMERICAL ANALYSIS}

This section discusses the numerical methodology followed by this study. This section will be divided into two parts. The first discusses the analysis methodology for active cooling (with fan) of the module (system level simulation) while the other discusses the analysis methodology of an isolated PQ 40/40 transformer (component level) in a natural convection scenario. All simulation results are for steady state.

\section{A. Active Cooling of Power Module}

The computational domain under study can be seen in Fig. 5. The numerical modelling and simulation is carried out using commercially available CFD software ANSYS Icepack. The dimensions of the domain are $0.08 \mathrm{~m}, 0.07596 \mathrm{~m}, 0.37935 \mathrm{~m}$ in the $x, y$ and $z$ direction respectively. The module is enclosed by an aluminum chassis. The fan can be seen in Fig. 5 and the flow rate depends on the fan curve, which is provided as an input to the solver. The other face highlighted in dark blue in Fig. 5 is open to the ambient.

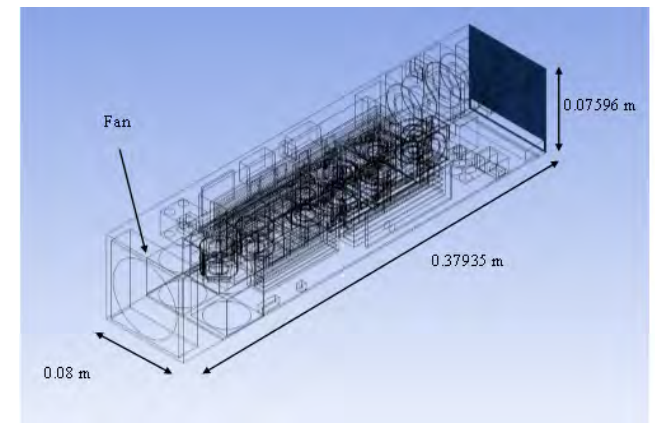

Figure 5: Active cooling computational domain, face open to ambient is highlighted in dark blue.

Convective boundary condition of $5 \mathrm{~W} / \mathrm{m}^{2} \cdot \mathrm{K}$ is applied to the external walls of the chassis, while the outlet (highlighted in Fig. 5) is open to ambient pressure (1 atm) and temperature $\left(2{ }^{\circ} \mathrm{C}\right)$. RNG $k-\varepsilon$ turbulence model is used to model the turbulent nature of the flow. No slip boundary condition is applied at solid-fluid interfaces.

A control volume method was used to discretize the domain and a semi-implicit method for pressure linked equation (SIMPLE) method was used for pressure-velocity coupling.
The discrete algebraic equations are solved by a point implicit (Gauss Seidel) method in conjugation with an Algebraic Multigrid (AMG) method. This helps reduce the computational time required for each simulation. The conservation equations being solved for are (1), (2) and (3) in the fluid domain and (3) in the solid domain (reduces to simple conduction equation).

$$
\begin{gathered}
\frac{\partial \rho}{\partial t}+\nabla(\rho \vec{v})=0 \\
\frac{\partial}{\partial t}(\rho \vec{v})+\nabla(\rho \vec{v} \vec{v})=-\nabla p+\nabla(\bar{\tau})+\rho \vec{g}+\vec{F} \\
\frac{\partial}{\partial t}(\rho h)+\nabla(\rho h \vec{v})=\nabla\left[\left(k+k_{\mathrm{t}}\right) \nabla T\right]+S_{h}
\end{gathered}
$$

Here, $\rho$ is density, $\overrightarrow{\mathrm{v}}$ is velocity vector, $\mathrm{P}$ is pressure, $\overline{\bar{\tau}}$ is the stress tensor, $\vec{F}$ is the source term, $h$ is enthalpy, $k$ is the molecular thermal conductivity, $\mathrm{k}_{\mathrm{t}}$ is the conductivity due to turbulent transport, $\mathrm{T}$ is temperature and $\mathrm{S}_{\mathrm{h}}$ is the source term like volumetric heat generation.

The convergence criteria is fixed at $10^{-4}$ for velocity and $10^{-7}$ for energy. As for the mesh, unstructured tetrahedral cells are generated by in house meshing software Mesher-HD. The mesh density is higher near the surface of solids to ensure resolution of boundary layer. The total mesh count is around 6531290 cells.

\section{B. Isolated $P Q 40 / 40$ transformer}

The computational domain used for this analysis can be seen in Fig. 6. The dimensions are $0.088 \mathrm{~m}, 0.053616 \mathrm{~m}, 0.2 \mathrm{~m}$ in the $x, y$ and $z$ directions respectively. The dimensions of the transformer itself can be readily found in manufacturer datasheets [12]. The transformer is mounted on a PCB (highlighted in yellow) and enclosed in an aluminum chassis. The domain is set such that it is representative of the small form factor characteristic of such problems.

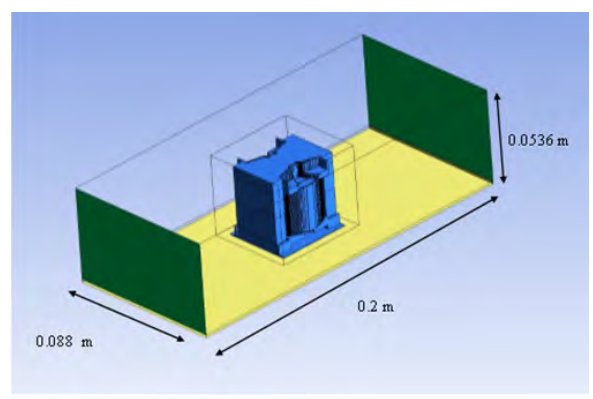

Figure 6: Computational domain for isolated PQ transformer under study, faces open to ambient highlighted in green.

A convective boundary condition of $5 \mathrm{~W} / \mathrm{m}^{2} \cdot \mathrm{K}$ is applied to the external walls of the chassis. The other two faces, as highlighted in green, are open to ambient. One of the key assumptions made in modelling such a transformer, is that the windings are approximated/modelled as a foil.

The same conservation equations and solvers have been used as the previous active cooling analysis. The only change is that, in this case the flow is entirely laminar and no turbulence model is employed. But to characterize natural convection, the Bossinesq approximation is used. The convergence criteria is 
again set as $10^{-4}$ for velocity and $10^{-7}$ for energy. The mesh element count is around 1841815 cells.

\section{RESULTS}

The organization of this section will follow the research methodology as described in Fig. 3.

\section{A. Active cooling: experimental vs numerical}

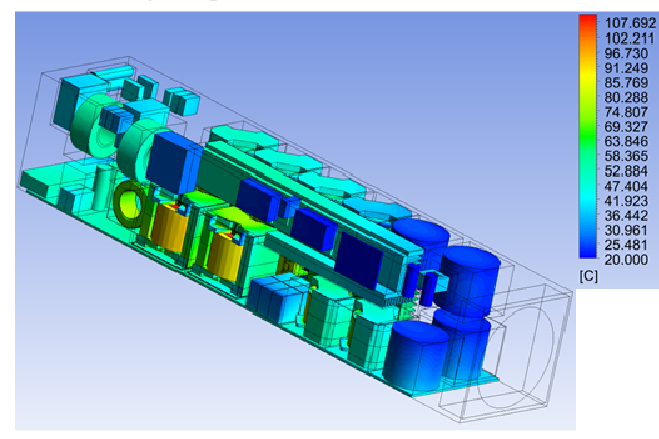

Figure 7: Temperature contour for active (fan cooled) operation of power module at $4.5 \mathrm{~kW}$. The thermal contours of the top PCB is not shown.

The experimental and numerical test was run for multiple power levels. Fig. 7 shows the global temperature contours for the module at a power level of $4.5 \mathrm{~kW}$. Table. 1 shows the comparison between the experimental and numerical results at $4.5 \mathrm{~kW}$. The model can predict temperature within $10-15 \%$ of its experimental values. The results for other power levels were excluded for the sake of brevity. Given the complexity of the problem, we were satisfied with our modelling methodology and progressed to the next step of the analysis.

Table 1: Comparison of experimental vs numerical results for a few components at $4.5 \mathrm{~kW}$ for active cooling condition.

\begin{tabular}{|l|l|l|l|}
\hline Component & $\begin{array}{l}\text { Experimental } \\
\left({ }^{\circ} \mathbf{C}\right)\end{array}$ & $\begin{array}{l}\text { Numerical } \\
\left({ }^{\circ} \mathbf{C}\right)\end{array}$ & Error \\
\hline Transformer winding inside $\left(1^{\text {st }}\right)$ & 112 & 107 & -4.46 \\
\hline Transformer Core & 56 & 53 & -5.35 \\
\hline Primary MOSFET & 50 & 55 & 10 \\
\hline Secondary MOSFET & 57 & 62 & 8.77 \\
\hline Resonant inductor inside & 93 & 85 & -8.6 \\
\hline Resonant capacitor & 44 & 40 & -9 \\
\hline
\end{tabular}

\section{B. Experimental passive cooling $(0.5 \mathrm{~kW})$}

Fig. 8 shows the temperature versus time plot for passive (fan off) testing of the module at $0.5 \mathrm{~kW}$. From Fig. 8 we can see that the curve has not yet reached steady state as the test was shut off as specified in the previous sections. The components with the poorest thermal performance are the transformers and the MOSFETs on the DC-DC side. The components on the PFC side of the rectifier show relatively good thermal performance. The temperature within the primary windings of the first transformer (first from the end open to ambient) is the global hot spot in this system. Given the slope of the curve we can assume that the system would have failed if run for longer. The test is clearly indictive of the transformer being the most critical component in this system.

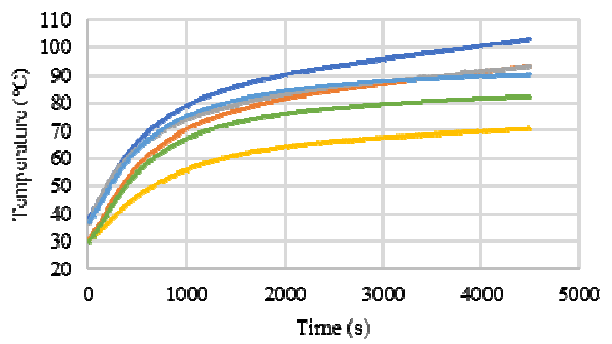

$$
\begin{array}{ll}
\text { - lst Transformer primary winding } & \text { - lst Transformer secondary winding } \\
\text {-Transformer core (1st) } & \text { Secondary MOSFET } \\
\text {-2nd Transformer primary winding } & \text {-2nd Transformer secodary winding }
\end{array}
$$

Figure 8: Thermocouple data for fan off testing condition at $0.5 \mathrm{~kW}$. Only critical components are shown.

\section{Isolated $P Q 40 / 40$ transfomer (Component Level)}

Now that we have established that the transformer is the most critical component in a passive (fan off scenario). We study the results of our component level thermal analysis.

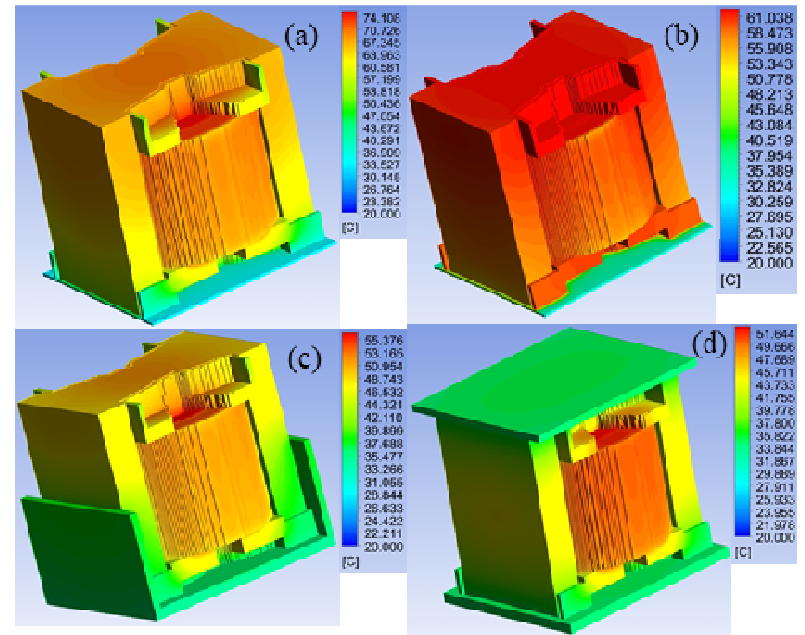

Figure 9. (a) Thermal contour of conventional PQ transformer (5 W loss). (b) Thermal contour of transformer with internal modification. (c) Thermal contour of transformer with external modification 1 (U clamp). (d) Thermal contour of transformer with external modification 2 (double sided cooling).

Fig. 9(a) shows the thermal contour of a conventional PQ 40/40 transformer dissipating a total of $5 \mathrm{~W}$ (4 W copper loss and $1 \mathrm{~W}$ iron loss). We can observe that the hot spot is predicted to be inside the primary winding. Further we observe a large temperature gradient between the internal structure of the transformer and the core side leg. This is indicative of a large internal thermal resistance.

Now, let us highlight the modifications and their corresponding effects on thermal performance.

1) Internal Modification : Change of bobbin material from thermosetting plastic to Ceramic (ALN).

The role of bobbin in a transformer is to provide mechanical support to the winding structure whilst also providing electrical insulation. Bobbins are generally made 
from plastics like Polytetrafluoroethylene (PTFE) which are characteristic of a low thermal conductivity $(k \sim 0.25 \mathrm{~W} / \mathrm{m} \cdot \mathrm{K})$. Ceramic materials like Aluminum Nitride (ALN) has similar electrical insulating characteristics as plastics (insulation of around $15 \mathrm{kV} / \mathrm{mm}$ ), while possessing superior thermal characteristics $(k \sim 150 \mathrm{~W} / \mathrm{mK})$. The first modification we study is changing the bobbin material from plastic (PTFE) to ALN. Fig. 9 (b) shows the temperature contour for this modification, we can see that heat spreading is greatly improved and the transformer almost behaves like a lumped thermal mass. The hot spot is also reduced by around $13{ }^{\circ} \mathrm{C}$. An interesting property of ferrite cores (3C96 in this case) is lower iron loss and better thermal performance at elevated temperatures (around $100{ }^{\circ} \mathrm{C}$ ). Hence, providing a heat transfer path between the core and windings is beneficial not just to thermal performance but also the magnetic performance of the core.

2) External Modification 1: U clamp made of ceramic, bonded to the bottom of the transformer to provide a conduction path to the chassis of the module. The PCB layer is cut through to provide a direct path to the chassis

Liu et al. [13] observed that providing ceramic inserts within the ferrite core is beneficial to heat transfer in a natural conduction scenario. We similarly extended this finding to our case by bonding a U-clamp (base plate: $0.038 \mathrm{~m} * 0.0445$ $\mathrm{m} * 0.002 \mathrm{~m}$, side legs: $0.038 \mathrm{~m} * 0.021076 \mathrm{~m} * 0.001 \mathrm{~m})$ to the bottom of the transformer and connecting it directly to the chassis by cutting through the PCB layer. The thermal benefit of such a modification can be seen in Fig. 9(c), we can observe a reduction in hot spot temperature of around $20{ }^{\circ} \mathrm{C}$. Due to the small factor characteristic of such transformers and low heat transfer coefficient (HTC) in natural convection scenario (around $10 \mathrm{~W} / \mathrm{m}^{2} \cdot \mathrm{K}$ for the core side leg,) the convective resistance to air within the module is very high and providing a purely conductive heat transfer path to the ambient is greatly beneficial to heat transfer.

3) External Modification 2: Double sided cooling to provide a conduction path from the transfomer to the chassis on both top and bottom sides.

In this modification, we provide a ceramic interface $(0.038$ $\mathrm{m} * 0.0445 \mathrm{~m} * 2 \mathrm{~m}$ ) between both the bottom and top surface of the transformer and the chassis. Generally, most electronic packaging methodologies have a large air gap between the components and the top of the chassis. This air gap is not very beneficial to heat transfer in a natural convection scenario (low HTC, around $5 \mathrm{~W} / \mathrm{m}^{2} \cdot \mathrm{K}$ ). We circumvent this by directly bonding it the top of the transformer to a ceramic interface. This modification provides a conduction path to the ambient in two directions. The benefit of this modification can be seen in Fig. 9 (d), with a reduction in hot spot temperature of around $24{ }^{\circ} \mathrm{C}$. This holds good with our finding that the heat transfer for a PQ transformer in a natural convection scenario is convection limited and providing a minimum resistance conduction path to the ambient is the best practice for thermal management.

Although the modifications we study are an ideal case, as there is no conjugate heat transfer between different components and there is a large dedicated chassis area for heat dissipation to ambient, we can clearly see that providing a minimum resistance path from the transformer to the chassis is the best thermal management practice to realize passive cooling.

\section{CONCLUSION}

In this study, experimental and numerical analysis was carried out for a $5 \mathrm{~kW}$ rectifier power module. Critical components in this system were identified to be magnetic components like transformers. Numerical model for the fan cooled operation was validated with experimental results. Further, numerical model of a PQ 40/40 transformer was developed and studied from a passive cooling point of view. The heat transfer was observed to be convection limited and providing a minimum resistance conduction path to ambient was observed to be the best practice to realize passive cooling. Modifications of the bobbin material was also studied and the importance of providing a heat transfer path between the core and coil was observed to be beneficial for both heat transfer and general operation of the core. The effect of these changes is yet to be evaluated experimentally.

\section{REFERENCES}

[1] US MIL-HDBK 217F, Military Handbook: Reliability Prediction of Electronic Equipment, U.S. Dept. of Defense, Washington, D.C., Dec. 1991.

[2] G. E. Moore, "Cramming more components onto integrated circuits," Proc. IEEE, vol. 86, no. 1, pp. 82-85, Jan. 1998. doi: 10.1109/JPROC.1998.658762

[3] M. Marz, "Thermal management in high-density power converters," in IEEE International Conference on Industrial Technology, Maribor, SI, 2003, pp. 1196-1201. doi: 10.1109/ICIT.2003.1290835

[4] I. Mudawar, "Assessment of high-heat-flux thermal management schemes," IEEE Trans. Compon. Packag. Technol., vol. 24, no. 2, pp. 122-141, June 2001. doi: 10.1109/6144.926375

[5] D. C. Kilper et al., "Power trends in communication networks," IEEE J. Sel. Top. Quantum Electron., vol. 17, no. 2, pp. 275-284, Mar.-Apr. 2011. doi: 10.1109/JSTQE.2010.2074187

[6] S. Moghaddam, M. Rada, A. Shooshtari, M. Ohadi and Y. Joshi, "Evaluation of analytical models for thermal analysis and design of electronic packages," Microelectron. J., vol. 34, no. 3, pp. 223-230, Mar. 2003. doi: 10.1016/S0026-2692(02)00192-1

[7] X. Luo, Z. Mao, S. Liu and J. Liu, "An analytical thermal resistance model for calculating mean die temperature of a typical BGA packaging," Thermochim. Acta, vol. 512, no. 1-2, pp. 208-216, Jan. 2011. doi: 10.1016/j.tca.2010.10.009

[8] J. Biela and J. W. Kolar, "Cooling concepts for high power density magnetic devices," in 2007 Power Conversion Conference, Nagoya, JP, 2007, pp. 1-8. doi: 10.1109/PCCON.2007.372915

[9] M. Pavlovsky, de Haan, S. W. H and J.A. Ferreira, "High-performance thermal management for high-power high-frequency transformers," EPE Journal, vol. 19, no. 2, pp. 20-27, June 2009. doi: 10.1080/09398368.2009.11463713

[10] G. Swift, T.S. Molinski and W. Lehn, "A fundamental approach to transformer thermal modeling. I. Theory and equivalent circuit," IEEE Trans. Power Delivery, vol. 16, no. 2, pp. 171-175, Apr. 2001. doi: $10.1109 / 61.915478$

[11] A. Skillen, A. Revell, H. Iacovides and W. Wu, "Numerical prediction of local hot-spot phenomena in transformer windings," Appl. Therm. Eng., vol. 36, pp. 96-105, Apr. 2012. doi: 10.1016/j.applthermaleng.2011.11.054 
[12] TDK. (2009). Ferrites and Accessories [Online]. Available: https://en.tdk.eu/inf/80/ds/b65883a.pdf

[13] W. Liu, J. Dirker, and J.D. van Wyk, "Power density improvement in integrated electromagnetic passive modules with embedded heat extractors," IEEE Trans. Power Electron, vol. 23, no. 6, pp. 3142-3150, Nov. 2008. doi: 10.1109/TPEL.2008.2005367 


\title{
Analytical heat conduction model of annular composite fins
}

\author{
Chen Feng, Subramaniam Yugeswaran, Michael Gibbons, Sanjeev Chandra \\ Mechanical and Industrial Engineering, \\ University of Toronto \\ Toronto, ON, Canada
}

\begin{abstract}
A two-dimensional analytical heat conduction model of an annular composite fin has been carried out. The composite fins composed of a porous polyethylene core, a square aluminum insert, and metallic zinc coating layers, was fabricated using wire-arc spraying technology. Analytical solutions of temperature distribution, energy dissipation and fin efficiency through the fins at natural convection condition have been proposed.
\end{abstract}

Keywords - composite fins; heat conduction; analytical model.

\section{INTRODUCTION}

Composite fins are used in industrial applications such as heat exchangers and heat sinks [1]. In such composite fins the thermal conductivities of the coating $\left(k_{c}\right)$ and substrate $\left(k_{s}\right)$ materials differ and the total heat transfer depends on the thermal conductivity ratio $\left(k_{c} / k_{s}\right)$. If this ratio is less than unity the coating material is less conductive than the substrate and insulates the fin, whereas a ratio greater than unity means that the coating enhances heat transfer.

Cortes [2] developed analytical solutions for heat transfer in composite fins with variable thickness varying the $k_{c} / k_{s}$ from 0.04 to 13.6 , which reflects combinations of metals commonly used in practical applications. Luna-Abad and Alhama [3] optimized the dimensions of composite rectangular fins while varying thermal conductivity ratios and convective heat transfer coefficients. Tu [4] derived analytical solutions for the efficiency of composite fins made of a metallic core and a coating with lower thermal conductivity.

In this paper we carry out an analytical model of heat conduction through an annular composite fin, consisting of a polymer substrate on which a thin metal coating was applied by wire arc spraying to enhance heat conduction. This model was developed assuming a two-dimensional conduction in the thicker polymer core and one-dimensional conduction in the thin coating layer because of their transverse thermal resistances. The model was validated by comparing predicted temperature variations with experimental measurements. The model was used to examine the effect of varying coating thickness and fin dimensions on heat dissipation and efficiency of the fins.

\section{FABRICATION AND EXPERIMENTAL SETUP}

\section{A. Fabrication of composite fins}

Wire-arc spraying was used in this study, in which an electric arc is struck between the tips of two continuously fed wires to melt them. A high velocity air jet strips the molten metal from the wires and creates a spray of droplets that impact on the substrate. This was used to coat a polyethylene sheet with zinc, which has a relatively low melting point $\left(420^{\circ} \mathrm{C}\right)$ and did not damage the substrate during coating.

A composite fin was made by laser cutting a $148 \mathrm{~mm}$ diameter disk from a $3.2 \mathrm{~mm}$ thick sheet of polyethylene. An aluminum block, $3.2 \mathrm{~mm}$ thick and $22 \mathrm{~mm}$ square was inserted into the center of the disk as shown in Fig. 1a and bonded to it using thermally conductive epoxy (DP270, 3M, St. Paul, USA). The relatively high thermal conductivity of the aluminum $(200 \mathrm{~W} / \mathrm{mK})$ ensured a uniform temperature that was used as a boundary condition in the analytical model. The model assumed radial symmetry and the fin was assumed to be an annulus with an internal radius of $11 \mathrm{~mm}$ and an outer radius of $74 \mathrm{~mm}$.

An electric wire arc spray system (ValuArc, Sulzer Metro Inc., Westbury, NY), mounted on a robot arm, was used to melt and spray high purity zinc wires (Catalog\#

1031592, Sulzer Metro Inc., Westbury, NY) onto the disk and form thin, dense zinc layers on both sides. The average coating thickness was kept at $400 \mu \mathrm{m}$. The thermal conductivity of the polyethylene $\left(k_{1}\right)$ was $0.3 \mathrm{~W} / \mathrm{mK}$ and that of the zinc $\left(k_{2}\right)$ was $60(\mathrm{~W} / \mathrm{mK})$. The final composite fin after coating is shown in Fig. 1b.

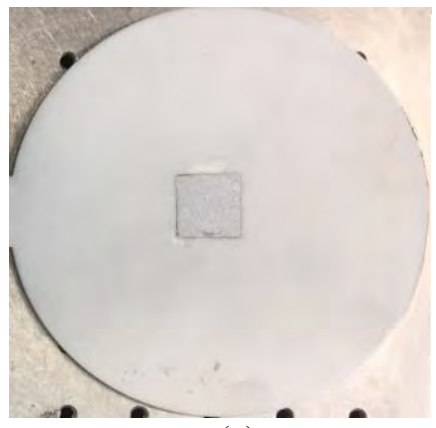

(a)

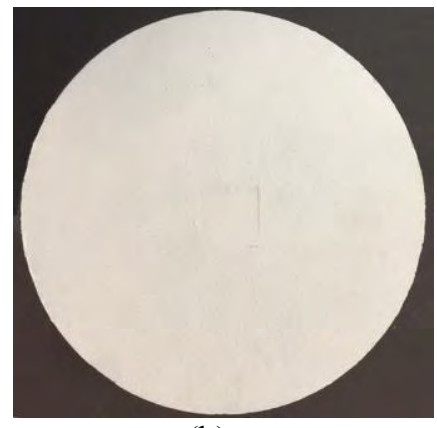

(b)
Figure 1. Pictures of the annular composite fin, (a) before spraying, and (b) after spraying. 


\section{B. Experimental setup}

The centre of the fin was heated using a flexible, silicone rubber heater, $23 \mathrm{~mm}$ square, (35765K126, McMaster Carr, Santa Fe Springs, California, USA) attached to the aluminum insert using thermal tape (TAPE-T404, Aavid Thermalloy, San Jose, California, USA). The other side of the heater was insulated using fiber glass insulation so that the heat lost from it was negligible. Moreover, the temperature variation on the fin surfaces was measured using an infra-red camera. The fin was mounted horizontally with an infrared camera (ThermaCAM SC 5000, FLIR, USA) pointing downwards to record the temperature of the upper surface.

The power supplied to the heart was varied to $1.6,2.5,4.0$ and $9.0 \mathrm{~W}$, and the fin allowed to come to steady state which took approximately $30 \mathrm{~min}$, and the temperature on the top fin surface recorded.

\section{ANALYTICAL MODELING}

The composite fin was circular with a rectangular crosssection, and the geometry can be simplified for purposes of modelling as shown in Fig. 2. The fin was constructed as a sandwich structure with a polymer core with thickness $2 H$, an outer radius $r_{0}$ and inner radius $x_{1}$. Heat conduction in the twodimensional polymer core is governed by the following partial differential equation,

$$
\frac{\partial^{2} T_{1}}{\partial r^{2}}+\frac{1}{r} \frac{\partial T_{1}}{\partial r}+\frac{\partial^{2} T_{1}}{\partial y^{2}}=0
$$

Defining the following non-dimensional variable,

$$
r^{*}=\frac{\gamma-T_{i}}{L} ; y^{*}=\frac{y}{H} ; \quad \theta_{1}=\frac{T_{1}-T_{\text {mir }}}{T_{b}-T_{\text {mir }}} ; \quad \theta_{2}=\frac{T_{2}-T_{\text {mîr }}}{T_{b}-T_{\text {mîr }}}
$$

Where $\mathrm{L}$ is the characteristic length of the fin, defined as $r_{0}-r_{\mathrm{l}}, T_{\mathrm{b}}$ is the wall temperature of the aluminum insert and $T_{\text {air }}$ is the ambient air temperature, and $T_{1}$ and $T_{2}$ the temperature distributions in the polymer core and coating respectively. Substituting these dimensionless variable into equation (1) gives,

$$
\frac{\partial^{2} \theta_{1}}{\partial y^{2}}+\frac{L}{y} \frac{\partial \theta_{1}}{\partial y^{2}}+\frac{z^{2}}{H^{2}} \frac{\partial^{2} \theta_{1}}{\partial y^{2}}=0
$$

The following boundary conditions can be applied for the core,

$$
\begin{gathered}
r^{*}=0, \theta_{1}=1 ; \quad r^{*}=1, \frac{\partial \theta_{1}}{\partial r^{*}}=0 \\
y^{*}=0, \frac{\partial \theta_{1}}{\partial y^{*}}=0 \quad ; \quad y^{*}=1, \frac{\partial \theta_{1}}{\partial y^{*}}=\frac{k_{2}}{k_{1}} \frac{\partial \theta_{2}}{\partial y^{*}}
\end{gathered}
$$

Where $k_{1}$ and $k_{2}$ are thermal conductivity of the polymer core and the metallic coating layers, respectively. h, $\mathbf{T}_{\text {air }}$

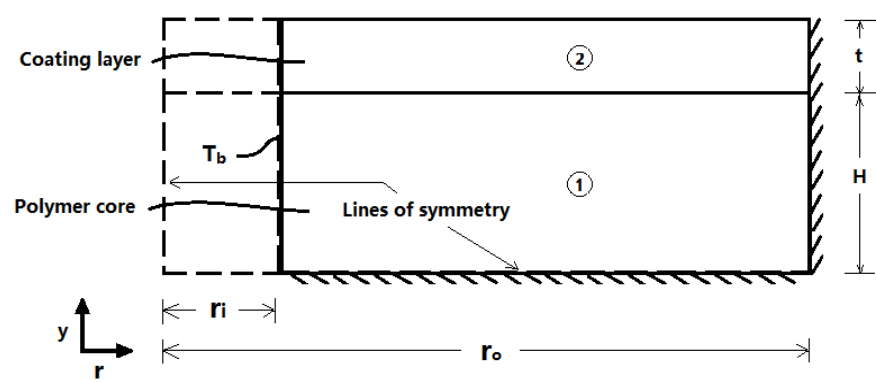

Figure 2. Analytical heat conduction model of the composite fin consisting of two domains.

Since heat transfer in the coating is assumed to onedimensional, with a temperature gradient only in the radial direction [5], an energy balance gives,

$k_{2} t\left(\frac{\partial^{2} T_{2}}{\partial r^{2}}+\frac{1}{r} \frac{\partial T_{2}}{\partial r}\right)-h\left(T_{2}-T_{\text {äY }}\right)-\varepsilon \sigma\left(T_{2}^{4}-T_{\text {QUY }}{ }^{4}\right)=\left.k_{1} \frac{\partial T_{1}}{\partial y}\right|_{y=H}$

The three terms on the left-hand side of equation (3) represent heat conduction, natural convection, and radiation in heat transfer, respectively. The term $T_{2}^{4}$ can be expanded in a Taylor series [6], and the new expression substitute into equation (3) gives,

$$
k_{2} t\left(\frac{\partial^{2} T_{2}}{\partial r^{2}}+\frac{1}{r} \frac{\partial T_{2}}{\partial r}\right)-h_{e}\left(T_{2}-T_{a i r}\right)=\left.k_{1} \frac{\partial T_{1}}{\partial y}\right|_{y=H}
$$

Where $h_{e}=h+4 T_{\text {air }}{ }^{a} \varepsilon \sigma$ is an effective heat transfer coefficient that combines convection and radiation effects. Non-dimensionalizing equation (4) gives,

$$
k_{2} t\left(\frac{\partial^{2} \theta_{2}}{\partial y^{2}}+\frac{L}{r} \frac{\partial \theta_{2}}{\partial y^{m}}\right)-L^{2} h \theta_{2}-\left.\frac{L^{2}}{H} k_{1} \frac{\partial \theta_{1}}{\partial y^{y}}\right|_{y^{n}=1}=0
$$

With the boundary conditions,

$$
r^{*}=0, \theta_{2}=1 \quad ; \quad r^{*}=1, \frac{\partial \theta_{2}}{\partial \mathrm{r}^{\omega}}=0
$$

Using separation of variables by assuming that we can write $\theta_{1}$ as a product of two variable $R(r)$ and $Y(y)$ so that $\theta_{1}\left(r^{*}{ }_{0} y^{*}\right)=R\left(r^{*}\right) Y\left(y^{*}\right)$ and substituting in equation (2), gives two equations

$$
\begin{gathered}
Y^{s}+m^{2} Y=0 \\
R^{m}+\frac{L}{\gamma} R^{s}-\frac{m^{2} L^{2}}{H^{2}} \mathrm{R}=0
\end{gathered}
$$

Where $m$ is an arbitrary separation constant. The general solutions of equation (6) and (7) are given [7],

$$
Y=c_{1} \cos \left(m y^{*}\right)+c_{2} \sin \left(m y^{*}\right)
$$




$$
R=c_{3} I_{0}\left(\frac{m L}{H} r^{*}+\frac{m}{H} \gamma_{\mathrm{i}}\right)+c_{4} K_{0}\left(\frac{m L}{H} r^{*}+\frac{m}{H} \gamma_{i}\right)
$$

Where $I_{n}(x)$ and $K_{n}(x)$ are the solutions to the modified Bessel differential function of the first and second kinds [8], [9]. At the interface between the core and the coating $(y=H)$ the temperatures in the core and coating are equal $\left(\theta_{1}\left(r^{*}, 1\right)=\right.$ $\left.\theta_{2}\left(r^{*}\right)\right)$. Substituting $\theta_{2}\left(r^{*}\right)=R\left(r^{*}\right) Y(1)$ into equation (5), we obtain,

$$
Y^{v}+\frac{H^{2} \hat{n}_{-}-k_{2} t m^{2}}{k_{1} H} Y=0
$$

The boundary condition of $y^{*}=0, Y^{s}=0$ gives $c_{2}=0$ in equation (8). Substituting $Y=c_{1} \cos \left(m y^{*}\right)$ into equation (10), we obtain,

$$
\tan (m)=\frac{H^{2} \hbar_{-}-k_{2} t m^{2}}{k_{1} H m}
$$

In our experiment $m$ is in a range of 0.028 to 0.032 , so we can use the approximation that for small $m$, $\tan (m) \approx m$ and introducing the fin parameter,

$$
M=\sqrt{\frac{\hbar}{k_{1} H+k_{2} t}}
$$

gives

$$
m=M H
$$

The coefficients $c_{3}$ and $c_{4}$ in equation (9) can be found by applying the boundary condition at $r^{*}=1_{3} \frac{\partial \theta_{1}}{\partial r^{*}}=0$. Then,

$$
c_{4}=\frac{I_{1}\left(M r_{0}\right)}{K_{1}\left(M r_{T_{Q}}\right)} c_{3}
$$

Combining the solutions for $R\left(r^{*}\right)$ and $Y\left(y^{*}\right)$ gives,

$$
\begin{aligned}
& \theta_{1}\left(r^{*}, y^{*}\right)=c_{5}\left[I_{0}\left(M L r^{*}+M r_{1}\right)+\right. \\
& \left.\frac{I_{1}\left(M r_{0}\right) K_{0}\left(M L r^{*}+M r_{1}\right)}{K_{1} \backslash\left(M r_{0}\right)}\right] \cos \left(M H y^{*}\right)
\end{aligned}
$$

Where the only unknown parameter, $c_{5}$, can be obtained from the boundary condition that at $r^{*}=0, \theta_{1}=1$.

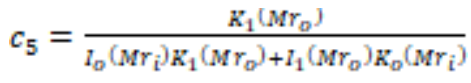

Then,

$$
\begin{aligned}
& \theta_{1}\left(r^{*}, y^{*}\right)=
\end{aligned}
$$

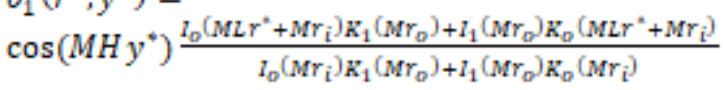

The radial temperatures of the fin core and coating must be the same at their interface so that $\theta_{2}(r)=\theta_{1}\left(r_{v} H\right)$. The radial variation of $\theta_{2}$ is, therefore,

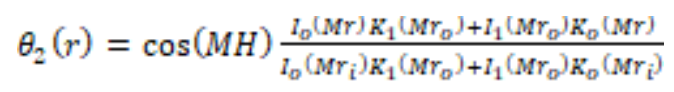

And

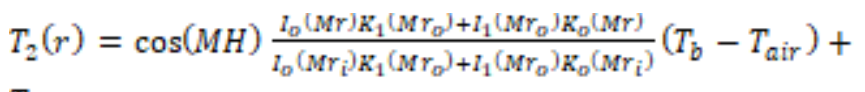
$T_{\text {air }}$

The heat conduction through the fin can be derived from,

$$
Q=\left.k A \frac{d r_{2}}{d y}\right|_{r=r_{i}}
$$

Where $A$ is the fin cross-section area at $r=\gamma_{\mathbb{i}}$. Evaluating equation (17) gives,

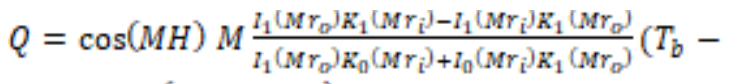

$$
\begin{aligned}
& \left.T_{\text {Lir }}\right) \cdot 2 \pi r_{1}\left(k_{1} H+k_{2} t\right)
\end{aligned}
$$

The fin efficiency can be defined as,

$$
\begin{aligned}
& \eta_{\text {fin }}=\frac{Q_{\text {fin }}}{Q_{\text {fin }} \text { mux }}
\end{aligned}
$$

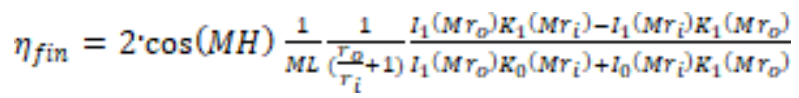

\section{REsults}

Fig. 3 shows both measured and calculated radial temperature variations on the top surface of the fin for four values of the heater power. The temperature is maximum closest to the heater and decreases radially. Increasing the heater power raises the temperature. Predictions from the analytical model agree well with experimental measurements. In the analytical model the natural convection coefficients are evaluated using the experimental correlation [10], which are $4.3,5.1,5.5$ and $6.6 \mathrm{~W} / \mathrm{m}^{2} \mathrm{~K}$ at the base temperature of 30,39 , 44 and $70^{\circ} \mathrm{C}$, respectively, and the radiation heat transfer coefficient, $4 T_{\text {aif }}{ }^{3} \varepsilon \sigma_{s}$ is $5.1 \mathrm{~W} / \mathrm{m}^{2} \mathrm{~K}$. Radiation therefore plays a significant role in heat transfer from the fin. 


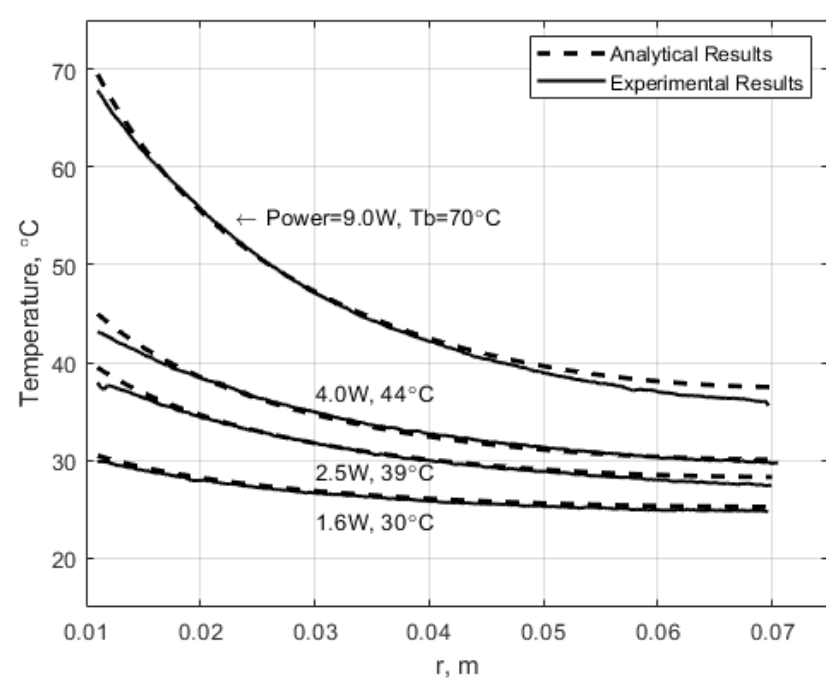

Figure 3. Analytical temperature curves of the composite annular fin (Equation 16) fits the experimental results at four heat input conditions, 1.6, $2.5,4.0$ and $9.0 \mathrm{~W}$.

Fig. 4 shows the variation of the base temperature of the fin, measured at the interface between the aluminium insert and the polymer fin, with the applied heater power. The base temperature increases with heater power in both the experimental and analytical results. The thermal resistance of natural convection and radiation is reciprocal to the effective heat transfer coefficient,

$$
R=\frac{1}{h_{e}}
$$

Known $h_{e}$ equals $h+4 T_{\text {air }}{ }^{a}$ हa and is a function of $R a$ and the ambinent temperature. $R a$ number depends on the fin temperature and increases with the base temperature. The natural convection coefficient increases with the power input, so the overall thermal resistance of natural convection and radiation reduces.

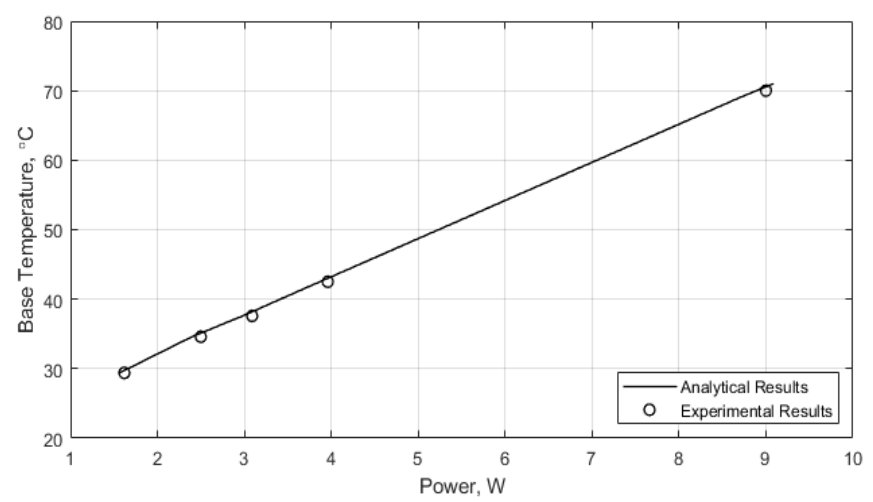

Figure 4. Analytical base temperature result of the composite annular fin (Equation 16) fits the experimental results at four heat input conditions, 1.6, $2.5,4.0$ and $9.0 \mathrm{~W}$.

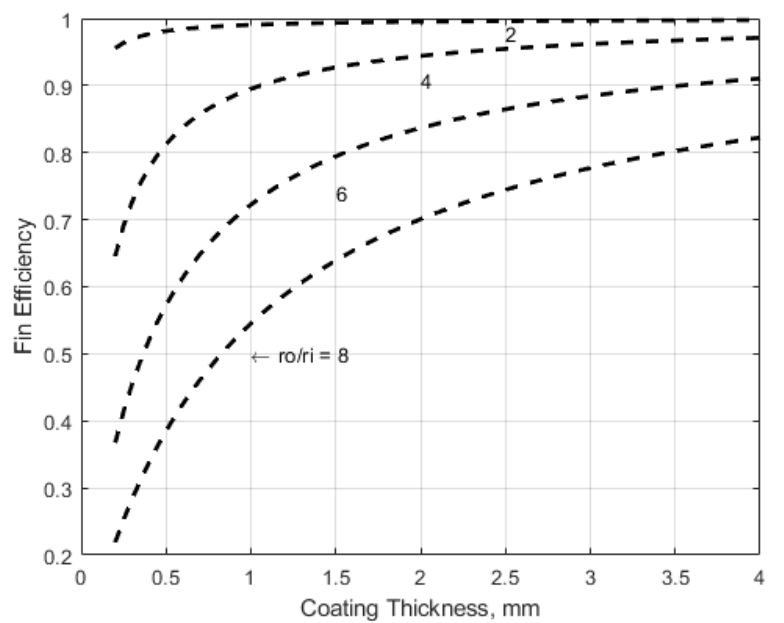

Figure 5. Fin efficiency of the composite annular fin (Equation 20) varies with the coating thickness at different ro/ri ratios.

Fig. 5 shows the variation in fin efficiency of annular composite (polymer/zinc) fins with coating thickness at four different $r_{o} / r_{i}$ ratios. Polymer core thickness, the inner fin radius $r_{i}$ and all thermal properties were assumed to be the same as those in our experiment. Fin efficiency increases with coating thickness in all cases because, as seen in Eqn 20, fin efficiency is a function of the fin parameter $M$ which decreases as coating thickness increases. For the annular fin that was tested in experiments, $r_{o} / r_{i}=6.7$ and the coating thickness was $0.4 \mathrm{~mm}$, giving a fin efficiency of approximately $50 \%$. Fin efficiency can be improved by reducing the fin radius since heat transfer decreases with distance from the center of the fin, so reducing $r_{o} / r_{i}$ or increasing the coating thickness would both improve efficiency.

\section{CONCLUSIONS}

An annular composite fin composed of a porous polyethylene core, a square aluminum insert, and metallic zinc coating layers, has been fabricated using wire-arc spraying technique. The analytical solutions of two-dimensional heat conduction model show an excellent prediction with the experimental results. The solutions indicate that the coating thickness has highly influence on the fin efficiency, and the fin efficiency can be accurately predicted by a single thermal length (ML), Eqn 20. The proper size of the fin can be determined by following the heat dissipation and fin efficiency curves of the fins in Figs 4 and 5. The solutions of this analytical model provide criteria in the annular composite fin design. 


\section{REFERENCES}

[1] R-T Wang and J-C Wang, "Analyzing the structural designs and thermal performance of nonmetal lighting devices of LED bulbs," Int. J. Heat Mass Transfer, vol 99, pp. 750-761, Aug. 2016.

doi: 10.1016/j.ijheatmasstransfer.2016.03.112.

[2] C. Cortés, L. I. Díez, A. Campo, "Efficiency of composite fins of variable thickness," Int. J. Heat Mass Transfer. vol 51, no. 9-10, pp. 2153-2166, 2008. doi: 10.1016/j.ijheatmasstransfer.2007.11.004.

[3] J.P. Luna-Abad, F. Alhama, "Design and optimization of composite rectangular fins using the relative inverse thermal admittance," $J$. Heat Transfer, vo. 135, no. 8, pp. 084504, 2013. doi: 10.1115/1.4024016.

[4] P. Tu, H. Inaba, A. Horibe, Z. Li, N. Haruki, "Fin efficiency of an annular fin composed of a substrate metallic fin and a coating layer," J. Heat Transfer, vol. 128, no. 8, pp. 851, 2006. doi: 10.1115/1.2227043.

[4] P. Tu, H. Inaba, A. Horibe, Z. Li, N. Haruki, "Fin efficiency of an annular fin composed of a substrate metallic fin and a coating layer," J. Heat Transfer, vol. 128, no. 8, pp. 851, 2006. doi: 10.1115/1.2227043.

[6] Weisstein EW. Taylor Series. MathWorld--A Wolfram Web Resource.

[7] Salas, Hille, Etgen, Calculus: One and Several Variables-Second order linear differential equations. 2006 10th Ed., John Wiley \& Sons Inc.

[8] G. Arfken, "Neumann Functions, Bessel Functions of the Second Kind, Nv(x),".Mathematical Methods for Physicists, pp. 596-604, 1985.

[9] G. Arfken, "Bessel Functions of the First Kind, JV(x)" and "Orthogonality," Mathematical Methods for Physicists, pp. 573-91, 91-9, 1985.

[10] P D. DeWitt. Fundamentals of heat and mass transfer, 2002. New York: J. Wiley. 


\title{
Continuous Fiber Polymer Composites for Thermal Applications
}

\author{
Yehia Elsayed, Ahmed Elkholy, Garrett Melenka and Roger Kempers \\ Department of Mechanical Engineering \\ York University \\ Toronto, Canada \\ kempers@yorku.ca
}

\begin{abstract}
This paper presents an analytical investigation into the effective thermal conductivity of 3D printed continuous fibre polymer composites (CFPCs) using rule of mixture micro-structural analysis. Two fused deposition modelling techniques were utilized using a off-the-shelf printer and a low-cost modified printer. Results demonstrate significant improvement in the effective thermal conductivity of the composite compared to the base polymer. One samples was experimentally tested to examine the veracity of the model predictions.
\end{abstract}

Keywords-component; Continuous fibre; continuous wire Conductive polymers; Thermal Properties, $3 D$ printing

\section{BACKGROUND AND LITERATURE}

Conductive polymer composites (PC) are utilized in many applications such as heat exchangers because they can offer relatively moderate thermal conductivity in addition to the advantages of light weight, corrosion resistance and low cost. These advantages result in a convenient choice for water desalination and electronic cooling applications [1],[2]. The performance of heat exchangers can be enhanced by increasing the thermal conductivity of the material, adding convective surface area, or increasing the convective heat transfer coefficient through shape complexity and turbulent generators.

Additive manufacturing (AM) techniques such as fused deposition modeling (FDM) can be employed in conjunction with PC to create more complex and effective heat exchangers [3],[4]. The addition of metal and reinforcement or other reinforcing materials such as carbon fiber, graphite and carbon nanotubes can increase the thermal conductivity of the material [5],[6]. However, the discontinuous nature of the reinforcement materials is believed to limit conductivity improvement where the interfacial resistances become dominant specifically at low reinforcement percentages[7],[8].

Recently, continuous fiber AM gained more attention due to the improved stiffness that can be achieved [9],[10]. Modified and commercial printers were used to investigate the mechanical properties of continuous fiber polymer composites (CFPCs) structures printed with reinforcement materials such as carbon, Kevlar ${ }^{\circledR}$ and glass fibers [11]-[13]. Presently, the thermal properties of CFPCs have not been studied despite the advantages that can be offered.

This study analytically investigates the thermal conductivity of AM CFPCs using two different printing techniques. The first method uses a commercial continuous fiber $3 \mathrm{D}$ printer to examine the effect of carbon fiber layers on overall thermal conductivity of the produced composite material. Secondly, a modified conventional FDM 3D printer was used which embeds continuous fibers within the printed structure. In the current stage, continuous metal wires are used as the reinforcing material. The goal of this work is to use high conductivity fibers such as continuous pitch-based carbon fibers and carbon nanotube yarns to produce highly conductive polymer composite structures.

\section{METHODOLOGY}

Two 3D printing machines were used in this work. The first 3D printer is the commercially available printer (MarkOne, MarkForged, Somerville, MA) which can print CFPCs using dual extruders, one for the polymer matrix and the other for continuous reinforcing fibers. Although Markforged printers can print different types of fibers such as carbon fibers, Kevlar and glass fibers with high reinforcement volumes, it is believed that the thermal conductivity of these fibers is relatively low compared to ultra-high conductive fibers such as the pitch-based carbon fibers. This will degrade the overall thermal conductivity of the CFPCs due to the existence of the polymer. On the other hand, the method described by Elsayed et al. [14] utilize an open-source, modified 3D printer with a single extruder combines polymer with more conductive continuous reinforcements such as Nickel chromium and copper wires within the printed structure. It may be possible to achieve higher overall thermal conductivity with the CFPCs printed with modified printer than the MarkForged components even with lower reinforcement volumes. 


\section{A. Samples Description}

\section{1) MarkForged samples}

To evaluate the thermal conductivity of CFPCs, six samples of different carbon fiber reinforcement volume fractions samples were designed and printed using a commercial CFPC printer (MarkOne, MarkForged, Somerville, MA) and Nylon as the base matrix. Figure 1 shows and example sample geometry, dimensions and fibers layer configuration. Sample geometry was created using computer aided design software (SolidWorks 2017 SP4.0, Dassault Systems, and uploaded to the online based slicing software (Eiger 1.2, MarkForged, Somerville, MA). The software provides information about the total number of fibers and nylon layers in the printed samples which can be used to deduce fiber volume fraction $\left(v_{f}\right)$. Table 1 shows sample dimensions and predicted fiber volume fraction by the software. Fibers printing angle was set to zero degree while two build directions were used to investigate the thermal conductivity in the axial and transverse directions of the fibers (Table 1). The physical properties of the $0.375 \mathrm{~mm}$ carbon fiber filament manufactured by MarkForged were not provided by the supplier. However, it is assumed that the fiber filament is a combination of polyacrylonitrile (PAN) based carbon fibers and polymeric material that give the filament the apparent stiffness. With this assumption, the mechanical properties and thermal conductivity of these fibers can be inferred.

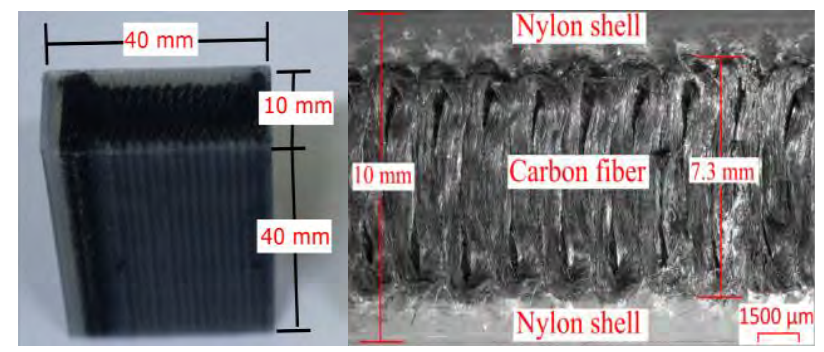

Figure 1. Carbon fiber reinforced sample (0-degree fiber printing angle) Left: sample with dimensions 40x40x25mm. Right: microscope image showing the continuous fiber.

TABLE 1 SAMPLES DIMENSION AND FIBER VOLUME FRACTION

\begin{tabular}{|c|c|c|c|}
\hline $\begin{array}{c}\text { Sample } \\
\text { No. }\end{array}$ & $\begin{array}{c}\text { Sample dimensions } \\
(\mathbf{L} \times \mathbf{W} \times \mathbf{~ H})(\mathbf{m m})\end{array}$ & $\begin{array}{c}\text { Fiber printing } \\
\text { angle }\end{array}$ & $\begin{array}{c}\text { Fiber volume } \\
\text { fraction }\left(\boldsymbol{v}_{\boldsymbol{f}}\right) \mathbf{\%}\end{array}$ \\
\hline 1 & $40 \times 10 \times 40$ & 0 & 79 \\
\hline 2 & $40 \times 10 \times 40$ & 0 & 55 \\
\hline 3 & $40 \times 10 \times 40$ & 0 & 31 \\
\hline 4 & $40 \times 40 \times 10$ & 0 & 76 \\
\hline 5 & $40 \times 40 \times 10$ & 0 & 63 \\
\hline 6 & $40 \times 40 \times 10$ & 0 & 51 \\
\hline
\end{tabular}

\section{2) Modified printer samples}

A FDM printer (Prusa i3, Prusa Research, Czech Republic) was modified to create CFPC components. Six samples of CFPCs were designed using the method described by Elsayed et al [14] where Polylactic acid (PLA) filament $(1.75 \mathrm{~mm}$, Spool $3 \mathrm{~d}$, Canada) was fused through the printing nozzle and combined with Nickel-chromium wires (75 um, McMaster Carr, Ohio, USA), copper wires (75 um, Remington Industries, Canada) and Pitch carbon fiber (Mitsubishi Chemicals Carbon Fiber and Composites, California, USA) to create CFPC samples. Figure 2 shows a Nickel chromium reinforced prototype sample with the geometry, dimensions and reinforcement configuration. The prototype sample was printed using $0.6 \mathrm{~mm}$ diameter nozzle, $0.4 \mathrm{~mm}$ layer height and 180 degrees Celsius printing temperature. Sample printing code was generated using a custom script (MATLAB, Mathworks, Natick, MA), where the printing angle was set to zero to investigate the thermal conductivity in the axial and transverse directions by changing the build direction of the samples.

Table 2 shows samples dimensions and the nominal reinforcement volume fraction calculated from the input printing parameters.

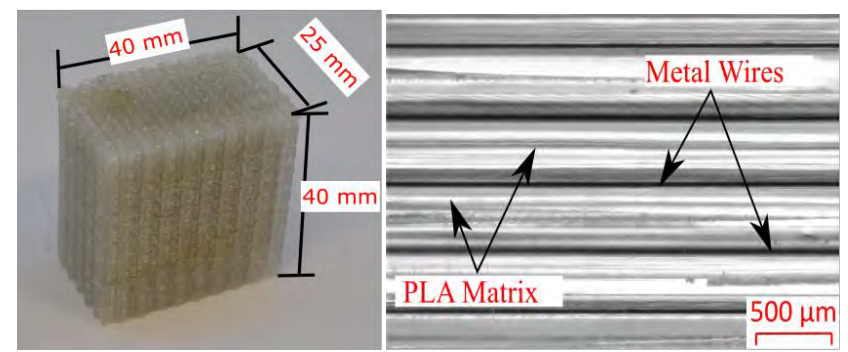

Figure 2. Prototype sample for the modified printer Left: sample with dimensions 40x40x25mm. Right: microscope image showing the continuous reinforced metal wires.

TABLE 2 SAMPLES DIMENSIONS AND REINFORCEMENT VOLUME FRACTION

\begin{tabular}{|c|c|c|c|c|}
\hline $\begin{array}{c}\text { Sample } \\
\text { No. }\end{array}$ & $\begin{array}{c}\text { Sample } \\
\text { Dimensions } \\
(\mathrm{L} \times \mathrm{W} \times \mathrm{H}) \\
(\mathrm{mm})\end{array}$ & $\begin{array}{c}\text { Reinforcement } \\
\text { material }\end{array}$ & $\begin{array}{c}\text { Reinforcement } \\
\text { printing angle }\end{array}$ & $\begin{array}{c}\text { Reinforcement } \\
\text { volume } \\
\text { fraction }\left(v_{f}\right) \%\end{array}$ \\
\hline 7 & $40 \times 10 \times 40$ & $\begin{array}{c}\text { Nickel } \\
\text { chromium }\end{array}$ & 0 & 1.7 \\
\hline 8 & $40 \times 10 \times 40$ & $\begin{array}{c}\text { Copper } \\
\text { fiber }\end{array}$ & 0 & 1.7 \\
\hline 9 & $40 \times 10 \times 40$ & $\begin{array}{c}\text { Pitch carbon } \\
\text { Nickel } \\
\text { chromium }\end{array}$ & 0 & 1.7 \\
\hline 7 & $40 \times 40 \times 10$ & $\begin{array}{c}\text { Copper } \\
40 \times 40 \times 10\end{array}$ & 0 & 1.7 \\
\hline 12 & $40 \times 40 \times 10$ & $\begin{array}{c}\text { Pitch carbon } \\
\text { fiber }\end{array}$ & 0 & 1.7 \\
\hline
\end{tabular}

\section{B. Experimental setup}

Different apparatuses are being used for characterizing materials thermal conductivity based on different operating concepts. The steady state and the transient apparatuses families are the most commonly used. Based on the nature of the material being measured and the rang of the thermal conductivity, a convenient apparatus can be chosen. A steady state thermal test apparatus was used to assess the thermal conductivity of the CFPC samples. The employed test rig belongs to the steady state category which mainly depends on 
transferring heat through the sample and measuring the resultant temperature difference across the sample at steady state conditions. Similar apparatuses were used for measuring thermal conductivity of thermal interface materials[15] and conductive polymers [16]. Fig. 3 shows that the apparatus consists of two sides; one for supplying the heat and the other one represents the sink. Each side consists of two isothermal blocks; one is the main one while the other working as a guard for it. The main block of the hot side has a dimension of 40 $\mathrm{mm} \times 40 \mathrm{~mm} \times 6.35 \mathrm{~mm}$ while the guard one is $70 \mathrm{~mm} \times 70$ $\mathrm{mm} \times 15.7 \mathrm{~mm}$ and has a groove which allows the main to be fixed inside. Its function is to guarantee that all the heat coming from the main heater is going into the sample and eliminate any losses from the main heater sides. This was done by tuning its power until reaching a temperature that follow the main heater temperature. Isothermal conditions for all parts were achieved by manufacturing them from copper material, $\mathrm{k}=391.2 \mathrm{w} / \mathrm{m} \mathrm{k}$, to produce a uniform temperature field inside it. Moreover, this was validated using numerical simulation. The temperature of both blocks was observed at three different locations where each sensor from the main block is opposite to its analogous one from the guard block. It was postulated that the secondary power is convenient when the temperature difference between each pair did not exceed $0.001{ }^{\circ} \mathrm{C}$ at steady-state. Also, it was assumed that the steady state conditions were reached when the time gradient of the temperature for each sensor is less than 0.00001 .

The cold side main block has a dimension of $40 \mathrm{~mm} \times 40$ $\mathrm{mm} \times 12$, while the secondary one is $80 \mathrm{~mm} \times 80 \mathrm{~mm} \times 12$ $\mathrm{mm}$. All the blocks and the sample are clamped together against a force gauge. The specimen was manufactured with small thickness to have a negligible heat loss from its side and to verify that the heat flow is one-dimensional. Furthermore, all parts were wrapped by silica aerogel insulating material having a thermal conductivity of $0.014 \mathrm{w} / \mathrm{m} \mathrm{k}$.

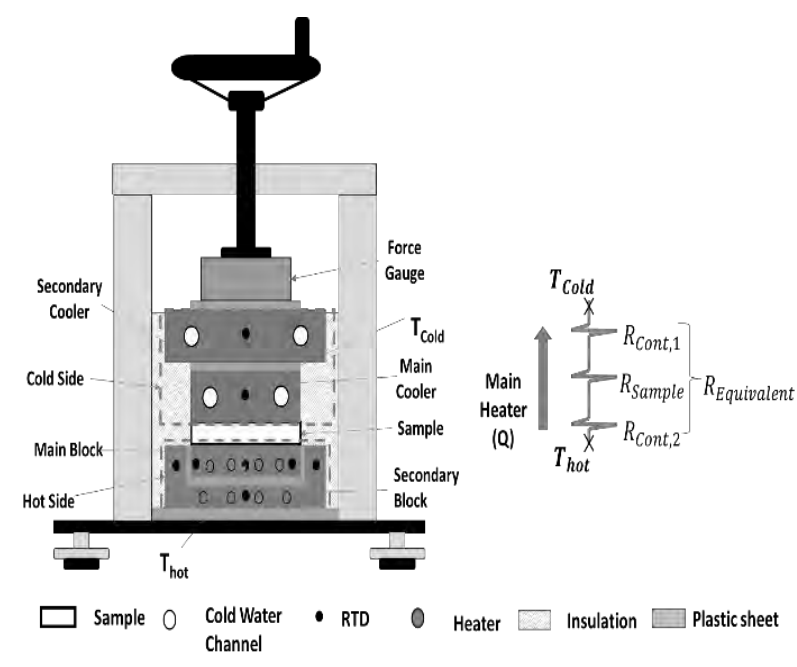

Figure 3. Experimental setup for measuring the thermal conductivity.

The thermal conductivity is calculated using the Fourier conduction equation as follows;

$$
k=\frac{Q \Delta t}{A_{c}\left(T_{\text {hot }}-T_{\text {cold }}\right)}
$$

where $k$ is the tested thermal conductivity, $\Delta t$ is the sample thickness, $A_{c}$ is the cross-section area and $\left(T_{h}-T_{c}\right)$ is the temperature difference across the sample.

\section{MICROSCOPY}

The internal structure of the CFPC samples was examined using stereo-microscope (MZ10 F, Leica, Concord, Ontario). Samples produced using the MarkForged 3D printer and modified printer were examined to investigate the difference between the reinforcement volume fraction given by the software and the actual reinforcement fraction assessed by image processing. Fiber volume fraction measurements are necessary for accurate predication of the thermal and mechanical properties. The samples were sectioned perpendicular to the reinforcement axis using a high-speed abrasive cutting wheel. Thereafter, the samples went through four steps of grinding and polishing using 240, 400, 600 and $1200 / 4000$ - grit silicon carbide discs to get a convenient surface for microscopy. Figure 4 shows the cross-sectional microscopy of the two samples showing reinforcement and polymer layers with the air voids existing due to the nature of FDM printing technique. Image processing was performed using image analysis software (ImageJ, National Institutes of Health, Bethesda, Maryland, USA) to measure reinforcement volume fraction across the two samples. For the MarkForged Sample no. 3 the analysis showed $46.7 \%$ of fiber volume fraction exist in the sample compared to $31 \%$ calculated using the Eiger software. For the custom metal reinforced samples, the analysis determined $1.48 \%$ fiber volume fraction compared to $1.7 \%$ calculated from the input printing parameters for the modified printer prototype sample.

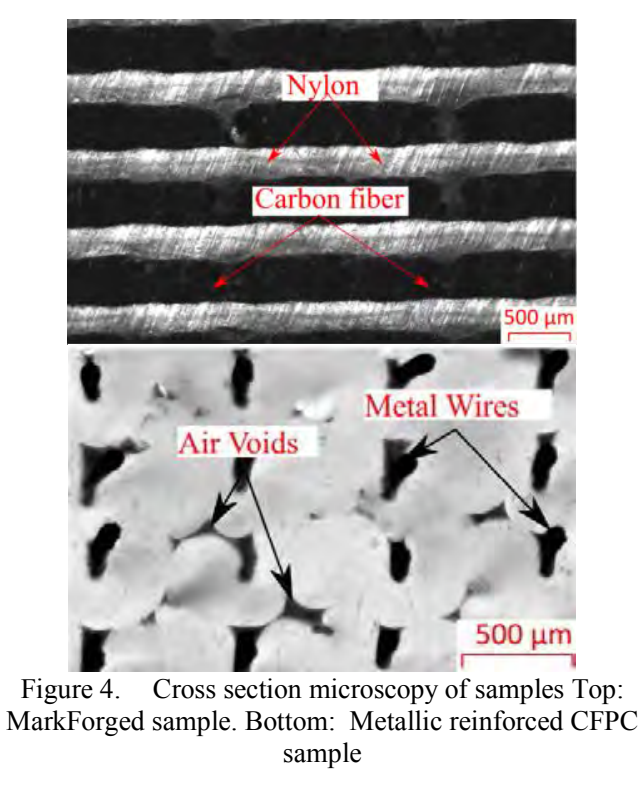


Additionally, a single fiber filament (Carbon Fiber CFF, Markforged, Somerville, MA) used for printing the fiber layers in the Markforged samples was examined under the microscope to evaluate the percentage of the carbon fibers and the polymeric material. Figure 5 shows the filament cross-section at three different positions. It was noticed that the filament was not completely filled with fibers and that the fibers configuration within the filament is not fixed which strengthen the possibility that the fibers are not fully continuous inside the filament. Image processing was performed again and a value of $35.5 \%$ of fibers was found within the fiber filament. The value obtained reduces the overall fiber percentage predicted by the software and the one from the image processing of the sample cross section.

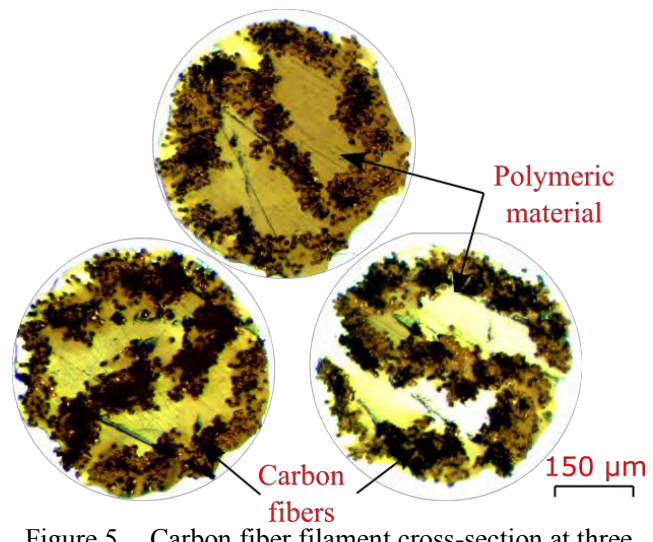

Figure 5. Carbon fiber filament cross-section at three different positions

\section{ANALYTICAL MODEL}

Different analytical models are available for the prediction of composites material thermal conductivity [17]. Depending on the reinforcement shape, orientation and measurement direction, considerably accurate models can be deduced. One basic model for composites properties description is the rule of mixture.

The reinforcement configurations addressed here are shown in Fig. 6. Here the samples consist of continuous unidirectional reinforcements which allows the straightforward rule of mixture model to be used. This model describes composite properties as a function of constitutes properties and reinforcement volume fraction. For CFPCs, rule of mixture can take simple forms of parallel and series models shown in Eqs. 2 and 3, respectively. Where $\mathrm{k}_{c}, \mathrm{k}_{\mathrm{p}}$ and $\mathrm{kf}_{\mathrm{f}}$ are composite, polymer and fiber thermal conductivity respectively while $\emptyset$ is fiber volume fraction. The main limitation for the rule of mixture model in this case is over estimation that can occur due to the neglection of the interfacial resistances especially for the series model.

$$
\begin{gathered}
k_{c}=(1-\emptyset) k_{p}+\emptyset k_{f} \\
k_{c}=\left(\frac{1-\emptyset}{k_{p}}+\frac{\emptyset}{k_{f}}\right)^{-1}
\end{gathered}
$$

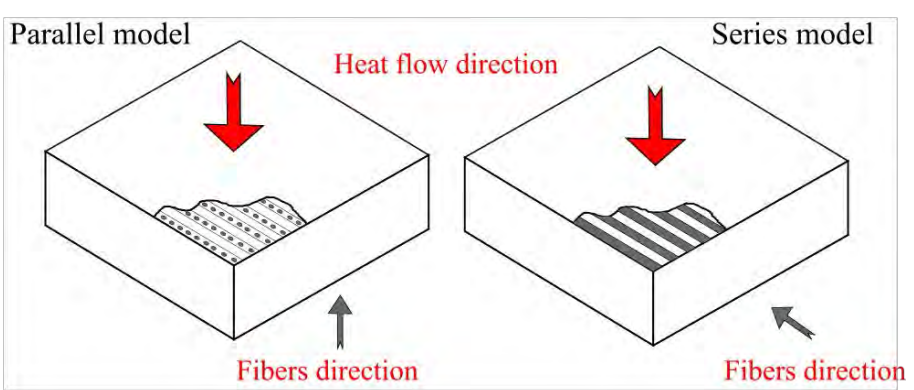

Figure 6. Parallel and series models

Constituents' properties can greatly affect CFPCs overall thermal conductivity. Different reinforcement materials are applied to the model to study the effect of ultra-high conductive reinforcements such as copper and Pitch based carbon fiber on the overall thermal conductivity. Again, the carbon fiber provided by Markforged is believed to be PAN based with relatively low thermal conductivity compared to metal reinforcements. Assumed values for thermal properties provided from [18], [19] shown in Table 3 are used in the thermal conductivity evaluation as there is insufficient information about the thermal properties from the suppliers.

TABLE 3 MATERIALS THERMAL PROPERTIES [18], [19]

\begin{tabular}{|c|c|}
\hline Material & Thermal Conductivity (W/m·K) \\
\hline Nylon & $0.3[18]$ \\
\hline PAN Carbon fiber & $8[19]$ \\
\hline PLA & $0.29[18]$ \\
\hline Nickel chromium & $13[18]$ \\
\hline Copper & $385[18]$ \\
\hline Pitch carbon fiber & $500[19]$ \\
\hline
\end{tabular}

\section{RESULTS AND DISCUSSION}

Using the rule of mixture model described in Section IV, analytically predicted values for the overall thermal conductivity were obtained for both the commercial and modified 3D printers. Samples 1-3 and 7-9 thermal conductivity was predicted using the parallel model in "(2)" as the continuous reinforcement is parallel to the heat flow direction while the series model in "(3)" was utilized for samples $4: 6$ and 10:12 as the heat flows transversely through the reinforcement. Figure 6 shows the results for the Markforged samples described in Table 1. A maximum value of $6.42 \mathrm{~W} / \mathrm{m} \cdot \mathrm{K}$ was found for sample no. 1 with $79 \%$ nominal fibers content when the thermal conductivity is predicted in the axial direction of the fibers. However, this value drops to $2.8 \mathrm{~W} / \mathrm{m} \cdot \mathrm{K}$ when the actual fiber content obtained from the fiber filament microscopy is used assuming that the matrix material in the filament has the same thermal conductivity as Nylon. As well, the thermal conductivity is predicted in the transverse direction where low thermal conductivity (1.13 $\mathrm{W} / \mathrm{m} \cdot \mathrm{K}$ ) was obtained even with $76 \%$ nominal fibers content 
in sample no. 4. The modified printer samples, described in Table 2, showed higher thermal conductivity using copper wires and Pitch carbon fibers (samples 8 and 9) even with as low reinforcement volume fraction as $1.7 \%$ (Figure 7). This means that the modified printer is more convenient for creating increasingly conductive CFPCs. The overall thermal conductivity of the CFPCs made by the Markforged printers can be significantly improved if Pitch-based carbon fibers are used due to their high thermal conductivity. Meanwhile for the customized printer, further modification that can increase the reinforcement volume fraction will boost the overall thermal conductivity of the prints. For the experimental results, sample no. 1 was tested using the previously mentioned setup. A value of $1.84 \mathrm{~W} / \mathrm{m} \cdot \mathrm{K}$ was found for the thermal conductivity. The deviation between the measured value and the value predicted by the model with actual fiber content is believed to be caused by uncertainty in the fibers grade and conductivity. Additionally, the possibility that the fibers are not fully continuous within the samples inferred from the filament cross-section in Figure 5 can cause the thermal conductivity to drop. Future work will focus on experimental testing of the rest of the samples to examine the compatibility of the analytical models with the printed samples and increasing fiber volume fraction of the modified printer to achieve higher thermal conductivity.

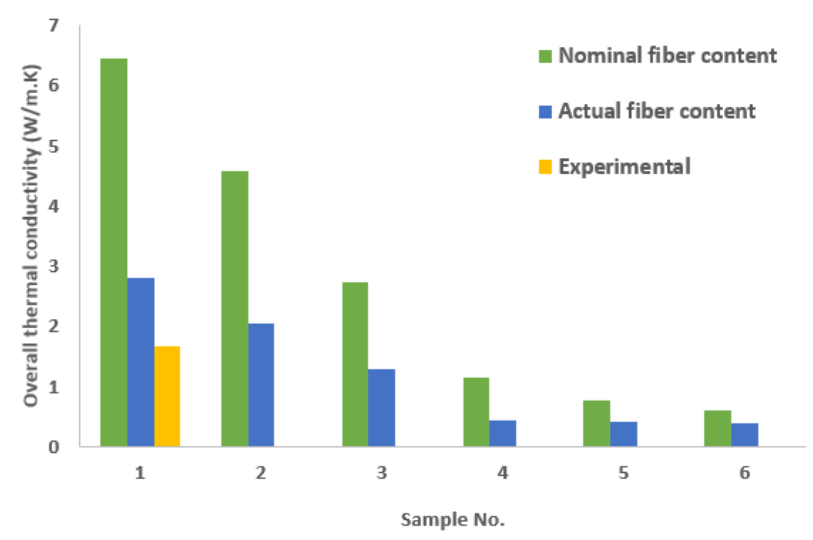

Figure 6. Overall thermal conductivity of Markforged samples

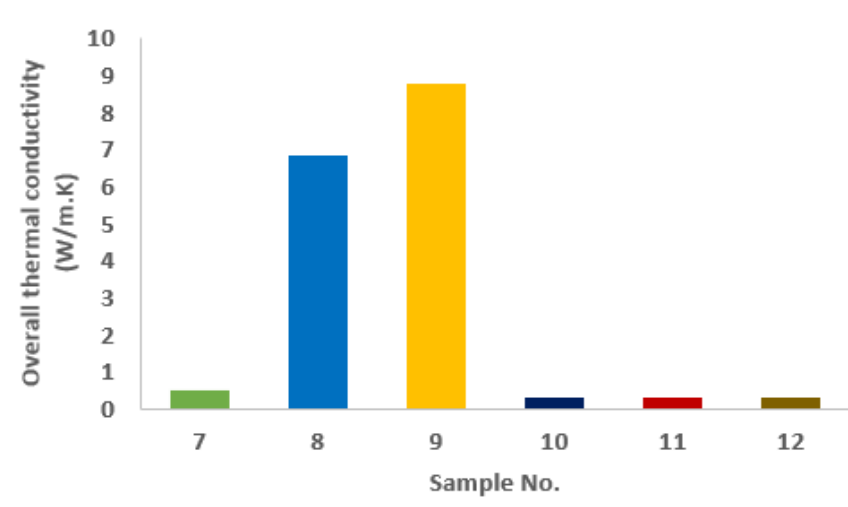

Figure 7. Overall thermal conductivity of the modified printer samples

\section{CONCLUSIONS}

CFPCs can be an attractive choice for thermal applications like heat exchangers by utilizing conductive fibers. Additive manufacturing combined with fiber reinforcement can be used to generates sufficient heat transfer coefficients for thermal applications. The high overall thermal conductivity values showed in this study makes CFPCs comparable to moderate conductive metals such as stainless steel which make it convenient for heat exchanging applications especially for corrosive fluids.

\section{REFERENCES}

[1] Q. Wang, X. H. Han, A. Sommers, Y. Park, C. T'Joen, and A. Jacobi, "A review on application of carbonaceous materials and carbon matrix composites for heat exchangers and heat sinks," Int. J. Refrig., vol. 35, no. 1, pp. 7-26, 2012. doi: 10.1016/j.ijrefrig.2011.09.001.

[2] T. B. Scheffler and A. J. Leao, "Fabrication of polymer film heat transfer elements for energy efficient multi-effect distillation," Desalination, vol. 222, no. 1-3, pp. 696-710, 2008. doi: 10.1016/j.desal.2007.02.076.

[3] J. H. K. Haertel and G. F. Nellis, "A fully developed flow thermofluid model for topology optimization of 3D-printed air-cooled heat exchangers," Appl. Therm. Eng., vol. 119 , pp. 10-24, 2017. doi: 10.1016/j.applthermaleng.2017.03.030.

[4] J. Cevallos, A. Bar-Cohen, and D. C. Deisenroth, "Thermal performance of a polymer composite webbed-tube heat exchanger," Int. J. Heat Mass Transf., vol. 98, pp. 845-856, 2016. doi: 10.1016/j.ijheatmasstransfer.2016.03.075.

[5] U. Kalsoom, A. Peristyy, P. N. Nesterenko, and B. Paull, "A 3D printable diamond polymer composite: a novel material for fabrication of low cost thermally conducting devices," RSC Adv., vol. 6, no. 44, pp. 38140-38147, 2016.

doi: 10.1039/C6RA05261D.

[6] M. Nikzad, S. H. Masood, and I. Sbarski, "Thermo-mechanical properties of a highly filled polymeric composites for Fused Deposition Modeling," Mater. Des., vol. 32, no. 6, pp. 3448-3456, 2011. doi:10.1016/j.matdes.2011.01.056.

[7] T. Flaata, G. J. Michna, and T. Letcher, "Thermal Conductivity Testing Apparatus for 3D Printed Materials," in ASME 2017 Summer Heat Transfer Conference, 2017, no. August, p. HT2017-4856. doi: 10.1115/HT2017-4856.

[8] C. Shemelya et al., "Anisotropy of thermal conductivity in 3D printed polymer matrix composites for space based cube satellites," Addit. Manuf., vol. 16, pp. 186-196, 2017. doi: 10.1016/j.addma.2017.05.012.

[9] C. Yang, X. Tian, T. Liu, Y. Cao, and D. Li, "3D printing for continuous fiber reinforced thermoplastic composites: mechanism and performance," Rapid Prototyp. J., vol. 23, no. 1, pp. 209-215, 2017. doi: 10.1108/RPJ-08-2015-0098.

[10] T. H. J. Vaneker, "Material Extrusion of Continuous Fiber Reinforced Plastics Using Commingled Yarn," Procedia CIRP, vol. 66, pp. 317-322, 2017. doi: 10.1016/j.procir.2017.03.367.

[11] G. W. Melenka, B. K. O. Cheung, J. S. Schofield, M. R. Dawson, and J. P. Carey, "Evaluation and prediction of the tensile properties of continuous fiber-reinforced 3D printed structures," Compos. Struct., vol. 153, pp. 866-875, 2016. doi: 10.1016/j.compstruct.2016.07.018.

[12] A. N. Dickson, J. N. Barry, K. A. Mcdonnell, and D. P. Dowling, "Fabrication of continuous carbon, glass and Kevlar fibre reinforced polymer composites using additive manufacturing," Addit. Manuf., vol. 16, pp. 146-152, 2017. doi: 10.1016/j.addma.2017.06.004. 
[13] Q. Hu, Y. Duan, H. Zhang, D. Liu, B. Yan, and F. Peng, "Manufacturing and 3D printing of continuous carbon fiber prepreg filament," J. Mater. Sci., vol. 53, no. 3, pp. 1-12, 2017. doi: 10.1007/s10853-017-1624-2.

[14] Y. Elsayed, Garrett W. Melenka, Roger Kempers, "Fabrication and Tensile Testing of 3D Printed Continuous Wire Polymer Composites," Rapid Prototyping J., vol. 24, no. 7, pp.1131-1141, 2017. doi: 10.1108/RPJ-11-2017-0222.

[15] R. Kempers, P. Kolodner, A. Lyons, and A. J. Robinson, "A high-precision apparatus for the characterization of thermal interface materials," Rev. Sci. Instrum., vol. 80, no. 9, pp. 1-11, 2009. doi: 10.1063/1.3193715.

[16] T. Letcher, T. Flaata, G. L. Michna, "Thermal Conductivity Testing Apparatus for 3D Printed Materials," ASME 2017 HEat Transfer Summer Conference, pp. V002T15A006, July 2017, doi: 10.1115/HT2017-4856.

[17] H. Chen et al., "Thermal conductivity of polymer-based composites: Fundamentals and applications," Prog. Polym. Sci., vol. 59, pp. 41-85, 2015. doi: 10.1016/j.progpolymsci.2016.03.001.

[18] MATWEB, "Material properties by MATWEB," 2017. [Online]. Available: http://www.matweb.com.

[19] M. Srinivasan, P. Maettig, K. W. Glitza, B. Sanny, and A. Schumacher, "Out of Plane Thermal Conductivity of Carbon Fiber Reinforced Composite Filled with Diamond Powder," Open J. Compos. Mater., vol. 6, pp. 41-57, 2016. doi: 10.4236/ojcm.2016.62005. 


\title{
Thermal Conductivity of $\beta$-Arsenene Under iaxial Tensile Strain: a First rinciple Study
}

\author{
Armin Taheri, Carolos da Silva, Christina H. Amon \\ Department of Mechanical and Industrial Engineering \\ University of Toronto \\ Toronto, Canada \\ armin.taheri@mail.utoronto.ca, carlos.dasilva@utoronto.ca \\ cristina.amon@utoronto.ca
}

\begin{abstract}
A first principle study is conducted to explore the phonon thermal transport in a buckled arsenene monolayer $(\boldsymbol{\beta}$-As) subjected to tensile strain. The results showed that the thermal conductivity first decreases with strains from $0 \%$ to $1 \%$, then it increases with strains from $1 \%$ to $5 \%$, and finally it decreases with strains from $5 \%$ to $9 \%$. The maximum thermal conductivity occurs at strain of $5 \%$, which is 1.45 times higher than that of unstrained arsenene. Phonon properties are investigated to understand the causes of this thermal response to strain.
\end{abstract}

First Principle; Arsenene; Strain; Thermal conductivity Phonon Properties

\section{INTRODUCTION}

Since the discovery of graphene, two-dimensional (2D) materials have attracted a great deal of attention. The appealing thermal [1] and electrical [2] properties of graphene have motivated the scientific community to explore for other 2D materials. This effort has resulted in many other nanomaterials added to the 2D family such as silicene, germanene, boron nitride $(\mathrm{BN})$ and transition metal dichalcogenides (TMDCs) [3,4]. Very recently, group-VA monolayers such as phosphorene (P), arsenene (As), antimonene $(\mathrm{Sb})$ and bismuthene (Bi) have attracted significant attention. As opposed to graphene that does not have a band gap, group-VA monolayers have band gaps in the range of $1-2.5 \mathrm{eV}$ [5,6,7], making them intriguing for electronic and optoelectronic applications. From a thermal point of view, materials of this family have a much lower thermal conductivity than graphene [8], making them more suitable for thermoelectric applications. There are two possible atomic structures for these materials: buckled (known as $\boldsymbol{\beta}$ ) and puckered (known as $\boldsymbol{\alpha}$ ). The thermal conductivity of buckled structure is isotropic while the puckered structure has a highly anisotropic thermal conductivity in the zigzag and the armchair directions [8]. The focus of this paper is on buckled structures. Figure 1 shows a schematic of the group-VA buckled structure, in which atoms are arranged on a honeycomb lattice similar to graphene, with two atoms per unit cell. What makes these structures different from graphene is the non-zero buckling height $d$ between two atomic planes

The authors would like to acknowledge support from the Natural Science and Engineering Research Council (NSERC) of Canada.
(Fig. 1). The minimum and maximum value of $d$ are exhibited by $\boldsymbol{\beta}$-phosphorene and $\boldsymbol{\beta}$ - bismuthene, respectively.

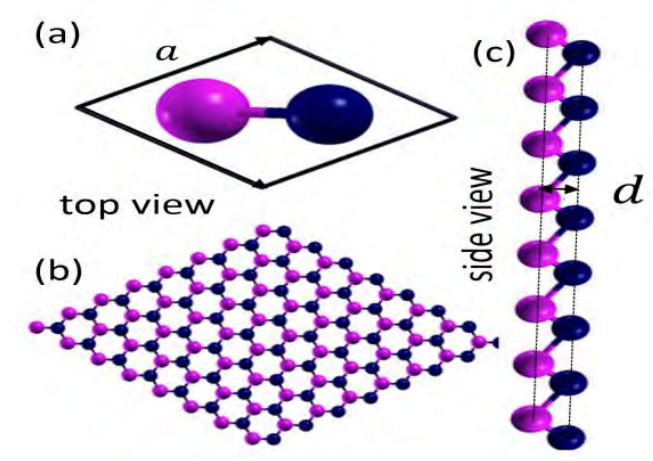

Figure 1. Lattice structure of group-VA monolayers: (a) unit cell (b) a $7 \times 7 \times 1$ supercell (c) side view of the supercell. All atoms are the same kind, any of the group-VA elements ( $\mathrm{P}, \mathrm{As}, \mathrm{Sb}$ or $\mathrm{Bi})$. Different colors are used to show atoms in the upper and lower planes.

Apart from the value of accurately predicting the thermal conductivity of these novel materials, it is of paramount importance to understand how to tune the thermal conductivity. For that, it is necessary to investigate the thermal transport from a phonon transport perspective. 2D materials are commonly subjected to residual strains in real applications, and it is well known that strain has a considerable effect on the phonon properties and the thermal conductivity of $2 \mathrm{D}$ materials. Many studies have been conducted on the effects of strain on 2D materials such as graphene, stanene, germanene and silicene [1,9]. These studies revealed that tensile strain might increase or decrease the thermal conductivity. Also, tensile strain increases the dependency of the thermal conductivity to sample size. Zhang et al. [10] studied the effect of strain on phonon transport of $\boldsymbol{\beta}$-Sb based on Boltzmann transport equation (BTE) under single relaxation time approximation (RTA) [11]. They considered strains ranging from $-6 \%$ to $6 \%$. Based on their results, the buckled structure of $\boldsymbol{\beta}-\mathrm{Sb}$ is not stable for strains less than $-1 \%$. For strains from $-1 \%$ to $6 \%$, they found that the thermal conductivity increases by increasing strain. For instance, the thermal conductivity of $\boldsymbol{\beta}$-Sb under tensile strain of $6 \%$ is about three times higher 
than that of unstrained structure. However, there are still intriguing questions that need to be answered. For example, is the variation of the thermal conductivity with strain similar in other group-VA 2D materials? What will be the effect of higher tensile strain values on the phonon properties and thermal conductivity of other members of the group-VA family? In this work, we address these questions investigating the thermal properties of $\boldsymbol{\beta}$-As, using a rigorous first-principle density functional theory (DFT) approach. We considered the effect of tensile strain $(0-9 \%)$ on the thermal conductivity and phonon propertied of $\boldsymbol{\beta}$-As. The rest of this paper is organized as follows: Section II discusses the computational approach, Section III presents the harmonic and anharmonic phonon properties for different strain levels. Finally, Section IV summarizes the key findings.

\section{COMPUTATIONAL APPROACH}

The methodology here is based on a full solution of the BTE [11]. Having the harmonic and anharmonic interatomic force constants (IFCs), the BTE can be iteratively solved for the three-phonon scattering lifetime $\tau_{\lambda}$ of different phonons. Then, the thermal conductivity along the $\alpha$ direction, $\kappa_{\alpha \alpha}$, is

$$
\left.\kappa_{\alpha \alpha}=\frac{1}{A h} \sum_{\lambda(\boldsymbol{k}, p)} \hbar \omega_{\lambda} \frac{\partial n_{\lambda}^{0}}{\partial T} v_{\lambda}^{\alpha} v_{\lambda}^{\alpha} \tau_{\lambda}\right) 1(
$$

where $\lambda(\boldsymbol{k}, p)$ shows a phonon mode with $\boldsymbol{k}$ being the phonon wave vector and $p$ the polarization branch, $\mathrm{A}$ is the unit cell area, $h$ is the thickness of the material, $\omega$ is the phonon frequency, $T$ is temperature, $v_{\lambda}{ }^{\alpha}$ is the phonon group velocity in the direction $\alpha$, and $n_{\lambda}^{0}$ is the phonon occupation number given by the equilibrium Bose-Einstein distribution.

The DFT calculations were conducted by the QUANTUM ESPRESSO (QE) package [12] with projected augmented wave (PAW) pseudopotential and generalized gradient approximation (GGA) exchange-correlation. First, the structure is fully optimized to obtain the relaxed lattice constant. We used an electronic wave-vector grid of $25 \times 25 \times 1$ for the Brillouin zone (BZ) integration, and the plane-wave cutoff was set to $70 \mathrm{Ry}$. We also considered a vacuum of $20 \AA$ between $\boldsymbol{\beta}$-As monolayers to avoid interaction between adjacent layers. The relaxed lattice constant and buckling height of $\boldsymbol{\beta}$-As is found to be $a_{0}=3.61 \AA$ and $d=1.40 \AA$, respectively (Fig 1 ). These values are in excellent agreement with previously reported values in $[8,13]$. Then, the percentage of applied strain is defined as

$$
\epsilon=\frac{a-a_{0}}{a_{0}} \times 100
$$

where $a$ is the lattice constant of the strained structure. Strains values studied here are $0 \%, 1 \%, 3 \%, 5 \%, 7 \%$ and $9 \%$. For each value of strain, the phonon calculations are performed on a phonon wave-vector grid of $7 \times 7 \times 1$. The anharmonic IFCs were calculated based on supercells, using a finite-difference method within the thirdorder package[11]. We used a $5 \times 5 \times 1$ supercell, and atomic interactions were considered up to the tenth nearest neighbor. Finally, the thermal conductivities are calculated using ShengBTE code [11] on an $80 \times 80 \times 1$ phonon wave-grid vector. Rigorous convergence tests with respect to all DFT settings implemented here showed that using the parameters described above led to fully converged phonon properties and thermal conductivities.

Based on Eq. (1), the thermal conductivity depends on the thickness $h$ of the unit cell. However, the definition of $h$ is controversial among 2D materials. Here, the value of $h$ is considered the same as the length of the unit cell along the $z$ direction, i.e., $20 \AA$, the same strategy as previously used in [10].

\section{RESULTS AND DISCUSSION}

Figure 2 shows the variation of the lattice thermal conductivity of $\boldsymbol{\beta}$-As with strain $(0-9 \%)$ at $300 \mathrm{~K}$. The thermal conductivity first decreases with strains from $0 \%$ to $1 \%$, and then it increases with strains from $1 \%$ to $5 \%$. For $5 \%$ of strain, the thermal conductivity exhibited its maximum value, which was 1.45 times higher than that of strain $0 \%$. This critical value of strain that resulted in a maximum conductivity, was also observed in silicene, germanene and stanene [9]. Thermal conductivity reduction occurs for strain values higher than $5 \%$. In contrast to what was found in Ref. [10], an increase in the thermal conductivity of $\boldsymbol{\beta}$-Sb from $0 \%$ to $1 \%$ of strain, we found here that the thermal conductivity of $\boldsymbol{\beta}$-As slightly decreases from $0 \%$ to $1 \%$ of strain. Moreover, in Ref. [10], the authors did not consider strain percentages higher that $6 \%$. Based on our results, we conclude that the thermal conductivity response of group-VA 2D materials to strain may differ although they are similar in structure. Also, considering a wider range of strain (in the elastic region) is necessary to obtain a comprehensive picture of the thermal response of 2D materials to strain. Figure 3 shows the variation of the thermal conductivity of $\boldsymbol{\beta}$-As for temperatures in the range of 200 to $1000 \mathrm{~K}$. The relative difference of the thermal conductivity between $0 \%$ and $5 \%$ of strain decreases from $49 \%$ at $200 \mathrm{~K}$ to $43 \%$ at $1000 \mathrm{~K}$.

Now, we analyze the harmonic and anharmonic phonon properties to shed light into the mechanism responsible for the thermal conductivity variation of $\boldsymbol{\beta}$-As with strain. Harmonic properties such as phonon dispersion and phonon group velocity govern the thermal transport in nanomaterials. As an example, the anisotropic thermal conductivity of puckered phosphorene and arsenene ( $\alpha$-P and $\alpha$-As) is directly related to the anisotropic group velocities near the center of BZ $[14,15]$. Fig. 4 illustrates the phonon dispersion curve along highly 


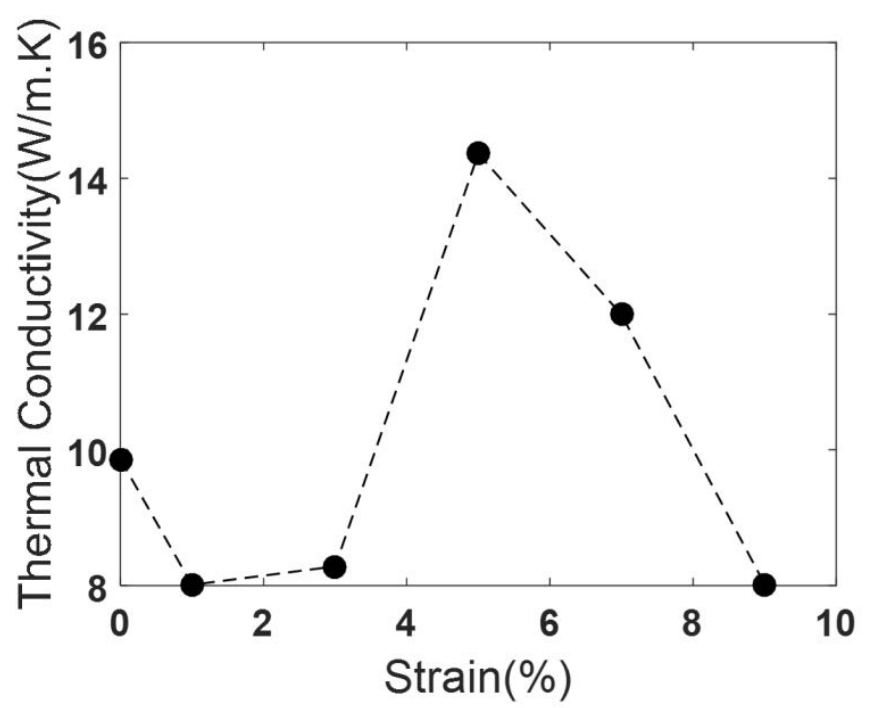

Figure 2. Strain variation of the thermal conductivity of $\boldsymbol{\beta}$-As with strain at $300 \mathrm{~K}$.

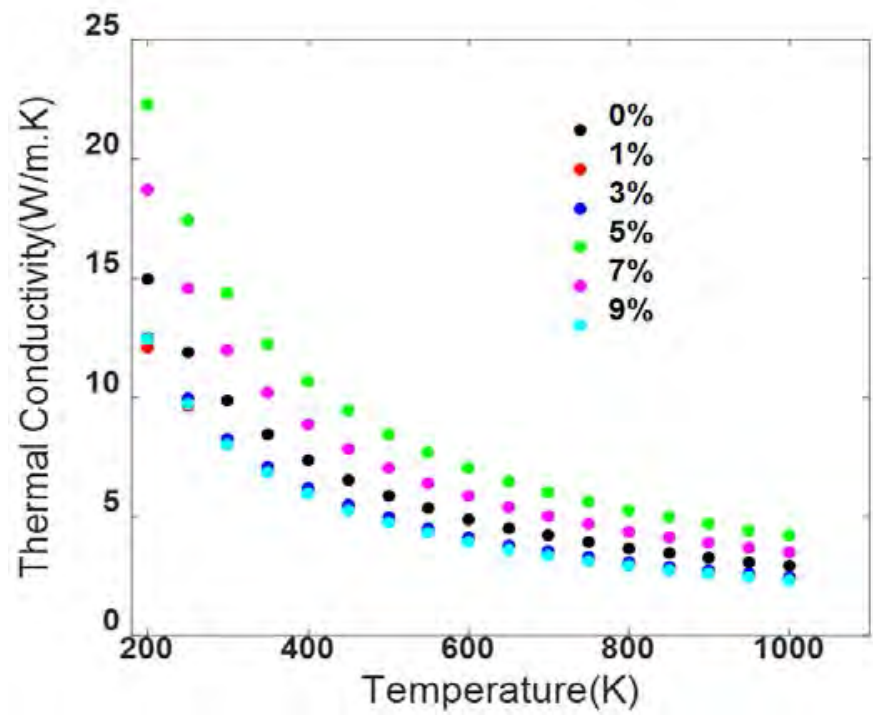

Figure 3. Variation of the thermal conductivity of $\boldsymbol{\beta}$-As with temperature for different strain levels.

symmetric directions of the Brillouin zone for strains from $0 \%$ to $6 \%$. There are two atoms in the unit cell $\boldsymbol{\beta}$-As. Thus, there are six phonon branches: three acoustic modes called ZA, TA and LA; and three optical modes. Fig. 4 shows that increasing strain has not identical effects on different phonon modes. For example, as strain increases the ZA mode becomes more linear (higher phonon frequencies), and the LA mode exhibits a lower slope (lower phonon frequencies). The TA mode is less affected by strain compared to LA and ZA. Optical modes are also affected by strain. All optical modes become lower for higher strains. The same effect was also observed in graphene, silicene, stanene, germanene, and $\boldsymbol{\beta}$-Sb $[1,9,10]$.
The group velocity of a phonon mode is defined as the derivative of the phonon frequency with respect to the wave vector. Based on Eq. (1), the thermal conductivity has a strong dependence on the group velocity. We present the group velocity of all phonon modes for strain values of $0 \%, 3 \%, 5 \%$ and $7 \%$ in Fig. 5. In correlation with the responses of the phonon dispersion branches, the strain does not have the same impact on the group velocities of different phonon modes. Regarding the ZA mode, the group velocity near the center of the BZ increases with increasing strain. The group velocity of the ZA near the $\Gamma$-point is $0.36 \mathrm{~km} / \mathrm{s}$ for strain $0 \%$ and increases to $1.30 \mathrm{~km} / \mathrm{s}$ as strain increases to $7 \%$. However, this is not the case for higher phonon frequencies of the ZA mode. As shown in Fig. 5, phonon group velocities of the ZA mode decreases as strain increases from $0 \%$ to $7 \%$. Based on Eq. (1), this effect reduces the thermal conductivity. Considering the TA mode, the group velocity decreases near the $\Gamma$-point as strain increases. The group velocity is $3.07 \mathrm{~km} / \mathrm{s}$ in unstrained $\boldsymbol{\beta}$-As, and it is $2.68 \mathrm{~km} / \mathrm{s}$ for strain of $7 \%$. For other frequencies of the TA mode, considerable changes are not observed.

The reduction in group velocities as strain increases is also observed in the LA modes in Fig. 5. Unstrained $\boldsymbol{\beta}$-As has the highest group velocities which decrease with increasing strain. In consequence, one could infer that the trend observed in group velocities of the acoustic modes leads to smaller thermal conductivities as strain increases. Group velocities of the three optical modes generally increases as strain increases, which is more obvious in the first two optical modes. However, since the group velocities of optical modes are smaller in magnitude than those of acoustic modes, it is predicted that the most important contribution in thermal conductivity of $\boldsymbol{\beta}$-As comes from acoustic phonon modes. Therefore, regarding the group velocities, strain has a decreasing effect on thermal conductivity, as previously reported for $\boldsymbol{\beta}-\mathrm{Sb}[10]$.

The other phonon property that has a crucial role in determining the thermal conductivity of a material is the phonon lifetime. Based on Eq. (1), higher phonon lifetimes result in higher thermal conductivities. The phonon lifetime of the acoustic and optical modes obtained from the iterative solution of BTE is plotted in Fig. 6. Since optical modes have smaller phonon lifetimes and group velocities than acoustic phonon modes, here we limit our discussion to the acoustic modes. Considering the ZA mode, the phonon lifetimes of the low-frequency phonons at $0 \%$ of strain are the highest. As shown in Fig. 6, the ZA mode of the unstrained structure has higher phonon lifetimes in the low-frequency region while the phonon lifetimes for $3 \%, 5 \%$ and $7 \%$ of strain in the same region are almost the same. However, the situation is different in the high-frequency region. As the strain increases from $3 \%$ to $5 \%$, the phonon lifetimes of the ZA mode increases. To summarize, in the low-frequency region, the unstrained structure has the highest lifetimes among all strains considered. In the high-frequency region, $7 \%$ and $5 \%$ strains 


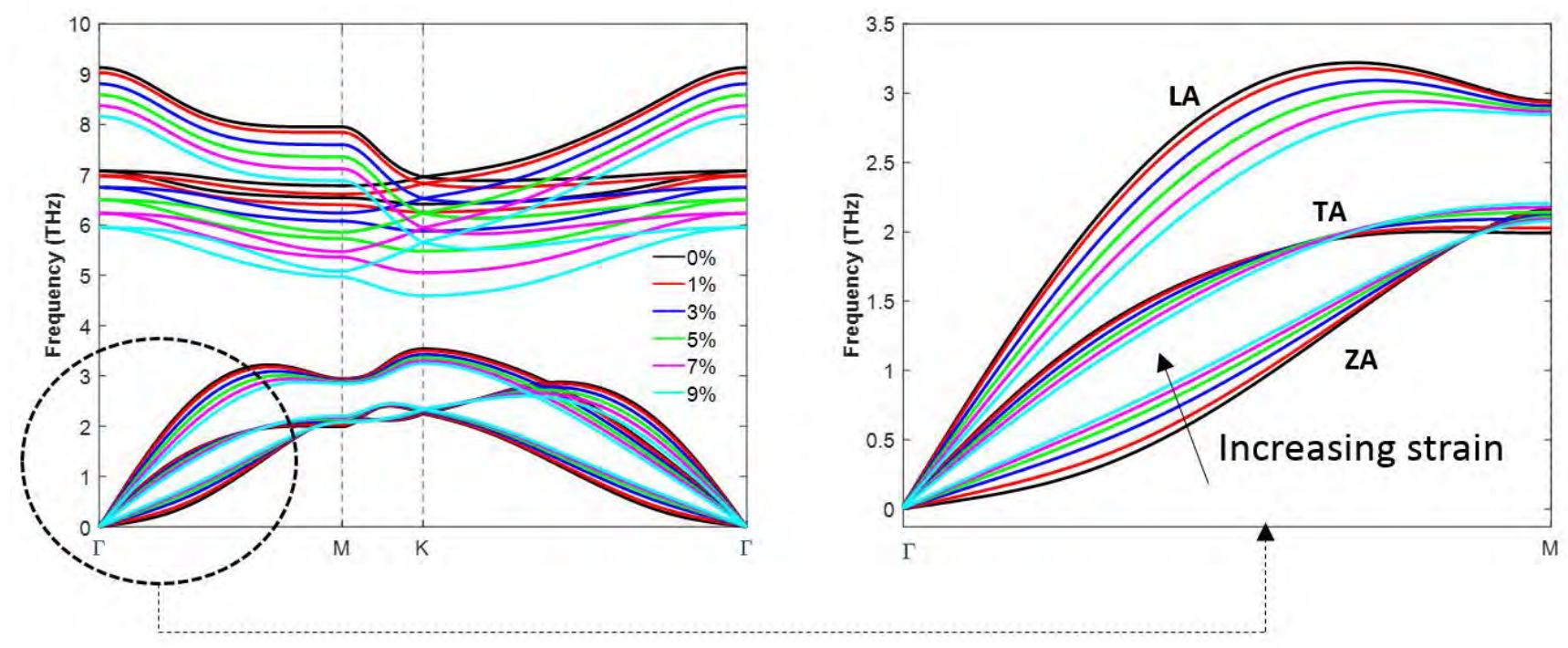

Figure 4. The phonon dispersion of buckled arsenene for different values of tensile strain.

result in higher phonon lifetimes than $1 \%$, which in turn exhibits higher lifetimes than $3 \%$. The situation is different in $\boldsymbol{\beta}$-Sb [10], were the phonon lifetimes of the ZA mode were found to increase as strain increases from $-1 \%$ to $6 \%$, both in the low- and the high-frequency region. Regarding the TA and LA phonon modes, the phonon lifetimes increased as strain increased, except for the $0 \%$ and $3 \%$ strains, with $3 \%$ resulting in lower lifetimes than $1 \%$. Overall, for all strain levels considered here, as strain increases, the phonon lifetimes of TA, LA and high-frequency ZA modes also increase. The only exception is for $3 \%$ strain, which resulted in lower lifetimes than those for $0 \%$ in all acoustic modes. Based on what has been discussed in this section, there is an interplay between group velocities and phonon lifetimes as strain increases. Group velocities are lower at higher strains, while phonon lifetime increases with increasing strain. This interplay is the main reason for the up and down variation of the thermal conductivity versus strain in buckled Arsenene.

\section{CONCLUSION}

In this work, strain effects on the phonon properties and thermal conductivities of buckled arsenene have been investigated. Six different strains have been considered ranging from $0 \%$ to $9 \%$. We found that thermal conductivity decreases as strain increases from $0 \%$ to $1 \%$, then it increases for strains in the range of $1 \%$ to a critical value of $5 \%$. Afterward, it decreases for strains between $5 \%$ and $9 \%$. Harmonic and anharmonic phonon properties have been analyzed to understand the causes of this variation. We observed that the cause of this variation is the interplay between the responses of the group velocities and phonon lifetimes of the acoustic modes to strain. The variation of the thermal conductivity of buckled arsenene with strain is different than that observed in other materials of the same family, such as buckled antimonene. This study revealed that tensile strain can have different effects on different 2D materials, even if they belong to the same family with similar structure.

\section{ACKNOWLEDGMENT}

This work was supported by the Natural Science and Engineering Research Council of Canada (NSERC), through the Discovery and the Collaboration Research and Development (CRD) grant program. Computations were performed on the GPC supercomputer at the SciNet HPC Consortium. SciNet is funded by the Canada Foundation for innovation under the auspices of Compute Canada; the Government of Ontario; Ontario Research fund- Research Excellence; and the University of Toronto. 

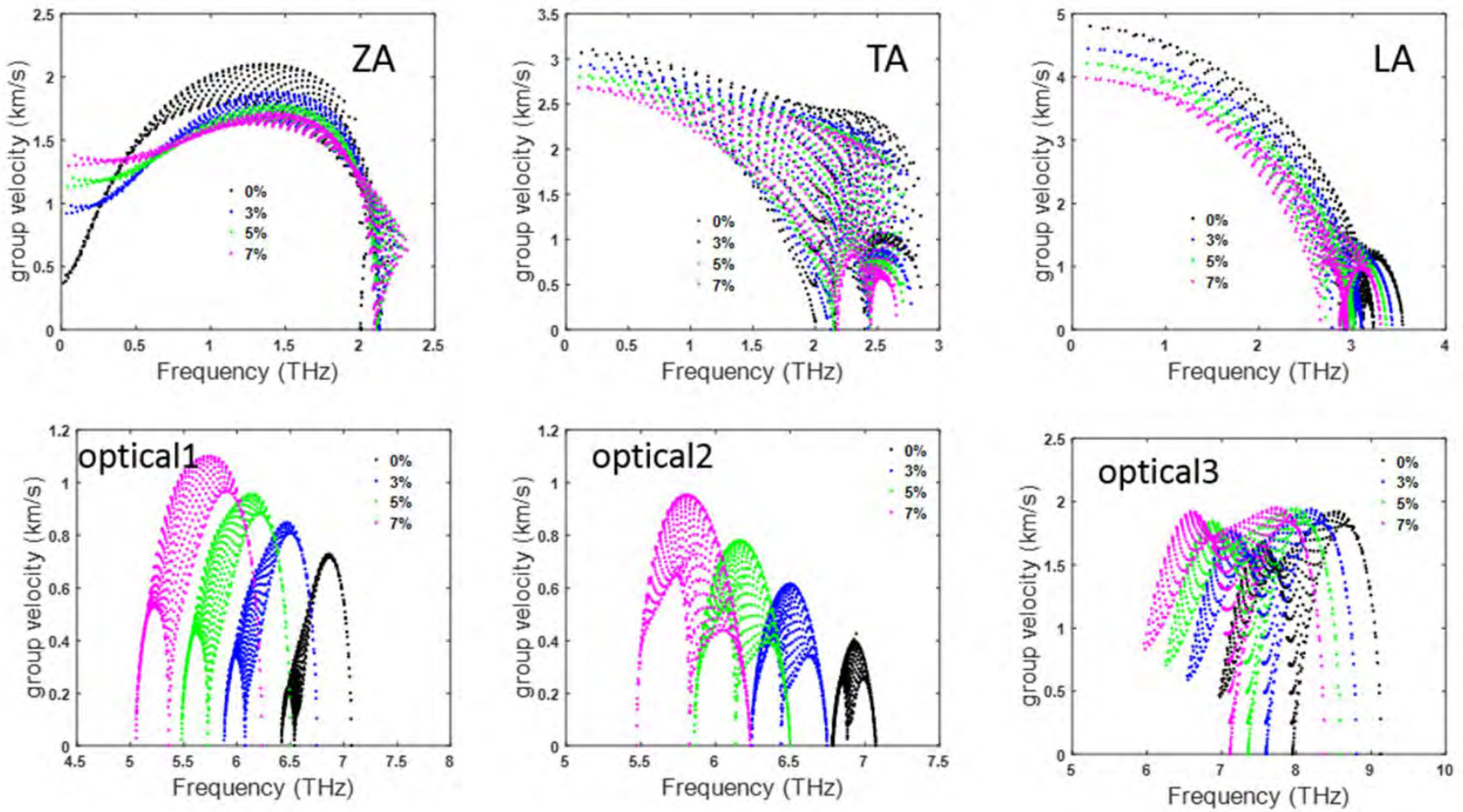

Figure 5. The phonon group velocity of acoustic and optical modes for strains of $0 \%, 3 \%, 5 \%$ and $7 \%$.
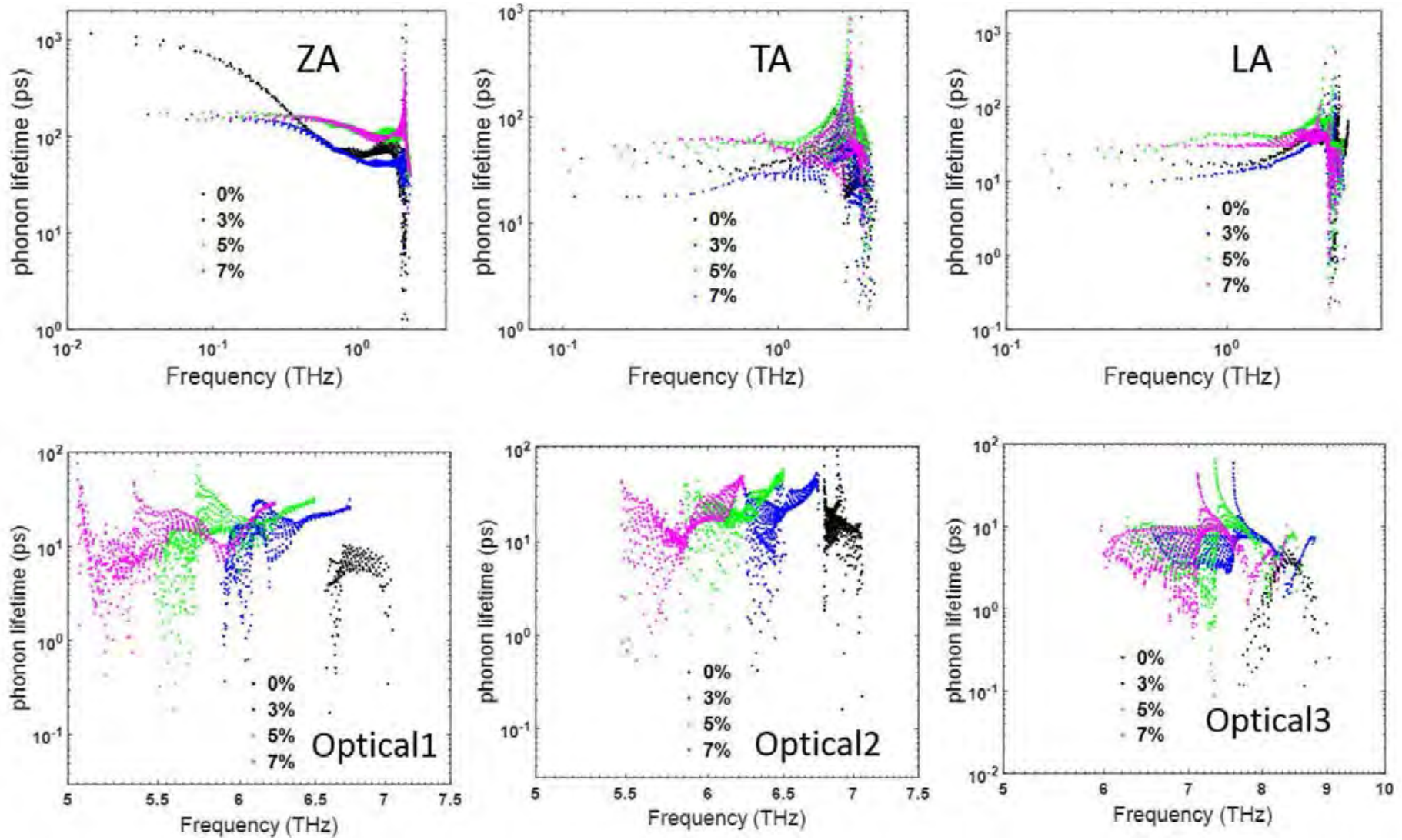

Figure 6. The phonon lifetime of acoustic and optical modes for strains of $0 \%, 3 \%, 5 \%$ and 7 


\section{REFERENCES}

[1] Y. Kuang, L. Lindsay, S. Shi, X. Wang, and B. Huang, "Thermal conductivity of graphene mediated by strain and size," Int. J. Heat Mass Transf., vol. 101, pp. 772-778, 2016.

doi: 10.1016/j.ijheatmasstransfer.2016.05.072.

[2] K. S. Novoselov, S. V. Morozov, D. Jiang, Y. Zhang, S. V. Dubonos, I. V. Grigorieva, A. A. Firsov, and A. K. Geim, "Electric Field Effect in Atomically Thin Carbon Films," Science (80-.) ., vol. 306, no. 5696, pp. 666-669, 2004.

[3] C. Da Silva, F. Saiz, D. A. Romero, and C. H. Amon, "Coherent phonon transport in short-period two-dimensional superlattices of graphene and boron nitride," Phys. Rev. B, vol. 93, no. 12, pp. 1-10, 2016. doi: 10.1103/PhysRevB.93.125427

[4] C. da Silva, J. Sborz, D. A. Romero, and C. H. Amon, "Thermal response of boron nitride and boron nitride-graphene nanosheets to uniaxial tensile strain," in Proceedings of the Canadian Society for Mechanical Engineering International Conference (CSME), 2014, pp. 1-5.

[5] S. Zhang, M. Xie, F. Li, Z. Yan, Y. Li, E. Kan, W. Liu, Z. Chen, and H. Zeng, "Semiconducting Group 15 Monolayers: A Broad Range of Band Gaps and High Carrier Mobilities," Angew. Chemie - Int. Ed., vol. 55, no. 5, pp. 1666-1669, 2016. doi: 10.1002/anie.201507568.

[6] C. Kamal and M. Ezawa, "Arsenene: Two-dimensional buckled and puckered honeycomb arsenic systems," Phys. Rev. B - Condens. Matter Mater. Phys., vol. 91, no. 8, 2015.

[7] H. Liu, A. T. Neal, Z. Zhu, Z. Luo, X. Xu, D. Tománek, and P. D. Ye, "Phosphorene: An unexplored 2D semiconductor with a high hole mobility," ACS Nano, vol. 8, no. 4, pp. 4033-4041, 2014.

doi: $10.1021 / \mathrm{nn} 501226 \mathrm{z}$.

[8] G. Zheng, Y. Jia, S. Gao, and S. H. Ke, "Comparative study of thermal properties of group-VA monolayers with buckled and puckered honeycomb structures," Phys. Rev. B, vol. 94, no. 15, pp. 1-6, 2016. doi: 10.1103/PhysRevB.94.155448.

[9] Y. D. Kuang, L. Lindsay, S. Q. Shi, and G. P. Zheng, "Tensile strains give rise to strong size effects for thermal conductivities of silicene, germanene and stanene," Nanoscale, vol. 8, no. 6, pp. 3760-3767, 2016. doi: 10.1039/C5NR08231E.

[10] A.-X. Zhang, J.-T. Liu, and S.-D. Guo, "Strain effects on phonon transport in antimonene from a first-principles study," Phys. Chem. Chem. Phys., vol. 19, pp. 14520-14526, 2017. doi: 10.1039/C7CP02486J.

[11] W. Li, J. Carrete, N. A. Katcho, and N. Mingo, "ShengBTE: A solver of the Boltzmann transport equation for phonons," Comput. Phys. Commun., vol. 185, no. 6, pp. 1747-1758, 2014.

doi: 10.1016/j.cpc.2014.02.015.
[12] P. Giannozzi, S. Baroni, N. Bonini, M. Calandra, R. Car, C. Cavazzoni, D. Ceresoli, G. L. Chiarotti, M. Cococcioni, I. Dabo, A. Dal Corso, S. De Gironcoli, S. Fabris, G. Fratesi, R. Gebauer, U. Gerstmann, C. Gougoussis, A. Kokalj, M. Lazzeri, L. Martin-Samos, . Marzari, F. Mauri, R. Mazzarello, S. Paolini, A. Pasquarello, L. Paulatto, C. Sbraccia, S. Scandolo, G. Sclauzero, A. P. Seitsonen, A. Smogunov, P. Umari, and R. M. Wentzcovitch, "QUANTUM ESPRESSO: A modular and open-source software project for quantum simulations of materials," J. Phys. Condens. Matter, vol. 21, no. 39, 2009. doi: 10.1088/0953-8984/21/39/395502.

[13] B. Peng, D. Zhang, H. Zhang, H. Shao, G. Ni, Y. Zhu, and H. Zhu, "The conflicting role of buckled structure in phonon transport of 2D group-IV and group-V materials," Nanoscale, vol. 9, no. 22, pp. 7397-7407, 2017. doi: $10.1039 / \mathrm{C} 7 \mathrm{NR} 00838 \mathrm{D}$.

[14] A. Jain and A. J. H. McGaughey, "Strongly anisotropic in-plane thermal transport in single-layer black phosphorene," Sci. Rep., vol. 5, no. 1, p. 8501, 2015. doi: 10.1038/srep08501.

[15] M. Zeraati, S. M. Vaez Allaei, I. Abdolhosseini Sarsari, M. Pourfath, and D. Donadio, "Highly anisotropic thermal conductivity of arsenene: An ab initio study," Phys. Rev. B, vol. 93, no. 8, pp. 1-5, 2016.

doi: 10.1103/PhysRevB.93.085424. 


\title{
The Design of Infrared Mirror Coatings for the Enhanced Performance of Incandescent Lighting
}

\author{
Mohsen Rostami, Nima Talebzadeh, Paul G. O’Brien \\ Department of Mechanical Engineering \\ Lassonde School of Engineering, York University \\ Toronto, Canada \\ msnros@yorku.ca, paul.obrien@lassonde.yorku.ca
}

\begin{abstract}
Herein we present the design of infrared mirror coatings for the enhanced performance of incandescent lighting. We consider single and stacked dielectric mirrors comprised of alternating layers of $\mathrm{TCO}$ and $\mathrm{SiO}_{2}$ nanoparticle films to function as infrared mirrors that reduce heat losses in incandescent lights. In this work, thin-film theory was employed to develop MATLAB code that calculates the reflectance and transmittance spectra of dielectric mirrors. In order to validate the MATLAB code, we compared our results to experimental results reported in the literature as well as results calculated using COMSOL Multiphysics software. Our results show that an infrared dielectric mirror coated onto the glass bulb of an incandescent light can increase its efficiency by $\sim 32 \%$. However, stacked dielectric mirror coatings prevent a significant portion of visible light from transmitting through the glass bulb, and consequently decrease the efficiency of incandescent lights by $\sim 6 \%$.
\end{abstract}

Keywords- Infrared Reflectors; Thin-films; Nanoparticle Films; Lighting Technology; Optical films

\section{INTRODUCTION}

Since the invention of early commercial light bulbs in 1880 by Thomas Edison ${ }^{1}$, they have been used widely as a main source of illumination. By heating a filament wire covered by a glass bulb, the incandescent light bulb produces light. Usually the glass bulb is occupied by an inert gas to prevent the filament from burning. ${ }^{2}$ However, incandescent lights are being replaced by a new generation of more efficient lights, such as light-emitting diodes. ${ }^{3}$ One way to improve the efficiency of incandescent light bulbs is to reduce their radiative heat losses by preventing them from emitting near-infrared light. Herein we consider the design of an optical coating that achieves high reflectance in the near-infrared region and high transmittance in visible region. This can be achieved by using either a dielectric/metal film stack, ${ }^{4,5}$ a dielectric multi-layered film, ${ }^{6}$ or metallic photonic band gap (PBG) structures ${ }^{7}$. In this study, we focus on a dielectric mirror (often referred to as a Braggreflector or one-dimensional photonic crystal) comprised of alternating layers of transparent conducting oxide (TCO) and $\mathrm{SiO}_{2}$ nanoparticle (NP) films with indices of refraction of $\mathrm{n}=2$ and $\mathrm{n}=1.3$, respectively. In previous work dielectric mirrors comprised of alternating layers of TCO and $\mathrm{SiO}_{2} \mathrm{NP}$ films have been designed to selectively reflect light strongly over one spectral region while being highly transmissive over other spectral regions. These dielectric mirrors have been used as semi-transparent reflectors in "see-through" and multi-junction silicon-based photovoltaic cells. ${ }^{8,9}$ Furthermore, in order to expand the width of the spectral region over which the dielectric mirror reflects light, one can fabricate a coating comprised of two stacked dielectric mirrors. ${ }^{10}$ In this work we calculate and compare the efficiency of incandescent mirrors for the case in which the glass bulb is (1) bare, (2) coated with a dielectric mirror with a peak reflection in the infrared region and (3) coated with stacked dielectric mirrors that exhibit an extended reflection peak in the infrared region.

\section{METHOD}

A dielectric mirror is comprised of alternating films with a higher (denoted as $\mathrm{H}$ ) and lower (denoted as L) index of refraction. Thin-film theory can be used to calculate the infrared reflectance and visible light transmittance for a dielectric mirror ${ }^{11}$ using the refractive indices and layer thickness of the films within the dielectric stack as input. The theory uses matching boundary conditions for Maxwell's equations, and more information is available in the literature ${ }^{12}$. Using thin-film theory, and with reference to Figure 1, the reflectance, $\mathrm{R}$, and transmittance, $\mathrm{T}$, at a surface are provided by Equations 1 and 2, respectively.

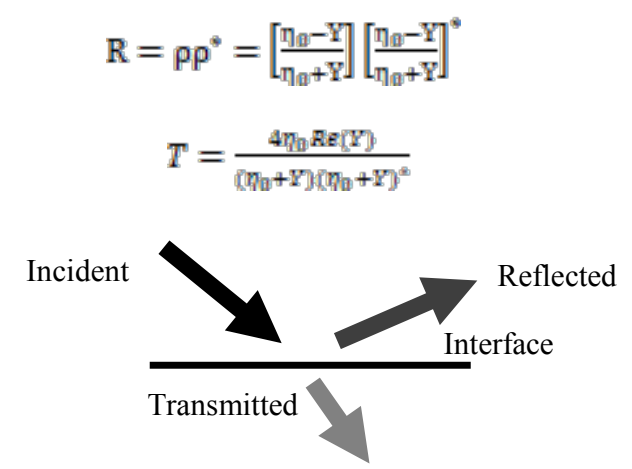

Figure 1. Transmittance and Reflection at an interface between two media 
Where $\rho$ is the amplitude of the reflection coefficient, $\eta_{0}$ represents the admittance of the incident medium and $\mathrm{Y}$ is the admittance of the medium. Also, $R e$ denotes the real part of $\mathrm{Y}$ in the Equation 2. However, Equations 1 and 2 are valid only for normal incidence. For the case of oblique incidence, one can define p-polarized and s-polarized light which are electric vectors parallel and perpendicular to the plane of incidence, respectively (Figure 2). Consequently, the new expressions will be defined with the tilted admittance for s-polarized and ppolarized light as in Equations 3 and 4:

$$
\begin{array}{ll}
\eta_{s}=y \cos \theta=(n-i k) \cos \theta & \text { for s-polarized light } \\
\eta_{p}=y / \cos \theta=(n-i k) / \cos \theta & \text { for p-polarized light }
\end{array}
$$

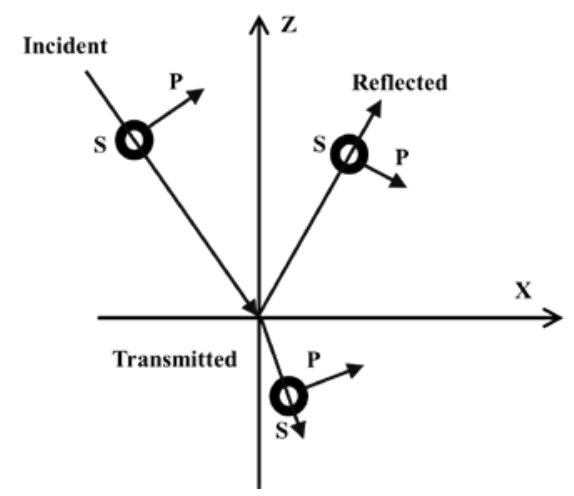

Figure 2. P-polarized and s-polarized light based for oblique incidence.

\section{A. Matlab Code Validation}

We developed a MATLAB code, based on the methods described in the previous section, that calculates the reflectance and transmittance spectra of dielectric mirrors. To validate the code we calculated and compared the reflectance and transmittance of dielectric mirrors to those studied in the literature. For example, we calculated the reflectance from a dielectric mirror comprised of nine layers of polystyrene and tellurium and the results are compared to experimentally measured results ${ }^{12}$ in Figure 3. We also calculated the transmittance of dielectric mirrors comprised of alternating layers of indium-tin oxide (ITO) and $\mathrm{SiO}_{2}$ nanoparticle films and compared our results to experimentally measured results ${ }^{10}$. As shown in Figure 4, the reflectance and transmittance spectra calculated using our MATLAB code provide a good approximation to those reported in the literature. Discrepancies between the calculated and experimentally measured spectra shown in Figures 3 and 4 are attributed to differences between the thickness or index of refraction values used in the experiment as compared to the values assigned to parameters in the analytical method. We also compared reflectance and transmittance spectra calculated using our MATLAB code and COMSOL Multiphysics software (version 5.3), which offers two separate modules that can be used for thin-film calculations (Ray Optics and Wave Optics). The results from our MATLAB code are compared with those from COMSOL in Figures 5 and 6 . The results from both ray optics and wave optics modules agree well with those of the MATLAB code.

\section{DIELECTRIC MIRROR DESIGN}

We design a one-dimensional dielectric mirror (otherwise referred to as a Bragg-reflector) to function as a coating for an incandescent light bulb that internally reflects infrared radiation back towards the light filament to reduce radiative heat losses. The term "one-dimensional" in our case refers to the fact that there is no variation in the dielectric function, $\varepsilon$, along directions excluding (z). ${ }^{14}$ In this report, TCO $(n=2)$ is assumed to be the high index of refraction film, represented by $\mathrm{H}$, and a $\mathrm{SiO}_{2} \mathrm{NP}$ film $(\mathrm{n}=1.3)$ is assumed to be the low index of refraction film, which is represented as $\mathrm{L}$ in the stacking sequence shown in Figure 7.

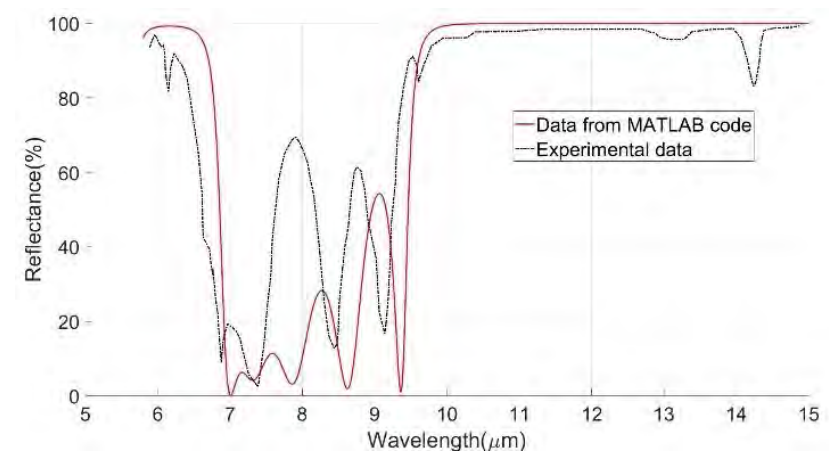

Figure 3. Experimentally measured and calculated (MATLAB code) reflectance spectra of a dielectric mirror comprised of nine layers of polystyrene and tellurium ${ }^{11}$.

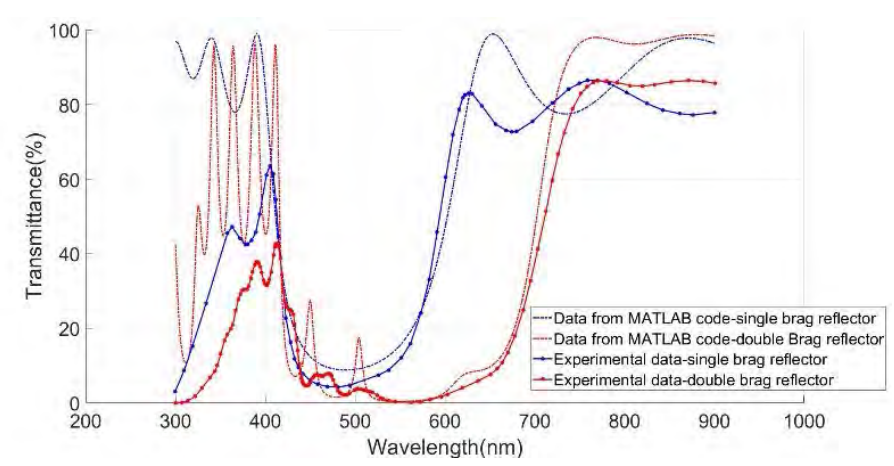

Figure 4. Experimentally measured and calculated (MATLAB code) transmittance spectra of dielectric mirrors comprised of alternating layers of ITO and $\mathrm{SiO}_{2}$ nanoparticle films ${ }^{8}$.

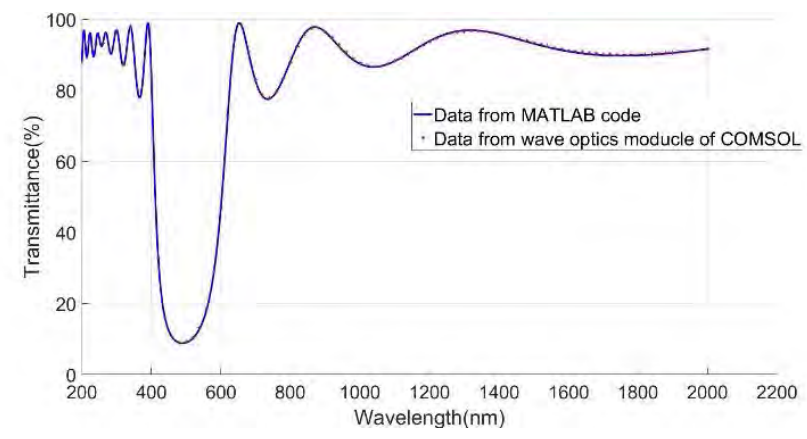

Figure 5. Transmittance of a dielectric mirror comprised of alternating layers of TCO and $\mathrm{SiO}_{2}$ nanoparticle films calculated using our MATLAB code and

COMSOL Multiphysics software (five layers of TCO and $\mathrm{SiO}_{2}$ with thicknesses of 60 and $90 \mathrm{~nm}$, respectively ). 
Figure 7 shows a dielectric mirror with a stacking sequence of $[\mathrm{HLH}]^{10}$ where $\mathrm{H}$ and $\mathrm{L}$ represent the TCO and $\mathrm{SiO}_{2} \mathrm{NP}$ films, respectively. As discussed in the following section, the thicknesses of the TCO and $\mathrm{SiO}_{2}$ films were chosen such that the spectral position of the reflectance peak minimizes the radiative heat losses from the incandescent bulb.

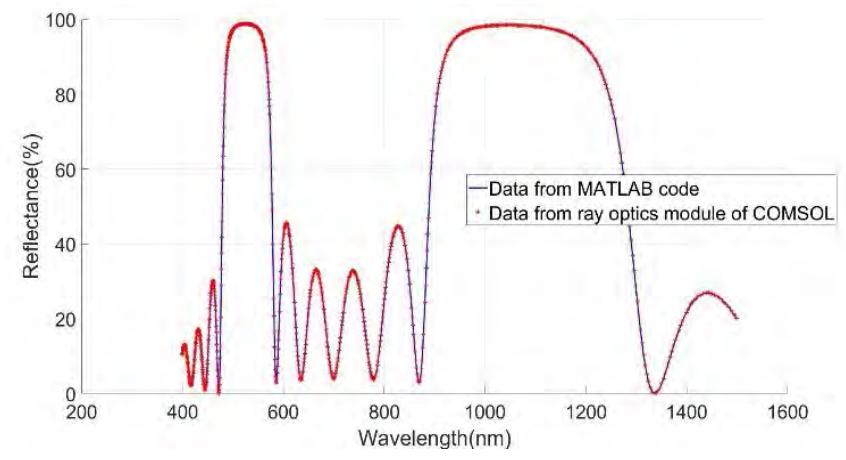

Figure 6. Reflectance spectra from a dielectric mirror comprised of alternating layers of $\mathrm{TCO}$ and $\mathrm{SiO}_{2}$ nanoparticle films calculated using our MATLAB code and COMSOL Multiphysics software (five layers of TCO and

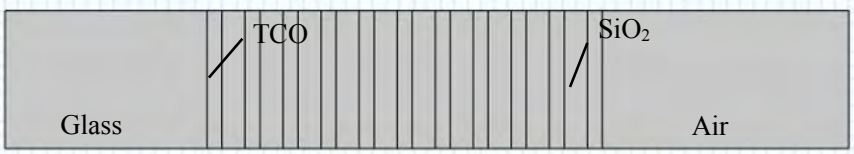

$\mathrm{SiO}_{2}$ with thicknesses of 73 and $257 \mathrm{~nm}$, respectively ).

Figure 7. A one-dimensional dielectric mirror comprised of alternating layers of $\mathrm{TCO}$ and $\mathrm{SiO}_{2}$ films. This dielectric mirror is coated onto glass.

\section{A. Light Bulb Blackbody Radiation Spectrum}

Herein we assume the spectral distribution of radiation emitted from an incandescent bulb approximates that of a blackbody at a temperature of $3300 \mathrm{~K}$, which is the operating temperature of an incandescent light bulb filament ${ }^{15}$. As can be seen in the Figure 8 , the highest intensity emitted from a blackbody at a temperature of $3300 \mathrm{~K}$ occurs at a wavelength of $880 \mathrm{~nm}$, and therefor we choose this as the reference wavelength for the Bragg-peak position of the dielectric mirror. It follows that the thicknesses of the TCO and $\mathrm{SiO}_{2}$ nanoparticle films in a quarter-wave stack with a reflectance peak wavelength of $880 \mathrm{~nm}$ is $110 \mathrm{~nm}$ and $169.5 \mathrm{~nm}$, respectively. Considering the dielectric mirror with a stacking sequence of $[\mathrm{HLH}]^{10}$ shown in Figure 7 , the values of $\mathrm{H}$ and $\mathrm{L}$ would be $55 \mathrm{~nm}$ and $169.5 \mathrm{~nm}$, respectively. The superscript ' 10 ' indicates that the $[\mathrm{HLH}]$ stacking sequence is repeated 10 times; in this case the uppermost and bottommost films are 55 $\mathrm{nm}$ thick TCO films, which act as optical matching layers that reduce reflection outside the Bragg-reflection peak.

\section{B. Results}

The reflectance spectrum for the $[\mathrm{HLH}]^{10}$ stack comprised of $110 \mathrm{~nm}$ thick TCO films and $169.5 \mathrm{~nm}$ thick $\mathrm{SiO}_{2} \mathrm{NP}$ films is shown in Figure 9. This reflectance spectrum exhibits a strong reflectance peak with a full-width at half-maximum of
$360 \mathrm{~nm}$, starting at $770 \mathrm{~nm}$ and ending at $1030 \mathrm{~nm}$, and a maximum reflectance value of $\sim 100 \%$ in the spectral vicinity of $880 \mathrm{~nm}$. The other spectral regions, including the visible and infrared, show some fluctuations with the reflectance value below $30 \%$ over most of these regions. As shown in Figure 8, a large portion of the radiation emitted from a blackbody at a temperature of $3300 \mathrm{~K}$ is emitted with a wavelength greater than $1030 \mathrm{~nm}$. In order to extend the high reflectance values for the quarter wave stack shown in Figure 9 to wavelengths beyond $1030 \mathrm{~nm}$, one can stack a second dielectric mirror onto the original dielectric mirror, which will be discussed in the next section.

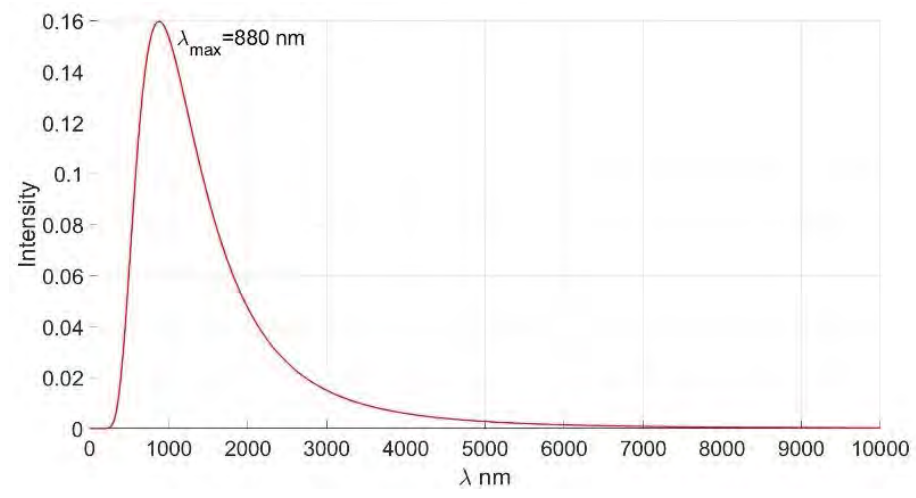

Figure 8. Radiation spectrum for a blackbody at a temperature of $3300 \mathrm{~K}$.

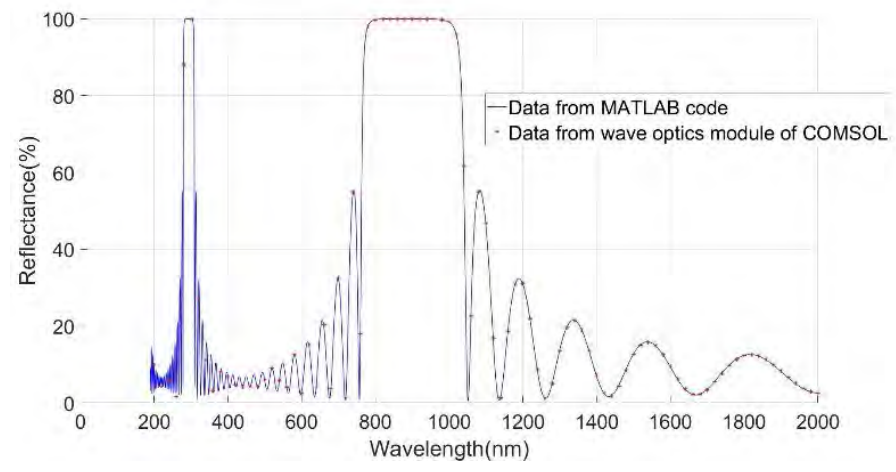

Figure 9. Reflectance spectrum for a quarter-wave stack comprised of alternating layers of TCO and $\mathrm{SiO}_{2} \mathrm{NP}$ films with thicknesses of $110 \mathrm{~nm}$ and $169.5 \mathrm{~nm}$, respectively.

\section{DOUBLE BRAGG REFLECTOR DESIGN AND CORRESPONDING RESULTS}

The reflectance spectra shown in Figure 9 can be extended to wavelengths greater than $1030 \mathrm{~nm}$ by stacking the initial dielectric mirror, which has a peak reflection wavelength of $880 \mathrm{~nm}$, with a second dielectric mirror that has a different reflection peak position. The stacked dielectric mirrors, shown in Figure 10 and referred to as a 'double stack', can be designed with a layered configuration denoted as $[\mathrm{hLh}]^{10}[\mathrm{HLH}]^{10}$. In this notation $[\mathrm{HLH}]^{10}$ represents the initial quarter-wave stack comprising 10 bilayers with $\mathrm{L}=169.5 \mathrm{~nm}$ thick $\mathrm{SiO}_{2} \mathrm{NP}$ films and $\mathrm{H}=55 \mathrm{~nm}$ thick TCO films. The second dielectric stack, denoted as $[\mathrm{hLh}]^{10}$, is also comprised of TCO and $\mathrm{SiO}_{2}$ films, although the thickness of the TCO films 
have been increased to $169.5 \mathrm{~nm}$ (with $\mathrm{h}=84.75 \mathrm{~nm}$ ) to move the reflection peak position further into the infrared spectral region. The maximum Bragg-peak reflectance wavelength for the $[\mathrm{hLh}]^{10}$ stack is $\lambda_{\mathrm{p}}=2 .\left(\mathrm{t}_{\mathrm{L}} \cdot \mathrm{n}_{\mathrm{L}}+\mathrm{t}_{\mathrm{H}} \cdot \mathrm{n}_{\mathrm{h}}\right) \approx$ $2 \times(169.5 \mathrm{~nm} \cdot 2+169.5 \mathrm{~nm} \cdot 1.3) \approx 1119 \mathrm{~nm}$.

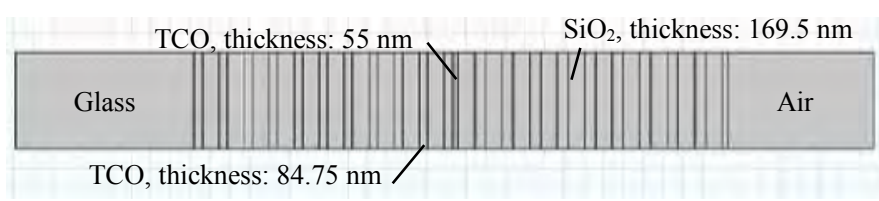

Figure 10. The one-dimensional multilayer film used for the single Bragg reflector design

The reflectance spectra calculated using our MATLAB code for the single and double-stacked dielectric mirrors are shown in Figure 11. The double-stacked dielectric mirror is strongly reflecting over a broad spectral region extending beyond 800 to $1200 \mathrm{~nm}$. We determine the amount of radiant power emitted from the incandescent light by multiplying the blackbody radiation spectra from the light filament with the transmission spectra of the glass bulb for the cases in which it is bare, coated with a single dielectric mirror and coated with the stacked dielectric mirror, and the results are shown in Figure 12.

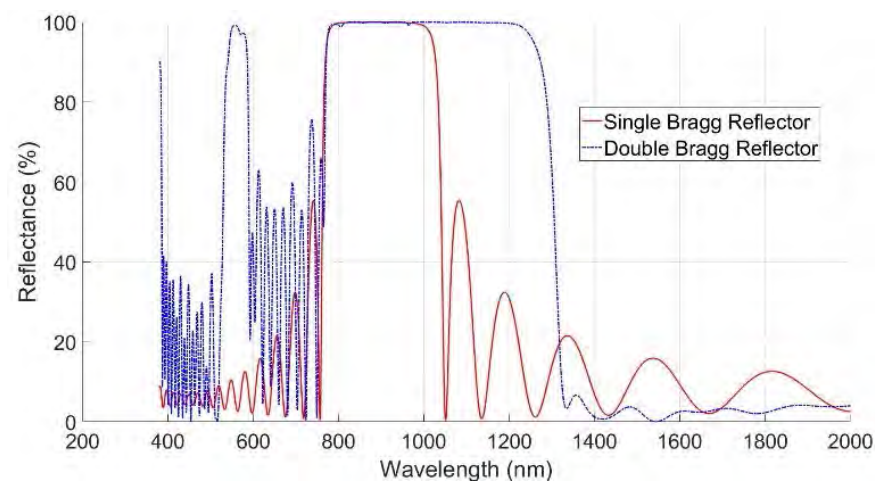

Figure 11. Reflectance spectrum for the "double stack" comprised of alternating layers of TCO and $\mathrm{SiO}_{2} \mathrm{NP}$ films with a $[\mathrm{hLh}]^{10}[\mathrm{HLH}]^{10}$ stacking sequence

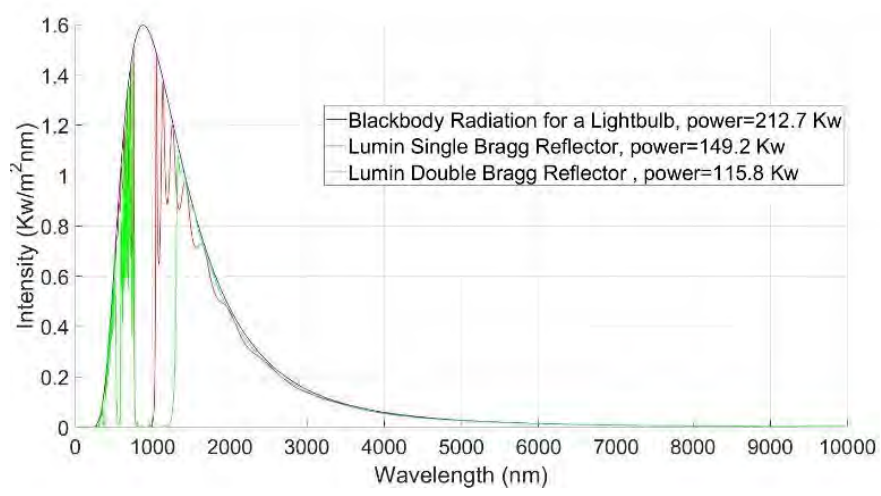

Figure 12. The radiation spectra for a blackbody at a temperature of $3300 \mathrm{~K}$ (blue curve) and the intensity of radiation emitted from a blackbody that is transmitted through the $[\mathrm{HLH}]^{10}$ Bragg reflector and the "double-stack" with a $[\mathrm{hLh}]^{10}[\mathrm{HLH}]^{10}$ stacking sequence.

\section{LIGHT BULB EFFICIENCY}

The lumens emitted from a light bulb with a filament at a temperature of $3300 \mathrm{~K}$ for the case in which the glass bulb is: bare, coated with the $[\mathrm{HLH}]^{10}$ quarter wave stack, or coated with the "double-stack" with a $[\mathrm{hLh}]^{10}[\mathrm{HLH}]^{10}$ stacking sequence, are shown in Figure 13. For each of these cases, the transmitted irradiance of the light bulb was multiplied by the CIE 1978 eye sensitivity function ${ }^{17}$ to determine the transmitted lumens ${ }^{18}$. The lumens transmitted for the case of the stacked dielectric mirror is a lot less than the lumens transmitted for the case of the bare glass bulb or single dielectric mirror coating. The corresponding values are equal to $9.51,8.785$ and $3.44 \mathrm{Klm} / \mathrm{m}^{2}$ for the case the bare lightbulb, the single dielectric mirror and the stacked mirror, respectively.

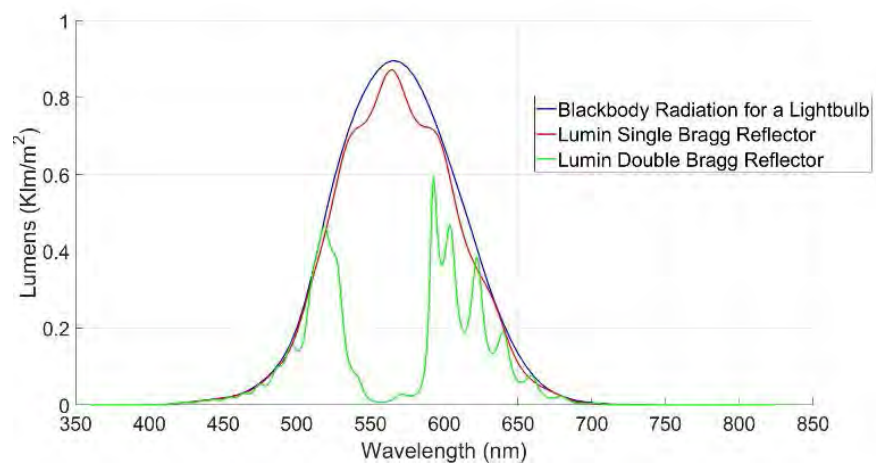

Figure 13. The lumens emitted from a light bulb with a filament at a tempeature of $3300 \mathrm{~K}$ for the case in which the glass bulb is: bare (blue curve), coated with the $[\mathrm{HLH}]^{10}$ quarter wave stack (red curve), or coated with the "double-stack" with a $[\mathrm{hLh}]^{10}[\mathrm{HLH}]^{10}$ stacking sequence.

In this work we assume the temperature of the filament in the incandescent light is $3300 \mathrm{~K}$, regardless of what coating is applied to the bulb. However, the power consumed by the filament to reach a temperature of $3300 \mathrm{~K}$ depends on the optical coating applied to the glass bulb. The efficiency of the bulb can be estimated using Equation 5.

$$
\eta=\frac{\Phi}{\text { power }}
$$

Where the power in the denominator is the total radiant power emitted by the light over all spectral regions, and $\Phi$ is the power of light emitted in the visible spectral region. It follows that the efficiency of the incandescent light bulb for the case in which the glass bulb is bare is $4.47 \%$. Moreover, this efficiency can be increased to $5.89 \%$ by coating the glass bulb with the dielectric mirror comprised of $\mathrm{TCO}$ and $\mathrm{SiO}_{2} \mathrm{NP}$ films with a $[\mathrm{HLH}]^{10}$ stacking sequence and a Bragg-reflectance peak position of $880 \mathrm{~nm}$. However, the efficiency decreases to $2.97 \%$ if the glass bulb is coated with the "double-stack" with the $[\mathrm{hLh}]^{10}[\mathrm{HLH}]^{10}$ stacking sequence.

\section{CONCLUSION}

In this work we presented the design of a dielectric mirror coating that improves the efficiency of incandescent light bulbs. The dielectric mirror is comprised of alternating layers of TCO and $\mathrm{SiO}_{2} \mathrm{NP}$ films with indices of refraction of 2 and 1.3 , respectively. The thicknesses of these $\mathrm{TCO}$ and $\mathrm{SiO}_{2}$ are 
set such that the dielectric mirror has a peak reflectance value at the same wavelength as the maximum of the radiation spectrum of the filament within the incandescent bulb. For example, we considered a filament operating temperature of $3300 \mathrm{~K}$, which has a radiation spectrum peak value of $880 \mathrm{~nm}$, and the dielectric mirror was designed such that its peak reflectance value is also at $880 \mathrm{~nm}$. Our results show that the dielectric mirror coating increases the efficiency of the incandescent light by $\sim 32 \%$.

\section{ACKNOWLEDGEMENTS}

This research was supported by the Natural Sciences and Engineering Research Council of Canada

\section{REFERENCES}

[1] F. Robert and P. Israel, Edison's Electric Light: Biography of an Invention. New Brunswick, New Jersey: Rutgers University Press, 1986.

[2] M. K. Giridharan, "Chapter 3," in Electrical Systems Design, I .K. International Pvt, 2010.

[3] O. Ilic, G. Chen, I. Celanovic, P. Bermel, J. D. Joannopoulos, and M. Soljacic, "Tailoring high-temperature radiation and the resurrection of the incandescent source, Nature Nanotechnology, vol. 11, no. 4, pp. 320-324, Jan. 2016. doi: 10.1038/nnano.2015.309.

[4] J. Brett, R. Fontana, P. Walsh, S. Spura, L. Parascandola, W. Thouret, and L. Thorington, "Development of high energy-conserving incandescent lamps," J. Illuminating Eng. Soc. vol. 10 no. 4, pp. 214-218, Sept. 1980. doi: 10.1080/00994480.1980.10748614.

[5] I. S. Goldstein, R. P. Fontana and L. Thorington, "The design, construction and performance of an incandescent light source with a transparent heat mirror," Lighting Research Tech., vol. 18, pp. 93-97, June 1986. doi: 10.1177/096032718601800206.
[6] Y.-S. Kim, A. Chang, S.H. Lin, and J.-H. Lee, "Analysis of photon recycling using metallic photonic crystal," J. Appl. Phys., vol. 102, no. 6, p. 063107, Oct. 2007. doi: $10.1063 / 1.2779271$.

[7] S.-Y. Lin and Y.-S. Kim, A cool light bulb, SPIE, 2008.

[8] Y. Yang, P. G. O'Brien, G. A. Ozin, N. P. Kherani, "See-through amorphous silicon solar cells with selectively transparent and conducting photonic crystal back reflectors for building integrated photovoltaics," Appl. Phys. Lett., vol. 103, 221109. doi: 10.1063/1.4833542.

[9] B. Ramautarsingh, P. O'Brien, A. Flood, and N. Kherani, "Quantum efficiency enhancement in multi-junction solar cells with spectrally selective and conducting 1D photonic crystals" J. Mater. Chem. C, vol.4, pp. 9276-9286, 2016. doi: 10.1039/C6TC02898E.

[10] P. G. O'Brien et al., "Selectively transparent and conducting photonic crystal solar spectrum splitters made of alternating sputtered indium-tin oxide and spincoated silica nanoparticle layers for enhanced photovoltaics," Sol. Energy Mater. Sol. Cells, vol. 102, pp. 173-183, 2012. doi: 10.1016/j.solmat.2012.03.005.

[11] J. Zhang, "Hyper-NA optical systems and applications in sample measurement," Appendix F, Thesis Report, University of Arizona, 2010.

[12] H. A. Macleod, Thin Films Optical Filters, Fourth Edition, Taylor and Francis Group, 2010. doi: 10.1201/9781420073034.

[13] Y. Fink, J. N. Winn, S. Fan, C. Chen. J. Michel, J. D. Joannopoulos, and E. L. Thomas, "A Dielectric Omnidirectional Reflector," Science, vol. 282, is.5394, pp. 1679-1682. 1998. doi: 10.1126/science.282.5394.1679.

[14] J. D. Joannopoulos, S. G. Johnson, J. N. Winn, and R. D. Meade, "Chapter 4 - The Multilayer Film: A One-Dimensional Photonic Crystal," in PhotonicCrystals: Molding the Flow of Light, Princeton University Press, 2008.

[15] A. C. Wilbraham, et. al., Prentice Hall Chemistry, Pearson Prentice Hall, 2006.

[16] G. B. Rybicki, A. P. Lightman, Radiative Processes in Astrophysics, John Wiley \& Sons, 1979.

[17] E. F. Schubert, "Ch. 16. Human eye sensitivity and photometric qualities," in Light-Emitting Diodes, 2nd edition, Cambridge University Press, 2006. doi: 10.1017/CBO9780511790546.

[18] P. G. O'Brien, A. Chutinan, P. Mahtani, K. Leong, G. A. Ozin, and N. P. Kherani, "Selectively transparent and conducting photonic crystal rear-contacts for thin-film silicon-based building integrated photovoltaics," Opt. Exp., vol. 19, no. 18, 2011. doi: 10.1364/OE.19.017040. 


\title{
Composite eat Sink LED Cooling
}

\author{
M. J. Gibbons, C. Feng, S. Chandra \\ Department of Mechanical and Industrial Engineering, University of Toronto, Toronto, ON, Canada
}

\begin{abstract}
Metal coating of 3D printed polymers is an attractive proposition for thermal dissipation of light emitting diodes, due to its high efficiency and markedly lower material costs than conventional aluminum heat sinks. Efficient thermal cooling of light emitting diodes is essential in maintaining electronic and optical performance. The thermal performance of three heat sink designs were experimentally investigated for three applied heat fluxes $\left(0.5-1.5 \mathrm{~W} \mathrm{~cm}^{-2}\right)$. The results show that metal coating of the polymer heat sinks enables significant heat transfer enhancement of $37 \%$ over the uncoated case. The inclusion of an aluminum insert into the base of the composite heat sink design, in tandem with the zinc coating reduced on chip temperatures by $27 \%$ over the zinc coated case for the greatest applied heat flux.
\end{abstract}

Keywords: Light emitting diode; Heat transfer; Metal spray coating; Composite heat sink

\section{INTRODUCTION}

Light emitting diodes (LEDs) are a fast devloping technology due to their high energy efficiency, long lifecycle, environmental benefits and optical performance [1-5]. LED lighting uses $75 \%$ less energy their incandescent or fluorescent counterparts [6]. $70 \%$ of the total energy consumed by an LED light is emmitted as heat. Effective thermal dissipation of LED devices is crucial in maintaining luminous performance and extending a LEDs lifespan [6,9-12]. Numerous authors haven investigated the thermal disipation of LED devices both experimentally [3, 10, 13-15] and numerically [16-21].

Conventional aluminum heat sinks that are used to passively cool LED chips are thermally inefficient. A thermal resistance bottleneck exist at the gas-solid interface $\left(\mathrm{R}_{2}\right)$ (Figure 1) which

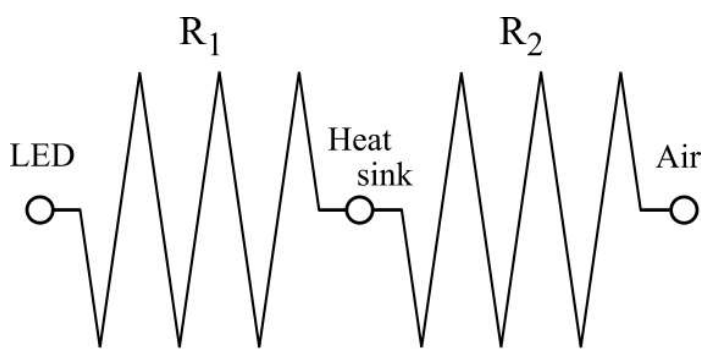

Figure 1. LED cooling thermal resistance diagram. is significantly larger than the thermal resistance from the LED through the aluminium solid $\left(\mathrm{R}_{1}\right)$. Metal coating of $3 \mathrm{D}$ printed polymers enables the matching of thermal resistances $\left(R_{1}=R_{2}\right)$ by controlling the thickness of the coated metal, with the polymer substrate acting as a structural component. $3 \mathrm{D}$ printing also enables inovative heat sink design, which is not limited by the manufacturing processes of traditional heat sinks.

This research endevours to be the first to experimentally investigate low power LED cooling using composite heat sinks. This was achieved using three heat sink designs tested across three applied heat fluxes $\left(\mathrm{q}^{\prime \prime}\right.$ gen $)$. This methodology was employed to characterise the thermal dissipation enhancement due to the zinc coating and to demonstrate the technology viability for future adaptation in cooling low power LED devices.

\section{EXPERIMENTAL APPARATUS AND DATA REDUCTION}

The experimental apparatus consists of three primary components; the composite heat sink, pseudo LED heater pad and the data acquisition system. A schematic of the apparatus is shown in Figure 2.

\section{A. Composite heat sink}

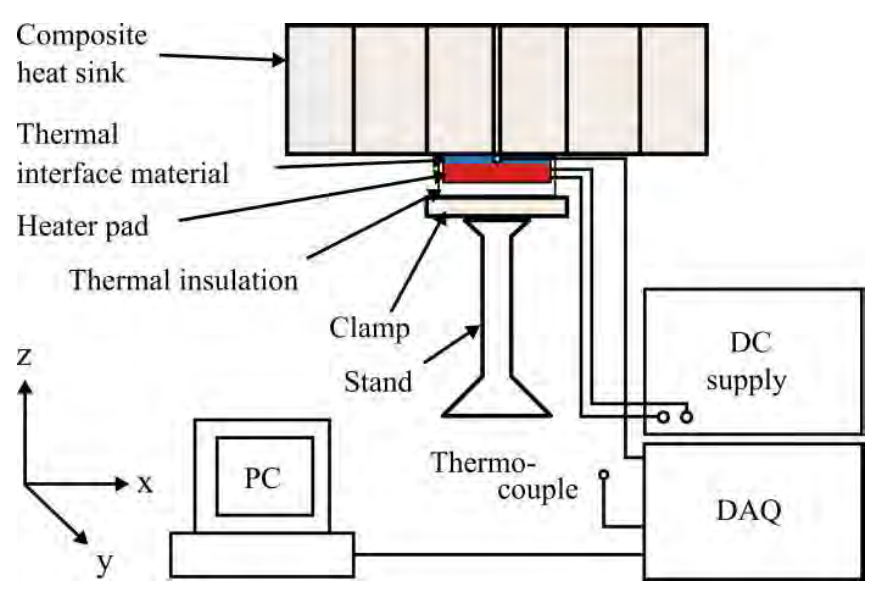

Figure 2. Schematic of the experimental facility. 


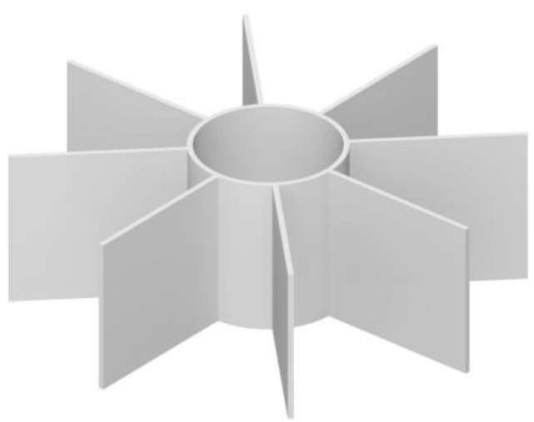

(a)

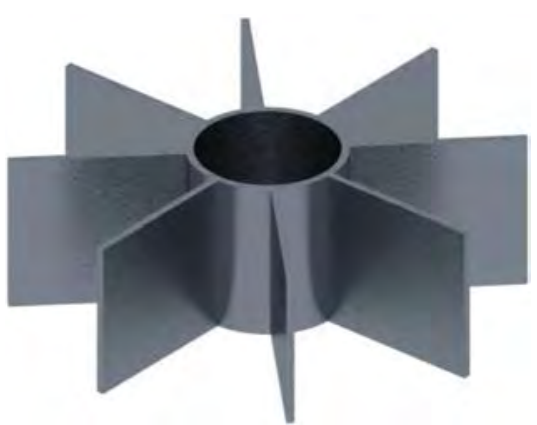

(b)

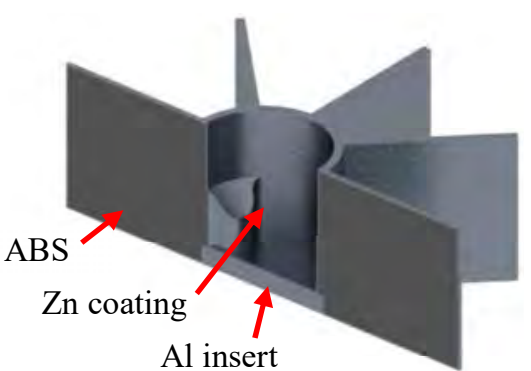

(c)

Figure 3. Heat sink design. (a) ABS heat sink, (b) zinc coated heat sink and (c) zinc coated heat sink with aluminum insert.

The three heat sink designs are shown in Figure 3. The design is based on a commercial LED heat sink (Aavid, P/N: NX300159) and they were fabricated using a Stratasys uPrint $3 \mathrm{D}$ printer $(\mathrm{P} / \mathrm{N}$ : 680-50105-D). The heat sinks have a maximum diameter of $132 \mathrm{~mm}$ and a height of $42 \mathrm{~mm}$. An eight fins design was implemented to optimize the uniformity of the zinc coating thickness. Each fin measures 41.4 x $42 \times 1.5 \mathrm{~mm}^{3}$ before the zinc coating is applied. The first heat sink (Figure 3a) is an uncoated Acrylonitrile Butadiene Styrene (ABS, $\mathrm{k}=0.25$ $\mathrm{W} \mathrm{m} \mathrm{m}^{-1} \mathrm{~K}^{-1}, \rho=1040 \mathrm{~kg} \mathrm{~m}^{-3}$, T P/N: P430) design. The second (Figure $3 \mathrm{~b}$ ) heat sink consists of ABS coated in zinc. The final design is zinc coated ABS with an aluminum insert ( $5 \mathrm{~mm}$ thick, $41.3 \mathrm{~mm}$ in diameter) incorporated into the base of the heat sink (Figure 3c).

A Wire-arc spraying system (ValuArc, Sulzer Metro Inc.) was employed in coating the 3D printed heat sink designs with zinc (Sulzer Metro Inc., $\mathrm{k}=116 \mathrm{~W} \mathrm{~m}^{-1} \mathrm{~K}^{-1}, \rho=7140 \mathrm{~kg} \mathrm{~m}^{-3}$, $\mathrm{P} / \mathrm{N}$ : 1031592). In wire-arc spraying an electric arc is struck between the tips of two continuously fed wires to melt them. A high velocity air jet strips the molten metal from the wires and create a spray of droplets that impact on the substrate. Zinc was chosen due its high thermal conductivity and its relatively low melting pointing $\left(420^{\circ} \mathrm{C}\right)$. This ensured that the polymer heat sinks were not damaged during the coating process. A list of the relevant heat sink properties are outlined in Table 1.

Table 1. Heat sink properties

\begin{tabular}{|l|c|c|c|}
\hline Heat sink design & ABS & ABS-Zn & $\begin{array}{c}\text { ABS-Al- } \\
\text { Zn }\end{array}$ \\
\hline Initial mass [g] & 32.4 & 32.5 & 50.3 \\
\hline Coated mass [g] & 32.4 & 117.1 & 140.9 \\
\hline $\begin{array}{l}\text { Average coating } \\
\text { thickness [um] }\end{array}$ & 0 & 268 & 271 \\
\hline
\end{tabular}

\section{B. Experimental facility}

The applied heat flux was generated using a thermal heating pad (Mcmaster-Carr, 25.4 x 25.4 x $5 \mathrm{~mm}^{3}, \mathrm{P} / \mathrm{N}$ : $35765 \mathrm{~K} 14$ ) (See Figure 1). The thermal pad is attached to the base of each heat sink using thermally conductive tape $(3 \mathrm{M}, \mathrm{P} / \mathrm{N}: 1-5-8810)$ and is clamped to a fixed pressure of $265 \mathrm{kPa}$ using a torque screwdriver (Mcmaster-Carr, P/N: 5716A21). The thermal pad is insulated from the clamp using $10 \mathrm{~mm}$ thick Cryogel $\mathrm{z}$ (Aspen aerogel, $25.4 \times 25.4 \times 5 \mathrm{~mm}^{3}, \mathrm{k}=0.014 \mathrm{~W} \mathrm{~m}^{-1} \mathrm{~K}^{-1}$ ) as shown in Figure 1. The heat sink assembly is mounted $150 \mathrm{~mm}$ from the ground using a 3D printed stand to ensure unobstructed air flow during testing. A $1.6 \mathrm{~mm}$ hole is drilled in the center of the base each heat sink, to facilitate measurement of the heat sink base temperature $\left(\mathrm{T}_{\mathrm{b}}\right)$ during testing. T-type thermocouples (Omega, $\mathrm{P} / \mathrm{N}$ : FF-TI-20) are used to measure the base temperature and the ambient surrounding temperature $\left(\mathrm{T}_{\infty}\right)$. Temperature measurement are acquired using an Omega DAQ (P/N: OMBDAQ-56).

\section{Experimental testing and analysis}

Experiments were conducted at atmospheric pressure and room temperature after steady state conditions were reached. The applied heat flux ranged between $0.5-1.5 \mathrm{~W} \mathrm{~cm}^{-2}$ in increments of $0.5 \mathrm{~W} \mathrm{~cm}^{-2}$. It is assume that all of the generated theral energy is dissipated by the composite heat sink. Temperature data was averaged over a 15 minute period once steady state conditions had been achieved.

\section{Experimental uncretainty}

The experimental uncertainty for all parameters was implemented using the methodology outlined by Kirkup and Frenkel [22]. A list of the relevant parameter and their associated percentage uncertainty (PU) is outlined in Table 2. All listed values are to a $95 \%$ confidence level.

Table 2. Experimental uncertainty

\begin{tabular}{|l|c|c|}
\hline Parameter & & PU [\%] \\
\hline $\mathrm{q}^{\prime \prime}$ gen & $+/-$ & 6 \\
\hline $\mathrm{T}_{\mathrm{b}}$ & $+/-$ & 2.6 \\
\hline $\mathrm{T}_{\infty}$ & $+/-$ & 4.4 \\
\hline
\end{tabular}




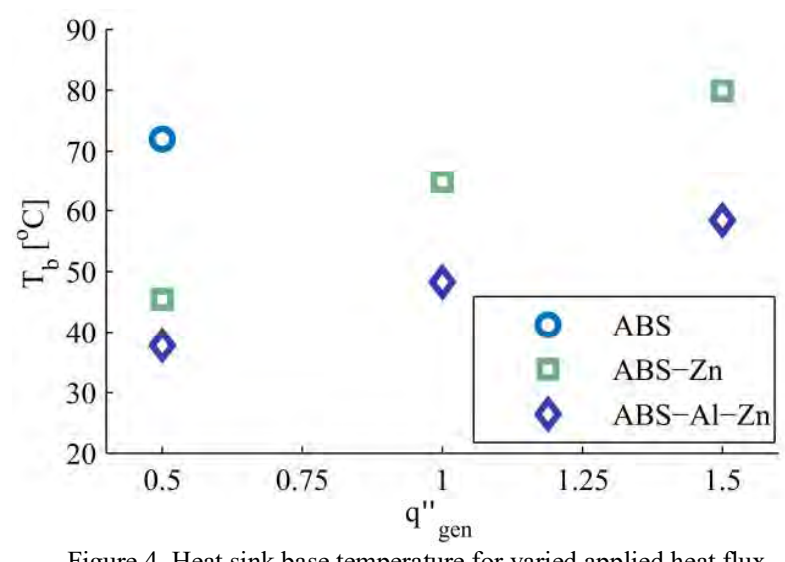

Figure 4. Heat sink base temperature for varied applied heat flux.

\section{RESULTS}

Figure 4. shows how the base temperature of each heat sink design varies with the applied heat flux. For the lowest $\mathrm{q}^{\prime \prime}$ gen the uncoated $\mathrm{ABS}$ heat sink reached a steady state temperature of $72^{\circ} \mathrm{C}$. As $\mathrm{q}^{\prime \prime}$ gen is increased to $1 \mathrm{~W} \mathrm{~cm}^{-2}$ the base temperature exceeded the glass trnasition temperature of the $\operatorname{ABS}\left(105^{\circ} \mathrm{C}\right)$. The zinc coating on the second heat sink (ABS-Zn) reduces the base temperature by $37 \%$ over the uncoated case to $45.4^{\circ} \mathrm{C}$. As $\mathrm{q}^{\prime \prime}$ gen increases the base temperature increases linearly up to $79.9^{\circ} \mathrm{C}$ at the maximum applied heat flux.

The final heat sink (ABS-Al-Zn) incorporates an aluminium insert into it base, this enables a much lower thermal resistance path between the circumfrential fins and the thermal pad. The thermal resistance between the inner surface of the inner cylinder and the thermal pad is also significantly decreased by the insert. This results in a greater cooling than the ABS-Zn heat sink. A $48 \%$ and $17 \%$ decrease in the base temperature is observed over the ABS and ABS-Zn heat sinks respectively, at the lowest applied heat flux. For the largest $\mathrm{q}_{\text {gen }}^{\prime \prime}$ a base temperature of $58.4^{\circ} \mathrm{C}$ was noted, this corresponds to a $27 \%$ decrease in comparison with the ABS-Zn heat sink.

\section{CONCLUSIONS}

The thermal performance of three low power LED heat sinks have been characterized. Optimum cooling was achieved by the zinc coated ABS heat sink which incorporated an aluminum insert into its base. The zinc coating was shown to significantly increases cooling performance over the uncoated case. Most importantly this research has demonstrated the potential of metal coated polymer heat sinks in cooling low power LED devices. Future work will focus on varying the coating material, numerical simulation to investigate optimum geometry and coating thickness, and direct comparison with an aluminum heat sink of a similar design.

\section{REFERENCES}

[1] C-J. Weng, "Advanced thermal enhancement and management of LED packages," Int. Commun. Heat Mass Transfer, vol. 36, no. 3, pp. 245-248, 2009. doi: 10.1016/j.icheatmasstransfer.2008.11.015.

[2] C. H. Huang, G. J. Wang, "A design problem to estimate the optimal fin shape of LED lighting heat sinks,"Int. Commun. Heat Mass Transfer, vol. 106, pp. 1205-1217, 2017, doi: 10.1016/j.ijheatmasstransfer.2016.10.101.

[3] S. H. Yu, D. Jang, K. S. Lee, Effect of radiation in a radial heat sink under natural convection. Int. J. Heat Mass Transfer, vol. 55, no. 1-3, pp. 505-509, 2012 doi: 10.1016/j.ijheatmasstransfer.2011.09.026.
[4] S. Tarashioon, A. Baiano, H. Van Zeijl, C. Guo, S. W. Koh, W. D. Van, "An approach to "Design for Reliability" in solid state lighting systems at high temperatures," Microelectron. Reliab., vol. 52, no. 5, pp. 783-793, 2012. doi: 10.1016/j.microrel.2011.06.029.

[5] C. Qian, X. J. Fan, J. J. Fan, C. A. Yuan, G. Q. Zhang, "An accelerated test method of luminous flux depreciation for LED luminaires and lamps," Reliability Engineering \& System Safety, vol. 147, pp. 84-92, 2016. doi: 10.1016/j.ress.2015.11.009.

[6] D. Jang, S. J. Yook, K. S. Lee, "Optimum design of a radial heat sink with a fin-height profile for high-power LED lighting applications," Appl. Energy, vol. 116, pp. 260-268, 2014. doi: 10.1016/j.apenergy.2013.11.063.

[7] J. K. Park, C. H. Kim, S. H. Park, H. D. Park, S. Y. Choi, "Application of strontium silicate yellow phosphor for white light-emitting diodes,"Appl. Phys Lett., vol. 84, no. 10, pp. 1647-1649, 2004. doi: 10.1063/1.1667620.

[8] C. J. Weng, "Advanced thermal enhancement and management of LED packages," Int. Commun. Heat Mass Transfer, vol. 36, no. 3, pp. 245-248, 2009. doi:10.1016/j.icheatmasstransfer.2008.11.015.

[9] C. H. Huang, G. J. Wang, "A design problem to estimate the optimal fin shape of LED lighting heat sinks," Int. J. Heat Mass Transfer, vol. 106, pp. 1205-1217, 2017. doi: 10.1016/j.ijheatmasstransfer.2016.10.101.

[10] S. H. Yu, K. S. Lee, S. J. Yook, "Optimum design of a radial heat sink under natural convection," Int. J. Heat Mass Transfer, vol. 54, no. 11-12, pp. 2499-2505, 2011. doi: 10.1016/j.ijheatmasstransfer.2011.02.012.

[11] B. Sun, X. Fan, H. Ye, J. Fan, C. Qian, W. van Driel, G. Zhang, "A novel lifetime prediction for integrated LED lamps by electronic-thermal simulation," Reliability Engineering \& System Safety, vol. 163, pp.14-21, 2017. doi: 10.1016/j.ress.2017.01.017.

[12] M. Ha, S. Graham, "Development of a thermal resistance model for chipon- board packaging of high power LED arrays," Microelectron. Reliab., vol. 52, no. 5, pp. 836-844, 2012. doi:10.1016/j.microrel.2012.02.005.

[13] S. J. Park, D. Jang, S. J. Yook, K. S. Lee, "Optimization of a staggered pinfin for a radial heat sink under free convection, " Int. J. Heat Mass Transfer, vol. 87, pp. 184-188, 2015. doi: 10.1016/j.ijheatmasstransfer.2015.03.089.

[14] D. Jang, S. H. Yu, K. S. Lee, "Multidisciplinary optimization of a pin-fin radial heat sink for LED lighting applications," Int. J. Heat Mass Transfer, vol. 55, no. 4, pp. 515-521, 2012. doi: 10.1016/j.ijheatmasstransfer.2011.11.016.

[15] D. Jang, S. J. Yook, K. S. Lee, "Optimum design of a radial heat sink with a fin-height profile for high-power LED lighting applications," Appl. Energy, vol. 116, pp. 206-268, 2014. doi: 10.1016/j.apenergy.2013.11.063.

[16] H. Najafi, B. Najafi, P. Hoseinpoori, "Energy and cost optimization of a plate and fin heat exchanger using genetic algorithm," Appl. Therm. Eng., vol. 31, no.10, pp. 1839-1847, 2011. doi: 10.1016/j.applthermaleng.2011.02.031.

[17] R-T Wang, J-C Wang, "Analyzing the structural designs and thermal performance of nonmetal lighting devices of LED bulbs," Int. J. Heat Mass Transfer, vol. 99, pp. 750-761, 2016. doi: 10.1016/j.ijheatmasstransfer.2016.03.112.

[18] G. Xie, Y. Song, M. Asadi, G. Lorenzini, "Optimization of pin-fins for a heat exchanger by entropy generation minimization and constructal law," $J$. Heat Transfer, vol. 137, no. 6, pp. 061901, 2015. doi: 10.1115/1.4029851.

[19] Y. T. Yang, H. S. Peng, "Numerical study of pin-fin heat sink with ununiform fin height design," Int. J. Heat Mass Transfer, vol. 51, no. 19-20, pp. 4788-4796, 2008. doi: 10.1016/j.ijheatmasstransfer.2008.02.017.

[20] T. Bello-Ochende, J. P. Meyer, A. Bejan, "Constructal multi-scale pin fins," Int. J. Heat Mass Transfer, vol. 53, no. 13-14, pp. 2773- 2779, 2010. doi: 10.1016/j.ijheatmasstransfer.2010.02.021.

[21] D. H. Park, D. B. Lee, E. R. Seo, Y. J. Park, "A parametric study on heat dissipation from a LED-lamp," Applied Thermal Engineering, vol. 108, pp. 1261-1267, 2016. doi: 10.1016/j.applthermaleng.2016.08.032.

[22] L. Kirkup, R.B. Frenkel, An introduction to uncertainty in measurement: using the GUM (Guide to the Expression of Uncertainty in Measurement), Cambridge University Press, 2006. 
BIOMECHANICS \&

BIOMEDICAL

ENGINEERING 


\section{Integration and Simulation of Microfluidic Platforms and Terahertz Time-Domain Spectroscopy Systems}

\author{
Rasha Al-Hujazy \\ School of Engineering \\ University of Guelph \\ Guelph, Canada \\ ralhujaz@uoguelph.ca
}

\author{
Christopher M. Collier \\ School of Engineering \\ University of Guelph \\ Guelph, Canada \\ ccollier@uoguelph.ca
}

\begin{abstract}
This work serves as a design tool for the development of integrated terahertz time-domain spectroscopy systems with microfluidic platforms. The emission of terahertz radiation from a GaAs $\mathrm{THz}$ antenna is simulated. This simulation involves a semi-classical computational model with white noise effects used to control the dynamic range of the system. The maximum measurable frequency for the overall integrated system is strongly influenced by the sample thickness of the microfluidic platform and the dynamic range of the terahertz time-domain spectroscopy system.
\end{abstract}

\section{Keywords-biosensing; microfluidics; terahertz}

\section{INTRODUCTION}

Ultrafast science and measurement of terahertz (THz) electromagnetic radiation is a rapidly-growing research area with many applications [1-7]. Terahertz electromagnetic radiation occupies a unique section of the electromagnetic spectrum, being 0.1-10 THz. This $\mathrm{THz}$ electromagnetic radiation has important applications in the fields of security [8], communications [9], quality control [10], and biomedical spectroscopy [11].

One of the most important tools that has been enabled through $\mathrm{THz}$ electromagnetic radiation is $\mathrm{THz}$ time-domain spectroscopy (THz-TDS). Terahertz time-domain spectroscopy [12] has been applied to various biomedical applications, including genome analysis [13], oncology [14], and proteomics [11]. In THz-TDS, absorptive and refractive material properties are detected in pulses of $\mathrm{THz}$ radiation with extremely short duration $(<1 \mathrm{ps})$ through changes to amplitude and phase, respectively. Measurements are performed with and without a sample and compared. For analyses of vibrational and rotational modes, THz-TDS performance is at or beyond that of traditional methods such as Fourier transform spectroscopy [15]. Additionally, $\mathrm{THz}$ electromagnetic radiation is non-ionizing, making THz-TDS an appealing option compared to X-ray and other spectra [16].

Despite the above advantages, THz-TDS systems have critical design parameters which should be taken into account for use in modern biomedical devices. This is especially true for integration with microfluidic devices [17-20]. These limitations come from the strong absorption of liquids at $\mathrm{THz}$ frequencies. With this strong absorption in liquids, THz-TDS microfluidic systems must be made with sufficiently small microfluidic platform thickness and sufficiently high dynamic range, to ensure that spectroscopy is properly performed over the full bandwidth of the THz-TDS system [21].

This paper addressed the knowledge gap between microfluidic platforms and THz-TDS systems. We present a comprehensive investigation of microfluidic platform thickness and dynamic range for a THz-TDS microfluidic system. Data analysis is implemented through a semi-classical computational method [22] with white noise incorporated into the simulation. An increasing noise amplitude is used to examine the effects of THz-TDS dynamic range. The maximum measurable frequency, $f_{\max }$, of the system is found for numerous combinations of input parameters, being dynamic range and microfluidic platform thickness.

\section{THEORY}

A THz-TDS microfluidic system is shown in Fig. 1. Here, an ultrafast laser pulse pumps a $\mathrm{THz}$ emitter to generate a reference THz pulse. Upon passing through the microfluidic platform, the reference THz pulse, $E_{\mathrm{R}}(f)$ becomes the THz sample pulse, $E_{\mathrm{S}}(f)$.

The electric field of the $\mathrm{THz}$ pulse in the far-field can be expressed [23] as

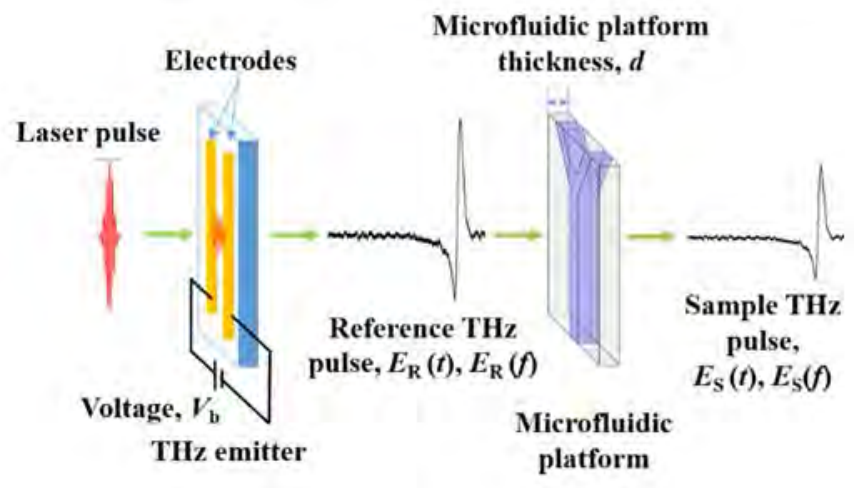

Figure 1. Microfluidic-based THz-TDS system 


$$
E(z, t)=\frac{L_{y}}{8 \pi \varepsilon_{o} c^{2} z} \frac{d}{d t} \int_{0}^{L_{x}} k_{n}(x, t) d x
$$

where $L_{y}$ is the gap width in the $y$-direction, $L_{x}$ is the gap width in the $x$-direction, $\varepsilon_{0}$ is the permittivity of free space, $c$ is the speed of light, and $k_{n}(x, t)$ is the surface current of electrons. $=$ (The electric field is simulated according to the semi-classical computational method of Rodriguez and Taylor [22].)

The semiconductor electromagnetic equations for the $\mathrm{THz}$ emitter accomplishes a noise free radiation, the white noise (which is dominant over other noise sources at high $\mathrm{THz}$ frequencies) is added and defines the noise floor of the $\mathrm{THz}$ emitter measurements [24]. The equation of the reference $\mathrm{THz}$ pulse is

$$
E_{R}(t)=E(z, t)+A_{n} n(t)
$$

where $A_{n}$ is the noise amplitude, and $n(t)$ is the uniform random noise signal (centered about zero, peak-to-peak spread made the same as $\mathrm{THz}$ pulse amplitude). A Fourier transform of the generated pulse can be used to find the dynamic range for different noise conditions. The maximum absorption coefficient for a THz-TDS system is

$$
\alpha=\frac{1}{d} \ln \left[D R \frac{4 n}{(n+1)^{2}}\right]
$$

for refractive index of $n$ [25].

\section{RESULTS}

The simulation is run with standard operation conditions of $50 \mathrm{~mW}$ average power in the laser pulse and external bias of 0.17 $\mathrm{V} / \mu \mathrm{m}$. The effect of noise amplitude, $A_{n}$, on the reference $\mathrm{THz}-$ TDS pulse is investigated by increasing the noise amplitude from zero to $1 \mathrm{E}-3,1 \mathrm{E}-2,1 \mathrm{E}-1,1 \mathrm{E} 0$, and $1 \mathrm{E} 1$. The resulting reference THz pulses in the time domain are shown in Fig. 2 for progressively increasing $A_{\mathrm{n}}$ values. The same $\mathrm{THz}$ pulses in the frequency domain are shown in Fig. 3 for progressively decreasing dynamic range values of $\mathrm{DR}=5.8 \mathrm{E} 3,5.7 \mathrm{E} 3,2.4 \mathrm{E} 3$, $3.0 \mathrm{E} 2,2.8 \mathrm{E} 1$, and $2.8 \mathrm{E} 0$. It is clear that as noise amplitude increases, dynamic range decreases.

By finding the intersection of the maximum absorption coefficient with a known water absorption coefficient curve, $\alpha_{\mathrm{W}}$, the maximum measurable frequency can be found. This is shown in Fig. 4(a) for $d=120 \mu \mathrm{m}$ and DR $=2.4 \mathrm{E} 3$. For decreased DR or increased $d$, the $\alpha$ curve will shift down, forcing maximum measurable frequency to a lower value. For increased DR or decreased $d$, the $\alpha$ curve will shift up, forcing maximum measurable frequency to a higher value. Given this behaviour, the various dynamic ranges shown in Fig. 3 can be used in conjunction with the absorption coefficient of water, being $\alpha_{\mathrm{W}}=$ $61 f+54 \mathrm{~cm}^{-1}$, to find the corresponding maximum measurable frequency.

Ultimately, the maximum measurable frequencies can be collected for a variety of simulated conditions for microfluidic platform thickness and dynamic range. This is shown in Fig. 4(b) which displays a three-dimensional surface plot that shows the maximum measurable frequency versus dynamic range and microfluidic platform thickness. As described previously, one

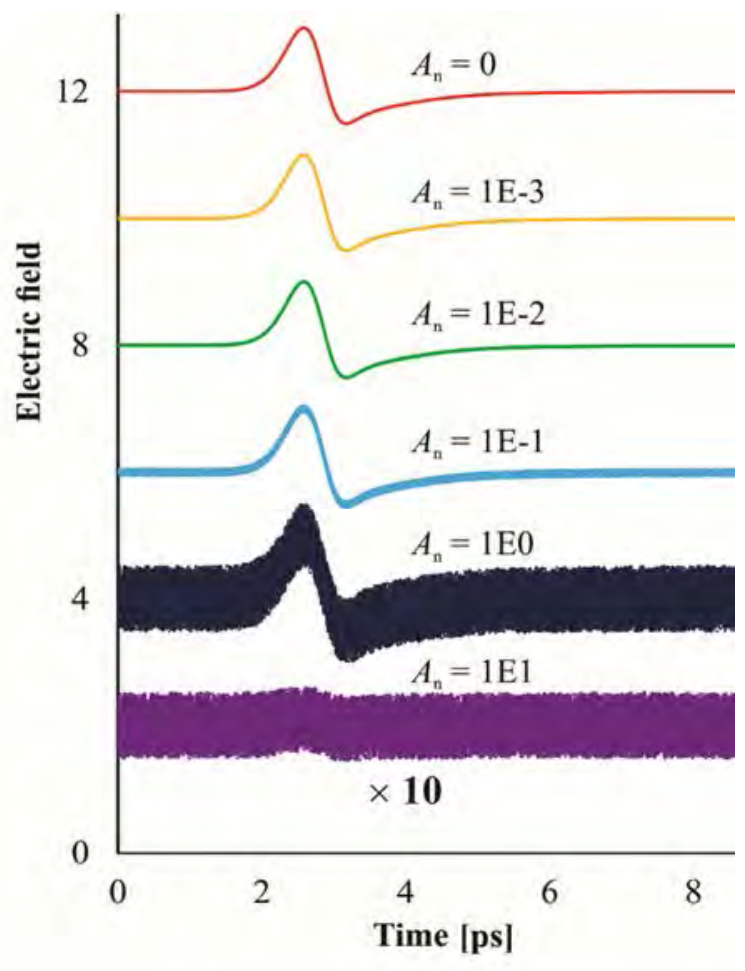

Figure 2. Simulated $\mathrm{THz}$ pulses in the time domain with different noise amplitudes.

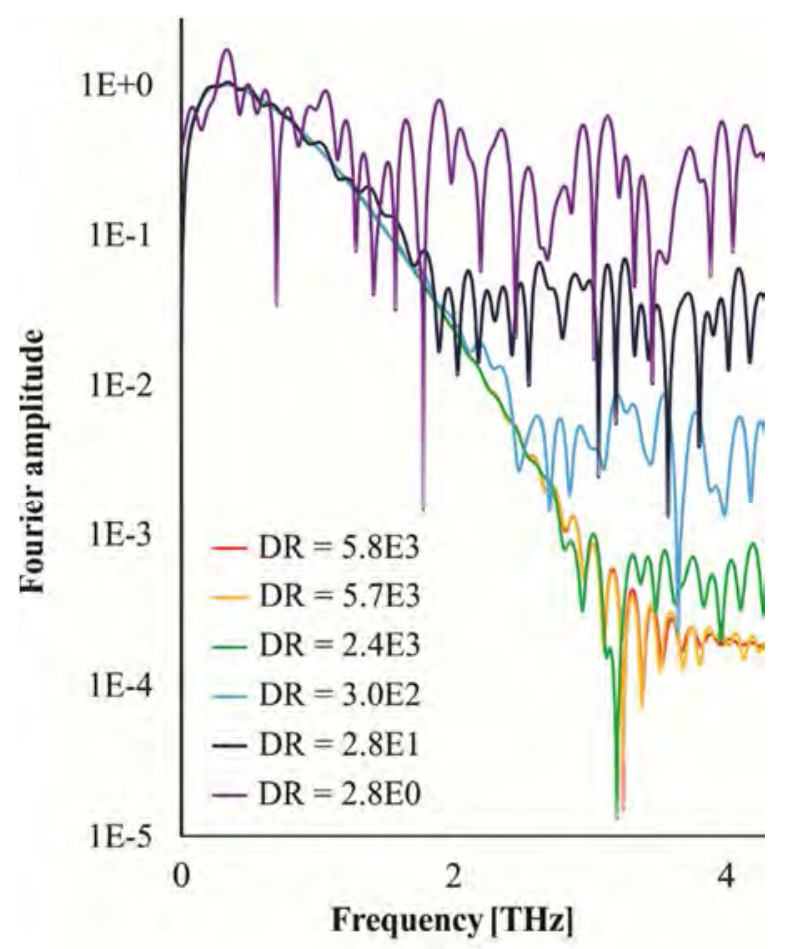

Figure 3. Simulated $\mathrm{THz}$ pulses in the frequency domain with different dynamic ranges (corresponding to the noise amplitudes from Fig. 2). 


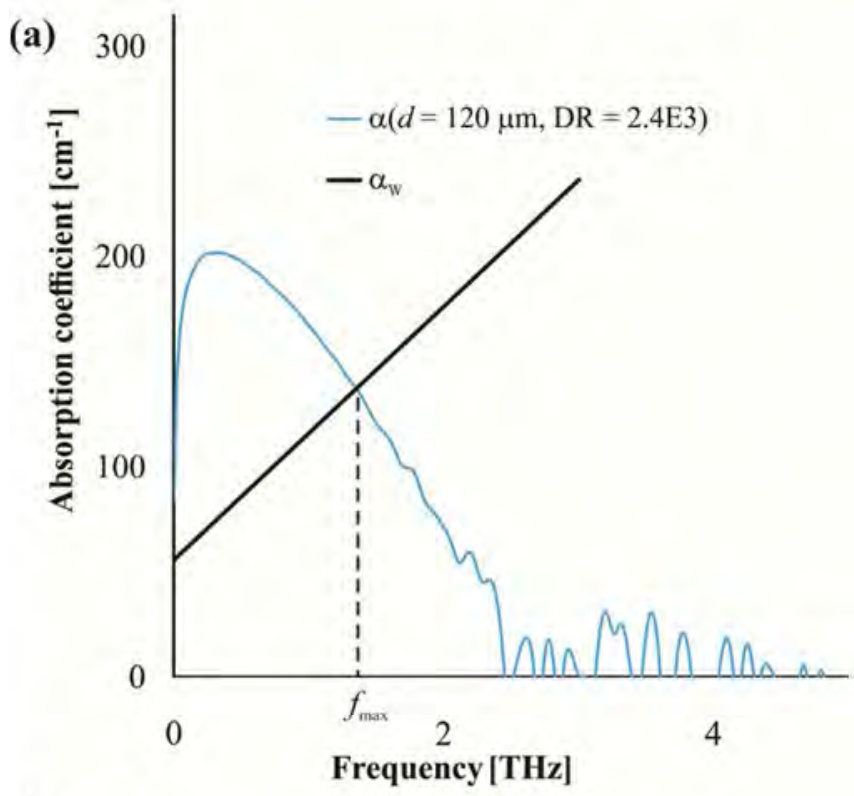

(b)

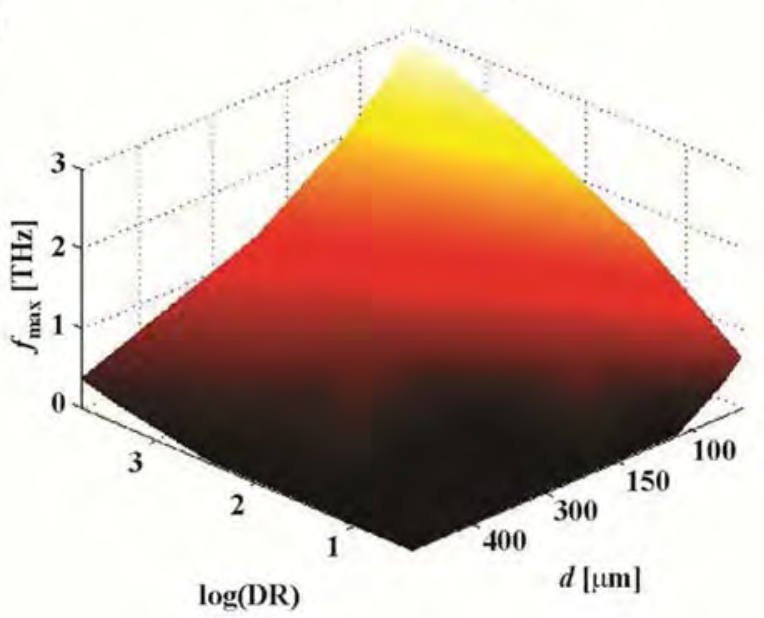

Figure 4. (a) The absorption coefficient versus frequency is shown for a representative case of $120 \mu \mathrm{m}$ microfluidic platform thickness and 2.4E3 dynamic range. (b) The maximum measurable frequency is plotted against the log of the dynamic range and the microfluidic platform thickness. The maximum measurable frequency has a maximum value of approximately 3 $\mathrm{THz}$ and decreases with decreasing dynamic range and increasing microfluidic platform thickness.

can observe an increase of the maximum measurable frequency with decreased $d$ and increased DR. These results can be used to inform future designs.

\section{CONCLUSIONS}

In conclusion, for THz-TDS microfluidic systems, the microfluidic platform thickness and dynamic range of the THzTDS system play a critical rule in defining the maximum measurable frequency. It was found that the maximum measurable frequency decrease with decreasing the dynamic range and increasing sample thickness. These fundamental results can be used in the design of future microfluidic THz-TDS systems for biomedical applications.

\section{ACKNOWLEDGMENT}

The authors would like to acknowledge financial support through the Natural Science and Engineering Research Council of Canada.

\section{REFERENCES}

[1] H. Pahlevaninezhad, B. Heshmat, and T. E. Darcie, "Efficient terahertz slot-line waveguides," Opt. Express, vol. 19, no. 26, pp. B47-B55, 2011. doi: 10.1364/OE.19.000B47.

[2] X. Jin, C. M. Collier, J. J. A. Garbowski, B. Born, and J. F. Holzman, "Ultrafast transient responses of optical wireless communication detectors," Appl. Opt., vol. 52, no. 20, pp. 5042-5049, 2013. doi:10.1364/AO.52.005042.

[3] C. M. Collier, B. Born, X. Jin, and J. F. Holzman, "Ultrafast charge carrier and phonon dynamics in GaP," Appl. Phys. Lett., vol. 103, no. 7 , 072106(1-4), 2013.

[4] S.-W. Huang et al., "Globally stable microresonator turing pattern formation for coherent high-power THz radiation on-chip," Phys. Rev. X, vol. 7, no. 4, 041002(1-10), 2017.

[5] C. M. Collier and J. F. Holzman, "Ultrafast photoconductivity of crystalline, polycrystalline and nanocomposite $\mathrm{ZnSe}$ material systems for terahertz applications," Appl. Phys. Lett., vol. 104, no. 4, 042101(1- 5), 2014.

[6] M. Reid and R. Fedosejevs, "Terahertz birefringence and attenuation properties of wood and paper," Appl. Opt., vol. 45, no. 12, pp. 2766- 2772, 2006. doi: 10.1364/AO.45.002766.

[7] C. M. Collier, T. J. Stirling, I. R. Hristovski, J. D. A. Krupa, and J. F. Holzman, "Photoconductive terahertz generation from textured semiconductor materials," Sci. Rep., vol. 6, 23185(1-10), 2016.

[8] J. F. Federici et al, "THz imaging and sensing for security applications -explosives, weapons and drugs," Semicon. Sci. Technol., vol.20, no.7, pp. S266-S280, June 2005. doi: 10.1088/0268-1242/20/7/018.

[9] J. Federici, and L. Moeller, "Review of terahertz and subterahertz wireless communications," J. Appl. Phys., vol. 107, pp 111101-22, 2010. doi: 10.1063/1.3386413.

[10] B. B. Hu, and M. C. Nuss, "Imaging with terahertz waves," Opt. Lett., vol. 16, pp. 1716-1719, August 1995. doi: 10.1364/OL.20.001716.

[11] A. Markelz, S. Whitmire, J. Hillebrecht, and R. Birge, "THz time domain spectroscopy of biomolecular conformational modes," Phys. Med. Biol., vol. 47, iss. 21, pp. 3797-3805, October 2002. doi: $10.1088 / 0031-9155 / 47 / 21 / 318$.

[12] C. M. Collier, M. H. Bergen, T. J. Stirling, M. A. DeWachter, and J. F. Holzman, "Optimization processes for pulsed terahertz systems," Appl. Opt., vol. 54, no. 3, pp. 535-545, 2015. doi:10.1364/AO.54.000535.

[13] A. N. Bogomazova et al., "No DNA damage response and negligible genome-wide transcriptional changes in human embryonic stem cells exposed to terahertz radiation," Sci. Rep., vol. 5, 7749, 2015. doi: $10.1038 /$ srep07749.

[14] E. Pickwell et al., "In vivo study of human skin using pulsed terahertz radiation," Phys. Med. Biol., vol. 49, pp. 1595-1607, 2004. doi: 10.1088/0031-9155/49/9/001

[15] P. Y. Han, M. Usman, and S. Kono, "A direct comparison between terahertz time-domain spectroscopy and far-infrared Fourier transform spectroscopy," J. App. Phys., vol. 89, no. 4, pp. 2357-2363, February 2001. doi: $10.1063 / 1.1343522$.

[16] C.-C. Chen, D.-J. Lee, T. Pollock, and J. F. Whitaker, "Pulsedterahertz reflectometry for health monitoring of ceramic thermal barrier coatings," Opt. Express, vol.18, iss.4, pp. 3477-3486, 2010. doi: 10.1364/OE.18.003477.

[17] P. A. George et al, "Microfluidic devices for terahertz spectroscopy of biomolecules," Opt. Express, vol. 16, no. 3, pp. 1577-1582, January 2008. doi: 10.1364/OE.16.001577.

[18] C. M. Collier, M. Wiltshire, J. Nichols, B. Born, E. L. Landry, and J. F. Holzman, "Nonlinear dual-phase multiplexing in digital microfluidic architectures," Micromachines, vol. 2, pp. 369-384, 2011. doi: $10.3390 / \mathrm{mi} 2040369$. 
[19] J. Nichols, C. M. Collier, E. L. Landry, M. Wiltshire, B. Born, and J. F. Holzman, "On-chip digital microfluidic architectures for enhanced actuation and sensing," J. Biomed. Opt., vol. 17, no. 6, 067005(1-7), 2012.

[20] J. Nichols, E. L. Landry, B. Born, M. Wiltshire, C. M. Collier, and J. F. Holzman, "Optical sensing for on-chip digital microfluidics," Proceedings of SPIE Photonics West, San Francisco, USA, January, 2012.

doi: $10.1117 / 12.909390$.

[21] M. Naftaly, and R. Dudley, "Methodologies for determining the dynamic ranges and signal-to-noise ratios of terahertz time-domain spectrometers," Opt. Lett., vol. 34, iss. 8, pp. 1213-1215, April 2009.

doi: 10.1364/OL.34.001213.

[22] G. Rodriguez and A. J. Taylor, "Screening of the bias field in terahertz generation from photoconductors," Opt. Lett., vol. 21, no. 14, pp. 1046-1048, July 1996. doi: 10.1364/OL.21.001046.

[23] C. M. Collier, T. J. Stirling, S. Dekock-Kruger, and J. F. Holzman, "Spectral response tuning of photoconductive terahertz emitters with binary phase masks," IEEE J. Quantum Electron, vol. 23, iss. 4, July- August 2017.

[24] P. Shumyatsky, and R. R. Alfano, "Terahertz sources," J. Biomed. Opt., vol. 16, iss. 3, 0330011, March 2011. doi: 10.1117/1.3554742.

[25] P. U. Jepsen, and B. M. Fischer, "Dynamic range in terahertz timedomain transmission and reflection spectroscopy," Opt. Lett., vol. 30, iss. 1, pp. 29-31, January 2005. doi: 10.1364/OL.30.000029. 


\section{In Pursuit of an Optimum Optical Imaging Technology For Eearly Detection of Dental Caries}

\author{
Elnaz Baradaran Shokouhi \\ Department of Mechanical Engineering \\ York University, Hybrid Biomedical Optics Lab \\ 4700 Keele Street, Toronto, Ontario M3J 1P3, Canada \\ ebaradar@yorku.ca
}

\author{
Nima Tabatabaei \\ Department of Mechanical Engineering \\ York University, Hybrid Biomedical Optics Lab \\ 4700 Keele Street, Toronto, Ontario M3J 1P3, Canada \\ Nima.Tabatabaei@lassonde.yorku.ca
}

\begin{abstract}
In the last two decades, majority of the newly developed dental caries detection techniques have been opticsbased, relying either on enhancement of light scattering in early carious lesion (e.g. optical coherent tomography or OCT) or enhancement of light absorption in early caries (e.g. thermophotonic lock-in imaging or TPLI). This paper aims to explore the detection threshold capabilities between light scattering and light absorption based dental caries detection methods. With this intention, the experiments will be conducted through examination of controlled artificiallyinduced early caries. It is anticipated that the molecularcontrast TPLI imaging technology outperforms OCT due to the more specific nature of light absorption contrast mechanism.
\end{abstract}

Keywords-component; early dental caries; thermophotonic lock-in imaging; spectral-domain optical coherence tomography; artificial demineralization.

\section{INTRODUCTION}

Dental caries continues to be a major public health challenge in both children and adults [1]. Over the past years, the state of the art in preventive dentistry has provided promising therapeutic techniques for preventing the progression of early dental caries to cavities and complete tooth decay [2]. However, the effectiveness of these preventive provisions rely on detection of caries at very early stages. But, unfortunately, clinical detection methods such as x-ray radiography and visual-tactile inspection lack sufficient sensitivity for detection of early stages of carious legions due to morphology of tooth structure and large surface area of healthy enamel around the carious legion [3]. In this paper, a quantification of detection threshold for mainstream optical imaging modalities, optical coherence tomography (OCT) and thermophotonic lock-in imaging (TPLI) (as representative techniques based on light scattering and absorption, respectively), is performed in early detection of dental caries. Optical coherence tomography (OCT) is a promising modality capable of imaging samples based on back reflection of light, and is capable of providing 3-dimensional sub-surface morphology of biological tissue microstructure with micronscale resolution [4]. The ability of OCT in providing high- resolution 3-dimensional images has led to its popularity in dentistry especially in diagnosis and screening of dental diseases [5][6].

While OCT provides three dimensional maps of lightreflecting interfaces in biological tissues, such as void interfaces introduced by demineralization in tooth, light absorption-based technologies, such as TPLI, produce maps of subsurface features absorbing the specific incident laser radiation (e.g., Calcium and phosphate as the byproducts of demineralization). The working principle of lock-in thermography involves an incorporation of active thermography along with lock-in demodulation signal processing in turbid samples to detect thermal waves emitted back from subsurface molecular absorption sites (e.g. early dental caries) by focusing the infrared camera on the sample [7]. As such, lock-in thermography is a very advantageous tool in electronic device testing and failure analysis of materials [8], but it also has important applications in early pre-clinical diagnosis and control during treatment in medical fields such as imaging of early caries [9]. Therefore, the motivation behind this study is to evaluate the detection threshold of two emerging medical imaging technologies, spectral domain-OCT (OCT) and TPLI, in diagnosis of early dental caries.

\section{MATERIALS AND METHODS}

\section{A. Thermophotonic Lock-in Imaging}

The experimental setup of TPLI (Fig. 1(a)) [10] consists of a continuous wave $808 \mathrm{~nm}$ near-infrared laser diode $(808 \mathrm{~nm}$; Jenoptik, Jena, Germany) which is regulated by a laser controller unit (Ostech, Berlin, Germany) in order to allow for modulation of laser intensity. Samples are put on LEGO blocks and positioned at the focal plane of the camera. The LWIR camera (Gobi 640; Xenics, Leuven, Belgium) used has a spectral range of $(8-14 \mu \mathrm{m})$ and maximum frame rate of 50 frames per second. The detector of the camera consists of $640 \times 480$ elements with pixel pitch of $25 \mu \mathrm{m}$. A custom made extension tube and an $18 \mathrm{~mm}$ focal-length objective lens are used to obtain a magnification of one from the interrogated surface of the sample.

The multifunctional data acquisition board synchronously generates three signals: reference pulse train, in-phase, and 
quadrature reference signals. The reference pulse train finds the beginning of each modulation cycle for averaging. The inphase reference signal is also sent to the laser controller to modulate the intensity of the laser beam. The in-phase reference signal is in-phase with the laser modulation while there is a $90^{\circ}$ phase lead between the in-phase and quadrature reference signals. The images are then taken with a long-wave infrared camera and then weighed and averaged using the instantaneous readings of the two reference signals to yield inphase $\left(\mathrm{S}^{0}\right)$ and quadrate $\left(\mathrm{S}^{90}\right)$ images [10]. The amplitude and phase for each pixel are then calculated by applying equation 1 :

$$
\mathrm{A}=\left[\left(S^{0}\right)^{2}+\left(S^{90}\right)^{2}\right]^{0.5} \text { and } \varphi=\arctan \left(S^{90} / S^{0}\right)
$$

In this paper, we only present and discuss the phase images as they are known to be of superior sensitivity due to the emissivity normalized nature of phase channel [11].

\section{B. Spectral Domain Optical Coherence Tomography}

Fig. 1(b) depicts a schematic of the developed OCT system. OCT laser is a broadband source of $30 \mathrm{~mW}$ superluminiscent diode (SLD; Exalos) centered at wavelength of $1315 \mathrm{~nm}$ and bandwidth of approximately $110 \mathrm{~nm}$. Spectrometer is a 2048pixel high speed camera with line scan/acquisition rate of 147 $\mathrm{kHz}$. The theoretical axial resolution of the system is approximately $4.5 \mu \mathrm{m}$ in tooth and the theoretical lateral resolution is $10 \mu \mathrm{m}$. The experimental axial resolution in air and tissue were measured as 12 and $8.6 \mu \mathrm{m}$, respectively. Optical power at sample is $\sim 7 \mathrm{~mW}$ and the signal-to-noise (SNR) of the built OCT system is $>100 \mathrm{~dB}$. The measured imaging depth of the system is $5 \mathrm{~mm}$ in air. The OCT beam in scanned along the smooth surface of the tooth using the two galvo scanners (GS) and performs the inverse Fourier transform operation on the Fourier-domain signals registered by the spectrometer, thus creating a 3D tomographic image of early caries. For OCT, the backscattered light in a form of integrated en-face images are measured and displayed.

\section{Controlled Demineralization Protocol}

Following the bio- and laser safety regulations at York University, anonymous extracted human teeth were collected from local oral surgeons and teeth with no visible stains or white spot lesions were selected for the study. In controlled demineralization of dental samples, a lactic acid-based solution is prepared. The solution is an acidified gel, consisting of $0.1 \mathrm{M}$ lactic acid and $0.1 \mathrm{M} \mathrm{NaOH}$ which is gelled with addition of $6 \% \mathrm{w} / \mathrm{v}$ hydroxyethylcellulose [10][11]. As such, teeth with no visible defect are selected and the surface of the tooth is covered by nail polish except for a rectangular treatment window. Sample is then submerged upside down in the acidic gel for certain number of days. Finally, sample is removed from the gel, and the nail polish is removed for imaging.

\section{Data Processing and Analysis}

To quantify the detection threshold of TPLI and OCT in progression of early caries, the thermophotonic phase images at

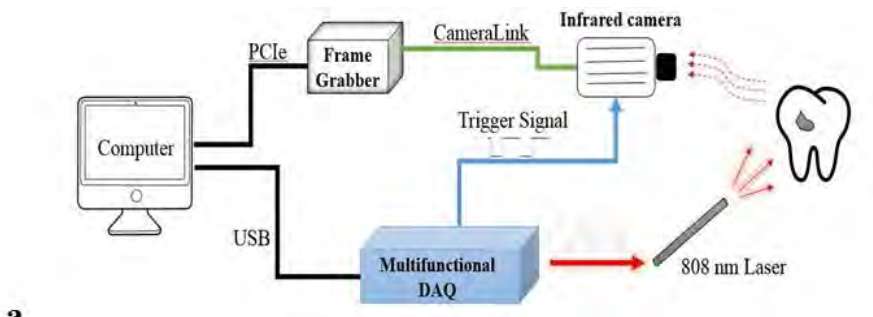

a

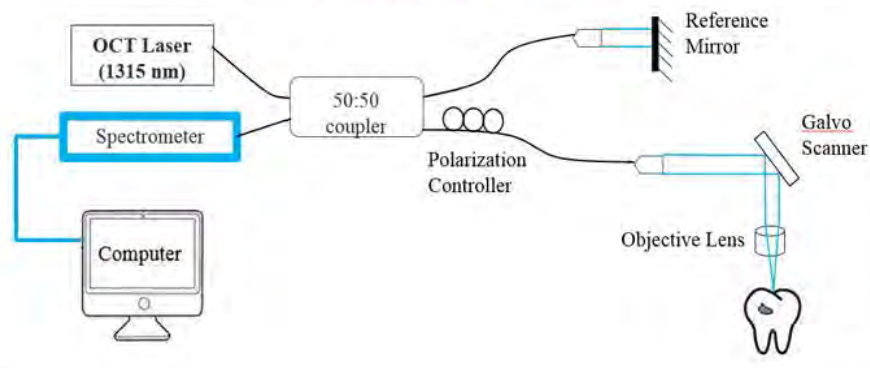

b

Figure 1. Schematic representation of (a) TPLI system and (b) spectraldomain OCT system.

$2 \mathrm{~Hz}$ and integrated en-face images derived from OCT were normalized [11]. For TPLI phase images, the pixel values were normalized by subtracting the phase value of pixels by the average pixel value of an intact reference area from the same image. For integrated en-face images, normalization was performed by dividing the amplitude values of pixels by the average amplitude values of an intact reference area from the same image. To compare and monitor the progression of artificial tooth demineralization in OCT and TPLI, the normalized phase and integrated en-face images were averaged in the regions of interest and the standard deviation of pixel values was calculated.

\section{RESULTS AND DISCUSSION}

Detection threshold is an important parameter of a diagnostic modality, i.e. how early the diagnostic system can detect caries.

\section{A. Detection Threshold of OCT and TPLI}

To this end, a time-dependent controlled demineralization procedure was followed on smooth surface of a tooth sample. Fig. 2(a) shows the visual photograph of the sample and the dashed rectangle shows the location of the treatment window.

In integrated en-face OCT (Fig. 2(b)-(1)), it can be observed that as the treatment time increases, the contrast between the treatment window and the intact region gradually increases as a result of progression of artificially-induced early caries which results in enhancement of light scattering. The maximum contrast occurs at day 15 of demineralization. Similarly, visual inspection of TPLI phase images (Fig. 3(a)(k)) shows that TPLI starts to show very small change in contrast with progression of early caries starting from day 1 of demineralization. This change becomes fully visible at day 2 of demineralization. While the contrast from day 2 to day 10 

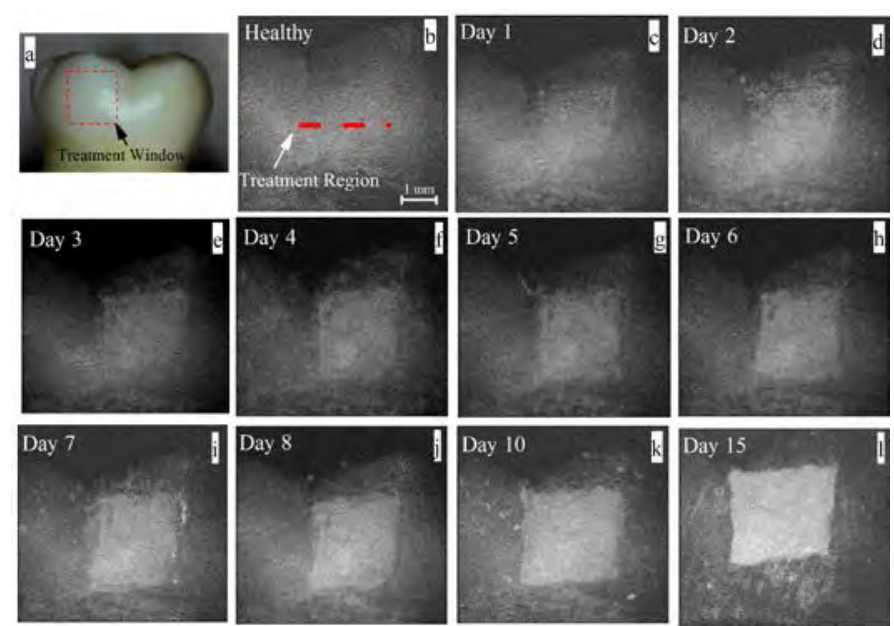

Figure 2. Image of the sample (a) before demineralization and after (b) 0 days, (c) 1 day, (d) 2 days, (e) 3 days (f) 4 days (g) 5 days (h) 6 days (i) 7 days (j) 8 days (k) 10 days, and (h) 15 days of demineralization

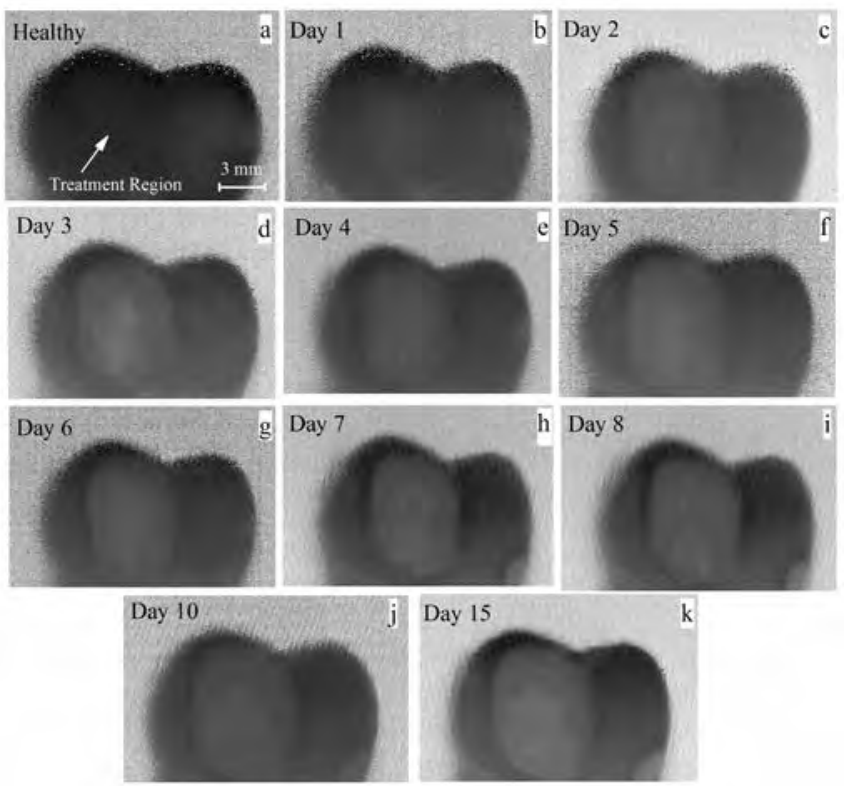

Figure 3. TPLI phase images at 2-Hz modulation frequency at (a) 0 days, (b) 1 day, (c) 2 days, (d) 3 days (e) 4 days (f) 5 days (g) 6 days (h) 7 days (i) 8 days (j) 10 days, and (k) 15 days of demineralization

increases gradually, at day 15 of demineralization the maximum contrast occurs. In addition to visual observations, statistical analysis was conducted on images of Fig. 2 and Fig. 3. The bar plots of Fig. 4 confirm the superiority of detection threshold of TPLI over OCT. In integrated en-face OCT bar plots, it can be seen that in each demineralization step, the system tends to show higher standard deviation compared to the bar plots of TPLI. The relatively large standard deviation present in OCT is due to its non-specific nature of light scattering mechanism. It is observed that with progression of artificially-induced early caries, there are gradual enhancements in average integrated intensities. However, there also exists some instability in the average integrated intensity values between the demineralization steps of day 4 to 10 . Consequently, it can be concluded that after application of demineralization for 15 days or more, OCT could differentiate between healthy and early carious lesions as the standard deviation of treatment steps up to day 10 contain intensity values which lie within the standard deviation of the healthy region. Thus, at 15-day of treatment step the detection threshold of the system can be defined. Bar plots of TPLI show an increase in the average phase values between each treatment interval. Due to smaller standard deviation of average phase value obtained from the healthy region of the sample, the detection threshold of TPLI can be distinguished at day 2 of demineralization. More comprehensive comparison between TPLI and OCT is tabulated in Table 1. From these comparison parameters it can be concluded that TPLI is an optimal choice for detection of early dental caries.

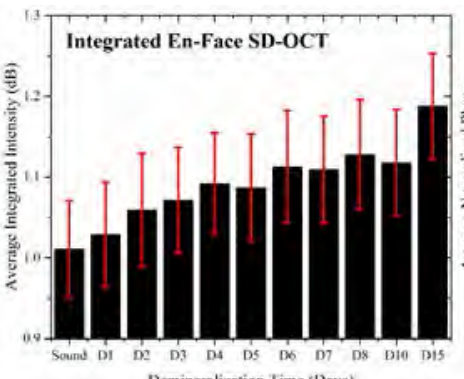

Demineralization Time (Days)

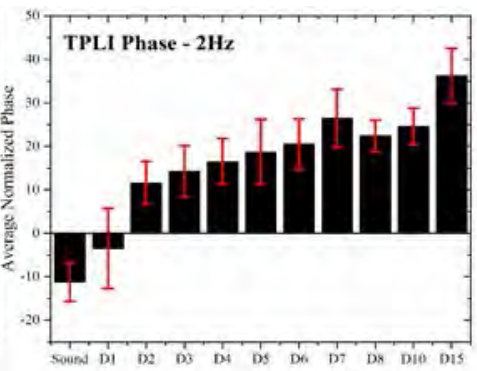

Demineralization Time (Days)
Figure 4. Bar plots of (left) spectral-domain optical coherence tomography (OCT) and thermophotonic lock-in imaging (TPLI) (right) with their standard deviations.

\begin{tabular}{|c|c|c|}
\hline Parameters & TPLI & OCT \\
\hline Source of Contrast & Absorption & Scattering \\
\hline Imaging Depth & few mm-cm & $1-2 \mathrm{~mm}$ \\
\hline Resolution & Low $(\sim 100 \mu \mathrm{m})$ & $\operatorname{High}(\sim 10 \mu \mathrm{m})$ \\
\hline Field of view & $\begin{array}{c}\text { Wide } \\
\text { (whole tooth) }\end{array}$ & Small \\
\hline Detection Sensitivity & Very High & Low \\
\hline Detection Threshold & 2 Days of Demin & 15 Days of Demin \\
\hline 3D Imaging & No & Yes \\
\hline Clinical Translation & Difficult & Straight Forward \\
\hline Cost (\$) & $\$$ & $\$ \$$ \\
\hline
\end{tabular}

\section{CONCLUSION}

In this study, we present a study that quantifies the detection threshold between spectral domain optical coherence tomography and thermophotonic lock-in imaging in detection of early dental caries. These systems used for diagnosis of early caries and their progression would significantly improve caries management decisions with respect to preventive care. Results suggest that the detection threshold for detection of early dental caries were found to be better in TPLI images compared to OCT due to its light absorption contrast mechanism compared to light scattering contrast mechanism in OCT. 


\section{REFERENCES}

[1] R. A. Bagramian, F. Garcia-Godoy, and A.R. Volpe, "The global increase in dental caries. A pending public health crisis," Am. $J$. Dent., vol. 22, no. 1, pp. 3-8, Feb. 2009.

[2] M. Mohanraj, V. R. Prabhu, R. Senthil, "Diagnostic methods for early detection of dental caries- A review," Int. J. Pedod. Rehabil., vol. 1, no. 1, pp. 29-36, 2016.

[3] N. Garg, and A. Garg, Textbook of Operative Dentistry, 2nd ed. New Delhi, IN: Jaypee, 2013.

[4] Y. S. Hsieh, Y. C. Ho, S. Y. Lee, C. C. Chuang, J. Tsai, K. F. Lin, and C.W. Sun, "Dental Optical Coherence Tomography," Sensors, vol. 13, no. 7, pp. 8928-8949, 2013. doi: 10.3390/s130708928.

[5] M. T. Tsai et al. "Effective indicators for diagnosis of oral cancer using optical coherence tomography," Optics Express, vol. 16, no. 20, pp. 15847-15862, Sep. 2008. doi: 10.1364/OE.16.015847.

[6] Y. Shimada, A. Sadr, M. Burrow, J. Tagami, N. Ozawa, and Y. Sumi, "Validation of swept-source optical coherence tomography (SSOCT) for the diagnosis of occlusal caries," J. Dent., vol. 38, no. 8, pp. 655-665, Aug. 2010. doi: 10.1016/j.jdent.2010.05.004.

[7] O. Breitenstein, W. Wilhelm, and M. Langenkamp, Lock-in Thermography: Basics and Use for Evaluating Electronic Devices and Materials, 2nd ed. Heidelberg, DE: Springer-Verlag, 2010.

[8] Y.-K. An, J. Kim, and H. Sohn, "Laser lock-in thermography for detection of surface-breaking fatigue cracks on uncoated steel structures," NDT \& E Int., vol. 65, pp. 54-63, July 2014. doi: 10.1016/j.ndteint.2014.03.004.

[9] N. Tabatabaei, A. Mandelis, and B. Amaechi, "Thermophotonic lock-in imaging of early demineralized and carious lesions in human teeth," J. Biomed. Opt., vol. 16, no. 7, pp. 071402, July 2011. doi: $10.1117 / 1.3564890$.

[10] A. Ojaghi, A. Parkhimchyk, and N. Tabatabaei, "First step toward translation of thermophotonic lock-in imaging to dentistry as an early caries detection technology," J. Biomed. Opt., vol. 21, no. 9, pp. 096003, Sep. 2016. doi: 10.1117/1.JBO.21.9.096003.

[11] N. Tabatabaei, A. Mandelis, M. Dehghany, K. Michaelian, and B. Amaechi, "On the sensitivity of thermophotonic lock-in imaging and polarized Raman spectroscopy to early dental caries diagnosis," J. Biomed. Opt., vol. 17, no. 2, pp. 0250021, Feb. 2012. doi: $10.1117 / 1 . J B O .17 .2 .025002$. 


\title{
Helical Spring Design optimization for Endoscopic Devices Using a Design- of-Experiments and Response Surface Approach
}

\author{
Hamid Ebrahimi, PhD \\ Holland Musculoskeletal Research Program, \\ Sunnybrook Research Institute \\ IBBME, University of Toronto \\ Toronto, Canada \\ hamid.ebrahimi@mail.utoronto.ca
}

\author{
Cari Whyne, $\mathrm{PhD}$ \\ Holland Musculoskeletal Research Program, \\ Sunnybrook Research Institute \\ Dept of Surgery and IBBME, University of Toronto \\ Toronto, Canada \\ cari.whyne@sunnybrook.ca
}

\author{
Albert Yee, MSc, MD, FRCSC \\ Holland Musculoskeletal Research Program, \\ Sunnybrook Research Institute \\ Dept of Surgery and IBBME, University of Toronto \\ Toronto, Canada \\ albert.yee@sunnybrook.ca
}

\begin{abstract}
Flexible endoscopes require reliable advancement and steerability of the device tip. Helical spring design is critical to both endoscopic steerability and cable function. To characterize the impact of geometric and material factors on endoscopic function, a parametric helical spring and a cable assembly was modelled using the finite element method and analyzed using a design-of-experiments approach. Individual input parameters (height, modulus (E), force, and width) and two interactions (pitch/width and pitch/height/E/width) were found to significantly impact the radius of curvature (a measure of steerability). The force and pitch/width interaction had negative effects, in contrast to positive effects from height, E, width and the pitch/height/E/width interaction. This information provides critical geometric and material information to guide helical spring design for optimized endoscopic steerability.
\end{abstract}

Keywords: Endoscope, Helical Spring, Finite Element Analysis, Design-of-Experiments, Geometry and Material Parameters, Radius of Curvature

\section{INTRODUCTION}

Endoscopes are long thin tubular devices for non-invasive visualization of the interior of cavities, canals and vessels. The flexibility of articulated endoscopes is important in many clinical applications. Articulated endoscopes are generally controlled by cables and levers which bend the distal extremity of the device to enable route selection or to change the field of view.

Challenges associated with flexible endoscope design include reliable advancement and steerability of the device tip [1]. Reliable advancement can be addressed by utilization of relatively rigid helical springs within the device shaft. The helical geometry provides bending flexibility at the expense of some reduction in axial stiffness and tensile strength [2]. However, the device tip must be flexible for steerability to accommodate different bend radii. The device tip can be steered via a cable attached to a control mechanism within the endoscopic handle.

In order to achieve tight bends while preventing cable malfunctions [3] a thorough understanding of the helical spring stress distribution under specific load conditions is necessary. Multiple studies have developed analytical and numerical models to characterize stress distribution in helical springs under axial and pure bending loads [4]. However, to date studies have not considered the optimization of bend radii representative of helical spring and cable assemblies in traditional endoscopes. As such, the aim of this study is to understand the impact of endoscopic tip design (geometry and material properties) on the radius of curvature governing steerability in traditional endoscopes.

\section{METHODS}

\section{A. Experimental Design}

A two-level five-factor design-of-experiments methodology was utilized to understand the main geometric and material effects and their interactions on the attainable radius of curvature of an endoscopic device (outcome). The effects of width, height, pitch, Young's modulus (E) and applied force were assessed at combination of high and low factor levels, resulting in 32 experimental groups (Table 1). The range for width, height and pitch were based on commercially available endoscopic designs, with $\mathrm{E}$ representing metals from Aluminum $(\sim 70 \mathrm{GPa})$ to Steel $(\sim 200 \mathrm{GPa})$. Force ranged from $0.5 \mathrm{~N}$ (to allow the device tip in the most rigid configuration to 
displace at least $0.1 \mathrm{~mm}$ ) to $2 \mathrm{~N}$ (representing the maximum tensile force a $0.1 \mathrm{~mm}$ diameter steel cable can withstand before plastic deformation).

\begin{tabular}{|l|l|l|l|l|l|}
\hline $\begin{array}{c}\text { Factor } \\
\text { Level }\end{array}$ & $\begin{array}{c}\text { Width } \\
(\mathbf{m m})\end{array}$ & $\begin{array}{c}\text { Height } \\
(\mathbf{m m})\end{array}$ & $\begin{array}{c}\text { Pitch } \\
\mathbf{( m m )}\end{array}$ & $\begin{array}{c}\text { E } \\
(\mathbf{G P a})\end{array}$ & $\begin{array}{c}\text { Force } \\
(\mathbf{N})\end{array}$ \\
\hline Low & 0.1 & 1 & 3 & 70 & 0.5 \\
\hline High & 1 & 2.5 & 4 & 200 & 2 \\
\hline
\end{tabular}

TABLE I. HIGH AND LOW FACTOR LEVELS ARE PRESENTED FOR WIDTH, HEIGHT, PITCH, E AND FORCE USED TO INVESTIGATE THE ENDOSCOPIC GEOMETRIC AND MATERIAL EFFECTS AND THEIR INTERACTIONS ON THE ACHIEVABLE RADIUS OF CURVATURE

\section{B. Finite Element (FE) Model Generation}

An FE model of a traditional endoscope was generated that enabled parametric geometric and material property assignments (Table 1). The helical spring (rectangular cross section) was modelled using linear brick elements. The cable was modelled using linear 3-D truss elements. Once assembled the cable proximal end was connected to the helical spring proximal outer circumference (Fig. 1a) and the cable distal end was placed inside the helical spring (Fig. 1b). Interactions were modelled using a general contact algorithm. An ENCASTRE boundary condition was used to fix the distal end of the helical spring. The cable was connected to the helical spring tip using a tie constraint. A concentrated force was then applied to the cable distal end to simulate the pulling force from the controller unit and analysed in ABAQUS dynamic/explicit.

The radius of curvature was calculated based on spatial displacement outputs identified between a node defined at the cable attachment site on the helical spring tip and the most distal node with greater than zero displacement at the base of the helical spring. Mesh convergence analysis was performed by refining the mesh size and calculating the radius of curvature and maximum deformation in each iteration (to within 5\%). The final model contained 3692 nodes, 2135 elements and 10920 degrees of freedom.

An automated procedure through the ABAQUS Scripting Interface (ASI) was utilized to create parts, assign material properties, assemble parts, apply boundary conditions and force, and solve each generated model. The generated nodal displacement outputs and deformed geometry image data sets were analysed by a python script to calculate the radius of curvature, annotate images with their corresponding configuration parameters and organize the raw data to be used for design-of-experiments analysis (Fig. 1).

\section{Design-Of-Experiments}

Thirty-two configurations were analyzed in the two-level factorial design-of-experiments analysis (Design-Ease). Based on the Box-Cox plot, a base 10 Log transformation of the output response (radius of curvature) was used to stabilize the variance. A Sum-of-Squares chart with a $2 \%$ weighted contribution threshold was utilized to determine the model inclusion criteria. A Shapiro-Wilk test was also conducted to confirm data normality. An Analysis of Variance (ANOVA) with a Bonferroni correction was conducted to determine the significance of the model, effects and interactions.

\section{RESULTS}

All thirty-two models were solved successfully. The model, four of the input parameters (width, height, E, force) and two interactions (between pitch/width and pitch/height/E/width)
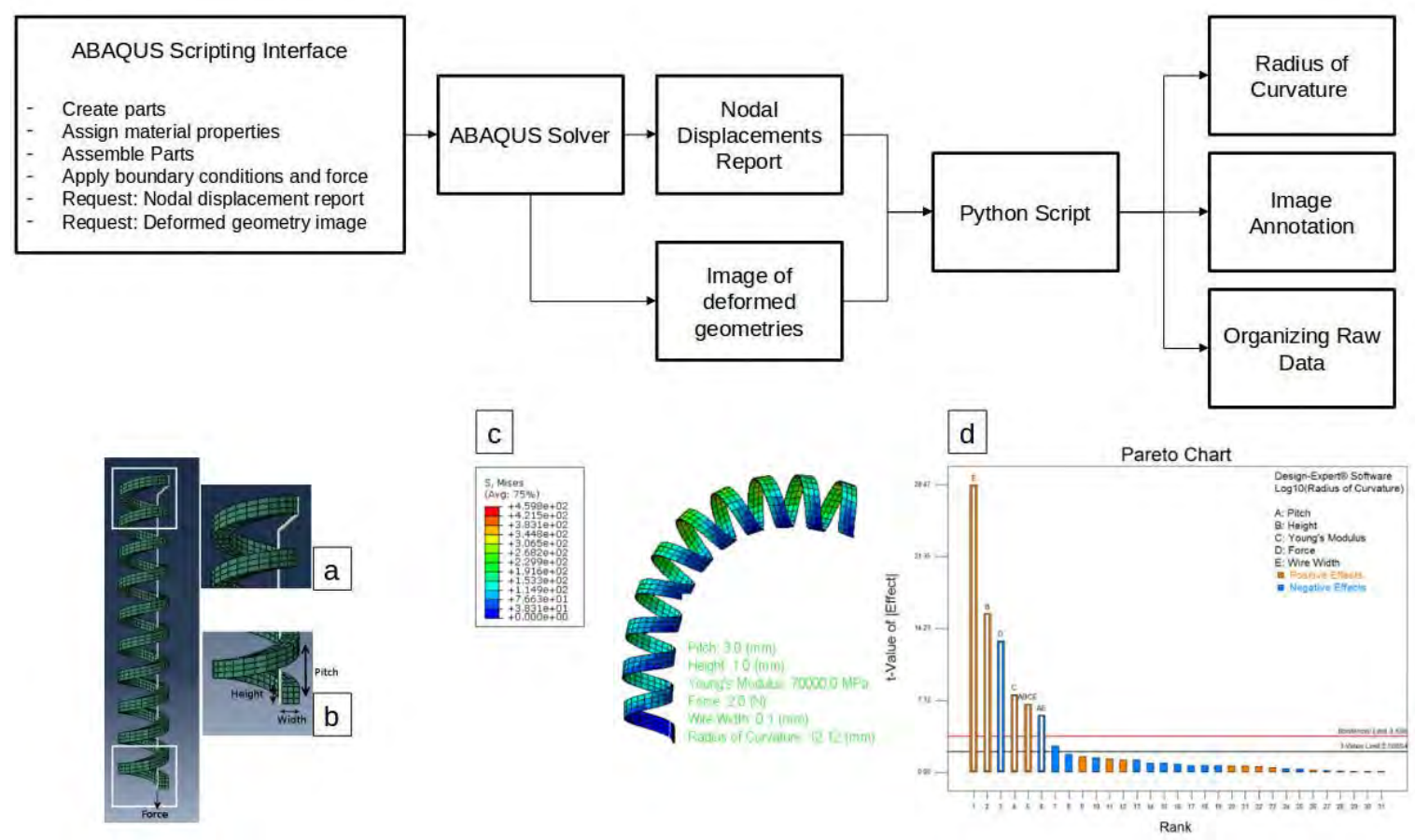

Figure 1. A flowchart describing the automation of the FE model creation and DOE analysis. The unloaded spring model and its parameters are shown (left, $\mathrm{a}$ and $\mathrm{b}$ ) with the lowest radius of curvature model presented (centre, c). The Pareto chart (right, d) identifies the significant effects and interactions. 
were found to be statistically significant $(\mathrm{P}<0.0001)$. The lowest radius of curvature achieved was $12.12 \mathrm{~mm}$ (Fig. 1c). A Pareto chart summarizing the main effects and model errors is shown in Fig. 1d.

The largest contribution to the model was the width of the spring (effect $=1.39,58.33 \%$ contribution). The force and pitch/width interaction each had a negative influence, whereas the height, E, width and pitch/height/E/width interaction showed a positive influence. A negative effect in which higher forces reduce the radius of curvature allows for tighter bends. The negative AE effect shows that pitch and width work in opposition with respect to their impact on radius of curvature, as evident in the response shown in Fig. 2. Pitch and all other interaction effects below the $2 \%$ contribution threshold were applied as model error. A summary of the statistical data as well as the final equation in terms of coded factors are presented in Table 2 .

\section{DISCUSSION AND CONCLUSION}

A robust computational model was developed using a design-of-experiments approach that allows parametric optimization of endoscopic tip design parameters for radius of curvature minimization. Based on this model some of the identified disadvantages of traditional endoscopes can be addressed by altering design parameters for different applications. A small pitch and width yield a radius of curvature optimal for acute bending radii. If due to design restrictions a larger width is necessary, an increase in pitch may employed to maintain a sufficiently small bending radius.

The challenges associated with endoscopic device advancement have been reported in multiple studies [5,6,7]. A study of FDA reports from 1985 to 2009 (Chen et al. 2010) reported that $21 \%$ of endoscopic mechanism failures are due to cable malfunctions. Based on our models, if advancement of
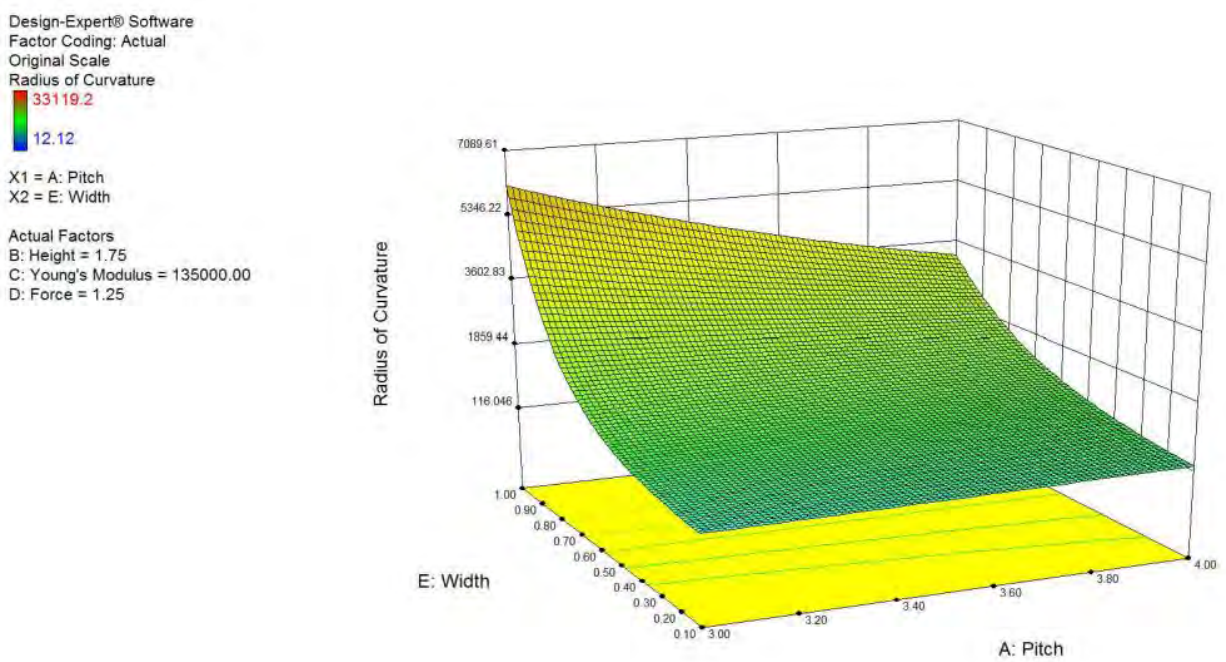

Figure 2. A response surface describing the negative interaction between helical spring pitch and width with respect to radius of curvature.

\begin{tabular}{|c|c|c|c|c|c|c|}
\hline $\begin{array}{c}\text { Applied } \\
\text { Transformation }\end{array}$ & Source & $\begin{array}{l}\text { Coded } \\
\text { Factors }\end{array}$ & Effect & Contribution (\%) & F Value & P Value \\
\hline \multirow{7}{*}{$\begin{array}{l}\text { Base } 10 \\
\text { Logarithm }\end{array}$} & Model & NA & NA & NA & 227.41 & $<0.0001$ \\
\hline & Width & $\mathrm{E}$ & 1.39 & 58.33 & 810.45 & $<0.0001$ \\
\hline & Height & B & 0.77 & 17.82 & 247.54 & $<0.0001$ \\
\hline & Force & $\mathrm{D}$ & -0.63 & 12.19 & 169.30 & $<0.0001$ \\
\hline & Young's Modulus (E) & $\mathrm{C}$ & 0.37 & 4.26 & 59.23 & $<0.0001$ \\
\hline & Pitch/Height/E/Width & $\mathrm{ABCE}$ & 0.33 & 3.29 & 45.72 & $<0.0001$ \\
\hline & Pitch/Width & $\mathrm{AE}$ & -0.28 & 2.32 & 32.20 & $<0.0001$ \\
\hline Final Equation & \multicolumn{6}{|c|}{$\log ($ radius of Curvature $)=2.96+0.38 * \mathrm{~B}+0.19 * \mathrm{C}-0.32 * \mathrm{D}+0.69 * \mathrm{E}-0.14 * \mathrm{~A} * \mathrm{E}+0.16 * \mathrm{~A} * \mathrm{~B} * \mathrm{C} * \mathrm{E}$} \\
\hline
\end{tabular}

TABLE II. 
the device requires high pushing forces, the device axial stiffness must be increased by increasing the cable width and /or modulus of elasticity and the acute bend radius can still be achieved by increasing the pitch, and/or lowering the height without increasing the force applied to the cables.

This study demonstrates the potential of a combined FE modelling DOE approach for optimization in biomedical instrumentation design. Through optimization of geometric and material properties, endoscopes can be optimized to meet specific tip design criteria and function safely and effectively within varied environmental conditions.

\section{ACKNOWLEDGMENT}

This work was supported by a CIHR Banting and Best scholarship.

\section{REFERENCES}

[1] A. Menciassi, J. H. Park, S. Lee, S. Gorini, P. Dario, and Jong-Oh Park, "Robotic solutions and mechanisms for a semi-autonomous endoscope," in IEEE/RSJ Int. Conf. Intelligent Robots and System, pp. 1379-1384, Oct. 2002. doi: 10.1109/IRDS.2002.1043947.

[2] M. Raoof and T. J. Davies, "Determination of the bending stiffness for a spiral strand," J. Strain Anal. Eng. Des., vol. 39, no. 1, pp. 1-13, Jan. 2004. doi: $10.1177 / 030932470403900101$.

[3] Y. Chen, S. Tanaka and I. W. Hunter, "Disposable endoscope tip actuation design and robotic platform," in Ann. Int. Conf. IEEE Eng. Med. Biol. Soc EMBC, 2010. doi: 10.1109/IEMBS.2010.5627677.

[4] S. G. Keller and A. P. Gordon, "Equivalent stress and strain distribution in helical compression springs subjected to bending," J. Strain Anal. Eng. Des., vol. 46, no. 6, pp. 405-415, Jun. 2011.

doi: $10.1177 / 0309324711410128$.

[5] D. K. Rex, M. Khashab, G. S. Raju, J. Pasricha, and R. Kozarek, "Insertability and Safety of a Shape-Locking Device for Colonoscopy," Am. J. Gastroenterol., vol. 100, no. 4, pp. 817-820, Apr. 2005. doi: 10.1111/j.1572-0241.2005.40801.x.

[6] A. J. Loeve, D. H. Plettenburg, P. Breedveld, and J. Dankelman, "Endoscope Shaft-Rigidity Control Mechanism: 'FORGUIDE,'” IEEE Trans. Biomed. Eng., vol. 59, no. 2, pp. 542-551, Feb. 2012. doi: 10.1109/TBME.2011.2175730.

[7] T. K. Adebar, J. D. Greer, P. F. Laeseke, G. L. Hwang, and A. M. Okamura, "Methods for Improving the Curvature of Steerable Needles in Biological Tissue," IEEE Trans. Biomed. Eng., vol. 63, no. 6, pp. 11671177, Jun. 2016. doi: 10.1109/TBME.2015.2484262. 


\section{Focused Ultrasound Surgery of Breast Cancer: A Computational Study on a Realistic Patient Model}

\author{
Moslem Sadeghi-Goughari; Soo Jeon; Hyock-Ju Kwon \\ Department of Mechanical and Mechatronics \\ Engineering, University of Waterloo \\ Waterloo, Canada. \\ M8sadegh@uwaterloo.ca, soojeon@uwaterloo.ca, \\ hjkwon@uwaterloo.ca
}

\begin{abstract}
Focused ultrasound surgery (FUS) is a new energybased therapeutic technique which has been recognized as a truly non-invasive surgical method. Since the ultrasonic energy is applied from an external transducer, FUS has the potential to improve both oncologic and cosmetic outcomes for breast cancer treatment. In the present study, we conducted a computational analysis on a realistic patient model to investigate the mechanism and the applicability of FUS in breast cancer treatment. Magnetic Resonance Imaging (MRI) data of a patient with breast tumor were used to provide a clinical model for finite element analysis (FEA) using COMSOL. Results from computational analysis clarify the acoustic and thermal mechanisms of FUS and suggest the way how this technique can be carried out during the breast cancer treatment.
\end{abstract}

Keywords-focused ultrasound surgery; breast cancer; finite element analysis;wave propagation; heat transfer.

\section{INTRODUCTION}

After the cardiovascular and cerebrovascular diseases, cancer is considered as the second leading cause of death in the world, with nearly 8.8 million deaths $(15.7 \%$ of total) each year. Breast cancer is the most common cancer among women, with an estimated 1.7 million new cases diagnosed each year in the world [1]. This represents about $25 \%$ of all cancer in women. The Canadian Cancer Society statistics show that about 26,300 new cases of breast cancer have been diagnosed in Canadian women in 2017, and about 5,000 of the cases will be developed to death [2]. The high mortality rate substantiates the importance of early detection and treatment of breast cancers.

Determinant factors in the treatment of a breast tumor are highly dependent on the type, size and location of the tumor [3]. For both benign and malignant cases, surgical techniques such as mastectomy and breast-conserving therapy (BCT) have been considered as the main methods of treatment. Mastectomy, which is the surgical removal of the entire breast, was first introduced by William Halstedt in 1894, and remained as the cornerstone of therapy for about 80 years [4]. Whereas $\mathrm{BCT}$ is achieved just by removing a discrete portion of breast

\author{
Toktam Beheshtian \\ Breast Disease Research Department, Breast Cancer \\ Research Center, Motamed Cancer Institute, ACECR, \\ Tehran, Iran \\ tbeheshtian@gmail.com
}

tissue containing the cancer, and can practically be followed by adjuvant treatment modalities such as chemotherapy and radiotherapy to get more efficient results. Despite the widespread use of surgery as a main treatment for cancer, it is invasive and unable to preserve the structure of the breast postoperatively.

The sensitivity of the human body to the temperature change has caused the development of new therapeutic methods for cancer. Thermal ablation therapy can be achieved by increasing the tissue temperature above a standard level to change the properties of targeted tissue [5]. Some of the energy-based methods used to achieve the thermal ablation of tumors are radiofrequency ablation, laser thermotherapy, and ultrasound therapy. Among them, ultrasound surgery is truly a non-invasive technique, since acoustic waves are emitted by an external transducer [6].

Ultrasound therapies can be split into two major categories, namely "ultrasound hyperthermia" and "high intensity focused ultrasound (HIFU) surgery". During the hyperthermia treatment, the targeted tissue is exposed to the acoustic energy at a low intensity level for a long period of time (10-60 minutes), such that the tumor temperature is evaluated and maintained at $41-45^{\circ} \mathrm{C}$. In contrast, HIFU uses the ultrasonic energy at a high intensity field to increase the temperature of focused area up to $56^{\circ} \mathrm{C}$ with a short ablation time (from 0.1 to 30 seconds) [7].

FUS is a truly non-invasive therapeutic technique which has been recognized for enormous medical applications ranging from cancer treatment to thrombolysis [6]. In principle, FUS allows the local treatment of a tumor by focusing acoustic energies to a targeted area from an extracorporeal source of ultrasound. This process induces tissue heating and corresponding temperature rise in the focal region that would be associated with irreversible biological effects such as necrosis and cell apoptosis.

FUS has been considered as a promising option for the thermal therapy of breast tumors due to its non-invasive mechanism. Since this technique is applied from an external source remotely, it does not cause any significant changes in patients' mammary shape, and has the potential to upgrade both oncologic and cosmetic outcomes for breast cancer 
treatment [8]. In response to the potential benefits of HIFU to patients with breast cancer, many researches have been carried out on the different aspects of HIFU including acoustic-heating characteristics of ultrasound transducer, effects of blood perfusion, and physiological effects of HIFU ablation. During the current decade, the clinical researches on the HIFU treatment of breast cancer were promoted by development of monitoring techniques [8].

In the present paper, we conducted a computational analysis to simulate the wave propagation and heat transfer mechanisms in the FUS of the breast cancer. Finite element model of breast tissue including a tumor was constructed from MRI images of a patient. Using COMSOL software, thermal mechanical results are presented in graphical form to investigate the performance of FUS in the treatment of breast cancer.

\section{MATHERIALS AND METHOD}

\section{A. FUS Mechanism}

Figure 1 shows a schematic drawing of the FUS system to treat breast cancer. When the acoustic waves propagate through the tissue, the medium particles start to vibrate, resulting in alternating cycles of compression and rarefaction pressure inside the tissue [9]. The ultrasound beams then converge into the focal area where the resulting acoustic pressure reaches the highest amplitude. This process leads to temperature rise in the focal region that would be associated with biological ablation and therapeutic results[7].

A single HIFU exposure ablates a small volume of the tumor, so in order to achieve the complete necrosis, the ablating treatment should be applied to the entire volume of tumor [10]. In practice, many of ablated regions must be placed side by side to paint out the entire tumor. The size of each lesion depends on many factors such as the characteristics of transducer and the acoustic properties of targeted tissue, but typically a single insonation covers a cigar shape region with approximate dimensions in the order of 8-15 $\mathrm{mm}$ (along beam axis) $\times 1-3 \mathrm{~mm}$ (transverse direction). The postoperative imaging data show that two weeks following FUS, the periphery of the ablated regions would be replaced by proliferative repair tissue [10].

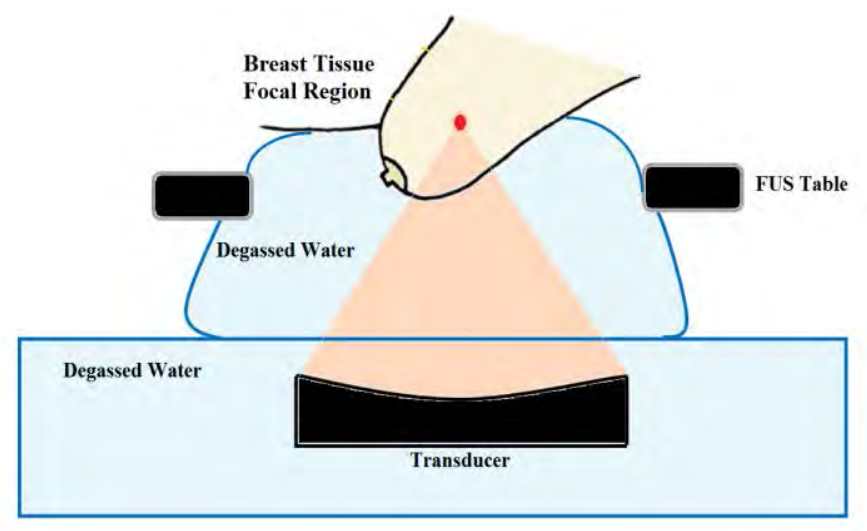

Figure 1. schematic drawing of FUS system used to treat breast cancer

\section{B. Physics and Mathermatics}

The main operation of FUS, is the ablation of a targeted region inside the body by focusing ultrasound beams to that area. [7]. The two predominant factors of FUS, which lead to the therapeutic results, are the conversion of acoustic energy to heat (thermal effect), and the cavitation (mechanical effect). In principle, the role of each effect depends on the dosage parameters, i.e. the intensity at the focal area and the exposure time. If the targeted tissue is exposed to an ultrasound field at a low intensity level for a long period of time, the thermal effect will be the only mechanism of action. However, for applying mechanical effects, a focused energy at a high intensity level for a short time is required [9].

For the mathematical modeling of the focused ultrasound induced heating, the mathematical theories of continuum physics can be used to model the thermal effects of FUS at a relatively low intensity level and before the cavitation threshold [11]. To this end, a set of mathematical equations that describes the wave propagation, absorption and heat transfer mechanisms should be combined together. Accordingly, the ultrasound wave propagation can be characterized with Helmholtz equation, which represents the time independent form of wave equation [12]:

$$
\nabla^{2} P+\left(\frac{\omega}{C_{c}}\right)^{2} P=0
$$

where $P$ is the acoustic pressure, $\omega$ is the angular frequency, and $C_{c}$ is the local speed of sound. To model the heat transfer during FUS, the absorbed acoustic energy can be considered as a heat source for the Pennes bioheat transfer equation [12]:

$$
\rho C_{p} \frac{\partial T}{\partial t}=\nabla \cdot(k \nabla T)-\rho_{b} C_{b} w_{b}\left(T-T_{b}\right)+2 \alpha_{A B S} I,
$$

where $T, \rho, C_{p}$ and $k$ denote the temperature distribution, density, specific heat and thermal conductivity of breast tissue, respectively. $\rho_{b}$ refers to the density of blood, $C_{b}$ is the specific heat of blood, $w_{b}$ is the blood perfusion rate, and $T_{b}$ is the temperature of the blood. $\alpha$ is the acoustic absorption coefficient, and $I$ is the local acoustic intensity which can be determined as a function of the acoustic pressure.

\section{Finite element analysis}

In order to simulate the heating mechanism induced by ultrasound energy and to calculate the acoustic pressure and consequent temperature rise during the FUS process, a FEA using COMSOL software was performed on a realistic patient model. In order to obtain the precise information on the breast tissue and tumor geometries, MRI of a patient with breast tumor was utilized. According to the Helsinki agreement, the whole study was thoroughly explained to the patient. Bilateral MR mammography was performed on a 42 years old lady in the "Erfan imaging center" in Tehran, Iran. The patient was a known case of recently diagnosed breast cancer in the right breast. The images show that there is a $26 \times 17 \mathrm{~mm}$ ill-defined low signal intensity mass in T1W in the right LIQ middle zone. Figure 2 displays the MRI images and consequent 3D solid model of the breast tissue and tumors. 


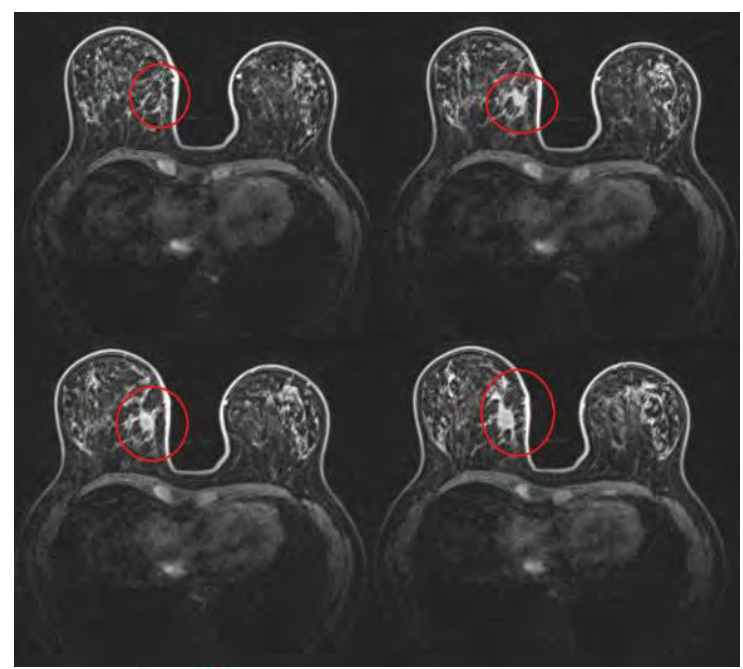

(a)

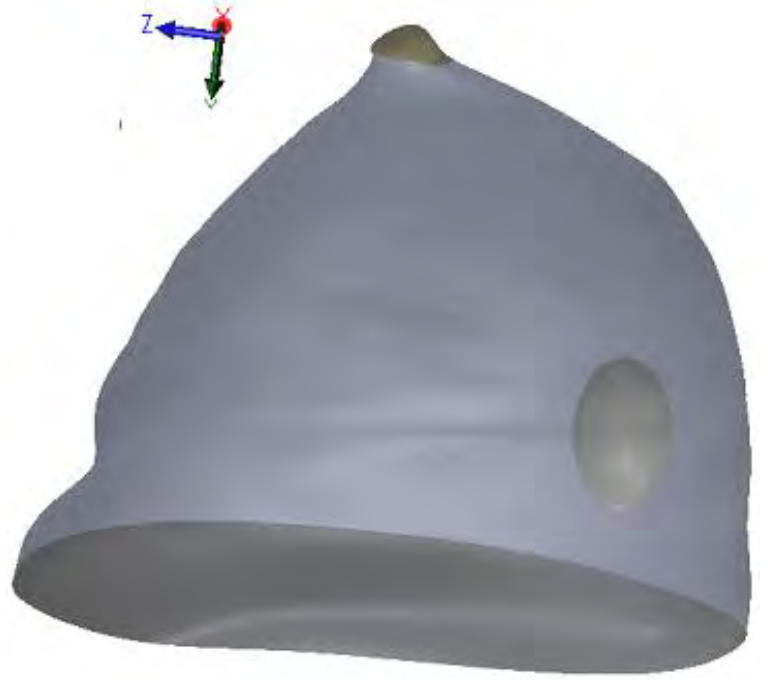

Figure 2. Geometrical features of breast and tumor location: (a) MRI data; (b) Solidworks Model

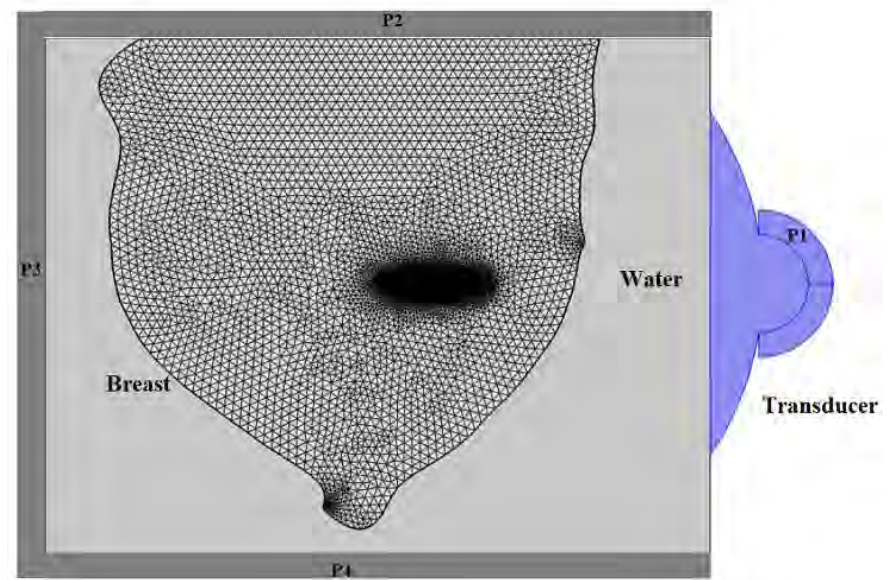

Figure 3. Breast and transducer model for FEA od FUS

A FUS simulation model composed of breast tissue, water and a transducer was developed, as shown in Figure 3. The transducer was bowl-shaped having a focal length of 62.64 $\mathrm{mm}$, an aperture of $35 \mathrm{~mm}$ in radius, and a hole of $10 \mathrm{~mm}$ in radius in the center [9]. A medium of water was considered between the breast tissue and the transducer to act as a coupling material. Four perfectly matched layers (P1-P4) were defined to absorb the outgoing waves. The pressure acoustics simulation was applied to all domains while the thermal analysis was performed only in the breast tissue domain.

The transducer was driven at the frequency, $f$, turned on for 15 seconds, and then turned off to let the breast tissue cool down. For meshing procedure, quadratic (2nd order) elements were used to discretize the temperature in the breast domain and quartic (4th order) elements were used to discretize the acoustic pressure in all domains including breast tissue, water, transducer and PMLs. The acoustic and thermal properties of the water and breast phantom used in the model simulation were derived from Ref [9].

\section{RESULTS AND DISCUSSION}

\section{A. Validation of numerical results}

First, the numerical analysis was developed for a simplified model of water domain to validate the numerical results with those achieved from the experiments [9]. In Figure 4, the normalized pressure profiles in the focal plane are presented, where the results from FEA are compared with those from experiments acquired in water at $30{ }^{\circ} \mathrm{C}$ and at source frequency of $1 \mathrm{MHz}$. The pressure profile from FEA in the present study shows good agreement with the experimental result, particularly in the focal region.

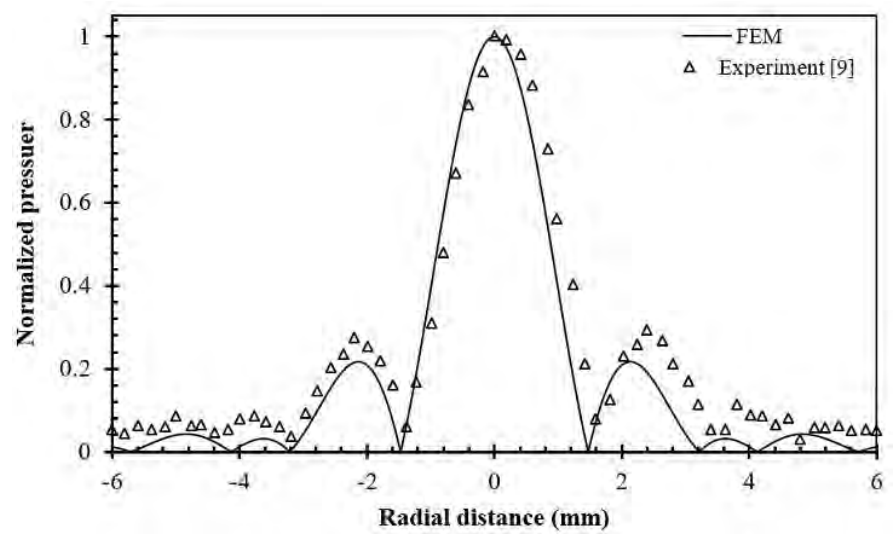

Figure 4. Validation of numerical data with experimental model

\section{B. Acoustic effect}

In this section, the results of wave propagation analysis in the water and the breast domains are presented when the transducer was driven at the frequency of $1 \mathrm{MHz}$. Figure 5 shows the acoustic pressure profile generated by the ultrasound waves propagated through the domains. The figure shows compression and rarefaction regions insides the breast tissue caused by the wave propagation. Here, the waves are focused into the tumor area which lead to a region with high acoustic pressures. A focal region with approximate size of $10 \times 2 \mathrm{~mm}$ is noticeable in the parts of breast which contains cancerous cells. 


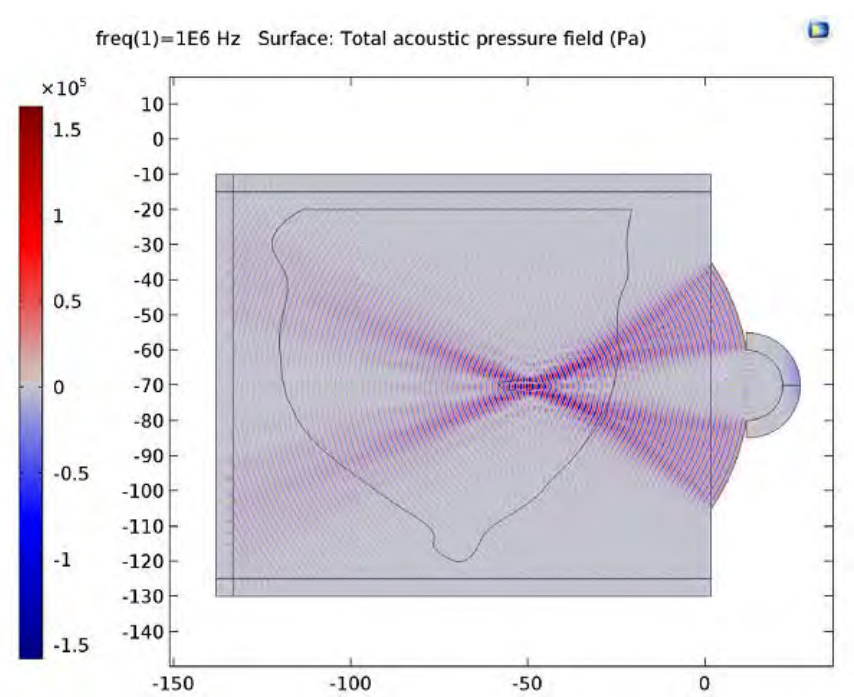

Figure 5. Total acoustic pressure field in breast and water domains.

\section{Thermal effect}

After presenting the acoustic pressure distribution in the breast tissue and water domains, the heat transfer mechanism generated by the absorption of acoustic energy in the breast tissue was determined. Figure 6 shows the temperature distribution in the breast tissue at the different moments of time during the thermal ablation treatment. The figure shows how the temperature rises in the tumor area that needs to be treated. According to the temperature distribution, the maximum temperature rise happened in the cigar-shaped focal region with an approximate length of $10 \mathrm{~mm}$ and a width of $2 \mathrm{~mm}$.
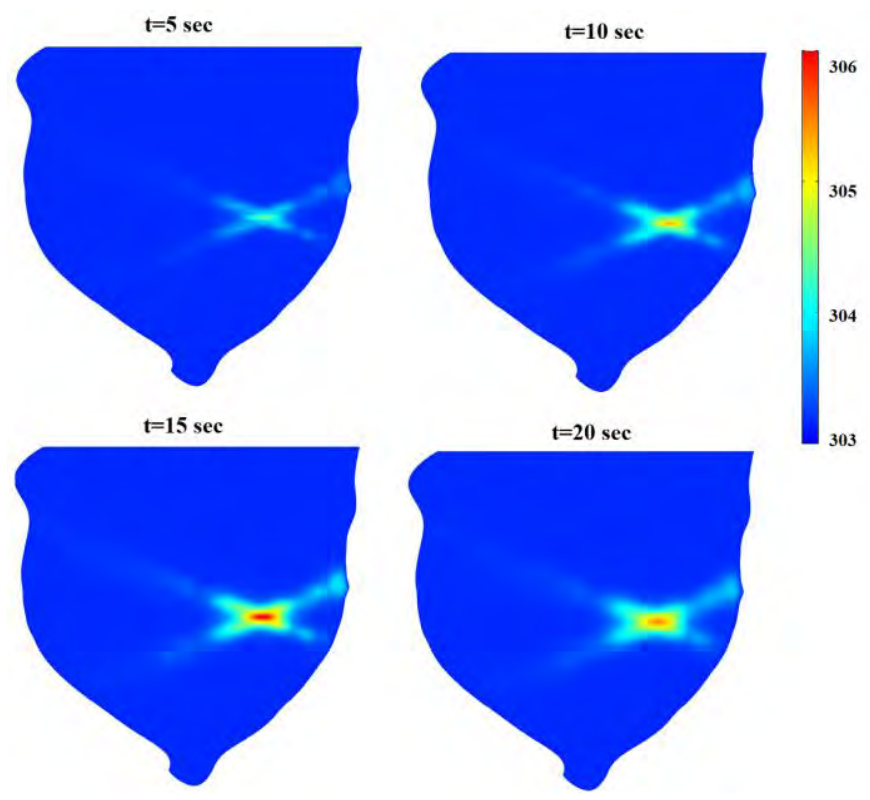

\section{CONCLUSION}

In the present study, a computational study on a realistic patient model was performed to examine the mechanism and the applicability of FUS in the treatment of breast cancer. To this end, the MRI data of a patient with breast tumor were used to mimic the breast tissue and tumor geometry for the finite element analysis of acoustic wave propagation and heat transfer. The results form acoustic and thermal analyses enabled us to understand the mechanism of wave propagation and heat transfer in the breast tissue during the FUS of breast cancer.

\section{ACKNOWLEDGMENT}

This research was supported in part by Natural Sciences and Engineering Research Council of Canada (NSERC), and Government of Ontario under Ontario Trillium Scholarships.

\section{REFERENCES}

[1] R. L. Siegel, K. D. Miller, and A. Jemal, "Cancer statistics, 2016," CA. Cancer J. Clin., vol. 66, no. 1, pp. 7-30, 2016. doi: $10.3322 /$ caac. 21332 .

[2] www.cancer.ca

[3] G. N. Hortobagyi, "Treatment of breast cancer," N. Engl. J. Med., vol. 339, no. 14, pp. 974-984, 1998. doi: 10.1056/NEJM199810013391407.

[4] W. S. Halsted, "I. The results of radical operations for the cure of carcinoma of the breast," Ann. Surg., vol. 46, no. 1, p. 1, 1907. doi: 10.1097/00000658-190707000-00001.

[5] S. N. Goldberg, G. S. Gazelle, and P. R. Mueller, "Thermal ablation therapy for focal malignancy: a unified approach to underlying principles, techniques, and diagnostic imaging guidance," Am. J. Roentgenol., vol. 174, no. 2, pp. 323-331, 2000.

doi: 10.2214/ajr.174.2.1740323.

[6] J. E. Kennedy, "High-intensity focused ultrasound in the treatment of solid tumours," Nat. Rev. Cancer, vol. 5, no. 4, p. 321, 2005. doi: doi.org/10.1038/nrc1591.

[7] T. J. Dubinsky, C. Cuevas, M. K. Dighe, O. Kolokythas, and J. H. Hwang, "High-intensity focused ultrasound: current potential and oncologic applications," Am. J. Roentgenol., vol. 190, no. 1, pp. 191-199, 2008. doi: 10.2214/AJR.07.2671.

[8] S. Li and P.-H. Wu, "Magnetic resonance image-guided versus ultrasound-guided high-intensity focused ultrasound in the treatment of breast cancer," Chin. J. Cancer, vol. 32, no. 8, p. 441, 2013. doi: $10.5732 / \mathrm{cjc} .012 .10104$.

[9] J. Huang, R. G. Holt, R. O. Cleveland, and R. A. Roy, "Experimental validation of a tractable numerical model for focused ultrasound heating in flow-through tissue phantoms," J. Acoust. Soc. Am., vol. 116, no. 4, pp. 2451-2458, 2004. doi: 10.1121/1.1787124.

[10] J. E. Kennedy et al., "High-intensity focused ultrasound for the treatment of liver tumours," Ultrasonics, vol. 42, no. 1, pp. 931935, 2004. doi: 10.1016/j.ultras.2004.01.089.

[11] J. Huang, "Heating in vascular tissue and flow-through tissue phantoms induced by focused ultrasound." Boston University, 2002.

[12] R. Martínez, A. Vera, and L. Leija, "HIFU induced heating modelling by using the finite element method," Phys. Procedia, vol. 63, pp. 127-133, 2015. doi: 10.1016/j.phpro.2015.03.021. 


\section{Controlled Deflection of Diamagnetic Biocompatible Aqueous Droplets}

\author{
Stephanie Buryk \\ Graduate Program in Biomedical Engineering, \\ Ryerson University \\ Toronto, Canada \\ sburyk@ryerson.ca \\ Jennifer Kieda \\ Department of Electrical and Computer Engineering \\ Ryerson University \\ Toronto, Canada \\ jennifer.kieda@ryerson.ca
}

\author{
Scott S. H. Tsai \\ Department of Mechanical and Industrial Engineering \\ Ryerson University, Toronto, Canada. \\ Keenan Research Centre for Biomedical Science - St. \\ Michael's Hospital, Toronto, Canada. \\ Institute for Biomedical Engineering, Science and \\ Technology (iBEST), Toronto, Canada. \\ scott.tsai@ryerson.ca
}

\begin{abstract}
We present a microfluidic platform that is capable of controlled diamagnetic droplet displacement. In this method, monodisperse aqueous droplets are produced in a continuous phase of hydrophobic ferrofluid. Both phases are exposed to magnetic field. Precise deflection of the aqueous droplets is then achieved in a single step by adjusting the flowrate of the disperse phase.
\end{abstract}

\section{Microfluidics, diamagnetics, droplet control, biocompatible.}

\section{INTRODUCTION}

Microfluidically generated droplets are readily used for the storing of biomaterials and biological analysis [1]. Control of these droplets using magentophoresis has emerged as a key technology in microbiology. Currently, the leading methods of droplet control by magentophoresis require the synthesis of biocompatible fluids and magnetic labeling, which is often time-intensive and costly [2].

Here, we present a fast, low-cost, and label-free alternative for the precise control of biocompatible droplets using negative magnetophoresis. We describe a ferro-hydrodynamic microfluidic system requiring only microchannels and a permanent magnet.

\section{EXPERIMENTAL AND RESULTS}

\section{A. Devcie fabricaiton}

We fabricated microfluidic polydimethylsiloxane (PDMS) channels based on a standard soft-lithography method. Then we introduced a permanent magnet parallel to the main channel of the device.
A magnetic fluid phase and a water phase were introduced to the system through the device inlets. Syringe pumps were used to control flow-rates of the fluids.

\section{B. Fluid properties}

The magnetic phase consists of oil-based ferrofluid, mineral oil, and a surfactant. The ferrofluid used contains magnetite nanoparticles with a $10 \mathrm{~nm}$ diameter [3].

\section{Droplet Production and Control}

A monodispersed stream of aqueous water droplets is achieved in the magnetic phase using a jetting regime. The external magnetic field attracts the magnetic medium of ferrofluid in the continuous phase. The attraction of this phase preferentially deflects the water droplets from their laminar flow. By simply adjusting the flow-rate of the dispersed aqueous phase, the degree of droplet deflection is precisely controlled.

\section{REFERENCES}

[1] W. Zhao et. al. "Label-free and continuous-flow feerodydrodynamic separation of HeLa cells and blood cells in biocompatible ferrofluids," Adv. Funct. Mater., vol. 26, no. 22, pp. 3990-3998, 2016. doi: $10.1002 / \mathrm{adfm} .201503838$.

[2] K. Zhang et al., "On-demand microfluidic droplet manipulation using hydrophobic ferrofluid as a continuous-phase," Lab Chip, vol. 11, no. 7, pp. 1271-1275, 2011. doi: 10.1039/c01c00484g.

[3] Ferrotec. (2018). Ferrofluid Safety Data Sheet [Online]. Available: http://ferrofluid.ferrotec.com. [Accessed 19 Jan 2018]. 


\title{
Characterization of the Electrodes of DEP-based Micro-separator
}

\author{
Arash Dalili, Erfan Taatizadeh, Mina Hoorfar \\ School of Engineering \\ University of British Columbia \\ Kelowna, Canada \\ mina.hoorfar@ubc.ca
}

\begin{abstract}
In recent years, advances in lab-on-a-chip (LOC) devices has led to separation, sorting and manipulation of cells and particles on miniaturized devices. Among the different mechanisms that have been used in this regard, dielectrophoresis (DEP) offers high controllability on the particles, provides high throughput, and is tunable. Due to these advantages, DEP is used in this paper for the design of a microseparator. To optimize the geometry of such a separator, COMSOL Multiphysics ${ }^{\circledR}$ is used to simulate the electric field with the goal of achieving the highest performance in cell separation. For a DEP-based micro-separator, two inclined rectangle planar electrodes are considered. The effect of the width of each one of these electrodes as well as the gap between them on the DEP force is investigated to find the optimum design.
\end{abstract}

Keywords- DEP; COMSOL modeling; Optimization, Microfluidics

\section{INTRODUCTION}

The separation of cells and particles in a micro scale has attracted the attention of researchers in the fields of microfluidics, bio-engineering, biology, chemistry, etc. The researchers are using different methods, which can be categorized into passive and active. The passive methods include micro-filters, micro-devices based on inertial forces, deterministic lateral displacement and the pinched flow fractionation. While the passive operate on their own, the active methods rely on an external force to manipulate the cells or particles, which can lead to more accurate and specific separation. Acoustic, magnetic, optical and electrical forces are among the forces that are used for cell/particle manipulation. The electric force can manipulate charged particles, which is called electrophoresis, or it can manipulate uncharged particles, which is called dielectrophoresis (DEP). DEP has been used by researchers for different applications such as cell patterning [1], trapping [2,3], focusing [4], enrichment [5], separation [6] and isolation $[7,8]$. DEP can separate the cells based on their electrical properties (electrical conductivity and permittivity) and size. Although some studies have used biomarkers to change the electrical properties of the cells [9], in general, DEP is a marker-free method depending on the intrinsic properties of the cells. Eliminating the labeling of the cells and biomarkers makes the preparation for DEP-based separation much faster and cheaper than some other methods such as fluorescenceactivated cell sorter (FACS) and magnetic-activated cell sorter (MACS). Another advantage of DEP over other marker-free techniques is its relative ease of fabrication compared to other techniques such as those based on acoustic forces.

Different configureations of electordes such as interdigitated [10], castellated [11], top-bottom paterned [12] and sidewall patterned [13] have been used for DEP-based devices. Among these configurations, planar slanted electrodes are easy to fabricate and operate, and are suitable for continuous cell separation. The slanted electrodes can continuously move the cells laterally toward the target outlet.

To apply an effective DEP force for cell/particle separation, the design needs to be optimized. This optimized design can lead to higher throughput and lower voltage (which means lower joul heating and higher cell viability). In this study, slanted electrodes are chosen and simulation is used to find the optimum width of the electrodes and the gap between them for achieving a maximum DEP force and hence effective cell separation.

\section{THEORY OF DIELECTROPHORETIC FORCE}

When a particle is in a non-uniform electric field, it is polarized and experiences a force from the electric field. This phenomenon is referred to as DEP. The DEP force can be calculated as:

$$
F_{D E P}=2 \pi \varepsilon_{m} R^{3} C M \nabla E^{2}
$$

where $\varepsilon_{m}$ is the absolute permittivity of the medium, $R$ is the radius of the particle, $C M$ is the Clausius Mossotti factor and $E$ is the electric field. The $C M$ factor depends on the relative permittivity of the particle and medium, and it is the factor that determines the sign of the DEP force as described below:

$$
C M=\frac{\varepsilon_{p}-\varepsilon_{m}}{\varepsilon_{p}+2 \varepsilon_{m}}
$$

Based on the sign of the $C M$ factor, there are two categories of DEP: negative dielectrophoresis (nDEP) and positive dielectrophoresis (pDEP). The pDEP happens when the particles are more polarizable than the medium and the DEP force on the particles is towards the highest intensity of the electric field regions. On the other hand, the higher polarizability of the medium in comparison to the particle generates an $\mathrm{nDEP}$ effect, 
pushing the particles towards the regions with the lowest intensity of gradient of the electric field square.

\section{COMPUTATIONAL MODELING}

The electric current module of COMSOL Multiphysics software have been used for the simulation of our design.

Figure 1 shows the geometry (the cross section along width of the channel) which has been used for studying the effect of DEP on cell separation. The geometry consists of two rectanglar electrodes, which are positioned at the bottom of the microfluidics channel. The channel is $1 \mathrm{~mm} \times 75 \mu \mathrm{m}$ and height of the electrodes are $100 \mathrm{~nm}$. The width of the electrodes varies from 10 to $200 \mu \mathrm{m}$ during simulation to investigate the effect of the electrode size on the DEP force.

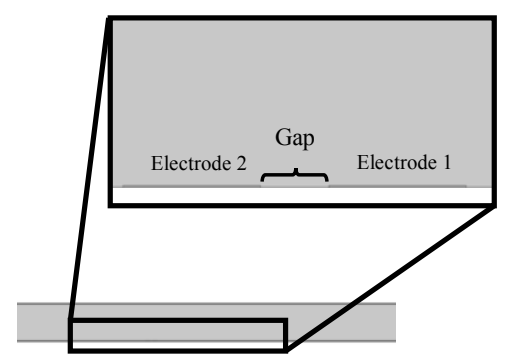

Figure 1. The geometry used for simulation

The minimum feature size that we can fabricate in our clean room is $5 \mu \mathrm{m}$. Therefore, this is the minimum gap (see Fig 1) that is used in this study. The simulation has been done for gaps ranging from 5 to $85 \mu \mathrm{m}$ and electode width ranging from 10 to $300 \mu \mathrm{m}$. 1 volt AC electric current with a frequency of $100 \mathrm{kHz}$ is applied to the electrodes and the other boundaries of the channel is assumed to be isolated.

Electrical potential of 1 and -1 voltage were used as boundary conditions for electrode 1 and 2 , respectively. In addition, at the interface of the electodes and fluid, continuity boundary condition was assumed. Furthermore, water and copper are used as the materials for the fluid and electrodes, respectively.

In order to evaluate the changes in DEP force exerted on particles, one cut line across the top of the channel is drawn. As can be seen in Figure 2 and Figure 3, the variation of the forces across this line have been evaluated.

The electric current, electrical potential, and electrical field can be evaluated by solving the Ohm's law. Fundamental equations from Ohm's law are shown from equation (3) to equation (5).

$$
\begin{gathered}
\mathrm{E}=-\nabla \mathrm{V} \\
J=\sigma E+j \omega D \\
D=\varepsilon_{0} \varepsilon_{r} E
\end{gathered}
$$

$J, \sigma, E, \omega$ and $V$ are electric current density, electrical conductivity, electrical filed, angular frequency, and electrical voltage, respectively. $D$ is the corrected electrical field in different mediums, $\varepsilon_{0}$ is the relative permittivity of air and $\varepsilon_{r}$ is the relative permittivity of the used medium. AC voltage is applied as an input, both real and imaginary terms are created in the electrical flux $J$ and will be changed by the input frequency.

In this study, only the variable $E$ is important as it has a direct influence on amount of DEP force, as demonstrated in equation (1). Furthermore, all other terms of equation (1) are constant for this specific study, except $\nabla E^{2}$ term. Consequently, we focus on this term as it has a direct impact on the DEP force. The widths of electrode 1 and electrode 2 and also the width of the gap between the two electrodes. play a vital role on the magnitude and direction of the $\nabla E^{2}$ term and the DEP force. Therefore, these parameters were used in this study.

\section{RESULTS AND DISCUSSION}

As mentioned before, the parameter that affects the DEP force in Equation (1) is $\nabla E^{2}$, which depends on the geometry of the device. The other parameters in this equation rely on the particle, medium, and frequency of the applied electric field. As for $\nabla E^{2}$, only its horizontal component affects the lateral displacement of the particles. Thus, the horizontal component of $\nabla E^{2}$ is used to optimize the electrodes geometry. As shown in Figure 2, the value of $\nabla E^{2}$ is minimum on the top of the channel, resulting in the minimum DEP force. To make sure that all the particles are affected by DEP, the $\nabla E^{2}$ term must be maximized on the top of the channel.

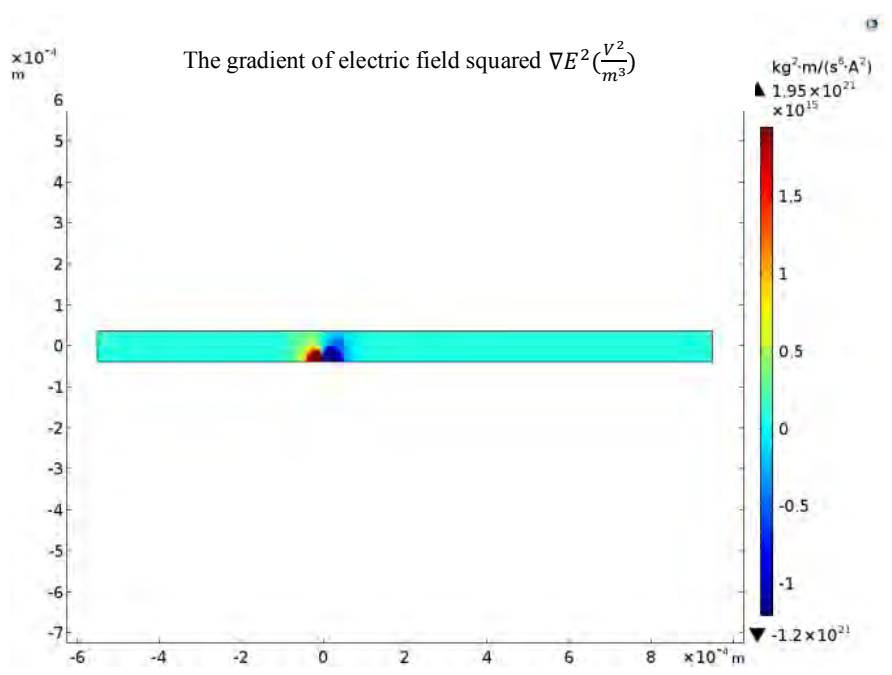

Figure 2. The contour of $\nabla E^{2}$ inside a channel with the width of the electrodes of bot $W_{1}=W_{2}=300 \mu \mathrm{m}$ and $G=5 \mu \mathrm{m}$. 
First, the effect of the gap between the electordes is studied. For this, the electrodes are assumed to have the same size. Then, the gap is changed to find the optimum gap size resulting in the maximum value of $\nabla E^{2}$ on the top of the channel. The curves in

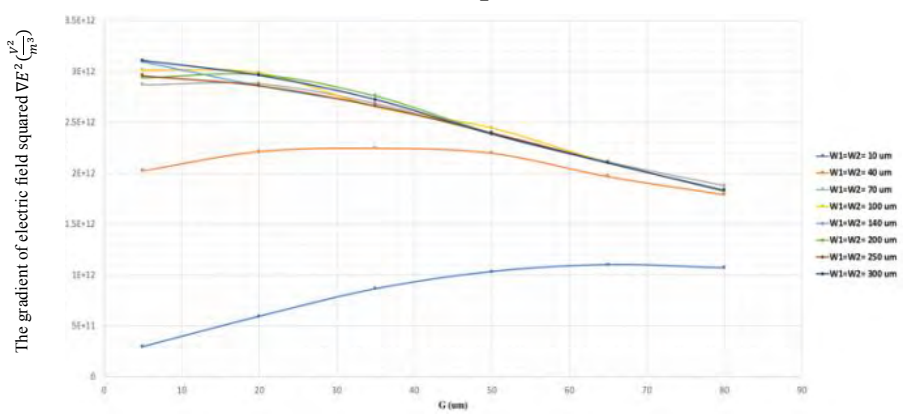

Figure 3. Maximum value of $\nabla E^{2}$ along the top of the channel as a function of the gap size.
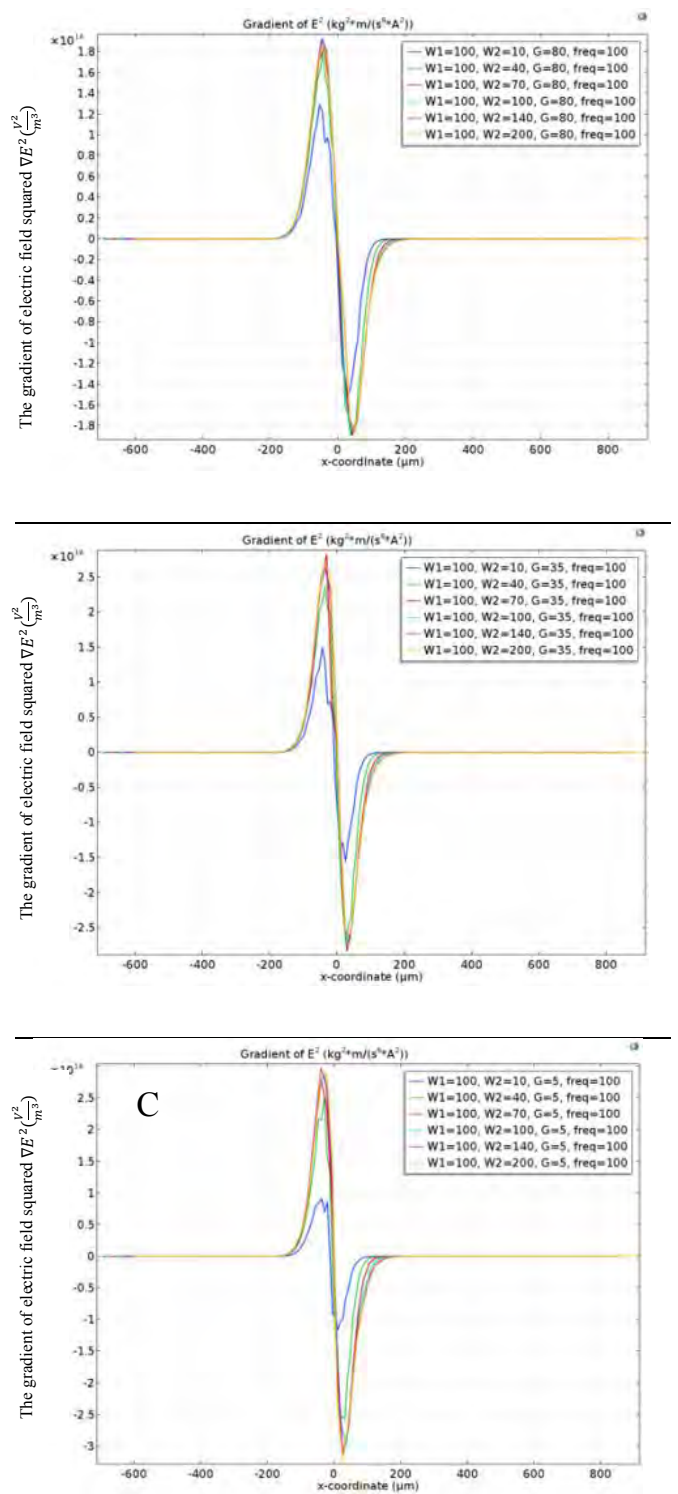

Figure 4. Variations of the exerted force on the particles as afucntion of the distance across the cut line. $W_{1}$ (th width of electrode 1) is considered as $10 \mu \mathrm{m}$ while $W_{2}$ (the width of electrode 2) is varied from 10 to $200 \mu \mathrm{m}$. $G$ (gap between two electrodes) is A) $80 \mu \mathrm{m}$, B) $35 \mu \mathrm{m}$ and C) $5 \mu \mathrm{m}$.

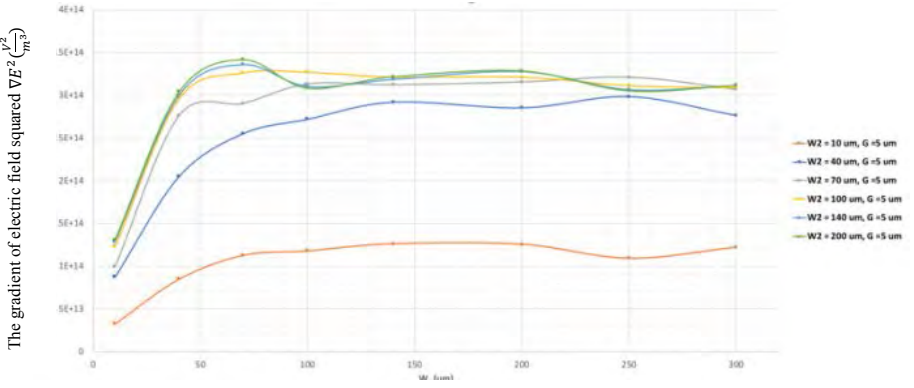

Figure 5. Maximum value of $\nabla E^{2}$ on the top of the channel for the case that the gap is $5 \mu \mathrm{m}$ and different combinations of the electrodes

Figure 3 are each associated with a certain value of the electrode width. The results show that all the curves have a similar trend (as the gap is reduced the value of $\nabla E^{2}$ increases), except for the electrode widths of 10 and $40 \mu \mathrm{m}$. In order to make sure that this result is not just for the case of the same size electrodes, assuming that the first electrode is $100 \mu \mathrm{m}$ wide, the second electrode's size is changed for three different gaps, and the results are provided in Figure 4. Comparing Figure 4 (A), (B) and $(\mathrm{C})$ shows that even if the electrodes' sizes are not the same, as the gap decreases the DEP force increases. Hence, the optimum gap size is $5 \mu \mathrm{m}$.

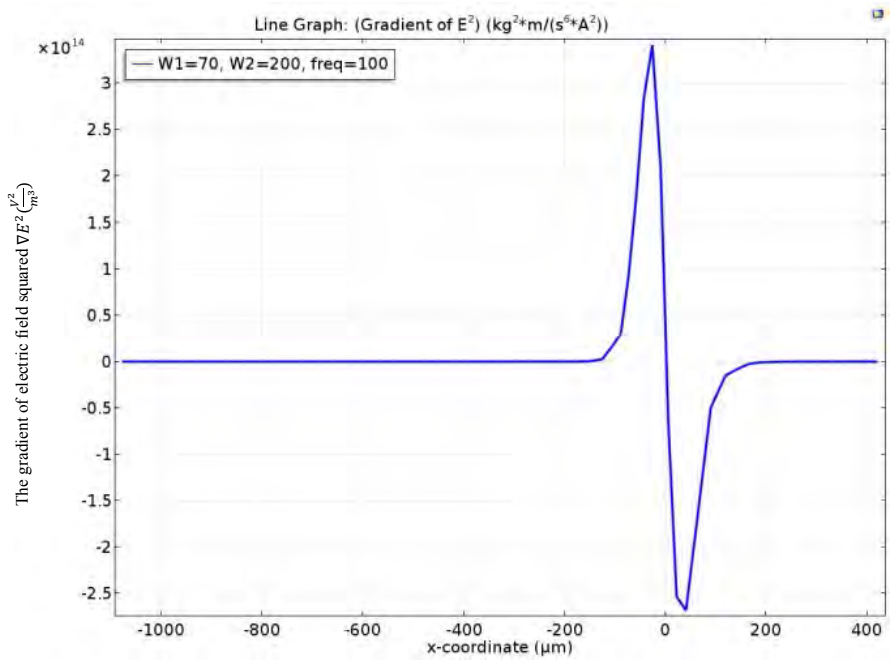

Figure 6. Variation of $\nabla E^{2}$ along the top of the channel for a width of $\mathrm{W} 1=70 \mu \mathrm{m}$ and $\mathrm{W} 2=200 \mu \mathrm{m}$ and gap size of $5 \mu \mathrm{m}$.

To find the optimum electrode width, the simulation was run with the gap size of $5 \mu \mathrm{m}$ and the electrode size combination of of $10 \mu \mathrm{m}, 40 \mu \mathrm{m}, 70 \mu \mathrm{m}, 100 \mu \mathrm{m}, 140 \mu \mathrm{m}, 200 \mu \mathrm{m}, 250 \mu \mathrm{m}$ and $300 \mu \mathrm{m}$. The results in Figure 5 show that the electrodes smaller than $70 \mu \mathrm{m}$ wide, lead to significantly lower $\nabla E^{2}$. This means that electrodes smaller than $70 \mu \mathrm{m}$ wide should be avoided in this design. However, increasing the width after $70 \mu \mathrm{m}$ does not have a signigicant effect on $\nabla E^{2}$. A closer look to the results shows that a slightly higher $\nabla E^{2}$ (and DEP force) can be generated by using a combination of $70 \mu \mathrm{m}$ and $200 \mu \mathrm{m}$ wide electrodes. 
With the optimum values of the gap and the electrode widths obtained, the variations in $\nabla E^{2}$ are calculated and shown in Figure 6. This graph shows that the maximum value of the $\nabla E^{2}$ term occurs on the side of the smaller electrode (blue line). Thus, the particle motion will be from the side of the large to the small electrodes. Based on these optimum values, the design shown in Figure 7 is used for a DEP-based micro-separator.

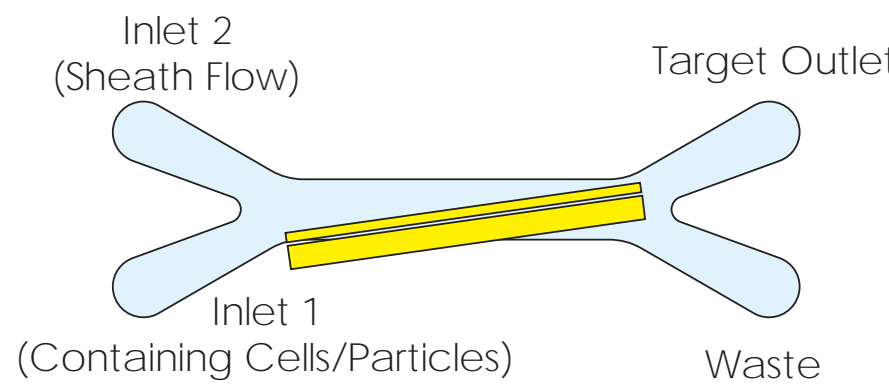

Figure 7. A schematic of the proposed DEP-based micro-seperator

\section{CONCLUSIONS}

In this study, COMSOL Multiphysics ${ }^{\circledR}$ tool was used to simulate the electric field created by two electrodes for a DEPbased cell/particle micro-separator. Different geometries in terms of the width of the electrodes and the gap between them have been investigated to find an optimum design, which leads to the maximum value of the DEP force on the top of the channel. The results show that in order to get the best outcome, the gap size must be as low as possible (which is $5 \mu \mathrm{m}$ in our lab) and the width of the electrodes must be larger than $70 \mu \mathrm{m}$. Further investigation shows that the best results are obtained when the width of one of the electrodes is $75 \mu \mathrm{m}$ and the other is $200 \mu \mathrm{m}$ wide. Based on these results, a DEP-based microseparator design is optimized.

\section{REFERENCES}

[1] B. Nestor et al., "Digital microfluidic platform for dielectrophoretic patterning of cells encapsulated in hydrogel droplets," RSC $A d v$., vol. 6, no. 62, pp. 57409-57416, 2016. doi: 10.1039/C6RA10412F.

[2] K. Park, S. Kabiri, and S. Sonkusale, "Dielectrophoretic lab-on-CMOS platform for trapping and manipulation of cells," Biomed. Microdevices, vol. 18, no. 6, pp. 1-11, Feb. 2016. doi: 10.1007/s10544-016-0030-x.

[3] S. H. Kim et al., "Highly efficient single cell arraying by integrating acoustophoretic cell pre-concentration and dielectrophoretic cell trapping," Lab Chip, vol. 15, no. 22, pp. 4356-4363, Oct. 2015. doi: 10.1039/C5LC01065A.

[4] Y. C. Kung et al., "Tunable dielectrophoresis for sheathless 3D focusing," in 2015 28th IEEE Int. Conf. MEMS, pp. 196-199.

[5] R. Wang et al., "An integrated microsystem with dielectrophoresis enrichment and impedance detection for detection of Escherichia coli," Biomed. Microdevices, vol. 19, no. 2, pp. 1-10, June 2017. doi: 10.1007/s10544-017-0167-2.

[6] A. Menachery et al., "Dielectrophoretic characterization and separation of metastatic variants of small cell lung cancer cells," $U N E$, vol. 3, pp. 386-389, 2016.

[7] J. Cemažar et al., "Enhanced contactless dielectrophoresis enrichment and isolation platform via cell-scale microstructures", Biomicrofluidics, vol. 10, no. 1, p. 014109, 2016.

doi: 10.1063/1.4939947.
[8] L. D'Amico et al., "Isolation and concentration of bacteria from blood using microfluidic membraneless dialysis and dielectrophoresis," Lab Chip, vol. 17, no. 7, pp. 1340-1348, Feb. 2017. doi: 10.1039/C6LC01277A.

[9] B .J. Sanghavi et al., "Ultrafast immunoassays by coupling dielectrophoretic biomarker enrichment in nanoslit channel with electrochemical detection on graphene," Lab Chip, vol. 15, no. 24, pp. 4563-4570, Oct. 2015. doi: 10.1039/C5LC00840A.

[10] V. V. Swaminathan et al., "Enhanced sub-micron colloidal particle separation with interdigitated microelectrode arrays using mixed AC/DC dielectrophoretic scheme," Biomed. Microdevices, vol. 17, no. 2, p. 29, Apr. 2015. doi: 10.1007/s10544-015-9935-z.

[11] H. Zhu et al., "Screen-printed microfluidic dielectrophoresis chip for cell separation," Biosens. Bioelectron., vol. 63, pp. 371-378, Jan. 2015. doi: 10.1016/j.bios.2014.07.072.

[12] S. H. Ling et al., "Continuous cell separation using dielectrophoresis through asymmetric and periodic microelectrode array, "Anal. Chem., vol. 84, no. 15, pp. 6463-6470, Jul. 2012. doi: $10.1021 / \mathrm{ac} 300079 \mathrm{q}$.

[13] S. Li et al., "A novel method to construct 3D electrodes at the sidewall of microfluidic channel," Microfluid. Nanofluid., vol. 14, no. 3-4, pp. 499-508, Mar. 2013. doi: 10.1007/s10404-012-1068-6. 


\title{
Automation in high throughput/content screening for cancer stem cell drug discovery
}

\author{
Eleftherios Sachlos \\ Department of Mechanical Engineering Lassonde \\ School of Engineering, York University
}

Mounting evidence suggests that cancer development is due to a rare population of cancer stem cells (CSC) uniquely able to initiate and sustain the disease (Al-Hajj et al., 2003; Bonnet and Dick, 1997; Lapidot et al., 1994; Li and Ren, 2008; Singh et al., 2003; Smalley and Ashworth, 2003). Conventional chemotherapeutics which inhibit cell proliferation are however ineffective against quiescent CSCs capable of re-initiating the disease (Bao et al., 2006; Dean et al., 2005; Diehn et al., 2009; Diehn and Clarke, 2006; Eyler and Rich, 2008; Li et al., 2008; Woodward et al., 2007). Instead, the indiscriminate cytotoxicity of these drugs often affects normal stem cells and progenitor populations, leading to dosage restrictions and necessitating supportive treatment (Smith et al., 2006). This traditional therapy has defined current patient survival rates, but these rates remain largely unchanged during the past 3 decades (Estey and Dohner, 2006; Visvader and Lindeman, 2008) implying that novel approaches are required to fight cancer. Recently, agents that selectively induce CSC apoptosis have been identified (Gupta et al., 2009) but their impact on normal stem cells has yet to be validated.

Stem cells, whether normal or CSC, are defined by an equilibrium between 1) self-renewal and 2) differentiation. In the case of CSCs, this equilibrium shifts towards enhanced self-renewal and limited differentiation capacity. A deviation in equilibrium however leads to eventual stem cell exhaustion (Duncan et al., 2005). One approach to eradicate CSCs is to tilt the equilibrium in favour of terminal differentiation in an effort to exhaust the CSC population. Eliminating cancer by inducing differentiation was first proposed in the 1970s (Fibach et al., 1973; Friend et al., 1971; 
Sachs, 1978a; Sachs, 1978b). This led to the identification of all-trans-retinoic acid (ATRA) (Breitman et al., 1981; Breitman et al., 1980) and then arsenic trioxide (ATO) (Niu et al., 1999; Raffoux et al., 2003) as differentiation-inducing agents for the treatment of acute promyelocytic leukemia (APL), an acute myeloid leukemia (AML) subtype. If left untreateted, APL causes death within weeks. ATRA/ATO treatment of APL currently demonstrates remission rates in excess of 93\% with 5year overall patient survival rates approaching 100\% (Sanz, 2006; Sanz et al., 2009; Wang and Chen, 2008) and exemplifies how differentiation therapy can be used to transformed a fatal, non-resectable, cancer to one that is essentially curable.

Cancer differentiation therapy has not however been translated to the treatment of other cancer types, let alone other AML subtypes (Burnett et al., 2010; Estey et al., 1999). This failure is, in part, due to the absence of robust in vitro assays which can interrogate CSC differentiation.

To address these issues, an overview of the application of automation for cancer stem cell screening will be presented. 


\section{REFERENCES}

[1] M. Al-Hajj, M.S. Wicha, A. Benito-Hernandez, S.J. Morrison, and M.R. Clarke, "Prospective identification of tumorigenic breast cancer cells," Proc Natl Acad Sci U S A. vol. 100, pp. 3983-3988, 2003. doi: 10.1073/pnas.0530291100.

[2] S. Bao, Q.Wu, R.E. McLendon, Y. Hao, Q. Shi, A. B. Hjelmeland, M.W. Dewhirst, D. D. Bigner, and J.N. Rich, "Glioma stem cells promote radioresistance by preferential activation of the DNA damage response," Nature vol. 444, pp.756-760, 2006. doi: 10.1038/nature05236.

[3] D. Bonnet and J. E. Dick,"Human acute myeloid leukemia is organized as a hierarchy that originates from a primitive hematopoietic cell," Nat. Med., vol 3, pp. 730-737, 1997. doi: 10.1038/nm0797-730.

[4] T. R. Breitman, S.J. Collins, and B.R. Keene, " "Terminal differentiation of human promyelocytic leukemic cells in primary culture in response to retinoic acid," Blood, vol. 57 , pp. 1000-1004, 1981.

[5] T.R. Breitman, S.E. Selonick, and S. J. Collins, (1980). "Induction of differentiation of the human promyelocytic leukemia cell line (HL-60) by retinoic acid," Proc Natl Acad Sci U S A vol. 77, pp.2936-2940, 1981. doi: 10.1073/pnas.77.5.2936.

[6] A.K. Burnett, R.K. Hills, C. Green, S. Jenkinson, K. Koo, Y. Patel, C. Guy, A. Gilkes, D.W. Milligan, A. H. Goldstone, et al., "The impact on outcome of the addition of all-trans retinoic acid to intensive chemotherapy in younger patients with nonacute promyelocytic acute myeloid leukemia: overall results and results in genotypic subgroups defined by mutations in NPM1, FLT3, and CEBPA. Blood," vol. 115, pp. 948-956, 2010. doi: 10.1182/blood-2009-08-236588.

[7] M. Dean, T. Fojo, and S. Bates, (2005). Tumour stem cells and drug resistance," Nat. Rev. Cancer., vol. 5, pp. 275-284, 2005. doi: 10.1038/nrc1590.

[8] Diehn, M., Cho, R. W., Lobo, N. A., Kalisky, T., Dorie, M. J., Kulp, A. N., Qian, D., Lam, J. S., Ailles, L. E., Wong, M., et al. (2009). Association of reactive oxygen species levels and radioresistance in cancer stem cells. Nature 458, 780-783.doi:10.1038/nature07733

[9] M. Diehn and M.F. Clarke, "Cancer stem cells and radiotherapy: new insights into tumor radioresistance,"J. Natl. Cancer. Inst., vol 98, pp. 1755-1757, 2006. doi: $10.1093 / \mathrm{jnci} / \mathrm{djj} 505$.

[10] A. W. Duncan, F. M. Rattis, L. N. DiMascio, K. L. Congdon, G. Pazianos, C. Zhao, K. Yoon, J.M. Cook, K. Willert, N. Gaiano and T. Reya,"Integration of Notch and Wnt signaling in hematopoietic stem cell maintenance," Nat. Immunol., vol. 6, pp. 314-322, 2005. doi: 10.1038/ni1164.

[11] E. Estey and H. Dohner, "Acute myeloid leukaemia," Lancet vol. 368, pp.1894-1907, 2006. doi: 10.1016/S0140-6736(06)69780-8.

[12] E. H. Estey, P. F. Thall, S. Pierce, J. Cortes, M. Beran, H. Kantarjian, M. J. Keating, M. Andreeff and E. Freireich,"Randomized phase II study of fludarabine + cytosine arabinoside + idarubicin +/- all-trans retinoic acid +/- granulocyte colony-stimulating factor in poor prognosis newly diagnosed acute myeloid leukemia and myelodysplastic syndrome," Blood, vol. 93, pp. 2478-2484, 1999.

[13] Eyler, C. E., and Rich, J. N. (2008). Survival of the fittest: cancer stem cells in therapeutic resistance and angiogenesis. J.Clin. Oncol., vol. 26, pp. $2839-2845$, 2008. doi: 10.1200/JCO.2007.15.1829.

[14] E. Fibach, M. Hayashi and L. Sachs, "Control of normal differentiation of myeloid leukemic cells to macrophages and granulocytes," Proc. Natl. Acad. Sci. U S A, vol 70, pp. 343-346, 1973. doi: 10.1073/pnas.70.2.343.

[15] C. Friend, W. Scher, J. G. Holland and T. Sato, " "Hemoglobin synthesis in murine virus-induced leukemic cells in vitro: stimulation of erythroid differentiation by dimethyl sulfoxide," Proc. Natl. Acad. Sci. US A, vol. 68, pp. 378-382, 1971. doi: 10.1073/pnas.68.2.378.

[16] P. B. Gupta, T. T. Onder, G. Jiang, K. Tao, C. Kuperwasser, R. A. Weinberg and E. S. Lander, "Identification of selective inhibitors of cancer stem cells by highthroughput screening," Cell, vol. 138, pp. 645-659, 2009. doi: 10.1016/j.cell.2009.06.034.

[17] T. Lapidot, C. Sirard, J. Vormoor, B. Murdoch, T. Hoang, J. Caceres-Cortes, M. Minden, B. Paterson, M. A. Caligiuri and J. E. Dick, "A cell initiating human acute myeloid leukaemia after transplantation into SCID mice," Nature, vol. 367, pp. 645-648, 1994. doi: 10.1038/367645a0.

[18] X. Li, M.T. Lewis, J. Huang, C. Gutierrez, C. K. Osborne, M. F. Wu, S. G. Hilsenbeck, A. Pavlick, X. Zhang, G. C. Chamness et al., "Intrinsic resistance of tumorigenic breast cancer cells to chemotherapy," J. Natl. Cancer. Inst., vol. 100, pp. 672-679, 2008. doi:b10.1093/jnci/djn123.

[19] X. Li and J. Ren, "Isolation of CD44+/CD24 -/low and side population cells from MDA-MB-453 cells and the analysis of their activation of Wnt and Notch pathway," Beijing Da Xue Xue Bao, vol. 40, pp. 471-475, 2008.

[20] C. Niu, H. Yan, T. Yu, H. P. Sun, J. X. Liu, X. S. Li, W. Wu, F. Q. Zhang, Y. Chen, L. Zhou, et al., " (1999). Studies on treatment of acute promyelocytic leukemia with arsenic trioxide: remission induction, follow-up, and molecular monitoring in 11 newly diagnosed and 47 relapsed acute promyelocytic leukemia patients," Blood, vol. 94, pp. 3315-3324, 1999.

[21] E. Raffoux, P. Rousselot, J. Poupon, M. T. Daniel, B. Cassinat, R. Delarue, A. L. Taksin, D. Rea, A. Buzyn, A. Tibi, et al. , "Combined treatment with arsenic trioxide and all-trans-retinoic acid in patients with relapsed acute promyelocytic leukemia," J. Clin. Oncol., vol. 21, pp. 2326-2334, 2003.

doi: 10.1200/JCO.2003.01.149.

[22] L. Sachs, "Control of normal cell differentiation and the phenotypic reversion of malignancy in myeloid leukaemia."Nature, vol. 274, pp. 535-539, 1978b. doi: $10.1038 / 274535 \mathrm{a} 0$.

[23] L. Sachs, "The differentiation of myeloid leukaemia cells: new possibilities for therapy," Br. J. Haematol., vol. 40, pp. 509-517, 1978. doi:10.1111/j.1365-2141.1978.tb05826.x

[24] M.A. Sanz,"Treatment of acute promyelocytic leukemia," Hematology Am. Soc. Hematol. Educ. Program, pp. 147-155, 2016. doi: 10.1182/asheducation-2006.1.147.

[25] M. A. Sanz, D. Grimwade, M. S. Tallman, B. Lowenberg, P. Fenaux, E. H. Estey, T. Naoe, E. Lengfelder, T. Buchner, H. Dohner, et al., "Management of acute promyelocytic leukemia: recommendations from an expert panel on behalf of the European Leukemia Net," Blood, vol. 113, pp. 1875-1891, 2009.

doi: 10.1182/blood-2008-04-150250. 
[26] S. K. Singh, I. D. Clarke, M. Terasaki, V. E. Bonn, C. Hawkins, J. Squire and P. B. Dirks, "Identification of a cancer stem cell in human brain tumors,: Cancer Res., vol. 63, pp. 5821-5828, 2003.

[27] M. Smalley and A. Ashworth, "Stem cells and breast cancer: A field in transit," Nat. Rev. Cancer, vol. 3, pp. 832-844, 2003. doi: 10.1038/nrc1212.

[28] T. J. Smith, J. Khatcheressian, G. H. Lyman, H. Ozer, J. O. Armitage,L. Balducci, C. L. Bennett, S. B. Cantor, J. Crawford, S. J. Cross et al. , "2006 update of recommendations for the use of white blood cell growth factors: an evidence-based clinical practice guideline," J. Clin. Oncol., vol. 24, pp. 3187-3205.2006. doi: 10.1200/JCO.2006.06.4451.

[29] J. E. Visvader and G. J. Lindeman, "Cancer stem cells in solid tumours: accumulating evidence and unresolved questions," Nat. Rev. Cancer, vol. 8, pp. 755-768, 2008. doi: $10.1038 /$ nrc2499.

[30] Z. Y. Wang and Z. Chen, "Acute promyelocytic leukemia: from highly fatal to highly curable," Blood, vol. 111, pp. 2505-2515, 2008. doi: 10.1182/blood-2007-07-102798

[31] W. A. Woodward, M. S. Chen, F. Behbod, M. P. Alfaro, T. A. Buchholz and J. M. Rosen,"WNT/beta-catenin mediates radiation resistance of mouse mammary progenitor cells," Proc. Natl. Acad. Sci. U S A, vol. 104, pp.618-623, 2007. doi: 10.1073/pnas.0606599104. 


\section{Investigation of Epithelial-to-Mesenchymal Transition through Microcontact Printing}

\author{
Huma Inayat \\ Graduate program in Biomedical Engineering \\ Ryerson University \\ Toronto, Canada \\ inayathu@ryerson.ca
}

\author{
Scott S. H. Tsai* \\ Department of Mechanical and Industrial \\ Engineering, Ryerson University \\ Toronto, Canada \\ scott.tsai@ryerson.ca
}

\author{
Andras Kapus* \\ Keenan Research Centre for Biomedical \\ Science, St. Michael's Hospital \\ Toronto, Canada \\ kapusA@smh.ca
}

\begin{abstract}
We have used microcontact printing as a means of simulating injury or wounds in a healthy monolayer of LLC PK1 cells to study the expression of Epithelial-to-Mesenchymal Transition (EMT) signature proteins. Cells were patterned on PDMS substrates using stamps that were prepared in the cleanroom using conventional soft lithography techniques. Patterned cells were exposed to an external stimulus of Transformation Growth Factor $\beta$ (TGF $\beta$ ) and analyzed further for the presence and upregulation of EMT signature proteins that are indicative of the progression of EMT.
\end{abstract}

Microcontact Printing, Epithelial-to-Mesenchymal Transition, LLC PK1 cells, TGFB

\section{Introduction}

Organ fibrosis is considered a failed or dysregulated wound healing process, triggered by prolonged and repetitive injury to epithelium and endothelium. It was first discovered in 1995, that fibroblasts that contribute to fibrosis often originate from the local conversion of epithelium. ${ }^{1}$ This discovery set forward a slurry of research activity around the phenomenon of Epithelialto-Mesenchymal transition or EMT, and its role in organ fibrosis. ${ }^{2}$ EMT is a process through which epithelial cells lose their strong intercellular contacts and polarity, and transition to migratory and often invasive mesenchymal cell types. ${ }^{3}$ These

mesenchymal cells then mediate either physiological functions (e.g. tissue differentiation during embryogenesis or normal wound healing), or participate in pathological processes (fibrosis, cancer). ${ }^{3}$
Our goal is to investigate the concept that epithelial wounding or injury (which can be simulated in vitro by the absence of cell-cell contacts), predisposes the injured cells for various features of EMT, including the expression of EMT signature proteins (such as alpha-Smooth Muscle Actin). We also aim to investigate the proportional secretory response (termed profibrotic epithelial phenotype or PEP) caused by the loss of cell-cell contacts (or injury) which holds true even if EMT is just partial. We hypothesize that the length of free epithelial edges in a monolayer is proportional to the propensity of the epithelium to acquire a profibrotic epithelial phenotype.

To address this hypothesis, we have used microfabrication to establish a model in which the proportionality of the response can be investigated. To establish our gold standard, initially we investigated SMA expression., the hallmark of full-blown EMT. The next step will be the investigation of the secretory phenotype.

\section{EXPERIMENTAL SETUP}

\section{A. Cell Culture and treatment}

We use porcine kidney proximal tubule cell line (LLC-PK1) used to conduct all experiments as described in previous studies. ${ }^{4}$ Cells are cultured using DMEM supplemented with $10 \%$ fetal bovine serum and $1 \%$ penicillin/streptomycin. After patterning, cells are treated with $5 \mathrm{ng} / \mathrm{ml}$ TGF $\beta$ in serum free medium for 48 hours. Cells are washed with phosphate- buffered saline (PBS) prior to analysis.

\footnotetext{
* Co-corresponding authors.
} 


\section{B. Mold fabrication for Microcontact printing}

The stamps used for patterning cells are prepared using conventional soft lithography techniques in a cleanroom. Stamps are incubated with $80 \mathrm{ug} / \mathrm{ml}$ Fibronectin prior to stamping. Polydimethylsiloxane (PDMS) is used to line 6-well plates to prepare surface for cell patterning.

\section{REFERENCES}

[1] F. Strutz et al., "Identification and characterization of a fibroblast marker: FSP1," J. Cell Biol. vol. 130, pp. 393-405, 1995. doi: $10.1083 /$ jcb.130.2.393.

[2] S. E. Quaggin and A. Kapus, "Scar wars: mapping the fate of epithelial-mesenchymal-myofibroblast transition," Kidney Int. vol 80, pp. 41-50, 2011. doi: 10.1038/ki.2011.77.

[3] J. P. Thiery, H. Acloque, R. Y. J. Huang and M. A. Nieto, "Epithelial-Mesenchymal Transitions in Development and Disease," Cell, vol. 139, pp. 871-890, 2009.

doi: 10.1016/j.cell.2009.11.007.

[4] A. Kapus et al., "Differential Topical Susceptibility to TGFß in Intact and Injured Regions of the Epithelium: Key Role in Myofibroblast Transition," Molecular Biology of the Cell, vol 21, pp. 3326-3336, 2013.

[5] G. M. Whitesides et al., "Soft Lithography," Annual review of Materials Science, vol. 28, pp. 153-184, 1998.

doi: 10.1146/annurev.matsci.28.1.153. 


\section{Kinetics Analysis of Multi-Segment Trunk After Experimental Errors Minimization}

\author{
Alireza Noamani, Hossein Rouhani \\ Department of Mechanical Engineering \\ University of Alberta \\ Edmonton, Alberta, Canada \\ noamani@ualberta.ca,hrouhani@ualberta.ca \\ Albert H. Vette \\ Department of Mechanical Engineering \\ University of Alberta \\ Edmonton, Alberta, Canada \\ albert.vette@ualberta.ca
}

\author{
Richard Preuss \\ School of Physical \& Occupational Therapy, \\ McGill University, \\ Montreal, Quebec, Canada \\ richard.preuss@mcgill.ca \\ Milos R. Popovic \\ Institute of Biomaterials and Biomedical Engineering \\ University of Toronto \\ Toronto, Ontario, Canada \\ milos.popovic@utoronto.ca
}

\begin{abstract}
Linked-segment models of the head-arms-trunk (HAT) along with an inverse dynamics approach can be used for estimating inter-vertebral moments. Several studies estimated the lumbo-sacral inter-vertebral moment using onesegment HAT models during execution of different functional tasks. However, methods for estimating inter-vertebral moments at different levels of the spinal column have rarely been investigated due to the propagation of the experimental errors. This study quantified multi-segment HAT kinetics during multi-directional trunk-bending after minimizing the experimental errors. Eleven healthy individuals participated in a multi-directional trunk-bending experiment in five directions with self-comfortable pace. We used a reconstructed sevensegment HAT model for each participant along with a bottomup inverse dynamics approach to estimate intervertebral moments after minimizing the effect of experimental errors. Our results indicate a significant effect of joint level and trunkbending direction as well as interaction effects. Our results revealed complex patterns for three-dimensional (3D) intervertebral moments which can only be obtained via a multisegment model and error minimization. Inter-vertebral moment patterns along the spinal column after minimizing the errors can play a significant role in objective clinical evaluations and in designing pre- and post-treatment strategies.
\end{abstract}

Keywords-component; Inverse dynamics; Joint moments; Multisegment model; Trunk kinetics

\section{INTRODUCTION}

Estimation of the inter-vertebral interactions such as motions and loads is useful for clinical evaluation of several pathological conditions such as low-back pain [1] and spinal cord injury [2]. Moreover, injury prevention strategies, as well as the risk of injury assessment, can benefit from the accurate estimation of inter-vertebral loads [3]. As a mathematical technique, linkedsegment models of the body have been extensively used for invivo studying of spine kinematics [4]. Previous studies have addressed the three-dimensional (3D) kinematics of the upper body using single-segment and multi-segment models of the head-arms-trunk (HAT). Estimating joint moments and forces using a linked-segment model of the HAT along with an inverse dynamics approach requires inter-segmental kinematics, accurate estimation of individual-specific body segment parameters (BSPs), and force plate measurements of the external forces. However, the accuracy of an inverse dynamics approach can be affected by experimental errors such as inaccuracies in (a) kinematics, (b) estimation of BSPs, and (c) force plate measurements. These inaccuracies could cause errors $6 \%$ to $232 \%$ of the estimated peak moment [5] implying that minimizing the effect of experimental errors is of great importance.

Previous studies addressed the significant effect of the relative motion between the skin-mounted markers and actual bony anatomical landmarks, soft tissue artifacts (STA), on the kinematics and kinetics of the lower limb [6], [7]. The effect of STA on the kinematics of the spinal column has been investigated [8]. However, No study has addressed the effect of STA on the estimating inter-segmental moments of a multisegment HAT.

In addition, estimating inter-segmental moments using a linked-segment model and an inverse dynamics approach requires accurate estimation of BSPs including mass, the center of mass (COM), moments of inertia, and joint centers of rotation (JCRs). Medical imaging techniques can provide an accurate estimation of BSPs for each individual; however, radiation exposure [9] make them non-practical for routine clinical motion analyses. Some studies reported BSPs for a single cadaver [10], other studies proposed regression equations based on cadaveric data [9] for estimating individual-specific BSPs based on body weight and height. However, estimating BSPs based on cadaveric data may have error larger than 40\% [11]. As a different approach, some studies used optimization techniques to minimize the effect of inaccuracies in BSPs on joint moment estimation at lower limb joints with assuming HAT as a single rigid segment [11]. However, there is currently no study that 
estimated joint moments in a multi-segment HAT based on optimized individual-specific BSPs.

Several studies have calculated the $3 \mathrm{D}$ joint moment at the $L 5 / S 1$ joint using a single-segment HAT model based on BSPs estimated via regression equations [12], [13] and for clinical evaluation of low-back pain [14] and lower-limb amputation [15]. However, none of these studies obtained 3D joint moments at different levels of the spinal column using a multi-segment model due to several technical challenges such as estimating individual-specific BSPs. Our team has recently proposed (a) a method for compensating the effect of STA on kinematics of a multi-segment HAT, and (b) an optimization-based method for estimating individual-specific BSPs for each HAT segment. Based on our previous studies, this study presents 3D joint moments in a multi-segment HAT model using optimized individual-specific BSPs and STA error compensation during trunk-bending tasks at different directions. The effect of joint levels and trunk-bending directions have also been investigated.

\section{METHODS}

\section{A. Experimental Protocol}

The experimental protocol was described in detail in our previous study [4] and thus, are only briefly described here. Eleven non-disabled individuals ( 4 females; age: $28.5 \pm 3.3$ years; trunk height: $0.75 \pm 0.04 \mathrm{~m}$ ) with no history of spine-related neuromuscular or musculoskeletal impairments or recurrent back pain take part in this study. All participants provided written consent prior to participating in the experiment. Research Ethics Board approval was received from the local ethics committee.

Participants sat on a rigid force plate in an upright natural posture with no backrest or foot support. The arms were crossed motionless over the chest. Five targets were placed anterior to the participant. The distance and height of each target were adjusted to represent the trunk-bending angle of $45^{\circ}$. Each participant was asked to lean toward the target, touch the target with the head, and return to the initial upright position. Each trunk-bending trial was performed three times at selfcomfortable pace. To avoid a counterweight effect of the lower legs during trunk movement, participants were asked to keep their legs vertically downwards throughout the experiment (Figure 1).

\section{B. Data Acquisition and HAT Model}

Twenty-three reflective markers were placed on the spinous processes and around them for each participant to constitute a seven-segment HAT model. The instantaneous position of markers was captured via six motion tracker cameras (Vicon, Oxford, UK) at the sampling rate of $120 \mathrm{~Hz}$. A force-plate (AMTI, Watertown, MA, USA) was used to measure ground reaction forces (GRFs) and center of pressure position (COP) at the frequency of $1000 \mathrm{~Hz}$. The time-series of the marker's trajectory were filtered via an $8^{\text {th }}$-order dual-pass Butterworth low-pass filter with a cut-off frequency of $2 \mathrm{~Hz}$.

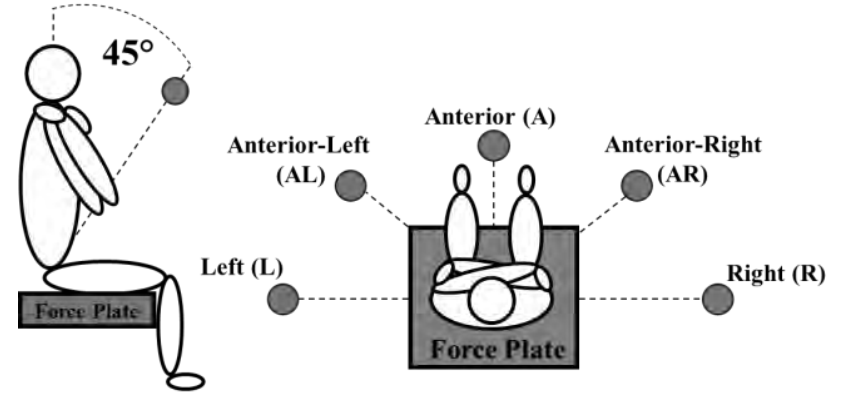

Figure 1. Targets were placed for each participant at the distance and height which represent the trunk-bending angle of $45^{\circ}$. Targets were placed in the transverse plane at $45^{\circ}$ intervals, anteriorly and laterally of the participant.

\section{Seven-Segment HAT Modeling}

We reconstructed a seven-segment model for each participant consisting of two segments for lumbar spine: upper lumbar (UL: T12-L3), lower lumbar (LL: L3-S1). Four segments for thoracic spine: upper thoracic (UT: C7-T3), midupper thoracic (MUT: T3-T6), mid-lower thoracic (MLT: T6T9), lower thoracic (LT: T9-T12), and one segment for head and neck (HD). The segments were assumed to be rigid and connected to each other by $3 \mathrm{D}$ revolute joints located at the center of respective inter-vertebral discs (Figure 2). Each segment was defined by a cluster of three markers: one marker placed centrally on the spinous process of the caudal vertebra of that segment, and two markers placed laterally at $5 \mathrm{~cm}$ distance from the spinous process of the rostral vertebra of that segment (Figure 2).

Based on the three markers of each segment, a segment-fixed frame was defined for: The X-axis pointing from left to right, parallel to the two rostral markers, the Z-axis pointing superiorly, parallel to the line between the caudal marker and the mid-point of two rostral markers, and the $\mathrm{Y}$-axis pointing anteriorly, as the cross-product of the $\mathrm{Z}$ and $\mathrm{X}$ axes. Therefore, the $\mathrm{X}-, \mathrm{Y}-$, and $\mathrm{Z}$-axes represented flexion/extension, lateral bending, and axial rotation, respectively. A pelvis-fixed frame was defined based on the markers placed on the left and right anterior superior iliac spine (ASIS) and the midpoint between the posterior superior iliac spines (PSIS).

\section{Inverse Dynamics}

A custom-made Newton-Euler iterative algorithm was developed for calculating the inter-segmental moments based on bottom-up and top-down inverse dynamic approaches. Using kinematic data, BSPs for each segment as well as force plate measurements, the bottom-up approach calculates joint moments from the bottom-most joint and proceeds superiorly with applying force plate measurements as the boundary condition of the inferior segment. The top-down approach uses kinematic data and BSPs to calculate joint moments from the top-most joint and proceeds downward with assuming zero loads at the top-most joint.

\section{E. Minimizing the Effect of Experimental Errors}

\section{1) Soft Tissue Artifacts (STA)}

STA is defined as the relative displacement between the skin-mounted marker and actual underlying bony anatomical 
landmark. The effect of STA induced error on kinematic measurements of the multi-segment HAT was compensated based on the model introduced in our previous study [8]. This model assumed that the relative displacement between the skinmounted marker and actual bony anatomical landmark is minimum (zero) at initial upright posture and proportionally increases with the trunk-bending angle, and thus, it is maximum at the maximum trunk-bending posture. $\theta_{t}$, instantaneous trunk, was defined as the angle between the line from $S 1$ and $C 7$ markers at each instant of time $(t)$, and the initial upright posture. Soft tissue artifact of marker $i$ in segment $j$ was calculated in the segment's local frame as defined in equation (1):

$$
\operatorname{STA} A_{i}^{j}\left(\theta_{t}\right)=S T A_{i}^{j}\left(\theta_{\max }\right) \frac{\theta_{t}}{\theta_{\max }}
$$

where $\theta_{\max }$ and $\operatorname{STA} A_{i}^{j}\left(\theta_{\max }\right)$ are the maximum trunk-bending angle, soft tissue artifacts, respectively. The instantaneous position of the markers were then corrected:

$$
C_{i}^{j}(t)={ }_{L}^{G} R_{j}(t)\left[{ }_{L}^{G} R_{j}{ }^{-1}(t) \cdot P_{i}^{j}(t)-S T A_{i}^{j}\left(\theta_{t}\right)\right]
$$

Figure 2. Markers were placed over the spinal column to form a seven-segment trunk model: Head and neck (HD), upper thoracic (UT), mid-upper thoracic (MUT), mid-lower thoracic (MLT), lower thoracic (LT), upper lumbar (UL), lower lumbar (LL), and sacral (SC) segments.

where $P_{i}^{j}(t)$ and $C_{i}^{j}(t)$ are the preliminary and corrected trajectory of marker $i$ in segment $j$ at the time index $t$, respectively, and ${ }_{L}^{G} R_{j}(t)$ is the instantaneous rotation matrix from the segment-fixed frame to the lab-fixed frame.

\section{2) Optimized Individual-Specific BSPs and COP Offsets}

Calculating 3D joint moments requires individual-specific estimation of BSPs for each segment including mass, COM, moments of inertia, and JCRs. Vette et al. [10] calculated upper body BSPs for a 38-year-old white male cadaver (height: $1.80 \mathrm{~m}$ and weight: $90 \mathrm{~kg}$ ). We estimated the initial guess of BSPs for each individual by scaling cadaveric data based on participant's trunk height and body weight. We assumed that the body is symmetric in the frontal plane and, thus, that all COMs and JCRs lie in the body's sagittal plane. However, scaling method based on cadaveric data induces inaccuracies in estimating BSPs when the individual falls outside the originally studied population for which the BSPs were estimated in terms of age, gender, body type, and ethnicity [16]. As a result of induced inaccuracies, the value of the joint moments calculated via bottom-up and topdown approaches differ mostly due to inaccurate estimation of individual-specific BSPs. In our previous study, we developed a nonlinear constrained multi-step optimization-based method to find an optimized set of individual-specific BSPs for each HAT segment as well as compensating the effect of COP offsets that minimizes the difference between the joint moments calculated via bottom-up and top-down inverse dynamics at all intersegmental levels. We used the optimized individual-specific BSPs and COP offsets obtained via our optimization-based method to quantify multi-segment trunk kinetics after minimizing the effect of inaccuracies in BSPs, COP offsets, STA to obtain a less erroneous estimation of inter-segmental moments.

\section{F. Data Analysis}

The Kolmogorov-Smirnov test and Levene's test showed that the absolute peak values of the 3D moments came from a normal distribution with equal variances. We conducted a two-way analysis of variance (ANOVA) on the absolute peak moments in the sagittal, coronal, and transverse planes to investigate the effect of joint levels (SC $\sim$ LL to UT $\sim \mathrm{HD}$ ), and trunk-bending directions. Moreover, we investigate the effect of STA compensation on estimating joint moments. We performed a two-way ANOVA on the root-mean-square (RMS) difference between the net joint moments before and after STA error compensation and investigated the effect of joint level and trunkbending directions for both bottom-up and top-down inverse dynamic approaches. For all statistical analyses, the significance level was set at 0.01 by considering Bonferroni correction. We performed a multi-comparison post hoc test for interpreting the main effects of joint level and trunk-bending direction as well as their interaction effect.

\section{RESULTS}

Figure 3 represents the RMS difference between the net joint moments calculated with and without compensating the effect of STA. Results are presented as $c$ at all inter-segmental levels, for five trunk-bending directions, calculated via both bottom-up and top-down approaches. Statistical analysis revealed significant main effects of joint level and trunk-bending direction and twoway interaction effect on the RMS difference between the joint moments calculated with and without STA error compensation. We also observed a significant difference of inverse dynamic approaches.

Figure 4 represents the absolute peak joint moments in the sagittal, coronal, and transverse planes as well as the plane of movement (net sagittal-coronal) after minimizing the effect of inaccuracies due to COP offsets, BSPs, and STA. Results are presented as a mean \pm standard error at all inter-segmental levels and for five trunk-bending directions. The main effect of the joint level, trunk-bending direction, as well as two-way interaction effect.

The main effect of joint level revealed that the sagittal moments of the lumbar joints were significantly larger $(p<0.01)$ than thoracic and cervical joints. Among the lumbar joints, the LL UL joint tended to have the largest sagittal moment. Among the thoracic and cervical joints, the sagittal moment at each inferior joint was significantly larger than superior joints implying that the sagittal moment decreased from inferior joints to superior joints. A similar trend was observed for the coronal moment, and it decreased from the inferior joints to the superior joints except for SC $\sim$ LL joint which was significantly smaller $(p<0.01)$ than the LL $\sim \mathrm{UL}$ joint. The transverse moment at the 
most superior joint (UT $\sim$ HD) was significantly larger than all other joints while no significant difference was observed among all other joints.

The main effect of trunk-bending direction reflected that the sagittal moment across different joint significantly decreased with more lateral directions and it was maximum at the anterior direction $(\mathrm{p}<0.01)$. No significant bilateral asymmetry effect was observed $(\mathrm{p}=1.00)$. The opposite pattern was observed for the coronal moment. The coronal moments across different joints increased significantly with more lateral trunk-bending directions $(\mathrm{p}<0.01)$, and it was maximum for bending towards left and right direction and minimum for the anterior direction. No bilateral asymmetry was observed $(p=1.00)$. No effect of direction was observed for the transverse moment.

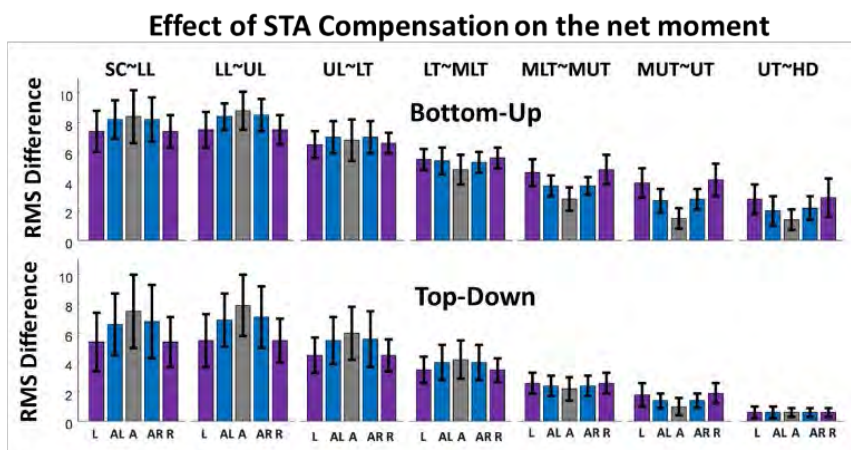

Figure 3. RMS difference between the inter-segmental net joint moments calculated before and after STA error compensation at each joint level of the proposed HAT model (Figure 2) for five trunk-bending directions (Figure 1). Results are expressed as mean \pm standard deviation among all participants and obtained through both bottom-up and top-down inverse dynamic approaches.

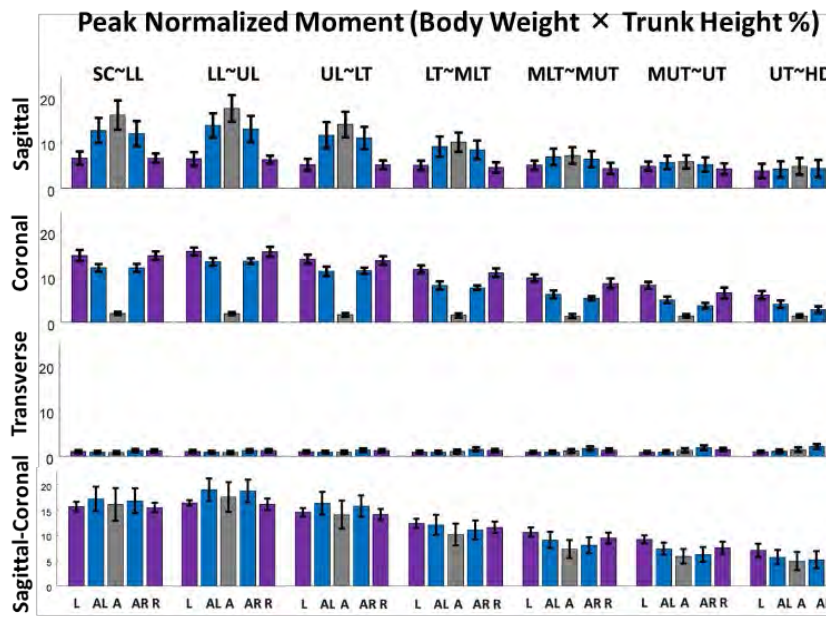

Figure 4. Peak joint moment in sagittal, coronal, and transverse planes and in the plane of movement (net sagittal-coronal) calculated via bottom-up approach using optimized individual-specific BSPs and STA compensation for different joint levels, and trunk-bending directions. Results are presented as bar and error bar plots. Moments (N.m) were normalized by participant's body weight and trunk height. (L: left, AL: anterior-left, A: anterior, AR: anterior-right, R: right).

\section{DISCUSSIONS}

Estimating inter-vertebral moments requires: (a) the capture of inter-segmental kinematics, (b) accurate measurement of GRFs, and (c) individual-specific estimation of BSPs. Using regression and scaling equations [12], [13] along with singlesegment trunk models, many studies have investigated lumbosacral (L5-S1) joint moments. The accuracy of the estimated joint moments is dependent on the accuracy of the kinematics, BSPs estimation, and force plate measurements. Therefore, methods that compensate for the above-mentioned inaccuracies can result in less erroneous estimation of joint moments. Although previous studies demonstrated the influence of STA on estimating lower limb joint moments, its effect on the joint moment estimation of the multi-segment HAT is yet to be studied. Our team has recently proposed a method to compensate for STA induced error on the kinematics of the multi-segment HAT model. We employed the same approach in the current study to investigate the effect of STA on the multi-segment HAT kinetics. Moreover, Inaccuracy in estimating individual-specific BSPs and its effect on the lower limb kinetics with assuming the trunk as a single rigid body has been addressed by previous studies. In fact, due to the high inter-participant variability of BSPs for HAT segments, multi-segment HAT kinetics have been rarely investigated. We have recently proposed a nonlinear, constrained, multi-step optimization-based method for estimating individual-specific BSPs for each HAT segment in the proposed model (Figure 2). In the current study, we used our STA error compensation method along with the optimized individual-specific BSPs to obtain a less erroneous estimation of inter-segmental moments in the proposed multi-segment HAT model. We subsequently investigated the effect of the joint level, and trunk-bending directions in the sagittal, coronal, and transverse planes.

\section{A. Effect of STA on Net Joint Moments}

The results (Figure 3) revealed that for both inverse dynamic approaches, the RMS difference between the net joint moments calculated before and after STA error compensation was significantly larger at the two bottom-most joints $(\mathrm{SC} \sim \mathrm{LL}$ and LL UL) compared to all other superior joints. Moreover, The RMS difference significantly decreased from the inferior joints to the superior joints. This implies that kinetics of the inferior joints can be significantly more affected by STA and thus STA compensation is more required for the kinetics of the inferior joints compared to the superior joints.

In addition, The RMS difference between the net moments calculated with and without STA compensation tended to decrease with more later directions at SC $\sim$ LL and LL $\sim$ UL joints. The opposite was observed for MLT $\sim$ MUT and MUT $\sim$ UT joints where the RMS difference tended to increase with more lateral directions. This is due to the fact that trapezius muscles in this region are involved in bending toward lateral directions which increases STA.

\section{B. Effect of Joint Levels and Bending Directions}

Both sagittal and coronal components of the 3D moments, decreased from LL $\sim$ UL joint to UT $\sim$ HD joint, going superiorly. This is due to the fact that inferior joints bear more weight during the trunk-bending task. Although no significant difference was observed between the sagittal moment at SC $\sim \mathrm{LL}$ and $\mathrm{LL} \sim \mathrm{UL}$ joints, this component tended to be larger at the $\mathrm{LL} \sim \mathrm{UL}$ joint. However, the coronal component at the LL UL joint was 
significantly larger. This is justified as the maximum curvature of the lumbar spine occurs at this joint when the trunk bends. [12] A. Plamondon, M. Gagnon, and P. Desjardins, "Validation of two 3-D

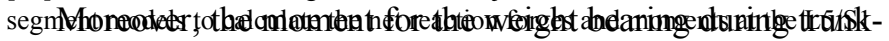

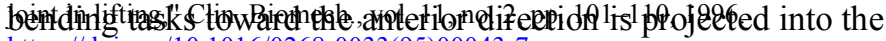

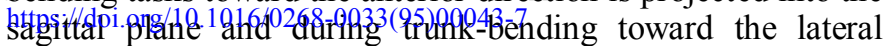
directions is projected into the coronal plane which can be observed in Figure 4. The two-way interaction effect between the joint level and trunk-bending direction revealed no significant difference among the sagittal moments across different joint for the lateral directions. However, the sagittal moment at the mid-lower joints $(\mathrm{SC} \sim \mathrm{LL}, \mathrm{LL} \sim \mathrm{UL}, \mathrm{UL} \sim \mathrm{LT}$, and $\mathrm{LT} \sim \mathrm{MLT}$ ) for anterior direction was significantly larger compared to more lateral bending directions while sagittal moment at the mid-upper joints (MLT $\sim$ MUT, MUT $\sim$ UT, $\mathrm{UT} \sim \mathrm{HD})$ was not significantly affected by trunk-bending direction. This finding suggests that even though the sagittal moment significantly decreased from inferior joints to the superior joint for anterior direction, this is not valid for bending in lateral directions. No significant difference was observed among the coronal moments across different joints for the anterior direction while for the lateral direction this component significantly decreased from the inferior joints to the superior joints. Results also indicate that the transverse component was significantly smaller and negligible compared to the sagittal and coronal components at each joint level and trunk-bending direction. This is due to the fact that the participants were asked to preserve their spine's torsional direction during bending trials.

We calculated the net sagittal-coronal moment to investigate whether these components reflect the upper body weight bearing moment projected into the sagittal and coronal planes (Figure 4). The effect of the joint level was similar to the sagittal and coronal moments. Net sagittal-coronal moment significantly increased inferiorly from $\mathrm{UT} \sim \mathrm{HD}$ to $\mathrm{SC} \sim \mathrm{LL}$ except for the LL UL which was the largest. Interestingly, no significant effect of trunk-bending direction was observed while the contribution of the sagittal and coronal moments to upper body weight bearing increased with more anterior and lateral directions, respectively. No significant two-way interaction effect was observed for the net sagittal-coronal moment. These findings imply that the sagittal and coronal moments are the weightbearing moments projected into the sagittal and coronal planes during trunk-bending tasks. The results of the joint moments obtained for the multi-segment HAT model reflect the complex, task-specific patterns across joint levels and trunk-bending directions which cannot be observed using a single-segment HAT model or without compensating inaccuracies in input parameters. The bilateral symmetrical moment patterns found in this study could be useful for objective clinical assessments to recognize any asymmetrical patterns at different levels of the spinal column. Note that this study demonstrates the results for a mix-gender relatively small population which can be a general representation of neither male nor female non-disabled populations. Larger datasets could be useful for identifying clinical meaningful moment patterns for clinical evaluations.

In conclusion, this study presented a less erroneous estimation of the 3D inter-segmental moments at different levels of the spinal column during bending toward different directions after minimizing the effect of experimental errors using a multisegment HAT model. The error minimization was achieved by using STA error compensation method, optimized individualspecific BSPs and COP offsets from our previous studies. Our results showed complex and task-specific patterns for 3D moments at different levels of the spinal column which could not be captured by a one-segment HAT model. Accurate estimation of the joint moments can be useful for objective clinical evaluation, rehabilitation, as well as designing pre- and postsurgery treatments.

\section{REFERENCES}

[1] T. Lund, T. Nydegger, D. Schlenzka, and T. R. Oxland, "ThreeDimensional Motion Patterns During Active Bending in Patients with Chronic Low Back Pain," Spine (Phila. Pa. 1976)., vol. 27, no. 17, pp. 1865-1874, 2002. doi:10.1097/00007632-200209010-00012.

[2] M. Lalumiere, D. H. Gagnon, F. Routhier, L. Bouyer, and G. Desroches, "Upper Extremity Kinematics and Kinetics During the Performance of a Stationary Wheelie in Manual Wheelchair Users With a Spinal Cord Injury," J. Appl. Biomech., vol. 30, pp. 574-580, 2014. doi: 10.1123/jab.2013-0333.

[3] N. Arjmand, D. Gagnon, A. Plamondon, A. Shirazi-Adl, and C. Larivière, "Comparison of trunk muscle forces and spinal loads estimated by two biomechanical models," Clin. Biomech., vol. 24, no. 7, pp. 533-541, 2009. doi: 10.1016/j.clinbiomech.2009.05.008.

[4] R. A. Preuss and M. R. Popovic, "Three-dimensional spine kinematics during multidirectional, target-directed trunk movement in sitting," $J$. Electromyogr. Kinesiol., vol. 20, no. 5, pp. 823-832, 2010. doi: 10.1016/j.jelekin.2009.07.005.

[5] R. Riemer, E. T. Hsiao-Wecksler, and X. Zhang, "Uncertainties in inverse dynamics solutions: A comprehensive analysis and an application to gait," Gait Posture, vol. 27, no. 4, pp. 578-588, 2008.

doi: 10.1016/j.gaitpost.2007.07.012.

[6] K. B. Smale, B. M. Potvin, M. S. Shourijeh, and D. L. Benoit, "Knee joint kinematics and kinetics during the hop and cut after soft tissue artifact suppression: Time to reconsider ACL injury mechanisms?," J. Biomech., pp. 1-8, 2017. doi:10.1016/j.jbiomech.2017.06.049.

[7] M. Y. Kuo, T. Y. Tsai, C. C. Lin, T. W. Lu, H. C. Hsu, and W. C. Shen, "Influence of soft tissue artifacts on the calculated kinematics and kinetics of total knee replacements during sit-to-stand," Gait Posture, vol. 33, no. 3, pp. 379-384, 2011. doi: 10.1016/j.gaitpost.2010.12.007.

[8] S. Mahallati, H. Rouhani, R. Preuss, K. Masani, and M. R. Popovic, "Multisegment Kinematics of the Spinal Column: Soft Tissue Artifacts Assessment," J. Biomech. Eng., vol. 138, no. 7, p. 71003, 2016. doi: $10.1115 / 1.4033545$.

[9] J. L. Durkin and J. J. Dowling, "Analysis of Body Segment Parameter Differences Between Four Human Populations and the Estimation Errors of Four Popular Mathematical Models," J. Biomech. Eng. ASME, vol. 125, no. August 2003, pp. 515-522, 2003.

[10] A. H. Vette, T. Yoshida, T. A. Thrasher, K. Masani, and M. R. Popovic, "A complete, non-lumped, and verifiable set of upper body segment parameters for three-dimensional dynamic modeling," Med. Eng. Phys., vol. 33, no. 1, pp. 70-79, 2011. doi: 10.1016/j.medengphy.2010.09.008.

[11] R. Riemer and E. T. Hsiao-Wecksler, "Improving Net Joint Torque Calculations Through a Two-Step Optimization Method for Estimating Body Segment Parameters," J. Biomech. Eng., vol. 131, no. 1, p. 11007, 2009.

[12] A. Plamondon, M. Gagnon, and P. Desjardins, "Validation of two 3-D segment models to calculate the net reaction forces and moments at the L5/S1 joint in lifting," Clin. Biomech., vol. 11, no. 2, pp. 101-110, 1996. doi: 10.1016/0268-0033(95)00043-7. 
[13] I. Kingma, M. P. De Looze, H. M. Toussaint, H. G. Klijnsma, and T. B. M. Bruijnen, "Validation of a full body 3-D dynamic linked segment model," Hum. Mov. Sci., vol. 15, no. 6, pp. 833-860, 1996.

doi: 10.1016/S0167-9457(96)00034-6.

[14] C. Larivière, D. Gagnon, and P. Loisel, "A biomechanical comparison of lifting techniques between subjects with and without chronic low back pain during freestyle lifting and lowering tasks," Clin. Biomech., vol. 17, no. 2, pp. 89-98, 2002. doi: 10.1016/S0268-0033(01)00106-1.

[15] B. D. Hendershot and E. J. Wolf, "Three-dimensional joint reaction forces and moments at the low back during over-ground walking in persons with unilateral lower-extremity amputation," Clin. Biomech., vol. 29, no. 3, pp. 235242, 2014. doi: 10.1016/j.clinbiomech.2013.12.005.

[16] R. N. Hinrichs, "Regression Equations to Predict Segmental Moments Inertia From Anthropometric Measurements: Extension Of The Data Of Chandler Et Al. (1975)," J. Biomech., vol. 18, no. 8, pp. 621-624, 1985. doi: 10.1016/0021-9290(85)90016-8. 


\section{Development of a Dynamic, Reconfigurable Finger-Hand Skeletal Structure}

\author{
Shreya Patki \\ Mechanical Automotive, and Materials Engineering \\ University of Windsor \\ Windsor, Canada \\ patki1@uwindsor.ca
}

\author{
R. Jill Urbanic \\ Mechanical Automotive, and Materials Engineering \\ University of Windsor \\ Windsor, Canada \\ jurbanic@uwindsor.ca
}

\begin{abstract}
A finger or hand brace may be required to be worn for people who require physical support or 'controlled motion' support. Ehlers Danlos Syndrome (EDS) patients have connective tissue disorders, and wear braces to support and limit motion. Manual assembly operators, carpal tunnel sufferers, and the elderly may also benefit from wearing finger or hand braces. Additive manufacturing (AM) solutions provide flexible manufacturing options for customized braces; however, a CAD model must be developed to facilitate a manufacturing solution. The goal of this research is to develop a readily adaptable CAD model for a hand, which would link to a flexible brace model. Rhinoceros ${ }^{\circledR}$ and Grasshopper ${ }^{\circledR}$ modelling tools are employed to develop a solution that can be dynamically manipulated to adjust to a specific hand size, and/or localized finger-hand configurations. The final CAD model (hand or brace) can then be built using an AM process.
\end{abstract}

Keywords-human skeletal modeling; dynamic CAD model; finger; hand; additive manufacturing

\section{INTRODUCTION}

The human hand is an incredible tool. It is capable of up to $47 \mathrm{~kg}$ grip strength ${ }^{1}$ (with a $9.5-9.7 \mathrm{~kg}$ standard deviation for men aged $20-49$ ) [1] as well as controlling extremely fine motor movements. However, 'tools' can wear. Complex motions, such as securing a button, require dexterity and precise motor control. Strength and fine motor control may prove to be difficult for people with Ehlers Dowler Syndrome (EDS), a connective tissue disorder, for people who encounter repetitive strain injury when performing tasks that are prone to developing carpal tunnel syndrome (CTS), and the elderly.

For the people who suffer from EDS, everyday activities are problematic [2]. The braces for the fingers and/or hand are required to provide support, the needed limits for motion, and to allow for some precise motion control [3]. CTS is condition that causes pressure on the median nerve in the hand, which results in numbness in the hand, tingling, or itching. In severe cases, there is pain. CTS can occur as a result of repetitive motion activities where the wrist is overextended repeatedly. Other conditions linked with CTS are diabetes, high blood pressure, or fracture or trauma to the wrist. CTS is the most common nerve compression syndrome, affecting 3 to $6 \%$ of the American public [4]. As shown in Fig. 1, the manufacturing sector typically has $2-3$ times more injury occurrences compared to most other industries [5]. Well-designed custom braces would allow workers to perform their tasks with the needed dexterity and the required control while keeping the wrists in a neutral position and reducing the pressure on the nerves. People in the older demographic may also have lower dexterity [6] and have trouble with daily tasks such as holding cutlery and opening containers. Proper brace designs can assist with support and motion control, similar to EDS patients, although the physical conditions are different.

Although hand braces may be useful for many situations, 'general' sized hand braces may not allow for the needed flexibility or comfort, and customized braces are costly. Localized customization may be required if a person has had broken bones or arthritis. The introduction of additive manufacturing (AM), or 3D printing, processes can lead to a better 'quality of life' solution for the various scenarios described here, as complex patient-specific designs can be readily fabricated. However, prior to fabricating a solution, a manifold or 'watertight' CAD model must be created. The brace model should be linked to a personalized hand model, which is challenging to create. Reverse engineering techniques may be employed to collect point cloud data, but many scans would have to be performed to collect all the shape data, and then point cloud registration, filtering, mesh development refinement, and the final surface modeling must be performed.

Blending surface patches is time consuming and may require several iterations for representing the base geometry [78]. Alternatively, a parametric or reconfigurable model can be created, and then adapted to suit based on selected measurements, which is the goal of this research. Creating an adaptable joint - finger - hand model, which allows for either global or local modifications without executing advanced surface or solid modelling commands manually, will facilitate the development of a buildable model, and any necessary design modifications determined from field testing or usage. Coupling the reconfigurable hand model to a brace model, and leveraging AM processes, will provide a platform for 
customizable, yet affordable, hand braces for the general public.

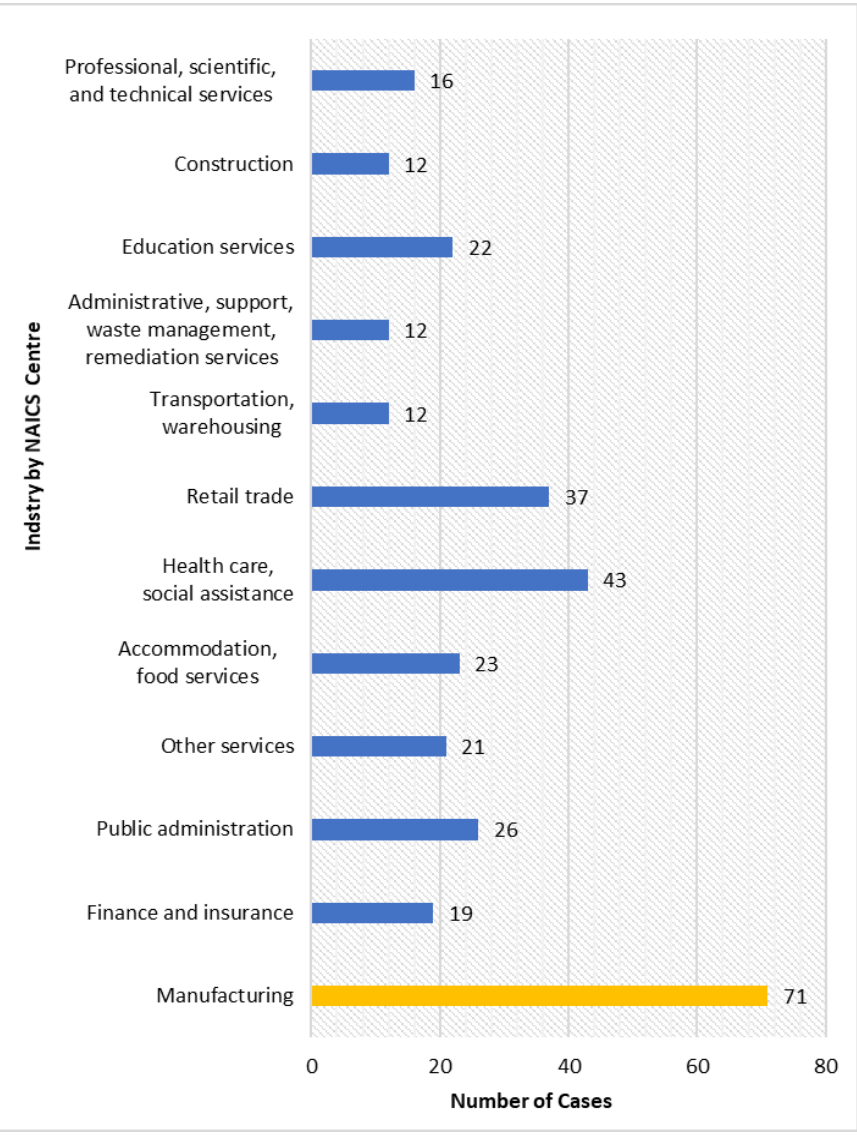

Figure 1. Carpal tunnel cases by North American Industry Classification System (NAICS) [5]

\section{BACKGROUND/LITERATURE}

Variations in the human hand/fingers size and shape are due to gender, age, body mass index (BMI), activity levels, and differences in body symmetry (i.e. the left and right hand and fingers will have differences). As illustrated by the anthropological data for the thumb and index finger (Table 1), there are significant variations from person to person (up to $35 \%$ for the thumb breadth), and these variations need to be considered when developing a customizable brace solution.

TABLE I. AVERAGE VALUES FOR SELECTED PERCENTILE DATA FOR THE THUMB AND INDEX FINGER, ADAPTED FROM [9]

\begin{tabular}{|l|l|c|c|c|c|c|}
\hline \multirow{2}{*}{ Dimension } & Gender & $\begin{array}{c}\text { 5th } \\
\text { percentile } \\
\text { (mm) }\end{array}$ & $\begin{array}{c}\text { 50th } \\
\text { percentile } \\
\text { (mm) }\end{array}$ & $\begin{array}{c}\text { 95th } \\
\text { percentile } \\
\text { (mm) }\end{array}$ & $\begin{array}{c}\Delta \text { 5th - } \\
\text { 95th } \\
\text { percentile }\end{array}$ & $\begin{array}{c}\text { Var. } \\
\text { from } \\
\text { mean }\end{array}$ \\
\hline \multirow{2}{*}{$\begin{array}{l}\text { Thumb } \\
\text { length }\end{array}$} & Male & 44 & 51 & 58 & $\mathbf{1 4}$ & $\mathbf{1 4 \%}$ \\
\cline { 2 - 8 } $\begin{array}{l}\text { Thumb } \\
\text { breadth }\end{array}$ & Female & 40 & 47 & 53 & $\mathbf{1 3}$ & $\mathbf{1 4 \%}$ \\
\cline { 2 - 8 } $\begin{array}{l}\text { Index finger } \\
\text { length }\end{array}$ & Female & $11-12$ & 23 & $26-27$ & $\mathbf{1 6}$ & $\mathbf{3 5 \%}$ \\
\cline { 2 - 8 } & Male & 64 & 72 & 79 & $\mathbf{1 4}$ & $\mathbf{3 4 \%}$ \\
\hline
\end{tabular}

Presently, the standardized models that have been created to date are constructed for one hand [10], and for the majority of the population - irregular or damaged hand types are not taken into consideration. However, using advanced reverse engineering techniques, specialized models can be very accurate when modeled to fit a custom hand type [11].

Ideally, a complex and detailed model, which can be easily modified, should be constructed. There are general 'structural' relationships that can be exploited linking finger geometry to hand length and width relationships (Fig. 2, Table II and III), and establishing a robust general model that allows for local modifications that could allow for rapid changes, would minimize the reverse engineering data collection.

The goal of this research is to leverage advanced CAD modeling techniques to create a dynamically reconfigurable hand model that can be animated to test finger movement, and how a brace would behave for different positions (ex. A fist or gripping position).

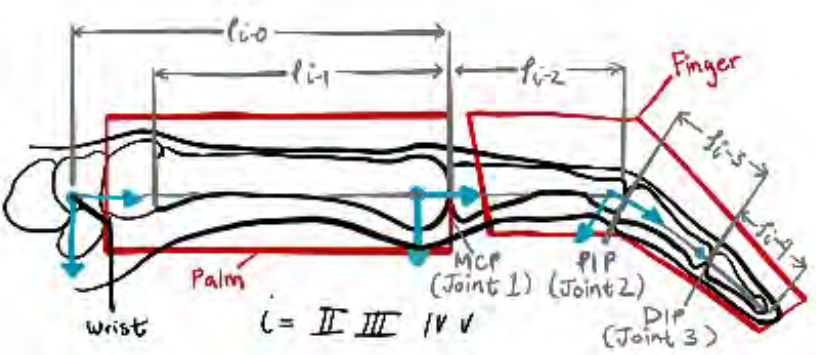

Figure 2. The 'i' represents each finger, the thumb being I and pinky V.

TABLE II. TABLE OF METACARPAL RELATIONSHIPS, WHERE $\mathrm{HL}=\mathrm{HAND}$ LENGTH [12]

\begin{tabular}{|c|l|}
\hline \multicolumn{2}{|l|}{ Metacarpal Bones } \\
\hline Thumb & $0.251 * \mathrm{HL}$ \\
\hline Index & $\left((0.374 * \mathrm{HL})^{2}+(0.126 * \mathrm{HB})^{2}\right)^{0.5}$ \\
\hline Middle & $0.373 * \mathrm{HL}$ \\
\hline Ring & $\left((0.336 * \mathrm{HL})^{2}+(0.077 * \mathrm{HB})^{2}\right)^{0.5}$ \\
\hline Pinky & $\left((0.295 * \mathrm{HL})^{2}+(0.179 * \mathrm{HB})^{2}\right)^{0.5}$ \\
\hline
\end{tabular}

TABLE III. TABLE OF FINGER PARAMETRIC RELATIONSHIPS FOR THE PHALANGEAL BONES[12]

\begin{tabular}{|c|c|c|c|}
\hline & Proximal & Middle & Distal \\
\hline Thumb & $0.196 * \mathrm{HL}$ & & $0.158 * \mathrm{HL}$ \\
\hline Index & $0.265 * \mathrm{HL}$ & $0.143 * \mathrm{HL}$ & $0.097 * \mathrm{HL}$ \\
\hline Middle & $0.277 * \mathrm{HL}$ & $0.170 * \mathrm{HL}$ & $0.108 * \mathrm{HL}$ \\
\hline Ring & $0.259 * \mathrm{HL}$ & $0.165 * \mathrm{HL}$ & $0.107 * \mathrm{HL}$ \\
\hline Pinky & $0.206 * \mathrm{HL}$ & $0.117 * \mathrm{HL}$ & $0.093 * \mathrm{HL}$ \\
\hline
\end{tabular}

\section{RESEARCH METHODOLOGY}

Initial research has been performed to design, fabricate, and test specialized designs for finger and hand braces [13]. Different parametric modelling approaches are being explored for readily accommodating special conditions. The modelling approach should be flexible, adaptable, easy to use by nondesigners, and require a minimal amount of rendering time. For this research, the 64-bit version of Rhinoceros ${ }^{\circledR}$ is employed with the Grasshopper ${ }^{\circledR}$ graphical programming and Bongo ${ }^{\circledR}$ animation add-ons. The Grasshopper ${ }^{\circledR}$ graphical programming tool can leverage known structural relationships and can

${ }^{1}$ Hand strength is measured by the quantity of static force a hand can squeeze around a dynamometer, and is measured in kg or $1 \mathrm{~b}$. 
propagate changes throughout the whole design simultaneously. Local modifications can be performed using the Rhinoceros ${ }^{\circledR}$ CAD tools, and joint / motion relationships for any design can be readily modeled for virtual testing. The general process flow is shown in Fig. 3. The methodology presented is an overview. The details are not included due to space constraints.

The basic finger bone modelling is established for the thumb as it has the simplest structure (Table III). A simple model of the proximal thumb bone with flat ends and a tapered intermediate section was developed using multiple lofted circles. Since every hand is unique, the lengths of the fingers need to be calculated using the parametric relationships shown in Table I and II (i.e., using the hand length as the key input). This static model was updated to include the distal bone.

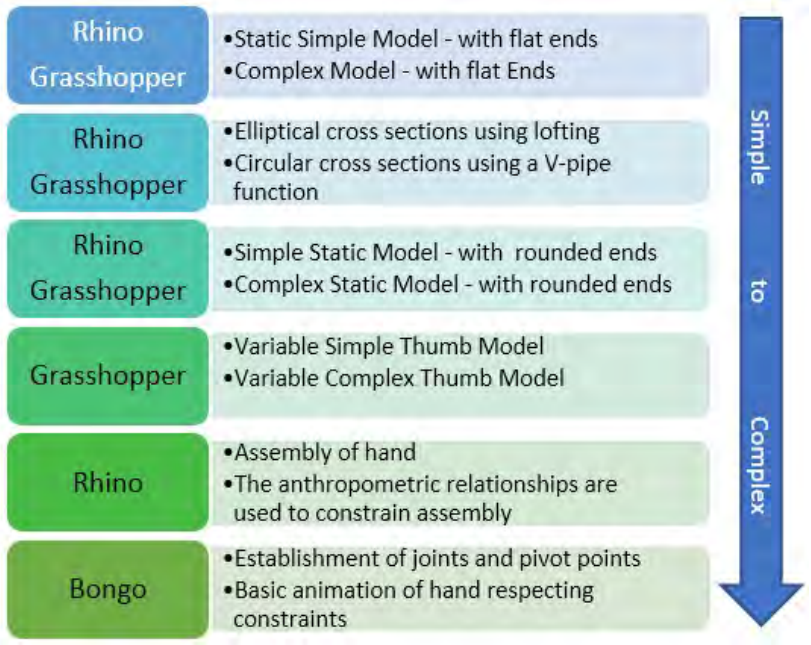

Figure 3. Steps in creating the final adaptable model

Once basic geometric relationships were established, elliptical cross-sections, with specialty Grasshopper ${ }^{\circledR}$ programming commands (V-Pipe or variable pipe), are employed to make this model more realistic. Sets of ellipses, and their control planes, are established. The model with the elliptical cross sections is more customizable as there are variable radii in two directions, and the ellipse positions may be altered using the input to the parametric relationships in the model (Fig. 4). Radii are controlled by a 'slider bar' input, as shown in Fig.8. This modeling approach leverages known relationships yet can accommodate of a wider variety of hands or localized variations, i.e., an arthritic hand, as shown Fig. 5.

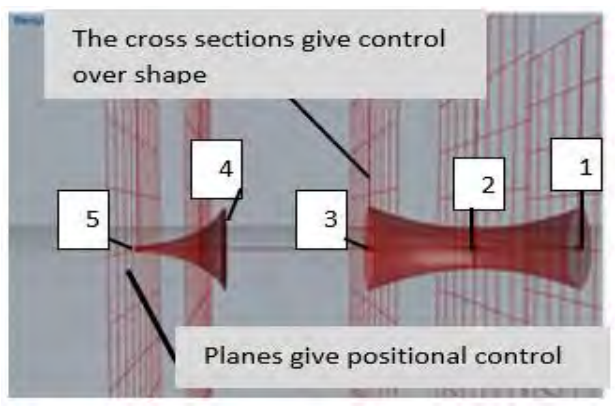

Figure 4. Elliptical cross sections and control planes illustrating the local control of the finger shapes. The 5 cross sections are labelled.

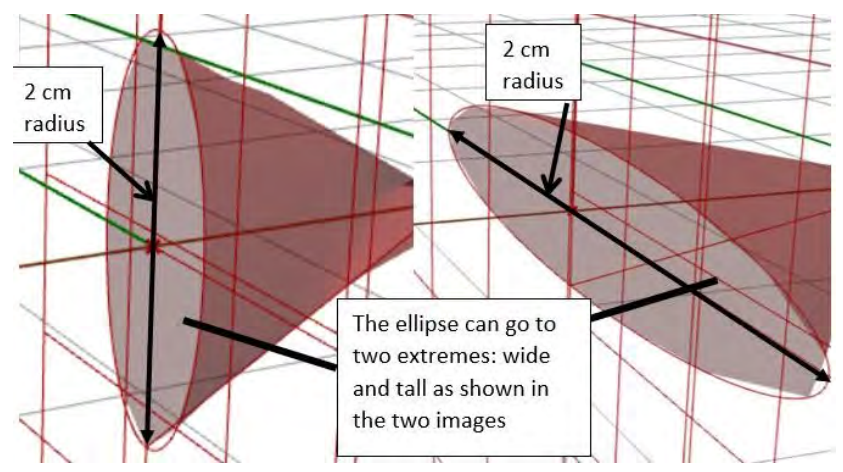

Figure 5. Another angle of the bone cross section and its flexability

Using the elliptical model as a base line, the rounded ends to the proximal bone are added via ellipsoids, where the control geometry is linked to the bone geometry. The endcaps model can also be personalized, as necessary. The progression from a bone to a finger is shown from right-top counter clockwise to right bottom (labelled 1-4) in Fig. 6. The semi-circular ring at the end of the finger is optional for now.

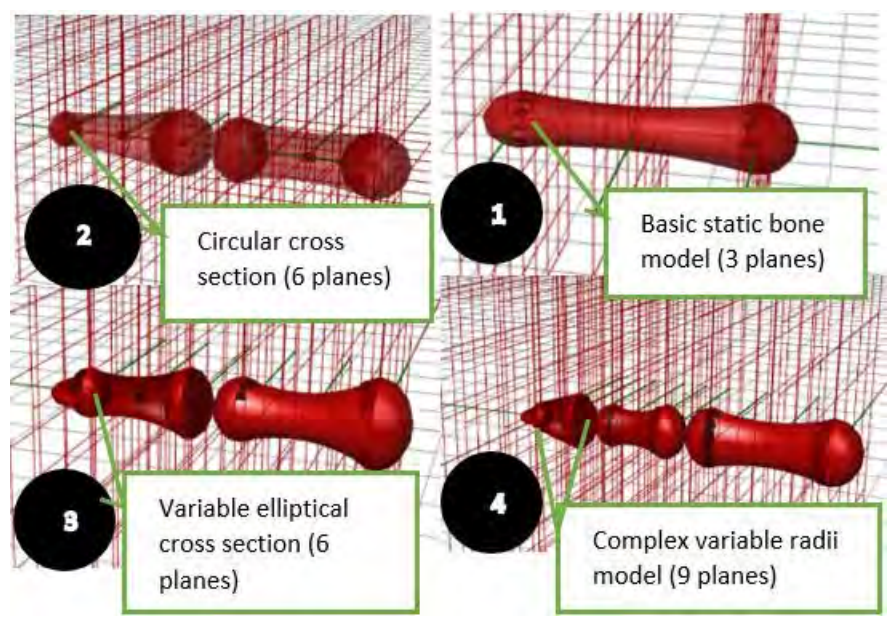

Figure 6. The build of the finger bones ordered from simple to complex (and order of creation)

Using the relationships and design approach, the bonefinger model is updated to become automatically adaptable. Instead of using the fixed numbers with the formulas and parametric relationships, 'slider bar' inputs or variables, which are controlled by the user, are utilized. The flexibility provided by the slider bars is shown in Fig. 7, 8 and 9.

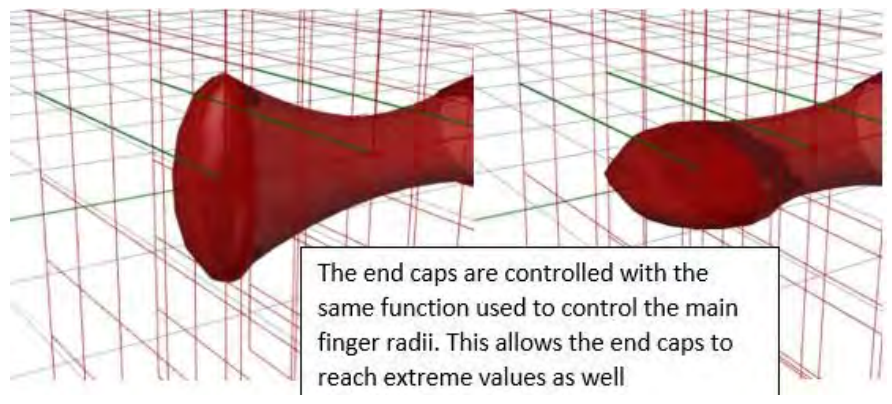

Figure 7. The adaptability of the end caps demonstrated 


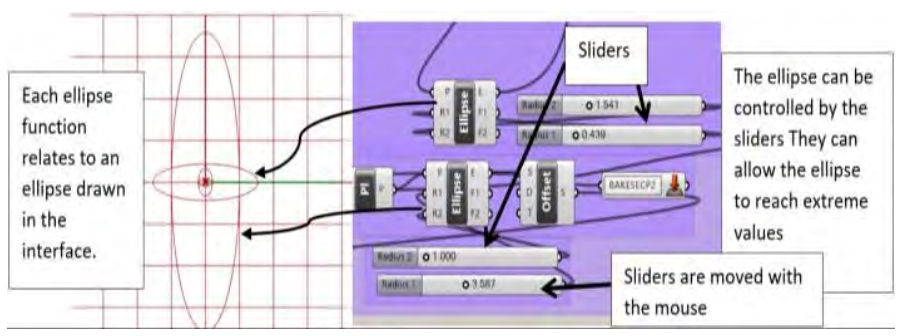

Figure 8. The simplicty of changing the finger radii is shown with the radii if the finger bone

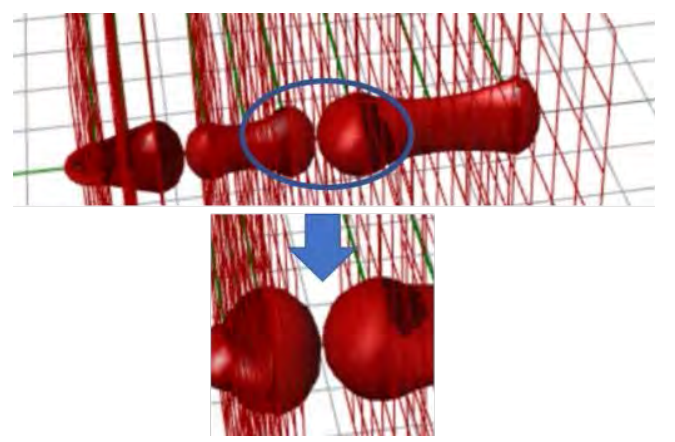

Figure 9. The wide range of the radii is demonstrated through the extreme sizes of the end caps shown.

The interface of the Grasshopper ${ }^{\circledR}$ algorithm is shown in Fig. 10. The parts are connected to each other using 'wires' in the program. This feature allows the input data to be transformed using mathematical functions, and the programmable control shapes to create surface, mesh, or solid model geometry in the Rhinoceros ${ }^{\circledR}$ environment.

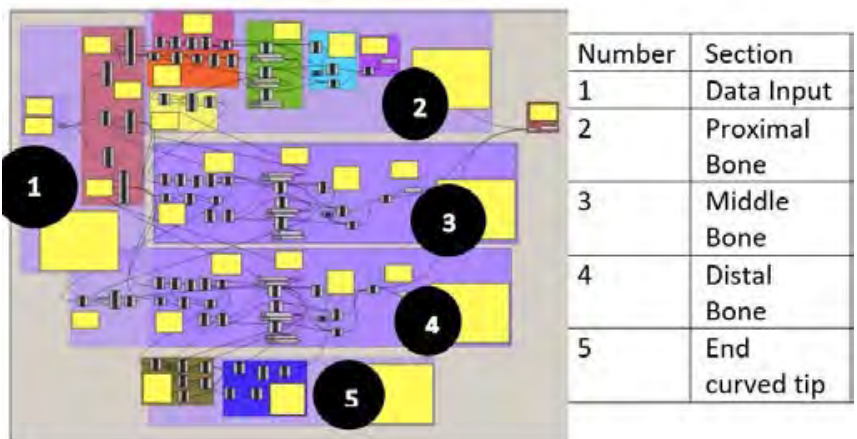

Figure 10. Labelled Grasshopper ${ }^{\circledR}$ interface shown (the behind the scenes of the finger model).

These finger models can easily be assembled into the individuals hand model (in the Rhino ${ }^{\circledR}$ environment) using anthropometric relationships and constraints to link the individual components into a final kinematic assembly. This bone-finger-hand model can then be animated for different situations such as hand gripping using the Bongo ${ }^{\circledR}$ add-on. Once the appropriate relationships are finalized, custom finger braces or hand brace models can be built on top of this.

\section{RESUlTS AND DISCUSSION}

The result of this process is a customizable bone-fingerhand model that can be adaptable for a variety of shapes instantaneously. The programming elements of this model allows a hand configuration to be programmed using parametric relationships established by other researchers. The hand length and breadth are measured. They are then used as an input to establish the bone lengths for the metacarpal and phalangeal bones each finger. Example solutions are shown in Figure 11 and 12. They are hand of a young female and young male. On the left is the wireframes printout of the model, center the hand, and on the right the 3D shaded model of the hand from Rhinoceros ${ }^{\circledR}$. The reconfigurable reverse engineered hand models are created.

Both hands shown have different hand lengths, breadths and finger radii, and this model was able to conform to the measurements and create a customized model of the hand.

A preliminary 'proof of concept' animation of these reconfigurable models has been completed. Examples of two animations with developed with the Bongo ${ }^{\circledR}$ add-on are shown in the links below (and Fig. 13 and 14).

https://www.screencast.com/t/m0Fec2dG2mE

https://www.screencast.com/t/qOnf6SetqA

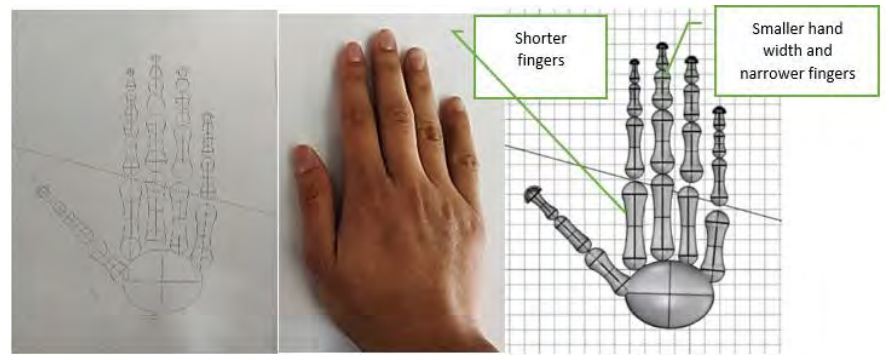

Figure 11. Female hand model.

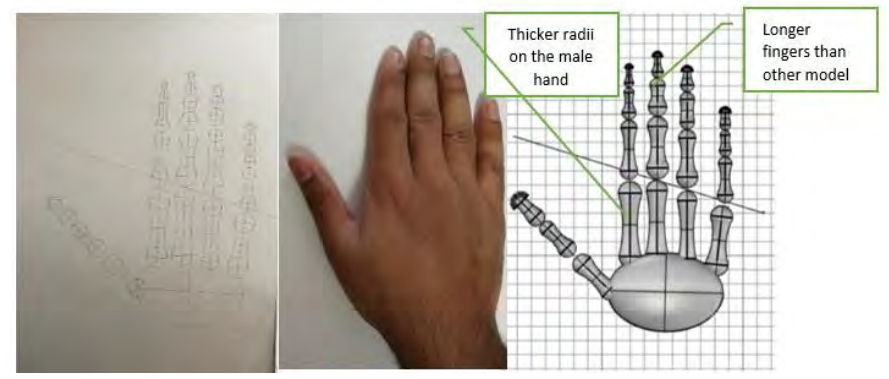

Figure 12. Male hand model

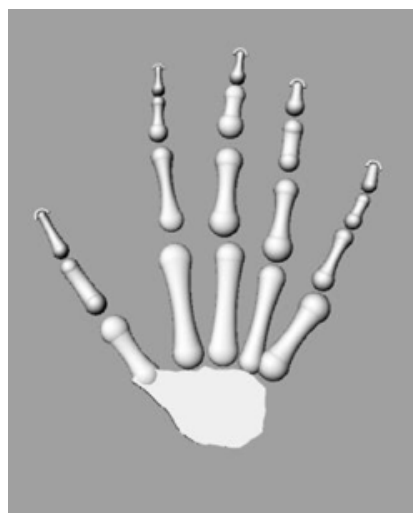

Figure 13. The hand model with moderately spread apart fingers 


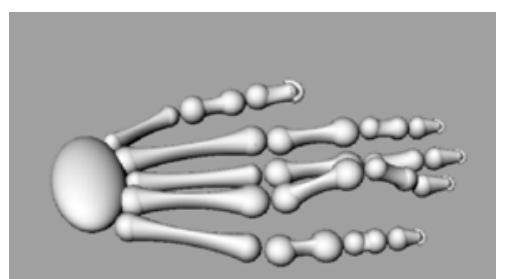

Figure 14. Still from a hand model animation of finger movement

\section{SUMMARY AND CONCLUSIONS}

Reverse engineering a hand to create an editable surface or solid model is a time intensive task and requires highly skilled designers familiar with surface modeling strategies. The goal to develop a dynamically reconfigurable bone-finger-hand model was realized using a set of modeling tools typically used in animation environments. Developing organic parametric models in CATIA is problematic due to linking the many bonefinger-hand constraints [14].

As human anatomy has general known relationships (anthropometric data), but individual variants, the Grasshopper ${ }^{\circledR}$ visual programming language is used to leverage the relationships to create editable models in the Rhinoceros environment. This foundational research allows for the creation a customized hand brace without a significant amount of measurements or data manipulation for developing a personalizing CAD model. This CAD model can then be used as an input for an AM process.

Throughout this process, the main goal was to add enough detail to represent the uniqueness of the individual's hand but not to be overly complex or have the data entry be a timeconsuming task. Due to the model's ability to have full control over the shape of the finger, (essentially the thickness), muscles and skin will be added in the future. The overall process for creating specialty braces shown in Fig. 15.

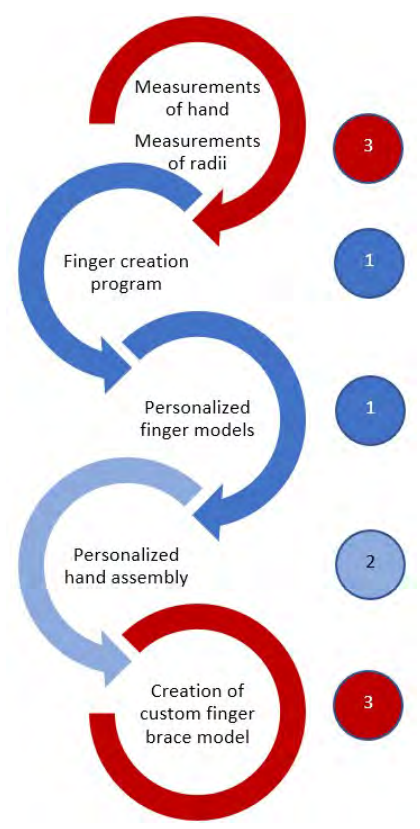

Figure 15. Steps for creating final adaptable hand and braces models (1 present research, 2 - on-going research, 3 - future work)

\section{FUTURE STUDIES}

Localized 'non-elliptical' geometry solutions will be explored. The animation models will be improved to include anthropometric relationships, as there are unique motion conditions for our hands. For example, all joints in the ring finger cannot be fully rotated inwards without creating motion in the middle or pinky fingers. The Grasshopper ${ }^{\circledR}$ model will be streamlined to add a user interface, data collection strategies determined, and brace design solutions will be designed, fabricated (using an AM process) and tested.

\section{ACKNOWLEDGMENTS}

This research is partially funded by the NSERC Discovery Grant and the University of Windsor Outstanding Scholars programs. Special thanks to hand model Swapnil Patki.

[1] N. M Massy-Westropp et al., "Hand grip strength: age and gender stratified normative data in a population-based study," BMC Res. Notes, vol. 4, pp. 127-127, Apr. 2011. doi: 10.1186/1756-0500-4-127.

[2] Mayo Clinic (2017, Oct. 13). Ehlers-Danlos syndrome [Online]. Available: https:// www.mayoclinic.org/diseases-conditions/ehlersdanlos-syndrome/doctors-departments/ ddc-20362171

[3] J. R. Gravlee and D. J. Van Durme, "Braces and splints for musculoskeletal conditions," Am. Fam. Physician, vol. 75, no. 1, pp. 342-348, Feb. 2011.

[4] K. E. LeBlanc and W. Cestia, "Carpal tunnel syndrome," Am. Fam. Physician, vol. 83, no. 8, pp. 952-958, Apr. 2011.

[5] S. E. Luckhaupt et al, "Prevalence and work-relatedness of carpal tunnel syndrome in the working population, United States, 2010 national health interview survey," Am. J. Ind. Med., vol. 56, no. 6, pp. 615-624, Apr. 2012. doi: 10.1002/ajim.22048.

[6] E. Carmeli, H. Patish, and R. Coleman, "The aging hand," J. Gerontol., Ser. $A$, vol. 58, no. 2, pp. 146-152, Feb. 2003.

[7] M. Attene and M. Spagnuolo, "Automatic Surface Reconstruction from Point Sets in Space", Comput. Graphics Forum, vol. 19, pp. 457-465, 2000. doi: $10.1111 / 1467-8659.00438$.

[8] L. M Galantucci, G. Percoco, and R. Spina, "An artificial intelligence approach to registration of free-form shapes," CIRP Ann., vol. 53, no. 1, pp. 139-142, 2004. doi: 10.1016/S0007-8506(07)60663-5.

[9] Georgia Tech Research Institute (2007). Ease of use assistant: Hand anthropometry [Online]. Available: https://usability.gtri.gatech.edu/ eou_info/hand_anthro.php

[10] M. I. Saludares and R. Atienza, "Parametric 3D hand model," in Proc. 7th Int. Conf.on Intelligent Systems, Modelling and Simulation, Bangkok, Thailand, 2016, pp. 307-311. doi: 10.1109/ISMS.2016.54.

[11] T. Rhee, U. Neumann, and J.P. Lewis, "Human hand modelling from surface anatomy," in Proc. 2006 ACM SIGGRAPH Symposium on Interactive $3 D$ Graphics and Games, Redwood City, CA, pp. 27-34

[12] E. P. C. C. Pitarch, "Virtual human hand: grasping strategy and simulation," Ph.D. dissertation, Universitat Polit' ecnica de Catalunya (UPC), Barcelona, Spain, 2007.

[13] H. Kalemi, A Khayat, and R. J. Urbanic, "Investigating exo-skeleton design and fabrication challenges for the hand region using material extrusion processes," IFAC-PapersOnline, vol. 49, no. 31, pp. 30-35, 2016. doi: 10.1016/j.ifacol.2016.12.157.

[14] R. J. Urbanic and L. DiCecco, "Development of adaptable light weighting methods for material extrusion processes," in Proc. ASME 2015 Int. Mechanical Engineering Congress and Exposition, Houston, TX, vol. 2A, pp. 1-3. doi: 10.1115/IMECE2015-51174. 


\title{
Development of a Rehabilitation Mobility Assistive Device
}

\author{
Xueqiao Han, Reza Fotouhi*, Doug Bitner \\ Department of Mechanical Engineering \\ University of Saskatchewan \\ Saskatoon, Canada \\ *Corresponding author Reza.fotouhi@usask.ca
}

\begin{abstract}
- the aim of the overall research presented here was to investigate the motion of a wheeled mobile robot in an indoor (structured) setting while following a pre-set trajectory. An example of an application for this research would be automated maneuvering of a smart power wheelchair in a health care setting, such as a hospital, which would benefit the aging Canadian population. For the specific research reported here, the aim was to investigate the mobility of an assistive device for Sit-to-Stand (STS) operation and walking. A rehab robot was developed and was attached to a wheeled mobile robot to accomplish STS operation and to help walk a patient for rehabilitation. Four major phases of this research are: (i) design of the rehab robot, (ii) development of the control algorithm, (iii) experimentation, and (iv) navigation of the mobile robot and the rehab walker (robot). This research project can be extended to lower limb rehabilitation and design of a smart walker. It intensively studied current research and projects, designed a rehab robot and four control algorithms to help people in both STS and walking processes. Experiments were implemented, and the results indicated the effectiveness of the control algorithms and the prescribed navigation algorithm. According to the results, this project achieved the original goals to assist people in standing up and walking, and to navigate to a pre-set location.
\end{abstract}

Keywords: mobility assistive device; rehab robot; navigation

\section{BACKGROUND AND MOTIVATION}

As the aging population increases, more and more countries require a larger workforce to take care of the elderly. Meanwhile, the gradually soaring cost and investment in healthcare and nursing have become a global issue. Taking care of people with impaired lower limb functions is one of the trickiest problems. A number of elderly have problems with their knees, hips, and lower limb strength, which affects their mobility. They have difficulties in walking and are not able to cope well at home. People recovering from stroke, lower limb fracture, and some other illnesses suffer similar problems as well. Rehabilitation services and nursing are available, but access is limited due to constrained medical resources and their high cost. As a result, some of these people miss the best time for recovery, and their limited activity keeps them in their home and hence, they suffer from more health issues caused by lack of movement and being alone. One of the motivations for this research is to provide easy standing and walking assistance for these people and an alternative solution for nursing care as well. This is also expected to help in lower limb rehabilitation and patients' autonomy.

A variety of walkers are available on the market that help in walking, such as the rollator walker and the adjustable standard walker [15]. However, most traditional walkers are designed for people with a high self-care ability level. For people with poor health conditions, such as weakened leg strength, limited hand and finger motion, and hemiplegia sequelae, merely operating the walker would be a huge challenge. Traditional walkers do not provide support for Sitto-Stand (STS), which is one of the toughest challenges for self-autonomy. Hence, it leads to a second motivation for this research, to design a device that helps a broader range of people.

Motivated by medical needs as stated above, this project is focused on research of lower limb rehabilitation and assistance by devising a machine with the following capabilities:

1. Have basic walker functions.

2. Help in the STS process (the major goal of this design).

3. Provide assistance in standing and walking.

4. Be smart in navigation (this feature will provide care, convenience, and assistance to users, and allow patients for rehabilitation by walking in a pre-defined path).

5. Have a good level of adaptability (it should be able to help a wide range of people having different height, weight and health conditions, and be useful for home/hospital).

\section{A. Literature Review}

Several researchers proposed interesting designs and ideas, and also studied some related topics in this area.

Morbi et al. [1] designed a system called Gait-Enable, which is used in over the ground walking and balance training. This design integrated the Bungee Mobility Trainer, which is a patented elastic passive body weight support system that 
consists of a 3-DOF linkage that supports the patients' pelvis from below. The test results show that the Gait-Enable system will reduce pelvis and torso motion constraints. Ahmed et al. [2] designed an assistive device named E-Just Assistive Device (EJAD), a low cost intelligent assistive device to help elders in walking and sit-to-stand activities. This is a mechanical design based on the imitation of the caregivers' motion during the support task, which is mainly composed of back support, a robot arm and an active walker. Bauzel et al. [4, 5] analyzed current research on smart walkers and proposed three required significant features: provide dynamic support, demand little or no effort to use, be user-friendly. Also, a new user interface which allows users to control the walker intuitively was designed and implemented on a ASBGO walker, which is an active robotic walker that assists the elderly and disabled people in walking. Measures have been adopted to assure safety and customer comfort, such as infrared rays gesture detection, force detection of falls, and limit wheels' orientation and speed. Bulea et al. [6] proposed a new design with adapted gas spring actuators to enable auto lifting, utilizing both handles and platforms for arm support. The gas spring provides lifting forces and a steel shaft is designed to help users moving freely. The experimental results from a partially paralyzed male show that the lift walker significantly shortened the transition time of the sit-to-stand process and also resulted in a steadier rise than with a standard walker. Jorge et. al. [7] reviewed all current robotic systems for lower-limb rehabilitation in 2011 and pointed out that research in this field has grown exponentially during the last decade. About 43 robotic systems were mentioned that can be classified into the following five categories: Treadmill Gait Trainers, Foot-Plate-Based Gait Trainers, Overground Gait Trainers, Stationary Gait Trainers and Ankle and Knee Rehabilitations Systems made up of Stationary Systems and Active Foot Orthoses. Of these, more than half of the systems have neither been marketed nor applied for home use. This is due to their bulky size, elevated cost and the lack of high clinical improvement evidence. Research specifically in the STS area has also been investigated and has further contributed to this work. It has helped in developing and designing the motion control algorithms of the walker used in this project.

Other research work has been carried out to help the development of smart robotic walkers. Pasqui et. al. [8] found that the global shape of a patient's trajectory is an "s-like" curve. This was discovered through experiments participated by patients. Wang et al. [9] presented a fuzzy controller design for a lower limb rehabilitation robot, which is more effective than a traditional PID controller. Pasqui et al. [10] proposed a method to assess the efficiency of smart-walkers by measuring the time cost of different walkers when users were required to complete a series of movements. Other types of robotics to assist walking and lower limb rehabilitation have also been studied these include a new design of an exoskeleton system presented by Castro et al. [11], a new robot design for children with cerebral palsy presented by Giergiel et al. [12] and a mechanism model of a lower limb passive rehabilitation system built by Zhang et al. [13]. In addition, some researchers have also studied the psychological aspects of lower limb rehabilitation. For example, Koenig et al. [14] discussed a psychological measurement method which may help in robotic-assisted gait rehabilitation.

To summarize, some of the work done in this area has been reported here. However, most of the existing rehabilitation systems are not good enough for commercialization. Most of them have issues in cost, safety and portability; and also lack reliable testing. Unfortunately, none of the intelligent devices available can help both STS and the walking process. Hence, a mature, smart, and affordable rehabilitation system for STS and walking has yet to be designed.

\section{B. Research Objective}

The objectives of this research can be summarized as follows: 1- Investigate the state-of-the-art for the existence and needs of an assistive device for the sit-to-stand (STS) and walking operation. Develop such a device and its relevant motion control algorithms to help patients with rehabilitation.

2- Investigate and test the STS operation and the interaction between the user and the device. Test control algorithms for the rehab robot and evaluate its effectiveness in helping people with lower limbs rehabilitation

3- Investigate/examine navigation algorithms in a medical setting that may help in walking for rehabilitation.

\section{Research Methodology}

A literature review was performed and a device (rehab robot) was designed based on conclusions and ideas drawn from it. After a prototype of the rehab robot was manufactured, control algorithms for three different means of STS: arm-pad support, handlebar support, and belt support, were developed. Following this, practical tests with different control algorithms were implemented and analyzed. Also, a questionnaire was handed out to participants and the results were analyzed qualitatively. Lastly, the smart walker was attached to an Autonomous Ground Vehicle (AGV) which was used as the driver, and an existing navigation algorithm was tested to assess its feasibility for this project.

\section{DESIGN OF THE REHAB ROBOT}

Since this project is an attempt to develop an affordable mature smart walker, the AGV was adopted as a driver in the experiments. A rehab robot was designed to attach to the AGV and complete the major functions of a smart walker. This rehab robot has basic walker functions and adjusts its height according to a control algorithm or a user's commands, hence it helps in the STS process and walking.

There are three main parts in the design process: function analysis, mechanical design, and electrical design. Function analysis was applied first in order to guide both the mechanical and electrical design. Six major functions were determined as follows: help the STS process, be capable of moving, be automatic, be safe, be comfortable to various 
users, and be cost effective. Each function was also analyzed in detail [16], which assured that the design met the requirements.

After function analysis, a mechanical prototype was designed since it not only was needed to meet all requirements, but also had to provide sufficient convenience for electrical design and its functions. For example, a battery box was designed for the storage of batteries and other electrical components. There are several parts in the mechanical design section: conceptual design, detailed design, Finite Element Analysis and material selection. The rehab robot prototype is shown in Figure 1.

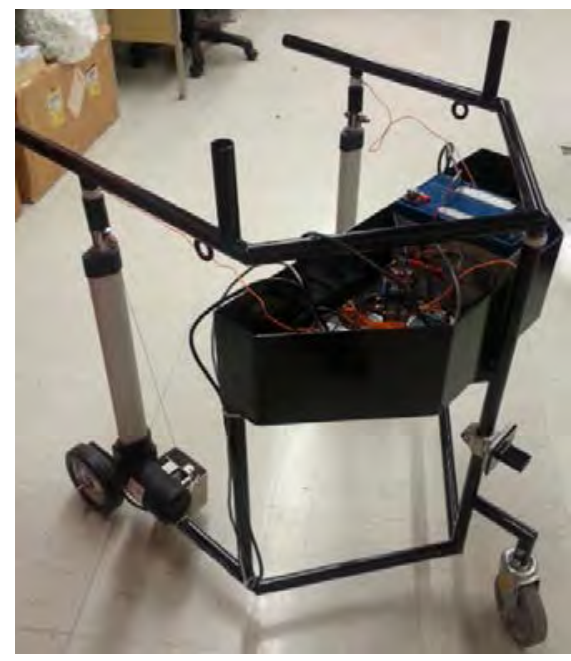

Figure 1. Prototype of the Rehab Robot

The electrical component design was developed for motion and control. First, the goal and requirements for the electrical design were clarified and then appropriate electrical components were selected (after extensive research). The electrical flowchart of the system is shown in Fig. 2. The basic idea is that microcontrollers (two Arduino Mega 2560) collect data from real-time transducers including, force sensors, position sensors, keypad inputs and as options, a sound detector and voice recognition module. Then the microcontrollers process the data through a programmed control algorithm downloaded to the microcontrollers and output the corresponding signals to a motor controller. Next, the motor controller, that uses a constant power supply from batteries and a voltage regulator, outputs a voltage that governs the motion of the actuators. Transducers collect data at one second intervals. Hence, the closed-loop control algorithm is able to control the motion of the actuators quickly and effectively.

Transducers in the prototype collect position and force data that mainly helps in detecting the users' status, such as sitting, standing, walking and falling. Keypad, screen, voice recognition module, sound detector, and speaker were designed as the user interface. This enabled the rehab robot to take in data that reflects user information and preference, and to react to real-time commands.

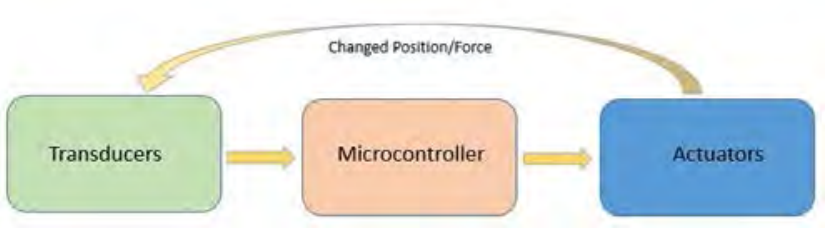

Figure 2. Electronics Connection Schematic Diagram

\section{CONTROL ALGORITHM DESIGN AND RESEARCH}

The algorithms developed in this project utilized three different techniques to help in the STS process: 1-arm-pad support, 2-handlebar support, and 3-belt support, as shown in Fig. 3. There are two algorithms (Test 3 and Test 2-1) for armpad support, one algorithm (Test 4-2) for handlebar support, and one for belt support (Test 5). More algorithms and corresponding test information can be found in Table 1.

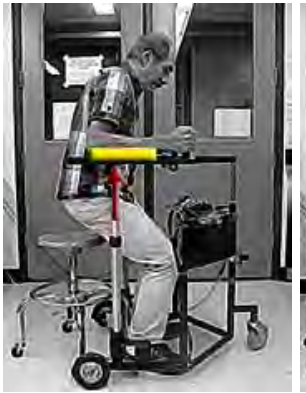

(a) Arm-pad Support

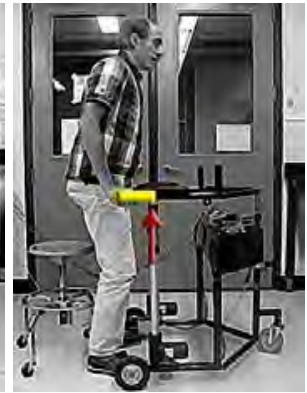

(b) Handlebar Support

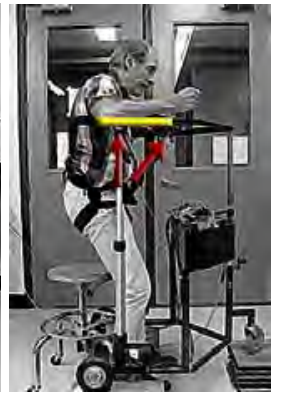

(c) Belt Support
Figure 3. Three Lifting Methods

These algorithms were used to first detect the status of the user and then react appropriately for each status. For the arm-pad support algorithms, once the device detects a user, it triggers the upper frame with the support arm-pad to lift to a theoretical best height, calculated from the applied force on the actuators, user's height and weight. Once it reaches the theoretical height, it enters into a height adjustment phase, where the user will be able to adjust the height automatically or manually. When the adjusting phase is completed, the rehab robot goes into a steady state mode and maintains the same height for the upcoming walking process. When no input is received from the user after a specified period, the actuators will retract to the initial position and wait for the next round of usage. The schematic diagram of the control algorithm is shown in Fig. 4.

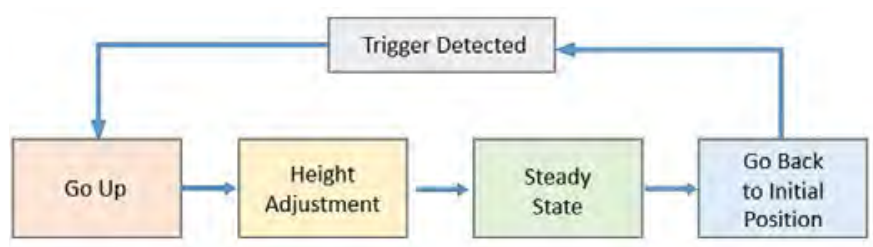

Figure 4. General Control Algorithm Concept Diagram 
The belt support algorithm is very similar to the arm-pad support algorithm, except that the actuators are lifted to a higher position first to enable the belt to help lift the user to a fully upright position. It will then be lowered to the theoretical best height before entering into the height adjustment phase.

The handlebar support algorithm is a simple algorithm that controls the height of the rehab robot to follow the trajectory of the desired hand height of the user during the STS process. Abnormal cases have been considered in all algorithms, and voice control is also available. A flowchart for each algorithm and a detailed introduction to these can be found in section 3.1 of [16].

\section{RESULTS AND ANALYSIS}

This section discusses the test process for the control algorithms, provides results, analysis and comparison with all experiments conducted. Seven experiments were conducted with the help of three participants. Table 1 shows a summary of these experiments. In general, the tests demonstrated in [16, Chapter 4] that they can also be categorized into three classes: arm-pad support lift tests (Test 1-1, Test 1-2, Test 2-1, Test 3), handlebar support lift tests (Test 2-2, Test 4-2), and belt support lift test (Test 5). Also Natural STS is Test 4-1.

TABLE 1. TEST List of ReHAB RoBot (ALL ALGORITHMS USE FORCE AND DISPLACEMENT FEEDBACK)

\begin{tabular}{|c|c|c|c|}
\hline Test No. & Description & Controller Type & Importance \\
\hline Test 1-1 & ift the upper frame to preset practical best height for a particular use & Open Loop & Secondary \\
\hline Test 1-2 & Lift the upper frame to highest position & Open Loop & Secondary \\
\hline Test 2-1 & Controlled by Applied Force Based Arm-pad Lifting Methond & Closed Loop & Primary \\
\hline Test 2-2 & Controlled by Simple Handlebar Lifting Method & Closed Loop & Secondary \\
\hline Test 3 & Controlled by Body Weight Based Arm-pad Lifting Methond & Closed Loop & Primary \\
\hline Test 4-1 & Natural STS process & No Device Required & Secondary \\
\hline Test 4-2 & Lift the handlebar to preset practical best height for a particular user & Open Loop & Secondary \\
\hline Test 5 & Controlled by Belt Support Lifing Method & Closed Loop & Primary \\
\hline
\end{tabular}

The purpose of discussing the eight tests in this project is to compare the three different support lifting methods, and also compare the effectiveness of the different algorithms for the same lifting method. In general, all three lifting methods provide assistance to the STS process, and require less leg strength, compared with the natural STS process. The belt support lifting method provides the most assistance, which can be concluded from Comparison I: Arm Force Comparison [16, section 4.4]. As the force on arm settles down quicker than other approaches. The other two lifting methods provide a similar amount of support assistance during the STS process. Fig. 5 is a typical result of arm force comparison for different algorithms. For overall lower limb assistance, the arm-pad support lifting method could be the best, because it also carries the upper limb weight during the walking process, and maybe some upper trunk weight as well. The results can be found in detail in Comparison II: Leg Force Comparison [16, section 4.6]. Fig. 6 shows the averaged test results comparison.

In the two control algorithms designed for the arm-pad support lifting method, the Applied Force Based control algorithm was found to be the better algorithm, according to Comparison I: Arm Force Comparison [16, section 4.4]. Perhaps, this is because this algorithm is more stable, and it takes less time for the participants to find their best arm height.

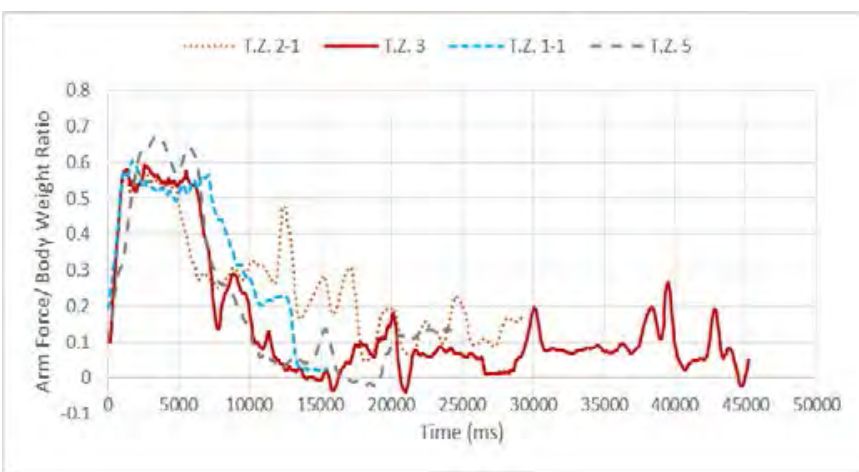

Figure 5. Test Results of the Participant TZ (Force on Arms Comparison), arm-pad support (Tests, 1-1, 2-1, 3), belt support (Test 5).

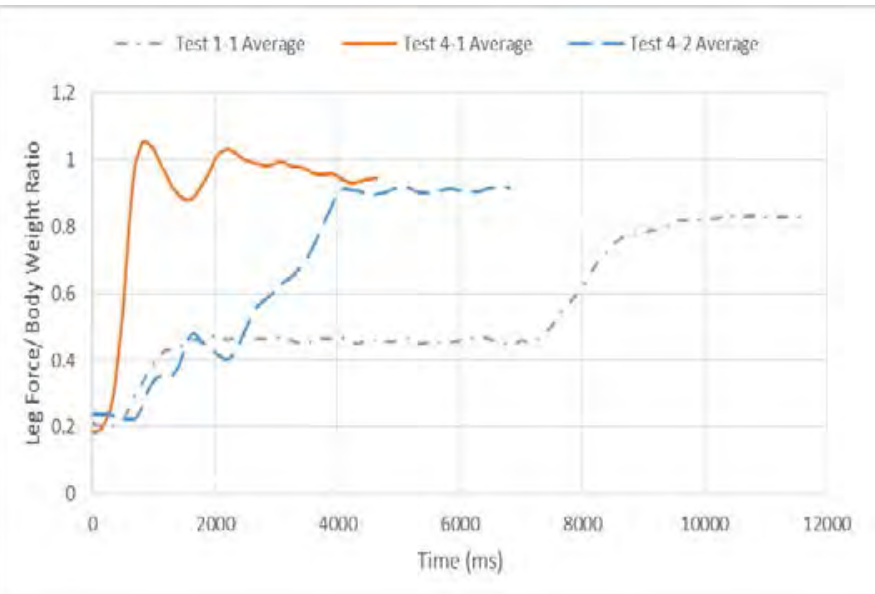

Figure 6. Comparing Different Methods of STS (Leg Force), average results of three participants. Arm-pad support (Test 1-1), Natural STS (Test 4-1), Handlebar support (Test 4-2).

\section{NAVIGATION ALGORITHM AND TESTS}

To demonstrate the walking aspect of the rehab robot, a Fuzzy-Logic-Based (FLB) algorithm [18] was adopted for navigation of the $\mathrm{AGV}$ driving the rehab robot in an indoor medical setting. Using this algorithm, the AGV was able to successfully reach several goals while avoiding obstacles during its motion.

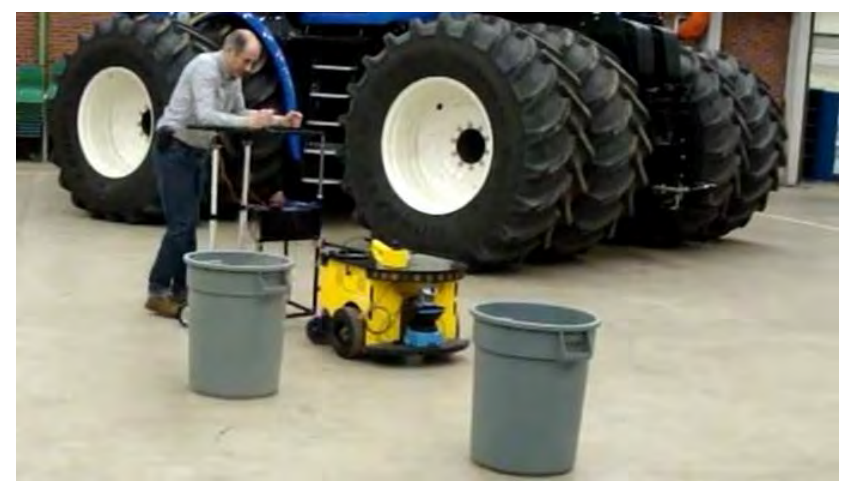

Figure 7. Test of rehab-robot Avoiding Obstacles 
The rehab robot was attached to the AGV and tested using the FLB algorithm (see Fig. 7). Based on H. Barden's gait speed classification [17], three speed tests were implemented: 0.9 $\mathrm{m} / \mathrm{s}, 0.65 \mathrm{~m} / \mathrm{s}, 0.4 \mathrm{~m} / \mathrm{s}$, and one-obstacle and two-obstacle cases were tested for each speed. During the tests, the device successfully reached preassigned goals and avoided obstacles, however, it made sharp turns, and the user experience was not ideal for practical applications. Fig. 8 shows a typical example of navigation of the rehab robot while avoiding a two-obstacle situation. Detailed test results and findings can be found in [16, section 5.3].

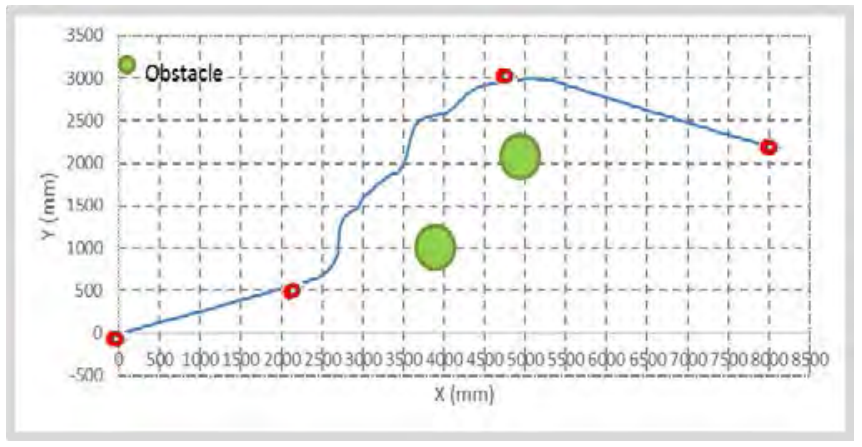

Figure 8. Typical Test Results of Avoiding Two Obstacles (Path). (X: Horizontal Displacement, Y: Vertical Displacement, Goal Position: (8000, 2200))

\section{CONCLUSIONS}

The design of the mechanical and electrical parts, development and comparison of the algorithms, and tests of the navigation algorithm were addressed here. Also, several participants tested the algorithms. The results showed that the designed algorithms helped in the STS process and aided users in finding a comfortable walking position within a reasonable time. According to the results of experiments and questionnaires, the four developed algorithms successfully helped participants to complete the STS process and provided a relatively good user experience at the same time. Hence, it can be concluded that major design goals for the rehab robot have been achieved. However, for future research and possible commercialization, some design details have to be improved and large-scale test data is needed for optimizing the control algorithm. After the rehab robot was fully developed, it was attached to a wheeled mobile robot and went through a set of tests. For navigation, the FLB algorithm adopted in this project, helped in reaching the goal position while avoiding obstacles. However, the user experience was not ideal and hence the navigation aspect of this research needs to be modified for use in any future medical applications.

\section{REFERENCES}

[1] A. Morbi, M. Ahmadi, and A. Nativ, "GaitEnable: An omnidirectionalrobotic system for gait rehabilitation," Mechatronics and Automation (ICMA) International Conference, pp.936,941, 5-8 Aug. 2012. doi: 10.1109/ICMA.2012.6283269.
[2] S. Koenig et al., "A Systematic Approach for Design a Low-Cost Mobility Assistive Device for Elderly People," Int. J. Medical, Health, Biomedical and Pharmaceutical Engineering, vol. 5, no.11, pp.597,602, 2011.

[3] S. Omar, A. A. Ramadan, S. Sessa, A. A. Ismail, M. Fujie, A. Takanishi, "ANFIS-based Sensor Fusion System of Sit-to-stand for Elderly People Assistive Device Protocol," Int. J. Automation and Computing, vol. 10, no. 5, pp. 405-413, 2013. doi: 10.1007/s11633-013-0737-6.

[4] M. Martins, C. Santos, E. Seabra, A. Frizera and R. Ceres, "Design, implementation and testing of a new user interface for a smart walker," 2014 IEEE Int. Conf. on Autonomous Robot Systems and Competitions (ICARSC), Espinho, pp. 217,222, 14-15 May, 2014

doi: 10.1109/ICARSC.2014.6849789.

[5] M. Martins, C. Santos, A. Frizera and R. Ceres, "Real time control of the ASBGo walker through a physical human-robot interface," Measurement, vol. 48, no. 2, pp.77, 86, 2014. doi: 10.1016/j.measurement.2013.10.031.

[6] T. Bulea and R. Triolo, "Design and Experimental Evaluation of a Vertical Lift Walker for Sit-to-Stand Transition Assistance," J. Med. Device, vol. 6, no. 1, 2012. doi: 10.1115/1.4005786.

[7] I. Díaz, J. Gil and E. Sánchez, "Lower-Limb Robotic Rehabilitation: Literature Review and Challenges," J. Robotics, vol. 11, 11 pp., 2011. doi: $10.1155 / 2011 / 759764$.

[8] V. Pasqui, L. Saint-Bauzel and O. Sigaud, "Characterization of a Least Effort User-Centered Trajectory for Sit-to-Stand Assistance," In: Stephan G, L. Kovacs L. and A. Toth. IUTAM Symposium on Dynamics Modeling and Interaction Control in Virtual and Real Environments, Springer Netherlands, vol. 30, pp. 197-204, 2011. doi: 10.1007/978-94-007-1643-8 22.

[9] H. Guo, Z. Wang, "Design of Fuzzy Controller Used in Lower Limbs Rehabilitative Robot Drive System Based on Genetic Algorithm," Fuzzy Systems and Knowledge Discovery, Fourth International Conf., vol.4, pp. 168,171,24-27 Aug. 2007. doi: 10.1109/FSKD.2007.245.

[10] V. Pasqui, L. Saint-Bauzel, P. Rumeau, N. Vigouroux, "Proposed Generic Method to Assess Efficiency of Smart-Walkers" Lecture Notes in Computer Science, vol. 7251, pp. 218-221, 2012. doi: 10.1007/978-3-642-30779-9_29.

[11] S. Castro, E. Lugo, P. P. Cruz, and A. Molina, "Assistive Robotic Exoskeleton for Helping Limb Girdle Muscular Dystrophy," Int. Conf. on Electron. and Automot. Eng.(ICMEAE), Morelos, Mexico, pp. 27, 32, 19-22, Nov. 2013. doi: 10.1109/ICMEAE.2013.9.

[12] M. Giergiel, A. Budzinski, G. Piatek, M. Waclawski, "Personal Lower Limb Rehabilitation Robot for Childre," in Mechatronics - Ideas for Industrial Application, J. Awrejcewicz, R. Szewczyk, M. Trojnacki, and M. Kaliczynska. Springer International Publishing, 2015, pp. 169-176.

[13] M. Zhang and X.Li, "A Design of the Mechanism for Lower Limbs Passive Rehabilitation and Kinematics Analysis," 2010 Fifth Int. Conf. Frontier of Comp. Sci. and Tech. (FCST), Changchun, Jilin Province, pp.603,607, 18-22 Aug. 2010. doi: 10.1109/FCST.2010.94.

[14] S. Koenig et al., "Psychological state estimation from physiological recordings during robot-assisted gait rehabilitation," J. Rehab. Res. Dev., vol. 48, 367-386, 2011. doi: 10.1682/JRRD.2010.03.0044.

[15] Drive Medical, Four Wheel Walker Rollator with Fold Up Removable Back Support, 2017. [Online] Available: https://www.amazon.ca/Wheel-WalkerRollator-Removable-Support/dp/B005S1CHKC/, Accessed Apr 22, 2017.

[16] X.Han, "Development of a Mobility Assistive Device for Sit-to-Stand," M.Sc. thesis, Dept. Mech. Eng., Univ. Saskatchewan, Saskatoon, Canada, 2017.

[17] H. Braden, "Self-selected gait speed: A critical clinical outcome," 2012. [Online]. Available: http://lermagazine.com/article/self-selected-gait-speed-acritical-clinical-outcome. Accessed May 26, 2017.

[18] F. Heidari, M. Vakil and R. Fotouhi, "Sensor-based navigation of agricultural autonomous mobile robots," ASME Int. Design Engineering Technical Conf, Chicago, United States, Aug 12-15, 2012. DETC2012-71348. 


\section{Design and Rapid prototyping of Novel Ventricular Assist device}

\author{
Sayedali Mousavi \\ Department of Mechanical Engineering, Njafabad Branch, \\ Islamic Azad University, Najafabad, Iran
}

\begin{abstract}
According to statistics coronary heart disease is one of the most common causes of death in the world. Heart failure patients with these diseases are the most common one. External assisted Pumps help ventricular thrust can treat this condition. Novel designed pump were developed in this research due to the expensive production techniques prices by using rapid prototyping stereo-lithography .This device were fabricated and experimental flow parameters were tested by artificial blood .The obtained experimental results were compared with software results . Significant collaboration were obtained and the minor discrepancies in the results were indicated because of the lack of impeller surface finishing defects in stereo lithography. Future research could be conducted to evaluate the parameters with other rapid prototyping techniques such as Selective laser sintering.
\end{abstract}

Keywords- ventricular assist device, Pump, Rapid prototyping, Automatic

\section{INTRODUCTION}

According to statistics released by coronary heart disease and one of the most common causes of death in the world.[1] Congestive heart failure is one of the most common diseases with Sander recent decades due to the great need for these patients to a heart transplant and a shortage of hearts for transplantation, doctors and engineers together have tried using mechanical pumps; devices help ventricular for this disease and produce. [2,3] . Different pumps for ventricular assist thrust were developed in several researches and several fabrication methods were presented.[4,5,6]

These pumps were divided into different categories, one of the divisions based on fluid flow through the pump into two general types Axial and Centrifugal.[7] Axial pumps have more advantages in comparison to the pumps. Axial flow blood pumps due to the fluid flow during pumping will not redirect any the fluid were pumped in the direction of the axis ;it resulted that these blood pumps has with less damage than others.[8,9] Axial pumps are consist of four important part of the building is (Inducer, Impeller, diffuser, split Straightener) as it illustrated in figure 1 . It is an important principle in the design of pumps blood to be pumped with less noise and turbulence Because of turbulence in fluids such as blood can

\author{
Afshin .Enteshari \\ Department of Mechanical Engineering, Njafabad Branch, \\ Islamic Azad University, Najafabad,Iran
}

damage blood components that cause clotting of red blood cells in the blood.[10]

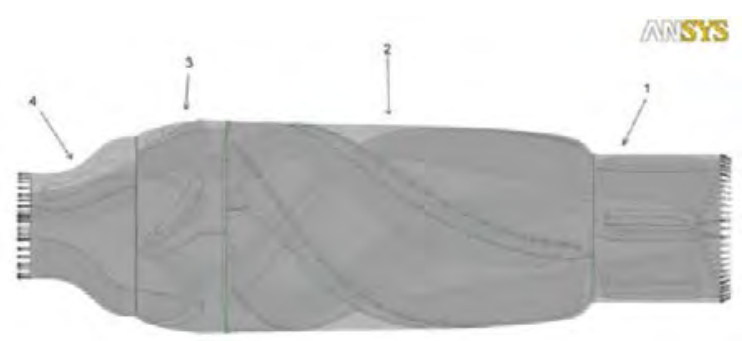

Figure 1: Main components of axial pumps.

Recently, by rapid development of CAD technology in detailed design $\mathrm{s}$, modification forms data in $3 \mathrm{D}$ through tranquil. As a result of this data in programming $\mathrm{CNC}$ and $\mathrm{RP}$ (rapid prototyping), by CAD / CAM, automatic tangible progress is remarkable. Increase the number of common parts and advanced design makes increasing use of CAD design is more complex levels. To reduce the time and costs involved as well as new developments in industrial production, rapid prototyping is known as a great way to prototyping.[11] The purpose of this paper is to present a method of rapid prototyping (RP) used in the development of a of ventricular assist pump. stereo lithography technology was used to create complex impeller blade profiles for testing as part of a this device to optimization fluid convection process.

\section{Methodology}

\section{Design and Construction of Vane Pump}

The Ventricular Assist pump and the environment by using software were designed. As mentioned, the pump is composed of four blades, each blade of the specific task they are doing at the pump. The four blades, three blades and other moving Impeller are fixed In order to reduce the time to design and simulate blades of any part of the blade is a blade design and simulation The output of the simulation and analysis for a complete blade. So when output from any part of a pre-existing 
ANSYS. The output file that can be acceptable for CATIA software for design and ANSYS for analytical analyzed experiments and the final output file is in IGS format. The output files are in the software CATIA as a shell First it should be map the output and then turned to the other parts and details should be added. Which these output would be used with ANSYS for experimental result simulation. (Figure 2 )

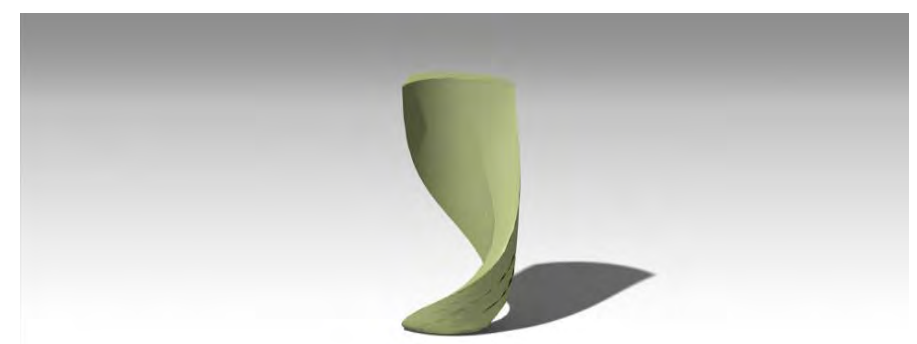

Figure 2: a blade of Impeller

IGS file output from part design were converted using ANSYS software from CATIA environment module, by using the multimedia section. The volume created for the following blade with 4 blades creates. Thus, we repeat the above steps for the other Vane Pump.(Figure 3)

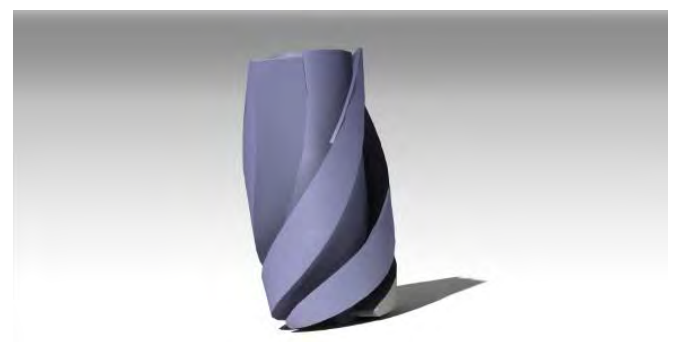

Figure 3: Impeller completed

\section{Design and construction of the pump casing}

After the turn of the pump casing is designed blades. Designed pump casing is designed according to the shape of the blades. As already mentioned, only with rotary vane pump is Impeller. The distance (clearance) Impeller the body of each side is $0.2 \mathrm{~mm}$. The following figure Impeller with preImpeller body inside it shows.

The body design Impeller a groove at both ends, for male and female for the purpose of making and assembling other parts of the body are embedded. In real examples of the different parts of the fuselage on both the threads and the $\mathrm{O}$ rings are used to seal off these parts. Due to the method of stereo lithography construction on the project it should be designed housing for threaded connections there. The possibility of making the thickness of less than $0.8 \mathrm{~mm}$ is not possible for us and because of the thickness and depth of less than 0.8 is threaded on the connection. In this project we have used male and female groove. After Impeller body design, body design Inducer we start. Inducer first blades from the inlet pump. The blades rotate and move not because any Within the body and is fixed for any clearance between the blades there Inducer body fluid passes only through the vane pump. In other words, in addition to covering the body Inducer duty blade holder also holds Inducer. (Figure 4)

In the actual case, the pump inlet in parallel with the axis of the blade and shift into the pumped fluid without any In the prototype system driving the pump electrically and fails Impeller pump rotor and a winding around the exterior Impeller, and by passing an electric current through the coil, rotor, which Impeller be induced and began to spin and causes the fluid motion forward.

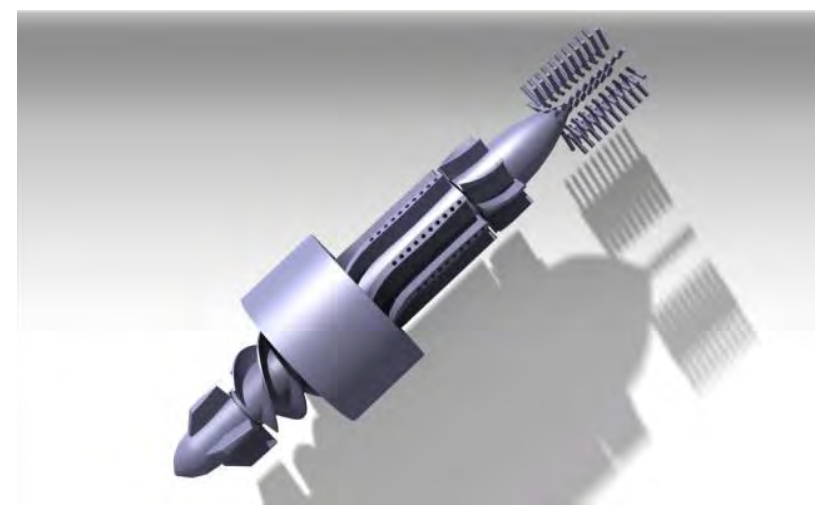

Figure 4: Body Inducer with pre Inducer inside

In these pumps because the steel blades of titanium and titanium as well as magnetism, no holes on the blades Impeller create and sticks very small magnetic inserted up to a coil around it could bars inspire and create spin in Impeller But the project of a power transmission systems outside the pumps have been used. In the following sections we will refer to it because of the design of the pump inlet to discuss the case of 90 degrees. After Inducer body design, body design diffusers have begun. Like Inducer a fixed diffuser blades and chassis in addition to covering the diffuser blades, it also has the duty holder. At the beginning and end of the groove body in order to get on Impeller body and the body is designed Straightener. The last part of the pump body, the body is Straightener. This body should also fully compatible with the external form Straightener blades because the body in addition to covering the blade, blade holder also plays a role. This part of the system, like other parts of the body to the groove of the male and female together. It is located in the pump outlet pipe. Outlet pipe to the inlet pipe diameter is the same.

\section{Transmission}

As mentioned in the previous sections due to laboratory use of the pump system for the external drive. This pump were installed with two mm shaft rotor diameter and the hole on the side of Inducer and pre Inducer and pre Impeller had crossed in front, and inside the bearings inside the body of the diffuser of the bearings packed and in the end Inducer body by bearings inside the bearing are sealed. Because the system driving the 
pump externally and by an axis of rotation of the pump to Impeller transmitted, so unlike real examples pump axial inlet hose pump with the pump shaft angle of 90 degrees to find which in turn caused a slight difference in pump efficiency compared to the simulated data. Compliance is centered inside the Impeller for the referendum. Sliding bearings used in the pump within Inducer body and diffuser are asking for compliance and the final fabrication were illustrated in figure5.

The modified impeller blade profiles, relative to the standard radial configuration, were evaluated with the use of computational fluid dynamics (CFD) and experimental testing. Rapid prototype impellers were needed for experimental validation and comparison of results with software (CFD) results.

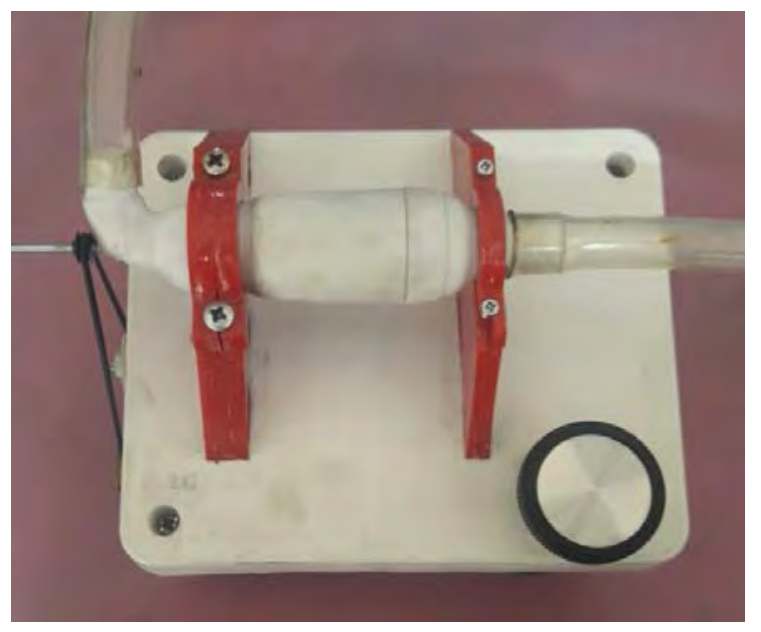

Figure 5: Sample Laboratory pumps

\section{RESULTS}

We have chosen. Then the pump inlet pipe placed inside the container. In the direction of the inlet pipe to connect a tap water tested. Pump outlet pipe into an empty container with a capacity of 6 liters made. the path is not output, the pumps are turned on In the direction of the outlet pipe pump, a pressure gauge and a pressure relief valve to measure and control the output pressure placed on the inlet port of the pump and the valve at the outlet is also open to any pressure The path is not output, First, we tested the pump with 4000rpm rotational speed, the speed water pump with flow rate of $3 \mathrm{~L} / \mathrm{min}$ and a pressure of $40 \mathrm{mmHg}$ the pump. Next, set the pump on a rotational speed of $6000 \mathrm{rpm}$ and we turn it on. The speed of the fluid pump is proceeding with a flow rate of 5 liters per minute and a pressure of $80 \mathrm{mmHg}$ pump. (figue.6) During testing the pressure reducing valve is slowly closed until pressure is $100 \mathrm{mmHg}$. The pump discharge pressure to $4.8 \mathrm{~L} \mathrm{/} \mathrm{min}$ reduced. In the final stage pump with ideal rotational speed that simulate the speed of $6250 \mathrm{rpm}$, the test was conducted At this rate observed at $100 \mathrm{ml}$ mercury pressure, pump flow is equal to 7.5 liters per minute.(table1).

Table 1: Results of testing pumps

\begin{tabular}{|l|l|l|l|} 
Experimen & output fluid & rotationa & (liters \\
\hline
\end{tabular}

\begin{tabular}{|c|c|c|c|}
\hline $\mathrm{t}$ & $\begin{array}{l}\text { pressure (mm } \\
\mathrm{Hg})\end{array}$ & $\begin{array}{l}1 \text { speed } \\
(\mathrm{rpm})\end{array}$ & per minute) \\
\hline $\begin{array}{c}\text { The first } \\
\text { stage }\end{array}$ & 04 & 0444 & 3 \\
\hline $\begin{array}{c}\text { The second } \\
\text { stage }\end{array}$ & 04 & 0444 & 5 \\
\hline $\begin{array}{c}\text { The third } \\
\text { stage } \\
\text { The fourth } \\
\text { stage }\end{array}$ & 044 & 0554 & $0 / 0$ \\
\hline
\end{tabular}
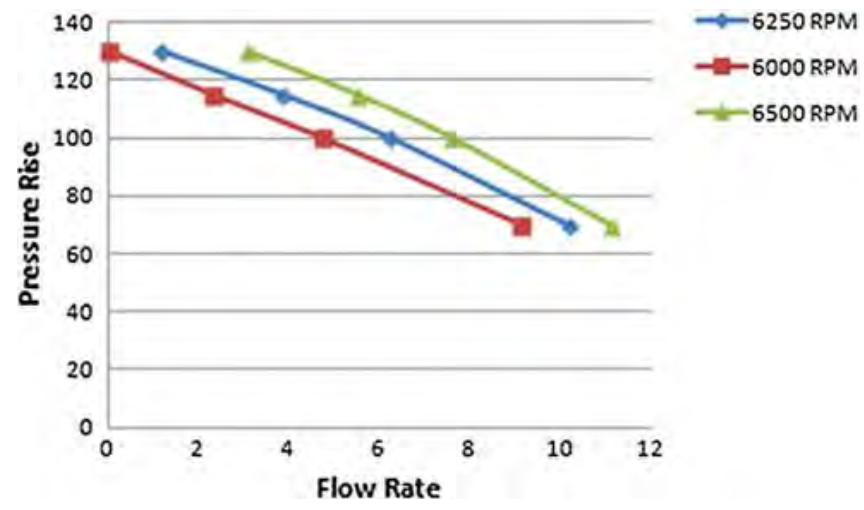

Figure 6: The results of the simulation software

\section{DISCUSSION AND CONCLUSION}

In fabrication of such a complex and sensitive products, the product development process should be done gradually. The product is designed, for example, then the simulation is done, then built and tested prototypes of the product were developed gradually. Actual samples of products were fabricated and the processes were repeated to justify test condition environment. After making quality assurance of product were obtained, the product mass-produced and used. The aim of the project is the construction of a ventricular assist pump test. This pump using actual environment was designed and simulated. In order to ensure the results of the simulation, we decided to build a prototype of the pump. All sizes according to the size of the pump is powered by software. After making the prototype pump were manufactured with rapid prototyping and the experiments were conducted to test it by flowing artificial blood.

The significant results were obtained with slight difference with comparison with the results of the simulation. The reasons for this minor difference between the results of the simulation results were analyzed. The ability to manufacture complex blade profiles that are robust enough for testing, in a rapid and cost effective manner is proving essential in the overall design optimization process for this device.

Finally, the main clues were obtained for the rapid prototyping method of making practices such as, low cost and suitability of accuracy of this method to manufacture prototype 
of the pump. Construction of the pump with other rapid prototyping methods such as FDM ,SLS or SLA would be next phase of this research, which at this stage is a real sample of the product will be produced by selective laser sintering with bio metal material such as tantalum..

\section{REFERENCES}

[1] M. Gibber et al., "In Vivo Experience of the Child-Size Pediatric Jarvik 2000 Heart: Update," ASAIO Journal, p. 1, May 2010.

doi: 10.1097/MAT.0b013e3181dbe55e.

[2] S. M. Patel, A. L. Throckmorton, A. Untaroiu, P. E. Allaire, H. G. Wood, and D. B. Olsen, "The Status of Failure and Reliability Testing of Artificial Blood Pumps," ASAIO Journal, vol. 51, no. 4, pp. 440-451, Jul. 2005. doi: 10.1097/01.mat.0000169083.90253.3c.

[3] M. S. Slaughter et al., "Transapical miniaturized ventricular assist device: Design and initial testing," J. Thorac. Cardiovasc. Surg., vol. 142, no. 3, pp. 668-674, Sep. 2011. doi: 10.1016/j.jtcvs.2011.01.011.

[4] M J. Yazdi and R. K. Moghaddam, "Improvement of Left Ventricular Assist Device (LVAD) in Artificial Heart Using Particle Swarm Optimization," J. Arti. Intell. Electr. Eng., vol 1, no. 4, March 2013.

[5] M. Koochaki and H. Niroomand-Oscuii, "A new design and computational fluid dynamics study of an implantable axial blood pump," Australas. Phys. Eng. Sci. Med., vol. 36, no. 4, pp. 417-422, Nov. 2013. doi: 10.1007/s13246-013-0225-x.

[6] D. J. Goldstein, M. C. Oz, and E. A. Rose, "Implantable Left Ventricular Assist Devices," N. Engl. J. Med., vol. 339, no. 21, pp. 1522-1533, Nov. 1998. doi: 10.1056/NEJM199811193392107.

[7] T. Lieneke, V. Denzer, G. A. O. Adam, and D. Zimmer, "Dimensional Tolerances for Additive Manufacturing: Experimental Investigation for Fused Deposition Modeling," Procedia CIRP, vol. 43, pp. 286-291, 2016. doi: 10.1016/j.procir.2016.02.361.

[8] E. A. Rose et al., "Long-Term Use of a Left Ventricular Assist Device for End-Stage Heart Failure," N. Engl. J. Med., vol. 345, no. 20, pp. 14351443, Nov. 2001. doi: 10.1056/NEJMoa012175.

[9] X. Song, H. G. Wood, and D. Olsen, "Computational Fluid Dynamics (CFD) Study of the 4th Generation Prototype of a Continuous Flow Ventricular Assist Device (VAD)," J. Biomech. Eng., vol. 126, no. 2, p. 180, doi: 2004.10.1115/1.1688776.

[10] S. G. Chopski, "Experimental and Computational Assessment of Mechanical Circulatory Assistance of a Patient-Specific Fontan Vessel Configuration," Dissertation, Ph.D., Virginia Commonwealth University Richmond, Virginia October 2013 .

[11] M. Foroghi, S. Mosuavi, Rapid prototyping technology with medical science approach, Jahad University book, Iran 2016. 


\section{The Ergonomics of Syringe Operation During the Injection of Fluid Into Tissue Expanders}

\author{
Sarah A. Horton, Marc Doumit \\ Department of Mechanical Engineering \\ University of Ottawa \\ Ottawa, ON, Canada \\ marc.doumit@uottawa.ca
}

\author{
Gloria Rockwell \\ Division of Plastic Surgery Ottawa Hospital General \\ University of Ottawa \\ Ottawa, ON, Canada \\ office@plasticsurgerymd.ca
}

\begin{abstract}
The reconstruction process following a mastectomy procedure may require the use of a tissue expander(s) to accommodate an implant. Over several months, the expanders are routinely filled with sterile saline solution to gradually stretch the skin and muscle around the subpectoral pocket. The saline solution is injected manually by the surgeon or nurse into the expander using a syringe, catheter and needle. The injection process is physically demanding and repetitive; it has been reported to pose negative long-term effects on the muscles and joints of the hand, causing repetitive strain injuries for the syringe operator. Thus, the purpose of this study is to analyze the injection process and provide a comprehensive understanding of the factors that can be intervened upon to make this process more ergonomic and safe. To achieve this understanding, a laboratory testing setup is developed to mechanically simulate and analyze the fluid injection process into tissue expanders. Experimental results show that the magnitude of the syringe force required to inject the fluid is significantly correlated to the rate of compression, the size of the syringe and the resistance produced by the stretching of tissue expander. Moreover, the magnitude of forces measured during testing are found to be well above the recommended values to prevent repetitive stress injuries.
\end{abstract}

Keywords- Breast Tissue Expander, Breast Implant, Biomechanics, Ergonomics, Mastectomy.

\section{INTRODUCTION}

The mastectomy procedure is a common prophylactic management for breast cancer, wherein some or all the breast tissue and overlying skin is removed. The breast implant involves the insertion of a synthetic sac-like prosthesis under the skin and muscle structures of the chest wall to mimic the natural breast mound. In this process, a tissue expander can be utilized prior to implant insertion to reduce discomfort and maximize the aesthetic outcome. The expander is a flexible shell that is inserted into the subpectoral pocket immediately postmastectomy or later. It is gradually injected with a saline solution, which increases the volume of the expander shell to create a breast mound, and consequently stretch the involved skin and muscle tissue. Saline solution is introduced through the skin of the patient into the expander through a syringe, catheter and needle, via a fill port located within the expander [1]. Figure 1 shows these elements.

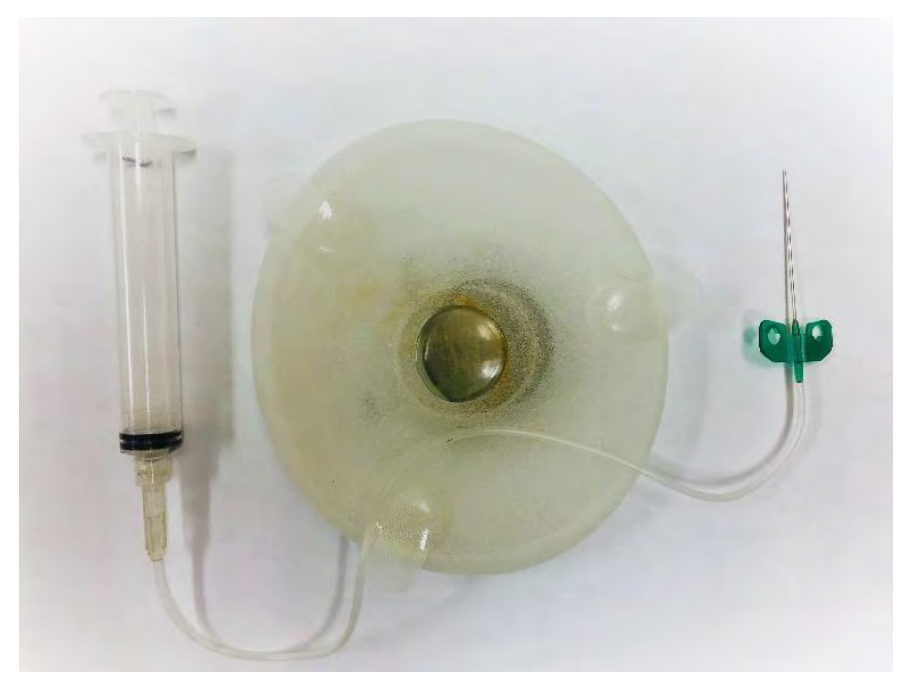

Figure 1: Syringe, catheter, needle and breast implant expander

Repetitive hand strain injuries are an occupational hazard that can cause long-term disability and impact quality of life of operators. Actions such as applying force to a syringe plunger for a prolonged period when filling a tissue expander can cause joint pain and even cause cumulative trauma disorders, such as carpal tunnel syndrome [2]. It is believed that the trauma is inflicted by repetitive tension in the flexor tendons of the hand, which over time lead to inflammation and compression of the median nerve within the carpal tunnel [3].

The force applied on a syringe by the operator resembles a chuck pinch, or a pinch implicating the thumb, the second and third digits. A study conducted on eight female and eight male participants found that a thumb in hyperextended position can achieve a maximum pinch force of $49.49 \mathrm{~N}$ and $73.7 \mathrm{~N}$, respectively, when the wrist was in a neutral position [4-5]. However, pinch strength appears to decrease significantly, as the wrist is flexed as well as with the use of the non-dominant hand 
[6-7]. Moreover, if the thumb is not hyperextended, the resulting force is inferior and pinch force is inversely proportional to the pinch width [8]. Other studies also reported that the maximum acceptable force for tasks completed two times per week at a rate of two repetitions per minute involving repetitive flexion pinch grips is $6.35 \mathrm{~N}$ [9], which is considerably less than the maximum pinch force abilities of the human hand. This is comparable to the frequency that a surgeon or nurse operates a syringe during the expansion procedure on a weekly basis.

Through the literature review, no studies have analyzed the mechanical behaviour of the injection process for tissue expanders and its human factor implications. Consequently, it is unclear to what extent different factors germane to the expander expansion process can influence comfort and ergonomics for the operator. Thus, this study aims to achieve a comprehensive understanding of this mechanical procedure and determine the optimal ergonomic method for filling the breast tissue expanders.

\section{METHOD}

In this study, the mechanical behavior of tissue expanders during the expansion process is experimentally characterized using an Instron ${ }^{\mathrm{TM}}$ universal testing machine. The magnitude of the syringe force required to inject the fluid into the expander is analyzed while altering key variables. Five variables that are believed to have an impact on the syringe injection mechanism and its ergonomics are selected and presented as follow:

\section{Syringe size:}

Different operators use distinct syringe sizes to inject fluid. A varying syringe size corresponds to a changing plunger surface area and thus a change in the force required to create the same fluid pressure. In this study, three common syringe sizes are tested to evaluate their impact: 10,20 and $60 \mathrm{~mL}$.

\section{Syringe compression speed:}

The operator can only inject the fluid at a limited speed before feeling a substantial syringe force resistance. It is believed that the injection speed affects the plunger dynamics, fluid resistance through the needle and catheter as well as the resistance caused by the mechanical properties of the tissue expander. For this variable, syringe compression speeds of 20 , 25 and $30 \mathrm{~mm} / \mathrm{min}$ are selected and tested.

\section{Expander size:}

The size of the tissue expander varies between patients such to adapt to their needs. A different expander size equates to a different volume and surface and thus alters the resistive behavior of the tissue expander especially when inserted in the subpectoral pocket. For these tests, expanders from Naturelle [10] in common volume sizes of 250, 300, 400, 500, 600, 700, 750 and $850 \mathrm{~mL}$ are selected and tested.

\section{Expander resistance:}

The skin and muscle of the patient creates a resistance against the expansion process. The pressure applied to the tissue expander is hypothesized to restrict the flow of fluid coming from the syringe, thus increasing the required syringe force applied by the operator. Two constant weights of 1 and $2 \mathrm{lbs}$ are placed on the tissue expanders while testing to simulate constant skin resistance load.

\section{Injected fluid volume:}

A limited amount of fluid may be injected per visit to minimize discomfort. Expanders with larger volumes tend to have a larger diameter, which may cause an unsymmetrical or incorrect breast shape for patients with smaller chest widths. In these cases, smaller expanders with the correct diameter are overfilled (filling the expander beyond the recommended fluid amount) to further stretch the skin and give a more natural appearance. Consequently, this process amplifies the effort required to inject the saline solution and leads to hand fatigue and discomfort. This scenario is mechanically simulated to analyze the extent of the impact of filling the expander past the rated volume.

To achieve the tests and evaluate the impact of the five selected variables, an experimental apparatus is developed in laboratory to facilitate standardized measurements of the tissue expanders expansion mechanism. As shown in Figure 2, a supporting jig is fabricated using a PVC tube with three openings: one at the top to observe the amount of liquid dispensed, and two at opposite sides of the tube's base to facilitate access to the expanders, catheter and needle. A plexiglass circle with a centered hole was attached to the top of the tube using mechanical fasteners to accommodate the placement of the syringes. The expanders were elevated to help keep the catheter straight and minimize additional resistance.

All data is collected using an Instron ${ }^{\mathrm{TM}}$ universal testing machine with a $1 \mathrm{kN}$ load cell with an accuracy of $+/-0.25 \%$. Tap water is used as a substitute to saline fluid for simplicity.

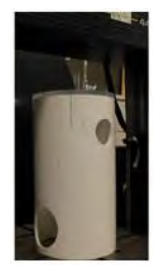

a)

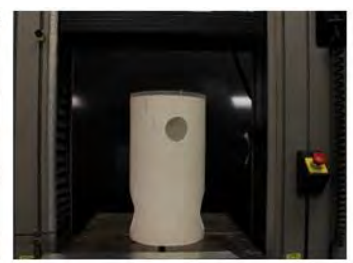

b)

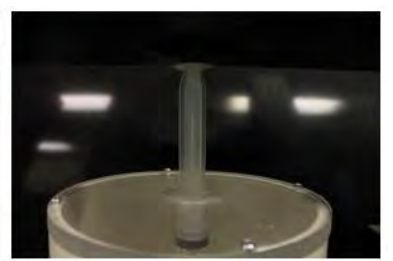

c)
Figure 2: (a) Front view b) Side view c) Top view of the plastic tube (with $60 \mathrm{~mL}$ syringe)

\section{RESULTS}

The introduction of the five selected variables throughout testing had shown a substantial impact on the syringe force required to inject the fluid into the tissue expanders. A first test is conducted, without the use of a tissue expander where the fluid is simply injected into an open container. This is achieved to determine the mechanical behaviour attributed to the syringe plunger, catheter and needle mechanisms. The data from this test is presented in Figure 3. This graph also shows a general profile of the curve found in all the achieved tests. The curve displays the transient and steady state response of the syringe force (yaxis, load) when the plunger is moved by the Instron machine with a uniform displacement speed. For clarity and simplicity, only the average of the syringe force will be presented as a bar plot for all subsequent presented results. 


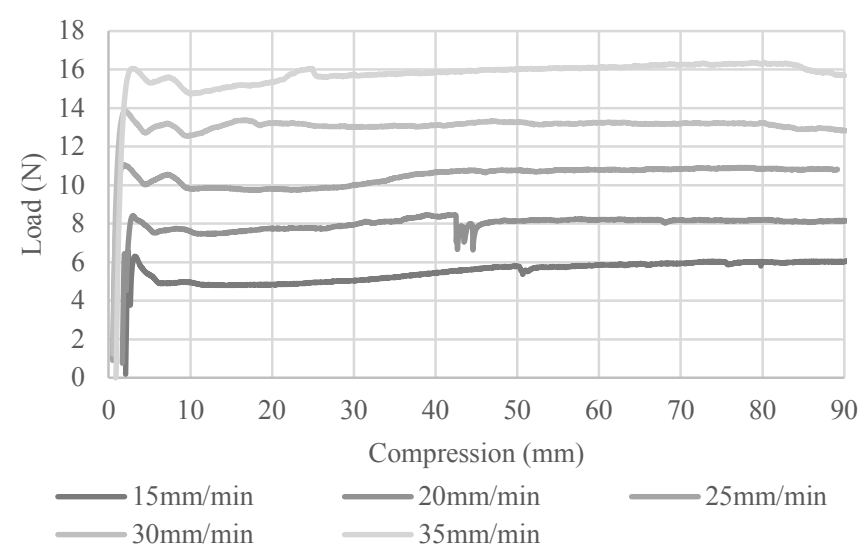

Figure 3: Compressive syringe force (Load) vs compression for a $60 \mathrm{~mL}$ syringe subjected to compression rates of $15,20,25,30$ and $35 \mathrm{~mm} / \mathrm{min}$

Preliminary experimental tests showed that the results are relatively consistent as the tissue expander is progressively filled until it reaches its recommended volume (without being overfilled). Thus, the following results and analysis will only consider the overfill scenarios such to demonstrate the effects of this practice on the required syringe force.

\section{Syringe size and syringe compression speed rate}

Figure 4 shows the results for the average compression force for three distinct syringe sizes $(10,20$ and $60 \mathrm{~mL})$ while varying the compression speed rate $(20,25$ and $30 \mathrm{~mm} / \mathrm{min})$. The test is achieved using a $250 \mathrm{~mL}$ expander and being overfilled with an additional volume of $60 \mathrm{~mL}$.

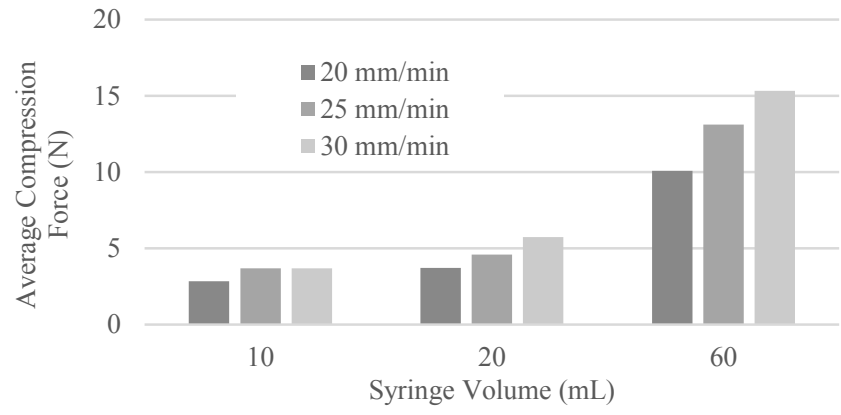

Figure 4: Average compression force of various syringe sizes subjected to a varying compression rate using a $250 \mathrm{~mL}$ expander

\section{Expander size}

Figure 5 shows the results for the average compression force for eight distinct tissue expanders $(250,300,400,500,600,700$, $750,850 \mathrm{~mL}$. The tests are conducted at a speed of $25 \mathrm{~mm} / \mathrm{min}$ while being overfilled with an additional volume of $60 \mathrm{~mL}$ using a $60 \mathrm{~mL}$ syringe.

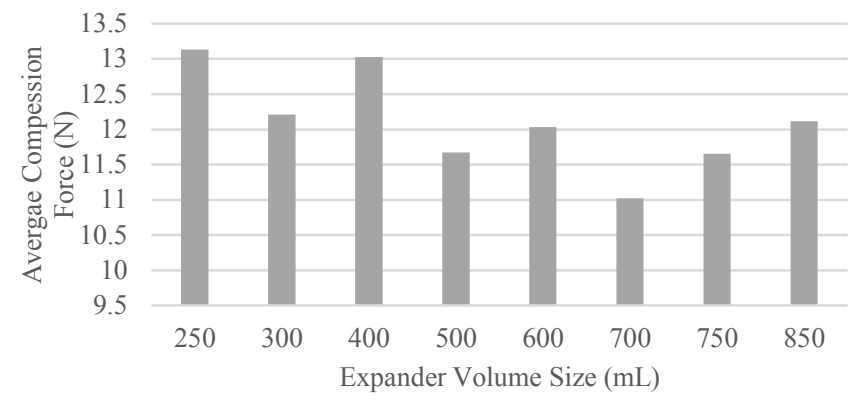

Figure 5: Average compression force for a $60 \mathrm{~mL}$ syringe subjected to various expander sizes at a compression rate of $25 \mathrm{~mm} / \mathrm{min}$

\section{Expander resistance}

Figure 6 shows the results for the average compression force for three distinct resistance weights (1,2 and $3 \mathrm{lbs}$.). The tests are conducted at a speed of $25 \mathrm{~mm} / \mathrm{min}$ while being overfilled with an additional volume of $60 \mathrm{~mL}$ using a $60 \mathrm{~mL}$ syringe.

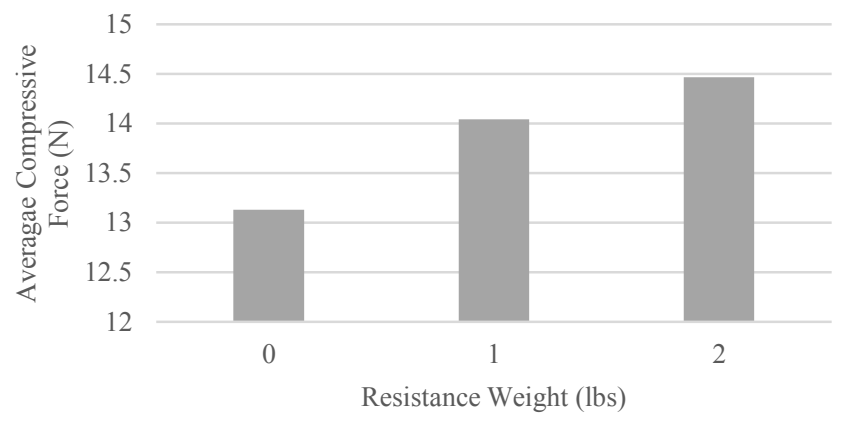

Figure 6: Average compression force for a $250 \mathrm{~mL}$ expander subjected to various exterior resistance with a compression rate of $25 \mathrm{~mm} / \mathrm{min}$ using a 60 $\mathrm{mL}$ syringe

\section{Injected fluid volume}

Figure 7 shows the average compression force for the seven sequences that are required to fill a $250 \mathrm{~mL}$ expander using a 60 $\mathrm{mL}$ syringe at a speed rate of $25 \mathrm{~mm} / \mathrm{min}$.

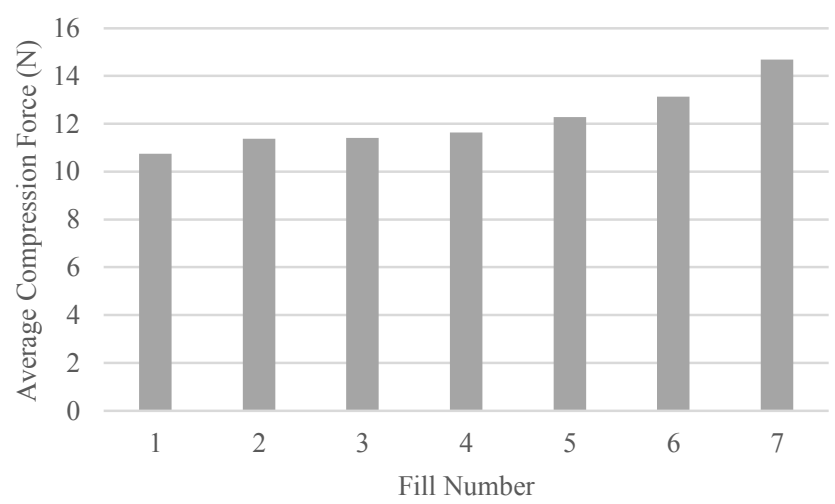

Figure 7: Average compression force of the seven sequences required to fill a $250 \mathrm{~mL}$ expander with a $60 \mathrm{~mL}$ syringe at a compression rate of $25 \mathrm{~mm} / \mathrm{min}$

\section{DISCUSSION}

The first results presented in Figure 3 display the transient and steady state response of the syringe force upon the Instron 
machine moving the syringe plunger with a uniform compression speed. Depending on the speed, the measured force varied between 5 and $16 \mathrm{~N}$ for a speed range of 15 to $35 \mathrm{~mm} / \mathrm{min}$. These represent significant force magnitudes given the absence of the tissue expander and patient skin resistance properties in these tests. Moreover, these results also demonstrate the viscoelastic behaviour of the syringe, catheter and needle mechanism.

\section{Syringe size}

The impact of syringe size on the compression force is demonstrated in Figure 4. It has been shown that even while varying the speed of compression, the magnitude of force measured remains proportional to the syringe size. Relatively low force magnitudes of $5 \mathrm{~N}$ or less were measured for the syringes of 10 and $20 \mathrm{~mL}$ while large force magnitudes of about $15 \mathrm{~N}$ were measured for the $60 \mathrm{~mL}$ syringe. Based on these results, a smaller syringe size would be ideal to achieve smooth and small syringe forces during the injection process. However, a smaller syringe size may be less desirable since it prolongs the expansion process and consequently the overall surgical procedure.

\section{Syringe compression speed}

Compression speed refers to the rate at which the syringe plunger is compressed $(\mathrm{mm} / \mathrm{min})$. Figure 4 shows how the compression speed affects the required compressive force while the rate varies from 20 to 25 to $30 \mathrm{~mm} / \mathrm{min}$. On average, a 5 $\mathrm{mm} / \mathrm{min}$ increase in speeds equates to a force increase of $2.65 \mathrm{~N}$ without an expander, and $2.54 \mathrm{~N}$ with an expander. The results demonstrate high viscoelastic behaviour and such when the compressive speed is increased; the compression force is also increased. Therefore, the use of a high compressive force to drive the syringe faster is counterproductive as intuitively experienced by operators. A slower and consistent syringe compression speed results in a smaller compression force and a safer process for the operator.

\section{Expander size}

Figure 5 shows the compression force while filling various expander sizes $(250,300,400,500,600,700,750,850 \mathrm{~mL})$. Given the absence of the skin resistance properties, there is no clear correlation between the compression force and the expander size. However, the two largest forces were found when filling the smaller expanders $(13.13 \mathrm{~N}$ for the $250 \mathrm{~mL}$ expander and $13.03 \mathrm{~N}$ for the $400 \mathrm{~mL}$ expander). Some limitations of the study may provide an explanation for this phenomenon.

\section{Expander resistance}

As seen in Figure 6, an increase in the external resistance simulated by the addition of the free weights (1 and $2 \mathrm{lbs}$.) resulted in an increase in the magnitude of the compression force. The addition of the $1 \mathrm{lbs}$. weight increased the average compression force by $0.91 \mathrm{~N}$, whereas the $2 \mathrm{lbs}$. weight caused an increase of $1.34 \mathrm{lbs}$. Similar but amplified resistance is expected by the skin and over-lying tissues given its nonuniform and increasing stiffness properties throughout deformation. The procedure may be more strenuous on patients with lower tissue elasticities (i.e. patients that have undergone radiation).

\section{Injected fluid volume}

Figure 7 shows data spanning the entire process of filling one $250 \mathrm{~mL}$ expander with a $60 \mathrm{~mL}$ syringe, at a compression rate of $25 \mathrm{~mm} / \mathrm{min}$. Data is divided into seven sequences, wherein 50 $\mathrm{mL}$ of saline was injected per sequence. The first five sequences represented complete filling of a $250 \mathrm{~mL}$ expander, whereas the last two sequences recorded the effects of overfilling the expander. The compression force required to push the syringe plunger remains relatively the same as the expander is being filled, with a small gradual increase of $0.39 \mathrm{~N}$ on average. However, the force tends to increase more rapidly as it nears full capacity and when the expander is being overfilled. The force increases by $0.85 \mathrm{~N}$ between the $5^{\text {th }}$ and $6^{\text {th }}$ trials, and then by $1.55 \mathrm{~N}$ between the $6^{\text {th }}$ and $7^{\text {th }}$ trails.

\section{Limitations}

The substitution of tap water for saline may have affected the magnitude of the measured forces since saline solution is denser than tap water and would therefore require larger force. However, the magnitude of the force difference is considered negligible.

Instability of the weights on the expander during the experiment may have caused inconsistencies during data acquisition. The variation in the placement of the weights could also have an impact on the magnitude of the compression force.

The absence of a correlation between the compression force and the expander size during overfill could be explained by the selected overfill amount. The overfill volume was chosen to be a constant $100 \mathrm{~mL}$ for all the expanders. This means that during the overfill phase of the experiment, a $250 \mathrm{~mL}$ expander was overfilled by $40 \%$ whereas a $500 \mathrm{~mL}$ expander was only overfilled by $20 \%$. Therefore, the smaller the expander, the higher the percentage of overfill. Since a higher percentage of overfill creates more pressure in the expander, it increases the magnitude of the compression force (as seen in the high force observed for the $250 \mathrm{~mL}$ and $400 \mathrm{~mL}$ expander in Figure. 5).

\section{CONCLUSION}

The measured magnitudes of force required to fill a breast tissue expander in a mechanical setup were mostly above the recommended value for preventing repetitive stress injuries given in [9]. The data showed quantifiable trends among most of the selected tested variables. It was observed that a slower compression speed required a smaller compressive force and was a more ergonomic alternative. The magnitude of the compressive force also decreased when the size of the syringe decreased. Therefore, a smaller syringe is more ergonomic as opposed to a larger one. An array of expander sizes was tested; however, the size of the expander did not display any definite effect on the magnitude of the compressive force. External resistance was also applied to the expander using free weights to mimic the resistance applied on the expander by the skin in a real case scenario. As the magnitude of the external resistance was increased, the magnitude of the compressive force also increased. Incorporation of the observed principles from this study (i.e. smaller syringe sizes and a slower rate of compression) into clinical practice may influence the incidence of repetitive strain injuries for surgeons and nursing staff who participate in tissue expansion on a routine basis. Other factors 
such as the use of a larger needle could facilitate the task but may cause additional discomfort to the patient. Further clinical research is needed to determine the significance of these adjustments.

\section{REFERENCES}

[1] S. Ahmed, A. Snelling, M. Bains, and I. H. Whitworth,"Breast reconstruction," BMJ, vol. 330, no. 7497, pp. 943-948, Apr. 2005. doi: $10.1136 / \mathrm{bmj} .330 .7497 .943$.

[2] V. Putz-Anderson, Cumulative Trauma Disorders. A Manual for Musculoskeletal Disorders of the Upper Extremity. London: Taylor \& Francis, 1988.

[3] E. M. Smith, D. A. Sonstergard, and W. H. Anderson, "Carpal tunnel syndrome: contribution of flexor tendons.," Arch. Phys. Med. Rehabil., vol. 58, no. 9, pp. 379-385, Sep. 1977.

[4] P. G. Dempsey and M. M. Ayoub, "The influence of gender, grasp type, pinch width and wrist position on sustained pinch strength - ScienceDirect," Science Direct, Mar-1996. [Online]. Available: http://www.sciencedirect.com/ science/article/pii/0169814194001081. Accessed: 14-Jul-2017.

[5] R. Terrell and J. L. Purswell, "The Influence of Forearm and Wrist Orientation on Static Grip Strength as a Design Criterion for Hand Tools," Proc. Hum. Factors Soc. Annu. Meet., vol. 20, no. 1, pp. 28-32, Jul. 1976. doi: $10.1177 / 154193127602000115$.

[6] J. E. Fernandez, J. B. Dahaian, C. A. Halpern, and V. Viswanath, "The effect of wrist posture on pinch strength," Proc Hum. Factors Soc. 35th Annu. Meet. St. Monica CA, pp. 748-752, 1991.

[7] A. Swanson B., I. B. Matev, and G. De Goot, "The strength of the hand," Bull Prosthet, no. Res 10.14, pp. 145-153, 1970.

[8] S. N. Imrhan and R. Rahman, "The effects of pinch width on pinch strengths of adult males using realistic pinch-handle coupling," Int. J. Ind. Ergon., vol. 16, no. 2, pp. 123-134, Aug. 1995.

doi: 10.1016/0169-8141(94)00090-P

[9] S. H. Snook, D. R. Vaillancourt, V. M. Cirello, and B. S. Webster, "Psychophysical studies of repetitive wrist flexion and extension," Ergonomics, vol. 38, no. 7, pp. 1488-1507, Jul. 1995.

doi: $10.1080 / 00140139508925204$

[10] Natrellesurgeon.com, '133 Shaped Tissue Expanders', 2018. [Online]. Available: https://www.natrellesurgeon.com/shaped-tissue-expanders/133. Accessed 14-Feb-2018 


\section{Analysis of a Low-cost Sensor Towards an EMG-based Robotic Exoskeleton Controller}

\author{
Georgeta Bauer, Ya-Jun Pan \\ Department of Mechanical Engineering \\ Dalhousie University \\ Halifax, Nova Scotia, Canada \\ georgeta.bauer@dal.ca,yajun.pan@dal.ca
}

\author{
Robert Adamson \\ School of Biomedical Engineering \\ Dalhousie University \\ Halifax, Nova Scotia, Canada \\ rob.adamson@dal.ca
}

\begin{abstract}
This paper describes the evaluation of the MyoWare Electromyographic (EMG) sensor performance during a typical end-use application to help determine if it could be used for an EMG-based controller of an upper-limb robotic exoskeleton. Tests were conducted to study the signalto-noise ratio (SNR) and a series of experiments were performed to determine the sensor's capability of capturing key EMG signal features while a subject performed bicep curls. LabVIEW was used for data collection and processing, and Matlab was used for statistical analysis. The results revealed that the SNR was between $10 \mathrm{~dB}$ and $33 \mathrm{~dB}$ for the average peak root mean square (RMS) EMG, and between $1 \mathrm{~dB}$ and $27 \mathrm{~dB}$ for the average voluntary contraction (AVC) EMG which - except for one case - were all above the acceptable level in the field. The validation of the sensor performance showed a correlation consistent with literature between the force exerted and the RMS EMG signal under both dynamic and static loading. These initial results indicate that the MyoWare EMG sensor could be used in a more advanced robotic exoskeleton EMG-based controller beyond its current popular use as an EMG-level threshold-based ON/OFF switch.
\end{abstract}

Keywords- electromyography, EMG, muscle, myosignals, upper limb, robotic exoskeleton.

\section{INTRODUCTION}

With the expected surge in the global aging population and the inevitable motor impairment in the elderly, it is anticipated that the already overloaded medical workforce will not be able to keep up with the onset of anticipated rehabilitation demands [1]. To address this issue, the field of Robotic Exoskeletons is growing rapidly, though most of the existing systems are at the research level or are too cost-prohibitive to be widely available. It is pertinent for the technology to be accessible now to educate future researchers and developers in the field. Consequently, Beyond Robotics GmbH released the EduExo Robotic Exoskeleton kit in October of 2017. The kit contains all the major subsystems relevant to a Robotic Exoskeleton including an Electromyography (EMG) sensor - the MyoWare EMG sensor (AT-04-001) from Advancer Technologies. This sensor reads a wearer's EMG signals which are indicative of muscle activation levels [2]. The controller implemented with this kit activates the exoskeleton elbow joint motor when the measured EMG signal surpasses a set threshold value. In this paper the present authors investigate the performance of the sensor in an application to determine the possibility of using it in a future-build robotic exoskeleton controller to, for example, provide proportional control of the motors to assist the user in achieving the desired motion. A brief background is provided on EMG controllers to justify why studying the sensor's SNR and its degree of linearity are important for the intended application. Factors which influence the fidelity of the signal will also be discussed, followed by a description of the sensor specifications. The experimental setup and procedure will then be presented, the results will be shown and discussed, after which conclusions will be drawn.

\section{BACKGROUND}

There are numerous factors that can influence the quality and amplitude of EMG measurements. On the apparatus side, De Luca [3] states that the "main issue of concern that influences the fidelity of the [EMG] signal is the signal to noise ratio." Additionally, the design of the electrodes, the distance between them, and the placement and the orientation of them along the muscle affect signal fidelity. Furthermore, the quality of the contact between the electrodes and the skin, the wire length to the amplifier, the characteristics between the electrodes [4], the amplifier unit, and the signal processing methods are also important. There are many factors which can influence the EMG signal on the subject side as well, such as the conditions of the muscles. For example, EMG measurements can look very different depending on whether or not a muscle has been warmed up or fatigued. Other factors that could affect the EMG levels are age, sex, quality of the skin, body mass index (BMI) level (which is indicative of the thickness of the fat layer over the muscle), and quality and health of the muscle. EMG readings can also vary from day to day [5] even if the subject is performing the same tests as on the previous day. It is also to be noted that the subject's psychophysiological factors, such as emotions, play a factor in the produced levels of the EMG signal [6]. 
Numerous existing Robotic Exoskeleton Systems [7,8,9] use these EMG signals as the primary command signal to robot controllers. The EMG signal is typically captured using electrodes on the body that are connected to commercial amplifiers, such as those from Biopack Systems [10]. These controllers incorporate muscle models (myoprocessors) which rely on a correlation between the EMG signal amplitude and dynamic or static exerted force. This information can enable either position control or torque control of the joints with the goal of providing seamless assist-as-needed support to the user. An example of an EMG high-level position controller is shown in Fig. 1 where an EMG sensor is used to determine the desired position of a joint. It is, therefore, critical to identify the correlation between the EMG signal under different loads. Perry [7] points out that a great advantage of EMG controllers is that, due to the "electromechanical delay in the human neuromusculoskeletal physiology, the system can predict the operator's intention". The EMG signal changes in magnitude $20-80 \mathrm{~ms}$ before the actual contraction of the muscle [11]. As a result, the controller can provide smooth motion coordination between the user and the exoskeleton [7] before the muscles have even begun to move.

An EMG controller "needs to provide the correct amount of support to the user with correct timing" [12]. Control systems rely on sensor feedback updates - sometimes hundreds of times per second in motor control applications. When discussing applications that depend on EMG signals, De Luca [2] emphasizes that the EMG signal should be detected and recorded with maximum fidelity as it is an indicator of the initiation of muscle activation and it has a relationship to the force produced by the muscle. If the signal is noisy and not properly filtered, the resulting exoskeleton motion could be unstable and unsafe. High EMG signal SNR is thus crucial.

The question at hand is: Is the MyoWare EMG sensor able to collect EMG data that is high-fidelity and relatable to the amount of effort exerted by the user so that it could be used in a future-build Robotic Exoskeleton EMG controller for more sophisticated control strategies than just ON/OFF?

\section{EMG SENSOR AND SYSTEM DESCRIPTION}

Table I summarizes the MyoWare EMG sensor characteristics that will be studied in this paper, along with what they indicate, their relevance, how they will be characterized, and the location from where they are calculated as shown on a sample EMG signal (Fig. 2).
The MyoWare EMG sensor, shown in Fig. 3, measures electrical signals which are detected on the skin just before and during muscle contraction. These signals are usually on the order of $\mu$ Volts to low mVolts [5] and need to be amplified so that they can be digitized, recorded, analyzed and utilized. The MyoWare EMG sensor is an all-in-one device which amplifies, rectifies and filters the raw EMG signal, and provides the RMS EMG envelope as an output with an amplitude between 0 and the supply voltage $(2.9-5.7 \mathrm{~V})$. Some of the advantages of the sensor are that it is inexpensive, the raw signal is also available, and the electrode connectors, which are placed $3 \mathrm{~cm}$ apart, are embedded on the sensor board to minimize noise.

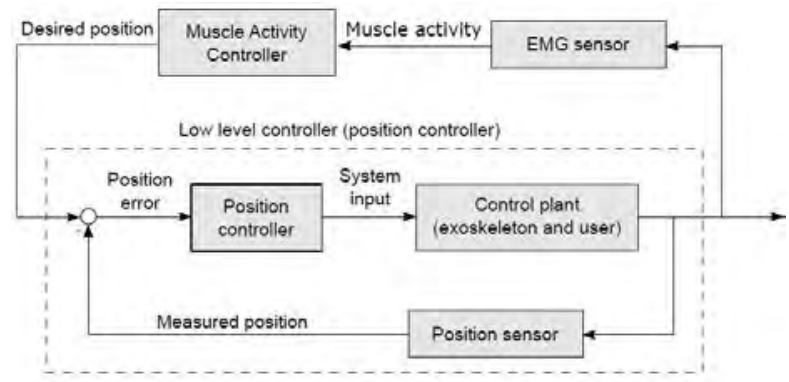

Fig. 1 High-level EMG-Controller implemented on top of the Low-level Position Controller (used with permission from [12])

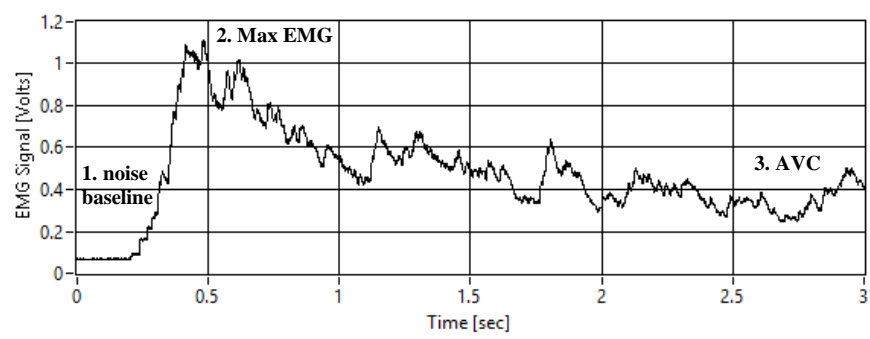

Fig. 2 Sample EMG Signal while subject curled a $5 \mathrm{lbs}$ weight

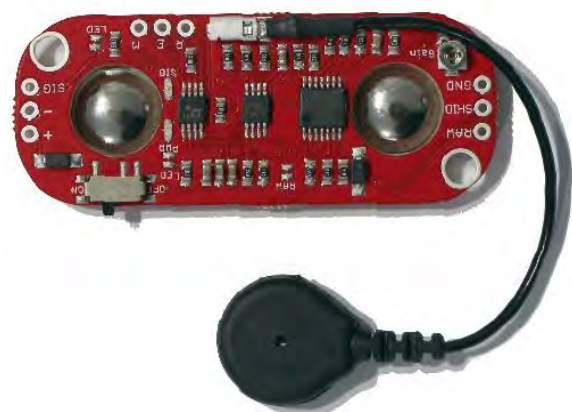

Fig. 3 The MyoWare EMG Muscle Sensor (shown at full scale)

TABLE I

SENSOR Behaviour Characteristics Pertinent to EMG CONTROLlers

\begin{tabular}{|c|c|c|c|c|}
\hline $\begin{array}{l}\text { Characteristic to } \\
\text { be measured }\end{array}$ & Indicator & $\begin{array}{c}\text { Relevance to Robotic Exoskeleton EMG } \\
\text { Controllers }\end{array}$ & Characterization & Location in Fig. 2 \\
\hline SNR & $\begin{array}{l}\text { Fidelity of the } \\
\text { signal }\end{array}$ & $\begin{array}{l}\text { High-fidelity data is crucial for timely and } \\
\text { appropriate motor commands }\end{array}$ & $\begin{array}{l}\text { Compare the EMG signal amplitude } \\
\text { between rest and Max EMG signal, } \\
\text { and rest and static loading EMG signal }\end{array}$ & $\begin{array}{l}1 \text { vs } 2 \text { (dyn. loading) } \\
\text { and } 1 \text { vs } 3 \text { (static } \\
\text { loading) }\end{array}$ \\
\hline $\begin{array}{c}\text { Linearity } \\
\text { Max RMS EMG } \\
\text { (dynamic loading) }\end{array}$ & $\begin{array}{l}\text { Indication of the } \\
\text { intended exerted } \\
\text { force }\end{array}$ & $\begin{array}{l}\text { The EMG signal is used as control input to the } \\
\text { EMG controller to prepare the assist-as-need } \\
\text { routine before the muscles have even moved }\end{array}$ & $\begin{array}{c}\text { Compare the Max EMG signals from } \\
200 \text { tests performed with different } \\
\text { weights }\end{array}$ & 2 \\
\hline $\begin{array}{c}\text { Linearity } \\
\text { AVC RMS EMG } \\
\text { (static loading) }\end{array}$ & $\begin{array}{l}\text { Indication of the } \\
\text { sustained force }\end{array}$ & $\begin{array}{l}\text { The EMG signal is used as control input to the } \\
\text { EMG controller to command required torque or } \\
\text { position command to the low-level controller }\end{array}$ & $\begin{array}{l}\text { Compare the Average EMG signals } \\
\text { during static loading from } 200 \text { tests } \\
\text { performed with different weights }\end{array}$ & 3 \\
\hline
\end{tabular}




\section{Testing Methodology}

The evaluation process consisted of initial tests to verify the sensor's SNR after which a series of extensive tests were performed by a subject to validate the linearity between exerted force and the EMG signal.

\section{A. SNR Tests}

To measure the baseline noise level of the sensor and verify the SNR of the MyoWare EMG sensor, the EMG signal from the sensor was collected while the two parallel electrodes were connected to the reference potential and when the electrodes were connected to the subject's arm and the bicep muscle was relaxed. The data was captured using the National Instruments USB-6361 Analog/Digital Converter (A/DC) and a LabVIEW program which sampled the data for $500 \mathrm{~ms}$ at a sampling frequency of $2000 \mathrm{~Hz}$.

\section{B. Linearity Tests}

A series of tests were then carried out to observe the performance of the sensor and to determine the degree of linearity during a typical end-goal application scenario, such as those performed during upper-limb robotic exoskeleton assisted rehabilitation sessions.

The tests were performed on a healthy female with no history of bicep pathology, neuromuscular conditions or cardiac disorder. As the SNR is determined almost "exclusively by the electrodes, and more specifically, the properties of the electrode-electrolyte-skin contact" $[6,13]$, great effort was taken to ensure optimum contact. In particular, the subject's skin at the contact site was exfoliated, cleaned and dried. Next, tape was applied and swiftly pulled from the skin to remove any remnant dry skin at the site. Two $3 \mathrm{M}$ RedDot bi-polar surface $\mathrm{Ag} / \mathrm{AgCl}$ monitoring electrodes with soft cloth tape and solid gel were placed parallel on the subject's dominant (left) belly of the bicep branchii in accordance with the sensor manufacturer's instructions.

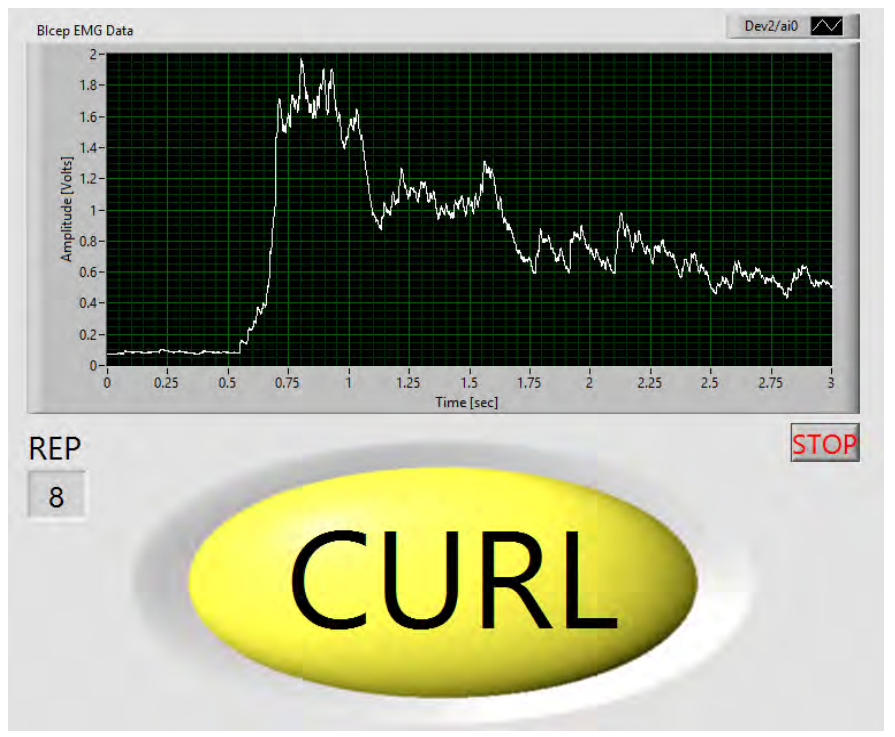

Fig. 4 LabVIEW User Interface for Curling Tests.
Furthermore, as suggested by De Luca [3], the subject flexed her bicep to find the optimum placement of the electrodes to minimize cross-talk noise from neighbouring muscles and increase SNR. A third electrode was placed as far as possible from the bicep branchii, which in this case was limited by an 8 $\mathrm{cm}$ length of wire which connected the reference electrode snap to the sensor board. During the tests, the subject sat on a chair and watched the LabVIEW User Interface shown in Fig. 4. Each test lasted 3 seconds.

At the beginning of the test, the subject had her arm down holding the weight at full extension $\left(0^{\circ}\right)$ with the bicep muscle relaxed. When the 'CURL' indicator on the LabVIEW User Interface turned yellow, the subject lifted the current weight to full elbow flexion $\left(90^{\circ}\right)$. The subject then maintained a static hold at $90^{\circ}$ for the remainder of the 3 seconds. Then there was a 3 second rest period after which the next repetition started. This procedure was carried out 40 times for a specific weight. When completed, the subject was given a 5-minute rest period. The set of 40 repetitions were performed consecutively with 5 different weights: $01 \mathrm{lbs}, 3 \mathrm{lbs}, 5 \mathrm{lbs}, 10 \mathrm{lbs}$, and $15 \mathrm{lbs}$. A total of 200 tests were, therefore, performed by the subject. Note that the maximum weight was limited to the $15 \mathrm{lbs}$ dumbbell since the amplifier sometimes saturated during tests with heavier weights and the subject could also not perform 40 tests in a row using the larger weights. The data was collected using the National Instruments USB-6361 A/DC and a LabVIEW program with a sampling rate of $2000 \mathrm{~Hz}$ which was consistent or faster than tests in the literature $[6,10]$. The experimental setup diagram in Fig. 5 shows the computer running the LabVIEW program, the Arduino Uno microprocessor which was used in this case to power the EMG sensor, the EMG sensor placed on the bicep, and the EMG sensor's connection to the NI A/DC which digitized the data that was then collected by the LabVIEW program running on the computer.

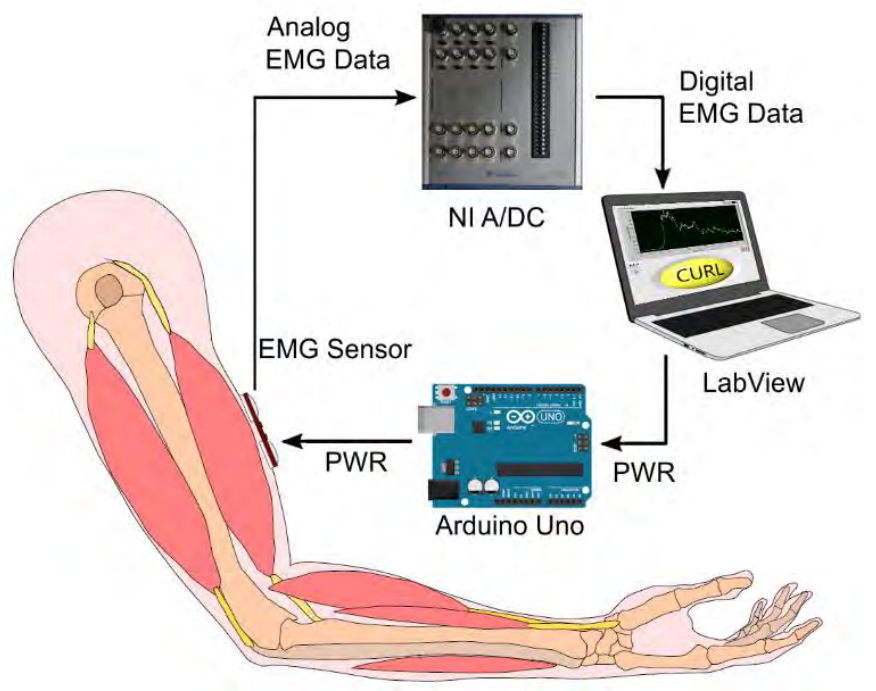

Fig.5 Experimental Setup Diagram for the Curl Tests (modified and used with permission from [12]) 


\section{RESULTS AND DISCUSSION}

LabVIEW was used to process the EMG data and Matlab was used to perform statistical analysis and plot the results.

A. SNR Tests

The measured (amplified and processed) baseline noise Vrms noise was $65 \mathrm{mV}$ (for both when the electrodes were connected to the reference electrode and when the muscle was relaxed). The SNR was then calculated as follows $[14,15]$ :

$$
\mathrm{SNR}=20 \log _{10}\left(\mathrm{Vrms}_{\text {signal }} / \mathrm{Vrms}_{\text {noise }}\right)
$$

Table II shows the calculated average maximum RMS EMG (dynamic loading) values for the groups of tests performed with each weight, while Table III shows the Average Voluntary Contraction (AVC) RMS EMG (static loading) values for the groups of tests performed with each weight. As can be seen in these tables, as the amount of effort increased, the EMG signal amplitude also increased resulting in a greater SNR. Delysis Inc [16], an industry leader in wearable sensors, states that SNR values above 1.2 are acceptable. Except for the SNR of the AVC averages at 0lbs, the SNR for the MyoWare EMG sensor is very good.

One important value to note in the noise baseline of the EMG signal when the muscle is relaxed. Florimond [5] has pointed out that it is generally accepted that the surface EMG of a muscle at rest should be below $5 \mu \mathrm{V}$, while De Luca [2] indicated that it should be less than $2 \mu \mathrm{V}$. The voltages measured during the tests carried out in this paper are amplified. Thus, to determine the relaxed muscle (noise) voltage, it was necessary to divide the measured EMG voltage at the skin by the amplification factor of 9,306.3 [17] resulting in $65 \mathrm{mV} / 9,306.3=7 \mu \mathrm{V}$ which is consistent with the literature. This value is satisfactory.

TABLE II

SNR: AVERAGE MAX RMS EMG (DYNAMIC LOADING)

\begin{tabular}{|c|c|c|c|}
\hline $\begin{array}{c}\text { Weight } \\
\text { (lbs) }\end{array}$ & $\begin{array}{c}\text { Avg Max RMS EMG } \\
\text { at this weight [mV] }\end{array}$ & SNR & SNR (dB) \\
\hline 0 & 0.2150 & 3.26 & 10 \\
\hline 3 & 0.5599 & 8.48 & 19 \\
\hline 5 & 0.8816 & 13.36 & 23 \\
\hline 10 & 1.5280 & 23.15 & 27 \\
\hline 15 & 2.7970 & 42.38 & 33 \\
\hline
\end{tabular}

TABLE III

SNR: AVC RMS EMG (STATIC LOADING)

\begin{tabular}{|c|c|c|c|}
\hline $\begin{array}{c}\text { Weight } \\
\text { (lbs) }\end{array}$ & $\begin{array}{c}\text { AVC RMS EMG at } \\
\text { this weight }[\mathbf{m V}]\end{array}$ & SNR & SNR (dB) \\
\hline 0 & 0.0748 & 1.13 & 1 \\
\hline 3 & 0.1693 & 2.57 & 8 \\
\hline 5 & 0.3299 & 5.00 & 14 \\
\hline 10 & 0.6328 & 9.59 & 20 \\
\hline 15 & 1.4142 & 21.43 & 27 \\
\hline
\end{tabular}

\section{B. Linearity Tests for Dynamic Loading}

Fig. 6 plots the maximum RMS EMG signal for all 200 tests (40 tests at each of the 5 weights). As can be seen in the figure, the Max RMS EMG signal became progressively larger as the exerted force increased. Fig. 7 plots the average maximum RMS EMG signal as a function of the weights lifted along with the corresponding $95 \%$ confidence intervals. A straight line and a second-order polynomial were fit to the data, with $\mathrm{R}^{2}$ values of 0.9728 and 0.9949 (shown), respectively.

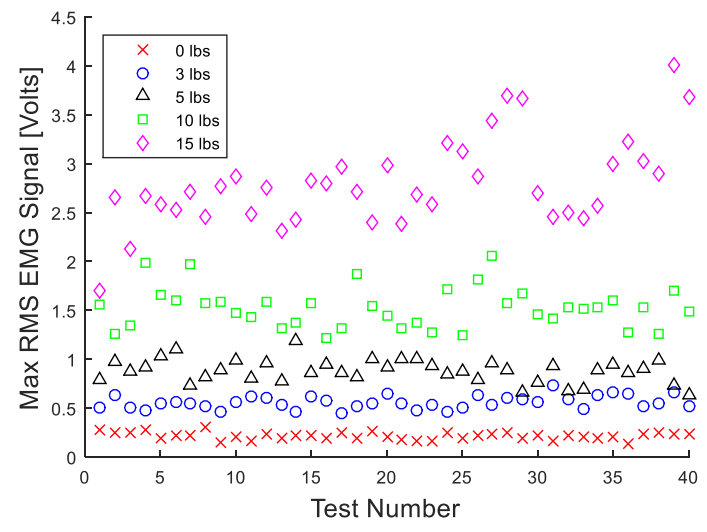

Fig. 6 Maximum RMS EMG Signal for Tests with Different Weights (Dynamic Loading)

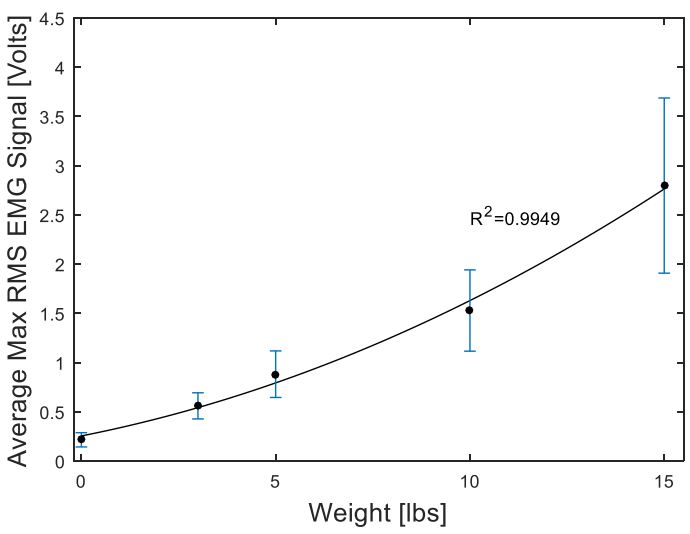

Fig. 7 Average Max. RMS EMGS for Different Weights with 95\% Confidence Interval (Dynamic Loading)

\section{Linearity Tests for Static Loading}

Fig. 8 plots the Average Voluntary Contraction RMS EMG (Static Loading) signal for the last $500 \mathrm{~ms}$ of each of the 200 tests (40 tests for each of the 5 weights). Similar to the Max RMS EMG signal, the AVC RMS EMG signal also became progressively larger as the exerted muscle force increased, but with roughly half of the amplitude. Fig. 9 plots the average AVC RMS EMG signal as a function of the weights lifted along with the corresponding 95\% confidence intervals. A straight line and a second-order polynomial (shown) were fit for the static loading as was done with the dynamic loading plots. $\mathrm{R}^{2}$ values of 0.9311 and 0.9921 , were calculated for the straight line and the second-order polynomial fits, 
respectively. Like with the dynamic loading, a correlation between the exerted force and the measured AVC RMS EMG signal was observed and are both consistent with the curves attained in literature $[18,19]$ for 61 subjects and 10 subjects respectively. Since the results show a characterizable relationship, the sensor would likely be suitable for a futurebuild robotic exoskeleton EMG-based controller.

A correlation was also observed between the variability of the EMG signal and the applied force for both types of loading - up to $50 \%$ for the $15 \mathrm{lbs}$ dynamic loading tests. Similar observations were presented in [20]. Some possible solutions to minimize variability could be to apply sensor-side or controller-side processing or limit the number of repetitions in each test and the payload to 5lbs or less - where the spread is significantly smaller.

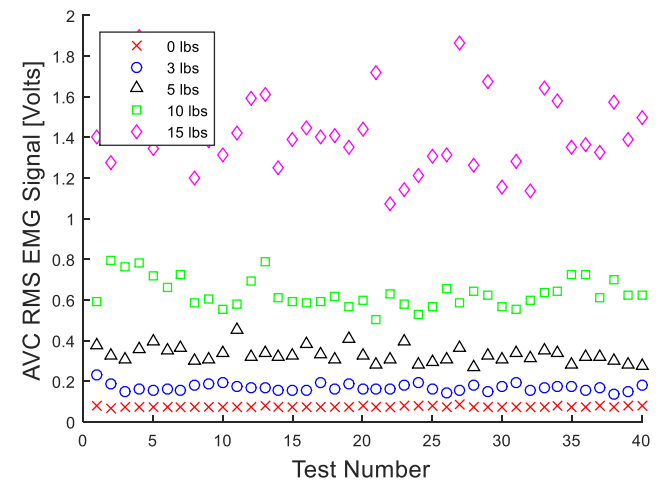

Fig. 8 Average AVC RMS EMGS for Different Weights with 95\% Confidence Interval (Static Loading)

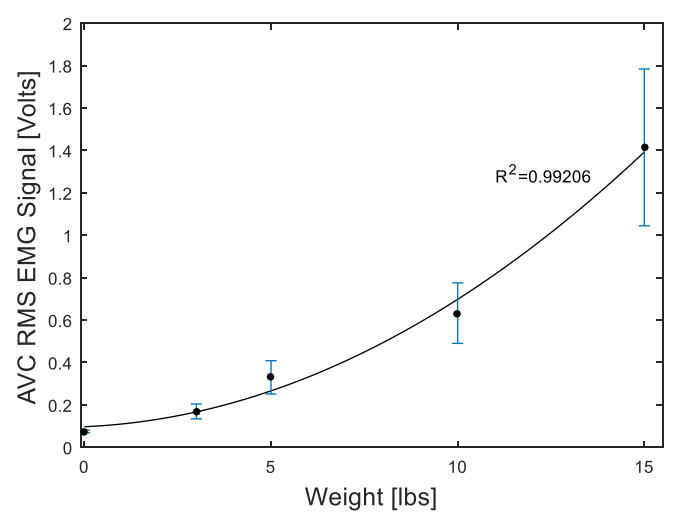

Fig. 9 Average AVC RMS EMGS for Different Weights with 95\% Confidence Interval (Static Loading)

\section{CONCLUSION}

Experimental tests were performed on a subject using the MyoWare EMG sensor to evaluate its use for future robotic exoskeleton applications. Verification test showed acceptable SNR values. The degree of linearity of the sensor was studied while it was utilized in a typical end-use application and the results showed expected correlation between the exerted force and both the dynamic and static load EMG signals. Although these results look promising and suggest that the sensor would be a good candidate for a proportional robotic exoskeleton EMG controller, further investigation would be beneficial to verify the linearity of the sensor itself without the human in the loop. It would also be interesting to perform the same tests on more subjects, and with other EMG sensors. Additionally, it would be beneficial to examine the sensor's raw EMG signal and explore its performance in the frequency domain.

\section{ACKNOWLEDGMENT}

We would like to thank Dr. Bartenbach, Dr. Kesner, and Mr. Kaminski for their help.

\section{REFERENCES}

[1] T. M. Dall, et al., "An aging population and growing disease burden will require a large and specialized healthcare workforce by 2025," Health Affairs vol. 32, no. 11, 2013.

[2] C. De Luca, "The use of surface electromyography in biomechanics," Journal of Applied Biomechanics, 1997. doi: 10.1123/jab.13.2.135.

[3] C. De Luca "Surface Electromyography: Detection and Recording," DelSys Inc., 2002.

[4] B. S. Rajaratnam, et al., "A Comparison of EMG signals from surface and finewire electrodes during shoulder abduction," Int. Journal of Physical Med. \& Rehab., 2014. doi: 10.4172/2329-9096.1000206.

[5] V. Florimond, "Basics of surface electromyography applied to physical rehab. and biomechanics," Belgrave Ave, 2010.

[6] V. Florimond, "Basics of Surface Electromyography Applied to Psychophysiology," Thought Technology Ltd, Doc Number MAR900, 2008.

[7] J.C. Perry, J. Rosen. "Design of a 7 degree-of-freedom upper-limb powered exoskeleton," Biomed. Rob. Biomechatronics, 2006. doi: 10.1109/BIOROB.2006.1639189.

[8] http://myomo.com [Online]. Accessed: Dec, 2017.

[9] https://www.cyberdyne.jp [Online]. Accessed: Dec, 2017.

[10] J. Rosen, et al. "A myosignal-based powered exoskeleton system," IEEE Trans. Sys., Man, Cybern., vol. 31, no. 3, pp. 210-222, May 2001. doi: $10.1109 / 3468.925661$.

[11] V. Krasin, V. Gandhi, Z. Yang, and M. Karamanoglu, "EMG based elbow joint powered exoskeleton for biceps brachii strength augmentation," in 2015 Int. Jt. Conf. Neural Networks, Proc., 2015. doi: 10.1109/IJCNN.2015.7280643.

[12] Beyond Robotics GmbH, "EduExo - The Robotic Exoskeleton Kit Muscle Control Extension Handbook and Tutorial," 2017.

[13] S. Day, "Important factors in surface EMG measurement," Bortec Biomedical Ltd publishers, 2002.

[14] R. Adamson, BMNG5210 Course, Module 2, Example Problems, 2016.

[15] P. Zhou and X. Zhang, "A novel technique for muscle onset detection using surface EMG signals without removal of ECG artifacts," Physiol. Meas., vol. 35, no. 1, pp. 45-54, Dec. 2013. doi: 10.1088/0967-3334/35/1/45.

[16] http://www.delsys.com. [Online]. Accessed: Nov, 2017.

[17] Advancer Technologies, "3-lead Muscle/Electromyography Sensor for Microcontroller Applications", Datasheet, 2015.

[18] M. B. I. Reaz, M. S. Hussain, and F. Mohd-Yasin, "Techniques of EMG signal analysis: detection, processing, classification and applications," Biol. Proced. Online, vol. 8, no. 1, pp. 11-35, Dec. 2006. doi: 10.1251/bpo115. 
[19] H. Uliam, F. M. de Azevedo, L. S. Ota Takahashi, E. Moraes, R. de F. Negrao Filho, and N. Alves, "The Relationship Between Electromyography and Muscle Force," in EMG Methods for Eval. Muscle and Nerve Function, InTech, 2012. doi: 10.5772/25381.

[20] R. Lo Martire, K. Gladh, A. Westman, and B. O. Äng, "Neck Muscle EMG-Force Relationship and Its Reliability During Isometric Contractions," Sports Med. - Open, vol. 3, no. 1, Apr. 2017.

doi: 10.1186/s40798-017-0083-2. 


\title{
Smelling through Microfluidic Olfaction Technology
}

\author{
Pouria Mehrabi, Justin Hui, Mahyar Mohaghegh Montazeri, Kim Tien Nguyen, Abigail Logel, Allen O’Brien, \\ Mina Hoorfar \\ School of Engineering \\ University of British Columbia \\ Kelowna, Canada \\ mina.hoorfar@ubc.ca
}

\begin{abstract}
Detection of volatile organic compounds (VOCs) in the exhaled breath is found to be a promising method to diagnose different diseases. The amount of alcohol or drugs absorbed/inhaled in the body can also be measured using gas sensors. Oral habits can affect the composition and also concentration of VOCs produced as a result of cellular metabolic reactions inside our body. Recognition of exhaled breath patterns including composition and concentration of VOCs provides useful information regarding how the breath affects the artificial olfaction systems. This can provide a powerful tool to calibrate gas sensors and detect VOCs associated with different diseases.

In the following study, the breath signatures are extracted after different activities including fasting, brushing teeth, and drinking coffee. The results are normalized and implemented into a feature extraction model that extracts principal features for each regime. This will determine the breath signature of each regime. The results show that the effect of these activities on the breath is consistent between different subjects. This study provides the base signature of the exhaled breath which can be used in a clinical setting to identify other target VOCs that are considered the biomarkers of diseases.
\end{abstract}

Keywords- breath analyzer, gas sensor, microfluidic artificial olfaction

\section{INTRODUCTION}

Breath analyzers have been considered as a non-invasive method for detection of chemicals or mixtures of chemicals that are either produced as part of biological metabolism or are absorbed from exogenous sources [1]. The exhaled breath can contain volatile organic compounds (VOCs) (e.g., acetone, ethanol, and isoprene), inorganic compounds (e.g., carbon dioxide and nitrogen), and non-volatile substances (e.g., isoprostanes and peroxynitrite) [2]. The "smell-print" can be used as an indicator of the patient physical condition and lifestyle. Despite its great potential, breath analysis still has several practical challenges. A mixture of inorganic gases (e.g. $\mathrm{CO}_{2}$, ethanol, and $\mathrm{N}_{2}$ ) and VOCs in the exhaled breath of a normal healthy person can be identified (many of these chemicals are absorbed from environment such as smoking, food consumption, bacterial microflora, work environments and medication [3]). However, The exact composition is influenced by diet, age, body mass index (BMI), and gender, and hence, varies between individuals [4]. Although the majority of VOCs in exhaled breath have external sources, they still should be distinguished from the endogenous species.

Another challenge associate with breath analysis is calibration of the sensor based on standard samples. For example, for detection of the cannabis use, it is necessary to control temperature and humidity, and breath samples must be collected from the lower airways (alveolar air). Also, in order to quantify impairment, one must discriminate between THC (the psychoactive component) and CBD.

Finally, identifying a particular health condition is not achievable by detection of a single target gas and instead requires detection of a pattern of compounds [5]. For example, monitoring the level of methane and hydrogen is a promising technique for diagnosis of Small intestinal bacterial overgrowth (SIBO), Irritable bowel syndrome (IBS), and diabetes [6]. However, these gases could be produced as a result of normal digestion and respiration. Therefore, the rate and concentration of these species in exhaled breath should be determined base on each metabolic reaction [7]. Also, malabsorption of lactose and glucose causes huge production of hydrogen and methane in breath [8]. Discovery of the breath pattern as a result of this process will prevent mixing up this malfunction of metabolic system with another disease like diabetes at first stages [9].

The technology presented here has high sensitivity and selectivity, and it uses a single sensor embedded in a microfluidic channel [10]. The latter adds several benefits (such as less calibration complexity, smaller size, and lower cost) compared to traditional e-noses [11]. This technology is used to detect VOCs in the exhaled breath of several subjects after different activities. The purpose of this study is to develop base 
signatures of the breath that can be considered while it is used for detection of a certain biomarker for a disease.

\section{EXPERIMENTAL}

\section{A. Device description}

This technology is portable and easy to assemble, making it ideal for a hand-held device (see Fig. 1). The air chamber is built into the main cartridge, eliminating the need for a separate chamber to be filled with the sample. Both humidity filters (i.e., potassium hydroxide and zeolite) can be easily installed/replaced simply by sliding each piece into the cartridge. A total of three pieces are needed to complete the two filters. The last piece of the filter design includes slots for three springs to be inserted. These are used to make a one-way valve. A rubber stopper is attached to the end of the three springs to prevent a backflow of the air sample. When the breath is blown into the device, the force of the air will compress the springs allowing the air to flow pass the filters and into the sensing chamber. The one way valve housing is bolted to the cartridge to ensure an air right seal and easy assembly. The opening at the end of the one way valve housing allows a disposable mouthpiece to be attached for each new sample.

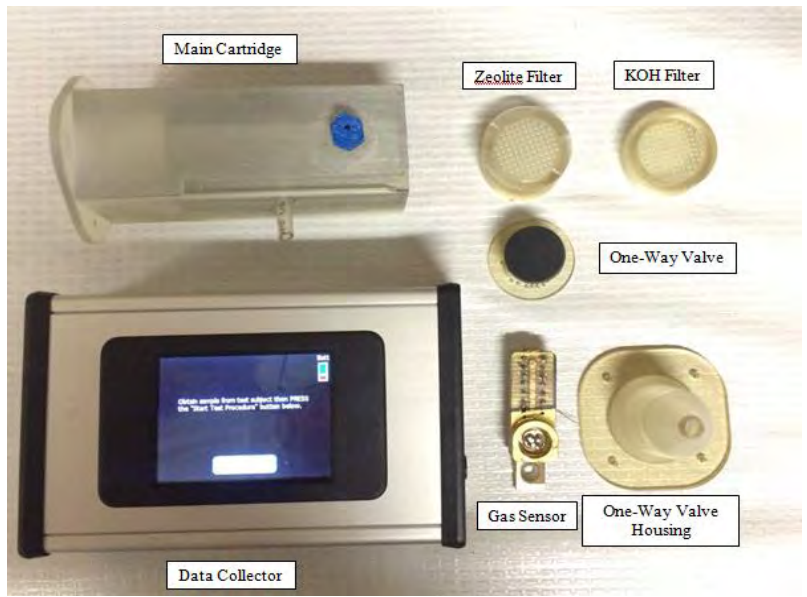

Fig. 1 - Layout of the breathalyzer including each component

A box containing the sensor and electronics is connected to the cartridge and sensing chamber with a push valve. A large bracket will then slide into place following guides imbedded into the cartridge. It can hold the sensing device firmly in place. A schematic of the device including all components and also the test setup is shown in Fig. 2.

\section{B. Materials}

The entire device is $3 \mathrm{D}$ printed to reduce machining costs. The cartridge, filters, a one-way valve housing are made of veroclear (UBCO Star 3D printing facility) to allow intricate geometries. Before testing, the cartridge and all of the parts are coated with Parylene $\mathrm{C}$ to prevent diffusion of the gases into the plastic.

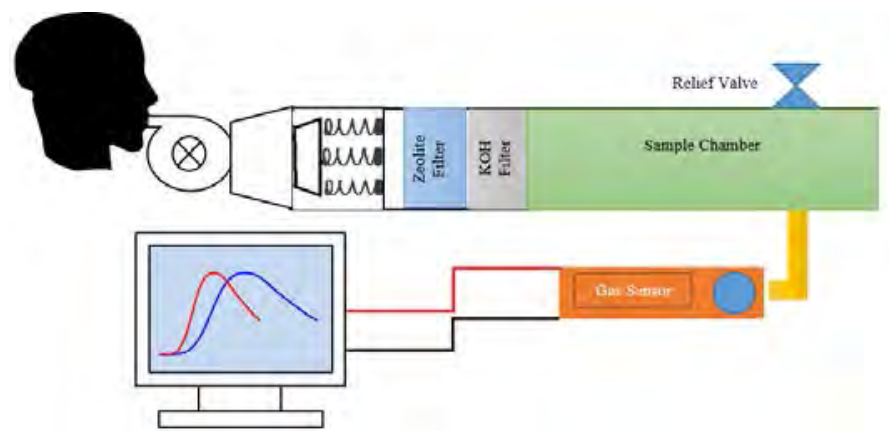

Fig. 2 - Schematic of the device and test setup

\section{Testing procedure}

Over the period of testing, the subjects started the experiment by fasting for 12 hours. Following the collection of the breath after fasting, the experiment was carried out by collecting the breath samples after certain activities including drinking black coffee, brushing the teeth (Colgate cavity protection winter fresh), and washing using a mouthwash (Listerine original). After performing each activity, the subject blows into the device twice, giving two samples to ensure the results reproducibility.

\section{RESULTS}

Fig. 3 shows the sensor response (referred to as the "smell print") after each activity for each subject. For every subject, the response was the strongest after using mouthwash. The second strongest response curve is after fasting for 12 hours, which is followed by the signal of the breath sample collected after drinking coffee and finally brushing the teeth. The sensor's strong response after the use of mouthwash is due to the presence of various alcohols $(26.9 \%)$ and also organic compounds (e.g. Thymol and Menthol) in the mouthwash solution. Also, since the concentration of ethanol and acetone in the exhaled breath of a person after fasting for 12 hours increases [12], the sensor's second largest response is for the samples collected after fasting. It is worth to mention that regardless of the subject the trend of the response curves associated with each activity is similar. This proves that the "smell print" of each activity is unique in the breath.

The pattern of breath signature across different activities looks similar for that particular activity between different subjects. This similarity can be observed in the slopes of the curve during the rising period (i.e., for the exposure process) and decaying part (i.e., for the recovery stage).

Fig. shows the results of the feature extraction method (performed based on three features including: the area under each curve, the maximum response, and response at $\mathrm{t}=200 \mathrm{~s}$ for the breath samples collected after using the mouthwash. The results show that each subject has a unique breath signature after rinsing the mouthwash. Also, the two replications conducted for the breath of each subject are in agreement in 3dimensional space, showing reproducibility of the results. 

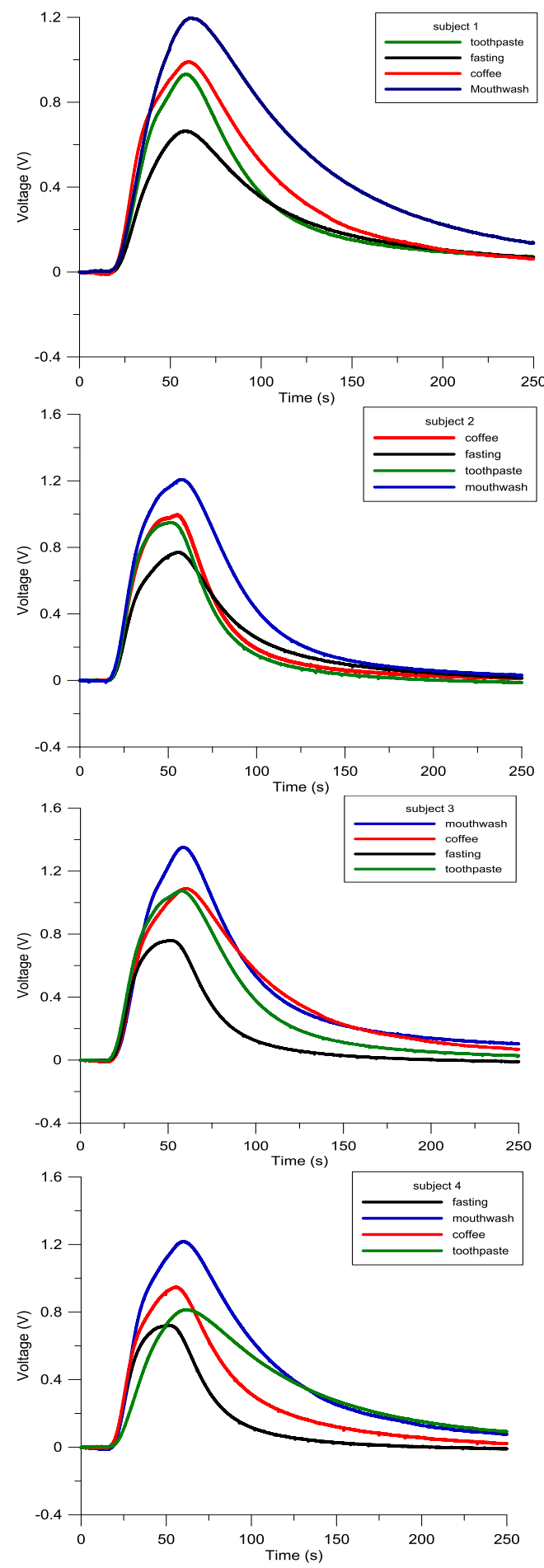

Fig. 3 - Results of the experiment for each subject. The trend in each graph for each activity is similar for different subjects.

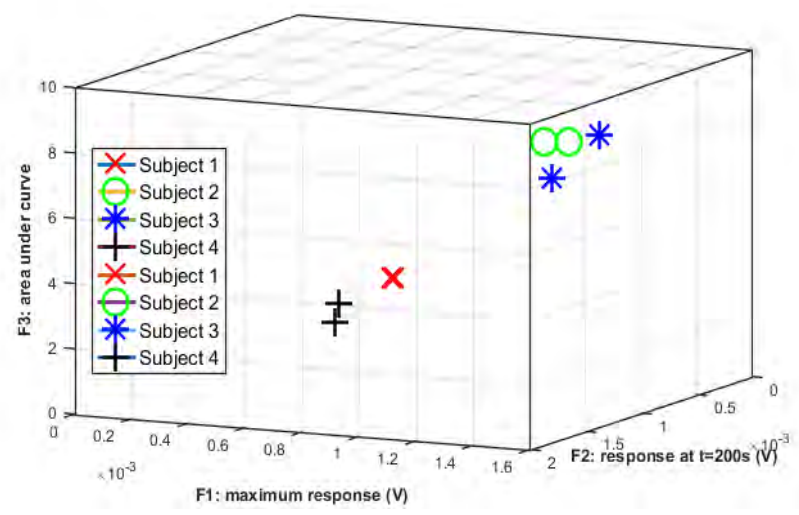

Fig. 4- Feature extraction of results for the breath samples collected after using the mouthwash. These features are based on the area underneath the curve, the maximum response, and response at $\mathrm{t}=200 \mathrm{~s}$.

\section{CONCLUSION}

The response of the microfluidic olfaction breath analyzer showed that the trend for the breath samples collected after different activities is consistent regardless of the subject. The response of the sensor ("smell print") is much stronger after the use of mouthwash and fasting due to high amount of alcohol existing in exhaled breath in both of these activities. The experimental results were reproducible for each subject as the signal magnitude trend between different activities was the same in all cases. For the future study, a diverse range of subjects will be tested to observe and extract biomarker patterns in a wide variety of cases.

\section{AKNOWLEDGMENT}

We would like acknowledge the Natural Sciences and Engineering Research Council of Canada (NSERC) for funding this research.

\section{REFERENCES}

[1] K.-H. Kim, S. A. Jahan, and E. Kabir, "A review of breath analysis for diagnosis of human health," TrAC Trends Anal. Chem., vol. 33, pp. 1-8, Mar. 2012. doi: 10.1016/j.trac.2011.09.013.

[2] P. Kintz, P. Mura, C. Jamey, and J.-S. Raul, "Detection of (Delta) 9tetrahydrocannabinol in exhaled breath after cannabis smoking and comparison with oral fluid," Forensic Toxicol., vol. 35, no. 1, pp. 173- 178, Jan. 2017. doi: 10.1007/s11419-016-0333-x.

[3] L. Blanchet et al., "Factors that influence the volatile organic compound content in human breath," J. Breath Res., vol. 11, no. 1, p. 016013, Feb. 2017. doi: 10.1088/1752-7163/aa5cc5.

[4] C. Di Natale et al., "Solid-state gas sensors for breath analysis: A review," Anal. Chim. Acta, vol. 824, pp. 1-17, May 2014. doi: 10.1016/j.aca.2014.03.014.

[5] K. Arshak et al., "A review of gas sensors employed in electronic nose applications," Sens. Rev., vol. 24, no. 2, pp. 181-198, Jun. 2004. doi: 10.1108/02602280410525977. 
[6] M. Ledochowski, "Fructose malabsorption is associated with early signs of mental depression," Eur. J. Med. Res., vol. 3, no. 6, pp. 295-298, June 1998.

[7] B. P. de Lacy Costello, M. Ledochowski, and N. M. Ratcliffe, "The importance of methane breath testing: a review," J. Breath Res., vol. 7, no. 2, p. 024001, June 2013. doi: 10.1088/1752-7155/7/2/024001.

[8] V. Ruzsanyi et al., "Diagnosing lactose malabsorption in children: difficulties in interpreting hydrogen breath test results," J. Breath Res., vol. 10, no. 1, p. 016015, Mar. 2016. doi: 10.1088/1752-7155/10/1/016015.

[9] A. Eisenmann et al., "Implementation and interpretation of hydrogen breath tests," J. Breath Res., vol. 2, no. 4, p. 046002, July 2008. doi: 10.1088/1752-7155/2/4/046002.

[10] M. Paknahad et al., "Highly selective multi-target 3D-printed microfluidic-based breath analyzer," in Proc. IEEE 29th Int. Conf. Micro Electro Mechanical Systems (MEMS), 2016, pp. 905-908.

doi: 10.1109/MEMSYS.2016.7421777.

[11] A. D. Wilson and M. Baietto, "Advances in electronic-nose technologies developed for biomedical applications", Sensors, vol. 11, no. 1, pp. 1105-1176, Jan. 2011. doi: 10.3390/s110101105.

[12] M. P. Kalapos, "Possible physiological roles of acetone metabolism in humans," Med. Hypotheses, vol. 53, no. 3, pp. 236-242, Sept. 1999. doi: 10.1054/mehy.1998.0752. 


\title{
In Vitro Investigation of the Effect of a Dysfunctional Bileaflet Mechanical Aortic Valve on Flow Characteristics in the Ascending Aorta
}

\author{
Ahmed Darwish $^{1 *}$, Wael Saleh ${ }^{1,2}$, Giuseppe Di Labbio ${ }^{1}$, Lyes Kadem ${ }^{1}$ \\ ${ }^{1}$ Mechanical, Industrial, and Aerospace Engineering Department \\ Concordia University \\ Montreal, QC, Canada \\ *email: 1cfd@encs.concordia.ca \\ ${ }^{2}$ Mechanical Engineering Department \\ Assiut University, Egypt
}

\begin{abstract}
Heart valve replacement is still the optimal solution in young patients with severe symptomatic heart valve disease. In those patients, bileaflet mechanical heart valves (BMHV) are preferred to biological valves because of their higher durability. However, thrombus formation has been reported as a common complication following BMHV implantation despite a permanent anticoagulation therapy. Thrombus formation can lead to valve leaflet dysfunction, a lifethreatening event that requires immediate surgical intervention. This study aims to investigate the fluid dynamics downstream of a dysfunctional bileaflet mechanical aortic valve. A bileaflet ON-X mechanical aortic valve is used with different dysfunctional configurations. In this study, partially and totally blocked configurations of one of the leaflets is investigated relative to its orientation with the sinus of Valsalva. Time-resolved two-dimensional particle image velocimetry measurements are performed to investigate flow field characteristics in the ascending aorta in terms of velocity and vorticity fields and circulation. Vorticity and circulation in the aorta are significantly affected by the valve orientation with respect to the sinus of Valsalva.
\end{abstract}

\section{Bileaflet Mechanical Heart Valve; Particle Image Velocimetry; Hemodynamics.}

\section{INTRODUCTION}

Heart valve failure is a known cardiovascular disease where valves fail to open or close properly. A report from the American Heart Association mentioned that the prevalence of heart valve diseases in the United States is $2.5 \%$ in 2013[1].

Treatment of valvular diseases ranges from adopting healthy lifestyle up to valve replacement [2] which is recommended for severe symptomatic cases.

Bileaflet Mechanical Heart Valve (BMHV) is a preferable choice for valve replacement in young patients because of its reported longer durability compared to prosthetic biological heart valves (BHV) [3], [4]. However, BMHV can be affected by thrombus of pannus formation [5]-[7]. Thrombosis formation has been shown to be related to non-physiological flow characteristics at the vicinity of the valve leaflets [5], where the shear stress levels may cause blood cell damage that would lead to platelet activation and clot formation [5], [8], [9]. Thrombus formation is more anticipated in BMHV than in $\mathrm{BHV}$ [6]. The incidence rate of prosthetic valve thrombus ranges between $0.03 \%-5.7 \%$ per year [7]. Moreover, its occurrence is as high as $24 \%$ within the first year of the valve replacement [7]. This highly anticipated thrombus formation is expected to cause a dysfunction of one or both BMHV leaflets which will inevitably affect the fluid dynamics and hemodynamics in the ascending aorta [5].

Few previous studies investigated the flow past a dysfunctional BMHV. They considered the fluid dynamics and hemodynamics changes induced by the dysfunctional valve. In addition, they investigated the ability of current diagnostic tools to detect BMHV dysfunction.

Smadi et al. [10] first investigated numerically the steady flow through a defective BMHV at different flow and pathological conditions. They reported that Doppler echocardiography assessment of the dysfunctional valve is suboptimal mostly in the evaluation of transvalvular pressure gradients. The same authors [11] further investigated experimentally and in vivo the flow downstream of a dysfunctional BMHV. They showed that current clinical guidelines cannot accurately detect the presence of a dysfunctional BMHV and suggested new clinical parameters. Finally, Shahriari et al. [12] investigated numerically the shear stress accumulation on blood components in a dysfunctional BMHV by using smoothed particle hemodynamics. Their study was the first using a fully mesh-free method to cardiovascular flows. They reported that a dysfunctional BMHV leads to significantly more damage to blood components compared to a normal valve. 
In the previous studies, the orientation of the dysfunctional leaflet with respect to the sinus of Valsalva (SV) was not investigated. Despite the fact that bileaflet valves are usually installed with one leaflet facing one sinus and the other leaflet being shared by the other two sinuses [13]-[16]. This is an important issue since the development of the sinus vortex (washout vortex) and the coronary flow are both linked to the leaflet position relative to the sinus [5], [17]-[19]. Therefore, it is important to investigate the direct impact of BMHV dysfunction on flow characteristics downstream of the valve but also in the sinus region. This study aims to investigate experimentally using time-resolved two-dimensional (2D) particle image velocimetry the effect of a dysfunctional BMHV on flow characteristics in the ascending aorta.

\section{METHODOLOGY}

\section{A. Silicone molding}

An anatomically accurate aorta, left atrium and a simplified symmetric left ventricle are constructed using silicone molding. The molding process is done by adding a silicone rubber base to a curing catalyst (XIAMETER RTV-4234-T4 Base and XIAMETER T4/T4 O Curing Agent), with a ratio of 10:1 by mass respectively. The cured silicone has a Young modulus of 1.675 $\mathrm{MPa}$. The silicone is coated on a three-dimensional printed core mold of the parts. Four layers are coated, then, the parts are mounted to a rotating arrangement (speed of rotation $=10 \mathrm{rpm}$ ) to ensure a homogenous distribution of silicone over the core mold surface. The final thickness of the silicone layers ranges between $2-3 \mathrm{~mm}$.

\section{B. In vitro left heart simulator}

The left heart is assembled and mounted to a custom-made double activation in vitro heart simulator which is shown in Fig.1. The heart simulator has an activation box where the left ventricle is placed. The activation box is made of plexiglass (with index of reflection $=1.49$ ) which is filled with blood mimicking fluid and is connected to a cylinder which contains a compliance chamber to adjust the left ventricle compliance. A piston is sliding inside the cylinder to transfer the stroke from a linear motor to the left ventricle. Above the activation box, both aorta and left atrium are connected to the left ventricle. The aorta is placed inside a plexiglass box and is connected to the top of the open reservoir. The four pulmonary veins branching from the left atrium are all connected to the bottom of the reservoir. On the system, the left atrium is also activated by using a cam-follower arrangement mounted on a servomotor which transfers its motion to the left atrium. Both linear and servomotor are controlled with custom-made LabView interface which controls the timing and stroke of both motors. The liquid used is a mixture of water/glycerol with a volumetric ratio of $60 \% / 40 \%$, respectively. The mixture has an index of reflection $=1.4$ at $20^{\circ} \mathrm{C}$, while its dynamics viscosity is $=4 \mathrm{cP}$ at $23^{\circ} \mathrm{C}$ and its density is $=1080 \mathrm{~kg} / \mathrm{m}^{3}$. The flow rate is recorded for each case by using a magnetic inductive flow sensor with a flow range from 0.03-6.6 g/min and with a resolution of $0.01 \mathrm{~g} / \mathrm{min}$ (Prosense FMM50-102, Germany), while the pressure is recorded by using a fiber optic pressure sensor with a pressure range up to $300 \mathrm{mmHg}$ and with a resolution of $<0.3 \mathrm{mmHg}$ (FISO FOP-M260, Canada).

\section{Mechanical aortic valve}

The mechanical aortic valve used in this study is a bileaflet mechanical heart valve (On-XA-25, (manufactured by CryoLife, Inc, GA, USA)) with an inside diameter of $24.9 \mathrm{~mm}$. The valve is assembled and placed inside the aorta model. The leaflets orientation relative to the sinuses of Valsalva is selected based on previous studies [13]-[16].

The investigated dysfunctional cases are summarized in Table 1. In order to induce partial valve leaflet dysfunction, a plastic wire of $0.7 \mathrm{~mm}$ diameter is placed at accurately

calculated positions relative to the leaflets to work as an obstacle to leaflet opening. The level of leaflet dysfunction is confirmed by analyzing the opening angle of each leaflet and comparing it to the reported values by the manufacturer. The small sized plastic wire has the advantage of not interfering with the flow upstream of the valves. Complete valve leaflet dysfunction cases (C and D) are achieved by gluing the leaflet with the valve's inner casing.

\section{Particle image velocimetry measurements}

Time-resolved 2D Particle image velocimetry (PIV) measurements are carried out using a YLF laser with a $10 \mathrm{~mJ}$ output energy at $1 \mathrm{kHz}$ at $527 \mathrm{~nm}$ wave length (Litron Laser, UK). The laser sheet is placed as shown in Fig. 2. Images are captured using a Phantom V9.1 high speed camera with 1000 fps at a full resolution of $1632 \times 1200$ pixels (Vision Research, Inc., USA). Both laser and camera are controlled using a highspeed controller (Lavision $\mathrm{GmbH}$, Germany). The number of recorded frames is 400 and the recording duration is $1 \mathrm{~s}$. The fluid inside the heart simulator is seeded with polyamide seeding particles with a mean particle diameter of $50 \mu \mathrm{m}$ and an index of reflection of 1.5 (Dantec Dynamics A/S, Denmark).

Each captured frame contains two pairs of images where the time interval $\Delta \mathrm{t}$ between the two pairs is $600 \mu$ s for cases $\mathrm{N}$, $\mathrm{B}$, and $\mathrm{C}$, while $\Delta \mathrm{t}$ is $400 \mu \mathrm{s}$ for $\mathrm{A}, \mathrm{D}$, and $\mathrm{E}$.

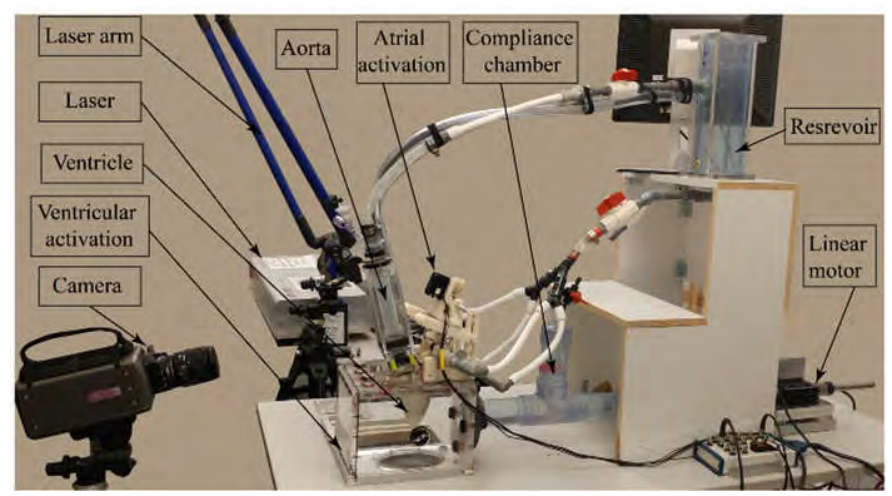

Figure 1 Experimental setup showing the camera, the laser, and the custommade heart simulator components. 


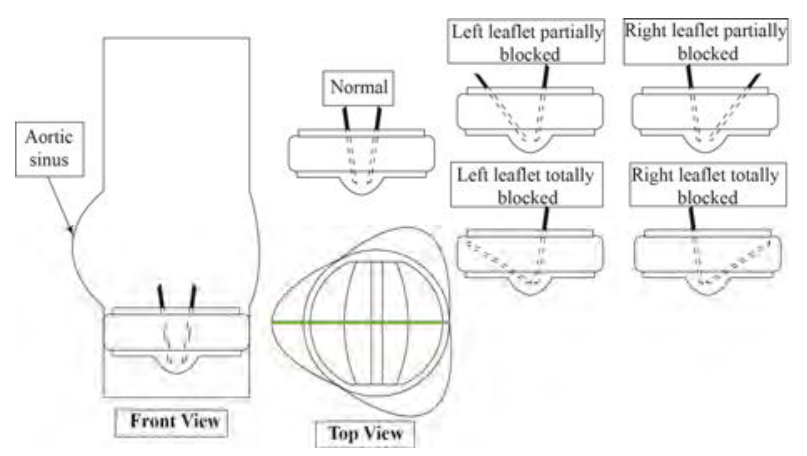

Figure 2 Front and top views of aorta showing the position of the aortic valve and the leaflets orientation with respect to the sinuses of Valsalva, beside the different dysfunction configurations of valve's leaflets. In the top view, green line represents the particle image velocimetry measurement plane.

TABLE 1. SUMMARY OF INVESTIGATED CASES AND MAXIMAL VELOCITY RECORED AT THE PEAK OF SYSTOLE

\begin{tabular}{|c|c|c|c|c|}
\hline Symbol & Case & $\begin{array}{l}\text { Dysfunction } \\
(\%)\end{array}$ & $\begin{array}{l}\text { Maximum } \\
\text { flow } \\
\text { velocity } \\
(\mathrm{m} / \mathrm{s})\end{array}$ & $\begin{array}{l}\text { Percentage of } \\
\text { velocity } \\
\text { increase relative } \\
\text { to normal } \\
\text { operation (\%) }\end{array}$ \\
\hline $\mathbf{N}$ & Normal & Null & 1.26 & Null \\
\hline $\mathbf{A}$ & $\begin{array}{l}\text { Left leaflet } \\
\text { partially } \\
\text { dysfunctional }\end{array}$ & $40-45 \%$ & 1.41 & $11.9 \%$ \\
\hline B & $\begin{array}{l}\text { Right leaflet } \\
\text { partially } \\
\text { dysfunctional }\end{array}$ & $40-45 \%$ & 1.39 & $10.3 \%$ \\
\hline $\mathbf{C}$ & $\begin{array}{l}\text { Left leaflet } \\
\text { totally } \\
\text { dysfunctional }\end{array}$ & $100 \%$ & 2.37 & $88.8 \%$ \\
\hline D & $\begin{array}{l}\text { Left leaf leaflet } \\
\text { totally } \\
\text { dysfunctional }\end{array}$ & $100 \%$ & 2.48 & $96.8 \%$ \\
\hline
\end{tabular}

The difference in the time interval between the investigated cases is due to the difference in the expected maximum flow velocity and to fulfill the requirements of the " $1 / 4$ th law"

DaVis 7.2 software (Lavision GmbH, Germany) is used to post-process the recorded images where it calculates the velocity vectors from the raw images by using a fast Fourier transform cross-correlation with an initial 64x64 squared interrogation window (with three passes) with $50 \%$ overlap down to a $32 \times 32$ circular window (with two passes) with $50 \%$ overlap. A linear filter is then being applied to filter the noisy vectors.

A trigger is used between the heart simulator and DaVis7.2 so that each recording starts at the same time during the cardiac cycle (start of left ventricle systole). The recording is taken after 20 cycles lapse to ensure that the flow has reached its normal operation conditions.

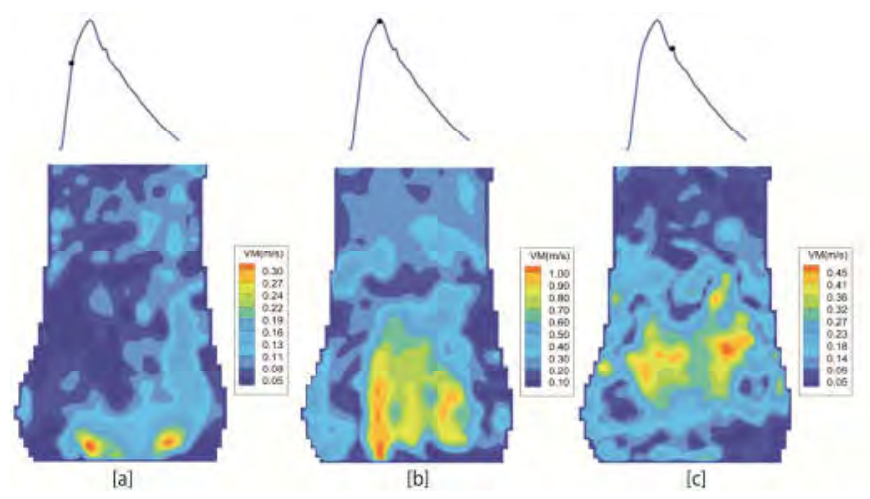

Figure 3 Velocity contours for normal case at different instants, [a] when the leaflets open, [b] at the peak of systole, [c] when the leaflets close.

\section{RESULTS}

\section{A. Velocity field downstream of the BMHV}

The maximal velocity at the peak of systole is summarized for all cases in TABLE 1. Figure 3 shows the velocity contours at the measurement plane at different phases during the cardiac cycle for the normal case. The normal maximum velocity for the valve is consistent with the reported values in [8], [17] by taking into consideration that the mean flow rate in this study is $3.8 \mathrm{l} / \mathrm{min}$. The velocities are much higher for the totally blocked cases which is directly related to the reduction in the effective orifice area (EOA) of the valve. The velocities obtained for cases $\mathrm{C}$ and $\mathrm{D}$ also agree with the values reported by [2].

\section{B. Vorticity and circulation}

To evaluate the effect of valve dysfunction on the flow field in the ascending aorta, vorticity contours are shown in Fig. 4 for each case at the peak of systole. The vorticity is calculated using (1), using a $4^{\text {th }}$ order compact Richardson scheme:

$$
\omega_{\mathrm{z}}=0.5(\delta v / \delta x-\delta u / \mathrm{d} y)
$$

where $\omega_{z}$ is the vorticity in z plane, $\delta v / \delta x$ is the derivative of $\mathrm{y}$-velocity component in $\mathrm{x}$ direction, and $\delta u / \mathrm{d} y$ is the derivative of $\mathrm{x}$-velocity component in $\mathrm{y}$ direction.

In the normal case, vorticity values in both directions are almost the same despite a higher value at the left side.

For a dysfunctional left leaflet or totally blocked (cases A, C), higher negative vorticity values are noticed, however the positive vorticity (counter clockwise rotation) dominates the measurements plane as shown in Fig. 4 a, b. The opposite behavior is noticed when the right leaflet is blocked, where clockwise rotation (negative sign vorticity) dominated the flow field as shown in Fig. 4 c, d. Therefore, the magnitude and distribution of circulation regions inside aorta is dependent on the position of the blocked leaflet. 


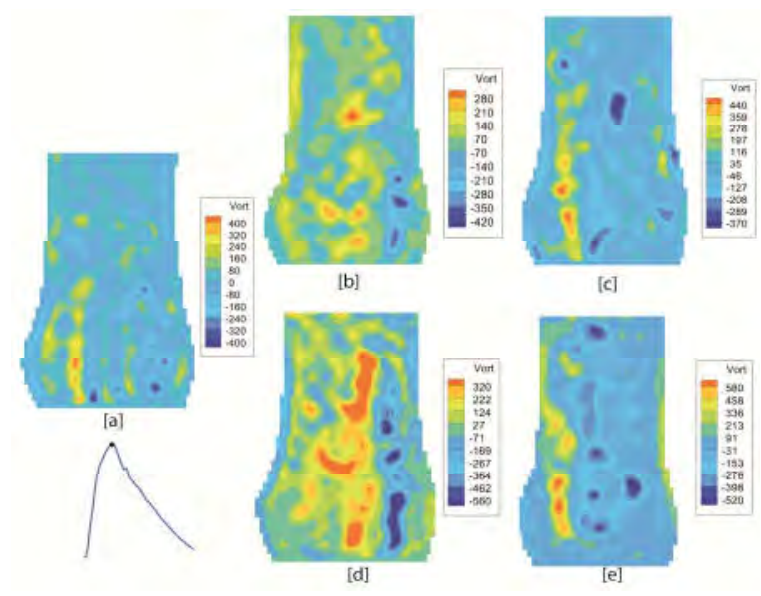

Figure 4 Vorticity contours $(1 / s)$ at the peak systole for [a] normal case, [b]left leaflet partially blocked, [c] right leaflet partially blocked, [d] left leaflet totally blocked, and [e] right leaflet totally blocked

Circulation, $\Gamma$ is calculated by integrating the vorticity over the $2 \mathrm{D}$ area in the sinus of Valsalva (SV) using (2) and is plotted against time for all cases as shown in Figure 5.

$$
\Gamma=\iint_{\mathrm{A}(\mathrm{x}, \mathrm{y})} \omega_{\mathrm{z}} d x d y
$$

As shown in Fig.5, circulation magnitude and direction inside the SV is dependent on the position of the blocked leaflet relative to the SV. In the normal case, the circulation inside the SV is positive during systole. When the right leaflet is blocked, positive circulation is also noticed in both $\mathrm{B}$ and $\mathrm{D}$, however at A when the left leaflet is partially blocked, we still can notice a positive circulation which is of a lower value compared to the normal case $\mathrm{N}$. Case $\mathrm{C}$ caused a negative circulation inside the SV, which is also noticed to be lower in magnitude compared to case $\mathrm{D}$.

The effect of circulation inside the SV has been reported to be related to coronary flow and to wall shear stresses by [18], [19]. Cao and Sucosky [19] described the interaction between the circulation inside SV and the coronary flow. While Stein et al.[18] noticed that the function of SV is to reduce turbulence near the entry of the coronary artery. Therefore, future studies have to investigate the impact of BMHV dysfunction on coronary flow circulation.

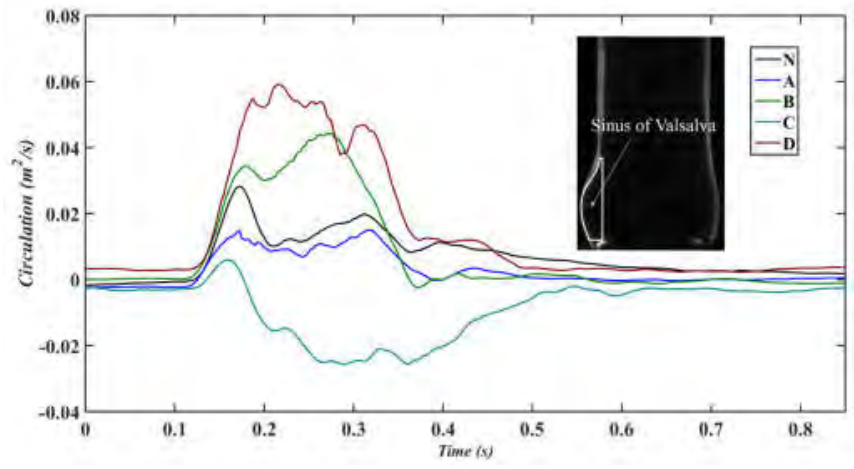

Figure 5 Temporal evolution of circulation -for (N) Normal case, (A) left leaflet partially blocked, (B) right leaflet partially blocked, (C) left leaflet totally blocked, and (D) right leaflet totally blocked-over the $2 D$ area of the sinus of Valsalva (in white).

\section{LIMITATIONS}

In this study, time-resolved 2D PIV measurements are performed while the flow pattern is expected to be of a 3D nature. Time-resolved 3D PIV measurement is one of our recommendations for future studies. Another limitation is using only one type of BMHVs. Future studies have to consider testing other types of valves and different valve sizes.

\section{CONCLUSION}

In this study we investigated the fluid dynamics downstream of a dysfunctional bileaflet mechanical aortic valve. An ON-X BMHV is used with different dysfunctional configurations. Both partially and totally blocked leaflet configurations are investigated relative to the orientation with the sinus of Valsalva. Time-resolved 2D particle image velocimetry measurements are performed to determine the flow field characteristics in the ascending aorta. Vorticity and circulation inside the aorta is significantly affected by the valve orientation with respect to the SV. The magnitude and direction of flow circulation inside the SV were also affected by the position of the dysfunctional leaflet relative to the SV. This observation should be considered in future studies dealing with the flow dynamics downstream of dysfunctional BMHVs. Further studies are required to fully understand the effect of a dysfunctional leaflet on flow topology and stability.

\section{REFERENCES}

[1] D. Mozaffarian et al., "Heart disease and stroke statistics - 2016 update: A report from the American Heart Association," Circulation, vol. 133, no. 4, pp. e38-e48, 2015.

[2] R. A. Nishimura et al., "2014 AHA/ACC guideline for the management of patients with valvular heart disease : A report of the American College of Cardiology/American Heart Association task force on practice guidelines," Circulation, vol. 129, no. 23, 2014.

[3] R. Bagur, P. Pibarot, and C. M. Otto, "Importance of the valve durabilitylife expectancy ratio in selection of a prosthetic aortic valve," Heart, vol. 103, no. 22, pp. 1756-1759, 2017. doi:10.1136/heartjnl-2017-312348.

[4] Y. Misawa, A. Muraoka, K. Aizawa, and S. Ohki, "Recurrent dysfunction after open-heart valve surgery," J. Cardiovasc. Disord., vol. 3, no. 2, pp. 3-5, 2016.

[5] L. P. Dasi et al., "Fluid mechanics of artificial heart valves," Clin. Exp. Pharmacol. Physiol., vol. 36, no. 2, pp. 225-237, 2009.

doi: 10.1111/j.1440-1681.2008.05099.x.

[6] R. Roudaut, K. Serri, and S. Lafitte, "Thrombosis of prosthetic heart valves: Diagnosis and therapeutic considerations," Heart, vol. 93, no. 1, pp. 137-142, 2007. doi: 10.1136/hrt.2005.071183.

[7] J. Salamon et al., "Mechanical valve obstruction: Review of diagnostic and treatment strategies," World J. Cardiol., vol. 7, no. 12, pp. 875-881, 2015. doi: 10.4330/wjc.v7.i12.875.

[8] F. Sotiropoulos, T. B. Le, and A. Gilmanov, "Fluid Mechanics of Heart Valves and Their Replacements," Annu. Rev. Fluid Mech., vol. 48, no. 1, pp. 259-283, 2016. doi: 10.1146/annurev-fluid-122414-034314.

[9] A. P. Yoganathan, K. B. Chandran, and F. Sotiropoulos, "Flow in Prosthetic Heart Valves: State-of-the-Art and Future Directions," Ann. Biomed. Eng., vol. 33, no. 12, SPEC. ISS., pp. 1689-1694, 2005. 
[10] O. Smadi et al., "Flow through a defective mechanical heart valve: A steady flow analysis," Med. Eng. Phys., vol. 31, no. 3, pp. 295-305, 2009. doi: 10.1016/j.medengphy.2008.07.003.

[11] O. Smadi et al., "Accuracy of doppler-echocardiographic parameters for the detection of aortic bileaflet mechanical prosthetic valve dysfunction," Eur. Heart J. Cardiovasc. Imaging, vol. 15, no. 2, pp. 142-151, 2014. doi: 10.1093/ehjci/jet059.

[12] S. Shahriari et al., "Evaluation of shear stress accumulation on blood components in normal and dysfunctional bileaflet mechanical heart valves using smoothed particle hydrodynamics," J. Biomech., vol. 45, no. 15, pp. 2637-2644, 2012. doi: 10.1016/j.jbiomech.2012.08.009.

[13] I. Borazjani and F. Sotiropoulos, "The effect of implantation orientation of a bileaflet mechanical heart valve on kinematics and hemodynamics in an anatomic aorta," J. Biomech. Eng., vol. 132, no. 11, p. 111005, 2010. doi: 10.1115/1.4002491.

[14] L. Haya and S. Tavoularis, "Effects of bileaflet mechanical heart valve orientation on fluid stresses and coronary flow," J. Fluid Mech., vol. 806, pp. 129-164, 2016. doi: $10.1017 / \mathrm{jfm} .2016 .582$.

[15] P. Kleine et al., "Effect of mechanical aortic valve orientation on coronary artery flow: Comparison of tilting disc versus bileaflet prostheses in pigs," J. Thorac. Cardiovasc. Surg., vol. 124, no. 5, pp. 925-932, 2002. doi: $10.1067 / \mathrm{mtc} .2002 .126046$.

[16] J. Laas et al., "Orientation of tilting disc and bileaflet aortic valve substitutes for optimal hemodynamics," Ann. Thorac. Surg., vol. 68, no. 3, pp. 1096-1099, 1999. doi: 10.1016/S0003-4975(99)00780-8.

[17] A. P. Yoganathan, Z. He, and S. Casey Jones, "Fluid Mechanics of Heart Valves," Аnnu. Rev. Biomed. Eng., vol. 6, no. 1, pp. 331-362, 2004. doi: 10.1146/annurev.bioeng.6.040803.140111.

[18] P. D. Stein et al., "Sinus of Valsalva: A Converging Nozzle that Contributes to Stable Flow in the Coronary Arteries," J. Anat., vol. 225, no. 1, pp. 94-97, 2014. doi: 10.1111/joa.12192.

[19] K. Cao and P. Sucosky, "Aortic Valve Leaflet Wall Shear Stress Characterization Revisited: Impact of Coronary Flow," Comput. Methods Biomech. Biomed. Engin., vol. 20, no. 5, pp. 468-470, 2017. doi: 10.1080/10255842.2016.1244266.

[20] M. Raffel et al., Particle Image Velocimetry, Berlin, Germany: Springer, 2007. 


\section{Experimental Measurements of Far Field Cough Airflows Produced by Healthly and Influenza-Infected Subjects}

\author{
N. Dudalski, A. Mohamed, E. Savory \\ Dept. Mechanical. \& Materials Engineering \\ The University of Western Ontario \\ London, Canada
}

\author{
S. Mubareka \\ Dept. Biological Sciences \\ Sunnybrook Research Institute \\ Toronto, Canada
}

\begin{abstract}
An experimental study of cough airflow fields produced by subjects who had influenza-like illness was conducted. Particle image velocimetry (PIV) and hot wire anemometry (HWA) measurements were taken in the far-field downstream of the mouth of a participant. Droplet sampling was performed at two locations within a large cough chamber, and a nasal swab confirmed the presence of an infection. The present work analyzes data from two separate cohorts, and modest differences were observed between coughs from sick and convalescent participants. The results are also compared to data obtained from a large eddy simulation (LES) which seeks to model the transient behaviour of a human cough.
\end{abstract}

Keywords-Cough; Influenza; Cold; Airflow; Particle image velocimetry; Hot wire anemometry; Bioaerosol; Fluid mechanics

\section{INTRODUCTION}

Surprisingly little is known about the mechanism of respiratory virus transmission and the dispersion of viral bioaerosols, even though such information is critical in healthcare settings during viral outbreaks. Presumed safe separation distances are not based on scientific evidence, yet there is a widespread adoption of the " $3 \mathrm{ft} / 1 \mathrm{~m}$ rule" and the " $6 \mathrm{ft} / 2 \mathrm{~m}$ rule" [1]. Several studies have conducted measurements of cough strength at the mouth and in the near-field region $(\mathrm{x}<$ $60 \mathrm{~mm}$ downstream) [2-7], but such small separation distances between people are atypical and so significant experimental data in the far field region $(x \geq 1 \mathrm{~m})$, while lacking, could provide useful information in the development of infection prevention measures and protocols.

The current project seeks to (1) Assess the " $3 \mathrm{ft} / 1 \mathrm{~m}$ rule" by analyzing velocity measurements taken $1 \mathrm{~m}$ downstream, (2) Estimate and quantify the exposure to airborne viruses and (3) develop a computational fluid dynamics model of cough flow and particle transport based on Large Eddy Simulation (LES) $[8,9]$. The objective is to recruit 50 participants, naturally infected with influenza, conduct laboratory experiments when they are presumed ill ("sick trials") and to repeat the experiments later when they have recovered ("convalescent trials"). The project also aims to recruit a reference cohort of up to 50 healthy volunteers. Participant recruitment and data analysis will continue throughout 2018, therefore, the current paper represents a preliminary report.

\section{METHODOLOGY}

\section{A. Experimental facility (The FLUGIE cough chamber)}

The Fluids from Undergraduates Influenza Enclosure (FLUGIE) consists of a $1.81 \mathrm{~m} \times 1.78 \mathrm{~m} \times 1.81 \mathrm{~m}$ rectangular wooden box (Fig. 1). A pear-shaped opening allows the participant to cough into the chamber with their nose and mouth unobstructed, while a padded head rest and chin rest ensure that the position of participant's head remains constant between individual trials. The walls of the chamber are painted black, except for a glass side wall allowing optical access, and a glass insert on the bottom of the chamber, allowing a thin laser sheet $(\mathrm{t}=1 \mathrm{~mm})$ to pass through base. Titanium dioxide $\left(\mathrm{TiO}_{2}\right)$ particles are aerosolized before they fill the chamber for Particle Image Velocimetry (PIV) measurements.

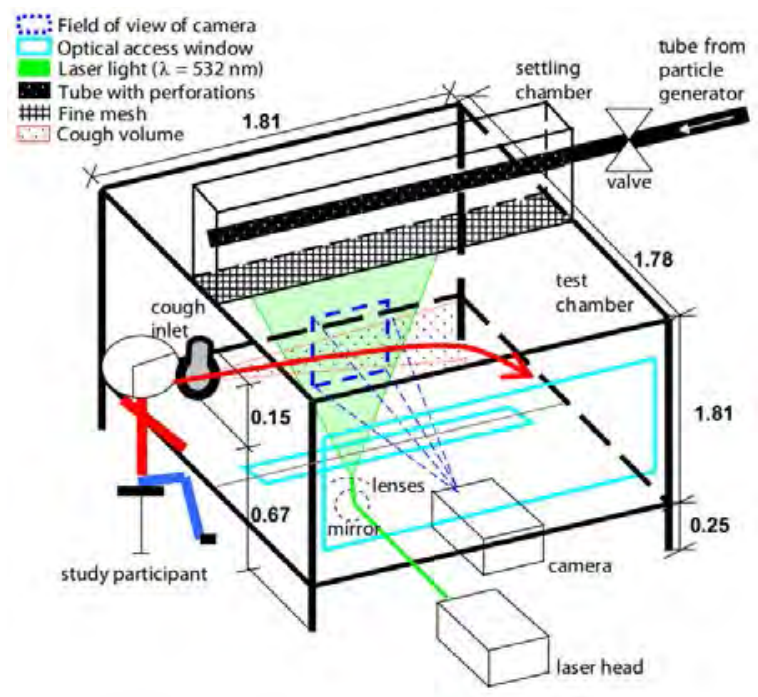

Figure 1: FLUGIE Schematic (All dimensions in m) 


\section{B. Experimental measurements}

After the chamber has been seeded with $\mathrm{TiO}_{2}$ particles, a double-pulsed Nd-Yag laser, operating at $15 \mathrm{~Hz}$, generates a vertical light sheet. The $120 \mathrm{~mJ}$ pulses have a duration of $3-5 \mathrm{~ns}$, and the camera operates with a PIV exposure of 400us. The measured field of view (Fig. 2) is $0.48 \mathrm{~m}$ height $\mathrm{x} 0.89 \mathrm{~m}$ width, and it is centered $1 \mathrm{~m}$ downstream from the participant. Image pairs are captured by a CCD camera (TSI Inc) with a spatial resolution of $6.87 \mathrm{pixels} / \mathrm{mm}$. Eighty image pairs per cough, are then processed into $102 \times 18$ vector arrays using Insight 4G software. In separate trials, a single hot-wire anemometer (HWA) probe samples velocity readings at $1 \mathrm{kHz}$, at a location $1 \mathrm{~m}$ downstream and $0.170 \mathrm{~m}$ below the inlet centre-line. This probe is calibrated using a separate facility for low air velocity measurements. To assess the presence of viral bioaerosols produced by the violent expiratory events, two low-flow air sampling pumps draw aerosols within the air onto PTFE membrane filters at a constant rate of $4000 \pm 40 \mathrm{~mL} / \mathrm{min}$. The membranes are transferred into individual tubes of UTM viral transport medium. A mid-turbinate swab (MTS) is also collected, to identify the pathogen causing the respiratory infection. All of the sampling tubes are shaken by a vortex shaker before they are stored at $-80^{\circ} \mathrm{C}$. The samples are shipped on dry ice and analyzed by the Dept. of Microbiology at Sunnybrook Health Sciences Centre (Toronto).

\section{Procedure}

Influenza-infected participants are recruited from the clinic at Western Student Health Services after the physicians determine their symptoms to be consistent with an influenza infection. The recruitment procedures are approved by Western's Research Ethics Board (REB approval no. 108945). The inclusion criteria are: age 18-35 and presenting with fever and cough and/or sore throat in the absence of another known cause of illness (e.g. allergies), while exclusion criteria include: immunocompromised, underlying cardiopulmonary disease, pregnancy and smoking. The referred participant is asked to cough 3 times, while aerosol sampling occurs and hot-wire measurements are recorded. There is sufficient time between

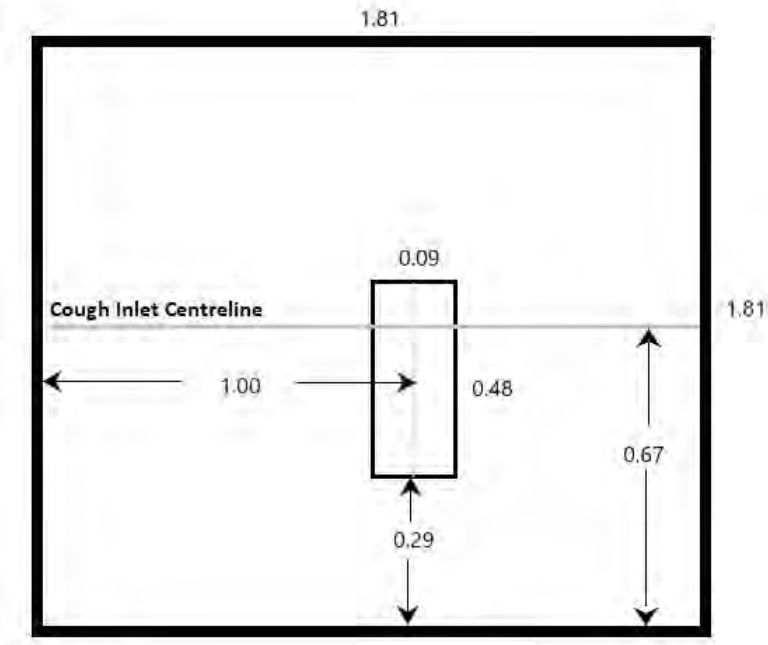

Figure 2: PIV Field of View (All dimensions in m) trials to ensure that residual air motion within the trials is minimal. The hot-wire and sampling cassettes are then removed from the chamber so that the chamber can be seeded for PIV measurements. This is necessary since the seeding particles would damage the hot-wire upon contact. Three additional coughs are then recorded by the PIV system after an even distribution of particles is present within the chamber. Subjects then self-collect a MTS before the experiments are completed. The participant returns after at least 4 weeks, to repeat the hotwire and PIV measurements in their convalescent state. The same procedure is followed for the healthy cohort, but MTS are not collected, and there is no aerosol sampling. Healthy participants also do not return for a second laboratory visit.

\section{RESULTS AND DISCUSSION}

Throughout the 2018 flu season, 7 participants have been recruited to date. From the 7 participants recruited this year, we have collected quality PIV data for 14 coughs. Coughs were excluded from this analysis if the entire width of the expired jet was not contained vertically within the field of view, and if the bulk velocity within the jet was lower than $0.2 \mathrm{~m} / \mathrm{s}$, since in this scenario, it is difficult to distinguish the jet from ambient fluid motion within the chamber.

Each cough was examined, and boundaries that contain the entire jet were specified. Velocity profiles at the midpoint of these boundaries, where the highest velocities are noticed, were extracted at $1 \mathrm{~m}$ downstream. Fig. 3 shows these profiles plotted with respect to time. The velocity obtained from the LES simulation is shown on the same plot for comparison. Boundary conditions for the LES were selected according to conditions of a representative cough based on [10]. A mouth diameter of $3 \mathrm{~cm}$ was specified, with a maximum, transient inlet velocity of $20 \mathrm{~m} / \mathrm{s}$. The average peak velocity for the PIV results is $1.4 \mathrm{~m} / \mathrm{s}$, at $1 \mathrm{~m}$ downstream, which is considerably higher than the $1.1 \mathrm{~m} / \mathrm{s}$ obtained from the LES. The difference may be attributed to the selection of the LES conditions as the average of coughs from 12 female and 13 male participants, whereas the experimental coughs each had different inlet velocities. The presence of

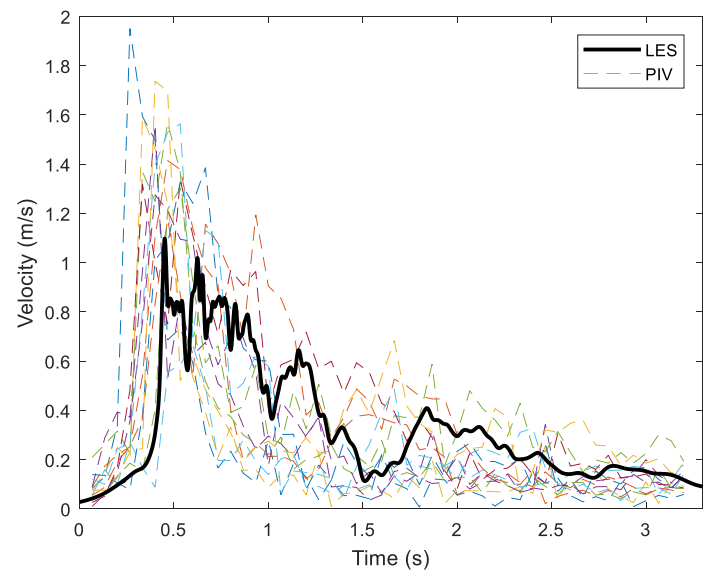

Figure 3: PIV Jet Centre 2D Velocity Magnitudes for Individual Trials 


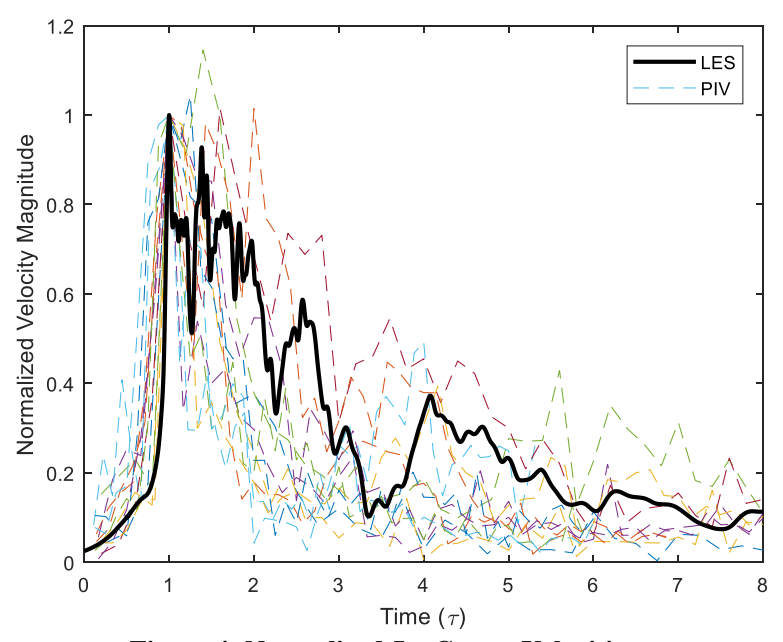

Figure 4: Normalized Jet Centre Velocities

several very strong coughs produced by a couple of participants has influenced the mean, and it is believed that as more participants are recruited, the average maximum velocity at this location will more closely align with the LES. The velocity profiles were normalized by the initial peak $2 \mathrm{D}$ velocity magnitude $\left(\left\langle\mathrm{V}^{\prime}\right\rangle=\langle\mathrm{V}\rangle \mid\langle\mathrm{V}\rangle\right.$ peak $)$, and the time that the peak occurs $\left(\tau=\mathrm{t} / \mathrm{t}_{\text {peak }}\right)$ (Fig. 4). Following normalization, it may be seen that the LES profile more closely resembles those obtained experimentally, but there are data available for many more time steps within the LES. The LES exhibits a secondary peak, which is observed experimentally as well, but this secondary peak is closer in velocity magnitude to the initial peak, and in some trials, it surpasses the velocity of the first peak. The velocity magnitude within the jet was spatially averaged and normalized (Fig. 5). The plot, again, shows a reasonable agreement between the LES and experimental data, although a large variability was observed experimentally.

In order to obtain a better understanding of the spread, and angle of entry of the studied cough jets, U-component velocity measurements were averaged across all $\mathrm{x}$-values, for each $\mathrm{y}$ value $\left(\left\langle\mathrm{U}_{\mathrm{x}}{ }^{\prime}\right\rangle\right)$. These profiles were then normalized by the maximum average $U$ velocity and the time that it occurs (Fig. 6). These directionally-averaged profiles were translated so that the jet was centered on the inlet axis. The average distance that the profiles were translated was $0.179 \mathrm{~m}$, indicating that the hot-wire anemometer was appropriately placed to measure velocities at the centre of the jet. An average jet entry angle of 10.2 degrees below the horizontal centreline was calculated from basic trigonometric ratios. Gupta et al. demonstrated that the average cough angle was 40 degrees [10], thus the padded headrest and chin rest successfully reduced the angle, so that the cough enters the chamber roughly horizontally.

The hot-wire data obtained from this cohort requires further analysis before the results can be presented, but hot-wire data was collected from 9 subjects who were recruited during the 2017 flu season. From these, 4 subjects had laboratory confirmed illnesses from the MTS results. Considering the recruitment methods, this is overall a good result. While the other 5 participants exhibited influenza-like symptoms, they might have had bacterial infections like pneumonia or streptococcus, although the presence of these infections was not assessed. The four etiological agents that were found were: coronavirus (CoV) NL63, CoV OC43, Influenza A (H3N2) and respiratory syncytial virus (RSV). Typical velocity time histories are shown for one participant in Fig. 7. The coughs were processed using a moving average filter, with a window size of 299 samples $(0.299 \mathrm{~s})$, so that residual turbulence fluctuations could be separated from the mean cough speed. The peak moving average cough speeds were ranked (Fig. 8), and negligible far field velocities were obtained for about a third of the coughs, in both the sick and convalescent cases. Fig. 9 shows the cumulative average of peak moving average velocity and turbulence intensity of coughs ordered from smallest to largest. The results show that for this cohort, convalescent coughs were slightly stronger but a minimal difference in turbulence intensity was noticed.

\section{CONCLUSION}

At the time of writing 44 participants have been recruited since the study began in 2014. Of these 44, 21 have exhibited influenza-like symptoms and were included in the "sick" cohort. Altogether, HWA data were collected for 48 "sick" coughs, 24 "convalescent" coughs, and 33 "healthy" coughs. PIV data were collected for 29 "sick" coughs, 9 "convalescent" coughs and 15 "healthy" coughs, though much of the data requires further analysis, and were not included in the present paper. MTS and filter cassettes from the most recent cohort of "sick" participants will soon be assessed for viral content. When comparing the preliminary data with the LES model there is reasonable agreement. As more data are collected, it is expected that the agreement will improve, and that it should then be possible to better quantify the differences between coughs from sick and healthy subjects.

\section{ACKNOWLEDGMENTS}

This work is funded by the CIHR/NSERC Collaborative Health Research Program, Canada. Initial funding was provided

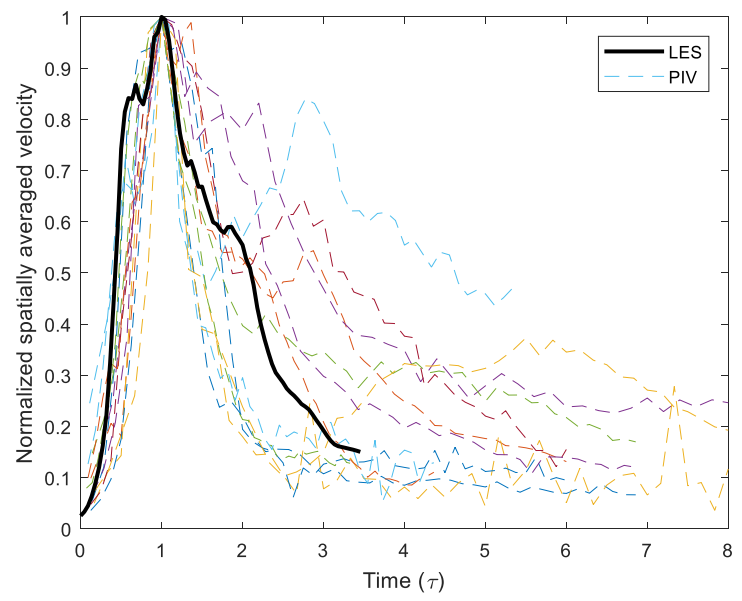

Figure 5: Normalized PIV Spatially Averaged Velocity Magnitudes 
through the Ontario Thoracic Society and the NSERC Engage program. We would also like to express gratitude to the staff at Western Student Health Services for their assistance in participant recruitment, and to Dr. William Lin for his work in conducting previous experiments, and his work in the construction of the experimental facility.
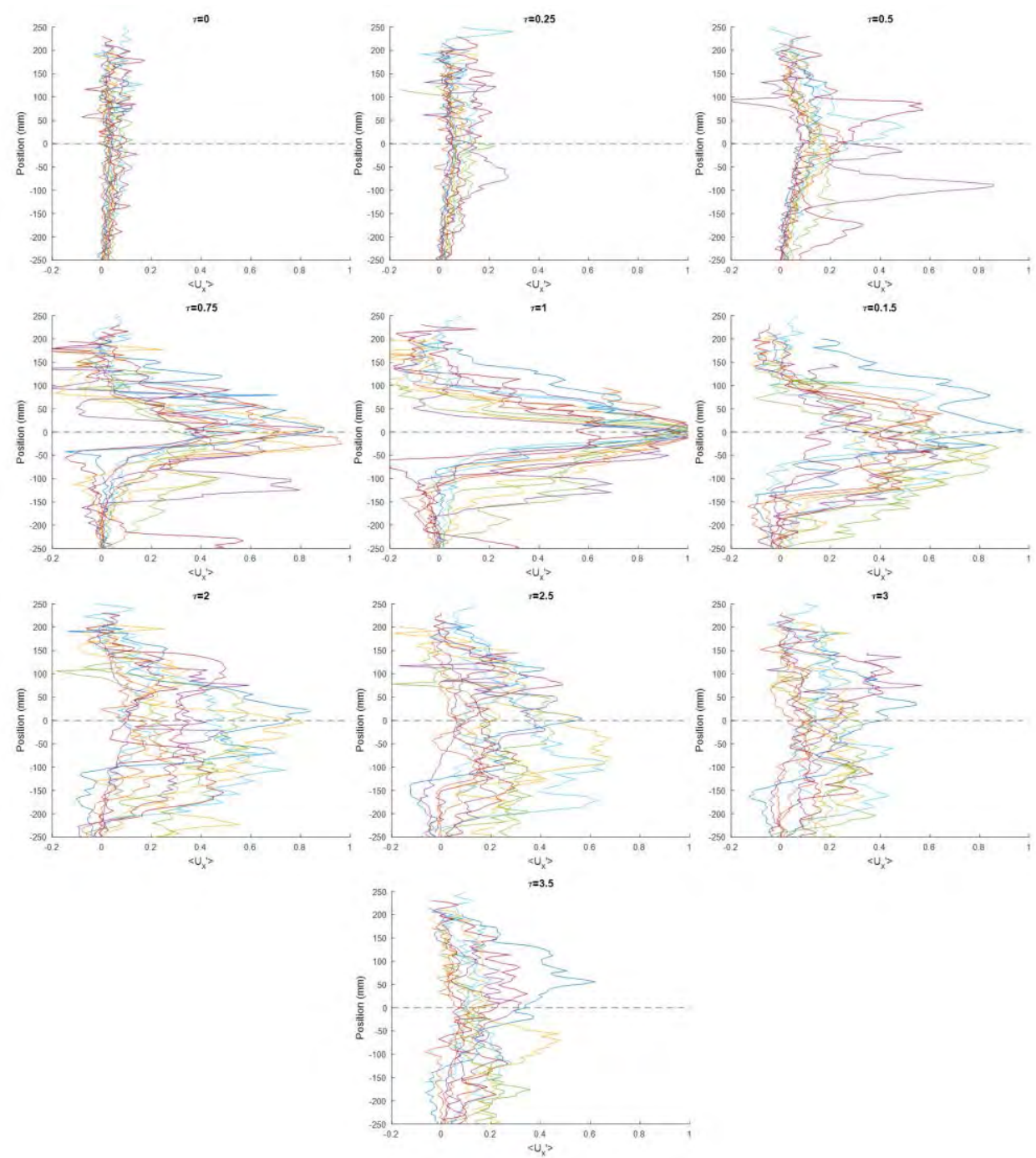

Figure 6: PIV U component velocity averaged across $x$, plotted against $y$ 


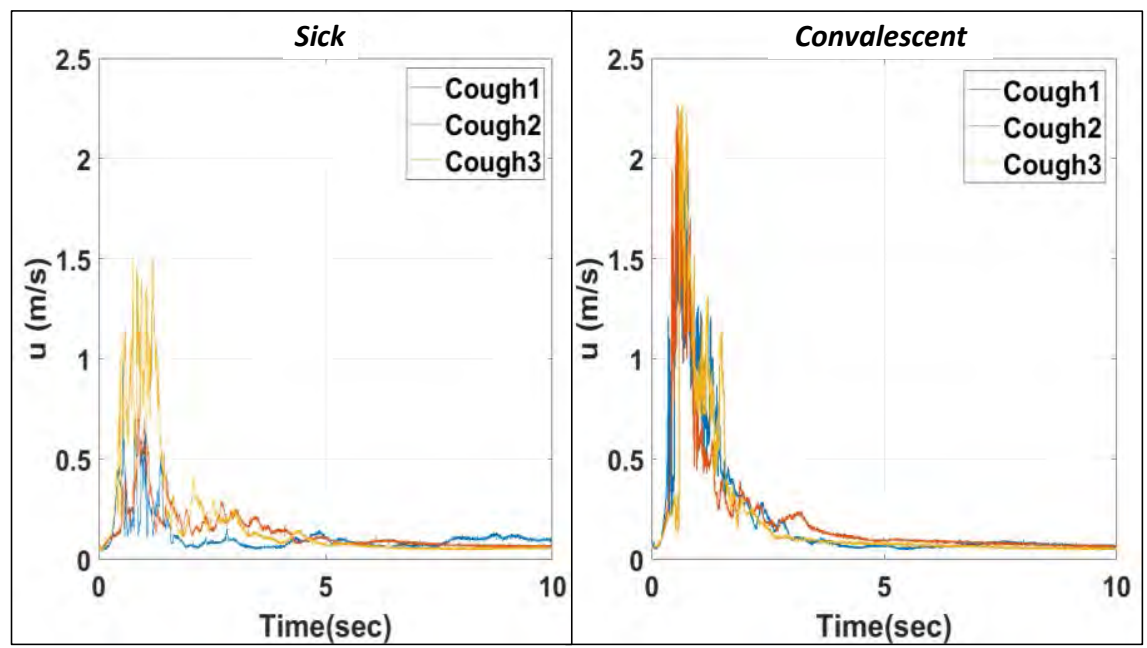

Figure 8: HWA Velocity Profiles

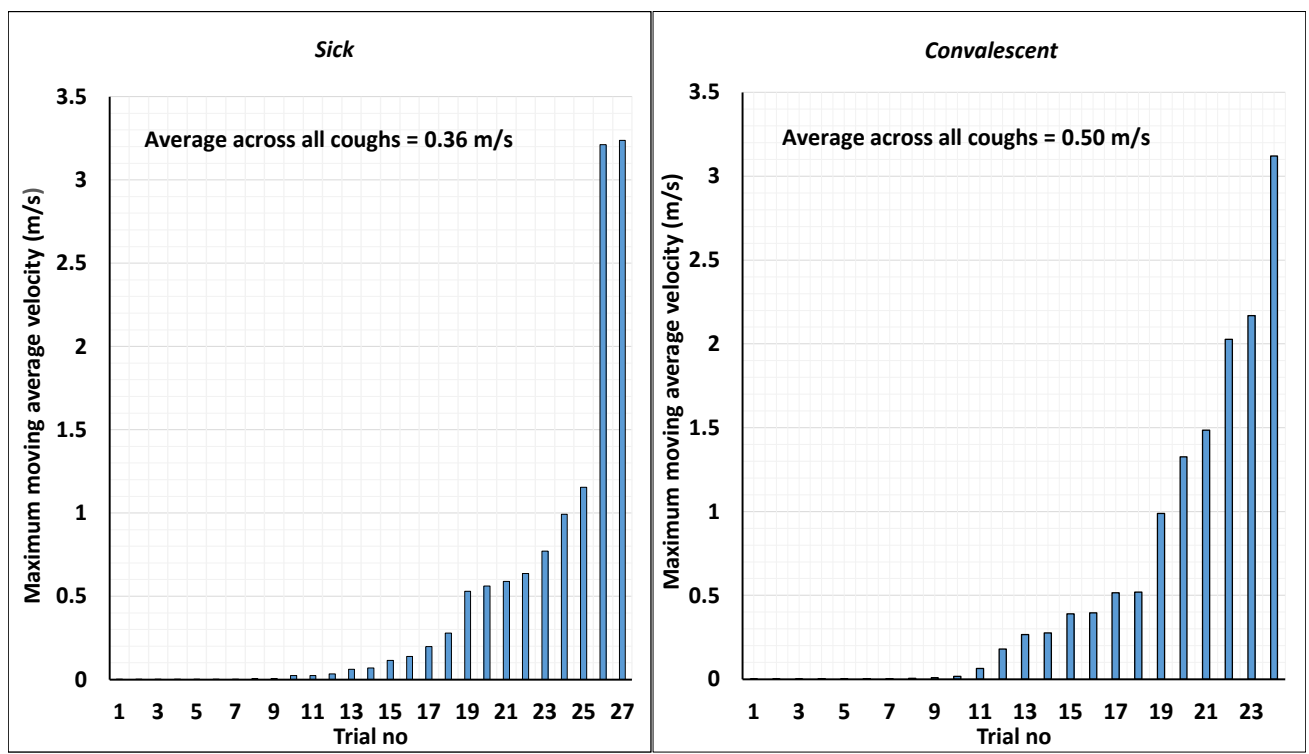

Figure 7: Ranked moving average velocities

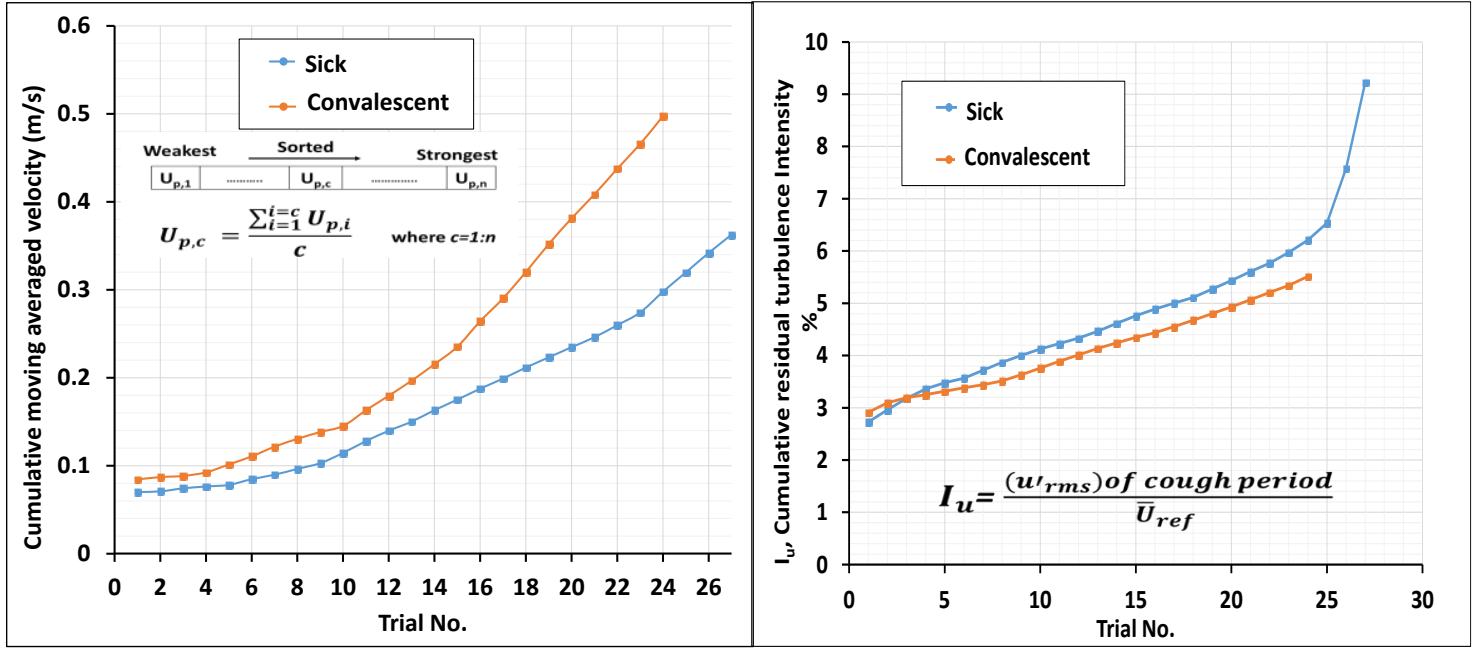

Figure 9: Cumulative averaged velocities and turbulence intensities 


\section{REFERENCES}

[1] M. Kennamer, Basic infection control for health care providers. 2nd ed. Albany: Thomson Delmar Learning, 2007.

[2] S. Zhu, S. Kato, and J. H. Yang, "Study on transport characteristics of saliva droplets produced by coughing in a calm indoor environment," Build Environ., vol. 41, no. 12, pp. 1691-1702, Dec. 2006. doi: 10.1016/j.buildenv.2005.06.024.

[3] J. W. Tang et al., "A Schlieren optical study of the human cough with and without wearing masks for aerosol infection control," $J$. R. Soc., Interface, vol. 6, no. Suppl 6, pp. S727-S736, Sept. 2009. doi: 10.1098/rsif.2009.0295.focus.

[4] S.-B. Kwon et al., "Study on the initial velocity distribution of exhaled air from coughing and speaking," Chemosphere, vol. 87, no. 11, pp. 1260-1264, June 2012. doi: 10.1016/j.chemosphere.2012.01.032.

[5] H. Nishimura, S. Sakata, and A. Kaga, "A new methodology for studying dynamics of aerosol particles in sneeze and cough using a digital high-vision, high-speed video system and vector analyses," PLoS One, vol. 8, p. e80244, Nov. 2013. doi: 10.1371/journal.pone.0080244.

[6] C. Y. H. Chao et al., "Characterization of expiration air jets and droplet size distributions immediately at the mouth opening," $J$. Aerosol. Sci., vol. 40, no. 2, pp. 122-133, Feb. 2009. doi: 10.1016/j.jaerosci.2008.10.003.

[7] M. VanSciver, S. Miller, and J. Hertzberg, "Particle image velocimetry of human cough," Aero. Sci. Tech., vol. 45, pp. 415-422. Jan. 2011. doi: 10.1080/02786826.2010.542785.

[8] E. Savory et al., "Western cold and flu (WeCoF) aerosol study preliminary results," BMC Res. Notes, vol. 7, no. 1, p. 563, 2014. doi: 10.1186/1756-0500-7-563.

[9] R. Bi et al., "Computational Fluid Dynamics modeling of human coughs," in Proc 25th Annual Conf. of CFD Soc. of Canada, Windsor, Ontario, June 2017.

[10] J. K. Gupta, C.-H. Lin, and Q. Chen, "Flow dynamics and characterization of a cough," Indoor Air, vol. 19, no. 6, pp. 517525, Nov. 2009. doi: 10.1111/j.1600-0668.2009.00619.x. 


\section{On the Validity Extent of Linear Viscoelastic Models of Human Brain}

\author{
Hesam Hoursan \\ Ph.D Student, School of Mechanical Engineering \\ Sharif University of Technology \\ Tehran, Iran \\ Hesam.hoursan74@student.sharif.edu
}

\author{
Reyhaneh Kazemiasfeh \\ Taleghani Hospital \& Medical Center \\ Tehran, Iran \\ rkazemi68@gmail.com
}

\author{
Mohammad Taghi Ahmadian \\ Professor, School of Mechanical Engineering \\ Sharif University of Technology \\ Tehran, Iran \\ Ahmadian@sharif.edu
}

\begin{abstract}
Characterization of human brain material properties in the form of computationally feasible mathematical models is a complex problem; especially when the models are used in complicated Finite Element simulations. Various models have been proposed to include the tissue's hyper-viscoelasticity, most of which are quite complicated and therefore only suited to Software-based Finite Element methods. Use of linear material models simplifies the problem and saves much time and effort, allowing the researcher to verify the results of more sophisticated models with lower computational cost. However, the preciseness of the results from such models is subject to special conditions. This study proposes and validates a Generalized Maxwell linear viscoelastic model with five constants to be used as an acceptable computational method to simulate brain's viscoelastic behavior at low strain rates. To this end, an explicit numerical integration scheme is used to simulate the single-DOF tissue response with a Generalized Maxwell viscoelastic model. Using the material constants of a previous hyper-viscoelastic model, the results are compared with those obtained from a previous experiment. The comparison shows that the linear GM viscoelastic model is predicting the low-strain-rate behavior of the brain tissue with acceptable error.
\end{abstract}

Keywords- Viscoelasticity; Finite Element; Generalized Maxwell, Linear Viscoelastic Model; Strain Rate

\section{1- INTRODUCTION}

The head is often considered as the most critical region of the human body for life-threatening injuries as result of accidents. The cost incurred by the accidents is estimated to be 160 billion Euros per year in the European Union alone [1]. In a 6-year period, an average of 1.4 million cases of traumatic brain injury occurred in the United States each year, of which $20 \%$ resulted from motor vehicles accidents. In order to develop effective protective measures, a better understanding of the process of injury development in the brain is required. [2-5]

Over the past three decades, several researchers have investigated the mechanical properties of brain tissue in order to establish constitutive relationships over a wide range of loading conditions. In experimental methods, dynamic oscillatory shear tests $[4,8,9]$ and unconfined compression tests [10] have been conducted more frequently. The resulting constitutive models have been introduced in Finite Element (FE) analyses to simulate the actual response of the tissue to external stimuli. Among these modes, most of them use hyperviscoelastic constitutive material assumption which, when combined with the complex geometry of brain parts, lead to complicated analyses [11]. The nonlinearity included in such models may lead to distorted results if the convergence problem is not handled. Therefore, simpler linear models such as the one presented here can serve as useful tools for verification of these models.

In current FE head models, brain tissue is commonly assumed to display hyper-viscoelastic material behavior. The tissue behaves like a non-linear viscoelastic solid for shear strains above $1 \%$. The modelling of the tissue behavior with these assumptions leads to somewhat more accurate results than linear models. However, it maintains the big drawback of high 
computational cost and time-consuming nature of these constitutive nonlinear models. [5, 6, 12]. Simpler computational frameworks such as the one presented herein serve as useful tools for engineering approximations of the resulting values of stress and strain fields.

This study seeks to investigate the extent of validity of a linear viscoelastic numerical model for modelling the response of the brain tissue to single-DOF loading-unloading cycles of shear strain. In order to evaluate the model, the results are compared, on various levels, with those of an experimental study.

\section{2- METHODOLOGY}

\section{2-1- Derivation of Mathematical Models}

\section{Generalized Maxwell Model}

In order to derive a computational framework for solving the Generalized Maxwell (GM) model (figure 1), one may derive the equation for a single mode spring and damper in series and then sum over the number of modes. The effect of infinity spring is finally added to complete the model.

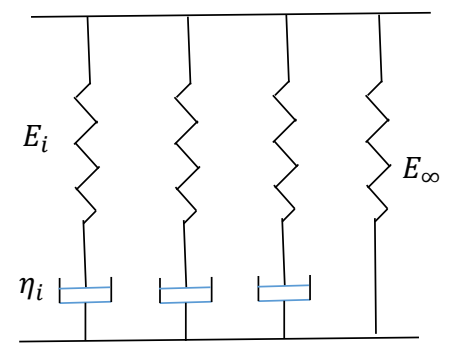

Figure 1- The Generalized Maxwell (GM) model for viscoelastic materials. $E_{i}$ and $\eta_{i}$ are serial spring and damping constants and $E_{\infty}$ is the parallel spring constant

Considering a single mode spring and damper in series and the infinity spring in parallel, the stress can be written in terms of strain and strain rate in the following form,

$\sigma(t)=E_{\infty} \epsilon(t)+\sigma^{*}$

where

$\sigma^{*}=\boldsymbol{\eta} \frac{\mathrm{d} \epsilon^{*}}{\mathrm{dt}}$

Solving and integrating the equation above for $\epsilon(t)$, the integral equation for two consecutive steps is obtained in the following form,

$\sigma_{n+1}^{*}-\sigma_{n}^{*}=\frac{-1}{\lambda} \int_{t_{n}}^{t_{n+1}} \sigma^{*}(t) d t+E\left(\epsilon_{n+1}^{*}-\epsilon_{n}^{*}\right)$

Considering that the integral is from $t_{n}$ to $t_{n+1}$, it's possible to approximate it using Implicit or Explicit method (Forward Euler and Backward Euler)
Substituting,

$\sigma^{*}(t)=E \epsilon(t)-y(t)$

Where,

$\frac{d y(t)}{d t}=\frac{-1}{\lambda} y(t)+\frac{E}{\lambda} \epsilon(t)$

An explicit numerical method can be obtained to approximate the integral in the following form:

$$
\sigma_{n+1}^{*}=e^{\frac{-\Delta t}{\lambda}} \sigma_{n}^{*}+e^{\frac{-\Delta t}{\lambda}} E \Delta \epsilon_{n}
$$

Now one can add the effects of all the parallel units the single-mode model. $\sigma_{n}^{*}$ is calculated for each unit and finally summed to obtain the total $\sigma^{*}$.

$$
\sigma^{*}=\sigma_{1}^{*}+\sigma_{2}^{*}+\cdots+\sigma_{n}^{*}
$$

The total $\sigma^{*}$ is subsequently added to the effect of infinity spring to yield the total stress by substituting into equation 1 . In the above equations, $\mathrm{n}$ is the order of GM model denoting the number of parallel modes. Time constants need to be calculated for every parallel Maxwell unit and considered separately in the corresponding stress update algorithm:

$$
\lambda_{i}=\frac{\eta_{i}}{E_{i}}
$$

\section{2-2- Application of Load}

Two load scenarios were considered as input, namely Load Scenario A and Load Scenario B. The load scenarios were chosen to replicate a previous experiment on samples from human head $(\operatorname{Ref}[4])$

\section{2-2-1- Load Scenario A}

In this load scenario, triangular pulses with constant strain rate of $1.5 / \mathrm{s}$ were applied to the viscoelastic single-dimensional model. The input pulses were obtained from [4] and simulated as triangular pulses (Fig. 2). The strain was applied as input by using explicit finite difference method with 600 steps and time constant of $1.1 \mathrm{~s}$. The strain rate was kept constant at the value of $1.5 / \mathrm{s}$ (Table 1$)$. 


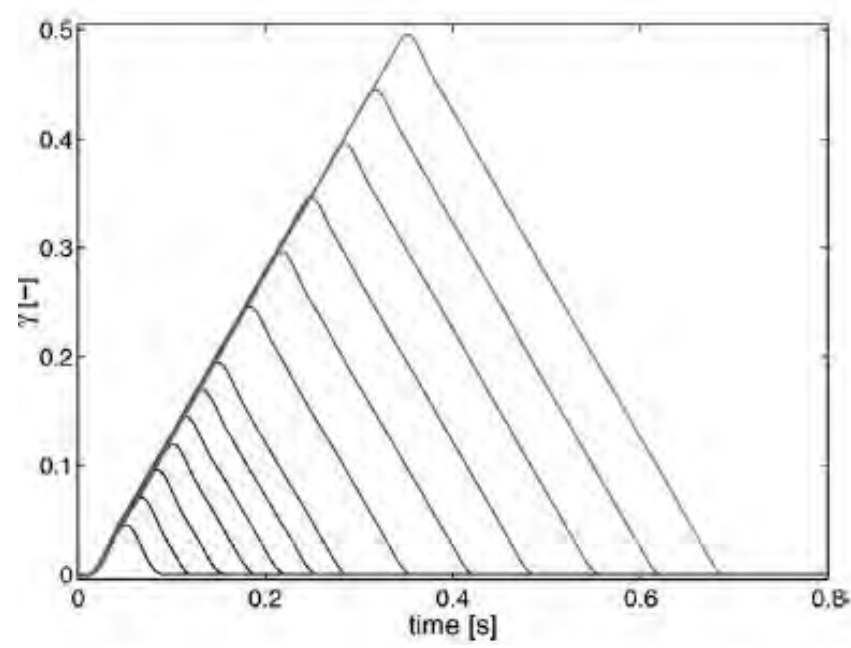

Figure 2- Triangular pulses (constant shear rate in loading and unloading) of Scenario A [4]

Table 1- Characteristics of the Triangular pulses of Scenario A [4]

\begin{tabular}{|c|c|c|c|}
\hline $\begin{array}{c}\text { Pulse } \\
\text { number }\end{array}$ & $\begin{array}{c}\text { Max. } \\
\text { Strain }\end{array}$ & $\begin{array}{c}\text { Pulse } \\
\text { Duration (s) }\end{array}$ & $\begin{array}{c}\text { strain Rate } \\
(\mathbf{s})\end{array}$ \\
\hline $\mathbf{1}$ & 0.05 & 0.06 & 1.50 \\
\hline $\mathbf{2}$ & 0.10 & 0.13 & 1.50 \\
\hline $\mathbf{3}$ & 0.15 & 0.20 & 1.50 \\
\hline $\mathbf{4}$ & 0.20 & 0.26 & 1.50 \\
\hline $\mathbf{5}$ & 0.30 & 0.40 & 1.50 \\
\hline $\mathbf{6}$ & 0.50 & 0.66 & 1.50 \\
\hline
\end{tabular}

\section{2-2-2- Load Scenario B}

In this load scenario, triangular pulses with various strain rates of $1 / \mathrm{s}, 0.1 / \mathrm{s}$ and $0.01 / \mathrm{s}$ were applied to the viscoelastic single-dimensional model. Table 2 shows the characteristics of the input strain pulses applied to the computational model.

Table 2- Characteristics of the Triangular pulses of Scenario B

\begin{tabular}{|c|c|c|c|}
\hline $\begin{array}{c}\text { Pulse } \\
\text { number }\end{array}$ & $\begin{array}{c}\text { Max. } \\
\text { Strain }\end{array}$ & $\begin{array}{c}\text { Pulse end } \\
\text { time (s) }\end{array}$ & $\begin{array}{c}\text { Strain rate } \\
(/ \mathbf{s})\end{array}$ \\
\hline $\mathbf{1}$ & 0.1 & 0.2000 & 1 \\
\hline $\mathbf{2}$ & 0.1 & 2.0000 & 0.1 \\
\hline $\mathbf{3}$ & 0.1 & 20.0000 & 0.01 \\
\hline
\end{tabular}

\section{3- Material constants}

For the 5-branch GM linear viscoelastic model, 5 sets of material data including the instantaneous and equilibrium shear moduli, time constant, and viscous damping coefficient was obtained from Ref [4]. The input data was included in the GM model in the form of Prony Series (Table 3)

Table 3-constants of the GM model obtained from curve-fitting of a hyperviscoelastic model on the experimental results of Ref [4]

\begin{tabular}{|c|c|c|c|c|}
\hline $\begin{array}{c}\text { GM } \\
\text { Mode } \\
\text { Number }\end{array}$ & $\begin{array}{c}\text { G } \\
\text { (Modulus) } \\
\text { (Pa) }\end{array}$ & $\begin{array}{c}\boldsymbol{\tau} \text { (time } \\
\text { constant) } \\
\text { (S) }\end{array}$ & $\begin{array}{c}\text { ๆ (Viscous } \\
\text { damping } \\
\text { coefficient) }\end{array}$ & $\begin{array}{c}\text { Ge } \\
\text { (Equilibrium } \\
\text { Modulus) } \\
\text { (pa) }\end{array}$ \\
\hline Mode 1 & 835.50 & 0.012 & 10.02 & 182.90 \\
\hline Mode 2 & 231.20 & 0.35 & 80.92 & 182.90 \\
\hline Mode 3 & 67.10 & 4.62 & 310.00 & 182.90 \\
\hline Mode 4 & 3.610 & 12.13 & 43.68 & 182.90 \\
\hline Mode 5 & 2.79 & 54.31 & 151.49 & 182.90 \\
\hline
\end{tabular}

\section{4- Results}

The results of the application of nine strain pulses from two scenarios to the GM model are shown in figures 3 and 4. Fig. 3 indicates the resulting stress from input strain of load scenario A with constant strain rate, and Fig. 4 shows the effect of changing the strain rate (load scenario B)

In all calculations, in order to reach better convergence and considering a recovery time as two times a full pulse, explicit algorithm has been used with 600 steps. 


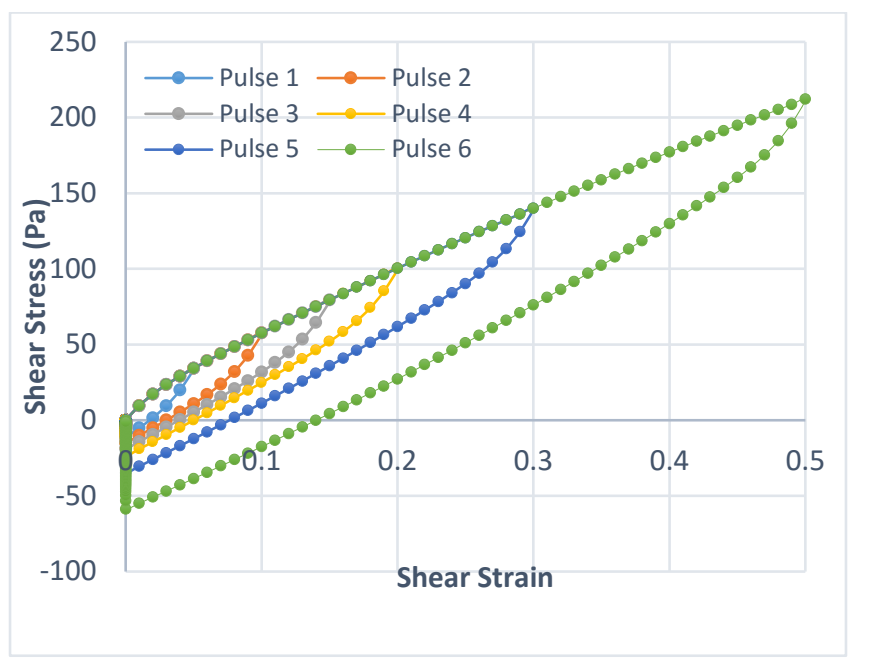

Figure 3 -Stress-Strain results obtained from applying the input pulses of Scenario A to the Generalized Maxwell Model (5 branches)

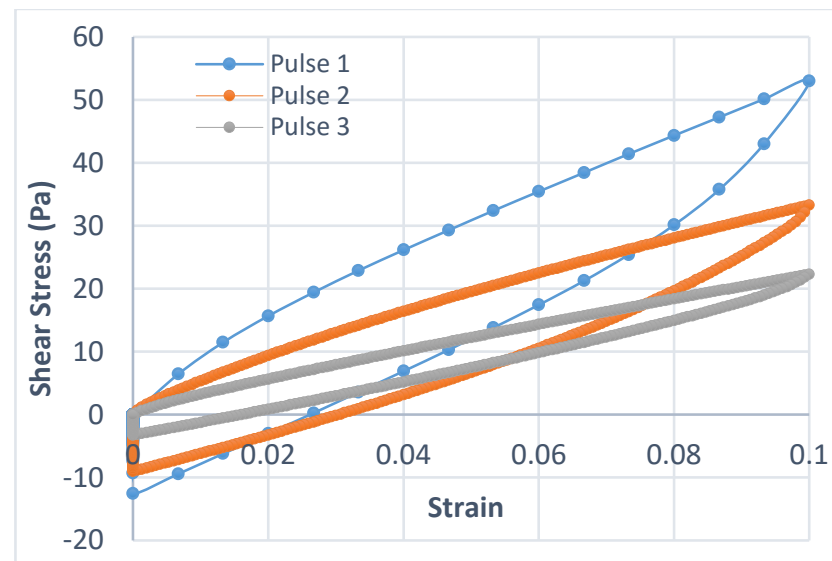

Figure 4 -Stress-Strain results obtained from applying the input pulses of Scenario B to the Generalized Maxwell Model (5 branches)

\section{4- Discussion of Results}

Nine input strain pulses were applied to a single-DOF linear GM viscoelastic model in two load scenarios. The resulting stress was calculated by using a finite difference integration scheme and plotted against the stress for all pulses (Fig. 3, 4). The input strain and material constants were obtained from curve-fitting of a hyper-viscoelastic model on a set of experimental curves from the literature [4].

The results from the application of the same load pulses in the referenced experiment are shown in Fig 5 and 6. As it can be observed, the Generalized Maxwell of order 5 shows acceptable qualitative agreement with those of the experiment. As it was predicted, the similarity of the trends is more noticeable at lower strain rates. The relative error at the end of the loading cycle (i.e. at maximum strain) for pulse 3 is $3 \%$ with respect to the experimental value. This can be observed by comparison of Fig. 3 and 5. The linearity of the model leads to differences with the experimental results which are more pronounced at higher input strains and strain rates. At the strain of 0.5 , the result of stress shows $10 \%$ deviation from the experimental results (pulse 6 from load scenario A). In addition, the quantitative comparison of the results reveals the incapability of the linear model in prediction of the material behavior at high strain rates and high strains (by comparison of the stress in pulse 1 from scenario B and the corresponding curve in Fig. 6). At the strain rate of 0.1/s and strain of 0.1 , the relative error of the linear model with respect to experiment results is $33 \%$ which is more than the one reported by the hyper-viscoelastic model of reference [4] $(21 \%)$.

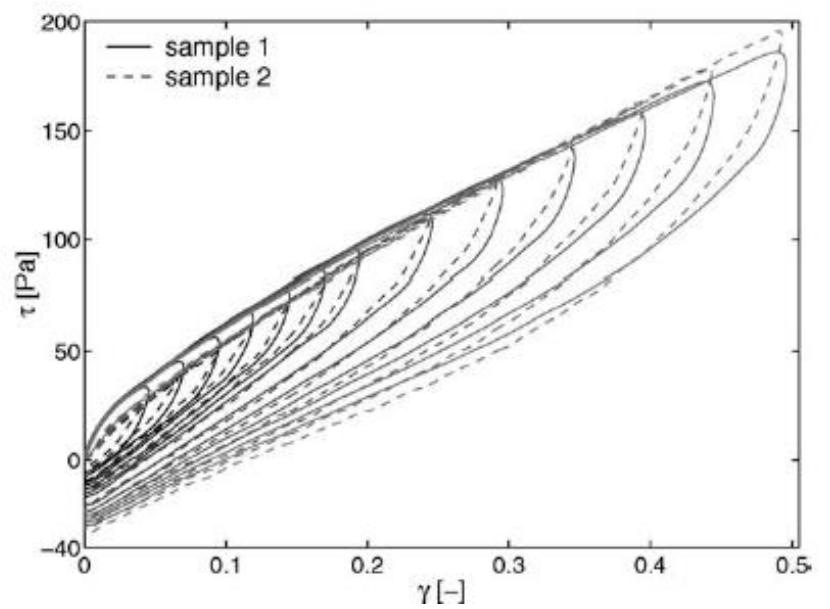

Figure 5-Stress-Strain curve (Curve-fitted) obtained from applying the input pulses of Scenario A (constant shear rate) in the experiment of Ref [4] for two samples.

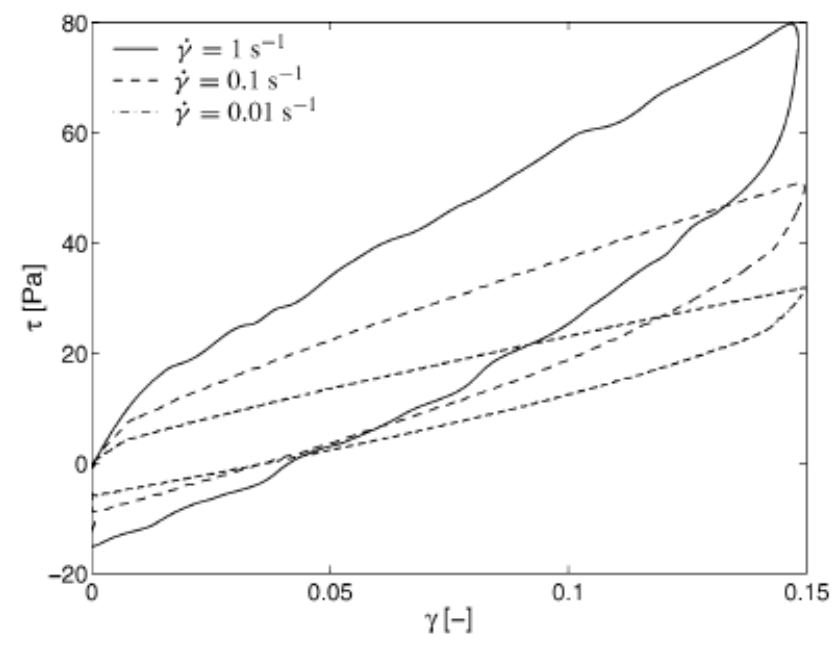

Figure 6-Stress-Strain curve obtained from applying the input pulses of Scenario B (constant shear rate) in the experiment of Ref [4] 


\section{5- Conclusions}

In this study, a linear viscoelastic model was used to assess the capability of linear computational models to model the complex behavior of brain material. 9 input strain pulses were replicated from a previous experiment and applied to the model and the resulting stress was compared with the ones reported in the literature.

It was observed that the results of our model show good qualitative agreement with those of the experiment. In terms of quantity, however, the agreement of the results is restricted to lower strain values and strain rates. Also, the impact of strain rate on the difference seems to be more noticeable than the absolute value of strain input. However, it seems that the mentioned deviation does not justify using a nonlinear hyperviscoelastic model in some studies instead of a linear viscoelastic one which is simpler and has a lower computational cost. It should also be noted that, in order to use the linear viscoelastic model, the parameters of the model must be obtained through direct comparison with experimental results, which may lead to improvements in the accuracy of the present model.

Finally, the comparison of the results reveals that the linear model, although not capable to fully trace the hyper-viscoelastic nature of the phenomena, displays a maximum relative error of $10 \%$, which can be justified given the lower computational cost. This can especially be useful in real-time simulations of surgical procedures, tumor growth and other applications involving change in brain tissue with low strain rates. The GUI used in this study to simulate and obtain the results can be an example of a useful tool for such applications (Figure 7).

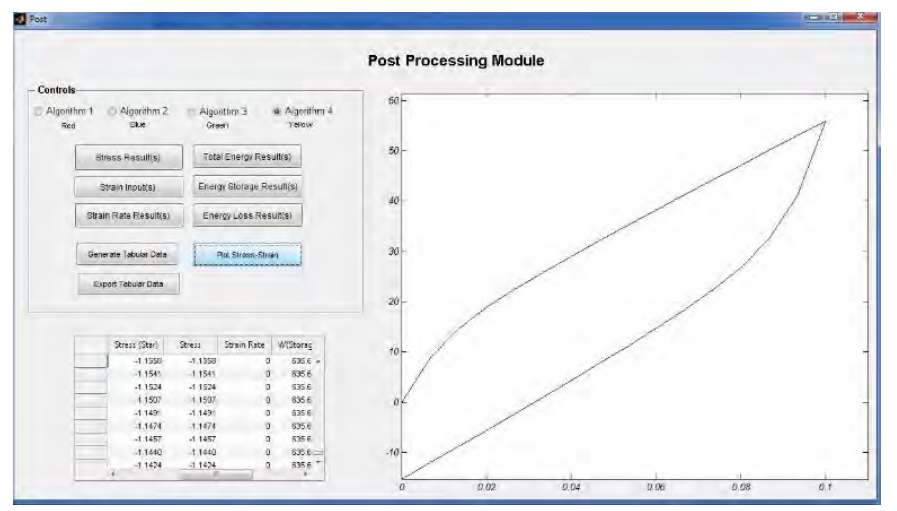

Figure 7- Stress-strain result of the application of pulse No. 2 from Scenario $B$ to the model and visualization of the results via Matlab GUI. The GUI was developed based on the calculations presented in this paper to better visualize the results.

\section{6- References}

[1] J.Ivarsson, D.C,Viano, P.Lovsund, B.Aldman, "Strain relief from the cerebral ventricles during head impact: experimental studies on natural protection of the brain,".Journal of Biomechanics, vol. 33, issue 2, pp.181-189, 2000, doi:10.1016/S0021-9290(99)00144-X.

[2] S.S.Margulies, L.E.Thibault and T.AGennarelli, "Physical model simulation of brain injury in the primate". J.Biomech, vol 23, pp. 823-836, 1990, doi:10.1016/0021-9290(90)90029-3.

[3] S.S.Margulies and L.E.Thibault. "A proposed tolerance criterion for diffuse axonal injury in man". J.Biomech, vol. 25, issue 8, pp: 917-923, 1992, doi:10.1016/0021-9290(92)90231-O.

[4] M.I. Hrapko, J.A. van Dommelen, G. W. Peters, J. S. Wismans, "The mechanical behaviour of brain tissue: large strain response and constitutive modelling," Biorheology. vol. 43, issue 5, pp.623-36, 2006.

[5] M.Prange, S.Margulies, "Tissue strain thresholds for axonal injury in the infant brain". Proc. ASME Bioeng. Conf. BED vol. 50, pp. 833-834, Snowbird, UT: Am. Soc. Mech. Eng.

[6] W.Hardy, C.D. Foster, M.J. Mason, K.H. Yang and A.I. King, "Investigation of head injury mechanisms using neutral density technology and high speed biplanar x-Ray," Stapp Car Crash Journal, November 2001, vol 45, paper no: 2001-22-0016.

[7] S.A.Lippert, M.J.Grimm, "Estimating the material properties of brain tissues at impact frequencies: a curve fitting solution", Summer Bioengineering conference 2003, June 25-29, Sonesta beach resort in key Biscayne, Florida.

[8] K.K. Darvish and J.R. Crandall, "Nonlinear viscoelastic effects in oscillatory shear deformation of brain tissue," Med. Eng.\& Phys., vol. 23, pp. 633-645, 2001, doi: 10.1016/S1350-4533(01)00101-1.

[9] B.R. Donnelly and J. Medige, "Shear properties of human brain tissue," J. Biomech. Eng. vol., 119, pp. 423-432, 1997, doi: $10.1115 / 1.2798289$.

[10] M.S. Estes and J.H. McElhaney, "Response of brain tissue of compressive loading," in Proceedings of the 4th ASME J.Biomechanics Conf., 70-BHF-13, 1970.

[11] K. Miller, "Constitutive modeling of brain tissue: Experiment and theory," J. Biomech., vol. 30, pp. 1115-1121, 1997, doi: 10.1016/S0021-9290(97)00092-4.

[12] B. Rashid, M. Destrade, M. Gilchrist, "Mechanical characterization of brain tissue in tension at dynamic strain rates," $J$. Mech. Behav. Biomed. Mater., vol. 10, pp. 23-38, 2012, doi: 10.1016/j.jmbbm.2012.01.022.

[13] A. Goriely, M.G.D. Geers, G.A. Holzapfel, et al., "Mechanics of the brain: perspectives, challenges, and opportunities," Biomech. Model. Mechanobiol., vol. 14, pp. 931-965, 2015, doi: 10.1007/s10237-015-0662-4. 
ENGINEERING ANALYSIS

$\&$ DESIGN 


\section{Vibration Isolation of a Carbon Nanotube Filled with a Mass Chain}

\author{
$\mathrm{Lu} \mathrm{Lu}$ \\ Department of Mechanical Engineering \\ University of Alberta \\ Edmonton, AB T6G 2G8, Canada \\ Shanghai Institute of Applied Mathematics and Mechanics \\ Shanghai University \\ Shanghai 200072, China E-mail address: 1lu1@ualberta.ca
}

\author{
C.Q. $\mathrm{Ru}$ \\ Department of Mechanical Engineering \\ University of Alberta \\ Edmonton, AB T6G 2G8, Canada
}

\begin{abstract}
Vibration isolation is one of the most prominent characteristics of elastic metamaterial with negative effective mass. In this work, we show that a carbon nanotube filled with a linear mass chain can behave like an elastic metamaterial with negative effective mass density and exhibit remarkable vibration isolation phenomena within a certain frequency range.
\end{abstract}

Keywords - carbon nanotube; metamaterial; negative effective mass; mass chain; vibration isolation.

\section{INTRODUCTION}

Elastic metamaterials characterized by negative effective mass and/or negative effective modulus have attracted considerable attention [1-3]. It has been shown that the negative effective mass/modulus of elastic metamaterials are essentially attributed to their internal degrees of freedom, particularly so-called "locally resonant" microstructure $[4,5]$. Inspired by an idea that a carbon nanotube (CNT) filled with a mass chain [6-8] could offer an already fabricated and available metamaterial with locally resonant microstructure, the present work aims to investigate filled carbon nanotubes as a potential new kind of elastic metamaterials. Unlike the known elastic metamaterials proposed in literature which are all based on often complicated artificial design of their microstructure, the carbon nanotube-based metamaterials proposed in the present work can exhibit negative effective mass and achieve desirable metamaterial dynamic properties even without any artificial design of their microstructure.

\section{CONTINUUM MODELING}

Fig. 1 shows the schematic of a single-walled CNT filled with a linear C-chain. The CNT is modeled as an elastic beam of length $L$, diameter $d$, bending rigidity $E I$, mass density $\rho_{1}$ and cross section area $A_{1}$. The inserted C-chain is treated as an elastic string of mass density $\rho_{2}$ and cross section area $A_{2}$ but with no meaningful bending rigidity. Thus, the coupled governing equations for the filled CNT can be written as [7]

$$
\begin{gathered}
E I \frac{\partial^{4} w_{1}}{\partial x^{4}}+\rho_{1} A_{1} \frac{\partial^{2} w_{1}}{\partial t^{2}}=c\left(w_{2}-w_{1}\right) \\
\rho_{2} A_{2} \frac{\partial^{2} w_{2}}{\partial t^{2}}=c\left(w_{1}-w_{2}\right)
\end{gathered}
$$

where $w_{1}$ and $w_{2}$ are the transverse deflection of the CNT and the $\mathrm{C}$-chain, respectively, $t$ is the time and $c$ is the van der Waals interaction coefficient (per unit length) between the mass chain and the CNT.

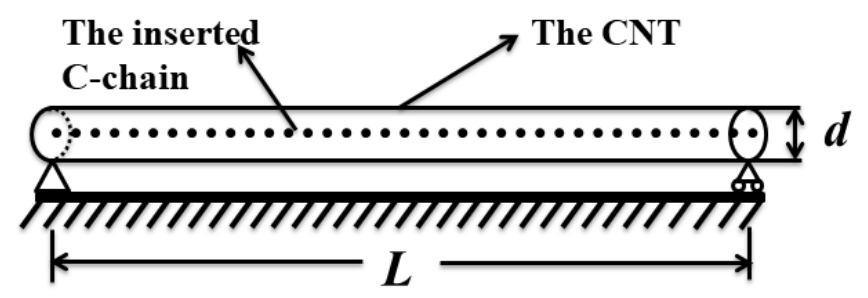

Figure 1 schematic of a single-walled CNT filled with a linear C-chain

Substituting Eq. (1) into Eq. (2), the governing equation of the filled CNT for the deflection $w_{1}$ can be obtained as

$$
\begin{aligned}
& E I\left(1+\frac{\rho_{2} A_{2}}{c} \frac{\partial^{2}}{\partial t^{2}}\right) \frac{\partial^{4} w_{1}}{\partial x^{4}}+\left(\rho_{1} A_{1}+\rho_{2} A_{2}\right) \\
& \times\left[1+\frac{\rho_{1} \rho_{2} A_{1} A_{2}}{c\left(\rho_{1} A_{1}+\rho_{2} A_{2}\right)} \frac{\partial^{2}}{\partial t^{2}}\right] \frac{\partial^{2} w_{1}}{\partial t^{2}}=0
\end{aligned}
$$

which is of the standard form of dynamic equation for a metamaterial elastic beam with negative effective mass density within a certain range of frequencies. To see this, let us consider a periodic harmonic motion $w_{1}(x, t)=f(x) \exp (i \omega t)$, where $f(x)$ is the mode shape function, $i$ denotes the imaginary unit and $\omega$ represents the frequency. Substituting $w_{1}$ into Eq. 
(3), the effective mass density of the filled CNT can be obtained as the following form:

$$
\rho_{\text {eff }}=\frac{1-\frac{\rho_{1} \rho_{2} A_{1} A_{2}}{\left.c \rho_{1} A_{1}+\rho_{2} A_{2}\right)} \omega^{2}}{1-\frac{\rho_{2} A_{2}}{c} \omega^{2}} \rho_{\text {avg }}
$$

It is clearly seen that the effective mass density become negative when the frequency is within the bandgap

$$
\sqrt{\frac{c}{{ }_{2} A_{2}}}=\omega_{0}<\omega<\omega \sqrt{1+\frac{\rho_{2} A_{2}}{\rho_{1} A_{1}}}
$$

It is noticed that the values and order of magnitude of this "metamaterial" bandgap are determined by the van der Waals interaction coefficient $c$ and the mass of the inserted mass chain, and the bandgap can be wider if the mass of inserted chain is not very small as compared to the mass of the CNT.

\section{FORCED VIBRATION DRIVEN BY PERIODICALLY VIBRATING ENDS}

Consider forced vibration of a hinged filled CNT driven by periodically vibrating ends: $w_{1}=\delta \sin \omega t$ at $x=0$ and $x=L$, where $\delta$ is the amplitude of the vibrating ends. The stimulated steady state forced vibration of the filled CNT is of the form

$$
w_{1}(x) \quad\left(1+\sum_{k=1}^{\infty} a_{k} \sin \frac{k \pi x}{L}\right) \sin \omega t
$$

where $a_{k}(k=1,2,3, \cdots)$ are some real constants. Substituting Eq. (6) into Eq. (3), and using the Fourier series expansion

$$
1=\sum_{k=1}^{\infty} \frac{2[1-\cos (k \pi)]}{k \pi} \sin \frac{k x}{L}, 0 \leq x \leq L
$$

where the coefficients $a_{k}$ are to be determined. Thus, forced vibration of the hinged filled CNT driven by two periodically vibrating ends can be evaluated. To demonstrate this, a $(5,5)$ CNT filled with a linear C-chain is considered. The material and geometrical properties of the CNT are taken as: Young's modulus $E=1 \mathrm{TPa}$, mass density $\rho_{1}=2200 \mathrm{~kg} / \mathrm{m}^{3}$, and singlewalled thickness $h=0.34 \mathrm{~nm}$.

The bandgap of the C-chain filled $(5,5)$ CNT is $\left(\omega_{0}\right.$, $\left.1.05 \omega_{0}\right)$, where $\omega_{0}$ is the lower-edge frequency $=3.09 \mathrm{THz}$. What plotted in Fig. 2 is forced vibrational modes of a C-chain filled-CNT driven by two periodically vibrating ends for excitation frequencies within the bandgap. It is seen that when the excitation frequency is within the bandgap, the forced vibrational mode is highly localized near the two vibrating ends but is vanishingly small in all other parts of the filled CNT, in sharp contrast to other excitation frequencies out of the bandgap for which the forced vibrational mode always spreads into the entire filled CNT. Therefore, a filled CNT indeed exhibits remarkable vibration isolation when it is excited by its periodically vibrating ends.

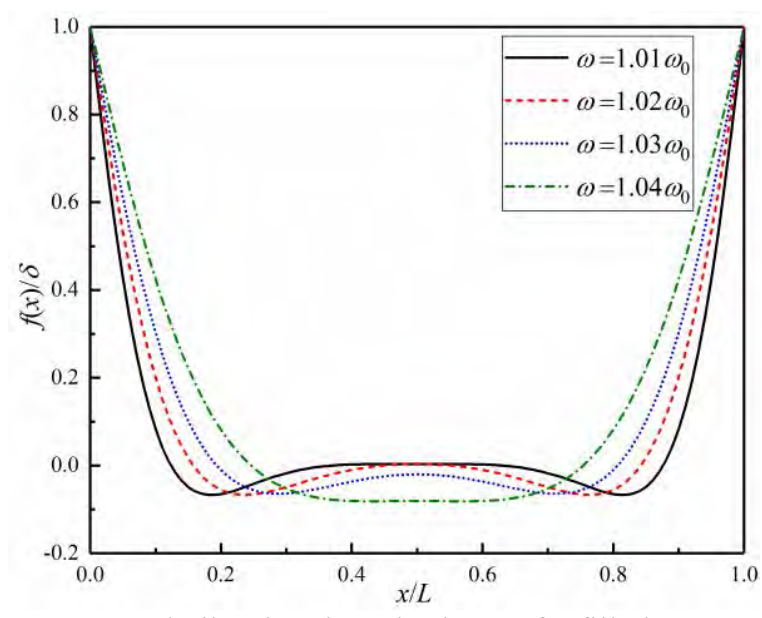

Figure 2 Forced vibrational mode shape of a filled CNT under different excitation frequencies $(L=10 d)$.

\section{CONCLUSIONS}

A carbon nanotube filled with a mass chain can behave like a metamaterial with negative effective mass and exhibit remarkable vibration isolation within a certain frequency range without any artificial design of their microstructure. When the excitation frequency is within the bandgap, the forced vibrational mode of a filled carbon nanotube driven by periodically vibrating ends is highly localized near the two vibrating ends but is vanishingly small in all other parts of the filled CNT.

\section{ACKNOWLEDGMENT}

Lu thanks the support from the China Scholarship Council (CSC) and $\mathrm{Ru}$ appreciates the support from the Natural Science and Engineering Research Council of Canada (NSERC-RGPIN204992).

\section{REFERENCES}

[1] Z.Y. Liu et. al.,"Locally resonant sonic materials," Science, vol. 289, issue 5485, pp. 1734-1736, 2000. doi: 10.1126/science.289.5485.1734.

[2] Y. Q. Liu, X. Y. Su, C.T. Sun, "Broadband elastic metamaterial with single negativity by mimicking lattice systems," J Mech Phys Solids, vol. 74, pp. 158-74, Jan. 2015. doi: 10.1016/j.jmps.2014.09.011.

[3] Y.Y. Chen, G.K. Hu, G.L. Huang, "A hybrid elastic metamaterial with negative mass density and tunable bending stiffness," J Mech Phys Solids, vol. 105, pp.179-198, Aug. 2017. doi: 10.1016/j.jmps.2017.05.009.

[4] Z. Y. Liu, C.T. Chan, S. Sheng, "Analytic model of phononic crystals with local resonances," Phys. Rev. B, vol. 71, pp. 014103, Jan. 2005. doi: 10.1103/PhysRevB.71.014103.

[5] G. W. Milton, J. R. Willis, "On modifications of Newton's second law and linear continuum elastodynamics," Proc. R. Soc. A, vol. 463, issue 2079, pp. 855-880, 2007. doi: $10.1098 /$ rspa.2006.1795.

[6] X. L. Zhao, Y. Ando, Y. Liu, M. Jinno, T. Suzuki, "Carbon nanowire made of a long linear carbon chain inserted inside a multiwalled carbon nanotube," Phys. Rev. Lett., vol. 90, 187401, 2003. doi: 10.1103/PhysRevLett.90.187401.

[7] Z. L. Hu, X. M. Guo, C.Q. Ru, "The effects of an inserted linear carbon chain on the vibration of a carbon nanotube," Nanotechnology, vol. 18, 485712. doi: 10.1088/0957-4484/18/48/485712.

[8] Z. L. Hu, X. M. Guo, C. Q. Ru, "Enhanced critical pressure for buckling of carbon nanotubes due to an inserted linear carbon chain," Nanotechnology, vol. 19, 305703. 802 doi: 10.1088/957-4484/19/30/305403. 


\section{Vibration Control of Downtown Toronto High-Rise Development}

\author{
Ali Noormohamed, Oya Mercan \\ Department of Civil Engineering \\ University of Toronto \\ Toronto, ON, Canada
}

\author{
Amirahmad Fathieh \\ Stephenson Engineering \\ Toronto, ON, Canada
}

\begin{abstract}
In this industry-academia collaboration, a multiplatform hybrid numerical simulation was performed to assess the effectiveness of a tuned liquid damper (TLD) to be installed in a high-rise condominium located in Downtown Toronto. The structure was modeled using OpenSees, while the nonlinear TLD model was developed in MATLAB. A dynamic analysis was performed using statistically-generated dynamic wind loads. It was determined that the TLD improved the performance of the structure (storey acceleration, roof drift) for various levels of wind loading, improving the comfort of the residents of the building. Minimal benefit was observed for reducing base shear as well as storey shear demands on the structure.
\end{abstract}

Keywords-tuned liquid damper; vibration control; structural control; hybrid simulation; wind engineering

\section{INTRODUCTION}

High-rise structures are flexible structures, and are highly sensitive to dynamic excitation - even vibration caused by ordinary wind loading may be problematic from the viewpoint of serviceability and comfort of occupants. Tuned-liquid dampers (TLDs) are passive vibration absorbers which are used to control wind-induced vibrations of tall structures. The TLD's properties are tuned to the system's fundamental oscillation frequency such that the liquids sloshing action dampens the building's oscillations. In this collaboration, a multi-platform tool was developed to accurately and precisely model the components required to understand the structure-liquid interaction. The structure was modeled using OpenSees [1], while the TLD was modeled in MATLAB [2] using Yu's model. Lastly, the dynamic wind loading applied to the structure was developed using NatHaz Online Wind Simulator (NOWS) webbased tool [3]. The details of each portion of the multi-platform tool are given below.

\section{Development of Multi-Platform ToOL}

\section{A. Structural Model}

OpenSees, an object-oriented open source software framework, was used to model the tower. The structural model which was developed for the central tower of the 90 Harbour Street development from the 6th storey upwards - since the podium levels and underground parking garages are shared between the two towers, they were assumed to provide a rigid base for each of the individual towers. As such, the base nodes of the structure were modeled as fixed nodes in OpenSees.

The structure was modeled using fibre section elements, wherein the sections were varied based on cross-sectional area, level of horizontal and vertical reinforcement, and concrete strength. Due to the complexity of the structure, and the large number of individual element properties to consider, the tower was split into seven categories (by storey), varying along the height of the structure. The individual member properties were assumed to remain constant in each section of the building.

Lastly, the floor slabs were not modeled directly in OpenSees. Rather, they were assumed to provide a rigid support at each floor level. The EqualDOF command in OpenSees was used, wherein each node at a given floor level is forced to have the same displacements as the designated master node at that floor. While the structural properties of the floor slab were not modeled, the mass of the slabs was still accounted for, with the mass being distributed to the nodes by tributary areas.

\section{B. TLD Model}

TLDs dissipate energy through liquid boundary layer friction, free surface contamination, and wave breaking. With the horizontal component of the liquid velocity related to the wave motion, wave crests descend as amplitude of motion increases, and simple linear models are no longer adequately able to describe the liquid behaviour [4].

1) Theory of Yu's Model: Using shallow water wave theory, the TLD can be modeled as an equivalent nonlinear-stiffnessdamping (NSD) system [5][6]. The behaviour of the NSD system is matched to that of the real TLD using an energy dissipation matching procedure, where the NSD parameters $m_{d}, c_{d}, k_{d}$ are determined by introducing the interaction force created by the TLD liquid sloshing inside the tank.

The energy dissipation inside the tank can be found using
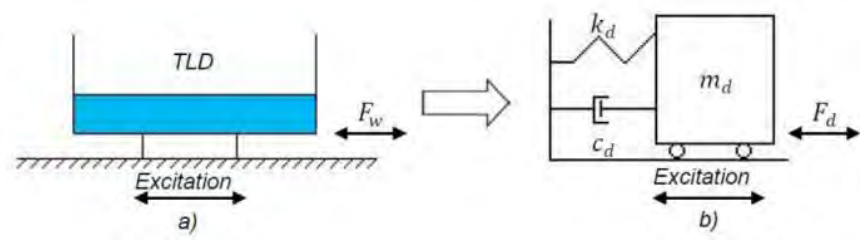

Figure 1: Schematic representation of (a) TLD and (b) equivalent NSD model.

Sponsored by NSERC Engage Grant EGP 486047-15. 


$$
E_{w}=\int_{T_{s}} F_{w} d x
$$

where $F_{w}$ is the force generated by the liquid sloshing inside the tank. The non-dimensionalized energy can be determined:

$$
E_{w}=\frac{E_{w}}{0.5 m_{w}(A \omega)^{2}}
$$

The non-dimensionalized energy dissipation parameter for the NSD model is determined using the behaviour when subjected to base excitation with frequency $\beta$ :

$$
E_{d}=2 \pi\left|F_{d}\right| \sin \theta
$$

where the non-dimensionalized magnitude $\left|F_{d}\right|$ and phase angle $\theta$ can be found using:

$$
\begin{aligned}
\left|F_{d}\right| & =\frac{\sqrt{\left(1+\left(4 \zeta_{d}^{2}-1\right) \beta^{2}\right)^{2}+4 \zeta_{d}^{2} \beta^{6}}}{1+\left(4 \zeta_{d}^{2}-2\right) \beta^{2}+\beta^{4}} \\
\theta & =\tan ^{-1}\left(\frac{2 \zeta_{d} \beta^{3}}{-1+\left(1-4 \zeta_{d}^{2}\right) \beta^{2}}\right)
\end{aligned}
$$

where $\zeta_{d}=\frac{c_{d}}{c_{c r}}$ is the damping ratio of the NSD model. The nondimensionalized energy dissipation parameters are matched together using a least-squares method, analyzing the results through two parameters: the frequency shift ratio $\xi=\frac{f_{d}}{f_{w}}$ and the stiffness hardening ratio $\kappa=\frac{k_{d}}{k_{w}}$. The frequency of the water in the tank is determined using:

$$
f_{w}=\frac{1}{2 \pi} \sqrt{\frac{\pi g}{L} \tanh \left(\frac{\pi h_{w}}{L}\right)}
$$

The stiffness of the water in the tank can then be determined:

$$
k_{w}=m_{w}\left(2 \pi f_{w}\right)^{2}
$$

In order to calculate the restoring force that the TLD applies to the structure, the following equations were used:

$$
F_{T L D}=k_{d} x_{T L D}+c_{d} \dot{x}_{T L D}
$$

where $x_{T L D}$ and $\dot{x}_{T L D}$ are the displacement and velocity of the TLD determined from the OpenSees model, and $k_{d}$ and $c_{d}$ are the NSD stiffness and damping parameters, determined from the energy matching procedure as follows:

$$
\begin{aligned}
k_{d} & =2.52\left(\frac{x_{T L D}}{L}\right)^{0.25} m_{d}\left(2 \pi f_{w}\right)^{2} \\
c_{d} & =0.52\left(\frac{x_{T L D}}{L}\right)^{0.35} 2\left(k_{d} m_{d}\right)^{2}
\end{aligned}
$$

2) Verification of Yu's Model: To justify the use of Yu's model in this simulation, results from the MATLAB model were compared with previously performed experiments on TLDs [4]. The TLD which was tested had measurements of $464 \mathrm{~mm} \times 305$ $\mathrm{mm}$, and a water height of $40 \mathrm{~mm}$. The corresponding tank frequency was $0.667 \mathrm{~Hz}$, and the weight of the water in the tank was $5.64 \mathrm{~kg}$. The TLD was subjected to a sinusoidal displacement history with amplitude of $20 \mathrm{~mm}$, and the results are shown in Figure 2.

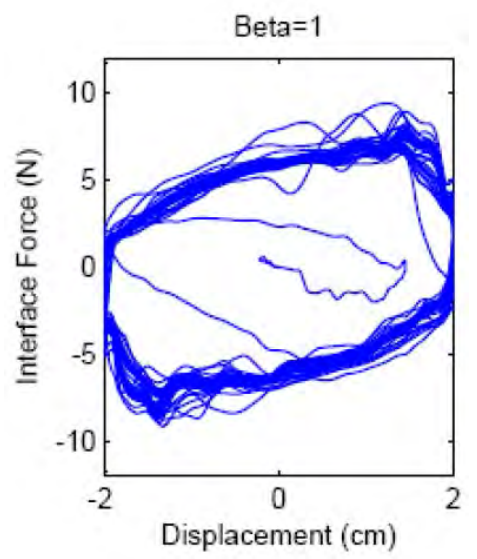

Figure 2: Experimental results for TLD force-displacement behaviour. TLD dimensions: $464 \mathrm{~mm} \times 305 \mathrm{~mm}$, water height $40 \mathrm{~mm}$.

The same TLD was then modeled in Matlab using Yu's model. In this implementation, a simple SDOF structure (portal frame) was modeled with the TLD at the roof level. A sinusoidal force was applied to the portal frame which resulted in a TLD displacement of $20 \mathrm{~mm}$, as in the experimental setup. This system was analyzed using the Newmark-beta method, and results are shown in Figure 3.

The results show that the MATLAB model matches the experimental data well - the shape of the force-displacement curve is similar, and the magnitude of the force is similar as well. Since the shallow water limit $\left(\frac{h_{w}}{L}<0.1\right)$ is met for both experimental and numerical TLD models, we can conclude that Yu's model is able to accurately capture the experimental TLD behaviour for the shallow water scenario.

\section{Dynamic Wind Loading Simulator}

The dynamic wind loading patterns for the structure were obtained using the NatHaz Online Wind Simulator (NOWS) web-based interface. The tool provides users with an on-line simulation of stationary Gaussian multivariate wind fields.

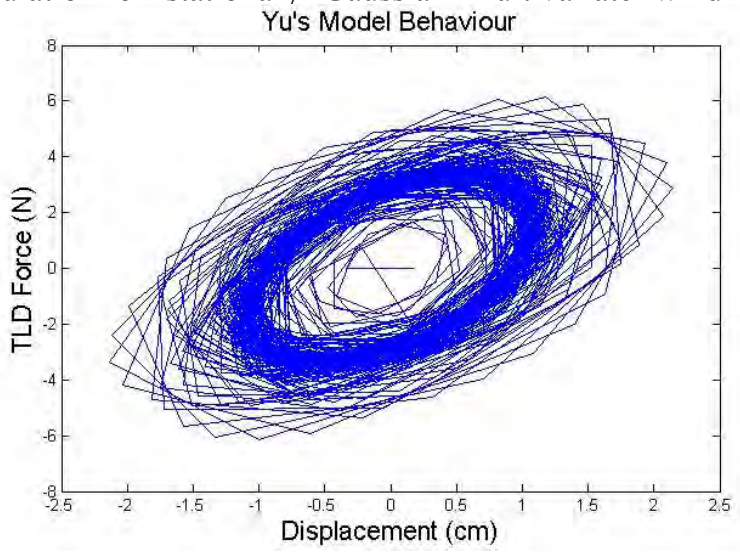

Figure 3: Matlab model results for TLD behaviour. TLD dimensions same as for experimental specimen in Figure 2. 
Random wind fields of varying intensities, corresponding to various National Building Code of Canada 2010 (NBCC) limit states [7], were generated using a discrete frequency function with Cholesky decomposition and Fast Fourier Transform (FFT). The outputs of the tool were a time history of wind speeds at the various heights of the structure - a sample output from the tool is shown in Figure 4. These wind speeds were then converted to equivalent forces using the guidelines given in ISO19902, which gives:

$$
F_{w}=\frac{1}{2} \rho_{a}\left(U_{w}\right)^{2} C_{s} A
$$

where $\rho_{a}$ is the mass density of air, $U_{w}$ is the given wind speed in $\frac{m}{s}, C_{s}$ is the shape coefficient of the structure, $A$ is the tributary cross-sectional area of the wind-facing surface, and $F_{w}$ is the equivalent lateral force.

\section{Multi-Platform Model Algorithm}

The methodology and algorithm that was used to define the interaction between all components of the tool is shown in Figure 5. This interaction loop was repeated for each time step that the wind loading was simulated. The outputs of interest were taken from the Matlab model and the OpenSees model, and included a time history of TLD force as well as structural displacements, acceleration, storey and base shears, as well as element forces.

\section{E. Solution Procedure}

The model was analyzed using a transient analysis method, and the Newton numerical method. While the details of this solution method are not given in this paper, further details can be found in literature [8].
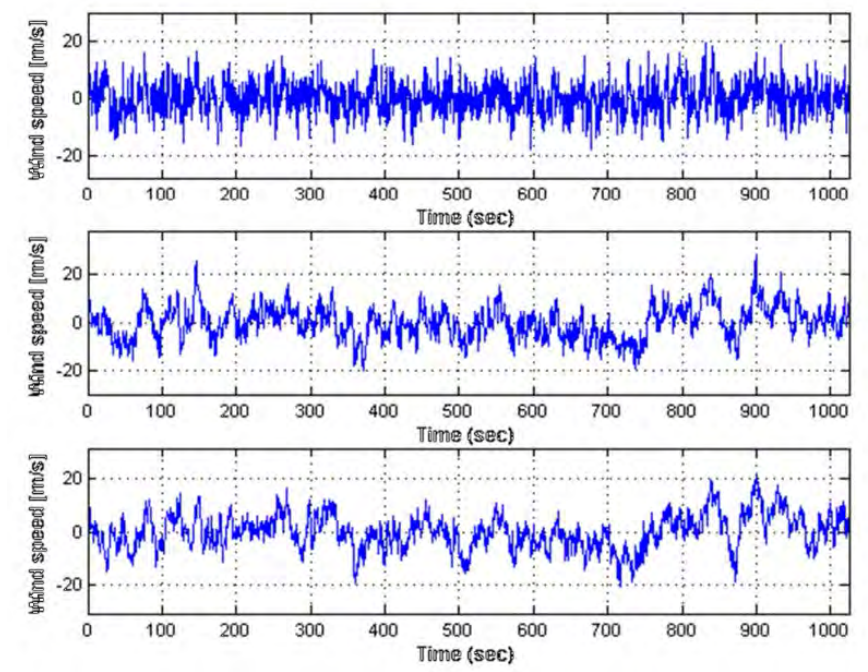

Figure 4: Fluctuating wind speed (about mean wind speed) at Floors 1, 34, and 67 for a sample wind loading scenario obtained from NOWS web tool.

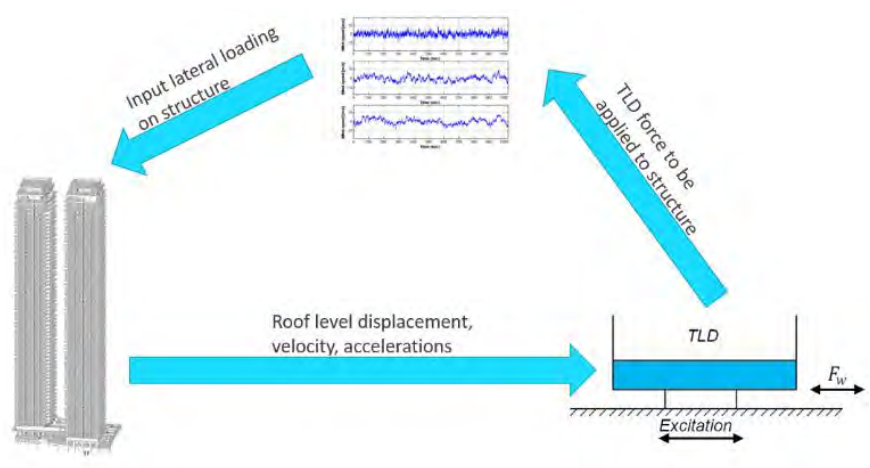

Figure 5: Schematic representation of interaction algorithm used in TLDstructure simulations.

Table 1: Comparison of periods of vibration (ETABS vs. OpenSees) for dominant modes of vibration.

\begin{tabular}{|l|l|l|}
\hline & ETABS Model & OpenSees Model \\
\hline Mode 1 Period & $6.166 \mathrm{sec}$ & $6.204 \mathrm{sec}$ \\
\hline Mode 1 Direction & E-W & E-W \\
\hline Mode 2 Period & $4.970 \mathrm{sec}$ & $3.738 \mathrm{sec}$ \\
\hline Mode 2 Direction & N-S & N-S \\
\hline
\end{tabular}

The results show that the first mode period is quite wellmatched between the two models. This is important as the structure was analyzed in the direction of the first mode (E-W).

\section{F. Description of Wind Loading Profiles}

Table 2 shows the three wind loading profiles which were chosen for analyzing the structure with and without the TLD. These wind loading profiles were selected in order to analyze the structure and be able to compare the results to NBCC 2010 human comfort and structural integrity checks. Scenario 1 corresponds to a 1/10 level of wind, which is recommended by the NBCC 2010 for checking serviceability comfort levels as well as structural performance. Scenario 2 corresponds to the recommended 1/50 wind level, and Scenario 3 represents a more severe wind storm.

Table 2: Summary of wind loading conditions.

\begin{tabular}{|l|l|l|l|}
\hline & Scenario 1 & Scenario 2 & Scenario 3 \\
\hline $\begin{array}{l}6^{\text {th }} \text { floor } \\
\text { wind speed }\end{array}$ & $12.1 \mathrm{~m} / \mathrm{s}$ & $35.5 \mathrm{~m} / \mathrm{s}$ & $53.2 \mathrm{~m} / \mathrm{s}$ \\
\hline $\begin{array}{l}30^{\text {th }} \\
\text { wind speed }\end{array}$ & $20.4 \mathrm{~m} / \mathrm{s}$ & $45.4 \mathrm{~m} / \mathrm{s}$ & $68.2 \mathrm{~m} / \mathrm{s}$ \\
\hline $\begin{array}{l}\text { Roof wind } \\
\text { speed }\end{array}$ & $23.1 \mathrm{~m} / \mathrm{s}$ & $51.4 \mathrm{~m} / \mathrm{s}$ & $77.2 \mathrm{~m} / \mathrm{s}$ \\
\hline
\end{tabular}

The structure was analyzed under various performance indicators, including peak storey accelerations. These values were compared to the NBCC 2010 acceleration limit for human comfort, as well as the limit suggested by the Council for Tall Buildings and Urban Habitat (CTBUH).

In NBCC 2010, several levels of service for the performance of the building under wind loading are considered - specifically, Commentary I sentence 78 requires that the maximum acceleration be less than $1.5 \%$ of $\mathrm{g}$, as any higher level of acceleration is perceptible to occupants (barring additional effects such as visual cues, body position, and orientation). This 
acceleration guideline is the same in CTBUH, with the added restriction that the Root-Mean-Square (RMS) acceleration should be less than $0.5 \%$ of g. Furthermore, NBCC 2010 Commentary I sentence 74 states that the lateral deflection of the building should be no more than 1/500 of the building height [10].

\section{G. Discussion of Results}

Table 3: Summary of key structural performance parameters with TLD (and \% change compared to uncontrolled structure).

\begin{tabular}{|l|l|l|l|}
\hline Parameter & Scenario 1 & Scenario 2 & Scenario 3 \\
\hline $\begin{array}{l}\text { Peak roof } \\
\text { drift (m) }\end{array}$ & $16.8(-12 \%)$ & $172(-20 \%)$ & $395(-21 \%)$ \\
\hline $\begin{array}{l}\text { Peak storey } \\
\text { acceleration } \\
(\% \text { g) }\end{array}$ & $0.69(-12 \%)$ & $9.22(-2 \%)$ & $24.8(-0 \%)$ \\
\hline $\begin{array}{l}\text { Peak Base } \\
\text { Shear (MN) }\end{array}$ & $1.7(-1.1 \%)$ & $24.1(-0.2 \%)$ & $65.6(-0.1 \%)$ \\
\hline
\end{tabular}

Using the multi-platform tool previously described, it was determined that for the $1 / 10$ level of wind, the structure met the performance criteria set out in both NBCC 2010 as well as CTBUH - the storey accelerations were well below the NBCC $20101.5 \%$ of g guideline, and the RMS acceleration was $0.507 \%$ of $\mathrm{g}$. It is important to note that in the CTBUH guidelines, a 6year level of wind was recommended when checking RMS acceleration limits, whereas a 10 -year level of wind was used in the simulations for consistency with NBCC 2010. Furthermore, the roof drift was well below the $400 \mathrm{~mm}$ limit prescribed by NBCC 2010, with a maximum roof drift of $19 \mathrm{~mm}$ without the TLD and $16 \mathrm{~mm}$ with the TLD.

The roof drift limits are similarly met for Scenario 2, which represents a more severe wind level. While the peak storey acceleration limits are not met for Scenario 2, the wind level represents a more severe loading than suggested for serviceability checks (Scenario 1). The mean storey displacements for Scenario 2 are shown in Figure 6, and a marked reduction in storey displacements can be observed, with more significant improvements to be seen at the higher storeys of the structure.
Table 4: Summary of improvement in mena storey displacement/shear results (top \& bottom 20 storeys) with TLD vs without TLD.

\begin{tabular}{|l|l|l|l|}
\hline Parameter & Scenario 1 & Scenario 2 & Scenario 3 \\
\hline \multicolumn{4}{|c|}{ Top 20 Storeys } \\
\hline $\begin{array}{l}\text { \% Change in } \\
\text { mean storey } \\
\text { displacement }\end{array}$ & $-8.27 \%$ & $-7.51 \%$ & $-6.90 \%$ \\
\hline $\begin{array}{l}\text { \% Change in } \\
\text { mean storey } \\
\text { shear }\end{array}$ & $+5.71 \%$ & $+2.06 \%$ & $+0.62 \%$ \\
\hline \multicolumn{3}{|c|}{ Bottom 20 Storeys } \\
\hline $\begin{array}{l}\text { \% Change in } \\
\text { mean storey } \\
\text { displacement }\end{array}$ & $-0.25 \%$ & $+0.98 \%$ & $+0.65 \%$ \\
\hline $\begin{array}{l}\text { \% Change in } \\
\text { mean storey } \\
\text { shear }\end{array}$ & $-1.10 \%$ & $-0.36 \%$ & $-0.15 \%$ \\
\hline
\end{tabular}

Figure 7 also shows the time history of roof displacement under the most severe wind storm, Scenario 3. The results show that even for such a high level of wind, the TLD can provide significant reductions in roof drift, keeping the structure within the NBCC 2010 limit for roof deflection.

Table 4 summarizes some key parameters for structural performance, namely storey displacements and storey shear, but for the upper 20 storeys of the structure only. This is to understand the benefits that the TLD provides to the most flexible parts of the structure. For all three wind loading scenarios, the TLD can reduce the storey displacement demands at the upper storeys of the structure - this is critical for the comfort of the occupants, as these storeys typically experience the largest displacements.

Across all three wind loading scenarios, a couple of trends can be seen for the structural performance with and without TLD. The first is that there is a negligible change in base shear for the structure with the addition of the TLD; slightly higher storey shears are observed at the upper levels of the structure, whereas slightly lower storey shears are observed at the lower levels of the structure. While the TLD can dissipate vibration energy through non-linear liquid sloshing, the addition of a significant mass $(1.5 \%$ of the structural mass $)$ at the roof level of

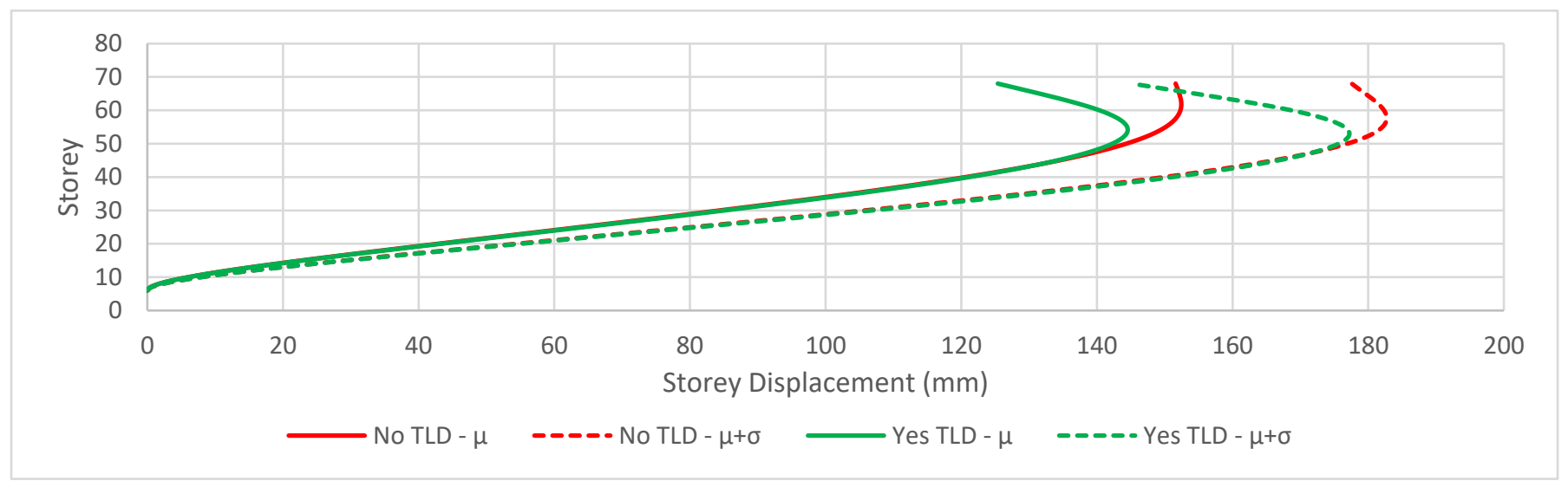

Figure 6: Mean storey displacement for wind loading scenario 2. 


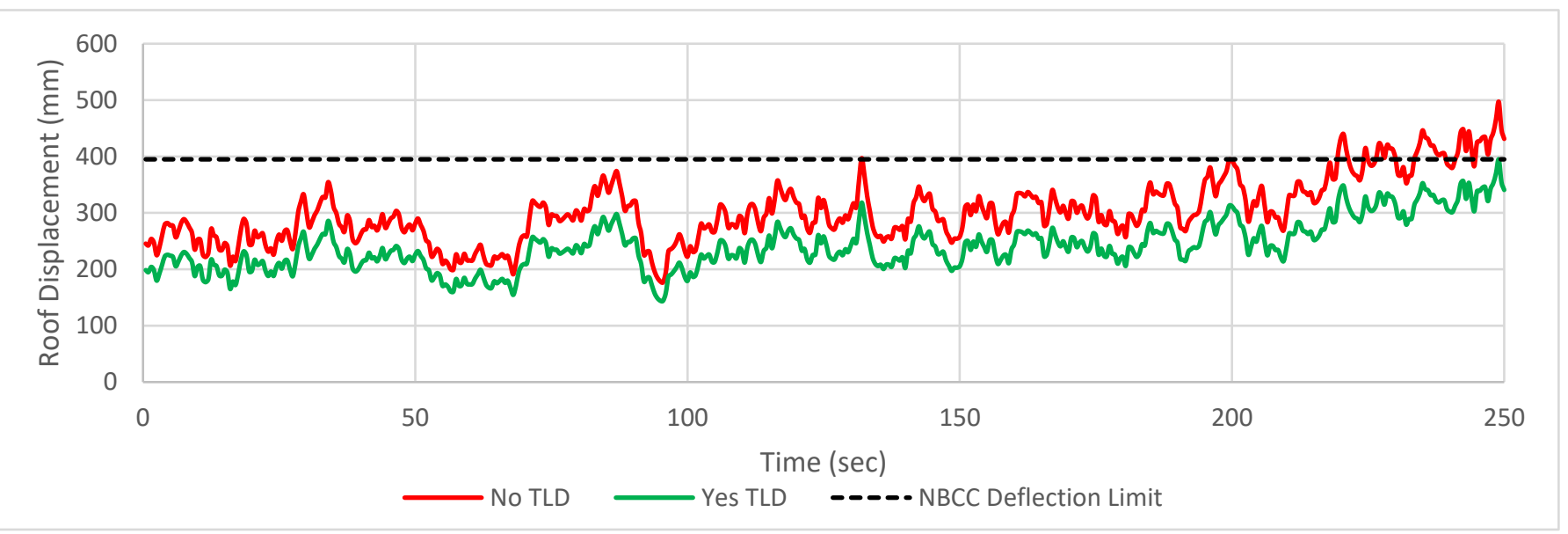

Figure 7: Roof displacement time history for wind loading scenario 3.

the structure imposes large forces on the upper storeys of the structure.

Secondly, a noticeable improvement in structural performance is observed with regards to roof displacement and global mean storey displacement, while a smaller improvement is seen with regards to storey accelerations. As was the case with the storey shears, the TLD provides more benefits at the upper storeys of the structure than at the lower storeys. This is still a good result, as these measures are critical for human comfort and serviceability checks, and the upper levels of the structure are where accelerations and displacements are the greatest - and hence the need for improved performance is also greatest.

\section{CONCLUSION AND RECOMMENDATIONS}

The objective of this collaboration was to analyze structural performance of a real high-rise structure both with and without TLD under various levels of wind loading to better understand the effects and potential benefits the TLD could provide to the structure. Structural performance was then to be compared to the limits set in NBCC 2010 and CTBUH for human comfort and safety, including maximum lateral deflection of the structure and maximum storey accelerations.

The TLD model which was built was based on Yu's model, where the TLD is modeled as an equivalent non-linear-stiffnessdamping oscillator. The TLD properties were tuned to the first mode of vibration of the structure, which was determined in OpenSees and cross-checked and verified with existing linear models in ETABS developed by the team at Stephenson Engineering, as well as third-party wind tunnel testing results.

The structure was analyzed under dynamic wind loading using a transient, multi-platform simulation algorithm. Three wind loading scenarios were considered, including NBCC 2010 service-level wind loads, and two more severe wind loading cases. The structure was found to remain in the elastic range for all three cases, and the structure met the NBCC 2010 and CTBUH human comfort limits. At higher levels of wind, the TLD effects were more pronounced, with benefits in terms of storey displacements and accelerations observed especially at the upper storeys of the structure. However, improvements to the structure with respect to storey and base shear were negligible with the TLD addition, due to the large additional mass imposed by the TLD at the roof level of the structure.

It is recommended that the TLD and structure are monitored together, with the TLD being modified such that its properties match that of the structure. TLD performance is highly correlated with the tuning of its frequency to the structure - as the structure ages and concrete cracks, it is likely that its vibration characteristics will change, and hence the TLD will likely need to be adjusted (e.g. changing water levels) to match the structure over time.

\section{ACKNOWLEDGEMENTS}

The authors would like to thank the several members of the team at Stephenson Engineering who assisted on the project, including Senior Associate Xi Xin (Fisher) Li, Senior Associate Dr. Sobhy Masoud, Principal Saundra Cullen, and Bahar Zavarei.

\section{REFERENCES}

[1] The Regents of the University of California, OpenSees (2000) [Online]. Available: http://opensees.berkeley.edu/index.php.

[2] The MathWorks, Inc, Matlab (2013a), Natick, Massachusetts, United States.

[3] NatHaz Modeling Laboratory, "NatHaz Online Wind Simulator," 2007. [Online]. Available: http://windsim.ce.nd.edu/gew_init2.html.

[4] A. Ashasi-Sorkhabi, H. Malekghasemi, A. Ghaemmaghami and O. Mercan, "Experimental investigations of tuned liquid damper-structure interactions in resonance considering multiple parameters," Journal of Sound and Vibration, vol. 388, pp. 141-153, 2017. doi: 10.1016/j.jsv.2016.10.036.

[5] J.-K. Yu, "Nonlinear characteristics of tuned liquid dampers," PhD thesis, Dept. of Civil Engineering, University of Washington, Seattle, Wa., 1997.

[6] J.-K. Yu, Wakahara, Toshihiro and D. A. Reed, "A non-linear numerical model of the tuned liquid damper," Earthquake Engineering and Structural Dynamics, pp. 671-686, 1999. doi: 10.1002/(SICI)1096-9845(199906)28:6<671::AIDEQE835>3.0.CO;2-X.

[7] Canadian Commission on Building and Fire Codes, NBCC 2010 Part 4 Division B - Structural Design, Ottawa: National Research Council of Canada, 2011.

[8] A. K. Chopra, Dynamics of Structures: Theory and Applications to Earthquake Engineering, Englewood Cliffs: Prentice Hall, 1995.

[9] Computers and Structures, Inc. Structural and Earthquake Software (2015), ETABS, [Online]. Available: https://www.csiamerica.com/content/etabs-2015-151032.

[10] Canadian Commission on Building and Fire Codes, User's Guide - NBC 2010

Structural Commentaries (Part 4 of Division B), Ottawa: National Research Council of 807 Canada, 2011. 


\section{Modelling Thermal Conductivity of Porous Thermal Barrier Coatings for High-Temperature Aero Engines Using Five Phase Model}

\author{
Ramandeep Singh Ghai \\ Department of Mechanical Engineering \\ University of Ottawa \\ Ottawa, Canada \\ rghai036@uottawa.ca
}

\author{
Dr. Kuiying Chen \\ Aerospace Portfolio \\ National Research Council Canada \\ Ottawa, Canada
}

\author{
Dr. Natalie Baddour \\ Department of Mechanical Engineering \\ University of Ottawa \\ Ottawa, Canada
}

\begin{abstract}
Thermal Barrier Coatings (TBC) are used to shield hot sections of gas turbine engines, helping to prevent the melting of metallic surfaces. This paper proposes a Five-phase model to calculate the effective thermal conductivity of a TBC, taking into consideration the effect of various defects. By comparing the predicted values with experimental results, it was shown that the proposed five-phase model can predict the thermal conductivity of ceramic coatings closer to the actual values.
\end{abstract}

Keywords- TBC, Thermal Conductivity, High-temperature coatings, Modelling, Porosity and Defects.

\section{INTRODUCTION}

The advancement in high-temperature engineering alloys led to the design and development of the present era turbine engines. The laws of thermodynamics suggest that with an increase in inlet operating temperature, the performance and efficiency of an engine can be increased [1].

The components of a turbine engine are exposed to elevated temperatures as well as to an oxidative and corrosive gaseous environment. In certain cases, there are some impacts from high-velocity foreign particles during operation [2]-[4]. The components used at elevated temperatures are coated with a ceramic coating to have protection against high-temperature degradation. A Thermal Barrier Coating (TBC) provides thermal shock resistance, creep resistance, strain tolerance, protection against hot corrosion and stability to the substrate at higher temperatures [5] [6].

The simple structure of the TBC system consists of the ceramic top coat, bond coat and superalloy substrate. During service life, a thermally grown oxide (TGO) layer (mostly $\alpha$ $\mathrm{Al}_{2} \mathrm{O}_{3}$ ) forms on the bond coat surface and reduces the rate of oxidation [7]. The objective of the top ceramic coating is to reduce the metal temperature. A metallic bond coat is used to enhance the bonding between the top coat and the underlying super-alloy and to protect the super-alloy from oxidation and hot corrosion [8]. Yttria-stabilized Zirconia [YSZ] containing
$6-8 \% \mathrm{Y}_{2} \mathrm{O}_{3}$ is known as a state of the art thermal barrier coating topcoat [9].

The key properties of YSZ TBC are low thermal conductivity, high melting point, phase compatibility with alpha alumina, and the combination of good resistance to corrosion and damage from large particle impacts [10]. The microstructure of ceramic coatings is highly heterogeneous as it consists of imperfections such as pores, voids, and vacancies, along with cracks of different shapes and sizes. Overall, the thermal conductivity of the coating is highly affected by the presence of such defects [11], [12] and spraying parameters [13]. The extent of change in the thermal and mechanical properties depends on the amount, size, and morphology of the defects present in the coating.

Defects lead to a lower value for thermal conductivity and a lower thermal conductivity implies a longer service life, as heat transfer to the substrate is reduced. Lower heat transfer into the substrate also leads to lower damage to the coating interface, where most of the failure occurs [8]. To achieve lower values for thermal conductivity, better strain tolerance and higher lifetime, the distribution of cracks and pores in the coating needs to be optimized [14]. It thus becomes essential to understand the fundamental microstructural properties of the $\mathrm{TBC}$ in order to produce an optimized coating. Hence, one of the aims of TBC design is to design coatings with a lower thermal conductivity. Therefore, modelling provides an economical way to develop and understand the coating microstructure that will have lower thermal conductivity. This paper proposed a five-phase model for calculating the effective thermal conductivity of a TBC with the goal to improve the accuracy of modelling.

\section{MODELLING THERMAL CONDUCTIVITY}

\section{A. Modelling approach}

Modelling or/and simulation is a cost-effective and flexible approach to optimize and understand the coating microstructure. Development of new types of coatings design or new structures can easily be performed. Modification of the

Special Thanks to Dr. Natalie Baddour for sponsoring this paper. 
parameters is simple, and the analysis can be performed quickly compared to a traditional experimental approach. Simulation can provide analysis of different microstructural parameters and their individual effect, as well as the combined effect on the TBC.

The present study is based on the different types of defects (pores, voids and cracks) that are present in the coating during the fabrication of the coating. The effect of various defects needs to be included in the model to better predict the thermal conductivity of coating and to have a better understanding of the coating microstructure. Many researchers have presented models that can predict and calculate the thermal conductivity of the porous coatings. Out of those models, Bruggeman's formula provides a model that takes into consideration the shape, orientation and volumetric fraction of pores. The details can be found in next section.

\section{B. Two-phase model for thermal conductivity}

Thermal conductivity $(k)$, is the measure of heat transfer from one surface to another that is having a cross-sectional area $A$ and are separated by a distance $L$. There are many formulas to calculate thermal conductivity depending on the coatings and its parameters. The thermal conductivity of free-standing materials can be determined by [15]

$$
k=\alpha C_{p} \rho
$$

where $\rho$ is the density of the free-standing material $(\mathrm{kg} / \mathrm{m} 3), C_{p}$ indicates the heat capacity of materials at constant pressure $(\mathrm{J} /(\mathrm{kg} \mathrm{K}))$, and $\alpha$ is the thermal diffusion rate $\left(\mathrm{m}^{2} / \mathrm{s}\right)$.

Bruggeman provided a model to predict the thermal conductivity of porous coatings [16]. Bruggeman extended the Maxwell model to systems having random dispersions of spherical particles of several sizes. He proposed a model assuming that if a relatively large spherical particle is introduced into a dispersion containing much smaller particles, there will be a negligible disturbance of the field around the large sphere due to the small spheres. With this model, he showed that the limitation on a volumetric fraction of dilute dispersion can be removed. The Maxwell model is extended to [17]

$$
\frac{k-k_{m}}{k+2 k_{m}}=f \frac{k_{d}-k_{m}}{k_{d}+2 k_{m}}
$$

where $k$ is the thermal conductivity of the composite, $k_{m}$ is the thermal conductivity of the matrix, $k_{d}$ is the thermal conductivity of the dispersed phase, and $f$ is the volumetric fraction of the $i^{\text {th }}$ phase. A change in conductivity $d M$, with the change in volume fraction of the dispersed phase, is expressed as

$$
\frac{d M}{3 M}=\frac{d P}{1+P}\left(\frac{k_{d}-M}{k_{d}+2 M}\right)
$$

Integrating $P$ from 0 to $f /(1+f)$ and $M$ from $k_{\mathrm{m}}$ to $k$. leads to Bruggeman's two-phase model given by

$$
\left(\frac{\frac{k}{k_{m}}-\frac{k_{d}}{k_{m}}}{\left(\frac{k}{k_{m}}\right)^{\frac{1}{3}}\left(1-\frac{k_{d}}{k_{m}}\right)}\right)=(1-f)
$$

An example for the two-phase coating can be seen in Figure 1. Also, in this case, it is possible to generalise the modelling to a solute dispersion of randomly oriented ellipsoids.

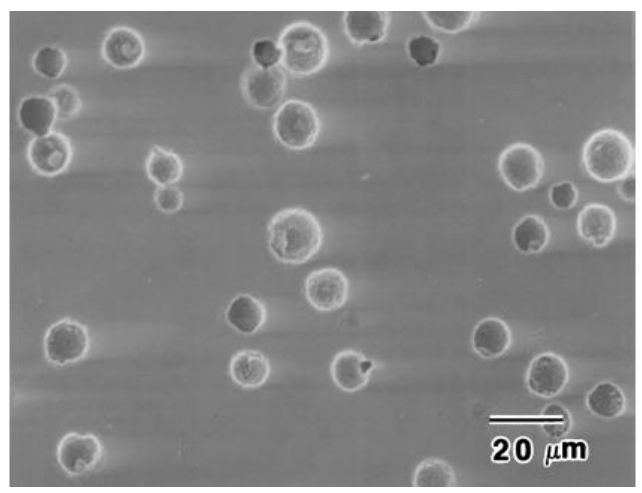

Figure 1 Scanning Electron Microscopic Image of spherical pores in continuous matrix [18]

Some assumptions in this work are as follows:

- The thermal conductivity of the dispersed phase or of the defect is assumed to be negligible.

- Heat transfer is along the thickness of the coating only (or perpendicular to bond coat-substrate interface). No lateral heat transfer is assumed.

- The effect of connected pores is neglected.

- There is a linear relation between the porosity and thermal conductivity.

\section{Five Phase MOdEl For Thermal CONDUCTIVITY}

\section{A. Simplification of Bruggeman two-phase model}

Bruggeman's model is further simplified by assuming the thermal conductivity of pores/defects is negligible i.e. $k_{d}=0$. Under the condition of non-radiating pores, the equation (4) reduces to

$$
\frac{k}{k_{m}}=(1-f)^{\frac{3}{2}}
$$

This equation is the special case when the pores are of spherical shape. The Bruggeman model is based on ellipsoids of revolution, hence in general, for the dispersion of an ellipsoid, the Bruggeman model is a modified version of the equation (5) and is given by [19]

$$
\frac{k}{k_{m}}=(1-f)^{X}
$$


where the value of $X$ depends on certain factors such as the shape factor of the ellipsoid $(F)$ and $\alpha$, which is the angle between the heat flux and the axis of revolution. The value of $X$ can be described by [19]

$$
X=\frac{1-\cos ^{2} \alpha}{1-F}+\frac{\cos ^{2} \alpha}{2 F}
$$

The value $X$ is set to $3 / 2$ when there are only spherical pores in a continuous matrix. The assumption of non-conducting pores/defects is valid for a certain temperature limit, however, its primary purpose is to simplify the model [16].

In reality, coatings contain several types of defects [11], [20]-[22]. Therefore, for more realistic modelling of coatings, superposition of the contributions of different defects types on the overall thermal conductivity is required. One approach is to iterate the Bruggeman's two-phase model to higher levels of porosities. This approach is explained in detail in subsequent paragraphs.

\section{B. Iteration Approach}

This approach works in two steps, first of all, type 1 porosity is added in the continuous matrix so that the average thermal conductivity of a binary mixture is obtained. Then, for the second step, the binary mixture is considered as a continuous matrix, and subsequently, type 2 porosity is added in the same manner. This gives the combination of two different types of porosities in a continuous matrix. Suppose that $f_{l}$ and $f_{2}$ are the final percentages of type 1 and type 2 porosity, respectively, then the total porosity in the coating is given by $f$, which is given as the sum of the different types of porosities. Therefore, $f=f_{1}+f_{2}$. There can be two ways of adding the defects in a continuous matrix. Consider if we add a Type 1 porosity first into the continuous matrix and then add a Type 2 porosity. This will lead to an equation given by

$$
\left\{\Phi\left[\frac{f_{2}}{1-f_{1}}\right] \Psi\left(f_{1}\right)\right\}
$$

Now consider if we first add Type 2 porosity in the continuous matrix and then Type 1, this will generate the formula as-

$$
\left\{\Psi\left[\frac{f_{1}}{1-f_{2}}\right] \Phi\left(f_{2}\right)\right\}
$$

The model to calculate thermal conductivity is developed by averaging the multiple values of the constituents that directly make up the composite material. Therefore, when we average the two possible cases we can have the thermal conductivity of the three-phase mixture [19] as

$$
k=\frac{k_{0}}{2}\left\{\Psi\left\lfloor\frac{f_{1}}{\left(1-f_{2}\right)}\right\rfloor \Phi\left(f_{2}\right)+\Phi\left\lfloor\frac{f_{2}}{\left(1-f_{1}\right)}\right\rfloor \Psi\left(f_{1}\right)\right\}
$$

where $k_{0}$ is the thermal conductivity of the matrix, $\Psi(f)$ and $\Phi(f)$ are functions describing the effect of defects on the thermal conductivity of the coating. This process is also known as an averaging technique. This process of averaging the all possible ways in which different types of defects can be added will provide the formula for $n$ number of defects under consideration. A five-phase model will have 24 different equations that will be averaged to obtain thermal conductivity of coating. Therefore, for the five-phase model, there are four different types of defects that are assumed to be embedded in a continuous matrix.

\section{Five-phase model}

The volumetric fractions of different types of defects are given by $f_{1}, f_{2}, f_{3}$ and $f_{4}$. The effect of each defect on thermal conductivity is given by functions $\Phi(f), \Psi(f), \Theta(f)$ and $\beta(f)$, respectively. The values of volumetric fraction are obtained from image analysis using Image $\mathrm{J}$ and from references using MIP (Mercury Intrusion Porosimetry). The functions are all defined by the equation (6). The five-phase model can be expressed as

$$
k=\frac{k_{0}}{24}(A+B+C+D)
$$

where $A, B, C$ and $D$ provides simplification of the formula. The formula averages all the possible conditions in which the four different types of defects can be added in different sequences. The details regarding the $\mathrm{A}, \mathrm{B}, \mathrm{C}$ and $\mathrm{D}$ can be found somewhere else [23].

\section{DATA RESOURCES}

The data for the modelling work is obtained from several references, Image analysis and from MIP. The image analysis and MIP provide the details regarding the volumetric fraction of various kinds of defects present in the coating. In this work, a spheroidal shape is used to model various kinds of defects. This shape can cover a large number of real-life defects that are present in the coating.

Image $\mathrm{J}$ provides the details regarding the porosity content present in the coatings. The four types of defects that are under consideration are open randomly oriented cracks, microcracks, non-flat spheroids porosity and defects having revolution axis oriented perpendicular to heat flux (penny-shaped defects). The equation (6) is used to define the functions and equation (7) is used to obtain the values of $\mathrm{X}$. The $\mathrm{X}$-factors obtained during this study are listed below in Table 1 . Overall porosity content can be seen in Table 2 that are obtained from image analysis.

Table $1 \mathrm{X}$ factor for different defects [23]

\begin{tabular}{|c|l|}
\hline X-Factor & \multicolumn{1}{|c|}{ Functions } \\
\hline 1.66 & Open Randomly Oriented \\
\hline 7 & Microcracks \\
\hline 2 & Penny shaped $\left(\alpha=90^{\circ}\right)$ \\
\hline 1.7 & Non-flat porosity \\
\hline
\end{tabular}


Table 2 Overall Porosity Content of various coatings

\begin{tabular}{|l|c|}
\hline \multicolumn{1}{|c|}{ Coatings } & Overall Porosity (\%) \\
\hline 8YSZ (As-sprayed) & 24.5 \\
\hline 8YSZ (Annealed) & 20.7 \\
\hline 22MSZ (As-sprayed) & 18.9 \\
\hline 22MSZ (Annealed) & 16.8 \\
\hline 25CSZ (As-sprayed) & 23.7 \\
\hline 25CSZ (Annealed) & 13.9 \\
\hline F\&C (As-sprayed) & 21.3 \\
\hline F\&C (Annealed) & 16.9 \\
\hline A\&S (As-sprayed) & 17.9 \\
\hline A\&S (Annealed) & 16.1 \\
\hline HOSP (As-sprayed) & 19 \\
\hline HOSP (Annealed) & 14.4 \\
\hline
\end{tabular}

\section{RESULT AND DISCUSSION}

\section{A. Obtained Thermal Conductivity}

The five-phase model is used to calculate the thermal conductivity of the coating using the porosity content, volumetric fraction and the $X$ values. The values of thermal conductivity for various coatings can be obtained from Table 3 .

Table 3 Thermal conductivity values obtained using five-phase model

\begin{tabular}{|l|c|}
\hline \multicolumn{1}{|c|}{ Coatings } & $\begin{array}{c}\text { Thermal Conductivity } \\
\text { (W/mK) }\end{array}$ \\
\hline 8YSZ (As-sprayed) & 1.18 \\
\hline 8YSZ (Annealed) & 1.60 \\
\hline 22MSZ (As-sprayed) & 1.14 \\
\hline 22MSZ (Annealed) & 1.35 \\
\hline 25CSZ (As-sprayed) & 1.175 \\
\hline 25CSZ (Annealed) & 1.93 \\
\hline F\&C (As-sprayed) & 1.2 \\
\hline F\&C (Annealed) & 1.79 \\
\hline A\&S (As-sprayed) & 1.33 \\
\hline A\&S (Annealed) & 1.56 \\
\hline HOSP (As-sprayed) & 1.12 \\
\hline HOSP (Annealed) & 1.36 \\
\hline
\end{tabular}

\section{B. Validation of Results}

The results obtained from the five-phase model are validated against experimental results. Thermal conductivity of different coatings obtained from the five-phase model, four-phase model and experimental results are compared in Figure 2. Thermal conductivity values of different Yttria Stabilized Zirconia (YSZ) obtained from the five-phase model, experimental and FEA model are compared in Figure 3.

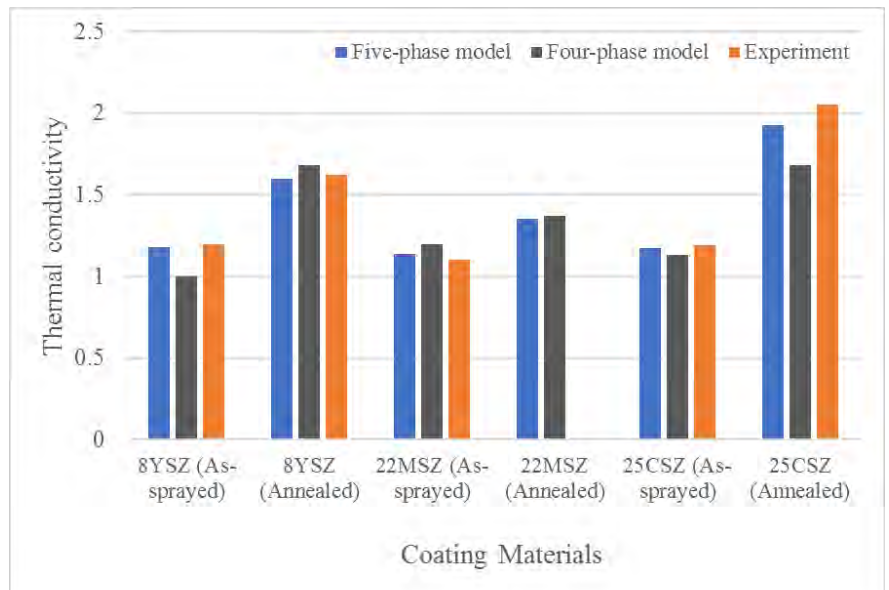

Figure 2 Comparison of thermal conductivity values.

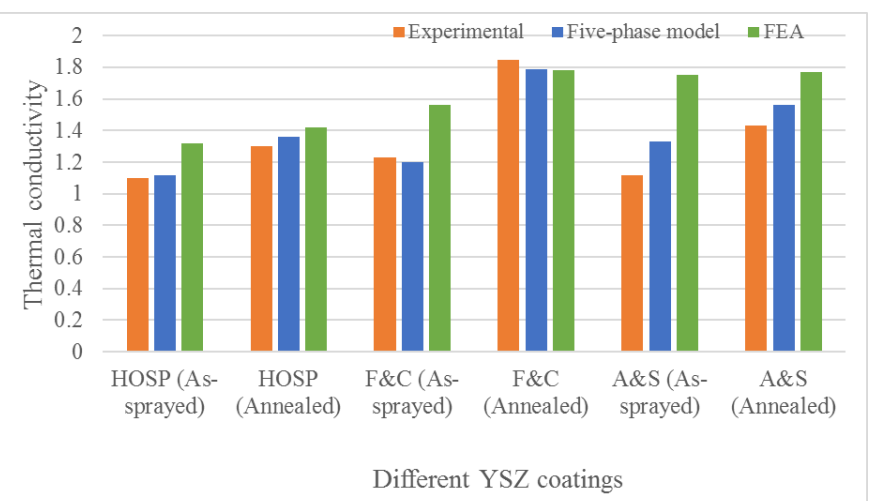

Figure 3 Comparison of thermal conductivity values for various YSZ coatings

\section{CONCLUSION}

A five-phase model to predict thermal conductivity of thermal barrier coatings was developed in this work and validated against the results from the four-phase model, FEA model and experimental results. The presented model takes into consideration the different types of pores that are mostly present in a topcoat. The parameters used in the model were obtained from previous models and fitting parameters. The results obtained with the new proposed model were then validated against the reference data.

By comparing the predicted values with experimental results, it was shown that the proposed five-phase model can predict the thermal conductivity of ceramic coatings closer to the actual values. The five-phase model can predict the values of thermal conductivity within $6 \%$ of the experimental results. The proposed model uses real microstructure images and MIP results to obtain porosity content in the coatings to better predict the thermal conductivity. The proposed model has the potential to predict microstructure-property relationships. 
The presence of different types of pores and cracks influences the overall thermal conductivity of the coatings. Microcracks present in the coating's microstructure influence the thermal conductivity. The density of microcracks is affected by heat treatment due to the expansion of the coating material. Smaller cracks disappear in the coating due to sintering and lead to lower porosity content, which ultimately leads to an increase in thermal conductivity.

\section{ACKNOWLEDGEMENT}

This research is supported by the Air Defence System Program of the National Research Council Canada and the Natural Sciences and Engineering Research Council of Canada.

\section{REFERENCES}

[1] R. C. Reed, The Superalloys: Fundamentals and Applications by Roger C. Reed. Cambridge University Press, 1742.

[2] A. K. Ray and R. W. Steinbrech, "Crack propagation studies of thermal barrier coatings under bending," J. Eur. Ceram. Soc., vol. 19, no. 12, pp. 2097-2109, Oct. 1999. doi: 10.1016/S0955-2219(99)00028-X.

[3] W. J. Brindley and R. A. Miller, "Thermal barrier coating life and isothermal oxidation of low-pressure plasma-sprayed bond coat alloys," Surf. Coat. Technol., vol. 43-44, no. 1-3, pp. 446-457, 1990. doi: 10.1016/0257-8972(90)90096-U.

[4] K. Kokini and Y. R. Takeuchi, "Initiation of surface cracks in multilayer ceramic thermal barrier coatings under thermal loads," Mater. Sci. Eng. A, vol. 189, no. 1, pp. 301-309, Dec. 1994. doi: 10.1016/0921-5093(94)90428-6.

[5] J. R. Brandon and R. Taylor, "Thermal properties of ceria and yttria partially stabilized zirconia thermal barrier coatings," Surf. Coat. Technol., vol. 39-40, pp. 143-151, 1989. doi: 10.1016/0257-8972(89)90049-2.

[6] A. Vardelle et al., "The 2016 thermal spray roadmap," J. Therm. Spray Technol., vol. 25, no. 8, pp. 1376-1440, Dec. 2016.

doi: 10.1007/s11666-016-0473-x.

[7] A. Fahr, B. Rogé, and J. Thornton, "Detection of thermally grown oxides in thermal barrier coatings by nondestructive evaluation," J. Therm. Spray Technol., vol. 15, no. 1, pp. 46-52, Mar. 2006.

doi: $10.1361 / 105996306 \times 92587$.

[8] H. M. Tawancy, A. I. Mohammad, L. M. Al-Hadhrami, H. Dafalla, and F. K. Alyousf, "On the performance and failure mechanism of thermal barrier coating systems used in gas turbine blade applications: Influence of bond coat/superalloy combination," Eng. Fail. Anal., vol. 57, pp. 1-20, Nov. 2015. doi: 10.1016/j.engfailanal.2015.07.023.

[9] R. A. Miller, "Thermal barrier coatings for aircraft engines: history and directions," J. Therm. Spray Technol., vol. 6, no. 1, p. 35, Mar. 1997. doi: 10.1007/BF02646310.
[10] X. F. Zhang et al., "Enhanced properties of Al-modified EB-PVD 7YSZ thermal barrier coatings," Ceram. Int., vol. 42, no. 12, pp. 13969-13975, 2016. doi: 10.1016/j.ceramint.2016.05.210.

[11] L. Wang et al., "Influence of pores on the thermal insulation behavior of thermal barrier coatings prepared by atmospheric plasma spray," Mater. Des., vol. 32, no. 1, pp. 36-47, 2011. doi: 10.1016/j.matdes.2010.06.040.

[12] J. Zhang and V. Desai, "Evaluation of thickness, porosity and pore shape of plasma sprayed TBC by electrochemical impedance spectroscopy," Surf. Coat. Technol., vol. 190, no. 1, pp. 98-109, 2005. doi: 10.1016/j.surfcoat.2004.06.019.

[13] J. Wang et al., "Effect of spraying power on microstructure and property of nanostructured YSZ thermal barrier coatings," J. Alloys Compd., vol. 730, no. Supplement C, pp. 471-482, Jan. 2018. doi: 10.1016/j.jallcom.2017.09.323.

[14] G.-R. Li, G.-J. Yang, C.-X. Li, and C.-J. Li, "A comprehensive mechanism for the sintering of plasma-sprayed nanostructured thermal barrier coatings," Ceram. Int., vol. 43, no. 13, pp. 9600-9615, Sep. 2017. doi:10.1016/j.ceramint.2017.04.083.

[15] L. Liu, H. Zhang, X. Lei, and Y. Zheng, "Dependence of microstructure and thermal conductivity of EB-PVD thermal barrier coatings on the substrate rotation speed," Phys. Procedia, vol. 18, no. Complete, pp. 206-210, 2011.

doi: 10.1016/j.phpro.2011.06.082.

[16] D. A. G. Bruggeman, "Berechnung verschiedener physikalischer Konstanten von heterogenen Substanzen. I. Dielektrizitätskonstanten und Leitfähigkeiten der Mischkörper aus isotropen Substanzen," Ann. Phys., vol. 416, pp. 636-664, 1935. doi: 10.1002/andp.19354160802.

[17] S. Banisi, J. A. Finch, and A. R. Laplante, "Electrical conductivity of dispersions: A review," Miner. Eng., vol. 6, no. 4, pp. 369-385, Apr. 1993.

doi: 10.1016/0892-6875(93)90016-G.

[18] D. R. Clarke, M. Oechsner, and N. P. Padture, "Thermal-barrier coatings for more efficient gas-turbine engines," MRS Bull., vol. 37, no. 10, pp. 891-898, Oct. 2012. doi: $10.1557 / \mathrm{mrs} .2012 .232$.

[19] F. Cernuschi, S. Ahmaniemi, P. Vuoristo, and T. Mäntylä, "Modelling of thermal conductivity of porous materials: application to thick thermal barrier coatings," $J$. Eur. Ceram. Soc., vol. 24, no. 9, pp. 2657-2667, Aug. 2004. doi: 10.1016/j.jeurceramsoc.2003.09.012.

[20] Z. Wang, A. Kulkarni, S. Deshpande, T. Nakamura, and H. Herman, "Effects of pores and interfaces on effective properties of plasma sprayed zirconia coatings," Acta Mater., vol. 51, no. 18, pp. 5319-5334, Oct. 2003.

doi: 10.1016/S1359-6454(03)00390-2.

[21] S. Wei, W. Fu-chi, F. Qun-Bo, and M. Zhuang, "Effects of defects on the effective thermal conductivity of thermal barrier coatings," Appl. Math. Model., vol. 36, no. 5, pp. 1995-2002, May 2012. doi: 10.1016/j.apm.2011.08.018.

[22] M. Zhao, W. Pan, C. Wan, Z. Qu, Z. Li, and J. Yang, "Defect engineering in development of low thermal conductivity materials: A review," J. Eur. Ceram. Soc., vol. 37, no. 1, pp. 1-13, Jan. 2017. doi: 10.1016/j.jeurceramsoc.2016.07.036.

[23] R. S. Ghai, "Modelling thermal conductivity of porous thermal barrier coatings for high-temperature aero engines," M.Eng. thesis, Dept. of Mech. Eng., Université d'Ottawa / University of Ottawa, Canada, 2017. 


\title{
Inter-laminar Strain Energy Continuity in Orthotropic Face Sandwich and Composite Plate Analysis Using Improved Higher-order Theory
}

\author{
${ }^{1,2}$ Temesgen Takele Kasa ${ }^{\mathrm{a}^{*}}$ \\ ${ }^{I}$ Addis Ababa science and Technology University, \\ Department of Electro-Mechanical and Manufacturing \\ Engineering, Addis Ababa, Ethiopia \\ Department of Mechanical Engineering, Pukyong \\ 2 National University, Busan-south Korea ${ }^{\text {a }}$ \\ ttkh1@pukyong.ac.kr
}

\begin{abstract}
The main goal of this paper is, to suggest improved higher order refined theory to the analysis of perfectly bonded stack sandwich and composite laminates with usual type lamination configurations. The analysis incorporates continuous flexural and in-plane displacements in the interface. Furthermore, the transverse shear stress is continuous and also constrained with the Lagrange multiplier technique by introducing new fourteen unknown variables. The unknown variables expressed in terms of interfacial strain energy; assuming the interfacial strain energy is continuous throughout the thickness of the laminate. To determine the newly introduced flexural and in-plane unknowns' variables, total potential energy (TPE) is minimized using varational calculus. The numerical results are compared with existing reliable published papers. In general, the aforementioned approach is sufficient enough to analyze sandwich and laminate structures with the required accuracy.
\end{abstract}

Keywords- Refined theory; Flexural displacement; In-plane displacement; Constraint; Strain Energy; TPE

\section{INTRODUCTION}

Nowadays, light weight sandwich and composite structures are widely used in the aeronautical/aerospace and marine industries to gain superior safety, higher payload and good fatigue resistance property. To achieve the above appropriate properties, multilayered laminate structure should requires advanced understanding of statics and dynamical behavior of composite materials[1]. Likewise, sandwich and composite structures are complicated in analysis and design aspect; because some unforeseen failure modes like delamination's are considered [2-4].To overcome the aforementioned challenges inter-laminar strain energy continuity assumption based refined modeling has suggested. This approach gives limitless ways to design and optimize the laminates in accordance with the applied external load.
In general, there is no exact mathematical assumption to analyze sandwich and laminated composite plate with the required accuracy. To plug in the gap, this paper play a vital role by considering layer by layer refined theory analytical approach with assumption of perfect bond between layers and the interfacial strain energy continuity throughout the thickness of the laminate.

The energy in the interfaces to estimate the mechanical behavior of the laminate structures. The theory is implemented on layer by layer technique; in which total potential energy of each layer has minimized by Lagrange multiplier, in-plane and flexural displacement using varaitional calculus. To maintain the principle of continuous uniform deformation theory the plies are bounded perfectly and the continuity conditions constrained by the Lagrange multipliers to satisfy the boundary conditions in terms of in-plane and flexural displacements. Using the above approach, the in plane and flexural stresses can be easily analyzed in each layer of the lamina [5-7].

In general, we considered a refined composite and sandwich plate theory that incorporates continuous strain energy within the interfaces to calculate the mechanical behavior of laminate structures. Our theory is implemented using a layer-by-layer technique in which the total potential energy of each layer is minimized via the Lagrange multiplier to obtain in-plane and flexural displacements using variational calculus. To maintain the principles behind the continuous uniform deformation theory, the plies are perfectly bonded and the continuity conditions are constrained by the Lagrange multipliers to satisfy the governing equilibrium equation using boundary conditions to determine the unknown variables. By applying this approach, the flexural and in-plane stresses are easily analyzed at each layer of the lamina. Further, these formulations are calculated to yield easier plate configurations in two dimensions. 


\section{POTENTIAL ENERGY MINIMIZATION}

In order to determine the natural boundary conditions and governing equilibrium equations in flexural and in-plane form, we can use minimization of total potential energy. In ord er to analyze the above geometry of plate we have been made $t$ he following fundamental assumptions.(1) The laminate have uniform thickness and symmetrical at the mid-plane; to simplif $\mathrm{y}$ the governing equilibrium equations; (2)The core is compres sible;(3) The global axes does not coincide with the local axes of symmetry; (4) The skin plies bonds to core perfectly; (5) Th e core and skin layers are linear elastic (6) The face and core 1 ayers are considered as 2D and 3D structures respectively; (7) The plate assumed to be flat and naturally it has no curve. Under this assumption, all odd polynomial superscript coeffici ents become zero in the equilibrium equations. Therefore, the nine governing equilibrium equations of the laminate can be se parated into five flexural and four in-plane components. By incorporating the constraints, the total potential energy minimized as follows.

$$
\begin{array}{lll}
\frac{\partial\left(\Pi+U_{\text {int }}\right)}{\partial u_{i}^{o}}=0 & , & \frac{\partial\left(\Pi+U_{\text {int }}\right)}{\partial \phi_{i}}=0 \\
\frac{\partial\left(\Pi+U_{\text {int }}\right)}{\partial \psi_{i}}=0 & , & \frac{\partial\left(\Pi+U_{\text {int }}\right)}{\partial \Lambda_{u i}^{a}}=0 \\
\frac{\partial\left(\Pi+U_{\text {int }}\right)}{\partial \theta_{i}}=0 & , & \frac{\partial\left(\Pi+U_{\text {int }}\right)}{\partial \Lambda_{i j}^{a}}=0
\end{array}
$$

The interfacial strain energy formulated as follows

$$
U_{\text {int }}=\frac{1}{2} \int_{A_{\text {int }}}\left(\begin{array}{l}
\left(\Lambda_{u_{1}}^{T o p} u_{1}^{(1)}-\Lambda_{u_{1}}^{T o p} u_{1}^{(2)}\right) \\
+\left(\Lambda_{u_{2}}^{T o p} u_{2}^{(1)}-\Lambda_{u_{2}}^{T o p} u_{2}^{(2)}\right) \\
+\left(\Lambda_{u_{3}}^{T o p} u_{3}^{(1)}-\Lambda_{u_{3}}^{T o p} u_{3}^{(2)}\right) \\
+\left(\Lambda_{13}^{C T} \tau_{13}^{(2)}-\Lambda_{13}^{C T} \tau_{13}^{(3)}\right) \\
+\left(\Lambda_{23}^{C T} \tau_{23}^{(2)}-\Lambda_{23}^{C T} \tau_{23}^{(3)}\right) \\
+\left(\Lambda_{13}^{T} \tau_{13}^{(3)}+\Lambda_{23}^{(1)} \tau_{23}^{(3)}\right) \\
+\left(\Lambda_{u_{2}}^{B o t} u_{2}^{(1)}-\Lambda_{u_{1}}^{B o t} u_{1}^{(2)}\right) \\
+\left(\Lambda_{u_{3}}^{B o t} u_{3}^{(1)}-\Lambda_{u_{3}}^{B o t} u_{3}^{(2)}\right) \\
+\left(\Lambda_{13}^{B C} \tau_{13}^{(1)}-\Lambda_{13}^{B C} \tau_{13}^{(2)}\right) \\
+\left(\Lambda_{23}^{B C} \tau_{23}^{(1)}-\Lambda_{23}^{B C} \tau_{23}^{(2)}\right) \\
+\left(\Lambda_{13}^{B} \tau_{13}^{(1)}+\Lambda_{23}^{B} \tau_{23}^{(2)}\right)
\end{array}\right) d x_{1} d x_{2}
$$

\section{ANALYTICAL CLOSED FORM SOLUTIONS}

The aim of analytical closed form solutions is, to determine the unknown variables analytically. The unknown variables can be obtained from the displacement and interlaminar strain energy continuity formulas. For this approach the Lagrange, in-plane and flexural displacements equations are expanded as Fourier series based on boundary conditions. Considering a load:

$$
q=Q_{m n}^{o} \sin \alpha x_{1} \sin \beta x_{2}
$$

\section{RESULTS}

Symmetric three layered simply supported square composite and sandwich plate with height is equal to $h=10 t$ be used. Applying various aspect $\operatorname{ratios}(\mathrm{S})$ the numerical results of flexural displacement, inter-laminar shear stress and in plane stress are shown in the Table-1 and 2.Table-1 2 and 3 shows that, the percentage error obtained from the suggested approach is in a good agreement with exact elasticity solution[8] and in conclusion while the error percentages decreasing as the plate aspect ratio increase. For S, equal to 4, 10 and 20, the new approach gives a better estimate of inplane stress and inter-laminar shear stress than other approaches for the given aspect ratios. Furthermore, for very thin ply $(\mathrm{a} / \mathrm{h}=50$ and above) the suggested theory contributes a better accuracy for in-plane stresses as compared to other classical models.

The results in the tables report are obtained by the following non-dimensional formulas:

$$
\begin{aligned}
& \bar{u}_{1}=u_{1}\left(\frac{100 * h^{3} E_{22}}{q a^{4}}\right), \bar{u}_{2}=u_{2}\left(\frac{100 * h^{3} E_{22}}{q a^{4}}\right), \\
& \bar{\sigma}_{22}=\sigma_{22}\left(\frac{h^{2}}{q a^{2}}\right) \\
& \bar{u}_{3}=u_{3}\left(\frac{100 * h^{3} E_{22}}{q a^{4}}\right), \bar{\sigma}_{11}=\sigma_{11}\left(\frac{h^{2}}{q a^{2}}\right) \bar{\tau}_{12}=\tau_{12}\left(\frac{h^{2}}{q a^{2}}\right),
\end{aligned}
$$

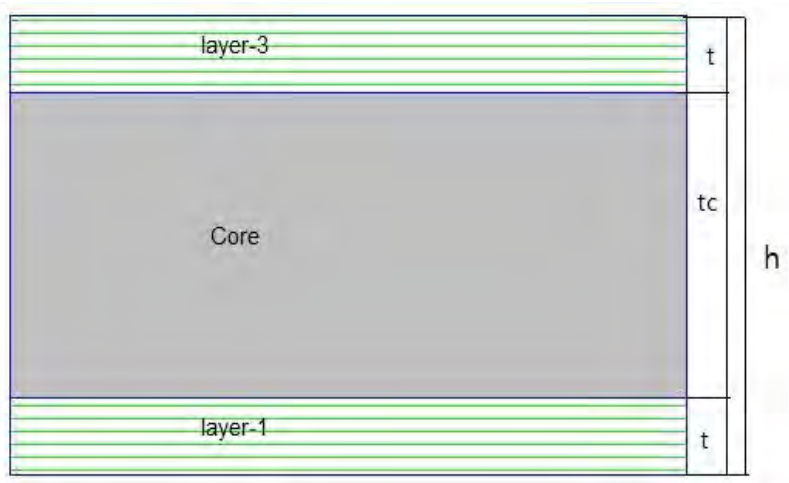

Figure-1. Sandwich plate 
Table: 1 Error Percentage of maximum stress in nondimensional form $(0 /$ core $/ 0)$

\begin{tabular}{c|ccccr}
\hline $\mathrm{S}$ & source & $\bar{\sigma}_{11}$ & $\bar{\sigma}_{22}$ & $\bar{\tau}_{12}$ & \multicolumn{1}{c}{$\bar{u}_{3}$} \\
$\mathbf{2}$ & Elasticity[8] & \pm 2.653 & \pm 0.3919 & \pm 0.2338 & 0.1402 \\
& New & \pm 2.6593 & \pm 0.3917 & \pm 0.2337 & 0.1405 \\
& Error & $\mathbf{0 . 2 3 7 5}$ & $\mathbf{0 . 0 5 1 0 3}$ & $\mathbf{0 . 0 4 2 7 7}$ & $\mathbf{0 . 0 7 1 3 3}$ \\
$\mathbf{4}$ & Elasticity[8] & \pm 1.512 & \pm 0.2533 & \pm 0.1481 & 0.1072 \\
& New & \pm 1.5139 & \pm 0.25314 & \pm 0.1479 & 0.1075 \\
& Error & $\mathbf{0 . 1 2 5 7}$ & $\mathbf{0 . 0 6 3 1 6 6}$ & $\mathbf{0 . 0 2 0 5 7}$ & $\mathbf{0 . 2 7 8 9}$ \\
$\mathbf{1 0}$ & Elasticity[8] & \pm 1.152 & \pm 0.1099 & \pm 0.0707 & 0.0527 \\
& New & \pm 1.153 & \pm 0.10976 & \pm 0.07059 & 0.0529 \\
$\mathbf{2 0}$ & Error & $\mathbf{0 . 0 8 6 8}$ & $\mathbf{0 . 0 3 6 3 9}$ & $\mathbf{0 . 1 5 5 5 8}$ & $\mathbf{0 . 3 7 9 5}$ \\
& Elasticity[8] & \pm 1.110 & \pm 0.070 & \pm 0.0511 & 0.0361 \\
& New & \pm 1.112 & \pm 0.070017 & \pm 0.05118 & 0.0364 \\
$\mathbf{5 0}$ & Error & $\mathbf{0 . 0 1 8 0 1}$ & $\mathbf{0 . 0 1 4 2}$ & $\mathbf{0 . 1 5 2 4}$ & $\mathbf{0 . 5 2 8}$ \\
& Elasticity[8] & \pm 1.099 & \pm 0.0569 & \pm 0.0446 & 0.0306 \\
& New & \pm 1.0991 & \pm 0.056905 & \pm 0.04463 & 0.03064 \\
$\mathbf{1 0 0}$ & Error & $\mathbf{0 . 0 1 8}$ & $\mathbf{0 . 0 0 8 7 8}$ & $\mathbf{0 . 0 8 2 6}$ & $\mathbf{0 . 6 1 5}$ \\
& Elasticity[8] & \pm 1.098 & \pm 0.0550 & \pm 0.0437 & 0.0297 \\
& New & \pm 1.099 & \pm 0.0551 & \pm 0.0438 & 0.02982 \\
& Error & $\mathbf{0 . 0 1 5}$ & $\mathbf{0 . 0 0 5 9}$ & $\mathbf{0 . 0 7 6}$ & $\mathbf{0 . 7 4 0 3}$
\end{tabular}

Table-2:Error percentage for orthotropic face sandwich

\begin{tabular}{|c|c|c|c|c|c|}
\hline $\mathrm{S}$ & source & $\bar{\sigma}_{11}$ & $\bar{\sigma}_{22}$ & $\bar{\tau}_{12}$ & $\bar{u}_{3}$ \\
\hline \multirow[t]{3}{*}{2} & Kant $-2[5]$ & \pm 4.0665 & \pm 0.531 & \pm 0.5184 & 39.0218 \\
\hline & New & \pm 4.0668 & \pm 0.5340 & \pm 0.5171 & 39.042 \\
\hline & Error & 0.0074 & 0.0073 & 0.2505 & 0.0518 \\
\hline \multirow[t]{3}{*}{4} & Kant -2[5] & \pm 1.7931 & \pm 0.2128 & \pm 0.2702 & 14.4949 \\
\hline & New & \pm 1.7704 & \pm 0.2138 & \pm 0.2725 & 14.471 \\
\hline & Error & 5.79 & 5.46 & 4.76 & 6.0985 \\
\hline \multirow[t]{3}{*}{10} & Kant -2[5] & \pm 0.8344 & \pm 0.1527 & \pm 0.1352 & 3.8899 \\
\hline & New & \pm 0.8345 & \pm 0.1533 & \pm 0.1354 & 3.8925 \\
\hline & Error & 0.0046 & 0.052 & 0.1479 & 0.0668 \\
\hline \multirow[t]{3}{*}{100} & Kant -2[5] & \pm 0.6647 & \pm 0.0642 & \pm 0.0699 & 1.0806 \\
\hline & New & \pm 0.6648 & \pm 0.0641 & \pm 0.0700 & 1.0827 \\
\hline & Error & 0.015 & 0.1558 & 0.0121 & 0.628 \\
\hline
\end{tabular}

Table-3 Error percentage for composite plate

\begin{tabular}{|c|c|c|c|c|c|}
\hline $\mathbf{S}$ & source & $\bar{\sigma}_{11}$ & $\bar{\sigma}_{22}$ & $\bar{\tau}_{12}$ & $\bar{u}_{3}$ \\
\hline \multirow[t]{3}{*}{2} & Elasticity[8] & 0.937 & 0.669 & 0.0859 & 4.9362 \\
\hline & New & 0.93814 & 0.670096 & 0.086099 & 4.93853 \\
\hline & Error & 0.12166 & 0.16442 & 0.2328 & 0.0474 \\
\hline \multirow[t]{3}{*}{4} & Elasticity[8] & 0.755 & 0.556 & 0.0505 & 1.7287 \\
\hline & New & 0.7558698 & 0.556799 & 0.0505627 & 1.72907 \\
\hline & Error & 0.1152 & 0.14388 & 0.12475 & 0.021735 \\
\hline \multirow[t]{3}{*}{10} & Elasticity[8] & 0.59 & 0.285 & 0.0289 & \\
\hline & New & 0.590631 & 0.285359 & 0.028932 & 0.7171 \\
\hline & Error & 0.1069 & 0.1263 & 0.1136 & \\
\hline \multirow[t]{3}{*}{20} & Elasticity[8] & 0.552 & 0.21 & 0.0289 & \\
\hline & New & 0.552563 & 0.210242 & 0.028930 & 0.5427 \\
\hline & Error & 0.1021 & 0.1154 & 0.1045 & \\
\hline \multirow[t]{3}{*}{50} & Elasticity[8] & 0.541 & 0.185 & 0.0216 & 0.4432 \\
\hline & New & 0.541471 & 0.185172 & 0.0216209 & 0.443282 \\
\hline & Error & 0.087 & 0.093 & 0.0968 & 0.0187 \\
\hline \multirow[t]{3}{*}{100} & Elasticity[8] & 0.539 & 0.181 & 0.0213 & \\
\hline & New & 0.539442 & 0.181164 & 0.021319 & 0.4347 \\
\hline & Error & 0.082 & 0.0906 & 0.0932 & \\
\hline
\end{tabular}

\section{SUMMARY}

In this paper, we explored and analyzed the accuracy of a layer-by-layer refined theory model with flexural and in-plane displacement continuity in a laminate composite and sandwich plate. The improved higher-order theory was applied for efficient execution of the refined theory. Inter-laminar continuity equations were also constrained using Lagrange multipliers by introducing new variables. The existing and newly introduced variables were then solved through a total potential energy minimization technique. Here, Navier-type analytical closed-form solutions were adopted for analyses. Considering simply supported boundary conditions, we analyzed higher and lower-aspect ratio sandwich plates. Further, the sandwich plate was subjected to sinusoidal distributed loading on the top face. Given this, we calculated all flexural displacements, in-plane displacements, in-plane stress, and interfacial shear stresses and then compared them with exact values presented in previous studies. Further, we performed some parametric tests, with results showing that the aspect ratio increases the accuracy of the analysis also increases for the above approach. We also found that each displacement or stress component requires its own plate model (which differs according to the change in outputs) to obtain exact results. Further, the accuracy of the solution also depends on thickness coordinate $x_{3}$. The key advantage of our proposed approach is the ability to obtain remarkably accurate results for all ranges of aspect and modular ratios. Further, if $\mathrm{n}$ eeded, it is possible to enhance our proposed approach by deco mposing the lower-aspect ratio layer into a number of higher a spect ratio layers.

\section{ACKNOWLEDGMENT}

I acknowledge NIIDE and Pukyong National University for their material support of this research.

\section{REFERENCES}

[1] J. R. Vinson, The Behavior of Sandwich Structures of Isotropic and Composite Materials: CRC Press, 1999.

[2] A. C. Garg, "Delamination-a damage mode in composite structures," Engineering Fracture Mechanics, vol. 29, no. 5, pp. 557-584, 1988. doi: 10.1016/0013-7944(88)90181-6.

[3] F. Crossman, W. Warren, A. Wang, and G. Law Jr, "Initiation and growth of transverse cracks and edge delamination in composite laminates Part 2.Experimental correlation," Journal of Composite Materials, vol. 14, no. 1, pp. 88-108, 1980.doi: 10.1177/002199838001400107. 
[4] P. P. Camanho, and C. G. Dávila, "Mixed-mode decohesion finite elements for the simulation of delamination in composite materials," NASA/ TM, No. 211737, 2002

[5] T. Kant, and K. Swaminathan, "Analytical solutions for the static analysis of laminated composite and sandwich plates based on a higher order refined theory," Composite Structures, vol. 56, no. 4, pp. 329-344, 2002.

doi:10.1016/S0263-8223(02)00017-X.

[6] T. Kant, and K. Swaminathan, "Estimation of transverse/interlaminar stresses in laminated composites-a selective review and survey of current developments," Composite Structures, vol. 49, no. 1, pp. 65-75, 2000. doi:10.1016/S0263-8223(99)00126-9.

[7] J. N. Reddy, "A simple higher-order theory for laminated composite plates," Journal of Applied Mechanics, vol. 51, no. 4, pp. 745-752, 1984. doi:10.1115/1.3167719.

[8] N. J. Pagano, "Exact solutions for rectangular bidirectional composites and sandwich plates," in Mechanics of Composite Materials, Reddy J.N. (eds) Dordrecht: Springer, 1994, pp. 86-101. doi: 10.1007/978-94-017-2233-9_8. 


\section{The Libration Stabilization of a Partial Space Elevator System Using Analytical Reel Rate Control}

\author{
Gefei Shi \\ Northwestern Polytechnical University \\ Xi'an, 710072, PR China \\ gefeishi@mail.nwpu.edu.cn \\ Zhanxia Zhu \\ Northwestern Polytechnical University
}

\author{
Xi'an, 710072, PR China \\ zhuzhanxia@nwpu.edu.cn \\ Zheng H. Zhu \\ Department of Mechanical Engineering, York University \\ Toronto, Canada \\ gzhu@yorku.ca
}

\begin{abstract}
This paper investigates the libration stabilization control of a partial space elevator system with a moving climber in circular orbits. The system is described by a modified two-piece dumbbell model. The model consists of one main satellite, one climber and one end-body connected by two straight, massless and inextensible tethers. The climber and the end body can move along the tether. The libration motion and the tether reeling motion are separated. And a reel rate function is obtained by analyzing the equilibrium state of the libration motion, based on the which an analytical control scheme is designed. Using the sliding mode control law, the proposed control scheme can be implemented effectively and robustly. The results of numerical simulations show that the proposed control scheme has good performance in keeping the stable of the climber's transfer of a partial space elevator satellite system. Furthermore, the proposed libration suppression control can be realized by using tension control only.
\end{abstract}

Keywords: Partial space elevator system; libration stabilization; stable transfer; analytical reel rate control

\section{INTRODUCTION}

Partial space elevator system is a partial space elevator system which is a typical tethered satellite system (TTS) [1-6] where a middle body (climber) can move between the main satellite and end body. One difficulty associated with such system is to suppress the libration of the climber and the end body [7 - 9]. The libration is intrinsically unstable due to the Coriolis force produced by the moving climber. The Coriolis force could lead to the tumbling of the partial space elevator system $[9,10]$. Moreover, by the end of the transfer period, the magnitude of the final libration angles of the climber and the end body apart from the equilibrium point should be limited so that the system can work in a general stable condition after transferring. Otherwise, additional energy consumption is required to stable the system [7]. Thus, the suppression of such system is critical for a successful climber transfer mission and making sure of the stable working state [10 - 12].

In this study, we aim to keep the climber at a desired constant libration angle in the transfer period. By analyzing the system dynamic, the proposed climbing speed of the climber is obtained. The proposed transfer speed is demonstrated by numerical simulations with a modifying control. The control can be realized by controlling the reel rate of the tether. The results show that the newly developed control method can be used to kept the climber at the desired angle. The simulation results also present that using the proposed control method, the end-body motion is also suppressed in the transfer period. Moreover, the control input is smooth overall, this is a good condition for the practical use.

\section{DYNAMICS OF A PARTIAL SPACE ELEVATOR SYSTEM}

Consider a partial space elevator system in an orbital plane of a circular orbit as shown in figure. 1 .

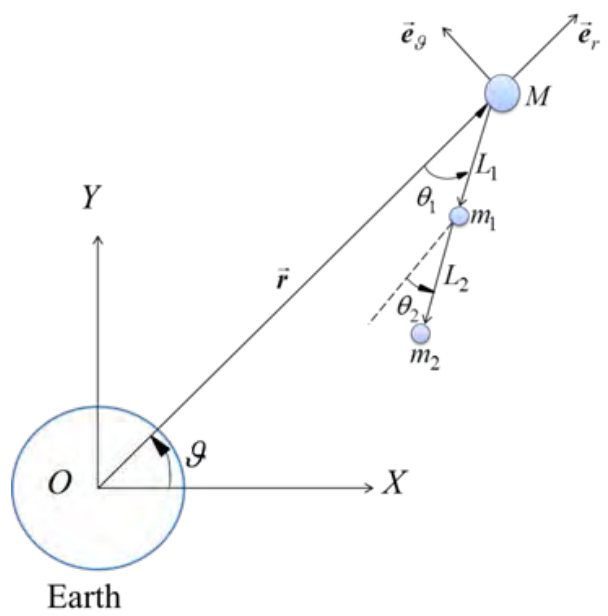

Figure 1 Partial space elevator system.

The dimensionless math model of the partial space elevator system can be expressed as [9]

$$
M_{1}=m_{1} / m_{t o t}, M_{2}=m_{2} / m_{t o t}, l_{1}=L_{1} / L_{0}, l_{2}=L_{2} / L_{0}
$$

where $\tau=\omega t$ is the dimensionless time, $M_{1}$ and $M_{2}$ are the dimensionless mas of the climber and the end-body, respectively. $l_{1}$ and $l_{2}$ are the dimensionless length of the tether 1 and tether 2 , respectively. 


$$
\begin{aligned}
\theta_{1}^{\prime \prime}= & \frac{-3}{2} \sin \left(2 \theta_{1}\right)-\frac{3 M_{2} \cos ^{2}\left(\theta_{1}\right) \tan \left(\theta_{1}-\theta_{2}\right)}{-M_{2}+\sec ^{2}\left(\theta_{1}-\theta_{2}\right)}-\frac{2\left(1+\theta_{1}^{\prime}\right) l_{1}^{\prime}}{l_{1}} \\
& -\frac{2 M_{2}\left(1-l_{1}\right) \sec \left(\theta_{1}-\theta_{2}\right) \tan \left(\theta_{1}-\theta_{2}\right) \theta_{2}^{\prime}}{l_{1}\left[-M_{2}+\sec ^{2}\left(\theta_{1}-\theta_{2}\right)\right]} \\
& -\frac{M_{2}\left(1-l_{1}\right) \sec \left(\theta_{1}-\theta_{2}\right) \tan \left(\theta_{1}-\theta_{2}\right)\left(\theta_{2}^{\prime}\right)^{2}}{l_{1}\left[-M_{2}+\sec ^{2}\left(\theta_{1}-\theta_{2}\right)\right]} \\
- & \frac{M_{2} \tan \left(\theta_{1}-\theta_{2}\right)\left(\theta_{1}^{\prime}\right)^{2}}{-M_{2}+\sec ^{2}\left(\theta_{1}-\theta_{2}\right)}-\frac{2 M_{2} \tan \left(\theta_{1}-\theta_{2}\right) \theta_{1}^{\prime}}{-M_{2}+\sec ^{2}\left(\theta_{1}-\theta_{2}\right)} \\
- & \frac{3 M_{2}\left(1-l_{1}\right) \cos ^{2}\left(\theta_{2}\right) \sec \left(\theta_{1}-\theta_{2}\right) \tan \left(\theta_{1}-\theta_{2}\right)}{l_{1}\left[-M_{2}+\sec ^{2}\left(\theta_{1}-\theta_{2}\right)\right]}+t o l_{1} \\
\theta_{2}^{\prime \prime}= & \frac{-3}{2} \sin \left(2 \theta_{2}\right)+\frac{3 l_{1} \cos ^{2}\left(\theta_{1}\right) \sin \left(\theta_{1}-\theta_{2}\right)}{\left(1-l_{1}\right)\left[M_{1}+M_{2} \sin { }^{2}\left(\theta_{1}-\theta_{2}\right)\right]} \\
& +\frac{2 \sin \left(\theta_{1}-\theta_{2}\right) l_{1} \theta_{1}^{\prime}}{\left(1-l_{1}\right)\left[M_{1}+M_{2} \sin ^{2}\left(\theta_{1}-\theta_{2}\right)\right]}+\frac{2\left(1+\theta_{2}^{\prime}\right) l_{1}^{\prime}}{1-l_{1}} \\
& +\frac{2 M_{2} \cos \left(\theta_{1}-\theta_{2}\right) \sin ^{2}\left(\theta_{1}-\theta_{2}\right) \theta_{2}^{\prime}}{M_{1}+M_{2} \sin ^{2}\left(\theta_{1}-\theta_{2}\right)} \\
& +\frac{l_{1} \sin \left(\theta_{1}-\theta_{2}\right)\left(\theta_{1}^{\prime}\right)^{2}}{\left(1-l_{1}\right)\left[M_{1}+M_{2} \sin ^{2}\left(\theta_{1}-\theta_{2}\right)\right]} \\
& +\frac{M_{2} \cos \left(\theta_{1}-\theta_{2}\right) \sin ^{2}\left(\theta_{1}-\theta_{2}\right)\left(\theta_{2}^{\prime}\right)^{2}}{M_{1}+M_{2} \sin ^{2}\left(\theta_{1}-\theta_{2}\right)} \\
& +\frac{3 M_{2} \cos { }^{2}\left(\theta_{2}\right) \cos ^{2}\left(\theta_{1}-\theta_{2}\right) \sin \left(\theta_{1}-\theta_{2}\right)}{M_{1}+M_{2} \sin ^{2}\left(\theta_{1}-\theta_{2}\right)}+t o l_{2} \\
&
\end{aligned}
$$

where

$$
\begin{aligned}
& t o l_{1}=\frac{M_{2} \sin \left(\theta_{1}-\theta_{2}\right)\left[\cos \left(\theta_{1}-\theta_{2}\right)-1\right] l_{1}^{\prime \prime}}{l_{1}\left[M_{1}+M_{2} \sin ^{2}\left(\theta_{1}-\theta_{2}\right)\right]} \\
& t o l_{2}=-\frac{\sin \left(\theta_{1}-\theta_{2}\right)\left[1-M_{2} \cos \left(\theta_{1}-\theta_{2}\right)\right] l_{1}^{\prime \prime}}{\left(1-l_{1}\right)\left[M_{1}+M_{2} \sin ^{2}\left(\theta_{1}-\theta_{2}\right)\right]}
\end{aligned}
$$

Eq. (4) is the length accelerating section that denotes the impacts of the tensions along tethers on the changes of $\theta_{1}$ and $\theta_{2}$. In the analysis, these two parts are regarded as system noise and ignored, thus, they are the main disturbances source that can be deal with the modifying control.

\section{DYNAMIC ANALYSIS AND ANALYTICAL REEL RATE CONTROL DESIGN}

\section{A. Steady state during the moving of the climber}

The steady state solution of the system can be simply obtained by setting all first and second order derivatives to zero, such that,

$$
\theta_{1}^{\prime}=0, \theta_{2}^{\prime}=0, \theta_{1}^{\prime \prime}=0, \theta_{2}^{\prime \prime}=0
$$

To ensure climber's stable climbing with constant pitch angles, the stable libration angles can be obtained from the simple dynamic equations with ignoring the length accelerating sections, such as

$$
\theta_{1}=\theta_{1 e}=-\frac{2 M_{1} l_{1 e}^{\prime}}{3\left(l_{1} M_{1}+M_{2}\right)} \text { and } \theta_{2}=\theta_{2 e}=0
$$

For constant pitch angles $\theta_{1 e}$ and $\theta_{2 e}$, the stable climbing is dependent on $l_{1 e}^{\prime}$, which can be derived from Eq. (16) as follows

$$
l_{1 e}^{\prime}=-\frac{3\left(l_{1} M_{1}+M_{2}\right)}{2 M_{1}} \theta_{1 e}
$$

\section{B. Control law design based on stable climbing function}

To stable the libration angle during the transfer phase of the climber, control law is needed to compensate the possible disturbances. In this paper, we only aim to obtain the stable transfer of the climber, thus, the desired state for the climber in partial space elevator is $\theta_{1}=\theta_{1 e}$ and $\theta_{2}=0$ is not required. To achieve this state, define a sliding mode manifold that drives both $\theta_{1}$ and $\theta_{1}^{\prime}$ to $\theta_{1 e}$ and zero, respectively, such that,

$$
s=c\left(\theta_{1}-\theta_{1 e}\right)+\theta_{1}^{\prime}=0
$$

where $c$ is a positive constant that defines the bandwidth of error dynamics of $s$.

The error dynamics can be derived by taking derivative with respect to $\tau$ at both sides of Eq. (23)

$$
s^{\prime}=\frac{d s}{d \tau}=c \theta_{1}^{\prime}+\theta_{1}^{\prime \prime}
$$

Assume the control input is the velocity function of the climber. Define $\theta_{1}^{\prime \prime}=f_{1}+b_{1} u$ where $f_{1}$ is the nonlinear function of $\theta_{1}^{\prime \prime}, b_{1}$ is a gain function depending on $l_{1}$ and $\theta_{1}^{\prime}$, and the control input $u$ is a modification velocity of the obtained function $l_{1 e}^{\prime}$, thus, the real velocity of the climber becomes $l_{1}^{\prime}=u+l_{1 e}^{\prime}$.

Substituting $\theta_{1}^{\prime \prime}$ into to Eq. (24) yield

$$
s^{\prime}=c \theta_{1}^{\prime}+f_{1}+b_{1} u
$$

Thus, the sliding mode control law is derived from Eq. (23) as,

$$
\left\{\begin{array}{l}
u=u_{e q}+u_{s w} \\
u_{e q}=-b_{1}^{-1}\left(f_{1}+c \theta_{1}^{\prime}\right) \\
u_{s w}=-b_{1}^{-1} k \cdot \operatorname{sign}(s)
\end{array}\right.
$$

where $k$ is a positive control gain, $u_{\text {eq }}$ is the equivalent control input and $u_{\text {sw }}$ is the switching input.

For the sake of the avoidance of chattering in the sign function of Eq. (26), $\operatorname{sign}(s)$ is replaced by the saturation function

$$
\operatorname{sat}(s)=\left\{\begin{array}{cl}
s & \text { if }\|s\| \geq \varepsilon \\
s / \varepsilon & \text { if }\|s\|<\varepsilon
\end{array}\right.
$$

where $\varepsilon \in \mathbb{R}_{+}$is a small constant.

$$
\begin{aligned}
& \text { Next, consider a candidate Lyapunov function as } \\
& V=s \cdot s / 2
\end{aligned}
$$

Taking the derivative of $V$ with respect to $\tau$ yields

$$
V^{\prime}=s \cdot s^{\prime}=-k s \cdot \operatorname{sign}(s) \leq 0
$$


Furthermore, if there is a bonded disturbance $d$ in the system, Eq. (12) is revised as,

$$
f_{d}=f_{1}+d
$$

Submitting Eq. (32) in to Eq. (31) yields

$$
V^{\prime}=s \cdot s^{\prime}=[d-k \cdot \operatorname{sign}(s)] s \leq\left. 0\right|_{\text {if } k \geq|d|}
$$

Thus, the system is robust when subjected to a bonded disturbance.

\section{CASE STUDIE}

In this work, $t_{0} l_{1}$ and $t_{0} l_{2}$ are set zero, this equals to add dynamic errors, - tol $_{1}$ and - tol $_{2}$, to the dynamic Eqs. (12) and (13). Two cases are studied $\theta_{1 e}$, the desired stability angles of $\theta_{1}$ are set 0.2 and 0.4 , respectively. The climber is moving upwards, such that $l_{1}^{\prime}<0$. The dimensionless masses $M_{1}=1 / 3$ and $M_{1}=2 / 3$. The results are shown in Fig. $2-$ Fig. 6.

Under the affection of the dynamic errors, the libration angles do not follow the obtained steady state even when the climbing speed follows the designed function, see figure 2 and 3 . The libration angle of the climber increases continuously from the beginning, see figure 2. After 0.02 orbit, $\theta_{1}$ is suppressed, and it converges to the desired magnitude. Meanwhile, $\theta_{2}$ is also suppressed although the control law is not designed to control it exactly. Figure 4 shows the control input. In the whole transfer period, the change of the reel rate is smooth generally, this is very good for the realization of the practical condition. The moving path of the climber is shown in figure 6 . In the mission program, the libration angle of the climber is kept at the desired angle generally, this matches the result shown in figure 2 .

\section{A. Case 1:}

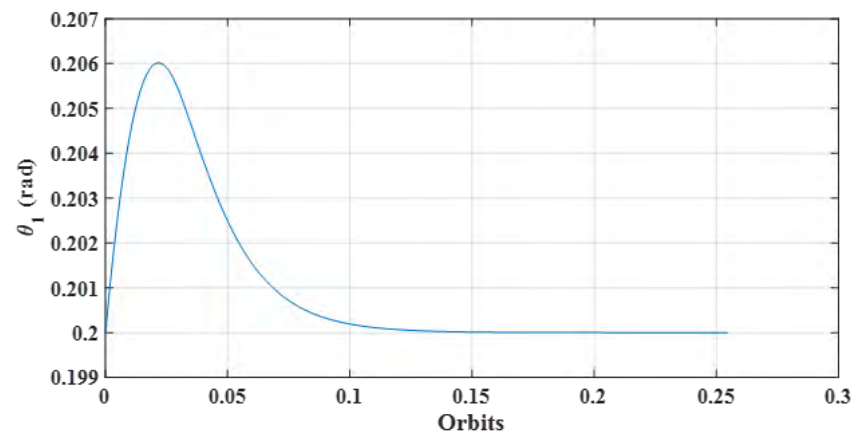

Figure 2 Libration motion of $\theta_{1}$ versus orbits when $\theta_{1 e}=0.2$

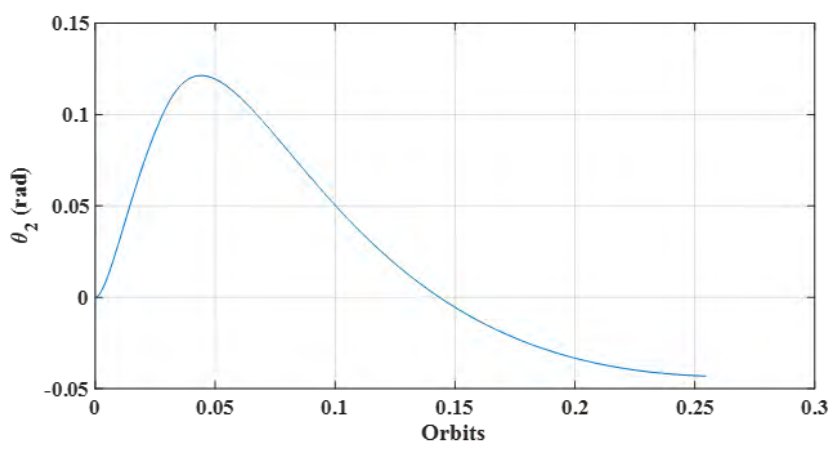

Figure 3 Libration motion of $\theta_{2}$ versus orbits when $\theta_{1 e}=0.2$

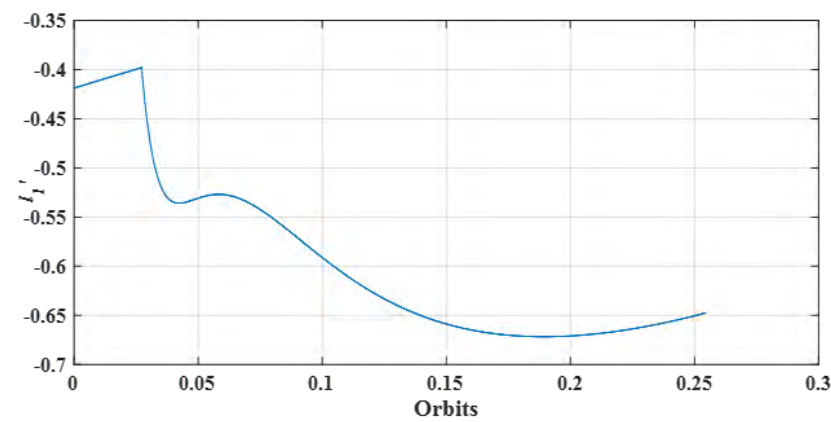

Figure 4 Dimensionless velocity of the climber along $l_{1}$ when

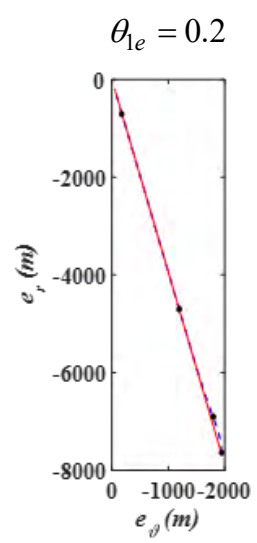

Figure 6 Trajectory of the climber when $\theta_{1 e}=0.2$

\section{CONCLUSION}

The dynamic and stable control of a partial space elevator in the transfer period is studied. By analyzing the system dynamic, the proposed climbing speed of the climber is obtained. The proposed transfer speed is demonstrated by numerical simulations with a sliding mode control with considering the input charting. The control can be realized by controlling the reel rate of the tether. The simulation results show that the newly developed control method can be used to kept the climber at the desired angle. The simulation results also present that using the proposed control method, the endbody motion is also suppressed in the transfer period. Moreover, the control input is smooth overall, this is a good condition for the practical use. 


\section{REFERENCES}

[1] A. K. Misra, Z. Amier, and V. J. Modi, "Attitude dynamics of three-body tethered systems," Acta Astronautica, vol. 17, no. 10, pp. 1059-1068, Oct. 1988. doi: 10.1016/0094-5765(88)90189-0.

[2] K. D. Kumar, "Review on Dynamics and Control of Nonelectrodynamic Tethered Satellite Systems," J. Spacecr. Rockets, vol. 43, no. 4, pp. 705-720, Jul. 2006. doi: 10.2514/1.5479.

[3] C. Lorenzini, M. Cosmo, S. Vetrella, and A. Moccia, "Dynamics and control of the tether elevator/crawler system," J. Guidance, Control, and Dynamics, vol. 12, no. 3, pp. 404-411, May 1989.

doi: $10.2514 / 3.20422$

[4] H. A. Fujii, T. Watanabe, T. Kusagaya, D. Sato, and M. Ohta, "Dynamics of a Flexible Space Tether Equipped with a Crawler Mass," J. Guidance, Control, and Dynamics, vol. 31, no. 2, pp. 436-440, Mar. 2008. doi: $10.2514 / 1.26240$

[5] P. Woo and A. K. Misra, "Dynamics of a partial space elevator with multiple climbers," Acta Astronautica, vol. 67, no. 7-8, pp. 753-763, Oct. 2010. doi: 10.1016/j.actaastro.2010.04.023.

[6] Y. Yamagiwa et al., "Space experiments on basic technologies for a space elevator using microsatellites," Acta Astronautica, vol. 138, pp. 570-578, Sep. 2017. doi: 10.1016/j.actaastro.2016.12.022.

[7] H. Kojima, M. Iwasaki, H. A. Fujii, C. Blanksby, and P. Trivailo, "Nonlinear Control of Librational Motion of Tethered Satellites in Elliptic Orbits," J. Guidance, Control, and Dynamics, vol. 27, no. 2, pp. 229-239, Mar. 2004. doi: 10.2514/1.9166.

[8] H. Kojima, K. Fukatsu, and P. M. Trivailo, "Mission-function control of tethered satellite/climber system," Acta Astronautica, vol. 106, pp. 24-32, Jan. 2015. doi: 10.1016/j.actaastro.2014.10.024.

[9] G. Shi, Z. Zhu, and Z. H. Zhu, "Libration suppression of tethered space system with a moving climber in circular orbit," Nonlinear Dyn. vol. 91, no. 2, pp. 923-937, Nov. 2017. doi: 10.1007/s11071-017-3919-x.

[10] S. Aslanov and A. S. Ledkov, Dynamics of Tethered Satellite Systems, Woodhead Publishing, 2012. doi: 10.1533/9780857096005.

[11] P. Huang, Z. Hu, and F. Zhang, "Dynamic modelling and coordinated controller designing for the manoeuvrable tether-net space robot system," Multibody System Dynamics, vol. 36, no. 2, pp. 115-141, Nov. 2015. doi 10.1007/s11044-015-9478-3.

[12] Y. Lu, P. Huang, Z. Meng, Y. Hu, F. Zhang, and Y. Zhang, "Finite time attitude takeover control for combination via tethered space robot," Acta Astronautica, vol. 136, pp. 9-21, Jul. 2017.

doi: 10.1016/j.actaastro.2017.02.022. 


\section{Design, Analysis and 3D Printing of Architected Sandwich Panels}

\author{
H. Yazdani Sarvestani \\ AM3L Laboratory, Department of Bioresource \\ Engineering \\ McGill University \\ Island of Montreal, QC H9X 3V9 Canada \\ H. Niknam \\ AM3L Laboratory, Department of Bioresource \\ Engineering \\ McGill University \\ Island of Montreal, QC H9X 3V9 Canada
}

\author{
A.H. Akbarzadeh \\ AM3L Laboratory, Department of Bioresource \\ Engineering \\ McGill University \\ Island of Montreal, QC H9X 3V9 Canada \\ Email: hamid.akbarzaeh@mcgill.ca
}

\author{
K. Hermenean \\ MACHINA Corp \\ Edmonton, AB T6H 2H3 \\ Canada
}

\begin{abstract}
In this study, we implement a finite element approach and conduct experimental impact tests to evaluate the performance of $3 \mathrm{D}$ printed lightweight sandwich panels with architected cellular cores of programmable six-sided cells. The standard mechanics homogenization technique is implemented through a finite element modelling to accurately predict the effective mechanical properties of architected cellular cores. We implement an explicit large deformation finite element simulation using ANSYS to analyze the elastoplastic behavior of sandwich panels under a low-velocity impact. To experimentally corroborate the developed computational model and to evaluate the manufacturability of architected sandwich panels, we use the fused deposition modeling to 3D print samples of polylactic acid biopolymers. We conduct low-velocity impact experimental tests on the 3D printed panels to investigate their energy absorption capabilities. The results show that the auxetic sandwich panel is potentially an appropriate candidate for energy absorption applications due to its high energy absorption capability.
\end{abstract}

Keywords-component: Architected 3D printed panels; Cellular solids; Energy absorption; Low-velocity impact.

\section{INTRODUCTION}

Sandwich panels are nowadays extensively used in aerospace, marine, automotive, and windmills industries due to their high flexural stiffness-to-weight ratio, excellent thermal insulation, and high energy absorption capability [1]. Sandwich panels consist of two thin but stiff face-sheets at the top and bottom of the panels separated by a lightweight and relatively thick core. The lightweight core connects the face-sheets with small increase in weight but provides sandwich panels with a high bending stiffness and buckling resistance [2] as well as superb shear stiffness and energy absorption capability [3]. The lightweight core can be made of alternative materials but it is usually in the form of cellular materials, e.g. foams or periodic lattices. In a sandwich panel, the face-sheets carry most of inplane and bending loads while the core mainly carries transverse shear [1].

The energy absorption capability of an architected sandwich panel mainly depends on the material properties and geometrical features of solid sheets and the cellular core. Among all cell topologies for the core of architected sandwich panels, hexagonal honeycomb has been commonly used and analyzed as a cellular core $[4,5]$. Sandwich panels with conventional honeycomb cellular cores are stiff and lightweight, while they absorb high energy under impact and shockwaves for applications in sports goods, automotive, and aerospace $[6,7]$. However, they have some issues due to their closed-cell architectures including gas retention, leading to low thermal conductivity, and moisture trapping [1]. An alternative sandwich core is open-cell cores such as truss-like structures $[8,9]$. It has been shown that truss-like cellular cores, with low relative densities, have significantly higher buckling resistance and energy absorption capability compared to hexagonal honeycomb cellular cores $[10,11]$.

More recently, auxetic sandwich cores have been examined due to their unusual deformation mechanisms, which is negative Poisson's ratio. Auxetic cores possess promising properties in the aforementioned applications compared to other topologies of cellular solids. Due to the auxetic behavior, auxetic cellular cores reveal unique mechanical properties such as increased indentation resistance [12], shear resistance [13], fracture toughness $[14,15]$, and energy absorption capacity $[16,17]$. Although a few investigations have been performed on architected sandwich panels, e.g. flexural behavior [18-20] and out-of-plane compressive strength [21,22], less attention has been paid to explore the energy absorption capabilities of 3D printed sandwich panels with architected cellular cores.

The current study focuses on the effect of core's cell topology and relative density on the energy absorption 
capability and structural design of 3D printed sandwich panels with an architected cellular core.

\section{SANDWICH PANEL AND EXPERIMENTAL TEST CONFIGURATION}

Dimensions of the sandwich panel including length $(a)$, width $(b)$, and total thickness $(h)$ are shown in Fig. 1. The coordinate system $(x, y, z)$ is located at the midplane of the sandwich panel. The selected dimensions are listed in Table 1, which are chosen based on ASTM standard D3763 [23] and testing limitations.

TABLE I. GEOMETRIC PARAMETERS OF SANDWICH PANELS (UNIT: MM).

\begin{tabular}{|c|c|c|c|c|}
\hline Length (a) & Width (b) & $\begin{array}{c}\text { Total } \\
\text { thickness (h) }\end{array}$ & $\begin{array}{c}\text { Core } \\
\text { thickness } \\
\text { (tc) }\end{array}$ & $\begin{array}{c}\text { Face-sheet } \\
\text { thickness } \\
\text { (ts) }\end{array}$ \\
\hline 100 & 100 & 50 & 40 & 5 \\
\hline
\end{tabular}

\section{A. Sample preparation}

MK2 3D printer of MACHINA Corp. with the layer height of $0.25 \mathrm{~mm}$ manufactures the architected sandwich panels. MK2 3D printer uses fused deposition modeling technology (FDM) to additively manufacture architected samples of PLA polymers. Two types of sandwich panels (vertical or horizontal cells) with three different topologies of cellular cores (hexagonal, rectangular, and auxetic) are 3D printed.

\section{B. Experimental low-velocity impact test}

The low-velocity impact tests are performed on sandwich panels with different 3D printed cores using a drop weight machine based on the guidelines given in the ASTM standard

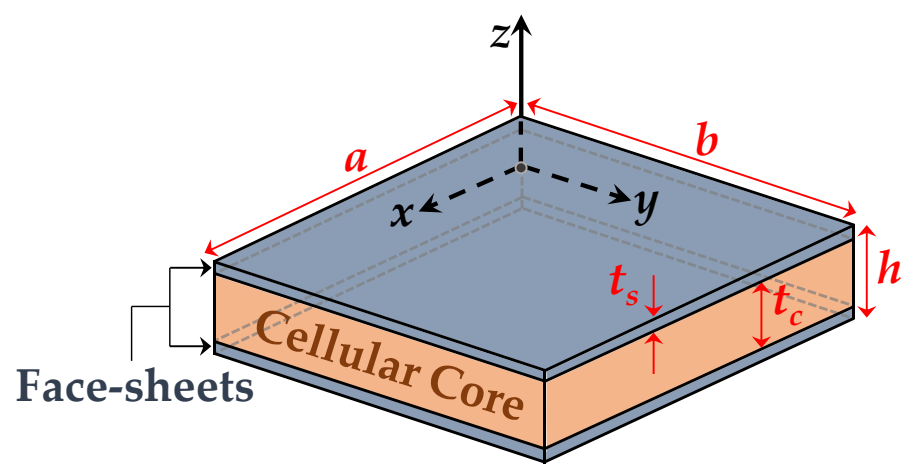

Figure 1. Geometry of an architected sandwich panel and the considered coordinate system.

\section{FINITE ELEMENT MODELING}

The explicit large deformation 3D finite element modeling (FEM) is conducted using the commercial software ANSYS Workbench 18.2. Fig. 3 presents the model developed in ANSYS for conducting the low velocity impact analysis. The face-sheets and the core are meshed with quadrilateral and triangular elements and a convergence study is conducted to avoid mesh size dependency of FEM results. The spherical impactor is defined as a rigid body and the dynamic load is applied by assuming an initial velocity for the rigid impactor. The stress-strain curve of PLA is assumed as an elastoperfectly plastic model for FEM analysis.

\section{RESULTS AND DISCUSSION}

In this section, we examine the effects of the topology and relative density of cellular core of architected sandwich panels on energy absorption when the sandwich panel is subjected to a low-velocity impact. For experimental study, the sandwich panel is subjected to a $3 \mathrm{~J}$ low-velocity impact test.

\section{A. Energy-time history}

Fig. 4 presents the experimental and numerical results for the energy-time history for alternative cell configuration, cell topology, and cell relative density. There is a good agreement between the results of experimental tests and numerical analyses. In the energy absorption versus time history res, the amounts of absorbed and returned (released) energies during the impact test can be observed. The absorbed energy is the energy mostly dissipated by various failure mechanisms such as delamination and cracking [24]. The returned (released) energy is the elastic energy. Herein, we define the energy performance based on the ratio of absorbed energy / maximum impact energy [24] (see Fig. 4).

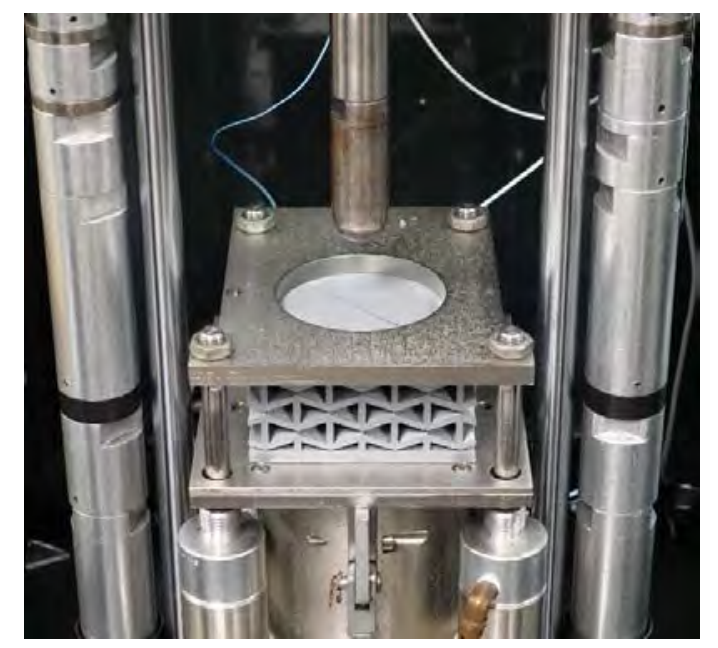

Figure 2. Low-velocity impact test configuration of 3D printed architected sandwich panels. 


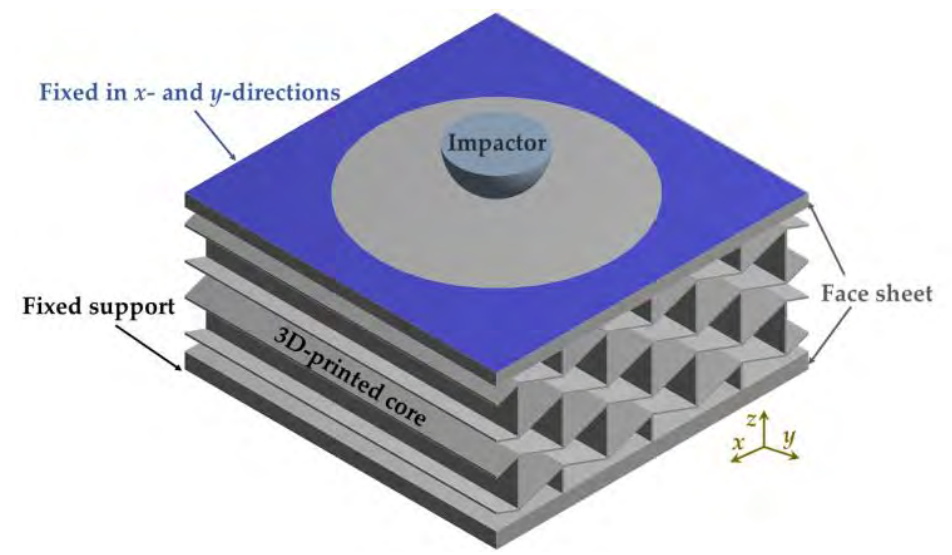

Figure 3. Model developed in ANSYS for the low-velocity impact analysis.
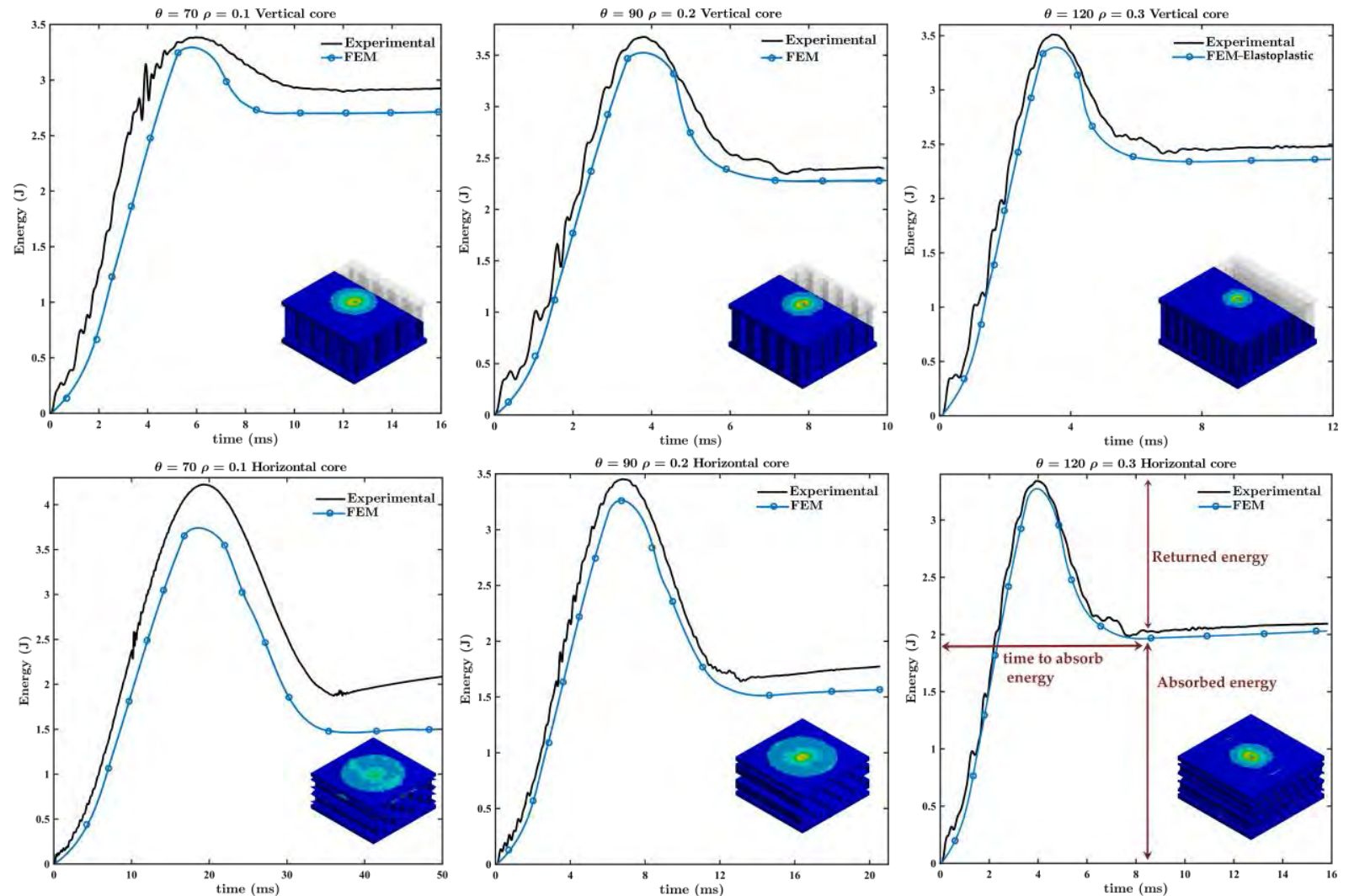

Figure 4. Energy-time history obtained from the experimental impact test on 3D printed architected sandwich panels of alternative cellular core configuration, cell topology, and cell relative density compared with FEM results 

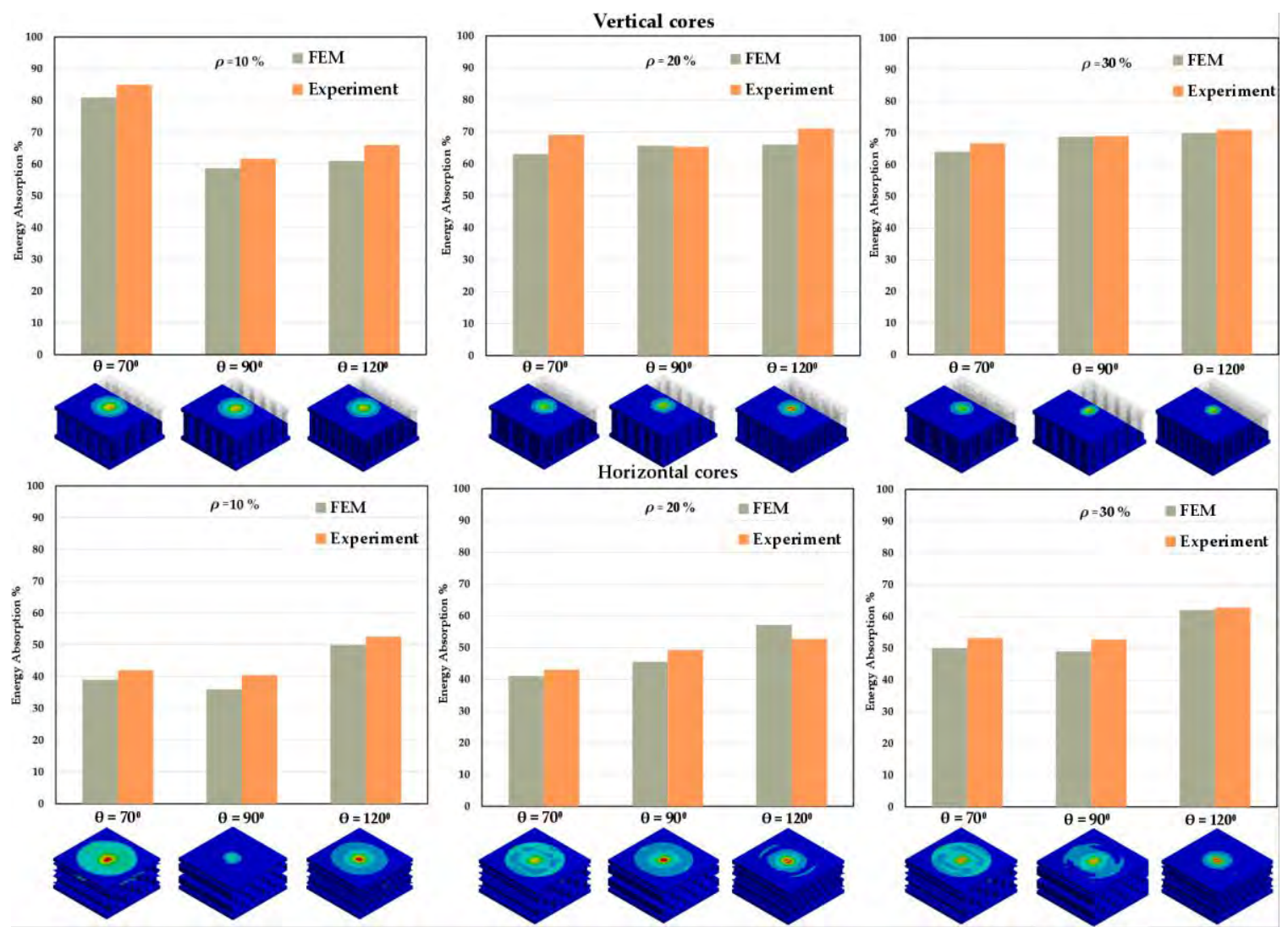

Figure 5. Experimental and FEM results for the energy absorption capability of 3D printed architected sandwich panels of alternative cellular core configuration, cell topology, and cell relative density.

\section{B. Energy absorption capability}

Fig. 5 shows the experimental and numerical energy absorption capability of 18 3D printed sandwich panels with different core topologies including the horizontal and vertical auxetic, rectangular, and hexagonal cores (cell wall angle $\theta=$ $70^{\circ}, 90^{\circ}$, and $120^{\circ}$, respectively); and the relative densities of $\rho$ $=0.1,0.2$, and 0.3 . There is a good agreement between the results of experimental tests and numerical analyses. For all considered relative densities of horizontal/vertical cellular cores, the rectangular and hexagonal cellular cores provide a slightly higher energy absorption capability than the auxetic cellular core except for the auxetic cellular core with the relative density of $\rho=0.1$. It should be noted that the auxetic cellular core with the relative density of $\rho=0.1$ in the vertical configuration is the optimum cellular core, in terms of energy absorption, for the architected sandwich panels subjected to specific impact energy. $25 \%$ and $29 \%$ improvements for energy absorption are observed during the experimental tests by using auxetic cellular cores for architected sandwich panels in comparison with hexagonal and rectangular cores, respectively.

\section{CONCLUDING REMARKS}

We develop a numerical and experimental study to determine the optimum geometry of architected 3D printed sandwich panels with a periodic cellular core. We 3D print architected sandwich panels of three different cell topologies made of biopolymers and conduct experimental low-velocity impact tests on samples to estimate their energy absorption capability. The results show that if relative density of the auxetic cellular core is selected appropriately for a specific value of impact energy, the sandwich panels with auxetic cellular cores can have a higher level of energy absorption capability up to $33 \%$ compared to the rectangular and hexagonal cellular sandwich panels.

\section{ACKNOWLEDGMENT}

A.H. Akbarzadeh acknowledges the financial supports provided by McGill University and Natural Science and Engineering Research Council of Canada (NSERC) through NSERC Discovery Grant RGPIN-2016-04716 and NSERC Engage Grant EGP 507009-16.

\section{REFERENCES}

[1] T. A. Schaedler and W. B. Carter, "Architected Cellular Materials," Annu. Rev. Mater. Res., vol. 46, no. 1, pp. 187-210, Jul. 2016. doi: 10.1146/annurev-matsci-070115-031624.

[2] L. J. Gibson and M. F. Ashby, Cellular Solids, Cambridge University Press, 1997. doi: 10.1017/CBO9781139878326.

[3] H. G. Analysis and Design of Structural Sandwich Panels: A volume in the Commonwealth and International library: structures and Solid Body Mechanics Division, Elsevier, 2013 (1969). doi: 10.1016/C2013-0-02134-2.

[4] H. J. Rathbun et al., "Performance of metallic honeycomb-core sandwich beams under shock loading," Int. J. Solids Struct., vol. 43, no. 6, pp. 1746 1763, Mar. 2006. doi: 10.1016/j.ijsolstr.2005.06.079. 
[5] B. L. Buitrago, C. Santiuste, S. Sánchez-Sáez, E. Barbero, and C. Navarro, "Modelling of composite sandwich structures with honeycomb core subjected to high-velocity impact," Composite Structures, vol. 92, no. 9, pp. 2090-2096, Aug. 2010. doi: 10.1016/j.compstruct.2009.10.013.

[6] I. G. Masters and K. E. Evans, "Models for the elastic deformation of honeycombs," Composite Structures, vol. 35, no. 4, pp. 403-422, Aug. 1996. doi: 10.1016/S0263-8223(96)00054-2.

[7] W. Becker, "Closed-form analysis of the thickness effect of regular honeycomb core material," Composite Structures, vol. 48, no. 1-3, pp. 6770, Jan. 2000.The copyright for the paper content remains with the author. doi: 10.1016/S0263-8223(99)00074-4.

[8] D. J. Sypeck, "Cellular Truss Core Sandwich Structures," Appl. Compos. Mater., vol. 12, no. 3-4, pp. 229-246, May 2005. doi: $10.1007 / \mathrm{s} 10443-005-1129-\mathrm{z}$.

[9] Y. Hu, W. Li, X. An, and H. Fan, "Fabrication and mechanical behaviors of corrugated lattice truss composite sandwich panels," Compos. Sci. Technol., vol. 125, pp. 114-122, Mar. 2016. doi: 10.1016/j.compscitech.2016.02.003.

[10] Wadley, H.N., N.A. Fleck, and A.G. Evans, "Fabrication and structural performance of periodic cellular metal sandwich structures," Compos. Sci. Technol., vol. 63, no. 16, pp. 2331-2343, Dec. 2003. doi: $10.1016 / \mathrm{S} 0266-3538(03) 00266-5$.

[11] J. Xiong, L. Ma, S. Pan, L. Wu, J. Papadopoulos, and A. Vaziri, "Shear and bending performance of carbon fiber composite sandwich panels with pyramidal truss cores," Acta Mater., vol. 60, no. 4, pp. 1455-1466, Feb. 2012. doi: 10.1016/j.actamat.2011.11.028.

[12] R. S. Lakes and K. Elms, "Indentability of Conventional and Negative Poisson's Ratio Foams," J. Compos. Mater., vol. 27, no. 12, pp. 1193 1202, Dec. 1993. doi: 10.1177/002199839302701203.

[13] R. Lakes, "Foam Structures with a Negative Poisson's Ratio," Science, vol. 235 , no. 4792 , pp. $1038-1040$, Feb. 1987.

doi: $10.1126 /$ science. 235.4792 .1038

[14] W. Yang, Z.-M. Li, W. Shi, B.-H. Xie, and M.-B. Yang, "Review on auxetic materials," J. Mater. Sci., vol. 39, no. 10, pp. 3269-3279, May 2004. doi: 10.1023/B:JMSC.0000026928.93231.e0.

[15] J. N. Grima and K. E. Evans, "Auxetic behaviour from rotating squares," J. Mater. Sci. Let., vol. 19, no. 17, pp. 1563-1565, 2000. doi: 10.1023/A:1006781224002.

[16] A. Alderson and K. L. Alderson, "Auxetic materials," Proc. Inst. Mech. Eng., Part G, vol. 221, no. 4, pp. 565-575, Apr. 2007. doi: 10.1243/09544100JAERO185.
[17] S. Hou, T. Liu, Z. Zhang, X. Han, and Q. Li, "How does negative Poisson's ratio of foam filler affect crashworthiness?" Mater. Des., vol. 82, pp. 247-259, Oct. 2015. doi: 10.1016/j.matdes.2015.05.050.

[18] G. AltaThe copyright for the paper content remains with the author.n and V. Kovan, "Flexural behavior of 3D printed honeycomb sandwich structures with waste filler material," Mater. Testing, vol. 58, no. 10, pp. 833-838, Oct. 2016. doi: $10.3139 / 120.110927$

[19] T. Li and L. Wang, "Bending behavior of sandwich composite structures with tunable 3D-printed core materials," Compos. Struct., vol. 175, pp. 46-57, Sep. 2017. doi: 10.1016/j.compstruct.2017.05.001.

[20] J. B. Berger, H. N. G. Wadley, and R. M. McMeeking, "Mechanical metamaterials at the theoretical limit of isotropic elastic stiffness," Nature, vol. 543, no. 7646, pp. 533-537, Feb. 2017. doi: 10.1038/nature21075.

[21] D. K. Jayashankar and N. A. Prasanth, "Out of plane compressive strength of 3D printed vertical pillared corrugated core structure," Proc. 2nd Inter. Conf. Prog. Add. Manu., Singapore, 2016.

[22] A. J. Turner, Low-Velocity Impact Behavior of Sandwich Panels with 3D Printed Polymer Core Structures, MSME, 2017, Wright State University.

[23] Association, U.S., ASTM D3763-2006 Standard Test Method for High Speed Puncture Properties of Plastics using Load and Displacement Sensor. USA Standards Association International, USA, 2006.

[24] R. Ouadday, A. Marouene, G. Morada, A. Kaabi, R. Boukhili, and A. Vadean, "Experimental and numerical investigation on the impact behavior of dual-core composite sandwich panels designed for hydraulic turbine applications," Composite Structures, vol. 185, pp. 254-263, Feb. 2018. doi: 10.1016/j.compstruct.2017.11.007. 


\title{
The Evalutation of Modelling Techniques for Lubricant Cavitation in the application of Squeeze Film Damplers
}

\author{
Tieshu Fan, Kamram Behdinan \\ Department of Mechanical and Industrial Engineering \\ University of Toronto \\ Toronto, Canada
}

\begin{abstract}
Squeeze film damper (SFD) is widely adopted in turbo-engines to suppress the rotor vibration. However, the prediction of SFD performance is complicated due to the inevitable occurrence of lubricant cavitation. This paper shows the application of three different cavitation algorithms for SFD with sealed conditions. In particular, the linear complementarity problem (LCP) method, which is advanced from a previous research study, is applied to compare results from the well-known methods, i.e. the $\pi$-film model and the Elrod cavitation method, for SFD executing circular centered orbits with fully degassed lubricant in the absence of oil feeding. Moreover, numerical models are developed incorporating the mentioned algorithms to predict the hydrodynamic pressure distribution over the cavitated fluid film. Results show that the conventional $\pi$-film model overestimates the cavitation region but under-estimates the reaction force.
\end{abstract} model

Keywords: SFD; Cavitation; LCP; Elrod Algorithm; $\pi$-film

\section{INTRODUCTION}

SFD is commonly integrated into aircraft turbine engines to attenuate the level of vibration. It helps to improve the rotor stability and decrease the engine vibration leading to a reduction in the cabin noise. The design parameter of an SFD is the damping capacity. There is an optimum damping value in each application, given the fact that the rotor-bearing system has multiple degrees of freedom and too much or too little damping leads to the overall damping being less effective [1]. The dynamic performance of an SFD is affected by factors such as the damper geometry, the lubricant property and the operating condition. Development of a model that accurately predicts the dynamic behavior and the damping capacity requires integrating the effects of sophisticated lubricant characteristics including the lubricant cavitation effect.

Lubricant cavitation is an ever-present phenomenon in hydrodynamic journal bearings. Typically, cavitation reduces the lubricant density, resulting in the degradation of damping capacity especially at high whirl frequencies and large journal eccentricities [2]. Cavitation in the SFD arises under three scenarios, including the entrapment of surrounding air at low hydrodynamic pressure, the release of dissolved gas in the lubricant due to the rapid change of the oscillating pressure, and the liquid vaporization at vapor pressure. Air entrapment is usually prevented by placing seals on the SFD [3]. In addition, the lubricant is regarded as well-degassed for application in a rotor-SFD system operating under steady-state conditions [4]. Consequently, only the vapor cavitation is considered for sealed SFDs under periodic motions. Vapor cavitation significantly influences the SFD load capacity, especially at high operating speeds.

For several decades, researchers have been working on the improvement of modelling techniques to accurately simulate the lubricant cavitation. The main challenge in SFDs is the identification of the cavitation boundaries, which determines the hydrodynamic pressure distribution and the hydrodynamic fluid film reaction forces. In conventional SFD models, the fluid cavitation is represented by using the $\pi$-film model (i.e. half-Sommerfeld model) [5], where the full-film extends for $\pi$ radians in the region of positive pressure. Furthermore, Gumbel suggested that the negative pressure values based on the flow equations correspond to cavitation, while the positive pressure region is invulnerable to cavitation [6]. While the Gumbel condition is readily incorporated into pressure calculations, it violates the conservation of mass in the cavitation boundary. Swift [7] and Stieber [8] suggested an enhanced boundary condition to simulate the cavitation. This condition, which is referred to as the Reynolds boundary condition, satisfies the flow continuity at the onset of the lubricant cavitation. The more sophisticated Jakobsson-Floberg-Olsson (JFO) boundary conditions $[9,10]$ provide an accurate representation of the cavitation boundaries, while maintaining the conservation of mass; however, this condition has limited application due to its computational inefficiency. The computational deficiency corresponding to the cavitation models is addressed by the Elrod algorithm [11, 12]. This model transforms the governing lubricant cavitation equation from elliptic form into parabolic form for both full film and cavitation regions. It firstly calculates the lubricant mixture density in the cavitation zone and subsequently, uses the density values to determine the hydrodynamic lubricant pressure distribution. The Elrod algorithm provides accelerated computational efficiency; however, the calculation of the film pressure and the cavitation extent substantially depend on the liquid bulk modulus. A novel lubricant cavitation evaluation technique has been 
recently introduced based on the principle of complementarity [13]. This method is further elaborated to successfully solve the cavitation problem for SFD with open-ended conditions [14].

This paper will evaluate three cavitation algorithms, i.e. the $\pi$-film model, the Elrod cavitation algorithm and the LCP method, for sealed SFD executing circular centered orbits with fully degassed lubricant in the absence of oil feeding. Firstly, the lubricant cavitation algorithms are briefly introduced, which will then be incorporated into a mathematical model to generate simulation results. Several different scenarios are subsequently studied and the results are presented for discussion.

\section{NOMENCLATURE}

\begin{tabular}{|c|c|c|}
\hline Symbol & Quantity & Unit \\
\hline$c$ & SFD Radial Clearance & $m$ \\
\hline$F_{r}$ & Radial Force & $N$ \\
\hline$F_{t}$ & Tangential Force & $N$ \\
\hline$g$ & Switch Function & \\
\hline$h=c(1+\varepsilon \cos \theta)$ & Fluid Film Thickness & $m$ \\
\hline$L$ & SFD Length & $m$ \\
\hline$p$ & Fluid Pressure & $P a$ \\
\hline$p_{0}$ & $\begin{array}{l}\text { Pressure at Maximum } \\
\text { Film Thickness }\end{array}$ & $P a$ \\
\hline$p_{c a v}$ & Cavitation Pressure & $P a$ \\
\hline$R$ & SFD Radius & $m$ \\
\hline $\operatorname{Re}=\rho \omega \mathrm{c}^{2} / \mu$ & Squeeze-Film Reynold Number & \\
\hline$t$ & Time & $s$ \\
\hline$\beta$ & Fluid Bulk Modulus & $P a$ \\
\hline$\varepsilon$ & Eccentricity Ratio & \\
\hline$\theta$ & Angular Coordinate & $\mathrm{rad}$ \\
\hline$\rho$ & Fluid Density & $\mathrm{kg} / \mathrm{m}^{3}$ \\
\hline$\rho_{c}$ & $\begin{array}{l}\text { Liquid Density at } \\
\text { Cavitation Pressure }\end{array}$ & $\mathrm{kg} / \mathrm{m}^{3}$ \\
\hline $\bar{\rho}=\rho / \rho_{c}$ & Density Ratio & \\
\hline$\mu$ & Fluid Viscosity & $P a \cdot s$ \\
\hline$\omega$ & Whirling Velocity & $\mathrm{rad} / \mathrm{s}$ \\
\hline$\xi$ & Complementarity Variable & $\mathrm{kg} / \mathrm{m}^{3}$ \\
\hline$\eta$ & Complementarity Variable & $\mathrm{kg} / \mathrm{m}^{3}$ \\
\hline
\end{tabular}

\section{Simulation Method}

This section describes the governing equations for the hydrodynamic pressure and the fluid film reaction forces in SFDs. Furthermore, different lubricant cavitation models are presented.

Tightly sealed SFDs allow little lubricant flow in the axial direction, such that the pressure gradient along the bearing length becomes negligible. Conventionally, the long bearing approximation (LBA) [15] is applicable to tightly sealed SFDs. Moreover, the Reynolds equation is typically applied to describe the pressure distribution in the thin film [16]. The Reynolds equation corresponding to LBA for the SFD is

$$
\frac{1}{R^{2}} \frac{\partial}{\partial \theta}\left(\frac{\rho h^{3}}{12 \mu} \frac{\partial p}{\partial \theta}\right)=\frac{\partial(\rho h)}{\partial t}
$$

Integrating (1) twice in the axial direction and applying the Sommerfeld boundary condition [16] at the maximum lubricant thickness $(\theta=0)$ yields an analytical solution for the fluid pressure. Assuming that the journal center executes circularcentered orbits (CCOs), the pressure expression becomes

$$
p=-\frac{12 \mu R^{2} \omega}{c^{2}} \frac{\varepsilon \sin \theta(2+\varepsilon \cos \theta)}{(1+\varepsilon \cos \theta)^{2}\left(2+\varepsilon^{2}\right)}+p_{0} .
$$

The subsequent cavitation models will be developed based on the assumption that $p_{0}=0$.

\section{A. $\pi$-film Model}

The $\pi$-film model [5] applies the Gumbel condition which assumes that the negative pressure that is calculated from (2) corresponds to the cavitation pressure. Accordingly, the pressure distribution is described as follows

$$
p=\left\{\begin{array}{cc}
-\frac{12 \mu R^{2} \omega}{c^{2}} \frac{\varepsilon \sin \theta(2+\varepsilon \cos \theta)}{(1+\varepsilon \cos \theta)^{2}\left(2+\varepsilon^{2}\right)} & \pi<\theta \leq 2 \pi \\
p_{c a v} & 0<\theta \leq \pi
\end{array} .\right.
$$

Furthermore, the fluid force components are determined by integrating the fluid pressure over the journal surface as

$$
\begin{aligned}
F_{r} & =\int_{\pi}^{2 \pi} p \cos \theta R L d \theta=-\frac{12 \mu R^{3} L \omega}{c^{2}} \frac{2 \varepsilon^{2}}{\left(2+\varepsilon^{2}\right)\left(1-\varepsilon^{2}\right)} \\
F_{t} & =\int_{\pi}^{2 \pi} p \sin \theta R L d \theta \\
& =2 p_{c a v} R L-\frac{12 \mu R^{3} L \omega}{c^{2}} \frac{\pi \varepsilon}{\left(2+\varepsilon^{2}\right) \sqrt{1-\varepsilon^{2}}} .
\end{aligned}
$$

\section{B. Elrod Caviation Model}

The Elrod cavitation algorithm is widely employed to calculate the effect of cavitation. Firstly, (1) is modified as

$$
\frac{1}{R^{2}} \frac{\partial}{\partial \theta}\left(\frac{\beta h^{3}}{12 \mu} g \frac{\partial \bar{\rho}}{\partial \theta}\right)=\frac{\partial(\bar{\rho} h)}{\partial t},
$$

where $\beta$ is the fluid bulk modulus defined as

$$
\beta=\rho \frac{\partial p}{\partial \rho}
$$

$\bar{\rho}$ is a dimensionless parameter, which represents the ratio between the density of fluid and the liquid density at the cavitation pressure, i.e.

$$
\bar{\rho}=\frac{\rho}{\rho_{c}} .
$$


Note that $\bar{\rho}$ is less than 1 in the cavitation zone due to the reduced density by the generated cavitation bubbles.

$g$ is a switch function defined as

$$
g=\left\{\begin{array}{lll}
0 & \text { in cavitation } & \text { zone } \\
1 & \text { in full-film zone }
\end{array}\right\} .
$$

Furthermore, the switch function can be expressed by the relative density as follows to meet the required condition in both flow regions

$$
g=\frac{1}{2}+\frac{1}{2}\left(\frac{|\bar{\rho}-1|}{\bar{\rho}-1}\right) .
$$

Subsequently, numerical techniques are applied to iteratively solve $\bar{\rho}$ and $g$. The pressure distribution is calculated based on the direct integration of the bulk modulus, i.e.

$$
p=p_{c a v}+g \beta \ln \bar{\rho} .
$$

Accordingly, the reaction forces of the squeezed film are determined by integrating the hydrodynamic pressure over the journal center, i.e.

$$
\left\{\begin{array}{l}
F_{r} \\
F_{t}
\end{array}\right\}=\int_{0}^{2 \pi} p\left\{\begin{array}{l}
\cos \theta \\
\sin \theta
\end{array}\right\} L R d \theta d z
$$

\section{LCP Method}

The LCP method provides a linear complementarity equation set that can be used to efficiently find the pressure distribution.

Define two variables $\xi$ and $\eta$ as

$$
\begin{aligned}
& \xi=\rho_{c} e^{\left(p-p_{c}\right) / \beta}-\rho \\
& \eta=\rho_{c}\left[e^{\left(p-p_{c}\right) / \beta}-1\right] .
\end{aligned}
$$

It has been proved that $\xi$ and $\eta$ are non-negative and complementarity to each other in the fluid domain regardless of film rupture [14], i.e.

$$
\left\{\begin{array}{l}
\xi \geq 0 \\
\eta \geq 0 \\
\xi \eta=0 \\
\xi \frac{\partial \eta}{\partial \theta}=0
\end{array} .\right.
$$

Based on (13) \& (14), the fluid density and pressure can be described in terms of $\xi$ and $\eta$ as

$$
\begin{gathered}
\rho=\rho_{c}+\eta-\xi \\
p=p_{c}+\beta \ln \left(1+\frac{\eta}{\rho_{c}}\right) .
\end{gathered}
$$

Accordingly, the pressure gradient can also be derived from (17) as

$$
\frac{\partial p}{\partial \theta}=\frac{\partial p}{\partial \eta} \frac{\partial \eta}{\partial \theta}=\frac{\beta}{\rho_{c}+\eta} \frac{\partial \eta}{\partial \theta} .
$$

Substitution of (16)-(18) to (1) yields the following:

$$
\frac{\partial}{R^{2} \partial \theta}\left[\frac{\beta h^{3}\left(\rho_{c}+\eta-\xi\right)}{12 \mu\left(\rho_{c}+\eta\right)} \frac{\partial \eta}{\partial \theta}\right]=\frac{\partial\left(\rho_{c}+\eta-\xi\right) h}{\partial t} .
$$

The above equation can be reduced to the following after applying the complementarity condition described in (15):

$$
\frac{\partial}{R^{2} \partial \theta}\left[\frac{\beta h^{3}}{12 \mu} \frac{\partial \eta}{\partial \theta}\right]=\frac{\partial(\eta-\xi) h}{\partial t}+\rho_{c} \frac{\partial h}{\partial t} .
$$

Consequently, an LCP equation set is formulated with respect to the introduced complementarity variables after applying the finite difference method, i.e.

$$
\left\{\begin{array}{l}
\{\eta\}=[M]\{\xi\}+\{q\} \\
\{\eta\} \geq 0 \\
\{\xi\} \geq 0 \\
\{\eta\}\{\xi\}^{T}=0
\end{array},\right.
$$

where the detailed description of (21) is provided in the Appendix.

A conventional technique for solving (21) is the Lemke's pivoting algorithm [17]. Once the pressure distribution is calculated, the fluid film reaction forces are determined based on (12).

\section{Case Study and Results}

This section represents results for the three lubricant cavitation models that were discussed in the previous section. The simulation condition is described as follows: the bearing diameter is $104.9 \mathrm{~mm}$; the damper length is $110.2 \mathrm{~mm}$; the radial clearance is $0.127 \mathrm{~mm}$; the lubricant viscosity is $0.005 \mathrm{pa} \cdot \mathrm{s}$; the fluid bulk modulus is $0.069 \mathrm{GPa}$; the cavitation pressure is $1 \mathrm{kPa}$; the journal executes $\mathrm{CCO}$ and the whirling speed is $3000 \mathrm{rpm}$. Based on the values of the system parameters, the squeeze Reynolds number is at 0.9935. Large squeeze Reynolds number (i.e. $\mathrm{Re}>1$ ) requires extra nonlinear expressions to modify the Reynolds equation to address the fluid flow, which opens the gate to study the effect of the fluid inertia. In our study scenario, this number is less than 1 , thus the effect of fluid inertia is neglected so that the Reynolds equation is applied [16]. Subsequently, the three cavitation algorithms are incorporated into a MATLAB simulation model using the finite difference method.

Fig. 1-4 compares the pressure distribution for the three cavitation models under different eccentricity ratios. In general, the $\pi$-film model predicts the largest cavitation zones and the smallest peak pressure amplitudes, while the LCP method provides the largest full-film regions and the highest peak pressures. As the eccentricity ratio increases, the extent of 
cavitation based on the $\pi$-film model remains unchanged due to the corresponding assumption for the cavitation boundary condition; however, the extent of the cavitation deteriorates for the other two models. Moreover, the discrepancy between Elrod algorithm and LCP method results becomes smaller at high eccentricity ratios (i.e. $\varepsilon=0.7$ ).

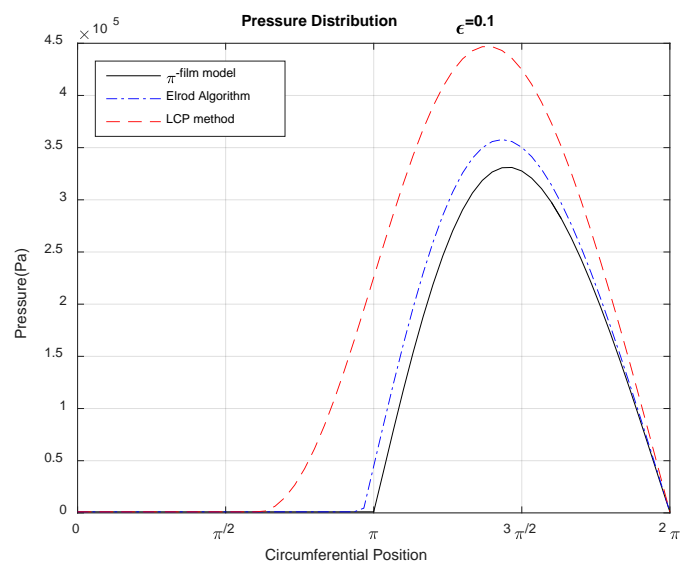

Figure 1. Comparison between the pressure distributions for the three cavitation models at eccentricity ratio 0.1

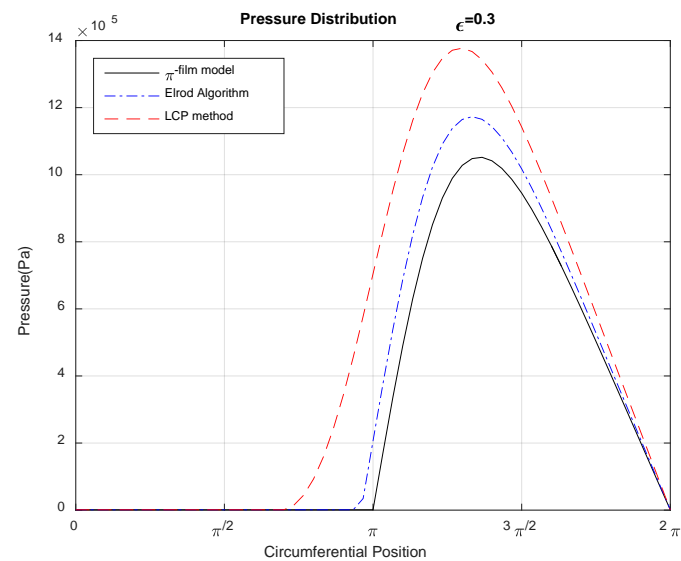

Figure 2. Comparison between the pressure distributions for the three cavitation models at eccentricity ratio 0.3

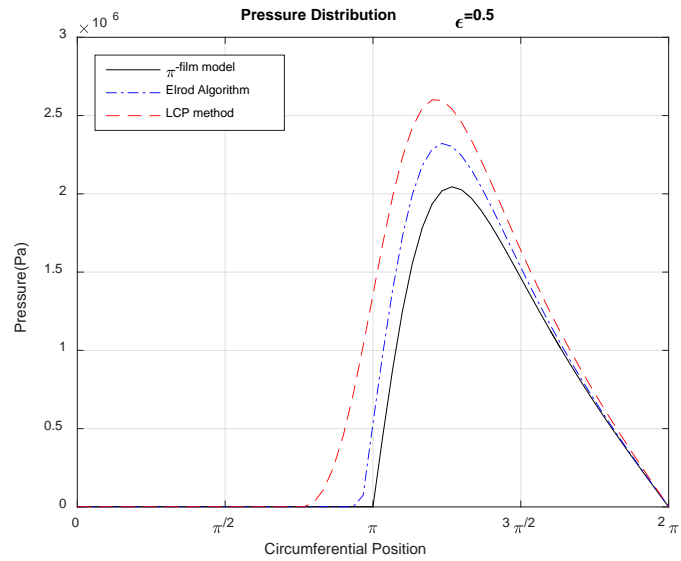

Figure 3. Comparison between the pressure distributions for the three cavitation models at eccentricity ratio 0.5

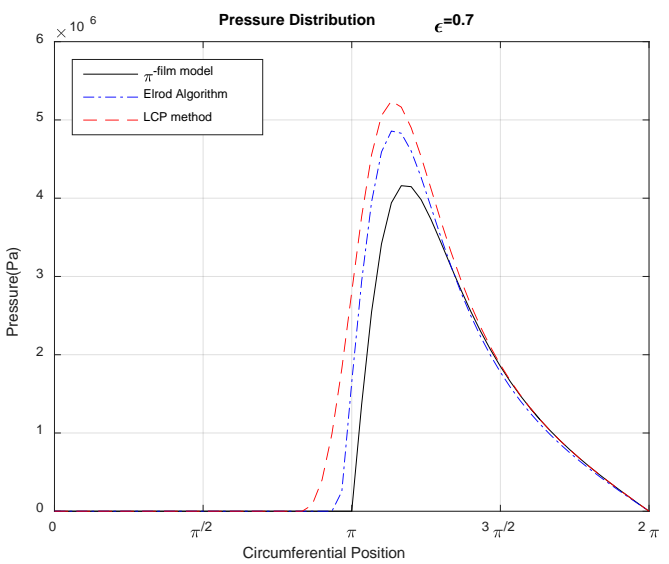

Figure 4. Comparison between the pressure distributions for the three cavitation models at eccentricity ratio 0.7

Fig. 5 and Fig. 6 compare the fluid film reaction force components at different journal eccentricity ratios. Fig. 5 shows that the magnitude of the radial force increases nonlinearly with the eccentricity ratio. Meanwhile, the $\pi$-film model predicts smaller magnitudes of the radial forces, while the LCP method presents the largest force components. Furthermore, Fig. 6 indicates a quasi-linear increase of the magnitude for the tangential force with the eccentricity ratio. The $\pi$-film model maintains the lowest predicted force magnitudes and the LCP method shows the largest force magnitudes. The discrepancies among the models are the result of differences in the pressure prediction, which can be attributed to the different assumptions about the cavitation boundary.

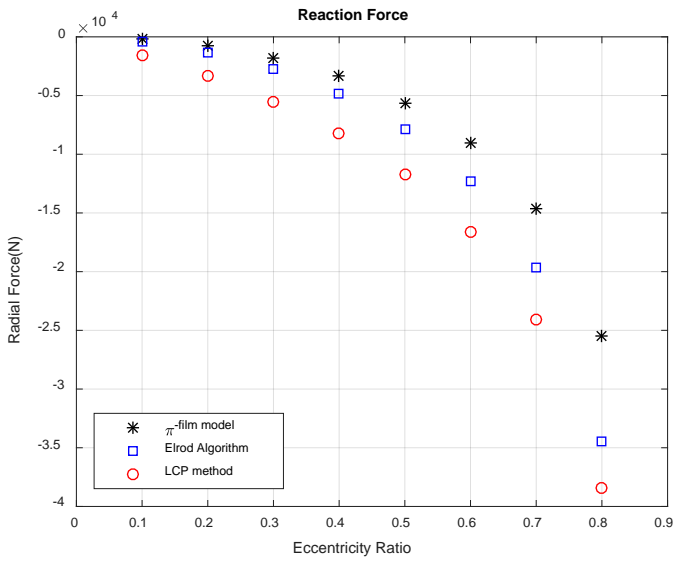

Figure 5. Comparison between the radial fluid film reaction forces for the three cavitation models at different eccentricity ratios 


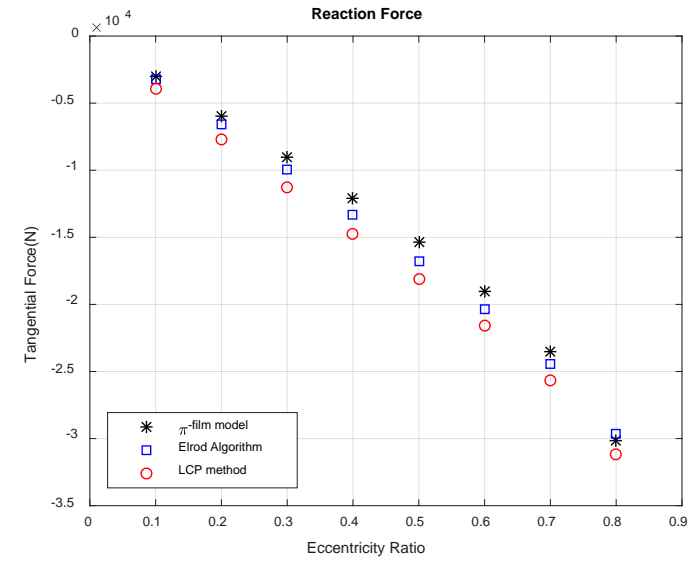

Figure 6. Comparison between the tangential fluid film reaction forces for the three cavitation models at different eccentricity ratios

Fig. 7 compares the attitude angle for the cavitation models. The attitude angel is calculated as:

$$
\theta=\arctan \left(-F_{t} / F_{r}\right) .
$$

In general, the attitude angle declines as the journal eccentricity increases. The LCP method provides the prediction of the smallest angle. Furthermore, the discrepancy between the models decreases at higher amplitude motions.

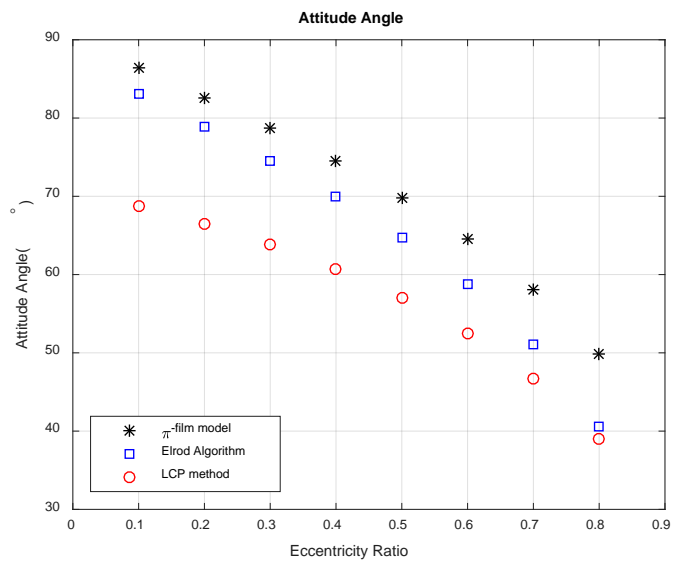

Figure 7. Comparison between the attitude angles for the three cavitation models at different eccentricity ratios

\section{CONCLUSION}

This work compares three cavitation models for sealed SFD, namely the $\pi$-film model, the Elrod algorithm, and the LCP method. Simulation shows that the extent of cavitation region is superior at large eccentricities, which is associated with the rapid buildup of larger magnitude pressure. Furthermore, the Elrod algorithm and the LCP method provide larger reaction forces but smaller attitude angles than that from the simple $\pi$ film model. These models are computationally efficient so they have the potential to be integrated into rotordynamic studies.

\section{ACKNOWLEDGMENT}

This work is supported by grants from Natural Science and Engineering Research Council (NSERC) and Pratt and Whitney Canada.

\section{REFERENCES}

[1] L. San Andres, "Squeeze film dampers: operation, models, and technical issues", Modern Lubrication Theroy, Notes 13, Texas A\&M University Digital Libraries, College Station, TX, 2010.

[2] S. E. Diaz and L. A. San Andrés, "Reduction of the Dynamic Load Capacity in a Squeeze Film Damper Operating With a Bubbly Lubricant," J. Eng. Gas Turbines Power, vol. 121, no. 4, p. 703, 1999. doi: 10.1115/1.2818530.

[3] L. San Andrés and A. Delgado, "Identification of Force Coefficients in a Squeeze Film Damper With a Mechanical End Seal—Centered Circular Orbit Tests," $J$. Tribol., vol. 129, no. 3, p. 660, 2007. doi: 10.1115/1.2736708.

[4] F. Y. Zeidan and J. M. Vance, "Cavitation Regimes in Squeeze Film Dampers and Their Effect on the Pressure Distribution," Tribol. Trans., vol. 33, no. 3, pp. 447-453, Jan. 1990. doi: 10.1080/10402009008981975.

[5] A. Sommerfeld, A, "Zur Hydrodynamischen theorie der Schmiermittelreibung (On the hydrodynamic theory of lubrication)", Zeit. Math. Phys., vol. 50, pp. 97-155, 1904.

[6] L. Gumbel, "Monatsblatter Berliner Bezirksver", VDI, vol. 5, pp. 87-104, 1914.

[7] H. W. Swift, "The stability of lubricating film in journal bearings", Min. Proc. Inst. Civil Engineers, London, vol. 233, pp. 267-288, 1932. doi: 10.1680/imotp.1932.13239.

[8] W. Stieber, "Das Schwimmlager", VDI., Berlin, 1933.

[9] B. Jakobsso and L. Floberg, The Fnite Journal Bearing, Considering Vaporization, Chalmers Tekniska Hogskolas Handlingar, Goteborg, Sweden, vol. 189, pp. 1-116, 1957.

[10] K. O. Olsson, Cavitation in Dynamically Loaded Bearings, Chalmers Tekniska Hogskolas Handlingar, Goteborg, Sweden, vol. 308, pp. 1-60, 1965.

[11] H. G. Elrod and M. Adams, "A computer program for cavitation and starvation problems", Cavitation and Related Phenomena in Lubrication, ImechE, England, vol. 103, pp. 37-42, 1974.

[12] H. G. Elrod, "A cavitation algorithm", J. Lubr. Technol., vol. 103, pp. 350-354, 1981. doi: 10.1115/1.3251669.

[13] ]M. Giacopini, M. T. Fowell, D. Dini, and A. Strozzi, "A Mass-Conserving Complementarity Formulation to Study Lubricant Films in the Presence of Cavitation," J. Trib., vol. 132, no. 4, p. 41702, 2010. doi: 10.1115/1.4002215.

[14] T. Fan and K. Behdinan, "The evaluation of linear complementarity problem method in modeling the fluid cavitation for squeeze film damper with off-centered whirling motion," Lubricants, vol. 5, pp. 46, 2017.

doi: 10.3390/lubricants5040046.

[15] M. M. Dede, M. Dogan and R. Holmes, "The damping capacity of a sealed squeeze film bearing", J. Trib., vol. 107, pp. 411-418, 1985. doi: $10.1115 / 1.3261097$.

[16] A. Z. Szeri, Fluid film lubricant: theory and design, Cambridge University Press, 2005.

[17] R. W. Cottle, J. S. Pang, and R. E. Stone, "The linear complementarity problem", Report No. 60, SIAM, Philadelphia, PA, 2009. 


\section{APPENDIX}

The details of (21) are described as follows.

Since the journal executes CCOs, the time variation can be expressed as the partial variation in the circumferential direction, i.e.

$$
\frac{\partial f}{\partial t}=-\omega \frac{\partial f}{\partial \theta}
$$

where $f$ represents a function. Accordingly, (20) can be written as

$\frac{\partial}{R^{2} \partial \theta}\left[\frac{\beta h^{3}}{12 \mu} \frac{\partial \eta}{\partial \theta}\right]=-\omega \frac{\partial h}{\partial \theta}(\eta-\xi)-\omega h \frac{\partial(\eta-\xi)}{\partial \theta}-\omega \rho_{c} \frac{\partial h}{\partial \theta}$

After applying the finite difference method to the above, the LCP equation is formulated as (21), where

$$
\begin{gathered}
\{\eta\}=\left\{\begin{array}{lll}
\eta_{1} & \ldots & \eta_{N}
\end{array}\right\}^{T} \\
\{\xi\}=\left\{\begin{array}{lll}
\xi_{1} & \ldots & \xi_{N}
\end{array}\right\}^{T} \\
{[M]=\left[\begin{array}{ll}
L & ]^{-1}[N]
\end{array}\right.} \\
\{q\}=[L]^{-1}\{s\} \\
\{s\}=\left\{\begin{array}{c}
\rho_{c}\left(e^{\left(p_{0}-p_{c a v}\right) / \beta}-1\right) \\
-\omega \rho_{c} \frac{\partial h_{2}}{\partial \theta} \\
\vdots \\
-\omega \rho_{c} \frac{\partial h_{n}}{\partial \theta} \\
\rho_{c}\left(e^{\left(p_{0}-p_{c a v}\right) / \beta}-1\right)
\end{array}\right\}
\end{gathered}
$$

$$
[L]=\left[\begin{array}{ccccc}
1 & 0 & & & \\
a_{2}-b_{2}-d_{2} & -2 a_{2}+b_{2}+c_{2}+d_{2} & a_{2} & & \\
& \ddots & \ddots & \ddots & \\
& & a_{n}-b_{n}-d_{n} & -2 a_{n}+b_{n}+c_{n}+d_{n} & a_{n} \\
& & & 0 & 1
\end{array}\right]
$$

$$
\begin{aligned}
& {[N]=\left[\begin{array}{ccccc}
1 & 0 & & & \\
-d_{2} & c_{2}+d_{2} & 0 & & \\
& \ddots & \ddots & \ddots & \\
& & -d_{n} & c_{n}+d_{n} & 0 \\
& & & 0 & 1
\end{array}\right]} \\
& a_{i}=\frac{\beta\left(h_{i}\right)^{3}}{12 \mu R^{2}(\Delta \theta)^{2}} \\
& b_{i}=\frac{\beta\left(h_{i}\right)^{2}}{4 \mu R^{2} \Delta \theta} \frac{\partial h_{i}}{\partial \theta} \\
& c_{i}=\omega \frac{\partial h_{i}}{\partial \theta} \\
& d_{i}=\frac{\omega h_{i}}{\Delta \theta} \\
& e_{i}=\omega \rho_{c} \frac{\partial h_{i}}{\partial \theta}
\end{aligned}
$$

The subscript $i(i=1,2, \ldots, \mathrm{n})$ denotes the nodal position in the discretized circumferential domain.

The first row and the last row in the LCP equation represent the boundary condition given the pressure at the maximum film thickness. 


\section{Design and Optimization of a Novel Beam-based Shimmy Damper}

\author{
Mohsen Rahmani \\ Graduate Student, ARL-MLS Lab \\ MIE Department, University of Toronto \\ Toronto, Canada \\ mohsen.rahmani@mail.utoronto.ca
}

\author{
Kamran Behdinan \\ Professor, ARL-MLS Lab \\ MIE Department, University of Toronto \\ Toronto, Canada \\ behdinan@mie.utoronto.ca
}

\begin{abstract}
Shimmy damper is a passive solution for undesirable oscillations in landing gears. Although it mitigates shimmy to an allowable degree, it can introduce weight, cost, and reliability penalties especially when retrofitted to existing gears. In this report, early investigations into a novel shimmy damper are presented. This damper is suitable for existing and new landing gears and is based on replacing a torque link member with a combination of springs and dampers to suppress the shimmy. The damper is then optimized for a given geometry to deliver the maximum damping force.
\end{abstract}

Keywords- shimmy damper; landing gear; optimization; design;

\section{INTRODUCTION}

Nose Landing Gear (NLG) shimmy is known as selfinduced simultaneous torsional and lateral vibrations arising from the coupling of gear structure, elastic tire(s), and the fuselage structure during ground operations. The oscillations are typically in the range of $10-30 \mathrm{~Hz}$. Shimmy impairs the pilot's visibility and control and causes passenger discomfort, structural damage, as well as sudden failure of the gear [1]. This phenomenon can occur during landing, take-off, and taxying operations. Worn parts contribute to shimmy; however, it also happens in new aircrafts due to the resonance between the gear and the airframe [2]. It is understood that shimmy mode is excited due to transfer of kinetic energy from the moving aircraft to the wheels [3], acting as the energy source for the undesired oscillations. Close to $60 \%$ of aircraft failures are related to the landing gear systems with fatigue due to multi-axial loads (e.g. case of shimmy) playing the number-one role in these accidents [4]. Examples of accidents due to shimmy can be found in [5] and [6]. The latter involved failure of the shimmy damper in addition to the landing gear.

Shimmy needs to be prevented through adequate design during the aircraft development. In practice, shimmy is not discovered until the aircraft is flown and shimmy tests are performed on the ground. In the event of observing shimmy, it is often too late to alter the well-established gear and aircraft design, hence a damper may be designed and prescribed to remedy it. The UTAS (Goodrich) shimmy damper was presented in 2012 [7] which consisted of a damper on one side and a beam with adequate stiffness and strength on the other,

The authors gratefully acknowledge the research grant provided by SPPCA and Natural Sciences and Engineering Research Council of Canada (NSERC) in support of this work. the combination of which makes the shimmy damper. Early studies on the UTAS damper revealed a major issue due to its unsymmetrical design [3]. Although performing better than the flagship Boeing damper [8] in most of the cases, the UTAS damper led to unsymmetrical oscillations which degrades the lifespan of components and tires due to imbalanced loading and uneven wear. The Boeing damper has drawbacks such as adding extra freeplay and weight to the system. Both existing dampers are shown in Fig. 1 along the NLG schematic.
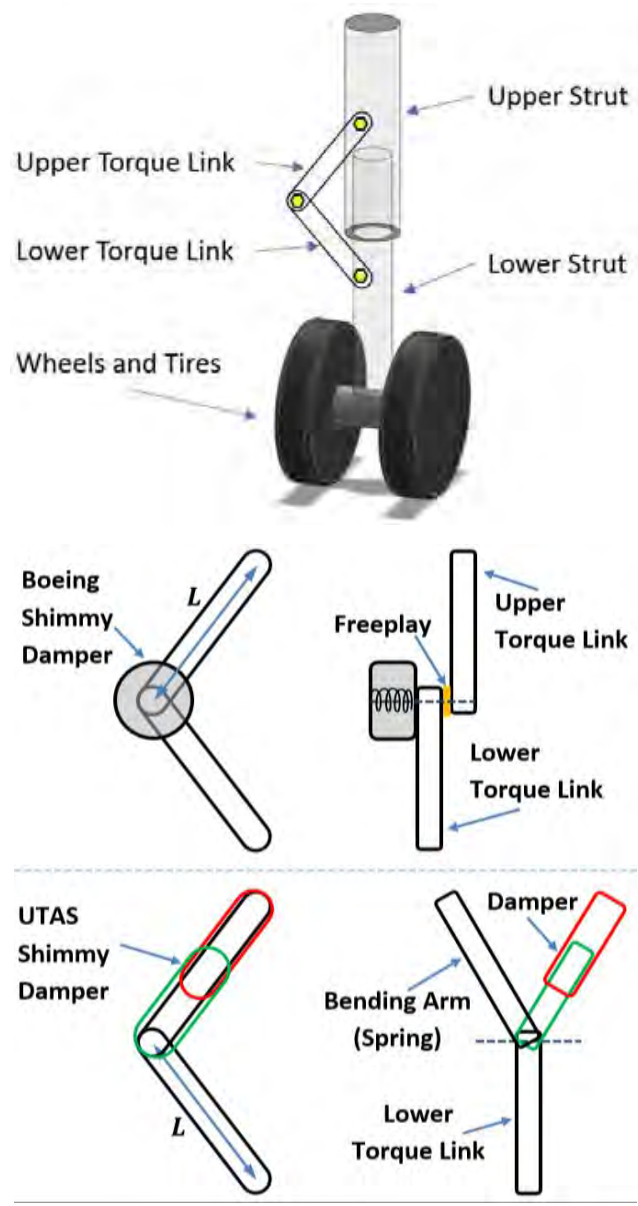

Figure 1. Schematic of the Nose Landing Gear (top), side and front views of Boeing damper (middle) and UTAS damper (bottom) 
Inspired by the UTAS damper and aiming to address its shortcomings, the idea of the present design is to replace one of the torque links (here the upper one) by a flexible beam and accompanying dampers to help stabilize the NLG, while no unsymmetrical behavior is induced in the system.

\section{THE SYMmetric BeAM-BASEd SHIMMY DAMPER}

As shown in Fig. 2, the upper torque link in the proposed design includes a beam (shaded) at the center and two identical damping devices (here pure dampers) symmetrically arranged on either side. The lower torque link is assumed to be rigid. The beam is the load-bearing element of the upper torque link which acts as a spring in parallel to the side dampers as well.

In the event of shimmy, the lower part of the landing gear (tire and lower strut) tends to vibrate rotationally, hence deflecting the upper torque link. The dampers on either side experience a displacement as a result of the beam deflection. Fig. 3 depicts this effect in which the deflection of the beam at the torque link apex and its rate are designated as $\varepsilon$ and $\dot{\varepsilon}$. The beam deflection and its rate at the damper attachment location are shown as $\varepsilon_{a}$ and $\dot{\varepsilon}_{a}$ respectively. Displacement of the right and left damping devices (measured along the device axis) are $\lambda_{1}$ and $\lambda_{2}$ with rates of $\dot{\lambda}_{1}$ and $\dot{\lambda}_{2}$ respectively.
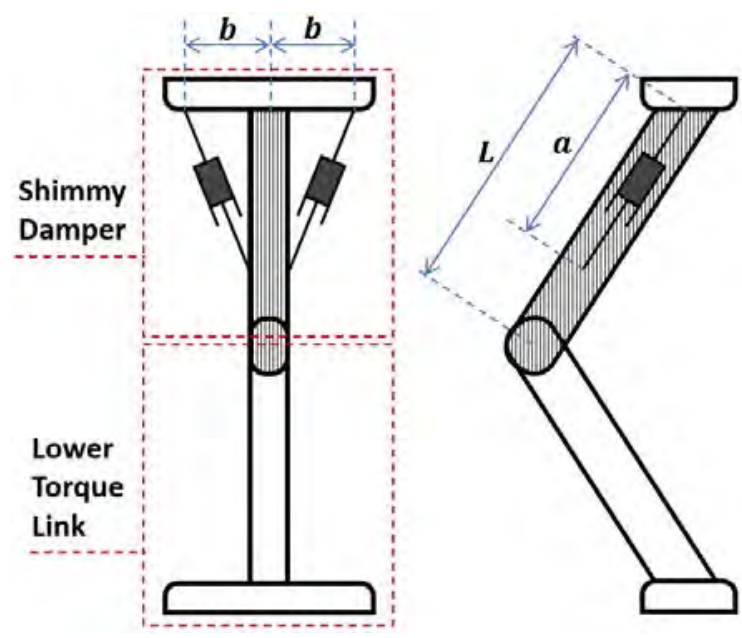

Figure 2. Schematic of the novel shimmy damper and lower torque link. Left: front view, Right: side view

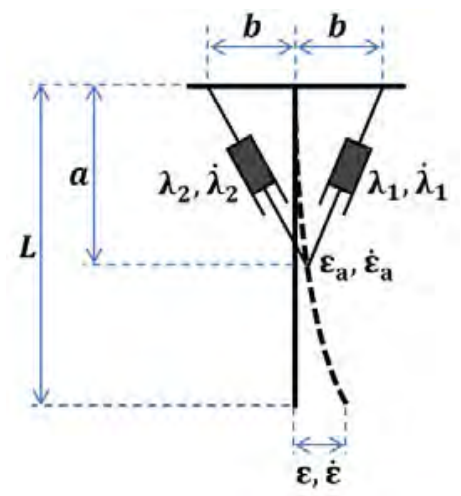

Figure 3. Schematic of the damper beam deflection and damper displacements
Here, the goal is to express the net damping force as a function of the system inputs which are $\varepsilon$ and $\dot{\varepsilon}$. These two inputs are a function of the landing gear dynamics but here are treated as knowns. From the geometry of the design one can write:

$$
\begin{aligned}
& \lambda_{1}=\sqrt{a^{2}+b^{2}-2 b \varepsilon_{a}}-\sqrt{a^{2}+b^{2}} \\
& \lambda_{2}=\sqrt{a^{2}+b^{2}+2 b \varepsilon_{a}}-\sqrt{a^{2}+b^{2}}
\end{aligned}
$$

and hence:

$$
\begin{aligned}
& \dot{\lambda}_{1}=-b \dot{\varepsilon}_{a} / \sqrt{a^{2}+b^{2}-2 b \varepsilon_{a}} \\
& \dot{\lambda}_{2}=b \dot{\varepsilon}_{a} / \sqrt{a^{2}+b^{2}+2 b \varepsilon_{a}} .
\end{aligned}
$$

The dampers generate forces along the damper axis which are proportional to $\dot{\lambda}_{1}$ and $\dot{\lambda}_{2}$. However, only the components of these forces which are perpendicular to the beam are going to resist the beam deflection and hence alleviate shimmy. Therefore, we need to isolate the components of $\dot{\lambda}_{1}$ and $\dot{\lambda}_{2}$ which are perpendicular to the beam. These are designated as $\dot{\bar{\lambda}}_{1}$ and $\dot{\bar{\lambda}}_{2}$. To find them we can write:

$$
\begin{gathered}
\bar{\lambda}_{1}=-\lambda_{1}\left(b-\varepsilon_{a}\right) / \sqrt{a^{2}+\left(b-\varepsilon_{a}\right)^{2}} \\
\bar{\lambda}_{2}=\lambda_{2}\left(b+\varepsilon_{a}\right) \sqrt{a^{2}+\left(b+\varepsilon_{a}\right)^{2}} .
\end{gathered}
$$

After taking derivatives and simplifying:

$$
\begin{aligned}
& \dot{\bar{\lambda}}_{1}=-\dot{\lambda}_{1} \frac{b-\varepsilon_{a}}{\sqrt{a^{2}+\left(b-\varepsilon_{a}\right)^{2}}}+\lambda_{1} \frac{a^{2} \dot{\varepsilon}_{a}}{\left(a^{2}+\left(b-\varepsilon_{a}\right)^{2}\right)^{3 / 2}} \\
& \dot{\bar{\lambda}}_{2}=\dot{\lambda}_{2} \frac{b+\varepsilon_{a}}{\sqrt{a^{2}+\left(b+\varepsilon_{a}\right)^{2}}}+\lambda_{2} \frac{a^{2} \dot{\varepsilon}_{a}}{\left(a^{2}+\left(b+\varepsilon_{a}\right)^{2}\right)^{3 / 2}} .
\end{aligned}
$$

Assuming a viscous damper with the coefficient of $C_{d}$ on each side, the total damping force can the expressed as:

$$
Q=C_{d}\left(\dot{\bar{\lambda}}_{1}+\dot{\bar{\lambda}}_{2}\right)
$$


The $L$ and $b$ dimensions are dictated by the geometry and strength of the torque links and existing gear design. However, $a$ needs to be decided by the damper designer in a way to ensure maximum efficiency during the ground operations. Smaller $a$ means the damping devices will be more perpendicular to the beam, but they will experience a smaller beam deflection. The opposite holds for a bigger $a$. Therefore, the optimal value of this parameter need to be determined for the range of inputs generated from the operation of the landing gear, which is the focus of the current study.

It is important to note that the beam deflection is affected by the damper force as well, as depicted in Fig. 4. Hence, the relationship between $\varepsilon$ and $\varepsilon_{a}$ (and their rates) is a function of both $P$ and $Q$ forces, where $P$ is the force applied by the lower torque link. Through governing relationships of a beam with modulus of $E$ and area moment $I$ and assuming small deflections we can write:

$$
\begin{gathered}
E I \varepsilon=\frac{P L^{3}}{3}-\frac{Q a^{2}}{6}(3 L-a) \\
E I \varepsilon_{a}=\frac{P a^{2}}{6}(3 L-a)-\frac{Q a^{3}}{3} .
\end{gathered}
$$

Defining $R_{1}=\varepsilon_{a} / \varepsilon$ and $R_{2}=Q / P$ we will have:

$$
R_{1}=\frac{a^{2}(3 L-a)-2 a^{3} R_{2}}{2 L^{3}-a^{2}(3 L-a) R_{2}} .
$$

Hence, the relationship between the beam deflection at the torque link apex and damper attachment location can be expressed through $\varepsilon_{a}=R_{1} \varepsilon$. For simplicity of the analysis, it is assumed here that the deflection rates are also related through the same ratio, i.e. $\dot{\varepsilon}_{a}=R_{1} \dot{\varepsilon}$, which is to assume that $R_{2}$ does not vary significantly with time. Introducing $R_{1}$ and $R_{2}$ parameters allows us to optimize the performance while considering the effect of the damping force on the beam deflection, and without the need to directly estimate $P$, which is dictated by the NLG dynamics.

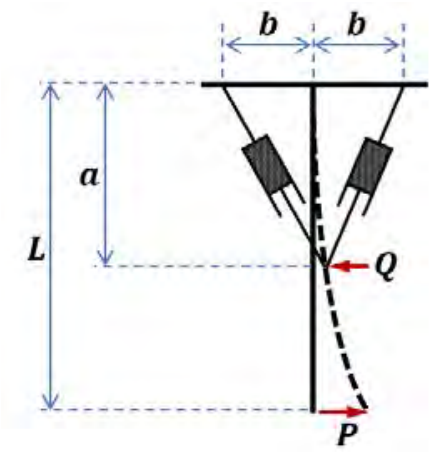

Figure 4. The forces applied to the beam

\section{PERFORMANCE ANALYSIS}

The analysis is commenced by looking at the simpler case when the influence of damping force on the beam deflection is neglected, i.e. $R_{2}=0$. Later, we quantify the effect of this simplifying assumption. The geometry features of $L=0.4 \mathrm{~m}$ and $b=0.25 L$ are assumed as our demonstration case and will be used throughout the paper unless otherwise stated. The net damping force is depicted as a function of $a / L$ in Fig. 5 with the optimal location (corresponding to the maximum damping force) marked as a star on each curve. For all cases that follow, unit damper coefficient and apex displacement rate $\left(C_{d}=1\right.$, $\dot{\varepsilon}=1$ ) are used since the net damping force is a linear function of these parameters. From Fig. 5 one observes that the optimal damper location is a function of $\varepsilon$. However, for the practical range of $0 \leq \varepsilon \leq 0.05 \mathrm{~m}$ the optimal $a / L$ only changes from 0.63 to 0.58 . Therefore, one may choose $a / L \approx 0.6$ for this case as a value which provides near-optimal performance for the intended range of operation.

Next, the effect of $b$ parameter is demonstrated for a fixed apex deflection of $\varepsilon=0.01 \mathrm{~m}$. Again, $R_{2}=0$ and damping force is shown in Fig. 6. Typically, $b$ is not a design variable since it is dictated by the design of the gear to which the shimmy damper will be retrofitted. Nevertheless, it is beneficial to understand that a bigger $b$ leads to a bigger optimal $a / L$ and a larger damping force as shown in Fig. 6.

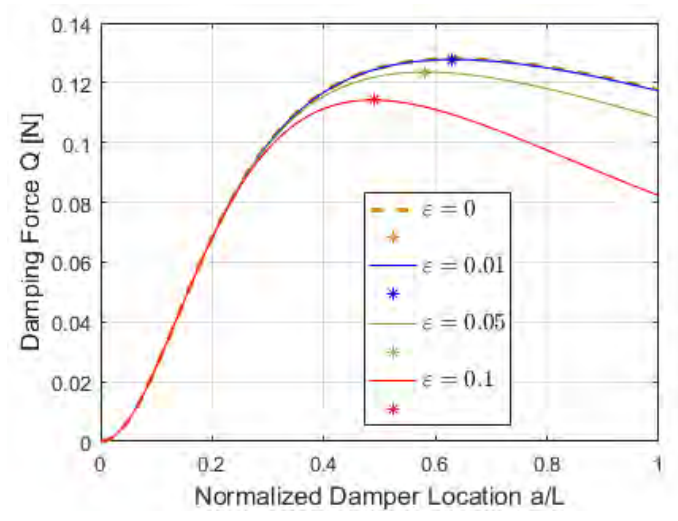

Figure 5. Damping force versus damper location for $R_{2}=0$ and various $\varepsilon$

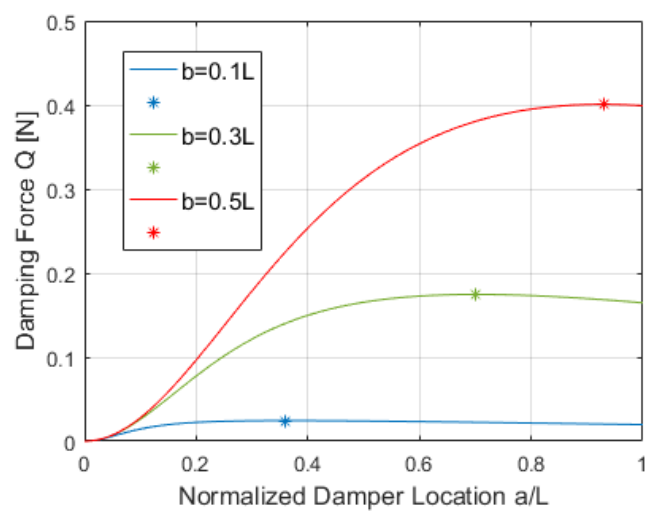

Figure 6. Damping force versus damper location for $R_{2}=0$ and various $b$ 


\section{A. Effect of $R_{2}=0$ Assumption}

To account for the effect of damping force on the beam deflection we need to understand the influence of $R_{2}$ parameter on the predicted response. In practice, this value is non-zero due to presence of the damping force $Q$. In fact, we are dealing with a loop here: the calculation of damping force needs the accurate beam deformation and the beam deformation can be obtained by knowing the damping force. Hence, one may go through an iterative scheme to get the solution. Instead, here we calculate the solution for different values of $R_{2}$ parameter which indicates how sensitive to the damping force the exact solution is.

In Fig. 7, the damping force and optimal damper location are depicted for various values of $R_{2}$ for both small $(\varepsilon=0.01 \mathrm{~m})$ and large $(\varepsilon=0.1 \mathrm{~m})$ apex displacement values. As expected, it is observed that a non-zero $R_{2}$ alters the estimated damping force curve and the optimal damper location. Hence, the optimal damper location is underpredicted when assuming $R_{2}=0$. However, for small values of $R_{2}$ the difference is negligible, and one can proceed with the $R_{2}=0$ assumption for initial concept design studies. Furthermore, the influence of $R_{2}$ on the optimal damper location decreases as $\varepsilon$ grows. This is key since performance at larger $\varepsilon$ values should drive the design as it corresponds to severe shimmy situations.
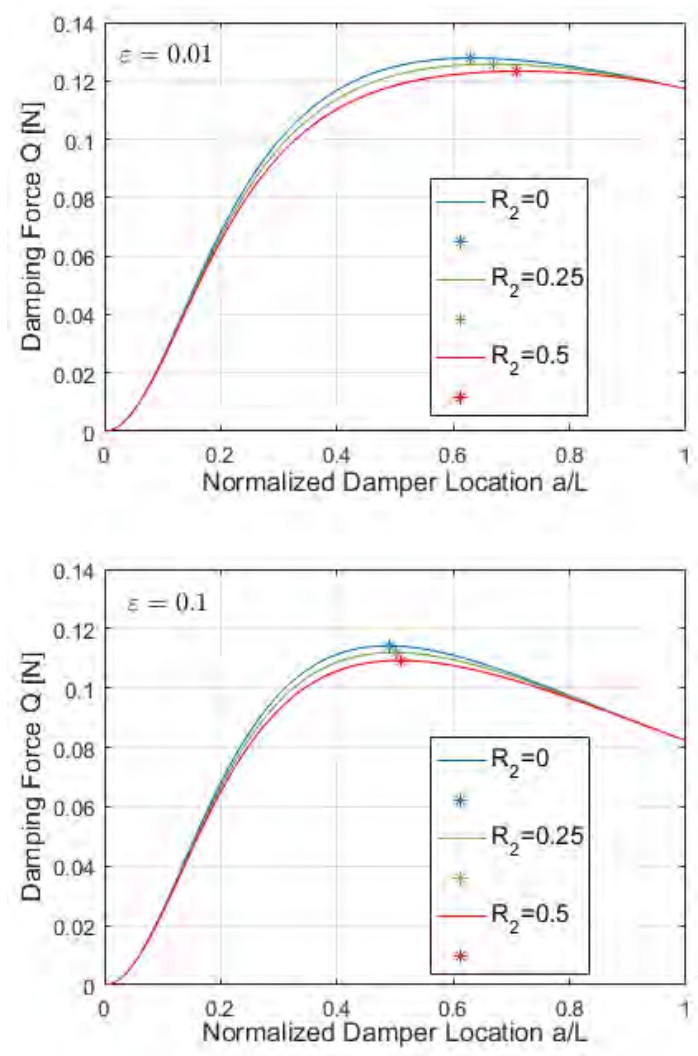

Figure 7. Damping force versus damper location for various $R_{2}$. Top: $\varepsilon=0.01 \mathrm{~m}$, bottom: $\varepsilon=0.1 \mathrm{~m}$

\section{OPTIMIZATION}

Using the analyses presented so far, we can arrive at a more complete picture of the optimal damper location for a given geometry and range of operation. Here the torque link dimensions are given as $L=0.4 \mathrm{~m}$ and $b=0.25 L$. The torque link apex displacement $\varepsilon$ is assumed to be in the $0 \leq \varepsilon \leq 0.1 \mathrm{~m}$ range. Fig. 8-top shows the optimal location versus $\varepsilon$ for different values of $R_{2}$. Assuming $R_{2}=0$ results in a smaller $a_{\mathrm{opt}} / L$ but the error can be acceptable considering the curves are converging as $\varepsilon$ grows. For instance, in the case of a given shimmy-prone landing gear with maximum apex displacement of $\varepsilon=0.04 \mathrm{~m}$ one can choose $a_{\text {opt }} / L=0.6$ (assuming $R_{2}=0$ ) according to Fig. 8-top to ensure the damper performance is optimal for the maximum $\varepsilon$ and near optimal (since the lines are not steep) for smaller values. Similar curves are plotted for different $b$ values in Fig. 8-bottom. The predicted trend is that the $a_{\text {opt }} / L$ is a function of $\varepsilon$ but may be fixed at the value corresponding to the maximum $\varepsilon$ in the operation range to ensure effective shimmy suppression. This embodies a worstcase scenario design strategy where the performance of the system is optimal for the severe shimmy case and near optimal for other cases. Although this decision is adequate for the initial concept design, one needs to perform more thorough analysis involving real inputs from the NLG to ensure shimmy is suppressed effectively.
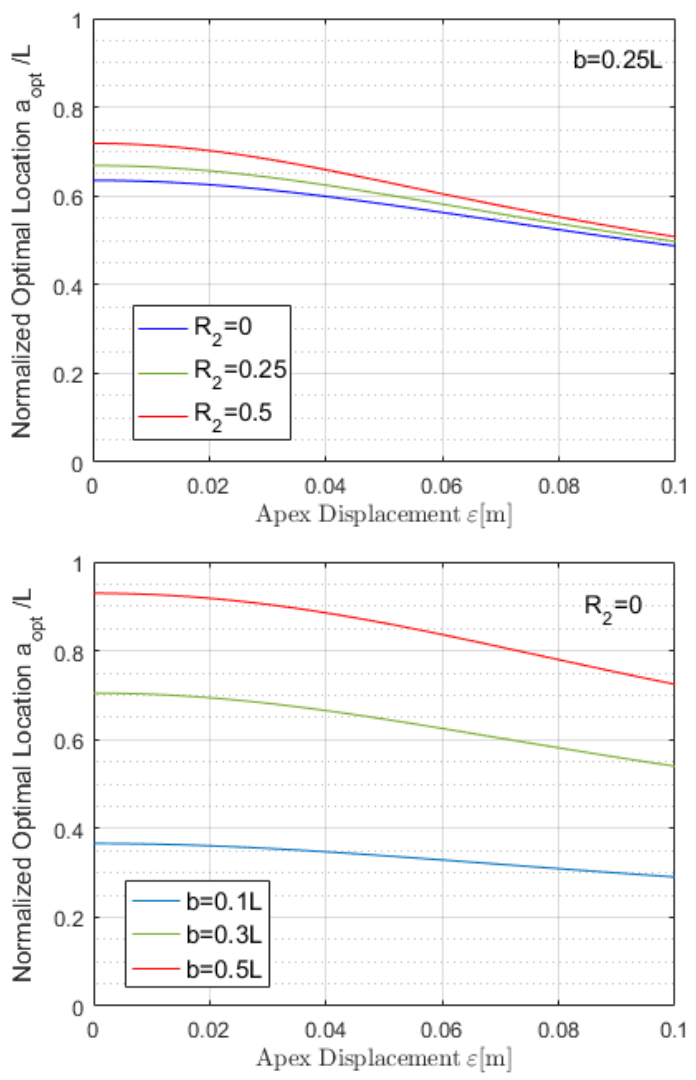

Figure 8. Optimal damper location versus $\varepsilon$ for various $R_{2}$ values (top) and various $b$ values (bottom) 


\section{CONCLUSIONS}

A novel shimmy damper concept is featured in the current work and initial optimization studies are presented to ensure maximum efficiency of the damper for the most critical shimmy scenario. This damper is superior to the Boeing one in that it does not add freeplay and is easily retrofitted to existing gears. It also addresses the unsymmetrical response issue of the UTAS damper. The evidence of symmetric response of the damper based on dynamic simulation of the NLG equipped with the damper remains to be obtained, although the symmetric design of the device promises such performance.

The detail design of the beam and damping devices, as well as multi-disciplinary optimization studies require a computational dynamic model of the NLG such as [9] and [10]. The exact damping force can be estimated by adding the present damper model to the landing gear dynamic model which can provide the inputs in real-time. The damping force should also be fed back to the NLG system and the performance of the damper needs to be evaluated in ground operation simulations such as a landing scenario. Furthermore, more advanced combinations of springs, dampers, and inerters will be considered as damping devices to arrive at a more efficient and compact design. These ideas are under investigation by the authors and comprise the next steps of the present work.

\section{REFERENCES}

[1] W. Krüger et al., "Aircraft Landing Gear Dynamics: Simulation and Control," Vehicle Syst. Dyn., vol. 28, no. 2-3, pp. 119158, Aug. 1997. doi: 10.1080/00423119708969352.

[2] W. E. Luce, "Torque Link with Shimmy Damper," U.S. Patent 8448 899, May 28, 2013.

[3] C. Arreaza, K. Behdinan, and J. W. Zu, "Linear Stability Analysis and Dynamic Response of Shimmy Dampers for Main Landing Gears," J. Appl. Mech., vol. 83, no. 8, pp. 081002-081002-10, May. 2016. doi: 10.1115/1.4033482.

[4] S. K. Lok, J. M. Paul, and V. Upendranath, "Prescience Life of Landing Gear Using Multiaxial Fatigue Numerical Analysis," Procedia Eng., vol. 86, pp. 775-779, Jan. 2014. doi: 10.1016/j.proeng.2014.11.097.

[5] P. Eret, J. Kennedy, and G. J. Bennett, "Effect of Noise Reducing Components on Nose Landing Gear Stability for a Mid-Size Aircraft Coupled with Vortex Shedding and Freeplay," J. Sound Vib., vol. 354, pp. 91-103, Oct. 2015. doi: $10.1016 /$ j.jsv.2015.06.022.

[6] "Accident of Aircraft Fokker MK-100, Registration I-ALPL, at Barcelona Airport (Barcelona), on 7 November 1999," CIAIAC., Barcelona., CAT, A-068/1999, 1999

[7] W. E. Luce, "Torque Link with Shimmy Damper," U.S. Patent 8292 218 , Oct 23, 2012.

[8] W. E. Boehringer, "Torque Linkage Damper," U.S. Patent 5224 668, Jul 6, 1993.

[9] M. Rahmani and K. Behdinan, "Investigation on the Effect of Coulomb Friction on Nose Landing Gear Shimmy," J. Vib. Control, vol. 25, no. 2, pp. 255-272, Jan. 2019. doi: $10.1177 / 1077546318774440$

[10] P. Thota, B. Krauskopf, and M. Lowenberg, "Bifurcation Analysis of Nose-Landing-Gear Shimmy with Lateral and Longitudinal Bending," J. Aircraft, vol. 47, no. 1, pp. 87-95, Jan. 2010. doi: $10.2514 / 1.43507$. 


\section{Gas Thruster Calibration for Satellite Simulators on an Air Bearing Table}

\author{
Joshua Cookson \\ Department of Earth and Space Sciences and Engineering \\ York University, M.Sc. Candidate \\ Toronto, Canada
}

\author{
Zheng H. Zhu \\ Department of Mechanical Engineering \\ York University \\ Toronto, Canada
}

\begin{abstract}
This paper covers a simple method of calibration for gas thrusters for the application of simulated space flight. Gas thrusters are a common means of controlling spacecraft, and proper calibration of such thrusters is essential to ensure accurate and stable control of the spacecraft. Satellite simulators attempt to replicate the behaviours of satellites in flight using a 3 degree of freedom air bearing table, which allows for frictionless flight, and gas thrusters to emulate the flight dynamics.
\end{abstract}

Keywords-gas; thruster; calibration; force; spacecraft; satellite; simulator

\section{INTRODUCTION}

In the following we will cover the process of calibrating gas thrusters which are used to control satellite simulators on an air bearing table. The purpose of this is not to precisely determine the output force of the thrusters, but rather to ensure that each thruster outputs the same force. These satellite simulators utilize gas thrusters as their primary means of positional and attitude control. As the thrusters are only able to be toggled on or off during operations, with no adjustments to the amount of output force, the specific output of each thruster must be calibrated to be equal to allow for reliable control under continuous thrust output.

The simulator is a cube with 8 thrusters, each aligned to the horizontal plane, with two thrusters on each vertical face pointing outwards. In this configuration, motion in a straight line relative to a face is controlled by the two thrusters which are positioned in the opposite direction, and rotational maneuvers possible by firing alternating thrusters on each face. The thrusters utilize simple compressed air as propellant. The onboard air tank is charged to 20 Megapascals, this pressure is then reduced through two regulator valves to 0.4 Megapascals which is sent to the thrusters. If the two thrusters do not output the same force, then there will be a torque exerted on the simulator which causes it to turn.

\section{INSTRUMENTATION}

To calibrate the thrusters, a means of detecting the output force is required. For this we utilized a digital force sensor with a magnetic mounting plate to attach the thruster and connected it via USB to a PC to record the measurements. The force sensor used here can resolve forces to an accuracy of 0.001 Newtons.
The thrusters themselves consist of a housing, which contains the actuator to toggle the output, and has a set of power cables running into the base, a set screw which allows the output to be adjusted, and an output nozzle. The set screw has an air inlet coupled with its connection to the housing.

The sensor is placed on a level surface, here using the air bearing platform which is precisely leveled. The sensor is oriented vertically, with the magnetic mount at the top, the thruster is then attached with the nozzle output oriented to either push or pull against the force sensor. Either orientation of the output will provide the same magnitude measurements, with reversed sign of the value. As the thrusters require the power and air lines to be connected to operate, additional lengths of both are added to remove tensional forces. The air line is then charged to the operating pressure as the line may move while it pressurizes. This configuration on the level surface allows us to easily consider the force due to gravity acting on the thruster and the connected lines and assume the only change in force measured is due to the thruster output. The sensor is then zeroed in this position.

\section{MEASUREMENTS}

The sample rate for the sensor was set to 200 samples per second for a 10 second duration. Force measurements were taken for a 1-3 second duration with the thruster off, and it is then switched on for the remainder of the test. Fig. 1 shows a single set of data. There is a large spike in the measured force

when the thruster is switched from off to on, this is due to the actuation of the control valve in the thruster. This region of data is not included in the force determinations as it does not

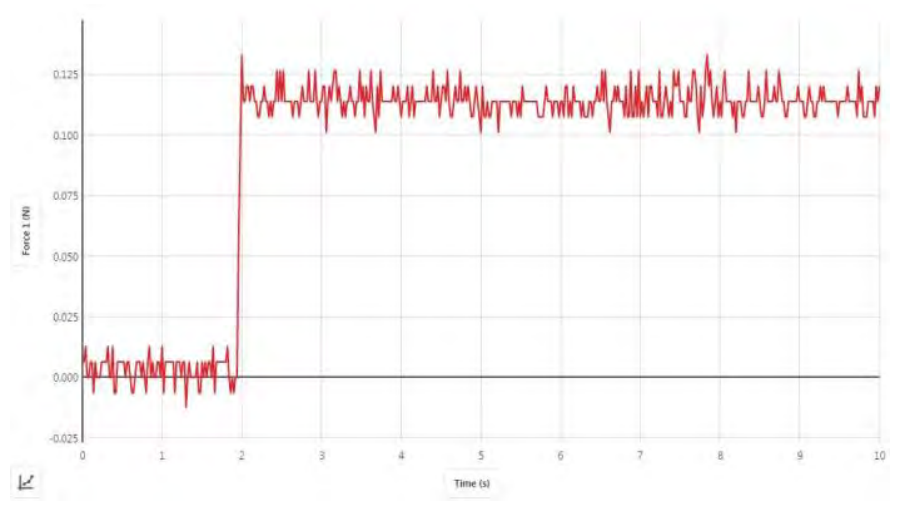

Figure 1. Force Output Data 
represent the steady state output of the thruster.

The data is then averaged for both the off and on positions of the thruster, the averaging process reduces the contributions of noise and vibrations in the system due to the air flow generated by the thruster, thereby providing a more accurate representation of the steady state characteristics of the system. The average value for the off state of the thruster, representing the zeroing error and noise of the system, is then subtracted from the average value of the on state of the thruster. This final value represents the steady state output force of the thruster.

To calibrate each thruster to the same force, the performance envelope for each thruster nozzle first needs to be determined. Each nozzle is hand made and therefore has unique performance characteristics. The output force is measured with the setscrew at maximum and minimum in turn, which gives the range of pressures which are attainable with that thruster.

Following the determination of the performance envelopes and selection of thrusters to calibrate, an overlapping force is selected for each to be calibrated to, here being 0.065 Newtons. To reach this output each thruster is again mounted on the force sensor, the setscrew is adjusted slightly, and another set of measurements is taken. This process is repeated until the desired output force is reached, the setscrew is then locked in place with a nut. Table 1 shows the resulting calibration measurements.

TABLE I. ForCE CALIBRATION RESUlts

\begin{tabular}{|l|l|l|l|}
\hline \multirow{2}{*}{ Thruster } & \multicolumn{3}{|c|}{ Force Measurement } \\
\cline { 2 - 4 } & Measured $(\boldsymbol{N})$ & Zero Error $(\boldsymbol{N})$ & Force Output $(\boldsymbol{N})$ \\
\hline 1 & 0.065 & 0 & 0.065 \\
\hline 2 & 0.074 & 0.009 & 0.065 \\
\hline 3 & 0.060 & -0.005 & 0.065 \\
\hline 4 & 0.068 & 0.003 & 0.065 \\
\hline 5 & 0.066 & 0.001 & 0.065 \\
\hline 6 & 0.073 & 0.008 & 0.065 \\
\hline 7 & 0.065 & 0 & 0.065 \\
\hline 8 & 0.067 & 0.002 & 0.065 \\
\hline
\end{tabular}

\section{VERIFICATION}

The final calibration of the thrusters is then able to be verified through a flight test of the satellite simulator system. The simulator is tasked to travel in a straight line and then stop. This requires 2 thrusters to be fired to begin moving, and then fire the 2 alternate thrusters on the same faces to bring the simulator to a stop. With the thrusters properly calibrated the simulator can perform this maneuver with no rotation occurring.

\section{CONCLUSION}

Gas thrusters are a common means of spacecraft control. Using this method of thruster calibration, the satellite simulator system can reliably demonstrate the dynamics of space flight, with stable control using gas thrusters. This method of thruster calibration proves to be simple and reliable for this application.

\section{REFERENCES}

[1] J. Jarrige et al., "Thrust Measurements of the Gaia Mission Flight-Model Cold Gas Thrusters," J. Propul. Power, vol. 30, no. 4, pp. 934 943, May 2014. doi: 10.2514/1.B35091.

[2] S.-F. Wu, W. H. Steyn, and R. E. Bordany, "In-orbit Thruster Calibration Techniques and Experiment Results with UoSAT-12," Control Eng. Pract., vol. 12, no. 1, pp. 87-98, Jan. 2004. doi: 10.1016/S0967-0661(02)00317-9.

[3] J. Lun and C. Law, "Direct Thrust Measurement Stand with Improved Operation and Force Calibration Technique for Performance Testing of Pulsed Micro-Thrusters," Meas. Sci. Technol., vol. 25, no. 9, p. 095009, Aug. 2014. doi: 10.1088/0957-0233/25/9/095009. 


\section{Predicting the Thermal Conductivity of Epoxy/MWCNT Composites Using Analytical Modelling}

\author{
Mohammed Ghuzi \\ Department of Chemical and Materials Engineering. \\ University of Alberta \\ Edmonton, AB, Canada \\ ghuzi@ualberta.ca
}

\author{
Pierre Mertiny \\ Department of Mechanical Engineering. \\ University of Alberta \\ Edmonton, AB, Canada \\ pmertiny@ualberta.ca
}

\begin{abstract}
Polymer nanocomposites have opened a new path for multifunctional materials. In particular, carbon nanotubes have the potential to be used in various applications. This study focused on the evaluation of thermal conductivity of epoxy/carbon nanotube composites using analytical modeling. The influence of the filler content, the geometry, the size, and the aspect ratio on thermal conductivity of the composite were discussed within the context of the studied models.
\end{abstract}

Keywords - polymer nanocomposites; multi-walled carbon nanotubes; thermal condcutivity; analytical modelling

\section{INTRODUCTION}

Polymer-based composites (PC) have received significant attention due to their promising potential in various applications. They are rapidly replacing traditional inorganic materials such as metals, and natural materials such as wood due to their superior physical and mechanical properties [1]. One of the promising applications involves the use of PC materials (e.g. thermoset or thermoplastic matrices with glass or carbon fiber reinforcement) in different structural applications such as aircrafts, ships and automobiles [2]. However, PC are usually flammable and have comparatively low resistance to fire. As such, a critical safety issue may arise when PC get exposed to fire resulting in failure and collapse of a structure, and hence causing injuries or even fatalities. Therefore, a research thrust has been immerging to improve the flammability properties of PC using nanofillers such as carbon nanotubes (CNT). Nanofillers with suitable morphology, distribution and dispersion act in the condensed phase akin to char forming flame retardants, that is, they create a thermal insulation and mass transport barrier for the underlying polymer, hence mitigating the creation and escape of gaseous fuel for combustion. In addition, nanofillers may greatly increase the viscosity of the polymer decomposition phase, thus inhibiting flow and drip-off, reducing the decomposition area available for combustion. Kashiwagi et al. [3] used multiwalled carbon nanotubes (MWCNT) as a fire retardant additive in polypropylene. They found that MWCNT significantly enhance the thermal stability of polypropylene in nitrogen atmosphere at high temperatures by greatly reducing the heat release rate of the polypropylene. However, in a different study, it was reported that an increase in MWCNT concentration resulted in an increase in a peak heat release rate due to an increase in thermal conductivity of the nanocomposites [4]. While polymers have low thermal conductivity ranging approximately from $0.2 \mathrm{~W}^{-1} \cdot \mathrm{K}^{-1}$ for amorphous polymers to $0.5 \mathrm{Wm}^{-1} \mathrm{~K}^{-1}$ for highly crystalline polymers, CNT possess a thermal conductivity that is four order of magnitude higher $\left(2000-6000 \mathrm{~W}^{-1} \mathrm{~K}^{-1}\right)$ [5]. Reducing the thermal insulation properties inherent to polymers may thus lead to reduced ignition times [4]. It is therefore necessary to gain a thorough understanding of the thermal response of nanocomposites, such as heat release rate, time of ignition and heat conduction, to evaluate their flammability and thus safety [2]. One of the main thermalphysical properties that controls the thermal response is thermal conductivity.

Many studies reported that the incorporation of CNT into a polymer matrix can lead to a certain enhancement of thermal conductivity of the CNT/polymer nanocomposites. Thermal conductivity is a phonon based mechanism influenced by many factors such as the thermal conductivity of each constituent, the shape, volume fraction, and the dispersion of nanofillers into the polymer matrix, as well as the interfacial thermal resistance between the filler and the polymer [6]. Manipulating one or more of these factors to observe the effect on the composite thermal conductivity might require a multitude of experiments. It is therefore imperative to find methods for estimating the thermal conductivity of the CNT/polymer nanocomposites and hence limit the number of experiments. Among these methods, analytical models supply closed-form expressions for the composite thermal conductivity. However, numerous analytical models have been proposed since the $19^{\text {th }}$ century, and selecting the right model is not always straightforward.

The present paper reports on the modelling the thermal conductivity of epoxy composites filled with low content of MWCNT. Analytical models available in the technical literature were employed for this purpose, and their associated results were contrasted to experimental data from published studies. The aim of this work is explore the effectiveness of selected thermal conductivity prediction models and provide a perspective on the aforementioned factors and their significance when estimating the thermal conductivity of epoxy/MWCNT composites. 


\section{REVIEW OF LITERARTURE MODELS}

A variety of analytical models have been developed to predict the effective thermal conductivity of a composite material. They range from simple expressions (e.g. rule of mixture models) to rather complex expressions, depending on the factors taken into consideration. In this section, the most expedient models, based on the authors' point of view, are discussed. The following notations are used: $K_{\mathrm{c}}, K_{\mathrm{m}}, K_{\mathrm{f}}$ are correspondingly the thermal conductivities (through-thickness direction) of the composite, polymer matrix and filler; and $V_{\mathrm{f}}$ is the filler volume fraction.

\section{A. Maxwell model [7]:}

The Maxwell expression is one of the early models proposed to predict the thermal conductivity of a composite material. The model was developed in 1904 and it describes a system of dilute spherical particles embedded in a continuous matrix. The model neglects the thermal interactions between the filler particles and only accounts for the thermal conductivity of the constituents and the volume fraction of the dispersed spherical fillers as follows:

$$
K_{\mathrm{c}}=K_{\mathrm{m}}\left(1-\frac{3 V_{\mathrm{f}}}{\frac{K_{\mathrm{f}}-2 K_{\mathrm{m}}}{K_{\mathrm{f}}-K_{\mathrm{m}}}-V_{\mathrm{f}}}\right)
$$

\section{B. Hamilton and Crosser model [8]:}

Hamilton and Crosser improved the Maxwell expression by taking into account the effect of the geometry of the filler. They showed that for non-spherical fillers, there is a drastic increase in thermal conductivity of the composite provided that the discrepancy in thermal conductivity of the continuous phase and the dispersed phase is an order of 100 or more. Thus, they introduced an empirical shape factor, $n$, that accounts for such effect as follows:

$$
K_{\mathrm{c}}=K_{\mathrm{m}}\left[\frac{K_{\mathrm{f}}+(n-1) K_{\mathrm{m}}-(n-1) V_{\mathrm{f}}\left(K_{\mathrm{m}}-K_{\mathrm{f}}\right)}{K_{\mathrm{f}}+(n-1) K_{\mathrm{m}}+V_{\mathrm{f}}\left(K_{\mathrm{m}}-K_{\mathrm{f}}\right)}\right]
$$

The shape factor, $n$, can be calculated using the following:

$$
n=\frac{3}{\gamma}
$$

where $\gamma$ is the sphericity and it is defined as the ratio of the surface area of a sphere (with the same volume as the given particle) to the surface area of the particle.

\section{Lewis-Nielsen model [9]:}

Even though the Lewis-Nielsen model does not consider the Kapitza or interface thermal resistance (ITR) between a filler particle and its surrounding polymer matrix, it was found to yield results that are in good agreement with measurements [9]. The model accounts for a wide range of filler and nanocomposite morphologies and can be described as follows:

$$
K_{\mathrm{c}}=\frac{1+\left(\frac{K_{\mathrm{f}} / K_{\mathrm{m}}-1}{K_{\mathrm{f}} / K_{\mathrm{m}}+A}\right) A V_{\mathrm{f}}}{1-\left(\frac{K_{\mathrm{f}} / K_{\mathrm{m}}-1}{K_{\mathrm{f}} / K_{\mathrm{m}}+A}\right) \beta V_{\mathrm{f}}}
$$

where $A$ is the shape coefficient of the filler particles and it depends on the particle's aspect ratio; $\beta$ accounts for the type of packing of the particles and can be calculated by Eq.(5).

$$
\beta=1+\left(\frac{1-V_{\mathrm{m}}}{V_{\mathrm{m}}^{2}}\right) V_{\mathrm{f}}
$$

where $V_{m}$ is the maximum packing fraction of the filler.

\section{Model by Deng et al. [10]:}

Deng et al. developed a specific analytical model for predicting the thermal conductivity of CNT composites with low CNT loading. The model takes into consideration the effects of the thermal conductivity anisotropy, aspect ratio, non-straightness, interfacial thermal resistance, interaction, and either a random or aligned CNT distribution. The model can be written in the following form:

$$
K_{\mathrm{c}}=K_{\mathrm{m}}\left[\left(\frac{1}{3} \frac{\theta}{\frac{K_{\mathrm{m}}}{\theta K_{33}^{\mathrm{cs}}}+H(\theta p)}\right) V_{\mathrm{f}}+1\right]
$$

where

$$
\begin{gathered}
K_{33}^{\mathrm{cs}}=\frac{K_{33}^{\mathrm{c}}}{\left(1+\frac{2 R_{\mathrm{k}} K_{33}^{\mathrm{c}}}{L}\right)} \\
H(\theta p)=\frac{1}{(\theta p)^{2}-1}\left[\frac{\theta p}{\sqrt{(\theta p)^{2}-1}} \ln (\theta p\right. \\
\left.+\sqrt{\left.(\theta p)^{2}-1\right)}-1\right]
\end{gathered}
$$

where is $\theta$ is the straightness ratio and is given by $\theta=L^{\text {ce }} / L$; $L^{\text {ce }}$ is the equivalent average straight length of carbon nanotubes; $L$ is the average twisted length of carbon nanotubes; $p$ is the aspect ratio and is given by $p=L / d$ where $d$ is the average carbon nanotube diameter; $K_{33}^{\text {cs }}$ is the equivalent longitudinal thermal conductivity; $K_{33}^{\mathrm{c}}$ is the axial thermal conductivity of the CNTs; $R_{\mathrm{k}}$ is the ITR.

\section{VALIDATION AND VERFICATION OF MODELS}

For the present study, experimentally determined thermal conductivity data for epoxy/MWCNT nanocomposites as a function of the filler volume fraction were obtained from the technical literature. The literature data collected for epoxy/MWCNT nanocomposites varied in their properties due to differences in the constituent materials used in composite fabrication and possibly also due to the measurement methods, the thermal conductivities of the composite were normalized with their respective thermal conductivity of neat epoxy. Table 1 lists the different epoxy/MWCNT nanocomposite systems that were adopted for this study. 
TABLE 1. SUMMARY OF EPOXY/MWCNT NANOCOMPOSITES TAKEN FROM TECHNICAL LITERATURE AND USED IN THIS STUDY.

\begin{tabular}{|c|c|c|c|c|c|c|c|c|}
\hline \multirow[b]{2}{*}{ Reference } & \multicolumn{4}{|c|}{ MWCNTs properties } & \multicolumn{2}{|c|}{ Epoxy properties } & \multirow{2}{*}{$\begin{array}{l}\text { Measurement } \\
\text { device for } K_{c}\end{array}$} & \multirow{2}{*}{$\underset{(\%)}{\text { Range of } V_{f}}$} \\
\hline & $\begin{array}{l}\text { Density } \\
\left(\mathrm{g} / \mathrm{cm}^{3}\right)\end{array}$ & $\begin{array}{c}K_{\mathrm{f}} \\
\left(\mathrm{W} \cdot \mathrm{m}^{-1} \cdot \mathrm{K}^{-1}\right)\end{array}$ & $\begin{array}{c}\text { Length } \\
(\mu \mathrm{m})\end{array}$ & $\begin{array}{l}\text { Diameter } \\
(\mathrm{nm})\end{array}$ & $\begin{array}{c}K_{\mathrm{m}} \\
\left(\mathrm{W} \cdot \mathrm{m}^{-1} \cdot \mathrm{K}^{-1}\right)\end{array}$ & $\begin{array}{l}\text { Density } \\
\left(\mathrm{g} / \mathrm{cm}^{3}\right)\end{array}$ & & \\
\hline [11] & 2.09 & 3000 & $0.1-10$ & 10 & 0.22 & 1.24 & Hot Disk TPS 2500 & $0.01-0.59$ \\
\hline [12] & 2.09 & 3000 & 5 & $10-15$ & 0.29 & 1.15 & TCi Mathis Analyzer & $0.55-0.17$ \\
\hline [13] & - & - & $10-50$ & 20 & 0.12 & - & ASTM-E12 25-87 & $0.30-0.89$ \\
\hline [14] & - & - & $20-30$ & $10-20$ & 0.24 & - & Hot Disk TPS 2500 & $0.20-1.20$ \\
\hline [15] & - & - & - & - & 0.24 & - & Hot Disk AB & $0.06-0.17$ \\
\hline
\end{tabular}

\section{RESULTS AND DISCUSSION}

Figure 1 depicts the normalized experimental thermal conductivity data for epoxy/MWCNT nanocomposites that were taken from the technical literature and used in the present study. The data is shown as a function of filler volume fraction. Notably, data from [11], [13] and [15], and the initial data point from [14] appear to collapse approximately onto a single straight line.

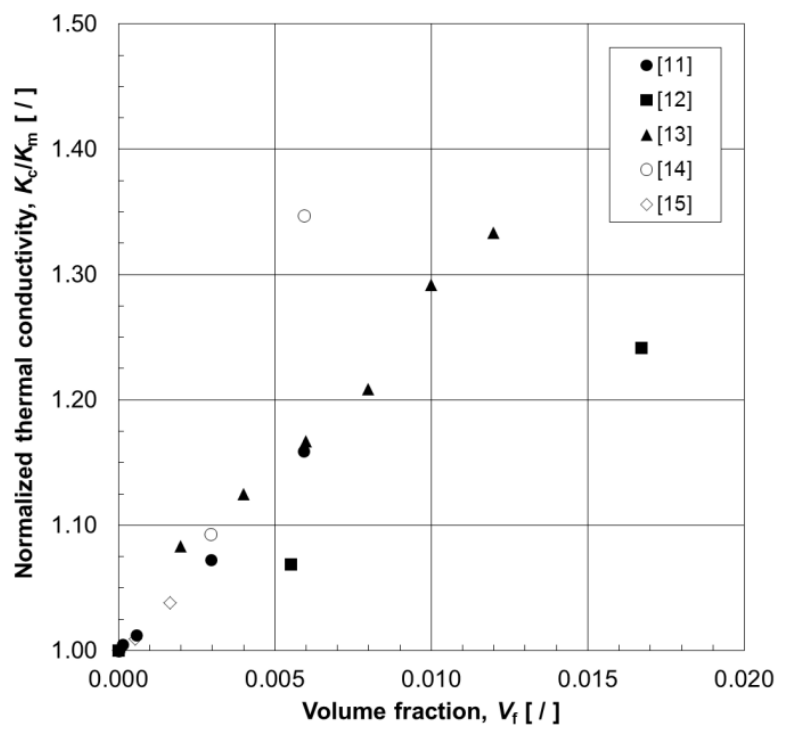

Figure 1. Normalized thermal conductivity of epoxy/MWCNT nanocomposites taken from technical literature.

An attempt was made to predict the experimental thermal conductivity data of nanocomposites shown in Fig.1. The models proposed by Maxwell [7], Hamilton and Crosser [8], Lewis-Nielsen [9] and Deng et al. [10] were employed for the predictions. Table 2 summarizes the parameter values that were used in this context. Figure 2 depicts the predicted values in terms of normalized thermal conductivity for the above models. Note that due to a lack of information on the filler morphology a filler straightness ratio $\theta$ of 0.7 was specified for the analysis, which assumes a MWCNT with moderate curvature.

Figure 2 indicates that the Hamilton and Crosser model overestimates the experimental data. This behavior can be explained by analyzing the empirical shape factor function, Eq.(3), which was derived for geometries that deviate from sphericity $(\gamma)$ by as low as 0.5 . In this study, the sphericity value was calculated to be 0.012 , which clearly indicates that
Eq. (3) was used outside of their study range. However, the Hamilton and Crosser model can be modified by determining an empirical shape factor expression that accounts for the high aspect ratio of CNT. The Deng et al. model predicts thermal conductivity values that are higher than the experimental data from literature. Nevertheless, the Deng et al. model yielded predictions that are closer to experimental data than the Hamilton and Crosser model. It should be noted that the model by Deng et al. is rather comprehensive in the sense that it accounts for various nanocomposite morphology factors, i.e., ITR, aspect ratio, size and geometry.

In contrast, the Maxwell and the Lewis-Nielsen models underestimate the literature data. The Maxwell model was developed for spherical fillers and ignored the effect of the ITR, the filler shape and aspect ratio. According to Nan et al. [18], the aspect ratio of CNT has a strong impact on the thermal conductivity of a composite. A large aspect ratio of CNT will minimize the amount of polymer matrix in between fillers, which results in an increased thermal conductivity by reducing the ITR as the probability of direct CNT contact increases [18]. Therefore, the Maxwell model cannot effectively predict fillers with high aspect ratios especially at higher volume fractions.

An attempt was made to adjust the predictive models for the two models closest to the experimental data, i.e. Deng et al. model and the Lewis-Nielsen model. The Deng et al. model prediction was modified by adjusting the filler straightness ratio $\theta$ while keeping other parameters constant. A straightness ratio of $\theta=0.4$ was implemented, which is lower than the initially assumed value of 0.7 . A lower $\theta$ signifies that the CNT dispersed in the polymer matrix are not straight but rather curved. In an experimental study, an effort could be made to quantify the CNT straightness in nanocomposite samples using e.g. transmission electron microscopy.

TABLE 2. PARAMETERS USED FOR THERMAL CONDUCTIVITY PREDICTIONS.

\begin{tabular}{llc}
\hline \multicolumn{1}{c}{ Parameter } & \multicolumn{1}{c}{ Value } & Reference \\
\hline Average $K_{\mathrm{m}}\left(\mathrm{W} \cdot \mathrm{m}^{-1} \cdot \mathrm{K}^{-1}\right)$ & 0.22 & \\
$K_{\mathrm{f}}\left(\mathrm{W} \cdot \mathrm{m}^{-1} \cdot \mathrm{K}^{-1}\right)$ & 3000 & Table 1 \\
Average length $(\mu \mathrm{m})$ & 17 & \\
Average diameter $(\mathrm{nm})$ & 16 & \\
Shape coefficient $A$ & 8.38 & {$[9]$} \\
$V_{\mathrm{m}}$ & 0.52 & {$[9]$} \\
$K_{\mathrm{c}}^{33}$ & 3000 & {$[16]$} \\
$R_{\mathrm{k}}\left(\mathrm{m}^{2} \cdot \mathrm{K} \cdot \mathrm{W}^{-1}\right)$ & $8.30 \mathrm{E}-08$ & {$[17]$} \\
$\theta$ & 0.7 & Assumption \\
\hline
\end{tabular}




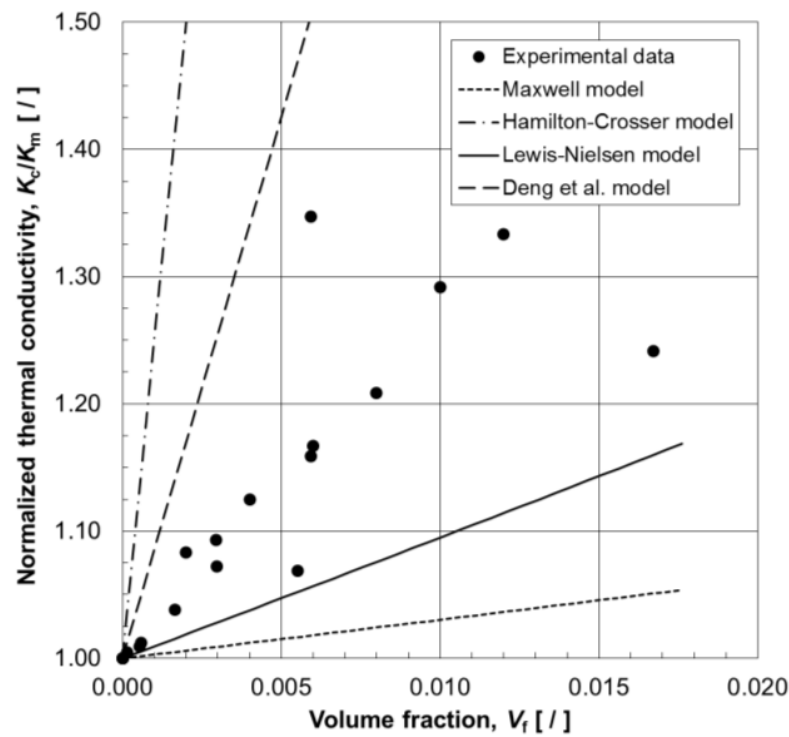

Figure 2. Normalized data for predicted thermal conductivity and experimental values as a function of filler volume fraction.

Similarly, the Lewis-Nielsen model prediction was adapted by changing the shape coefficient, $A$, as it is the most sensitive parameter in the model. Setting $A=26$ yielded values that matched the adjusted prediction based on the Deng et al. model. However, for the sake of clarity, $A$ was set to 24 so that the reader can distinguish between the two models shown in Fig.3. For the considered filler volume fraction, these findings indicate that the filler geometry and aspect ratio play a critical role for the accurate prediction of thermal conductivity of polymer nanocomposites as demonstrated by adjusting respective parameters for both the Deng et al. and LewisNielsen models.

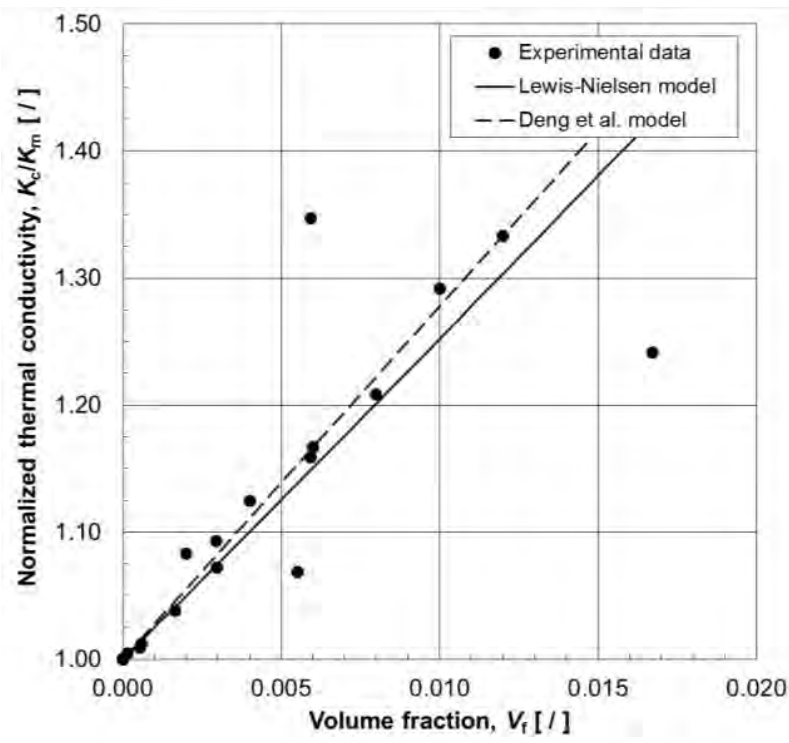

Figure 3. Normalized thermal conductivity predictions from the LewisNielsen and Deng et al. models with adjusted filler morphology parameters, compared to experimental data.

\section{CONCLUSIONS AND RECOMMENDATIONS}

In the present study, models for the prediction of the thermal conductivity of epoxy/MWCNT nanocomposites were examined. A set of prevalent analytical models and experimental data were selected from the technical literature. All models indicated an increase in thermal conductivity with increasing filler loading. For the range of filler volume fractions studied the following conclusions were derived.

- The Maxwell model strongly underestimated the thermal conductivity for epoxy/MWCNT nanocomposites. This model was developed for spherical fillers and is therefore limited in its ability to predict the thermal conductivity of high aspect ratio fillers.

- The Hamilton and Crosser model strongly overestimated the thermal conductivity of epoxy/MWCNT nanocomposites, which is due to a shape factor function that is incompatible with high aspect ratio CNT fillers.

- The Lewis-Nielsen model and the Deng et al. model yielded the most favorable predictions. Results from models could further be improved by adjusting the shape factor and filler straightness ratio, respectively.

- In general, it was observed that the parameters related to the filler morphology (e.g. aspect ratio, curvature) have a strong influence on thermal conductivity predictions. Predictions are less sensitive to the physical properties of the constituents such as their individual thermal conductivities.

\section{REFERENCES}

[1] T. Kashiwagi, F. Du, J. F. Douglas, K. I. Winey, R. H. Harris, and J. R. Shields, "Nanoparticle networks reduce the flammability of polymer nanocomposites," Nat. Mater., vol. 4, no. 12, pp. 928-933, Oct. 2005. doi: 10.1038/nmat1502.

[2] A.P. Mouritz et al., "Review of fire structural modelling of polymer composites," Composites, Part A, vol. 40, no. 12, pp. 1800-1814, Dec. 2009. doi: 10.1016/j.compositesa.2009.09.001.

[3] T. Kashiwagi, E. Grulke, J. Hilding, R. Harris, W. Awad, J. Douglas, "Thermal degradation and flammability properties of poly(propylene)/ carbon nanotube composites," Macromol. Rapid Comm., vol. 23, no. 13, pp. 761-765, Jan. 2002.

[4] Q. Wu, W. Zhu, C. Zhang, Z. Liang and B. Wang, "Study of fire retardant behavior of carbon nanotube membranes and carbon nanofiber paper in carbon fiber reinforced epoxy composites," Carbon, vol. 48, no. 6, pp. 1799-1806, May 2010. doi: 10.1016/j.carbon.2010.01.023.

[5] Z. Han, A. Fina, "Thermal conductivity of carbon nanotubes and their polymer nanocomposites: A review," Prog. Polym. Sci., vol. 36, no. 7, pp. 914-944, Jul. 2011. doi: 10.1016/j.progpolymsci.2010.11.004.

[6] S. J. Jafari Nejad "A review on modeling of the thermal conductivity polymeric nanocomposites," e-Polymers, vol. 12, no. 1, Jan. 2012. doi: 10.1515/epoly.2012.12.1.253.

[7] Y. Zhang, Y. Wang, C.M. Wang and Y. Gu, "Thermal conductivity of graphene and its polymer nanocomposites: A Review", in Advanced Computational Nanomechanics, Chichester, UK: John Wiley \& Sons Ltd., 2016, pp. 1-28.

[8] R.L. Hamilton and O.K. Crosser, "Thermal conductivity of heterogeneous two component systems," Ind. Eng. Chem. Fundamen., vol. 1, no. 3, pp. 187-191, Aug. 1962. doi: 10.1021/1160003a005.

[9] K. Pietrak and T.S. Wiśniewski, "A review of models for effective thermal conductivity of composite materials. J. Power Technol., vol. 95, no. 1 , pp. 14-24, 2015. 
[10] L. Wang, C. Nan, F. Deng and Q. Zheng, "Effects of anisotropy, aspect ratio, and nonstraightness of carbon nanotubes on thermal conductivity of carbon nanotube composites," Appl. Phys. Lett., vol. 90, no. 2, p. 21914, Jan. 2007. doi: 10.1063/1.2430914.

[11] V. Romano, C. Naddeo, L. Guadagno, and L. Vertuccio, "Thermal conductivity of epoxy resins filled with MWCNT and hydrotalcite clay: Experimental data and theoretical predictive modeling," Polym. Composites, vol. 36, no. 6, pp. 1118-1123, Dec. 2014. doi: $10.1002 / p c .23355$.

[12] C. Kostagiannakopoulou, E. Fiamegkou, G. Sotiriadis and V. Kostopoulos, "Thermal conductivity of carbon nanoreinforced epoxy composites," J. Nanomater., vol. 2016, pp. 1-12, 2016. doi: $10.1155 / 2016 / 1847325$.

[13] J. Jiao, Y. Cui and Y. Xia, "Improved thermal conductivity of epoxy composites prepared with a mixed filler of multiwalled carbon nanotubes and aluminum nitride particles," High. Perform. Polym., vol. 29, no. 4, pp. 484-492, Apr. 2016. doi: 10.1177/0954008316644036.

[14] Y.S. Song and J.R. Youn, "Influence of dispersion states of carbon nanotubes on physical properties of epoxy nanocomposites," Carbon, vol. 43, no. 7, pp. 1378-1385, Jun. 2005. doi: 10.1016/j.carbon.2005.01.007

[15] F.H. Gojny, M.H.G. Wichmann, B. Fiedler, I.A. Kinloch, W. Bauhofer, A.H. Windle and K. Schulte, "Evaluation and identification of electrical and thermal conduction mechanisms in carbon nanotube/epoxy composites," Polymer, vol. 47, no. 6, pp. 2036-2045, Mar. 2006. doi: 10.1016/j.polymer.2006.01.029.

[16] P. Kim, L. Shi, A. Majumdar and P.L. McEuen, "Thermal transport measurements of individual multiwalled nanotubes," Phys. Rev. Lett., vol. 87 , no. 21 , pp. 215502 , Oct. 2001 doi: 10.1103/PhysRevLett.87.215502.

[17] S. Huxtable et al., "Interfacial heat flow in carbon nanotube suspensions", Nat. Mater., vol. 2, no. 11, pp. 731-734, Oct. 2003. doi: $10.1038 /$ nmat 996.

[18] C.W. Nan, G. Liu, Y. Lin, and M. Li, "Interface effect on thermal conductivity of carbon nanotube composites," Appl. Phys. Lett., vol. 85, no. 16, pp. 3549-3551, Oct. 2004. doi: 10.1063/1.1808874. 


\title{
Application of FBG Optical Sensors to In-situ Monitoring the Thermo-mechanical Behaviour of Cold Spray Coated Samples
}

\author{
Bahareh Marzbanrad, Farid Ahmed, Hamid Jahed, Ehsan Toyserkani \\ MME Department, University of Waterloo \\ Waterloo, Canada
}

\begin{abstract}
In this research, a fiber Bragg grating (FBG) sensor is employed for monitoring thermal and mechanical strain induced by severe plastic deformation during high thermal and mechanical strain rate of cold spray technique. The FBG sensors are embedded in magnesium alloy substrates and the strain evolutions of the substrates are recorded during the cold gas spray coating process. In these experiments, the localized transient thermo-mechanical strain induced in the close vicinity of the substrate surface is monitored. Qualitative analysis of the complicated spectra shapes obtained during coating and cooling processes demonstrates the repeatability and sensitivity of the sensors in this condition. In addition, the obtained result from FBG sensors reveals the existence of compressive strain in the substrate near the interface during peening; however, it is released after a few second because of the high impact temperature of cold spray coating.
\end{abstract}

Keywords- FBG Sensor; Cold Gas Spray; Magnesium alloy

\section{INTRODUCTION}

The FBG sensors have been employed for sensing the different stimulus such as strain, temperature, pressure, and vibration, because of the unique advantages (small size, immunity to external interferences, ability to operate in harsh environmental conditions, etc.) over other commercial sensors. The optical measurement is safe and superior over electrical one for wide range of applications in harsh environments such as lightening, high electromagnetic fields and in temperature ranging from cryogenic environments to high temperatures of up to $1000^{\circ} \mathrm{C}[1-3]$. FBG is typically constructed by writing periodic index modulation in the core of an optical fiber, which may later be coated with acrylate, polyimide, or organic modulated ceramic for specific application [4]. The grating works as an invisible reflector that reflects only certain wavelengths of light [5]. Deformation of a grating device in presence of external stimulus is represented by wavelength shifts, which can be recorded by an interrogator. If the deformation across the grating part is not uniform (e.g. localized loading), the reflected wavelength shape appears to be asymmetric. Such asymmetric behavior of FBG spectrum can be analyzed to quantify the thermal/mechanical strains. With the recent advancement in optical data acquisition systems, FBG sensors are able to record data (the wavelength changes) with high frequency. Hence, the FBG sensors are ideal candidates for the measurement of localized high temperature and high strain rate; a new application for FBG sensor that needs to be addressed.

Cold gas spray is a method of coating in which, high velocity particles impact a surface and create a layer of solidstate coating $[6,7]$. Adiabatic shear deformation of the particles upon impact generates the localized heat and shear deformation at the contact area of the powder and substrate, which leads to mechanical and lor metallurgical bonding. Based on the cold spray mechanism, thermal softening and work hardening are in competition; since $90 \%$ of impact kinetic energy is dissipated as a heat in less than $100 \mathrm{~ns}$, thermal softening may occur rather than work hardening [8]. Although the cold spray should be a low temperature coating method, the generated heat during impact process may cause to relive the beneficial residual stress due to peening process and microstructural changes, which needs to be considered. However, it may depend on coating and substrate material characteristics. This is a special localized high temperature and fast deformation phenomenon that can be observed in situ by using a FBG sensor.

In this study, we investigate the capability of FBG sensor for revealing the localized residual stress development in magnesium during the cold spray coating process. A FBG with grating length of $4 \mathrm{~mm}$ was embedded in the magnesium sample; however, the phenomena happen over a few hundred microns below the substrate surface. Therefore, only a small part of the FBG was under stress. The goal of this research is to study the dynamic deformation of the substrate as well as temperature changes simultaneously. They determine the equilibrium situation of the magnesium grains, which may cause compressive or tensile residual stress and localized heat effects such as stress relief.

\section{A. Response of FBG sensor to the localized external stimulus}

During the experiments, any mechanical and $\backslash$ or thermal strain changes can shift the reflected wavelengths of the FBG sensors, which can be recorded by an interrogator. In this research, Sm125-200 with one optical channel, and the wavelength accuracy of $10 \mathrm{pm}$ was employed.

The relationship between the wavelength shifts, strain, and temperature changes is specified according to the following equations: 


$$
\begin{aligned}
& \varepsilon=\frac{1}{K}\left(\frac{\Delta \lambda}{\lambda_{0}}-\alpha_{\delta} \times \Delta T\right) \\
& \varepsilon=\varepsilon_{m}+\varepsilon_{T} \\
& \varepsilon_{T}=\alpha_{s p} \times \Delta T \\
& \alpha_{\delta}=\frac{\delta n / n}{\delta T}
\end{aligned}
$$

where, $\Delta \lambda$ is the wavelength shift, $\lambda_{0}$ is the base wavelength at test start, $\mathrm{k}$ is the gauge factor, $\varepsilon$ is the total strain caused by force $\left(\varepsilon_{\mathrm{m}}\right)$ and temperature $\left(\varepsilon_{\mathrm{T}}\right), \alpha_{\delta}$ is defined by the change of the refractive index with temperature, $\alpha_{\mathrm{sp}}$ is the expansion coefficient of the sample $\left(1 /{ }^{\circ} \mathrm{K}\right)$, and $\Delta \mathrm{T}$ is the temperature change $\left({ }^{\circ} \mathrm{K}\right)$. For measuring temperature, a type $\mathrm{K}$ thermocouple (300 $\mu \mathrm{m}$ wires) was attached to each sample; hence, it would be able to detect the temperature of substrate surface during coating process. The FBG was used to record the strain developed in the sample. However, if the FBG sensor experiences a non-uniform strain over the grating length, the response of the sensor will be different. For instance, if only a part of the grating experiences the strain while the rest remains unresponsive to the phenomenon, the spectral response comes only from the sensor length that is exposed to the strain. In this case, the sensor works as two individual in-line FBGs with different grating periods. Based on the optomechanical model proposed by Alemohammad et al. [9], the spectra of the FBG sensor was simulated when distributed strain was applied over the grating length of FBG sensor. The optomechanical model was employed to illustrate the response of the FBGs to localized strain. For instance, it was assumed that an external stimulus applied tensile stains of 0.001 and 0.002 over $200 \mu \mathrm{m}$ length of the $10 \mathrm{~mm}$ grating (Fig 1). Based on the simulation, the main wavelength peak of the sensor before applying the strain was a single peak with high intensity (black spectrum) (Fig 1-b). However, the induced strain created a lopsided peak at the right side of the main peak, while the intensity of the original peak was decreased. Increasing the strain from 0.001 to 0.002 led to significant lopsided peak shifts compared to the previous case. Therefore, it is predicted that in case of the localized strain on a small part of the grating, the main spectrum will be spilt to lopsided peaks and the position of the peaks is proportional to strain amplitude.

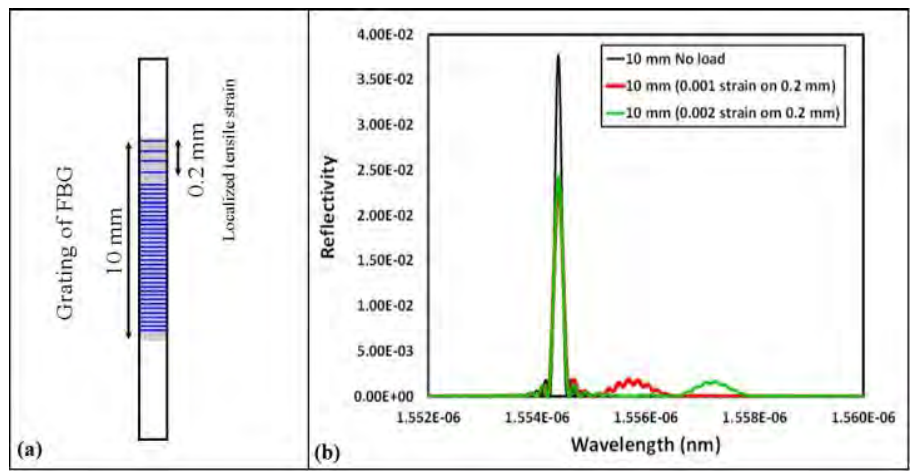

Fig 1: a) A schematic of grating part of FBG sensor under localized tensile loading; b) the spectra obtained from optomechanical model in different strain amplitudes

\section{EXPERIMENTAL PROCEDURES}

In this study, three AZ31B-H24 Mg alloy samples with $4 \mathrm{~mm}$ thickness were used for in-situ strain and temperature measurement. Properties of the Mg alloy can be found in [10]. The shape of samples is shown in Fig 2-a. For these tests, the specimens were finely polished to minimize surface roughness.

\section{A. Sample preparation}

An initial step in developing a smart $\mathrm{Mg}$ alloy structure is specimen layout in a way that it would be enabled to accommodate the FBG sensors, respecting to the location of sensing the strain and temperature in the sample during the cold spray tests. Since the maximum strains are induced around 100 $\mu \mathrm{m}$ below the substrate surface due to peening process, the grating needs to be embedded very close to the surface for optimum outcome. For this, two different embedding positions of the optical sensors were considered so that the propagation axis of the fiber makes an angle of $90^{\circ}$ or $45^{\circ}$ with the substrate surface. Three different holes were drilled in the samples. Two holes with a diameter of $700 \mu \mathrm{m}$ was drilled at the angle of $90^{\circ}$ and $45^{\circ}$ relative to the substrate surface for embedding the FBGs, while the $1 \mathrm{~mm}$ hole was drilled in the sample for embedding the thermocouple. Fig 2-a shows the positions of thermocouple and FBG sensors in the samples. After drilling the holes, all the samples were heat treated in order to relieve the induced stress during manufacturing and preparation of the samples. The heat treatment process was conducted at a temperature of under $260^{\circ} \mathrm{C}\left(500^{\circ} \mathrm{F}\right)$ for 15 minutes, followed by air-cooling [11].

\section{B. Preparation of the FBG sensors}

In this work, $10 \mathrm{~mm}$ long FBG sensors inscribed in Corning SMF-28 optical fiber with initial center wavelengths of 1540 $\mathrm{nm}$ and $1560 \mathrm{~nm}$ were utilized (Fig 2-b). To have more precise results, the polyimide jacket was removed from the grating part of the FBG sensors before embedding inside the magnesium alloy sample's holes.

Then, the bare FBG sensors were accommodated in such a way that the $4 \mathrm{~mm}$ length of grating was placed inside the holes and the rest was out of the sample. High temperature EPO-TEK 353ND adhesive was used for embedding the sensors and thermocouple. To cure the epoxy resin, the samples were heated at $150^{\circ} \mathrm{C}$ for an hour using an electric furnace. Fig 2-c shows the setup of the sample with embedded sensors and thermocouple. After embedding the sensors and curing the resin, the $6 \mathrm{~mm}$ extra part of the grating were cut and gently polished with 800 -grit sandpaper.

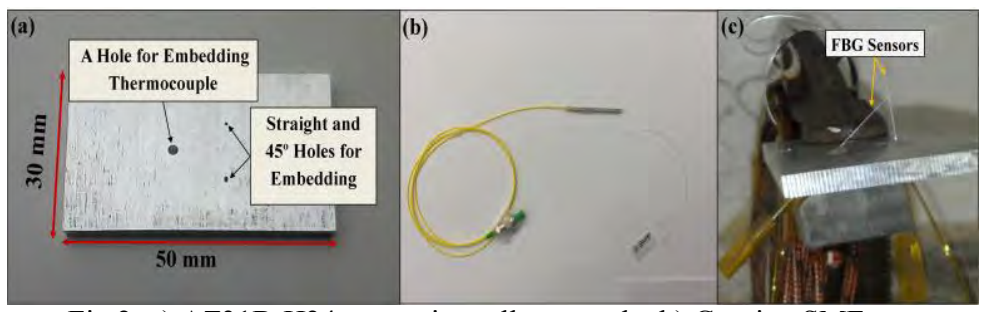

Fig 2: a) AZ31B-H24magnesium alloy sample; b) Corning SMF28 FBG sensor; c) Test sample setup of embedding the sensor with thermal epoxy 
Fig 3-a illustrates the reflected spectrum of one of the sensors, which demonstrates the initial center wavelength of $1560 \mathrm{~nm}$ before embedding the sensor. After embedding, the shape of spectrum was completely rough-and-tumble and the peak broadening was also observed (Fig 3-b). Moreover, the intensity of the peak was changed after curing process. Fig 3-c shows the spectrum changes after cutting and polishing the extra part of the sensor. As seen, the spectrum became narrower after cutting in comparison to the same sensor after embedding.

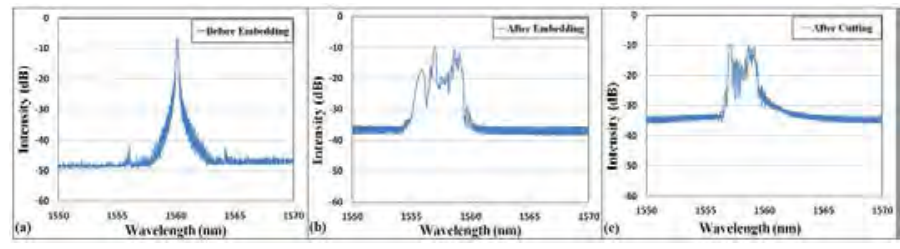

Fig 3: FBG spectrum, a) before embedding; b) after embedding with thermal epoxy; c) after cutting and polishing the sensor

\section{Cold spray process}

A commercial Cold Spray system (SST Series P) manufactured by Centerline was used to deposit spherical commercial Al7075 powder (supplied by Centerline) with the mean particle size of $23 \mu \mathrm{m}$ (measured by Retsch technology, Camsizer XT) on the smart magnesium alloy samples. The chemical compositions of Al7075 powders are listed in Table 1. A17075 powders were accelerated through the De Laval UltiLife TM Nozzle with reciprocating movement on the samples. To create a coating layer of aluminium with $300 \mu \mathrm{m}$ thickness, low porosity and high hardness, the Nitrogen gas was used with stagnation pressures of 200 Psi and temperatures of $400^{\circ} \mathrm{C}$ to produce the suitable particles velocity. For more clarification, all requirement parameters for conducting the relatively high quality of coating by cold spray deposition are listed in Table 2.

Table 1: Chemical compositions of A17075 coating powders

\begin{tabular}{|l|c|c|c|c|c|c|c|c|}
\hline Composition & $\begin{array}{c}\text { Aluminum } \\
(\mathrm{Al})\end{array}$ & $\begin{array}{c}\text { Zinc } \\
(\mathrm{Zn})\end{array}$ & $\begin{array}{c}\text { Iron } \\
(\mathrm{Fe})\end{array}$ & $\begin{array}{c}\text { Nickel } \\
(\mathrm{Ni})\end{array}$ & $\begin{array}{c}\text { Chromium } \\
(\mathrm{Cr})\end{array}$ & $\begin{array}{c}\text { Copper } \\
(\mathrm{Cu})\end{array}$ & $\begin{array}{c}\text { Magnesium } \\
(\mathrm{Mg})\end{array}$ & $\begin{array}{c}\text { Other } \\
\text { Elements }\end{array}$ \\
\hline Weight $\%$ & 90 & 5.20 & 0.35 & 0.005 & 0.25 & 1.55 & 2.35 & 0.30 \\
\hline
\end{tabular}

Table 2: Cold Spray Coating Parameters [12]

\begin{tabular}{|c|c|}
\hline Flow Gas & Nitrogen \\
\hline Gas Temperature & $400^{\circ} \mathrm{C}$ \\
\hline Gas Pressure & $200 \mathrm{Psi}(1.4 \mathrm{MPa})$ \\
\hline Powder Feed Rate & $8 \mathrm{gr} / \mathrm{min}(5 \mathrm{rm})$ \\
\hline Nozzle Speed & $2 \mathrm{~mm} / \mathrm{s}$ \\
\hline Step Over & $1.2 \mathrm{~mm}$ \\
\hline Stand-off Distance & $12 \mathrm{~mm}$ \\
\hline Nozzle Type & De Laval UltiLife TM \\
\hline Nozzle Length & $120 \mathrm{~mm}$ \\
\hline Nozzle Orifice & $2 \mathrm{~mm}$ \\
\hline Nozzle Exit Diameter & $6.3 \mathrm{~mm}$ \\
\hline
\end{tabular}

\section{RESULTS AND DISCUSSION}

In-situ monitoring of mechanical and thermal strain was implemented during the cold spray coating process through embedded FBG sensors in Mg alloy substrates. Response of FBG sensors to axial deformation (extension or contraction of the gratings) due to thermal and/or mechanical stimulus is a linear wavelength change; an increase in grating period due to elongation of the fiber causes a red shift (higher wavelength) of the central wavelength and vice versa $[13,14]$. Fig 4 shows a set of critical spectrums obtained at different nozzle positions during cold spraying for three different embedded samples. It should be noted that during the cold spray experiments, only the responses of sensors embedded at a $45^{\circ}$ directions were recorded. In all of the tests as shown in Fig 4-a, the first curves reveal the response of the sensors after embedding (before running the cold spray tests). When the nozzle approached the sensors, peak broadening and spectral red shift were observed (Fig 4-b). In this condition, thermal strain due to the carrier gas temperature leads to the shifting and broadening of the spectrums. The asymmetric spectra of the sensors were generated because of the non-uniform strains induced along the grating length. The spectra were expanded towards higher wavelengths, which emphasized on elastic strains accumulation close to the substrate surface. With decreasing the distance between nozzle and sensors, more stretching and shifting of spectrums expected. However, with placing the nozzle exactly above the sensors, in spite of higher predicted temperature due to the adiabatic shear instability for particles/substrate bonding, the peaks narrowing were observed while the main peaks still showed more transition to the right (Fig 4-c). This shape change of spectrum uncovers that a significant compressive residual stress is developed in the surface and as a consequence, a compressive strain is induced by peening and severe plastic deformation during the deposition, diminishing the effect of thermal expansion. This observation clarified the contrast between the peening effect and temperature deviation. After passing the nozzle the peaks became broaden and shifted even further to the right where the sign of compressive strains was no more observable. This indicates that the induced stress weakened in the substrate in a few second. Fig 4-e shows the shape of the spectrums after the cold spray tests were completed and the $\mathrm{Mg}$ alloy samples reached to the room temperature. The shapes of spectrums became narrow; however, the peaks were shifted to the right compare to the very initial spectrums (Fig 4-a). For Test \#2 before recording the spectrum after cold spray at room temperature, the FBG failed; hence, the spectrum was not recorded. It should be noted that during the experiments the temperature of samples were recorded by thermocouples, simultaneously. The results obtained from the thermocouples showed that the substrates' temperature was increased up to $330^{\circ} \mathrm{C}$ [15], while the annealing temperature of magnesium alloy AZ31B is about $260^{\circ} \mathrm{C}$ that confirmed the results obtained from FBG sensors, which demonstrated the relieving stress. Fig 5 shows the magnesium alloy sample embedded with two FBG sensors and thermocouple after conducting the cold spray coating tests. 


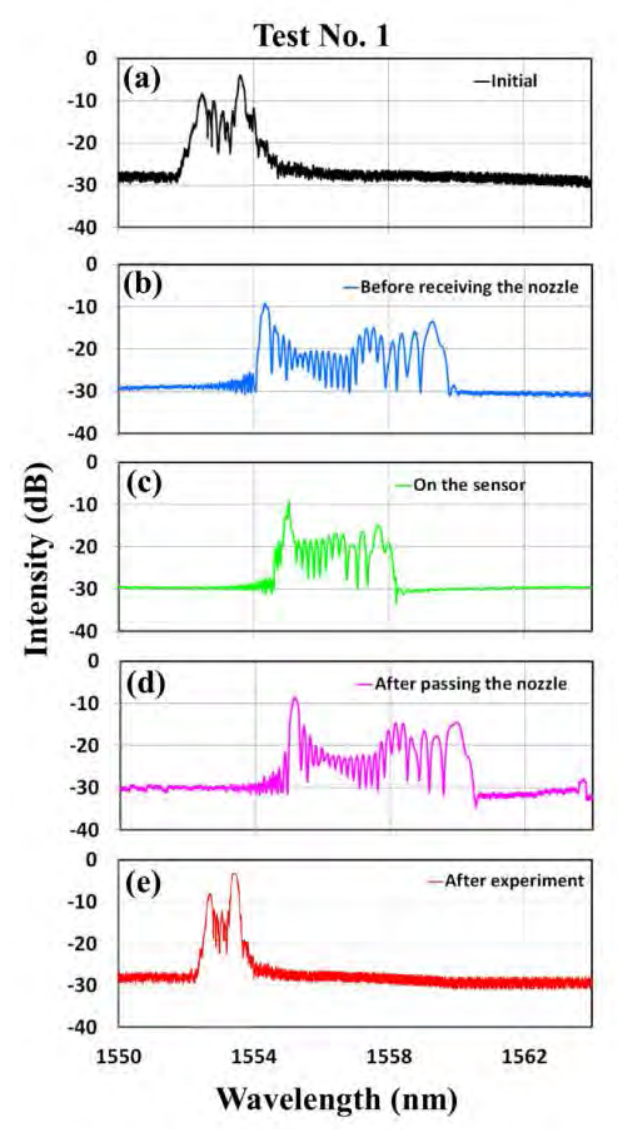

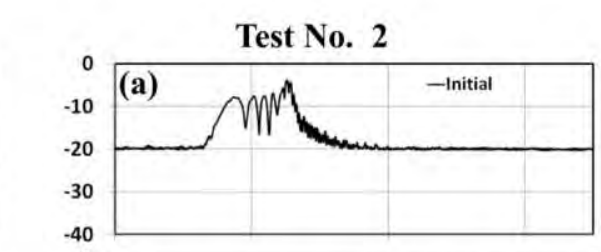
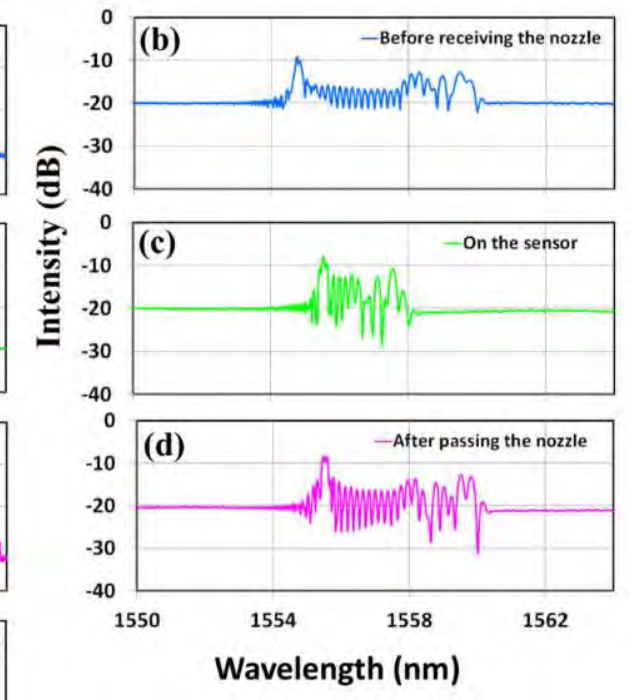

Test No. 5
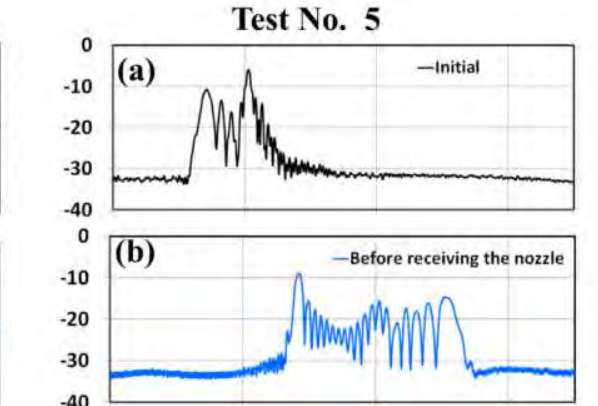

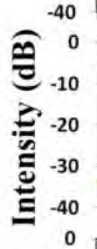
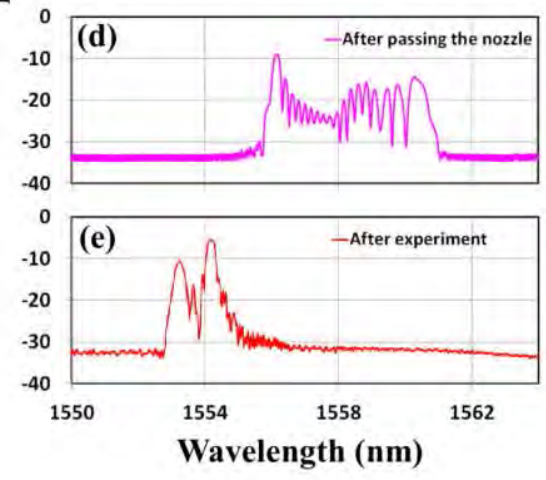

Fig 4: The spectrums of FBG sensors for three different tests in five different situations: a) after embedding a sensor; b) during coating at $10 \mathrm{~mm}$ distance from the nozzle; c) coating on the sensor; d) at $10 \mathrm{~mm}$ after passing the nozzle; e) after coating at RT.

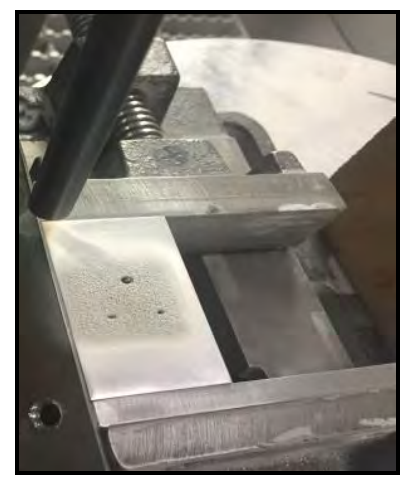

Fig 5: Smart magnesium alloy tested sample after cold spray coating test

For more clarification, the bandwidths and shifts of spectrums at different locations were measured: 1) before conducting the cold spray tests; 2 ) before receiving the nozzle to the tip of the sensor location; 3) where the nozzle was on the tip of sensors; 4) after passing the nozzle; and, 5) after the cold spray tests were completed and the part arrived at room temperature. The bar graphs in Fig 6-a depict the changes of spectrum bandwidth during the experiments. Usually, the bandwidth of the reflection peak is defined by Full-Width-Half-
Maximum (FWHM), which is the width of $50 \%$ of the main peak. In addition, the averages of the widths for all experiments in each situation were added to the bar graphs. Based on the measurements, for all experiments when the nozzle was on top of the sensor the bandwidths of spectra were decreased, significantly, while before and after receiving the nozzle during the cold spray the bandwidths were increased due to the raise in temperature.

In addition, the wavelength shifts during the experiments and after the cold spray tests at room temperature were measured (see Fig 6-b). Based on the observations, the wavelengths shifted to the right noticeably, during the experiments because of increased temperature; however, subtle shifts occurred at different situations during the tests. After conducting the cold spray tests, a significant amount of wavelengths shifts observed in all samples (yellow bars in Fig 6-b), which indicates the existence of tensile strains. These results highlight the effect of temperature due to carrier gas and adiabatic shear deformation on the evolution of tensile strains in the coated samples. It is noticeable that the stress relief process releases the residual elastic energy of the material while in these cases, not only the compressive stresses due to peening process were relieved, but also tensile residual strains were imposed in the substrates. This can be explained by 
considering the thermal mismatch strain of the Al7075 coating and AZ31B-H24 substrate, which created tensile residual strain during the cooling process (after coating).

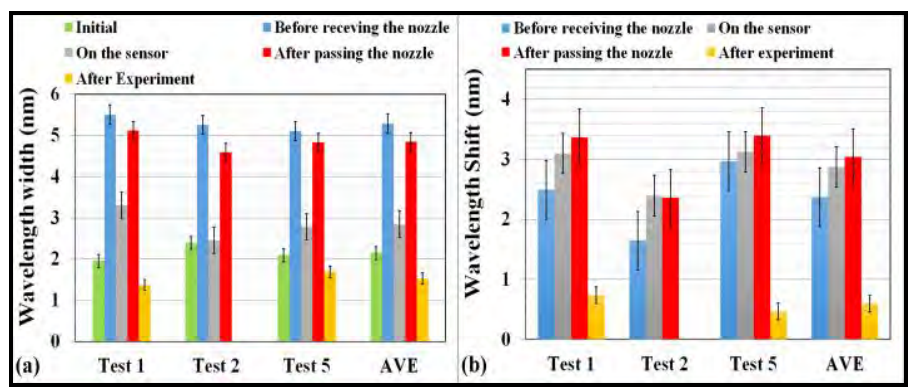

Fig 6: a) Wavelength width for different situations in three tests; b) Wavelength shifts for different situations in three tests

\section{CONCLUSION}

In this research, the capability of FBG sensors for detecting the localized thermal and mechanical strain induced in cold spray coated magnesium alloy is investigated. Localized increase in temperature and distributed deformation in a small area below the surface was implemented through the cold spray coating process. To this end, the FBG sensors were embedded in a magnesium alloy substrates and responses of the sensor recorded during the coating process. This observation revealed that the FBG sensor is able to tolerate the thermal and mechanical impact of the cold spray coating and detect the high mechanical and thermal strain rate during the process. In addition these experiments demonstrated that a compressive residual stress was induced in the substrate during coating, but it was relived after a few second, which could be an evidence for stress relief of the magnesium alloy substrate due to the coating temperature.

\section{REFERENCES}

[1] M. Fokin, "Underlying mechanisms, applications, and limitations of chemical composition gratings in silica based fibers," J. Non-Cryst. Solids, vol. 349, pp. 98-104, Dec. 2004. doi: 10.1016/j.jnoncrysol.2004.08.208.

[2] V. P. Wnuk, A. Mendez, S. Ferguson, and T. Graver, "Process for mounting and packaging of Fiber Bragg grating strain sensors for use in harsh environment applications," Proc. of SPIE, vol. 5758, pp.46-53, May 2005. doi: 10.1117/12.601971.

[3] J.H. Ha, P. Muralidharan, and D. K. Kim, "Hydrothermal synthesis and characterization of self-assembled h-WO3 nanowires/nanorods using EDTA salts," J. Alloys Compd., vol. 475, no. 1-2, pp. 446-451, May 2009. doi: 10.1016/j.jallcom.2008.07.048.

[4] M. M., R. C. S. B. Allil, B. A., and F. V. B. de Nazar, "A guide to Fiber Bragg grating sensors," in Current Trends in Short-and Long-period Fiber Gratings, InTech, 2013. doi: 10.5772/54682

[5] E. Udd, W.B. Spillman, Eds. Fiber Optic Sensors: An Introduction to Engineer and Scientists, 2nd ed., Hoboken, NJ: Wiley, 2011.

[6] A. Papyrin, V. Kosarev, S. Klinkov, A. Alkhimov, V. Fomin, Cold Spray Technology, 1st ed., Amsterdam: Elsevier, 2007.

[7] G. Shayegan et al., "Residual stress induced by cold spray coating of magnesium AZ31B extrusion," Mater. Des., vol. 60, pp. 72-84, Aug. 2014. doi: 10.1016/j.matdes.2014.03.054.

[8] H. Assadi, H. Kreye, F. Gärtner, and T. Klassen, 'Cold spraying - A materials perspective', Acta Mater., vol. 116, pp. 382-407, Sep. 2016. doi: 10.1016/j.actamat.2016.06.034.
[9] H. Alemohammad, E. Toyserkani, "Modeling and analysis of fiber optic Bragg Grating shape sensors," in ASME 2007 International Mechanical Engineering Congress and Exposition, Seattle, WA, 2007, pp. 907-916. doi: 10.1115/IMECE2007-42178.

[10] S. B. Behravesh, H. Jahed, S. Lambert, "Characterization of magnesium spot welds under tensile and cyclic loadings," Mater. Des., vol. 32, no. 10, pp. 4890-4900, Dec. 2011. doi: 10.1016/j.matdes.2011.06.001 .

[11] ASM Handbook, ASM Int., Materials Park, OH, 1991.

[12] S. B. Dayani, S. K. Shaha, R. Ghelichi, J. F. Wang, and H. Jahed, "The impact of AA7075 cold spray coating on the fatigue life of AZ31B cast alloy," Surf. Coat. Technol., vol. 337, pp. 150-158, Mar. 2018. doi: 10.1016/j.surfcoat.2018.01.008.

[13] B. Marzbanrad, H. Jahed, and E. Toyserkani, "On the sensitivity and repeatability of fiber Bragg grating sensors used in strain and material degradation measurement of magnesium alloys under cyclic loads," Int. $J$. Adv. Manuf. Technol., vol. 86, no. 9-12, pp. 3453-3461, Feb. 2016. doi: 10.1007/s00170-016-8485-8.

[14] R. Kashyap, Fiber Bragg Gratings, 2nd ed., Burlington, MA: Academic Press, 2010.

[15] B. Marzbanrad, H. Jahed, and E. Toyserkani, "On the evolution of substrate's residual stress during cold spray process: A parametric study," Mater. Des., vol. 138, pp. 90-102, Jan. 2018. doi: 10.1016/j.matdes.2017.10.062 . 


\title{
Buckling Paradox and Anisotropic Plastic Plate Bifurcation
}

\author{
Suresh Shrivastava \\ Department of Civil Engineering and Applied Mechanics McGill University \\ Montreal, Quebec, Canada H3A 2K6 \\ Email: suresh.shrivastava@mcgill.ca
}

\begin{abstract}
Anisotropic elastic/plastic plate bifurcation is investigated. The pre-bifurcation loading consists of biaxial stresses along $\xi v$ axes parallel to the sides of a simply supported rectangular plate: $\sigma_{v v}=\alpha \sigma_{\xi \xi},-1 \leq \alpha \leq 1$. The $\xi v$ axes are at an angle $\beta$ with the $x y$ principal axes of anisotropy. Analytical variational method is used together with Hill's theory of anisotropic strain-hardening plasticity. Bifurcation stresses are determined for the anisotropic, and the classical isotropic incremental and deformation theories of plasticity, for equibiaxial compression $(\alpha=1)$, equal compression and tension $(\alpha=-1)$, and uniaxial $(\alpha=0)$ cases. The plastic plate buckling paradox is examined for each of the cases.
\end{abstract}

Keywords-anisotropic plastic bifurcation, isotropic plastic bifurcation (incremental, deformation), buckling paradox

\section{INTRODUCTION}

The plate plastic buckling paradox originated from the work of Handelman and Prager [1]. They found that the bifurcation stresses predicted by the isotropic Mises incremental theory, the "correct" theory of strain hardening plasticity, were absurdly higher than those from the "incorrect" deformation theory of plasticity. Experiments generally favour the predictions of the deformation theory. Hence, the plastic plate buckling paradox: a correct theory yields the wrong results, while an incorrect one gives the right results. Onat and Drucker [2] explained the paradox by showing that by taking "unavoidable" out-of-plane geometric imperfection into account in a nonlinear growth analysis of a plate, the incremental theory gives maximum loads matching the deformation theory bifurcation loads. The growth analysis is quite complicated, and can only be done numerically; efforts have been made to lower the buckling loads of the incremental theory by various means, but without success.
Here, a bifurcation analysis for plate buckling is performed by considering the anisotropic behaviour of the plate material. For this purpose Hill's incremental theory [3] for anisotropic strain hardening of sheet metals is used. The $x$ principal axis of anisotropy is taken as the rolling direction; $y$ is the transverse principal axis. These anisotropy axes are assumed to remain fixed, uninfluenced by the loading of the plate for bifurcation. Plane stress conditions are assumed to prevail. The loading is biaxial $\sigma_{v v}=\alpha \sigma_{\xi \xi},-1 \leq \alpha \leq 1$, with $\xi v$ axes parallel to the sides of the rectangular plate, simply supported at $\xi=0, a$ and at $v=0, b$. The $\xi v$ axes are at an angle $\beta$ (which may or may not be zero) with respect to the $x y$ principal axes of anisotropy. Three cases are considered: (1) equibiaxial, (2) equal compression tension, and (3) uniaxial compression. The anisotropic bifurcation stresses are compared with those from the isotropic incremental and deformation theories of plasticity.

\section{ANISOTROPIC CONSTITUTIVE RELATIONS}

The stresses along the anisotropy axes are

$$
\begin{aligned}
\sigma_{x x} & =\sigma_{\xi \xi} \cos ^{2} \beta+\sigma_{v v} \sin ^{2} \beta \\
\sigma_{y y} & =\sigma_{\xi \xi} \sin ^{2} \beta+\sigma_{v v} \cos ^{2} \beta \\
\sigma_{x y} & =\left(\sigma_{\xi \xi 1}-\sigma_{v v}\right) \cos \beta \sin \beta \\
\sigma_{y x} & =\left(\sigma_{\xi \xi}-\sigma_{v v}\right) \cos \beta \sin \beta
\end{aligned}
$$

The anisotropic yield criterion of Hill [3] is

$$
2 f\left(\sigma_{i j}\right)=(G+H) \sigma_{x x}^{2}+(F+H) \sigma_{y y}^{2}-2 H \sigma_{x x} \sigma_{y y}+2 N \sigma_{x y}^{2}=1
$$


where

$G+H=\frac{1}{X^{2}}, F+H=\frac{1}{Y^{2}}, 2 H=\frac{1}{X^{2}}+\frac{1}{Y^{2}}-\frac{1}{Z^{2}}, 2 N=\frac{1}{T^{2}}$.

$X, Y, Z$ and $T$ are the current direct and shear yield stresses.

For isotropy $Z=Y=\sqrt{3} T=X$.

Normality rule gives the plastic strain increments: $d \varepsilon_{i j}^{P}=d \lambda \frac{\partial f}{\partial \sigma_{i j}}$ where $d \lambda=d W^{P}=\sigma_{i j} d \varepsilon_{i j}^{P}$. Explicitly:

$d \varepsilon_{x x}^{P}=d W^{P}\left\{\left(G \sigma_{x x}+H\left(\sigma_{x x}-\sigma_{y y}\right)\right\}\right.$,

$d \varepsilon_{y y}^{P}=d W^{P}\left\{\left(F \sigma_{y y}-H\left(\sigma_{x x}-\sigma_{y y}\right)\right\}\right.$,

$d \varepsilon_{z z}^{P}=-d W^{P}\left(G \sigma_{x x}+F \sigma_{y y}\right)$

$d \varepsilon_{x y}^{P}=2 d W^{P} N \sigma_{x y}, d \varepsilon_{y z}^{P}=0, d \varepsilon_{z x}^{P}=0$

The elastic increments, assumed isotropic, are

$$
\begin{aligned}
d \varepsilon_{i j}^{E} & =\frac{1+v}{E} d \sigma_{i j}-\frac{v}{E} d \sigma_{k k} \delta_{i j}, d \varepsilon_{i i}^{E}=\frac{1-2 v}{E} d \sigma_{k k} \\
d \sigma_{i j} & =\frac{E}{1+v}\left\{d \varepsilon_{i j}^{E}+\frac{v}{(1-2 v)} d \varepsilon_{k k}^{E} \delta_{i j}\right\}
\end{aligned}
$$

The total strain increments, elastic plus plastic, are

$d \varepsilon_{i j}=\frac{1+v}{E} d \sigma_{i j}-\frac{v}{E} d \sigma_{k k} \delta_{i j}+d W^{P} \frac{\partial f}{\partial \sigma_{i j}}$

Now, as defined by Hill, the equivalent stress and the equivalent plastic strain increments are:

$\sigma_{e q}=\sqrt{\frac{3}{2} \frac{h^{2}}{\left(F_{0}+G_{0}+H_{0}\right)}}, d \varepsilon_{e q}^{P}=\sqrt{\frac{2}{3} \frac{\left(F_{0}+G_{0}+H_{0}\right)}{h^{2}}} d W^{P}$

where $F_{0}, G_{0}, H_{0}$ and $N_{0}$ are related to the initial yield stresses in direct and shear stresses, and

$$
h=\sqrt{\left(F_{0}+H_{0}\right) \sigma_{y y}^{2}+\left(G_{0}+H_{0}\right) \sigma_{x x}^{2}-2 H_{0} \sigma_{x x} \sigma_{y y}+2 N_{0} \sigma_{x y}^{2}} \geq 1
$$

is the non-dimensional hardening function, assumed common for all yield stresses:

$$
\begin{aligned}
& X=h X_{0}, Y=h Y_{0}, Z=h Z_{0}, T=h T_{0} \text { or, } \\
& F_{0}=h^{2} F, G_{0}=h^{2} G, H_{0}=h^{2} H, N_{0}=h^{2} N .
\end{aligned}
$$

Also

$d W^{P}=\sigma_{m n} d \varepsilon_{m n}^{P}=\sigma_{e q} d \sigma_{e q} \frac{d \varepsilon_{e q}^{P}}{d \sigma_{e q}}=\frac{d\left(\sigma_{e q}^{2}\right)}{2} \frac{(\lambda-1)}{E}$

where $\lambda=E / E_{t}, E$ and $E_{t}$ are the elastic and tangent moduli from a uniaxial stress-strain curve of the material. Hence

$$
d \varepsilon_{i j}=\frac{(1+v) d \sigma_{i j}-v d \sigma_{k k} \delta_{i j}}{E}+\frac{3(\lambda-1) d h^{2}}{8 E h^{2}\left(F_{0}+G_{0}+H_{0}\right)} \frac{\partial h^{2}}{\partial \sigma_{i j}}
$$

where $\frac{\partial f}{\partial \sigma_{i j}}=\frac{1}{2 h^{2}} \frac{\partial h^{2}}{\partial \sigma_{i j}}$ has been substituted.

For plane stress behaviour $d \sigma_{z z}=0$, one has:

$d \varepsilon_{z z}=-\frac{v}{E}\left(d \sigma_{x x}+d \sigma_{y y}\right)-\left(d \varepsilon_{x x}^{P}+d \varepsilon_{y y}^{P}\right)$.

The above relations can be inverted to express $d \sigma_{i j}$ in terms of $d \varepsilon_{i j}$. The results can be expressed as:

$$
\left\{\begin{array}{l}
d \sigma_{x x} \\
d \sigma_{y y} \\
d \sigma_{x y} \\
d \sigma_{y x}
\end{array}\right\}=\left[\begin{array}{llll}
C_{11} & C_{12} & C_{13} & C_{14} \\
C_{21} & C_{22} & C_{23} & C_{24} \\
C_{31} & C_{32} & C_{33} & C_{34} \\
C_{41} & C_{42} & C_{43} & C_{44}
\end{array}\right]\left\{\begin{array}{l}
d \sigma_{x x} \\
d \sigma_{y y} \\
d \sigma_{x y} \\
d \sigma_{y x}
\end{array}\right\}, C_{j i}=C_{i j}
$$

The incremental moduli $C_{i j}$ depend on the stresses at bifurcation, $\lambda=E / E_{t}$, and the anisotropic parameters $F_{0}, G_{0}, H_{0}, N_{0}$. Referred to the $\xi v$ axes:

$$
\left\{\begin{array}{l}
d \sigma_{\xi \xi} \\
d \sigma_{v v} \\
d \sigma_{\xi v} \\
d \sigma_{v \xi}
\end{array}\right\}=[R]\left\{\begin{array}{l}
d \sigma_{x x} \\
d \sigma_{y y} \\
d \sigma_{x y} \\
d \sigma_{y x}
\end{array}\right\},[R]=[R(\beta)],[R]^{-1}=[R]^{T}=[R(-\beta)],
$$

$[R]=\left[\begin{array}{cccc}\cos ^{2} \beta & \sin ^{2} \beta & \cos \beta \sin \beta & \cos \beta \sin \beta \\ \sin ^{2} \beta & \cos ^{2} \beta & -\cos \beta \sin \beta & -\cos \beta \sin \beta \\ -\cos \beta \sin \beta & \cos \beta \sin \beta & \cos ^{2} \beta & \sin ^{2} \beta \\ -\cos \beta \sin \beta & \cos \beta \sin \beta & \sin ^{2} \beta & \cos ^{2} \beta\end{array}\right]$

where, as defined before, $\beta$ is the angle between the $\xi$ axis of loading and the $x$ principal axis of anisotropy. Thus

$$
\left\{\begin{array}{l}
d \sigma_{\xi \xi} \\
d \sigma_{v v} \\
d \sigma_{\xi v} \\
d \sigma_{v \xi}
\end{array}\right\}=\left[\begin{array}{llll}
B_{11} & B_{12} & B_{13} & B_{14} \\
B_{12} & B_{12} & B_{12} & B_{12} \\
B_{12} & B_{12} & B_{12} & B_{12} \\
B_{12} & B_{12} & B_{12} & B_{12}
\end{array}\right]\left\{\begin{array}{l}
d \varepsilon_{\xi \xi} \\
d \varepsilon_{v v} \\
d \varepsilon_{\xi v} \\
d \varepsilon_{v \xi}
\end{array}\right\},[B]=[R]^{T}[C][R]
$$

$B_{j i}=B_{i j}$ are too complicated to express explicitly. 


\section{CLASSICAL ISOTROPIC THEORIES}

The incremental stress strain relations based upon the $J_{2}$ (Mises) yield criterion are given by [4]:

$\sigma_{\xi \xi}=B^{\prime} \varepsilon_{\xi \xi}+C^{\prime} \varepsilon_{v v} \quad \sigma_{v v}=C^{\prime} \varepsilon_{\xi \xi}+D^{\prime} \varepsilon_{v v}$

$\sigma_{\xi v}=2 F^{\prime} \varepsilon_{\xi v}, \sigma_{v \xi}=2 F^{\prime} \varepsilon_{v \xi}$

where

$$
\begin{aligned}
& B^{\prime}=\frac{E\{(\lambda+3+3 e+4 \lambda \alpha(\alpha-1)\}}{\Delta}, \\
& D^{\prime}=\frac{E\left\{\left(\alpha^{2}(\lambda+3+3 e)-4 \lambda(\alpha-1)\right\}\right.}{\Delta}, \\
& C^{\prime}=\frac{E\left\{2\left(1+\alpha^{2}\right)(\lambda-1+2 v)+\alpha(5+3 e-5 \lambda-4 v)\right\}}{\Delta}, \\
& F^{\prime}=\frac{E}{(2+2 v+3 e)},
\end{aligned}
$$$$
\Delta=\left(1+\alpha^{2}-\alpha\right)\left\{\lambda(5+3 e-4 v)-(1-2 v)^{2}\right\}+
$$$$
3 \alpha(1-\lambda+e)(1-2 v) \text {. }
$$

The parameters $\lambda=E / E_{t}, e=E / E_{s}-1 \quad$ where $E_{t}=d \sigma / d \varepsilon$ and $E_{s}=\sigma / \varepsilon$ are the tangent and secant moduli at the equivalent stress level $\sigma_{e q}^{\prime}$ which for the biaxial state of stress $\sigma^{\prime}{ }_{v V}=\alpha \sigma_{\xi \xi}^{\prime}$, is

$$
\begin{aligned}
& \sigma_{e q}^{\prime}=\sqrt{3 J_{2}}=\sqrt{\left({\sigma^{\prime}}_{\xi \xi}\right)^{2}-\sigma_{\xi \xi}^{\prime}{\sigma^{\prime}}_{v V}+\left(\sigma^{\prime}{ }_{v V}\right)^{2}} \\
& =\sqrt{1-\alpha+\alpha^{2}} \times{\sigma^{\prime}{ }_{\xi \xi}}^{\prime}
\end{aligned}
$$

Above are the current moduli of the $J_{2}$ deformation theory. The current moduli of the $J_{2}$ incremental theory follow by substituting $e=0$ in the above equations. Moduli for the elastic theory are obtained by substituting $e=0$ and $\lambda=1$. Thus, the constitutive relations incorporate the two isotropic strainhardening plasticity theories, and also the purely elastic behaviour. We write:

$$
\left\{\begin{array}{l}
d \sigma_{\xi \xi} \\
d \sigma_{v v} \\
d \sigma_{\xi_{v}} \\
d \sigma_{v^{\xi}}
\end{array}\right\}=\left[\begin{array}{llll}
B_{11}^{\prime} & B_{12}^{\prime} & B_{13}^{\prime} & B^{\prime}{ }_{14} \\
B_{21}^{\prime} & B_{22}^{\prime} & B_{23}^{\prime} & B^{\prime}{ }_{24} \\
B_{32}^{\prime} & B_{32}^{\prime} & B^{\prime}{ }_{33} & B_{34}^{\prime} \\
B^{\prime}{ }_{41} & B^{\prime}{ }_{42} & B^{\prime}{ }_{43} & B^{\prime}{ }_{44}
\end{array}\right]\left\{\begin{array}{l}
d \varepsilon_{\xi \xi} \\
d \varepsilon_{v v} \\
d \varepsilon_{\xi_{v}} \\
d \varepsilon_{v_{\xi}}
\end{array}\right\}
$$

where $B^{\prime}{ }_{j i}=B^{\prime}{ }_{i j}$ are the isotropic moduli in (19) in lieu of the anisotropic moduli in (15).

\section{STRESS STRAIN CURVE}

The material is taken to be 2024S-T3 Aluminum alloy, used in aerospace, with a stress strain curve:

$\varepsilon=\frac{\sigma}{76,500}+0.002\left(\frac{\sigma}{300}\right)^{7}$

where $\sigma$ is in MPa units [5] and $E=76,500 \mathrm{MPa}$. This is taken as the required relation between $\sigma_{e q}=\sigma$ and $\varepsilon_{e q}^{P}=\int d \varepsilon_{e q}^{p}$. Yielding begins around $170 \mathrm{MPa}$. The representation is experimentally valid up to $\sigma=310 \mathrm{MPa}$. Poisson's ratio is taken as 0.33 .

For accounting anisotropic plasticity $X_{0}=170 \mathrm{MPa}$ is taken. Other yield stresses $Y_{0}, Z_{0}, T_{0}$ are given appropriate values in terms of $X_{0}$. The angle $\beta$ between the loading and anisotropy axes is taken here to be just $0^{0}$ and $22.5^{0}$.

\section{FORMULATION FOR BIFURCAION}

Let $w(\xi, v)$ be the bifurcation transverse $z$ displacement. Invoking the Kirchhoff kinematic hypothesis for thin plates, the incremental strains (symbol $d$ omitted hereon) due to bifurcation are

$\varepsilon_{\xi \xi}=-z w_{\xi \xi}, \varepsilon_{v v}=-z w_{v v}, \varepsilon_{\xi v}=-z w_{\xi v}, \varepsilon_{v \xi}=-z w_{v \xi}$

The stress increments are therefore

$$
\begin{aligned}
& \sigma_{\xi \xi}=-z\left(B_{11} \varepsilon_{\xi \xi}+B_{12} \varepsilon_{v v}+B_{13} \varepsilon_{\xi v}+B_{14} \varepsilon_{v \xi}\right) \\
& \sigma_{v v}=-z\left(B_{21} \varepsilon_{\xi \xi}+B_{22} \varepsilon_{v v}+B_{23} \varepsilon_{\xi v}+B_{24} \varepsilon_{v \xi}\right) \\
& \sigma_{\xi v}=-z\left(B_{31} \varepsilon_{\xi \xi}+B_{32} \varepsilon_{v v}+B_{33} \varepsilon_{\xi v}+B_{34} \varepsilon_{v \xi}\right) \\
& \sigma_{v \xi}=-z\left(B_{41} \varepsilon_{\xi \xi}+B_{42} \varepsilon_{v v}+B_{43} \varepsilon_{\xi v}+B_{44} \varepsilon_{v \xi}\right)
\end{aligned}
$$

Plastic bifurcation under increasing load (Shanley's concept) requires $B_{i j}$ to be constants over the thickness. Hence

$$
\begin{aligned}
& M_{\xi \xi}=\frac{t^{3}}{12}\left(B_{11} w_{\xi \xi}+B_{12} w_{v v}+B_{13} w_{\xi v}+B_{14} w_{v \xi}\right), \\
& M_{v v}=\frac{t^{3}}{12}\left(B_{21} w_{\xi \xi}+B_{22} w_{v v}+B_{23} w_{\xi v}+B_{24} w_{v \xi}\right), \\
& M_{\xi v}=\frac{t^{3}}{12}\left(B_{31} w_{\xi \xi}+B_{32} w_{v v}+B_{33} w_{\xi v}+B_{34} w_{v \xi}\right) . \\
& M_{v \xi}=\frac{t^{3}}{12}\left(B_{41} w_{\xi \xi}+B_{42} w_{v v}+B_{43} w_{\xi v}+B_{44} w_{v \xi}\right) .
\end{aligned}
$$


Now, the principle of virtual work requires

$$
\begin{aligned}
& \int_{0}^{a} \int_{0}^{b}\left(M_{\xi \xi} \delta w_{\xi \xi}+M_{v v} \delta w_{v v}+M_{\xi v} \delta w_{\xi v}+M_{v \xi} \delta w_{v \xi}-\right. \\
& \left.P_{1} w_{\xi} \delta w_{\xi}-\alpha P_{1} w_{v} \delta w_{v}\right) d \xi d v=0
\end{aligned}
$$

where virtual work of axial loads $P_{1}$ and $P_{2}=\alpha P_{1}$ per unit length has been accounted. Substitution of $M_{\xi \xi}$ etc. renders the virtual work equation as

$$
\begin{aligned}
& \frac{t^{3}}{12} \int_{0}^{a} \int_{0}^{b}\left\{\left(B_{11} w_{\xi \xi}+B_{12} w_{v v}+B_{13} w_{\xi v}\right) \delta w_{\xi \xi}+\left(B_{21} w_{\xi \xi}+B_{22} w_{v v}+\right.\right. \\
& \left.B_{23} w_{\xi v}+B_{24} w_{v \xi}\right) \delta w_{v v}+\left(B_{31} w_{\xi \xi}+B_{32} w_{v v}+B_{33} w_{\xi v}+\right. \\
& \left.B_{34} w_{v \xi}\right) \delta w_{\xi v}+\left(B_{41} w_{\xi \xi}+B_{42} w_{v v}+B_{43} w_{\xi v}+B_{44} w_{v \xi}\right) \delta w_{v \xi} \\
& \left.-P_{1}\left(w_{\xi} \delta w_{\xi}+\alpha w_{v} \delta w_{v}\right)\right\} d \xi d v=0
\end{aligned}
$$

\section{DEERMINING THE BIFURCATION STRESS}

Galerikin procedure is used. The displacement $w$ and its variation $\delta w$ are taken of the same form, satisfying the simple support conditions around the edges. The functions

$$
\begin{aligned}
& w=\sum A_{i} \sin \frac{m_{i} \pi \xi}{a} \sin \frac{n_{i} \pi v}{b}, \\
& \delta w=\Sigma \delta A_{i} \sin \frac{m_{i} \pi \xi}{a} \sin \frac{n_{i} \pi v}{b}
\end{aligned}
$$

are such functions, where $m_{i}, n_{i}$ are the number of half waves in $\xi, v$ directions. The terms are such that $m_{i}+n_{i}$ is equal to $2,3,4,5,6,7,8$, giving 28 terms, a number assumed to provide sufficiently accurate values of the bifurcation stresses.

When substituted in the above virtual work equation and integrated, the arbitrary character of $\delta A_{i}$ yields a system of linear homogeneous equations of a generalized eigenvalue problem

$$
\frac{t^{3}}{12}\left[K_{1}\right]\{A\}-P_{1}\left[K_{2}\right]\{A\}=\{0\}
$$

For a chosen $\sigma_{\xi \xi}$, the problem can be cast as

$\left[\left[K_{1}^{\prime}\right]-\frac{b^{2}}{t^{2}}\left[K_{2}\right]\right]\{A\}=\{0\}$

where $\left[K_{1}^{\prime}\right]=\frac{b^{2}}{12 \sigma_{\xi \xi}}\left[K_{1}\right]$ depends on $\sigma_{\varsigma \xi}$, moduli $B_{i j}$ or $B_{i j}^{\prime}$ and $r=\frac{a}{b}$. Thus, the eigenvalue is $\frac{b^{2}}{t^{2}}$.
Mathematica programs were constructed to obtain the critical values of $\frac{t}{b}$ versus $\sigma_{\xi \xi}$ for the present incremental anisotropic theory of plasticity of Hill, and for the two classical isotropic theories.

\section{EQUIBIAXIAL COMPRESSION CASE}

The results are given for rectangular plates with $a / b=5$. The equibiaxial compressive stresses result in zero shear stress everywhere. The graphs below show gradual divergence between the incremental and deformation theory. At $t / b \approx 0.0927$ the critical stress is $310 \mathrm{MPa}$ for the incremental theory, whereas for the deformation theory it is around $286 \mathrm{MPa}$ which is lower by $7.7 \%$. This is not large difference and can be made still smaller by taking the transverse shear effects in the incremental theory. For circular SS plate [6] this difference is about $1 \%$. This means that for this equibiaxial loading the paradox is practically non-existent. For a slight anisotropy $X_{0}=Y_{0}=170 \mathrm{MPa} Z_{0}=0.9 X_{0}$ the critical stress is around $290 \mathrm{MPa}$, somewhat higher than that from the deformation theory and lower than the incremental theory. No experimental data are available for this loading.

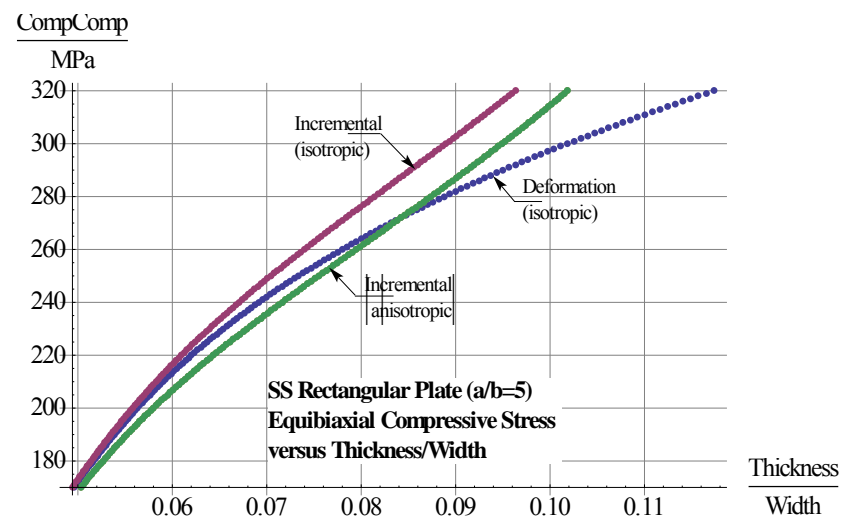

Fig 1: Critical Stress $\sigma_{\xi \xi}$ in Equibiaxial Loading

\section{EQUAL COMPRESSION AND TENSION LOADING}

Figure 2 below shows, for $a / b=1$ square plates, the extreme divergence between the bifurcation stresses predicted by the isotropic incremental and and deformation theories, confirming the plastic plate buckling paradox for this case of equal compression and tension loading. The results of the incremental theory exhibit increasing stiffness with increasing $t / b$ ratio, while those of the deformation theory show markedly softening behaviour. It seems that the result of the incremental theory are too high and those of the deformation theory too low. In the absence of any experimental results for 
such loading, it is conjectured that the true values are midway between the results of the two theories.

Graphs obtained by choosing

$X_{0}=170, Y_{0}=1.3 \times X_{0}, Z_{0}=1.35 \times X_{0}, T_{0}=X_{0} / \sqrt{3} \mathrm{MPa}$

are shown for $\beta=0$ and $\beta=22.5^{\circ}$. These graphs lie between the results of the two other theories.

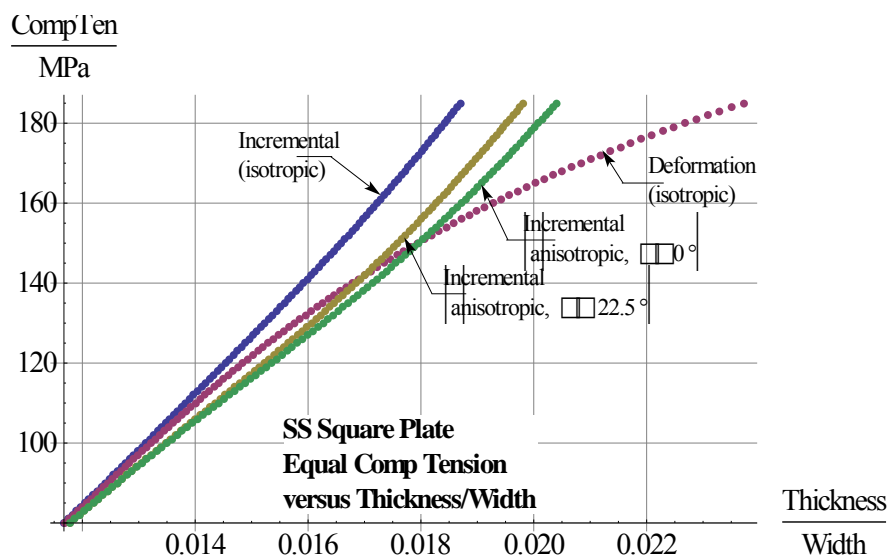

Fig. 2: Critical Stress $\sigma_{\xi \xi}$ in Equal Comp-Tension

The above anisotropic values are not unique. They were chosen by trial and error to obtain graphs midway between the graphs of the two isotropic theories, keeping in mind that the yield stresses should not be very dissimilar.

\section{UNIAXIAL COMPRESSION LOADING}

This case, for long plates, is the one most discussed, and has many experimental studies generally favoring the results of the deformation theory. It was also the case dealt by Handleman and Prager [1], and the paradox was discovered.

Figure 3 , drawn for $a / b=5$, shows the wide divergence between the results of the incremental and the deformation theories for the uniaxial loading. The anisotropic graphs are for

$X_{0}=170, Y_{0}=1.6 \times 170, Z_{0}=1 \times 170, T_{0}=1.35 \times 170 \quad \mathrm{MPa}$, and $\beta=0^{\circ}, \beta=22.5^{\circ}$.

The graphs for these anisotropic values are quite close to the critical stresses predicted by the deformation theory, especially for $\beta=0^{\circ}$.
This holds true for the entire range of stress in plastic strainhardening behaviour. For $\beta=22.5^{\circ}$ a slightly stiffer behaviour is predicted than for $\beta=0$.

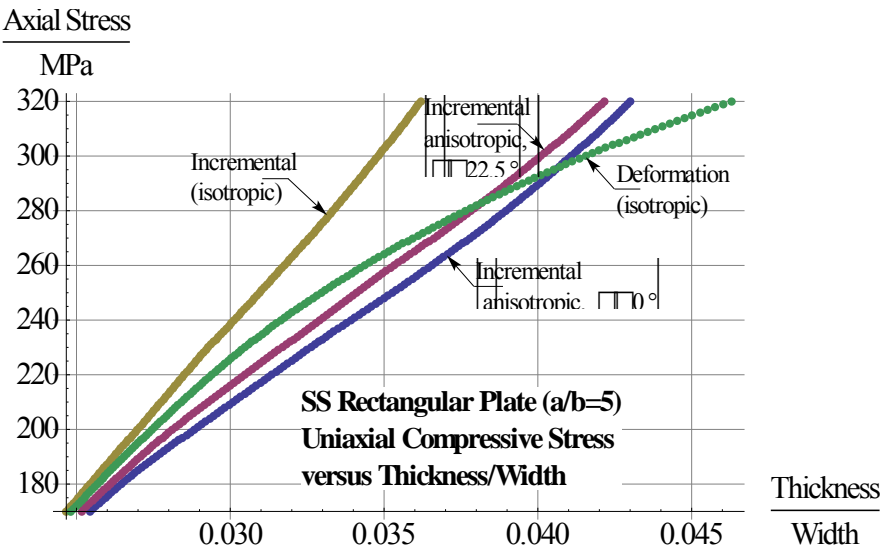

Fig. 3: Critical Stress $\sigma_{\xi \xi}$ in Uniaxial Compression

\section{CONCLUSION}

It is clear that the incremental theory, by considering the anisotropic plastic behaviour of the plate material, predicts bifurcation stresses which are close to the isotropic deformation theory results, which in turn are close to the available experimental values.

\section{REFERENCES}

[1] G. H. Handleman and W. Prager, "Plastic buckling of a rectangular plate under edge thrusts," NACA., Washington D.C., 1949.

[2] E. T. Onat, D. C. Drucker, "Inelastic Instability and Incremental Theories of Plasticity, J. Aeronaut. Sci. vol. 20, no. 3, pp. 181186, Mar. 1953. doi: 10.2514/8.2585.

[3] R. Hill, The Mathematical Theory of Plasticity, London, England: Oxford University press, 1950.

[4] S. Shrivastava and B. M. Mayenba. "Plastic Buckling Paradox in Biaxial Loading," in 25th Cancam, Western University, London, ON. 2013.

[5] P. Seide and E. Z. Stowell, "Elastic and Plastic Buckling of Simply Supported Solid-Core Sandwich Plates in Compression," NACA., Washington D.C., 1948.

[6] A. Bahous, "Plastic Buckling of Circular Plates on a Partial Elastic Foundation," M.S. thesis, Eng., McGill U., Montreal, QC., 2014. 


\title{
Effect of loading strain rates on unloading behavior of shot peened materials
}

\author{
Amir Yazdanmehr, Hamid Jahed \\ Fatigue and Stress Analysis Laboratory \\ University of Waterloo \\ Waterloo, Canada \\ amir.yazdanmehr@uwaterloo.ca, hamid.jahed@uwaterloo.ca
}

\begin{abstract}
Shot peening is a process widely used in industry to improve the fatigue life of materials through induced compressive residual stresses that retard crack initiation and growth. In the peening process, there are two stages: 1) loading: shot penetrating into target; and 2) unloading: shot rebounding from the target. The strain rates in the loading process are known to be in $10^{5}-10^{6} 1 / \mathrm{s}$ range, having heavy impact on the materials' properties. However, the effect of the loading strain rates on the rebounding stage is not well studied. This paper aims to determine the effects of the loading strain rates on the unloading behavior of a material using FEM method. First, to better understand the material behavior, this study evaluates the loading-unloading responses of one element at high strain rates in different scenarios. Then, it obtains the strain rates during the loading and unloading for the different elements of a material being impinged by one shot. The results show that the unloading behavior of a material depends only on the loading equivalent plastic strain and the strain rate of the unloading step.
\end{abstract}

Keywords: Shot peening, high strain rate, loading-unloading

\section{INTRODUCTION}

Shot peening is the process of impinging small spherical shots on the surface of a target material. These impacts create plastic deformation that leads to the development of compressive residual stress over the few-hundred micrometers layer of a material within the vicinity of the shot. As fatigue cracks are generally initiated from the surface of a material, this compressive stress delay crack initiation and retards crack growth, resulting in improved fatigue life of the material. Rateindependent analytical/numerical methods for calculation of residual stresses [1], with applications in many different engineering fields [2]-[4], and for multiple applications of the process [5] have been established. However, strain rate effects in shot peening cannot be ignored.

Analytical and finite element (FE) modeling has been employed to estimate residual stresses and/or model the process of shot peening. The analytical method was developed based on quasi-static response of a target material to single impingement [6], [7]. It was further developed to consider dynamic effects [8],
[9]. The FE evaluation of residual stress distribution has been established in 2D [10] and 3D modeling [11], [12].

A major issue in modeling is the incorporation of strain rate effect. The strain rates in the shot peening process can be as high as $10^{5}-10^{6} 1 / \mathrm{s}$ [13][11]. Several studies have shown the effect of strain rates in modeling residual stress distribution [11], [13]. Kim et al. [12] showed that the magnitude of the compressive residual stress increases when considering strain rate effect. Meguid et al. [11] showed that strain rate has a significant role in the modeling of residual stress profiles in the peening of AISI 4340. Attempts have also been made to extend quasi-static based analytical solutions [7] to strain rate-dependent solutions [13]. However, a comprehensive review of the literature reveals that there is no study on the effect of loading strain rates on the unloading behavior of a material and its effect on modeling the shot peening process. This paper discusses different loadingunloading scenarios to provide a simple evaluation of material response at different loading and unloading strain rates. The impact of one shot is modeled to capture the strain rate history of the different elements through the depth of a material. Also, necessary modifications for modeling the residual stress distribution have been identified.

\section{MATERIALS AND METHODS}

This paper includes two parts. In part A, the effect of loading strain rates on the unloading behavior of a material is discussed. For better understanding of this effect in general, a virtual material property with an exaggerated strain rate effect has been considered using a one-element FE model. In part B, the actual one shot impact on a steel alloy has been modeled to illustrate the application of findings of the part $\mathrm{A}$ in the shot peening process.

\section{A. Effect of loading strain rate on unloading behavior of materials, using one-element simulation}

To figure out the effect of loading strain rates on the unloading behavior of a material, this section discusses oneelement simulation of a virtual material with an exaggerated dependency on strain rates. The one-element modeling is performed because the loading and unloading conditions can be simply applied and the response of the material can be obtained. The virtual flow curves of the material assigned to the single 
element modeling is shown in Figure 1 for strain rates of 0.1 and $51 / \mathrm{s}$.

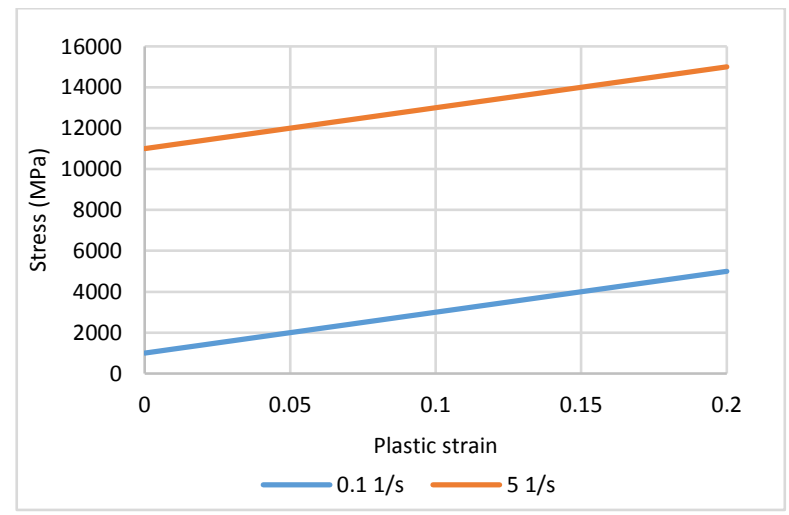

Figure 1. Virtual flow curves of the material used in one-element simulation

The FE analysis is performed using Abaqus software. One element with symmetric boundary condition at $\mathrm{xz}$ plane for its bottom side has been used. In this case, the strain and stress in the y-axis are the equivalent strain and stress of the element, respectively. The element size is $1 \times 1 \times 1 \mathrm{~m}$. Figure 2 shows the geometry of one element modeling as well as the boundary, and loading conditions.

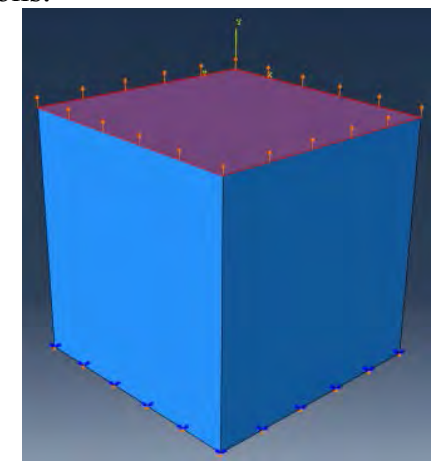

Figure 2. One element modeling geometry

To study the effect of loading and unloading strain rates on the stress-strain response of the material, a simulation using five different strain rate scenarios is considered. Table 1 and Figure 3 describes these different scenarios and represents the strain histories, respectively.

TABLE 1. FIVE LOADING AND UNLOADING SCENARIOS

\begin{tabular}{|c|l|}
\hline Scenario & Description \\
\hline 0 & Quasi-static loading and unloading \\
\hline 1 & Load fast and keep the strain constant \\
\hline 2 & Load fast and then continue loading slowly \\
\hline 3 & $\begin{array}{l}\text { High strain rate loading followed by quasi } \\
\text { static unloading }\end{array}$ \\
\hline 4 & $\begin{array}{l}\text { High strain rate loading followed by } \\
\text { unloading with the same rates as loading }\end{array}$ \\
\hline 5 & $\begin{array}{l}\text { High strain rate loading followed by } \\
\text { unloading with high rates different from } \\
\text { loading rate }\end{array}$ \\
\hline
\end{tabular}

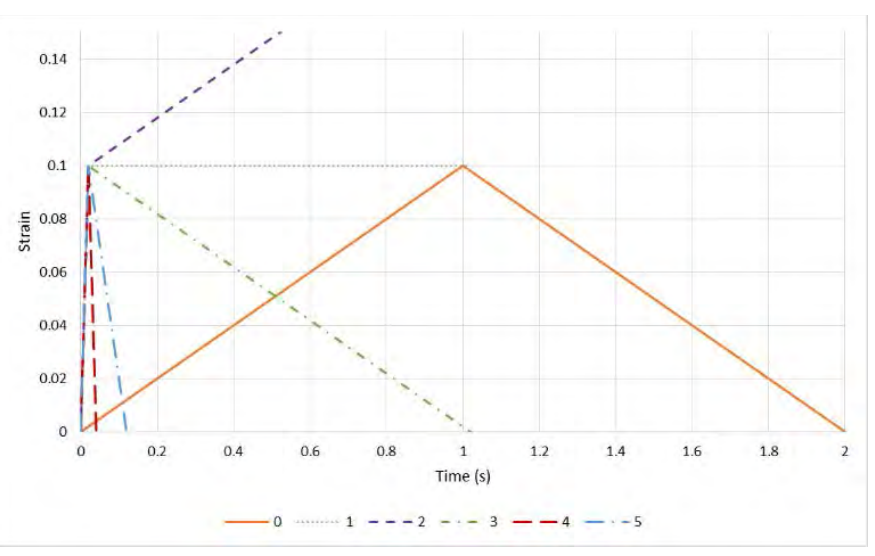

Figure 3. Strain history for the different scenarios shown in Table 1

\section{B. Application on modeling the shot peening process}

As noted, shot peening is a process whereby loading strain rates are high and unloading behavior can be affected by these high loading rates. To determine these effects, this section discusses the finite element modeling of one shot impingement. Abaqus/Explicit was used for modeling the shot peening process. SAE1070 spring steel with dimensions of $76 \mathrm{~mm} \times 19$ $\mathrm{mm} \times 1.29 \mathrm{~mm}$ has been used as the peened material. The stressstrain curve is assumed to be bilinear. Figure 4 presents the stress-strain curve of the SAE 1070 spring steel [14], known as Almen strip type A. The Young's modulus and Poisson's ratio are $200 \mathrm{GPa}$ and 0.31 , respectively. The strain rate effect on the stress-strain curve of this material has been modeled using the Johnston-Cook model with $\mathrm{C}=0.0134$ and a reference strain rate of $75001 / \mathrm{s}[13][15]$.

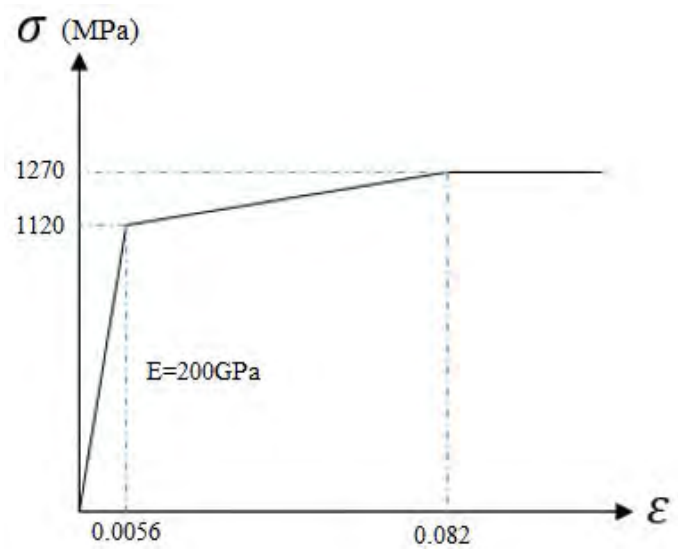

Figure 4. Stress-strain curve of SAE1070 spring steel

Figure 5 shows the flow curves of the material at different strain rates [13]. Table 2 shows the shot properties in the modeling. The shot velocity of $45 \mathrm{~m} / \mathrm{s}$ has been used in this modeling. The steel shot is assumed to be elastic. The target width and height of $5 R$ and $6 R$ are used as suggested in [11], [16]-[18], respectively, where R is the radius of the shot. Eightnode linear brick elements with reduced integration (C3D8R) and a variable element size with minimum size of $5 \times 5 \times 5 \mu \mathrm{m}$ at the impact point are employed for the modeling. 


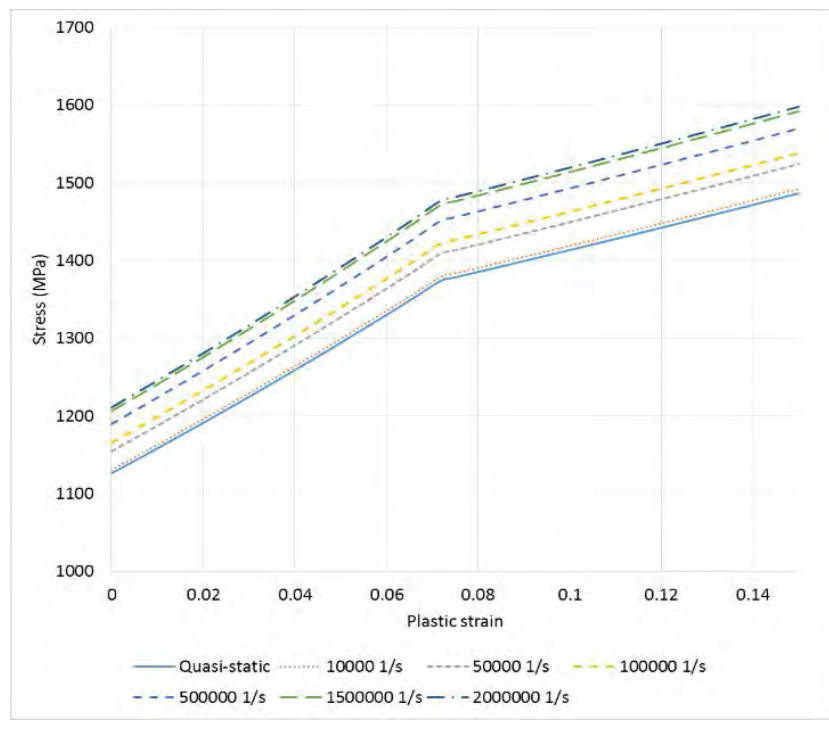

Figure 5. Effect of strain rates on the flow curve of SAE 1070 spring steel [13]

\begin{tabular}{|c|c|c|c|c|}
\multicolumn{7}{c}{ TABLE 2. SHOT PROPERTIES } \\
\hline $\begin{array}{c}\text { Shot } \\
\text { material }\end{array}$ & $\begin{array}{c}\mathrm{E} \\
(\mathrm{GPa})\end{array}$ & $v$ & $\begin{array}{c}\text { Density }(\mathrm{Kg} . \mathrm{m}- \\
3)\end{array}$ & $\begin{array}{c}\mathrm{D} \\
(\mathrm{mm})\end{array}$ \\
\hline Steel shot & 210 & 0.31 & 7800 & 0.4 \\
\hline
\end{tabular}

All degrees of freedom at the bottom side of the target are fixed, and symmetry conditions are applied at the $x z$ and $y z$ planes. The damping model of [11] is used to remove unnecessary oscillations. The penalty contact algorithm with an isotropic coulomb friction coefficient of 0.2 is used [19][20]. Figure 6 shows the single shot geometry and meshed model.

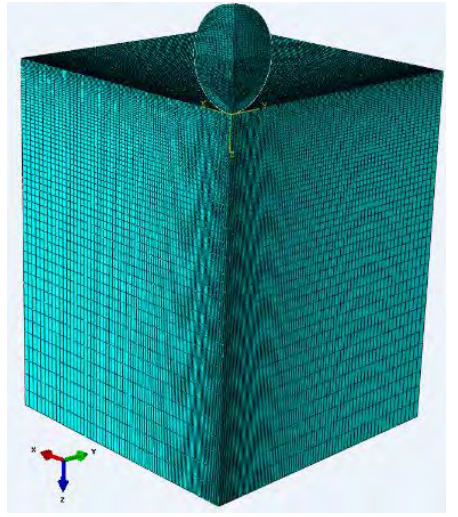

Figure 6. Single shot geometry and meshing

\section{RESULTS AND DISCUSSION}

In this section, the results of one-element and one-shot impingement are discussed.

\section{A. Effect of loading strain rate on unloading behavior of materials, using one-element simulation}

The stress-strain response of one-element model in the loading and unloading with the properties mentioned in Figure 4 are shown in Figure 7. For scenario 0, which is quasi-static loading and unloading, the material follows the given flow curve at the lowest strain rate. In scenario 1 , in which the material is loaded fast and then the loading surface is fixed, the material follows the high strain rate behavior in the loading step, and at the end of the loading, the stress drops to the equivalent stress in the quasi-static stress-strain curve. The equivalent stress at each moment is shown to be a function of equivalent strain and the strain rate at that moment. Scenario 2, in which the material is loaded fast and then the application of tensile strain continues at a low strain rate, reveals that the material follows the stressstrain curve at the high strain rate, then the stress is dropped to follow the rest of the quasi-static loading behavior. In scenario 3 , in which fast loading and then slow unloading happens, the loading behavior of the material is the same as the stress-strain behavior at the high strain rate, but the unloading curve fits the unloading curve of the quasi-static condition. Thus, if the unloading is quasi-static, its stress-strain curve, independent from the loading strain rate, follows the quasi-static unloading curve (scenario 0). Scenario 4 discusses the situation in which both the loading and unloading happen at the high strain rate. In this state, as the strain rates of both the loading and unloading steps are the same, reverse yield happens at the reverse value of the maximum loading stress if the material has isotropic hardening behavior. Finally, scenario 5, in which the loading and unloading strain rates are high but different, shows that the unloading behavior is the same as the response of a material when the unloading strain rate is applied for both loading and unloading steps.

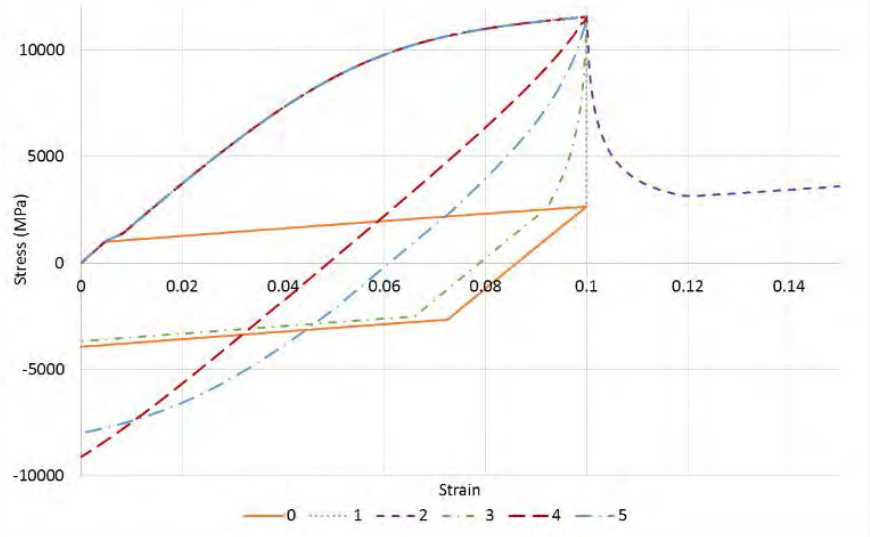

Figure 7. Stress-strain response of one-element in different loadingunloading scenarios

\section{B. Application on modeling the shot peening process}

This part examines the loading and unloading strain rates in the shot peening process, as well as the considerations necessary in modeling of this process, which requires evaluation of the loading-unloading and equivalent stress histories. The strain rates' histories in one-shot impingement for different elements through the thickness are shown in Figure 8. This figure shows that the history of the strain rate is a combination of the different scenarios discussed in the previous section. It also confirms that the unloading strain rates are much lower than the loading ones. The end of the loading step is defined as the moment when the shot is at its maximum penetration depth through the target; after that the shot will rebound. First, as shown, the maximum strain rate happens when a load is applied to an element. After that, the 
strain rates are still high and vanish as the modeling time increases. The time of the unloading is specified in Figure 8. Although the strain rates in the unloading steps are much lower than those in the loading step, their values are still considerable.

Figure 9 shows the variation of equivalent stress for various elements through the depth of the material during the simulation time. The equivalent stresses suddenly increase, at high rates initially, and then continue to increase gradually when the shot is still pressing the target. At the end of the loading step, equivalent stresses drop elastically, with lower strain rates than during the loading step.

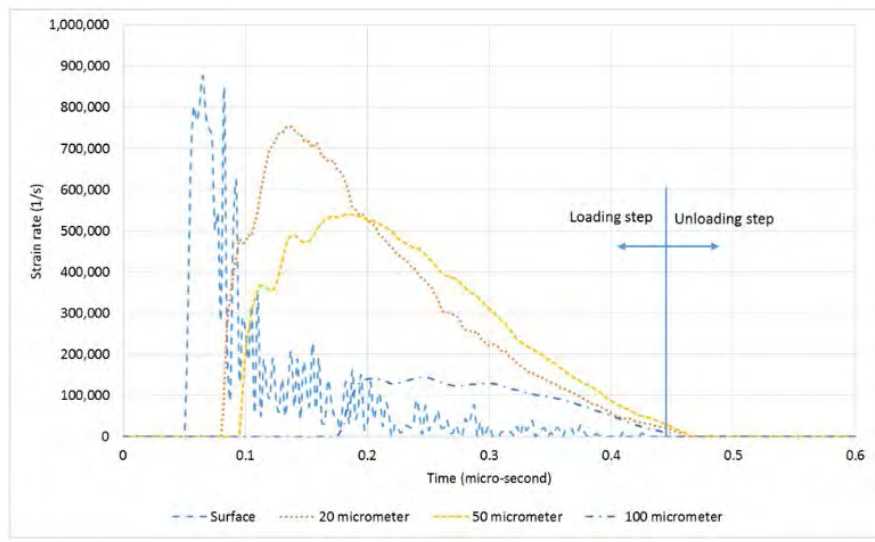

Figure 8. Strain rates of different elements through the thickness elements vs the time of modeling

Figure 10 shows the maximum unloading strain rates of the different elements through the depth of the material. For this peening example, the maximum strain rate in the unloading step occurs for the element that is 50 micrometers below the surface. Although the level of strain rates is one order of magnitude lower than those in the loading step, the strain rate values are in the order of $10^{5} 1 / \mathrm{s}$. Unloading strain rates vanish quickly because when the unloading is ended, the element's strains will stabilized. Thus, the material will follow scenario 3 of the oneelement simulations, in which the unloading curves are fitted to the unloading stress-strain curve of the quasi-static loadingunloading.

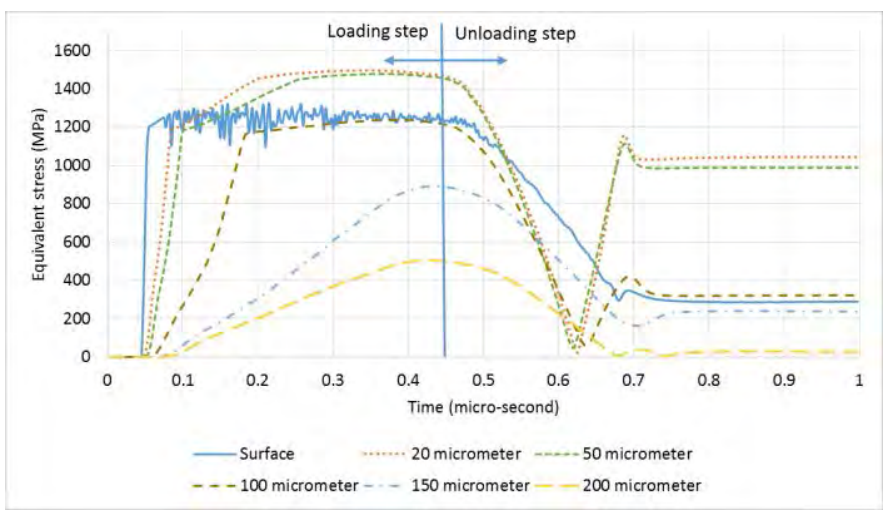

Figure 9. Equivalent stress histories of different elements through the depth of the material during the modeling time

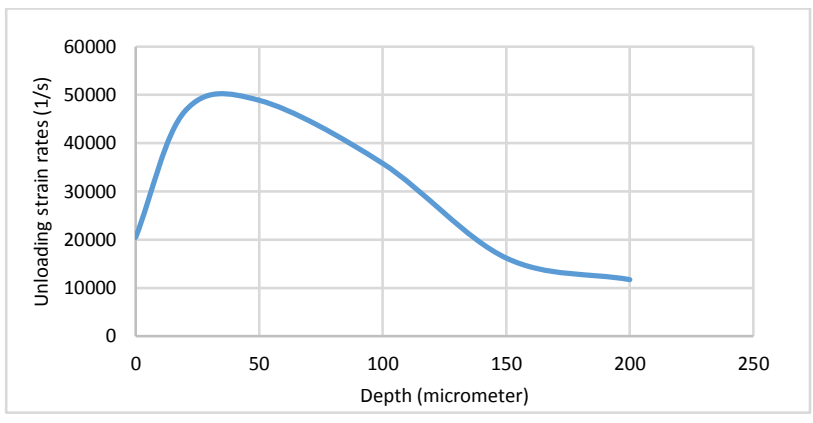

Figure 10. Elastic unloading strain rates of different elements through the depth of the material

\section{CONCLUSION}

The effect of loading strain rates on the unloading behavior of materials has been discussed. The equivalent stress at each moment has been shown to be a function of the equivalent plastic strain and the strain rate of that moment. Also, unloading behavior was found to depend only on the loading equivalent plastic strain and the strain rate in the unloading step, meaning the unloading behavior is independent of the loading strain rate. The fact of considering just the loading strain rates, and scaling the unloading curves based on the loading rates, can lead to an error in modeling the residual stress distribution.

\section{REFERENCES}

H. Jahed and R. N. Dubey, "An Axisymmetric Method of ElasticPlastic Analysis Capable of Predicting Residual Stress Field," J. Press. Vessel Technol., vol. 119, no. 3, p. 264, 1997. doi: $10.1115 / 1.2842303$.

[2] H. Jahed, S. B. Lambert, and R. N. Dubey, "Variable material property method in the analysis of cold-worked fastener holes," J. Strain Anal. Eng. Des., vol. 35, no. 2, pp. 137-142, Feb. 2000. doi: 10.1243/0309324001514080.

[3] H. Jahed and S. Sherkatti, "Thermoplastic analysis of inhomogeneous rotating disk with variable thickness," in $E M A S$ Conference of Fatigue, 2000.

[4] E. Kalatehmollaei, H. Mahmoudi-Asl, and H. Jahed, "An asymmetric elastic-plastic analysis of the load-controlled rotating bending test and its application in the fatigue life estimation of wrought magnesium AZ31B," Int. J. Fatigue, vol. 64, pp. 33-41, 2014. doi: 10.1016/j.ijfatigue.2014.02.012.

[5] H. Jahed, B. A. Moghadam, and M. Shambooli, "ReAutofrettage," J. Press. Vessel Technol., vol. 128, no. 2, p. 223, 2006. doi: $10.1115 / 1.2172619$.

[6] Al-Hassani, "Mechanical aspect of residual stress development in shot peening," lst International Conference on Shot Peening. pp. 583-602, 1981.

[7] J. K. Li, Y. Mei, W. Duo, and W. Renzhi, "Mechanical approach to the residual stress field induced by shot peening," Mater. Sci. Eng., vol. 147, pp. 167-173, 1991. doi: $10.1016 / 0921-5093(91) 90843-C$

[8] A. S. Franchim, V. S. de Campos, D. N. Travessa, and C. de M. Neto, "Analytical modelling for residual stresses produced by shot peening," Mater. Des., vol. 30, no. 5, pp. 15561560, 2009. doi: 10.1016/j.matdes.2008.07.040. 
[9] H. Y. Miao, S. Larose, C. Perron, and M. Levesque, "An analytical approach to relate shot peening parameters to Almen intensity," Surf. Coatings Technol., vol. 205, no. 7, pp. 2055-2066, 2010. doi: 10.1016/j.surfcoat.2010.08.105.

[10] K. ichiro Mori, K. Osakada, and N. Matsuoka, "Finite element analysis of peening process with plasticity deforming shot," $J$. Mater. Process. Tech., vol. 45, no. 1-4, pp. 607-612, 1994.

[11] S. A. Meguid, G. Shagal, and J. C. Stranart, "3D FE analysis of peening of strain-rate sensitive materials using multiple impingement model," Int. J. Impact Eng., vol. 27, no. 2, pp. 119 134, 2002. doi: 10.1016/S0734-743X(01)00043-4.

[12] T. Kim, H. Lee, H. C. Hyun, and S. Jung, "Effects of Rayleigh damping, friction and rate-dependency on 3D residual stress simulation of angled shot peening," Mater. Des., vol. 46, pp. 2637, 2013. doi: 10.1016/j.matdes.2012.09.030.

[13] B. Bhuvaraghan, S. M. Srinivasan, B. Maffeo, and O. Prakash, "Analytical solution for single and multiple impacts with strain-rate effects for shot peening," C. -Comput. Model. Eng. Sci., vol. 57, no. 2, pp. 137-158, 2010.

[14] J. . Almen and P. . Black, Residual stresses and fatigue in metals. New york: McGrow Hill, 1963.

[15] T. Özel and Y. Karpat, "Identification of constitutive material model parameters for high-strain rate metal cutting conditions using evolutionary computational algorithms," Mater. Manuf. Process., vol. 22, no. 5, pp. 659-667, 2007. doi: $10.1080 / 10426910701323631$
[16] A. Gariépy, S. Larose, C. Perron, and M. Lévesque, "Erratum: Shot peening and peen forming finite element modelling Towards a quantitative method," Int. J. Solids Struct., vol. 83, p. 183 , 2016. doi: 10.1016/j.ijsolstr.2016.01.012.

[17] A. Ghasemi, S. M. Hassani-Gangaraj, A. H. Mahmoudi, G. H. Farrahi, and M. Guagliano, "Shot peening coverage effect on residual stress profile by FE random impact analysis," Surf. Eng., vol. 32, no. 11, pp. 861-870, 2016. doi: 10.1080/02670844.2016.1192336

[18] K. Murugaratnam, S. Utili, and N. Petrinic, "A combined DEM-FEM numerical method for Shot Peening parameter optimisation," Adv. Eng. Softw., vol. 79, pp. 13-26, 2015. doi: 10.1016/j.advengsoft.2014.09.001.

[19] M. Jebahi, A. Gakwaya, J. Levesque, O. Mechri, and K. Ba, "Robust methodology to simulate real shot peening process using discretecontinuum coupling method," Int. J. Mech. Sci., vol. 107, pp. 21-33, 2016. doi: 10.1016/j.ijmecsci.2016.01.005.

[20] H. Y. Miao, S. Larose, C. Perron, and M. Levesque, "On the potential applications of a 3D random finite element model for the simulation of shot peening," Adv. Eng. Softw., vol. 40, no. 10, pp. 1023-1038, 2009. doi: 10.1016/j.advengsoft.2009.03.013. 


\section{The Design and Validations of the Ultrasonic Tactile Sensor}

\author{
Yanjun Qian \\ Department of Mechanical and Mechatronics \\ University of Waterloo \\ Waterloo, Canada \\ y32qian@uwaterloo.ca
}

\author{
Hyock Ju Kwon \\ Department of Mechanical and Mechatronics \\ University of Waterloo \\ Waterloo, Canada \\ hjkwon@uwaterloo.ca
}

\begin{abstract}
An ultrasonic tactile sensor that can measure the stiffness of the tissue was developed. By combining analytical and numerical approaches, efficient design methodology was presented. The electrical and mechanical performance of developed sensor was experimentally validated.
\end{abstract}

\section{Ultrasonics; Tactile sensing; Equivalent circuit}

\section{INTRODUCTION}

Pathological changes in the tissues, such as tumors, affect the tissue stiffness. It has been reported that malignant tissues are several times stiffer than normal ones [1]. In clinical diagnosis, palpation is the oldest, but still the most commonly used technique by physicians $[2,3]$ to determine the stiffness of target regions. While palpation is simple, it is just a qualitative assessment and the results are widely open to user interpretation. To overcome these shortcomings, various species of tactile sensors have been developed. By integrating different types of the sensing mechanisms onto the tactile probe, one can gather haptic responses from the object in standard forms and digitize them into quantitative measurement with the help of modern signal processing techniques [2, 3].

Being a direct imitation of palpation, static-force based tactile sensors are the most common types of tactile sensors. A typical design of the force-based tactile sensor can be found in the work by Sangpradit et al. [4]. They used a robotic positioning mechanism to control the sensing position and compression depths and measured the static forces with a load cell to estimate the stiffness of inclusions inside a softer phantom. The sensing mechanism is straight-forward, and the raw data is easy to comprehend. However, it can induce severe deformations on the surface of the object, which makes the method inappropriate for deformation-sensitive operations like neurosurgery. Besides, unlike the lab phantom, the real geometry of the object is usually irregular and rough, which brings tremendous challenges to the precise displacement control.

On the other hand, vibro-tactile sensors utilize resonance phenomenon, thus can be applied to force-sensitive surfaces without inducing excessive deformations. Since the vibration characteristics are very sensitive to the environment in contact with the sensor, only minimal level of force to secure the contact with the object needs be applied; hence, no excessive pressure on the target surfaces is required. One of the examples of vibro-tactile sensors is the one developed by O.A. Lindahl et al. [5]. The device was intended to measure the stiffness of the human skin. The incident vibration signal is provided by the resonant circuit and the computer collects the filtered system response gathered by the piezoelectric pick-up. Instead of controlling the compression depths, the tactile behavior is bounded by the static force, which is done mechanically by limiting the deformation of the spring.

Based on the same principle as the Lindahl's device, our vibro-tactile sensor also works at ultrasonic frequency, but with an additional horn attached to increase the quality factor of the device. The enhanced frequency selectivity of the new design gave us a chance to use other parameters, such as quality factor and/or the sharpness of the impedance sweep response to assess the change in material properties of the target.

In this paper, the design procedures of our ultrasonic tactile sensor are presented, followed by experimental validations of mechanical and electrical performance.

\section{SENSOR DESIGN}

Fig. 1 shows the assembly of our ultrasonic tactile sensor.

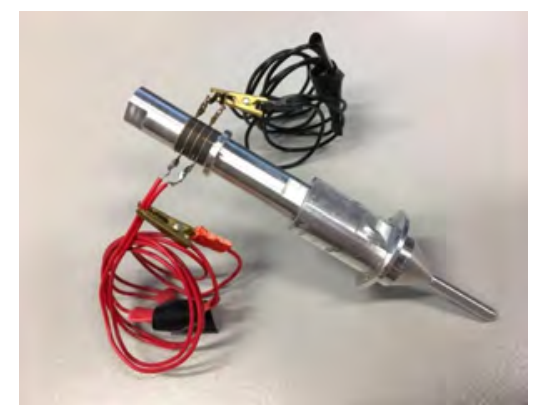

Figure 1. Full assembly of the ultrasonic tactile sensor

A commercially available Langevin-type piezoelectric transducer was employed as the vibrator. Usually, such transducer is not operated independently, but with a deliberately designed structure attached to the output end of the transducer with a stressed bolt connection. In the meantime, the additional attachment includes a design feature at its nodal section that permits contact with a peripheral structure without affecting the vibration behavior of the system. 
In tactile sensing application of the ultrasonic transducer, a vibration concentrator, a horn with a decreasing radius towards the output end, can greatly improve the sensing performance. The concentrator not only reduces the contact area of the output end (gives an edge in precise measurement), but also increases the quality factor $\mathrm{Q}$ of the current system. As a result, it enhanced the frequency selectivity of the device and made the load-induced response changes in frequency domain obvious. Therefore, an aluminum horn that resonates longitudinally at transducer's resonance frequency $(40 \mathrm{kHz})$ was fabricated and attached to the transducer with a bolt connection.

Last but not the least, as an interface between the electrical driving circuit and electro-mechanical transducer, two sets of wires were soldered onto the copper electrodes and were later clamped to the terminals of the impedance analyzer with clippers.

\section{A. Ultrasonic Transducer}

The transducer used in this research was a commercially available device designed to operate at $40 \mathrm{kHz}$ (Model: HEC1340P4BF, Honda Electronics, Japan). Technical drawings are available on their website. The typical application for this unit is ultrasonic machining where mechanical losses are the major concerns. This ensures that this type of piezoelectric transducer has a higher quality factor over its comparators. The power is not a critical design factor in this project, but the size is. So, the most compact model with the smallest power was selected.

\section{B. Horn Design}

The horn has a combinational shape of a step and a taper as shown in Fig. 1. Analytical derivation of precise design parameters for the horn is mathematically very challenging [68]. Instead, a hybrid approach was adopted in this study, i.e. initial guesses were extracted from a simple analytical model and then refine these parameters through iterative processes with the aid of FEA models.

In the initial guess stage, the horn was sketched out as a half-wavelength long stepped horn, which is a successive combination of two equally long cylinders of different diameters. Note that although a stepped horn design can be adopted for tactile sensor, it may introduce a large error between the model predictions and the actual behaviors because the continuum assumption in the pure analytical model does not hold well. To smooth the transition shape, a tapered feature was introduced to replace the step. To simplify the problem and simultaneously reduce the difficulty in machining, we have tried to avoid placing the fixture feature, the flange, at the tapered part. Next, in order to incorporate the tapered feature, we can replace the smaller end of the original design with a new cantilever stepped horn and smooth out the step with the slope afterwards (Fig. 2).

These dimensions from the analytical derivations of the simple 2D model were found according to the equivalent circuit theory based on impedance analogies as shown in Fig. 3 [9]. The electrical components' values can be derived as in (1). A MATLAB code was developed to solve for them numerically.

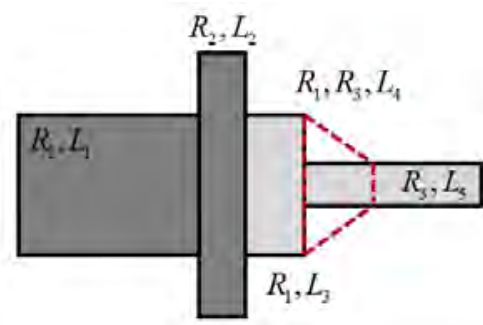

Figure 2. Design sketch for the horn: The darker grey part marks the initially designed half-wavelength (L1+L2) horn with larger diameter (R1) with the flange (R2) for fixation; The lighter grey part is the cantilever design output end (R3) with a total length of L3+L4+L5; And, finally the red dotted line shows the slope (smoothing transition from R1 to R3 in a length of L4)

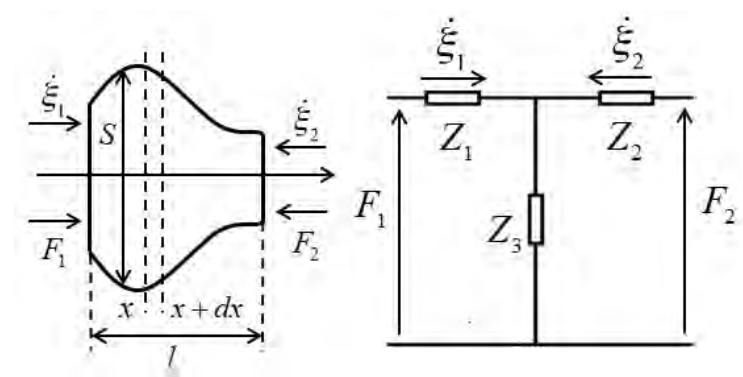

Figure 3. The analogy between the mechanical system and the corresponding electrical circuit (the impedance analogy)

$\left\{\begin{array}{l}Z_{1}=\frac{\rho c}{2 j k}\left(\frac{\partial S}{\partial x}\right)_{x=0}+\frac{\rho c K S_{1}}{j k} \cot K l-\frac{\rho c K \sqrt{S_{1} S_{2}}}{j k \sin K l} \\ Z_{2}=-\frac{\rho c}{2 j k}\left(\frac{\partial S}{\partial x}\right)_{x=0}+\frac{\rho c K S_{2}}{j k} \cot K l-\frac{\rho c K \sqrt{S_{1} S_{2}}}{j k \sin K l} \\ Z_{3}=\frac{\rho c K \sqrt{S_{1} S_{2}}}{j k \sin K l}\end{array}\right.$

where $K=\sqrt{k^{2}-\frac{1}{\sqrt{S}} \frac{\partial^{2} \sqrt{S}}{\partial x^{2}}}$ is the generalized wave number.

In the iterative FEA simulation stage, a parametric model was constructed with a commercially validated Multiphysics package COMSOL to finalize the design with parameter sweeps. The lengthwise dimensions were tuned accordingly until the resonance frequency of the design converged within 1 $\mathrm{kHz}$ offset from $40 \mathrm{kHz}$. Fig. 4 shows the final design of the horn.

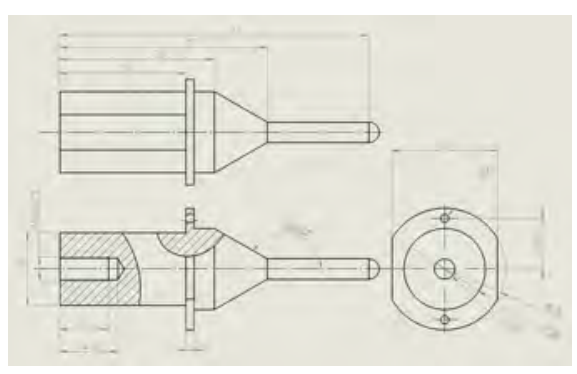

Figure 4. Final design for the horn 


\section{EXPERIMENTAL VALIDATIONS}

\section{A. Experiment Setups}

The first experiment was conducted to examine the mechanical behavior of fabricated horn. The test schematics is exhibited in Fig. 5.

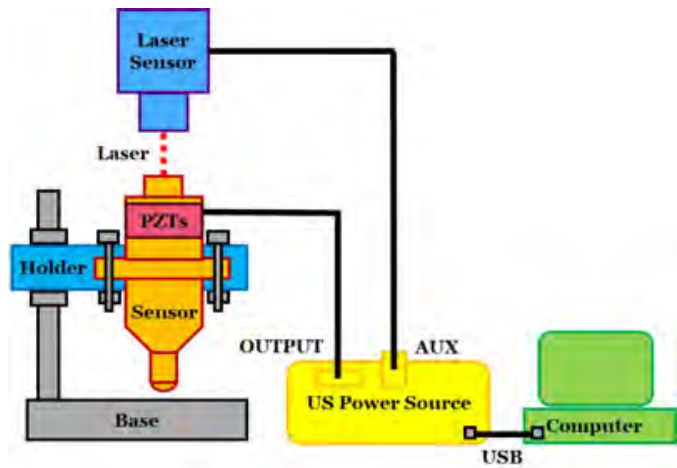

Figure 5. Test setup schematics for the design validation

A temporary holding mechanism was implemented to secure the measurement positions for the laser doppler vibrometer (Polytec OFV-505) in the experiment. The base was a bulky part made of steel, which is not sensitive to the incident vibrations generated by our sensor. The holder, made of aluminum, was fixed on the threaded pole with a set of nuts so that the height of the holder is adjustable. The sensor was fixed to the holder at its flange with two bolts. The active elements, PZTs in the transducer, were connected to the output LEMO connector on the ultrasonic power source (PDUS200, Micromechatronics, USA). The vibrometer was also connected to the power source via its auxiliary input $\mathrm{BNC}$ connector. The data were collected with a host computer using the controlling program for the power source via the USB connection. The actual equipment and layout are shown in Fig. 6.
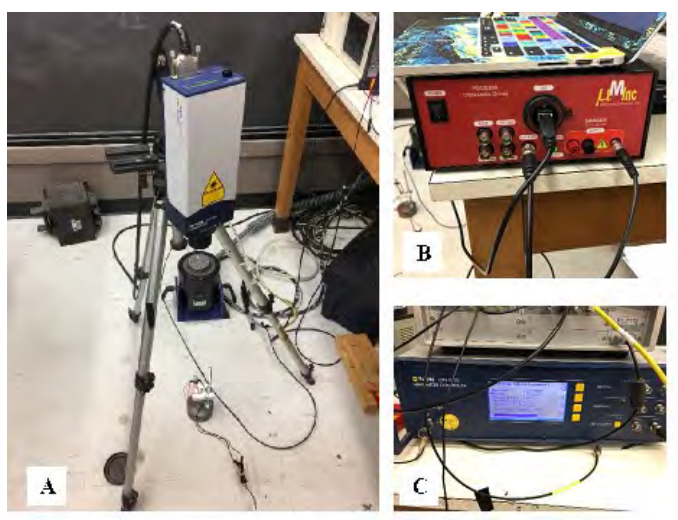

Figure 6. Actual equipment and layout of the test: A) The vibrometer head and the sensor assembly; B) The signal-collecting laptop and the ultrasonic power source; C) The vibrometer controller box

The AC signal generated by the ultrasonic power source was at the level of $20 \mathrm{Vpp}$, and the sensitivity of the laser doppler vibrometer was tuned to $1 \mathrm{um} / \mathrm{V}$. For each measurement, a complete cycle sweep was conducted, i.e., it swept and recorded from $38 \mathrm{kHz}$ to $42 \mathrm{kHz}$ and then backwards. The measurement was taken on both tip end and the back of the horn as in Fig. 7.

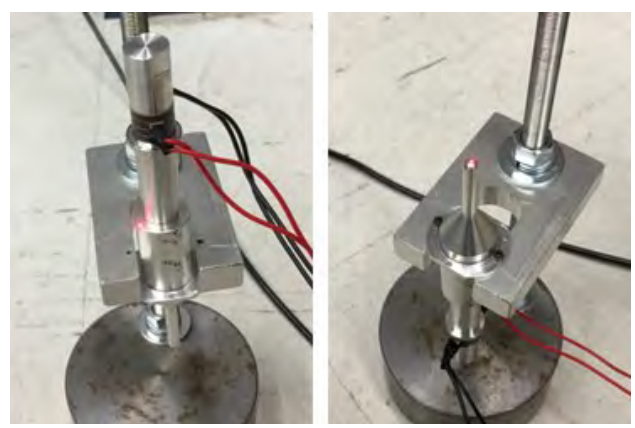

Figure 7. The laser measurement positions on the horn

The second experiment was conducted to investigate the electrical behavior of the total assembly. As discussed in the introduction, part of the designing purpose of the horn was to increase the quality factor of the sensor and thus increase the frequency selectivity. Therefore, frequency sweeps were conducted with the impedance analyzer (HP4194A) under the conditions with and without the horn.

\section{B. Mechanical Vibration Measurement Results}

The data collected from the experiments are plotted in Fig. 8(a) and the amplification ratio derived from Fig. 8(a) is plotted in Fig. 8(b).
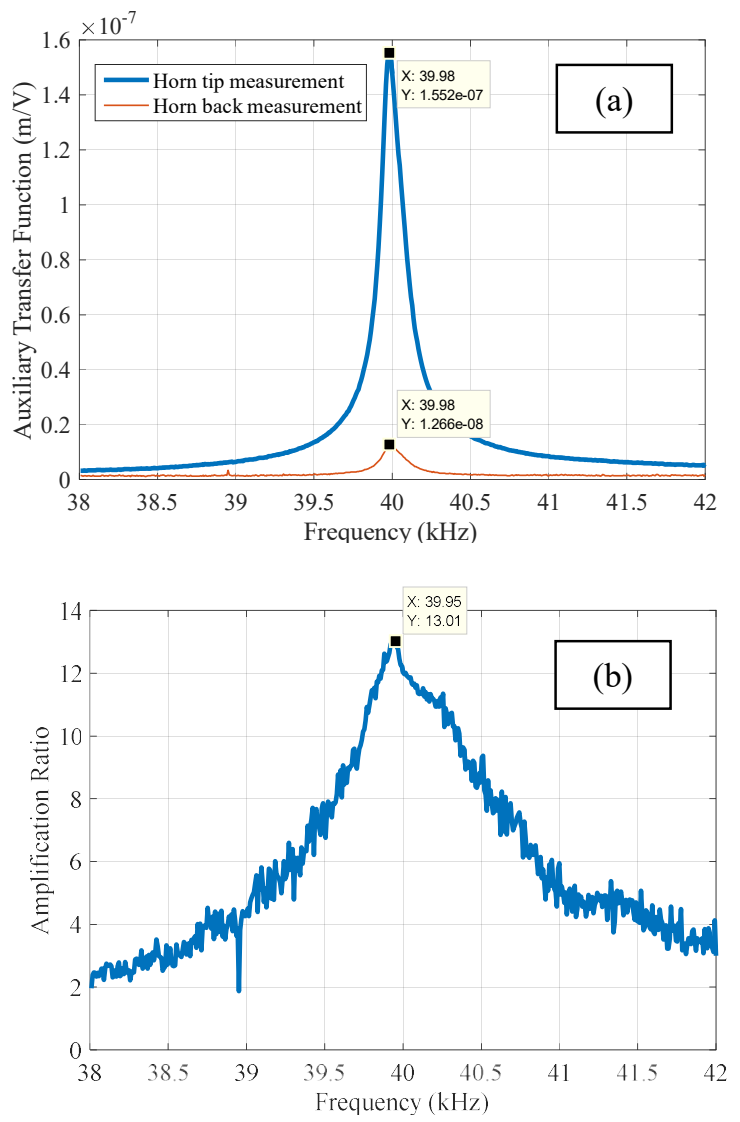

Figure 8. (a) experimental data; (b) The derived amplification ratio 
The auxiliary transfer function (ratio between output voltage and auxiliary input voltage) was also automatically recorded by the embedded function in the ultrasonic power source controller program. Combining the calibration information from the laser doppler vibrometer controller box, we can find the actual transfer function ratio between the vibration magnitude (in [m]) and the input AC voltage (in [V]). It can be inferred from Fig. 8 that the system has a clear mechanical resonance at $\sim 40 \mathrm{kHz}(39.98 \mathrm{kHz}$ to be exact).

Amplification ratio shown in Fig. 8(b) followed the similar trend to the transfer function in Fig. 8(a), and the maximum amplification ratio was 13.01 at $39.95 \mathrm{kHz}$.

\section{Electrical Measurement Results}

Fig. 9 shows the impedance and phase angle responses of the ultrasonic device with and without the horn attached.
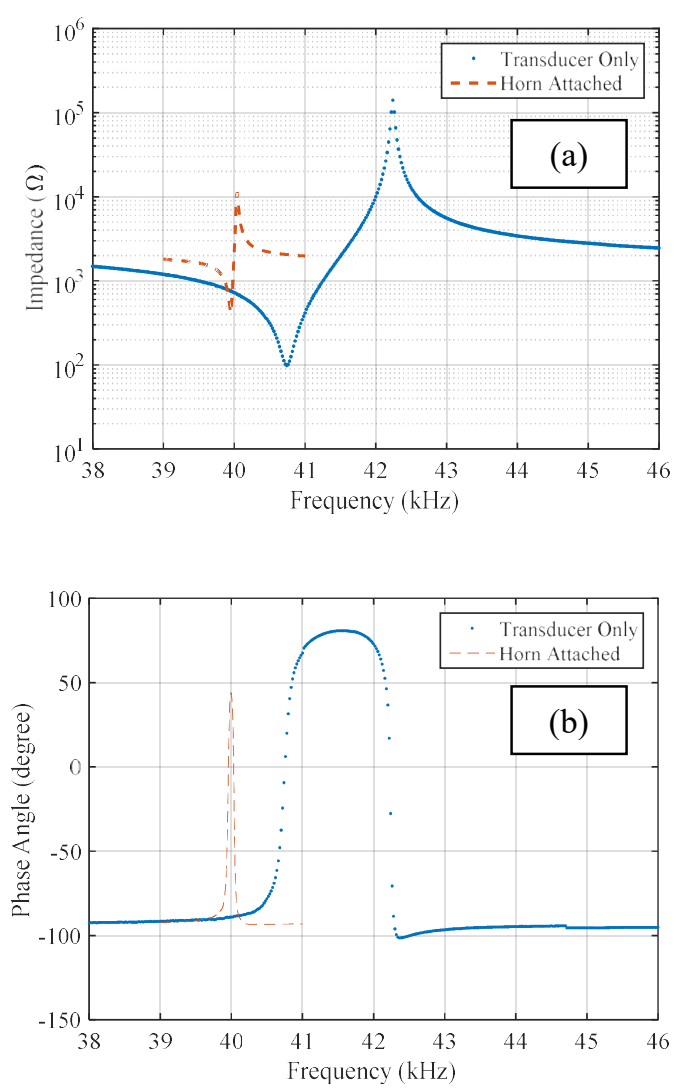

Figure 9. (a) The impedance response of the device; (b) The phase response of the device

Different ranges of frequency were used for the sweeps due to the obvious discrepancies in both responses. Both the series resonance frequency (close to the frequency where the impedance reaches minimum in Fig. 9(a) and the lower frequency where the phase angle is 0 in Fig. 9(b)) and the parallel resonance frequency (close to the frequency where the impedance reaches maximum in Fig. 9(a) and the higher frequency where the phase angle is 0 in Fig. 9(b)) shifted lower and became closer to each other (reduced difference from $2 \mathrm{kHz}$ to less than $1 \mathrm{kHz}$ ) around $40 \mathrm{kHz}$ when the horn was attached, which resulted in a steeper change from the lowest impedance to the highest impedance in Fig. 9(a) and a sharper response in the phase angle in Fig. 9(b).

\section{Comparisons and Discussions}

Table I presents the results from the analytical derivation, numerical simulation in COMSOL, and experimental measurement.

TABLE I. DATA COMPARISONS

\begin{tabular}{|c|c|c|}
\hline \multirow{2}{*}{ Source } & \multicolumn{2}{|c|}{ Results } \\
\cline { 2 - 3 } & Resonance Frequency $(\boldsymbol{k H z})$ & Amplification Ratio \\
\hline Analytical & 40.00 & $>7.2$ \\
\hline Numerical & 40.61 & 11.4 \\
\hline Experimental & 39.95 & 13.01 \\
\hline
\end{tabular}

The experimental results show good agreement with the analytical and the numerical results. This clearly indicates that the "guess and optimize" approach is a valid way to design the current device, and mechanically, the ultrasonic sensor was working as expected.

As for the electrical responses, significant changes can be observed in Fig. 9. The responses were much "narrower" when the horn was attached to the transducer, which is a strong indication of the increase of quality factor $Q$, as pursued by our design.

In conclusion, the ultrasonic horn design was successfully performed through the hybrid approach by taking advantage of the modern computing power and the programable software package. By integrating the ultrasonic horn into the system, the gap between both electrical resonance frequencies was significantly reduced, which made the identification of the mechanical resonance frequency (located somewhere between two electrical resonance frequencies) more accurate and easier. The increased Q factor suggests the feasibility of using Q factor deductions as a supplementary parameter to determine the mechanical property changes in the target objects.

\section{ACKNOWLEDGMENT}

This research was supported by Natural Sciences and Engineering Research Council of Canada (NSERC).

\section{REFERENCES}

[1] E. L. Baker, J. Lu, D. Yu, R. T. Bonnecaze, and M. H. Zaman, "Cancer cell stiffness: Integrated roles of three-dimensional matrix stiffness and transforming potential," Biophys. J., vol. 99, no. 7, pp. 2048-2057, 2010. doi: 10.1016/j.bpj.2010.07.051.

[2] M. I. Tiwana, S. J. Redmond, and N. H. Lovell, "A review of tactile sensing technologies with applications in biomedical engineering," Sensors Actuators, A Phys., vol. 179, pp. 17-31, 2012. doi:10.1016/j.sna.2012.02.051.

[3] M. Sadeghi-Goughari, A. Mojra, and S. Sadeghi, "Parameter estimation of brain tumors using intraoperative thermal imaging based on artificial tactile sensing in conjunction with artificial neural network," J. Phys. D. Appl. Phys., vol. 49, no. 7, 2016. doi: 10.1088/0022-3727/49/7/075404. 
[4] K. Sangpradit, H. Liu, P. Dasgupta, K. Althoefer, and L. D. Seneviratne, "Finite-element modeling of soft tissue rolling indentation," IEEE Trans.

Biomed. Eng., vol. 58, no. 12 PART 1, pp. 3319-3327, 2011.

[5] O. A. Lindahl, S. Omata, and K.-A. Ängquist, "A tactile sensor for detection of physical properties of human skin in vivo," J. Med. Eng. Technol., vol. 22, no. 4, pp. 147-153, 1998.

doi: 10.3109/03091909809032532.

[6] A. Mohammed, "Equivalent circuits of solid horns undergoing longitudinal vibrations," J. Acoust. Soc. Am., vol. 38, no. 5, pp. 862- 866, 1965. doi: 10.1121/1.1909817.

[7] S. Sherrit, B. P. Dolgin, Y. Bar-Cohen, D. Pal, J. Kroh, and T. Peterson, Modeling of horns for sonic/ultrasonic appications, 1999 IEEE Ultrasonics Symposium. Proceedings International Symposium, 199. doi: 10.1109/ULTSYM.1999.849482.

[8] S. Lin, "Study on the longitudinal-torsional composite mode exponential ultrasonic horns," Ultrasonics, vol. 34, no. 7, pp. 757-762, 1996.

doi:10.1016/0041-624X(96)00062-5.

[9] Y. J. Qian, S. W. Han, and H. J. Kwon, "Development of Ultrasonic Surface Treatment Device," Applied Mechanics and Materials, 2016, vol. 835 , pp. 620-625. 


\title{
Investigating the Effect of a Speckle Pattern on Measurement Uncertainty in a Three-Dimensional Digital Image Correlation (3D-DIC) System
}

\author{
Calvin C. Jee, Gabriel B. P. Salata, David S. Nobes, Kajsa K. Duke \\ Department of Mechanical Engineering \\ University of Alberta \\ Edmonton, Alberta \\ kkduke@ualberta.ca
}

\begin{abstract}
Three-dimensional digital image correlation (3D-DIC) is an imaging technique that uses cameras to measure the surface displacement of a speckled specimen under test loading from which surface strains can be derived. This study aims to investigate the effect of the speckle pattern on the uncertainty in the measurement system. A Monte-Carlo experimental approach is used by uniformly displacing a known speckle pattern by a prescribed amount. This allows the coupled influence of the image collection system, processing and post-processing to be investigated. To minimize the uncertainty of a speckle pattern, it was determined that uniform speckle size of 5-pixel diameter speckles at a density of one speckle per 20 square-pixels is optimal. The methods used to measure and analyze the speckle pattern effects on measurement uncertainty are presented.
\end{abstract}

Keywords-digital image correlation; measurement uncertainty; speckle pattern; strain measurement

\section{INTRODUCTION}

Three-dimensional digital image correlation (3D-DIC) is a measurement technique that uses two cameras in a stereo configuration to measure all three surface displacement components of a specimen [1]. This includes bulk movement of the specimen as well as local movement generated by load and deformation of the sample. Each camera captures two images of a specimen at different stages of the experimental testing. The stereo pairs are used to locate the position of the surface and the difference between successive image pairs is used to calculate the surface displacement.

Some surfaces are intrinsically difficult to track with cameras as they may be smooth, featureless or reflective. This is typically addressed by introducing a random speckle pattern to the surface of the specimen. The approach generates visibly contrasting surface features within the images to be tracked in a manner that is minimally invasive to the specimen surface properties. However, there may be large variations in different speckle patterns due to the difficulty in controlling the application of the speckle. This potentially results in different qualities of data between speckled specimens and even within a single specimen that has varying speckle quality. The objective of this investigation is to determine which characteristics of a speckle pattern most significantly contribute to uncertainty in a 3D-DIC measurement system [2].

\section{Methodology}

\section{A. Materials}

The image data are captured using a pair of typical commercial machine vision cameras (GX-3300, Allied Vision Prosillica) mounted in a stereo configuration with $\mathrm{a} \pm 40^{\circ}$ angle offset from the viewing region-of-interest (ROI) or target, as shown in Figure 1. These are high resolution cameras, each capturing an image $3296 \times 2472$ pixels with an 8-bit resolution. The cameras were equipped with $80 \mathrm{~mm}$ fixed length commercial SLR lenses (\#2.8, Arax) with a $5^{\circ}$ tilt angle. The tilt allows rotation and overlapping of the focal planes of both cameras to the same ROI. An LED white light source (CN-600SA, Nanguang Photo \& Video Systems Co.) was used for illumination. Using in-house software, cameras were controlled and images were collected and stored for later post processing.

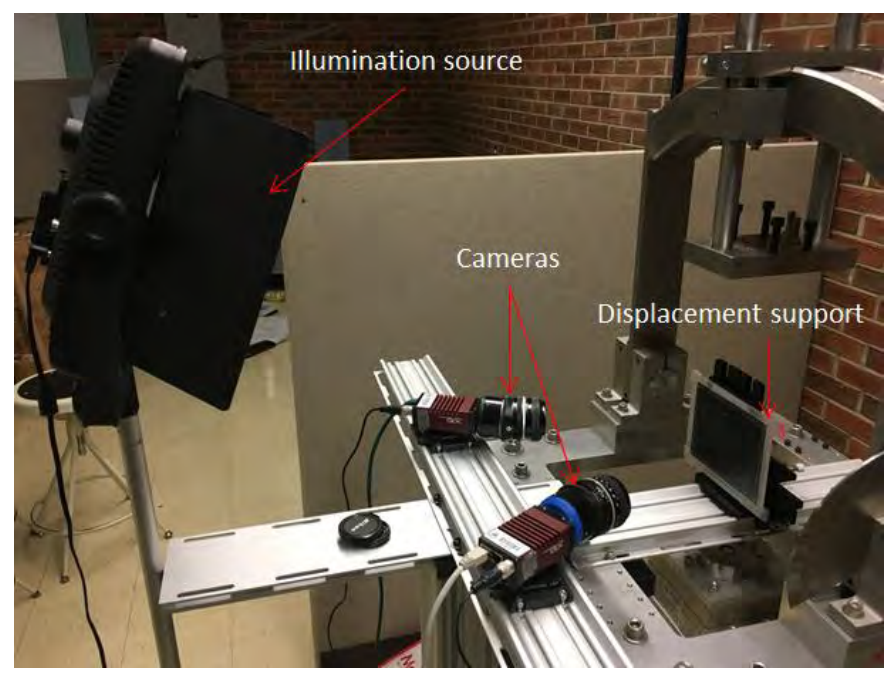

Figure 1. The 3D-DIC measurement system setup for sample testing.

\footnotetext{
Sponsors: NSERC, CFI.
} 
The test specimens were speckle patterns that were generated by a widely used open source software [3] and printed on paper. The software allowed randomized speckle patterns of prescribed speckle densities, speckle sizes and speckle size variations to be generated. Speckle patterns of varying characteristics were printed on printing paper at 1440 dots per inch (dpi). An example comparing the file images of two of the different speckle patterns tested is shown in Figure 2 . Note that these images are the same physical size.

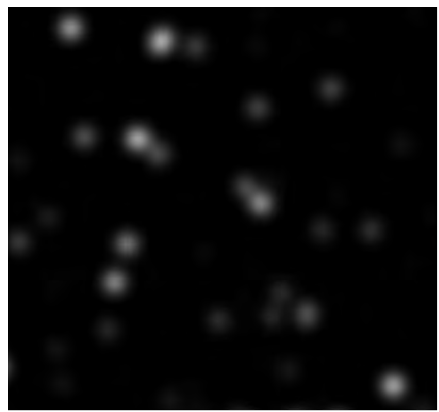

(a)

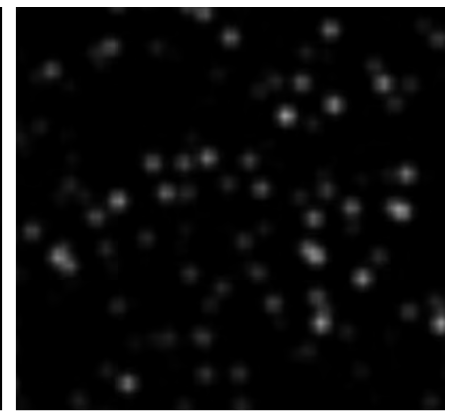

(b)
Figure 2. Comparison of file images of two different speckle patterns: (a) larger speckles at low density, and (b) smaller speckles at high density.

The printed speckle specimens were mounted onto a flat target that was connected to an optomechanical fixture that included a traversing stage. The movements of the stage were controlled and measured by a micrometer allowing movement in only one direction, as shown below in Figure 3. This allowed for the speckle sample to be displaced perpendicularly towards and away from the cameras in a general out-of-plane direction.

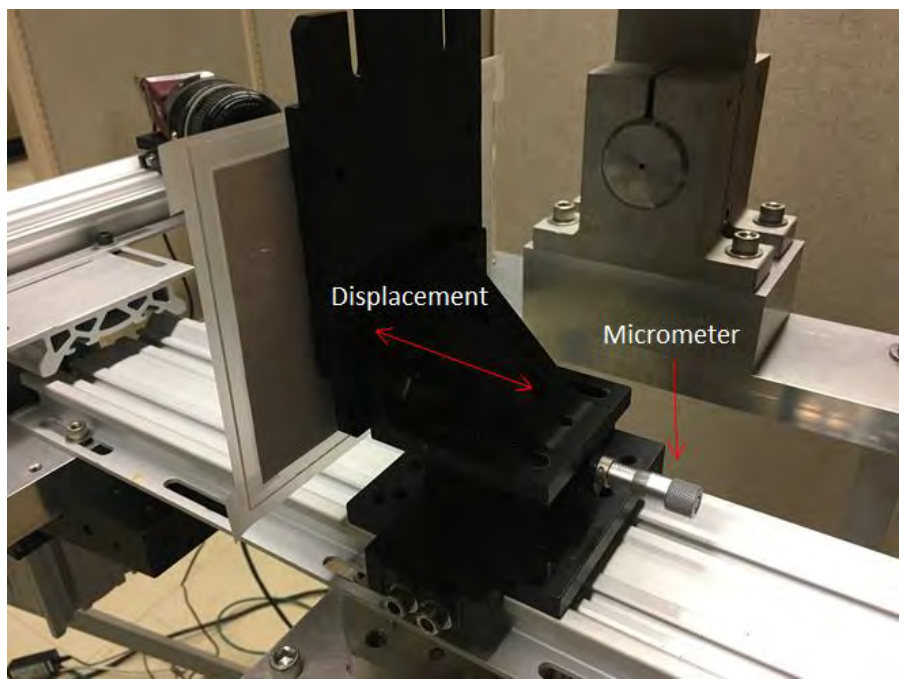

Figure 3. Displacement of micrometer-controlled stage.

\section{B. Digital Image Correlation}

Commercial DIC software (DaVis 8.4 , LaVision $\mathrm{GmbH}$ ) was used to analyze the image data. Camera calibration was conducted with a 3D calibration plate (Type 11, LaVision $\mathrm{GmbH}$ ) that contains a known rectangular dot pattern of known dot size and dot spacing at two different surface heights. Each camera captured an image of the calibration plate from their respective fixed positions. The DIC software used these two images to spatially locate the cameras, allowing for the conversion of image data to physical space. The stereo cameras were located in a coordinate system associated with the location and orientation of the calibration target. The calibration target was generally aligned with the coordinate system of the experiment. Images captured post calibration were collected in the coordinate system of cameras, then dewarped and overlapped into the same coordinate system as the calibration target.

The following procedure was used to measure each speckle test specimen. First, a reference image was taken at the position of best focus relative to the cameras. All subsequent displacements were measured in relation to this image. Displacements of $1 \mathrm{~mm}$ steps were made from this point in the out-of-plane direction towards the cameras up to $5 \mathrm{~mm}$. This direction was in the coordinate system of the experiment for which the misalignment with a coordinate system of the cameras was corrected.

After the data were collected, 3D-DIC processing was conducted to extract physical displacements from the image data. To do this, a sub-region, or window, of the image was observed and an image cross-correlation algorithm was conducted between the same window of two different images: a measured displacement image and the reference image. The window of the displaced image was then deformed and compared with the reference image to in an attempt to find a better correlation between the windows of both images. This reoccurred until a better match between the windows could not be found - the final window transformation was the resultant displacement vector of the window. The process was repeated using equally sized and equally spaced windows across the whole image. For all sample displacement images, the result was a displacement field spanning the entire image. The displacement field was then analyzed to determine the uncertainty of the measurement as detailed below.

\section{Design of Experiments}

A $2^{3}$ full factorial experiment based on design of experiments (DOE) was the approach used to quantify how individual characteristics of a speckle pattern affect measurement uncertainty. The responses of this experiment were the two properties used characterize uncertainty: precision and accuracy. The precision was defined as the width of the distribution of measurements in a displacement field, whereas the accuracy was defined as a measure of the difference between the expected displacement, defined by the movement of the micrometer in the experiments, and the average value of all displacement vectors in a displacement field. Thus, the highest quality speckle pattern (i.e., the one with the least uncertainty) was quantifiably the most precise and accurate [4].

The factor combinations for each sample are shown in Table 1. The three input variables were the speckle density, the speckle size and variation in the speckle size. The $2^{3}$ experimental design allowed both main effects and effects of interactions between two factors to be observed. Each input was tested at two levels, and the samples were different combinations of input levels. 
Table 1. Experiment sample factor combinations

\begin{tabular}{|c|c|c|c|}
\hline $\begin{array}{c}\text { Sample } \\
\text { no. }\end{array}$ & $\begin{array}{c}\text { Speckle } \\
\text { density } \\
\left(\# / \text { pixel }^{2} \text { ) }\right.\end{array}$ & $\begin{array}{c}\text { Speckle } \\
\text { diameter } \\
\text { (pixel) }\end{array}$ & $\begin{array}{c}\text { Speckle size } \\
\text { variation } \\
( \pm \text { pixel) }\end{array}$ \\
\hline 1 & 0.20 & 10 & 0 \\
\hline 2 & 0.20 & 10 & 3 \\
\hline 3 & 0.20 & 5 & 0 \\
\hline 4 & 0.20 & 5 & 3 \\
\hline 5 & 0.05 & 10 & 0 \\
\hline 6 & 0.05 & 10 & 3 \\
\hline 7 & 0.05 & 5 & 0 \\
\hline 8 & 0.05 & 5 & 3 \\
\hline
\end{tabular}

As the focus of this study was to investigate the effect of the speckle pattern on the derived displacement, all other parameters were held constant. These included the camera components and positions, the optomechanical components used and the illumination source. The constant parameters of the speckle specimen were the type of paper used and the print settings.

\section{RESULTS AND DISCUSSION}

From the DIC measurements, displacement fields were generated for each measured sample displacements. Because there was no material or local deformation occurring due to loading of the sample, the displacement was expected to be uniform across the entire region and equal to that physically determined by the micrometer. However, this was not the case such as in Figure 4. The measured out-of-plane displacement field, shown in Figure 4(a), shows that the displacement vectors generated by the $3 \mathrm{D}$-DIC processing for a single speckle pattern were not be identical throughout the entire field. From all measured displacement vectors in the entire field, the probability density function (PDF) in Figure 4 (b) was generated. The difference between the mean and the prescribed displacement was the accuracy of the measurement. The spread of the measurement, quantified by the standard deviation, was the precision. The combination of the two characterize and quantify the uncertainty of the measurement. This measurement procedure was used for every displacement of each speckle sample to compare the uncertainties between speckle patterns of known varying parameters.

After calculating the precision and accuracy of all measurements, the DOE $2^{3}$ full factorial experiment data was analyzed using commercial software (Minitab 18, Minitab Inc.) as summarized in Table 2 below. This statistical analysis used a standard $p$-value $<0.05$ to determine if a main effect or an interaction effect was significant. The analysis showed that for accuracy, all factors had a $p$-value that suggested significance. However, for precision the most significant factors were speckle size variation followed by the effect of the interaction between speckle density and speckle size.

Table 2. Significance of speckle pattern parameters

\begin{tabular}{|c|c|c|}
\hline \multirow{2}{*}{ Factor } & \multicolumn{2}{|c|}{ P-value } \\
\cline { 2 - 3 } & Accuracy & Precision \\
\hline Speckle density & $<\mathbf{0 . 0 0 1}$ & 0.395 \\
\hline Speckle diameter & $\mathbf{0 . 0 0 7}$ & 0.588 \\
\hline Speckle size variation & $<\mathbf{0 . 0 0 1}$ & $\mathbf{0 . 0 2 3}$ \\
\hline $\begin{array}{c}\text { Interaction } \\
\text { (density/diameter) }\end{array}$ & $<\mathbf{0 . 0 0 1}$ & $<\mathbf{0 . 0 0 1}$ \\
\hline $\begin{array}{c}\text { Interaction } \\
\text { (density/size variation) }\end{array}$ & $<\mathbf{0 . 0 0 1}$ & 0.690 \\
\hline $\begin{array}{c}\text { Interaction } \\
\text { (diameter/size variation) }\end{array}$ & $<\mathbf{0 . 0 0 1}$ & 0.454 \\
\hline
\end{tabular}
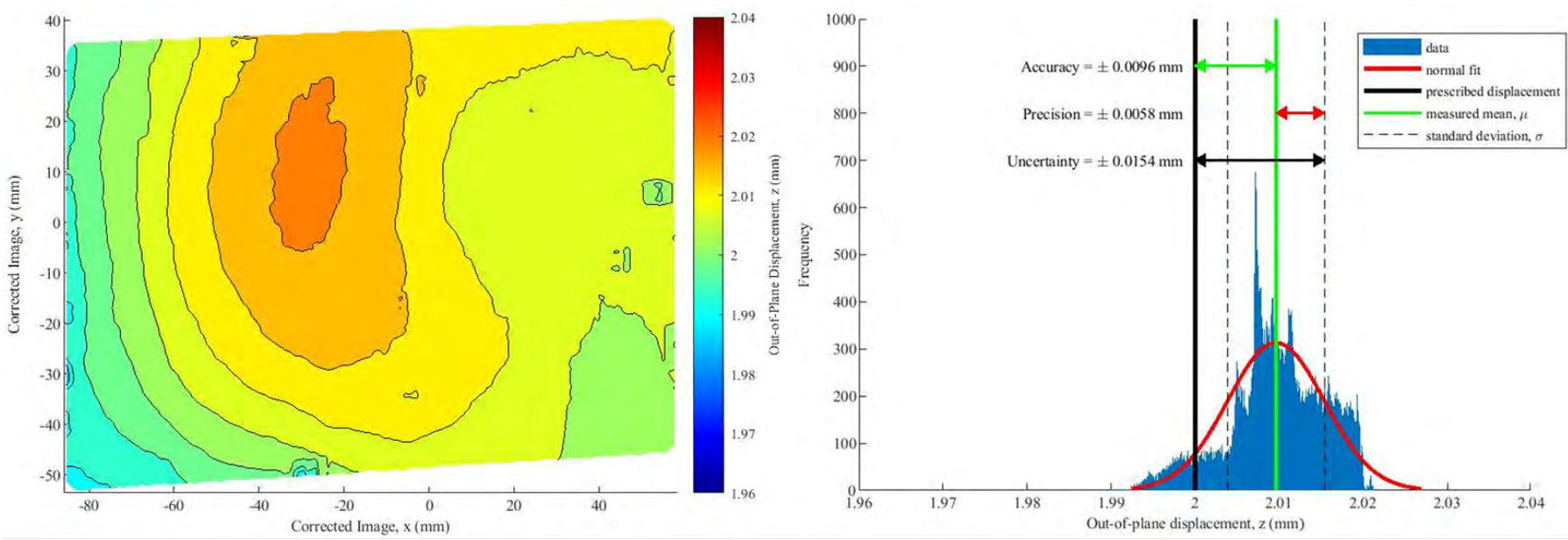

(b)

Figure 4. Displacement of a speckle sample 1 by $2 \mathrm{~mm}$, showing (a) measured displacement vector field and (b) resultant PDF used to determine accuracy and precision of measurement and resultant uncertainty. 
From the means plots of the factors significant to the precision in Figure 5, the most precise speckle pattern had speckle size uniformity and either larger speckles with higher density or smaller speckles with lower density. The difference between either interaction settings was minimal at these levels.

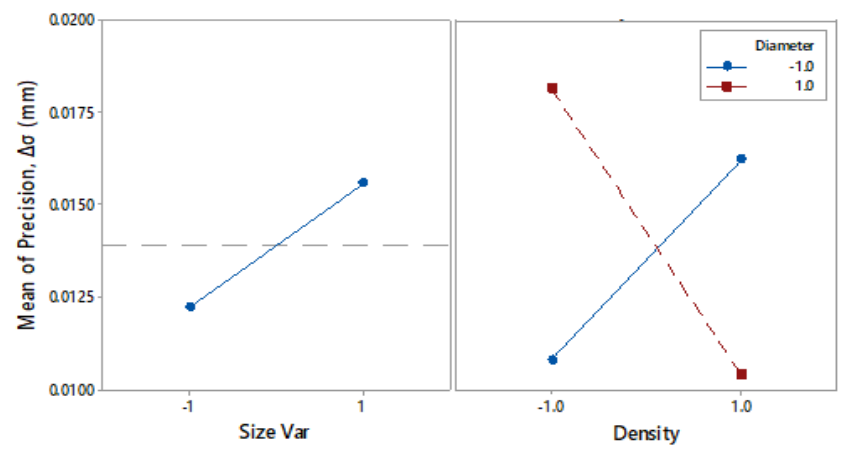

Figure 5. Means plots of effects significant to precision measurement (left speckle size variation; right - speckle density and diameter interaction).

The reason for the reduction of precision from speckle size variation may be due to larger speckles (or larger empty spaces) physically dominating the correlation window. As a result, the smaller speckles have a less significant effect in the DIC process. This means that the displacement measurement is more heavily influenced by fewer particles, resulting in an effectively lower sample size and increasing the standard deviation of the total measurement.

The interaction between speckle density and speckle diameter was the most significant factor regarding precision. Larger speckles at low density may reduce precision similarly to the speckle size variation effect; single large speckles may be too dominant in a single window. Regarding smaller speckles at too high a density, there may be too many speckles in a single correlation window such that they appear as noise that is difficult to track. It also may be a limitation of using speckles printed on paper. The details of a small speckle may be too fine to capture on the prints and in the camera image data. Consequentially, they can appear blurry and may not represent the speckles as accurately as intended. A comparison of a camera image from the experiment and its respective original file is shown in Figure 6.

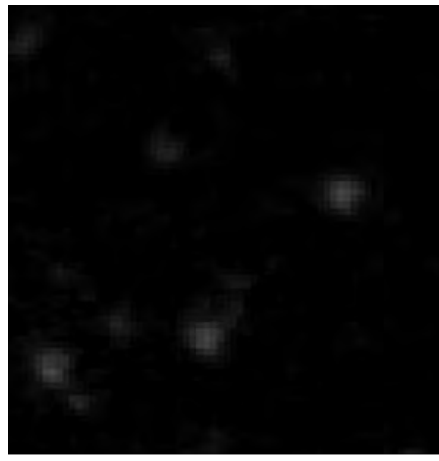

(a)

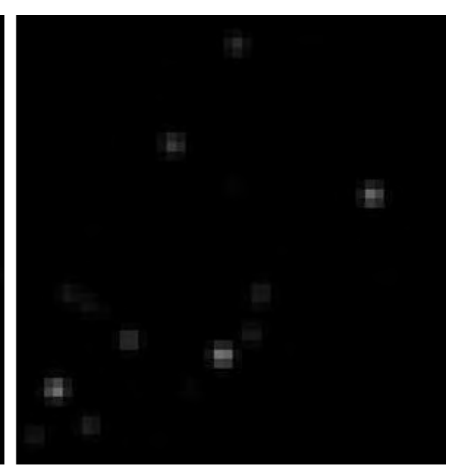

(b)
Figure 6. Comparison of (a) camera image data to (b) original file.
Because all factors were observed as statistically significant regarding accuracy, the pareto chart shown in Figure 7 was used to determine if any factors were more significant than others. The size variation and the interaction between the speckle diameter and density were the most significant in this scenario as well. Because the interactions involving speckle size variation and the other two factors were more significant than the main effects, the remaining factors may be significant by association only. Therefore, heavier emphasis was placed on the main effect of the speckle size variation and the interaction effect of the speckle diameter and density interaction.

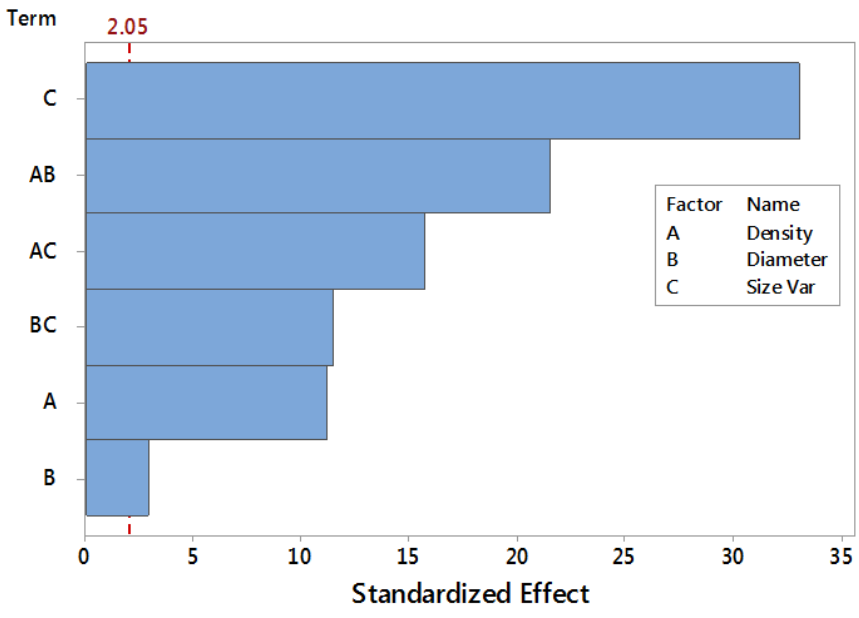

Figure 7. Pareto chart showing factor effects with respect to accuracy.

From Figure 8, the means plots for accuracy showed that the smallest uncertainty resulted from the speckle pattern with lower density, smaller speckle diameters, and speckle size uniformity. Like the statistical analysis of measurement precision, the interaction effect of speckle density and speckle diameter yielded higher accuracy with larger speckles at high density or smaller speckles at low density. However, greater accuracy resulted from the latter. This suggests that the optimal speckle characteristics for minimizing uncertainty (regarding both precision and accuracy) are 5-pixel diameter speckles at a density of 0.05 speckles per square-pixel with no variation in speckle size.

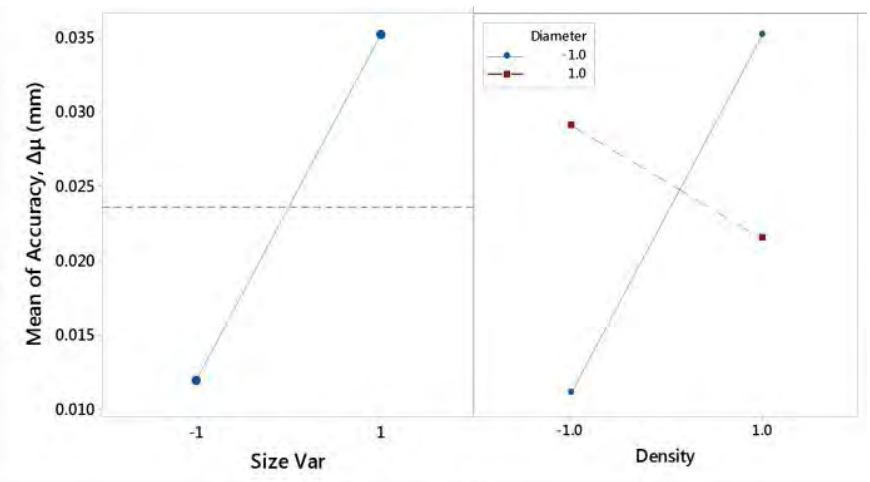

Figure 8. Means plots of effects significant to accuracy measurement (left speckle size variation; right - speckle density and diameter interaction). 
The next step would be to verify the results with physically air-brushed speckled specimens. Along with validating the results, this process would also further investigate what can physically be reproduced and what can practically be applied in an experiment setting using DIC. Additionally, it may be easier to acquire a less blurry image of a speckle pattern with the airbrush method as opposed to the printed paper method, potentially resolving this issue. Through these measures, a greater understanding of the sources of uncertainty and the approaches to limitations within a DIC measurement system can be established.

A major limitation of this study is that the specimen is flat. This serves as a necessary control in the study, but most 3D-DIC applications observe contoured surfaces. 3D surface profiles will be investigated in a future study. Also, this study only measures out-of-plane movement as in-plane movement is expected to be less significant in the overall uncertainty of the measurement of displacement in a stereo setting [5].

\section{CONCLUSION}

This study examined how the characteristics of a speckle pattern affect measurement uncertainty. Using DOE, the effects of input variables and interactions between variables were observed and it was shown that speckle size variation and the effect of the interaction between speckle density and speckle diameter are the most significant effects regarding DIC measurement uncertainty. Within the bounds of this experiment, the optimal speckle pattern was determined to have 5-pixel diameter speckles, a density of one speckle per 20 square-pixel area, and speckle size uniformity. The knowledge from this investigation is significant in understanding the sources of uncertainty within a DIC measurement system, as well as knowing how to effectively approach future applications of DIC.

\section{ACKNOWLEDGMENTS}

The authors acknowledge financial support from Natural Sciences and Engineering Research Council (NSERC) of Canada, the Alberta Ingenuity Fund and the Canadian Foundation for Innovation (CFI).

\section{REFERENCES}

[1] M. N. Helfrick, C. Niezrecki, P. Avitabile, and T. Schmidt, “3D digital image correlation methods for full-field vibration measurement," Mech. Sys. Sig. Processing, vol. 25, no. 3, pp. 917-927, Apr. 2011. doi: 10.1016/j.ymssp.2010.08.013.

[2] S. Yaofeng and J. H. L. Pang, "Study of optimal subset size in digital image correlation of speckle pattern images," Optics and Lasers in Engineering, vol. 45, no. 9, pp. 967-974, Sep. 2007. doi: 10.1016/j.optlaseng.2007.01.012.

[3] W. Thielicke, "PIVlab - particle image velocimetry (PIV) tool," 7 February 2018. [Online]. Available: https://www.mathworks.com/matlabcentral/fileexchange/27659pivlab-particle-image-velocimetry--piv--tool?s_tid=gn_loc_drop. [Accessed 8 February 2018.

[4] H. Haddadi and S. Belhabib, "Use of rigid-body motion for the investigation and estimation of the measurement errors related to digital image correlation technique," Optics and Lasers in Engineering, vol. 46, no. 2, pp. 185-196, Feb. 2008. doi: 10.1016/j.optlaseng.2007.05.008.

[5] N. J. Lawson and J. Wu, "Three-dimensional particle image velocimetry: experimental error analysis of a digital angular stereoscopic system," Measurement Science and Technology, vol. 8, no. 12, pp. 1455-1464, Dec. 1997. doi: $10.1088 / 0957-0233 / 8 / 12 / 009$. 


\section{Natural Frequencies of Internally-Doubly-Tapered Laminated Composite Beams}

\author{
Amin Fazili \\ Department of Mechanical, Industrial \& Aerospace \\ Engineering \\ Concordia University \\ Montreal, Canada
}

\author{
Rajamohan Ganesan \\ Department of Mechanical, Industrial \& Aerospace \\ Engineering \\ Concordia University \\ Montreal, Canada
}

\begin{abstract}
In the present study, the free vibration response of symmetric thickness-and-width-tapered (doubly-tapered) laminated composite beams is investigated. Considering the continuous-plies-interspersed ply drop-off configuration, called herein as taper configuration $\mathrm{D}$, both conventional and hierarchical finite element formulations are developed based on the cylindrical bending theory of laminated beam. Natural frequencies of different types of internally-tapered composite beams are determined. Comparison of the hierarchical finite element solution with the Rayleigh-Ritz solution and a higherorder finite element solution is performed. A parametric study is conducted to investigate the effects of width-taper-ratio and the thickness-taper-ratio (or equivalently thickness-tapering angle) on the free vibration response of doubly-tapered laminated composite beams.
\end{abstract}

Keywords: Tapered Composite Beam, Free Vibration, Hierarchical Finite Element Method, Natural Frequencies

\section{INTRODUCTION}

Composite material refers to material that is created by the synthetic assembly of two or more organic or inorganic materials in order to obtain specific material properties such as high strength and stiffness to weight ratios, corrosion resistance, thermal properties, fatigue life, wear resistance and increased tolerance to damage [1]. In some specific applications of composite structures such as helicopter yoke or turbine blades, the laminate needs to be stiff at one location and flexible at another location. This type of structure is created by terminating or dropping-off selected plies at specific locations to reduce the stiffness, and is called as the tapered composite structure [2].

Finite Element Method (FEM) is one of the most accurate and powerful tools used to study the response of complex mechanical structures such as the vibration of laminated composite beams. The convergence and accuracy of the results determined using finite element formulation depend strongly on the selected type of element. The type of element that is considered for the finite element formulation in this study has two degrees of freedom per node (deflection and rotation) and two nodes per element. In Conventional Finite Element Method (CFEM), interpolation or shape functions are determined based solely on these degrees of freedom. In the Hierarchical Finite Element Method (HFEM), a number of trigonometric terms are added to these interpolation functions in a hierarchical manner.

There are several studies on the free vibration of laminated composite beams, mostly on the subject of uniform-thickness uniform-width beams. Free vibration of uniform laminated composite beams was studied by Abarcar and Caniff [3], Chandrashekhara et al. [4], Marur and Kant [5] and Miller and Adams [6]. Reddy [7], Berthelot [8], Whitney [9], and Jones [10] have given the exact solutions for the free vibrations of uniform laminated composite beams. Vinson and Sierakowski [11] determined the exact natural frequencies of a simply-supported uniform composite beam based on classical laminate theory.

There are selectively few studies conducted on tapered composite beams. He et al. [12] have conducted a review of the works on tapered laminated composite structures. Ganesan and Zabihollah have studied thickness-tapered laminated composite beams $[13,14]$ using a higher-order but conventional finite element formulation and performed a parametric study. Ahmed [2] has studied and conducted experiments on free and forced vibration response of thickness-tapered composite beams including the effects of axial force and damping. Badagi [15] conducted the free and forced vibration analysis of thicknessand-width-tapered laminated composite beams using RayleighRitz method. Farghaly and Gadelrab [16] have studied the free vibration of stepped uniform-width thickness-tapered Timoshenko composite beams. Salajegheh and Ganesan [17] have studied the vibrations of thickness-and-width-tapered laminated composite beams with rigid and elastic supports using a higher-order but conventional finite element.

Hierarchical Finite Element Method (HFEM) has been used in the following works. Zienkiewicz [18], Cook [19] and Reddy [20] have described the use of HFEM to analyze the vibration of metallic and composite beams. Ganesan and Chen [21] have studied the free vibration response of thickness-tapered composite beams using HFEM and Rayleigh-Ritz method. Lees and Thomas [22] conducted a modal analysis on a clampedclamped Timoshenko beam using HFEM. Bardell [23] conducted a free vibration analysis of a rectangular plate using the HFEM. Yu et al. [24] studied a multivariable HFEM for static and vibration analysis of beams. Ribeiro and Petyt [25] 
studied the non-linear free and forced vibrations of composite laminated plates using HFEM.

It is learnt that although there are several works on the study of uniform composite beams, works on the vibration of tapered composite beams are scarce to find in the literature. Also, due to its accuracy and efficiency, HFEM seems the appropriate choice to employ for vibration analysis of composite beams. In the present work, doubly-tapered composite beam is studied for its free vibration response using HFEM.

\section{FINITE ELEMENT FORMULATION}

The composite beam has a large length-to-thickness ratio as shown in Fig. 1, and therefore by applying the cylindrical bending theory and considering classical laminate theory, one can get the equation of motion in variational form as:

$\int_{0}^{L} b(x) D_{11}(x)\left(\frac{\mathrm{d}^{2} w}{\mathrm{~d} x^{2}}\right)\left(\frac{d^{2} \delta w}{\mathrm{~d} x^{2}}\right) d x-\int_{0}^{L} b(x) N_{x}\left(\frac{\mathrm{d} w}{\mathrm{dx}}\right)\left(\frac{\mathrm{d} \delta w}{\mathrm{dx}}\right) d x$

$-\omega^{2} \int_{0}^{L} \int_{-H / 2}^{H / 2} \rho b(x) w \delta w d x d z=0$

In which it is assumed that the deflection in (transverse) $\mathrm{z}$ direction can be expressed as:

$w(x, t)=W(x) e^{i \omega t}$

In (1), $L$ is the length of the beam, $b(x)$ is the width of the beam at each cross section along the length of the tapered part of the beam $\left(b(x)=\left(b_{R}-b_{L}\right) x / L+b_{L}\right), N_{x}$ is the compressive axial force (if present), $H$ is the thickness of the beam, $\rho$ is the beam density. $D_{11}(x)$ is the coefficient of bending stiffness of the laminated composite beam [8] and it can be calculated as:

$D_{11}(x)=\sum_{k=1}^{n}\left[t_{k} z_{k}^{2}-\frac{t_{k}^{3}}{12}\right]\left(\bar{Q}_{11}\right)_{k}$

where $t_{k}$ denotes the thickness of $k^{\text {th }}$ ply and $\left(\bar{Q}_{11}\right)_{k}$ denotes the transformed reduced stiffness coefficient of the $k^{\text {th }}$ ply.

\section{A. CFEM formulation}

In Conventional Finite Element Method (CFEM), the structure is divided into a number of Euler-Bernoulli beam elements. Each element can have a number of nodes, which are the critical points. In this study, two nodes per element and two degrees of freedom per node are used in the formulation as shown in Fig. 1.

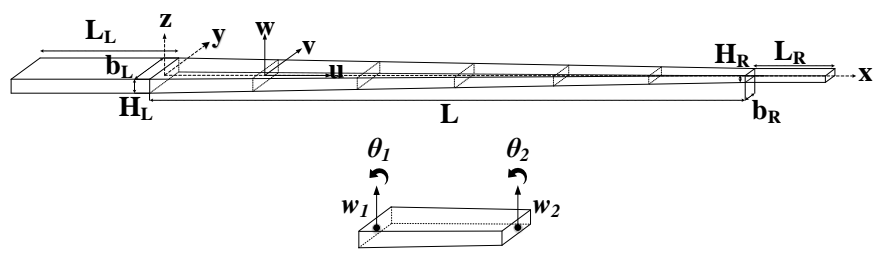

Figure 1. Global and local co-ordinates (top), and nodal displacements and rotations (bottom)
Having four degrees of freedom per element, a third-order polynomial is required for the expression of deflection to satisfy the boundary conditions as below:

$W(x)=c_{1}+c_{2} x+c_{3} x^{2}+c_{4} x^{3}$

After determining and using corresponding interpolation functions and following the CFEM, the equation of motion becomes:

$$
\begin{aligned}
& {\left[\int_{0}^{l_{e}} b(x)\left[D_{11}\left[N^{M}\right]^{T}\left[N^{M}\right]-N_{x}\left[N^{d}\right]^{T}\left[N^{d}\right]\right] d x\right.} \\
& \left.-\omega^{2} \int_{0}^{l_{e}} b(x)\left(\rho_{p} H_{p}+\rho_{r} H_{r}\right)\left[N^{w}\right]^{T}\left[N^{w}\right] d x\right]\{u\}=0
\end{aligned}
$$

In (5), $\left[N^{w}\right]$ is the shape function matrix and $\left[N^{d}\right]$ and $\left[N^{M}\right]$ are the first and second derivatives of $\left[N^{w}\right]$, respectively. $\rho_{p}$ is the density of the ply, $\rho_{r}$ is the density of resin, $l_{e}$ is the length of the element and $H_{p}$ and $H_{r}$ are the equivalent thicknesses of the resin and ply in each element, respectively. For example, if an element has a cross-sectional area of $A=A_{P l y}+A_{\text {Resin }}$ and element length of $l_{e}$, therefore $H_{p}=A_{P l y} / l_{e}$ and $H_{r}=A_{\text {Resin }} /$ $l_{e}$.

Stiffness $[k]$ and mass $[m]$ matrices are defined for each element as:

$[k]=\int_{0}^{l_{e}} b(x)\left[D_{11}(x)\left[N^{M}\right]^{T}\left[N^{M}\right]-N_{x}\left[N^{d}\right]^{T}\left[N^{d}\right]\right] d x$
$[m]=\int_{0}^{L} b(x)\left(\rho_{p} H_{p}+\rho_{r} H_{r}\right)\left[N^{w}\right]^{T}\left[N^{w}\right] d x$

One can assemble global stiffness $[K]$ and mass $[M]$ matrices of the beam using element matrices by enforcing deflection and rotation continuity conditions. As a result, (5) transforms into:

$\left[[K]-\omega^{2}[M]\right]\{u\}=0$

Equation (8) is an eigenvalue problem and can be solved to determine the natural frequencies and mode shapes of the beam.

\section{B. HFEM formulation}

In the CFEM formulation, a cubical displacement function was assumed in Equation (4). In the Hierarchical Finite Element Method (HFEM) the displacement function is modified by adding trigonometric or polynomial functions [7]. In this study, the trigonometric hierarchical functions are used as:

$W(x)=c_{1}+c_{2} x+c_{3} x^{2}+c_{4} x^{3}+\sum_{i=1}^{N} c_{i+4} \sin \frac{i \pi x}{l_{e}}, \quad i=1,2,3, \ldots$

in which $l_{e}$ is the length of the element and $N$ is the number of hierarchical terms. Equation (9) can be expressed as:

$$
\begin{aligned}
& W=\left[K_{w}\right][c] \\
& K_{w}=\left[\begin{array}{llllll}
1 & x & x^{2} & x^{3} \sin \frac{\pi x}{l_{e}} \ldots \sin \frac{N \pi x}{l_{e}}
\end{array}\right]
\end{aligned}
$$


$[c]=\left[\begin{array}{c}c_{1} \\ c_{2} \\ c_{3} \\ c_{4} \\ c_{5} \\ \vdots \\ c_{N+4}\end{array}\right]$

In the same manner, rotation $(\theta)$ can be expressed as:

$\theta=\left[K_{\theta}\right][c]$

$K_{\theta}=\frac{d\left(K_{w}\right)}{d x}=\left[\begin{array}{llllll}0 & 1 & 2 x & 3 x^{2} & \frac{\pi}{l_{e}} \cos \frac{\pi x}{l_{e}} \ldots \frac{N \pi}{l_{e}} \cos \frac{N \pi x}{l_{e}}\end{array}\right]$

The displacement matrix in local coordinate system is:

$\{u\}=\left\{\begin{array}{c}w_{1} \\ \theta_{1} \\ w_{2} \\ \theta_{2} \\ A_{1} \\ \vdots \\ A_{N}\end{array}\right\}=\left[K_{u}\right]\{c\}$

in which $A_{\mathrm{i}}$ are the hierarchical degrees of freedom corresponding to hierarchical terms and $\left[K_{u}\right]$ can be written as [21]:

$\left[K_{u}\right]=\left[\begin{array}{ccccccc}1 & 0 & 0 & 0 & 0 & \ldots & 0 \\ 0 & 1 & 0 & 0 & \frac{\pi}{l_{e}} & \ldots & \frac{N \pi}{l_{e}} \\ 1 & l_{e} & l_{e}^{2} & l_{e}^{3} & 0 & \ldots & 0 \\ 0 & 1 & 2 l_{e} & 3 l_{e}^{2} & -\frac{\pi}{l_{e}} & \ldots & (-1)^{N} \frac{N \pi}{l_{e}} \\ 0 & 0 & 0 & 0 & 1 & \ldots & 0 \\ \vdots & \vdots & \vdots & \vdots & \vdots & \ddots & \vdots \\ 0 & 0 & 0 & 0 & 0 & \ldots & 1\end{array}\right]$

The procedure to determine the stiffness [k] and mass [m] matrices for each element from the variational statement of equations of motion, (1), is analogous to that described for CFEM. The algorithm to assemble the global stiffness $[K]$ and global mass $[M]$ matrices is described in the Appendix.

Table I. Material Properties

\begin{tabular}{|c|c|c|c|}
\hline \multicolumn{2}{|c|}{ Mechanical properties of ply } & \multicolumn{2}{|c|}{ Mechanical properties of resin } \\
\hline Longitudinal modulus $\left(\mathrm{E}_{1}\right)$ & $113.9 \mathrm{GPa}$ & Elastic Modulus (E) & $3.93 \mathrm{GPa}$ \\
\hline Transverse modulus $\left(\mathrm{E}_{2}\right)$ & $7.985 \mathrm{GPa}$ & Shear modulus (G) & $1.03 \mathrm{GPa}$ \\
\hline $\mathrm{E}_{3}=\mathrm{E}_{2}$ & $7.985 \mathrm{GPa}$ & Poisson's ratio $(v)$ & 0.37 \\
\hline In-plane shear modulus $\left(\mathrm{G}_{12}\right)$ & $3.138 \mathrm{GPa}$ & Density of resin ( $\rho r)$ & $1000 \mathrm{~kg} / \mathrm{m}^{3}$ \\
\hline Major Poisson's ratio $\left(v_{12}\right)$ & 0.288 & & \\
\hline Minor Poisson's ratio $\left(v_{21}\right)$ & 0.178 & & \\
\hline Density of ply $\left(\rho_{p}\right)$ & $1480 \mathrm{~kg} / \mathrm{m}^{3}$ & & \\
\hline
\end{tabular}

\section{FREE VIBRATION ANALYSIS AND DISCUSSION}

\section{A. Validation and convergence of HFEM solution}

The material chosen in this study is NCT-301 graphite-epoxy prepreg which is available in the laboratory of Concordia Centre for Composites (CONCOM). The mechanical properties of the ply and the resin are given in the Table I. The beam has [0/90]gs laminate configuration unless specified otherwise.

In order to validate the HFEM formulation, a uniformthickness uniform-width laminated composite beam is considered. The beam is composed of 36 plies. Individual ply thickness $\left(t_{k}\right)$ is $0.125 \mathrm{~mm}$ and the beam thickness $(H)$ is $4.5 \mathrm{~mm}$. Free vibration analysis is carried out using both CFEM and HFEM for clamped-free boundary condition.
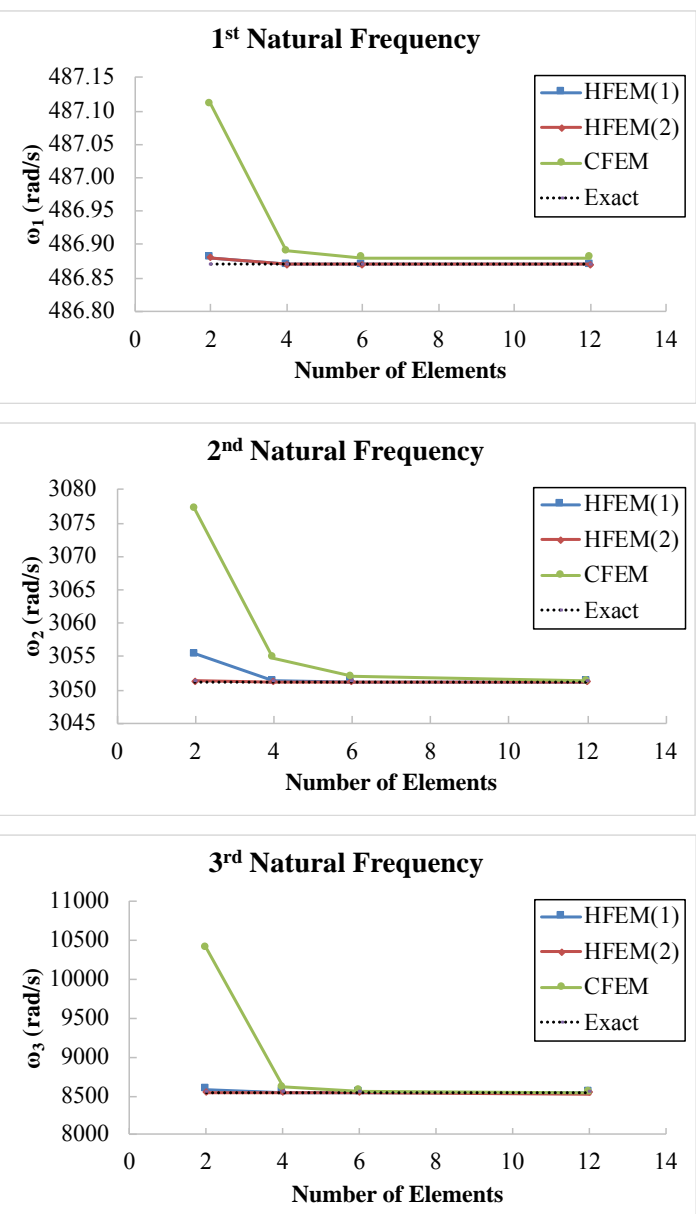

Figure 2. The comparison between results obtained using CFEM and HFEM

The first three natural frequencies are given in Fig. 2 for CFEM, HFEM with one hierarchical term $(\operatorname{HFEM}(1))$ and HFEM with two hierarchical term $(\operatorname{HFEM}(2))$ alongside the exact values of the natural frequencies [8].

As it is shown in Fig. 2, HFEM provides a better accuracy than CFEM with less number of elements that will significantly reduce the time required for the computations and reduce significantly the discontinuities in stress distributions across the nodes. In addition, there is no significant difference between the 
results when the second hierarchical term is added. As a result, in this study HFEM with one trigonometric hierarchical term is considered.

In order to validate the accuracy of the HFEM model, a comparison with the results obtained using R-R (Rayleigh-Ritz method) [15] and a HOFEM (Higher-Order Finite Element Method) [17] is performed. The values of the first three natural frequencies using HFEM are presented alongside the results obtained using R-R and HOFEM methods in Table A.I in Appendix.

The comparison shows that HFEM provides accurate results within $0.2 \%$ of difference from the above-mentioned methods, which is very acceptable and appealing.

\section{B. Parametric Study}

As shown in Figure 3 (not to scale), a thickness-and-widthtapered composite beam composed of 36 plies at the thick (left) side and 12 plies at the thin (right) side, is considered. Thickness of each ply is $0.125 \mathrm{~mm}$ and therefore the thick side beam thickness is $4.5 \mathrm{~mm}$ as opposed to $1.5 \mathrm{~mm}$ beam thickness at the thin side. The beam has a width of $15 \mathrm{~mm}$ at the wide section $\left(b_{L}\right)$ and $7.5 \mathrm{~mm}$ at the narrow section $\left(b_{R}\right)$, which leads to a width-taper-ratio $\left(b_{R} / b_{L}\right)$ of 0.5 . The beam has a length of $25 \mathrm{~cm}$ and $[0 / 90]_{9 s}$ laminate configuration. The material properties are given in Table I. The beam has a simply supported boundary condition.

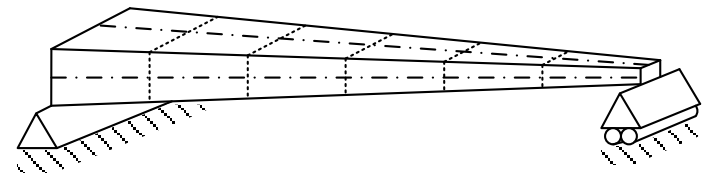

Figure 3. Doubly-tapered composite beam in simply-supported boundary condition

The following is a parametric study to determine the effect tapering on natural frequencies. In each section one variable is considered while all other variables remain constant with the values mentioned above as the base values.

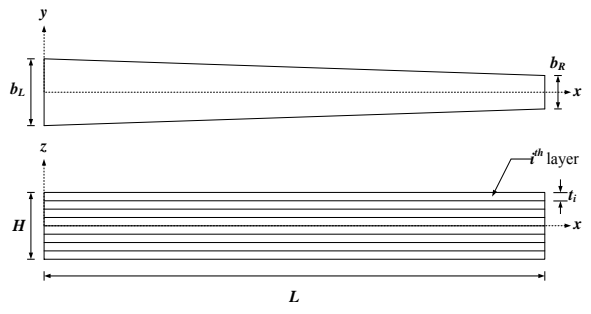

Figure 4. Uniform-thickness and width-tapered composite beam

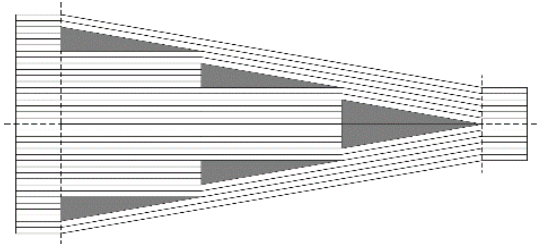

Figure 5. Thickness-taper configuration D
First, the effect of width-tapering, as shown in Figure 4 (not to scale), on the first three natural frequencies is considered. Then, the effect of thickness-tapering for taper configuration $\mathrm{D}$, as shown in Figure 5 (not to scale), on the first three natural frequencies is investigated. Finally, the first three natural frequencies are calculated for a doubly-tapered composite beam.

\section{Discussion}

Figure 6 shows the effect of width-taper-ratio on the natural frequency ratio of a uniform-thickness and width-tapered composite beam, for the first three natural frequencies. Natural frequency change is the ratio of the corresponding natural frequency for a given width-taper-ratio minus natural frequency of the uniform composite beam over the natural frequency of the uniform composite beam. As it is shown in Figure 6, The first natural frequency slightly decreases as the width-taper-ratio decreases. However, even by having a width-taper-ratio of $1 / 3$, only $1.06 \%$ decrease is observed. However, For the second and third natural frequencies, by decreasing the width-taper-ratio, the natural frequencies slightly increase (less than $0.2 \%$ ).

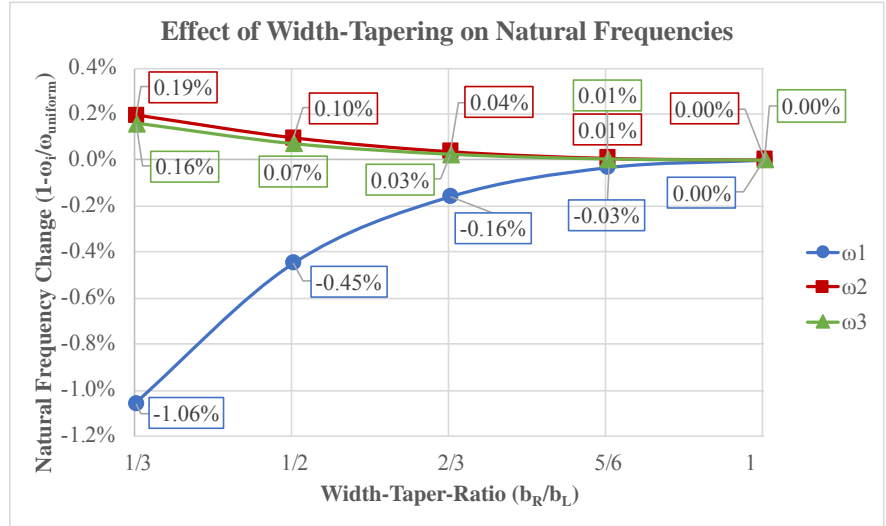

Figure 6. Effect of width-taper-ratio on the first three natural frequencies

In Figure 7, the effect of thickness-tapering on the natural frequencies of thickness-tapered composite beams is plotted. Here, the natural frequency change is the ratio of the corresponding natural frequency for a given thickness-ratio minus natural frequency of the uniform composite beam over the natural frequency of the uniform composite beam. Thicknessratio is the ratio of the thickness of the thin side over the thickness of the thick side. For this study thickness ratios of $1 / 3$, $1 / 2,2 / 3,5 / 6$ and 1 are considered which corresponds to thickness-taper angles of $0.344^{\circ}, 0.258^{\circ}, 0.172^{\circ}, 0.086^{\circ}$ and $0^{\circ}$, respectively. As it is shown in Figure 7, as the thickness ratio decreases, the first natural frequency decreases drastically. For a thickness ratio of $1 / 3$ (or equivalently thickness-taper angle of $0.344^{\circ}$ ), the first natural frequency decreases by $39.82 \%$. The second and the third natural frequencies show similar drastic reductions. This can be interpreted by the fact that by removing ply material along the thickness of the composite beam, the value of $\mathrm{D}_{11}$ decreases cubically, which results in the drastic reduction of stiffness of the beam. Moreover, thickness-tapering introduces packets of resin along the thickness of the beam which would also result in the decrease in stiffness of the beam and consequently the decrease of the natural frequencies. 


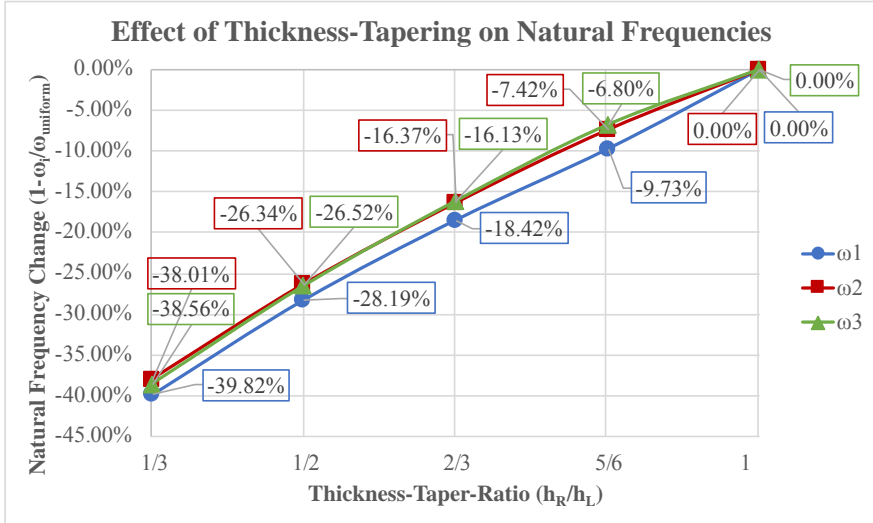

Figure 7. Effect of thickness-taper-ratio (or equivalently thickness-taperangle) on the first three natural frequencies

Figure 8, shows the effect of width-tapering on a thicknesstapered composite beam with the thickness ratio of $1 / 3$ (or equivalently thickness-taper angle of $0.344^{\circ}$ ). It can be seen from Figure 8 that by having no width-tapering (width-taperingratio $=1$ ) the reduction of $39.82 \%$ in the first natural frequency is present. By decreasing the width-tapering-ratio we see an additional decrease in natural frequencies (up to $3.27 \%$ for width-taper-ratio $=1 / 3$ ). However, as it was observed before, the second and the third natural frequencies are slightly increased (less than $0.9 \%$ compared to the case of thicknesstapered-only) by decreasing the width-taper-ratio.

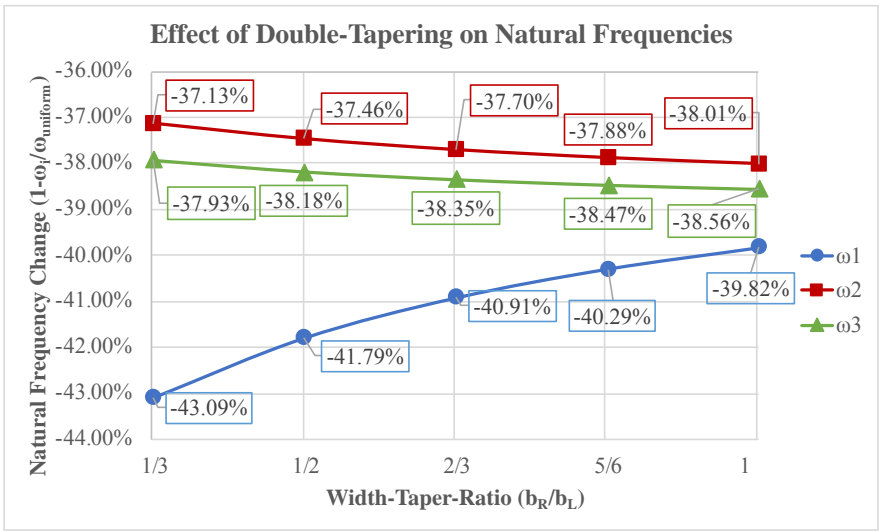

Figure 8. Effect of double-tapering on the first three natural frequencies thickness-taper-ratio $=1 / 3$

\section{CONCLUSION}

Hierarchical Finite Element Method (HFEM) provides a better accuracy than Conventional Finite Element Method (CFEM) with less number of elements and this will significantly reduce especially the stress/strain discontinuities across nodes. In addition, there is no significant difference between the results when the second hierarchical term is added. Moreover, a comparison with other published results obtained using R-R (Rayleigh-Ritz method) and a HOFEM (HigherOrder Finite Element Method) shows that HFEM provides accurate results within $0.2 \%$ of difference from the abovementioned methods, which is very acceptable.

A parametric study on the effect of boundary conditions, width-taper-ratio and thickness-taper-ratio (or equivalently thickness-taper angle on the free vibration response of doublytapered laminated composite beams is conducted and the following are concluded:

- Width-tapering has a small effect $(1 \%$ decrease for a width-taper-ratio of 1/3) on first natural frequency of a simply supported composite beam. This effect is even less significant for the second and the third natural frequencies (less than $0.2 \%$ increase).

- Thickness-tapering on the other hand has a significant effect $(9.73 \%$ decrease for thickness-taper-ratio of $5 / 6$ $39.82 \%$ decrease for thickness-taper-ratio of $1 / 3$ ) on the first natural frequency of a simply supported composite beam. This effect is similar for the second and the third natural frequencies.

- Addition of width-tapering to a thickness-tapered composite beam has a slight effect on its first natural frequency $(1.97 \%$ decrease for width-taper-ratio of $1 / 2)$. However, this effect is insignificant on the second and the third natural frequencies $(0.54 \%$ and $0.38 \%$ increase, respectively).

\section{REFERENCES}

[1] O. O. Ochoa and J. N. Reddy, "Finite element analysis of composite laminates," Dordrecht; Boston: Kluwer Academic Publishers, 1992. doi: 10.1007/978-94-015-7995-7.

[2] H. E. U. Ahmed, "Free and forced vibrations of tapered composite beams including the effects of axial force and damping," Montreal: Concordia Universty, 2008.

[3] R. B. Abarcar and P. F. Cunniff, "The vibration of cantilever beams of fiber reinforced material," Journal of Composite Materials, vol. 6, pp. 504-517, 1972.doi: $10.1177 / 002199837200600406$.

[4] K. Chandrashekhara, K. Krishnamurthy, and S. Roy, "Free vibration of composite beams including rotary inertia and shear deformation," Composite Structures, vol. 14, pp. 269-279, 1990. doi: 10.1016/0263-8223(90)90010-C.

[5] S. R. Marur and T. Kant, "Free vibration analysis of fiber reinforced composite beams using higher order theories and finite element modelling," Journal of Sound and Vibration, vol. 194, pp. 337-351, 1996. doi: $10.1006 /$ jsvi.1996.0362

[6] A. K. Miller and D.F. Adams, "An analytic means of determining the flexural and torsional resonant frequencies of generally orthotropic beams," Journal of Sound and Vibration, vol. 41, pp. 433-449, 1975. doi: 10.1016/S0022-460X(75)80107-6

[7] J. N. Reddy, "Mechanics of laminated composite plates and shells : theory and analysis," Boca Raton: CRC Press, 2004.doi: 10.1201/b12409.

[8] J. M. Bertholet, "Composite materials : mechanical behavior and structural analysis," New York: Springer, 1999.doi: 10.1007/978-1-4612-0527-2.

[9] J. M. Whitney and J. E. Ashton, "Structural analysis of laminated anisotropic plates," Lancaster, Pa., U.S.A.: Technomic Pub. Co., 1987.

[10] R. M. Jones, "Mechanics of composite materials," Philadelphia, PA: Taylor \& Francis, 1999.

[11] Vinson, Jack R., and Robert L. Sierakowski. "The behavior of structures composed of composite materials", vol. 105. Springer Science \& Business Media, 2006. 
[12] K. He, S. V. Hoa, and R. Ganesan, "The study of tapered laminated composite structures: a review," Composites Science and Technology, vol. 60, pp. $2643-2657,11 / / 2000$

[13] R. Ganesan, A. Zabihollah, "Vibration analysis of tapered composite beams using a higher-order finite element. Part I: Formulation," Composite Structures, vol. 77, pp. 306-318, 2007.

doi: 10.1016/j.compstruct.2005.07.018.

[14] R. Ganesan, A. Zabihollah, "Vibration analysis of tapered composite beams using a higher-order finite element. Part II: parametric study,"

Composite Structures, vol. 77, pp. 319-330, 2007.

doi: 10.1016/j.compstruct.2005.07.017.

[15] V. K. Badagi, "Dynamic response of width- and thickness-tapered composite beams using Rayleigh- Ritz method and modal testing," Montreal: Concordia University, 2012..

[16] S. H. Farghaly, R. M. Gadelrab, "Free vibration of a stepped composite timoshenko cantilever beam," Journal of Sound and Vibration, vol. 187, pp. 886-896, 1995. doi: 10.1006/jsvi.1995.0571.

[17] P. Salajegheh, R. Ganesan, "Finite element vibration analysis of variablethickness variable-width laminated composite beams," 27th Annual Technical Conference of the American Society for Composites, Arlington, TX, United States: pp. 1307-1320, 2012.

[18] O. C. Zienkiewicz, The Finite Method, London; New York: McGrawHill, 1977.
[19] R. D. Cook, D. S. Malkus, and M. E. Plesha, "Concepts and applications of finite element analysis," New York: Wiley, 1989. [20] J. N. Reddy, "An introduction to the finite element method," New York: McGraw-Hill, 1993.

[21] R. Ganesan, L. Chen, "Vibration analysis of tapered composite beams using hierarchical finite element and three-dimensional ply stiffness analysis," International Conference on Resource Utilisation and Intelligent Systems (INCRUIS-2006), Erode, India 2006

[22] A. W. Lees, D. L. Thomas, "Modal hierarchical Timoshenko beam finite elements," Journal of Sound and Vibration, vol. 99, pp. 455-461, 1985. doi: 10.1016/0022-460X(85)90533-4.

[23] N. S. Bardell, "Free vibration analysis of a flat plate using the hierarchical finite element method," Journal of Sound and Vibration, vol. 151, pp. 263- 289, 1991. doi: 10.1016/0022-460X(91)90855-E.

[24] Z. Yu, X. Guo, F. Chu, "A multivariable hierarchical finite element for static and vibration analysis of beams," Finite Elements in Analysis and Design, vol. 46, pp. 625-631, 2010. doi: 10.1016/j.finel.2010.03.002.

[25] P. Ribeiro, M. Petyt, "Non-linear vibration of composite laminated plates by the hierarchical finite element method," Composite Structures, vol. 46, pp. 197-208, 1999.

doi: 10.1016/S0263-8223(99)00055-0 


\section{APPENDIX}

Table A.I. Comparison between the natural frequencies obtained using HFEM, HOFEM and R-R method for different width-taper-ratios $\left(b_{L}=1.66 \mathrm{~cm}\right)$

\begin{tabular}{|c|c|c|c|c|c|c|c|c|c|c|c|}
\hline & & \multicolumn{5}{|c|}{$\mathrm{b}_{\mathrm{R}} / \mathrm{b}_{\mathrm{L}}=0.4$} & \multicolumn{5}{|c|}{$\mathrm{b}_{\mathrm{R}} / \mathrm{b}_{\mathrm{L}}=0.6$} \\
\hline & & R-R [15] & $\begin{array}{c}\text { HOFEM } \\
{[17]}\end{array}$ & HFEM & $\begin{array}{c}\text { Difference } \\
\text { with } \\
\text { R-R }(\%)\end{array}$ & $\begin{array}{c}\text { Difference } \\
\text { with } \\
\text { HOFEM }(\%)\end{array}$ & $\mathrm{R}-\mathrm{R}$ & HOFEM & HFEM & $\begin{array}{c}\text { Difference } \\
\text { with } \\
\text { R-R }(\%)\end{array}$ & $\begin{array}{c}\text { Difference } \\
\text { with } \\
\text { HOFEM }(\%)\end{array}$ \\
\hline \multirow{3}{*}{ S-S } & $\omega_{1}(\mathrm{rad} / \mathrm{s})$ & 1259.9 & 1261.39 & 1261.4 & 0.12 & 0.00 & 1266.5 & 1267.88 & 1267.88 & 0.11 & 0.00 \\
\hline & $\omega_{2}(\mathrm{rad} / \mathrm{s})$ & 5085.7 & 5091.9 & 5092.02 & 0.12 & 0.00 & 5081.5 & 5087.07 & 5087.18 & 0.11 & 0.00 \\
\hline & $\omega_{3}(\mathrm{rad} / \mathrm{s})$ & 11439 & 11453.15 & 11455.45 & 0.14 & 0.02 & 11432 & 11444.15 & 11446.53 & 0.13 & 0.02 \\
\hline \multirow{3}{*}{$\mathrm{C}-\mathrm{C}$} & $\omega_{1}(\mathrm{rad} / \mathrm{s})$ & 2835.8 & 2839.29 & 2839.27 & 0.12 & 0.00 & 2864.6 & 2867.75 & 2867.75 & 0.11 & 0.00 \\
\hline & $\omega_{2}(\mathrm{rad} / \mathrm{s})$ & 7873.7 & 7883.45 & 7883.8 & 0.13 & 0.00 & 7915.2 & 7923.87 & 7924.32 & 0.12 & 0.01 \\
\hline & $\omega_{3}(\mathrm{rad} / \mathrm{s})$ & 15485 & 15504.47 & 15511.12 & 0.17 & 0.04 & 15533 & 15549.98 & 15557.16 & 0.16 & 0.05 \\
\hline \multirow{3}{*}{$\mathrm{C}-\mathrm{F}$} & $\omega_{1}(\mathrm{rad} / \mathrm{s})$ & 589.8 & 590.5 & 590.52 & 0.12 & 0.00 & 527.05 & 527.62 & 527.63 & 0.11 & 0.00 \\
\hline & $\omega_{2}(\mathrm{rad} / \mathrm{s})$ & 3089.8 & 3093.44 & 3093.58 & 0.12 & 0.00 & 2974.1 & 2977.28 & 2977.36 & 0.11 & 0.00 \\
\hline & $\omega_{3}(\mathrm{rad} / \mathrm{s})$ & 8200.2 & 8209.93 & 8211.02 & 0.13 & 0.01 & 8075.8 & 8084.5 & 8085.48 & 0.12 & 0.01 \\
\hline \multirow{3}{*}{$\mathrm{F}-\mathrm{C}$} & $\omega_{1}(\mathrm{rad} / \mathrm{s})$ & 341.34 & 341.77 & 341.76 & 0.12 & 0.00 & 386.35 & 386.79 & 386.77 & 0.11 & 0.01 \\
\hline & $\omega_{2}(\mathrm{rad} / \mathrm{s})$ & 2599.3 & 2602.53 & 2602.46 & 0.12 & 0.00 & 2701.1 & 2704.12 & 2704.08 & 0.11 & 0.00 \\
\hline & $\omega_{3}(\mathrm{rad} / \mathrm{s})$ & 7708.9 & 7718.54 & 7718.95 & 0.13 & 0.01 & 7809.6 & 7818.32 & 7818.89 & 0.12 & 0.01 \\
\hline & & \multicolumn{5}{|c|}{$\mathrm{b}_{\mathrm{R}} / \mathrm{b}_{\mathrm{L}}=0.8$} & \multicolumn{5}{|c|}{$\mathrm{b}_{\mathrm{R}} / \mathrm{b}_{\mathrm{L}}=1$} \\
\hline & & R-R & HOFEM & HFEM & $\begin{array}{c}\text { Difference } \\
\text { with } \\
\text { R-R }(\%) \\
\end{array}$ & $\begin{array}{c}\text { Difference } \\
\text { with } \\
\text { HOFEM (\%) }\end{array}$ & R-R & HOFEM & HFEM & $\begin{array}{c}\text { Difference } \\
\text { with } \\
\text { R-R }(\%) \\
\end{array}$ & $\begin{array}{c}\text { Difference } \\
\text { with } \\
\text { HOFEM (\%) }\end{array}$ \\
\hline \multirow{3}{*}{ S-S } & $\omega_{1}(\mathrm{rad} / \mathrm{s})$ & 1269.2 & 1270.44 & 1270.44 & 0.10 & 0.00 & 1270 & 1271.06 & 1271.06 & 0.08 & 0.00 \\
\hline & $\omega_{2}(\mathrm{rad} / \mathrm{s})$ & 5079.9 & 5084.81 & 5084.92 & 0.10 & 0.00 & 5080 & 5084.23 & 5084.34 & 0.09 & 0.00 \\
\hline & $\omega_{3}(\mathrm{rad} / \mathrm{s})$ & 11429 & 11440.43 & 11442.84 & 0.12 & 0.02 & 11430 & 11439.52 & 11441.93 & 0.10 & 0.02 \\
\hline \multirow{3}{*}{$\mathrm{C}-\mathrm{C}$} & $\omega_{1}(\mathrm{rad} / \mathrm{s})$ & 2875.9 & 2878.71 & 2878.72 & 0.10 & 0.00 & 2879 & 2881.34 & 2881.35 & 0.08 & 0.00 \\
\hline & $\omega_{2}(\mathrm{rad} / \mathrm{s})$ & 7931.3 & 7938.96 & 7939.44 & 0.10 & 0.01 & 7936 & 7942.54 & 7943.03 & 0.09 & 0.01 \\
\hline & $\omega_{3}(\mathrm{rad} / \mathrm{s})$ & 15552 & 15566.62 & 15573.99 & 0.14 & 0.05 & 15558 & 15570.55 & 15577.96 & 0.13 & 0.05 \\
\hline \multirow{3}{*}{$\mathrm{C}-\mathrm{F}$} & $\omega_{1}(\mathrm{rad} / \mathrm{s})$ & 484.13 & 484.6 & 484.6 & 0.10 & 0.00 & 452.44 & 452.81 & 452.81 & 0.08 & 0.00 \\
\hline & $\omega_{2}(\mathrm{rad} / \mathrm{s})$ & 2895.1 & 2897.87 & 2897.91 & 0.10 & 0.00 & 2835.4 & 2837.71 & 2837.73 & 0.08 & 0.00 \\
\hline & $\omega_{3}(\mathrm{rad} / \mathrm{s})$ & 7996.6 & 8004.25 & 8005.11 & 0.11 & 0.01 & 7939.1 & 7945.68 & 7946.46 & 0.09 & 0.01 \\
\hline \multirow{3}{*}{$\mathrm{F}-\mathrm{C}$} & $\omega_{1}(\mathrm{rad} / \mathrm{s})$ & 422.33 & 422.74 & 422.73 & 0.09 & 0.00 & 452.44 & 452.81 & 452.81 & 0.08 & 0.00 \\
\hline & $\omega_{2}(\mathrm{rad} / \mathrm{s})$ & 2776 & 2778.65 & 2778.65 & 0.10 & 0.00 & 2835.4 & 2837.71 & 2837.73 & 0.08 & 0.00 \\
\hline & $\omega_{3}(\mathrm{rad} / \mathrm{s})$ & 7881.6 & 7889.28 & 7889.97 & 0.11 & 0.01 & 7939.1 & 7945.68 & 7946.46 & 0.09 & 0.01 \\
\hline
\end{tabular}


Table A.II shows the assembly algorithm of $\mathrm{K}$ and $\mathrm{M}$ matrices in CFEM for a composite beam divided into 6 elements. Each element has 2 nodes and each node has 2 degrees of freedom (wi and $\theta i$ ). Each color represents an element's $k$ or $m$ matrix. In the areas where two matrices overlap, the two corresponding matrix elements are being added together.

Table A.II. Assembly algorithm of $\mathrm{K}$ and $\mathrm{M}$ matrices in CFEM

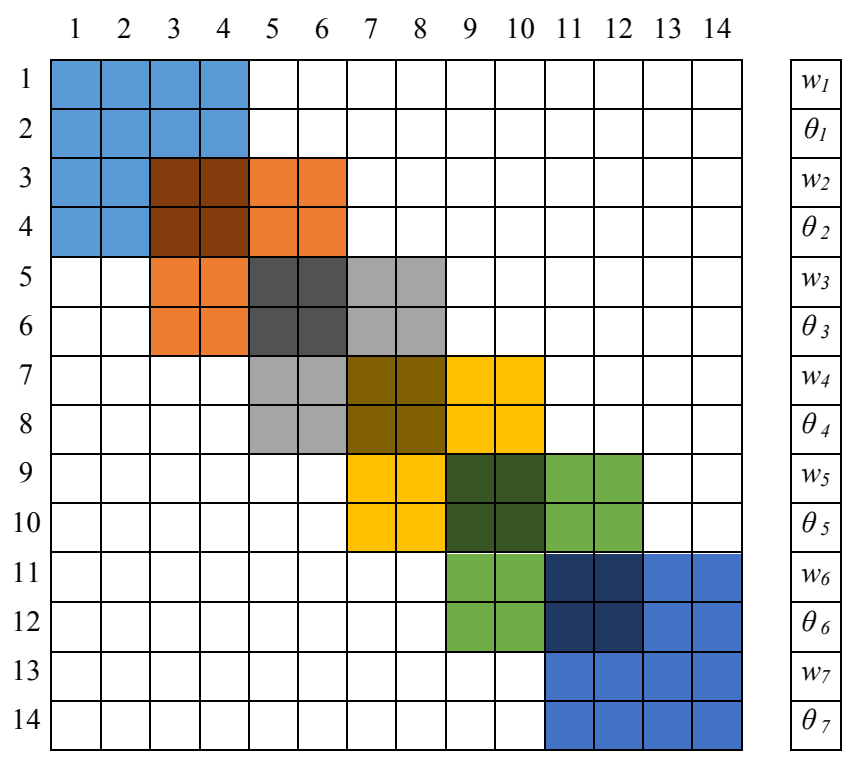

In HFEM with one trigonometric term, each element's stiffness $(\mathrm{k})$ or mass $(\mathrm{m})$ matrix is a $5 \times 5$ matrix. The fifth row and the fifth column are the hierarchical terms corresponding to hierarchical non-physical degree of freedom (Ai) to complete the form of a square matrix. As it is shown in Table A.III, the global stiffness (K) and mass (M) matrices are assembled in the same manner as CFEM for the first four rows and columns and the hierarchical terms fill the rest of the matrix.

Table A.III. Assembly algorithm of K and M matrices in HFEM with one hierarchical term
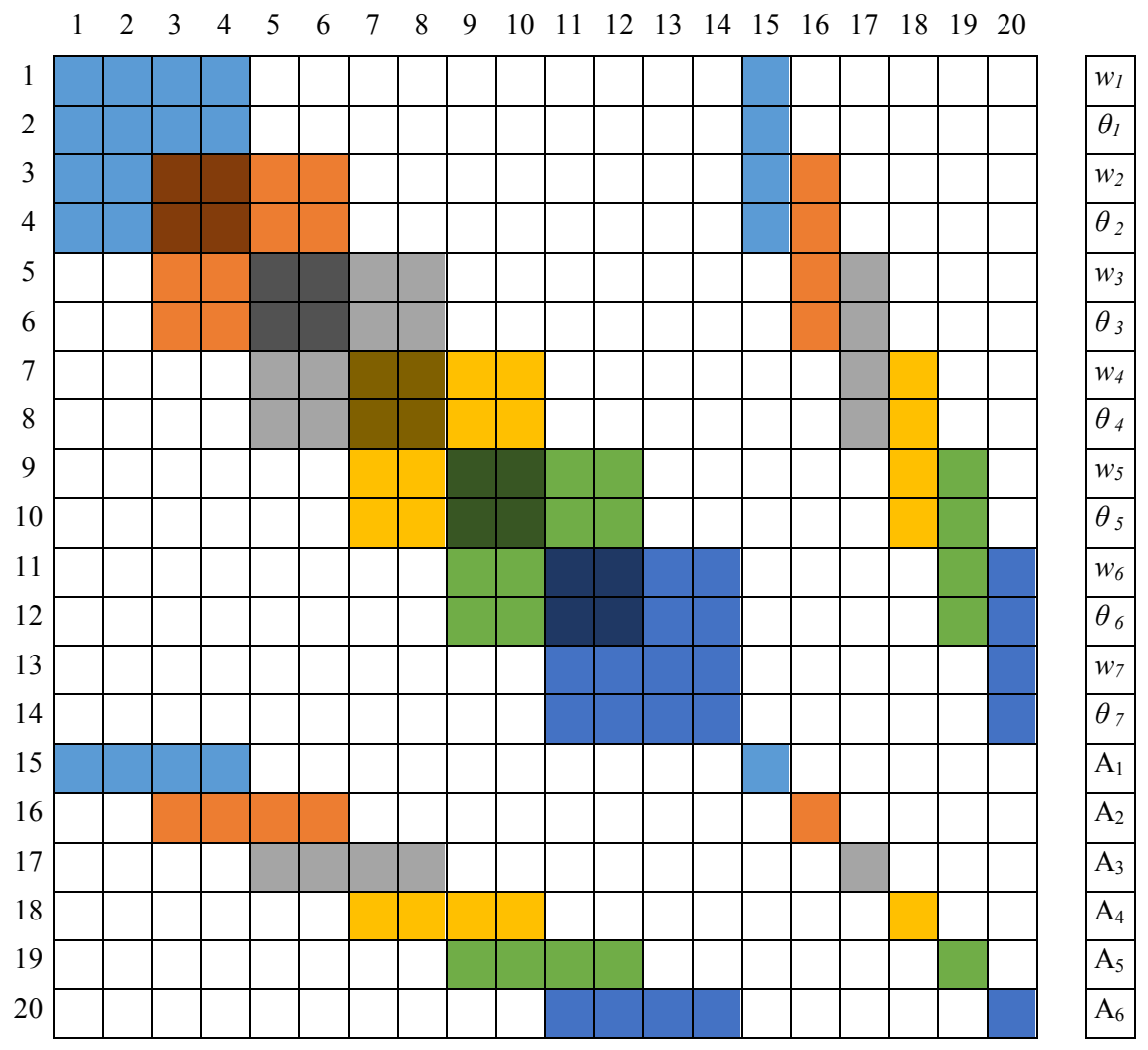


\title{
Modeling of Magneto-Mechanical Response of Magnetorhological Elastomers having Different Arrangement of Magnetic Particles
}

\author{
Mostafa Asadi Khanouki and Ramin Sedaghati \\ Department of Mechanical, Industrial and Aerospace Engineering \\ Concordia University \\ Montreal, QC, Canada
}

\begin{abstract}
A model describing the magneto-mechanical properties of magnetorheological elastomers (MREs) under an external magnetic field and a mechanical shear deformation is presented. The main purpose of the present study is to demonstrate the effect of particle distribution and applied magnetic field on the MRE mechanical properties. Four types of rectangular lattice models are considered as the representation of spatial distribution of magnetic particles in the matrix. Using the energy method, shear modulus is obtained and numerically calculated as function of the strength of the external magnetic field and the shear strain. The results show a high sensitivity of shear modulus on the spatial distribution of particles. Depending on the lattice type, shear modulus exhibits an increasing or decreasing behavior with the increase of magnetic field intensity.
\end{abstract}

Keywords- MR elastomers; microstructure modeling; magnetomechanical properties; particle distribution

\section{INTRODUCTION}

Among different types of smart materials, those that are responsive to an external magnetic field are called magnetically activated smart materials or simply magneto-active materials. Basically, magneto-active materials are composed of two main components: magnetizable particles and nonmagnetic matrix. The host nonmagnetic matrix can be a solid or a liquid, which are then impregnated with micron-sized ferromagnetic particles. Once a magnetic field is applied, the rheology of the material will change as a result of magnetic interaction between particles. Therefore, a common name for the magneto-active materials is magnetorheological materials or in abbreviation, MR materials. Among the MR materials, those with a solid elastomeric matrix are called MR elastomer or simply MRE.

MREs can provide field-dependent elastic modulus making them ideal for many applications such as adaptive spring elements in seismic isolators, vibration absorbers, automotive suspension and artificial human body limbs to name a few [1]. Development of physic-based models capable of predicting the mechanical properties of MRES is of paramount importance for design of MRE-based adaptive devices and also development of control strategies. Many theoretical models have been presented to predict magneto-mechanical properties of MREs [2-5]. One of the promising modeling approaches is based on the magnetophysical properties of components of the MREs. The method considers microstructure of the MRE and examines magnetic interaction between magnetic particles and their corresponding effect on elastomeric matrix that causes change of properties under an applied magnetic field [6-9]. Jolly et al. [6] presented a quasi-static dipole model to predict magneto-viscoelastic behavior of MREs with anisotropic (chain-like) distribution of particles. They only considered the interaction of two adjacent dipoles in a chain of particles in their model.

Based on the previous progresses in modeling mechanical behavior of MR fluids [10, 11], Davis [7] has also worked on a dipole model to predict the maximum possible increase of shear modulus (once saturation of MRE is occurred) as a function of saturation magnetization and volume fraction of filler particles. Later, some authors tried to present models to encircle the interactions of all particles in a chain [9] or in the whole structure [12]. As a recent progress in this field, Ivaneyko et al. [8] developed a model that can predict magneto-mechanical behavior of MREs under an applied magnetic field by considering interactions between all particles in the network. They discussed the case of a MRE with isotropic spatial distribution of magnetic particles and finally presented the MRE's modulus of elasticity as a function of magnetic field for tensile deformations.

In the present work, the model of Ivaneyko et al. [8] is extended for the case of static shear deformations in the framework of a nonlinear elasticity approach. Magnetic particles are supposed to be located on the sites of four lattice types namely simple cubic, body centered cubic, face centered cubic and edge centered cubic lattices. The two former lattices were also used in [8] but the two latter ones are utilized here for the first time. In the following, first, the mathematical formulation of the model is introduced. This is followed by the derivation of total potential energy of the deformed MRE in a simple shear test under an applied magnetic field. The field dependent shear modulus is then extracted from the derived energy function. The shear modulus depends on the strength of the magnetic field, shear strain, mechanical and magnetic properties of the matrix and particles and finally on the particles' lattice type. Finally, 
numerical results for the shear modulus are provided and discussed.

\section{MATHEMATICAL FORMULATIONS}

In order to model the mechanical response of a MRE that has a particular spatial distribution of the magnetic particles, the total energy of a MRE sample is obtained in this section. Total energy of the elastomer is a function of the external applied shear deformation, applied magnetic field intensity, lattice model of the magnetic particles and mechanical or magnetic properties of the material. In this study, a MRE sample under a simple shear loading in one direction ( $\mathrm{x}$-axis) which is normal to the direction of an applied magnetic field (y-axis) is considered. Fig. 1 illustrates this situation schematically that the simple shear happens in the $x-y$ plane and the magnetic field $(H)$ is in the y direction. Magnetic particles inside the sample have magnetic dipoles in the direction of the external field.

\section{A. Total Potential Energy}

Total potential energy of a deformed MRE in the presence of a magnetic field is composed of two parts. One is the elastic energy of the deformed MRE due to the entropic elasticity of polymer chains $\left(e_{e l}\right)$ and the other is due to the potential energy of the magnetic particles placed in an external magnetic field $\left(e_{m}\right)$. Thus, the total potential energy of the deformed $\operatorname{MRE}\left(e_{T}\right)$ under external magnetic field can be written as:

$$
e_{T}=e_{e l}+e_{m}
$$

Here first, the elastic part of the energy $\left(e_{e l}\right)$ is calculated for the case of a simple shear test on a continuous medium. Ogden strain potential is extensively used for such applications which is a nonlinear deformation model and valid for the large strain situations [13]. Here, a simplified form of the Ogden model with known coefficients is utilized. Based on this simplified model, the elastic energy of a MRE sample under nonlinear deformations can be presented with the Neo-Hooke law of the following general form [8]:

$$
e_{e l}=\frac{G_{0}}{2}\left(\lambda_{x}^{2}+\lambda_{y}^{2}+\lambda_{z}^{2}-3\right)
$$

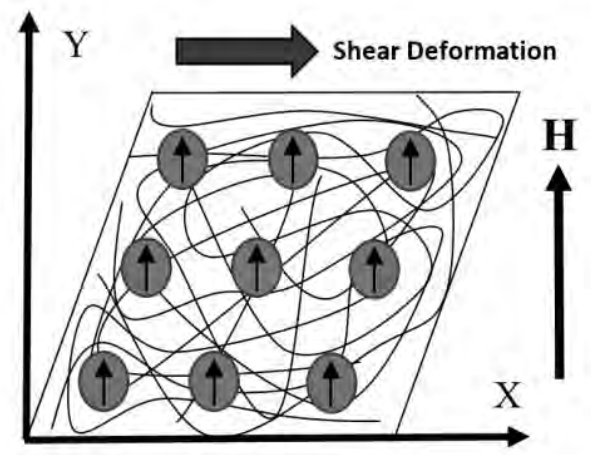

Figure 1. Schematic representation of a MRE sample under simple shear deformation normal to the applied magnetic field direction where $\lambda_{x}, \lambda_{y}, \lambda_{z}$ are the principal extension ratios, i.e. the ratio of the current length to original length, in the principal directions. $G_{0}$ is the shear modulus of the MRE at zero field which is usually obtained experimentally. This material parameter introduces the effect of different complex phenomena into the elastic energy of the sample such as matrix reinforcement by the hard particles, adhesion of the matrix onto the surface of magnetic particles and so forth.

The magneto-mechanical response of the deformed MRE under the applied magnetic field is characterized based on the value of the shear strain $\gamma$ in the $x-y$ plane. For the case of large shear strains, the relation between the extension ratio in $x$ direction and the shear strain is [14]:

$$
\gamma=\lambda_{x}-\frac{1}{\lambda_{x}}
$$

Assuming the material to be incompressible $\left(\lambda_{x} \lambda_{y} \lambda_{z}=1\right)$, and considering a plane strain state $\left(\lambda_{y}=1\right)$ the extension ratios are characterized based on the shear strain as:

$$
\begin{gathered}
\lambda_{x}=\frac{\gamma+\sqrt{4+\gamma^{2}}}{2}, \lambda_{y}=1, \\
\lambda_{z}=\frac{1}{\lambda_{x}}=\frac{2}{\gamma+\sqrt{4+\gamma^{2}}}
\end{gathered}
$$

Using relations ( 4 ) into ( 2 ), the elastic energy of the elastomer as a function of $\gamma$ can be expressed as:

$$
e_{e l}=\frac{G_{0}}{2}\left(\left(\frac{\gamma+\sqrt{4+\gamma^{2}}}{2}\right)^{2}+\left(\frac{2}{\gamma+\sqrt{4+\gamma^{2}}}\right)^{2}-2\right)
$$

The second part of the energy in (2) is due to the fieldinduced interaction of magnetic particles inside the elastomer. Applying a magnetic field on the MRE activates the elementary atomic magnetic dipoles in the magnetic particles. The induced dipole moment of each particle has an interaction with the other dipoles in the particle network and is known as the dipoledipole interaction. The magnetic energy of the material which is the dipole-dipole interaction energy per unit volume can be expressed as $[8,15]$ :

$$
\begin{aligned}
e_{m}=-\frac{1}{V} \frac{\mu_{r} \mu_{0}}{4 \pi} \sum_{i j}\left[\frac{3\left(\vec{m}_{i} \cdot \vec{R}_{i j}\right)\left(\vec{m}_{j} \cdot \vec{R}_{i j}\right)}{\left|\vec{R}_{i j}\right|^{5}}\right. \\
\left.-\frac{\left(\vec{m}_{i} \cdot \vec{m}_{j}\right)}{\left|\vec{R}_{i j}\right|^{3}}\right]
\end{aligned}
$$

where $V$ is the volume of the sample, $\mu_{0}$ is vacuum permeability, and $\mu_{r}$ is the relative permeability of the medium. Since we assume the elastomeric matrix to be non-magnetic, the relative permeability of the medium is unity $\left(\mu_{r}=1\right)$. Here $\vec{m}_{i}$ and $\vec{m}_{j}$ are average induced dipole moments of the $i$-th and $j$-th magnetic particles, $\vec{R}_{i j}$ is the radius vector between the $i$-th and $j$-th magnetic particles. The summation in ( 6 ) accounts for the superposition of mutual interaction between all pairs of 
particles in the network. In order to simplify the calculation of the summation, we consider some lattice networks as the representation of the particle's distribution in the matrix. In this work, besides the two lattice models of simple cubic (SC) and body centered cubic (BCC) which have been used in the work of Ivaneyko et al. 2012 [8], face centered cubic (FCC) lattice and edge centered cubic (ECC) have also been investigated. A unit cell of these four lattice networks as models of spatial distribution of the particles is illustrated in Fig. 2.

For the sake of simplicity, all the particles are assumed to have the same spherical shape and with an average radius of $r$. The volume fraction of the magnetic particles dispersed in the matrix is given by:

$$
\phi=\lambda \frac{v_{0}}{a^{3}}
$$

where $v_{0}=\frac{4}{3} \pi r^{3}$ is a particle volume and $\lambda$ is a factor depends on the lattice type which considering Fig. 2 are found to be:

$$
\lambda_{S C}=1, \quad \lambda_{B C C}=2, \lambda_{F C C}=4, \lambda_{E C C}=4
$$

As stated before, application of a magnetic field on a MRE, causes each of the magnetic particles to have a magnetic dipole moment. The induced magnetic dipoles $\vec{m}_{i}$ and $\vec{m}_{j}$ in (6) are directed along the direction of applied magnetic field, $\vec{H}$, toward y-axis as shown in Fig. 1. Because of the symmetry of the lattice models with regard to the magnetic field, the absolute values of dipole moments are identical and related to the magnetization $(M)$ of each particle as $m_{i}=m_{j}=v_{0} M$.

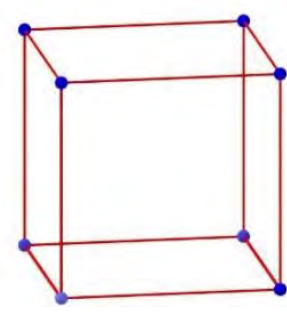

Simple cubic (SC)

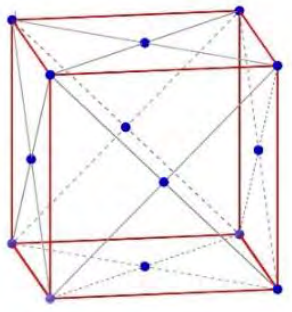

Face centered cubic (FCC)

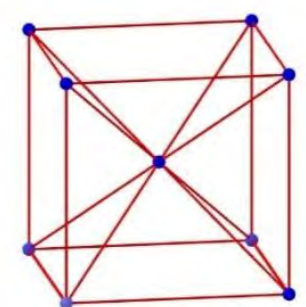

Body centered cubic (BCC)

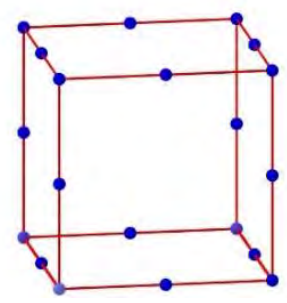

Edge centered cubic (ECC)
Figure 2. Schematic illustration of different lattice networks as representation for spatial distribution of magnetic particles in the elastomeric matrix

The relationship between the particle magnetization $M$ and the applied field strength, $H$, can be approximated by the Frohlish-Kennely equation as $[8,16]$ :

$$
M=\frac{M_{s}\left(\mu_{i n i}-1\right) H}{M_{s}+\left(\mu_{i n i}-1\right)|H|}
$$

where $M_{s}$ is the saturation magnetization and $\mu_{\text {ini }}$ is the initial magnetic permeability of the particles. To have more compact and simpler formulation, (9) can be transferred into a dimensionless form as:

$$
\frac{M}{M_{S}}=\frac{h}{1+|h|}
$$

where $h=\left(\mu_{\text {ini }}-1\right) H / M_{s}$ is called reduced magnetic field and $M / M_{s}$ the reduced magnetization. Equation (9) can predict saturation of a magnetic material. Indeed, the value of magnetization $M$ increases with an increasing magnetic field and reaches to saturation magnetization, $M_{s}$, when $H \rightarrow \infty$.

By expressing the spatial distribution of particles with simplified arrangements as the lattice models presented in Fig. 2 , the summation over indices $i$ and $j$ in ( 6 ) can be simplified. The $j$-th particle is taken and the summation is performed over index $i$. Since there is a translational symmetry in the infinite lattice with regards to the magnetic field, the dipole-dipole interaction of a given $j$-th particle with adjacent particles around it does not depend on the number $j$. Therefore, the double sum over indices $i$ and $j$ is reduced to a simple sum over index $i$ multiplied by the number of particles $N$. Thus, ( 6 ) can be simplified as:

$$
e_{m}=-u_{0} v_{0}^{2} c\left(\frac{M}{M_{s}}\right)^{2} \sum_{i}\left[\frac{3\left(\vec{R}_{i}\right)_{x}^{2}-\left|\vec{R}_{i}\right|^{2}}{\left|\vec{R}_{i}\right|^{5}}\right]
$$

where $c=N / V$ is the concentration of particles. The vector $\vec{R}_{i j}$ is replaced by $\vec{R}_{i}$ for convenience, since the vector is calculated from the origin of a lattice. The parameter $u_{0}$ is defined as

$$
u_{0}=\frac{\mu_{0} M_{s}^{2}}{4 \pi}
$$

which is defined as the characteristic energy density for magnetic interaction [8]. The radius vector $\overrightarrow{R_{l}}$ in ( 11 ) depends on the macroscopic shear strain $\gamma$. The components of the radius vector after deformation can be defined as

$$
\begin{aligned}
&\left(R_{i}\right)_{x}=\left(R_{i}^{0}\right)_{x} \lambda_{x}=\left(R_{i}^{0}\right)_{x} \frac{\left(\gamma+\sqrt{4+\gamma^{2}}\right)}{2} \\
&\left(R_{i}\right)_{y}=\left(R_{i}^{0}\right)_{y} \lambda_{y}=\left(R_{i}^{0}\right)_{y} \\
&\left(R_{i}\right)_{z}=\left(R_{i}^{0}\right)_{z} \lambda_{z}=\left(R_{i}^{0}\right)_{z} \frac{2}{\left(\gamma+\sqrt{4+\gamma^{2}}\right)}
\end{aligned}
$$

where $\left(\vec{R}_{i}^{0}\right)_{\xi}$ are the components of the radius vectors before deformation, $(\xi=x, y, z)$. In a non-deformed MRE, the radius vector $\vec{R}_{i}^{0}$ is defined based on the assumed lattice models in Fig. 2 and can be presented as, 


$$
\vec{R}_{i}^{0}=a \cdot \vec{r}_{l}
$$

where the dimensionless vector $\vec{r}_{l}$ runs over all particles on the sites of all lattices with the edge length $a=1$. Using (10), and (13-16), the magnetic energy $e_{m}$ as a function of a reduced magnetic field $h$ and shear strain $\gamma$ can be expressed as:

$$
e_{m}=u_{0} \phi^{2}\left(\frac{h}{1+|h|}\right)^{2} f(\gamma),
$$

where the dimensionless function of strain $f(\gamma)$ is in the following form:

$$
\begin{aligned}
& f(\gamma) \\
& =-\frac{8}{\lambda}(\Gamma)^{3} \sum_{i}\left[\frac{2 r_{i x}^{2}(\Gamma)^{4}-4 r_{i y}^{2}(\Gamma)^{2}-16 r_{i z}^{2}}{\left(r_{i x}^{2}(\Gamma)^{4}+4 r_{i y}^{2}(\Gamma)^{2}+16 r_{i z}^{2}\right)^{\frac{5}{2}}}\right]
\end{aligned}
$$

and $\Gamma=\gamma+\sqrt{4+\gamma^{2}}$.

The components of vector $\vec{r}_{l}=\left(r_{i x}, r_{i y}, r_{i z}\right)$ represent the location of all particles in the lattices which depend on the lattice type. Accordingly, the value of the function $f(\gamma)$ also depends on the lattice type. In order to solve the problem numerically in the next section, vector $\vec{r}_{l}$ should be programmed as such to locate the particles arranged in the four considered lattice networks. This would be done through nested loops in the coding for each of the lattice types. The number of loops depend on the configuration of particles in the lattice.

Finally, using ( 5 ) for elastic energy and ( 17 ) for magnetic energy, the total potential energy of the deformed MRE under application of the external magnetic field, can be obtained as:

$$
e=\frac{G_{0}}{2}\left(\left(\frac{\Gamma}{2}\right)^{2}+\left(\frac{2}{\Gamma}\right)^{2}-2\right)+u_{0} \phi^{2}\left(\frac{h}{1+|h|}\right)^{2} f(\gamma)
$$

where the value of function $f(\gamma)$ presented in ( 18 ) depends on the lattice types shown in Fig. 2. Having the total energy as a combination of elastic and magnetic contributions, the magneto-mechanical properties of the MRE can be investigated. In the next section, elastic shear modulus of the elastomer will be obtained from the energy function and will be presented on graphs as a function of the applied magnetic field intensity.

\section{B. Elastic Shear Modulus}

The interesting feature of magnetorheological elastomers is their adaptability and possibility of changing their modulus of elasticity by application of an external magnetic field. Shear modulus of a MRE sample having different idealized distribution of particles can be derived from the total potential energy of the material formulated in the previous section. Considering the case of a MRE sample under shear deformation normal to the direction of external magnetic field as shown in Fig. 1, the elastic shear modulus can be obtained as the second derivative of total energy with respect to $\gamma$, at equilibrium state as:

$$
G=\left.\frac{\partial^{2} e}{\partial \gamma^{2}}\right|_{\gamma=0},
$$

where $G$ is the shear modulus of the MRE. Here, the ratio of modulus $G / G_{0}$ is going to be depicted as a function of magnetic field magnitude $H$ for each of the considered types of particle networks shown in Fig. 2. This dimensionless ratio also depends on the parameter $\phi$ and the dimensionless ratio of $G_{0} / u_{0}$.

\section{RESULTS AND DISCUSSIONS}

By programming in MATLAB $^{\circledR}$ environment, shear modulus is calculated numerically. Fig. 3 shows the shear modulus ratio $G / G_{0}$ as a function of the applied magnetic field, $H$, at different volume fractions $\phi=0,0.05,0.1,0.15$ and constant value of $G_{0} / u_{0}=1.5$ for all types of lattices considered in this work i.e. simple cubic, body centered cubic, face centered cubic, and edge centered cubic. Fig. 4 presents the same results at the fixed value of volume fraction $\phi=0.1$ but different values of parameter $G_{0} / u_{0}=1,1.5,5,10$. It is noted that numerical values of $M_{s}=1990 \mathrm{kA} / \mathrm{m}$, and $\mu_{\text {ini }}=132$ as in [16] are utilized for obtaining numerical results.

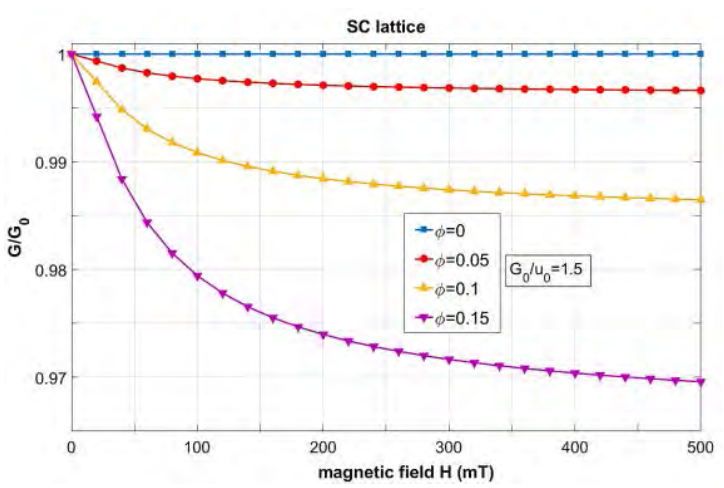

(a)

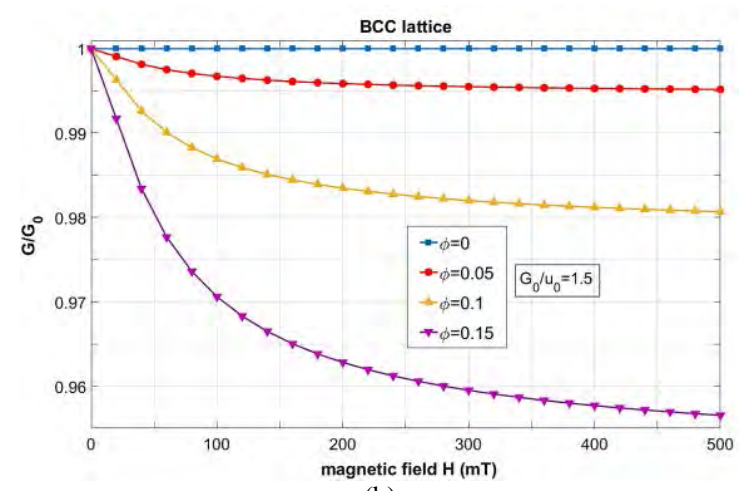

(b) 


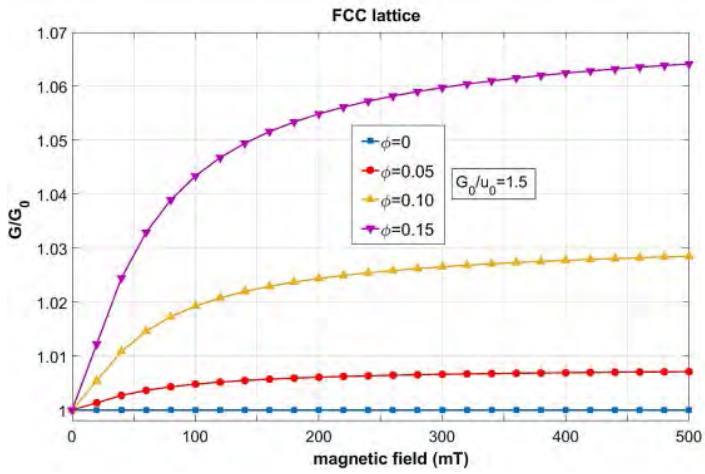

(c)

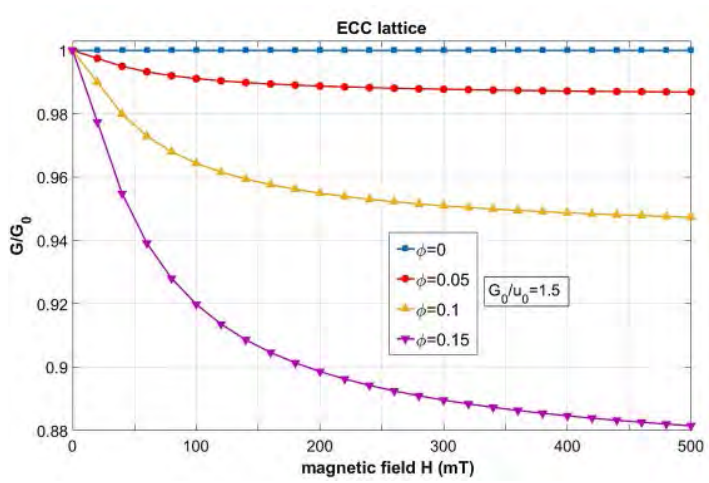

(d)

Figure 3. Shear modulus ratio $\boldsymbol{G} / \boldsymbol{G}_{\mathbf{0}}$ versus magnetic field intensity $\boldsymbol{H}(\mathrm{mT})$ at different volume fractions $\boldsymbol{\phi}$, obtained for four different lattice networks: simple cubic lattice (a), body-centered cubic lattice (b), face centered cubic lattice (c), and edge centered cubic lattice (d).

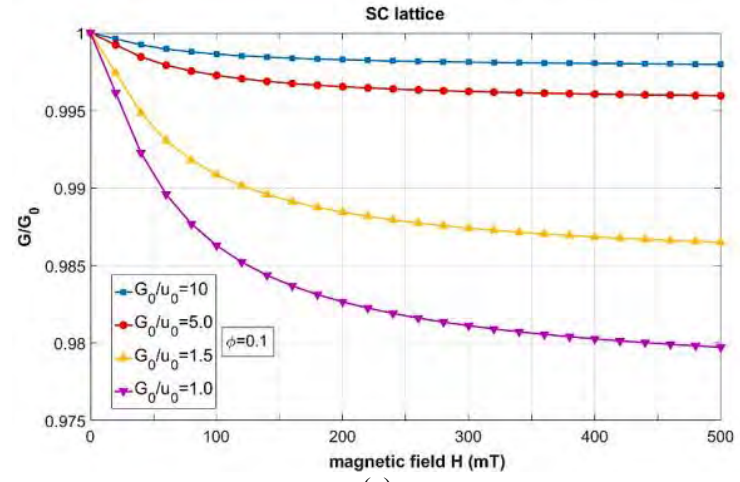

(a)

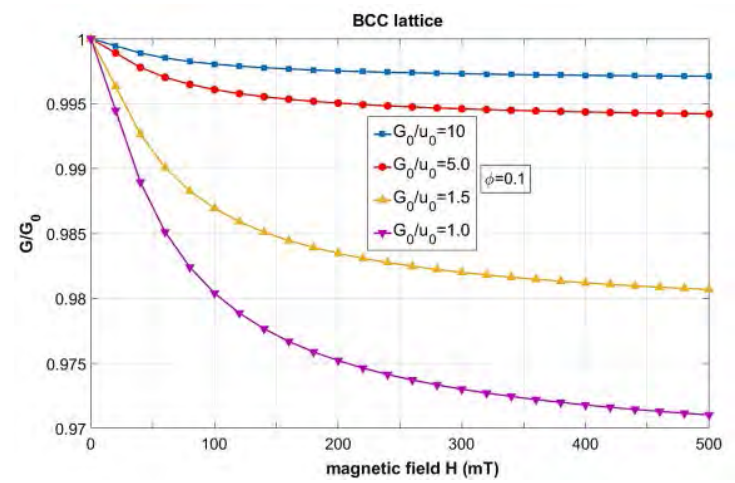

(b)

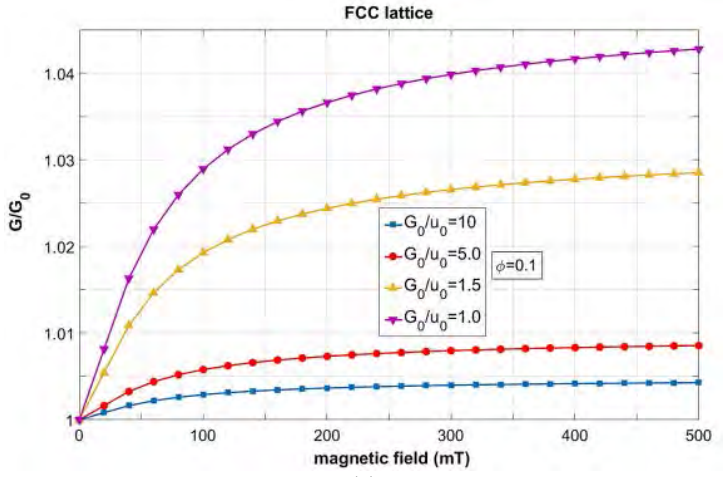

(c)

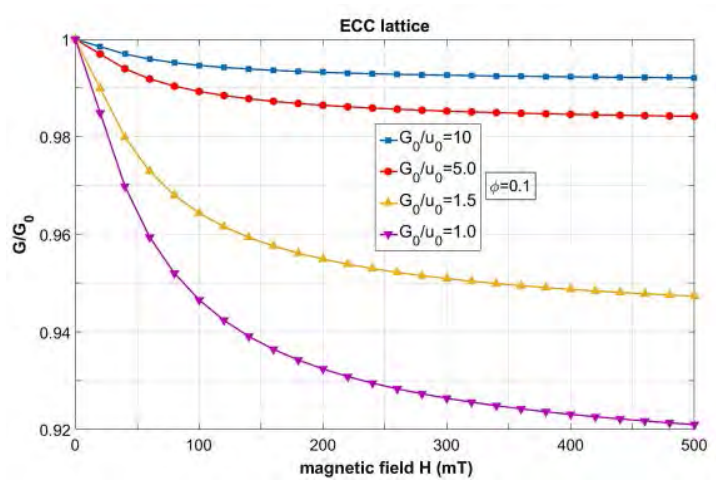

(d)

Figure 4. Shear modulus ratio $G / G_{0}$ versus magnetic field intensity $H(\mathrm{mT})$ at different values of $G_{0} / u_{0}$ and fixed volume fraction $\phi=0.1$, obtained for four types of lattice networks same as Fig. 3

Examination of Figs. 3 and 4 reveals that, shear modulus of a magnetorheological elastomer decreases with magnetic field for the simple cubic, body centered cubic, and edge centered cubic lattices and increases for the face centered cubic lattice. These observations obviously show the impact of particles' network type on the magneto-mechanical behavior of MR elastomers. Different responses of lattice types to the applied magnetic field is due to the special arrangement of particles in each network which causes a different interaction between magnetic particles. Since the mutual interaction of all particles in the network is considered and summed for the calculation of magnetic energy and shear modulus, it is very difficult to evaluate the differences in reciprocal actions of particles for each lattice type. Among the considered lattices, edge centered cubic lattice has the largest change in the absolute value of the change of the shear modulus $\left|G-G_{0}\right|$ with magnetic field. This may be due to the more compact arrangement of particles in this lattice type in comparison to the others.

In addition, it can be seen from Fig. 3 that an increase of the volume fraction $\phi$ results in the increase of the absolute value of the change of the modulus $\left|G-G_{0}\right|$ for all lattice types. That means the more number of particles participated in the material, the bigger the contribution of magnetic energy in the shear modulus by the application of a magnetic field. Furthermore, according to Fig. 4 , increase of the parameter $G_{0} / u_{0}$ decreases the absolute value of the change of the modulus $\left|G-G_{0}\right|$ for all distributions of particles. Considering that the parameter $G_{0} / u_{0}$ 
is in fact the ratio of the characteristic values of the elastic energy to the magnetic energy, increase of this parameter decreases the contribution of magnetic interaction on the shear modulus of the material. The results of this paper are compatible with those reported in [8] for the Young's modulus of a magneto-sensitive elastomer.

\section{CONCLUSIONS}

In this work, a microstructure model of mechanical properties of MR elastomer materials was employed and extended to predict the static shear modulus of a MRE under an external applied magnetic field. To identify the effect of magnetic particles distribution on the magneto-mechanical behavior of a MRE, four idealized homogeneous arrangements of particles called lattice networks were considered. The utilized lattice types are simple cubic, body centered cubic, face centered cubic, and edge centered cubic. First the mathematical formulation of the total potential energy of a MRE sample under a shear deformation and also an external magnetic field was derived. The total energy has two parts of the elastic energy and the magnetic energy. The latter one depends on the interaction of magnetic particles and so on the lattice type. Second the shear modulus of the MR elastomer was extracted from the total energy. Then, using numerical calculations, the shear modulus was obtained as a function of magnetic field and presented for samples having different content of particles and initial properties.

According to the results presented in this work, the shear modulus decreases with increase of the magnetic field for three of the considered lattice types including simple cubic, body centered cubic, and edge centered cubic. However, the shear modulus increases with the magnetic field for the face centered cubic lattice. The variety of the results of the lattice types to the magnetic field is an obvious indication for the impact of magnetic particles distribution on the magneto-mechanical properties of the material. Besides, the effect of volume fraction of particles $\phi$ and the ratio $G_{0} / u_{0}$ on the shear modulus is also investigated. The absolute value of the change of shear modulus from the initial modulus $\left|G-G_{0}\right|$ increases with an increase of volume fraction and decreases with an increase of the ratio $G_{0} / u_{0}$.

In order to know which of the assumed particle networks can be a more realistic model of particles distribution in homogeneous MRE materials, these theoretical results should be compared with experimental tests. Looking through the literature, the static shear modulus of a MR elastomer normally should increases under an applied magnetic field [7, 9, 17]. Accordingly, the results of the face centered cubic (FCC) lattice can be of higher validity. Comparing the theoretical results of the investigated model in the present work with the results of experimental tests can be as an objective of another work in this subject.

\section{REFERENCES}

[1] Ubaidillah, J. Sutrisno, A. Purwanto, and S. A. Mazlan, "Recent Progress on Magnetorheological Solids: Materials, Fabrication, Testing, and applications," Adv. Eng. Mater., vol. 17, no. 5, pp. 563-597, Sep. 2014. doi: 10.1002/adem.201400258.

[2] M. A. Cantera, M. Behrooz, R. F. Gibson, and F. Gordaninejad, "Modeling of magneto-mechanical response of magnetorheological elastomers (MRE) and MRE-based systems: a review," Smart Mater. Struct, vol. 26, no. 2, p. 23001, Jan. 2017. doi: 10.1088/1361-665X/aa549c.

[3] I. A. Brigadnov and A. Dorfmann, "Mathematical modeling of magnetosensitive elastomers," Int. J. Solids Struct., vol. 40, no. 18, pp. 4659-4674, Sep. 2003. doi: 10.1016/S0020-7683(03)00265-8.

[4] I. Agirre-Olabide, A. Lion, and M. J. Elejabarrieta, "A new threedimensional magneto-viscoelastic model for isotropic magnetorheological elastomers," Smart Mater. Struct., vol. 26, no. 3, p. 35021, Feb. 2017. doi: $10.1088 / 1361-665 X / 26 / 3 / 035021$.

[5] M. Norouzi, S. M. Sajjadi Alehashem, H. Vatandoost, Y. Q. Ni, and M. M. Shahmardan, "A new approach for modeling of magnetorheological elastomers," J. Intell. Mater. Syst. Struct., vol. 27, no. 8, pp. 1121-1135, Nov. 2015. doi: 10.1177/1045389X15615966.

[6] M. R. Jolly, J. D. Carlson, B. C. Muñoz, and T. A. Bullions, "The Magnetoviscoelastic Response of Elastomer Composites Consisting of Ferrous Particles Embedded in a Polymer Matrix," J. Intell. Mater. Syst. Struct., vol. 7, no. 6, pp. 613-622, Nov. 1996.

doi: $10.1177 / 1045389 \times 9600700601$.

[7] L. C. Davis, "Model of magnetorheological elastomers," J. App. Phys., vol. 85 , no. 6, pp. 3348-3351, Mar. 1999. doi: 10.1063/1.369682.

[8] D. Ivaneyko, V. Toshchevikov, M. Saphiannikova, and G. Heinrich, "Effects of particle distribution on mechanical properties of magnetosensitive elastomers in a homogeneous magnetic field," arXiv preprint arXiv:1210.1401, 2012.

[9] Y. Shen, M. F. Golnaraghi, and G. R. Heppler, "Experimental Research and Modeling of Magnetorheological Elastomers," J. Intell. Mater. Syst. Struct., vol. 15, no. 1, pp. 27-35, Jan. 2004. doi: $10.1177 / 1045389 X 04039264$.

[10] J. M. Ginder and L. C. Davis, "Shear stresses in magnetorheological fluids: Role of magnetic saturation," Appl. Phys. Lett., vol. 65, no. 26, pp. 3410-3412, Dec. 1994. doi: 10.1063/1.112408.

[11] J. Ginder, L. Davis, and L. Elie, "Rheology of magnetorheological fluids: models and measurements," Int. J. Mod. Phys. B, vol. 10, no. 23n24, pp. 3293-3303, Oct. 1996. doi: 10.1142/S0217979296001744.

[12] Y. Zhu, X. Gong, H. Dang, X. Zhang, and P. Zhang, "Numerical Analysis on Magnetic-induced Shear Modulus of Magnetorheological Elastomers Based on Multi-chain Model," Chin. J. Chem. Phys., vol. 19, no. 2, pp. 126-130, Aug. 2006. doi: 10.1360/cjcp2006.19(2).126.5.

[13] L.R.G. Treloar, The Physics of Rubber Elasticity, Oxford University Press, 1975.

[14] R.W. Ogden, Non-linear elastic deformations, Courier Corporation, 1997.

[15] R.E. Rosensweig, Ferrohydrodynamics, Courier Corporation, 2013.

[16] G. Bossis, C. Abbo, S. Cutillas, S. Lacis, and C. Métayer, "Electroactive and Electrostructured Elastomers," Int. J. Mod. Phys. B, vol. 15, no. 06n07, pp. 564-573, Mar. 2001. doi: 10.1142/S0217979201005027.

[17] C. Bellan and G. Bossis, "Field dependence of viscoelastic properties of MR elastomers," Int. J. Mod. Phys. B, vol. 16, no. 17n18, pp. 2447-2453, Jul.2002. doi: 10.1142/S0217979202012499. 


\title{
On-line Determination of a Go-NoGo State Using a Contin ous Estimation of the System Response
}

\author{
Johanna McPhee and Rishad A. Irani \\ Dept. of Mechanical and Aerospace Engineering \\ Carleton University \\ Ottawa, Canada \\ Johanna.McPhee3@,Carleton.ca, Rishad.Irani@,Carleton.ca
}

\begin{abstract}
The formulation, tuning and performance of a signal prediction algorithm as applied to the determination of a Go-NoGo state are discussed. Simulations were used to tune and assess the performance of the signal prediction algorithm. The paper describes the development of useful criteria, based on the mean and standard deviation of the predicted signal, used for producing the Go-NoGo state. A latching algorithm was used to improve the output of the Go-NoGo state.
\end{abstract}

Keywords-Signal prediction; Go-NoGo state; control system state estimation

\section{INTRODUCTION}

In marine applications, the determination of the ship motion and the relative motion between two independent bodies is a challenging task. The motion of the mothership often defines the availability for load transfer, aircraft landing opportunities and the launch and recovery of marine craft. Sophisticated and established methods do exist that determine the motion of a ship in a seaway through panel or finite element methods [1],[2]; however, these methods do not lend themselves to real-time estimation. Moreover, these methods cannot be extrapolated to determine any generalized signal or motion. Kuchler et al. [3] presented an implementation of a wave-prediction algorithm, which combats transport delays between actual ship motion and measurement of ship motion. Woodacre et al. [4] was able to build upon Kuchler et al. [3] and use a version of the prediction algorithm as a previewing function for a Model-Predictive Control scheme (MPC). The current authors are building upon these works to generalize a signal prediction algorithm (SPA) so that it can predict any sinusoidal signal and, given a set of criteria, determine if a process has a high likelihood of success.

This paper presents the formulation of the signal prediction algorithm (SPA) in the following section, followed by an investigation of the tuning parameters used for detecting the modes of the signal. Simulation results are analyzed in Section III and useful criteria for defining a "GO" scenario are developed for the purpose of producing a Go-NoGo command signal. In Section IV, a latching algorithm is proposed to remove fluctuations in the Go-NoGo command signal. The paper ends with concluding remarks and future work. In general, the methods proposed can be used for fault detection and avoidance of unmanned systems.

\section{Signal Prediction Algorithim}

\section{A. Signal Prediction Formulation}

The signal prediction method based on the work of Kuchler et al [3] and Woodacre et al. [4],[5] is composed of three distinct parts: mode detection, estimation, and prediction. To predict the wave motion, the periodic components, or modes, must be identified. These modes are determined by decomposing the measured signal $s(t)$ into a set of $N$ sine waves expressed as

$$
s(t)=\sum_{i=1}^{N} A_{i} \sin \left(2 \pi f_{i} t+\varphi_{i}\right)+v(t)
$$

where the amplitude $A$, frequency $f$, and phase $\varphi$ of each mode $i$ are obtained by taking the Fast Fourier Transform (FFT) of past measured data over a specified time interval. Additionally, the static signal offset is denoted by $v(t)$. The specified time window for the FFT, referred to as $T_{F F T}$, and sampling frequency are set depending on the frequency content of the known wave spectrum. The time window $T_{F F T}$ is chosen such that the highest frequency of the wave spectrum can be detected, while also preventing aliasing. The sampling frequency of the FFT is selected such that a desired resolution in the frequency domain is achieved.

As conditions change, the number of modes $N$ and associated frequencies $f$ change with time; therefore, to predict the periodic motion, a peak detection algorithm is performed at every $T_{F F T}$ interval on the latest set of measured data. This peak detection algorithm determines the amplitude $A_{F F T}$ and frequency $f_{F F T}$ of each mode based on the dominant peaks in the FFT spectrum. A peak in the FFT spectrum is determined to be 
dominate when it exceeds a peak detection sensitivity, $\eta$, which is set by the user. When a new set of modes is identified, it is used to initialize an observer model with a new set of parameters: $A_{F F T}, f_{F F T}, \varphi_{F F T}$, and $N$.

The observer model is used to continuously estimate the mode parameters $A_{F F T}$ and $\varphi_{F F T}$ such that the latest signal measurements are used to adapt $A_{F F T}$ and $\varphi_{F F T}$ at each time step. For the prediction algorithm, a discrete Kalman filter is implemented to estimate the system states and has the form

$$
\begin{gathered}
\hat{x}_{k+1}=\boldsymbol{\Phi} \hat{x}_{k}+\boldsymbol{L}\left(w_{k}-\widehat{w}_{k}\right), \hat{x}_{0}=x_{0} \\
\widehat{w}_{k}=\boldsymbol{C} \hat{x}_{k}
\end{gathered}
$$

where $k$ is the current time step, $\boldsymbol{\Phi}$ is the discrete system matrix, $\widehat{\boldsymbol{x}}$ is the vector of observed states, $\boldsymbol{L}$ is the observer gains matrix, $\hat{w}$ and $w$ are the estimated motion and measured motion respectively, and $\boldsymbol{C}$ is the system output matrix. The discrete system matrix, observed states and system output matrix were derived following the work of Kuchler et al. [3].

For the current study, a static observer gain matrix was selected via manual tuning and has the form

$$
\boldsymbol{L}=\left[\begin{array}{llllllll}
0.2 & 3 & 0.2 & 3 & \ldots & 0.2 & 3 & 0.1
\end{array}\right]
$$

From each detected mode, two observer states $x_{i, 1}$ and $x_{i, 2}$ are estimated and then rearranged to solve for the adapted observer parameters $\varphi_{o b s}$ and $A_{o b s}$ at the current time $t_{k}$, such that:

$$
\begin{gathered}
\varphi_{o b s}=\arctan \left(\frac{2 \pi f_{i} \hat{x}_{i, 1}}{\hat{x}_{i, 2}}\right)-2 \pi f_{i} t_{k} \\
A_{o b s}=\frac{\hat{x}_{i, 1}}{\sin \left(2 \pi f_{i} t_{k}+\varphi_{o b s}\right)}
\end{gathered}
$$

Finally, the prediction algorithm can be used to forecast the motion sequence over a prediction horizon $T_{\text {Pred }}$, i.e. at the time $t_{k}+T_{\text {Pred }}$. Referring to Equation 1, the predicted motion at $t_{k}+$ $T_{\text {Pred }}$ is

$$
\begin{aligned}
& S_{\text {Pred }}(t)= \\
& \sum_{i}^{N} A_{\text {obs }, i, k} \sin \left(2 \pi f_{i}\left(t_{k}+T_{\text {Pred }}\right)+\varphi_{\text {obs }, k, i}\right)+v(t)
\end{aligned}
$$

To test the SPA and develop the Go-NoGo discriminator, a benchmark data set was used.

\section{B. Benchmark Data}

Figure 1 shows the benchmark test case used as the input signal $s(t)$ throughout this paper. This test case originates from a set of digitized ship motion data from the Australian DSTO report [6]. The signal $s(t)$ is the resolved roll motion of the data, in degrees.

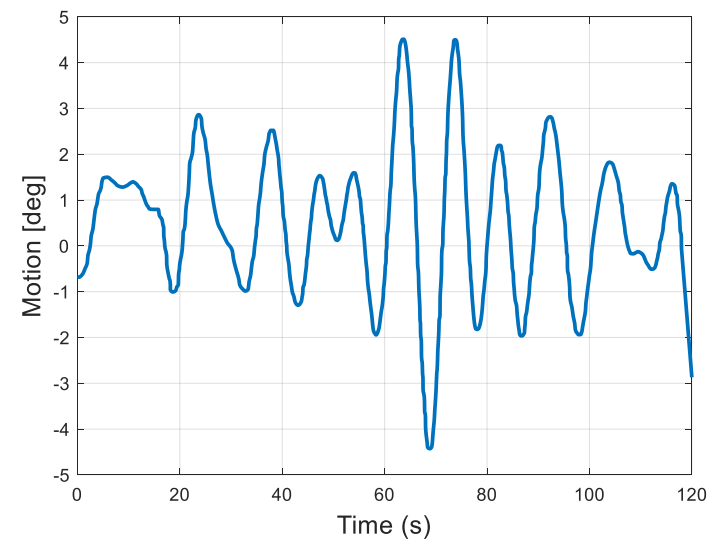

Figure 1. Benchmark test case of resolved roll motion from the Australian DSTO report [6].

\section{Mode Detection Tuning}

In the SPA formulation section above, it was discussed that to detect the dominant modes of the incoming signal it is necessary to select an appropriate $T_{F F T}$ and peak detection sensitivity, $\eta$. The selection of an inappropriate $T_{F F T}$ can result in two undesirable scenarios: the first being too short an interval that results in no modes detected, and the second being too long an interval that results in modes detected at a rate that does not keep up with changing conditions, yielding aliasing. To examine these issues, Figure 2 plots the input signal $s(t)$, as a solid blue line, and the prediction $s_{\text {Pred }}(t)$, as a dashed red line, at $0.25 \mathrm{~s}$ into the future. $s_{\text {Pred }}(t)$ is shifted back 0.25 seconds, i.e. 25 time steps, to place both the input signal and the prediction in the same time frame. The dotted green line between $70 \mathrm{~s}$ and $80 \mathrm{~s}$ in Figure 2 depicts the scenario where there is no predicted signal due to no modes detected during the previous $T_{F F T}$ interval.

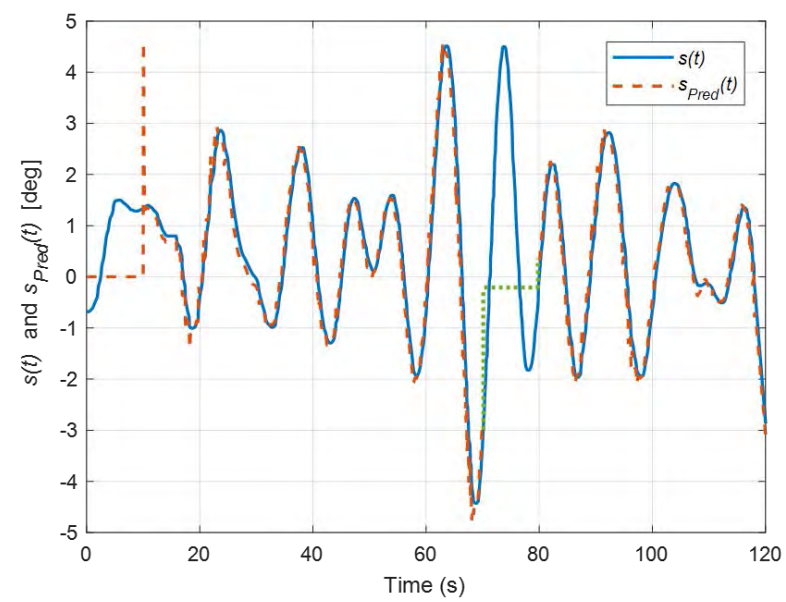

Figure 2. Measured and predicted signal together, with prediction signal time shifted to the input signal timeframe. The prediction discontinuity due to no modes detected is shown in green.

To address the issue of discontinuities in mode detection, the detection algorithm was amended such that in the case where zero modes are detected, the set of data over which the 
FFT is performed is expanded every time step until at least one new mode is detected. The next mode detection is then performed after another $T_{F F T}$ window, starting where the new set of modes was detected. In this way, the mode detection algorithm can handle slower than expected modes that may exist in the signal $s(t)$. In addition to the latter, the proposed amendments stated above also eliminate the second undesirable scenario, as the user can set a shorter $T_{F F T}$ to avoid aliasing, without needing to consider the $T_{F F T}$ length required to ensure continuous mode detection.

The peak sensitivity $\eta$ describes the minimum peak height; a threshold value used by the peak detection algorithm to determine the dominant peaks in the FFT spectrum. Figure 3 shows the amplitude-frequency spectrum of the first $10 \mathrm{~s}$ of the benchmark test case in Figure 1 with $\eta=0.01$, indicated by a dashed red line. For this $\eta$, the number of peaks detected is 6 , denoted by the triangle markers. By inspection of the first $10 \mathrm{~s}$ of the benchmark test case in Figure 1, to capture the dominate motion at most two peaks should be detected. Any other peaks in the FFT spectrum is attributed to sensor noise. By increasing the value of $\eta$, the sensor noise can be rejected. Shown as a dashed red line in Figure 4, $\eta$ has been increased by a factor of $10(\eta=0.1)$ and the number of peaks detected is two. The selection of peak detection sensitivity $\eta$ must therefore be set based on sensor noise and is case specific. Future work could examine a recursive methodology to determine if a peak is valid or estimate how many peaks should be identified.

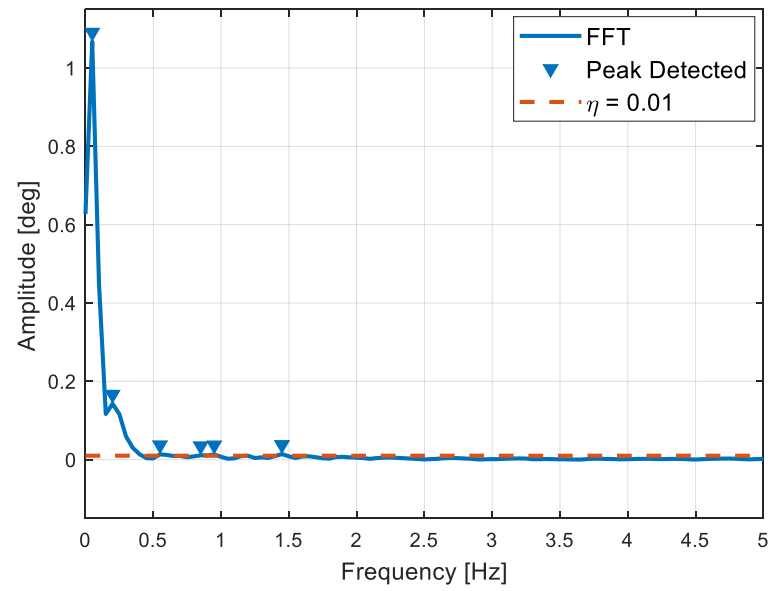

Figure 3. Dominant peaks detected from the FFT spectrum of the first $10 \mathrm{~s}$ of the benchmark case for $\eta=0.01$

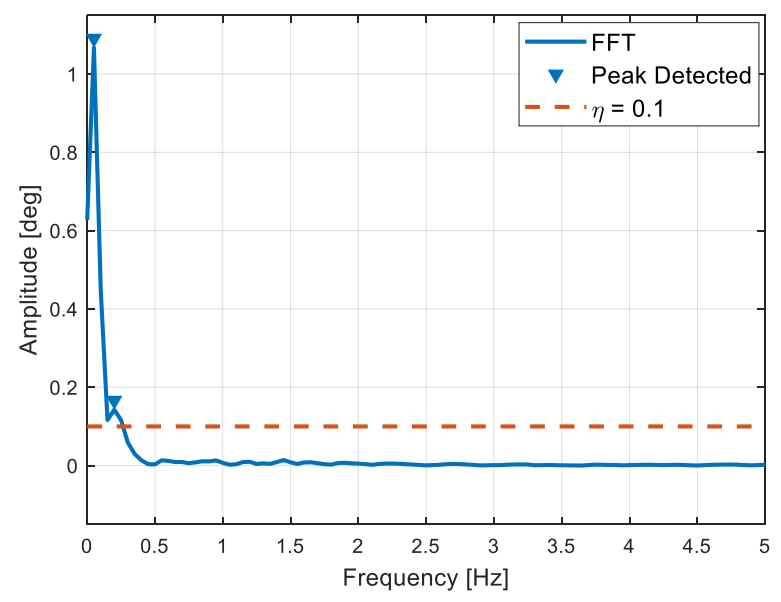

Figure 4. Dominant peaks detected from the FFT spectrum of the first $10 \mathrm{~s}$ of the benchmark case for $\eta=0.1$

\section{RESULTS}

\section{A. Simulation}

The SPA detailed in Section II was implemented in MATLAB and simulated in the Simulink environment. Figure 5 depicts the Simulink block that calls on the SPA function. The input variables include input signal $s(t)$; the GO criteria; tuning parameters $T_{F F T}$ and $\eta$; and the prediction horizon $T_{P r e d}$. The SPA block outputs the predicted signal $s\left(t+T_{\text {Pred }}\right)$ and the Go/NoGo command signal. A fixed step, discrete solver was used with fixed step $d t=0.01 \mathrm{~s}$. For the benchmark case in Figure 1, the simulation runtime is approximately $11 \mathrm{~s}$ for the full $118 \mathrm{~s}$ of the benchmark signal.

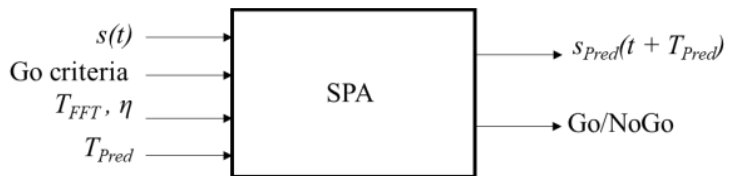

Figure 5. SPA function block implemented in the Simulink environment

\section{B. Prediction Assessment}

Figure 6 displays the predicted signal, solid red line, over a continuous prediction horizon of $10 \mathrm{~s}$ for the benchmark input signal, solid blue line, at time $t=38 \mathrm{~s}$. The parameters used for the prediction were: $T_{F F T}=10 \mathrm{~s}$ and $\eta=0.1$. In Figure 6, good accordance is observed between the input signal and predicted curve until $T_{\text {Pred }} \approx 3 \mathrm{~s}$. To investigate this further, the predicted signal is examined for $1 \mathrm{~s}, 3 \mathrm{~s}$ and $10 \mathrm{~s}$ prediction horizons.

Figure $7 \mathrm{a}$ depicts the prediction error for the prediction at 1 $\mathrm{s}$ into the future at every point in time. Similarly, Figure $7 \mathrm{~b}$ and $\mathrm{c}$ respectively depict the prediction error at $3 \mathrm{~s}$ and $10 \mathrm{~s}$ into the future at every point in time. Note that the first $11 \mathrm{~s}$ are omitted to ignore the initialization period of the SPA. The maximum, minimum, mean and standard deviation of the error for each case are tabulated in Table I for $t=11 \mathrm{~s}$ to $110 \mathrm{~s}$. From Table I, both the mean and standard deviation of the absolute error are significantly larger, by an order of magnitude, for the longest 
prediction horizon of $10 \mathrm{~s}$. The latter is consistent with what was qualitatively observed in Figure 6 at $t=38 \mathrm{~s}$. The results in Table I suggest that determining the likelihood of success of a process to produce a Go-NoGo command becomes less accurate for longer prediction horizons as the mean error increases significantly over this time interval. Furthermore, defining the GO scenario based on the maximum error observed over $T_{\text {Pred }}$ alone is not representative of true future events for longer predictions. The following section will develop useful GO criteria that considers the error that exists in the prediction.

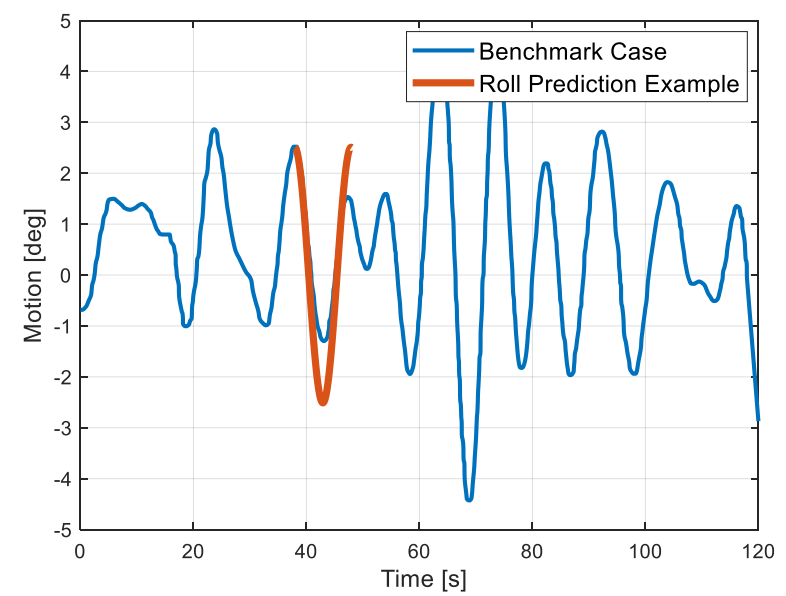

Figure 6. Benchmark data with prediction curve at $t=38 \mathrm{~s}$ plotted over a continuous time horizon of $10 \mathrm{~s}$ into the future.

TABLE I. ERROR METRICS FOR VARIOUS PREDICTION HORIZONS

\begin{tabular}{|l|l|l|l|}
\hline \multirow{2}{*}{$\mid$ Error| [deg] } & \multicolumn{3}{|l|}{ Prediction Horizon, $\boldsymbol{T}_{\text {Pred }}$} \\
\cline { 2 - 4 } & $\boldsymbol{T}_{\text {Pred }}=\mathbf{1} \boldsymbol{s}$ & $\boldsymbol{T}_{\text {Pred }}=\mathbf{3} \boldsymbol{s}$ & $\boldsymbol{T}_{\text {Pred }}=\mathbf{1 0} \boldsymbol{s}$ \\
\hline Maximum & 1.74 & 3.72 & 3.88 \\
\hline Minimum & $4.57 \times 10^{-6}$ & $4.41 \times 10^{-4}$ & $2.93 \times 10^{-4}$ \\
\hline Mean & 0.29 & 0.89 & 1.41 \\
\hline $\begin{array}{l}\text { Standard } \\
\text { Deviation }\end{array}$ & 0.35 & 0.92 & 1.69 \\
\hline
\end{tabular}
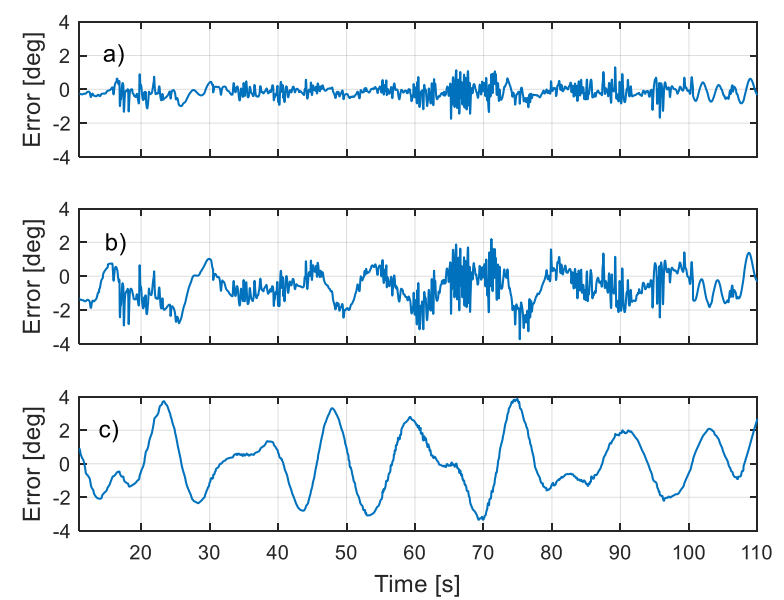

Figure 7. Error between benchmark data and prediction at a) $1 \mathrm{~s}$ into the future, b) $3 \mathrm{~s}$ into the future and c) $10 \mathrm{~s}$ into the future.

\section{Developing the Go/NoGo Criteria}

For the purposes of this paper, the SPA is used to output a Go-NoGo command based on given GO criteria. The GO criteria could be one or several parameters used to define the acceptable conditions for a GO scenario. The criteria are compared with the predicted signal over the continuous prediction horizon $T_{\text {Pred }}$ at each time step. If over the prediction horizon, the predicted signal meets the GO criteria, a GO command is output. Conversely, if the GO criteria are not met by the predicted signal over the prediction horizon, a NoGo command is output. In this section, the development of useful definitions of the GO scenario, to be identified based on the GO criteria, are investigated.

The first definition of the GO scenario to be investigated is the simple Maximum Prediction Threshold (MPT) approach. This approach identifies a GO scenario if over the entire prediction horizon, the maximum value of the predicted signal is below the given threshold value. In other words, only one point of the predicted signal must to be above the threshold to output a NoGo command. The results in Table I suggest that this simple Maximum Prediction Threshold approach would not provide a useful definition of the GO scenario for longer $T_{\text {Pred }}$, as there exists more error with the predicted signal as the prediction horizon increases. Two more GO criteria are suggested below that are defined such that the error that exists in the prediction is considered.

If instead, the GO scenario is defined based on the statistical spread of the predicted signal, it is possible to relax how the GO criteria identify a GO scenario. The spread of 1 Standard Deviation (1-SD) approach outputs a GO command when the prediction mean, evaluated over the prediction horizon, plus 1 standard deviation of the prediction is below a threshold value. The 1-SD approach is expressed empirically in Equation 6 as

$$
\text { Go Criteria }>\overline{\text { Pred }, k_{1}}+\sigma_{k}
$$

where $\overline{S_{\text {Pred }}}$ is the prediction mean over $T_{\text {Pred }}$ at time step $k$, and $\sigma$ is the standard deviation of the prediction over $T_{\text {Pred }}$ at time step $k$. Equation 6 is equivalent to stating that for any randomly selected sample of the predicted signal over $T_{\text {Pred }}$, a GO command is output if $68 \%$ of the sampled points fall below the threshold.

The spread of 1.645 Standard Deviations (1.645-SD) approach defines the GO scenario to occur when the prediction mean plus 1.645 standard deviations is below the threshold value. The 1.645-SD approach is expressed empirically in Equation 7 as

$$
\text { Go Criteria }>\overline{S_{\text {Pred }, k}}+1.645 \sigma_{k}
$$

Equation 7 is equivalent to stating that for any randomly selected sample of the predicted signal over $T_{\text {Pred, }}$ a GO command is output if $90 \%$ of the sampled points fall below the threshold. Both methods described in Equations 6 and 7 are based on the same principle but differ in how conservatively they evaluate the predicted signal. 
To evaluate the three definitions of the GO scenario MPT, 1$\mathrm{SD}$ and 1.645-SD, the SPA was run for each case with the benchmark data and compared with the desired Go-NoGo command signal. The desired command signal was determined by setting the command as GO when the benchmark case is below the threshold value and setting the command as NoGo when the benchmark case is above the threshold value. Figure 8 a shows the input signal, left axis, as a solid blue line with the GO criteria threshold, set as $2.5^{\circ}$, as a dashed red line. In Figure $8 \mathrm{a}$, the desired Go-NoGo command signal, right axis, is also shown for $t=50 \mathrm{~s}$ to $t=90 \mathrm{~s}$ of the simulation. Here GO $=1$ and $\mathrm{NoGo}=0$. Figure 8 part $\mathrm{b}, \mathrm{c}$ and $\mathrm{d}$ depict the Go-NoGo command signal for the MPT, 1-SD and 1.645-SD approaches respectively. The three GO scenario definitions were evaluated based on the number of seconds that a GO command is output compared to the amount of GO time registered by the desired command signal. When obtaining the GO time, the first $11 \mathrm{~s}$ of data are omitted to ignore the SPA initialization period. In Table II, the GO time for the benchmark case from $t=11 \mathrm{~s}$ to $t=118$ $\mathrm{s}$ is tabulated for each approach.

TABLE II. EVALUATION OF GO SCENARIO DEFINITIONS

\begin{tabular}{|l|c|c|c|c|}
\hline \multirow{2}{*}{$\begin{array}{l}\text { "GO" } \\
\text { metric }\end{array}$} & \multicolumn{4}{|c|}{ “GO" scenario definition [11 s-118 s] } \\
\cline { 2 - 5 } & Desired & $\boldsymbol{M P T}$ & $\mathbf{1 - S D}$ & $\mathbf{1 . 6 4 5 - S D}$ \\
\hline $\begin{array}{l}\text { "GO" } \\
\text { time [s] }\end{array}$ & \multirow{2}{*}{98.98} & 87.71 & 94.78 & 77.94 \\
\cline { 1 - 3 } Error [s] & & 11.27 & 4.20 & 21.04 \\
\hline
\end{tabular}

Due to its close agreement with the desired command signal, the best definition of the GO scenario, based on a given threshold value, is the 1-SD method. Moreover, with the least amount of GO time, the 1.645-SD method was found to be the most conservative method for defining the GO scenario.
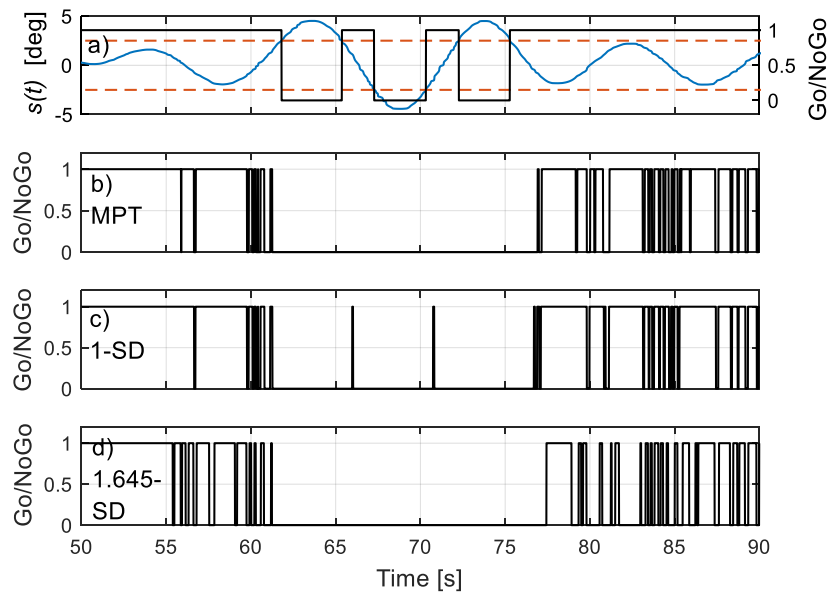

Figure 8. Go-NoGo command signals with desired command signal in a), and resulting command outputs for approaches b) MPT, c) 1-SD and d) 1.645-SD. For display purposes, the results are shown for $t=50 \mathrm{~s}$ to $t=90 \mathrm{~s}$.

\section{Go-NoGo Command Latching}

Observing the Go-NoGo signals in Figure 8b, c and d at 60 $\mathrm{s}, 80 \mathrm{~s}$ and $90 \mathrm{~s}$, there is rapid fluctuation in the command signal.
For a physical implementation of the SPA, it is desired that the SPA latches on to a GO or NoGo command to meet the responsivity of the physical system components. A method for Go-NoGo command latching is proposed below to limit this undesirable fluctuation.

Figure 9 shows the logic flowchart for the latching algorithm. To start, the latching algorithm receives the Go-NoGo state determined from the predicted signal. Next, the algorithm looks to see if a target state has been set. If no target state has been set, the current Go-NoGo state is set as the target state. The algorithm must then determine whether it will commit to this target by watching the incoming Go-NoGo state over an evaluation period $T_{\text {eval }}$. During this evaluation period, if at any point the incoming Go-NoGo state differs from the target state, the current Go-NoGo state is set as a new target state and $T_{\text {eval }}$ is reset. However, if all Go-NoGo states received during the evaluation period match the target state, the algorithm will "latch" onto the target state and output this command over the runtime interval $T_{\text {run }}$. After $T_{\text {run }}$ is complete, a new evaluation period begins.

Figure 10 shows the results of the latching algorithm where the Go-NoGo command signal without latching is shown in $10 \mathrm{a}$, and the Go-NoGo command signal with latching is shown in $10 \mathrm{~b}$. For illustrative purposes, $T_{\text {eval }}$ was set as $0.1 \mathrm{~s}$ and $T_{\text {run }}$ was set as $2 \mathrm{~s}$, i.e. if a GO condition is sustained for $0.1 \mathrm{~s}$ the system will latch in a GO condition for at least 2 seconds. The command signals are output for the benchmark test case for $t=$ $50 \mathrm{~s}$ to $t=90 \mathrm{~s}$. It is observed that for the selected $T_{\text {eval }}$ and $T_{\text {run }}$, all fluctuation was removed from the original command signal in Figure 10 a. $T_{\text {eval }}$ and $T_{\text {run }}$ are case specific and should be based on the physical systems involved.

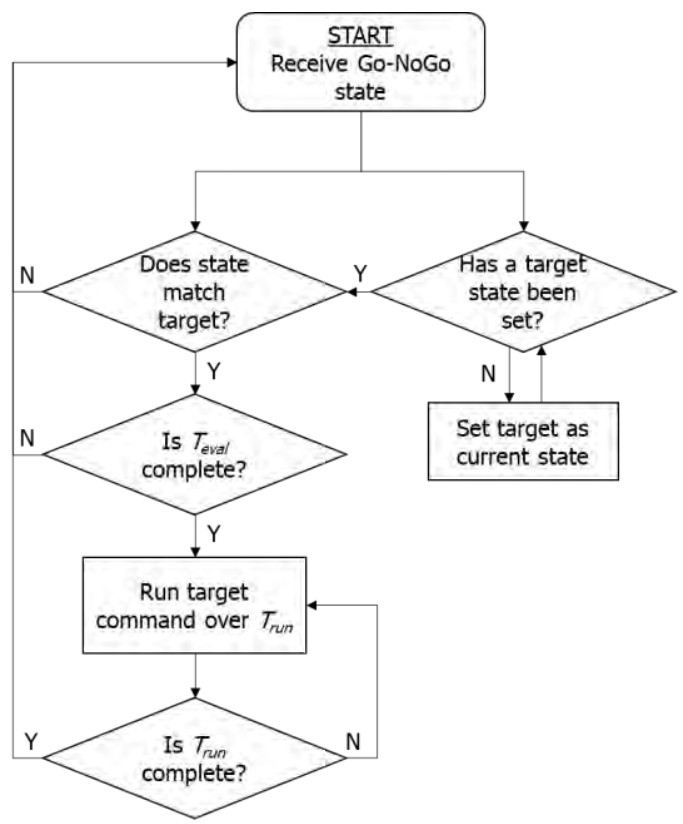

Figure 9. Flow chart of the logic used in the command signal latching algorithm. 

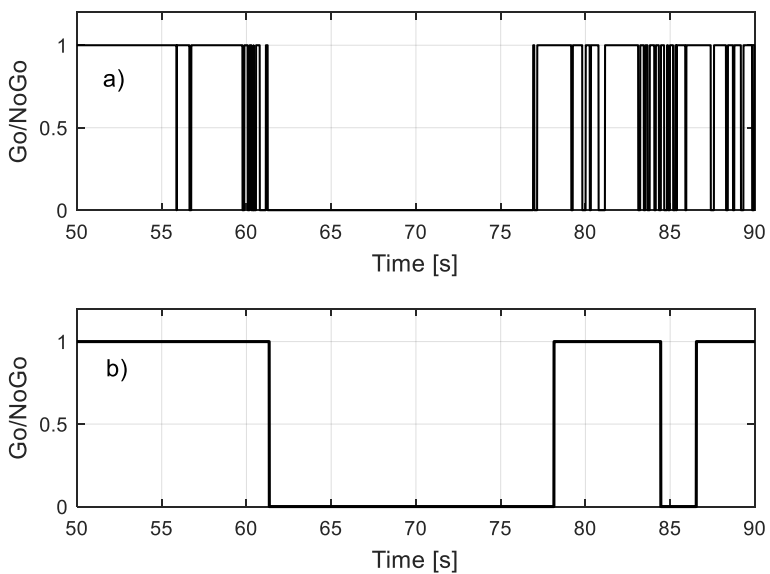

Figure 10. Go-NoGo command signals for the benchmark test case without latching in a) and with command latching in b). For display purposes, the results are shown for $t=50 \mathrm{~s}$ to $t=90 \mathrm{~s}$.

\section{CONCLUSIONS}

This paper describes the generalized formulation and tuning of a signal prediction algorithm as it applies to the determination of a Go-NoGo state. A key modification made to the SPA was the implementation of continuous mode detection that enables the algorithm to dynamically handle changing conditions of the input signal. Tuning the SPA also included setting a peak height sensitivity parameter that is used for determining the dominant peaks in the FFT spectrum during mode detection. By investigating the FFT spectrum of one identification period of the input signal, it was determined that the peak height sensitivity should be set such that it ignores sensor noise in the measured signal and is therefore case specific.

The paper also described the development of useful GO criteria used for producing the Go-NoGo state. Because prediction error increases over the prediction horizon, it was determined that a GO scenario identified by the maximum point of the prediction should not be used, as this approach can lead to erroneous conclusions regarding the Go-NoGo state. Instead, a GO scenario defined by the mean and one standard deviation of the predicted signal over the prediction horizon was found to produce a Go-NoGo signal that agreed the most with the desired Go-NoGo signal.

Performance of the SPA was further improved with the implementation of a latching algorithm that successfully removed undesirable fluctuation in the Go-NoGo signal.

It is anticipated that several practical industrial applications can benefit from the proposed methods of determining a GoNoGo state. In general, the methods proposed can be used for fault detection and avoidance of unmanned systems. Specifically, with UAV ship landing operations the SPA can be employed to estimate the 'quiescent period', where the roll and pitch angles of the ship are below critical threshold values. From the estimated quiescent period, a Go-NoGo state is inferred that can be used to increase the effectiveness of autonomous vertical landings on transient platforms.

\section{ACKNOWLEDGEMENT}

The authors would like to thank the Natural Sciences and Engineering Research Council of Canada (NSERC), Kraken Robotic Systems Inc. and Carleton University for their support of this research.

\section{REFERENCES}

[1] K. McTaggart, "ShipMo3D version 3.0 user manual for creating ship models," Tech. Rep. TM 2011-308, Defence Research and Development Canada, 2012.

[2] G. D. Weymouh, R. V. Wilson, and F. Stern. "RANS computational fluid dynamics predictions of pitch and heave ship motions in head seas." Journal of Ship Research vol. 49, no. 2, pp. 80-97, 2005.

[3] S. Kuchler, T. Mahl, J. Neupert, K. Schneider, and O. Sawodny, "Active control for an offshore crane using prediction of the vessels motion," Mechatronics, IEEE/ASME Transactions, vol. 16, no. 2, pp. 297-309, 2011.

[4] J. K. Woodacre, R .J. Bauer, R. A. Irani, "Hydraulic valve-based activeheave compensation using a model-predictive controller with non-linear valve compensations," Ocean Engineering, vol. 152, pp. 47- 56, 2018. doi: 10.1016/j.oceaneng.2018.01.030.

[5] J. Woodacre, W. Wahidi, R. Bauer and R. Irani, "Coupling a Standard Hydraulic Valve and Advanced Control to Achieve a Motion Compensation System," in ASNE Launch and Recovery Symposium, Nov. 16-17, 2016.

[6] A. Arney, FFG-7 ship motion and airwake trial. part II: removal of ship motion effects from measured airwake data. Air Operations Division Aeronautical and Maritime Research Laboratory, Melbourne Victoria, Australia. Oct. 1994. 


\title{
Application of Fiber Bragg Grating Sensor for Strain Measurement at the Notch Tip under Cyclic Loading
}

\author{
Amirhossein Pahlevanpour, Bahareh Marzbanrad, Seyed Behzad Behravesh, Hamid Jahed \\ Mechanical and Mechatronics Engineering Department, University of Waterloo \\ Waterloo, Canada
}

\begin{abstract}
Notches are inevitable in many components and structures due to design limitations. In addition, they are the locations for stress concentration and are susceptible to fatigue failure. As a result, the cyclic stress/strain response at a notch is of key importance. Fiber Bragg Grating (FBG) sensors have been successfully utilized for mechanical and thermomechanical strain measurement in many cases; nevertheless, their capability of measuring strain at spots with intensive stress/strain has not yet been explored. In this research, FBG sensors are employed for strain measurement at the notch tip. A verification test was designed to substantiate the FBG measurements. The test involves a rectangular magnesium sheet with a center hole, subjected to uniaxial cyclic loading while the strain was measured at the notch tip using three different methods: strain gage, digital image correlation (DIC), and FBG. There were good agreements between the three measurements.
\end{abstract}

Keywords- Notch; strain measurement; FBG; DIC

\section{INTRODUCTION}

Fatigue is the dominant failure mode for components under cyclic loading, particularly in notched members [1]. To assess fatigue life, the cyclic stress/strain response at the notch tip is of key importance; however, strain measurement at this location has been challenging due to the high gradient of strain over a small area in the vicinity of the notch.

The common experimental approaches for notch stress/strain assessment are photoelasticity, thermoelasticity, brittle coatings, strain gages, and digital image correlation (DIC). Photoelasticity was initially devised by Brewster [2]. More than a century later, Coker and Filon developed this method as a tool for stress distribution assessment [3]. In this technique, a transparent material is utilized for fabricating a specimen with geometry similar to that of the notched member, and stress distribution is obtained by monitoring changes in the optical properties of the loaded transparent specimen via a polariscope. Photoelasticity can be adapted to both static and dynamic loading scenarios, even though doing so requires model preparation, and sometimes, tedious post-calculation [4].
The thermoelastic effect in metals was first recognized by Weber [5]. Belgen developed the first thermoelasticity application for contactless experimental stress analysis [6]. In thermoelasticity, local small temperature alterations of the loaded specimen indicate the stress distribution, allowing fullfield stress map to be made of the surface. In this method, the minimum detectable stress is restricted by the sensitivity of the employed apparatus; therefore, high precision requires costly experimental setup [7].

The brittle coating method begins with spraying a brittle coating on the surface of the notched member and letting it dry. The applied load will form crack patterns in the coating. The region and magnitude of stress concentrations can be indicated by comparing the crack patterns with the intact surface of the calibration coating. The coating's vulnerability to humidity and temperature, and the qualitative rather than quantitative results are two disadvantages of this method [8].

The strain gage technique is the most common experimental method for local strain measurement. The strain gage consists of a metallic foil pattern that acts as electrical resistance, supported by an insulating backing. It is attached to a prepared surface in the region of interest by a suitable adhesive. The dimensional change caused by the applied load results in electrical resistance alternation of the foil, which is used for strain measurement [9]. Initial calibration, nonlinearity, and pointwise measurement are among strain gage's main drawbacks [7].

Digital image correlation (DIC), is a non-contact optical technique for strain measurement, employs image tracking and registration techniques [10]. One of the first applications of DIC for deformation quantification was suggested by Peters and Ranson [11]. The DIC's accuracy has been widely substantiated through comparison with finite element and other verified methods [12]-[17]. Two DIC algorithms were used by Gonzáles and co-workers [10] for elastoplastic strain distribution measurement in shallow and deep notches. They verified their results with finite element method (FEM). Kotousov et al. [18] employed DIC for displacement- and strain-fields quantification near a sharp notch and compared the measurements with analytical and numerical solutions. 
The Fiber Bragg grating sensor is another advanced optical method for gauging external stimulants like strain. The Bragg gratings are written into the germanium-doped core of an optical fiber by periodic ultraviolet exposure leading to peculiar wavelengths reflection [19]. Any externally applied load and/or change in temperature increases or decreases the initial wavelength that is recorded by an interrogator during the experiment. The magnitude of this alternation can be converted into strain values by means of the mathematical expressions [19]. Even though the merit of FBG sensors for mechanical strain quantification has been verified extensively in the literature [20]-[24], its capability for intense notch tip strain measurement is still not well understood.

Because of the high resolution of the grating in the FBG sensor, it could be a proper candidate for mechanical strain measurement at spots with an intensive stress/strain gradient, e.g., at notches. To explore the applicability of the FBG, they are employed in this work for strain measurements at the notch tip. Variable amplitude cyclic loading was applied to the specimen, and the strain was measured utilizing three different measurement methods: strain gage, DIC, and FBG.

\section{Measurement And Test Procedures}

\section{A. Material and Experimental Setup}

The material under investigation was $4 \mathrm{~mm}$ thick AZ31BH24 hot-rolled magnesium sheet, provided by Magnesium Elektron of North America (MENA). Wrought magnesium has different yields in tension and compression [25] similar to the strength-differential effect seen in high strength steels [26]. The specimen was machined along the rolling direction with the dimensions shown in Fig. 1.

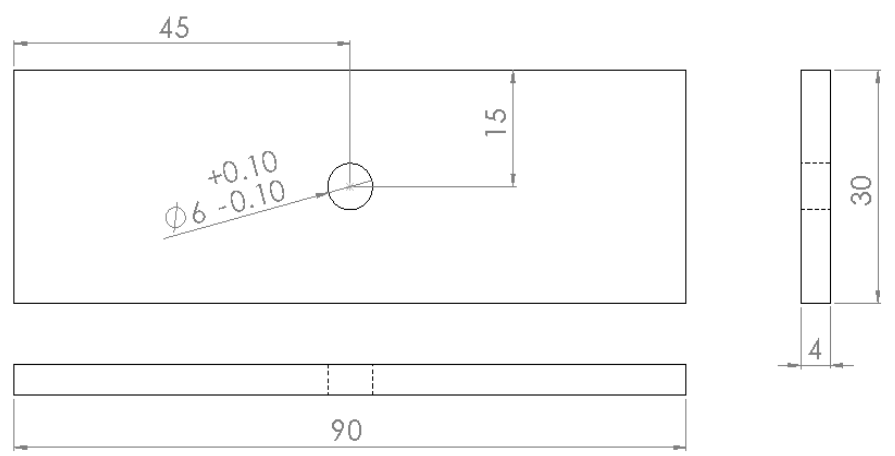

Figure 1. Notched specimen geometry (dimensions in millimeters)

Miniature strain gage C2A-06-015LW-120 from Micro Measurement Co. was selected for parallel strain measurement. The geometry of the strain gage is shown in Fig. 2. DAQDQ430 data-acquisition was employed, and one dummy gage was linked to the device to support quarter bridge gage configuration.

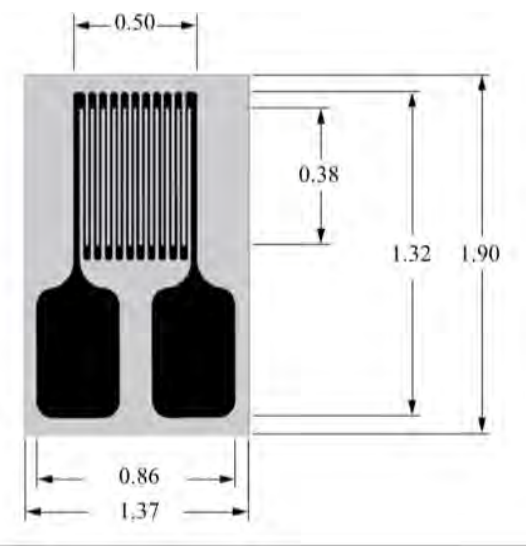

Figure 2. C2A-06-015LW-120 Strain Gage

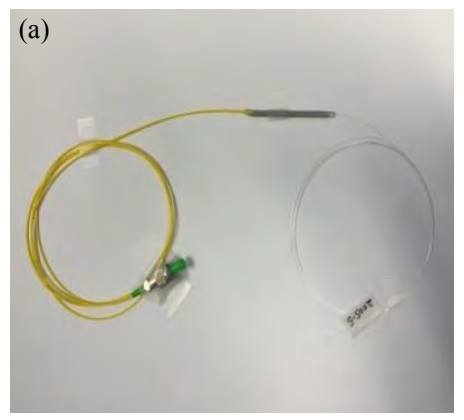

(b)

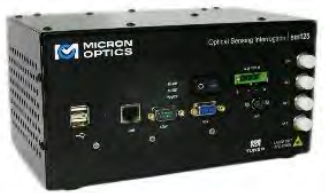

Figure 3. FBG equipment a) Corning SMF-28 with Bragg Grating b) Micron Optic SM125-200 Interrogator

Corning SMF-28 FBG with $5 \mathrm{~mm}$ effective length and 125 $\mu m$ dimeter were used in this research. The fiber optic sensor is illustrated in Fig. 3 (a). The initial wavelength was measured to be $1550 \mathrm{~nm}$. The grating modulation is Gaussian-apodized for this sensor, and the Bragg grating (1 $\mathrm{mm}$ length) had been written on a single mode of the optical fiber. The utilized optical interrogator is a Micron Optic SM125-200 with $5 \mathrm{pm}$ resolution and an accuracy specification of $10 \mathrm{pm}$, as shown in Fig. 3 (b). It was connected to one side of the fiber optic cable by a FC/APC fiber connector.

For DIC strain measurement, the ARAMIS 3D System with two 5 megapixels CCD cameras, capable of 15 fps readout at full resolution has been employed. This stereo system delivers precise $3 \mathrm{D}$ coordinates by using stochastic patterns or reference point markers.

Three different methods- DIC, strain gage, and FBG- were utilized for notch tip strain measurement. The schematic configuration indicating the devoted area to each technique is illustrated in Fig. 4 (a). To obtain comparable results from the different techniques, measurement was performed at the same distance from the notch tip. Due to the installation limitations enforced by the strain gage dimensions, the strain gage was attached to the specimen as close to the notch tip as was technically possible. Then, its location was used as a reference for the FBG sensor and DIC measurements. The FBG sensor was connected to the other side of the notch, as shown in Fig. 4 (b). For simultaneous strain measurement, LabVIEW was used. 


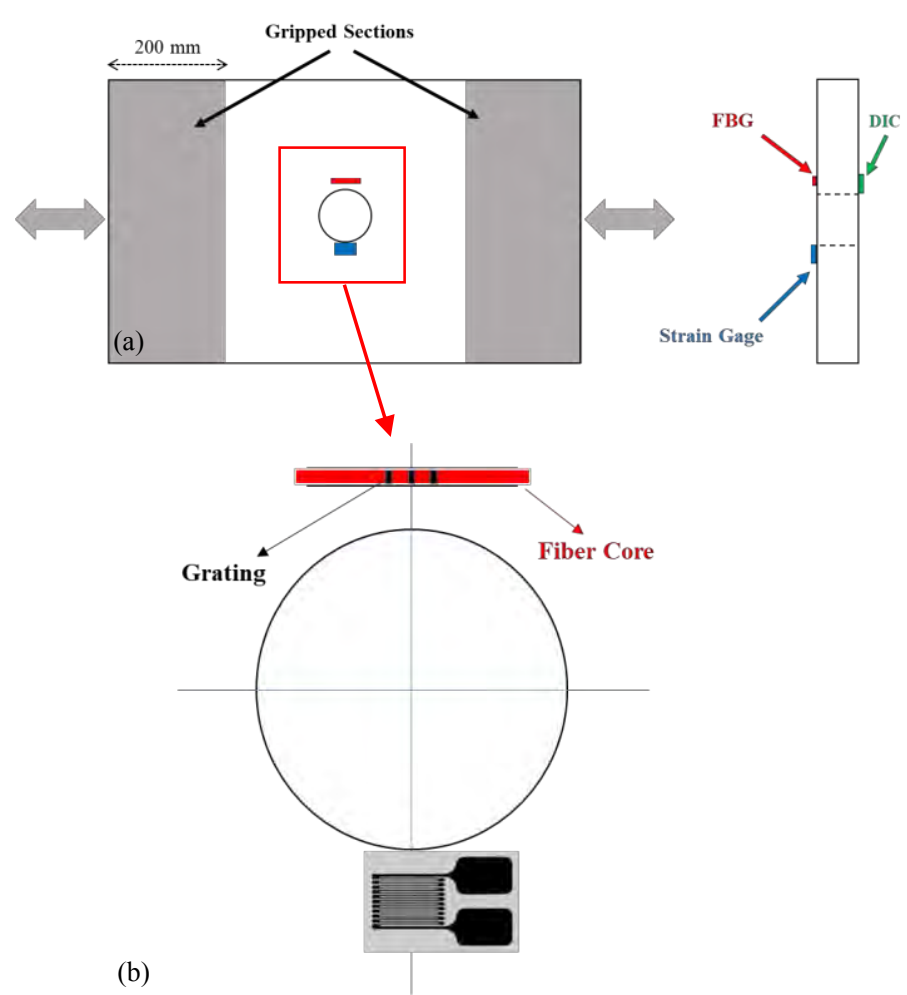

Figure 4. Schematic configurations of a) techniques used for notch tip strain measurement b) FBG and strain gage positions relative to the notch

\section{B. Test Procedures}

The specimen was loaded using the 8874 Instron servohydraulic test frame with $25 \mathrm{kN}$ load capacity and $100 \mathrm{~N} . \mathrm{m}$ torque capacity. The test was run in load-control mode, and a variable amplitude load (VAL) pattern was applied. The peak force was $13 \mathrm{KN}$ in accordance with the notch tip stress concentration to compel plastic deformation at the notch tip, while the far field remained elastic. Given the specimen geometry, shown in Fig.1, and the mode of loading, Fig. 4 (a), the stress concentration factor is equal to 2.5 [27]. The VAL loading scenario is depicted in Fig. 5.

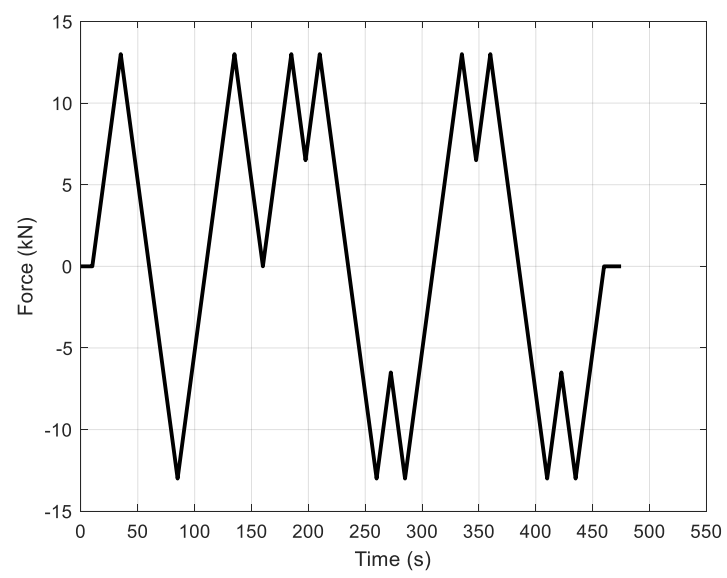

Figure 5. VAL scenario for FBG evaluation

\section{RESULTS AND DISCUSSION}

Fig. 6 shows the test setup. Measured Peak and valley strains are tabulated in Table. I, and the engineering strain as a function of time is plotted in Fig. 7. All three methods follow the VAL pattern closely, with DIC having more noise in the measurements. This issue can possibly be resolved by utilizing higher resolution cameras. Fig 8. manifests the strain distribution around the notch tip for the first peak of loading captured by DIC.
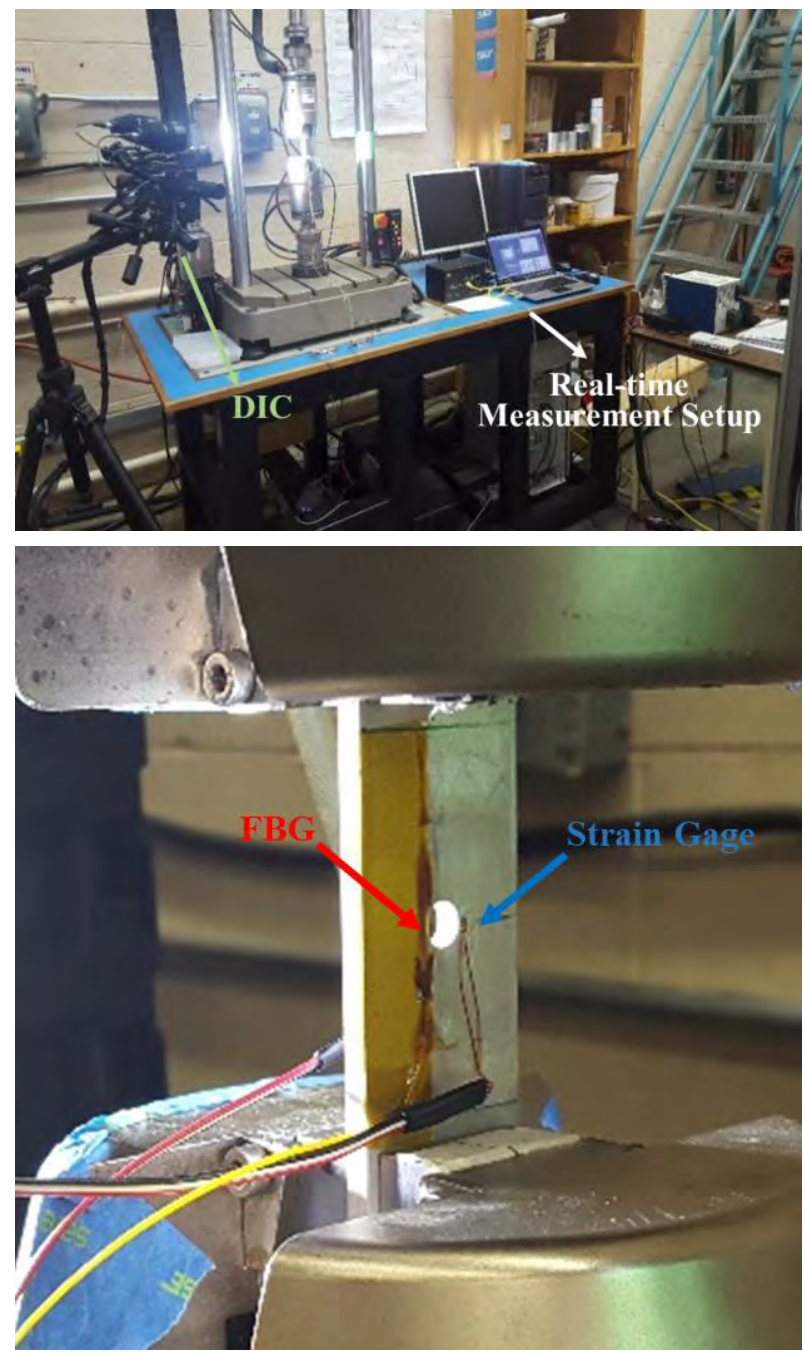

Figure 6. Final setup for simultaneous strain measurement

TABLE I. First Peak AND VAlLey Of Strain (\%) MEASured By THREE DIFFERENT TECHNIQUES

\begin{tabular}{ccc} 
Techniques & Peak & Valley \\
\hline Strain Gage & 0.587 & -0.497 \\
\hline FBG & 0.601 & -0.575 \\
\hline DIC & 0.599 & -0.523
\end{tabular}




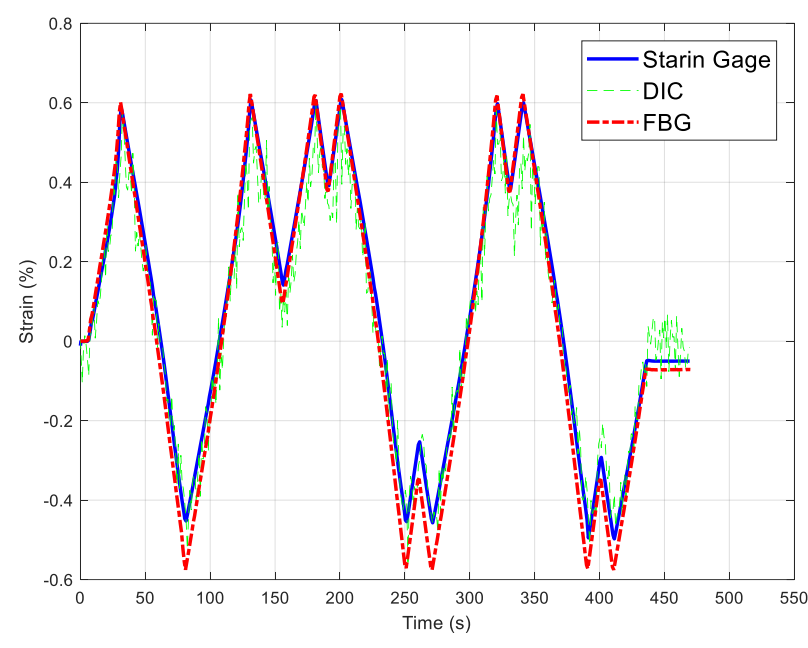

Figure 7. Notch strain measured by three experimental approaches

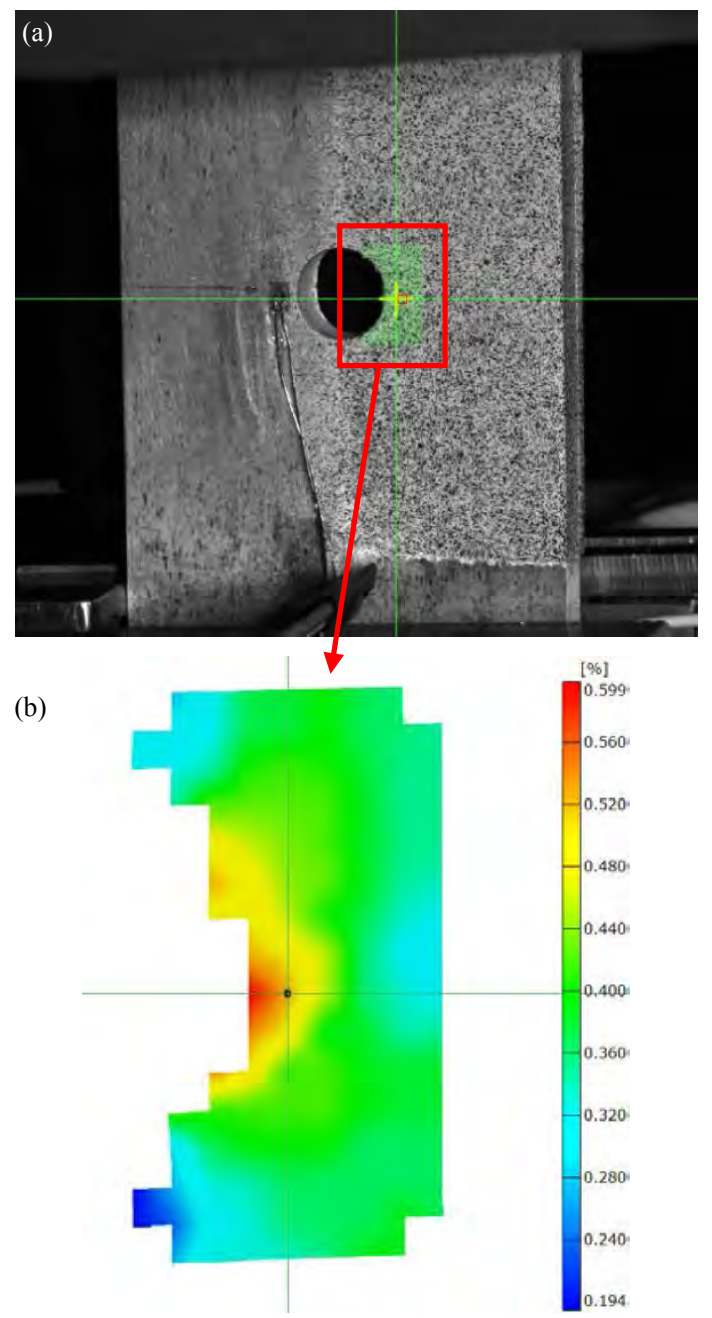

Figure 8. Peak strain distribution at the notch tip captured by DIC a) sample with speckle pattern b) strain map
The FBG sensor was capable of monitoring real-time strain during the experiment, whereas for DIC, the obtained images had to be analyzed after the test. In addition, the FBG pointwise resolution was higher compared to the strain gage. Overall, this study demonstrates the promising capability of FBG for fine strain measurement at the notch tip. Major sources for the slight difference in results are attributed to the following:

- Due to the very high strain gradient in the area near the notch root, even a few microns difference in the measurement position can affect the results significantly.

- All the implemented techniques average the strain over a specific area, e.g., the resistor area $\left(0.38 \times 0.5 \mathrm{~mm}^{2}\right)$ of the strain gage, although efforts have been made to keep it as small as possible.

\section{REFERENCES}

[1] R. I. Stephens, A. Fatemi, R. R. Stephens and H. O. Fuchs, Metal Fatigue in Engineering (second Edition), vol. 103, no. 4. 2001.

[2] D. Brewster, Experiments on the Depolarization of Light as Exhibited by Various Mineral, Animal, and Vegetable Bodies, with a Reference of the Phenomena to the General Principles of Polarization, vol. 2. 1815.

[3] E. G. Coker and L. Filon, Treatise on Photoelasticity. 1930.

[4] J. F. Doyle and J. W. Phillips, Manual on experimental stress analysis, vol. 5. 1989.

[5] W. Weber, "Über Die Spezifische Wärme Fester Körper Insbesondere Der Metalle," Ann. Phys. Chem, no. 96, pp. 177-213, 1830.

[6] M. H. Belgen, Infrared radiometric stress instrumentation application range study. 1968.

[7] W. N. J. Sharpe, Springer Handbook of Experimental Solid Mechanics, 2008. doi: 10.1007/978-0-387-30877-7.

[8] W. A. M. Brekelmans, H. W. Poort, and T. J. J. H. Slooff, "A new method to analyse the mechanical behaviour of skeletal parts," Acta Orthop., vol. 43, no. 5, pp. 301-317, 1972. doi: 10.3109/17453677208998949.

[9] M. Kutz, Handbook of measurement in science and engineering, vol. 3.2016. doi: 10.1002/9781119244752.

[10] H. Schreier, J. J. Orteu, and M. A. Sutton, Image correlation for shape, motion and deformation measurements: Basic concepts, theory and applications. 2009.

[11] W. H. Peters and W. F. Ranson, "Digital Imaging Techniques In Experimental Stress Analysis," Opt. Eng., vol. 21, no. 3, pp. 427-431, 1982. doi: $10.1117 / 12.7972925$.

[12] B. W. Smith, X. Li, and W. Tong, "Error Assessment for Strain Mapping by Digital Image Correlation," Exp. Tech., vol. 22, no. 4, pp. 19-21, 1998. doi: 10.1111/j.1747-1567.1998.tb02332.x.

[13] W. Tong, "An Evaluation of Digital Image Correlation Criteria for Strain Mapping Applications," pp. 167-175, 2005.

[14] H. W. Schreier, J. R. Braasch, and M. A. Sutton, "Systematic errors in digital image correlation caused by intensity interpolation," Opt. Eng., vol. 39, no. November, p. 2915, 2000. doi: 10.1117/1.1314593.

[15] Y. Q. Wang, M. A. Sutton, H. A. Bruck, and H. W. Schreier, "Quantitative error assessment in pattern matching: Effects of intensity pattern noise, interpolation, strain and image contrast on motion measurements," Strain, vol. 45, no. 2, pp. 160-178, 2009. doi: 10.1111/j.1475-1305.2008.00592.x. 
[16] B. Pan, A. Asundi, H. Xie, and J. Gao, "Digital image correlation using iterative least squares and pointwise least squares for displacement field and strain field measurements," Opt. Lasers Eng., vol. 47, no. 7-8, pp. 865-874, 2009.

doi: 10.1016/j.optlaseng.2008.10.014.

[17] Z. Y. Wang, H. Q. Li, J. W. Tong, and J. T. Ruan, "Statistical analysis of the effect of intensity pattern noise on the displacement measurement precision of digital image correlation using self-correlated images," Exp. Mech., vol. 47, no. 5, pp. 701-707, 2007. doi: 10.1007/s11340-006-9005-9.

[18] A. Kotousov, Z. He, and A. Fanciulli, "Application of digital image correlation technique for investigation of the displacement and strain fields within a sharp notch," Theor. Appl. Fract. Mech., vol. 79, pp. 51-57, 2015.

doi: 10.1016/j.tafmec.2015.05.012.

[19] M. M. Werneck, R. C. Allil, and F. V. . Nazare, Fiber Bragg Gratings:

Theory, Fabrication, and Applications, 2017.

[20] B. Marzbanrad, H. Jahed, and E. Toyserkani, "On the evolution of substrate's residual stress during cold spray process: A parametric study," Mater. Des., vol. 138, pp. 90-102, 2018. doi: 10.1016/j.matdes.2017.10.062.

[21] B. Marzbanrad, E. Toyserkani, and H. Jahed, "Cyclic hysteresis of AZ31B extrusion under load-control tests using embedded sensor technology," Fatigue Fract. Eng. Mater. Struct., vol. 40, no. 2, pp. 221-232, Feb. 2017. https://doi.org/10.1111/ffe.12488

[22] B. Marzbanrad, H. Jahed, and E. Toyserkani, "On the sensitivity and repeatability of fiber Bragg grating sensors used in strain and material degradation measurement of magnesium alloys under cyclic loads," Int. J. Adv. Manuf. Technol., vol. 86, no. 9-12, pp. 3453-3461, 2016. doi: 10.1007/s00170-016-8485-8.

[23] B. Marzbanrad, "Behaviour of Magnesium Alloy Under Load-Control Cyclic Testing," 2015.

[24] A. Feng, D. Chen, C. Li, and X. Gu, "Flat-cladding fiber Bragg grating sensors for large strain amplitude fatigue tests," Sensors, vol. 10, no. 8, pp. 7674-7680, Aug. 2010. doi: $10.3390 / \mathrm{s} 100807674$.

[25] S. B. Behravesh, H. Jahed, and S. Lambert, "Characterization of magnesium spot welds under tensile and cyclic loadings," Mater. Des., vol. 32, no. 10, pp. 4890-4900, 2011. doi: 10.1016/j.matdes.2011.06.001.

[26] J. Casey and H. Jahedmotlagh, "The strength-differential effect in plasticity," Int. J. Solids Struct., vol. 20, no. 4, pp. 377-393, 1984.

doi: 10.1016/0020-7683(84)90047-7.

[27] W. C. Young and R. G. Budynas, Roark's Formulas for Stress and Strain, vol. 7, no. 7th Edition. 2002. 


\title{
Evaluation of Energy Efficient Propulsion Technologies for Unamnned ia i
}

\author{
Jay Matlock, Stephen Warwick, Phillip Sharikov, Jenner Richards, Afzal Suleman \\ Mechanical Engineering \\ University of Victoria \\ Victoria, Canada
}

\begin{abstract}
The transition to cleaner, more efficient and longerendurance aircraft is at the forefront of current research and development in air transportation systems. The focus of this research is to experimentally evaluate Hybrid Propulsion and Energy Harvesting Systems in Unmanned Aerial Vehicles (UAV). Hybrid systems offer several potential benefits over more conventional gasoline and electric systems including lower environmental impacts, reduced fuel consumption, longer endurance, redundancy and distributed propulsion. Additional energy efficiency can be achieved by harvesting some of the thermal energy of the exhaust gases. By using the Seebeck effect, the temperature gradient between ambient air and the exhaust can be used to generate electric power, making it possible to eliminate costly mechanical systems such as alternators and reduce fuel consumption.
\end{abstract}

The development and experimental evaluation of a hybridpropulsion UAV was carried out at the University of Victoria Center for Aerospace Research (UVIC-CfAR) in the framework of the Green Aviation Research \& Development Network (GARDN) grant. The work involved the development of a framework to evaluate UAV hybrid propulsion efficiency, as well as to predict the amount of power harvestable from thermoelectric generators (TEG). The hybrid propulsion framework was used to investigate the trade-offs between different hybrid architectures against conventional electric and internal combustion propulsion systems. The energy harvesting module was designed to evaluate the trade-off between energy harvested, implementation costs and weight.

In order to validate the computational results, experimental testing was performed. First, an apparatus was designed to collect performance data of a triple-TEG system connected to a 4-stroke Saito internal combustion engine. Thermal performance of the system was evaluated at eleven different test points, and a number of variables were modified to simulate real flight profiles. Next, another apparatus was designed to characterize the performance of a parallel hybrid-electric propulsion system in a UAV. This apparatus allows for different mission profiles that closely match the flight test data from other propulsion types.

Green Aviation Research \& Development Network

(GARDN)
Keywords: hybrid propulsion, series and parallel architecture; energy harvesting; thermoelectric generators

\section{INTRODUCTION}

Conventional aircraft are powered by fossil fuels used in different types of internal combustion engines, which produce a substantial amount of pollutants in the form of carbon dioxide. With new regulations and increase of public interest there has been an effort to transition to green transportation. Hybridelectric propulsion and energy harvesting technologies are potential solutions to mitigate some of the issues identified. There is substantial pressure on the industry to maximize the efficiency of propulsion systems and minimize environmental impact. Gasoline internal combustion engines and electric motors are the two main architectures for UAVs, but each system has disadvantages.

Internal combustion engines tend to have higher endurance than electric systems, but this comes at the cost of high vibration and noise pollution, as well as high levels of emissions. Typically, gasoline engines are oversized for cruise in order to satisfy climb requirements. Electric propulsion systems have the benefit of lower noise, vibration and emissions and can supply a high torque across their operating range. However, the power density of battery packs is much lower than that of hydrocarbons, and the additional weight means that electric propulsion systems suffer from low endurance. A hybrid propulsion system that offers the benefits from both gasoline and electric configurations could prove to be a viable candidate for UAVs. The potential advantages of a hybrid propulsion system include the reduction in fuel burn and emissions, lower acoustic signature when operating in electric-only mode, lower-vibration operations, and the possibility of novel configurations through distributed propulsion. The efficiency of the overall system could be increased even further if some energy harvesting techniques are applied to the exhaust gas using thermoelectric generators. A hybrid propulsion system could also include some intangible benefits such as redundancy in the case of engine failure.

The two types of hybrid-electric configurations used in this project are series and parallel, as shown in Fig. 1. In a series configuration, the combustion engine and electric motor are not mechanically coupled. Instead, the combustion engine generates power using a separate generator which can be used to drive the electric motor or charge the battery packs. In a parallel 
configuration, the combustion engine and electric motor are connected using some sort of mechanical coupling, and together drive the propeller. In a parallel configuration, a clutch is required to disconnect either the electric motor or combustion engine to operate in isolated modes.

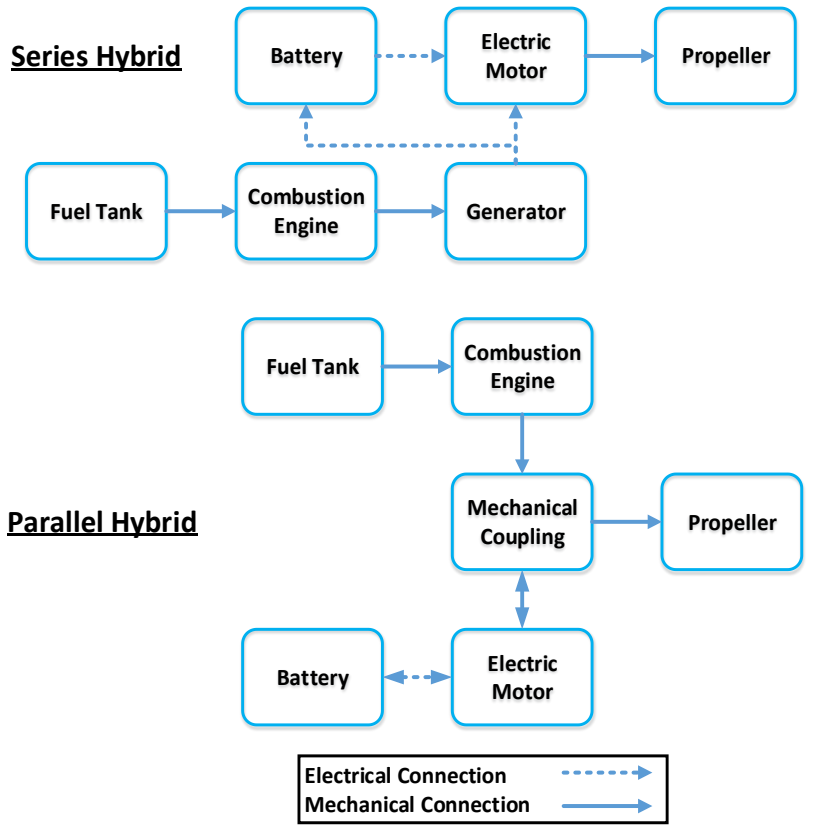

Figure 1. Series and Parallel hybrid-electric configurations.

One of the first major hybrid-electric propulsion UAV projects began in 2010 with the Air Force Institute of Technology's 'Project Condor'[1], [2]. This design involved conceptual design work, simulation, and control strategy development by several groups and predicted efficiencies of 94\% [3]-[5]. The University of Colorado has shown success with their project HELIOS which also made use of a parallel hybrid architecture in their UAV [6]. Further, parallel hybrid research by Glassock, Hung, Gonzalez and Walker at Queensland University have suggested that an increase of power of $35 \%$ can be achieved with only a $5 \%$ weight penalty for a UAV [7].

In a study conducted by Harmon, Frank and Joshi [4] it was estimated that a parallel configuration on a small UAV would be approximately $8 \%$ lighter than a series configuration. This estimate is due to the large electric motor and generator requirement of the series configuration. Although series hybrid configurations suffer substantial energy-conversion losses, they are simpler to control and offer features that are suitable for intelligence, surveillance and reconnaissance (ISR) missions such as distributed propulsion or electric-only operation. These features are possible for a series configuration because the internal combustion engine is decoupled from the electric motor that provides the propulsion.

Energy harvesting is the practice of generating energy from ambient energy sources, such as solar, vibrational, acoustic or thermal energy. Many applications for energy harvesting can be found in aviation, from structural health monitoring (SHM) systems requiring in-situ power production to electric-based systems harvesting energy to increase endurance. Research into energy harvesting at UVIC CfAR has focused on recovering thermal energy to provide auxiliary power to an aircraft's electrical systems.

Thermoelectric generators utilize the Seebeck effect to convert thermal energy to electrical power when subjected to a temperature gradient. TEG efficiency depends on both the materials used and the temperature difference between the two sides of the module, as TEGs commonly operate at approximately 20\% Carnot efficiency [8]. Commercial units have up to $12 \%$ conversion efficiency, with a $5 \%$ efficiency being common.

As mentioned previously, reciprocating internal combustion engines are a popular choice for UAV propulsion due to the fuel high energy density; however, this comes with a tradeoff of low thermal efficiency. Only $30-45 \%$ of the fuel energy is utilized to produce mechanical power, and this figure is even lower for small-scale engines [9]. The remaining energy is lost to the environment, with $40 \%$ of the total energy lost as exhaust gas kinetic and thermal energy [10]. Exhaust temperatures commonly reach as high as 800 degrees Celsius. Therefore, the high temperature of the exhaust relative to the environment makes the exhaust system a potential candidate for thermoelectric energy harvesting.

Thus far, thermoelectric energy harvesting research has largely been concentrated in the automotive sector. Generally, TEGs are placed on a specially designed exhaust pipe and are cooled with engine coolant; systems using heat pipes have been explored but are limited to low-temperature applications [8]. As weight is a secondary concern in the industry compared to aviation, a number of high-power energy recovery systems have thus been created and tested by companies such as BMW, Ford and Honda. Research has indicated that thermoelectric systems are capable of meeting the electrical requirements of vehicles, with experimental systems exceeding $700 \mathrm{~W}$ power generation during bench testing [10]. An increase in fuel efficiency of up to $10 \%$ has been claimed, [11] though real-world efficiency gains were closer to $3 \%$ [9].

In contrast, thermoelectric energy harvesting for UAV applications has only received limited attention. Fleming, Ng and Ghamaty investigated thermoelectric power systems for micro air vehicle applications, [12] and Langley, Taylor, Wagner and Morris conducted theoretical modelling, design and optimization, and subsequent flight testing of a thermoelectric power system [13]. As system weight is of much higher concern in aerospace, thermoelectric energy harvesting is largely prevented from employing the liquid-cooled heat exchangers prevalent in automotive research; ambient air becomes a much more feasible option for cooling. Previous research on the subject has largely been limited to low-temperature, smallgradient applications [14].

\section{HYBRID PROPULSION SYSTEM}

The purpose of the framework is to compare conventional electric and gasoline UAV propulsion systems against various hybrid-electric and energy efficient systems. As new hybrid UAV projects emerge, it is difficult to quantify their performance against other designs as there is a wide range of 
designs and applications. This framework was developed using MathWorks MATLAB and includes all of the aircraft dynamics independent of the propulsion module so that propulsion systems can be used interchangeably. Once the models are validated with real flight test data, this framework can be used to compare theoretical systems or prototype systems still in development. Since all of the aircraft components are modelled parametrically, the design space can be thoroughly explored and optimized through various component sweeps and trade studies. The results from this exercise can be used to observe tradeoffs and impacts between different propulsion architectures and will drive design decisions for future UAVs.

The series and parallel hybrid architectures were evaluated for several mission profiles. These missions provide a variety of different operating points that UAVs are typically used for, rather than steady-state comparison between propulsion systems. These profiles include a high-speed Interception Mission, a maximum-endurance Communications Relay Mission, a terrain following Pipeline Inspection Mission, and finally a flight path optimization mission for LIDAR Data Collection. These missions are depicted in Fig. 2.

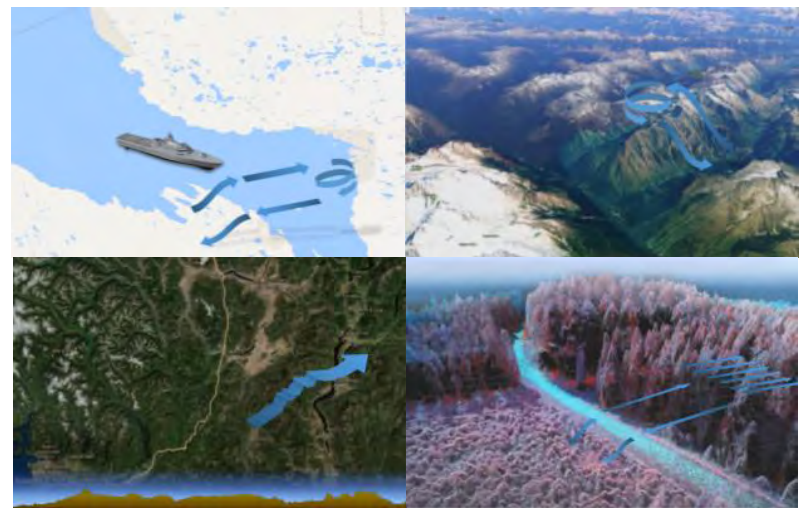

Figure 2. Interceptor Mission (top left), Communications Relay Mission (top right), Pipeline Inspection Mission (bottom left), and LIDAR Data Collection (bottom right) profiles.

Several of the individual components of the aircraft, such as the propeller, lithium polymer ( $\mathrm{LiPo})$ batteries, electric motors and the internal combustion engines were modelled from existing architectures and combined in the framework. The propeller model includes a parametric model to morph measured geometry for a range of sizes. For the LiPo batteries, an equivalent circuit was used to model both the discharge and charge characteristics of a battery in order to accurately estimate the voltage drop. This model was used in combination with experimental data of voltage curves collected by discharging a LiPo battery at different rates. A thrust test stand was developed to determine the performance of these LiPo batteries with several electric motors. This thrust test stand allowed for any combination of batteries, electronic speed controller and motor to be tested together to collect experimental data, and included safety cut-offs for thermal, voltage or current thresholds. In order to validate these models, component-level bench tests were conducted.
Fig. 3 depicts the results of an example exercise using the framework. Here, the coefficient of lift parameter was swept from 0.55 to 0.90 to observe the trade-offs of fuel burn between the gasoline configuration, and both series and parallel hybrid configurations for a sample aircraft. As can be seen, the series hybrid architecture will burn less fuel than the gasoline-only configuration at higher $C_{L}$ values, and the parallel hybrid is the most efficient with the lowest fuel burn for the full $C_{L}$ range. The ability to observe these trends and analyze the tradeoffs between different propulsion systems attests to the power and novelty of this framework.

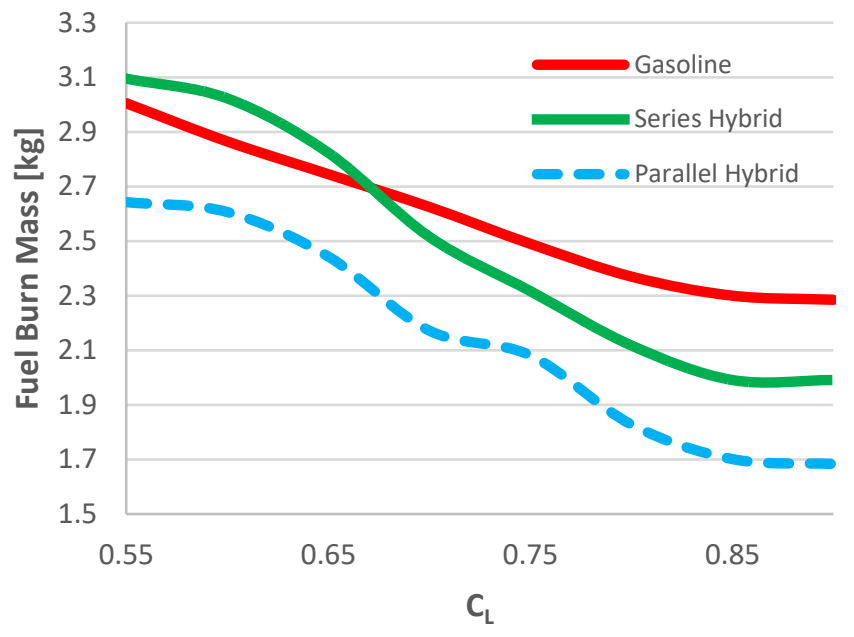

Figure 3. Framework design study example: Pipeline Inspection Mission $C_{L}$ versus Fuel Consumption.

In future work, all of the components will be combined for system-level bench tests for both series and parallel hybrid architectures. The system-level bench tests will provide the opportunity to observe the performance of the propulsion system in a controlled environment and provide repeatable results. As with the component level testing, the results from the hybrid bench tests will be updated back into the framework to increase the accuracy of the theoretical models and lead to the design and integration into an airframe.

\section{THERMOELECTRIC ENERGY HARVESTING}

To validate the theoretical model of an exhaust energy harvester, a test apparatus was designed and built. The energy harvester was designed to use the exhaust flow from a commercial-grade $50 \mathrm{~cm}^{3} 4$-stroke Saito FG36 gas engine to provide heat to 3 Marlow Industries $30 \mathrm{~mm}$ x 30mm TG12-4 thermoelectric generators. The cooling was provided by forced air. An Advanced Thermal Solutions ATS-EXL68-300-R0 heat sink was modified to provide two $15 \mathrm{~cm}$-long profiles, and the TEG modules were fixed between them using Arctic Silver Ceramique 2 thermal paste to maximize thermal contact. The heat sinks were enclosed in aluminum shrouds and the two sides were clamped together. To simulate external airflow, an electric ducted fan unit was integrated to simulate an aircraft speed of up to approximately $200 \mathrm{~km} / \mathrm{hr}$ at ground level. The cold air 
flow and the exhaust flow were counter-current to each other. The system was connected to the engine exhaust using a corrugated metal hose. Removable fiberglass insulation was also installed to control heat loss from the apparatus.

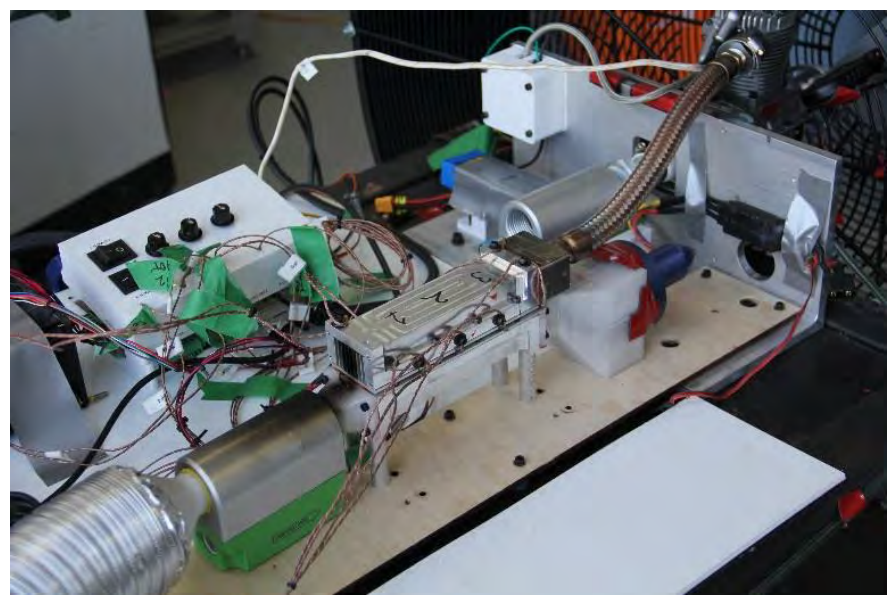

Figure 4. Energy Harvesting Apparatus Setup.

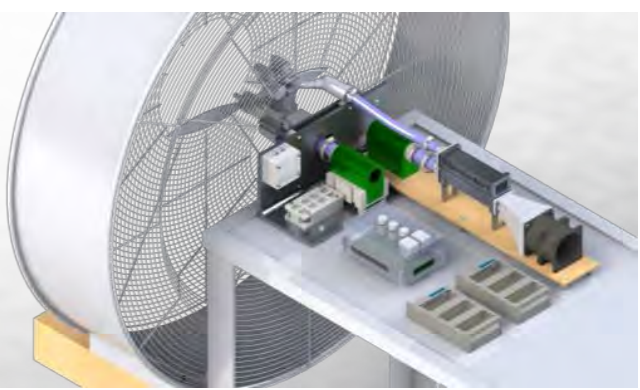

Figure 5. Energy Harvesting Apparatus.

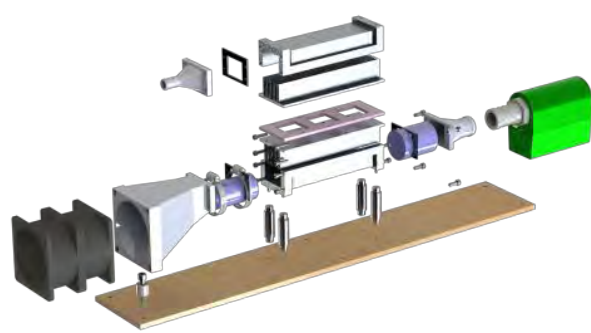

Figure 6. TEG module exploded view.

The system was instrumented to record 5 design variables (engine throttle control, cold air flow rate control, and resistive load control for each of the 3 TEGs). The temperature gradient across each TEG was measured using two Digilent K-type thermocouples, and the exhaust temperature was recorded at both the exhaust manifold and the entrance to the heat exchanger. Ambient air flow temperature was tracked at the inlet and the outlet of the heat exchanger, and ambient air flow rate was measured at the heat exchanger inlet using an $\mathrm{E}+\mathrm{E}$ Elektronik EE741 flow meter. For the engine control, air flow was tracked at the carburetor inlet and fuel mass was tracked using a load cell. Data collection and system control was accomplished using LabVIEW software.

An energy harvesting module for the MATLAB framework was developed to predict thermoelectric power generation based on the operational state of the aircraft. Engine parameters were used as inputs to predict exhaust temperature, and the aircraft's speed is used to determine the cold air flow. Load resistance was set to optimize power production throughout the mission, simulating the overall power generation. This power was directly applied to a battery module in the framework, and can be used to recharge the flight packs or supply power to a payload. Due to the relatively low operating temperature range, the energy harvesting module makes the assumption that the TEG material properties are temperature independent.

Extensive component and system-level testing was performed. The performance of TEG modules was quantified experimentally as shown in Fig. 7. TEG power generation was changed by sweeping load resistance at constant airspeed and engine power. The ability to control load resistance ensures optimal power generation at any flight profile. The relationship between engine power, flight speed, and TEG temperatures was defined empirically and used to refine the energy harvesting module.

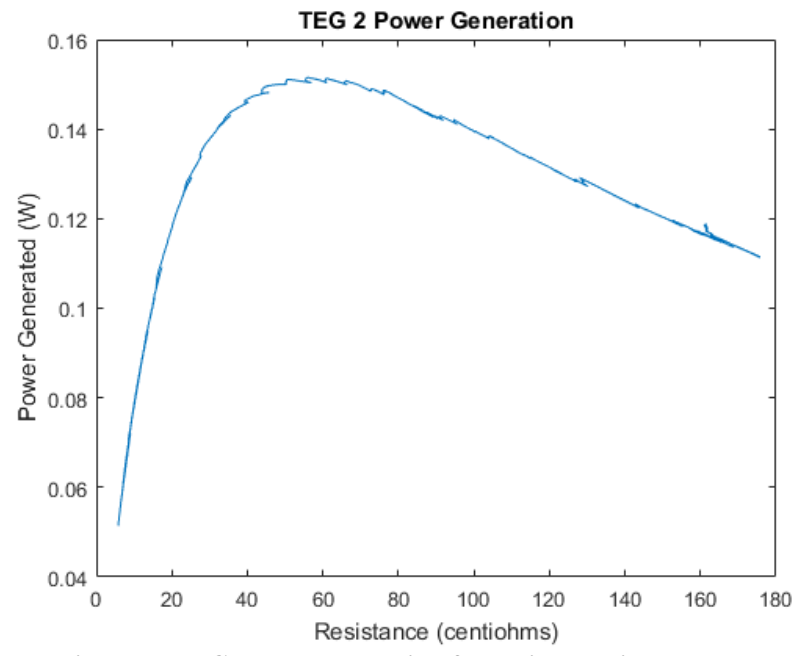

Figure 7. TEG power generation for a given resistance.

\section{CONCLUSION/RECOMMENDATIONS}

A hybrid propulsion system framework has been implemented and evaluated for multiple mission scenarios. This framework allows the user to define component information and sizing, aircraft parameters, and complex mission profiles. From this setup, design trade-offs between UAV propulsion systems were identified and quantified to drive engineering design decisions. Specific experiments can be conducted to observe very precise and slight changes to the overall performance of an aircraft. As hybrid-electric aircraft increase in popularity, the aeronautical industry will benefit from such an analysis tool to optimize designs. As new and more efficient propulsion technologies are created, this framework will allow users to benchmark new designs.

Using the framework, mission profiles were designed to simulate realistic missions such as Interceptor, Communications Relay, Pipeline Inspection and LIDAR Data Collection. Since UAVs are used in a variety of applications, it is a useful exercise 
to test the designs across multiple mission profiles to determine the optimal design. Together with the energy harvesting module, design decisions can be made on the trade-offs between different component sizes and optimized.

Future work will include the construction of more detailed and complex mission profiles to further test the performance of aircraft. The ability to test the designs in real-world missions will not only allow for realistic performance estimates, but also open the opportunity in future work to tune the model using actual flight test results. Furthermore, experimental data from the component bench testing and the energy harvesting apparatus will be used to validate the framework and produce more accurate results.

This project clearly demonstrates the need and novelty of the hybrid propulsion design tool to compare the performance of UAV propulsion types, and proves that hybrid UAVs are viable candidates for a variety of missions. Green aviation will likely be achieved by hybrid technology, and the framework provides valuable insight into engineering design decisions. Results from this project will be used to optimize the hybrid propulsion system for UAVs.

\section{ACKNOWLEDGMENT}

This research was supported by a grant from GARDN (Green Aviation Research and Development Network).

\section{REFERENCES}

[1] F. G. Harmon, A. A. Frank, and J.-J. Chattot, "Conceptual Design and Simulation of a Small Hybrid-Electric Unmanned Aerial Vehicle," J. Aircr., vol. 43, no. 5, pp. 1490-1498, Sep. 2006. doi: 10.2514/1.15816.

[2] J. K. (USAF) Ausserer, "Integration, Testing, and Validation of a Small Hybrid-Electric Remotely-Piloted Aircraft," Wright-Patterson Air Force Base, 2012.

[3] R. M. Hiserote, "Analysis of Hybrid-Electric Propulsion System Designs for Small Unmanned Aircraft Systems," 2010.

[4] F. G. Harmon, A. A. Frank, and S. S. Joshi, "Application of a CMAC neural network to the control of a parallel hybrid-electric propulsion system for a small unmanned aerial vehicle," Neural Networks, 2005. IJCNN '05. Proceedings. 2005 IEEE Int. Jt. Conf., vol. 1, pp. 355-360 vol. 1, 2005. doi: 10.1109/IJCNN.2005.1555856.

[5] R. M. Hiserote and F. Harmon, "Analysis of Hybrid-Electric Propulsion System Designs for Small Unmanned Aircraft Systems," 8th Annu. Int. Energy Convers. Eng. Conf., no. July, pp. 1-148, Jul. 2010. doi: $10.2514 / 6.2010-6687$.

[6] J. Koster, C. Humbargar, E. Serani, A. Velazco, D. Hillery, and L. Makepeace, "Hybrid Electric Integrated Optimized System (HELIOS) Design of a Hybrid Propulsion System for Aircraft," Am. Inst. Aeronaut. Astronaut. AIAA Meet. Pap., no. January, 2011.

doi: $10.2514 / 6.2011-1011$.

[7] R. Glassock, J. Y. Hung, L. F. Gonzalez, and R. A. Walker, "Design, modelling and measurement of a hybrid powerplant for unmanned aerial systems," Aust. J. Mech. Eng., vol. 6, no. 2, pp. 69-78, 2008. doi: 10.1080/14484846.2008.11464559.

[8] B. Orr, A. Akbarzadeh, M. Mochizuki, and R. Singh, "A review of car waste heat recovery systems utilising thermoelectric generators and heat pipes," Appl. Therm. Eng., vol. 101, pp. 490-495, 2016.

doi: 10.1016/j.applthermaleng.2015.10.081.
[9] J. Fairbanks, "Automotive Thermoelectric Generators and HVAC," 2013 Annu. Merit Rev. Peer Eval. Meet., 2013.

[10] S. Kumar, S. D. Heister, X. Xu, J. R. Salvador, and G. P. Meisner, "Thermoelectric generators for automotive waste heat recovery systems part I: Numerical modeling and baseline model analysis," J. Electron. Mater., vol. 42, no. 4, pp. 665-674, 2013. doi: 10.1007/s11664-013-2471-9.

[11] J. Lagrandeur, D. Crane, S. Hung, B. Mazar, and A. Eder, "Automotive Waste Heat Conversion to Electric Power using Skutterudites, TAGS, PbTe and Bi2Te3," Thermoelectr. 2006. ICT '06. 25th Int. Conf., pp. 343-348, 2006.

[12] J. Fleming, W. Ng, and S. Ghamaty, "Thermoelectric-Based Power System for Unmanned-Air-Vehicle/ Microair-Vehicle Applications," J. Aircr., vol. 41, no. 3, pp. 674-676, 2004. doi: $10.2514 / 1.11486$.

[13] J. Langley, M. Taylor, G. Wagner, and S. Morris, Thermoelectric Energy Harvesting from Small Aircraft Engines. 2009

[14] C. T. Hsu, G. Y. Huang, H. S. Chu, B. Yu, and D. J. Yao, "Experiments and simulations on low-temperature waste heat harvesting system by thermoelectric power generators," Appl. Energy, vol. 88, no. 4, pp. 1291-1297, 2011. doi: 10.1016/j.apenergy.2010.10.005. 


\title{
Dynamic Testing System for Rocks Under In Situ Stresses
}

\author{
Wei Yao, Kaiwen Xia \\ Department of Civil Engineering \\ University of Toronto \\ Toronto, Canada \\ wei.yao@mail.utoronto.ca, kaiwen.xia@utoronto.ca
}

\begin{abstract}
Rocks may be subjected to dynamic disturbances while under high in situ stresses. When disturbed by dynamic loads from blasting, seismicity or rockbursts, the underground structures would be vulnerable to failure. Depending on the distance from the underground opening, the in situ stress states change from hydrostatic in the far-field, to triaxial in the intermediate distance, and to the pre-tension nearby the opening. Thus, SHPB testing system is further adjusted with confining pressure system into dynamic testing system of rocks under different in situ states. In the experiment with this dynamic testing system, the Brazilian disc rock specimens are first subjected to pre-stresses simulating in-situ stresses underground (including pre-tension, hydrostatic confinement, and triaxial confinement) and then loaded dynamically using the modified SHPB system. The dependence of dynamic tensile strength of the rock material on the static pre-stress and loading rate is investigated. These experimental results will be of great importance in the design and safety of underground rock engineering projects.
\end{abstract}

Keywords-SHPB; Pre-stress; Hydrostatic Confinement; Triaxial Stress; Rate dependence

\section{INTRODUCTION}

With the development of underground space, it is necessary to study the mechanical properties of underground rocks, which are subjected to high lithostatic and tectonic stress. Problems involving underground rocks are common in many important economic fields including hydropower, transportation, mining and defense infrastructure. Deep rock structures are prone to dynamic loads such as blast mining, rock burst and small seismic events, so it is necessary to simulate the underground stress state and conduct designed dynamic experiments to investigate the dynamic properties of underground rocks. Depending on the distance from the underground opening, the in situ stress states change from hydrostatic in the far-field, to triaxial in the intermediate distance, and to the pre-tension nearby the opening, as shown in Fig. 1.

Dynamic mechanical properties of engineering materials have been investigated for many years and various devices are used to conduct experiments on these materials. One of the most frequently used techniques is the split Hopkinson pressure bar (SHPB) system. Using the SHPB technique, dynamic mechanical properties such as compressive strength, tensile strength and fracture toughness have been studied on engineering materials, mostly under uniaxial loading conditions [1]. However, underground rocks are subjected to in situ stresses and occasional dynamic loadings. Most research on axial compression properties of rock-like materials under the preloading situations are conducted on ceramics and concretes under lateral confinement, either passive or active [2]. Gong and Malvern [3] proposed a way to provide passive confinement on concrete specimens in the SHPB system. Chen and Ravichandran [4] adopted the passive confinement method to investigate the dynamic properties of ceramics. Song and Chen et al. [5] studied confinement effects on the dynamic compressive properties of an epoxy syntactic foam, considering the effect of both the loading rate and passive lateral confinement. Christen and Swanson et al. [6] designed dynamic mechanical experiments under active confinement. Gary and Bailly [7] adopted the technique and found the confining pressure more stable with air as the medium. They conducted experiments on concrete and proposed a mesomechanical model. Lindholm and Yeakley et al. [8] proposed the experimental method under hydrostatic confining pressure in 1974. Li and Zhou et al. [9] and Zuo and Li et al. [10] investigated the dynamic response of rocks under both axial and active lateral confinement. Frew and Akers et al. [11] improved Lindholm's device and developed a hydrostatic apparatus based on SHPB system.

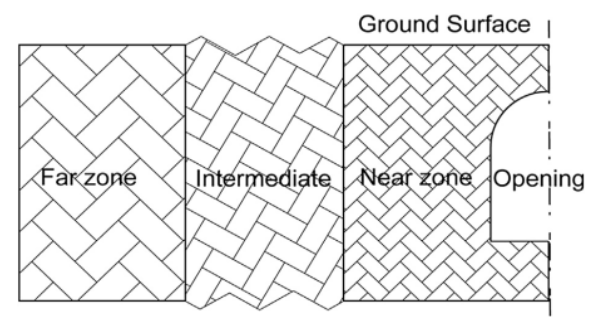

Figure 1. Zoning of the confining stress states around an underground opening

It is worth noting that, in previous research, there are not many results of tensile experiments on pre-stressed rocks, and 
if so, all of them are under quasi-static stress states. Some researchers conducted Brazilian disc (BD), shear and torsion tests on rocks under hydrostatic pressure $[12,13]$ and they found that the strength of rock increases with the hydrostatic pressure. Vasarhelyi [14] investigated the influence of confinement on the mode I fracture of Gneiss. Al-Shayea and Khan et al. [15] tested straight notched Brazilian disk (SNBD) specimens under diametrical compression to study the influence of confinement on fracture toughness of limestone. Chen and Zhang [16] studied the influence of confinement on rock fracture toughness using notch-hole combined Brazilian disc specimens and Funatsu and Seto et al. [17] did the same tests on notched semi-circular bend (NSCB) specimens and they found the same increasing trend with confining pressure.

Therefore, there have been many improvements in dynamic testing of rocks with the static stress states; however, the research and testing system concerning various dynamic response (e.g. compression and tension strength, fracture toughness) of rocks subjected to different pre-stresses (i.e. pretension, hydrostatic confinement, and triaxial confinement) are still deficient. In this paper, a SHPB testing system is adjusted with confining pressure system for dynamic response of rocks under different pre-stress states. To calibrate this testing system, the Brazilian disc rock specimens are first subjected to simulating in-situ stresses underground (including pre-tension, hydrostatic confinement, and triaxial confinement) and then loaded dynamically using the modified SHPB system. The dependence of dynamic tensile strength of the rock material on the static pre-stress and loading rate is investigated. The calibrated testing system will be importantly applied to the design and safety of underground rock engineering projects.

\section{SHPB SYSTEM FOR ROCKS UNDER PRE- TENSION}

\section{A. The Pre-tension SHPB System}

The modified SHPB system for pre-tension test includes three bars (a striker bar, an incident bar, and a transmitted bar) [18] and the pre-tension system (Fig. 2). The elastic bars are made of high strength maraging steel. The pre-tension system is mainly composed of a pressure chamber that provides axial preload to the bars and specimen, and a rigid mass at the incident bar end that is connected to the chamber by tie-rods. The pre-tension system is similar to that innovated by Frew et al. [11], who developed a modified SHPB system for dynamic tests under hydrostatic confinement. However, there is a main difference between the current design and those of Few et al. [11] and Zhou et al. [19]. In their designs, the bars are connected by the tie-rods from the impact end of the incident bar to the free end of the transmitted bar, while in the current design, the bars are connected near the other end of the incident bar through a flange (Fig. 2). The total length of the compressed bars in the current design is much shorter and thus is less prone to buckling.

The recording system consists of the foil strain gauges, a signal conditioner, and an oscilloscope. There are two strains gauges on each bar attached at the symmetrical position, and they are connected to a signal conditioner through a Wheatstone bridge. The oscilloscope is connected to the signal conditioner using two channels, one for the signal on the incident bar and the other for the signal on the transmitted bar.

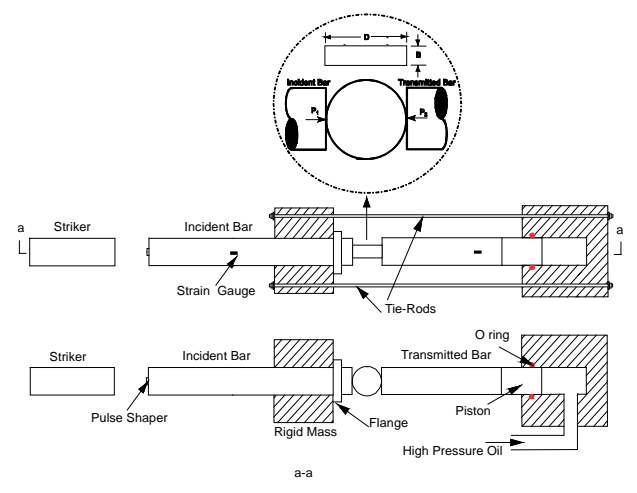

Figure 2. Schematics of the pre-tension SHPB system

\section{B. The Validity of Dynamic Test with Pre-tension}

During the tests, the static pre-tension is applied to the specimen by the pressure loading unit attached to the end of the transmitted bar through the elastic bars and flange supported by a rigid mass. When the desired pre-tension is achieved, dynamic loading is applied from the impact of the striker bar on the free end of the incident bar. The incident pulse propagates along the incident bar before it hits the specimen, leading to a reflected stress wave and a transmitted stress wave that are recorded by the strain gauges attached on the incident and transmitted bar surfaces. The motion induced by the incident wave is to the right and thus the flange has no effect on the wave propagation. The strains of incident wave, reflected wave and transmitted wave are denoted by $\varepsilon_{i}, \varepsilon_{r}$ and $\varepsilon_{t}$, respectively.

Based on the one dimensional stress wave theory, and assuming stress equilibrium during loading [18] (i.e., $\varepsilon_{i}+\varepsilon_{r}=\varepsilon_{t}$ ), the history of the force on the specimen is:

$$
P(t)=P_{0}+P_{d}(t)
$$

where $P_{0}$ is the static preload on the bars, $P_{d}(t)$ is the dynamic force history on the bars after the impact. The tensile stress history at the center of the disc specimen can be determined as:

$$
\sigma(t)=\sigma_{0}+\sigma_{d}(t)=\frac{A_{0} E_{0} \varepsilon_{t}(t)}{\pi R B}
$$

where $\sigma_{0}$ is the pre-tension at the center of the disc, and

$$
\sigma_{0}=\frac{P_{0}}{\pi R B}
$$

where $\sigma_{d}(t)$ is the dynamic tensile stress, $E_{0}$ is the Young's Modulus of the bars, $A_{0}$ is the cross-sectional area of the bars; $R$ is the radius of the specimen and $B$ is the thickness of the specimen. The tensile strength is the maximum value of the tensile stress when the rock specimen is damaged. There is an 
approximately linear region in $\sigma_{d}(t)$ (Fig. 3), and its slope is taken as the loading rate.

Similar to the traditional dynamic rock tension experiments in the SHPB system, the validity of dynamic test with pretension is achieved by the force balance in the specimen during the experiments. This can be researched by using pulse shaper technique [18]. Fig. 3 shows the force history according to the strain gauges attached on the incident bar and the transmitted bar. The force on one side of the specimen is the sum of forces by the incident and reflected stress waves (marked as In.+Re), and the force on the other side of the specimen is by the transmitted stress wave (marked as Tr). The initial force on the transmitted bar corresponds to the preload on the specimen, which is $P_{0}$ as in Eq. (1). It can be seen from Fig. 3 that the dynamic forces on both sides of the specimens are almost identical during $80 \mu \mathrm{s}$ to $160 \mu \mathrm{s}$, which is the main dynamic loading period. It is also noted that the forces on the two ends of the specimen are different before $80 \mu \mathrm{s}$. The reason is that before the dynamic impact, the specimen is subject to the static preload through the transmitted bar, which is already compressed and thus the strain gauge on the transmitted bar measured the preload. However, the incident bar is free before impact loading and thus there is no deformation measured by the strain gauge on the incident bar at the beginning. The force difference at time zero in Fig. 3 is the static preload applied on the rock specimen, which is $10.07 \mathrm{KN}$ in this case, corresponding to the pre-tension of $10 \mathrm{MPa}$ at the specimen center.

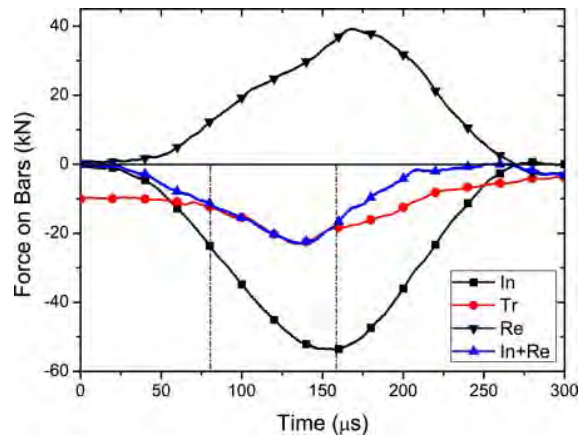

Figure 3. Dynamic force balance in a typical pre-tension SHPB test

\section{Dynamic Brazilian Test with Pre-tension}

Five groups of $\mathrm{BD}$ rock specimens (with static tensile strength of $12.8 \mathrm{MPa}$ ) under the pre-tension of $0 \mathrm{MPa}, 2 \mathrm{MPa}$, $4 \mathrm{MPa}, 8 \mathrm{MPa}$, and $10 \mathrm{MPa}$ are tested under different loading rates.

During each test, the tensile strength is the maximum value of the tensile stress history. Fig. 4 illustrates the dynamic tensile strength versus loading rate. It is obvious that the dynamic strength increases with the loading rate, revealing the phenomenon of rate dependency that is common for engineering materials, such as rock [20], concrete [21, 22], ceramic $[23,24]$.

Apart from the rate dependency mentioned above, what can be seen from Fig. 4 is that the dynamic tensile strength of the rock decreases with the increase of the pre-tension when subjected to the same loading rate. For example, when the loading rate is approximately $190 \mathrm{GPa} / \mathrm{s}$, the dynamic tensile strength is $19.8 \mathrm{MPa}$ when the specimen is loaded stress-free being subjected to dynamic loading. However, the dynamic tensile strength of the one with $10 \mathrm{MPa}$ pre-tension is 11.7 $\mathrm{MPa}$, which is $8.1 \mathrm{MPa}$ lower than the former. The decrease of dynamic tensile strength is caused by the opening of microcracks when the specimen bears the pre-tension stress, which is consistent to the results reported by Xia et al. that the microstructures affect the dynamic stress of rock specimens $[2]$.

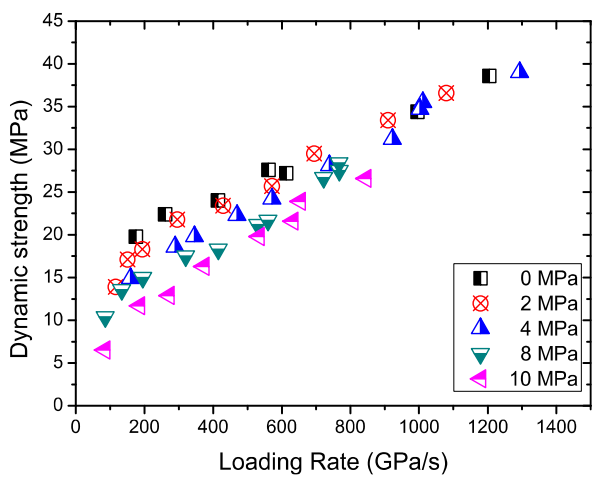

Figure 4. The dynamic strength versus loading rate for different pre-tensions

\section{SHPB SYSTEM FOR ROCKS UNDER HYDROSTATIC STRESS}

\section{A. The SHPB System with Hydrostatic Confinement}

The modified SHPB apparatus for tri-axial stress state includes the three bars (a striker bar, an incident bar, and a transmitted bar) [18] and a hydraulic system, as shown in Fig. 5. The elastic bars are made of the same material as that for the pre-tension test. The hydraulic system is mainly composed of a cylinder that applies lateral confinement to the rock specimens (Cylinder 1), and a pressure chamber that provides axial preload (Cylinder 2) to the bars and specimen. The only difference between the apparatus in Fig. 2 and the apparatus in Fig. 5 is Cylinder 1 that provides the lateral confining pressure. The recording system is same as the pre-tension SHPB system (Fig. 5).

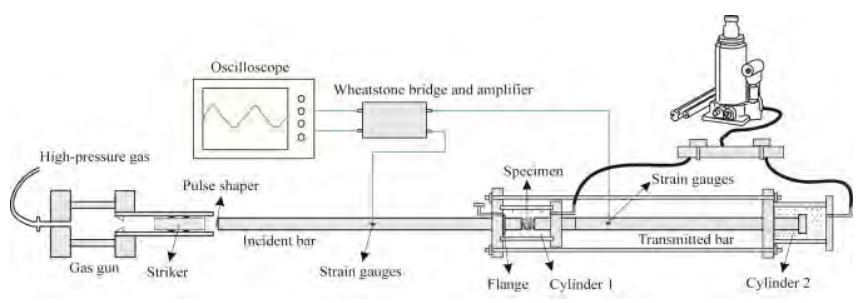

Figure 5. Schematics of the modified SHPB system for Tri-axial test

As shown in Fig. 6, the two cylinders are connected to the same hydraulic press by separate valves. When the two valves are open at the same time, the rock specimen would be in hydrostatic stress state, regardless of the shape of the rock specimen. Denote $A_{b}$ as the cross area of the bars, $A_{s}$ as the 
contact area between the specimen and the transmitted bar; denote $\sigma_{0}$ as the oil pressure provided by the pump, $\sigma_{1}$ and $\sigma_{2}$ as the stresses of the specimen at the transmitted bar end and the incident bar end, respectively.

When the two valves are open at the same time, both the lateral confining stress on the specimen and the stress on the transmitted bar are equal to the oil pressure $\sigma_{0}$. Since the transmitted bar is force balanced, the force on the transmitted bar at the specimen end equals that at the pump end. The force at the specimen end is composed of two parts, the one by the pressure oil $\sigma_{0}\left(A_{b^{-}} A_{s}\right)$ and the one by the specimen $\sigma_{1} A_{s}$; the force at the pump end is $\sigma_{0} A_{b}$. So, the force balance of the transmitted bar leads to:

$$
\sigma_{0}\left(A_{b}-A_{\mathrm{s}}\right)+\sigma_{1} A_{\mathrm{s}}=\sigma_{0} A_{b}
$$

solve Eq. (4) we can get

$$
\sigma_{1}=\sigma_{0}
$$

similarly, we can get

$$
\sigma_{0}=\sigma_{1}=\sigma_{2}
$$

Hence the specimen is in hydrostatic stress state when the two valves are both open at the same time. It can be shown in the similar way that for a specimen with arbitrary shape, as long as it is symmetric with respect to the loading axis, the specimen is under hydrostatic confinement if the pressure in both cylinders is identical.

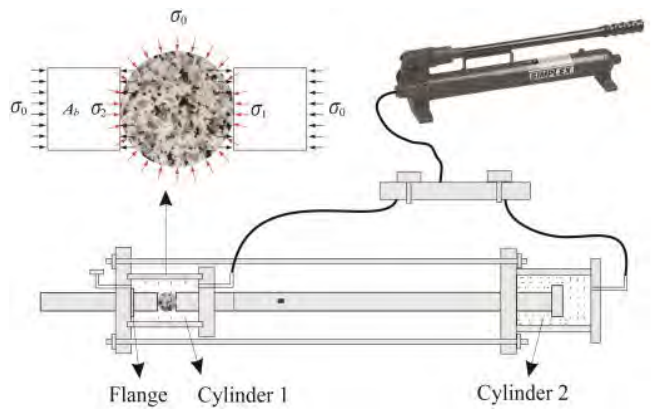

Figure 6. Hydrostatic stress of rock materials in a SHPB test

\section{B. The Validity of Dynamic Test with Hydrostatic Confinement}

Similar to the dynamic test with pre-tension in SHPB system, the dynamic test with hydrostatic confinement in SHPB system is valid when the force balance in the specimen during the experiments is achieved. This is also facilitated by using pulse shaper technique. In addition, enough loads are required to break the confined specimen so the striker is launched with sufficient air pressure every time with different pulse shapers. Different dimensions of the shapers made of different materials lead to different loading rates on the rock specimens.

Fig. 7 shows a typical force history of the two loading ends of the specimen measured by the strain gauges. On one side of the specimen is the sum of forces by the incident and reflected stress waves, which is marked as In.+Re. in the figure, and on the other side is the force by the transmitted stress wave, marked as Tr. It can be seen that the dynamic forces on both sides of the specimens are identical during the loading and unloading period, demonstrating the validity of the test. It also shows that although the incident force is as high as about 200 $\mathrm{KN}$, the force on the specimen is only about $50 \mathrm{KN}$, which is 25 percent of the incident force, and this is why the striker is launched at high air pressure every time to make sure the load is high enough to break the confined specimens.

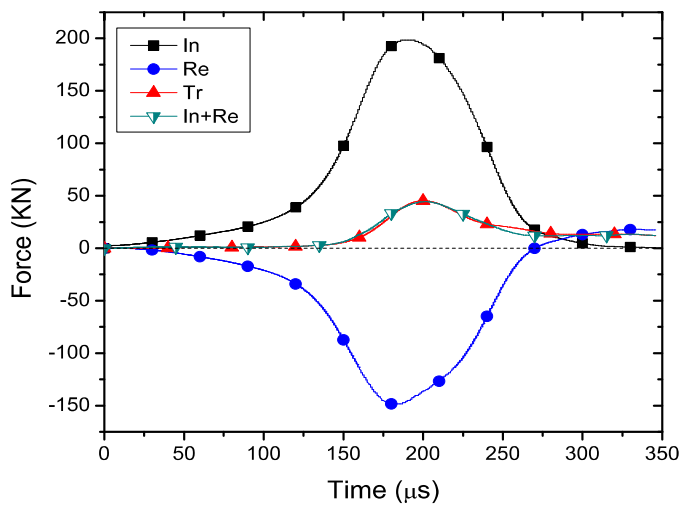

Figure 7. Dynamic force balance in a typical SHPB test with confined Brazilian disc specimen

\section{Dynamic Brazilian Test with Hydrostatic Confinement}

Five groups of specimens are tested under the hydrostatic confinements of $0 \mathrm{MPa}, 5 \mathrm{MPa}, 10 \mathrm{MPa}, 15 \mathrm{MPa}$, and $20 \mathrm{MPa}$. The tensile stress history at the center can be determined as:

$$
\sigma(t)=\frac{2 A_{b} E_{b} \varepsilon_{t}(t)}{\pi B D}
$$

where $E_{b}$ is the Young's Modulus of the bars, $A_{b}$ is the crosssectional area of the bars; $B$ and $D$ are the thickness and the diameter of the specimen, respectively. The tensile strength is the maximum value of the tensile stress when the confined rock specimen is damaged.

Fig. 8 illustrates the dynamic tensile strength versus loading rate. It is obvious that the dynamic strength increases with the loading rate.

Based on the fitted curves in Fig. 8. It is obvious that the dynamic tensile strength increases with both the hydrostatic stress and the loading rate. It can be also seen that the increment of the dynamic tensile strength decreases with the loading rate and the hydrostatic stress. This indicates that when the confining pressure reaches a certain value, or at a certain underground depth, the dynamic tensile strength of rock materials would mainly depend on the loading rate, it does not 
change much as the confinement increases further under the same loading rate.

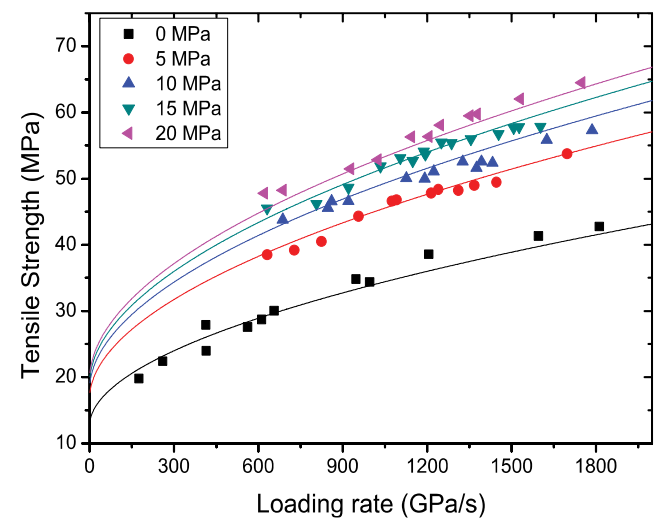

Figure 8. Dynamic tensile testing results under different loading rates and hydrostatic pre-stress, and data fitting of the results

\section{SHPB SYSTEM FOR ROCKS UNDER TRI-AXIAL STRESS STATES}

\section{A. The SHPB System for Tri-axial Stress State}

The modified SHPB apparatus for tri-axial stress state is the same as that for hydrostatic confinement test, as shown in Fig. 5. The experimental design is that we first exert a hydrostatic stress to the BD specimen through the cylinders and the press, by opening the two valves at the same time. When the hydrostatic stress is achieved, the valve controlling the lateral confinement is closed, and the cylinder at the transmitted bar end is used to provide more axial load to the specimen for pretension so that the specimen is under triaxial stress conditions (Fig. 9).

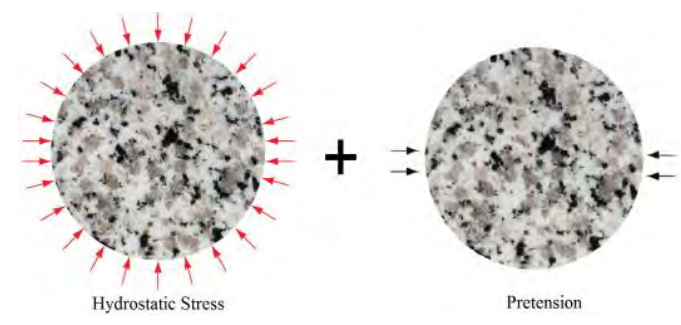

Figure 9. Experimental design with different hydrostatic stress and pretension

After the calculation of the stress on the specimen, the stress exerted on the bar is then determined to achieve the prestress conditions before the impact is launched. For example, when the hydrostatic stress is $5 \mathrm{MPa}$, then the Brazilian tensile strength of the specimen is $17.8 \mathrm{MPa}$, which is the sum of the hydrostatic stress and the BD strength without any confinement. Then with the design that the pretension is $20 \%$ of the strength, which is $3.56 \mathrm{MPa}$ on the specimen. Through the correlation between the tensile stress on the specimen and the stress at the bar specimen interface, the load needed on the bar can be determined.
Denote $\sigma_{t}$ as the tensile stress at the specimen center, through the correlation between the tensile stress at the specimen center and the stress at the specimen-bar interface,

$$
\Delta \sigma_{\mathrm{t}}=\frac{2 P}{\pi B D}=\frac{1}{2} \frac{\pi D_{b}^{2} \sigma_{b}}{\pi B D}=\frac{D_{b}^{2} \sigma_{b}}{2 B D} .
$$

where $P$ is the force on the bar, $B$ and $D$ are the thickness and diameter of the specimen, while $\sigma_{b}$ is the stress on the bar, and $D_{b}$ is the diameter of the bars. So, the stress on the bar should be calculated from Eq. (9) to achieve the desired pre-stress conditions.

$$
\sigma_{b}=\frac{2 B D \sigma_{t}}{D_{b}^{2}}
$$

With hydrostatic confinement, the axial stress applied on transmitted bar is:

$$
\sigma_{b}=\frac{2 B D \sigma_{t}}{D_{b}^{2}}+\sigma_{0}
$$

where $\sigma_{0}$ is the hydrostatic stress on the specimen.

\section{B. Dynamic Brazilian Test under Tri-axial Stress State}

Similar to the dynamic test with hydrostatic confinement in SHPB system, the dynamic test for the tri-axial stress state is valid by using the achievement of the force balance in the specimen during the experiments. This is also facilitated by using pulse shaper technique.

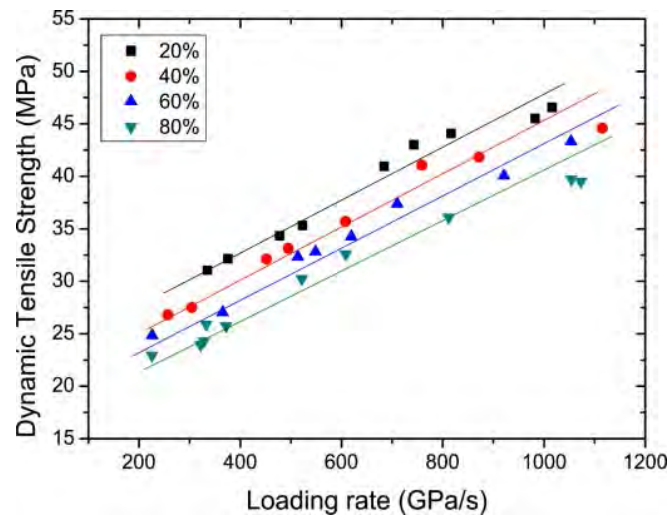

Figure 10. Total strength of rock specimen under 5MPa hydrostatic stress and pre-tension

The tensile stress history at the center can be determined as:

$$
\sigma(t)=\frac{2 A_{b} E_{b} \varepsilon_{t}(t)}{\pi B D}
$$


where $E_{b}$ is the Young's Modulus of the bars, $A_{b}$ is the crosssectional area of the bars; $B$ and $D$ are the thickness and the diameter of the specimen, respectively. The tensile strength is the maximum value of the tensile stress when the confined rock specimen is damaged.

Four groups of specimens are tested under the hydrostatic confinements of $5 \mathrm{MPa}$ and the pretensions are $20 \%, 40 \%$, $60 \%$ and $80 \%$ of the tensile strength under the corresponding hydrostatic stress state, as shown in Fig. 10. Fig. 10 illustrates the correlation between tensile strength and the loading rate when the rock specimens are under $5 \mathrm{MPa}$ hydrostatic stress and various pretensions, respectively. It can be observed that the tensile strength increases with the loading rate almost linearly, revealing the phenomenon of rate dependency that is common for engineering materials. Besides the rate dependency mentioned above, it is also obvious from Fig. 10 that the dynamic tensile strength of the rock decreases with triaxial stress states, which is also observed from the pre-tension tests in Section II.

\section{CONCLUSIONS}

When the underground rocks under high in situ stresses are disturbed by dynamic loads from blasting, seismicity or rockbursts, the underground structures would be vulnerable to failure. In situ stress states of the underground openings change from hydrostatic in the far-field, to triaxial in the intermediate distance, and to the pre-tension nearby the opening. In order to investigate the dynamic behaviors (e.g. compression and tension strength, fracture toughness) of rocks under in situ states, a dynamic testing system of rocks under different in situ states (i.e. pre-tension, hydrostatic confinement, and triaxial confinement) is modified with the SHPB testing system and confining pressure system.

To calibrate the dynamic testing system of rocks under in situ states, the Brazilian disc rock specimens are first subjected to simulating in-situ stresses underground (including pretension, hydrostatic confinement, and triaxial confinement) and then loaded dynamically through the modified SHPB system. The advantages of the design for the dynamic testing system is given and the validity of dynamic BD rock tests performed in this dynamic testing system was discussed. Based on this system, the dependence of dynamic tensile strength of the rock material on the static pre-stress and loading rate is investigated. The calibrated testing system will be importantly applied to the design and safety of underground rock engineering projects.

\section{REFERENCES}

[1] K. Xia and W. Yao. "Dynamic rock tests using split Hopkinson (Kolsky) bar system-A review," Int. J. Rock Mech. Min., vol. 7, pp. 27-59, 2015. doi: 10.1016/j.jrmge.2014.07.008.

[2] K. Xia et al., "Effects of microstructures on dynamic compression of Barre granite," Int. J. Rock Mech. Min., vol. 45, pp. 879-887, 2008. doi:10.1016/j.ijrmms.2007.09.013.
[3] J. C. Gong and L. E. Malvern. "Passively Confined Tests of Axial Dynamic Compressive Strength of Concrete," Exp. Mech., vol. 30, pp. 55-59, 1990. doi: 10.1007/BF02322703.

[4] W. Chen and G. Ravichandran. "An experimental technique for imposing dynamic multiaxial-compression with mechanical confinement," Exp. Mech., vol. 36, pp. 155-158, 1996. doi: 10.1007/BF02328712.

[5] B. Song et al., "Confinement effects on the dynamic compressive properties of an epoxy syntactic foam," Compos. Struct., vol. 67, pp. 279-287, 2005. doi: 10.1016/j.compstruct.2004.07.011.

[6] R. J. Christen, S. R. Swanson, W. S. Brown. "Split-HopkinsonBar Tests on Rock under Confining Pressure," Exp. Mech., vol. 12, pp. 508-513, 1972. doi: 10.1007/BF02320747.

[7] G. Gary, P. Bailly. "Behaviour of quasi-brittle material at high strain rate. Experiment and modelling," Eur. J. Mech. A/Solids, vol. 17, pp. 403- 420, 1998. doi: 10.1016/S0997-7538(98)80052-1.

[8] U. S. Lindholm, L. M. Yeakley, and A. Nagy. "Dynamic Strength and Fracture Properties of Dresser Basalt," Int .J. Rock. Mech. Min., vol. 11, pp. 181-191, 1974. doi: 10.1016/0148-9062(74)90885-7.

[9] X. B. Li et al., "Innovative testing technique of rock subjected to coupled static and dynamic loads," Int. J. Rock. Mech. Min., vol. 45, pp. 739-748, 2008. doi: 10.1016/j.ijrmms.2007.08.013.

[10] Y. J. Zuo et al., "Damage and failure rule of rock undergoing uniaxial compressive load and dynamic load," J. Cent. South. Univ. T., vol. 12, pp. 742-748, 2005. doi: 10.1007/s11771-005-0080-3.

[11] D. J. Frew et al., "Development of a dynamic triaxial Kolsky bar," Meas. Sci. Technol., vol. 21, pp. 105704(105710), 2010.

[12] E. C. Robertson, "Experimental Study of the Strength of Rocks," Geol. Soc. Am. Bull., vol. 66, pp. 1275-1314, 1955. doi: 10.1130/0016-7606(1955)66[1275:ESOTSO]2.0.CO;2.

[13] J. C. Jaeger and E. R. Hoskins, "Rock Failure under Confined Brazilian Test," J. Geophys. Res., vol. 71, pp. 2651-2659, 1966. doi: 10.1029/JZ071i010p02651.

[14] B. Vasarhelyi. "Influence of pressure on the crack propagation under mode I loading in anisotropic gneiss," Rock. Mech. Rock. Eng., vol. 30, pp. 59-64, 1997. doi: 10.1007/BF01020113.

[15] N. A. Al-Shayea, K. Khan, and S. N. Abduljauwad, "Effects of confining pressure and temperature on mixed-mode (I-II) fracture toughness of a limestone rode," Int. J. Rock. Mech. Min., vol. 37, pp. 629-643, 2000. doi: 10.1016/S1365-1609(00)00003-4.

[16] M. Chen and G. Q. Zhang, "Laboratory measurement and interpretation of the fracture toughness of formation rocks at great depth," J. Petrol. Sci. Eng., vol. 41, pp. 221-231, 2004.

doi: 10.1016/S0920-4105(03)00156-6.

[17] T. Funatsu et al., "Combined effects of increasing temperature and confining pressure on the fracture toughness of clay bearing rocks," Int. J. Rock. Mech. Min., vol. 41, pp. 927-938, 2004. doi: 10.1016/j.ijrmms.2004.02.008.

[18] Y. X. Zhou et al., "Suggested methods for determining the dynamic strength parameters and mode-I fracture toughness of rock materials," Int. J. Rock Mech. Min., vol. 49, pp. 105-112, 2012. doi: 10.1016/j.jirmms.2011.10.004.

[19] Z. Zhou et al., "Dynamic Brazilian tests of granite under coupled static and dynamic loads," Rock. Mech. Rock Eng., vol. 47, pp. 495-505, 2014. doi: 10.1007/s00603-013-0441-4. 
[20] Q. B. Zhang and J. Zhao, "A Review of Dynamic Experimental Techniques and Mechanical Behavior of Rock Materials," Rock Mech. Rock Eng., vol. 47, pp. 1411-1478, 2013. doi: 10.1007/s00603-013-0463-y.

[21] G. Cusatis, "Strain-rate effects on concrete behavior," Int. J. Impact Eng., vol. 38, pp. 162-170, 2011. doi: 10.1016/j.ijimpeng.2010.10.030.

[22] K. Fujikake et al., "Effects of Strain Rate on Tensile Behavior of Reactive Powder Concrete," J. Adv. Concr. Technol., vol. 4, pp. 79-84, 2006. doi: 10.3151/jact.4.79.

[23] N. S. Brar and Z. Rosenberg. "Brittle Failure of Ceramic Rods under Dynamic Compression," J. Phys-Paris, vol. 49, pp. 607-612, 1988.

[24] J. T. Zhang, L. S. Liu, P. C. Zhai, Q. J. Zhang. "Experimental and numerical researches of dynamic failure of a high strength alumina/boride ceramic composite," High-Performance Ceramics V, Pts 1 and 2, vol. 368-372, pp. 713-716, 2008. doi: 10.4028/www.scientific.net/KEM.368-372.713. 


\section{A two-stage conversion process for AZ31B corrosion}

\author{
Jie Wang ${ }^{1}$ \\ ${ }^{1}$ Department of Mechanical \& Mechatronics Engineering \\ University of Waterloo \\ Waterloo, Canada \\ j756wang@uwaterloo.ca
}

\author{
Hamid Jahed ${ }^{2}$ \\ ${ }^{2}$ Department of Mechanical \& Mechatronics Engineering \\ University of Waterloo \\ Waterloo, Canada \\ hjahedmotlagh@uwaterloo.ca
}

\author{
Xin Pang ${ }^{3}$ \\ ${ }^{3}$ CanmetMATERIALS \\ Natural Resources Canada \\ Hamilton, Canada \\ xin.pang@canada.ca
}

\begin{abstract}
Permanganate phosphate coating is considered as one of the most promising alternative coatings to chromate conversion coating because of the similarity between permanganate and chromate. However, the permanganate phosphate coating always has net-work cracks. In this paper, a thick and crack-free permanganate phosphate coating was developed by a two-stage conversion process. Results of scanning electron microscope (SEM) with X-ray diffraction (XRD) analysis showed that some more corrosion resistant compounds appeared in the coating after a two-stage conversion treatment, which were not found in the single conversion coating. Moreover, the two-stage conversion coating has a higher thickness with few cracks. Furthermore, electrochemical tests and salt spray test further exhibited that the two-stage conversion coating had a better corrosion performance than either of the two single conversion coatings.
\end{abstract}

Keywords-AZ31; Conversion coating; Two stage process; Corrosion

\section{INTRODUCTION}

Two major obstacles have limited the expansion of Mg alloy use in transportation industry. The limited slip systems at room temperature leads to activation of deformation twinning which causes different yield strengths in tension and compression [1-2] (which is different from hydrostatic stress dependent strength different effect [3] seen in high strength steel). The high corrosion susceptibility of magnesium alloys extremely restricts their further application in automotive, aerospace industries and electronics [4]. Presently, the most widely used method in industries to provide the protection on magnesium and its alloys is generally the chromate conversion coating. However, the inevitable environmental and health problems caused by hexavalent chromate leads to a real necessity of developing alternative conversion coatings. During the past 20 years, a few environmental friendly conversion coatings were proposed to be aimed at replacing chromate conversion coating, including metal phosphate coatings, rare earth coating, stannate coating, etc [5-8].

Compared to chromate conversion coating, permanganate phosphate coatings are more environmental friendly and could provide equivalent corrosion resistance and paint adhesion. In recent years, varieties of research related to phosphate permanganate coating have been made on magnesium alloys. Hawke et al. [9] developed a coating consisting of an agglomerate of well-formed crystals with the coating thickness of 4-6 $\mu \mathrm{m}$ using a conversion bath containing $\mathrm{KMnO}_{4}$ and $\mathrm{NH}_{4} \mathrm{H}_{2} \mathrm{PO}_{4}$, which showed good corrosion resistance and paint base performance. Typically, permanganate phosphate coatings have severe cracks, through which corrosive spices could readily reach the Mg substrate. Jian, etc. [10] reported that a phosphate-permanganate conversion coating without cracks was able to be fabricated in a strongly acidic solution which exhibited excellent corrosion resistance. However, this coating is too thin and too weak. To date, there are no work on magnesium alloys reporting a permanganate phosphate conversion coating that is both thick and crack-free.

In the present study, a two-stage conversion process is proposed to form a dense and thick phosphate-permanganate coating. The microstructure and corrosion resistance of this two-stage conversion coating was evaluated by SEM-EDS, electrochemical testing. Results showed that this two-stage conversion coating were both dense and thick that the corrosion resistance was improved compared to the single-step conversion coating. 


\section{MATERIALS AND EXPERIMENTS}

\section{A. Materials and surface preparation}

Specimens of AZ31B [11-12] cast alloy with a size of $3 \times 25.4 \times 50.8 \mathrm{~mm}$ taken from a cylindrical ingot were utilized as the test material. These coupons were mechanically grinded to $1200 \#$ using emery paper, ultrasonic degreased in acetone, washed in ethanol and dried in air stream.

\section{B. Conversion coating treatment and characterization methods}

Potassium permanganate (15g/L KMnO4), potassium dihydrogen phosphate $(3 \mathrm{~g} / \mathrm{L}$ KH2PO4), manganese sulfate $(4 \mathrm{~g} / \mathrm{L} \mathrm{MnSO} 4)$ and ethylenediaminetetraacetic acid, namely EDTA (1g/L C10H16N2O8) were used to prepare the aqueous conversion bath. The two-stage conversion process was firstly conducted in a low $\mathrm{pH}$ bath and then was immediately treated by a high $\mathrm{pH}$ solution Another two single stage coatings were also separately obtained as a comparison by a single low $\mathrm{pH}$ solution treatment (marked as LP treatment) and a single high $\mathrm{pH}$ solution treatment (marked as HP treatment).

The surface morphology, microstructure and chemical composition of the conversion coating were characterized using scanning electron microscopy (SEM) and energy-dispersive spectrometry (EDS). The potentiodynamic polarization and electrochemical impedance spectroscopy (EIS) measurements were performed in $3.5 \%$ sodium chloride $(\mathrm{NaCl})$ using a potentiostat/galvanostat electrochemical system (Solartron SI 1287 and 1255B). Prior to the polarization scan and EIS measurement, each sample was immersed in the testing solution for $30 \mathrm{~min}$ to reach a steady open circuit potential (OCP). The potentiodynamic polarization measurement was conducted by sweeping the potential from $-100 \mathrm{mV}$ to $+500 \mathrm{mV}$ vs the OCP at a scan rate of $1 \mathrm{mV} / \mathrm{s}$. For EIS analysis, each spectrum was recorded at the OCP in frequencies ranging from $100 \mathrm{kHz}$ to $10 \mathrm{mHz}$, with a sinusoidal signal amplitude of $10 \mathrm{mV}$. All experimental data were presented in the form of the average \pm the standard deviation of three measurements.

The salt spray chamber test was performed according to the SAE J2334 standard. In this test, the coupons were placed at an angle of $15^{\circ}-30^{\circ}$ from the vertical direction in a chamber. One 24-hour cycle includes a 6 hours exposure in $100 \%$ condensing humidity at around $50^{\circ} \mathrm{C}$, a 15 minutes exposure at $25^{\circ} \mathrm{C}$ to a fog containing 0.5 wt. $\% \mathrm{NaCl}, 0.1 \% \mathrm{CaCl} 2,0.075 \% \mathrm{NaHCO} 3$ and a 17.75 hours exposure in a dry environment at $60^{\circ} \mathrm{C}(50 \%$ relative humidity).

\section{RESULTS AND DISCUSSION}

\section{A. Surface morphology and coating composition analysis}

Figure 1.1 shows the surface morphology of the coating obtained by the two-stage conversion process described above. Another two coatings, i.e. the most corrosion resistant from the low $\mathrm{pH}$ treatment and the most corrosion resistant from the high $\mathrm{pH}$ treatment, are also presented in the figure together with the bare alloy for comparison. In general, all the coatings displayed similar surface morphology with networks of shallow cracks except that some cracks in the two-stage coating was slightly widened. The ESD analysis suggested that the second phase particles in the bare alloy were oxidized after the conversion process, as shown in the Table. And it can be seen from Figure that more oxidized second phase particles were seen in the coating from the high $\mathrm{pH}$ treatment (HP coating) than that from the low $\mathrm{pH}$ bath (LP coating). The fact that the second phase particles can be seen on the coating surface indicates that the coating was quite thin. The most interesting aspect of EDS results is that some manganese oxides were also detected in the two-stage conversion coating, as shown by the spot $\mathrm{D}$ in Figure (d). Manganese oxides $\left(\mathrm{MnO} / \mathrm{MnO}_{2} / \mathrm{Mn}_{2} \mathrm{O}_{3}\right)$ are generally more corrosion resistant than $\mathrm{MgO}$ and $\mathrm{Al}_{2} \mathrm{O}_{3}$. Therefore, this two-stage conversion process has the potential to further improve corrosion resistance of the Mn-P coating, as compared to a single conversion process. Additionally, the thickness of each coating could be evaluated from the weight gain of the coated samples, as presented in Figure . It distinctly demonstrated that the two-stage conversion coating had the highest thickness and the HP coating film was the thinnest, indicating that the two-stage conversion process can be used to grow thick and corrosion resistant $\mathrm{Mn}-\mathrm{P}$ coating on $\mathrm{Mg}$ alloys.
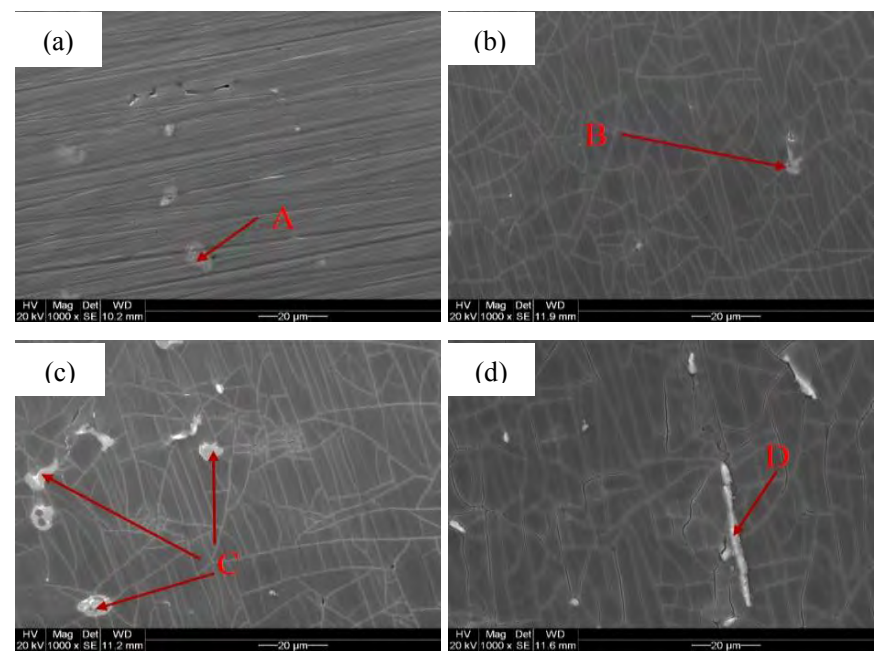

Figure 1.1 The surface morphology of (a) the bare alloy; (b) the coating obtained by low $\mathrm{pH}$ treatment (LP coating); (c) the coating obtained by high $\mathrm{pH}$ treatment (HP coating); (d) Two-stage coating.

Table 1 Chemical composition (atm.\%) of the compounds as indicated in

\begin{tabular}{cccccc}
\multicolumn{7}{c}{ Figure 1.1 } \\
\hline Spot & $\mathrm{Mg}$ & $\mathrm{Al}$ & $\mathrm{Zn}$ & $\mathrm{Mn}$ & $\mathrm{O}$ \\
\hline $\mathrm{A}$ & 90.65 & 6.88 & 2.47 & & \\
$\mathrm{~B}$ & 90.50 & 3.69 & 3.81 & & 2.00 \\
$\mathrm{C}$ & 67.64 & 19.01 & 12.18 & & 1.16 \\
$\mathrm{D}$ & 50.45 & 9.45 & 2.31 & 25.04 & 9.79 \\
\hline
\end{tabular}




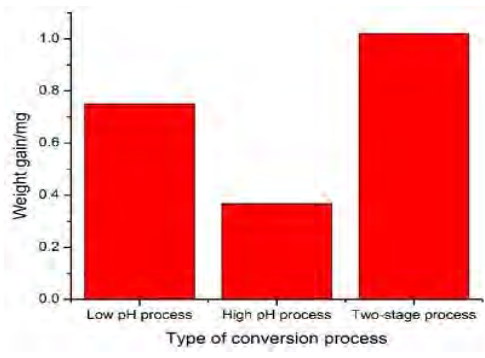

Figure 1.2 Weight gain of the samples after being treated by different conversion process

\section{B. Electrochemical test}

Figure shows the potentiodynamic polarization curves of the various conversion coated $\mathrm{AZ} 31 \mathrm{~B}$ in $3.5 \% \mathrm{NaCl}$ solution. Generally, the cathodic polarization curve is related to the evolution of hydrogen and the anodic polarization curve represents the corrosion resistance of the coating. As can be seen in the figure below, both the anodic and cathodic polarization curves of the conversion coatings shifted toward the lower current direction (left) significantly compared to those of the bare alloy, indicating that both the anodic reaction and cathodic reactions were inhibited and hence the corrosion resistance of the AZ31B was effectively improved by the conversion coating. Moreover, it can be observed that a passive region (defined as the potential difference between the corrosion potential $\mathrm{E}_{\text {corr }}$ and the breakdown potential $\mathrm{E}_{\text {break }}$ ) occurred in the anodic polarization branch of the coated samples. Among all coated samples, the HP coating exhibited the largest passive region of $432.2 \mathrm{mV}$. From the polarization test results we can see that the HP coating and two-stage coating showed similar corrosion behavior and both were more corrosion resistant than the LP coating.

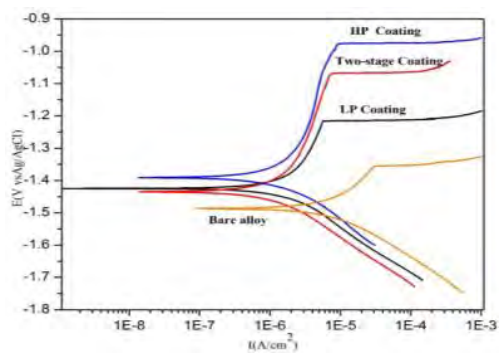

Figure 1.3 Polarization curves of the bare alloy and different conversion coated samples

Electrochemical impedance spectroscopy (EIS) was then used to further study the corrosion resistance of the coated samples. As can be clearly observed from the Bode plots (Figure ) of various conversion coatings and the bare alloy, the two-stage conversion coating exhibited the highest impedance values in the low frequency range. The impedance generally represents the ability of the coating to resist the flow of electrical current. Higher impedance suggests a lower anodic dissolution rate. Therefore, from the point view of EIS results, the two-stage conversion coating shows the highest corrosion resistance.

From the results of the electrochemical tests, it can be concluded that the two-stage conversion process resulted in a thick and dense coating with better corrosion resistance than the coating obtained by a single conversion process (LP coating and HP coating).

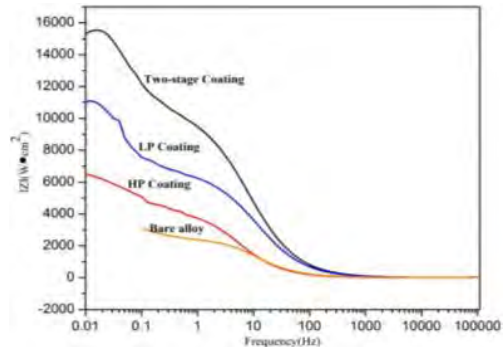

Figure 1.4 Bode plots of the bare alloy and different conversion coated samples

\section{Salt spray test}

To characterize long-term corrosion performances of the coated specimens, salt spray chamber test according to SAE J2334 standard was conducted for up to 168 hours. The edge of the examined samples were all sealed with epoxy to avoid the edge effect in the corrosion process. By visual examination of the conversion coated samples as shown in Figure 1.5, we observed that a few corrosion pits were seen on the surface of LP coated and HP coated samples after a test duration of 96 hours. In contrast, only several small corrosion pits were found on the surface of the two-stage conversion coated specimen. Furthermore, as the testing duration went up to 168 $\mathrm{h}$, corrosion pitting on the LP coated and HP coated coupons became more severe with an increased number of pits. However, the corrosion of two-stage conversion coated coupon did not deteriorate so much with the increasing testing time.
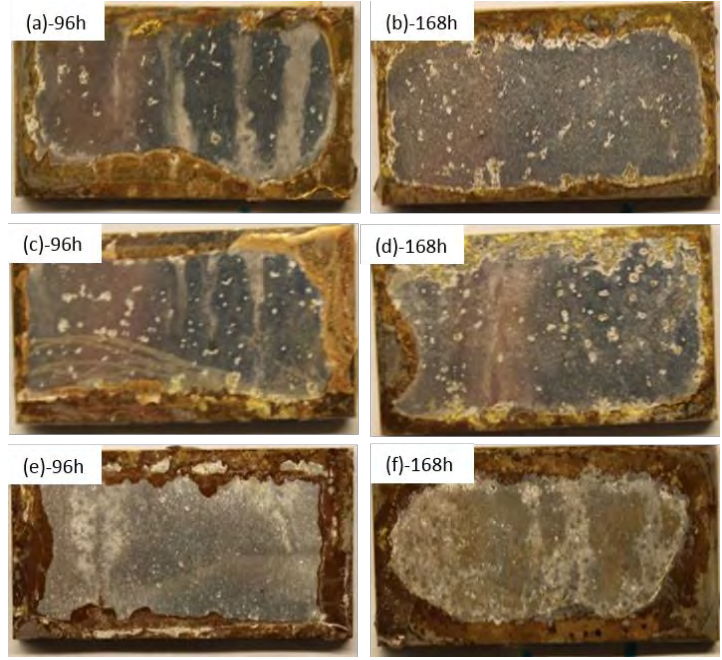

Figure 1.5 Salt spray test results conversion coated samples: (a)(b) LP coating; (c)(d) HP coating; (e)(f) Two-stage coating

\section{CONCLUSIONS}

A two-stage conversion process, namely, the treatment in a low $\mathrm{pH}$ solution followed by an immediate treatment in a high $\mathrm{pH}$ solution, was developed to deposit a thick and dense protective surface coating on $\mathrm{Mg}$ alloy AZ31B. Results of SEM analysis, electrochemical tests and salt spray test exhibited that the two-stage conversion coating had better 
corrosion performance than either of the two single conversion coatings.

\section{REFERENCES}

[1] A. A. Roostaei and H. Jahed, "Role of loading direction on cyclic behaviour characteristics of AM30 extrusion and its fatigue damage modelling," Mater. Sci. Eng., A, vol. 670, pp. 26-40, 2016.

doi: 10.1016/j.msea.2016.05.116.

[2] H. Jahed and J. Albinmousa, "Multiaxial behaviour of wrought magnesium alloys-A review and suitability of energy-based fatigue life model," Theor. Appl. Fract. Mech., vol. 73, pp. 97-108, 2014.

doi: 10.1016/j.tafmec.2014.08.004.

[3] J. Casey and H. Jahedmotlagh, "The strength-differential effect in plasticity," International Journal of Solids and Structures, vol. 20, no. 4, pp. 377-393, 1984. doi: 10.1016/0020-7683(84)90047-7.

[4] A. Atrens et al,. "Review of recent developments in the field of magnesium corrosion," Adv. Eng. Mater., vol. 17, no. 4, pp. 400-453, 2015. doi: 10.1002/adem.201400434.

[5] J. E. Gray and B. Luan, "Protective coatings on magnesium and its alloys —a critical review," J. Alloys Compd., vol. 336, no. 1-2, pp. 88-113, 2002. doi: 10.1016/S0925-8388(01)01899-0.

[6] X . B. Chen, N. Birbilis, and T. B. Abbott, "Review of corrosionresistant conversion coatings for magnesium and its alloys," Corrosion, vol. 67, no. 3, pp. 035005-1, 2011. doi: 10.5006/1.3563639.

[7] S. K. Shaha, S. B. Dayani, and H. Jahed, "Influence of cold spray on the enhancement of corrosion fatigue of the AZ31B cast Mg alloy," TMS Annu. Meet. Exhib., pp. 541-550, Feb. 2018. doi: 10.1007/978-3-319-72526-0_51.

[8] D. Mohammad, X. Pang, and H. Jahed, "The effect of pure aluminum cold spray coating on corrosion and corrosion fatigue of magnesium $(3 \% \mathrm{Al}-1 \%$ Zn) extrusion," Surf. Coat. Technol., vol. 309, pp. 423-435, 2016.

doi: 10.1016/j.surfcoat.2016.11.014.

[9] D. Hawke and D. L. Albright, "A phosphate-permanganate conversion coating for magnesium," Met. Finish., vol. 93, no. 10, pp. 34-38, 1995. doi: 10.1016/0026-0576(96)80428-4.

[10] J. Shun-Yi, Y.-R. Chu, and C.-S. Lin, "Permanganate conversion coating on AZ31 magnesium alloys with enhanced corrosion resistance," Corros. Sci., vol. 93, pp. 301-309, 2015. doi: 10.1016/j.corsci.2015.01.040.

[11] D. Toscano et al., "Effect of forging on the low cycle fatigue behavior of cast AZ31B alloy," Mater. Sci. Eng., A., vol. 706, pp. 342-356, 2017. doi: 10.1016/j.msea.2017.08.086.

[12] S. B. Behravesh, H. Jahed, and S. Lambert, "Characterization of magnesium spot welds under tensile and cyclic loadings," Mater. Des., vol. 32, no. 10, pp. 4890-4900, 2011. doi: 10.1016/j.matdes.2011.06.001. 


\section{Development of a Ground Based Testbed for Studying the Libration Dynamics of Orbiting Tethered Satellite Systems}

\author{
Udai Bindra \\ Department of Earth and Space Science \\ York University \\ Toronto \\ bindra.udai@gmail.com
}

\author{
Zheng Hong Zhu \\ Department of Mechanical Engineering \\ York University \\ Toronto \\ gzhu@yorku.ca
}

\begin{abstract}
This paper discusses the development of a ground based experimental environment that can mimic the libration dynamics of tethered satellite systems. The setup consists of an Air-Bearing Inclinable Turntable (A-BIT), whose inclination and rotation rate can be adjusted independently. Appropriate scaling factors help compare the tethered system in the experiment to that in orbit, both of which experience vastly different magnitudes of forces. The two scenarios also differ in their physical parameters including the length of the tether, the mass of the satellite, and the tension along the tether. The scaling factors are used to compare the behavior of these two tethered satellite systems.
\end{abstract}

Keywords- space tethers; air bearing table; inclinable turntable; ground based testing; tether deployment; scaling tether deployment; tether dynamics.

\section{INTRODUCTION}

Tethers are long ropes or tapes that connect two satellites orbiting at different altitudes in space. They are usually constructed using a bare metal like aluminum, however, depending on their application they may have a part or the entire tether covered with insulating material. Tethers have numerous applications in space including power generation, attitude stabilization, momentum transfer, and de-orbiting the satellite at the end of its mission [1] [2] [3] [4]. When a tethered satellite is launched, the tether is folded and stowed inside of the satellites. Once the satellites are in orbit, the tether is deployed and stabilized in the desired orientation. In fact, all successful tethered satellites rely on the successful deployment and stabilization of the tether, while the missions that fail to achieve this struggle to complete their objectives. This fact is evidenced by missions such as the Tethered Payload Experiment (TPE) I \& II, launched in 1980 and 1981, respectively. The TPE I mission deployed only $38 \mathrm{~m}$, instead of the planned $400 \mathrm{~m}$ tether deployment, while the TPE II faired marginally better, deploying $103 \mathrm{~m}$ out of its 500m long tether [2] [4]. Onboard cameras showed that the under-deployed tether wasn't under tension all the time, which resulted in the tether coiling up. Similarly, the Small Expendable Deployer System (SEDS-I), launched in 1993, failed to slow down the deployment of the tether, which caused the satellites to recoil at the end of deployment [1] [2] [3]. The unsuccessful deployment of the tether in all such missions inhibited the payload onboard from completing the mission objectives. This presents a serious challenge for tethered satellite missions, as a tremendous amount of resources are wasted if the tether does not deploy properly. Although it is not possible to completely eliminate the possibility of mechanical failure of the deployment and stabilization mechanism, an experimental verification of the behavior of the satellite, given the mission parameters, can help fine-tune the deployment process in order to ensure a smooth and complete deployment of the tether.

This research focuses on the development of a testbed that is capable of imitating the behavior of a tethered satellite system orbiting in a circular Low Earth Orbit. The testbed utilizes an Air-Bearing Inclinable Turntable or $A-B I T$, whose inclination and rotation rate can be adjusted independently. A similar experimental technique was employed by [5] [6], focusing on the dynamics of a climber attached to a pre-deployed tether. The $A-B I T$ aims to provide insight into the libration dynamics of a tethered satellite and provide a platform to test control strategies that stabilize the libration of the tethered satellite system.

\section{EXPERIMENTAL SETUP}

The proposed experimental setup of the $A-B I T$ is shown in Fig. (1). The table is $2 \mathrm{~m}$ long and $1 \mathrm{~m}$ wide. There are two dummy CubeSats on the table. The mother satellite is anchored near the edge of the table furthest from the rotation axis, while the daughter satellite is free to move across the table. There are three air blowers mounted on the rotating frame that allow the satellite to float on the testbed. The mother and daughter satellite are connected by an aluminum tape tether which has a width of $1 \mathrm{~cm}$ and a thickness of $50 \mu \mathrm{m}$.

Prior to deployment, the tether is folded and stored in a stowage box placed above the daughter satellite [7], and both satellites are held together by an electromagnet mounted on the mother satellite. The electromagnet latches on to a threaded steel rod which passes through a spring mounted on the daughter satellite. As the daughter satellite is pushed together with the mother satellite, this spring get compressed between the two satellites. To deploy the daughter satellite, the electromagnet is remotely deactivated, converting the potential energy of the spring into the kinetic energy of the daughter satellite. The stowage box of the tether includes a passive braking mechanism 


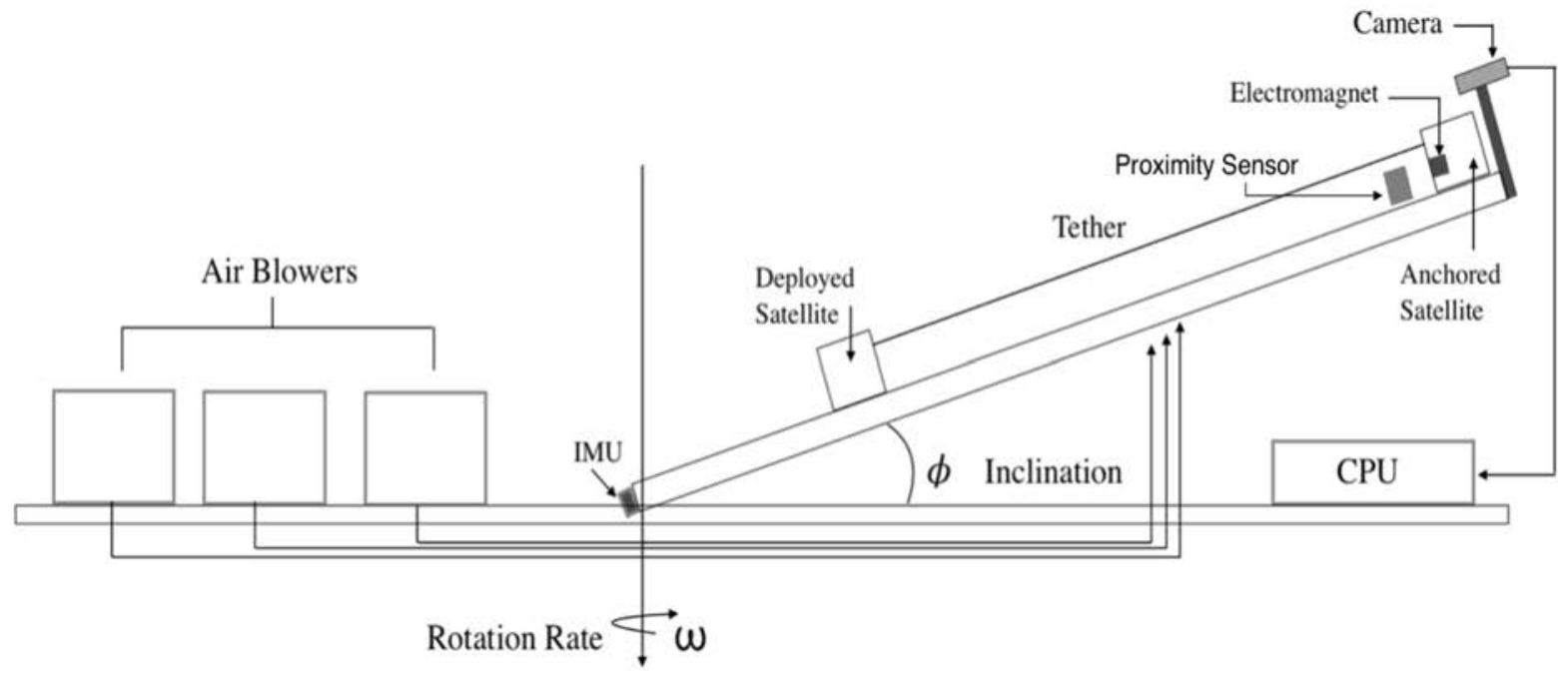

Figure 1: An Overview of the Experimental Setup

which uses friction to slow down the deployment process and avoid recoil. There are two cameras mounted on the table, one on either side of the mother satellite in order to track the motion of the daughter satellite. A proximity sensor is placed on the table near the mother satellite to measure the deployment speed of the daughter satellite.

The inclination of the $A-B I T$ with respect to the local horizontal $\Phi$, and its rotation rate $\omega$ can both be adjusted independently. An Inertial Measurement Unit (IMU) is mounted on the turntable to measure the values of $\Phi$ and $\omega$. The inclination of the table emulates the gravity gradient along the tether, while the table's rotation emulates the centrifugal and Coriolis forces acting on the daughter satellite.

\section{SCALING EXPERIMENTAL PARAMETERS}

In order to emulate the behavior of an orbiting tether using the $A-B I T$ experiment properly, the forces acting on the daughter satellite in the experiment should be similar to those in orbit, namely, the gravitational gradient, the centrifugal force and the Coriolis force. Since the magnitude of these forces are not the same as those in orbit, they need to be appropriately scaled. This can be achieved by equating the net torque and the net angular momentum of the two systems. This provides a relationship between the libration angle of the tether in the experiment to the same in orbit, as expressed in Eq. (1)

$$
\sin \theta_{e}=\frac{3 L^{2} \Omega^{2} M}{2 m l\left(g \sin \emptyset-\omega^{2} r \cos ^{2} \emptyset\right)} \sin 2 \theta_{o}
$$

where $\theta_{e}$ is the libration angle of the tether on the $A$-BIT, $\theta_{o}$ is the libration angle of tether in orbit, $g$ is the acceleration due to gravity, $r$ is the distance of the mother satellite to the rotation axis, and $\Phi$ is the inclination of the $A-B I T$ as defined before. The terms $M, L$, and $\Omega$ represent the mass of the daughter satellite, the length of the tether and orbital rate of the tethered satellite system respectively in orbit, while the terms $m, l$, and $\omega$ represent the same, but in the experiment.
Similarly, the relation between the tension along the tether in orbit and the tension along tether in the experiment, is expressed in Eq. (2) [8]:

$$
\begin{gathered}
T_{o}=\frac{M L}{I_{o}^{2}}\left[\Omega^{2}+f \pm 2 \Omega \sqrt{f}+2 \Omega^{2} I_{o}^{2}\right] \\
f=\frac{I_{e}^{2}}{m l}\left[T_{e}-m g \sin \Phi+m k \omega^{2} \cos ^{2} \Phi(r-l)\right]
\end{gathered}
$$

where $T_{e}$ and $T_{o}$ are the tensions along the tether in the experiment and in orbit respectively, while $I_{e}$ and $I_{o}$ are the moments of inertia of the system in the experiment and in orbit respectively. The moment of inertia is measured about the center of mass of the mother satellite.

The following expression is introduced in order to relate the orbital rate of the tethered satellite system with the rotation rate of the air-bearing table [6] [8]:

$$
\omega=\sqrt{\frac{M L^{2} \Omega^{2}}{k m l^{2}}}
$$

where $k$ is a dimensionless constant used to limit the inclination and rotation rate of the $A-B I T$. It should be noted that this expression holds true for small libration angles only.

As with the forces, the timescale of the tether libration in orbit and in the experiment, is not the comparable. In order to overcome this, both timescales can be resolved into dimensionless time by using the orbital rates as a normalizing factor.

\section{EXPERIMENTAL RESULTS AND ANALYSIS}

Once the orbital parameters to be simulated have been decided, the experiment is setup using the parameters developed in the previous section. Figures (2a) and (2b) show the experimental results along with the numerical simulation of an $800 \mathrm{~m}$ long tether orbiting at $1440 \mathrm{Km}$ and $1450 \mathrm{Km}$ above the Earth's surface, respectively. The results show that the time periods of libration, in orbit and in the experimental setup, are comparable. The results of the experiment show a 3\% longer time period than the simulation. This is most likely caused by 
the damping experienced by the system in the experiment, but not in orbit. However, this damping can be measured and its effects can be accounted for. As mentioned in the previous section, the scaling factors have been developed such that the amplitude of libration matches only for small libration angles. Since no control law has been applied to the simulation of the tether in the orbit, the amplitude of libration is very high and thus does not match up to that in the experiment.

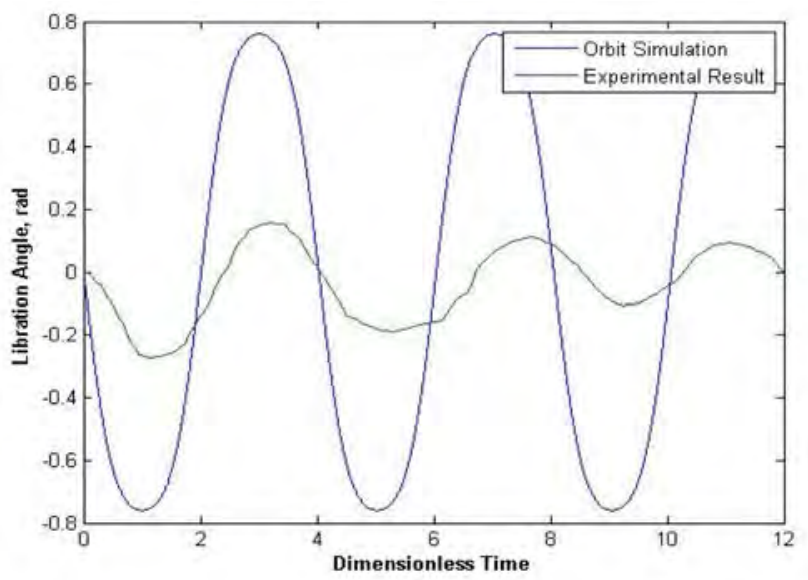

(a)

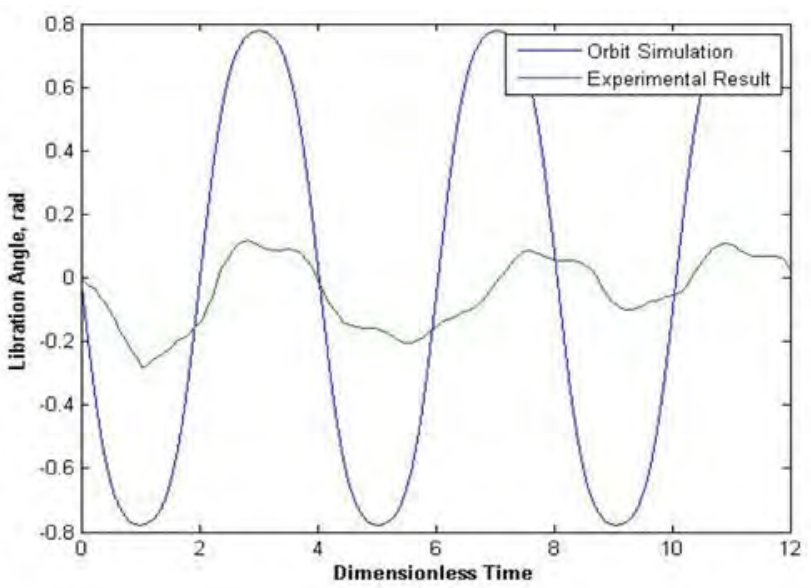

(b)

Figure 2: Tether Libration Dynamics. Emulated orbital height (a) $1440 \mathrm{Km}$ (b) $1450 \mathrm{Km}$

\section{CONCLUSION}

The results serve as a preliminary indicator that the $A-B I T$ can be utilized to study the behavior of the tethered satellites in orbit. They show that the scaling factors have been implemented effectively and that these factors can be used to scale up the libration dynamics measured in the experiment to those expected to be observed in orbit. Future work on the experimental setup would include measurement of the extent of damping experienced by the system and model it out of the resultant motion observed. Sensors would be put in place to measure the tension along the tether. A wide-angle camera would be mounted on top of the table. This would provide better position estimation and reduce the drag experienced by the daughter satellite due to the change in orientation of the mounted target that is being tracked. The experimental setup will be modified to include various control strategies, whose behavior in orbit is known. Implementing these control laws will reduce the libration angle of the tether. Comparing the behavior of the tethered satellites in the experiment to that in orbit should provide details about the effectiveness of the experimental setup to measure amplitude of the libration as well, instead of just its time period. A successful imitation of both these parameters in the experiment will allow this setup to be used for validating the effectiveness of new control strategies to stabilize tethers in orbit.

\section{REFERENCES}

[1] J. A. Carroll and J. C. Oldson, "Tethers for Small Satellite Applications," in AIAA/ USU Small Satellite Conf., Logan, 1995.

[2] Y. Chen, R. Huang, X. Ren, L. He and Y. He, "History of the Tether Concept and Tether Missions: A Review," ISRN Astron. Astrophys., vol. 2013, pp. 1-7, 2013. https://doi.org/10.1155/2013/502973

[3] M. L. Cosmo and E. C. Lorenzini, "Tethers in Space Handbook," NASA Marshall Space Flight Center, 1997.

[4] M. V. Pelt, Space Tethers and Space Elevators, Berlin: Springer, 2009. https://doi.org/10.1007/978-0-387-76556-3

[5] H. Kojima and Y. Furukawa, "Experimental Verification of Chaotic Librational Motion of Tethered Satellite System in Elliptic Orbit," in AIAA Modeling and Simulation Technologies Conf., Chicago, 2009. https://doi.org/10.2514/6.2009-5815

[6] H. Kojima, Y. Sugimoto and Y. Furukawa, "Experimental Study on Dynamics and Control of Tethered Satellite Systems with Climber," Acta Astronaut., vol. 69, no. 1-2, pp. 96-108, 2011. https://doi.org/10.1016/j.actaastro.2011.02.009

[7] H. A. Fujii and e. al., "Space Demonstration of Bare Electrodynamic Tape-Tether Technology on the Sounding Rocket S520-25," in AIAA Guidance, Navigation, and Control Conf., Portland, 2011.

[8] U. Bindra and Z. H. Zhu, "Development of an Air-Bearing Inclinable Turntable for Testing Tether Deployment," in AIAA Guidance, Navigation, and Control Conf., San Diego, 2016. 


\title{
Anisotropic Fatigue Modeling of Wrought Magnesium Alloys
}

\author{
Amirhossein Pahlevanpour, Seyed Behzad Behravesh, Hamid Jahed \\ Mechanical and Mechatronics Engineering Department, University of Waterloo \\ Waterloo, Canada
}

\begin{abstract}
The merit of Jahed-Varvani (JV) as an energy-based model and Smith-Watson-Toper (SWT) as a critical plane fatigue model are assessed for three wrought magnesium alloys. The raw data, including the stabilized strain-stress hysteresis loops and strain-life curves, was collected from the literature. The SWT model provided more scattered predictions than the JV model, which suggests that the JV model is more appropriate for fatigue modeling of wrought magnesium alloys, that exhibit anisotropic and asymmetric behavior. A discussion justifies the differences between the predictions of the two models. Eventually, the life of the materials in different directions is predicted by one set of JV parameters, which was previously extracted for AM30 in the transverse direction. The promising results provide supplementary support for the auspicious capability of strain energy density as a damage parameter for wrought magnesium alloys.
\end{abstract}

Keywords- Magnesium; wrought; anisotropy; fatigue; modeling

\section{INTRODUCTION}

The profound impact of the automotive industries on greenhouse gas emission and global warming has led them into light-weighting of their products [1]. Magnesium (Mg) alloys with roughly $35 \%$ and $75 \%$ less density than aluminum and steel, respectively are the lightest available structural metals. This low density and high specific strength of the $\mathrm{Mg}$ alloys have made them highly attractive for the transportation industries.

Load-bearing components in vehicles undergo cyclic loadings; therefore, fatigue analysis is inevitable in their design process. However, finding a reliable model to mimic the fatigue behavior of wrought $\mathrm{Mg}$ alloys has been a challenge due to their asymmetric and anisotropic characteristics.

Numerous approaches have been developed and evaluated for the life prediction of wrought $\mathrm{Mg}$ alloys including, the energy-, strain-, stress-, and fracture-mechanic-based models. These efforts can be categorized into either model that consider just one material direction [2]-[6] or the ones that account for the anisotropic characteristic of wrought $\mathrm{Mg}$ alloys [7]-[11].

Lugo et. al [3] developed a microstructure-sensitive model that successfully imitates strain-controlled fatigue experimental observations along the working direction. The model is based on the crack initiation and growth stage constitution, and was justified for three AZ31 initial conditions, i.e., extrusion, rolled plate, and rolled sheet. Castro and Jiang [5] assessed three critical plane approaches: Smith-Watson-Toper (SWT) [12], Fatemi-Socie, and Jiang [14] models, during tensioncompression, torsion, and combined axial-torsion loading on AZ31B along the extrusion direction. The Fatemi-Socie damage parameter provided reasonable fatigue life prediction, but the SWT failed to follow torsion and tension-compression experimental data. The mixed mode cracking was suggested as the source of inaccurate crack orientation prediction for both the SWT and Fatemi-Socie models. The results of Jiang's model were substantiated for all loading conditions, predicting both the fatigue life and the cracking orientation. Xiong and Jiang [2] noticed the SWT parameter's inability to correlate the fatigue life of ZK60 extrusion while scrutinizing its cyclic behavior with intense texture under high strain amplitudes up to $6 \%$ and also stress-controlled condition. Specimen failure under compression for strain amplitudes larger than or equal to $3.5 \%$ was implied to be the cause of the inaccurate SWT predictions, based on the fact that SWT is founded on tensile cracking as the failure mechanism. In contrast, SWT exhibits promising life prediction in the range of $0.2 \%$ to $1.5 \%$ strain amplitudes [6].

Concerning anisotropic fatigue modeling, Lin et al. [4], [8] modified the conventional Basquin's model to account for the mean stress effect and applied the model to hot-rolled AZ91 under cyclic asymmetric stress-controlled loading for rolling and transverse directions. Park et al. [10] evaluated the fatigue life prediction capability of the Ellyin energy-based model [15] in conjunction with stress and plastic strain amplitudes as two damage parameters for rolled AZ31. Loads were applied parallel and perpendicular to the rolling direction. This study concluded that the energy model performs better than the other two models. By employing the modified total strain energy density model proposed by Jahed and Varvani (JV) [16], Roostaei and Jahed [9] achieved satisfactory fatigue life prediction for AM30 extrusion independent of material orientation. They reported that the predicted lives by the SWT model are more scattered and more conservative than the JV model. This behavior was attributed to intricate maximum stress variation at different strain amplitudes [9].

In spite of several attempts to model the fatigue behavior of $\mathrm{Mg}$ alloys, a phenomenological study in search of suitable fatigue criterion capable of capturing the asymmetric and anisotropic behavior of wrought $\mathrm{Mg}$ alloys is still lacking. This paper examines SWT and JV models for anisotropic life 
prediction of wrought $\mathrm{Mg}$ alloys using data available in the literature. Then, the idea of employing a single set of parameters for life estimation of wrought $\mathrm{Mg}$ alloys in various loading directions is evaluated. For this study, the set of parameters for the JV model, already been extracted for AM30 extrusion in the transverse direction, was utilized to predict the fatigue life of $\mathrm{AZ31B}$ and AZ80 in different directions.

\section{EXPERIMENTAL SETUP}

Only a few published works present the strain-life curves and stabilized hysteresis loops for wrought $\mathrm{Mg}$ alloys in different directions. The studies conducted by $\mathrm{L}$. $\mathrm{Wu}$ et al. on rolled AZ31B-H24 [17], F. Lv et al. on rolled AZ31B [11], Xiong and Jiang on rolled AZ80 [7] and Roostaei and Jahed on AM30 extrusion [9] are among those few published results. The details of the investigated materials are presented in Table. I. In what follows, rolling direction (RD) will be assigned as the reference direction for AZ31-H24, AZ31B, and AZ80 in two fatigue models and the life in the other directions will be predicted based on the reference parameters.

TABLE I. INVESTIGATED WROUGHT MG ALLOYS

\begin{tabular}{c|c|c|c|c|c} 
Material & Process & Directions & $\begin{array}{c}\text { Strain } \\
\text { ratio }\end{array}$ & Environment & Reference \\
\hline $\begin{array}{c}\text { AZ31B- } \\
\text { H24 }\end{array}$ & Rolled & $\begin{array}{c}\text { Rolling (RD) } \\
\text { Transverse (TD) } \\
\text { Normal (ND) }\end{array}$ & -1 & Ambient & {$[17]$} \\
\hline AZ31B & Rolled & $\begin{array}{c}\text { Rolling (RD) } \\
\text { Normal (ND) }\end{array}$ & -1 & Ambient & {$[11]$} \\
\hline AZ80 & $\begin{array}{c}\text { Hot- } \\
\text { rolled }\end{array}$ & $\begin{array}{c}\text { Rolling (RD) } \\
\text { Normal (ND) } \\
\text { to normal (ND30) } \\
60^{\circ} \text { to normal (ND60) }\end{array}$ & -1 & Ambient & {$[7]$} \\
\hline AM30 & Extrusion & $\begin{array}{c}\text { Extrusion (ED) } \\
\text { Transverse (TD) }\end{array}$ & -1 & Ambient & {$[9]$}
\end{tabular}

\section{MODELING}

\section{A. Smith-Watson-Topper (SWT)}

SWT is a critical plane fatigue model, that takes into account mean stress effect through the maximum stress term as shown in )1( [12]:

$$
S W T=\sigma_{n, \max } \frac{\Delta \varepsilon_{1}}{2}
$$

where $\sigma_{n, \max }$ and $\Delta \varepsilon_{1}$ are the maximum normal stress and the principal strain range on the principal strain plane, respectively. The SWT parameter has been correlated to the fatigue life of $\mathrm{Mg}$ alloys by either [2], [6],

$$
\left(S W T-F P_{0}\right)^{v} N_{f}=D
$$

where $F P_{0}, v$, and $D$ are fitting coefficients; or by CoffinManson parameters [9], [18]-[20] employed in this study as well,

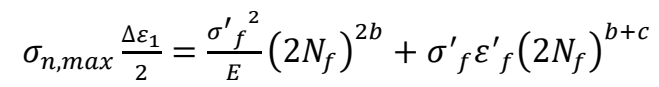

where:

$\sigma_{f}^{\prime}$ : Fatigue strength coefficient

$\varepsilon_{f}^{\prime}$ : Fatigue toughness coefficient

$b$ : Fatigue strength exponent

$c$ : Fatigue toughness exponent

and $E$ is the modulus of elasticity. The parameters in the SWT model are essentially the same as those in the Coffin-Manson model [21], [22],

$$
\frac{\Delta \varepsilon}{2}=\frac{{\sigma_{f}^{\prime}}_{E}}{E}\left(2 N_{f}\right)^{b}+\varepsilon_{f}^{\prime}\left(2 N_{f}\right)^{c}
$$

Therefore, the parameters are obtained by the decomposition of strain range into elastic and plastic strain ranges, as shown in Fig. 1.

The Coffin-Manson parameters for the three different materials were extracted in the reference direction, $\mathrm{RD}$, and are tabulated in Table. II. Adopting the SWT criteria and using the Coffin-Manson parameters, fatigue lives in all directions were calculated using a single set of parameters per material. Fig. 2 depicts the predicted life versus the experimental life for the three materials in various directions. The solid line denotes the perfect estimation, whereas the dashed and dashed-dot lines enclose the factor of 2 and 3 boundaries, respectively.

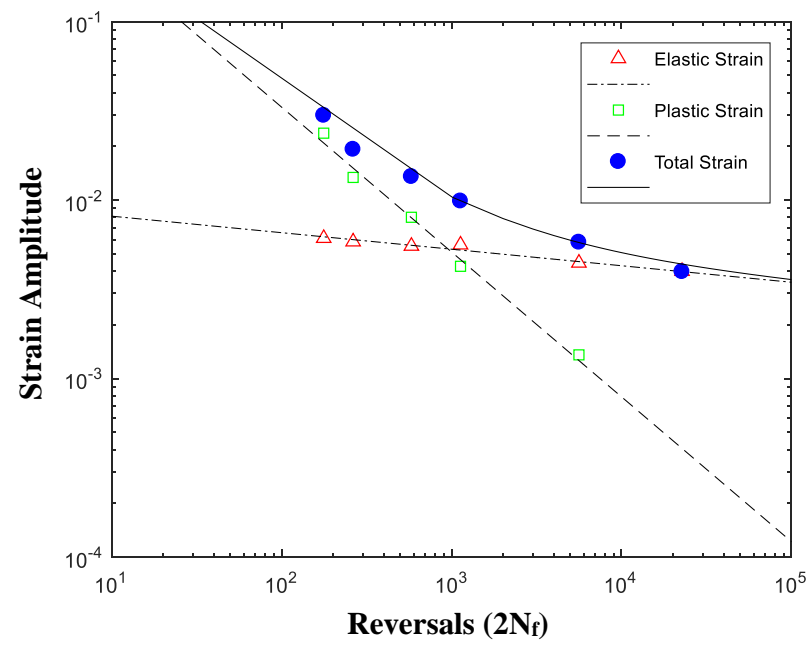

Figure 1. Strain range decomposition for the Coffin-Manson model; the typical result for AZ31B-H24 along RD

TABLE II. COFFIN-MANSON PARAMETERS ALONG RD FOR INVESTIGATED MG ALLOYS

\begin{tabular}{c|c|c|c}
\multicolumn{2}{c|}{ AZ31-H24 } & AZ31B & AZ80 \\
\hline $\boldsymbol{\sigma}_{\boldsymbol{f}}^{\prime}$ (MPa) & 442.49 & 749.75 & 422.07 \\
\hline $\boldsymbol{\varepsilon}_{\boldsymbol{f}}^{\prime}$ & 1.393 & 0.185 & 0.103 \\
\hline $\boldsymbol{b}$ & -0.092 & -0.179 & -0.071 \\
\hline $\boldsymbol{c}$ & -0.811 & -0.604 & -0.545
\end{tabular}


As expected, the life estimation for $\mathrm{RD}$ in all materials is satisfactory, demonstrating that the mathematical fitting was conducted correctly. However, the fatigue lives in other directions were overpredicted for AZ31B-H24 and AZ80, and underpredicted in AZ31B. In particular, the predicted life deviated from the factor of 3 bound for ND samples of AZ80 and AZ31-H24. This deviation is more intense for AZ80 in ND30 specimens, which will be discussed later. In contrast, the predicted lives for AZ31B, being conservative, fall within the factor of 2 bound lines.
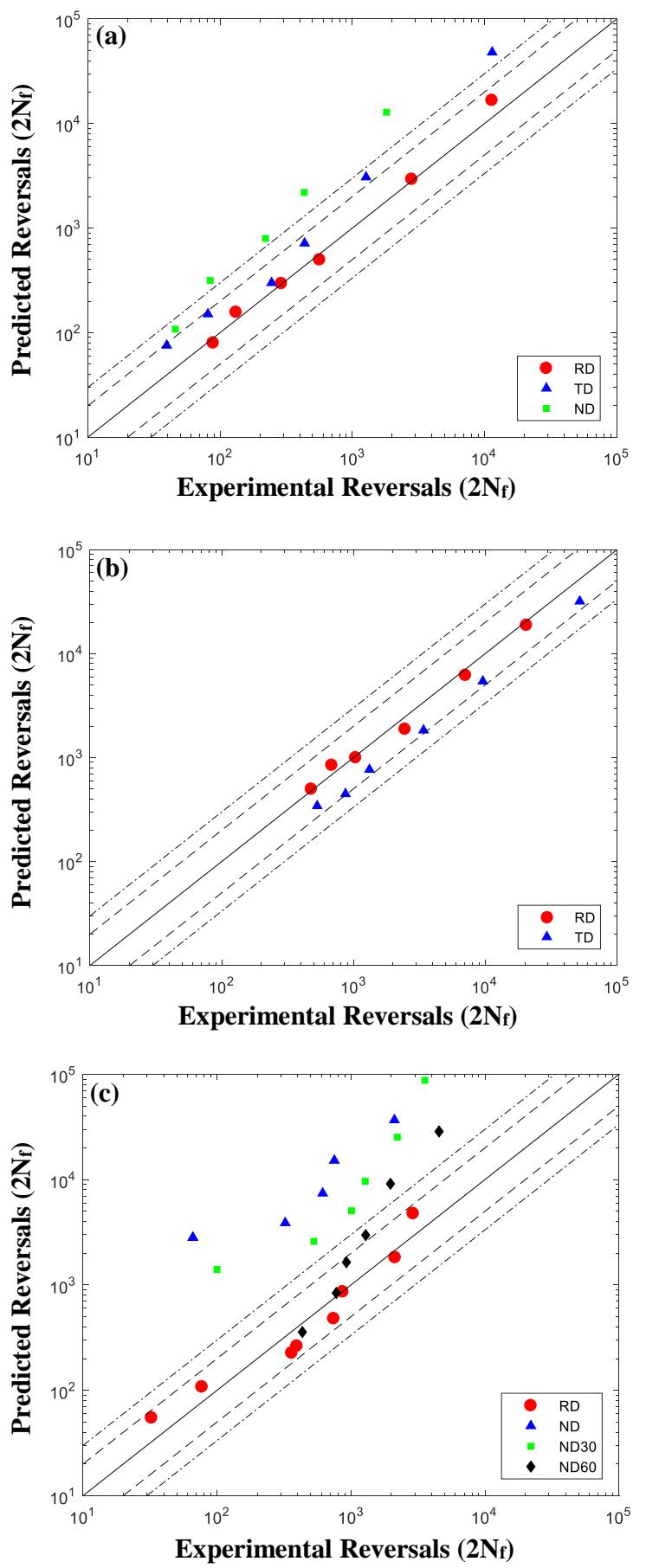

Figure 2. SWT fatigue life prediction for a) AZ31B-H24, b) AZ31B, and c) AZ80

\section{B. Jahed-Varvani (JV)}

The invariant nature of energy makes energy-based fatigue models appropriate candidates for $\mathrm{Mg}$ alloys with asymmetric and anisotropic characteristics, and they have been employed for the life estimation of many Mg alloys [5], [9], [10], [18], [20], [23]-[27]. Jahed and Varvani [16] employed total strain energy density as the damage parameter by incorporating energy-based fatigue properties,

$$
\underbrace{\Delta E}=\Delta E_{e}^{+}+\Delta E_{p}
$$

JV parameter

where $\Delta E_{p}$ is plastic strain energy density and defined as the area enveloped by the stabilized hysteresis loops and $\Delta E_{e}^{+}$is the positive elastic strain energy density, and is calculated as follows:

$$
\Delta E_{e}^{+}=\frac{\sigma_{\max }^{2}}{2 E}
$$

where $\sigma_{\max }$ is the maximum tensile stress of the stabilized hysteresis loop. The JV parameter is correlated to the life through:

$$
J V=E_{e}^{\prime}\left(2 N_{f}\right)^{B}+E_{f}^{\prime}\left(2 N_{f}\right)^{C}
$$

where

$E_{e}^{\prime}$ : Fatigue strength coefficient

$E_{f}^{\prime}$ : Fatigue toughness coefficient

$B$ : Fatigue strength exponent

$C$ : Fatigue toughness exponent

are the material constants extracted from the curves fitted to the elastic and plastic strain energy versus life, as depicted in Fig. 3. This figure presents the typical strain energy decomposition for AZ31B-H24 along the reference direction, RD. The JV model parameters for the three different materials along $\mathrm{RD}$ are summarized in Table. III.

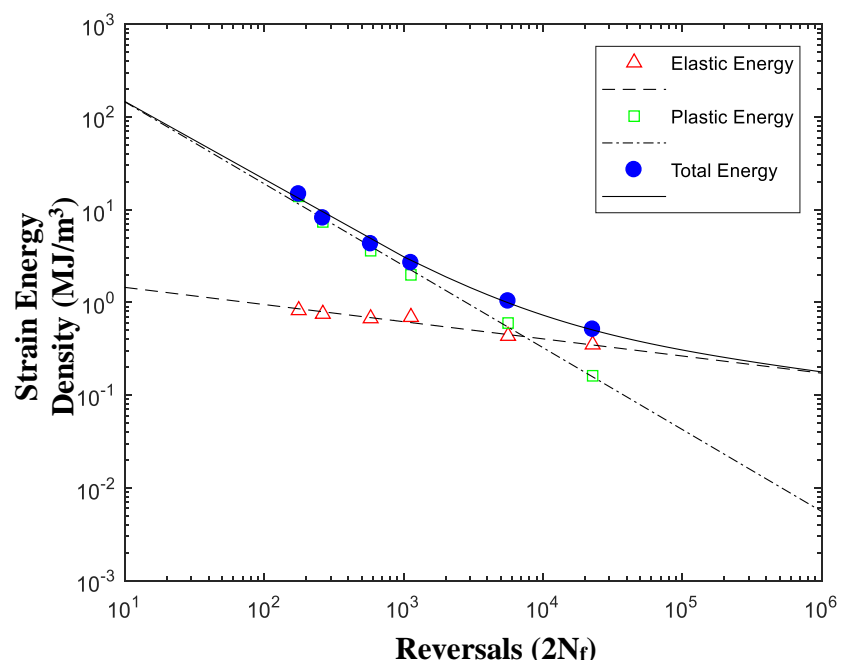

Figure 3. Decomposition of strain energy for the JV model; the typical result for AZ31B-H24 along RD 
TABLE III. JV MODEL PARAMETERS ALONG RD FOR DIFFERENT MG ALLOYS

\begin{tabular}{c|c|c|c} 
& AZ31-H24 & AZ31B & AZ80 \\
\hline $\boldsymbol{E}^{\prime}{ }_{\boldsymbol{e}}\left(\mathbf{M J} / \mathbf{m}^{\mathbf{3}}\right)$ & 2.225 & 6.387 & 2.024 \\
\hline $\boldsymbol{E}_{\boldsymbol{f}}{ }_{\boldsymbol{f}}\left(\mathbf{M J} / \mathbf{m}^{\mathbf{3}}\right)$ & 1115.85 & 746.37 & 64.26 \\
\hline $\boldsymbol{B}$ & -0.185 & 0.357 & -0.142 \\
\hline $\boldsymbol{C}$ & -0.884 & 0.911 & -0.598
\end{tabular}

By employing the JV model in conjunction with a single set of parameters per material, fatigue lives in different directions were predicted and plotted against the experimental lives in Fig. 4. The vast majority of data points for all subject materials are bounded within the factor of 3 , independent of loading direction. Modest overprediction is perceived for AZ31-H24; in contrast, the lives congregate tightly about the ideal estimation with slight conservative predictions for AZ31B. The life of AZ80 samples in ND is highly overpredicted at the two extremes of the experimental life range, whereas estimated life is almost uniformly distributed over and under the ideal prediction in RD, ND30, and ND60 directions, besides laying within the bounds of \pm 3 factor. The observed results demonstrate the competency of the JV model for anisotropic and asymmetric wrought $\mathrm{Mg}$ alloys.
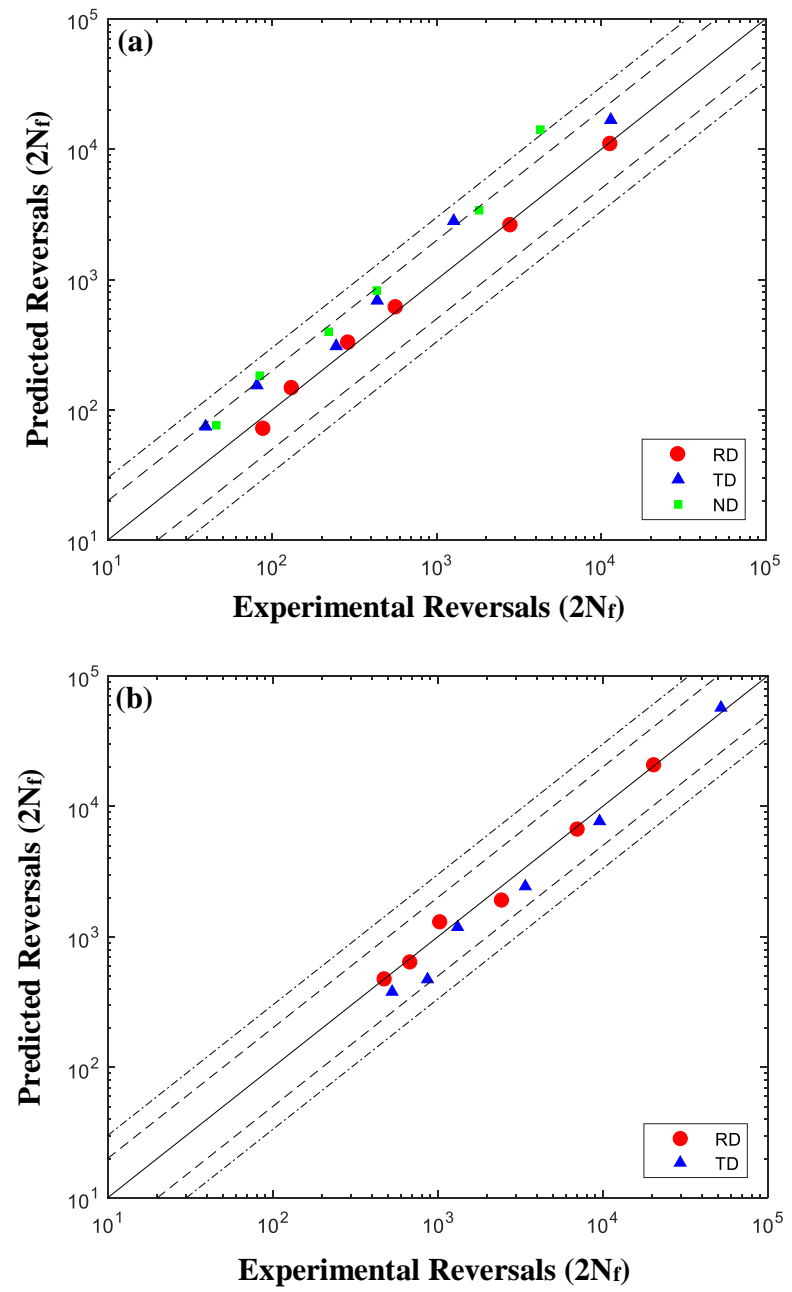

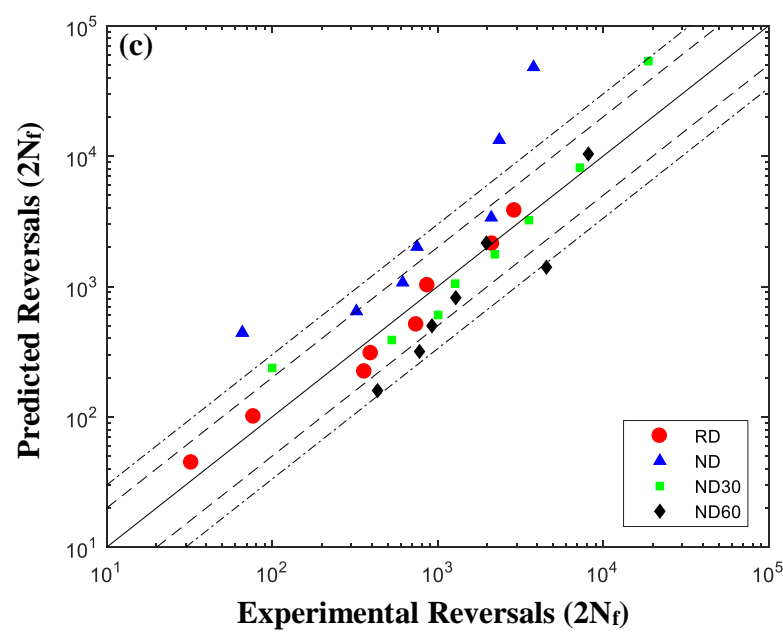

Figure 4. JV fatigue life prediction for a) AZ31B-H24, b) AZ31B, and c) AZ80

\section{Discussion}

In general, the two models provide a good fatigue-life prediction performance. The SWT model's shortcomings for some of the fatigue data are attributed to two factors [2]:

1. Many wrought $\mathrm{Mg}$ alloys show nonlinear elastoplastic strain responses in the strain amplitudes higher than $1 \%$ which could not be captured by linear Coffin-Manson fitting embedded in the employed SWT method [2], [7], [11].

2. Deformation mechanism alternation from slip to twinning/detwinning at some certain strain level introduces a kink point in the strain-life curve [2], [5], [6], which can considerably affect the fatigue resistance. Also, the change in the deformation mechanism is reflected in the hysteresis-loop shape and the enclosed area, which is not accounted for in the SWT model.

To further investigate the life prediction of SWT under large strain amplitudes, the stabilized hysteresis loops for AZ31B$\mathrm{H} 24$ under $\varepsilon_{\mathrm{a}}=1.4 \%$ in different directions are provided in Fig. 5 . The life reported for these tests in all directions are roughly the same and equal to 250 cycles [17]; however, their hysteresis loops are substantially different. Also, a significant mean stress is developed that is not accounted for, in the life model parameters of SWT. Table. IV compares the JV and its components in addition to SWT damage parameter for RD and ND. In spite of the fact that both RD and ND samples had nearly the same life, the SWT parameter values in these directions differ by $42 \%$, whereas the difference for the JV parameter is $14 \%$. The pronounced elastic strain divergence in comparison with plastic strain could explain the SWT's unsatisfactory life prediction in HCF where the elastic energy is dominant. The minimum difference belongs to the plastic portion of the strain energy density, which has been proven to be a robust damage parameter for the LCF regime [7], although its precision will 
decrease in the HCF regime, where less energy is dissipated in each cycle.

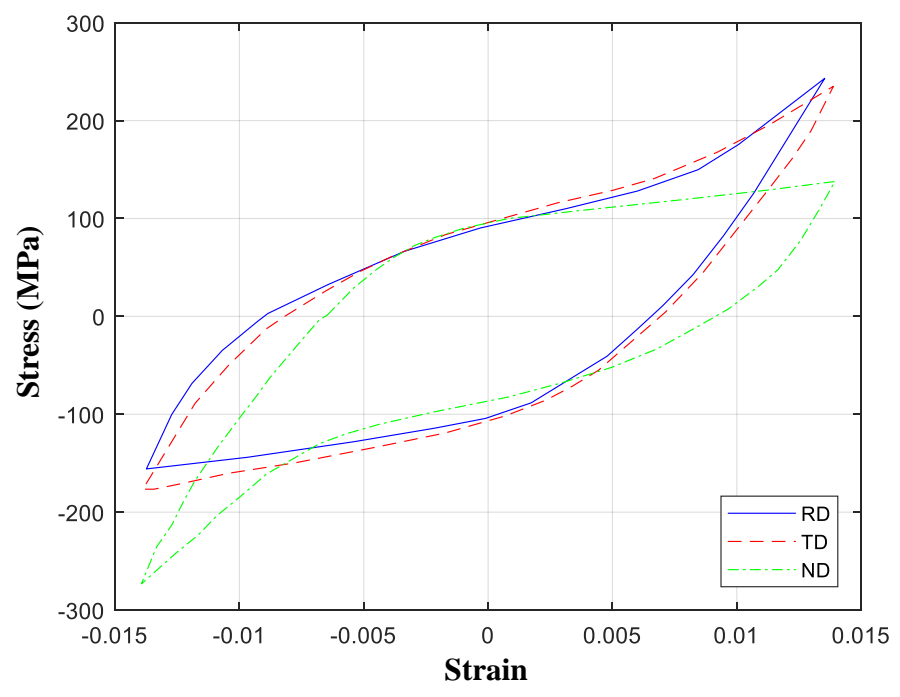

Figure 5. AZ31B-H24 stabilized hysteresis loops for $1.4 \%$ strain

TABLE IV. AZ31B-H24 FATIGUE MODEL PARAMETERS FOR AZ31B-H24 UNDER $\varepsilon_{\mathrm{A}}=1.4 \%$

\begin{tabular}{c|c|c|c} 
& RD & ND & $\begin{array}{c}\text { Difference } \\
(\%)\end{array}$ \\
\hline $\begin{array}{c}\text { Elastic Energy } \\
\text { Density }\end{array}$ & 0.672 & 0.215 & 68.0 \\
\hline $\begin{array}{c}\text { Plastic Energy } \\
\text { Density }\end{array}$ & 3.612 & 3.475 & 3.8 \\
\hline JV & 4.284 & 3.690 & 13.9 \\
\hline $\boldsymbol{\varepsilon}_{\text {elastic }}$ & 0.0055 & 0.0031 & 43.6 \\
\hline $\boldsymbol{\varepsilon}_{\text {plastic }}$ & 0.0085 & 0.0109 & 28.2 \\
\hline SWT & 3.292 & 1.921 & 41.6
\end{tabular}

In order to evaluate $\mathrm{JV}$ as a universal fatigue model for $\mathrm{Mg}$ alloys, the JV parameters extracted for AM30 in TD, which are tabulated in Table. V [9], were employed to predict the life of the three materials under investigation. Fig. 6 shows predicted versus experimental fatigue lives, where most of the points are condensed within the factor of 3 boundaries. The present research further supports the findings of previous studies [5], [9], [10], [18], [20], [23]-[26] in which the strain energy density with its corresponding life model is an appropriate damage parameter for wrought $\mathrm{Mg}$ alloys with asymmetric and anisotropic behavior.

TABLE V. JV PARAMETERS FOR AM30 EXTRUSION ALONG TD [9]

\begin{tabular}{c|c}
$\boldsymbol{E}_{\boldsymbol{e}}^{\prime}\left(\mathbf{M J} / \mathbf{m}^{\mathbf{3}}\right)$ & 2.995 \\
\hline $\boldsymbol{E}_{\boldsymbol{f}}^{\prime}\left(\mathbf{M J} / \mathbf{m}^{\mathbf{3}}\right)$ & 1710.690 \\
\hline $\boldsymbol{B}$ & -0.281 \\
\hline $\boldsymbol{C}$ & -0.975
\end{tabular}

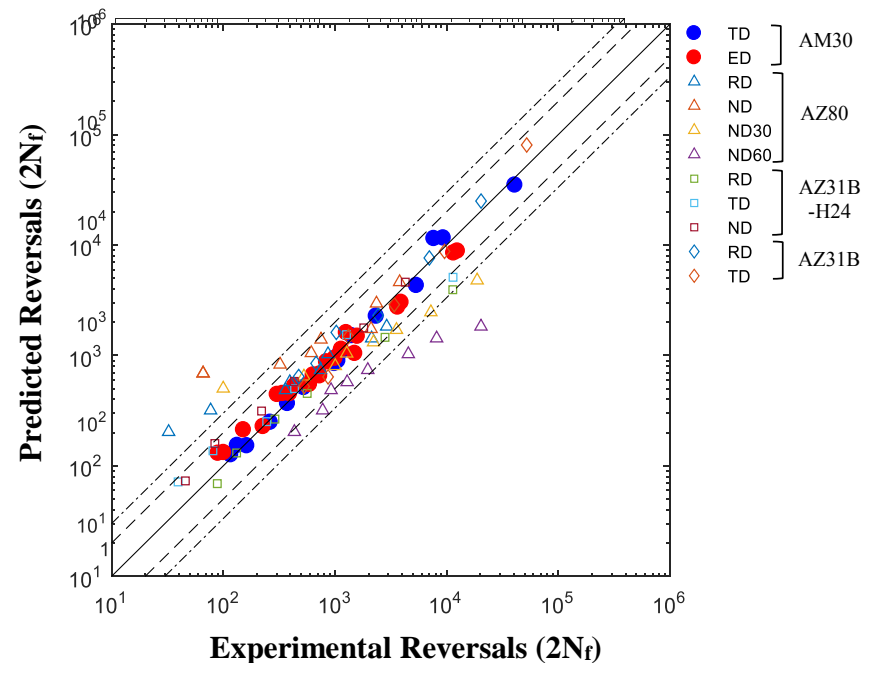

Figure 6. JV fatigue life estimation

REFERENCES

[1] H. Friedrich, "Research for a 'new age of magnesium' in the automotive industry," J. Mater. Process. Technol., vol. 117, pp. 276-281, 2001. doi: 10.1016/S0924-0136(01)00780-4.

[2] Y. Xiong and Y. Jiang, "Fatigue of ZK60 magnesium alloy under uniaxial loading," Int. J. Fatigue, vol. 64, pp. 74-83, 2014. doi: 10.1016/j.ijfatigue.2014.02.019.

[3] M. Lugo et al., "Role of different material processing methods on the fatigue behavior of an AZ31 magnesium alloy," Int. J. Fatigue, vol. 52, pp. 131-143, 2013. doi: 10.1016/j.ijfatigue.2013.02.017.

[4] X. M. Chen, Y. C. Lin, and J. Chen, "Low-cycle fatigue behaviors of hotrolled AZ91 magnesium alloy under asymmetrical stress-controlled cyclic loadings," $J$. Alloys Compd., vol. 579, pp. 540-548, 2013. doi: 10.1016/j.jallcom.2013.07.049.

[5] F. Castro and Y. Jiang, "Fatigue life and early cracking predictions of extruded AZ31B magnesium alloy using critical plane approaches," Int. J. Fatigue, vol. 88, pp. 236-246, 2016. doi: 10.1016/j.ijfatigue.2016.04.002.

[6] Q. Yu, J. Zhang, Y. Jiang, and Q. Li, "An experimental study on cyclic deformation and fatigue of extruded ZK60 magnesium alloy," Int. J. Fatigue, vol. 36, no. 1, pp. 47-58, 2012. doi: 10.1016/j.ijfatigue.2011.08.016.

[7] Y. Xiong and Y. Jiang, "Cyclic deformation and fatigue of rolled AZ80 magnesium alloy along different material orientations," Mater. Sci. Eng. A, vol. 677, pp. 58-67, 2016. doi: 10.1016/j.msea.2016.09.031.

[8] Y. C. Lin, X.-M. Chen, Z.-H. Liu, and J. Chen, "Investigation of uniaxial lowcycle fatigue failure behavior of hot-rolled AZ91 magnesium alloy," Int. J.

Fatigue, vol. 48, no. C, pp. 122-132, 2013. doi: 10.1016/j.ijfatigue.2012.10.010.

[9] A. A. Roostaei and H. Jahed, "Role of loading direction on cyclic behaviour characteristics of AM30 extrusion and its fatigue damage modelling," Mater. Sci. Eng. A, vol. 670, pp. 26-40, 2016. doi: 10.1016/j.msea.2016.05.116.

[10] S. H. Park, S. G. Hong, W. Bang, and C. S. Lee, "Effect of anisotropy on the low-cycle fatigue behavior of rolled AZ31 magnesium alloy," Mater. Sci. Eng. A, vol. 527, no. 3, pp. 417-423, 2010. doi: 10.1016/j.msea.2009.08.044.

[11] F. Lv, F. Yang, Q. Q. Duan, Y. S. Yang, S. D. Wu, S. X. Li, and Z. F. Zhang, "Fatigue properties of rolled magnesium alloy (AZ31) sheet: Influence of specimen orientation," Int. J. Fatigue, vol. 33, no. 5, pp. 672-682, 2011. doi: $10.1016 /$ j.ijfatigue.2010.10.013.

[12] Kn. Smith, T. H. Topper, and P. Watson, "A stress-strain function for the fatigue of metals(Stress-strain function for metal fatigue including mean stress effect)," J. Mater., vol. 5, no. January 1970, pp. 767-778, 1970. 
[13] A. Fatemi and D. F. Socie, "A critical plane approach to multiaxial fatigue damage including out-of-phase loading," Fatigue Fract. Eng. Mater. Struct., vol. 11, no. 3, pp. 149-165, 1988. doi: 10.1111/j.1460-2695.1988.tb01169.x.

[14] Y. Jiang, "Fatigue criterion for general multiaxial loading,"

Fatigue Fract.Eng. Mater. Struct., vol. 23, no. 1, pp. 19-32, 2000. doi: 10.1046/j.1460-2695.2000.00247.x.

[15] F. Ellyin, "Fatigue Damage, Crack Growth and Life Prediction," p. XVI-470, 1996

[16] H. Jahed and a. Varvani-Farahani, "Upper and lower fatigue life limits model using energy-based fatigue properties," Int. J. Fatigue, vol. 28, no. 56, pp. 467-473, 2006

[17] L. Wu et al., "The effects of texture and extension twinning on the low-cycle fatigue behavior of a rolled magnesium alloy, AZ31B," Mater. Sci. Eng. A, vol. 527, no. 26, pp. 7057-7067, 2010. doi: 10.1016/j.msea.2010.07.047

[18] D. Toscano, S. K. Shaha, B. Behravesh, H. Jahed, and B. Williams, "Effect of forging on the low cycle fatigue behavior of cast AZ31B alloy," Mater. Sci. Eng. A, vol. 706, no. August, pp. 342-356, 2017.

doi: 10.1016/j.msea.2017.08.086.

[19] A. Gryguc, S. K. Shaha, S. B. Behravesh, H. Jahed, M. Wells, B. Williams, and X. Su, "Monotonic and cyclic behaviour of cast and cast-forged AZ80 Mg," Int. J. Fatigue, vol. 104, pp. 136-149, 2017.

doi: $10.1016 /$ j.ijfatigue.2017.06.038

[20] J. Albinmousa and H. Jahed, "Multiaxial effects on LCF behaviour and fatigue failure of AZ31B magnesium extrusion," Int. J. Fatigue, vol. 67, pp. 103-116, 2014. doi: 10.1016/j.ijfatigue.2014.01.025.

[21] J. F. Tavernelli and L. F. Coffin, "Experimental Support for Generalized Equation Predicting Low Cycle Fatigue," J. Basic Eng., vol. 84, no. 4, p. 537, 1962. doi: 10.1115/1.3658701

[22] S. Manson, "Discussion on JF. Tavernelli and LF. Coffin "Experimental support for generalized equation predicting low cycle fatigue"," J. Basic Eng., vol. 84, no. 4, p. 537, 1962. doi: 10.1115/1.3658702.

[23] S. H. Park, S.-G. Hong, J. Yoon, and C. S. Lee, "Influence of loading direction on the anisotropic fatigue properties of rolled magnesium alloy," Int. J. Fatigue, vol. 87, pp. 210-215, 2016. doi: 10.1016/j.ijfatigue.2016.01.026.

[24] A. Gryguc, S. K. Shaha, H. Jahed, M. Wells, B. Williams, and J. McKinley, "Tensile and fatigue behaviour of as-forged AZ31B extrusion," Frat. ed Integrita Strutt., vol. 10, no. 38, pp. 251-258, 2016.

[25] J. Albinmousa, H. Jahed, and S. Lambert, "Cyclic behaviour of wrought magnesium alloy under multiaxial load," Int. J. Fatigue, vol. 33, no. 8, pp. 1127-1139, 2011. doi: 10.1016/j.ijfatigue.2011.01.009.

[26] F. Castro and Y. Jiang, "Fatigue of extruded AZ31B magnesium alloy under stress- and strain-controlled conditions including step loading," Mech. Mater., vol. 108, pp. 77-86, 2017. doi: 10.1016/j.mechmat.2017.03.002.

[27] H. Jahed and J. Albinmousa, "Multiaxial behaviour of wrought magnesium alloys - A review and suitability of energy-based fatigue life model," Theor. Appl. Fract. Mech., vol. 73, pp. 97-108, 2014.

doi: 10.1016/j.tafmec.2014.08.004. 


\title{
Finite Element Analysis of Modificed Conebolt Under Static and na $i$ a in
}

\author{
Benjamin Nguyen, Ming Cai, Krishna Challagulla \\ Bharti School of Engineering \\ Laurentian University \\ Sudbury, Canada \\ bv_nguyen@laurentian.ca \\ mcai@laurentian.ca \\ kchallagulla@laurentian.ca
}

\begin{abstract}
Axisymmetric finite element models are developed to simulate static pull test and dynamic drop test of MCB33 (modified conebolt with full dedonding) using ABAQUS. Results from the numerical models are in reasonable agreement with the test results. A parametric study is performed considering various variables (i.e. friction, cone angle, material strength, etc.) to analyze the performance of MCB33. The results demonstrate that friction between the steel and resin, cone angle, and the Poisson's ratio of the resin affect the static and dynamic behaviors of the rockbolt. These parameters can be modified to improve the current design and enhance the overall performance of the rockbolt.
\end{abstract}

Keywords: finite element analysis; rockbolt; modified conebolt; static pull test; dyanmic drop test

\section{INTRODUCTION}

As depth of mining and underground construction increases, stress-induced rock fracturing is inevitable due to high in-situ stress and complex geological and geometrical conditions in underground hard rock mines. In some cases, rock can fail violently, leading to seismic events and rockbursts [1, 3, 6,7 and 8].

Rockbursts can cause fatalities and injuries to workers, damage mine infrastructures and equipment, disrupt production, thus increase investment risk. To minimize the rockburst risk, design measures will be required. As an important line of defense, ground control support systems are used to prevent or minimize rockburst damage to excavation and enhance workplace safety.

In some deep hard rock mines, the modified conebolt (MCB) [2] are used for dynamic rock support. To further improve the performance of the MCB, design modification and subsequent static and dynamic testing is required. However, laboratory and field-testing of a new product is time-consuming and costly. To complete one design modification, several iterations of prototype manufacturing and testing are required. Hence computer-aided product design and testing is required to reduce the product development cycle and cost.

\section{A. Modified conebolt}

The modified conebolt shown in Fig. 1 is a long slender steel bar (17.3 mm diameter) with a cone-shaped anchorage, resin mixing blade and a plastic sleeve on the shank to fully debond the bolt from the resin grout and apply all loads to the bolt to facilitate cone plough. As the cone ploughs through grout in a controlled fashion, the bolt absorbs energy. The MCB33 (modified conebolt with $33 \mathrm{~mm}$ borehole size) attributes mostly to cone resistance in resin without the frictional forces of the shank. As a result, the cone plough is more predictable.

Once a borehole (e.g. diameter $33 \mathrm{~mm}$ ) is drilled deep into the stable hard rock, resin packs are inserted. The MCB33 is then drilled into the borehole while the resin-mixing blade mixes the resin, thus the bolt is encapsulated with resin. The plastic sleeve on the shank fully debonds the shank from the resin. When the resin is cured in a few minutes, the MCB33 is ready to take load and deform. If a rockburst is significant and impose a large dynamic loading to the rock, the cone will plough through the resin and aid in dissipating energy. The act of cone ploughing creates displacement without significant yielding of the bolt.

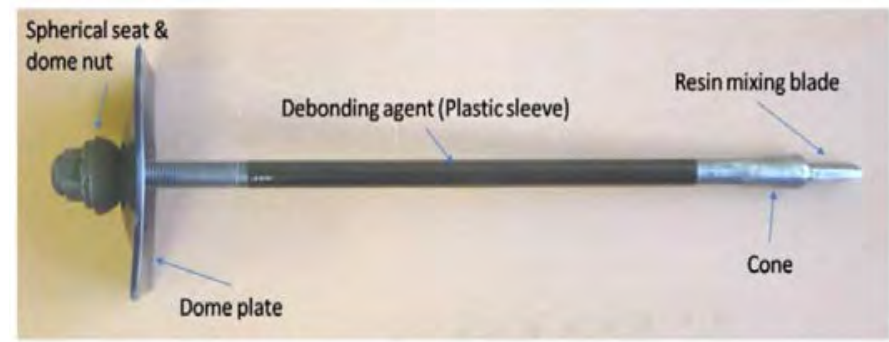

Figure 1. Schematic of a MCB33 [2]. 


\section{TESTING METHODS}

\section{A. Static pull test}

The static pull test is used to determine the loaddisplacement curve of a bolt. A hydraulic jack is used to apply a force on the end plate. The applied force on the bolt plate will deform the bolt and if load and displacement are measured, the load-displacement curve can be obtained.

\section{B. Dynamic drop test}

The dynamic drop test is used to evaluate energy absorbing capacities of rockbolts. A rockbolt is encapsulated in a thick wall steel pipe with the use of grout - resin or cement. A weight free falls onto the end plate to load the rockbolt dynamically at a pre-determined impact energy. Monitor systems are used to measure load and displacements at both ends of the rockbolt so that strains can be calculated. Fig. 2 shows the drop test facility at CANMET, Canada.

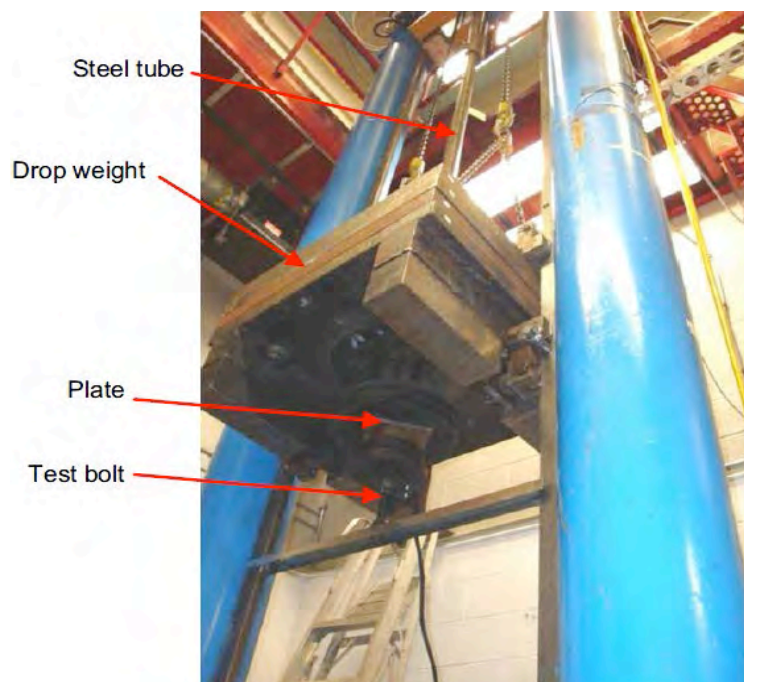

Figure 2. Drop test setup for MCB rockbolt testing at CANMET, Canada.

\section{MODELLING METHODOLOGY}

\section{A. Static pull test}

An axisymmetric finite element model of MCB33 (Fig. 3) is developed to simulate the in-situ pull test. The conebolt is fully encapsulated in resin in a $33 \mathrm{~mm}$ borehole in hard rock. Model dimensions of the rockbolt follow typical dimension of the modified conebolt for borehole sizes of $33 \mathrm{~mm}$, and the rock surrounding the borehole is large enough for using a fixed boundary condition. The axisymmetric model has four components: (a) modified conebolt for borehole size $33 \mathrm{~mm}$, (b) resin bonding the modified conebolt to the surround hard rock, (c) surrounding hard rock, and (d) interface between the bolt and the resin.

The following assumptions are considered to simplify the static pull test model:

- The rockbolt has no threads
- No head nut and plate for the rockbolt

- Rock-resin interface is assumed to be tied

- Friction interface is assumed for the bolt-resin interface.

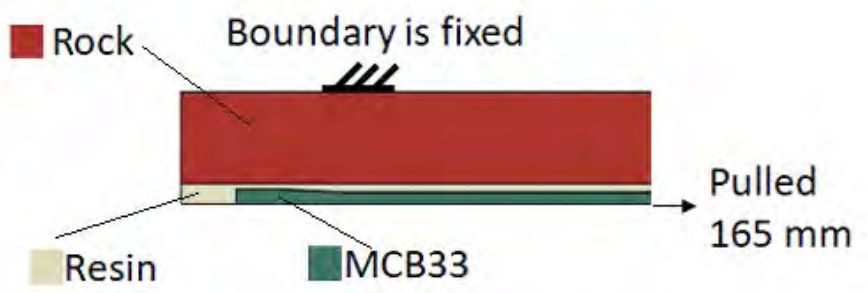

Figure 3. Schematic of the axisymmetric static pull test model (not to scale).

\section{B. Dynamic drop test}

An axisymmetric finite element model of the modified conebolt is developed to simulate the dynamic drop test. A conebolt is fully encapsulated in resin in a steel pipe with 33 $\mathrm{mm}$ inner diameter. The pipe wall is $13.5 \mathrm{~mm}$ thick. A drop weight around the pipe is dropped from $1.5 \mathrm{~m}$ height to impact the plate. The model has five components: (a) a modified conebolt for borehole size $33 \mathrm{~mm}$ with a plate, (b) resin bonding the modified conebolt and the pipe, (c) surrounding pipe, (d) drop weight, and (d) interface between the bolt and the resin.

The following assumptions are considered to simplify the dynamic drop test model:

- The rockbolt has no threads

- No head nut for the rockbolt

- Resin-pipe interface is assumed to be tied

- Friction interface is assumed for bolt-resin interface.

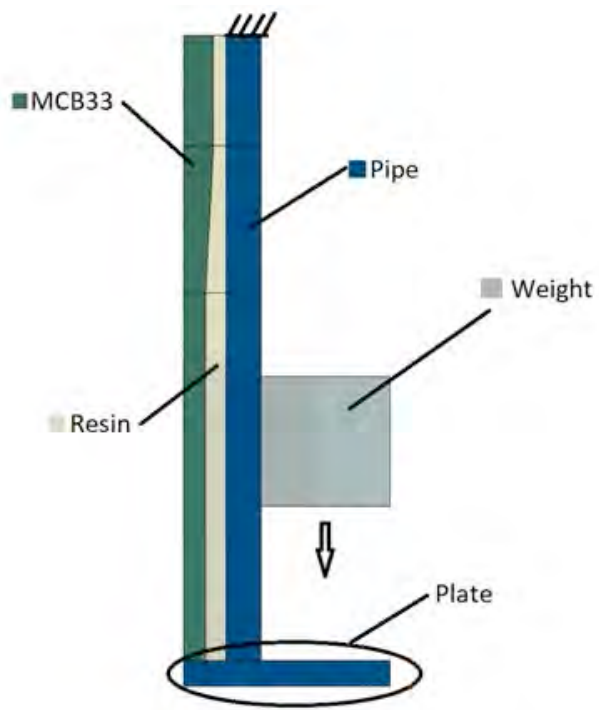

Figure 4. Schematic of the axisymmetric dynamic drop test model (not to scale). 


\section{Finite element model properties}

All finite element models developed in this study are analyzed using dynamic explicit analysis. Axisymmetric stress quad (CAX4R) element in ABAQUS is utilized to simulate the static pull test and the dynamic drop test. Because computational time is less for this type of analysis, a fine mesh with an aspect ratio between 1 and 1.5 is used.

As mentioned above the MCB has a debonded shank. The coefficient of friction affects the contact behaviors of the shank-to-resin interface and the cone-to-resin interface. We assume frictionless for the shank-to-resin interface due to perfect debonding, but the friction of the cone-to-resin interface is unknown thus a parametric study is needed to investigate the effect of the coefficient of friction of the coneto-resin interface on the performance of MCB.

\section{Material properties}

For initial models the material properties are either measured through lab testing or obtained from available values in literature. The aim is to choose a property that best represents each component. The two main components of concerns are the encapsulate resin and the rockbolt. All other parts (e.g. rock, pipe, plate and weight) are simplified to isotropic elastic homogenous materials.

To represent the rockbolt, the model must be able to represent pre- and post-failure. The steel's (C1055) tensile and compressive behaviors are the same; thus the selection of an appropriate material model is based on elasticity, plasticity and ductile damage consideration. Experiments were conducted to determine the deformation behavior of the steel. The forcedisplacement curve obtained from the experiments was converted into true stress-true strain curve for input into ABAQUS.

The encapsulated resin is difficult to represent in the model because of the plastic softening behavior. Laboratory experiments were conducted to determine the Faslok resin behavior. It is found that the behavior of the resin can be captured using Concrete Damage Plasticity [5] model.

The simulation results are compared with the experimental results [2] to calibrate the parameters of the static pull test model. Once the final model of fully calibrated data best represents the static pull test from the field experiments, the parameters (i.e. resin material properties and coefficient of friction) are used in the dynamic drop test.

Parameters obtained from the static pull test are utilized for the initial dynamic drop test model with an impact energy of $16 \mathrm{~kJ}$. The results from the dynamic drop test model are compared with the experiment ones [2]. The finite element model is further calibrated to replicate the experimental results for all drop tests with an impact energy of $16 \mathrm{~kJ}$. Then those parameters are used to simulate the drop test with $26 \mathrm{~kJ}$ impact energy.

\section{INPUT DATA USED IN ANALYSIS}

Table 1 presents the material properties used for each part of the simulation model. Table 2 presents the coefficients of friction between different interfaces for the static and dynamic tests. Fig. 5 shows the converted nominal stress-nominal strain curve from laboratory testing of tensile samples of the rockbolt material (C1055). Faslok resin is used for the encapsulating a bolt in a borehole or a steel pipe (for dynamic drop test). The pipe, plate, and weight are simulated using a general steel property. The density of the drop weight is varied to obtain the desired weight for each drop test. The rock material is for the surround rock and the Young's modulus can be varied to consider soft and hard rocks.

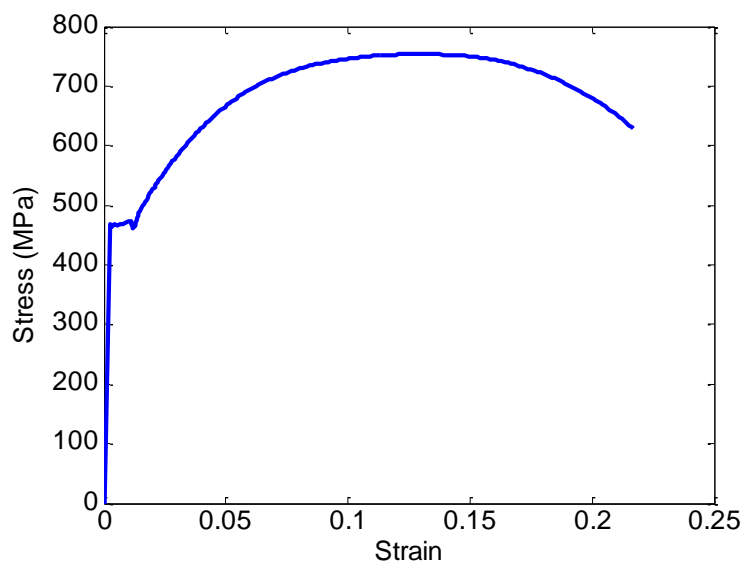

Figure 5. Stress-strain curve of C1055 steel obtained from lab test.

TABLE I. MATERIAL PROPERTIES FROM EXPERIMENTS AND LITERATURE [4]. * DEPENDING ON THE TEST AND THE GEOMETRY OF THE WEIGHT THE DENSITY IS CALCULATED TO GET 16 KJ AND 26 KJ IMPACT ENERGY

\begin{tabular}{|c|c|c|c|c|}
\hline \multirow{2}{*}{ Material } & \multicolumn{4}{|c|}{ Properties } \\
\cline { 2 - 5 } & $\begin{array}{c}\text { Young's } \\
\text { modulus } \\
(\mathbf{G P a})\end{array}$ & $\begin{array}{c}\text { Poisson's } \\
\text { ratio }\end{array}$ & $\begin{array}{c}\text { Density } \\
\left(\mathbf{K g} / \mathbf{m}^{3}\right)\end{array}$ & $\begin{array}{c}\text { Uniaxial } \\
\text { compressive } \\
\text { strength } \\
(\mathbf{M P a})\end{array}$ \\
\hline C1055 & 200 & 0.3 & 7500 & - \\
\hline $\begin{array}{c}\text { Faslok } \\
\text { resin }\end{array}$ & $10-30$ & $0.15-0.38$ & 2050 & 30 \\
\hline Steel & 210 & 0.3 & $*$ & - \\
\hline Rock & $5-50$ & 0.25 & $2440-2700$ & - \\
\hline
\end{tabular}

TABLE II. COEFFICIENTS OF FRICTION BETWEEN DIFFERENT INTERFACES

\begin{tabular}{|c|c|c|}
\hline \multirow{2}{*}{ Test } & \multicolumn{2}{|c|}{ Interface } \\
\cline { 2 - 3 } & Cone-resin & Shank-resin \\
\hline Static pull & $0.1-0.3$ & 0 \\
\hline Dynamic drop & $0.5-0.1$ & 0 \\
\hline
\end{tabular}




\section{SimUlation RESUltS}

\section{A. MCB33 static pull test simulation results}

With the axisymmetric models, preliminary design parameters are used to get a general idea of the trends and behavior of the MCB33 rockbolt under static loading. The model is further calibrated to give satisfactory results. Fig. 6 shows the stress in the y-direction (parallel to the bolt's long axis) when the conebolt is pulled $165 \mathrm{~mm}$. Fig. 7 presents the modeled load-displacement relation superimposed with the experiment results [2], showing a good agreement between the two.

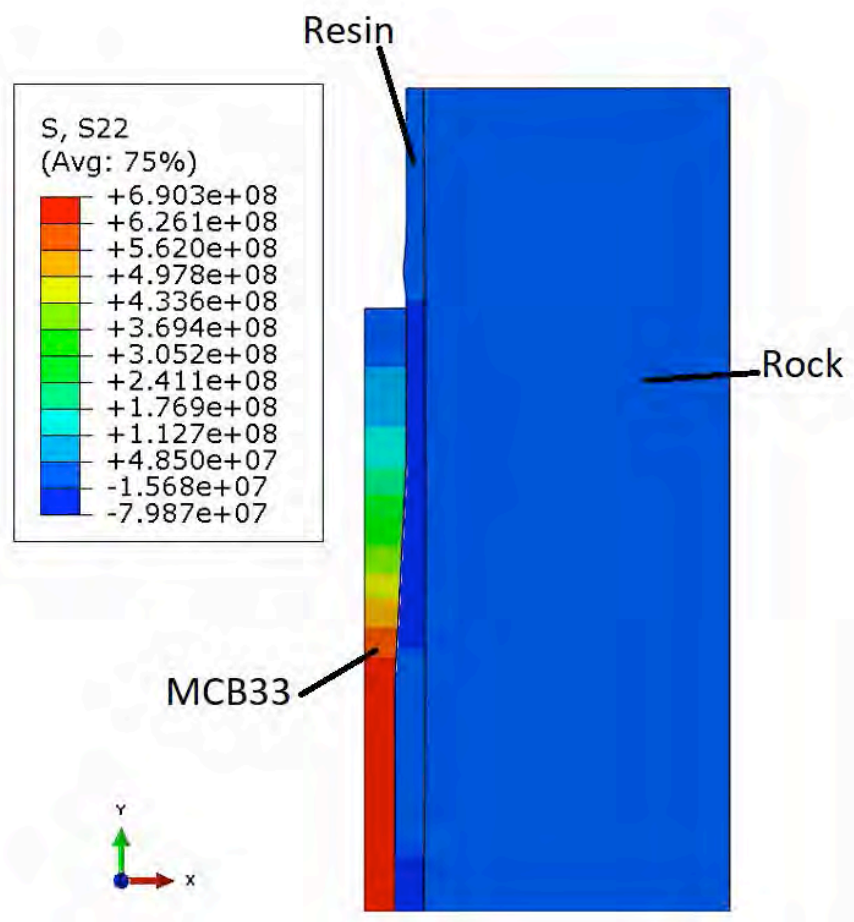

Figure 6. Top section of the MCB33 finite element model for the static pull test showing the stress $(\mathrm{Pa})$ distribution along the y-direction (S22).

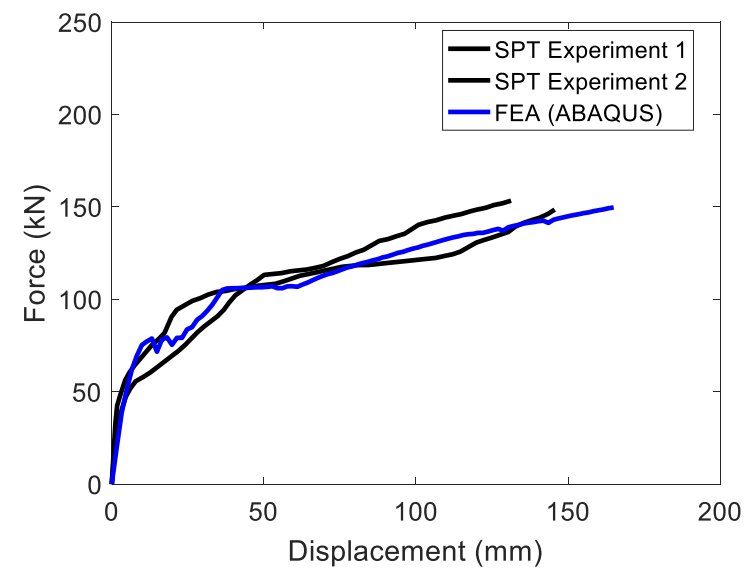

Figure 7. Static pull test (SPT) results [2] superimpossed with the results from numerical modeling (FEA).

\section{B. MCB33 dynamic drop test simulation results}

With the calibrated parameters using the static pull test results, dynamic drop test simulations were conducted. Fig. 8 shows the y-stress distribution at time $0.57 \mathrm{~s}$ from a $16 \mathrm{~kJ}$ drop test simulation. Figs. 9 and 10 show the plate displacements from the numerical simulations for $16 \mathrm{~kJ}$ and $26 \mathrm{~kJ}$ impact energy, respectively, superimposed with the experiment results [2]. It is seen that the numerical modeling captures the test results very well.
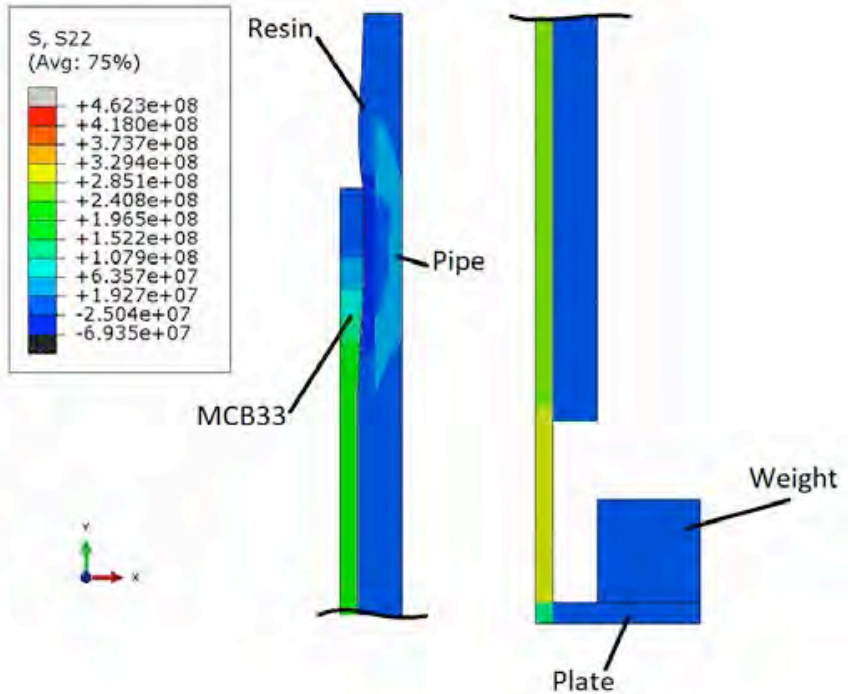

Figure 8. Top section (left side) and bottom section (right side) of the MCB33 dynamic drop test model showing stress (Pa) distribution in the ydirection (S22).

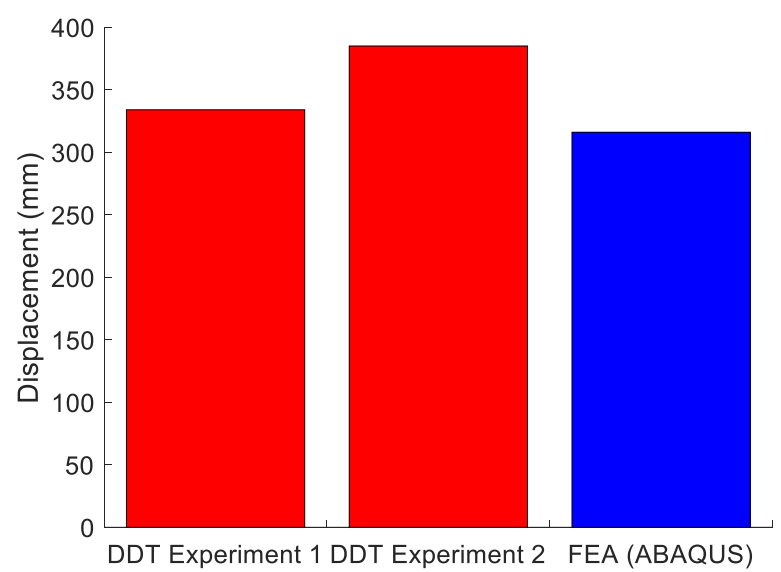

Figure 9. Dynamic drop test results for plate displacement at $16 \mathrm{~kJ}$ impact energy [2] superimposed with the numerical modeling results. 


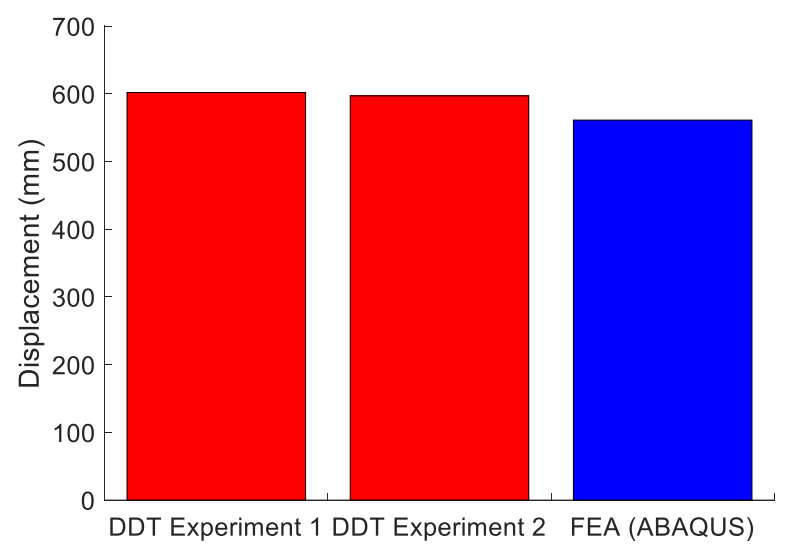

Figure 10. Dynamic drop test results for plate displacement at $26 \mathrm{~kJ}$ impact energy [2] superimposed with the numerical modeling results.

A further parametric study was conducted for both static and dynamic tests. The rockbolt material's strength and geometry, the resin material's strength and geometry, and the coefficient of friction between the bolt-resin interfaces are varied to study the performance of the bolt. It is seen from the static pull test and drop test simulation results that increasing the rockbolt material strength will increase performance of the MCB33. The resin material properties have a large influence on the performance of the MCB33. Higher Young's modulus of the resin grout can lead to less cone plough. Smaller coefficients between the bolt and the resin lead to more cone plough and increasing the cone angle reduces cone plough. Hence, it is possible to use numerical tools such as ABAQUS to calibrate available test results and refine design to optimize the performance of the MCB33 rockbolts.

\section{CONCLUSION}

Static and dynamic test results of MCB33 rockbolts have been simulated using an explicit FEM tool. The modeling results agree well with field and lab test results. Dynamic rock support is needed for mining in highly stressed ground to reduce rockburst damage risk and increase workplace safety. Further development of yielding rockbolts such as refinement of MCB33 and proofing other new support technologies can be assisted with the use of advanced numerical modeling to reduce product development cycle and cost.

\section{ACKNOLEDGEMENTS}

Financial supports from the Natural Sciences and Engineering Research Council (NSERC, CRDPJ 461108 - 13) of Canada and Mansour Mining Technologies Inc. for this work are gratefully acknowledged.

\section{REFERENCES}

[1] M. Cai and D. Champaigne, "The art of tock support in bursttroneground," in Proc. 7th Int. Symp.on Rockburst and Seismicity in Mines (RaSIM 7): Controlling Seismic Hazard and Sustainable Development of Deep Mines, Dalian, China, 2009, pp. 33-46.

[2] M. Cai and D. Champaigne, "Influence of bolt-grout bonding on MCB conebolt performance," Int. J. Rock Mech. Min. Sci., vol. 49, pp. 165-175, Jan. 2012. doi: 10.1016/j.ijrmms.2011.11.006.

[3] M. Cai, "Principles of rock support in burst-prone ground," Tunnelling and Underground Space Technol., vol. 36, pp. 46-56, June 2013. doi: 10.1016/j.tust.2013.02.003.

[4] L. Chen, G. Chen, and G. Sheng, "Numerical modeling of rock boltssubject to dynamic loading," in Proc. 46th US Rock Mechanics/ Geomechanics Symp., American Rock Mechanics Association (ARMA), 2012, pp. 277-283.

[5] U. Cicekli, G. Z. Voyiadjis, and R. K. Abu al-rub, "A plasticity andanisotropic damage model for plain concrete," Int. J. Plast., vol. 23, no. 10-11, pp. 1874-1900, Oct.-Nov. 2012. doi: 10.1016/j.ijplas.2007.03.006.

[6] P. K. Kaiser, D. R. Mccreath, and D. D. Tannant, Canadian rockburst support handbook. Geomechanics Research Centre, 1996.

[7] P. K. Kaiser and M. Cai, "Design of rock support system under rock burst condition," J. Rock Mech. Geotech. Eng., vol. 4, no. 3, pp. 215-227, Sept. 2012. doi: 10.3724/SP.J.1235.2012.00215.

[8] D. D. Tannant, R. K. Brummer and X. Yi, "Rock bolt behaviour under dynamic loading: field tests and modelling," Int. J. Rock Mech. Min. Sci. Geomech. Abstr., vol. 32, no. 6, pp. 537-550, Sept. 1995. doi: 10.1016/0148-9062(95)00024-B. 


\title{
Effect of the Error Propagation Rates in Developing a Skin Model of Measured Surfaces in Coordinate Metrology
}

\author{
Cody Berry, Lillian Goodwin, and Ahmad Barari \\ Faculty of Engineering and Applied Science \\ UOIT \\ Oshawa, Canada \\ ahmad.barari@uoit.ca
}

\begin{abstract}
Coordinate metrology is the most commonly used inspection method to analyze surfaces for manufacturing errors and imperfections. The computational in today's coordinate metrology are composed of a series of extensive computational tasks. Developing a triangulated skin model using a finite element method is an approach to construct a surface based on the discrete measured points, which can be used for integrated computational algorithms reducing the uncertainty of the inspection process. Reducing the errors in developing the skin model by calibrating the two main parameters of the algorithm, which specify the propagation of the geometric deviations in two orthogonal directions, is the objective in this work. By varying theses propagation parameters and tracking the overall error of the constructed skin model, their effects are evaluated. Various cases are studied and the resulting patterns of the errors for different surfaces are used to make suggestions on selection of the two parameters resulting accurate construction of the skin model of the measured work pieces based on the coordinate metrology data. This will provide a reliable technique to develop the skin model of the manufactured products for quality control, manufacturing process control, or repair and finishing of the products.
\end{abstract}

Keywords: coordinate metrology; skin model; error propagation rates; Deviation Zone Evaluation (DZE); Point Measurement Planning (PMP)

\section{INTRODUCTION}

Coordinate metrology incorporates the use of three different processes, namely, Point Measurement Planning (PMP), Substitute Geometry Estimation (SGE), and Deviation Zone Evaluation (DZE). In such systems, the inspection accuracy is subject to the issue of uncertainties due to estimating some aspects of a probability distribution on the basis of sampling which adversely affects the accuracy and reliability of the inspection.

Selection of location, distribution, and number of sample points using the available information is the objective of PMP

Sponsored by NSERC process. Finding the best ideal substitute geometry that represent the selected sample points using a desired fitting criteria is performed by SGE process. Developing a comprehensive representation of geometric deviation for any point of the measured surface including the points that are not physically measured, is the goal of DZE process. These three computational tasks were traditionally conducted in a sequential manner. All three tasks have access to three sets of external data including: the desired geometric features which are typically represented by Computer Aided Design (CAD) models, data and information from the corresponding manufacturing operations used to fabricate the work piece, and the objective of the inspection process corresponding to the downstream processes that are planned for the measured work piece [1].

Output of each task is used as the input for the next task in the PMP-SGE-DZE sequential order. The typical output of the measurement instruction developed by PMP is used for capturing a limited number of discrete 3D points from the work piece. This data set is used as an input to SGE to fit the best representative substitute geometry. The external data regarding the objective of the inspection process is used to define the fitting criteria in SGE process. The geometric deviations of the measured sample points are calculated using the results of SGE and are input to the finishing computational task, i.e., DZE. DZE is responsible to analyze the distribution of geometric deviations and represent a detailed deviation zone for the entire measured surface. This result is the ultimate output of the coordinate metrology process which can be represented by statistical characteristics of the distribution and/or a full skin model of the measured surface. It is worth mentioning that, in order to develop the full skin model, DZE should be capable to estimate the geometric deviation function for the entire measured surface including infinite points that are not physically measured [1].

The concept of skin model is introduced in the field of tolerance analysis [2]. Tolerances are specified during the design process to meet the desired form, fit, and functionality of the products. Today's design and manufacturing paradigms do not allow the design tolerance analysis being performed without considering the manufacturing uncertainties. A 
detailed representation of the geometric deviations from the best fitted desired geometry, which are mainly caused by the inherent errors in the employed manufacturing processes is needed to complete an integrated tolerance analyses. This detailed model of geometric deviations is a non-ideal Surface, which is also referred as a Skin Model of the part in Geometrical Product Specification (GPS) standards [2]. The need for developing a Skin Models is addressed by the standards and is the new focus of research [3]. However, methodologies to develop these models need further research.

A skin model is a discretized surface generated from a point cloud. The advantage of this method is its ability to reflect the deviation of the surface at any location, even those that were not sampled from. This data can be used to allow for virtual sampling of the piece. In a previous work, Lalehpour and Barari developed a finite element method to develop the skin model using the coordinate metrology discrete 3D points [1]. However, the developed method was not fine-tuned to provide the most accurate results. This paper will seek to finetune that method. The accuracy of the developed skin model

is of paramount importance in design and manufacturing

systems. The skin model is used directly for any possible downstream design and manufacturing processes that need the results of coordinate metrology process, this includes planning for precise finishing operations, manufacturing error compensation, closed-loop of manufacturing and inspection, dynamic process control, or for any tolerance evaluation required for the assembly and interaction of the work piece with the other mechanical components. Therefore, the added value in developing an accurate skin model of the work piece to the design and manufacturing processes is highly significant.

It is shown in several recent research [4-8] that integration of PMP, SGE, and DZE tasks will result in reducing the computational uncertainties in coordinate metrology. Another interesting application of a full skin model is to reduce the uncertainties of coordinate metrology process by conducting mutual closed loops between the computational tasks. The developed skin model by DZE is dynamically feed-backed to SGE and PMP to improve the processes in integrated inspection system using DZE-SGE and DZE-PMP closedloops.

\section{THEORETICAL BACKGROUND}

Lalehpour and Barari [1] developed the finite element method utilized in this paper. In this method, the data set that is obtained from the surface is first randomly pruned down. This is done as the entire sample set should not be required to accurately create a skin model. These points that are removed from the skin model data set will be used to validate the skin model once it is generated.

The new sample set is then transformed from real space, where $\mathrm{x}, \mathrm{y}$ and $\mathrm{z}$ coordinates are used, to a parametric space. In this space, $\mathrm{u}, \mathrm{v}$, and $\mathrm{d}$ become the axes. The two largest principal axes of the data set become the $u$ and $v$ axes, while the smallest principal axis becomes $d$, or the geometric deviations. The data set is then divided into windows. Each of on the size of the data set is selected from each window. These will become non-moving vertices of the skin model. This skin model relies on triangular elements, so a Delaunay triangulation is used on these non-moving vertices to create the triangular model.

This triangular surface becomes the basis for the rest of the skin model. The areas of each triangle are checked for size, with large areas being subdivided into smaller ones with the addition of another point at the centroid. This process repeats until all triangles are under the size threshold defined by the overall size of the data set. This increase in density causes an increase in accuracy for the finished skin model. These additional points do not initially have the correct geometric deviation to accurately represent the measured surface. In order to determine the correct placement of these vertices, a finite element method was used using Poisson Equation as follows:

$$
\frac{\partial}{\partial u}\left(K_{u u} \frac{\partial S}{\partial u}\right)+\frac{\partial}{\partial v}\left(K_{v v} \frac{\partial S}{\partial v}\right)+E=0
$$

where, $\mathrm{u}$ and $\mathrm{v}$ are two orthogonal directions on the measured surface, $\mathrm{K}_{\mathrm{uu}}$ and $\mathrm{K}_{\mathrm{vv}}$ are the error propagation rates in $\mathrm{u}$ and $\mathrm{v}$ directions, $\mathrm{E}$ is the determined geometric deviation, and $\mathrm{S}$ is the skin model. The $\mathrm{K}$ in [1], these $\mathrm{K}$ values were both assumed to be equal to unity for simplification and validation purpose. This paper will focus on determining the optimal $\mathrm{K}$ values. The effect of these two parameters on the accuracy of the skin model is studied in this paper. This resulting information can be used to produce more accurate skin model or to be used as the initial condition for an optimization process. Particle swarm optimization algorithm [9] is suggested for this case which is one of many algorithms that optimize based on different patterns found in nature.

\section{METHODOLOGY}

A large amount of data is measured from the work-pieces using a coordinate measuring machine (CMM). The PMP process is then deployed to virtually sample from the given datasets. Once the smaller datasets were chosen, SGE is performed to find the closest representative plane to fit the given dataset. The method utilized for this purpose is total least squares (TLS) fitting to find the best fit plane for the given surfaces. This can be utilized for the SGE process because of the use of solely planar geometry.

The propagation rates of geometric deviations (PRGD), both in the $u$ and $v$ directions, used to construct the skin model are examined using the following process. A $40 \times 40$ matrix of PRGD values in the $u$ and $v$ directions ranging from -20 to 20 discrete values. This constructs a total of 1600 different skin models for each given dataset. After the skin model is constructed and the deviation of each point is known, the total error for each skin model is calculated by taking the difference from the generated original chosen data points to the generated skin model. The total error is the cumulative sum of square of the errors for each skin model. 
The effect of the fitting parameters (PRGDs) is analyzed by graphing the total error for each skin model to show the total error for the given matrix of values. The results are then optimized using an algorithm to find the lowest values and removing them recursively to find a trend line of values. This trend line is then analyzed for patterns involving different topologies.

\section{RESULTS AND DISCUSSION}

In order to determine the area for optimization, the results of the error value for the 1600 skin models was graphed for each case study. Since the observed pattern were consistent, the graphs corresponding a typical case study is used for the rest of this paper.

Fig. 1 presents the error values corresponding to 1600 skin models developed for the case study, with a random sample piece for illustrative purposes. With this data, it was easy to determine problem values for the error propagation rate values. The worst results were consistently those where the error propagation rates had signs that differed from one another. With this in mind, a filter was applied to remove a large number of the error samples. For the first pass, any value larger than $0.000001 \%$ of the minimum value was removed. This resulted in Fig. 2.

In Fig. 2, the sections with the smallest error can be seen. Those areas where the error propagation rates had differing signs have been completely removed. Another interesting characteristic is the symmetry developed between the two remaining segments. This means the optimization algorithm can be configured to only search one small segment of the overall data set, and it will be likely to achieve an acceptable result in a short amount of time.

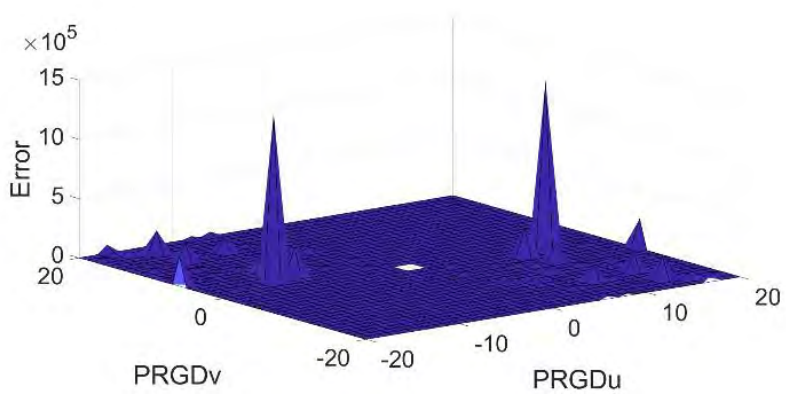

Figure 1. Error Results for 1600 Skin Models

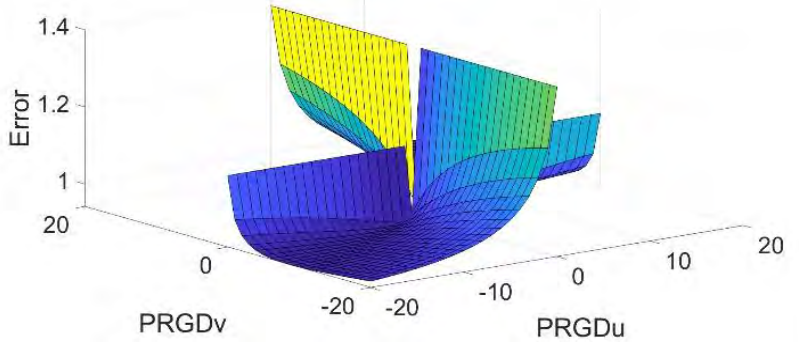

Figure 2. $99.999999 \%$ of the Range Removed

Another factor looked at was the density of points in different locations. This was examined to see if there was a correlation between high density zones of points and the lowest error results. With this information, the optimization area could be further refined. To test this, a data set was generated with equally spaced points. Then, a section of the data set was populated with a much higher density data set. Finally, the error graph generated was examined to determine where the lowest points were found. These points had a line fit to them, and the slope of the line was examined. Fig. 3 shows the first of these data sets.

Data Set 1 is heavily weighted to the positive $\mathrm{x}$ direction, and is shown in Fig. 3. Fig. 4 shows the results of the error analysis. The final graph has the errors of the opposing signed error propagation rates removed like in Fig. 2. There is also a large decrease in error between these two figures as data set 1 is very planar. None of the minimum error values were found in either of those regions, which was to be expected.

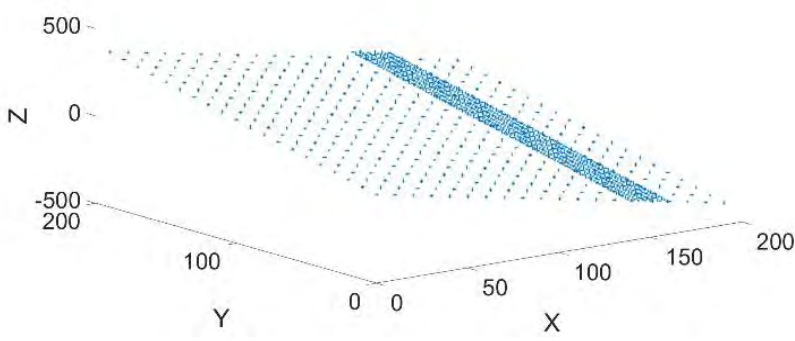

Figure 3. Data Set 1

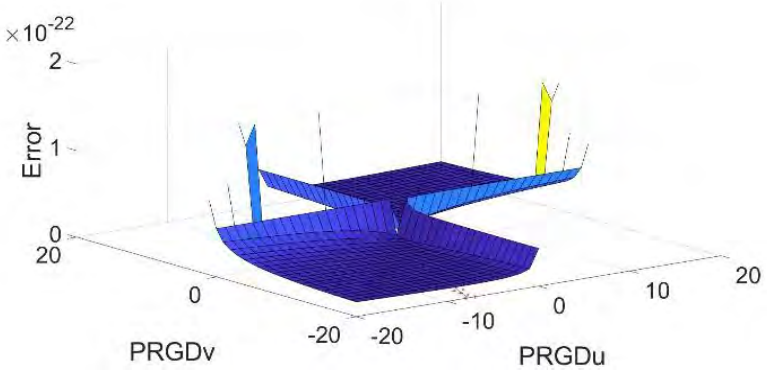

Figure 4. Data Set 1 - Error Modeling Results 
The red line on the bottom of the graph is the best fit line for the lowest error values. It has a very large slope, which shows that the error propagation rate in the $\mathrm{v}$ axis on the refit data set dominates, corresponding with the increase in point density. This result is reflected in Fig. 5 and Fig. 6.

As the high density area is moved along the $\mathrm{x}$-axis in the negative $\mathrm{x}$ direction, there is a corresponding shift in the slope of the line in the results. This test was repeated with the high density section moving along the entire u-axis. In each case, the slope of the line continued to shift in the same direction. This meant that the location of the points had an effect on the location of the minimum values, and therefore the start conditions for the optimization algorithm could be selected more intelligently, increasing the accuracy of the skin model as it would be more likely to find a better solution.

\section{CONCLUSION}

The goal of this paper was to determine the area of optimization for the two main parameters involved in the skin model process, i.e. the geometric error propagation rates in the two orthogonal directions. A series of tests are conducted to model the effect of the error propagation rates on the accuracy of the resulting skin model. It was found that when the PRGD values had different signs from each other, the error was greatly increased. This allowed for the removal of those regions from analysis. Further examination showed that the remaining regions were symmetric, and therefore only one of them needed to be used to find the best PRGD values. Next, the effect of the density of sample points on the error values was examined.

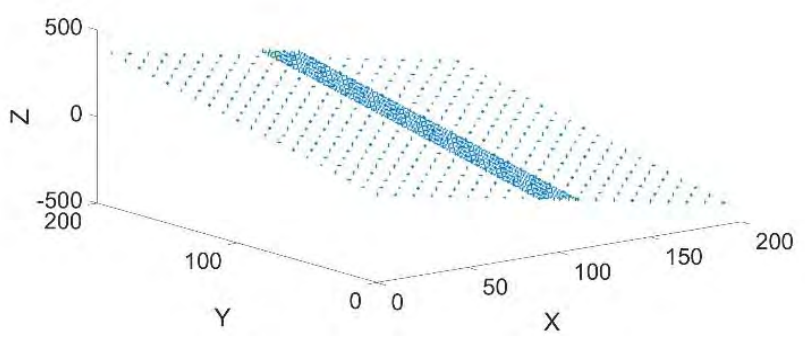

Figure 5. Data Set 2

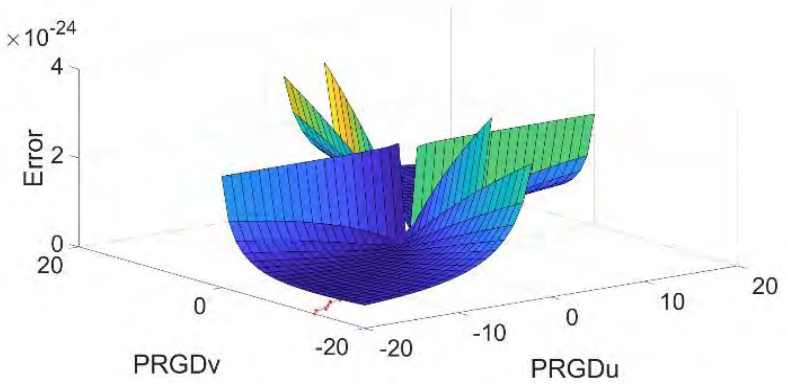

Figure 6. Data Set 2 - Error Modeling Results
The results showed that this density did have a large effect on the error. This effect was also fairly systematic, and therefore could be predicted. With this information, the area of optimization can be further refined. Both of these results can be used to better customize the skin model algorithms and achieve more accurate results using the skin model method.

\section{ACKNOWLEDGMENT}

The research support provided by the Natural Science and Engineering Research Council of Canada (NSERC) is greatly appreciated.

\section{REFERENCE}

[1] A. Lalehpour and A. Barari, "Developing skin model in coordinate metrology using a finite element method," Meas., vol. 109, pp. 149-159, Oct. 2017. doi: 10.1016/j.measurement.2017.05.056.

[2] EN ISO 17450-1:2011, Geometrical product specifications (GPS) General concepts - Part 1: Model for geometrical specification and verification (2011).

[3] B. Schleich et al., "Skin model shapes: A new paradigm shift for geometric variations modelling in mechanical engineering," Comput.-Aided Des., vol. 15, pp. 1-15, 2014. doi: 10.1016/j.cad.2014.01.001.

[4] A. Barari and S. Jamiolahmadi, "Convergence of a finite difference approach for detailed deviation zone estimation in coordinate metrology," Acta IMEKO, Vol. 4, no. 4, pp. $2025,2015$.

[5] A. Barari and F. Kaji,"The uncertainty of coordinate metrology as a function of sampling strategy," J. CMSC, vol. 9, no 1, pp. 22- 27, 2014.

[6] S. Jamiolahmadi and A. Barari, "Study of detailed deviation zone considering coordinate metrology uncertainty," Meas., vol. 126, pp. 433-457, Oct. 2018.

doi: 10.1016/j.measurement.2016.12.032.

[7] H. Gohari and A. Barari, "A quick deviation zone fitting in coordinate metrology of NURBS surfaces using principle component analysis," Meas., vol. 92, pp. 352-364, Oct. 2016. doi: 10.1016/j.measurement.2016.05.050.

[8] A. Barari, "Inspection of the machined surfaces using manufacturing data," $J$. Manuf. Syst., vol. 32, no. 1, pp. 107-113, Jan. 2013. doi: 10.1016/j.jmsy.2012.07.011.

[9] T. Zeugmann et al., "Particle swarm optimization," in Encyclopedia of Machine Learning. Boston, MA: Springer, 2011, pp. 760-766. 


\title{
Constrained Topology Optimization for Additive Manufacturing of Structural Components in ANSYS ${ }^{\circledR}$
}

\author{
Davin Jankovics, Hossein Gohari, Ahmad Barari \\ Faculty of Engineering and Applied Science \\ University of Ontario Institute of Technology \\ Oshawa, Ontario \\ davin.jankovics@uoit.ca, hossein.goharibahabadi@uoit.ca, ahmad.barari@uoit.ca
}

\begin{abstract}
Topology Optimization is currently the main technique to optimize an objects structural design. This method commonly produces parts that have exceedingly complex geometry. Additive manufacturing (AM) is the main manufacturing process to produce these optimized designs due to the flexibility and speed it offers. However, results of topology optimization without considering manufacturing process limits, even AM ones, may result in designs that are expensive and difficult to build. This paper presents a topology optimization filter that minimizes the effect of overhang structures. These structures are very difficult to manufacture using conventional AM techniques. In order to constrain the gradient compliances with respect to densities and converge the results towards a structure with the least amount of overhang structures, sensitivities are modified using the proposed filter. To implement the proposed filter and the base topology optimization methods ESO and SIMP, ANSYS Parametric Design Language (APDL) is employed within the ANSYS $^{\circledR}$ Workbench $^{\mathrm{TM}}$ environment. The results of a case study using the different topology optimization methods are investigated. Finally, an implementation of the proposed AM filter is used to solve an MBB-beam problem. The result is a structure that needs the least amount of support structure.
\end{abstract}

Keywords-Additive Manufacturing; 3D Printing; Topology Optimization; Finite Element Analysis; ANSYS $^{\circledR}$; ANSYS Parametric Design Language (APDL)

\section{INTRODUCTION}

Topology optimization is a subset of structural optimization with the goal of finding an optimal design for a certain set of constraints. It can be described as an iterative process where the results of a finite element analysis (FEA) are used to determine the optimal geometry and locations of voids in a solid object [1].

In the context of engineering, finite element analysis is a mathematical method of solving a system which cannot easily be described using closed form equations. Instead it breaks the system into smaller, easier to solve or approximate, parts using what are known as elements [2]. One popular FEA software for engineering is ANSYS $^{\circledR}$. Released in 1971 and still being actively developed, ANSYS ${ }^{\circledR}$ can be used to solve a diverse field of engineering problems including static structural, dynamic, heat transfer, fluid flow, and electromagnetism. Currently, the user facing software of ANSYS ${ }^{\circledR}$ is Workbench ${ }^{\mathrm{TM}}$, from which a coding language called ANSYS Parametric Design Language (APDL) is called when simulations are run. This language executes the FEA, calculating all necessary parameters to solve the analysis. For advanced users, APDL is user accessible through custom command scripts that can be injected at any point during the analysis.

Creating a topology optimizer from scratch would include the development of a topology optimization algorithm and the development of a complete FEA solver. Additionally, if the creation and editing of geometry was also required, a computer aided design software would also be required. Instead, the accessible interface of ANSYS ${ }^{\circledR}$ and its mature FEA solver can be utilized to create a more extensive and easy to use topology optimizer through custom injected APDL scripts. Since ANSYS $^{\circledR}$ includes Multiphysics simulations, the ability to add additional constraints and objectives, including manufacturing constraints and coupled analyses may become possible.

Additive manufacturing (AM) is a fairly young and quickly developing technology that overcomes many traditional manufacturing limits for complex geometries. However, all additive manufacturing methods still have some constraints that need to be considered during the design process. The use of disposable support material is one method to work around these constraints, but it is not a perfect solution as it often leads to a poor surface quality finish [3]. This support material is used to prevent failure during the manufacturing procedure by preventing overhang structures in the part geometry from collapsing, or otherwise ruining the final parts finish [4]. Since most AM processes use a manufacturing method where material is added layer by layer, each being fused to the previous, it can be said that there exists an angle where supports will be required.

Other approaches to minimize the prevalence of overhangs in topology optimized parts have been developed [5]. In this paper, a 3D implementation of the evolutionary structural optimization (ESO) and solid isotropic material with penalisation (SIMP) methods are presented along with a new 
filtering method to reduce the amount of material needed for supports, all in ANSYS $^{\circledR}$.

\section{BACKGROUND}

\section{A. Evolutionary Structural Optimization (ESO) Method}

A topology optimization problem seeks to minimize compliance while satisfying various constraints such as a given amount of material, weight, manufacturing requirement, and cost [1]. The measure of stiffness of a structure can be calculated using a finite element analysis. In literature, instead of stiffness, mean compliance $\mathrm{C}$, is considered as the objective for topology optimization. The evolutionary procedure based on stiffness as the objective function can be described as follows:

1) Discretize the body for FEA.

2) FEA determines sensitivity number for each element.

3) Remove elements with lowest sensitivity number based on element removal ratio.

4) Repeat the procedure to reach termination term (maximum displacement, stress, etc).

\section{B. Solid Isotropic Material with Penalization (SIMP)}

Minimizing compliance is the most used objective in topology optimization, shown in (1).

$$
\begin{gathered}
\min _{x}: c(\rho)=U^{T} K U=\sum_{e=1}^{N}\left(\rho^{e}\right)^{p} u_{e}^{T} k^{e} u_{e} \\
\text { Subject to: } \quad \frac{V(\rho)}{V_{0}}=f \\
; K U=F \\
; 0<\rho_{\min } \leq x \leq 1
\end{gathered}
$$

Where the stiffness matrix for each element can be found from (2).

$$
\begin{gathered}
k^{e}=\int_{\Omega} H^{T} D H d \Omega \\
=\sum_{i=1}^{2} \sum_{j=1}^{2} w_{i} w_{j}|J(\xi, \eta)| H(\xi, \eta)^{T} D H(\xi, \eta)
\end{gathered}
$$

Here, the starting Young's modulus of an object does not influence the optimal results of topology optimization, since any changes will be relative. The general solution of the topology optimization is constructed to minimize compliance using a Lagrange multiplier method as shown in (3).

$$
L(\rho, \lambda)=f^{T} d(\rho)+\lambda^{T}(f-K(\rho) d(\rho))
$$

By setting $\lambda=d$ and $k^{e}=\int_{\Omega} H^{T}\left(E_{0}+\left(\rho^{e}\right)^{p} E_{1} D^{*} H d \Omega\right.$, the derivative of the lagrangian equation with respect to the design variables can be determined as (4) [6].

$$
\frac{\partial L(\rho, \lambda)}{\partial \rho^{e}}=-P\left(\rho^{e}\right)^{p-1} d^{e T} K_{f}^{e} d^{e}
$$

Updating of element densities heuristically can be achieved using the bi-section algorithm. A heuristic algorithm is used here based on the Optimality Criteria (OC) method shown in (5) [7]. A numerical constraint has been considered to limit the update for densities, $m$.

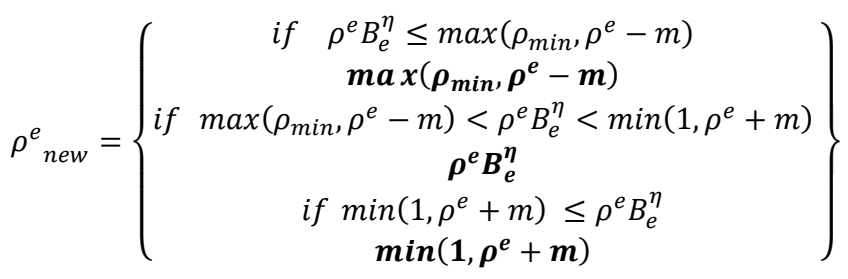

Where $B_{e}^{\eta}=-\frac{1}{\lambda} \frac{\partial c}{\partial \rho^{e}}$ and $\eta(=1 / 2)$ is a numerical damping coefficient.

\section{Implementation of Overhang Constraint}

Applications of developed topology optimization methods have been very restricted due to the limitations in manufacturing and inspection of the resulting complex geometries. A successful design process needs to be conducted by considering all of the manufacturing and inspection requirements [8]. Although additive manufacturing processes are still not able to produce high surface qualities [9], [10], they are highly flexible in producing the complex geometries produced by topology optimization [11], [12]. Also, the advances in coordinate metrology algorithms allow relatively fast inspection of the complex topologies resulting by the topologically optimized designs [13].

These normal topology optimization methods converge to a computationally stiff structure, but without regard for the difficulty of manufacturing. Here is where another constraint is added that limits the surface area of elements to be less than a specified value for the optimal solution. This approach can be used to implement an overhang constraint. In the methodology section a new scheme is developed to estimate the sensitivities of overhanging elements in 3D space. These elements can then be penalized with respect to their overall sensitivity.

\section{Using APDL in ANSYS ${ }^{\circledR}$}

All the simulation and FEA functionality of ANSYS ${ }^{\circledR}$ comes from APDL commands [2]. Any FEA parameter that is calculated in ANSYS $^{\circledR}$ can be read, and as logic applies, can be modified.

The syntax for an APDL commands starts with the name of the commands, followed by a comma, and then the operators of the commands, all separated by commas. There are also logical operators such as do, if, ifelse, and while.

The actual FEA solver in ANSYS ${ }^{\circledR}$ operates much like other FEA solvers [14]. There are three overall levels within the solver, the first being the preprocessor. This is where the mesh of the geometry is defined, as well as the loads on the body and boundary conditions. Next, the solver processes the generated simultaneous equations, which results in the values for degrees of freedom for the nodes on each element. This data is then taken into the final level, the postprocessor, where all other results are 
calculated, such as stress and strain. It is important to note that only certain APDL commands can be executed in certain levels of the FEA, limiting how one can interact with the program. However, with a large selection of pre-defined commands combined with logical operators, essentially anything related to the FEA process can be modified and manipulated.

\section{MethodOLOGY}

\section{A. Implementing the Topology Optimization Overhang Constraint}

Using a similar method to that Sigmund used to solve the optimality criteria, the area of an element can be considered as a new constraint. A method has been developed to calculate the derivative of the surface area of each element with respect to its density [15]. Using the same approach shown in (6), the approximate surface area sensitives are calculated for the bottom elements, which are multiplied by a constant coefficient $\gamma$, as shown in Fig. 1.

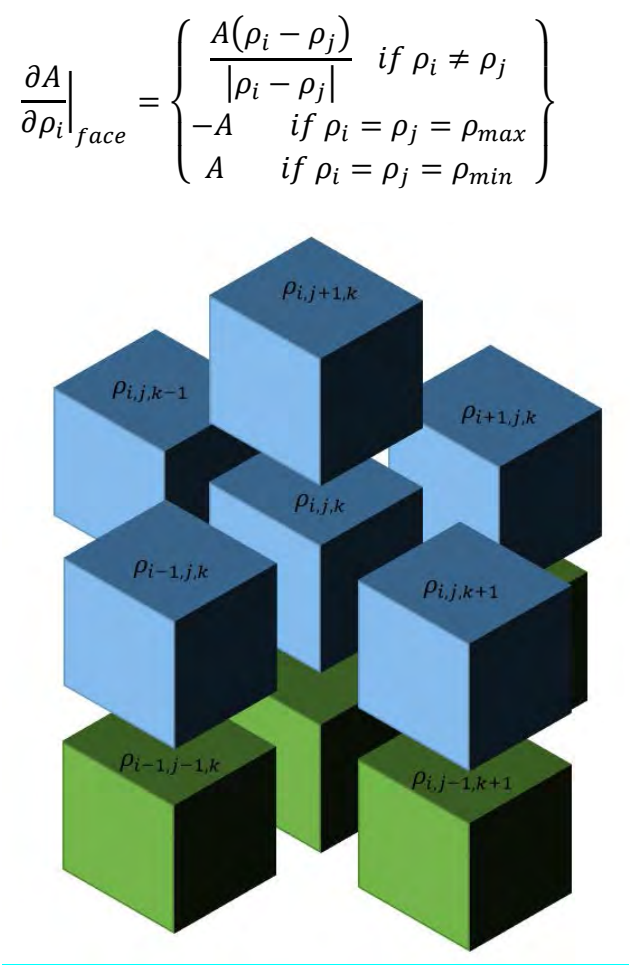

Figure 1. Elements that are considered in the modification of perimeter sensitives based on each element connectivity

For the estimation of the overhang sensitivities, a scheme used to penalize overhang structures shown in (7) is developed. Individual element connectivity's are found using a predefined function inside ANSYS $^{\circledR}$ that finds the six elements connected to the element.

$$
\begin{gathered}
\frac{\partial \mathrm{A}}{\partial \rho^{e}}=\left\{\left.\frac{\partial \mathrm{A}}{\partial \rho_{\mathrm{i}}}\right|_{\mathrm{face}(i, j+1, k)}+\left.\frac{\partial \mathrm{A}}{\partial \rho_{\mathrm{i}}}\right|_{\mathrm{face}(i-1, j, k)}+\left.\frac{\partial \mathrm{A}}{\partial \rho_{\mathrm{i}}}\right|_{\mathrm{face}(i-1, j, k+1)}+\right. \\
\left.\frac{\partial \mathrm{A}}{\partial \rho_{\mathrm{i}}}\right|_{\mathrm{face}(i+1, j, k)}+\gamma\left[\left.\frac{\partial \mathrm{A}}{\partial \rho_{\mathrm{i}}}\right|_{\mathrm{face}(i-1, j-1, k)}+\right. \\
\left.\frac{\partial \mathrm{A}}{\partial \rho_{\mathrm{i}}}\right|_{\mathrm{face}(i-1, j, k-1)}+\left.\frac{\partial \mathrm{A}}{\partial \rho_{\mathrm{i}}}\right|_{\mathrm{face}(i+1, j-1, k)}+ \\
\left.\left.\left.\frac{\partial \mathrm{A}}{\partial \rho_{\mathrm{i}}}\right|_{\mathrm{face}(i, j-1, k)}+\left.\frac{\partial \mathrm{A}}{\partial \rho_{\mathrm{i}}}\right|_{\mathrm{face}(i, j-1, k-1)}\right]\right\}
\end{gathered}
$$

From this, the modified sensitivities are shown in (8).

$$
C_{e}^{\eta}=-\frac{1}{\lambda}\left[\frac{\partial c}{\partial \rho^{e}}+\delta \frac{\partial A}{\partial \rho^{e}}\right]
$$

Now, the update scheme shown in (5) is used for the topology optimization procedure, except the modified sensitivities are used instead of the compliance sensitivities to converge the densities away from overhang structures, (9).

$$
\rho_{n e w}^{e}=\left\{\begin{array}{c}
\text { if } \rho^{e} C_{e}^{\eta} \leq \max \left(\rho_{\min }, \rho^{e}-m\right) \\
\boldsymbol{m a x}\left(\boldsymbol{\rho}_{\min }, \boldsymbol{\rho}^{\boldsymbol{e}}-\boldsymbol{m}\right) \\
\text { if } \max \left(\rho_{\min }, \rho^{e}-m\right)<\rho^{e} C_{e}^{\eta}<\min \left(1, \rho^{e}+m\right) \\
\boldsymbol{\rho}^{e} \boldsymbol{C}_{\boldsymbol{e}}^{\eta} \\
\text { if } \min \left(1, \rho^{e}+m\right) \leq \rho^{e} C_{e}^{\eta} \\
\min \left(\mathbf{1}, \boldsymbol{\rho}^{\boldsymbol{e}}+\boldsymbol{m}\right)
\end{array}\right\}
$$

\section{B. Topology Optimization using APDL}

Building the topology optimizer on top of ANSYS ${ }^{\circledR}$ allows for a more flexible design platform. Using the methodologies just established, three APDL topology optimizers were coded. One based on the ESO method, one based on SIMP, and the last one adding the overhang constraint in the SIMP program. In each of the programs, the material property APDL command was used to modify the elements young's modulus, therefore directly affecting its stiffness. Overall each script solves the initial FEA problem and gets the initial element values. Then it retrieves each elements sensitivity value, and uses the corresponding methodology to choose which ones to modify. Once all elements are looped through, the script re-solves the FEA and repeats until the convergence criteria are met.

\section{RESULTS AND DISCUSSION}

\section{A. Topology Optimization - Case Study 1}

As an example, a bell crank was considered. There are two forces and a fixed hole which define the boundary conditions for this analysis. The setup in ANSYS $^{\circledR}$ is shown in Fig. 2. The element size is $2 \mathrm{~mm}$. The topology optimization results using ESO and SIMP without any additional constraints are shown in Fig. 3 and Fig. 4 at iterations 60 and 30 respectively. It can be seen that this structure may be difficult to produce using AM processes. 


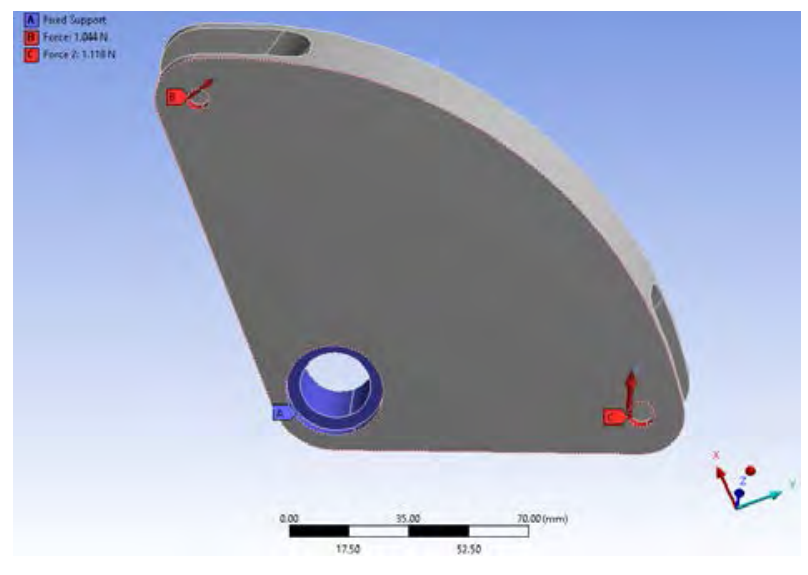

Figure 2. Case Study 1 Setup

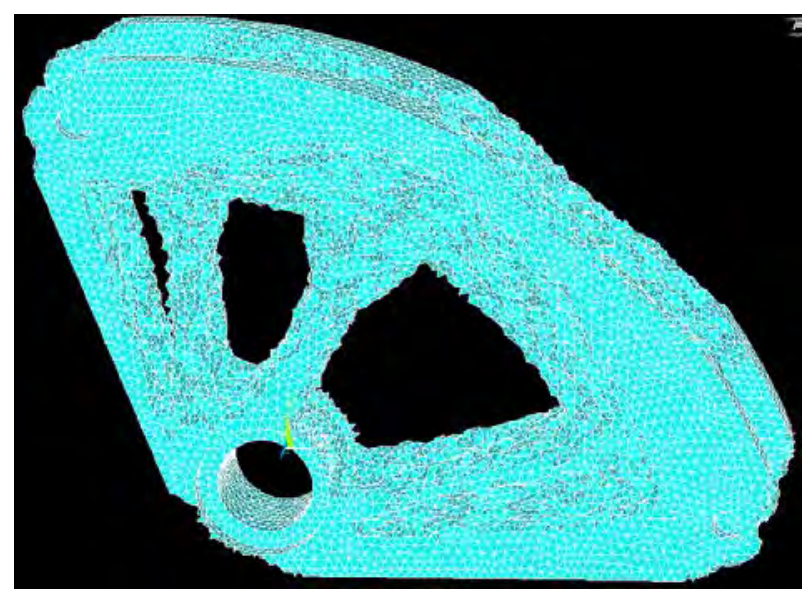

Figure 3. Case Study 1 Results after 60 Iterations using ESO

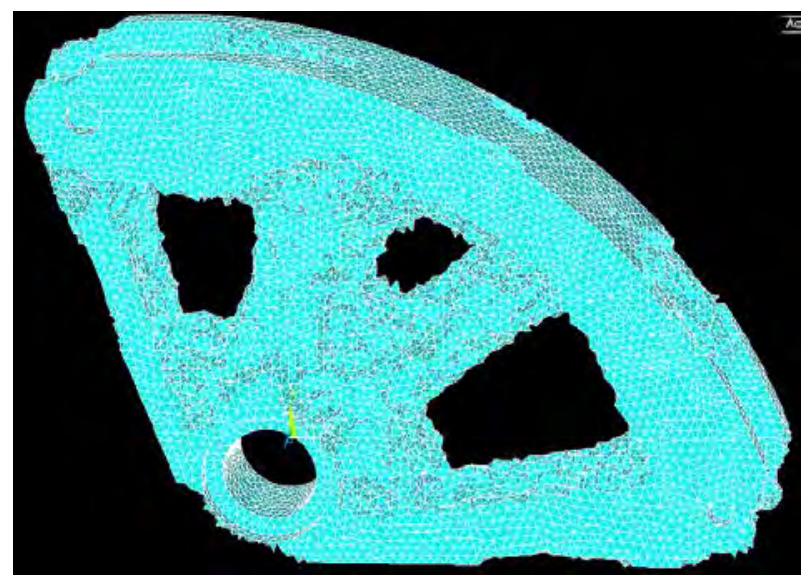

Figure 4. Case Study 1 Results after 30 Iterations using SIMP

\section{B. Topology Optimization-Case Study 2: Overhang Constraint}

Next, an MBB-beam as shown in Fig. 5 was considered. It has a fixed constraint at the bottom corner of one end and a rolling constraint at the other end, with a $1000 \mathrm{~N}$ force applied at the middle point of the top surface. It has a mesh size of 40x180. The setup in ANSYS $^{\circledR}$ is shown in Fig. 6. The SIMP method topology optimization results using half design domain with symmetry are shown in Fig. 7. The results in Fig. 8 and Fig. 9 are with the overhang constraint with varying $\delta$ values from (8). The blue line at the bottom represents the build platform, with the blue arrow specifying the build direction. By inspection it appears that this structure does indeed have less overhanging structures than the normal SIMP method.

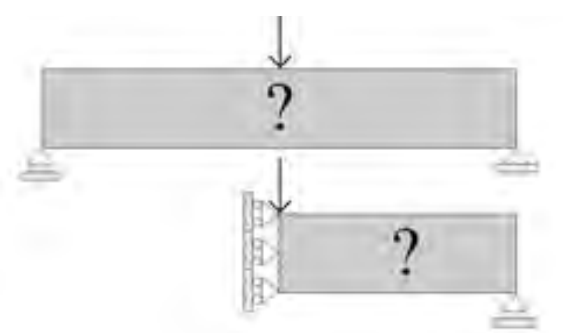

Figure 5. MBB-beam. Top: full design domain, bottom: half design domain with symmetry [7].

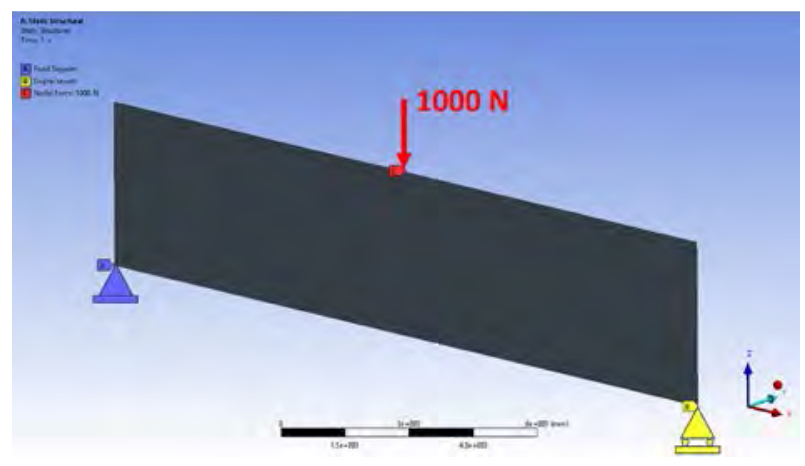

Figure 6. Case Study 2 Setup

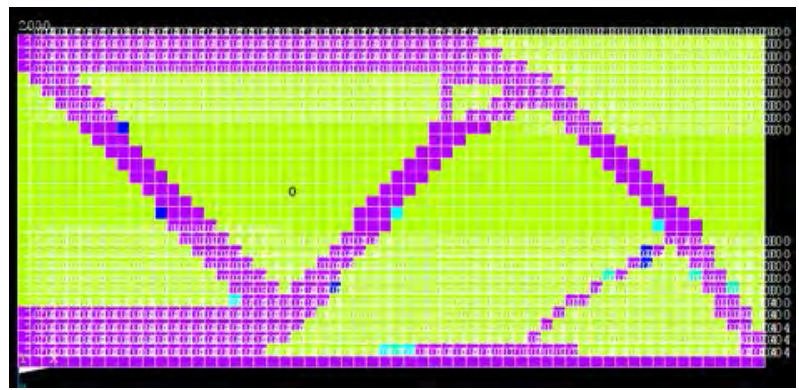

Figure 7. Case Study 2 Half Design Domain Results after 50 Iterations using SIMP (Purple elements are solid, green elements are a void)

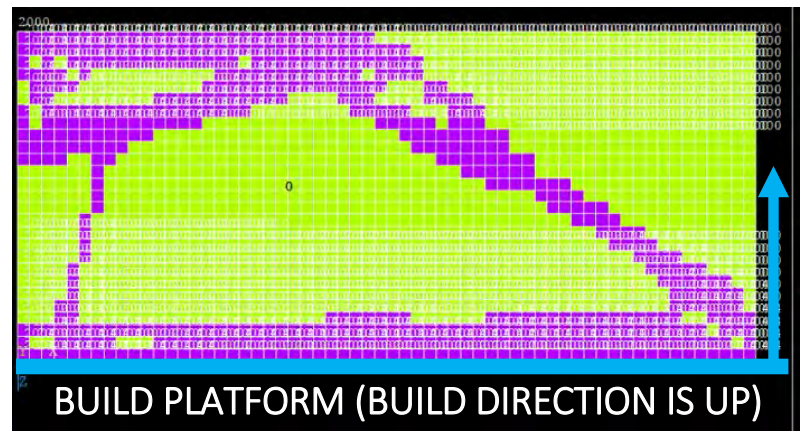

Figure 8. Case Study 2 Half Design Domain Results using Overhang Constraints, $\delta=0.00005$ (Purple elements are solid, green elements are a void) 


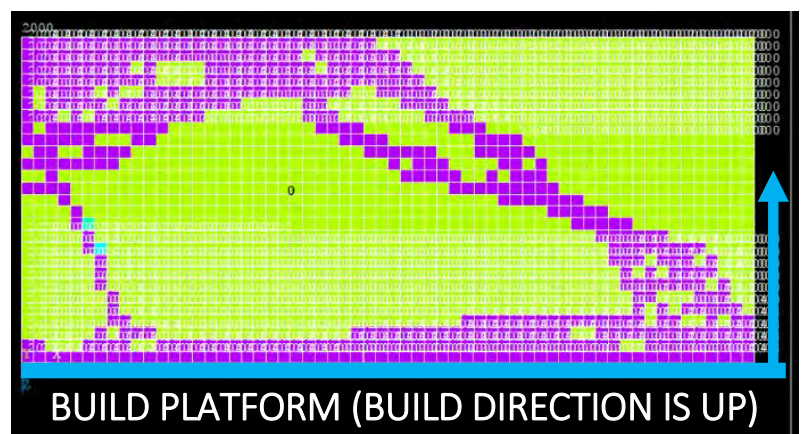

Figure 9. Case Study 2 Half Design Domain Results using Overhang Constraints, $\delta=0.0001$ (Purple elements are solid, green elements are a void)

\section{CONCLUSION}

In this paper, three topology optimization codes were developed. Two based on previous methodologies, the ESO method and the SIMP method, and the final attempted to optimize the topology for AM processes by reducing the need for support material. All were implemented in ANSYS ${ }^{\circledR}$ directly, using APDL scripts, in order to achieve a flexible and reliable development platform. The first case study looked at the implementation of the ESO and SIMP methods, and the last one looked at the implementation of the overhang constraint. The proposed topology optimization filter modifies the element sensitivities to converge the optimization towards the optimal structure that needs the least amount of material for support structures. This will reduce the manufacturing cost and time for preprocessing and post-processing operations, and reduce material waste. The established methodology can be easily developed for various additive manufacturing technologies in various industrial applications.

\section{ACKNOWLEDGMENT}

The research support provided by the Natural Science and Engineering Research Council of Canada (NSERC) and the research collaboration of SimuTech Group, Inc. are greatly appreciated.

\section{REFERENCES}

[1] X. Huang and M. Xie, Evolutionary topology optimization of continuum structures: methods and applications. Chichester, UK: John Wiley \& Sons, 2010.

[2] M. K. Thompson and J. M. Thompson, ANSYS Mechanical APDL for Finite Element Analysis. Cambridge, MA: Butterworth-Heinemann, 2017.

[3] A. Lalehpour and A. Barari, "Post processing for fused deposition modeling parts with acetone vapour bath," IFAC-Pap., vol. 49, no. 31, pp. 42-48, 2016. doi: 10.1016/j.ifacol.2016.12.159.

[4] J. Vanek, J. A. G. Galicia, and B. Benes, "Clever support: Efficient support structure generation for digital fabrication," Comput. Graph. Forum, vol. 33, no. 5, pp. 117-125, Aug. 2014. doi: 10.1111/cgf.12437.

[5] M. Langelaar, "An additive manufacturing filter for topology optimization of print-ready designs," Struct. Multidiscip. Optim., vol. 55, no. 3, pp. 871-883, Mar. 2017. doi: 10.1007/s00158-016-1522-2.

[6] M. P. Bendsøe and O. Sigmund, "Topology optimization by distribution of isotropic material," in Topology Optimization, Berlin, Germany: Springer, 2004, pp. 1-69.
[7] O. Sigmund, "A 99 line topology optimization code written in Matlab," Struct. Multidiscip. Optim., vol. 21, no. 2, pp. 120-127, Apr. 2001. doi: $10.1007 / \mathrm{s} 001580050176$

[8] A. Barari, "Inspection of the machined surfaces using manufacturing data," $J$. Manuf. Syst., vol. 32, no. 1, pp. 107-113, Jan. 2013.

doi: 10.1016/j.jmsy.2012.07.011.

[9] F. Kaji and A. Barari, "Evaluation of the surface roughness of additive manufacturing parts based on the modelling of cusp geometry," IFAC-Pap., vol. 48, no. 3, pp. 658-663, 2015. doi: 10.1016/j.ifacol.2015.06.157.

[10] A. Barari et al., "On the surface quality of additive manufactured parts," Int J. Adv. Manuf. Technol., vol. 89, no. 5-8, pp. 1969-1974, Mar. 2017.

doi: 10.1007/s00170-016-9215-y.

[11] S. Sikder, A. Barari, and H. A. Kishawy, "Effect of adaptive slicing on surface integrity in additive manufacturing," in Proc. ASME IDETC/CIE, Buffalo, NY, USA, Aug. 2014, pp. V01AT02A052.

doi: 10.1115/DETC2014-35559.

[12] H. Gohari, A. Barari, and H. Kishawy, "Using multistep methods in slicing $21 / 2$ dimensional parametric surfaces for additive manufacturing applications," IFAC-Pap., vol. 49, no. 31, pp. 67-72, 2016. doi: 10.1016/j.ifacol.2016.12.163.

[13] H. Gohari and A. Barari, "A quick deviation zone fitting in coordinate metrology of NURBS surfaces using principle component analysis,"

Measurement, vol. 92, pp. 352-364, Oct. 2016.

doi: 10.1016/j.measurement.2016.05.050.

[14] S. Moaveni, Finite element analysis theory and application with ANSYS, 3rd ed. Upper Saddle River, NJ: Pearson Higher Education, 2011.

[15] C. S. Jog, "Topology design of structures using a dual algorithm and a constraint on the perimeter," Int. J. Numer. Methods Eng., vol. 54, no. 7, pp. 1007-1019, Apr. 2002. doi: 10.1002/nme.457. 


\section{Vision-based elative State and nertia atio Estimation of Un-cooperative Targets}

\author{
Qian Feng, Quan Pan, Xiaolei Hou, Yong Liu \\ School of Automation \\ Northwestern Polytechnical University \\ Xi'an, Shannxi, 710072, China \\ qianfeng.nwpu@gmail.com,quanpan@nwpu.edu.cn
}

\author{
Zheng H. Zhu \\ Department of Mechanical Engineering \\ York University \\ Toronto, Ontario M3J 1P3 \\ gzhu@yorku.ca
}

\begin{abstract}
This paper develops an algorithm to estimate the relative pose, motion, and inertia ratio of an unknown target using stereoscopic vision. First, the positions and velocities of detected feature points on the spacecraft are estimated. Second, the angular velocity and attitude of the target are estimated by the least square method and q-method, respectively. Third, the position and velocity of the center of mass of the spacecraft are recovered. Finally, the inertia ratio is estimated based on the angular momentum conversation using the estimated angular velocity and attitude of the target. Numerical simulations are conducted to demonstrate the proposed algorithm.
\end{abstract}

Keywords-relative state; inertia ratio; un-cooperative spacecraft; stereoscopic vision; 3-D reconstruction; constrains.

\section{INTRODUCTION (HEADING 1)}

Estimating the dynamical parameters (pose and motion) and inertia property of an unknown target is one of the critical technologies during on-orbit autonomous close proximity operation missions [1], such as spacecraft rescuing, repairing, and capturing. These unknown targets are usually noncooperative without prior information about their structural and inertia parameters, nor artificial markers located known positions on the target. Therefore, the relative state and inertia properties are complete unknown. For a safe close proximity operation mission, such like approach to and capture the target, the relative state can provide important navigation information for the control strategy. Besides, the knowledge of inertia properties of the target is necessary to propagate its state and can be used in the motion prediction before capture and despun control design after capture.

Numerous vision-based estimation methods were proposed in the past [2-6] for non-cooperative targets. Terui et al. [7] proposed a 3D model matching method to estimate the relative state with the prior known structure of the target. Dehann et al. [8] adopted the stereo vision to measure the geometric center of the target as an approximation to its center of mass (CM). Du et al. [9] determined the pose of a large non-cooperative target based on two collaborative cameras by recognizing a rectangular feature on the target. Dong and Zhu $[10,11]$ estimated the pose and motion of a non-cooperative target with a monocular camera using some known feature point. Lichter and Dubowsky [12] presented a method to estimate the state using thousands of 3D points acquired from several cooperative 3D sensors, which leads to heavy computational efforts. Tweddle [13] proposed a Simultaneous Localization and Mapping (SLAM) solution to estimate the full state of a spinning non-cooperative target, with high computation complexity. Shay Segal [14] calculated the pose and motion with image position and velocity measurements of feature points on the target. By applying torque on the target produced from reaction wheels or a robotic arm, the inertia parameters are in-flight estimated $[15,16]$.

The purpose of this paper is to develop an innovative stereo vision-based algorithm to estimate the relative pose, motion, and inertia ratio of a non-cooperative target without prior knowledge about the positions of the feature points. The inertia ratio is estimated based on the conservation of angular momentum, assuming that the target is freely tumbling without any external torque on it.

The remainder of this paper is organized as follows. Section II provides the observation model. Section III gives the details of the proposed estimation algorithm. In Section IV, the newly proposed algorithm is validated by numerical simulation. Finally, Section V concludes the paper.

\section{OBSERVATION MODEL}

As depicted in Fig. 1, assume that two cameras mounted on the chaser are identical with parallel image planes and separated by a baseline $b$, the distance between the right camera's center of projection $\mathbf{C O P}_{R}$ and the left camera's center of projection $\mathbf{C O P}_{L}$. The target is in the field of view (FOV) of the both cameras. Two coordinate frames are used to describe the relative pose and motion of the unknown target: a) the target body frame $\mathcal{T}$ is a Cartesian frame with its origin at the $\mathrm{CM}$ of the target; b) the camera frame $\mathcal{C}$ is a Cartesian frame with its origin attached to $\mathbf{C O P}_{R}, \hat{\mathbf{x}}_{C}$ and $\hat{\mathbf{z}}_{C}$ axes parallel to the image plane and $\hat{\mathbf{y}}_{\mathcal{C}}$ axis pointing towards to the target. Here the frame $\mathcal{C}$ is assumed to be aligned with the inertial frame. The position and attitude of the target are defined by the vector $\boldsymbol{\rho}_{0}$ in the frame $\mathcal{C}$ and the direction cosine matrix $\mathbf{R}_{c}^{t}$ 
converting vectors from the frame $\mathcal{C}$ to the frame $\mathcal{T}$. The corresponding translational and rotational velocities are denoted as $\dot{\boldsymbol{\rho}}_{0}$ and $\boldsymbol{\omega}_{t c}^{c}$, respectively.

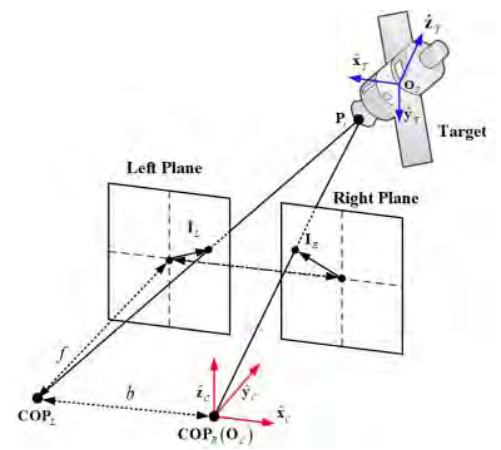

Fig. 1. Stereo vision measurement system.

Assuming that an arbitrary point $P_{i}$ on the target has the 3D coordinates $\boldsymbol{\rho}_{i}=\left[\begin{array}{lll}\rho_{i x} & \rho_{i y} & \rho_{i z}\end{array}\right]^{T}$ in the frame $\mathcal{C}$ and the $2 \mathrm{D}$ images coordinates $\mathbf{I}_{R}=\left[\begin{array}{ll}u_{i R} & v_{i R}\end{array}\right]^{T}$ and $\mathbf{I}_{L}=\left[\begin{array}{ll}u_{i L} & v_{i L}\end{array}\right]^{T}$ in the right and left image frames, respectively. According to the ideal pinhole camera model, the perspective projection transforms the feature point $P_{i}$ from the 3-D space onto the 2$\mathrm{D}$ image plan, such that

$$
\boldsymbol{\eta}_{i}=\left[\begin{array}{c}
u_{i R} \\
v_{i R} \\
u_{i L} \\
v_{i L}
\end{array}\right]=\left[\begin{array}{c}
f \frac{\rho_{i x}}{\rho_{i y}} \\
f \frac{\rho_{i z}}{\rho_{i y}} \\
f \frac{\rho_{i x}+b}{\rho_{i y}} \\
f \frac{\rho_{i z}}{\rho_{i y}}
\end{array}\right]
$$

where $t$ is the focal length.

Define the disparity as

$$
d_{i} \triangleq u_{i L}-u_{i R}
$$

The image velocity of the feature point is defined as the time derivative of its image coordinates,

$$
\left[\begin{array}{c}
\dot{u}_{i R} \\
\dot{v}_{i R} \\
\dot{u}_{i L} \\
\dot{v}_{i L}
\end{array}\right]=\frac{1}{\rho_{i y}}\left[\begin{array}{ccc}
f & -u_{i R} & 0 \\
0 & -v_{i R} & f \\
f & -u_{i L} & 0 \\
0 & -v_{i L} & f
\end{array}\right] \dot{\boldsymbol{\rho}}_{i}
$$

Considering the image noise, denoted by $\boldsymbol{\varepsilon}$, the images coordinates with noise could be modeled as

$$
\overline{\boldsymbol{\eta}}=\boldsymbol{\eta}+\boldsymbol{\varepsilon}
$$

where $\overline{\boldsymbol{\eta}}=\left[\bar{u}_{i R}, \bar{v}_{i R}, \bar{u}_{i L}, \bar{v}_{i L}\right]^{T}$ is the image measurement and $\boldsymbol{\varepsilon}_{i}$ is a zero-mean white noise with covariance matrix $\mathbf{R}_{i}$, i.e., $\boldsymbol{\varepsilon}_{i} \sim \mathcal{N}\left(0, \mathbf{R}_{i}\right)$.

\section{DESIGN AND IMPLEMENTATION OF STATE AND STRUCTURE ESTIMATION OF TARGET}

\section{A. Positions and velocities of feature points}

The positions of feature points are estimated by

$$
\overline{\boldsymbol{\rho}}_{i}=\left[\begin{array}{c}
\bar{\rho}_{i x} \\
\bar{\rho}_{i y} \\
\bar{\rho}_{i z}
\end{array}\right]=\left[\begin{array}{c}
\frac{b\left(\bar{u}_{i L}+\bar{u}_{i R}\right)}{2 \bar{d}_{i}}-\frac{b}{2} \\
\frac{b f}{\bar{d}_{i}} \\
\frac{b\left(\bar{v}_{i L}+\bar{v}_{i R}\right)}{2 \bar{d}_{i}}
\end{array}\right]
$$

The velocities of feature points are determined by

$$
\overline{\boldsymbol{\rho}}_{i}=C_{i}^{+} \boldsymbol{\xi}_{i}
$$

where the superscript "+" denotes the Moore-Penrose inversion of a matrix, and

$$
C_{i}=\frac{1}{\bar{\rho}_{i y}}\left[\begin{array}{ccc}
f & -\bar{u}_{i R} & 0 \\
0 & -\bar{v}_{i R} & f \\
f & -\bar{u}_{i L} & 0 \\
0 & -\bar{v}_{i L} & f
\end{array}\right], \xi_{i}=\left[\begin{array}{c}
\overline{\dot{u}}_{i R} \\
\dot{\bar{v}}_{i R} \\
\dot{\bar{u}}_{i L} \\
\dot{\bar{v}}_{i L}
\end{array}\right] .
$$

\section{B. Pose and motion estimation of the target}

At time instant $t$, the relationship between $\boldsymbol{\rho}_{i}$ and $\mathbf{r}_{i}$ is expressed as:

$$
\boldsymbol{\rho}_{i}(t)=\boldsymbol{\rho}_{0}(t)+\mathbf{R}_{t}^{c}(t) \mathbf{r}_{i}
$$

where $\mathbf{R}_{t}^{c}=\left(\mathbf{R}_{c}^{t}\right)^{T}$ is the rotation matrix that transforms vectors from the frame $\mathcal{T}$ to the frame $\mathcal{C}$. Taking the time derivative of (7) yields

$$
\dot{\boldsymbol{\rho}}_{i}(t)=\dot{\boldsymbol{\rho}}_{0}(t)+\boldsymbol{\omega}_{t c}^{c}(t) \times\left[\mathbf{R}_{t}^{c}(t) \mathbf{r}_{i}\right]
$$

Defining $\delta \boldsymbol{\rho}_{i}(t)=\boldsymbol{\rho}_{i}(t)-\boldsymbol{\rho}_{1}(t)$ and $\delta \mathbf{r}_{i}=\mathbf{r}_{i}-\mathbf{r}_{1}$ yield

$$
\left\{\begin{array}{l}
\delta \boldsymbol{\rho}_{i}(t)=\mathbf{R}_{t}^{c}(t) \delta \mathbf{r}_{i} \\
\delta \dot{\boldsymbol{\rho}}_{i}(t)=\boldsymbol{\omega}_{t c}^{t}(t) \times\left[\mathbf{R}_{\mathrm{t}}^{c}(t) \delta \mathbf{r}_{i}\right]
\end{array}\right.
$$

Eliminating $\mathbf{R}_{t}^{c}(t) \delta \mathbf{r}_{i}^{t}$ in (9) leads to

$$
\left[\delta \boldsymbol{\rho}_{i}(t) \times\right] \boldsymbol{\omega}_{t c}^{c}(t)=-\delta \dot{\boldsymbol{\rho}}_{i}(t)
$$

where $[\mathbf{a} \times]$ is the cross-product matrix expressed as

$$
[\mathbf{a} \times]=\left[\begin{array}{ccc}
0 & -a_{3} & a_{2} \\
a_{3} & 0 & -a_{1} \\
-a_{2} & a_{1} & 0
\end{array}\right]
$$

The angular velocity of the target is estimated using LS method by

$$
\overline{\mathbf{\omega}}_{t c}^{c}(t)=\left[\mathbf{J}(t) \mathbf{J}(t)^{T}\right]^{-1} \mathbf{J}(t)^{T} \mathbf{b}(t)
$$

where $N$ is the number of feature points, and 


$$
\mathbf{J}(t)=\left[\begin{array}{c}
{\left[\delta \boldsymbol{\rho}_{1}(t) \times\right]} \\
\vdots \\
{\left[\delta \boldsymbol{\rho}_{N-1}(t) \times\right]}
\end{array}\right], \mathbf{b}(t)=\left[\begin{array}{c}
-\delta \dot{\boldsymbol{\rho}}_{1}(t) \\
\vdots \\
-\delta \dot{\boldsymbol{\rho}}_{N-1}(t)
\end{array}\right] .
$$

According to (9), the following relationship holds,

$$
\left\{\begin{array}{l}
\delta \boldsymbol{\rho}_{i}\left(t_{k}\right)=\mathbf{R}_{t}^{c}\left(t_{k}\right) \delta \mathbf{r}_{i} \\
\delta \boldsymbol{\rho}_{i}\left(t_{0}\right)=\mathbf{R}_{t}^{c}\left(t_{0}\right) \delta \mathbf{r}_{i}
\end{array}\right.
$$

where $t_{k}=t_{0}+k \Delta t, \Delta t$ is the sample interval time, and $k$ is a positive integer.

Eliminating $\delta \mathbf{r}_{i}^{t}$ in (13) gives

$$
\delta \mathbf{\rho}_{i}\left(t_{k}\right)=\mathbf{R}_{t}^{c}\left(t_{k}\right)\left[\mathbf{R}_{t}^{c}\left(t_{0}\right)\right]^{T} \delta \mathbf{\rho}_{i}\left(t_{0}\right), 1 \leq i \leq N-1
$$

Define $\delta \mathbf{R}_{t}^{c}\left(t_{k, 0}\right)=\mathbf{R}_{t}^{c}\left(t_{k}\right)\left[\mathbf{R}_{t}^{c}\left(t_{0}\right)\right]^{T}$ as the attitude change from $t_{0}$ to $t_{k}$, then (14) is rewritten as

$$
\delta \boldsymbol{\rho}_{i}\left(t_{k}\right)=\delta \mathbf{R}_{t}^{c}\left(t_{k, 0}\right) \delta \boldsymbol{\rho}_{i}\left(t_{0}\right), 1 \leq i \leq N-1
$$

Equation (15) is solved by the q-method. Choose a set of nonnegative weights $\left\{a_{i}\right\}, i=1,2 \ldots, N-1$ and define

$$
\mathbf{B}=\sum_{i=1}^{N-1} a_{i} \delta \boldsymbol{\rho}_{i}\left(t_{k}\right)\left[\delta \mathbf{\rho}_{i}\left(t_{0}\right)\right]^{T}, \quad \mathbf{L}(\mathbf{B})=\left[\begin{array}{cc}
\mathbf{B}+\mathbf{B}^{\mathrm{T}}-(\operatorname{tr} \mathbf{B}) \mathbf{I}_{3} & \mathbf{z} \\
\mathbf{z}^{\mathbf{T}} & \operatorname{tr} \mathbf{B}
\end{array}\right] .
$$

where $\mathbf{z}=\sum_{i=1}^{N-1} a_{i}\left[\delta \boldsymbol{\rho}_{i}\left(t_{k}\right) \times \delta \boldsymbol{\rho}_{i}\left(t_{0}\right)\right]$.

The optimal estimation $\delta \hat{\mathbf{q}}_{c t}\left(t_{k, 0}\right)$ is the eigenvector corresponding to the largest eigenvalue of $\mathbf{L}(\mathbf{B})$. Given the initial relative attitude, the relative attitude quaternion of the frame $\mathcal{C}$ relative to the frame $\mathcal{T}, \hat{\mathbf{q}}_{c t}\left(t_{k}\right)$, can be calculated at any time. Accordingly, the estimated quaternion of the frame $\mathcal{T}$ relative to the frame $\mathcal{C}$ is

$$
\hat{\mathbf{q}}_{t c}\left(t_{k}\right)=\left[\begin{array}{ll}
-\hat{\varrho}_{c t}^{T}\left(t_{k}\right) & \hat{q}_{c t 4}\left(t_{k}\right)
\end{array}\right]^{T}
$$

\section{Position and velocity of the CM estimation of the target}

Assume the $\mathrm{CM}$ of the target moves approximately at a constant velocity in a short sampling interval $\Delta t$, such that

$$
\left\{\begin{array}{l}
\boldsymbol{\rho}_{0}\left(t_{k}\right)=\boldsymbol{\rho}_{0}\left(t_{k}-\Delta t\right)+\dot{\boldsymbol{\rho}}_{0}\left(t_{k}-\Delta t\right) \cdot \Delta t \\
\dot{\boldsymbol{\rho}}_{0}\left(t_{k}\right)=\dot{\boldsymbol{\rho}}_{0}\left(t_{k}-\Delta t\right)
\end{array}\right.
$$

Define state as $\mathbf{X}=\left[\begin{array}{lll}\mathbf{r}_{i}^{T} & \boldsymbol{\rho}_{0}^{T} & \dot{\boldsymbol{\rho}}_{0}^{T}\end{array}\right]^{T}$, which satisfies

$$
\mathbf{X}\left(t_{k}-j \cdot \Delta t\right)=\mathbf{A}^{-j} \mathbf{X}\left(t_{k}\right), k-c \leq j<k
$$

where $j$ is a positive integer, $c$ is a time period, and

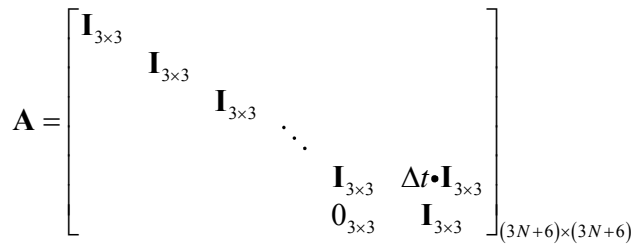

The measurement model can be written as

$$
\mathbf{Y}\left(t_{k}-j \cdot \Delta t\right)=\mathbf{C}\left(t_{k}-j \cdot \Delta t\right) \mathbf{A}^{-j} \mathbf{X}\left(t_{k}\right), k-c \leq j<k
$$

with

$$
\begin{gathered}
\mathbf{Y}\left(t_{k}\right)=\left[\begin{array}{lcc}
\mathbf{Y}_{1}\left(t_{k}\right)^{T} & \ldots & \mathbf{Y}_{i}\left(t_{k}\right)^{T}
\end{array}\right]^{T}, 1 \leq i \leq N \\
\mathbf{C}\left(t_{k}\right)=\left[\begin{array}{ccc}
\hat{\mathbf{R}}_{t}^{c}\left(t_{k}\right) & I_{3 \times 3} \\
\vdots & I_{3 \times 3} \\
& \hat{\mathbf{R}}_{t}^{c}\left(t_{k}\right) & I_{3 \times 3} \\
{\left[\hat{\boldsymbol{\omega}}_{t c}^{\mathrm{c}}\left(t_{k}\right) \times\right]} & \hat{\mathbf{R}}_{t}^{c}\left(t_{k}\right) & I_{3 \times 3} \\
\vdots & & I_{3 \times 3} \\
& {\left[\hat{\boldsymbol{\omega}}_{t c}^{\mathrm{c}}\left(t_{k}\right) \times\right] \hat{\mathbf{R}}_{t}^{c}\left(t_{k}\right)} & I_{3 \times 3}
\end{array}\right]
\end{gathered}
$$

The state $\mathbf{X}$ can be estimated by LS given as

$$
\hat{\mathbf{X}}\left(t_{k}\right)=\left(\mathbf{H H}^{T}\right)^{-1} \mathbf{H}^{T} \mathbf{Y}
$$

where

$$
\mathbf{H}=\left[\begin{array}{c}
\mathbf{C}\left(t_{k}\right) \\
\mathbf{C}\left(t_{k}-\Delta t\right) \mathbf{A}^{-1} \\
\vdots \\
\mathbf{C}\left(t_{k}-j \cdot \Delta t\right) \mathbf{A}^{-1} \\
\vdots \\
\mathbf{C}\left(t_{k}-c \cdot \Delta t\right) \mathbf{A}^{-1}
\end{array}\right], \mathbf{Y}=\left[\begin{array}{c}
\mathbf{Y}\left(t_{k}\right) \\
\mathbf{Y}\left(t_{k}-\Delta t\right) \\
\vdots \\
\mathbf{Y}\left(t_{k}-j \cdot \Delta t\right) \\
\vdots \\
\mathbf{Y}\left(t_{k}-c \cdot \Delta t\right)
\end{array}\right]
$$

\section{Inertia ratio estimation}

In the absence of external torques, the angular momentum of the target is constant in the inertial frame. Since the frame $\mathcal{C}$ is assumed to be aligned with the inertial frame in this paper, the angular momentum of the target is constant in the frame $\mathcal{C}$, which is denoted as $\mathbf{h}^{c}$. At time instant $t$,

$$
\mathbf{R}_{c}^{t}(t) \mathbf{h}^{c}(t)=\mathbf{I} \omega_{t c}^{t}(t)
$$

where $\boldsymbol{\omega}_{t c}^{t}(t)=\mathbf{R}_{c}^{t}(t) \boldsymbol{\omega}_{t c}^{c}(t)$

Define the inertia vector as

$$
\mathbf{x}=\left[\begin{array}{llll}
\mathbf{I}^{* T} & h_{x}^{c} & h_{y}^{c} & h_{y}^{c}
\end{array}\right]^{T}
$$

with $\mathbf{I}^{*}=\left[\begin{array}{llllll}I_{x x} & I_{x y} & I_{x z} & I_{y y} & I_{y z} & I_{z z}\end{array}\right]^{T},(21)$ can be rewritten as

$$
\mathbf{A x}=\mathbf{0}
$$

where

$$
\mathbf{A}=\left(\begin{array}{c|c}
\Omega^{t}\left(t_{1}\right) & \mathbf{R}_{c}^{t}\left(t_{1}\right) \\
\vdots & \vdots \\
\Omega^{t}\left(t_{M}\right) & \mathbf{R}_{c}^{t}\left(t_{M}\right)
\end{array}\right)
$$


with

$$
\Omega^{b}(t)=\left(\begin{array}{cccccc}
\boldsymbol{\omega}_{t c x}^{t}(t) & \boldsymbol{\omega}_{t c y}^{t}(t) & \boldsymbol{\omega}_{t c z}^{t}(t) & 0 & 0 & 0 \\
0 & \boldsymbol{\omega}_{t c x}^{t}(t) & 0 & \boldsymbol{\omega}_{t c y}^{t}(t) & \boldsymbol{\omega}_{t c}^{t}(t) & 0 \\
0 & 0 & \boldsymbol{\omega}_{t c x}^{t}(t) & 0 & \boldsymbol{\omega}_{t c y}^{t}(t) & \boldsymbol{\omega}_{t c z}^{t}(t)
\end{array}\right)
$$

Equation (23) can be solved in the least square, which means that the solution should minimize

$$
f(\mathbf{x})=\|\mathbf{A} \mathbf{x}\|_{2}^{2}=(\mathbf{A} \mathbf{x})^{T} \mathbf{A} \mathbf{x}=\mathbf{x}^{T} \mathbf{A}^{T} \mathbf{A} \mathbf{x}
$$

Obviously $f(\mathbf{x})$ has the minimum when

$$
\mathbf{B x}=\mathbf{0}
$$

with $\mathbf{B}=\mathbf{A}^{T} \mathbf{A} \in \mathbb{R}^{9 \times 9}$.

Reordering such that

$$
\mathbf{B}=\left[\begin{array}{ll}
b_{11} & \mathbf{b}_{1}^{T} \\
\mathbf{b}_{1} & \mathbf{B}_{r}
\end{array}\right]
$$

where $b_{11} \in \mathbb{R}^{+}, \mathbf{b}_{1} \in \mathbb{R}^{8 \times 1}$, and $\mathbf{B}_{r} \in \mathbb{R}^{8 \times 8}$. Setting the first variable in $\mathbf{x}$ to 1, i.e. $\mathbf{x}=\left[\begin{array}{ll}1 & \mathbf{x}_{r}^{T}\end{array}\right]^{T},(26)$ is reduced to

$$
\mathbf{B}_{r} \mathbf{x}_{r}=-\mathbf{b}_{1}
$$

However, there are physical constraints between the elements of the inertia tensor $\mathbf{I}$. Assuming that $I_{x x}$ is the largest diagonal element of $\mathbf{I}$, the following constraints hold,

$$
\left\{\begin{array}{l}
0<I_{y y}<I_{x x} \\
0<I_{z z}<I_{x x} \\
I_{y y}+I_{z z}>I_{x x} \\
2\left|I_{x y}+I_{x z}+I_{y z}\right|<I_{x x}+I_{y y}+I_{z z}
\end{array}\right.
$$

Since $\mathbf{B}_{r}$ is also a positive-definite matrix. Therefore, the problem is a convex quadratic programming problem with convex quadratic function $q\left(\mathbf{x}_{r}\right)=\frac{1}{2} \mathbf{x}_{r}^{T} \mathbf{B}_{\mathbf{r}} \mathbf{x}_{r}+\mathbf{b}_{1}^{T} \mathbf{x}_{r} \quad$ and inequality constraints (29) by setting the first variable in $\mathbf{x}$ to 1 . The interior-point-convex method is adopted here to solve this convex quadratic programming problem.

\section{Numerical Simulations}

In this section, numerical simulations are performed to verify the proposed estimation algorithm. The estimation errors are defined as:

$$
\begin{gathered}
e_{\rho_{0}}=\frac{\left\|\hat{\boldsymbol{\rho}}_{0}-\boldsymbol{\rho}_{0}\right\|_{2}}{\left\|\boldsymbol{\rho}_{0}\right\|_{2}}, e_{\dot{\rho}_{0}}=\frac{\left\|\hat{\boldsymbol{\rho}}_{0}-\dot{\boldsymbol{\rho}}_{0}\right\|_{2}}{\left\|\dot{\boldsymbol{\rho}}_{0}\right\|_{2}}, e_{\boldsymbol{\omega}_{t c}^{c}}=\frac{\left\|\hat{\boldsymbol{\omega}}_{t c}^{c}-\boldsymbol{\omega}_{t c}^{c}\right\|_{2}}{\left\|\boldsymbol{\omega}_{t c}^{c}\right\|_{2}} \\
e_{\mathbf{r}_{i}}=\frac{\left\|\hat{\mathbf{r}}_{i}-\mathbf{r}_{i}\right\|_{2}}{D}, e_{\mathbf{I}_{i}}=\frac{\left\|\hat{\mathbf{I}}_{i}^{*}-\mathbf{I}_{i}^{*}\right\|}{\left\|\mathbf{I}_{i}^{*}\right\|}
\end{gathered}
$$

where the superscripts " $\bullet$ " and "." denote the estimated value and true value, respectively, \|\|$_{2}$ is the norm of a vector, $\mathbf{I}_{i}^{*}$ is the component of the normalization of $\mathbf{I}^{*}$, and $D$ is a reference dimension. The relative attitude estimation error is defined as

$$
e_{\theta}=2 \cos ^{-1}\left(q_{e 4}\right)
$$

\begin{tabular}{|c|c|c|}
\hline \multicolumn{2}{|c|}{ Simulation parameter } & Values \\
\hline \multirow{4}{*}{$\begin{array}{c}\text { Camera } \\
\text { parameters }\end{array}$} & Baseline length & $1 \mathrm{~m}$ \\
\hline & Focal length & $0.1 \mathrm{~m}$ \\
\hline & Image noise & 0.2 pixel \\
\hline & Pixel Size & $2.55 \mu \mathrm{m}$ \\
\hline \multirow{4}{*}{$\begin{array}{c}\text { Initial } \\
\text { state }\end{array}$} & $\begin{array}{l}\text { Initial relative angular } \\
\text { velocity }\end{array}$ & {$\left[\begin{array}{llll}1.5 & 2.3 & -1.9\end{array}\right] \mathrm{rad} / \mathrm{s}$} \\
\hline & Initial relative attitude & {$\left[\begin{array}{lllll}0 & 0 & 0 & 1\end{array}\right]^{\mathrm{T}}$} \\
\hline & Initial position & {$\left[\begin{array}{llll}20 & 60 & 30\end{array}\right] \mathrm{m}$} \\
\hline & Initial velocity & {$\left[\begin{array}{lll}-0.01 & -0.01 & -0.02] \mathrm{m} / \mathrm{s}\end{array}\right.$} \\
\hline
\end{tabular}

where $q_{e 4}$ is the scalar part of the error quaternion defined by $\mathbf{q}_{e}=\mathbf{q}_{c c} \otimes \hat{\mathbf{q}}_{t c}$.

In the simulation, the sample time step is assumed to be $1 \mathrm{~s}$, the total simulation period is $3,000 \mathrm{~s}$, and the time period is 10 , the number of feature points is 4 and the reference dimension is $3 \mathrm{~m}$, the other conditions are listed in Table I.

Table I Simulation parameters.
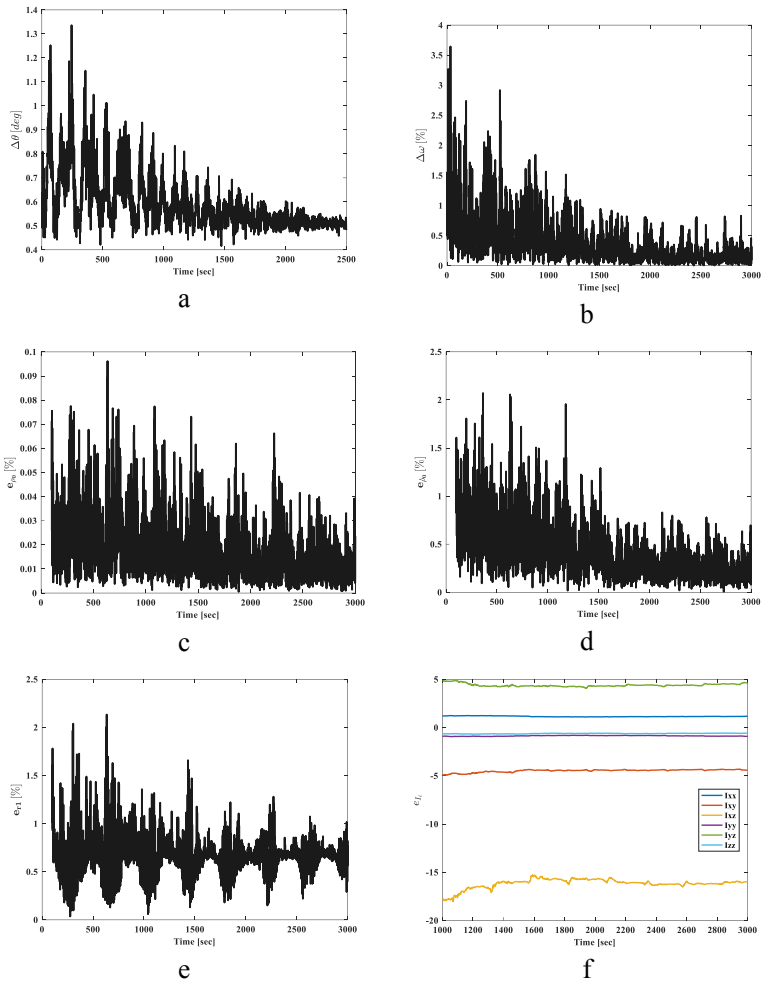

Fig. 2. Error of estimation. a) error of relative attitude, b) error of relative angular velocity, c) error of position for $\mathrm{CM}$ of the target, d) error of velocity for $\mathrm{CM}$ of the target, e) error of position estimation of one feature point, $\mathrm{f}$ ) error of inertia ratio estimation.

As shown in Fig. 2, the relative state and inertia ratio estimation can get high accuracy by the designed algorithm. The relative attitude error is less than $2.5 \%$, the relative angular velocity error is no more than $4 \%$, and the position and velocity errors of the $\mathrm{CM}$ are less than $0.1 \%$ and $2.5 \%$, respectively, and the position error of one feature relative the reference dimension is less than $2.5 \%$, the error of the normalized moments of inertia is of magnitude $18 \%$. In fact, the number of the attitudes and angular velocities influence the accuracy of inertia ratio which is based on a least square optimization. 


\section{CONCLUSIONS}

This paper develops an effective algorithm to estimate the relative pose, motion, $\mathrm{CM}$ and inertia ratio of an unknown target based on the consecutive images obtained from stereoscopic vision. The positions and velocities of the detected feature points on the target are estimated first, and then the estimation of the pose, motion, CM and inertia ratio. The advantage of this algorithm is its ability to estimate the CM, inertia ratio and the constellation of feature points of the target simultaneously without prior knowledge of the target. The simulation results show that the proposed algorithm can get a good estimation accuracy in acceptable measurement noise level.

\section{ACKNOWLEDGMENT}

The work of this paper is by National Natural Science Foundation of China under grant 61503304, grant 61790552, grant 61703343 , and the Discovery Grant of the Natural Sciences and Engineering Research Council of Canada (NSERC).

\section{REFERENCES}

[1] David Barnhart et al., "XSS-10 micro-satellite demonstration," in AIAA Defense and Civil Space Programs Conference and Exhibit 2013.

[2] S. I. Nishida, S. Kawamoto, Y. Okawa, F. Terui, and S. Kitamura, "Space debris removal system using a small satellite," Acta Astronaut., vol. 65, no. 12, pp. 95-102, Jul. 2009. doi: 10.1016/j.actaastro.2009.01.041.

[3] L. Zhang, S. Zhang, H. Yang, H. Cai, and S. Qian, "Relative attitude and position estimation for a tumbling spacecraft," Aerosp. Sci. Technol., vol. 42, pp. 97-105, Apr. 2015.doi: 10.1016/j.ast.2014.12.025.

[4] C. Ma, H. Dai, and J. Yuan, "Estimation of inertial characteristics of tumbling spacecraft using constant state filter," Adv. Space Res., vol. 60, no. 3, pp. 513-530, Aug. 2017. doi: 10.1016/j.asr.2017.03.032.

[5] S. Segal, A. Carmi, and P. Gurfil, "Stereovision-Based Estimation of Relative Dynamics Between Noncooperative Satellites: Theory and Experiments," IEEE Transact. Control Syst. Technol., vol. 22, no. 2, pp. 568584, Mar. 2014. doi: 10.1109/TCST.2013.2255288.

[6] W. Xu, B. Liang, C. Li, and Y. Xu, "Autonomous rendezvous and robotic capturing of non-cooperative target in space," Robotica, vol. 28, no. 5, pp. 705-718, Aug. 2009. doi: 10.1017/S0263574709990397.

[7] F. Terui, H. Kamimura, and S. ichiro Nishida, "Motion Estimation to a Failed Satellite on Orbit using Stereo Vision and 3D Model Matching," in 2006 9th International Conference on Control, Automation, Robotics and Vision, 2006. doi: 10.1109/ICARCV.2006.345305.

[8] F. Dehann, T. Brent, U. Steve, and O. Alvar Saenz, "Vision-Based Relative Navigation and Control for Autonomous Spacecraft Inspection of an Unknown Object," in AIAA Guidance, Navigation, and Control (GNC) Conference. American Institute of Aeronautics and Astronautics, 2013.

[9] X. Du, B. Liang, W. Xu, and Y. Qiu, "Pose measurement of large noncooperative satellite based on collaborative cameras," Acta Astronaut., vol. 68, no. 11-12, pp. 2047-2065, Jun. 2011.

doi: $10.1016 /$ j.actaastro.2010.10.021.
[10] G. Dong and Z. H. Zhu, "Autonomous robotic capture of non-cooperative target by adaptive extended Kalman filter based visual servo," Acta Astronaut., vol. 122, pp. 209-218, May 2016. doi: 10.1016/j.actaastro.2016.02.003.

[11] G. Dong and Z. H. Zhu, "Incremental inverse kinematics based vision servo for autonomous robotic capture of non-cooperative space debris," Adv. Space Res., vol. 57, no. 7, pp. 1508-1514, Apr. 2016. doi: 10.1016/j.asr.2016.01.011.

[12] M. D. Lichter and S. Dubowsky, "State, shape, and parameter estimation of space objects from range images," in IEEE International Conference on Robotics and Automation, 2004. Proceedings. ICRA '04. 2004, 2004.

doi: 0.1109/ROBOT.2004.1307513.

[13] B. E. Tweddle, "Computer Vision-Based Localization and Mapping of an Unknown, Uncooperative and Spinning Target for Spacecraft Proximity Operations," $\mathrm{Ph} . \mathrm{D}$. dissertation, Massachusetts Institute of Technology, 2013.

[14] S. Shay, and G. Pini, "Stereoscopic Vision-Based Spacecraft Relative State Estimation," AIAA Guidance, Navigation, and Control Conference, 2009.

[15] O. Ma, H. Dang, and K. Pham, "On-Orbit Identification of Inertia Properties of Spacecraft Using a Robotic Arm,” J. Guid., Control, Dyn., vol. 31, no. 6, pp. 17611771, Nov. 2008.doi: 10.2514/1.35188.

[16] Kim, Dong-Hun, Choi, Dae-Gyun, and Oh, Hwasuk, "Inertia Estimation of Spacecraft Based on Modified Law of Conservation of Angular Momentum," Journal of Astron. Space Sci., vol. 27, no. 4, pp. 353-357, Dec. 2010. doi: 10.5140/JASS.2010.27.4.353. 


\section{Generating Reuse Concepts for Retired Wind-Turbine Blades}

\author{
S.R.X. Jiang \\ Mechanical \& Industrial Engineering \\ University of Toronto \\ Toronto, Canada
}

\author{
David Inkermann \& Thomas Vietor \\ Institute for Engineering Design \\ Technische Universität Braunschweig \\ Braunschweig, Germany
}

\author{
L.H. Shu* \\ Mechanical \& Industrial Engineering \\ University of Toronto \\ Toronto, Canada \\ *shu@mie.utoronto.ca
}

\begin{abstract}
This work examines identifying reuses for wind-turbine blades that are retired when they reach the end of their technical life. Wind-turbine blades are made from fibercomposite materials, for which effective material-based recycling technologies are extremely limited. An approach to avoid or postpone recycling is to repurpose wind-turbine blades in other applications, as they should not be reused as windturbine blades for safety reasons.
\end{abstract}

However, identifying promising reuses for wind-turbine blades is challenging due to their specific shape properties. In addition to issues of functional fixedness, wind-turbine blades are physically much larger than everyday objects with which people typically reason. Following a series of studies where engineering students were asked to identify wind-turbine-blade reuses, a method involving perspective-taking was developed and applied. The effects of this method on concept generation are reported and compared to SCAMPER, an existing design method. To better understand and motivate human designers, this work also incorporates psychological concepts, including Need for Closure and Regulatory Focus Theory.

Keywords: Sustainability, Component reuse, Wind-turbine blades, Concept generation, Functional fixedness

\section{INTRODUCTION}

Global installation of wind-turbine technology is expected to continue to increase, decreasing reliance on energy sources that contribute to climate change. However, wind-turbine blades of up to 100 meters in length are typically manufactured using glass-fiber reinforced polymer (GFRP). While GFRP provides structural integrity, it is also comprised of thermoset resins, which is difficult to reform or recycle. Although wind turbines are maintained over their use life for effective operation, they are eventually retired based on durability and prediction models. Ramirez-Tejeda et al. (2017) estimates that by 2028, retired wind-turbine blades will comprise 330,000 tons/year of composite waste. Recycling of retired wind-turbine blades for their scrap material is challenging since GFRP can only be reformed through machining, which destroys reinforcing fibers and compromises the material's integrity.

Funded by Natural Sciences and Engineering Research Council of Canada (NSERC)
For the above reasons, the current work explores the process of identifying possible repurposes or reuses for retired windturbine blades. In contrast to other cases of reuse, wind-turbine blades should not be reused in the same application, i.e., as windturbine blades nor aircraft components for safety reasons. While retired wind turbines have been repurposed as children's play structures and benches for public seating, such reuses alone will not likely fully absorb the supply of retired wind-turbine blades.

\section{BACKGROUND}

This work combines design-related and social-psychology concepts to better understand and motivate designers. Thus, we begin with background on the seemingly disparate concepts before describing how they are incorporated in the current work.

\section{A. Functional Fixedness}

Adamson (1952) described functional fixedness as a tendency to focus on an object's previously known function, which inhibits discovering appropriate new uses for this object. Related to design fixation, functional fixedness often leads designers to repeat the same set of solutions they identified, unable to see more creative solutions. Specific to the reuse of wind-turbine blades, individuals who are strongly affected by functional fixedness may have trouble identifying reuses other than as wind-turbine blades.

\section{B. SCAMPER}

SCAMPER is an acronym that corresponds to: Substitute, Combine, Adapt, Modify, Put to other use, Eliminate, and Reverse. SCAMPER is often applied to make modifications in configuration design, but it also has potential to help identify reuses for products. That is, each word or phrase of the acronym can be applied to a product or part to trigger potential reuses. Moreno et al. (2016) observed SCAMPER's effectiveness in generating novel concepts, since reflecting on each corresponding word or phrase could provide novel insights.

\section{Regulatory Focus Theory (RFT)}

A motivational framework developed by social psychologist Higgins (2000), Regulatory Focus Theory (RFT) has two independent self-regulatory orientations: prevention and promotion. This framework adds a dimension to the hedonistic model of approaching pleasure and avoiding pain. In RFT, a prevention-focused individual would approach safety and security (non-losses) and avoid losses. In contrast, a promotion- 
focused individual would approach hopes and accomplishments (gains) and avoid stagnation (non-gains).

In a variety of applications over the past two decades, Higgins et al. have demonstrated that increased task engagement results when individuals' regulatory focus and task orientation align. Thus, appropriate framing of a design task may improve the engagement of designers with different regulatory foci.

\section{Need for Closure (NFC)}

Individuals in general have different needs when it comes to obtaining firm answers to questions. Webster and Kruglanski (1994) developed a scale, called Need for Closure (NFC) that aims to measure these needs for cognitive closure and aversion to ambiguity. This scale has the following five subscales and corresponding sample statements: (1) order and structure: I enjoy having a clear and structured mode of life; (2) ambiguity: I'd rather know bad news than stay in a state of uncertainty; (3) decisiveness: I usually make important decisions quickly and confidently; (4) predictability: I don't like to go into a situation without knowing what I can expect from it; (5) closed mindedness: I do not usually consult many different options before forming my own view. To such statements, respondents select from choices that range from strongly disagree to strongly agree, with each choice contributing to their NFC score.

\section{E. Authors' Related Past Work}

Lai and Shu (2016) found that individuals with high NFC scores are more likely to fixate on a provided example when asked to develop a new concept. Olteteanu and Shu (2018) also linked subscales of the NFC to functional fixedness.

Olteteanu and Shu (2018) presented objects in various orientations, i.e., turned 0,90 and 180 degrees from a default orientation, and asked participants to identify alternative uses for the shown objects. Analysis of participant responses revealed that participants who were more receptive to reorientation cues were more likely to identify more, and more varied, uses for the objects. Thus, reorientation was shown to be an effective strategy to overcome functional fixedness in identifying alternative uses for everyday objects. These findings are consistent with those of Kudrowitz and Dippo (2013), whose Alternative-Uses-Test participants with more responses had more original responses.

Relating to the current study, wind-turbine blades are much larger than the everyday objects used in past AUT studies. In addition, people do not tend to interact with wind-turbine blades as they would with common everyday objects. Wind-turbine blades also rotate, i.e., change orientation during use, which may further reduce the effectiveness of using reorientation in the same way to decrease functional fixedness.

\section{METHODS}

A series of studies were conducted to gain insight into the process of generating reuse concepts for wind-turbine blades. This paper focuses on the fourth and fifth iterations, but major changes between iterations are summarized below to clarify previously encountered obstacles and how they were addressed. These studies serve to demonstrate the challenge in developing reuse concepts for a sustainable-energy object, which currently has few sustainable end-of-life options.

\section{A. Overview of Studies}

In each iteration of the study, engineering students were asked to identify reuses for parts of wind-turbine blades. They were shown drawings of four wind-turbine blade sections in at least one isometric and three orthogonal views. For parts with hollow sections, an additional isometric view was provided to show the section cut in half. The wind-turbine blades were presented as sections, since they are often cut into smaller pieces after being decommissioned for easier transportation. To convey part size, the first three iterations of the study used male and female CAD models as human scales. Since no difference in concepts were observed as a result of the different gendered models, the scale was replaced with a neutral stick figure to facilitate the introduction of other interventions. Fig. 1 shows the isometric views of all four sections, using this neutral figure to convey scale. Fig. 2 shows how the first part was presented to participants.

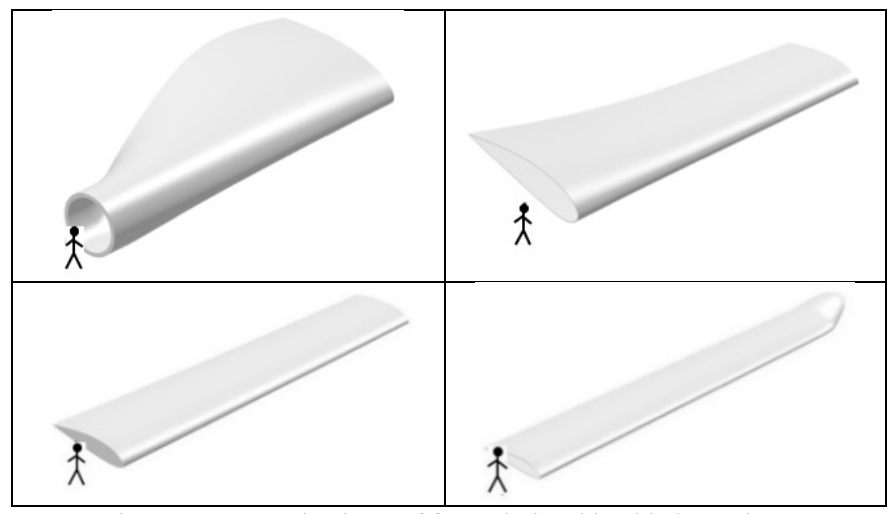

Figure 1. Isometric views of four wind-turbine blade sections.

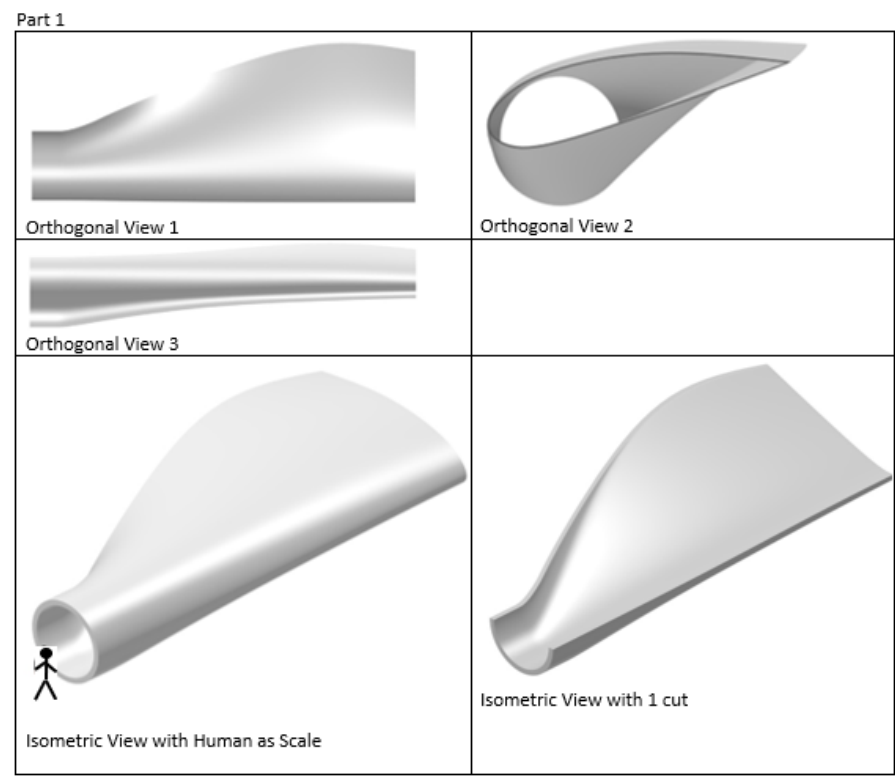

Figure 2. Different views of wind-turbine-blade Part 1.

\section{B. Previous Iterations of Study}

The first iteration of the study was conducted at Oldenburg University in Germany, in a graduate-level engineering course. To reduce functional fixedness, participants were not told that the parts were wind-turbine blades. For the same reason, they 
were not specifically prohibited from identifying reuses as windturbine blades. However, many engineering-student participants recognized the air-foil cross-section (in parts 2 and 3), and thus identified reuses as wind-turbine or airplane parts. Since the parts could not be reused in wind turbines, much less airplanes for safety reasons, these reuses were not feasible.

Thus, the second iteration, conducted in an engineering course at Bremen University in Germany, revealed that the parts were wind-turbine blades, and prohibited their reuse as the same or as airplane components. Although this restriction succeeded in reducing these specific reuse applications, the resulting reuse concepts either already exist, e.g., as children's play structures, or were impractical, e.g., as giant industrial mixing paddles.

Personal communication with a life-cycle engineering expert (Hauschild, 2017) towards more promising reuses led to the additional constraint that concepts address climate change. As this constraint could be expressed in multiple ways, they were formulated in line with Higgins' promotion versus prevention foci. Thus, third-iteration participants were asked to develop concepts that either "Enable pro-environmental behaviors to decrease the rate of climate change" (promotion focused), or "Help people cope with the inevitable results of climate change" (prevention focused). While this additional constraint provided direction and focus for concept generation, third-iteration participants in a 4th year undergraduate design course at the University of Toronto were observed to struggle when developing concepts. Thus, subsequent iterations explored existing (SCAMPER) and new (perspective-taking) methods to support this concept-generation activity.

\section{ITERATION FOUR USING SCAMPER}

The fourth iteration of the study was conducted at the Technische Universität Braunschweig in Germany.

\section{A. Methods}

Sixty-five (65) participants were recruited from a course on the basics of engineering design and product development, in a bachelor degree program. Of the 65,29 were asked to use SCAMPER, and the remaining 36 comprised the control group. However, the participants were known to be unfamiliar with SCAMPER. Therefore, those who were asked to use SCAMPER were given a four-page instructional aid, demonstrating how it could be applied towards concepts that reuse plastic bottles. Viswanathan et al. (2016) showed that examples with familiar features caused more fixation. Thus, examples in plastic-bottle reuse were chosen as they were dissimilar to wind-turbine blades in both function and scale. Fig. 3 shows examples for SCAMPER's "adapt" (similar features). In the top example, the cylindrical portion of the bottle is adapted for use as cylindrical magazine holders. In the bottom example, threads on the mouth of the bottle are adapted to fit with threads on a garden hose.

As was the case for the third iteration, participants were asked to complete the Need for Closure (NFC) questionnaire, and to identify which of two climate-change related goals their concepts addressed. They were specifically asked to clarify how each of their concepts can be used to either decrease the rate of climate change, or cope with the results of climate change.

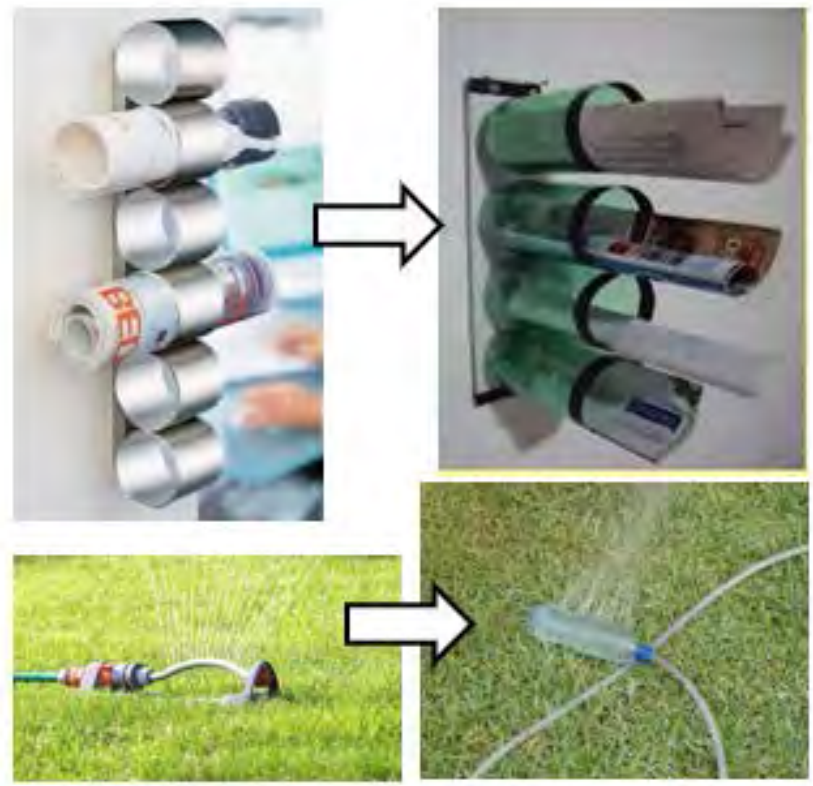

Figure 3. SCAMPER plastic-bottle reuse examples for Adapt (Top left image used with permission of APlaceForEverything.co.uk; Bottom right image used under https://creativecommons.org/licenses/by-nc-nd/2.0/).

To reduce instances of inaccurate claims, participants were reminded that reusing the material alone, e.g., as art, count as neither preventing nor coping with climate change.

\section{B. Results}

Table 1 compares by condition the average number of concepts generated per participant (including those who did not generate any concepts), as well as per concept-generating participant. The SCAMPER intervention led to fewer concepts compared to the control condition for both groups of participants, but this difference is significant for neither.

TABle 1. Average Number of CONCEPTS Per PARTICIPANT

\begin{tabular}{|c|c|c|c|}
\hline & SCAMPER & Control & $\mathbf{p}$ \\
\hline $\begin{array}{c}\text { Concept-generating } \\
\text { participants }\end{array}$ & 2.54 & 2.95 & 0.12 \\
\hline All participants & 1.14 & 1.64 & 0.10 \\
\hline
\end{tabular}

Fig. 4 compares between conditions, the percentage of participants who generated concepts for none, to all four, of the four wind-turbine-blade parts. The first pair of bar graphs correspond to the number of participants who did not generate any concepts: $16 / 36(44.4 \%)$ control and 16/29 (55.2\%) SCAMPER participants. Combining the latter four bar graphs for the SCAMPER condition, 13 of 29 participants (44.8\%) were able to generate reuse concepts for one or more of the four windturbine parts. In contrast, 20 of $36(55.6 \%)$ control-condition participants generated concepts for one or more of the four parts. The $10.7 \%$ difference between conditions in the proportion of participants who were able generate concepts for at least one part is insignificant $(\mathrm{p}=0.38)$. Thus, the SCAMPER condition was not successful in increasing the proportion of participants who were able to generate at least one reuse concept, and in fact decreased the proportion. 


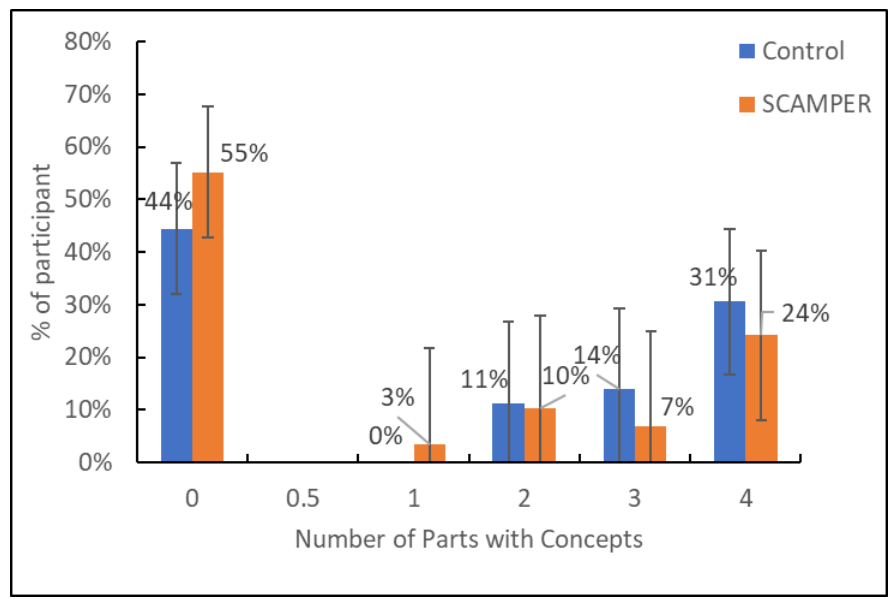

Figure 4. Proportion of participants who generated concepts for 0 to 4 of the four wind-turbine blade parts.

After removing the participants who produced zero concepts, Fig. 5 compares the proportion of participants who produced concepts for just one, up to all four of the parts. On average, each concept-generating participant in the control condition developed concepts for $3.35 / 4$ parts, while SCAMPERcondition participants generated concepts for $3.15 / 4$ parts, which is an insignificant difference.

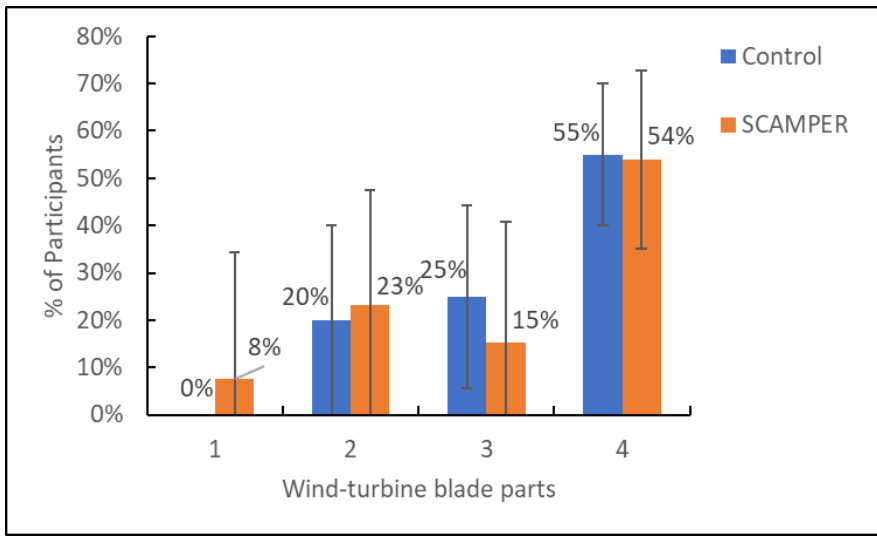

Figure 5. Proportion of concept-producing participants who generated concepts for just 1 up to all 4 of the four wind-turbine blade parts.

Fig. 6 compares the proportion of concepts generated for Parts 1 through 4 of the wind-turbine blade, which were always presented in the same order. As expected, more concepts are generally produced for the first versus last part presented, likely due to participant fatigue.

Table 1 compared by condition the average number of unique concepts generated per participant. However, participants often identified similar reuses for different windturbine parts, e.g., used both Parts 2 and 3 as fences. For concepts repeated by a participant, the one count for that same concept was allocated between the parts where the concept appears.

The SCAMPER method increased neither the number of concepts generated to reuse the wind-turbine blade parts, nor the proportion of participants who were able to generate concepts. However, the reuse concepts generated both with and without SCAMPER were categorized towards another possible method to support concept generation, as described in the next section.

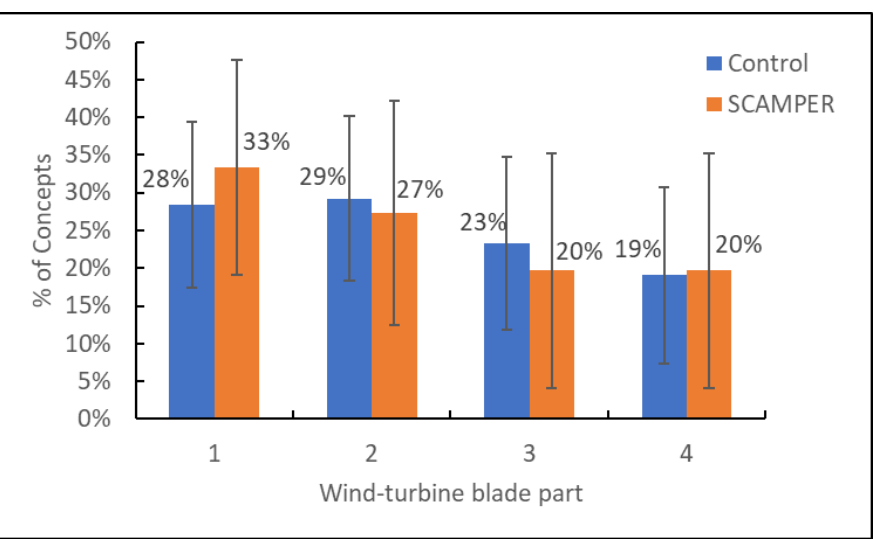

Figure 6. Proportion of concepts generated for wind-turbine-blade Parts 1-4.

\section{Iteration Five Using Perspective TAKing}

Building on previous iterations, a fifth iteration was conducted at Technische Universität Braunschweig in Germany.

\section{A. Methods}

Several approaches to categorizing the viable concepts from previous iterations were considered. One approach that emerged as promising for not just categorization, but also concept generation, involved perspective taking. This approach was then developed and tested in the current iteration on whether it can support participants to develop reuses for wind-turbine blades.

Participant responses in the fourth iteration also suggested a language barrier as non-English notes were found on the worksheets. To reduce this possible language barrier in the fifth iteration, the worksheets were translated to German.

\section{1) Participants}

Two hundred and four (204) participants were recruited from a second-year machine-elements course in mechanical engineering at TU Braunschweig. Demographic information, e.g., gender, native language and program of study, were collected along with their concepts and NFC responses. Of the participants who generated concepts, $85 \%$ identified as male, $10 \%$ identified as female, and $5 \%$ did not select from these choices. With respect to language proficiency, $87 \%$ were native speakers of German. Since all participants had attended a German university for at least one year, they were assumed to be able to understand and follow all the instructions in German.

\section{2) Intervention: Perspective-Taking Method}

In a way, the reorientation cue developed by Olteteanu and Shu (2018) for everyday objects was modified to support participants in developing reuses for much-larger wind-turbine parts. Rather than ask participants to reorient wind-turbine blade parts, they were asked to imagine the part on six different sides of them (above, below, front, back, left, right). In other words, participants were asked to mentally place the parts around themselves and view the parts from different perspectives. The perspective-taking intervention will also be referred to as the perspective method or condition below.

Fig. 7 shows how the perspective-taking intervention was conveyed graphically to participants, using "Teil", the German 
word for part, in a single-page aid. Compared to the four-page aid for SCAMPER in the fourth iteration, the perspective aid sheet is concise and requires minimal reading. The figures shown in Fig. 7 were revised multiple times to maximize neutrality and reduce suggestion of specific concepts.

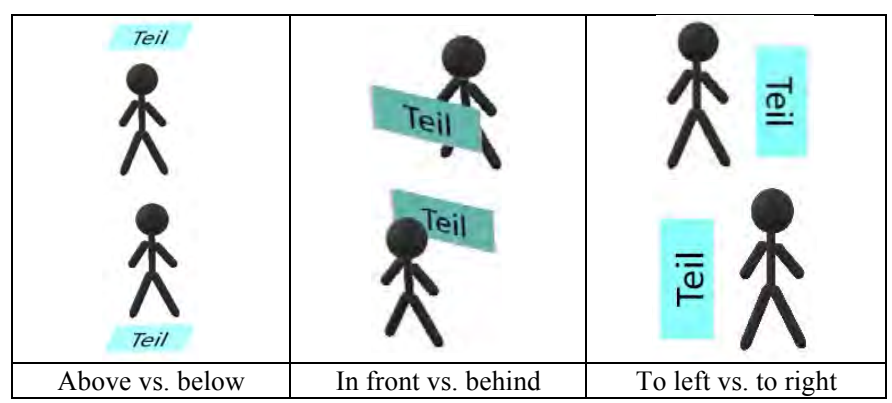

Figure 7. How the perspective-taking intervention was conveyed.

\section{3) Instructions to Participants}

The importance of wind-turbine-blade reuse was first conveyed through a presentation that highlighted the expected amount of waste generated from retired wind-turbine blades. Participants were then provided with worksheets that showed views of the four wind-turbine parts, with one part on each page. As in the two previous iterations, the worksheets asked participants to choose between and explain how their concepts either allow people to reduce the rate of climate change, or protect people from the effects of climate change.

Participants were asked to represent their concepts by simple sketches and minimize required cutting to the parts. They were also reminded that reusing the material alone counts towards neither preventing nor coping with climate change.

\section{4) Distribution of Worksheets}

Of the 204 participants, 98 were given the perspectivevisualization method shown in Fig. 7 as the intervention, or perspective condition. The remaining 106 were not given the intervention and comprise the control group or condition. Worksheets with the two conditions were alternatively distributed to participants. That is, participants should have received a condition that is different from their immediate neighbors. Finally, worksheets were collected at the end of the 45-minute limit given for generating and explaining concepts.

\section{B. Results}

To evaluate participants' concepts, their responses were first translated to English. The generated reuse concepts were then analyzed and classified. For concepts repeated by the same participant, the count for that same concept is allocated between the parts for which the concept appears, as demonstrated in Table 2 . Table 3 compares by condition, the average number of unique concepts generated per participant by all participants (including those who did not generate any concepts), versus only the participants who generated at least one concept.
TABLE 2: EXAMPLE CONCEPT ALLOCATION TO WIND-TURBINE-BLADE PARTS

\begin{tabular}{|c|c|c|}
\hline Part & Concepts & Points \\
\hline Part 1 & Roof for a house & 1 \\
\hline Part 2 & Wind barrier, ship's hull & 1.5 \\
\hline Part 3 & Wind barrier & 0.5 \\
\hline Part 4 & Dike reinforcement & 1 \\
\hline
\end{tabular}

TABle 3: Average Number of Concepts Generated Per Participant

\begin{tabular}{|l|c|c|c|}
\hline & Perspective & Control & p \\
\hline $\begin{array}{l}\text { Concept generating } \\
\text { participants }\end{array}$ & 2.81 & 3.10 & 0.29 \\
\hline All participants & 1.91 & 1.55 & 0.043 \\
\hline
\end{tabular}

Considering only the concept-generating participants, the perspective group produced fewer concepts compared to the control condition, although this difference is insignificant. This non-intuitive trend could be because participants who persisted (instead of abandoned) the control condition were intrinsically stronger designers, and thus able to generate more concepts.

What was significant, is that the perspective group produced more concepts compared to the control condition when averaged over all participants. That is, when including all participants, the perspective group has a 0.36 higher average number of concepts than the control group. This difference is significant with a 1tailed t-test at $\mathrm{p}=0.043$, supporting the hypothesis that perspective-taking helps participants to generate concepts.

Furthermore, in the perspective condition, 72 of 106 participants $(67.9 \%)$ were able to generate reuse concepts for one or more of the four wind-turbine parts. In contrast, 49 of 98 $(50.0 \%)$ control-condition participants generated concepts for one or more of the four parts. The $17.9 \%$ difference between the proportion of the two groups who were able generate concepts for at least one part is significant ( $\mathrm{p}=0.0095,95 \%$ Confidence Interval: $4.40 \%$ to $30.6 \%$ ). Thus, the perspective method was successful in increasing the proportion of engineering-student participants who were able to generate at least one reuse concept.

Fig. 8 compares by condition, the proportion of participants who generated concepts for zero up to four of the wind-turbineblade parts. Consistent with the above results, a higher number of participants in the control condition returned worksheets with no concepts. However, when analyzing participants who did generate at least one concept, the gap between conditions reduces. Fig. 9 compares by condition the number of windturbine parts for which concept-generating participants identified reuses. On average, each concept-generating participant in the control condition identified reuses for 2.80/4 parts while perspective-condition participants identified reuses for $2.72 / 4$ parts, an insignificant difference.

The difference between the average number of parts with concepts and the average number of unique concepts (Table 3) is due to a combination of repetition and multiple concepts per part. Participants would generate similar concepts for different wind-turbine parts, or multiple concepts per part. 


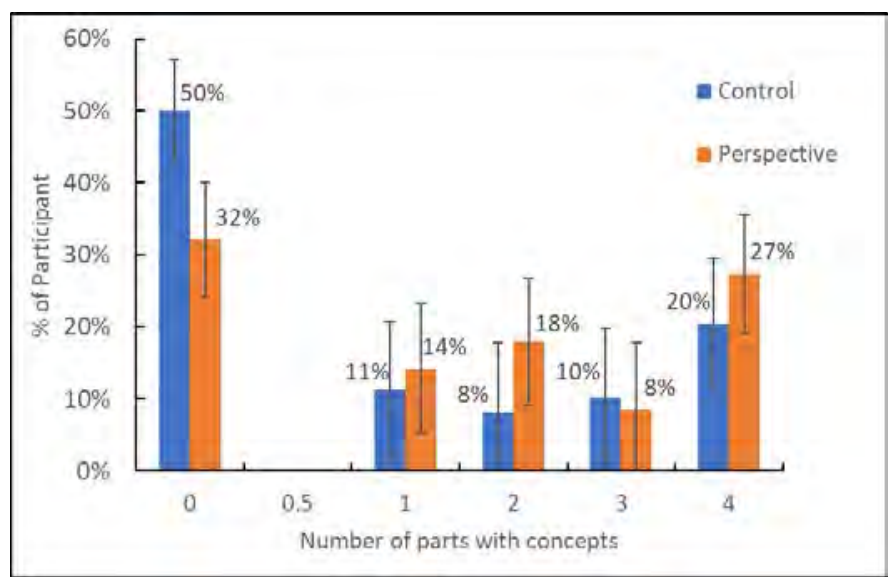

Figure 8. Proportion of participants who generated concepts for 0 to 4 of the four wind-turbine blade parts.

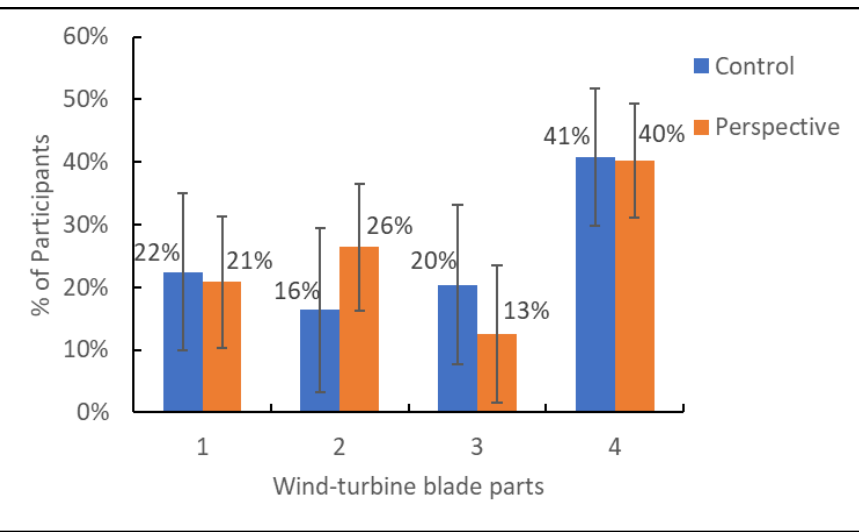

Figure 9. Proportion of concept-producing participants who generated concepts for just 1 to all 4 of the four wind-turbine-blade parts.

Similar to the previous (fourth) iteration, since participants went through worksheet pages ordered corresponding to windturbine-blade Parts 1 to 4 , the number of concepts generated for each subsequent part decreased as shown in Fig. 10. This result is likely due to participant fatigue. Again, for concepts repeated by the same participant, the count for that same concept is allocated between the parts where the concept appears.

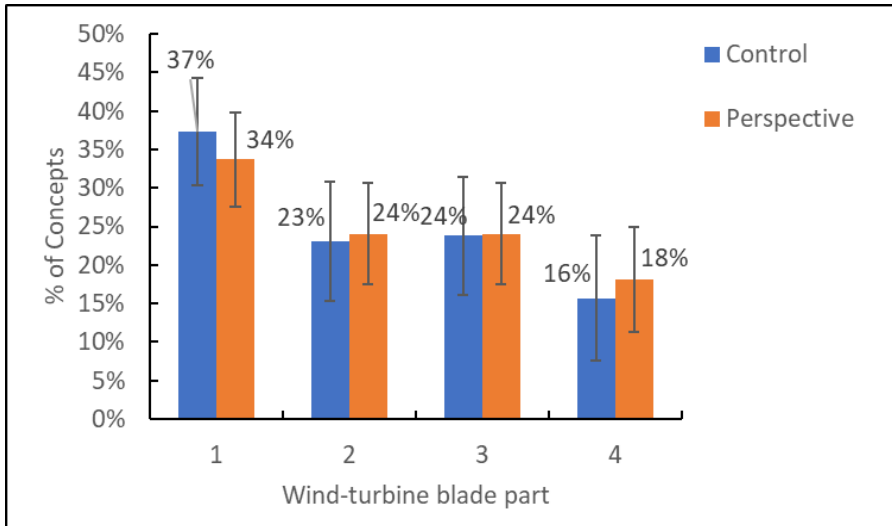

Figure 10. Proportion of concepts generated for wind-turbine-blade Parts 1-4.

\section{DISCUSSION}

\section{A. SCAMPER vs. Perspective-Taking Conditions}

Comparing the SCAMPER group of the fourth iteration and the perspective-taking group of the fifth iteration yields a $23 \%$ difference in proportion of participants who produced no concepts ( $\mathrm{p}=0.024,95 \%$ Confidence Interval: $3.05 \%$ to $41.3 \%$ ). This significant difference suggests the benefit of the perspective-taking method by comparing it against an existing method as an intervention. Furthermore, the corresponding proportions for the control condition in the fourth iteration that used SCAMPER was $44 \%$, versus $50 \%$ for the control condition in the fifth iteration that used the perspective-taking method. The difference of only $6 \%$ in the control-group participants between iterations of the study that used different interventions is insignificant $(\mathrm{p}=0.54)$. Fig. 11 shows the proportion of participants who produced no concepts for both conditions of the fourth and fifth iterations of the study.

Limitations of this comparison include the following. While both the SCAMPER and perspective-taking iterations were performed at TU Braunschweig's mechanical engineering department, participants were from different classes and years of study. Moreover, the SCAMPER materials were provided in English vs. German for the perspective-taking materials. Finally, the length of the 4-page SCAMPER aid sheets was considerably longer than the 1-page perspective-taking aid sheet.

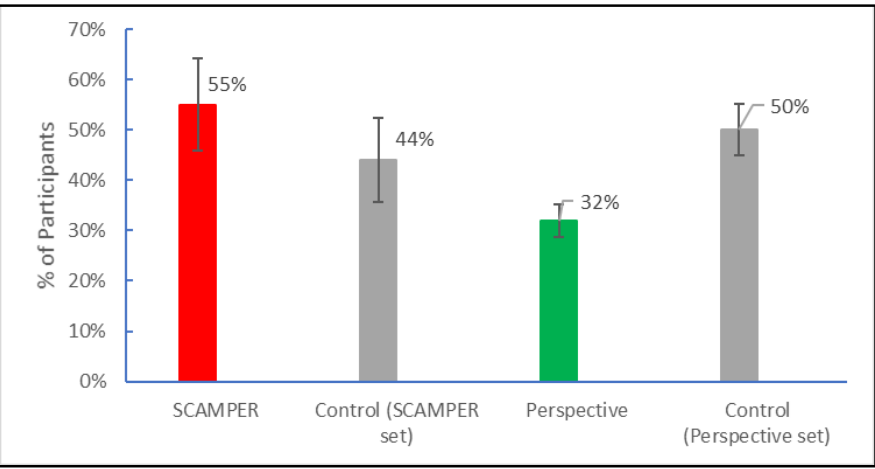

Figure 11. Proportion of participants who produced no concepts in fourth iteration using SCAMPER and fifth iteration using perspective-taking.

\section{B. Regulatory Focus Theory and Need for Closure}

As previously mentioned, participants were asked to develop concepts that either 1) Allow people to reduce the rate of climate change, or 2) Protect people from the effects of climate change.

The first choice corresponds to the promotion focus of Regulatory Focus Theory (RFT) by approaching hopes and gains, e.g., improvement of the environment. The second choice corresponds to the prevention focus of RFT, which is about preventing losses, e.g., protecting people.

Common promotion-focused concepts included: turbines for hydroelectric power stations; artificial coral reefs; and solar panel mounts. Concepts generated under the prevention-focus (protect people) category included: flood and wind barriers; dike reinforcement or erosion protection; sun covering; and emergency shelters.

In the perspective condition of the fifth iteration, 65 (of 106) participants completed the Need for Closure (NFC) 
questionnaire. Linear regression yielded a negative relationship between the ambiguity subscale of NFC and the proportion of promotion-focused concepts. That is, the higher a participant's desire to avoid ambiguity, the fewer promotion-focused concepts they produced as a proportion of all the concepts they generated. Shown in Table 4, a medium negative correlation ( $\mathrm{r}$ $=-0.31$ ) was found between participants' ambiguity subscale and their tendency to produce promotion-based concepts. This finding is related to the work of Toh and Miller (2014), who found that creativity is affected by varying tolerance levels for ambiguity.

TABLE 4: REGRESSION RESULTS FOR PERSPECTIVE-CONDITION PARTICIPANTS' NFC AMBIGUITY SUBSCALE AND PROPORTION OF PROMOTION-FOCUSED CONCEPTS

\begin{tabular}{|c|c|c|c|}
\hline Coefficients & Estimate & $\begin{array}{c}\text { 95\% Confidence } \\
\text { Interval }\end{array}$ & $\mathbf{p}$ \\
\hline Intercept & 1.38 & 0.633 to 2.13 & 0.0005 \\
\hline Ambiguity & -0.0237 & -0.0431 to -0.00424 & 0.018 \\
\hline
\end{tabular}

$\mathrm{F}=5.96, \mathrm{R}^{2}=0.0961, \mathrm{r}=-0.31$

This same trend was not seen in the control condition of the fifth iteration. Instead, the proportion of promotion-based concepts correlated with the decisiveness subscale of the NFC. That is, the more decisive the participant, the more promotionfocused concepts they generated. Analysis of the 46 participants with complete NFC scores yielded a medium positive correlation between their decisiveness subscale and the proportion of promotion-based concepts they generated, as shown in Table 5.

TABLE 5: REGRESSION RESULTS FOR CONTROL-CONDITION PARTICIPANTS' NFC DECISIVENESS SUBSCALE AND PROPORTION OF PROMOTION-FOCUSED CONCEPTS

\begin{tabular}{|c|c|c|c|}
\hline Coefficients & Estimate & $\begin{array}{c}\text { 95\% Confidence } \\
\text { Interval }\end{array}$ & $\mathbf{p}$ \\
\hline Intercept & -0.336 & -1.133 to 0.463 & 0.40 \\
\hline Decisiveness & 0.0284 & -0.0004 to -0.0572 & 0.053 \\
\hline
\end{tabular}

$\mathrm{F}=4.84, \mathrm{R}^{2}=0.131, \mathrm{r}=0.36$

Overall, only 75 of 204 (48 perspective- and 27 controlcondition) participants explicitly identified whether their concepts aimed to achieve promotion-focused versus prevention-focused goals. Some uncategorized concepts were analyzed and added to the two categories. Overall, the control group generated 42 prevention-based concepts and 25 promotion-based concepts, and the perspective group generated 65 prevention-based concepts and 67 promotion-based concepts. Therefore, the perspective condition appeared to help balance the categories.

\section{Effects of Conditions on Shredding Concepts}

While participants were discouraged from making excessive cuts to wind-turbine blade parts, some participants suggested incineration or shredding the parts for use as insulation.

In the fifth iteration, $2 \%$ of the perspective-taking participants generated concepts which required shredding compared to $7 \%$ in the control condition. This $5 \%$ decrease $(\mathrm{p}=$
0.011) suggests that the perspective-taking method encouraged reuses that utilize the overall shape of the part, by asking participants to imagine the parts around them.

In the fourth iteration using the SCAMPER intervention, too few (3) concepts that involve shredding were observed over both conditions combined; therefore, no conclusions can be drawn.

\section{Prevention-Based vs. Promotion-Based Example Reuses}

Using wind-turbine-blade parts in shelter construction is a common participant-generated concept. Fig. 12 shows a busshelter concept generated by a participant in the perspectivetaking group. The participant selected the box indicating that this concept is for protecting people from climate change, i.e., the prevention-focus choice. In the perspective-taking condition, 27 of $76(35.5 \%)$ concept-generating participants produced ideas involving shelter or roofing. In the control condition, 16 of 49 $(32.6 \%)$ participants produced similar concepts.

Fig. 13 presents a unique (control-condition) participant's concept of a vertical planter for plants to absorb carbon dioxide. The participant selected the box indicating that this concept is for enabling people to reduce the rate of climate change, i.e., the promotion-focus choice.

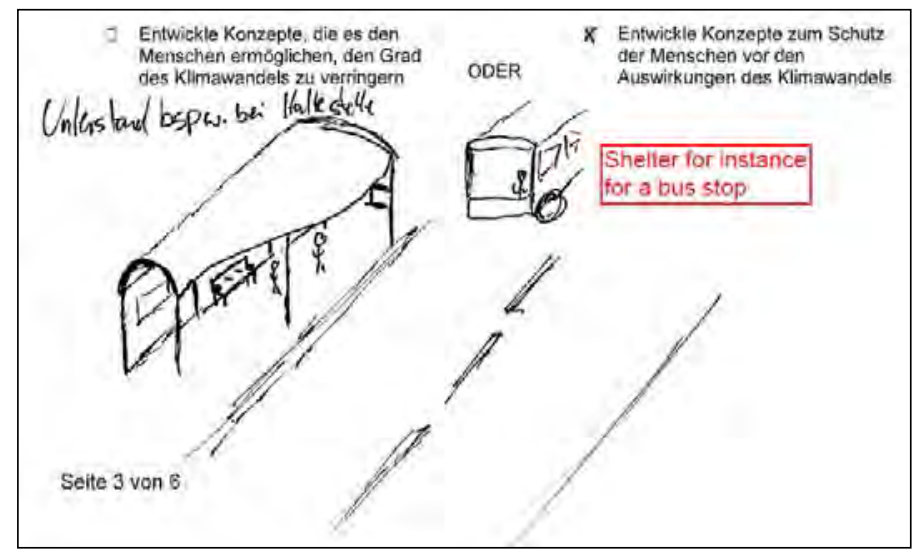

Figure 12. Participant-generated bus shelter concept for Part 1.

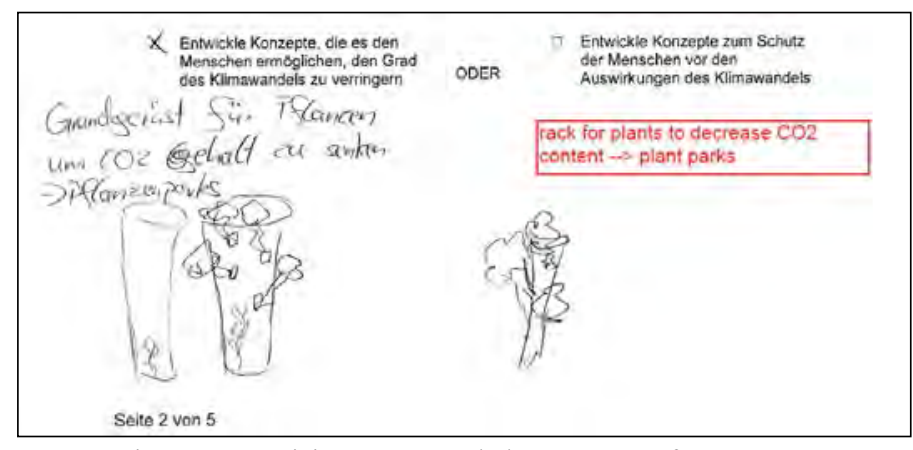

Figure 13. Participant-generated planter concept for Part 1.

\section{SUMMARY AND FUTURE WORK}

Five iterations of a study asked engineering-student participants to generate reuse concepts for wind-turbine blades. With focus on the fourth and fifth iterations, this paper examined the effect of different methods to support this challenging concept-generation activity. 
To reduce functional fixedness, the first iteration did not reveal that the parts shown were wind-turbine blades, nor were participants specifically prohibited from identifying reuses as wind-turbine blades. However, many participants identified reuses as wind-turbine blades or airplane parts, which are excluded for safety reasons. Thus, the second iteration revealed that the parts were wind-turbine blades, and prohibited their reuse in the same or airplane applications. While this restriction reduced these specific reuse applications, the resulting reuse concepts either already exist or were impractical. Kwon et al. (2018) report in more detail the first two iterations.

To help guide possible concepts, third-iteration participants were asked to develop concepts to address climate change. While this additional constraint provided direction and focus for concept generation, participants were observed to struggle to develop solutions. Thus, subsequent iterations explored methods to support this concept-generation activity.

The fourth iteration used SCAMPER, an existing method, to support engineering-student designers in concept generation. However, SCAMPER did not appear to aid in reuse-concept generation. Thus, the perspective-taking method was developed for the fifth iteration. This method was observed to reduce the number of participants who produced no concepts, but did not affect the number of parts for which concepts were generated, nor the number of concepts generated by participants who persisted in the study. However, participants were not specifically encouraged to maximize the number of concepts they generated.

While participants were asked to generate reuse concepts to address climate change, they were not given guidance to increase the environmental benefits of their concepts. Therefore, future work could also incorporate aspects to guide concepts towards those that maximize environmental benefits. Metrics to evaluate how well reuse concepts contribute to sustainability include manufacturing cost, waste management, and energy consumption (Jawahir \& Dillon, 2007).

This work aims to gain insights on the process of finding potential alternative uses of wind-turbine blades, which currently lack sustainable end-of-life solutions. The lack of sustainable post-use opportunities is recognized as a major impact area in sustainable manufacturing (Haapala et al., 2013). While attention to recycling and remanufacturing contributes to environmentally conscious product design, end-of-life options for already-designed and installed wind-turbine blades are required. Typical product end-of-life options include: direct reuse, repair, remanufacturing, recycling, and disposal (Ramani et al., 2010; Ilgin \& Gupta, 2010), with Sabbaghi \& Behdad (2018) noting that repair decisions are highly price dependent. Missing from these options is the opportunity to design for alternative uses of products at end-of-life. The role of product design in sustainable manufacturing has long focused on designing for the environment (Allen et al., 2002). In the case of closing the loop for wind-turbine blades, product design methods could also be leveraged towards alternative reuses of these parts, in the absence of other viable end-of-life solutions.

\section{ACKNOWLEDGMENTS}

The authors thank: Drs. Alexandra Pehlken (Oldenburg Univ.) and KlausDieter Thoben (Bremen Univ.); Elisa Kwon (Univ. of Toronto); Study participants and colleagues at TU Braunschweig; Prof. Gernot von Collani (Univ. of Leipzig) for his German translation of NFC questionnaires.

\section{REFERENCES}

[1] R. Adamson, "Functional fixedness as related to problem solving: A repetition of three experiments," J. Exp. Psychol., vol. 44, no. 4, pp. 288-291, 1952. doi: 10.21236/AD0006119.

[2] D. Allen et al., "Environmentally benign manufacturing: Trends in Europe, Japan, and the USA," J. Manuf. Sci. Eng., vol. 124, no. 4, pp. 908-920, Oct. 2002. doi:10.1115/1.1505855.

[3] M. Dinar et al., "Empirical studies of designer thinking: past, present, and future," J. Mech. Des., vol. 137, no. 2, pp. 021101-021101-13, Feb. 2015, doi: $10.1115 / 1.4029025$.

[4] Global Wind Energy Council, "Global wind report: Annual market update," Global Wind Energy Council, Brussels, Belgium, 2016. Available: https:/gwec.net/publications/global-wind-report-2/global-wind-report-2016/

[5] M. Hauschild, personal communication, Aug. 22, 2017. CIRP GA.

[6] K. Haapala et al., "A review of engineering research in sustainable manufacturing," J. Manuf. Sci. Eng., vol. 135, no. 4, pp. 041013-041013-16, July 2013. doi: 10.1115/1.4024040.

[7] E. Higgins, "Making a good decision: Value from fit," Am. Psychol., vol. 55, no. 11 , pp. 1217-1230, 2000. doi:10.1037/0003-066X.55.11.1217.

[8] M. A. Ilgin and S. M. Gupta, "Environmentally conscious manufacturing and product recovery (ECMPRO): A review of the state of the art," J. Environ. Manage., vol. 91, no. 3, pp. 563-591, Jan.-Feb. 2010.

doi: 10.1016/j.jenvman.2009.09.037.

[9] D. Jansson and S. Smith, "Design fixation," Des. Stud., vol. 12, no. 1, pp. 3-11, Jan. 1991. doi: 10.1016/0142-694X(91)90003-F.

[10] I. Jawahir and O, Dillon, "Sustainable manufacturing processes: New challenges for developing predictive models and optimization techniques," in Proc. 1st Int. Conf. on Sustainable Manuf., 2007, pp. 1-19.

[11] B. Kudrowitz and C. Dippo, "When does a paper clip become a sundial? Exploring the progression of novelty in the alternative uses test," J. Integr. Des. Process Sci., vol. 17, no. 4, pp. 3-18, Oct. 2013.

[12] E. Kwon et al., "Visual similarity to aid alternative-use concept generation for retired wind-turbine blades," in Proc. ASME IDETC/CIE, Quebec City, QC, Aug. 2018, pp. V007T06A005.

doi: 10.1115/DETC2018-85670.

[13] S. Lai and L. Shu, "Individual differences in tendency for design fixation," Int. Conf. DCC, Evanston, IL, USA, June 2016, pp. 321-338.

[14] D. Moreno et al., "Overcoming design fixation: Design by analogy studies and nonintuitive findings," AI EDAM, vol. 30, no. 2, pp. 185-199, Apr. 2016. doi: $10.1017 / \mathrm{S} 0890060416000068$.

[15] A. Olteteanu and L. Shu, "Object reorientation and creative performance," J. Mech. Des. vol. 140, no. 3, pp. 031102-031102-9, 2018.

doi:10.1115/1.4038264.

[16] K. Ramani et al., "Integrated sustainable life cycle design: a review," $J$. Mech. Des., vol. 132, no. 9, pp. 1-15, Sept. 2010. doi: 10.1115/1.4002308.

[17] D. Ramanujan et al., "Visual analytic tools for sustainable lifecycle design: current strategies, challenges, and future opportunities," J Mech. Des., vol. 139, no. 11, pp. 1-19, 2017. doi: 10.1115/1.4037479.

[18] M. Sabbaghi and S. Behdad, "Consumer decisions to repair mobile phones and manufacturer pricing policies: The concept of value leakage," Resour. Conserv. Recycl., vol. 133, pp. 101-111, June 2018. doi: 10.1016/j.resconrec.2018.01.015. 
[19] K. Ramirez-Tejeda, D. Turcotte, and S. Pike, "Unsustainable wind turbine blade disposal practices in the United States: A case for policy intervention and technological innovation," New Solutions: J. Environ. Occup. Health Policy, vol. 26, no. 4, pp. 581-598, Oct. 2016.

doi: $10.1177 / 1048291116676098$

[20] C. Toh and S. Miller, "The role of individual risk attitudes on the selection of creative concepts in engineering design," in Proc. ASME IDETC/CIE, Buffalo, NY, USA, Aug. 2014, pp. V007T07A027. doi: 10.1115/DETC2014-35106.

[21] V. Viswanathan, M. Tomko, and J. Linsey, "A study on the effects of example familiarity and modality on design fixation," AI EDAM. vol. 30, no. 2, pp. 71-184, May 2016. doi: $10.1017 /$ S0890060416000056.

[22] J. Wang et al., "Durability and prediction models of fiberreinforced polymer composites under various environmental conditions: A critical review," J Reinf. Plast. Compos., vol. 35, no. 3, pp. 179-211, Nov. 2015. doi: 10.1177/0731684415610920.

[23] D. Webster and A. Kruglanski, "Individual Differences in need for cognitive closure," J. Pers. Soc. Psychol., vol. 67, no. 6, pp.

1049-1062, 1994. doi: 10.1037/0022-3514.67.6.1049. 


\section{Collapse Surfaces of the Octet-Truss Lattice at Different Lattice Angles}

\author{
Mohamed Abdelhamid \\ Department of Mechanical Engineering \\ York University \\ Toronto, Canada \\ mahamid@yorku.ca
}

\author{
Aleksander Czekanski \\ Department of Mechanical Engineering \\ York University \\ Toronto, Canada \\ alex.czekanski@lassonde.yorku.ca
}

\begin{abstract}
Cellular materials are found extensively in nature such as wood, honeycomb, butterfly wings and foam-like structures like trabecular bone and sponge. This class of materials proves to be structurally-efficient by combining low weight with superior mechanical properties. Recent studies have shown there are coupling relations between the mechanical properties of cellular materials and their relative density. Due to its favorable stretching-dominated behavior, continuum models of the octet-truss were developed to describe its effective mechanical properties. However, previous studies were only performed for the cubic symmetry case where the lattice angle $\theta=45^{\circ}$. In this work, we study the impact of the lattice angle on the effective strength of the octet-truss. A graphical method is utilized to plot the collapse surfaces for plastic yielding and elastic buckling for different loading combinations at different lattice angles. Generally, the $\left(\bar{\sigma}_{x z}, \bar{\sigma}_{z z}\right)$ loading space is preferable for lattice angles greater than $45^{\circ}$, while the $\left(\bar{\sigma}_{x x}, \bar{\sigma}_{y y}\right)$ loading space is preferable for lattice angles lower than $45^{\circ}$.
\end{abstract}

Keywords - octet-truss; collapse surfaces; lattice angle; effective strength

\section{INTRODUCTION}

In the last few decades there has been a growing interest in lightweight load-bearing structures. Inspiration from nature can be found in natural cellular materials like wood, honeycomb, butterfly wings, and foam-like structures such as trabecular bones and sponge [1]. Architectural cellular materials have been used to create mechanically-efficient engineering structures such as the Eiffel Tower and the Garabit Viaduct [2]. This class of materials combines the benefits of low density as it only occupies a fraction of the monolithic bulk solid, and strength by arranging its elements efficiently to carry the loads. Previous studies have shown that the macroscopic mechanical properties of cellular materials depend on three parameters: the constituent material properties, the deformation mechanism, and the relative density $\bar{\rho}$ (defined as the solid volume within the unit cell divided by the total volume of the unit cell). Cellular-solids theory predicts scaling relationships between the macroscopic stiffness and strength vs. the relative density, namely $E_{s} \alpha \bar{\rho}^{m}$ and $\sigma_{y} \alpha \bar{\rho}^{n}$ respectively, where the dimensionless parameters $m$ and $n$ depend on the unit cell geometry [3].
For a 3D structure to be rigid (i.e. statically and kinematically determinate), a minimum nodal connectivity of $Z=6$ is required. A connectivity of $Z=12$ categorizes the structure as stretching-dominated where the lattice members deform by tension/compression. Bending-dominated structures that deform through the bending of their members, has a connectivity of $6 \leq$ $Z<12$ [4], [5]. For stretching-dominated structures such as the octet-truss lattice, these scaling relationships are linear. On the other hand, for bending-dominated structures such as honeycombs or the octahedral lattice, they are quadratic or stronger [6], [7].

When the dimensions of the lattice members are scaled down below the micron length scale, they exhibit different mechanical behavior. Examples of these size-dependent changes include strengthening in single crystalline metals and transition from brittle to ductile behavior in metallic glasses and ceramics [8], [9]. Recent advances in additive manufacturing techniques have made it possible to manufacture lattice structures with more geometrical and dimensional freedom. Certain AM techniques like self-propagating photopolymer waveguides [10], projection micro stereolithography [1], and two-photon lithography [11], [12] have been utilized to produce micro and nanolattices within the length scales required to activate material size effects. This is in addition to the structural effects activated by changing the various geometric parameters of the lattice unit cell [13].

Continuum constitutive models have been developed to describe the effective mechanical properties of the octet-truss lattice structure. A common assumption amongst these models is that the lattice members are pin-jointed at all nodes, hence the contribution from the bending resistance of the members and nodes is negligible compared to the axial tensile/compressive stiffness of the members [14]. Deshpande et al. (2001) checked the accuracy of the pin-jointed assumption by comparing FE calculations of rigid-jointed structures against analytical values of pin-jointed models for relative densities $\bar{\rho}$ ranging 0.01 to 0.5 , the results showed excellent agreement between the FE and analytical values proving the validity of this assumption [4]. Generally, symmetry considerations could be employed to deduce the number of independent constants in the macroscopic stiffness tensor. Following the pin-jointed assumption, these elastic constants are determined by averaging the contribution from each element to the macroscopic stiffness, which is achieved through 3D coordinate transformations [15], [16]. 
Nayfeh and Hefzy (1978) derived a first order approximation of the relative density of the octet-truss lattice by dividing the solid volume within the unit cell by the total volume of the unit cell [15]. They employed 3D coordinate transformation and volume averaging in order to obtain the macroscopic stiffness matrix. Lake (1992) constructed a strength tensor by converting applied stresses to strains for each parallel group of members using the macroscopic compliance matrix [17]. Failure would occur in a member if its axial strain exceeded a critical value based on an elastic buckling limit. The choice of elastic buckling over plastic yielding is somehow justified given that space structures, the typical application of lattice structures at that time, usually compose of slender members. Lake's strength tensor could easily accommodate multiaxial loading as well as different loading directions through coordinate transformation. The author also developed a 3D plot of the uniaxial compression strength in cartesian coordinates, from which he concluded the direction and value of the maximum strength of the octet-truss lattice for the case of cubic symmetry where the lattice angle $\theta$ equals $45^{\circ}$ (the angle between the individual members and the horizontal midplane as shown in Fig. 1). Deshpande et al. (2001) investigated the effective properties of the octet-truss lattice structure both theoretically and experimentally [4]. They validated the analytically-predicted elastic modulus and strength using FEM and experimental uniaxial compression of octet-truss lattice made from a casting aluminum alloy. They also explored the collapse criteria of two competing mechanisms (plastic yielding and elastic Euler buckling) and plotted the collapse surfaces for these two mechanisms under different loading conditions. The macroscopic collapse stress was evaluated by equating the external work for the kinematically admissible modes of collapse to: (i) the plastic dissipation in stretching the struts for the case of plastic yielding, (ii) and the internal work in buckling the struts for the case of elastic buckling. In addition, they proposed a third-order approximate formula for the relative density that includes a parameter dependent on the nodes' detailed geometry.

It is important to note that the previous studies were performed only for the case of cubic symmetry. At this angle, the octet-truss is considered to be at the highest attainable level of symmetry. However, potential applications of metamaterials (e.g. thin-walled pressure vessels) necessitates the use of anisotropic lattice structures in order to achieve the optimal combination of low density and high load-carrying capacity.

\section{EFFECTIVE STRENGTH}

The macroscopic strength of the octet-truss lattice is defined as the maximum stress the lattice can sustain without any of its members reaching a critical stress limit. This limit can be defined according to two main modes of failure: (i) yielding for ductile materials or fracture for brittle materials (tensile or compressive), (ii) and Euler beam buckling (compressive). An additional mode of failure for hollow-tube lattices is the shell buckling (compressive). Dominance of either one of these types depends on the loading conditions and the geometry of the lattice members, namely their aspect ratio and cross-section [18].

In order to relate the effective strength of the octet-truss lattice to the local strength of its individual members, the effective macroscopic strains are transformed from the global coordinates to the local member coordinates as follows:

$\varepsilon^{(k)}=N_{i j}^{(k)} \bar{\varepsilon}_{i j}=n_{i}^{(k)} n_{j}^{(k)} \bar{\varepsilon}_{i j}$

where $\varepsilon^{(k)}$ is the axial strain in the $k^{t h}$ member, $N_{i j}^{(k)}$ is a linear transformation operator that can be reduced to the product of the $k^{t h}$ member's direction cosines $n_{i}^{(k)}$ and $n_{j}^{(k)}$, and $\bar{\varepsilon}_{i j}$ is the macroscopic strain, which can be related to a general stress tensor $\left[\bar{\sigma}^{\prime \prime}\right]$ applied through a general direction defined by the angles $\varphi$ and $\alpha$ as shown in Fig. 1 through the following relation:

$$
\left\{\bar{\varepsilon}_{i j}\right\}=[S]\left[T_{1}\right]\left[T_{3}\right]\left\{\bar{\sigma}^{\prime \prime}\right\}
$$

where $[S]$ is the macroscopic compliance matrix, $\left[T_{1}\right]$ and $\left[T_{3}\right]$ are the first and second strain transformation matrices respectively. The reader is referred to [19], [20] for details on the coordinate transformations. By considering the tetrahedron substructure selected as the structural basis in the continuumbased modelling of the octet-truss, the stress in its six members can be expressed as follows:

$\sigma^{(k)}=E_{s} n_{i}^{(k)} n_{j}^{(k)}[S]\left[T_{1}\right]\left[T_{3}\right]\left\{\bar{\sigma}^{\prime \prime}\right\}$

where $i=1,2,3, j=1,2,3$, and $k=1,2 . .6$.

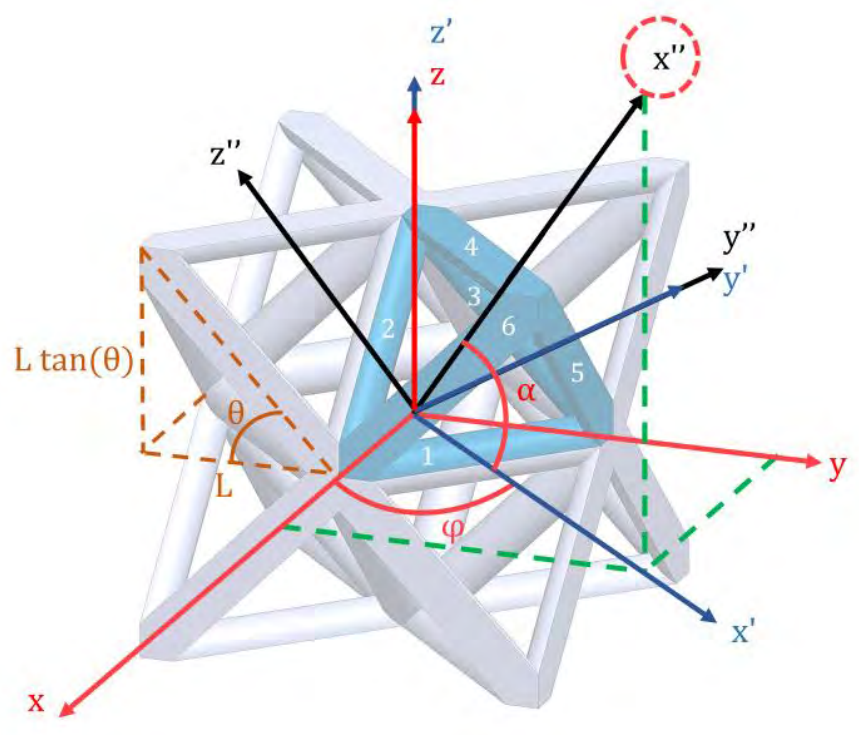

Figure 1. The octet-truss unit cell with the tetrahedron substructure geometry (shown in blue) and the transformation coordinate systems (numbers shown in white identify the six tetrahedron truss members).

\section{COLlaPSE SuRfaces}

The collapse surfaces of the octet-truss lattice due to plastic yielding and elastic buckling are calculated for two combinations of loading, namely $\left(\bar{\sigma}_{x z}, \bar{\sigma}_{z z}\right)$ and $\left(\bar{\sigma}_{x x}, \bar{\sigma}_{y y}\right)$ for different lattice angles. The tetrahedron substructure is used in the analysis in both cases, along with the pin-jointed assumption employed in the continuum-based modelling of the octet-truss. The collapse surface for each combination of loading can be 
categorized into a number of modes depending on the governing collapse equation and which members would reach the stress limit (i.e. buckling or yielding) with reference to Fig. 1. Unlike the analytical methods used by Deshpande et. al (2001) [4], we utilized a graphical method to plot the collapse surfaces. Collapse lines are plotted according to the collapse criteria of each of the six tetrahedron members, the inner-most area is then isolated, and the surrounding collapse lines are used to plot the collapse surface. Hence, fundamentally each of the following governing equation is the collapse criteria of one (or more) of the six tetrahedron members.

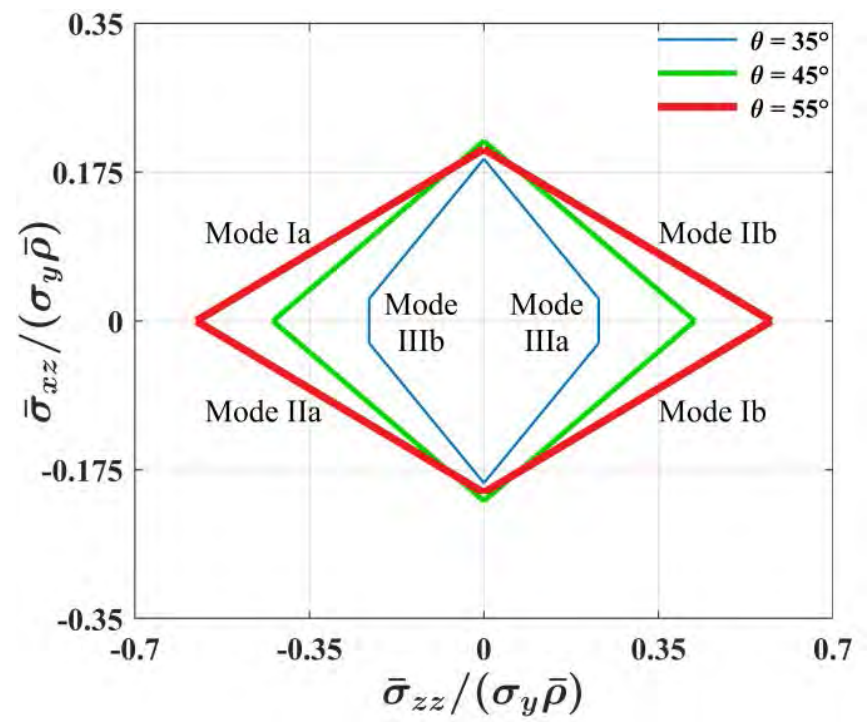

(a)

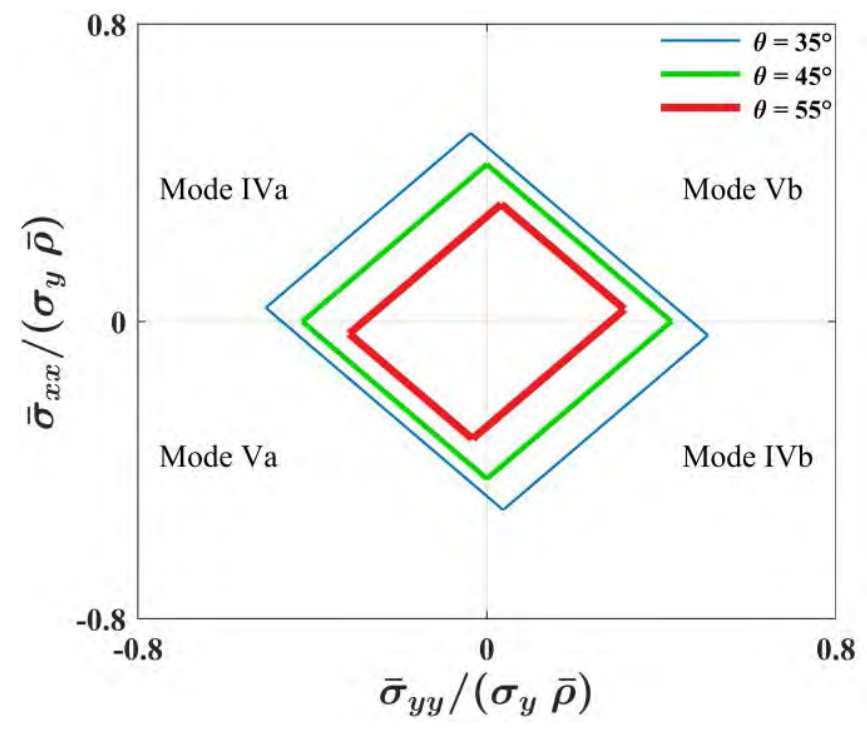

(b)

Figure 2. Collapse surface of the octet-truss due to plastic yielding in $\left(\bar{\sigma}_{x z}, \bar{\sigma}_{z z}\right)$ space (top) and $\left(\bar{\sigma}_{x x}, \bar{\sigma}_{y y}\right)$ space (bottom) in specific strength formulation at an aspect ratio of $r / L=0.14$.

\section{A. $\left(\bar{\sigma}_{x z}, \bar{\sigma}_{z z}\right)$ space due to plastic yielding}

The collapse surface under the applied loads $\left(\bar{\sigma}_{x z}, \bar{\sigma}_{z z}\right)$ for three different lattice angles is shown in Fig. 2a using the specific formulation $\bar{\sigma} /\left(\sigma_{\mathrm{y}} \bar{\rho}\right)$.

In Modes $I a$ and $I b$, member no. 2 has reached the yield stress $\sigma_{y}$ (under compression in Mode Ia and tension in Mode $I b)$. The governing equations are as follows:

Mode Ia: $\quad \frac{\bar{\sigma}_{x z}}{\sigma_{y}}=\frac{\bar{\sigma}_{z z}}{2 \tan (\theta) \sigma_{y}}+\cos (\theta) \frac{A_{c}}{L^{2}}$

Mode $I b: \quad \frac{\bar{\sigma}_{x z}}{\sigma_{y}}=\frac{\bar{\sigma}_{z z}}{2 \tan (\theta) \sigma_{y}}-\cos (\theta) \frac{A_{c}}{L^{2}}$

As for Modes $I I a$ and $I I b$, member no. 5 has reached the yield stress $\sigma_{y}$ (under compression in Mode IIa and tension in Mode $I I b$ ). The governing equations are as follows:

Mode IIa: $\quad \frac{\bar{\sigma}_{x z}}{\sigma_{y}}=\frac{-\bar{\sigma}_{z z}}{2 \tan (\theta) \sigma_{y}}-\cos (\theta) \frac{A_{c}}{L^{2}}$

Mode $I I b: \quad \frac{\bar{\sigma}_{x z}}{\sigma_{y}}=\frac{-\bar{\sigma}_{z z}}{2 \tan (\theta) \sigma_{y}}+\cos (\theta) \frac{A_{c}}{L^{2}}$

For lattice angles $\theta<45^{\circ}$, there exists two additional Modes, III $a$ and $I I I b$, where members no. $1 \& 4$ have reached the yield stress $\sigma_{y}$ (under compression in Mode IIIa and tension in Mode $I I I b$ ). The governing equations are as follows:

Mode IIIa: $\frac{\bar{\sigma}_{z z}}{\sigma_{y}}=+\sqrt{2} \tan (\theta) \frac{A_{c}}{L^{2}}$

Mode $I I I b: \quad \frac{\bar{\sigma}_{z z}}{\sigma_{y}}=-\sqrt{2} \tan (\theta) \frac{A_{c}}{L^{2}}$

\section{B. $\left(\bar{\sigma}_{x x}, \bar{\sigma}_{y y}\right)$ space due to plastic yielding}

The collapse surface under the applied loads $\left(\bar{\sigma}_{x x}, \bar{\sigma}_{y y}\right)$ for three different lattice angles is shown in Fig. $2 \mathrm{~b}$ using the specific formulation $\bar{\sigma} /\left(\sigma_{y} \bar{\rho}\right)$.

In Modes IVa and IVb, members no. 2, 3,5 \& 6 have reached the yield stress $\sigma_{y}$ (albeit $2 \& 5$ are under tension and $3 \& 6$ are under compression in Mode $I V a$ and the opposite in Mode $I V b$ ). The governing equations are as follows:

Mode IVa: $\quad \frac{\bar{\sigma}_{x x}}{\sigma_{y}}=\frac{\bar{\sigma}_{y y}}{\sigma_{y}}-\frac{2 \cos (\theta) A_{c}}{\tan (\theta) L^{2}}$

Mode $I V b: \quad \frac{\bar{\sigma}_{x x}}{\sigma_{y}}=\frac{\bar{\sigma}_{y y}}{\sigma_{y}}+\frac{2 \cos (\theta) A_{c}}{\tan (\theta) L^{2}}$

As for Modes $V a$ and $V b$, members no. $1 \& 4$ have reached the yield stress $\sigma_{y}$ (under compression in Mode $V a$ and under tension in Mode $V b$ ). The governing equations are as follows: 
Mode $V a: \quad \frac{\bar{\sigma}_{x x}}{\sigma_{y}}=\frac{-\bar{\sigma}_{y y}}{\sigma_{y}}-\frac{\sqrt{2} A_{c}}{\tan (\theta) L^{2}}$

Mode $V b: \quad \frac{\bar{\sigma}_{x x}}{\sigma_{y}}=\frac{-\bar{\sigma}_{y y}}{\sigma_{y}}+\frac{\sqrt{2} A_{c}}{\tan (\theta) L^{2}}$

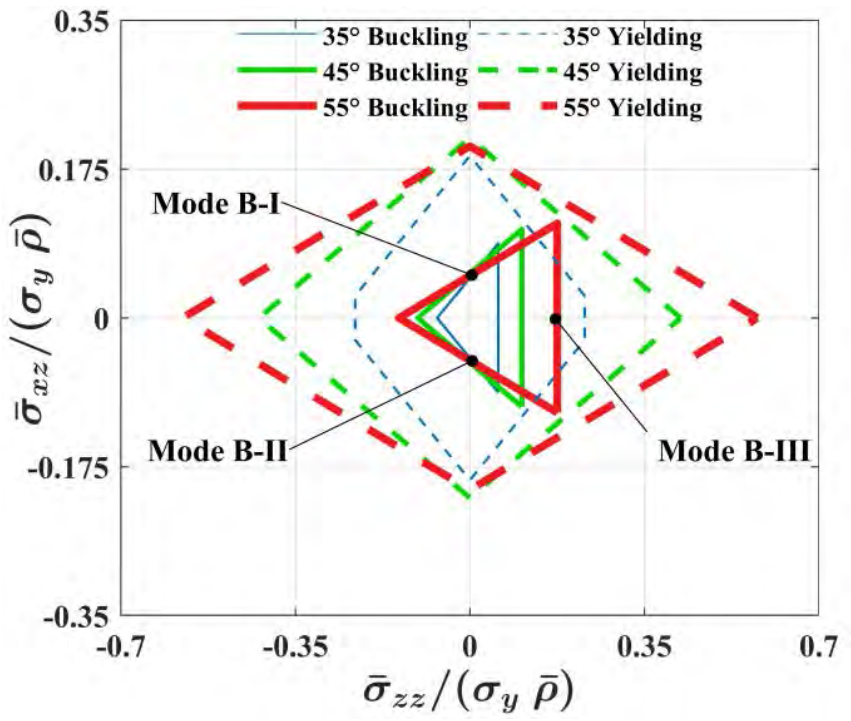

(a)

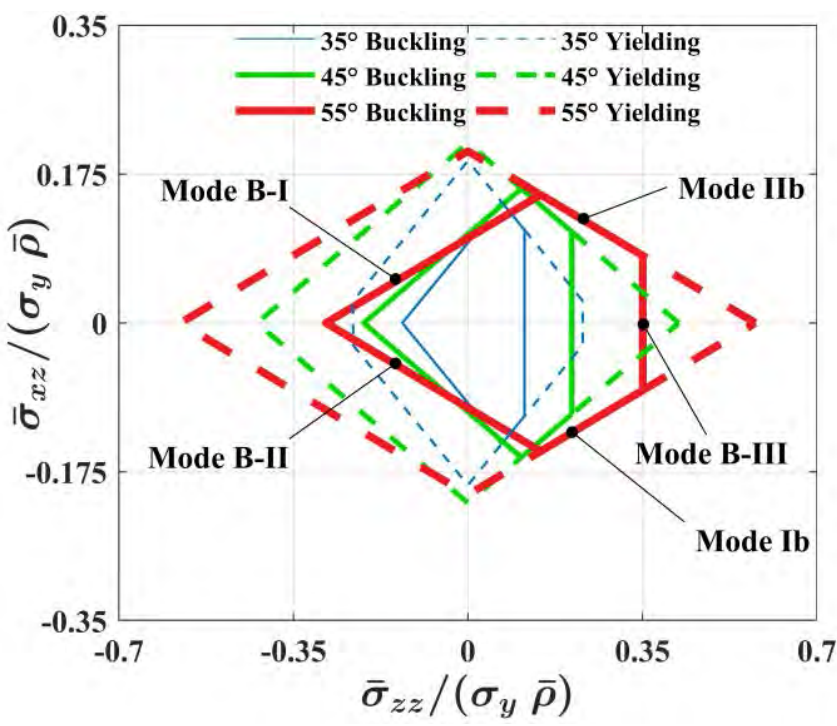

(b)

Figure 3. Collapse surface of the octet-truss due to elastic buckling in $\left(\bar{\sigma}_{x z}, \bar{\sigma}_{z z}\right)$ space at an aspect ratio of $r / L=0.14$ and a yield strain of $\varepsilon_{y}=0.1$ (top) and $\varepsilon_{y}=0.05$ (bottom).

\section{C. $\left(\bar{\sigma}_{x z}, \bar{\sigma}_{z z}\right)$ space due to elastic buckling}

The collapse surface under the applied loads $\left(\bar{\sigma}_{x z}, \bar{\sigma}_{z z}\right)$ for three different lattice angles is shown in Fig. 3 using the specific formulation $\bar{\sigma} /\left(\sigma_{\mathrm{y}} \bar{\rho}\right)$. A yield strain has to be used in order to show both elastic buckling and plastic yielding collapse lines in the same plot. Yield strains of $\varepsilon_{y}=0.1$ and 0.05 are utilized in Fig. $3 \mathrm{a}$ and $3 \mathrm{~b}$ respectively. It can be seen that the yield strain value has no effect on the yielding collapse lines.

In Mode $B-I$, member no. 2 has reached its elastic buckling limit. As for Mode $B-I I$, member no. 5 has reached its elastic buckling limit. In Mode $B-I I I$, members no. $1 \& 4$ have reached their elastic buckling limit. The governing equations are as follows:

Mode $B-I: \quad \frac{\bar{\sigma}_{x z}}{\sigma_{y}}=\frac{+\bar{\sigma}_{z z}}{2 \sigma_{y} \tan (\theta)}+\frac{\pi A_{c}^{2} \cos (\theta)}{8 \varepsilon_{y} L^{4}}$

Mode $B-I I: \quad \frac{\bar{\sigma}_{x z}}{\sigma_{y}}=\frac{-\bar{\sigma}_{z z}}{2 \sigma_{y} \tan (\theta)}-\frac{\pi A_{c}^{2} \cos (\theta)}{8 \varepsilon_{y} L^{4}}$

Mode $B-I I I: \frac{\bar{\sigma}_{z z}}{\sigma_{y}}=\frac{\pi A_{c}^{2} \tan (\theta)}{4 \sqrt{2} \varepsilon_{y} L^{4}}$

\section{D. $\left(\bar{\sigma}_{x x}, \bar{\sigma}_{y y}\right)$ space due to elastic buckling}

The collapse surface under the applied loads $\left(\bar{\sigma}_{x x}, \bar{\sigma}_{y y}\right)$ for three different lattice angles is shown in Fig. 4 using the specific formulation $\bar{\sigma} /\left(\sigma_{y} \bar{\rho}\right)$. Similar to Fig. 3, a yield strain of $\varepsilon_{y}=$ 0.1 and 0.05 are utilized in Fig. $4 \mathrm{a}$ and $4 \mathrm{~b}$ respectively.

In Mode $B-I V$, members no. $2 \& 5$ have reached their elastic buckling limit. As for Mode $B-V$, member no. $3 \& 6$ have reached their elastic buckling limit. In Mode $B-V I$, members no. $1 \& 4$ have reached their elastic buckling limit. The governing equations are as follows:

Mode $B-I V: \frac{\bar{\sigma}_{x x}}{\sigma_{y}}=\frac{+\bar{\sigma}_{y y}}{\sigma_{y}}-\frac{\pi A_{c}^{2} \cos (\theta)}{4 L^{4} \varepsilon_{y} \tan (\theta)}$

Mode $B-V: \quad \frac{\bar{\sigma}_{x x}}{\sigma_{y}}=\frac{+\bar{\sigma}_{y y}}{\sigma_{y}}+\frac{\pi A_{c}^{2} \cos (\theta)}{4 L^{4} \varepsilon_{y} \tan (\theta)}$

Mode $B-V I: \quad \frac{\bar{\sigma}_{x x}}{\sigma_{y}}=\frac{-\bar{\sigma}_{y y}}{\sigma_{y}}-\frac{\pi A_{c}^{2}}{4 \sqrt{2} L^{4} \varepsilon_{y} \tan (\theta)}$

Upon comparison, the collapse surfaces at the lattice angle of $\theta=45^{\circ}$ under the two loading combinations of $\left(\bar{\sigma}_{x z}, \bar{\sigma}_{z z}\right)$ and $\left(\bar{\sigma}_{x x}, \bar{\sigma}_{y y}\right)$ for both plastic yielding and elastic buckling clearly agree with those developed by Deshpande et al. (2001) [4].

\section{CONCLUSIONS}

The purpose of the present research is to investigate the effect of the lattice angle on the effective strength of the octettruss lattice structure. A graphical method is utilized to plot the collapse surfaces for plastic yielding and plastic buckling for different loading conditions and different lattice angles. Generally, the $\left(\bar{\sigma}_{x z}, \bar{\sigma}_{z z}\right)$ loading space is preferable for lattice 
angles greater than $45^{\circ}$, while the $\left(\bar{\sigma}_{x x}, \bar{\sigma}_{y y}\right)$ loading space is preferable for lattice angles lower than $45^{\circ}$.

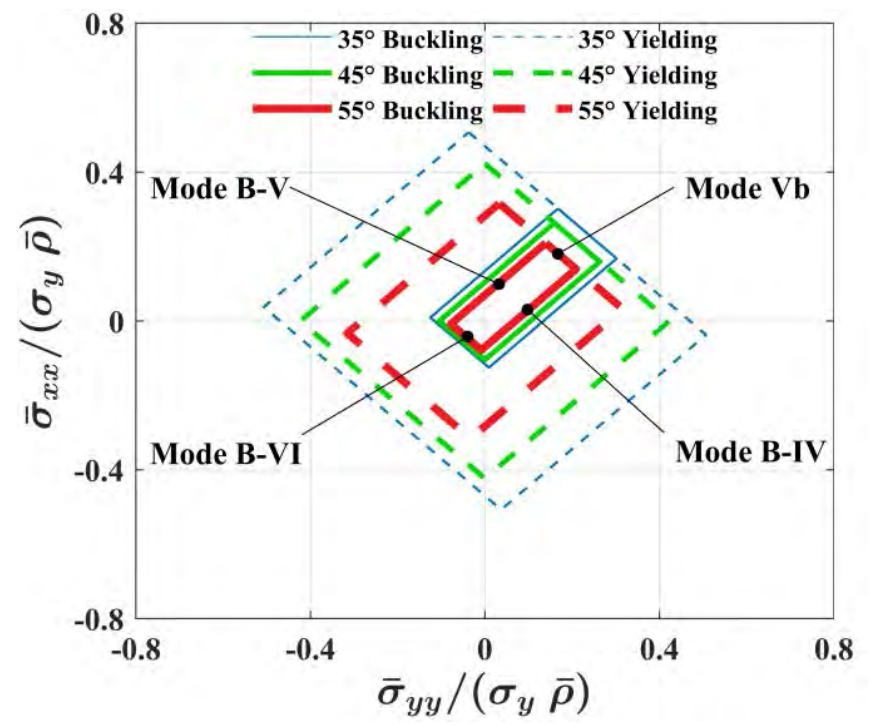

(a)

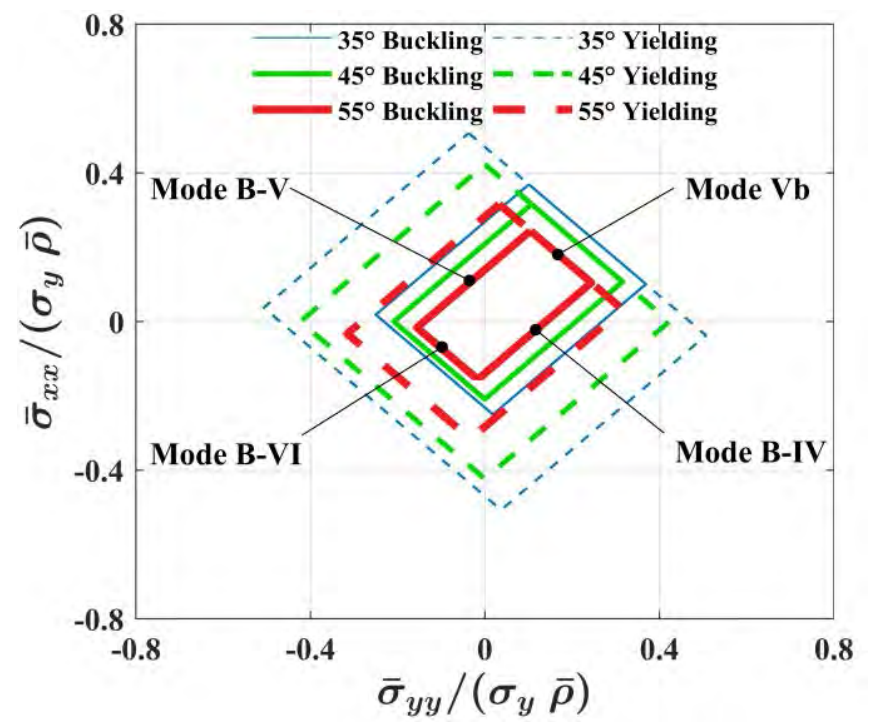

(b)

Figure 4. Collapse surface of the octet-truss due to elastic buckling in $\left(\bar{\sigma}_{x x}, \bar{\sigma}_{y y}\right)$ space at an aspect ratio of $r / L=0.14$ and a yield strain of $\varepsilon_{y}=$ 0.1 (top) and $\varepsilon_{y}=0.05$ (bottom).

\section{REFERENCES}

[1] X. Zheng et al., "Ultralight, ultrastiff mechanical metamaterials.," Science, vol. 344, no. 6190, pp. 1373-7, 2014. doi: 10.1126/science.1252291.

[2] R. Lakes, "Materials with structural hierarchy," Nature, vol. 361, no. 6412, pp. 511-515, Feb. 1993. doi: 10.1038/361511a0.

[3] L. J. Gibson and M. F. Ashby, Cellular solids: structure and properties, Second. Cambridge: Cambridge UP, 1997. doi:10.1017/CBO9781139878326.

[4] V. S. Deshpande, N. A. Fleck, and M. F. Ashby, "Effective properties of the octettruss lattice material," J. Mech. Phys. Solids, vol. 49, no. 8, pp. 1747-1769, Aug. 2001. doi: 10.1016/S0022-5096(01)00010-2.

[5] V. S. Deshpande, M. F. Ashby, and N. A. Fleck, "Foam topology: bending versus stretching dominated architectures," Acta Mater., vol. 49, no. 6, pp. 1035-1040, 2001. doi: 10.1016/S1359-6454(00)00379-7.

[6] N. A. Fleck, V. S. Deshpande, and M. F. Ashby, "Micro-architectured materials: past, present and future," Proc. R. Soc. A, vol. 466, no. 2121, pp. 2495-2516, Jun. 2010. doi: 10.1098/rspa.2010.0215.

[7] M. F. Ashby, T. Evans, N. Fleck, J. W. Hutchinson, H. N. G. Wadley, and L. J. Gibson, Metal foams: a design guide, First. Oxford: Butterworth-Heinemann, 2000.

[8] D. Jang, L. R. Meza, F. Greer, and J. R. Greer, "Fabrication and deformation of three-dimensional hollow ceramic nanostructures.," Nat. Mater., vol. 12, no. 10, pp. 893-898, Oct. 2013. doi: 10.1038/nmat3738.

[9] J. Wang, J. Lian, J. R. Greer, W. D. Nix, and K.-S. Kim, "Size effect in contact compression of nano- and microscale pyramid structures," Acta Mater., vol. 54, no. 15, pp. 3973-3982, Sept. 2006. doi: 10.1016/j.actamat.2006.04.030.

[10] T. A. Schaedler et al., "Ultralight metallic microlattices," Science, vol. 334, no. 6058, pp. 962-965, Nov. 2011. doi: 10.1126/science.1211649.

[11] L. R. Meza and J. R. Greer, "Mechanical characterization of hollow ceramic nanolattices," J. Mater. Sci., vol. 49, no. 6, pp. 2496-2508, Dec. 2013. doi: 10.1007/s10853-013-7945-x.

[12] J. Bauer, S. Hengsbach, I. Tesari, R. Schwaiger, and O. Kraft, "High-strength cellular ceramic composites with 3D microarchitecture.," Proc. Natl. Acad. Sci. U. S. A., vol. 111, no. 7, pp. 2453-8, Feb. 2014. doi: 10.1073/pnas.1315147111.

[13] L. C. Montemayor and J. R. Greer, "Mechanical response of hollow metallic nanolattices: combining structural and material size effects," J. Appl. Mech., vol. 82, no. 7, pp. 1-10, 2015. doi: 10.1115/1.4030361.

[14] M. M. Mikulas, Jr., H. G. Bush, and M. F. Card, "Structural stiffness, strength and dynamic characteristics of large tetrahedral space truss structures," NASA tech. memo, 1977.

[15] A. H. Nayfeh and M. S. Hefzy, "Continuum modeling of three-dimensional truss-like space structures," AIAA J., vol. 16, no. 8, pp. 779-787, Aug. 1978. doi: $10.2514 / 3.7581$.

[16] K. Heki, "On the effective rigidities of lattice plates," Recent Res. Struct. Mech., pp. 31-46, 1968.

[17] M. S. Lake, "Stiffness and strength tailoring in uniform space-filling truss structures," Nasa Tech. Pap. 3210, pp. 1-28, Apr. 1992.

[18] L. R. Meza, S. Das, and J. R. Greer, "Strong, lightweight, and recoverable threedimensional ceramic nanolattices," Science, vol. 345, no. 6202, pp. 1322-1326, Sep. 2014. doi: 10.1126/science. 1255908 .

[19] M. Abdelhamid and A. Czekanski, "On the effective properties of 3D metamaterials," Proc. ASME Int. Mech. Eng. Congr. Expo. 2016. doi: 10.1115/IMECE2016-67407.

[20] M. Abdelhamid and A. Czekanski, "Impact of the Lattice Angle on The Effective Properties of The Octet-truss Lattice Structure," J. Eng. Mater. Technol., vol. 140, no. 4, June 2018. doi: 10.1115/1.4040409. 


\section{New Experimental Setup Design for Applying Physical Blowing Agent in Rapid Rotational Foam Molding Technology}

\author{
Isha Raktim \\ Faculty of Engineering and Applied Science \\ University of Ontario Institute of Technology \\ Oshawa, Canada
}

\author{
Remon Pop-Iliev \\ Faculty of Engineering and Applied Science \\ University of Ontario Institute of Technology \\ Oshawa, Canada
}

\begin{abstract}
This paper presents a manufacturing process for extrusion assisted foam production with a physical blowing agent (PBA) for Rapid Rotational Foam Molding. A completely new experimental setup was designed to identify, conceive, establish, and develop, the necessary processing concepts and conditions for fabricating a new class of rotationally molded integral-skin cellular composite having low-density foamed core. The new design incorporates a helical static mixer that can operate at $31 \mathrm{MPa}$ in $200^{\circ} \mathrm{C}$ environment. Supercritical $\mathrm{CO}_{2}$ will be injected into the pressurized static mixer to thoroughly disassociate into the polymer melt to create a single-phase solution. The process temperature is maintained by PID controlled band heaters and an integral liquid cooling jacket. The system can facilitate rapid pressure drop as high as $30 \mathrm{MPa}$ to produce polyolefin foam.
\end{abstract}

Keywords; helical; static mixer; RRFM; PBA; Polyolefin; supercritical fluid; QFD; carbon dioxide

\section{INTRODUCTION}

Rotational molding technology has been in use for the last few decades to produce light weight structural foam composites with a distinct foamed core encapsulated by an unfoamed outer skin. This type of roto-molded articles are in high demand in the automotive, construction and marine industries for their enhanced strength-to-weight ratio $[1,2]$. However, the manufacturing process associated with rotational molding is lengthy and highly energy intensive. In recent years a newly patented rotational foam molding process, referred to as Rapid Rotational Foam Molding (RRFM) was developed to reduce the lengthy production time and to improve the overall quality of the foamed composites $[3,4]$.

The RRFM technology by design produces the outer skin independent from the foaming process. This novel processing concept takes advantage of an extruder to produce the polymer foam, which is directly injected inside the hot mold cavity containing the soft outer skin. The foamed core encapsulated by the outer skin is gradually cooled to room temperature before extracting the final part from the mold. Through greater control over the manufacturing process, RRFM technology has reduced the cycle time and energy consumption compared with traditional rotational foam molding process [3].

In the current art, RRFM process is open to further improvement. The technology can be modified in two areas, rotational molding process, or the foam extrusion process.
However, the foam extrusion process shows greater potential for improvement. Currently the polymer foam is created using chemical blowing agents (CBA). The foamable resin is mixed in with the CBA before introducing the mixture into the extruder. In the extruder the CBA activates to produce the polymer foam. An alternative approach to this process is to produce the foam using a physical blowing agent (PBA). Polymer foaming with a PBA for rotational foam molding has never been attempted. Plastic foam produced with PBA have higher cell density which can further improve the strength-to- weight ratio [5]. Therefore, it is the next logical step in development to replace CBA with PBA for processing polyolefin integral-skin composites with RRFM.

The principal objective of the presently presented research is to design and develop an experimental setup that would enable testing and verification of novel processing concepts that would improve further melt extrusion-assisted rotational foam molding operations. In this context, the existing lab-scale rapid rotational foam molding experimental setup will have to be modified to introduce a physical blowing agent.

\section{CONCEPT GENERATION}

The RRFM experimental setup consists of a rotational arm assembly; mold translation system; convection oven and the extruder assembly (Fig. 1). The new modification will be applied to the extruder assembly to introduce PBA into the polymer melt. In the subsequent experiments, super critical carbon dioxide will be used as the blowing agent. $\mathrm{CO}_{2}$ is ideal for foaming application for having higher solubility in polymer melt compared to nitrogen gas. In this context, the foam produced from carbon dioxide is of a higher quality [6].

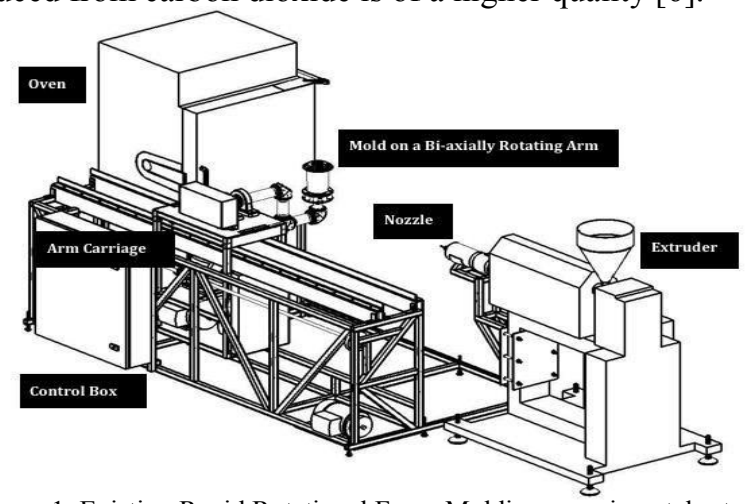

Figure 1. Existing Rapid Rotational Foam Molding experimental setup. 


\section{A. Design Selection Methodology}

It is important to identify the engineering specifications that are necessary for a good design. In the current state, the experimental setup is not equipped for PBA introduction. The goal is to design a system that can work efficiently with the existing extruder and produce foamed articles that are of equal or of a higher quality than RRFM foam composites. To identify the design requirements, a quality function deployment (QFD) tool was used. It translates general qualitative (non-specific) customer needs into engineering specifications for a new system design. Utilizing the house of quality (QFD tool), the most important engineering specifications were identified from the prospective of a customer. In this QFD assessment the primary customer is identified as a researcher in polymer processing industry. The strongest relationship between a customer requirement and the design parameters are identified in table I.

The identified design parameters are implemented in the concept generation phase. A decision matrix is used to select a system design from one of these concepts. The criteria for selection is based on the overall operation of each concept, rather than on each individual component. The importance of each criteria is assigned a value from 1 to 10 , where 10 is the most important criteria. One of the concept is selected as the datum to compare against the alternatives. A positive or a negative symbol is given on how well a criterion is satisfied under each concept against the datum (table II). The weighted total is tabulated for each concept by multiplying the symbol to each criteria rating. The concept with the highest weighted total is selected for the new system design.

\section{B. Concept Selection}

Each concept is represented in a simplified block diagram where all major components are identified in Fig. 2. The functional requirement for every concept is to achieve high degree of mixing between the polymer melt and supercritical $\mathrm{CO}_{2}$. It is through the mixing process where $\mathrm{CO}_{2}$ gas is dissolved into the polymer melt to produce a single-phase solution [7]. This process is controlled through manipulating the chamber pressure, temperature, and the flow rate of both gas and molten polymer. The dissolved $\mathrm{CO}_{2}$ gas within the molten polymer will produce the foam after experiencing a rapid pressure drop at the die exit. In order to understand the necessary processing condition for PBA application in RRFM technology, each concept presents a different foaming strategy for feasibility analysis.

a) Concept 1: In this experimental setup, the molten polymer is fed into a static mixer from the extruder. The melt pressure and the polymer flowrate are controlled by the screw RPM. The $\mathrm{CO}_{2}$ gas is injected into the static mixer chamber to begin the mixing process. The gas laden polymer melt moves down the static mixer barrel toward stationary mixing blades. These blades provide the mixing action through splitting the polymer flow and then recombining it to disassociate the $\mathrm{CO}_{2}$ gas into the polymer matrix. The temperature of the mixture is controlled by band heaters and an oil-based cooling jacket placed on the exterior shell. The final polymer solution is extruded out of the die where rapid drop in pressure produces the foam.

b) Concept 2: In this configuration, two extruders are placed in tandem seen in Fig. 2. The first extruder plasticizes the polymer resin and the second extruder is used to mix the $\mathrm{CO}_{2}$ gas. It is through the rotation of the second screw where vigorous shear mixing can be achieved [7]. The polymer/gas solution pressure and flowrate are controlled from the second extruder. However, the feedrate into the second extruder is controlled by the first one. Temperature of the gas laden polymer melt is maintained by PID controlled band heaters on the extruder barrel.

c) Concept 3: The final proposal is a modified version of concept 1 . In this setup, a gear pump is used to compensate for pressure loss incurred in the static mixer because of frictional losses at the blade surface. The pressure and flow rate can be controlled by both the gear pump and the extruder screw RPM. In the cooling system, water is used as the cooling fluid rather than oil.

The weighted total from the decision matrix is negative for concepts $2 \& 3$. Therefore, concept 1 will be used as the experimental setup for PBA application. Although concept 1 has lower degree of process control, it is more attractive for having fewer design and fabrication complexity than the alternatives. In regards to safety, concept 2 is better for having an integrated heating element housed within the extruder and thereby lowering the risk of contact with the operator. However this setup requires more lab space which makes it difficult for

TABLE I. QUALity Function Deployment ANALysis

\begin{tabular}{|c|c|c|}
\hline \multirow{4}{*}{ Customer } & \multicolumn{2}{|c|}{$\begin{array}{c}\text { Design Parameters from House of Quality } \\
\text { Assessment }\end{array}$} \\
\cline { 2 - 3 } & $\begin{array}{c}\text { Customer } \\
\text { Requirements }\end{array}$ & $\begin{array}{c}\text { Engineering } \\
\text { Specifications }\end{array}$ \\
\hline \multirow{7}{*}{ Researcher } & $\begin{array}{c}\text { Homogenize } \\
\text { Mixing }\end{array}$ & $\begin{array}{c}\text { Gas solubility in polymer } \\
\text { melt; Heat Input; Screw } \\
\text { RPM; Blade geometry \& } \\
\text { length }\end{array}$ \\
\cline { 2 - 3 } & $\begin{array}{c}\text { Microcellular } \\
\text { bubble formation }\end{array}$ & $\begin{array}{c}\text { Screw RPM; Gas injection } \\
\text { pressure; Pressure drop }\end{array}$ \\
\cline { 2 - 3 } & Process Control & $\begin{array}{c}\text { Heat Input; Gas injection } \\
\text { pressure; Screw RPM; } \\
\text { Cooling fluid flowrate }\end{array}$ \\
\hline
\end{tabular}

TABLE II. CONCEPT FeAsIbILITy OF PBA APPLICATION

\begin{tabular}{|c|c|c|c|c|}
\hline \multirow[b]{2}{*}{ Criteria } & \multirow[b]{2}{*}{ Importance } & & \multicolumn{2}{|c|}{ Alternative } \\
\hline & & $\begin{array}{c}\text { Concept } \\
1\end{array}$ & $\begin{array}{c}\text { Concept } \\
2\end{array}$ & $\begin{array}{c}\text { Concept } \\
3\end{array}$ \\
\hline $\begin{array}{c}\text { Design } \\
\text { Complexity }\end{array}$ & 10 & \multirow{5}{*}{ Datum } & - & - \\
\hline $\begin{array}{c}\text { Process } \\
\text { Controllability }\end{array}$ & 9 & & + & + \\
\hline $\begin{array}{l}\text { Space Required } \\
\text { for Components }\end{array}$ & 7 & & - & - \\
\hline Safety & 8 & & + & - \\
\hline $\begin{array}{l}\text { Fabrication } \\
\text { Complexity }\end{array}$ & 9 & & - & - \\
\hline \multicolumn{2}{|c|}{ Weighted Total } & 0 & -9 & -25 \\
\hline
\end{tabular}


the operator to move around and use other equipment. Concept 2 is the least desirable in term of safety for having a water cooling system, where steam might be produced. Which will pose as a hazard for the operator by leaking out through small clearances in the pipefittings and connecting junctions. Steam has to be safely vented to stop pipe rupture. Also, when water turns to steam a liquid pump cannot pump it. Therefore, a cooling fluid with a high boiling point must be selected to prevent this problem. An oil-based cooling system will be implemented to provide all the cooling needs for the foam extrusion process.

\section{FINAL PBA EXPERIMENTAL SETUP DESIGN}

The newly modified lab-scale custom-build experimental setup must accomplish high degree of mixing between the $\mathrm{CO}_{2}$ gas and the polyolefin melt. A single-phase polymer-gas solution with homogeneity greater than $80 \%$ is desired. The pressure drop across the length of the mixing apparatus must not exceed the burst pressure of the extruder. The maximum allowable pressure drop should be limited 7 to $10 \mathrm{MPa}$.

\section{A. Design Guidelines}

The physical blowing agent $\left(\mathrm{CO}_{2}\right)$ will be injected directly into the static mixer at pressures between 20-30 MPa to mix with the melted polyolefin resin. For instance, $\mathrm{CO}_{2}$ is soluble in molten polyethylene at high pressure and it can act as a plasticizer. Static mixers have to be attached after the extruder to completely dissolve the gas solution into the polymer melt. The blade design of the static mixer must generate shear field to enhance gas disassociation. Rapid pressure drop will be initiated at the die exit to cause phase separation between the gas and polymer solution. This will cause the gas to bubble out of the solution creating polymer foam. The foam will be injected into the open mold to fill the mold's cavity on top of the hot skin as the mold is rotated in a single axis.

An optimal cooling rate must be established to ensure the generation of microcells and even cell distribution. A cooling system on the static mixer wall will be used to cool the mixture temperature near the die inlet. This will lower the gas solubility within the polymer matrix and this will initiate phase separation and cell nucleation.

\section{B. Static Mixer Chamber Design}

Static mixers provide advantages and disadvantages in polymer foam processing. Static mixers have static blades that replicate shear fields necessary for high degree of mixing. The geometry of the mixing elements reorients the flow in radial direction to enhance the mixing. Through this mixing process radial temperature gradient is eliminated, and wall-creep material is incorporated into the bulk polymer flow. A major disadvantage is the pressure loss across the length of the static mixer. The static mixer type and size are crucial to determine if the extruder can provide enough force to push the material through the mixing elements. Static mixers are installed in front of the extruder. Many types of arrangements are possible which depends on the specific application. Some industrial applications include, extrusion blow molding, foam sheet production, coextrusion and for blow film [8].
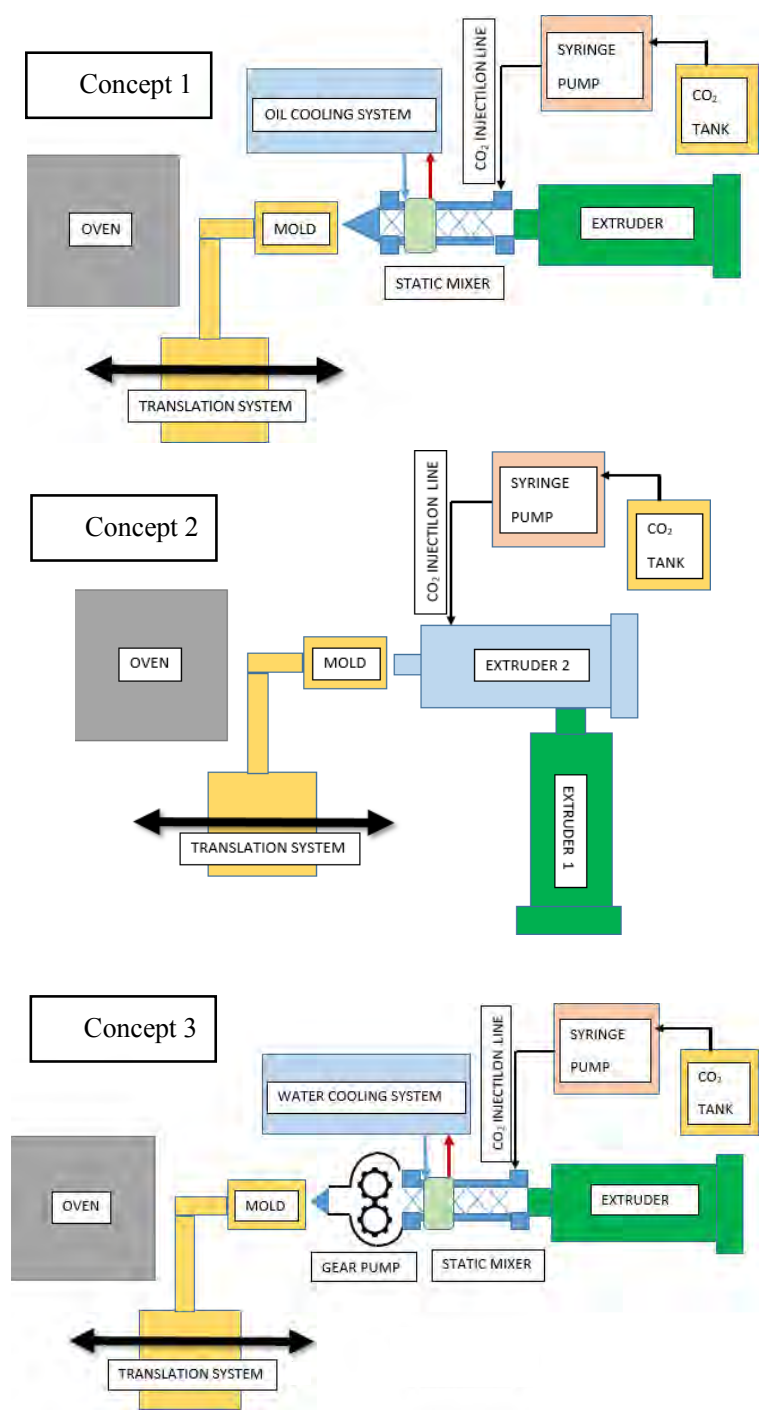

Figure 2. Possible Experimental setup for PBA Application.

The main purpose of the static mixer is to homogenize the melt upstream from the die exit. This is achieved through splitting the flow toward the radial direction and later rearranging the two split streams. The mixing elements are offset by $90^{\circ}$ to ensure uniform distribution. The pressure loss across the length of the static mixer is dependent on the polymer-melt viscosity, Reynolds number, mixing element geometry, and process temperature and polymer grade [8].

$$
\begin{gathered}
\Delta P=\left(\frac{4}{\pi}\right) \operatorname{NeRe}_{D}\left(\frac{\dot{V}}{D^{3}}\right) \eta\left(\frac{n_{M E}}{2}\right) . \\
\eta=f\left(\dot{\gamma}, T, \text { polymer }(\text { type }, \text { grade }), p_{o p} .\right. \\
\dot{\gamma}=f\left(\dot{V}, D_{M E}, \text { geometry }\right) .
\end{gathered}
$$

The pressure loss inside a static mixer is obtained from "(1)", where "NeRe $e_{D}$ " is the Reynolds number and " $\dot{V}$ " is the volumetric flow rate and " $n_{M E}$ " is the number of mixing elements and " $\eta$ " is the melt viscosity. The melt viscosity is obtained from "(2)", which is dependent on operating pressure, temperature, polymer type and shear rate " $\dot{\gamma}$ ". The shear rate "(3)" is a function of flow rate, and the mixing blade geometry. 
In relation to a grid mesh mixer, the Reynolds number can be between $1200 \& 2000$. However, inside a helical type mixer the Reynolds number is between $200 \& 400$.

Engineering Equation Solver (EES) software has been used to perform parametric analysis on different types of static mixers. A range of viscosity values was used to calculate the pressure drop across the length of the static mixer. It is important to note here that the gas/polymer solution will have a lower viscosity than a pure polymer in the actual experiment. The pressure loss is highest for GX-type static mixer sold by Stamixco.inc shown in Fig. 3. A higher degree of mixing is possible with the GX-type static mixer compared to a helical static mixer. The minimum length of the static mixer cannot be less than $20 \mathrm{~cm}$. As multiple ports need to be drilled into the wall of the static mixer chamber, shorter tubes are more likely to warp during the drilling process. The extruder's maximum pressure limit is $45 \mathrm{MPa}$ and the maximum pressure loss for a $16.5 \mathrm{~cm}$ helical static mixer is $5 \mathrm{MPa}$ at 3000 Pa.s viscosity (Fig. 3). Hence, the maximum possible pressure loss for a helical static mixer is within acceptable range. The acceptable pressure loss range for the GX type mixers is at viscosity levels below 1000 $\mathrm{Pa}-\mathrm{s}$. Helical static mixers can be safely operated at higher polymer viscosity composites without rupturing the rupture disc on the extruder. Therefore, the static mixer will be $16.5 \mathrm{~cm}$ long with twelve helical mixing elements [8].

Stamixco manufactured the static mixer chamber. It was designed to meet all the engineering requirements for PBAbased extrusion foaming (Fig. 4). The static mixer assembly comprises of three sections: gas injection; homogenization; foam extrusion (Fig. 5). The static mixer pipe thickness was determined according to ASME section 8 division II pressure vessel code. The maximum operating temperature is $200^{\circ} \mathrm{C}$, which serves well for polypropylene (PP) foam production. The maximum allowable pressure according to Stamixco's analysis is $31 \mathrm{MPa}$. The pipe is made of $17-4 \mathrm{PH}$ stainless steel with ID = $1.9 \mathrm{~cm}$. The static mixer is $13.5 \mathrm{~cm}$ long with 12 helical mixing blades.

a) Gas Injection Section: In the aft section of the static mixing chamber, supercritical $\mathrm{CO}_{2}$ gas will be injected into the polymer melt through an injection port. The port was designed for a 1/8 NPT check valve. The spring activated check valve will be screwed into the injection port to prevent back flow. This will stop molten polymer from getting into the injection tube and "freezing". The check valve cracking pressure will be set higher than the maximum operating pressure of the extruder to eliminate the possibility for back flow into the injection pipe.

b) Homogenizing Section: In this section of the static mixer chamber, twelve helical blades are used to dissolve the $\mathrm{CO}_{2}$ gas into the polymer melt. A single-phase polymer-gas solution is necessary for obtaining fine-celled microcellular foam. Helical blades are a popular design with lower pressure drop compared to more complex shaped mixing blades. The helical blades are manufactured by twisting metal strips or by machining a single piece of solid rod. Advanced machining techniques has allowed for smaller diameter helical blades, which are ideal for high-pressure applications. Each blade is $1.65 \mathrm{~cm}$ wide and $2.86 \mathrm{~cm}$ long. The blades are welded together in a series, and each blade is oriented at a $90^{\circ}$ offset from the blades on either side. This offset splits the polymer flow toward the radial direction increasing the degree of mixing. The contours of the helical blades generate shear fields to dissolve the gas bubbles into the polymer matrix.

The temperature within the barrel of the static mixer must be maintained above the melting temperature of the polymer. In real test conditions, the temperature within the barrel of the mixer will be kept at the $\mathrm{CO}_{2}$ saturation point for a given mixing pressure. This will ensure that the maximum possible amount of $\mathrm{CO}_{2}$ can be dissolved into the polymer. Higher concentration of $\mathrm{CO}_{2}$ dissolved within the mixture creates higher cell density in the final foamed product. Three Mica band heaters with an output of 200 watts can maintain the surface temperature of the static mixture above $400^{\circ} \mathrm{C}$. Band hater output will be controlled using a $J$ type thermocouple in a closed-loop feedback. One inch thick insulation will be placed between the band heaters to maintain the correct temperature profile. The insulating material is made from silica aerogel with thermal conductivity value of $35(\mathrm{~mW} / \mathrm{m} . \mathrm{K})$ at $300^{\circ} \mathrm{C}$.

c) Cooling Section: The cooling shell on the static mixer is an integral part of the overall cooling system design. The inlet and outlet ports are positioned in a manner to make the cooling shell a parallel flow heat exchanger shown in (Fig. 6). Channels on the static mixer wall (ribbed section) increase the overall surface area to promote high rate of heat transfer. The cooling fluid flows from the inlet port and gradually flows through each of the channel before exiting through the outlet port. It is not necessary for the cooling fluid to be pressurized because the flash point of engine oil is over $200^{\circ} \mathrm{C}$. The operating temperature of the oil will be well below this point minimizing the presence of any combustible vapor.

The polymer melt cooler consists of an air-cooled radiator, hydraulic oil pump, throttling valve, 1/4" hydraulic tubing, and two 12 V DC power supply (Fig. 6). The two power supply unit provide the same voltage but different current according to the maximum current rating of the radiator fan and the oil pump. The pump can supply cooling oil at a maximum rate of $1.5 \mathrm{l} / \mathrm{min}$ to the cooling shell. The flow rate can be reduced with the aid of a throttling valve if needed. The air cooled radiator can cool the return fluid from the static mixer as high as $100^{\circ} \mathrm{C}$ using a fan capacity of 266 CFM. Two thermocouples will be used to

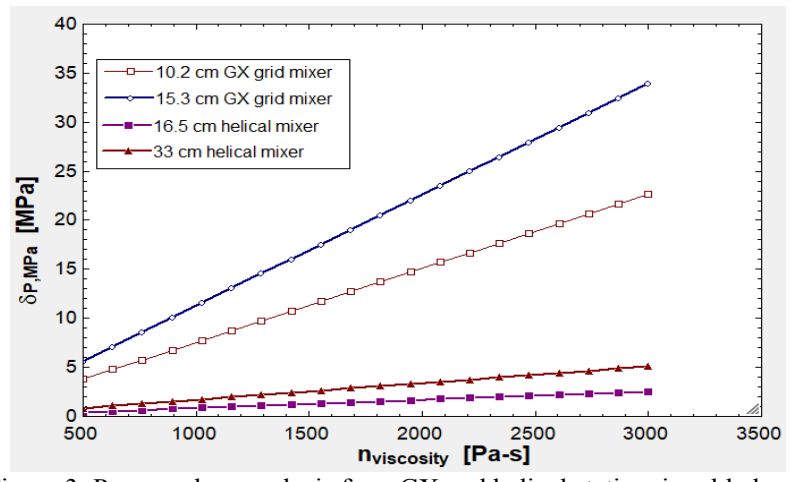

Figure 3. Pressure loss analysis for a GX and helical static mixer blades. 


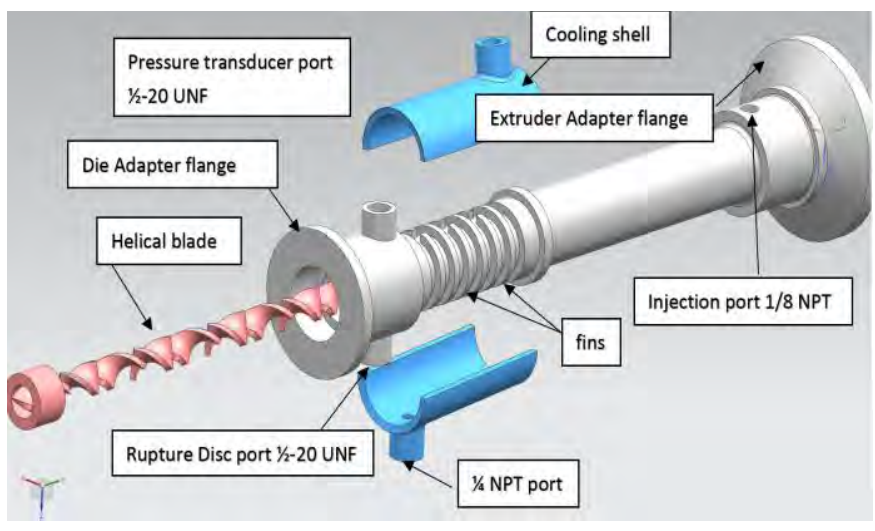

Figure 4. Exploded view of the static mixer chamber assembly.

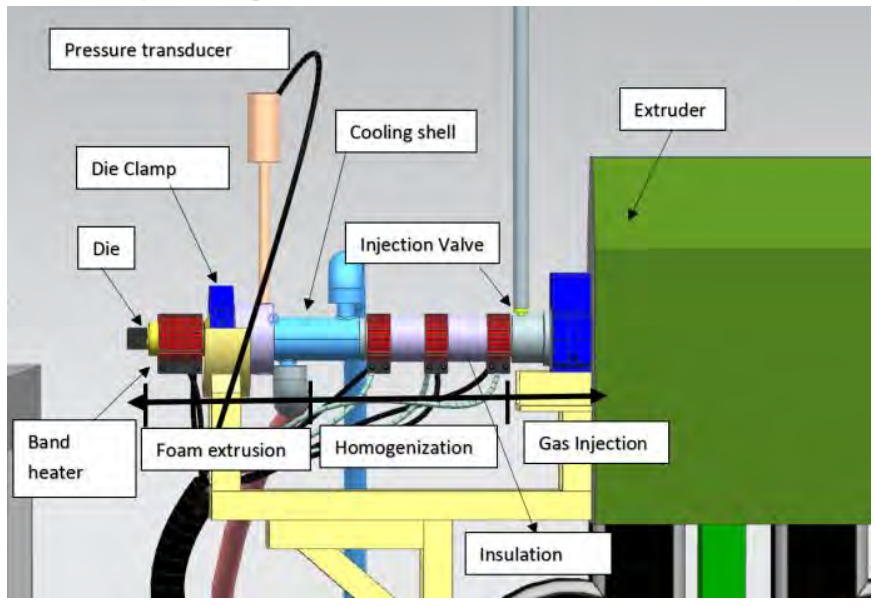

Figure 5. Full assembly arrangement of the static mixer chamber.

monitor the oil temperature at the inlet and the outlet ports. If the return fluid temperature is above $110^{\circ} \mathrm{C}$, a safety switch will be triggered to shut down the pump. A manual emergency switch has been integrated into the system to shutdown operation if a leak is present. The whole system can be turned on and off using the green switch during normal operation (Fig. $6)$.

a) Extrusion Section: The single-phase solution will experience rapid pressure drop as it flows out of the die nozzle opening. This will initiate phase separation within the mixture and the polymer melt will start to foam and fill the mold cavity. The interior contours of the die channel the gas laden polymer melt toward the opening seen in Fig. 7. The die opening is $3 \mathrm{~mm}$ and the length is $100 \mathrm{~mm}$. It has been designed to deliver the polymer foam inside a cylindrical mold. The overall pressure drop inside the die was determined from ANSYS Polyflow software package for polyethylene melt. It was determined from the analysis that with a maximum melt flow rate of $5 \mathrm{~kg} / \mathrm{h}$ and $1000 \mathrm{~Pa}$.s die inlet viscosity, the maximum pressure drop from the inlet to the outlet is $30 \mathrm{MPa}$ as seen in Fig. 8.

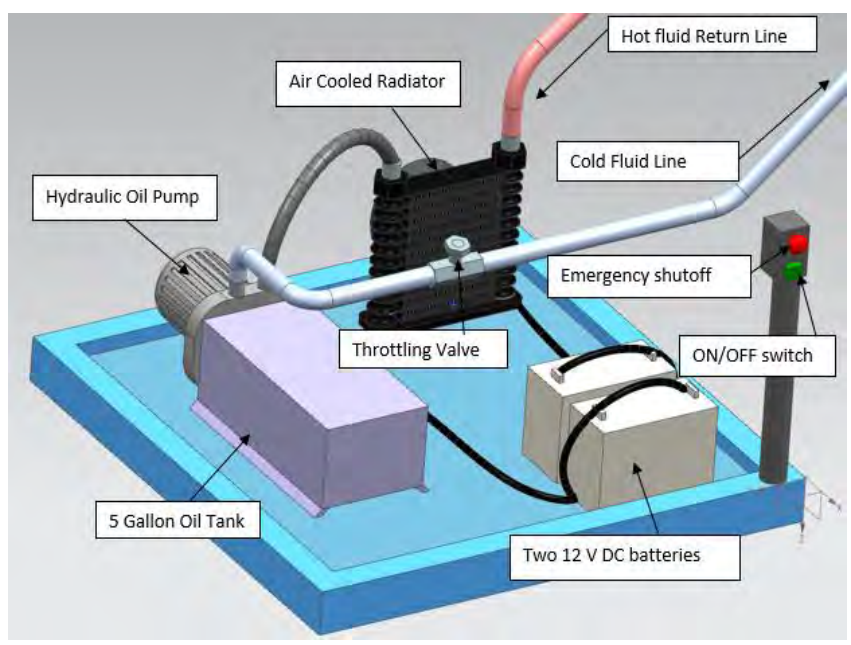

Figure. 6. Polymer melt cooler assembly.

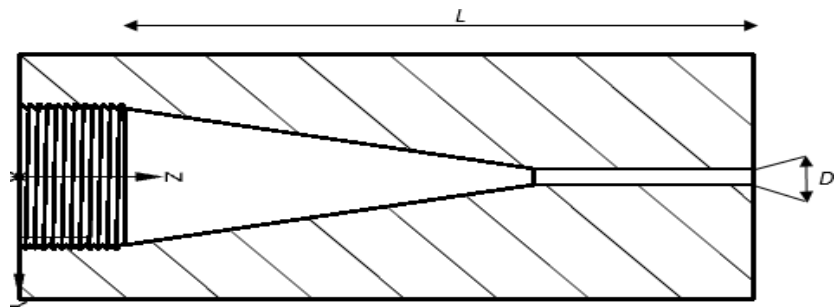

Figure. 7. Die geometry for PBA foam extrusion.

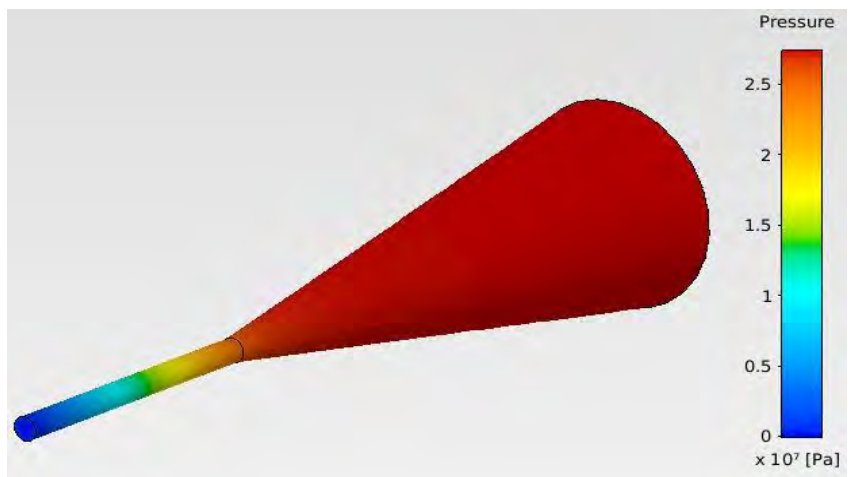

Figure. 8. Pressure loss along the die length.

\section{CONCLUSION}

The presented experimental setup was designed to produce polyolefin foam with PBA for RRFM process. The new setup was selected for having fewer design and fabrication complexities compared against the alternative proposals. In this unique design, the PBA is directly injected into the mixing chamber rather than the extruder barrel. The gas/polymer solution will be produced inside the static mixer. It has been shown, that helical static mixers are suitable for polymer foaming application for having significantly lower pressure loss. The new experimental setup could lead to developing breakthrough processing concepts that would be capable of fabricating new classes of ultra-low-density rotationally molded integral-skin cellular composites. 


\section{REFERENCES}

[1] G. L. Beall, "The rotational molding industry," in Rotational Molding Design Materials, Tooling, and Processing, Cincinnati: Hanser/Gradner Publications, 1998, ch. 1, pp 22-23.

[2] S. Semerdjiev, "Structural foam," Society of Plastics Engineer, 1982, ch 1 pp.1-2.

[3] R. Pop-Iliev, "Processing of integral-skin cellular polymeric composites in rapid rotational foam molding," Acta Phys. Pol., A, vol. 120, No. 2, pp. 292-297, Aug. 2011. doi: 10.12693/APhysPolA.120.292.

[4] R. Pop-Iliev, et. al., "Rapid rotational foam molding process," U. S. Patent 8,628,704 B., 2014.

[5] S. Siripurapu, Y. Gay, J. Royer, J. DeSimone, R. Spontak, and S. Khan, "Generation of mirocellular foams of PVDF and its blends using supercritical carbon dioxide in a continuous process," Polymer, vol. 43, no. 20, pp.

5511-5520, 2002. doi: 10.1016/S0032-3861(02)00407-X.

[6] M. Sauceau, J. Fages, A. Common, C. Nikitine, and E. Rodier, "New challenges in polymer foaming: A review of extrusion processes assisted by supercritical carbon dioxide," Prog. Polym. Sci., vol. 36, no. 6, pp. 749-766, 2011. doi: 10.1016/j.progpolymsci.2010.12.004.

[7] C. Park, and N. Suh, "Rapid polymer/gas solution formation for continuous production of microcellular plastics," J. Manuf. Sci. Eng., vol. 118, no. 4, pp. 639-645, November 1996. doi: 10.1115/1.2831079.

[8] Gottlieb Schneider, "Static Mixing Technology For Extrusion and Injection Molding," Stamixco Ltd., Switzerland, CH-8404 Winterthur / SWITZERL. 


\section{Material Properties of 3D Printed Parts: Challenges in Design and Analysis}

\author{
Madhukar Somireddy \\ Department of Mechanical Engineering \\ York University, Toronto, Canada
}

\author{
Aleksander Czekanski \\ Department of Mechanical Engineering \\ York University, Toronto, Canada
}

\begin{abstract}
The present paper addresses the challenges in design and analysis of 3D printed structures with anisotropic material properties. In recent years the additive manufacturing technologies have been used in most of the industries because of their freedom in fabrication of any complex geometry part. However, the final properties of the 3D printed parts are anisotropic though the material used is isotropic. Further, the properties of printed part are not homogeneous. This change in the properties is due to process parameters and build orientation of the parts. Therefore, this calls special attention of the designer for effective design and analysis of such parts. In this paper, design consideration of the parts for 3D printing and also, their structural analysis for effective design of parts for 3D printing are discussed. Further, different steps involved from design to $3 \mathrm{D}$ printing of a product are discussed.
\end{abstract}

Keywords-3D printing; Material properties; Design; Analysis;

\section{INTRODUCTION}

Additive manufacturing, also known 3D printing, fabricates 3D part by deposition of material layer upon layer. This layer by layer fabrication technology can build a part of any kind of geometry. It means that the technology facilitates more freedom for designing of any intricate shape of parts. The ASTM [1] classifications of AM processes based on process methodology are binder jetting, direct energy deposition, material extrusion, material jetting, powder bed fusion and sheet lamination and photo-polymerization. The other main benefits are rapid prototyping, fabrication of product with minimum part count, better structural integrity of product, no special tooling, flexibility for fabrication of wide range of products, no need of skilled operator, eliminates different sizes of stock materials and minimal or no wastage of processing materials. Furthermore, the AM techniques allow tailoring the material properties of the part by printing desired mesostructure during material deposition and also building functional graded parts much easier. The design of part for $3 \mathrm{D}$ printing is not same as traditional design for machining methods and the design rule for additive manufacturing is discussed by [2]. The material properties of the printed parts are differing form their initial properties and the effect of anisotropic material properties on structural behavior of the printed parts was addressed in [3]. Effect of process parameters on the material microstructure and design principle for additive manufacturing a part is available in [4]. The design of a part for 3D printing involves much more than just fabricating a three dimensional part. It involves design of microstructure, selection of printing direction, process parameters and build orientation. This paper addresses the effect of anisotropic material properties on the design of a part. Also, challenges in design and analysis of part for $3 \mathrm{D}$ printing are discussed. Then the different stages and the factors that need to be considered during design stage of a part for additive manufacturing process are presented.

\section{CHALLENGES IN DESIGN AND ANALYSIS}

The 3D printing of any complex geometry allows the designer for designing of parts of size microstructural (micrometers) to structural level (meter level). At the same time the designer needs to pay attention to the fabrication process and its process parameters while designing the part for $3 \mathrm{D}$ printing. The physical phenomena that take place during the layer upon deposition of the material have strong impact on the quality of the printed parts that includes mechanical properties. This is due to printing the microstructure of the part during layer upon layer material deposition and the mesostructure is mainly governed by the process parameters. This introduces the anisotropy in the material properties even though the isotropic material used for printing. Also, the degree of anisotropy depends on the type of AM process employed for printing the part. Furthermore, the material of the printed part is not homogeneous in different sections of the part. This inconsistency in the material properties of the printed part is major challenge to designer to specify the final material properties of the part. To account this change in the material behavior, the parts have to be designed not just based on geometrical analysis but also based on the phenomena that is taking places during printing. Therefore, it is essential to consider type of AM process and its process parameters while designing the parts for $3 \mathrm{D}$ printing.

The limitations of AM processes are range of materials for printing, dimensional accuracy, build time, support structures, surface finish, mass production, post processing and consistency in the material properties. These are mainly governed by the type of AM process and its process parameters. Also, the build orientation of the parts influences the build time, quality of the part and material properties. So far, the design of parts for printing is based on geometrical analysis, reduction in build time and cost, minimal support structures and for better surface finish. The anisotropy in the material properties of the printed part via different AM 


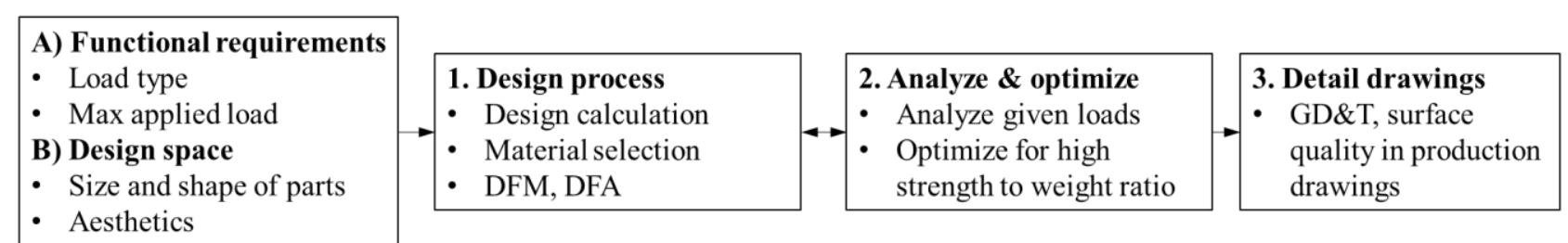

Figure 1. Stages in the product design, development and fabrication

processes was addressed [5]. Effect of the build orientation of the part and process parameters on the mechanical properties of the printed part via FDM was discussed in [6]. The anisotropic material behavior of printed parts via SLA [7]. The variation in the material properties of the printed part via SLM are discussed in [8]. Therefore, while designing a part for 3D printing the designer need to consider the influence of AM process and its process parameters on the final printed part. Further, the limitation of the process and influence of build orientation on quality and performance of the part are to be considered while designing. It means that for effective design and analysis of parts for 3D printing the designer need to have idea about the relationship between process-structure-propertyperformance.

The parts subject to certain loads are designed and analyzed for a selected material to get the its geometrical parameters. It means the geometrical features (size, shape) of the part derived from design and analysis calculations for desired strength to sustain the applied load on it. Different stages involved in the design and development of a product for traditional machining methods are shown in Fig. 1. The main inputs for the product design are its functional requirements and design space. The functional requirements of product include its purpose, load type and maximum applied load. Then the design space defines the maximum allowed size and shape of the product. Then the geometry of the parts of the product are designed for the applied load and selected material. This involves the design calculation that the stresses of the parts produced due to applied load lower than the strength of the selected material. Simultaneously during design stage, the design for manufacturability (DFM) and design for assembly (DFA) are considered for fabrication and assembly and disassembly of the parts of the product. Then in the next stage, the product is analyzed and optimized for higher strength to weight ratio. Finally, the detail drawing of the optimized parts are produced with the tolerances, surface quality requirements and suggestions of machining methods for fabrication of the parts. For instance, a part is to be designed for holding two shafts and subjects to load (P). The Fig. 2 explains different stages involved in design and analysis of the part.

The design of a part for 3D printing could not follow the same stages, because the challenges in designing and their limitations of AM processes as mentioned earlier. Anisotropy and inconsistent material properties of printed part are the main challenges during the design. Let us consider a $3 \mathrm{D}$ printed $\mathrm{L}$ bracket via FDM process. The bracket has two plates; horizontal and vertical plate and a cylindrical section to each of them. The horizontal plate is oriented on the substrate of the printer and the orientation of the vertical plate is perpendicular to the horizontal. As discussed earlier that the properties of the printed parts depend on process parameters and it build orientation. The material properties and behavior of the two plate would not be same because of their build orientation. The behavior of printed horizontal plate would be orthotropic and vertical is transverse isotropic. The $3 \mathrm{D}$ printing of $\mathrm{L}$ bracket with different build orientations is shown in Fig. 3 and their influence is presented in Table I. The other process parameters of FDM such as layer thickness, printing direction, percentage of infill, infill pattern, air gap between the fibers, temperature of build area and printing speed influence the mechanical properties of the printed part. Because of change in the material properties, the initial properties used in design calculations of a part for 3D printing do not provide reliable design output. That includes the geometrical feature of the part and expected material behavior of the part for applied loads. Besides that, results of analysis and optimization of the part based on initial properties are not correct. Because of such challenges, the design of a product for additive manufacturing (DFAM) is much more than the traditional design process for machining methods. The DFAM requires the designer to take care of type of AM process, material properties, build orientation, support structures, build time, cost and quality of the parts.
Input

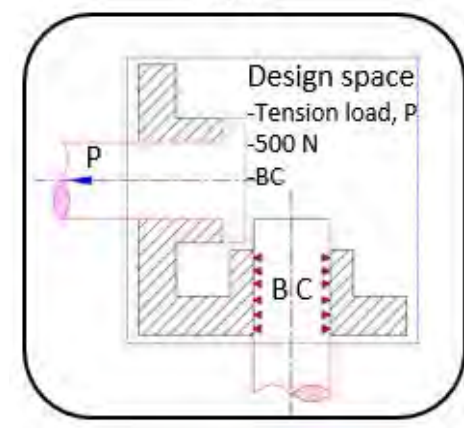

1. Design Process

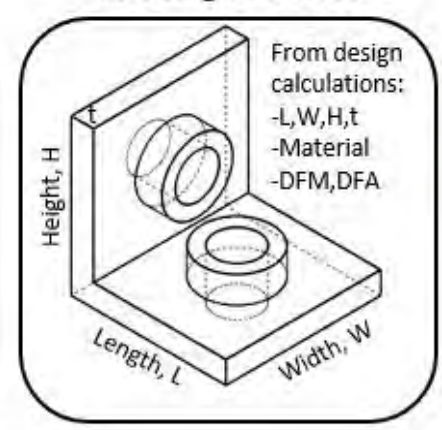

2. Analyze \& optimize

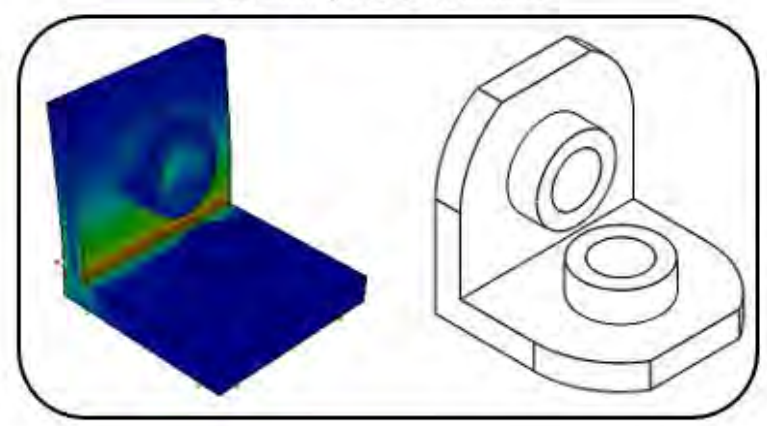

Figure 2. Stages in design and analysis of a part. 


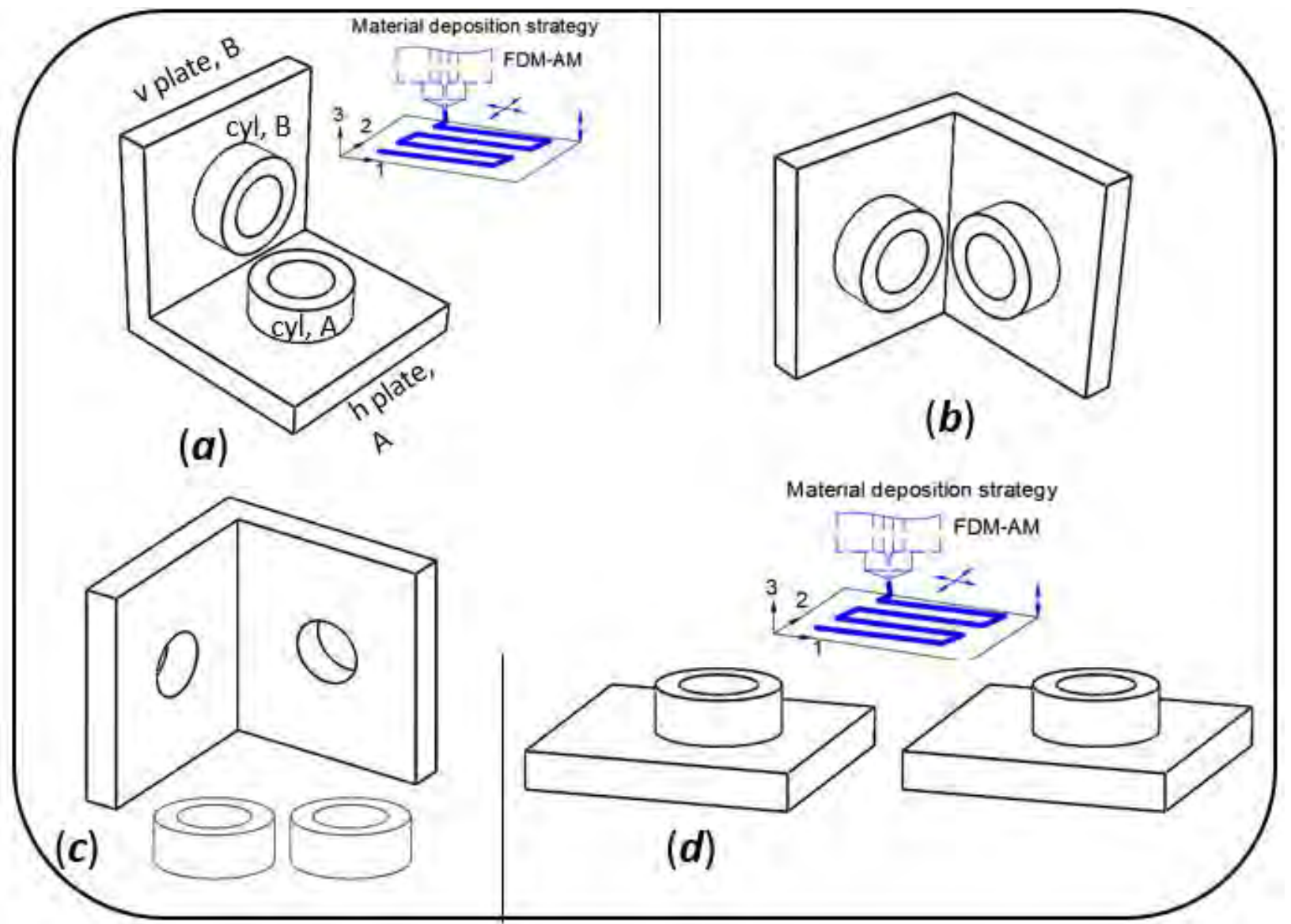

Figure 3. Different build orientations of the L bracket for 3D printing.

TABLE I. INFLUENCE OF BUILD ORIENTATION OF A PART IN 3D PRINTING.

\begin{tabular}{|l|c|c|c|c|}
\hline & $\mathbf{3}(\mathbf{a})$ & $\mathbf{3}(\mathbf{b})$ & $\mathbf{3}(\mathbf{c})$ & $\mathbf{3}(\mathbf{d})$ \\
\hline Properties of A,B & $\neq$ & $\neq$ & $=$ & $=$ \\
\hline Homogeneity in A, B & No & No & Yes & Yes \\
\hline Support structures & Cyl B & Cyl A, B & None & None \\
\hline Build time & Higher & Highest & Lower & Lower \\
\hline Assembly & None & None & Yes & Yes \\
\hline Structural integrity & Strong & Strong & Weak & Weak \\
\hline Post processing & Yes & Yes & No & No \\
\hline
\end{tabular}

A modified design process and the special design tools that consider the flow of information between the processstructure-property-performance could help the designer for effective design and analysis of the parts for $3 \mathrm{D}$ printing. The modified design process and steps involved during design of part for $3 \mathrm{D}$ printing is presented in Table II. The designer tools for 3D printing guides the designer based on the type of $\mathrm{AM}$ process and its process parameters for fabricating the parts. More importantly material data of the final printed parts based on the process parameters and build orientation for effective design and analysis of parts for 3D printing.
TABLE II. STEPS IN DESIGN OF A PART FOR 3D PRINTING.

\begin{tabular}{|c|c|c|}
\hline \multicolumn{3}{|c|}{ 1. Inputs; functional requirements, design space of product } \\
\hline \multicolumn{3}{|c|}{ 2. Preliminary design-design calculations based on initial material } \\
\hline \multicolumn{3}{|c|}{ 3. Design for additive manufacturing } \\
\hline $\begin{array}{l}\text { Build time, support } \\
\text { structure, quality, DFA, } \\
\text { build orientation }\end{array}$ & $\begin{array}{l}\text { Material properties, } \\
\text { structural integrity }\end{array}$ & $\begin{array}{l}\text { AM Process and its } \\
\text { parameters }\end{array}$ \\
\hline \multicolumn{3}{|c|}{ 4. Material mesostructure modeling and AM process modeling } \\
\hline \multicolumn{3}{|c|}{ 5. Analyze design } \\
\hline \multicolumn{3}{|c|}{ 6. Redesign with actual material properties } \\
\hline \multicolumn{3}{|c|}{ 7. Optimize } \\
\hline \multicolumn{3}{|c|}{ 8. 3D print } \\
\hline
\end{tabular}

\section{CONCLUSIONS}

The additive manufacturing technologies facilitated more freedom for fabrication of any shape of the part and with additional benefits such as rapid prototyping, no tooling and minimal or no wastage of material. However, the changes in the material properties of a part due to the physical phenomena that is taking places during layer upon layer deposition of a material need to be addressed. The change in the material behavior greatly influences the design and analysis of the parts for 3D printing. The anisotropy and inhomogeneity in the material properties do not allow the designer for effective design of the parts. Also, designing of a part for 3D printing requires to consider influence of other factors that include type of AM process and its parameters and build orientation on build time, cost, support structure 
and surface quality of the parts. Further, the relation between the process-structure-property-performance is need to be considered during the design stage of a part for 3D printing for effective design and analysis.

\section{REFERENCES}

[1] Standard Terminology for Additive Manufacturing Technologies 1,2, (n.d.). doi: 10.1520/F2792-12A.

[2] G. A. Adam and D. Zimmer, "On design for additive manufacturing: evaluating geometrical limitations". Rapid Prototyping J., vol. 21(6), pp. 662-670, 2015. doi: 10.1108/RPJ-06-2013-0060.

[3] C. Mueller, A. Irani and B. Jenett, "Additive manufacturing of structural prototypes for conceptual design", In Proc. of the IASS-SLTE Symp., Shells, Membranes and Spatial Structures: Footprints, Brasil and Pauletti (Eds.), Brasilia, Brazil 2014 Sep 15.
[4] D. W. Rosen, "Research supporting principles for design for additive manufacturing: This paper provides a comprehensive review on current design principles and strategies for AM". Virtual and Physical Prototyping, vol. 9, no. 4, pp.225-232, 2014. doi: $10.1080 / 17452759.2014 .951530$.

[5] J. Kotlinski, "Mechanical properties of commercial rapid prototyping materials", Rapid Prototyping J., vol. 20, no.6, pp.499-510, 2014. doi: 10.1108/RPJ-06-2012-0052.

[6] A. Bagsik, V. Schöppner, and E. Klemp, "FDM part quality manufactured with Ultem* 9085", In 14th Inter. Scientific Conf. on Poly. Mater., vol. 15, pp. 307-315, 2010.

[7] R. Hague, S. Mansour, N. Saleh, and R. Harris, "Materials analysis of stereolithography resins for use in rapid manufacturing," J. Mater. Sci., vol. 39, no. 7, pp.2457-2464, 2004

doi: 10.1023/B:JMSC.0000020010.73768.4a.

[8] D. Agius, K.I. Kourousis, and C. Wallbrink, "A Review of the As-Built SLM Ti-6Al-4V Mechanical Properties towards Achieving Fatigue Resistant Designs," Metals, vo. 8, no.1, p.75, 2018. doi: 10.3390/met8010075. 
MICROTECHNOLOGY \& NANOTECHNOLOGY 


\title{
A Procedure for Performance Experimental Analysis of a Globe Control Valve
}

\author{
Hesam Hoursan \\ Ph.D Student, School of Mechanical Engineering \\ Sharif University of Technology \\ Researcher at Rasta Group Industrial Valve Co. \\ Tehran, Iran \\ Hesam.hoursan74@student.sharif.edu \\ Mohammad Omid Hadjiazim \\ Graduate, Civil Engineering \\ University of Tehran \\ Researcher at Rasta Group Industrial Valve Co. \\ Tehran, Iran \\ Omid.azimi@rastagroup.net
}

\author{
Mohammad J. Moradi \\ M.S. Graduate, Petroleum Engineering Department, \\ Amirkabir University of Tech \\ Researcher at Rasta Group Industrial Valve Co. \\ Tehran, Iran \\ Mohamad.moradi89@yahoo.com
}

\author{
Mohammad Taghi Ahmadian \\ Professor, School of Mechanical Engineering \\ Sharif University of Technology \\ Tehran, Iran \\ Ahmadian@sharif.edu
}

\author{
Ahmad Barari \\ Faculty of Engineering and Applied Science \\ University of Ontario Institute of Technology \\ Oshawa, Ontario, Canada \\ Ahmad.barari@uoit.ca
}

\begin{abstract}
Control valves are known as the final control element in hydraulic closed/open loops of modern process industries around the world. Proper selection of control valve leads to enhanced performance curve of the hydraulic systems and therefore increases the efficiency, reliability, profitability and safety of the system. Flow coefficient (CV) of a control valve describes the relation between the pressure drop across the valve and the flow passing through it. Despite many computational efforts for calculating the exact value and curve of $\mathrm{CV}$, the experimental procedure of the $\mathrm{CV}$ test has not been documented well. We used a control valve test-set up designed based on the standards ANSI/ISA-75.02-1996 and IEC 605342-3 (2013) to evaluate the performance of a $3 \mathrm{in}$. control valve. Upon extracting the results in terms of inlet, and outlet pressure and flow, the characteristic parameters such as $\mathrm{CV}$ and opening percentage were derived and compared with an ideal curve. Error analysis was performed to account for the tolerance of the measured parameters by the measuring devices. The results show acceptable agreement within the criteria of a reference standard approving the validity of the design method.
\end{abstract}

Keywords- Control valve; Flow coefficient, Ideal curve, Test setup, Performance

\section{1- INTRODUCTION}

Control valves have long been used as the final control elements in various types of process lines. They are typically known to be in charge of one-third of the total pressure drop along the hydraulic line which represents a high impact on the regulation of total line performance curve. The coefficient of valve $(\mathrm{CV})$ is related to the surface area through which the fluid can throttle (pass). Such area is controlled via moving (linear) or rotating (rotary) a closure element relative to a fixed housing (seat). The position of the closure element is controlled by various types of actuators including pneumatic, electric, electrohydraulic and hydraulic actuators; among which pneumatic actuators are simpler and more common.

A Globe valve with linear actuator is composed of body, bonnet, plug, seat, stem, and for the case where more pressure drop is required at the price of less $\mathrm{CV}$, various types of cage are used. Flow coefficient $(\mathrm{CV})$ of a control valve describes the relation between the pressure drop across the valve and the flow passing through it. The definition of Control Valve Coefficient was soon accepted and applied universally after its first introduction to industry by Masoneilan in 1944. [1] In more practical terms, the flow coefficient $\mathrm{CV}$ is the volume (in US gallons) of water at $60^{\circ} \mathrm{F}$ that will flow per minute through a 
valve with a pressure drop of 1 psi across the valve. [2, 3] A comprehensive laboratorial set-up is required for fine measurement of variables such as flow and pressure drop, from which the $\mathrm{CV}$ can be calculated and plotted. The specifications of such set-up for incompressible flow measurement are mentioned in ANSI/ISA-75.02-1996 and IEC 60534-2-3 (2013) standards $[4,5]$. Previous researchers have implemented the testing process such as that of Vikas et al., in which a 4 inch globe control valve is tested experimentally for its equal percentage and linear performance curves. [6] Computational methods have been presented by Guy Borden [7] to calculate and plot the ideal performance characteristics for throttling valves which include linear and equal percentage characteristics of the valve. Also, Aragon et al. have presented a method for experimental determination of valve capacity with compressible flow [8].

Despite the achievements of previous publications, a full procedure for testing and analysis of a control valve performance curve has not been proposed. Since such analysis can serve as a validation method for the control valve design and on-site performance, it is of critical importance to consider the effects of various variables such as hysteresis in sequential opening and closing of the valve, error analysis and considering the existing criteria [9] for acceptance of the curve, on which few documents have been published. The current study aims to analyze the results of a performance test on a 3 inch commercial Globe Control Valve with equal percentage trim to represent a nonlinear characteristic behavior.

\section{2- METHODOLOGY}

\section{2-1- Valve Characteristics}

A 3 inch. globe control valve manufactured with modern technology was tested. The specifications of the test valve are shown in Table 1.

The trim of the top guided valve is a contoured plug head with equal percentage characteristics. The contoured plug head of the valve is designed based on the computations of [7] for a $3 * 2.5$ in valve with $40 \mathrm{~mm}$ of stroke. A computational code in MATLAB was used to estimate the curve of the contoured plug (Figure 1). The control valve is equipped with a single acting pneumatic actuator and a Yamatake positioner with $0.1 \%$ travel accuracy for fine positioning of the stem during opening and closing cycles.
Table 1- Type test characteristics and dimensions

\begin{tabular}{|l|c|}
\hline Valve Type \& Rating & Globe Top Guided GS1000- ANSI 300 \\
\hline Valve Size & 3 in. Manifold *2.5 in Plug \\
\hline Valve Rated CV (Fully Open) & 72 \\
\hline Valve Characteristic & $\begin{array}{c}\text { Equal percentage with 0.05 of Rated CV } \\
\text { at Min. }\end{array}$ \\
\hline Actuator Type & \begin{tabular}{c} 
Pneumatic Single Act. \\
\hline Positioning
\end{tabular} \\
\hline
\end{tabular}
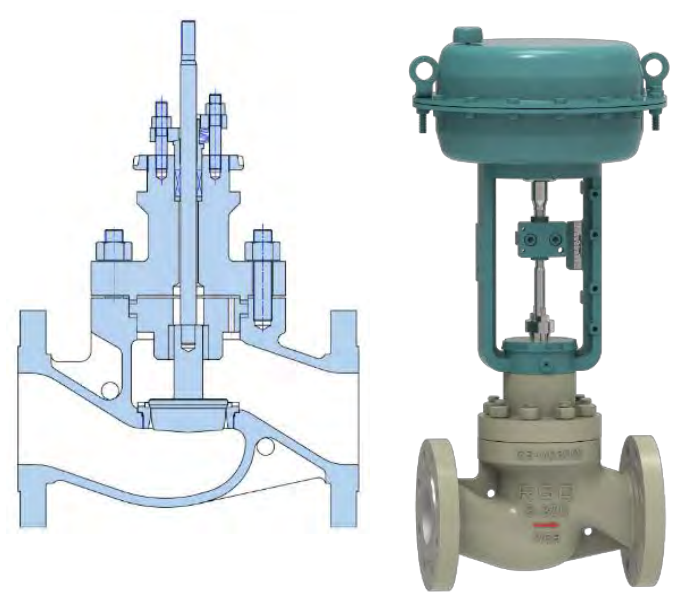

Figure 1- Top Guided Globe Valve Trim Style (Courtesy of Rasta Group Industrial Valve Co.)
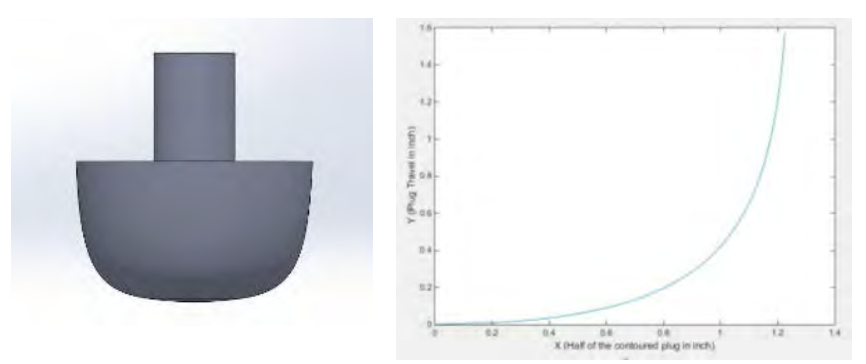

Figure 2- Left: Plug head curve (half of the contoured plug) designed for the $3 * 2.5$ in valve with $40 \mathrm{~mm}$ stroke and recovery factor of 0.9 Right: a MATLAB code is written for computation of the curve based on [7] (courtesy of Rasta Group Industrial valve Co.)

\section{2-2- Laboratory Test Set-up}

A laboratorial test set-up which has been designed for 3 in. valves was prepared for the study. (Figure 4) The set-up was verified for dimensional agreement with ANSI/ISA 75.021996. [9] (Figure 3). The standard ANSI/ISA-75.01.01-2002 (2002) was used for sizing calculations which defines equations 
for sizing control valves and showing the result as a flow capacity coefficient, CV. $[1,2]$

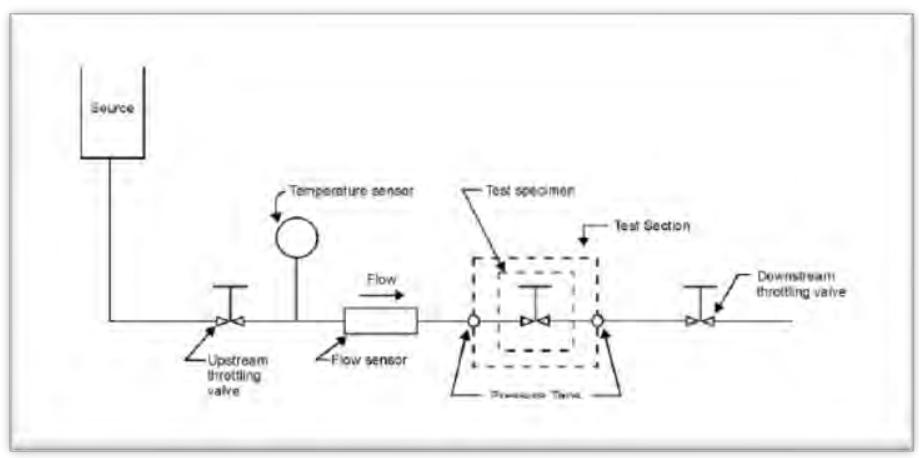

Figure 3- Test Circuit Suggested by ANSI/ASME 75.02 for testing the performance characteristic of control valves $[3,4]$

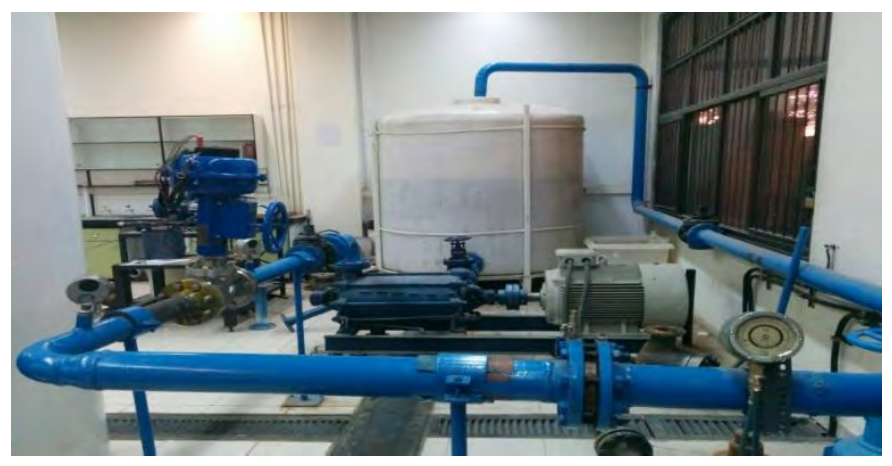

Figure 4- The test bench used for the experiment (Courtesy of Thermohydraulic Laboratory of the Iranian Ministry of Power)

The test bench instrumentation was chosen to meet the specifications of standards ANSI/ISA-75.02-1996 (1996) and ANSI/ISA-75.01.01-2002 (2002). [4, 5] Three physical variables were obtained at each run: pressure, temperature and flow rate, with the following characteristics,

Pressure: All pressure measurements are made with an error not exceeding $\pm 2 \%$ of the actual value. [4, 5] Rosemount 3051 Pressure Transmitters are used to meet the specified accuracy. (Figure 5)

Temperature: The flow temperature should be measured within an error not exceeding $\pm 1^{\circ} \mathrm{C}\left( \pm 2^{\circ} \mathrm{F}\right)$ of actual value. [4, 5] A standard temperature sensor is used to make sure the temperature of water is within acceptable limits.

Flow: The flow rate instrumentation may be any device that meets specified accuracy. This instrument is used to determine the true time average flow rate within an error not exceeding \pm $2 \%$ of the actual value. $[4,5]$ The resolution and repeatability of the instrument must be within $\pm 0.5 \%$. A Magnetic MAXIFLO flow-meter with the accuracy of $2 \%$ has been used to measure the flow. Three readings are made each time and the average is calculated and reported as data.

Pump: A centrifugal Pump with maximum flow capacity of $120 \mathrm{~m} 3 / \mathrm{h}$ and suitable pressure limit for 4 in piping is used.
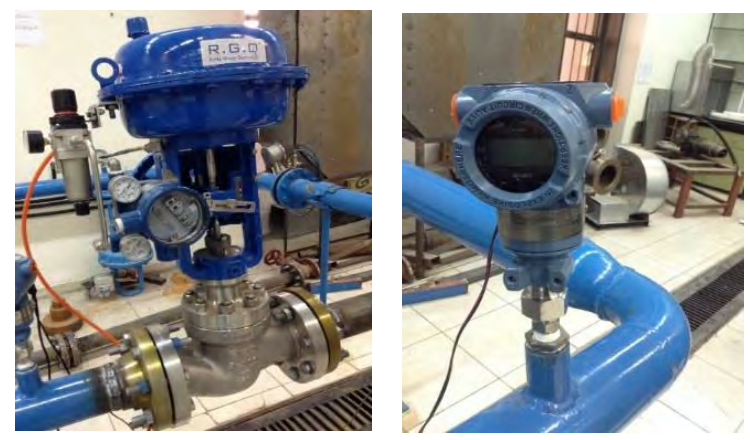

Figure 5- Valve installed in 3-in pipeline (left), Pressure transducers installed on pressure taps (right)

\section{2-3- Test procedure}

The set-up was used to test the valve through stepwise closing and opening of the valve and recording the measured parameters including differential pressure across the valve and flow. The test was repeated in three cycles of closing-openingclosing of the valve and during the steps, data was recorded on every $6.25 \%$ change in stem travel (This is equivalent to $1 \mathrm{~mA}$ change in positioner signal input in the range of 4-20 mA current).

Scenario A- Closing the valve from fully-open condition (maximum $\mathrm{Cv}$ ) to minimum flow at 0.05 of maximum $\mathrm{Cv}$. And recording data including: 1- Inlet and Outlet static pressure using digital pressure gauges. 2- Flow passing through valve measured by a magnetic flow-meter. Results were plotted as calculated $\mathrm{Cv}$ vs. travel percentage to characterize the valve. Attempt was made to avoid choking conditions which correspond to maximum allowable pressure drop.

Scenario B- The above step was repeated from fully-closed condition to fully open.

Scenario C- The above step was repeated once more from fully-open condition to fully-closed.

Calculation of flow coefficient based on the standard ISA 75.01 and IEC 60534 was performed as follows [2, 3]:

$C_{V}=\frac{w}{27.3 \times \sqrt{\rho \times \Delta P}}$

, where $\mathrm{w}$ is the mass flow rate in $\mathrm{kg} / \mathrm{h}, \rho$ is fluid density in $\mathrm{kg} / \mathrm{m} 3$, and $\Delta P$ stands for the pressure drop across the valve in 
bar. The equal percentage theoretical characteristic of valve is calculated from the formula of equation (2). [7]

$q=a^{1-h}$

, where

$q=\frac{C V \text { at current opening }}{C V \text { at fully-open }(\text { Rated } C V)}$

$a=\frac{C V \text { at Minimum opening }}{C V \text { at fully-open }(\text { Rated } C V)}=0.03$

$h=$ Travel Percentage $=\frac{\text { Plug Distance From Seat }(\text { Travel })}{\text { Travel at fully-open }(\text { Stroke })}$

(5)

The resulting curve was compared with the ideal equal percentage curve based on the criteria suggested by ANSI/ISA75.11.01-1985 (R2002) [9] which provides limits for acceptable deviations of actual inherent characteristic from the characteristic reported by manufacturer. (Figure 6)

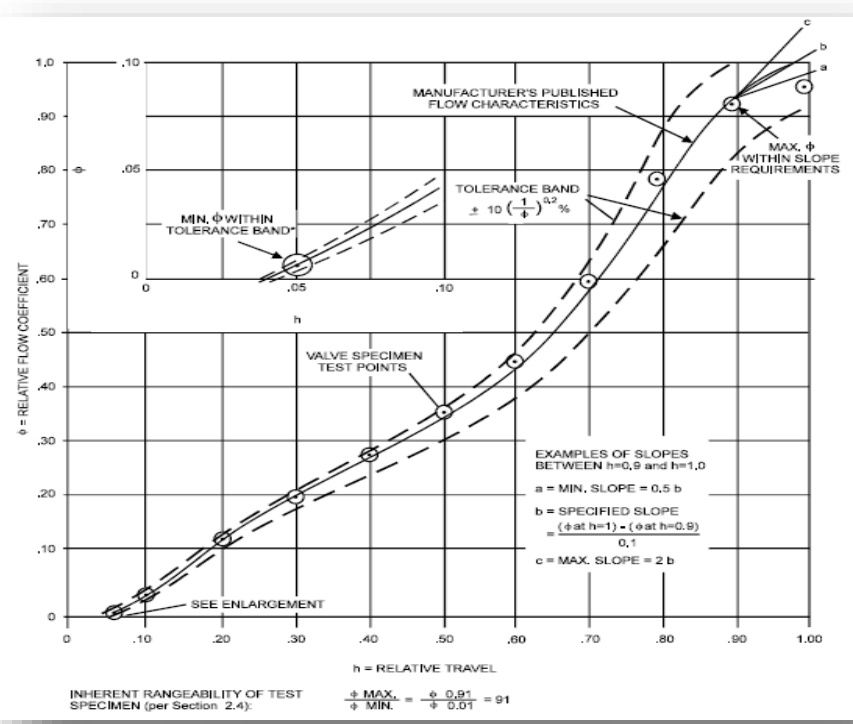

Figure 6- Acceptable range of deviations from catalogue curve by ANSI/ISA75.11.01-1985 (R2002) [9]

\section{3- RESULTS}

The obtained results - in terms of valve coefficient $(\mathrm{CV})$ versus travel percentage of valve stem - from the first scenarios (Fully-open to Fully-closed) are shown and compared to the theoretical equal percentage characteristic from equation (3) in Figure 7. As it was expected, the equal percentage characteristic was properly followed by the performance curve of the trim. However, there seemed to be minor deviations around the opening of $80 \%$.
Additionally, the results of the other two scenarios were demonstrated in Fig. 8. It seems that the cyclic hysteresis effect is more considerable in the range $60 \%-80 \%$ opening where it caused fluctuations and minor discrepancy in the results of the three scenarios. $\mathrm{R}^{2}$ value from statistical analyses of six pairs of results are shown in Table 2. Based on the values, the first scenario is the closest to the ideal characteristic and the closing cycles (scenarios $1 \& 3$ ) seem to be more similar in pattern than the other pairs.

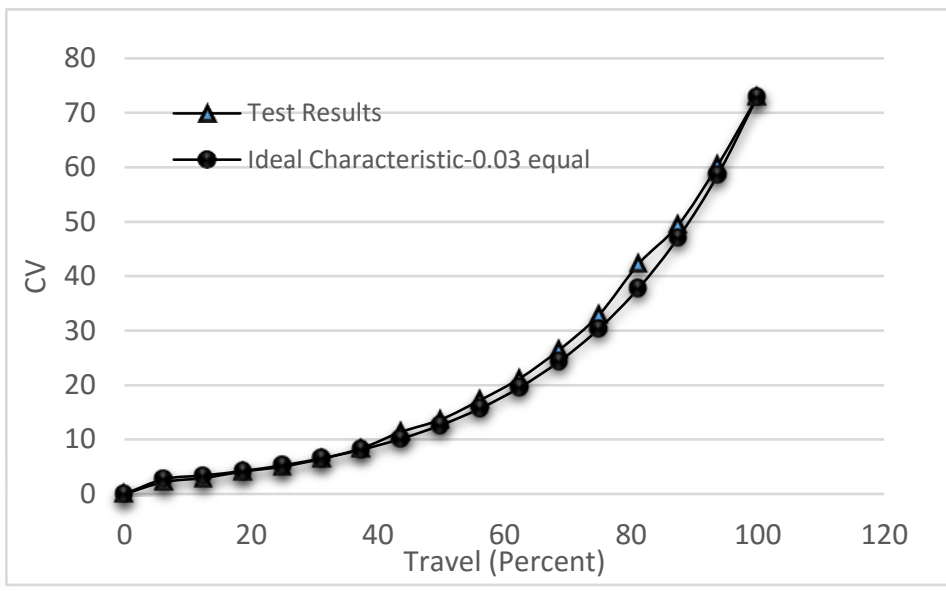

Figure 7- Flow characteristic obtained from the test vs. the ideal equal percentage flow characteristic

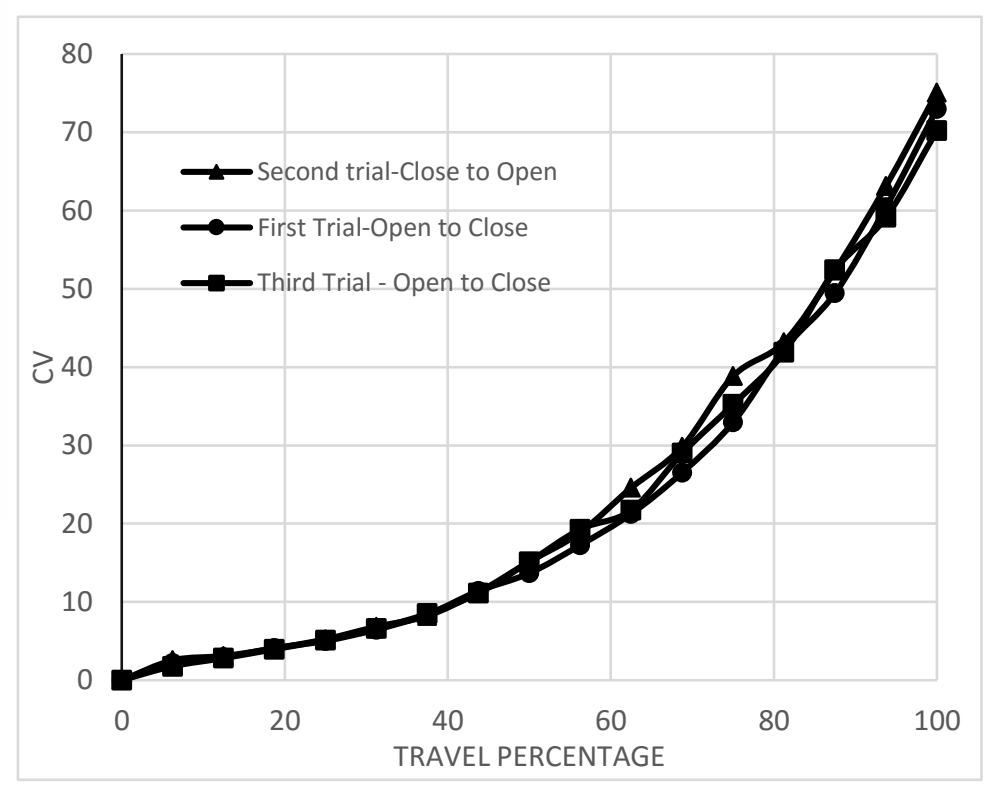

Figure 8- Flow characteristic obtained from the test vs. the ideal equal percentage flow 
Table 2- R2 values for trend lines of the three test scenarios with theoretical curve. Ideal characteristic is considered equal percentage with Min CV of 3\%

\begin{tabular}{|c|c|c|c|c|c|}
\hline $\begin{array}{c}\text { Ideal- } \\
\text { Test1 }\end{array}$ & $\begin{array}{c}\text { Ideal- } \\
\text { Test2 }\end{array}$ & $\begin{array}{c}\text { Ideal- } \\
\text { Test3 }\end{array}$ & $\begin{array}{c}\text { Test1- } \\
\text { Test2 }\end{array}$ & $\begin{array}{c}\text { Test1- } \\
\text { Test3 }\end{array}$ & $\begin{array}{c}\text { Test2- } \\
\text { Test3 }\end{array}$ \\
\hline 0.991 & 0.963 & 0.975 & 0.985 & 0.990 & 0.984 \\
\hline
\end{tabular}

\section{4- DISCUSSION OF RESULTS}

The three recorded equal percentage characteristic curves are shown and compared for hysteresis of the hydraulic system denote fluctuations in the measurements at some points along the valve full stroke. The data of the first scenario (fully-open to fully closed condition) are compared to the ideal equal percentage characteristic of reference [7] shown in Equation (2). This is displayed in Figure 7 where the deviations from ideal equal percentage characteristic are almost negligible for the first scenario. However, the deviation becomes more noticeable in the two remaining repetitions of the scenario (scenarios 2 and 3). This can be attributed partly to measurement and reading errors.

The comparison of the trend of test results in terms of Rsquared in Table 2 shows that the test results are acceptable valid regression models of the theoretical data. However, the similarity of the trend line is more noticeable in opening curves (higher R-squared value for test1-test3 comparison). For better evaluation of the quantitative validity of test results, a statistical T-test was performed. (Table 3 ) In this table, the average of the three values for each test point in terms of $\mathrm{CV}$ has been considered as test results, which is compared to the ideal characteristic. From equation (6), the result of the T-test show that the averages of the two sets of data do not differ significantly. As it can be seen, the statistical analysis including the qualitative $\left(\mathrm{R}^{2}\right)$ and quantitative (T-test) results demonstrate acceptable results.

Table 3- T-Test comparison for ideal characteristic and test data

\begin{tabular}{|l|l|l|}
\hline \multicolumn{1}{|c|}{ Parameter } & \multicolumn{1}{|c|}{ Ideal } & \multicolumn{1}{c|}{ Test Result } \\
\hline Mean & 21.16 & 22.807055 \\
Variance & 461.74 & 500.219218 \\
Observations & 17 & 17 \\
t Stat & 0.21 & \\
P(T<=t) one-tail & 0.41 & \\
t Critical one-tail & 1.69 & \\
P $(T<=t)$ two-tail & 0.82 & \\
t Critical two-tail & 2.03 & \\
\hline
\end{tabular}

,the requirement of the T-test acceptability is as follows:

$$
\begin{gathered}
-\mathrm{t} \text { Critical two }- \text { tail } \leq \mathrm{t} \text { Stat } \leq+\mathrm{t} \text { Critical two }- \text { tail } \\
-2.03 \leq 0.2 \leq+2.03
\end{gathered}
$$

More precise analysis can be made through the method of ANSI/ISA-75.11.01-1985 (R2002) which provides limits for acceptable deviations of actual inherent characteristic from the characteristic reported by manufacturer. The Ensemble CV in Equation (7) at fully-open condition is average for the three scenarios at this condition. As it is observed, the maximum relative error is $4.3 \%$ at fully-open condition for the third scenario where the calculated $\mathrm{CV}$ is 75.09 while the nominal $\mathrm{CV}$ equals 72. In addition, the changes of relative error threshold from reference [9] is compared to the actual relative error from ideal equal percentage trim (the average of three scenarios at each control point has been used) in Figure 9. It can be inferred from the Figure that, although the average values of $\mathrm{CV}$ do not exceed the threshold level, the values of CV tend to slightly fall beyond the levels at some points of the threefold scenarios. The highest relative errors, which are accompanied by slightly exceeding the threshold, occur when the valve stem is $6.25 \%, 43.75 \%$ and $81.25 \%$ open.

$$
\text { Average Ensemble CV at full Travel }=\frac{73+70.23+75.09}{3}=72.77
$$

Nominal Rated $C V=72$

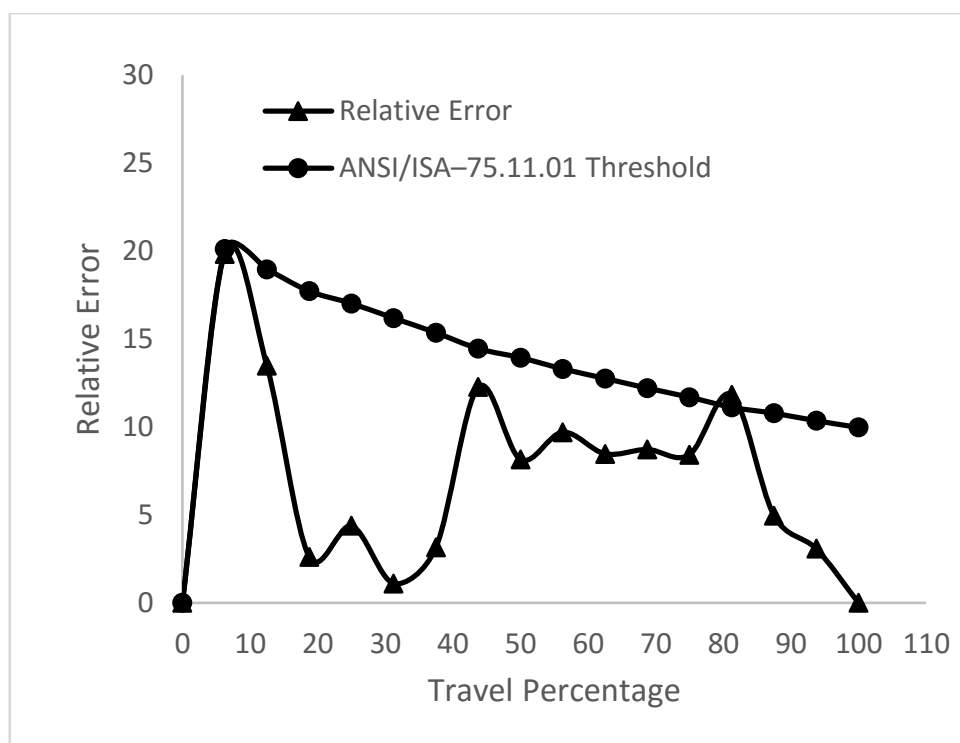

Figure 9- Comparison between experiment relative error (average of three scenarios at each control point has been used) and allowable limit of error from [9]

The compound error of $\mathrm{CV}$ calculation from the measured parameters ( $\mathrm{w}$ and pressure drop) is presented in Equation (8),

$$
\delta(C V)=\left[\frac{1}{27.3 \times \sqrt{\rho \times \Delta P}} \times \delta(w)\right]+\left[\frac{w}{27.3 \times 2 * \times \sqrt{\rho \times \Delta P} \times \Delta P} \times \delta(\Delta P)\right]
$$

,where $\delta(C V)$ is the ensemble error of $\mathrm{CV}, \delta w$ error of flow measurement which is $2 \%$ for based on the specifications of the measurement device, and $\delta(\Delta P)$ represents the error of pressure drop measurements which equals two times the error 
of one reading (totally $4 \%$ since the pressures are read at input and output and then they are subtracted).

As it can be inferred from Figure 10, the measurement compound error grows as the calculated $\mathrm{CV}$ is increased. This occurs due to higher contribution of flow (than pressure drop) to $\mathrm{CV}$ measurements in control valve. As a result of the proportionality of flow and $\mathrm{CV}$, and given the somewhat constant pressure drop throughout the test, the width of the error bars grow exponentially similar to the equal percentage characteristic of valve. By adding the values of measurement compound relative error to the relative errors of the experiment results from ideal characteristic (Figure 9), the threshold is surpassed only at $81.5 \%$ travel by the amount of $3 \%$ relative error which can be considered negligible.

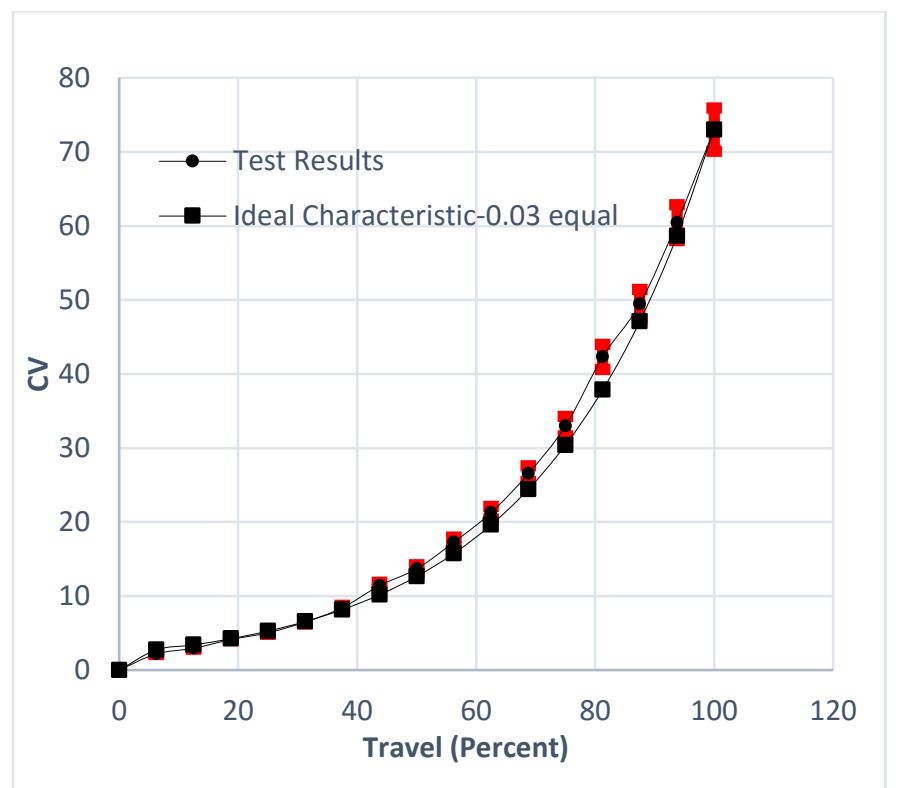

Figure 10- Compound error of CV measurement based on the accuracy of measurement devices shown for the results of the first scenario (fully-open to fully-closed) compared with exact ideal equal percentage characteristic (with minimum relative $\mathrm{CV}$ of 0.03 )

\section{5- CONCLUSIONS}

A 3 in. globe control valve with top guided contoured trim was tested experimentally for its performance curve on a standard 3 in. control loop set-up. The performance test was repeated three times starting with a fully-open to fully-closed condition of valve and the data of water flow and pressure drop across the valve were recorded after each $1 \mathrm{~mA}$ change in positioner input signal (equivalent to $6.25 \%$ change in valve stem travel after calibration of positioner).

The tests were carried out in three various scenarios including successive opening and closing cycles. The results show negligible hysteresis existing between the cycles with opposite moving directions of valve stem. The valve characteristic was compared to the ideal characteristic of reference [7] which demonstrated acceptable results not exceeding the threshold of relative error addressed by reference [9]. However, it was later shown that the compound measurement error of $\mathrm{CV}$ can push the values slightly beyond the acceptable limits of reference [9]. Although, the overall results of the experiment are acceptable, In order to further reduce the compound errors of measurements, there are a number of methods including use of a pressure subtractor measurement device and performing sensitivity analysis.

\section{6- ACKNOWLEDGEMENTS}

The authors would like to express their appreciations to Rasta Group Industrial Valve Co. for financially supporting the project. Instrumentation specialists $\mathrm{Mr}$. Zargaran and $\mathrm{Mr}$. Arbabi for accompanying the project to the very end. In addition, the cooperation of the Iranian Ministry of Power is appreciated for making the necessary arrangements for valve set-up and installation.

\section{7- REFERENCES}

[1] J. Love, "Valve Sizing," in Process Automation Handbook, Berlin, Germany: Springer, pp. 133-141. doi:10.1007/978-1-84628-282-9_20.

[2] Industrial-process control valves. Flow capacity. Flow capacity. Sizing equations for fluid flow under installed conditions, BS EN 60534-2-1:1999, 1999. doi: 10.3403/02604354.

[3] Industrial-process control valves. Flow capacity. Sizing equations for fluid flow under installed conditions, BS EN 60534-2-1:2011, 2011. doi: $10.3403 / 30178780$.

[4] Control Valve Capacity Test Procedures, ANSI/ISA-75.02.01-2008, 2008.

[5] Industrial-Process Control Valves, Part 2: Flow Capacity, Section 3: Test Procedures, IS/IEC 60534-2-3, 1997.

[6] V. L. Karade, A. B. Shinde, and P. V. Pol, "Control Valve Coefficient Testing," Int. J. Innov. Res. Technol., vol. 2, no. 6, pp. 5, Nov. 2015.

[7] G. Borden, P. G. Friedmann. Control valves. NC: Instrument Society of America, 1998.

[8] G. Aragon-Camarasa, G. Aragon-Gonzalez, A. Canales-Palma, and A. Leon-Galicia, "Test Bench for Process Control Valves.," in Electronics, Robotics and Automotive Mechanics Conference (CERMA 2007), 2007, pp. 139-144. doi: 10.1109/CERMA.2007.4367675.

[9] Inherent flow characteristic and rangeability of control valves, ANSI/ ISA-75.11.01, 1985. 


\section{Graphene Oxide Reinforced Bio-epoxy Polymers}

\author{
Anthony Loeffen \\ Mechanical Engineering \\ University of Saskatchewan \\ Saskatoon, Canada \\ aal771@mail.usask.ca
}

\author{
Mina Sabzevari \\ Mechanical Engineering \\ University of Saskatchewan \\ Saskatoon, Canada \\ mina.sab@usask.ca
}

\author{
Duncan Cree \\ Mechanical Engineering \\ University of Saskatchewan \\ Saskatoon, Canada \\ duncan.cree@usask.ca
}

\author{
Lee Wilson \\ Department of Chemistry \\ University of Saskatchewan \\ Saskatoon, Canada \\ lee.wilson@usask.ca
}

\begin{abstract}
The majority of epoxy/graphene oxide (GO) composite studies have been conducted on synthetic epoxies. This work presents the results on GO filler loadings of $0.1,0.2$ and 0.3 wt. $\%$ to a green bio-epoxy polymer. GO was synthesized from oxidation of graphite flakes. The epoxy/GO composites were prepared using a solution mixing route. Scanning electron microscopy (SEM) was used to examine the graphite and GO powder morphology and composite fractured surfaces. Fourier transform infrared (FTIR) spectroscopy was used to identify functional groups on the produced GO material. Tensile strength of pure and modified bio-epoxy composites was evaluated. SEM showed differences in fractured surfaces which implies the GO material was able to modify the bio-epoxy polymer. The FTIR results confirmed oxidation of the graphite was successful. The tensile strength and modulus improved by $23 \%$ and $35 \%$, respectively as compared to the pure bio-epoxy with only $0.3 \mathrm{wt}$. $\% \mathrm{GO}$ filler. Additions of GO to bio-epoxy revealed a significant enhancement in tensile strength and stiffness could be achieved with considerable lower filler loadings than traditional fillers.
\end{abstract}

Keywords-graphene oxide; bio-epoxy; composite; mechanical properties

\section{INTRODUCTION}

Synthetic epoxy polymers are used in a number of industries such as aerospace, automotive and consumer products due to their lightweight, corrosion and chemical resistance. Cured epoxy composites tend to be brittle and have low impact strengths which can have limitations on certain applications. Fillers are added to polymers to reduce component costs. Mineral fillers in thermoset polymers help to improve impact toughness, modulus and hardness of the composites. However, these fillers tend to decrease the tensile strengths of the polymer composites. It has been reported much less graphene oxide (GO) fillers are required compared to traditional fillers to improve mechanical properties [1].

Graphite, a carbon based three-dimensional crystalline material was discovered by KW Scheele in 1779. Each layer of carbon atoms are covalently bonded, while the bonding between the layers or sheet are weakly bonded with Van der Waals bonds [2]. By exfoliating the sheets of graphite, graphene a two-dimensional material is formed. Graphene was discovered in 2004 and is defined as a single flat sheet of graphite where its carbon atoms are arranged in a hexagon structure [3]. Although studies have shown graphene can improve properties of polymer composites, graphene has a smooth, non-reactive surface which prevents a good bond to form, limiting the load transfer from the polymer matrix to the graphene sheets [4]. Compared to graphene, $\mathrm{GO}$ obtained from oxidizing graphite contains a number of GO sheets stacked on top of each other. The GO sheet can be exfoliated into single sheets by sonication in water, solvents and polymers. The advantage of using GO in polymers is the presence of functional groups on the surface and edges of the GO sheets. Significant improvements in mechanical properties can be obtained by adding GO to epoxy resin. For example, when GO was added to a synthetic epoxy in loadings of $0.1 \mathrm{wt} . \%, 0.2$ wt. $\%$ and 0.5 wt. $\%$, tensile strength improved by $7 \%, 9 \%$ and $11 \%$, respectively and tensile modulus increased by $8 \%$, $20 \%$ and $24 \%$, respectively [1].

This study investigates the mechanical tensile strength of a green bio-epoxy composite containing GO fillers in loadings of $0.1-0.3$ wt. $\%$. The aim of the work was to evaluate the morphology of the GO filler and fractured surfaces of the 
modified and unmodified composites and verify appropriate oxidation of the graphite. The initial results of this work are provided.

\section{EXPERIMENTAL}

\section{A. Materials}

Super Sap CPM/CPL a bio-based epoxy was purchased from Entropy Resins Inc., San Antonio, USA. The resin was mixed with a ratio of 10:4 by weight, bio-epoxy to hardener. Natural graphite flakes, -325 mesh $(44 \mu \mathrm{m}), 99.8 \%$ (metals basis) were provided by Alfa Aesar Thermo Fisher Scientific.

\section{B. Graphene Oxide Preparation}

GO was prepared according to the modified Hummer's method which oxidizes graphite using sulfuric acid, sodium nitrate and potassium permanganate. The solution was purified with multiple rinsing using millipore water, $30 \%$ hydrochloric acid and ethanol until a $\mathrm{pH}$ of $\sim 7$ was reached. The product was finally vacuum dried at $40{ }^{\circ} \mathrm{C}$ for 12 hours to produce a GO film. The film was then made into powder using a mortar and pestle.

\section{GO/Epoxy Resin Composite Preparation}

The GO powder was added to acetone in separate batches in weight fractions of 0.1 wt. $\%, 0.2$ wt. $\%$ and 0.3 wt. $\%$. The GO powder was homogeneously dispersed and exfoliated in acetone by sonication for $1 \mathrm{~h}$. The GO/acetone mixture was added to the bio-epoxy resin and mechanically stirred for another $1 \mathrm{~h}$. The resin was placed in an oven at $40{ }^{\circ} \mathrm{C}$ under vacuum overnight to evaporate the acetone. The hardener was added and mechanically stirred for $10 \mathrm{~min}$. followed by vacuum degassing at room temperature for $30 \mathrm{~min}$. The mixture was then poured into silicone molds and cured for 24 $\mathrm{h}$ at room temperature followed by a post-cure at $82^{\circ} \mathrm{C}$ for $1 \mathrm{~h}$. Tensile specimens are shown in Fig.1.

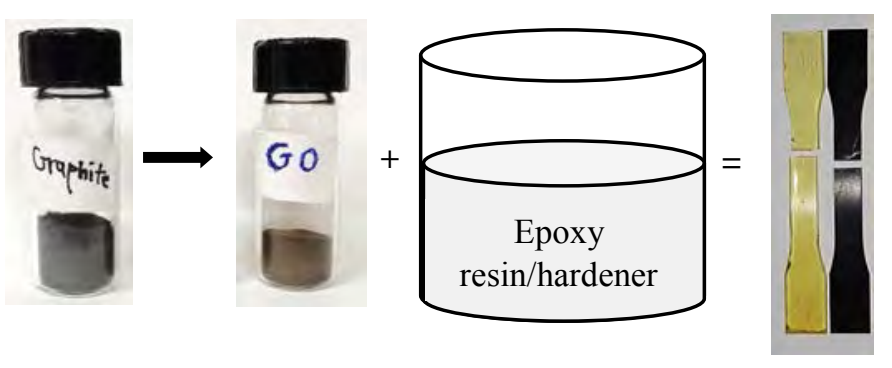

Figure 1. Schematic route for preparation of epoxy and epoxy/GO composites.

\section{Measurements and Characterization}

Graphite and GO powder morphology and tensile fractured surfaces were observed by scanning electron microscopy (SEM) using a JEOL JSM-6010 LV (Tokyo, Japan) operated at $10-15 \mathrm{kV}$. The samples were coated with a thin layer of gold. Fourier transform infrared (FTIR) spectroscopy was performed on as received graphite and as synthesized GO. The FTIR spectra were conducted on a Bio-Rad FTS-40 spectrophotometer. The spectra for each analysis was obtain in reflectance mode with a resolution of $4 \mathrm{~cm}^{-1}$ over the spectral range between 500 and $4000 \mathrm{~cm}^{-1}$. The samples were made into powdered form and compressed into tablets of $\mathrm{KBr}$ in a weight ratio of 1:10 (sample powder: $\mathrm{KBr}$ ). Tensile strength tests were conducted using an Instron 1137 universal testing machine with a $10 \mathrm{kN}$ load cell. Tensile strength was evaluated at a strain rate of $5 \mathrm{~mm} / \mathrm{min}$ according to ASTM D638-14 on dog-bone specimens measuring $165 \times 13.0 \times 3.3$ $\mathrm{mm}^{3}(1 \mathrm{x} \quad \mathrm{w} \times \mathrm{t})$. Five specimens were tested for each composite and averaged.

\section{RESULTS AND DISCUSSION}

\section{A. Scanning Electron Microscopy Analysis}

The different morphologies of the starting graphite and synthesized GO sheets are shown in Fig. 2. The majority of natural graphite has a flake-like structure, with some having smooth, rounded edges stacked together forming solid dispersed materials as shown in Fig. 2 (a). The graphite flakes also show a flat plate-like morphology with sizes less than $44 \mu \mathrm{m}$ and thicknesses of several micrometers. After chemical oxidization, the GO material has an irregular, layered, platelike structure as shown in Fig. 2 (b). The flakes appear to be thinner with fewer layers than the initial graphite flakes possibly due to exfoliation from the sonication process.
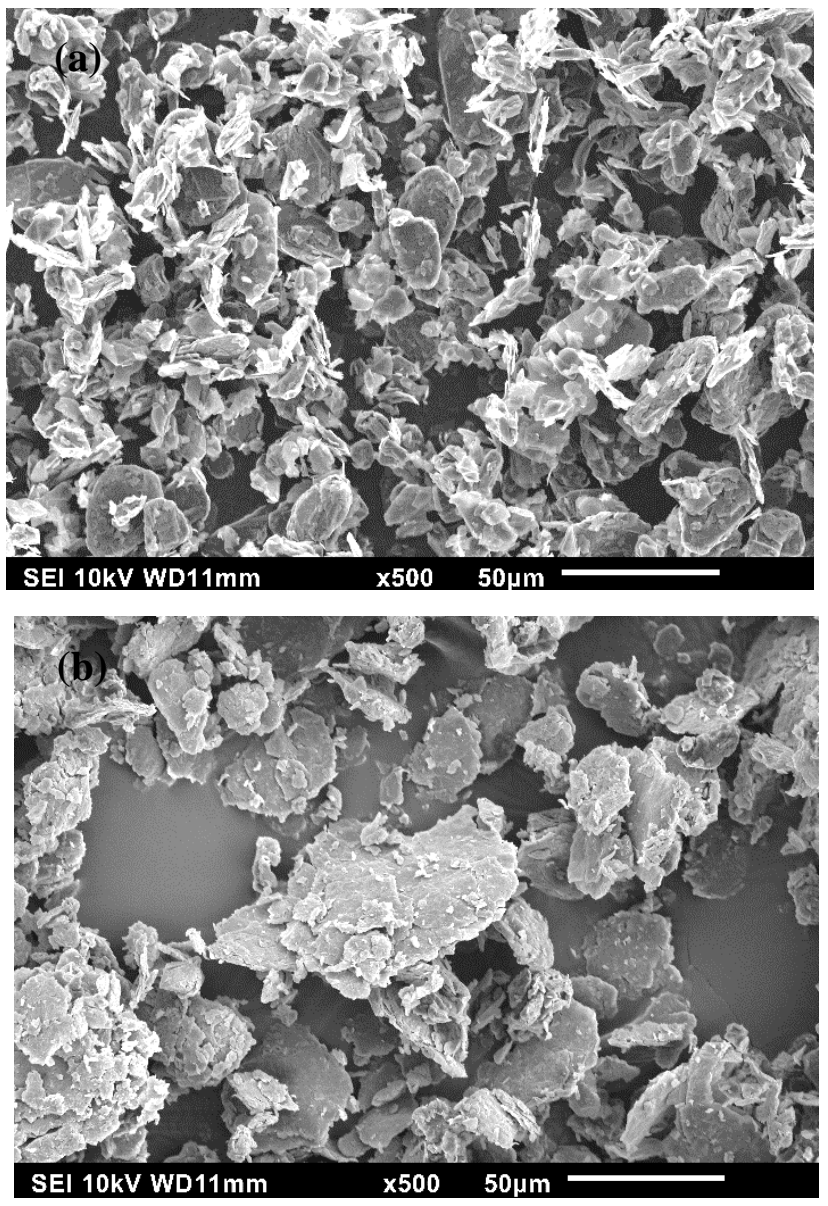

Figure 2. SEM image of (a) graphite powder and (b) GO powder. 
The SEM images of the fractured tensile surfaces are shown in Fig. 3. Pure epoxy is shown in Fig. 3 (a) with its typical smooth plate-like featureless surface. Some cracks are present indicating brittle fracture (low ductility). With the addition of 0.3 wt. \% GO reinforcement into the bio-epoxy polymer, the fractured surface becomes altered as shown in Fig. 3 (b). The surface appears to be rougher and more irregular with smaller failure surfaces. The image shows additional white regions which exhibits a more ductile fracture. The dispersion and possible agglomeration of GO sheets in the bio-epoxy matrix are difficult to visualize using SEM. One proposed method would be to etch the composite in sulfuric acid to reveal the GO sheets and their locations [6].
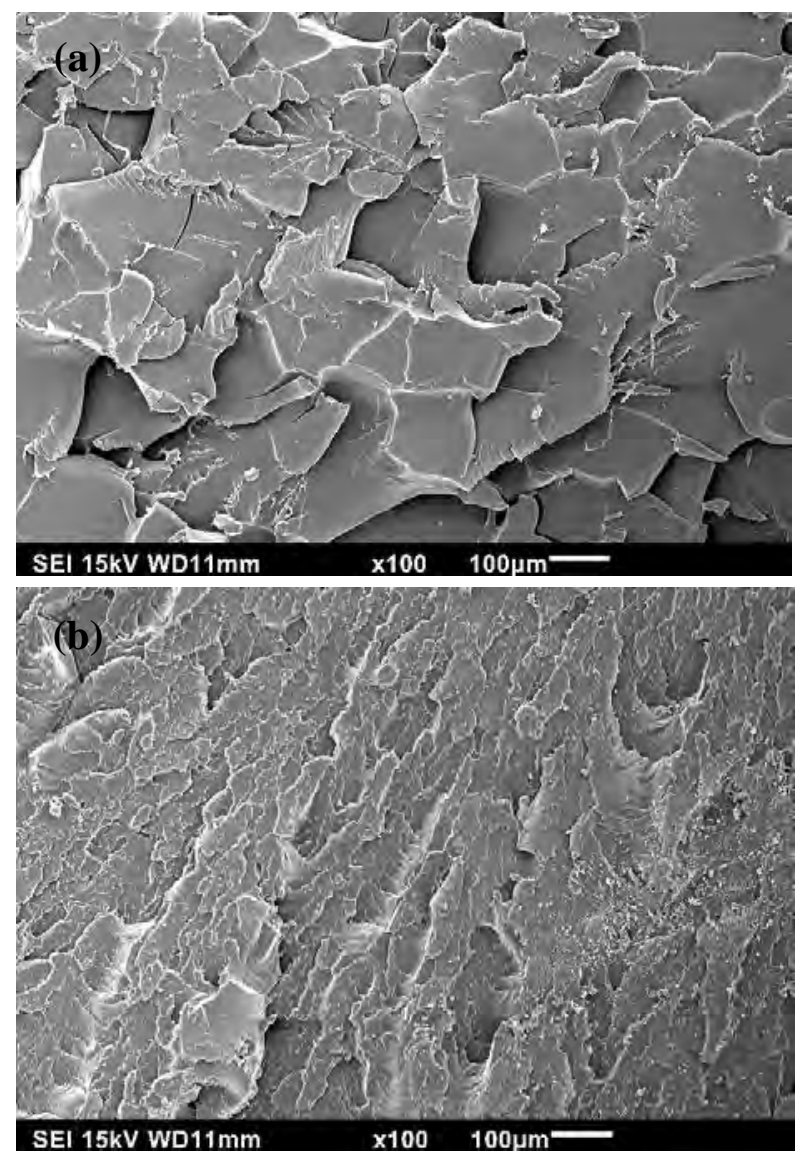

Figure 3. SEM images of fractured tensile surfaces of (a) pure epoxy and (b) epoxy $/ 0.3$ wt. $\%$ GO composite.

\section{B. FTIR Analysis}

FTIR tests were carried to confirm successful oxidation of graphite as shown in Fig. 4. Natural graphite powder did not show any significant peaks. In contrast, GO contains different functional groups as observed by characteristic feature peaks in the FTIR spectrum. A broad peak at $3400-3600 \mathrm{~cm}^{-1}$ signifies O-H stretching vibration of the hydroxyl groups [1] and water absorption at $3200 \mathrm{~cm}^{-1}$ [5]. The absorption peak at $1720-1732 \mathrm{~cm}^{-1}$ is attributed to stretching vibration of the carbonyl $(\mathrm{C}=\mathrm{O}) /$ carboxyl $(\mathrm{COOH})$ groups $[1,5]$. The peak at $1620-1622 \mathrm{~cm}^{-1}$ is attributed to the vibration of adsorbed water and vibrations from skeletal un-oxidized graphitic domain (some regions did not oxidize) which can be assigned to the aromatic $\mathrm{C}=\mathrm{C}$ bonds [5]. The $1200-1237 \mathrm{~cm}^{-1}$ peak is the $\mathrm{C}$ $\mathrm{OH}$ stretching from carboxylic groups [5], while the peak at $1050-1057 \mathrm{~cm}^{-1}$ is attributed to the skeletal C-O or C-C stretching from carbonyl, carboxylic and epoxy groups [5]. FTIR confirmed GO was successfully synthesized based on the presence of the oxygen-containing functional groups $(\mathrm{O}-\mathrm{H}$, $\mathrm{C}=\mathrm{O}, \mathrm{COOH}, \mathrm{C}-\mathrm{OH}$ and $\mathrm{C}-\mathrm{O}$ ).

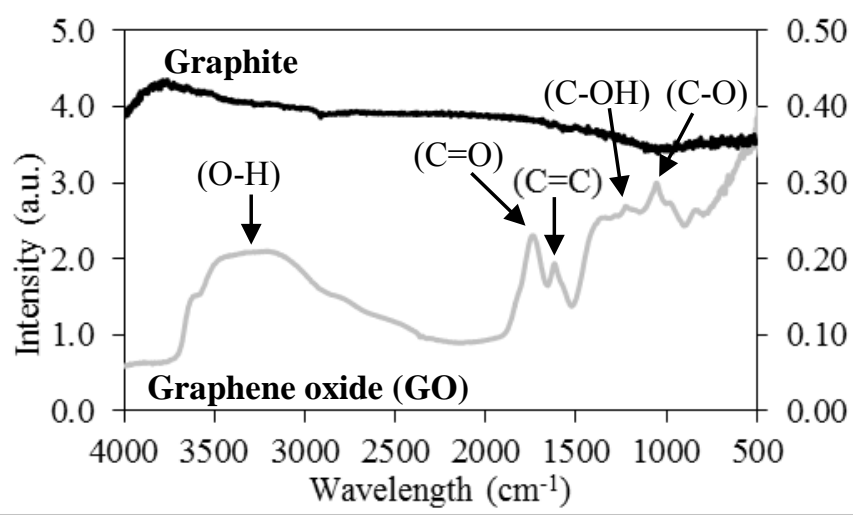

Figure 4. FTIR results for graphite (left scale) and graphene oxide (right scale).

\section{Mechanical Properties}

When GO was mixed into the bio-epoxy, the composites turned black in color as shown in Fig.1. This occurred for all bio-epoxy/GO composite loadings, even with 0.1 wt. \% GO. This change in color was also observed for epoxy composites containing 0.5-2 wt. \% GO and was attributed to the addition of the GO material [6].

The tensile strength and modulus results are shown in Fig. 5 with error bars representing the standard deviations. The pure bio-epoxy had a tensile strength and modulus of $59.68 \mathrm{MPa}$ and $2.6 \mathrm{GPa}$, respectively. The composites showed an improvement compared to the pure bio-epoxy for all GO filler loadings. The most significant improvement was at a loading of 0.3 wt. $\%$ GO where the tensile strength and modulus reached $73.55 \mathrm{MPa}$ and 3.5 $\mathrm{GPa}$, respectively which are increases of $23 \%$ and $35 \%$. The large surface area and functional groups on the surface and edges of the GO sheets have an affinity to the bio-epoxy matrix [7]. This improved interaction translates to a strong interfacial adhesion and load transfer between the matrix and the GO reinforcements.

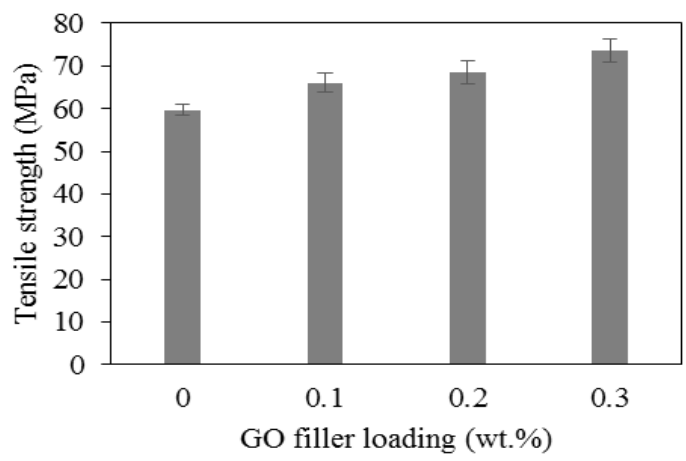




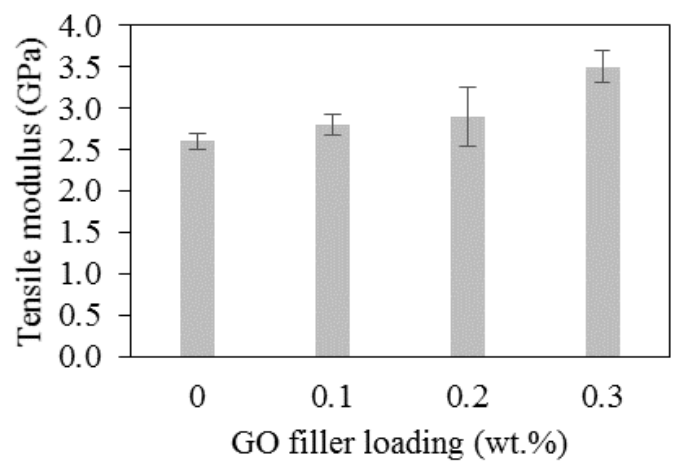

Figure 5. Tensile strengths and tensile modulus results for pure epoxy and epoxy/GO composites.

\section{CONCLUSION}

Epoxy/GO composites have successfully been produced using a simple solution mixing method. SEM showed the fractured surfaces of the tensile samples were altered from the addition of GO. FTIR verified the presence of oxygen based functional groups on the surface of the GO material and confirmed successful oxidation of graphite using the modified Hummer's method. Both tensile strength and tensile modulus showed a steady increase with the addition of GO. With a 0.3 wt. \% loading, the tensile strength improved by $23 \%$ whereas the modulus increased by $35 \%$ over the pure bio-epoxy. Therefore, a reinforcing effect has been observed with the addition of very small amounts of synthesized GO material. Based on the results, adding GO to a green bio-epoxy polymer is seen as a positive approach to enhance its tensile strength properties much more than traditional fillers of the same weight percentages. This composite would be able to compete in industries where synthetic epoxies are currently used.

\section{ACKNOWLEDGMENT}

The authors would like to acknowledge financial support of the Natural Sciences and Engineering Research Council of Canada (NSERC) under the Discovery Grant (418729-2012 RGPIN) and the University of Saskatchewan Undergraduate Student Research Assistantship.

\section{REFERENCES}

[1] Z. Li et al., "The role of functional groups on graphene oxide in epoxy nanocomposites," Polym., vol. 54, no. 21, pp. 5821-5829, Oct. 2013. doi: 10.1016/j.polymer.2013.08.026.

[2] J.-C. Charlier, X. Gonze, and J.-P. Michenaud, "Graphite interplanar bonding: Electronic delocalization and van der Waals interaction," EPL, vol. 28, no. 6, pp. 403-408, Nov. 1994. doi: $10.1209 / 0295-5075 / 28 / 6 / 005$.

[3] I. Ali et al., "Water treatment by new-generation graphene materials: hope for bright future," Environ. Sci. Pollut. Res., vol. 25, no. 8, pp. 7315-7329, Mar. 2018. doi: 10.1007/s11356-018-1315-9.

[4] J. Yan et al., "Functionalized graphene oxide with ethylenediamine and 1,6-hexanediamine," New Carbon Mater., vol. 27, no. 5, pp. 370-376, Oct. 2012. doi: 10.1016/S1872-5805(12)60022-5.
[5] D. Galpaya et al., "Preparation of graphene oxide/epoxy nanocomposites with significantly improved mechanical properties," J. Appl. Phys., vol. 116, no. 5, p. 053518, Aug. 2014. doi: $10.1063 / 1.4892089$.

[6] W. Xiao, Y. Liu, and S. Guo, "Composites of graphene oxide and epoxy resin assuming a uniform 3D graphene oxide network structure," RSC Adv., vol. 6, no. 90, pp. 8690486908, Sep. 2016. doi: 10.1039/C6RA16335A.

[7] S. Chatterjee et al., "Mechanical Reinforcement and Thermal Conductivity in Expanded Graphene Nanoplatelets Reinforced Epoxy Composites," Chem. Phys. Lett., vol. 531, pp. 6-10, Apr. 2012. doi: 10.1016/j.cplett.2012.02.006. 


\section{High Density Polyethylene/Carbon Nanotube Nanocomposite Foams: Electrical Conductivity and Percolation Threshold}

\author{
Linh T. Hoang \\ Department of Physics \& Astronomy \\ York University \\ Toronto, ON, Canada M3J 1P3 \\ linhhlp@yorku.ca
}

\author{
Mohammadmehdi Aghelinejad, Siu N. Leung, \\ and Zheng $\mathrm{H}$. Zhu \\ Department of Mechanical Engineering \\ Lassonde School of Engineering, York University \\ Toronto, ON, Canada M3J 1P3 \\ sunny.leung@lassonde.yorku.ca
}

\begin{abstract}
Electrically conductive polymer/carbon nanotube nanocomposites have drawn attention for a wide range of applications because of their light weight, good chemical resistance, ease of processing, and tunable multifunctional properties. High density polyethylene (HDPE)/multiwalled carbon nanotube (MWCNT) nanocomposites and their foams were fabricated, and their electrical properties were explored in this research. Parametric studies were conducted to investigate the effects of initial MWCNT loading and processing conditions on the foaming behaviors of HDPE/MWCNT nanocomposites. Experimental results revealed that both the initial MWCNT loading and foam morphology were governing factors to tune the electrical conductivity and the percolation threshold of HDPE/MWCNT nanocomposites.
\end{abstract}

Keywords-electrical conductivity; foam morphology; multiwalled carbon nanotubes; percolation threshold; polymer nanocomposites

\section{INTRODUCTION}

Polymer/carbon nanotube (CNT) nanocomposites have drawn extensive interests from researchers and industries because of their low density, superior chemical resistance, good manufacturability, and tunable multifunctional properties. As a result, this versatile material system has found a wide spectrum of applications including electromagnetic interference shielding [1], energy harvesting [2], energy storage [3], sensors [4-6], and thermal management [7]. Recent studies have suggested foaming polymer composites and nanocomposites as a promising fabrication strategy to tailor their micro-andnanostructures. Through biaxial stress fields induced by foam expansion, it is possible to align both one-dimensional (1D) and two-dimensional (2D) multifunctional fillers and thereby promote the material system's mechanical [8], electrical [9], and thermal [10] properties.

The electrical conductivity of a polymer/CNT nanocomposite can be drastically improved once its CNT loading has increased above a critical level, denoted as percolation threshold $\left(p_{c}\right)$. While high CNT loading promotes nanocomposites' electrical conductivity, it is detrimental to the weight, processability, and mechanical properties of
polymer/CNT nanocomposites. In this context, extensive studies were conducted to develop strategies for promoting polymer/CNT nanocomposite's electrical conductivity with lower CNT loading. These includes the utilization of hybrid conductive fillers with different types, sizes, and/or shapes as well as using immiscible polymer blends. Park et al. [11] investigated the effect of hybrid fillers on the electrical conductivity of the polypropylene (PP) composite. In their investigation, the inclusion of both MWCNT and carbon fiber in the PP matrix led to better conductivity than the case filled with only carbon fiber. In another study [12], acrylonitrile butadiene styrene (ABS) was melt-blended in polycarbonate (PC)/ MWCNT nanocomposite. Due to the partial solubility of ABS in PC, the selective dispersion of the MWCNTs in the ABS phase significantly promoted the electrical conductivity while reducing $p_{c}$.

This paper reports the application of supercritical carbon dioxide $\left(\mathrm{ScCO}_{2}\right)$ foaming to tailor the nanostructure of high density polyethylene (HDPE)/multiwalled carbon nanotube (MWCNT) nanocomposites. Parametric studies were conducted to elucidate the processing-structure-property relationships of HDPE/MWCNT nanocomposites and their foams. The effects of initial MWCNT loading and foaming temperature on the foam morphology and MWCNT networking of HDPE/MWCNT nanocomposites were studied. Furthermore, the interrelations among HDPE/MWCNT nanocomposite's foam morphology, MWCNT localization and networking, as well as the material system's electrical conductivity and percolation threshold were systematically studied. This work provides important guidelines to design and fabricate polymer/carbon nanotube nanocomposites with enhanced electrical conductivity and suppressed percolation threshold.

\section{EXPERIMENTAL}

\section{A. Materials}

Commercially available HDPE/MWCNT nanocomposite masterbatch (Nanocyl, Plasticyl HDPE1501), loaded with 15 wt\% MWCNT (Nanocyl, NC7000 ${ }^{\mathrm{TM}}$ ), and neat HDPE (NOVA Chemicals, SCLAIR ${ }^{\circledR}$ 2710) were used to prepare HDPE/MWCNT nanocomposites of different MWCNT 
loadings. The constituent MWCNT had an average length of 1.5 $\mu \mathrm{m}$ and an average diameter of $9.5 \mathrm{~nm}$. The physical foaming agent used in this study was carbon dioxide $\left(\mathrm{CO}_{2}\right)$ (Linde Gas Inc., $99.8 \%$ purity). Tables I through III summarize the physical properties of HDPE/MWCNT masterbatch, HDPE, and MWCNT, respectively. All materials were used as received without any modification.

\section{B. Sample Preparation}

Calculated amounts of HDPE/MWCNT nanocomposite masterbatch were weighed and the MWCNT content were reduced to various levels by adding different amounts of neat HDPE. The dilution was done by melt compounding the mixtures using a micro-compounder (HAAKETM MiniCTW Micro-Conical Twin Screw Compounder) in the circulating mode for 5 minutes at $215^{\circ} \mathrm{C}$, with the screw speed set at $75 \mathrm{rpm}$. The extrudates were pelletized and compression molded into a circular disc sample of $115 \mathrm{~mm}$ in diameter and $0.50 \mathrm{~mm}$ in thickness using a compression molding machine (Craver Press,

TABLE I. PHYSICAL PROPERTIES OF HDPE/MWCNT NANOCOMPOSITE MASTERBATCH

\begin{tabular}{|l|c|c|}
\hline \multicolumn{1}{|c|}{ Property } & Value & Unit \\
\hline MWCNT Loading & 15 & $\mathrm{wt} \%$ \\
\hline Density & 977 & $\mathrm{~kg} / \mathrm{m}^{3}$ \\
\hline Melting Temperature & 135 & ${ }^{\circ} \mathrm{C}$ \\
\hline Resistivity & $1.0(\max )$ & $\mathrm{k} \Omega$ \\
\hline
\end{tabular}

TABLE II. Physical Properties of NeAt HDPE

\begin{tabular}{|l|c|c|}
\hline \multicolumn{1}{|c|}{ Property } & Value & Unit \\
\hline Density & 951 & $\mathrm{~kg} / \mathrm{m}^{3}$ \\
\hline Vicat Softening Temperature & 125 & ${ }^{\circ} \mathrm{C}$ \\
\hline Resistivity & $1.0(\max )$ & $\mathrm{k} \Omega$ \\
\hline
\end{tabular}

TABLE III. PHYSICAL PROPERTIES OF MWCNT

\begin{tabular}{|l|c|c|}
\hline \multicolumn{1}{|c|}{ Property } & Value & Unit \\
\hline Average Diameter & 9.5 & $\mathrm{~nm}$ \\
\hline Average Length & 1.5 & $\mu \mathrm{m}$ \\
\hline Carbon Purity & 90 & $\%$ \\
\hline Surface Area & $250-300$ & $\mathrm{~m}^{2} / \mathrm{g}$ \\
\hline Volume Resistivity & $10^{-4}$ & $\Omega \cdot \mathrm{cm}$ \\
\hline
\end{tabular}

TABLE IV. PARAMETERs STUdied In Physcial FoAming EXPERIMENTS

\begin{tabular}{|l|c|c|}
\hline \multicolumn{1}{|c|}{ Parameter } & Value & Unit \\
\hline MWCNT Loading & $1,2,3,5,7$, and 10 & $\mathrm{wt} \%$ \\
\hline$P_{\text {sat }}$ & 1,200 (fixed) & $\mathrm{psi}$ \\
\hline$T_{\text {sat }}$ & $123-135$ & ${ }^{\circ} \mathrm{C}$ \\
\hline Saturation Time & 30 (fixed) & $\min$ \\
\hline
\end{tabular}

$4386 \mathrm{CH})$. The compression molding procedure is summarized below:

STEP 1. Weighed amount of HDPE/MWCNT nanocomposite pellets were loaded into a circular disc mold of $115 \mathrm{~mm}$ in diameter and $0.50 \mathrm{~mm}$ in thickness.

STEP 2. The compression molding machine was equilibrated at $155^{\circ} \mathrm{C}$.

STEP 3. The heat platens of the compression molding machine were brought into contact with the mold, which loaded with the nanocomposite pellets, for 5 minutes to ensure the complete melting of the nanocomposite pellets.

STEP 4. The molding pressure was ramped up to 4,000 psi and held at this level for 6 minutes.

STEP 5. The disc samples, which were still inside the mold, were transferred to a cooling module with flowing water channels to solidify the disc samples.

STEP 6. The samples were cut into rectangular specimens with dimensions of $12 \mathrm{~mm} \times 10 \mathrm{~mm} \times 0.50 \mathrm{~mm}$. The specimens would then be characterized using an electrical conductivity analyzer or be foamed by $\mathrm{ScCO}_{2}$.

HDPE/MWCNT nanocomposite foams were fabricated by a batch foaming chamber using $\mathrm{ScCO}_{2}$ as the physical foaming agent. In the physical foaming process, nanocomposite specimens were saturated with $\mathrm{ScCO}_{2}$ for 30 minutes at preset combinations of saturation pressure $\left(P_{\text {sat }}\right)$ and saturation temperature $\left(T_{\text {sat }}\right)$. Subsequently, an outlet valve of the batch foaming chamber was opened to rapidly depressurize it. This led to thermodynamic instability in the HDPE/MWCNT/ScCO system, and thereby induced cell nucleation and subsequent cell growth in the HDPE matrices. Table IV summarizes the key parameters being studied in the physical foaming experiments.

\section{Sample Characterization}

The apparent density of MWCNT/HDPE nanocomposite foams, were determined in accordance with ASTM D792. After measuring their masses in air and in water, the apparent density of a foam sample $\left(\rho_{f}\right)$ and the volume expansion ratio $(\phi)$ can be determined by Equations (1) and (2), respectively.

$$
\rho_{f}=\frac{m_{\text {air }} \rho_{\text {water }}}{m_{\text {air }}-m_{\text {water }}}
$$

where $m_{\text {air }}$ and $m_{\text {water }}$ are the masses of samples measured in air and in water, respectively; and $\rho_{\text {water }}$ is the density of water.

$$
\phi=\frac{\rho_{s}}{\rho_{f}}
$$

where $\rho_{s}$ is the density of the solid HDPE/MWCNT nanocomposite sample.

The phase and foam morphologies of the solid and foamed HDPE/MWCNT nanocomposite samples were characterized by scanning electron microscopy (SEM) (FEI Company Quanta 3D FEG). The cross-sections of all samples were exposed by cryo-fracturing the samples under liquid nitrogen. The fractured 
surfaces were then sputter-coated with gold (Denton Vacuum, Desk V Sputter Coater). The cell size and cell population density of selected foam samples were obtained by analyzing the SEM micrographs of the foams using Image J (National Institute of Health). The cell population density $\left(N_{0}\right)$ with respect to the unfoamed volume was determined by Equation (3).

$$
N_{0}=\phi \times\left[\frac{n M^{2}}{A}\right]^{\frac{3}{2}}
$$

where $n$ is the number of cells in the SEM micrograph; $M$ is the magnification factor; and $A$ is the area of the micrograph.

The electrical conductivity of solid HDPE/MWCNT nanocomposite samples were measured by the two-wire technique using a multifunctional source meter (Keithley, 2450 Source Meter). The two-wire technique was performed in accordance with the ASTM D257-07 standard. By printing conductive silver-epoxy paste as electrodes on both ends of a sample, the voltage difference was introduced through the sample and the flowing current was recorded to calculate the electrical conductivity of the nanocomposite sample. Depending on the volume expansion ratios, the thicknesses of foamed nanocomposite samples varied from 0.50 to $2.5 \mathrm{~mm}$. Fig. 1 illustrates the schematic of a nanocomposite sample prepared for the two-wire technique.

\section{RESUlts AND DISCUSSION}

Parametric studies were conducted to elucidate the processing-structure-property relationships of HDPE/MWCNT nanocomposite foams. On the one hand, it was expected that the presence of MWCNT would influence the $\mathrm{ScCO}_{2}$ foaming of HDPE. On the other hand, the biaxial stress fields induced by foam expansion would affect the alignment and localization of MWCNT in the HDPE matrix.

\section{A. Micro-and-Nanostructures of HDPE/MWCNT \\ Nanocomposites and Their Foams}

Fig. 2 (a) and (b) illustrate representative SEM micrographs of HDPE/MWCNT nanocomposites loaded with $3 \mathrm{wt} \%$ and 10 $\mathrm{wt} \%$ MWCNT, respectively. While increasing MWCNT loadings would promote the interconnectivity of MWCNT in HDPE matrices, it can be observed that continuous networks of MWCNT were omnipresent in the HDPE matrix even at $3 \mathrm{wt} \%$ MWCNT loading.

Fig. 3 (a) through (f) show the SEM micrographs of HDPE/MWCNT loaded with $3 \mathrm{wt} \%$ MWCNT before subjected to $\mathrm{ScCO}_{2}$ foaming at $P_{\text {sat }}$ of $1,200 \mathrm{psi}$ and different $T_{\text {sat }}$. Experimental observation revealed that foam morphologies of HDPE/MWCNT nanocomposite foams had strong dependence on $T_{\text {sat }}$. At $123^{\circ} \mathrm{C}$, the HDPE matrix, reinforced by MWCNT, was too stiff to achieve observable foam expansion. As $T_{\text {sat }}$ gradually increased, the foam morphologies evolved from having a high cell density with fine cells to a reduced cell density with larger cells. The foam structure was finally deteriorated when $T_{\text {sat }}$ was increased to $135^{\circ} \mathrm{C}$. In other words, within the processing window, varying $T_{\text {sat }}$ would be an effective approach to tune the cell morphologies and thereby the MWCNT network of HDPE/MWCNT nanocomposite foams.

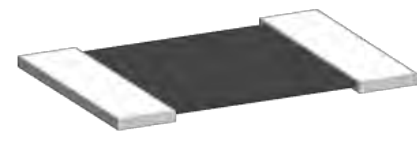

Figure 1. A Schematic of a Nanocomposite Sample Prepared for Electrical Conductivity Measurement by Two-Wire Technique

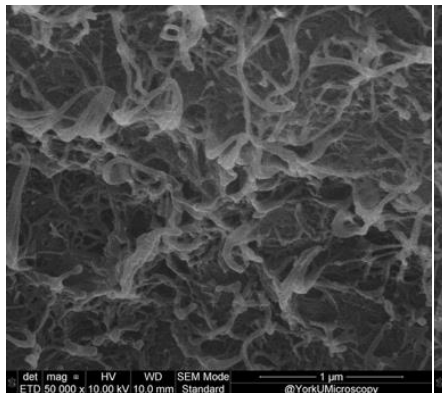

(a)

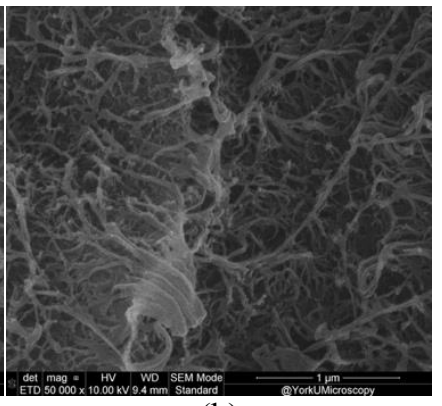

(b)
Figure 2. SEM Micrographs of HDPE/MWCNT Nanocomposites Loaded with (a) $3 \mathrm{wt} \%$ and (b) $10 \mathrm{wt} \%$ MWCNT

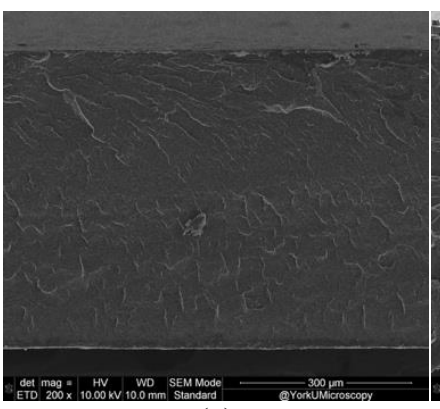

(a)

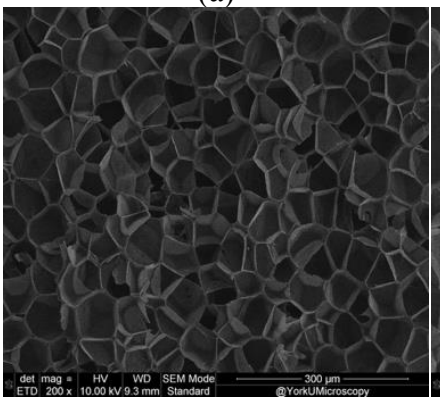

(c)

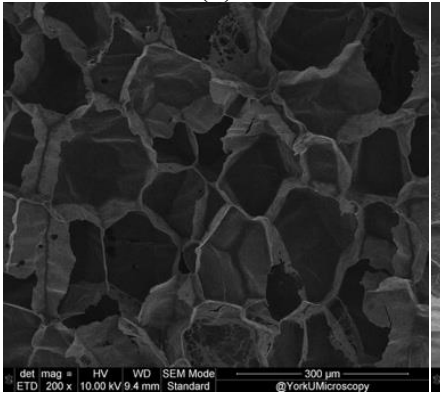

(e)

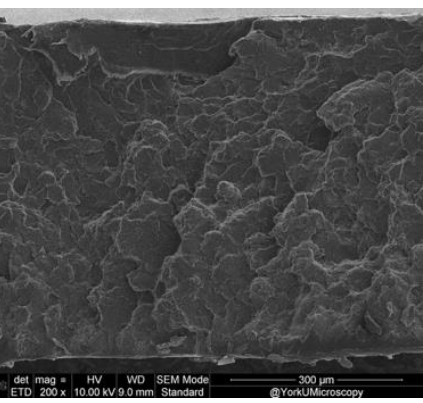

(b)

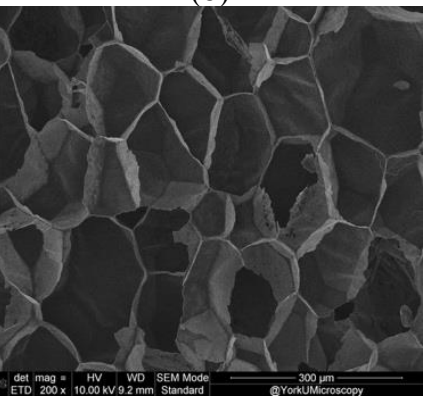

(d)

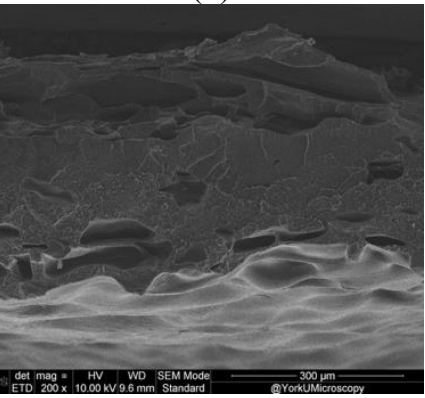

(f)
Figure 3. SEM Micrographs of HDPE/MWCNT Nanocomposite and Foams Loaded with $3 \mathrm{wt} \%$ Fabricated at $P_{\text {sat }}$ of $1,200 \mathrm{psi}$ and different $T_{\text {sat }}$ (a) Solid Nanocomposite; (b) $123^{\circ} \mathrm{C}$; (c) $125^{\circ} \mathrm{C}$; (d) $127^{\circ} \mathrm{C}$; (e) $130^{\circ} \mathrm{C}$; and (f) $135^{\circ} \mathrm{C}$ 


\section{B. Foaming Behaviours of HDPE/MWCNT Nanocomposites}

Experimental observation revealed that the optimal $T_{\text {sat }}$, to maximize the volume expansion of HDPE/MWCNT nanocomposite foams, depended on the initial loading of MWCNT. According to Fig. 4, by increasing the MWCNT content of the nanocomposites from 1 to $10 \mathrm{wt} \%$, their optimal $T_{\text {sat }}$ value also increases as the nanocomposite's melting temperature rises by increasing the MWCNT loadings. This could be attributed to the increased melt strength of the cell wall and reduced $\mathrm{CO}_{2}$ diffusivity as MWCNT loading increased.

\section{Electrical Conductivity and Percolation Thresholds of HDPE/MWCNT Nanocomposites and Their Foams}

To compare solid and foamed composites, the loadings of MWCNT were converted from weight fraction to volume fraction by considering the MWCNT density to be $1.75 \mathrm{~g} / \mathrm{cm}^{3}$ [9]. The volume fraction of MWCNT in foamed samples were calculated with respect to the total volumes of the solid part and the voids. Fig. 5 illustrates the effects of volume fractions of MWCNT on the electrical conductivity of HDPE/MWCNT nanocomposites and their foams at different initial MWCNT loadings (i.e., 3, 5, and $10 \mathrm{wt} \%$ ). Regarding of the initial MWCNT loading, the electrical conductivity of all nanocomposites increased with the final volume fraction of MWCNT. However, depending on the initial MWCNT content, nanocomposite foams loaded with the same final volume fraction of MWCNT showed significantly different electrical conductivity values. Experimental results also indicated that at low volume fraction of MWCNT, the electrical conductivity of foamed samples was remarkably higher than the solid samples. This increase was more significant for nanocomposites with higher MWCNT loading. As an example, the electrical conductivity of HDPE/MWCNT foam samples initially loaded with $10 \mathrm{wt} \%$ MWCNT was two orders of magnitude higher than the solid samples at $0.5 \mathrm{vol} \%$ (about $1 \mathrm{wt} \%$ ) and seven orders of magnitude higher at $0.3 \mathrm{vol} \%$ (about $0.5 \mathrm{wt} \%$ ).

The value of $p_{c}$ of the HDPE/MWCNT nanocomposites, which is the minimum MWCNT loading that would transform the nanocomposites from insulating to conductive, was estimated by fitting the percolation model (i.e., Equation (4)) to the experimentally obtained electrical conductivity data.

$$
\sigma=\sigma_{0}\left(p-p_{c}\right)^{t}
$$

where $\sigma$ is the electrical conductivity of the nanocomposite; $p$ is the loading of MWCNT; and $\sigma_{0}, p_{c}$ and $t$ were fitting constants and achieved by curve fitting using the root-mean-square-error method.

Fig. 6 presents the effect of foaming process on reducing $p_{c}$ of the solid HDPE/MWCNT nanocomposites. Experimental results indicated that $p_{c}$ of HDPE/MWCNT nanocomposites effectively decreased after foaming. The initial MWCNT content was a critical factor on suppressing the percolation threshold of the nanocomposite foams. By increasing the initial MWCNT loading of the nanocomposite samples from $3 \mathrm{wt} \%$ to $10 \mathrm{wt} \%$, the percolation threshold of the nanocomposite decreased from $0.55 \mathrm{vol} \%$ to $0.36 \mathrm{vol} \%$.

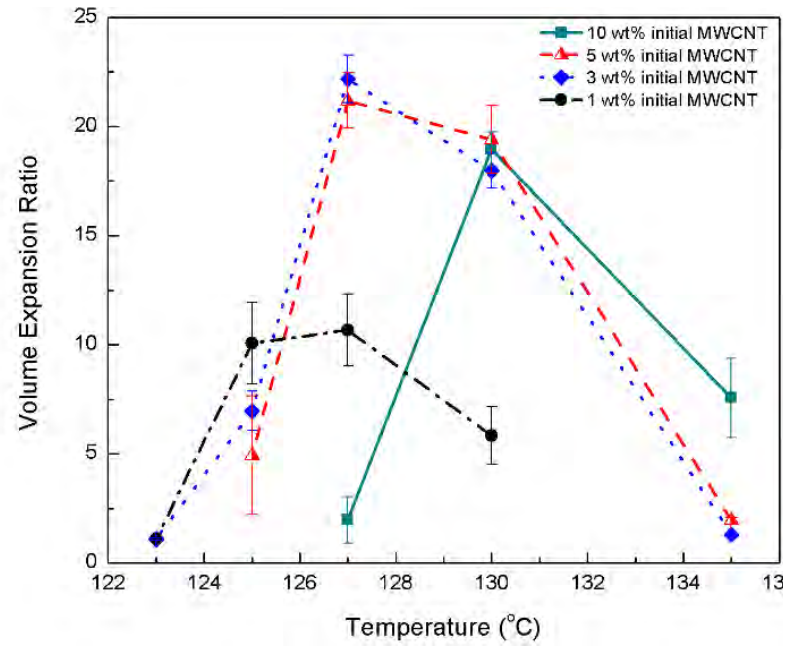

Figure 4. Effects of Tsat on Volume Expansion of HDPE/MWCNT Nanocomposite Foams with Different Initial MWCNT Loadings

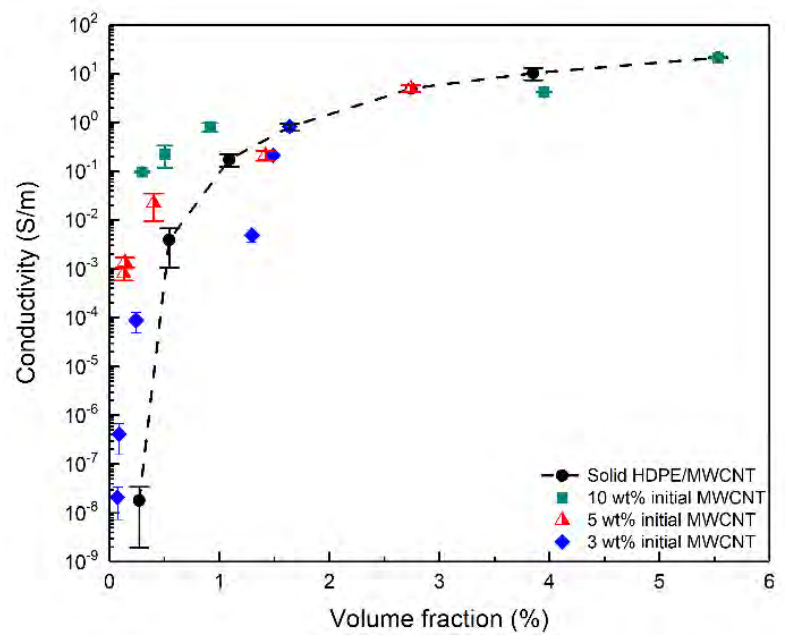

Figure 5. Effects of Volume Fractions of MWCNT on the Electrical Conductivity of HDPE/MWCNT Nanocomposites and Their Foams at different initial MWCNT loadings

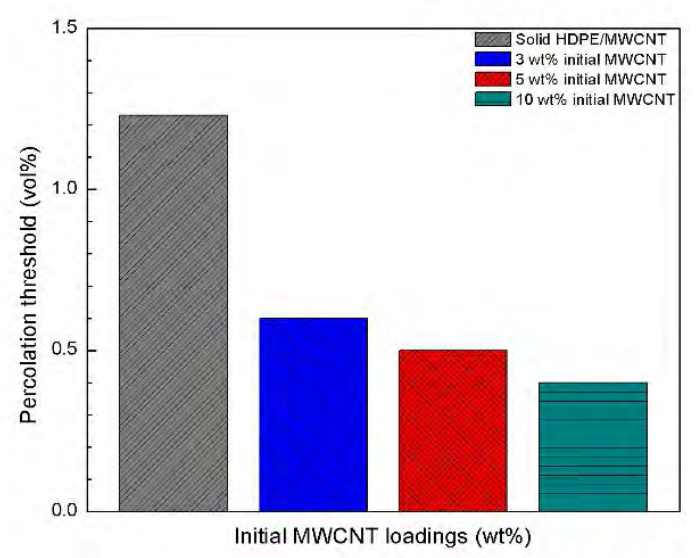

Figure 6. Percolation threshold for foams prepared with different initial MWCNT loadings vs that of solid nanocomposite 


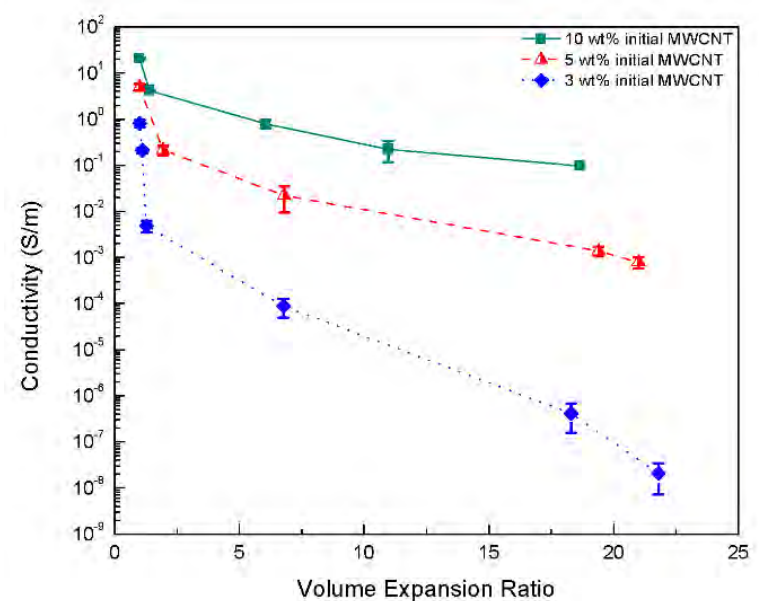

Figure 7. Effect of Volume Expansion Ratio on the Electrical Conductivity of HDPE/MWCNT Nanocomposites and Their Foams at different initial MWCNT loadings

Fig. 7 shows the dependence of electrical conductivity on the volume expansion ratio of HDPE/MWCNT nanocomposite foams. At the same initial MWCNT content, the higher expansion ratio would lead to smaller volume fractions of MWCNT, which decreased the electrical conductivity value for the foamed samples. Similar behavior was observed for all nanocomposite samples with different MWCNT initial loadings. However, it must be noted that larger volume expansion would also reduce the effective MWCNT loading in the nanocomposite foam. Overall, experimental results demonstrated that foaming was a promising strategy to increase the electrical conductivity of HDPE/MWCNT nanocomposites and to reduce the percolation threshold.

\section{CONCLUSION}

In this study, the effects of physical foaming on the electrical conductivity and the percolation threshold of HDPE/MWCNT nanocomposites were investigated. By changing the saturation temperature, one of the governing physical foaming parameters, the effects of foam morphology and volume expansion ratio on the electrical conductivity of the foamed samples were also explored in this research. The foam morphology and volume expansion ratio of nanocomposite foams were crucially dependent on both saturation temperature and initial loadings of MWCNTs in nanocomposites. The electrical conductivity of foamed samples was significantly higher than their solid counterparts, especially at low MWCNT volume fraction. The enhancement in the electrical conductivity was more pronounce for nanocomposites with higher initial MWCNT loadings. This also led to lower percolation thresholds than their solid nanocomposites.

\section{ACKNOWLEDGMENT}

The authors are grateful about the financial support by the Natural Sciences and Engineering Research Council of Canada.

\section{REFERENCES}

[1] J. M. Thomassin et al., "Foams of polycaprolactone/MWNT nanocomposites for efficient EMI reduction," J. Mater. Chem., vol. 18, pp. 792-796, 2008. doi: 10.1039/b709864b.

[2] M. Aghelinejad and S. N. Leung, "Enhancement of thermoelectric conversion efficiency of polymer/carbon nanotube nanocomposites through foaming-induced microstructuring," J. Appl. Polym. Sci., vol. 134, pp. 45073, 2017. doi: $10.1002 / a p p .45073$.

[3] C. Peng et al., "Carbon nanotube and conducting polymer composites for supercapacitors," Prog. Nat. Sci., vol. 18, pp. 777-788, 2008. doi: 10.1016/j.pnsc.2008.03.002.

[4] S. Gong, Z. H. Zhu, and Z. Li, "Electron tunnelling and hopping effects on the temperature coefficient of resistance of carbon nanotube/polymer nanocomposites," PCCP, vol. 19, pp. 5113-5120, 2017. doi: $10.1039 / \mathrm{C} 6 \mathrm{CP} 08115 \mathrm{~K}$.

[5] S. Gong and Z. H. Zhu, "On the mechanism of piezoresistivity of carbon nanotube polymer composites," Polymer, vol. 55, pp. 4136-4149, 2014. doi: 10.1016/j.polymer.2014.06.024.

[6] N. H. Quang et al., "Effect of NH3 gas on the electrical properties of single-walled carbon nanotube bundles," Sens. Actuators, B, vol. 113, pp. 341-346, 2006. doi: 10.1016/j.snb.2005.03.089.

[7] S. N. Leung et al., "Synergistic effects of hybrid fillers on the development of thermally conductive polyphenylene sulfide composites," J. Appl. Polym. Sci., vol. 127, pp. 3293-3301, 2013. doi: 10.1002/app.37941.

[8] M. Okamoto et al., "Biaxial Flow-Induced Alignment of Silicate Layers in Polypropylene/Clay Nanocomposite Foam," Nano Lett., vol. 1, pp. 503-505, 2001. doi: 10.1021/n1010051+.

[9] A. Ameli et al., "Polypropylene/carbon nanotube nano/microcellular structures with high dielectric permittivity, low dielectric loss, and low percolation threshold," Carbon, vol. 71, pp. 206-217, 2014. doi: 10.1016/j.carbon.2014.01.031.

[10] H. Ding, Y. Guo, and S. N. Leung, "Development of thermally conductive polymer matrix composites by foaming-assisted networking of micron- and submicron-scale hexagonal boron nitride," J. Appl. Polym. Sci., vol. 133, 2016. doi: 10.1002/app.42910.

[11] D. H. Park et al., "Effects of hybrid fillers on the electrical conductivity and EMI shielding efficiency of polypropylene/conductive filler composites," Macromol. Res., vol. 21, pp. 905-910, 2013. doi: 10.1007/s13233-013-1104-8.

[12] S. Maiti, N. K. Shrivastava, and B. B. Khatua, "Reduction of percolation threshold through double percolation in melt-blended polycarbonate/ acrylonitrile butadiene styrene/multiwall carbon nanotubes elastomer nanocomposites," Polym. Compos., vol. 34, pp. 570-579, 2013. doi: $10.1002 / \mathrm{pc} .22462$. 
MATERIALS TECHNOLOGY 


\section{A Finite Element Model to Predict the Effect of Porosity on Elastic Modulus in Low Porosity Materials}

\author{
Liam Morrissey \\ Department of Mechanical Engineering \\ Memorial University of Newfoundland \\ St. John's, Canada
}

\author{
Sam Nakhla \\ Department of Mechanical Engineering \\ Memorial University of Newfoundland \\ St. John's, Canada
}

I. Abstract - The effect of porosity on elastic modulus in low porosity materials is investigated. First, several models used to predict the reduction in elastic modulus due to porosity are compared with a compilation of experimental data to determine their ranges of validity and accuracy. The overlapping solid spheres model is found to be most accurate with the experimental data and valid between $3-10 \%$ porosity. Next, a FEM is developed with the objective of demonstrating that a macroscale plate with a center hole can be used to model the effect of microscale porosity on elastic modulus. The FEM agrees best with the overlapping solid spheres model and shows higher accuracy with experimental data than the overlapping solid spheres model.

Keywords-porosity, elastic modulus, FEA

\section{INTRODUCTION}

\section{A. Background}

Porosity can cause changes to a material's behaviour under loading that is different than predicted. In the extreme case, these changes, if unaccounted for, could lead to major safety and economic concerns. Therefore, the ability to accurately and easily model the effect of porosity is of paramount importance during the design and testing stages. While metals are typically not intended to contain significant pores, micro pores can be present due to manufacturing defects. These pores, which at first glance may appear inconspicuous, can increase in size during service in harsh environmental conditions. For example, in oil and gas pipelines and in hydrogen fuel tanks (typically found in aerospace), materials can be subject to high concentrations of hydrogen. Over time, hydrogen can diffuse into the material and recombine into $\mathrm{H}_{2}$, increasing the size of the initial voids and thus raising the porosity to a level that may effect the elastic modulus of the material [1-2]. However, while the effect of low levels of porosity on the yield stress has been comprehensively studied and captured in finite element software, the effect on the elastic modulus requires further research [3].

\section{B. Porosity and its Effect on Modulus}

Significant research has been completed on the relationship between porosity and elastic modulus. Models typically agree that the relationship falls into categories based on the level of porosity. For example, the work of Zhang and Wang in [4] has recommend subdividing porosity levels into low, medium and high porosity. Where low porosity is less than $10 \%$, medium porosity is $10-70 \%$ and, finally, high porosity is greater than $70 \%$. Therefore the approach taken to predict the effect of porosity on elastic modulus depends first on the degree of porosity in the material. It is important to remember that the numbers in these ranges are not necessarily exact and could possibly be better described as extremely low porosity, extremely high porosity, and then the large middle level [5]. In low porosity materials $(<10 \%$ porosity) there are several suggested relationships between porosity and elastic modulus that depend only on porosity. However, they do not predict the same influence of porosity and create a potential confusion when attempting to model a porous material. Therefore, this study attempted to clarify this confusion by evaluating these models and then demonstrating the applicability of a finite element model (FEM) to accurately predict the change in elastic modulus due to porosity.

1) Low Porosity Materials

For materials of low porosity there have been both analytical and experimental studies that developed a relationship to predict the elastic modulus in terms of the porosity. In this porosity level the elastic modulus is typically determined based on the assumption that voids are not interacting and that void fraction, and not void orientation, effects the materials behaviour [6]. As a result, models in the low porosity level are independent of the material and depend only on the degree of porosity.

For example, it has been suggested that a more simplified approach to modelling the elastic modulus of a porous material can be to consider the rule of mixtures used in composites to relate the modulus of two phases based on a volumetric fraction. In this case, one of the phases is considered as the solid non porous material and the other phase is the empty voids $[5,7]$. However, there is a lack of research on the validity of this model or its ranges of accuracy as compared to experimental data. As another example, Coble and Kingery in [8] developed a model to describe materials with spherical pores through testing of cast alumina with porosity from 5$50 \%$. Elastic modulus was then measured using samples in transverse bending and a model was generated that best fit experimental findings. Similarly, Maiti et al. in [9] developed a relationship between modulus and porosity by measuring the mechanical properties of three types of cellular solids as a 
function of density. An analytical model was then developed based on the concept that the loading of foam first causes deflection in the cells walls, which can be calculated by relating the densities of the foams to the cell walls. While this study was initially developed to model properties as a function of density, the ratio of the densities can also be related to a porosity ratio; allowing for the subsequent development of a model to predict the behaviour of porous materials. Finally, in [10] $\mathrm{Lu}$ et al. studied the effect of porosity on common industrial materials such as carbon rods, woods, ceramics and foams. Based on these experimental results a micromechanical theoretical model was then developed that related the elastic modulus to the porosity for porosity less than $30 \%$. This model considered the material as an isotropic matrix with $n$ spherical voids of varying sizes. Average shear strains were then calculated for a given shear stress, allowing the shear and elastic modulus to be calculated as a function of porosity. The theoretical model showed good agreement with the experimental data presented.

\section{2) Intermediate Porosity}

It is important to note that some models developed for the intermediate porosity level have also shown accuracy with experimental values in the low porosity level. As a result, some of these analytical models were also considered for their accuracy in predicting the elastic modulus in low porosity materials. In medium porosity materials pores can interact and lead to stress redistribution in the material. Moreover, there exists a critical porosity fraction that, when reached, the material loses all stiffness and load carrying capability. Accounting for this critical porosity value, data Bert et. al. in [11] presented (1) to predict the elastic modulus of medium porosity materials through an empirical observation of trends in experimental data. This model was developed based on the assumption that the ratio of porous material property to the solid material property is proportional to the ratio of minimum solid contact area to total cross-section area in the porous material [12]. The parameters $\phi_{0}$ and $\mathbf{n}$ must be determined for each model/material by best fitting to experimental/analytical results.

$$
\mathrm{E}(\phi)=\mathrm{E}_{0}\left(1-\left(\phi / \phi_{0}\right)\right)^{\mathrm{n}}
$$

Where $E_{0}$ is the elastic modulus of the material without pores, $\phi_{0}$ is the critical porosity value for the given material (between .37 and .97), $\phi$ is the porosity of the material, and $n$ is a power exponent that must be determined for each model/material.

Building on this model, in [13] Roberts and Garboczi used finite element analysis to study the influence of porosity and pore shape on the elastic properties of model porous ceramics. The study developed three different FEMs by placing spheres/ellipses in a unit cell to create various pore shapes and then best fitting the results to the model suggested by Bert in [5] to determine the $\phi_{0}$ and $n$ parameters. Predicted elastic moduli agreed well with experimental data and several models also accurately predicted the elastic modulus of materials with porosity below $10 \%$. Therefore these models will also be considered as potential models for the low porosity level.

Similarly, Hardin and Beckermann in [5] used a FEM to determine the best fit parameters for cast steel. First, a simple tension test and radiographic measurement was performed and a FE mesh was mapped onto an object in Abaqus. However, due to the microscopic nature of the pores, a small representative volume with a defined porosity fraction was used. A relationship for porosity was then determined by minimizing the difference between the FEM and experimental values and best fitting the results to the model suggested by Bert in [5].Results demonstrated that this method was accurate in the prediction of strains but had significant error in predicting the elastic modulus. The paper concluded that there was no correlation between average porosity and elastic modulus and that the reason for this error in modulus was because the FEM was a representative sample of the total porosity [5]. However, while the results may not be as useful in the prediction of elastic modulus, they serve to shed light on the importance of a FEM fully accounting for variations in porosity in the material.

\section{Finite Element Models}

Perhaps the most commonly referenced FEM for porous metals is the porous plasticity model found in Abaqus [14]. This model is based on the Gurson-Needleman-Tvergaard (GTN) model originally developed by Gurson in [15] and later extended by Tvergaard in [3] for the failure of ductile materials due to void coalescence. This model defines a yield potential based on an isolated spherical void and forms the basis of the porous plasticity model currently used in Abaqus to predict the behaviour of mildly voided materials. However, while the model is able to accurately predict micromechanical parameters such as yield stress, it is unable to account for the macro scale effects of the micro scale pores. For example, Hardin and Beckermann in [16] investigated using the porous plasticity model to model the influence of porosity on the yielding of cast steel. As porosity was changed the only observable difference in the stress strain curves was a change in yield stress [16]. Therefore, because there was no change in the slope of linear portion of the plot, the model does not predict a change in elastic modulus due to porosity. This finding serves to highlight the confusion at hand. While studies and intuition dictate that porosity should effect the modulus, the most commonly used models appear to ignore or not account for the effect on elastic modulus.

\section{Purpose of Study}

As can be seen from the above review, several models exist to predict the influence of porosity on elastic modulus. However, because there is no study on the ranges of validity and relative accuracy of these models there is a potential confusion when attempting to predict the elastic modulus of a low porosity material. Moreover, the GTN model, a popular microscale model in Abaqus for low porosity materials, only adds to the confusion by predicting a change in the yield stress and no change in the elastic modulus. As a result, Abaqus does not have the capability to accurately predict the elastic modulus of a porous material. To help eliminate this gap in knowledge this study focused on the evaluation of models used for materials in the low porosity level. The first objective of this study was to compare the present models and to clearly identify their validity when compared to experimental data. Next, a FEM was developed with the objective of demonstrating that a 
macroscale plate with a center hole can be used to the effect of microscale porosity on elastic modulus.

\section{PROCEDURES}

\section{A. Evaluation of Existing Models}

The first step in this study was to examine experimental data to verify that that there was indeed an effect of porosity on elastic modulus. This was achieved by compiling a set of previously conducted experimental data on the elastic modulus of materials with porosity ranging from $0-10 \%$. Next, this experimental data was used to evaluate the previously suggested relationships between porosity and elastic modulus to determine their relative accuracy.

\section{B. Finite Element Model Study}

After determining which relationship best predicted the effect of porosity on elastic modulus a simplified FEM was then developed using the finite element software Abaqus. First, a large 2D stainless steel plate in plane stress was developed and a pressure load was applied on its right edge. The plate was fixed in the U1 direction on its left edge to model a tension test. A simulation was then run to produce a stress strain curve using the applied pressure and change in length of the plate. Finally, the slope of this curve was used to obtain an effective elastic modulus of $200 \mathrm{GPa}$; matching the inputted value and therefore verifying the model.

Next, python scripting was used to investigate the effect of pore orientations on elastic modulus. The pores simulated various degrees of porosity in the material. The above test was replicated for a range of pore orientations and stress strain curves were used to calculate the elastic modulus at 2,4,6 8 and $10 \%$ porosity. A summary of the pore orientations tested is shown in Figure 1.

Finally, based on the findings of pore orientation, python scripting was used to test plates with a center pore accounting for $1-10 \%$ of the total surface area of the plate. The center pore simulated various degrees of porosity in the plate based on the assumption that total pore volume, and not pore orientation, effects the modulus for low porosity materials. The slope of the stress strain curve was then used to obtain the effective elastic modulus for the steel at various degrees of porosity. These results were then compared with experimental data and the most accurate models from above to demonstrate that a macroscale FEM accurately predicted the influence of porosity on elastic modulus.

\section{RESULTS AND DisCUSSION}

\section{A. Effect of Porosity on Elastic Modulus}

While several models exist to predict the change in elastic modulus due to porosity, their relative accuracy and ranges of validity remains unknown. To close this knowledge gap a comparison between several models and experimental data was conducted. Figure 3 compiles a set of experimental data on the percent reduction in elastic modulus for a range of porous materials. The data observed a linear trend of increasing percent reduction in elastic modulus with increasing porosity $\left(\mathrm{R}^{2}=.77\right)$. Therefore, it is clear that porosity has a direct effect on the elastic modulus of a material. The wide range of materials and preparation techniques for the experimental data also shed light upon the variables that should be considered when modelling porosity. For example, alumina values were obtained from two different sources [17-18]. In [18], Asmani et. al shaped alumina pellets via uniaxial pressing and then sintered these pellets at various temperatures to produce closed porosity shapes. The elastic modulus was then measured using a pulse echo method that calculated the transit time and longitudinal/transverse wave velocities. In contrast, the data provided by Knudsen in [17] was a compilation for a range of tests on the elastic modulus of alumina. Samples were prepared via cold and hot pressing and elastic modulus was determined both statically and dynamically. Therefore, given that both data sets closely follow the same trend, it appears that preparation/measuring techniques do not influence the reduction in elastic modulus. While the majority of materials closely followed this trend, porosity in $\mathrm{HfO}_{2}$ resulted in a lower reduction in elastic modulus than other materials. The $\mathrm{HfO}_{2}$ samples were prepared using a dried powder that was formed calcined, and then stabilized with approximately $30 \% \quad \mathrm{Er}_{2} \mathrm{O}_{3}$ [19]. However, this additive was shown to change the microstructure and effect the baseline elastic modulus [19]. As a result, the stabilizer may have changed the porosity of the material and lead to less of a reduction in elastic modulus. Moreover, when the data for $\mathrm{HfO}_{2}$ is ignored the linearity of the experimental values raises to and R-squared value of .87 . Overall, because the data for several different materials followed the same linear trend it supports the conclusion that models for low porosity materials do not need to be material specific and that the pore volume is critical parameter.

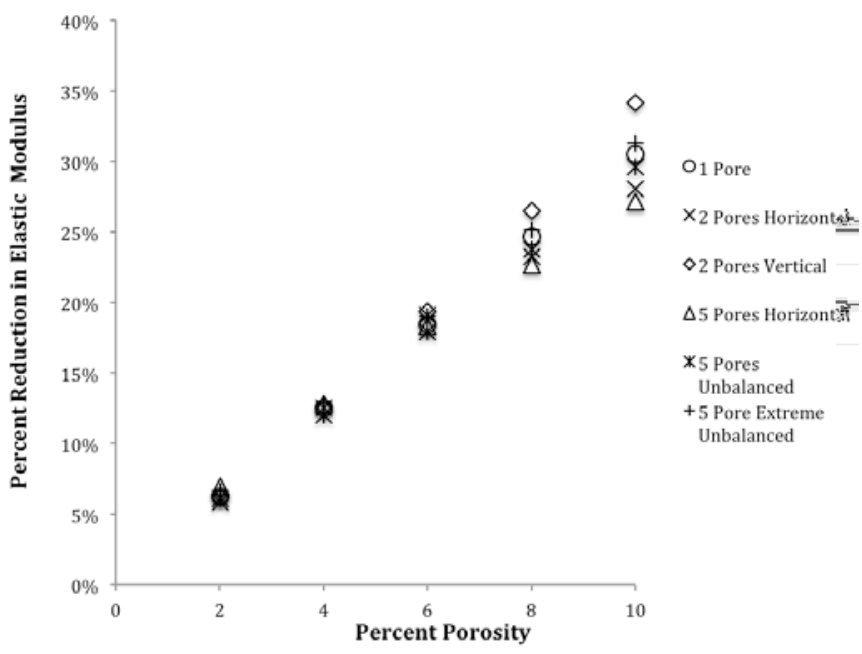

Fig. 1 - A compilation of experimental data for the percent reduction in elastic for various materials with porosity between 1-10\% (Alumina 1 [17], Alumina 2[18], thermoset polyester resin [20], $\mathrm{HfO}_{2}$ [19], sintered Iron [21], $\mathrm{MgAl}_{2} \mathrm{O}_{4}$ ([22])

\section{B. Evaluation of Existing Models}

Next, 6 models for low porosity materials were evaluated against the experimental data above. First, the three 
relationships modelled by Roberts and Garboczi in[13] based on various pore orientations were considered (2-4). These equations were developed by placing either spheres or ellipses into a unit cell such that the orientations of these pores created various pore shapes. Results were then best fit to (1) to determine the $\phi_{0}$ and 12 parameters.

$$
\begin{aligned}
& \mathrm{E}(\phi)=\mathrm{E}_{0}(1-(\phi / 0.652))^{2.23} \\
& \mathrm{E}(\phi)=\mathrm{E}_{0}(1-(\phi / 0.818))^{1.65} \\
& \mathrm{E}(\phi)=\mathrm{E}_{0}(1-(\phi / 0.798))^{2.23}
\end{aligned}
$$

Where (2) was developed for overlapping solid spheres by placing solid spheres at random points in a unit cell, (3) was developed for overlapping spherical pores by interchanging the roles of the solid and pore phase of the overlapping solid sphere model and (4) was developed for overlapping ellipsoidal pores by changing to spherical pores to overlapping oblate ellipsoidal pores bound by a surface.

In addition to these models, the rule of mixtures (5) in [7], the model for materials with spherical holes developed by Coble and Kingery (6) in [8], the model for closed cell porous materials developed by Maiti et al. (7) in [9], and the micromechanical model suggested by Lu et al. (8) in [10] were also considered.

$$
\begin{aligned}
& \mathrm{E}(\phi)=\mathrm{E}_{0}(1-\phi) \\
& \mathrm{E}(\phi)=\mathrm{E}_{0}\left(1-1.86 \phi+0.86 \phi^{2}\right) \\
& \mathrm{E}(\phi)=\mathrm{E}_{0}(1-(\phi))^{3} \\
& \mathrm{E}(\phi)=\mathrm{E}_{0}(1-2 \phi)\left(1+4 \phi^{2}\right)
\end{aligned}
$$

Figure 4 presents the predicted percent reduction in elastic modulus for each model as compared to the experimental data for low porosity materials. First from an overall perspective, all analytical models considered captured the trend of increasing percent reduction in elastic modulus with increasing porosity demonstrated by the experimental values. Between $0-2 \%$ porosity (Figure $2 \mathrm{~A}$ ) it is difficult to discern a clear trend and the range of predicted values was closely clustered between a $2-6 \%$ reduction in elastic modulus. However, as the porosity increased (Figure $2 \mathrm{~B}$ ) the range of predicted percent reduction grew to over $20 \%$ and there were stark differences in the models.

First, for the FEMs presented by Roberts and Garboczi in [13] it can be seen that the overlapping solid spheres model and ellipsoidal pores model both fell within the range of experimental values and were more accurate than the spherical pores model. Moreover, when compared to line of best fit of the experimental data the overlapping solid spheres model was the most accurate model tested. However, the micromechanical geometries of these models create potential limitations to the respective ranges of validity. For example, in the overlapping spherical pores model pores only become macroscopically connected at porosities above $30 \%$. Therefore, an overlapping solid spheres microstructure is not applicable for low porosity materials, potentially explaining the inaccuracy with the experimental. Similarly, pores in the overlapping ellipsoidal pores model only become macroscopically connected at porosities above $20 \%$. However, while the range of validity for this model was also outside the porosity level in question, it's validity was closer to the $0-10 \%$ level than the overlapping solid spheres model. This may help explain why it followed the experimental line of best fit closer than the overlapping solid spheres model. Finally, the overlapping spherical pores model was the most accurate of the three and, unlike the other models, has a microstructure than becomes macroscopically connected at porosities above $3 \%$. Therefore, this model is valid within the majority of the low porosity range and was most accurate when compared to the experimental line of best fit.

When examining the other models considered it can clearly be seen that (5), the rule of mixtures model, drastically under predicted the percent reduction in elastic modulus and did not agree with any experimental data. Similarly, the models suggested by Coble and Kingery (6) and Lu (8) both under predicted the reduction in elastic modulus and agreed only with the experimental data for $\mathrm{HfO}_{2}$ presented by Dole et al. in [19]. Therefore, these models are not recommended to predict the effect of porosity on elastic modulus. In contrast, (7), the model for closed cell porous materials developed by Maiti et al., also closely followed the experimental data and was the second most accurate model when compared to the line of best fit. This model was actually derived during the development of a model to predict mechanical properties of a cellular material in compression. The initial model predicted the elastic modulus of a foam through the ratio of the relative densities and the elastic modulus of the solid cell wall. (7) was then derived by replacing the ratio of the densities of foam to solid by one minus the porosity, and by then replacing the elastic modulus of the cell wall with the elastic modulus of the solid material.

\section{Finite Element Model}

\section{1) Effect of Pore Orientation on Elastic Modulus}

After comparing and evaluating the existing models, the next step was to examine the applicability of a FEM for low porosity materials in Abaqus. Although porosity is a microscale phenomenon, its effect can be seen on both the yield stress (a microscale property) and on elastic modulus (a macroscale property). Therefore, the fundamental question asked by this FEM was whether microscopic pores can be approximated as one bulk macroscopic hole and then be used to accurately obtain the elastic modulus, a macroscopic property. The first step to answering this question was to investigate the effect of pore orientation on elastic modulus. This was achieved by measuring the slope of the stress strain curve of a macroscopic plate in tension in Abaqus with various pore orientations accounting for $0-10 \%$ porosity. 

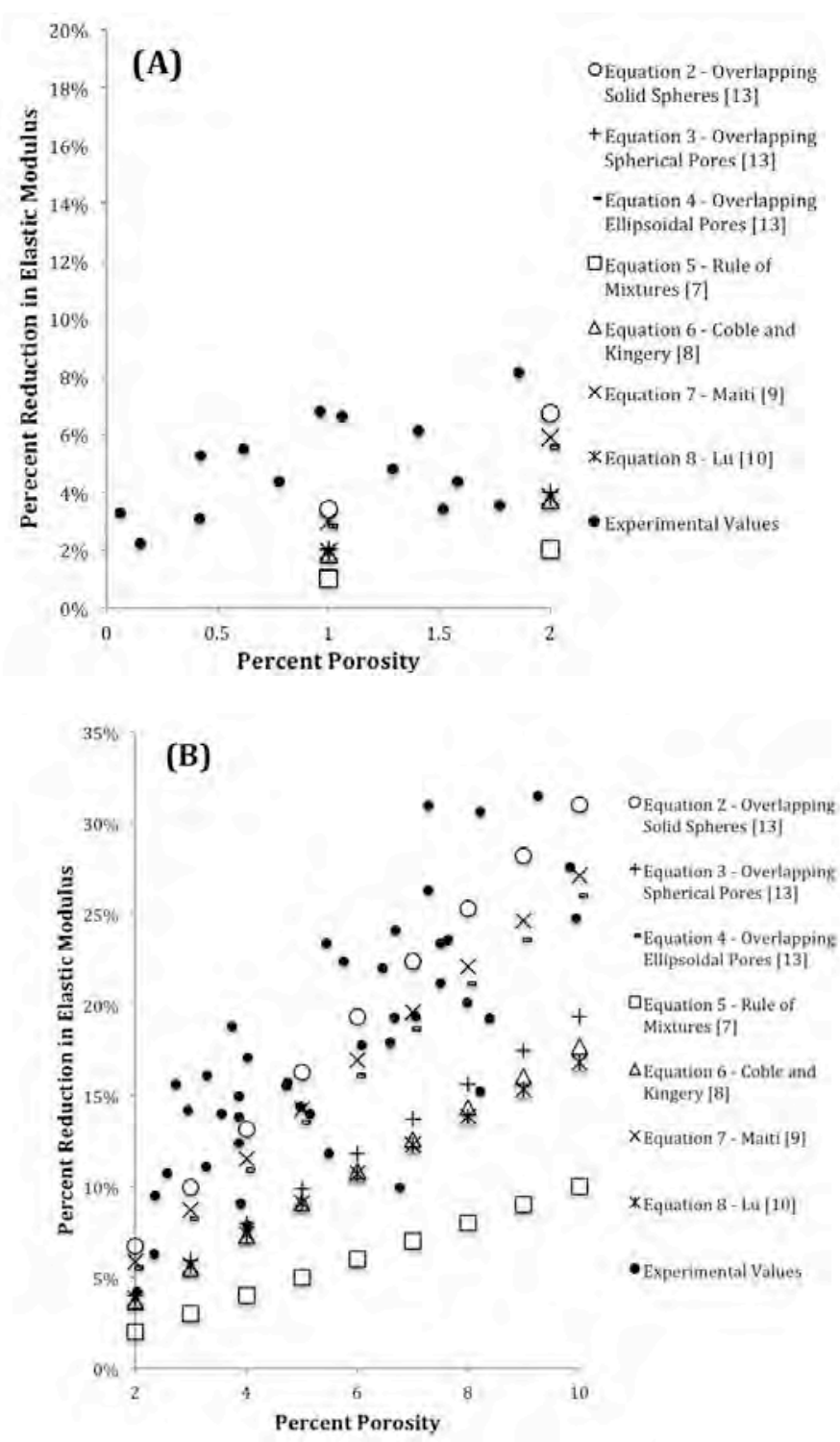

Fig. 2 - Predicted reduction in elastic modulus for various analytical models compared with experimental data for materials with porosity between $0-2 \%$ (A) and $2-10 \%$ (B)

The effect of porosity on elastic modulus for various pore orientations was compared in Figure 3. Several different pore orientations were considered that varied the size, number, and location of the pores. The maximum error between orientations occurred at $10 \%$ porosity $(8.32 \%)$, while all other errors were below $6 \%$. Therefore, changing pore orientation did not appear to cause a significant effect of the percent reduction of elastic modulus. However, it is important to note that as porosity increased the standard deviation between the various orientations also increased. For example, at $2 \%$ porosity the standard deviation between all orientations was only 0.004 and the range in predicted reduction in elastic modulus was $5.9 \%-6.9 \%$. However, as the porosities increased so did the gap in the predicted reduction of elastic modulus. For example, by simply looking at Figure 3 it is clear that data at $10 \%$ porosity the results are significantly

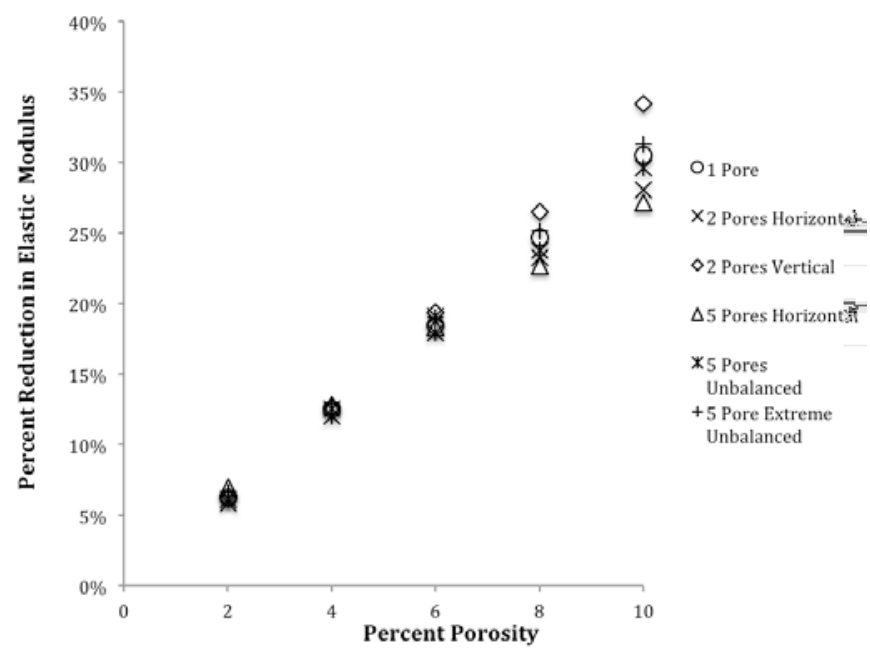

Fig. 3 - Effect of pore orientation on reduction of elastic modulus for low porosity materials.

more spread out than the at the lower porosities. At $10 \%$ porosity the standard deviation was raised by approximately seven times to 0.025 and range in values was between 27 $34 \%$. Therefore, while the assumption that pore orientation does not effect change in elastic modulus for low porosity materials was supported, there is more error in the assumption as the material nears $10 \%$ porosity. In addition, it can be seen that the 1 center pore always fell within the middle of the ranges of percent reduction in elastic modulus. Therefore, to ensure that the results were not biased by orientation, the center pore was used for comparison with analytical and experimental results.

\section{2) Comparison of Finite Element Model with Analytical} Models and Experimental Values

After investigating the effect of pore orientation in a FEM, the next step was to compare the FEM with analytical and experimental results. First, Figure 4 compares the predicted reduction in elastic modulus for porosities between $0-10 \%$ for the proposed FEM with the analytical models. The FEM produced a highly linear relationship between porosity and elastic modulus between $1-10 \%$ porosity $\left(\mathrm{R}^{2}=.9983\right)$. Over the entire porosity range the overlapping solid spheres model had the lowest average error when compared to the FEM (average error of $-4.38 \%$ with standard deviation of .0216) followed by (7) (average error of $8.67 \%$ with standard deviation of .0199). Therefore, the two models shown to be most accurate to the experimental data also matched best with the proposed FEM.

Next, Figure 5 compares the results of the FEM with experimental data from several different low porosity materials. The experimental data and FEM both closely followed a similar linear trend with the FEM falling within the range determined by the various experiments. When comparing the FEM to a linear trend line of experimental data it can be seen that the FEM followed the experimental data 
better than all other models for porosities between 5-10\% and was second only to the overlapping solid spheres model for all other porosities. Moreover, because the overlapping solid spheres model is not valid between $0-2 \%$, the FEM was both more accurate and applicable over a larger range of validity. Therefore, a macroscale plate with a hole was shown to successfully predict the elastic modulus of materials with micro pores.

\section{CONCLUSIONS}

An accurate relationship between elastic modulus and porosity is critical to safely using materials with porosity. However, while several models exist to predict the effect of porosity on elastic modulus for low porosity materials, these relationships predict varying effects of porosity. This can create confusion for engineers looking to predict the behaviour of a porous material. Therefore, this study compared commonly used models against a compilation of experimental values. While several models did not match well with experimental values, the overlapping solid spheres model (2) best agreed well with the data. Next, this paper considered a large plate with a center pore in tension to demonstrate the validity of a using a macroscale model to predict the effect of microscale pores on the elastic modulus using FEA. The FEM matched with experimental data better than the overlapping solid spheres model and, unlike this model, was applicable for the entire low porosity range. Therefore, this study successfully demonstrated the most accurate relationship between porosity and elastic modulus and then used a FEM to improve upon this model.

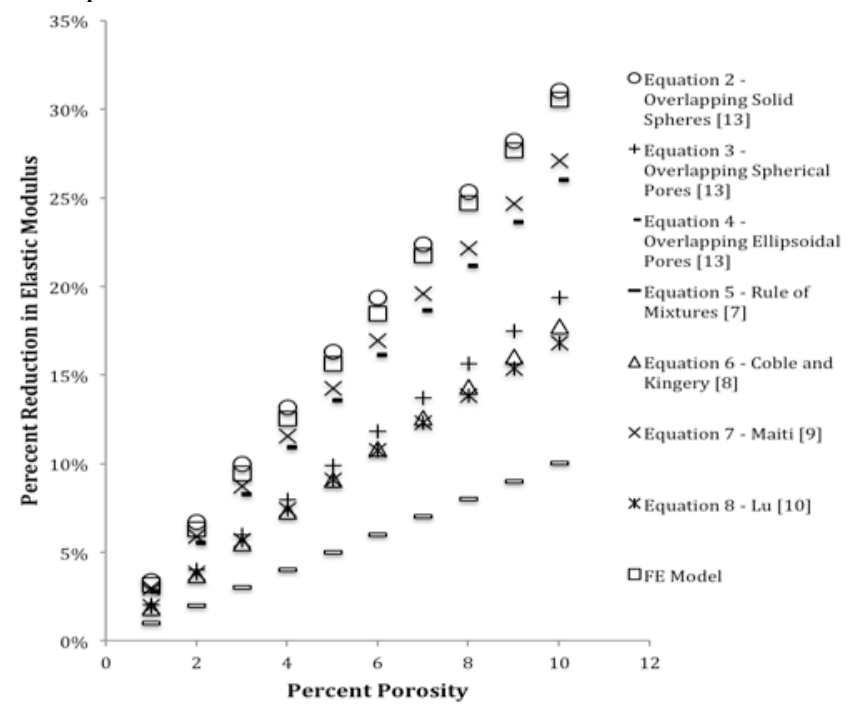

Fig. 4 - The predicted reduction in elastic modulus for porosities between $0-10 \%$ for the proposed Finite Element model as compared to analytical models.

\section{REFERENCES}

[1] Y. Liang, D. C. Ahn, P. Sofronis, R. H. Dodds Jr and D. Bammann, "Effect of hydrogen trapping on void growth and coalescence in metals and alloys," Mech. Mater., vol. 40, issue 3, pp. 115-132, 2008. doi: 10.1016/j.mechmat.2007.07.001.

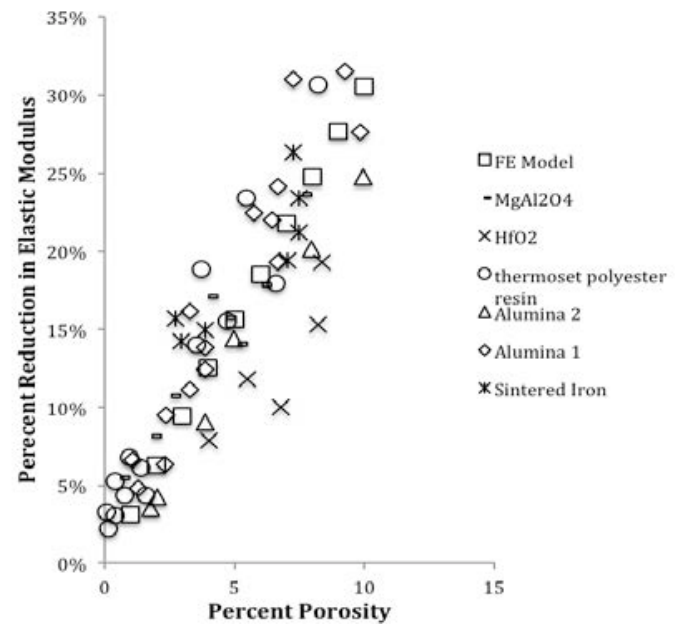

Fig. 5 - Reduction in modulus for FEM and experimental data (Alumina 1 [17], Alumina 2[18], thermoset polyester resin [20], $\mathrm{HfO}_{2}$ [19], sintered Iron [21], $\mathrm{MgAl}_{2} \mathrm{O}_{4}$ ([22])

[2] M. Nagumo, "Hydrogen related failure of steels - a new aspect," Mater. Sci. Technol., vol. 20, issue 8, pp. 940-950, 2004. doi: $10.1179 / 026708304225019687$.

[3] V. Tvergaard, "Influence of voides on shear band instabilities under plane strain conditions, Int. J. Fract., vol.17, pp. 389-407, 1981, doi: 10.1007/BF00036191.

[4] E. Zhang and B. Wang, " On the compressive behaviour of sintered porous coppers with low to medium porosities-Part I: Experimental study," Int. J. Mech. Sci., vol. 47, issues 4-5, pp. 744-756, 2005. doi: 10.1016/j.ijmecsci.2004.12.011

[5] R. A. Hardin and C. Beckermann, "Effect of Porosity on the Stiffness of Cast Steel," Metall. Mater. Trans. A, vol. 38, pp. 2992-3006, 2007. doi: 10.1007/s11661-007-9390-4.

[6] J. M. Dewey, "The Elastic Constants of Materials Loaded with Non - Rigid Fillers," J. Appl. Phys. (Melville, NY, U. S.), vol. 18, pp. 578-581, 1947. doi: 10.1063/1.1697691.

[7] T. Sumitomo, C. H. Cáceres and M. Veidt, "The elastic modulus of cast Mg-Al-Zn alloys," Journal of Light Metals 2002, vol. 2, issue 1, pp. 49-56, 2002. doi: 10.1016/S1471-5317(02)00013-5.

[8] R. L. Coble and W. D. Kingery, "Effect of Porosity on Physical Properties of Sintered Alumina," J. Am. Ceram. Soc., vol. 39, pp. 377-385. doi: 10.1111/j.1151-2916.1956.tb15608.x.

[9] S. K. Maiti, L. J. Gibson and M. F. Ashby, "Deformation and energy absorption diagrams for cellular solids," Acta Metall., vol. 32, issue 11, pp. 1963-1975, 1982. doi: 10.1016/0001-6160(84)90177-9.

[10] G. Lu, G. Q. Lu and Z. M. Xiao, "Mechanical Properties of Porous Materials," J. Porous Mater., vol. 6, pp. 359-368, 1999. doi: 10.1023/A:1009669730778.

[11] C. W. Bert, "Prediction of elastic moduli of solids with oriented porosity," J. Mater. Sci., vol. 20, pp. 2220-2224, 1985. doi: 10.1007/BF01112307.

[12] R. W. Rice, "Use of normalized porosity in models for the porosity dependence of mechanical properties," J. Mater. Sci., vol. 40, pp. 983-989, 2005. doi: 10.1007/s10853-005-6517-0. 
[13] A. P. Roberts and E. J. Garboczi, "Elastic Properties of Model Porous Ceramics," J. Am. Ceram. Soc.,vol. 83, issue 12, pp. 3041-3048, 2000. doi: 10.1111/j.1151-2916.2000.tb01680.x.

[14] K. S. Hibbit, Inc.: Abaqus Theory Manual V 6.5. (2004).

[15] A. L. Gurson, "Continuum theory of ductile rupture by void nucleation and growth: part I-yield criteria and flow rules for porous ductile ,edia," J. Eng. Mater. Technol., vol. 99, issue 1, pp. 2-15, 1977. doi: $10.1115 / 1.3443401$.

[16] R. A. Hardin and C. Beckermann, "Measurement and prediction of mechanical behavior of cast steel plates with centerline porosity," In Proceedings of the 65th SFSA Technical and Operating Conference, Paper No. 5.4. Steel Founders' Society of America, Chicago, IL, 2011.

[17] F. P. Knudsen, "Effect of Porosity on Young's Modulus of Alumina," J. Am. Ceram. Soc., vol. 45, issue 2, pp. 94-95, 1962. doi: 10.1111/j.1151-2916.1962.tb11089.x.

[18] M. Asmani, C. Kermel, A. Leriche and M. Ourak, "Influence of porosity on Young's modulus and Poisson's ratio in alumina ceramics," J. Eur. Ceram. Soc., 2001, vol. 21, pp. 1081-1086. doi: 10.1016/S0955-2219(00)00314-9.

[19] S. L. Dole, O. Hunter and F. W. Calderwood, "Elastic properties of stabilized $\mathrm{HfO}^{2}$ compositions," J. Am. Ceram. Soc., vol. 63, pp. 136-139, 1980. doi: 10.1111/j.1151-2916.1980.tb10677.x.

[20] K. K. Phani and R. N. Mukerjee,"Elastic properties of porous thermosetting polymers ," J. Mater. Sci., vol. 22, issue 10, pp. 3453-3458, 1987. doi: 10.1007/BF01161441.

[21] J. P. Panakkal, H. Willems and W. Arnold, "Nondestructive evaluation of elastic parameters of sintered iron powder compacts," J. Mater. Sci., vol. 25, issue 2, pp. 1397-1402, 1995. doi: 10.1007/BF00585456.

[22] D. F. Porter, J. S. Reed and D. Lewis III, "Elastic moduli of refractory spinels," J. Am. Ceram. Soc., 1977, vol. 60, issue 7-8, pp. 345-349, 1977, doi: 10.1111/j.1151-2916.1977.tb14128.x. 


\section{Evaluation of Anti-Icing Polymer Coatings}

\author{
Nick Tepylo and Xiao Huang \\ Carleton University, Dept. of Mechanical and \\ Aerospace Engineering
}

\author{
Marc Budinger \\ University of Toulouse, Institut Clément Ader (ICA), \\ INSA \\ Pierrick Rouset and Valérie Pommier-Budinger \\ University of Toulouse, ISAE SUPAERO
}

\begin{abstract}
Ice accumulation on aircraft, wind turbines and power lines can have detrimental effects, including efficiency reduction, mechanical failures and the creation of safety hazards. The aim of this study is to investigate the ice adhesion and wear resistance of three hydrophobic and icephobic coatings applied onto an aluminum substrate. Ethylene-tetrafluoroethylene (ETFE) coating was deposited using a plasma spray method while advanced liquid glass (ALG) and silicone R-2180 were both applied using dipping followed by furnace curing. Water was applied and frozen between both bare and coated surfaces using a custom built jig at $-20{ }^{\circ} \mathrm{C}$ for 24 hours. The ice adhesion strength was measured using a lap shear test done inside an insulated chamber. The results showed low ice adhesion strengths for both ALG and silicone R-2180 coatings when compared to the bare surface. It was also found the silicone R-2180 coating had a higher wear rate than both the ETFE and ALG coatings. By combining icephobic coatings with an ultrasonic de-icing system, the power required by the system can be reduced, creating a low-powered active approach to the de-icing problem.
\end{abstract}

Keywords - anti-icing, coatings, ultrasonic de-icing system, ice adhesion

\section{INTRODUCTION}

Ice accumulation and wet-snow adhesion to solid outdoor surfaces can cause severe accidents and large economic losses. Such problems are present in many fields, from aeronautics [1] to off-shore oil platforms [2] and from power lines [3] to wind turbines [4]. On aircraft, ice accumulation can result in decreased lift, increased drag, decreased thrust, reduced stall angle, altered stall characteristics and even engine failure due to ice shedding. Aircraft icing can occur both during flight and on the ground. It has led to many reported aircraft accidents including Air Florida Boeing 737 (1982) and the American Eagle ATR 72 (1997). To ensure aircraft safety, regulatory bodies such as the FAA, JAA and EASA have established regulations for anti-icing and de-icing measures.

Present-day anti-icing and de-icing strategies can be chemical, thermal, electrical, and mechanical. Chemical methods belong to the passive techniques and can be used for anti-icing and de-icing. They can be categorized into freezing point depressants or icephobic/hydrophobic coatings. Freezing point depressants lower the freezing point of water which prevents the freezing of supercooled water droplets or creating a thin film of water between the aircraft surface/ice interface to assist ice-shedding. Viscous icephobic/hydrophobic coatings adhere to the surface, creating a thin film promoting ice-shedding. Chemicals can only be employed as an effective anti-icing strategy when being applied just prior to takeoff and reapplied for whenever the possibility of ice accretion exists on the ground. Corrosion becomes an issue with sodium chloride based inorganic freezing point depressants while organic antiicing and de-icing chemicals (such as propylene glycol) have the potential to provide better snow and ice control performance and are less corrosive. However, organic materials are generally more costly and may be subject to dilution and pose environmental concerns.

The active methods depend on an external action being applied such as thermal, electro-thermal, electro-mechanical, or electromagnetic, and, as for passive methods, rely on the physical properties of the solid surface [5]. Thermal methods are based on the extraction of hot air from the engine, but this bleed reduces the efficiency of the engine. An electro-thermal de-icing system was adopted by Boeing in the B787 but this solution is energy-intensive. Mechanical devices such as pneumatic boots which break the accumulated ice by inflating can be implemented but these boots have a significant impact on the aerodynamics of the aircraft and a low durability. Finally, electromagnetic technologies based on the deformation of a winding are penalized by the weight of the power supply.

All of these existing methods entail disadvantages (cost, environmental concerns, large amount of power required). As such, the aviation industry is searching for a more efficient and cost-effective means for de-icing and anti-icing [6].

The authors advocate the use of hydrophobic and icephobic coatings in conjunction with electro-mechanical ice protection systems. The active ice protection systems generate shear stresses at the interface between the ice and the structure to break the accumulated ice and this shear stress required for ice shedding is reduced by the coating, which reduces the power 
required by the actuators, and also the size and weight of the overall system. Based on the published literature, two main coating types can lessen the effect of ice accumulation. The first type contributes to the so called icephobic materials [710]. Such material reduces the shear forces required to break the accumulated layer of ice on the solid surface. The second type focuses on hydrophobic features. Surfaces coated with hydrophobic materials have a high tendency in repelling water due to the high contact angle between the water droplet and the solid surface. Hence this method eliminates the accumulation of water before water freezes and ice formation occurs. In the present study, two hydrophobic coatings (Ethylene-tetrafluoroethylene (ETFE), advanced liquid glass (ALG)) and one icephobic coating (silicone R-2180) were applied onto the aluminum (Al) 2024 substrate and tested for ice adhesion strength and wear resistance. These coatings were selected to experiment with different coating application methods and surface properties.

\section{MATERIALS AND EXPERIMENTAL PROCEDURES}

\section{A. Sample Preparation}

The substrate material used in this study was Al 2024-T3 cut to a size of $75 \mathrm{~mm}$ by $25 \mathrm{~mm}$ for ice adhesion testing and a 25 $\mathrm{mm}$ by $25 \mathrm{~mm}$ section for the wear test. Four different surface conditions were investigated. A sandblasted (SB) coupon with no coating, a SB coupon with ethylene-tetrafluoroethylene (ETFE from Dupont) plasma sprayed coating, a SB coupon with an Advanced Liquid Glass (ALG, from Liquid Glass Shield) coating, and a SB coupon with silicone R-2180 (Nusil Technology). ETFE is similar to PTFE as they are both hydrophobic and composed of a carbon chain with fluorine reaction groups while ALG is a hydrophobic low friction coating with added silica particles. For ice adhesion tests, coating was applied only to $25 \mathrm{~mm}$ by $25 \mathrm{~mm}$ section of the coupon.

During the plasma spraying of ETFE, a mixture of $20 \%$ nitrogen gas and $80 \%$ argon was used as the carrier gas. The aluminum coupons were preheated for two minutes by the plasma stream before spraying. Other spraying parameters for ETFE coupons consisted of 190 A arc current, $220 \mathrm{slm}$ argon gas flow rate and $100 \mathrm{~mm}$ offset distance between the nozzle and the coupons. After coating, all ETFE coupons were baked at $274^{\circ} \mathrm{C}$ for 20 minutes as recommended by the supplier [11].

The other two coatings, ALG and silicone R-2180, were applied using dipping application. Samples were dipped into a beaker containing ALG for 5 seconds and were cured in an air furnace at $250{ }^{\circ} \mathrm{C}$ for 1 hour. For the silicone R-2180 coating, the samples were dip coated and allowed to cure at room temperature for 30 minutes, then were placed in a furnace at $75{ }^{\circ} \mathrm{C}$ for 45 minutes and subsequently the temperature was increased to $150{ }^{\circ} \mathrm{C}$ and cured for another 135 minutes.

\section{B. Ice Adhesion Test}

A freezing jig was manufactured to hold the coupons in place during the freezing process. The jig was designed such that the gap clearance between the top and bottom coupons remains constant at $1 \mathrm{~mm}$. The jig shown in Figure 1 uses slots to keep the coupons aligned and can hold six test specimens at once (a total of 12 coupons). A layer of parafilm was placed on top of the jig to prevent the coupons from sticking to the surface of the jig during the freezing process. Using a $1 \mathrm{~mL}$ syringe, 0.6 $\mathrm{mL}$ of deionized water was then injected into the hole in the lap shear joint ( $25 \mathrm{~mm}$ overlap) and the jig was placed in a freezer for 24 hours. This was done to ensure a complete transition of water to ice between the two coated surfaces. After 24 hours, each specimen was removed and inspected to ensure complete formation of ice between the coated surfaces.

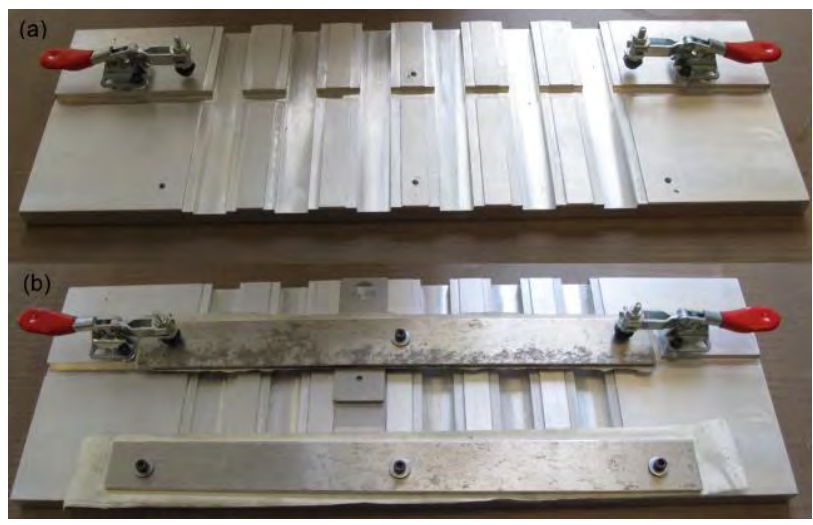

Figure 1: (a) As fabricated jig used to align lap shear samples during freezing and (b) jig with sample loaded.

The ice adhesion test was carried out using a Material Testing System (MTS) to measure the force required to break the lap joint. From the measured force, the shear stress at which ice is detached from the surface can be determined. The frozen specimen was placed inside an insulated chamber and gripped by the MTS. The chamber kept the specimens at a temperature of approximately $-20{ }^{\circ} \mathrm{C}$ for the duration of the test. The test setup can be found in Figure 2(a) while the inside of the chamber is shown in Figure 2(b). An axial displacement rate of $1 \mathrm{~mm} / \mathrm{min}$ was used to pull the joint apart. The maximum load was then recorded for each sample.

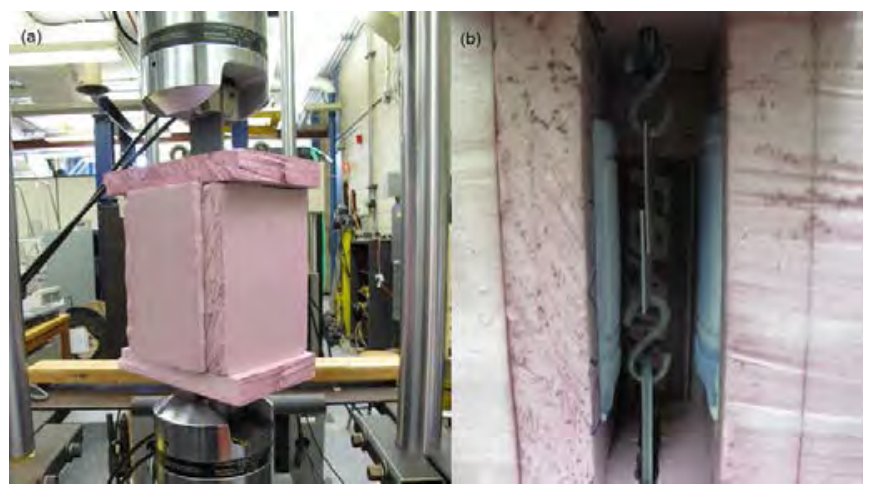

Figure 2: (a) Insulated chamber shown in the MTS and (b) cross-section of the insulated chamber with a loaded sample. 
In order to compare the results obtained, the adhesion reduction factor (ARF) was used and is calculated using the following equation:

$\mathrm{ARF}=\left(\tau_{\text {aluminum }} / \tau_{\text {coating }}\right)$

where $\tau_{\text {aluminum }}$ is the shear stress of the bare aluminum sample and $\tau_{\text {coating }}$ is the shear stress in the coated sample being investigated. A high ARF value corresponds to a coating that has a low ice adhesion strength when compared to aluminum [12].

\section{Maintaining the Integrity of the Specifications}

A pin-on-disk wear test was carried out to compare the coating's resistance to wear. It was performed under the dry sliding condition, according to ASTM 99-05 using a wear tester with model No: NEO-TRIBO MPW110 provided by NEOPLUS. This apparatus uses a rotating pin pressed under a normal force of $24.5 \mathrm{~N}$ against a static coating sample. The pin used was a Teflon ball with a radius of $2.5 \mathrm{~mm}$. During the test, the specimen was placed horizontally with its center at a distance of $5 \mathrm{~mm}$ away from the vertical axis of the pin shaft. The pin (ball) was spinning at a constant speed of 50 RPM. As the result of friction/wear, a $10 \mathrm{~mm}$ diameter circular wear track was generated on the specimen surface. Wear loss of the coating material was measured based on the track depth. A new Teflon ball was used for each test to eliminate cross contamination between samples.

\section{RESULTS}

\section{A. Ice Adhesion Strength Results}

The lap shear test results are presented in Table 1. The bare, sandblasted aluminum samples required on average $447 \mathrm{kPa}$ to shear the ice between the two coupons. This indicates that the bare sand blasted coupons exhibited a stronger adhesion to ice than the other coatings tested in this study.

\section{Table 1: Adhesion strength for tested coatings}

\begin{tabular}{|l|c|c|}
\hline Coating & Adhesion Strength $(\mathrm{kPa})$ & ARF \\
\hline Sandblasted Al & $447 \pm 71$ & - \\
\hline ALG & $151 \pm 69$ & 2.96 \\
\hline ETFE & $357 \pm 72$ & 1.25 \\
\hline R-2180 & $187 \pm 26$ & 2.39 \\
\hline
\end{tabular}

A total of six samples were shear tested for each type of the coatings. A large variation was observed in the results obtained from this study. However, these variations in adhesion strength for each coating group are similar and also comparable that from previous tests conducted by other researchers. The ice adhesion strength of aluminum has been measured as low as $242 \mathrm{kPa}$ [13] and sandblasted aluminum has been measured at $610 \mathrm{kPa}$ [14]. In a similar experiment carried out using a lap joint shear test conducted in an MTS, the ice adhesion strength of the bare aluminum 2024 sample was $399.7 \mathrm{kPa}$ [15] which closely resembles the $447 \mathrm{kPa}$ obtained in this study. Although the results of this study do not closely match previous work [2], the ARF instead acts as a comparison tool for the tested coatings. ALG had the highest tested ARF of 2.96, followed by R-2180 (2.39) and ETFE (1.25).

There are several variables that could not be controlled during the testing and lead to deviations in the results. Any deviation in terms of actual ice temperature would have an impact on the required shear force. Another variable is the clamping pressure used during freezing. While the position of the clamps was constant for freezing each sample, the pressure exerted on each sample could be different due to the effects of the surface tension exerted by the water. Since each surface is different in the surface tension can vary significantly and cause a difference in the adhesion of ice to the sample.

\section{B. Wear Resistance Results}

A pin on disc wear test was used to evaluate the wear resistance of the three coatings using the procedure described previously. After the wear testing was conducted, SEM images were obtained to assess the damage to the coatings. The amount of wear was dependent on the type of coating and the duration of the test. Each coating was tested for durations of 1, 2, 4 and 8 minutes. A graph showing the wear loss, in terms of wear depth tracked by the location of the pin, of the three coatings plotted against the number of cycles can be found in Figure 3. ETFE wears at a rate of approximately twice the rate of the ALG samples while the silicone R-2180 samples wear out approximately six times more than the ALG samples.

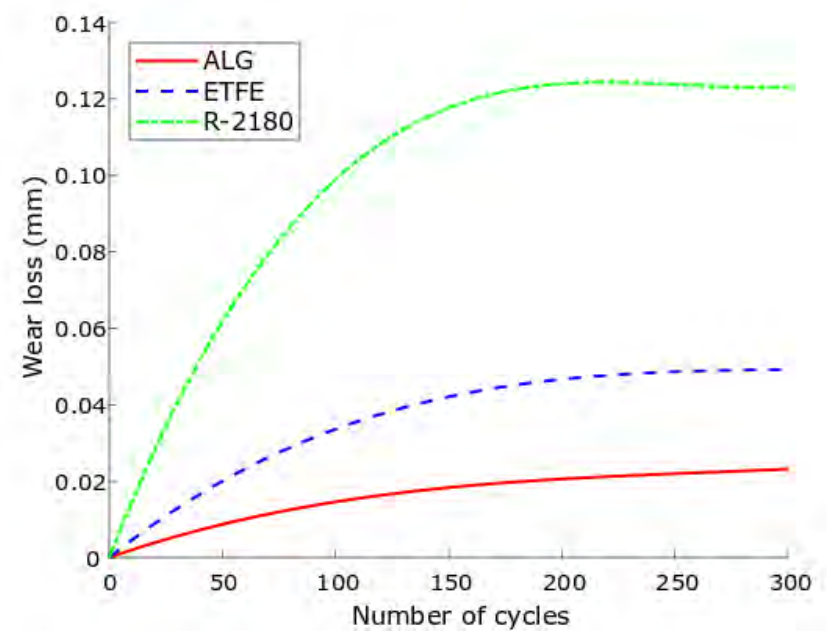

Figure 3: Wear loss for the ALG (red), ETFE (blue) and silicone R-2180 (green) samples.

As the test duration increased, the wear rate of each coating decreased. This can be expected as the longer the test lasts, the Teflon ball comes into contact with more coating surface area and a larger force is required to displace the coating. ALG coating, being a primarily $\mathrm{SiO}_{2}$ based, was the most resistive to wear. The silicone R-2180 is an elastomer-based coating 
and is fairly soft so the ball could easily displace the coating along the wear track. There was also a net mass increase in each sample due to the deposition of Teflon flakes from the pin onto the sample. As the surface of the pin was in contact with the coating, flakes wore off from the tip and were left along the wear track. Figure 4 shows the flakes that were deposited into R-2180 coating that was tested for 4 minutes.

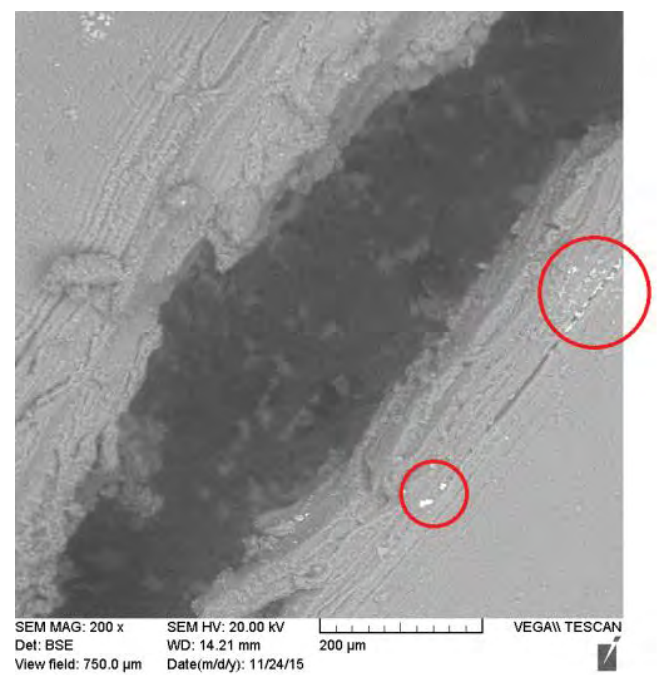

Figure 4: Teflon flakes were deposited into the wear track.

\section{Feasibility for anti-icing applications}

A composite plot was created in order to aid the selection process of anti-icing coatings for industrial applications. This plot is shown in Figure 5. As can be seen on the plot, ALG has the combination of the highest ARF and the lowest wear loss making it the most suitable coating for anti-icing applications. Coatings in the contained region possess a high ARF and low wear rate relative to other tested coatings. Coatings outside of this region are not suitable for aircraft as they possess either an ARF that is low when compared to other coatings or can wear quickly, exposing the surface of the aircraft.

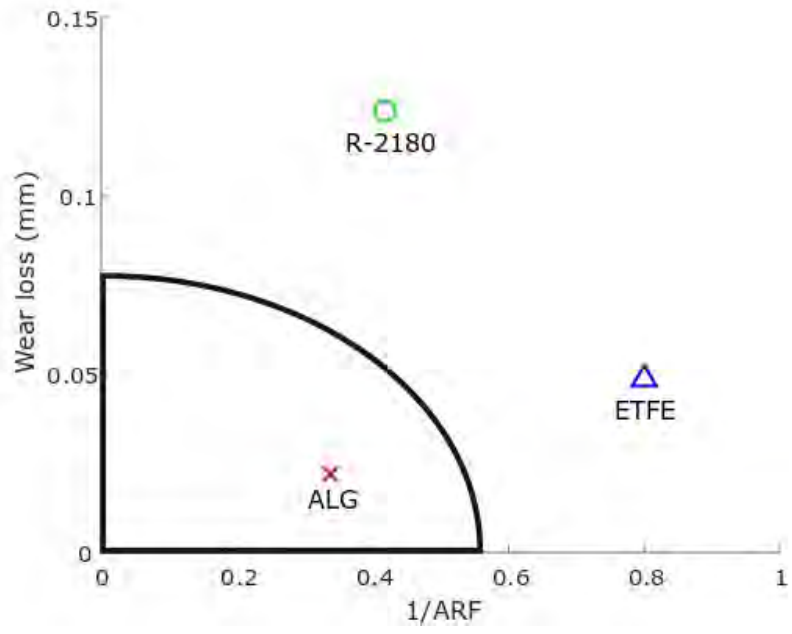

Figure 5: Composite plot of ARF and wear loss for the tested coatings.
D. Potential for combined anti-icing coating and ultrasonic de-icing systems

The potential exists to combine icephobic coatings with preexisting de-icing systems to reduce the ice adhesion strength on the surface, which results in lower power consumption. Budinger et al. experimented with ultrasonic de-icing systems and developed a model to calculate the stress in the piezoceramic and the required voltage and current needed to delaminate a sheet of ice [16]. It was shown that the voltage and current required for delamination are linearly related to the ice adhesion strength [6]. Thus, the power required to delaminate a sheet of ice is proportional to the square of the ice adhesion strength, which means that an ARF of two would cause the power to decrease by a factor of four, reducing the size of actuator needed for the system.

Listed in Table 2 are the ARF of the tested coatings and the theoretical reduction in power due to the coating being used in conjunction with the ultrasonic de-icing system. Further decreases in the power can be achieved using icephobic coatings with a higher ARF.

Table 2: Power reduction factor when the coating is combined with an ultrasonic de-icing system

\begin{tabular}{|l|c|c|}
\hline Coating & ARF & Power Reduction Factor \\
\hline ALG & 2.96 & 8.76 \\
\hline R-2180 & 2.39 & 5.71 \\
\hline ETFE & 1.25 & 1.56 \\
\hline
\end{tabular}

The proposed system is shown in Figure 6. Several piezoelectric actuators are positioned on the inside of the leading edge of the aircraft while an icephobic coating is applied on the exterior. The size and quantity of the actuators is dependent on the ARF of the selected coating. The system is similar to the Low Frequency De-Icing system (LFDI) proposed by Endres et al. [17] with the surface having a lower ice adhesion strength due to the icephobic coating.

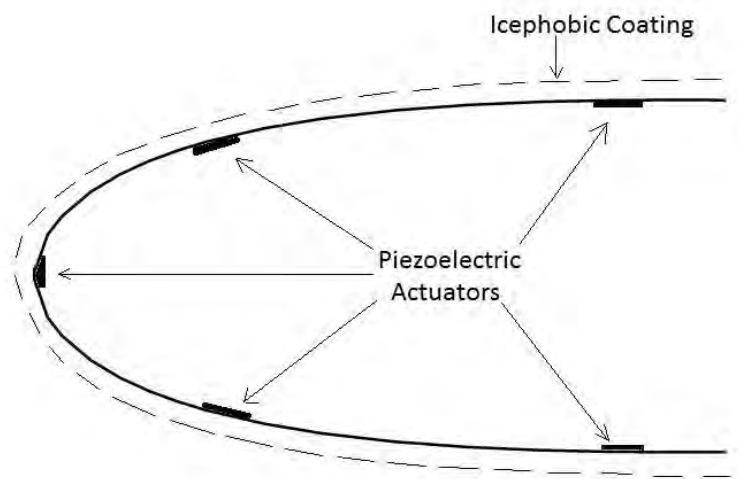

Figure 6: Proposed ice protection using both icephobic coating and piezoelectric actuators. 


\section{CONCLUSIONS}

Ice accretion on aircraft can have catastrophic consequences and the aviation industry is looking for both passive and active approaches to prevent and eliminate ice accretion. Ice adhesion was studied on four types of surfaces with different characteristics in order to analyze their icephobic properties. Ice adhesion strength measurements were obtained using an MTS and a constant axial displacement rate. The ALG coating decreased the ice adhesion by a factor of 2.96 and the silicone R-2180 reduced the ice adhesion strength by a factor of 2.39 when compared to an uncoated aluminum substrate. In addition to analyzing the icephobic characteristics of coatings, the durability in terms of wear was also assessed to validate its continuous effectiveness against ice. The R-2180 coating wore off easily compared to the ETFE and ALG coatings. As such, ALG coating was found to be a more suitable coating for the anti-icing application as it would not need to be consistently reapplied while having the highest ARF.

By selecting a coating with a high ARF and a low wear rate, the coating can be coupled with ultrasonic de-icing systems and provide an effective solution to de-icing. The relationship between the ARF and reduction in power is exponential, which makes the ARF a critical factor when selecting a coating.

\section{ACKNOWLEDGMENT}

The authors would like to acknowledge Ayman Ibrahim and Fred Barrett for developing the plasma spray process used to coat ETFE samples.

\section{REFERENCES}

[1] A. Heinrich, et. al, Aircraft Icing Handbook - Volume 3 of 3. Gates Learjet Corporation, 1991.

[2] C. C. Ryerson,"Assessment of superstructure ice protection as applied to offshore oil operations safety," Tech. Rep. CRREL TR-09-4, U.S. Army Cold Regions Research and Engineering Laboratory, Hanover, New Hampshire 03755, April 2009.

[3] J. Laforte, M. Allaire, and J. Laflamme, "State-of-the-art on power line de-icing," Atmospheric Research, vol. 46, pp. 143-158, 1998. doi:10.1016/S0169-8095(97)00057-4.

[4] R. Karmouch, et, al., "Icephobic PTFE coatings for wind turbines operating in cold climate conditions," 2009 IEEE Electrical Power and Energy Conf., 22-23 Oct., 2009.

[5] N. Dalili, A. Edrisy, and R. Carriveau, "A review of surface engineering issue critical to wind turbine performance," Renewable and Sustainable Energy Rev., vol. 13, pp. 428-438, 2009. doi: 10.1016/j.rser.2007.11.009.

[6] M. Budinger et al., "Ultrasonic ice protection systems: Analytical and numerical models for architecture tradeoff," J. Aircr., vol. 68, pp. 91-98, 2016. doi: $10.2514 / 1 . C 033625$.

[7] KISS Polymers LLC. Product Summary, 2008 [Online]. Available: http://www.kisspolymers.com/index.htm. Accessed on: 12-June-2016.

[8] N. D. Mulherin and R. Haehnel, "Progress in Evaluating Surface Coatings for Icing Control at Corps Hydraulic Structures," Tech. Rep. Ice Engineering Information Exchange Bulletin, TN-03-4, U.S. Army Cold Regions Research and Engineering Laboratory, Hanover, New Hampshire 03755, 2003.

[9] R. Menini and M. Farzaneh, "Elaboration of Al2O3/PTFE icephobic coatings for protecting aluminum surfaces," Surf. Coat. Technol., vol. 203, pp. 1941-1946, 2009. doi:10.1016/j.surfcoat.2009.01.030.

[10] J. E. Mark, "Some interesting things about polysiloxanes," Acc. Chem. Res., vol. 37, pp. 946-953, 2004. doi: 10.1021/ar030279z.
[11] DuPont Teflon ${ }^{\circledR}$. Non-stick and Industrial Coatings, 2015. [Online]. Available: http://www.intechservices.com/TeflonProduct-Overview. Accessed: 12-June-2016.

[12] M. Susoff et al., "Evaluation of ice-phobic coatingsscreening of different coatings and influence of roughness," App. Surf. Sci., vol. 282, pp. 870-879, 2013. doi: 10.1016/j.apsusc.2013.06.073.

[13] F. Arianpour, M. Farzaneh, and R. Jafari, "Hydrophobic and ice-phobic properties of self-assembled monolayers (SAMs) coatings on AA6061," Progress in Organic Coatings, vol. 93, pp. 41-45, 2016. doi: 10.1016/j.porgcoat.2015.12.008.

[14] M. Zou, et al., "Effects of surface roughness and energy on ice adhesion strength," App. Surf. Sci., vol. 257, pp. 3786-3792, 2011. doi: 10.1016/j.apsusc.2010.11.149.

[15] B. Dixon et al., "Novel phase change material icephobic coating for ice mitigation in marine environments," in Green Ships, Eco Shipping, Clean Seas, The 12th Annual General Assembly of IAMU, 2014.

[16] V. Pommier-Budinger et al., "Analysis of Piezoelectric Ice Protection Systems Combined with Ice-Phobic Coatings," in 8th AIAA Atmospheric and Space Environments Conference, pp. 623-628, 2016. doi: 10.2514/6.2016-3442.

[17] M. Endres, et al., "Experimental Study of two Mechanical De-Icing Systems Applied on a Wing Section tested in an Icing Wind Tunnel," German Aerospace Congress 2016, (Braunschweig, Germany), September 2016. 


\section{Low-Velocity Impact Response and Experimental Optimization of Modified Fiber Metal Laminates with Integrated Mechanical Interlock Bonding System}

\author{
Tran-Vu Nong, M.S.A ElSayed* \\ Dept. of Mechanical and Aerospace Engineering \\ Carleton University \\ Ottawa, Canada \\ *Mostafa.elsayed@carleton.ca
}

\begin{abstract}
This paper presents a modified version of fiber metal laminates with integrated mechanical interlock bonding system for aerospace applications. Sheet metals of Al 2024-T3 with surface machined infinitesimal hooks are used along with impregnated glass fiber composites to manufacture a modified version of GLAss REinforced aluminum (GLARE). Low-velocity impact responses of the modified GLARE is examined using a drop weight impact testing machine at an impact energy of 7.5 J. To optimize the geometry of the machined hooks to maximize the modified GLARE low-velocity impact resistance, we developed and tested four configurations of modified GLARE with four variants of hooks' geometry, including two hook sizes, namely, nano and micro and two hook profiles, namely, curved and straight. Impact tests show that modified GLARE with Straight Nano Hooks (SNH) have comparable dynamic responses to the standard GLARE (without hooks), while experiencing much less delamination and fiber damage. Microscopic inspection of the four configurations of modified GLARE also illustrates that SNHs generate modified GLARE with minimal manufacturing defects. The results obtained indicate that SNH is the optimum hook geometry for the development of modified GLARE. It can be considered as an alternative surface treatment for sheet metals in FML development process as it offers a modified version of the material with comparable impact responses to those manufactured by the industrial standard methodology but at a fraction of production cost.
\end{abstract}

Keywords: Fiber Metal Laminates, Experimental Optimization, Surface Topology, Structured Materials, Aerospace Materials, GLARE

\section{INTRODUCTION}

Increasing demands in aerospace industries for highperformance and lightweight structures with cost-effective manufacturability have stimulated a strong trend towards the development of refined models of hybrid materials such as Fiber-metal laminates (FMLs). While FMLs possess better specific mechanical properties compared to traditional aerospace materials, it is also several times more expensive.

\author{
Thomas Letellier \\ École Nationale Supérieure de Mécanique et \\ d'Aérotechnique (ISAE-ENSMA) \\ Poitou-Charentes, France \\ thomas.letellier@etu.isae-ensma.fr
}

This can be attributed to the costly surface treatments and manufacturing processes designed to improve the adhesion bonding between FML layers to minimize its consistency failures. Particularly, surface topology optimization is a key element to maximize adhesion bonding between FML layers.

Developed during the late 1980s at Delft University, GLARE was the second generation of FMLs. Through its high-strength glass Fiber Reinforced Polymers (FRP) layers, GLARE possesses better impact behavior compared to monolithic aluminum alloys and FRPs [1, 2, 3]. GLARE also possesses superior tensile strength compared to monolithic aluminum alloys [3]. In addition, GLARE is extremely resistant to environmental factors such as moisture, fire, corrosion, and lightning, and can be machined and repaired using common techniques used for aluminum alloys $[4,5,6]$. With its low density compared to monolithic aluminum alloys, GLARE can offer weight savings of up to $25 \%[1,7,8]$. Given these promising properties, GLARE has been used in a variety of applications in the aerospace industry, such as in the cargo floor of the Boeing B777, the upper fuselage of the Airbus A380, and as bonded repair patches $[3,4,8,9]$.

Given that low-velocity impacts are common in aerospace structures during operation, improving the low-velocity impact response of GLARE is very important [10]. This can be done by preventing the common modes of failure that occur during impact. From existing literature [11, 12], delamination and debonding tend to be among the most important failure modes for laminated structures such as GLARE [11]. Studies have been done to prevent this type of failure by improving the adhesion at the internal interfaces between layers through metal surface treatments. Botelho et al. [12] investigated the effects of sulphuric chromic acid etching and chromic acid anodizing on the interfacial adhesion of GLARE and found that both surface treatments improved the adhesion between the aluminum and the FRP layers compared to non-treated aluminum. Ostapiuk et al. [13] found similar results with regards to sulphuric acid anodizing as well. However, these surface treatments are 
expensive and/or require specialized equipment. Since one of the main disadvantages of

GLARE is its high unit cost compared to aluminum, a more cost-effective solution should be found $[7,8,9]$.

In this study, the low-velocity impact behavior of a novel GLARE variant using a low-cost technology, developed by our industrial partner, is investigated. Here, machined hooks are created on the surface of sheet metal aluminum. During the curing cycle of the GLARE, those hooks penetrate the FRP layers creating an integrated mechanical interlock bonding system. While the modified GLARE possesses mechanical properties similar or comparable to those of GLARE build using the standard methodology available in the aerospace industry, the hook formation process is less costly compared to other surface treatment methods used to improve consistency failure. Moreover, we used the hotpress method to build our samples which is very cheap compared to the infrastructure required for the autoclave manufacturing process which further reduces the cost.

This paper is organized in five sections. After this introduction, the manufacturing process of the modified GLARE is explained in section 2. The test setup, procedures and results for impact testing are presented in section 3 . Mechanical characterization results obtained through tensile testing are presented in section 4. The paper is concluded in section 5 .

\section{MODIFIED GLARE}

\section{A. Lay-up}

We based the lay-up of the modified GLARE on those of GLARE-3 [14]. The lay-up of the modified GLARE is formed of three layers of aluminum 2024-T3 (two external layers with single-sided hooks and one middle layer with double-sided hooks) alternating with two pre-impregnated (pregregs) glass fiber layers. We used fiber layers consisting of $0.008 "(0.20 \mathrm{~mm})$ thick of $8 \mathrm{H}$ satin glass fiber weaved prepregs supplied by ACP Composites [15]. This is chosen due to availability, cost, and storage requirements.

To optimize the geometry of the hooks, four configurations of modified GLARE are considered in this study. The different configurations are based on geometry variants of the machined hooks including two hook sizes as well as two hook profiles. Table 1 summarizes the lay-up specifications of the four configurations of modified GLARE considered as well as those of the standard GLARE (with no-hooks).

\section{B. Hooks Development}

0.016" (0.40 mm) thick ALCLAD 2024-T3 aluminum sheet metals are used. Nano and micro hooks with straight and curved profiles are machined on the surface of sheet metals using the manufacturing services at our industrial partner. Due to tooling limitations, we are able to machine the hooks on only a 6" wide sheet metal strips. The sheet metal strips are cut into 12 " in length to fit within the hot-press available for the manufacturing of GLARE. Fig. 1 shows microscopic images of the machined sheet metal with straight and curved hooks.

TABLE I. LAY-UP SUMMARY OF THE STANDARD AND MODIFIED GLARE

\begin{tabular}{|c|c|c|c|c|c|}
\hline & \multirow[b]{2}{*}{$\begin{array}{c}\text { Standard } \\
\text { (No-Hooks) }\end{array}$} & \multicolumn{4}{|c|}{ Modified GLARE } \\
\hline & & $\begin{array}{c}\text { Straight } \\
\text { Nano } \\
\text { Hooks } \\
\text { (SNH) }\end{array}$ & $\begin{array}{l}\text { Straight } \\
\text { Micro } \\
\text { Hooks } \\
\text { (SMH) }\end{array}$ & $\begin{array}{c}\text { Curved } \\
\text { Nano } \\
\text { Hooks } \\
\text { (CNH) }\end{array}$ & $\begin{array}{l}\text { Curved } \\
\text { Micro } \\
\text { Hooks } \\
\text { (CMH) }\end{array}$ \\
\hline Metal Layer & $\begin{array}{l}\text { 2024-T3 } \\
\text { ALCLAD } \\
\text { aluminum }\end{array}$ & $\begin{array}{l}2024-T 3 \\
\text { ALCLAD } \\
\text { aluminum }\end{array}$ & $\begin{array}{l}2024-T 3 \\
\text { ALCLAD } \\
\text { aluminum }\end{array}$ & $\begin{array}{l}2024-T 3 \\
\text { ALCLAD } \\
\text { aluminum }\end{array}$ & $\begin{array}{l}2024-T 3 \\
\text { ALCLAD } \\
\text { aluminum }\end{array}$ \\
\hline $\begin{array}{c}\text { Metal } \\
\text { Thickness }\end{array}$ & $\begin{array}{c}0.016 "(0.40 \\
\mathrm{mm})\end{array}$ & $\begin{array}{c}0.016 " \\
(0.40 \mathrm{~mm})\end{array}$ & $\begin{array}{c}0.016 " \\
(0.40 \mathrm{~mm})\end{array}$ & $\begin{array}{c}0.016 " \\
(0.40 \mathrm{~mm})\end{array}$ & $\begin{array}{c}0.016 " \\
(0.40 \mathrm{~mm})\end{array}$ \\
\hline $\begin{array}{l}\text { No. of No- } \\
\text { Hooks } \\
\text { Layer }\end{array}$ & 3 sheets & - & - & - & - \\
\hline $\begin{array}{l}\text { No. of } \\
\text { Hooked } \\
\text { Layer }\end{array}$ & - & $\begin{array}{l}2 \text { single- } \\
\text { sided, } \\
1 \text { double- } \\
\text { sided }\end{array}$ & $\begin{array}{l}2 \text { single- } \\
\text { sided, } \\
1 \text { double- } \\
\text { sided }\end{array}$ & $\begin{array}{l}2 \text { single- } \\
\text { sided, } \\
1 \text { double- } \\
\text { sided }\end{array}$ & $\begin{array}{l}2 \text { single- } \\
\text { sided, } \\
1 \text { double- } \\
\text { sided }\end{array}$ \\
\hline Hook Type & - & $\begin{array}{l}\text { Straight- } \\
\text { profile } \\
\text { nano } \\
\text { hooks }\end{array}$ & $\begin{array}{l}\text { Straight- } \\
\text { profile } \\
\text { micro } \\
\text { hooks }\end{array}$ & $\begin{array}{l}\text { Curved- } \\
\text { profile } \\
\text { nano } \\
\text { hooks }\end{array}$ & $\begin{array}{l}\text { Curved- } \\
\text { profile } \\
\text { micro } \\
\text { hooks }\end{array}$ \\
\hline $\begin{array}{c}\text { Hook } \\
\text { Height }\end{array}$ & - & $\begin{array}{c}0.008 " \\
(0.20 \mathrm{~mm})\end{array}$ & $\begin{array}{c}0.028 " \\
(0.70 \mathrm{~mm})\end{array}$ & $\begin{array}{c}0.008 " \\
(0.20 \mathrm{~mm})\end{array}$ & $\begin{array}{c}0.028 " \\
(0.70 \mathrm{~mm})\end{array}$ \\
\hline Hook Width & - & $\begin{array}{c}0.0012 ” \\
(0.03 \mathrm{~mm})\end{array}$ & $\begin{array}{c}0.0042 ” \\
(0.11 \mathrm{~mm})\end{array}$ & $\begin{array}{c}0.0012 ” \\
(0.03 \mathrm{~mm})\end{array}$ & $\begin{array}{c}0.0042 ” \\
(0.11 \mathrm{~mm})\end{array}$ \\
\hline $\begin{array}{c}\text { Hook } \\
\text { Density }\end{array}$ & - & $\begin{array}{c}250-260 \\
\text { hooks/in }{ }^{2} \\
\text { (single- } \\
\text { sided), } \\
240-250 \\
\text { hooks/in }{ }^{2} \\
\text { per side } \\
\text { (double- } \\
\text { sided) }\end{array}$ & $\begin{array}{c}250-260 \\
\text { hooks/in }{ }^{2} \\
\text { (single- } \\
\text { sided), } \\
240-250 \\
\text { hooks/in }{ }^{2} \\
\text { per side } \\
\text { (double- } \\
\text { sided) }\end{array}$ & $\begin{array}{c}250-260 \\
\text { hooks/in }{ }^{2} \\
\text { (single- } \\
\text { sided), } \\
240-250 \\
\text { hooks/in }{ }^{2} \\
\text { per side } \\
\text { (double- } \\
\text { sided) }\end{array}$ & $\begin{array}{c}250-260 \\
\text { hooks/in } \\
\text { (single- } \\
\text { sided), } \\
240-250 \\
\text { hooks/in } \\
\text { per side } \\
\text { (double- } \\
\text { sided) }\end{array}$ \\
\hline $\begin{array}{c}\text { Prepreg } \\
\text { Layer } \\
\text { (ACP } \\
\text { Composite) }\end{array}$ & $\begin{array}{l}\text { E-glass fabric } \\
\text { impregnated } \\
\text { with a } \\
\text { thermosetting } \\
\text { epoxy resin } \\
\text { system }\end{array}$ & $\begin{array}{c}\text { E-glass } \\
\text { fabric } \\
\text { impregnate } \\
\text { d with a } \\
\text { thermosetti } \\
\text { ng epoxy } \\
\text { resin } \\
\text { system }\end{array}$ & $\begin{array}{l}\text { E-glass } \\
\text { fabric } \\
\text { impregnate } \\
\mathrm{d} \text { with a } \\
\text { thermosetti } \\
\text { ng epoxy } \\
\text { resin } \\
\text { system }\end{array}$ & $\begin{array}{c}\text { E-glass } \\
\text { fabric } \\
\text { impregnate } \\
\mathrm{d} \text { with a } \\
\text { thermosetti } \\
\text { ng epoxy } \\
\text { resin } \\
\text { system }\end{array}$ & $\begin{array}{c}\text { E-glass } \\
\text { fabric } \\
\text { impregnate } \\
\text { d with a } \\
\text { thermosetti } \\
\text { ng epoxy } \\
\text { resin } \\
\text { system }\end{array}$ \\
\hline $\begin{array}{c}\text { Prepreg } \\
\text { Thickness }\end{array}$ & $\begin{array}{c}0.008 "(0.20 \\
\mathrm{mm})\end{array}$ & $\begin{array}{c}0.008 ” \\
(0.20 \mathrm{~mm})\end{array}$ & $\begin{array}{c}0.008 " \\
(0.20 \mathrm{~mm})\end{array}$ & $\begin{array}{c}0.008 ” \\
(0.20 \mathrm{~mm})\end{array}$ & $\begin{array}{c}0.008 " \\
(0.20 \mathrm{~mm})\end{array}$ \\
\hline $\begin{array}{c}\text { Prepreg } \\
\text { Type }\end{array}$ & $\begin{array}{c}57 \times 548 \mathrm{H} \\
\text { Satin Weave }\end{array}$ & $\begin{array}{c}57 \times 548 \mathrm{H} \\
\text { Satin } \\
\text { Weave }\end{array}$ & $\begin{array}{c}57 \times 548 \mathrm{H} \\
\text { Satin } \\
\text { Weave }\end{array}$ & $\begin{array}{c}57 \times 548 \mathrm{H} \\
\text { Satin } \\
\text { Weave }\end{array}$ & $\begin{array}{c}57 \times 548 \mathrm{H} \\
\text { Satin } \\
\text { Weave }\end{array}$ \\
\hline $\begin{array}{c}\text { Resin } \\
\text { Content }\end{array}$ & $30 \%+/-3 \%$ & $\begin{array}{c}30 \%+/- \\
3 \%\end{array}$ & $\begin{array}{c}30 \%+/- \\
3 \%\end{array}$ & $\begin{array}{c}30 \%+/- \\
3 \%\end{array}$ & $\begin{array}{c}30 \%+/- \\
3 \%\end{array}$ \\
\hline $\begin{array}{c}\text { Surface } \\
\text { Treatments }\end{array}$ & $\begin{array}{c}\text { Grit blasting, } \\
\text { AC-130-2 } \\
\text { sol-gel by } \\
\text { 3M }\end{array}$ & - & 一 & - & - \\
\hline
\end{tabular}

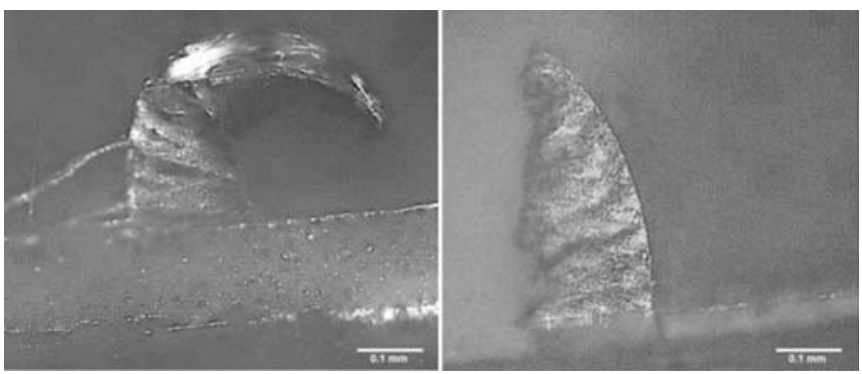

Figure 1. Microscopic view of CMH (left) and SMH (right) using AmScope Inverted Trinocular Metallurgical Microscope 


\section{Manufacturing}

The GLARE manufacturing process can be summarized into four distinct stages, namely, cleaning, lay-up, curing, and cutting. Prior to lay-up and curing, the prepreg layers are cut to $152 \times 304 \mathrm{~mm}^{2}\left(6 \times 12 \mathrm{in}^{2}\right)$ and debulked in a cold hydraulic press at 1.4 metric tons of pressure for 12 hours to remove any air pockets before lay-up.

The sheet metals are then degreased with methyl ethyl ketone (MEK) followed by cleaning in an ultrasonic bath using $100 \mathrm{~mL}$ of soap and $20 \mathrm{~L}$ of water for 15 minutes, before being thoroughly rinsed with tap water and wiped down with isopropyl alcohol, as shown in Fig. 4.

Following industry standards for the manufacturing of standard GLARE, prior to lay-up, no-hook sheet metal layers, used to manufacture the standard GLARE, are further treated with grit blasting using silica sand at 110 psi and then sprayed with $40 \mathrm{~mL}$ of AC-130-2 Sol-Gel, produced by $3 \mathrm{M}$ [16]. The Sol-Gel is then left to dry for 1 hour before beginning the lay-up of the standard GLARE. Sol-Gel forms an organic layer which promotes bonding between layers.

Prior to curing, the laid-up panels are debulked once again in a hydraulic press at 1.4 metric tons of pressure for 1 hour at room temperature to promote adhesion and to remove any air trapped during lay-up. Curing was done in house using a heated Carver pneumatic press at a pressure of 1.6 tons, as shown in Fig. 4. The temperature was increased from room temperature to $310^{\circ} \mathrm{F}$ at an approximate rate of $5^{\circ} \mathrm{F} / \mathrm{min}$, then cured for an hour at $310^{\circ} \mathrm{F}$ before being left to cool to room temperature. Cured samples are then removed from the press once their temperature fell below $140{ }^{\circ} \mathrm{F}$ (after approximately four hours).

Test samples are cut from the manufactured GLARE panels using a UHAP Smart Cut 6100 composite wet saw to the dimensions described in Section 3.2. The saw uses a nickel bond diamond wafering blade lubricated with distilled water to accurately cut samples to size with a precision of 0.13 $\mathrm{mm}(0.05 \mathrm{in})$.

\section{Microscopic Investigation}

To check the quality of the modified GLARE panels, pretesting microscopic analysis is conducted. Cross-sections are cut from a random selection of the manufactured panels and inspected using an optical microscope, as shown in Figs 2-5.

As shown in Fig. 2, GLARE with SNH showed the best manufacturing quality. It is observed that SNHs are able to penetrate well into the fiberglass composite which resulted in a very good integration at the interface of the different layers of the material. However, in few locations the hooks are found not penetrating the fibers and instead, the fibers are bending around the hooks creating potential gaps and fiber breakage, as shown in Fig. 2. A potential solution to this includes using a fiberglass weave with lower fiber density as well as redesigning the hooks with straight, sharper, pin profile.

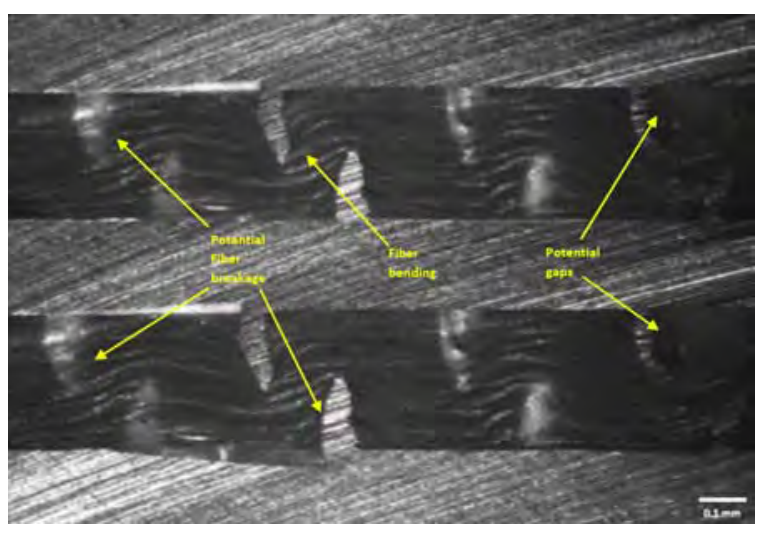

Figure 2. Microscopic view of modified GLARE with SNH using AmScope Inverted Trinocular Metallurgical Microscope

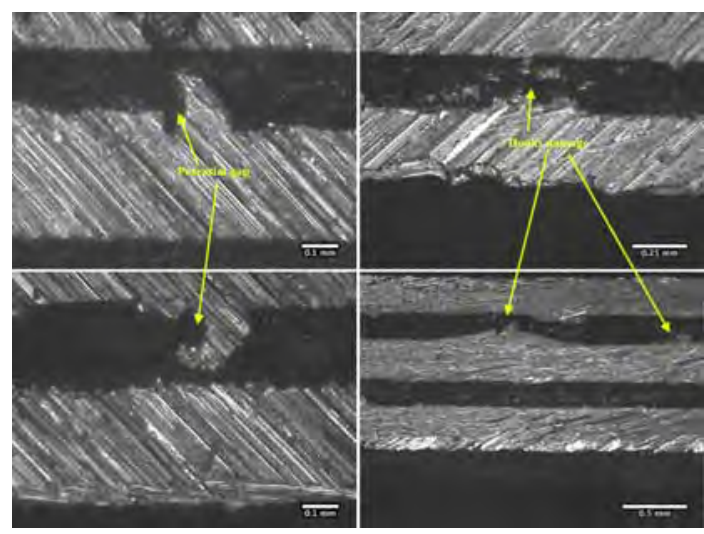

Figure 3. Four microscopic views of modified GLARE with $\mathrm{CNH}$ using AmScope Inverted Trinocular Metallurgical Microscope

As shown in Fig. 3, microscopic inspection of modified GLARE with CNH showed several manufacturing flaws including hooks crushing and breakage as well as development of gaps and fiber breakage. This can be attributed to the curved hook profile which significantly reduce the penetration capability of the hooks into the composite layers.

Modified GLARE with micro hooks showed the worst manufacturing quality. As shown in Fig. 4 and 5, most of the $\mathrm{SMH}$ and $\mathrm{CMH}$ are either crushed under pressure during curing or resulted in fiber breakage and development of several gaps.

\section{LOW-VELOCITY IMPACT TESTING}

\section{A. $\quad$ Test Setup}

Low velocity impact tests were performed using an Instron Dynatup 8200 drop weight impact tower following ASTM D7136/D7136-M [17]. A hemispherical impactor with a diameter of $25.4 \mathrm{~mm}$ and a mass of $10.1 \mathrm{~kg}$ was used in all tests. Samples were clamped using a custom-built impact fixture modelled after the impact fixture used by Laliberte et al. [18], shown in Fig. 6. The impact fixture had a filleted circular opening with a diameter of $112 \mathrm{~mm}(4.4 \mathrm{in})$ to 
prevent corner and edge deformations. Three samples of each GLARE configuration are tested at 7.5J energy level, including one configuration of standard GLARE with nohooks and four configurations of modified GLARE. Samples were cross-sectioned and examined under an optical microscope post-impact to determine internal damages.

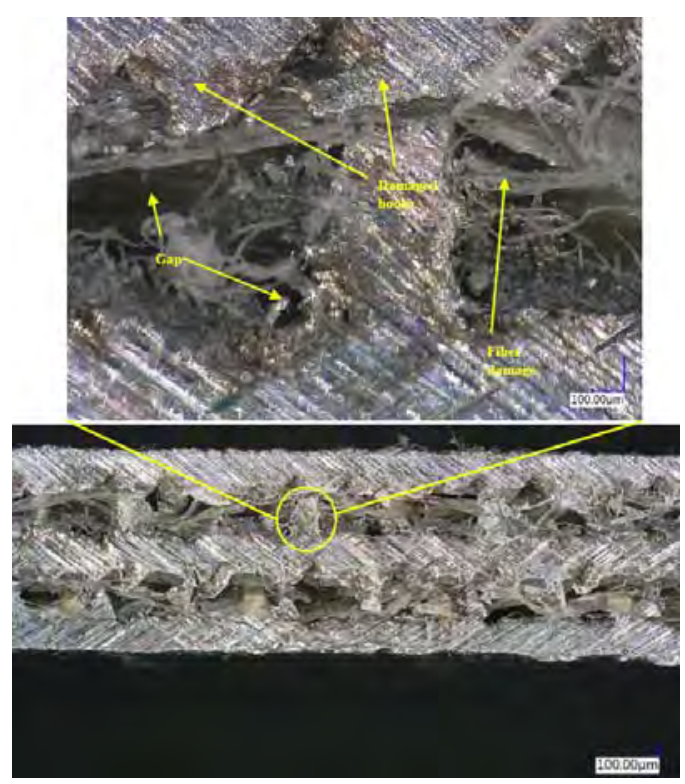

Figure 4. Microscopic views of modified GLARE with SMH using Keyence Digital Microscope VHX-6000 Series

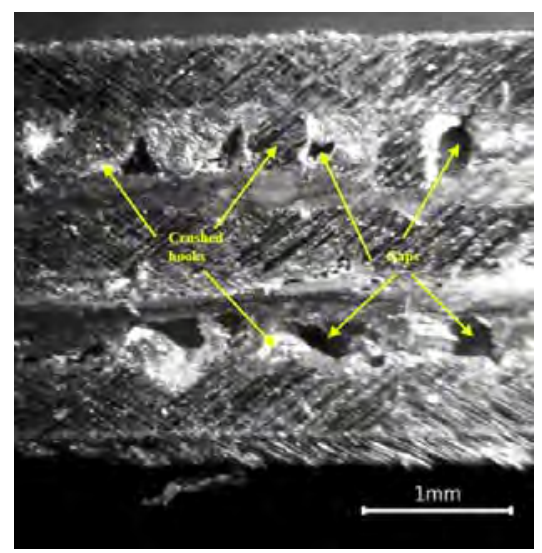

Figure 5. Microscopic view of modified GLARE with CMH using AmScope Inverted Trinocular Metallurgical Microscope

\section{B. Impact Response}

The averaged load versus time curves for each test configuration are shown in Fig. 7. The averaged maximum load of each test configuration is shown in Table 2. As shown in Fig. 7, samples of modified GLARE with nano hooks have similar impact responses to those of standard GLARE. While the modified GLARE with SNH supports a lower maximum load than standard GLARE, as shown in
Table 2, a drop of $2 \%$ is deemed insignificant compared to cost savings during metal sheet surface preparation.

Modified GLARE with micro hooks had worse impact responses compared to modified GLARE with nano hooks and standard GLARE. Despite supporting similar maximum loads to the other GLARE configurations, modified GLARE with micro hooks experienced significant internal failure during impact, as shown by the sharp drop in supported load in Fig. 7. This is attributed to the significant presence of voids and internal defects in the micro-hooked samples, as shown in Figs. 4 and 5.

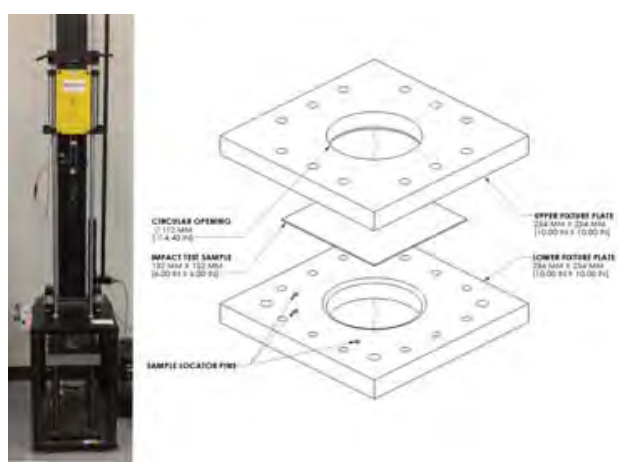

Figure 6. Drop tower (left) and custom impact fixture (right)

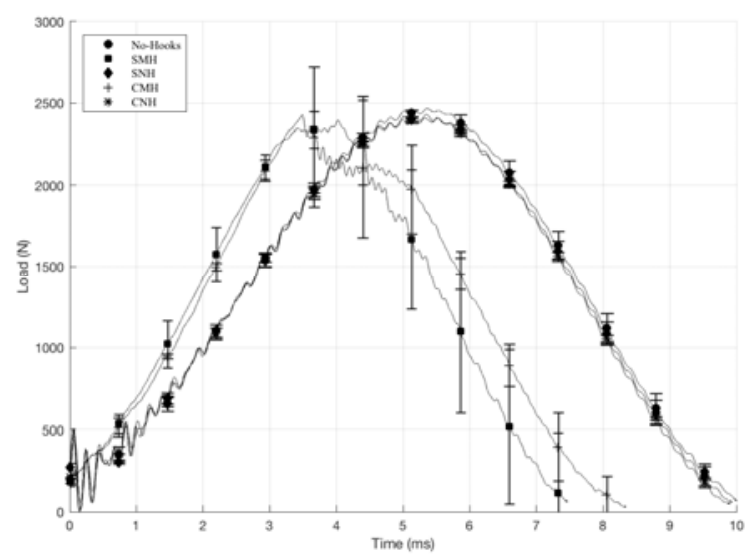

Figure 7. Averaged load v. time curves for each test configuration

TABLE II. AVERAGED MAXIMUM LOAD OF GLARE

\begin{tabular}{|c|c|}
\hline Specimen & $\begin{array}{c}\text { Averaged Maximum Load } \\
(\mathbf{N})\end{array}$ \\
\hline No-hooks & $2571.7 \pm 19.6$ \\
\hline SNH & $2256.5 \pm 0.8$ \\
\hline SMH & $2552.0 \pm 19.6$ \\
\hline CNH & $2529.6 \pm 21.0$ \\
\hline CMH & $2588.3 \pm 156.4$ \\
\hline
\end{tabular}

\section{Damage Assessment}

Fig. 8 shows a representative internal cross-section of each test configuration. As shown in Fig. 8, modified GLARE 
with nano hooks experienced significantly less delamination and fiber breakage than standard GLARE.

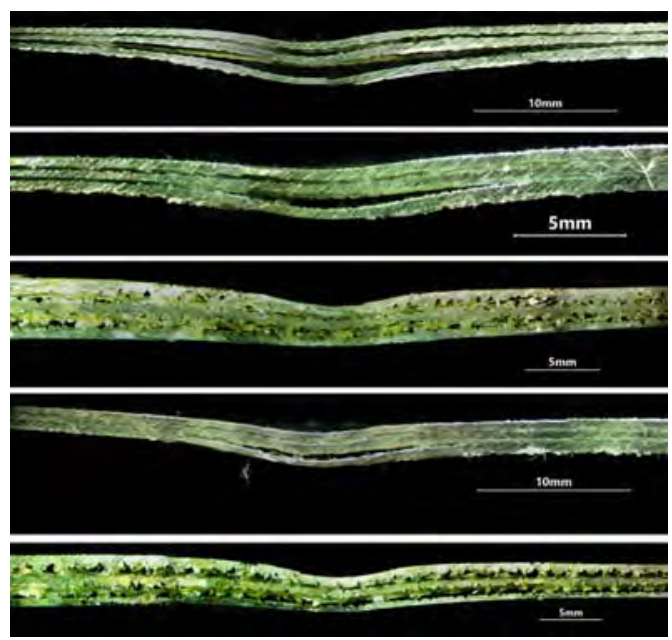

Figure 8. Internal cross sections of samples at $7.5 \mathrm{~J}$, in the following order from top to bottom: standard GLARE, SNH, SMH, CNH, CMH

Standard GLARE samples also possess a larger internal damage area, as internal damages extend far beyond the impactor dent. This can be attributed to the superior interlocking behavior of the nano hooks. The modified GLARE with micro hooks experience significantly more damage than the other test configurations. In addition to experiencing major fiber breakage near the impactor dent, the modified GLARE with micro hooks are the only test configurations that experience observable cracks in the metal surfaces, as shown in Fig. 8. This is due to the reduction in strength in the metal layers due to hooks machining. Since the hooks are made directly from the sheet metal surfaces, larger hooks would remove more material, reducing its strength. Since nano hooks have smaller hooks, it would not experience as significant of an issue.

\section{MECHANICAL CHARACTERIZATION}

Tensile tests were performed to mechanically characterize the modified GLARE. Tensile tests are performed using 810 Materials Testing System (MTS), shown in Fig. 9. Rectangular test samples are manufactured following ASTM D3039/D3039M [19] with dimensions of $216 \times 22 \mathrm{~mm}^{2}(8.5$ $\mathrm{x} 0.85 \mathrm{in}^{2}$ ) and grip lengths of $47 \mathrm{~mm}$ (1.85 in). Five samples of each GLARE configuration are tested including one configuration of standard GLARE with no-hooks and four configurations of modified GLARE. Samples are tested at a constant load rate of $2.0 \mathrm{~mm} / \mathrm{min}$. Test data was recorded at a frequency of $5.0 \mathrm{~Hz}$.

The averaged stress versus strain curves for each test configuration are shown in Fig. 10. The averaged elastic modulus, yield strength, ultimate tensile strength, and elongation to fracture of each test configuration are shown in Table 3.
All tensile samples of modified GLARE with micro hooks as well as those of standard GLARE experienced significant fiber breakage and edge delamination upon tensile failure, as shown in Fig. 11.
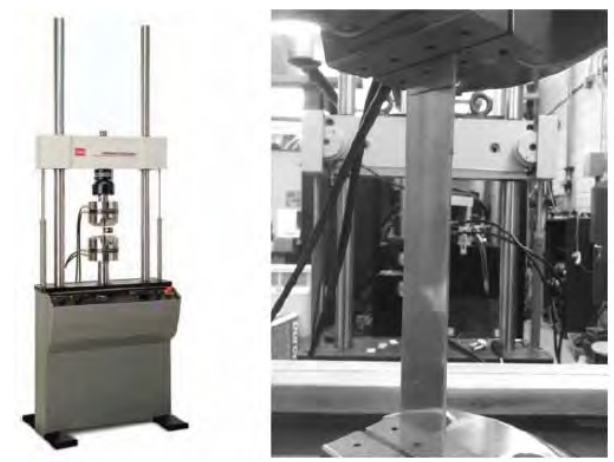

Figure 9. 810 Material Testing System (MTS) (left) and a mounted tensile test sample (right)

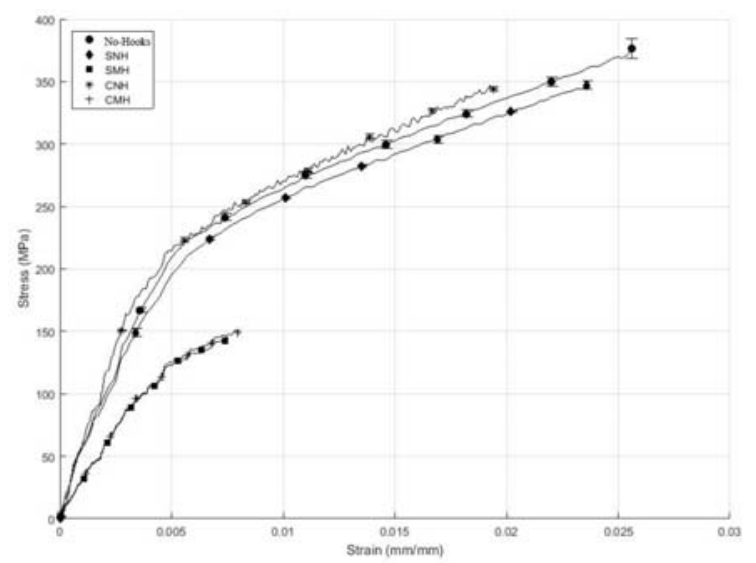

Figure 10. Tensile test results of GLARE

Also, their samples experienced full rupture upon failure, as shown in Fig. 12. Samples of modified GLARE with nano hooks tended to partially fracture, leaving the back metal sheet intact, as shown in Fig. 13. Despite earlier failure of modified GLARE samples with $\mathrm{CNH}$ and at lower ultimate stress level compared to standard GLARE and modified GLARE with SNH, the difference in strength between them lie only within $10 \%-20 \%$. As shown in Fig. 10 and Table 2, modified GLARE with SNH experienced drop of $4.5 \%$ and $3 \%$ in elastic modulus and yield strength, respectively, compared to the standard GLARE.

TABLE III. Tensile Mechanical Properties of GLARE

\begin{tabular}{|c|c|c|c|c|}
\hline Specimen & $\begin{array}{c}\text { Elastic } \\
\text { Modulus } \\
\text { (GPa) }\end{array}$ & $\begin{array}{c}\text { Yield } \\
\text { Strength } \\
\text { (MPa) }\end{array}$ & $\begin{array}{c}\text { Ultimate } \\
\text { Strength } \\
\text { (MPa) }\end{array}$ & $\begin{array}{c}\text { Elongation } \\
\text { to Break } \\
\text { (\%) }\end{array}$ \\
\hline No-hooks & $42.3 \pm 0.3$ & $243.4 \pm 2.3$ & $378.5 \pm 8.8$ & $2.69 \pm 0.1$ \\
\hline SNH & $40.4 \pm 1.6$ & $236.8 \pm 4.6$ & $362.3 \pm 16.8$ & $2.63 \pm 0.29$ \\
\hline SMH & $28.0 \pm 0.02$ & $141.5 \pm 2.3$ & $143.2 \pm 2.6$ & $0.75 \pm 0.02$ \\
\hline $\mathrm{CNH}$ & $53.1 \pm 1.4$ & $229.9 \pm 0.8$ & $352.2 \pm 2.8$ & $2.02 \pm 0.07$ \\
\hline $\mathrm{CMH}$ & $28.3 \pm 0.5$ & $145.7 \pm 1.8$ & $156.0 \pm 6.0$ & $0.93 \pm 0.15$ \\
\hline
\end{tabular}



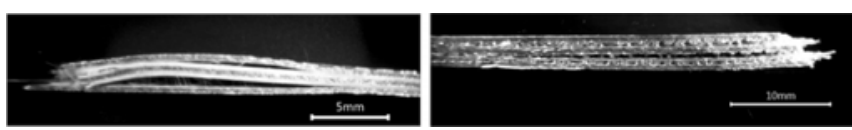

Figure 11. Fiber breakage and delamination in standard GLARE (left) and modified GLARE with CMH (right)
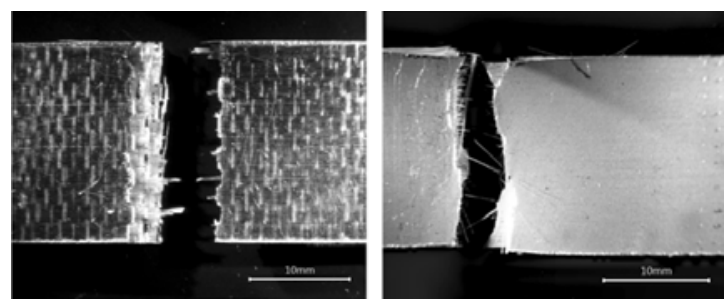

Figure 12. Top view of fractured samples of modified GLARE with $\mathrm{CMH}$ (left) and standard GLARE (right)
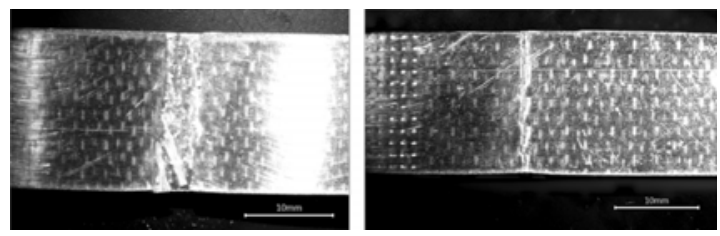

Figure 13. Partial fracture in samples of modified GLARE with SNH (left) and $\mathrm{CNH}$ (right)

The results from the tensile tests agree with the conclusions from the impact tests, namely comparable mechanical responses between the modified GLARE with SNH and standard GLARE, and the superior internal damage resistance of modified GLARE with SNH.

\section{CONCLUSION}

The low-velocity impact response of GLARE samples with and without hooks are experimentally investigated to determine whether machined hooks can be considered as a low-cost alternative to expensive industry-standard methods of improving adhesion in FML manufacturing. Hooks of different sizes and profiles are tested to determine the optimal hook size and profile. The following conclusions are made:

Modified GLARE with SNH samples had comparable dynamic impact responses to standard GLARE samples, while outperforming the standard GLARE samples in terms of the severity and extent of internal damages. While modified GLARE with CNH had comparable behavior to that with $\mathrm{SNH}$, microscopic analysis revealed that $\mathrm{CNHs}$ do not penetrate the fibers in the composite layer due to the shape of its profile.

Micro-hooked samples perform significantly worse compared to all other test configurations in terms of both dynamic response and internal damages. This is due to the high number of voids and internal defects in the microhooked samples, which stem from the composite layer being too thin to fully encompass the larger hooks. In addition, the micro hooks are deformed or damaged due to the clamping pressure exerted on it during manufacturing.

Based on these conclusions, modified GLARE with SNH has the potential to be a viable, low-cost alternative to traditional methods of improving consistency failure in FMLs.

\section{REFERENCES}

[1] A. Vlot, "Impact loading on fibre metal laminates," International Journal of Impact Engineering, vol. 18, no. 3, pp. 291-307, 1996.

[2] J. Bieniaś, B. Surowska and P. Jakubczak, "The Comparison of LowVelocity Impact Resistance of Aluminum/Carbon and Glass Fiber Metal Laminates," Polymer Composites, vol. 37, no. 4, p. 1056-1063, 2016.

[3] G. Wu and J.-M. Yang, "The Mechanical Behavior of GLARE Laminates for Aircraft Structures," JOM, vol. 57, no. 1, pp. 72-79, 2005.

[4] L. Vogelesang and A. Vlot, "Development of fibre metal laminates for advanced aerospace structures," Journal of Materials Processing Technology, vol. 103, no. 1, pp. 1-5, 2000.

[5] A. Vlot, L. Vogelesang and T. de Vries, "Towards application of fibre metal laminates in large aircraft," Aircraft Engineering and Aerospace Technology, vol. 71, no. 6, pp. 558-570, 1999.

[6] R. Alderliesten and R. Benedictus, "Fiber/Metal Composite Technology for Future Primary Aircraft Structures," Journal of Aircraft, vol. 45, no. 4, pp. 1182-1189, 2008.

[7] A. Asundi and A. Choi, "Fiber Metal Laminates: An Advanced Material for Future Aircraft," Journal of Materials Processing Technology, vol. 63, no. 1-3, pp. 384-394, 1997.

[8] C. Vermeeren, "An Historic Overview of the Development of Fibre Metal Laminates," Applied Composite Materials, vol. 10, no. 4, p. 189-205, 2003.

[9] T. Sinmazçelik, E. Avcu, M. Bora and O. Çoban, "A review: Fibre metal laminates, background, bonding types and applied test methods," Materials and Design, vol. 32, no. 7, p. 3671-3685, 2011.

[10] M. Sadighi, R. Alderliesten and R. Benedictus, "Impact resistance of fiber-metal laminates: A review," International Journal of Impact Engineering, vol. 49, pp. 77-90, 2012.

[11] R. Starikov, "Assessment of impact response of fiber metal laminates," International Journal of Impact Engineering, vol. 59, pp. 38-45, 2013.

[12] E. Botelho, R. Silva, P. L.C. and M. Rezende, "A Review on the Development and Properties of Continuous Fiber/epoxy/aluminum Hybrid Composites for Aircraft Structures," Materials Research, vol. 9, no. 3, pp. 247-256, 2006.

[13] M. Ostapiuk, B. Surowska and J. Bieniaś, "Interface analysis of fiber metal laminates," Composite Interfaces, vol. 21, no. 4, p. 309-318, 2014.

[14] A. Vlot and J. Gunnick, Fiber metal laminates: an introduction, Springer Science \& Business Media, 2001.

[15] "8.8 oz. 8H Satin Weave Fiberglass RTS Prepreg 50"," [Online]. Available: https://store.acpsales.com/products/3261/8-8-oz-8h-satinweave-fiberglass-rts-prepreg-50. [Accessed 4 December 2017].

[16] "3М $\mathrm{M}^{\mathrm{TM}}$ Surface Pre-Treatment AC-130-2," [Online]. Available: https://www.3m.com/3M/en_US/company-us/all-3m-products/ /3MSurface-Pre-Treatment-AC-130-2/?N=5002385+3292667587\&rt=rud. [Accessed 4 December 2017].

[17] Standard Test Method for Measuring the Damage Resistance of a Fiber-Reinforced Polymer Matrix Composite to a Drop-Weight Impact Event, ASTM D7136/D713M-15, 2015.

[18] J. Laliberté, P. Straznicky and C. Poon, "Impact Damage in Fiber Metal Laminates, Part 1: Experiment," AIAA Journal, vol. 43, no. 11, pp. 2445-2453, 2005.

[19] Standard Test Method for Tensile Properties of Polymer Matrix Composite Materials, ASTM D3039/D3039-M-14, 2014. 


\section{Corrosion Performances of Micro-arc Oxidation Coatings on AZ31B, AZ80, and ZK60 cast Mg Alloys}

\author{
Yuna Xue ${ }^{1,3}$ \\ ${ }^{1}$ School of Materials Science and Engineering \\ Xi'an University of Technology \\ Xi'an, China \\ xynlina@gmail.com \\ Bailing Jiang ${ }^{1}$ \\ jiangbail@vip.163.com
}

\author{
Xin Pang ${ }^{2}$ \\ ${ }^{2}$ CanmetMATERIALS \\ Natural Resources Canada \\ Hamilton, Canada \\ xin.pang@canada.ca \\ Hamid Jahed ${ }^{3}$ \\ ${ }^{3}$ Department of Mechanical \& Mechatronics Engineering \\ University of Waterloo \\ Waterloo, Canada \\ hjahedmotlagh@uwaterloo.ca
}

\begin{abstract}
Micro-arc oxidation (MAO) technology is attractive for the surface treatment of Mg alloys for diverse applications because it can be used to form adherent coatings with superior wear and corrosion resistance. In the present study, the corrosion protective MAO coatings were prepared on the AZ31B, AZ80 and ZK60 cast magnesium alloy at a constant current mode in an alkaline silicate electrolyte. Analysis of the surface and cross-section morphology, phase composition and microstructures of the MAO coatings and these three kinds of substrates was conducted utilizing scanning electron microscope (SEM) and X-ray diffraction (XRD) techniques. The corrosion properties of the uncoated and MAO coated Mg alloys were investigated using advanced electrochemical methods including the open circuit potential (OCP) measurement and electrochemical impedance spectroscopy (EIS). To further characterize the protectiveness of the MAO coating, the scribed MAO coated AZ31B and ZK60 alloy coupons were exposed to the continuous salt spray corrosion testing (SSCT) for $168 \mathrm{~h}$. The experimental results show that the phase composition, the thickness and the microstructure of MAO coatings on different kinds of $\mathrm{Mg}$ alloys did not show any differences between them. While the MAO coating provides the best robust protection on the ZK60 $\mathrm{Mg}$ alloy substrate in the aqueous salt solution and continuous salt spray environments, the corrosion resistance increased by nearly three orders of magnitude as compared to uncoated $\mathrm{Mg}$ alloy.
\end{abstract}

Keywords-Cast magnesium alloys; Micro-arc oxidation; Electrochemical kinetic parameters; Scribe testing

\section{INTRODUCTION}

In the automotive industry, magnesium and magnesium alloys have been given priority to be the structural components to reduce the weight and decrease the fuel emissions [1]. As the application of magnesium alloys becoming mature, they are not only applied in the internal and unloading components, but also serviced in the external and loading parts [2,3]. However, magnesium alloy is prone to corrosion during services, which is a major factor in obstructing its application $[4,5]$.

This paper discusses a promising surface treatment, microarc oxidation (MAO), to form the ceramic coatings on $\mathrm{Mg}$ and their alloys [6]. Up to now, the MAO coatings are widely used on different kinds of the magnesium alloy, which makes the perfect combination of the base material [7]. In this work, we chose two Mg-Al alloys (AZ31B and AZ80) and one Mg-Zn alloy (ZK60) as the substrate. Inclusion of Al can improve the corrosion resistance of magnesium alloy[8], and the zinc element with zirconium in $\mathrm{Mg}$ alloy has successful grain refinement and better anti-corrosion behavior [9]. For a better understanding of the correlations of MAO coatings, different substrate element alloys, corrosion kinetics in simulated service environment of automotive applications, the microstructure and corrosion properties of uncoated and MAO coated AZ31B, AZ80 and ZK60 were studied by the microscopic analysis and corrosion methods.

\section{MATERIALS AND EXPERIMENTS}

\section{A. Materials}

The materials were commercially available AZ31B [10,11], AZ80 [12] and ZK60 [9] cast Mg alloys (see Table 1). These alloys were cut into flat coupons with the dimensions of 50 $\mathrm{mm} * 25 \mathrm{~mm} * 3 \mathrm{~mm}$. And then all the specimens were ground with emery paper to achieve lower surface roughness.

The processing parameters for the MAO treatment are listed in Table 2. All MAO coatings were performed at Xi'an University of Technology. After the MAO treatment, the 
specimens were first cleaned with distilled water and then air dried for further analysis.

TABLE 1 Chemical composition of the substrate alloys

\begin{tabular}{|l|ccccccc|}
\hline \multirow{2}{*}{ Alloys } & \multicolumn{7}{|c|}{ Compositions (wt.\%) } \\
\cline { 2 - 8 } & $\boldsymbol{A} \boldsymbol{Z}$ & $\boldsymbol{Z n}$ & $\boldsymbol{Z r}$ & $\boldsymbol{M n}$ & $\boldsymbol{S i}$ & $\boldsymbol{C u}$ & $\boldsymbol{M g}$ \\
\hline AZ31B & 3.1 & 1.05 & 0.54 & 0.02 & 0.0008 & Balance \\
AZ80 & 8.4 & 0.48 & & 0.2 & 0.026 & 0.0026 & Balance \\
ZK60 & 5.2 & & 0.47 & 0.09 & 0.03 & 0.005 & Balance \\
\hline
\end{tabular}

TABLE 2 Processing parameters for the MAO treatment

\begin{tabular}{|c|c|}
\hline Electrolyte composition & $\begin{array}{l}\mathrm{Na}_{2} \mathrm{SiO}_{3}-5 \mathrm{~g} / \mathrm{L} ; \\
\mathrm{KF}-8 \mathrm{~g} / \mathrm{L} ; \\
\mathrm{KOH}-10 \mathrm{~g} / \mathrm{L}\end{array}$ \\
\hline Electrolyte $\mathrm{pH}$ & 13 \\
\hline Current density $(\mathrm{mA} / \mathrm{cm} 2)$ & 34 \\
\hline Power supply pulse width $(\mu \mathrm{s})$ & 80 \\
\hline Processing time (min) & 5 \\
\hline Power supply average current(A) & 1 \\
\hline
\end{tabular}

\section{B. Specimen examination and test methods}

For metallographic characterization of the base alloys, optical microstructure of the specimens were studied using the etching reagents, and the etching procedure was immersing specimen face up with gentle agitation until face turns brown. The surface and cross-section morphologies of the MAO coated different substrate were investigated by XL30 SEM. And phase composition of uncoated and MAO coated specimens was identified by XRD analysis.

The electrochemical measurements including open-current potential (OCP) and electrochemical impedance spectroscopy (EIS) were obtained using a Solartron Analytical 1287A potentiostat/galvanostat and Solartron 1255B Frequency Response Analyzer controlled by a personal computer at room temperature in the corrosive medium $3.5 \mathrm{wt} \% \% \mathrm{NaCl}$ solution.

The scribe test were conducted in the Singleton Corp.SCCH-salt fog chamber to a static environment of continuous salt spray with a concentration of $5 \mathrm{wt} . \% \mathrm{NaCl}$ at $36{ }^{\circ} \mathrm{C}, 100 \%$ relative humidity according to ASTM B117 [13]. MAO coated AZ31B and ZK60 contained a vertical scribe parallel to major long axis (about $3.5 \mathrm{~cm}$ in length) down the centerline. Scribing was performed by rounded tip scribing tool to make sure that fresh $\mathrm{Mg}$ alloy substrate metal was exposed. The rust creepage along the scribe after corrosion testing was evaluated.

\section{RESULTS AND DISCUSSION}

\section{A. Materials characterization}

Fig.1 shows the optical micrographs of the internal structure for the AZ31B, AZ80 and ZK60 cast magnesium alloys. For $\mathrm{AZ31B}$, the volume fraction ratio of the $\beta-\mathrm{Mg}_{17} \mathrm{Al}_{12}$ is low and it is diffused in the matrix $\alpha-\mathrm{Mg}$ because of the low aluminum content as shown in the Fig.1a. In the AZ80 alloy, the higher content of $\mathrm{Al}$ element, the richer $\beta$-phase formed a continuous network and precipitated along the grain boundaries. There are $\mathrm{MgZn}_{2}$ and $\mathrm{Zn}_{2} \mathrm{Zr}$ intermetallic compound in the
ZK60 alloy (shown in Fig.1c,d). The zirconium in ZK60 magnesium alloys can refine grain and purify the composition of alloy [14].
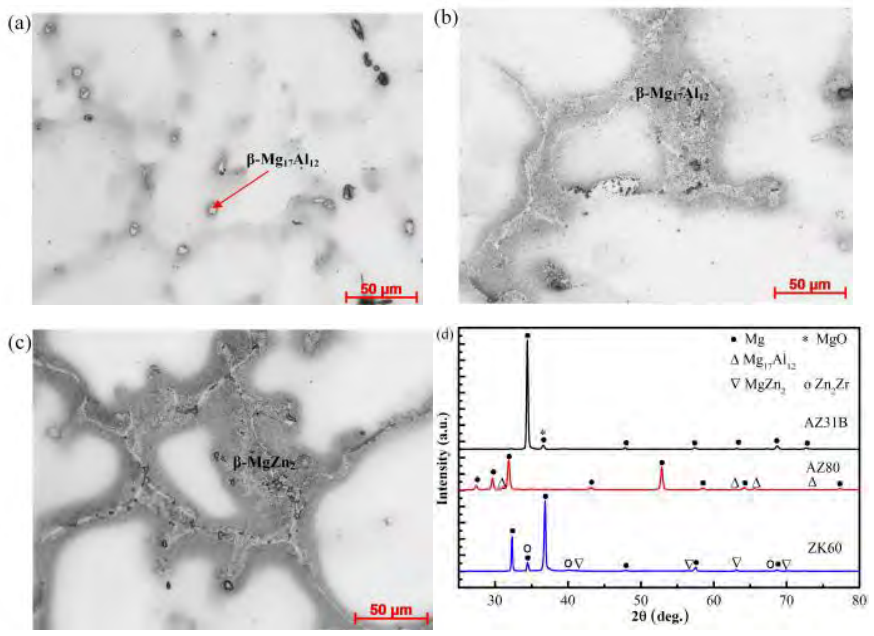

Figure 1. Optical micrographs (a,b,c) and XRD patterns (d) of the AZ31B, AZ80 and ZK60 Mg alloys

SEM images and phase compositions of the typical surface of the MAO coated magnesium alloys are shown in Fig.2. The surface of the MAO treated different $\mathrm{Mg}$ alloys under same processing parameters were featured by a porous morphology with lots of volcano top-like micro-pores which diameter is about $1 \mu \mathrm{m}$, dense and uniform ceramic coating with strong adhesion to substrate are formed on the substrates. There is no obvious difference between the surface micrographs of these three specimens. The thickness of the MAO coatings is about $5.5 \mu \mathrm{m}$, and a typical amount of micro-pores and micro-cracks can still be found in the coatings. Seen from the XRD spectra, it is clear that the surface of all treated specimens are mainly composed of $\mathrm{Mg}$ phase, $\mathrm{MgO}, \mathrm{MgF}_{2}$ and $\mathrm{Mg}_{2} \mathrm{SiO}_{4}$. And they also contained some complex silicate compounds $\mathrm{Mg}_{3} \mathrm{Al}_{2}\left(\mathrm{SiO}_{4}\right)_{3}$ and $\mathrm{Mg}_{\mathrm{x}} \mathrm{F}_{\mathrm{y}}\left(\mathrm{SiO}_{4}\right)_{\mathrm{z}}$.
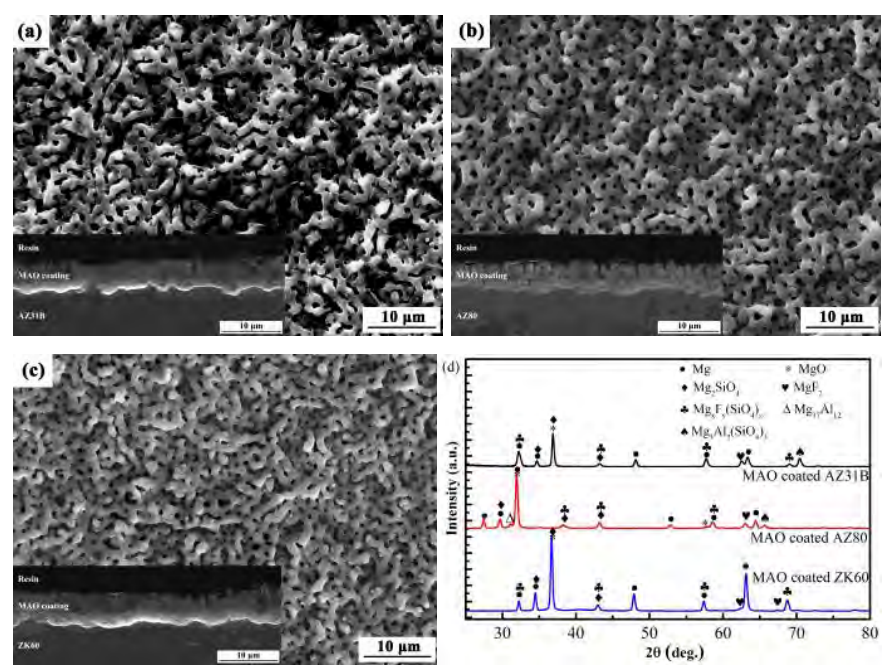

Figure 2. SEM images of the surface, cross-sectional morphology and the corresponding phase compositions (d) of the MAO coated (a) AZ31B, (b) AZ80 and (c) ZK60 Mg alloys 


\section{B. Electrochemical corrosion test}

\section{1) $\mathrm{OCP}$}

Fig. 3 shows the OCPs of these different specimens. After $30 \mathrm{~min}$ of immersion, all these specimens were reached to the relatively stable values of electrode potentials. Compared with the bare substrate, the OCPs of the specimens with MAO coated Mg alloys were shifted towards the noble direction. The uncoated $\mathrm{Mg}$ specimens had a low average OCP value of -1.54 , -1.52 and $-1.49 \mathrm{~V}$ for AZ31B, AZ80 and ZK60 Mg alloy, respectively. In contrast, all the MAO coated AZ31B, AZ80 and ZK60 specimens showed more noble average OCP values, which were $-1.29,-1.28$ and $-1.26 \mathrm{~V}$, respectively. These results suggest that MAO deposited on magnesium alloys showed a better thermodynamically stable and can improve the corrosion resistance of magnesium alloys in a dilute chloride solution.

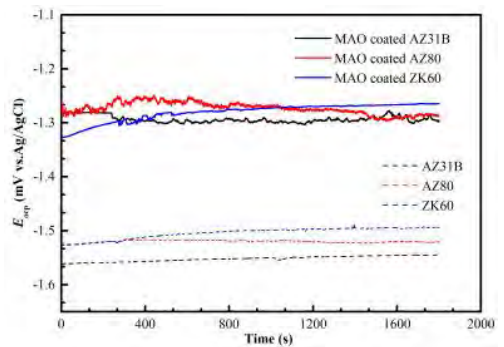

Figure 3. OCPs vs. immersion time curves in $3.5 \mathrm{wt} \% \mathrm{NaCl}$ solution for uncoated and MAO coated different $\mathrm{Mg}$ alloys

\section{2) $E I S$}

Fig.4 shows the plots of impedance modulus vs. frequency and phase angle vs. frequency measured after the OCP test for the MAO coated and bare magnesium alloys. The equivalent circuits seen in Fig.5 were used to simulate the measured impedance data of the uncoated and MAO coated Mg alloys, and it shows the charge transfer between the reference electrode and working electrode during the corrosion process.
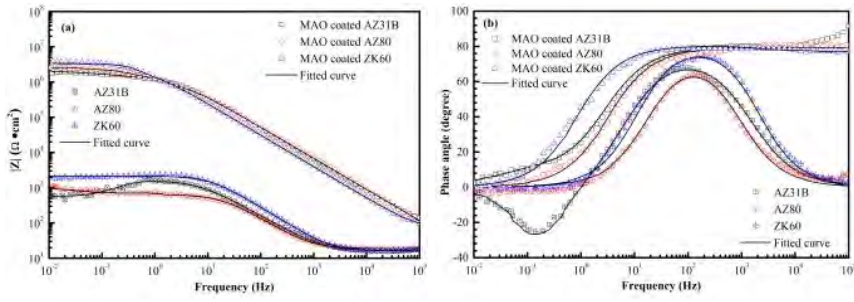

Figure 4. EIS experimental data and simulated curves for coated and uncoated Mg alloys specimens in the form of Bode plots: (a) impedance modulus vs. frequency, and (b) phase angle vs. frequency plots
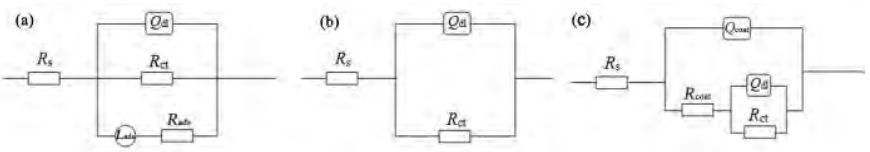

Figure 5. Equivalent electrical circuits for (a) AZ31B, (b) AZ80 and ZK60 and (c) MAO coated AZ31B, AZ80 and ZK60 alloys

For the substrate AZ31B Mg alloy, Fig.5 shows the adsorption inductance behavior in the low frequency range (less than $100 \mathrm{~Hz}$ ) of the spectrum. Its equivalent circuit (Fig.5a) is made up of the electrolyte resistance $\left(R_{\mathrm{S}}\right)$, charge transfer resistance $\left(R_{\mathrm{ct}}\right)$ of the double-layer capacitor and double-layer capacitance $\left(Q_{\mathrm{dl}}\right)$ which are attributed to the Faradaic processes [15], adsorption resistance $\left(R_{\mathrm{ads}}\right)$ and adsorption inductance $\left(L_{\mathrm{ads}}\right)$ which are associated with either the corrosion products such as $\mathrm{MgO} / \mathrm{Mg}(\mathrm{OH})_{2}$ formed on the surface of $\mathrm{Mg}$ alloy or $\mathrm{Mg}$ dissolution in chloride containing solution. In AZ80 and ZK60 alloy, their equivalent circuit includes $R_{\mathrm{s}}, R_{\mathrm{ct}}$ and $Q_{\mathrm{dl}}$. The proposed equivalent circuit of MAO coated Mg alloys is shown in Fig.5c. It consists of $R_{\mathrm{s}}, R_{\mathrm{ct}}$ and $Q_{\mathrm{dl}}$, coating resistance $\left(R_{\text {coat }}\right)$ and coating capacitance $\left(Q_{\text {coat }}\right)$ which is the virtual properties of MAO coating [16]. It can be seen from Fig.4 that the simulated curves fit very well with the experiment data. The fitting results are summarized in Table 3.

TABLE 3 Results of fitting EIS data for the uncoated and MAO coated Mg alloys specimens

\begin{tabular}{|c|c|c|c|c|c|c|c|}
\hline Specimen & $\begin{array}{l}R_{\mathrm{ct}} \\
\left(\Omega \cdot \mathrm{cm}^{2}\right)\end{array}$ & $\begin{array}{l}Q_{\mathrm{dl}} \\
\left(\mu \mathrm{F} / \mathrm{cm}^{2} \cdot \mathrm{s}^{1-\mathrm{n}}\right)\end{array}$ & & $\begin{array}{l}R_{\text {ads }} \\
\left(\Omega \cdot \mathrm{cm}^{2}\right)\end{array}$ & $\begin{array}{l}L_{\text {ads }} \\
\left(\mathrm{H} / \mathrm{cm}^{2}\right)\end{array}$ & & $\begin{array}{l}R_{\text {total }} \\
\left(\Omega \cdot \mathrm{cm}^{2}\right)\end{array}$ \\
\hline AZ31B & $1.59 \times 10^{3}$ & 23.59 & 0.864 & 870 & 1415 & & $2.459 \times 10^{3}$ \\
\hline AZ80 & $3.31 \times 10^{3}$ & 18.92 & 0.901 & & & & $3.307 \times 10^{3}$ \\
\hline ZK60 & $6.34 \times 10^{3}$ & 8.72 & 0.938 & & & & $6.335 \times 10^{3}$ \\
\hline Specimen & $\begin{array}{l}R_{\text {coat }} \\
\left(\Omega \cdot \mathrm{cm}^{2}\right)\end{array}$ & $\begin{array}{l}Q_{\text {coat }} \\
\left(\mu \mathrm{F} / \mathrm{cm}^{2} \cdot \mathrm{s}^{1-\mathrm{n}}\right)\end{array}$ & $\mathrm{n}_{\text {coat }}$ & $\begin{array}{l}R_{\mathrm{ct}} \\
\left(\Omega \cdot \mathrm{cm}^{2}\right)\end{array}$ & $\begin{array}{l}Q_{\mathrm{dl}} \\
\left(\mu \mathrm{F} / \mathrm{cm}^{2} \cdot \mathrm{s}^{1-\mathrm{n}}\right)\end{array}$ & & $\begin{array}{l}R_{\text {total }} \\
\left(\Omega \cdot \mathrm{cm}^{2}\right)\end{array}$ \\
\hline $\begin{array}{l}\text { MAO } \\
\text { coated } \\
\text { AZ31B }\end{array}$ & $7.97 \times 10^{5}$ & 0.066 & 0.885 & $1.34 \times 10^{6}$ & 0.186 & 0.856 & $2.141 \times 10^{6}$ \\
\hline $\begin{array}{l}\text { MAO } \\
\text { coated } \\
\text { AZ80 }\end{array}$ & $8.28 \times 10^{5}$ & 0.040 & 0.859 & $2.10 \times 10^{6}$ & 0.103 & 0.883 & $2.929 \times 10^{6}$ \\
\hline $\begin{array}{l}\text { MAO } \\
\text { coated } \\
\text { ZK60 }\end{array}$ & $2.10 \times 10^{6}$ & 0.010 & 0.847 & $3.54 \times 10^{6}$ & 0.068 & 0.895 & $5.645 \times 10^{6}$ \\
\hline
\end{tabular}

In this work, a $R_{\text {ads }}$ of $870 \Omega \cdot \mathrm{cm}^{2}$ was obtained for the uncoated AZ31B alloy specimen. In comparison, 3-4 orders of magnitude higher coating resistance $R_{\text {coat }}\left(7.97 \times 10^{5} \sim 2.10 \times 10^{6}\right.$ $\Omega \cdot \mathrm{cm}^{2}$ ) than the $R_{\mathrm{ads}}$ of uncoated AZ31B Mg alloy were obtained for the MAO coated specimens. It is suggested that the formation of complex compounds consisting of mainly $\mathrm{Mg}_{2} \mathrm{SiO}_{4}$ and $\mathrm{MgF}_{2}$ on the alloy surface during the MAO process improved the corrosion resistance of the alloy significantly. The high $R_{\text {coat }}$ and low $Q_{\text {coat }}$ indicate a small number of ionic conducting pathways (pores and micro-cracks) in the MAO coating, especially for the MAO coated ZK60 Mg alloy. It should be noted that for all coated specimens the charge transfer resistance $R_{\text {ct }}$ obtained is always higher than coating resistance $R_{\text {coat }}$, indicating that the charge transfer through the electrical double-layer at the coating/substrate interface is also pronouncedly impeded by the MAO coating, leading to improve the corrosion resistance of the MAO coated $\mathrm{Mg}$ alloy specimens.

For the charge transfer resistance $\left(R_{\mathrm{ct}}\right)$, it can be acquired from the high-frequency range and correspond to the dissolution of magnesium alloy and can directly characterize the corrosion resistance of the specimens. As shown in Table 3, the MAO coated ZK60 Mg alloy specimen has a very high $R_{\mathrm{ct}}$ $\left(3.54 \times 10^{6} \Omega \cdot \mathrm{cm}^{2}\right)$ and low $Q_{\mathrm{dl}}\left(0.068 \mu \mathrm{F} / \mathrm{cm}^{2} \cdot \mathrm{s}^{1-\mathrm{n}}\right)$ compared with other coated specimens. This can be attributed to the more compact coating microstructure and elements with more positive potential obtained under such a processing condition. It is worth noticing that the polarisation resistance $R_{\text {total }}$ 
$\left(R_{\text {total }}=R_{\text {coat }}+R_{\mathrm{ct}}\right.$ for MAO coated $\mathrm{Mg}$ alloys specimens, $R_{\text {total }}=R_{\text {ads }}+R_{\text {ct }}$ for uncoated AZ31B specimen, and $R_{\text {total }}=R_{\mathrm{ct}}$ for uncoated AZ80 and ZK60 specimens) measured by EIS, as listed in Table 3, follows such an order: MAO coated ZK60 > MAO coated AZ80 > MAO coated AZ31B; for bare alloys: ZK60 $>$ AZ80 $>$ AZ31B. The polarisation resistance from EIS has been widely used to account for the kinetics of electrochemical corrosion process [17]. The higher polarisation resistance, the better corrosion resistance the coated specimen exhibits.

\section{Scribe testing}

Fig. 6 shows the photographs of the MAO coated AZ31B and ZK60 specimens after $0 \mathrm{~h}, 72 \mathrm{~h}$ and $168 \mathrm{~h}$ exposure in the ASTM B117 test environment. After $72 \mathrm{~h}$, visual corrosion damage associated with the scribe was observed on the MAO coated AZ31B alloy. None of the MAO coated ZK60 alloy exhibited any visual corrosion damage associated with the scribe. As the corrosion continues (168 h), MAO coated AZ31B specimen exhibited significantly more corrosion products relative to the MAO coated ZK60 alloy. And there are some corrosion areas occurred on the MAO coated AZ31B alloy. The visual extent of the adherent corrosion product was significantly less than the MAO coated ZK60.

(a) MAO coated AZ31B

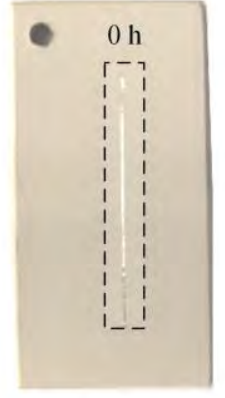

(b) MAO coated $\mathrm{ZK} 60$

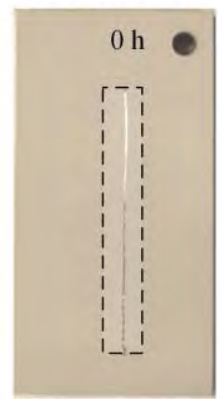

Figure 6. Photographs of the scribed MAO coated (a) AZ31B and (b) ZK60 specimens before corrosion and after ASTM B117 testing for $72 \mathrm{~h}$ and $168 \mathrm{~h}$

It seems that the spread of corrosion from scribe was influenced more by the difference in the coating substrate. Combining the microstructure and electrochemical corrosion results, in AZ series and ZK60 alloys, the corrosion mechanism was more influenced by the $\mathrm{Al}, \mathrm{Zn}$ distribution and morphology of the formed $\beta$-phase. The highest corrosion resistance of ZK60 among these three alloys is related to $\mathrm{Zn}$ and $\mathrm{Zr}$ elements which can form the compounds of $\mathrm{MgZn}_{2}$ and $\mathrm{Zn}_{2} \mathrm{Zr}$ [18]. For MAO coating, the MAO coated $\mathrm{Mg}$ alloys exhibited excellent corrosion performances due to the protection of the MAO coatings containing $\mathrm{Mg}_{2} \mathrm{SiO}_{4}$ and $\mathrm{MgF}_{2}$ phases that have relatively stable chemical properties [19]. Under the same MAO processing parameters, there is not much difference between the thickness and density of the coating on different substrate. When the coating is broken down, its corrosion is mainly depended on the substrate properties. After the scribe testing, the MAO coating on ZK60 specimen exhibited better corrosion protection in the continuous slat spray environment than that synthesized on the AZ31B alloy.

\section{CONCLUSIONS}

In this work, the significance of the microstructure and corrosion properties of the MAO coated different kinds of magnesium alloys were evaluated using advance microstructural and surface analysis techniques, electrochemical testing method and scribe testing exposure in the ASTM B117 test environment. The conclusions can be summarized as:

1) The corrosion behavior of the three kinds of cast magnesium alloy of AZ31B, AZ80 and ZK60 has shown that the ZK60 Mg alloy is better than AZ80 and AZ31B.

2) Adherent, dense and uniform MAO coatings are grown on AZ31B, AZ80 and ZK60 Mg alloy substrates in an alkaline silicate electrolyte. SEM and XRD analysis has shown that the MAO coating is a complex mixture of compounds including $\mathrm{MgO}, \mathrm{Mg}_{2} \mathrm{SiO}_{4}$ and $\mathrm{MgF}_{2}$, etc. The variation of different substrate materials did not result in any marked change in the compositional or phase composition and the thickness, microstructure of the MAO coating.

3) MAO coatings provide robust protection of the AZ31B, AZ80 and ZK60 Mg alloys substrate in both electrochemical and scribe salt spray corrosion tests. The MAO coated ZK60 exhibited better anti-corrosion performance among these specimens.

\section{ACKNOWLEDGMENT}

The technical support of Chao Shi of CanmetMATERIALS, Natural Resources Canada, Dr. Sugrib Shaha and Jie Wang of the University of Waterloo during the course of this work is gratefully acknowledged.

\section{REFERENCES}

[1] A. A. Luo, "Magnesium: Current and potential automotive applications," JOM., vol. 54, no. 2, pp. 42-48, Feb. 2002. doi: 10.1007/BF02701073

[2] S. Dayani, S. Shaha, R. Ghelichi, J. Wang, H. Jahed, "The impact of AA7075 cold spray coating on the fatigue life of AZ31B cast alloy," Surf. Coat. Technol., vol. 337, pp.150-158, Mar. 2018. doi: 10.1016/j.surfcoat.2018.01.008.

[3] D. Toscano, S. Shaha, B. Behravesh, H. Jahed, B. Williams, "Effect of forging on microstructure, texture, and uniaxial properties of cast AZ31B alloy," J. Mater. Eng. Perform., vol. 26, no. 7, pp. 3090-3103, May 2017. doi: 10.1007/s11665-017-2743-2

[4] I.J. Polmear, "Magnesium alloys and applications," Mater. Sci. Technol., vol. 10, no. 1, pp. 1-16, Jan. 1994. doi: 10.1179/026708394790163401.

[5] M. Diab, X. Pang, H. Jahed, "The effect of pure aluminum cold spray coating on corrosion and corrosion fatigue of magnesium ( $3 \% \mathrm{Al}-1 \% \mathrm{Zn})$ extrusion," Surf. Coat. Technol., vol. 309, pp.423-435, Jan. 2017. doi: 10.1016/j.surfcoat.2016.11.014. 
[6] F.C Walsh et al., "Plasma electrolytic oxidation (PEO) for production of anodised coatings on lightweight metal (Al, Mg, Ti) alloys," Trans. of the IMF, vol. 87, no. 3, pp. 122-135. May 2009. doi: $10.1179 / 174591908 \times 372482$.

[7] B. Vladimirov et al., "Microarc oxidation of magnesium alloys: A review," Surf. Eng. Appl. Electrochem., vol. 50, no. 3, pp.195-232. May 2014. doi: $10.3103 / \mathrm{S} 1068375514030090$.

[8] A. Pardo, M. C. Merino, A. E. Coy, F. Viejo, R. Arrabal, and S. Feliú Jr. "Influence of microstructure and composition on the corrosion behaviour of $\mathrm{Mg} / \mathrm{Al}$ alloys in chloride media," Electrochim. Acta., vol. 53, no. 27, pp.7890- 7902, Nov. 2008. doi: 10.1016/j.electacta.2008.06.001.

[9] S. Karparvarfard, S. Shaha, S. Behravesh, H. Jahed, B. Williams, "Microstructure, texture and mechanical behavior characterization of hot forged cast ZK60 magnesium alloy," J. Mater. Sci. Technol., vol. 33, no. 9, pp. 907-918, Sep. 2017. doi: 10.1016/j.jmst.2017.04.004.

[10] D. Toscano, S. Shaha, B. Behravesh, H. Jahed, B. Williams, "Effect of forging on the low cycle fatigue behavior of cast AZ31B alloy," Mater. Sci. Eng. A, vol. 706, pp.342-356, Oct. 2017. doi: 10.1016/j.msea.2017.08.086.

[11] S. Behravesh, H. Jahed, S. Lambert, "Characterization of magnesium spot welds under tensile and cyclic loadings," Mater. Des., vol. 32, no. 10, pp. 4890-4900, Dec. 2011. doi: 10.1016/j.matdes.2011.06.001.

[12] A. Gryguc, S. Shaha, S. Behravesh, H. Jahed, M. Wells, B. Williams, X. $\mathrm{Su}$, "Monotonic and cyclic behaviour of cast and cast-forged AZ80 Mg," Int. J. Fatigue, vol. 104, pp. 136-149, Nov. 2017. doi: 10.1016/j.ijfatigue.2017.06.038.
[13] Standard Practice for Operating Salt Spray (Fog) Apparatus, ASTM, B117-18, 2011.

[14] J. Robson, C. Paa-Rai, "The interaction of grain refinement and ageing in magnesium-zinc-zirconium (ZK) alloys," Acta Mater., vol. 95, pp. 10-19, Aug. 2015. doi: 10.1016/j.actamat.2015.05.012.

[15] V. Ezhilselvi, J. Nithin, J.N. Balaraju, S. Subramanian, "The influence of current density on the morphology and corrosion properties of MAO coatings on AZ31B magnesium alloy," Surf. Coat. Technol., vol. 288 pp. 221-229, Feb. 2016. doi: 10.1016/j.surfcoat.2016.01.040.

[16] H.S. Ryu, J. Ryu, D. Park, S. Hong, "Electrochemical corrosion properties of nanostructured YSZ coated AZ31 magnesium alloy prepared by aerosol deposition," J. Electrochem. Soc., vol. 158, no. 2, pp. 23-28, 2011. doi: 10.1149/1.3525271.

[17] J. Jorcin, M. Orazem, N. Pébère, B. Tribollet, "CPE analysis by local electrochemical impedance spectroscopy," Electrochim. Acta., vol.51, no. 8-9, pp. 1473-1479, Jan. 2006. doi: 10.1016/j.electacta.2005.02.128.

[18] G. Song, A. Atrens, "Corrosion Mechanisms of Magnesium Alloys," Adv. Eng. Mater., vol. 1, no. 1, pp. 11-33, Sep. 1999. doi: 10.1002/(SICI)1527-2648(199909)1:1<11::AID-ADEM11>3.0.CO;2-N.

[19] Y. Wang et al., "Corrosion behaviors and effects of corrosion products of plasma electrolytic oxidation coated AZ31 magnesium alloy under the salt spray corrosion test," Appl. Surf. Sci., vol. 378, pp.435-442. Aug. 2016. doi: 10.1016/j.apsusc.2016.04.011. 


\section{Influence of Porosity and Alloy Addition on the Wear Behaviour of a Sinter- Forged C45 Steel using Taguchi Method}

\author{
E. Naveen ${ }^{1}$ \\ Department of Mechanical Engineering, \\ Sri Sairam Engineering College, \\ Chennai, Tamilnadu, India \\ irumbalayar@gmail.com ${ }^{1}$
}

\author{
Sripada Raghavendra $\mathrm{K} \mathrm{N}^{2}$ \\ Department of Mechanical, Industrial and Aerospace \\ Engineering, \\ Concordia University \\ Montreal, QC, Canada \\ srirajji@gmail.com²
}

\begin{abstract}
Elemental powders of Atomized Iron (Fe), Carbon (C) and Molybdenum (Mo) were weighed and mixed in a pot mill to yield the composition of $\mathrm{C} 45, \mathrm{C} 45-1 \% \mathrm{Mo}$ and $\mathrm{C} 45-$ $2 \%$ Mo Steels, then compacted and sintered. The Sintered preforms had a density- $75 \%$ of the Theoretical Density. Then the Sintered preforms were subjected to densification to get two densities- $80 \%$ and $85 \%$ of the theoretical density through Forging. The sintered and densified preforms of alloy steels are subsequently machined to get the required test specimens. The experiments were conducted on a pin-on-disc Tribometer, conforming to ASTM G99 standards, on a rotating EN32 disc. Using Minitab 16 software, the Dry Sliding wear experiments were planned using L27 Orthogonal Array. The \% Theoretical Density of the Specimens, \% Mo Addition, Load and Sliding Speed were taken as input parameters, mass loss was the output parameter. It was observed that the increasing density of alloy steels adversely affects the wear resistance of the alloy steels and mass loss is increased. It was found that the addition of Mo significantly improves the wear resistance of the alloy steels irrespective of the densities due to hard phase carbides present in the microstructure. Empirical correlations for mass loss with respect to input parameters had been developed using Regression Analysis. In the case of Mo added alloy, Mo particulates embedded between the ferrite-pearlite grains are observed from the microstructure.
\end{abstract}

Keywords- Powder Metallurgy, Wear, Sintered Steels

\section{INTRODUCTION}

Tribology is a field of science which applies an operational analysis to problems of great economic significance such as reliability, maintenance and wear of technical equipment ranging from household appliances to aircraft. Derived from the Greek word 'Tribos', which means sliding or rubbing, the term deals with friction, wear and lubrication of the interacting surfaces in relative motion. Wear is an inevitable phenomenon and occurs at all places where there exist interacting surfaces in relative motion such as gears, bearing, coupling, sealing, cams, clutches, etc. Wear is the major cause of material wastage and loss of mechanical performance and any reduction of which can result in considerable savings. Friction is the principle cause of wear and energy dissipation. It is estimated that one third of world's energy resources in present use is needed to overcome friction in one form or another. One effective way to friction and wear is lubrication. Another convenient method is to devise special Tribo materials, which is more wear resistant as well as economically affordable

\section{LITERATURE SURVEY}

Molinari and Straffelini [1] investigated the wear behaviour of $\mathrm{Fe}-\mathrm{C}-\mathrm{Ni}-\mathrm{Mo}$ and $\mathrm{Fe}-\mathrm{C}-\mathrm{Mo}$ steels in three states namely steam treated, sintered and heat treated (oil quenching and stress relieving). Due to their high resistance to plastic flow, the heat-treated steels exhibited lesser wear rates. Oxidative wear is commonly observed in all the three states. The Molybdenum alloyed steel had a higher wear resistance compared to the other steels due to the evenly distributed bainite structure. Molinari and Straffelini [2] investigated the dry sliding wear behaviour of sintered and steam treated $\mathrm{Fe}-2 \% \mathrm{Cu}$ and $\mathrm{Fe}-$ $0.3 \% \mathrm{C}$ alloy steels. They found that the wear rate is independent of the chemical composition of steels as long as the oxide layer forms on the outer surface and the wear rate is lesser. The Copper and Carbon alloyed steels exhibit improved wear resistance because to the domination of delamination wear mechanism. Wang and Danninger [3] found that the Sintering temperature affects the wear behaviour of sintered steels with $1 \%$ Carbon and 3.5\% Molybdenum addition. At a Lesser sintering temperature $(11200 \mathrm{C})$ the wear rate of the steels is lesser when compared with Steels subjected to the higher sintering temperature $(12200 \mathrm{C})$. Razavizadch and Davies [4] reported that the addition of copper as alloying element to iron based alloys significantly improves the wear resistance of the alloy steels due to predominant delamination wear process. Ceschini et al [5] have found that the increase in Molybdenum addition to $\mathrm{Fe}-\mathrm{C}$ steels and higher a sintering temperature lead to significant improvement in the sliding wear resistance of $\mathrm{Fe}-\mathrm{C}-\mathrm{Mo}$ because to the formation of bainite microstructure. Gopinath [6] studied the influence of speed on the wear of sintered iron-based materials. The wear rates of sintered $\mathrm{Fe}-\mathrm{Cu}$ alloys got reduced with increasing 
sliding speed upto a particular threshold value, after which the trend got reversed. Zhang et al [7] observed that the addition of Sulphur (S) to Fe-Mo alloy steels contributes to reduction of the wear rate due to the chemical reactions of Sulphur with Molybdenum, which leads to the formation of Sulphide of Molybdenum, which forms adherent film on the wear surface. Murakami et al [8] observed that the Fe-Mo Steel specimen exhibited higher wear resistance at the non-annealed state when compared to the annealed specimen of the same alloy steel because of higher fracture toughness and hardness. The formation of oxides of Molybdenum during the wear test lead to lower coefficient of friction. Adhesive wear mechanism was identified as the predominant in the wear of alloy steel. Tekeli et al [9] experimentally found that the wear rate of Annealed $\mathrm{Fe}-0.3 \% \mathrm{C}$ P/M steel, followed by water quenching was lower compared to that of as sintered state. This is due to the transformation of pearlite into martensite microstructure during quenching process. The wear mechanism found in the alloy specimen is a mixture of adhesive and abrasive wear due to high volume fraction of martensite in microstructure. Tekeli and Gural [10] have investigated the effect of microstructure and heat treatment on dry sliding wear behaviour of Sintered steels with $0.3 \%$ Carbon and 2\% Nickel additions. They have experimentally found that the TemperedQuenched specimens had lower wear resistance when compared to the Inter-Critically Annealed specimens of same composition. It was due to the detachment of surface platelets (wear debris) by micro ploughing and subsequently the predominant abrasive wear mechanism is taking place on the surface. The specimens subjected to Lower Inter-Critical annealing temperature $(7280 \mathrm{C})$ exhibited lesser wear rate when compared to the specimens annealed at the higher InterCritical Annealing temperature $(9000$ C) due to the transformation of martensite from the rich austenite microstructure. Caloco et at [11] experimentally found that though the Laser Surface Melted- Sintered AISI M42 exhibited higher hardness when compared with as sintered AISI M42 specimens. The Laser Surface Melted- Sintered specimens exhibited poor wear resistance compared to sintered specimens. It was due to the complete elimination of residual porosity and formation of super saturated martensite that lead to the dominance of abrasive wear. Anton et al [12] found that the addition of $0.7 \%$ Carbon to plain carbon steels enhances their wear resistance when compared with $0.3 \%$ Carbon addition. They have also found that the addition of Manganese (Mn) alone to the $0.7 \%$ Carbon steels showed improved wear behaviour than combined Nickel-Manganese addition, due to the formation of higher percentage of martensitic-bainitic structure in a ferritic-pearlitic matrix. Abrasion-adhesion wear mechanism was found to be dominant in these alloy steels, that lead to higher wear rates. Leheup et al [13] investigated the effect of density on wear behaviour of sintered iron specimens. They have experimentally found that the wear behaviour of the sintered preforms is dependent upon the sliding amplitude rather than the density. At the higher sliding amplitude, the higher density sintered preforms are subjected to the higher wear loss compared to the lower density specimens. Lim and Brunton [14] investigated the predominant wear mechanism of the Sintered and NonSintered steels were ploughing and delamination. The open pores on the sliding surfaces of sintered iron preforms acted as a site for the generation and collection of wear debris. Lim et al [15] have investigated the wear properties of unlubricated specimens of sintered and non-sintered medium and low carbon steels. They found that the wear behaviour of both the non-sintered and sintered steels were similar. Mild oxidation wear producing fine oxide debris and oxide plateau, with delamination as a secondary mechanism at higher load and speed has been reported. Kang [16] has investigated the wear behaviour of Copper based and Iron based sintered alloy materials. At mild sliding conditions $\mathrm{Cu}$-based alloy had better frictional properties. On the other hand, for the severe sliding conditions Fe-based sintered material was better. At the lower load condition, the softening effect of $\mathrm{Cu}$ in the $\mathrm{Cu}$-based alloy could prevent the wear without material transfer and the delamination wear is found predominant at the higher load and speed conditions. Dhanasekharan and Gnanamoorthy [17] found that the addition of Molybdenum Disulphide (MoS2) to $\mathrm{Fe}-\mathrm{C}-\mathrm{Cu}$ and $\mathrm{Fe}-\mathrm{C}-\mathrm{Cu}-\mathrm{Ni}$ Sintered Steels improves the wear behaviour of alloys.

\section{SPECIMEN PREPARATION}

Due to their high strength and hardness, the P/M Low alloy steels under the present study could be potential candidates for high-wear applications. Therefore, the need for studying their wear behaviour was strongly felt. Atomized powders of Iron (Fe), Carbon (Graphite Form) and Molybdenum (Mo) were weighed separately in a 4 decimal Mitutoyo Digital Balance. Then the powders were mixed according to their weight composition in pot mill and allowed to mix well for about 8 hours. Few drops of Liquid Paraffin was added as a binder to these powders. After 8 hours, the powders were taken out, weighed separately and packed for the preparation of each specimen. Compaction of premix was carried out at different pressures from $400 \mathrm{Mpa}$ to $600 \mathrm{Mpa}$ by using Hydraulic compacting press of capacity 100 tons. Aspect ratio for samples was maintained less than $1(\mathrm{~L} / \mathrm{D}<1)$. The Die \& Punch design and fabrication were carried accordingly, such that they should withstand the sufficient Load. A High Chromium High Carbon Steel Die was selected for the Compaction process as shown in Figure 3.1. The Die must me fixed and does not escape from the base plate while applying load. Initially the lower punch was inserted in the die case and placed properly in the base plate of hydraulic press. Zinc Stearate was applied over the die cavity, upper punch and lower punch. Powder was poured into the die cavity carefully. Load was applied after the contact has been made between the upper punch and upper plate of hydraulic press for the compaction of powders as shown in Figure 3.2. The load was applied gradually until required pressure has attained. After that the upper plate is moved upside and carefully the upper punch were removed for obtaining the Green Compact as shown in Figure 3.3. The Green Compact was given a coating 
to prevent it from oxidation. Sintering of the coated samples was carried out in a muffle furnace. Sintering furnace of rectangular cross section $\left(\max\right.$ Temp. $\left.1250^{\circ} \mathrm{C}\right)$ with a provision for necessary reducing atmosphere was chosen for Sintering, where the loosely packed Green Compact will be transformed into metallurgically bonded sample. Samples were placed in heating zone and heated to its sintering temperature ( $\mathrm{Ts}=0.7-0.8$ Melting Point) as shown in Figure 3.4. In the first step of Sintering, the coating was removed and burned. This step is popularly known as 'de lube' or 'dewax' step. In the Second step the powder particles fuse together. The Third Step of the sintering cycle is the cooling step. Both sintering and cooling were done in controlled atmosphere. The details of the Sintering Parameters is given in Table 3.1. The Sintered Specimens had a Density about $75 \%$ of the theoretical density. Figure 3.5 shows the Sintered Specimens. Then the sintered specimens were subjected to densification through Forging. The specimens were subjected to two different loads in order to get two different densities for each alloy, namely $80 \%$ and $85 \%$ of theoretical density. The theoretical and the actual densities obtained, along with the Vicker's Hardness is given in Table 3.2

\begin{tabular}{|c|c|c|}
\hline S.No. & Sintering Parameters & Values \\
\hline 1 & $\begin{array}{c}\text { De-Lube/ De-Wax } \\
\text { temperature }\end{array}$ & $475 \pm 30^{\circ} \mathrm{C}$ \\
\hline 2 & Sintering temperature & $1100 \mathrm{C} \pm 10^{\circ} \mathrm{C}$ \\
\hline 3 & Sintering time & $120 \mathrm{~min}$ \\
\hline 4 & Cooling way & Furnace cooled \\
\hline 5 & Atmosphere used & Argon \\
\hline 6 & Gas Flow Rate & $0.4 \mathrm{~m}^{3} / \mathrm{hr}$ \\
\hline
\end{tabular}

Table 3.1: Sintering Parameters

\begin{tabular}{|c|c|c|c|c|}
\hline Specimen & $\begin{array}{c}\text { Theor } \\
\text { etical } \\
\text { Densit } \\
\text { y } \\
\text { (g/cc) }\end{array}$ & $\begin{array}{c}\text { Actual } \\
\text { Density } \\
\text { (g/cc) }\end{array}$ & $\begin{array}{c}\% \\
\text { Densified }\end{array}$ & $\begin{array}{c}\text { Vicker's } \\
\text { Hardness } \\
\text { (HV) }\end{array}$ \\
\hline \multirow{3}{*}{ C45 } & \multirow[b]{3}{*}{7.7148} & 5.7861 & 75 & 87 \\
\hline & & 6.1718 & 80 & 120 \\
\hline & & 6.5575 & 85 & 170 \\
\hline \multirow[b]{2}{*}{ C45- } & \multirow[b]{2}{*}{7.7328} & 5.7996 & 75 & 94 \\
\hline & & 6.1862 & 80 & 134 \\
\hline
\end{tabular}

\begin{tabular}{|c|c|c|c|c|}
\hline $1 \%$ Mo & & 6.5728 & 85 & 154 \\
\hline \multirow{2}{*}{$\begin{array}{c}\text { C45- } \\
2 \% \mathrm{M} o\end{array}$} & & 5.8150 & 75 & 100 \\
\cline { 3 - 5 } & 7.7534 & 6.2027 & 80 & 140 \\
\cline { 3 - 5 } & & 6.5903 & 85 & 180 \\
\hline
\end{tabular}

Table 3.2: Theoretical, Actual Density \& Hardness of the Specimens

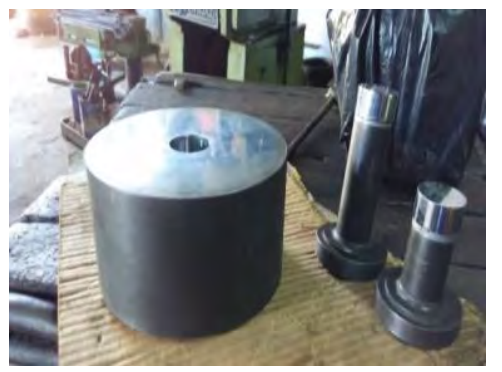

Fig 3.1 Die and Punch for Compaction

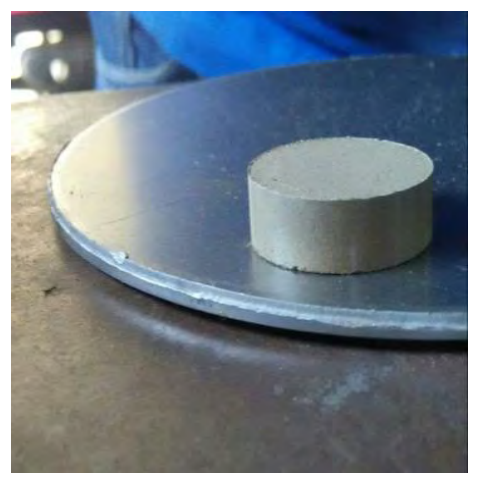

Fig 3.3: Green Compact

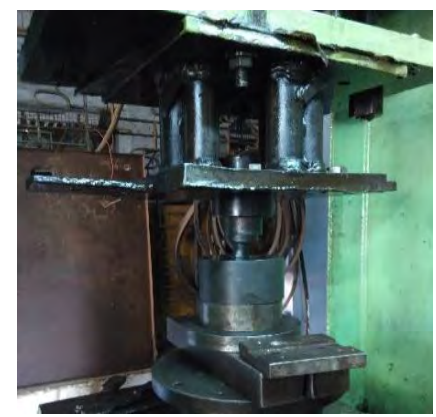

Fig 3.2: Load applied during Compaction

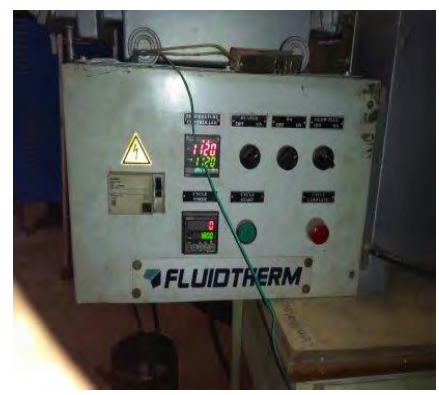

Fig 3.4: Sintering of Green Compact

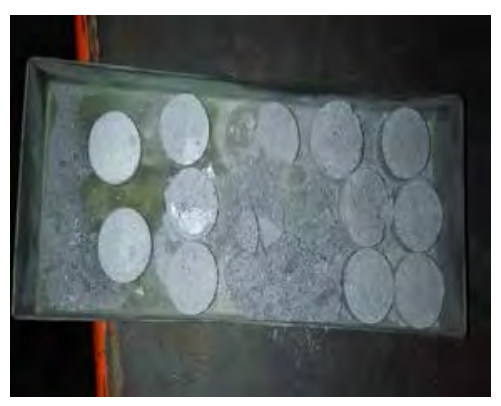

Fig 3.5: Sintered Specimens 


\section{WEAR TESTING}

In this work, a dry sliding wear behavior of $\mathrm{P} / \mathrm{M}$ Steels were studied using a pin on-disc apparatus (DUCOM ${ }^{\mathrm{TM}}$ make). Figure 4.1 shows the arrangement of pin-on-disc apparatus. The disc material was made of EN-32 steel with a hardness of 65 HRC. The pin specimen is pressed against disc at a specified load usually by means of an arm and attached weights. The dry sliding wear tests were carried out at room temperature $\left(30^{\circ} \mathrm{C} \pm\right.$ $3{ }^{\circ} \mathrm{C}$, RH $55 \% \pm 5 \%$ ) under dry sliding condition in accordance with the ASTM G 99 standard. Cylindrical pins of $10 \mathrm{~mm}$ diameter and $25 \mathrm{~mm}$ long were machined and polished by metallographic method. Immediately prior to testing, were cleaned and dried using acetone to remove all dirt and foreign matter from the specimens. Initial and final weight of the specimen was measured using a Mitutoyo ${ }^{\mathrm{TM}}$ make electronic weighing machine with an accuracy of 0.0001 grams. Wear measurement is carried out to determine the amount of materials removed (or worn away) after a wear test, (and in reality after a part in service for a period of time). The material worn away can be expressed either as weight (mass) loss, volume loss, or linear dimension change depending on the purpose of the test, the type of wear, the geometry and size of the test specimens, and sometimes on the availability of a measurement facility [4]. The specimen was fixed in the arm and the disc was made to rotate below the pin. Mass of the specimen before and after the test is measured using digital balance and mass loss is found out for each set of input parameters. Wear track images and SEM images were taken to analyze the maximum wear.
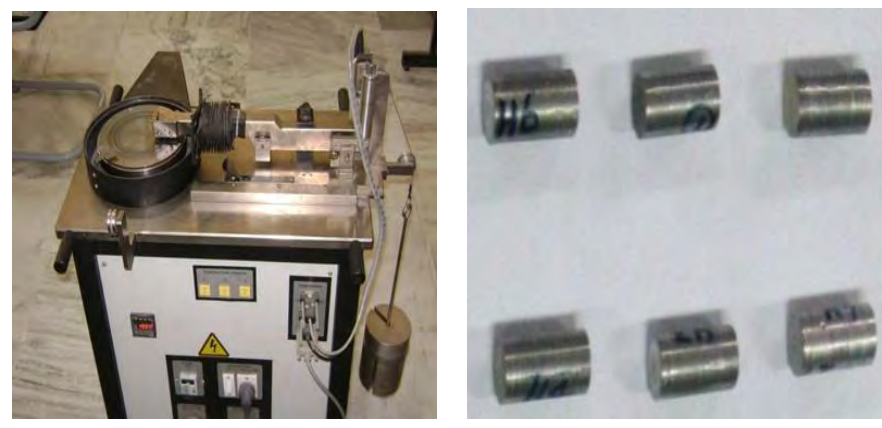

Fig 4.1 Pin-on-Disc Tribometer Fig 4.2: Wear Test Specimens Setup

\section{RESULTS AND DISCUSSIONS}

The Experiments were conducted according to the Taguchi's L27 Orthogonal Array- 3 level and 4 Factors. The Input Parameters and their Levels is given in Table 5.1. The result of the output parameter Mass loss is given in Table 5.2. Minitab 16 software was used for further analysis.

\begin{tabular}{|l|c|c|c|}
\hline $\begin{array}{c}\text { Input } \\
\text { Parameter }\end{array}$ & Level 1 & Level 2 & Level 3 \\
\hline $\begin{array}{l}\text { \% } \\
\text { Theoretical } \\
\text { Density }\end{array}$ & 75 & 80 & 85 \\
\hline $\begin{array}{l}\text { \% Mo } \\
\text { Addition }\end{array}$ & 0 & 1 & 2 \\
\hline Load (N) & 10 & 20 & 30 \\
\hline $\begin{array}{l}\text { Sliding } \\
\text { Speed (m/s) }\end{array}$ & 1 & 2 & 3 \\
\hline $\begin{array}{l}\text { Sliding } \\
\text { Distance (m) }\end{array}$ & \multicolumn{3}{|l|}{$3000 \mathrm{~m}$} \\
\hline
\end{tabular}

Table 5.1 Input parameters for Wear Test

Calculating the Signal-to-Noise ratio $(\eta)$ [5]: Mass Loss has to be minimized; it is smaller- the better type of quality characteristics. Hence, $\mathrm{S} / \mathrm{N}$ ratio for Mass Loss is computed from the following equation:

Smaller the better $\eta=-10 * \log \left(\frac{1}{n} * \sum_{i=1}^{n} y_{i j}^{2}\right)$

\begin{tabular}{|c|c|c|c|c|c|c|}
\hline S.No & $\begin{array}{c}\% \\
\text { Theoretical } \\
\text { Density }\end{array}$ & $\begin{array}{c}\% \text { Mo } \\
\text { Addition }\end{array}$ & $\begin{array}{l}\text { Load } \\
\text { (N) }\end{array}$ & \begin{tabular}{|} 
Sliding \\
Velocity \\
$(\mathrm{m} / \mathrm{s})$
\end{tabular} & $\begin{array}{l}\text { Mass Loss } \\
\text { (g) }\end{array}$ & S/N Ratio \\
\hline 1 & 75 & 0 & 10 & 1 & 0.4709 & 6.541426 \\
\hline 2 & 75 & 0 & 20 & 2 & 0.5351 & 5.431301 \\
\hline 3 & 75 & 0 & 30 & 3 & 0.5545 & 5.121969 \\
\hline 4 & 75 & 1 & 10 & 2 & 0.3369 & 9.44998 \\
\hline 5 & 75 & 1 & 20 & 3 & 0.3452 & 9.238584 \\
\hline 6 & 75 & 1 & 30 & 1 & 0.3943 & 8.083464 \\
\hline 7 & 75 & 2 & 10 & 3 & 0.2251 & 12.95249 \\
\hline 8 & 75 & 2 & 20 & 1 & 0.2542 & 11.89649 \\
\hline 9 & 75 & 2 & 30 & 2 & 0.2842 & 10.92752 \\
\hline 10 & 80 & 0 & 10 & 2 & 0.5724 & 4.846007 \\
\hline 11 & 80 & 0 & 20 & 3 & 0.5786 & 4.752431 \\
\hline 12 & 80 & 0 & 30 & 1 & 0.5839 & 4.67323 \\
\hline 13 & 80 & 1 & 10 & 3 & 0.4035 & 7.883129 \\
\hline 14 & 80 & 1 & 20 & 1 & 0.4556 & 6.828326 \\
\hline 15 & 80 & 1 & 30 & 2 & 0.4951 & 6.106141 \\
\hline 16 & 80 & 2 & 10 & 1 & 0.3320 & 9.577238 \\
\hline 17 & 80 & 2 & 20 & 2 & 0.3564 & 8.961246 \\
\hline 18 & 80 & 2 & 30 & 3 & 0.3800 & 8.404328 \\
\hline 19 & 85 & 0 & 10 & 3 & 0.6875 & 3.254546 \\
\hline 20 & 85 & 0 & 20 & 1 & 0.8944 & 0.969364 \\
\hline 21 & 85 & 0 & 30 & 2 & 0.9563 & 0.388117 \\
\hline 22 & 85 & 1 & 10 & 1 & 0.4249 & 7.434265 \\
\hline 23 & 85 & 1 & 20 & 2 & 0.4322 & 7.286305 \\
\hline 24 & 85 & 1 & 30 & 3 & 0.5481 & 5.222804 \\
\hline
\end{tabular}




\begin{tabular}{|l|l|l|l|l|l|l|}
\hline 25 & 85 & 2 & 10 & 2 & 0.4021 & 7.913319 \\
\hline 26 & 85 & 2 & 20 & 3 & 0.4263 & 7.405693 \\
\hline 27 & 85 & 2 & 30 & 1 & 0.4600 & 6.744843 \\
\hline
\end{tabular}

Table 5.2 Results of the Wear Test

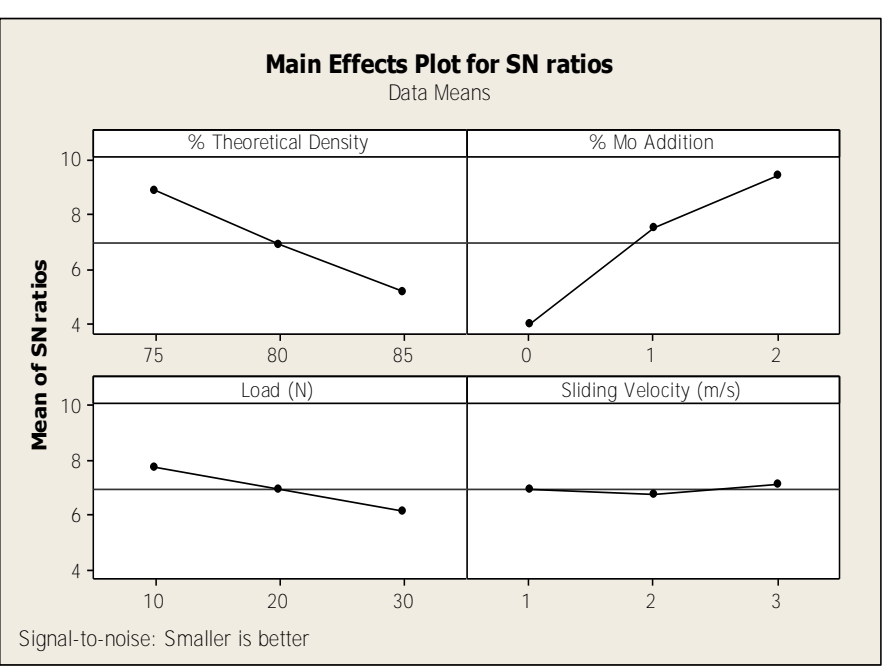

Fig 5.1: Main Effect Plots for S/N Ratios

\section{A. The regression equation}

Mass Loss $=3.17363-(0.0898507 * \%$ TD $)+(0.35593 \%$ Mo $)-(0.0257828 *$ Load $)+(0.151944 * \mathrm{SV})+(0.000704889 *$ \%TD* \%TD $)+(0.0712389 * \% \mathrm{Mo} \% \mathrm{Mo})-(2.46111 \mathrm{e}-005 *$ Load*Load $)-(0.0179111 * \mathrm{SV} * \mathrm{SV})-(90.00785467 *$ $\% \mathrm{TD} * \% \mathrm{Mo})+(0.000393511 * \% \mathrm{TD} * \mathrm{Load})$ $(0.00129911 * \% \mathrm{TD} * \mathrm{SV})-(0.00149511 * \% \mathrm{Mo} * \mathrm{Load})+$ $(0.00456444 * \% \mathrm{Mo} * \mathrm{SV})+(0.000616$ Load*SV $)$

\section{$\mathrm{S}=\mathbf{0 . 0 7 0 0 9 1 2} \mathrm{R}-\mathrm{Sq}=\mathbf{9 2 . 1 6} \% \quad \mathrm{R}-\mathrm{Sq}(\operatorname{adj})=\mathbf{8 3 . 0 1} \%$}

The lowering in wear rate of $\mathrm{P} / \mathrm{M}$ Steels can be raised due to the addition of Molybdenum and which reduces the wear rate of the P/M Steels. The decrease in the Mass loss can also be correlated by role of Molybdenum as spacers that prevent direct contact between rough surfaces. The thermal mismatch between the Iron and Molybdenum increase the dislocation density that increases the resistance of the materials to plastic deformation. The formation of solid film Oxide and Sulphides of Molybdenum, makes the C45 Steel wear resistant. At a higher loads, the thermal softening and the plastic deformation tends to prevail. From the Table 5.3, it is very clear that the \% porosity (Inverse of \% Theoretical Density) also plays a significant role in reducing the Wear rate of both the Steels. It is due to the fact that the pores acts as site, where there are no material present, thereby reducing the Mass Loss considerably. For the lower density preforms, the plastic deformation of material can be accommodated by the pores by reducing the volume and subsequently the effective number of asperities is getting reduced and makes the surface smooth. This in turn leads to the reduction in wear loss. In contrast, the plastic deformation with fewer pores in higher density preforms with asperities opposing on the surfaces, which in turn makes the specimen subjected to higher wear loss.

\begin{tabular}{|c|c|c|c|c|c|}
\hline Source & DF & Adj Ss & F & P & $\begin{array}{c}\text { \% } \\
\text { Contribution }\end{array}$ \\
\hline \%TD & 1 & 0.001177 & 0.2395 & 0.633379 & 24.79 \\
\hline \%Mo & 1 & 0.005237 & 1.0661 & 0.322195 & 54.41 \\
\hline Load & 1 & 0.002564 & 0.522 & 0.483838 & 4.74 \\
\hline SV & 1 & 0.000891 & 0.1813 & 0.677805 & 0.11 \\
\hline \%TD*\%TD & 1 & 0.001863 & 0.3793 & 0.549498 & 0.25 \\
\hline \%Mo*\%Mo & 1 & 0.03045 & 6.1981 & 0.028453 & 4.05 \\
\hline Load*Load & 1 & 0.000036 & 0.0074 & 0.932878 & 0 \\
\hline SV*SV & 1 & 0.001925 & 0.3918 & 0.543075 & 0.26 \\
\hline \%TD*\%Mo & 1 & 0.017352 & 3.532 & 0.08469 & 2.27 \\
\hline \%TD*Load & 1 & 0.004355 & 0.8865 & 0.364996 & 0.69 \\
\hline \%TD*SV & 1 & 0.000475 & 0.0966 & 0.761259 & 0.17 \\
\hline \%Mo*Load & 1 & 0.002515 & 0.5119 & 0.488012 & 0.33 \\
\hline \%Mo*SV & 1 & 0.000234 & 0.0477 & 0.830769 & 0.03 \\
\hline Load*SV & 1 & 0.000427 & 0.0869 & 0.773202 & 0.06 \\
\hline Error & 12 & 0.058953 & & & 7.84 \\
\hline Total & 26 & & & & 100 \\
\hline
\end{tabular}

Table 5.3: ANOVA table for $\%$ contribution of inputs for Mass Loss

\section{B. SEM Analysis Wear surface morphology of C45 Steels}

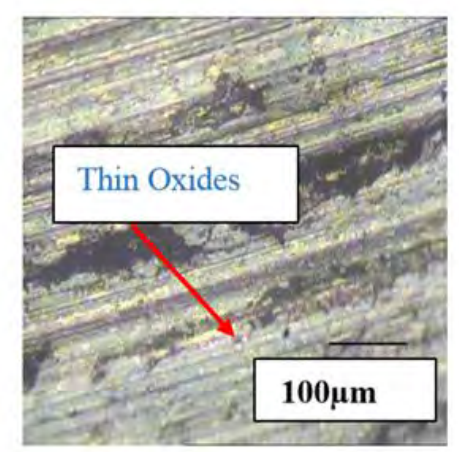

Fig 5.2.a C45 Steel-75\%TD

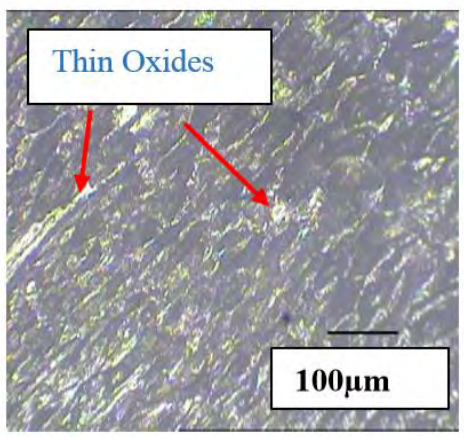

Fig 5.2.b C45 Steel-80\%TD 


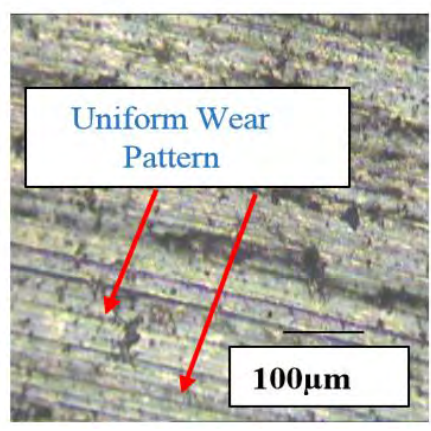

Fig 5.2.c C45 Steel-85\%TD

Figure 5.2.a, $\mathrm{b}$ and $\mathrm{c}$ shows the Optical Microscope images of Maximum Worn surfaces of C45 Steel at 75\%, 80\% and $85 \%$ Theoretical Density (TD).

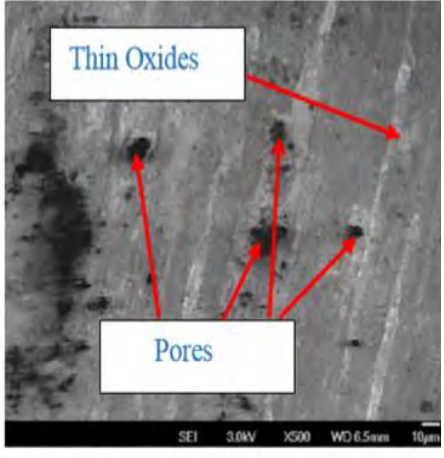

Fig 5.3.a C45 Steel-75\%TD

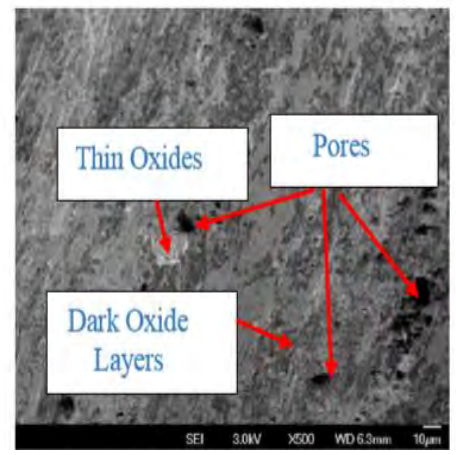

Fig 5.3.b C45 Steel-80\%TD

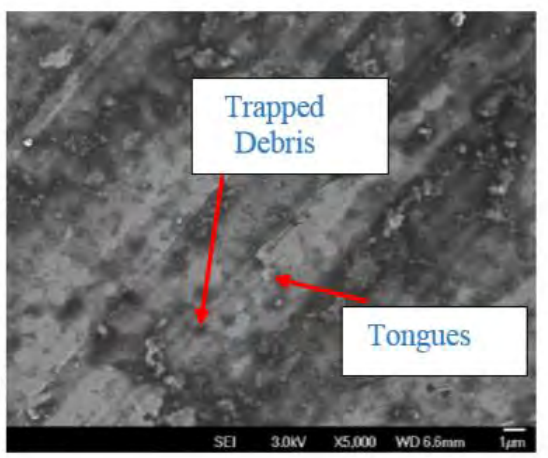

Fig 5.3.c C45 Steel-85\%TD

Figure 5.3.a, $\mathrm{b}$ and $\mathrm{c}$ shows the SEM images of Maximum Worn surfaces of C45 Steel at $75 \%, 80 \%$ and $85 \%$ Theoretical Density(TD). It is observed that the C45 steel is subjected to uniform wear throughout the wear region and makes it uniform mass loss over the contact surface. The bright areas of thin oxide layer are visible throughout the entire wear surface micrograph and the dark areas are probably the areas fully covered by oxide [9]. The alternate white and dark areas may be the hill and groove of the wear track [10]. Few bright

particles are also observed in the image and this could be the metallic debris embedded in the pores [11]. As the plain carbon steels consists of soft ferritic-pearlitic structure, the wear loss is observed to be uniform [12]. This is evident from the uniform wear track of the surface. The wear loss is observed to be uniform. This is evident from the uniform wear track of the surface.

\section{SEM Analysis Wear surface morphology of C45-2\% Mo Steels}

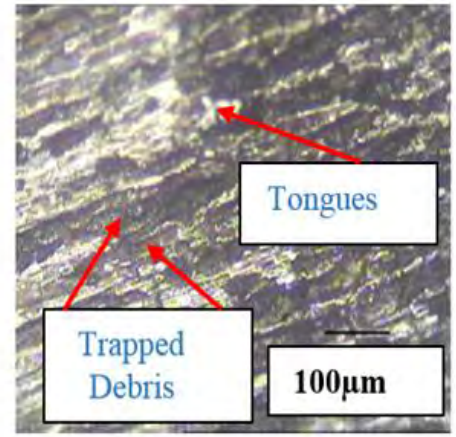

Fig 5.4.a C45-2\% Mo Steel Steel $75 \% \mathrm{TD}$

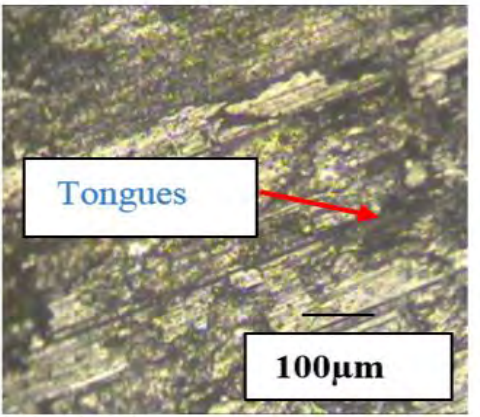

Fig 5.4.c C45-2\% Mo Steel-85\%TD

Figure 5.4.a, $\mathrm{b}$ and $\mathrm{c}$ shows the Optical Microscope images of Maximum Worn surfaces of C45-2\% Mo Steel at 75\%, 80\% and $85 \%$ Theoretical Density (TD).

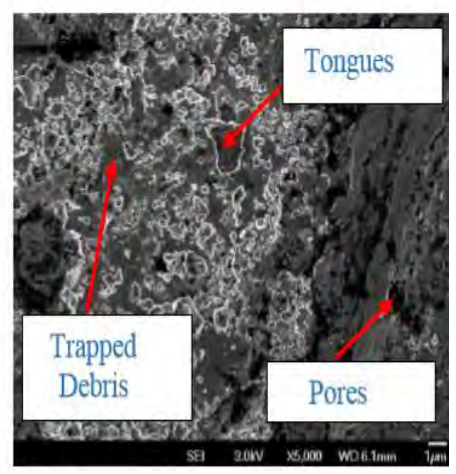

Fig 5.5.a C45-2\% Mo Steel $75 \%$ TD

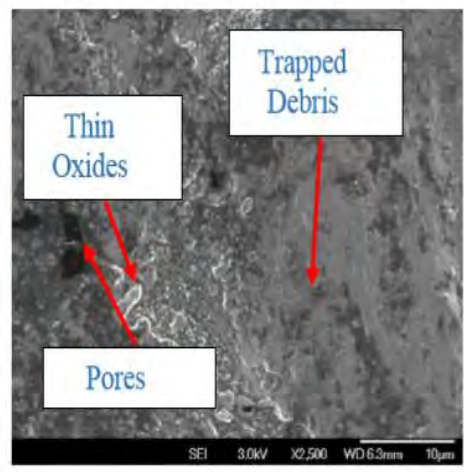

Fig 5.5.b C45-2\%Mo Steel $80 \%$ TD 


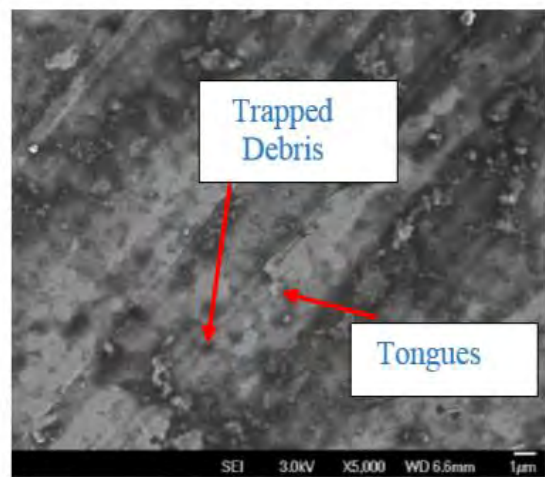

Fig 5.5.c C45-2\% Mo Steel-85\% TD

Figure $5.5 \mathrm{a}, \mathrm{b}$ and $\mathrm{c}$ shows the SEM images of Maximum Worn surfaces of C45-2\% Mo Steel at $75 \%, 80 \%$ and $85 \%$ Theoretical Density(TD). It is clearly evident from the SEM image of Mo alloyed steels that the layered appearance shows the removal of flakes due to delamination process [14]. The trapped wear asperities in the open pores, found in some places, significantly enhance the wear resistance of the alloy steel [14]. Appearance of tongues at some places in the SEM image could be the effect of deep metallic flow of steel due to deformation, which could further contribute to the reduction of wear rate of alloy steel and consequently enhance the friction at the interface [13]. The Wear of Mo alloyed steels, irrespective of densities is Non-Uniform.

\section{CONCLUSION}

The wear resistance is increased by addition of Molybdenum to C45 Steel. Irrespective of alloy steels as density increases wear resistance decreases and mass loss increases. The C45 Steel is found to undergo uniform wear due to the soft nature of the ferritic-pearlitic micro structure, while Mo alloyed steel is subjected to non-uniform surface wear due to the presence of hard particulates. Delamination is found to be a common mode of wear for carbon and alloy steels. Addition of Mo to plain carbon steel increases the hardness [16]. Hardness of As Sintered preforms is found to be decreased when compared to densified preforms. In the case of As Sintered preforms large no. of pores are observed when compared to densified preforms [17]. Confirmation Experiments were carried with the Empirical Equation obtained. The deviation from the Actual Readings were around 20\%.

\section{ACKNOWEDGEMENT}

The Authors are thankful to Mr.N.Ramanan, Senior EngineerR\&D Division, Sharda Motors Industries Ltd, ChengalpattuTamil Nadu State, India, for his valuable support and guidance throughout this work.

\section{REFERENCES}

[1] Giovanni Straffelini, Alberto Molinari, "Dry sliding wear behaviour of steam treated sintered iron alloys", Wear vol. 159, pp. 127-134, 1992. doi: 10.1016/0043-1648(92)90294-I.

[2] Giovanni Straffelini, Alberto Molinari, "Wear processes in high-strength sinteredalloys under dry rolling-sliding", Wear 1994: vol. 173, pp. 121-128, 1994. doi: 10.1016/0043-1648(94)90264-X.

[3] Junan Wang, Herbert Danninger, "Dry sliding behaviour of Molybdenum alloyedsintered steels", Wear, vol. 222, pp. 49-56, 1998. doi: 10.1016/S0043-1648(98)00279-8.

[4] K. Razavizadch, B. L. Davies, "The effects of steam treatment on the wear resistance of sintered iron and Fe-Cu alloys", Wear, vol. 69, no. 113, pp. 355-367, 1981.

[5] Lorella Ceschini, Giuseppe Palombarini, Giuliano sambogna, Donato Firrao, Giorgio Scavino, Graziano Ubertalli, "Friction and wear behaviour of sintered steels subjected to sliding and abrasion tests", Tribol. Int.. vol. 37 , pp. $748-755$, 2006. doi: 10.1016/j.triboint.2005.07.003.

[6] K. Gopinath, "The influence of speed on the wear of sintered iron-based materials," Wear, vol. 71, pp. 161-178, 1981.

doi:10.1016/0043-1648(81)90336-7.

[7] Zhang Guowei, Ouyang Jinlin, Meng Xiukun, Ma Li, Qi Shang Kui, Meng Xiukun et al., "Reactions during preparation and sliding of sintered FeMo-S wear resistant materials," Wear, vol. 162-164, pp. 450-457, 1993. doi: 10.1016/0043-1648(93)90529-U.

[8] T. Murakami, K. Kaneda, N. Nakano, H. Mano, A. Korenaga, S. Sasak, "Friction and wear properties of Fe-Mo intermetallic compounds under oil lubrication", Intermetallics, vol. 15, pp.1573-1581, 2007. doi: 10.1016/j.intermet.2007.06.008.

[9] S. Tekeli, A. Gural, D. Ozyurek, "Dry sliding wear behaviour of low carbon dual phase powder metallurgy steels", Mater. Des., vol. 28, pp. 1685-1688, 2007. doi: 10.1016/j.matdes.2006.03.013.

[10] S. Tekeli, A. Gural, "Dry sliding wear behavior of heat treated iron-based powder metallurgy steels with $0.3 \%$ Graphite $+2 \% \mathrm{Ni}$ additions", Mater. Des., vol. 28, pp. 1923-1927, 2007. doi: 10.1016/j.matdes.2006.05.009.

[11] R. Colaco, E. Gordo, E. M. Ruiz-Navas, M. Otasevic, R. Vilar, "A comparative study of the wear behavior of sintered and laser surface melted AISI M42 high speed steel diluted with iron," Wear, vol.260, pp. 949-956, 2006. doi: 10.1016/j.wear.2005.06.006.

[12] N. Anton, J. L. Delgodo, F. Velosco, J. M. Torralba, "Influence of alloying element additions on tribological behavior of sintered steels with high content in manganese-nickel", J. Mater. Process. Technol., vol. 143-144, pp. 475-480, 2003. doi: 10.1016/S0924-0136(03)00299-1.

[13] E. R. Leheup, Deen Zhang, J. R. Moon, "The effect of density on fretting wear of sintered iron", Wear, vol. 176, pp. 111-119, 1994. doi: 10.1016/0043-1648(94)90204-6.

[14] S. C. Lim, J. H. Brunton, "The unlubricated wear of sintered iron", Wear, vol. 113, pp. 371-382, 1986. doi: 10.1016/0043-1648(86)90035-9.

[15] S. C. Lim, D.C. Isaacs, R. H. McClean, J.H. Bruntont, "The unlubricated wear of sintered steels", Tribol. Int., vol. 20, no.3, 144-149, 1987. doi: 10.1016/0301-679X(87)90044-2.

[16] Suckchoon Kang, "A study of friction and wear characteristics of Copper-and iron- based sintered materials", Wear, vol. 162-164, pp. 1123-1128, 1993 doi: 10.1016/0043-1648(93)90131-5.

[17] S. Dhanasekaran, R. Gnanamoorthy, "Abrasive wear behavior of sintered steels prepared with MoS2 addition", Wear, vol. 262, pp. 617-623, 2007. doi: 10.1016/j.wear.2006.07.006. 


\title{
Effect of the the Interlacing Pattern on the Compaction Behavior of 3D Carbon Fibre Textile Reinforcements
}

\author{
R. Salekrostam, F. Robitaille \\ Department of Mechanical Engineering \\ University of Ottawa \\ Ottawa, Canada \\ $\underline{\text { Rsale051@uottawa.ca }}$
}

\begin{abstract}
D reinforcements were introduced to mitigate and overcome limitations arising from the use of traditional textile reinforcements in the construction of polymer matrix composites (PMC), in terms of their resistance to impact and inter-laminar shear strength (ILSS), both resulting from delamination.

Many thick interlaced textile structures were developed towards that end but many more possibilities exist in terms of yarn interlacing patterns, leading to different types of $3 \mathrm{D}$ textiles that may potentially be built. The effect of different interlacing patterns on the compaction behaviour of such interlaced 3D textiles is probed in this paper. Trends and differences in the compaction behaviour were observed for 5 series of 3 consecutive compaction cycles applied to 16-layers interlaced 3D reinforcements. Those trends and differences are presented and discussed.

Overall, the maximum recorded volume fraction ranged from 0.58 to 0.86 . Values of the volume fraction reached in the first cycle $C_{l}$ were always lower than in following cycles $C_{2}$ and $C_{3}$. Although some elements of behaviour were common to all samples and tests, differences also emerged between samples featuring different interlacing patterns.
\end{abstract}

Keywords- $3 D$ reinforcement fabrics; compaction; interlacing pattern; volume fraction

\section{INTRODUCTION}

3D textile reinforcements featuring relatively few throughthickness yarns may be constructed from 2D fabrics, stitching them together using structural thread to enable some level of local load transfer between layers in addition to assembling the 2D layers into a 3D textile. 2D textile layers can also be held together using technologies such as tufting or z-pins [1]. Advantages of such methods for producing simple 3D textile reinforcements include reduced labour and level of uniformity that are largely maintained, without the need for complex textile manufacturing processes. However, a suitable joining method for assembling different layers together is critical for handling of the fabrics, and for the mechanical properties of the final PMC part [2]. For example, Quan et al. reported a multi-layer 3D textile reinforcement consisting of 2D layers of plain weave, $0^{\circ}$ unidirectional non-crimp fabric, $90^{\circ}$ unidirectional non-crimp fabric, and plain weave laid either side of a foam core and held together by 4 pins [3].

3D reinforcements featuring more interlacing can be produced through dedicated weaving, braiding and knitting processes using specially designed automated looms. These reinforcements are better in the sense that yarn interlacing between layers is more pervasive, more consistent, and it is effectively part of the textile design. Many interlaced fabrics featuring different yarn interlacing patterns can be designed, resulting in numerous types of $3 \mathrm{D}$ woven fabrics that could theoretically be built. The University of Ottawa composites group has developed its Steered Preforming Technology (uOSPT) which enables the design and manufacturing of thick, netshape, flat drapable 3D reinforcement fabrics featuring variable spacing between either straight or curved yarns [4, 5]. Different yarn interlacing patterns in uO-SPT reinforcements result in different properties in the dry fabrics and final composite parts made from them [3,5]. Comparing with 2D woven fabrics, different yarn interlacing patterns have led to a number of classic patterns becoming standard constructions including plain weaves, 4-harness, 5-harness and 8-harness satin weaves, and twill weaves. These patterns are used routinely in composites construction and constitute the vast majority of 2D weaves used, even as many other patterns could be created. 3D reinforcements can also be built based on a large array of possible interlacing patterns [6]. Some 3D interlacing patterns are referred to more frequently in the literature such as orthogonal and interlock constructions.

The behaviour of different reinforcements subjected to compaction normal to their plane is very important to numerous aspects of the processing of reinforcements into composite parts. Compaction controls the permeability which largely dictates how resin flows through the reinforcement during processing, and it also controls the fibre volume fraction which influences the mechanical properties of the composite parts. A number of $3 \mathrm{D}$ reinforcements featuring different 
interlacing patterns were manufactured and their compaction behaviour are reported and compared.

\section{MATERIALS}

The uO-SPT was used for manufacturing 16-layers 3D carbon fibre reinforcements featuring 3 different interlacing patterns. The aim of choosing different interlacing patterns was to probe the effect of yarn interlacing on the compaction behaviour of the reinforcements: one of the reinforcements featured limited interlacing, one featured a high level of interlacing, and one was between these extremes.

Diagrams representing these fabrics were produced using textile modeling software TexGen from the University of Nottingham [7]. It should be noted that vertical dimensions in computer models were magnified for clarity. Stitching lines do not feature in the diagrams. The interlacing patterns of the 3D reinforcements were explained through illustrations of the yarn paths in the reinforcements, also generated using TexGen.

The reinforcement shown in "Fig 1" was labelled preform $\# 1$. Preform \#1 features no interlacing. Yarn paths for preform \#1 are shown in "Fig 2". "Fig 3" illustrates the second 16layers 3D reinforcement which features highly interlaced yarns. The reinforcement shown in "Fig 3" was labelled preform \#2. The yarn paths of preform\#2 are shown in "Fig. 4". The third reinforcement, which features moderate interlacing is labelled preform\#3 and is shown in "Fig. 5". "Fig. 6" illustrates the yarn paths for preform\#3.

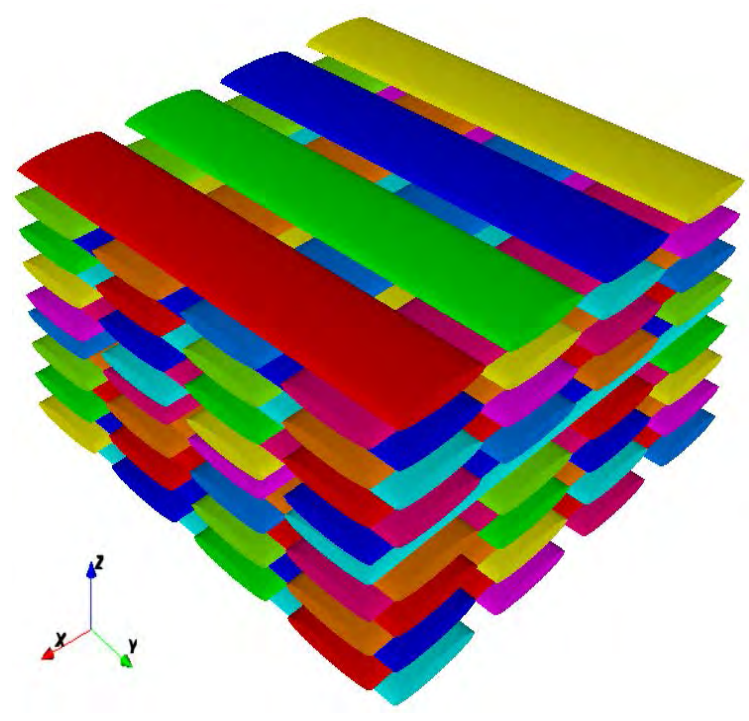

Figure 1. Preform\#1, 16-layers, no interlacing

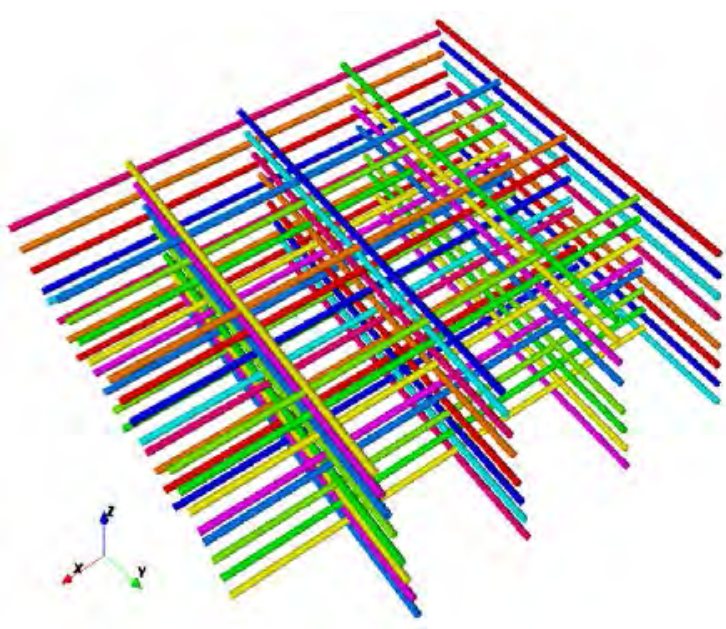

Figure 2. Yarn paths, preform \#1

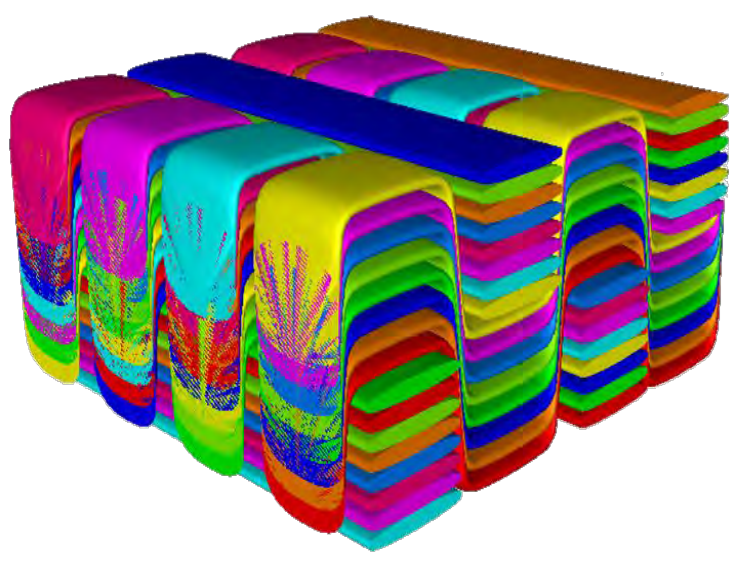

Figure 3. Preform\#2, 16-layers, high interlacing

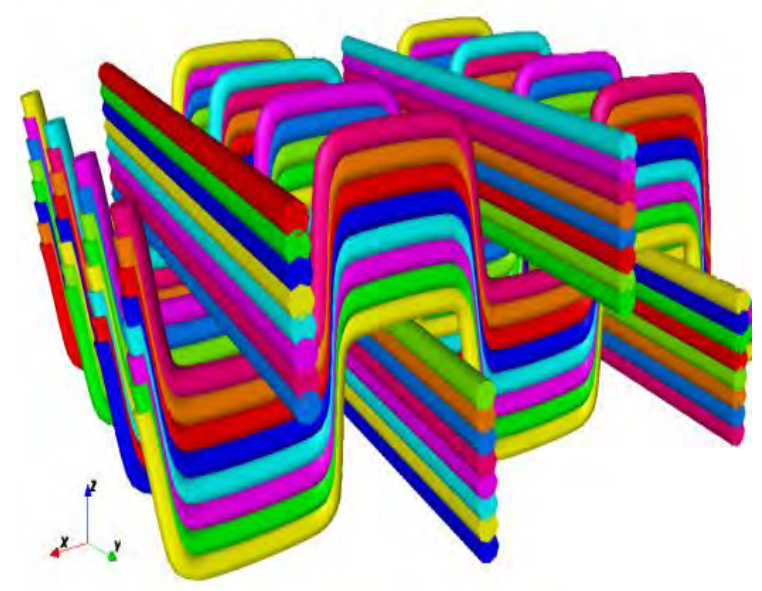

Figure 4. Yarn paths, preform \#2 


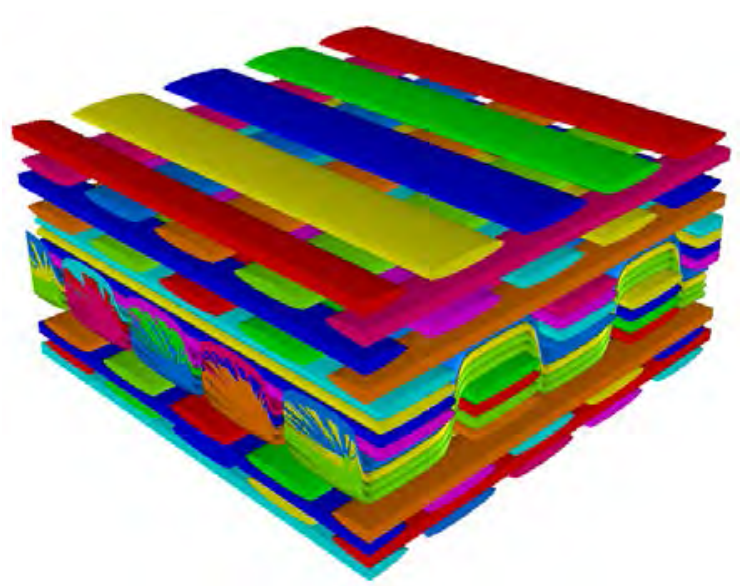

Figure 5. Preform\#3, 16-layers, moderate interlacing

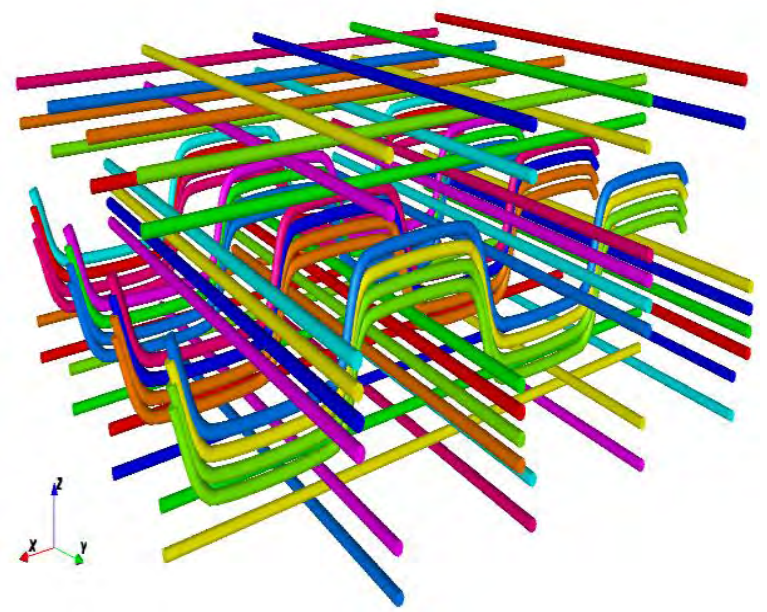

Figure 6. Yarn paths, preform \#3

\section{Experimental}

All compaction tests were conducted using an Instron 4482 universal testing frame equipped with a $100 \mathrm{KN}$ load cell, with an accuracy of $0.25 \%$ of maximum load. Compaction tests were conducted using a circular cross-section test rig. "Fig 7" shows the comaction test rig throughout compaction test of preform \#2. The test rig includes a lower platen and an upper platen with a diameter of $50 \mathrm{~mm}$. Tests were conducted up to a pressure of $0.12 \mathrm{Mpas}$. All compaction tests were performed by moving the upper platen onto the surface of the reinforcement at a fixed displacement rate of $0.1 \mathrm{~mm} / \mathrm{min}$, recording the reaction force applied by the compacted reinforcement on the platen.

Successive compaction cycles were conducted in each test, to investigate the effect of repeated loading cycles on the compaction behaviour of all 3D preforms. Each series of cycles was repeated 4 times to quantify the reproducibility of the data. Recorded values were converted to fibre volume fractions as a function of the compaction pressure, and graphs of average curves were produced. All compaction tests are conducted in room temperature.

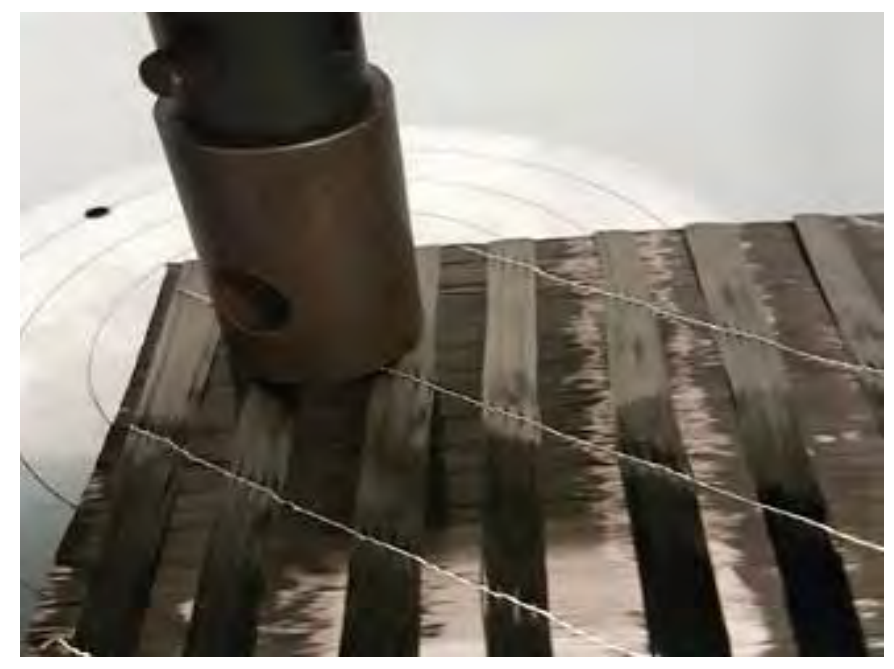

Figure 7. Compaction test on preform\#2

\section{RESULTS AND DISCUSSION}

Compaction graphs showing the behavioir of 3D reinforcements in different successive compaction cycles are derived . "Fig 8" shows the compaction behaviour of preforms $\# 1, \# 2$ and \#3 in the first compaction cycle. "Fig 9" and "Fig 10 " show the compaction behaviour of all three 3D reinforcements in the second and third successive cycles. "P\#1 / C1" means total average of data obtained for preform\#1 in the first cycle. The error bars on the graphs show the vsariability of the data.

Cycle 1

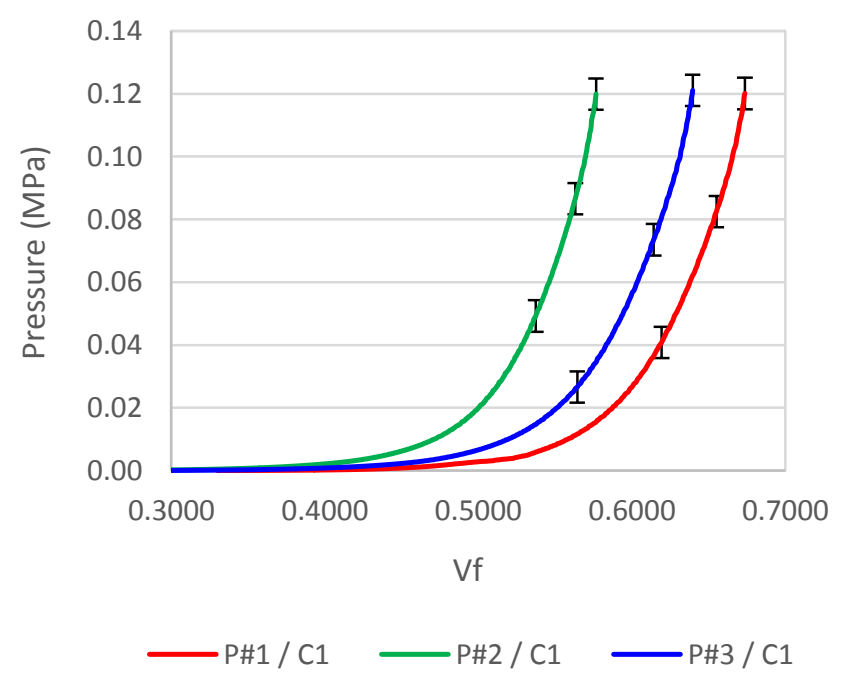

Figure 8. Compaction behaviour, 3 fabrics, cycle 1 
Cycle 2

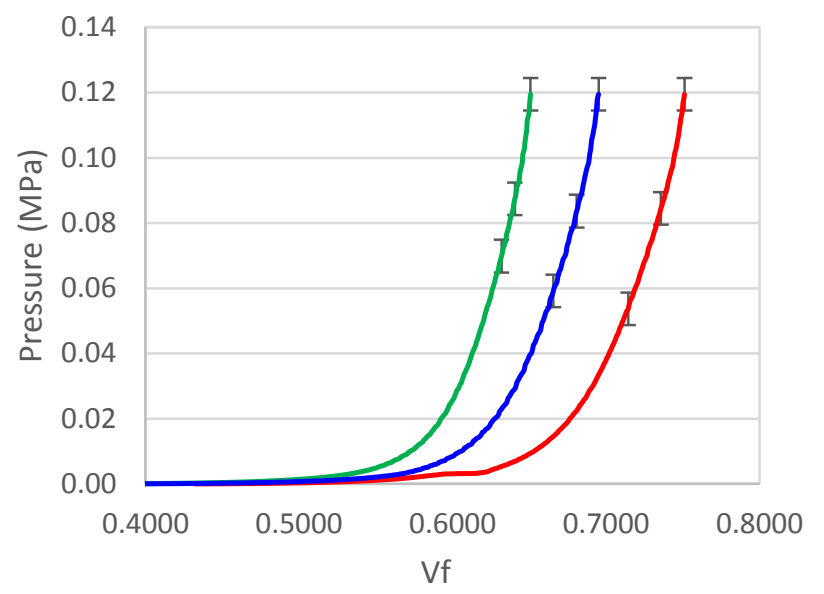

P\#1/C2 - $P \# 2 / C 2-P \# 3 / C 2$

Figure 9. Compaction behaviour, 3 fabrics, cycle 2

\section{Cycle 3}

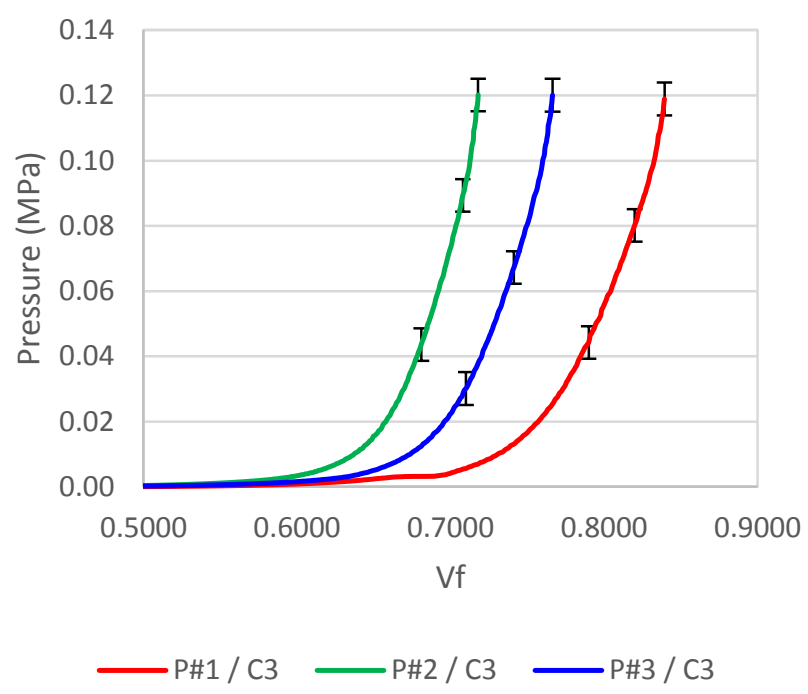

Figure 10. Compaction behavior, 3 fabrics, cycle 3

Different internal interlacing patterns in the reinforcements affect their compaction behaviour and volume fraction.

"Fig 11", "Fig 12" and "Fig 13" show the effect of compaction cycles on the maximum fibre volume fraction.

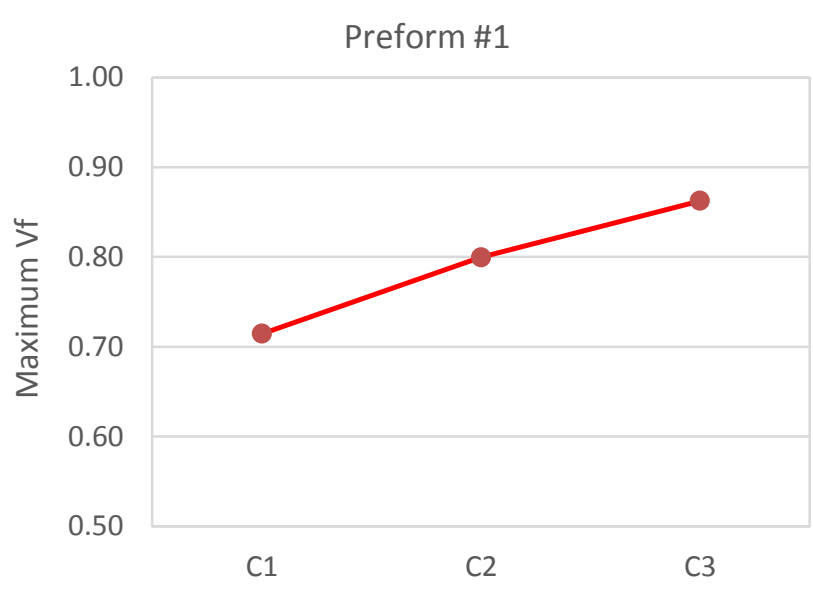

Figure 11. Effect of number of cycles on compaction behaviour, preform \#1

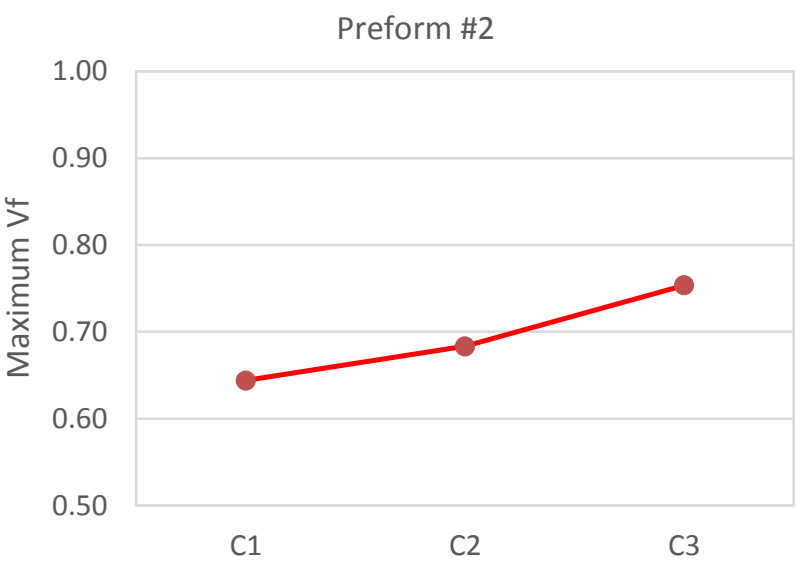

Figure 12. Effect of number of cycles on compaction behaviour, preform \#2

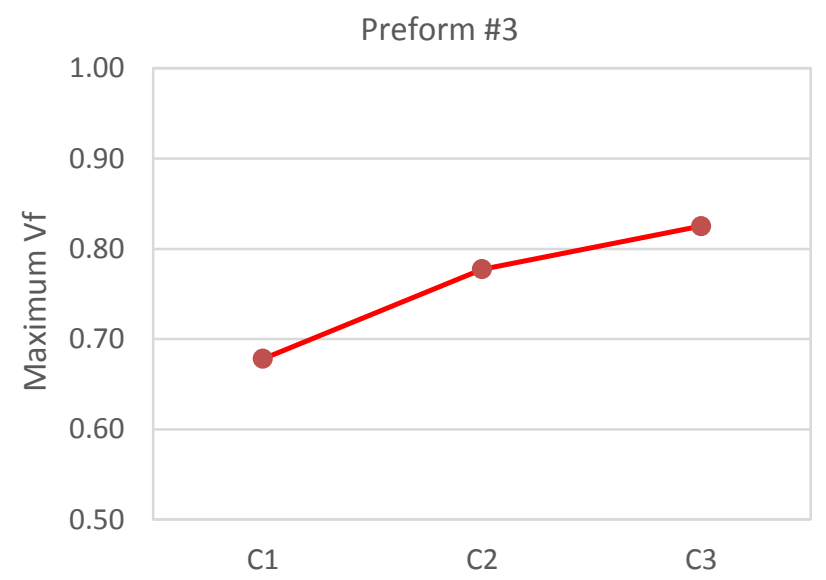

Figure 13. Effect of number of cycles on compaction behaviour, preform \#3 
An increase in the $V_{f}$ of all three preforms was observed from conducting successive compaction cycles as seen in "Fig 11 ", "Fig 12" and "Fig 13".

\section{CONCLUSIONS}

An experimental study of the compaction behaviour of three different 3D carbon fibre reinforcements with different interlacing patterns is presented. The influence of interlacing on the final volume fraction of the reinforcements was analysed.

3D reinforcements featuring more interlacing show lower volume fractions. Their volume fraction at any pressure is lower than 3D reinforcements fraturing lower interlacing. This shows direct effect of the quantity of the interlacing of 3D reinforcements on their compaction behaviour and volume fraction.

The effect of repeated compaction cycles on the different interlaced 3D reinforcements was also probed. The volume fraction of all 3D reinforcements increased for higher compaction cycles.

\section{REFERENCES}

A. P. Mouritz and P. Chang, "Tension fatigue of fibre-dominated and matrix-dominated laminates reinforced with z-pins," Int. J.

Fatigue, vol. 32, no. 4, pp. 650-658, 2010.

doi: 10.1016/j.ijfatigue.2009.09.001
[2] D. Heider, H. Deffor, M. Reuter, J. W. Gillespie, M. Mohamed, and R. Crane, "Large-scale joint fabrication using 3-D fabric preforms, sandwich core structure and VARTM processing," Sampe J., vol. 44, no. 5, pp. 29-34, 2008 .

[3] Z. Quan et al., "Additive manufacturing of multi-directional preforms for composites: Opportunities and challenges," Materials Today, vol. 18, no. 9. pp. 503-512, 2015. doi: 10.1016/j.mattod.2015.05.001.

[4] N. Burnford, "Development of Drape Simulation Software and the Optimisation of Variable-Length Textiles," MASc thesis, University of Ottawa, 2011

[5] T. Drivas, "Manufacturing Three-dimensional Carbon-fibre Preforms for Aerospace Composites", MASc thesis, University of Ottawa 2013

[6] R. Salekrostam, D. Muzar and F. Robitaille,, "Interlacing patterns for steered yarn near net-shape 3D reinforcements", SAMPE Conference Proceedings. Seattle, WA, May 22-25, 2017. Society for the Advancement of Material and Process Engineering - North America.

[7] A. C. Long and L. P. Brown, "Modelling the geometry of textile reinforcements for composites: TexGen", Woodhead Publishing Limited, 2011. doi: 10.1533/9780857093714.2.239. 


\title{
Investigation of Fraying in Textile Reinforcements for Composites
}

\author{
Yousef Badripour, François Robitaille \\ Department of Mechanical Engineering \\ University of Ottawa \\ Ottawa, Canada
}

\begin{abstract}
This paper discusses the fraying of textile reinforcements upon preforming. The paper also introduces a method for quantifying the fraying characteristics of fabrics. Six different fabrics including three carbon fabrics and three glass fabrics were tested. Digital microscopy was also used for measuring the structures of the fabrics tested. The relation between the structures and fraying is discussed.
\end{abstract}

Keywords- textiles; composites; fraying; yarn loss

\section{INTRODUCTION}

Fibre reinforced polymer matrix composites are increasingly used in numerous applications. However, a lack of established standard characterization methods for the dry fabrics used in manufacturing these composites preclude the use of quantitative methods for analyzing and predicting the manufacturing operations. Upon preforming, different fabrics behave in different ways, making it difficult to plan manufacturing efficiently. Each element of the behaviour of these textiles demands a reproducible testing method. Some elements have been investigated thoroughly [1]; however, others still lack proper testing methods. Knowledge of the fraying characteristics of carbon and glass fabrics upon cutting and handling is important in manufacturing; however, no established testing method exists for assessing it. Although some work was done towards that aim, leading to the yarn pull-out test [2] or the inter-yarn friction test [3], such tests mostly measure friction between yarns when subject to pulling or shear; however, yarns fraying from the edges upon draping on moulds is a recurring phenomenon. This paper introduces a reproducible test method for measuring fraying in industrial reinforcement fabrics.

\section{INDUSTRIAL CONTEXT}

When draped, industrial fabrics can show fraying around the edges. The amount of fraying can vary with the fabric type, fabric architecture and mould geometry. A typical situation when fraying can occur is the darting of a fabric, which is done to facilitate the draping of a mould of complex geometry [4]. "Fig. 1" and "Fig. 2" show two different fabrics after being cut and draped on a mould. More complex mould geometries result in higher shear and more fraying, eventually making the draping process more problematic.

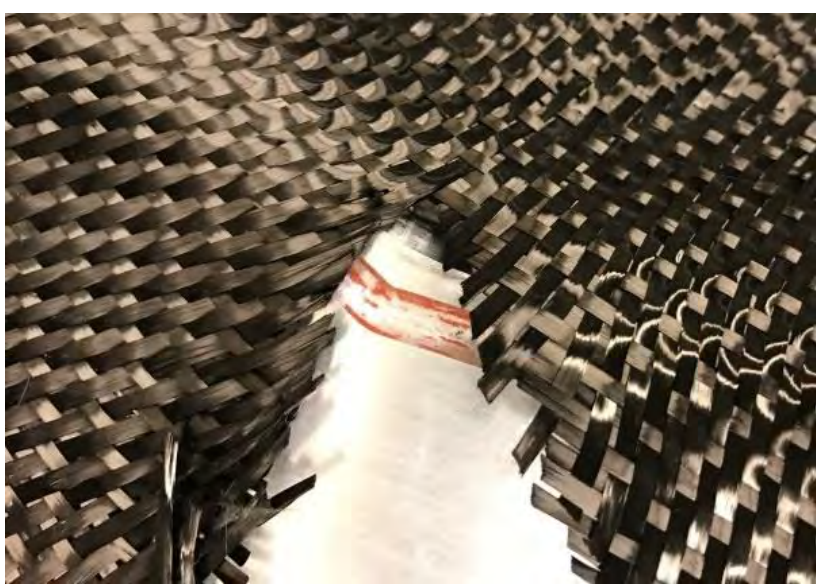

Figure 1. Fraying around edges after cutting and draping, 5-harness carbon fabric

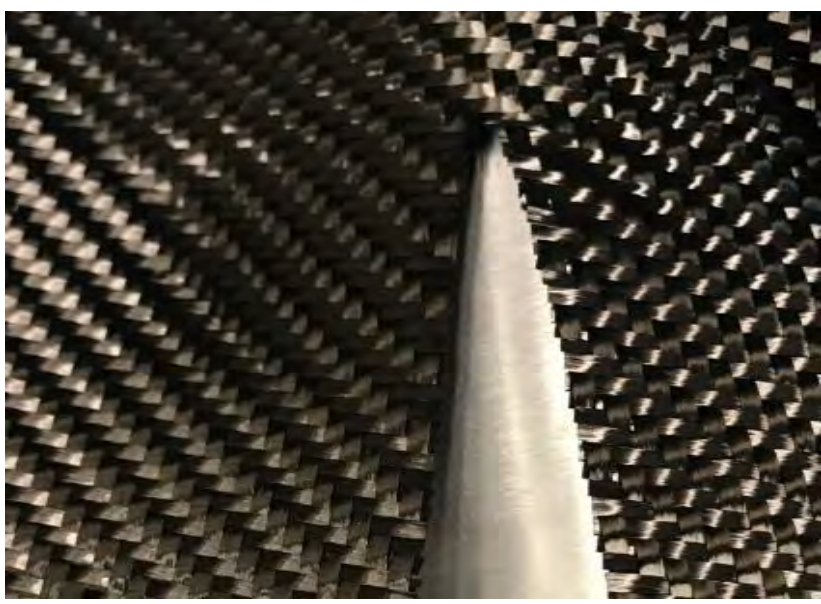

Figure 2. Fraying around edges after cutting and draping, twill carbon fabric 


\section{APPARATUS AND METHODOLOGY}

A testing rig featuring a $3.81 \mathrm{~cm}(1.5 \mathrm{in})$ circular platen supported by a $0.95 \mathrm{~cm}(3 / 8 \mathrm{in})$ rod is mounted into a spinner with an adjustable speed. Tests reported in this paper were conducted at a spinning speed of 1500 RPM. Circular fabric specimens measuring $15.24 \mathrm{~cm}$ (6 in) in diameter were placed on the platen. Double-sided General Sealants Inc tape covered the whole surface of the circular platen and held the fabric onto it. Samples were spun at the aforementioned speed for 3 seconds. Inertial forces caused some yarns to dislodge from the edges. Lost yarns were quantified using mass and area loss. Tests were run 5 times for each fabric and average losses are reported in Table 1 and Table 2.

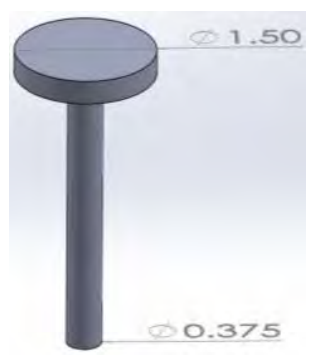

(a)

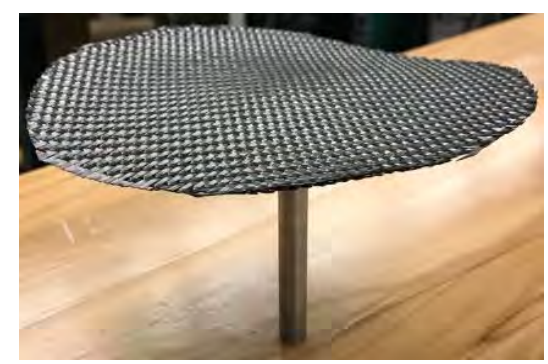

(b)
Figure 3. Test rig (a) and fabric specimen mounted on the rig (b)

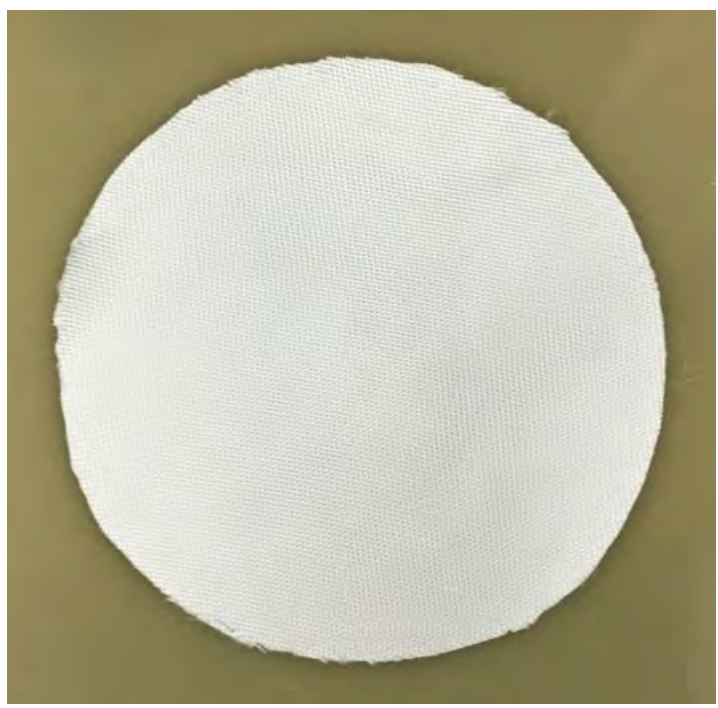

Figure 4. 8-harness glass fabric specimen, before test

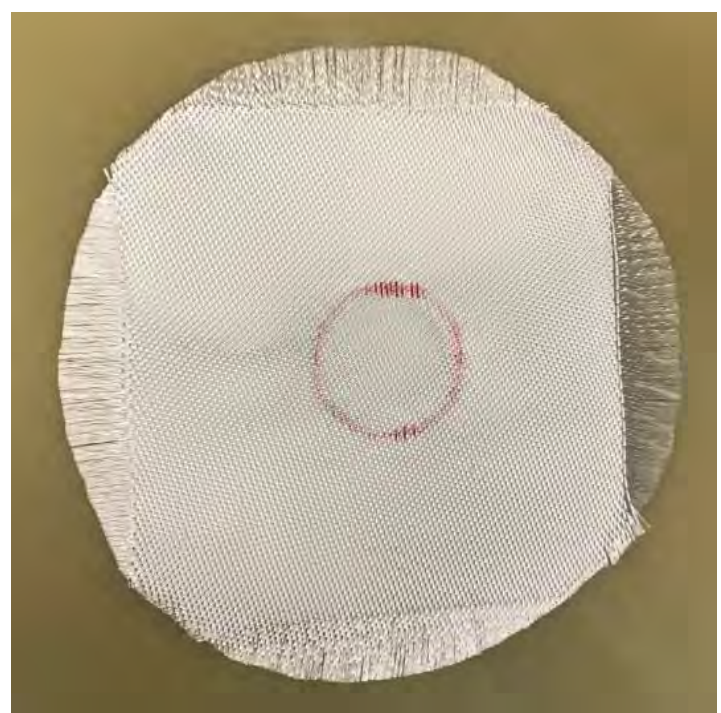

Figure 5. 8-harness glass fabric, after test

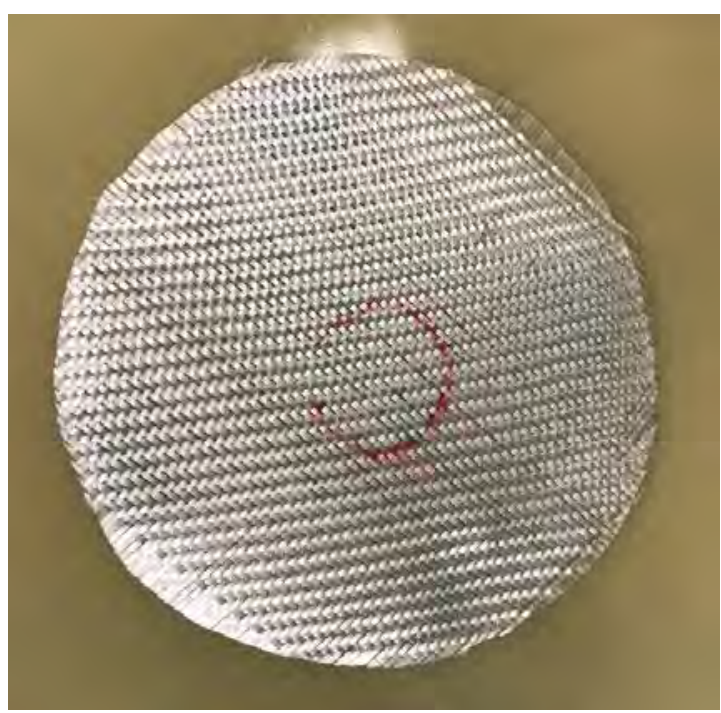

Figure 6. Twill glass fabric after test

The mass of each specimen was measured before and after the test, using a Smart weight dual platform scale with $200 \mathrm{~g}$ capacity and $\pm 0.001 \mathrm{~g}$ accuracy. Mass loss is reported as an average.

The area of the specimen before the test and the nonfrayed area after the test were measured using the ImageJ software. Area loss is reported as an average for each fabric.

Topographies were determined using Keyence VHX-6000 series digital microscope. Effects of maximum height and textiles patterns are assessed. 


\section{RESULTS}

Table 1 and Table 2 give fabrics information such as architecture, surface density as well as yarn count. It also lists results for mass loss, area loss and standard deviations for both measurements.

"Fig. 7" to "Fig. 12" illustrate topographies for the fabrics tested. These topographies returned maximum value of 192 $\mu \mathrm{m}$ in the twill glass and $195 \mu \mathrm{m}$ in the twill carbon with regard to their surface pattern range in the $\mathrm{z}$ axis direction. The value was $237.2 \mu \mathrm{m}$ for the 8 -harness glass fabric. Higher values indicated a higher crimp factor for a given fabric.

TABLE 1. TEST RESULTS FOR GLASS FABRICS

\begin{tabular}{|c|ccc|}
\hline Fabric & Glass & Glass & Glass \\
Architecture & Plain & Twill & 8-harness \\
$\begin{array}{c}\text { Surface density } \\
\text { (g/m } \mathbf{m}^{\mathbf{2}}\end{array}$ & 304 & 300 & 296 \\
$\begin{array}{c}\text { Yarn count } \\
\text { (yarn/cm) }\end{array}$ & 5.55 & 6.45 & 22.42 \\
$\begin{array}{c}\text { Mass loss (\%) } \\
\text { Area loss (\%) }\end{array}$ & 43.15 & 1.39 & 11.23 \\
$\begin{array}{c}\text { Mass loss standard } \\
\text { deviation (\%) }\end{array}$ & 77.07 & 6.04 & 20.68 \\
$\begin{array}{c}\text { Area loss standard } \\
\text { deviation (\%) }\end{array}$ & 6.63 & 15.83 & 10.24 \\
\hline
\end{tabular}

TABLE 2. TEST RESULTS FOR CARBON FABRICS

\begin{tabular}{|c|ccc|}
\hline Fabric & Carbon & Carbon & Carbon \\
Architecture & Twill & $\begin{array}{c}\text { 5-harness with } \\
\text { binder }\end{array}$ & Stitched \\
$\begin{array}{c}\text { Surface density } \\
\text { (g/cm } \mathbf{c m}^{2}\end{array}$ & 408 & 368 & 288 \\
$\begin{array}{c}\text { Yarn count } \\
\text { (yarn/cm) }\end{array}$ & 5 & 4.54 & 2 \\
$\begin{array}{c}\text { Mass loss (\%) } \\
\text { Area loss (\%) }\end{array}$ & 3.03 & 1.84 & 1.09 \\
$\begin{array}{c}\text { Mass loss standard } \\
\text { deviation (\%) }\end{array}$ & 17.82 & 4.75 & 3.39 \\
$\begin{array}{c}\text { Area loss standard } \\
\text { deviation (\%) }\end{array}$ & 18.89 & 33.68 & 26.84 \\
\hline
\end{tabular}

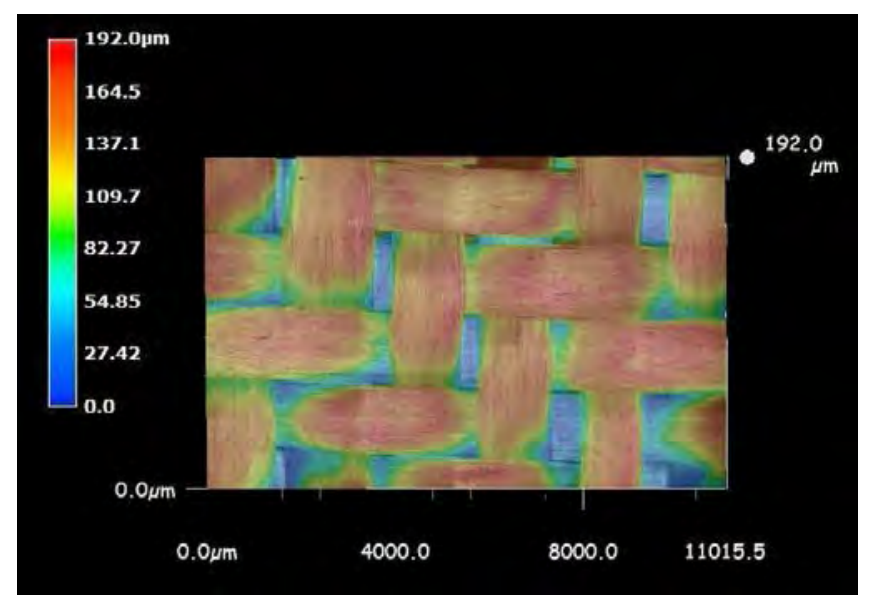

Figure 7. Topography of twill glass fabric

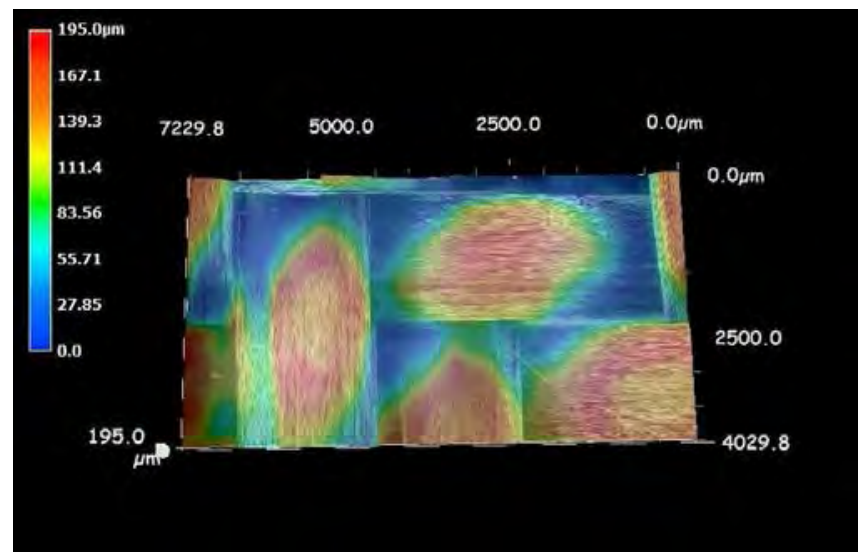

Figure 8. Topography of twill carbon fabric

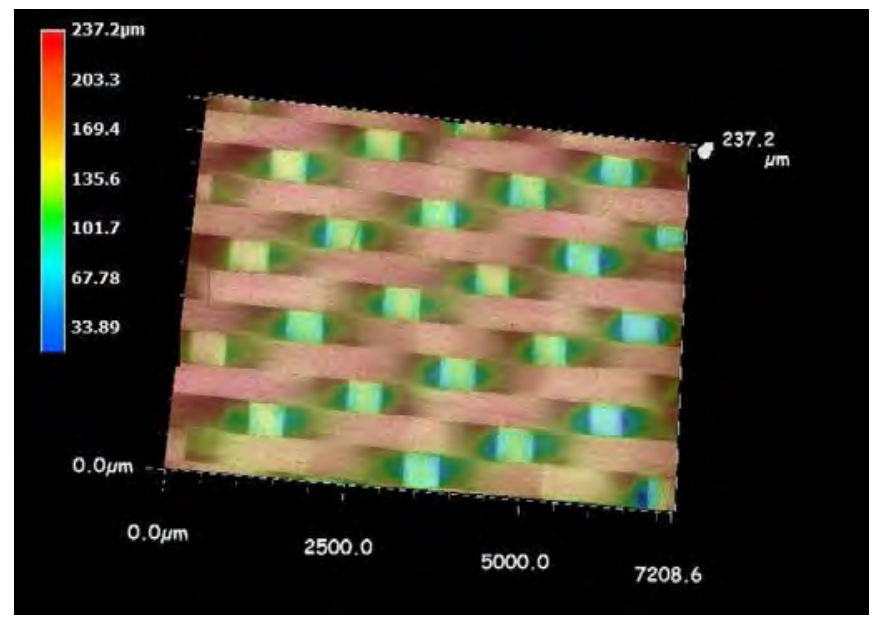

Figure 9. Topography of 8-harness glass fabric 


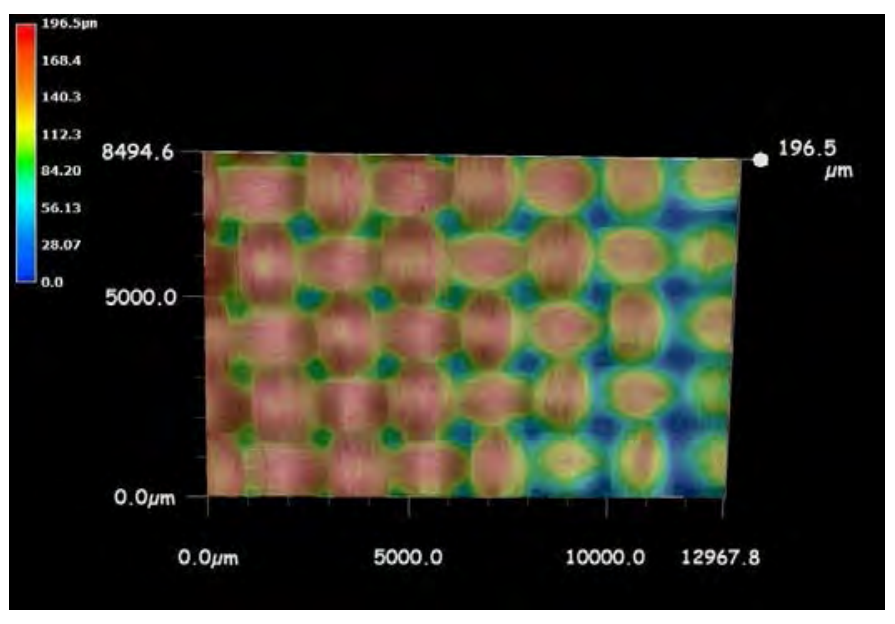

Figure 10. Topography of plain glass fabric

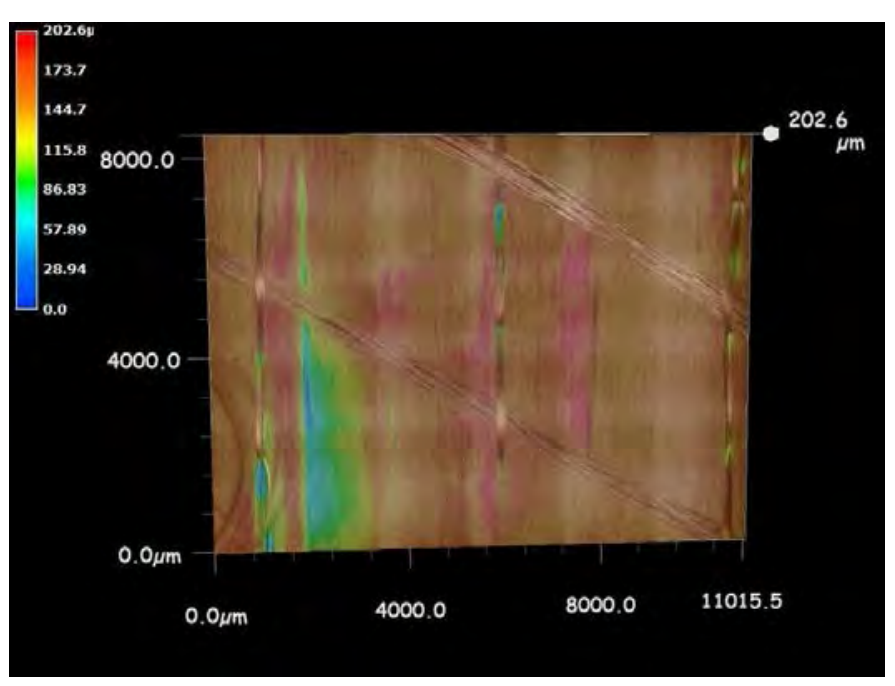

Figure 11. Topography of stitched carbon fabric

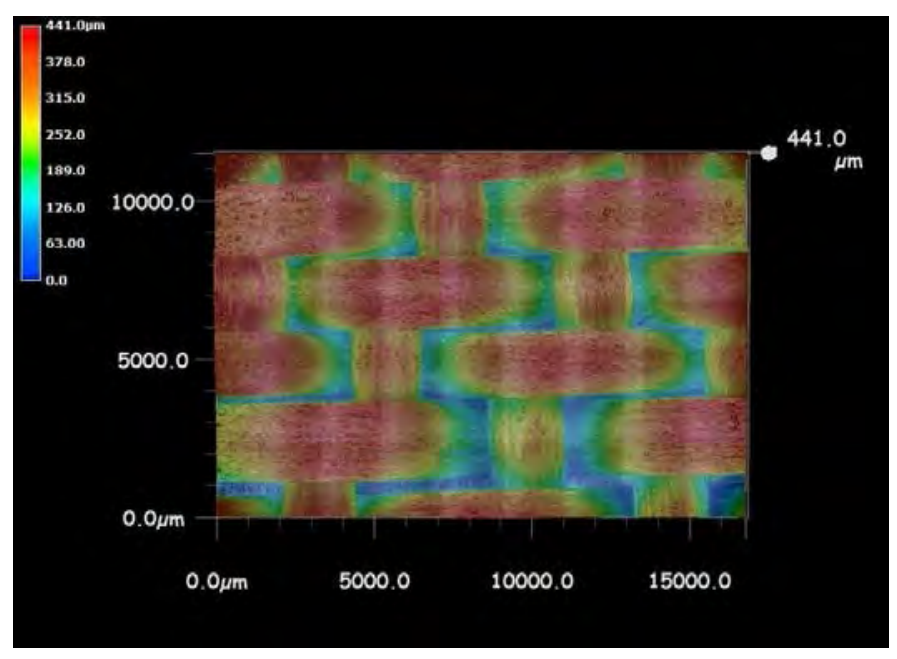

Figure 12. Topography of 5-harness carbon fabric with binder
"Fig. 13" shows the results from Table 1 and Table 2 comparing the mass loss (\%) and area loss (\%) for all fabrics.

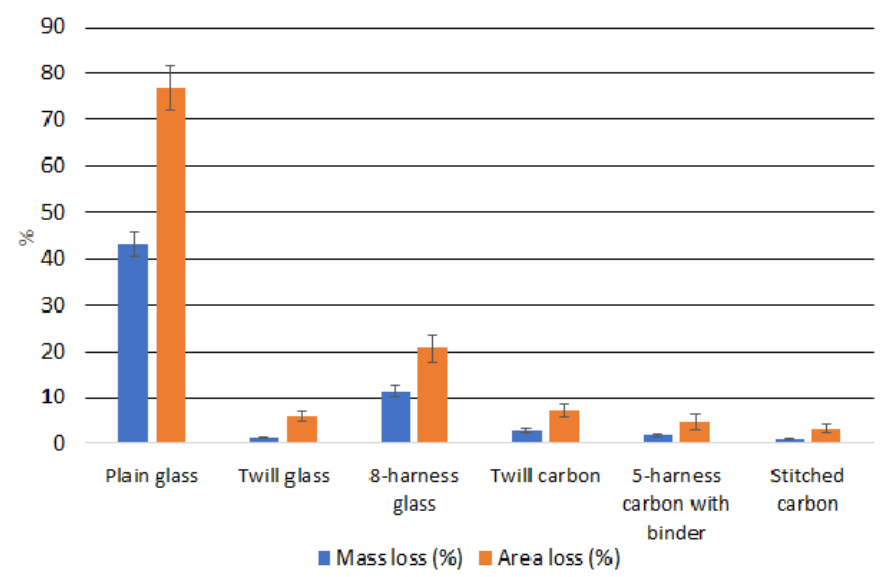

Figure 13. Mass loss and area loss chart for all tested fabrics

\section{DISCUSSION}

Test results for twill glass fabric, twill carbon fabric, 5harness carbon fabric with binder and stitched carbon fabric show limited yarn loss. On the other hand, results for the 8harness glass fabric, showed moderate amounts of yarn loss, and the plain glass fabric showed high amounts of yarn loss. This high amount of yarn loss in plain glass fabric could be due to low amount of inter-yarn friction as well as the plain structure itself. It can be concluded that twill fabrics show a high resistance to fraying. Also, the use of a binder on the fabric and the presence of stitching in fabrics prevent edge fraying efficiently.

The stitched carbon fabric used in testing had fibers extending along $45^{\circ}$ and $-45^{\circ}$ directions with a stitch along the $0^{\circ}$ direction. This fabric predictably returned the minimum amount of yarn loss, confirming that stitching can be a potent solution to the occurrence of fraying in some cases [5].

The analysis of fabric topography delivered information regarding each fabric crimp factor. Twill structured fabrics showed less crimp than the 8-harness one. Varying amounts of crimp can lead to differences in triggering of yarns sliding relatively to each other [6]. Tables 1 and 2 support this claim, as higher mass and area losses are seen for the 8-harness fabric compared with the twills, and similar figures were seen for both twill carbon and twill glass fabrics.

"Fig. 1", which illustrates noticeable fraying for the 5harness carbon fabric, and Table 1 which reports the second highest yarn loss for the 8-harness glass fabric, it can show that yarns in satin fabrics are more likely to slip from the edge.

Results for each fabric showed limited variability notably for the mass loss measurements. However, using an automated 
cutting machine [7] for preparing the specimens, would lead to more accurate specimens resulting in more repeatable results.

\section{CONCLUSION}

This work introduced a reproducible testing method for measuring yarn loss in carbon and glass fabrics with minimum variability in results aiming at quantifying fraying. The impact of some fabrics attributes such as architecture, stitching and presence of a binder on yarn loss was observed and discussed.

\section{REFERENCES}

[1] J. Cao et al., "Characterization of mechanical behavior of woven fabrics: Experimental methods and benchmark results," Compos. Part A Appl. Sci. Manuf., vol. 39, no. 6, pp. 1037-1053, 2008.

doi: 10.1016/j.compositesa.2008.02.016.

[2] Z. Dong and C. T. Sun, "Testing and modeling of yarn pull-out in plain woven Kevlar fabrics," Compos. Part A Appl. Sci. Manuf., vol. 40, no. 12, pp. 1863-1869, 2009. doi: 10.1016/j.compositesa.2009.04.019.

[3] X. S. Zeng, V. B. C. Tan, and V. P. W. Shim, "Modelling inter-yarn friction in woven fabric armour," Int. J. Numer. Methods Eng., vol. 66, no. 8, pp. 1309-1330, 2006.doi: 10.1002/nme.1596.

[4] N. Metaaphanon, Y. Bando, B. Y. Chen, and T. Nishita, "Simulation of tearing cloth with frayed edges," Comput. Graph. Forum, vol. 28, no. 7, pp. 1837-1844, 2009. doi: 10.1111/j.1467-8659.2009.01561.x.

[5] K. Bilisik and M. Korkmaz, "Multilayered and Multidirectionally-stitched aramid Woven Fabric Structures: Experimental Characterization of Ballistic Performance by Considering the Yarn Pull-out Test," Text. Res. J., vol. 80, no. 16, pp. 1697-1720, 2010. doi: 10.1177/0040517510365954.

[6] S. Backert, "The Relationship Between the Structural Geometry of a Textile Fabric and Its Physical Properties: Part II: The Mechanics of Fabric Abrasion," Text. Res. J., vol. 21, no. 7, pp. 453-468, 1951.

doi: 10.1177/004051755102100701.

[7] A. Angerer, C. Ehinger, A. Hoffmann, W. Reif, G. Reinhart, and G. Strasser, "Automated cutting and handling of carbon fiber fabrics in aerospace industries," 2010 IEEE Int. Conf. Autom. Sci. Eng. CASE 2010, pp. 861-866, 2010. doi: 10.1109/COASE.2010.5584262. 


\title{
Modelling Viscoelastomers With Nonlinear Viscosity
}

\author{
Jianyou Zhou, Liying Jiang and Roger E. Khayat \\ Department of Mechanical and Materials Engineering \\ The University of Western Ontario \\ London, Ontario N6A 6B9, Canada \\ lyjiang@eng.uwo.ca
}

\begin{abstract}
Consisting of highly mobile and flexible polymer chains, elastomers are known to exhibit viscoelastic behavior. Adopting concepts from the theory of polymer dynamics and finite-deformation viscoelasticity, this work presents a micromacro constitutive model to investigate the viscoelastic behavior of elastomers, in which the material viscosity varies with the macroscopic deformation. The developed model is then applied to study the stress response of elastomers. From the simulation results, it is observed that the developed model exhibits strong capability of capturing the typical response behaviors of elastomers (e.g., strain-softening behavior). A comparison of the stress responses between linear and nonlinear viscosity is also considered in this work. The modeling framework in this paper is expected to provide a general approach and a platform to analyze the viscoelastic behavior of rubber-like materials with nonlinear viscosity.
\end{abstract}

Elastomers; viscoelasticity; finite-deformation; nonlinear viscosity;

\section{INTRODUCTION}

Elastomers, capable of sustaining exceptionally large deformation, have extensive applications in engineering and industrial fields, such as flexible joints, automotive products, soft robots, artificial muscles and vibration isolators [1]. Formed by the cross-linking of highly mobile and flexible polymer chains, elastomers are hyperelastic and highly viscoelastic. These two major characteristics strongly affect the response of elastomers and have attracted much interest from the research community.

In the literature, numbers of constitutive models have been developed to capture the hyperelastic behavior of elastomers. These available models are established mainly based on two approaches: continuum mechanics treatments and statistical mechanics treatments. Developed through continuum mechanics framework, the phenomenological models assume that the hyperelastic properties of the material can be described by a strain energy function [2-3]. On the other hand, the hyperelastic constitutive models based on statistical treatments link the macroscopic response of the materials to their microstructure [4-5].

This work is supported by Natural Sciences and Engineering Research Council of Canada (NSERC).
As for modeling the viscosity of elastomers, extensive research is also available in the literature. For example, phenomenological models that adopt thermodynamics evolution laws have been developed to tackle the inelastic deformation and viscous effect of elastomers, where the timedependent strain variables are determined by the evolution laws [6-7]. Nevertheless, in these phenomenological models, details of the microstructure of the material related to the viscosity are not involved. Therefore, to reveal the physical mechanisms of the viscosity of the material, concepts from polymer dynamics [8] have been adopted later to develop the micromechanism inspired viscoelasticity models [9-10]. Although both the phenomenological models and the micromechanism inspired viscoelasticity models work well for fitting certain experimental data, they cannot capture the nonlinear viscosity of the material (viscosity varies with the deformation).

This motivates us to revisit the theory of polymer dynamics and the state-of-the-art theoretical framework of finitedeformation viscoelasticity to develop a micro-macro constitutive model, which aims to capture the viscoelastic deformation of elastomers with consideration of their nonlinear viscosity.

\section{THEORETICAL FRAMEWORK}

\section{A. Continuum Mechanics Framework}

Following the pioneering works of Sidoroff [11], and Reese and Govindjee [7], it is assumed that the elastomer comprises an elastic polymer ground network and a viscous polymer subnetwork, which can be represented by the rheological model shown in Figure 1. Polymer network A is a purely elastic network, while network $\mathrm{B}$ is a viscous network. Consider a material point $\mathrm{P}$ in the reference configuration denoted by its position vector $\mathbf{X}$. When the elastomer is subject to external loads, point $\mathrm{P}$ moves to position $\mathbf{x}$ in the current configuration. Then the total deformation gradient tensor $\mathbf{F}$ is defined as $\mathbf{F}=\nabla \mathbf{x}(\mathbf{X}, t)$. Since the deformation is applied to both polymer networks, $\mathbf{F}_{\mathrm{A}}=\mathbf{F}_{\mathrm{B}}=\mathbf{F}$. Borrowing a concept from finitedeformation plasticity, the deformation gradient tensor of network B can be further multiplicatively split into two parts, i.e., $\mathbf{F}_{\mathrm{B}}=\mathbf{F}_{\mathrm{B}}^{\mathrm{e}} \mathbf{F}_{\mathrm{B}}^{\mathrm{i}}$. Here, $\mathbf{F}_{\mathrm{B}}^{\mathrm{e}}$ represents the deformation gradient of 
the spring and $\mathbf{F}_{\mathrm{B}}^{\mathrm{i}}$ denotes the deformation gradient of the dashpot. Adopting the rheological model in Fig. 1, the total Helmholtz free energy density of the elastomer is expressed as

$$
W\left(\mathbf{F}_{\mathrm{A}}, \mathbf{F}_{\mathrm{B}}^{\mathrm{e}}\right)=W_{\mathrm{A}}\left(\mathbf{F}_{\mathrm{A}}\right)+W_{\mathrm{B}}\left(\mathbf{F}_{\mathrm{B}}^{\mathrm{e}}\right),
$$

where $W_{\mathrm{A}}$ and $W_{\mathrm{B}}$ are the strain energy densities of the springs of network A and B, respectively [12-13]. Then the Cauchy stress tensor $\boldsymbol{\sigma}$ and the first Piola-Kirchhoff stress tensor $\mathbf{P}$ are given as

$$
\mathbf{T}=J^{-1} \frac{\partial W_{\mathrm{A}}\left(\mathbf{F}_{\mathrm{A}}\right)}{\partial \mathbf{F}} \mathbf{F}^{\mathrm{T}}+J^{-1} \frac{\partial W_{\mathrm{B}}\left(\mathbf{F}_{\mathrm{B}}^{\mathrm{e}}\right)}{\partial \mathbf{F}} \mathbf{F}^{\mathrm{T}}
$$

and

$$
\mathbf{P}=J \mathbf{T F}^{-\mathrm{T}} .
$$

Moreover, the internal variables $\mathbf{F}_{\mathrm{B}}^{\mathrm{e}}$ and $\mathbf{F}_{\mathrm{B}}^{\mathrm{i}}$ must satisfy the following thermodynamic evolution equation, i.e.,

$$
-\frac{1}{2} \mathbf{F}_{\mathrm{B}} \frac{d\left[\left(\mathbf{C}_{\mathrm{B}}^{\mathrm{i}}\right)^{-1}\right]}{d t}\left(\mathbf{F}_{\mathrm{B}}\right)^{\mathrm{T}}\left(\mathbf{b}_{\mathrm{B}}^{\mathrm{e}}\right)^{-1}=\gamma^{-1}: \boldsymbol{\tau}_{\mathrm{B}} .
$$

Here, $\boldsymbol{\tau}_{\mathrm{B}}=2 \mathbf{F}_{\mathrm{B}}^{\mathrm{e}} \frac{\partial W}{\partial \mathbf{C}_{\mathrm{B}}^{\mathrm{e}}}\left(\mathbf{F}_{\mathrm{B}}^{\mathrm{e}}\right)^{\mathrm{T}}, \mathbf{b}_{\mathrm{B}}^{\mathrm{e}}=\mathbf{F}_{\mathrm{B}}^{\mathrm{e}}\left(\mathbf{F}_{\mathrm{B}}^{\mathrm{e}}\right)^{\mathrm{T}}, \mathbf{C}_{\mathrm{B}}^{\mathrm{i}}=\left(\mathbf{F}_{\mathrm{B}}^{\mathrm{i}}\right)^{\mathrm{T}} \mathbf{F}_{\mathrm{B}}^{\mathrm{i}}$ and $\gamma^{-1}$ takes the form

$$
\gamma^{-1}=\frac{1}{2 \eta_{\mathrm{B}}}\left(\mathbf{I}^{4}-\frac{1}{3} \mathbf{I} \otimes \mathbf{I}\right),
$$

where $\eta_{\mathrm{B}}$ is the viscosity of the viscous polymer subnetwork $\mathrm{B}$, $\mathbf{I}^{4}$ is the fourth order symmetric identity tensor and $\mathbf{I}$ is the second order identity tensor [7].

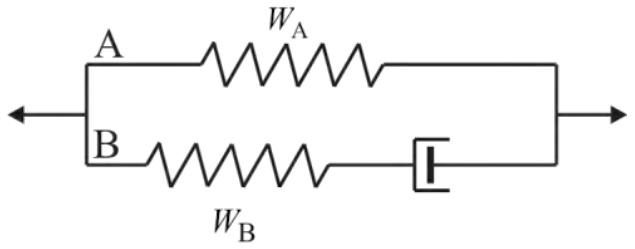

Figure 1. The rheological model of viscoelastic elastomers under finite-deformation.

\section{B. Nonlinear Viscosity}

To obtain the stress with (2), (3), (4) and (5), the viscosity $\eta_{\mathrm{B}}$ of the viscous network must be constitutively prescribed first. Following Doi and Edwards [8], $\eta_{\mathrm{B}}$ can be expressed in terms of the shear relaxation modulus $G_{\mathrm{r}}$, i.e,

$$
\eta_{\mathrm{B}}=\int_{0}^{\infty} G_{\mathrm{r}} d t
$$

In the short time-scale $\left(t<\tau^{\mathrm{e}}\right), G_{\mathrm{r}}$ is related to the microstructural parameters of the polymer network as

$$
G_{\mathrm{r}} \cong n_{\mathrm{B}} k_{\mathrm{B}} T\left(\frac{\tau^{\mathrm{e}}}{\tau^{\mathrm{R}}}\right)^{1 / 2}
$$

where $n_{\mathrm{B}}$ is the number of chains per unit volume in subnetwork $\mathrm{B}, k_{\mathrm{B}}$ is the Boltzmann constant, $T$ is the temperature, $\tau^{\mathrm{R}}$ is the relaxation time of the contour length of the primitive chain, and $\tau^{\mathrm{e}}$ is the critical time that the Brownian motion is restricted by the topological constraints (tube-like region) of the polymer network.

However, in the long time-scale $\left(t>\tau^{\mathrm{e}}\right)$, the polymer chains may reptate out of the topological constraints (the tube). When reptation occurs, the shear relaxation modulus $G_{\mathrm{r}}$ is proportional to the fraction $\varphi(t)$ of the chain still in the tube, i.e.,

$$
G_{\mathrm{r}} \cong n_{\mathrm{B}} k_{\mathrm{B}} T\left(\frac{\tau^{\mathrm{e}}}{\tau^{\mathrm{R}}}\right)^{1 / 2} \varphi(t)
$$

By solving the one-dimensional diffusion equation to obtain $\varphi(t)$ and integrating the shear relaxation modulus in (6),

$$
\eta_{\mathrm{B}}=\frac{\zeta n_{\mathrm{B}}^{4} b_{0}^{4}}{12\langle a\rangle^{2}}\left(\frac{\tau^{\mathrm{e}}}{\tau^{\mathrm{R}}}\right)^{1 / 2},
$$

where $a$ is the tube diameter in the current configuration, $\zeta$ is the monomer friction constant, $b_{0}$ is the effective bond length between monomers, and $\left.<^{*}\right\rangle$ is the expectation operation of parameter $*[13]$.

Considering nonlinear viscosity, $\langle a\rangle$ should vary with the deformation. Moreover, the mean diameter $\langle a\rangle$ is linked to the square end-to-end distance of the primitive chain $\mathbf{R}_{\mathrm{ee}}^{2}$ and the primitive chain length $L$ by $\langle a\rangle=\left\langle\mathbf{R}_{\mathrm{ee}}^{2}\right\rangle /\langle L\rangle$. Following Li et al. [14], $\left\langle\mathbf{R}_{\mathrm{ee}}^{2}\right\rangle$ is obtained as

$$
\left\langle\mathbf{R}_{\mathrm{ee}}^{2}\right\rangle=\int|\mathbf{F} \cdot \mathbf{R}|^{2} f_{0}(\mathbf{R}) d^{3} \mathbf{R},
$$

where $f_{0}(\mathbf{R})$ is the statistical distribution function of the end-toend vector. Also, the mean primitive chain length $\langle L\rangle$ is given as

$$
\langle L\rangle=\int \frac{\left|\mathbf{F} \cdot \mathbf{u}_{0}\right|}{4 \pi} L_{0} \mathrm{~d}^{2} \mathbf{u}_{0},
$$

where $\mathbf{u}_{0}$ is the initial tangent vector of the primitive chain in the reference configuration. Then the ratio of the tube diameter between the current configuration and the reference configuration is expressed as

$$
\frac{\left\langle a_{n}\right\rangle}{a_{0}}=\frac{\int|\mathbf{F} \cdot \mathbf{R}|^{2} f_{0}(\mathbf{R}) d^{3} \mathbf{R}}{\left\langle\mathbf{R}_{\mathrm{ee}}^{2}\right\rangle_{0} \int \frac{\left|\mathbf{F} \cdot \mathbf{u}_{0}\right|}{4 \pi} \mathrm{d}^{2} \mathbf{u}_{0}} .
$$

Here, $a_{0}$ is the tube diameter in the reference configuration. Therefore, the deformation-dependent viscosity is given as 


$$
\eta_{\mathrm{B}}=\frac{\eta}{\alpha(\mathbf{F})^{2}}
$$

where $\eta=\frac{\zeta n_{\mathrm{B}}^{4} b_{0}^{4}}{12 a_{0}^{2}}\left(\frac{\tau^{\mathrm{R}}}{\tau^{\mathrm{e}}}\right)^{1 / 2}$ is the viscosity of the elastomer in the reference configuration and $\alpha(\mathbf{F})=\frac{\int|\mathbf{F} \cdot \mathbf{R}|^{2} f_{0}(\mathbf{R}) d^{3} \mathbf{R}}{\left\langle\mathbf{R}_{\mathrm{ee}}^{2}\right\rangle_{0} \int \frac{\left|\mathbf{F} \cdot \mathbf{u}_{0}\right|}{4 \pi} \mathrm{d}^{2} \mathbf{u}_{0}}$ indicates the deformation-dependency.

\section{NUMERICAL SIMULATIONS}

To test the modeling capacity of the theoretical framework developed above, the material models (the strain energy densities $W_{\mathrm{A}}$ and $W_{\mathrm{B}}$ in (1)) need to be prescribed first. In this work, the Gent strain energy density function [3] is chosen as $W_{\mathrm{A}}$, i.e.,

$$
W_{\mathrm{A}}=-\frac{G^{\mathrm{EQ}} J_{\lim }}{2} \ln \left(\frac{J_{\lim }-\lambda_{1}^{2}-\lambda_{2}^{2}-\lambda_{3}^{2}}{J_{\lim }}\right) .
$$

Here, $G^{\mathrm{EQ}}$ is the shear modulus of polymer network A and $J_{\mathrm{lim}}$ is the material extensibility parameter. Also, $\lambda_{1}, \lambda_{2}$ and $\lambda_{3}$ are the stretch ratios in three directions. We assume that $W_{\mathrm{B}}$ takes the same form as $W_{\mathrm{A}}$, which gives

$$
W_{\mathrm{B}}=-\frac{G^{\mathrm{NEQ}} J_{\lim }}{2} \ln \left[\frac{J_{\lim }-\left(\lambda_{1}^{\mathrm{e}}\right)^{2}-\left(\lambda_{2}^{\mathrm{e}}\right)^{2}-\left(\lambda_{3}^{\mathrm{e}}\right)^{2}}{J_{\lim }}\right],
$$

where $G^{\mathrm{NEQ}}$ is the shear modulus of polymer network B.

Considering uniaxial tension, $\lambda_{1}=\lambda, \lambda_{2}=\lambda_{3}=1 / \sqrt{\lambda}$, $\lambda_{1}^{\mathrm{e}}=\lambda^{\mathrm{e}}$ and $\lambda_{2}^{\mathrm{e}}=\lambda_{3}^{\mathrm{e}}=1 / \sqrt{\lambda^{\mathrm{e}}}$. From (2) and (3), the dimensionless nominal stress is obtained as

$$
\frac{P_{11}}{G}=\frac{\chi J_{\lim }\left(\lambda-\lambda^{-2}\right)}{J_{\lim }-2 \lambda^{-1}-\lambda^{2}+3}+\frac{(1-\chi) J_{\lim }\left[\lambda\left(\lambda^{\mathrm{i}}\right)^{-2}-\lambda^{-2} \lambda^{\mathrm{i}}\right]}{J_{\lim }-2 \lambda^{-1} \lambda^{\mathrm{i}}-\lambda^{2}\left(\lambda^{\mathrm{i}}\right)^{-2}+3}
$$

where $G=G^{\mathrm{EQ}}+G^{\mathrm{NEQ}}$ and $\chi=G^{\mathrm{EQ}} / G$. Also, the thermodynamic evolution equation gives that

$$
\frac{d \lambda^{\mathrm{i}}}{d t}=\frac{J_{\lim } \lambda^{\mathrm{i}} \alpha(\mathbf{F})^{2}}{3 \tau\left[J_{\lim }-2 \lambda^{\mathrm{i}} \lambda^{-1}-\lambda^{2}\left(\lambda^{\mathrm{i}}\right)^{-2}+3\right]}\left[\lambda^{2}\left(\lambda^{\mathrm{i}}\right)^{-2}-\lambda^{\mathrm{i}} \lambda^{-1}\right] .
$$

Here, $\tau=\eta / G^{\mathrm{NEQ}}$ is the relaxation time.

Fig. 2 depicts the dimensionless nominal stress $P_{11} / G$ as a function of the stretch ratio of the elastomer at four different stretching rates, i.e., $|\dot{\lambda}|=0.01 / \mathrm{s},|\dot{\lambda}|=0.03 / \mathrm{s},|\dot{\lambda}|=0.05 / \mathrm{s}$ and $|\dot{\lambda}|=1 / \mathrm{s}$. It can be seen that the loading and unloading curves overlap at large stretch ratios when the stretching rates are relatively low $(0.01 / \mathrm{s} \sim 0.05 / \mathrm{s})$. According to (13), the viscosity $\eta_{\mathrm{B}}$ is inversely proportional to the second power of
$\alpha(\mathbf{F})$. Therefore, the viscosity of the elastomer is exceptionally low at large stretch ratios, which leads to much faster relaxation of the polymer chains, thus causing the loading and unloading curves to overlap. However, when the stretching rate is high (e.g., 1/s), a wider gap between the loading and unloading curves appears since the elastomer has less time to relax during the loading path. To further examine the effect of the nonlinear viscosity on the stress response of the elastomer, Fig. 3 depicts the dimensionless nominal stress $P_{11} / G$ obtained with $\eta_{\mathrm{B}}$ and $\eta$, respectively. A higher degree of strain-softening behavior is observed from the nonlinear viscosity case. Moreover, as the stretching rate increases, the stress difference between two cases becomes larger. Therefore, it is essential to consider the nonlinear behavior of the material viscosity in analyzing the stress response of elastomers, or it may lead to significant error in calculation. In Fig. 2 and Fig. 3, the material parameters are selected as $\chi=0.5, J_{\lim }=110$ and $\tau=500 \mathrm{~s}$ [12-13].

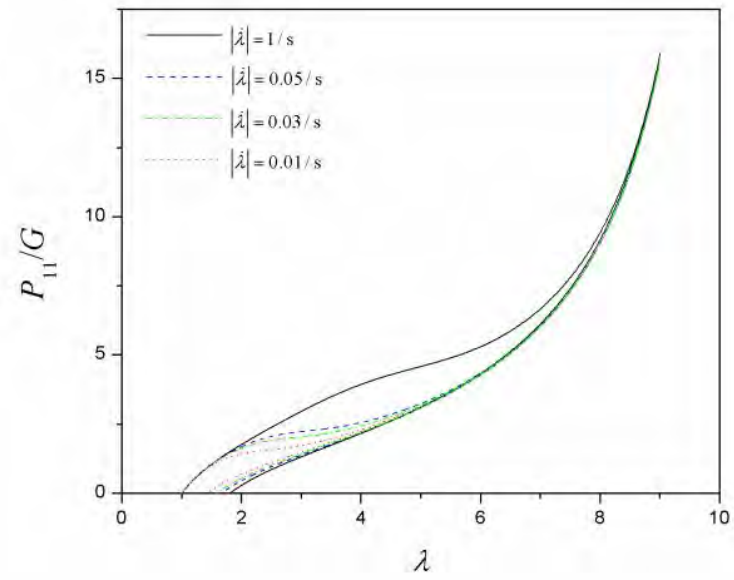

Figure 2. Stress response of the elastomer at different stretching rates.

(a)

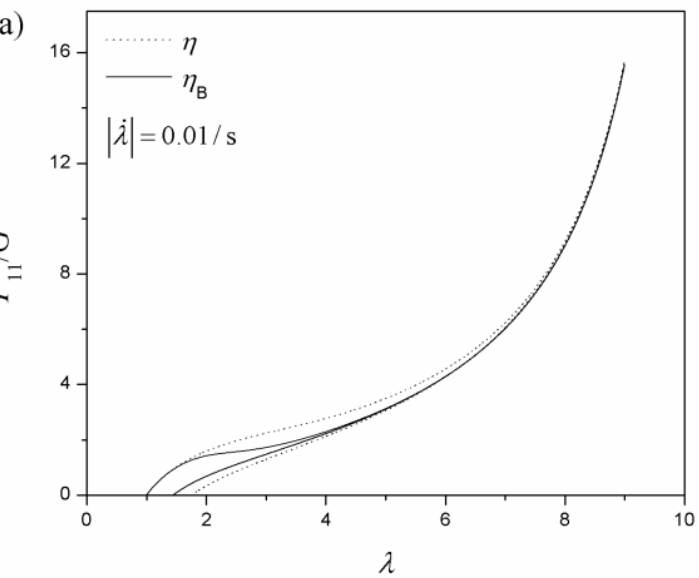



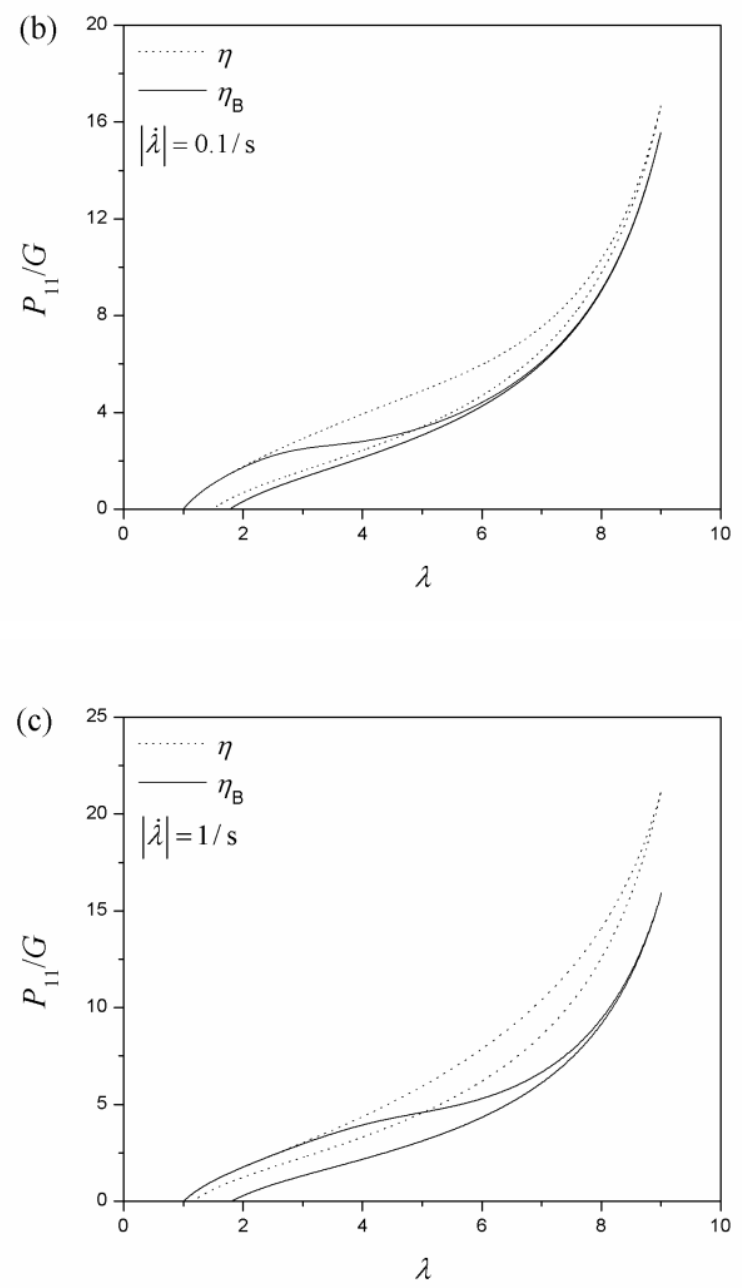

Figure 3. Comparison of the stress response between linear and nonlinear viscosity. (a) $|\dot{\lambda}|=0.01 / \mathrm{s}$, (b) $|\dot{\lambda}|=0.1 / \mathrm{s}$ and (c) $|\dot{\lambda}|=1 / \mathrm{s}$.

\section{CONCLUSION}

Based on the theory of polymer dynamics and finitedeformation viscoelasticity, a micro-macro constitutive model is developed to investigate the stress response and relaxation of elastomers under large deformation. For the developed model, all the material parameters have a microscopic foundation or physical meanings. Moreover, incorporating the nonlinear material viscosity into the continuum mechanics framework for finite-deformation viscoelasticity, the developed model can adopt most of strain energy density functions for hyperelastic solids and thermodynamics evolution laws of viscoelastic materials. From our simulation results, it is found that the developed model can better capture the strain-softening behavior of elastomers, which could be explained by the nonlinear viscosity of the material. In summary, the developed modeling framework is anticipated to provide significant guidelines for studying the viscoelastic behavior of elastomers.

\section{ACKNOWLEDGMENT}

This work is supported by Natural Sciences and Engineering Research Council of Canada (NSERC).

\section{REFERENCES}

[1] D. Rus, and M. T. Tolly, "Design, fabrication and control of soft robots," Nature, vol. 521, no. 7553, pp. 467-475, May 2015. doi: $10.1038 /$ nature14543.

[2] M. C. Boyce, and E. M. Arruda, "Constitutive models of rubber elasticity: a review," Rubber Chem. Technol., vol. 73, no. 3, pp. 504-523, Jul. 2000. doi: 10.5254/1.3547602.

[3] A. N. Gent, "A new constitutive relation for rubber," Rubber Chem. Technol., vol. 69, no. 1, pp. 59-61, Mar. 1996. doi: 10.5254/1.3538357.

[4] E. M. Arruda, and M. C. Boyce, "A three-dimensional constitutive model for the large stretch behavior of rubber elastic materials," $J$. Mech. Phys. Solids, vol. 41, no. 2, pp. 389-412, Feb. 1993. doi: 10.1016/0022-5096(93)90013-6.

[5] J. D. Davidson, N. C. Goulbourne, "A nonaffine network model for elastomers undergoing finite deformations," J. Mech. Phys. Solids, vol. 61, no. 8, pp. 1784-1797, Aug. 2013. doi: 10.1016/j.jmps.2013.03.009.

[6] J. Lubliner, "A model of rubber viscoelasticity," Mech. Res. Commun., vol. 12, no. 2, pp. 93-99, Mar.-Apr. 1985. doi: 10.1016/0093-6413(85)90075-8.

[7] S. Reese, and S. Govindjee, "A theory of finite viscoelasticity and numerical aspects," Int. J. Solids Struct., vol. 35, no. 26-27, pp. 3455-3482, Sep. 1998. doi: 10.1016/S0020-7683(97)00217-5.

[8] M. Doi, and S. F. Edwards, The Theory of Polymer Dynamics, Oxford: Clarendon, 1986.

[9] C. Miehe, and S. Göktepe, "A micro-macro approach to rubber-like material. Part II: the micro-sphere model of finite rubber viscoelasticity," J. Mech. Phys. Solids, vol. 53, no. 10, pp. 2231-2258, Oct. 2005. doi: 10.1016/j.jmps.2005.04.006.

[10] C. Linder, M. Tkachuk, and C. Miehe, "A micromechanically motivated diffusion-based transient network model and its incorporation into finite rubber viscoelasticity," J. Mech. Phys. Solids, vol. 59, no. 10, pp. 2134-2156, Oct. 2011. doi: 10.1016/j.jmps.2011.05.005.

[11] F. Sidoroff, "Un modèle viscoélastique non linéaire avec configuration intermédiaire," J. Méc., vol. 13, no. 4, pp. 679-713, 1974.

[12] W. Hong, "Modeling viscoelastic dielectrics," J. Mech. Phys. Solids, vol. 59, no. 3, pp. 637-650, Mar. 2011. doi: 10.1016/j.jmps.2010.12.003.

[13] J. Zhou, L. Jiang, and R. E. Khayat, "A micro-macro constitutive model for finite-deformation viscoelasticity of elastomers with nonlinear viscosity," J. Mech. Phys. Solids, vol. 110, pp. 137-154, Jan. 2018. doi: 10.1016/j.jmps.2017.09.016.

[14] Y. Li, S. Tang, M. Kroger, and W. K. Liu, "Molecular simulation guided constitutive modeling on finite strain viscoelasticity of elastomers," $J$. Mech. Phys. Solids, vol. 88, pp. 204-226, Mar. 2016. doi: 10.1016/j.jmps.2015.12.007. 


\section{Strategies for Improving Electrospinability of Polysulfone}

\author{
Iman Azarian Borojeni, Biao Zhou ${ }^{1}$ \\ Department of Mechanical, Automotive, and Materials \\ Engineering \\ University of Windsor, Windsor, Canada
}

\author{
S. Holger Eichhorn \\ Department of Chemistry and Biochemistry \\ University of Windsor, Windsor, Canada
}

\begin{abstract}
The overall objective of this study is the fabrication of defect-free electrospun mats of polysulfone (PSU) for applications as a porous support for proton exchange membranes. Electrospinning of PSU from dimethylformamide (DMF) solution generated mats free of cast formation and over-wetting, but unspun droplets were frequently observed in the mats because the electrospun jet was unstable. A stable jet and a large reduction of beads in the electrospun mats were obtained with a mixture of 20 vol.\% acetone in DMF. Subsequent optomization of the flow rate to $1.5 \mathrm{~mL} / \mathrm{h}$ and the applied voltage to $14 \mathrm{kV}$ generated electrospun mats free of any beads based on SEM analysis.
\end{abstract}

Keywords-component; Electrospinning; polysulfone (PSU); Dimethylformamide (DMF); Proton Exchange Membrane Fuel Cell (PEMFC)

\section{INTRODUCTION}

Proton Proton Exchange Membrane Fuel Cell (PEMFC) is one of the proposed effective methods to improve the energy conversion in vehicles due to their high efficiency and no hazardous emission [1]. However, there are still several barriers, such as high production cost and low durability, that need to be addressed for commercial development. Several deficiencies are rooted in the perfomance of the membranes PEMFCs, which is why the improvement of these membranes is a present focus of fuel cell related research [2].

The most broadly applied membrane materials are perflourosulfonic acids (PFSA), which show high proton conductivity at a fully hydrated state. However, they suffer from a high price and low dimensional stability during the starting up and shutting down of a PEMFC when the amount of humidity is varied widely $[2,3]$. To overcome this issue, the commercial membranes have relatively high thickness (50$175 \mu \mathrm{m})$ to show enough strength to overcome the changes in dimensions. However, thicker membranes negatively impact the performance of the fuel cell due to their decreased proton conductance [3].

${ }^{1}$ Corresponding Author and Principal Investigator: Dr. Biao Zhou, bzhou@uwindsor.ca, 1-519-253-3000 ext. 2630
One of the proposed methods for reducing the thickness of the membrane without decreasing the durability of the fuel cell is to build a porous inert reinforcement into the membrane to limit its expansion and contraction when its water content is changed. The inforcement porpous support would also improve the mechanical properties of the membrane and is expected to reduce the overall cost of production. The overall cost is likely reduced despite extra processing steps because the porous enforcment material is much less expensive than PFSA and the overall amount of required PFSA is significantly reduced [2].

Among different methods for the fabrication of porous support layers, electrospinning attracts great attention due to its ability to fabricate submicron fibers mats with different morphologies [4]. Typically, a high voltage power supply (usually in the $\mathrm{kV}$ range) connects to a collector and the conductive needle of a syringe. The syringe is filled with a viscous liquid (a polymer solution or a molten polymer) to charge it. At a certain critical voltage, the repulsive force of the charged liquid offsets its surface tension and the liquid ejects from the nozzle to the collector. If the polymer solution has enough entanglement, the ejected solution forms fibers and the process is called electrospinning; otherwise, the ejected solution turns into small droplets and the process is called electrospraying $[5,6]$. Simplicity, ability to control morphology at different levels makes this method attractive to researchers [4]. Moreover, the uniaxial alignment of polymer chain fibers can improve the mechanical properties of the membrane [7]. Finally, the low packing density of generated electrospun mats and their interconnected pore structure make them excellent substrates for filling with other materials to fabricate a dense membrane. Several polymers, such as polyvinylidene fluoride (PVDF), polyvinyl alcohol (PVA), Polystyrene (PS), and polysulfone (PSU) have been successfully electrospun as the porous support for proton exchange membranes [7]. However, more investigations are required to improve the electrospinning condition of these polymers for their application in a proton exchange membrane. 
Reported here is the electrospinnig behaviour of PSU in dimethylformamide-acetone mixtures and the effect of the electrospinning parameters on the morphology of the produced mats. Specifically, adjutments of the acetone content, flow rate, and voltage successfully suppressed the formation of macroscopic and microscopic defects observed for not optimized spinning conditions..

\section{EXPERIMENTAL PROCEDURE}

N,N-Dimethylformamide (DMF, anhydrous, 99.8\%) and Polysulfone (PSU, average $\mathrm{Mn} \approx 22,000$ ) were purchased from Sigma-Aldrich. Acetone (ACS grade) was also provided from VWR. Solvents with different volume ratios of acetone in DMF $(0 \%, 20 \%$, and $40 \%)$ were prepared and used for the preparation of PSU solutions at a concentration of $20 \mathrm{wt} \%$. The mixtures were stirred via a magnet stirrer for 6 hours to generate a homogeneous solution at room temperature. The solutions were kept overnight at room temperature to ensure no phase separation occurs. Only solutions that show no macroscopic phase separation were selected for electrospinning test.

Before electrospinning, the solution was stirred for 2 hours to homogenise again. A $5 \mathrm{cc}$ plastic syringe was filled bubble free and connected to a 22 gauge stainless steel blunt needle. The syringe was loaded onto a syringe pump (KDS, Legato $200)$ to control the flow rate $(0.2$ to $1.5 \mathrm{~mL} / \mathrm{h})$. To charge up the solution for electropsinning, a power supply (ES50, Gamma high voltage research) was connected to the needle and a grounded static steel plate covered by an aluminum foil that served as the current collector. The current collector was placed 10 to $20 \mathrm{~cm}$ from the needle tip and the applied voltage was varied between $10 \mathrm{kV}$ and $30 \mathrm{kV}$. The applied voltage was usually selected based on the critical voltage for ejection of the fluid from the Taylor cone (1 to $2 \mathrm{kV}$ higher than the critical voltage), but in a few cases voltages $7 \mathrm{kV}$ higher than the critical voltage were also examined to stabilize the process and avoid the formation of unspun droplets.

The electrospun samples were dried at $65{ }^{\circ} \mathrm{C}$ overnight to remove any possible residual solvent and then coated with gold by sputtering for the observation by SEM (Field Emission Scanning Electron Microscope Quanta FEG 200).

\section{RESULT AND DISCUSSION}

Before In our first experiments, pure DMF was used as the solvent for PSU. PSU showed appropriate solubility in DMF and clear solutions were obtained easily by stirring at room temperature. Electrospiining of these solutions was also straightforward and no cracking, cast formation, or peeling off was observed in the electrospun mats. However, the electrospun jet was not stable and unspun droplets were frequently observed during the process, which led to the formation of dotted patterns in the electrospun mats (Fig. 1-a). Although the problem was supposed to be addressed by reducing the flow rate [8], it became worse in our hands (Fig. $1-b)$.
The microstructure of the electrospun mats also suffered from the formation of beads. Fig. 2 shows the microstructure of the fabricated mats when the distance between the needle's tip and the collector was 20 and $15 \mathrm{~cm}$, respectively. Although the number of beads can be reduced effectively by optimizing the tip collector distance (d) the formation of unspun droplets still persisted. To address this issue, DMF-acetone mixtures were applied as the solvent for PSU to reduce the surface tension of the solvent. If the surface tension is reduced, the beads problem can be addressed effectively and the formation of unspun droplets is reduced because the electrospun jet is more stable.

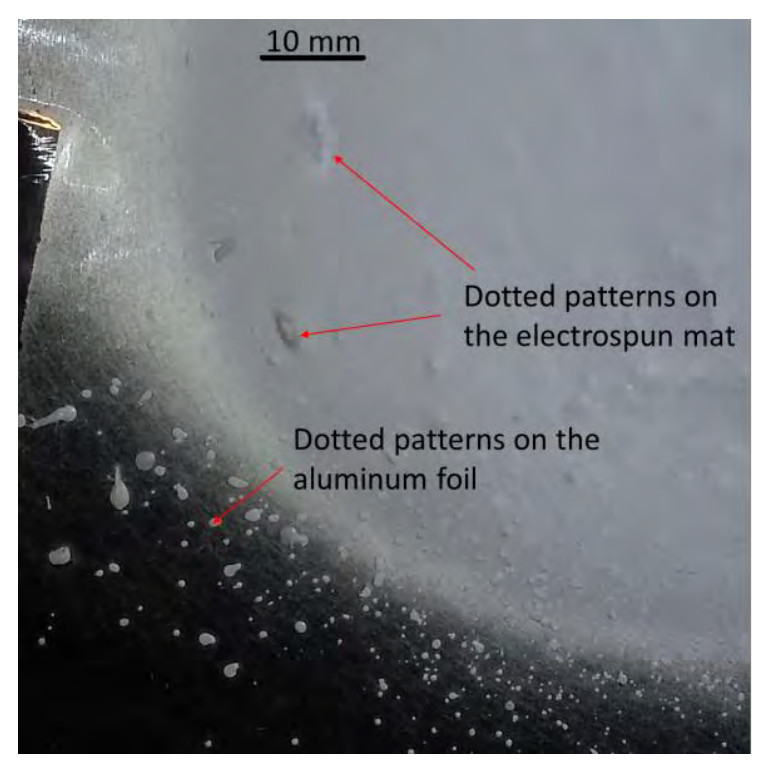

(a)

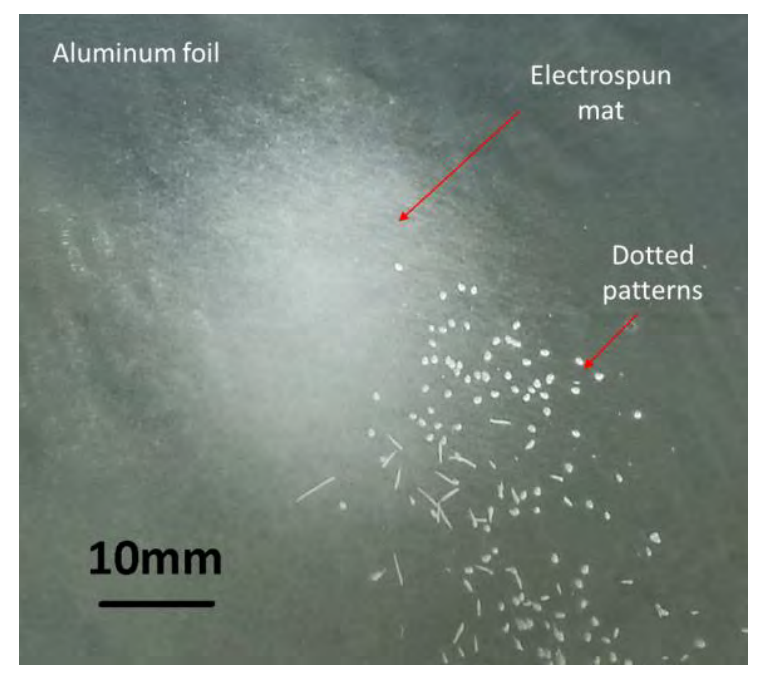

(b)

Figure 1. The PSU electrospun mat from DMF solution when the needle tip to collector distance is $15 \mathrm{~cm}$ : (a) flow rate $=0.5 \mathrm{~mL} / \mathrm{h}$ (b) flow rate $=0.2$ $\mathrm{mL} / \mathrm{h}$. 


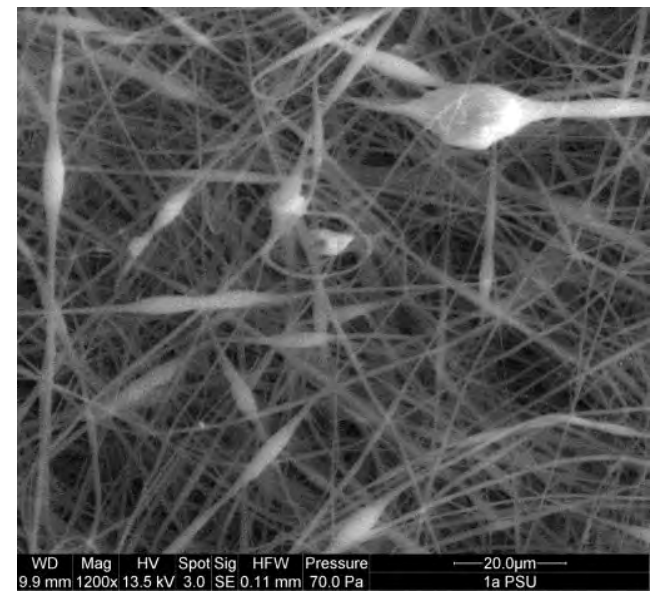

(a)

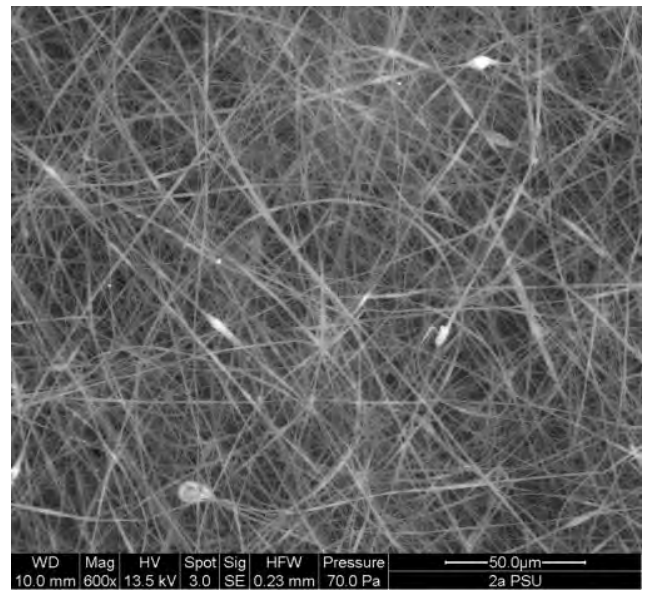

(b)

Figure 2. SEM images of the PSU electrospun fibers when DMF is applied as the solvent and the flow rate is $0.5 \mathrm{~mL} / \mathrm{h}$ : (a) $\mathrm{d}=20 \mathrm{~cm}$ (b) $\mathrm{d}=15 \mathrm{~cm}$.

PSU did not show proper solubility in 60\%DMF-40\% acetone (by Vol.) mixture. The solution was not clear and a macroscopic phase separation was observed after one day (a clear solution was on top and a milky solution was in bottom). By increasing the percentage of DMF to $80 \%$, the solubility improved remarkably. Although the prepared solution was milky again, no macroscopic phase separation was observed even within a week.

By replacing the solvent from pure DMF to a mixture of $80 \%$ DMF and 20\% acetone, some differences were observed in the electrospinning process. Firstly, the critical voltage for the Taylor cone was reduced significantly. The critical applied voltage for pure DMF was around $29 \mathrm{kV}$ when the needle tip collector distance was $15 \mathrm{~cm}$ while this value for a $4: 1$ DMF/acetone mixture was just $8 \mathrm{kV}$. Since in many mathematical models the critical voltage mostly depends on the surface tension (among other solution parameters such as viscosity and conductivity) [6], reduction of the surface tension by the addition of acetone seemed to play an important role in the reduction of the critical voltage.

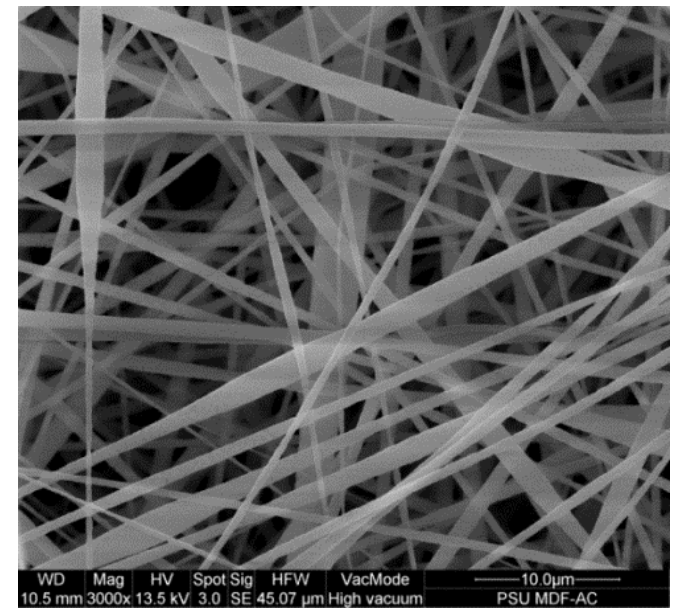

(a)

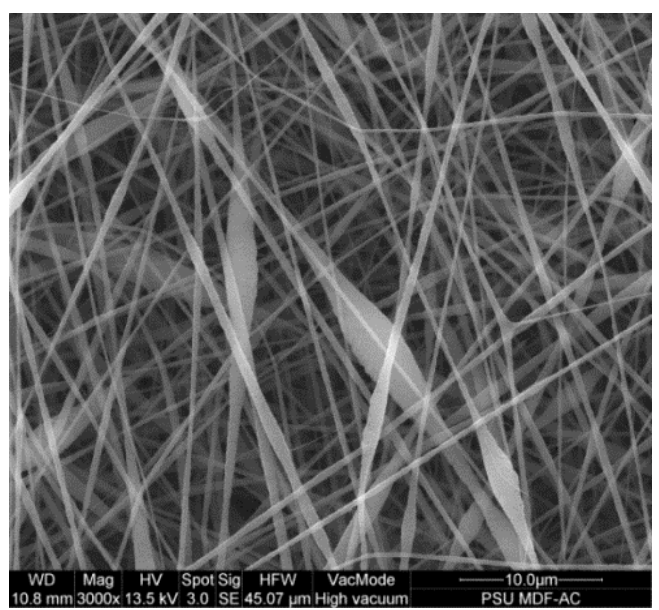

(b)

Figure 3. The SEM images of PSU electrospun fibers when $80 \%$ DMF$20 \%$ acetone was used as the solvent, the applied voltage was $10 \mathrm{kV}$ and $\mathrm{d}=15 \mathrm{~cm}$ : (a) flow rate $=1.2 \mathrm{~mL} / \mathrm{h}$, (b) flow rate $=0.2 \mathrm{~mL} / \mathrm{h}$.

Addition of acetone also affected the microstructure of the electrospun mats. The beads were almost removed in the microstructure due to reduction of the surface tension of the solution (Fig. 3-a). It should be mentioned that the driving force for the bead formation is the high surface energy of the electrspun fibers during the electrospinning process [9]. Therefore, reduction of the surface energy can address this problem significantly.

Additionally, the electrospun jet became more stable when acetone was added to the solution. It was reported that the jet become more stable when the surface tension of the solution was reduced [9]. The unspun droplets were still persistence, but the number of them was cut down effectively. To diminish this problem totally other electrospinning parameters should be tuned. Reduction of the flow rate is one of the effective strategies that has been recommended to address the formation of unspun droplets [8]. However, similar to the case of using pure DMF, reduction of the flow rate to $0.2 \mathrm{~mL} / \mathrm{h}$ was not only unable to improve the stability of the electrospun jet, but made 


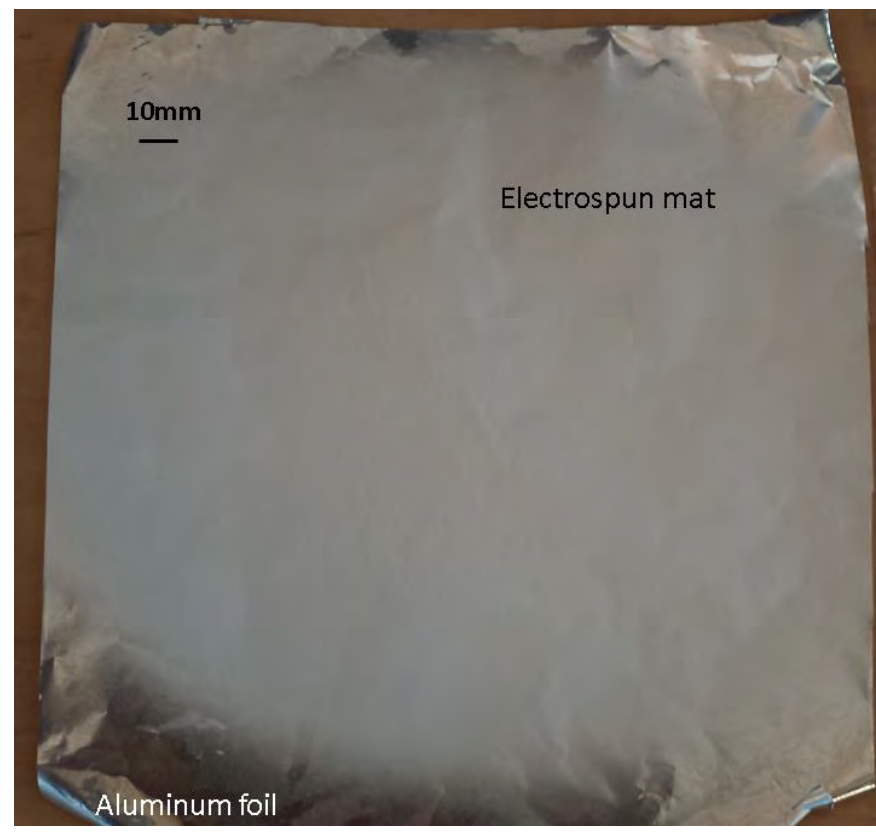

Figure 4. The PSU electrospun mat from $80 \%$ DMF-20\% acetone solvent when the applied voltage was $14 \mathrm{kV}$, flow rate was $1.5 \mathrm{~mL} / \mathrm{h}$ and $\mathrm{d}=15 \mathrm{~cm}$.

it less stable. This generated more unspun droplets and more beads appeared in the microstructures (Fig. 3-b).

Surprisingly, the higher flow rate $(1.5 \mathrm{~mL} / \mathrm{h})$ remarkably improved the stability of the jet. To achieve a fully stable jet and completely avoid the formation of unspun droplets, the applied voltage was increased to $14 \mathrm{kV}$. Only under this condition we were able to fully suppress the formation of unspun droplets and avoid the formation of dotted pattern of (Fig. 4). Fig. 5 illustrates the microstructures of the electrospun mats when the needle tip collector distance (d) was varied from 10 to $20 \mathrm{~cm}$ and the mentioned adjustment on the flow rate and the voltage were applied. The microstructures of the electrospun mats for $\mathrm{d}=15 \mathrm{~cm}$ or $20 \mathrm{~cm}$ were almost the same, although very few beads can be spotted for $\mathrm{d}=20 \mathrm{~cm}$. The beads were diminished when $\mathrm{d}=15 \mathrm{~cm}$. However, by reduction of $\mathrm{d}$ to $10 \mathrm{~cm}$, the surface of the fibers became rough and some particles like structure were formed, which had different structure from the electrospun fibers (Fig. 5-c and Fig. 6).

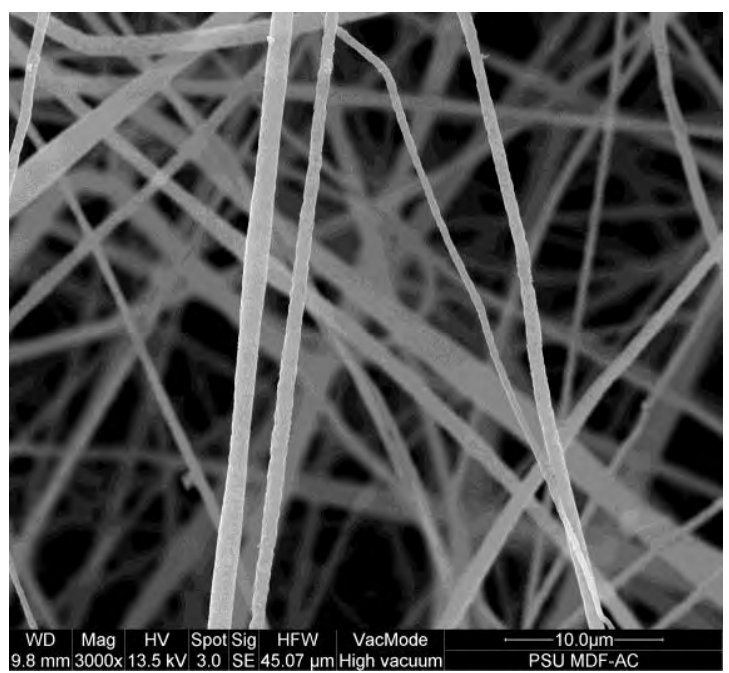

(a)

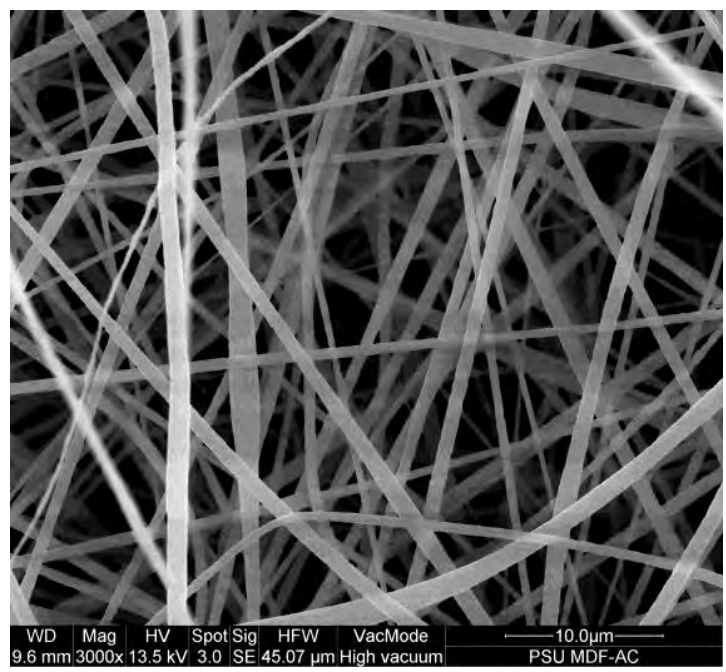

(b)

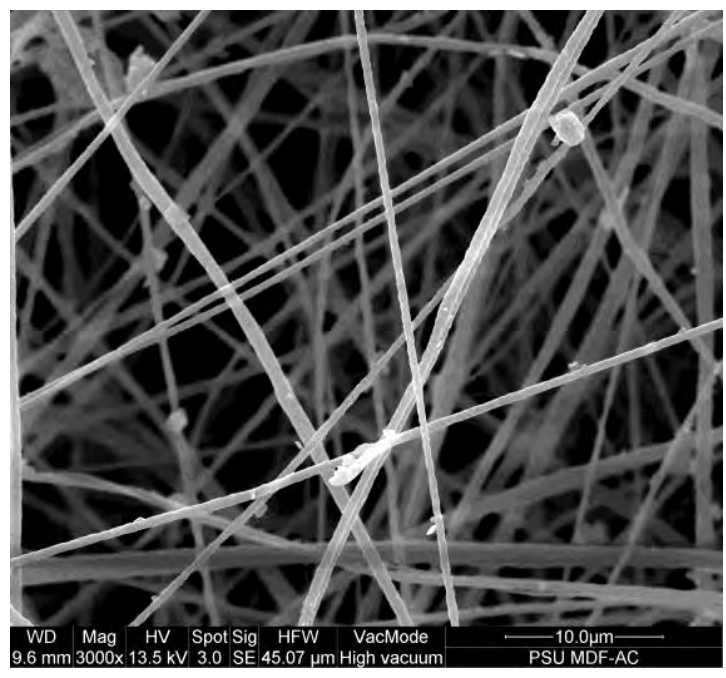

(c)

Figure 5. The SEM micrograph of the PSU electrospun fibers when $80 \%$ DMF-20\% acetone solvent when the applied voltage was $14 \mathrm{kV}$, flow rate was $1.5 \mathrm{~mL} / \mathrm{h}$ : (a) $\mathrm{d}=20 \mathrm{~cm}$, (b) $\mathrm{d}=15 \mathrm{~cm}$, , (c) $\mathrm{d}=10 \mathrm{~cm}$. 


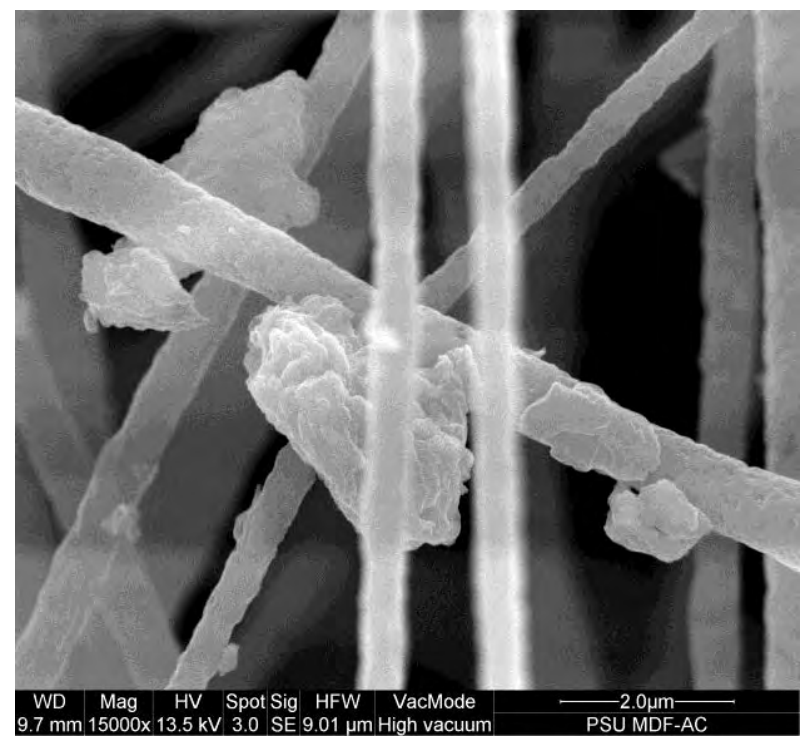

Figure 6 The SEM image of particle-like defect when $80 \%$ DMF- $20 \%$ acetone solvent when the applied voltage was $14 \mathrm{kV}$, flow rate was $1.5 \mathrm{~mL} / \mathrm{h}$ and $\mathrm{d}=10 \mathrm{~cm}$.

\section{SUMMARY}

We were not successful in the optimization of electrospinning parameters to generate defect-free mats with PSU solutions in DMF. This was mainly caused by an unstable jet that resulted in the formation of unspun droplets and a dotted pattern on the collector. Another manifestation of unsuitable spinning parameters was the formation of large numbers of beads in the mats. A stable jet and defect free electrospun mats were obtained with a solvent mixture of DMF/acetone 4:1 that has a drastically reduced surface tension. The collector distance, applied voltage, and flow rate were optimized to $15 \mathrm{~cm}, 1.5 \mathrm{~mL} / \mathrm{h}$, and $14 \mathrm{kV}$, respectively.

\section{ACKNOWLEDGMENT}

The authors are grateful for the support from the Natural Sciences and Engineering Research Council of Canada (NSERC) and the University of Windsor.

\section{REFERENCES}

[1] D. J. Kim, M. J. Jo, and S. Y. Nam, "A review of polymer-nanocomposite electrolyte membranes for fuel cell application," J. Ind. Eng. Chem., vol. 21, pp. 36-52, Jan. 2015. doi: 10.1016/j.jiec.2014.04.030.

[2] C. Houchins et al., "U.S. DOE progress towards developing low-cost, high performance, durable polymer electrolyte membranes for fuel cell applications," Membr., vol. 2, no. 4, pp. 855-878, Dec. 2012. doi: $10.3390 /$ membranes2040855.

[3] S. Banerjee, D. N. Prugh, and S. Frisk, "Advances in proton exchange membrane technology," ECS Trans., vol. 50, no. 2, pp. 887-895, 2013. doi: 10.1149/05002.0887ecst.

[4] Y. Si et al., "Electrospun nanofibers: Solving global issues," in Electrospun Nanofibers for Energy and Environmental Applications, B. Ding and J. Yu, Eds., 1st ed. Berlin, Germany: Springer, 2014, pp.3-38. doi: 10.1007/978-3-642-54160-5.
[5] A. Greiner and J. H. Wendroff, "Electrospinning: A fascinating method for the preparation of ultrathin fibers," ChemInform, vol. 38, no. 42, Sept. 2007. doi: 10.1002/chin.200742269.

[6] M. Yousefzadeh and S. Ramakrishna, "Modeling performance of electrospun nanofibers and nanofibrous assemblies" in Electrospun nanofibers, M. Afshari, Ed., 1st ed. Amsterdam, Netherlands: Elsevier, 2017, pp. 303-337.

[7] R. Sood et al., "Electrospun nanofibre composite polymer electrolyte fuel cell and electrolysis membranes," Nano Energy, vol. 26, pp. 729-745, Aug. 2016. doi: 10.1016/j.nanoen.2016.06.027.

[8] A. Haider, S. Haider, and I.-K. Kang, "A comprehensive review summarizing the effect of electrospinning parameters and potential applications of nanofibers in biomedical and biotechnology," Arabian. J. Chem., vol. 11, no. 8, pp. 1165-1188, Dec. 2018. doi: 10.1016/j.arabjc.2015.11.015.

[9] N. Bhardwaj and S. C. Kundu, "Electrospinning: A fascinating fiber fabrication technique," Biotechnol. Adv., vol. 28, no. 3, pp. 325-347, May-June 2010. doi: 10.1016/j.biotechadv.2010.01.004. 


\section{A Hybrid Manufacturing Approach for Low Volume High Temperature Thermoplastic / Thermoset Material Molds}

\author{
Hamed Kalami \\ Mechanical Automotive, and Materials Engineering \\ University of Windsor \\ Windsor, Canada \\ kalami@uwindsor.ca
}

\author{
R. Jill Urbanic \\ Mechanical Automotive, and Materials Engineering \\ University of Windsor \\ Windsor, Canada \\ jurbanic@uwindsor.ca
}

\begin{abstract}
The mold costs for low volume production molds can be expensive due to the mold material, the process planning time, and the fabrication costs. The focus of this research is to develop a methodology to fabricate molds for low volume production, where the production quantities vary between $1-200$ components. For this application, the cycle time is not an issue. Employing an additive manufacturing solution could reduce the required amount of materials and the processing planning costs, but there are cost, or technology feasibility issues related to constructing a mold directly from a CAD file. Consequently, a hybrid manufacturing approach is taken where an AM process (material extrusion based) is used to create a sacrificial pattern for specialty, low cost, interchangeable inserts manufactured using an epoxy (Aremco 805). An over molding case study is carried out using a high temperature molding material - Technomelt-PA 7846 black. The pattern, insert, coating, and mold fabrication is discussed, as well as the initial results. The initial material cost estimates to fabricate this over molding solution is approximately $\$ 140$ US. The durability of the RTV coating and the inserts needs to be determined to yield the final solution costs.
\end{abstract}

Keywords-mold fabrication; low volume; high temperature plastic materials; additive manufacturing

\section{INTRODUCTION}

The competitive landscape in the manufacturing domain is increasing in our global economy. The plastic mold making industry, which is a multi-million industry consisting of mainly small and medium size enterprises (502 establishments with 5,300 employees and $\$ 926$ million in shipments [1]), is actively pursuing opportunities to reduce tooling costs and processing time. The design solutions depend on the production volumes and planning horizons, and different mold materials and fabrication strategies are required for low volume, medium volume, and high-volume production. The focus of this research is on low volume production, where the production quantities vary between $1-200$ components, the cycle time is not an issue, and the target application is 'over molding' for specialty applications, or prototype low volume production.
Low volume production molds are utilized in many industrial sectors, and can be used to test product functionality, or employed to create sample components for assembly automation 'test and tune' applications for automotive high production systems. 'Soft' tooling or temporary molds are fabricated to produce a limited number of products. If the design is not optimal, another mold can be fabricated for the updated design. The over molding process involves the use of two separate materials to form one cohesive component. The most common type of over molding is insert molding. Insert over molding is an injection molding process where one material is molded "over" a secondary "substrate" material.

Low volume molds are less costly than medium or high volume molds, but the material, process planning, and fabrication costs may still be thousands of dollars. Additive Manufacturing (AM) approaches have the potential to reduce process planning time, and to reduce the both the time and manpower associated with the mold fabrication process.

There are several AM processes that have been developed: vat photopolymerization, binder jetting, material extrusion, powder bed fusion, directed energy deposition, material jetting, and sheet lamination. The AM process family is capable of producing complex geometries without any in-depth process planning, and are used in the automotive, aerospace domains, as well as in the medical and dental domains [2]. The common element to all these processes is that a three-dimensional (3D) part is developed from layering two dimensional cross sections successively to create the final solid. Undercuts, free form geometry, and blind features are manufactured "easily", especially compared to traditional machining processes. Presently, there are limited choices of material available for AM processes and anisotropic properties are exhibited due to the material bonding [3], [4]. There are compatibility issues with respect to the AM build process material with the molding material, and also limitations with respect to their cost effectiveness for 'lower' to intermediate production volume quantities as the product fabrication times may be long. Therefore, a rapid tooling design and fabrication strategy needs to be developed [5], which is the focus of this research. There are two solution paths being proposed: (i) employing an AM process to fabricate a mold (with the addition of a coating if 
there are material compatibility issues), and (ii) employing hybrid mold fabrication strategies.

The goal of this research is to develop solutions to fabricate a $200 \mathrm{~mm} \times 150 \mathrm{~mm} \times 50 \mathrm{~mm}$ core and cavity mold set that can produce up to 200 parts for $\leq \$ 1000$. In this study, the molding material is Technomelt-PA 7846 black which is a polyamide plastic. Its mechanical properties are shown in Table I. Technomelt is a high melting temperature polyamide (application temperature: 200 to $240{ }^{\circ} \mathrm{C}$ [6]), which limits the usable mold materials.

TABLE I. PROPERTIES OF TECHNOMELT-PA 7846 BLACK

\begin{tabular}{|l|l|}
\hline \multicolumn{1}{|c|}{ Mechanical property } & \multicolumn{1}{c|}{ Value } \\
\hline Density, $\mathrm{g} / \mathrm{cm}^{3}$ & 0.98 \\
\hline Melting Viscosity at $230{ }^{\circ} \mathrm{C}, \mathrm{mPas}$ & 3,000 \\
\hline Glass Transition, ${ }^{\circ} \mathrm{C}$ & -30 \\
\hline Working Temperature, ${ }^{\circ} \mathrm{C}$ & -40 to 130 \\
\hline Softening point, ${ }^{\circ} \mathrm{C}$ & 170 to 180 \\
\hline
\end{tabular}

\section{LITERATURE REVIEW}

Rodet et al. used the Stereoligthography (SLA) [7] process to fabricate molds using the DSM Somos 7110 resin and SL 7510 materials, and SLA-250 and SLA-3500 machines. Variable layer thicknesses were explored and correlated to a surface roughness. The SLA tools had limitations due to their low thermal and mechanical properties $\left(60-70^{\circ} \mathrm{C}\right.$ - little strength left). Hopkinson et al. utilized an epoxy resin SL-5170 with a SLA-500 machine. The operating conditions were at a higher temperature $\left(185^{\circ} \mathrm{C}\right)$. It was necessary to use different processing parameters for the molding compared to an aluminum mold solution, and there was difficulty ejecting the components (polypropylene material). Kovács et al. analyzed the warpage issues related to mold thermal conductivity, and explored using an epoxy resin filled with metal powder to increase stiffness and heat conductivity [8]. The SLA process family was not feasible for directly fabricating a mold set for components to be molded with the Technomelt-PA 7846 black material at this time.

The binder jetting process is an additive manufacturing process that fabricates components and assemblies by layering powered material and applying a binder where a 'solid interior' should be. The binder jetting family of technologies is capable of printing variety of powder materials, which includes polymers, metals, ceramics, sand, and plasters. However, the raw material costs for metals [9] are high compared to the raw stock costs used for machining; consequently, this process may be more suited to a medium volume production application. This is also true for the powder bed fusion processes.

The material extrusion family for AM processes consists of placing individual beads side by side to build up a layer. A contour boundary curve is extruded, and a raster fill strategy is used to fill the appropriate interior regions. The material is extruded through a nozzle. Typically, the build material is a thermoplastic such as Acrylonitrile Butadiene Styrene (ABS) or Polylactic Acid (PLA), although a polycarbonate can be employed. Both this process and the readily available material selections are problematic. Fabrication issues exist with the surface finish, anisotropic mechanical properties, and voids within the tool paths [10], but these issues are secondary. The glass transition temperature for ABS is approximately $105^{\circ} \mathrm{C}$, and the melting point for PLA 150 to $160{ }^{\circ} \mathrm{C}$. Polycarbonate has a glass transition temperature at approximately $147^{\circ} \mathrm{C}$. The softening point for the Technomelt-PA 7846 black is 170 to $180^{\circ}$. Thus, the material extrusion processes do not lend themselves to a high temperature mold fabrication application.

Consequently, directly printing a mold set from a Computer Aided Design (CAD) model is not feasible due to cost issues (binder jetting and powder bed fusion), or due to the available build materials (SLA and material extrusion). Therefore, a 'rapid tooling' hybrid mold manufacturing approach is to be explored.

S. Ma et al. investigated the feasibility of making reinforced epoxy molds. The metal, ceramic and mineral powders were mixed with epoxy to increase its mechanical properties. Different ratios of the powders were studied to find the best mixture to optimize mechanical and thermal properties. $20 \%$ of alumina powder found to be the most suitable additive [11]. T. Tabi et al compared thermal, mechanical and thermomechanical properties of injection molding of PLA material into epoxy-based PolyJet and conventional steel (P20) mold. They found that it is possible to mimic the thermomechanical properties of nucleated PLA which is injected into hot steel mold by injecting it into an epoxy-based PolyJet mold [12]. Therefore, using a metallic-resin mix for controlling the heat transfer and providing structural integrity is a valid solution approach for a mold, and is a focus of the solution presented here.

\section{METHODOLOGY}

For the hybrid manufacturing strategy, a disposable pattern for the core and cavity mold components will be manufactured using an AM process (rapid tooling), and the balance of the components are machined or cast to create permanent and interchangeable components. Two hybrid options are explored: (i) create a shell of each core and cavity mold surfaces, and back fill the shell with a filler material, and (ii) create a pattern, and use the pattern to create an interchangeable insert with a suitable resin material. For this paper, option 2 is explained. The process flow is presented in Fig. 1.

\section{A. Mold Design}

The mold base consists of two aluminum blocks sized to accommodate a variety of inserts. There is a $35 \mathrm{~mm}$ border around the machined rectangular cavities that are to be used for the mold inserts. Fig. 2 shows the mold base blocks and its sections, where: the A features ( 2 per side) are entrance holes specifically designed for the epoxy filling operation; the B features (4 per side) are to allow the users to quickly interchange epoxy inserts for new production runs; the $\mathrm{C}$ feature is the insert cavity, which includes a $30^{\circ}$ draft angle and $15 \mathrm{~mm}$ corner fillet radii for ease of interchangeably; and the D features ( 2 per side) are used to align the core and cavity blocks. These D features are also used to align the match plates to the mold set. The ejector features are threaded to fix the insert into position, and work as an ejector mechanism that pushes the used insert out of mold base cavities. The epoxy 
insert contains nuts for stable location. To reduce bonding of the epoxy material to the aluminum block, an RTV silicone material (Mold Star ${ }^{\circledR} 30$ [13]) is applied to the cavity surface before pouring the epoxy.

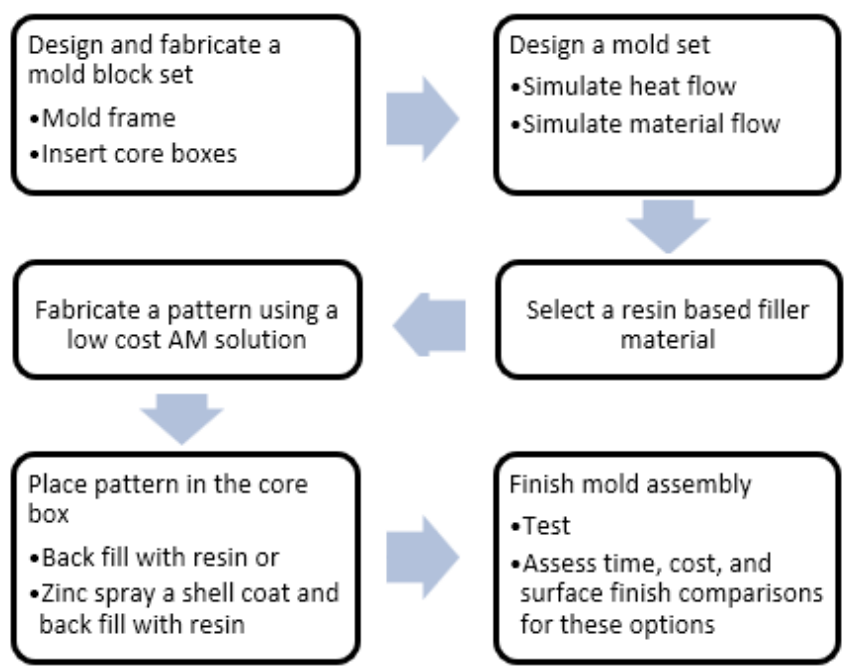

Figure 1. Hybrid mold design process flow.

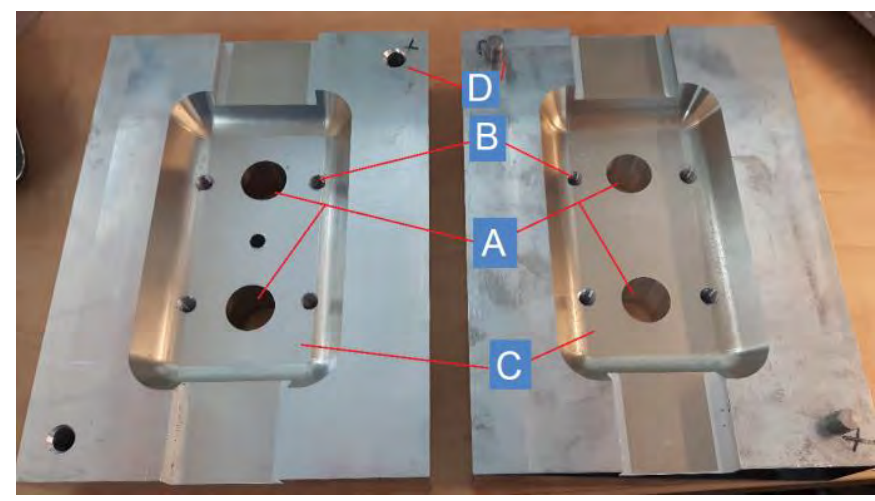

Figure 2. The mold bases- (A) the holes that epoxy is poured into the epoxy cavities (B) the ejector bolt of the epoxy block (C) epoxy filled cavities (D) hole-pin locators.

\section{B. Mold Making Process}

To fabricate the molding cavities, a pattern is needed. As the pattern may have shape complexity, but does not need to have strength, low cost additive manufacturing processes and materials are ideal for this 'rapid tooling' application. Pattern design characteristics will vary depending on the final product requirements. The parting line and features will be unique for a general product as compared to an over molding solution. Once the sample is divided from the parting line, each segment is attached to each side of a match plate. The locating pins (feature D) of the mold base align pattern, the match plate, and the mold bases. Due to space constraints, the analyses for the various epoxy materials are not included. However, from the experimental activities, is was found that the Aremco 805 (Table II) had the low shrinkage, viscosity, mechanical properties at room temperature and $200^{\circ} \mathrm{C}$, the temperature resistance, and the desired heat transfer characteristics.
TABLE II. PROPERTIES OF AREMCO 805 EPOXY [14]

\begin{tabular}{|l|l|}
\hline \multicolumn{1}{|c|}{ Mechanical property } & \multicolumn{1}{c|}{ Value } \\
\hline Thermal Conductivity $(\mathrm{W} / \mathrm{m} 2 . \mathrm{K})$ & 70.98 \\
\hline Tensile shear $(\mathrm{Pa})$ & $1.2 \mathrm{e}+7$ \\
\hline Flexural strengths $(\mathrm{Pa})$ & $1.06 \mathrm{e}+8$ \\
\hline Linear shrinkage $(\mathrm{m} / \mathrm{m})$ & 0.003 \\
\hline Shore D Hardness & 87 \\
\hline
\end{tabular}

In Fig. 3 (a) the build layers for the pattern are shown as visualized in the Insight ${ }^{\circledR}$ processing planning software (used for fused deposition modelling machines [15], in Fig. 3 (b) the original built pattern and an RTV coated pattern show the different surface textures, and in Fig. 3 (c), an over molding pattern is displayed, where the pattern includes representative over molding elements, the over molded region, and the match plate. RTV silicone rubber is used to coat the pattern to smooth the surface and to provide an easy part release. Surface roughness is an issue with the AM process family and researchers engaged in applying AM solutions for mold manufacturing have had ejection issues connected with the build surface finish. Post processing operations are typically required if a smooth surface is desired. Here, a material extrusion process and $\mathrm{ABS}$ material is employed to create the pattern, and in lieu of chemical smoothing or finish sanding, the RTV is employed to smooth the surface and be a releasant.

The patterns were easily removed from the epoxy. The final inserts are placed in the mold halves, as shown in Fig. 4. The over molded components have pockets for their placement in the insert. Additional end seals are also fabricated from the AM pattern for flash control.

The interior cavity surfaces are smooth. The process planning for the pattern was less than 1 minute, the assembly time for the pattern and match plate was approximately $1 / 2$ hour, and the cure time for the epoxy and the silicone was approximately $26 \mathrm{hrs}$. The time where an employee directly interacts with the fabrication process is short, and the required skill level for this insert solution is very low.

\section{Injection molding analysis}

The injection parameters need to be determined for the injection molding operation. Here, Autodesk Mold Flow Advisor 2017 is applied to determine the fill time, and the injection pressure using the input data provided in Table III.

TABLE III. MOLD FLOW SIMULATION INPUT DATA

\begin{tabular}{|l|l|}
\hline \multicolumn{1}{|c|}{ Injection property } & \multicolumn{1}{c|}{ Value } \\
\hline Injection Temperature $\left({ }^{\circ} \mathrm{C}\right)$ & 225 \\
\hline Mold Temperature $\left({ }^{\circ} \mathrm{C}\right)$ & 40 \\
\hline Gate Diameter $(\mathrm{mm})$ & 3 \\
\hline Maximum machine injection pressure $(\mathrm{MPa})$ & 1 \\
\hline
\end{tabular}

As shown in Fig. 4, the material injection gate is centrally located; consequently, the maximum fill time is 0.8 seconds along the longitudinal axis, but there are variants in the fill time, as shown in Fig. 5a. The temperature contours and cooling times are dependent on the system configuration, and are not presented here. The maximum injection pressure is at 
the gateway, and $0.11 \mathrm{MPa}$. The average injection pressure is very low and is almost homogenized (Fig. 5b).

(a)

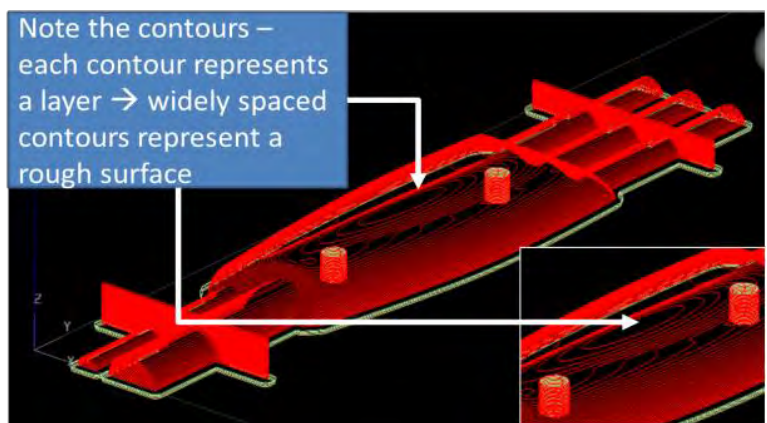

(b)
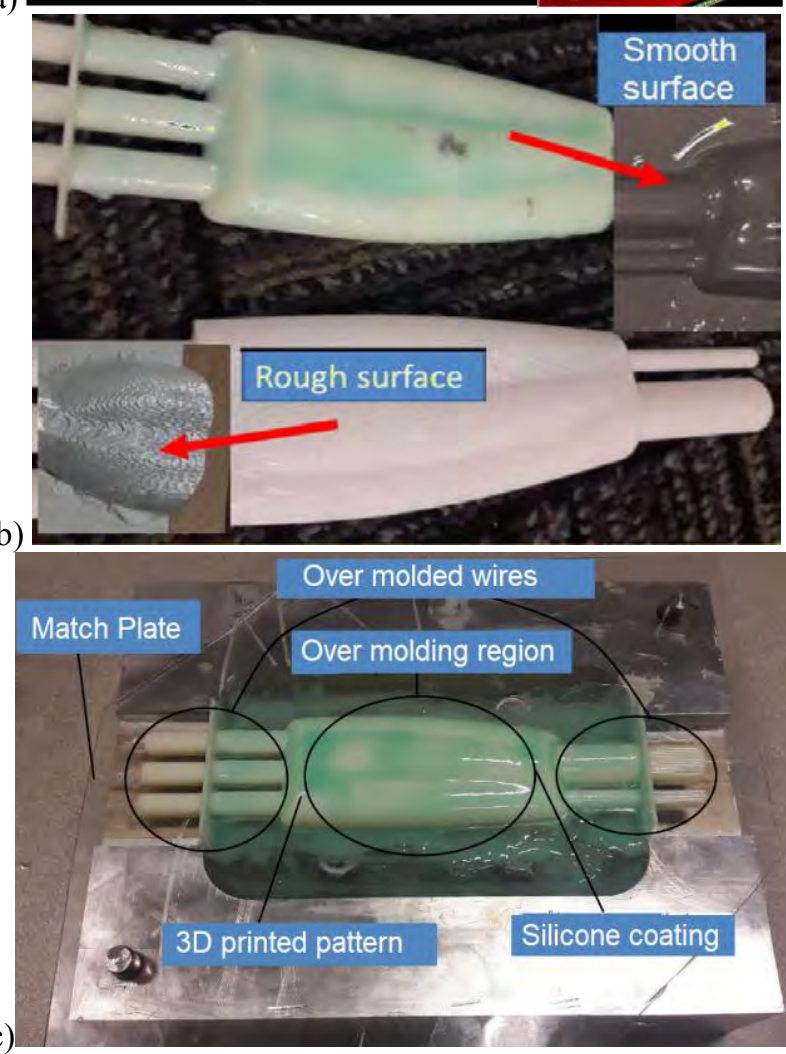

Figure 3. (a) The build layers as reprsented by the Insight ${ }^{\circledR}$ process planning software, (b) the built and coated patterns, and (c) The parts of the epoxy mold making process

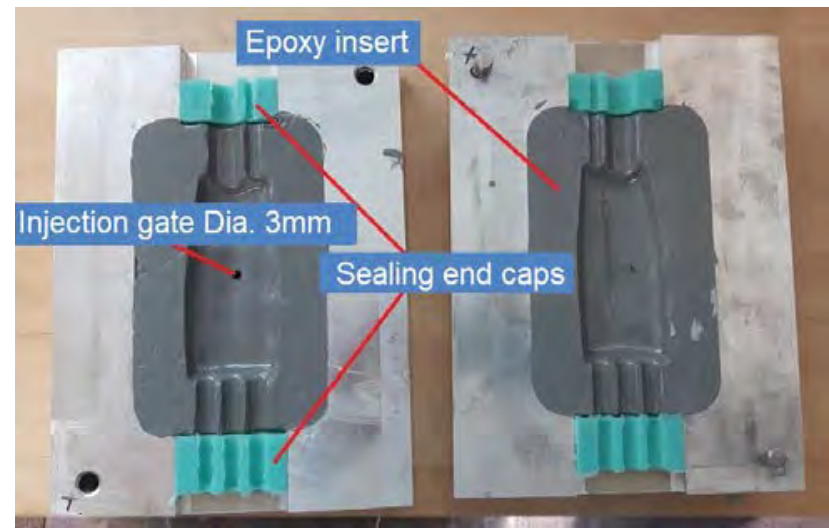

Figure 4. Epoxy inserts, and sealing end caps for the mold set.

\section{EXPERIMENTAL RESULTS}

In order to test the mold, the test part (Fig. 6) was placed inside the mold. The over molding region is encircled in the picture. The wires fit into the sealing end caps. The injected Technomelt-PA 7846 black covered the wire splice completely, and as shown in Fig. 7, the over molded product has a very smooth surface. The resulting product did not adhere to the mold cavity surface, and was removed easily. The material costs for this solution are divided into 2 categories: capital costs and consumable cost. (Table IV). The overall cost is approximately $\$ 140 \mathrm{US}$, which is much less than the cost of making a permanent aluminum mold using conventional machining processes (estimated to be $\$ 840$ US, using a 3 axis mill, and a final polishing operation, Table V).

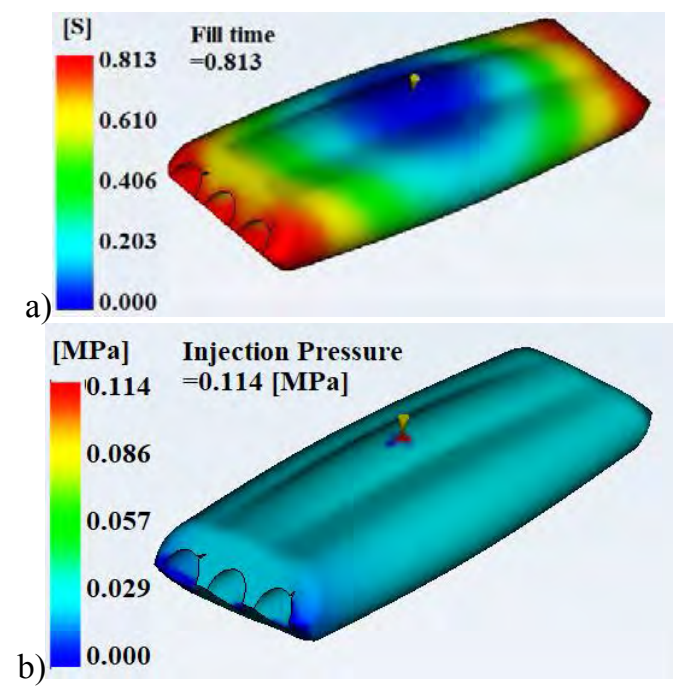

Figure 5. a)Fill time contours. B) Injection pressure contours.

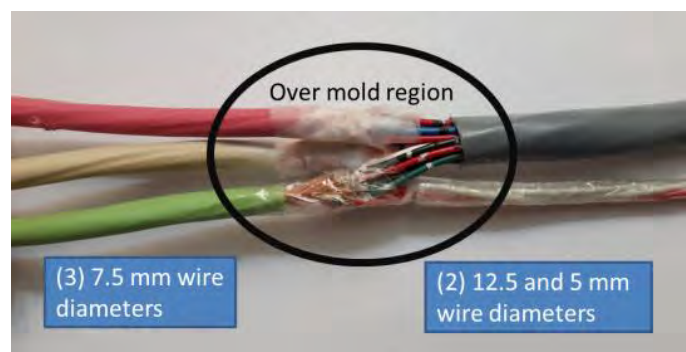

Figure 6. Over molding test application.

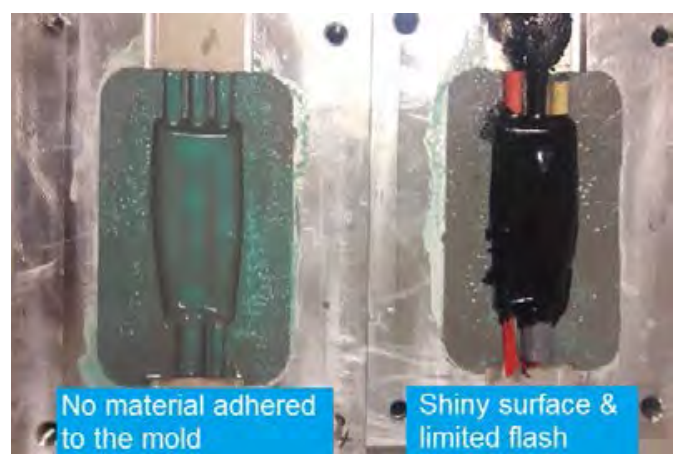

Figure 7. The over molded product in the mold. Note that there is no residual material in the left cavity and minimal flash in the right cavity. 
For machining a permanent aluminum mold, the Mastercam verification analysis indicates that 4 hours machining is required to fabricate the two mold blocks (Fig. 8). The machining process includes a face milling, two rough machining operations, followed by a finishing pass with a large step over. In Table V, a detailed cost breakdown is presented.

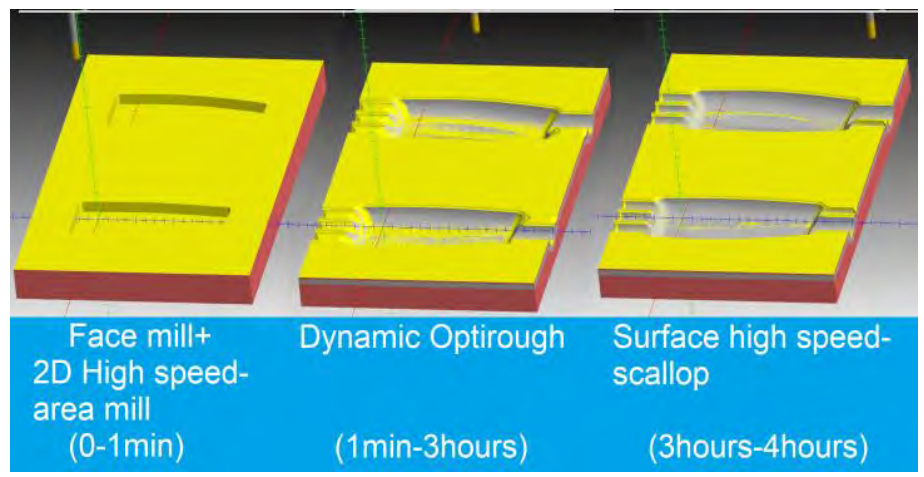

Figure 8. Permanent mold machining strategies and verification model.

TABLE IV. MATERIAL COSTS FOR FABRICATING THE EPOXY MOLD

\begin{tabular}{|l|l|c|}
\hline \multirow{2}{*}{ Capital costs } & Aluminum blocks & $\$ 70$ \\
\cline { 2 - 3 } & Match plate material & $\$ 5$ \\
\hline \multirow{3}{*}{ Consumables } & AM pattern printing & $\$ 16$ \\
\cline { 2 - 3 } & Aremco 805 & $\$ 44$ \\
\cline { 2 - 3 } & RTV Silicone & $\$ 6$ \\
\hline Total (US Dollar) & & $\mathbf{\$ 1 4 1}$ \\
\hline
\end{tabular}

TABLE V. COSTS FOR MACHINING A PERMANENT MOLD (NOTE: THIS DOES NOT INCLUDE MATERIAL COSTS)

\begin{tabular}{|l|c|c|}
\hline \multicolumn{1}{|c|}{ Process } & Time & Cost \\
\hline Process planning & 6 hours & $\$ 300$ \\
\hline Set up time & 1 hour & $\$ 80$ \\
\hline Machine run & 4 hours & $\$ 400$ \\
\hline Polishing & 16.2 inch $^{2}-2 \mu \mathrm{m}$ Rough & $\$ 60[16]$ \\
\hline Total (US Dollar) & & $\mathbf{\$ 8 4 0}$ \\
\hline
\end{tabular}

The final product is shown in Fig. 9. There is some flash; consequently, the sealing end cap solution will be redesigned.

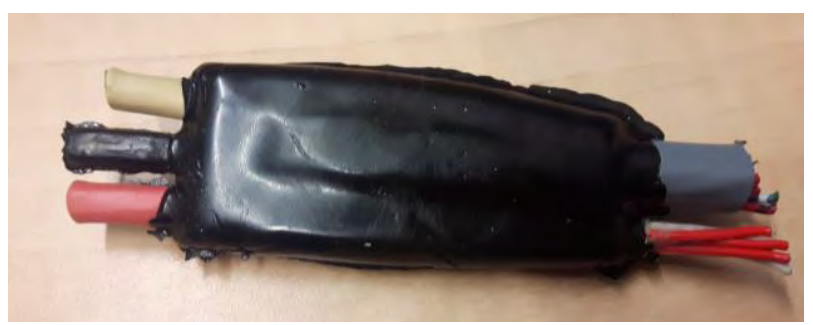

Figure 9. Final over molded product. The production quantity limitation for this mold solution will be caused by the wear of epoxy insert edges, the durability of the epoxy material from thermal shocks, and the durability of RTV silicone coating. Future experiments will be performed to determine the mold tool life.

Although the durability is important, epoxy insert replicates for a specific product will not increase the overall cost significantly. Therefore, if there are tool life issues, the RTV silicone coating can be applied as needed, and several epoxy inserts can be produced with the AM pattern.

\section{SUMMARY AND CONCLUSIONS}

A lower cost mold fabrication solution is presented for low volume, high temperature molding materials. AM processes cannot directly be used to fabrication a low-cost solution, but AM processes can be utilized to manufacture a sacrificial intermediary pattern. This pattern is employed to create an insert (or inserts - as required) made from an epoxy. Further experiments need to be performed to determine the tool life. Once this is completed, a comprehensive cost analysis can be developed to determine the overall costs of this build strategy.

\section{ACKNOWLEDGMENTS}

This research is partially funded by the NSERC Engage Grant, and the Ontario Centers of Excellent VIP programs. Special thanks to the industrial partner for their support.

\section{REFERENCES}

[1] Government of Canada (2012, Jan. 9). Archived - Plastics machinery and moulds [Online]. Available: https://www.ic.gc.ca/eic/site/plastics-plastiques.nsf/ eng/p101409.html.

[2] T. T. Wohlers, "Wohlers Report 2017: 3D printing and additive manufacturing state of the industry, Annual worldwide progress report," Denver, CO: Wohlers Associates, 2017.

[3] C. O. Balderrama-Armendariz et al., "Torsion analysis of the anisotropic behavior of FDM technology," Int. J. Adv. Manuf. Technol., vol. 96, no. 1-4, pp. 307-317, Apr. 2018.

[4] A. Bellini and S. Güçeri, "Mechanical characterization of parts fabricated using fused deposition modeling," Rapid Prototyping J., vol. 9, no. 4, pp. 252-264, 2003. doi: $10.1108 / 13552540310489631$.

[5] P. Dunne et al., "Some demands on rapid prototypes used as master patterns in rapid tooling for injection moulding," J. Mater. Process. Technol., vol. 150, no. 3, pp. 201-207, Jul. 2004. doi: 10.1016/S0924-0136(03)00571-5.

[6] Henkel (2018). TECHNOMELT PA 646 BLACK [Online]. Available: http:// na.henkel-adhesives.com/product-search-1554.htm?nodeid=8797758029825.

[7] V. Rodet and J. S. Colton, "Properties of rapid prototype injection mold tooling materials," Polym. Eng. Sci., vol. 43, no. 1, pp. 125-138, 2003.

doi: 10.1002/pen. 10011 .

[8] J. G. Kovács et al., "Thermal simulations and measurements for rapid tool inserts in injection molding applications," Appl. Therm. Eng., vol. 85, pp. 44-51, June 2015. doi: 10.1016/j.applthermaleng.2015.03.075.

[9] ExOne (2018). 3D Resources [Online]. Available: http://www.exone.com/ Resources/Materials.

[10] S-H. Ahn et al., "Anisotropic Material Properties of Fused Deposition Modeling ABS," Rapid Prototyping J., vol. 8, no. 4, pp. 248-257, 2002. doi: $10.1108 / 13552540210441166$.

[11] S. Ma et al., "Development of epoxy matrix composites for rapid tooling applications," J. Mater. Process. Technol., vol. 192-193, pp. 75-82, Oct. 2007. doi: 10.1016/j.jmatprotec.2007.04.086.

[12] T. Tabi et al., "Comparison of thermal, mechanical and thermomechanical properties of poly(lactic acid) injection-molded into epoxy-based Rapid Prototyped (PolyJet) and conventional steel mold," J. Therm. Anal. Calorim., vol. 123, no. 1, pp. 349+361, Jan. 2016.

[13] Smooth-On (2018). Mold Star 30 [Online]. Available: https://www.smoothon.com/products/mold-star-30/

[14] Aremco (2013, Oct. 25). Aremco-Bond 805 [Online]. Available: https:// www.aremco.com/news-item/aremco-bond-805-high-temp-thermallyconductive-epoxy-now-available/

[15] SMG 3D (2018). Insigt 3D Printing Software [Online]. Available: http:// www.smg3d.co.uk/3d_design_software/insight_software

[16] K. J. Fisher et al., "Development of a cost model for manual tool polishing," Int. J. Mod. Eng., vol. 6, no. 2, 2006. 


\title{
Influence of Flow Velocity on Erosion-corrosion Performance of 90응 Carbon Steel Elbows in Potash Brine
}

\author{
Raheem Elemuren, Richard Evitts, Ikechukwuka Oguocha, Glyn Kennell, Akindele Odeshi, Regan Gerspacher \\ Mechanical Engineering, Chemical and Biological Engineering \\ University of Saskatchewan \\ Saskatoon, Canada
}

\begin{abstract}
In this study, the effects of flow velocity and solid concentration on erosion-corrosion behavior of AISI 1018 long-radius steel elbows (Schedule 40) in saturated potash brine were investigated. Potash brine containing 10 and $30 \mathrm{wt} \%$ of silica sands flowing at 2.5, 3.0, 3.5 and 4.0 $\mathrm{m} / \mathrm{s}$ through a continuous loop with pipe internal diameter of $2.54 \mathrm{~cm}$ was used as the slurry. All experiments were conducted at $30{ }^{\circ} \mathrm{C}$. The surface damage on elbows was evaluated using scanning electron microscopy (SEM). It was found that material loss per unit area was greater at high slurry velocity and solid concentration. Corrosion pits were visible on the elbow surfaces at low slurry velocities, but pits were not formed at higher velocities. Mechanical damage was concluded to be the dominant degradation mechanism at high flow velocities.
\end{abstract}

Keywords- wear; erosion-corrosion; potash brine; pit; carbon steel

\section{INTRODUCTION}

The common occurrence of pipeline damage in the mineral processing industry is often attributed to erosion-corrosion. It is a process that occurs when abrasive solids suspended in a flowing corrosive liquid interact with the pipe internal wall resulting in significant material loss that may cause the pipe to leak or fail catastrophically. Pipe locations that experience abrupt changes in the flow direction, such as elbows, are subjected to severe hydrodynamic intensities occasioned by high angle impact by the particles in the moving fluid as well as change in flow regime at varying positions in the elbow [1].

It is widely acknowledged that the flow velocity and solid concentration are some of the key parameters that influence the wear behavior of pipeline materials subjected to erosioncorrosion [2]-[9]. Liu et al. [10] studied the effect of fluid flow velocity on the erosion-corrosion behavior of carbon steel elbows in $3.5 \mathrm{wt} \%$ sodium chloride solution containing quartz sand particles as erodent. They discovered that the erosioncorrosion rate of elbow samples increased with increasing flow velocity. Zeng, Zhang and Guo [11] investigated the erosion-corrosion performance at different locations in a stainless steel elbow using a flow loop and reported that the maximum erosion-corrosion rate occurred at the outer wall of the tested elbow outlet. Khayatan, Ghasemi and Abedini [12] compared the erosion-corrosion performance of pure titanium at different impingement angles. Their data showed that maximum degradation rates occurred at an impingement angle of $40^{\circ}$.

It is well known that the minimum material loss rate during erosion-corrosion of elbow occurs at the inlet section [10], [11]. The aim of this study was to investigate the effect of slurry flow velocity on the exit section of AISI 1018 steel elbow during erosion-corrosion in a slurry consisting of saturated potash brine and silica sand.

\section{EXPERIMENTAL PROCEDURE}

In this study, long radius $90^{\circ} 1018$ steel elbows (schedule 40) were used. The chemical composition range (in wt $\%$ ) of the steel is presented in Table 1. A typical optical image of the microstructure of the as-received elbows is shown in Fig. 1. The specimen for microstructure observation was prepared using standard metallographic methods. It was cut from one of the procured elbows and ground using SiC papers of 320, 400, 600,800 and 1200 grit sizes. This was followed by polishing with $3 \mu \mathrm{m}$ MD-Dac and $1 \mu \mathrm{m}$ MD-Nap polishing clothes and finally etched for $30 \mathrm{~s}$ using Nital solution consisting of $2 \%$ nitric acid and 98\% ethanol. A Nikon eclipse MA-100 inverted optical microscope was used to examine the microstructure which consists of a mixture of $82 \%$ ferrite and $18 \%$ pearlite.

Erosion-corrosion tests were conducted at $30{ }^{\circ} \mathrm{C}$ for $120 \mathrm{~h}$ in a flow loop (diameter of $2.54 \mathrm{~cm}$ ) using ASTM G119-09 [13]. Solid concentrations of 10 and $30 \mathrm{wt} \%$ and slurry flow velocities ranging from $2.5 \mathrm{~m} / \mathrm{s}$ to $4 \mathrm{~m} / \mathrm{s}$ were used. Fig. 3 shows a diagram of the flow loop used in this study. The operating temperature was controlled with the aid of a heat exchanger placed within the slurry tank.

TABLE I. CHEMICAL COMPOSITION OF 1018 STEEL

\begin{tabular}{|l|l|l|l|l|l|}
\hline $\mathrm{Fe}$ & $\mathrm{C}$ & $\mathrm{Mn}$ & $\mathrm{Mo}$ & $\mathrm{P}$ & $\mathrm{S}$ \\
\hline Balance & $0.19-0.21$ & $0.42-0.45$ & 0.001 & $0.012-0.018$ & $0.005-0.01$ \\
\hline
\end{tabular}




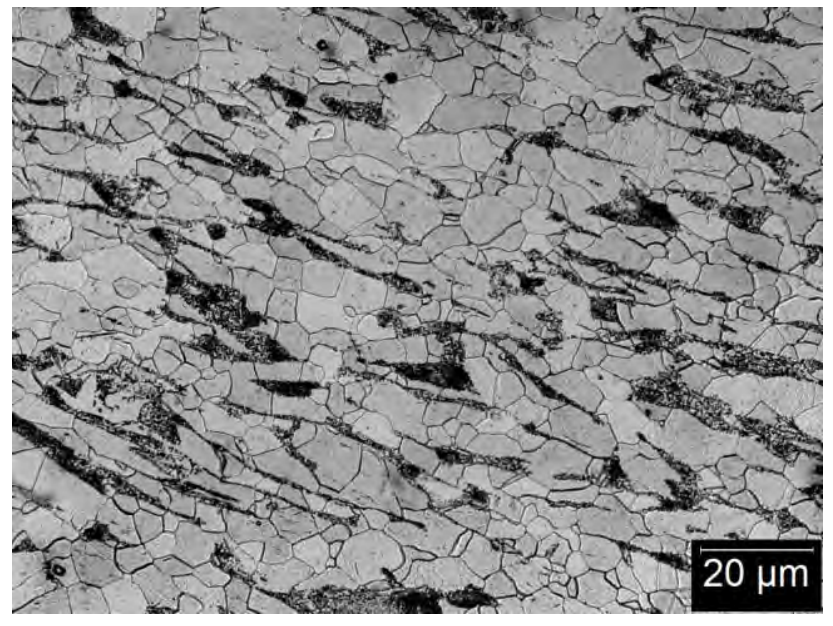

Fig. 1. Optical micrograph of 1018 steel elbow

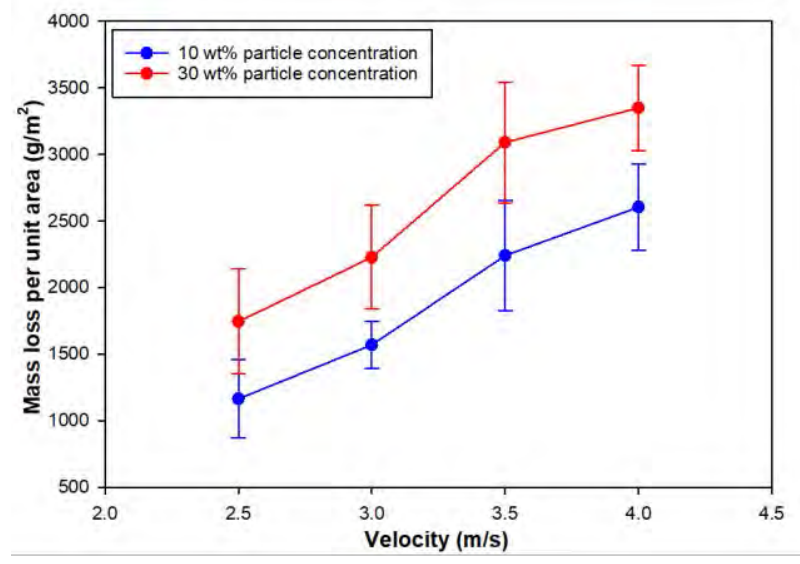

Fig. 2. Mass loss per unit area of elbows at different flow velocities after

$120 \mathrm{~h}$.
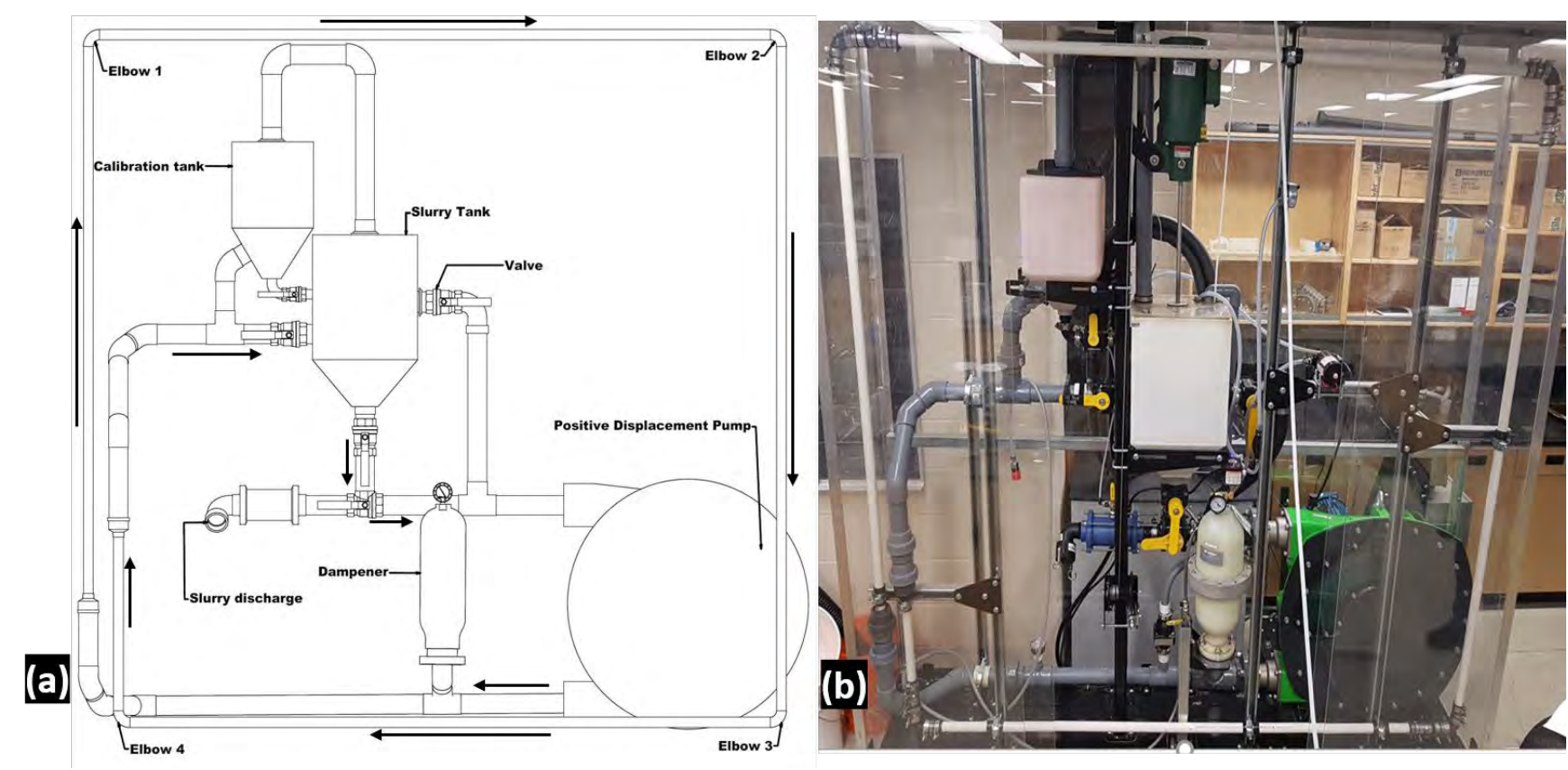

Fig. 3. Diagram of the experimental erosion-corrosion flow loop: (a) schematic (b) pictogram

The average weight loss of four 1018 steel elbows placed strategically at different locations in the loop was determined. The wear surfaces of tested elbows were evaluated using JEOL JSM-6010LA scanning electron microscope (SEM) to understand the dominant damage mechanisms.

\section{RESULTS AND DISCUSSION}

\section{A. Weight Loss Measurement}

The mass loss per unit area obtained for the tested elbows under different velocities and solid concentrations is presented in Fig. 2. Mass loss per unit area was affected by changes in both flow velocity and solid concentration. It increased with increasing slurry flow velocity and solid concentration. This is probably due to increase in mechanical interaction between the elbow surface and sand particles as slurry flow velocity and sand concentration increased.

\section{B. Surface Characterization}

Fig. 4 shows typical SEM micrographs obtained for the exit sections of tested elbows after $120 \mathrm{~h}$ in the loop. Fig. 4(a) shows the presence of corrosion pit and erosion-corrosion wear 

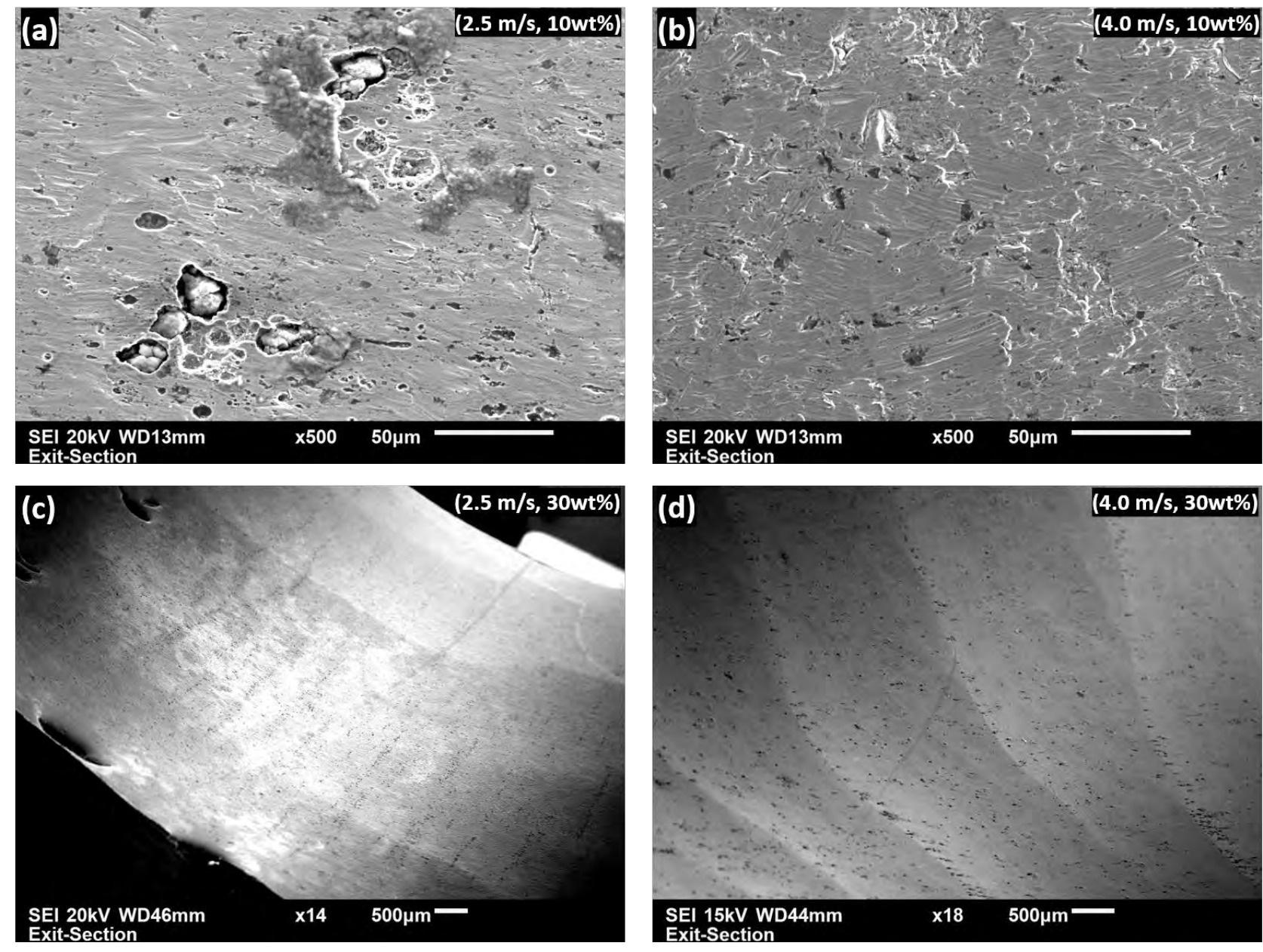

Fig. 4. Typical SEM micrographs of the elbows subjected to erosion-corrosion after $120 \mathrm{~h}$ : (a) and (c) exit section at $2.5 \mathrm{~m} / \mathrm{s}$; (b) and (d) exit section at $4.0 \mathrm{~m} / \mathrm{s}$.

scars (grooves) for elbows tested in a potash slurry containing $10 \mathrm{wt} \%$ flowing at $2.5 \mathrm{~m} / \mathrm{s}$. However, corrosion pits are not observed in the elbow subjected to a slurry flow velocity of 4.0 $\mathrm{m} / \mathrm{s}$ shown in Fig. 4(b). The presence of grooves is an indication that mechanical erosion contributed to the material degradation process, while the formation of corrosion pits indicates that corrosion played a role during erosion-corrosion of elbows at low velocities. At high slurry velocities, mechanical damage appears to have played a more prominent role in material degradation than corrosion. This can be due to the fact that more particles strike the elbow surface and subsequently increase the depth of particle impingement into the surface. Fig 4(c) and 4(d) shows the surface morphologies of elbow tested at $2.5 \mathrm{~m} / \mathrm{s}$ and $4.0 \mathrm{~m} / \mathrm{s}$ using a particle concentration of $30 \mathrm{wt} \%$. It can be observed that ridges formed on the elbow surface due to interactions with the sand particles over the test period. Few corrosion pits were observed on the surface of elbows tested at a slurry flow velocity of $2.5 \mathrm{~m} / \mathrm{s}$, which is consistent with what was observed for elbows tested with a slurry containing $10 \mathrm{wt} \%$ solid concentration.

\section{CONCLUSIONS}

The mass loss per unit area of $90^{\circ}$ steel elbows due to erosion-corrosion in saturated potash brine solutions containing silica sand were determined and the wear surface of the inner section of the elbows was investigated using a scanning electron microscope. The conclusions below are drawn from the experimental results.

1. Degradation of the tested elbows at low velocities was dominated by corrosion attack as evidenced by the presence of corrosion pits on the elbow surface. On the other hand, erosive wear due to particle impingement was the dominant damage mechanism at high slurry velocities.

2. Material loss per unit area increased with increasing flow velocity and solid concentration. 


\section{REFERENCES}

[1] L. Zeng, X. P. Guo, and G. A. Zhang, "Inhibition of the erosion-corrosion of a $90^{\circ}$ low alloy steel bend," J. Alloys Compd., vol. 724, pp. 827-840, 2017. doi: 10.1016/j.jallcom.2017.07.083.

[2] H. S. Grewal, H. Singh, and E. S. Yoon, "Interplay between erodent concentration and impingement angle for erosion in dilute water-sand flows," Wear, vol. 332-333, pp. 1111-1119, 2015.

doi: 10.1016/j.wear.2015.02.039.

[3] M. M. Stack and T. M. Abd El-Badia, "Some comments on mapping the combined effects of slurry concentration, impact velocity and electrochemical potential on the erosion-corrosion of WC/Co-Cr coatings," Wear, vol. 264, no. 9-10, pp. 826-837, 2008. doi: 10.1016/j.wear.2007.02.025.

[4] C. G. Telfer, M. M. Stack, and B. D. Jana, "Particle concentration and size effects on the erosion-corrosion of pure metals in aqueous slurries," Tribol. Int., vol. 53, pp. 35-44, 2012. doi: 10.1016/j.triboint.2012.04.010.

[5] M. Abedini and H. Ghasemi, "Erosion and erosion-corrosion of Al-brass alloy: Effects of jet velocity, sand concentration and impingement angle on surface roughness," Transactions of Nonferrous Metals Society of China, vol. 27, no. 11, pp. 2371-2380, 2017. doi: 10.1016/S1003-6326(17)60263-2.

[6] M. M. Stack, N. Corlett, and S. Turgoose, "Some thoughts on modelling the effects of oxygen and particle concentration on the erosion-corrosion of steels in aqueous slurries," Wear, vol. 255, no. 1-6, pp. 225-236, 2003. doi: 10/1016/S0043-1648(03)00205-9.

[7] H. X. Hu and Y. G. Zheng, "The effect of sand particle concentrations on the vibratory cavitation erosion," Wear, vol. 384-385, pp. 95-105, 2017. doi: 10.1016/j.wear.2017.05.003.

[8] M. M. Stack and S. M. Abdelrahman, "A CFD model of particle concentration effects on erosion-corrosion of $\mathrm{Fe}$ in aqueous conditions," Wear, vol. 273, no. 1, pp. 38-42, 2011.

doi: 10.1016/j.wear.2011.06.024.

[9] Y. P. Purandare, M. M. Stack, and P. E. Hovsepian, "Velocity effects on erosion-corrosion of CrN/NbN 'superlattice' PVD coatings," Surf. Coat. Technol., vol. 201, no. 1-2, pp. 361-370, 2006.

doi: $10.1016 /$ j.surfcoat.2005.11.143.

[10] J. G. Liu, W. Bakedashi, Z. Li, Y. Xu, W. Ji, C. Zhang, G. Cui, R. Zhang, "Effect of flow velocity on erosion-corrosion of 90-degree horizontal elbow," Wear, vol. 376-377, pp. 516-525, 2017.

doi: 10.1016/j.wear.2016.11.015.

[11] L. Zeng, G. A. Zhang, and X. P. Guo, "Erosion-corrosion of stainless steel at different locations of a 90 elbow," Corrosion Science, vol. 85, pp. 318-330, 2014. doi: 10.1016/j.corsci.2014.04.045.

[12] N. Khayatan, H. M. Ghasemi, and M. Abedini, "Synergistic erosioncorrosion behavior of commercially pure titanium at various impingement angles," Wear, vol. 380-381, pp. 154-162, 2017.

doi: 10.1016/j.wear.2017.03.016

[13] ASTM G119-93, "Standard guide for determining synergism between wear and corrosion," Wear erosion, Metal Corrosion, vol. 93, pp. 1-7, 1994. 


\section{Prepartion and Characterization of Graphene Oxide Cross-Linked Composites}

\author{
Mina Sabzevari, Duncan Cree \\ Department of Mechanical Engineering \\ University of Saskatchewan \\ Saskatoon, Canada \\ ( mina.sab@usask.ca ), ( duncan.cree@usask.ca )
}

\author{
Lee Wilson \\ Department of Chemistry \\ University of Saskatchewan \\ Saskatoon, Canada \\ ( lee.wilson@usask.ca )
}

\begin{abstract}
Graphene oxide (GO)-based materials have been studied for applications in adsorption and water treatment. Experimental results revealed that $\mathrm{GO}$ is a promising adsorbent due to its low-cost production, large surface area, and strong interaction with a wide range of dyes in an aqueous phase. GO chemical structure has the potential to be tuned using chemical methods such as cross-linking to produce a framework material. Therefore, in this study, cross-linking of GO structure using chitosan biopolymer as a cross-linker agent was investigated. Cross-linked GO composites were prepared through a green solution-based chemistry approach. Chemical structural, morphological and thermal changes in the crosslinked GO composites were investigated using Fourier transform infrared (FTIR) spectroscopy, scanning electron microscopy (SEM), and thermal gravimetric analysis (TGA). Also, adsorption properties of samples were obtained using methylene blue (MB) as a cationic probe, in solution phase. According to the spectroscopy results, cross-linked composites suggested interaction between the GO sheets with chitosan through the formation of amide linkages. SEM results showed irregular layer shapes connected to each other with higher surface roughness and porosity in cross-linked samples. Changes in the thermal stability of cross-linked samples can be ascribed to the cross-linking effect. Kinetic adsorption studies indicated higher sorption capacity of cross-linked samples toward $\mathrm{MB}$ in aqueous phase compare to pure GO and chitosan.
\end{abstract}

Keywords ; graphene oxide, chitosan, cross-link, composite.

\section{INTRODUCTION}

Removal of dyes and organic contaminants originating from printing, food and pharmaceutical industries throughout the world has greatly impacted materials engineering and chemistry. Many of the dye molecules are known to be carcinogenic and can affect air and water quality resulting in various undesirable human and ecosystem health issues [1]. Among the processes used for removal of dyes and contaminants, using the method of adsorption by solid adsorbents is considered as an efficient remediation technique due to its simplicity, low-cost and possibility of recycling of the adsorbent in the adsorption process [2].

Recently, graphene and graphene oxide (GO)-based materials have been investigated as potential adsorbents for applications in wastewater treatment [3]. Graphene comprises of $\mathrm{sp}^{2}$-hybridized carbon atoms arranged in a two-dimensional honeycomb lattice. Compared to graphene, which has a low aqueous solubility, GO is highly dispersible in organic solvents due to the presence of different oxygenated functional groups on its structure. The highly negative charge density of GO in aqueous solutions serves as an effective adsorption site for cationic dyes [3]. Thus, this material could be valuable for adsorption applications targeting cationic species in aqueous phases (such as methylene blue (MB) cation) and gas (such as $\mathrm{N}_{2}$ ) capturing applications [4]. Furthermore, the chemical structure and properties of GO can be further tuned using chemical methods [5]. Fabrication of framework structures from individual GO sheets was studied using chemical interactions to fabricate GO self-assembled structures [6]. Therefore, developing a stable framework from interconnected GO layers is attractive for the successful and practical use of these materials which remains the main challenge. It was found that a GO framework structure can be achieved by crosslinking GO sheets through covalent or non-covalent bond using bio-polymers such as chitosan as a cross-linker [7]. Due to the presence of amino groups, chitosan is considered as a positively charged polysaccharide, which can strongly attract GO sheets with a negative charge through electrostatic interactions. In addition, chitosan amino groups can covalently bond to the carboxyl groups of GO to form a homogenous and well-dispersed GO composite [7]. Therefore, chitosan is an efficient cross-linker which promotes the formation of the GO composite.

This study investigates the use of chitosan as a bio-polymer to produce green GO-chitosan cross-linked composite materials. The aim of this work was to develop a GO framework material with enhanced structural and thermal stability and to evaluate the chemical structure, morphology, thermal stability, and MB adsorption properties of GO crosslinked composites with chitosan for wastewater treatment and adsorption applications. 


\section{EXPERIMENTAL}

\section{A. Materials}

Medium molecular weight chitosan ( $\mathrm{Mw}=95,0000$ $310,000 \sim 95 \%$ deacetylation), sodium nitride $\left(\mathrm{NaNO}_{3}\right)$, potassium permanganate $\left(\mathrm{KMnO}_{4}\right)$, sulfuric acid $(98 \%$, ACS grade), methylene blue (high purity, biological stain), hydrogen peroxide $(30 \% \mathrm{v} / \mathrm{v})$ were obtained from Sigma-Aldrich Canada Ltd. Graphite flake, natural, -325 mesh, 99.8\% (metals basis) were obtained from Alfa Aesar Thermo Fisher Scientific.

\section{B. Synthesis of Graphene Oxide}

GO was synthesized from graphite flakes using the modified Hummer's method [8]. Briefly, $100 \mathrm{~mL}$ of concentrated $\mathrm{H}_{2} \mathrm{SO}_{4}$ was added into a $500 \mathrm{~mL}$ flask filled with $4 \mathrm{~g}$ of graphite in an ice bath, followed by the addition of $2 \mathrm{~g}$ of $\mathrm{NaNO}_{3}$ and stirred for $4 \mathrm{~h}$. Then, $12 \mathrm{~g}$ of $\mathrm{KMnO}_{4}$ was gradually added while the mixture was stirred for $2 \mathrm{~h}$. The ice-bath was then removed, and the system was heated at $35^{\circ} \mathrm{C}$ for another 30 minutes. Subsequently, $240 \mathrm{~mL}$ distilled water was slowly added to the system and continued to stir for another 30 minutes. Then $160 \mathrm{~mL}$ of water and $30 \% \mathrm{v} / \mathrm{v} \mathrm{H}_{2} \mathrm{O}_{2}$ were added to terminate the reaction. The solution was stirred overnight and purified using multiple washing with millipore water, $\mathrm{HCl}$ (30\%) and ethanol until it reached $\mathrm{pH} 7$. After multiple washings, the solid $\mathrm{GO}$ was vacuum-dried at $40^{\circ} \mathrm{C}$ to obtain completely dried GO powder.

\section{GO-Chitosan Cross-Linked Composite Preparation}

Preparing of the GO-chitosan composite materials was carried out by preparing the GO solution with a concentration of $1 \mathrm{mg} / \mathrm{mL}$. Also, $1 \% \mathrm{w} / \mathrm{v}$ chitosan solution was prepared by dissolving $5 \mathrm{~g}$ of chitosan in $500 \mathrm{~mL}$ of $1 \% \mathrm{v} / \mathrm{v}$ glacial acetic acid. The resulting chitosan solution was added dropwise to the GO solution with continuous stirring for about $4 \mathrm{~h}$. The mixture was neutralized to $\mathrm{pH} \sim 7$ followed by stirring for $12 \mathrm{~h}$. The precipitate was separated from the supernatant via mediumspeed centrifuge $(5000 \mathrm{rpm})$ followed by washing with millipore water and drying at ambient temperature for $48 \mathrm{~h}$. Separately, pure GO solution with concentration of $3 \mathrm{mg} / \mathrm{mL}$ was also prepared for comparison purposes. The resulting composites were ground in a mortar and pestle followed by sieving through a 40-mesh sieve. An outline of the experimental procedure is illustrated in Fig. 1.

\begin{tabular}{|c|c|c|c|c|}
\hline \begin{tabular}{l|l|l|l|} 
Graphite \\
Powder
\end{tabular} & $\begin{array}{c}\text { GO } \\
\text { Powder }\end{array}$ & $\begin{array}{c}\text { GO } \\
\text { Solution }\end{array}$ & $\begin{array}{c}\text { GO-Chitosan } \\
\text { Solution }\end{array}$ & $\begin{array}{c}\text { GO-Chitosan } \\
\text { Composite }\end{array}$ \\
\hline
\end{tabular}

Figure 1. Synthetic procedure for GO-based composite materials.

\section{Measurements and Characterization}

Fourier transform infrared (FTIR) spectra were obtained to confirm successful oxidation of graphite by observing the appearance of different functional groups and to monitor changes in these functional groups upon cross-linking. A Bio-
RAD FTS-40 IR spectrophotometer was used to obtain the IR spectra of the composite materials. The sample powder was mixed with pure spectroscopic grade $\mathrm{KBr}$ (weight ratio: 1:10). The FTIR spectra was obtained in reflectance mode with a resolution of $4 \mathrm{~cm}^{-1}$ over the spectral range of $500-4000 \mathrm{~cm}^{-1}$ using $71 \mathrm{kHz}$ SPINAL-64 decoupling during acquisition with external reference to adamantane at $\delta=38.48 \mathrm{ppm}$. The surface morphology and surface topography of non-cross-linked and cross-linked composite materials were studied using scanning electron microscopy (SEM) (Hitachi Model SU8010). Images from samples were collected under accelerating voltage $(3 \mathrm{kV})$. Thermal stability and decomposition temperature of the obtained composite materials were measured using a TA Instruments Q50000IR thermal gravimetric analysis (TGA) system which was operated from room temperature to $500^{\circ} \mathrm{C}$ at a heating rate of $5^{\circ} \mathrm{C} \mathrm{min}^{-1}$ under nitrogen atmosphere.

\section{E. Kinetic Sorption Studies of Methylene Blue}

Study of the kinetics of sorption of cross-linked material with chitosan toward MB was carried out by means of the onepot method [9], where $\sim 100 \mathrm{mg}$ of a powdered sample was added to a folded filter paper with both ends sealed before adding to the sorbate solution. The sealed filter paper was immersed in a fixed volume $(250 \mathrm{~mL})$ of a $5 \mu \mathrm{M}$ MB solution. The MB solution aliquots were pipetted $(3 \mathrm{~mL})$ at selected time intervals and the residual supernatant $\mathrm{MB}$ concentration estimated by UV-vis absorbance of MB $(\lambda \max =664 \mathrm{~nm})$. The kinetic uptake of pure GO, chitosan and its cross-linked composite at each sampling time interval $(\mathrm{t})$ was determined using (1), where $\mathrm{C}_{0}(\mathrm{mM})$ refers to the initial dye concentration at $\mathrm{t}=0, \mathrm{C}_{\mathrm{t}}(\mathrm{mM})$ is the residual amount of $\mathrm{MB}$ at variable time $(\mathrm{t}), \mathrm{V}(\mathrm{L})$ is the volume of the MB solution, and $\mathrm{m}(\mathrm{g})$ is the weight of the adsorbent. $C_{o}$ and $C_{t}$ refer to the dye concentration at $\mathrm{t}=0$ and variable times.

$$
\mathrm{Q}_{\mathrm{t}}=\frac{\left(\mathrm{C}_{0}-\mathrm{C}_{\mathrm{t}}\right)}{\mathrm{m}} \mathrm{V}
$$

To show and compare sorption characteristics of GO and its cross-linked form with chitosan the pseudo-second-order (PSO) model was used to obtain sorption parameters (such as $\mathrm{Q}_{\mathrm{e}}$ which is the adsorbate quantity in the solid phase $(\mathrm{mg} / \mathrm{g})$ ) and kinetic parameters (such as $\mathrm{K}$ which represents the sorption affinity constant). Kinetic isotherm profiles were obtained by plotting $\mathrm{Q}_{t}$ vs $\mathrm{t}$. The PSO kinetic models (2) was used to evaluate parameters of the isotherm, where $\mathrm{Q}_{\mathrm{e}}$ is the amount of solute adsorbed at a pseudo-equilibrium $(\mathrm{mg} / \mathrm{g})$ condition, $\mathrm{Q}_{\mathrm{t}}$ is the amount of solute adsorbed at time $\mathrm{t}(\mathrm{mg} / \mathrm{g})$ and $\mathrm{K}_{2}$ is the rate constant of PSO adsorption model.

$$
Q_{t}=\frac{Q_{e}^{2} K_{2} t}{1+K_{2} t Q_{e}}
$$

\section{RESULTS AND DISCUSSION}

Various GO samples were cross-linked with chitosan, where GO was obtained from graphite powder as shown in Fig. 2. 


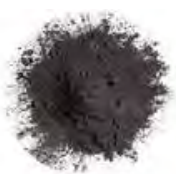

Graphite Powder

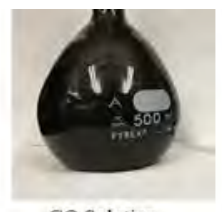

GO Solution

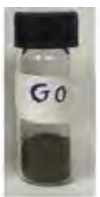

GO Powder

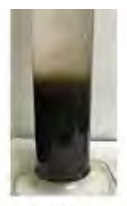

GO-Chitosan
Solution
Composite Powd
Figure 2. Image of the produced GO-based cross-linked composite materials

\section{A. Fourier Transform Infrared (FTIR) Spectroscopy}

Fig. 3 shows the FTIR spectra of the cross-linked composite materials versus pure GO and chitosan as precursors. Firstly, the successful GO preparation (oxidation of graphite) was validated using FTIR by the characteristic peaks located at 3200-3700 $\mathrm{cm}^{-1}$ ascribed to the stretching of the hydroxyl groups $(\mathrm{O}-\mathrm{H}), \sim 1680-1730 \mathrm{~cm}^{-1}$ and $1550-1650 \mathrm{~cm}^{-1}$ as characteristics of the carbonyl groups $(\mathrm{C}=\mathrm{O})$ and $\mathrm{sp}^{2}$ hybridized $\mathrm{C}=\mathrm{C}$, and the bands at $1230-1350 \mathrm{~cm}^{-1}$ corresponds to epoxy groups. Also, in the spectrum of chitosan, the main characteristic peaks are centred at $1650 \mathrm{~cm}^{-1}$ and 1590 $\mathrm{cm}^{-1}$; these correspond to the $\mathrm{C}=\mathrm{O}$ stretching vibration of $\mathrm{NHCO}$ and the $\mathrm{N}-\mathrm{H}$ bending of $\mathrm{NH}_{2}$, respectively. The presence of the peak of both precursors (GO and chitosan) are supported by similar spectral features of cross-linked composite materials. However, some bands for the cross-linked GO composites are absent or their intensity increase when compared to similar bands for the non-cross-linked GO. Formation of covalent bonds between GO and chitosan can be demonstrated by elimination of some of the original GO bands and rise of new ones. The absence of a peak at $1730 \mathrm{~cm}^{-1}$, is attributed to $\mathrm{C}=\mathrm{O}$ in carboxylic acid moieties in $\mathrm{GO}$, and the greater intensity of the peak at $1595 \mathrm{~cm}^{-1}$ corresponds to the formation of covalent bond in cross-linked GO composites. This information provides support for the formation of amide linkages between GO and chitosan as the linker and is in agreement with a previous report [10].

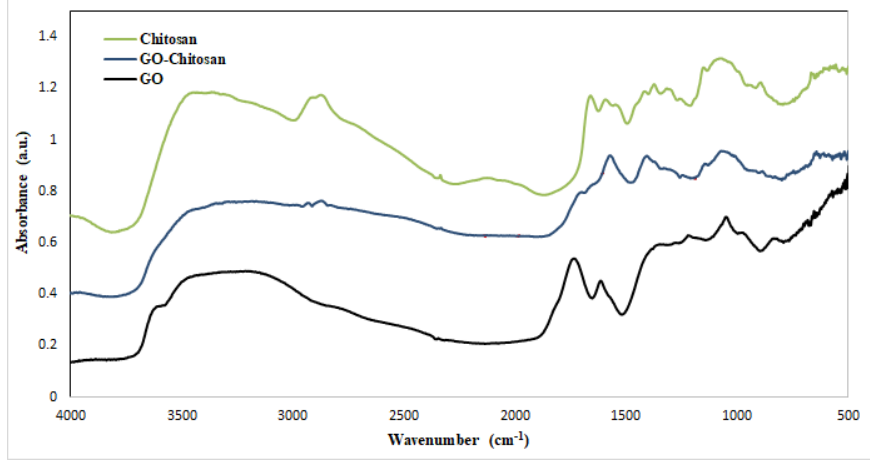

Figure 3. FTIR spectra of the precursors and GO-based cross-linked composite.

\section{B. Scanning Electron Microscopy}

Fig. 4 shows SEM micrographs of non-cross-linked and cross-linked GO composite materials. Fig. 4 (a) depicts graphite as a starting material, while Fig. 4 (b) is GO both made up of multiple layers stacked on top of each other. By comparison, the cross-linked GO composites Fig. 4 (c) and (d) indicate wrinkled ledges with irregular shape of layers connected to each other. The micrographs revealed that crosslinking of the GO alters its morphology and surface roughness according to the cross-linker type and content. The obtained results herein indicate the composites possess higher surface roughness and porosity when compared with pristine graphite or GO. These variations in morphological and textural changes provide support for the self-assembly cross-linking between GO and chitosan.
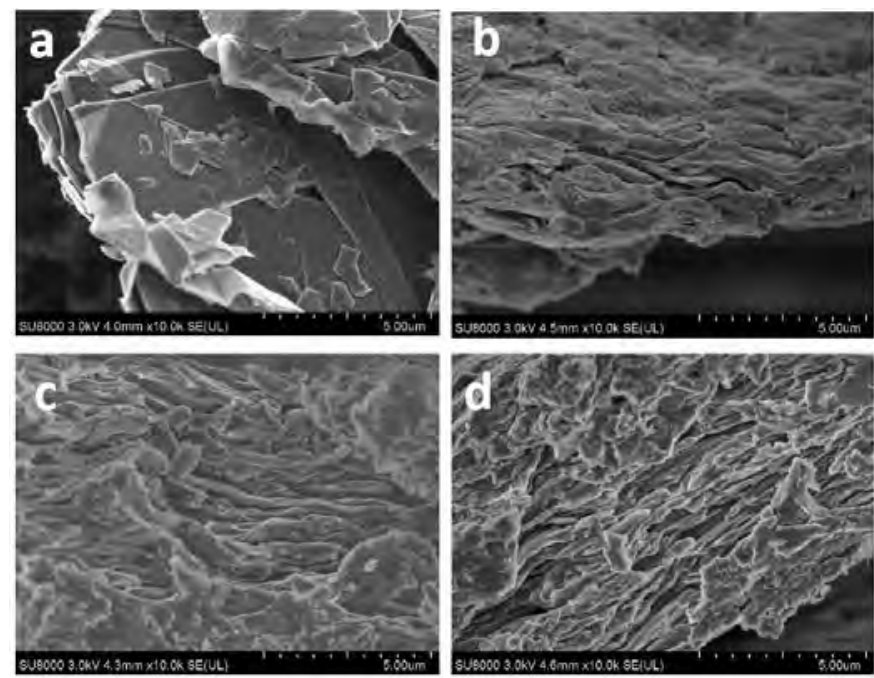

Figure 4. SEM micrographs of graphite (a), GO (b), GO-based cross-linked composite materials (c) and (d).

\section{Thermal Gravimetric Analysis}

The TGA results for the cross-linked GO composite materials are shown in Fig. 5. A rapid thermal decomposition occurred at $\sim 200^{\circ} \mathrm{C}$ for $\mathrm{GO}$, while the cross-linked $\mathrm{GO}$ displayed two thermal events at approximately $250^{\circ} \mathrm{C}$, and $\sim 455^{\circ} \mathrm{C}$. These thermal events for the $\mathrm{GO}$ composites correlate to decomposition of $\mathrm{GO}$ oxygen functionalities and its framework structure, respectively. The change in the thermal stability of the cross-linked GO is due to framework effects arising from formation of covalent (amide) linkage and crosslinking with chitosan in agreement with earlier FTIR band result (Fig. 3).

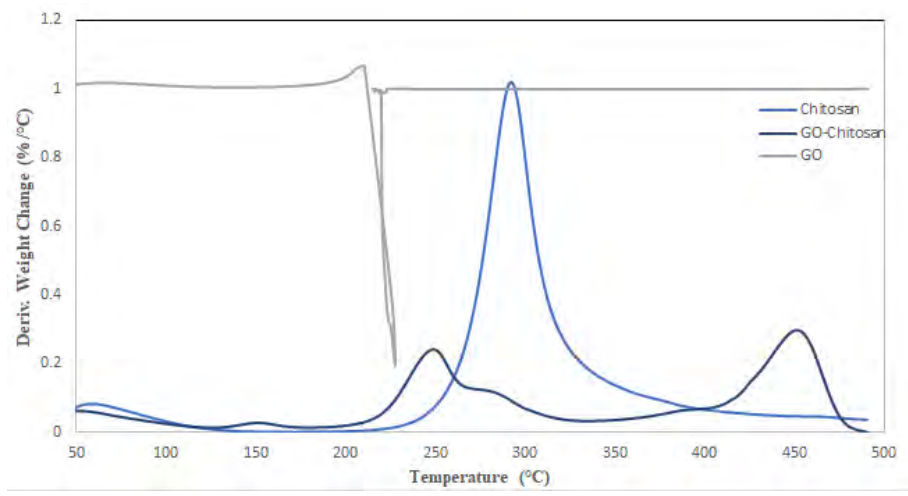




\section{Kinetic Uptake Studies of Methylene Blue}

Kinetic uptake studies of GO cross-linked chitosan systems can provide valuable insights on the reflection of cross-linking effect on adsorption properties GO composites. The surface functionality and sorption properties are affected by structural changes in the GO cross-linked material [11]. Fig. 6 presents the kinetic adsorption profiles with $\mathrm{MB}$ over a 240 minute interval, in which the PSO kinetic model provided reasonable fitting results. According to the obtained kinetic parameters $(\mathrm{k}$ and q) as listed in table 1, the GO cross-linked composite displays enhanced uptake capacity for MB which is related to the cross-linking effect. The $\mathrm{Q}_{\mathrm{e}}$ and the PSO rate constant values ( $\mathrm{k}$ and $\mathrm{q})$ for the three sorbent materials increased upon cross-linking: chitosan $(4.175)<\mathrm{GO}(5.059)<\mathrm{GO}$-chitosan $(5.107)$ and, chitosan $(0.003)<\mathrm{GO}(0.006)<$ GO-chitosan (0.019), respectively. It was shown that the intra-particle diffusion and external diffusion play an important role in the adsorption kinetics [12]. The greater kinetic uptake of the composite material recommends there are more active adsorption sites according to the increasing $\mathrm{Q}_{\mathrm{e}}$ values as the GO becomes cross-linked. The decreasing kinetic trend of all sorbent materials suggests diffusion of MB through the pore network of the sorbent materials decreased with increasing contact time between sorbent and MB. This may be related to the decreasing number of available adsorption sites as time increases.

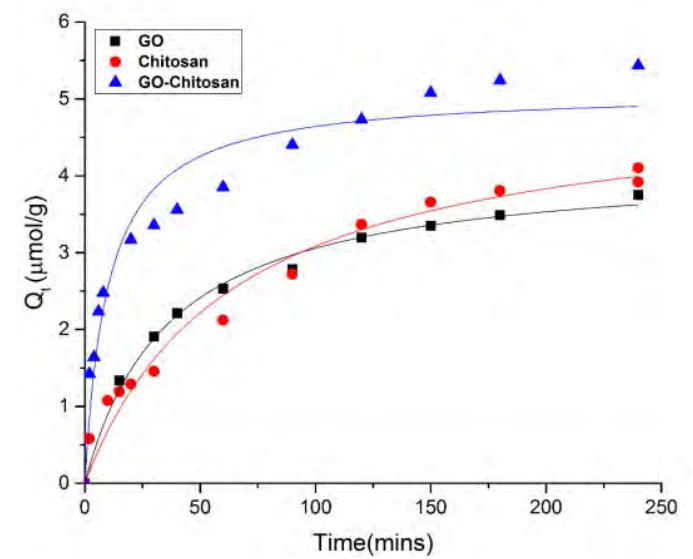

Figure 6. Uptake kinetic profile of $M B$ by $G O$ and GO-based cross-linked composite material, where the fitted lines are associated to the second-PSO model (see (2)).
TABle 1. PSO Kinetic Model Values for, GO, Chitosan and Its CrossLINKED COMPOSITE

\begin{tabular}{|l|l|c|l|}
\hline \multicolumn{1}{c|}{ model } & \multicolumn{3}{c|}{ pseudo-second order } \\
\hline \multirow{2}{*}{ equation } & \multicolumn{3}{c|}{$Q_{t}=\frac{Q_{e}^{2} K_{2} t}{1+K_{2} t Q_{e}}$} \\
\hline \multirow{2}{*}{ adj. R-Square } & 0.99779 & 0.981663 & 0.96183 \\
\hline \multirow{2}{*}{$\mathrm{GO}$} & & value & standard error \\
\hline \multirow{2}{*}{ chitosan } & $\mathrm{q}(\mathrm{mg} / \mathrm{g})$ & 4.17552 & 0.07646 \\
\cline { 2 - 4 } & $\mathrm{k}(\mathrm{g} \mathrm{mg} / \mathrm{min})$ & 0.00655 & $5.21701 \mathrm{E}-4$ \\
\cline { 2 - 4 } & $\mathrm{q}(\mathrm{mg} / \mathrm{g})$ & 5.05941 & 0.28235 \\
\hline \multirow{2}{*}{ GO-chitosan $(\mathrm{g} \mathrm{mg} / \mathrm{min})$} & 0.00308 & $6.54771 \mathrm{E}-4$ \\
\cline { 2 - 4 } & $\mathrm{q}(\mathrm{mg} / \mathrm{g})$ & 5.10796 & 0.19656 \\
\cline { 2 - 4 } & $\mathrm{k}(\mathrm{g} \mathrm{mg} / \mathrm{min})$ & 0.01948 & $6.64771 \mathrm{E}-4$ \\
\hline
\end{tabular}

\section{CONCLUSIONS AND FUTURE WORK}

In this study, GO-based cross-linked composite materials were synthesized with various surface properties and morphologies that arise from cross-linking with chitosan at various compositions. FTIR was conducted on the cross-linked composites and compared to pure GO to support the formation of amide linkages. The greater surface roughness of the GO cross-linked composite materials parallel the variable morphology revealed by SEM. TGA results revealed evidence of cross-linking between GO and chitosan according to changes in the thermal stability of the composite materials. In conclusion, GO-based composites could be used as stable framework structures for potential applications in the removal of dyes and pesticides in wastewater environments.

Future testing to support this study will involve BET nitrogen adsorption to obtain information on gas sorption capacity and estimate the average pore size and surface area of samples using a Micromeritics ASAP 2020 (Norcross, GA) instrument. Also, mechanical properties of GO composite thin films will be measured by Dynamic Mechanical Analyzer (DMA) 2890.

\section{ACKNOWLEDGMENTS}

The authors would like to acknowledge the financial support of the Natural Sciences and Engineering Research Council of Canada (NSERC) under the Discovery Grant (418729-2012 RGPIN). We would like to thank support and expert technical assistance of Ms. Leila Dehabadi in performing IR spectroscopy, and TGA analysis.

\section{REFERENCES}

[1] G. L. Dotto et al., "Application of chitosan films for the removal of food dyes from aqueous solutions by adsorption," Chem. Eng. J., vol. 214, pp. 8-16, 2013. doi: 10.1016/j.cej.2012.10.027.

[2] I. Ali, M. Asim, and T. A. Khan, "Low cost adsorbents for the removal of organic pollutants from wastewater," J. Enviro. Manag., vol. 113. pp. 170-183, 2012. doi: 10.1016/j.jenvman.2012.08.028.

[3] G. K. Ramesha et al., "Graphene and graphene oxide as effective adsorbents toward anionicand cationic dyes," J. Colloid Interface Sci., vol. 361, no. 1, pp. 270 277, 2011. doi: 10.1016/j.jcis.2011.05.050. 
[4] W. Li et al., "Molecular dynamics simulations of CO2/N2 separation through two-dimensional graphene oxide membranes," J. Phys. Chem. C, vol. 120, no. 45, pp. 26061-26066, 2016. doi: 10.1021/acs.jpcc.6b06940.

[5] S. Pei and H.-M. Cheng, "The reduction of graphene oxide," Carbon N. Y., vol. 50, no. 9, pp. 3210-3228, 2012. doi: 10.1016/j.carbon.2011.11.010.

[6] W. Chen et al., "Self-assembly and embedding of nanoparticles by in situ reduced graphene for preparation of a 3D graphene/nanoparticle aerogel," Adv. Mater., vol. 23, no. 47, pp. 5679-5683, 2011. doi: 10.1002/adma.201102838.

[7] Y. Chen et al., "Graphene oxide-chitosan composite hydrogels as broad-spectrum adsorbents for water purification," J. Mater. Chem. A, vol. 1, no. 6, pp. 1992-2001, 2013. doi: 10.1039/C2TA00406B.

[8] W. S. Hummers and R. E. Offeman, "Preparation of graphitic oxide," J. Am. Chem. Soc., vol. 80, no. 6, pp. 1339-1339, 1958. doi: 10.1021/ja01539a017.

[9] M. Mohamed and L. Wilson, "Kinetic uptake studies of powdered materials in solution," Nanomaterials, vol. 5, no. 2, pp. 969-980, 2015.

doi: 10.3390/nano5020969.

[10] P.-P. Zuo et al., "Fabrication of biocompatible and mechanically reinforced graphene oxide-chitosan nanocomposite films," Chem. Cent. J., vol. 7, no. 1, p. 39, 2013. doi: 10.1186/1752-153X-7-39.

[11] M. H. Mohamed et al., "Investigation of templated and supported polyaniline adsorbent materials," RSC Adv., vol. 5, no. 9, pp. 6976-6984, 2015. doi: 10.1039/C4RA07412B.

[12] L. Chen et al., "Removal of methylene blue from water by cellulose/graphene oxide fibres," J. Exp. Nanosci., vol. 11, no. 14, pp. 1156-1170, 2016. doi: 10.1080/17458080.2016.1198499. 


\section{On Microstructure and Corrosion Properties of Selective Laser Melted 316L Stainless Steel}

\author{
Mostafa Kazemipour ${ }^{1 *}$, Ali Nasiri ${ }^{1}$ \\ ${ }^{1}$ Faculty of Engineering and Applied Science, Memorial \\ University of Newfoundland, St. John's, NL, Canada \\ *mkazemipour@mun.ca
}

\author{
Mohsen Mohammadi ${ }^{2}$ \\ ${ }^{2}$ Marine Additive Manufacturing Centre of Excellence \\ (MAMCE), University of New Brunswick, Fredericton, NB, \\ Canada
}

\begin{abstract}
In this study, a laser additive manufacturing method, known as selective laser melting (SLM), was applied to produce cube blocks of $316 \mathrm{~L}$ stainless steel. The microstructure and corrosion properties of the produced samples were analyzed using scanning electron microscopy, cyclic potentiodynamic polarization testing, and electrochemical impedance spectroscopy. The results were also compared with the properties of a conventional wrought 316L stainless steel sample. The microstructural studies showed that the SLM-manufactured samples have a regular network of melt pools containing austenite grains along with elongated or equiaxed cellular sub-grains. The potentiodynamic polarization results depicted that the SLM fabricated samples had higher positive pitting potential and a wider passivation range than those of the wrought sample, corresponding to their better corrosion resistance. However, the SLM fabricated samples showed a weaker re-passivation property, which possibly is attributed to the presence of preexisting porosities in the structure of the SLM sample formed during the fabrication process. The EIS data also confirmed a larger capacitive arc for the SLM fabricated samples than its wrought counterpart, indicating a higher charge transfer impedance and a better corrosion resistance.
\end{abstract}

Keywords: Selective laser melting, Additive manufacturing, $316 \mathrm{~L}$ Stainless steel, Microstructure, Corrosion properties

\section{INTRODUCTION}

Nowadays, innovative and advanced 3-dimentional printing, also named additive manufacturing (AM), as a bottom-up method, is rapidly growing and has drawn many attentions from both academia and industry due to its capabilities in the production of near-net shaped components. Comparing with conventional manufacturing processes, such as casting or forming, this technology provides many advantages including fabrication of metallic parts with more complex shapes, less time from design stage to manufacturing, no need to post processing, and lower wastage precursors. In this technology, without the usage of specialized molds or tools, in a single step process, 3D components are fabricated through layer-wise addition of melted/sintered precursors powder on the substrate or previous layers, based on their digitally defined Computer Aided Design (CAD) data [1-6]. In recent years, various laser-based additive manufacturing methods for fabrication of metallic components have been developed, such as laser engineered net shaping (Lenz), direct metal deposition (DMD), laser solid forming (LSF), direct laser fabrication (DLF), laser metal deposition shaping (LMDS), direct metal laser sintering (DMLS), and selective laser melting (SLM) $[1,5,7]$.

316L austenite stainless steel (316L SS) with excellent corrosion resistivity, decent mechanical properties, and good weldability is widely used for various applications in many industries, such as oil and gas, marine, and biomedical sectors. Currently, 316L components are mainly produced using conventional manufacturing methods, which do not allow for the production of complex shapes and therefore, final parts need to be welded or machined, consequently both fabrication time and cost increase. Additive manufacturing has emerged as an appropriate solution to resolve this issue. Nevertheless, the components built through these techniques have different microstructures and properties than those fabricated through conventional methods, which makes it crucial to study and research their as-printed properties $[5,6,8]$. In spite of existing comprehensive works on investigating various properties of SLM fabricated parts, there is very limited literature on corrosion related characteristics and electrochemical properties of AM components [9]. In the present study, the microstructure and corrosion performance of a $316 \mathrm{~L}$ stainless steel (SS) produced through SLM technique were studied and the results were compared with a conventional wrought $316 \mathrm{~L} \mathrm{SS}$.

\section{MATERIALS AND METHODS}

Several cube blocks of 316L SS with the edge length of 15 $\mathrm{mm}$ were printed through SLM technique using a Renishaw AM 250 3D printer machine. The SLM processing parameters included powder layer thickness of $40 \mu \mathrm{m}$, laser power of 180 Watt, hatch distance of $0.08 \mathrm{~mm}$, hatch offset of $0.18 \mathrm{~mm}$, exposure time of $65 \mu \mathrm{s}$, and the bed temperature of $80^{\circ} \mathrm{C}$. The precursor powder particle size for SLM was maximum $63 \mu \mathrm{m}$ in diameter. 
Table 1. Nominal chemical composition of the used wrought and SLM-316L SS (wt. \%)

\begin{tabular}{|c|c|c|c|c|c|c|c|c|c|}
\hline Material & $\mathbf{C}$ & $\mathbf{P}$ & $\mathbf{S i}$ & $\mathbf{N i}$ & $\mathbf{M n}$ & $\mathbf{S}$ & $\mathbf{C r}$ & $\mathbf{M o}$ & $\mathbf{F e}$ \\
\hline Wrought 316L SS & 0.017 & 0.032 & 0.540 & 10.090 & 1.570 & 0.025 & 16.890 & 2.040 & Bal. \\
\hline SLM-316L SS & 0.030 & 0.025 & 0.750 & 14.000 & 2.000 & 0.010 & 18.000 & 2.620 & Bal. \\
\hline
\end{tabular}

The chemical composition of SLM-316L SS and its wrought counterpart are presented in Table 1. To investigate the impact of the building direction, the SLM produced samples were cross sectioned both perpendicular and parallel to the building direction, provided the top and the side views, respectively. To study corrosion resistivity of the samples, Cyclic Potentiodynamic Polarization (CPP) and Electrochemical Impedance Spectroscopy (EIS) tests were carried out. Before the electrochemical testing, all samples were cold mounted into an epoxy resin to limit the exposed area of the sample to only one face, while a wire was connected to the back side of the sample through the resin, providing the electrical connection during the electrochemical tests. This was followed by standard grinding and polishing of the surface to a mirror-like surface finish. All the tests were performed using an IVIUM Potentiostat/Galvanostat instrument in a three electrodes cell system containing the sample as the working electrode, a platinum plate as the auxiliary electrode, and $\mathrm{Ag} / \mathrm{AgCl}$ as the reference electrode. In all experiments, aerated $3.5 \mathrm{wt} . \%$ $\mathrm{NaCl}$ solution at room temperature $\left(25 \pm 1{ }^{\circ} \mathrm{C}\right)$ was used as the electrolyte. Before every test, open circuit potential (OCP) was measured for $3600 \mathrm{~s}$ to ensure the samples reached to the stability. The CPP test was conducted at scan rate of $1 \mathrm{mV} / \mathrm{s}$, starting from $-0.2 \mathrm{~V}$ versus the OCP up to the vertex current of $10 \mathrm{~mA}$, in which the scanning direction was then reversed. The EIS test was performed in an $\mathrm{AC}$ current with the amplitude perturbation of $\pm 10 \mathrm{mV}$ with respect to the OCP in a frequency range from $10^{5} \mathrm{~Hz}$ to $10^{-2}$ $\mathrm{Hz}$ in the 3.5 wt. $\% \mathrm{NaCl}$ solution at $25{ }^{\circ} \mathrm{C}$. For microstructural study, the polished samples were electroetched in $20 \%$ Nitric acid solution at the voltage of $1.7 \mathrm{~V}$ for $15 \mathrm{~s}$.

\section{RESULTS AND DISCUSSION}

Fig. 1 shows the microstructures of electro-etched wrought and SLM-316L samples. It can be seen that the SLM sample possesses a woven network of well-defined melt pools (Fig.1 a-d). This special structure has been resulted from the scanning action of the laser beam, where the long axis of the elliptical shaped melt pools corresponds to the direction of the laser scan in the SLM process (Fig. 1a-top view). Fig. 1d shows the cross-section of the printed sample across the plane parallel to the building direction (side view), revealing overlapped melt pools with semi-circular shapes. Clearly, the side view (Fig. 1d) contains higher density of the melt pool boundaries than the top view (Fig. 1a). At higher magnification, the austenite grains were appeared inside each melt pool and in each of the grains, elongated or equiaxed cellular sub-grains structure were revealed (Fig. $1 \mathrm{a}, \mathrm{c})$. The size of the sub-grains is around $1 \mu \mathrm{m}$ in diameter. These microstructural features are ascribed to extremely rapid solidification rate of the process, which in turn induces the elemental segregation and enrichment of sub-grain boundaries [7]. Trelewicz et al. [7] have shown that because of $\mathrm{Mo}$ and $\mathrm{Cr}$ segregation along the cell boundaries of SLM-316L, the corrosion characteristics of the intercellular regions are different, associated with rapid solidification and non-equilibrium nature of the process. Consequently, when the surface was electro-etched, the cell boundaries were not corroded as severe as the interior of the cells, resulting in unveiling the sub-grain structure. As expected, the microstructure of the wrought sample (Fig. 1e) contains annealed equiaxed grains along with recrystallized regions formed during annealing.

The cyclic potentioadynamic polarization curves of the SLM (both top and side views) and wrought samples obtained in aerated 3.5 wt. $\% \mathrm{NaCl}$ solution at room temperature are presented in Fig. 2. Electrochemical corrosion parameters including corrosion potential ( $\left.\mathrm{E}_{\mathrm{Corr}}\right)$, corrosion current $\left(\mathrm{i}_{\mathrm{Corr}}\right)$, pitting potential $\left(\mathrm{E}_{\mathrm{pit}}\right)$, defined as the inflection point in the anodic polarization curve, where the current density rapidly increases, and re-passivation potential $\left(E_{\text {rep }}\right)$, where the reverse scan intersects the forward scan, extracted from the CPP curves, are shown in Table 2. A combination of higher $\mathrm{E}_{\mathrm{Corr}}$ and lower $\mathrm{i}_{\text {Corr }}$ corresponds to a better corrosion performance. Comparing the corrosion data reveals that the top side of the SLM-sample has the highest corrosion resistance. All samples exhibited a clear passive region. Similarly, the top surface of the SLM sample possessed the widest passive range with the highest $\mathrm{E}_{\mathrm{pit}}$ contributing to a better corrosion properties of the sample. Using Tafel equation and tangent lines slopes on the cathodic and anodic regions of the curves, corrosion rates of the samples were derived that were found to be consistent with the above-mentioned results. In all samples, sharp fluctuations in current density can be seen in passive region showing the pitting initiation, so called metastable pitting. In fact, metastable pitting represents the start, growth and repassivation of a micro-pit on the surface of the samples. However, when the critical value of the potential is reached, the micro-pit can be transformed to a stable pit and then the passive layer on the surface is permanently ruined [10]. Comparing the $\mathrm{E}_{\text {rep }}$ of the samples, it is evidenced that SLM sample has weaker re-passivation property. 


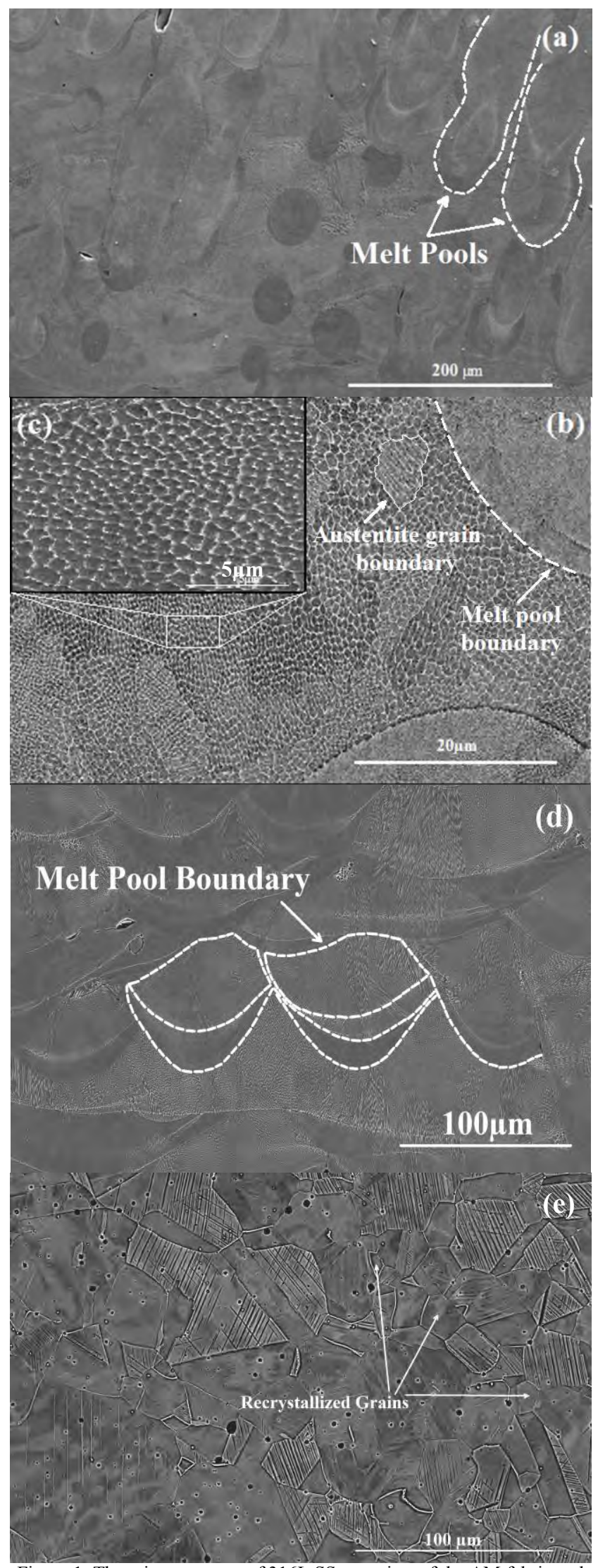

Figure 1. The microstructure of 316L SS: top view of the AM fabricated sample (a-c), side view of the AM fabricated sample (d), wrought sample (e).

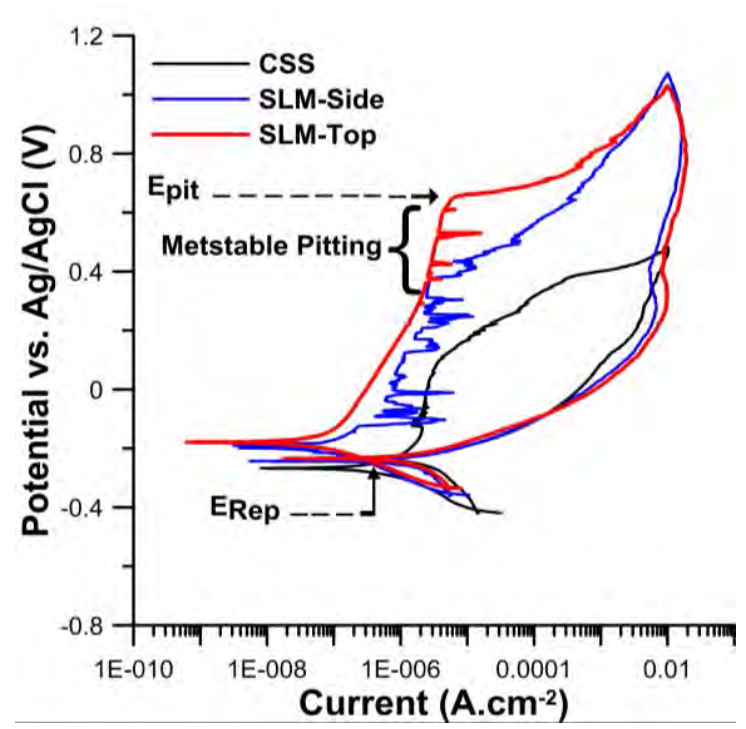

Figure 2. Cyclic potentiodynamic polarization curves of the studied samples.

No clear dependency so far has been reported between $E_{\text {Corr }}$, $\mathrm{i}_{\text {Corr, and }} \mathrm{E}_{\mathrm{pit}}$ of additively manufactured products with porosity level of the sample [11]. However, it has been shown that the amount of $E_{\text {rep }}$ in additively manufactured products is dependent on their porosity level [11]. Therefore, the improved re-passivation behavior of the wrought sample can be justified if the porosity level of the sample is considered zero relative to the SLM sample containing numerous pre-existing porosities in its structure (see Fig. 1d). The SEM micrographs of the SLM samples after the CPP test are depicted in Fig. 3. It can be seen that there is no preferred position for pit formation on the surface and the specimen exhibits isolated corrosion pits.

Additional experiment on the corrosion behavior of the samples were done by EIS tests to investigate the protective nature of the formed passive film on the 316L SS surface. The Nyquist plots in Fig. 4 depict a single semi-circle capacitive arc trend that corresponds to double layer and passive film formation on the samples' surfaces. This indicates that the corrosion mechanism of all three samples were identical and typical of stainless steel material. The EIS data showed a larger capacitive arc, an indication of improved corrosion resistance, for the SLM samples (both top and side views) than that of the wrought $316 \mathrm{~L}$ SS. It confirms the aforementioned CPP results of the corrosion properties. Comparing the results of the top and side views of the SLM sample reveals that the top view is covered by a more protective passive layer that can be correlated to the lower density of the melt pool boundaries on this face than that on the side view.

\section{Conclusion}

In the present work, the corrosion properties of the selective laser melted 316L stainless steel and its wrought counterpart were studied. Microstructural analysis of the samples 
revealed that the SLM processed stainless steel contained very fine cellular sub-grain structure resulted from scanning action of the laser beam combined with rapid solidification of the melt pools, contributing to elemental segregation along the intercellular boundaries. Through electrochemical tests, it was found that SLM-316L SS has higher pitting resistance, lower corrosion rate, and more noble corrosion potential than those of the wrought sample. However, its repassivation potential was degraded, which was primarily attributed to the pre-existing porosities in its structure formed during the fabrication process.

Table2. Corrosion characteristics of the studies samples

\begin{tabular}{|c|c|c|c|c|c|c|}
\hline Sample & $\mathbf{i}_{\text {Corr }}\left(\boldsymbol{\mu} \mathbf{A} / \mathbf{c m}^{\mathbf{2}}\right)$ & $\mathbf{E}_{\text {Corr }}(\mathbf{V})$ & $\mathbf{i}_{\text {Pitting }}\left(\boldsymbol{\mu A} \mathbf{A} / \mathbf{c m}^{\mathbf{2}}\right)$ & $\mathbf{E}_{\text {Pitting }}(\mathbf{V})$ & Corr. Rate $(\mathbf{m m} / \mathbf{y})$ & $\mathbf{E}_{\text {Rep }}(\mathbf{V})$ \\
\hline Wrought SS & 1.7957 & -0.3 & 3.630 & 0.0922 & 0.01824 & -0.2258 \\
\hline SLM-SS-Top & 0.1788 & -0.1923 & 7.026 & 0.6542 & 0.002165 & -0.2316 \\
\hline SLM-SS-Side & 0.1843 & -0.2 & 2.723 & 0.3656 & 0.002059 & -0.2412 \\
\hline
\end{tabular}

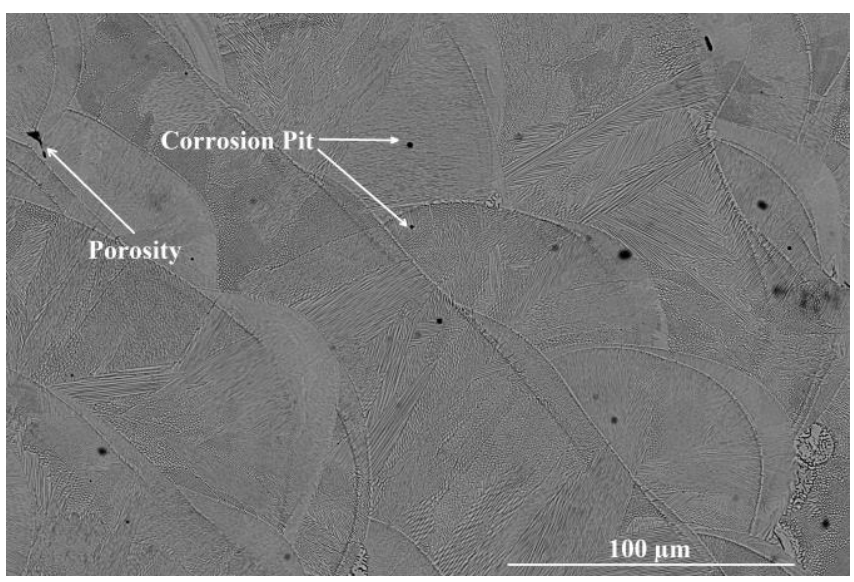

Figure 3. SEM micrograph of the AM sample after corrosion (side view).

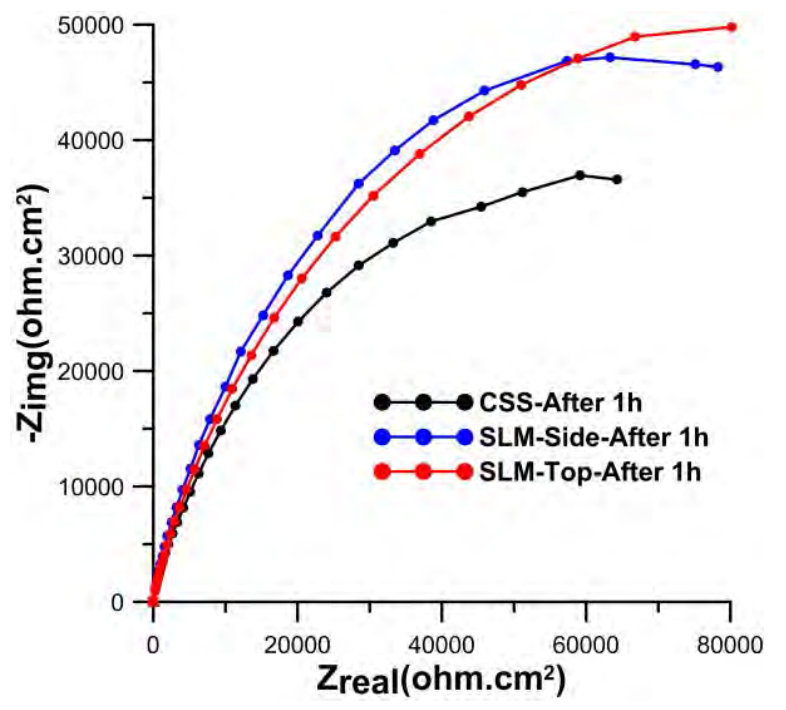

Figure 4. EIS measurement plots of the experimental samples.

\section{Acknowledgment}

The authors would like to thank the support of Natural Sciences and Engineering Research Council of Canada (NSERC) [grant number RGPIN-2017-04368], for sponsoring this work. M.M. would like to specially thank
NSERC [Grant number RGPIN-2016-04221] and New Brunswick Innovation Foundation (NBIF) [Grant number RIF2017-071] for providing enough funding to conduct this research work.

\section{References}

[1] M. Zietala et al., "The microstructure, mechanical properties and corrosion resistance of $316 \mathrm{~L}$ Stainless steel fabricated using laser engineered net shaping," Mater. Sci. Eng., A, vol. 677, pp. 1-10, Nov. 2016. doi: 10.1016/j.msea.2016.09.028.

[2] M. Ma, Z. Wang, and X. Zeng, "A comparison on metallurgical behaviors of $316 \mathrm{~L}$ stainless steel by selective laser melting and laser cladding deposition," Mater. Sci. Eng., A, vol. 685, pp. 265-273, Feb. 2017. doi: 10.1016/j.msea.2016.12.112.

[3] Y. Zhang et al, "Additive manufacturing of 316L stainless steel by electron beam melting for nuclear fusion applications," J. Nucl. Mater., vol. 486, pp. 234-245, Apr, 2017. doi: 10.1016/j.jnucmat.2016.12.042.

[4] J. Yu, M. Rombouts, and G. Maes, "Cracking behavior and mechanical properties of the austentitec stainless steel parts produced by laser metal deposition," Mater. Des., vol. 45, pp. 228-235, Mar. 2013. doi: 10.1016/j.matdes.2012.08.078.

[5] Y. Zhang et al., "Effects of surface quality on corrosion resistance of 316L stainless steel parts manufactured via SLM," J. Laser Appl., vol. 29, p. 022306, May 2017. doi: 10.2351/1.4983263.

[6] S. M. Yusuf et al.,"Investigation on porosity and microhardness of 316L stainless steel fabricated by selective laser melting," Met., vol. 7, no. 2, p. 64, Feb. 2017. doi: 10.3390/met7020064.

[7] J. R. Trelewicz et al., "Microstructure and corrosion resistance of laser additively manufactured 316L stainless steel," JOM, vol.68, no. 3, pp. 850-859, Mar. 2016.

[8] B. A. Almangour, "Additive manufacturing of highperformance 316L stainless steel nanocomposites via selective laser melting," Ph.D. dissertation, UCLA, 2017.

[9] X. Lou, M. A. Othon, and R. B. Rebak, "Corrosion fatigue crack growth of laser additively- manufactured 316L stainless steel in high temperature," Corros. Sci., vol. 127, pp. 120-130, Oct. 2017. doi: 10.1016/j.corsci.2017.08.023.

[10] P. Ganesh et al., "Studies on pitting corrosion and sensitization in laser rapid manufactured specimens of type $316 \mathrm{~L}$ stainless steel," Mater. Des., vol. 39, pp. 509-521, Aug. 2012. doi: 10.1016/j.matdes.2012.03.011.

[11] G. Sander et al., "On the corrosion and metstable pitting characteristics of 316L stainless steel produced by selective laser melting," J. Electrochem. Soc., vol. 164, no. 6, pp. 250-257, 2017. doi: $10.1149 / 2.0551706$ jes. 


\section{High Strain Rate Behavior of Mechanoluminescent Material Dispersed in a Soft Polymer Matrix}

\author{
Chukwubuikem Johnson Omeziri \\ Department of Mechanical Engineering, \\ Lassonde School of Engineering, York University \\ Toronto, ON, Canada \\ omeziri.johnson@gmail.com
}

\author{
Aleksander Czekanski \\ Department of Mechanical Engineering, \\ Lassonde School of Engineering, York University \\ Toronto, ON, Canada \\ alex.czekanski@lassonde.yorku.ca
}

\begin{abstract}
Mechanoluminescence (ML) is the emission of light from a solid material in response to mechanical stimuli [1]. Mechanoluminescent materials are classified based on the types of stress activation required for luminescence behaviour. In this paper, we propose the study of light emission characteristics of an elastico-mechanoluminescent (EML) material dispersed in a soft polymer matrix, under high-strain compressive loading for a qualitative and quantitative testing of onsite strain. We developed a strain sensor by creating a composite material through the combination of a polydimethylsiloxane (PDMS) matrix structure and strontium aluminate, europium, and dysprosium doped (SAOED) crystals. SAOED crystals possess a strong luminescence behavior, which has led to their proliferation as a viable optical sensor. Composite materials were tested using a modified Kolsky bar and high-speed camera. The light-emitting characteristics of the SAOED crystals in response to mechanical stimuli allows for the quantification of stress-strain experienced by the composite material. The light intensity, which is measured by a complementary metal-oxide-semiconductor (CMOS) sensor in a high-speed digital camera, provides a quantitative measure of the strain-rate. Light-emitting characteristics of the EML material and the strain behavior of the material were studied.
\end{abstract}

Keywords: mechanoluminescent crystals, high strain-rate, elasticomechanoluminescence, strain sensor, SAOED

\section{INTRODUCTION}

Measurement of stress and strain can be carried out via noncontact or contact methods such as digital image correlation (DIC) [2] and piezoresistive sensing [3]. In recent years, there has been concerted effort to develop noncontact strain-sensing technology for real-time monitoring of structures such as electronic speckle pattern interferometry (EPSI). However, this process requires tedious data processing techniques for stress and strain characterization of a material [4]. Non-contact strain sensing for structural health monitoring (SHM) of mechanical, civil, and aerospace structures has garnered interest in recent years [5-6]. High-impact forces, which are currently detected by SHM, have posed a great threat to the reliability of aerospace structures for over 50 years [7]. This problem has led to advancement in sensor technologies that allow for the monitoring and immediate measurement of stress-strain on these structures. These advanced sensors pose a significant challenge, as only trained professionals can fully interpret the data acquired from these measurements. Therefore, there is a need for an easy-to-use qualitative and quantitative stress-strain sensor for SHM.

Material that possesses the unique characteristic of mechanoluminescence (ML) makes it suitable for qualitative and quantitative stress-strain measurements. ML material emits light in response to mechanical stimuli such as tension, pressure, bending, compression, etc. ML material can be grouped into three categories: elastico-ML (EML), plastico-ML (PML), and fracto-ML (FML). These categories correspond to luminescence induced by elastic deformation, plastic deformation, and fracture, respectively [1]. The stress-induced luminescence behavior of fracto-ML and plastico-ML materials pose a severe challenge, as light is emitted post yielding. Hence, EML has been widely adopted for strain-sensing purposes, as it allows for non-destructive testing. This mechanical behavior of EML has led to its widespread use in sensing applications, such as a uniaxial tensile test for full-field strain measurement [8]. The mechanical and luminescent properties of EML materials provide both qualitative and quantitative measurements of strain.

Recently, Ryu et al. [9] proposed an ML composite based on polydimethylsiloxane (PDMS) for the measurement of high strain rate. They reported the light characteristics of the PDMSML composite europium tetrakis (dibenzoylmethide)triethylammonium (EuD4TEA) crystals under high-strain compressive loading using a Kolsky bar and an image processing technique. EuD $\mathrm{D}_{4} \mathrm{TEA}$ crystals are reportedly one of the brightest ML materials [10], and hence are used as a viable optical sensor. However, EuD 4 TEA crystals exhibit FML properties, which limit their application for non-destructive testing.

In this paper, we characterize the light-emitting properties of a PDMS-based EML composite for the direct visualization of stress and strain under high-strain compressive loading using a 
Kolsky bar. The EML crystal used is strontium aluminate, europium, and dysprosium doped (SAOED), which possesses a green phosphor. The SAOED crystal is uniformly dispersed in a PDMS matrix. A Split-Hopkinson pressure bar or Kolsky bar is widely used to measure the stress-strain relation of material under high-impact load. Strain and strain rate of the sample are derived from the pulse data generated by strain gauges on the input and output bar, assuming homogeneous deformation of the sample. A complementary metal-oxide-semiconductor (CMOS) sensor in a high-speed digital camera is used to measure the light emitted from the composite material. Image processing of the acquired 24-bit RGB images from the highspeed camera is used to quantify the light emission characteristics of SAOED crystals in the polymer matrix.

We began this study by creating a composite material made of SAOED crystals uniformly dispersed in a PDMS matrix. The composite material was then subjected to high-strain compressive loading. The strain data acquired from the Kolsky bar and images produced by the high-speed camera were then processed to obtain a quantitative relationship between strain and light intensity.

\section{EXPERIMENTAL METHODOLOGY}

\section{A. Materials}

PDMS (Sylgard 184 kit) was acquired from Dow Corning Corp., and SAOED crystals were purchased from SigmaAldrich and used as received.

\section{B. Composite Fabrication}

The PDMS mixture was first made by mixing a 10:1 weight ratio of PDMS base to curing agent. Then a 1:1 weight ratio of PDMS mixture to SAOED crystals was mixed and stirred. The combined mixture of PDMS and SAOED crystals was then heated in a polyethylene terephthalate (PETE) container for $\sim 40$ min on a heated bed at $52{ }^{\circ} \mathrm{C}$ (Fig. 1). The initial heating of the combined mixture increases the viscosity of the PDMS mixture and prevents the sedimentation of the SAOED crystals. Dispersion of the EML crystal was verified using an inverted microscope (Fig. 2A). The mixture was then degassed in a desiccator to remove air bulbs and poured into a circular aluminum mold with an inner circular diameter of $15 \mathrm{~mm}$ and a height of $4 \mathrm{~mm}$. The combined mixture of SAOED crystals and PDMS mixture was then cured over a heated bed at $100^{\circ} \mathrm{C}$ (Fig. 1). Six composite samples were made for each test.

\section{Experimental Setup}

A high-speed CMOS sensor camera (Phantom V1611) was used to photograph the ML emission during loading, and a SplitHopkinson pressure bar or Kolsky bar, supplied high-strain compressive loading. The high-speed camera, set with consistent exposure time, was positioned perpendicular to the Kolsky bar $\sim 30 \mathrm{~cm}$ from the sample. The Kolsky bar consists of a striker, input bar, and output bar, with strain gauges attached on the input and output bars. Two strain gauges were placed on the input bar and output bar, which were located close to the centre of the bar. The strain gauges were positioned $180^{\circ}$ from each other to negate bending waves when connected to a half-bridge circuit. Compressed nitrogen gas was used to drive the striker of the Kolsky bar. The pressure of the gas released is controlled by a valve on the compressed gas tank (Fig. 3). A hollow output bar

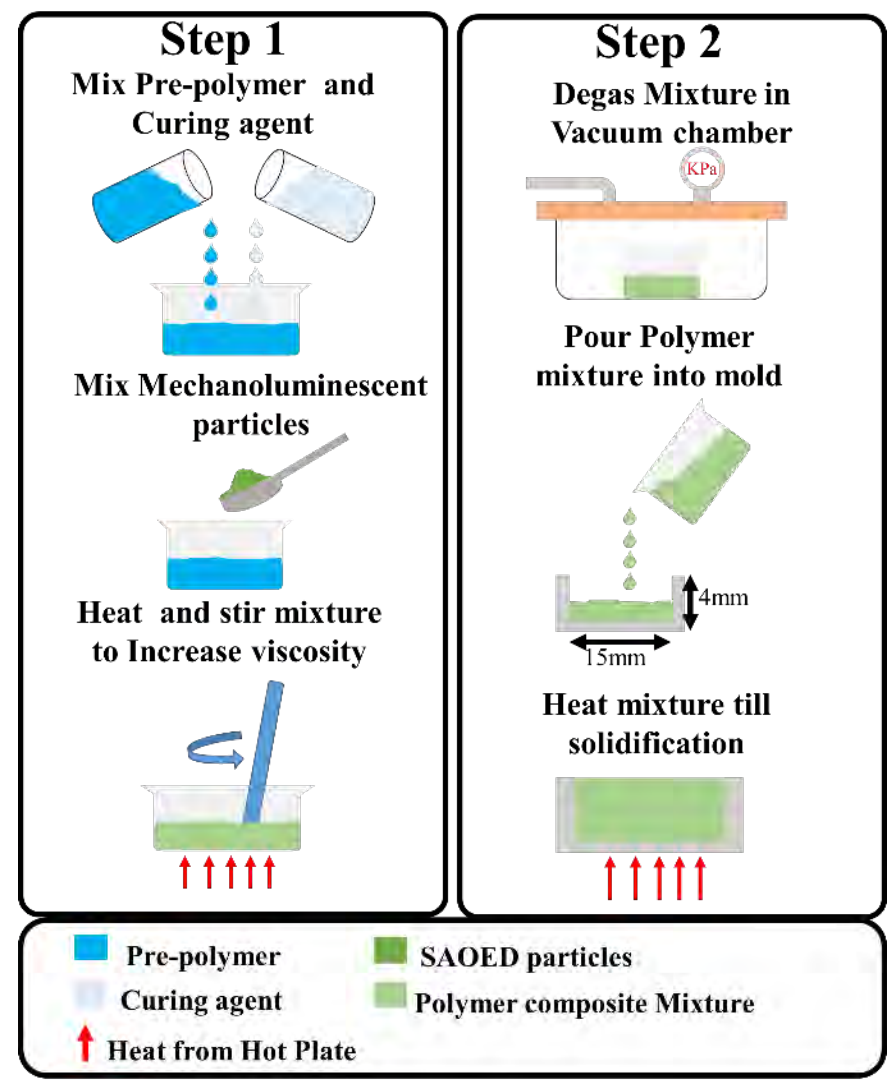

Figure 1. Fabrication process of strontium aluminate, europium, and dysprosium doped composite.

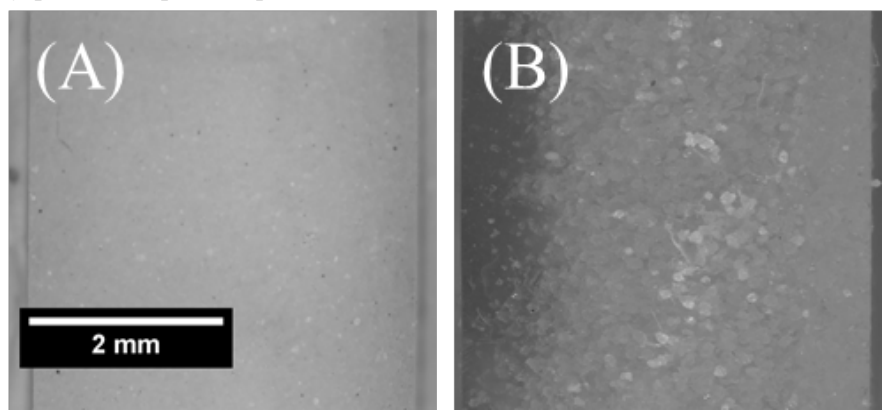

Figure 2. Mechanoluminescent crystals in polymer matrix. (A) Crystals suspended in the polymer matrix. (B) Initial result showing sedimentation of strontium aluminate, europium, and dysprosium doped (SAOED) crystals.

was used to increase load sensitivity [11]. National instrument USB-6341 was used for data acquisition from the strain gauges, and data acquisition occurred at $100 \mathrm{kHz}$. A relay was used to accurately synchronize the start time of the high-speed camera and the Kolsky bar (Fig. 3).

To begin testing, the PDMS-EML composite was consistently photo-excited by an $80 \mathrm{~W}$ LED lamp with a wavelength of 400-700 $\mathrm{nm}$ (white light) for $\sim 2 \mathrm{~min}$. This step ensured that the sample was fully saturated with the same photoexcitation power. An aging time of $\sim 2$ min immediately 


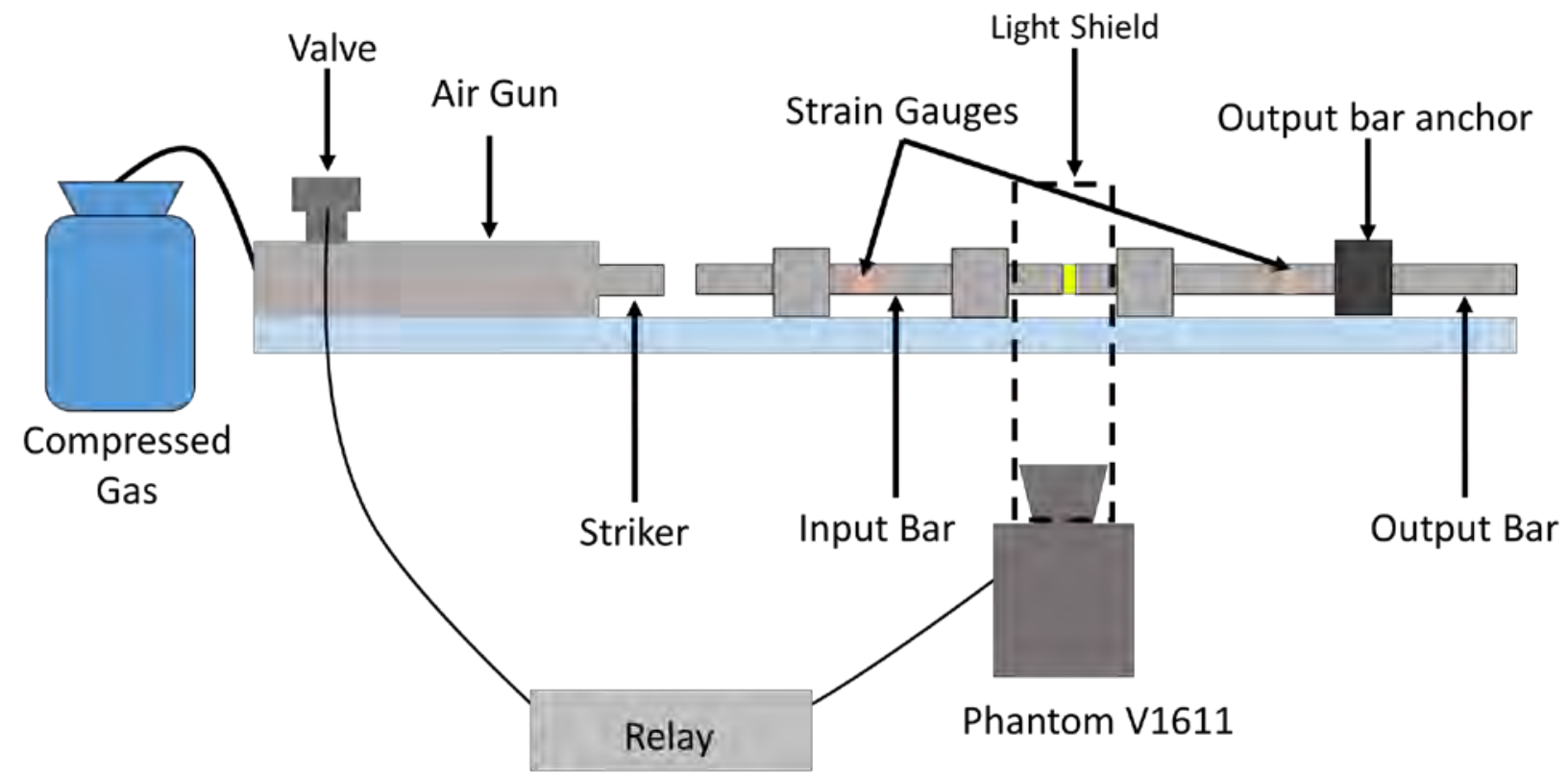

Figure 3. Experimental setup consisting of a Kolsky pressure bar and high-speed camera.
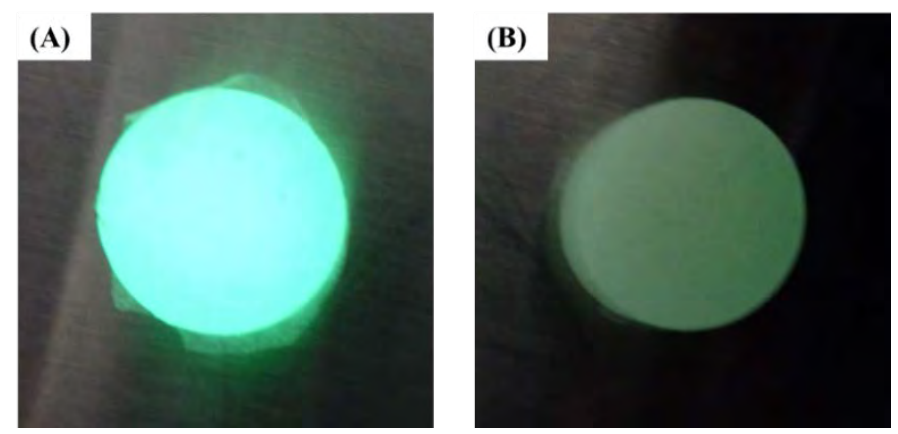

Figure 4. Photo-excitation of PDMS-EML composite. (A) Photo-excitation of PDMS-EML composite before aging. (B) Photo-luminescent stress-free decay of PDMS-EML composite.

followed this step, to minimize the effects of stress-free photoluminescent decay to maximize ML sensitivity [8]. The PDMSEML composite was aged in a dark environment before the loading was applied (Fig. 4).

\section{RESULTS AND DISCUSSION}

The light emission characteristics of the PDMS-EML composite were studied first, followed by derivation of strain from the pulse generated in the Kolsky bar. After aging the composite, the material was subjected to a high-impact compressive load from the Kolsky bar. The initial test was performed by controlling the shot pressure $(520 \mathrm{kPa})$ from the air gun (Fig. 3). Image data acquired from the high-speed camera at a frame rate of 50,000 frames/s and data from the strain gauges which were acquired at higher frequency were synchronized with timestamps. Image processing techniques were then used to characterize the light emission characteristics of the PDMS-EML composite.

Image processing involved exporting the video file from the camera in 24-bit AVI (Audio Video Interleave) format, with a custom image resolution of $64 \times 200$. From the RGB data collected green pixel values were chosen, as SAOED crystals possess a green phosphor. The green pixel values of the images were extracted, and an arithmetic mean of the values was used to quantify the intensity of light emitted. Green pixels could attain a mean value of 255 for very bright green and 0 for dark green. A second-order, low-pass filter and normalization were applied to the mean green pixel data to understand the emitting characteristics of the PDMS-EML composite (Fig. 4). The filtered and normalized mean green pixel data (hereinafter mean green pixel intensity, MGPI) were then plotted with respect to time (Fig. 5).

Fig. 5 shows the luminescence behavior of the PDMS-EML

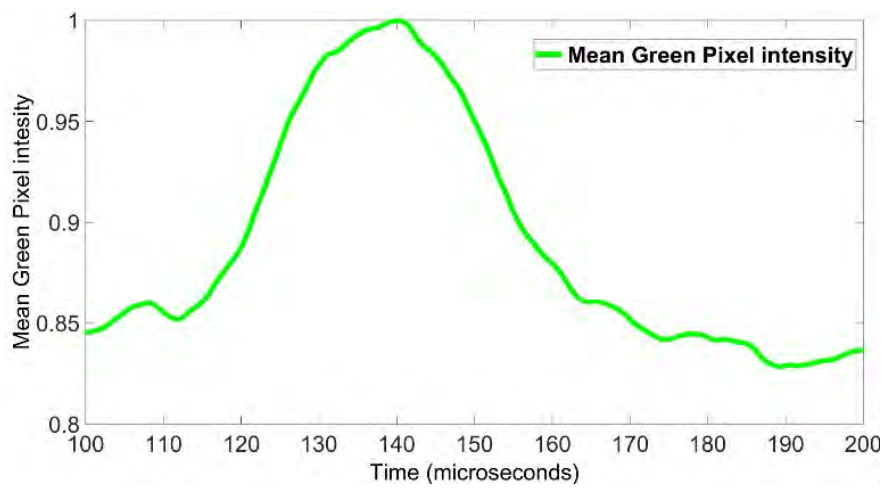

Figure 5. Quantification of light-emitting characteristics of SAOED crystals under compressive load.

composite during high-impact loading. The luminescence behavior displayed by the composite was similar to the pulse generated by the Kolsky bar.

The strain data were then correlated with the MGPI data with the aid of timestamps (Fig. 6). The luminescence behavior of the PDMS-EML composite does not provide a 1:1 correlation with the strain. However, has an instantaneous response to the applied strain. 


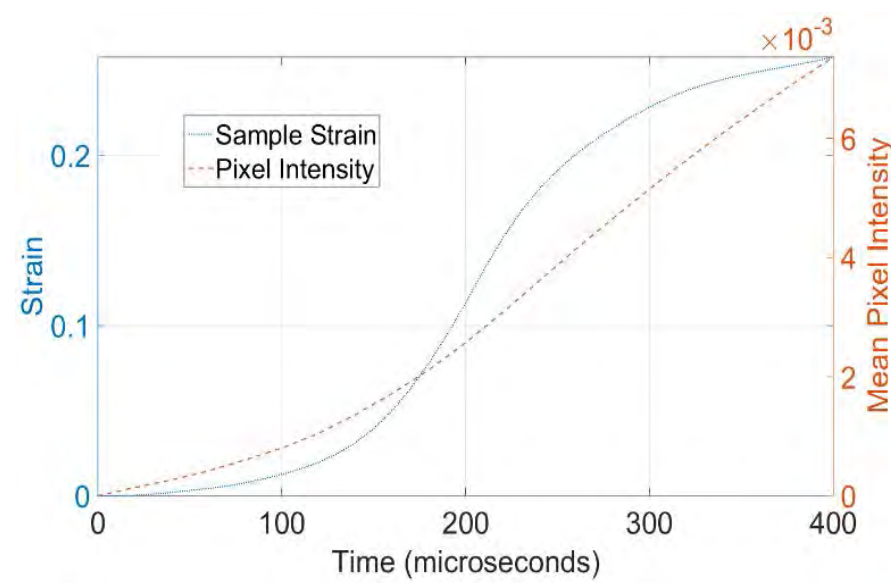

Figure 6. Strain and MGPI of PDMS-EML composite at a shot pressure of $520 \mathrm{kPa}$.

\section{CONCLUSION}

In this paper, we studied the behavior of an EML material dispersed in a PDMS matrix under high-strain compressive loading. For this purpose, a PDMS-EML composite was created using a clear silicone elastomer and SAOED EML crystals. The crystal dispersion in the polymer matrix was also studied to verify its uniform distribution within the matrix. To carry out mechanical testing, a Split-Hopkinson pressure bar or Kolsky bar was used to produce a high-strain compressive load on the composite material. A CMOS sensor high-speed camera was used to collect images of the light-emitting characteristics of the PDMS-EML composite. Image and data processing techniques were used to quantify the light-emitting characteristics of SAOED crystals in a soft polymer matrix as well as the mechanical behavior under a high strain rate.

Preliminary results of the PDMS-EML composite show great promise. However, the transmittance of the load to the crystals is limited in a soft polymer matrix, as the strain transmittance is low. Hence, the magnitude of light intensity observed was limited.

\section{ACKNOWLEDGMENT}

This research was partially supported by the Natural Sciences and Engineering Research Council Undergraduate Student Research Award and the Lassonde School of Engineering, York University. The authors are grateful for their financial support. We also thank Chaudhry Salman for his work and inputs on the Kolsky bar.

\section{REFERENCES}

[1] B. P. Chandra, "Mechanoluminescence," in Luminescence of Solids, D. R. Vij, Ed. Boston, MA: Springer US, 1998, pp. 361-389. doi: 10.1007/978-1-4615-5361-8 10.

[2] B. Pan and Z. Wang, "Recent progress in digital image correlation," in Application of Imaging Techniques to Mechanics of Materials and Structures, Volume 4, 2013, pp. 317-326.

[3] Y. Sun, T. Nishida and S. E. Thompson, "Piezoresistive Strain Sensors," in Strain Effect in Semiconductors: Theory and Device Applications, Boston, MA: Springer US, 2010, pp. 267-290. doi: 10.1007/978-1-4419-0552-9_8.

[4] C. Li, C. Xu, Y. Imai, W. Wang, L. Zhang and H. Yamada, "Real-time Monitoring of Dynamic Stress Concentration by Mechanoluminescent Sensing Film," Appl. Mech. Mater., vol. 13-14, no. 3, pp. 247-250, 2008.

[5] M. Fang, J. M. Niedzwecki, M. C. Ozbey, and T. J. Cummis, "A Brief Review of Structural Health Monitoring with Special Focus on Damage Detection and Sensor Optimization," Encyclopedia of Maritime and Offshore Engineering, John Wiley \& Sons, Ltd, pp. 1-8, 20-Apr-2017. doi: 10.1002/9781118476406.emoe328.

[6] H. Sohn, C. R. Farrar, F. Hemez, and J. Czarnecki, "A Review of Structural Health Monitoring Literature 1996 - 2001," Third World Conf. Struct. Control, Jan., pp. 1-7, 2002.

[7] C. A. Belk, J. H. Robinson, M. B. Alexander, W. J.Cooke and S. D. Pavelitz, "Meteoroids and Orbital Debris: Effects on Spacecraft," NASA Technical Report NRP-1408(1997).

[8] A. I. Azad, M. R. Rahimi, and G. J. Yun, "Quantitative full-field strain measurements by SAOED (SrAl2O4:Eu2+, Dy3+) mechanoluminescent materials," Smart Mater. Struct., vol. 25, no. 9, p. 095032, Oct. 2016. doi: 10.1088/0964-1726/25/9/095032.

[9] D. Ryu, N. Casta-o, R. Bhakta, and J. Kimberley, "Fractomechanoluminescent light emission of EuD 4 TEA-PDMS composites subjected to high strain-rate compressive loading." Smart Mater. Struc., vol. 26, no. 8, p.085006, Jul. 2017. doi: 10.1088/1361-665X/aa6fde.

[10] R. S. Fontenot, W. A. Hollerman, K. N. Bhat, and M. D. Aggarwal, "Effects of crystallite grain size on the triboluminescent emision for EuD4TEA," $A d v$. Mat. Lett., vol. 4, no. 8, pp. 605-609, 2013. doi: 10.5185/amlett.2012.12486.

[11] W. Chen, B. Zhang, and M. J. Forrestal, "A Split Hopkinson Bar Technique for Low Impedance Materials," Exp. Mech., vol. 39, no. 2, pp. 81-85, 1999. doi: 10.1007/BF02331109. 


\title{
Effects of Unit-Cell Boundary Type on the Electromechanical Properties of Randomly Distributed Multifunctional Composite Structures
}

\author{
Joshua Martin, Krishna Challagulla \\ Bharti School of Engineering, Laurentian University \\ Sudbury, Canada \\ Email: krishna.challagulla@laurentian.ca
}

\begin{abstract}
The unit-cell composition of three-dimensional finite element models for 3-0 and 3-1 type polymer (PVDF) ceramic (BaTiO3) and ceramic (PZT-7A) - ceramic (BaTiO3) structures are compared to determine the effects of fiber interaction at the surface of the unit-cell on the effective elastic, piezoelectric and dielectric properties of the multifunctional composite systems. The first unit-cell type examined has enclosed fibers that are completely contained within its boundaries, the second type has fibers that are contained within the sides of the unit-cell but can be cut at the top and bottom surfaces, and the third type has fibers that can be cut on the top, bottom and side surfaces of the unit-cell. All cut fibers are matched on opposing surfaces for continuity. Randomly distributed and aligned circular fibers, randomly distributed and randomly oriented circular fibers, and one central enclosed fiber with varying volume fractions and aspect ratios are compared with these three unit-cell structures. Results show that fiber models display greater or equal values of $\mathrm{C}_{22}$ when compared to aligned or randomly oriented fibers for all cases except aspect ratio 1 polymer-ceramic structures. The third type of unit-cell shows the highest $\mathrm{e}_{22}$ values for single, aligned and randomly oriented fiber structures, except for the aspect ratio 10 polymerceramic case where the second type of unit-cell has greater results for aligned and single fibers. Finally, it can generally be seen that randomly oriented fibers have smaller values than similar aligned and single fiber structures with the exception being $\mathrm{C}_{22}$ of the ceramic-ceramic structures.
\end{abstract}

Keywords-component; Multifunctional composites, finite element analysis, piezoelectricity, random fibers, smart structures

\section{INTRODUCTION}

Piezoelectric materials are being used more commonly for commercial and industrial applications due to their electromechanical properties. In products such as sensors, actuators and hydrophones their unique behavior makes them useful even as monolithic materials. Their properties, however, can be enhanced by combining multiple of these materials in a multifunctional composite structure. These composite systems can include many different types of materials, though the ones being examined in this study will be of the polymer-ceramic and ceramic-ceramic type.
Structured piezoelectric multifunctional composites have been studied fairly extensively from an analytical, numerical and experimental perspective. Kar-Gupta and Venkatesh determined the effects of geometry and grain-size on 3-0, 3-1 and 3-3 fibrous structures in [1]. Wu et al. examine the actuation performance of barium titanate nanoparticles in an epoxy resin in [2]. Yavarow and Erturk [3] develop a nonlinear elastodynamic model for fibrous piezoelectric composites, validating their mathematical model against experimental results.

This being said, randomly distributed and randomly oriented multifunctional composite structures have not been nearly as established. There has been some research done such as Berger et al. in [4], however a complete understanding of the effects of different structures on the effective electromechanical properties of random fiber multifunctional composites is not currently available. This study will try and determine how the interaction between fibers and the surface of a unit-cell will affect the overall properties. Thus, the objectives are:

(i) To develop a unit-cell based finite element model that will predict the elastic, dielectric and piezoelectric properties of several 3-0 and 3-1 type multifunctional composites with different boundary types at varying aspect ratios, volume fractions and cross-sectional shapes for aligned and randomly oriented fibers.

(ii) To systematically characterize the effects of unit-cell boundary structure on the effective electromechanical properties of multifunctional composites.

\section{Piezoelectric MATERIAls}

A total of three piezoelectric materials are used in this study: barium titanate $\left(\mathrm{BaTiO}_{3}\right)$, polyvinylidene fluoride (PVDF) and lead-zirconate titanate (PZT-7A). Their properties are outlined in table 1 on the next page. PVDF is used as the matrix polymer in the polymer-ceramic models, while PZT-7A is the matrix ceramic in the ceramic-ceramic case. $\mathrm{BaTiO}_{3}$ acts as the fiber in all composite structures. For this study, the matrix was poled in the 2-direction while the fibers were poled along their longitudinal axis for maximum effect, this is recognized as being an ideal case. 


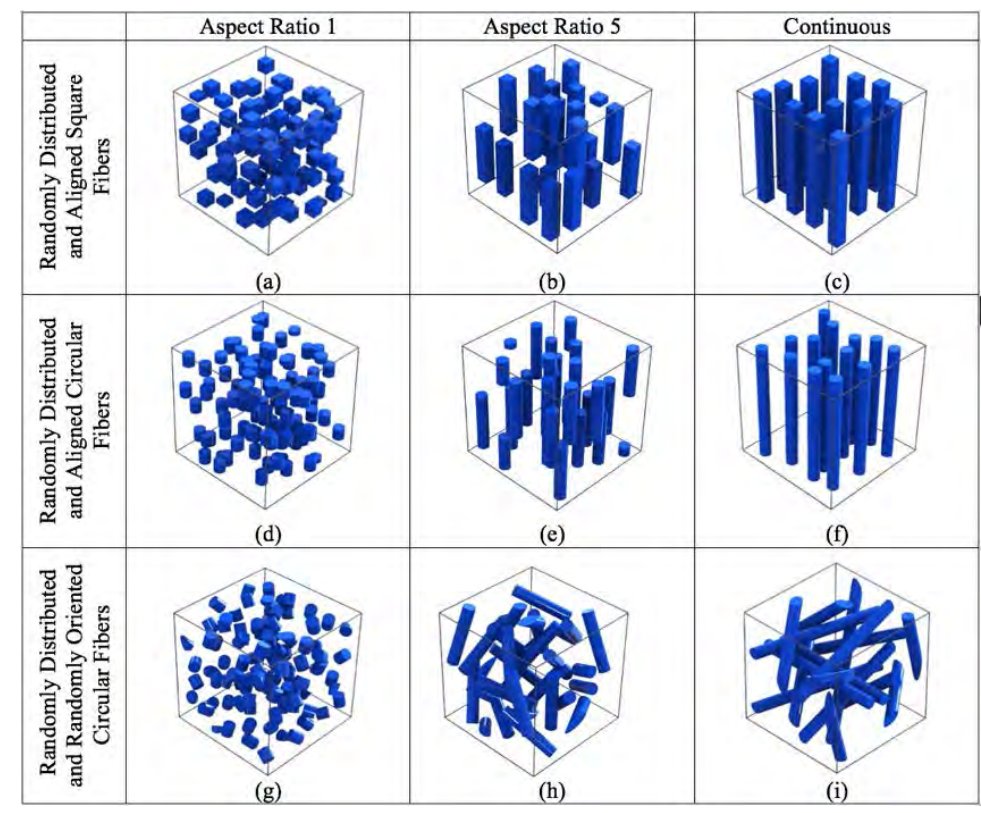

Figure 1: Schematic illustrating nine multifunctional composite structures based on fiber aspect ratio, cross-sectional shape, distribution and orientation.

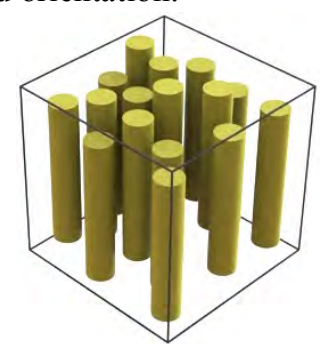

(a)

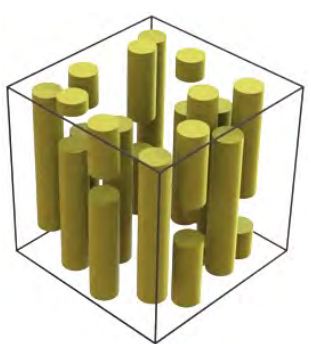

(b)

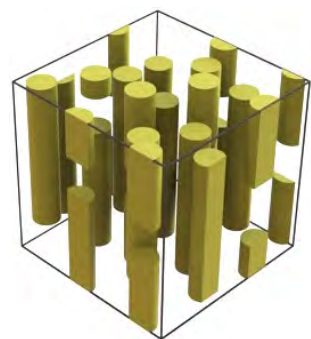

(c)

Figure 2: Unit-cell boundary types: (a) type 1 - fibers completely enclosed; (b) type 2 - fibers enclosed within the sides and not the top; (c) type 3 - fibers not enclosed within the surfaces.

TABLE I. MATERIAL Properties OF $\mathrm{BATIO}_{3}$, PZT-7A AND PVDF

\begin{tabular}{ccccc}
\hline Material Properties & & $\mathrm{BaTiO}_{3}$ & PZT-7A & PVDF \\
\hline $\mathrm{C}_{11}^{\mathrm{E}}=\mathrm{C}_{33}^{\mathrm{E}}$ & {$[\mathrm{GPa}]$} & 150.40 & 148.00 & 4.840 \\
$\mathrm{C}_{12}^{\mathrm{E}}=\mathrm{C}_{23}^{\mathrm{E}}$ & {$[\mathrm{GPa}]$} & 65.94 & 74.20 & 2.220 \\
$\mathrm{C}_{13}^{\mathrm{E}}$ & {$[\mathrm{GPa}]$} & 65.63 & 76.20 & 2.720 \\
$\mathrm{C}_{22}^{\mathrm{E}}$ & {$[\mathrm{GPa}]$} & 145.50 & 131.00 & 4.630 \\
$\mathrm{C}_{44}^{\mathrm{E}}=\mathrm{C}_{66}^{\mathrm{E}}$ & {$[\mathrm{GPa}]$} & 43.86 & 25.40 & 0.052 \\
$\mathrm{C}_{55}^{\mathrm{E}}$ & {$[\mathrm{GPa}]$} & 42.37 & 35.90 & 1.060 \\
$\mathrm{~K}_{11}=\mathrm{\kappa}_{33}$ & {$[\mathrm{nC} / \mathrm{Vm}]$} & 12.8 & 3.984 & 0.0664 \\
$\mathrm{\kappa}_{22}$ & {$[\mathrm{nC} / \mathrm{Vm}]$} & 15.1 & 2.081 & 0.0708 \\
$\mathrm{e}_{16}=\mathrm{e}_{34}$ & {$\left[\mathrm{C} / \mathrm{m}^{2}\right]$} & 11.40 & 9.31 & -0.001999 \\
$\mathrm{e}_{21}=\mathrm{e}_{23}$ & {$\left[\mathrm{C} / \mathrm{m}^{2}\right]$} & -4.32 & -2.324 & 0.004344 \\
$\mathrm{e}_{22}$ & {$\left[\mathrm{C} / \mathrm{m}^{2}\right]$} & 17.36 & 10.9 & 0.1099 \\
\hline & & & & \\
\hline
\end{tabular}

Piezoelectric materials are governed and fully defined by 21 elastic, 18 piezoelectric and 6 permittivity constants [5]. In the first equation, $\sigma$ represents a second order stress tensor, $C^{E}$ a fourth order elasticity tensor (at zero electric field), $\varepsilon$ a second order strain tensor, $\mathrm{E}$ an electric field vector, D an electric displacement vector, e a third order coupling tensor and $\kappa^{\varepsilon}$ a second order permittivity tensor (at zero strain). The indices $\mathrm{i}, \mathrm{j}, \mathrm{k}$ and $\mathrm{l}$ are remapped in the following way: $11 \rightarrow$ $1,22 \rightarrow 2,33 \rightarrow 3,23 \rightarrow 4,13 \rightarrow 5$ and $12 \rightarrow 6$.

$$
\begin{gathered}
\sigma_{i j}=C_{i j k l}^{E} \varepsilon_{k l}-e_{i j k} E_{k} \\
D_{i}=e_{i k l} \varepsilon_{k l}+\kappa_{i j}^{\varepsilon} E_{j}
\end{gathered}
$$

\section{Composite Unit-Cell Structure}

The multifunctional composite structures analyzed in this study were generated using python scripts which were then run using the commercially available finite element software, ABAQUS. The code developed created fibers with specified cross-sectional shapes and aspect ratios, then placed them randomly in a non-intersecting fashion within a unit-cell until a desired volume fraction was reached. Fibers were also constrained in relation to the boundary of the unit-cell in three ways, as shown in figure 2 :

- Type 1: fibers are completely enclosed within the boundary of the unit-cell. No fiber gets cut at or even touches any surface of the unit-cell.

- Type 2: fibers are enclosed within the four sides of the unit cell but can be cut on the top and bottom surfaces.

- Type 3: fibers can be cut on any surface of the unit-cell. 
All fibers cut on one surface have a complementary fiber on the opposing surface to have continuity if multiple unit-cells were to be attached, shown below in figure 3 .

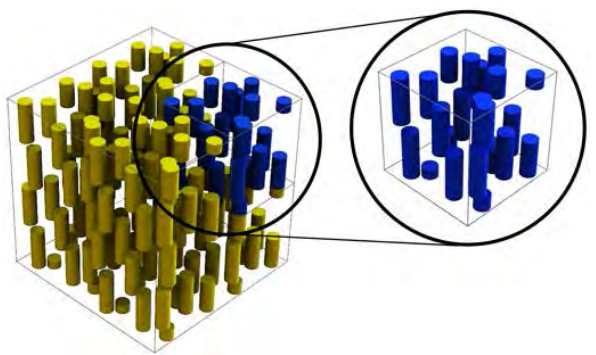

Figure 3: Continuity at unit-cell boundaries using complementary fibers.

The finite element models were run using threedimensional four-node linear piezoelectric tetrahedron elements (C3D4E). Nodes were given four degrees of freedom, each of the three directions and one electric potential $(1,2,3$ and 9, respectively). Fiber diameter and length was also kept constant in order to maintain aspect ratio. The number of fibers within a unit-cell could have been increased by keeping aspect ratio constant and reducing the diameter, however this was not done in this study to keep fibers consistent, which limited the volume fraction to approximately $25 \%$ [6].

\section{RESUlTS}

Finite element methods were used to analyze the various composite structures described in section 3 in order to determine effective elastic, dielectric and piezoelectric

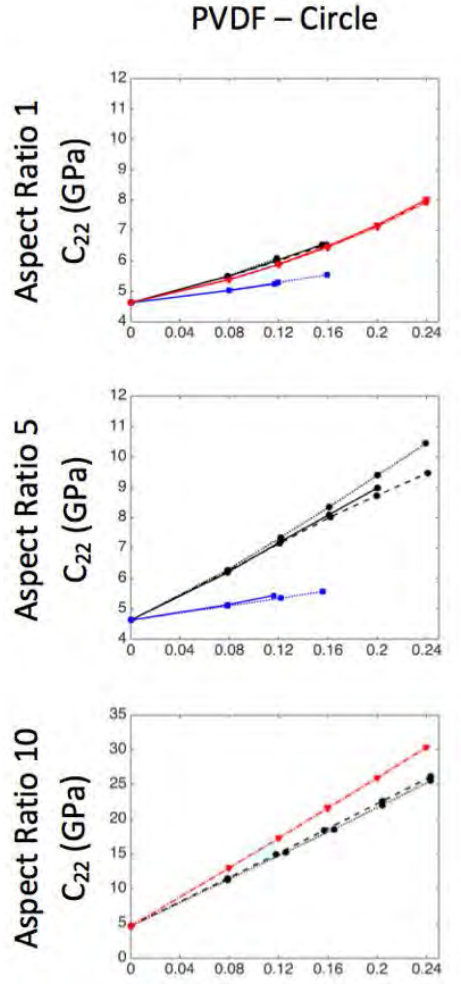

(a)
PVDF - Square
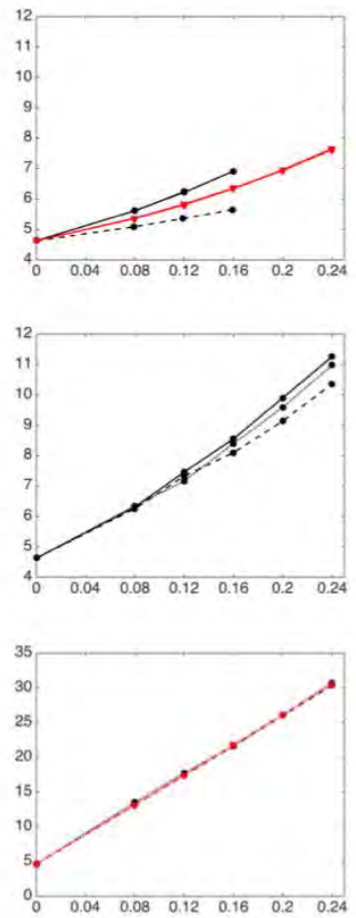

(b)

properties. The results plotted in the graphs of figure 4 show these effective properties for each structure at a given aspect ratio, volume fraction, cross-sectional shape, material and boundary type. Results displayed for any randomly distributed fiber structures are averages calculated from multiple iterations of the model. The cross-sectional fiber shapes examined are circular and square, while the material compositions studied are polymer-ceramic and ceramic-ceramic. Aspect ratio 1, 5 and 10 (short, medium and long fibers, respectively) are also compared with the three types of boundary structures.

The random fiber results from this study are initially compared to single fiber results with comparable structures to determine their accuracy. The circular single fiber results were verified against Kar-Gupta and Venkatesh [1], as well as Bowen et al [7], and Brito-Santana [8]. Single fiber results with the first type of boundary structure are shown to have nearly identical values and trends as those in [1,7 and 8], which is as expected since the structures are the same. There is some variance with different boundary structures and with randomly distributed fibers, however the models are overall in good agreement.

Square fibers were also compared with Kar Gupta and Venkatesh [9]. The aspect ratio 10 randomly distributed and single fiber structures were compared with those of [9] and showed similar trends. Though both studies examined polymerceramic and ceramic-ceramic structures with the same materials, the combinations of materials were different so an exact verification could not be made.

PZT - Circle
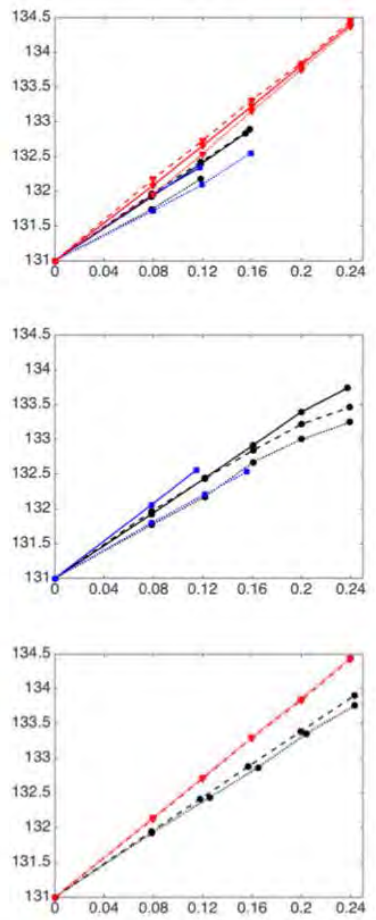

(c)
PZT - Square
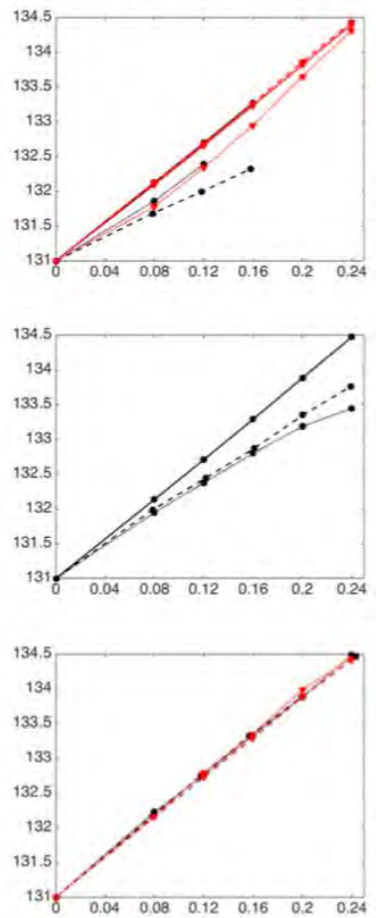

(d) 

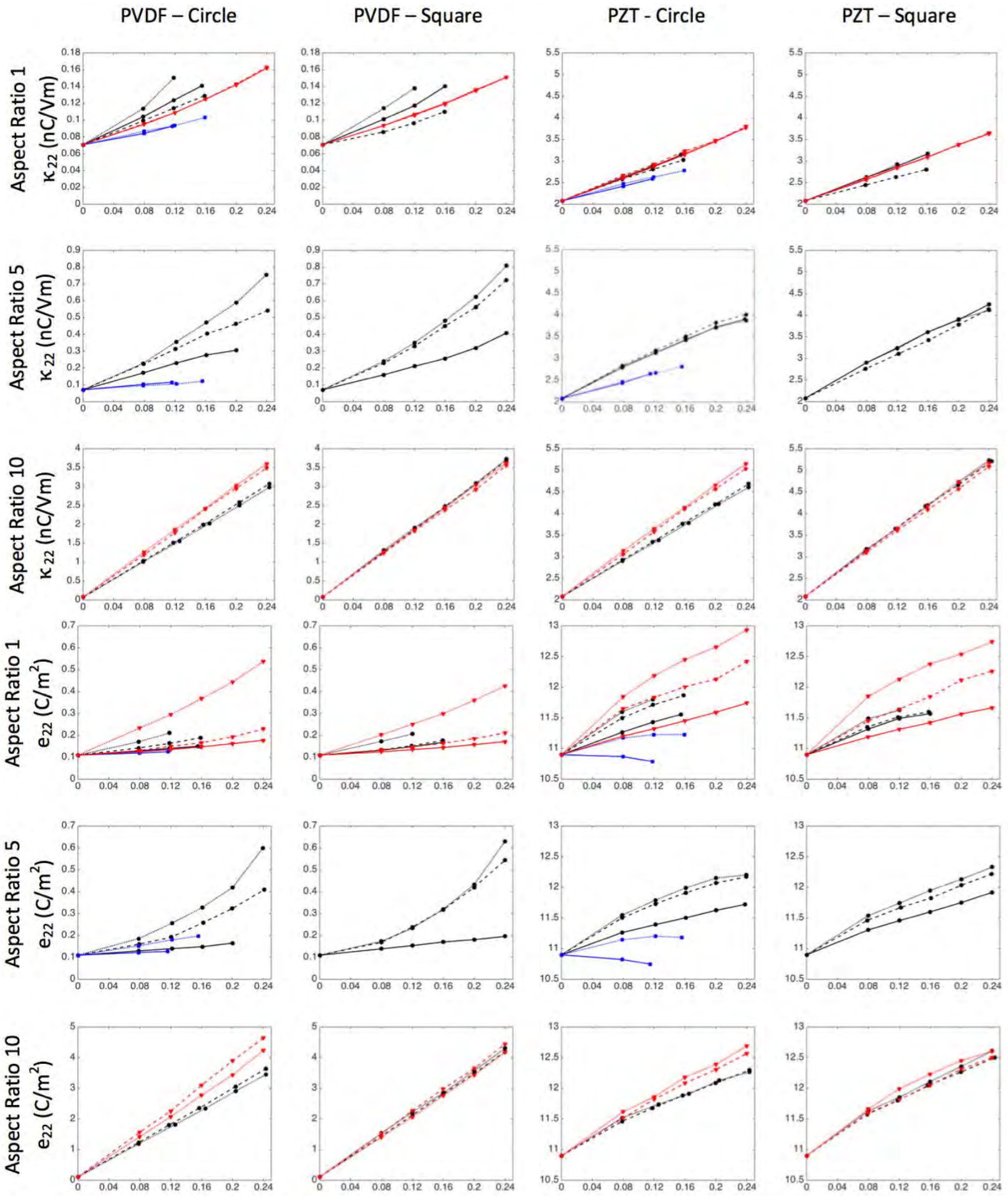

(a)

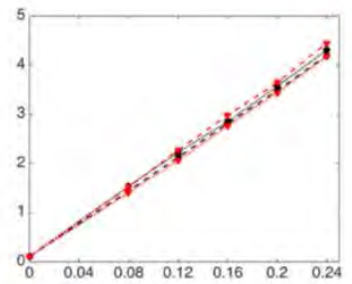

(b)

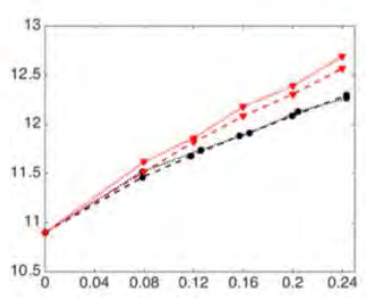

(c)

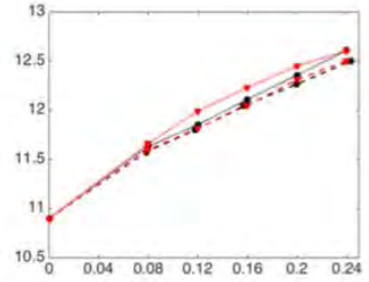

(d)

Figure 4: Variation of effective properties with respect to fiber volume fraction in (a) polymer (PVDF) - ceramic (BaTiO3) system with aligned and randomly distributed circular fibers, randomly oriented and randomly distributed circular fibers, and single aligned fibers (b) polymer-ceramic system with aligned, randomly distributed and single square fibers, (c) ceramic (PZT-7A) - ceramic (BaTiO3) system with aligned and randomly distributed circular fibers, randomly oriented and randomly distributed, and single aligned circular fibers, and (d) ceramic-ceramic system with aligned, randomly distributed and single square fibers - multifunctional composite structures. 
For $\mathrm{C}_{22}$, single fiber models display greater or equal values compared to aligned and randomly oriented fibers in all cases but aspect ratio 1 polymer-ceramic structures. In the longitudinal direction, it appears that the length and continuity of the fiber plays a more significant role in increasing mechanical properties than boundary type. It can, however, also be seen that the first type of boundary structure has the highest $\mathrm{C}_{22}$ value for a given structure when compared to the second and third boundary types with the exception of aspect ratio 1 ceramic-ceramic circular structures and aspect ratio 5 polymerceramic circular structures.

Randomly oriented fibers are generally seen to have the lowest values for $\mathrm{C}_{22}, \mathrm{e}_{22}$ and $\kappa_{22}$ when compared to aligned and single fibers with the exception being $\mathrm{C}_{22}$ of the ceramicceramic structures. Aligned fiber multifunctional composite systems have several distinct advantages when compared to these randomly oriented structures. They can be placed more efficiently in the unit-cell, leading to higher packing factors as well as providing generally flexible structures [10] with low acoustic impedance values [11]. Randomly oriented fiber structures, however, can provide higher values in transverse directions compared to aligned structures and can be easier to manufacture [12-15].

\section{CONCLUSION}

Random fiber multifunctional composites have a wide variety of advantages compared to structured and single fiber structures. Their ease in manufacturing and higher values in transverse directions make them useful and often less expensive. However, as the applications for these composites becomes more pronounced, their complexity will increase and the numerical models used to predict their behaviour will need to be improved. The purpose of this study was specifically to determine how the effective electromechanical properties of multifunctional composites might be affected by changes in the interaction between fibers and the surface of the unit-cell. At given volume fractions of fibers, aspect ratios, materials and cross-sectional shapes, the effects of the boundary structure of a unit-cell were found to be the following:

(i) $\mathrm{C}_{22}$ values were more commonly larger as a result of longer and continuous fibers rather than based on a unitcell boundary type, however the first type of boundary displays generally higher values compared to the second and third type except for aspect ratio 1 ceramic-ceramic circular structures and aspect ratio 5 polymer-ceramic circular structures.

(ii) The highest $e_{22}$ values for single, aligned and randomly oriented fiber structures except for the aspect ratio 10 polymer-ceramic case was the third type of unit-cell.

(iii) Randomly oriented fibers have the lowest values in general when compared to aligned and single fiber structures, with the exceptions of $\mathrm{C}_{22}$ of the ceramicceramic structures.

This study presents an overview of the effects different fiber-surface interactions can have on the overall properties of a unit-cell. When creating models to study the behaviour of random fiber multifunctional composite structures, the conclusions drawn here can help identify discrepancies in electromechanical properties between models that appear to be the same. As is shown, the same structure with different surface-fiber interactions can have varying effective mechanical, piezoelectric and dielectric properties without having a different core structure. The large variety of structures being compared in this study with varying aspect ratios, volume fractions, materials and cross-sectional shapes provides a baseline for future numerical models with more complex structures and unitcell surfaces.

\section{REFERENCES}

[1] R. Kar-Gupta and T.A. Venkatesh, "Electromechanical response of piezoelectric composites: Effects of geometric connectivity and grain size," Acta Mater., vol. 56, no. 15, pp. 3810-3823, 2008.

doi: 10.1016/j.actamat.2008.04.031.

[2] M. Wu, X. Yuan, H. Luo, H. Chen, K. Zhou, and D. Zhang, "Enhanced actuation performance of piezoelectric fiber composites induced by incorporated BaTiO3 nanoparticles in epoxy resin," Phys. Lett. A, vol. 381, no. 19, pp. 1641-1647, 2017.doi:10.1016/j.physleta.2017.02.025.

[3] T. P. Yavarow and A. Erturk, "Nonlinear elastodynamics of piezoelectric macro-fiber composites with interdigitated electrodes for resonant actuation," Compos. Struct., vol. 187, pp. 137-143, 2018. doi: 10.1016/j.compstruct.2017.12.056 .

[4] H. Berger, S. Kari, U. Gabbert, R. Rodriguez-Ramos, J. BravoCastillero, and R. Guinovart-Diaz, "Calculation of effective coefficients for piezoelectric fiber composites based on a general numerical homogenization technique," Compos. Struct., vol. 71, pp. 397- 400, 2005.

doi: 10.1016/j.compstruct.2005.09.038.

[5] P.W. Bosse, K.S. Challagulla., and T.A. Venkatesh, "Effects of foam shape and porosity aspect ratio on the electromechanical properties of 3-3 piezoelectric foams," Acta Mater., vol. 60, pp. 6464-6475, 2012. doi: 10.1016/j.actamat.2012.07.051.

[6] S. Kari, H. Berger, and U. Gabbert, "Numerical evaluation of effective material properties of randomly distributed short cylindrical fibre composites," Comput. Mater. Sci., vol. 39, pp. 198-204, 2007. doi: 10.1016/j.commatsci.2006.02.024 .

[7] R. Kar-Gupta and T.A. Venkatesh, "Electromechanical response of 1-3 piezoelectric composites: A numerical model to assess the effects of fiber distribution," Acta Mater., vol. 55, no. 4, pp. 1275-1292, 2007. doi: 10.1016/j.actamat.2006.09.042.

[8] X.J. Lin, K.C. Zhou, X.Y. Zhang, and D. Zhang, "Development, modeling and application of piezoelectric fiber composites," Trans. Nonferrous Met. Soc. China, vol. 23, pp. 98-107, 2013. doi:10.1016/S1003-6326(13)62435-8 .

[9] Y. Ismail, Y. Sheng, D. Yang, and J. Ye, "Discrete element modelling of unidirectional fibre-reinforced polymers under transverse tension," Composites Part B, vol. 73, pp. 118-125, 2015.

doi: 10.1016/j.compositesb.2014.12.024

[10] M. Chalh, S. Vedraine, B. Lucas, and B. Ratier, "Plasmonic Ag nanowire network embedded in zinc oxide nanoparticles for inverted organic solar cells electrode," Sol. Energy Mater. Sol. Cells, vol. 152, pp. 34-41, 2016. doi: 10.1016/j.solmat.2016.03.021.

[11] S. Kari, H. Berger, R. Rodriguez-Ramos, and U. Gabbert, "Computational evaluation of effective material properties of composites reinforced by randomly distributed spherical particles," Compos. Struct., vol. 77, no. 2, pp. 223-231, 2007.doi: 10.1016/j.compstruct.2005.07.003. 
[12] T. Vaughan and C. McCarthy, "A micromechanical study on the effect of intra-ply properties on transverse shear fracture in fibre reinforced composites," Compos. A Appl. Sci. Manuf., vol. 42, pp. 121728, 2011. doi: 10.1016/j.compositesa.2011.05.004.

[13] T. Vaughan and C. McCarthy, "Micromechanical modelling of the transverse damage behaviour in fibre reinforced composites," Compos. Sci. Technol., vol. 71, pp. 388-96, 2011.

doi: 10.1016/j.compscitech.2010.12.006.

[14] C.R. Bowen, A. Perry, H. Kara, and S.W. Mahon, "Analytical modelling of 3-3 piezoelectric composites," J. Eur. Ceram. Soc., vol. 21, no. 10-11, pp. 1463-1467, 2001. doi:10.1016/S0955-2219(01)00042-5.

[15] H. Brito-Santana, R. de Medeiros, R. Rodriguez-Ramos, anf V. Tita, "Different interface models for calculating the effective properties

inpiezoelectric composite materials with imperfect fiber-matrix adhesion," Compos. Struct., vol. 151, pp. 70-80, 2016.

doi: $10.1016 /$ j.compstruct.2016.02.003 


\section{ENVIRONMENTAL}

ENGINEERING 


\title{
WAVE INTERACTION OF PARTIALLY IMMERSED SEMICIRCULAR BREAKWATER SUSPENDED ON PILES USING FLOW-3D
}

\author{
A. A. Abo Zaid ${ }^{1}$, W. E. Mahmod ${ }^{2}$, A. S. Koraim ${ }^{3}$, E. M. Heikal ${ }^{4}$, H. E. Fath ${ }^{5}$ \\ ${ }^{I}$ Ph.D. student in Egypt-Japan University of Science and Technology (E-JUST), Egypt, ahmed.abozaid@ejust.edu.eg \\ ${ }^{2}$ Egypt-Japan University of Science and Technology (E-JUST), Egypt, wael.elham@ejust.edu.eg \\ ${ }^{3}$ Professor of harbor and marine structures, Faculty of Engineering, Zagazig University, Egypt, aymankoraim2004@yahoo.com. \\ ${ }^{4}$ Professor of harbor and marine structures, Faculty of Engineering, Zagazig University, Egypt, Elsadekheikal@yahoo.com. \\ ${ }^{5}$ Egypt-Japan University of Science and Technology (E-JUST), Egypt, hassan.fath@ejust.edu.eg
}

\begin{abstract}
This paper experimentally investigates the wave transmission through partially immersed semicircular breakwater suspended on two rows of circular piles. Different wave and structure parameters have been investigated such as; incident wave height, wave length, wave period, breakwater draft and the clear gap between the supporting piles. Multiple Polynomial Regression (MPR) model is used to develop an empirical equation to calculate the transmission coefficient $\left(\mathrm{k}_{\mathrm{t}}\right)$ as a representative of the transmitted wave energy. In addition, a Flow-3D numerical study is carried out to simulate the transmitted wave hydrodynamics and verified against the experimental results. The numerical analysis shows good agreement with experimental results with an acceptable value of NRMSE. The results show that the transmission coefficient $\left(\mathrm{k}_{\mathrm{t}}\right)$ decreases by about $31 \%$ with increasing the relative wave length from 0.12 to 0.22 and wave steepness from 0.006 to 0.021 . Also, the transmitted coefficient $\left(\mathrm{k}_{\mathrm{t}}\right)$ decreases by about $25 \%$ with increasing the relative immersion depths from $0.25 \mathrm{~m}$ to $0.5 \mathrm{~m}$ and increases by about $16 \%$ for increasing of pile porosity value from 0.5 to 0.83 respectively.
\end{abstract}

KEWORDS: Semicircular, Suspended Breakwater, Wave Transmission, Flow-3D, MPR.

\section{INTRODUCTION}

Breakwaters are used for coastal protection from incident wave attack by reducing part of the wave energy before it reaches the shoreline to enhance safe vessel berthing and maneuvering. Different types of breakwater are widely used and developed around the world. The conventional types of these breakwater are such as Rubble mound and bottom seated concrete types. When water depth increases $(>4.0 \mathrm{~m})$, these structures become more expensive and difficult to build and need high seabed bearing capacity. In addition, these breakwater types interrupt alongshore sediment transport and cause erosion to beaches downcoast of the breakwater. These conventional types of breakwater stop the seawater exchange that is essential for fish migration and for maintaining the water quality. The increased wave reflection in front of breakwaters causes also navigation problems. On the other hand, floating breakwater have the drawbacks that; i-they are ineffective for long waves range of 4 to 6 seconds [1], ii-vulnerable to structural failure during storms, iii-require a high amount of maintenance, large roll and iv-sway motions may affect its performance to be used as a berth or a pier. In order to avoid these disadvantages, the floating breakwater are suspended on the pile system (Suspended breakwater) which give it advantages such as: a-Low construction cost and require less material; bease of construction, c-applicability in poor soil foundation and complex bathymetry, d-less interference to the ecosystem, e-allow flow exchange between water body and open sea, f-enabling fish migration and g-preservation of water quality and sediment transport activity. They can also be dismantled and relocated with minimum effort and without leaving permanent damage to the environment and reduce visual impact. They can also sustain and preserve the natural beauty of the beach and therefore they favorable to the beach users.

In recent years, research has been conducted to develop new configurations of a breakwater; the partial breakwater. This new concept consists of a solid body, completely or partially immersed, suspended on concrete piles or floating and fixed by cables. The total height of such caissons is far smaller than the water depth. So, this type of breakwater is partially permeable to the incoming ocean waves, especially for long waves which can be transmitted below the structure. Since most of the wave energy is concentrated close to the water surface in deep water, a structure located near the free surface or intersecting it can dissipate part of this energy or reflect it by diffraction. Several investigations were carried out on the rectangular caisson, the most classical shape, for example: (Drimer et al., 1992),(Tolba, 1998),(Koutandos et al., 2004) and (Koutandos et al., 2005). These studies showed that this structure can reflect the incident wave energy for small wave periods. More complex configuration, named BYBOP proposed by (Duclos et al., 2004), is more efficient in reducing transmitted wave. Table 1 summarizes the different shapes of semi immersed breakwater investigated by previous researchers. 
Table 1.Characteristics of different models of semi immersed breakwater studied by previous researchers.

\begin{tabular}{|c|c|c|c|c|}
\hline Reference & Structure type & Modeling type & Model shape & Main parameters ranges \\
\hline $\begin{array}{l}\text { (Neelamani and Vedagiri, } \\
\text { 2002) }\end{array}$ & $\begin{array}{l}\text { Partially immersed } \\
\text { twin vertical } \\
\text { barriers }\end{array}$ & $\begin{array}{l}\text { Experimental } \\
\text { (Regular and } \\
\text { random waves) }\end{array}$ & & $\begin{array}{c}\mathrm{d} / \mathrm{h}=0.285, \mathrm{~B} / \mathrm{h}=1, \mathrm{~h} / \mathrm{L}=0.12- \\
0.45, \mathrm{Hi} / \mathrm{h}=0.067-0.102 \\
\mathrm{~B}=0.7, \mathrm{~d}=0.2, \mathrm{~h}=0.7\end{array}$ \\
\hline $\begin{array}{l}\text { (Sundar and Subba Rao, } \\
\text { 2002) }\end{array}$ & $\begin{array}{l}\text { Quadrant front } \\
\text { face pile supported } \\
\text { breakwater }\end{array}$ & $\begin{array}{l}\text { Experimental } \\
\text { (Regular waves) }\end{array}$ & & $\begin{array}{l}\mathrm{d} / \mathrm{h}=0.31-0.45, \mathrm{P}=0.5-0.83 \\
\mathrm{~B}=1, \phi=0.06, \mathrm{~h} / \mathrm{L}=0.16-0.6\end{array}$ \\
\hline (Koutandos et al., 2005) & & $\begin{array}{l}\text { Experimental } \\
\text { regular/irregular }\end{array}$ & & $\mathrm{d} / \mathrm{h}=0.2-0.33, \mathrm{~B} / \mathrm{h}=1, \mathrm{Hi} / \mathrm{h}=0.1$ \\
\hline (Koutandos et al., 2004) & $\begin{array}{l}\text { Single fixed } \\
\text { (restrained) }\end{array}$ & $\begin{array}{l}\text { Mathematical } \\
\text { Boussinesq Equ. }\end{array}$ & & \\
\hline (Tolba, 1998) & $\begin{array}{c}\text { floating } \\
\text { breakwater }\end{array}$ & $\begin{array}{l}\text { theoretically and } \\
\text { experimentally }\end{array}$ & & $\begin{array}{c}\mathrm{d} / \mathrm{h}=0.25, \mathrm{~B} / \mathrm{h}=0.5 \\
\mathrm{~h} / \mathrm{L}=0.1-0.5\end{array}$ \\
\hline (Drimer et al., 1992) & & Analytical & & \\
\hline (Koutandos, 2009) & $\begin{array}{l}\text { vertical semi- } \\
\text { immersed slotted } \\
\text { barrier }\end{array}$ & Numerical & ain & $\begin{array}{l}\mathrm{d} / \mathrm{h}=0.5 \\
\mathrm{~h}=2.0\end{array}$ \\
\hline (Teh et al., 2011) & $\begin{array}{l}\text { Free surface } \\
\text { semicircular }\end{array}$ & Experimental & & $\mathrm{d} / \mathrm{h}=0.071-0.214 \mathrm{~h}=0.7$ \\
\hline $\begin{array}{l}\text { (Koraim and Salem, } \\
\text { 2012) }\end{array}$ & $\begin{array}{l}\text { Single semi- } \\
\text { immersed } \\
\text { horizontal half } \\
\text { nines }\end{array}$ & Experimental & & $\begin{array}{c}\mathrm{d} / \mathrm{h}=0.5 \mathrm{~h} / \mathrm{L}=0.12-0.30 \\
\mathrm{~d}=0.1, \theta=90 \mathrm{o}, \mathrm{h}=0.20\end{array}$ \\
\hline (Duclos et al., 2004) & $\begin{array}{l}\text { Rectangular } \\
\text { caisson and } \\
\text { BYBOP }\end{array}$ & $\begin{array}{l}\text { Mathematical } \\
\text { RANS, Non Linear } \\
\text { Potential }\end{array}$ & & $\begin{array}{l}\mathrm{d} / \mathrm{h}=0.19 \\
\mathrm{~h}=0.8 \mathrm{~m}\end{array}$ \\
\hline (He and Huang, 2014) & $\begin{array}{c}\text { Suspended } \\
\text { oscillating water } \\
\text { column }\end{array}$ & Experimental & & $\begin{array}{c}\mathrm{d} / \mathrm{h}=0.25-0.5 ; \mathrm{Hi} / \mathrm{h}=0.0875 \\
\mathrm{Hi} / \mathrm{L}=0.01-0.03 \mathrm{~B}=\mathrm{h}=40\end{array}$ \\
\hline Present work & $\begin{array}{l}\text { Partially-immersed } \\
\text { semicircular } \\
\text { suspended on piles }\end{array}$ & $\begin{array}{l}\text { Experimental } \\
\text { and Numerical } \\
\text { (Flow 3d) }\end{array}$ & & $\begin{array}{c}\mathrm{d} / \mathrm{h}=0.25,0.5, \mathrm{~h}=0.4, \mathrm{~B}=0.1 \\
\mathrm{P}=0.5,0.83, \mathrm{~h} / \mathrm{L}=0.12-0.22 \\
\mathrm{Hi} / \mathrm{L}=0.006-0.021\end{array}$ \\
\hline
\end{tabular}

\section{EXPERIMENTAL TESTING}

\section{Experimental set-up}

The tested breakwater model was installed in the middle of the wave flume and situated between the inclined wave absorber and the wave generator. The module consists of a half-pipe manufactured from poly-vinyl chloride (PVC), with an outer diameter of $0.2 \mathrm{~m}$ and $0.01 \mathrm{~m}$ thickness. Vertical wood plate with a thickness of $0.01 \mathrm{~m}$ was fixed with the upper edge of the half pipe and the plate height was sufficient to prevent the wave overtopping on the breakwater. Two rows of PVC pipes with $0.05 \mathrm{~m}$ diameter $(\phi)$ and clear gap $(\mathrm{G})$ of 0.25 and $0.05 \mathrm{~m}$ were used as a supporter of the breakwater module as shown in Figure 1. The breakwater width (B) was kept constant at a value of $0.10 \mathrm{~m}$ and the tested breakwater drafts $(\mathrm{d})$ were 0.20 and $0.1 \mathrm{~m}$.

\section{Tests conditions}

Table 2 summarizes the tested values for different wave and breakwater parameters. Eight wave values were conducted with two breakwater draft (d) of 10 and $20 \mathrm{~cm}$ and two piles gap (G) of 5 and $25 \mathrm{~cm}$. The number of the experimental tests are 32runs.

\section{Wave gauge calibration}

Standard conductivity-type wave probe was used to measure the variations of water level with time. The wave probe comprises of two thin parallel stainless steel electrodes $(0.0015 \mathrm{~m}$ diameter, space $0.0125 \mathrm{~m}$ and length of $0.3 \mathrm{~m})$. The probe was connected to wave monitor module in the electronic console by a twin core flexible cable and delivered the output signals in the form of voltage data.Static calibration of the wave probe was carried out at the beginning of each set of experiments. Figure2 shows a linear relation between water level and output voltage resulting coefficient of determination $\left(\mathrm{R}^{2}=0.998\right)$. The linear equation was used in the programming of data acquisition card.The collected data 
during recording period converted into the water level by a simple computer program, resulting in the variation of water surface with time.

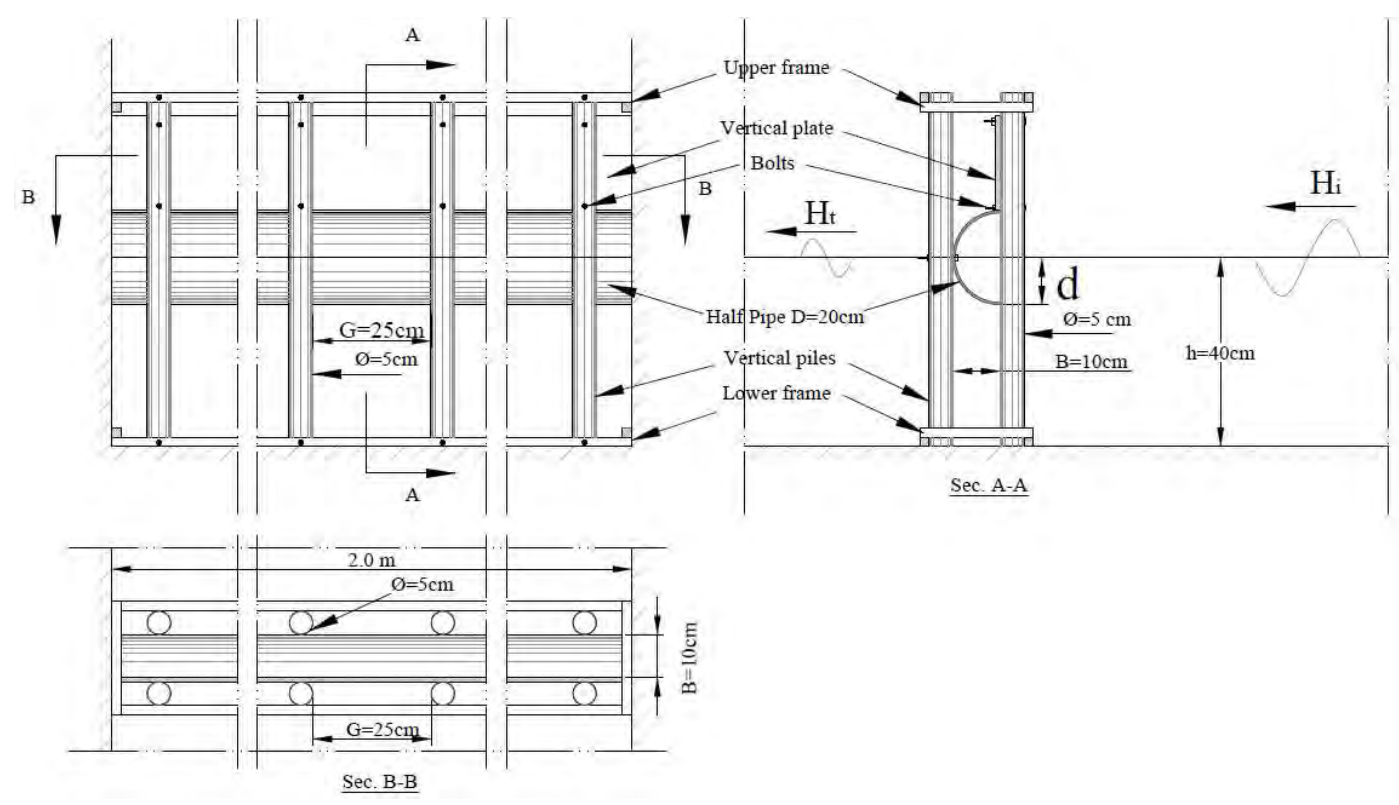

Figure 1. Different configuration of the tested breakwater.

Table 2.Experimental setup parameters of the breakwater model.

\begin{tabular}{ccc}
\hline Parameters & Unit & Ranges \\
\hline Wave periods (T) & $\mathrm{s}$ & $1.15-1.8$ \\
Wave length (L) & $\mathrm{m}$ & $1.81-3.35$ \\
Wave height (Hi) & $\mathrm{m}$ & $0.0207-0.038$ \\
Pile space (G) & $\mathrm{m}$ & $0.05-0.25$ \\
water depth (h) & $\mathrm{m}$ & 0.40 \\
breakwater width (B) & $\mathrm{m}$ & 0.10 \\
pile diameter ( $\phi)$ & $\mathrm{m}$ & 0.05 \\
outer pipe diameter (D) & $\mathrm{m}$ & 0.2 \\
Breakwater immersion depth (draft) (d) & $\mathrm{m}$ & $0.1-0.2$ \\
Relative wave length (h/L) & - & $0.12-0.22$ \\
Wave steepness (Hi/L) & - & $0.006-0.021$ \\
Relative immersion depth $(\mathrm{d} / \mathrm{h})$ & - & $0.25-0.5$ \\
Porosity of the lower part of breakwater $(\mathrm{P})$ & - & $0.5-0.83$ \\
\hline
\end{tabular}

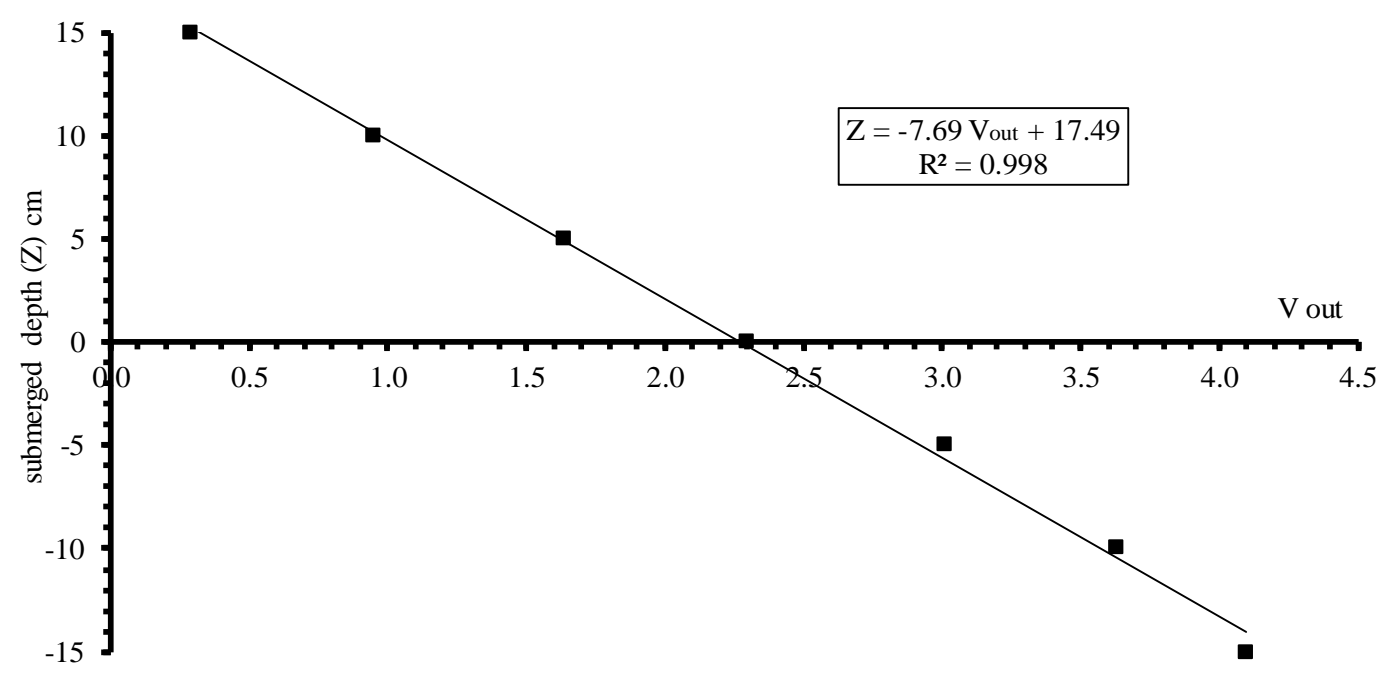

Figure 2.Calibration of the wave gauge. 


\section{Dimensional analysis}

The parameters affecting the transmission coefficient $\left(\mathrm{k}_{\mathrm{t}}=\mathrm{H}_{\mathrm{t}} / \mathrm{H}_{\mathrm{i}}\right)$ were investigated. These parameters are still water depth $(\mathrm{h})$, the unit weight of water $\left(\gamma_{\mathrm{W}}\right)$, the incident wave length $(\mathrm{L})$, the incident wave height $\left(\mathrm{H}_{\mathrm{i}}\right)$, the transmitted wave height $\left(\mathrm{H}_{\mathrm{t}}\right)$, the reflected wave height $\left(\mathrm{H}_{\mathrm{r}}\right)$, the acceleration of gravity $(\mathrm{g})$, the breakwater porosity $(\mathrm{P})$, breakwater immersion depth -draft (d) and the breakwater width (B). The relationship between these variables for the studied models is expressed as follows:

$$
\mathrm{f}\left(\mathrm{H}_{\mathrm{i}}, \mathrm{H}_{\mathrm{t}}, \mathrm{h}, \gamma_{\mathrm{w}}, \mathrm{L}, \mathrm{B}, \mathrm{d}, \mathrm{P}, \mathrm{g}\right)=0
$$

As the $\left(\gamma_{\mathrm{w}}\right),(\mathrm{g})$ and (B) are constant, then the number of variables $=6$, selecting $\mathrm{H}_{\mathrm{i}}$ as repeating variable, number of dimensionless parameter $=6-1=5$ as follows:

$$
\pi_{5}=\mathrm{f}\left(\pi_{1}, \pi_{2}, \pi_{3}, \pi_{4}\right)(2)
$$

Then, $k_{t}=f\left(h / L, H_{i} / L, P, d / h\right)$

The effect of $\mathrm{h} / \mathrm{Land} \mathrm{H}_{\mathrm{i}} / \mathrm{L}$ on $\mathrm{k}_{\mathrm{t}}$ is essentially need to investigate the hydrodynamic characteristics of the breakwater for coastal and deep-water regions and understand the performance of the breakwater for normal and extreme wave actions. Moreover, studying the effect of $\mathrm{P}$ and $\mathrm{d} / \mathrm{h}$ on $\mathrm{k}_{\mathrm{t}}$ is required to select the suitable structures configuration.

\section{Incident and transmitted wave heights measurement}

The dimensionless parameters were estimated from measuring the following variables in each individual experimental test. The incident wave heights $\left(\mathrm{H}_{\mathrm{i}}\right)$ was determined in the middle of the flume at the model location using the wave probe $\mathrm{P}_{1}$ (without breakwater model).The wavelength $(\mathrm{L})$ is calculated using the dispersion relationship based on the linear wave theory as follows: -

$$
L=\frac{g}{2 \pi} T^{2} \tanh (k h)(4)
$$

Where $\mathrm{k}$ is the wave number $(\mathrm{k}=2 \pi / \mathrm{L})$

The transmitted wave heights $\left(\mathrm{H}_{\mathrm{t}}\right)$ from the breakwater was measuredat the probe $\mathrm{P}_{2}$ behind the breakwater model at a distance of $1.5 \mathrm{~m}$ avoiding the effect of the turbulence caused by the wave breaking on the breakwater surface.(P1 and P2) locatins are shown in Figure 3 The transmission $\left(\mathrm{k}_{\mathrm{t}}\right)$ coefficient can be calculated as:

$$
\mathrm{k}_{\mathrm{t}}=\mathrm{H}_{\mathrm{t}} / \mathrm{H}_{\mathrm{i}}
$$

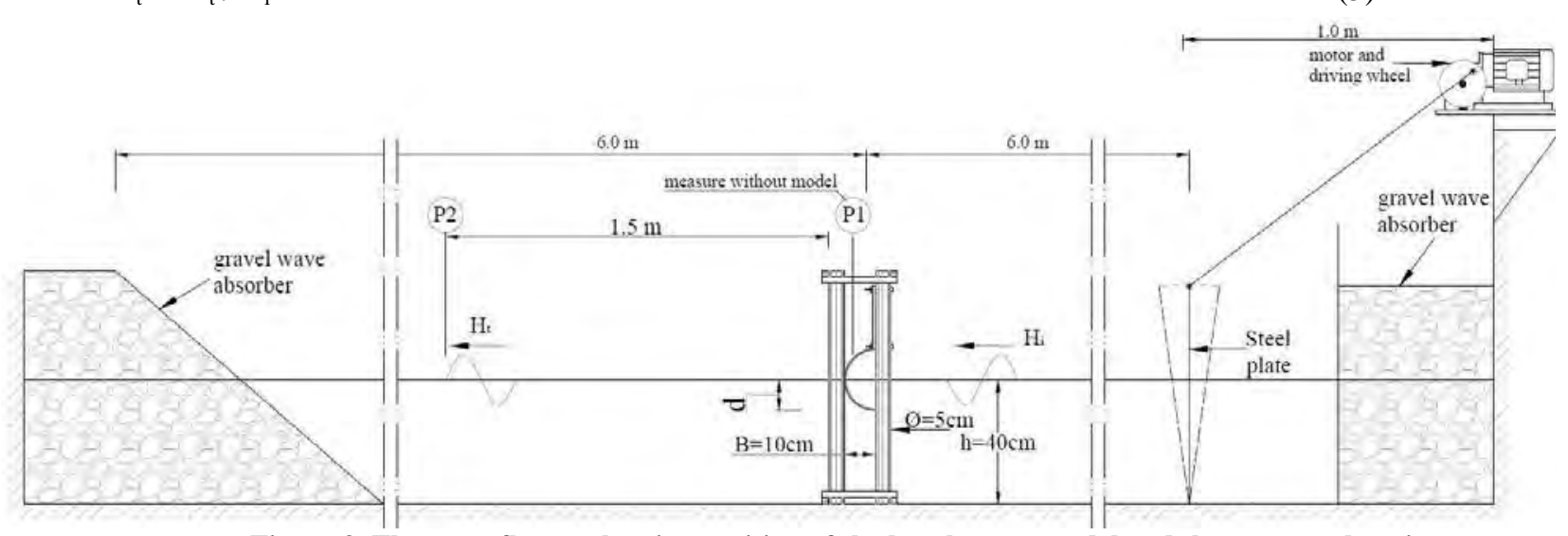

Figure 3. The wave flumes showing position of the breakwater model and the wave probes sites.

\section{Multiple Polynomial Regression Model (MPR)}

Using the above dimensionless parameters (See Equation 3), the regression analysis and the measured data, simple empirical equations for estimating the transmission coefficient for the different cases are developed. A Multiple Polynomial Regression (MPR) method was used to describe the behavior of a dependent variables $\left(\mathrm{k}_{\mathrm{t}}\right)$ that is related to the four independent variables $\mathrm{h} / \mathrm{L}, \mathrm{H}_{\mathrm{i}} / \mathrm{L}, \mathrm{P}, \mathrm{d} / \mathrm{h}\left(\pi_{1}, \pi_{2}, \pi_{3}, \pi_{4}\right)$ (Teh and Venugopal, 2013). The statistical program (SPSS Statistics 24 by IBM) was used to develop the empirical equations. The MPR technique was adopted to account for the nonlinearity of the data set and is expressed as:

$$
k_{t}=\left[\begin{array}{l}
a+a_{1} \Pi_{1}+a_{2} \Pi_{2}+a_{3} \Pi_{3}+a_{4} \Pi_{4} \\
a_{11} \Pi_{1}^{2}+a_{22} \Pi_{2}^{2}+a_{33} \Pi_{3}^{2}+a_{44} \Pi_{4}^{2}+ \\
a_{11} \Pi_{1} \Pi_{2}+a_{13} \Pi_{1} \Pi_{3}+a_{14} \Pi_{1} \Pi_{4}+ \\
a_{23} \Pi_{2} \Pi_{3}+a_{24} \Pi_{2} \Pi_{4}+ \\
a_{34} \Pi_{3} \Pi_{4}
\end{array}\right]
$$


Linear Regression analysis was usedand the predictors were selected by Enter method. The developed MPR model estimates the independent values $\left(\mathrm{k}_{\mathrm{t}}\right)$ by 14 predictors, shown in table 3 .

\section{Flow-3D numerical model}

Flow-3D is well known computational fluid dynamics(CFD) software, where the equations of motion are solved by the method of finite volume/finite differences in a Cartesian, staggered grid. The gravitational acceleration was set to $981\left[\mathrm{~cm} / \mathrm{s}^{2}\right]$. The viscosity and turbulence properties were set to be Newtonian Laminar flow which its result was more accurate and robust model available in the software.

\section{Meshing and geometry}

The semicircular model was exported in a stereo lithographic (stl) format to Flow-3D where the appropriate mesh could be generated. The accuracy of the results and the simulation time are effected by the cell size. So, it is important to minimize the amount of cells while including enough resolution to capture the important features of the geometry as well as sufficient flow details. For these reasons, multi-block meshwas used in the simulations, where bigger size cells were usedat less geometric details domain, and the smaller size cells near more geometric complexity as shown in Figure4.

\subsubsection{Boundary and initial conditions}

The upstream boundary condition in $\mathrm{x}$ direction was set to wave boundary and the downstream boundary was a wall boundary with wave absorbing block. All other open boundaries were specified as symmetric. The Stock wave theory of $5^{\text {th }}$ order has been used in Flow-3D; the wave is characterized by the wave height $\left(\mathrm{H}_{\mathrm{i}}\right)$, wave length $(\mathrm{L})$ and wave period $(\mathrm{T})$. In addition, at initial $(\mathrm{t}=0)$ a fluid region with height of $40 \mathrm{~cm}$ was simulated.

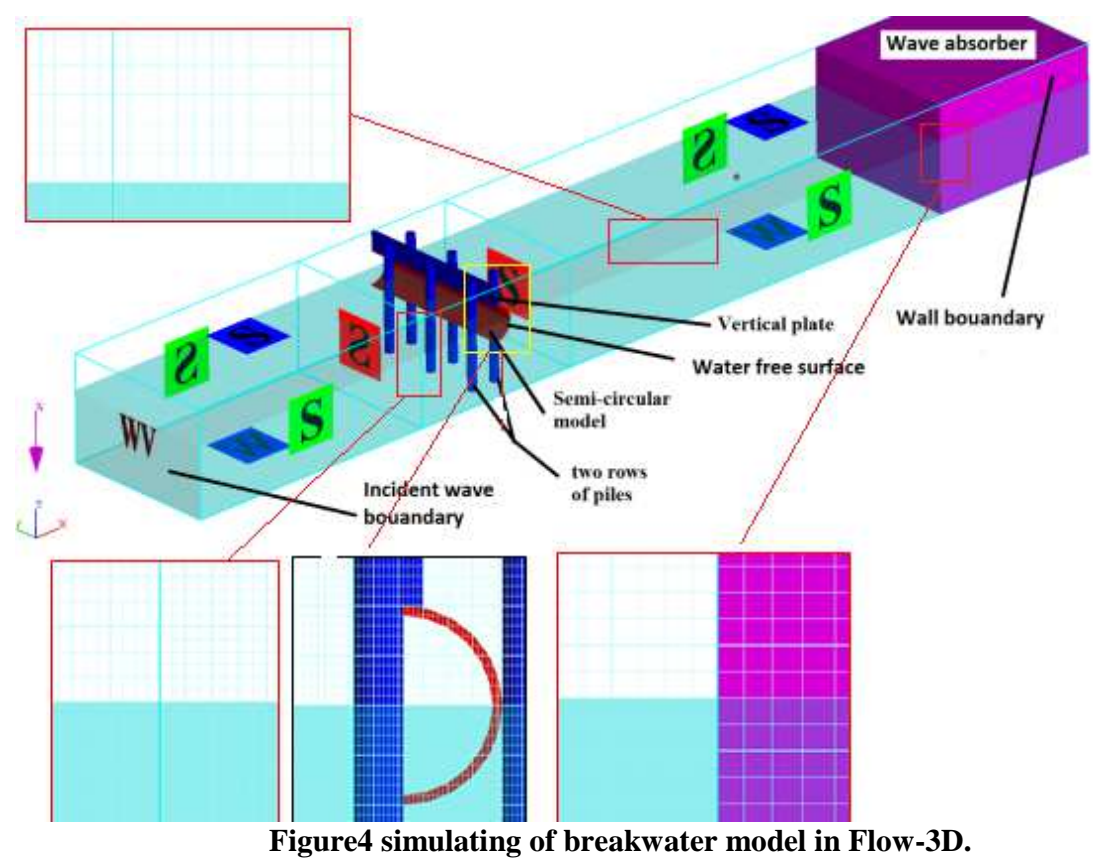

\section{RESULTS AND DISCUSSION}

the hydrodynamic efficiency of the partially immersed breakwater is affected by the wave and structure characteristics. The wave parameters are; wave length and height $\left(\mathrm{L}\right.$ and $\left.\mathrm{H}_{\mathrm{i}}\right)$, and the structural parameters are; the breakwater draft (d) and the space between piles (G). The dimensional analysis presents the hydrodynamic performance of the breakwater in the form of relationships between transmission coefficient $\left(\mathrm{k}_{\mathrm{t}}\right)$ and dimensionless parameters representing the wave and structure characteristics as presented in Equation 3. 


\section{Effect of Relative wave length $(\mathrm{h} / \mathrm{L})$ and Relative immersion depth $(\mathrm{d} / \mathrm{h})$}

Wave length is one of the most dominant parameters in the wave interaction with breakwater. In present study, the relative wave length $(\mathrm{h} / \mathrm{L})$ represents more the effect of wave length since the still water depth (h) was kept constant throughout the experiments. The variation of transmission coefficient $\left(k_{t}\right)$ as a function of $(h / L)$, is presented in Figure5 for relative immersion depth $(\mathrm{d} / \mathrm{h})$ ranges from 0.25 to 0.5 at piles porosity $(\mathrm{P})$ equal 0.5 and 0.83 . Figure 5 shows that by increasing of $(\mathrm{h} / \mathrm{L}), \mathrm{k}_{\mathrm{t}}$ decreases for all values of $(\mathrm{d} / \mathrm{h})$. For example, at $\mathrm{d} / \mathrm{h}=0.25$ and $\mathrm{P}=0.5, \mathrm{k}_{\mathrm{t}}$ decreases by $36 \%$ with $(\mathrm{h} / \mathrm{L})$ increases from 0.12 to 0.22 .Thisis presumably explained by considering the water particle motions. For shorter wave length, h/L increases, the water particles velocity and acceleration increase and after interaction with the breakwater. The water particles velocity and acceleration suddenly change then the resulted turbulence causes low wave transmission. While the breakwater width is not enough to effectively disturb the orbital cycle for longer wave length. Then, its hydrodynamic performance reduces for the lower value of $\mathrm{h} / \mathrm{L}$.

The variation of transmission coefficient, $k_{t}$, with respect to $(d / h)$ is also shown in Figure5 for relative wave length $(\mathrm{h} / \mathrm{L})$ ranges from 0.12 to 0.22 at piles porosity $(\mathrm{P})$ equal 0.5 and 0.83 . For all values of $(\mathrm{h} / \mathrm{L})$, by increasing of $(\mathrm{d} / \mathrm{h}), \mathrm{k}_{\mathrm{t}}$ decreases. For example, at $\mathrm{h} / \mathrm{L}=0.12$ and $\mathrm{P}=0.5, \mathrm{k}_{\mathrm{t}}$ decreases by $14 \%$ with $(\mathrm{d} / \mathrm{h})$ increases from 0.25 to 0.5 . This may be attributed to the increase of $(\mathrm{d} / \mathrm{h})$, the area that the water passes through decreases then the transmitted wave energydecreases.

\section{Effect of piles porosity (P)and Wave steepness (Hi/L)}

The variation of the transmission coefficient, $k_{t}$ with respect to $(P)$ is shown in Figure6 for wave steepness $\left(\mathrm{H}_{\mathrm{i}} / \mathrm{L}\right)$ ranges from 0.006 to 0.021 at $(\mathrm{d} / \mathrm{h})$ equal 0.5 and 0.25 . Figure6 shows that by increasing of $(\mathrm{P}), \mathrm{k}_{\mathrm{t}}$ increases for all values of $\left(\mathrm{H}_{\mathrm{i}} / \mathrm{L}\right)$. For example, at $\mathrm{H}_{\mathrm{i}} / \mathrm{L}=0.006$ and with $(\mathrm{P})$ increases from 0.5 to $0.83, \mathrm{k}_{\mathrm{t}}$ increases by $18 \%$ for $\mathrm{d} / \mathrm{h}=0.5$. This may be attributed to the increase of $(\mathrm{P})$, the area that the water passes through increases then the transmitted wave energy increases.

The variation of the transmission coefficients, $k_{t}$ a function of wave steepness $\left(H_{i} / L\right)$, is presented in Figure6 for piles porosity $(\mathrm{P})$ ranges from 0.5 to 0.83 at relative immersion depth $(\mathrm{d} / \mathrm{h})$ equal 0.5 and 0.25 . Figure6 shows that by increasing of $\left(H_{i} / L\right), \mathrm{k}_{\mathrm{t}}$ decreases for all values of $(\mathrm{d} / \mathrm{h})$. For example, at $\mathrm{d} / \mathrm{h}=0.5$ and with $\left(H_{i} / L\right)$ increases from 0.006 to $0.021, \mathrm{k}_{\mathrm{t}}$ decreases by $58 \%$ for $\mathrm{P}=0.5$.
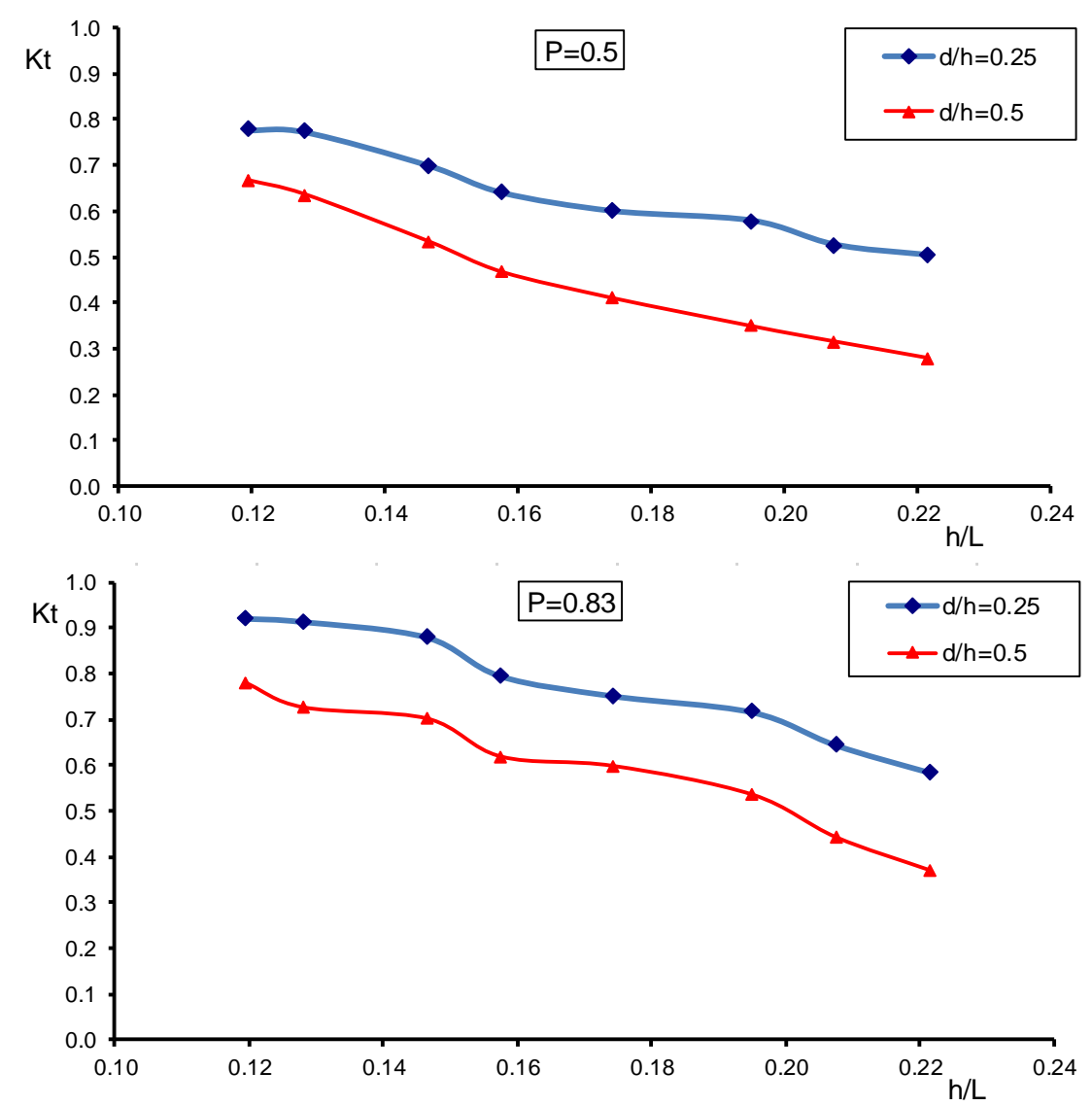

Figure5. Effect of Breakwater draft ratio $(\mathrm{d} / \mathrm{h})$ on the transmission coefficients as a function of relative wave length (h/L) when $P=0.5$ and 0.83 . 

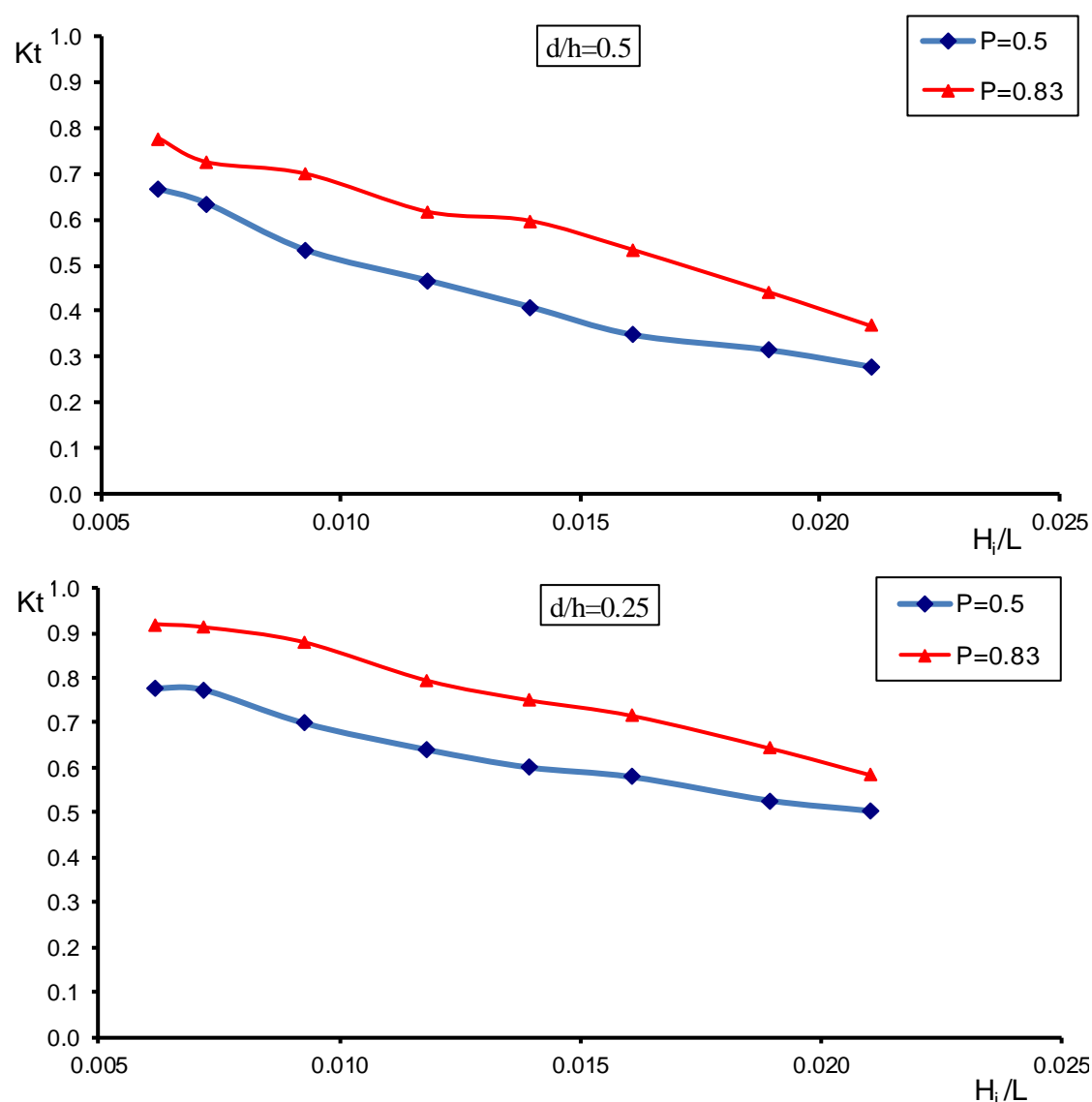

Figure6. Effect of pile porosity $(P)$ on the different hydrodynamic coefficients as a function of relative wave length $(\mathrm{h} / \mathrm{L})$ for a) $d / h=0.25$ and $b) d / h=0.5$.

\section{CALIBRATION OF MPR MODELS}

Table 3 presents the evaluation of coefficients values for MPR model using Stepwise method. while, Table 4 shows assessment of residuals analysis of transmission coefficient between calculated by MPR model and measured values.It can be observed that the equations over-predict and under-predict the transmission coefficient by values $\leq$ \pm 0.03 . Figure 7 shows the comparison between the measured and the calculated transmission coefficient $\left(k_{t}\right)$ of the breakwater using MPR model. A good agreement is obtained between the measured and the calculated transmission coefficient.

Table 3. Evaluation of coefficients values of parameters for MPR model

\begin{tabular}{ccc} 
Coefficient & parameter & value \\
\hline a & constant & 1.39 \\
a1 & h/L & -6.19 \\
a2 & Hi /L & 36.5 \\
a3 & d/h & - \\
a4 & $\mathrm{P}$ & -1.04 \\
a11 & $(\mathrm{h} / \mathrm{L}) 2$ & -0.27 \\
a22 & $(\mathrm{Hi} / \mathrm{L}) 2$ & 1329 \\
a33 & $(\mathrm{d} / \mathrm{h}) 2$ & 0.64 \\
$\mathrm{a} 44$ & $(\mathrm{P}) 2$ & - \\
$\mathrm{a} 12$ & $(\mathrm{~h} / \mathrm{L})(\mathrm{Hi} / \mathrm{L})$ & -154 \\
$\mathrm{a} 13$ & $(\mathrm{~h} / \mathrm{L})(\mathrm{d} / \mathrm{h})$ & -12.07 \\
$\mathrm{a} 14$ & $(\mathrm{~h} / \mathrm{L})(\mathrm{P})$ & 18.95 \\
$\mathrm{a} 23$ & $(\mathrm{Hi} / \mathrm{L})(\mathrm{d} / \mathrm{h})$ & 60 \\
$\mathrm{a} 24$ & $(\mathrm{Hi} / \mathrm{L})(\mathrm{P})$ & -136 \\
$\mathrm{a} 34$ & $(\mathrm{~d} / \mathrm{h})(\mathrm{P})$ & 0.09 \\
\hline
\end{tabular}


Table 4.Residuals analysis of MPR model.

\begin{tabular}{ccccc}
\hline Residual $=$ & \multicolumn{2}{c}{$\mathrm{d} / \mathrm{h}=0.5$} & \multicolumn{2}{c}{$\mathrm{d} / \mathrm{h}=0.25$} \\
measured-calculated & $\mathrm{P}=0.83$ & $\mathrm{P}=0.5$ & $\mathrm{P}=0.83$ & $\mathrm{P}=0.5$ \\
\hline RMSE x $\left(10^{-2}\right)$ & 1.601 & 1.674 & 0.961 & 1.317 \\
Min Res. & -0.02 & -0.028 & -0.016 & -0.019 \\
Max Res. & 0.03 & 0.024 & 0.013 & 0.02 \\
NRMSE & 0.04 & 0.045 & 0.03 & 0.046 \\
\hline
\end{tabular}

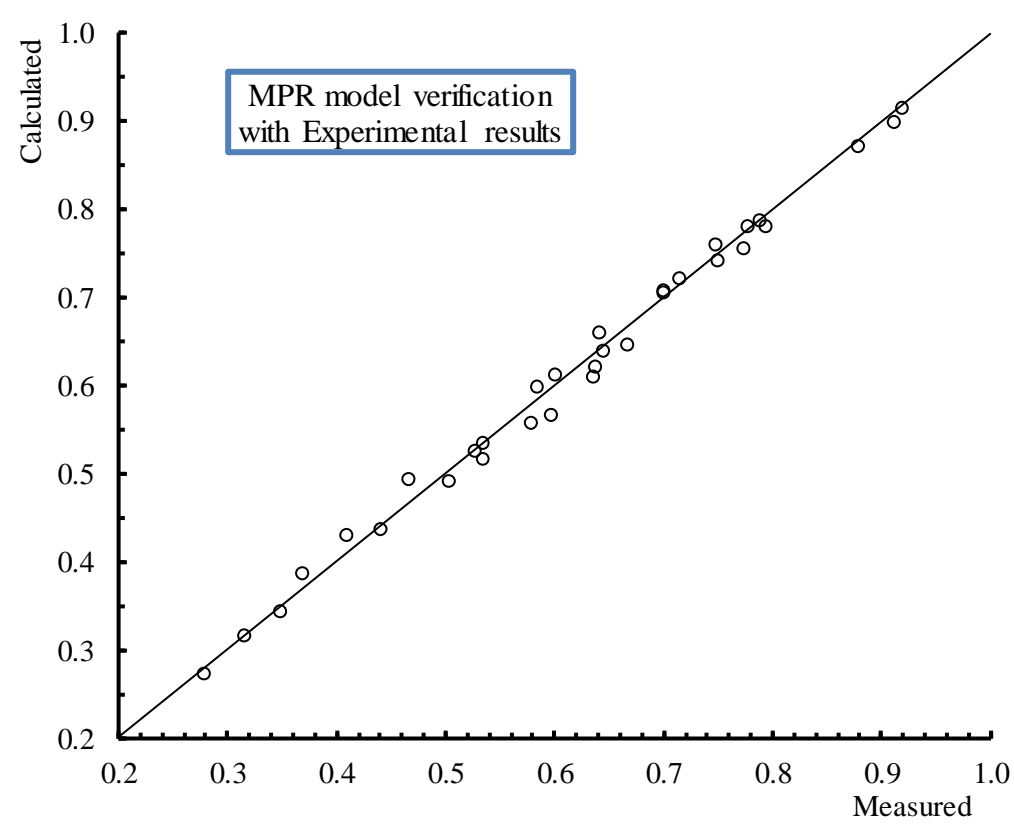

Figure 7.Comparison between calculated and experimental transmission coefficients results for MPR model.

\section{VALIDATION OF NUMERICAL RESULTS}

Figure8 shows data sample of the water elevation variation at location (P2) which represent the transmitted wave $\left(\mathrm{H}_{\mathrm{t}}\right)$. The figure presents the validation between experimental and numerical results for $\mathrm{d} / \mathrm{h}=0.25, \mathrm{P}=0.83, \mathrm{Hi}=0.038 \mathrm{~m}$ and $\mathrm{T}=1.15 \mathrm{~s}$. An acceptable agreement, between Flow-3D numerical and experimental results, can be observed.

Figure 9 presents a comparison between the measured and the calculated transmission coefficient $\left(\mathrm{k}_{\mathrm{t}}\right)$ of the breakwater using Flow-3D model. A good agreement is obtained between the measured and the calculated transmission coefficient.

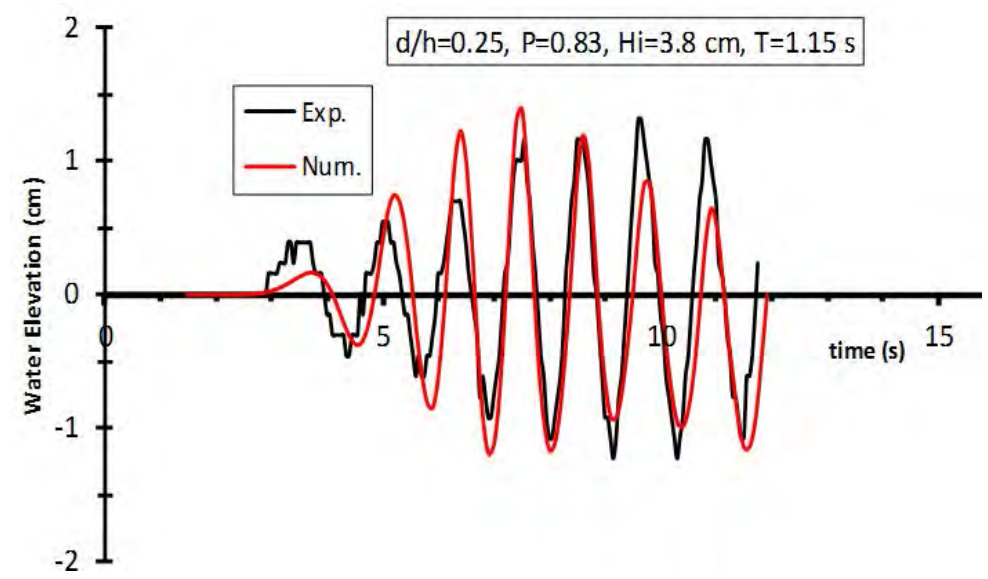

Figure8. variation of water elevation with time @ P2 for experimental and numerical results. 


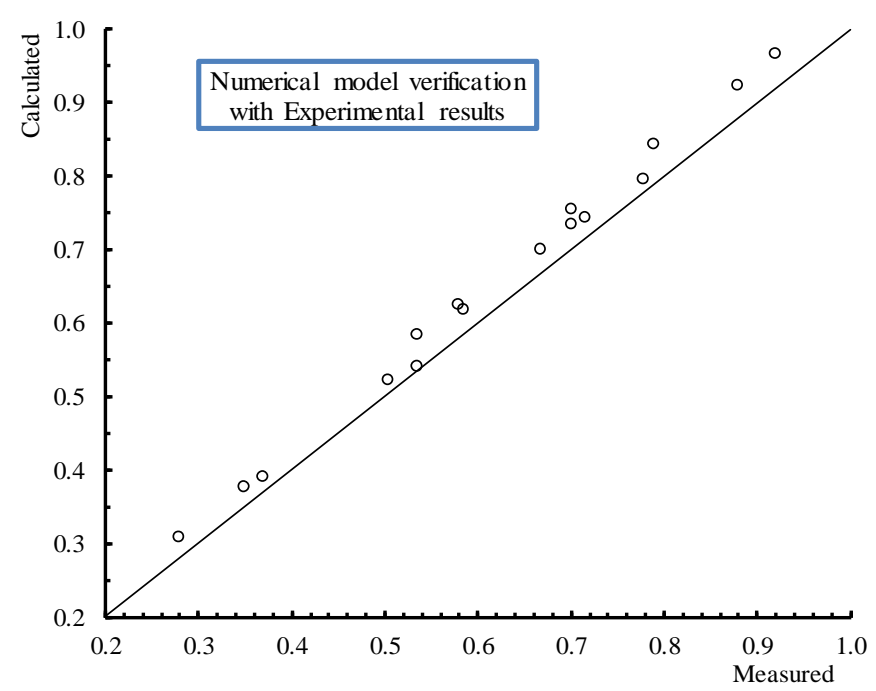

Figure9. comparison between numerical and experimental transmission coefficients results for Flow-3D model.

Table 5 shows assessment of residuals analysis of transmission coefficient between calculated Flow-3D and experimental values.It can be observed from the figure that Flow-3D model partially over-predict the transmission coefficient by values less than -0.06 .

Table 5.Residuals analysis of Flow-3D model.

\begin{tabular}{ccccc}
\hline Residual $=$ & \multicolumn{2}{c}{$\mathrm{d} / \mathrm{h}=0.5$} & \multicolumn{2}{c}{$\mathrm{d} / \mathrm{h}=0.25$} \\
measured-calculated & $\mathrm{P}=0.83$ & $\mathrm{P}=0.5$ & $\mathrm{P}=0.83$ & $\mathrm{P}=0.5$ \\
\hline RMSE x $\left(10^{-2}\right)$ & 3.63 & 3.775 & 4.084 & 3.946 \\
Min Res. & -0.058 & -0.051 & -0.048 & -0.055 \\
Max Res. & -0.009 & -0.030 & -0.030 & -0.019 \\
NRMSE & 0.087 & 0.097 & 0.122 & 0.143 \\
\hline
\end{tabular}

\section{COMPARISON WITH PREVIOUS STUDIES}

The experimental and numerical results of the present study as well as the empirical equation of transmission coefficientare compared with previous results for the studied semi-immersed breakwater.

Figures 10 presents the comparison between the results of the developed equation from MPR model, numerical and experimental results of the present study, and the different experimental and numerical results obtained by different published work presented in Table (1), as a function of $\mathrm{h} / \mathrm{L}$ for the suspended breakwater with $\mathrm{d} / \mathrm{h}=0.25$. The figure show that all calculated and measured $\mathrm{k}_{\mathrm{t}}$ coefficient decrease with $\mathrm{h} / \mathrm{L}$ increasing. Moreover, the MPR model and Flow$3 \mathrm{D}$ predicts the transmission coefficient with acceptable degree of accuracy.Figure 10presents the hydrodynamic performance of the different semi-immersed models, investigated by other published work of (Teh et al., 2011),(Suh et al., 2006), (Sundar and Subba Rao, 2002), (Koutandos et al., 2005), (He and Huang, 2014), (Duclos et al., 2004) and (Neelamani and Rajendran, 2002). The performance of the different models $\left(\mathrm{k}_{\mathrm{t}}\right)$ is plotted as a function of $\mathrm{h} / \mathrm{L}$. The characteristics of these experimental studies are shown in Table (1). The figure shows that high scatter in the performance of the different compared models. This can be attributed to the difference in the geometry and cross sections shape used in each case. The results trend shows that all the $\mathrm{k}_{\mathrm{t}}$ decrease with increasing $\mathrm{h} / \mathrm{L}$. Moreover, the present model gives smaller transmission values than (Suh et al., 2006) and(Teh et al., 2011) and larger values compared with others.

\section{CONCLUSIONS}

The wave transmission characteristic of a new shape of partially immersed breakwater is experimentally an numerically studied by using physical and Numerical models. The breakwater consists of a semicircular cross section suspended on two rows of circular piles, it was tested under different wave and structural parameters. In general, the proposed breakwater shows low transmission coefficients with increasing of the relative immersion depth $(\mathrm{d} / \mathrm{h})$ and decreasing of piles porosity $(\mathrm{P})$. Furthermore, increasing of both relative wave length $(\mathrm{h} / \mathrm{L})$ and wave steepness $\left(\mathrm{H}_{\mathrm{i}} / \mathrm{L}\right)$ cause lower transmission coefficients. Semicircular shape reduce the transmission slightly than rectangular shape especially for $\mathrm{h} / \mathrm{L}>0.16$. The results are compared with experimental and theoretical published work and shows a reasonable agreement. In addition, an empirical equation is developed for estimating the transmission coefficient of proposed breakwater. The results of this equation shows reasonable agreement with the experimental results. More investigations with different partially immersed breakwater shapes, under irregular, and obliquely incident waves are 
still required.

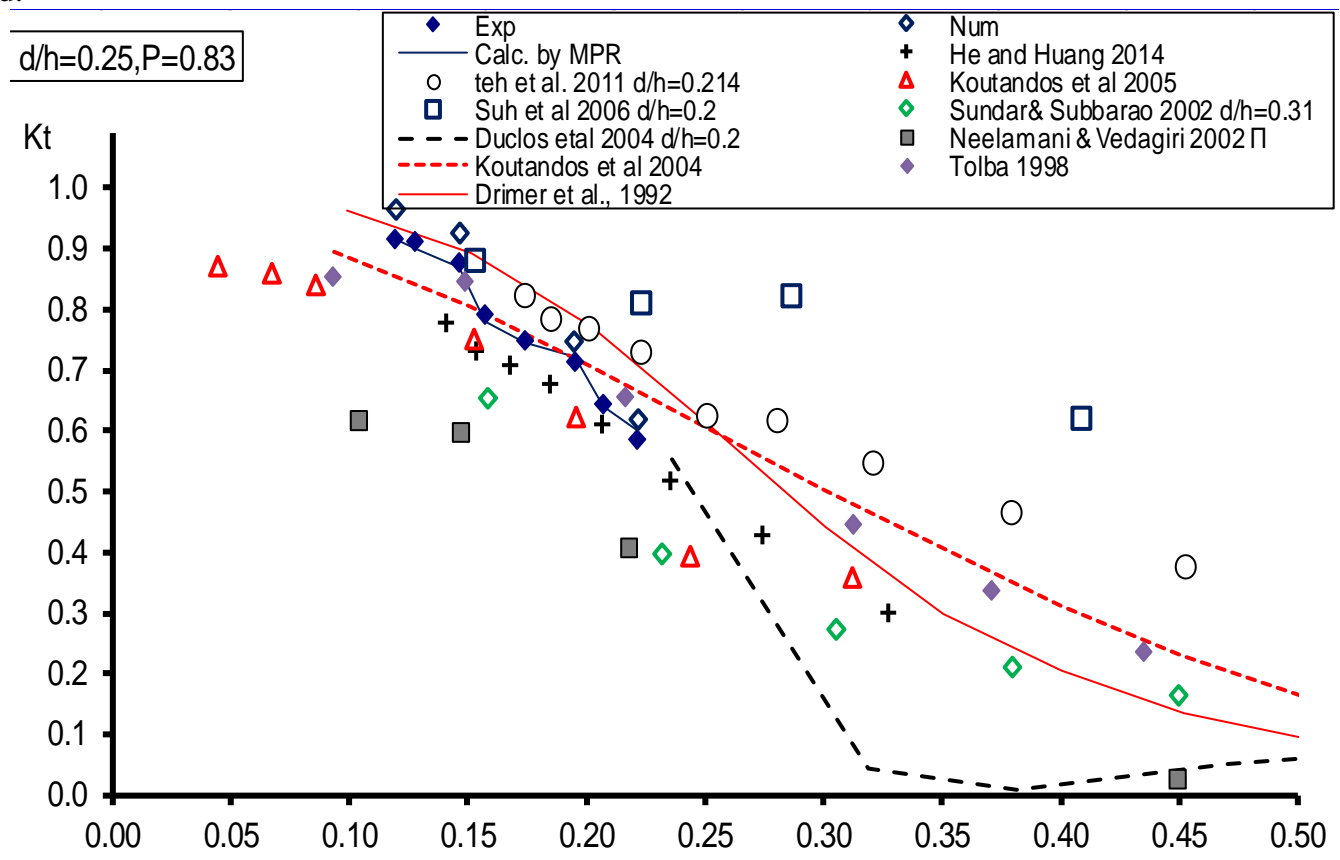

$h / L$

Figure 10. Comparison between the results of the present suspended breakwater model and the different results obtained from previous works when $\mathrm{d} / \mathrm{h}=\mathbf{0 . 2 5}$ and $\mathrm{P}=\mathbf{0 . 8 3}$.

\section{ACKNOWLEDGEMENT}

The first researcher is supported by a scholarship from the Mission Department for the Government of Egypt which is gratefully acknowledged. Second appreciative acknowledge to Harbor Engineering Lab. In water and water Engineering Dpt.- Zagazig University for permitting the author to carry out the experimental work of this paper. Last, but not least I am thankful to Egypt-Japan University of Science and Technology (E-JUST) and Japan International Cooperation Agency (JICA) for offering the tools and equipment needed for the research work.

\section{REFERENCES}

N. Drimer, Y. Agnon, M. Stiassnie, "A simplified analytical model for a floating breakwater in water of finite depth," Appl. Ocean Res. vol. 14, pp. 33-41, 1992. doi: 10.1016/0141-1187(92)90005-5.

G. Duclos, C. Josset, A. H. Clément, L. Gentaz, C. Colmard, 2004, "Hydrodynamic efficiency of a new design of half-submerged breakwater compared to a rectangular caisson, J. Waterw. Port, Coastal, Ocean Eng. , vol. 130, pp. 127-133, 2004.

doi: 10.1061/(ASCE)0733-950X(2004)130:3(127).

F. He, Z. Huang, "Hydrodynamic performance of pile-supported OWC-type structures as breakwaters: An experimental study," Ocean Eng., vol. 88, pp. 618-626, 2014. doi: 10.1016/j.oceaneng.2014.04.023.

A.S. Koraim, T.N. Salem,"The hydrodynamic characteristics of a single suspended row of half pipes under regular waves," Ocean Eng., vol. 50, pp. 1-9, 2012. doi: 10.1016/j.oceaneng.2012.04.008.

E. Koutandos, P. Prinos, X. Gironella,2005."Floating breakwaters under regular and irregular wave forcing: reflection and irregular wave forcing; reflection and transmission characeristics," Journal of Hydraulic Research, vol. 43, issue 2, pp. 174-188, 2005. doi:10.1080/00221686.2005.9641234.

E.V. Koutandos,"Hydrodynamics of vertical semi-immersed slotted barrier. WSEAS Trans. Fluid Mech. vol. 4, pp. 85-96, 2009.

E.V. Koutandos, T.V. Karambas, C.G. Koutitas, "Floating breakwater response to waves action using a Boussinesq model coupled with a 2DV elliptic solver," J. Waterw. Port, Coastal, Ocean Eng. vol. 130, pp. 243-255, 2004. doi: 10.1061/(ASCE)0733-950X(2004)130:5(243).

B.L. McCartney,"Floating Breakwater Design," J. Waterw. Port, Coastal, Ocean Eng., vol. 111, pp. 304-318, 1985. doi: 10.1061/(ASCE)0733-950X(1985)111:2(304).

S. Neelamani, R. Rajendran, R., "Wave interaction with " $\perp$ "-type breakwaters," Ocean Eng. vol. 29, issue 5, pp. 561-589, 2002. doi: 10.1016/S0029-8018(01)00030-0.

S. Neelamani, M. Vedagiri, M., 2002. "Wave interaction with partially immersed twin vertical barriers," Ocean Eng. vol. 29, pp. 215-238, 2002. doi: 10.1016/S0029-8018(00)00061-5.

K. D. Suh, S. Shin, D.T. Cox, "Hydrodynamic characteristics of pile-supported vertical wall breakwaters," J. Waterw. Port, Coastal, Ocean Eng., vol. 132, 83-96, 2006. doi: 10.1061/(ASCE)0733-950X(2006)132:2(83).

V. Sundar, B.V. Subba Rao, "Hydrodynamic pressure and forces on quadrant front face pile supported breakwater," Ocean Eng., vol. 29, pp. 193214. doi: 10.1016/S0029-8018(01)00009-9.

H. M. Teh, V. Venugopal, "Performance evaluation of a semicircular breakwater with truncated wave screens," Ocean Eng. 70, 160-176, 2013.

doi: 10.1016/j.oceaneng.2013.05.035. 


\section{The Spanwise Structure of the Roof-Level Turbulence in a Street Canyon Flow}

\author{
Tomek Jaroslawski \& Eric Savory \\ Department of Mechanical \& Materials Engineering \\ The University of Western Ontario \\ London, Canada \\ tjarosla@uwo.ca
}

\author{
Laurent Perret \& Karin Blackman \\ LHEEA UMR 6598 CNRS \\ Ecole Centrale de Nantes \\ Nantes, France
}

\begin{abstract}
The effect of upstream roughness on turbulent street canyon flow is presented. Horizontal measurements were conducted at near roof level of a street canyon using particle image velocimetry in a wind tunnel. Three upstream roughness arrays and two canyon width to height aspect ratios $(\mathrm{W} / \mathrm{h}=1$ and 3) were investigated. The arrays consisted of 3D cubes (plan area density, $\lambda_{\mathrm{p}}=25 \%$ ), $1 \mathrm{~h}$ spaced $2 \mathrm{D}$ bars (skimming flow, $\lambda_{\mathrm{p}}=50 \%$ ) and $3 \mathrm{~h}$ spaced $2 \mathrm{D}$ bars (wake interference flow, $\lambda_{\mathrm{p}}=25 \%$ ). Two-point correlations and integral length scales are computed over the different configurations. The results show a significant effect of upstream roughness on these quantities. It was found that a $3 \mathrm{D}$ upstream roughness has a significantly weaker correlation in the spanwise direction. A similar trend is observed in spanwise integral length scales. The shear layer boundary was found to be related to the strength of the correlations near roof level of the street canyon.
\end{abstract}

Keywords: Boundary layer - Street canyon - Particle Image Velocimetry - Wind tunnel

\section{INTRODUCTION}

Air quality in urban environments is a critical issue in present times with the resulting socioeconomic implications being of great concern. Pollution in the earth's atmosphere leads to human death, disease and harm to our natural resources [1]. The simple street canyon model is a useful representation of most urban street configurations. The effects of canyon aspect ratio AR (streamwise width W / canyon height h) and upstream roughness on street canyon flow is well studied. Grimmond and Oke [2] defined three regimes of flow in urban environments; skimming flow, wake interference flow and isolated roughness flow. They also found that the plan area packing density $\left(\lambda_{p}\right)$ of the upstream roughness and whether it was $2 \mathrm{D}$ or $3 \mathrm{D}$ had an impact on the flow within the canyon. Experimental wind tunnel studies on street canyons frequently use Particle Image Velocity (PIV) to measure the flow field, and mostly conduct measurements in a single vertical plane, such as Salizzoni Marro, Soulhac, Grosjean \& Perkins [3], Savory, Perret \& Rivet [4] and Blackman, Perret \& Savory [5]. When studying this configuration in a wind tunnel, Savory, Perret \& Rivet [4] noted that it is crucial to match the roughness length scale $\left(\mathrm{z}_{\mathrm{o}}\right)$ and the integral length scale $\left(\mathrm{L}_{\mathrm{uu}}\right)$ within factors of 2-3 to ensure that full and model scale are matched properly. It was also noted that the geometry of the upstream roughness significantly effects the structure of the oncoming boundary layer, where 3D roughness is found to generate $3 \mathrm{D}$ turbulent structures which resembles a more realistic boundary layer found in nature. Suggestions by Savory, Perret \& Rivet [4] have been employed in the present work.

Turbulence is a 3D phenomenon and thus to understand turbulent street canyon flow properly one must investigate how the flow behaves in both the spanwise (y) direction and the vertical (z) direction. Shaw, Brunet, Finnigan \& Raupach [6] conducted a wind tunnel study of air flow over waving wheat. Two-point, space-time correlations of streamwise (x) and vertical velocity components were computed from the wind tunnel simulation of an atmospheric boundary layer, with the wheat canopy model constructed of flexible nylon stalks. It was concluded that it was not appropriate to apply Taylor's hypothesis of frozen turbulence in the region of the canopy and, therefore, the integral length scales were computed directly from two-point statistics. It was also found that the lateral integral length scales were smaller than those computed in the streamwise direction by factors ranging from 1.9 to 4.2 . Raupach, Finnigan \& Brunet [7] stated that the approach of applying the Taylor's hypothesis is fraught with difficulty as high turbulence intensities $\left(\mathrm{u}^{\prime} / \overline{\mathrm{U}}>1\right)$ are present within the canopy. Inagaki, Kanda, Sato \& Michioka [8] conducted multipoint measurements (3D velocities and temperatures) in a cubical array using multiple sonic anemometers aligned at equal heights in the streamwise and spanwise directions. Using twopoint correlations, they reported that the correlation of the fluctuating streamwise velocity, the difference between instantaneous and mean velocities $\left(u^{\prime}=u-\bar{U}\right)$ was higher along the streamwise direction than for an equal magnitude of spanwise separation. The result was attributed to the existence of coherent structures elongated along the streamwise direction. It was postulated that the coherent structure of $\mathrm{u}^{\prime}$ is geometrically similar irrespective of the type of roughness. 
Raupach, Finnigan \& Brunet [7] conducted two-point correlations of various model and field canopies in the $\mathrm{x}-\mathrm{Z}$ and $\mathrm{y}-\mathrm{z}$ planes. In the $\mathrm{x}-\mathrm{z}$ plane the streamwise fluctuating velocity correlation produced nearly elliptical stretched correlation contours, with a tilt angle of approximately 18 degrees, an effect that was reported to diminish within the canopy. Shaw, Brunet, Finnigan \& Raupach [6] suggest that this more rapid reduction of correlation within the canopy is due to the creation of small scale motion and the breakdown of large scale flow by canopy elements. In contrast, the correlation of the vertical fluctuating velocity revealed nearly circular correlation contours and was found to decay much more rapidly with spatial separation. The correlations in the $y-z$ plane produced contours which were nearly circular for both streamwise and vertical fluctuating velocities. Raupach, Finnigan \& Brunet [7] concluded that in a time-averaged sense, fluid motions near the top of the canopy are well correlated over length scales of the order of $h$. It is also suggested that zones where the sign of the correlation reverses could be a direct consequence of the formation of dominant flow structures [6]. The effect of the roughness and canyon geometry on the mean turbulent statistics near roof level in the horizontal plane is investigated in this work. The questions to be addressed in the present paper are:

- What is the impact of $2 \mathrm{D}$ versus $3 \mathrm{D}$ arrays and $\lambda_{\mathrm{p}}$ on two-point correlations and integral length scales in the streamwise and spanwise direction in a horizontal plane near roof-level of the canyon?

- What does this tell us about the mean structure of the flow at this location of the street canyon?

The objective of this present research is to establish how the oncoming boundary layer flow interacts with the street canyon over a range of configurations. The flow will be characterized in the spanwise direction to complement the work of Blackman, Perret \& Savory [5], who characterized the vertical plane. The correlations and scales of turbulence will also be investigated to further understand the dynamics of the flow and structure of turbulence in the spanwise direction. The present results provide a clearer picture on the nature of $3 \mathrm{D}$ turbulent flow in street canyons.

\section{EXPERIMENTAL SETUP}

The experiments were conducted in the low-speed, suckdown boundary layer wind tunnel in the LHEEA Laboratory (École Centrale Nantes, France). The dimensions of the working section were $2 \mathrm{~m}$ (width) $\times 2 \mathrm{~m}$ (height) $\times 24 \mathrm{~m}$ (length) and the wind tunnel had a 5:1 ratio inlet contraction. Boundary layer development was initiated by five $800 \mathrm{~mm}$ high vertical tapered spires located immediately downstream of the contraction and a $200 \mathrm{~mm}$ high solid fence across the working section positioned $750 \mathrm{~mm}$ downstream of the spires. The street canyon flow measurements were taken $5.5 \mathrm{~m}$ downstream of this initial development region. Two canyon aspect ratios were studied for three upstream roughness conditions. The street canyon was constructed using two square cross section rectangular bars with a height of $50 \mathrm{~mm}(\mathrm{~h})$ and a lateral length of $1500 \mathrm{~mm}$. The upstream roughness conditions consisted of a staggered cubical array $\left(\lambda_{\mathrm{p}}=25 \%\right)$ or $2 \mathrm{D}$ square cross section rectangular bars with a spacing of $1 \mathrm{~h}$ or $3 \mathrm{~h}\left(\lambda_{\mathrm{p}}=25 \%\right.$ and $\lambda_{\mathrm{p}}=$ $50 \%$ ). The height of the cubes and 2D bars was $50 \mathrm{~mm}$. It should be noted that the experimental conditions in this present work are the same as in Blackman, Perret \& Savory [5]. A schematic of the experimental setup can be found in figure 1 .

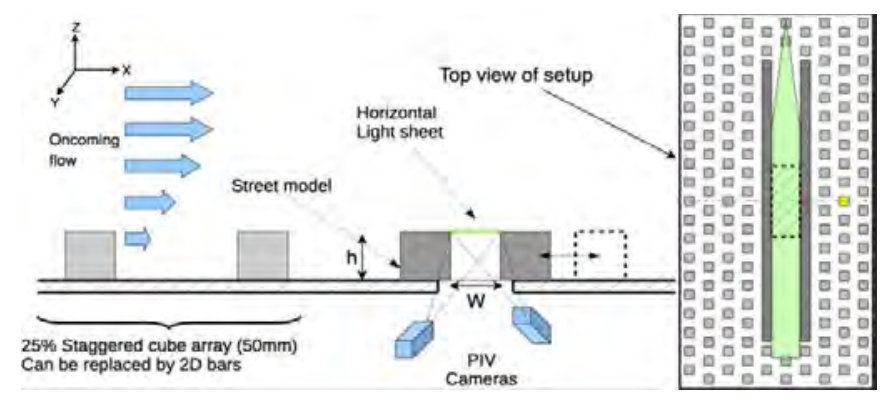

Figure 1. Experimental Setup

Stereoscopic PIV measurements were conducted in the horizontal plane located at a height of $0.9+/-0.05 \mathrm{~h}$ and aligned with the free stream flow. This height was verified by comparing turbulent statistics to those from Blackman, Perret \& Savory [5] which consisted of measurements in the vertical plane. The PIV setup was located beneath the wind tunnel floor and images were taken through a glass floor. The final spatial resolution of the vector field was $1.6 \mathrm{~mm}(0.032 \mathrm{~h})$ and a temporal sampling frequency of $7 \mathrm{~Hz}$ was used. The fields of view were $1 \mathrm{~h} \times 6 \mathrm{~h}$ and $3 \mathrm{~h} \times 6 \mathrm{~h}$ for the $1 \mathrm{~h}$ and $3 \mathrm{~h}$ canyons, respectively. A Litron double cavity $2 \times 200 \mathrm{~mJ} \mathrm{Nd}$-Yag laser was used to illuminate the measurement region. The flow was seeded with glycol/water droplets (average diameter of $1 \mu \mathrm{m}$ ) via a fog generator. An iterative cross-correlation analysis was performed which consisted of an initial window size of 64 x 64 pixels with a final interrogation of $32 \times 32$ pixels and the overlap of the analysis window was $50 \%$. A pulse interval of $500 \mu$ s was used in the computation of the velocity vector fields. A pitot-static tube located at $\mathrm{x}=15 \mathrm{~m}, \mathrm{y}=0 \mathrm{~m}, \mathrm{z}=1.5 \mathrm{~m}$ was used to measure dynamic pressure from which the velocity was determined to be $5.9 \mathrm{~m} / \mathrm{s}$. This velocity was used for all of the experiments in this present work giving a Reynolds number of $1.9 \times 10^{4}$, based on this velocity, $\mathrm{U}_{\mathrm{e}}$ and canyon height, $\mathrm{h}$.

\section{RESUlTS AND DiscuSSION}

In this section, two-point statistics are discussed in part (a) and computed integral length scales are assessed in (b).

\section{a) Two-Point Statistics}

Two-point space and time fluctuating velocity correlations offer important information regarding the nature of the flow field which single point measurements cannot provide. A two-point spatial correlation was conducted using the middle of the street canyon $\left(\mathrm{x}_{\mathrm{ref}}=0 \mathrm{~h}, \mathrm{y}_{\mathrm{ref}}=0 \mathrm{~h}\right)$ as the reference point. The two-point correlation coefficient was computed using (1).

$$
R_{\mathrm{uu}}\left(\mathrm{x}_{\text {ref }}, \mathrm{y}_{\text {ref }}, \mathrm{x}, \mathrm{y}\right)=\frac{\overline{\mathrm{u}^{\prime}\left(\mathrm{x}_{\text {ref }}, \mathrm{y}_{\text {ref }}\right) \mathrm{u}^{\prime}(\mathrm{x}, \mathrm{y})}}{\sqrt{\frac{\mathrm{u}^{\prime 2}\left(\mathrm{x}_{\mathrm{ref}}, \mathrm{y}_{\text {ref }}\right)}{\sqrt{\mathrm{u}^{2}(\mathrm{x}, \mathrm{y})}}}} .
$$


Contours of the correlation fields are presented in figure 2 and figure 3 for $1 \mathrm{~h}$ and $3 \mathrm{~h}$ canyon widths, respectively. Plots of the correlations along the $\mathrm{x}$ and $\mathrm{y}$ centrelines for streamwise $\left(\mathrm{u}^{\prime}\right)$ and vertical $\left(\mathrm{w}^{\prime}\right)$ fluctuating velocities ( $w$ ith $\mathrm{x}_{\mathrm{ref}}=0 \mathrm{~h}, \mathrm{y}_{\mathrm{ref}}=0 \mathrm{~h}$ set as the reference point) can be found in figure 4 to figure 5 . It can be observed that the correlation is symmetrical for all quantities in the spanwise direction. Symmetry in the y-z plane for correlations of streamwise and vertical velocity fluctuations was also observed by Shaw, Brunet, Finnigan \& Raupach [6] and Raupach, Finnigan \& Brunet [7]. Raupach, Finnigan \& Brunet [7] noted that the correlation contours were roughly spherical with a radius of $0.8 \mathrm{~h}$. Within figure 2 and figure 3 , the effect of roughness on the flow can be seen qualitatively. The effect of roughness is more prominent in $\mathrm{C} 1 \mathrm{~h}$ than $\mathrm{C} 3 \mathrm{~h}$ configurations. The structure of the correlation is more elongated in the streamwise direction and is narrower for $\mathrm{C} 3 \mathrm{~h}$. The opposite is true for $\mathrm{C} 1 \mathrm{~h}$, with the structure of the correlation elongated in the spanwise direction. For the $\mathrm{AR}=1$ canyons the largest spherical radius of the correlation (region of the correlation field which is circular) was $0.3 \mathrm{~h}$ for the $\mathrm{C} 1 \mathrm{hR} 3 \mathrm{~h}$ configuration, whilst for $\mathrm{AR}=3$ the largest radius was found to be approximately $0.3 \mathrm{~h}$ for $\mathrm{C} 3 \mathrm{hR} 3 \mathrm{~h}$. This suggests that the size of the spherical radius of the correlations is independent of canyon geometry, and only dependent on roughness (other configurations had smaller spherical correlation radii, all within $0.1 \mathrm{~h}-0.2 \mathrm{~h}$ for both $\mathrm{R} 1 \mathrm{~h}$ and $\mathrm{Rcu}$ ). Although the spherical correlation radii are smaller than those found by Raupach, Finnigan \& Brunet [7], this could be attributed to the different flow regimes (flow over a model wheat field) and the measurement region being higher $(\mathrm{z}=1 \mathrm{~h}$ versus $0.9 \mathrm{~h}$ in the present work).
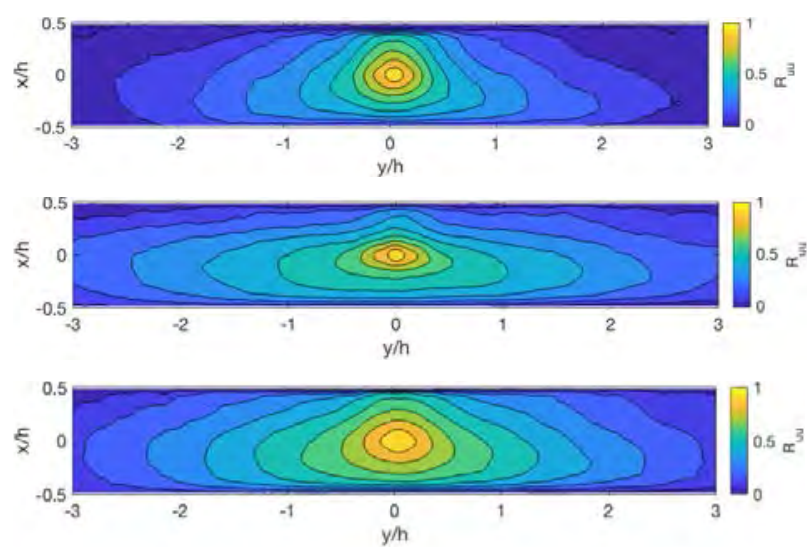

Figure 2. Two-point correlations: (top) $\mathrm{C} 1 \mathrm{hRcu}$ (middle) $\mathrm{C} 1 \mathrm{hR} 1 \mathrm{~h}$ and (bottom) C1hR3h

Raupach, Finnigan \& Brunet [7] defined a significant correlation to be $\mathrm{R}_{\mathrm{uu}}>0.2$ at height $\mathrm{h}$. Referring to figures 4 and 5 it can be found that that for the C1hR $1 \mathrm{~h}$ and $\mathrm{C} 1 \mathrm{hR} 3 \mathrm{~h}$ configurations there is a significant correlation for the entire spanwise width of the canyon ( $-3 \mathrm{~h}$ to $3 \mathrm{~h})$. The $\mathrm{C} 1 \mathrm{hRcu}$ configuration shows a significant spanwise correlation from $1.5 \mathrm{~h}$ to $1.5 \mathrm{~h}$. It is also observed that $\lambda_{\mathrm{p}}$ does not seem to effect the spanwise correlation for the $\mathrm{Clh}$ configurations. For the $\mathrm{C} 3 \mathrm{~h}$ configurations the effect of roughness on the correlations
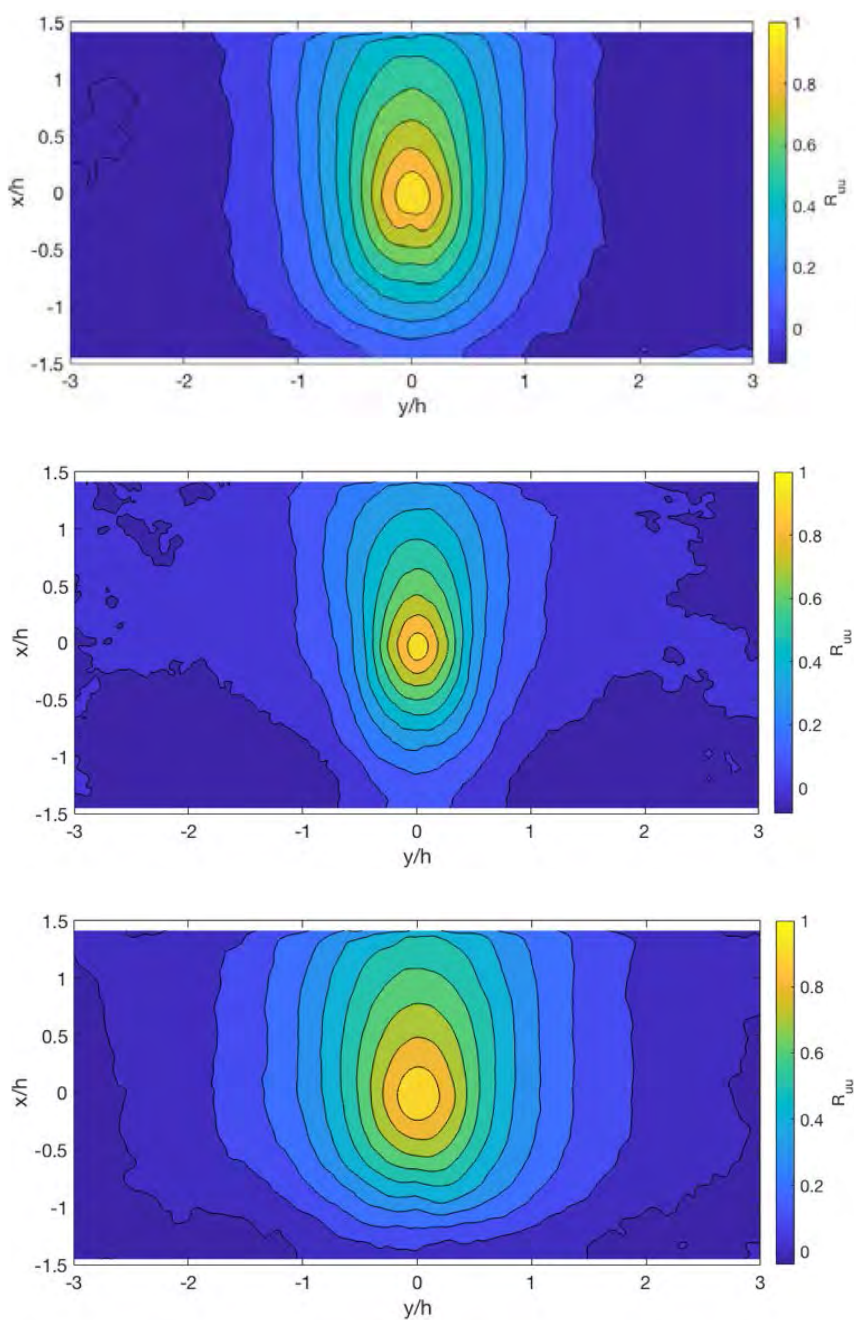

Figure 3. Two-point correlations: (top) $\mathrm{C} 3 \mathrm{hRcu}$ (middle) $\mathrm{C} 3 \mathrm{hR} 1 \mathrm{~h}$ and (bottom) C3hR3h

is less strong. The $\mathrm{C} 3 \mathrm{hR} 3 \mathrm{~h}$ configuration has a significant spanwise correlation from $-1.5 \mathrm{~h}$ to $1.5 \mathrm{~h}, \mathrm{C} 3 \mathrm{hR} 1 \mathrm{~h}$ and $\mathrm{C} 3 \mathrm{hRcu}$ are $-1 \mathrm{~h}$ to $1 \mathrm{~h}$ and $-0.8 \mathrm{~h}$ to $0.8 \mathrm{~h}$, respectively. It can be concluded that spanwise correlations decay much more rapidly when AR is increased. The smallest correlation for $\mathrm{C} 1 \mathrm{~h}$ was the largest for $\mathrm{C} 3 \mathrm{~h}$. An opposite trend is observed for correlations in the streamwise direction, with $\mathrm{C} 3 \mathrm{~h}$ configurations having a significant correlation for most the canyon width (-1h to $1.5 \mathrm{~h})$ and the $\mathrm{C} 1 \mathrm{~h}$ configurations having significant correlations from approximately $-0.4 \mathrm{~h}$ to $0.4 \mathrm{~h}$. The correlation could decay more rapidly for $\mathrm{C} 1 \mathrm{~h}$ than $\mathrm{C} 3 \mathrm{~h}$ because of a larger circulation region expected in a larger AR canyon. It is also quite possible that the shear layer has an impact on the correlation decay. Blackman, Perret \& Savory [5] computed the shear layer boundaries from vertical PIV data for the same configurations present in this work. The shear layer boundary was computed using turbulent kinetic energy in the shear layer [5]. They found that C3h had thicker shear layers than $\mathrm{C} 1 \mathrm{~h}$. A thicker and larger shear layer would oscillate at a lower frequency, resulting in the flow in the canyon to have a lower frequency as well, this would result in a stronger correlation near roof level of the canyon with the AR 
$=3$. On the contrary, $\mathrm{C} 1 \mathrm{~h}$ was found to have a smaller shear layer [5]. The faster moving and thinner shear layer would cause the correlation near-roof level to decay more rapidly. In the present work, the streamwise correlation at near-roof level for $\mathrm{C} 1 \mathrm{~h}$ was symmetrical but asymmetrical for $\mathrm{C} 3 \mathrm{~h}$ (see figures 4 and 5). This, again, can be explained by the shear layer boundaries computed by Blackman, Perret \& Savory [5]. Blackman, Perret \& Savory [5] found shear layer boundaries to be less symmetrical in the streamwise direction for $\mathrm{C} 3 \mathrm{~h}$ than $\mathrm{C} 1 \mathrm{~h}$. For $\mathrm{C} 3 \mathrm{~h}$ the thickness of the shear layer boundary was much thicker at the downstream wall than near the upstream wall (approximately 4x larger), this difference being significantly smaller for $\mathrm{C} 1 \mathrm{~h}$ (approximately $2 \mathrm{x}$ larger). There appears to be a relationship between streamwise correlation symmetry at roof level and the geometry of the shear layer. Finally, it was stated above that the effect of roughness is much more profound on the spanwise correlations near the roof level for canyons with an $\mathrm{AR}=1$ than 3 . This trend is, again, present in the shear layer boundaries computed by Blackman, Perret \& Savory [5], with the $\mathrm{C} 1 \mathrm{~h}$ configurations varying more with roughness. Correlation decay in the streamwise direction was found to be related to shear layer boundaries in that those configurations with the thickest shear layer had the strongest correlations at roof level.
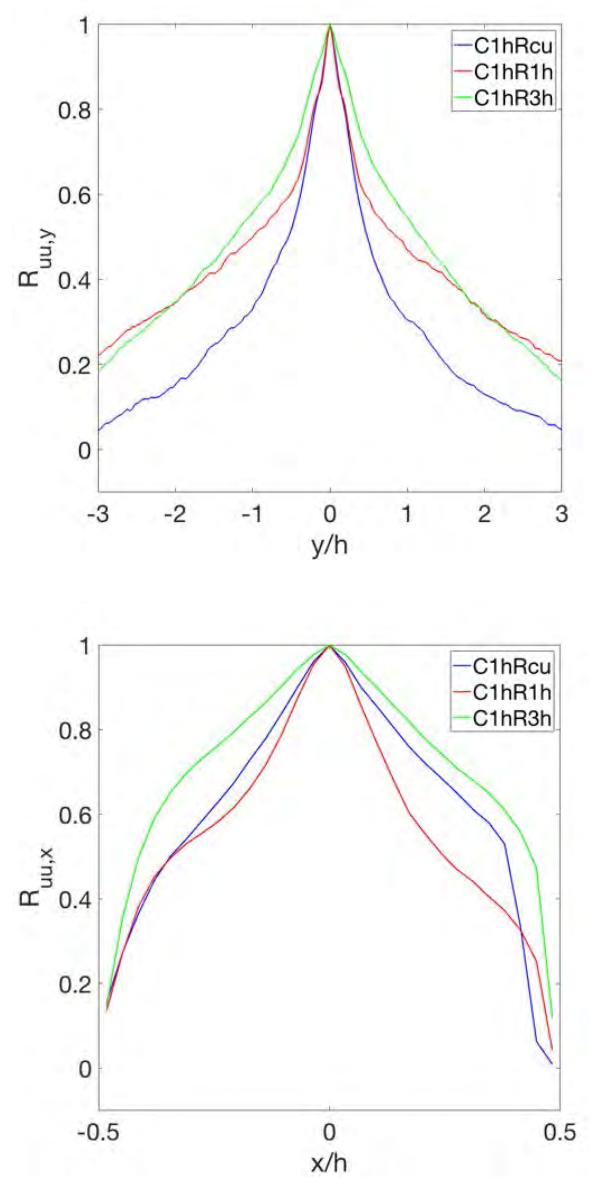

Figure 4. $\mathrm{R}_{\mathrm{uu}}$ two-point corelations for $\mathrm{C} 1 \mathrm{~h}$ (top: $\mathrm{y}$-direction, bottom: $\mathrm{x}$ direction)
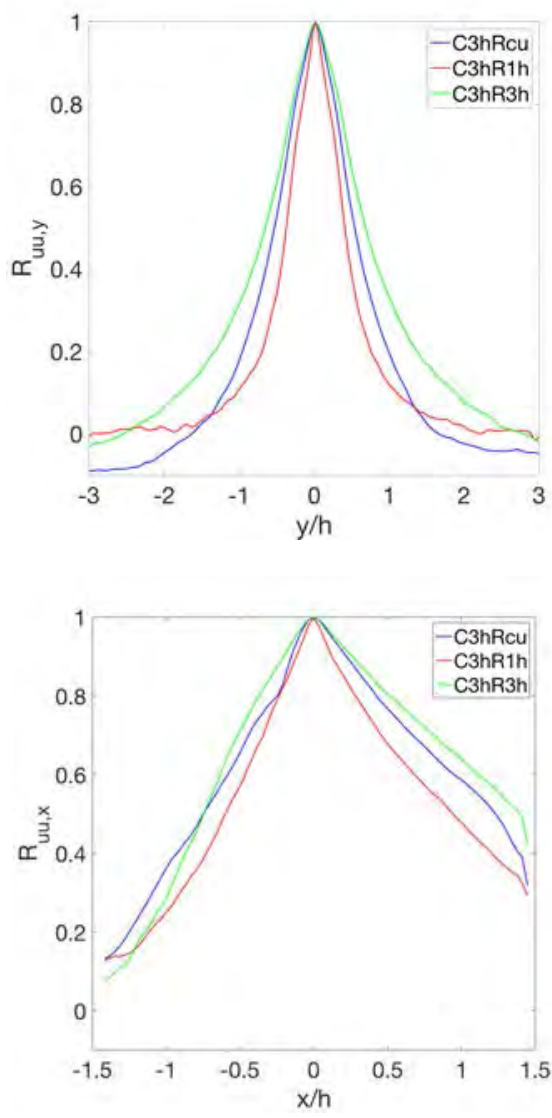

Figure 5. $\mathrm{R}_{\mathrm{uи}}$ two-point corelations for C3h (top: $\mathrm{y}$-direction, bottom: $\mathrm{x}-$ direction)

\section{b) Integral Length Scale $\left(L_{u u}\right)$}

The turbulence integral length scale can be defined as the size of the average-energy containing eddy. The turbulence integral length scales were calculated using (2) for the spanwise velocity fluctuations and (3) for the streamwise velocity fluctuations. The reference points used to compute $\mathrm{R}_{\mathrm{uu}}$ used in (2) and (3) were $x_{\text {ref }}=-0.5 h, y_{\text {ref }}=0 h$ for the streamwise $L_{u u}$ and $\mathrm{x}_{\text {ref }}=0 \mathrm{~h}, \mathrm{y}_{\mathrm{ref}}=-3 \mathrm{~h}$ for the spanwise $\mathrm{L}_{\mathrm{uu}}$. The integral length scale was computed by integrating until the first zero crossing of a two-point spatial correlation.

$$
\begin{aligned}
& \mathrm{L}_{\mathrm{uu}}(\mathrm{y})=\int\left(\mathrm{R}_{\mathrm{uu}}\right) d y \\
& \mathrm{~L}_{\mathrm{uu}}(\mathrm{x})=\int\left(\mathrm{R}_{\mathrm{uu}}\right) \mathrm{dx}
\end{aligned}
$$

The integral length scales for both the streamwise fluctuating velocity $\left(u^{\prime}\right)$ and vertical fluctuating velocity $\left(w^{\prime}\right)$ in the $x$ and $\mathrm{y}$ directions are presented in figure 6 . It may be seen that roughness and $A R$ have a significant effect on the spanwise integral length scale. $\mathrm{L}_{\mathrm{uu}, \mathrm{y}}$ for $\mathrm{C} 1 \mathrm{hRcu}$ was found to be almost $50 \%$ smaller than the $\mathrm{L}_{\text {uu,y }}$ for $\mathrm{C} 1 \mathrm{hR} 1 \mathrm{~h}$ and $\mathrm{C} 1 \mathrm{hR} 3 \mathrm{~h}$. This suggests whether the flow is $2 \mathrm{D}$ or $3 \mathrm{D}$ influences the magnitude of $\mathrm{L}_{\mathrm{uu}, \mathrm{y}}$ not $\lambda_{\mathrm{p}}$. Blackman, Perret \& Savory [5] found that the 
streamwise integral length scale was larger for the 2D case than the $3 \mathrm{D}$ case when packing density was equal. Volino, Schultz \& Flack [9] also observed that integral length scales where significantly smaller for 3D than for 2D cases. The results from Takimoto, Ignaki, Kanda, Sando \& Michioka [10] contradict this as they showed larger $\mathrm{L}_{\mathrm{uu}}$ in the $3 \mathrm{D}$ configuration. However, as suggested by Blackman, Perret \& Savory [5] this could be due the simulation method employed, as no spires were used in the experiment, resulting in a smaller boundary layer. Blackman, Perret \& Savory [5] found that, at roof-level, the streamwise $\mathrm{L}_{\mathrm{uu}}$ for Rcu was approximately $4 \mathrm{~h}$ but it is lower in the present work because the measurements were taken at $\mathrm{z} / \mathrm{h}=$ 0.9 . The present results show that that $\mathrm{L}_{\mathrm{uu}}$ is smaller for $3 \mathrm{D}$ than 2D flows in the spanwise direction, which was also found in previous work ([5] and [9]) for the streamwise direction. The integral length scales for vertical velocity fluctuations $\left(\mathrm{L}_{\mathrm{ww}}\right)$ were found to be approximately 3-4 times smaller than $\mathrm{L}_{\mathrm{uu}}$.
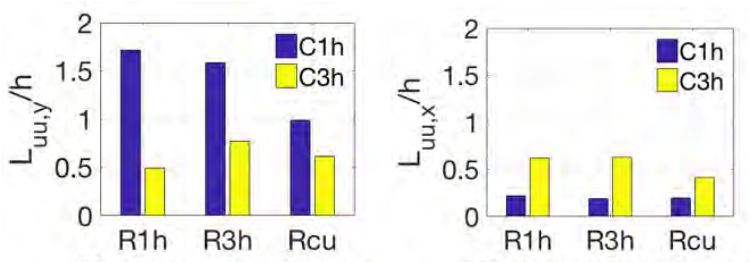

Upstream Roughness
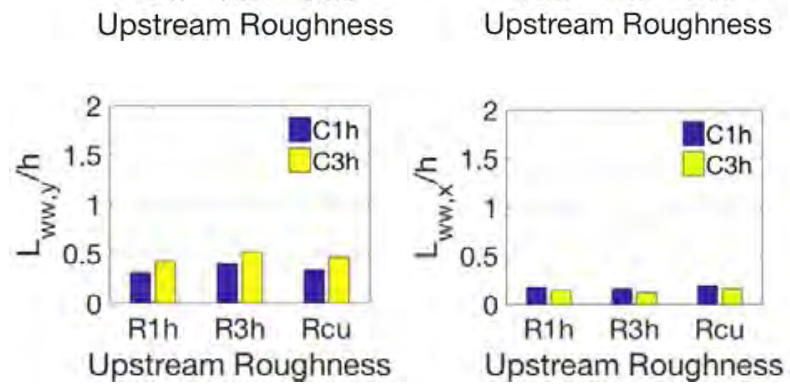

Figure 6. Impact of roughness on $\mathrm{L}_{\mathrm{uu}, \mathrm{y}}, \mathrm{L}_{\mathrm{uu}, \mathrm{x}}$ (top) and $\mathrm{L}_{\mathrm{ww}, \mathrm{y}}, \mathrm{L}_{\mathrm{ww}, \mathrm{x}}$ (bottom)

\section{CONCLUSION}

Horizontal measurements were conducted at near roof-level in a street canyon using particle image velocimetry in a wind tunnel, for 6 configurations of upstream roughness and canyon aspect ratio. It was observed that the two-point correlations were symmetrical for all quantities in the spanwise direction. Stronger correlations were found for 2D roughness arrays than in $3 \mathrm{D}$ arrays and the planform packing density was found to have no effect on spanwise correlations. It was found that the correlation decay in the streamwise direction was related to shear layer boundaries such that the configurations with the thickest shear layer had the strongest correlation. The $\mathrm{L}_{u u}$ was found to be smaller for $3 \mathrm{D}$ than $2 \mathrm{D}$ flows in the spanwise direction.

\section{ACKNOWLEDGMENT}

The authors would like to thank Mr Thibaut Piquet for his technical support during the experimental program and the LHEEA research group.

\section{REFERENCES}

[1] World Health Organization. (2014). 7 million premature deaths annually linked to air pollution [Online]. Available:

http://www.who.int/mediacentre/news/releases/2014/air-pollution/en/.

[2] C. S. B. Grimmond and T. R. Oke, "Aerodynamic Properties of Urban Areas Derived from Analysis of Surface Form," J. Appl. Meteorol., vol. 38, no. 9, pp. 1262-1292, Sep. 1999. doi:10.1175/1520-0450(1999)038<1262:APOUAD>2.0.CO;2.

[3] P. Salizzoni, M. Marro, L. Soulhac, N. Grosjean, and R. J. Perkins, "Turbulent Transfer Between Street Canyons and the Overlying Atmospheric Boundary Layer," Boundary-Layer Meteorol., vol. 141, no. 3, pp. 393-414, Aug. 2011. doi: 10.1007/s10546-011-9641-1.

[4] E. Savory, L. Perret, and C. Rivet, "Modelling considerations for examining the mean and unsteady flow in a simple urban-type street canyon," Meteorol. Atmos. Phys., vol. 121, no. 1-2, pp. 1-16, Apr. 2013. doi: $10.1007 / \mathrm{s} 00703-013-0254-8$.

[5] K. Blackman, L. Perret, and E. Savory, "Effect of upstream flow regime on street canyon flow mean turbulence statistics," Environ. Fluid Mech., vol. 15, no. 4, pp. 823-849, Oct. 2014. doi: 10.1007/s10652-014-9386-8.

[6] R. H. Shaw, Y. Brunet, J. J. Finnigan, and M. R. Raupach, "A wind tunnel study of air flow in waving wheat: Two-point velocity statistics," BoundaryLayer Meteorol., vol. 76, no. 4, pp. 349-376, Dec. 1995. doi: 10.1007/BF00709238.

[7] M. R. Raupach, J. J. Finnigan, and Y. Brunei, "Coherent eddies and turbulence in vegetation canopies: The mixing-layer analogy," Boundary-Layer Meteorol., vol. 78, no. 3-4, pp. 351-382, Mar. 1996. doi: 10.1007/BF00120941.

[8] A. Inagaki, A. Maruyama, and M. Kanda, "Spatial and temporal scales of coherent turbulence over outdoor reduced urban scale model," in 7th Int. Conf. Urban Climate, Yokohama, Japan, 2009.

[9] R. J. Volino, M. P. Schultz, and K. A. Flack, "Turbulence structure in a boundary layer with two-dimensional roughness," J. Fluid Mech., vol. 635, p. 75, Sep. 2009. doi: 10.1017/S0022112009007617.

[10] H. Takimoto, A. Inagaki, M. Kanda, A. Sato, and T. Michioka, "Lengthscale similarity of turbulent organized structures over surfaces with different roughness types," Boundary-Layer Meteorol., vol. 147, no. 2, pp. 217-236, Dec. 2012. doi: 10.1007/s10546-012-9790-x. 


\section{Development of Raw and Treated Flax Straw for Oil Spill Cleanup}

\author{
Pooya Ghasemi, Duncan Cree \\ Department of Mechanical Engineering \\ University of Saskatchewan \\ ( pooya.ghasemi@usask.ca),( duncan.cree@usask.ca )
}

\author{
Alvin Ulrich \\ Biolin Research Inc. \\ Saskatoon, Canada \\ ( aulrich@biolin.sk.ca)
}

\begin{abstract}
An oil spill is a critical problem and appears to have increased in the number of incidence over the past decade. There are several methods for oil spill cleanups. One method is called sorption using either synthetic or natural sorbents. Synthetic sorbents are harmful to the environment as they create another form of waste for nature. This gives rise to evaluate a more environmentally friendly natural sorbent. This research represents the results of study carried out to evaluate the effect of chemical treatment (acetylation) on properties of flax straw as a natural fiber. Sorption capacity of untreated and chemically treated flax straw was examined. It was found that performing the acetylation treatment on flax straw, significantly increases oil sorption capacity in both light and heavy oil by $41 \%$ and $22 \%$ respectively and also decreases their water uptake by $5 \%$. Moreover, by decreasing the particle size (to $0.6 \mathrm{~mm}$ ) of the chopped flax straw, the oil sorption capacity increases. This research contributes to a greater understanding of flax straw properties for potential remediation and reclamation applications, representing the possibility of turning the available waste material into a valuable oil sorbent.
\end{abstract}

Keywords ; flax straw, treated straw, oil spill, cleanup, remediation.

\section{INTRODUCTION}

Our planet has enormous resources of oil and gas deep under its surface which is economically important in all parts of our lives. Every day, a portion of this valuable material is drawn out of the earth using modern machinery by thousands of oil and gas companies. At the same time, the number of related oil spill incidents are rising and therefore more research is required in oil spill cleanup [1].

So far, various methods are used in different circumstances, including sorption methods. Sorbents are either synthetic or natural. Although manufactured sorbents are high in sorption rate, they create another form of waste after application [2]. This is the main reason why studying natural sorbents are important. Most of the plants consist of fibers which are found to be a reliable oil sorbent [3]. Among them, the flax plant which is widely available across the province of Saskatchewan consists of a large number of fibers [4]. In Canada, flax is mainly cultivated because of its linseed oil and after the extraction of the oil seed the remaining plant is left to decompose in the field or burned. To develop an oil sorbent using this biomass, the first approach can be to extract the fibers from the plant's stem. Based on studies, flax fiber can adsorb up to 30 grams of oil per gram of the fibers which is considerable [4]. However, the process of removing fibers from the plant straw is time consuming and costly which would not be suitable for a low-cost oil sorbent.

The second proposal, which is the basis of the current study, is to develop an oil sorbent using the stem of flax plant commonly referred to as flax straw. To improve oil sorption and reduce water uptake, a chemical acetylation treatment was performed on the sample and the change in oil sorption characteristics was observed. Based on observed results, the acetylation method was found to increase the oil sorption capacity of flax straw while decreasing the water uptake.

\section{EXPERIMENTAL}

\section{A. Materials}

Flax straw from bales was supplied by Biolin Research Inc., a local company researching on development and application of value-added products from flax plant in Saskatoon, Canada. Flax received from the manufacture had a packing density of 220-310 g/L. Two types of oil were selected and purchased from Fisher Scientific based on ASTM F726 - 17, and ASTM F716 - 07. Light oil had a density range of $0.820 \mathrm{~g} / \mathrm{cm}^{3}$ to $0.870 \mathrm{~g} / \mathrm{cm}^{3}$, while heavy oil had a density range of 0.930 $\mathrm{g} / \mathrm{cm}^{3}$ to $1.000 \mathrm{~g} / \mathrm{cm}^{3}$. For acetylation, acetic acid and $\mathrm{N}$ Bromosuccinimide (NBS) were provided by Fisher Scientific.

\section{B. Raw Flax Straw Oil Sorbent Preparation}

A batch of flax straw was washed with distilled water, weighted and put in the oven at $60{ }^{\circ} \mathrm{C}$ for $24 \mathrm{~h}$. After $24 \mathrm{~h}$, the samples were reweighted to calculate the moisture content. After oven drying the batch of flax straw was chopped using the Retsch knife milling machine. The gap between the knife 
and cutting bar was adjusted to $1 \mathrm{~mm}$ and the opening of the output screen was $8 \mathrm{~mm}$ (Fig. 1). An outline of the experimental procedure is illustrated in Fig. 2.

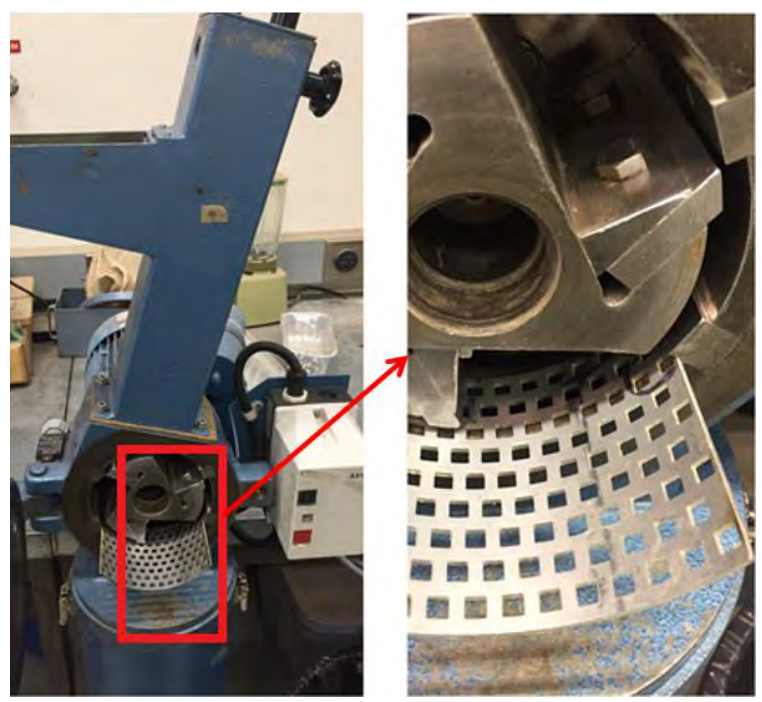

Figure 1. Chopping machine used for cutting the flax straw.

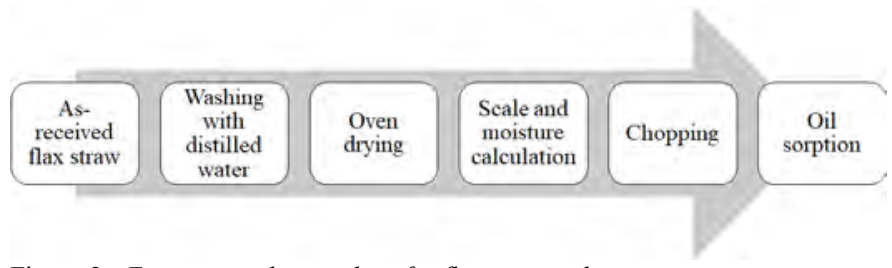

Figure 2. Experimental procedure for flax straw oil sorption test.

\section{Characterization of Flax Straw}

Three-dimensional imaging and viewing of the surface morphology for the as received flax straw was conducted using scanning electron microscopy (SEM) model JEOL JSM6010 LV (Tokyo, Japan). Images were taken at a low operating voltage of $5 \mathrm{kV}$ to prevent samples from burning (Fig. 3).

At this stage, a dried batch of flax straw was sieved based on the ASABE Standard S424.1: method of determining and expressing particle size of chopped forage material by screening. The opening size of the series of sieves used in this study was $3.3,2.3,1.6,1.1,0.8$ and $0.6 \mathrm{~mm}$.

\section{Acetylated Flax Straw Oil Sorbent Preparation}

For the acetylated samples, 15 grams of sieved material with a size less than $0.6 \mathrm{~mm}$ was chosen. The samples were placed in a flask containing $300 \mathrm{~mL}$ acetic anhydride and $1 \%$ NBS. The container was then placed in an oil bath at $100{ }^{\circ} \mathrm{C}$ for $1 \mathrm{~h}$ as was proposed for a similar natural plant sugarcane bagasse [5]. After the reaction was completed the samples were removed from the container, washed with ethanol and acetone multiple times to remove any remaining by-products. The samples were then oven dried at $60{ }^{\circ} \mathrm{C}$ for $12 \mathrm{~h}$.

\section{E. Oil Sorption Experiment}

The sorption characteristics of raw and treated flax straw were evaluated with light and heavy oil. To understand the relation of particle size and sorption capacity, the experiments were performed on light and heavy oil for different particle sizes. Based on ASTM F726 - 17 the sorbent was considered of Type II (loose sorbent). Each sample had an approximate minimum weight of 4 grams. To begin the test, flax straw samples were put in a rectangular stainless-steel Mesh No.20 ( $0.841 \mathrm{~mm}$ opening) basket having dimensions of $4 \times 9 \times 7 \mathrm{~cm}^{3}$ (length $\mathrm{x}$ width $\mathrm{x}$ depth). Since the sorbent is of the loose type, the basket should have enough space for sorbent to freely float within the test cell. The rectangular basket was then lowered into the $1000 \mathrm{~mL}$ beaker containing $200 \mathrm{~mL}$ light or heavy oil. After 15 minutes of soaking the basket was removed from the beaker and hung for 30 seconds allowing excessive oil to drain. The sample was then removed from the basket and the experiment weight was recorded. Standard deviation considered for this experiment was $15 \%$ and each experiment was repeated three times. The weight of adsorbed oil was calculated by subtracting the weight of dry sorbent before the experiment from its weight after the experiment. Oil sorption capacity of samples were calculated using (1).

$$
\text { oil sorption }=\frac{\text { weight of adsorbed oil }}{\text { weight of sorbent }}
$$

\section{F. Water Uptake Experiment}

The proposed experiment for water uptake calculations is as follows: each trial test utilized approximately 5 grams of raw or treated flax straw. Samples with different sizes were placed in a $200 \mathrm{~mL}$ beaker and distilled water was added until they were completely submerged for 10 minutes. The samples were removed from the water and placed into a basket to hang for 5 minutes in order to release excess water. Finally, the sample were weighed. The amount of water uptake was calculated by subtracting the dried sample weight from wet sample weight.

\section{RESULTS AND DISCUSSION}

SEM was used to observe the morphology of the flax straw. As shown in Fig. 3 (c) there are several layers in a single flax straw structure. Each of them consists of a primary thin layer which contain both cellulose and hemicellulose [6]. The flax stem consists of two main parts; fiber and non-fiber part. The non-fibrous, soft spongy part is also called shives.
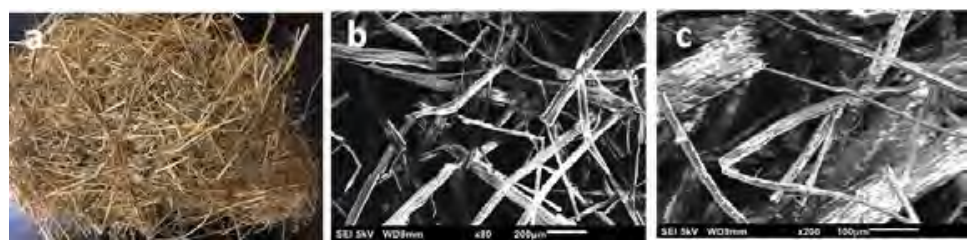

Figure 3. Images of purified and dried flax fiber or straw (a) macroscopic (b) 
and (c) SEM with magnification of $50 X$ and $200 X$, respectively.

In Fig. 4 the content of the sieved material with openings of $3.3 \mathrm{~mm}$ is compared to the sieve with $0.6 \mathrm{~mm}$ openings. The combination of shives and fiber is more homogenous in the smaller mesh size compared to the larger size.
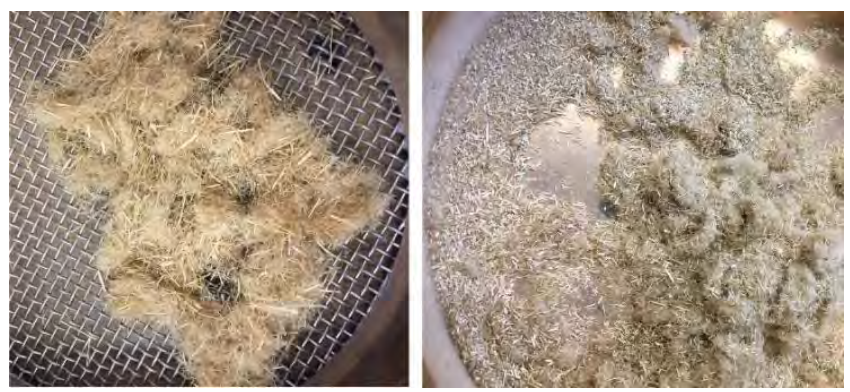

Figure 4. Comparison between the content of two different mesh sizes.

Different sieves used in this experiment are shown in Fig 5. The weight of particles trapped within each sieve is calculated as well as the mass ratio of each size category.

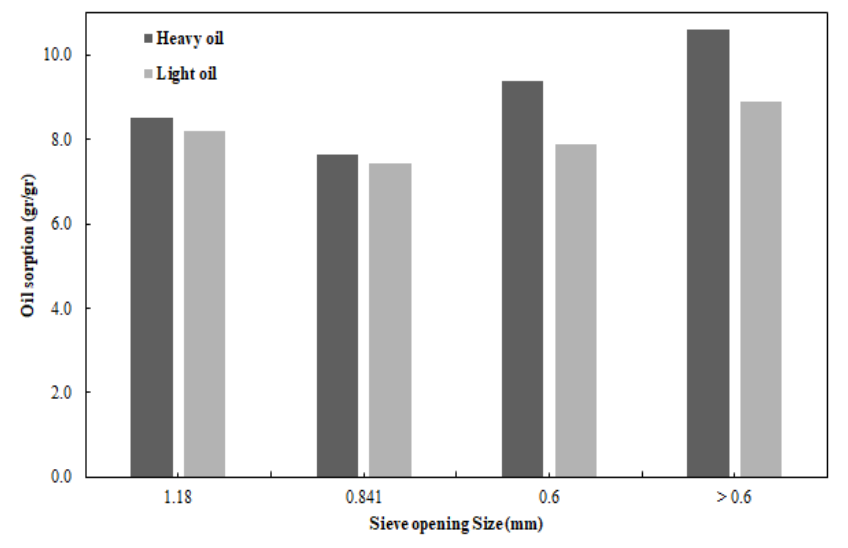

Figure 5. Comparison between oil sorption of the raw sample in light oil and heavy oil.

The result illustrates a steady increase in oil sorption capacity of raw flax from $7.6 \mathrm{~g} / \mathrm{g}$ to $10 \mathrm{~g} / \mathrm{g}$ while the particle size of the samples decreased from $0.841 \mathrm{~mm}$ to $0.6 \mathrm{~mm}$, respectively. Although decreasing the particle size of the flax straw increases oil sorption capacity, it is expected that decreasing the size to $0.6 \mathrm{~mm}$ or smaller, will decrease the capacity due to lack of void spaces [7]. However, for sizes larger than $1.6 \mathrm{~mm}$, the experiment did not successfully pass the test requirement of being in a range of $15 \%$ deviation and the acquired results had many fluctuations. This can be explained by having less homogenous combination of fibers and shives in higher sieve sizes compared with smaller sizes which agrees with results shown in Fig. 4.
Fig. 6 compares the oil sorption capacity of raw and treated sample for sizes $0.6 \mathrm{~mm}$. Acetylated samples show a slightly higher oil sorption capacity which is probably due to the higher capillary forces between plasticized cellulose fibers and oil. More importantly, acetylated samples revealed less water uptake compared to untreated samples. This is due to the chemical modifications where the hydroxyl groups on the surface of fiber are replaced with acetyl groups. Upon this modification the properties of the fiber changed, and they became hydrophobic.

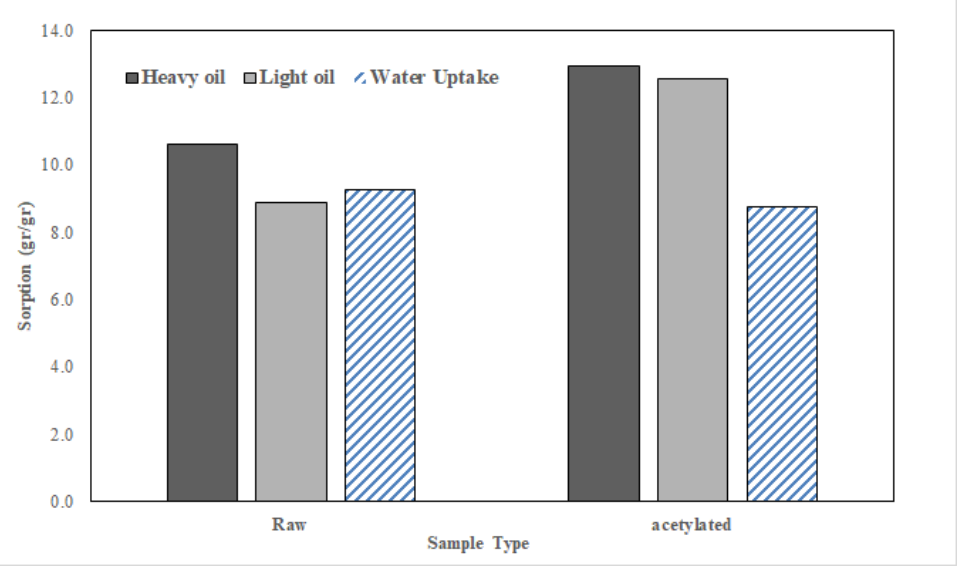

Figure 6. Comparison between the sorption capacity of the raw sample in light oil with heavy oil.

\section{CONCLUSIONS AND FUTURE WORK}

A new method for water and oil sorption was developed using flax straw where different physical and chemical treatment was performed on this material and the oil and water uptake properties was shown to improve.

1. It was found that the combination of shives and fiber is more homogenous in the smaller mesh size compared to the larger size which is an important consideration prior to performing oil sorption experiment.

2. The oil sorption results revealed an increase in oil sorption capacity up to $11 \mathrm{~g} / \mathrm{g}$ with decreasing particle size to $0.6 \mathrm{~mm}$.

3. Chemical modification changed the sorption properties of flax straw where the chemically treated samples showed an increase in oil sorption capacity by $41 \%$ and $22 \%$ in light oil and heavy oil, respectively. Also, the water uptake was reduced by $5 \%$ in chemically modified samples which is related to changes in surface properties (such as functional groups) of flax straw.

4. In conclusion, to address the use of sustainable sorbents, this study showed that a natural sorbent material made of waste flax straw obtained from the oil seed industry in Saskatchewan could be used as an oil spill cleanup material. 
Future testing to support this study will involve investigation of oil sorption properties of fibers in a media where both water and oil are presented at same time. Another important consideration would be investigation reusability and flammability of sorbent.

\section{ACKNOWLEDGMENTS}

The authors would like to acknowledge the support of the Department of Chemistry at University of Saskatchewan in assistant with performing acetylation setup. The authors express their gratitude to Alvin Ulrich for helpful discussions and the supply of the flax straw.

\section{REFERENCES}

[1] E. Quintana-Rizzo, Ester, J. J. Torres, S. W. Ross, I. Romero, K. Watson, E. Goddard, and D. Hollander., "d15N and d13C in deep-living fishes and shrimps after the deepwater horizon oil spill, Gulf of Mexico," Mar. Pollut. Bull., vol. 94, pp. 241-250, 2015. doi: 10.1016/j.marpolbul.2015.02.002.

[2] M. O. Adebajo, R. L. Frost, J. T. Kloprogge, O. Carmody, and S.Kokot, "Porous materials for oil spill cleanup: A review of synthesis and absorbing properties," J. Porous Mater., vol. 10, no. 3. pp. 159-170, 2003.

doi: 10.1023/A:1027484117065.

[3] H. M. Choi and R. M. Cloud, "Natural sorbents in oil spill cleanup," Environ. Sci. Technol., vol. 26, no. 4, pp. 772-776, 1992.

doi: 10.1021/es00028a016.

[4] I. A. Ansari, G. C. East, and D. J. Johnson, "Structure-property relationships in natural cellulosic fibres: Part III: Flax-an oil sorbent.," $J$. Text. Inst., vol. 94, no. 1-2, pp. 1-15, 2003.

doi: 10.1080/00405000308630590.

[5] X. F. Sun, R. C. Sun, and J. X. Sun, "Acetylation of sugarcane bagasse using NBS as a catalyst under mild reaction conditions for the production of oil sorption-active materials," Bioresour. Technol., vol. 95, no. 3, pp. $343-$ 350, 2004. doi: 10.1016/j.biortech.2004.02.025.

[6] M. Z. Rong, M. Q. Zhang, Y. Liu, G. C. Yang, and H. M. Zeng, "The effect of fiber treatment on the mechanical properties of unidirectional sisalreinforced epoxy composites," Compos. Sci. Technol., vol. 61, no. 10, pp. 1437-1447, 2001. doi: 10.1016/S0266-3538(01)00046-X.

[7] R. Behnood, B. Anvaripour, N. J. H. Fard, and M. Farasati, "Petroleum hydrocarbons adsorption from aqueous solution by raw sugarcane bagasse," Int. J. Emerg. Sci. Eng., vol. 1, no. 6, pp. 96-99, 2013. 
MECHANICAL

ENGINEERING

EDUCATION 


\section{Preparing and Coordinating Undergraduate Engineering Design Projects}

\author{
Roger Carrick \\ Department of Mechanical Engineering \\ Lassonde School of Engineering, York University \\ Toronto, Canada \\ roger.carrick@lassonde.yorku.ca
}

\author{
Aleksander Czekanski \\ Department of Mechanical Engineering \\ Lassonde, School of Engineering, York University \\ Toronto, Canada \\ alex.czekanski@lassonde.yorku.ca
}

\begin{abstract}
Design projects have become common in engineering classrooms. Earlier exposure to and training in the design engineering process hold much value for an enriched experience and an in-depth understanding of engineering design. Simultaneously, students in their earlier years require more guidance and frequent feedback to inform their own expectations of learning objectives, as well as develop effective learning strategies. In this paper, we will examine the considerations required to design and conduct an undergraduate engineering design course, with reflections from several years' experience with a second-year mechanical engineering design course.
\end{abstract}

Keywords- Design; Experiential Learning; Problem Based Learning; Collaborative Learning; Course Creation

\section{INTRODUCTION}

Design is the primary mode in which professional engineers operate, but until recently, there has not been a focus on teaching design and the associated soft skills. This has led to decades of engineering graduates that are excellent scientists, but lack design, analysis and professional skills [1-4]. Surveys of industry professionals and recent engineering graduates show that the attributes of design, teamwork, communication and problem solving are the most important in their daily work [3][5][6]. There have been efforts to improve these skills in undergraduate programs, beginning with the introduction of Capstone Design courses, and later, cornerstone design courses to give first year students an introduction to the design process.

This paper describes the process behind the development and coordination of a second-year mechanical engineering design project course with the goal of creating a bridge between the first year introduction to the engineering design process, and the more complex, open-ended Capstone Design projects.

Cornerstone Design courses have been introduced relatively recently and were created in response to the apparent disconnect that first and second year engineering students were feeling from engineering practice [4]. The outcomes from these Cornerstone Design courses can vary greatly depending on the constraints placed upon the course, such as available time, resources and class size. Additionally, the prior knowledge and training of first year students can vary greatly, limiting the potential subject matter and scale of the projects. Despite these limitations, Cornerstone Design courses have had great success, and their introduction has helped to increase student satisfaction with their early education [4].

\section{BACKGROUND}

\section{A. Experiential Learning}

Kolb's experiential learning cycle [7] is the model that is most commonly associated with teaching engineering design and the engineering design process. Kolb's cycle is composed of experimentation, concrete experience, reflective observation and abstract conceptualization. The iterative nature of this learning theory matches well with the engineering design process as a whole, where a product is designed, built, tested and analyzed, and lastly reflected upon for possible future design improvement. The experiential learning cycle can also be applied at a smaller scale to the individual steps of the design process. For example, during the conceptual design phase, students will generate potential concepts, evaluate the concept against design goals and criteria, and use this knowledge to iterate their designs if they do not satisfactorily meet their requirements.

Kolb's experiential learning cycle has been implemented into several pedagogical methods. Of interest when discussing engineering design projects are Problem or Project-Based Learning (PBL) and Cooperative Learning (CL). PBL is a student focused approach to aimed at improving the problemsolving skills of students. Students are encouraged to hypothesize solution methodologies and perform independent research to solve problems. Students then propose solutions, and test their appropriateness, followed by critical reflection on the process. Teachers act as facilitators to the process [8]. Cooperative Learning is a teaching methodology focused on improving how students work in teams, which is a very important part of design project experience. The most commonly implemented model of CL is that of Johnson et al. [9], which specifies 5 basic conditions under which students 
must work; positive interdependence, individual accountability, face-to-face promotive interaction, appropriate use of collaborative skills and regular self-assessment of group functioning. Research has shown that students taught in this method tend to have higher individual academic achievement and improvements in design skills, communication skills and group skills [10].

\section{B. Design Project Selection}

Choosing the correct subject for an engineering design project, particularly for first or second year students, can be difficult. The project should be challenging, pushing them the think beyond what they have seen in class. The project should be scoped in a way that is able to be completed within the timeline set by the course. The subject of the project should be well understood and documented and not include material from the fringes of scientific understanding. As these projects are part of an engineering education, they should emphasize the application of theory (mechanics, thermodynamics, etc). Finally, they should use the engineering design process as a framework.

Dutson et. al. [11] identified five potential sources for design projects for use in engineering curriculums. In hypothetical projects, course directors invent a project which can be tailored to meet the requirements of the class. Student selected projects, in which students, sometimes with guidance, choose the problem they wish to solve. Research related projects are recruited from professors and related to their research activities. Finally, industry sponsored projects are solicited from industry, ideally to solve real problems a company faces.

Each of these sources for design projects has their own advantages and disadvantages depending on the desired outcomes of the project, and each has been applied to Capstone projects. When working with Cornerstone design projects however, meeting the requirements of "understood subject matter" and "application of theory" is significantly more limiting. Searching for design projects from research, industry or student design competitions that can meet the scope requirements as well as contain only the theory and skills that first or second year students have been exposed to would be extremely difficult. Student selected projects are also difficult if the course developer would like to include the full design cycle (design, build and test) as students at that stage of their education have a poor understanding of the full scope of work required for projects. Hypothetical projects are the most convenient choice for project topics as all aspects of the scope, technical requirements and resources required can be tailored to meet the needs of the course. If properly contextualized, a hypothetical project can teach many of the same lessons as a "real" engineering problem.

\section{Teamwork}

Teamwork is often cited as the most desired outcome from team projects [12]. Teamwork is the primary mode in which professional engineers operate and encouraging students to work well within a diverse team environment is of great interest for course developers. There are many models of effective teams that have been introduced [13-15], and there are several key behaviors that each of them attempts to promote.

The first behavior encouraged is interdependence [12]. In order for an effective group to complete their task they must rely upon on the work of the individual members of the team; if one member of the team does not complete their assigned task, it prevents the entire group from completing the project. There are varying levels of interdependence that teams can operate under from pooled interdependence to intensive interdependence. In pooled interdependence, students divide tasks, and complete them in parallel often with poor levels of communication. Intensive interdependence is considered the more desirable form, and each of the team members' divided work relies upon input from other team members' work, which encourages communication and coordination.

Trust is another important factor for effective teams. Trust itself can be defined in many ways, but the definitions most closely related to team effectiveness is the students' confidence in the abilities and trustworthy intentions of their team members [12]. Trust can be encouraged in teams through team-building exercises that help to reveal the abilities and strengths of the team members, as well as share past teamwork experiences.

The most cited and common complaint from students when working is teams is the concept of social loafing [12]. Social loafing occurs when one or more member of the team refuses to complete their fair share of the team's work. Self and peer evaluation has been found to be the most effective method of reducing social loafing in teams. When the individual contributions made by team members can be quantified and reported, social loafing can be largely eliminated. Another method of reducing social loafing is to encourage each team member to have a unique contribution to the team. Unique contributions are easiest to encourage in multidisciplinary environments such as Capstone projects but can be more difficult in Cornerstone design projects as the students all have similar pre-existing skills.

\section{Feedback and Mentorship}

Compared to technical subjects, design can be much more ambiguous and subjective. Usually there can be multiple solutions to a problem, and the path taken to reach a solution can vary greatly. Coupled with the extensive amount of time required to complete one design project, students can become lost, lose motivation, or become stuck in non-productive modes of thinking. In industrial settings, projects will often follow a stage-gate process with milestones and checkpoints to ensure that projects remain on track. In a course context, student deliverables will match these milestones, and students will receive feedback in the form of marks and comments. Giving meaningful feedback can be difficult, particularly if student have difficulty articulating their thought process. Simply relying upon written feedback can lead to students continually making the same mistakes or failing to internalize corrections or suggestions. Often this is linked to "concept fixation" which is common for early designers [16]. 


\section{EXPERIENCES AND DISCUSSION}

\section{A. Course Context}

The courses described in the remainder of this paper is the second and mechanical engineering design project courses at York University's Lassonde School of Engineering. This course has been run for 3 years and has been updated each year based upon feedback and reflections from previous iterations.

At this point in their academic career, students who participated in this course had taken the prerequisite physics and math courses, as well as two first-year introduction to engineering design courses. The introduction to design courses included exposure to the design process, design-for-x, various creative thinking and innovative design heuristics. These courses also included a team-based design project; however, this project was taken only to the conceptual design phase, emphasizing needs analysis and brainstorming techniques. Additionally, students have been introduced to CAD modelling in SolidWorks ${ }^{\mathrm{TM}}$ and were given the chance to $3 \mathrm{D}$ print their designs. Additional relevant courses include an introduction to Machine Element Design which was run concurrently with the design project course and Mechanics of Materials.

The goal of this course is to give students an opportunity to experience the complete design process as well as promote good teamwork practices. Integrating as many of the skills and theories to which students had been introduced up to this point was one of the primary driving factors in the choice of the projects. By incorporating the various elements of the undergraduate curriculum into the project, students will be able to see practical applications of their theory as well as observe how interrelated their seemingly distinct core courses can be.

\section{B. Past Projects}

For the reasons mentioned previously, we chose to proceed with hypothetical projects for this course. We had specific goals of incorporating elements of physics, mechanics of materials, machine element design computer aided design (CAD) and rapid manufacturing. All student teams were given the same design challenge. A portion of the grade was based upon the relative performance compared to the other teams to promote friendly rivalry and ensure students move beyond a design that "just works" and optimize their design.

Each year the project changed, but some elements remained common. First, some form of energy transformation was included in all projects. Second, low strength raw materials were supplied to make the structural design non-trivial and ensure that students considered the material properties as a design variable. The variety of materials available was limited to encourage creativity in a constrained environment. Electronic components were excluded or extremely limited to place emphasis on the core mechanical principles desired, and to ensure that all students had an equal understanding of the theory.

Teams assumed the role of a design team competing for a design contract. They are given a design brief with a set of requirements and constraints, as well as the performance metrics by which they will be evaluated.
In the first iteration of the course, students were tasked with designing a scale version of a car that would store energy for locomotion in a spring. Limitations were placed upon the size of the vehicle, and the loading force; the loading force was much lower than the force required to extend the available springs. Performance was based upon vehicle weight and distance travelled on a single charge. Students applied principles of gear design, force and mechanical advantage, friction and strength of materials.

The second project had students design a small generator that would be powered by a raised weight of fixed mass. The context given was that they were designing a device that could be used as a cell phone charger, or small light source in remote communities. Students were given a small dc motor, and limited materials (high density fiberboard, metal rods, machine screws and nuts, glue). All designs had to mount to a standard fixture. Teams were evaluated on the value of the materials their design used and the length of time that they were able to sustain a $4 \mathrm{~V}$ output from the generator. Gear train design, strength of materials and motor characteristics were some of the knowledge emphasized.

The third iteration took inspiration from a news article that showed children making a dangerous river crossing suspended on a single rope. Students were tasked with designing a small, battery powered carriage that could carry a basket with a mass across a single rope. The rope was placed at various inclines, and the performance of the designs was evaluated on the speed of crossing the rope, and the total cost of the designs.

All of these design challenges appeared, to the students, to be simple, but as they began to apply their engineering knowledge to the analysis and design process, they found that even simple challenges can be complex.

\section{Team Creation and Teambuilding}

A team size of 4-5 was chosen for this course as the literature highlights that larger teams can be more susceptible to social loafing, and teams smaller than 3 may not have all of the skills required to complete the task [17]. Of the three possible group formation methods (self-selected, instructorselected or random), instructor-selected was chosen as it is generally regarded as the most effective method [17-18]. Students were required to complete a questionnaire at the beginning of the term, and students were distributed based on a number of criteria.

In the first iteration, the primary criteria by which students were assigned groups was personality based. Students completed an online Myers-Briggs personality assessment with the goal of distributing different personalities. While teams created with similar personality types tend to have better communication and reduced conflicts, teams with diverse personalities can have enhanced problem-solving skills as the weaknesses of one member can be complemented by the strengths of others [19]. In subsequent iterations, personality or creativity style profiles were used more as a teambuilding exercise than the primary grouping criteria.

Other criteria which were taken into consideration at a lower weighting during team formation were overall GPA, 
available free time, and previous skills. Subsequent iterations focused on distributing students primarily upon GPA and students comfort with hands-on activities as those were found to be better indicators of successful students.

The first two weeks of tutorials were dedicated to encouraging teams to work together effectively. During the first tutorial, a roundtable discussion of effective teamwork skills was conducted. This was aimed at address some of the common issues with teamwork, and highlighting techniques to overcome them. Students were then introduced to their teams, and asked to choose a team name. Students were asked to complete a team policies and expectations contract (modified from [17]). This contract outlined what was expected of each student when working in a team, such as communicating promptly and completing assigned work on time. The contract also contained a policy for dealing with social loafing or uncooperative members within teams. Students were encouraged to modify or add policies at their own discretion. The contract ensured that all students understood the policies set out by the course, and gave them a sense of ownership over the performance of their team. During the second tutorial, a teambuilding exercise was conducted using the LEGO Serious Play ${ }^{\mathrm{TM}}$ Platform. Students used a guided LEGOTM building experience to share previous team experiences, with a goal of helping to build trust between members of the group.

\section{Peer-Evaluation}

Peer- and self-evaluation was included as the major tool in reducing social loafing, monitoring conflicts within teams and promoting collaborative learning. Two online peer evaluation tools, CATME and TEAMMATES were used in various iterations of the course. Students preferred to use TEAMMATES as it was significantly less time required to complete an evaluation at the cost of accuracy and repeatability. The peer evaluation instrument was used to rate the performance of each team member in terms of contributing to the team, interaction with the team, keeping on track, quality of work, and more. Peer- and self-evaluation surveys were conducted after the submission of a major deliverable. Students were given participation marks for taking part in the surveys and based upon the results of the final peer evaluation survey, a modifier was applied to their final report mark. The results of each of the peer evaluations was released to the students anonymously so that students could receive feedback regarding how the rest of the team perceived their contributions and communication. The peer evaluation tools allowed students to confidentially communicate feedback to each other, and course directors. Intervention into poorly performing teams was done based upon these peer evaluations.

Despite the work on developing strong teams, and the rigorous peer evaluations, one of the major complaints with the course has been team dysfunction. Primarily it is related to social-loafing, real or perceived. Often, some students become very engrossed in the project, leading them to spend significantly more time on the project than other members of their group. While the other team members contributed in the form of ideas and workload at a level that, in another group, would be considered equal, their perceived contribution in that group is lower. Identifying these groups and working with them to normalize expectations or identify new ways of collaborating can help to prevent conflicts.

Another common complaint from students is that they feel their ideas and opinions are not respected by their team mates. This can lead to students taking a passive role in their teams, and eventually lower levels of perceived participation. Despite updated training on how to promote contribution from everyone, the issue still arose. These groups require early intervention and continual monitoring to prevent issues.

\section{E. Design Process and Deliverables}

The structure of the deliverables was set up to follow the engineering design process. The design process highlighted for this course was the popular VDI 2221, Systematic Approach to the Design of Technical Systems and Products [20].

Design fixation has led to students skipping major steps in the design process, or beginning with a final design in mind, and working backwards in the design process to tailor preliminary design steps to meet their desired design. This led to many students' designs failing to solve the desired problem, not meeting constraints, or arriving at a poorly optimized solution. To emphasize the importance of sequentially following the design process, reports corresponding to VDI 2221 design process "results" were collected at predetermined intervals during the term.

Following the submission and marking of each report, they were returned, and course instructors met with each team to discuss their reports, and any errors. Students were given time to reflect, ask questions, and make improvements to their ideas so that they could be used in subsequent design steps. These review sessions were essential in ensuring that students understood the subject matter and constantly worked to improve their designs. Frequent and face-to-face feedback and discussion was found to be by far the most important part in ensuring that students remained on track and internalized feedback from their deliverables.

Following the completion of the students' Definitive Layout, and 2 weeks before the final competition, students were required to build and test a prototype. The prototype was crucial in ensuring that students identified unforeseen errors in their design, limitations of the materials and/or manufacturing methods.

The final design report contained descriptions of the final design, CAD models, and testing results. It was also used as a final reflection on the overall design process. Students were asked to use the knowledge and experience that they had gained over the design process and reflect on how they would have approached each of the design phases differently.

\section{CONCLUSIONS}

Developing a design project course for first and second year students can be difficult, but the tools available to course developers can help create a successful program. By understanding the skills and knowledge that the students have developed, a project can be tailored to be challenging, yet attainable. 3D printing and other rapid prototyping tools can allow projects to be designed, built and tested, all during a 
single term. By leveraging freely available online tools, we were able to implement the major components of PBL and CL.

For most students, this is their first opportunity to fully design, analyze, build and test a design. Whether or not students are able to create a high performing design, they learn valuable lessons in design and manufacturing, as well as practical applications of the theory presented in their engineering courses. Most difficulties in running this course are due to teamwork problems. Special consideration for teamwork training, monitoring and interventions must be made to ensure students have a successful project.

Including design activities, particularly team-based design projects, during the early years of engineering curriculum can help to develop this skill and practices that industry demands.

\section{ACKNOWLEDGEMENTS}

The authors gratefully acknowledge the generous financial support of the Lassonde School of Engineering (York University) and the NSERC-Quanser Chair in Design Engineering grant.

\section{REFERENCES}

[1] R. Adams et al., "Multiple Perspectives on Engaging Future Engineers," J. Eng. Educ., vol. 100, no. 1, pp. 48-88, 2011. doi: 10.1002/j.2168-9830.2011.tb00004.x.

[2] D. S. Strong and W. Stiver, "Engineering Design Competency: Perceived Barriers to Effective Engineering Design Education," Proc. Can. Eng. Educ. Assoc., Aug. 2011. doi: 10.24908/pceea.v0i0.3944.

[3] L. M. Nicolai, "Viewpoint: An industry view of engineering design education," Int. J. Eng. Educ., vol. 14 no. 1 pp. 7-13, 1998.

[4] C. L. Dym, A. M Agogino, O. Eris, D. D. Frey, and L. J. Leifer, "Engineering design thinking, teaching, and learning," J. Eng. Educ. vol. 94, no. 1, pp. 103-120, 2005. doi: 10.1002/j.2168-9830.2005.tb00832.x.

H. J. Passow, "Which ABET competencies do engineering graduates find most important in their work," J. Eng. Educ., vol. 101 no. 1, pp. 95-118, 2012. doi: $10.1002 / j .2168-9830.2012 . t b 00043 . x$.

[6] J. S. Lamancusa, J. E. Jorgensen, and J. L. Zayas-Castro, "The learning factory-a new approach to integrating design and manufacturing into the engineering curriculum," J. Eng. Educ., vol. 86, no. 2, pp. 103-112, 1997. doi: 10.1002/j.2168-9830.1997.tb00272.x.

[7] Alice Y. Kolb and David A. Kolb, "Learning styles and learning spaces: Enhancing experiential learning in higher education," Acad. Management Learning \& Education, vol. 4, no. 2, pp. 193-212, 2005. doi: 10.5465/amle.2005.17268566.

[8] H.S Barrows, "Problem-based learning in medicine and beyond: A brief overview," New Directions for Teaching and Learning, vol. 68, pp. 3-12, 1996 doi: 10.1002/tl.37219966804.

[9] D. W. D. Johnson, R. T. R. Johnson, and M. B. M. Stanne, "Cooperative learning methods: A meta-analysis," October, 2000.

[10] C. L. Colbeck J. M. Parente S. A. Bjorklund P T. Terenzini, A F. Cabrera, "Collaborative learning vs. lecture/discussion: Students' report learning gains," J. Eng. Educ., vol. 90, no. 1, pp. 123-130, 2001. doi: 10.1002/j.2168-9830.2001.tb00579.x.

[11] Alan J. Dutson, Robert H. Todd, Spencer P. Magleby, and Carl D. Sorensen, "A review of literature on teaching engineering design through project-oriented capstone courses," J. Eng. Educ., vol. 86, no.1 pp. 1728, 1997. doi: 10.1002/j.2168-9830.1997.tb00260.x.
[12] M. Borrego, J. Karlin, L. D. McNair, and K. Beddoes, "Team Effectiveness Theory from Industrial and Organizational Psychology Applied to Engineering Student Project Teams: A Research Review," J. Eng. Educ., vol. 102 no. 4, pp. 472-512, 2013. doi: 10.1002/jee.20023.

[13] M. C. Yang, and J. Yan, "An examination of team effectiveness in distributed and co-located engineering teams," Int. J. Eng. Educ., vol. 24, no. 2, pp. 400-408, 2008.

[14] S. G. Adams, C. R. Zafft, M. C. Molano, and K. Rao, "Development of a protocol to measure team behavior in engineering education," J. STEM Educ.: Innovations \& Research, vol. 9, no. 1/2, pp. 13-20, 2008.

[15] R. Luechtefeld, D. Baca, and S. E. Watkins, "Training for self-managed student teams," Int. J. Eng. Educ., vol. 24, no. 6, pp. 1139-1147, 2008.

[16] J. S. Linsey, I. Tseng, K. Fu, J. Cagan, K. L. Wood, and C. Schunn, "A Study of Design Fixation, Its Mitigation and Perception in Engineering Design Faculty," J. Mech. Des., vol. 132, no. 4, p. 041003, 2010. doi: $10.1115 / 1.4001110$.

[17] B. O. Lakley, R. M. Felder, R. Brent, and I. Elhajj, "Turning Student Groups into Effective Teams," J. Student Centered Learning, vol. 2, no. 1, pp. 9-34, 2004

[18] R. A. Layton, M. L. Loughry, M. W. Ohland, and G. D. Ricco, "Design and validation of a web-based system for assigning members to teams using instructor-specified criteria," Adv. Eng. Educ., vol. 2, no.1, pp.1-9, 2010.

[19] C. H. Amato, and L. H. Amato, "Enhancing Student Team Effectiveness: Application of Myers-Briggs Personality Assessment in Business Courses," J. Marketing Education, vol. 27, no. 1, pp.41-51, 2005. doi: $10.1177 / 0273475304273350$.

[20] "VDI-2221: Systematic approach to the development and design of technical systems and products," Assoc. German Engineers, 1993-2005. 


\title{
A Practical Approach for Teaching Finite Element Analysis for Senior Undergraduate Mechanical Engineering Students
}

\author{
Khaled S. Al-Athel \\ Mechanical Engineering Department \\ King Fahd University of Petroleum \& Minerals \\ Dhahran, Saudi Arabia \\ kathel@kfupm.edu.sa
}

\begin{abstract}
This paper discusses the common trends in teaching finite element analysis (FEA) in mechanical engineering programs and then presents a fresh look into a practical approach to teaching FEA. A case study on teaching FEA at King Fahd University of Petroleum \& Minerals (KFUPM) is presented by discussing the syllabus, course structure, nature of exams, and the lab work.
\end{abstract}

Keywords: Finite element analysis, teaching, mechanical engineering, undergraduate students

\section{INTRODUCTION}

Finite Element Analysis (FEA) is one of the subjects where one would find some variations in the way it is taught in mechanical engineering departments. Most schools started teaching FEA at the graduate level due to the nature of the subject as it is heavily based on mathematics and programming. FEA taught at the graduate level usually focuses more on the theory and enable students to develop skills to write their own programs or codes rather than utilize a commercial software. In addition, such courses usually are based on research topics instead of practical engineering applications [1].

This was mostly true till the 90 s where many commercial software became available and the need to write an FEA code became less necessary. With that, many mechanical engineering departments started offering FEA at the undergraduate level as a senior elective course in addition to keeping graduate level FEA with the same objectives. Some engineering programs integrate some FEA aspects into engineering courses (structural mechanics, vibration, etc.) [1, 2]. This works well as an introduction to FEA, but without a dedicated course to FEA, students will not have the minimum needed theoretical background and hands-on practice on commercial codes.

Shaikh [3] presented a study on the role of introducing an FE commercial code in teaching an undergraduate FE course. This obviously had a positive impact but course still was based on theory and had the same simply supported beam project for all students. Zhuge and Mills [4] presented a project-based approach to teaching FEA for undergraduate students. The course covers the usually FE theory but focuses more on projects where students could pick a practical topic of their interest and go in detailed analysis using FEA. A simple module was presented by Hossain [5] but for a quarter module (instead of the traditional fall/winter semesters). This restricted the course to simple examples using the FE software due to the lack of time. Moazed et al. [6] presented a different course structure that is based heavily on lab session and less on theory. The course was offered for Mechanical Engineering Technology (BMET) program, hence the course being more practical. The course had two lab sessions per week, in addition to the lectures, and covered topics in structural mechanics, thermal analysis, and dynamics. The course has an excellent model but lacks in theory, as it is not intended for ME B.Sc.

In this paper, a practical approach to teaching FEA to undergraduate students is presented with details on the topics covered, course structure, and samples from exams and projects. The approach presented covers the minimum needed theory for mechanical engineers while focusing heavily on utilizing commercial FE software for engineering design and analysis.

\section{MINIMUM FE THEORY NEEDED}

Usually, the amount of theoretical topics needs to be covered in the course is what dictates how far someone can go in covering practical FE examples using FE commercial software. Table 1 lists the common theoretical topics usually covered in undergraduate FEA courses and discusses whether they should be covered or not (from the authors' perspective).

One of the main theoretical aspects of FEA is the derivation of the stiffness matrix and matrix equations. There are many different approaches used, commonly the direct stiffness method, potential energy approach, Galerkin's residual method. These methods serve the same purpose, regardless of the advantages/disadvantages in applying them. Graduate FEA courses might require students to learn how to derive their own elements to serve a certain behavior, and therefore they need to learn how to derive such matrices and equations. Undergraduate engineering students only need to learn how the equations were derived in order to understand how to link them with the physical behavior. Therefore, the direct stiffness method serves that purpose and is very easy for students to follow and understand. That being said, as the course moves 
from 1D elements to 2D and 3D elements, the direct stiffness method becomes tedious and lengthy, hence the need for one of the advanced methods. The potential energy approach also serves that purpose and is easy to follow and understand. Its only drawback is that it applies only to linear analysis. This is not an issue as the majority of FEA undergraduate course do not cover nonlinear topics (which is suggested). There is no need for undergraduate students to learn all the methods. The direct stiffness method is sufficient for lectures and HWs, whereas the potential energy approach is useful for more advanced topics to be covered in the lectures.

TABLE I. Theoretical topics to be covered in an FE course

\begin{tabular}{|c|c|}
\hline Topics & Comments \\
\hline $\begin{array}{l}\text { Spring, Bar (Truss) } \\
\text { Elements }\end{array}$ & Covered with direct stiffness method \\
\hline Beam Elements & $\begin{array}{l}\text { Covered with direct stiffness method and } \\
\text { potential energy }\end{array}$ \\
\hline Frames and Grids & Covered \\
\hline $\begin{array}{l}\text { Plane Stress \& Plane Strain } \\
\text { Using Rectangular } \\
\text { elements }\end{array}$ & Covered with potential energy \\
\hline $\begin{array}{l}\text { Plane Stress \& Plane Strain } \\
\text { Using Linear-Strain } \\
\text { Triangle (LST) }\end{array}$ & Covered \\
\hline Axisymmetric Elements & Covered with potential energy \\
\hline $\begin{array}{l}\text { Isoparametric } \\
\text { Formulations }\end{array}$ & $\begin{array}{l}\text { Not covered. This is only useful for code writing } \\
\text { and not useful for practical engineers when } \\
\text { using commercial codes }\end{array}$ \\
\hline 3D FEA & $\begin{array}{l}\text { Not covered. The formulation is very similar to } \\
2 \mathrm{D} \text { but very lengthy for hand calculations. } 3 \mathrm{D} \\
\text { can be covered in practical examples in lab } \\
\text { sessions }\end{array}$ \\
\hline Plates \& Shells & $\begin{array}{l}\text { Not covered. The theory is more advanced and } \\
\text { will require substantial time from the lecture } \\
\text { hours to cover it with adequate number of } \\
\text { examples. It can be covered in practical } \\
\text { examples in lab sessions }\end{array}$ \\
\hline Heat Transfer & $\begin{array}{l}\text { Covered without details on stiffness equation } \\
\text { derivation. The approach is already covered in } \\
\text { the mechanical part of the course. This could be } \\
\text { given as a read assignment for students if the } \\
\text { instructor deemed important. }\end{array}$ \\
\hline Thermal Analysis & Covered without derivation. \\
\hline Structural Dynamics & $\begin{array}{l}\text { This depends on the independent ME programs. } \\
\text { If structural dynamics or vibration is a core } \\
\text { course, then this could be covered. If not, then it } \\
\text { should not be covered, as it would require } \\
\text { teaching the basic vibration theory in addition to } \\
\text { the FEA theory. This could be considered as a } \\
\text { suggestion for additional topics. }\end{array}$ \\
\hline Additional Topics & $\begin{array}{l}\text { Every mechanical engineering department } \\
\text { should have additional topics based on the } \\
\text { industrial and research topics that are considered } \\
\text { relevant to the department and the region where } \\
\text { the university is located. } \\
\text { Examples for such topics: } \\
\text { - Coatings } \\
\text { - Composite materials } \\
\text { - Piezo electric materials (PZTs) } \\
\text { - Introduction to CFD }\end{array}$ \\
\hline
\end{tabular}

\section{SUGGESTED PRACTICAL APPROACH FOR TEACHING FEA}

The suggested practical way to teach FEA for undergraduate mechanical engineering students is to limit the theory to what is needed to understand how FE works. The rest should focus on how to utilize commercial FE software in design and analysis of practical applications. The link between the theory and practice comes from utilizing the understanding of how FE works and converting it into practical use of FE software on how to properly model the problem and apply boundary conditions (BCs) and loads (problem idealization is commonly used for to describe this).

Lab sessions should go in parallel with the suggested topics in Table I. It is important to discuss mesh dependency to students from the beginning to establish it as a regular part of the process. In addition, model validation should be done early on to teach students the concept of validation and verification of computational results. A special lab session with prepared examples should be given when going into $2 \mathrm{D}$ and $3 \mathrm{D}$ analysis to cover advanced topics such as types of symmetry, stress singularity, mesh convergence, etc.

Due to the nature of the course, hand calculations should be given in assignments, as they tend to be lengthy, while keeping the theory-based exam limited to simple theoretical discussion and short calculations. Final exam should be based on solving a practical problem including all the necessary steps in FE modeling and analysis. Lab assignments are based on the topics covered in a weekly basis. Course projects should be kept to the students to choose a topic of interest or choose a topic related to his/her capstone senior design project, or apply FEA to any other project from another course. Table II lists a suggested grading scheme. Students are encouraged to work in teams for their projects, but individually for their assignments. The course is designed such that assignment are used to test students' understanding of the FE theory, whereas the midterm is used to test the understanding of the basic concepts of FE.

TABLE II. FEA Course Grading Scheme

\begin{tabular}{|c|c|c|}
\hline Assessment & Weight & Comments \\
\hline Assignments & $25 \%$ & $\begin{array}{l}\text { Assignments are a mix of: } \\
\text { - Hand calculation problems (for lengthy problems } \\
\text { students are encouraged to use MATLAB or } \\
\text { other similar software) } \\
\text { - FE software problems. }\end{array}$ \\
\hline Project & $25 \%$ & $\begin{array}{l}\text { Students can work in groups of their choice. } \\
\text { Students should pick project topics based on: } \\
\text { - Their interests, or } \\
\text { - Their senior capstone projects, or } \\
\text { - Other courses projects }\end{array}$ \\
\hline Midterm & $20 \%$ & $\begin{array}{l}\text { Simple hand calculations, conceptual questions, } \\
\text { discussions, etc. }\end{array}$ \\
\hline Final Exam & $30 \%$ & $\begin{array}{l}\text { Practical engineering problem(s) where students } \\
\text { submit a soft copy of a report that would include: } \\
\text { - Problem idealization } \\
\text { - FE model details } \\
\text { - Requested results (figures) } \\
\text { - Results and Discussion } \\
\text { - Suggested validation approach }\end{array}$ \\
\hline
\end{tabular}




\section{CASE STUDY}

This section provides examples from when the FEA course was offered in the new structure in the fall semester 2017/2018 at the mechanical engineering department at King Fahd University of Petroleum \& Minerals. At the end, comments from the students are given to share students' perspective of the course structure. A sample home assignment including both hand calculations as well as FE software problems is given as an example to the assignment structure. A short version of the final exam problem is shown here for reference. And finally, some of the course projects are listed to highlight the how far students can reach with the suggested course structure.

\section{A. Assignment Problems}

Assignments are developed in a way that students would utilize an FE software to solve the problem and then be able to validate using hand calculations. A couple of typical assignments are listed in Table III for reference and so the reader would follow the structure suggested for the assignments. Assignments at the beginning of the course tend to have more hand calculations and less practical problems to be solved by the software as they are mostly truss and 1D beam problems. That being said, one can have practical 1D problems as the first example shown in Table I. As the course moved toward 2D and 3D problems, one can assign problems similar to examples 2 and 3 .

\section{B. Exam Problems}

As mentioned in Table II, there are two exams in this structure. The midterm is supposed to have short hand calculations and some conceptual questions. These usually the typical exams that are given in such courses. The focus here will be on the nature of the final exam using an FE software. The course is supposed to teach students the concepts of FEA to be applied to practical problems and analyzed using an FE software. Therefore, it is suggested in this structure that the final exam should be a practical problem were students would use their FE software skills to analyze it while utilizing their theoretical knowledge of FEA. Table IV lists a practical problem on thermal barrier coatings (TBCs) that was taken from an actual research [7] but simplified to for the course level and was offered in the final exam. Students are expected not only to solve the problem using the software, but include all the necessary steps that they have been doing in the lab and assignments (idealization, mesh sensitivity, validation approach, etc.)

\section{Course projects}

Students were asked from the second week to form groups of their own and choose topics (they can also work individually). Students were encouraged to utilize their capstone projects, or projects from other senior level courses. If neither is applicable, then they can choose a topic of their interest. Table V lists some of the projects that student worked on with sample FE results. Projects are worth $25 \%$, were $5 \%$ is evaluated by two other faculty members in a poster session, and $20 \%$ is evaluated based on the project report.
TABLE III. Examples of FEA course assignments

\begin{tabular}{|c|c|}
\hline Example & Description \\
\hline 1 & $\begin{array}{l}\text { As an engineer with background in FEA, your supervisor asked } \\
\text { you to design a beam made of ASTM A36 steel ( } E=210 \mathrm{GPa} \text { ) } \\
\text { with allowable stress of } 160 \mathrm{MPa} \text { to support the load as shown in } \\
\text { the initial design figure. An "I" shape (wide flange) beam is to be } \\
\text { used. Compare the stresses when using different W460 sections. } \\
\text { What is the safety factor of each case? Suggest one section to be } \\
\text { your final choice } \\
\text { 1. Discretize the problem using four elements (for hand } \\
\text { calculation validation). } \\
\text { 2. Show your FE model with the loads and BC. } \\
\text { 3. List the units you are using for the dimensions, load, and } \\
\text { material properties. } \\
\text { 4. Provide a table with columns for the W460 size, maximum } \sigma_{x} \text {, } \\
\text { and safety factor. }\end{array}$ \\
\hline 2 & $\begin{array}{l}\text { Stiffeners are common in engineering applications. The purpose of } \\
\text { using another material as an added beam layer, or as a stiffener, is } \\
\text { to increase certain properties or enhance the behavior, such as to } \\
\text { make the structure stiffer. Consider an aluminum beam }(E=70 \\
\text { GPa, } v=0.3) \text { with dimensions length } \mathrm{L}=200 \mathrm{~cm} \text {, width } \mathrm{W}=10 \\
\mathrm{~cm}, \text { and height } \mathrm{H}=5 \mathrm{~cm} \text {. A steel beam }(E=200 \mathrm{GPa}, v=0.3) \text { is } \\
\text { added on top of the aluminum beam with dimensions' length } \mathrm{L}= \\
\text { 200 cm, width } \mathrm{W}=10 \mathrm{~cm} \text {, and height } \mathrm{H}=2 \mathrm{~cm} \text {. The beam is } \\
\text { loaded with applied forces of } 1000 \mathrm{~N} \text { and } 2000 \mathrm{~N} \text { at the free end as } \\
\text { shown in the figure. } \\
\text { 1. Discretize the problem } \\
\text { 2. Provide mesh sensitivity analysis } \\
\text { 3. Evaluate the deflection at the tip of the aluminum beam with } \\
\text { and without the steel beam. Comment on the effect of adding } \\
\text { the thin steel beam on the deflection of the aluminum beam. } \\
\text { 4. Plot the contours of the von-Mises stress and the von Mises } \\
\text { strain and comment on the results }\end{array}$ \\
\hline & $\begin{array}{l}\text { Dimensions are given in } \\
\text { another detailed 2D figure }\end{array}$ \\
\hline 3 & $\begin{array}{l}\text { Heat sinks are used to enhance heat dissipation from electronic } \\
\text { devices. In this case study, a heat sink made of aluminum is used } \\
\text { for the study. A fan forces air over all surfaces of the heat sink } \\
\text { except for the bottom of base, where a heat flux } q^{\prime}=1000 \mathrm{~W} / \mathrm{m}^{2} \text { is } \\
\text { prescribed. The surrounding air is } 28^{\circ} \mathrm{C} \text { with a heat transfer } \\
\text { coefficient of } h=30 \mathrm{~W} /\left(\mathrm{m}^{2 \circ} \mathrm{C}\right) \text {. } \\
\text { 1. Idealize the problem } \\
\text { 2. Perform mesh sensitivity analysis } \\
\text { 3. Discuss your approach for validation of the model } \\
\text { 4. Study the steady-state and transient thermal response of the } \\
\text { heat sink }\end{array}$ \\
\hline
\end{tabular}


TABLE IV. Final exam problem

\begin{tabular}{|c|c|c|c|c|c|}
\hline Section & \multicolumn{5}{|c|}{ Description } \\
\hline Background & \multicolumn{5}{|c|}{$\begin{array}{l}\text { Supcralloy } \\
\text { Thermal barrier coatings (TBCs) are widely used in gas } \\
\text { turbines. They protect the engine blades from high } \\
\text { temperature. The TBC system, as shown in the figure, is used } \\
\text { as a coating for gas turbine blades and consists of a ceramic } \\
\text { top coat (TC) that reduces the metal temperature; a metallic } \\
\text { bond coat (BC) to enhance the bonding between the top coat } \\
\text { and the underlying superalloy, and to also protect the } \\
\text { superalloy from oxidation and hot corrosion. Due to hot } \\
\text { temperature corrosion, a thermally grown oxide (TGO) layer } \\
\text { develops at the top of the bond coat. The concern in TBC } \\
\text { systems is the fracture failure between the TC and the BC } \\
\text { (TGO) either by normal separation (vertical) or by shear } \\
\text { fracture }\end{array}$} \\
\hline \multirow[t]{6}{*}{ Problem } & $\begin{array}{l}\text { Consider a TBC } \\
\text { the substrate for } \\
\text { is assumed bet } \\
\text { metallic), and a } \\
\text { top coat, TGO } \\
\text { operated under a } \\
\text { superalloy has a } \\
\text { the effect of the } \\
\text { indicate if, and } \\
\text { interface betwee } \\
\text { MPa for tensil } \\
\text { Material propert }\end{array}$ & $\begin{array}{l}\text { ystem c } \\
\text { our ana } \\
\text { een the } \\
\text { sinusoid } \\
\text { and th } \\
\text { heat flux } \\
\text { emperat } \\
\text { thermal } \\
\text { here, lo } \\
\text { the TC } \\
\text { fractur } \\
\text { s are gi }\end{array}$ & 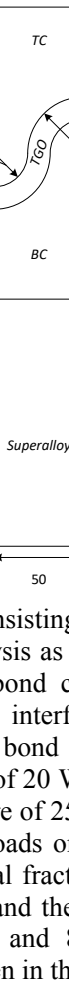 & 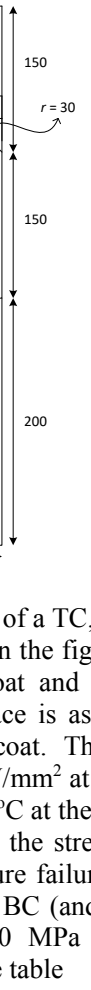 & $\begin{array}{l}\text { TGO, BC, an } \\
\text { tre. A flat int } \\
\text { he substrate } \\
\text { umed betwee } \\
\text { turbine bla } \\
\text { he surface, ar } \\
\text { bottom. Inves } \\
\text { ses develope } \\
\text { might occur } \\
\text { TGO) fails a } \\
\text { or shear fra }\end{array}$ \\
\hline & Material & $\begin{array}{c}E \\
(\mathrm{GPa})\end{array}$ & $v$ & $\begin{array}{c}\alpha \\
\left(1 /{ }^{\circ} \mathrm{C}\right)\end{array}$ & $\begin{array}{c}k \\
\left(\mathrm{~W} /\left(\mathrm{m} .{ }^{\circ} \mathrm{C}\right)\right)\end{array}$ \\
\hline & Top coat & 205 & 0.18 & $\begin{array}{l}12 \times \\
10^{-6} \\
\end{array}$ & 2.6 \\
\hline & TGO & 180 & 0.27 & $\begin{array}{c}8.3 \mathrm{x} \\
10^{-6}\end{array}$ & 5.5 \\
\hline & $\begin{array}{c}\text { Bond } \\
\text { coat }\end{array}$ & 150 & 0.30 & $\begin{array}{c}14.3 \\
\times 10^{-6} \\
\end{array}$ & 31.0 \\
\hline & Substrate & 203.4 & 0.35 & $\begin{array}{c}8.6 \times \\
10^{-6} \\
\end{array}$ & 21.9 \\
\hline
\end{tabular}

TABLE V. Samples of students' projects

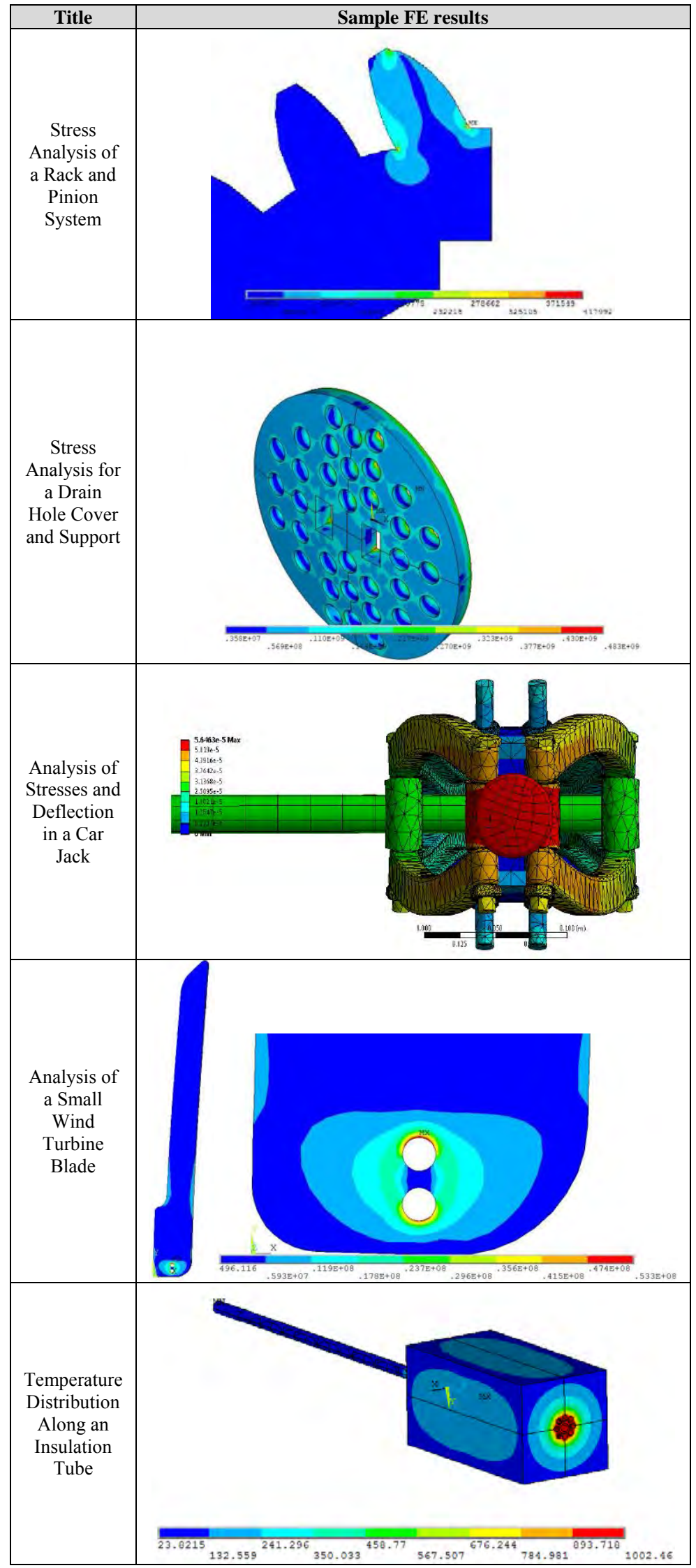




\section{Students' comments}

Students were asked towards the end of the course to submit anonymous feedback regarding the course structure and the way it was taught. It is important to note that most engineering and science courses at KFUPM tend to have two major exams instead of one midterm exam. Also assignments are usually worth between $10-15 \%$ of the overall grade.

- The method of making the homework worth $25 \%$ is a very good approach to make students learn, but the problem is that the students are not used to it. A recommendation that I have is to create a texting group for communications instead of blackboard discussion board which is not convenient.

- This is an effective way of teaching (especially the idea of giving conceptual short exam problems and heavy assignments where students can learn). I suggest teaching the software part should be $50 \%$ or more of the course because I think it is the most important part.

- The instructor changed the way of teaching for this course. He made it more about practicing and thinking. The assignments are the major part of that. I liked it, some did not. Maybe they don't like spending long hours on assignments.

- I suggest having video tutorials as they are much more effective than step by step guidelines for the software solved problems. We can learn listening and watching much better than just reading.

- The theory is lengthy and quite boring, he tried all he can to make it not.

- The teaching philosophy is awesome. He really cares about the students understanding. He teaches in a manner that is tailored to the conceptual understanding of the subject. The only complaint that I have is the difficulty in following in class. This is not because of the teacher, rather because of the nature of the subject. Outside of the class, when I have the ability to turn pages on a book, understanding the material is not a problem

There were other comments but related to topics other than the structure of the course and the link between theory and practice. Overall, students responded well to keeping the lengthy hand calculations for students to solve at home as part of the assignments, while focusing more on the use of an FE software in solving practical engineering problems in the lectures and the lab.

\section{CONCLUSION}

This paper presented a modified approach in teaching undergraduate FEA course with more focus on the practical use of FEA. This was based on offering the course as an elective for senior level students at the mechanical engineering department at King Fahd University of Petroleum \& Minerals.
A list of suggested FE theory topics is listed and discussed. Many of the classical topics were removed as they do not add anything to the practical knowledge needed for the students. Cutting many of the theoretical topics gave more room for the practical side of the course to expand and cover a wider range of practical examples. Around $50 \%$ of the lectures were offered in a PC lab were the lecture would focus on solving an engineering problem.

The assignments are carefully designed to ensure a good link between theory (and hand calculations) and utilization of an FE software. Students can solve the problems using the software, but then would use hand calculations for validation and verification of their results. Projects were left to students to work individually or as groups, and pick a topic of their interest. The topic has to be approved by the instructor to ensure that a substantial FE work can be done in the project. Final exam is where the real outcome of the course is tested. Students are given an actual and relevant engineering problem. They are asked to read a little background to understand the topic. Then they are asked to prepare a report on analyzing the problem from idealization till the discussion of the FE results.

Students' comments at the end of the course were mostly positive as they liked this approach. Most of the negative comments were not related to the course structure or the grading scheme.

\section{ACKNOWLEDGMENT}

The author would like to thank King Fahd University of Petroleum \& Minerals (KFUPM) for their support and providing the needed resources to teach the course in the new suggested format.

\section{REFERENCES}

[1] W. Gilewski, "To Teach or not to Teach Finite Elements: This is a Question", in 6th AECEF Symposium, Vilnius, 2008.

[2] S. Zhang, "Integrating Finite Element Analysis into Mechanical Engineering Undergraduate Courses.", in ASEE Annual Conference \& Exposition, Seattle, 2015.

[3] F. U. A. Shaikh, "Role of Commercial Software in Teaching Finite Element Analysis at Undergraduate Level: a Case Study," Eng. Educ., vol. 7, no. 2, pp. 2-6, Dec. 2012. doi: 10.11120/ened.2012.07020002.

[4] Y. Zhuge, and J.E. Mills, "Teaching Finite Element Modelling at the Undergraduate Level: A PBL Approach," in 2009 AaeE Conference, Adelaide, Austrailia, 2009.

[5] A. Hossain, "Teaching an Undergraduate Introductory Finite Element Analysis Course: Successful Implementation for Students Learning," in ASME International Mechanical Engineering Congress and Exposition, Houston, TX., 2015. doi: 10.1115/IMECE2015-50091.

[6] A. R. Moazed, R. Roberts, Z. Le and A. Duva, "Teaching Finite Element Analysis in Undergraduate Technology Curriculum," in ASEE Northeast Section Conference, Boston, MA, 2010.

[7] K. Al-Athel, K. Loeffel, H. Liu, and L. Anand, "Modeling Decohesion of a Top-Coat from Thermally-Growing Oxide in a Thermal Barrier Coating," Surf. Coat. Technol., vol. 222, pp. 68-78, May 2013. doi: 10.1016/j.surfcoat.2013.02.005. 


\section{Towards a Conceptual Model of Design Team Learning in Mechanical Engineering Education}

\author{
Minha R. Ha \\ Department of Mechanical Engineering \\ Lassonde School of Engineering, York University \\ Toronto, Canada \\ minhareo@yorku.ca
}

\author{
Aleksander Czekanski \\ Department of Mechanical Engineering \\ Lassonde, School of Engineering, York University \\ Toronto, Canada \\ alex.czekanski@lassonde.yorku.ca
}

\begin{abstract}
Design education is the backbone of the undergraduate Mechanical Engineering (ME) curriculum at Lassonde School of Engineering (LSE). ME students take project-based design courses every year of their program. Students in the design courses were surveyed and interviewed, in order to examine the key learning outcomes and their development over the undergraduate years. Early-stage coding and analysis on the interview data have resulted in (1) the identification of possible threshold concepts in each year of project-based design learning, (2) the variation and changes in the meaning of design across cohorts, and (3) the three-level factors to design team success. Recommendations are proposed for the instructional training and course structure to enhance their support for design team project experience and outcomes.
\end{abstract}

Keywords- design education; project teams; mental models; threshold concepts

\section{INTRODUCTION}

What is engineering design (ED)? Design engineering is embedded in the contexts of techno-scientific, socio-cultural, and econo-organizational realities; it also serves as a central convergence point for transdisciplinary knowledge integration to occur [1], [2]. As such, many scholars have made efforts to define the nature of engineering design [3]-[5], and the abilities of a design engineer [6]-[10].

How educators understand design abilities shapes curricular decisions that structure student learning. In the context of our design curriculum, an early and repeated engagement of engineering students with project-based learning in engineering design serves multiple purposes. First, the design projects closely resemble the practice environment, providing an opportunity to more accurately grasp the nature of the complex engineering work [10]. Projects involve change management, knowledge integration, and interpersonal work that require practice and feedback. Students "act out the role of designer" [6], the opportunity for which is not often available in individual, separately topic-based courses.
Second, the design projects empower students to take active ownership and receive recognition for their creative work. Producing tangible outcomes builds confidence, motivates engagement, and is expected to motivate prolonged engagement in the engineering field [11]. At the same time, because of the 'realness' students perceive in the project experience, design projects become an educational environment where students "come to care about the valuable things involved" (Peters 1965, quoted in [11]) - and reveal what things are actually valued in the institutional program. All such aspects are expected to enable reflection and deep changes in the way students understand and relate to their work in engineering design.

However, the preference for project-based learning for engineering design lacks in comparison a robust empirical and theoretical explanation of the actual learning processes that take place. How do students learn engineering design, or develop as design engineers? This is not a straightforward question; and as we have delved into the study of design learning in our curricular setting since 2015, we have undergone changes in our own 'mental models' of ED teaching and learning. Mental models are "our understanding of the causal structures of the system, the boundary we draw around the system, the time horizon we consider relevant, or our goals and values... our framing or articulation of a problem" [12]. Student perspective data from our longitudinal study has pushed us to articulate and have an honest look at our choices, assumptions, and expectations as educators.

This paper focuses on the unexpected findings from student interviews that link design project teams' interpersonal dimensions much more closely to the design performance than previously expected. Originally, teamwork skills were considered complementary but not core to the design team performance. We will demonstrate how students' experiencebased insights can be used to develop a useful framework of design team effectiveness that potentially enhances the teaching content and strategies for design projects. Design is indeed social and ethical in nature [13], [14]. The findings helps start to fill the gap in otherwise strongly cognitivefocused understanding of design education [15], and takes 
further the affective and moral attitudes into practical team competencies.

The paper also summarizes key findings in the potential threshold concepts in design learning. Threshold concepts are learning outcomes that are difficult to achieve, but once achieved, they are irreversible and transformative [16]. This fits well with the view of learning as experiential 'process of change' or 'process of becoming' [17]. The threshold concept theory allows the researcher to focus on difficult, transformative learning outcomes (rather than attempt to document all results of learning). Student perspectives offer much value in this research because they are highly self-aware of the transformative changes, and the struggles they experienced in that transformation.

Therefore, in order to build our understanding of how students learn engineering design, this paper addresses two questions: (1) What key changes do students experience? (2) What enables design project team success for students? The results on design learning trajectory and design team success are expected to contribute useful teaching and learning materials, and also inform course design decisions on the sequence and focus of training.

\section{CONTEXT OF THE STUDY PARTICIPANTS}

The undergraduate Mechanical Engineering (ME) curriculum at Lassonde School of Engineering (LSE) has a strong design focus. Students take a full project-based design courses every year in their winter semester. The general first year design course focuses on problem solving process and techniques (from problem identification to prototyping). Second and third year design courses are for ME students only. The capstone (final year) course is multidisciplinary, involving students from ME, Electrical Engineering and Computer Science (EECS), and Earth Sciences and Space and Engineering (ESSE).

Project-based format is chosen because of the expectation that is mimics real-life practice, and enables 'learning by doing.' Most importantly, spending time to move through one complete cycle of design process is of critical importance, for design abilities are expected to be demonstrated in practice theoretical knowledge of design methodologies must be applied in practice. There is a possible weakness that students are not asked to abstract the design methodology based on their experience, which may or may not contribute to their ability to perform in different methodological frameworks (or innovate their methodologies).

ME students are exposed to multiple design philosophies and approaches in their first year and capstone courses (which are common courses to LSE students, working with peers across departments). In $2^{\text {nd }}$ and $3^{\text {rd }}$ year ME design courses, however, the course deliverables closely follow the conventional design process developed by Pahl and Beitz [7], which is a well-known conventional design methodology in the ME context [18]: conceptual design, embodiment design, prototype report and final report are submitted. Earlier reports are especially important for facilitating frequent feedback, and managing project timelines. Recognizing the importance of teamwork skills, the following modules are integrated into the courses via lectures, assignments and tutorial activities: team contract, peer evaluation, problem-solving style assessment, conflict resolution skills, and personal reflection.

\section{METHODOLOGY}

\section{A. Data Collection}

Data were drawn from a larger longitudinal study on the program effectiveness and learning processes in engineering design. In particular, this paper draws data from second (UG2) to fourth (UG4) students in Mechanical Engineering, who participated in the In-Program Survey by and the in-depth interviews that followed. The survey participants were recruited through class announcement and an online link posted on the learning management system; in some courses some class time was allotted to conduct the survey in paper format. Research staff visited classes in a way that no researcher was also a teaching assistant or instructor in the class visited.

In-depth interviews included in this paper were conducted by two female members of the research team (including the first author). Both interviewers represent racialized minorities as well the female presence in an otherwise male-dominant undergraduate program in ME. Interview participants were recruited through the In-Program Survey (by indication of interest), and by posting recruitment message on the learning management system. The total numbers of participants in the In-Program Survey and in-depth interviews are included in Table I.

TABLE I. NUMBER OF RESEARCH PARTICIPANTS

\begin{tabular}{|l|l|c|}
\hline Data Collected in & \multicolumn{1}{|c|}{ In-Program Survey } & In-Depth Interview \\
\hline $\begin{array}{l}\text { Sept 2015-Aug } \\
2016\end{array}$ & Only Entrance & UG3: 3 male \\
\hline $\begin{array}{l}\text { Sept } 2016-\text { Aug } \\
2017\end{array}$ & UG2: 73 & UG2: 2 female, 4 male \\
Sept $2017-$ Aug & UG2: 32 & UG3 2 male \\
2018 & UG3: 36 & UG4: 2 male \\
& UG4: 17 & Recruitment ongoing \\
\hline
\end{tabular}

B. Data Analysis

Written survey responses were reviewed and categorized based on key themes that emerged. The category labels (open codes) were compared to identify possible properties and dimensions that would help relate and group the labels further.

Coding of interview data adapted the Grounded Theory analytic procedures outlined by Strauss and Corbin [19]. Interview transcripts were first coded by paragraphs (open coding), offering a quick summary of each transcript. Relationships between open codes, and hence specific sections of the data, were identified (axial coding). Several versions of potential categories emerged and guided the subsequent data collection. For example, in 2017, a potential model of design learning in team projects was developed. In 2018, a new focus on the factors/features of design team success resulted from added data analysis (Table III). Once the potential categories were chosen, student data were extracted and organized based on the categories. The summaries and category-based enabled volume reduction of the data to be analyzed. 
For some questions, student responses were simply summarized and organized for easy presentation (e.g. Fig. 1). However, interviewees in 2018 were asked to review the preliminary findings from 2017 as well, and invited to confirm/disconfirm, elaborate, and add nuances.

\section{FINDINGS}

\section{A. Key Outcomes in Undergraduate Design Learning}

Third and fourth year students were asked to identify key learning outcomes related to design projects in each year of their program. These are summarized below (Fig. 1):

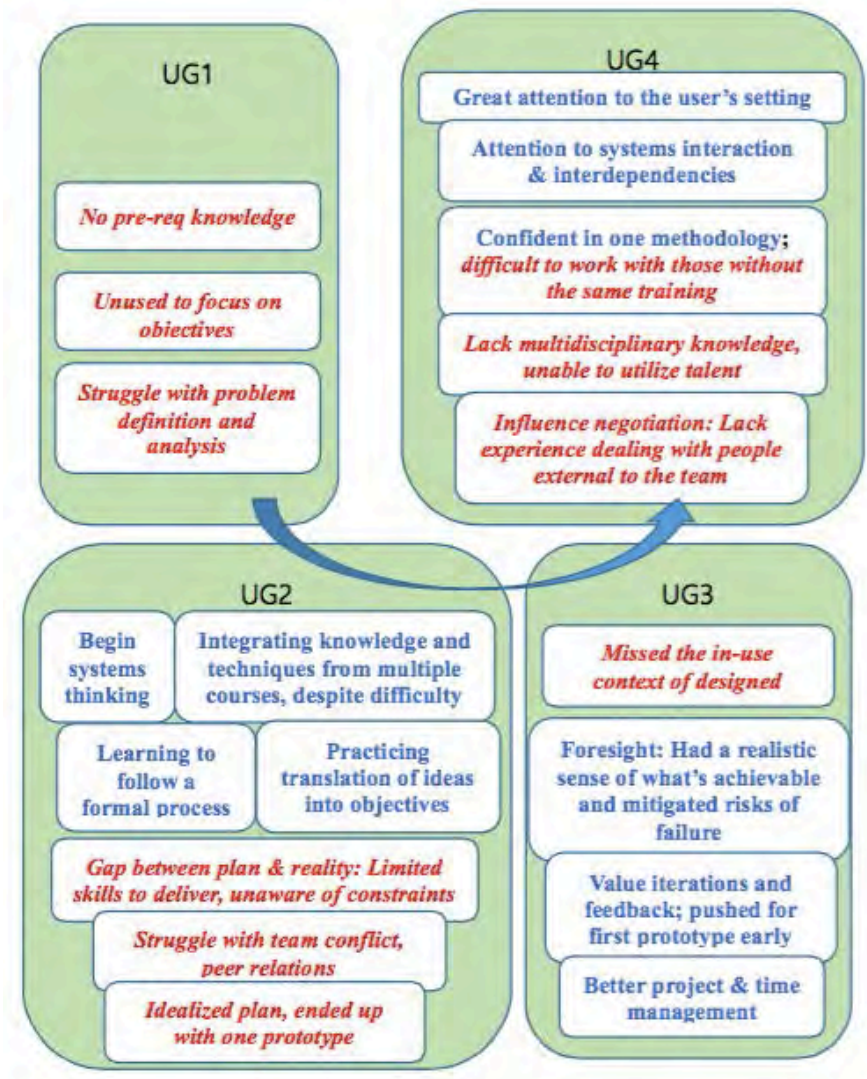

Figure 1. Transformative Outcomes in Design Learning

The italicized text represents self-identified evidence of struggles; bolded text represents self-recognized demonstration of newly achieved abilities. Both attest to the experiences of irreversible change suggesting possible threshold concepts in learning engineering design in our ME context.

UG1 was a general first year, with design projects that emphasized problem identification and did not require a final product beyond the first prototype. UG2 was the first time ME students had to complete the design cycle, testing prototypes and producing a final outcome. UG3 followed a similar project and course structure. UG4 introduced a new environment for ME students: working in teams with students trained 3 years in different disciplines. Repeat experience in a similar project environment demonstrated more achievements in previously identified challenges; Entering a new project environment identified more new challenges to work on.
Survey responses also confirm the importance of the formal methodology in training thinking habits (Table II). Students were asked to self-identify biggest changes in their approach to design projects. The following key categories emerged:

\section{TABLE II. Key Changes In APProAChing Design Projects, From} UG1 TO TIME OF SURVEY

\begin{tabular}{|c|}
\hline Categories and Sample Quotes from 2016-2017 Survey \\
\hline $\begin{array}{l}\text { From haste actions } \rightarrow \\
\text { To following a methodical, systematic process } \\
\text { "Headed straight for the problem solution" } \rightarrow \text { "Follow steps } \\
\text { to get the problem solution (word logical chart, friction block } \\
\text { diagram)" (\#31, male, UG2) }\end{array}$ \\
\hline $\begin{array}{l}\text { "try to come with solution on spot" } \rightarrow \text { "have a systematic } \\
\text { approach now" (\#8, male, UG2) }\end{array}$ \\
\hline $\begin{array}{l}\text { From lacking knowledge foundations } \rightarrow \\
\text { To developing deeper understanding } \\
\text { "getting used to so much material at once" } \rightarrow \text { "acquire a deep } \\
\text { understanding of the material" (\#1, female, UG2) }\end{array}$ \\
\hline $\begin{array}{l}\text { From unrealistic, arbitrariness } \rightarrow \\
\text { To realistic designs with complexity } \\
\text { "ambitious and impractical" } \rightarrow \text { "keep design simple and } \\
\text { functional" (\#28, male, UG2) }\end{array}$ \\
\hline $\begin{array}{l}\text { "fantasy world, did not understand limitations at all" } \rightarrow \text { "take } \\
\text { into account important aspects like cost, effectiveness, ease of } \\
\text { use, ergonomics and aesthetics" (\#4, female, UG3) }\end{array}$ \\
\hline
\end{tabular}

Students' description and explanation of their approaches to engineering design also allude to a 'mental model' of engineering design that guides their action [9]. A crucial part of evolving the mental model is the feedback one receives from real world experiences. An early and repeated exposure of to the experience of engineering design has given students opportunities to become aware of their own limitations and achievements, reflect and practice new habits and skills.

\section{B. The Meaning of Design in Engineering Context}

An important part of the mental model is the understanding of the nature of engineering design, and the students' relationship to the design activity. Design can be recognized as a formal process, an intermediary plan for an artefact (representing characteristics of an artefact), or an output product. One third year student captured the ambiguous use of the term 'design': "Design is both the process in which engineers create and test new ideas (aka the 'design process') but also can refer to a specific style of something ("a certain design was preferred" for example)" (O4, male, UG3, 20162017). Most students chose to describe 'design' in single definitions. Survey responses to the question, "What does 'design' mean, in engineering context?" were first categorized by the type of definition (e.g. design as process, as action, as product, as attribute). The results from 2016-2017 data are presented in Figure 2. 


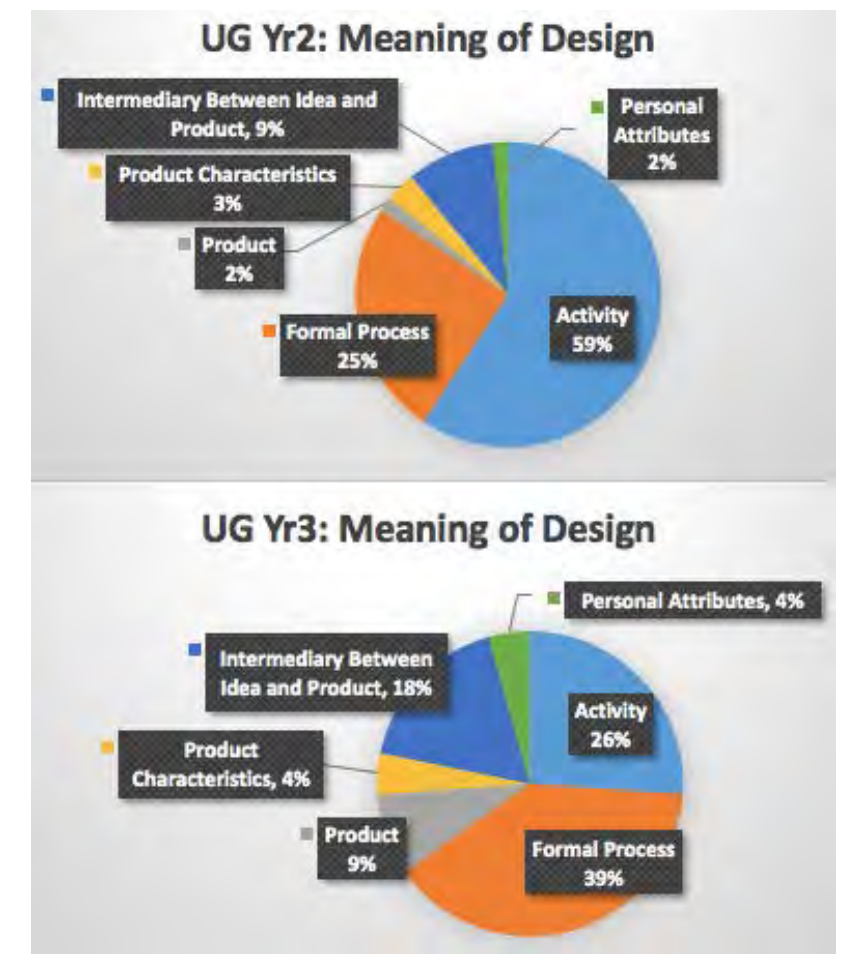

Figure 2. Comparison of UG2 and UG3 Meanings of Engineering Design

It is interesting to note that third year students have a more distributed understanding of what design typifies (a formal process, an output product, and an intermediary between the two). Interview data confirms that students gain a greater recognition of design as a formal process, rather than simply doing something (activity). However, the survey captured a much greater range of responses. For example, the appreciation of the intermediary artefact (that facilitates the translation from an idea to product) was not as clearly selfarticulated by the interviewees (even though the work producing the intermediary artefact was described in detail).

It is important to appreciate the diversity of meanings that persist despite sharing the same curriculum (which has a tendency to create conformity to a limited number of views). Using the term 'design' as a personal attribute, or a characteristic of oneself, suggests a different identity relationship to the work of design than viewing 'design' as a separate product. Translation of an idea into a physical reality, and creativity, remain prevalent goals of design for second and third year students. Referring to some of the interview data, there is a deeply motivating aspect in making something become a physical reality:

[I thought] maybe this isn't for me sometimes. Going into second year then, having the actual project, then something amazing happened. I kept doing it, and I think at this point [I] decided, I'm doing for myself. (S9, female, UG2, 4)

Purpose of engineering design was an embedded part of students' definition of engineering design. The same survey data were categorized based on the implied goals of design in the engineering context. The results from 2016-2017 data are presented in Figure 3.

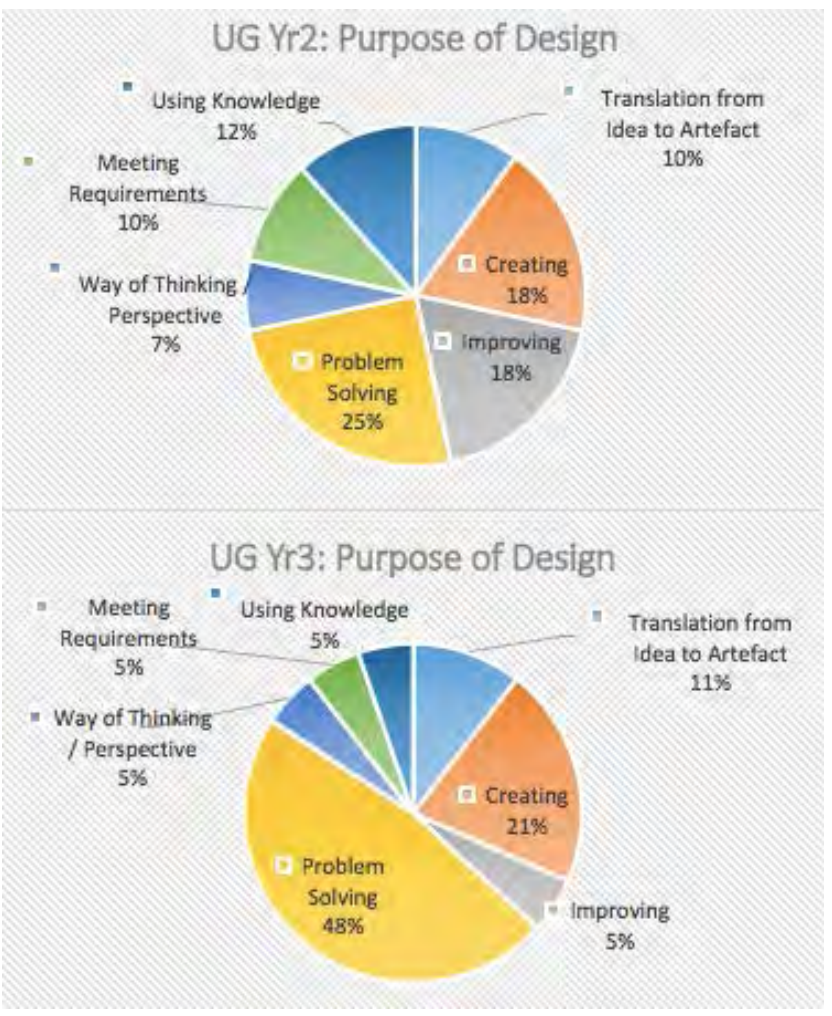

Figure 3. Comparison of UG2 and UG3 Goals of Engineering Design

The close connection between purpose and meaning of design suggests a further investigation into the motivations, aspirations, and values students bring to engineering design. It is also interesting to note that 'social impact' was mentioned in a few responses to the question about the meaning of design, but mentioned much more frequently as reasons to choosing engineering. This aspect is dealt much more effectively in survey and interview data relating to student motivation, and their desired future roles as engineers. These data are not included in this paper.

\section{Three Levels of Design Project Team Enablement}

A significant part of student responses regarding design projects involved experiencing and addressing challenges to team success. There were three distinct types of factors that attributed to team success, with the key concepts organized in the following table. This distinction offers increased clarity and a holistic view of the skills, values, and processes required for an effective team. This also helps explain why, despite separate efforts to establish, e.g. conflict resolution procedures or communication plans, teams might struggle.

Female students, despite fewer in number, offered much more breadth and precision in the use of terminology (Appendix I). Abstracted further by combining both male and female participant voices, the results are summarized in Table III. 
TABLE III. Key FeAtures OF EFFECtive Design TeAms

\begin{tabular}{|c|c|c|c|}
\hline & Level 1 & Level 2 & Level 3 \\
\hline $\begin{array}{l}\text { Descrip- } \\
\text { tion }\end{array}$ & $\begin{array}{l}\text { Individual's } \\
\text { Meaningful } \\
\text { Connection to } \\
\text { the Project }\end{array}$ & $\begin{array}{l}\text { Processes that } \\
\text { Enable Effective } \\
\text { Engagement and } \\
\text { Contribution }\end{array}$ & $\begin{array}{c}\text { Team Culture } \\
\text { and Values } \\
\text { that Foster } \\
\text { Effective } \\
\text { Levels } 1 \text { \& } 2\end{array}$ \\
\hline $\begin{array}{l}\text { Example } \\
\text { Features }\end{array}$ & $\begin{array}{l}\text { Commitment } \\
\text { Priority } \\
\text { Responsibility } \\
\text { Perceived } \\
\text { purpose } \\
\text { Motivation } \\
\text { Learning } \\
\text { Leading } \\
\text { Overall } \\
\text { understanding } \\
\text { of project }\end{array}$ & $\begin{array}{l}\text { Identification of } \\
\text { each member } \\
\text { strengths and } \\
\text { needs } \\
\text { Communication } \\
\text { management } \\
\text { Decision making } \\
\text { Task development } \\
\text { Task allocation } \\
\text { Knowledge } \\
\text { management } \\
\text { Expectation } \\
\text { management } \\
\text { Conflict resolution } \\
\text { Consensus building } \\
\text { Conversations for } \\
\text { understanding } \\
\text { Role clarification }\end{array}$ & $\begin{array}{l}\text { Mutual respect } \\
\text { Fairness in } \\
\text { recognition } \\
\text { Truthfulness } \\
\text { Value for the } \\
\text { person } \\
\text { Goal of shared } \\
\text { success } \\
\text { Inclusivity } \\
\text { Friendship and } \\
\text { belonging }\end{array}$ \\
\hline
\end{tabular}

\section{Level 1: Meaningful Connection to the Design Work}

From students' perspective, a team is successful in its most basic level if all individuals are contributing effectively. Individuals bring their own motivation, behaviour, attitudes, unique needs and strengths. They also carry multiple responsibilities and competing priorities outside the project. For a team working to its "maximum potential" (S9, female, $\mathrm{UG} 2,13$ ), where people want to do the work, and work hard, an individual must be connected meaningfully to the design project. "[T] hey're doing something more for themselves, and they want it, they're motivated to complete it and to do well in it" (A2, male, UG2, 131). A meaningful connection involves perceived value and importance of the project work, a willingness and desire to achieve a level of standard in that work. A shared understanding of the purpose, scope, and processes of the project is also an important part of that connection (e.g. A1, A2, A3). Openness to learning, and the willingness to collaborate with the team also demonstrate a meaningful connection (e.g. A1).

However, it is also possible to limit team success even when individuals come in with high motivation and ability. It is possible demotivate members, burn out responsible members with unequal work distribution, leave talents under-utilized, or fail to form a cohesive team. "[It is important] to have a friendly environment [so] that it's not something hostile [such] that they don't want to do something" (A2, male, UG2, 131). Each member needs to feel valued in the team, in order to stay connected to the project. The initial motivation and contribution levels can change by the team processes and the team culture. This leads to the Level 2 and Level 3 factors to design team success.

\section{Level 2: Practical Strategies to Enabling Level 1}

Level 2 deals with practical strategies to enable effective contribution and meaningful engagement of team members:
[You must] figure out a way where everyone is involved. Sometimes for team meetings, or brainstorming, you want to get everyone's opinion, you want everyone to be present and to be part of it. (A1, male, UG2, 117)

Such practical strategies to ensure member engagement, integration, and contribution included: identifying individual members' strengths and learning needs (e.g. A1, A3, S7), balancing autonomy and peer learning (S7, A2), structuring meetings for active input (S9), adapting communication strategies for members with different needs (S7), holding constructive conversations when Level 1 is not working (S9), adapting managerial styles (A2), managing expectations and building a common understanding of the team processes (S7, S9, A1), inviting input and feedback (S7, A3, A1), and checking into individual experiences and well-being (S7).

It is important to note that individuals may need different styles of onboarding or integration before they can engage comfortably with the rest of the team:

Two of our members were very quiet. I was really close with one of them and my friend was close with the other. We said, I'm in charge of this one, etc., we said we're going to make sure you are in the loop [...] That allowed us to have a conversation, and helped him express himself.

$$
\text { (S7, female, UG2, 25) }
$$

Disengaged members and team conflicts pose significant challenges to project teams, and students actively reflect on how they might encourage and enhance motivation and performance of individuals. Level 2 processes would include strategies to mitigate challenges and resolve conflicts:

If everyone is upset with that obviously they're going to something about it. And instead of making everyone upset, I could just go up to that guy and try to convince him - you need to start working or this project will not work as smoothly as it could. There would be problems, there would be conflicts, other people will start saying we're doing too much work and he's not doing any work, why do we have to do work? So, to avoid those conflicts, I would just approach the person ahead of time. (A1, male, UG2, 148)

[S]ometimes it takes people have to realize, you have try and show them, that that's what they want to do, that that's something they should focus on and be motivated to do." (A2, male, UG2, 150)

$[W]$ e had a member who thought they were smart and said they knew what they were doing ... it got better but it was a lot of work with him... the rest of the team project was making sure everyone had a chance to say. (S7, female, UG2, 12)

Students also commented on the factors that make each team and situation different. Part of being able to lead a team was to recognize the circumstantial or historical variations that require adapted responses to create effective team strategies:

I think language is a big one. Recognizing language is different to everyone. I may be saying one word but you might think it means something else. Your personal background and 
life has effect. If you value other things above this project and others value it, then that's going to be an issue. If you have any personal issue, let us know, don't tell us what, but we have the right to know. (S7, female, UG2, 74)

When we're working with teams, it's good, cause if you have people of different skill sets and different mindsets [...] the head project manager, you want that person to be less like nitty gritty details and more of a by the book, or figuring out the main of how the over arching things that you want to happen. I guess other people would be doing certain jobs and tasks and I guess they could be creative through it. (A2, male, UG2, 119).

It might depend on the project or the situation you're in. Usually there are many different ways to do things. But there are circumstances where there might just be one certain way, and [...] you'd like to be someone like that. That they'd be able

to focus and have their blinders on to that certain way. (A1, male, UG2, 120)

In general, male students spoke of Level 2 strategies in terms of convincing someone to do what one believes is correct. The diversity people brought to the team were given meaning only in terms of the project tasks. Factors outside the scope of the project activities were not considered. In contrast, S7, one of the two female interviewees, offered articulate insights on how an individual's behaviour can be interpreted, as information to help adapt Level 2 responses. Furthermore, an individual's ability to contribute was understood as an outcome of internal and external systems that shape a person's holistic well-being:

In [one] phase there might be a leader but it doesn't mean someone else can't take charge. Someone who will make sure things are going on time, and make sure everyone is doing ok, and making sure they're mentally and emotionally ok to do it.

I recognized, 'hey you're not behaving the way you usually

do, 'then they say, 'yeah, there's this thing that's causing me stress.' That's a leader's point of view, to make sure everyone's doing okay. If I am the leader, there are aspects that I don't know anything about so might say, 'hey why don't you take charge of this?' The leader is the role of... who initiates conversation, but they don't need to be in charge. (S7, female, UG2, 29-30)

A team leader, without a formal title, was seen as a person who recognizes the factors to individuals' effectiveness and wellbeing, and facilitated Level 2 processes. The leader's responses helped to correct maladaptive behaviours, meet individual needs, provide support, and create engagement. These interactions served as a catalyst to change individual motivation, behaviour, attitudes, strengths.

Students who establish effective Level 2 processes set certain standards and espouse particular values that help the team mature and adapt. At the same time, in spite of good intentions and known strategies, significant challenges have emerged that thwart the efforts at effective Level 2 processes. The contrast between Level 2 enablers and inhibitors led to the identification of Level 3 factors to team success, presented in the next section.
Level 3: Team Culture that Enables Effectiveness of Levels 1 and 2

Level 3 characterizes the team culture by its core values that are expressed in practical ways. Level 3 recognizes that the emotional and relational qualities of team experience have a significant impact on Level 2 and Level 1 effectiveness. For example, even in the case of a conflict, shared values for honest conversation and respect helped turn the conflict into an opportunity for team building: "[E]verybody spoke and said what they wanted to say in a very respectful way, everyone came out feeling more satisfied" (S9, 33). On the other hand, individuals who did not value communication impeded mitigation processes (Level 2) that left Level 1 issues unresolved: "[A] lot of people view communicating as a very fluffy and unnecessary thing, but it reaches a point where I can't have conversation with you because you don't think you need to have it" (S9, 31). Members can passively disengage as well as actively shut down conversations and hinder team effectiveness: "[T]he problem is that a conversation isn't an argument. To me, we had a lot more arguments than conversations, anytime a conversation was initiated it was shut down" (S7, 70).

Level 3 also directly sustained individuals' engagement and contribution levels in the project (Level 1):

[W] e had a lot of different ideas and different thought processes and that helped with problem solving, because we were friends, we didn't have to be fake nice so we could be honest and be productive. Because we were genuine, we got everything done on time, all that was really good.

(S7, female, UG2, 12a)

Trust, friendliness, inclusivity, and respect created a mutually empowering relational culture (Level 3), which enabled effective role integration and task-work facilitation (Level 2).

Students also experienced how disrespect could manifest in different ways to hinder team effectiveness:

So how some people think they're too good for the group this is a big problem, I work with people who have big egos so I don't feel for them, I don't give them credit for things they didn't do, I don't care, they didn't do the work, they don't get the credit, then the people who do the work, I give them the credit and I boost them. Ethics: people who don't follow those will never be successful in engineering. (S1, male, UG3, 122).

I didn't realize how important it was [to address the issue of disrespect or dysfunction]. I thought it just happened naturally... it was a really interesting learning [experience] [...] He took over, at one point you keep fighting for something, and get nothing [...] Sometimes he thought I was challenging him just because I was clarifying. Unnecessary animosity [...] him trying to prove me wrong, constantly competing with one another, the lack of communication, it was really bad [...] It got better but it was a lot of work with him [...] not accepting the random bits of shut-down, saying hey you're acting like a jerk, [making it clear that] criticism for no reason isn't acceptable. (S7, female, UG2, 55, 49, 59b, 12c, 59a). 
Disrespect can be based on unconscious biases that view peers as less than equal. S1 dealt with a male peer who showed off his material wealth, treated project tasks as not important enough for himself but expected of peers like S1. The confusion of socioeconomic status as merit to project credit, with simultaneous exemption from labour, belittled S1 and the importance of the project work itself. On the other hand, S7 dealt with hostility from a male peer who asserted his dominance over the team, and refused to take S7's input seriously, as legitimate. While this kind of disrespectful attitude has been seen between male peers, the subtle sexism was distinct in the personal offense one took as a challenge to the hierarchy (male dominance, has a right to speak without responsibility over behaviour; female submissiveness, has no right to speak up nor disagree with the dominant male).

Both S1 and S7 had very positive team experiences that emulated Level 3 qualities, of collaborative, mutually empowering culture. It only took single individuals - who took their privileged position to impose a hierarchical relationship to make Level 3 very difficult to achieve.

In contrasting high performance team experience and poor team experience, it is also important to note how each member's Level 1 contribution can affect the Level 3 characteristics of the team. Likewise, one person's poor contribution in Level 1 can affect Level 2 processes, Level 3 culture, and another person's Level 1 connection to the team. From S7's example:

$[W]$ e were able to say 'I trust that you're going to read the [meeting minutes] even if you couldn't make it', or 'I trust you'll finish your part of the project without me having to nag you.' [...] I would send a notice about the meeting an hour before, and they would all show up to the meeting, they were able to get started themselves if I was late. $(\mathrm{S} 7,28)$.

[W] e worked on a proposal, I said, 'hey I'll do this and this, but I don't know this, so can you do it? '[...] He didn't do it, it ended up not being done. It was really hard to communicate. We started on passive aggressively, sometimes I just didn't want to go to the meeting. (S7, 36).

Each team member can affect other members in important ways, whether negative or positive, whether intended or not. All three levels of team success are mutually reinforcing.

\section{DISCUSSION}

The sequence of the key learning outcomes, in the context of the ME curricular design, reveals interesting possibilities in structuring the learning experience to support the learning outcomes. Sometimes course directors receive feedback on what is not working, but much less on what is working. There are features about the second year design course that created a pivotal experience for many transformative learning outcomes. Many of these were solidified by the similar course experience in the third year. There were first year material that had a clear connection to the upper year learning outcomes, but could be presented and structured to make that connection clearer. Students felt much more challenged in the fourth year with a changed course environment, while they built much confidence through a familiar course environment that was repeated (albeit with greater complexity in the project content) in the third year experience. There may be a trade-off between building confidence (solidifying achievement in potential threshold concepts) and expanding students' perceived horizon of the learning that lies ahead (growth, awareness of the lacking capabilities, broadening the understanding of the field). Engineering design educators need to be conscious of the tradeoffs, diligently collecting evidence to confirm or disconfirm our beliefs about what achieves the intended learning objectives.

Listening to student perspectives also demonstrated a huge value in identifying learning outcomes that we had not anticipated. This is a direct feedback into our own mental models about what constitutes engineering design learning. Without recognizing such valuable learning outcomes, it may leave certain effective learning experiences or course design features to be dropped in the continuous improvement efforts. Sterman emphasized the importance of going beyond just the simplistic use of feedback to 'improve' decisions. He explained how learning must involve a better understanding of the complex systems that constitute an implicit picture of reality, or how the learner frames the problems in the first place. This indeed applies to the educators themselves, to be in continuous inquiry of what design education in engineering is, how it takes place, and what our roles are.

Evidence suggests that it is possible to lose motivated individuals, and that each member may need different procedures to become fully engaged in a team. Then it becomes important that, before a member becomes or continues to be someone "who doesn't want to do anything" (A3, 146), the team makes effort to adapt and establish Level 2 processes and Level 3 culture. "[R]elationship building should've been done since the beginning of the project. I always try to go out for lunch with the group before we work. I try it a lot... I think that's so important" (S7, 73). The importance of Level 1 contributions cannot be understated; however, an individual member's contribution to team success now includes - beyond task delivery - interactional and relational work that facilitates team procedures (Level 2) and establishes a sustainable team culture (Level 3). Because of the mutually reinforcing reflexivity between each of the three levels, the degree of team effectiveness may be a summative result of:

$$
\begin{gathered}
\text { Team Effectiveness }=a L 1+b L 2+c L 3+ \\
d(L 1 L 2)+e(L 2 L 3)+e(L 1 L 3)+f(L 1 L 2 L 3)
\end{gathered}
$$

where the weight of each level factor may vary between first to fourth year students.

Disrespect is considered a moral injury to the respect and worth of a person [20]. There is an overall inadequate language and problem identification within male-dominant culture for misbehaviour, disrespect, power dynamics, and subtle discrimination based on socioeconomic status and sex - this may extend to other minority experiences, such as ESL, race, personalities, and disability. Engineering design educators, especially as they are involved in developing team leaders, will need to be better equipped to model and raise the level of student proficiency in equity, diversity, and inclusion. 
The existing level of student leadership and excellence should not be ignored. The ethical sensitivities, creativity, relational competencies and proactive action students bring to team life are indeed impressive. Students offered profound insights on which meaningful educational problems exist, how they are engaging in them, and what resources they would like from the institution. Students are partners and co-creators of our educational program success, as this research has taught us.

\section{ACKNOWLEDGMENT}

For the larger study that embeds this paper, we gratefully acknowledge the financial support by Quanser, National Sciences and Engineering Research Council (NSERC), Lassonde School of Engineering and Queen Elizabeth II Graduate Scholarships in Science \& Technology (QEII-GSST) program.

\section{REFERENCES}

[1] W. E. Eder, "Design Engineering and Needs for Methodology," in International Conference on Engineering Design, ICED'09, 2009, p. 12

[2] M. Gibbons and H. Nowotny, "The Potential of Transdisciplinarity," in Transdisciplinarity: Joint Problem Solving among Science, Technology, and Society. Schwerpunktprogramm Umwelt / Programme Prioritaire Environnement / Priority Programme Environment, J. T. Kleinm, R. Häberli, R. W. Scholz, W. Grossenbacher-Mansuy, A. Bill, and M. Welti, Eds. Birkhäuser, Basel, 2001, pp. 67-80.

[3] P. E. Vermaas, P. Kroes, A. Light, and S. A. Moore, Eds., Philosophy and Design: From Engineering to Architecture. Springer, 2008.

[4] S. H. Christensen, C. Mitcham, B. Li, and Y. An, Eds., Engineering, Development and Philosophy: American, Chinese and European Perspectives, vol. 53, no. 9. New York: Springer, 2012.

[5] I. van de Poel and D. E. Goldberg, Eds., Philosophy and Engineering: An Emerging Agenda. New York: Springer, 2010. doi: 10.1007/978-90-481-2804-4 .

[6] N. Cross, Designerly Ways of Knowing. Heidelberg, Germany: Springer, 2006

[7] G. Pahl, W. Beitz, J. Feldhusen, and K.-H. Grote, Engineering Design: a Systematic Approach, 3rd ed. London: Springer, 2007. doi: 10.1007/978-1-84628-319-2 .
[8] C. Spitas, "Analysis of systematic engineering design paradigms in industrial practice: Scaled experiments," J. Eng. Des., vol. 22, no. 6, pp. 447-465, 2011. doi: 10.1080/09544820903437742.

[9] P. Godfrey, R. Deakin Crick, and S. Huang, "Systems thinking, systems design and learning power in engineering education," Int. J. Eng. Educ., vol. 30, no. 1, pp. 112-127, 2014.

[10] C. L. Dym, A. M. Agogino, E. Ozgur, D. D. Frey, and L. J. Leifer, "Engineering Design Thinking, Teaching, and Learning," J. Eng. Educ., vol. 94, no. 1, pp. 103-120, 2005. doi: $10.1002 / \mathrm{j} .2168-9830.2005 . t b 00832 . x$.

[11] E. Alpay, a. L. Ahearn, R. H. Graham, and a. M. J. Bull, "Student enthusiasm for engineering: Charting changes in student aspirations and motivation," Eur. J. Eng. Educ., vol. 33, no. 5, pp. 573-585, 2008. doi: 10.1080/03043790802585454.

[12] J. D. Sterman, "Learning in and about complex systems," Syst. Dyn. Rev., vol. 10, no. 2/3, pp. 291-330, 1994. doi: $10.1002 / \mathrm{sdr} .4260100214$.

[13] H. Plattner, C. Meinel, and L. Leifer, Eds., Design Thinking Research: Building Innovation Eco-Systems. Springer International Publishing, 2014.

[14] I. van de Poel and P.-P. Verbeek, "Ethics and engineering design," Sci. Technol. Hum. Values, vol. 31, no. 3, pp. 223-236, 2006. doi: $10.1177 / 0162243905285838$.

[15] C. Noweski, A. Scheer, N. Buttner, J. von Thienen, J. Erdmann, and C. Meinel, "Towards a paradigm shift in education practice: Developing twenty-first century skills with design thinking," in Design Thinking Research: Measuring Performance in Context, $\mathrm{H}$. Plattner, C. Meinel, and L. Leifer, Eds. New York: Springer, 2012, pp. 71-93.

[16] J. H. F. Meyer and R. Land, "Threshold concepts and troublesome knowledge : Practising within the disciplines," Oxford: OCSLD, 2003.

[17] C. Beard and J. P. Wilson, "Exploring Experiential Learning," in Experiential Learning: a best practice handbook for educators and trainers, 2006, pp. 15-44.

[18] T. Tomiyama, P. Gu, Y. Jin, D. Lutters, C. Kind, and F. Kimura, "Design methodologies: Industrial and educational applications," CIRP Ann. - Manuf. Technol., vol. 58, no. 2, pp. 543-565, 2009. doi: $10.1016 /$ j.cirp.2009.09.003

[19] J. Corbin and A. Strauss, "Grounded Theory Research: Procedures, canons and evaluative criteria," Z. Soziol., vol. 19, no. 6, pp. 418-427, 1990. doi: 10.1515/zfsoz-1990-0602.

[20] A. Honneth, Struggle for Recognition: the Moral Grammar of Social Conflicts. Cambridge, MA: MIT Press, 1996.

[21] S. R. Daly, R. S. Adams, and A. M. Bodner, "What does it mean to design? A qualitative investigation of design professionals' experiences," J. Eng. Educ., vol. 101, no. 2, pp. 187-219, 2012. doi: 10.1002/j.2168-9830.2012.tb00048.x. 


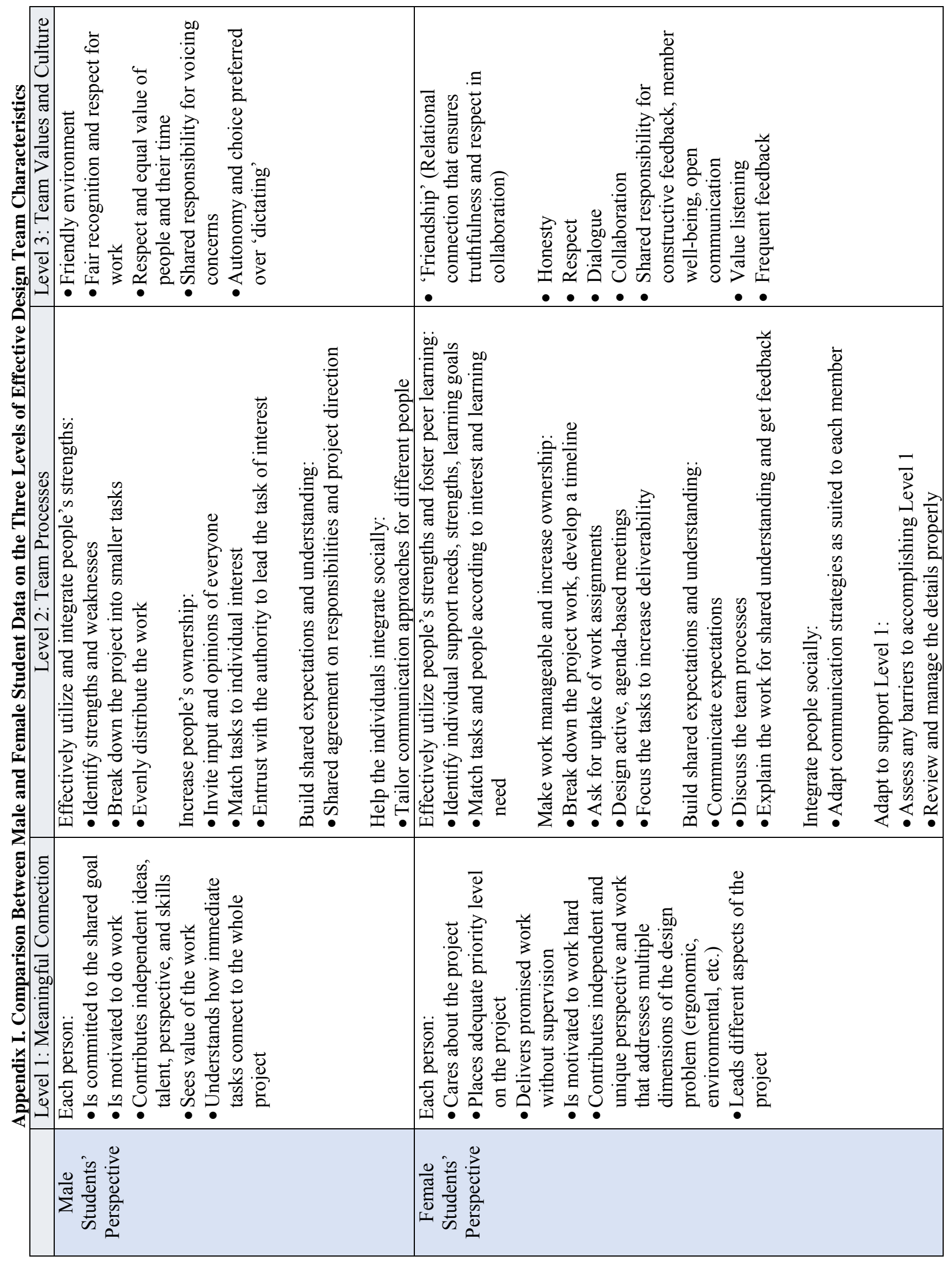




\section{Graduate Attributes and Threshold Concepts: Are We Measuring What's Important?}

\author{
Nancy Nelson \\ Electronic Systems Engineering / Engineering Education \\ Conestoga ITAL / University of Calgary \\ Cambridge / Calgary, Canada \\ Nancy.Nelson1@ucalgary.ca
}

\author{
Robert Brennan \\ Mechanical \& Manufacturing Engineering \\ University of Calgary \\ Calgary, Canada \\ rbrennan@ucalgary.ca
}

\begin{abstract}
A great deal of work has been done within the engineering community to identify the threshold concepts that students must master in order to transform from novice to practitioner. At the same time, engineering regulatory bodies have established a set of graduate attributes that help ensure graduating engineers are prepared to practice professional engineering. Students and recent graduates have identified that one area in which they felt lacking in skills and confidence was solving the complex and multi-faceted problems encountered in the engineering work place. This seems to indicate that students have not fully mastered one or more discipline-specific threshold concepts. This paper presents a framework engineering educators can use to identify, map, and monitor those concepts as key indicators used to track graduate attributes.
\end{abstract}

Keywords: threshold concepts; graduate attributes; engineering education; key indicators; benchmarks

\section{INTRODUCTION}

Engineers Canada reports that 69,015 undergraduate engineering degrees were conferred over the five year span from 2012 to 2016 [1]. This trend is expected to continue as the overall number of enrolments increases yearly. But are these graduates confident that they can apply their acquired knowledge and skills in the workplace?

Engineering education research has identified a number of threshold concepts that learners must master as they make their way from novice to practicing engineer. At the same time, engineering accrediting bodies, including the Canadian Engineering Accreditation Board (CEAB), have identified a set of graduate attributes to ensure graduates are prepared for a professional career. Each accredited engineering program is responsible for identifying and monitoring a set of benchmarks or key indicators for each of these graduate attributes.

This paper discusses the pilot test of a mapping framework used to determine the relationship between existing key indicators and established threshold concepts. This information can help shape the continual improvement process as programs work to strengthen the undergraduate engineering experience.

\section{BACKGROUND}

\section{A. Engineering Education}

Over the last 100 years engineering education has shifted from a hands-on practical approach to one that focuses on engineering science and analysis [2][3]. Currently there are moves toward outcomes-based curriculum, increased design experience in the undergraduate curriculum, and educating "engineers who can engineer" [4]. There are currently 43 schools offering 281 different accredited engineering programs in Canada. These programs range from the more traditional offerings of civil, mechanical, and electrical engineering, to disciplines as varied as aerospace, biomedical, environmental, sustainable design, petroleum, and software engineering [5].

Canadian engineering schools have been accredited since 1965. This process ensures that graduates of engineering programs meet the high standards necessary to become professional engineers. An accreditation review examines the depth and breadth of the science, mathematics, engineering science, engineering design, and complementary studies curriculum, as well as 12 graduate attributes that ensure proficiency in discipline-specific knowledge and skills, employability skills, and professional responsibility [6].

\section{B. Graduate Attributes}

All graduating engineers must be technically competent. They must have mathematical, scientific, and disciplinespecific knowledge, and be able to analyze and solve complex engineering problems. They must know how to conduct investigations in order reach valid conclusions. They must be able to design solutions and systems for open-ended problems, and use engineering tools appropriately. This professional body of knowledge forms the core of every engineering program.

Graduate attributes move beyond these technical abilities to include a set of qualities required of graduates as they become practicing engineers. In order to be employable graduates must be able to work both individually and as part of a team. They must be able to communicate effectively and continue to learn throughout their careers. Most importantly, engineers must be able to make professional, responsible, and ethical decisions for which they are accountable. The challenge for engineering 
programs is to choose appropriate indicators that demonstrate their students are meeting each of these graduate attributes.

\section{Threshold Concepts and Threshold Practices}

Long before Meyer and Land coined the term 'threshold concept' [7], educators were questioning whether students actually understood what was being taught in a class [8][9], and looking for ways to help overcome common bottlenecks in student learning[10].

Meyer and Land defined a threshold concept as a portal, a way of thinking about something in a "new and previously inaccessible way"[7]. They considered it a space between where a learner is and where that learner needs to be in order to make the transition from novice to experienced practitioner in a discipline. Some learners master threshold concepts quite easily, while others struggle in a transitional state called liminality. With new knowledge to learn and misconceptions and misunderstandings to unlearn, this liminal state can involve disorientation and ambiguity as a learner moves between a state of knowing and not knowing.

A threshold concept is identified by five key characteristics: it is (1) transformative, (2) troublesome, (3) irreversible, (4) integrative, and (5) bounded. Land nicely summarizes these in a 2013 interview for Tomorrow's Professor Newsletter [11]:

"New conceptual understandings pull together (integrate) various stands of understanding into a new understanding that fundamentally changes (transforms) the way students think about the subject. Because the process involves the loss of a familiar way of thinking and the security it provides, the process of crossing the threshold commonly causes some mental and emotional discomfort (troublesome). There's some debate about whether these new understandings are reversible or not, but once a student "gets it," it seems hard for them to "un-get it." "Bounded" seems the least obvious descriptor perhaps because its meaning derives more from contextual concerns than specifically conceptual ones."

Over the years, the definition of a threshold concept has expanded to include threshold skills and practices [12][13]. Like a threshold concept, a threshold skill is considered to be transformative, integrative, and troublesome. It is, however, considered only semi-irreversible, since learners tend to lose certain skills if they are not practiced, practice being the fifth characteristic. The idea of partial understanding exists for threshold concepts, where a learner can apply the concept, but not explain it in an abstract way. With threshold skills this is considered partial mastery where a learner can only demonstrate certain parts of the skill or practice, or knows that the skill should be used, but cannot to use it properly.

\section{Threshold Concepts in Engineering}

Much work has been done to identify threshold concepts within specific engineering disciplines, but a research study out of the University of Western Australia created the first Integrated Engineering Threshold Concept Inventory (IETCI) [13]. These concepts, which include a number of graduate attributes, are grouped into three main categories: (1) learning to become an engineer, (2) thinking and understanding like an engineer, and (3) shaping the world as an engineer.

Funded by National Sciences and Engineering Research Council of Canada (NSERC)
Learning to become an engineer includes recognizing the role an engineer plays in society, the values and responsibilities associated with being an engineer, and the engagement in and responsibility for one's own learning. It also includes the thresholds associated with teamwork and communicating. Thinking and understanding like an engineer encompasses the abstraction, modeling, and theories required to master the core concepts within one's discipline, and shaping the world as an engineer includes engineering design, and the approaches, thinking skills, and integration of concepts an engineer uses in his/her day-to-day practice.

Mastery of these engineering threshold concepts, skills, and practices should indicate graduates are well on their way to becoming capable engineers. It then seems logical that the graduate attributes identified and monitored as part of an accreditation's graduate attribute assessment should be linked to these thresholds.

This research established and piloted a framework for engineering educators to examine the level to which the graduate attributes identified for engineering accreditation measure mastery of the threshold concepts identified in the integrated engineering threshold concepts inventory.

\section{PROCESS}

The Graduate Attribute - Threshold Concepts (GATC) framework allows an engineering program to analyze the relationship between graduate attribute key indicators and the threshold concepts identified in the IETCI. These results can be used to make curriculum and accreditation tracking decisions during the required continual improvement process.

\section{A. Framework}

Analysis of program data using the GATC framework is a three stage process: (1) extraction of accreditation data, (2) mapping graduate attribute indicators and exemplars to threshold concepts, and (3) interpreting the results.

The first stage requires access to the program's most recent CEAB accreditation documentation. Fig. 1 shows the result of this stage where accreditation table 3.1.2, found in the EN_6C_vXX spreadsheet, is copied exactly as is into the CEABB312 worksheet of the GATC working document.

This data is used to populate the TC Analysis mapping worksheet used in phase 2. Fig. 2 shows this transfer of CEAB data into a form where each learning activity is separated out into its own mapping row. The person or team doing the analysis now needs to identify the exemplar (problem, assignment, exam question, lab, or project) associated with the learning activity listed in the Activity column. Fig. 2 also shows two exemplars, one for each of the first two activities.

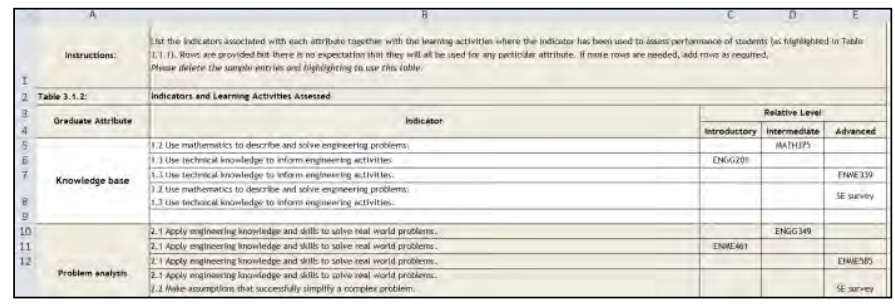

Figure 1: GATC phase 1 - CEAB data 


\begin{tabular}{|c|c|c|c|c|}
\hline Graduate Attribute & Indicator & Level & Activity & Exemplar \\
\hline \multirow[t]{5}{*}{ Knowledge base } & \multirow{3}{*}{$\begin{array}{l}1.2 \text { Use mathematics to describe and solve } \\
\text { engineering problems. }\end{array}$} & Introductory & & \\
\hline & & Intermediate & MATH375 & $\begin{array}{l}\text { Solve first order differential } \\
\text { equations with applications }\end{array}$ \\
\hline & & Advanced & & \\
\hline & \multirow[t]{2}{*}{$\begin{array}{l}1.3 \text { Use technical knowledge to inform } \\
\text { engineering activities. }\end{array}$} & Introductory & ENGG201 & $\begin{array}{l}\text { Perform hydrostatic pressure } \\
\text { calculations and basic fluid flow } \\
\text { calculations }\end{array}$ \\
\hline & & Intermediate & & \\
\hline
\end{tabular}

Figure 2: GATC phase 2 - TC analysis - exemplars

Mapping begins once an exemplar has been identified for each of the activities. Each of the threshold concepts included in the inventory is listed in a column across the top of the worksheet. Fig. 3 shows a few of the threshold concepts in the 'learning to become an engineer' and the 'thinking and understanding like an engineer' categories of the IETCI. The task for the analysis team is to determine if each exemplar maps to one or more threshold concept. It is expected that some exemplars will map, while others will not. If a map is found, the intersecting cell is marked with an ' $\mathrm{x}$ '. Resources including a concept map of IETCI, a summary table of the threshold concepts identified in the IETCI, and a suggested categorization and mapping of threshold concepts identified in other research studies, are provided in the spreadsheet to help analysts identify potential threshold concepts. Fig. 3 shows the first exemplar mapped to three of the threshold concepts in the abstraction, modelling, and theories section, and the second exemplar mapped to one. Once each exemplar has been examined, the mapping process is complete.

The GATC report is broken into two parts: an examination of the graduate attributes and threshold concepts, and the tracking of threshold concepts. The examination of graduate attributes and threshold concepts reports three things useful in determining if the exemplars they are tracking are ones that show the transformation learners make as they move from novice to practitioner: (1) the percentage of exemplars that are mapped to at least one threshold concept, (2) the number of threshold concepts mapped to each graduate attribute (see Fig. 4), and (3) a breakdown of which threshold concept categories (learn, think, shape) are mapped to each graduate attribute (see Fig. 5).

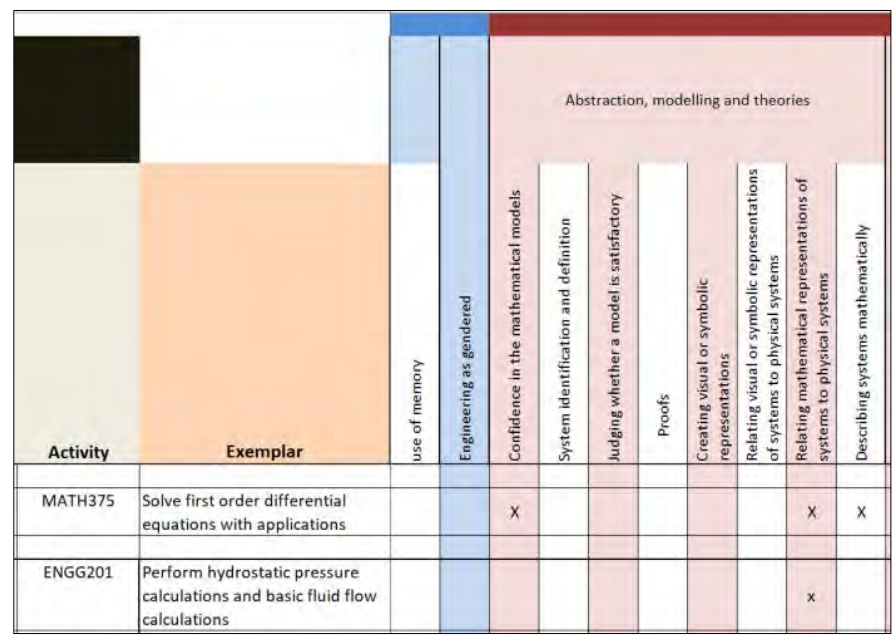

Figure 3: GATC phase 3 - TC analysis - mapping

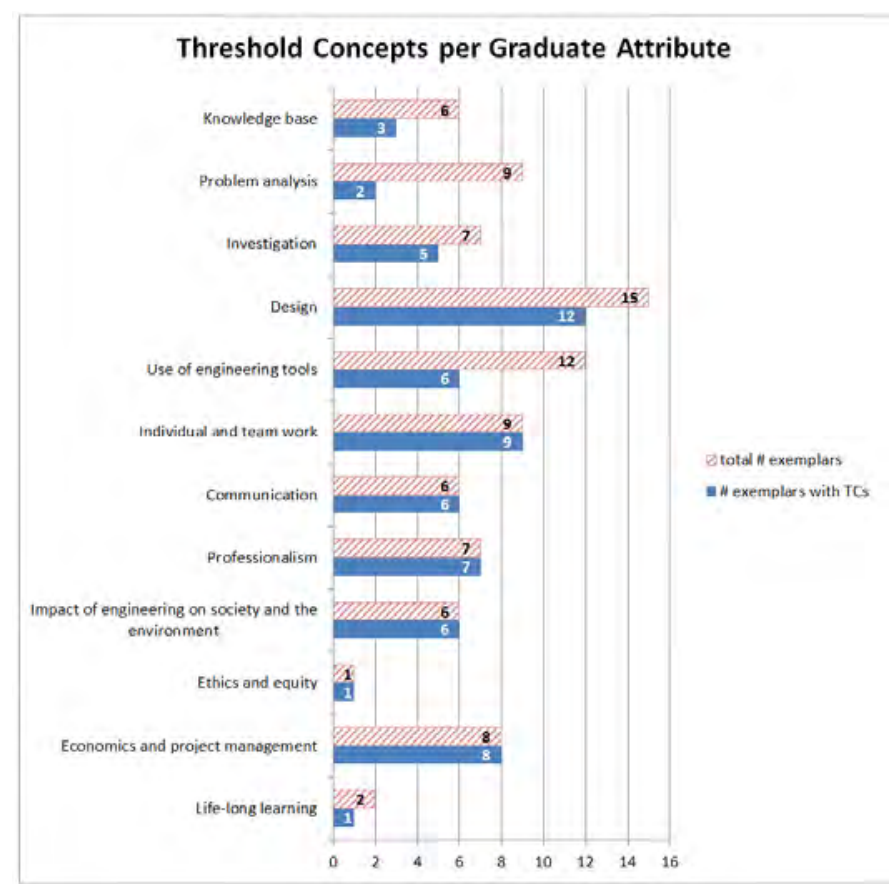

Figure 4: GATC report part 1 - grad attributes \& threshold concepts

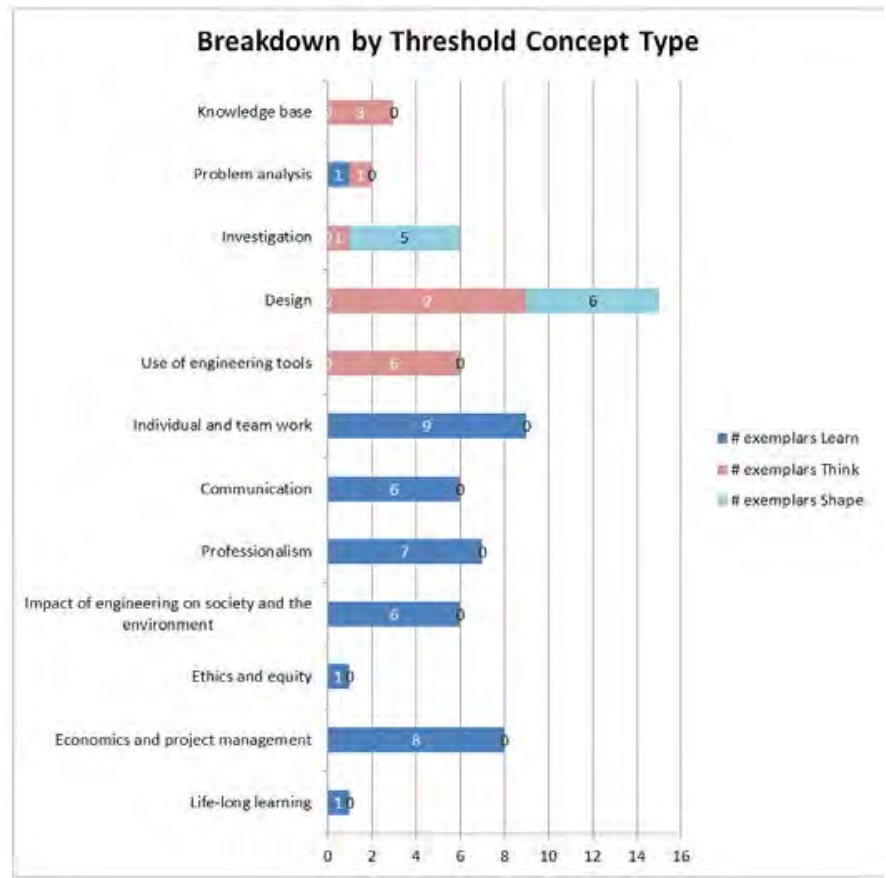

Figure 5: GATC report part 1 - breakdown by threshold concept type

The second part of the report specifies the percentage of threshold concepts that were mapped to at least one graduate attribute (see Fig. 6). It also identifies the number of mappings for each threshold concept. This allows the analysts to quickly identify any unmapped threshold concepts that are important to their discipline, and should be considered as potential exemplars. 


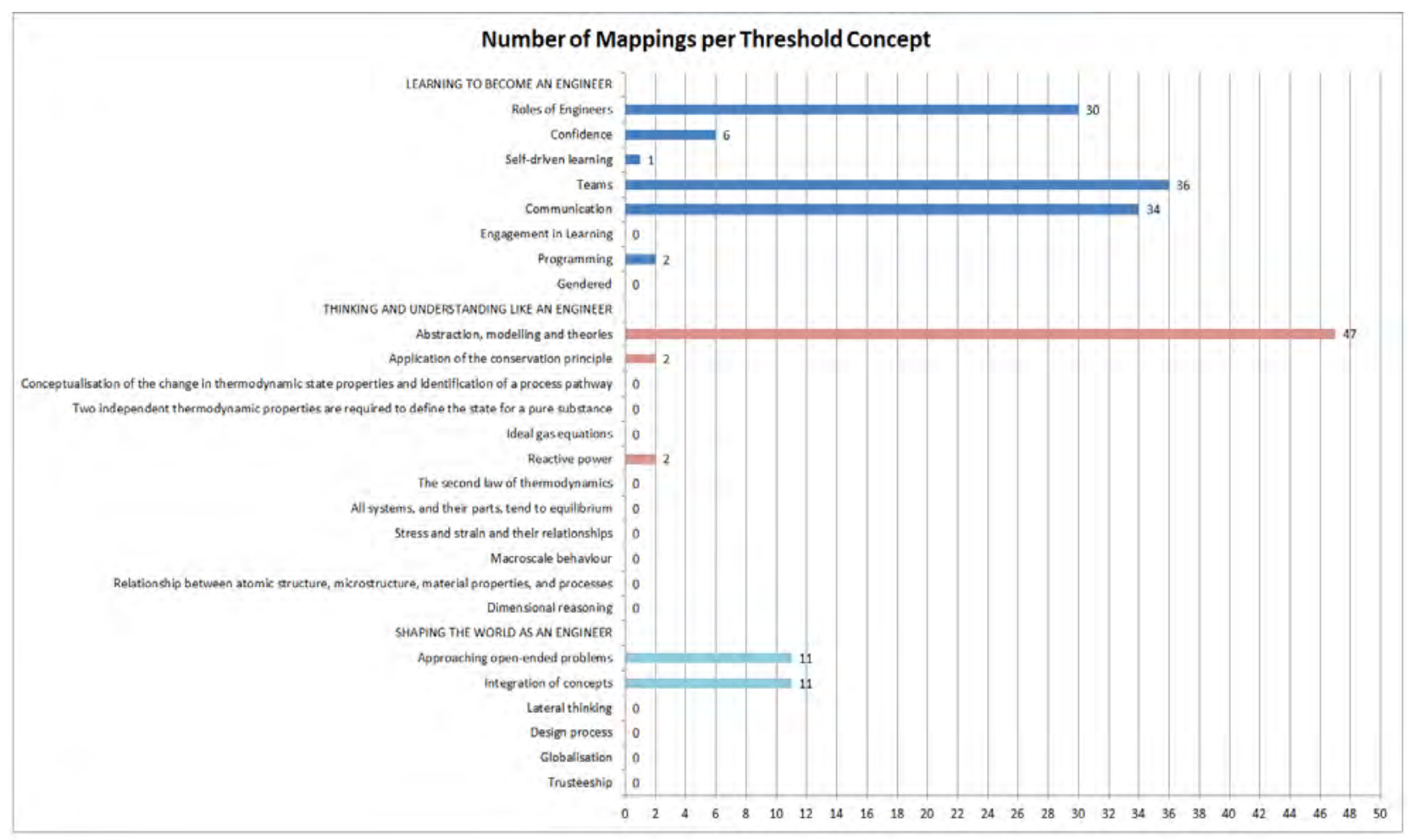

Figure 6: GATC report part 2 - number of mappings per threshold concept

\section{B. Application}

The GATC framework was piloted with two programs that underwent their $\mathrm{CEAB}$ accreditation reviews in 2017. It was reported to be easy to use, and in both cases took less than one hour to load the graduate attribute data, and enter and map the exemplars.

The first program had 47 exemplars with 46 mapped to threshold concepts (97.7\%), while the second had 88 exemplars with 66 mapped to threshold concepts $(75.0 \%)$. This percentage is not a reflection of the quality of the program or the choice of indicators. It is simply an indication of how many of the selected benchmarks are threshold concepts, and where they are focused. If a graduate attribute does not map to any of the threshold concepts, or there is an imbalance of mapping across the threshold concept types ('learn', 'think', 'shape'), then the program should consider modifying their choice of exemplar to one that tracks a significant point in a student's transformation from novice to practitioner.

The first program had 29 of the 57 threshold concepts mapped from its graduate attributes (59.9\%), and the second program had 32 of the 57 threshold concepts mapped from its graduate attributes $(56.1 \%)$. A higher percentage of mappings does not necessarily mean a "better" program. It simply identifies a threshold concept that has been associated with a number of graduate attribute exemplars. Programs are encouraged to look at the threshold concepts that have few or no mappings. If these are important concepts within the discipline, then consideration should be made to add graduate attribute exemplars to monitor student learning associated with these threshold concepts.

\section{DISCUSSION}

Users in both pilot tests recognized the value of the GATC framework as part of the continual improvement requirement of the accreditation process. It was easy to use, and helped identify areas where graduate attributes could be better mapped to discipline-specific threshold concepts. One program recognized that a critical graduate attribute indicator had inadvertently been omitted from their CEAB submission. Had the GATC framework analysis been done prior to submission this omission would have been caught. The other program identified gaps within the 'learn', 'think', and 'shape' categories of threshold concepts tracked and will reevaluate the choice of exemplars to provide broader coverage of these categories.

\section{FUTURE WORK}

Future work on the GATC framework will focus on two key areas: (1) expanding the pilot study to gather feedback from other interested programs, and (2) integrating the findings of discipline and topic specific threshold concept research into the GATC framework's topic search resource to help users more easily map their graduate attribute indicators.

\section{REFERENCES}

[1] Engineers Canada (2016). Canadian engineers for tomorrow: Trends in engineering enrolment and degrees awarded 2011-2015 [Online]. Available: https://engineerscanada.ca/reports/canadian-engineers-for-tomorrow.

[2] A. Cheville, "Defining Engineering Education," in 121st ASEE Annu. Conf. Expo., Indianapolis, IN, 2014.

[3] J. E. Froyd, P. C. Wankat, and K. A. Smith, "Five major shifts in 100 years of engineering education," in Proc. IEEE, vol. 100, pp. 1344-1360, 2012. doi: 10.1109/JPROC.2012.2190167. 
[4 ] E. F. Crawley et al., Rethinking Engineering Education, 2nd ed. Switzerland: Springer International Publishing, 2014. doi: 10.1007/978-3-319-05561-9.

[5] Engineers Canada, About accreditation, 2018. [Online]. Available: https://engineerscanada.ca/accreditation/aboutaccreditation.

[6] W. MacQuarrie et al., "Canadian Engineering Accreditation Board: 2017 Accreditation Criteria and Procedures Report," Engineers Canada, Ottawa, ON, pp. 1-126, 2017. [Online] Available: https://engineerscanada.ca/sites/default/files/ accreditation-criteria-procedures-2017.pdf.

[7] J. H. F. Meyer and R. Land, "Threshold concepts and troublesome knowledge: Linkages to ways of thinking and practising within the disciplines." ETL Project, Edinburgh, UK, 2003, pp.1-14.

[8] E. A. Marek, "They misunderstand, but they'll pass," Sci. Teach., vol. 53, no. 9, pp. 32-35, 1986.
[9] D. Perkins, "The many faces of constructivism," Educ. Leadersh., vol. 57, no. 3, pp. 6-11, 1999.

[10] A. Rugarcia et al., "The future of engineering education I. A vision for a new century," Chem. Eng. Educ., vol. 34, no. 1, pp. $16-25,2000$.

[11] J. Rhem, "Threshold concepts and troublesome knowledge," Tomorrow's Profr., vol. 22, no. 4, p. 2, 2013.

[12] L. Thomas et al., "In the liminal space : software design as a threshold skill," Pract. Evid. Scholarsh. Teach. Learn. High. Educ., vol. 12, no. 2008, pp. 333-351, 2015.

[13] S. A. Male, "Integrated engineering foundation threshold concept inventory," Sydney: Australian Government Office for Learning and Teaching, 2012. 
The CSME International Congress promotes a technical program that focus on topics and themes relevant to Mechanical Engineers. One of the primary roles of CSME is to train and update the mechanical engineering community on new advances and emerging fields. It is through the Congress that we provide a platform for community members to acquire and disseminate such information to each other as well as discuss recent research challenges and practical importance to the profession and its related field.

The papers in the proceedings are on the following topics:

- Fluid Mechanics

- Mechatronics, Robotics and Control

- Advance Energy Systems

- Manufacturing

- Computational Mechanics

- Student Paper Competition

- Heat Transfer

- Biomechanics and Biomedical Engineering

- Engineering Analysis \& Design

- Materials Technology

- Environmental Engineering

- Mechanical Engineering Education

\begin{abstract}
Any statements expressed in these materials are those of the individual authors and do not necessarily represent the views of CSME, which takes no responsibility for any statement made herein. No reference made in this publication to any specific method, product, process, or service constitutes or implies an endorsement, recommendation, or warranty thereof by CSME. The materials are for general information only and do not represent a standard of CSME, nor are they intended as a reference in purchase specifications, contracts, regulations, statutes, or any other legal document. CSME makes no representation or warranty of any kind, whether express or implied, concerning the accuracy, completeness, suitability, or utility of any information, apparatus, product, or process discussed in this publication, and assumes no liability therefor. The information contained in these materials should not be used without first securing competent advice with respect to its suitability for any general or specific application. Anyone utilizing such information assumes all liability arising from such use, including but not limited to infringement of any patent or patents.
\end{abstract}

Published by York University Libraries

Copyright (C) 2018 by the Canadian Society of Mechanical Engineering

All Rights Reserved

DOI: $10.25071 / 10315 / 35193$

ISBN (ebook) 978-1-77355-023-7

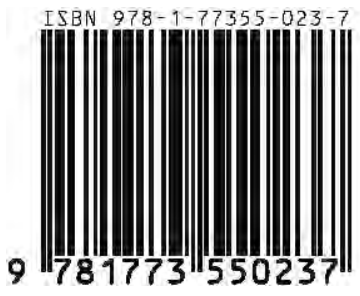

